\title{
Mechanistically Guided Workflow for Relating Complex Reactive Site Topologies to Catalyst Performance in $\mathbf{C}-\mathbf{H}$ Functionalization Reactions
}

\author{
Ryan C. Cammarota ${ }^{1 \ddagger}$, Wenbin $\mathrm{Liu}^{2 \ddagger}$, John Bacsa ${ }^{2}$, Huw M. L. Davies*,2 and Matthew S. \\ Sigman*,1
}

${ }^{1}$ Department of Chemistry, University of Utah, 315 South 1400 East, Salt Lake City, Utah 84112

${ }^{2}$ Department of Chemistry, Emory University, 1515 Dickey Drive, Atlanta, Georgia 30322

Email: matt.sigman@utah.edu

hmdavie@emory.edu

\section{Table of Contents}

1. General Considerations

Page

2. Acquisition and Preparation of Reported Compounds

3. Synthesis of New $\mathrm{Rh}_{2}(S-2-\mathrm{Cl}-4 / 5-\mathrm{RTPCP})_{4}$ Catalysts

4. General Procedure and Analysis for $\mathrm{C}-\mathrm{H}$ Functionalization Reactions

5. Crude ${ }^{1} \mathrm{H}$ NMR Spectra for Site-Selectivity Determination

S19

6. Computational Methods and Details

S20

7. Workflow and Considerations for Obtaining SMART Descriptors $\quad$ S68

8. Multivariate Linear Regression and Classification Model Development $\quad$ S74

9. References $\quad$ S89

10. Atomic Coordinates of Calculated Stationary Points $\quad$ S92

10.1. Dirhodium Catalyst Conformer Structures $\quad$ S92

10.2. Carboxylate Ligands, Rotational Transition States, and SMART Probe $\quad$ S269

11. NMR Spectra for Characterization S293

12. HPLC Spectra for Enantioselectivity Determination S306

13. X-Ray Crystallographic Data for Four Dirhodium Catalysts S323 


\section{General Considerations}

All solvents were purified and dried by a Glass Contour Solvent System unless otherwise stated. The dichloromethane used for $\mathrm{C}-\mathrm{H}$ functionalization reactions was dried and degassed at reflux over activated $4 \AA$ molecular sieves for 1 hour under argon, and subsequently stored with activated $4 \AA$ molecular sieves under an argon atmosphere and used directly.

Thin layer chromatographic (TLC) analysis was performed on Kieselgel 60 F254 aluminumbacked silica plates by visualizing with $\mathrm{UV}$ light and/or staining with an aqueous $\mathrm{KMnO}_{4}$ stain.

${ }^{1} \mathrm{H}$ and ${ }^{13} \mathrm{C}$ NMR spectra were recorded at $600 \mathrm{MHz}\left({ }^{13} \mathrm{C}\right.$ at $\left.151 \mathrm{MHz}\right)$ on a Bruker-600 spectrometer or IVONA-500 spectrometer. Unless otherwise stated, NMR spectra were collected in solutions of deuterated chloroform $\left(\mathrm{CDCl}_{3}\right)$ with tetramethylsilane (TMS) as an internal standard $\left(0 \mathrm{ppm}\right.$ for ${ }^{1} \mathrm{H}$, and $0 \mathrm{ppm}$ for $\left.{ }^{13} \mathrm{C}\right)$ or residual chloroform taken as an internal standard (7.26 ppm for ${ }^{1} \mathrm{H}$, and $77.16 \mathrm{ppm}$ for ${ }^{13} \mathrm{C}$ ), and chemical shifts were reported in parts per million (ppm). Abbreviations for signal multiplicity are as follows: $\mathrm{s}=$ singlet, $\mathrm{d}=$ doublet, $\mathrm{t}=$ triplet, $\mathrm{q}=$ quartet, $\mathrm{m}=$ multiplet, $\mathrm{dd}=$ doublet of doublet, etc. Coupling constants $(J$ values $)$ were calculated directly from the spectra.

IR spectra were collected on a Nicolet iS10 FT-IR spectrometer and reported in units of $\mathrm{cm}^{-1}$.

Mass spectra were taken on a Thermo Finnigan LTQ-FTMS spectrometer with APCI, ESI or NSI.

Melting points (mp) were measured in open capillary tubes with a Mel-Temp Electrothermal melting points apparatus and are uncorrected.

Optical rotations were measured on Jasco P-2000 polarimeters.

Analytical enantioselective chromatographs were measured on either Varian Prostar or Agilent1100 series instruments using an isopropanol/hexane gradient. Chiral HPLC conditions were determined by obtaining separation of the racemic products obtained from reactions catalyzed by a $50 / 50$ mixture of $R / S$ catalysts. 


\section{Acquisition and Preparation of Reported Compounds}

The following compounds were commercially available and used directly (unless otherwise stated):

1-Bromo-4-n-pentylbenzene, methyl formate, lithium chloride, copper (I) bromide, sodium methoxide solution $(0.5 \mathrm{M}$ in methanol), potassium trimethylsilanolate (used fresh from SigmaAldrich bottle or heated under vacuum before use for anhydrous powder), $\mathrm{Rh}_{2}(\mathrm{OAc})_{4}$, potassium phosphate tribasic, $\mathrm{Pd}(\mathrm{dppf}) \mathrm{Cl}_{2}$, (4-(tert-butyl)phenyl)boronic acid, phenylboronic acid, (4methoxy-3,5-dimethylphenyl)boronic acid, (4-(dimethylamino)phenyl)boronic acid, (3,5dimethylphenyl)boronic acid, (3,5-bis(trifluoromethyl)phenyl)boronic acid, and mesitylboronic acid.

The following substrates and catalysts were prepared by procedures adapted from previous literature:

2,2,2-Trichloroethyl 2-(4-bromophenyl)-2-diazoacetate, ${ }^{1}$ methyl $(S)$-1-(4-bromo-2chlorophenyl)-2,2-diphenylcyclopropane-1-carboxylate, ${ }^{2} \mathrm{Rh}_{2}(R \text {-TPCP })_{4},{ }^{3} \mathrm{Rh}_{2}\left(R-p-\mathrm{NO}_{2} \mathrm{TPCP}\right)_{4} ;{ }^{4}$ $\mathrm{Rh}_{2}\left(R-p-\mathrm{CF}_{3} \mathrm{TPCP}\right)_{4},{ }^{4} \quad \mathrm{Rh}_{2}(R-p-\mathrm{BrTPCP})_{4},{ }^{3} \quad \mathrm{Rh}_{2}(R-p-\mathrm{PhTPCP})_{4},{ }^{5} \quad \mathrm{Rh}_{2}\left(R-3,5-\mathrm{diCF}_{3} \mathrm{TPCP}\right)_{4},{ }^{4}$

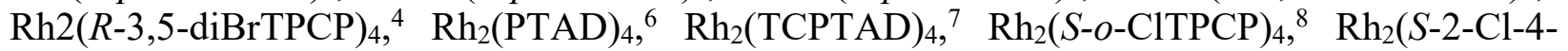
$\mathrm{BrTPCP})_{4},{ }^{2} \mathrm{Rh}_{2}(S-2-\mathrm{Cl}-5-\mathrm{BrTPCP})_{4},{ }^{2}$ and $\mathrm{Rh}_{2}\left(S-2-\mathrm{Cl}-5-\mathrm{CF}_{3} \mathrm{TPCP}\right)_{4} .{ }^{9}$ 


\section{Synthesis of New $\mathrm{Rh}_{2}(\mathrm{~S}-2-\mathrm{Cl}-4 / 5-\mathrm{RTPCP})_{4}$ Catalysts}

\section{$\mathrm{Rh}_{2}(\mathrm{~S} \text {-2-Cl-4-OMeTPCP })_{4}(\mathrm{C})$}

This methoxy derivative is derived from the enantiopure methyl ester form of the ligand for $\mathrm{Rh}_{2}(S-$ 2-Cl-4-BrTPCP) 4 , methyl (S)-1-(4-bromo-2-chlorophenyl)-2,2-diphenylcyclopropane-1carboxylate, and followed the same hydrolysis and ligand exchange procedures.
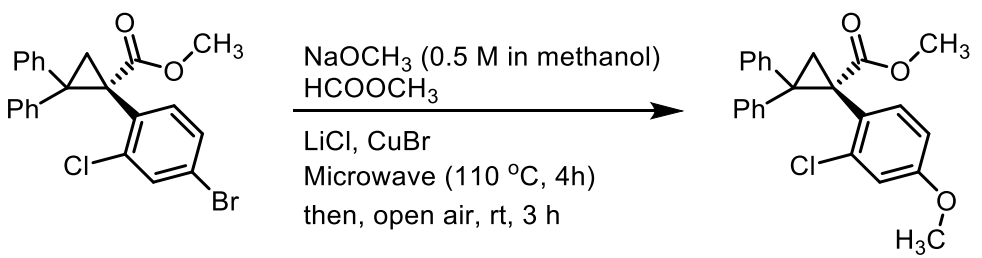

A flame-dried Microwave Vial (Anton Paar, G30) was charged with methyl (S)-1-(4-bromo-2chlorophenyl)-2,2-diphenylcyclopropane-1-carboxylate (2.2 g, $5 \mathrm{mmol}, 1.0$ equiv.), methyl formate ( $0.31 \mathrm{~mL}, 5 \mathrm{mmol}, 1.0$ equiv.), $\mathrm{LiCl}(212 \mathrm{mg}, 5 \mathrm{mmol}, 1.0$ equiv.) and $\mathrm{CuBr}(107.6 \mathrm{mg}$, $0.75 \mathrm{mmol}, 15 \mathrm{~mol} \%)$. After the vial was flushed with argon, $\mathrm{NaOCH}_{3}$ solution $\left(0.5 \mathrm{M}^{2} \mathrm{CH}_{3} \mathrm{OH}\right.$, $20 \mathrm{~mL}, 10 \mathrm{mmol}, 2.0$ equiv.) was added and the septum was replaced. The vial was inserted into the microwave reactor to react at $110^{\circ} \mathrm{C}$ for $4 \mathrm{~h}$, followed by stirring at room temperature for $3 \mathrm{~h}$ (open to air) until all copper was oxidized to the +2 state (green solution). The resulting mixture was diluted with $20 \mathrm{~mL}$ of $1 \mathrm{M} \mathrm{HCl}$ aqueous solution and extracted with methyl tert-butyl ether $(3 \times 20 \mathrm{~mL})$. The combined organic layer was dried over $\mathrm{Na}_{2} \mathrm{SO}_{4}$, concentrated under reduced pressure, and the residue was purified by flash column chromatography (hexane/ethyl acetate $=$ $33 / 1$ to $11 / 1$ ) to afford the product as white solid in $56 \%$ yield ( $66 \%$ conversion).

\section{(-)-Methyl (S)-1-(4-methoxy-2-chlorophenyl)-2,2-diphenylcyclopropane-1-carboxylate:}

mp: $128-130{ }^{\circ} \mathrm{C} ; \mathbf{R f}=0.40$ (hexane/ethyl acetate $=6 / 1$ );

$[\alpha]^{20} \mathrm{D}:-367.4^{\circ}\left(\mathrm{c}=1.00, \mathrm{CHCl}_{3},>99 \%\right.$ ee $)$;

${ }^{1} \mathbf{H}$ NMR (600 MHz, $\left.\mathbf{C D C l}_{3}\right) \boldsymbol{\delta} 7.56(\mathrm{~d}, J=7.0 \mathrm{~Hz}, 2 \mathrm{H}), 7.43-7.36(\mathrm{~m}, 2 \mathrm{H}), 7.32(\mathrm{tt}, J=7.2,2.3$

Hz, 1H), 7.03 (dt, $J=6.7,2.7 \mathrm{~Hz}, 4 \mathrm{H}), 6.91-6.80$ (m, 3H), 6.59 (d, $J=8.4 \mathrm{~Hz}, 1 \mathrm{H}), 3.73$ (s, 3H), 3.40 (s, 3H), 2.81 (s, br, 1H), 2.46 (s, br, 1H);

${ }^{13} \mathbf{C}$ NMR (151 MHz, $\left.\mathbf{C D C l}_{3}\right) \boldsymbol{\delta} 171.2,159.2,141.7,139.5,137.4,134.9,130.6,128.5,128.4$, $127.4,127.1,126.3,126.2,114.8,112.3,55.4,52.4,44.9,41.6,26.4$

IR (neat) 3058, 3024, 2948, 2838, 1727, 1605, 1497, 1291, 1218, 1043, 748, $705 \mathrm{~cm}^{-1}$;

FTMS (+p APCI) calcd for $\mathrm{C}_{24} \mathrm{H}_{22} \mathrm{ClO}_{3}(\mathrm{M}+\mathrm{H})^{+} 393.1252$ found 393.12571 . 


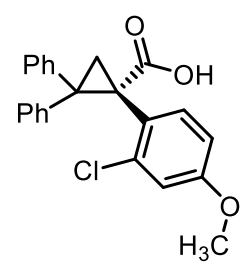

\section{(-)-(S)-1-(4-methoxy-2-chlorophenyl)-2,2-diphenylcyclopropane-1-carboxylic acid:}

This reaction was performed following the same procedure as the hydrolysis for the ligand of $\mathrm{Rh}_{2}(S-2-\mathrm{Cl}-4-\mathrm{BrTPCP})_{4}:^{2} \mathrm{~A} 100 \mathrm{~mL}$ round-bottom flask was charged with ester from the previous step (1.57 g, $4.0 \mathrm{mmol}, 1.0$ equiv.) and dissolved in $40 \mathrm{~mL}$ of dry DMSO. After the flask was flushed with argon gas (3 times), TMSOK ( $4.11 \mathrm{~g}, 32.0 \mathrm{mmol}, 8$ equiv.) was added in one portion. Then, the reaction mixture was stirred at room temperature and monitored by TLC until the starting material was consumed completely $(30 \mathrm{~h})$. The reacted mixture was quenched with $40 \mathrm{~mL}$ of 0.4 $\mathrm{M}$ aqueous citric acid by stirring for $1 \mathrm{~h}$. Then, the solution was extracted by ethyl acetate ( 3 times), washed with brine and dried over $\mathrm{Na}_{2} \mathrm{SO}_{4}$. The organic solution was concentrated under reduced pressure, and the residue was purified by flash column chromatography (hexane/ethyl acetate = $9 / 1$ to $2 / 1$ ) to afford the product as white solid in $96 \%$ yield.

mp: $105-107^{\circ} \mathrm{C}$;

$\mathbf{R f}=0.23$ (hexane/ethyl acetate $=5 / 1$ );

$[\alpha]^{20} \mathrm{D}:-395.6^{\circ}\left(\mathrm{c}=1.00, \mathrm{CHCl}_{3},>99 \%\right.$ ee $)$;

${ }^{1} \mathbf{H}$ NMR (600 MHz, CDCl$)_{3} \boldsymbol{\delta} 7.46(\mathrm{dd}, J=8.1,1.3 \mathrm{~Hz}, 2 \mathrm{H}), 7.33-7.26(\mathrm{~m}, 4 \mathrm{H}), 7.09$ (dd, $J=$ 8.5, 2.2 Hz, 1H), $7.04-6.94(\mathrm{~m}, 5 \mathrm{H}), 6.66(\mathrm{~d}, J=8.6 \mathrm{~Hz}, 1 \mathrm{H}), 3.80(\mathrm{~s}, 3 \mathrm{H}), 2.56(\mathrm{~d}, J=5.6 \mathrm{~Hz}$, $1 \mathrm{H}), 2.40(\mathrm{~d}, J=5.5 \mathrm{~Hz}, 1 \mathrm{H})$;

${ }^{13}$ C NMR (151 MHz, $\left.\mathbf{C D C l}_{3}\right) \boldsymbol{\delta}$ 176.0, 153.9, 141.4, 139.1, 133.5, 131.2, 129.8, 128.7, 128.5, $128.5,127.8,127.1,126.5,121.3,110.9,56.0,45.9,41.4,23.4$;

IR (neat) 3024, 1690, 1605, 1498, 1450, 1292, 1233, 1042, 864, 750, $704 \mathrm{~cm}^{-1}$;

FTMS (+p APCI) calcd for $\mathrm{C}_{23} \mathrm{H}_{20} \mathrm{ClO}_{3}(\mathrm{M}+\mathrm{H})^{+} 379.1905$ found 379.10985 . 


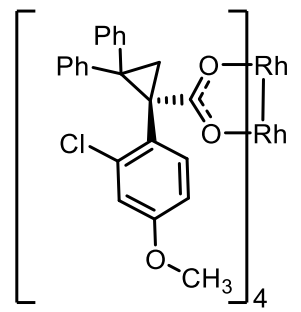

Dirhodium tetrakis((S)-1-(4-methoxy-2-chlorophenyl)-2,2-diphenylcyclopropanecarboxylate):

The reaction was performed following the same procedure as the ligand exchange reaction for $\mathrm{Rh}_{2}(\mathrm{~S} \text {-2-Cl-4-BrTPCP })_{4}:^{2}$ A $100 \mathrm{~mL}$ round-bottom flash was charged with carboxylic acid from the previous step (1.14 g, $3.0 \mathrm{mmol}, 8.0$ equiv.) and $\mathrm{Rh}_{2}(\mathrm{OAc})_{4}(165.8 \mathrm{mg}, 0.38 \mathrm{mmol}, 1.0$ equiv.). Then, approximately $70 \mathrm{~mL}$ toluene was added into the flask. A Soxhlet extractor with a suitable amount of $\mathrm{K}_{2} \mathrm{CO}_{3}$ in it was added on the top of that flask, as well as a condenser (with slow flow of water). The solution was heated to reflux for 3 days and monitored by the color of the solution and by TLC.

The reacted mixture was concentrated under reduced pressure, and the residue was purified by flash column chromatography (hexane/dichloromethane $=3 / 1$ to $2 / 1$ ) to afford the product as a green solid in $76 \%$ yield.

$\mathbf{R f}=0.17$ (hexane/ethyl acetate $=2 / 1)$;

${ }^{1}$ H NMR (600 MHz, CDCl $\left.)_{3}\right) \boldsymbol{\delta} 7.45$ - $7.40(\mathrm{~m}, 2 \mathrm{H}), 7.16$ - 7.07 (m, 5H), 7.01 (d, J=8.4 Hz, 1H), $6.96-6.87(\mathrm{~m}, 4 \mathrm{H}), 6.56(\mathrm{q}, J=2.6 \mathrm{~Hz}, 1 \mathrm{H}), 3.66(\mathrm{~s}, 3 \mathrm{H}), 2.63(\mathrm{~d}, J=4.8 \mathrm{~Hz}, 1 \mathrm{H}), 2.21(\mathrm{~d}, J=$ $4.8 \mathrm{~Hz}, 1 \mathrm{H})$;

${ }^{13}$ C NMR (151 MHz, CDCl $\left._{3}\right) \delta$ 188.4, 158.7, 143.0, 140.0, 137.3, 131.5, 131.4, 128.7, 128.2, $127.4,127.2,126.0,125.5,115.2,111.5,55.5,45.8,41.4,25.9$;

IR (neat) 3023, 2930, 1587, 1495, 1385, 1291, 735, $703 \mathrm{~cm}^{-1}$;

FTMS (+p NSI) calcd for $\mathrm{C}_{92} \mathrm{H}_{72} \mathrm{Cl}_{4} \mathrm{O}_{12} \mathrm{Rh}_{2}(\mathrm{M})^{+} 1714.18880$ found 1714.19342. 


\section{$\mathrm{Rh}_{2}(\mathrm{~S}-2-\mathrm{Cl}-4 / 5-\mathrm{ArTPCP})_{4}$}

In order to generate the $\mathrm{Rh}_{2}(S \text { - } O \text {-ClTPCP })_{4}$ derivatives more efficiently, the direct Suzuki coupling reaction on $\mathrm{Rh}_{2}(S-2-\mathrm{Cl}-4-\mathrm{BrTPCP})_{4}(\mathbf{B})$ and $\mathrm{Rh}_{2}(S-2-\mathrm{Cl}-5-\mathrm{BrTPCP})_{4}(\mathbf{I})$ complexes is preferred (utilizing the aryl bromides on these complexes). Conducting the Suzuki coupling reaction directly on the dirhodium (II) tetracarboxylate complexes was previously reported in the development of $\mathrm{Rh}_{2}\left[S-3,5-\operatorname{di}\left(p-{ }^{t} \mathrm{BuC}_{6} \mathrm{H}_{4}\right) \mathrm{TPCP}\right]_{4}(\mathbf{W})$, which was obtained from 8-fold Suzuki coupling of $\mathrm{Rh}_{2}(S$ 3,5-diBrTPCP $)_{4}(\mathbf{V}){ }^{4}$ The coupling reaction conditions were adapted and optimized first for the synthesis of $\mathrm{Rh}_{2}\left[S-2-\mathrm{Cl}-4-\left(p-{ }^{t} \mathrm{BuC}_{6} \mathrm{H}_{4}\right) \mathrm{TPCP}\right]_{4}(\mathbf{G})$ from $\mathbf{B}$ (Table S1). The initial attempt using the same conditions in the literature with conventional oil-bath heating and identical reagent loadings was found to be unreliable, giving mixtures of products with different degrees of coupling, as analyzed by high resolution mass spectroscopy (HRMS). The incomplete reaction mixture was re-subjected to the same reaction conditions and the desired product with 4-fold couplings was obtained in $58 \%$ yield. A second trial by adding a second batch of base, boronic acid and palladium catalyst after $6 \mathrm{~h}$ was also found to work with $62 \%$ yield. Optimal conditions were achieved with higher yield (70\%) when conducting the reaction using a microwave reactor at $100{ }^{\circ} \mathrm{C}$ for $2 \mathrm{~h}$.

Table S1. Conditions optimization for 4-fold Suzuki coupling on $\mathrm{Rh}_{2}(\mathrm{~S}-2-\mathrm{Cl}-4-\mathrm{BrTPCP})_{4}$

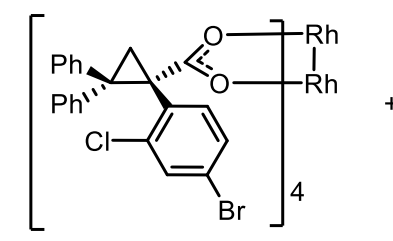

$\mathrm{Rh}_{2}(\mathrm{~S}-2-\mathrm{Cl}-4-\mathrm{BrTPCP})_{4}$

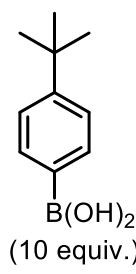

(10 equiv.)

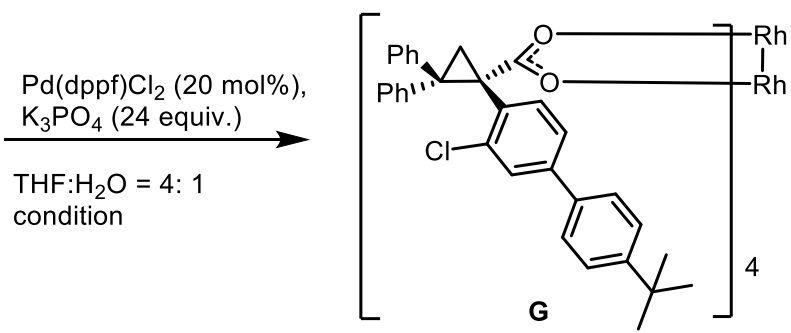

$\mathrm{Rh}_{2}\left[\right.$ S-2-Cl-4-(p- $\left.{ }^{t} \mathrm{BuC}_{6} \mathrm{H}_{4}\right) \mathrm{TPCP}_{4}$

\begin{tabular}{lll}
\hline Entry & Condition & yield, \% \\
\hline 1 & reflux (oil-bath), $12 \mathrm{~h}$ & -- (incompleted) \\
$1-2$ & reflux (oil-bath), $12 \mathrm{~h}$, rxn mixture from entry $1+\left[\right.$ boronic acid, $\left.\mathrm{Pd}(\mathrm{dppf}) \mathrm{Cl}_{2}, \mathrm{~K}_{3} \mathrm{PO}_{4}\right]$ & 58 \\
2 & reflux (oil-bath), $12 \mathrm{~h}+\left[\right.$ boronic acid, $\left.\mathrm{Pd}(\mathrm{dppf}) \mathrm{Cl}_{2}, \mathrm{~K}_{3} \mathrm{PO}_{4}\right]$ after $6 \mathrm{~h}$ & 62 \\
3 & microwave, $100^{\circ} \mathrm{C}, 2 \mathrm{~h}$ & 70 \\
\hline
\end{tabular}



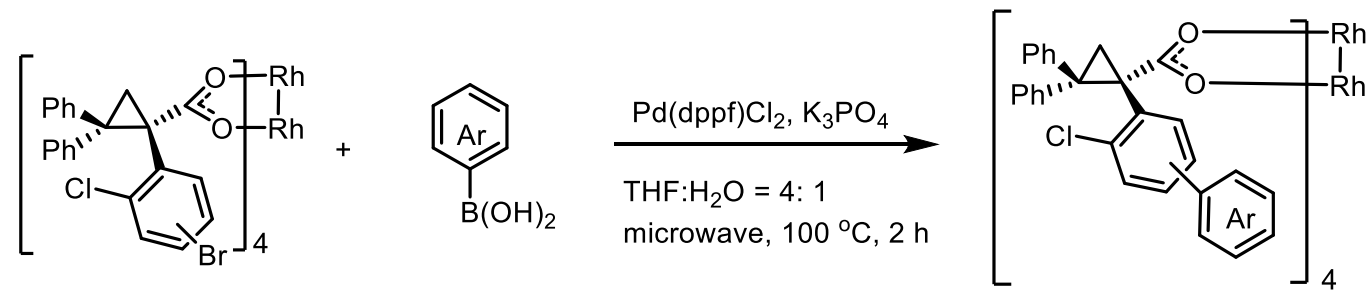

General procedure: An oven-dried microwave reaction tube (G30) was charged with $\mathrm{Rh}_{2}(S-2-\mathrm{Cl}$ 4-BrTPCP $)_{4}$ [or $\left.\mathrm{Rh}_{2}(S-2-\mathrm{Cl}-5-\mathrm{BrTPCP})_{4}\right]$ (95.6 mg, $0.05 \mathrm{mmol}, 1.0$ equiv.), the desired boronic acid ( $0.5 \mathrm{mmol}, 10$ equiv.), and $\mathrm{K}_{3} \mathrm{PO}_{4}(255 \mathrm{mg}, 1.2 \mathrm{mmol}, 24$ equiv.), followed by $6 \mathrm{~mL}$ of THF and $1.5 \mathrm{~mL}$ of DI $\mathrm{H}_{2} \mathrm{O}$. After the reaction vessel was purged with argon (bubbling argon gas until addition results in $\sim 1 \mathrm{~mL}$ THF evaporated), $\mathrm{Pd}(\mathrm{dppf}) \mathrm{Cl}_{2}(7.4 \mathrm{mg}, 0.01 \mathrm{mmol}, 20 \mathrm{~mol} \%$ ) was added and the septum was replaced with a new one. The reaction mixture was irradiated in a Biotage microwave to $100{ }^{\circ} \mathrm{C}$ for $2 \mathrm{~h}$. The resulting green solution was concentrated and extracted with DCM (3 times). The organic layer was concentrated and purified by flash column chromatography (hexane/dichloromethane $=4 / 1$ to $1 / 1$, or hexane/ethyl acetate $19 / 1$ to $5 / 1$ ) to give the desired product as a green solid. 


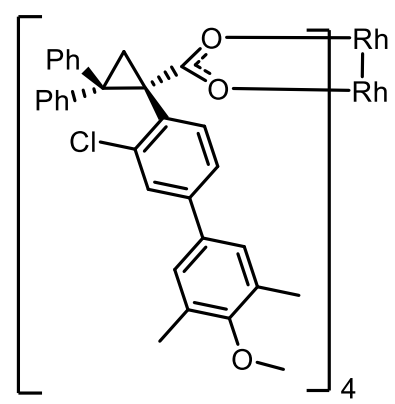

Dirhodium tetrakis((S)-1-(3-chloro-4'-methoxy-3',5'-dimethyl-[1,1'-biphenyl]-4-yl)-2,2diphenylcyclopropane-1-carboxylate) (D)

D was obtained in 54\% yield following the general procedure above using $\mathrm{Rh}_{2}(S-2-\mathrm{Cl}-4-\mathrm{BrTPCP})_{4}$ and (4-methoxy-3,5-dimethylphenyl)boronic acid (90 mg).

$\mathbf{R f}=0.59$ (hexane/dichloromethane $=2 / 3)$;

${ }^{1} \mathbf{H}$ NMR (600 MHz, CDCl $)$ ) $7.48(\mathrm{dt}, J=21.8,3.6 \mathrm{~Hz}, 2 \mathrm{H}), 7.22-7.07(\mathrm{~m}, 9 \mathrm{H}), 6.99(\mathrm{~s}, 1 \mathrm{H})$, $6.93-6.83(\mathrm{~m}, 3 \mathrm{H}), 3.65$ (d, $J=30.5 \mathrm{~Hz}, 3 \mathrm{H}), 2.73(\mathrm{~d}, J=4.2 \mathrm{~Hz}, 1 \mathrm{H}), 2.28(\mathrm{~d}, J=4.5 \mathrm{~Hz}, 1 \mathrm{H})$, $2.25(\mathrm{~s}, 3 \mathrm{H}), 2.18(\mathrm{~s}, 3 \mathrm{H})$;

${ }^{13}$ C NMR (151 MHz, $\left.\mathbf{C D C l}_{3}\right) \delta$ 187.3, 156.7, 143.5, 142.9, 140.5, 140.3, 139.8, 137.9, 137.1, 137.0, 135.3, 135.2, 134.5, 134.3, 131.4, 131.3, 131.2, 131.0, 130.9, 130.7, 129.0, 128.5, 128.4, $128.2,127.5,127.3,127.3,127.2,127.1,127.0,125.9,125.9,125.3,125.1,124.3,124.1,77.2$, $59.8,45.5,41.5,25.4,21.5,16.1$;

IR (neat) 3613, 3056, 2926, 2246, 1585, 1476, 1386, 1237, 1016, 909, 732, $694 \mathrm{~cm}^{-1}$;

FTMS (+p ESI) calcd for $\mathrm{C}_{124} \mathrm{H}_{104} \mathrm{O}_{12} \mathrm{Cl}_{4} \mathrm{Rh}_{2}(\mathrm{M})^{+} 2130.4392$ found 2130.43266. 


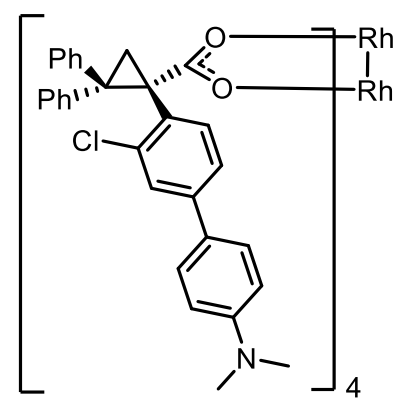

Dirhodium tetrakis((S)-1-(3-chloro-4'-(dimethylamino)-[1,1'-biphenyl]-4-yl)-2,2diphenylcyclopropane-1-carboxylate) (E)

E was obtained in 50\% yield following the general procedure above using $\mathrm{Rh}_{2}(S-2-\mathrm{Cl}-4-\mathrm{BrTPCP})_{4}$ and (4-(dimethylamino)phenyl)boronic acid (82.5 mg).

$\mathbf{R f}=0.57$ (hexane/dichloromethane $=2 / 3$ );

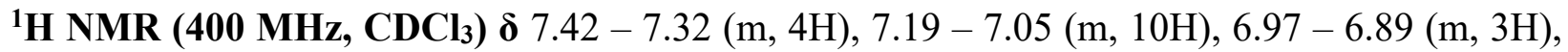
$3.05-2.62(\mathrm{~m}, 3 \mathrm{H}), 2.59(\mathrm{~d}, J=6.3 \mathrm{~Hz}, 1 \mathrm{H}), 2.22(\mathrm{~d}, J=6.1 \mathrm{~Hz}, 1 \mathrm{H}), 2.19-2.14(\mathrm{~m}, 1 \mathrm{H}), 1.99$ $-1.91(\mathrm{~m}, 2 \mathrm{H})$;

${ }^{13}$ C NMR (100 MHz, $\left.\mathbf{C D C l}_{3}\right) \boldsymbol{\delta}$ 187.4, 154.2, 143.0, 142.4, 142.2, 139.9, 139.3, 137.7, 136.5, $131.4,131.1,127.3,127.2,126.2,125.8,125.3,120.1,119.5,46.0,45.3,41.4,41.2,25.0$;

IR (neat) 3024, 2921, 2850, 1739, 1587, 1491, 1376, 1150, 1045, 812, 734, 692, $509 \mathrm{~cm}^{-1}$; FTMS (+p ESI) calcd for $\mathrm{C}_{120} \mathrm{H}_{100} \mathrm{O}_{8} \mathrm{~N}_{4} \mathrm{Cl}_{4} \mathrm{Rh}_{2}(\mathrm{M})^{+} 2070.4405$ found 2070.4368 . 


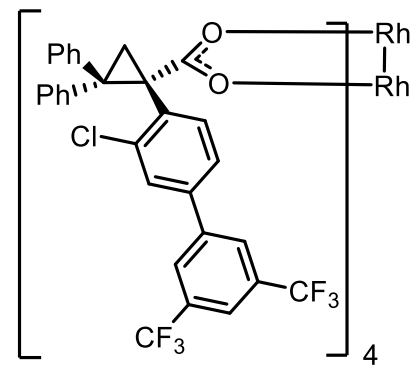

Dirhodium tetrakis((S)-1-(3-chloro-3',5'-bis(trifluoromethyl)-[1,1'-biphenyl]-4-yl)-2,2diphenylcyclopropane-1-carboxylate) (F)

F was obtained in $68 \%$ yield following the general procedure above using $\mathrm{Rh}_{2}(S-2-\mathrm{Cl}-4-\mathrm{BrTPCP})_{4}$ and (3,5-bis(trifluoromethyl)phenyl)boronic acid (129 mg).

$\mathbf{R f}=0.54$ (hexane/dichloromethane $=2 / 3)$;

${ }^{1}$ H NMR (600 MHz, CDCl $)$ ) 7.85 - 7.77 (m, 3H), $7.54-7.45$ (m, 2H), 7.32 - 7.27 (m, 2H), $7.25(\mathrm{~d}, J=1.8 \mathrm{~Hz}, 1 \mathrm{H}), 7.23-7.14(\mathrm{~m}, 5 \mathrm{H}), 6.98(\mathrm{dd}, J=8.5,6.9 \mathrm{~Hz}, 2 \mathrm{H}), 6.95-6.89(\mathrm{~m}, 1 \mathrm{H})$, $2.84(\mathrm{~d}, J=4.9 \mathrm{~Hz}, 1 \mathrm{H}), 2.38(\mathrm{~d}, J=4.8 \mathrm{~Hz}, 1 \mathrm{H})$;

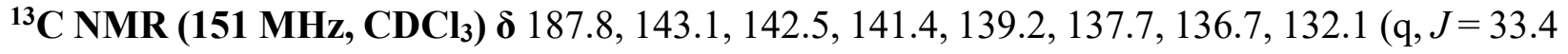
Hz), 131.5, 131.2, 128.4, 127.7, 127.4, 127.3, 126.9, 126.3, 125.9, 124.7, 123.2 (d, $J=272.7 \mathrm{~Hz})$, 121.2, 46.1, 41.6, 25.6;

IR (neat) 3618, 3058, 1738, 1585, 1496, 1383, 1279, 1185, 1136, $701 \mathrm{~cm}^{-1}$;

FTMS (+p ESI) calcd for $\mathrm{C}_{120} \mathrm{H}_{72} \mathrm{O}_{8} \mathrm{~F}_{24} \mathrm{Cl}_{4} \mathrm{Rh}_{2}(\mathrm{M})^{+} 2442.1708$ found 2442.16239 . 


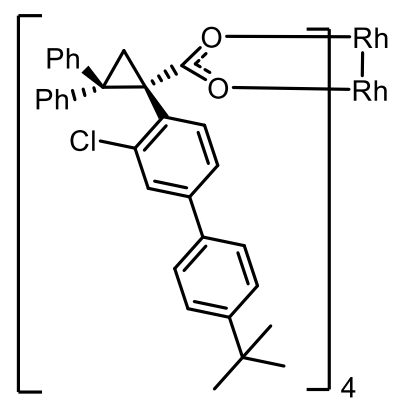

Dirhodium tetrakis((S)- 1-(4'-(tert-butyl)-3-chloro-[1,1'-biphenyl]-4-yl)-2,2diphenylcyclopropane-1-carboxylate) (G)

$\mathbf{G}$ was obtained in $70 \%$ yield following the general procedure above using $\mathrm{Rh}_{2}(\mathrm{~S}-2-\mathrm{Cl}-4-\mathrm{BrTPCP})_{4}$ and (4-(tert-butyl)phenyl)boronic acid ( $89 \mathrm{mg})$.

$\mathbf{R f}=0.71$ (hexane/ethyl acetate $=4 / 1)$;

${ }^{1} \mathbf{H}$ NMR (600 MHz, $\left.\mathbf{C D C l}_{3}\right) \boldsymbol{\delta} 7.48(\mathrm{td}, J=10.0,8.6,5.2 \mathrm{~Hz}, 7 \mathrm{H}), 7.23-7.13(\mathrm{~m}, 4 \mathrm{H}), 7.11-$ $7.06(\mathrm{~m}, 2 \mathrm{H}), 7.02$ (d, $J=8.3 \mathrm{~Hz}, 1 \mathrm{H}), 6.96-6.88(\mathrm{~m}, 3 \mathrm{H}), 2.75$ (d, $J=4.8 \mathrm{~Hz}, 1 \mathrm{H}), 2.34$ (d, $J=$ $4.8 \mathrm{~Hz}, 1 \mathrm{H}), 1.38(\mathrm{~s}, 9 \mathrm{H})$;

${ }^{13} \mathbf{C}$ NMR (151 MHz, $\left.\mathbf{C D C l}_{3}\right) \boldsymbol{\delta} 187.6,150.5,143.6,140.5,139.8,137.1,136.9,134.5,131.6$, 130.9, 128.6, 127.7, 127.4, 127.2, 126.7, 126.1, 125.7, 125.3, 124.4, 45.7, 41.7, 34.6, 31.5, 25.5;

IR (neat) 3028, 2960, 2867, 1726, 1574, 1494, 1386, 1271, 907, 731, 711, 694, $552 \mathrm{~cm}^{-1}$;

FTMS (+p ESI) calcd for $\mathrm{C}_{128} \mathrm{H}_{112} \mathrm{O}_{8} \mathrm{Cl}_{4} \mathrm{Rh}_{2}(\mathrm{M})^{+} 2122.5221$ found 2122.51721 . 


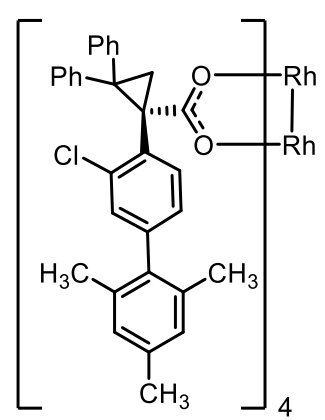

Dirhodium tetrakis((S)-1-(4-(2,4,6-trimethylphenyl)-2-chlorophenyl)-2,2-diphenylcyclopropanecarboxylate) $(\mathrm{H})$

$\mathbf{H}$ was obtained in $63 \%$ yield following the general procedure above using $\mathrm{Rh}_{2}(S \text {-2-Cl-4-BrTPCP })_{4}$ and mesitylboronic acid (89 $\mathrm{mg})$.

$\mathbf{R f}=0.62($ pentane/dichloromethane $=3 / 2) ;$

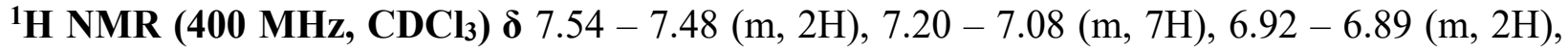
$6.87-6.84(\mathrm{~m}, 2 \mathrm{H}), 6.80(\mathrm{~d}, J=12.6 \mathrm{~Hz}, 1 \mathrm{H}), 6.75-6.71(\mathrm{~m}, 1 \mathrm{H}), 2.76(\mathrm{~d}, J=4.4 \mathrm{~Hz}, 1 \mathrm{H}), 2.29$ $-2.25(\mathrm{~m}, 4 \mathrm{H}), 1.97(\mathrm{~s}, 3 \mathrm{H}), 1.51(\mathrm{~s}, 3 \mathrm{H})$;

${ }^{13}$ C NMR (101 MHz, CDCl $)$ $\delta$ 187.7, 143.4, 142.7, 142.0, 140.6, 139.8, 137.7, 137.0, 136.8, 136.2, 134.3, 131.7, 131.4, 130.8, 130.3, 130.0, 128.7, 128.1, 127.4, 127.2, 126.7, 126.0, 125.7, $125.4,119.4,45.5,42.0,35.5,31.5,22.6,21.1$.

IR (neat) 3022, 1713, 1587, 1392, 754, $703 \mathrm{~cm}^{-1}$;

FTMS (+p ESI) calcd for $\mathrm{C}_{124} \mathrm{H}_{104} \mathrm{O}_{8} \mathrm{Cl}_{4} \mathrm{Rh}_{2}(\mathrm{M})^{+} 2066.49954$ found 2066.45488 . 


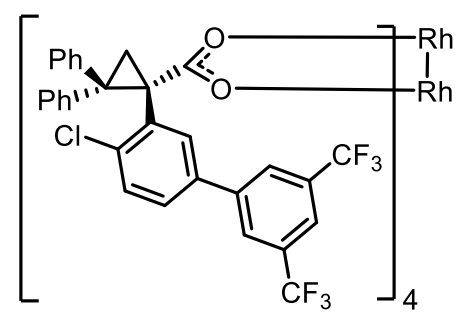

Dirhodium tetrakis((S)-1-(4-chloro-3',5'-bis(trifluoromethyl)-[1,1'-biphenyl]-3-yl)-2,2diphenylcyclopropane-1-carboxylate) (K)

$\mathbf{K}$ was obtained in $66 \%$ yield following the general procedure above using $\mathrm{Rh}_{2}(S-2-\mathrm{Cl}-5-\mathrm{BrTPCP})_{4}$ and (3,5-bis(trifluoromethyl)phenyl)boronic acid (129 mg).

$\mathbf{R f}=0.5$ (hexane/dichloromethane $=1 / 1)$;

${ }^{1} \mathbf{H}$ NMR (600 MHz, $\left.\mathbf{C D C l}_{3}\right) \boldsymbol{\delta} 7.92(\mathrm{~d}, J=1.8 \mathrm{~Hz}, 2 \mathrm{H}), 7.54-7.48(\mathrm{~m}, 2 \mathrm{H}), 7.37-7.29(\mathrm{~m}, 2 \mathrm{H})$, $7.22-7.10(\mathrm{~m}, 7 \mathrm{H}), 6.95$ (dddt, $J=12.6,8.4,7.2,2.7 \mathrm{~Hz}, 3 \mathrm{H}), 2.86$ (d, $J=4.9 \mathrm{~Hz}, 1 \mathrm{H}), 2.41$ (d, $J=4.9 \mathrm{~Hz}, 1 \mathrm{H})$;

${ }^{13}$ C NMR (151 MHz, $\left.\mathbf{C D C l}_{3}\right) \boldsymbol{\delta} 187.7,142.4,141.9,137.8,136.8,135.9,132.1$ (q, $\left.J=33.1 \mathrm{~Hz}\right)$, 131.2, 130.2, 130.0, 128.3, 128.0, 127.4, 127.0, 126.8, 126.4, 125.7, 124.3, 122.5, 121.0, 120.7, $119.2,46.2,41.9,35.3$

IR (neat) 3620, 3058, 2931, 1735, 1580, 1375, 1279, 1183, 1135, $704 \mathrm{~cm}^{-1}$;

FTMS (+p ESI) calcd for $\mathrm{C}_{120} \mathrm{H}_{72} \mathrm{O}_{8} \mathrm{~F}_{24} \mathrm{Cl}_{4} \mathrm{Rh}_{2}(\mathrm{M})^{+} 2442.1708$ found 2442.17346 . 


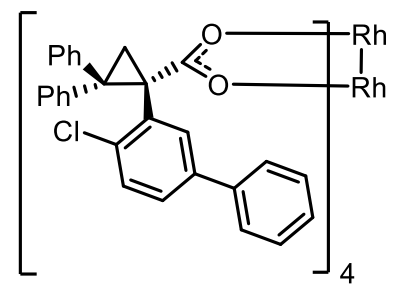

Dirhodium tetrakis((S)-1-(4-chloro-[1,1'-biphenyl]-3-yl)-2,2-diphenylcyclopropane-1carboxylate) (L)

$\mathbf{L}$ was obtained in $65 \%$ yield following the general procedure above using $\mathrm{Rh}_{2}(S-2-\mathrm{Cl}-5-\mathrm{BrTPCP})_{4}$ and phenylboronic acid (61 mg).

$\mathbf{R f}=0.40$ (hexane/ethyl acetate $=4 / 1)$;

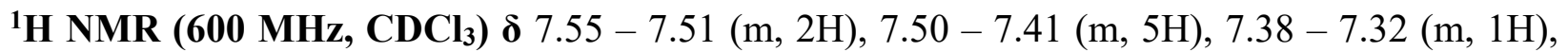
$7.22-7.13(\mathrm{~m}, 4 \mathrm{H}), 7.10-7.05(\mathrm{~m}, 2 \mathrm{H}), 6.98(\mathrm{~d}, J=8.2 \mathrm{~Hz}, 1 \mathrm{H}), 6.96-6.90(\mathrm{~m}, 3 \mathrm{H}), 2.75(\mathrm{~d}, J$ $=4.9 \mathrm{~Hz}, 1 \mathrm{H}), 2.35(\mathrm{~d}, J=4.8 \mathrm{~Hz}, 1 \mathrm{H})$;

${ }^{13}$ C NMR (151 MHz, CDCl $)$ $\delta$ 188.0, 142.5, 140.3, 139.2, 138.9, 136.3, 135.9, 131.5, 130.1, $129.1,128.7,128.5,127.3,127.3,127.0,127.0,126.4,125.9,125.6,45.3,42.4,25.8$;

IR (neat) 3609, 3086, 3057, 3028, 1740, 1585, 1470, 1400, 1380, 1042, 909, 760, $695 \mathrm{~cm}^{-1}$; FTMS (+p ESI) calcd for $\mathrm{C}_{112} \mathrm{H}_{80} \mathrm{O}_{8} \mathrm{Cl}_{4} \mathrm{Rh}_{2}(\mathrm{M})^{+} 1898.2717$ found 1898.26942. 


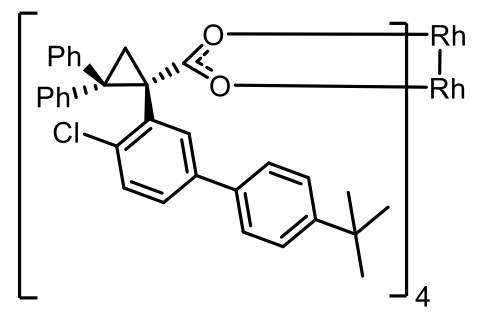

Dirhodium tetrakis((S)-1-(4'-(tert-butyl)-4-chloro-[1,1'-biphenyl]-3-yl)-2,2diphenylcyclopropane-1-carboxylate) (M)

M was obtained in $67 \%$ yield following the general procedure above using $\mathrm{Rh}_{2}(S$-2-Cl-4BrTPCP) 4 and (4-(tert-butyl)phenyl)boronic acid (89 mg).

$\mathbf{R f}=0.48$ (hexane/dichloromethane $=3 / 2$ );

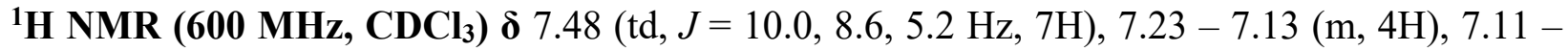
$7.06(\mathrm{~m}, 2 \mathrm{H}), 7.02(\mathrm{~d}, J=8.3 \mathrm{~Hz}, 1 \mathrm{H}), 6.96-6.88(\mathrm{~m}, 3 \mathrm{H}), 2.75(\mathrm{~d}, J=4.8 \mathrm{~Hz}, 1 \mathrm{H}), 2.34(\mathrm{~d}, J=$ $4.8 \mathrm{~Hz}, 1 \mathrm{H}), 1.38(\mathrm{~s}, 9 \mathrm{H})$;

${ }^{13} \mathbf{C}$ NMR (151 MHz, $\left.\mathbf{C D C l}_{3}\right) \boldsymbol{\delta}$ 188.0, 150.4, 142.6, 139.3, 138.6, 137.4, 136.1, 135.7, 131.5, 129.8, 129.2, 128.6, 128.3, 127.3, 127.0, 126.6, 126.2, 125.9, 125.6, 45.4, 42.4, 34.6, 31.4;

IR (neat) 3619, 3537, 3056, 2960, 2868, 1728, 1585, 1472, 1379, 1112, 909, 840, 731, $703 \mathrm{~cm}^{-1}$; FTMS (+p ESI) calcd for $\mathrm{C}_{128} \mathrm{H}_{112} \mathrm{O}_{8} \mathrm{Cl}_{4} \mathrm{Rh}_{2}(\mathrm{M})^{+} 2122.5221$ found 2122.51525 . 


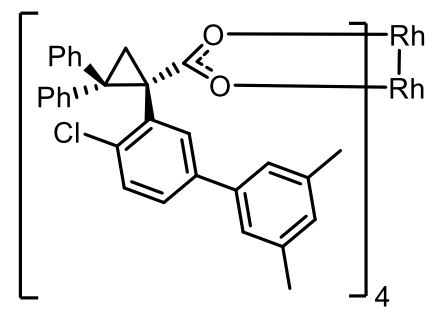

Dirhodium tetrakis((S)-1-(4-chloro-3',5'-dimethyl-[1,1'-biphenyl]-3-yl)-2,2diphenylcyclopropane-1-carboxylate) (N)

$\mathbf{N}$ was obtained in $66 \%$ yield following the general procedure above using $\mathrm{Rh}_{2}(S-2-\mathrm{Cl}-5-\mathrm{BrTPCP})_{4}$ and (3,5-dimethylphenyl)boronic acid (75 mg).

$\mathbf{R f}=0.54$ (hexane/ethyl acetate $=4 / 1)$;

${ }^{1}$ H NMR (600 MHz, CDCl $)$ ) $7.51-7.47$ (m, 2H), 7.40 (d, J=2.2 Hz, 1H), $7.21-7.08$ (m, 9H), 7.00 (dq, $J=1.7,0.9 \mathrm{~Hz}, 1 \mathrm{H}), 6.95-6.89(\mathrm{~m}, 3 \mathrm{H}), 2.79$ (d, $J=4.9 \mathrm{~Hz}, 1 \mathrm{H}), 2.40$ (s, 6H), 2.34 (d, $J=4.8 \mathrm{~Hz}, 1 \mathrm{H})$;

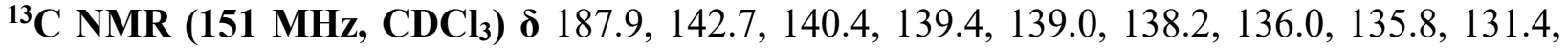
$129.8,129.1,128.9,128.5,127.3,127.0,126.5,125.9,125.5,124.9,77.2$, 45.5, 42.3, 25.7, 21.5;

IR (neat) 3619, 3055, 3024, 2929, 1735, 1588, 1376, 819, 732, $703 \mathrm{~cm}^{-1}$;

FTMS (+p ESI) calcd for $\mathrm{C}_{120} \mathrm{H}_{96} \mathrm{O}_{8} \mathrm{Cl}_{4} \mathrm{Rh}_{2}(\mathrm{M})^{+} 2010.3969$ found 2010.40020 . 


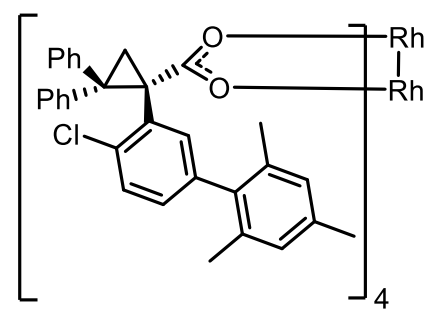

Dirhodium tetrakis((S)-1-(4-chloro-2',4',6'-trimethyl-[1,1'-biphenyl]-3-yl)-2,2diphenylcyclopropane-1-carboxylate) $(0)$

$\mathrm{O}$ was obtained in $67 \%$ yield following the general procedure above using $\mathrm{Rh}_{2}(S-2-\mathrm{Cl}-4-\mathrm{BrTPCP})_{4}$ and mesitylboronic acid $(82 \mathrm{mg})$.

$\mathbf{R f}=0.44$ (hexane/dichloromethane $=1 / 1)$;

${ }^{1} \mathbf{H}$ NMR (600 MHz, $\left.\mathbf{C D C l}_{3}\right) \boldsymbol{\delta} 7.50-7.42(\mathrm{~m}, 2 \mathrm{H}), 7.20-7.08(\mathrm{~m}, 5 \mathrm{H}), 6.95-6.84(\mathrm{~m}, 7 \mathrm{H})$, $6.70(\mathrm{dd}, J=8.0,2.1 \mathrm{~Hz}, 1 \mathrm{H}), 2.68(\mathrm{~d}, J=4.9 \mathrm{~Hz}, 1 \mathrm{H}), 2.33$ (s, 3H), $2.26(\mathrm{~d}, J=4.8 \mathrm{~Hz}, 1 \mathrm{H}), 1.98$ $(\mathrm{s}, 3 \mathrm{H}), 1.70(\mathrm{~s}, 3 \mathrm{H})$;

${ }^{13} \mathbf{C}$ NMR (151 MHz, $\left.\mathbf{C D C l}_{3}\right) \delta$ 187.6, 142.7, 139.7, 138.7, 138.1, 136.8, 136.4, 136.0, 135.2, $131.7,131.2,130.2,128.9,128.5,128.3,127.9,127.8,127.2,127.1,125.9,125.5,45.7,41.9,35.3$, 21.1, 20.9, 20.3;

IR (neat) 3618, 3056, 2921, 1734, 1586, 1468, 1400, 1380, 1044, 825, $703 \mathrm{~cm}^{-1}$;

FTMS (+p ESI) calcd for $\mathrm{C}_{124} \mathrm{H}_{104} \mathrm{O}_{8} \mathrm{Cl}_{4} \mathrm{Rh}_{2}(\mathrm{M})^{+} 2066.4595$ found 2066.46321. 


\section{General Procedure and Analysis for C-H Functionalization Reactions}

\subsection{General Procedure for C-H Functionalization Reactions}

A 16-mL reaction vial $(21 \times 70 \mathrm{~mm})$ with screw cap (open top with PTFE faced silicone septum) was charged with the $\mathrm{Rh}_{2} \mathrm{~L}_{4}$ complex of interest $(0.003 \mathrm{mmol}, 1 \mathrm{~mol} \%)$ and 1-bromo-4pentylbenzene ( $136.3 \mathrm{mg}, 0.6 \mathrm{mmol}, 2.0$ equiv.). The reaction vessel was then evacuated and backfilled with argon ( 3 times), followed by the addition of dry, degassed $\mathrm{CH}_{2} \mathrm{Cl}_{2}(3 \mathrm{~mL})$. The solution was heated to $39^{\circ} \mathrm{C}$ (b.p. of $\mathrm{CH}_{2} \mathrm{Cl}_{2}$ ) for $10 \mathrm{~min}$. 2,2,2-Trichloroethyl 2-(4-bromophenyl)2-diazoacetate (111.7 mg, $0.3 \mathrm{mmol}, 1.0$ equiv.) was weighed in a 20-mL scintillation vial and dissolved in $6 \mathrm{~mL}$ of dry, degassed $\mathrm{CH}_{2} \mathrm{Cl}_{2}$ under argon. Then, under reflux condition and an argon atmosphere, the diazo solution was added to the reaction vessel dropwise via syringe pump over 3 $\mathrm{h}$. The reaction mixture was stirred at $39^{\circ} \mathrm{C}$ for another $30 \mathrm{~min}$. Subsequently, the reaction mixture was concentrated under vacuum for obtaining a crude ${ }^{1} \mathrm{H}$ NMR. Finally, the crude product was purified by flash column chromatography to afford the product (mixture of regioisomers and diastereomers) as a colorless oil (gradient elution from pentane to $5 \%$ diethyl ether/pentane).

\subsection{Regioisomer and Diastereomer Ratios Determination}

The crude ${ }^{1} \mathrm{H}$ NMR in the general procedure was utilized for determining regioisomer and diastereomer ratios. ${ }^{1} \mathrm{H}$ NMR spectra were obtained using a Bruker-600 MHz spectrometer with an ID probe, and acquisition was conducted with 16 scans and a relaxation time of 1 second.

The crude ${ }^{1} \mathrm{H}$ NMR spectra were analyzed with MestReNova 11.0. Before integration, spectra were processed through a Segments Smoother baseline correction manually. The ratios were measured by integration of the peaks corresponding to the hydrogens indicated below (see crude ${ }^{1} \mathrm{H}$ NMR spectra).

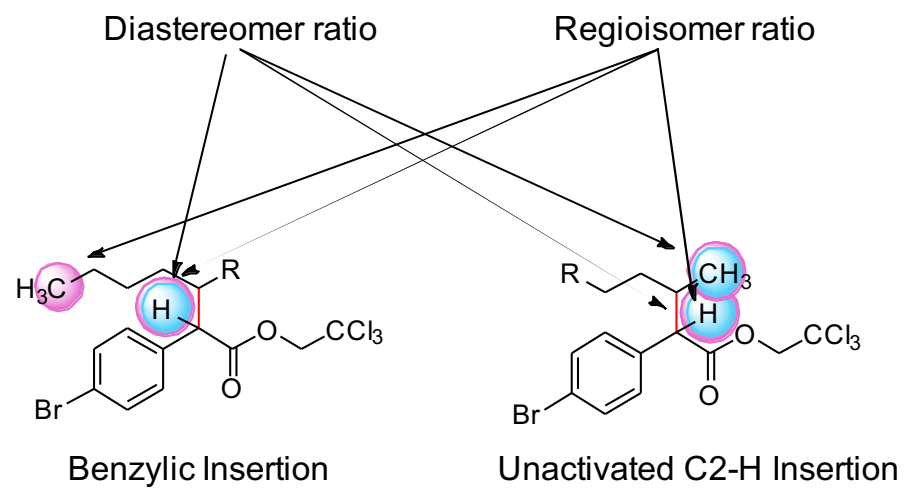




\section{Crude ${ }^{1}$ H NMR Spectra for Site-Selectivity Determination}

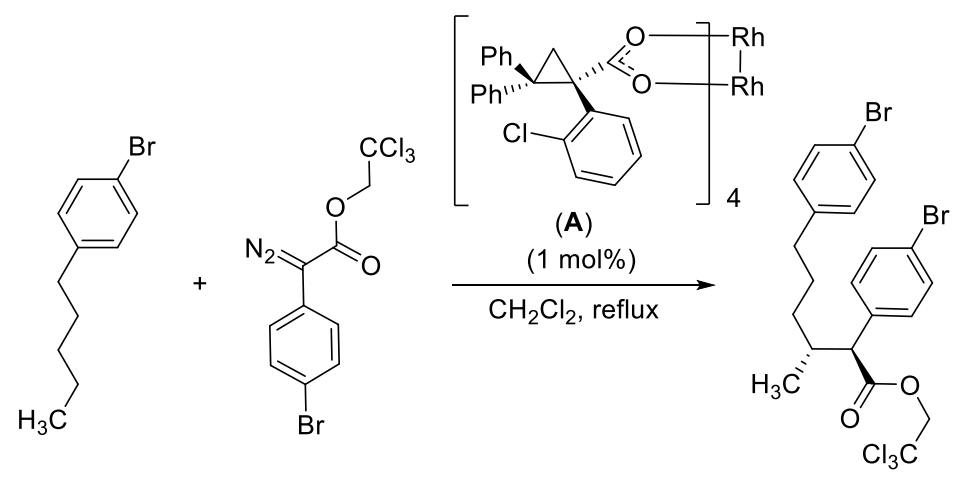

(Acetone- $d_{6}$ )
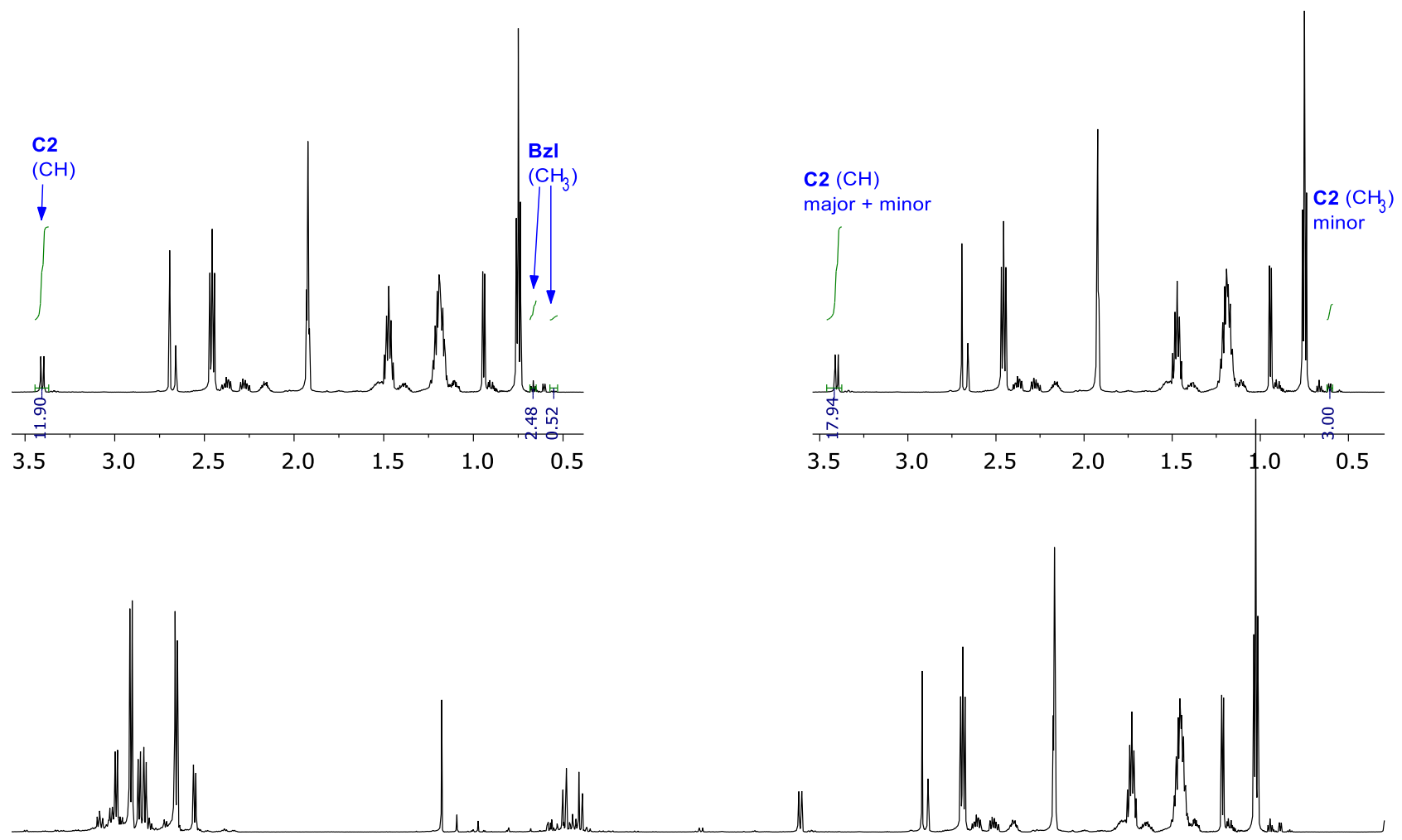

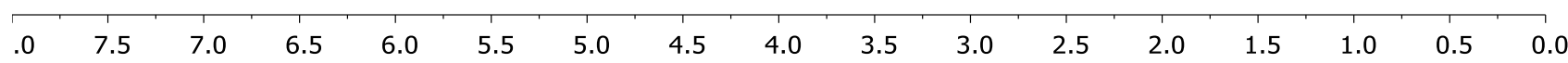




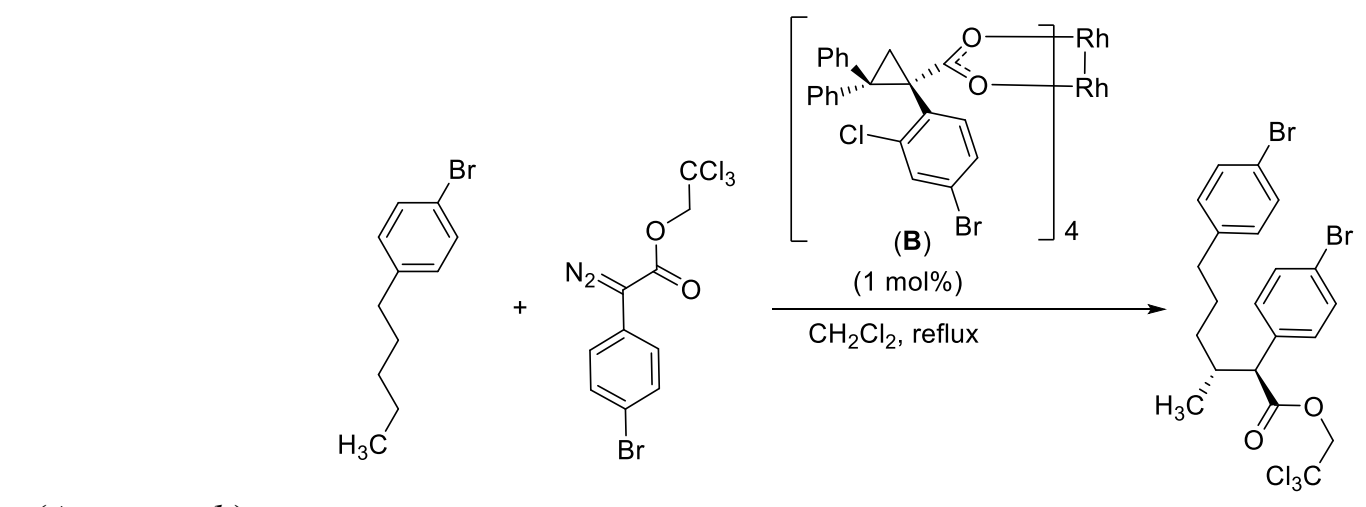

(Acetone- $\left.d_{6}\right)$
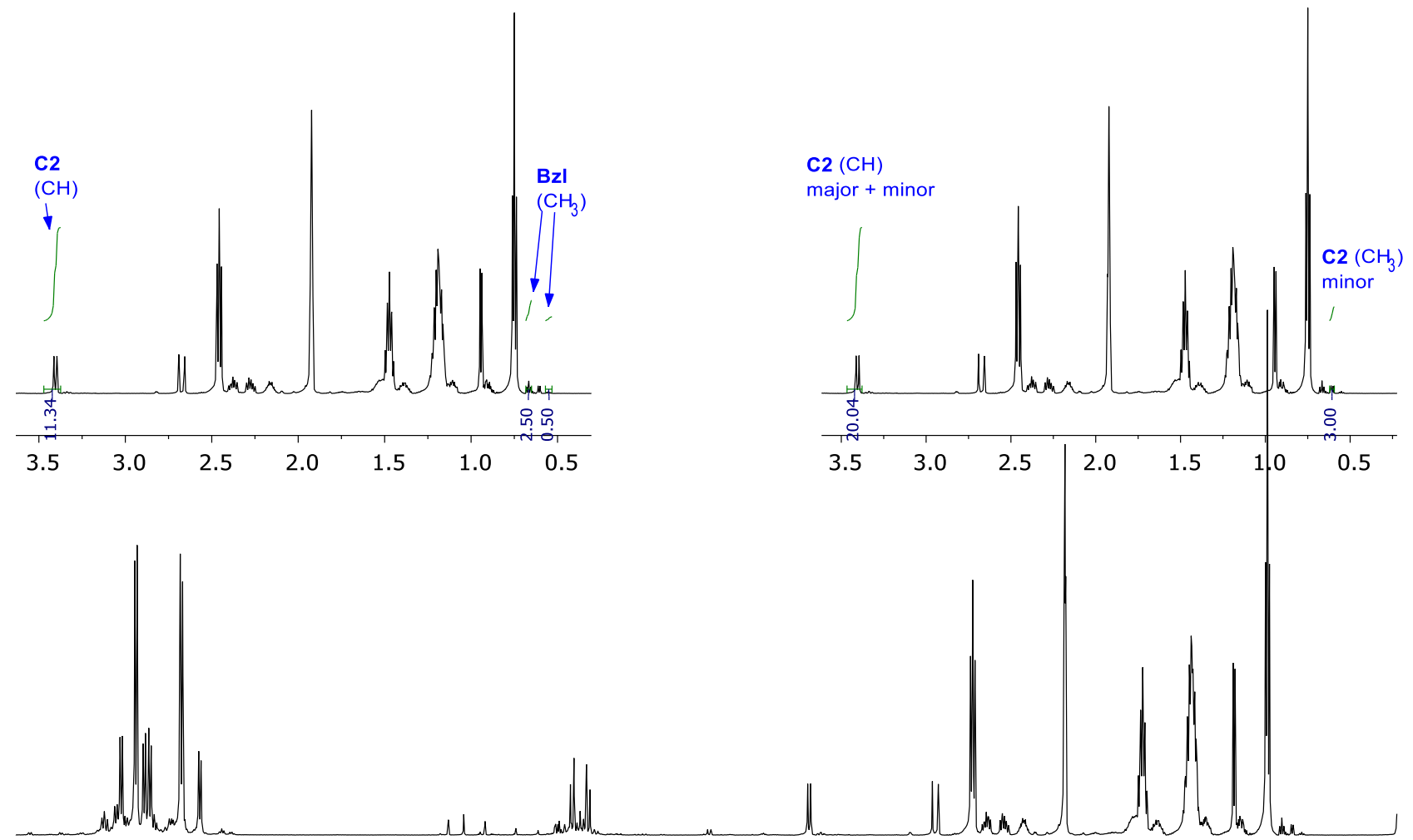

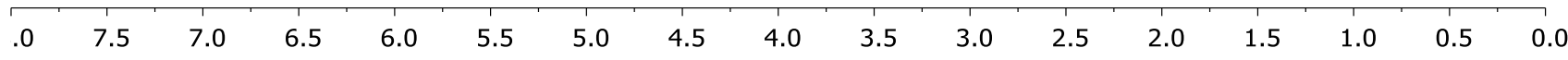



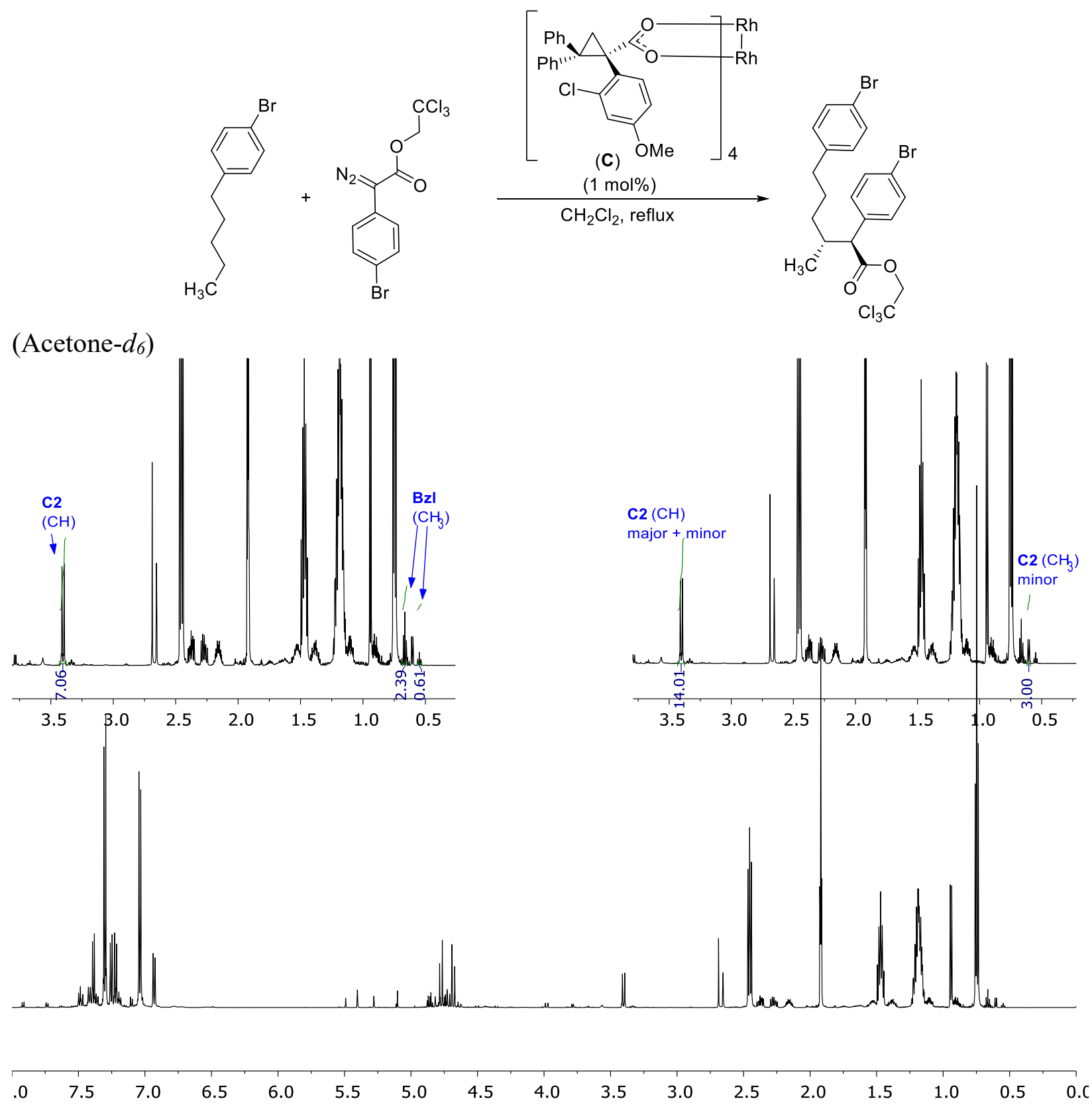

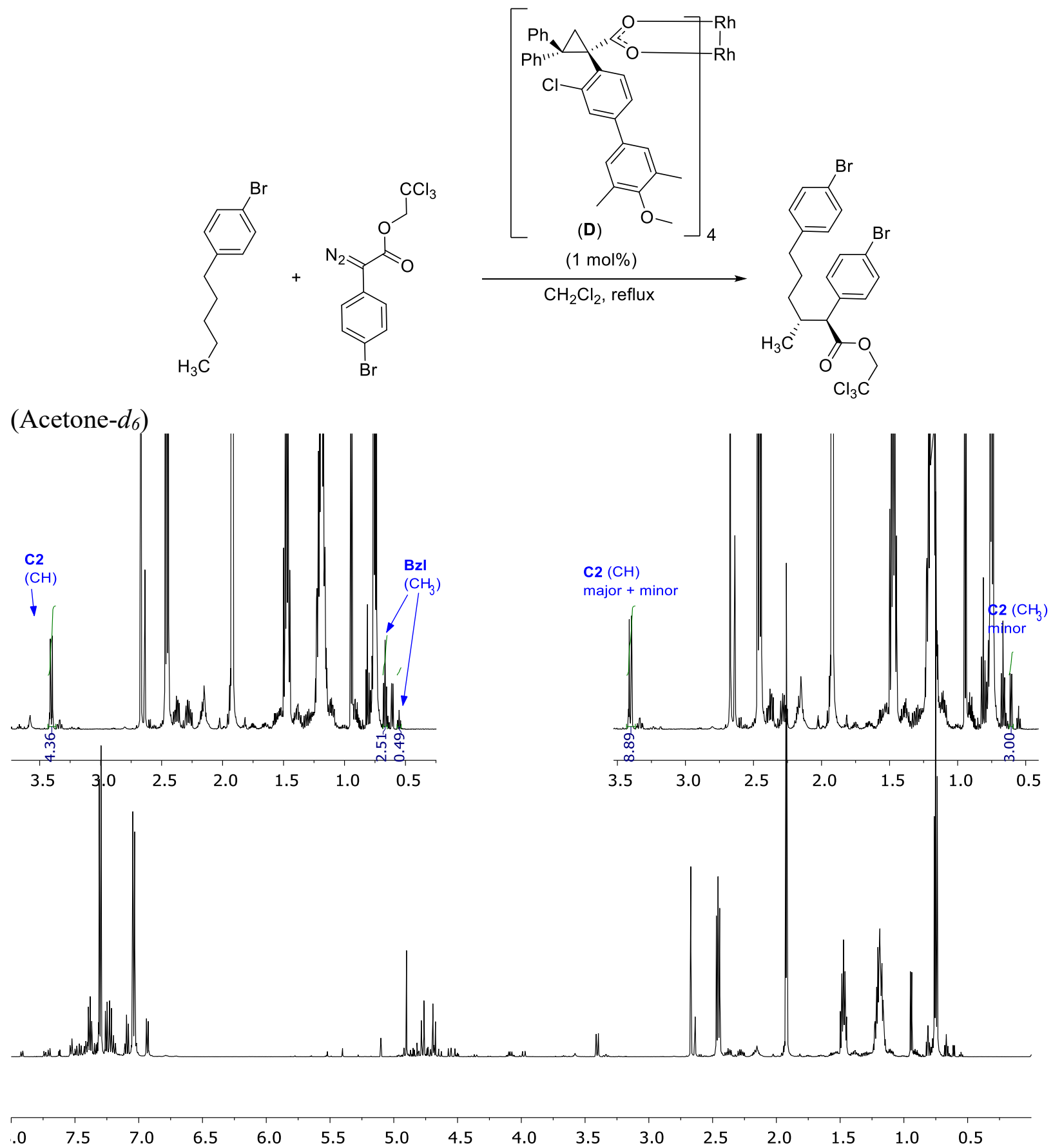

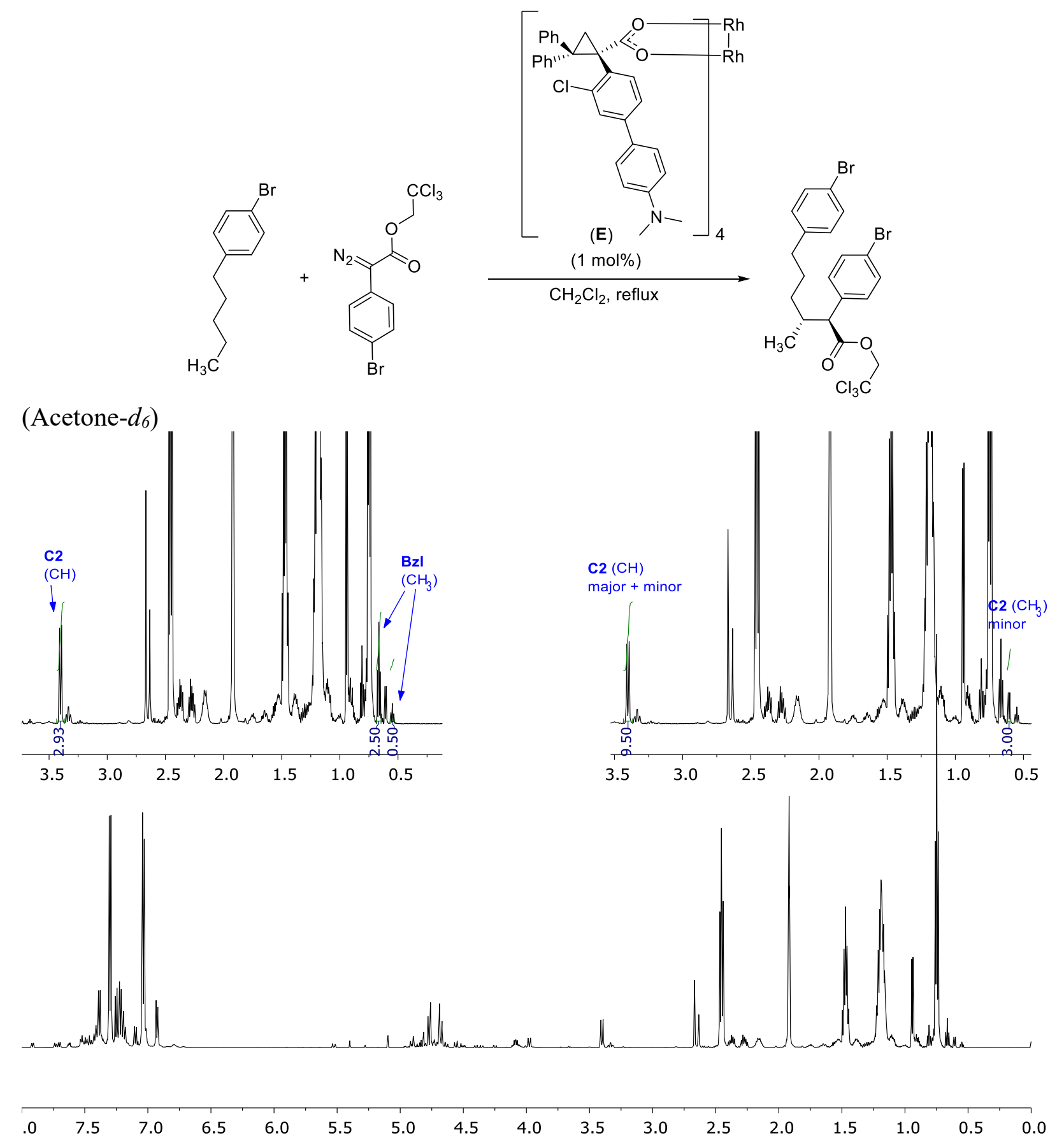

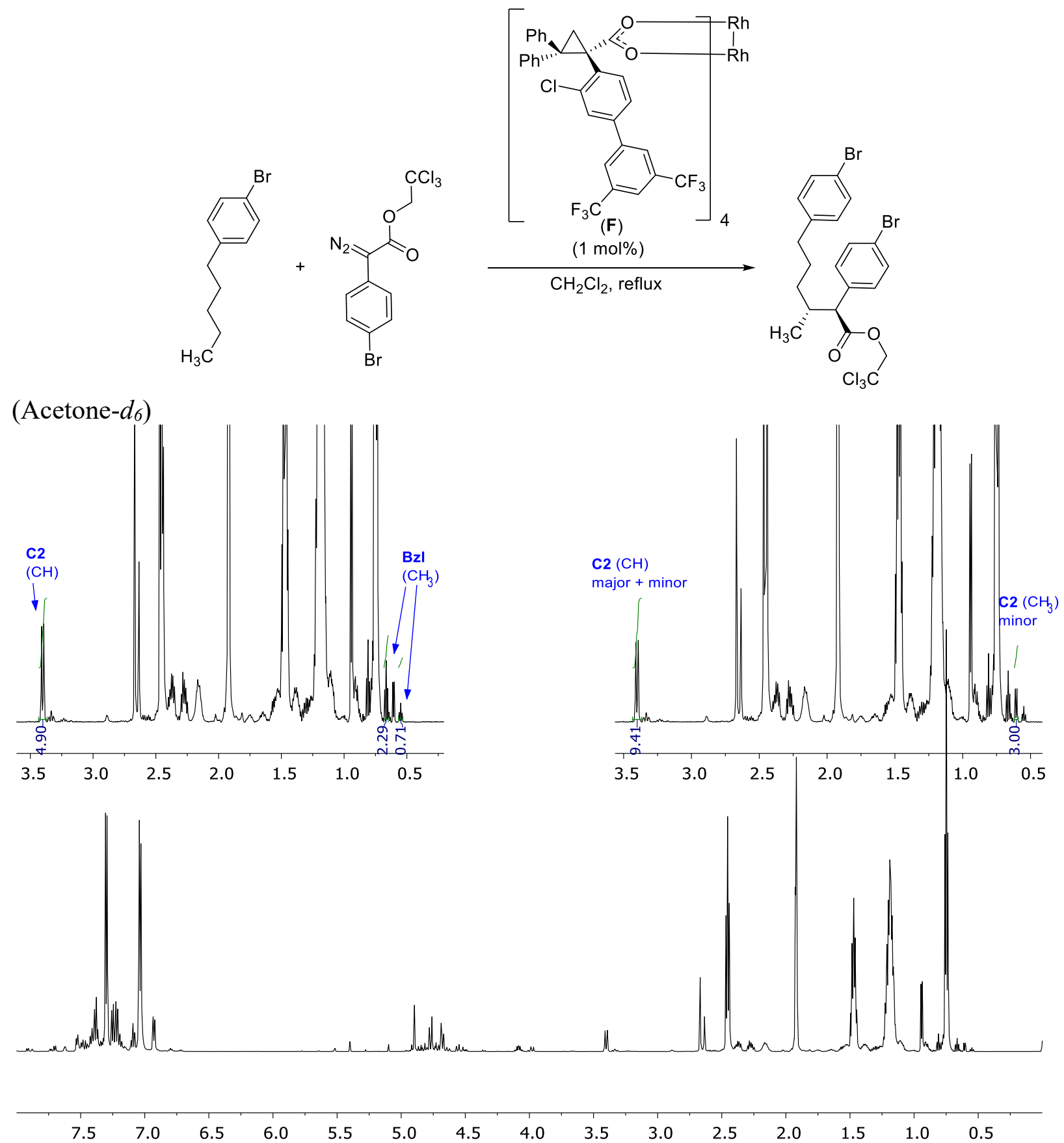

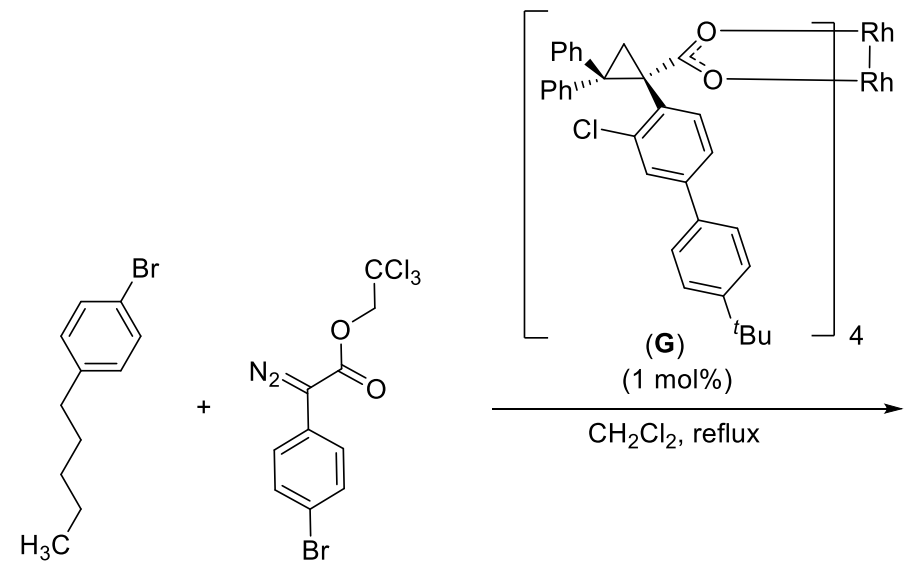

\section{(Acetone- $d_{6}$ )}
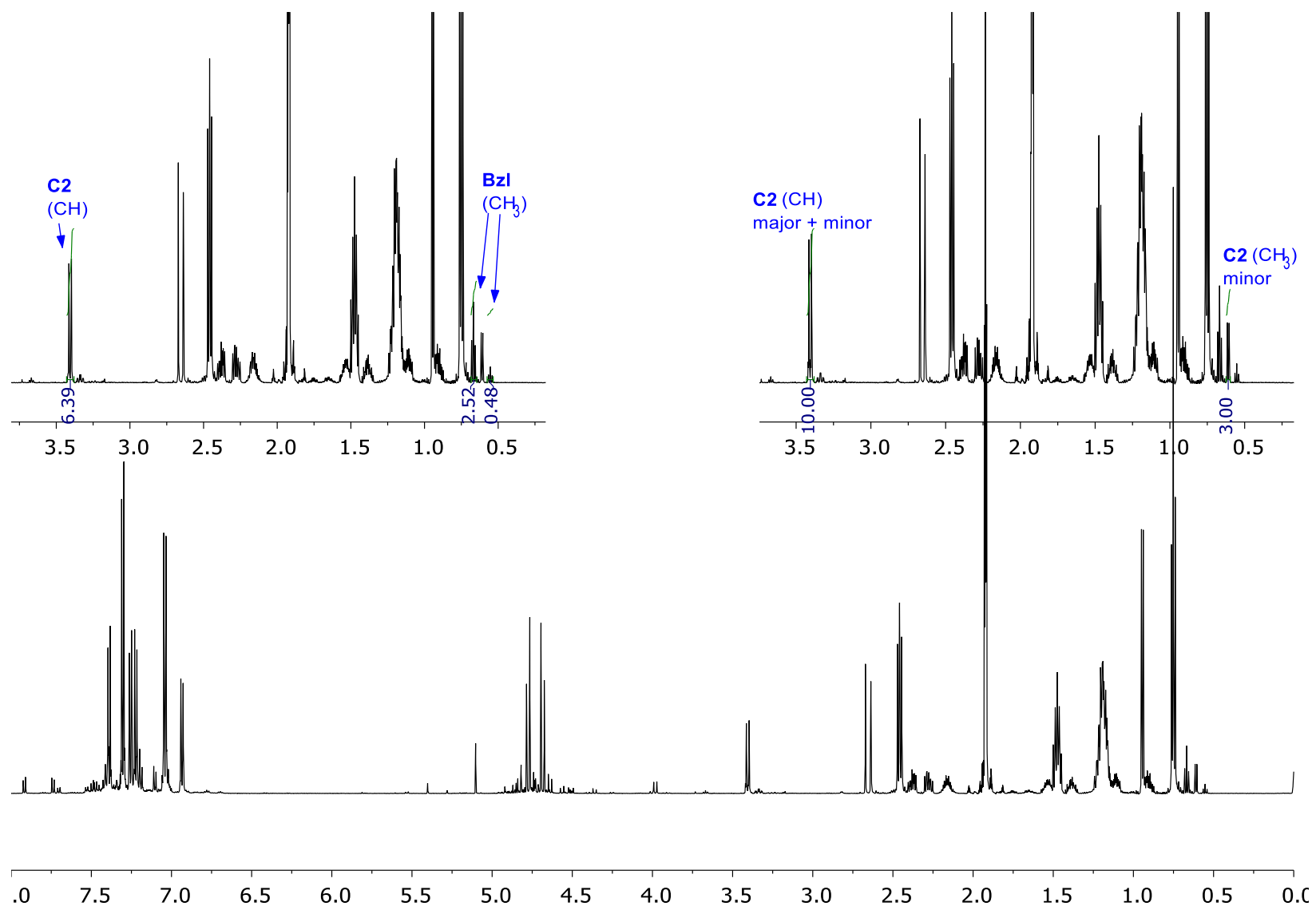

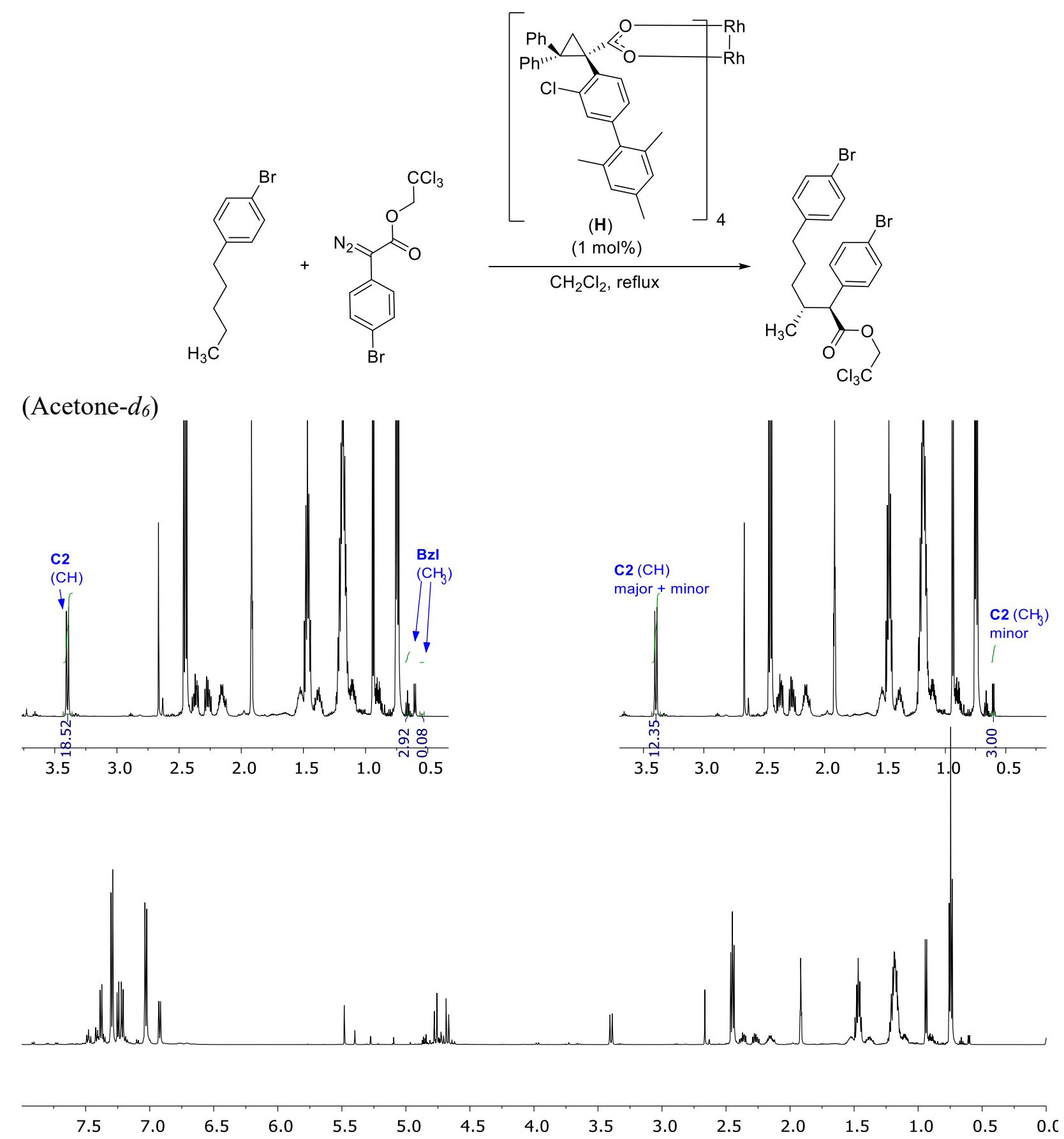


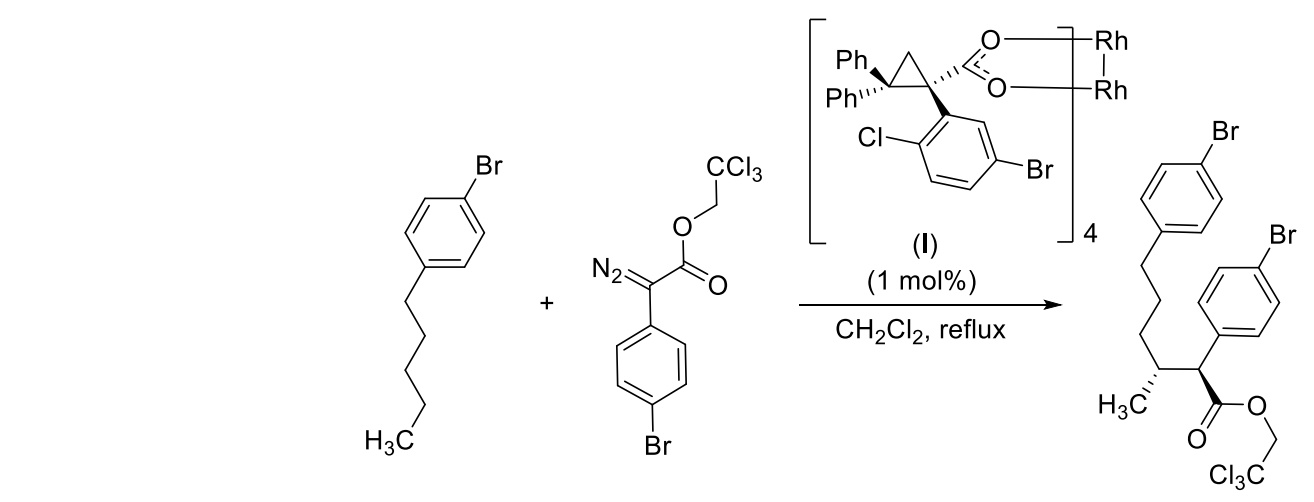

(Acetone- $d_{6}$ )
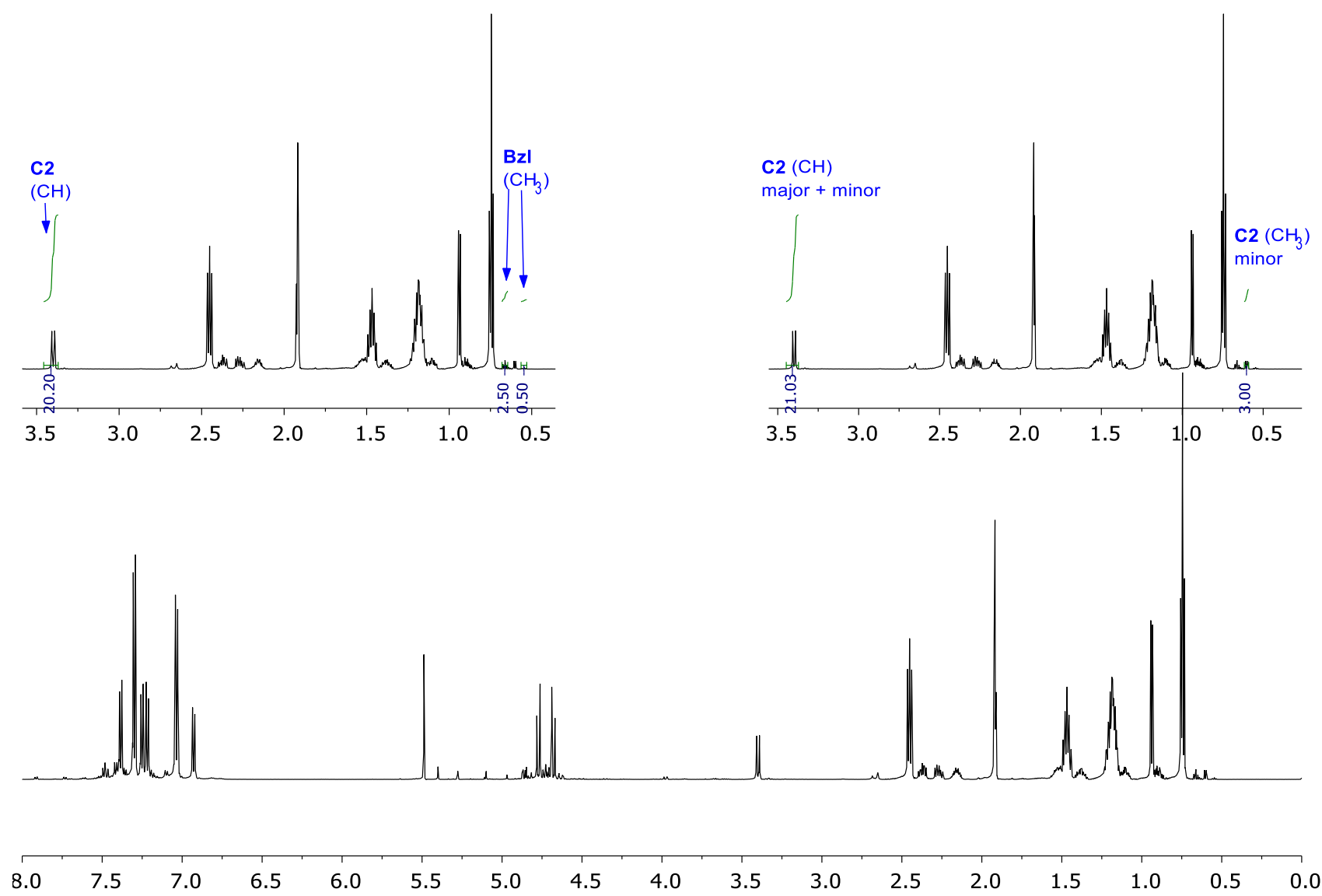

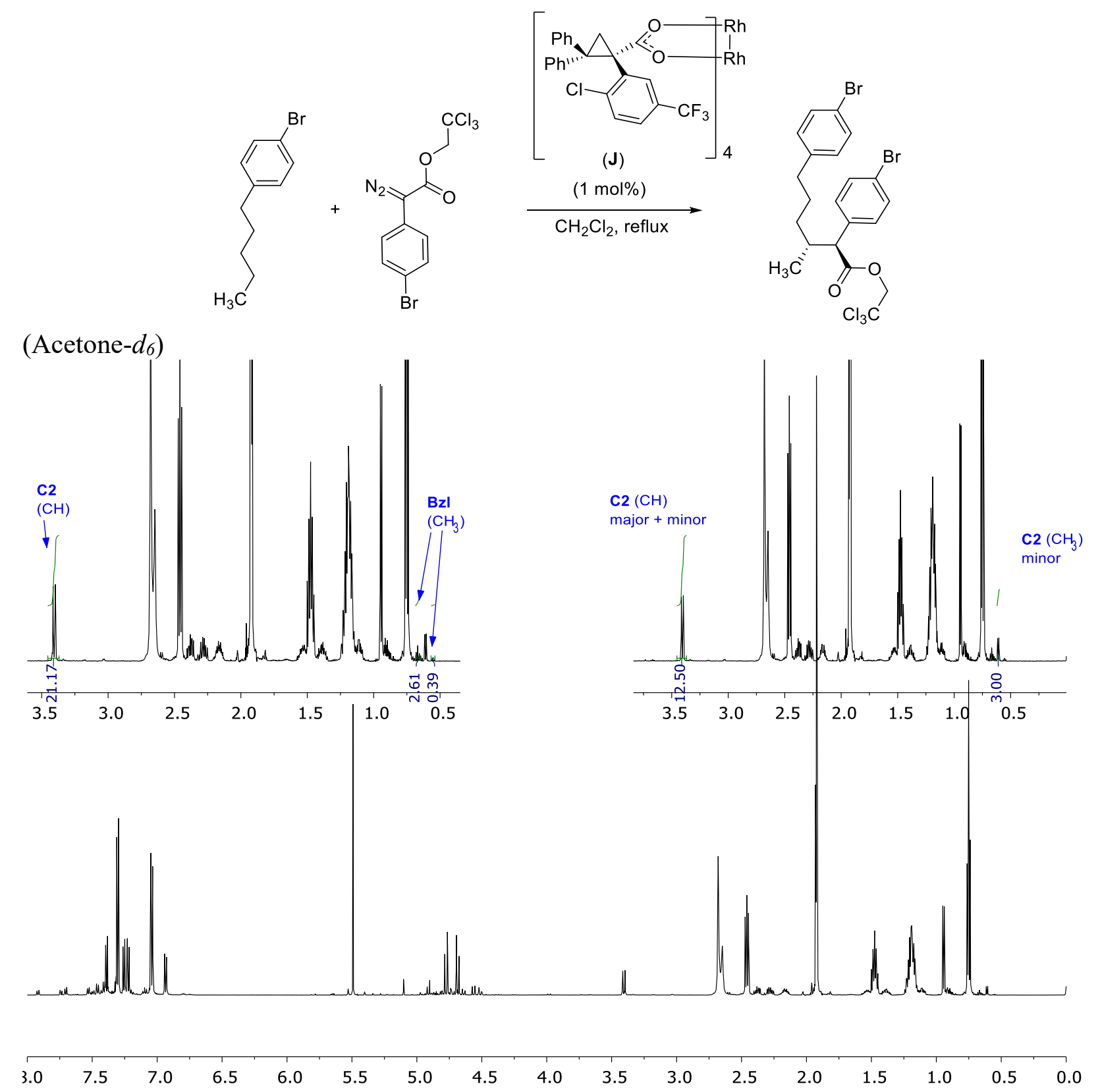


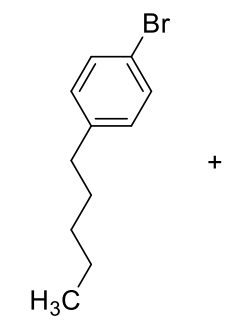

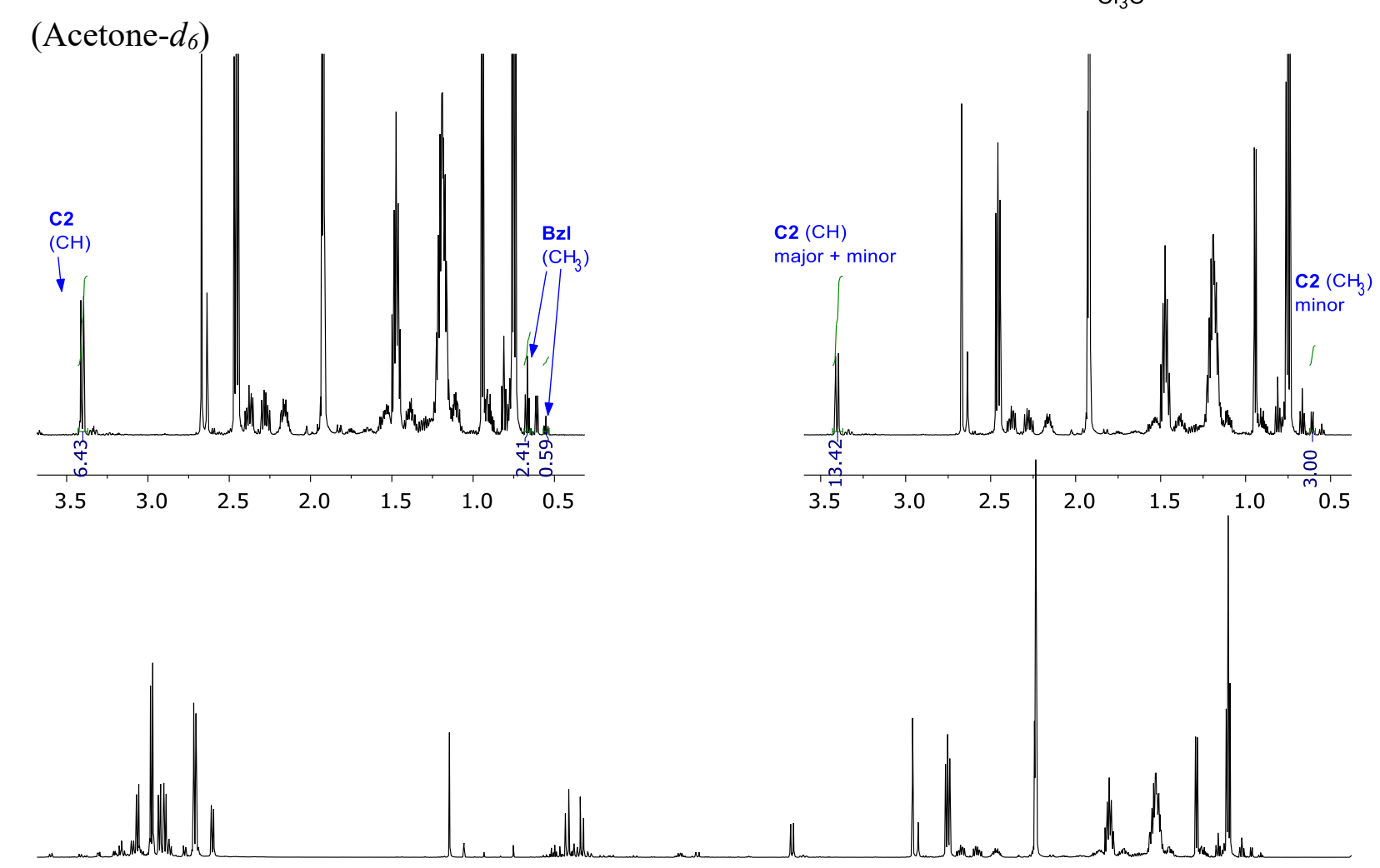

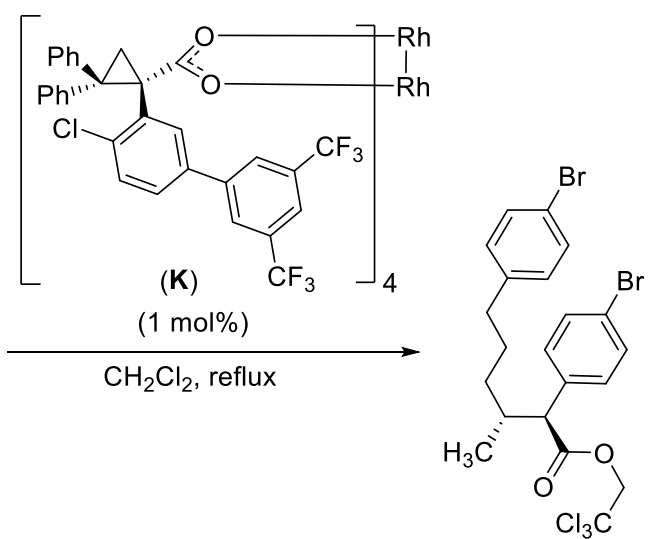

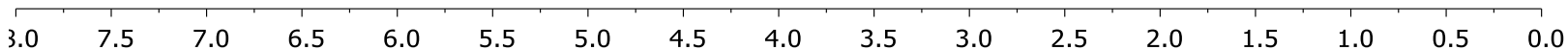




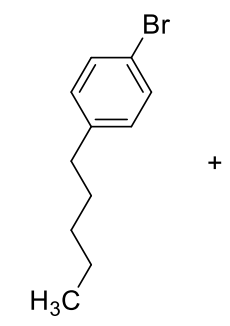

(Acetone- $d_{6}$ )
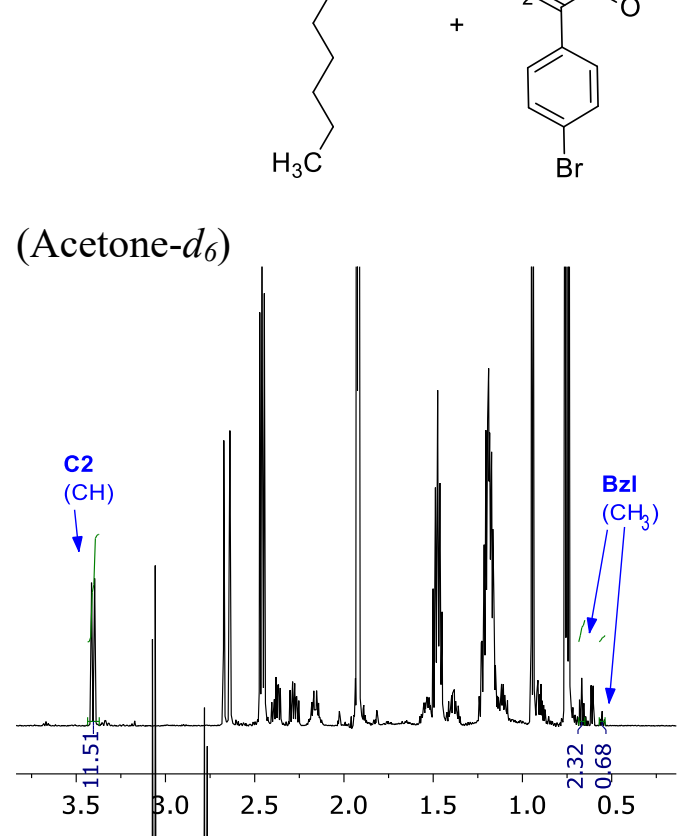

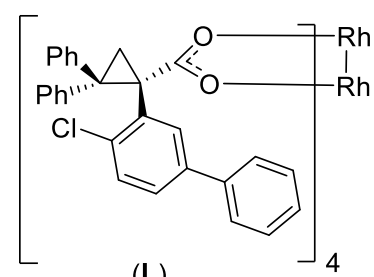

(L)

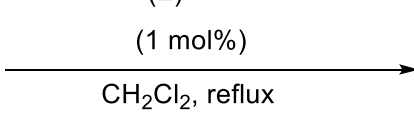

$\mathrm{H}_{2} \mathrm{Cl}_{2}$, reflux

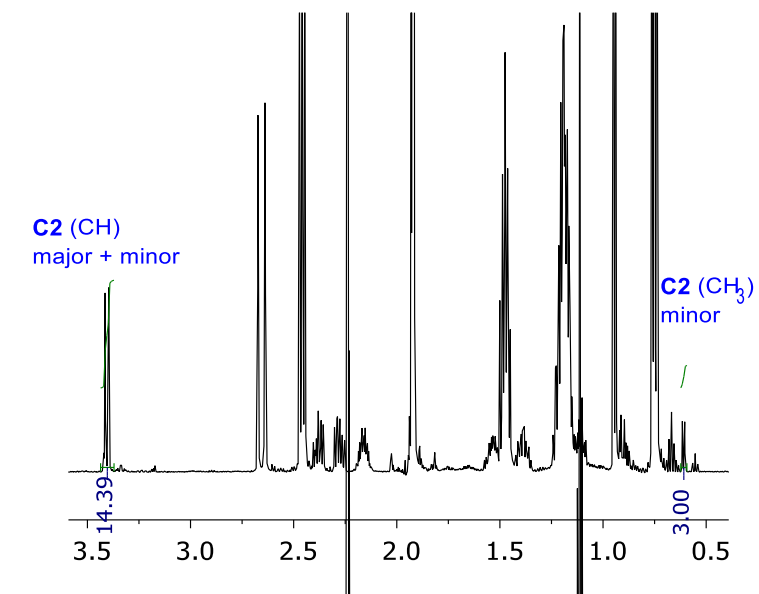

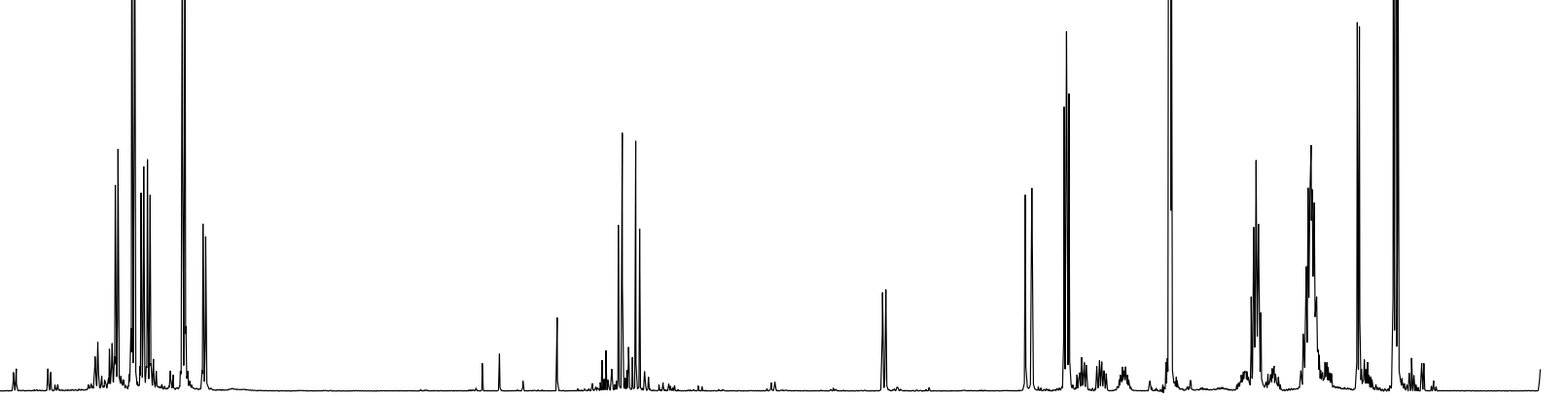

$\begin{array}{llllllllllllllll}7.5 & 7.0 & 6.5 & 6.0 & 5.5 & 5.0 & 4.5 & 4.0 & 3.5 & 3.0 & 2.5 & 2.0 & 1.5 & 1.0 & 0.5 & 0.0\end{array}$ 

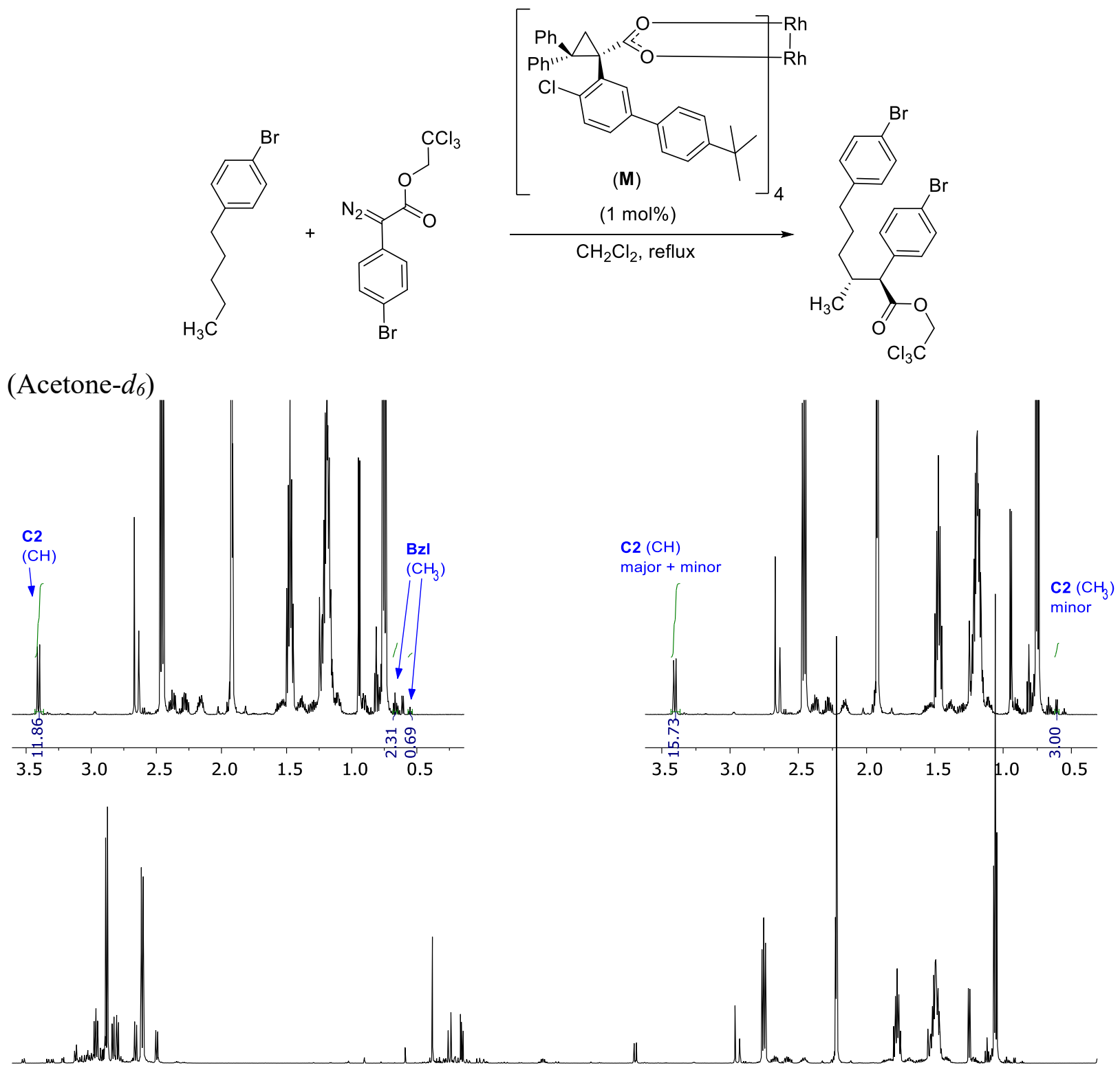

$$
3 .
$$
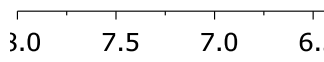

$6.0 \quad 5.5$

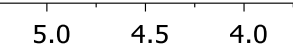

$\begin{array}{lllllll}3.5 & 3.0 & 2.5 & 2.0 & 1.5 & 1.0 & 0.5\end{array}$ 

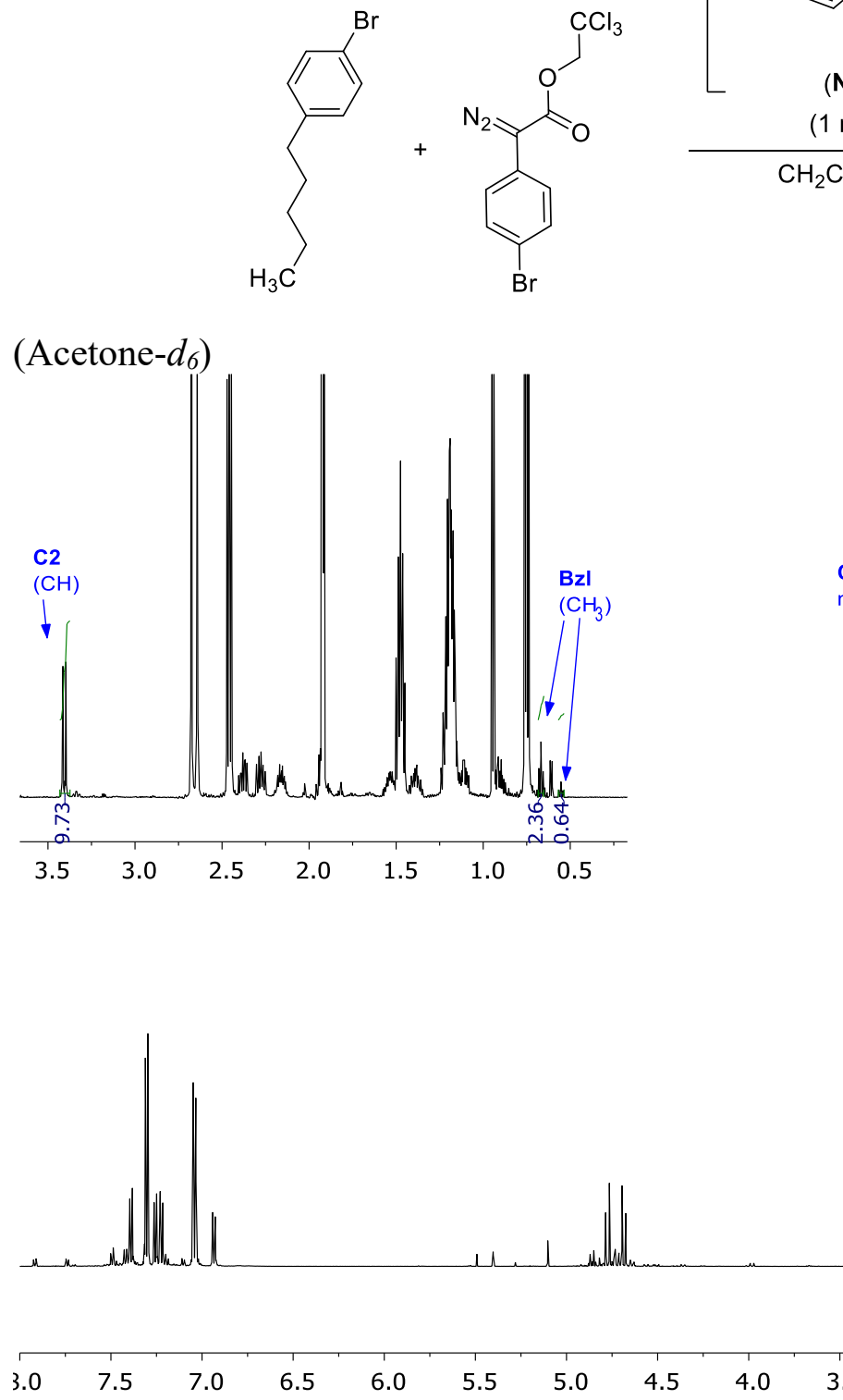
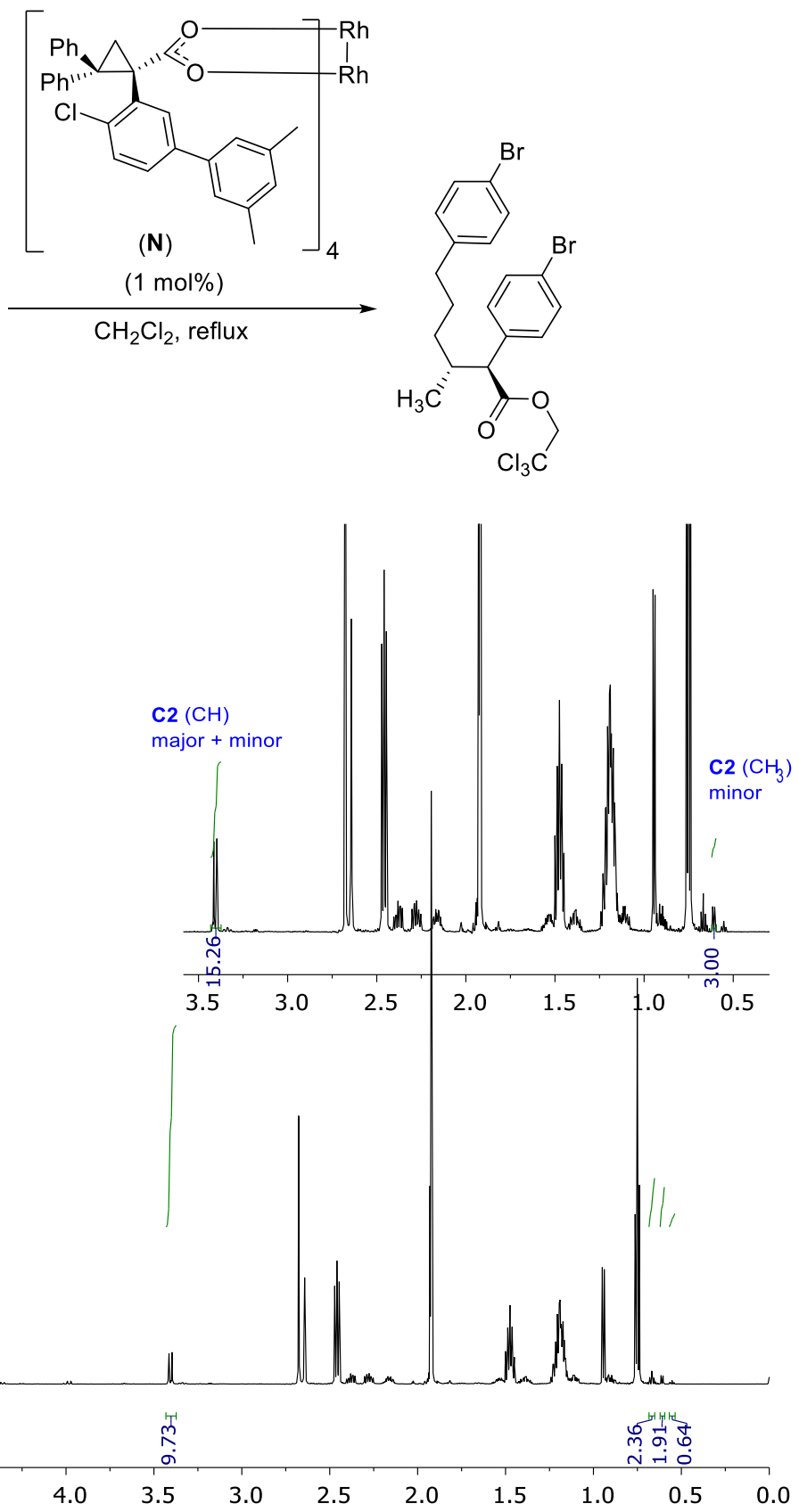


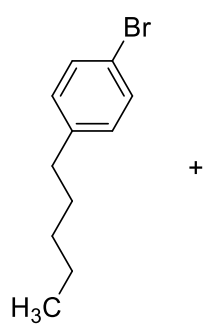

(Acetone- $d_{6}$ )
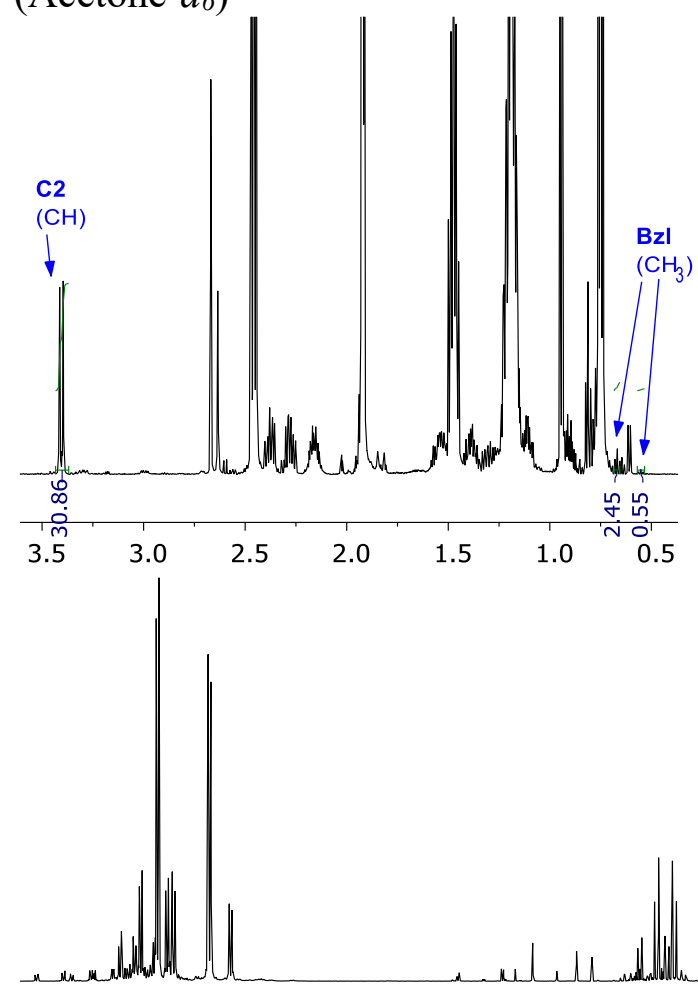
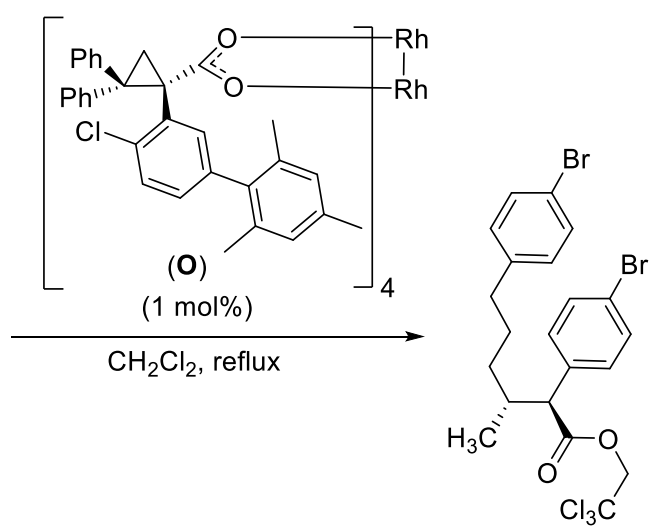

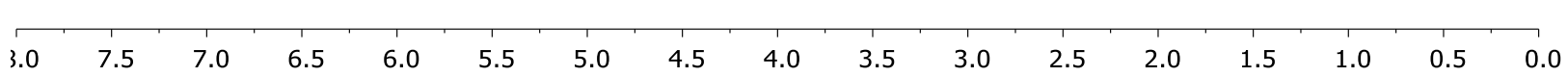




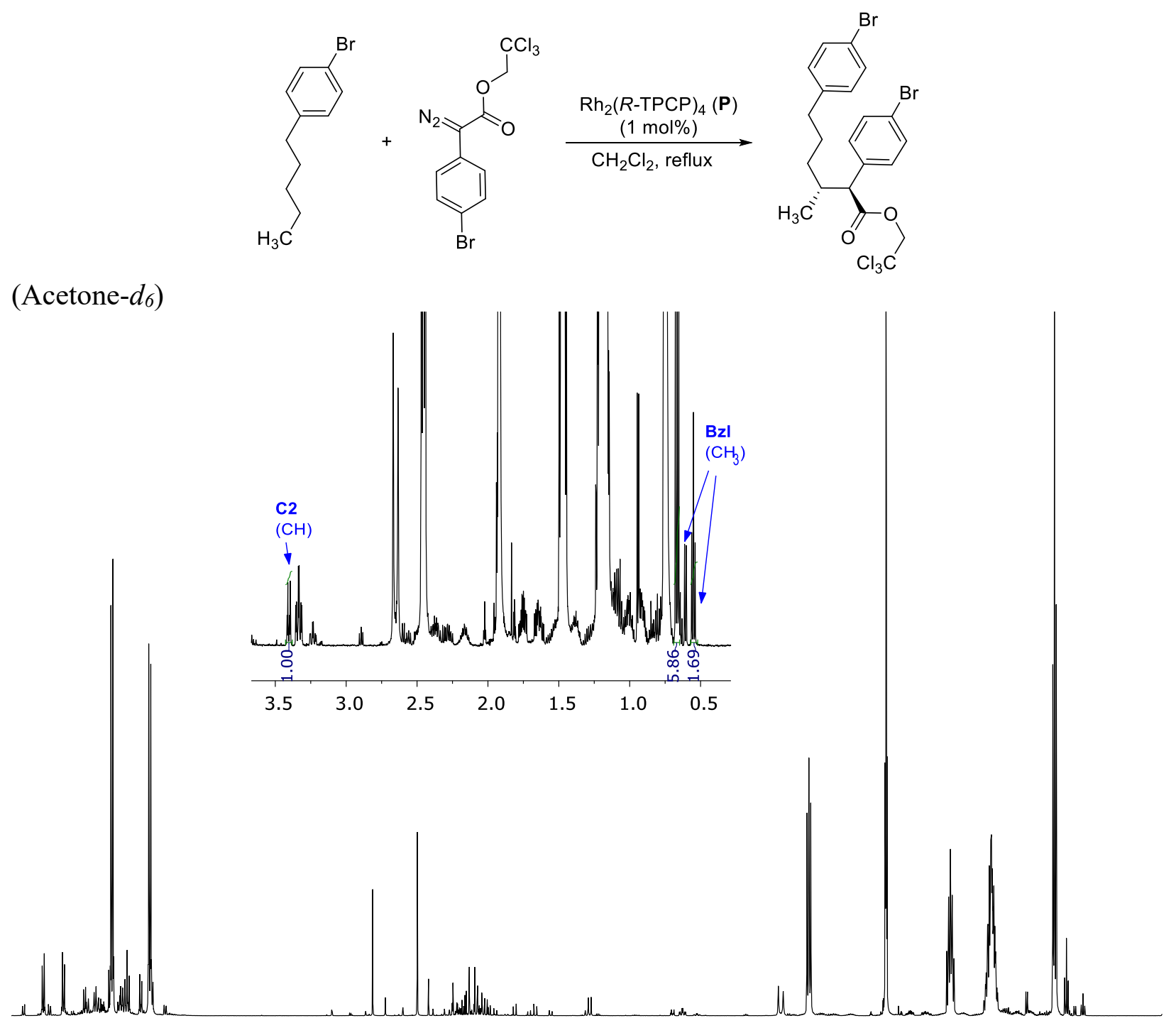

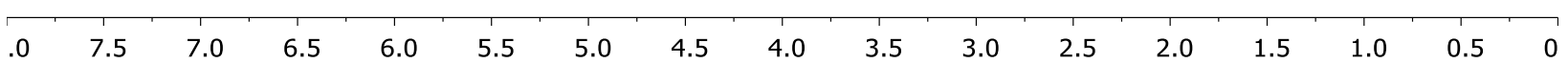




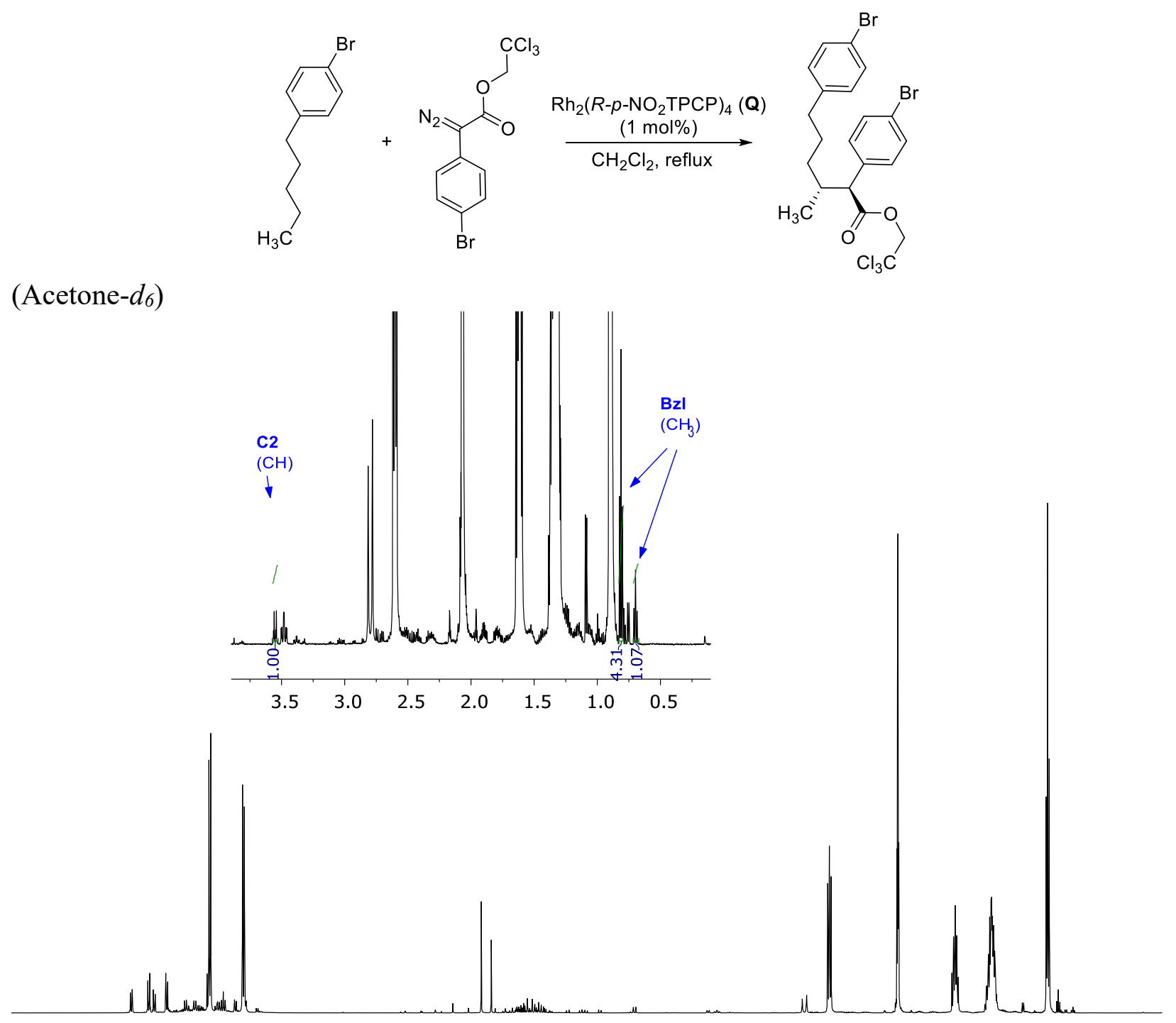

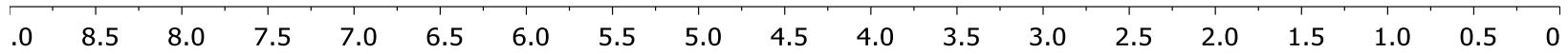



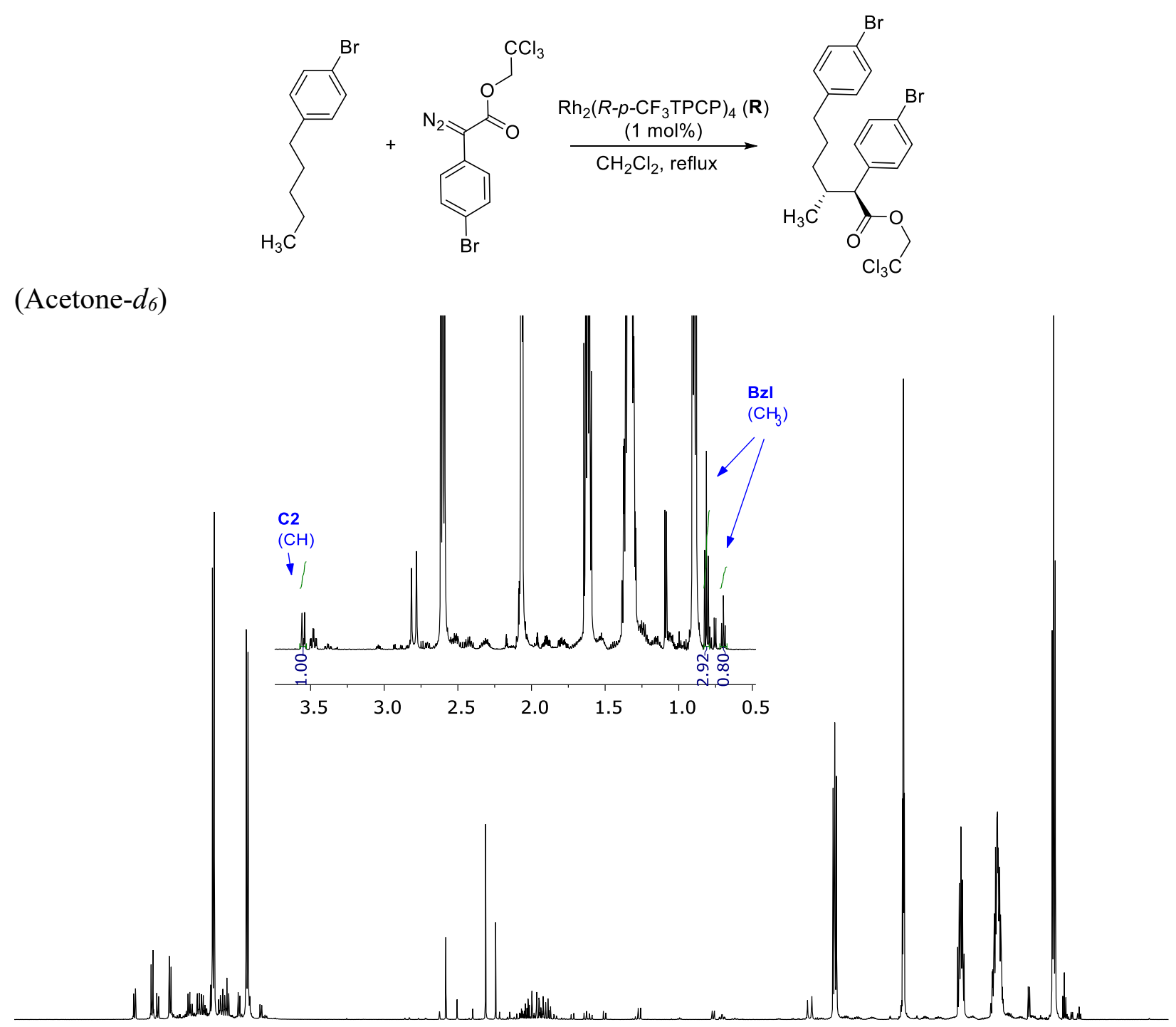

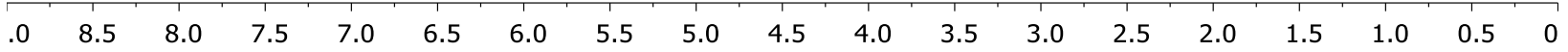




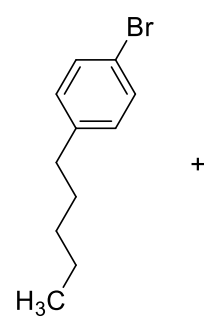

(Acetone- $\left.d_{6}\right)$

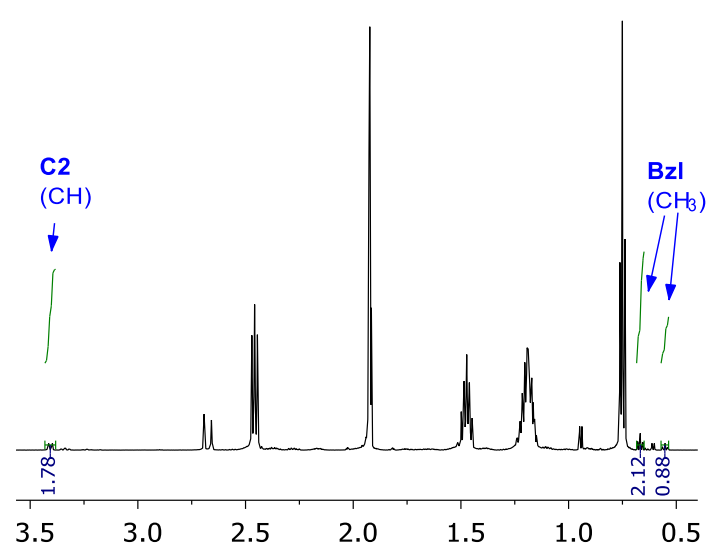

$\mathrm{Rh}_{2}(\mathrm{~S}-\mathrm{p}-\mathrm{BrTPCP})_{4}(\mathbf{S})$

$\underset{\mathrm{CH}_{2} \mathrm{Cl}_{2} \text {, reflux }}{\stackrel{(1 \mathrm{~mol} \%)}{\longrightarrow}}$

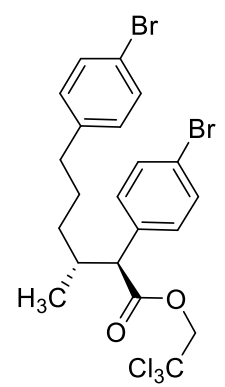

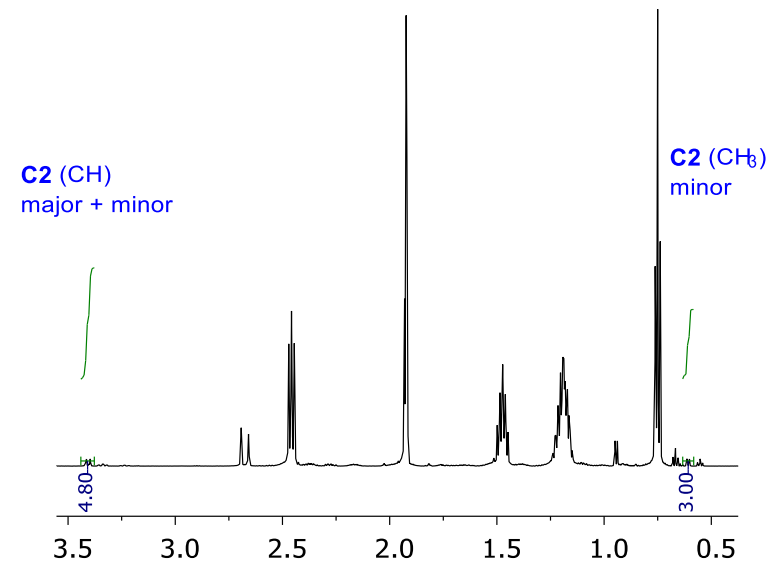

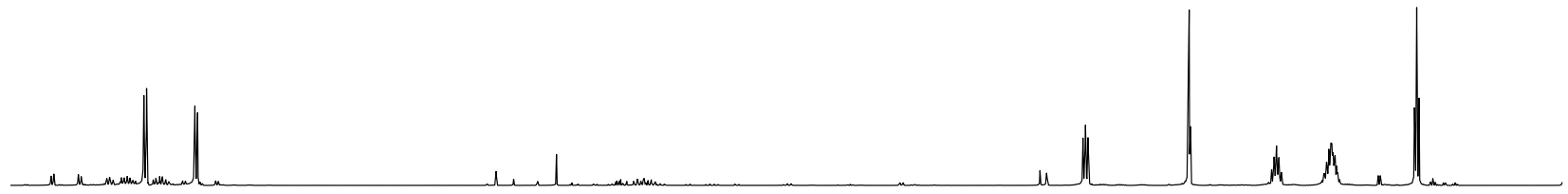

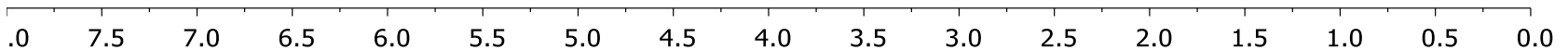




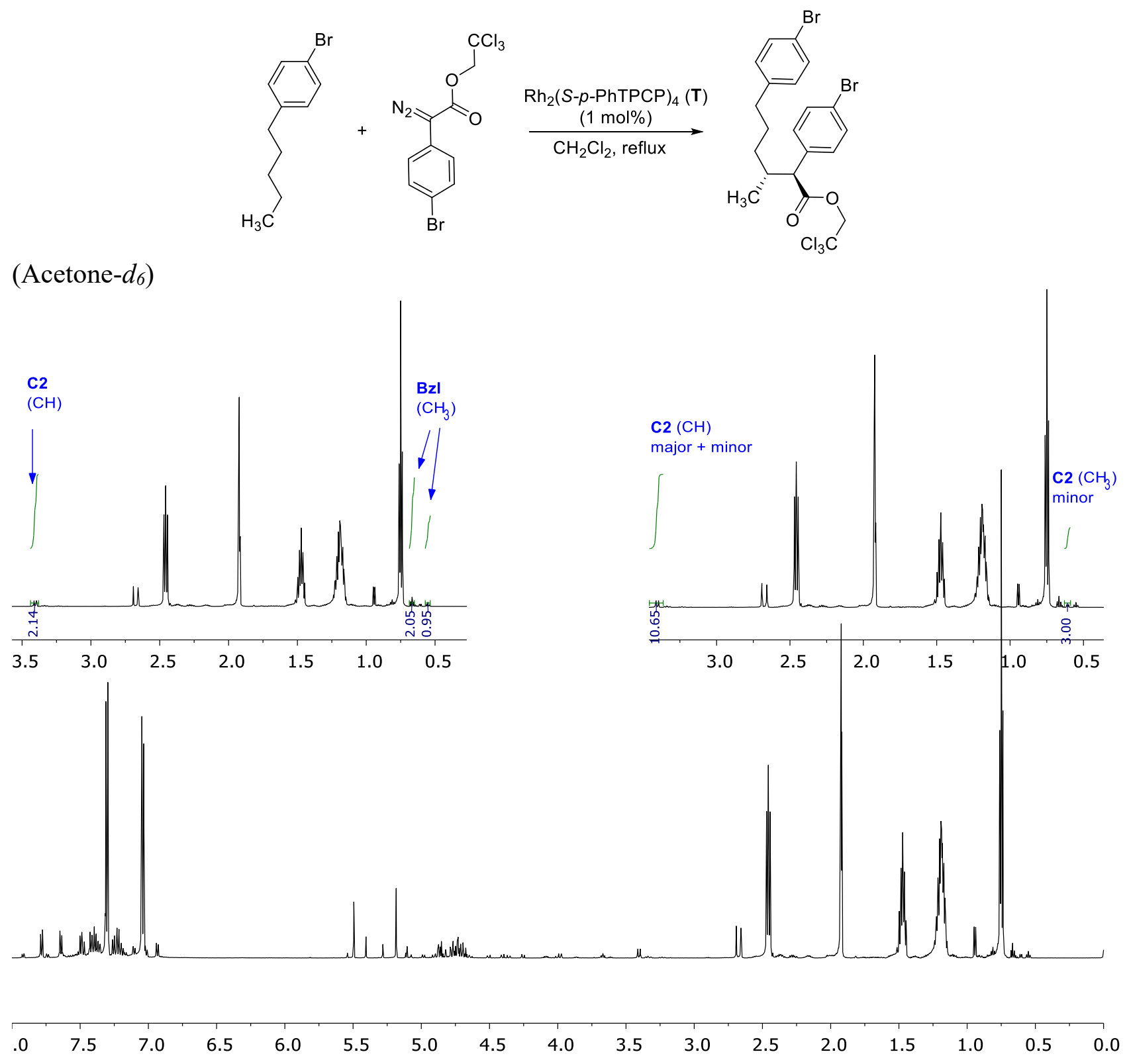




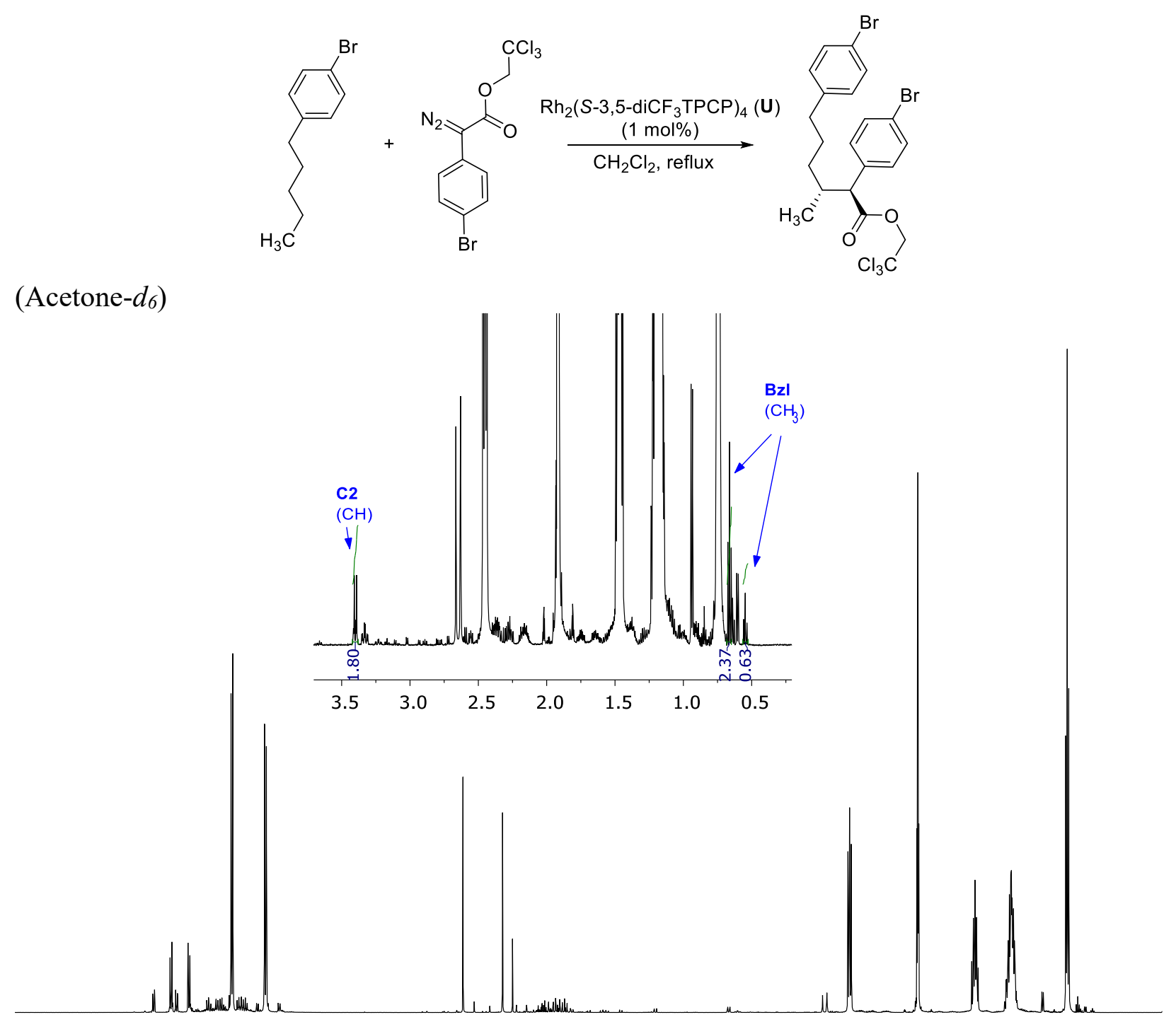

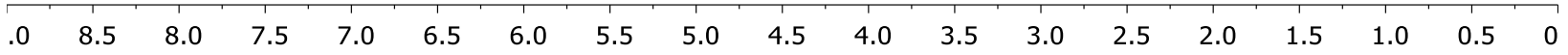



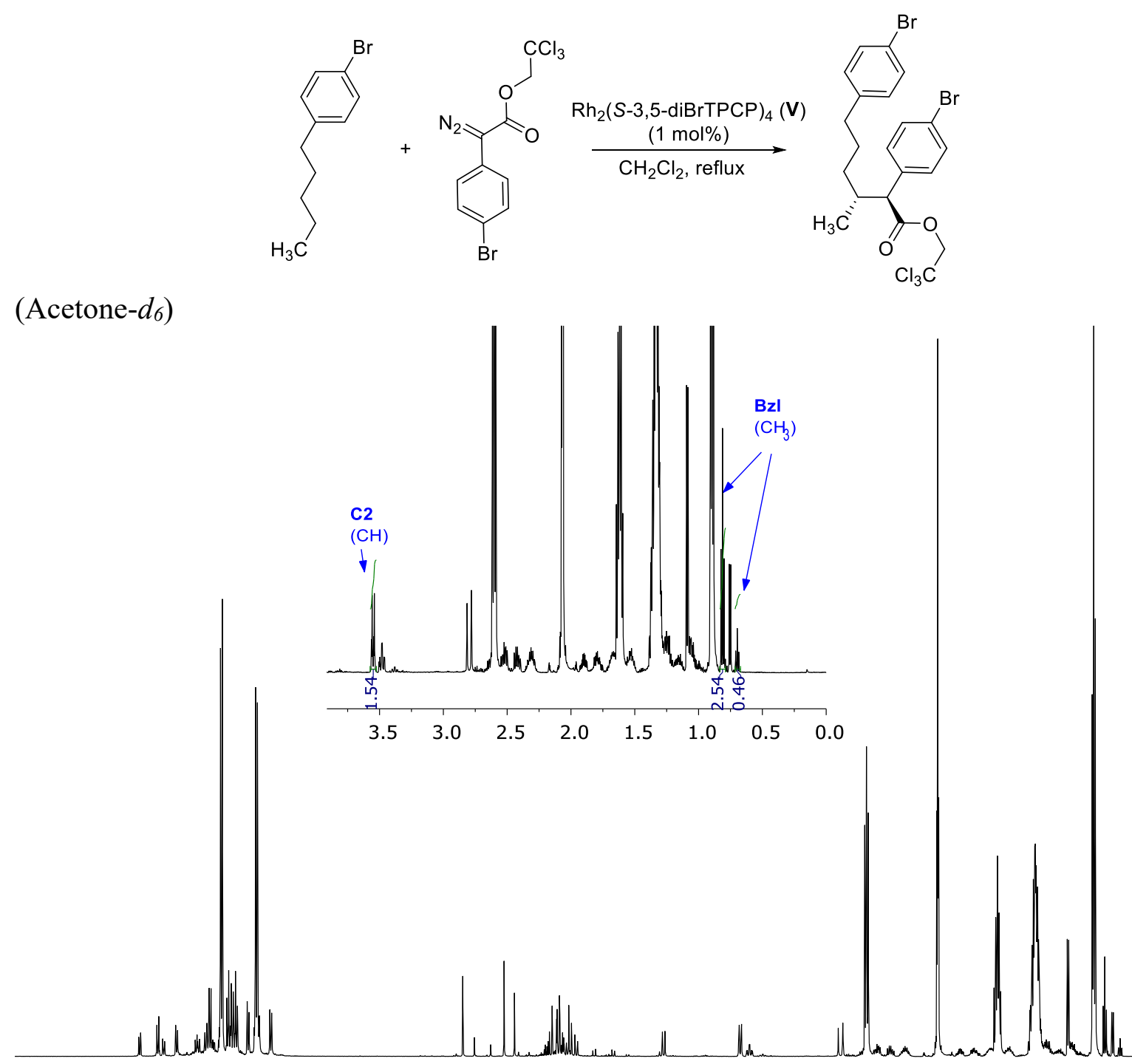

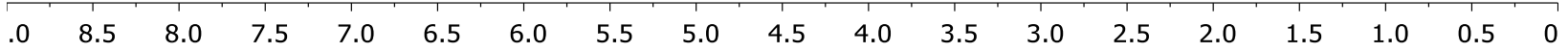




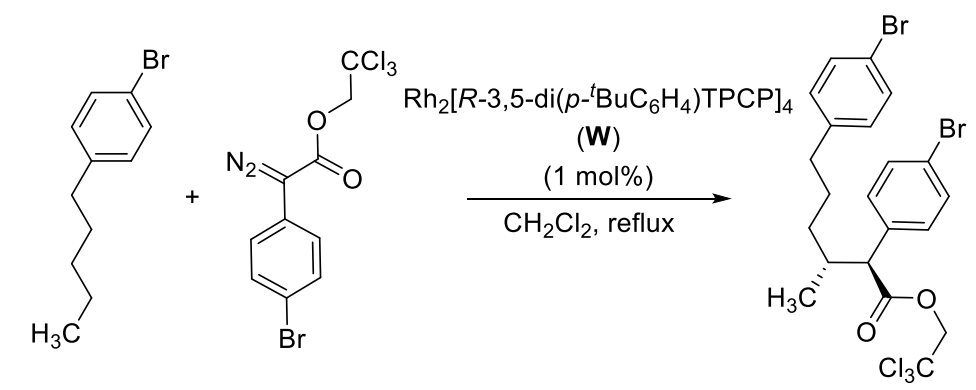

(Acetone- $d_{6}$ )
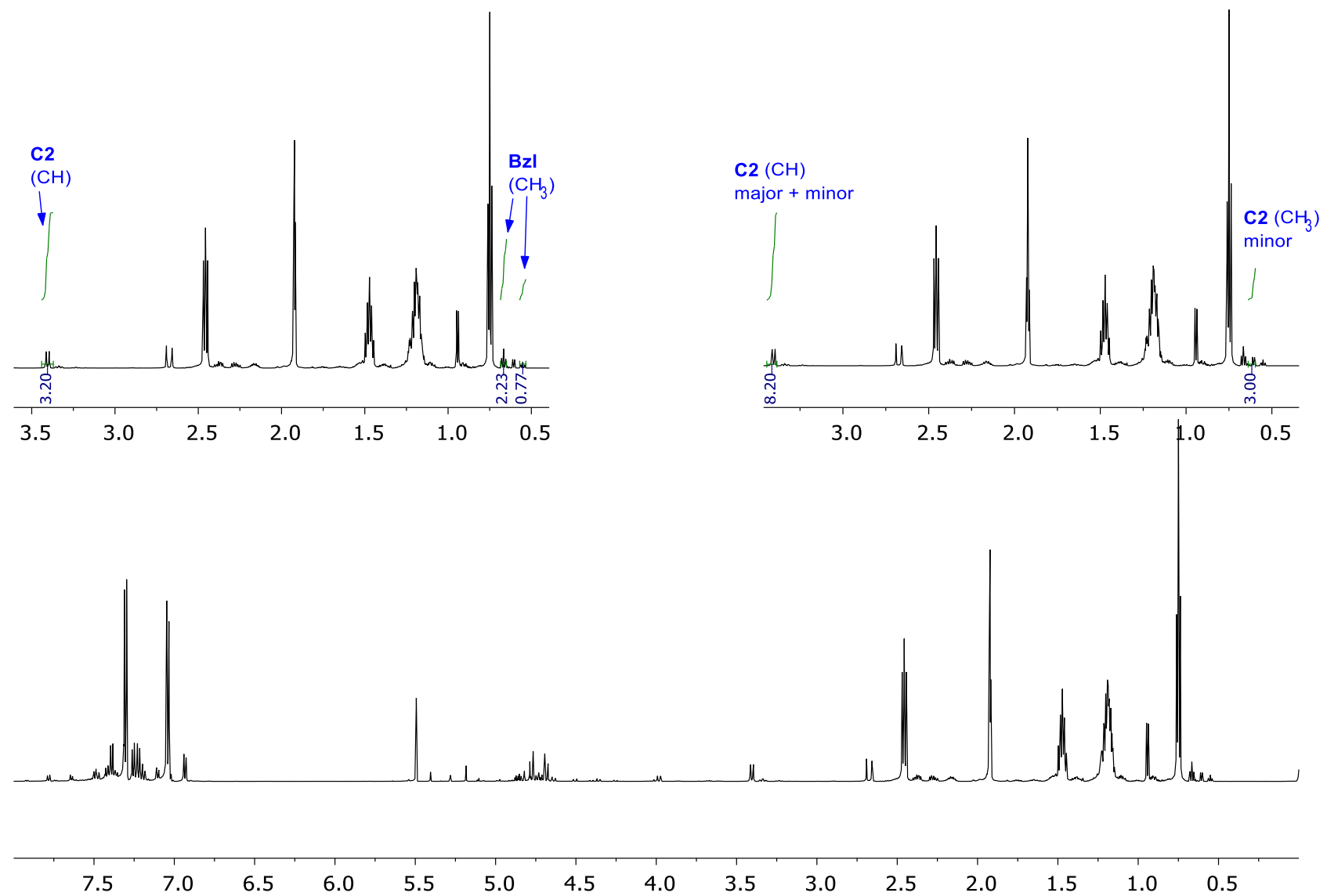


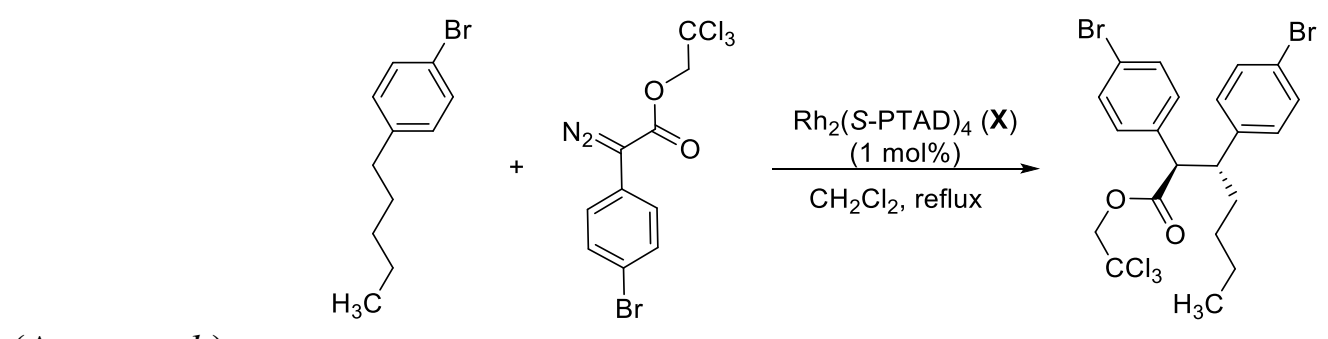

(Acetone- $\left.d_{6}\right)$

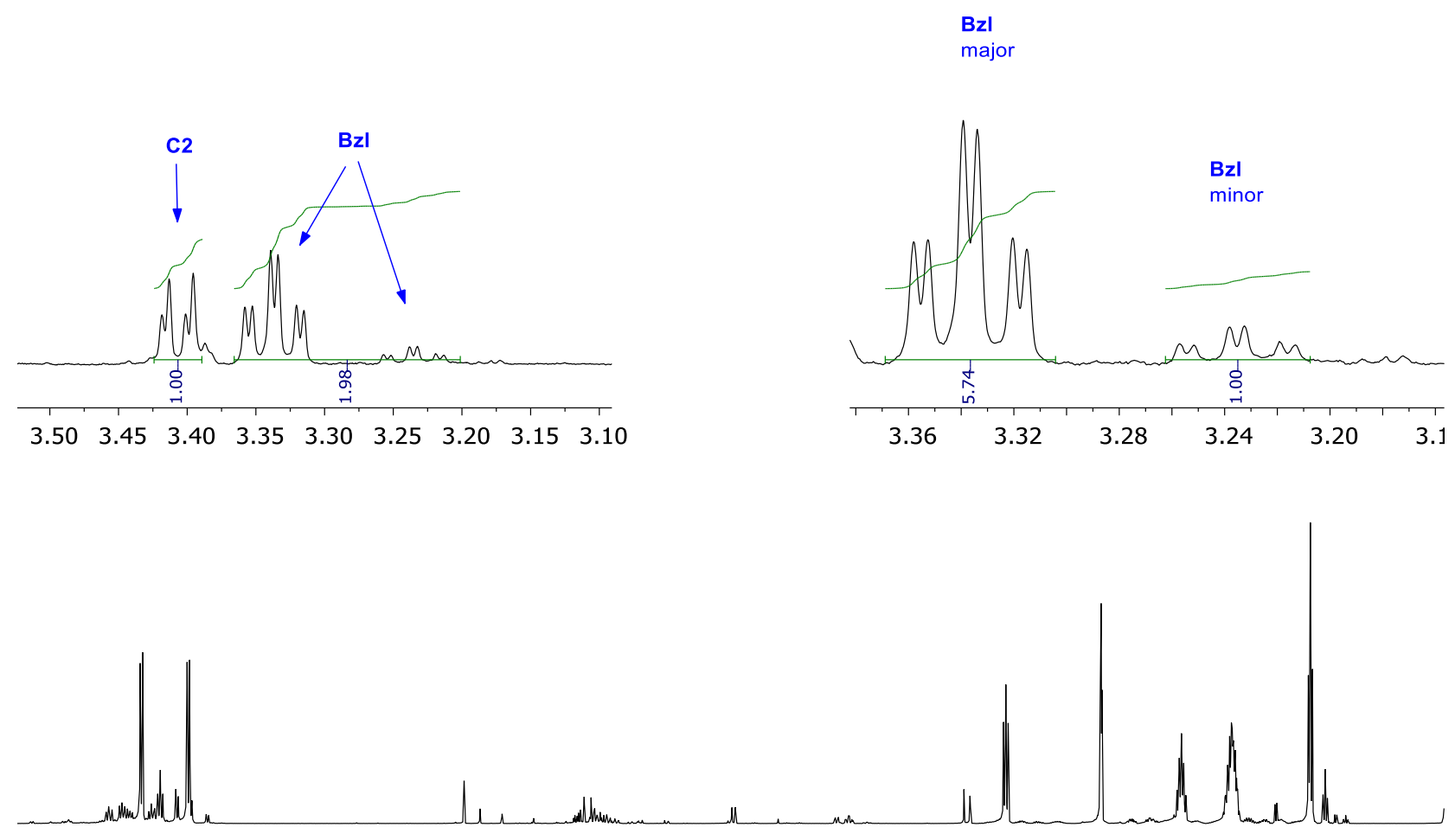

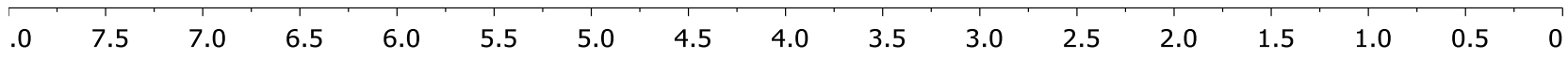




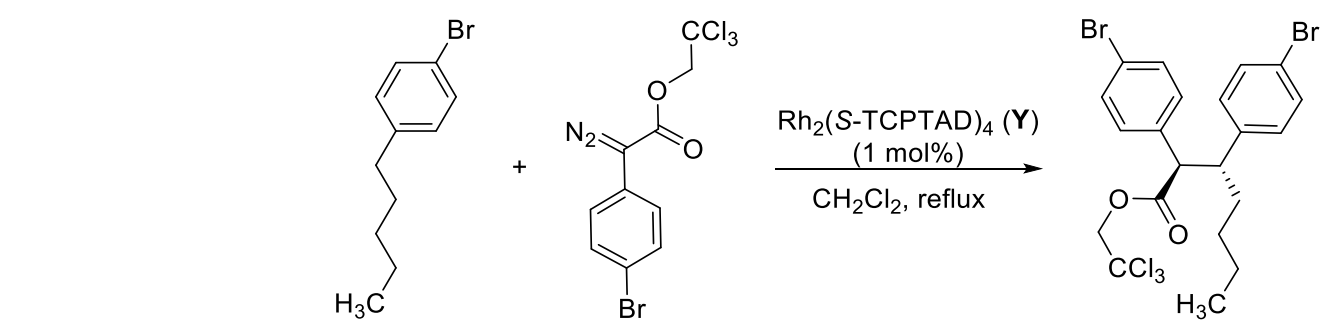

(Acetone- $\left.d_{6}\right)$
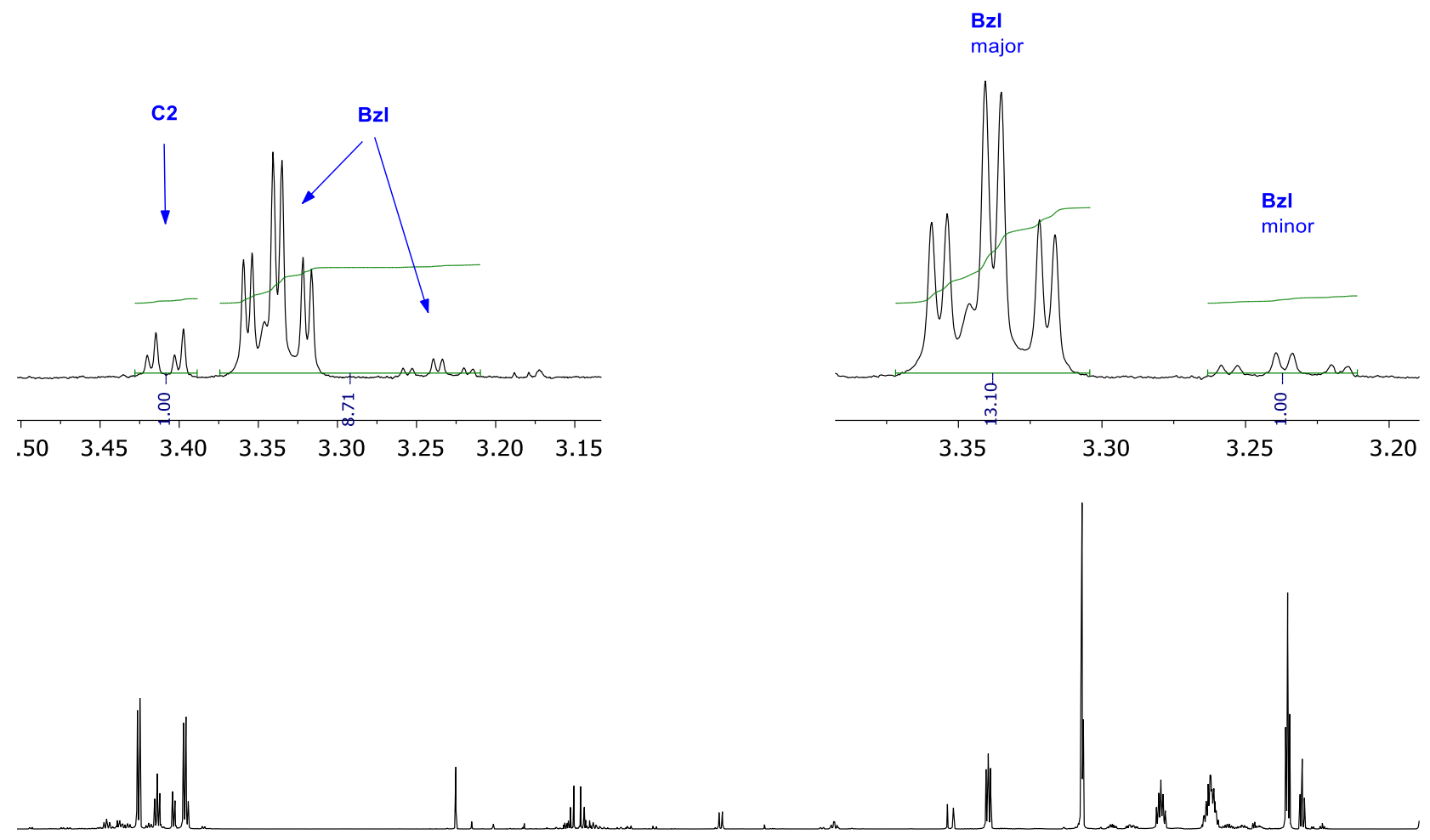

\begin{tabular}{llllllllllllll|l|l|l|l|l|l}
\hline .0 & 7.5 & 7.0 & 6.5 & 6.0 & 5.5 & 5.0 & 4.5 & 4.0 & 3.5 & 3.0 & 2.5 & 2.0 & 1.5 & 1.0 & 0.5 &
\end{tabular}




\section{Computational Methods and Details}

\subsection{Molecular Mechanics (MM) Methods for Catalyst Conformational Searches}

Dirhodium catalyst input structures for conformational searches were generated via adaptation from the most similar X-ray structure or previously calculated geometry. Conformational searches were performed using the OPLS3 force field implemented in MacroModel version 11.8. ${ }^{10-11}$

Given the inability of generic force fields to accurately describe the paddlewheel geometry of the dirhodium core, constrained conformational searches were performed for each input structure by using the substructure capability of MacroModel to freeze 14 core atoms (shown in blue in Figure S1), while allowing all other atoms to move freely to adopt energetically accessible conformations. Notably, free $\mathrm{C}-\mathrm{C}$ rotation about the carboxylate permits all possible catalyst configurations (e.g., $\alpha, \alpha, \alpha, \alpha ; \alpha, \alpha, \alpha, \beta ; \alpha, \alpha, \beta, \beta ;$ and $\alpha, \beta, \alpha, \beta)$ to be sampled during substructure conformational searching. Additionally, this search enables identification of irregular catalyst configurations that may not otherwise be considered as possible geometries $\left(\alpha, \alpha, \alpha{ }^{\prime}, \alpha{ }^{\prime}{ }^{\prime} ; \alpha, \alpha, \alpha, \gamma ; \sigma, \beta, \alpha, \gamma ;\right.$ etc.).

The search protocol is summarized in section 4.5 (pages 47-49) of the MacroModel Quick Start manual (https://www.chem.uci.edu/ jsnowick/groupweb/Maestro/macromodel_quick_start.pdf). Specifically, searches were performed with no solvent using Monte Carlo Multiple Minimum (MCMM) torsional sampling with automatic setup for defining MCMM search variables. Minimization to convergence was performed using the Polak-Ribier Conjugate Gradient (PRCG) with a threshold of 0.05 after 5000 maximum iterations. Intermediate sampling without retaining mirror-image conformers was performed with a maximum of 1000 steps (using 100 steps per rotatable bond). Redundant conformers in the conformational search were eliminated using a maximum atom deviation cutoff of $0.5 \AA$. A large energy window for saving structures of 41.9 $\mathrm{kJ} / \mathrm{mol}(10.01 \mathrm{kcal} / \mathrm{mol})$ was utilized to account for the inability of the force field to accurately determine relative conformer energetics. This treatment allows for rigorous examination of all possible catalyst shapes at the outset for further consideration using QM/MM methods (vide infra).

\subsection{Methods for Clustering Catalyst Conformations}

Typically, 200-400 conformations were obtained for each catalyst from the constrained conformational search. Fortunately, many catalyst conformers are very similar and need not be considered further. However, it is not trivial to identify the appropriate number of representative conformers to consider further based on the diversity of conformational space for a given catalyst.

To identify representative catalyst conformations to consider further using higher levels of theory, conformer clustering tools implemented in MacroModel were utilized to group similar conformers. A RMSD matrix was generated using root-mean-square deviation (RMSD) for all catalyst atomic positions, and hierarchical clustering was performed using the average-linkage method, which is recommended in cases where the optimal number of clusters is not known in advance. ${ }^{12}$ While there are many different methods for determining the optimal cluster count for a given data set, ${ }^{13}$ Kelley penalty minimization ${ }^{14}$ comes with the convenient advantage that it has already been implemented within MacroModel. ${ }^{10-11}$ The Kelley penalty criteria is an automated approach for identifying the optimal number of clusters for a given selection of $\mathrm{N}$ conformers. ${ }^{14}$ Clustering was performed in accordance with the number of clusters recommended by the Kelley penalty minimization criteria, and the catalyst structure nearest to the centroid for each cluster was exported for further optimization and energetic evaluation at higher levels of theory. 


\subsection{Evaluating Catalyst Energetics: Quantum Mechanics/Molecular Mechanics (QM/MM)}

Typically, 25 to 35 representative catalyst conformers were considered further for each dirhodium catalyst, with subsequent QM/MM optimization providing a relatively rapid means of efficiently evaluating the relative energetics across the full conformational space possible for each catalyst. Given the large number of atoms (166 to 342) and representative conformations for each of the 25 catalysts, a two-layer QM/MM approach was utilized to efficiently identify preferred catalyst conformations. QM/MM gas-phase optimizations were performed by adapting the methods previously used by Houk and Musaev (B3LYP-D3BJ/BS1: UFF), ${ }^{15}$ as implemented in Gaussian 16 (revision C.01). ${ }^{16}$ BS1 refers to the basis sets employed, which were LANL2DZ (with corresponding Hay-Wadt effective core potential) for $\mathrm{Rh}$ and $6-31 \mathrm{G}^{*}$ basis sets for all other atoms (refer to refs. 18-32 for computational methods details). The optimized geometries were verified as stationary points by frequency calculations (i.e., zero imaginary frequencies). Single-point calculations were performed for all optimized geometries at the M06/BS2 level of theory with solvation in dichloromethane using the SMD model, ${ }^{17}$ where BS2 employs SDD (with corresponding Hay-Wadt ECP) for Rh and def2TZVP basis sets for all other atoms. Reported relative free energies (in $\mathrm{kcal} / \mathrm{mol}$ ) utilize the solvated electronic energies to correct the gas phase free energies. Natural bond orbital (NBO) analysis using NBO 7.0 was conducted to obtain catalyst electronic descriptors, including $\mathrm{Rh}$ partial charges and $d$-orbital energies. ${ }^{33}$
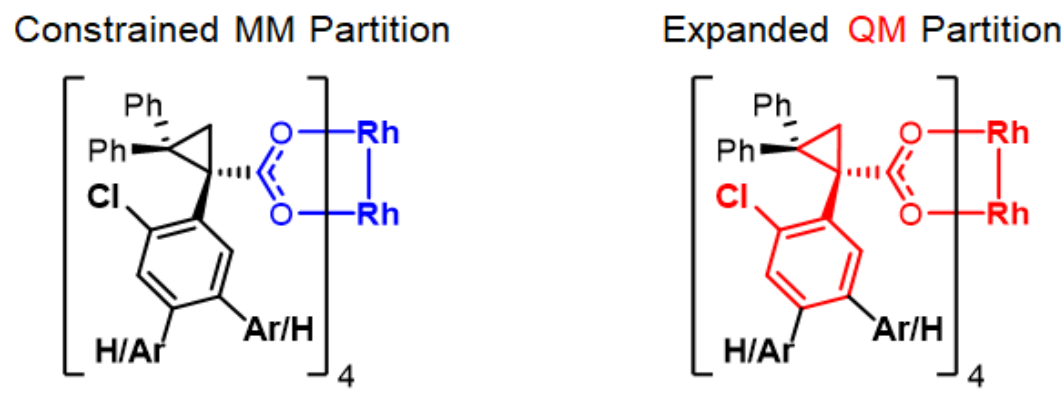

Figure S1. Layering schemes for constrained conformational searches (left) and QM/MM geometry optimizations (right). Atoms that were frozen are depicted in blue, atoms shown in black were treated at the MM level of theory, and atoms shown in red were treated at the QM level of theory. Note that conformers were considered redundant based on free energy $(<0.05 \mathrm{kcal} / \mathrm{mol})$ and dipole $(<0.1)$ cutoffs.

\subsection{Comparing QM/MM Approach to X-ray Crystal Structures}

Structural comparisons to X-ray crystal structures served as one means of validating our QM/MM approach for obtaining reliable catalyst geometries. X-ray crystal structures for catalysts $\mathbf{G}, \mathbf{H}, \mathbf{M}$, and $\mathbf{N}$ were obtained in this study, and crystal structures have previously been obtained for catalysts A, B, I, T, W, and Y..$^{2,4-5,7-8}$ Structural agreement between QM/MM calculated structures optimized using various layering partitions and X-ray structures was quantified in terms of atomic RMSD, and structural overlay visualizations were generated using UCSF ChimeraX 1.1. ${ }^{34-35}$

In particular, expanding the QM partition from the 14 core atoms (blue) considered by Houk and Musaev for related dirhodium-TPCP catalysts ${ }^{15}$ to also include the $\mathrm{C} 1$ aryl and cyclopropyl rings (QM partition shown in red in Figure S1) was found to be critical for closely matching the $\mathrm{C}_{4}$ $(\alpha, \alpha, \alpha, \alpha)$ bowl-shaped cavity orientations adopted by $\mathrm{Rh}_{2}(S \text { - } o \text {-ClTPCP })_{4}$ catalysts. ${ }^{8,36}$ This can be seen for $\mathrm{Rh}_{2}(S \text {-o-CITPCP })_{4}$, where optimization with an expanded QM partition (red) allows for much closer structural congruence with the experimental X-ray crystal structure ${ }^{8}$ (black) than does treating only the 14 dirhodium core atoms at the QM level of theory (blue; Figure S2). 

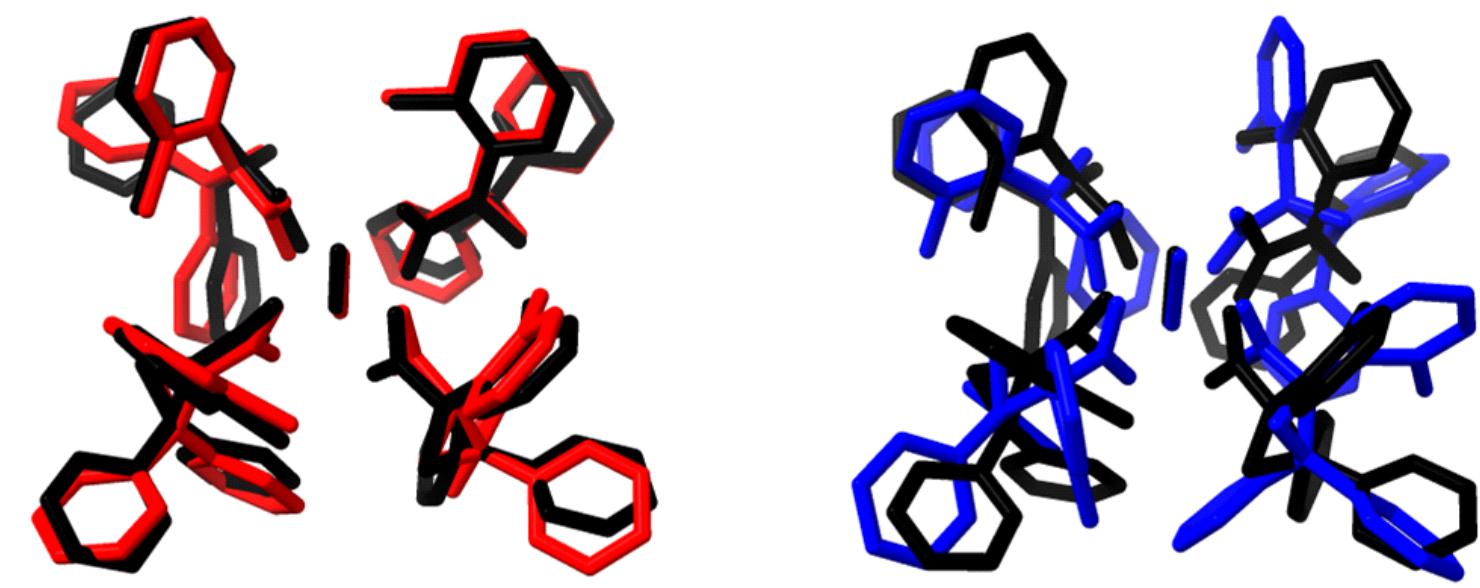

Figure S2. Structural overlays comparing the $\mathrm{X}$-ray crystal structure of $\mathrm{Rh}_{2}(\mathrm{~S} \text {-o-ClTPCP })_{4}$ (black) with $\mathrm{QM} / \mathrm{MM}$ calculated structures using two different QM partitions. Expanding the QM partition to include $\mathrm{C} 1$ aryl and cyclopropyl rings (red) was found to be necessary for reproducing the characteristic bowl shape (RMSD $=0.592 \AA$ ). Alternatively, considering only the 14 dirhodium core atoms at the QM level of theory (blue) results in a distorted bowl shape that is in poor agreement with the experimental solid-state structure $(\mathrm{RMSD}=1.803 \AA$ ). It should be noted that this simplified QM partition also was problematic in that convergence often could only be achieved to structures that were not stationary points on the QM/MM potential energy surface (i.e., one or more imaginary frequencies).

The described QM/MM approach also afforded structures that were in close agreement with the previously obtained experimental solid-state structures for four additional catalysts (Figure S3). This further validates the QM/MM approach for reliably approximating dirhodium structures for catalysts spanning disparate subclasses of tetracarboxylate ligands (i.e., ТРCP, $o-\mathrm{ClTPCP,}$ phthalimido). It should be noted that axially bound solvent molecules in X-ray structures of $\mathbf{B}$ $\left(\mathrm{H}_{2} \mathrm{O} / \mathrm{H}_{2} \mathrm{O}\right)$, I $\left(\mathrm{MeCN} / \mathrm{MeCN}\right.$ or $\left.\mathrm{MeCN} / \mathrm{H}_{2} \mathrm{O}\right), \mathbf{T}\left(\mathrm{Et}_{2} \mathrm{O} / \mathrm{Et}_{2} \mathrm{O}\right)$, and $\mathbf{Y}\left(\mathrm{MeCN} / \mathrm{H}_{2} \mathrm{O}\right)$ have been omitted for clarity, and they do not appear to necessitate significant catalyst shape perturbations.

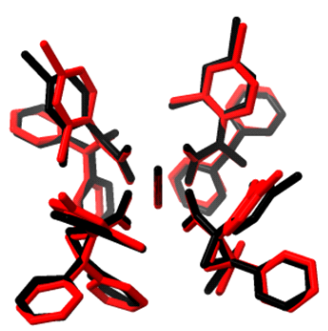

$\mathrm{Rh}_{2}(\mathrm{~S}-2-\mathrm{Cl}-4-\mathrm{BrTPCP})_{4}$ Catalyst B

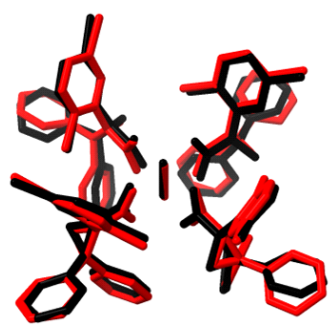

$\mathrm{Rh}_{2}(\mathrm{~S}-2-\mathrm{Cl}-5-\mathrm{BrTPCP})_{4}$ Catalyst I

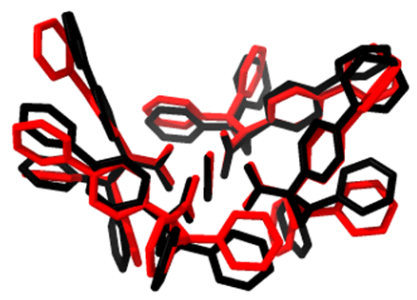

$\mathrm{Rh}_{2}(\mathrm{~S}-p-\mathrm{PhTPCP})_{4}$ Catalyst $\mathbf{T}$

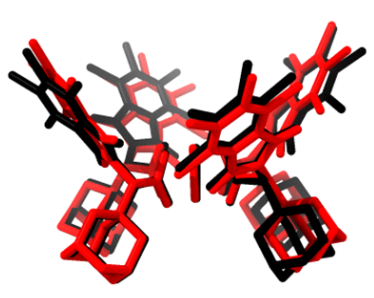

$\mathrm{Rh}_{2}(\mathrm{~S}-\mathrm{TCPTAD})_{4}$ Catalyst $Y$

Figure S3. Structural overlays comparing the X-ray crystal structures (black) with QM/MM calculated structures (red). QM/MM structures for the lowest energy conformers of catalysts $\mathbf{B}$ (RMSD = $0.404 / 0.596 \AA)$, I $(\mathrm{RMSD}=0.433 \AA)$, and $\mathbf{Y}(\mathrm{RMSD}=0.599 \AA)$ closely match the experimental solidstate structures. Note that two RMSD values are reported for $\mathbf{B}$ due to the presence of two independent molecules in the X-ray unit cell to which the computed structure was compared. Catalyst $\mathbf{T}$ is considerably more flexible and is computed to populate several different possible conformers similar to the $\alpha, \alpha^{\prime}, \alpha, \alpha^{\prime}$ configuration, many of which can easily interconvert via Ar-Ar torsional rotation (see Figure S16). Nevertheless, the second lowest-energy conformation computed for $\mathbf{T}(+0.3 \mathrm{kcal} / \mathrm{mol}$ in relative free energy) aligns reasonably well with the X-ray structure (RMSD $=0.900 \AA$ ). Note: $\mathbf{T}$ and $\mathbf{Y}$ were crystallized as the opposite enantiomer in prior studies and aligned by $x y z$-coordinate inversion. 
The four catalysts for which X-ray crystal structures have been obtained in this study are displayed aligned with their computed structures in Figure S4. Our QM/MM approach identifies low energy conformers that closely match the solid-state structures of $\mathrm{Rh}_{2}(S-2-\mathrm{Cl}-4-\mathrm{ArTPCP})_{4}$ catalysts $\mathbf{G}$ $(\mathrm{RMSD}=0.607 \AA)$ and $\mathbf{H}(\mathrm{RMSD}=0.640 \AA)$. For catalyst $\mathbf{G}$, different independent molecules in the unit cell feature different biaryl torsions that nominally break $\mathrm{C}_{4}$ symmetry and give rise to slightly different reactive site shapes that are likely undergoing facile interconversion under the reaction conditions $\left(\mathrm{CH}_{2} \mathrm{Cl}_{2}, 313 \mathrm{~K}\right) . \mathrm{Rh}_{2}(S-2-\mathrm{Cl}-4 \text {-ArTPCP })_{4}$ catalyst conformers in all feasible biaryl torsion combinations were considered as possible reactive conformers for MLR feature extraction (see Figure S11-S12 and Table S2 for details).

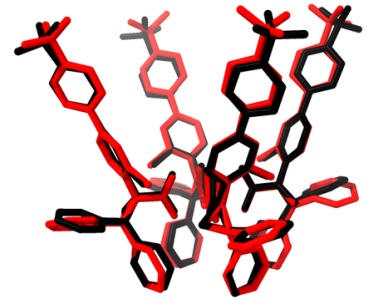

$\mathrm{Rh}_{2}\left(\mathrm{~S}-2-\mathrm{Cl}-4-\left(p-\mathrm{BuC}_{6} \mathrm{H}_{4}\right) \mathrm{TPCP}_{4}\right.$ Catalyst $\mathbf{G}$

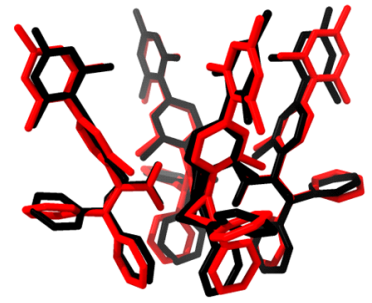

$\mathrm{Rh}_{2}(\mathrm{~S}-2-\mathrm{Cl}-4-\mathrm{MesTPCP})_{4}$ Catalyst $\mathbf{H}$

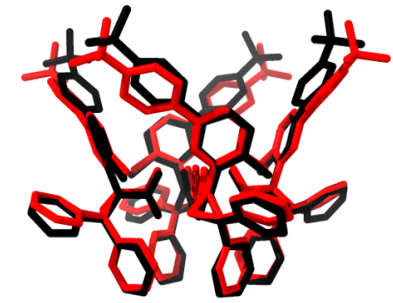

$\mathrm{Rh}_{2}\left(\mathrm{~S}-2-\mathrm{Cl}-5-\left(p-{ }^{-} \mathrm{BuC}_{6} \mathrm{H}_{4}\right) \mathrm{TPCP}\right)_{4}$ Catalyst $\mathbf{M}$

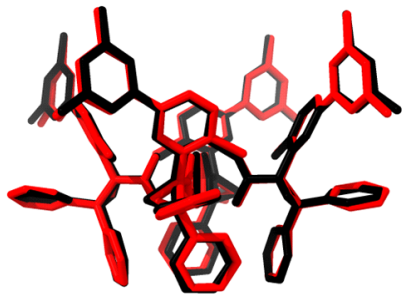

$\mathrm{Rh}_{2}\left(\mathrm{~S}-2-\mathrm{Cl}-5-\left(3,5-\mathrm{diMeC}_{6} \mathrm{H}_{3}\right) \mathrm{TPCP}\right)_{4}$ Catalyst $\mathbf{N}$

Figure S4. Structural overlays comparing the X-ray crystal structures (black) with optimized structures (red) calculated at the QM/MM $(\mathbf{G}, \mathbf{H})$, extended 5-Ar QM/MM (N), or QM level of theory $(\mathbf{M})$. Note that all five $\mathrm{Rh}_{2}(S-2-\mathrm{Cl}-5 \text {-ArTPCP })_{4}$ catalyst structures were re-optimized entirely at the QM level to account for dispersive effects between closely approaching neighboring ligands and better assess relative energetics between competing catalyst configurations of similar stability.

For $\mathrm{Rh}_{2}(S-2-\mathrm{Cl}-5-\mathrm{ArTPCP})_{4}$ catalysts like $\mathbf{M}$ and $\mathbf{N}$, atoms of neighboring ligands closely approach one another. To more accurately account for such dispersive interactions, the entire 5-Ar groups were initially treated at the QM level of theory in an extended QM/MM partitioning. This provided excellent structural agreement with the $\mathrm{C}_{4}$-symmetric bowl structure obtained in the solid-state, as shown for catalyst $\mathbf{N}(\mathrm{RMSD}=0.277 \AA$; Figure S4).

However, it was still ambiguous whether this structure was the thermodynamically preferred catalyst configuration, as several other configurations (i.e., $\alpha, \beta, \alpha, \beta ; \alpha, \alpha, \beta, \beta ; \alpha, \alpha, \alpha, \beta)$ were computed to be nearly isoenergetic at this extended QM/MM level of theory. Thus, low energy conformers of $\mathrm{Rh}_{2}(S-2-\mathrm{Cl}-5-\mathrm{ArTPCP})_{4}$ catalysts (K-O) were re-optimized entirely at the QM level to provide increased clarity on the relative energies of competing catalyst configurations (see Figure 3B). The QM structure obtained for $\mathbf{M}$ matches adequately (RMSD = 0.667/0.691/0.923 $\AA$ ) with the three independent catalyst molecules in the corresponding X-ray structure unit cell (Figure S4). For further discussion of the thermodynamic and kinetic considerations that influence the reactive conformations for K-O, see Figure S14 and Tables S3-S5.

Good agreement was also observed for $\mathrm{Rh}_{2}\left[R-3,5-\mathrm{di}-\left(p-{ }^{t} \mathrm{BuC}_{6} \mathrm{H}_{4}\right) \mathrm{TPCP}\right]_{4}(\mathbf{W})$, which adopts a $\mathrm{D}_{2}$ $(\alpha, \beta, \alpha, \beta)$ structure, albeit when an extended QM partition was employed (Figure S5). Due to the large number of atoms $(>300)$ in $\mathrm{Rh}_{2}\left[R-3,5-\mathrm{di}-\left(p-{ }^{t} \mathrm{BuC}_{6} \mathrm{H}_{4}\right) \mathrm{TPCP}\right]_{4}$ and the fact that $\alpha, \beta, \alpha, \beta$ is unambiguously the preferred configuration, ${ }^{4,37-38}$ it was deemed unnecessary to treat all atoms at the QM level. This enabled all possible reactive $\alpha, \beta, \alpha, \beta$ conformations to be efficiently surveyed for MLR feature extraction. Several other stable $\alpha, \beta, \alpha, \beta$ ' conformations where the reactive site is more spatially exposed to allow for diazo binding were also identified (e.g., W1 in Figure 3C and W2-W7), expanding on one such conformation (4B) reported in a prior QM study. ${ }^{37}$ 

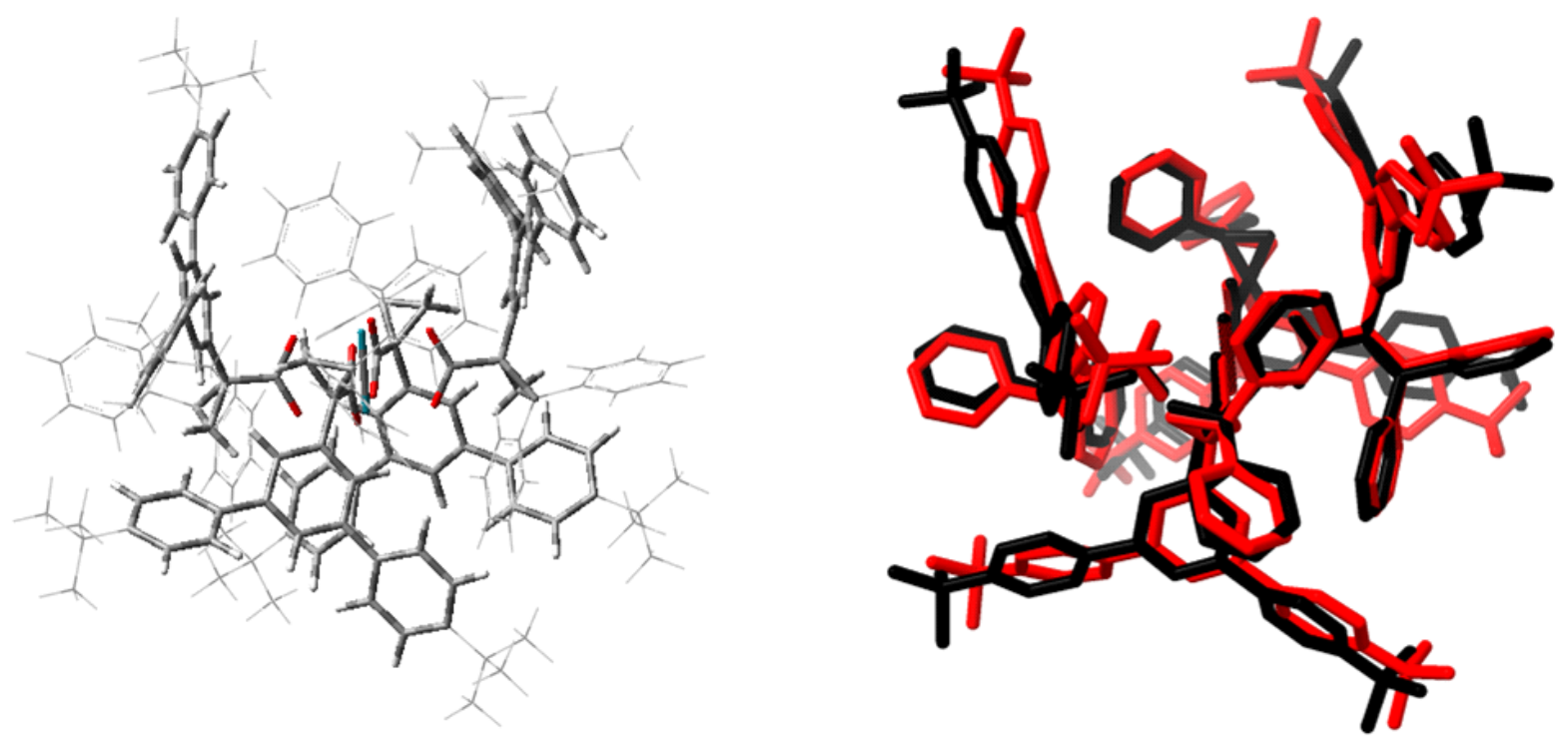

Figure S5. Extended $\mathrm{QM}$ partition (left) for $\mathrm{QM} / \mathrm{MM}$ geometry optimization of $\mathrm{Rh}_{2}[R-3,5-\mathrm{di}-(p$ $\left.\left.{ }^{t} \mathrm{BuC}_{6} \mathrm{H}_{4}\right) \mathrm{TPCP}\right]_{4}(\mathbf{W})$, which was necessary to energetically account for dispersion interactions between neighboring ligands within the $\mathrm{D}_{2}$ bowl structure. The structural overlay (right) comparing the X-ray crystal structure (black) with a low energy QM/MM calculated conformer (W'3 in red) shows adequate agreement $\left(\mathrm{RMSD}=0.924 \AA\right.$ ). Many other similar $\alpha, \beta, \alpha, \beta^{\prime}$ conformers potentially relevant to reactivity were identified that adopt different biaryl torsion combinations (W1-W7).

\subsection{Comparing QM/MM Approach to Previous QM-Optimized Structures}

While structural agreement with X-ray crystal structures was encouraging, recent computational studies of $\mathrm{Rh}_{2}(\mathrm{TPCP})_{4}$ catalysts have highlighted the potential for crystal packing effects to give rise to drastic differences between solid-state structures and those relevant for catalytic reactivity in solution. ${ }^{37}$ Thus, the accuracy of our QM/MM approach for describing catalyst conformational ensembles in $\mathrm{CH}_{2} \mathrm{Cl}_{2}$ was further verified via benchmarking against available structures and relative energetics from previous QM studies for select catalysts for which such conformational analysis is available.

An instructive representative example is $\mathrm{Rh}_{2}(p \text {-BrTPCP })_{4}$ (catalyst $\mathbf{S}$ ), which has been crystallized in the $D_{2}$-symmetric $\alpha, \beta, \alpha, \beta$ configuration. ${ }^{3}$ Recent $\mathrm{QM}$ studies have established that the $\mathrm{C}_{2}$ symmetric $\alpha, \alpha^{\prime}, \alpha, \alpha^{\prime}$ configuration is significantly more thermodynamically stable in $\mathrm{CH}_{2} \mathrm{Cl}_{2}$ solution than all other possible conformations. ${ }^{37}$ To verify that our QM/MM approach could accurately approximate the relative structures and energetics of competing catalyst configurations in $\mathrm{CH}_{2} \mathrm{Cl}_{2}$ solution, we compared various conformations of catalyst $\mathbf{S}$ on the respective QM (2A2G in original report ${ }^{37}$ ) and $\mathrm{QM} / \mathrm{MM}$ potential energy surfaces (S1-S11). It is notable that the QM structures were optimized with solvation (C-PCM), whereas the QM/MM structures were only subjected to less intensive gas phase optimizations and subsequently corrected using single-point electronic energies (see computational methods, vide supra).

Overall, the QM structures and relative energetics are generally reproduced well given the relatively low number of atoms being treated at the QM level of theory in our QM/MM approach. Of note, all configurations except $\mathrm{D}_{2}(\alpha, \beta, \alpha, \beta)$ closely match structurally (i.e., RMSD $\left.\leq 1\right)$, and even in that case its relative energy compared with the most stable $\alpha, \alpha^{\prime}, \alpha, \alpha^{\prime}$ configuration is still adequately assessed (Figure S6). 


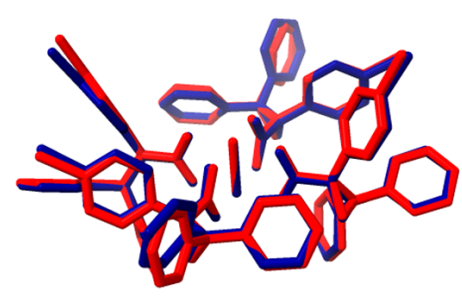

$\alpha, \alpha^{\prime}, \alpha, \alpha^{\prime}$ Conformer $2 \mathrm{~A}(+0.0)$ Conformer S1 $(+0.0)$ $\mathrm{RMSD}=0.443 \mathrm{~A}$

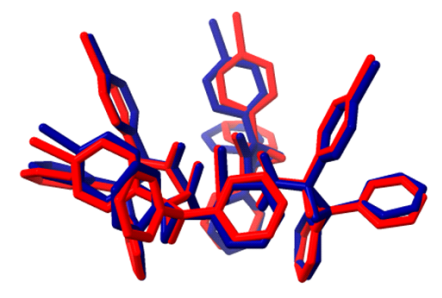

$a, a, a, y$ Conformer $2 \mathrm{~F}(+4.8)$ Conformer S3 $(+3.8)$ $\mathrm{RMSD}=0.471 \mathrm{~A}$

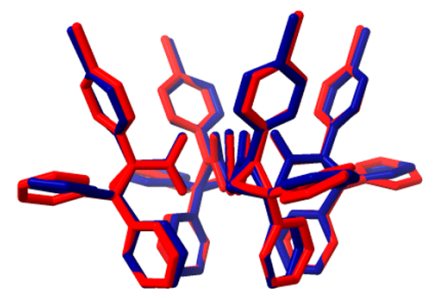

$\alpha, \alpha, \alpha, \alpha$ Conformer 2D $(+7.9)$ Conformer S11 (+8.1) $\mathrm{RMSD}=0.488 \mathrm{~A}$

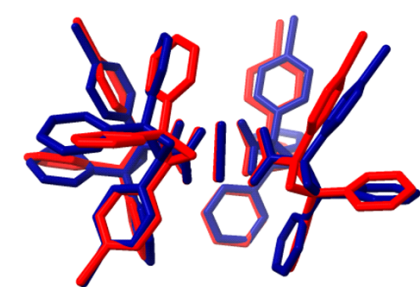

$\alpha, \alpha, \alpha, \beta$

Conformer $2 \mathrm{E}(+7.9)$

Conformer S10 (+7.9) $\mathrm{RMSD}=1.017 \mathrm{~A}$

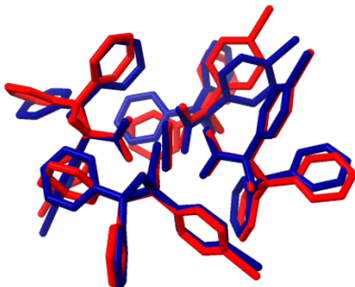

$\alpha, \alpha, \beta, \gamma$

Conformer $2 \mathrm{G}(+6.9)$

Conformer S5 $(+5.4)$ $\mathrm{RMSD}=0.819 \AA$

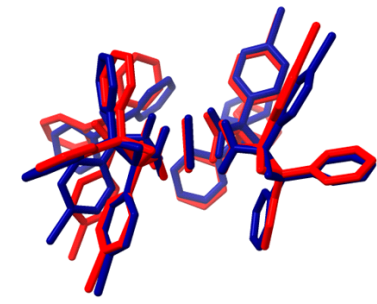

$\alpha, \alpha, \beta, \beta$ Conformer $2 \mathrm{~B}(+7.0)$ Conformer S7 $(+6.8)$ RMSD $=0.996 \AA$

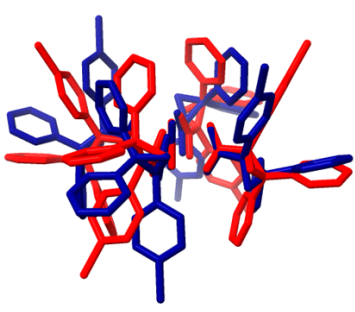

$\alpha, \beta, \alpha, \beta$

Conformer $2 \mathrm{C}(+9.1)$

Conformer S9 $(+7.9)$ $\mathrm{RMSD}=3.176 \AA$

Figure S6. Structural overlays for conformers of catalyst $\mathbf{S}$ comparing our QM/MM optimized structures (red) with previously reported QM structures (blue) optimized at the B3LYP-D3BJ/6-31G**$\operatorname{lanl} 2 \mathrm{dz}(\mathrm{Rh})$ level with C-PCM solvation. All energies are solvated free energies (in $\mathrm{kcal} / \mathrm{mol}$ ) relative to the most stable conformation of catalyst $\mathbf{S}$ on each potential energy surface. Note that the $R$ enantiomers were computed in the prior study (ref. 37) and aligned here via $x y z$ coordinate inversion.

We hypothesize that $\mathrm{D}_{2}(\alpha, \beta, \alpha, \beta)$ is more poorly reproduced structurally at the QM/MM level because it is the only configuration where no pairs of neighboring $\mathrm{C} 1$ aryl groups about each $\mathrm{Rh}$ face are treated at the QM level in our partitioning scheme. In contrast, all four core $\mathrm{C} 1$ aryl groups that closely approach about a single $\mathrm{Rh}$ face in the $\mathrm{C}_{4}(\alpha, \alpha, \alpha, \alpha)$ and $\mathrm{C}_{2}{ }^{\prime}\left(\alpha, \alpha^{\prime}, \alpha, \alpha^{\prime}\right)$ configurations are treated at the QM level. Even the $\mathrm{C}_{2}(\alpha, \alpha, \beta, \beta)$ configuration features two neighboring $\mathrm{C} 1$ aryl groups that are considered at the QM level, which acts to confine the remaining possible spatial positions of the other two ligand aryl groups being treated at the MM level toward their preferred positions. Fortunately, $\mathrm{D}_{2}(\alpha, \beta, \alpha, \beta)$ structures are either substantially thermodynamically unstable or predicted to be kinetically inaccessible for all catalysts except the $\mathrm{Rh}_{2}(3,5 \text {-diXTPCP })_{4}$ catalyst series $(\mathbf{U}-\mathbf{W})$, and thus this minor discrepancy is an acceptable concession to make given the large numbers of catalysts and atoms per catalyst being considered in this study. Furthermore, the $\mathrm{D}_{2}$ structure of catalyst $\mathbf{W}$ was approximated well in comparison to its X-ray structure (Figure S5).

In addition to the catalyst conformations originally reported for $\mathbf{S}$, the ability to rapidly survey the full conformational space at the QM/MM level of theory also allowed for several other relevant catalyst conformers that are of similar or lower energy to be identified (Figure S7). Of note, a slightly distorted $\alpha, \alpha^{\prime}, \alpha, \alpha^{\prime}$ conformation $(\mathbf{S 2})$ is found that is only marginally less stable than $\mathbf{S 1}$, along with a unique $\alpha, \beta, \alpha, \gamma$ conformation (S4). Additionally, conformers that are computed to be slightly more stable for $\alpha, \alpha, \alpha, \beta$ (S8) and $\alpha, \beta, \alpha, \beta$ (S6) configurations were identified. Note that only reactive conformations that are present under the reaction conditions $\left(\mathrm{CH}_{2} \mathrm{Cl}_{2}, 313 \mathrm{~K}\right)$ and spatially accessible enough to allow for facile carbene formation (in terms of $\mathrm{G} \%$ ) were considered for molecular descriptor extraction and MLR modeling (conformers S1 and S2 in this case; see SI section $\mathrm{X}$ for further details regarding this analysis for other catalysts). 


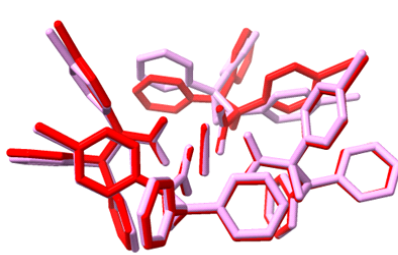

$\alpha, \alpha^{\prime}, \alpha, \alpha^{\prime}$

Conformer $\mathrm{S} 2(+1.4)$

Conformer S1 $(+0.0)$

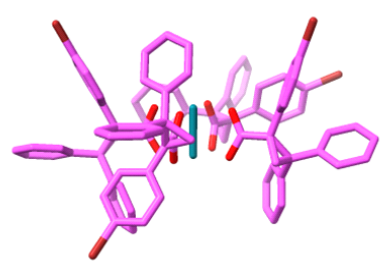

$\alpha, \beta, \alpha, y$

Conformer S4 (+4.0)

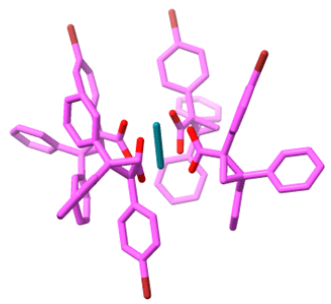

$\alpha, \alpha, \alpha, \beta$

Conformer S8 (+7.2)

Conformer S10 (+7.9)

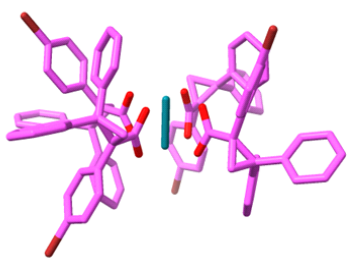

$\alpha, \beta, \alpha, \beta$

Conformer S6 (+6.7)

Conformer S9 (+7.9)

Figure S7. Additional conformers of catalyst $\mathbf{S}$ (pink) obtained from rigorous conformer searching protocol and $\mathrm{QM} / \mathrm{MM}$ optimization compared against the most similar conformation displayed in Figure S6 (red). All energies are solvated free energies (in kcal/mol) relative to major conformer $\mathbf{S 1}$.

Lastly, relative computed energetics in $\mathrm{CH}_{2} \mathrm{Cl}_{2}$ for $\mathrm{Rh}_{2}(\mathrm{~S} \text {-o-ClTPCP })_{4}(\mathrm{~A})$ were compared between our QM/MM approach and a previous QM study at the B3LYP-D3BJ/6-31G*-lanl2dz(Rh) level of theory (with C-PCM solvation). ${ }^{8}$ The potential energy surfaces match well, with the $\alpha, \alpha, \alpha, \alpha$ geometry unambiguously the most stable in each case, followed by the $\alpha, \alpha, \alpha, \beta(+4.6 /+3.4), \alpha, \alpha, \beta, \beta$ $(+5.2 /+8.3)$, and $\alpha, \beta, \alpha, \beta(+6.9 /+10.5)$ configurations, respectively, where solvated free energy values are reported as $\mathrm{kcal} / \mathrm{mol}$ relative to the $\alpha, \alpha, \alpha, \alpha$ conformer $(\mathrm{QM} /\{\mathrm{QM} / \mathrm{MM}\})$ ).

The reliability of our QM/MM approach for approximating catalyst structures and relative energetics in both solution and in the solid-state for disparate catalyst types validates its utility for broad application to dirhodium catalyst space. Importantly, implementing these methods using structural inputs generated based on standardized conformer searching and clustering protocols ensures that all representative catalyst reactive site shapes are examined to identify which are relevant to reactivity and should be further considered for SMART analysis and MLR modeling. Furthermore, part of the motivation behind identifying efficient QM/MM methods for approximating dirhodium catalyst structures and relative energetics is to enable facile future virtual screening now that sophisticated catalyst descriptors have been developed.

\subsection{Reactive Catalyst Conformer Classification for Extracting Descriptors for MLR}

To be relevant to reactivity and further considered for MLR analysis, a catalyst conformation must be: (1) sufficiently thermodynamically stable to be present in solution under the reaction conditions $\left(\mathrm{CH}_{2} \mathrm{Cl}_{2}, 313 \mathrm{~K}\right)$; (2) kinetically accessible via ligand flipping interconversion from the precatalyst configuration; and (3) classified as kinetically competent for carbene formation and reactivity based on spatial accessibility filtering (i.e., $\mathrm{G} \%$ classification).

A $3.5 \mathrm{kcal} / \mathrm{mol}$ relative energy cutoff was utilized to establish conformers stable enough to be present in solution for a given catalyst $\left(>0.2 \%\right.$ population at $313 \mathrm{~K}$ ). Catalysts with low $\Delta G^{\ddagger}$ rot barriers in the single node decision tree classification were permitted to populate all stable conformations (see Figure 3B), whereas $\mathrm{Rh}_{2}(S-o-\mathrm{ClTPCP})_{4}$ catalysts with hindered rotation were confined to the "all-up" conformations of the pre-catalysts (i.e., $\alpha, \alpha, \alpha, \alpha ; \alpha, \alpha, \alpha, \alpha$ '; etc.) due to prohibitive ligand flipping barriers. Spatial accessibility filtering was subsequently performed by assessing $\mathrm{G} \%$ for each $\mathrm{Rh}$ face of each conformer remaining using Solid-G. ${ }^{39}$ A ligand shielding cutoff was taken to be $7 / 8$ of the surrounding sphere $(\mathrm{G} \geq 87.5 \%)$, as these $\mathrm{Rh}$ faces are nearly completely inaccessible. Lastly, $\mathrm{Rh}$ faces with $\mathrm{G} \%>2.5+\mathrm{G} \% \min$ were considered too hindered to be kinetically competent for diazo binding and dirhodium-carbene formation (under non-CurtinHammett control), where $\mathrm{G} \%$ min represents the approachability of the most spatially accessible 
conformation present in solution for a given catalyst. Although 2.5\% may appear to be a small G\% difference, because of the large surrounding sphere and the relative nature of the $\mathrm{G} \%$ metric, ${ }^{39}$ it equates to a dramatic difference in absolute spatial accessibility.

This analysis for identifying reactive conformations is shown for each representative catalyst subclass. The pool of conformations for each catalyst are displayed as space-filling models in Figures S8-S20. Reactive conformations considered in MLR analysis are displayed as green spacefilling diagrams (labelled as [Letter\#]), with the axial Rh atom shown in yellow for visual clarity. Conformations that are thermodynamically stable but likely not present in solution under the reaction conditions due to prohibitive ligand flipping barriers are shown as gray space-filling diagrams (labelled as [Letter]' [\#]). Conformations present but too spatially inaccessible to be kinetically competent for diazo binding and carbene formation based on G\% filtering are shown as red space-filling diagrams (and labelled as [Letter]'[\#]). All conformers are numbered in ascending order within each classification according to calculated relative free energy.

The most accessible of the two Rh faces for a given conformer is shown as a larger picture in Figures S8-S20, with a smaller inset showing the topology of the other less accessible Rh face. Note that for $\alpha, \beta, \alpha, \beta$ and $\alpha, \alpha, \beta, \beta$ conformers, both faces may have very similar reactive site topology and spatial accessibility. G\% values are shown for each Rh face for green and red spacefilling diagrams to directly illustrate the reactive conformer classification process, with green $\mathrm{G} \%$ text indicating the accessible face of the reactive conformer and red text indicating a hindered $\mathrm{Rh}$ face that was not further considered for feature extraction. The calculated relative free energies in $\mathrm{CH}_{2} \mathrm{Cl}_{2}$ (in kcal/mol) for each catalyst conformer relative to the most stable catalyst conformation present in solution is provided, with the corresponding $x y z$ atomic coordinates listed at the end of the document. The \% equilibrium population values based on the relative free energies and the "reactive population \%" are both reported in Tables S3-S9. Reactive population \% is reported among reactive conformers only, so as to correspond with the weighting scheme used to tabulate the Boltzmann-averaged catalyst properties (see appended .xlsx file for descriptor values).
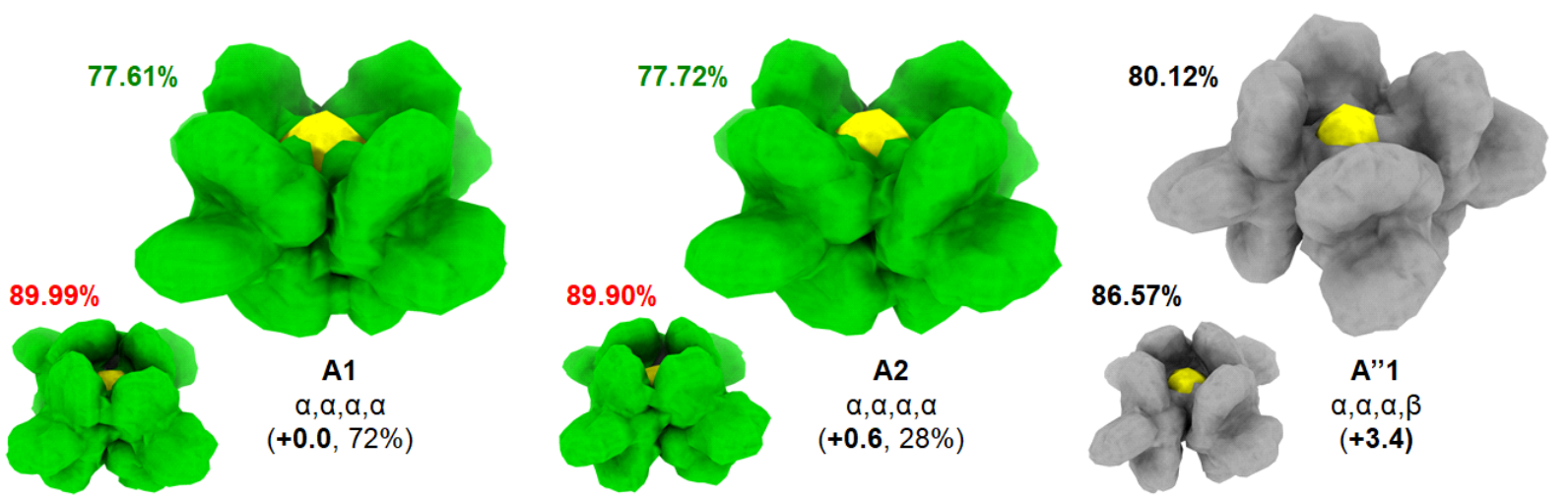

Figure S8. Conformations of $\mathrm{Rh}_{2}(S-o-C l T P C P)_{4}(\mathbf{A})$. Two very similar $\alpha, \alpha, \alpha, \alpha$ configurations were identified that differ very slightly in energy and bond metrics (A1 and A2). The only other thermodynamically stable conformer within the $3.5 \mathrm{kcal} / \mathrm{mol}$ cutoff is $\mathbf{A}$ ', $\mathbf{1}$, but its formation would require ligand flipping isomerization from the $\alpha, \alpha, \alpha, \alpha$ pre-catalyst that is kinetically unlikely under the reaction conditions. Among the reactive conformations, only the $\mathrm{C} 1$ aryl bowl face of each is spatially accessible, while the opposite face is nearly completely blocked ( $\mathrm{G} \sim 90 \%)$. Conformers $\mathbf{A 1}$ and $\mathbf{A 2}$ are analogous to those for the reactive $\alpha, \alpha, \alpha, \alpha$ conformations of $\mathrm{Rh}_{2}(S-2-\mathrm{Cl}-4-\mathrm{BrTPCP})_{4}(\mathbf{B 1}-\mathrm{B2})$. B1 $(+0.0$, $67 \%)$ and $\mathbf{B 2}(+0.4,33 \%)$ are also calculated to be much more stable than the most stable $\alpha, \alpha, \alpha, \beta(+4.0)$, $\alpha, \alpha, \beta, \beta(+8.4)$, and $\alpha, \alpha, \beta, \beta(+9.3)$ conformers of catalyst $\mathbf{B}$. 

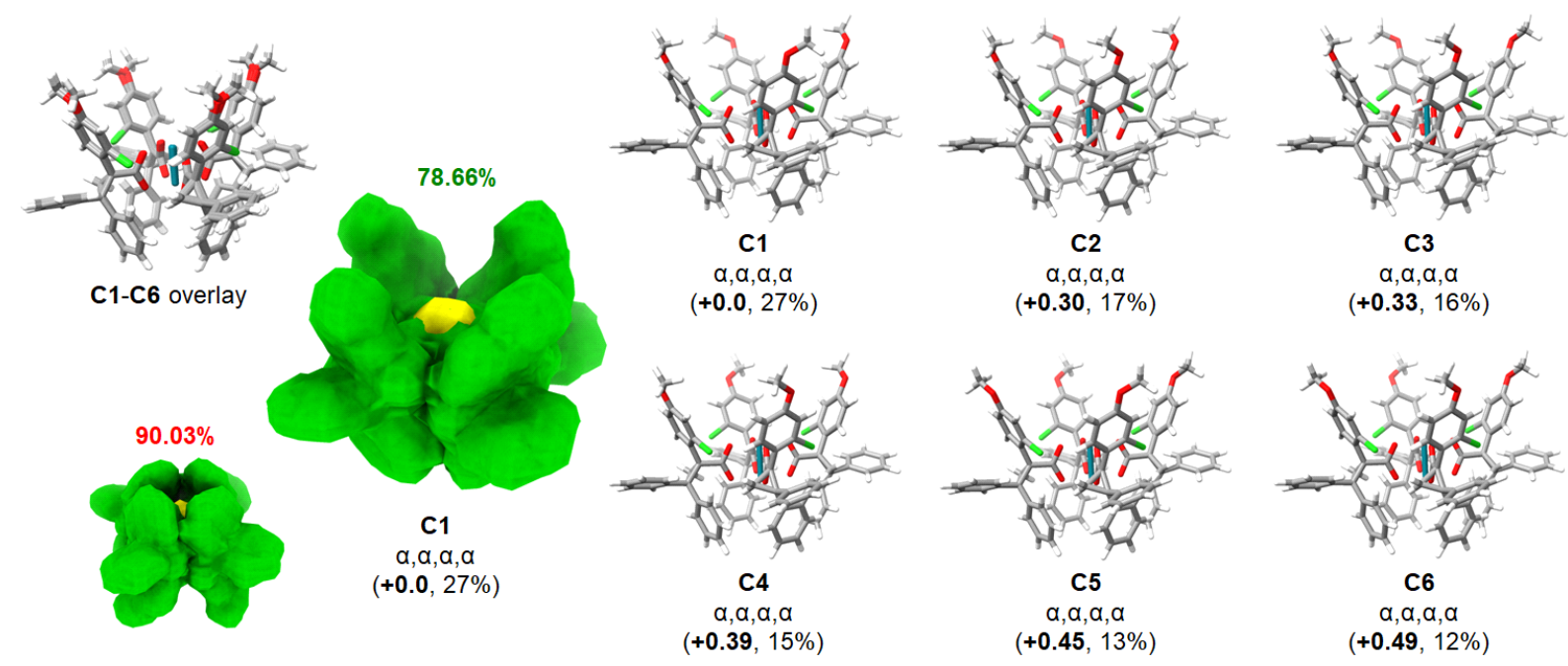

Figure S9. Conformations of $\mathrm{Rh}_{2}(S-2-\mathrm{Cl}-4-\mathrm{OMeTPCP})_{4}(\mathbf{C})$, which persists exclusively in the $\alpha, \alpha, \alpha, \alpha$ configuration under catalytic conditions but can adopt various methoxy group orientation combinations (C1-C6). This can be seen in the overlay of conformers C1-C6 (top left). The space-filling diagrams are very similar (as are their properties), so representative images for $\mathbf{C 1}$ are displayed. Much like other $\mathrm{Rh}_{2}(S \text {-o-CITPCP })_{4}$ catalysts, only the $\mathrm{C} 1$ aryl bowl face is spatially accessible, while the opposite face is nearly completely blocked $(\mathrm{G} \sim 90 \%)$ by four closely packed phenyl groups. $\mathbf{C 1}$ is computed to be much more stable than the most stable $\alpha, \alpha, \alpha, \beta(+4.5), \alpha, \beta, \alpha, \beta(+8.0)$, and $\alpha, \alpha, \beta, \beta(+11.0)$ conformations.
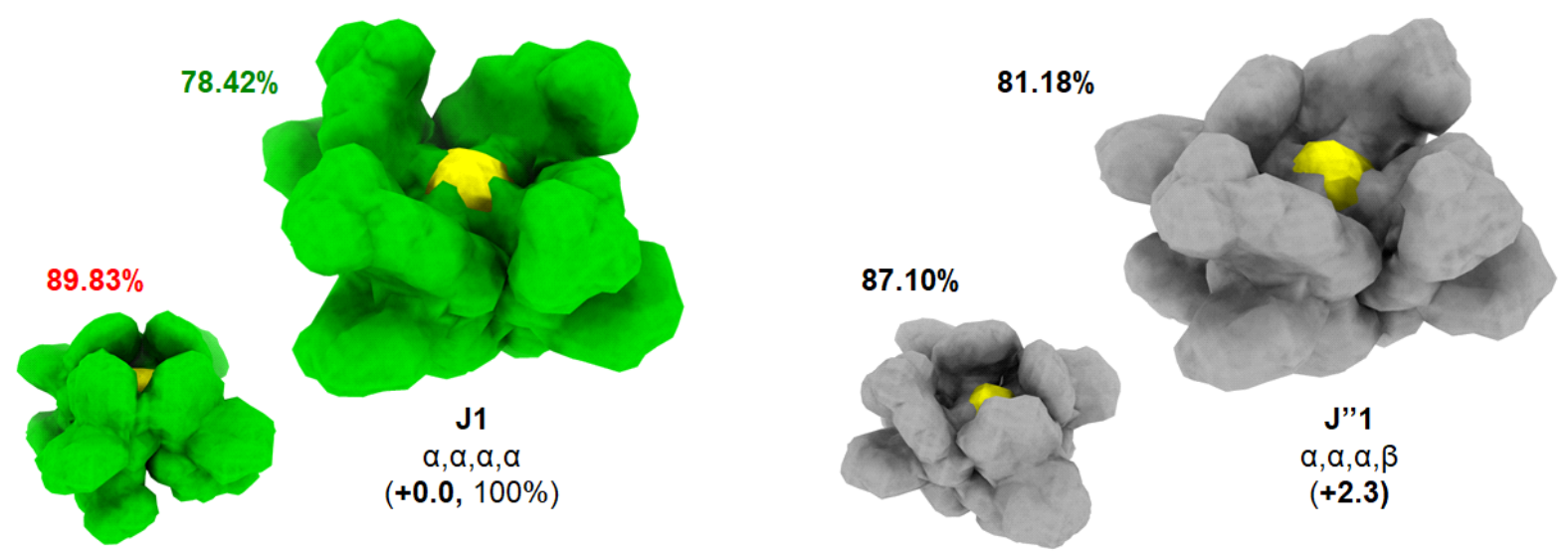

Figure S10. Conformations of $\mathrm{Rh}_{2}\left(S-2-\mathrm{Cl}-5-\mathrm{CF}_{3} \mathrm{TPCP}\right)_{4}(\mathbf{J})$, which persists exclusively in the $\alpha, \alpha, \alpha, \alpha$ configuration under catalytic conditions $(\mathbf{J 1})$. The only other thermodynamically stable conformer within the $3.5 \mathrm{kcal} / \mathrm{mol}$ cutoff is $\mathbf{J}$ ' 1 , but its formation would require ligand flipping isomerization from the $\alpha, \alpha, \alpha, \alpha$ pre-catalyst that is kinetically unlikely under the reaction conditions. $\mathbf{J 1}$ is also significantly more stable than the most stable $\alpha, \beta, \alpha, \beta(+6.2)$ and $\alpha, \alpha, \beta, \beta(+8.5)$ conformations of $\mathbf{J}$. For the lone reactive conformation, $\mathbf{J 1}$, only the $\mathrm{C} 1$ aryl bowl face is spatially accessible, while the opposite face is nearly completely blocked $(\mathrm{G} \sim 90 \%)$. Conformers $\mathbf{J 1}$ and $\mathbf{J}$ '' 1 are analogous to those for $\mathrm{Rh}_{2}(\mathrm{~S}-2-\mathrm{Cl}-$ 5 -BrTPCP) $)_{4}$ (I1 and I''1). I1 (+0.0) is significantly more stable than $\alpha, \alpha, \alpha, \beta(\mathbf{I}$ ' $1 ;+3.1), \alpha, \beta, \alpha, \beta(+6.7)$ and $\alpha, \alpha, \beta, \beta(+7.7)$ conformers. I1 has nearly identical reactive site topology to $\mathbf{J 1}$, with one accessible $\mathrm{C} 1$ aryl bowl face $(\mathrm{G}=77.68 \%)$ and one inaccessible $\mathrm{Rh}$ face $(\mathrm{G}=89.86 \%)$. 


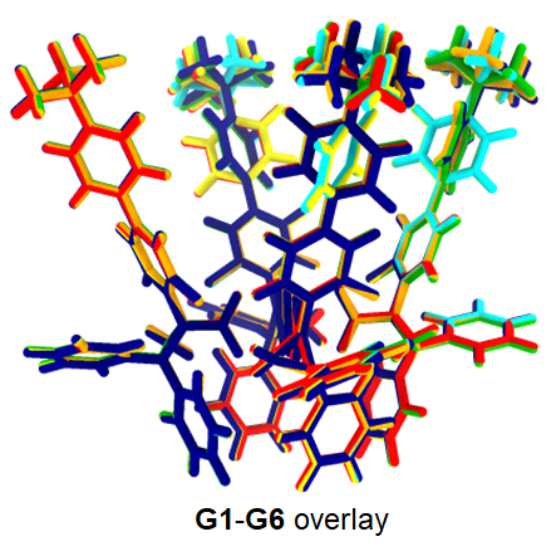

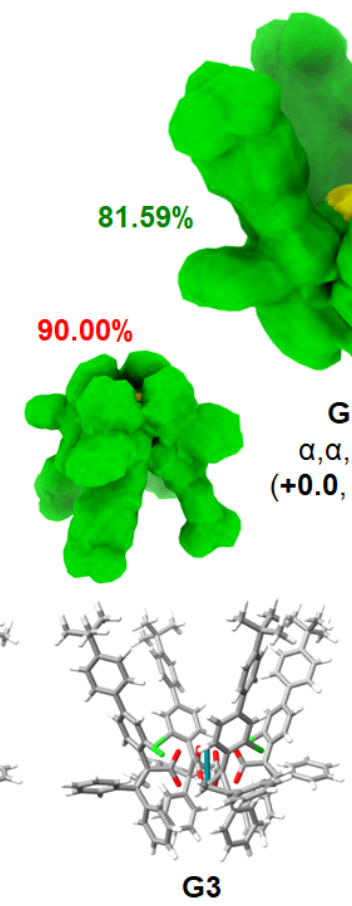

$\alpha, \alpha, \alpha, \alpha$

$(+0.21,17 \%)$

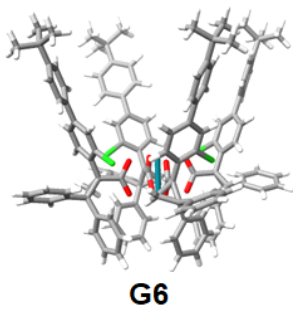

$\alpha, \alpha, \alpha, \alpha$

$(+0.55,10 \%)$

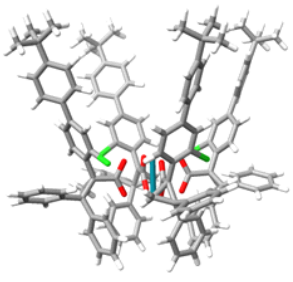

G5

$\alpha, \alpha, \alpha, \alpha$

$(+0.42,12 \%)$ $\alpha, \alpha, \alpha, \alpha$

$(+0.11,20 \%)$

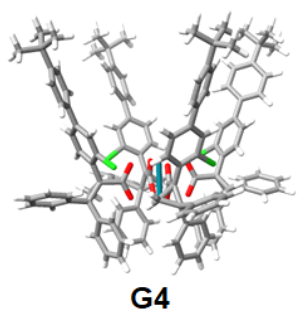

$\alpha, \alpha, \alpha, \alpha$

$(+0.22,17 \%)$

Figure S11. Conformations of $\mathrm{Rh}_{2}\left(S-2-\mathrm{Cl}-4-\left(p-{ }^{t} \mathrm{BuC}_{6} \mathrm{H}_{4}\right) \mathrm{TPCP}\right)_{4}(\mathbf{G})$, which persists exclusively in the $\alpha, \alpha, \alpha, \alpha$ configuration under catalytic conditions but can adopt various biaryl torsion combinations and ${ }^{t} \mathrm{Bu}$ rotamers (see overlay of conformers G1-G6; top left). G1 is calculated to be much more stable than the most stable $\alpha, \alpha, \alpha, \beta(+4.3), \alpha, \beta, \alpha, \beta(+5.7)$, and $\alpha, \alpha, \beta, \beta(+9.9)$ conformers. The space-filling diagrams for G1-G6 look similar in 2D, so representative images for G1 are displayed. However, the biaryl dihedrals adopted do change the peripheral reactive cavity shapes, so all were relevant to consider for parameterization. Only the $\mathrm{C} 1$ aryl bowl face is spatially accessible, while the opposite face is nearly completely blocked $(\mathrm{G} \sim 90 \%)$. Catalyst $\mathbf{G}$ was chosen as representative for $\mathrm{Rh}_{2}(S-2-\mathrm{Cl}-4-\mathrm{ArTPCP})_{4}$ catalysts (D-G), which each adopt 6-8 unique "all-up" conformations that are likely interconverting rapidly via biaryl dihedral rotations. For $\mathrm{Rh}_{2}(S-2-\mathrm{Cl}-4-\mathrm{ArTPCP})_{4}$ catalysts $\mathbf{D}-\mathbf{F}$, the most stable $\alpha, \alpha, \alpha, \alpha$ conformer is also unambiguously preferred to the most stable alternative configurations (Table S2).

Table S2. Relative conformer energetics for $\mathrm{Rh}_{2}(S-2-\mathrm{Cl}-4-\mathrm{ArTPCP})_{4}$ catalysts $\mathbf{D}-\mathbf{F}^{\mathrm{a}}$

\begin{tabular}{ccccc} 
Catalyst & $\begin{array}{c}\alpha, \alpha, \alpha, \alpha \\
(\mathrm{kcal} / \mathrm{mol})\end{array}$ & $\begin{array}{c}\alpha, \alpha, \alpha, \beta \\
(\mathrm{kcal} / \mathrm{mol})\end{array}$ & $\begin{array}{c}\alpha, \beta, \alpha, \beta \\
(\mathrm{kcal} / \mathrm{mol})\end{array}$ & $\begin{array}{c}\alpha, \alpha, \beta, \beta \\
(\mathrm{kcal} / \mathrm{mol})\end{array}$ \\
\hline $\mathbf{D}$ & $+0.0^{\mathrm{b}}$ & $>+20$ & $>+20$ & $>+20$ \\
$\mathbf{E}$ & $+0.0^{\mathrm{b}}$ & +4.3 & +6.0 & +10.7 \\
$\mathbf{F}$ & $+0.0^{\mathrm{b}}$ & +4.3 & +3.9 & +8.3 \\
\hline
\end{tabular}

${ }^{a}$ High energy irregular configurations (e.g., $\alpha, \alpha, \alpha, \gamma ; \alpha, \alpha, \beta, \gamma ; \alpha, \beta, \alpha, \gamma ;$ etc.) were also considered.

'Similar to G, multiple "all-up" (nominally $\alpha, \alpha, \alpha, \alpha)$ configurations were identified for each catalyst. For D: D1 (+0.0), D2 $(+0.03)$, D3 (+0.04), D4 (+0.36), D5 (+0.44), and D6 $(+1.56)$. For E: E1 $(+0.0)$, E2 $(+0.37)$, E3 $(+0.44), \mathbf{E 4}(+0.48)$, E5 $(+0.59)$, E6 $(+0.73)$, E7 $(+0.85)$, and E8 $(+0.93)$. For F: F1 $(+0.0)$, F2 $(+0.03)$, F3 $(+0.18)$, F4 $(+0.27)$, F5 $(+0.29)$, and $\mathbf{F 6}(+0.43)$. Several different conformations of the $\alpha, \alpha, \alpha, \beta, \alpha, \beta, \alpha, \beta$, and $\alpha, \alpha, \beta, \beta$ configurations were also identified, but only the relative energy of the lowest energy conformer of each is reported here for simplicity. 

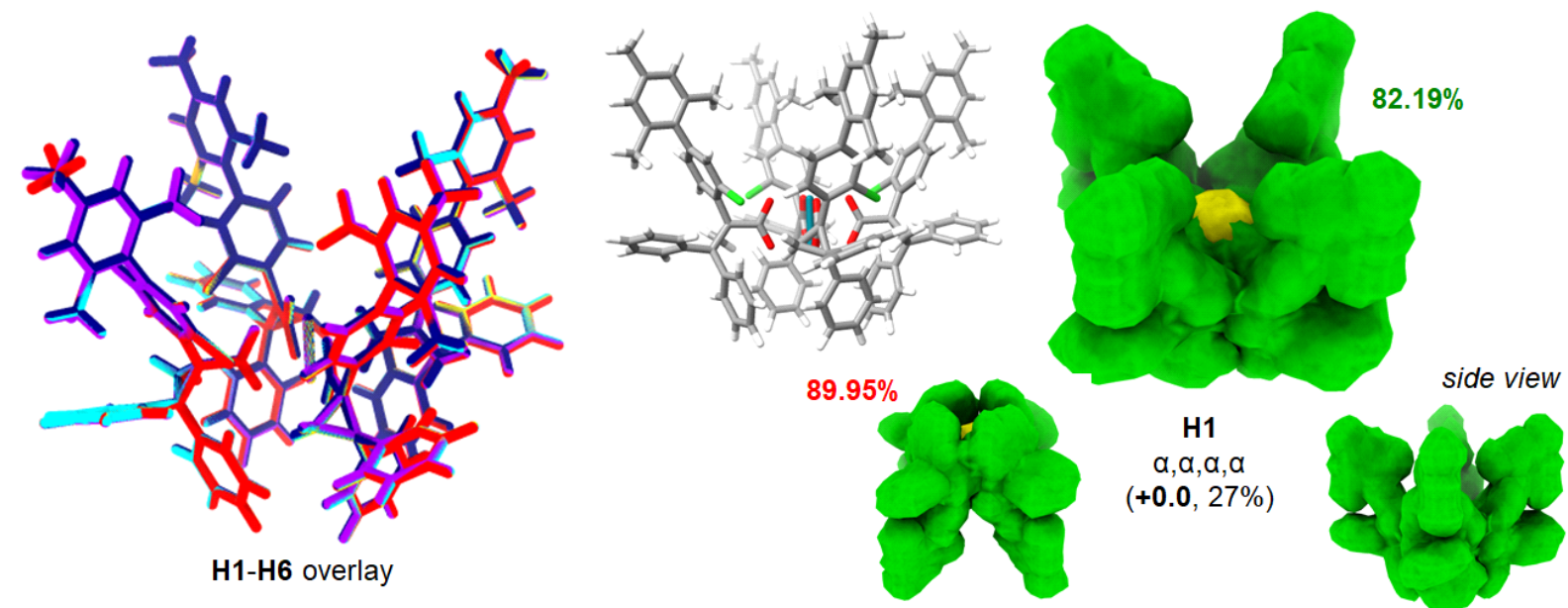

Figure S12. Conformations of $\mathrm{Rh}_{2}(S-2-\mathrm{Cl}-4-\mathrm{MesTPCP})_{4}(\mathbf{H})$, which persists exclusively in the "all-up" $\alpha, \alpha, \alpha, \alpha$ configuration under catalytic conditions but can adopt various conformers and rotamers (H1H6; left overlay). Conformers $\mathrm{H2}, \mathrm{H3}, \mathrm{H4}, \mathrm{H5}$, and $\mathrm{H6}$ are all very similar in structure and energy to H1, with relative free energies and equilibrium population $\%$ of $+0.10(23 \%),+0.19(20 \%),+0.49$ $(12 \%),+0.56(11 \%)$, and $+0.79(7 \%)$, respectively. The space-filling diagrams look very similar, so a representative image for $\mathbf{H 1}$ is displayed. Only the $\mathrm{C} 1$ aryl bowl face is spatially accessible, while the opposite face is nearly completely blocked $(\mathrm{G} \sim 90 \%)$. As with the other $\mathrm{Rh}_{2}(S-2-\mathrm{Cl}-4-\mathrm{ArTPCP})_{4}$ catalysts, $\mathbf{H 1}$ is significantly more stable than the most stable $\alpha, \alpha, \alpha, \gamma(+7.8), \alpha, \alpha, \alpha, \beta(+9.6), \alpha, \beta, \alpha, \beta$ $(+9.5)$, and $\alpha, \alpha, \beta, \beta(+15.2)$ conformers. The main difference in the structure of $\mathbf{H}$ compared with other $\mathrm{Rh}_{2}(S-2-\mathrm{Cl}-4-\mathrm{ArTPCP})_{4}$ catalysts is that the ortho-Me substituents of the mesityl groups significantly protrude into the reactive cavity to avoid steric clash with their respective neighboring aryl hydrogens.

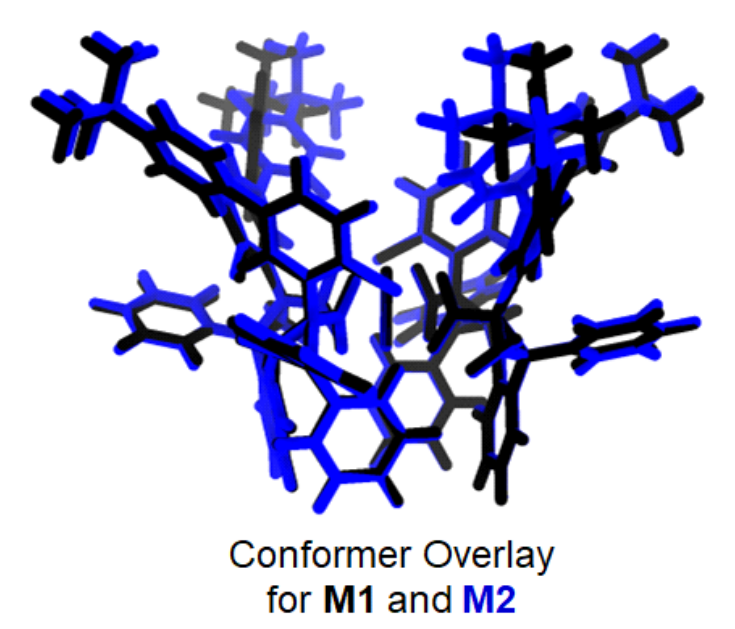

Figure S13. Structural overlay of reactive conformations for $\mathrm{Rh}_{2}\left(S-2-\mathrm{Cl}-5-\left(p-{ }^{t} \mathrm{BuC}_{6} \mathrm{H}_{4}\right) \mathrm{TPCP}\right)_{4}(\mathbf{M})$, which persists exclusively in the $\alpha, \alpha, \alpha, \alpha$ configuration under catalytic conditions but can adopt various biaryl torsion combinations (M1-M2). M2 is a more bowl-like structure, whereas two opposite biaryl torsions are adopted in M1 that slightly enlarges the reactive site cavity. This can be seen in the space-filling diagrams (Figure S14), where the lower G\% metric for M1 indicates that it is slightly more spatially accessible for diazo precursor approach. Both M1 and M2 are significantly more spatially accessible than M'1 and M'2 (see Figure S14 for details). 

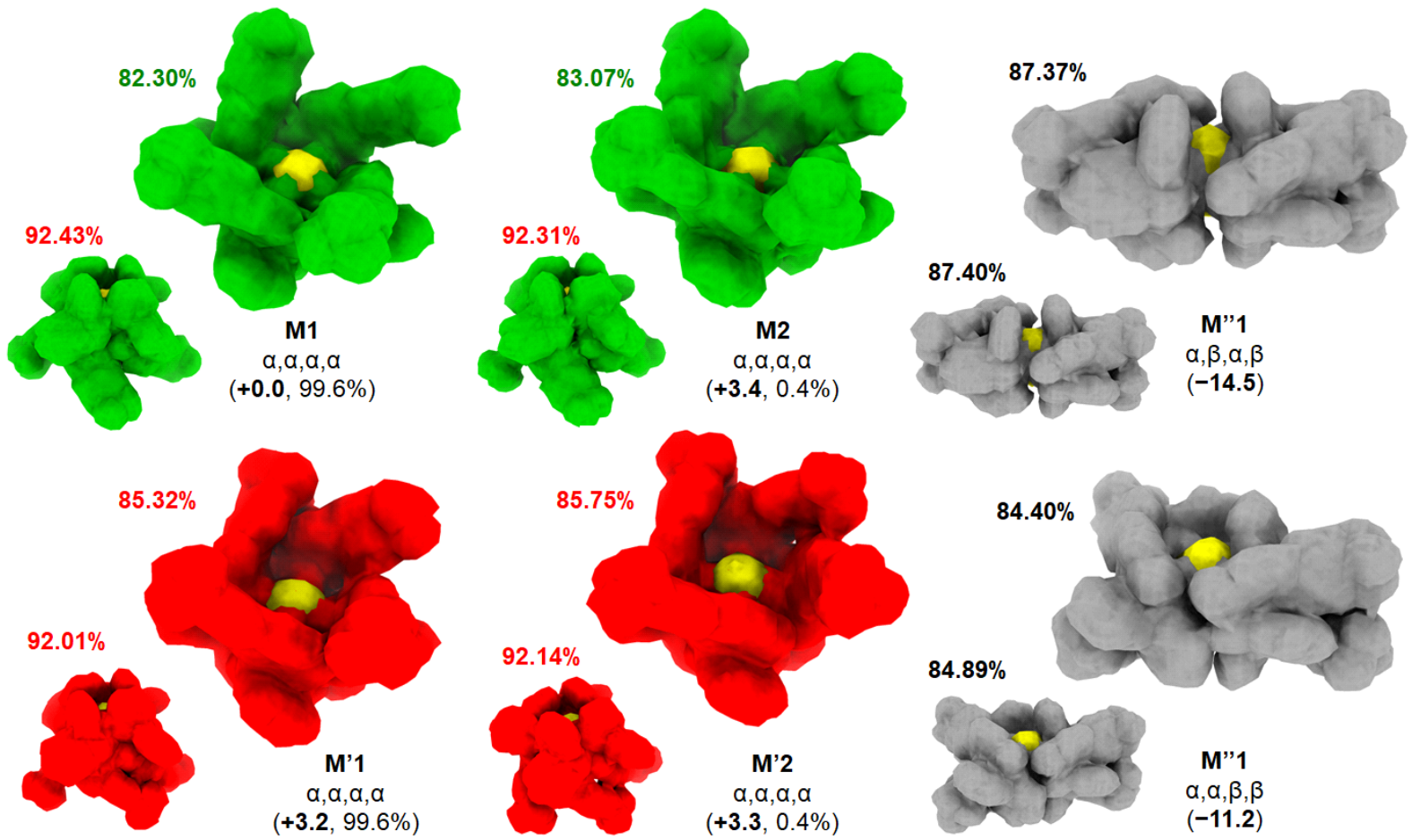

Figure S14. Conformations of $\mathrm{Rh}_{2}\left(S-2-\mathrm{Cl}-5-\left(p-{ }^{t} \mathrm{BuC}_{6} \mathrm{H}_{4}\right) \mathrm{TPCP}\right)_{4}(\mathbf{M})$, which persists exclusively in the $\alpha, \alpha, \alpha, \alpha$ configuration under catalytic conditions but can adopt various biaryl torsion combinations and ${ }^{t} \mathrm{Bu}$ rotamers (M1-M2 and M'1-M'2). M''1 and M'2 are calculated to be significantly more thermodynamically stable than M1 and M2, but their formation from the pre-catalyst $(\alpha, \alpha, \alpha, \alpha)$ is likely prohibited under catalytic conditions due to high ligand flipping isomerization barriers. Note that these stable but kinetically inaccessible alternative configurations are denoted as having negative relative free energy values (in comparison to the most stable $\alpha, \alpha, \alpha, \alpha$ conformer of the pre-catalyst). Conformers M'1 and M'2 are not spatially accessible enough to be kinetically relevant for diazo binding and carbene formation based on their significantly higher G\% values relative to M1 and M2. M'1 and M'2 both adopt biaryl torsion angles that result in one 5-Ar group protruding into the reactive site, so presumably these conformers would easily interconvert via biaryl dihedral rotation to more accessible conformers like M1 and M2 to accommodate the incoming diazo precursor. M1 and M2 also differ in the biaryl dihedral angle combinations adopted, which can be more easily seen in the structural overlay shown in Figure S13. Only the $\mathrm{C} 1$ aryl bowl face is spatially accessible, while the opposite face is nearly completely blocked $(\mathrm{G} \sim 92 \%)$. Catalyst $\mathbf{M}$ was chosen as representative for $\mathrm{Rh}_{2}(S \text {-2-Cl-5-ArTPCP })_{4}$ catalysts (K-N), which each adopt $\sim 6$ unique "all-up" conformations that are likely interconverting rapidly via biaryl dihedral rotations in solution. Similar patterns are observed with these other $\mathrm{Rh}_{2}(S-2-$ Cl-5-ArTPCP $)_{4}$ catalysts, where alternative configurations are more thermodynamically stable but likely kinetically inaccessible, as well as that some "all-up" conformers are too spatially inaccessible and are classified as not relevant to reactivity based on G\% (see Tables S3-S5). An example showing this filtering process comparing $\mathbf{N} \mathbf{1}$ to $\mathbf{N}$ '3 is shown in Figure 3D. 
Table S3. Relative energetics and accessibility for $\mathrm{Rh}_{2}\left(\mathrm{~S}-2-\mathrm{Cl}-5-\left(3,5-\mathrm{diCF}_{3} \mathrm{C}_{6} \mathrm{H}_{3}\right) \mathrm{TPCP}\right)_{4}(\mathbf{K})$

\begin{tabular}{|c|c|c|c|c|c|}
\hline $\begin{array}{l}\text { Catalyst } \\
\text { Conformer }\end{array}$ & $\begin{array}{c}\text { Catalyst } \\
\text { Configuration }\end{array}$ & $\begin{array}{c}\text { Free Energy } \\
(\mathrm{kcal} / \mathrm{mol} ; \%)\end{array}$ & $\begin{array}{c}\text { "Reactive" } \\
\text { Population \% }\end{array}$ & $\begin{array}{l}\mathrm{G} \%{ }_{\mathrm{RH}} \\
\text { face A }\end{array}$ & $\begin{array}{l}\mathrm{G} \% \mathrm{RH} \\
\text { face B }\end{array}$ \\
\hline K1 & $\alpha, \boldsymbol{\alpha}, \boldsymbol{\alpha}, \boldsymbol{\alpha}$ & $+1.7(6 \%)$ & $71 \%$ & $81.34 \%$ & $92.10 \%$ \\
\hline K2 & $\alpha, \alpha, \alpha, \alpha$ & $+2.3(2 \%)$ & $29 \%$ & $81.50 \%$ & $92.07 \%$ \\
\hline$K^{\prime} 1$ & $\alpha, \alpha, \alpha, \alpha$ & $+0.0(89 \%)$ & - & $86.57 \%$ & $91.74 \%$ \\
\hline$K^{\prime} 2$ & $\alpha, \alpha, \alpha, \alpha$ & $+2.4(2 \%)$ & - & $84.63 \%$ & $92.04 \%$ \\
\hline K'3 & $\alpha, \alpha, \alpha, \alpha$ & $+3.0(1 \%)$ & - & $84.68 \%$ & $92.08 \%$ \\
\hline K'1 & $\alpha, \alpha, \alpha, \beta$ & -3.0 & - & $87.73 \%$ & $88.53 \%$ \\
\hline K'2 & $\alpha, \beta, \alpha, \beta$ & -2.9 & - & $86.71 \%$ & $86.72 \%$ \\
\hline K'?3 & $\alpha, \beta, \alpha, \beta^{\prime}$ & -2.2 & - & $84.56 \%$ & $85.07 \%$ \\
\hline K'4 & $\alpha, \alpha, \beta, \beta$ & -1.7 & - & $87.39 \%$ & $87.39 \%$ \\
\hline K'5 & $\alpha, \alpha, \alpha^{\prime}, \beta$ & -0.9 & - & $83.09 \%$ & $83.92 \%$ \\
\hline K'\%6 & $\alpha, \beta, \alpha, \beta^{\prime}$ & +0.3 & - & $83.85 \%$ & $90.65 \%$ \\
\hline
\end{tabular}

Table S4. Relative energetics and accessibility for $\mathrm{Rh}_{2}(S-2-\mathrm{Cl}-5-\mathrm{PhTPCP})_{4}(\mathbf{L})$

\begin{tabular}{|c|c|c|c|c|c|}
\hline $\begin{array}{l}\text { Catalyst } \\
\text { Conformer }\end{array}$ & $\begin{array}{c}\text { Catalyst } \\
\text { Configuration }\end{array}$ & $\begin{array}{c}\text { Free Energy } \\
(\mathrm{kcal} / \mathrm{mol} ; \%)\end{array}$ & $\begin{array}{c}\text { "Reactive" } \\
\text { Population \% }\end{array}$ & $\begin{array}{l}\mathrm{G} \% \mathrm{RH} \\
\text { face A }\end{array}$ & $\begin{array}{l}\mathrm{G} \% \mathrm{RH} \\
\text { face B }\end{array}$ \\
\hline L1 & $\alpha, \boldsymbol{\alpha}, \boldsymbol{\alpha}, \boldsymbol{\alpha}$ & $+\mathbf{0 . 0}(83 \%)$ & $83 \%$ & $78.86 \%$ & $92.86 \%$ \\
\hline L2 & $\boldsymbol{\alpha}, \boldsymbol{\alpha}, \boldsymbol{\alpha}, \boldsymbol{\alpha}$ & $+1.4(9 \%)$ & $9 \%$ & $79.77 \%$ & $92.42 \%$ \\
\hline L3 & $\alpha, \alpha, \alpha, \alpha$ & $+1.6(6.4 \%)$ & $6.4 \%$ & $79.45 \%$ & $92.65 \%$ \\
\hline L4 & $\alpha, \alpha, \alpha, \alpha$ & $+2.7(1.0 \%)$ & $1.0 \%$ & $80.22 \%$ & $92.33 \%$ \\
\hline L5 & $\alpha, \alpha, \alpha, \alpha$ & $+3.1(0.6 \%)$ & $0.6 \%$ & $79.91 \%$ & $92.46 \%$ \\
\hline L'"1 & $\alpha, \alpha, \beta, \beta$ & -1.2 & - & $86.30 \%$ & $86.30 \%$ \\
\hline L'?2 & $\alpha, \alpha, \alpha^{\prime}, \beta$ & -0.5 & - & $82.80 \%$ & $92.86 \%$ \\
\hline L'?3 & $\alpha, \beta, \alpha, \beta^{\prime}$ & -0.3 & - & $85.45 \%$ & $90.40 \%$ \\
\hline L',4 & $\alpha, \beta, \alpha, \beta$ ' & -0.1 & - & $84.53 \%$ & $92.41 \%$ \\
\hline L'95 & $\alpha, \alpha^{\prime}, \alpha, \beta$ & +1.4 & - & $81.18 \%$ & $93.88 \%$ \\
\hline
\end{tabular}

Table S5. Relative energetics and accessibility for $\mathrm{Rh}_{2}\left(\mathrm{~S}-2-\mathrm{Cl}-5-\left(3,5-\mathrm{diMeC}_{6} \mathrm{H}_{3}\right) \mathrm{TPCP}\right)_{4}(\mathbf{N})$

\begin{tabular}{|c|c|c|c|c|c|}
\hline $\begin{array}{l}\text { Catalyst } \\
\text { Conformer }\end{array}$ & $\begin{array}{c}\text { Catalyst } \\
\text { Configuration }\end{array}$ & $\begin{array}{c}\text { Free Energy } \\
\text { (kcal/mol; \%) }\end{array}$ & $\begin{array}{c}\text { "Reactive" } \\
\text { Population \% }\end{array}$ & $\begin{array}{l}\mathrm{G} \% \mathrm{RH} \\
\text { face } \mathrm{A}\end{array}$ & $\begin{array}{l}\mathrm{G} \% \text { RH } \\
\text { face B }\end{array}$ \\
\hline N1 & $\alpha, \boldsymbol{\alpha}, \boldsymbol{\alpha}, \boldsymbol{\alpha}$ & $+\mathbf{0 . 3}(31 \%)$ & $100 \%$ & $81.18 \%$ & $92.14 \%$ \\
\hline N'1 & $\alpha, \alpha, \alpha, \alpha$ & $+0.0(50 \%)$ & - & $85.95 \%$ & $92.40 \%$ \\
\hline$N^{\prime} 2$ & $\alpha, \alpha, \alpha, \alpha$ & $+0.8(13 \%)$ & - & $86.99 \%$ & $91.94 \%$ \\
\hline N'3 & $\alpha, \alpha, \alpha, \alpha$ & $+1.9(3 \%)$ & - & $83.79 \%$ & $92.03 \%$ \\
\hline$N^{\prime} 4$ & $\alpha, \alpha, \alpha, \alpha$ & $+1.9(2 \%)$ & - & $86.52 \%$ & $91.57 \%$ \\
\hline N'5 & $\alpha, \alpha, \alpha, \alpha$ & $+2.6(1 \%)$ & - & $86.07 \%$ & $91.84 \%$ \\
\hline N'P1 & $\alpha, \alpha^{\prime}, \beta, \beta^{\prime}$ & -9.2 & - & $83.97 \%$ & $84.02 \%$ \\
\hline $\mathrm{N}, 2$ & $\alpha, \alpha, \beta, \beta$ & -9.2 & - & $86.90 \%$ & $86.90 \%$ \\
\hline N'?3 & $\alpha, \alpha, \beta, \beta$, & -8.2 & - & $84.19 \%$ & $86.07 \%$ \\
\hline N'94 & $\alpha, \beta, \alpha, \beta$ & -8.0 & - & $87.03 \%$ & $87.04 \%$ \\
\hline N'95 & $\alpha, \alpha, \alpha, \beta$ & -7.9 & - & $84.86 \%$ & $89.40 \%$ \\
\hline
\end{tabular}

Note: In Tables S3-S5, \% indicates relative population $\left(\mathrm{CH}_{2} \mathrm{Cl}_{2}, 313 \mathrm{~K}\right)$, whereas "reactive" \% is tabulated among reactive conformers only to correspond with Boltzmann-weighting for MLR catalyst property inputs. 

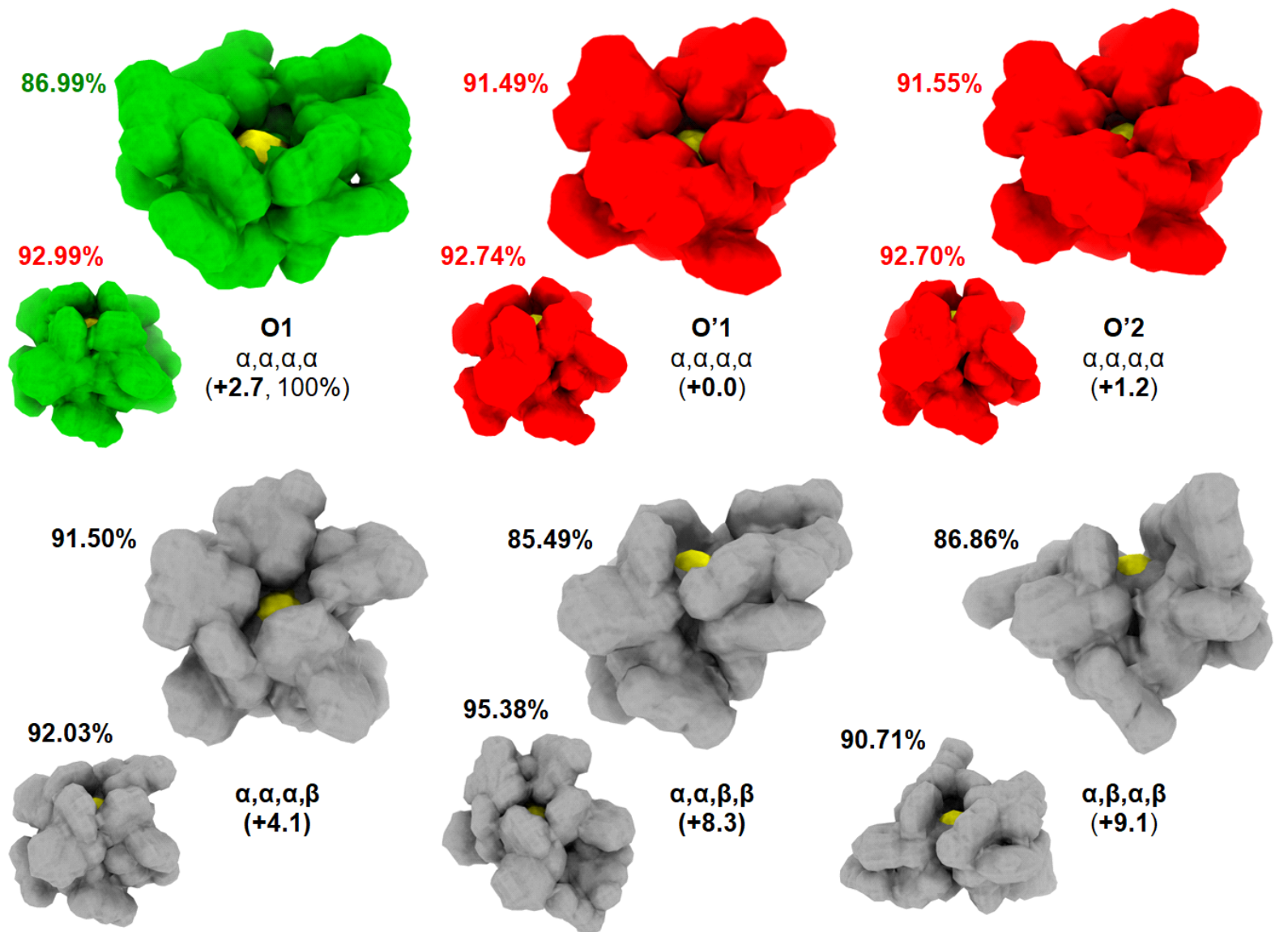

Figure S15. Conformations of $\mathrm{Rh}_{2}(S-2-\mathrm{Cl}-5-\mathrm{MesTPCP})_{4}(\mathbf{O})$, which persists exclusively in the "all-up" $\alpha, \alpha, \alpha, \alpha$ configuration under catalytic conditions. The most stable of the $\alpha, \alpha, \alpha, \alpha$ conformers are both nearly completely spatially inaccessible on both $\mathrm{Rh}$ faces (O'1-O'2). The lone reactive conformer $\mathbf{O 1}$ is also relatively inaccessible, and ligand flipping to generate other conformers is both kinetically and thermodynamically unfavorable in this case. Moreover, none of these other conformers have significantly greater spatial accessibility than $\mathbf{O 1}$ even if they were stable and kinetically accessible. While the hindered structure of $\mathbf{O 1}$ surely adapts to spatially accommodate the dirhodium-carbene, so too do the structures of other dirhodium catalysts, and so the relative differences in spatial confinement of the reactive site can still clearly be predictive. 


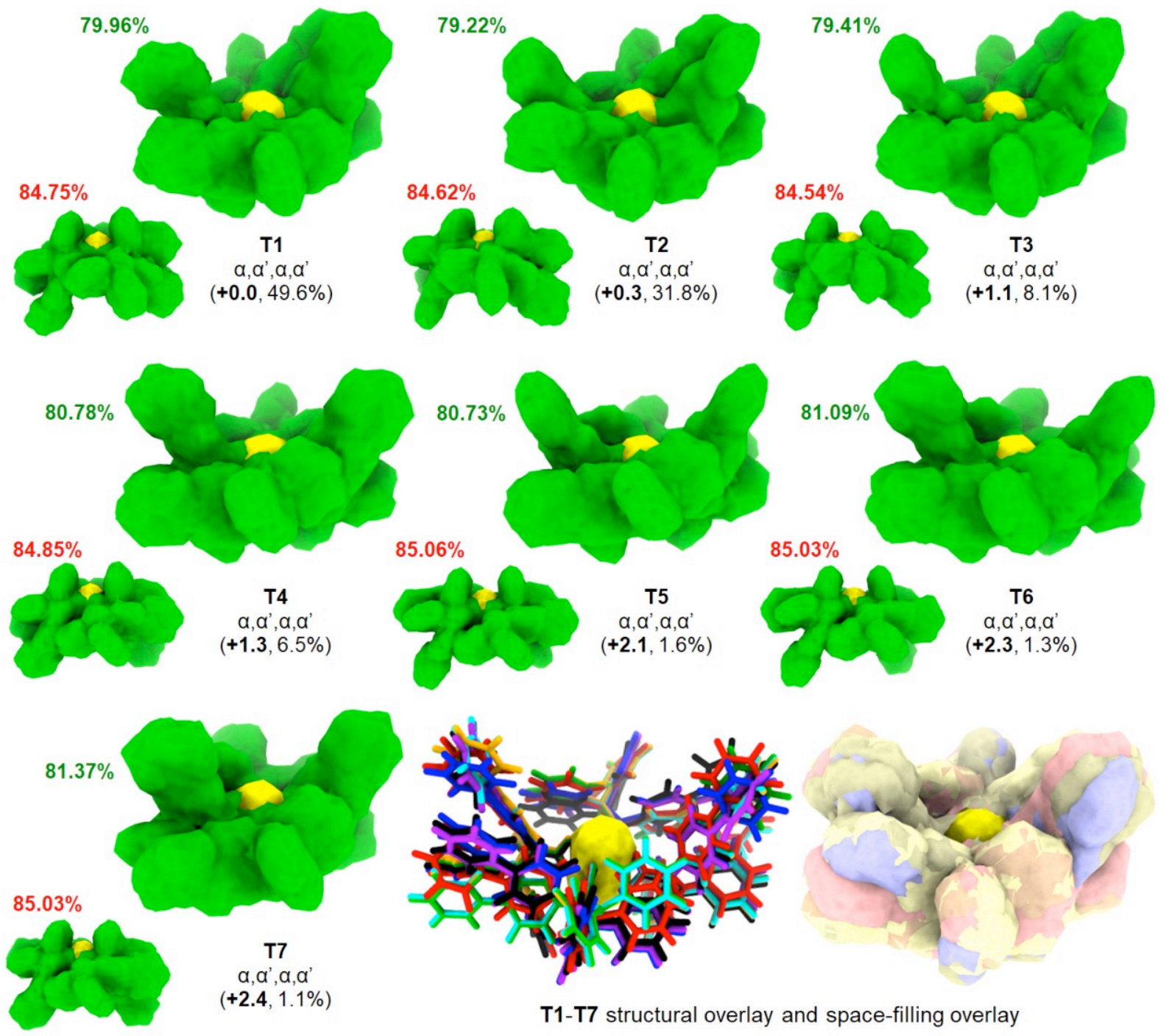

Figure S16. Conformations of $\mathrm{Rh}_{2}(S-p \text {-PhTPCP })_{4}(\mathbf{T})$, which persists exclusively in the $\alpha, \alpha^{\prime}, \alpha, \alpha^{\prime}$ configuration under catalytic conditions but can adopt various biaryl torsion combinations that permit varying degrees of non-covalent interactions (NCIs) between neighboring ligands (T1-T7). The structural variation across all possible reactive conformers can be more easily seen in the structural and space-filling overlays (bottom right). Namely, the preferred $\mathrm{C}_{2}$-symmetric $\alpha, \alpha^{\prime}, \alpha, \alpha^{\prime}$ configuration can be modestly perturbed as opposite ligands "slide" downward incrementally, weakening the NCIs between neighboring ligands and adopting orientations closer to perpendicular with the Rh-Rh vector. Additionally, there are several different possible combinations of biaryl torsions. For all conformers, the $\mathrm{C} 1$ aryl face $(\mathrm{G} \sim 80 \%)$ is substantially more spatially accessible than the opposite face $(\mathrm{G} \sim 85 \%)$. Catalyst $\mathbf{T}$ was chosen as representative for $\mathrm{Rh}_{2}(p-\mathrm{XTPCP})_{4}$ catalysts $(\mathbf{P}-\mathbf{T})$, which each adopt similar $\alpha, \alpha^{\prime}, \alpha, \alpha^{\prime}$ configurations, in accordance with the prior QM study (see Figure S6 and ref. 37). 
Table S6. Relative energetics and accessibility for other $\mathrm{Rh}_{2}(p-\mathrm{XTPCP})_{4}$ catalysts $^{\mathrm{a}}(\mathbf{P}-\mathbf{S})$

\begin{tabular}{|c|c|c|c|c|c|}
\hline $\begin{array}{c}\text { Catalyst } \\
\text { Conformer } \\
\end{array}$ & $\begin{array}{c}\text { Catalyst } \\
\text { Configuration } \\
\end{array}$ & $\begin{array}{c}\text { Free Energy } \\
(\mathrm{kcal} / \mathrm{mol})\end{array}$ & $\begin{array}{c}\text { Population \% } \\
(313 \mathrm{~K})\end{array}$ & $\begin{array}{l}\text { G\%RH } \\
\text { face } A\end{array}$ & $\begin{array}{l}\text { G\% } \% \text { RH } \\
\text { face B }\end{array}$ \\
\hline P1 & $\boldsymbol{\alpha}, \boldsymbol{\alpha}^{\prime}, \boldsymbol{\alpha}, \boldsymbol{\alpha}^{\prime}$ & +0.0 & $99.2 \%$ & $78.61 \%$ & $84.05 \%$ \\
\hline P2 & $\boldsymbol{\alpha}, \boldsymbol{\alpha}^{\prime}, \boldsymbol{\alpha}, \boldsymbol{\alpha}$, & +3.0 & $0.8 \%$ & $78.58 \%$ & $87.27 \%$ \\
\hline Q1 & $\boldsymbol{\alpha}, \boldsymbol{\alpha}^{\prime}, \boldsymbol{\alpha}, \boldsymbol{\alpha}^{\prime}$ & $+\mathbf{0 . 0}$ & $100 \%$ & $79.19 \%$ & $84.38 \%$ \\
\hline R1 & $\alpha, \alpha^{\prime}, \alpha, \alpha^{\prime}$ & +0.0 & $90.2 \%$ & $79.38 \%$ & $84.57 \%$ \\
\hline $\mathbf{R 2}$ & $\boldsymbol{\alpha}, \boldsymbol{\alpha}^{\prime}, \boldsymbol{\alpha}, \boldsymbol{\alpha}^{\prime}$ & +1.5 & $8.3 \%$ & $79.87 \%$ & $84.88 \%$ \\
\hline R3 & $\alpha, \alpha^{\prime}, \alpha, \alpha^{\prime}$ & +2.5 & $1.5 \%$ & $80.31 \%$ & $85.20 \%$ \\
\hline S1 & $\boldsymbol{\alpha}, \boldsymbol{\alpha}^{\prime}, \boldsymbol{\alpha}, \boldsymbol{\alpha}^{\prime}$ & +0.0 & $91.1 \%$ & $78.86 \%$ & $84.37 \%$ \\
\hline S2 & $\alpha, \alpha^{\prime}, \alpha, \alpha '$ & +1.4 & $8.9 \%$ & $79.42 \%$ & $84.86 \%$ \\
\hline
\end{tabular}

${ }^{\mathrm{a}} \overline{\mathrm{Other}}$ configurations are much less stable and have similar energies to those reported for $\mathbf{S}$ (Figure $\mathrm{S} 6$ ).
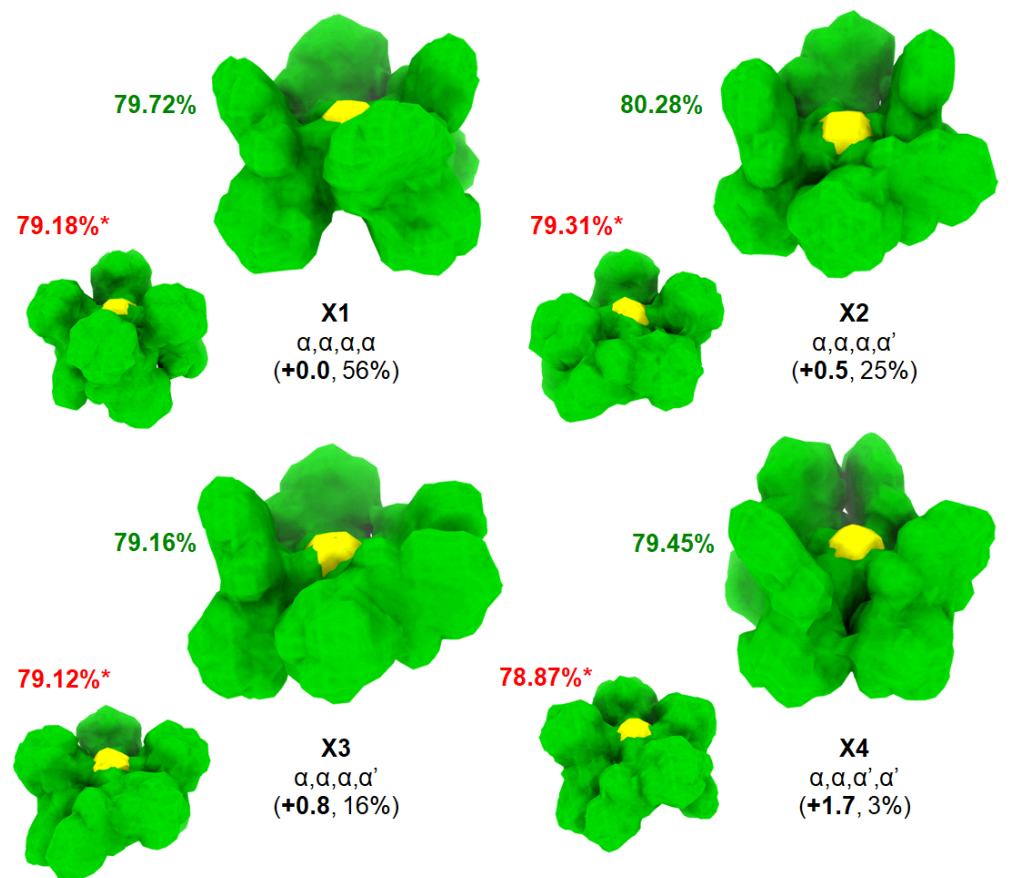

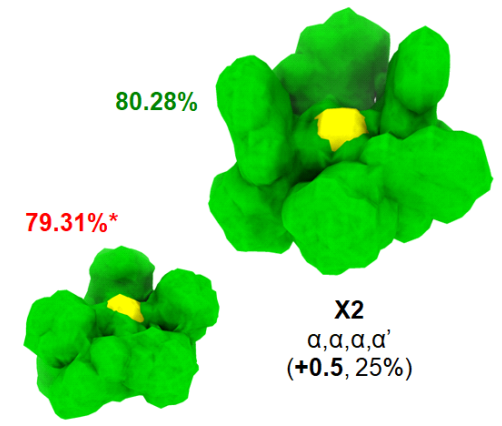

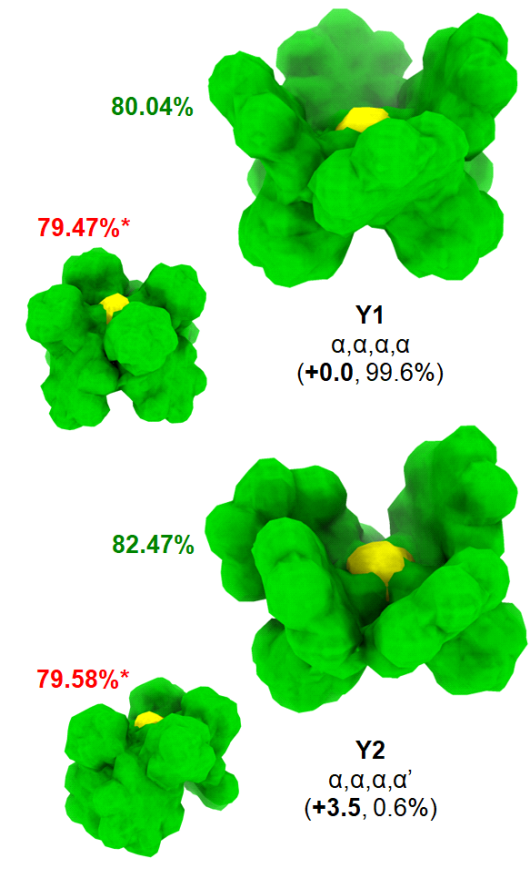

Figure S17. Space-filling diagrams of the reactive conformations of the phthalimido catalysts, $\mathrm{Rh}_{2}(S$ PTAD $)_{4}(\mathbf{X})$ and $\mathrm{Rh}_{2}(S \text {-TCPTAD })_{4}(\mathbf{Y})$. Both prefer $\alpha, \alpha, \alpha, \alpha$ configurations, but $\mathbf{X}$ is substantially less rigid and can access several stable conformations in which one of the four sides of the bowl "folds down." This may be relevant for opening additional reactive site space for accommodating the incoming diazo precursor and generating the dirhodium-carbene. An interesting artifact of $\mathrm{G} \%$ as a parameter is illustrated here, as light can escape the surrounding sphere through the crevices between bulky adamantly groups on the opposite $\mathrm{Rh}$ face even though these small channels are largely irrelevant to the effective space available for substrate(s) at the reactive site. The result is that both $\mathrm{Rh}$ faces have identical $\mathrm{G} \%$ values even though the phthalimido bowl face has much more space for the carbene to form. This situation is one that SMART parameters can remedy in future analyses by adjusting the probe size and flexibility. Based on literature precedent (refs. 7, 40-44), only the phthalimido face was considered further for feature extraction. $\mathbf{X} 1$ and $\mathbf{Y 1}$ are also calculated to be much more stable than the $\alpha, \alpha, \alpha, \beta(\mathbf{X}:+5.9 ; \mathbf{Y}:+8.1), \alpha, \beta, \alpha, \beta(\mathbf{X}:+11.5 ; \mathbf{Y}:+8.2)$ and $\alpha, \alpha, \beta, \beta(\mathbf{X}:+6.8 ; \mathbf{Y}:+11.2)$ conformations. 

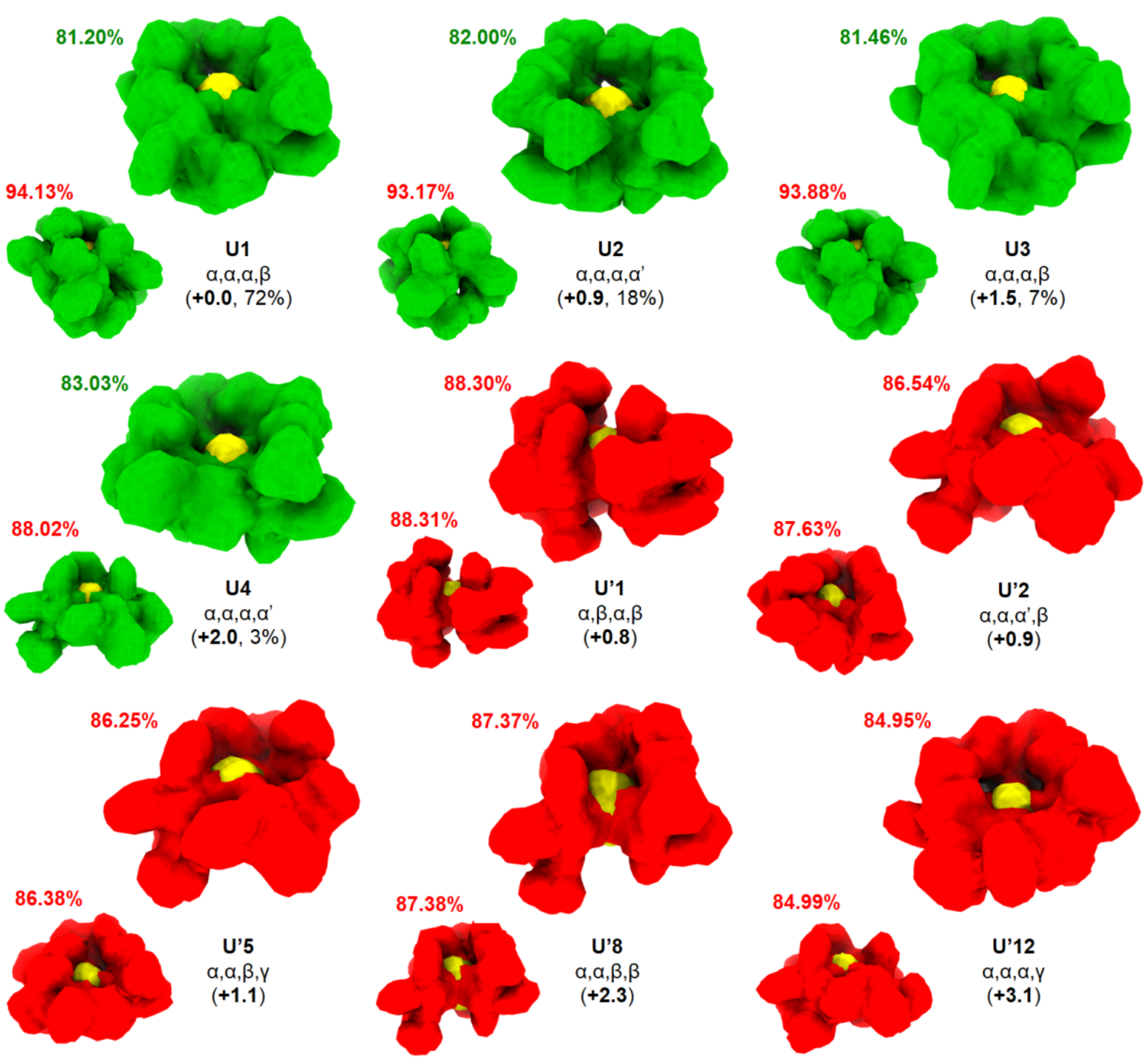

Figure S18. Space-filling diagrams of $\mathrm{Rh}_{2}\left(S-3,5-\mathrm{diCF}_{3} \mathrm{TPCP}\right)_{4}(\mathbf{U})$ showing all reactive conformations (green) and selected stable but spatially inaccessible configurations (red) that were deemed irrelevant for describing the relative dirhodium-carbene reactive site topology. Remarkably, QM-optimized structures for all possible catalyst configurations are predicted to be within $3.5 \mathrm{kcal} / \mathrm{mol}$ in relative thermodynamic stability, and the low $\Delta G^{\ddagger}$ rot values stemming from the lack of an ortho-Cl substituent suggest that all of these possible configurations can be accessed via ligand flipping in solution. Both $\alpha, \alpha, \alpha, \beta$ (U1, U3) and $\alpha, \alpha, \alpha, \alpha^{\prime}$ (U2, U4) conformers are classified as reactive, although the primary reactive conformations are $\alpha, \alpha, \alpha, \beta$ (79\% total) as discussed in the main text (U1 is shown in Figure 9B). Analogous to the preferred $\mathrm{D}_{2}(\alpha, \beta, \alpha, \beta)$ configuration of $\mathrm{Rh}_{2}\left[R-3,5-\operatorname{di}-\left(p-{ }^{t} \mathrm{BuC}_{6} \mathrm{H}_{4}\right) \mathrm{TPCP}\right]_{4}(\mathbf{W})$, U'1 is also very stable and is nearly isoenergetic with $\mathbf{U} \mathbf{1}$. However, it is highly spatially inaccessible and is predicted to be kinetically outcompeted for diazo binding and downstream dirhodium-carbene formation by U1-U4. Similarly, selected other possible configurations that were filtered out based on G\% spatial classification are shown (U'2, U'5, U'8, U'12), with the remainder of these stable but spatially inaccessible conformers listed in Table S7. 
Table S7. Relative energetics and accessibility for $\mathrm{Rh}_{2}\left(S-3,5-\mathrm{diCF}_{3} \mathrm{TPCP}\right)_{4}(\mathbf{U})$

\begin{tabular}{cccccc}
$\begin{array}{c}\text { Catalyst } \\
\text { Conformer }\end{array}$ & $\begin{array}{c}\text { Catalyst } \\
\text { Configuration }\end{array}$ & $\begin{array}{c}\text { Free Energy } \\
(\mathrm{kcal} / \mathrm{mol} ; \%)\end{array}$ & $\begin{array}{c}\text { "Reactive" } \\
\text { Population \% }\end{array}$ & $\begin{array}{c}\mathrm{G} \% \mathrm{RH} \\
\text { face A }\end{array}$ & $\begin{array}{c}\mathrm{G} \% \mathrm{RH} \\
\text { face B }\end{array}$ \\
\hline U1 & $\boldsymbol{\alpha}, \boldsymbol{\alpha , \boldsymbol { \alpha } , \boldsymbol { \beta }}$ & $\mathbf{+ 0 . 0}(38 \%)$ & $\mathbf{7 2 \%}$ & $\mathbf{8 1 . 2 0 \%}$ & $\mathbf{9 4 . 1 3 \%}$ \\
U2 & $\boldsymbol{\alpha , \alpha , \alpha , \boldsymbol { \alpha }}$ & $+\mathbf{0 . 9}(9.6 \%)$ & $\mathbf{1 8 \%}$ & $\mathbf{8 2 . 0 0 \%}$ & $\mathbf{9 3 . 1 7 \%}$ \\
U3 & $\boldsymbol{\alpha , \alpha , \alpha , \beta}$ & $+\mathbf{1 . 5}(3.7 \%)$ & $\mathbf{7 \%}$ & $\mathbf{8 1 . 4 6 \%}$ & $\mathbf{9 3 . 8 8 \%}$ \\
U4 & $\boldsymbol{\alpha , \alpha , \alpha , \alpha}$ & $+\mathbf{2 . 0}(1.6 \%)$ & $\mathbf{3 \%}$ & $\mathbf{8 3 . 0 3 \%}$ & $\mathbf{8 8 . 0 2 \%}$ \\
U'1 & $\alpha, \beta, \alpha, \beta$ & $+0.8(10.1 \%)$ & - & $\mathbf{8 8 . 3 0 \%}$ & $\mathbf{8 8 . 3 1 \%}$ \\
U'2 & $\alpha, \alpha, \alpha, \beta$ & $+0.9(8.8 \%)$ & - & $\mathbf{8 6 . 5 4 \%}$ & $\mathbf{8 7 . 6 3 \%}$ \\
U'3 & $\alpha, \alpha, \alpha, \beta$ & $+1.0(7.5 \%)$ & - & $\mathbf{8 4 . 0 8 \%}$ & $\mathbf{9 2 . 1 2 \%}$ \\
U'4 & $\alpha, \alpha, \alpha, \beta$ & $+1.0(7.1 \%)$ & - & $\mathbf{8 4 . 1 0 \%}$ & $\mathbf{9 2 . 1 3 \%}$ \\
U'5 & $\alpha, \alpha, \beta, \gamma$ & $+1.1(6.0 \%)$ & - & $\mathbf{8 6 . 2 5 \%}$ & $\mathbf{8 6 . 3 8 \%}$ \\
U'6 & $\alpha, \alpha, \beta, \gamma$ & $+1.6(2.8 \%)$ & - & $\mathbf{8 6 . 2 6 \%}$ & $\mathbf{8 6 . 3 8 \%}$ \\
U'7 & $\alpha, \alpha, \beta, \gamma$ & $+2.1(1.3 \%)$ & - & $\mathbf{8 4 . 0 7 \%}$ & $\mathbf{8 9 . 0 8 \%}$ \\
U'8 & $\alpha, \alpha, \beta, \beta$ & $+2.3(0.9 \%)$ & - & $\mathbf{8 7 . 3 7 \%}$ & $\mathbf{8 7 . 3 8 \%}$ \\
U'9 & $\alpha, \alpha, \beta, \beta$ & $+2.3(0.9 \%)$ & - & $\mathbf{8 7 . 3 9 \%}$ & $\mathbf{8 7 . 3 9 \%}$ \\
U'10 & $\alpha, \alpha, \beta, \gamma$ & $+2.4(0.7 \%)$ & - & $\mathbf{8 4 . 0 2 \%}$ & $\mathbf{8 9 . 2 5 \%}$ \\
U'11 & $\alpha, \alpha, \beta, \gamma$ & $+2.7(0.5 \%)$ & - & $\mathbf{8 4 . 0 8 \%}$ & $\mathbf{8 9 . 0 5 \%}$ \\
U'12 & $\alpha, \alpha, \alpha, \gamma$ & $+3.1(0.3 \%)$ & - & $\mathbf{8 4 . 9 5 \%}$ & $\mathbf{8 4 . 9 9 \%}$ \\
U'13 & $\alpha, \alpha, \alpha, \beta$ & $+3.4(0.2 \%)$ & - & $\mathbf{8 5 . 0 5 \%}$ & $\mathbf{8 8 . 0 0 \%}$ \\
\hline
\end{tabular}

Table S8. Relative energetics and accessibility for $\mathrm{Rh}_{2}(S-3,5-\operatorname{diBrTPCP})_{4}(\mathrm{~V})$

\begin{tabular}{|c|c|c|c|c|c|}
\hline $\begin{array}{c}\text { Catalyst } \\
\text { Conformer }\end{array}$ & $\begin{array}{c}\text { Catalyst } \\
\text { Configuration }\end{array}$ & $\begin{array}{l}\text { Free Energy } \\
(\mathrm{kcal} / \mathrm{mol} ; \%)\end{array}$ & $\begin{array}{c}\text { "Reactive" } \\
\text { Population \% }\end{array}$ & $\begin{array}{l}\text { G\% } \% \text { RH } \\
\text { face } A\end{array}$ & $\begin{array}{l}\text { G\%RH } \\
\text { face B }\end{array}$ \\
\hline V1 & $\boldsymbol{\alpha}, \boldsymbol{\alpha}, \boldsymbol{\alpha}, \boldsymbol{\alpha}$, & $+0.5(13.4 \%)$ & $47 \%$ & $81.41 \%$ & $87.95 \%$ \\
\hline V2 & $\boldsymbol{\alpha}, \boldsymbol{\beta}, \boldsymbol{\alpha}^{\prime}, \boldsymbol{\beta}$, & $+0.7(9.9 \%)$ & $35 \%$ & $83.10 \%$ & $88.17 \%$ \\
\hline V3 & $\boldsymbol{\alpha}, \boldsymbol{\alpha}, \boldsymbol{\alpha}, \gamma$ & $+1.2(4.3 \%)$ & $15 \%$ & $81.88 \%$ & $88.27 \%$ \\
\hline V4 & $\alpha, \alpha, \alpha, \alpha$ & $+2.3(0.7 \%)$ & $2 \%$ & $80.56 \%$ & $92.54 \%$ \\
\hline V5 & $\alpha, \boldsymbol{\alpha}, \boldsymbol{\alpha}^{\prime}, \beta$ & $+3.1(0.2 \%)$ & $1 \%$ & $83.11 \%$ & $86.99 \%$ \\
\hline$V^{\prime} 1$ & $\alpha, \beta, \alpha, \gamma$ & $+0.0(28.9 \%)$ & - & $83.47 \%$ & $85.10 \%$ \\
\hline$V^{\prime} 2$ & $\alpha, \alpha, \beta, \gamma$ & $+0.3(18.6 \%)$ & - & $84.46 \%$ & $85.97 \%$ \\
\hline$V^{\prime} \mathbf{3}$ & $\alpha, \alpha, \beta, \gamma$ & $+0.4(15.7 \%)$ & - & $83.19 \%$ & $87.88 \%$ \\
\hline$V^{\prime} 4$ & $\alpha, \gamma, \alpha, \gamma$ & $+1.3(3.6 \%)$ & - & $84.46 \%$ & $85.18 \%$ \\
\hline$V^{\prime} 5$ & $\alpha, \alpha, \beta, \beta$ & $+1.8(1.7 \%)$ & - & $86.48 \%$ & $86.48 \%$ \\
\hline$V^{\prime} 6$ & $\alpha, \beta, \alpha, \gamma$ & $+1.9(1.3 \%)$ & - & $84.07 \%$ & $85.53 \%$ \\
\hline$V^{\prime} 7$ & $\alpha, \alpha, \alpha, \beta$ & $+2.1(1.0 \%)$ & - & $83.49 \%$ & $89.72 \%$ \\
\hline$V^{\prime} 8$ & $\alpha, \beta, \alpha, \beta$ & $+2.4(0.6 \%)$ & - & $83.59 \%$ & $84.49 \%$ \\
\hline$V^{\prime} 9$ & $\alpha, \beta, \alpha^{\prime}, \beta^{\prime}$ & $+3.4(0.1 \%)$ & - & $85.47 \%$ & $85.84 \%$ \\
\hline
\end{tabular}

Note: In Tables $\mathrm{S} 7-\mathrm{S} 8, \%$ indicates relative population $\left(\mathrm{CH}_{2} \mathrm{Cl}_{2}, 313 \mathrm{~K}\right)$, whereas "reactive" \% is tabulated among reactive conformers only to correspond with Boltzmann-weighting for MLR catalyst property inputs. 

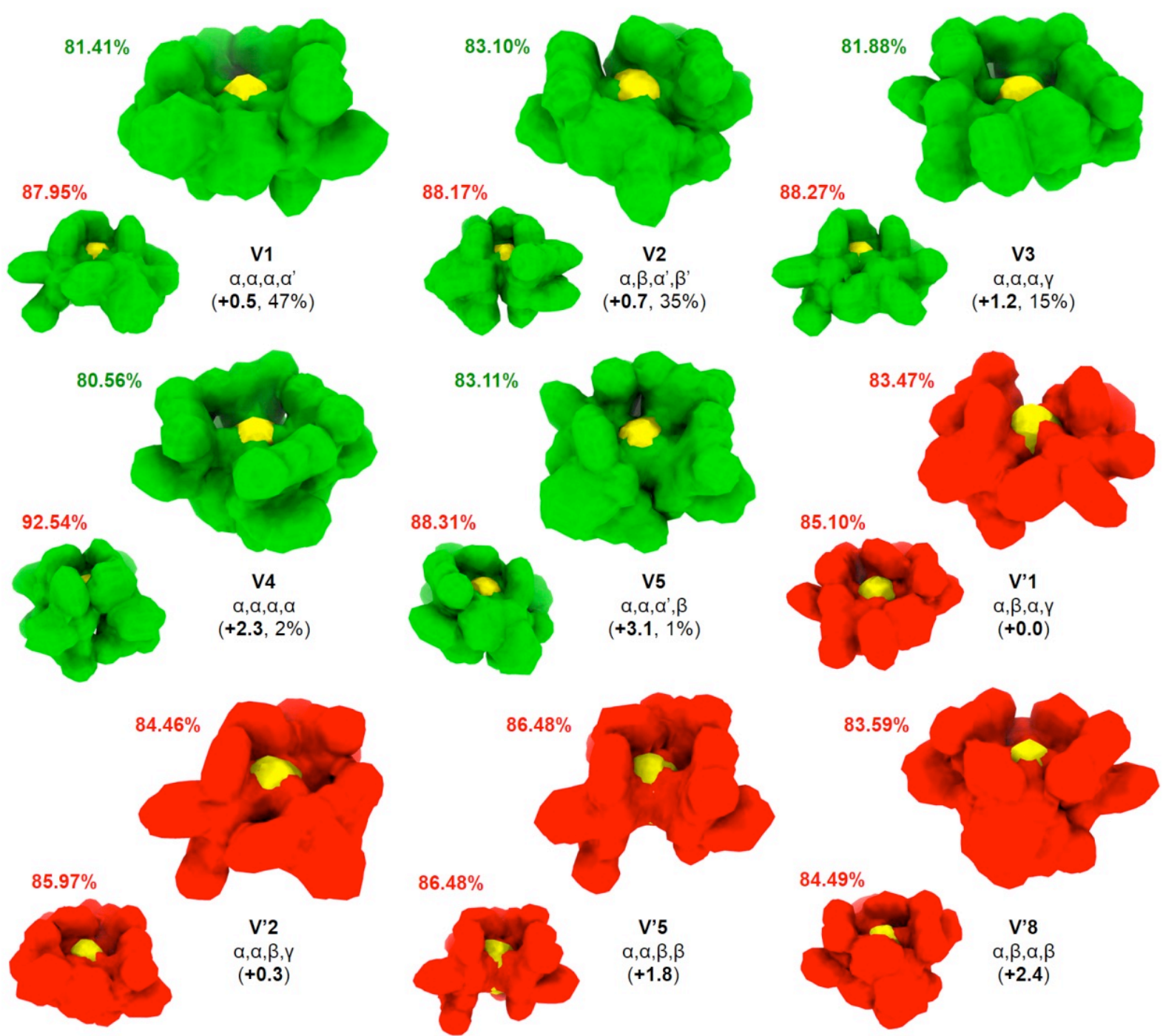

Figure S19. Space-filling diagrams of $\mathrm{Rh}_{2}(S-3,5-\mathrm{diBrTPCP})_{4}(\mathbf{V})$ showing all reactive conformations (green) and selected stable but spatially inaccessible configurations (red) that were deemed irrelevant for describing the relative dirhodium-carbene reactive site topology. Remarkably, QM-optimized structures for all possible catalyst configurations are predicted to be within $3.5 \mathrm{kcal} / \mathrm{mol}$ in thermodynamic stability, and the low $\Delta G^{\ddagger}$ rot values stemming from the lack of an ortho- $\mathrm{Cl}$ substituent suggest that all possible configurations may be accessed via ligand flipping in solution. Nominally "allup" conformations, including $\alpha, \alpha, \alpha, \alpha(\mathbf{V 4}), \alpha, \alpha, \alpha, \alpha^{\prime}(\mathbf{V 1})$, and $\alpha, \alpha, \alpha, \gamma(\mathbf{V 3})$ are classified as reactive, along with $\alpha, \alpha, \alpha^{\prime}, \beta$ (V5) and $\alpha, \beta, \alpha^{\prime}, \beta$ ' (V2) conformers. The primary reactive configurations are distorted "all-up" conformations (64\% total) as discussed in the main text (V1 is shown in Figure 9B). Selected other possible conformers that were filtered out based on G\% spatial classification are shown ( $\left.\mathbf{V}^{\prime} \mathbf{1}, \mathbf{V}^{\prime} \mathbf{2}, \mathbf{V}^{\prime} \mathbf{5}^{\prime}, \mathbf{V}^{\prime} \mathbf{8}\right)$, with the remainder of these stable but spatially inaccessible conformers listed in Table S8. 

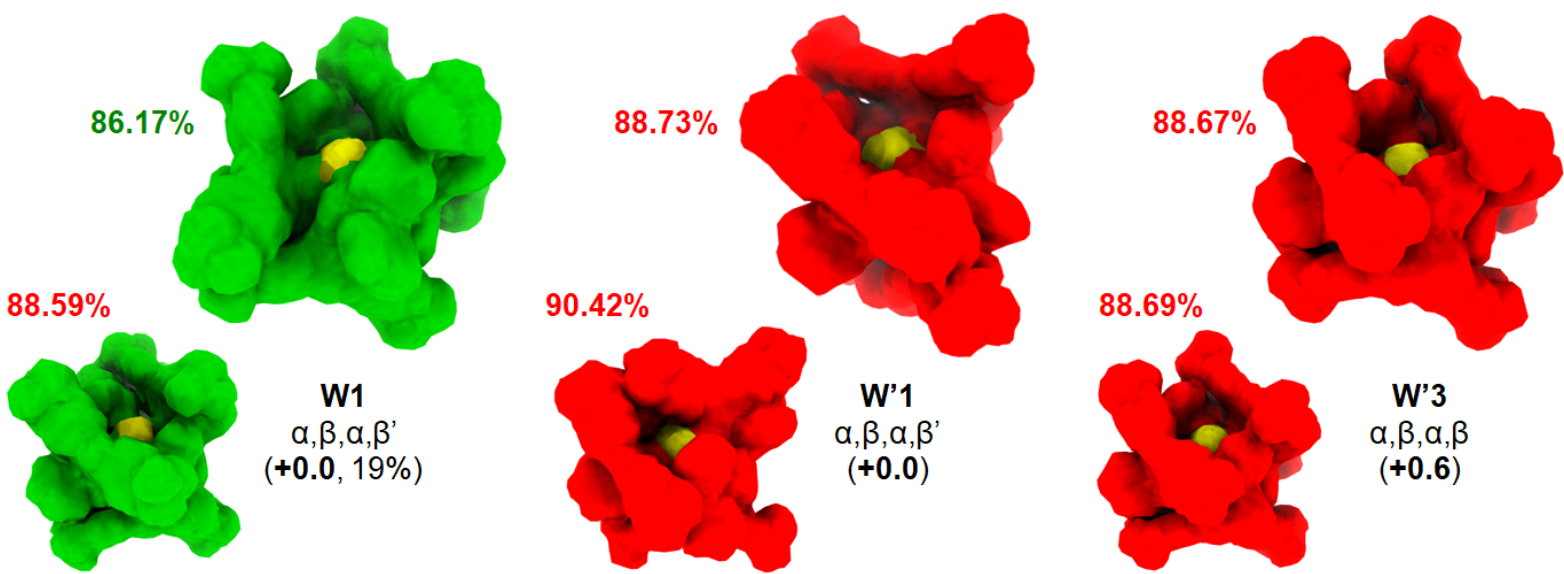

Figure S20. Space-filling diagrams of $\mathrm{Rh}_{2}\left[R-3,5-\mathrm{di}-\left(p-{ }^{t} \mathrm{BuC}_{6} \mathrm{H}_{4}\right) \mathrm{TPCP}\right]_{4}(\mathbf{W})$. Seven unique reactive conformers were found, all of which have $\alpha, \beta, \alpha, \beta$ ' geometry and relatively similar reactive site topology to $\mathbf{W 1}$ (i.e., $100 \%$ reactive population looks similar to face $\mathrm{A}$ of $\mathbf{W 1}$, which is also shown in Figure 3C). Stable $\alpha, \beta, \alpha, \beta$ ' (W'1) and $\alpha, \beta, \alpha, \beta$ (W'2-W'3) conformers with spatially inaccessible Rh faces were also identified. W'2 and $\mathbf{W}$ ' 3 are ${ }^{t} \mathrm{Bu}$ rotamers that exhibit global $\mathrm{D}_{2}$ symmetry and closely resemble the X-ray crystal structure (see Figure S5 for overlay of W'3 with X-ray structure). Facile biaryl dihedral rotation will presumably allow conformations like $\mathbf{W}^{\prime} \mathbf{1}-\mathbf{W}^{\prime} \mathbf{3}$ to interconvert to conformations like W1 in order to accommodate the incoming diazo precursor and open sufficient reactive site space to enable carbene formation. Thus, W1-W7 more closely describe the reactive site topology of the dirhodium-carbene species and were considered further for molecular descriptor extraction and MLR analysis. All conformations of $\mathbf{W}$ and their relative free energy and $\mathrm{G} \%$ values are shown in Table S9.

Table S9. Relative energetics and accessibility for $\mathrm{Rh}_{2}\left[R-3,5-\mathrm{di}-\left(p-{ }^{t} \mathrm{BuC}_{6} \mathrm{H}_{4}\right) \mathrm{TPCP}\right]_{4}(\mathbf{W})$

\begin{tabular}{|c|c|c|c|c|c|}
\hline $\begin{array}{l}\text { Catalyst } \\
\text { Conformer }\end{array}$ & $\begin{array}{c}\text { Catalyst } \\
\text { Configuration }\end{array}$ & $\begin{array}{l}\text { Free Energy } \\
(\mathrm{kcal} / \mathrm{mol} ; \%)\end{array}$ & $\begin{array}{c}\text { "Reactive" } \\
\text { Population \% }\end{array}$ & $\begin{array}{l}\mathrm{G} \% \mathrm{RH} \\
\text { face A }\end{array}$ & $\begin{array}{l}\text { G\% } \% \text { RH } \\
\text { face B }\end{array}$ \\
\hline W1 & $\boldsymbol{\alpha}, \boldsymbol{\beta}, \boldsymbol{\alpha}, \boldsymbol{\beta}$, & $+\mathbf{0 . 0}(19.1 \%)$ & $30.2 \%$ & $86.17 \%$ & $88.59 \%$ \\
\hline W2 & $\alpha, \boldsymbol{\beta}, \boldsymbol{\alpha}, \boldsymbol{\beta}$, & $+0.2(13.4 \%)$ & $21.1 \%$ & $86.00 \%$ & $88.01 \%$ \\
\hline W3 & $\alpha, \beta, \alpha, \beta$, & $+0.4(10.8 \%)$ & $17.1 \%$ & $86.38 \%$ & $88.56 \%$ \\
\hline W4 & $\boldsymbol{\alpha}, \boldsymbol{\beta}, \boldsymbol{\alpha}, \boldsymbol{\beta}$, & +0.5 (9.1\%) & $14.5 \%$ & $86.08 \%$ & $88.15 \%$ \\
\hline W5 & $\alpha, \beta, \alpha, \beta$, & $+0.7(6.2 \%)$ & $9.9 \%$ & $86.62 \%$ & $88.77 \%$ \\
\hline W6 & $\alpha, \beta, \alpha, \beta^{\prime}$ & $+0.9(4.5 \%)$ & $7.1 \%$ & $86.41 \%$ & $88.50 \%$ \\
\hline W7 & $\alpha, \beta, \alpha, \beta$, & $+3.5(0.1 \%)$ & $0.1 \%$ & $86.41 \%$ & $88.38 \%$ \\
\hline W'1 & $\alpha, \beta, \alpha, \beta^{\prime}$ & $+0.0(19.4 \%)$ & - & $88.73 \%$ & $90.42 \%$ \\
\hline W'2 & $\alpha, \beta, \alpha, \beta$ & $+0.4(9.8 \%)$ & - & $88.68 \%$ & $88.70 \%$ \\
\hline W'3 & $\alpha, \beta, \alpha, \beta$ & $+0.6(7.6 \%)$ & - & $88.67 \%$ & $88.69 \%$ \\
\hline
\end{tabular}

Note: \% indicates relative population $\left(\mathrm{CH}_{2} \mathrm{Cl}_{2}, 313 \mathrm{~K}\right)$, whereas "reactive" \% is tabulated among reactive conformers only to correspond with Boltzmann-weighting of catalyst input properties for MLR analysis. 


\subsection{Computational Methods for Free Ligands, $T_{\text {rot }}$ Geometries, and $\Delta G^{\ddagger}$ rot Barriers}

Free carboxylate ligand anion conformational searches were performed using the OPLS3 force field implemented in MacroModel version 11.8. ${ }^{10-11}$ Searches were performed with no solvent using mixed torsional/low-mode sampling, with minimum and maximum distances for a low-mode move of 3 and $6 \AA$, respectively. Minimization to convergence was performed with the default probability of torsion rotation/molecule translation of 0.5 . Intermediate sampling without retaining mirror-image conformers was performed with a maximum of 1000 steps (using 100 steps per rotatable bond). Redundant conformers in the conformational search were eliminated using a maximum atom deviation cutoff of $0.5 \AA$. A large energy window for saving structures of 21.0 $\mathrm{kJ} / \mathrm{mol}(5.02 \mathrm{kcal} / \mathrm{mol})$ was utilized.

Conformers obtained in the search were subsequently optimized in the gas phase at the M062X$\mathrm{D} 3 / 6-311++\mathrm{G}(\mathrm{d}, \mathrm{p})$ level of theory ${ }^{19,45-50}$ as implemented in Gaussian 16 (revision C.01). ${ }^{16}$ Diffuse functions were included in the basis sets to accurately describe the anionic charge state of the free carboxylate ligands. ${ }^{46-48}$ The optimized geometries were verified by frequency calculations as local minima (i.e., zero imaginary frequencies). Single-point calculations on optimized geometries were performed at the same level of theory with solvation modeled using SMD (solvent $\left.=\mathrm{CH}_{2} \mathrm{Cl}_{2}\right) .{ }^{17}$ Solvated free energies were obtained by correcting the gas phase free energies with the solvated electronic energies from the single-point calculations.

The lowest energy free ligand conformation was identified for each ligand, and rotational transition state input guess structures were obtained by adapting this structure. Previously computed protonated carboxylic acid transition state geometries for ligand $\mathbf{I}$ and its experimentally determined rotational barrier were utilized for guidance. ${ }^{36}$ Transition states were computed for free carboxylate anions to avoid complications arising from various conformations that differ in where the carboxylate proton is positioned (i.e., relative barriers across the ligand set are the primary interest here). Transition state structures were calculated at the same level of theory as the ground state carboxylate anion structures using the "noeigen,ts" Gaussian optimization keywords. Frequency calculations verified the presence of a single imaginary frequency, which was visually inspected to confirm that the correct rotational transition state had been found in each case.

For ortho-CITPCP ligands, two different transition states are possible for relating the $M$ and $P$ free ligand ground states, which differ in whether the ortho- $\mathrm{Cl}$ substituent passes by the carboxylate $\mathrm{CO}_{2}{ }^{-}$group $\left(\mathrm{TS}_{\mathrm{alt}}\right)$ or a Ph group (TS $\left.\mathrm{Tot}\right)$. This is illustrated for $S-2-\mathrm{Cl}-5-\mathrm{BrTPCP}$ (ligI) in Figure $\mathrm{S} 21$. The low energy ground state conformer that results from rotation was also identified among the free ligand conformers for each ligand. The $P$ isomer was slightly more stable (typically by 0.1 to $0.7 \mathrm{kcal} / \mathrm{mol}$ ) for all ortho-ClTPCP ligands except $S$-2-Cl-4-(3,5-diCF $\left.{ }_{3} \mathrm{C}_{6} \mathrm{H}_{3}\right) \mathrm{TPCP}$ (ligF) and $\mathrm{S}-2-\mathrm{Cl}-5-\mathrm{MesTPCP}$ (ligO). For consistency, the lowest energy conformer regardless of $M / P$ assignment was labeled as "lig[letter]1", the product conformer from C1 aryl rotation was labeled as "lig[letter]2", and the transition state barriers $\left(\Delta G^{\ddagger}\right.$ rot and $\Delta G^{\ddagger}$ alt $)$ were tabulated relative to the lowest energy free ligand conformer in all cases.

Although $\Delta G^{\ddagger}$ alt $(13-16 \mathrm{kcal} / \mathrm{mol})$ is lower energy than $\Delta G^{\dagger}$ rot $(17-20 \mathrm{kcal} / \mathrm{mol})$ for all orthoCITPCP ligands, $\mathrm{TS}_{\mathrm{alt}}$ involves a dramatic contortion of the carboxylate group to allow the ortho$\mathrm{Cl}$ substituent to pass by, which would likely interfere with dirhodium coordination and be too high in energy to occur for $\mathrm{Rh}_{2} \mathrm{~L}_{4}$ catalysts. Thus, $\Delta G^{\ddagger}$ rot barriers were taken to be most representative of the relative conformational rigidity of the dirhodium catalysts, as described in the single node decision tree analysis in Figure 3B. 
Note that for other TPCP ligands, only a single $\mathrm{TS}_{\text {rot }}$ exists for interconversion between the lowest energy ground state conformer with itself. For phthalimido ligands this is also true, with the caveat that the phthalimido group rotational barrier was computed in lieu of the $\mathrm{C} 1$ aryl rotational barrier for TPCP ligands (both form the bowl-shaped reactive cavities). Atomic coordinates are provided for $\mathrm{TS}_{\mathrm{rot}}$ and $\mathrm{TS}_{\mathrm{alt}}$, as well as for the low energy ligand ground state conformers between which these transition states facilitate interconversion. Calculated free energy barriers are reported relative to the lowest energy ligand conformer in each case, and the single negative vibrational frequency is reported for each TS.

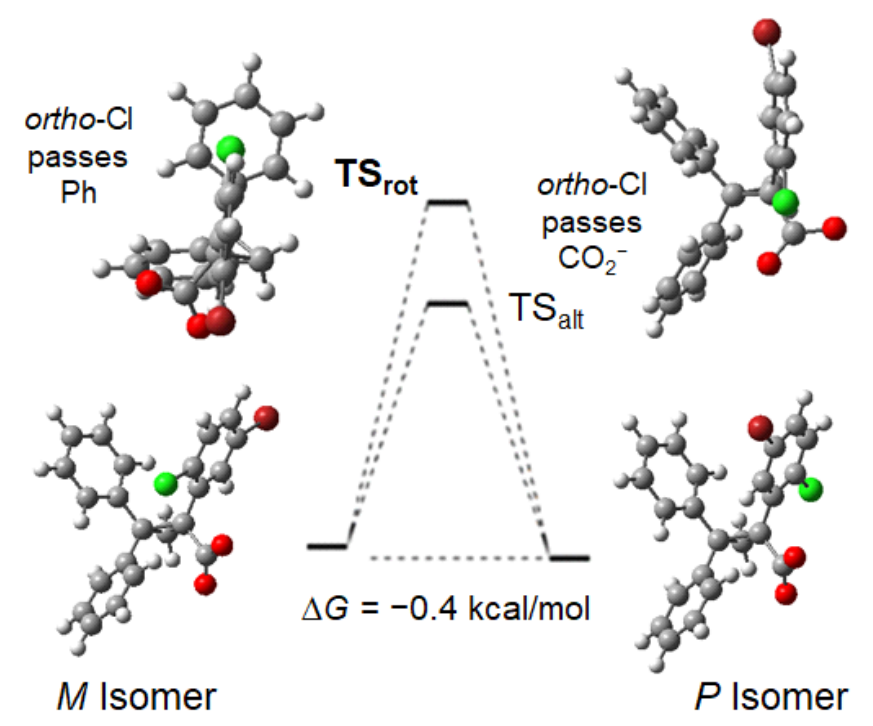

Figure S21. Rotational reaction coordinate for $M / P$ isomer interconversion of $S$-2-Cl-5-BrTPCP free ligand anions (ligI1 and ligI2) via either $\mathrm{TS}_{\text {rot }}$ (ligI_TSrot) or TS alt (ligI_TSalt).

Table S10. C1 aryl rotational transition state calculations for free ortho-CITPCP ligand anions ${ }^{\mathrm{a}}$

\begin{tabular}{ccccccc} 
Ligand & $\begin{array}{c}\text { lig1 } \\
(\text { hartrees })\end{array}$ & $\begin{array}{c}\Delta \boldsymbol{G}_{\text {rot }}^{\star} \\
(\mathrm{kcal} / \mathrm{mol})\end{array}$ & $\begin{array}{c}\Delta G^{\star} \text { alt } \\
(\mathrm{kcal} / \mathrm{mol})\end{array}$ & $\begin{array}{c}\text { lig2 } \\
(\mathrm{kcal} / \mathrm{mol})\end{array}$ & $\begin{array}{c}\mathrm{TS}_{\text {rot }} \text { freq. } \\
\left(\mathrm{cm}^{-1}\right)\end{array}$ & $\begin{array}{c}\mathrm{TS}_{\text {alt }} \text { freq. } \\
\left(\mathrm{cm}^{-1}\right)\end{array}$ \\
\hline ligA & -1458.37 & $\mathbf{1 7 . 8}$ & 14.2 & 0.3 & -40.93 & -44.15 \\
ligB & -4031.96 & $\mathbf{1 7 . 7}$ & 14.0 & 0.1 & -38.60 & -45.00 \\
ligC & -1572.85 & $\mathbf{1 7 . 6}$ & 13.8 & 0.3 & -40.65 & -44.12 \\
ligD & -1882.37 & $\mathbf{1 7 . 9}$ & 14.2 & 0.6 & -37.66 & -42.02 \\
ligE & -1823.20 & $\mathbf{1 7 . 7}$ & 14.0 & 0.3 & -36.84 & -42.38 \\
ligF & -2363.43 & $\mathbf{1 7 . 0}$ & 14.1 & 0.2 & -32.35 & -41.72 \\
ligG & -1846.44 & $\mathbf{1 7 . 8}$ & 13.9 & 0.2 & -36.81 & -43.54 \\
ligH & -1807.17 & $\mathbf{1 8 . 0}$ & 14.7 & 0.2 & -39.97 & -43.67 \\
ligI & -4031.96 & $\mathbf{1 8 . 6}$ & 14.1 & 0.4 & -30.68 & -42.83 \\
ligJ & -2363.43 & $\mathbf{1 8 . 8}$ & 14.6 & 1.2 & -31.19 & -43.49 \\
ligK & -1689.32 & $\mathbf{1 8 . 7}$ & 15.6 & 1.2 & -27.86 & -41.14 \\
ligL & -1846.44 & $\mathbf{1 8 . 8}$ & 14.1 & 0.9 & -28.62 & -43.20 \\
ligM & -1767.89 & $\mathbf{1 8 . 7}$ & 13.7 & 0.7 & -27.74 & -43.30 \\
ligN & -1807.17 & $\mathbf{1 8 . 5}$ & 12.9 & 0.4 & -26.05 & -41.60 \\
ligO & -1458.37 & $\mathbf{1 9 . 8}$ & 14.4 & 1.4 & -25.94 & -42.17 \\
\hline
\end{tabular}

${ }^{a}$ All energies reported relative to the free energy of lowest energy conformer (lig[letter]1). 
Table S11. Computed rotational transition state data for other TPCP and phthalimido ligands ${ }^{\mathrm{a}}$

\begin{tabular}{cccc} 
Ligand & $\begin{array}{c}\text { lig1 } \\
(\text { hartrees })\end{array}$ & $\begin{array}{c}\Delta \boldsymbol{G}^{\ddagger} \text { rot } \\
(\mathrm{kcal} / \mathrm{mol})\end{array}$ & $\begin{array}{c}\mathrm{TS}_{\text {rot }} \text { freq. } \\
\left(\mathrm{cm}^{-1}\right)\end{array}$ \\
\hline ligP & -998.756 & $\mathbf{5 . 8}$ & -39.68 \\
ligQ & -1203.26 & $\mathbf{5 . 9}$ & -36.06 \\
ligR & -1335.81 & $\mathbf{6 . 2}$ & -36.74 \\
ligS & -3572.35 & $\mathbf{5 . 7}$ & -37.00 \\
ligT & -1229.71 & $\mathbf{6 . 0}$ & -32.07 \\
ligU & -1672.87 & $\mathbf{6 . 6}$ & -16.85 \\
ligV & -6145.93 & $\mathbf{6 . 6}$ & -17.52 \\
ligW & -1774.89 & $\mathbf{6 . 5}$ & -17.23 \\
ligX & -1129.59 & $\mathbf{1 4 . 3}$ & -49.11 \\
ligY & -2968.02 & $\mathbf{1 2 . 8}^{\mathbf{b}}$ & -47.86 \\
\hline
\end{tabular}

a Free energy barriers reported relative to the free energy of lowest energy conformer (lig[letter]1).

${ }^{b}$ Note that $\mathrm{C} 1$ aryl rotation is reported for TPCP ligands and phthalimido rotation for ligX and ligY. 


\section{Workflow and Considerations for Obtaining SMART Descriptors}

\subsection{Considerations for Extracting SMART Descriptors for Reactive Catalyst Conformers}

The accompanying tutorial will explain in detail how SMART descriptors for reactive catalyst conformers were extracted using UCSF Chimera and ChimeraX programs. ${ }^{34-35,51}$ A fully automated workflow for the entire process using MacroModel (OPLS3e) ${ }^{10-11}$ and UCSF Chimera programs has been developed by Beck Miller in the Sigman group, and this workflow will be disclosed in a forthcoming publication.

To begin with, an optimized reactive site structure of interest is treated as an "approachable rigid target" to which a chosen probe molecule can be docked at a specified position of interest. For this reason, it is important to obtain all energetically accessible orientations that the reactive site may adopt and consider each. The ideal probe molecule will be highly sensitive to peripheral changes in the reactive site pocket. If the probe is too small, it may not reach the outer contours of the cavity in order to map it effectively. If the probe is too large, the entry surface area (ESA) metric will be less useful in that it will no longer represent surface area that is actually near the reactive site periphery. In our study of dirhodium catalysts, we tested macrocyclic silicon thioether probes with 10-, 11-, 12-, and 13-membered ring sizes and found that an 11-membered ring size probe is ideal for this purpose (probeA at the end of atomic coordinates section). The $\mathrm{S}$ atom of the probe was docked at $2 \AA$ distance from $\mathrm{Rh}$ along the $\mathrm{Rh}-\mathrm{Rh}$ vector on the catalyst face of interest to mimic the position of the dirhodium-carbene carbon (typically $\mathrm{Rh}-\mathrm{C}_{\text {carbene }}=2.0$ to $2.05 \AA$ ).

While a hypothetical silicon thioether macrocycle may seem like an esoteric probe choice, a macrocyclic probe was found to be ideal in that it cannot stray too far in any one direction but is still sufficiently flexible to explore and map the reactive site contours. Moving to a hypothetical "all Si" macrocycle rather than carbon was simply a matter of convenience to enable facile automated removal of the catalyst "mold" from the probe conformers in the early processing stages as the descriptor was being developed. Since none of the catalyst molecules had Si atoms, removing all carbon atoms to remove the catalyst and leave the probe was simpler, and Si can be tolerated in the revised automated workflow. Other probes may also be used, and in principle probes with different degrees of flexibility may give different measurements of the effective space available at the reactive site. Ideally, properly matching the flexibility of the probe to that of the substrates that are coming together in the relevant transition state inside the reactive site will give the best results. Similar to testing different sphere radii for $\% \mathrm{~V}_{\text {bur }}$ descriptors, ${ }^{52-54}$ different SMART descriptors may be tabulated for different probes (or the same probe at different positions in the cavity), and all may be useful in their own right as input parameters for MLR or machine learning. Even testing several $\% \mathrm{~V}_{\text {bur }}$ radii settings, there are still significant limitations for describing and differentiating the reactive site steric hindrance for dirhodium catalysts (and many other systems) that SMART descriptors can remedy, as illustrated in Figure S24.

After the docking of the probe molecule, the next step in obtaining SMART descriptors is a constrained substructure conformer search similar to that used for dirhodium catalysts in MacroModel (OPLS3), ${ }^{10-11}$ with the entire catalyst and the $\mathrm{S}$ atom of the probe frozen and all other atoms permitted to move freely. All conformers within $10.00 \mathrm{kcal} / \mathrm{mol}$ were considered, and the catalyst-probe conformers were exported as a single .mol2 file. Using UCSF ChimeraX, ${ }^{34-35}$ all C, $\mathrm{H}, \mathrm{N}, \mathrm{O}, \mathrm{F}, \mathrm{Cl}, \mathrm{Br}, \mathrm{Rh}$, and $\mathrm{S}$ atoms were removed from the catalyst-probe structures to leave only Si atoms from the overlaid probe conformers to define the pocket (see tutorial section for details). Enclosing these atoms in a 3D surface using ChimeraX provides the SMART pocket from which 
the various descriptors can be tabulated. Technical details for settings utilized in UCSF Chimera and ChimeraX programs are explained in the subsequent tutorial section.
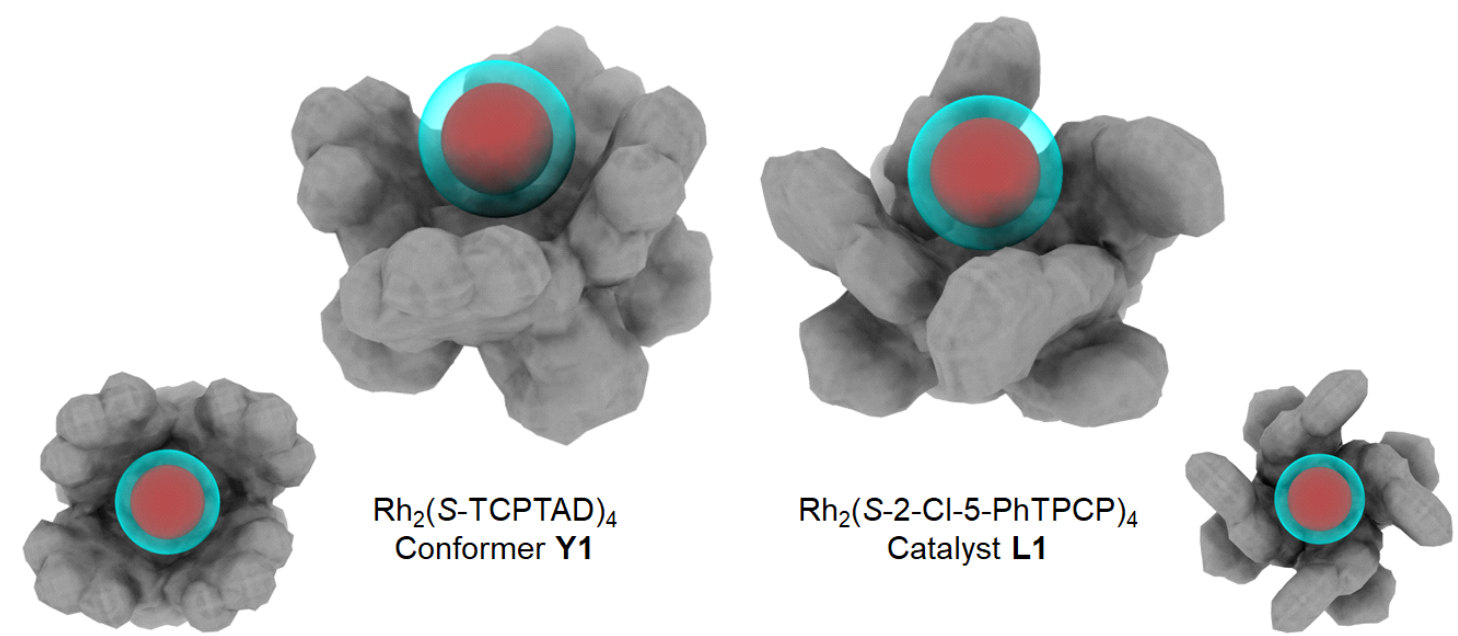

Figure S22. Illustrations of the limitations of $\% \mathrm{~V}_{\text {bur }}$ for describing the reactive site steric hindrance of dirhodium catalysts with different reactive site shapes. Even with various sphere radii settings tested (see SI section 8 specifically), $\% \mathrm{~V}_{\text {bur }}$ is similarly low for $14-20$ of the 25 catalysts (blue $=3.5 \AA$, red $=$ $2.5 \AA$, centered at 4 and $5 \AA$ distances along $\mathrm{Rh}-\mathrm{Rh}$ vector). Larger spheres of $4.5 \AA$ radius end up being optimal for starting to differentiate catalysts with low proximal steric congestion (see SI section 8 ), but this still results in 14 of the 25 catalysts having nearly identical $\% \mathrm{~V}_{\text {bur }}$ (Figures S39-S40). Larger spheres than this tend to encompass the entire catalyst face in non-intuitive and less chemically meaningful ways.

One challenging final consideration that must be addressed is that for catalysts with drastically different pocket sizes, it is more descriptive to combine overlaid probe conformer ensembles from multiple probe locations along the $\mathrm{Rh}-\mathrm{Rh}$ vector in order to obtain more representative SMART ESA and V VAVITY metrics in comparison to other catalysts. This is a benefit of the N descriptor, as it allows for simpler comparison of the more proximal reactive site constraints between catalysts of very different sizes. In this study, the catalyst bowl height was tabulated as the distance between the axial $\mathrm{Rh}$ atom of interest and the perpendicular mean plane calculated for the atom within each of the four carboxylate ligands with the largest $z$-coordinate (where $\mathrm{Rh}-\mathrm{Rh}$ is the $z$-axis). For most catalysts in this set, the bowl height is $\sim 5.5$ to $7.5 \AA$, but the $\mathrm{Rh}_{2}(S-2-\mathrm{Cl}$ 4-ArTPCP $)_{4}$ (D-H) and $\operatorname{Rh}_{2}\left[R-3,5-\operatorname{di}-\left(p-{ }^{t} \mathrm{BuC}_{6} \mathrm{H}_{4}\right) \mathrm{TPCP}\right]_{4}(\mathbf{W})$ catalysts feature much larger reactive site pockets. Thus, ESA for how much absolute surface area there is for a substrate to enter the reactive site pocket is not well represented by the overlaid probe conformers from the probe docked $2 \AA$ from Rh for these very large catalysts because the pocket does not reach the top of the bowl. To obtain comparable ESA and $\mathrm{V}_{\text {CAVITY }}$ in a standard manner, catalysts with bowl height $>7.5 \AA$ necessitated a pocket generated in combination with a probe conformer ensemble from a second probe location, where the $\mathrm{S}$ atom was docked at $5 \AA$ below the bowl height to match that for the typical catalyst in the set. Future studies will utilize proximal and distal volume classifications (vide supra) to minimize the need for relatively arbitrary distance cutoffs. The final MLR model presented (Figure 10) utilizes the more robust NPROBE descriptor type and thereby partially circumvents this issue (albeit while incorporating SMART ESA $\Delta$ instead). 


\subsection{Using UCSF Chimera(X) for Manually Extracting SMART Descriptors Tutorial}

\section{Helpful Links:}

- UCSF ChimeraX User Guide: https://www.rbvi.ucsf.edu/chimerax/docs/user/index.html

- ChimeraX Quick Start: https://www.rbvi.ucsf.edu/chimerax/docs/quickstart/index.html

\section{Extracting SMART descriptors:}

1. This tutorial covers using ChimeraX $\mathrm{X}^{34-35}$ and Chimera $^{51}$ to extract SMART descriptors, with many basic functionalities of Chimera $(\mathrm{X})$ demonstrated along the way. The first two steps in tabulating SMART descriptors are not covered here: (1) choosing and adding a probe file to all structure(s) of interest using an automated script developed by Iris Guo; and (2) running a constrained conformational search using the substructure capability in MacroModel. ${ }^{10-11}$ A full step-by-step for how to run constrained conformational searching using substructure with automatic setup is available in section 4.5 (pages 47-49: https://www.chem.uci.edu/ jjsnowick/groupweb/Maestro/macromodel quick_start.pdf). A fully automated workflow for extracting SMART descriptors has been developed by Beck Miller in the Sigman group and will be disclosed in a forthcoming publication.

2. Importing Files: $\mathrm{sdf}, \mathrm{pdb}, \& \mathrm{~mol} 2$ files can all be opened in ChimeraX. OPEN the mol2 file with overlaid probe conformers from the constrained conformer search (exported in one file from MacroModel). OPEN a separate file with the structure alone (without probe).

\section{Basic Interface/Control Panels (on PC):}

- LEFT CLICK + HOLD to rotate structure. RIGHT CLICK + HOLD to translate.

- MOUSE SCROLL WHEEL will adjust size of the structure.

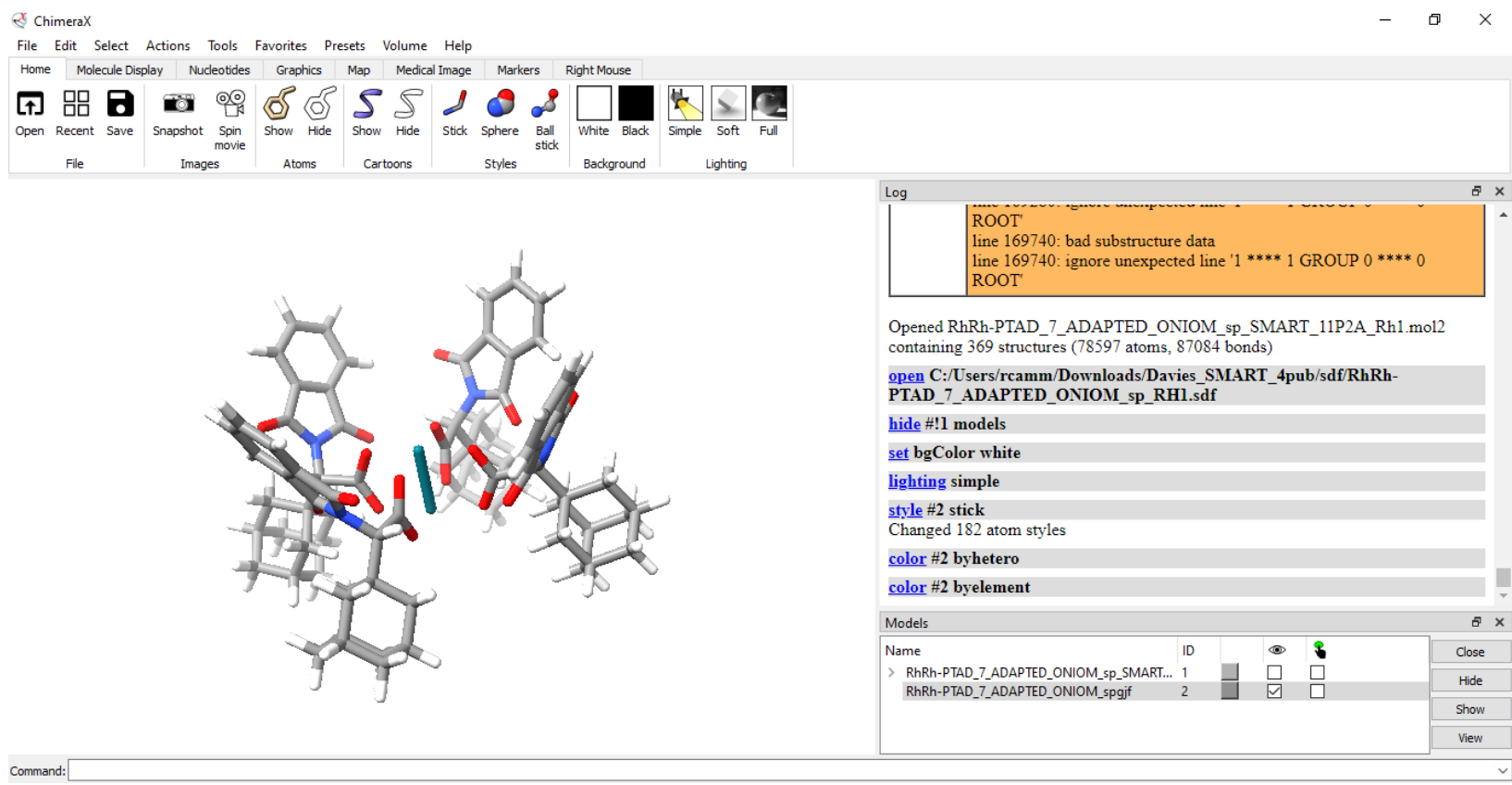

Right side panels - Log output and models panel:

- SELECT to only view "model 2" using the "eye" graphic in the model pane.

- $C H A N G E$ the color of the structure using the adjacent color button. 
Top toolbar and dropdown menus - most options for color, lighting, style, videos:

- CHANGE background color to white using the top toolbar.

- TOGGLE lighting options (simple, soft), and display styles (stick, ball stick).

- COLOR by heteroatom using dropdown options (Actions $<$ Color $<$ By Heteroatom).

- $C O L O R$ by element (Actions $<$ Color $<$ By Element).

Command line controls - convenient, easily can interface with python scripts:

- VIEW the log file for all commands for actions taken so far

- SELECT to see only model 1 using the models panel.

- Now repeat using the command line ('hide \#2 models', then 'show \#!1 models').

\section{Removing catalyst "mold":}

- SELECT all probe $\mathrm{S}$ atoms (Select $<$ Chemistry $<$ Element $<$ S) and DELETE them (Actions $<$ Atoms $/$ Bonds $<$ Delete).

- Now delete all catalyst atoms using the command line. The relevant syntax is: 'delete \#1.1-end@rh?,rh??,rh???,rh???,c?,c??,c???,c???,h?,h??,h???,h???,

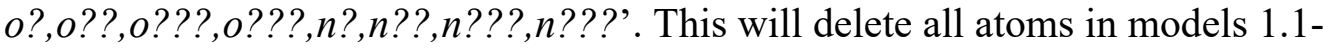
$1 . \mathrm{N}$ that begin with $\mathrm{rh}, \mathrm{c}, \mathrm{h}, \mathrm{o}$, and $\mathrm{n}$, where '?' indicates all possible characters and numbers for remainder of the atom IDs (i.e., rh1, rh2, c200, etc.).

\section{Enclosing "the brains":}

- GENERATE a 3D map for the intermeshed probe atoms (cavity) in model 1 by typing 'molmap \#1 2.8' into the command line. \#1 refers to the model \# and 2.8 is the map resolution. Play around with this value to see how it impacts the 3D map, but 2.8 is about the optimal value for matching the probe conformer ensemble. (https://www.rbvi.ucsf.edu/chimera/docs/UsersGuide/midas/molmap.html\#options)

- This map has now been added as model \#3 to the models panel, and now the volume viewer also appears. For a full explanation of the volume viewer, refer to: https://www.rbvi.ucsf.edu/chimerax/docs/user/tools/volumeviewer.html.

- $A D J U S T$ the threshold level to observe its impact on the 3D map. $\mathrm{V}_{\text {CAVITY }}$ and ESA descriptors in this paper were collected with a level of 4, but reasonable values for generating images and videos for a cavity could be anywhere from 2 to 20 .

- TOGGLE between surface and mesh display settings for optimal viewing.

- SELECT model \#2 and generate a map of the catalyst ('molmap \#2 2.8'). SET catalyst threshold level to 0.07 in volume viewer for model \#4.

- 3D surface color can be set using either the models panel, volume viewer, or command line. SET the colors of \#3 and \#4 to whatever you prefer.

- CHANGE \#3 to be a transparent catalyst pocket surface (Actions $<$ Surface $<$ Transparency $<70 \%$ ). VIEW \#3 and \#4 together.

- SAVE\#3 as filename_pocket.cmap and \#4 as filename_catalyst.cmap. Entire sessions can be saved to resume later by saving as .cxs (check 'maps included').

- RECORD a snapshot or spin movie by clicking those buttons in the top toolbar. 


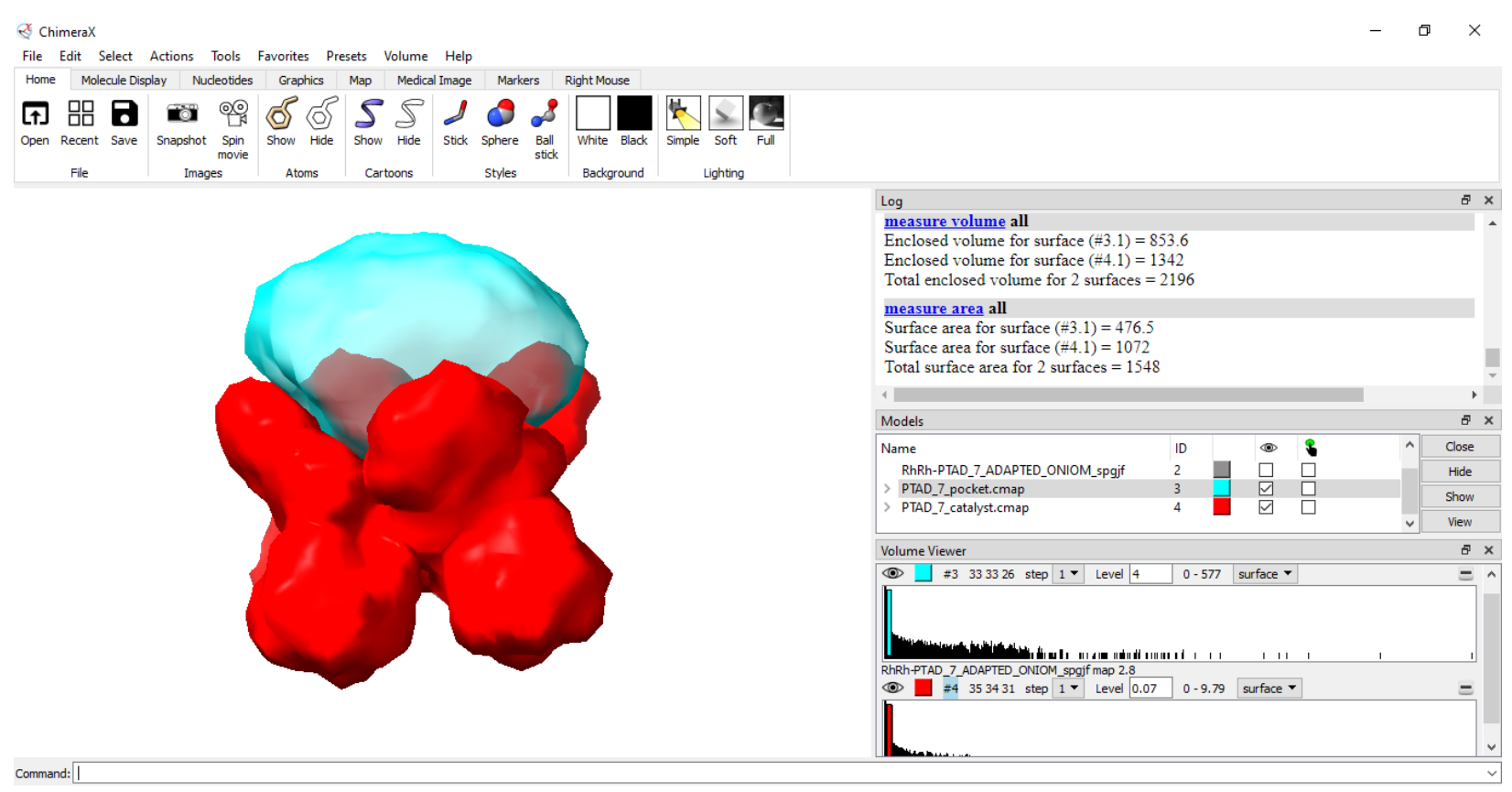

- TABULATE $\mathrm{V}_{\text {CAVITY }}$ and total cavity surface area (TSA) using 'measure volume all' and 'measure area all'. The volume and surface area results will appear in the $\log$ output pane for all catalyst and pockets of interest being viewed. Be aware that the measurement outputs are dependent on the threshold levels you set for each of the $3 \mathrm{D}$ surfaces in the volume viewer.

\section{Using Chimera to find contact surface area (CSA) and entry surface area (ESA):}

- UCSF ChimeraX encloses surfaces well without generating "holes" in the surfaces that cause errors and is a very user-friendly interface. However, Chimera has some additional capabilities, including the capability to more easily identify buried contact area between two surfaces.

- In UCSF Chimera, OPEN the .cmap files saved previously. OPEN filename pocket.cmap first, and SET the threshold level to 4 in the volume viewer that pops up. Without moving or rotating the surface, OPEN filename_catalyst.cmap and SET threshold level to 0.07. The volume viewer also allows for changing of surface colors.

- ROTATE, TRANSLATE, and RE-SIZE the surfaces. Basic controls are analogous to ChimeraX, except that translate and re-size are switched (on PC). CHANGE the background color to white (Actions $>$ Color $>$ All Options, click background, select white).

- ENABLE the model panel and the command line by using the dropdown menu (Tools $>$ General Controls $>$ Model Panel [Command Line]).

- MEASURE contact surface area (CSA) by typing 'measure contactArea \#0 \#1 1.1 color black offset 0 ' in the command line. The resulting CSA value appears below the command line (see picture). Contact distances of 1.0, 1.1, and 1.2 were tested, with 1.1 yielding the best visual (contiguous shading) and statistical results.

- Entry Surface Area (ESA) can be obtained by simply subtracting: SA - CSA = ESA. CSA may be important as well for catalyst surface area that could engage in NCIs near the reactive site pocket. 


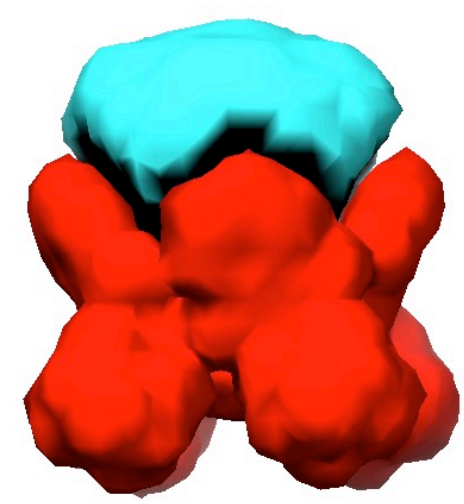

- Other composite descriptors can be obtained as well, like accessible volume $\left(\mathrm{SMART}\right.$ ACC VOL $=\mathrm{ESA} \% *\left(\mathrm{~V}_{\mathrm{CAVITY}}\right)$ or G ACC VOL $\left.=\mathrm{G} \% *\left(\mathrm{~V}_{\text {CAVITY }}\right)\right)$, and all descriptors can be tabulated as maximum, minimum, delta $(\Delta=$ maximum minimum), and/or weighted standard deviation values across a conformational ensemble. Another very useful descriptor is $\mathrm{N}_{\text {probe }}$ (or $\log \mathrm{N}_{\text {PROBE }}$ ) for the number of total probe conformers within a particular energy range from the constrained conformational search (easily obtained by exporting results table in MacroModel as .csv). This is another very generalizable way of detecting more local steric hindrance that is still too distal to capture effectively with $\% \mathrm{~V}_{\text {bur }}$ descriptors.

- RECORD a spin movie by typing 'movie record ; turn y 1360 ; wait ; movie encode' into the command line.

\section{Other useful functionality:}

- SLICING up surfaces into $\mathrm{V}_{\text {CAVITY }}$ by quadrant ("lobe" in Figure 6) can be accomplished crudely using ChimeraX:

http://plato.cgl.ucsf.edu/pipermail/chimera-users/2009-August/004211.html. This capability will be more robustly implemented in the forthcoming release of the automated SMART workflow developed by Beck Miller in the Sigman group.

- CLASSIFYING SMART V VAVITY as either proximal or distal reactive site volume can also be accomplished by finding the intersection volume of the SMART pocket with a sphere of all points within a specified distance of the axial $\mathrm{Rh}$ atom (or from the dirhodium-carbene carbon dummy atom along the $\mathrm{Rh}-\mathrm{Rh}$ vector). This too will be implemented in the forthcoming automated workflow.

- In general, the UCSF ChimeraX User Guide and [Chimera-Users] forums are great resources for learning about how more complex actions can be best executed. 


\section{Multivariate Linear Regression and Classification Model Development}

\subsection{General Considerations and Technical Details}

Single node decision tree classification was performed in a Jupyter notebook as implemented in the Scikit-learn package in Python. ${ }^{55-57}$ The $\Delta G^{\ddagger}$ rot threshold was defined by the algorithm to best divide the dirhodium catalysts based on site-selectivity performance, where $\Delta \Delta G^{*}=-0.9 \mathrm{kcal} / \mathrm{mol}$ divided catalysts as highly $\mathrm{C} 2$ selective. Enantioselectivity classification among the $15 \mathrm{Rh}_{2}(\mathrm{~S}$-oCITPCP) $)_{4}$ catalysts was performed similarly to divide catalysts that are highly enantioselective (er $\Delta \Delta G^{\ddagger}<-1.5 \mathrm{kcal} / \mathrm{mol}$ ). Spatial accessibility classification was performed using the Solid-G program, ${ }^{39}$ but subsequent automation for checking each $\mathrm{Rh}$ face was accomplished by Beck Miller by adapting a G\% script developed by Allen and co-workers. ${ }^{58}$

For MLR modeling, the experimentally observed site-selectivity $\left(\Delta \Delta G^{\ddagger}\right.$ in $\left.\mathrm{kcal} / \mathrm{mol}\right)$ was tabulated for each catalyst using the formula $\Delta \Delta G^{\sharp}=-\mathrm{RT} \ln (\mathrm{rr})$, where $\mathrm{R}$ is the gas constant, T is temperature $(312.8 \mathrm{~K})$, and $\mathrm{rr}$ is the regioselectivity ratio $(\mathrm{C} 2 / \mathrm{Bn})$. Multivariable model development for predicting $\Delta \Delta G^{\ddagger}$ as a function of calculated catalyst properties was performed using Python with previously reported algorithms ${ }^{55-57,59-60}$ based on forward stepwise linear regression that keeps sets of model candidates at each step based on leave-one-out (LOO) internal validation. ${ }^{61-62}$ An 80:20 training/validation set partitioning was performed by to yield either equidistant site-selectivity outputs or to split catalysts evenly based on their property space with the Kennard-Stone algorithm. ${ }^{63}$ Three TS/VS splits were tested for all models to ensure favorable performance was not largely the result of fortuitous training/validation partitioning, as well as to compare the sensitivity of the model coefficients obtained. These TS/VS splits were based on: (1) y-equidistant outputs (s1); (2) Kennard-Stone splits with an original more descriptive subset (s2; includes cat. $\mathbf{B}, \mathbf{C}, \mathbf{E}, \mathbf{J}$, and $\mathbf{Q}$ in VS); and (3) Kennard-Stone based on the full parameter set (s3).

Colinear descriptors that capture similar chemical features were not permitted to be in the same statistical model, with a collinearity threshold defined as 0.4 . Normalized descriptors were utilized (i.e., z-scores) to allow the model coefficients to reflect the relative importance of each catalyst property to its performance (i.e., site-selectivity). ${ }^{64}$ Models were evaluated in terms of their coefficient of determination, $\mathrm{R}^{2}$, where a high $\mathrm{R}^{2}$ value indicates that predicted $\Delta \Delta G^{\ddagger}$ closely matches the measured values. Models were further validated through internal cross validation. Leave-one-out (LOO) analysis, was performed to iteratively remove a single data point from the training set and predicting the observation that was left out based on the remaining data (hollow black squares are LOO predictions in Figure $7 \mathrm{~B}$ ). A $\mathrm{Q}^{2}$ value for $\mathrm{LOO}$ that is close to $\mathrm{R}^{2}$ is indicative that the model is not highly skewed by any single data point ${ }^{65}$ Repeated k-fold crossvalidation $(\mathrm{k}=4, \mathrm{n}=20 ! /\{5 ! \cdot 15 !\})$ was also performed, where $1 / \mathrm{k}$ of the training set data points were iteratively left out and predicted based on the remaining data. ${ }^{66-67} \mathrm{Q}^{2}$ and $\mathrm{k}$-fold values closely approach $\mathrm{R}^{2}$ for all models presented, indicating that the models are not highly skewed by any single data point or small group of data points. All models perform favorable with highly interpretable descriptors despite a high training set data-to-parameter ratio (20:3), suggesting robust models that are not the result of overfitting the training set data. ${ }^{68-69}$

Molecular descriptors calculated for each catalyst included 13 electronic parameters describing Rh partial charges and $d$-orbital energies obtained from Natural Bond Orbital (NBO 7.0) analysis. ${ }^{33}$ In addition, 18 spatial descriptors (SMART, Sterimol, $\% \mathrm{~V}_{\text {bur, }} \mathrm{G} \%$, etc.) were included. Each of these 31 descriptors was included as both a Boltzmann-averaged property across reactive catalyst conformers and as a Boltzmann-weighted standard deviation property, which resulted in 62 total 
descriptors. Additional parameters describing the maximum, minimum, and delta ( $\Delta=$ maximum - minimum) values for each of the 18 steric properties were also included ( $18 \times 3=54$ additional input parameters). The calculated free ligand $\Delta G^{\ddagger}$ rot values were included as a final descriptor (62 $+54+1=117$ total descriptors). An excel spreadsheet containing these 117 descriptors for each catalyst is included ("Dirhodium Catalyst Descriptors and Outputs for MLR Modeling.xlsx"), and this was utilized as the input for statistical modeling. A key listing each of the molecular descriptor abbreviations and meanings is displayed in Table S12. Descriptors without a specific label signify Boltzmann-averaged descriptors, where the given property is tabulated for each reactive catalyst conformer and a weighted average is computed based on the relative Boltzmann population of each reactive catalyst conformer under the reaction conditions $\left(313 \mathrm{~K}, \mathrm{CH}_{2} \mathrm{Cl}_{2}\right.$ ). It should be noted that original MLR modeling attempts using entirely free carboxylate ligand anion parameters (NBO charges, ${ }^{33}$ Sterimol values, ${ }^{70}$ etc.) were unsuccessful for achieving out-of-sample predictive power (Figure S32, Table S13). This prompted the approach outlined in this study for efficiently obtaining highly interpretable catalyst molecular descriptors and MLR models that would be more generalizable for extrapolation.

For brevity, only the y-equidistant split (s1) model plots themselves are shown for each model (mA-mO in Figures S30-S37). Alternative Kennard-Stone s2 split model plots are shown for the two models that are featured in the main text with s1 splits ( $\mathbf{m A}$ and $\mathbf{m B}$ in Figure S30). For all models $\mathbf{m A} \mathbf{A}-\mathbf{m O}$, all descriptors, coefficients, and statistical metrics $\left(\mathrm{R}^{2}, \mathrm{Q}^{2}, 4\right.$-fold, test $\left.\mathrm{R}^{2}\right)$ for each of the s1-s3 TS/VS splits are presented in Table S13. Importantly, the average statistical metrics across these three TS/VS splits were used for explicit quantitative comparisons between SMART and established steric descriptors. These average metrics and model coefficients are displayed in Figures S30-S37 (where the model plots themselves are specifically the s1 split unless otherwise noted). Note that models with slightly higher metrics are possible than those presented (both for SMART and for other steric descriptors); however, those we identified are similarly statistically robust and highly chemically interpretable, and thus are more likely to be generalizable when predicting reactivity for any dirhodium catalyst (rather than simply overfitting the training set data). 
Table S12. Catalyst molecular descriptor abbreviation key

\begin{tabular}{|c|c|}
\hline Descriptor & Explanation \\
\hline $\mathrm{G} \%$ & $\%$ of a surrounding sphere covered by ligand shadows with $\mathrm{Rh}$ as a light source \\
\hline Sterimol ${ }^{\text {a }}$ LR-Coo & length of ligand substituent along $\mathrm{C}_{\mathrm{COO}-\mathrm{R}}$ bond \\
\hline Sterimola $^{\mathrm{a}} \mathrm{B} 1_{\mathrm{R}-\mathrm{COO}}$ & minimum width of ligand substituent along $\mathrm{C}_{\mathrm{COO}-\mathrm{R}}$ bond \\
\hline Sterimol ${ }^{\mathrm{a}}$ B5R-Coo & maximum width ligand substituent along $\mathrm{C}_{\mathrm{COO}-\mathrm{R}}$ bond \\
\hline Sterimol ${ }^{\mathrm{a}} \mathbf{B 5}-\mathrm{B} 1_{\mathrm{R}-\mathrm{COO}}$ & difference in max and min widths for ligand substituent along CCOO-R bond \\
\hline$\% V_{\text {bur }, 4 c, 45 r^{b}}$ & percent buried volume for a sphere with $\mathrm{r}=4.5 \AA$ and $\mathrm{c}=4.0 \AA$ \\
\hline$\% V_{\text {bur }, 5 c, 45 r^{b}}$ & percent buried volume for a sphere with $\mathrm{r}=4.5 \AA$ and $\mathrm{c}=5.0 \AA$ \\
\hline CAT VOL & total volume of molecular surface enclosing the catalyst atoms \\
\hline CAT SA & total surface area of molecular surface enclosing the catalyst atoms \\
\hline SMART V VAVITY & total volume of reactive site cavity as defined by SMART probe method \\
\hline SMART SA & total surface area of reactive site cavity as defined by SMART probe method \\
\hline G\% ACC VOL & $\mathrm{G} \%$ accessible volume $=(\mathrm{G} \% / 100)^{*}$ SMART V VAVITY \\
\hline SMART CSA & total contact surface area between catalyst and reactive cavity surfaces \\
\hline SMART ESA & entry surface area $=\mathrm{SA}-\mathrm{CSA}$ \\
\hline SMART ESA\% & entry surface area $\%=(\mathrm{ESA} / \mathrm{SA}) \times 100$ \\
\hline SMART ACC VOL & SMART accessible volume $=(\text { ESA\%/100 })^{*}$ SMART V VAVITY \\
\hline SMART NPROBE & total number of probe conformers possible within cavity $(\leq 10 \mathrm{kcal} / \mathrm{mol})$ \\
\hline SMART NPROBE,LE & total number of low energy probe conformers $(\leq 5 \mathrm{kcal} / \mathrm{mol})$ \\
\hline Rh NBO & NBO partial charge of $\mathrm{Rh}$ atom on face of interest $\left(\mathrm{Rh}_{\mathrm{A}}\right)$ \\
\hline Rh NBO diff & Rh NBO partial charge difference $\left(\mathrm{Rh}_{\mathrm{A}}-\mathrm{Rh}_{\mathrm{B}}\right)$ \\
\hline Rh NBO sum & $\mathrm{Rh}$ NBO partial charge sum $\left(\mathrm{Rh}_{\mathrm{A}}+\mathrm{Rh}_{\mathrm{B}}\right)$ \\
\hline Rh d $\pi$ LPE & $\mathrm{Rh}_{\mathrm{A}} \mathrm{d} \pi \mathrm{NBO}$ lone-pair energy (average of $\mathrm{d}_{\mathrm{xz}} / \mathrm{d}_{\mathrm{yz}}$ ) \\
\hline Rh LPE $\mathbf{L}_{\mu}$ & average of all $\mathrm{Rh}_{\mathrm{A}}$ valence d-orbital NBO lone-pair energies \\
\hline $\operatorname{RhRh} d \pi \mathrm{LPE}_{\mu}$ & average of $\mathrm{Rh}_{\mathrm{A}}$ and $\mathrm{Rh} \mathrm{h}_{\mathrm{B}} \mathrm{d} \pi \mathrm{NBO}$ lone-pair energies \\
\hline RhRh LPE ${ }_{\mu}$ & average of all $R h_{A}$ and $R h_{B}$ valence d-orbital NBO lone-pair energies \\
\hline Rh d $\pi$ LPE diff & $\mathrm{Rh} d \pi$ NBO lone-pair energy difference $\left(\mathrm{Rh}_{\mathrm{A}}-\mathrm{R} \mathrm{h}_{\mathrm{B}}\right)$ \\
\hline Rh LPE $\mathbf{L}_{\mu}$ diff & difference in average $R h$ valence d-orbital NBO lone-pair energies $\left(R h_{A}-R h_{B}\right)$ \\
\hline RhRh $\sigma$ E & $\mathrm{Rh}-\mathrm{Rh} \sigma$ bonding orbital energy from NBO analysis \\
\hline RhRh $\sigma^{*} \mathbf{E}$ & $\mathrm{Rh}-\mathrm{Rh} \sigma^{*}$ anti-bonding orbital energy from NBO analysis \\
\hline RhRh dist & calculated $\mathrm{Rh}-\mathrm{Rh}$ bond distance \\
\hline $\operatorname{RhRh} \Delta \mathbf{E}_{\pi^{*} / \sigma^{*}} \mathbf{c}$ & represents energy gap between RhRh-based HOMO and LUMO \\
\hline$\Delta G^{\ddagger}$ rot & rotational transition state barrier relative to lowest energy ligand anion conformer \\
\hline
\end{tabular}

${ }^{a}$ Catalyst Sterimol values cannot be readily defined because each of four ligands would have unique values. ${ }^{b}$ Initially, 4 different $\% \mathrm{~V}_{\text {bur }}$ settings were tested ( $\mathrm{c}=4.0$ or $5.0 \AA$, with $\mathrm{r}=3.5$ and $4.5 \AA$ ), but these provided the best model statistics. ${ }^{\mathrm{c}}$ Added to 116 descriptors after initial model development. NBO does not consider localized Rh $\mathrm{d} \pi$ orbital mixing to engender RhRh $\pi$ and $\pi^{*}$ orbitals ${ }^{33}$ but higher energy $\mathrm{Rh} \mathrm{d} \pi$ will result in higher energy $\mathrm{RhRh} \pi^{*}$ orbitals and a lower $\Delta \mathrm{E}$ gap (see main text).

Descriptors are color coded to enable easier comparisons in Table S13 of SMART-derived descriptors with traditional steric and rigidity parameters. Catalyst electronic descriptors in the main text models are shown in green. Spatial descriptors are also denoted by color: SMART, Sterimol values, $\% \mathbf{V}_{\text {bur }}, \mathbf{G} \%$. Rigidity descriptors based on each of these steric parameter types are also colored analogously, along with $\Delta G^{\ddagger}$ rot. This allows Table S13 to be easily navigated as the steric and rigidity descriptor types are varied while the catalyst electronic descriptor(s) are held constant. Table S13 includes model IDs (mA-mO) and the molecular descriptors each model includes (x1-x117; see parameter spreadsheet or s1 split model figure labels). For each TS/VS split, Table S13 lists: (1) model coefficients (in descriptor order), followed by the y-intercept; (4) statistical metrics: $\mathrm{R}^{2}, \mathrm{Q}^{2}$, 4-fold, and test $\mathrm{R}^{2}$ (below in italics). To enable easy visual comparisons, average statistical metrics (across s1-s3) that are worse than those for SMART-derived $\mathbf{m A}$ by 0.10 or more are shown in dark red in both Table S13 and in the plots in Figures S30-S37. 


\subsection{Univariate Correlation Plots for Relevant Steric Input Descriptors with Site-Selectivity}

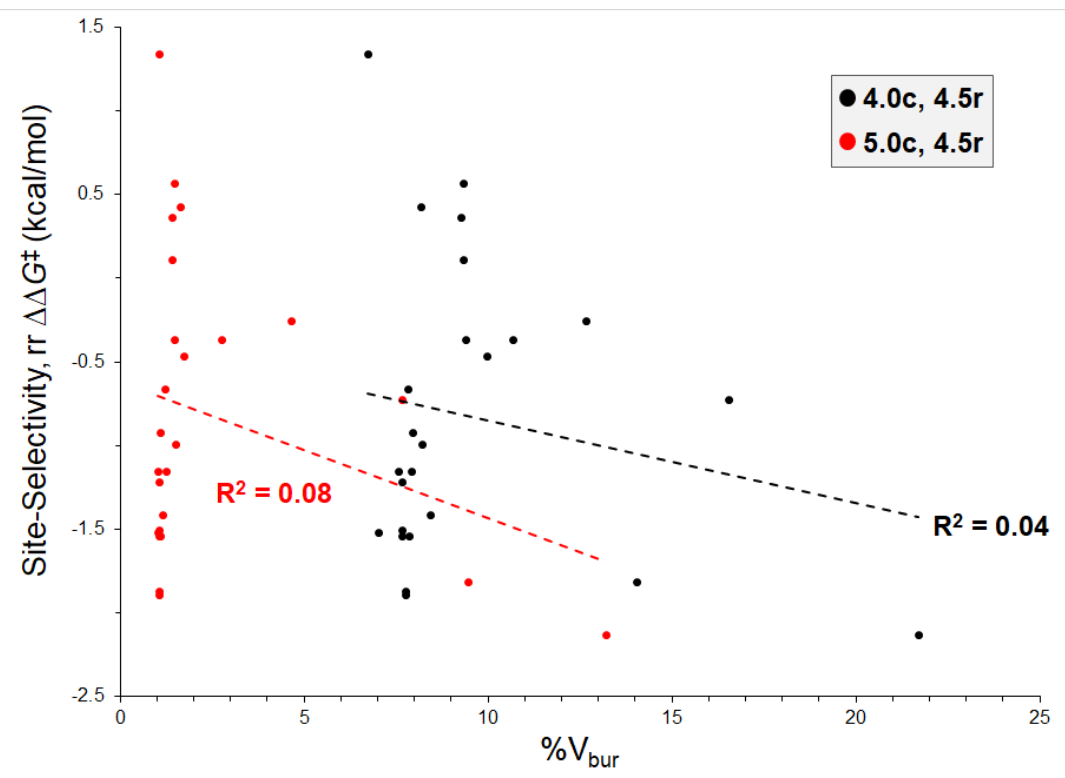

Figure S23. Little correlation was observed between C2:Bn site-selectivity ( $\operatorname{rr} \Delta \Delta G^{\ddagger}$ ) and \% $\mathrm{V}_{\text {bur }}$ descriptors. Note that high $\mathrm{C} 2: \mathrm{Bn}$ site-selectivity has arbitrarily been defined as larger negative $\Delta \Delta G^{*}$ values. $\% \mathrm{~V}_{\text {bur }}$ was initially defined for nine different settings: centered (c) either 3.0, 4.0, or 5.0 $\AA$ along the $\mathrm{Rh}-\mathrm{Rh}$ vector, with sphere radii ( $\mathrm{r}$ ) of $2.5,3.5$, or $4.5 \AA$. Many settings were not considered further because they gave virtually no $\% \mathrm{~V}_{\text {bur }}$ differences across the entire set of 25 catalysts. Even among the more descriptive $\% \mathrm{~V}_{\text {bur }}$ settings shown, differentiation of catalysts with low proximal steric hindrance (i.e., vertical lines on the plot with same $\% \mathrm{~V}_{\text {bur }}$ but divergent outputs) remains a major limitation.

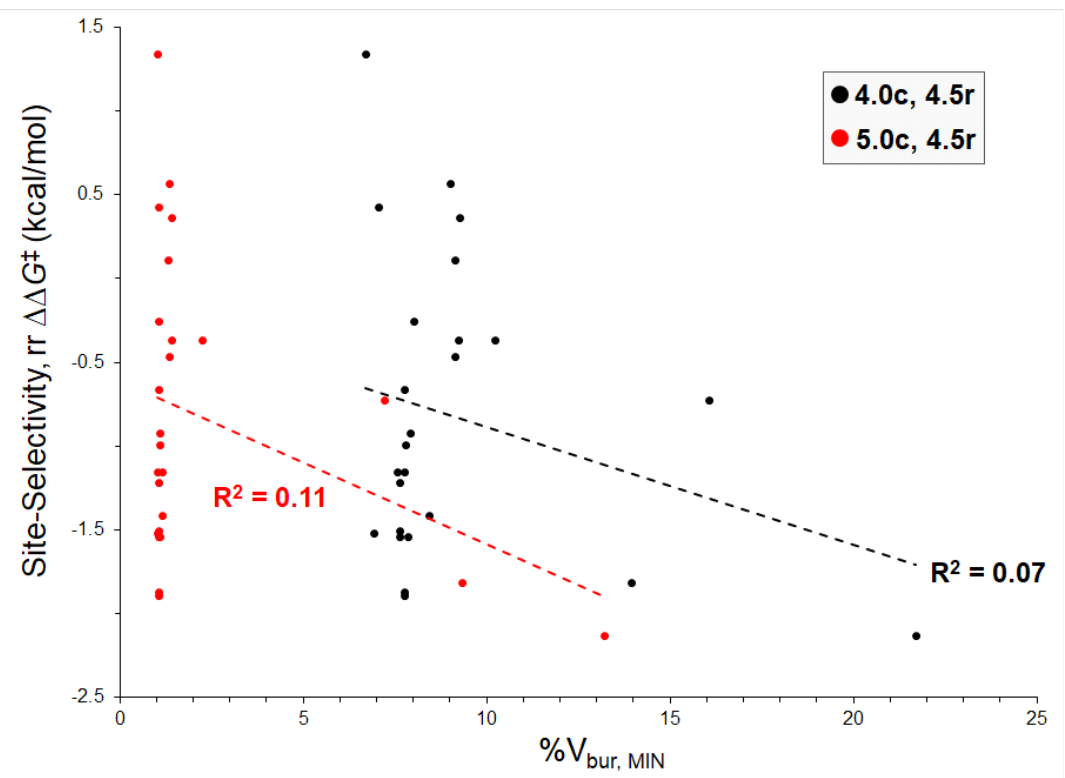

Figure S24. Little correlation was observed between C2:Bn site-selectivity ( $\operatorname{rr} \Delta \Delta G^{\ddagger}$ ) and $\% \mathrm{~V}_{\text {bur, MIN }}$ descriptors. Note that high $\mathrm{C} 2: \mathrm{Bn}$ site-selectivity has arbitrarily been defined as larger negative $\Delta \Delta G^{\ddagger}$ values. $\% \mathrm{~V}_{\text {bur,MIN }}$ was recently found to be highly descriptive for classifying how variations in phosphine ligand steric bulk impact ligation state to switch on or off the catalytic activity of Ni- and Pd-mediated cross coupling reactions. ${ }^{71}$ 


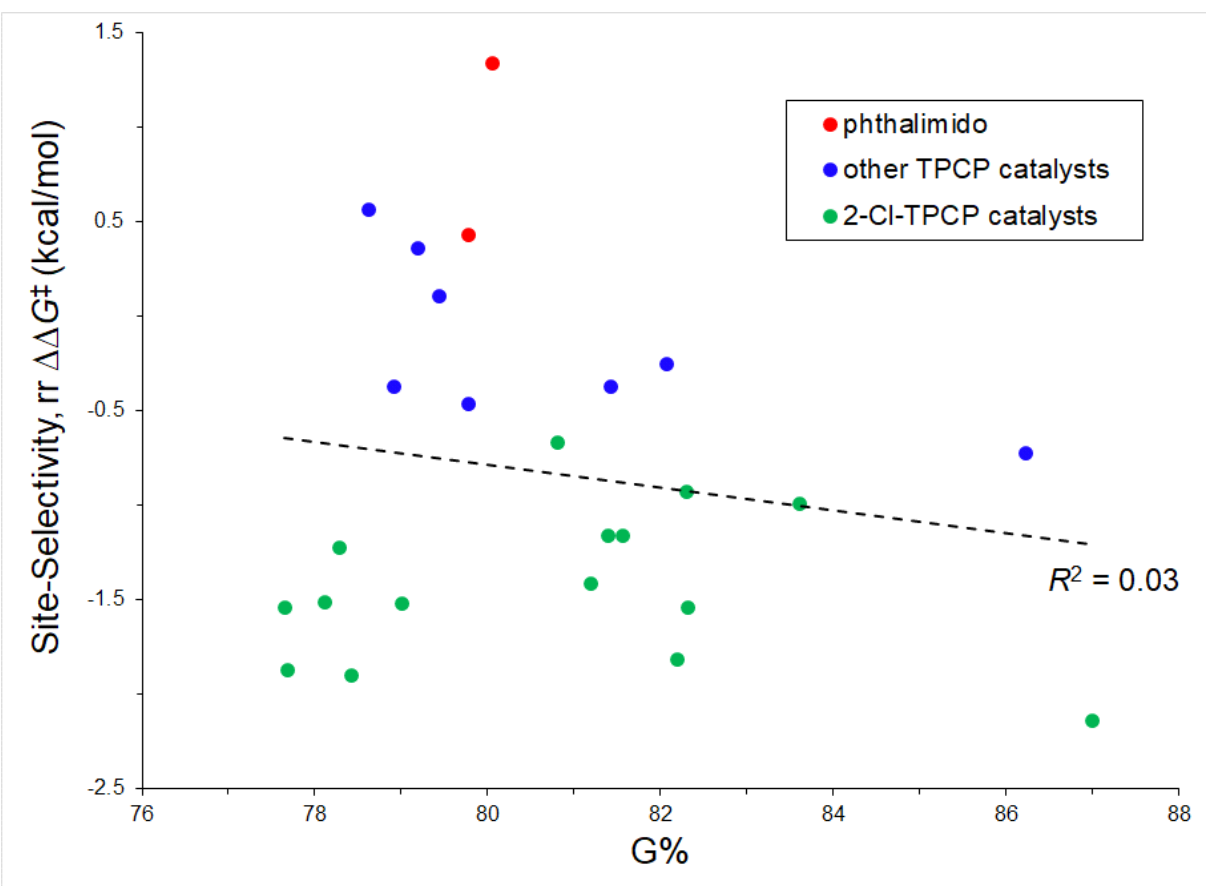

Figure S25. Little to no correlation was observed between C2:Bn site-selectivity (rr) and G\% for Rh. Note that high $\mathrm{C} 2$ :Bn site-selectivity has arbitrarily been defined as larger negative $\Delta \Delta G^{\ddagger}$ values, and $\mathrm{G} \%$ is expressed as the Boltzmann-averaged value across the reactive conformers for each catalyst.

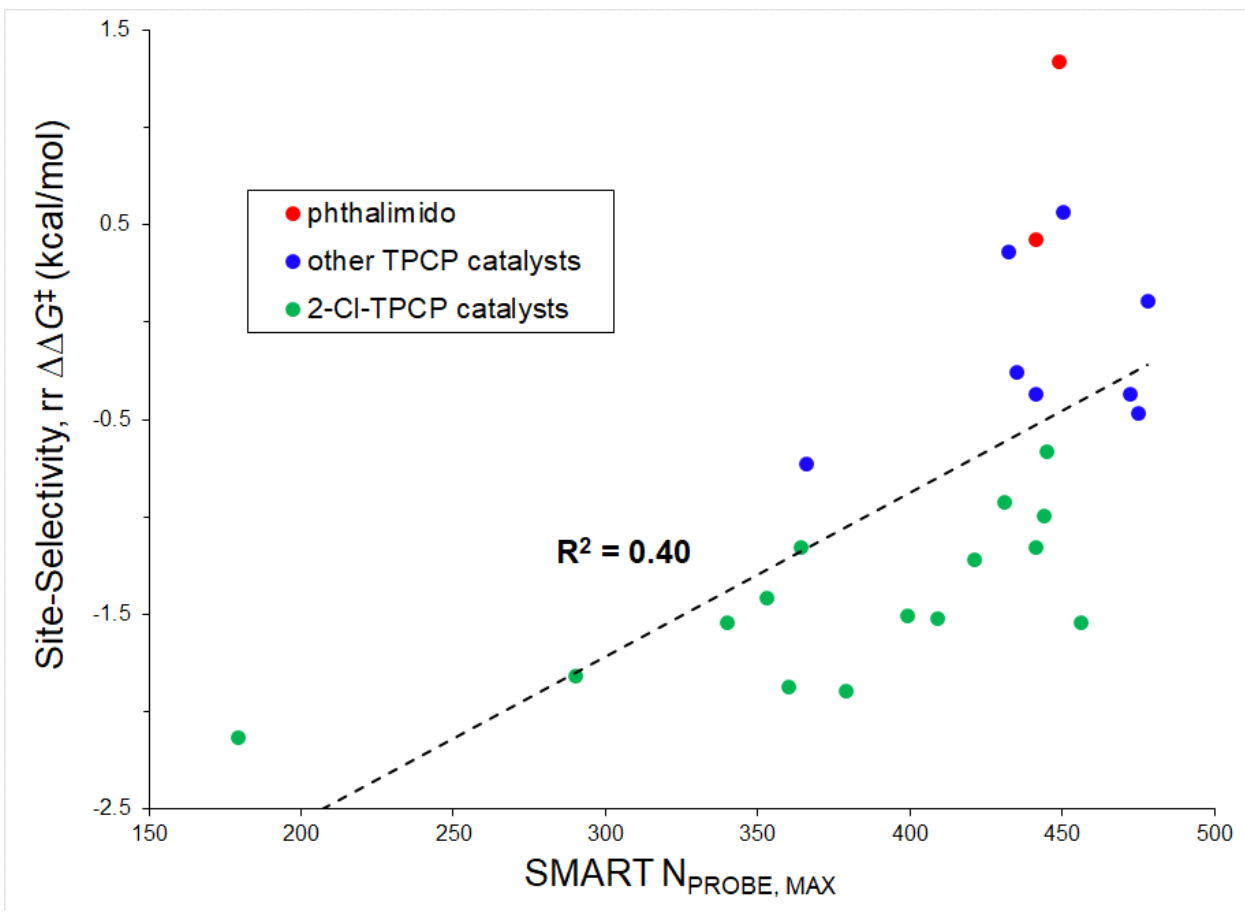

Figure S26. A moderate univariate correlation was observed between $\mathrm{C} 2: \mathrm{Bn}$ site-selectivity (rr) and

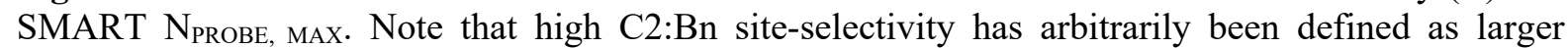
negative $\Delta \Delta G^{\ddagger}$ values. This is the highest correlation for any SMART descriptor (same $\mathrm{R}^{2}$ as $\mathrm{N}_{\mathrm{PROBE}}$ ). Other notable SMART univariate correlations include those for $\mathrm{N}_{\mathrm{PROBE}, \mathrm{LE}}\left(\mathrm{R}^{2}=0.28\right), \mathrm{V}_{\text {CAVITY, MAX }}\left(\mathrm{R}^{2}\right.$ $=0.13)$, and ESA $\Delta\left(\mathrm{R}^{2}=0.25\right)$. Interestingly, Sterimol $\mathrm{B} 1_{\mathrm{R}-\mathrm{COO}}$ also has a modest univariate correlation $\left(\mathrm{R}^{2}=0.36\right)$, but it performs poorly when included in MLR models (Table S13). 


\subsection{Univariate Correlation Plots Between Reaction Outputs}

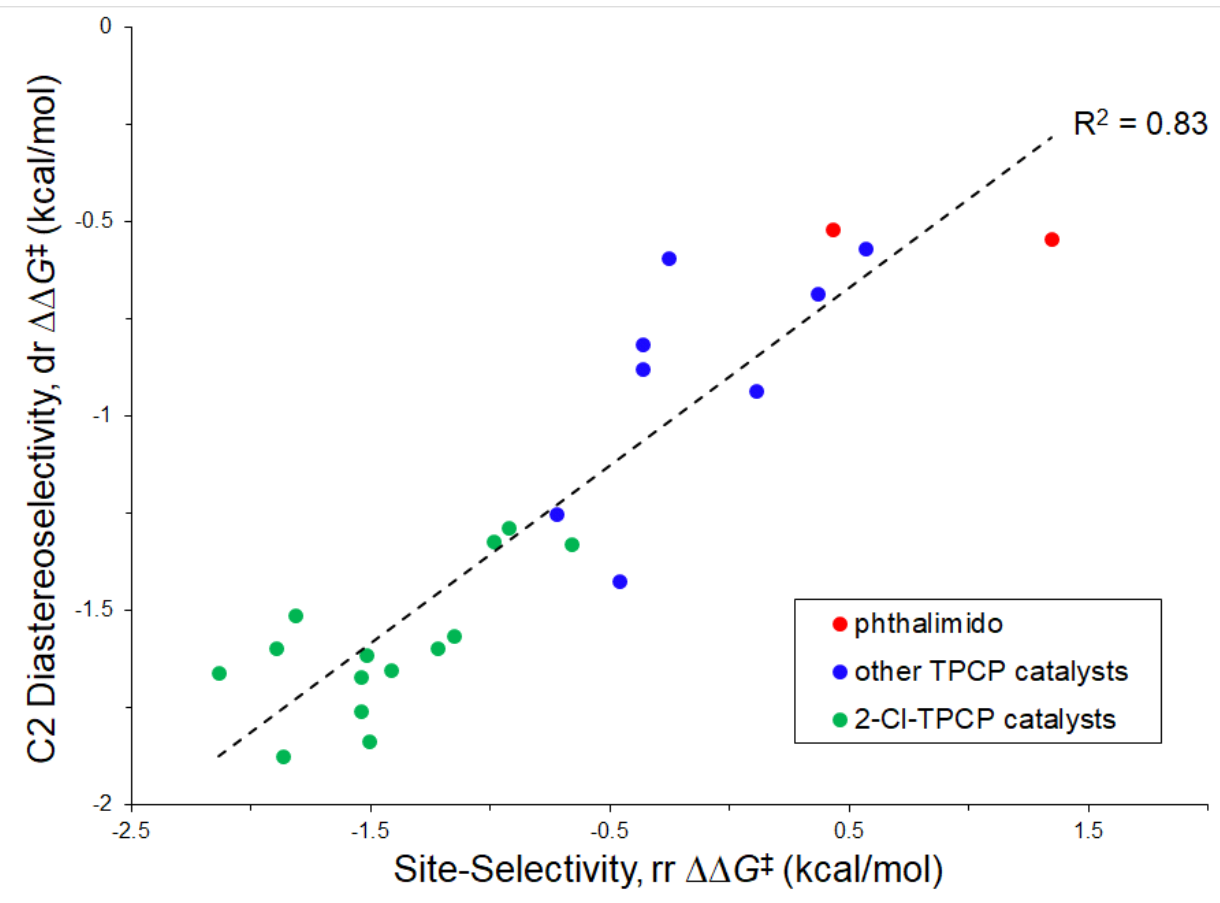

Figure S27. A strong correlation was observed between diastereoselectivity (dr) of the C2 functionalized product and $\mathrm{C} 2: \mathrm{Bn}$ site-selectivity (rr) when both are expressed as $\Delta \Delta G^{\ddagger}$ (in $\mathrm{kcal} / \mathrm{mol}$ ).

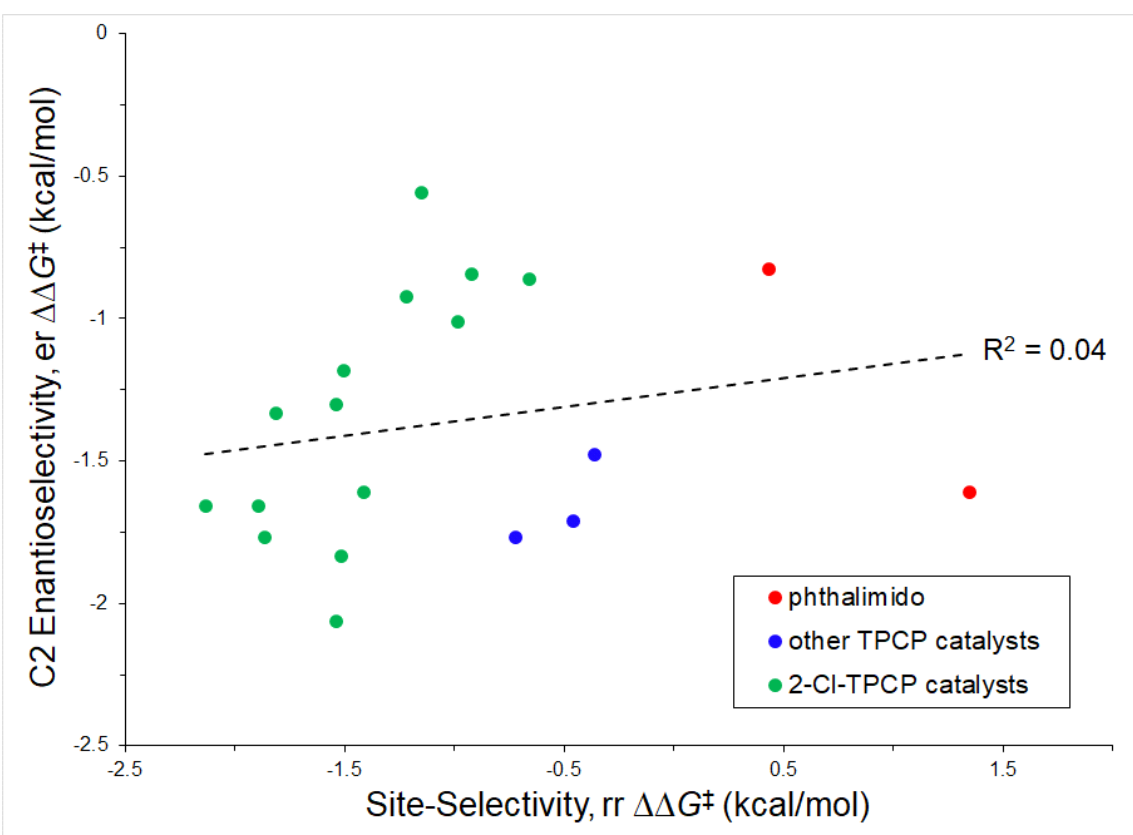

Figure S28. Little to no correlation was observed between enantioselectivity (er) of the C2 functionalized product and $\mathrm{C} 2: \mathrm{Bn}$ site-selectivity (rr) when both are expressed as $\Delta \Delta G^{\ddagger}$ (in $\mathrm{kcal} / \mathrm{mol}$ ). 


\subsection{Correlation Plots Between Relevant Catalyst Electronic Descriptor Inputs}

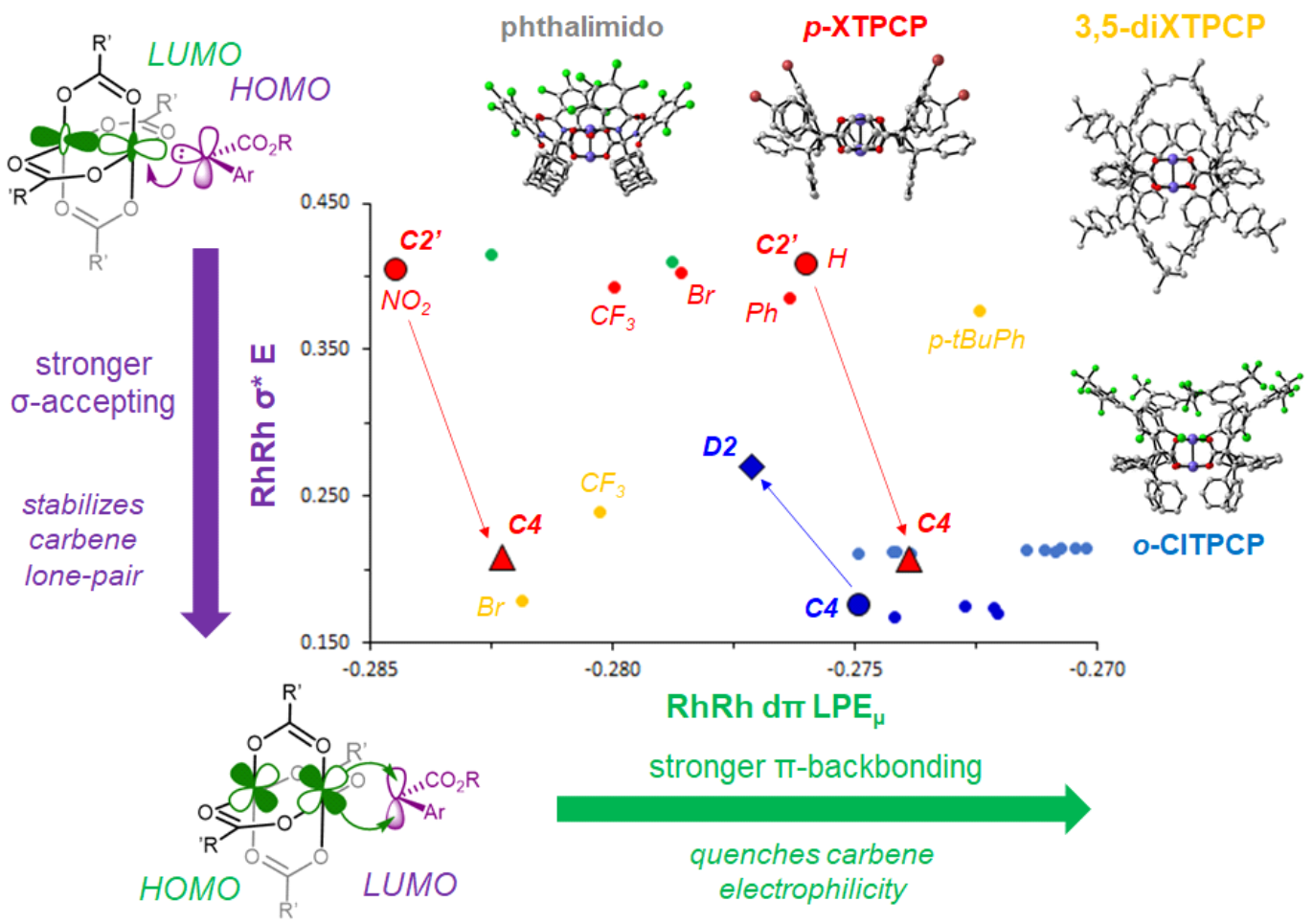

Figure S29. For a given ligand, the dirhodium catalyst position on the electronic properties map changes drastically depending on catalyst structural configuration. Perturbing $\mathrm{C}_{4}$ symmetric $(\alpha, \alpha, \alpha, \alpha)$ $\mathrm{Rh}_{2}(S \text {-o-CITPCP })_{4}$ catalysts to a $\mathrm{D}_{2}$ symmetric $(\alpha, \beta, \alpha, \beta)$ configuration with equivalent $\mathrm{Rh}$ faces increases the LUMO energy markedly, while more mild structural distortion of $\mathrm{Rh}_{2}(p \text {-XTPCP })_{4}$ from $\mathrm{C}_{2}{ }^{\prime}\left(\alpha, \alpha^{\prime}, \alpha, \alpha^{\prime}\right)$ to $\mathrm{C}_{4}(\alpha, \alpha, \alpha, \alpha)$ by breaking NCIs between neighboring ligands lowers the LUMO energy dramatically. These examples are strong evidence corroborating the proposed global structural effect on $\mathrm{Rh}-\mathrm{Rh}$ covalency and catalyst $\sigma$-accepting propensity, which collectively gives rise to significant changes in both dirhodium-carbene electrophilicity and $\mathrm{C}-\mathrm{H}$ functionalization site-selectivity. 


\subsection{Supplemental MLR Models and Decision Tree Classifications}

Table S13. MLR modeling results for explicit comparisons of SMART to other steric parameters

\begin{tabular}{|c|c|c|c|c|}
\hline $\begin{array}{l}\text { Model } \\
\text { ID }\end{array}$ & $\begin{array}{c}\text { Descriptors } \\
\text { Avg. Quality }\end{array}$ & $\begin{array}{l}\text { s1 coefficients, y-int. } \\
\text { s1 statistical metrics }\end{array}$ & $\begin{array}{l}\text { s2 coefficients, y-int. } \\
\text { s2 statistical metrics }\end{array}$ & $\begin{array}{l}\text { s3 coefficients, y-int. } \\
\text { s3 statistical metrics }\end{array}$ \\
\hline \multirow{2}{*}{$\mathbf{m A}$} & \multirow{2}{*}{$\begin{array}{c}\mathrm{x} 31, \mathrm{x} 112, \mathrm{x18} \\
0.88,0.81,0.79,0.75\end{array}$} & $0.57,0.39,0.15,-0.88$ & $0.55,0.38,0.20,-0.79$ & $0.60,0.35,0.22,-0.75$ \\
\hline & & $0.87,0.79,0.77,0.87$ & $0.89,0.81,0.80,0.77$ & $0.89,0.83,0.82,0.62$ \\
\hline \multirow{2}{*}{$\mathbf{m B}$} & \multirow{2}{*}{$\begin{array}{c}\mathrm{x} 24, \times 29, \times 72 \\
0.85,0.77,0.74,0.51\end{array}$} & $-0.50,0.34,0.19,-0.88$ & $-0.47,0.41,0.19,-0.79$ & $-0.46,0.46,0.17,-0.75$ \\
\hline & & $0.84,0.76,0.73,0.64$ & $0.84,0.77,0.74,0.56$ & $0.86,0.78,0.76,0.32$ \\
\hline \multirow{2}{*}{$\mathbf{m C}$} & \multirow{2}{*}{$\begin{array}{c}x 29, \times 24, x 79 \\
0.86,0.79,0.78,0.49\end{array}$} & $0.35,-0.40,0.23,-0.88$ & $0.41,-0.37,0.22,-0.79$ & $0.45,-0.36,0.25,-0.75$ \\
\hline & & $0.85,0.78,0.76,0.63$ & $0.85,0.78,0.76,0.57$ & $0.88,0.83,0.81,0.26$ \\
\hline \multirow{2}{*}{$\mathbf{m D}$} & \multirow{2}{*}{$\begin{array}{c}\mathrm{L}^{\mathrm{a}}, \mathrm{L} 2^{\mathrm{a}}, \mathrm{x} 3 \\
0.77,0.58,0.54,0.54\end{array}$} & $-0.55,-0.38,-0.19,-0.88$ & $-0.51,-0.51,-0.29,-0.79$ & $-0.44,-0.44,-0.31,-0.75$ \\
\hline & & $0.80,0.61,0.58,0.42$ & $0.77,0.62,0.56,0.43$ & $0.74,0.52,0.48,0.76$ \\
\hline \multirow{2}{*}{$\mathbf{m E}$} & \multirow{2}{*}{$\begin{array}{c}\mathrm{L}^{\mathrm{a}}, \mathrm{L}^{\mathrm{a}}, \mathrm{x} 3 \\
0.83,0.74,0.72,0.41\end{array}$} & $-0.57,0.53,-0.22,-0.88$ & $-0.62,0.40,-0.27,-0.79$ & $-0.63,0.51,-0.31,-0.75$ \\
\hline & & $0.83,0.73,0.70,0.50$ & $0.83,0.74,0.72,0.50$ & $0.84,0.76,0.74,0.22$ \\
\hline \multirow{2}{*}{$\mathbf{m F}$} & \multirow{2}{*}{$\begin{array}{c}\mathrm{x} 31, \mathrm{x} 3, \mathrm{x} 102 \\
0.74,0.63,0.60,0.71 \\
\end{array}$} & $0.68,-0.28,0.21,-0.88$ & $0.70,-0.29,0.20,-0.79$ & $0.73,-0.31,0.19,-0.75$ \\
\hline & & $0.71,0.59,0.55,0.85$ & $0.73,0.62,0.58,0.81$ & $0.78,0.68,0.66,0.46$ \\
\hline \multirow{2}{*}{ mG } & \multirow{2}{*}{$\begin{array}{c}\mathrm{x} 31, \mathrm{x} 104, \mathrm{x} 6 \\
0.83,0.73,0.68,0.61\end{array}$} & $0.55,0.34,-0.20,-0.88$ & $0.57,0.32,-0.21,-0.79$ & $0.66,0.28,-0.21,-0.75$ \\
\hline & & $0.82,0.72,0.66,0.69$ & $0.82,0.72,0.67,0.66$ & $0.84,0.75,0.70,0.48$ \\
\hline \multirow{2}{*}{$\mathbf{m H}$} & \multirow{2}{*}{$\begin{array}{c}x 31, x 104, x 3 \\
0.79,0.65,0.61,0.64\end{array}$} & $0.52,0.30,-0.11,-0.88$ & $0.54,0.28,-0.12,-0.79$ & $0.61,0.23,-0.16,-0.75$ \\
\hline & & $0.78,0.62,0.58,0.72$ & $0.78,0.64,0.59,0.71$ & $0.80,0.69,0.65,0.50$ \\
\hline \multirow{2}{*}{$\mathbf{m I}$} & \multirow{2}{*}{$\begin{array}{c}\mathrm{x31}, \mathrm{x6}, \mathrm{x} 102 \\
0.72,0.60,0.54,0.58\end{array}$} & $0.71,-0.18,0.04,-0.88$ & $0.72,-0.20,0.01,-0.79$ & $0.76,-0.20,-0.001,-0.75$ \\
\hline & & $0.69,0.56,0.49,0.78$ & $0.71,0.59,0.53,0.67$ & $0.76,0.65,0.60,0.30$ \\
\hline \multirow{2}{*}{$\mathbf{m J}$} & \multirow{2}{*}{$\begin{array}{c}x 31, x 99, x 6 \\
0.82,0.70,0.67,0.62\end{array}$} & $0.58,0.32,-0.16,-0.88$ & $0.63,0.29,-0.17,-0.79$ & $0.70,0.25,-0.18,-0.75$ \\
\hline & & $0.81,0.69,0.66,0.68$ & $0.81,0.69,0.66,0.69$ & $0.83,0.72,0.68,0.51$ \\
\hline \multirow{2}{*}{$\mathbf{m K}$} & \multirow{2}{*}{$\begin{array}{c}\mathrm{x} 31, \mathrm{x} 117, \mathrm{x6} \\
0.76,0.53,0.44,0.61\end{array}$} & $0.51,-0.25,-0.24,-0.88$ & $0.53,-0.25,-0.24,-0.79$ & $0.56,-0.28,-0.25,-0.75$ \\
\hline & & $0.73,0.47,0.35,0.81$ & $0.75,0.52,0.43,0.72$ & $0.80,0.60,0.53,0.30$ \\
\hline \multirow{2}{*}{$\mathbf{m L}$} & \multirow{2}{*}{$\begin{array}{c}\mathrm{x} 29, \times 24, \mathrm{x} 86 \\
0.86,0.79,0.77,0.36\end{array}$} & $0.43,-0.42,-0.20,-0.88$ & $0.50,-0.39,-0.20,-0.79$ & $0.53,-0.38,-0.21,-0.75$ \\
\hline & & $0.85,0.77,0.74,0.54$ & $0.86,0.79,0.77,0.42$ & $0.88,0.82,0.79,0.13$ \\
\hline \multirow{2}{*}{$\mathbf{m M}$} & \multirow{2}{*}{$\begin{array}{c}\mathrm{x} 31, \mathrm{x} 112, \mathrm{x} 6 \\
0.88,0.81,0.78,0.79\end{array}$} & $0.64,0.37,-0.13,-0.88$ & $0.67,0.37,-0.13,-0.79$ & $0.73,0.33,-0.15,-0.75$ \\
\hline & & $0.87,0.79,0.77,0.85$ & $0.88,0.81,0.79,0.80$ & $0.88,0.82,0.80,0.73$ \\
\hline \multirow{2}{*}{$\mathbf{m N}$} & \multirow{2}{*}{$\begin{array}{c}\mathrm{x} 31, \mathrm{x} 104, \mathrm{x} 18 \\
0.80,0.69,0.64,0.53\end{array}$} & $0.49,0.33,0.14,-0.88$ & $0.46,0.31,0.21,-0.79$ & $0.53,0.27,0.23,-0.75$ \\
\hline & & $0.79,0.65,0.60,0.71$ & $0.80,0.68,0.64,0.59$ & $0.82,0.73,0.69,0.29$ \\
\hline \multirow{2}{*}{ mO } & \multirow{2}{*}{$\begin{array}{c}\mathrm{x} 31, \mathrm{x} 99, \mathrm{x} 18 \\
0.81,0.70,0.67,0.53\end{array}$} & $0.51,0.34,0.14,-0.88$ & $0.50,0.31,0.21,-0.79$ & $0.56,0.27,0.24,-0.75$ \\
\hline & & $0.80,0.67,0.65,0.69$ & $0.81,0.69,0.67,0.59$ & $0.83,0.73,0.70,0.31$ \\
\hline
\end{tabular}

${ }^{a} \mathrm{~L} 1\left(\mathrm{HOMO}-\mathrm{LUMO} \Delta \mathrm{E}_{\text {lig }}\right.$ ) and L4 $\left(\mathrm{RCO}_{2}{ }^{-} \mathrm{V}_{\min }\right)$ are free ligand descriptors for electronics, while $\mathrm{L} 2\left(\mathrm{O}_{\mathrm{Coo}} \mathrm{NMR} \delta_{\Delta}\right)$ and L3 (IR $\mathrm{OCO}_{\text {ASYM }} v$ ) are ligand descriptors for electronic asymmetry. Free ligand modeling was initially performed with 55 descriptors based on NMR shifts, NBO charges, IR $v$, electrostatic potential minima $\left(\mathrm{V}_{\min }\right), \mathrm{HOMO} / \mathrm{LUMO}$ energies, and Sterimol values. Free ligand models (D-E) did not provide satisfactory statistical metrics and were more difficult to interpret chemically. 
$\Delta \Delta G^{\ddagger}=-0.81[7]+0.19[3]^{*}$ SMART N $N_{\text {PROBE, LE }}+$
$0.38[2]$ * SMART ESA $\Delta+0.57[3]^{*} \operatorname{RhRh~} \Delta \mathrm{E}_{\pi^{*} / \sigma^{*}}$

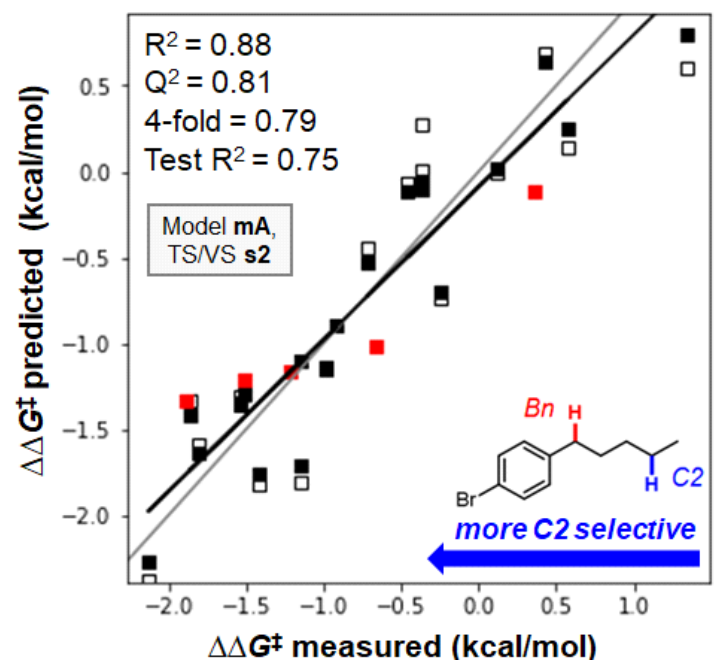

$\Delta \Delta \boldsymbol{G}^{\ddagger}=-0.81[7]+0.18[1] *$ SMART $\mathrm{V}_{\mathrm{CAVITY}, \mathrm{MAX}}$ $+0.40[6]^{*} \mathrm{RhRh} \sigma^{*} \mathrm{E}-0.48[2]^{*} \mathrm{RhRh} d \pi \mathrm{LPE}_{\mu}$

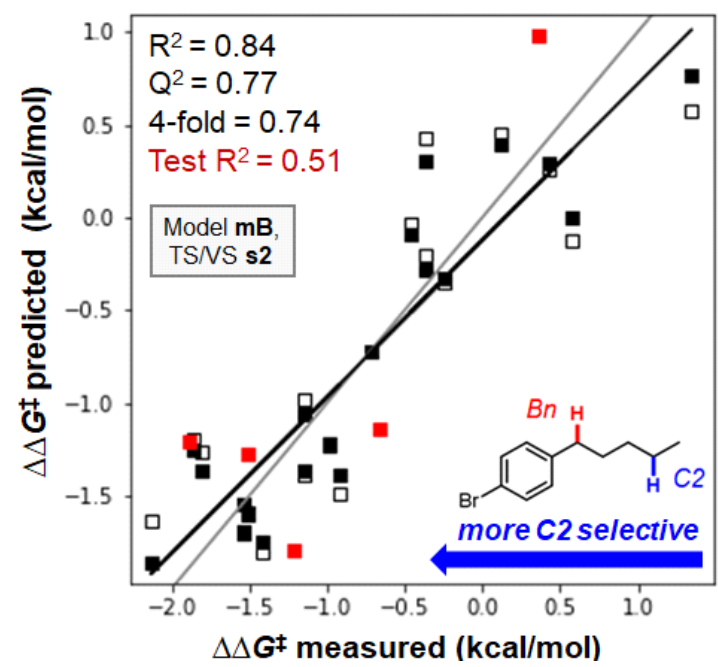

Figure S30. Alternative TS/VS split $\mathbf{s} 2$ model plots for main text $\mathbf{s} 1$ split models $\mathbf{m A}$ (Figure 10) and $\mathbf{m B}$ (Figure 7B). Note that statistical metrics listed are the average over s1-s3. The corresponding average descriptor coefficients (and y-intercept) are shown, along with standard deviations in the last digit [in brackets].

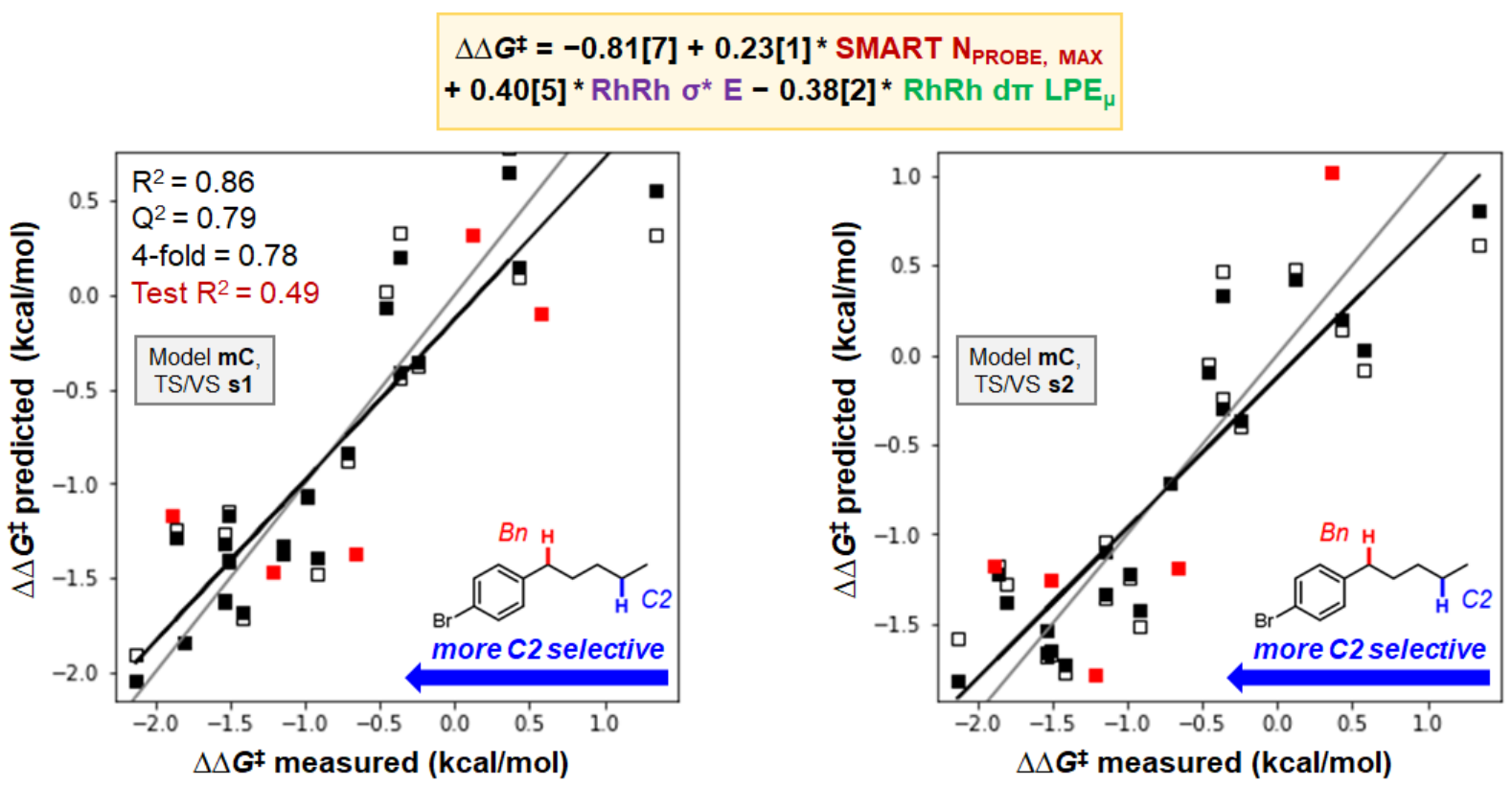

Figure S31. Alternative model $\mathbf{m C}$ for main text model $\mathbf{m B}$ (Figure 7B), where SMART V VAVITY, MAX has been replaced by SMART N NROBE, MAX. This shows that both of these SMART parameters are able to describe the reactive site, although SMART N pertaining to proximal steric hindrance (e.g., catalyst $\mathbf{H}$ ). Note that statistical metrics listed are the average over s1-s3. The corresponding average descriptor coefficients (and y-intercept) are shown, along with standard deviations in the last digit [in brackets]. 

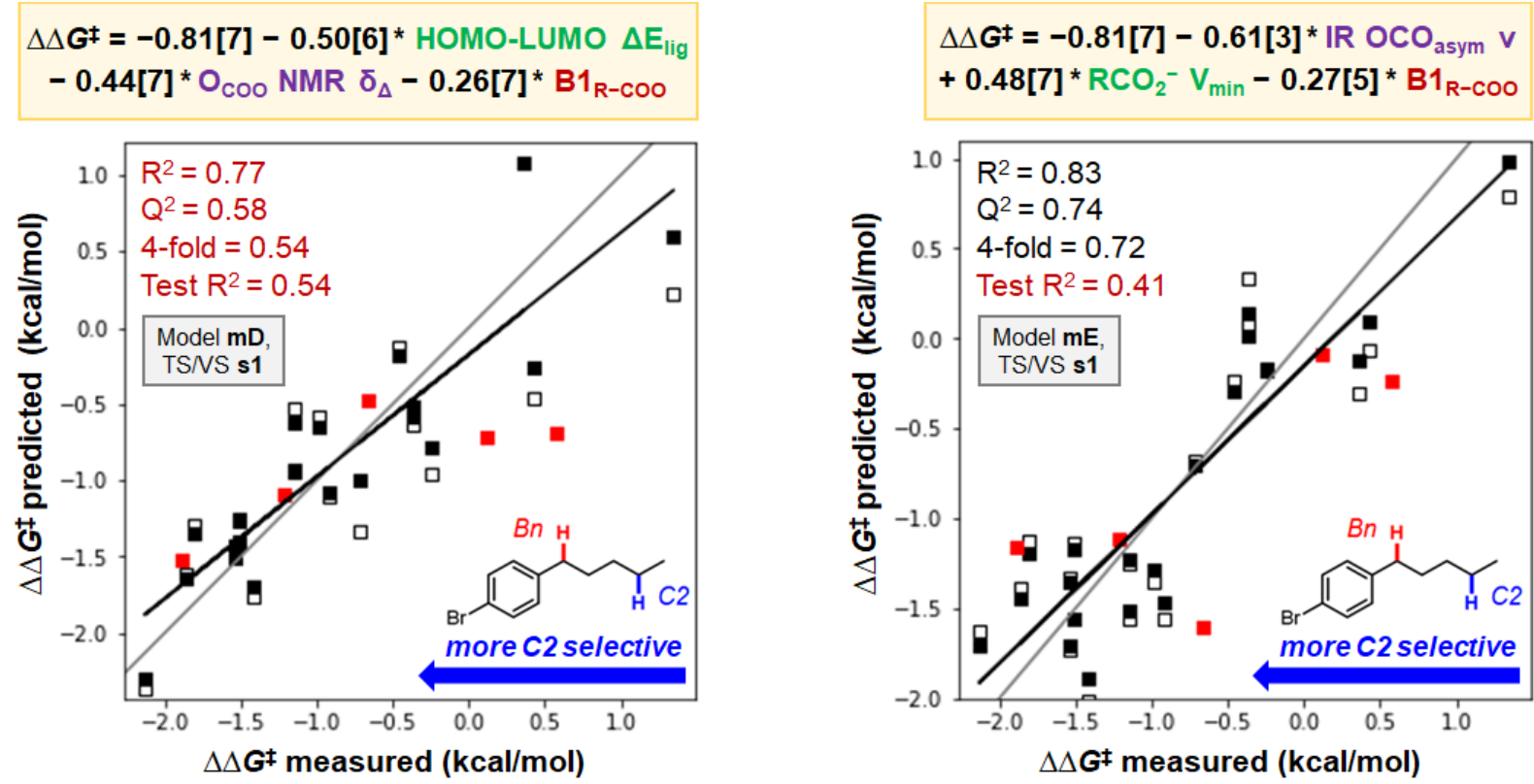

Figure S32. Models $\mathbf{m D}$ (left) and $\mathbf{m E}$ (right) based on free ligand descriptors. Model $\mathbf{m E}$ incorporates an IR frequency that is not as chemically interpretable (and may be an overfit of the training set data). The s1 TS/VS splits are pictured, but the statistical metrics listed are the average over s1-s3 (Table S13). The corresponding average descriptor coefficients (and y-intercept) are shown, along with standard deviations in the last digit [in brackets].
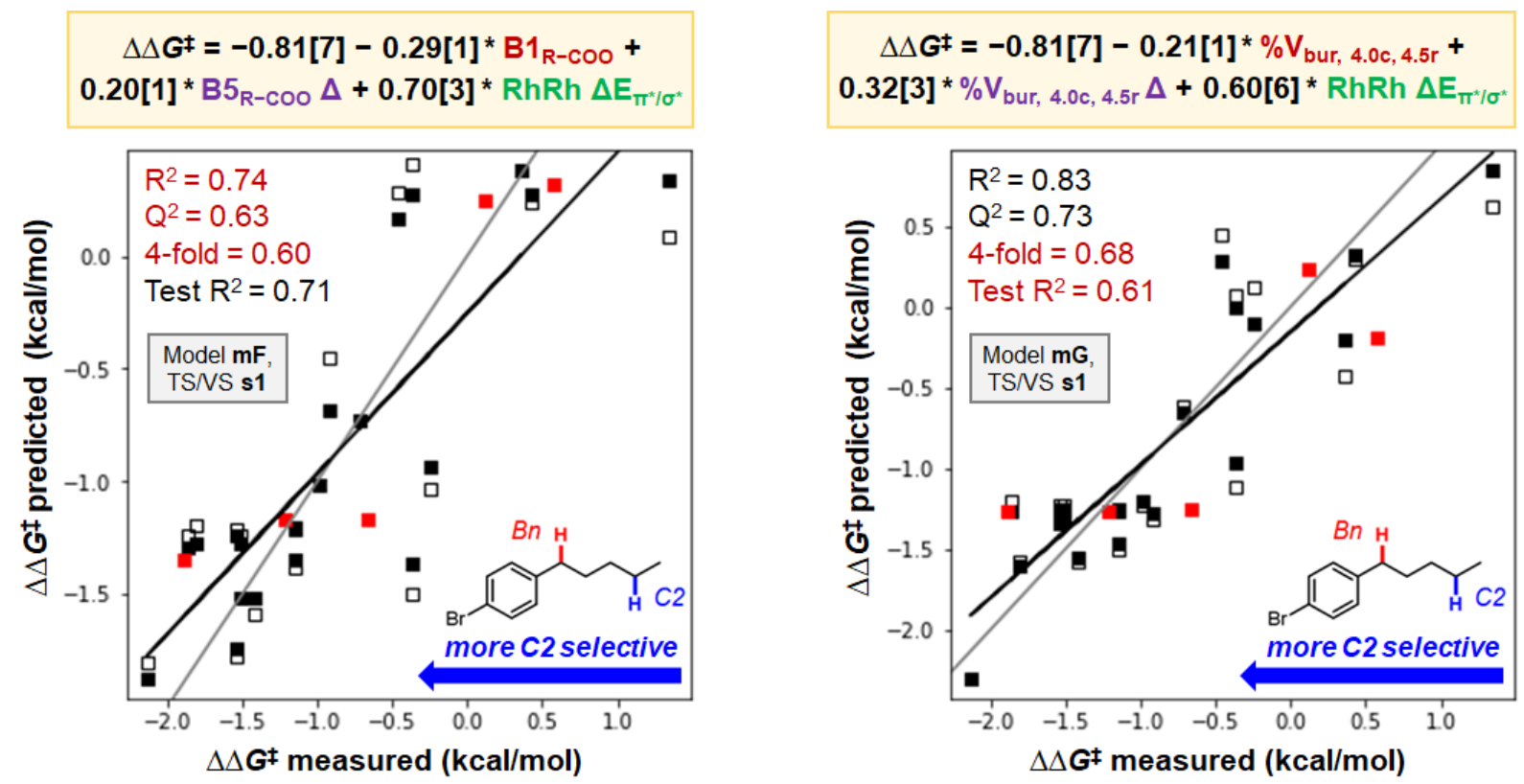

Figure S33. Models $\mathbf{m F}$ (left) and $\mathbf{m G}$ (right) based on the best steric and rigidity combinations of solely Sterimol and solely $\% \mathrm{~V}_{\text {bur }}$ descriptors, respectively. The s1 TS/VS splits are pictured, but the statistical metrics listed are the average over s1-s3 (Table S13). The corresponding average descriptor coefficients (and y-intercept) are shown, along with standard deviations in the last digit [in brackets]. 

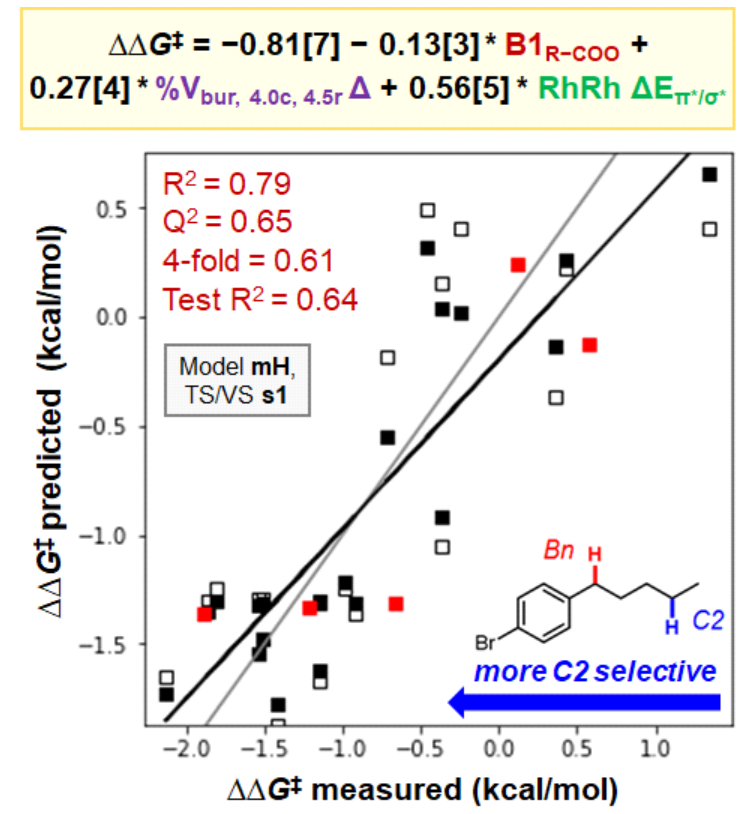

$\Delta \Delta \boldsymbol{G}^{\ddagger}=-0.81[7]-0.20[1]^{*} \% \mathrm{~V}_{\mathrm{bur}, 4.0 \mathrm{c}, 4.5 \mathrm{r}}+$ $0.02[2]^{*} B 5_{R-c o o} \Delta+0.73[3]^{*} \operatorname{RhRh} \Delta E_{\pi^{*} / \sigma^{*}}$

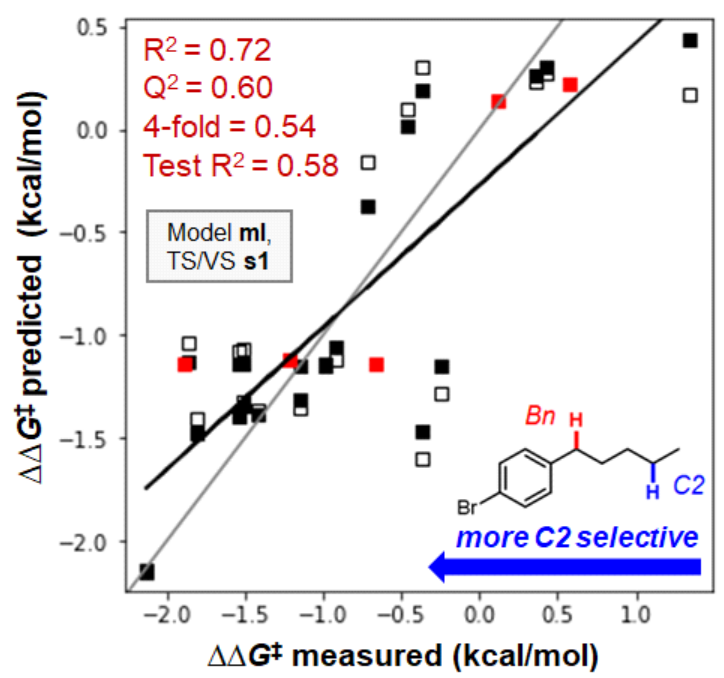

Figure S34. Models $\mathbf{~ m H}$ (left) and $\mathbf{m I}$ (right) based on the best steric and rigidity cross-combinations of Sterimol and $\% \mathrm{~V}_{\text {bur }}$ descriptors. The $\mathbf{s} 1 \mathrm{TS} / \mathrm{VS}$ splits are pictured, but the statistical metrics listed are the average over s1-s3 (Table S13). The corresponding average descriptor coefficients (and y-intercept) are shown, along with standard deviations in the last digit [in brackets].
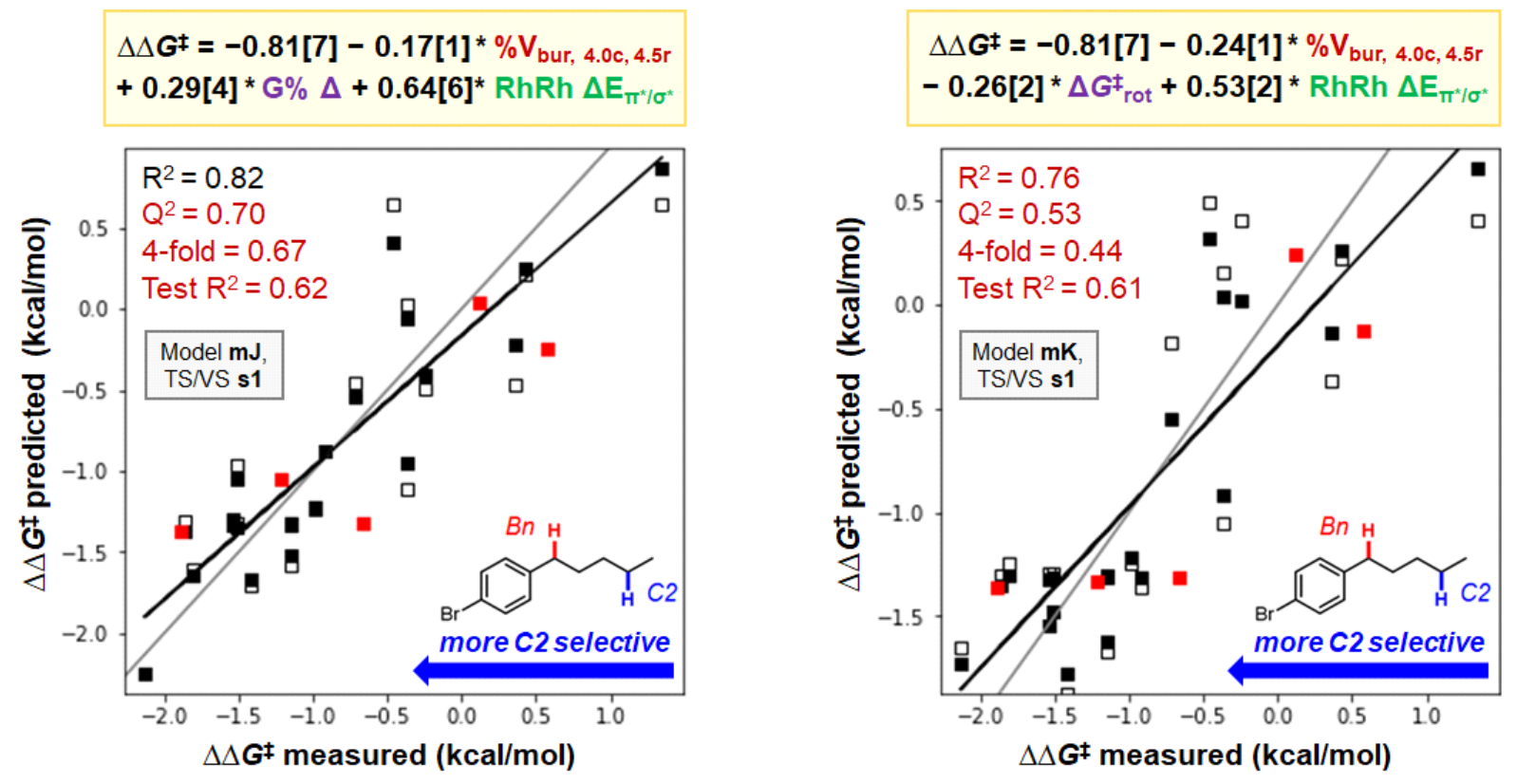

Figure S35. Models $\mathbf{m J}$ (left) and $\mathbf{m K}$ (right) based on the best combination $\% \mathrm{~V}_{\text {bur }}$ (sterics) and peripheral rigidity descriptors $\left(\mathrm{G} \% \Delta\right.$ or $\Delta \mathrm{G}^{\ddagger}$ rot). The s1 TS/VS splits are pictured, but the statistical metrics listed are the average over s1-s3 (Table S13). The corresponding average descriptor coefficients (and y-intercept) are shown, along with standard deviations in the last digit [in brackets]. 
$\Delta \Delta \boldsymbol{G}^{\ddagger}=-0.81[7]-0.20[1] * \% \mathrm{~V}_{\text {bur, } 4.0 \mathrm{c}, 4.5 \mathrm{r}, \mathrm{MIN}}+$

$0.48[5]{ }^{*}$ RhRh $\sigma^{*} E-0.39[2]$ * RhRh dT LPE $\mu$

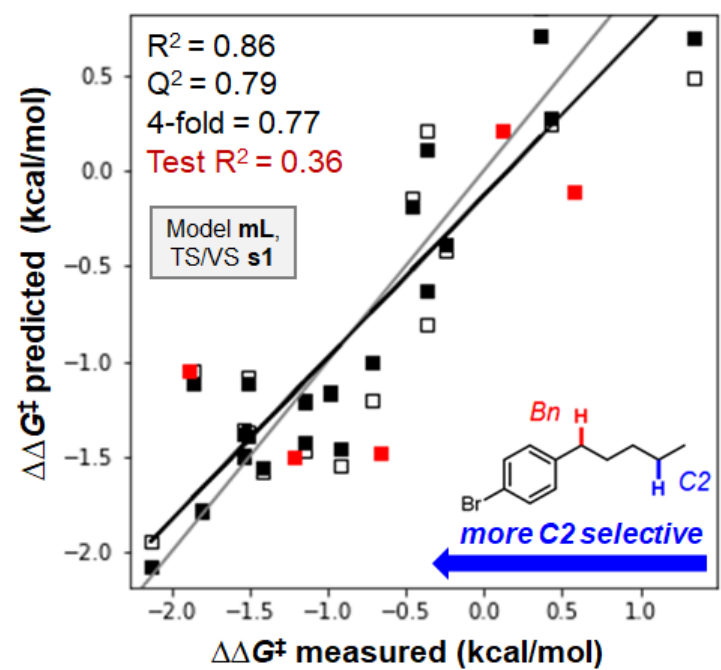

$\Delta \Delta \mathbf{G}^{\ddagger}=-0.81[7]-0.14[1]^{*} \% \mathrm{~V}_{\mathrm{bur}, 4.0 \mathrm{c}, 4.5 \mathrm{r}}+$ $0.36[2]^{*}$ SMART ESA $\Delta+0.68[5]^{*} \operatorname{RhRh} \Delta \mathrm{E}_{\pi^{*} / \sigma}$

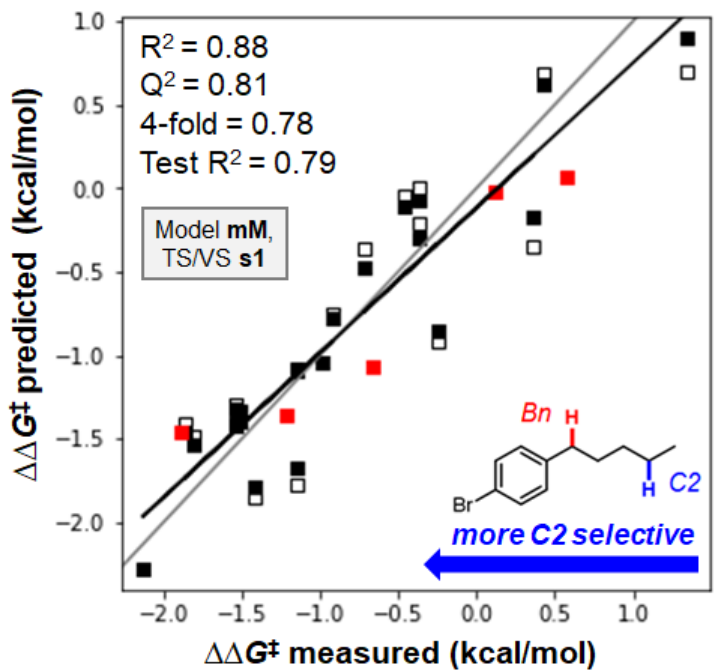

Figure S36. Models $\mathbf{~ m L}$ (left) and $\mathbf{m M}$ (right), where SMART steric descriptors are replaced with the best $\% \mathrm{~V}_{\text {bur }}$ parameters in models $\mathbf{m B}$ and $\mathbf{m A}$, respectively. Model $\mathbf{m L}$ shows a consistent drop in test $\mathrm{R}^{2}$ compared with model $\mathbf{m B}$, which arises from the inability of $\% \mathrm{~V}_{\text {bur }}$ to differentiate among $\mathrm{Rh}_{2}(o-$ CITPCP) 4 catalysts with little proximal hindrance (bottom left cluster). $\mathbf{m M}$ is the only model on par with model $\mathbf{m A}$, which illustrates the potential complementary utility of $\% \mathrm{~V}_{\text {bur }}$ for proximal sterics and SMART ESA $\Delta$ for peripheral reactive site rigidity. The s1 TS/VS splits are pictured, but the statistical metrics listed are the average over s1-s3 (Table S13). The corresponding average descriptor coefficients (and y-intercept) are shown, along with standard deviations in the last digit [in brackets].
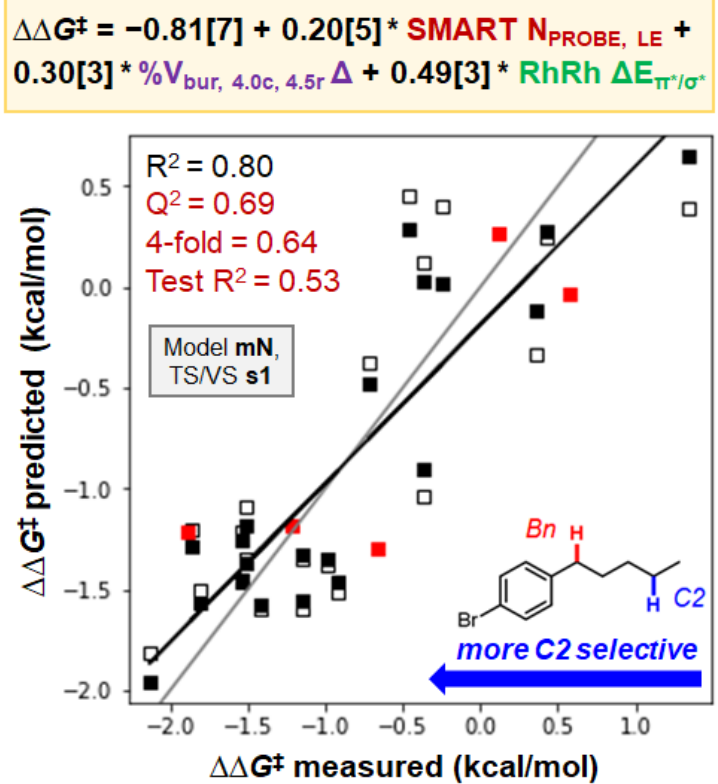

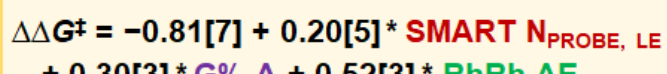

$+0.30[3]^{*} \mathrm{G} \% \Delta+0.52[3]^{*} \mathrm{RhRh} \Delta \mathrm{E}_{\pi^{*} / \sigma^{*}}$

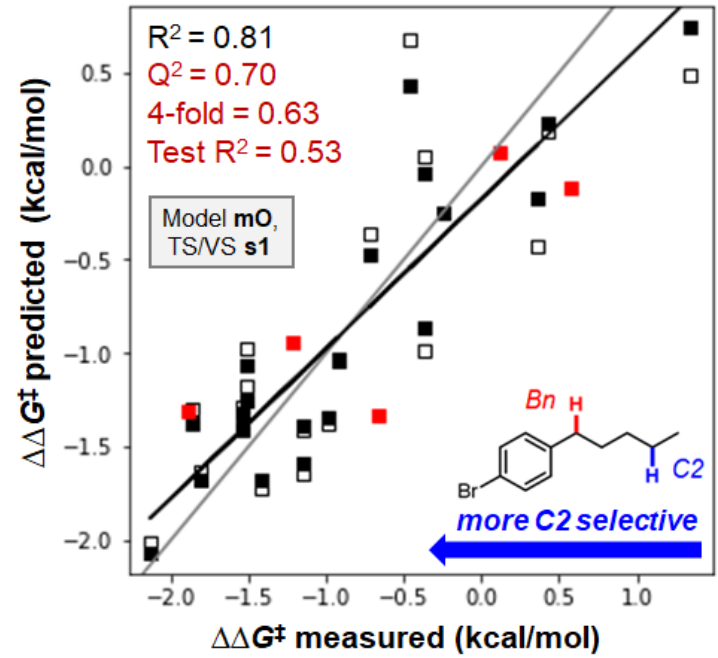

Figure S37. Models $\mathbf{m N}$ (left) and $\mathbf{m O}$ (right), where SMART ESA $\Delta$ in $\mathbf{m A}$ is replaced with the best alternative rigidity descriptors $\left(\% \mathrm{~V}_{\text {bur }} \Delta\right.$ or $\left.\mathrm{G} \% \Delta\right)$. The resulting statistical metrics are significantly worse, showcasing the uniqueness of SMART ESA $\Delta$. The s1 TS/VS splits are pictured, but the statistical metrics listed are the average over s1-s3 (Table S13). The corresponding average descriptor coefficients (and y-intercept) are shown, along with standard deviations in the last digit [in brackets]. 


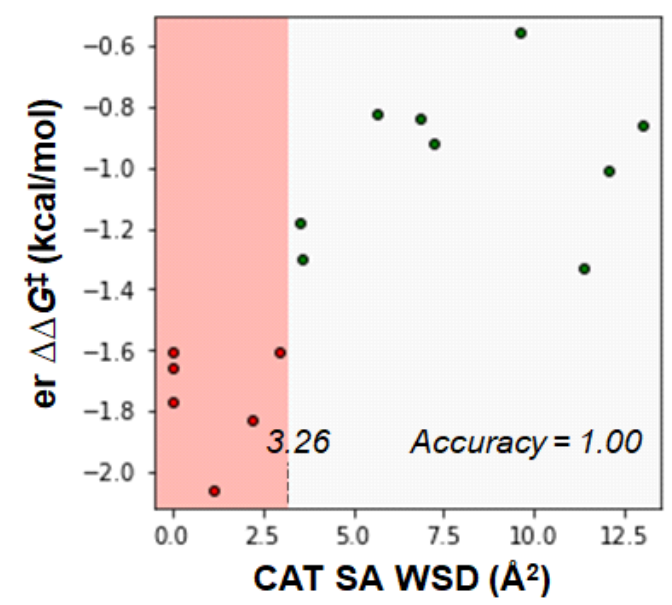

Figure S38. Single node decision tree for the $15 \mathrm{Rh}_{2}(S-o-C l T P C P)_{4}$ catalysts, which classifies catalysts that mediate highly enantioselective reactions (er $\Delta \Delta G^{\ddagger}<-1.5 \mathrm{kcal} / \mathrm{mol}$ ). CAT SA WSD is the Boltzmann-weighted standard deviation of molecular surface area across reactive catalyst conformers (x40 in descriptor spreadsheet). Among catalysts with similar steric and electronic properties, this shows that rigid catalysts with more consistently defined reactive site cavities facilitate more enantioselective $\mathrm{C}-\mathrm{H}$ functionalization reactions.

\subsection{Understanding Complementarity of SMART Descriptors Using Correlation Analysis}
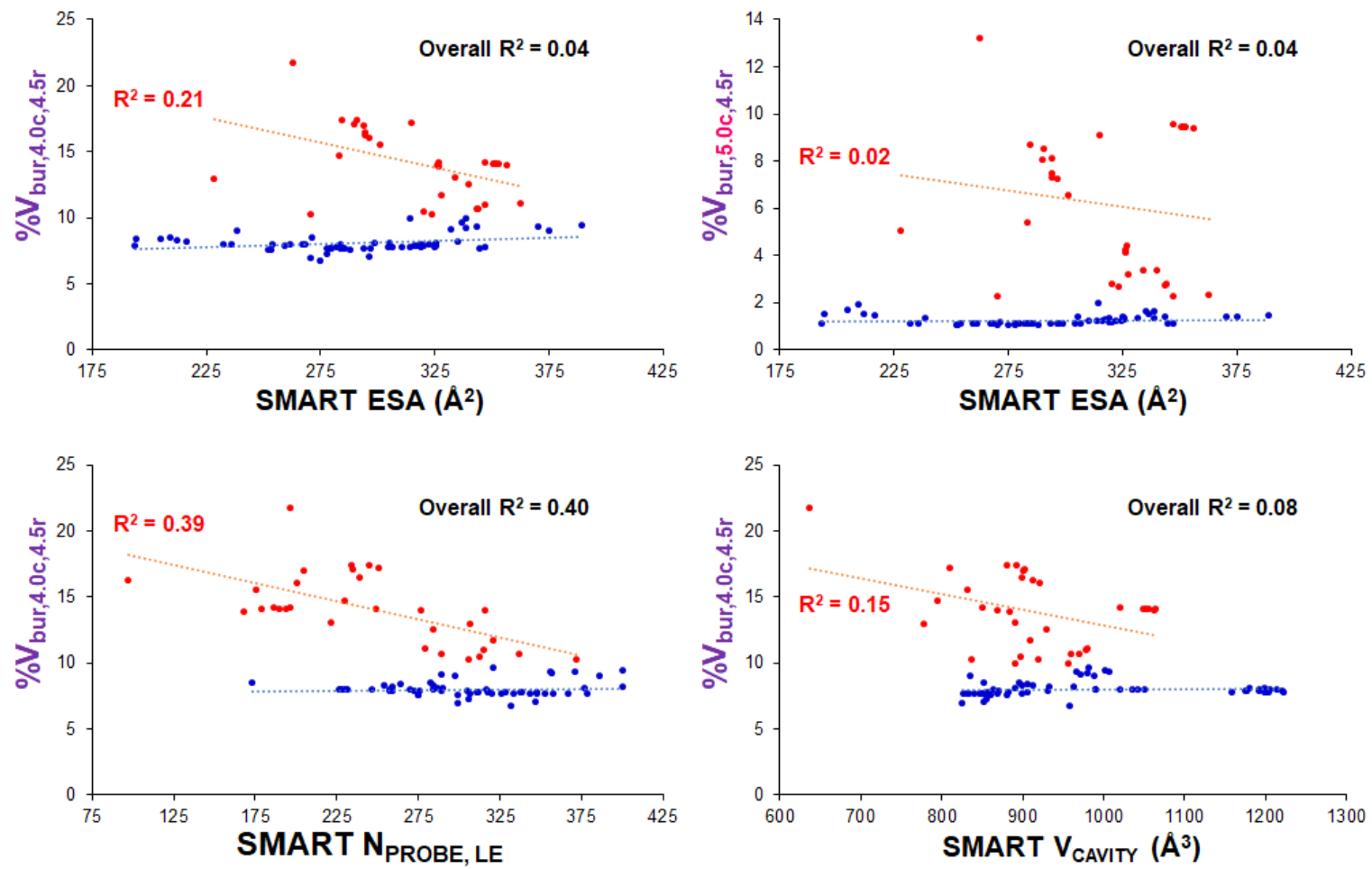

Figure S39. Correlations between $\% \mathrm{~V}_{\text {bur }}$ and SMART descriptors for all reactive catalyst conformers. Notably, SMART can differentiate, on the basis of peripheral spatial constraints, between reactive sites with minimal proximal congestion and similarly low (or identical) $\% \mathrm{~V}_{\text {bur }}(\sim 8-11 \%$; blue points). Even when removing these unhindered catalysts, SMART and $\% \mathrm{~V}_{\text {bur }}$ are not highly correlated (i.e., capture different fundamental steric properties). 

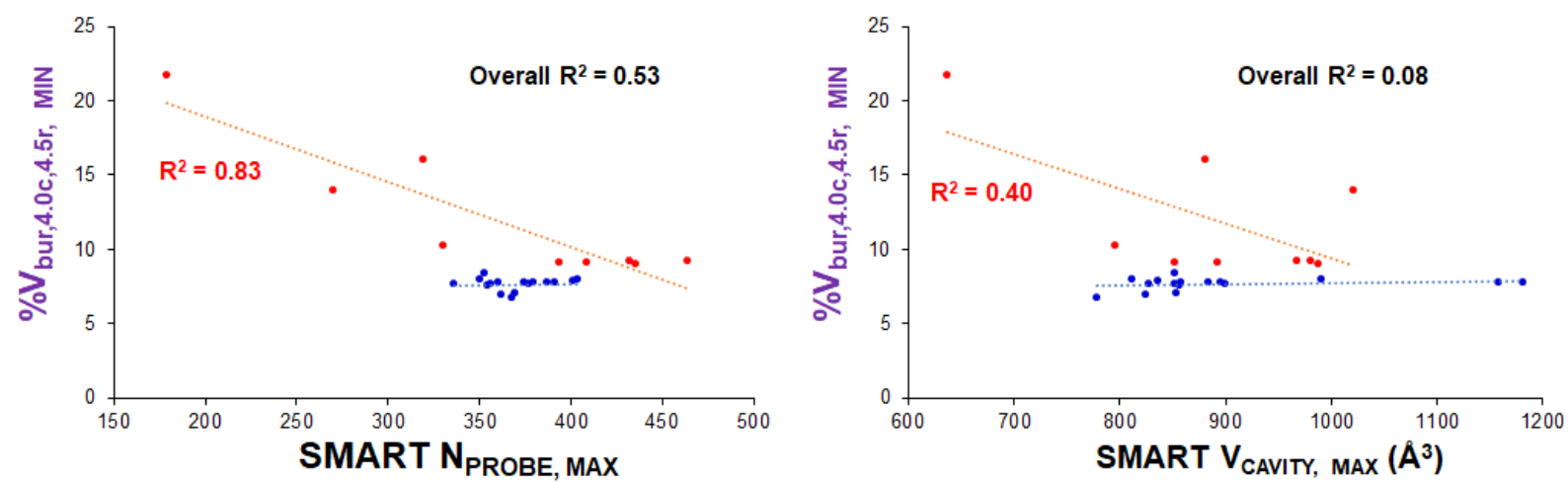

Figure S40. Plot showing the relationship between \% Vbur $_{M I N}$ and SMART $_{\text {MAX }}$ descriptors. Note that \% Vbur MIN $_{\text {and SMART }}$ MAX parameters both represent the steric hindrance for the least congested reactive conformer that can be adopted. Similarly to Boltzmann-averaged $\% \mathrm{~V}_{\text {bur }}$ comparisons in Figure S39, \% Vbur $_{\text {MIN }}$ is unable to differentiate catalysts with low proximal steric hindrance. This can be appreciated by looking at the horizontal lines of blue points, which have nearly identical \%Vbur MIN (7$9 \%$ ) but widely varying SMART $_{\mathrm{MAX}}$ descriptors. Low to moderate correlations are observed between \% Vbur MIN $_{\text {and SMART }}$ MAX descriptors, which do improve upon removing catalyst reactive sites with low proximal steric hindrance from consideration. Thus, SMART descriptors can be viewed as more descriptive than $\% \mathrm{~V}_{\text {bur }}$ over a wider, continuous-scale range of reactive site steric hindrance.
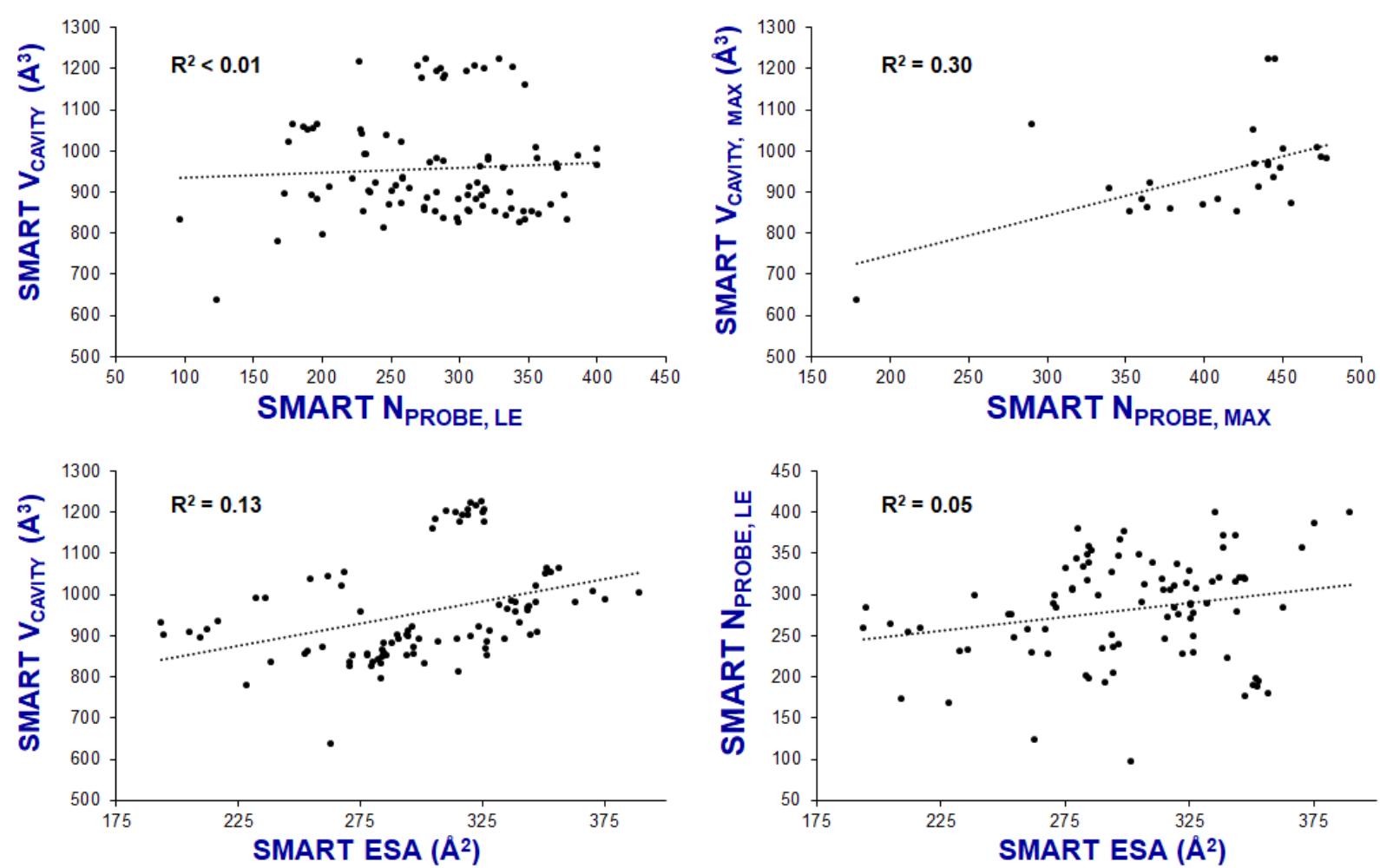

Figure S41. Low correlations between various SMART parameter types are shown. This illustrates the independent nature of the reactive site spatial properties captured by SMART V VAVITY, N NROBE, and ESA

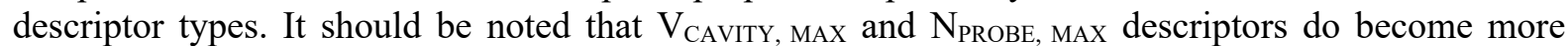
correlated save for a few outlier catalysts, which explains why replacing $\mathrm{V}_{\text {CAVITY, MAX }}$ with $\mathrm{N}_{\text {PROBE, MAX }}$ does result in similar MLR models (c.f., $\mathbf{m C}$ to $\mathbf{m B}$ in Figures S30-S31). 


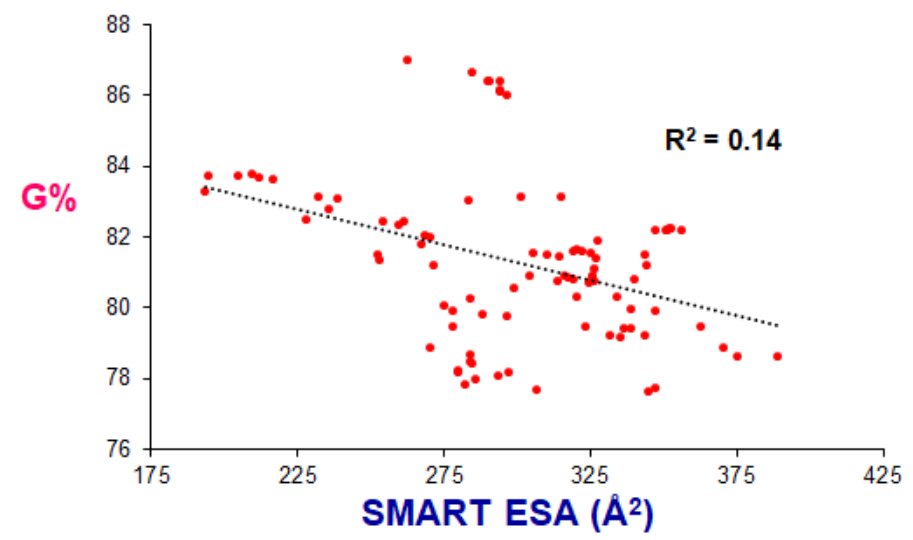

Figure S42. Plot showing the low correlation between SMART ESA and G\%. Indeed, SMART ESA is a distinctly different peripheral steric descriptor than $\mathrm{G} \%$, as we have designed it to capture absolute reactive site accessibility rather than relative $\%$ access. While $\mathrm{G} \%$ also captures accessibility, a larger catalyst requiring a surrounding sphere with a larger radius $(r)$ can have a larger absolute shadow projection area $(A)$ but still have an equivalent $\mathrm{G} \%$ to a smaller catalyst $\left(G \%=25 \pi \cdot \frac{A}{r^{2}}\right)$.
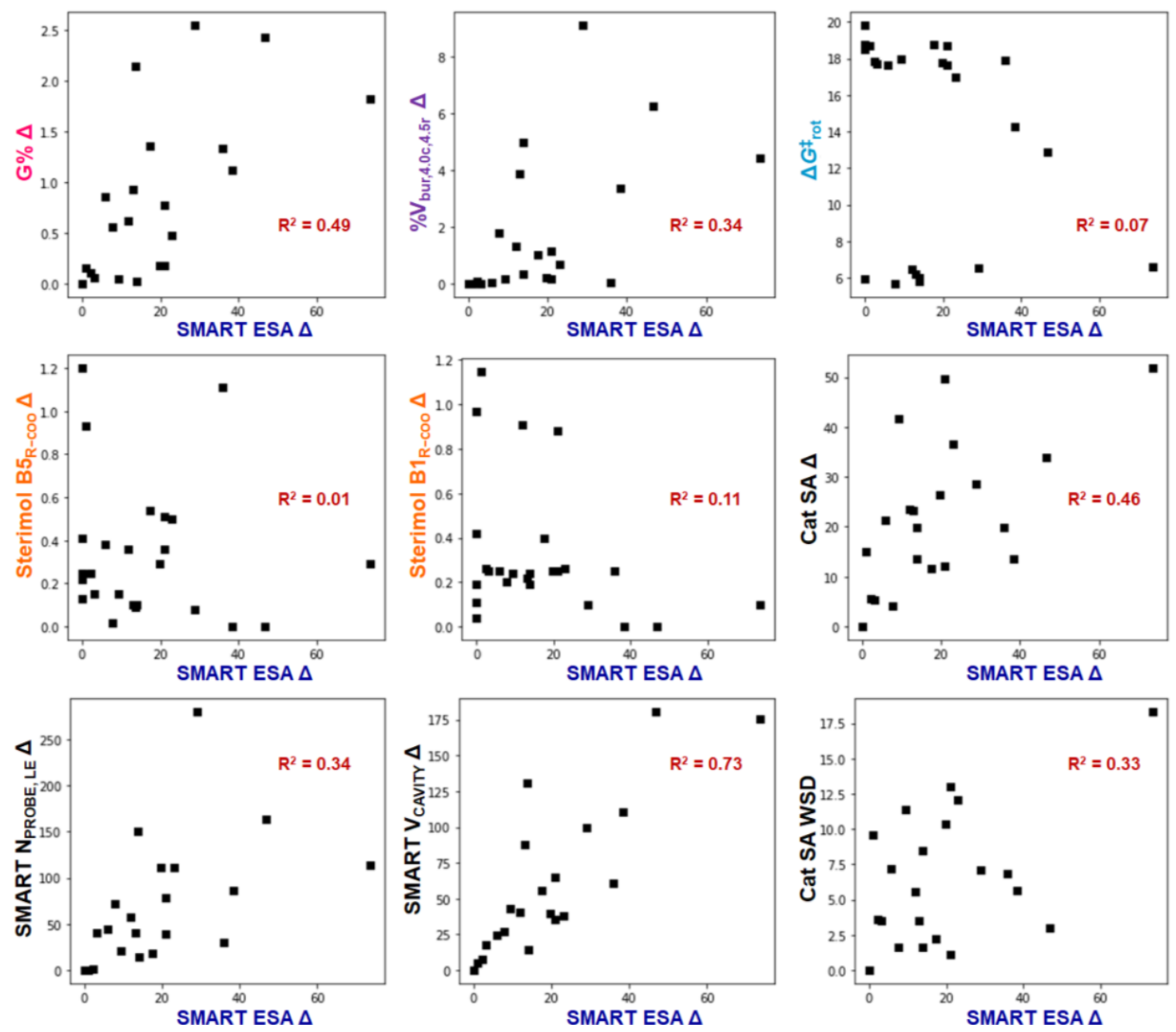

Figure S43. Univariate correlations of SMART ESA $\Delta$ with other descriptors for reactive site rigidity. No traditional descriptor is comparable, with the closest being $\mathrm{G} \% \Delta\left(\mathrm{R}^{2}=0.49\right)$. SMART $\mathrm{V}_{\text {CAVITY }} \Delta$ is among the most similar parameter types to ESA $\Delta$ within the SMART descriptor set $\left(\mathrm{R}^{2}=0.73\right)$. 


\section{References}

1. Guptill, D. M.; Davies, H. M. L. J. Am. Chem. Soc. 2014, 136, 17718.

2. Fu, L.; Hoang, K.; Tortoreto, C.; Liu, W.; Davies, H. M. L. Org. Lett. 2018, 20, 2399.

3. Qin, C; Boyarskikh, V.; Hansen,J. H.; Hardcastle, K. I.; Musaev, D. G.; Davies, H. M. L. J. Am. Chem. Soc. 2011, 133, 19198.

4. Liao, K.; Negretti, S; Musaev, D. G.; Bacsa, J.; Davies, H. M. L. Nature, 2016, 533, 230.

5. Qin, C.; Davies, H. M. L. J. Am. Chem. Soc. 2014, 136, 9792.

6. Reddy, R. P., Lee, G. H. and Davies, H. M. L., Org. Lett. 2006, 8, 3437.

7. Reddy, R. P. and Davies, H. M. L., Org. Lett. 2006, 8, 5013.

8. Liao, K.; Liu, W.; Niemeyer, Z. L.; Ren, Z.; Bacsa, J.; Musaev, D. G.; Davies, H. M. L. ACS Catal. 2018, 8, 678.

9. Hatridge, T. A.; Liu, W.; Yoo, C.-J.; Davies, H. M. L.; Jones, C. W. Angew. Chem. Int. Ed. 2020, 132, 19693.

10. MacroModel, version 11.8, Schrödinger, LLC: New York, NY 2017.

11. Harder, E.; Damm, W.; Maple, J.; Wu, C.; Reboul, M.; Xiang, J. Y.; Wang, L.; Lupyan, D.; Dahlgren, M. K.; Knight, J. L.; Kaus, J. W.; Cerutti, D. S.; Krilov, G.; Jorgenson, W. L.; Abel, R.; Friesner, R. A. J. Chem. Theory Comput. 2016, 12 (1), 281-296.

12. Abramyan, M. T.; Snyder, J. A.; Thyparambil, A. A.; Stuart, J. S.; Latour, R. A. J. Comput. Chem. 2016, 37, 1973-1982.

13. Fraccalvieri, D.; Pandini, A.; Stella, F.; Bonati, L. BMC Bioinform. 2011, 12, 158.

14. Kelley, L. A.; Gardner, S. P.; Sutcliffe, M. J. Protein Eng. Des. Sel. 1996, 9 (11), 1063-1065.

15. Liao, K.; Yang, Y.-F.; Li, Y.; Sanders, J. N.; Houk, K. N.; Musaev, D. G.; Davies, H. M. L. Nat. Chem. 2018, 10 (10), 1048-1055.

16. X. M. J. Frisch, G. W. Trucks, H. B. Schlegel, G. E. Scuseria, M. A. Robb, J. R. Cheeseman, G. Scalmani, V. Barone, G. A. Petersson, H. Nakatsuji, X. Li, M. Caricato, A. V. Marenich, J. Bloino, B. G. Janesko, R. Gomperts, B. Mennucci, H. P. Hratchian, J. V. Ortiz, A. F. Izmaylov, J. L. Sonnenberg, D. Williams-Young, F. Ding, F. Lipparini, F. Egidi, J. Goings, B. Peng, A. Petrone, T. Henderson, D. Ranasinghe, V. G. Zakrzewski, J. Gao, N. Rega, G. Zheng, W. Liang, M. Hada, M. Ehara, K. Toyota, R. Fukuda, J. Hasegawa, M. Ishida, T. Nakajima, Y. Honda, O. Kitao, H. Nakai, T. Vreven, K. Throssell, J. A. Montgomery, Jr., J. E. Peralta, F. Ogliaro, M. J. Bearpark, J. J. Heyd, E. N. Brothers, K. N. Kudin, V. N. Staroverov, T. A. Keith, R. Kobayashi, J. Normand, K. Raghavachari, A. P. Rendell, J. C. Burant, S. S. Iyengar, J. Tomasi, M. Cossi, J. M. Millam, M. Klene, C. Adamo, R. Cammi, J. W. Ochterski, R. L. Martin, K. Morokuma, O. Farkas, J. B. Foresman, and D. J. Fox, Gaussian 16, Revision C.01, Gaussian, Inc.: Wallingford, CT, 2016.

17. Marenich, A. V.; Cramer, C. J.; Truhlar, D. G. J. Phys. Chem. B 2009, 113 (18), 6378-6396.

18. Grimme, S.; Ehrlich, S.; Goerigk, L. J. Comput. Chem. 2011, 32 (7), 1456-1465.

19. Zhao, Y.; Truhlar, D. G. Theor. Chem. Acc. 2008, 120 (1), 215-241.

20. Johnson, E. R.; Becke, A. D. J. Chem. Phys. 2006, 124 (17), 174104.

21. Weigend, F.; Ahlrichs, R. Phys. Chem. Chem. Phys. 2005, 7 (18), 3297-3305.

22. Johnson, E. R.; Becke, A. D. J. Chem. Phys. 2005, 123 (2), 024101. 
23. Becke, A. D.; Johnson, E. R. J. Chem. Phys. 2005, 123 (15), 154101.

24. Rassolov, V. A.; Pople, J. A.; Ratner, M. A.; Windus, T. L. J. Chem. Phys. 1998, 109 (4), 1223-1229.

25. Stephens, P. J.; Devlin, F. J.; Chabalowski, C. F.; Frisch, M. J. J. Phys. Chem. 1994, 98 (45), 11623-11627.

26. Becke, A. D. J. Chem. Phys 1993, 98 (7), 5648.

27. Rappé, A. K.; Casewit, C. J.; Colwell, K. S.; Goddard III, W. A.; Skiff, W. M. J. Am. Chem. Soc. 1992, 114 (25), 10024-10035.

28. Andrae, D.; Häußermann, U.; Dolg, M.; Stoll, H.; Preuß, H. Theor. Chim. Acta 1990, 77 (2), 123-141.

29. Lee, C.; Yang, W.; Parr, R. G. Phys. Rev. B 1988, 37 (2), 785.

30. Hay, P. J.; Wadt, W. R. J. Chem. Phys. 1985, 82 (1), 270-283.

31. Vosko, S. H.; Wilk, L.; Nusair, M. Can. J. Phys. 1980, 58 (8), 1200-1211.

32. Grimme, S.; Antony, J.; Ehrlich, S.; Krieg, H. J. Chem. Phys. 2010, 132 (15), 154104.

33. E. D. Glendening, J., K. Badenhoop, A. E. Reed, J. E. Carpenter, J. A. Bohmann, C. M. Morales, P. Karafiloglou, C. R. Landis, and F. Weinhold NBO 7.0, Theoretical Chemistry Institute, University of Wisconsin, Madison, 2018.

34. Pettersen, E. F.; Goddard, T. D.; Huang, C. C.; Meng, E. C.; Couch, G. S.; Croll, T. I.; Morris, J. H.; Ferrin, T. E. Protein Sci. 2021, 30 (1), 70-82.

35. Goddard, T. D.; Huang, C. C.; Meng, E. C.; Pettersen, E. F.; Couch, G. S.; Morris, J. H.; Ferrin, T. E. Protein Sci. 2018, 27 (1), 14-25.

36. Liu, W.; Ren, Z.; Bosse, A. T.; Liao, K.; Goldstein, E. L.; Bacsa, J.; Musaev, D. G.; Stoltz, B. M.; Davies, H. M. L. J. Am. Chem. Soc. 2018, 140 (38), 12247-12255.

37. Ren, Z.; Musaev, D. G.; Davies, H. M. L. ChemCatChem 2021, 13 (1), 174-179.

38. Davies, H. M. L.; Liao, K. Nat. Rev. Chem. 2019, 3 (6), 347-360.

39. Guzei, I. A.; Wendt, M. Dalton Trans. 2006, 128 (33), 3991-3999.

40. Lindsay, V. N. G.; Lin, W.; Charette, A. B. J. Am. Chem. Soc. 2009, 131 (45), 16383-16385.

41. DeAngelis, A.; Dmitrenko, O.; Yap, G. P. A.; Fox, J. M. J. Am. Chem. Soc. 2009, 131 (21), 7230-7231.

42. Adly, F. G.; Gardiner, M. G.; Ghanem, A. Chem. Eur. J. 2016, 22 (10), 3447-3461.

43. Lindsay, V. N. G.; Charette, A. B. ACS Catal. 2012, 2 (6), 1221-1225.

44. DeAngelis, A.; Boruta, D. T.; Lubin, J.-B.; Plampin III, J. N.; Yap, G. P. A.; Fox, J. M. Chem. Commun. 2010, 46 (25), 4541-4543.

45. Pritchard, B. P.; Altarawy, D.; Didier, B.; Gibson, T. D.; Windus, T. L. J. Chem. Inf. Model. 2019, 59 (11), 4814-4820.

46. Spitznagel, G. W.; Clark, T.; von Ragué Schleyer, P.; Hehre, W. J. J. Comput. Chem. 1987, 8 (8), 1109-1116.

47. Clark, T.; Chandrasekhar, J.; Spitznagel, G. W.; von Ragué Schleyer, P. J. Comput. Chem. 1983, 4 (3), 294-301.

48. Francl, M. M.; Pietro, W. J.; Hehre, W. J.; Binkley, J. S.; Gordon, M. S.; DeFrees, D. J.; Pople, J. A. J. Chem. Phys. 1982, 77 (7), 3654-3665.

49. McLean, A. D.; Chandler, G. S. J. Chem. Phys. 1980, 72 (10), 5639-5648. 
50. Krishnan, R.; Binkley, J. S.; Seeger, R.; Pople, J. A. J. Chem. Phys. 1980, 72 (1), 650-654.

51. Pettersen, E. F.; Goddard, T. D.; Huang, C. C.; Couch, G. S.; Greenblatt, D. M.; Meng, E. C.; Ferrin, T. E. J. Comput. Chem. 2004, 25 (13), 1605-1612.

52. Clavier, H.; Nolan, S. P. Chem. Commun. 2010, 46 (6), 841-861.

53. Falivene, L.; Credendino, R.; Poater, A.; Petta, A.; Serra, L.; Oliva, R.; Scarano, V.; Cavallo, L. Organometallics 2016, 35 (13), 2286-2293.

54. Wu, K.; Doyle, A. G. Nat. Chem. 2017, 9 (8), 779-784.

55. Kluyver, T.; Ragan-Kelley, B.; Pérez, F.; Granger, B. E.; Bussonnier, M.; Frederic, J.; Kelley, K.; Hamrick, J. B.; Grout, J.; Corlay, S.; Ivanov, P.; Avila, D.; Abdalla, S.; Willing, C., Jupyter Notebooks-a publishing format for reproducible computational workflows. 2016; Vol. 2016.

56. Pedregosa, F.; Varoquaux, G.; Gramfort, A.; Michel, V.; Thirion, B.; Grisel, O.; Blondel, M.; Prettenhofer, P.; Weiss, R.; Dubourg, V.; Vanderplas, J.; Passos, A.; Cournapeau, D.; Brucher, M.; Perrot, M.; Duchesnay, É. J. Mach. Learn. Res. 2011, 12, 2825-2830.

57. Anaconda Software Distribution, Anaconda Inc.: 2020.

58. Bilbrey, J. A.; Kazez, A. H.; Locklin, J.; Allen, W. D. J. Chem. Theory Comput. 2013, 9 (12), 5734-5744.

59. Santiago, C. B.; Guo, J.-Y.; Sigman, M. S. Chem. Sci. 2018, 9 (9), 2398-2412.

60. Sigman, M. S.; Harper, K. C.; Bess, E. N.; Milo, A. Acc. Chem. Res. 2016, 49 (6), 1292-1301.

61. Bendel, R. B.; Afifi, A. A. J. Am. Stat. Assoc. 1977, 72 (357), 46-53.

62. Bursac, Z.; Gauss, C. H.; Williams, D. K.; Hosmer, D. W. Source Code Biol. Med. 2008, 3 (1), 1-8.

63. Kennard, R. W.; Stone, L. A. Technometrics 1969, 11 (1), 137-148.

64. Marquandt, D. J. Am. Stat. Assoc. 1980, 75 (369), 87-91.

65. Wold, S. Quant. Struct.-Act. Relat., in Wiley Online Library. 1991, 10, 191-193.

66. Golbraikh, A.; Tropsha, A. J. Mol. Graph. Model. 2002, 20 (4), 269-276.

67. Gramatica, P. QSAR Comb. Sci. 2007, 26 (5), 694-701.

68. Wold, S.; Dunn III, W. J. J. Chem. Inform. Comput. Sci. 1983, 23 (1), 6-13.

69. Hawkins, D. M. J. Chem. Inform. Comput. Sci. 2004, 44 (1), 1-12.

70. Verloop, A., In Drug Design, Ariens, E. J., Ed. Academic Press: 1976; Vol. III, p 133.

71. Newman-Stonebraker, S. H.; Smith, S. R.; Borowski, J. E.; Gensch, T.; Peters, E. B.; Johnson, H. C.; Sigman, M. S.; Doyle, A. G. Science. 2021, 374 (6565), 301-308.

Note: Refer to SI section 13 specifically for additional supporting information references pertaining to X-ray crystallographic data and analysis. 


\section{Atomic Coordinates of Calculated Stationary Points}

\subsection{Dirhodium Catalyst Conformer Structures}

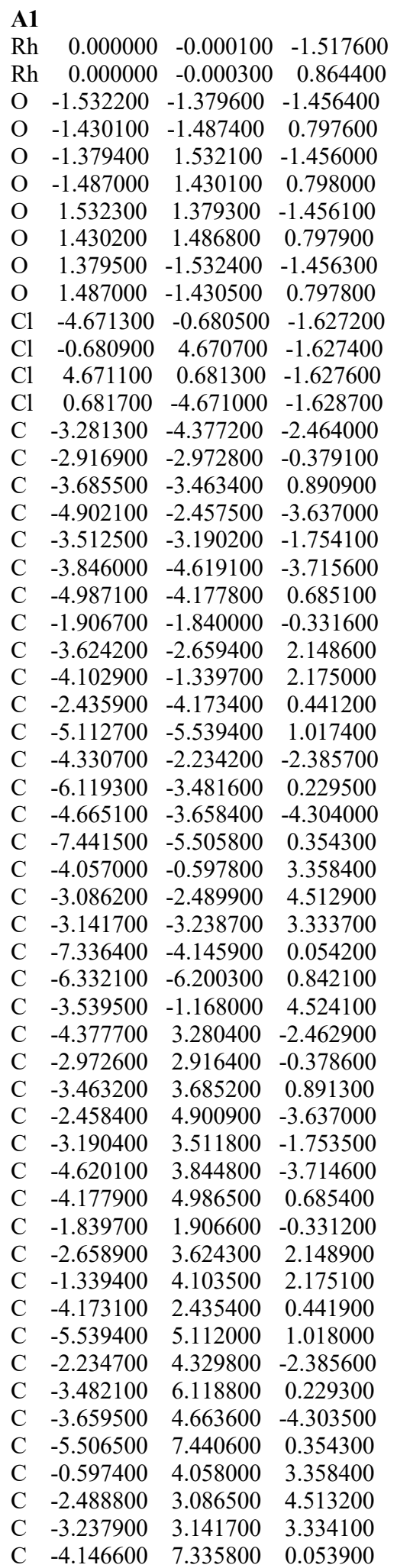

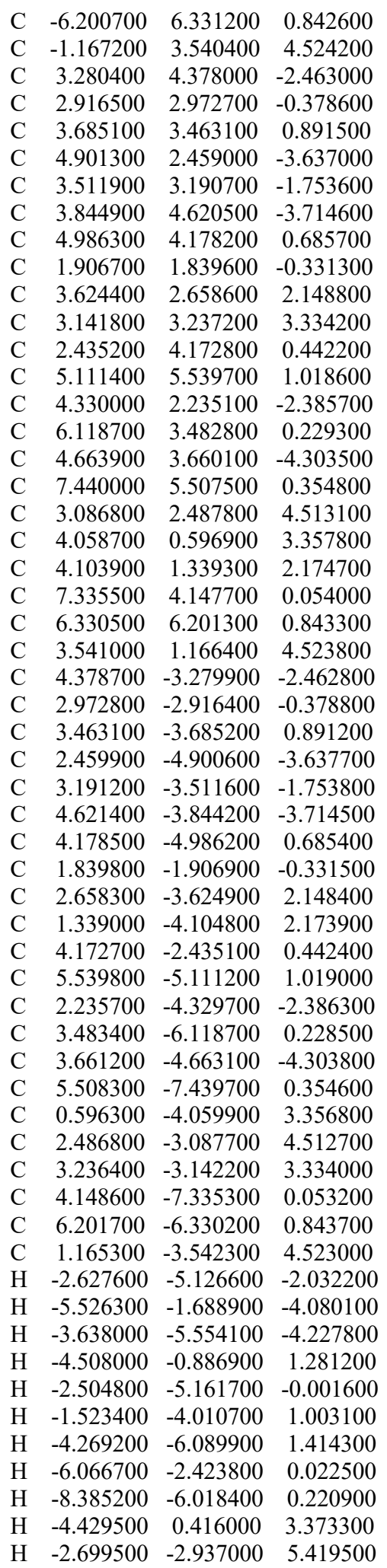

H $\quad-2.812700 \quad-4.269800 \quad 3.343200$

$\mathrm{H} \quad-8.201000 \quad-3.603600 \quad-0.306300$

$\mathrm{H} \quad-6.418300 \quad-7.250400 \quad 1.090000$

$\mathrm{H} \quad-3.501500 \quad-0.589900 \quad 5.438300$

$\mathrm{H} \quad-5.127000 \quad 2.626800 \quad-2.030500$

$\mathrm{H} \quad-1.689900 \quad 5.525000 \quad-4.080500$

$\mathrm{H} \quad-5.555200 \quad 3.636700 \quad-4.226400$

$\mathrm{H} \quad-0.886900 \quad 4.508700 \quad 1.281100$

$\mathrm{H} \quad-5.161300 \quad 2.504200 \quad-0.001000$

$\mathrm{H} \quad-4.010400 \quad 1.523100 \quad 1.004100$

$\mathrm{H} \quad-6.089600 \quad 4.268500 \quad 1.415300$

$\mathrm{H} \quad-2.424300 \quad 6.066300 \quad 0.022000$

H $\quad-6.019400 \quad 8.384200 \quad 0.220800$

$\mathrm{H} \quad 0.416300 \quad 4.431000 \quad 3.373100$

$\mathrm{H} \quad-2.935700 \quad 2.699800 \quad 5.419900$

$\mathrm{H} \quad-4.268900 \quad 2.812200 \quad 3.343800$

$\mathrm{H} \quad-3.604600 \quad 8.200300 \quad-0.307000$

$\mathrm{H} \quad-7.250600 \quad 6.417300 \quad 1.090800$

$\mathrm{H} \quad-0.589000 \quad 3.502800 \quad 5.438300$

$\mathrm{H} \quad 2.626600 \quad 5.127200 \quad-2.030800$

$\mathrm{H} \quad 5.525400 \quad 1.690500 \quad-4.080500$

H $\quad 3.636700 \quad 5.555600 \quad-4.226400$

$\mathrm{H} \quad 2.812000 \quad 4.268100 \quad 3.344200$

$\mathrm{H} \quad 2.503600 \quad 5.161300 \quad-0.000200$

$\mathrm{H} \quad 1.522900 \quad 4.009500 \quad 1.004200$

$\mathrm{H} \quad 4.267800 \quad 6.089700 \quad 1.416100$

$\mathrm{H} \quad 6.066400 \quad 2.425100 \quad 0.021700$

$\begin{array}{llll}\mathrm{H} & 8.383500 & 6.020600 & 0.221300\end{array}$

$\mathrm{H} \quad 2.700000 \quad 2.934400 \quad 5.419900$

$\mathrm{H} \quad 4.431900 \quad-0.416700 \quad 3.372300$

$\begin{array}{llll}\mathrm{H} & 4.509200 & 0.887000 & 1.280600\end{array}$

$\mathrm{H} \quad 8.200100 \quad 3.606000 \quad-0.307100$

$\begin{array}{llll}\mathrm{H} & 6.416300 & 7.251200 & 1.091800\end{array}$

$\mathrm{H} \quad 3.503600 \quad 0.587900 \quad 5.437800$

$\begin{array}{llll}\mathrm{H} & 5.127700 & -2.626300 & -2.030200\end{array}$

$\mathrm{H} \quad 1.691700 \quad-5.524700 \quad-4.081500$

H $\quad 5.556700 \quad-3.635900 \quad-4.226000$

$\mathrm{H} \quad 0.887100 \quad-4.510100 \quad 1.279600$

$\mathrm{H} \quad 5.161200 \quad-2.503400 \quad 0.000100$

$\mathrm{H} \quad 4.009200 \quad-1.523000 \quad 1.004400$

$\mathrm{H} \quad 6.089500 \quad-4.267600 \quad 1.416900$

H $\quad 2.425800 \quad-6.066500 \quad 0.020400$

H $\quad 6.021700 \quad-8.383000 \quad 0.221100$

$\mathrm{H} \quad-0.417100 \quad-4.433600 \quad 3.371000$

H $\quad 2.932900 \quad-2.700800 \quad 5.419700$

H $\quad 4.267200 \quad-2.812200 \quad 3.344300$

H $\quad 3.607200 \quad-8.199900 \quad-0.308400$

H $\quad 7.251500 \quad-6.415800 \quad 1.092600$

$\mathrm{H} \quad 0.586600 \quad-3.505100 \quad 5.436800$

$\mathrm{H} \quad 3.835300 \quad-5.111400 \quad-5.277900$

$\mathrm{H} \quad-3.833200 \quad 5.112200 \quad-5.277600$

H $\quad 5.112500 \quad 3.833900 \quad-5.277500$

$\mathrm{H} \quad-5.113700 \quad-3.831800 \quad-5.278000$

$\begin{array}{lrrr}\text { A2 } & & & \\ \text { Rh } & 0.000000 & 0.000000 & -1.511800 \\ \text { Rh } & 0.000000 & 0.000000 & 0.870500\end{array}$ 


\begin{tabular}{|c|c|c|c|}
\hline ) & 1.711900 & 1.151400 & 0.804700 \\
\hline$\Omega$ & 1.777600 & 1.044600 & -1.450800 \\
\hline O & -1.711800 & -1.151500 & 0.804700 \\
\hline $\mathrm{O}$ & -1.777600 & -1.044600 & -1.450800 \\
\hline $\mathrm{O}$ & 1.151500 & -1.711900 & 0.804700 \\
\hline $\mathrm{O}$ & 1.044600 & -1.777600 & -1.450900 \\
\hline $\mathrm{O}$ & -1.151400 & 1.711800 & 0.804700 \\
\hline ) & -1.044600 & 1.777600 & -1.450800 \\
\hline $\mathrm{Cl}$ & 4.700000 & -0.285300 & -1.648700 \\
\hline & -4.700000 & 0.285400 & -1.648400 \\
\hline & -0.285400 & -4.700000 & -1.648600 \\
\hline & 0.285300 & 4.700000 & -1.648600 \\
\hline & 2.244700 & 1.408300 & -0.325300 \\
\hline & 3.465500 & 2.309800 & -0.371500 \\
\hline & 4.086400 & 2.411300 & -1.748700 \\
\hline & 4.684900 & 1.311900 & -2.393100 \\
\hline & 5.283700 & 1.423200 & -3.646700 \\
\hline & 5.297700 & 2.653200 & -4.302700 \\
\hline & 4.699300 & 3.757800 & -3.701100 \\
\hline & 4.103200 & 3.626900 & -2.447500 \\
\hline & 3.244400 & 3.576600 & 0.460600 \\
\hline & 4.324200 & 2.622200 & 0.897200 \\
\hline & 4.106300 & 1.835900 & 2.148700 \\
\hline & 4.312100 & 0.447000 & 2.162600 \\
\hline & 4.123800 & -0.280500 & 3.340700 \\
\hline $\mathrm{C}$ & 3.734300 & 0.371500 & 4.51 \\
\hline & 3.553300 & 1.757100 & 4.514300 \\
\hline 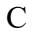 & 3.753000 & 2.489800 & 3.340600 \\
\hline $\mathrm{C}$ & 5.743200 & 3.057200 & 0.688100 \\
\hline C & 6.146300 & 4.360100 & 1.034100 \\
\hline $\mathrm{C}$ & 7.473500 & 4.760800 & 0.854700 \\
\hline $\mathrm{C}$ & 8.414400 & 3.861000 & 0.348700 \\
\hline $\mathrm{C}$ & 8.032200 & 2.554800 & 0.035000 \\
\hline $\mathrm{C}$ & 6.706500 & 2.150400 & 0.214400 \\
\hline $\mathrm{C}$ & -2.244700 & -1.408300 & -0.325300 \\
\hline $\mathrm{C}$ & -3.465600 & -2.309700 & -0.371500 \\
\hline $\mathrm{C}$ & -4.086500 & -2.411100 & -1.748700 \\
\hline $\mathrm{C}$ & -4.685000 & -1.311700 & -2.393000 \\
\hline $\mathrm{C}$ & -5.283800 & -1.422800 & -3.646600 \\
\hline $\mathrm{C}$ & -5.297900 & -2.652800 & -4.302700 \\
\hline $\mathrm{C}$ & -4.699500 & -3.757400 & -3.701100 \\
\hline $\mathrm{C}$ & -4.103300 & -3.626700 & -2.447600 \\
\hline $\mathrm{C}$ & -3.244400 & -3.576600 & 0.460500 \\
\hline $\mathrm{C}$ & -4.324200 & -2.622200 & 0.897200 \\
\hline $\mathrm{C}$ & -4.106200 & -1.836100 & 2.148800 \\
\hline $\mathrm{C}$ & -3.753000 & -2.490100 & 3.340600 \\
\hline $\mathrm{C}$ & -3.553300 & -1.757600 & 4.514400 \\
\hline $\mathrm{C}$ & -3.733900 & -0.372000 & 4.513500 \\
\hline $\mathrm{C}$ & -4.123400 & 0.280200 & 3.341000 \\
\hline $\mathrm{C}$ & -4.311700 & -0.447200 & 2.162800 \\
\hline $\mathrm{C}$ & -5.743200 & -3.057200 & 0.688100 \\
\hline $\mathrm{C}$ & -6.146300 & -4.360100 & 1.033900 \\
\hline $\mathrm{C}$ & -7.473600 & -4.760800 & 0.854500 \\
\hline $\mathrm{C}$ & -8.414500 & -3.860800 & 0.348700 \\
\hline $\mathrm{C}$ & -8.032300 & -2.554600 & 0.035200 \\
\hline $\mathrm{C}$ & -6.706400 & -2.150200 & 0.214600 \\
\hline $\mathrm{C}$ & 1.408300 & -2.244700 & -0.325400 \\
\hline & 2.309800 & -3.465500 & -0.371600 \\
\hline$C$ & 2.411200 & -4.086400 & -1.748700 \\
\hline $\mathrm{C}$ & 1.311800 & -4.684900 & -2.393100 \\
\hline $\mathrm{C}$ & 1.422900 & -5.283700 & -3.646700 \\
\hline & 2.652800 & -5.297800 & -4.302700 \\
\hline
\end{tabular}

\begin{tabular}{|c|c|c|c|}
\hline & 3.757500 & -4.699500 & -3.701200 \\
\hline & 3.626700 & -4.103300 & -2.447600 \\
\hline & 3.576600 & -3.244400 & 0.460400 \\
\hline & 2.622200 & -4.324200 & 0.897100 \\
\hline & 1.836000 & -4.106200 & 2.148700 \\
\hline & 0.447100 & -4.311900 & 2.162700 \\
\hline & -0.280400 & -4.123600 & 3.340800 \\
\hline & 0.371800 & -3.734100 & 4.513400 \\
\hline & 1.757400 & -3.553300 & 4.514300 \\
\hline & 2.490000 & -3.753100 & 3.340500 \\
\hline & 3.057200 & -5.743200 & 0.688000 \\
\hline & 4.360100 & -6.146300 & 1.033900 \\
\hline & 4.760800 & -7.473500 & 0.854500 \\
\hline & 3.860900 & -8.414400 & 0.348700 \\
\hline & 2.554700 & -8.032200 & 0.035000 \\
\hline & 2.150300 & -6.706400 & 0.214500 \\
\hline $\mathrm{C}$ & -1.408300 & 2.244700 & -0.325300 \\
\hline $\mathrm{C}$ & -2.309800 & 3.465500 & -0.371500 \\
\hline & -2.411300 & 4.086400 & -1.748600 \\
\hline 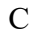 & -1.311900 & 4.685000 & -2.393000 \\
\hline $\mathrm{C}$ & -1.423100 & 5.283700 & -3.646600 \\
\hline $\mathrm{C}$ & -2.653100 & 5.297700 & -4.302600 \\
\hline $\mathrm{C}$ & -3.757600 & 4.699400 & -3.701100 \\
\hline $\mathrm{C}$ & -3.626900 & 4.103200 & -2.447500 \\
\hline & -3.576600 & 3.244400 & 0.460600 \\
\hline $\mathrm{C}$ & -2.622200 & 4.324200 & 0.897200 \\
\hline $\mathrm{C}$ & -3.057200 & 5.743200 & 0.688100 \\
\hline $\mathrm{C}$ & -4.360100 & 6.146300 & 1.034000 \\
\hline $\mathrm{C}$ & -4.760800 & 7.473500 & 0.854600 \\
\hline $\mathrm{C}$ & -3.860900 & 8.414400 & 0.348800 \\
\hline $\mathrm{C}$ & -2.554700 & 8.032200 & 0.035200 \\
\hline $\mathrm{C}$ & -2.150300 & 6.706400 & 0.214600 \\
\hline $\mathrm{C}$ & -1.836000 & 4.106200 & 2.148800 \\
\hline $\mathrm{C}$ & -0.447100 & 4.311900 & 2.162700 \\
\hline $\mathrm{C}$ & 0.280500 & 4.123600 & 3.340900 \\
\hline $\mathrm{C}$ & -0.371600 & 3.734100 & 4.513400 \\
\hline $\mathrm{C}$ & -1.757300 & 3.553300 & 4.514400 \\
\hline $\mathrm{C}$ & -2.489900 & 3.753000 & 3.340600 \\
\hline $\mathrm{H}$ & 5.732200 & 0.545900 & -4.100100 \\
\hline H & 5.767400 & 2.738300 & -5.278500 \\
\hline $\mathrm{H}$ & 4.687500 & 4.720200 & -4.204400 \\
\hline $\mathrm{H}$ & 3.622200 & 4.491900 & -2.005100 \\
\hline $\mathrm{H}$ & 3.512200 & 4.533800 & 0.025100 \\
\hline & 2.320300 & 3.598700 & 1.026300 \\
\hline H & 4.615800 & -0.069900 & 1.263300 \\
\hline $\mathrm{H}$ & 4.287800 & -1.348300 & 3.346400 \\
\hline $\mathrm{H}$ & 3.585400 & -0.195200 & 5.423300 \\
\hline & 3.267000 & 2.264800 & 5.426300 \\
\hline & 3.636700 & 3.565700 & 3.359900 \\
\hline H & 5.435300 & 5.065900 & 1.444700 \\
\hline $\mathrm{H}$ & 7.773700 & 5.768100 & 1.113200 \\
\hline 1 & 9.441900 & 4.172200 & 0.211800 \\
\hline & 8.765400 & 1.852400 & -0.339500 \\
\hline & 6.437500 & 1.128300 & -0.004300 \\
\hline $\mathrm{H}$ & -5.732400 & -0.545500 & -4.099900 \\
\hline $\mathrm{H}$ & -5.767600 & -2.737800 & -5.278400 \\
\hline & -4.687700 & -4.719800 & -4.204600 \\
\hline & -3.622300 & -4.491700 & -2.005200 \\
\hline & -3.512300 & -4.533800 & 0.024900 \\
\hline & -2.320300 & -3.599000 & 1.026100 \\
\hline & -3.636800 & -3.566000 & 3.359800 \\
\hline & -3.267000 & -2.265400 & 5.426300 \\
\hline
\end{tabular}

$\begin{array}{rrrr}\mathrm{H} & -3.584900 & 0.194600 & 5.423600 \\ \mathrm{H} & -4.287300 & 1.348000 & 3.346800 \\ \mathrm{H} & -4.615500 & 0.069900 & 1.263600 \\ \mathrm{H} & -5.435400 & -5.066000 & 1.444300 \\ \mathrm{H} & -7.773800 & -5.768100 & 1.112800 \\ \mathrm{H} & -9.442000 & -4.171900 & 0.211800 \\ \mathrm{H} & -8.765400 & -1.852000 & -0.339200 \\ \mathrm{H} & -6.437400 & -1.128200 & -0.003900 \\ \mathrm{H} & 0.545500 & -5.732300 & -4.100000 \\ \mathrm{H} & 2.737900 & -5.767500 & -5.278500 \\ \mathrm{H} & 4.720000 & -4.687600 & -4.204600 \\ \mathrm{H} & 4.491900 & -3.622300 & -2.005300 \\ \mathrm{H} & 4.533800 & -3.512200 & 0.024900 \\ \mathrm{H} & 3.598900 & -2.320300 & 1.026100 \\ \mathrm{H} & -0.069800 & -4.615600 & 1.263400 \\ \mathrm{H} & -1.348100 & -4.287500 & 3.346600 \\ \mathrm{H} & -0.194800 & -3.585100 & 5.423500 \\ \mathrm{H} & 2.265300 & -3.267000 & 5.426300 \\ \mathrm{H} & 3.565900 & -3.636700 & 3.359700 \\ \mathrm{H} & 5.066000 & -5.435300 & 1.444400 \\ \mathrm{H} & 5.768100 & -7.773700 & 1.112900 \\ \mathrm{H} & 4.172000 & -9.442000 & 0.211700 \\ \mathrm{H} & 1.852100 & -8.765400 & -0.339400 \\ \mathrm{H} & 1.128300 & -6.437500 & -0.004100 \\ \mathrm{H} & -0.545800 & 5.732300 & -4.100000 \\ \mathrm{H} & -2.738300 & 5.767400 & -5.278400 \\ \mathrm{H} & -4.720100 & 4.687500 & -4.204400 \\ \mathrm{H} & -4.491900 & 3.622200 & -2.005100 \\ \mathrm{H} & -4.533800 & 3.512200 & 0.025100 \\ \mathrm{H} & -3.598900 & 2.320300 & 1.026300 \\ \mathrm{H} & -5.066000 & 5.435300 & 1.444500 \\ \mathrm{H} & -5.768100 & 7.773700 & 1.113000 \\ \mathrm{H} & -4.172000 & 9.442000 & 0.211800 \\ \mathrm{H} & -1.852200 & 8.765300 & -0.339300 \\ \mathrm{H} & -1.128300 & 6.437500 & -0.004000 \\ \mathrm{H} & 0.069900 & 4.615600 & 1.263400 \\ \mathrm{H} & 1.348200 & 4.287500 & 3.346600 \\ \mathrm{H} & 0.195000 & 3.585100 & 5.423500 \\ \mathrm{H} & -2.265100 & 3.267000 & 5.426400 \\ \mathrm{H} & -3.565800 & 3.636700 & 3.359900\end{array}$

\section{A"1}

$\begin{array}{llll}\text { Rh } & -0.082300 & 0.066100 & -1.347100\end{array}$ $\begin{array}{llll}\text { Rh } & 0.108600 & 0.067600 & 1.029000\end{array}$ O $\quad-2.120400 \quad-0.148500-1.108300$ $\begin{array}{llll}\mathrm{O} & -1.936300 & -0.213100 & 1.142000\end{array}$ O $\quad-0.411100 \quad 2.110000 \quad-1.262500$ $\begin{array}{llll}\text { O } & -0.260700 & 2.099800 & 0.991600\end{array}$ $\begin{array}{llll}\mathrm{O} & 1.955500 & 0.366600 & -1.453900\end{array}$ $\begin{array}{llll}\text { O } & 2.120600 & 0.439900 & 0.797800\end{array}$ O $\quad 0.221300 \quad-1.967400 \quad-1.310000$ $\begin{array}{llll}\text { O } & 0.492000 & -1.949100 & 0.932200\end{array}$ $\mathrm{Cl} \quad-4.099000 \quad-2.787600 \quad 1.301300$ Cl $\quad 1.692600 \quad 4.495500 \quad-1.746000$ $\mathrm{Cl} \quad 4.238900 \quad-1.905200 \quad-1.803600$ $\mathrm{Cl} \quad-2.068600 \quad-4.275800 \quad-1.254300$ $\begin{array}{llll}\text { C } & -5.022100 & 0.959600 & 2.459800\end{array}$ $\begin{array}{llll}\text { C } & -4.096600 & 0.145400 & 0.234800\end{array}$ $\begin{array}{llll}\text { C } & -5.085500 & 0.183500 & -0.969200\end{array}$ C $\quad-5.101800 \quad-1.607900 \quad 3.505600$ $\begin{array}{llll}\text { C } & -4.593800 & -0.097000 & 1.643500\end{array}$ $\begin{array}{lrrr}\text { C } & -5.489600 & 0.755200 & 3.757500\end{array}$ 


\begin{tabular}{|c|c|c|c|}
\hline $\mathrm{C}$ & -6.545500 & 0.006300 & -0.680300 \\
\hline $\mathrm{C}$ & -2.613600 & -0.141200 & 0.064600 \\
\hline $\mathrm{C}$ & -4.654500 & -0.358800 & -2.293600 \\
\hline $\mathrm{C}$ & -4.599600 & 0.479800 & -3.420100 \\
\hline $\mathrm{C}$ & -4.448100 & 1.458700 & -0.478100 \\
\hline $\mathrm{C}$ & -7.043400 & -1.229200 & -0.233000 \\
\hline $\mathrm{C}$ & -4.640600 & -1.384600 & 2.209200 \\
\hline $\mathrm{C}$ & -7.449300 & 1.056700 & -0.924400 \\
\hline $\mathrm{C}$ & -5.533200 & -0.534500 & 4.283100 \\
\hline $\mathrm{C}$ & -9.292400 & -0.333500 & -0.194400 \\
\hline $\mathrm{C}$ & -4.204600 & -0.031200 & -4.660100 \\
\hline $\mathrm{C}$ & -3.951000 & -2.228900 & -3.678400 \\
\hline $\mathrm{C}$ & -4.345500 & -1.721500 & -2.436700 \\
\hline $\mathrm{C}$ & -8.814000 & 0.888600 & -0.672300 \\
\hline $\mathrm{C}$ & -8.408300 & -1.393400 & 0.019100 \\
\hline $\mathrm{C}$ & -3.876500 & -1.383500 & -4.788300 \\
\hline $\mathrm{C}$ & -2.287800 & 5.098700 & -2.047900 \\
\hline $\mathrm{C}$ & -0.965300 & 4.112600 & -0.116800 \\
\hline $\mathrm{C}$ & -0.853900 & 5.015100 & 1.152600 \\
\hline $\mathrm{C}$ & -0.005900 & 5.530900 & -3.562100 \\
\hline $\mathrm{C}$ & -1.053200 & 4.725300 & -1.497300 \\
\hline $\mathrm{C}$ & -2.395500 & 5.682200 & -3.309800 \\
\hline $\mathrm{C}$ & -0.890100 & 6.501100 & 0.959400 \\
\hline $\mathrm{C}$ & -0.470400 & 2.676400 & -0.124900 \\
\hline $\mathrm{C}$ & 0.003600 & 4.567600 & 2.292300 \\
\hline $\mathrm{C}$ & 1.392900 & 4.440800 & 2.126500 \\
\hline $\mathrm{C}$ & -2.129600 & 4.265900 & 0.869600 \\
\hline $\mathrm{C}$ & 0.159300 & 7.166400 & 0.303000 \\
\hline $\mathrm{C}$ & 0.082800 & 4.950400 & -2.298100 \\
\hline $\mathrm{C}$ & -1.945300 & 7.259200 & 1.499000 \\
\hline $\mathrm{C}$ & -1.249500 & 5.903900 & -4.069800 \\
\hline $\mathrm{C}$ & -0.942300 & 9.294000 & 0.652500 \\
\hline $\mathrm{C}$ & 2.197700 & 4.048100 & 3.199100 \\
\hline $\mathrm{C}$ & 0.244700 & 3.912200 & 4.621000 \\
\hline $\mathrm{C}$ & -0.563000 & 4.317600 & 3.554000 \\
\hline $\mathrm{C}$ & -1.974300 & 8.647400 & 1.336500 \\
\hline $\mathrm{C}$ & 0.126500 & 8.554400 & 0.141300 \\
\hline $\mathrm{C}$ & 1.624000 & 3.774700 & 4.443000 \\
\hline $\mathrm{C}$ & 4.952500 & 1.969200 & -2.681700 \\
\hline $\mathrm{C}$ & 4.057800 & 0.956800 & -0.530300 \\
\hline $\mathrm{C}$ & 5.052700 & 0.978400 & 0.674400 \\
\hline $\mathrm{C}$ & 5.217600 & -0.512300 & -3.891600 \\
\hline C & 4.574700 & 0.836000 & -1.947300 \\
\hline $\mathrm{C}$ & 5.462100 & 1.881300 & -3.976600 \\
\hline $\mathrm{C}$ & 6.518700 & 0.924400 & 0.368000 \\
\hline $\mathrm{C}$ & 2.612500 & 0.520800 & -0.375500 \\
\hline $\mathrm{C}$ & 4.672300 & 0.308400 & 1.955000 \\
\hline $\mathrm{C}$ & 4.443100 & -1.076800 & 1.992000 \\
\hline $\mathrm{C}$ & 4.320000 & 2.232900 & 0.276400 \\
\hline $\mathrm{C}$ & 7.349200 & 2.016800 & 0.678000 \\
\hline $\mathrm{C}$ & 4.712900 & -0.405100 & -2.597100 \\
\hline $\mathrm{C}$ & 7.096900 & -0.240800 & -0.163700 \\
\hline $\mathrm{C}$ & 5.600800 & 0.635500 & -4.584000 \\
\hline $\mathrm{C}$ & 9.277700 & 0.810600 & -0.152200 \\
\hline $\mathrm{C}$ & 4.099000 & -1.701600 & 3.193600 \\
\hline $\mathrm{C}$ & 4.234000 & 0.423200 & 4.343300 \\
\hline $\mathrm{C}$ & 4.590600 & 1.049800 & 3.145500 \\
\hline $\mathrm{C}$ & 8.467500 & -0.291900 & -0.432600 \\
\hline $\mathrm{C}$ & 8.720000 & 1.961800 & 0.409000 \\
\hline $\mathrm{C}$ & 3.984100 & -0.951500 & 4.366600 \\
\hline $\mathrm{C}$ & 1.706200 & -5.041100 & -2.443200 \\
\hline & 0.890200 & -3.992700 & -0.275300 \\
\hline
\end{tabular}

$\begin{array}{llll}\text { C } & 1.016500 & -4.897300 & 0.992500\end{array}$

C $\quad-0.875800 \quad-5.395200 \quad-3.392900$

C $\quad 0.643300 \quad-4.615800 \quad-1.633600$

$\begin{array}{llll}\text { C } & 1.501300 & -5.639700 & -3.685900\end{array}$

$\begin{array}{llll}\text { C } & 0.913100 & -6.379900 & 0.799800\end{array}$

C $\quad 0.470200 \quad-2.536600 \quad-0.198700$

$\begin{array}{llll}\text { C } & 0.493000 & -4.416000 & 2.307100\end{array}$

C $\quad-0.865700 \quad-4.097600 \quad 2.462900$

$\begin{array}{llll}\text { C } & 2.234100 & -4.213700 & 0.426500\end{array}$

C $\quad-0.317300 \quad-6.971400 \quad 0.467600$

C $\quad-0.652400 \quad-4.802400 \quad-2.151300$

$\begin{array}{llll}\text { C } & 2.028900 & -7.207700 & 1.022300\end{array}$

$\begin{array}{llll}\text { C } & 0.205100 & -5.822300 & -4.162500\end{array}$

C $\quad 0.706200 \quad-9.165300 \quad 0.491600$

C $\quad-1.347500 \quad-3.646900 \quad 3.695800$

$\begin{array}{llll}\text { C } & 0.866200 & -3.875800 & 4.647400\end{array}$

C $\quad 1.349700 \quad-4.329000 \quad 3.416700$

$\begin{array}{llll}\text { C } & 1.926200 & -8.592200 & 0.858000\end{array}$

C $\quad-0.416300 \quad-8.355900 \quad 0.303100$

C $\quad-0.480700 \quad-3.529600 \quad 4.785500$

$\mathrm{H} \quad-4.976300 \quad 1.972500 \quad 2.074500$

$\mathrm{H} \quad-5.117000 \quad-2.620000 \quad 3.895600$

$\mathrm{H} \quad-5.811300 \quad 1.603900 \quad 4.354200$

$\mathrm{H} \quad-4.859200 \quad 1.528000 \quad-3.337200$

$\mathrm{H} \quad-5.051500 \quad 2.185800 \quad 0.056600$

$\mathrm{H} \quad-3.651400 \quad 1.901200-1.067700$

$\mathrm{H} \quad-6.381600 \quad-2.069300 \quad-0.091200$

$\mathrm{H} \quad-7.099100 \quad 2.005500 \quad-1.311300$

$\mathrm{H} \quad-10.349400 \quad-0.462900 \quad-0.001100$

H $\quad-4.155300 \quad 0.621000 \quad-5.522400$

H $\quad-3.705200 \quad-3.277900 \quad-3.781000$

$\mathrm{H} \quad-4.404600 \quad-2.387500 \quad-1.587600$

$\mathrm{H} \quad-9.501600 \quad 1.704700 \quad-0.852700$

$\mathrm{H} \quad-8.781800 \quad-2.346000 \quad 0.372100$

$\mathrm{H} \quad-3.570900 \quad-1.777600 \quad-5.748800$

$\begin{array}{llll}\mathrm{H} & -3.192200 & 4.916800 & -1.477500\end{array}$

$\mathrm{H} \quad 0.899100 \quad 5.683700 \quad-4.140200$

$\mathrm{H} \quad-3.373700 \quad 5.954900 \quad-3.695400$

$\mathrm{H} \quad 1.852500 \quad 4.646500 \quad 1.169900$

$\mathrm{H} \quad-3.007900 \quad 4.816200 \quad 0.546200$

$\mathrm{H} \quad-2.357400 \quad 3.384300 \quad 1.460000$

$\mathrm{H} \quad 1.007700 \quad 6.617000 \quad-0.074200$

H $\quad-2.745400 \quad 6.776800 \quad 2.046600$

$\mathrm{H} \quad-0.965100 \quad 10.368900 \quad 0.528600$

H $\quad 3.267300 \quad 3.963100 \quad 3.066800$

$\mathrm{H} \quad-0.197900 \quad 3.710800 \quad 5.587900$

$\mathrm{H} \quad-1.628400 \quad 4.433400 \quad 3.710000$

$\mathrm{H} \quad-2.794700 \quad 9.222600 \quad 1.745700$

H $\quad 0.934600 \quad 9.057500 \quad-0.373700$

H $\quad 2.249200 \quad 3.465500 \quad 5.270600$

$\mathrm{H} \quad 4.830200 \quad 2.948800 \quad-2.233200$

H $\quad 5.305100 \quad-1.492500 \quad-4.347800$

$\mathrm{H} \quad 5.741800 \quad 2.786900 \quad-4.507200$

H $\quad 4.527800 \quad-1.670400 \quad 1.092600$

$\mathrm{H} \quad 4.861200 \quad 3.043500 \quad-0.201200$

$\mathrm{H} \quad 3.492200 \quad 2.565600 \quad 0.892200$

$\begin{array}{llll}\mathrm{H} & 6.937300 & 2.910800 & 1.129200\end{array}$

H $\quad 6.492800 \quad-1.113300 \quad-0.360200$

$\mathrm{H} \quad 10.339400 \quad 0.768700 \quad-0.358600$

H $\quad 3.928400 \quad-2.768400 \quad 3.216100$

$\mathrm{H} \quad 4.159000 \quad 1.002400 \quad 5.254600$

H $4.805000 \quad 2.110300 \quad 3.145300$
H $\quad 8.902900 \quad-1.190200 \quad-0.851000$

$\mathrm{H} \quad 9.350800 \quad 2.810100 \quad 0.640700$

H $\quad 3.712800 \quad-1.437400 \quad 5.294900$

H $\quad 2.722700 \quad-4.884300 \quad-2.100200$

$\mathrm{H} \quad-1.894200 \quad-5.515600 \quad-3.746700$

$\mathrm{H} \quad 2.355500 \quad-5.953500 \quad-4.279000$

$\mathrm{H} \quad-1.545200 \quad-4.188200 \quad 1.628000$

$\mathrm{H} \quad 2.993200 \quad-4.794500 \quad-0.087400$

H $\quad 2.628500 \quad-3.351200 \quad 0.952000$

H $\quad-1.203000 \quad-6.366000 \quad 0.349200$

H $\quad 2.978100 \quad-6.783800 \quad 1.325100$

$\mathrm{H} \quad 0.627600 \quad-10.237500 \quad 0.366400$

$\mathrm{H} \quad-2.392500 \quad-3.389800 \quad 3.808000$

H $\quad 1.534700 \quad-3.797600 \quad 5.495000$

H $\quad 2.389900 \quad-4.614600 \quad 3.327200$

$\begin{array}{llll}\mathrm{H} & 2.791600 & -9.221500 & 1.020400\end{array}$

H $\quad-1.366000 \quad-8.802000 \quad 0.037900$

$\mathrm{H} \quad-0.854400 \quad-3.179500 \quad 5.738900$

$\mathrm{H} \quad-5.894300 \quad-0.710500 \quad 5.292400$

H $\quad 0.029000 \quad-6.287100 \quad-5.128600$

H $\quad \begin{array}{llll}5.996900 & 0.549900 & -5.591800\end{array}$

H $\quad-1.315400 \quad 6.357700 \quad-5.054500$

\section{B1}

$\begin{array}{llll}\text { Rh } & 0.000000 & 0.000000 & 0.650900\end{array}$

$\begin{array}{lllll}\mathrm{Rh} & 0.000000 & 0.000000 & -1.731100\end{array}$

$\begin{array}{llll}\mathrm{O} & -1.503900 & 1.410100 & 0.589700\end{array}$

$\begin{array}{llll}\mathrm{O} & -1.404000 & 1.511800 & -1.664800\end{array}$

$\begin{array}{llll}\mathrm{O} & -1.410100 & -1.503900 & 0.589700\end{array}$

$\begin{array}{llll}\mathrm{O} & -1.511800 & -1.404000 & -1.664800\end{array}$

$\begin{array}{llll}\text { O } & 1.503900 & -1.410000 & 0.589700\end{array}$

$\begin{array}{llll}\mathrm{O} & 1.404000 & -1.511800 & -1.664800\end{array}$

$\begin{array}{llll}\mathrm{O} & 1.410100 & 1.503900 & 0.589700\end{array}$

O $\quad 1.511800 \quad 1.404000 \quad-1.664800$

$\begin{array}{llll}\mathrm{Cl} & -4.652600 & 0.767400 & 0.765000\end{array}$

$\begin{array}{llll}\mathrm{Cl} & -0.767400 & -4.652600 & 0.765000\end{array}$

$\begin{array}{llll}\mathrm{Cl} & 4.652600 & -0.767400 & 0.765000\end{array}$

$\begin{array}{llll}\mathrm{Cl} & 0.767400 & 4.652600 & 0.765000\end{array}$

$\mathrm{Br} \quad-5.375200 \quad 4.076000 \quad 5.151100$

$\mathrm{Br} \quad-4.076000 \quad-5.375200 \quad 5.151100$

$\mathrm{Br} \quad 5.375200 \quad-4.076100 \quad 5.151000$

$\mathrm{Br} \quad 4.076100 \quad 5.375300 \quad 5.151100$

$\begin{array}{llll}\text { C } & -3.197900 & 4.439500 & 1.596100\end{array}$

C $\quad-2.860100 \quad 3.026800 \quad-0.487600$

C $\quad-3.621400 \quad 3.531000 \quad-1.756700$

$\begin{array}{llll}\text { C } & -4.851500 & 2.549900 & 2.772700\end{array}$

C $\quad-3.449900 \quad 3.256100 \quad 0.887800$

$\begin{array}{llll}\text { C } & -3.757600 & 4.693700 & 2.847600\end{array}$

$\begin{array}{llll}\text { C } & -4.909000 & 4.269800 & -1.548800\end{array}$

C $\quad-1.871800 \quad 1.875200 \quad-0.535200$

$\begin{array}{llll}\text { C } & -3.577600 & 2.724600 & -3.013600\end{array}$

$\begin{array}{llll}\text { C } & -4.083100 & 1.414900 & -3.038300\end{array}$

$\begin{array}{llll}\text { C } & -2.357900 & 4.217600 & -1.309500\end{array}$

$\begin{array}{lrrr}\text { C } & -5.009400 & 5.633200 & -1.882100\end{array}$

$\begin{array}{llll}\text { C } & -4.284100 & 2.315800 & 1.521300\end{array}$

$\begin{array}{llll}\text { C } & -6.052900 & 3.596000 & -1.088500\end{array}$

C $\quad-4.593500 \quad 3.747800 \quad 3.437900$

$\begin{array}{llll}\text { C } & -7.335500 & 5.645800 & -1.209300\end{array}$

$\begin{array}{llll}\text { C } & -4.053000 & 0.671000 & -4.220900\end{array}$

$\begin{array}{llll}\text { C } & -3.044500 & 2.541500 & -5.378000\end{array}$

$\begin{array}{llll}\text { C } & -3.084200 & 3.292700 & -4.199600\end{array}$

$\begin{array}{llll}\text { C } & -7.256000 & 4.284300 & -0.908800\end{array}$ 


\begin{tabular}{|c|c|c|c|}
\hline C & -6.214700 & 6.318100 & -1.702500 \\
\hline C & -3.524500 & 1.229200 & -5.387500 \\
\hline $\mathrm{C}$ & -4.439500 & -3.197800 & 1.596100 \\
\hline $\mathrm{C}$ & -3.026800 & -2.860100 & -0.487600 \\
\hline $\mathrm{C}$ & -3.531000 & -3.621400 & -1.756700 \\
\hline $\mathrm{C}$ & -2.549900 & -4.851500 & 2.772700 \\
\hline $\mathrm{C}$ & -3.256000 & -3.449900 & 0.887800 \\
\hline $\mathrm{C}$ & -4.693700 & -3.757600 & 2.847600 \\
\hline $\mathrm{C}$ & -4.269800 & -4.908900 & -1.548800 \\
\hline $\mathrm{C}$ & -1.875200 & -1.871800 & -0.535200 \\
\hline $\mathrm{C}$ & -2.724600 & -3.577600 & -3.013600 \\
\hline $\mathrm{C}$ & -1.414900 & -4.083100 & -3.038300 \\
\hline $\mathrm{C}$ & -4.217600 & -2.357900 & -1.309500 \\
\hline $\mathrm{C}$ & -5.633200 & -5.009300 & -1.882100 \\
\hline $\mathrm{C}$ & -2.315800 & -4.284100 & 1.521300 \\
\hline C & -3.596100 & -6.052900 & -1.088500 \\
\hline $\mathrm{C}$ & -3.747800 & -4.593500 & 3.437800 \\
\hline $\mathrm{C}$ & -5.645900 & -7.335400 & -1.209400 \\
\hline $\mathrm{C}$ & -0.671000 & -4.052900 & -4.220900 \\
\hline $\mathrm{C}$ & -2.541500 & -3.044500 & -5.378000 \\
\hline $\mathrm{C}$ & -3.292700 & -3.084200 & -4.199600 \\
\hline $\mathrm{C}$ & -4.284300 & -7.255900 & -0.908800 \\
\hline $\mathrm{C}$ & -6.318100 & -6.214700 & -1.702500 \\
\hline $\mathrm{C}$ & -1.229200 & -3.524500 & -5.387500 \\
\hline $\mathrm{C}$ & 3.197800 & -4.439500 & 1.596100 \\
\hline $\mathrm{C}$ & 2.860100 & -3.026800 & -0.487600 \\
\hline $\mathrm{C}$ & 3.621400 & -3.531000 & -1.756800 \\
\hline $\mathrm{C}$ & 4.851500 & -2.550000 & 2.772700 \\
\hline $\mathrm{C}$ & 3.449900 & -3.256000 & 0.887700 \\
\hline $\mathrm{C}$ & 3.757600 & -4.693600 & 2.847600 \\
\hline $\mathrm{C}$ & 4.908900 & -4.269800 & -1.548800 \\
\hline $\mathrm{C}$ & 1.871800 & -1.875200 & -0.535200 \\
\hline $\mathrm{C}$ & 3.577600 & -2.724600 & -3.013600 \\
\hline $\mathrm{C}$ & 4.083100 & -1.414900 & -3.038300 \\
\hline $\mathrm{C}$ & 2.357900 & -4.217600 & -1.309500 \\
\hline $\mathrm{C}$ & 5.009300 & -5.633200 & -1.882100 \\
\hline $\mathrm{C}$ & 4.284100 & -2.315800 & 1.521300 \\
\hline $\mathrm{C}$ & 6.052900 & -3.596100 & -1.088500 \\
\hline $\mathrm{C}$ & 4.593500 & -3.747800 & 3.437800 \\
\hline $\mathrm{C}$ & 7.335400 & -5.645900 & -1.209400 \\
\hline $\mathrm{C}$ & 4.052900 & -0.670900 & -4.220900 \\
\hline $\mathrm{C}$ & 3.044500 & -2.541500 & -5.378000 \\
\hline $\mathrm{C}$ & 3.084200 & -3.292600 & -4.199600 \\
\hline $\mathrm{C}$ & 7.256000 & -4.284300 & -0.908800 \\
\hline $\mathrm{C}$ & 6.214700 & -6.318100 & -1.702500 \\
\hline $\mathrm{C}$ & 3.524500 & -1.229200 & -5.387500 \\
\hline $\mathrm{C}$ & 4.439500 & 3.197800 & 1.596100 \\
\hline $\mathrm{C}$ & 3.026800 & 2.860100 & -0.487600 \\
\hline $\mathrm{C}$ & 3.531000 & 3.621400 & -1.756700 \\
\hline $\mathrm{C}$ & 2.550000 & 4.851500 & 2.772700 \\
\hline $\mathrm{C}$ & 3.256000 & 3.449900 & 0.887800 \\
\hline $\mathrm{C}$ & 4.693700 & 3.757600 & 2.847600 \\
\hline $\mathrm{C}$ & 4.269800 & 4.908900 & -1.548800 \\
\hline $\mathrm{C}$ & 1.875200 & 1.871800 & -0.535200 \\
\hline $\mathrm{C}$ & 2.724600 & 3.577600 & -3.013600 \\
\hline $\mathrm{C}$ & 1.414900 & 4.083100 & -3.038300 \\
\hline $\mathrm{C}$ & 4.217600 & 2.358000 & -1.309500 \\
\hline $\mathrm{C}$ & 5.633200 & 5.009300 & -1.882200 \\
\hline $\mathrm{C}$ & 2.315800 & 4.284100 & 1.521300 \\
\hline $\mathrm{C}$ & 3.596100 & 6.052900 & -1.088500 \\
\hline $\mathrm{C}$ & 3.747800 & 4.593500 & 3.437800 \\
\hline & 5.645900 & 7.335400 & -1.209400 \\
\hline
\end{tabular}

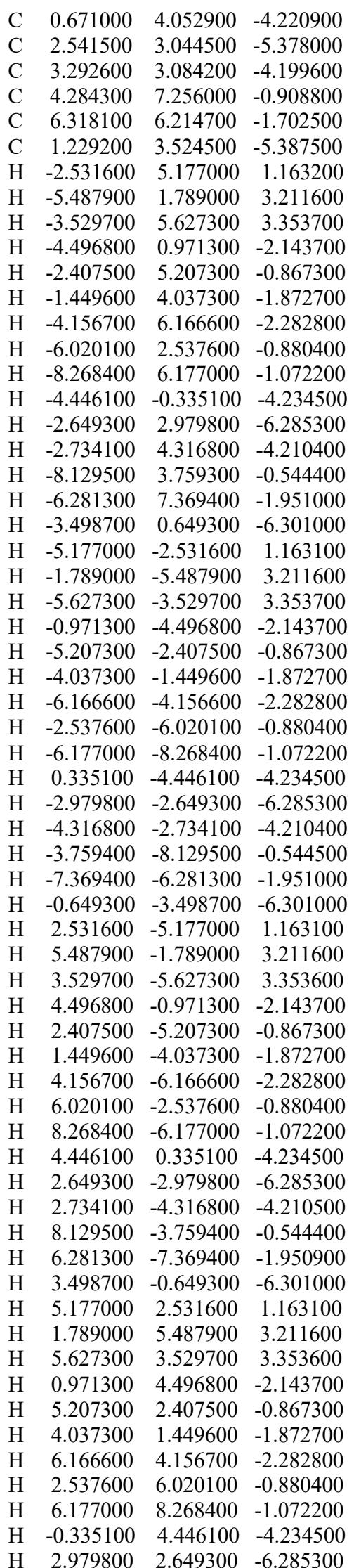

\begin{abstract}
H $\quad 4.316800 \quad 2.734100 \quad-4.210400$
H $\quad 3.759400 \quad 8.129500 \quad-0.544400$

$\mathrm{H} \quad 7.369400 \quad 6.281300 \quad-1.951000$

H $\quad 0.649300 \quad 3.498700 \quad-6.301000$
\end{abstract}

B2

$\begin{array}{llll}\text { Rh } & 0.000000 & 0.000000 & 0.651700\end{array}$

$\begin{array}{llll}\mathrm{Rh} & 0.000000 & -0.000100 & -1.730300\end{array}$

$\begin{array}{llll}\mathrm{O} & -1.445200 & 1.470400 & 0.590200\end{array}$

$\begin{array}{llll}\text { O } & -1.340900 & 1.568000 & -1.664300\end{array}$

$\begin{array}{llll}\text { O } & -1.470200 & -1.445100 & 0.590300\end{array}$

$\begin{array}{llll}\mathrm{O} & -1.568100 & -1.341000 & -1.664200\end{array}$

$\begin{array}{llll}\mathrm{O} & 1.445300 & -1.470300 & 0.590200\end{array}$

$\begin{array}{llll}\text { O } & 1.340800 & -1.568100 & -1.664300\end{array}$

$\begin{array}{llll}\mathrm{O} & 1.470400 & 1.445200 & 0.590100\end{array}$

$\begin{array}{llll}\text { O } & 1.568000 & 1.340900 & -1.664400\end{array}$

Cl $\quad-4.619100 \quad 0.957400 \quad 0.763500$

$\mathrm{Cl} \quad-0.957500 \quad-4.618900 \quad 0.763900$

Cl $\quad 4.619100 \quad-0.957400 \quad 0.763400$

$\begin{array}{llll}\mathrm{Cl} & 0.957500 & 4.619000 & 0.763500\end{array}$

$\mathrm{Br} \quad-5.205500 \quad 4.291000 \quad 5.151000$

$\mathrm{Br} \quad-4.290700 \quad-5.204900 \quad 5.151700$

$\begin{array}{llll}\mathrm{Br} & 5.205600 & -4.290800 & 5.151000\end{array}$

$\mathrm{Br} \quad 4.291100 \quad 5.205300 \quad 5.150900$

$\begin{array}{llll}\text { C } & -3.013500 & 4.565500 & 1.597000\end{array}$

C $\quad-2.733900 \quad 3.141200 \quad-0.487300$

C $\quad-3.473000 \quad 3.676700 \quad-1.756500$

$\begin{array}{llll}\text { C } & -4.744500 & 2.745700 & 2.772000\end{array}$

C $\quad-3.314200 \quad 3.394000 \quad 0.887900$

C $\quad-3.562600 \quad 4.842100 \quad 2.848500$

C $\quad-4.729000 \quad 4.468100 \quad-1.549000$

C $\quad-1.793600 \quad 1.950100 \quad-0.534800$

$\begin{array}{llll}\text { C } & -3.461800 & 2.869800 & -3.013700\end{array}$

C $\quad-4.020600 \quad 1.581900 \quad-3.039100$

$\begin{array}{llll}\text { C } & -2.182400 & 4.310500 & -1.308500\end{array}$

$\begin{array}{llll}\text { C } & -4.772800 & 5.834600 & -1.881800\end{array}$

$\begin{array}{llll}\text { C } & -4.186800 & 2.488900 & 1.520700\end{array}$

$\begin{array}{llll}\text { C } & -5.900100 & 3.842000 & -1.089700\end{array}$

C $\quad-4.437300 \quad 3.931500 \quad 3.437900$

$\begin{array}{llll}\text { C } & -7.096800 & 5.943200 & -1.210400\end{array}$

C $\quad-4.020400 \quad 0.837900 \quad-4.222000$

$\begin{array}{llll}\text { C } & -2.935800 & 2.666000 & -5.378000\end{array}$

C $\quad-2.945100 \quad 3.417700 \quad-4.199300$

C $\quad-7.073800 \quad 4.579300 \quad-0.910400$

$\begin{array}{llll}\text { C } & -5.949000 & 6.568600 & -1.702600\end{array}$

$\begin{array}{llll}\text { C } & -3.469000 & 1.374500 & -5.388200\end{array}$

$\begin{array}{llll}\text { C } & -4.565500 & -3.013300 & 1.597500\end{array}$

C $\quad-3.141300 \quad-2.733800 \quad-0.486900$

C $\quad-3.676900 \quad-3.473100 \quad-1.756000$

$\begin{array}{llll}\text { C } & -2.745600 & -4.744200 & 2.772500\end{array}$

C $\quad-3.394000 \quad-3.314100 \quad 0.888400$

C $\quad-4.841900 \quad-3.562300 \quad 2.849100$

$\begin{array}{llll}\text { C } & -4.468400 & -4.729000 & -1.548300\end{array}$

$\begin{array}{llll}\text { C } & -1.950100 & -1.793600 & -0.534700\end{array}$

C $\quad-2.870100 \quad-3.462100 \quad-3.013200$

C $\quad-1.582200 \quad-4.021000 \quad-3.038700$

$\begin{array}{llll}\text { C } & -4.310600 & -2.182400 & -1.308100\end{array}$

C $\quad-5.834900 \quad-4.772700 \quad-1.881000$

$\begin{array}{llll}\text { C } & -2.488900 & -4.186600 & 1.521200\end{array}$

C $\quad-3.842300 \quad-5.900000 \quad-1.088800$

C $\quad-3.931200 \quad-4.436800 \quad 3.438500$

$\begin{array}{lrrr}\text { C } & -5.943600 & -7.096500 & -1.209300\end{array}$ 


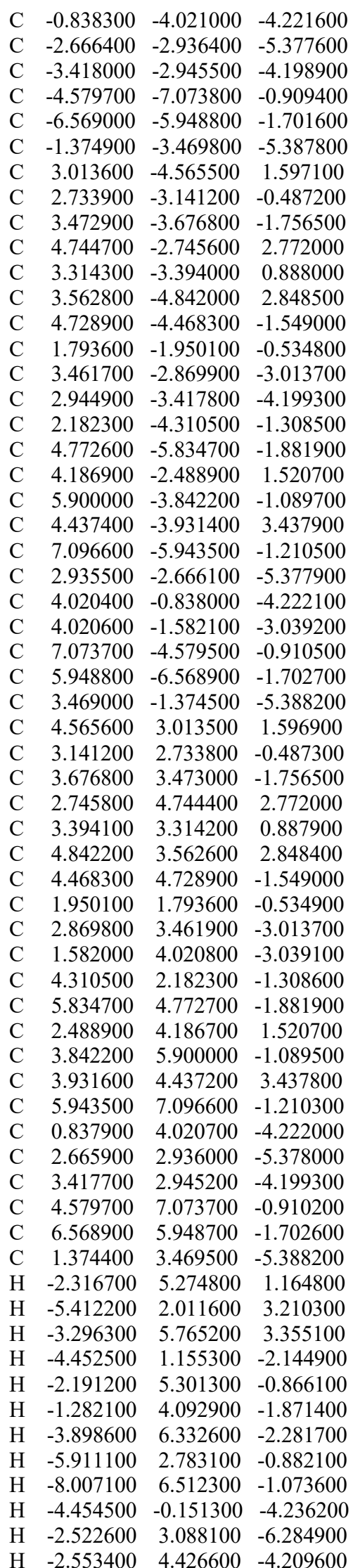

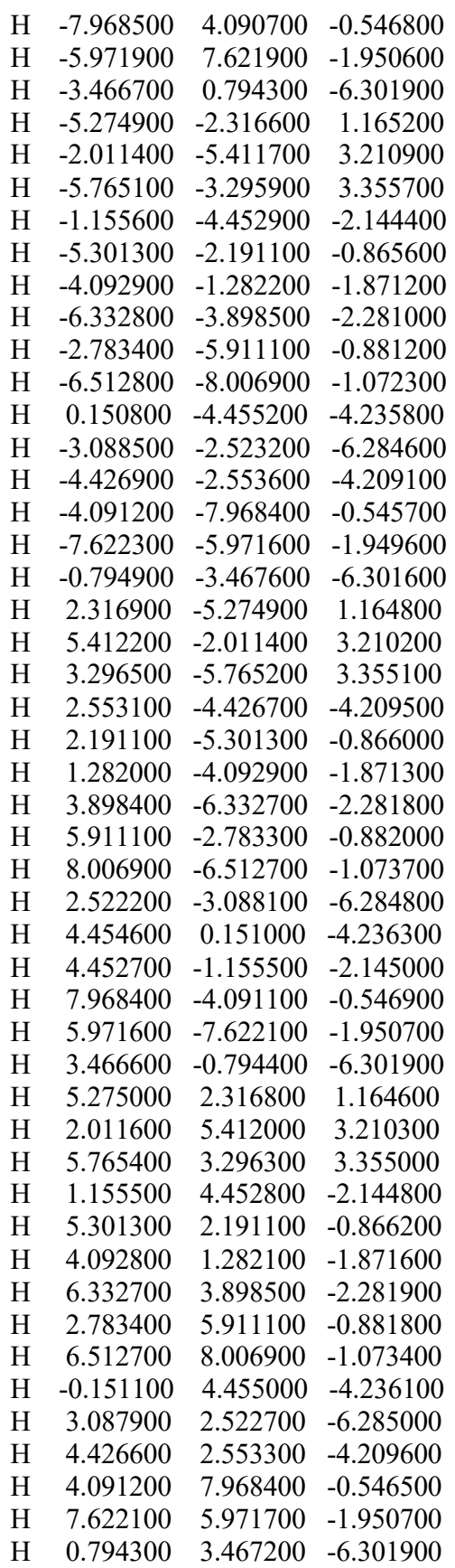

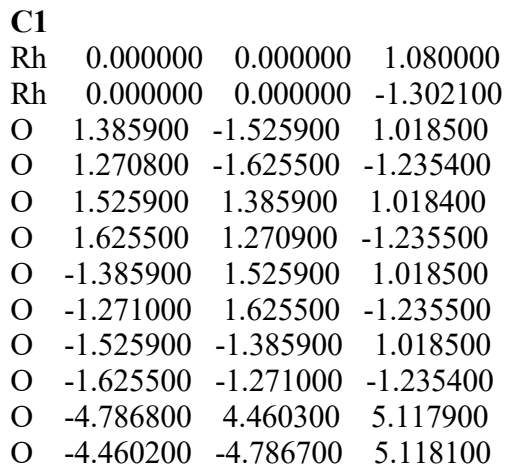

O $\quad 4.460200 \quad 4.786600 \quad 5.117900$

$\begin{array}{llll}\text { O } & 4.787200 & -4.459800 & 5.117900\end{array}$

Cl $\quad 4.574500 \quad-1.140100 \quad 1.189000$

$\mathrm{Cl} \quad 1.140300 \quad 4.574400 \quad 1.189300$

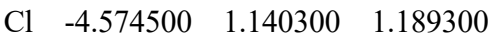

Cl $\quad-1.140300 \quad-4.574400 \quad 1.189400$

$\begin{array}{llll}\text { C } & 2.836900 & -4.674000 & 2.032200\end{array}$

$\begin{array}{llll}\text { C } & 2.604400 & -3.248800 & -0.057500\end{array}$

C $\quad 3.320400 \quad-3.816200 \quad-1.325900$

$\begin{array}{llll}\text { C } & 4.639300 & -2.919900 & 3.201700\end{array}$

$\begin{array}{llll}\text { C } & 3.176500 & -3.520500 & 1.316900\end{array}$

C $\quad 3.381800 \quad-4.960400 \quad 3.282300$

C $\quad 4.546100 \quad-4.653700 \quad-1.117900$

C $\quad 1.711400 \quad-2.022100-0.106200$

$\begin{array}{llll}\text { C } & 3.337700 & -3.012800 & -2.585300\end{array}$

$\begin{array}{llll}\text { C } & 3.943600 & -1.746400 & -2.614700\end{array}$

$\begin{array}{llll}\text { C } & 2.007400 & -4.398900 & -0.873800\end{array}$

C $\quad 4.537500 \quad-6.022100 \quad-1.445200$

$\begin{array}{llll}\text { C } & 4.083900 & -2.653500 & 1.951000\end{array}$

$\begin{array}{llll}\text { C } & 5.740800 & -4.070600 & -0.663000\end{array}$

$\begin{array}{llll}\text { C } & 4.296100 & -4.098400 & 3.892500\end{array}$

C $\quad 6.856900 \quad-6.215900 \quad-0.777300$

$\begin{array}{llll}\text { C } & 3.968800 & -1.005700 & -3.799400\end{array}$

$\begin{array}{llll}\text { C } & 2.816000 & -2.795600 & -4.949300\end{array}$

$\begin{array}{llll}\text { C } & 2.799400 & -3.544200 & -3.768700\end{array}$

$\begin{array}{llll}\text { C } & 6.886200 & -4.851000 & -0.482700\end{array}$

$\begin{array}{llll}\text { C } & 5.685400 & -6.799400 & -1.265100\end{array}$

C $\quad 3.396500 \quad-1.524500 \quad-4.963400$

$\begin{array}{llll}\text { C } & 4.674200 & 2.836700 & 2.032000\end{array}$

$\begin{array}{llll}\text { C } & 3.248800 & 2.604400 & -0.057500\end{array}$

C $\quad 3.816100 \quad 3.320400 \quad-1.326000$

$\begin{array}{llll}\text { C } & 2.920200 & 4.639000 & 3.201800\end{array}$

C $\quad 3.520600 \quad 3.176400 \quad 1.316800$

C $4.960800 \quad 3.381500 \quad 3.282100$

$\begin{array}{llll}\text { C } & 4.653800 & 4.546000 & -1.118000\end{array}$

$\begin{array}{llll}\text { C } & 2.022100 & 1.711400 & -0.106200\end{array}$

$\begin{array}{llll}\text { C } & 3.012600 & 3.337800 & -2.585300\end{array}$

$\begin{array}{llll}\text { C } & 1.746200 & 3.943900 & -2.614500\end{array}$

$\begin{array}{llll}\text { C } & 4.398800 & 2.007300 & -0.874000\end{array}$

$\begin{array}{llll}\text { C } & 6.022200 & 4.537200 & -1.445600\end{array}$

$\begin{array}{llll}\text { C } & 2.653700 & 4.083700 & 1.951100\end{array}$

$\begin{array}{llll}\text { C } & 4.070900 & 5.740700 & -0.662900\end{array}$

$\begin{array}{llll}\text { C } & 4.098700 & 4.295800 & 3.892500\end{array}$

$\begin{array}{llll}\text { C } & 6.216300 & 6.856600 & -0.777500\end{array}$

C $\quad 1.005400 \quad 3.969300 \quad-3.799100$

C $\quad 2.795000 \quad 2.816200 \quad-4.949300$

$\begin{array}{llll}\text { C } & 3.543800 & 2.799400 & -3.768800\end{array}$

$\begin{array}{llll}\text { C } & 4.851400 & 6.886000 & -0.482700\end{array}$

$\begin{array}{llll}\text { C } & 6.799600 & 5.685100 & -1.265500\end{array}$

C $\quad 1.524000 \quad 3.396800 \quad-4.963300$

$\begin{array}{lrrr}\text { C } & -2.836700 & 4.674200 & 2.032000\end{array}$

C $\quad-2.604400 \quad 3.248800-0.057500$

C $\quad-3.320400 \quad 3.816100 \quad-1.326000$

C $\quad-4.639100 \quad 2.920300 \quad 3.201800$

C $\quad-3.176400 \quad 3.520600 \quad 1.316800$

$\begin{array}{llll}\text { C } & -3.381600 & 4.960800 & 3.282100\end{array}$

C $\quad-4.546100 \quad 4.653700 \quad-1.118000$

$\begin{array}{llll}\text { C } & -1.711400 & 2.022100 & -0.106200\end{array}$

C $\quad-3.337800 \quad 3.012600 \quad-2.585300$

C $\quad-3.943900 \quad 1.746200 \quad-2.614500$

$\begin{array}{llll}\text { C } & -2.007300 & 4.398800 & -0.874000\end{array}$

$\begin{array}{llll}\text { C } & -4.537500 & 6.022100 & -1.445600\end{array}$ 


\begin{tabular}{|c|c|c|c|}
\hline $\mathrm{C}$ & -4.083800 & 2.653800 & 1.951100 \\
\hline $\mathrm{C}$ & -5.740800 & 4.070800 & -0.662900 \\
\hline $\mathrm{C}$ & -4.295900 & 4.098800 & 3.892500 \\
\hline $\mathrm{C}$ & -6.856700 & 6.216200 & -0.777500 \\
\hline & -3.969300 & 1.005300 & -3.799100 \\
\hline $\mathrm{C}$ & -2.816300 & 2.795000 & -4.949300 \\
\hline $\mathrm{C}$ & -2.799500 & 3.543800 & -3.768800 \\
\hline $\mathrm{C}$ & -6.886100 & 4.851300 & -0.482700 \\
\hline $\mathrm{C}$ & -5.685300 & 6.799500 & -1.265500 \\
\hline C & -3.396800 & 1.524000 & -4.963300 \\
\hline $\mathrm{C}$ & -4.674200 & -2.836700 & 2.032200 \\
\hline $\mathrm{C}$ & -3.248800 & -2.604400 & -0.057400 \\
\hline $\mathrm{C}$ & -3.816200 & -3.320500 & -1.325900 \\
\hline $\mathrm{C}$ & -2.920200 & -4.639000 & 3.202000 \\
\hline $\mathrm{C}$ & -3.520600 & -3.176400 & 1.316900 \\
\hline $\mathrm{C}$ & -4.960800 & -3.381500 & 3.282300 \\
\hline $\mathrm{C}$ & -4.653800 & -4.546100 & -1.117800 \\
\hline $\mathrm{C}$ & -2.022100 & -1.711400 & -0.106100 \\
\hline $\mathrm{C}$ & -3.012600 & -3.337900 & -2.585100 \\
\hline $\mathrm{C}$ & -1.746400 & -3.944000 & -2.614400 \\
\hline $\mathrm{C}$ & -4.398800 & -2.007400 & -0.873800 \\
\hline $\mathrm{C}$ & -6.022200 & -4.537400 & -1.445400 \\
\hline $\mathrm{C}$ & -2.653700 & -4.083700 & 1.951200 \\
\hline $\mathrm{C}$ & -4.070900 & -5.740800 & -0.662700 \\
\hline $\mathrm{C}$ & -4.098800 & -4.295800 & 3.892700 \\
\hline $\mathrm{C}$ & -6.216300 & -6.856700 & -0.777300 \\
\hline $\mathrm{C}$ & -1.005400 & -3.969400 & -3.798900 \\
\hline $\mathrm{C}$ & -2.795100 & -2.816400 & -4.949100 \\
\hline $\mathrm{C}$ & -3.543900 & -2.799600 & -3.768700 \\
\hline $\mathrm{C}$ & -4.851400 & -6.886000 & -0.482400 \\
\hline $\mathrm{C}$ & -6.799600 & -5.685200 & -1.265300 \\
\hline $\mathrm{C}$ & -1.524100 & -3.397000 & -4.963100 \\
\hline $\mathrm{C}$ & -5.721700 & 3.582200 & 5.748400 \\
\hline $\mathrm{C}$ & -3.582000 & -5.721600 & 5.748600 \\
\hline $\mathrm{C}$ & 3.582000 & 5.721600 & 5.748400 \\
\hline $\mathrm{C}$ & 5.722200 & -3.581600 & 5.748200 \\
\hline $\mathrm{H}$ & 2.113900 & -5.362800 & 1.609800 \\
\hline $\mathrm{H}$ & 5.327800 & -2.175700 & 3.586200 \\
\hline $\mathrm{H}$ & 3.076300 & -5.874700 & 3.785200 \\
\hline $\mathrm{H}$ & 4.392100 & -1.333800 & -1.722000 \\
\hline $\mathrm{H}$ & 1.979400 & -5.387500 & -0.427200 \\
\hline $\mathrm{H}$ & 1.115000 & -4.149500 & -1.436100 \\
\hline $\mathrm{H}$ & 3.644300 & -6.488200 & -1.841600 \\
\hline $\mathrm{H}$ & 5.792300 & -3.012000 & -0.459600 \\
\hline $\mathrm{H}$ & 7.745400 & -6.818600 & -0.639700 \\
\hline $\mathrm{H}$ & 4.438500 & -0.033000 & -3.816500 \\
\hline $\mathrm{H}$ & 2.386100 & -3.204300 & -5.854600 \\
\hline $\mathrm{H}$ & 2.370300 & -4.537900 & -3.775700 \\
\hline $\mathrm{H}$ & 7.799400 & -4.395300 & -0.122500 \\
\hline $\mathrm{H}$ & 5.668000 & -7.853800 & -1.509000 \\
\hline $\mathrm{H}$ & 3.414000 & -0.946900 & -5.878600 \\
\hline $\mathrm{H}$ & 5.363000 & 2.113800 & 1.609500 \\
\hline $\mathrm{H}$ & 2.176100 & 5.327400 & 3.586400 \\
\hline $\mathrm{H}$ & 5.875100 & 3.075900 & 3.785000 \\
\hline $\mathrm{H}$ & 1.333800 & 4.392400 & -1.721800 \\
\hline $\mathrm{H}$ & 5.387500 & 1.979200 & -0.427500 \\
\hline $\mathrm{H}$ & 4.149300 & 1.114900 & -1.436300 \\
\hline $\mathrm{H}$ & 6.488100 & 3.644100 & -1.842200 \\
\hline $\mathrm{H}$ & 3.012300 & 5.792300 & -0.459300 \\
\hline $\mathrm{H}$ & 6.819000 & 7.745000 & -0.639900 \\
\hline $\mathrm{H}$ & 0.032900 & 4.439100 & -3.816100 \\
\hline $\mathrm{H}$ & 3.203500 & 2.386300 & -5.854600 \\
\hline
\end{tabular}
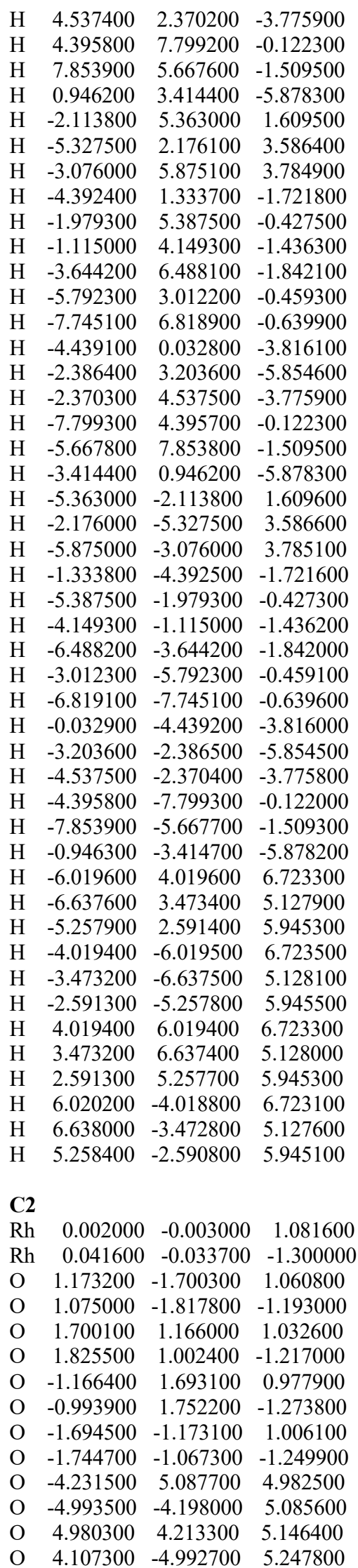

\begin{tabular}{|c|c|c|c|}
\hline & 4.383200 & -1.735100 & 1.271300 \\
\hline & 1.736100 & 4.379100 & 1.152900 \\
\hline & -4.380300 & 1.731400 & 1.082000 \\
\hline & -1.751100 & -4.383100 & 1.229900 \\
\hline & 2.183600 & -4.995500 & 2.138100 \\
\hline & 2.167000 & -3.584800 & 0.025900 \\
\hline & 2.818000 & -4.261100 & -1.224100 \\
\hline $\mathrm{C}$ & 4.187400 & -3.476700 & 3.308800 \\
\hline & 2.681300 & -3.908200 & 1.411800 \\
\hline $\mathrm{C}$ & 2.670600 & -5.332000 & 3.399600 \\
\hline & 3.919800 & -5.249300 & -0.986600 \\
\hline $\mathrm{C}$ & 1.444600 & -2.252100 & -0.052300 \\
\hline $\mathrm{C}$ & 2.957000 & -3.486800 & -2.494000 \\
\hline $\mathrm{C}$ & 3.722600 & -2.310300 & -2.531500 \\
\hline $\mathrm{C}$ & 1.433800 & -4.658800 & -0.783200 \\
\hline & 3.734800 & -6.609900 & -1.294100 \\
\hline $\mathrm{C}$ & 3.687400 & -3.158700 & 2.046800 \\
\hline $\mathrm{C}$ & 5.175500 & -4.821300 & -0.523600 \\
\hline & 3.683200 & -4.588800 & 4.010800 \\
\hline $\mathrm{C}$ & 6.000300 & -7.096400 & -0.590800 \\
\hline $\mathrm{C}$ & 3.859700 & -1.597800 & -3.725700 \\
\hline $\mathrm{C}$ & 2.500900 & -3.241400 & -4.868800 \\
\hline & 2.371000 & -3.962800 & -3.678300 \\
\hline & 6.205700 & -5.742600 & -0.315900 \\
\hline $\mathrm{C}$ & 4.768000 & -7.528600 & -1.086400 \\
\hline $\mathrm{C}$ & 3.241200 & -2.056500 & -4.891400 \\
\hline $\mathrm{C}$ & 4.993000 & 2.203900 & 2.091400 \\
\hline $\mathrm{C}$ & 3.588900 & 2.128400 & -0.024000 \\
\hline $\mathrm{C}$ & 4.269500 & 2.743500 & -1.289700 \\
\hline $\mathrm{C}$ & 3.470800 & 4.239800 & 3.200800 \\
\hline & 3.908100 & 2.681300 & 1.348000 \\
\hline $\mathrm{C}$ & 5.325500 & 2.725800 & 3.339900 \\
\hline $\mathrm{C}$ & 5.257600 & 3.851100 & -1.080400 \\
\hline $\mathrm{C}$ & 2.256100 & 1.404700 & -0.085900 \\
\hline $\mathrm{C}$ & 3.499000 & 2.847000 & -2.565300 \\
\hline & 2.324000 & 3.613200 & -2.628400 \\
\hline $\mathrm{C}$ & 4.665000 & 1.372300 & -0.808800 \\
\hline $\mathrm{C}$ & 6.618800 & 3.657000 & -1.379100 \\
\hline $\mathrm{C}$ & 3.156900 & 3.704900 & 1.952200 \\
\hline $\mathrm{C}$ & 4.829100 & 5.119400 & -0.653500 \\
\hline $\mathrm{C}$ & 4.580400 & 3.755100 & 3.920300 \\
\hline $\mathrm{C}$ & 7.104800 & 5.940900 & -0.737900 \\
\hline $\mathrm{C}$ & 1.615300 & 3.717200 & -3.828200 \\
\hline & 3.259500 & 2.322800 & -4.926600 \\
\hline & 3.977200 & 2.226000 & -3.730800 \\
\hline & 5.750400 & 6.154600 & -0.472200 \\
\hline $\mathrm{C}$ & 7.537500 & 4.695100 & -1.197900 \\
\hline & 2.076100 & 3.064400 & -4.974100 \\
\hline $\mathrm{C}$ & -2.209400 & 5.014100 & 1.936600 \\
\hline & -2.124600 & 3.549900 & -0.137300 \\
\hline & -2.734100 & 4.193700 & -1.424800 \\
\hline $\mathrm{C}$ & -4.249900 & 3.523700 & 3.080300 \\
\hline$C$ & -2.683600 & 3.908400 & 1.222400 \\
\hline & -2.736600 & 5.381900 & 3.173000 \\
\hline & -3.842600 & 5.187200 & -1.248900 \\
\hline $\mathrm{C}$ & -1.400700 & 2.215900 & -0.157400 \\
\hline $\mathrm{C}$ & -2.831700 & 3.387000 & -2.678400 \\
\hline & -3.597000 & 2.210200 & -2.711000 \\
\hline & -1.365000 & 4.603100 & -0.949400 \\
\hline & -3.647300 & 6.539300 & -1.585400 \\
\hline${ }^{2}$ & -3.709800 & 3.174500 & 1.843400 \\
\hline & -5.112800 & 4.770700 & -0.815800 \\
\hline
\end{tabular}




\begin{tabular}{|c|c|c|c|}
\hline C & -3.768300 & 4.653400 & 3.770000 \\
\hline $\mathrm{C}$ & -5.934100 & 7.042900 & -0.968800 \\
\hline $\mathrm{C}$ & -3.695100 & 1.467000 & -3.890400 \\
\hline $\mathrm{C}$ & -2.296900 & 3.080200 & -5.029400 \\
\hline C & -2.205900 & 3.832100 & -3.854300 \\
\hline C & -6.148800 & 5.696600 & -0.665500 \\
\hline $\mathrm{C}$ & -4.686300 & 7.462600 & -1.435300 \\
\hline $\mathrm{C}$ & -3.037400 & 1.895300 & -5.046200 \\
\hline $\mathrm{C}$ & -5.030600 & -2.165300 & 1.977900 \\
\hline $\mathrm{C}$ & -3.551300 & -2.156500 & -0.087900 \\
\hline $\mathrm{C}$ & -4.188000 & -2.806900 & -1.359000 \\
\hline $\mathrm{C}$ & -3.573400 & -4.157200 & 3.198500 \\
\hline $\mathrm{C}$ & -3.920700 & -2.668600 & 1.287200 \\
\hline $\mathrm{C}$ & -5.426400 & -2.634600 & 3.229600 \\
\hline $\mathrm{C}$ & -5.187000 & -3.905200 & -1.153100 \\
\hline $\mathrm{C}$ & -2.214100 & -1.439000 & -0.123700 \\
\hline $\mathrm{C}$ & -3.371500 & -2.950400 & -2.601700 \\
\hline $\mathrm{C}$ & -2.198500 & -3.722100 & -2.598600 \\
\hline $\mathrm{C}$ & -4.595500 & -1.421100 & -0.933300 \\
\hline $\mathrm{C}$ & -6.535800 & -3.716600 & -1.506800 \\
\hline $\mathrm{C}$ & -3.196600 & -3.674500 & 1.947100 \\
\hline $\mathrm{C}$ & -4.778900 & -5.161300 & -0.673600 \\
\hline $\mathrm{C}$ & -4.695700 & -3.656800 & 3.864500 \\
\hline $\mathrm{C}$ & -7.053000 & -5.979200 & -0.816500 \\
\hline $\mathrm{C}$ & -1.446800 & -3.864600 & -3.768000 \\
\hline $\mathrm{C}$ & -3.042800 & -2.496900 & -4.966900 \\
\hline $\mathrm{C}$ & -3.803500 & -2.362100 & -3.801700 \\
\hline $\mathrm{C}$ & -5.710000 & -6.188300 & -0.495400 \\
\hline $\mathrm{C}$ & -7.464300 & -4.746400 & -1.328600 \\
\hline $\mathrm{C}$ & -1.862100 & -3.244200 & -4.948900 \\
\hline $\mathrm{C}$ & -5.286100 & 4.346000 & 5.599300 \\
\hline $\mathrm{C}$ & -6.138100 & -3.693600 & 5.776900 \\
\hline $\mathrm{C}$ & 4.221500 & 5.265500 & 5.746300 \\
\hline $\mathrm{C}$ & 5.142300 & -4.235900 & 5.879200 \\
\hline $\mathrm{H}$ & 1.381300 & -5.589400 & 1.714900 \\
\hline $\mathrm{H}$ & 4.963400 & -2.823900 & 3.692800 \\
\hline $\mathrm{H}$ & 2.240900 & -6.190100 & 3.910600 \\
\hline $\mathrm{H}$ & 4.208500 & -1.945300 & -1.637800 \\
\hline $\mathrm{H}$ & 1.269900 & -5.628100 & -0.323500 \\
\hline $\mathrm{H}$ & 0.589200 & -4.302700 & -1.361800 \\
\hline $\mathrm{H}$ & 2.792800 & -6.960700 & -1.696200 \\
\hline $\mathrm{H}$ & 5.363700 & -3.775500 & -0.335100 \\
\hline $\mathrm{H}$ & 6.799700 & -7.808500 & -0.432000 \\
\hline $\mathrm{H}$ & 4.451600 & -0.694400 & -3.748900 \\
\hline $\mathrm{H}$ & 2.034200 & -3.605400 & -5.774900 \\
\hline $\mathrm{H}$ & 1.816900 & -4.892700 & -3.678200 \\
\hline $\mathrm{H}$ & 7.166600 & -5.405200 & 0.050400 \\
\hline $\mathrm{H}$ & 4.614600 & -8.575300 & -1.315100 \\
\hline $\mathrm{H}$ & 3.345900 & -1.500500 & -5.814100 \\
\hline $\mathrm{H}$ & 5.588000 & 1.390000 & 1.692800 \\
\hline $\mathrm{H}$ & 2.817000 & 5.026300 & 3.560900 \\
\hline $\mathrm{H}$ & 6.181700 & 2.310400 & 3.865600 \\
\hline $\mathrm{H}$ & 1.957300 & 4.125300 & -1.750100 \\
\hline $\mathrm{H}$ & 5.632800 & 1.221000 & -0.341800 \\
\hline $\mathrm{H}$ & 4.310300 & 0.512000 & -1.364700 \\
\hline $\mathrm{H}$ & 6.970200 & 2.704000 & -1.753900 \\
\hline $\mathrm{H}$ & 3.782900 & 5.313300 & -0.472900 \\
\hline П & 7.816900 & 6.744100 & -0.599500 \\
\hline $\mathrm{H}$ & 0.713300 & 4.310000 & -3.871100 \\
\hline $\mathrm{H}$ & 3.625200 & 1.829300 & -5.817800 \\
\hline $\mathrm{H}$ & 4.906000 & 1.670100 & -3.711900 \\
\hline $\mathrm{H}$ & 5.412600 & 7.125400 & -0.133600 \\
\hline
\end{tabular}

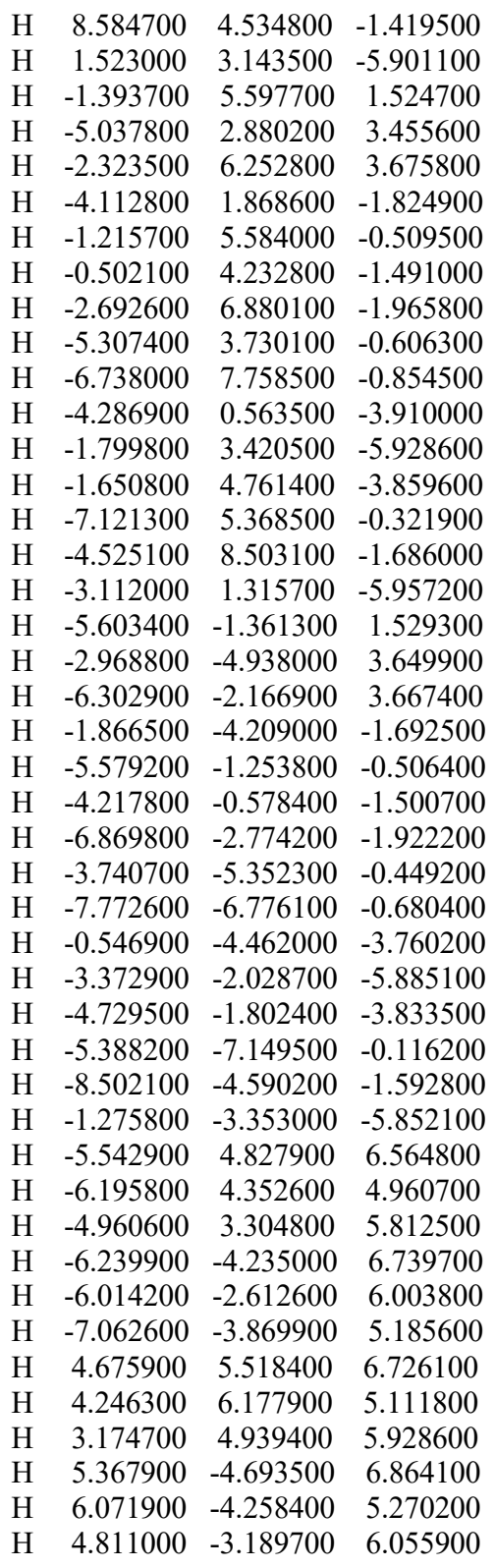

\section{C3}

$\begin{array}{llll}\text { Rh } & -0.003900 & 0.002100 & 1.076200\end{array}$ $\begin{array}{llll}\text { Rh } & -0.073200 & 0.002100 & -1.305300\end{array}$ $\begin{array}{llll}\mathrm{O} & -0.927200 & 1.845900 & 1.041000\end{array}$

$\begin{array}{llll}\mathrm{O} & -0.856000 & 1.910300 & -1.216100\end{array}$

$\begin{array}{llll}\mathrm{O} & -1.848800 & -0.919700 & 1.067700\end{array}$

$\begin{array}{lllll}\mathrm{O} & -1.978100 & -0.783800 & -1.183600\end{array}$

$\begin{array}{llll}\mathrm{O} & 0.915800 & -1.841700 & 0.987500\end{array}$

O $\quad 0.713600 \quad-1.905900 \quad-1.261600$

$\begin{array}{llll}\mathrm{O} & 1.837200 & 0.924100 & 0.960700\end{array}$

$\begin{array}{llll}\mathrm{O} & 1.836400 & 0.786700 & -1.294200\end{array}$

O $\quad 3.492200 \quad-5.593800 \quad 5.018100$

$\begin{array}{llll}\text { O } & 5.594700 & 3.499400 & 4.952200\end{array}$

$\begin{array}{llll}\mathrm{O} & -5.477900 & -3.385500 & 5.276900\end{array}$

$\begin{array}{llll}\text { O } & -3.389300 & 5.483600 & 5.211700\end{array}$

$\begin{array}{lllll}\mathrm{Cl} & -4.095300 & 2.348900 & 1.327200\end{array}$

$\mathrm{Cl} \quad-2.337500 \quad-4.092600 \quad 1.259400$
$\mathrm{Cl} \quad 4.090200 \quad-2.337500 \quad 1.075900$

$\mathrm{Cl} \quad 2.347500 \quad 4.097200 \quad 1.142600$

$\begin{array}{llll}\text { C } & -1.426200 & 5.274900 & 2.066300\end{array}$

C $\quad-1.661200 \quad 3.835800 \quad-0.014300$

$\begin{array}{llll}\text { C } & -2.237600 & 4.573800 & -1.266200\end{array}$

C $\quad-3.581300 \quad 4.096000 \quad 3.309800$

$\begin{array}{llll}\text { C } & -2.092400 & 4.255500 & 1.374000\end{array}$

$\begin{array}{llll}\text { C } & -1.813100 & 5.716600 & 3.330800\end{array}$

C $\quad-3.181200 \quad 5.714000 \quad-1.029100$

$\begin{array}{llll}\text { C } & -1.138400 & 2.412000 & -0.077900\end{array}$

C $\quad-2.515900 \quad 3.801500-2.514300$

$\begin{array}{llll}\text { C } & -1.893500 & 4.161700 & -3.720800\end{array}$

C $\quad-0.801300 \quad 4.777600 \quad-0.862300$

$\begin{array}{llll}\text { C } & -4.473700 & 5.479600 & -0.529900\end{array}$

$\begin{array}{llll}\text { C } & -3.178300 & 3.671300 & 2.045600\end{array}$

$\begin{array}{llll}\text { C } & -2.811500 & 7.027500 & -1.372300\end{array}$

C $\quad-2.916700 \quad 5.128400 \quad 3.977600$

$\begin{array}{llll}\text { C } & -4.966900 & 7.847600 & -0.633600\end{array}$

C $\quad-2.154500 \quad 3.442800 \quad-4.891000$

C $\quad-3.712700 \quad 2.040100 \quad-3.682900$

$\begin{array}{llll}\text { C } & -3.445300 & 2.749000 & -2.508800\end{array}$

C $\quad-3.697800 \quad 8.088700 \quad-1.164900$

$\begin{array}{llll}\text { C } & -5.356600 & 6.542900 & -0.322500\end{array}$

$\begin{array}{llll}C & -3.060400 & 2.379000 & -4.871000\end{array}$

C $\quad-5.243200 \quad-1.459500 \quad 2.177700$

$\begin{array}{llll}\text { C } & -3.867300 & -1.626800 & 0.049400\end{array}$

$\begin{array}{llll}\text { C } & -4.642600 & -2.164700 & -1.196900\end{array}$

$\begin{array}{llll}\text { C } & -4.010600 & -3.666300 & 3.319100\end{array}$

C $\quad-4.245200 \quad-2.100800 \quad 1.435900$

$\begin{array}{lrrr}\text { C } & -5.631300 & -1.902800 & 3.440400\end{array}$

C $\quad-5.775100 \quad-3.116400 \quad-0.955300$

C $\quad-2.446500 \quad-1.099700 \quad-0.040200$

C $\quad-3.908700 \quad-2.402600 \quad-2.476200$

C $\quad-4.306000 \quad-1.743300 \quad-3.651100$

$\begin{array}{llll}\text { C } & -4.834600 & -0.741900 & -0.742300\end{array}$

C $\quad-7.098300 \quad-2.737700 \quad-1.248200$

$\begin{array}{llll}\text { C } & -3.639000 & -3.207600 & 2.056100\end{array}$

C $\quad-5.525800 \quad-4.423400 \quad-0.503200$

C $\quad-5.032100 \quad-3.014600 \quad 4.037200$

$\begin{array}{llll}\text { C } & -7.895800 & -4.915600 & -0.552000\end{array}$

C $\quad-3.622700 \quad-1.966000 \quad-4.850200$

C $\quad-2.182900-3.559300 \quad-3.735200$

C $\quad-2.856000 \quad-3.330200 \quad-2.532100$

C $\quad-6.582500 \quad-5.313300 \quad-0.291700$

C $\quad-8.152600 \quad-3.630900-1.036800$

C $\quad-2.558200 \quad-2.870500 \quad-4.891300$

$\begin{array}{llll}\text { C } & 1.480600 & -5.268100 & 1.982100\end{array}$

$\begin{array}{llll}\text { C } & 1.589800 & -3.830400 & -0.108800\end{array}$

$\begin{array}{llll}\text { C } & 2.091300 & -4.568800 & -1.392100\end{array}$

$\begin{array}{llll}\text { C } & 3.719800 & -4.069900 & 3.096900\end{array}$

C $\quad 2.102000 \quad-4.249100 \quad 1.252100$

$\begin{array}{llll}\text { C } & 1.958500 & -5.693100 & 3.220100\end{array}$

$\begin{array}{llll}\text { C } & 3.048000 & -5.708300 & -1.210900\end{array}$

C $\quad 1.062200 \quad-2.407300 \quad-0.141900$

C $\quad 2.293800 \quad-3.797800 \quad-2.655500$

$\begin{array}{llll}\text { C } & 3.221200 & -2.744500 & -2.706900\end{array}$

C $\quad 0.681600 \quad-4.773500 \quad-0.903300$

$\begin{array}{llll}\text { C } & 4.367400 & -5.473000 & -0.788400\end{array}$

$\begin{array}{llll}\text { C } & 3.226400 & -3.661600 & 1.858600\end{array}$

$\begin{array}{llll}\text { C } & 2.659700 & -7.022000 & -1.532300\end{array}$

C $\quad 3.087300 \quad-5.111800 \quad 3.802800$

$\begin{array}{llll}\text { C } & 4.855200 & -7.840700 & -0.921600\end{array}$ 


\begin{tabular}{|c|c|c|c|}
\hline E & 3.416900 & -2.036500 & -3.895700 \\
\hline $\mathrm{C}$ & 1.790300 & -3.441800 & -5.006700 \\
\hline $\mathrm{C}$ & 1.600500 & -4.159800 & -3.822200 \\
\hline & 3.557300 & -8.082500 & -1.377500 \\
\hline & 5.261700 & -6.535900 & -0.633500 \\
\hline $\mathrm{C}$ & 2.694800 & -2.377200 & -5.042200 \\
\hline $\mathrm{C}$ & 5.295200 & 1.448900 & 1.870500 \\
\hline $\mathrm{C}$ & 3.795700 & 1.625700 & -0.172800 \\
\hline & 4.496100 & 2.166200 & -1.461800 \\
\hline & 4.152100 & 3.638400 & 3.087000 \\
\hline $\mathrm{C}$ & 4.255700 & 2.095800 & 1.189900 \\
\hline $\mathrm{C}$ & 5.773400 & 1.871100 & 3.110100 \\
\hline $\mathrm{C}$ & 5.642400 & 3.115800 & -1.285400 \\
\hline$c$ & 2.370700 & 1.101900 & -0.179800 \\
\hline & 3.687300 & 2.409100 & -2.694100 \\
\hline $\mathrm{C}$ & 4.011700 & 1.751900 & -3.892300 \\
\hline $\mathrm{C}$ & 4.712400 & 0.741900 & -1.023600 \\
\hline $\mathrm{C}$ & 5.422200 & 4.421700 & -0.816100 \\
\hline $\mathrm{C}$ & 3.690800 & 3.200300 & 1.847500 \\
\hline 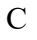 & 6.945300 & 2.736100 & -1.657100 \\
\hline $\mathrm{C}$ & 5.203900 & 2.992400 & 3.742700 \\
\hline $\mathrm{C}$ & 7.785700 & 4.911200 & -1.004300 \\
\hline $\mathrm{C}$ & 3.258500 & 1.979700 & -5.047700 \\
\hline $\mathrm{C}$ & 1.891800 & 3.573100 & -3.844100 \\
\hline C & 2.635100 & 3.338900 & -2.684100 \\
\hline $\mathrm{C}$ & 8.011500 & 3.627500 & 6500 \\
\hline $\mathrm{C}$ & 6.490800 & 5.309900 & -0.665500 \\
\hline $\mathrm{C}$ & 2.195700 & 2.886700 & -5.022400 \\
\hline $\mathrm{C}$ & 4.645600 & -5.002300 & 5.620700 \\
\hline $\mathrm{C}$ & 6.668500 & 2.846400 & 5.632800 \\
\hline $\mathrm{C}$ & -4.867900 & -4.521300 & 4100 \\
\hline $\mathrm{C}$ & -2.717100 & 6.537200 & 5.904900 \\
\hline $\mathrm{H}$ & -0.557700 & 5.734800 & 1.608300 \\
\hline $\mathrm{H}$ & -4.431300 & 3.601200 & 3.769800 \\
\hline $\mathrm{H}$ & -1.219300 & 6.513100 & 3.768400 \\
\hline $\mathrm{H}$ & -1.209900 & 5.000300 & -3.753900 \\
\hline $\mathrm{H}$ & -0.490300 & 5.722400 & -0.428500 \\
\hline $\mathrm{H}$ & -0.029800 & 4.292500 & -1.449000 \\
\hline $\mathrm{H}$ & -4.804400 & 4.475500 & -0.312700 \\
\hline $\mathrm{H}$ & -1.839100 & 7.231700 & -1.802200 \\
\hline $\mathrm{H}$ & -5.652500 & 8.669800 & -0.474800 \\
\hline $\mathrm{H}$ & -1.660500 & 3.715500 & -5.814600 \\
\hline $\mathrm{H}$ & -4.431100 & 1.233300 & -3.673200 \\
\hline $\mathrm{H}$ & -3.958500 & 2.477300 & -1.597100 \\
\hline $\mathrm{H}$ & -3.402300 & 9.097800 & -1.421300 \\
\hline $\mathrm{H}$ & -6.346700 & 6.354100 & 0.071700 \\
\hline $\mathrm{H}$ & -3.266600 & 1.825600 & -5.778100 \\
\hline $\mathrm{H}$ & -5.721700 & -0.578000 & 1.766000 \\
\hline $\mathrm{H}$ & -3.470100 & -4.529600 & 3.691100 \\
\hline $\mathrm{H}$ & -6.414100 & -1.359400 & 3.963900 \\
\hline $\mathrm{H}$ & -5.145600 & -1.060400 & -3.637100 \\
\hline $\mathrm{H}$ & -5.765900 & -0.445600 & -0.270500 \\
\hline $\mathrm{H}$ & -4.367600 & 0.048200 & -1.318900 \\
\hline $\mathrm{H}$ & -7.315200 & -1.752600 & -1.641700 \\
\hline $\mathrm{H}$ & -4.515800 & -4.759800 & -0.326100 \\
\hline $\mathrm{H}$ & -8.712900 & -5.606600 & -0.390200 \\
\hline $\mathrm{H}$ & -3.923800 & -1.443700 & -5.749100 \\
\hline $\mathrm{H}$ & -1.375600 & -4.276300 & -3.772600 \\
\hline $\mathrm{H}$ & -2.556700 & -3.871900 & -1.646000 \\
\hline $\mathrm{H}$ & -6.381900 & -6.315000 & 0.066300 \\
\hline $\mathrm{H}$ & -9.168900 & -3.328600 & -1.254200 \\
\hline 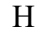 & -2.032400 & -3.047000 & -5.820800 \\
\hline
\end{tabular}
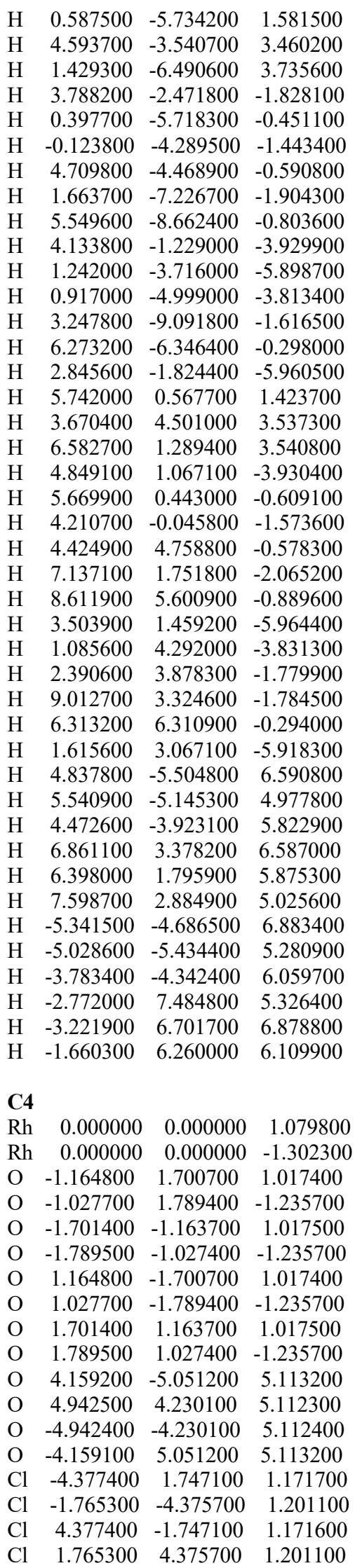

$\begin{array}{lrrr}\text { C } & -2.183200 & 5.011600 & 2.036600\end{array}$

C $\quad-2.134700 \quad 3.575000 \quad-0.057700$

$\begin{array}{llll}\text { C } & -2.762000 & 4.237800 & -1.326900\end{array}$

C $\quad-4.211100 \quad 3.512400 \quad 3.191200$

$\begin{array}{llll}\text { C } & -2.671700 & 3.916900 & 1.315100\end{array}$

C $\quad-2.690600 \quad 5.364600 \quad 3.285500$

C $\quad-3.864700 \quad 5.232000 \quad-1.120400$

$\begin{array}{llll}\text { C } & -1.415300 & 2.239300 & -0.107000\end{array}$

$\begin{array}{llll}\text { C } & -2.881600 & 3.448500 & -2.589600\end{array}$

$\begin{array}{llll}\text { C } & -3.649700 & 2.273600 & -2.625900\end{array}$

C $\quad-1.384400 \quad 4.636800 \quad-0.867200$

$\begin{array}{llll}\text { C } & -3.670300 & 6.588200 & -1.441100\end{array}$

$\begin{array}{llll}\text { C } & -3.690800 & 3.178000 & 1.941800\end{array}$

$\begin{array}{lrrr}\text { C } & -5.129500 & 4.813200 & -0.674000\end{array}$

$\begin{array}{llll}\text { C } & -3.715500 & 4.631400 & 3.888400\end{array}$

$\begin{array}{llll}\text { C } & -5.946000 & 7.089800 & -0.783000\end{array}$

C $\quad-3.768100 \quad 1.546700 \quad-3.813500$

$\begin{array}{llll}\text { C } & -2.385500 & 3.173000 & -4.953000\end{array}$

$\begin{array}{llll}\text { C } & -2.274100 & 3.908600 & -3.769400\end{array}$

C $\quad-6.160200 \quad 5.740000 \quad-0.495300$

$\begin{array}{llll}\text { C } & -4.704000 & 7.512300 & -1.262500\end{array}$

$\begin{array}{llll}\text { C } & -3.128300 & 1.989600 & -4.973800\end{array}$

$\begin{array}{llll}\text { C } & -5.024600 & -2.157600 & 2.031900\end{array}$

C $\quad-3.579800 \quad-2.126600 \quad-0.057900$

C $\quad-4.239600 \quad-2.758500 \quad-1.326400$

$\begin{array}{llll}\text { C } & -3.553800 & -4.169400 & 3.202500\end{array}$

$\begin{array}{llll}\text { C } & -3.928000 & -2.655300 & 1.316400\end{array}$

$\begin{array}{llll}\text { C } & -5.401100 & -2.641700 & 3.283900\end{array}$

C $\quad-5.238800 \quad-3.856300 \quad-1.118100$

C $\quad-2.241000 \quad-1.412700 \quad-0.106700$

C $\quad-3.444500 \quad-2.888500 \quad-2.584300$

C $\quad-2.274200 \quad-3.663900-2.610700$

C $\quad-4.635500 \quad-1.377100 \quad-0.876200$

C $\quad-6.592600 \quad-3.658900-1.446800$

$\begin{array}{llll}\text { C } & -3.196300 & -3.671800 & 1.951300\end{array}$

C $\quad-4.826900 \quad-5.119700 \quad-0.661500$

C $\quad-4.663200 \quad-3.674200 \quad 3.893500$

$\begin{array}{llll}\text { C } & -7.106000 & -5.928500 & -0.777000\end{array}$

C $\quad-1.542600 \quad-3.793700 \quad-3.794200$

C $\quad-3.153500 \quad-2.405500 \quad-4.948600$

C $\quad-3.894300 \quad-2.283300 \quad-3.769300$

C $\quad-5.758500 \quad-6.145900 \quad-0.481100$

C $\quad-7.521400 \quad-4.688000 \quad-1.266400$

$\begin{array}{llll}\text { C } & -1.975400 & -3.156800 & -4.959900\end{array}$

$\begin{array}{llll}\text { C } & 2.183300 & -5.011600 & 2.036600\end{array}$

$\begin{array}{llll}\text { C } & 2.134700 & -3.575100 & -0.057800\end{array}$

$\begin{array}{llll}\text { C } & 2.762000 & -4.237800 & -1.326900\end{array}$

C $\quad 4.211100 \quad-3.512400 \quad 3.191200$

$\begin{array}{llll}\text { C } & 2.671700 & -3.916800 & 1.315100\end{array}$

$\begin{array}{llll}\text { C } & 2.690600 & -5.364600 & 3.285500\end{array}$

C $\quad 3.864700 \quad-5.232000-1.120500$

C $\quad 1.415300 \quad-2.239300 \quad-0.107000$

$\begin{array}{llll}\text { C } & 2.881600 & -3.448500 & -2.589600\end{array}$

$\begin{array}{llll}\text { C } & 3.649700 & -2.273600 & -2.626000\end{array}$

C $\quad 1.384400 \quad-4.636800 \quad-0.867200$

$\begin{array}{llll}\text { C } & 3.670300 & -6.588200 & -1.441200\end{array}$

$\begin{array}{llll}\text { C } & 3.690800 & -3.178000 & 1.941800\end{array}$

C $\quad 5.129500 \quad-4.813200 \quad-0.674100$

$\begin{array}{llll}\text { C } & 3.715600 & -4.631400 & 3.888300\end{array}$

$\begin{array}{llll}\text { C } & 5.946000 & -7.089800 & -0.783100\end{array}$

C $\quad 3.768100 \quad-1.546700 \quad-3.813500$

$\begin{array}{llll}\text { C } & 2.385400 & -3.173000 & -4.953000\end{array}$ 


\begin{tabular}{|c|c|c|c|}
\hline E & 2.274000 & -3.908600 & -3.769400 \\
\hline $\mathrm{C}$ & 6.160200 & -5.740000 & -0.495300 \\
\hline $\mathrm{C}$ & 4.704000 & -7.512300 & -1.262500 \\
\hline & 3.128300 & -1.989600 & -4.973900 \\
\hline & 5.024600 & 2.157500 & 2.031900 \\
\hline $\mathrm{C}$ & 3.579700 & 2.126600 & -0.057900 \\
\hline $\mathrm{C}$ & 4.239600 & 2.758500 & -1.326500 \\
\hline $\mathrm{C}$ & 3.553900 & 4.169400 & 3.202500 \\
\hline & 3.928000 & 2.655300 & 1.316400 \\
\hline & 5.401100 & 2.641600 & 3.283900 \\
\hline $\mathrm{C}$ & 5.238800 & 3.856300 & -1.118100 \\
\hline $\mathrm{C}$ & 2.241000 & 1.412700 & -0.106700 \\
\hline $\mathrm{C}$ & 3.444400 & 2.888500 & -2.584300 \\
\hline$c$ & 2.274100 & 3.664000 & -2.610700 \\
\hline & 4.635400 & 1.377100 & -0.876300 \\
\hline $\mathrm{C}$ & 6.592600 & 3.658900 & -1.446900 \\
\hline $\mathrm{C}$ & 3.196300 & 3.671800 & 1.951300 \\
\hline $\mathrm{C}$ & 4.827000 & 5.119700 & -0.661500 \\
\hline $\mathrm{C}$ & 4.663300 & 3.674100 & 3.893400 \\
\hline & 7.106000 & 5.928500 & -0.777100 \\
\hline $\mathrm{C}$ & 1.542600 & 3.793800 & -3.794200 \\
\hline $\mathrm{C}$ & 3.153500 & 2.405600 & -4.948600 \\
\hline $\mathrm{C}$ & 3.894200 & 2.283400 & -3.769300 \\
\hline $\mathrm{C}$ & 5.758400 & 6.145900 & -0.481100 \\
\hline C & 7.521400 & 4.688000 & -1.266400 \\
\hline $\mathrm{C}$ & 1.975300 & 3.156900 & -4.959900 \\
\hline $\mathrm{C}$ & 5.206800 & -4.304700 & 5.736000 \\
\hline $\mathrm{C}$ & 6.073800 & 3.731000 & 5.829000 \\
\hline $\mathrm{C}$ & -6.073700 & -3.731200 & 5.829000 \\
\hline $\mathrm{C}$ & -5.206800 & 4.304700 & 5.736000 \\
\hline $\mathrm{H}$ & -1.372100 & 5.598100 & 1.620000 \\
\hline $\mathrm{H}$ & -4.995300 & 2.866400 & 3.569800 \\
\hline $\mathrm{H}$ & -2.267100 & 6.227600 & 3.793400 \\
\hline $\mathrm{H}$ & -4.151800 & 1.921000 & -1.736300 \\
\hline $\mathrm{H}$ & -1.225300 & 5.611200 & -0.416600 \\
\hline $\mathrm{H}$ & -0.531100 & 4.271200 & -1.426800 \\
\hline $\mathrm{H}$ & -2.720400 & 6.931400 & -1.831200 \\
\hline $\mathrm{H}$ & -5.324100 & 3.770400 & -0.476100 \\
\hline $\mathrm{H}$ & -6.745800 & 7.806000 & -0.646600 \\
\hline $\mathrm{H}$ & -4.361700 & 0.644500 & -3.835800 \\
\hline $\mathrm{H}$ & -1.902400 & 3.524700 & -5.855400 \\
\hline $\mathrm{H}$ & -1.717600 & 4.837100 & -3.771100 \\
\hline $\mathrm{H}$ & -7.128300 & 5.409800 & -0.141500 \\
\hline $\mathrm{H}$ & -4.543400 & 8.555700 & -1.501200 \\
\hline $\mathrm{H}$ & -3.218700 & 1.422600 & -5.891300 \\
\hline $\mathrm{H}$ & -5.602100 & -1.346100 & 1.603300 \\
\hline $\mathrm{H}$ & -2.944400 & -4.957700 & 3.633700 \\
\hline $\mathrm{H}$ & -6.268700 & -2.177100 & 3.742200 \\
\hline $\mathrm{H}$ & -1.928800 & -4.163500 & -1.716600 \\
\hline $\mathrm{H}$ & -5.611300 & -1.212200 & -0.431000 \\
\hline $\mathrm{H}$ & -4.264300 & -0.528400 & -1.439100 \\
\hline $\mathrm{H}$ & -6.930300 & -2.710200 & -1.844500 \\
\hline $\mathrm{H}$ & -3.785800 & -5.316800 & -0.456900 \\
\hline $\mathrm{H}$ & -7.825800 & -6.724900 & -0.639200 \\
\hline $\mathrm{H}$ & -0.644800 & -4.394200 & -3.809200 \\
\hline $\mathrm{H}$ & -3.497400 & -1.924400 & -5.855100 \\
\hline $\mathrm{H}$ & -4.818800 & -1.720200 & -3.778400 \\
\hline$\Pi$ & -5.433600 & -7.113000 & -0.119600 \\
\hline $\mathrm{H}$ & -8.563000 & -4.525100 & -1.511200 \\
\hline $\mathrm{H}$ & -1.404500 & -3.255800 & -5.874200 \\
\hline $\mathrm{H}$ & 1.372100 & -5.598100 & 1.620000 \\
\hline & 4.995400 & -2.866300 & 3.569800 \\
\hline
\end{tabular}
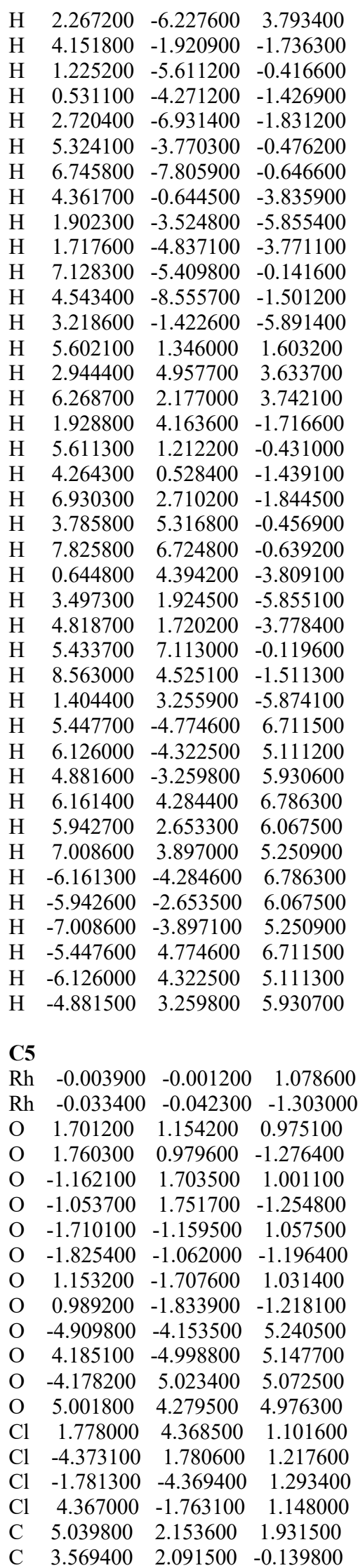

$\begin{array}{llll}\text { C } & 4.216200 & 2.698800 & -1.427000\end{array}$

C $\quad 3.589900 \quad 4.190600 \quad 3.084600$

$\begin{array}{llll}\text { C } & 3.936200 & 2.642700 & 1.220800\end{array}$

$\begin{array}{llll}\text { C } & 5.433000 & 2.658100 & 3.170300\end{array}$

$\begin{array}{llll}\text { C } & 5.221700 & 3.796200 & -1.249800\end{array}$

$\begin{array}{llll}\text { C } & 2.227400 & 1.381900 & -0.159900\end{array}$

$\begin{array}{llll}\text { C } & 3.406500 & 2.809900 & -2.677400\end{array}$

$\begin{array}{llll}\text { C } & 2.238900 & 3.589300 & -2.703500\end{array}$

$\begin{array}{llll}\text { C } & 4.612200 & 1.323900 & -0.957600\end{array}$

$\begin{array}{llll}\text { C } & 6.570700 & 3.587800 & -1.591300\end{array}$

$\begin{array}{llll}\text { C } & 3.215600 & 3.672600 & 1.846700\end{array}$

$\begin{array}{llll}\text { C } & 4.820000 & 5.068900 & -0.810200\end{array}$

$\begin{array}{llll}\text { C } & 4.706000 & 3.703500 & 3.770700\end{array}$

$\begin{array}{llll}\text { C } & 7.100400 & 5.866800 & -0.967100\end{array}$

$\begin{array}{llll}\text { C } & 1.493600 & 3.701300 & -3.880200\end{array}$

$\begin{array}{llll}\text { C } & 3.085500 & 2.286800 & -5.029200\end{array}$

C $\quad 3.839900 \quad 2.182400 \quad-3.856900$

$\begin{array}{llll}\text { C } & 5.757400 & 6.094400 & -0.658800\end{array}$

$\begin{array}{llll}\text { C } & 7.505400 & 4.616400 & -1.439900\end{array}$

$\begin{array}{llll}\text { C } & 1.910000 & 3.042300 & -5.039700\end{array}$

$\begin{array}{lllr}\text { C } & -2.136400 & 5.046100 & 1.970500\end{array}$

$\begin{array}{llll}C & -2.132000 & 3.566300 & -0.095000\end{array}$

$\begin{array}{llll}\text { C } & -2.775500 & 4.207500 & -1.367400\end{array}$

C $\quad-4.140900 \quad 3.602100 \quad 3.186300$

$\begin{array}{llll}\text { C } & -2.644900 & 3.939200 & 1.278900\end{array}$

C $\quad-2.606700 \quad 5.445400 \quad 3.220800$

C $\quad-3.867600 \quad 5.213700 \quad-1.163600$

C $\quad-1.423200 \quad 2.224200 \quad-0.129300$

$\begin{array}{llll}\text { C } & -2.921900 & 3.392400 & -2.610700\end{array}$

$\begin{array}{llll}\text { C } & -3.700200 & 2.223600 & -2.609200\end{array}$

C $\quad-1.387900 \quad 4.605500 \quad-0.938700$

C $\quad-3.669100 \quad 6.561400 \quad-1.516000$

$\begin{array}{llll}\text { C } & -3.657100 & 3.221700 & 1.936400\end{array}$

C $\quad-5.127500 \quad 4.813600 \quad-0.687200$

C $\quad-3.635300 \quad 4.721500 \quad 3.853100$

$\begin{array}{llll}\text { C } & -5.929800 & 7.093300 & -0.830600\end{array}$

C $\quad-3.845000 \quad 1.473100 \quad-3.779000$

C $\quad-2.467000 \quad 3.062200 \quad-4.975300$

C $\quad-2.329800 \quad 3.821800 \quad-3.809700$

C $\quad-6.148600 \quad 5.751500 \quad-0.510900$

$\begin{array}{llll}\text { C } & -4.693200 & 7.496500 & -1.339600\end{array}$

$\begin{array}{llll}\text { C } & -3.220600 & 1.885500 & -4.958900\end{array}$

$\begin{array}{llll}\text { C } & -5.023200 & -2.127900 & 2.130000\end{array}$

C $\quad-3.604600 \quad-2.134500 \quad 0.021900$

C $\quad-4.282800 \quad-2.784500 \quad-1.227700$

C $\quad-3.544200 \quad-4.126200 \quad 3.313300$

C $\quad-3.937000 \quad-2.640200 \quad 1.408800$

C $\quad-5.385700 \quad-2.591500 \quad 3.393800$

C $\quad-5.282700 \quad-3.875400 \quad-0.988700$

C $\quad-2.264200 \quad-1.426000 \quad-0.055500$

C $\quad-3.504500 \quad-2.937600 \quad-2.493500$

C $\quad-2.336400 \quad-3.716400 \quad-2.522200$

C $\quad-4.668100 \quad-1.394700 \quad-0.794800$

C $\quad-6.640100 \quad-3.678400 \quad-1.302700$

C $\quad-3.200600 \quad-3.649100 \quad 2.050300$

$\begin{array}{llll}\text { C } & -4.868900 & -5.132800 & -0.517700\end{array}$

$\begin{array}{llll}\text { C } & -4.643700 & -3.617000 & 4.010000\end{array}$

$\begin{array}{llll}\text { C } & -7.152000 & -5.935400 & -0.590200\end{array}$

C $\quad-1.620700 \quad-3.867900 \quad-3.712800$

C $\quad-3.243600 \quad-2.495200 \quad-4.869100$

C $\quad-3.968500 \quad-2.351400 \quad-3.682500$

$\begin{array}{llll}\text { C } & -5.801400 & -6.152800 & -0.308600\end{array}$ 


\begin{tabular}{|c|c|c|c|}
\hline $\mathrm{C}$ & -7.569800 & -4.701400 & -1.093700 \\
\hline $\mathrm{C}$ & -2.067200 & -3.249400 & -4.883200 \\
\hline $\mathrm{C}$ & 2.172600 & -5.004600 & 2.094700 \\
\hline $\mathrm{C}$ & 2.103600 & -3.603600 & -0.023100 \\
\hline $\mathrm{C}$ & 2.713200 & -4.290400 & -1.288100 \\
\hline $\mathrm{C}$ & 4.219000 & -3.493100 & 3.199300 \\
\hline $\mathrm{C}$ & 2.655800 & -3.924000 & 1.348900 \\
\hline $\mathrm{C}$ & 2.693700 & -5.338200 & 3.343200 \\
\hline $\mathrm{C}$ & 3.814600 & -5.285200 & -1.077800 \\
\hline $\mathrm{C}$ & 1.388400 & -2.266000 & -0.086600 \\
\hline $\mathrm{C}$ & 2.820500 & -3.523300 & -2.565400 \\
\hline $\mathrm{C}$ & 3.592900 & -2.352500 & -2.631200 \\
\hline $\mathrm{C}$ & 1.339800 & -4.676000 & -0.805300 \\
\hline $\mathrm{C}$ & 3.611300 & -6.645800 & -1.373200 \\
\hline $\mathrm{C}$ & 3.684800 & -3.178100 & 1.950700 \\
\hline $\mathrm{C}$ & 5.086100 & -4.863800 & -0.653400 \\
\hline $\mathrm{C}$ & 3.728200 & -4.598400 & 3.921200 \\
\hline $\mathrm{C}$ & 5.892800 & -7.145100 & -0.733400 \\
\hline $\mathrm{C}$ & 3.700200 & -1.646600 & -3.832400 \\
\hline $\mathrm{C}$ & 2.296900 & -3.286100 & -4.927000 \\
\hline $\mathrm{C}$ & 2.196800 & -4.000800 & -3.729700 \\
\hline $\mathrm{C}$ & 6.115400 & -5.791500 & -0.471100 \\
\hline $\mathrm{C}$ & 4.643700 & -7.570700 & -1.191100 \\
\hline $\mathrm{C}$ & 3.044400 & -2.106500 & -4.977200 \\
\hline $\mathrm{C}$ & -6.031000 & -3.640100 & 5.963000 \\
\hline $\mathrm{C}$ & 5.242600 & -4.245500 & 5100 \\
\hline $\mathrm{C}$ & -3.668600 & 6.165300 & 5.764500 \\
\hline $\mathrm{C}$ & 6.140000 & 3.788900 & 5.687800 \\
\hline $\mathrm{H}$ & 5.609200 & 1.332700 & 1.510100 \\
\hline $\mathrm{H}$ & 2.988300 & 4.988300 & 3.509400 \\
\hline $\mathrm{H}$ & 6.304600 & 2.198300 & 3.626000 \\
\hline $\mathrm{H}$ & 1.906300 & 4.106000 & -1.814200 \\
\hline $\mathrm{H}$ & 5.592800 & 1.162800 & -0.521300 \\
\hline $\mathrm{H}$ & 4.231200 & 0.467000 & -1.501100 \\
\hline $\mathrm{H}$ & 6.900200 & 2.631100 & -1.976500 \\
\hline $\mathrm{H}$ & 3.782100 & 5.273400 & -0.596400 \\
\hline $\mathrm{H}$ & 7.824700 & 6.662600 & -0.851800 \\
\hline $\mathrm{H}$ & 0.597700 & 4.304700 & -3.895000 \\
\hline $\mathrm{H}$ & 3.416500 & 1.788600 & -5.931200 \\
\hline $\mathrm{H}$ & 4.762100 & 1.615800 & -3.867200 \\
\hline $\mathrm{H}$ & 5.440500 & 7.068900 & -0.310200 \\
\hline $\mathrm{H}$ & 8.543300 & 4.445400 & -1.694500 \\
\hline $\mathrm{H}$ & 1.328500 & 3.127500 & -5.948600 \\
\hline $\mathrm{H}$ & -1.327600 & 5.613500 & 1.523900 \\
\hline $\mathrm{H}$ & -4.926600 & 3.002600 & 3.635700 \\
\hline $\mathrm{H}$ & -2.134500 & 6.319200 & 3.659500 \\
\hline $\mathrm{H}$ & -4.190400 & 1.894200 & -1.704000 \\
\hline $\mathrm{H}$ & -1.214800 & 5.587900 & -0.511300 \\
\hline $\mathrm{H}$ & -0.546500 & 4.222100 & -1.504200 \\
\hline $\mathrm{H}$ & -2.723600 & 6.889400 & -1.929000 \\
\hline $\mathrm{H}$ & -5.325900 & 3.776500 & -0.463800 \\
\hline $\mathrm{H}$ & -6.722300 & 7.818100 & -0.696000 \\
\hline $\mathrm{H}$ & -4.447000 & 0.576200 & -3.772500 \\
\hline $\mathrm{H}$ & -1.995800 & 3.390300 & -5.892800 \\
\hline $\mathrm{H}$ & -1.765500 & 4.744800 & -3.840400 \\
\hline $\mathrm{H}$ & -7.113000 & 5.435900 & -0.134100 \\
\hline $\mathrm{H}$ & -4.529500 & 8.533500 & -1.602900 \\
\hline $\mathrm{H}$ & -3.331200 & 1.300100 & -5.862500 \\
\hline $\mathrm{H}$ & -5.603300 & -1.321300 & 1.695900 \\
\hline $\mathrm{H}$ & -2.932000 & -4.909800 & 3.749100 \\
\hline $\mathrm{H}$ & -6.246300 & -2.116900 & 3.855400 \\
\hline П & -1.980600 & -4.201900 & -1.624400 \\
\hline
\end{tabular}
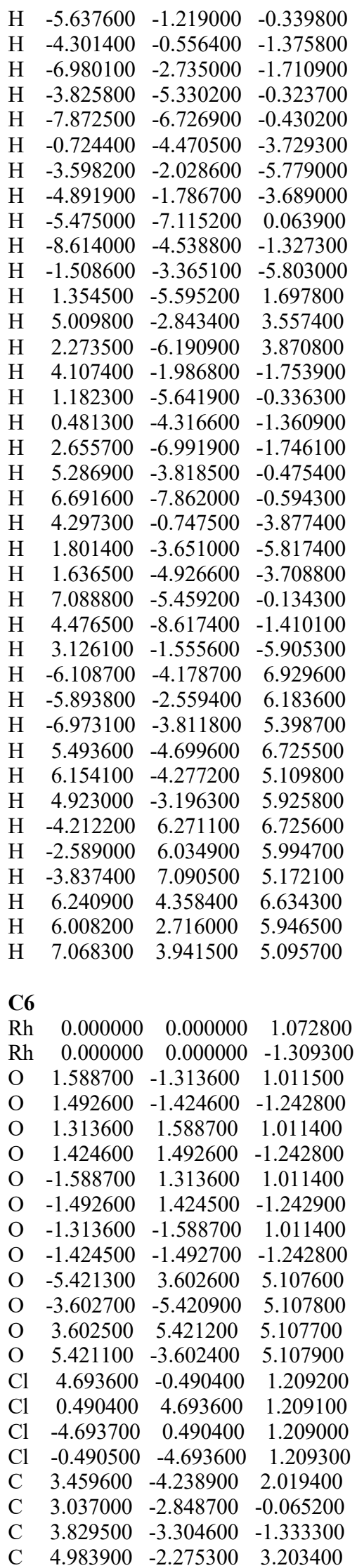

C $\quad 3.637000 \quad-3.044000 \quad 1.310100$

$\begin{array}{llll}\text { C } & 4.025800 & -4.473100 & 3.271700\end{array}$

$\begin{array}{llll}\text { C } & 5.158800 & -3.964700 & -1.123700\end{array}$

C $\quad 1.982200 \quad-1.758000 \quad-0.113200$

$\begin{array}{llll}\text { C } & 3.739900 & -2.499300 & -2.588400\end{array}$

C $\quad 4.165700 \quad-1.161500 \quad-2.608600$

$\begin{array}{llll}\text { C } & 2.608700 & -4.066400 & -0.889500\end{array}$

$\begin{array}{llll}\text { C } & 5.342100 & -5.318900 & -1.458800\end{array}$

$\begin{array}{llll}\text { C } & 4.410200 & -2.063200 & 1.951700\end{array}$

$\begin{array}{lrrr}\text { C } & 6.258900 & -3.223800 & -0.659700\end{array}$

C $\quad 4.812600 \quad-3.481700 \quad 3.888100$

$\begin{array}{llll}\text { C } & 7.663400 & -5.192000 & -0.781500\end{array}$

$\begin{array}{llll}\text { C } & 4.093700 & -0.417800 & -3.789600\end{array}$

C $\quad 3.202000 \quad-2.342600 \quad-4.953500$

$\begin{array}{llll}\text { C } & 3.283900 & -3.093000 & -3.776800\end{array}$

C $\quad 7.501200 \quad-3.838300 \quad-0.478800$

C $\quad 6.586400 \quad-5.930000 \quad-1.277800$

$\begin{array}{llll}\text { C } & 3.602500 & -1.003800 & -4.958800\end{array}$

$\begin{array}{llll}\text { C } & 4.238900 & 3.459600 & 2.019300\end{array}$

$\begin{array}{llll}\text { C } & 2.848700 & 3.037000 & -0.065300\end{array}$

$\begin{array}{llll}\text { C } & 3.304600 & 3.829400 & -1.333500\end{array}$

$\begin{array}{llll}\text { C } & 2.275300 & 4.984000 & 3.203300\end{array}$

$\begin{array}{llll}\text { C } & 3.044000 & 3.636900 & 1.310000\end{array}$

$\begin{array}{llll}\text { C } & 4.473100 & 4.025800 & 3.271600\end{array}$

C $\quad 3.964700 \quad 5.158700 \quad-1.123900$

$\begin{array}{llll}\text { C } & 1.758000 & 1.982200 & -0.113200\end{array}$

$\begin{array}{llll}\text { C } & 2.499400 & 3.739800 & -2.588500\end{array}$

$\begin{array}{llll}\text { C } & 1.161500 & 4.165700 & -2.608700\end{array}$

$\begin{array}{llll}\text { C } & 4.066400 & 2.608700 & -0.889600\end{array}$

C $\quad 5.318900 \quad 5.342000 \quad-1.459000$

$\begin{array}{llll}\text { C } & 2.063200 & 4.410300 & 1.951600\end{array}$

$\begin{array}{llll}\text { C } & 3.223800 & 6.258900 & -0.659900\end{array}$

$\begin{array}{llll}\text { C } & 3.481700 & 4.812700 & 3.888000\end{array}$

$\begin{array}{llll}\text { C } & 5.192000 & 7.663300 & -0.781700\end{array}$

$\begin{array}{llll}\text { C } & 0.417800 & 4.093700 & -3.789700\end{array}$

$\begin{array}{llll}\text { C } & 2.342600 & 3.201800 & -4.953600\end{array}$

C $\quad 3.093000 \quad 3.283800 \quad-3.776900$

C $\quad 3.838300 \quad 7.501100 \quad-0.479000$

$\begin{array}{llll}\text { C } & 5.930000 & 6.586300 & -1.278000\end{array}$

$\begin{array}{llll}\text { C } & 1.003800 & 3.602300 & -4.958900\end{array}$

C $\quad-3.459600 \quad 4.239000 \quad 2.019100$

$\begin{array}{llll}\text { C } & -3.037000 & 2.848700 & -0.065400\end{array}$

C $\quad-3.829400 \quad 3.304600-1.333600$

$\begin{array}{llll}\text { C } & -4.984000 & 2.275400 & 3.203200\end{array}$

$\begin{array}{llll}\text { C } & -3.637000 & 3.044100 & 1.309900\end{array}$

C $\quad-4.025800 \quad 4.473200 \quad 3.271400$

C $\quad-5.158800 \quad 3.964700 \quad-1.124000$

$\begin{array}{llll}\text { C } & -1.982200 & 1.757900 & -0.113300\end{array}$

$\begin{array}{llll}\text { C } & -3.739800 & 2.499300 & -2.588600\end{array}$

C $\quad-4.165700 \quad 1.161400 \quad-2.608800$

$\begin{array}{llll}\text { C } & -2.608700 & 4.066300 & -0.889800\end{array}$

$\begin{array}{llll}\text { C } & -5.342000 & 5.318800 & -1.459200\end{array}$

$\begin{array}{llll}\text { C } & -4.410300 & 2.063300 & 1.951500\end{array}$

C $\quad-6.258900 \quad 3.223800 \quad-0.660000$

$\begin{array}{llll}\text { C } & -4.812700 & 3.481800 & 3.887800\end{array}$

$\begin{array}{llll}\text { C } & -7.663300 & 5.192000 & -0.781900\end{array}$

$\begin{array}{llll}\text { C } & -4.093600 & 0.417700 & -3.789700\end{array}$

C $\quad-3.201700 \quad 2.342400 \quad-4.953700$

C $\quad-3.283800 \quad 3.092900 \quad-3.777100$

C $\quad-7.501200 \quad 3.838200 \quad-0.479200$

C $\quad-6.586200 \quad 5.929900 \quad-1.278200$

C $\quad-3.602300 \quad 1.003600 \quad-4.958900$ 


\begin{tabular}{|c|c|c|c|}
\hline & -4.239000 & -3.459500 & \\
\hline C & -2.848700 & -3.037000 & -0.065300 \\
\hline $\mathrm{C}$ & -3.304600 & -3.829500 & -1.333500 \\
\hline $\mathrm{C}$ & -2.275500 & -4.983800 & 3.203400 \\
\hline $\mathrm{C}$ & -3.044100 & -3.636900 & 1.310000 \\
\hline $\mathrm{C}$ & -4.473300 & -4.025600 & 3.271600 \\
\hline $\mathrm{C}$ & -3.964700 & -5.158800 & -1.123800 \\
\hline $\mathrm{C}$ & -1.757900 & -1.982200 & -0.113200 \\
\hline $\mathrm{C}$ & -2.499200 & -3.739900 & -2.588500 \\
\hline $\mathrm{C}$ & -1.161400 & -4.165800 & -2.608600 \\
\hline $\mathrm{C}$ & -4.066300 & -2.608700 & -0.889700 \\
\hline $\mathrm{C}$ & -5.318800 & -5.342000 & -1.459100 \\
\hline $\mathrm{C}$ & -2.063300 & -4.410200 & 1.951700 \\
\hline $\mathrm{C}$ & -3.223900 & -6.258900 & -0.659800 \\
\hline $\mathrm{C}$ & -3.481900 & -4.812600 & 3.888000 \\
\hline $\mathrm{C}$ & -5.192100 & -7.663300 & -0.781700 \\
\hline $\mathrm{C}$ & -0.417600 & -4.093900 & -3.789500 \\
\hline $\mathrm{C}$ & -2.342300 & -3.201900 & -4.953600 \\
\hline $\mathrm{C}$ & -3.092800 & -3.283900 & -3.777000 \\
\hline $\mathrm{C}$ & -3.838300 & -7.501100 & -0.478900 \\
\hline $\mathrm{C}$ & -5.930000 & -6.586300 & -1.278100 \\
\hline $\mathrm{C}$ & -1.003500 & -3.602600 & -4.958700 \\
\hline $\mathrm{C}$ & -5.252900 & 4.831500 & 5.817600 \\
\hline $\mathrm{C}$ & -4.831600 & -5.252500 & 5.817700 \\
\hline $\mathrm{C}$ & 4.831300 & 5.252800 & 5.817800 \\
\hline $\mathrm{C}$ & 5.252700 & -4.831300 & 5.817900 \\
\hline $\mathrm{H}$ & 2.840800 & -5.016200 & 1.585300 \\
\hline $\mathrm{H}$ & 5.572100 & -1.473900 & 3.640000 \\
\hline $\mathrm{H}$ & 3.817600 & -5.437500 & 3.724800 \\
\hline $\mathrm{H}$ & 4.549800 & -0.696100 & -1.711800 \\
\hline $\mathrm{H}$ & 2.716900 & -5.05 & 8100 \\
\hline $\mathrm{H}$ & 1.692200 & -3.940000 & -1.454100 \\
\hline $\mathrm{H}$ & 4.523900 & -5.902300 & -1.861900 \\
\hline $\mathrm{H}$ & 6.161500 & -2.169700 & -0.449600 \\
\hline $\mathrm{H}$ & 8.626600 & -5.665900 & -0.6 \\
\hline $\mathrm{H}$ & 4.425900 & 0.610000 & -3.800000 \\
\hline $\mathrm{H}$ & 2.835700 & -2.801400 & -5.862800 \\
\hline $\mathrm{H}$ & 2.995800 & -4.136200 & -3.790900 \\
\hline $\mathrm{H}$ & 8.340600 & -3.262100 & -0.111800 \\
\hline $\mathrm{H}$ & 6.716800 & -6.974900 & -1.527800 \\
\hline $\mathrm{H}$ & 3.544200 & -0.424300 & -5.871000 \\
\hline $\mathrm{H}$ & 5.016300 & 2.840800 & 1.585200 \\
\hline $\mathrm{H}$ & 1.473900 & 5.572200 & 3.639800 \\
\hline $\mathrm{H}$ & 5.437600 & 3.817700 & 3.724600 \\
\hline $\mathrm{H}$ & 0.696100 & 4.549800 & -1.711900 \\
\hline $\mathrm{H}$ & 5.051800 & 2.716900 & -0.448200 \\
\hline $\mathrm{H}$ & 3.940000 & 1.692200 & -1.454100 \\
\hline $\mathrm{H}$ & 5.902200 & 4.523900 & -1.862100 \\
\hline $\mathrm{H}$ & 2.169800 & 6.161500 & -0.449800 \\
\hline $\mathrm{H}$ & 5.665900 & 8.626600 & -0.643700 \\
\hline $\mathrm{H}$ & -0.610000 & 4.425900 & -3.800100 \\
\hline $\mathrm{H}$ & 2.801500 & 2.835500 & -5.862900 \\
\hline $\mathrm{H}$ & 4.136200 & 2.995700 & -3.791000 \\
\hline $\mathrm{H}$ & 3.262100 & 8.340600 & -0.112000 \\
\hline $\mathrm{H}$ & 6.975000 & 6.716800 & -1.528000 \\
\hline $\mathrm{H}$ & 0.424200 & 3.544000 & -5.871100 \\
\hline $\mathrm{H}$ & -2.840800 & 5.016200 & 1.585000 \\
\hline $\mathrm{H}$ & -5.572200 & 1.474000 & 3.639800 \\
\hline $\mathrm{H}$ & -3.817700 & 5.437700 & 3.724400 \\
\hline $\mathrm{H}$ & -4.549700 & 0.696000 & -1.711900 \\
\hline $\mathrm{H}$ & -2.716900 & 5.051800 & -0.448400 \\
\hline & -1.692200 & 3.940000 & -1.454300 \\
\hline
\end{tabular}
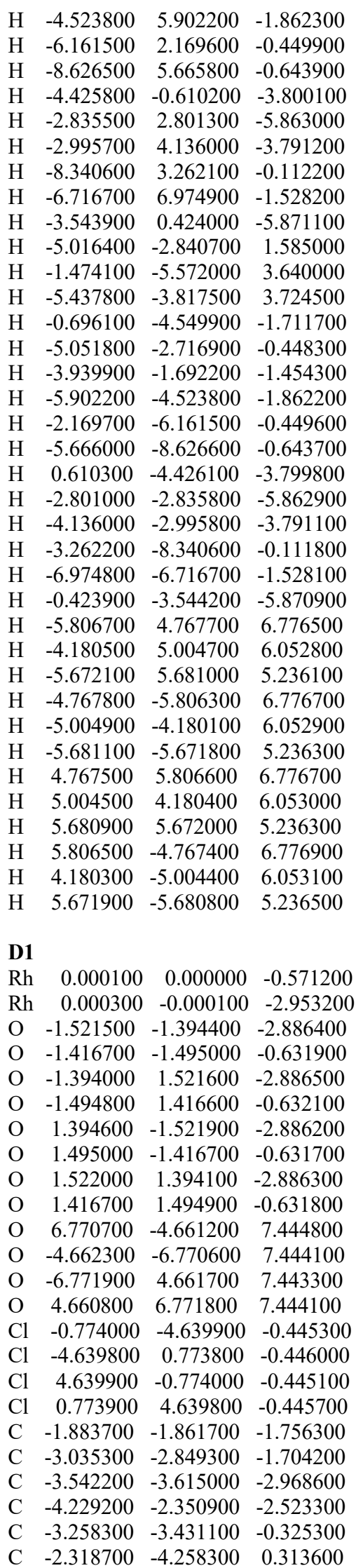

C $\quad-2.541900 \quad-4.807000 \quad 1.574400$

C $\quad-3.732100 \quad-4.546700 \quad 2.267700$

C $\quad-4.675200 \quad-3.712400 \quad 1.653700$

$\begin{array}{llll}\text { C } & -4.432700 & -3.172500 & 0.392100\end{array}$

$\begin{array}{llll}\text { C } & -3.974800 & -5.127500 & 3.615600\end{array}$

C $\quad-4.580000 \quad-4.358200 \quad 4.621700$

$\begin{array}{llll}\text { C } & -4.812400 & -4.895500 & 5.897000\end{array}$

$\begin{array}{llll}\text { C } & -4.459800 & -6.234500 & 6.164800\end{array}$

$\begin{array}{llll}\text { C } & -3.808600 & -6.998900 & 5.174500\end{array}$

C $\quad-3.583700 \quad-6.444100 \quad 3.905400$

C $\quad-2.738400 \quad-3.573500 \quad-4.227200$

C $\quad-1.427500 \quad-4.076100 \quad-4.252500$

C $\quad-0.684700 \quad-4.045500 \quad-5.435800$

C $\quad-1.245500 \quad-3.520000 \quad-6.602600$

$\begin{array}{llll}\text { C } & -2.558800 & -3.042700 & -6.592300\end{array}$

C $\quad-3.308700 \quad-3.082200 \quad-5.413000$

$\begin{array}{llll}\text { C } & -4.278000 & -4.903000 & -2.753300\end{array}$

C $\quad-5.642500 \quad-5.007000 \quad-3.080400$

C $\quad-6.325200 \quad-6.212100 \quad-2.890800$

$\begin{array}{llll}\text { C } & -5.649800 & -7.328900 & -2.393500\end{array}$

C $\quad-4.287000 \quad-7.246200 \quad-2.099000$

$\begin{array}{llll}\text { C } & -3.600900 & -6.043400 & -2.289000\end{array}$

$\begin{array}{llll}\text { C } & -1.861500 & 1.883800 & -1.756500\end{array}$

$\begin{array}{llll}C & -2.849100 & 3.035300 & -1.704400\end{array}$

C $\quad-3.614600 \quad 3.542300 \quad-2.969000$

C $\quad-2.350600 \quad 4.229300 \quad-2.523400$

C $\quad-3.431000 \quad 3.258300 \quad-0.325600$

$\begin{array}{llll}\text { C } & -4.258400 & 2.318600 & 0.313100\end{array}$

$\begin{array}{llll}\text { C } & -4.807300 & 2.541700 & 1.573800\end{array}$

$\begin{array}{llll}\text { C } & -4.547100 & 3.731900 & 2.267300\end{array}$

$\begin{array}{llll}\text { C } & -3.712600 & 4.675000 & 1.653400\end{array}$

$\begin{array}{llll}\text { C } & -3.172500 & 4.432600 & 0.391900\end{array}$

C $\quad-5.128100 \quad 3.974600 \quad 3.615000$

C $\quad-4.359000 \quad 4.579600 \quad 4.621400$

$\begin{array}{llll}\text { C } & -4.896400 & 4.811800 & 5.896600\end{array}$

$\begin{array}{llll}\text { C } & -6.235500 & 4.459300 & 6.164000\end{array}$

C $\quad-6.999800 \quad 3.808300 \quad 5.173500$

C $\quad-6.444900 \quad 3.583600 \quad 3.904500$

C $\quad-3.572900 \quad 2.738500 \quad-4.227500$

$\begin{array}{llll}\text { C } & -4.075500 & 1.427700 & -4.253000\end{array}$

$\begin{array}{llll}\text { C } & -4.044700 & 0.684900 & -5.436300\end{array}$

$\begin{array}{llll}\text { C } & -3.519000 & 1.245700 & -6.603000\end{array}$

C $\quad-3.041800 \quad 2.559100 \quad-6.592600$

$\begin{array}{llll}\text { C } & -3.081400 & 3.308800 & -5.413300\end{array}$

$\begin{array}{llll}\text { C } & -4.902600 & 4.278100 & -2.753700\end{array}$

C $\quad-5.006600 \quad 5.642600 \quad-3.080900$

C $\quad-6.211700 \quad 6.325300 \quad-2.891300$

$\begin{array}{llll}\text { C } & -7.328600 & 5.649900 & -2.394200\end{array}$

$\begin{array}{llll}\text { C } & -7.245900 & 4.287100 & -2.099800\end{array}$

$\begin{array}{llll}\text { C } & -6.043100 & 3.601000 & -2.289700\end{array}$

C $\quad 1.861800 \quad-1.884000 \quad-1.756000$

C $\quad 2.849300 \quad-3.035500 \quad-1.703700$

$\begin{array}{llll}\text { C } & 3.615100 & -3.542600 & -2.968000\end{array}$

C $\quad 2.351000 \quad-4.229500 \quad-2.522700$

C $\quad 3.431100 \quad-3.258400 \quad-0.324700$

$\begin{array}{llll}\text { C } & 4.258400 & -2.318600 & 0.314000\end{array}$

$\begin{array}{llll}\text { C } & 4.807100 & -2.541700 & 1.574900\end{array}$

$\begin{array}{llll}\text { C } & 4.546700 & -3.731800 & 2.268300\end{array}$

$\begin{array}{llll}\text { C } & 3.712300 & -4.675000 & 1.654400\end{array}$

C $\quad 3.172500 \quad-4.432600 \quad 0.392800$

$\begin{array}{llll}\text { C } & 5.127600 & -3.974300 & 3.616200\end{array}$

C $\quad 6.444200 \quad-3.583300 \quad 3.905900$ 


\begin{tabular}{|c|c|c|c|}
\hline E & 6.999000 & -3.808000 & 5.175000 \\
\hline $\mathrm{C}$ & 6.234500 & -4.458900 & 6.165500 \\
\hline $\mathrm{C}$ & 4.895500 & -4.811400 & 5.897800 \\
\hline $\mathrm{C}$ & 4.358200 & -4.579200 & 4.622500 \\
\hline $\mathrm{C}$ & 3.573700 & -2.738900 & -4.226700 \\
\hline $\mathrm{C}$ & 4.076200 & -1.428100 & -4.252200 \\
\hline $\mathrm{C}$ & 4.045700 & -0.685500 & -5.435600 \\
\hline $\mathrm{C}$ & 3.520200 & -1.246400 & -6.602300 \\
\hline & 3.042900 & -2.559700 & -6.591900 \\
\hline & 3.082400 & -3.309400 & -5.412500 \\
\hline $\mathrm{C}$ & 4.903100 & -4.278400 & -2.752500 \\
\hline $\mathrm{C}$ & 5.007100 & -5.643000 & -3.079500 \\
\hline $\mathrm{C}$ & 6.212200 & -6.325700 & -2.889800 \\
\hline 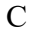 & 7.329000 & -5.650200 & -2.392500 \\
\hline & 7.246200 & -4.287300 & -2.098300 \\
\hline $\mathrm{C}$ & 6.043500 & -3.601300 & -2.288300 \\
\hline $\mathrm{C}$ & 1.884000 & 1.861500 & -1.756200 \\
\hline $\mathrm{C}$ & 3.035600 & 2.849100 & -1.704000 \\
\hline $\mathrm{C}$ & 3.542700 & 3.614700 & -2.968400 \\
\hline & 4.229600 & 2.350700 & -2.522800 \\
\hline $\mathrm{C}$ & 3.258400 & 3.431000 & -0.325000 \\
\hline $\mathrm{C}$ & 2.318600 & 4.258400 & 0.313600 \\
\hline $\mathrm{C}$ & 2.541600 & 4.807300 & 1.574300 \\
\hline $\mathrm{C}$ & 3.731700 & 4.547000 & 2.267900 \\
\hline C & 4.674900 & 3.712500 & 1.654100 \\
\hline $\mathrm{C}$ & 4.432600 & 3.172500 & 0.392600 \\
\hline $\mathrm{C}$ & 3.974200 & 5.128000 & 3.615700 \\
\hline $\mathrm{C}$ & 4.579100 & 4.358900 & 4.622100 \\
\hline $\mathrm{C}$ & 4.811200 & 4.896300 & 5.897300 \\
\hline $\mathrm{C}$ & 4.458600 & 6.235300 & 6.164800 \\
\hline $\mathrm{C}$ & 3.807700 & 6.999700 & 4200 \\
\hline $\mathrm{C}$ & 3.583100 & 6.444700 & 3.905100 \\
\hline $\mathrm{C}$ & 2.739100 & 3.573100 & -4.227000 \\
\hline $\mathrm{C}$ & 1.428200 & 4.075600 & -4.252700 \\
\hline $\mathrm{C}$ & 0.685600 & 4.044900 & -5.436100 \\
\hline $\mathrm{C}$ & 1.246600 & 3.519300 & -6.602700 \\
\hline $\mathrm{C}$ & 2.560000 & 3.042100 & -6.592100 \\
\hline $\mathrm{C}$ & 3.309500 & 3.081600 & -5.412800 \\
\hline $\mathrm{C}$ & 4.278500 & 4.902700 & -2.753000 \\
\hline $\mathrm{C}$ & 5.643000 & 5.006700 & -3.080000 \\
\hline $\mathrm{C}$ & 6.325700 & 6.211900 & -2.890300 \\
\hline $\mathrm{C}$ & 5.650200 & 7.328700 & -2.393200 \\
\hline $\mathrm{C}$ & 4.287400 & 7.246000 & -2.099000 \\
\hline $\mathrm{C}$ & 3.601400 & 6.043200 & -2.289000 \\
\hline $\mathrm{C}$ & -8.393500 & 3.327600 & 5.474400 \\
\hline $\mathrm{C}$ & -4.029200 & 5.400400 & 6.976200 \\
\hline $\mathrm{C}$ & -7.421600 & 5.925000 & 7.588000 \\
\hline $\mathrm{C}$ & 3.326900 & 8.393300 & 5.475100 \\
\hline $\mathrm{C}$ & 5.399600 & 4.029000 & 6.977000 \\
\hline $\mathrm{C}$ & 5.924100 & 7.421400 & 7.588900 \\
\hline $\mathrm{C}$ & -5.401200 & -4.028100 & 6.976300 \\
\hline $\mathrm{C}$ & -3.327700 & -8.392400 & 5.475700 \\
\hline $\mathrm{C}$ & -5.925500 & -7.420400 & 7.588700 \\
\hline $\mathrm{C}$ & 7.420300 & -5.924400 & 7.589700 \\
\hline $\mathrm{C}$ & 4.028100 & -5.399900 & 6.977300 \\
\hline $\mathrm{C}$ & 8.392600 & -3.327200 & 5.476100 \\
\hline $\mathrm{H}$ & -5.217100 & -2.400700 & -2.076900 \\
\hline $\mathrm{H}$ & -4.052400 & -1.444200 & -3.090100 \\
\hline $\mathrm{H}$ & -1.753000 & -5.417800 & 2.002300 \\
\hline $\mathrm{H}$ & -5.620000 & -3.475100 & 2.136100 \\
\hline $\mathrm{H}$ & -5.177200 & -2.513700 & -0.040400 \\
\hline $\mathrm{H}$ & -0.981900 & -4.487100 & -3.357700 \\
\hline
\end{tabular}

H $\quad 0.322700 \quad-4.435500 \quad-5.449800$

$\mathrm{H} \quad-0.666500 \quad-3.493800 \quad-7.516700$

$\mathrm{H} \quad-2.998700 \quad-2.649200 \quad-7.499500$

$\mathrm{H} \quad-4.333000 \quad-2.732700 \quad-5.422900$

H $\quad-6.178700 \quad-4.157100 \quad-3.483500$

$\mathrm{H} \quad-7.377500 \quad-6.281400 \quad-3.134400$

$\mathrm{H} \quad-6.179300 \quad-8.261600 \quad-2.248200$

H $\quad-3.759600 \quad-8.116800 \quad-1.731300$

$\mathrm{H} \quad-2.541800 \quad-6.008200 \quad-2.085400$

$\mathrm{H} \quad-2.400400 \quad 5.217200 \quad-2.077000$

$\mathrm{H} \quad-1.443800 \quad 4.052400 \quad-3.090100$

$\mathrm{H} \quad-5.418200 \quad 1.752800 \quad 2.001500$

$\mathrm{H} \quad-3.475400 \quad 5.619800 \quad 2.136000$

H $\quad-2.513600 \quad 5.177200 \quad-0.040500$

$\mathrm{H} \quad-4.486600 \quad 0.982000 \quad-3.358200$

$\mathrm{H} \quad-4.434700 \quad-0.322400 \quad-5.450400$

H $\quad-3.492800 \quad 0.666800 \quad-7.517100$

$\mathrm{H} \quad-2.648100 \quad 2.999000 \quad-7.499700$

$\mathrm{H} \quad-2.731900 \quad 4.333200 \quad-5.423100$

$\mathrm{H} \quad-4.156700 \quad 6.178900 \quad-3.483700$

$\mathrm{H} \quad-6.280900 \quad 7.377600 \quad-3.134900$

$\mathrm{H} \quad-8.261300 \quad 6.179400 \quad-2.249100$

$\mathrm{H} \quad-8.116500 \quad 3.759700 \quad-1.732200$

$\mathrm{H} \quad-6.007900 \quad 2.541900 \quad-2.086100$

$\mathrm{H} \quad 2.400700 \quad-5.217400 \quad-2.076100$

$\mathrm{H} \quad 1.444200 \quad-4.052800 \quad-3.089500$

H $\quad 5.417900 \quad-1.752800 \quad 2.002600$

$\mathrm{H} \quad 3.475000 \quad-5.619700 \quad 2.137000$

$\mathrm{H} \quad 2.513600 \quad-5.177200 \quad-0.039600$

H $\quad 4.487200 \quad-0.982300 \quad-3.357400$

H $\quad 4.435700 \quad 0.321900 \quad-5.449700$

$\mathrm{H} \quad 3.494000 \quad-0.667500 \quad-7.516500$

H $\quad 2.649400 \quad-2.999700 \quad-7.499000$

$\mathrm{H} \quad 2.732800 \quad-4.333700 \quad-5.422200$

$\mathrm{H} \quad 4.157300 \quad-6.179200 \quad-3.482500$

H $\quad 6.281400 \quad-7.378000 \quad-3.133200$

H $\quad 8.261700 \quad-6.179600 \quad-2.247200$

H $\quad 8.116800 \quad-3.759900 \quad-1.730500$

H $\quad 6.008200 \quad-2.542200 \quad-2.084900$

H $\quad 5.217500 \quad 2.400400 \quad-2.076200$

$\mathrm{H} \quad 4.052800 \quad 1.443800 \quad-3.089600$

$\mathrm{H} \quad 1.752600 \quad 5.418100 \quad 2.001900$

H $\quad 5.619600 \quad 3.475300 \quad 2.136800$

$\begin{array}{llll}\mathrm{H} & 5.177200 & 2.513600 & -0.039700\end{array}$

$\mathrm{H} \quad 0.982500 \quad 4.486700 \quad-3.357900$

$\mathrm{H} \quad-0.321700 \quad 4.434900 \quad-5.450300$

$\mathrm{H} \quad 0.667800 \quad 3.493000 \quad-7.516900$

H $2.999900 \quad 2.648400 \quad-7.499300$

H $\quad 4.334000 \quad 2.732000 \quad-5.422400$

$\mathrm{H} \quad 6.179300 \quad 4.156800 \quad-3.482800$

$\mathrm{H} \quad 7.378100 \quad 6.281000 \quad-3.133700$

$\begin{array}{llll}\mathrm{H} & 6.179700 & 8.261400 & -2.248000\end{array}$

$\mathrm{H} \quad 3.759900 \quad 8.116600 \quad-1.731400$

H $\quad 2.542200 \quad 6.007900 \quad-2.085500$

$\mathrm{H} \quad-7.045500 \quad 3.111500 \quad 3.136400$

$\mathrm{H} \quad-3.328400 \quad 4.846300 \quad 4.420400$

H $\quad-4.846900 \quad-3.327700 \quad 4.420500$

H $\quad-3.111600 \quad-7.044900 \quad 3.137400$

H $\quad 3.327700 \quad-4.846000 \quad 4.421400$

H $\quad 7.045100 \quad-3.111400 \quad 3.137800$

$\mathrm{H} \quad-8.376600 \quad 2.659900 \quad 6.361400$

H $\quad-9.059900 \quad 4.189600 \quad 5.677500$
$\mathrm{H} \quad-8.822700 \quad 2.757500 \quad 4.623100$

$\mathrm{H} \quad-4.418100 \quad 6.392600 \quad 7.280800$

$\mathrm{H} \quad-4.017100 \quad 4.724900 \quad 7.857400$

$\begin{array}{llll}\mathrm{H} & -2.981700 & 5.535800 & 6.632000\end{array}$

$\begin{array}{llll}\mathrm{H} & -8.272000 & 6.019500 & 6.881200\end{array}$

$\mathrm{H} \quad-7.816700 \quad 6.008900 \quad 8.620900$

H $\quad-6.711500 \quad 6.760700 \quad 7.417800$

H $\quad 2.659200 \quad 8.376400 \quad 6.362000$

H $\quad 4.188900 \quad 9.059700 \quad 5.678300$

H $\quad 2.757000 \quad 8.822600 \quad 4.623700$

$\mathrm{H} \quad 6.391700 \quad 4.417900 \quad 7.281800$

H $\quad 4.724000 \quad 4.016900 \quad 7.858100$

$\begin{array}{llll}\mathrm{H} & 5.535100 & 2.981700 & 6.632800\end{array}$

$\mathrm{H} \quad 6.018700 \quad 8.271800 \quad 6.882200$

H $\quad 6.007800 \quad 7.816400 \quad 8.621900$

$\begin{array}{llll}\mathrm{H} & 6.759700 & 6.711300 & 7.418800\end{array}$

H $\quad-6.393400 \quad-4.416900 \quad 7.280900$

$\mathrm{H} \quad-4.725900 \quad-4.015800 \quad 7.857600$

$\begin{array}{llll}\mathrm{H} & -5.536700 & -2.980700 & 6.631900\end{array}$

H $\quad-2.660200 \quad-8.375200 \quad 6.362800$

$\mathrm{H} \quad-4.189700 \quad-9.058900 \quad 5.678900$

$\mathrm{H} \quad-2.757500 \quad-8.821800 \quad 4.624500$

H $\quad-6.019900 \quad-8.271000 \quad 6.882000$

$\mathrm{H} \quad-6.009500 \quad-7.815300 \quad 8.621700$

H $\quad-6.761200 \quad-6.710400 \quad 7.418300$

H $\quad 8.270900 \quad-6.019000 \quad 6.883000$

H $\quad 7.815300 \quad-6.008200 \quad 8.622800$

$\mathrm{H} \quad 6.710200 \quad-6.760100 \quad 7.419500$

H $\quad 4.416900 \quad-6.392000 \quad 7.282100$

H $\quad 4.015800 \quad-4.724300 \quad 7.858400$

H $\quad 2.980700 \quad-5.535400 \quad 6.632900$

$\mathrm{H} \quad 8.375600 \quad-2.659500 \quad 6.363100$

H $\quad 9.059000 \quad-4.189200 \quad 5.679400$

H $\quad 8.822000 \quad-2.757200 \quad 4.624800$

$\mathrm{H} \quad 3.111200 \quad 7.045400 \quad 3.137000$

H $\quad 4.845800 \quad 3.328200 \quad 4.421100$

\section{D2}

Rh $\quad 0.077200 \quad-0.069800 \quad-0.583100$

$\begin{array}{llll}\mathrm{Rh} & 0.318100 & -0.324000 & -2.939600\end{array}$

O $\quad-0.076400 \quad 1.694000 \quad-3.130200$

$\begin{array}{llll}\mathrm{O} & -0.440000 & 1.897800 & -0.909900\end{array}$

$\begin{array}{llll}\mathrm{O} & 2.327900 & 0.092800 & -2.711800\end{array}$

$\begin{array}{llll}\mathrm{O} & 2.064400 & 0.467300 & -0.499500\end{array}$

$\begin{array}{llll}\mathrm{O} & -1.705000 & -0.726500 & -3.034800\end{array}$

$\begin{array}{llll}\mathrm{O} & -1.898500 & -0.618200 & -0.786600\end{array}$

$\begin{array}{llll}\mathrm{O} & 0.699400 & -2.328000 & -2.616900\end{array}$

$\begin{array}{llll}\mathrm{O} & 0.606700 & -2.050500 & -0.376700\end{array}$

$\begin{array}{llll}\mathrm{O} & -8.246500 & -2.522600 & 6.940900\end{array}$

$\begin{array}{llll}\mathrm{O} & -4.002100 & 8.217800 & 6.220800\end{array}$

$\begin{array}{llll}\mathrm{O} & 6.735300 & 4.139100 & 7.758200\end{array}$

$\begin{array}{llll}\text { O } & 2.583100 & -6.642200 & 8.486200\end{array}$

$\mathrm{Cl} \quad-3.453300 \quad 2.993400 \quad-1.136600$

Cl $3.150400 \quad 3.492600 \quad-0.515900$

Cl $\quad-3.027100 \quad-3.598200 \quad-0.374500$

$\begin{array}{lrrr}\mathrm{Cl} & 3.585100 & -3.095800 & 0.230800\end{array}$

C $\quad-0.393500 \quad 2.363700 \quad-2.092100$

C $\quad-0.629300 \quad 3.857300 \quad-2.225000$

C $\quad-0.886900 \quad 4.546500 \quad-3.603800$

C $\quad 0.499000 \quad 4.526600 \quad-3.014700$

C $\quad-1.138500 \quad 4.495400 \quad-0.951100$

$\begin{array}{llll}\text { C } & -2.393400 & 4.191400 & -0.395600\end{array}$ 


\begin{tabular}{|c|c|c|c|}
\hline C & -2.862300 & 4.799300 & 0.766600 \\
\hline $\mathrm{C}$ & -2.084800 & 5.751500 & 1.440700 \\
\hline $\mathrm{C}$ & -0.825100 & 6.058600 & 0.909900 \\
\hline $\mathrm{C}$ & -0.373700 & 5.438500 & -0.253600 \\
\hline $\mathrm{C}$ & -2.582200 & 6.398800 & 2.684400 \\
\hline C & -1.711100 & 6.644000 & 3.757600 \\
\hline $\mathrm{C}$ & -2.173900 & 7.247800 & 4.936700 \\
\hline $\mathrm{C}$ & -3.536800 & 7.589500 & 5.057200 \\
\hline $\mathrm{C}$ & -4.407800 & 7.391700 & 3.965700 \\
\hline $\mathrm{C}$ & -3.926900 & 6.786300 & 2.794800 \\
\hline $\mathrm{C}$ & -1.151400 & 3.706000 & -4.810200 \\
\hline $\mathrm{C}$ & -2.263900 & 2.850100 & -4.852600 \\
\hline $\mathrm{C}$ & -2.513200 & 2.075700 & -5.988900 \\
\hline $\mathrm{C}$ & -1.659600 & 2.153700 & -7.092100 \\
\hline $\mathrm{C}$ & -0.565700 & 3.023000 & -7.068200 \\
\hline $\mathrm{C}$ & -0.319800 & 3.807600 & -5.937600 \\
\hline $\mathrm{C}$ & -1.609800 & 5.859500 & -3.600800 \\
\hline $\mathrm{C}$ & -0.949000 & 7.037100 & -3.996300 \\
\hline $\mathrm{C}$ & -1.627200 & 8.259500 & -4.005100 \\
\hline $\mathrm{C}$ & -2.974300 & 8.315800 & -3.640200 \\
\hline $\mathrm{C}$ & -3.648900 & 7.147100 & -3.280000 \\
\hline $\mathrm{C}$ & -2.974400 & 5.923000 & -3.271700 \\
\hline $\mathrm{C}$ & 2.765100 & 0.421800 & -1.559700 \\
\hline $\mathrm{C}$ & 4.251400 & 0.674500 & -1.382400 \\
\hline $\mathrm{C}$ & 5.208300 & 0.933500 & -2.590400 \\
\hline $\mathrm{C}$ & 5.081300 & -0.449800 & -2.008700 \\
\hline $\mathrm{C}$ & 4.606800 & 1.197000 & -0.007500 \\
\hline $\mathrm{C}$ & 4.180600 & 2.450700 & 0.464300 \\
\hline $\mathrm{C}$ & 4.529900 & 2.932200 & 1.723800 \\
\hline $\mathrm{C}$ & 5.330900 & 2.169800 & 2.585400 \\
\hline $\mathrm{C}$ & 5.755400 & 0.911800 & 2.138200 \\
\hline $\mathrm{C}$ & 5.394100 & 0.447300 & 0.874900 \\
\hline $\mathrm{C}$ & 5.701700 & 2.680900 & 3.932500 \\
\hline $\mathrm{C}$ & 5.729800 & 1.817700 & 5.039200 \\
\hline $\mathrm{C}$ & 6.072000 & 2.293200 & 6.314300 \\
\hline $\mathrm{C}$ & 6.366000 & 3.660900 & 6.493200 \\
\hline $\mathrm{C}$ & 6.388100 & 4.524400 & 5.378400 \\
\hline $\mathrm{C}$ & 6.042900 & 4.030700 & 4.111100 \\
\hline $\mathrm{C}$ & 4.632900 & 1.182900 & -3.946400 \\
\hline $\mathrm{C}$ & 3.793100 & 2.285400 & -4.172600 \\
\hline $\mathrm{C}$ & 3.268100 & 2.520700 & -5.446200 \\
\hline $\mathrm{C}$ & 3.581300 & 1.662800 & -6.503400 \\
\hline $\mathrm{C}$ & 4.437900 & 0.579000 & -6.292700 \\
\hline $\mathrm{C}$ & 4.974000 & 0.347200 & -5.022600 \\
\hline $\mathrm{C}$ & 6.484600 & 1.671700 & -2.320800 \\
\hline $\mathrm{C}$ & 7.725400 & 1.022900 & -2.459900 \\
\hline $\mathrm{C}$ & 8.916100 & 1.715100 & -2.220300 \\
\hline $\mathrm{C}$ & 8.881600 & 3.064300 & -1.860500 \\
\hline $\mathrm{C}$ & 7.656800 & 3.726900 & -1.754100 \\
\hline $\mathrm{C}$ & 6.464500 & 3.038300 & -1.994600 \\
\hline $\mathrm{C}$ & -2.371400 & -0.812900 & -1.950800 \\
\hline $\mathrm{C}$ & -3.868300 & -1.053500 & -2.025500 \\
\hline $\mathrm{C}$ & -4.569000 & -1.584200 & -3.317600 \\
\hline $\mathrm{C}$ & -4.530400 & -0.106500 & -3.030200 \\
\hline $\mathrm{C}$ & -4.503800 & -1.278500 & -0.670900 \\
\hline $\mathrm{C}$ & -4.210000 & -2.393500 & 0.133000 \\
\hline $\mathrm{C}$ & -4.816800 & -2.603400 & 1.369300 \\
\hline $\mathrm{C}$ & -5.757600 & -1.692000 & 1.869000 \\
\hline $\mathrm{C}$ & -6.053200 & -0.566500 & 1.088700 \\
\hline $\mathrm{C}$ & -5.434500 & -0.374000 & -0.145000 \\
\hline $\mathrm{C}$ & -6.407000 & -1.909500 & 3.189700 \\
\hline $\mathrm{C}$ & -6.795400 & -3.197800 & 3.589600 \\
\hline
\end{tabular}

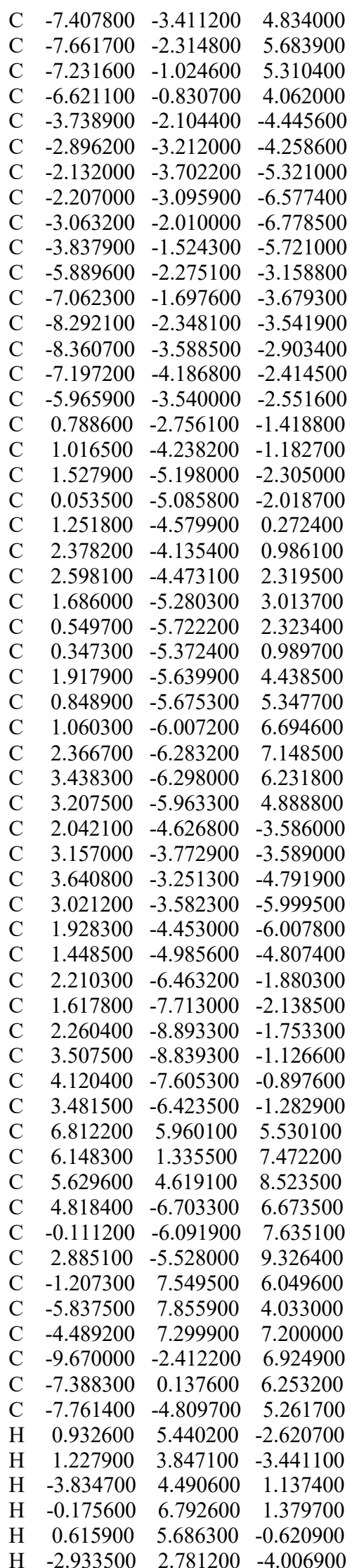

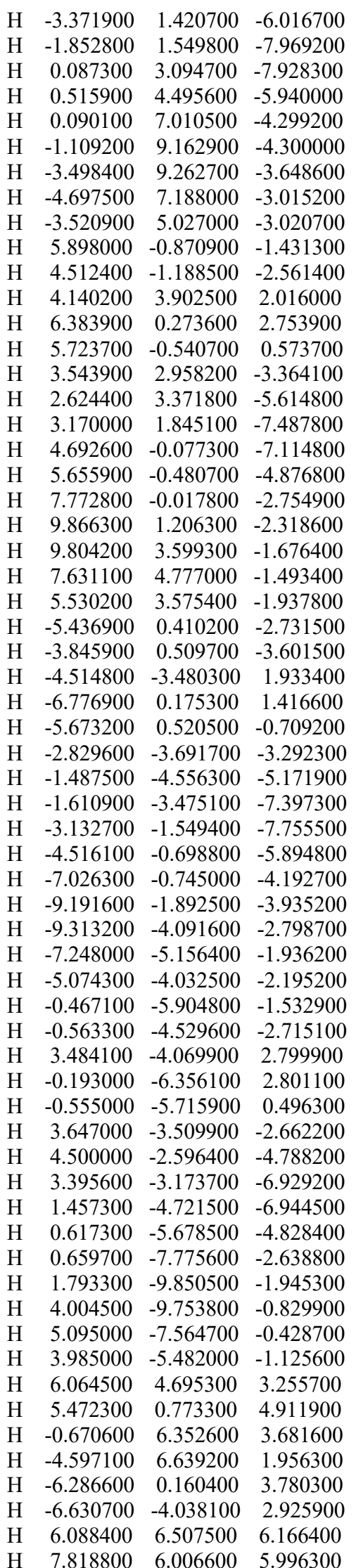




$\begin{array}{cccc}\mathrm{H} & 6.863000 & 6.478100 & 4.548800 \\ \mathrm{H} & 7.153600 & 1.392200 & 7.940200 \\ \mathrm{H} & 5.377800 & 1.587100 & 8.228200 \\ \mathrm{H} & 5.978500 & 0.286600 & 7.148000 \\ \mathrm{H} & 4.890100 & 3.811900 & 8.705600 \\ \mathrm{H} & 6.001600 & 4.978200 & 9.504800 \\ \mathrm{H} & 5.126900 & 5.465200 & 8.010800 \\ \mathrm{H} & 5.217100 & -5.970500 & 7.403300 \\ \mathrm{H} & 4.781400 & -7.707800 & 7.145300 \\ \mathrm{H} & 5.525000 & -6.750900 & 5.817700 \\ \mathrm{H} & -0.138500 & -7.094900 & 8.110600 \\ \mathrm{H} & -0.027000 & -5.315800 & 8.421900 \\ \mathrm{H} & -1.074500 & -5.936600 & 7.104300 \\ \mathrm{H} & 2.049100 & -4.798200 & 9.337000 \\ \mathrm{H} & 3.042400 & -5.892100 & 10.362200 \\ \mathrm{H} & 3.811600 & -5.016100 & 8.992800 \\ \mathrm{H} & -1.249400 & 8.630400 & 6.300200 \\ \mathrm{H} & -1.462300 & 6.954800 & 6.949500 \\ \mathrm{H} & -0.162700 & 7.303400 & 5.762800 \\ \mathrm{H} & -6.388600 & 7.285800 & 4.807500 \\ \mathrm{H} & -5.869400 & 8.937600 & 4.281600 \\ \mathrm{H} & -6.361700 & 7.709600 & 3.064700 \\ \mathrm{H} & -3.689100 & 6.603900 & 7.527300 \\ \mathrm{H} & -4.837200 & 7.870700 & 8.085000 \\ \mathrm{H} & -5.344500 & 6.712700 & 6.806000 \\ \mathrm{H} & -10.121100 & -3.168000 & 6.248900 \\ \mathrm{H} & -10.054900 & -2.588300 & 7.950000 \\ \mathrm{H} & -9.988000 & -1.398900 & 6.602800 \\ \mathrm{H} & -8.462800 & 0.349400 & 6.424000 \\ \mathrm{H} & -6.902300 & -0.098200 & 7.223300 \\ \mathrm{H} & -6.919400 & 1.059700 & 5.848400 \\ \mathrm{H} & -7.280200 & -5.036800 & 6.236300 \\ \mathrm{H} & -8.860400 & -4.912400 & 5.363200 \\ \mathrm{H} & -7.415000 & -5.563800 & 4.523200 \\ \mathrm{H} & 4.031800 & -5.979400 & 4.185800 \\ \mathrm{H} & -0.151100 & -5.431500 & 5.010100 \\ & & & \end{array}$

D3

$\begin{array}{llll}\text { Rh } & -0.116800 & 0.021800 & -0.572000\end{array}$

$\begin{array}{llll}\mathrm{Rh} & -0.482200 & 0.115000 & -2.924400\end{array}$

$\begin{array}{llll}\mathrm{O} & -2.420400 & 0.710700 & -2.532300\end{array}$

$\begin{array}{llll}\mathrm{O} & -2.108400 & 0.479500 & -0.306700\end{array}$

$\begin{array}{llll}\mathrm{O} & 0.131000 & 2.086000 & -2.873700\end{array}$

$\begin{array}{llll}\mathrm{O} & 0.337300 & 2.031200 & -0.624700\end{array}$

O $\quad-1.074500 \quad-1.861700 \quad-2.842900$

$\begin{array}{lllll}\mathrm{O} & -0.589700 & -1.982600 & -0.639800\end{array}$

$\begin{array}{llll}\mathrm{O} & 1.476800 & -0.484800 & -3.184000\end{array}$

O $\quad 1.855900 \quad-0.431800 \quad-0.957500$

$\begin{array}{llll}\text { O } & 0.509300 & -8.459600 & 7.125100\end{array}$

$\begin{array}{llll}\mathrm{O} & -6.983200 & 0.236900 & 8.621300\end{array}$

$\begin{array}{llll}\text { O } & 1.738300 & 7.933700 & 7.557600\end{array}$

$\begin{array}{llll}\text { O } & 9.215600 & -0.897300 & 6.061400\end{array}$

$\begin{array}{lllll}\mathrm{Cl} & -4.441900 & -1.679900 & 0.159700\end{array}$

$\mathrm{Cl} \quad-1.744100 \quad 4.401800 \quad-0.013000$

Cl $\quad 1.548700 \quad-4.370400 \quad-0.870200$

$\begin{array}{lllll}\mathrm{Cl} & 4.253800 & 1.705400 & -1.046400\end{array}$

C $\quad-2.826400 \quad 0.739600 \quad-1.323500$

C $\quad-4.234700 \quad 1.225300 \quad-1.032300$

C $\quad-5.334800 \quad 1.321200 \quad-2.138000$

C $\quad-4.529400 \quad 2.537600 \quad-1.764100$

$\begin{array}{llll}\text { C } & -4.630600 & 1.067000 & 0.419500\end{array}$

$\begin{array}{llll}\text { C } & -4.769900 & -0.187300 & 1.038500\end{array}$

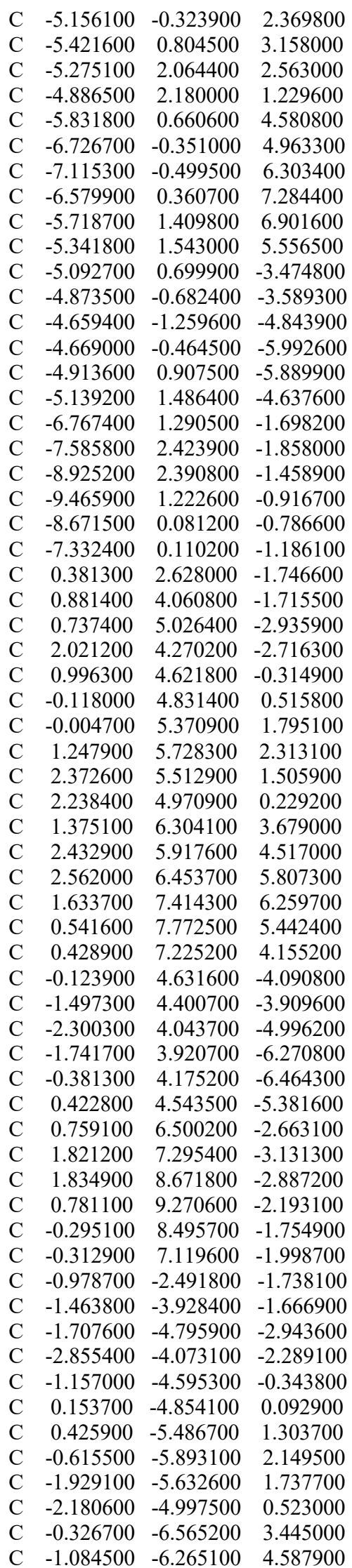

$\begin{array}{llll}\text { C } & -0.813900 & -6.889400 & 5.815100\end{array}$

$\begin{array}{llll}\text { C } & 0.249500 & -7.811000 & 5.909700\end{array}$

C $\quad 0.983600 \quad-8.154500 \quad 4.755600$

$\begin{array}{llll}\text { C } & 0.698700 & -7.519400 & 3.537200\end{array}$

C $\quad-1.230900 \quad-4.304000 \quad-4.271200$

C $\quad 0.135600 \quad-4.072800 \quad-4.496900$

C $\quad 0.577000 \quad-3.624500 \quad-5.744800$

C $\quad-0.339400 \quad-3.409800 \quad-6.777300$

C $\quad-1.697700 \quad-3.663400 \quad-6.569000$

C $\quad-2.141300 \quad-4.122800 \quad-5.325400$

C $\quad-1.664600 \quad-6.286400 \quad-2.790500$

$\begin{array}{llll}\text { C } & -2.828500 & -7.055000 & -2.975200\end{array}$

C $\quad-2.785200 \quad-8.446300 \quad-2.844300$

$\begin{array}{llll}\text { C } & -1.578600 & -9.084700 & -2.549100\end{array}$

C $\quad-0.410600 \quad-8.333900 \quad-2.398800$

$\begin{array}{llll}\text { C } & -0.449900 & -6.943100 & -2.530500\end{array}$

$\begin{array}{llll}\text { C } & 2.228900 & -0.603800 & -2.160900\end{array}$

$\begin{array}{llll}\text { C } & 3.652400 & -1.096300 & -2.349000\end{array}$

$\begin{array}{llll}\text { C } & 4.364800 & -1.091600 & -3.739900\end{array}$

C $\quad 3.693100 \quad-2.342500 \quad-3.237900$

C $\quad 4.471300 \quad-1.051500 \quad-1.077400$

$\begin{array}{llll}\text { C } & 4.810200 & 0.149900 & -0.431100\end{array}$

$\begin{array}{llll}\text { C } & 5.583300 & 0.182300 & 0.727300\end{array}$

$\begin{array}{llll}\text { C } & 6.058100 & -1.003700 & 1.304600\end{array}$

$\begin{array}{llll}\text { C } & 5.718700 & -2.212600 & 0.683100\end{array}$

$\begin{array}{llll}\text { C } & 4.943300 & -2.223900 & -0.474700\end{array}$

$\begin{array}{llll}\text { C } & 6.882000 & -0.975500 & 2.543000\end{array}$

$\begin{array}{llll}\text { C } & 7.856000 & 0.018100 & 2.728800\end{array}$

$\begin{array}{llll}\text { C } & 8.636500 & 0.051900 & 3.894400\end{array}$

C $\quad 8.460500 \quad-0.940200 \quad 4.881100$

$\begin{array}{llll}\text { C } & 7.451800 & -1.913300 & 4.723900\end{array}$

$\begin{array}{llll}\text { C } & 6.681300 & -1.931300 & 3.551400\end{array}$

$\begin{array}{llll}\text { C } & 3.738300 & -0.367500 & -4.886600\end{array}$

$\begin{array}{llll}\text { C } & 3.516200 & 1.017400 & -4.819100\end{array}$

$\begin{array}{llll}\text { C } & 2.941500 & 1.691600 & -5.899900\end{array}$

$\begin{array}{llll}\text { C } & 2.590100 & 0.991600 & -7.056700\end{array}$

C $\quad 2.832700 \quad-0.382100 \quad-7.142100$

$\begin{array}{llll}\text { C } & 3.418000 & -1.057600 & -6.067200\end{array}$

C $\quad 5.863700 \quad-1.081500 \quad-3.753700$

$\begin{array}{llll}\text { C } & 6.578700 & -2.189500 & -4.244400\end{array}$

C $\quad 7.976400 \quad-2.174700 \quad-4.268500$

$\begin{array}{llll}\text { C } & 8.672700 & -1.048600 & -3.823600\end{array}$

$\begin{array}{llll}\text { C } & 7.971700 & 0.070000 & -3.367800\end{array}$

$\begin{array}{llll}\text { C } & 6.574300 & 0.059300 & -3.343800\end{array}$

$\begin{array}{lrrr}C & -0.517900 & 8.713000 & 5.948900\end{array}$

C $\quad 3.666500 \quad 5.972400 \quad 6.708500$

$\begin{array}{llll}\text { C } & 2.511200 & 9.132400 & 7.623700\end{array}$

C $\quad 7.170500 \quad-2.908300 \quad 5.817100$

$\begin{array}{llll}\text { C } & 9.633000 & 1.161500 & 4.093400\end{array}$

C $\quad 10.421300 \quad-1.658400 \quad 5.986500$

$\begin{array}{llll}\text { C } & -8.125400 & -1.550700 & 6.675600\end{array}$

$\begin{array}{llll}\text { C } & -5.222800 & 2.403000 & 7.917400\end{array}$

$\begin{array}{llll}\text { C } & -6.138900 & -0.623300 & 9.386700\end{array}$

$\begin{array}{llll}\text { C } & 1.468700 & -7.777600 & 7.933400\end{array}$

$\begin{array}{lrrr}\text { C } & 2.044300 & -9.220100 & 4.811700\end{array}$

$\begin{array}{lrrr}\text { C } & -1.677200 & -6.590700 & 7.010700\end{array}$

$\mathrm{H} \quad-4.995100 \quad 3.344600 \quad-1.207700$

$\mathrm{H} \quad-3.754100 \quad 2.868900 \quad-2.445300$

$\mathrm{H} \quad-5.219600 \quad-1.329700 \quad 2.773300$

$\mathrm{H} \quad-5.471600 \quad 2.979100 \quad 3.116300$

$\mathrm{H} \quad-4.762700 \quad 3.172600 \quad 0.811400$

H $\quad-4.865200 \quad-1.309500 \quad-2.709100$ 


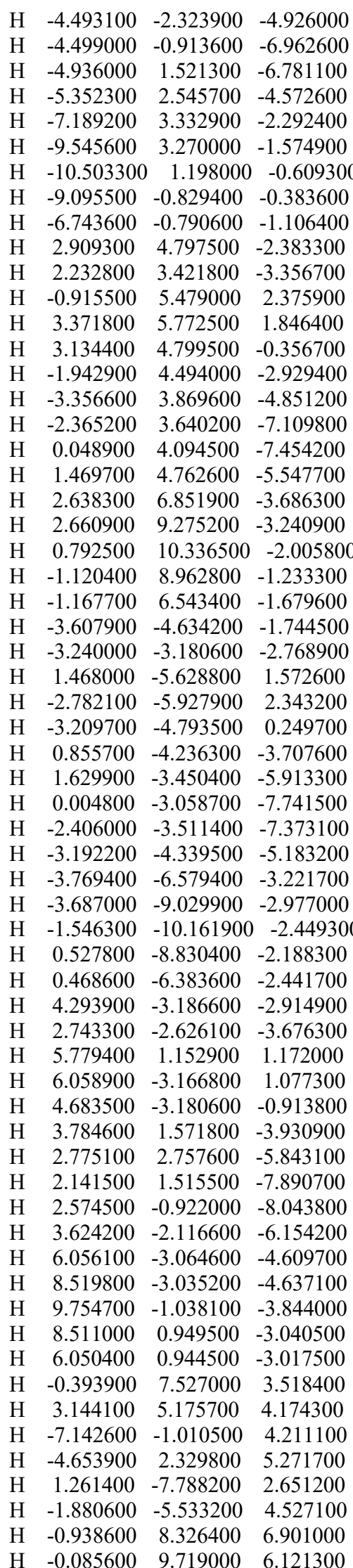

\begin{tabular}{|c|c|c|c|c|c|c|c|}
\hline & -1.351800 & 8.819900 & 5.222700 & $\mathrm{C}$ & -2.626700 & 4.837600 & 1.281600 \\
\hline & 3.235900 & 5.586000 & 7.656200 & $\mathrm{C}$ & -1.739500 & 5.758600 & 1.855800 \\
\hline & 4.252100 & 5.154100 & & $\mathrm{C}$ & -0.558900 & 6.048000 & 1.159000 \\
\hline & 4.364400 & 6.803000 & & $\mathrm{C}$ & -0.290500 & 5.443200 & -0.067400 \\
\hline & 2.055100 & 9.935700 & 7.008600 & C & -2.039400 & 6.394500 & 3.166900 \\
\hline & 2.547000 & 9.478400 & 8.676900 & & -1.021000 & 6.582900 & 4.114400 \\
\hline & 3.551500 & 8.955900 & 7.279600 & $\mathrm{C}$ & -1.292800 & 7.180800 & 4400 \\
\hline & 6.959500 & -2.372700 & 6.766600 & $\mathrm{C}$ & -2.600000 & 7.624300 & 5.643400 \\
\hline & 6.291100 & -3.543000 & 5.577200 & $\mathrm{C}$ & -3.638100 & 7.396100 & 4.716500 \\
\hline & 8.043300 & -3.576500 & 5.960000 & $\mathrm{C}$ & -3.347100 & 6.796100 & 81900 \\
\hline & 9.422700 & 1.687700 & 5.048300 & $c$ & -1.749700 & 3.838700 & -4.500800 \\
\hline & 10.662200 & 0.751100 & 4.120600 & $\mathrm{C}$ & -2.877900 & 3.008600 & 2000 \\
\hline & 9.584700 & 1.907600 & 3.271800 & $\mathrm{C}$ & -3.305900 & 2.268200 & 7200 \\
\hline & 11.085600 & -1.275400 & 5.184300 & $\mathrm{C}$ & -2.616500 & 2.355100 & 719300 \\
\hline & 10.958900 & -1.574600 & & $\mathrm{C}$ & & & -6.833500 \\
\hline & 10.205200 & -2.730800 & 5.800000 & $\mathrm{C}$ & -1.085100 & 3.950400 & 3200 \\
\hline & -7.663600 & -2.305600 & & $\mathrm{C}$ & -1.9 & 400 & 3000 \\
\hline & -8.984700 & -1.077700 & & $\mathrm{C}$ & -1.348400 & 400 & 3200 \\
\hline & -8.514500 & -2.080200 & 5.779900 & $\mathrm{C}$ & -1.989600 & 8.380500 & 39200 \\
\hline & -6.085300 & 2.866500 & 8.440900 & $\mathrm{C}$ & -3.269400 & 8.457700 & 5100 \\
\hline & -4.57 & 1.8 & & & -3.915 & & 7800 \\
\hline & -4.6 & 3.2 & & $\mathrm{C}$ & -3.2 & & 800 \\
\hline & -5.09 & -0.23 & & $\mathrm{C}$ & & & 100 \\
\hline & -6.52 & -0.66 & 6600 & $\mathrm{C}$ & 4.003 & 900 & \\
\hline & -6.13 & -1.6 & 8.9 & & 4.7 & 0.8 & 300 \\
\hline & 1.1 & -6.7 & & & 4.7 & & \\
\hline & 1.6 & -8.3 & & $\mathrm{C}$ & & & \\
\hline & 2.44 & -7.6 & & $\mathrm{C}$ & 4.25 & & \\
\hline & 2.87 & -8.8 & & C & & & \\
\hline & 1.61 & -10. & & & 5.6 & & \\
\hline & 2.46 & -9.4 & & & & & \\
\hline & -2.10 & -7.5 & & $\mathrm{C}$ & & & \\
\hline & $-1.0^{\circ}$ & -6.0 & & $\mathrm{C}$ & & & \\
\hline & -2.52 & & & C & & & \\
\hline & 5.903300 & -2.6 & & & 6.9 & & \\
\hline \multirow[t]{2}{*}{$\mathrm{H}$} & \multirow[t]{3}{*}{8.015900} & 0.76 & 1.9 & $\mathrm{C}$ & & & \\
\hline & & & & $\mathrm{C}$ & & & \\
\hline & & & & $\mathrm{C}$ & 6.6 & & 6300 \\
\hline & -0.031700 & & & $\mathrm{C}$ & & & \\
\hline & -0.1 & -0 . & & & & & \\
\hline & -0.50 & & -3.0 & C & & & \\
\hline & -0.5 & 1.9 & -0.7 & $\mathrm{C}$ & & & 100 \\
\hline & & 0.09 & -2.99 & $\mathrm{C}$ & & 0.6 & \\
\hline & 1.961200 & & -0.7 & & & & \\
\hline $\mathrm{O}$ & -2.1 & & & & & & \\
\hline & -2.0 & -0.5 & -0.4 & C & & & \\
\hline & 0.23 & -2.28 & -2.7 & $\mathrm{C}$ & & 1.5 & 000 \\
\hline $\mathrm{O}$ & 0.467600 & -2.063300 & -0.4 & & 1600 & 2.8 & 1000 \\
\hline $\mathrm{O}$ & -7.275200 & -2.5 & & & & & \\
\hline & -2.8 & & & & & & \\
\hline $\mathrm{O}$ & 7.916400 & 3.729300 & 6.80 & $\mathrm{C}$ & -2.670100 & -0.7 & 7400 \\
\hline $\mathrm{O}$ & 3.580400 & -6.915200 & & $\mathrm{C}$ & -4.167900 & -0.924600 & -1.451100 \\
\hline $\mathrm{Cl}$ & -3.523100 & 3.086200 & -0.5 & $\mathrm{C}$ & -5.060800 & -1.405200 & 40400 \\
\hline & & & -0.8 & $c$ & & & 7800 \\
\hline & -3.163400 & -3.529800 & & $\mathrm{C}$ & -4.608600 & & 4900 \\
\hline $\mathrm{Cl}$ & 3.473900 & -3.192000 & -0.335000 & $\mathrm{C}$ & -4.231700 & -2.315100 & 0.701300 \\
\hline & -0.648200 & 2.413300 & -1.947000 & $\mathrm{C}$ & -4.662900 & -2.546900 & 2.005500 \\
\hline & -0.862100 & 3.914600 & -2.013800 & $\mathrm{C}$ & -5.500800 & -1.630600 & 2.656100 \\
\hline & -1.294900 & & & & & & \\
\hline & 0.159600 & 4.577600 & -2.941900 & & -5.432400 & -0.263200 & 0.650300 \\
\hline & -1.169400 & 4.533200 & -0.667500 & $C$ & -5.963900 & -1.872400 & 4.049100 \\
\hline & -2.340500 & 4.244400 & 0.054000 & $\mathrm{C}$ & -6.324400 & -3.163400 & 4.466400 \\
\hline
\end{tabular}




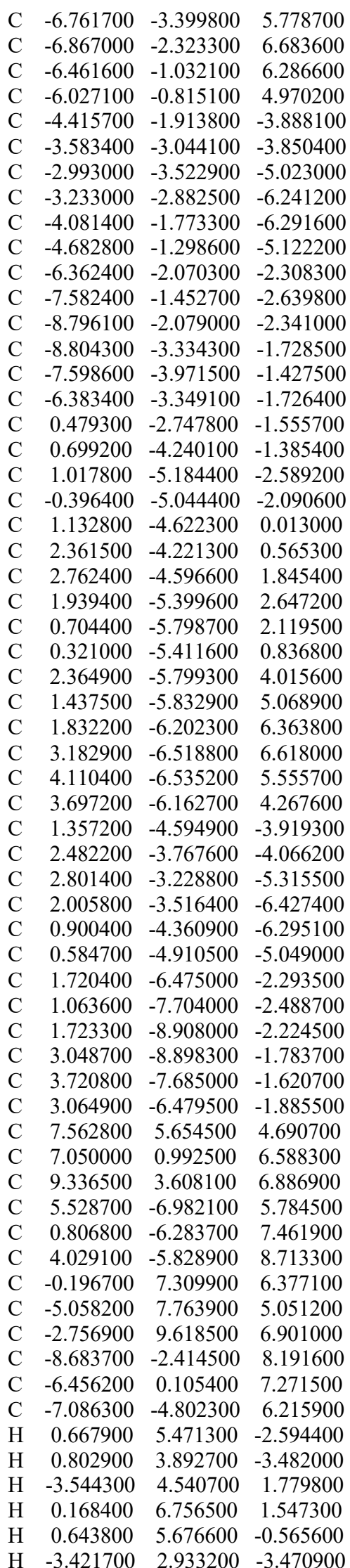

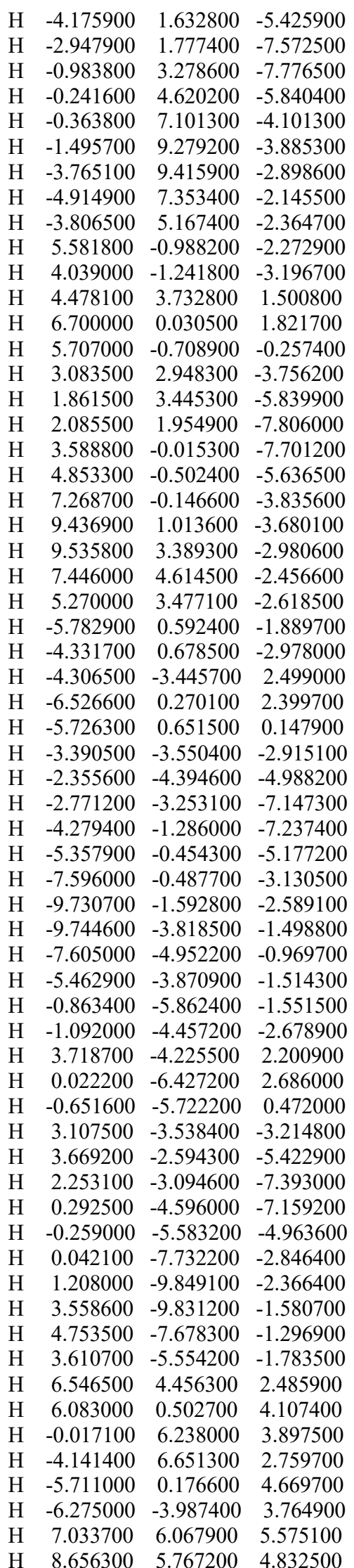

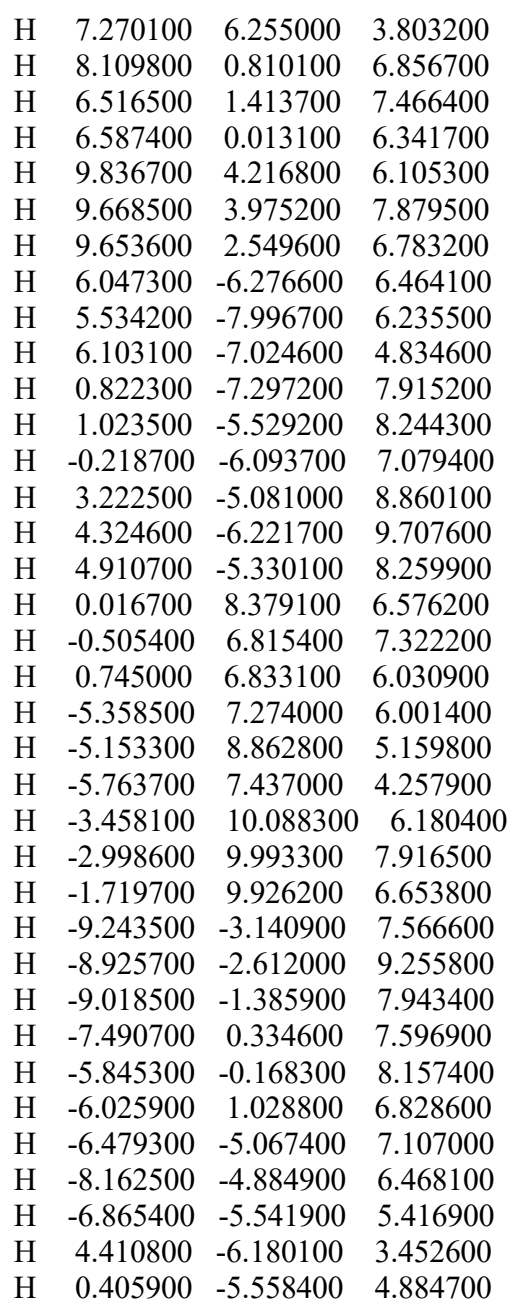

D5

$\begin{array}{llll}\mathrm{Rh} & -0.000100 & 0.000000 & -0.583600\end{array}$

$\begin{array}{llll}\mathrm{Rh} & -0.000400 & -0.000100 & -2.966000\end{array}$

$\begin{array}{llll}\mathrm{O} & 0.333300 & -2.036800 & -2.899200\end{array}$

$\begin{array}{llll}\mathrm{O} & 0.469000 & -2.005500 & -0.644300\end{array}$

$\begin{array}{llll}\mathrm{O} & -2.037100 & -0.334100 & -2.899000\end{array}$

$\begin{array}{llll}\mathrm{O} & -2.005800 & -0.468100 & -0.644000\end{array}$

$\begin{array}{llll}\mathrm{O} & 2.036200 & 0.333900 & -2.899600\end{array}$

$\begin{array}{llll}\mathrm{O} & 2.005700 & 0.468100 & -0.644600\end{array}$

O $\quad-0.334100 \quad 2.036600 \quad-2.899200$

$\begin{array}{llll}\mathrm{O} & -0.469200 & 2.005500 & -0.644300\end{array}$

$\begin{array}{llll}\text { O } & 7.643500 & 3.049900 & 7.431500\end{array}$

$\begin{array}{llll}\text { O } & 3.129500 & -7.603800 & 7.428000\end{array}$

$\begin{array}{llll}\mathrm{O} & -7.641300 & -3.049500 & 7.434000\end{array}$

$\begin{array}{llll}\text { O } & -3.127400 & 7.604300 & 7.428300\end{array}$

$\mathrm{Cl} \quad 3.451500 \quad-3.189700 \quad-0.455100$

Cl $\quad-3.197400 \quad-3.444300 \quad-0.440900$

Cl $3.197300 \quad 3.444200 \quad-0.442000$

$\mathrm{Cl} \quad-3.451600 \quad 3.189600 \quad-0.454300$

$\begin{array}{llll}\text { C } & 0.522400 & -2.596600 & -1.768900\end{array}$

C $\quad 0.716800 \quad-4.101200 \quad-1.717200$

$\begin{array}{llll}\text { C } & 1.080600 & -4.944600 & -2.981600\end{array}$

$\begin{array}{llll}\text { C } & -0.353700 & -4.825600 & -2.538000\end{array}$

$\begin{array}{llll}\text { C } & 1.080000 & -4.607800 & -0.338400\end{array}$

$\begin{array}{llll}\text { C } & 2.286300 & -4.274800 & 0.301700\end{array}$ 


\begin{tabular}{|c|c|c|c|}
\hline C & 2.622600 & -4.764200 & 1.561700 \\
\hline 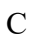 & 1.753400 & -5.619400 & 2.253300 \\
\hline & 0.539600 & -5.952100 & 1.637800 \\
\hline- & 0.221200 & -5.451200 & 0.376900 \\
\hline$C$ & 2.109800 & -6.141000 & 3.600200 \\
\hline & 1.134100 & -6.249400 & 4.603600 \\
\hline & 1.463500 & -6.734200 & 5.878600 \\
\hline$c$ & 2.796900 & -7.092900 & 6.165600 \\
\hline$c$ & 3.772700 & -7.033600 & 5.149000 \\
\hline 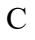 & 3.424400 & -6.545900 & 3.880200 \\
\hline 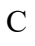 & 1.488600 & -4.249400 & -4.239500 \\
\hline$C$ & 2.628900 & -3.430200 & -4.263500 \\
\hline $\mathrm{C}$ & 3.012500 & -2.792200 & -5.446300 \\
\hline $\mathrm{C}$ & 2.266600 & -2.971900 & -6.613800 \\
\hline 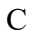 & 1.146400 & -3.807300 & -6.604900 \\
\hline $\mathrm{C}$ & 0.766200 & -4.455800 & -5.426200 \\
\hline $\mathrm{C}$ & 1.753600 & -6.266400 & -2.765900 \\
\hline $\mathrm{C}$ & 1.092600 & -7.464100 & -3.095400 \\
\hline $\mathrm{C}$ & 1.725300 & -8.696200 & -2.905400 \\
\hline $\mathrm{C}$ & 3.028700 & -8.744400 & -2.405500 \\
\hline $\mathrm{C}$ & 3.706300 & -7.559900 & -2.108700 \\
\hline $\mathrm{C}$ & 3.077500 & -6.326300 & -2.298900 \\
\hline $\mathrm{C}$ & -2.597100 & -0.521500 & -1.768500 \\
\hline $\mathrm{C}$ & -4.102100 & -0.712900 & -1.716900 \\
\hline $\mathrm{C}$ & -4.944900 & -1.080100 & -2.980700 \\
\hline $\mathrm{C}$ & -4.823800 & 0.355600 & -2.542700 \\
\hline $\mathrm{C}$ & -4.610600 & -1.069100 & -0.337000 \\
\hline $\mathrm{C}$ & -4.280900 & -2.273100 & 0.309200 \\
\hline $\mathrm{C}$ & -4.772100 & -2.602500 & 1.570500 \\
\hline $\mathrm{C}$ & -5.626300 & -1.728100 & 2.256900 \\
\hline $\mathrm{C}$ & -5.955000 & -0.516200 & 1.635300 \\
\hline $\mathrm{C}$ & -5.452400 & -0.204800 & 0.373500 \\
\hline $\mathrm{C}$ & -6.152200 & -2.073300 & 3.605000 \\
\hline $\mathrm{C}$ & -6.241600 & -1.092300 & 4.605000 \\
\hline $\mathrm{C}$ & -6.735100 & -1.407100 & 5.880300 \\
\hline $\mathrm{C}$ & -7.173700 & -2.719200 & 6.154300 \\
\hline $\mathrm{C}$ & -7.043100 & -3.721300 & 5.170600 \\
\hline $\mathrm{C}$ & -6.546600 & -3.387600 & 3.901200 \\
\hline $\mathrm{C}$ & -4.249000 & -1.494500 & -4.236100 \\
\hline $\mathrm{C}$ & -3.433700 & -2.637600 & -4.254800 \\
\hline $\mathrm{C}$ & -2.795800 & -3.027900 & -5.435500 \\
\hline $\mathrm{C}$ & -2.971000 & -2.285300 & -6.605800 \\
\hline $\mathrm{C}$ & -3.801900 & -1.161800 & -6.602000 \\
\hline $\mathrm{C}$ & -4.450800 & -0.775300 & -5.425500 \\
\hline $\mathrm{C}$ & -6.268200 & -1.749700 & -2.763800 \\
\hline $\mathrm{C}$ & -7.464200 & -1.088100 & -3.098000 \\
\hline $\mathrm{C}$ & -8.697700 & -1.717800 & -2.907000 \\
\hline $\mathrm{C}$ & -8.749100 & -3.018700 & -2.401100 \\
\hline $\mathrm{C}$ & -7.566200 & -3.697000 & -2.099300 \\
\hline $\mathrm{C}$ & -6.331200 & -3.071300 & -2.290600 \\
\hline $\mathrm{C}$ & 2.596600 & 0.521300 & -1.769300 \\
\hline $\mathrm{C}$ & 4.101600 & 0.712800 & -1.718100 \\
\hline $\mathrm{C}$ & 4.944000 & 1.079900 & -2.982200 \\
\hline $\mathrm{C}$ & 4.823100 & -0.355800 & -2.544100 \\
\hline $\mathrm{C}$ & 4.610500 & 1.069100 & -0.338400 \\
\hline $\mathrm{C}$ & 4.281000 & 2.273100 & 0.307800 \\
\hline $\mathrm{C}$ & 4.772500 & 2.602600 & 1.569000 \\
\hline $\mathrm{C}$ & 5.627000 & 1.728200 & 2.255100 \\
\hline $\mathrm{C}$ & 5.955500 & 0.516300 & 1.633600 \\
\hline $\mathrm{C}$ & 5.452500 & 0.204800 & 0.372000 \\
\hline $\mathrm{C}$ & 6.153300 & 2.073600 & 3.603100 \\
\hline$C$ & 6.547800 & 3.387900 & 3.899100 \\
\hline
\end{tabular}

C $\quad 7.044700 \quad 3.721600 \quad 5.168300$

$\begin{array}{llll}\text { C } & 7.175400 & 2.719600 & 6.152100\end{array}$

$\begin{array}{llll}\text { C } & 6.736900 & 1.407500 & 5.878200\end{array}$

$\begin{array}{llll}\text { C } & 6.243000 & 1.092600 & 4.603100\end{array}$

C $\quad 4.247800 \quad 1.494200 \quad-4.237400$

$\begin{array}{llll}\text { C } & 3.432300 & 2.637400 & -4.256000\end{array}$

$\begin{array}{llll}\text { C } & 2.794200 & 3.027600 & -5.436500\end{array}$

$\begin{array}{llll}\text { C } & 2.969100 & 2.284900 & -6.606800\end{array}$

$\begin{array}{llll}\text { C } & 3.800000 & 1.161400 & -6.603100\end{array}$

$\begin{array}{llll}\text { C } & 4.449200 & 0.774900 & -5.426900\end{array}$

$\begin{array}{llll}\text { C } & 6.267400 & 1.749600 & -2.765700\end{array}$

$\begin{array}{llll}\text { C } & 7.463300 & 1.087900 & -3.100300\end{array}$

C $\quad 8.696900 \quad 1.717500 \quad-2.909600$

$\begin{array}{llll}\text { C } & 8.748400 & 3.018500 & -2.403800\end{array}$

$\begin{array}{llll}\text { C } & 7.565600 & 3.696900 & -2.101700\end{array}$

C $\quad 6.330500 \quad 3.071200-2.292700$

C $\quad-0.523000 \quad 2.596500 \quad-1.768900$

$\begin{array}{llll}\text { C } & -0.717300 & 4.101100 & -1.717300\end{array}$

C $\quad-1.081500 \quad 4.944400 \quad-2.981700$

C $\quad 0.353000 \quad 4.825400 \quad-2.538400$

$\begin{array}{llll}\text { C } & -1.080100 & 4.607800 & -0.338400\end{array}$

$\begin{array}{llll}\text { C } & -2.286200 & 4.274800 & 0.302000\end{array}$

C $\quad-2.622100 \quad 4.764200 \quad 1.562100$

$\begin{array}{llll}\text { C } & -1.752800 & 5.619600 & 2.253500\end{array}$

C $\quad-0.539100 \quad 5.952300 \quad 1.637600$

$\begin{array}{llll}\text { C } & -0.221100 & 5.451300 & 0.376600\end{array}$

$\begin{array}{llll}\text { C } & -2.108800 & 6.141300 & 3.600400\end{array}$

C $\quad-1.132800 \quad 6.249700 \quad 4.603500$

$\begin{array}{llll}\text { C } & -1.461900 & 6.734600 & 5.878500\end{array}$

$\begin{array}{llll}\text { C } & -2.795200 & 7.093300 & 6.165900\end{array}$

C $\quad-3.771200 \quad 7.034000 \quad 5.149600$

$\begin{array}{llll}\text { C } & -3.423300 & 6.546200 & 3.880700\end{array}$

$\begin{array}{llll}\text { C } & -1.489800 & 4.249100 & -4.239300\end{array}$

C $\quad-2.630100 \quad 3.429800 \quad-4.263000$

C $\quad-3.014100 \quad 2.791900 \quad-5.445600$

C $\quad-2.268500 \quad 2.971500 \quad-6.613400$

C $\quad-1.148300 \quad 3.806900 \quad-6.604900$

C $\quad-0.767800 \quad 4.455400 \quad-5.426300$

$\begin{array}{llll}\text { C } & -1.754400 & 6.266200 & -2.765800\end{array}$

C $\quad-1.093500 \quad 7.463800 \quad-3.095500$

C $\quad-1.726200 \quad 8.696000 \quad-2.905500$

$\begin{array}{llll}\text { C } & -3.029400 & 8.744300 & -2.405200\end{array}$

C $\quad-3.707000 \quad 7.559700 \quad-2.108200$

$\begin{array}{llll}\text { C } & -3.078200 & 6.326100 & -2.298500\end{array}$

C $\quad-7.404200 \quad-5.148900 \quad 5.479100$

$\begin{array}{llll}\text { C } & -6.757600 & -0.352400 & 6.953200\end{array}$

$\begin{array}{llll}\text { C } & -9.054600 & -2.901600 & 7.572500\end{array}$

C $\quad-5.171800 \quad 7.521600 \quad 5.403500$

$\begin{array}{llll}\text { C } & -0.385300 & 6.892900 & 6.917600\end{array}$

$\begin{array}{llll}\text { C } & -3.550800 & 6.598600 & 8.349200\end{array}$

$\begin{array}{llll}\text { C } & 0.387200 & -6.892400 & 6.918000\end{array}$

$\begin{array}{llll}\text { C } & 5.173300 & -7.521200 & 5.402600\end{array}$

$\begin{array}{llll}\text { C } & 3.553100 & -6.598000 & 8.348700\end{array}$

$\begin{array}{llll}\text { C } & 9.056900 & 2.902100 & 7.569600\end{array}$

$\begin{array}{llll}\text { C } & 6.759700 & 0.352800 & 6.951200\end{array}$

C $\quad 7.405900 \quad 5.149300 \quad 5.476500$

$\mathrm{H} \quad-0.854900 \quad-5.679100 \quad-2.093000$

$\mathrm{H} \quad-1.014100 \quad-4.179700 \quad-3.105000$

H $\quad 3.565600 \quad-4.440000 \quad 1.990800$

H $\quad-0.175800 \quad-6.615600 \quad 2.116900$

H $\quad-0.737000 \quad-5.714300 \quad-0.056900$

H $\quad 3.216200 \quad-3.283800 \quad-3.368000$
H $\quad 3.891700 \quad-2.164600 \quad-5.459200$

$\mathrm{H} \quad 2.563600 \quad-2.473600 \quad-7.527500$

$\mathrm{H} \quad 0.576900 \quad-3.958500 \quad-7.512800$

$\mathrm{H} \quad-0.088300 \quad-5.120100 \quad-5.437300$

H $\quad 0.088700 \quad-7.445800 \quad-3.500400$

$\mathrm{H} \quad 1.206400 \quad-9.613700 \quad-3.150800$

H $\quad 3.517700 \quad-9.698900 \quad-2.260100$

$\mathrm{H} \quad 4.722800 \quad-7.596800 \quad-1.738800$

H $\quad 3.628500 \quad-5.421500 \quad-2.093300$

$\mathrm{H} \quad-5.677000 \quad 0.860000 \quad-2.100600$

$\mathrm{H} \quad-4.176200 \quad 1.012600 \quad-3.111600$

$\mathrm{H} \quad-4.449000 \quad-3.543700 \quad 2.004100$

$\mathrm{H} \quad-6.616200 \quad 0.203100 \quad 2.111800$

$\mathrm{H} \quad-5.712300 \quad 0.752200 \quad-0.064800$

$\mathrm{H} \quad-3.290600 \quad-3.222400 \quad-3.357100$

$\mathrm{H} \quad-2.171900 \quad-3.909800 \quad-5.444600$

$\mathrm{H} \quad-2.472500 \quad-2.587500 \quad-7.517800$

$\mathrm{H} \quad-3.949700 \quad-0.594900 \quad-7.512000$

$\begin{array}{llll}\mathrm{H} & -5.111600 & 0.081900 & -5.440500\end{array}$

H $\quad-7.443500 \quad-0.086100 \quad-3.507700$

$\mathrm{H} \quad-9.614000-1.198300 \quad-3.156200$

H $\quad-9.704700 \quad-3.505300 \quad-2.254800$

$\mathrm{H} \quad-7.605600 \quad-4.711700 \quad-1.724700$

$\mathrm{H} \quad-5.427800 \quad-3.623000 \quad-2.081000$

H $\quad 5.676400 \quad-0.860100 \quad-2.102200$

$\mathrm{H} \quad 4.175300 \quad-1.012800 \quad-3.112800$

H $\quad 4.449600 \quad 3.543800 \quad 2.002600$

H $\quad 6.616800 \quad-0.203000 \quad 2.109900$

$\mathrm{H} \quad 5.712300 \quad-0.752200 \quad-0.066400$

H $\quad 3.289600 \quad 3.222100 \quad-3.358200$

H $\quad 2.170400 \quad 3.909400 \quad-5.445500$

$\mathrm{H} \quad 2.470300 \quad 2.587000 \quad-7.518700$

H $\quad 3.947500 \quad 0.594400 \quad-7.513200$

H $\quad 5.110100 \quad-0.082200 \quad-5.442000$

H $\quad 7.442500 \quad 0.085900 \quad-3.509800$

H $\quad 9.613100 \quad 1.198100 \quad-3.159000$

H $\quad 9.704100 \quad 3.505100 \quad-2.257800$

H $\quad 7.605100 \quad 4.711600 \quad-1.727200$

H $\quad 5.427200 \quad 3.622900 \quad-2.082800$

$\mathrm{H} \quad 0.854300 \quad 5.679000 \quad-2.093600$

$\mathrm{H} \quad 1.013200 \quad 4.179500 \quad-3.105600$

$\mathrm{H} \quad-3.565100 \quad 4.440100 \quad 1.991500$

$\mathrm{H} \quad 0.176400 \quad 6.615700 \quad 2.116400$

$\mathrm{H} \quad 0.737000 \quad 5.714300 \quad-0.057500$

$\mathrm{H} \quad-3.217100 \quad 3.283600 \quad-3.367300$

$\mathrm{H} \quad-3.893300 \quad 2.164300 \quad-5.458300$

$\mathrm{H} \quad-2.565900 \quad 2.473000 \quad-7.526900$

$\mathrm{H} \quad-0.579100 \quad 3.958100 \quad-7.512900$

H $\quad 0.086700 \quad 5.119700 \quad-5.437700$

$\mathrm{H} \quad-0.089700 \quad 7.445600 \quad-3.500800$

$\mathrm{H} \quad-1.207300 \quad 9.613500 \quad-3.151100$

$\mathrm{H} \quad-3.518400 \quad 9.698800 \quad-2.259700$

$\mathrm{H} \quad-4.723300 \quad 7.596700 \quad-1.738000$

$\begin{array}{llll}\mathrm{H} & -3.629100 & 5.421400 & -2.092600\end{array}$

H $\quad-6.477500 \quad-4.153400 \quad 3.138100$

$\mathrm{H} \quad-5.900100 \quad-0.085000 \quad 4.399200$

H $\quad 0.115600 \quad-5.943600 \quad 4.397200$

H $\quad 4.174900 \quad-6.505300 \quad 3.099900$

$\mathrm{H} \quad 5.901400 \quad 0.085300 \quad 4.397500$

H $\quad 6.478500 \quad 4.153600 \quad 3.136000$

$\mathrm{H} \quad-6.839600 \quad-5.494400 \quad 6.370500$

H $\quad-8.491200 \quad-5.234100 \quad 5.678500$ 


$\begin{array}{rrrr}\mathrm{H} & -7.158500 & -5.825500 & 4.633000 \\ \mathrm{H} & -7.802100 & -0.133400 & 7.252400 \\ \mathrm{H} & -6.189000 & -0.706100 & 7.838900 \\ \mathrm{H} & -6.296800 & 0.596400 & 6.604800 \\ \mathrm{H} & -9.596200 & -3.566400 & 6.867900 \\ \mathrm{H} & -9.344700 & -3.179900 & 8.606200 \\ \mathrm{H} & -9.365300 & -1.851200 & 7.394400 \\ \mathrm{H} & -5.666400 & 6.885100 & 6.164300 \\ \mathrm{H} & -5.143900 & 8.571000 & 5.765400 \\ \mathrm{H} & -5.789500 & 7.492100 & 4.480700 \\ \mathrm{H} & -0.367300 & 7.941500 & 7.282300 \\ \mathrm{H} & -0.574300 & 6.211500 & 7.771100 \\ \mathrm{H} & 0.618800 & 6.654400 & 6.506600 \\ \mathrm{H} & -2.747300 & 5.853000 & 8.522900 \\ \mathrm{H} & -3.794500 & 7.079600 & 9.318500 \\ \mathrm{H} & -4.458600 & 6.077200 & 7.981100 \\ \mathrm{H} & 0.369300 & -7.940900 & 7.282800 \\ \mathrm{H} & 0.576400 & -6.210900 & 7.771400 \\ \mathrm{H} & -0.617000 & -6.654000 & 6.507200 \\ \mathrm{H} & 5.668100 & -6.884700 & 6.163200 \\ \mathrm{H} & 5.145500 & -8.570600 & 5.764600 \\ \mathrm{H} & 5.790700 & -7.491800 & 4.479600 \\ \mathrm{H} & 2.749700 & -5.852500 & 8.522500 \\ \mathrm{H} & 3.797100 & -7.078900 & 9.317900 \\ \mathrm{H} & 4.460800 & -6.076600 & 7.980300 \\ \mathrm{H} & 9.598300 & 3.566800 & 6.864800 \\ \mathrm{H} & 9.347300 & 3.180500 & 8.603200 \\ \mathrm{H} & 9.367500 & 1.851600 & 7.391600 \\ \mathrm{H} & 7.804300 & 0.133800 & 7.250100 \\ \mathrm{H} & 6.191300 & 0.706700 & 7.837000 \\ \mathrm{H} & 6.298700 & -0.596000 & 6.603000 \\ \mathrm{H} & 6.841500 & 5.494800 & 6.368200 \\ \mathrm{H} & 8.492900 & 5.234500 & 5.675600 \\ \mathrm{H} & 7.159900 & 5.825800 & 4.630500 \\ \mathrm{H} & -4.174100 & 6.505500 & 3.100700 \\ \mathrm{H} & -0.114400 & 5.943900 & 4.396800 \\ & & & \end{array}$

$\begin{array}{lccc}\text { D6 } & & & \\ \text { Rh } & -0.000100 & -0.000100 & -0.592600 \\ \text { Rh } & -0.000300 & -0.000300 & -2.975200 \\ \text { O } & 1.449200 & -1.469900 & -2.908000 \\ \text { O } & 1.548700 & -1.357700 & -0.653900 \\ \text { O } & -1.469900 & -1.449800 & -2.907700 \\ \text { O } & -1.357600 & -1.548800 & -0.653600 \\ \text { O } & 1.469300 & 1.449100 & -2.908300 \\ \text { O } & 1.357500 & 1.548700 & -0.654200 \\ \text { O } & -1.449800 & 1.469200 & -2.908000 \\ \mathrm{O} & -1.548800 & 1.357500 & -0.653900 \\ \mathrm{O} & 4.355500 & 6.915300 & 7.359200 \\ \mathrm{O} & 6.915300 & -4.353900 & 7.360100 \\ \mathrm{O} & -4.354100 & -6.913600 & 7.361600 \\ \mathrm{O} & -6.913800 & 4.355600 & 7.360600 \\ \mathrm{Cl} & 4.665700 & -0.594300 & -0.467200 \\ \mathrm{Cl} & -0.594300 & -4.665800 & -0.466200 \\ \mathrm{Cl} & 0.594200 & 4.665700 & -0.467400 \\ \mathrm{Cl} & -4.665800 & 0.594200 & -0.466400 \\ \mathrm{C} & 1.931600 & -1.812000 & -1.778100 \\ \mathrm{C} & 2.963300 & -2.924100 & -1.725400 \\ \mathrm{C} & 3.748200 & -3.401400 & -2.989600 \\ \mathrm{C} & 2.511900 & -4.137000 & -2.543900 \\ \mathrm{C} & 3.553100 & -3.123600 & -0.346200 \\ \mathrm{C} & 4.343000 & -2.152100 & 0.292400\end{array}$

\begin{tabular}{|c|c|c|c|}
\hline & 4.898600 & -2.353400 & 1.553800 \\
\hline C & 4.683700 & -3.551900 & 2.248300 \\
\hline & 3.887700 & -4.527700 & 1.634000 \\
\hline & 3.340300 & -4.306700 & 0.371600 \\
\hline & 5.272900 & -3.766700 & 3.597400 \\
\hline & 4.538100 & -4.417000 & 4.600800 \\
\hline & 5.083600 & -4.617500 & 5.877900 \\
\hline & 6.375400 & -4.127900 & 6.170900 \\
\hline & 7.143500 & -3.528700 & 5.148500 \\
\hline & 6.579300 & -3.337500 & 3.878000 \\
\hline & 3.675500 & -2.600200 & -4.248500 \\
\hline & 4.126700 & -1.270700 & -4.274400 \\
\hline & 4.067000 & -0.530100 & -5.457900 \\
\hline & 3.563700 & -1.111300 & -6.624400 \\
\hline & 3.138000 & -2.442300 & -6.613700 \\
\hline & 3.206700 & -3.189500 & -5.434100 \\
\hline & 5.064000 & -4.086100 & -2.774000 \\
\hline$C$ & 5.221000 & -5.445800 & -3.100500 \\
\hline & 6.451800 & -6.081000 & -2.910700 \\
\hline & 7.541600 & -5.362400 & -2.413800 \\
\hline & 7.405800 & -4.003700 & -2.120000 \\
\hline & 6.177300 & -3.365100 & -2.310100 \\
\hline $\mathrm{C}$ & -1.811900 & -1.932000 & -1.777700 \\
\hline & -2.924100 & -2.963700 & -1.724800 \\
\hline & -3.401300 & -3.748900 & -2.988900 \\
\hline & -4.136900 & -2.512400 & -2.543500 \\
\hline $\mathrm{C}$ & -3.123500 & -3.553200 & -0.345600 \\
\hline $\mathrm{C}$ & -2.152200 & -4.343000 & 0.293300 \\
\hline $\mathrm{C}$ & -2.353500 & -4.898300 & 1.554800 \\
\hline & -3.552000 & -4.683200 & 2.249300 \\
\hline & -4.527800 & -3.887400 & 1.634700 \\
\hline $\mathrm{C}$ & -4.306800 & -3.340200 & 0.372200 \\
\hline $\mathrm{C}$ & -3.766800 & -5.272100 & 3.598400 \\
\hline $\mathrm{C}$ & -4.417100 & -4.537000 & 4.601600 \\
\hline & -4.617700 & -5.082300 & 5.878900 \\
\hline & -4.128100 & -6.374000 & 6.172200 \\
\hline $\mathrm{C}$ & -3.528800 & -7.142300 & 5.150000 \\
\hline $\mathrm{C}$ & -3.337500 & -6.578400 & 3.879400 \\
\hline$C$ & -2.600100 & -3.676400 & -4.247700 \\
\hline & -1.270600 & -4.127700 & -4.273500 \\
\hline & -0.530000 & -4.068200 & -5.457000 \\
\hline $\mathrm{C}$ & -1.111200 & -3.565200 & -6.623700 \\
\hline $\mathrm{C}$ & -2.442100 & -3.139400 & -6.613000 \\
\hline & -3.189400 & -3.207800 & -5.433500 \\
\hline$C$ & -4.086100 & -5.064600 & -2.773000 \\
\hline 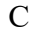 & -5.445700 & -5.221700 & -3.099600 \\
\hline $\mathrm{C}$ & -6.081000 & -6.452400 & -2.909500 \\
\hline & -5.362400 & -7.542100 & -2.412400 \\
\hline & -4.003800 & -7.406300 & -2.118500 \\
\hline $\mathrm{C}$ & -3.365100 & -6.177800 & -2.308900 \\
\hline & 1.811600 & 1.931600 & -1.778500 \\
\hline & 2.923700 & 2.963300 & -1.726000 \\
\hline & 3.400700 & 3.748200 & -2.990400 \\
\hline C & 4.136400 & 2.511800 & -2.544800 \\
\hline $\mathrm{C}$ & 3.123500 & 3.553100 & -0.346900 \\
\hline C & 2.152200 & 4.343000 & 0.291900 \\
\hline & 2.353800 & 4.898500 & 1.553300 \\
\hline & 3.552500 & 4.683700 & 2.247600 \\
\hline & 4.528100 & 3.887700 & 1.632900 \\
\hline $\mathrm{C}$ & 4.306900 & 3.340200 & 0.370700 \\
\hline$C$ & 3.767600 & 5.272900 & 3.596500 \\
\hline & 3.338400 & 6.579300 & 3.877200 \\
\hline
\end{tabular}

\begin{tabular}{|c|c|c|c|}
\hline $\mathrm{C}$ & 3.529900 & 7.143400 & 5.147700 \\
\hline $\mathrm{C}$ & 4.129300 & 6.375300 & 6.170000 \\
\hline & 4.618900 & 5.083600 & 5.876900 \\
\hline$c$ & 4.418100 & 4.538000 & 4.599800 \\
\hline 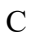 & 2.599300 & 3.675400 & -4.249000 \\
\hline & 1.269800 & 4.126800 & -4.274600 \\
\hline & 0.528900 & 4.067000 & -5.458000 \\
\hline $\mathrm{C}$ & 1.109900 & 3.563700 & -6.624600 \\
\hline $\mathrm{C}$ & 2.440800 & 3.138000 & -6.614200 \\
\hline $\mathrm{C}$ & 3.188300 & 3.206600 & -5.434800 \\
\hline 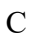 & 4.085600 & 5.064000 & -2.774900 \\
\hline $\mathrm{C}$ & 5.445100 & 5.221000 & -3.101700 \\
\hline $\mathrm{C}$ & 6.080400 & 6.451700 & -2.912000 \\
\hline $\mathrm{C}$ & 5.362000 & 7.541500 & -2.415000 \\
\hline $\mathrm{C}$ & 4.003400 & 7.405800 & -2.120900 \\
\hline $\mathrm{C}$ & 3.364600 & 6.177200 & -2.310900 \\
\hline $\mathrm{C}$ & -1.932000 & 1.811600 & -1.778100 \\
\hline $\mathrm{C}$ & -2.963700 & 2.923700 & -1.725500 \\
\hline $\mathrm{C}$ & -3.748800 & 3.400700 & -2.989700 \\
\hline $\mathrm{C}$ & -2.512400 & 4.136300 & -2.544400 \\
\hline $\mathrm{C}$ & -3.553200 & 3.123500 & -0.346200 \\
\hline $\mathrm{C}$ & -4.343000 & 2.152200 & 0.292800 \\
\hline $\mathrm{C}$ & -4.898300 & 2.353800 & 1.554300 \\
\hline $\mathrm{C}$ & -4.683300 & 3.552500 & 2.248500 \\
\hline $\mathrm{C}$ & -3.887500 & 4.528100 & 1.633700 \\
\hline $\mathrm{C}$ & -3.340200 & 4.306900 & 0.371300 \\
\hline $\mathrm{C}$ & -5.272200 & 3.767600 & 3.597600 \\
\hline $\mathrm{C}$ & -4.537200 & 4.418100 & 4.600600 \\
\hline $\mathrm{C}$ & -5.082400 & 4.619000 & 5.877900 \\
\hline $\mathrm{C}$ & -6.374200 & 4.129300 & 6.171300 \\
\hline $\mathrm{C}$ & -7.142400 & 3.529900 & 5.149100 \\
\hline $\mathrm{C}$ & -6.578500 & 3.338400 & 3.878600 \\
\hline $\mathrm{C}$ & -3.676300 & 2.599200 & -4.248300 \\
\hline $\mathrm{C}$ & -4.127600 & 1.269600 & -4.273800 \\
\hline $\mathrm{C}$ & -4.068100 & 0.528800 & -5.457200 \\
\hline $\mathrm{C}$ & -3.565100 & 1.109800 & -6.623900 \\
\hline $\mathrm{C}$ & -3.139300 & 2.440700 & -6.613600 \\
\hline $\mathrm{C}$ & -3.207800 & 3.188300 & -5.434200 \\
\hline $\mathrm{C}$ & -5.064600 & 4.085500 & -2.773900 \\
\hline $\mathrm{C}$ & -5.221700 & 5.445100 & -3.100800 \\
\hline $\mathrm{C}$ & -6.452400 & 6.080400 & -2.910900 \\
\hline $\mathrm{C}$ & -7.542100 & 5.361800 & -2.413600 \\
\hline $\mathrm{C}$ & -7.406300 & 4.003300 & -2.119500 \\
\hline $\mathrm{C}$ & -6.177800 & 3.364600 & -2.309700 \\
\hline $\mathrm{C}$ & -3.123600 & -8.571000 & 5.398100 \\
\hline $\mathrm{C}$ & -5.373600 & -4.292500 & 6.914200 \\
\hline $\mathrm{C}$ & -3.294900 & -6.692100 & 8.297000 \\
\hline $\mathrm{C}$ & -8.571100 & 3.124700 & 5.397200 \\
\hline $\mathrm{C}$ & -4.292700 & 5.375100 & 6.913000 \\
\hline $\mathrm{C}$ & -6.692300 & 3.296600 & 8.296100 \\
\hline $\mathrm{C}$ & 4.294100 & -5.373400 & 6.913400 \\
\hline $\mathrm{C}$ & 8.572200 & -3.123500 & 5.396100 \\
\hline $\mathrm{C}$ & 6.694100 & -3.294700 & 8.295500 \\
\hline $\mathrm{C}$ & 3.296600 & 6.694000 & 8.294800 \\
\hline $\mathrm{C}$ & 5.375000 & 4.294100 & 6.912200 \\
\hline $\mathrm{C}$ & 3.124700 & 8.572200 & 5.395500 \\
\hline $\mathrm{H}$ & 2.600100 & -5.121800 & -2.096900 \\
\hline $\mathrm{H}$ & 1.598700 & -3.995900 & -3.110500 \\
\hline $\mathrm{H}$ & 5.479300 & -1.542200 & 1.981900 \\
\hline $\mathrm{H}$ & 3.687600 & -5.481600 & 2.115100 \\
\hline $\mathrm{H}$ & 2.711500 & -5.076700 & -0.061200 \\
\hline $\mathrm{H}$ & 4.519900 & -0.809100 & -3.379700 \\
\hline
\end{tabular}




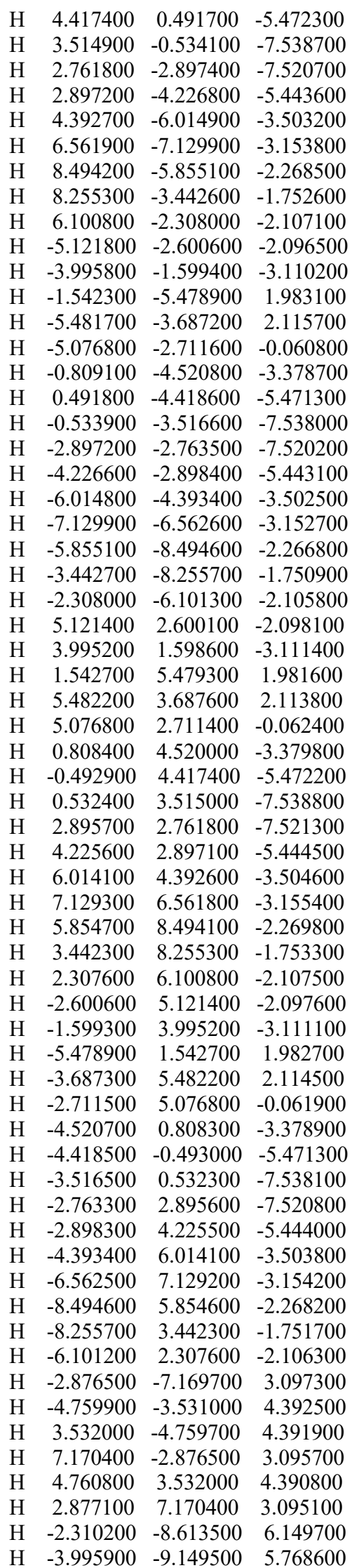

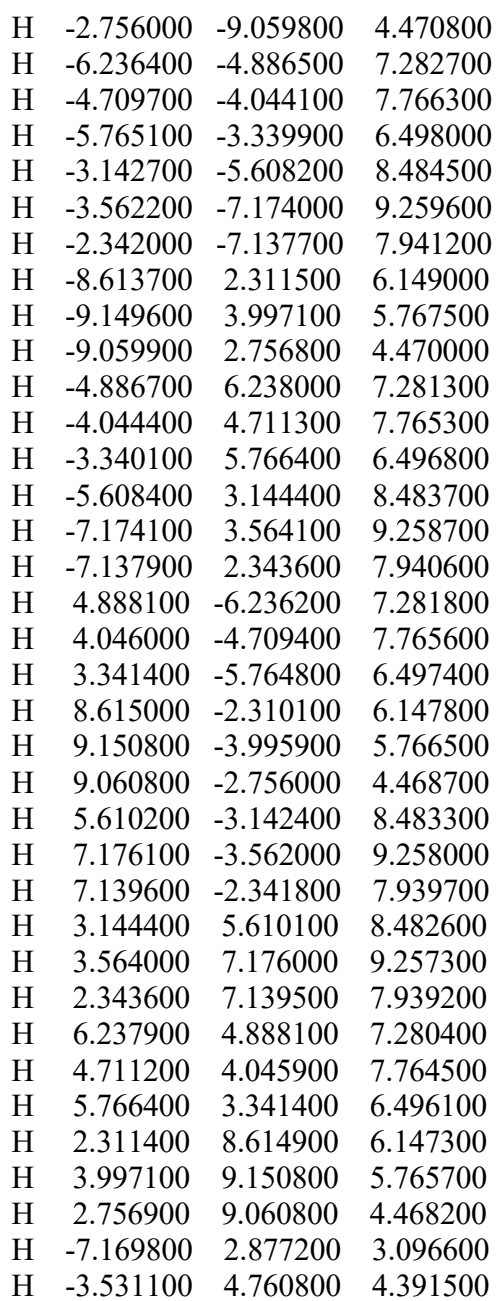

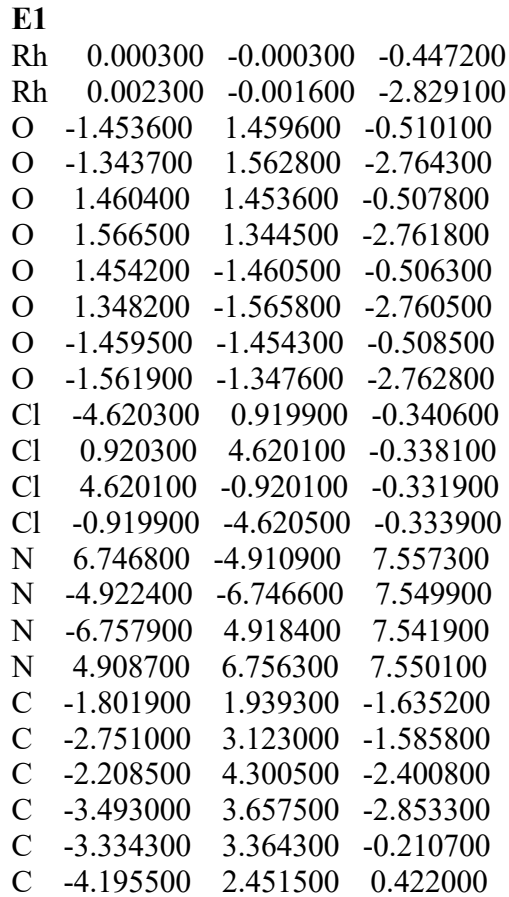

$\begin{array}{llll}\text { C } & -4.751100 & 2.694500 & 1.676200\end{array}$

$\begin{array}{llll}C & -4.464700 & 3.879100 & 2.369000\end{array}$

$\begin{array}{llll}\text { C } & -3.596200 & 4.795300 & 1.760900\end{array}$

$\begin{array}{llll}\text { C } & -3.048100 & 4.532000 & 0.507100\end{array}$

C $\quad-5.059100 \quad 4.146800 \quad 3.706300$

C $\quad-5.473200 \quad 5.443900 \quad 4.057100$

C $\quad-6.030200 \quad 5.696000 \quad 5.313800$

$\begin{array}{llll}\text { C } & -6.184300 & 4.657500 & 6.245700\end{array}$

$\begin{array}{llll}\text { C } & -5.771500 & 3.361800 & 5.898100\end{array}$

C $\quad-5.214700 \quad 3.108200 \quad 4.641600$

C $\quad-3.472700 \quad 2.855300 \quad-4.113400$

$\begin{array}{llll}\text { C } & -4.018200 & 1.561700 & -4.143700\end{array}$

$\begin{array}{llll}\text { C } & -4.007300 & 0.820900 & -5.328600\end{array}$

C $\quad-3.459000 \quad 1.366500 \quad-6.492000$

$\begin{array}{llll}\text { C } & -2.939100 & 2.663500 & -6.476900\end{array}$

C $\quad-2.958600 \quad 3.411600 \quad-5.296100$

C $\quad-4.756600 \quad 4.436000 \quad-2.643100$

$\begin{array}{llll}\text { C } & -5.922000 & 3.796800 & -2.187400\end{array}$

$\begin{array}{llll}\text { C } & -7.102000 & 4.522800 & -2.002600\end{array}$

C $\quad-7.136900 \quad 5.888200 \quad-2.293700$

$\begin{array}{llll}\text { C } & -5.994800 & 6.526800 & -2.782500\end{array}$

C $\quad-4.812500 \quad 5.804100 \quad-2.967100$

C $\quad 1.941500 \quad 1.802300 \quad-1.632100$

C $\quad 3.125200 \quad 2.751300 \quad-1.580900$

C $\quad 3.364600 \quad 3.334200 \quad-0.205300$

$\begin{array}{llll}\text { C } & 2.450900 & 4.195200 & 0.426400\end{array}$

$\begin{array}{llll}\text { C } & 2.692200 & 4.750700 & 1.681000\end{array}$

$\begin{array}{llll}\text { C } & 3.875900 & 4.464200 & 2.375300\end{array}$

$\begin{array}{llll}\text { C } & 4.792900 & 3.595900 & 1.768300\end{array}$

$\begin{array}{llll}\text { C } & 4.531400 & 3.048000 & 0.514000\end{array}$

C $\quad 4.141900 \quad 5.058500 \quad 3.713100$

$\begin{array}{llll}\text { C } & 5.438600 & 5.472600 & 4.065500\end{array}$

$\begin{array}{llll}\text { C } & 5.689100 & 6.029200 & 5.322700\end{array}$

$\begin{array}{llll}\text { C } & 4.649500 & 6.183000 & 6.253400\end{array}$

C $\quad 3.354100 \quad 5.770300 \quad 5.904000$

C $\quad 3.102100 \quad 5.214000 \quad 4.647100$

C $\quad 3.661400 \quad 3.493800 \quad-2.847400$

$\begin{array}{llll}\text { C } & 4.303700 & 2.209100 & -2.394500\end{array}$

C $\quad 2.860700 \quad 3.474100 \quad-4.108400$

$\begin{array}{llll}\text { C } & 3.418300 & 2.960200 & -5.290600\end{array}$

$\begin{array}{llll}\text { C } & 2.671600 & 2.941400 & -6.472300\end{array}$

$\begin{array}{llll}\text { C } & 1.374800 & 3.461700 & -6.488800\end{array}$

C $\quad 0.827800 \quad 4.009800 \quad-5.325900$

$\begin{array}{llll}\text { C } & 1.567300 & 4.020000 & -4.140100\end{array}$

$\begin{array}{llll}\text { C } & 4.439800 & 4.757100 & -2.635700\end{array}$

C $\quad 3.800300 \quad 5.922300 \quad-2.180100$

C $\quad 4.526200 \quad 7.102100 \quad-1.994000$

$\begin{array}{llll}\text { C } & 5.892000 & 7.137000 & -2.283500\end{array}$

C $\quad 6.531000 \quad 5.995100 \quad-2.772200$

$\begin{array}{llll}\text { C } & 5.808300 & 4.812900 & -2.958200\end{array}$

C $\quad 1.804500-1.941300 \quad-1.630300$

C $\quad 2.753300 \quad-3.125000 \quad-1.578100$

$\begin{array}{llll}\text { C } & 2.212300 & -4.303300 & -2.392800\end{array}$

$\begin{array}{llll}\text { C } & 3.497600 & -3.660900 & -2.843800\end{array}$

C $\quad 3.334500 \quad-3.364700 \quad-0.201800$

$\begin{array}{llll}\text { C } & 4.194300 & -2.451000 & 0.431400\end{array}$

$\begin{array}{llll}\text { C } & 4.748100 & -2.692700 & 1.686700\end{array}$

$\begin{array}{llll}\text { C } & 4.461100 & -3.876800 & 2.380100\end{array}$

$\begin{array}{llll}\text { C } & 3.593900 & -4.793900 & 1.771500\end{array}$

$\begin{array}{llll}\text { C } & 3.047600 & -4.531900 & 0.516600\end{array}$

C $\quad 5.053600 \quad-4.143100 \quad 3.718500$

$\begin{array}{llll}\text { C } & 5.468100 & -5.439700 & 4.070800\end{array}$ 


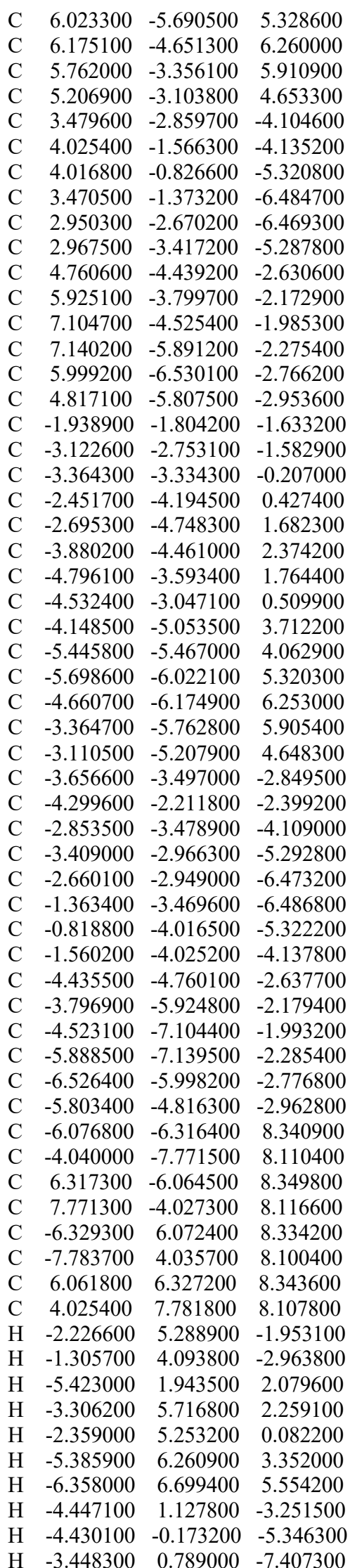

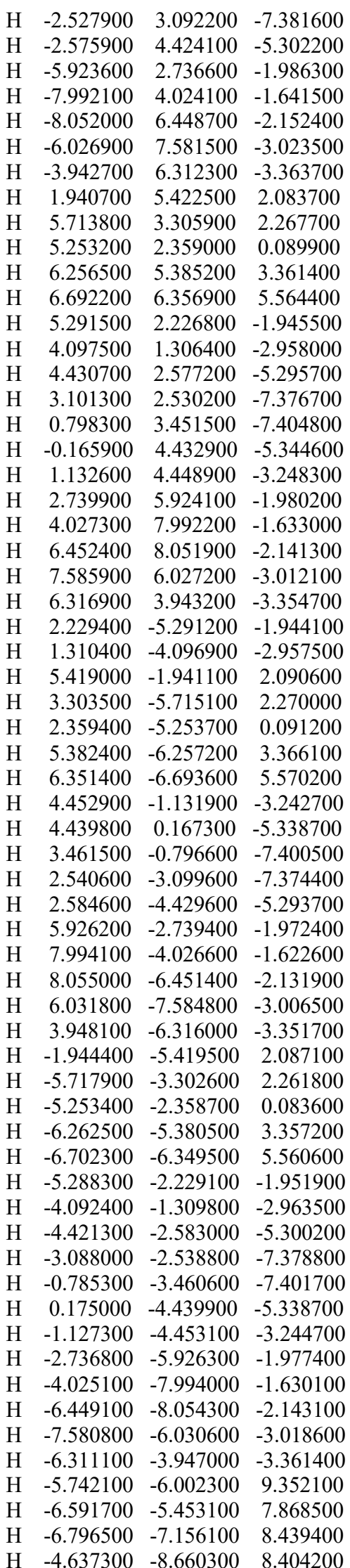

$\begin{array}{rrrr}\mathrm{H} & -3.280500 & -8.100500 & 7.369600 \\ \mathrm{H} & -3.520600 & -7.369200 & 9.005400 \\ \mathrm{H} & 6.003000 & -5.728700 & 9.360500 \\ \mathrm{H} & 5.454300 & -6.580600 & 7.878000 \\ \mathrm{H} & 7.157400 & -6.783600 & 8.449400 \\ \mathrm{H} & 8.099800 & -3.268400 & 7.374900 \\ \mathrm{H} & 7.368800 & -3.506900 & 9.011000 \\ \mathrm{H} & 8.660500 & -4.623700 & 8.411000 \\ \mathrm{H} & -6.016700 & 5.737300 & 9.345700 \\ \mathrm{H} & -5.465100 & 6.587700 & 7.863400 \\ \mathrm{H} & -7.169100 & 6.792000 & 8.431800 \\ \mathrm{H} & -8.111400 & 3.276400 & 7.358600 \\ \mathrm{H} & -7.382800 & 3.515700 & 8.995700 \\ \mathrm{H} & -8.673100 & 4.632700 & 8.393000 \\ \mathrm{H} & 5.725400 & 6.014400 & 9.354600 \\ \mathrm{H} & 6.577700 & 5.463300 & 7.873100 \\ \mathrm{H} & 6.781300 & 7.167100 & 8.442300 \\ \mathrm{H} & 4.622200 & 8.671100 & 8.401400 \\ \mathrm{H} & 3.267200 & 8.109800 & 7.365200 \\ \mathrm{H} & 3.504500 & 7.380700 & 9.002300 \\ \mathrm{H} & -2.552900 & -5.854400 & 6.615900 \\ \mathrm{H} & -2.105000 & -4.879200 & 4.417500 \\ \mathrm{H} & 5.852800 & -2.543400 & 6.620300 \\ \mathrm{H} & 4.877500 & -2.098800 & 4.421300 \\ \mathrm{H} & -5.863900 & 2.549600 & 6.608000 \\ \mathrm{H} & -4.885600 & 2.102900 & 4.410800 \\ \mathrm{H} & 2.541000 & 5.862800 & 6.612900 \\ \mathrm{H} & 2.097100 & 4.885000 & 4.414900\end{array}$

E2

$\begin{array}{llll}\text { Rh } & 0.016900 & -0.001500 & -0.449900\end{array}$

$\begin{array}{lllll}\mathrm{Rh} & 0.109700 & 0.000800 & -2.830400\end{array}$

O $\quad 1.503200 \quad-1.429300 \quad-0.454600$

O $\quad 1.484400-1.534800 \quad-2.711100$

O $\quad-1.407800 \quad-1.485200 \quad-0.568500$

$\begin{array}{llll}\mathrm{O} & -1.426700 & -1.378600 & -2.825100\end{array}$

O $\quad-1.464000 \quad 1.425900 \quad-0.567200$

$\begin{array}{llll}\mathrm{O} & -1.271400 & 1.536000 & -2.815300\end{array}$

$\begin{array}{llll}\mathrm{O} & 1.445600 & 1.483700 & -0.453300\end{array}$

$\begin{array}{llll}\text { O } & 1.640900 & 1.379900 & -2.701500\end{array}$

$\mathrm{Cl} \quad 4.646700 \quad-0.821600 \quad-0.149400$

Cl $\quad-0.807600 \quad-4.638400 \quad-0.362100$

$\mathrm{Cl} \quad-4.622500 \quad 0.810200 \quad-0.515600$

$\begin{array}{lllll}\mathrm{Cl} & 0.826600 & 4.638100 & -0.298500\end{array}$

$\begin{array}{llll}\mathrm{N} & -7.138300 & 4.738000 & 7.289500\end{array}$

$\begin{array}{llll}\mathrm{N} & 4.481400 & 6.873400 & 7.721500\end{array}$

$\begin{array}{lrrr}\mathrm{N} & 6.555400 & -4.789200 & 7.801700\end{array}$

$\mathrm{N} \quad-5.050900 \quad-6.811900 \quad 7.375700$

C $\quad 1.906100 \quad-1.901000 \quad-1.564500$

C $\quad 2.879100 \quad-3.062800 \quad-1.477400$

C $\quad 2.398300 \quad-4.250100 \quad-2.316600$

C $\quad 3.685300 \quad-3.576600 \quad-2.714000$

$\begin{array}{llll}\text { C } & 3.410200 & -3.294700 & -0.079600\end{array}$

$\begin{array}{llll}\text { C } & 4.227000 & -2.366600 & 0.589200\end{array}$

$\begin{array}{lrrr}\text { C } & 4.737200 & -2.603300 & 1.863600\end{array}$

$\begin{array}{llll}\text { C } & 4.446700 & -3.795300 & 2.541900\end{array}$

C $\quad 3.619500 \quad-4.725500 \quad 1.898600$

C $\quad 3.118500 \quad-4.469800 \quad 0.623700$

C $\quad 4.992100 \quad-4.053100 \quad 3.901900$

C $\quad 6.301400 \quad-3.661200 \quad 4.233300$

$\begin{array}{llll}\text { C } & 6.813100 & -3.902900 & 5.511100\end{array}$

$\begin{array}{llll}\text { C } & 6.027400 & -4.540800 & 6.483900\end{array}$ 


\begin{tabular}{|c|c|c|c|}
\hline $\mathrm{C}$ & 4.720100 & -4.933100 & 6.155600 \\
\hline$C$ & 4.207200 & -4.692300 & 4.878000 \\
\hline$C$ & 3.699300 & -2.771000 & -3.972000 \\
\hline & 4.217000 & -1.465700 & -3.976800 \\
\hline & 4.239400 & -0.721800 & -5.159500 \\
\hline & 3.752200 & -1.275700 & -6.345900 \\
\hline & 3.260500 & -2.583800 & -6.355300 \\
\hline & 3.247200 & -3.334900 & -5.176300 \\
\hline & 4.956500 & -4.326800 & -2.452900 \\
\hline & 6.086900 & -3.662800 & -1.947000 \\
\hline & 7.274400 & -4.362300 & -1.714500 \\
\hline $\mathrm{C}$ & 7.352700 & -5.725600 & -2.007400 \\
\hline $\mathrm{C}$ & 6.247200 & -6.388200 & -2.545500 \\
\hline $\mathrm{C}$ & 5.057300 & -5.692200 & -2.777800 \\
\hline & -1.836000 & -1.844600 & -1.710700 \\
\hline $\mathrm{C}$ & -2.998700 & -2.820400 & -1.705000 \\
\hline $\mathrm{C}$ & -3.282000 & -3.403100 & -0.337600 \\
\hline $\mathrm{C}$ & -2.378400 & -4.244100 & 0.334800 \\
\hline $\mathrm{C}$ & -2.660100 & -4.798100 & 1.581500 \\
\hline$C$ & -3.875400 & -4.529700 & 2.226800 \\
\hline $\mathrm{C}$ & -4.782500 & -3.680100 & 1.579700 \\
\hline $\mathrm{C}$ & -4.481800 & -3.135300 & 0.332800 \\
\hline $\mathrm{C}$ & -4.180400 & -5.120000 & 3.557900 \\
\hline $\mathrm{C}$ & -3.799900 & -6.440100 & 3.858900 \\
\hline $\mathrm{C}$ & -4.085700 & -6.993900 & 5.109800 \\
\hline $\mathrm{C}$ & -4.757100 & -6.240300 & 5600 \\
\hline $\mathrm{C}$ & -5.138100 & -4.922600 & 5.787600 \\
\hline $\mathrm{C}$ & -4.853200 & -4.367600 & 4.537000 \\
\hline $\mathrm{C}$ & -3.464300 & -3.578500 & -2.990000 \\
\hline $\mathrm{C}$ & -4.154600 & -2.308000 & -2.569100 \\
\hline $\mathrm{C}$ & -2.611700 & -3.543600 & -4.216200 \\
\hline $\mathrm{C}$ & -3.130000 & -3.044000 & -5.422300 \\
\hline $\mathrm{C}$ & -2.334700 & -3.011500 & -6.571600 \\
\hline $\mathrm{C}$ & -1.027600 & -3.504200 & -6.532100 \\
\hline $\mathrm{C}$ & -0.518600 & -4.038000 & -5.345700 \\
\hline $\mathrm{C}$ & -1.306800 & -4.061600 & -4.191900 \\
\hline $\mathrm{C}$ & -4.221900 & -4.858900 & -2.806400 \\
\hline $\mathrm{C}$ & -3.575800 & -6.007600 & -2.319100 \\
\hline $\mathrm{C}$ & -4.281700 & -7.203300 & -2.159200 \\
\hline $\mathrm{C}$ & -5.632700 & -7.270700 & -2.506800 \\
\hline $\mathrm{C}$ & -6.276100 & -6.145400 & -3.027100 \\
\hline $\mathrm{C}$ & -5.573600 & -4.947400 & -3.186800 \\
\hline $\mathrm{C}$ & -1.781000 & 1.899300 & -1.704100 \\
\hline $\mathrm{C}$ & -2.759900 & 3.059200 & -1.689000 \\
\hline $\mathrm{C}$ & -2.216600 & 4.251100 & -2.482300 \\
\hline $\mathrm{C}$ & -3.467200 & 3.577600 & -2.982700 \\
\hline $\mathrm{C}$ & -3.399000 & 3.283700 & -0.335900 \\
\hline $\mathrm{C}$ & -4.261200 & 2.349700 & 0.263800 \\
\hline C & -4.867000 & 2.576900 & 1.497600 \\
\hline $\mathrm{C}$ & -4.633200 & 3.766100 & 2.202300 \\
\hline $\mathrm{C}$ & -3.764900 & 4.703500 & 1.627200 \\
\hline $\mathrm{C}$ & -3.166000 & 4.456100 & 0.393500 \\
\hline $\mathrm{C}$ & -5.281200 & 4.016500 & 3.517900 \\
\hline $\mathrm{C}$ & -5.446300 & 2.972200 & 4.445300 \\
\hline $\mathrm{C}$ & -6.054900 & 3.209400 & 5.680800 \\
\hline $\mathrm{C}$ & -6.510700 & 4.494200 & 6.015200 \\
\hline $\mathrm{C}$ & -6.346700 & 5.538400 & 5.091500 \\
\hline $\mathrm{C}$ & -5.738100 & 5.302800 & 3.855600 \\
\hline $\mathrm{C}$ & -3.380600 & 2.777600 & -4.241500 \\
\hline $\mathrm{C}$ & -3.893100 & 1.471200 & -4.292600 \\
\hline $\mathrm{C}$ & -3.819900 & 0.732300 & -5.476400 \\
\hline$C$ & -3.242300 & 1.292600 & -6.618500 \\
\hline
\end{tabular}

$\begin{array}{llll}\text { C } & -2.755100 & 2.601900 & -6.583600\end{array}$

$\begin{array}{llll}\text { C } & -2.836700 & 3.347900 & -5.404100\end{array}$

C $\quad-4.756200 \quad 4.325000 \quad-2.819100$

$\begin{array}{llll}\text { C } & -5.921800 & 3.657100 & -2.406900\end{array}$

C $\quad-7.125100 \quad 4.353900 \quad-2.265600$

C $\quad-7.182400 \quad 5.718500 \quad-2.557300$

$\begin{array}{llll}\text { C } & -6.039000 & 6.385200 & -3.003400\end{array}$

C $\quad-4.833300 \quad 5.691700 \quad-3.144500$

$\begin{array}{llll}\text { C } & 1.961300 & 1.845000 & -1.558000\end{array}$

$\begin{array}{llll}\text { C } & 3.119200 & 2.821700 & -1.461400\end{array}$

C $\quad 3.293600 \quad 3.408000 \quad-0.077400$

$\begin{array}{llll}\text { C } & 2.338100 & 4.247500 & 0.520600\end{array}$

$\begin{array}{llll}\text { C } & 2.521700 & 4.807900 & 1.782800\end{array}$

$\begin{array}{llll}\text { C } & 3.686100 & 4.548900 & 2.519200\end{array}$

$\begin{array}{llll}\text { C } & 4.643700 & 3.701600 & 1.946100\end{array}$

C $4.439900 \quad 3.148000 \quad 0.683700$

C $\quad 3.890900 \quad 5.150000 \quad 3.864700$

$\begin{array}{llll}C & 5.164300 & 5.594800 & 4.261800\end{array}$

$\begin{array}{llll}\text { C } & 5.357100 & 6.159500 & 5.525600\end{array}$

$\begin{array}{llll}\text { C } & 4.281900 & 6.291100 & 6.418400\end{array}$

$\begin{array}{llll}\text { C } & 3.009700 & 5.847300 & 6.024700\end{array}$

C $\quad 2.815400 \quad 5.282600 \quad 4.761100$

$\begin{array}{llll}\text { C } & 3.684600 & 3.579000 & -2.706400\end{array}$

$\begin{array}{llll}C & 4.339700 & 2.309000 & -2.231100\end{array}$

C $\quad 2.931600 \quad 3.543200 \quad-3.996100$

$\begin{array}{llll}\text { C } & 3.544900 & 3.046000 & -5.157800\end{array}$

$\begin{array}{llll}C & 2.843200 & 3.012100 & -6.366400\end{array}$

C $\quad 1.535600 \quad 3.501100 \quad-6.430000$

$\begin{array}{llll}\text { C } & 0.932900 & 4.032800 & -5.287300\end{array}$

C $\quad 1.627500 \quad 4.057900 \quad-4.074900$

$\begin{array}{llll}\text { C } & 4.424700 & 4.859900 & -2.464500\end{array}$

$\begin{array}{llll}\text { C } & 3.741400 & 6.008900 & -2.031800\end{array}$

C $\quad 4.431800 \quad 7.205100 \quad-1.817400$

$\begin{array}{llll}\text { C } & 5.806300 & 7.272600 & -2.055900\end{array}$

$\begin{array}{llll}\text { C } & 6.489600 & 6.147000 & -2.521800\end{array}$

$\begin{array}{llll}\text { C } & 5.802500 & 4.948400 & -2.735800\end{array}$

$\begin{array}{llll}\text { C } & 3.555400 & 7.879800 & 8.243900\end{array}$

$\begin{array}{llll}\text { C } & 5.615200 & 6.473400 & 8.556900\end{array}$

$\begin{array}{llll}\text { C } & -8.163400 & 3.830200 & 7.807400\end{array}$

$\begin{array}{llll}\text { C } & -6.766700 & 5.899600 & 8.099400\end{array}$

$\begin{array}{llll}\text { C } & 7.278500 & -3.745200 & 8.529900\end{array}$

$\begin{array}{llll}\text { C } & 6.382400 & -6.092200 & 8.446400\end{array}$

$\begin{array}{llll}\text { C } & -4.032500 & -7.559800 & 8.115100\end{array}$

$\begin{array}{lrrr}\text { C } & -6.375500 & -6.659400 & 7.980300\end{array}$

H $\quad 2.420500 \quad-5.239200 \quad-1.870500$

H $\quad 1.514900 \quad-4.062300 \quad-2.915900$

$\mathrm{H} \quad 5.344000 \quad-1.823300 \quad 2.313000$

H $\quad 3.359400 \quad-5.671500 \quad 2.366300$

H $\quad 2.465600 \quad-5.205600 \quad 0.167600$

H $\quad 6.938500 \quad-3.186100 \quad 3.497900$

$\mathrm{H} \quad 7.830100 \quad-3.608100 \quad 5.737000$

H $\quad 4.598900 \quad-1.025400 \quad-3.066500$

$\mathrm{H} \quad 4.641100 \quad 0.281100 \quad-5.157600$

H $\quad 3.767000 \quad-0.695800 \quad-7.259700$

$\mathrm{H} \quad 2.897000 \quad-3.018600 \quad-7.277300$

$\mathrm{H} \quad 2.887800 \quad-4.355600 \quad-5.200700$

H $\quad 6.055900 \quad-2.603600 \quad-1.743400$

H $\quad 8.137000 \quad-3.844800 \quad-1.314900$

H $\quad 8.273700 \quad-6.265500 \quad-1.829200$

H $\quad 6.313500 \quad-7.441000 \quad-2.787700$

$\mathrm{H} \quad 4.216700 \quad-6.218600 \quad-3.211900$

H $\quad-1.896400 \quad-5.420600 \quad 2.037000$
$\mathrm{H} \quad-5.744600 \quad-3.435200 \quad 2.022300$

$\mathrm{H} \quad-5.200800 \quad-2.466000 \quad-0.126000$

H $\quad-3.299400 \quad-7.052700 \quad 3.119600$

$\mathrm{H} \quad-3.798700 \quad-8.018000 \quad 5.311700$

$\mathrm{H} \quad-5.159600 \quad-2.347800 \quad-2.161400$

$\mathrm{H} \quad-3.946400 \quad-1.402100 \quad-3.126700$

$\mathrm{H} \quad-4.149100 \quad-2.682600 \quad-5.471000$

$\mathrm{H} \quad-2.734700 \quad-2.611400 \quad-7.494300$

$\mathrm{H} \quad-0.413500 \quad-3.483500 \quad-7.423200$

$\mathrm{H} \quad 0.483800 \quad-4.440200 \quad-5.321600$

$\mathrm{H} \quad-0.900900 \quad-4.479400 \quad-3.281400$

$\mathrm{H} \quad-2.525200 \quad-5.984400 \quad-2.074200$

$\mathrm{H} \quad-3.778500 \quad-8.080500 \quad-1.773500$

$\mathrm{H} \quad-6.177500 \quad-8.197900 \quad-2.384800$

$\mathrm{H} \quad-7.318800 \quad-6.202600 \quad-3.311800$

$\mathrm{H} \quad-6.084500 \quad-4.090900 \quad-3.608300$

$\mathrm{H} \quad-2.275600 \quad 5.237900 \quad-2.034700$

$\mathrm{H} \quad-1.288400 \quad 4.067700 \quad-3.011300$

$\begin{array}{llll}\mathrm{H} & -5.535200 & 1.809400 & 1.875600\end{array}$

$\mathrm{H} \quad-3.514400 \quad 5.630300 \quad 2.137000$

$\mathrm{H} \quad-2.478900 \quad 5.193900 \quad-0.005200$

$\mathrm{H} \quad-5.085700 \quad 1.975400 \quad 4.225000$

$\mathrm{H} \quad-6.154500 \quad 2.393300 \quad 6.385300$

$\mathrm{H} \quad-4.344300 \quad 1.025900 \quad-3.417100$

H $\quad-4.217300 \quad-0.271900 \quad-5.510300$

$\mathrm{H} \quad-3.183400 \quad 0.716600 \quad-7.532900$

$\mathrm{H} \quad-2.321100 \quad 3.041600 \quad-7.472200$

$\mathrm{H} \quad-2.478800 \quad 4.369400 \quad-5.395600$

$\mathrm{H} \quad-5.905200 \quad 2.596900 \quad-2.206500$

$\mathrm{H} \quad-8.015600 \quad 3.833500 \quad-1.937800$

H $\quad-8.115400 \quad 6.256400 \quad-2.449800$

$\mathrm{H} \quad-6.087700 \quad 7.439000 \quad-3.245200$

$\mathrm{H} \quad-3.962000 \quad 6.221300 \quad-3.508600$

$\mathrm{H} \quad 1.740800 \quad 5.462100 \quad 2.157900$

H $\quad 5.552600 \quad 3.432700 \quad 2.478300$

H $\quad 5.192100 \quad 2.475900 \quad 0.286100$

$\mathrm{H} \quad 6.008300 \quad 5.525600 \quad 3.587200$

$\mathrm{H} \quad 6.343100 \quad 6.511200 \quad 5.801600$

H $\quad 5.309500 \quad 2.349300 \quad-1.745600$

$\mathrm{H} \quad 4.176100 \quad 1.402400 \quad-2.802400$

$\mathrm{H} \quad 4.565900 \quad 2.687800 \quad-5.126000$

H $\quad 3.315900 \quad 2.614000 \quad-7.254900$

H $\quad 0.993900 \quad 3.479300 \quad-7.366800$

H $\quad-0.069400 \quad 4.432000 \quad-5.342200$

H $\quad 1.149800 \quad 4.473900 \quad-3.199100$

$\mathrm{H} \quad 2.674500 \quad 5.985400 \quad-1.871500$

H $\quad 3.899100 \quad 8.082500 \quad-1.474200$

$\mathrm{H} \quad 6.339300 \quad 8.200100 \quad-1.891800$

H $\quad 7.551700 \quad 6.204300 \quad-2.722200$

H $\quad 6.345700 \quad 4.091700 \quad-3.114200$

$\begin{array}{llll}\mathrm{H} & 4.120700 & 8.783500 & 8.555200\end{array}$

H $\quad 2.816600 \quad 8.188000 \quad 7.473800$

$\mathrm{H} \quad 3.012500 \quad 7.469200 \quad 9.121000$

H $\quad 5.250500 \quad 6.155500 \quad 9.556400$

H $\quad 6.167400 \quad 5.620500 \quad 8.108000$

H $\quad 6.311100 \quad 7.330100 \quad 8.678200$

$\begin{array}{llll}\mathrm{H} & -9.076900 & 4.405600 & 8.067400\end{array}$

H $\quad-8.445200 \quad 3.065600 \quad 7.052600$

$\mathrm{H} \quad-7.784500 \quad 3.317400 \quad 8.716300$

$\begin{array}{llll}\mathrm{H} & -5.898000 & 6.436200 & 7.662200\end{array}$

$\mathrm{H} \quad-7.626200 \quad 6.599100 \quad 8.166700$

H $\quad-6.484500 \quad 5.569200 \quad 9.121300$ 


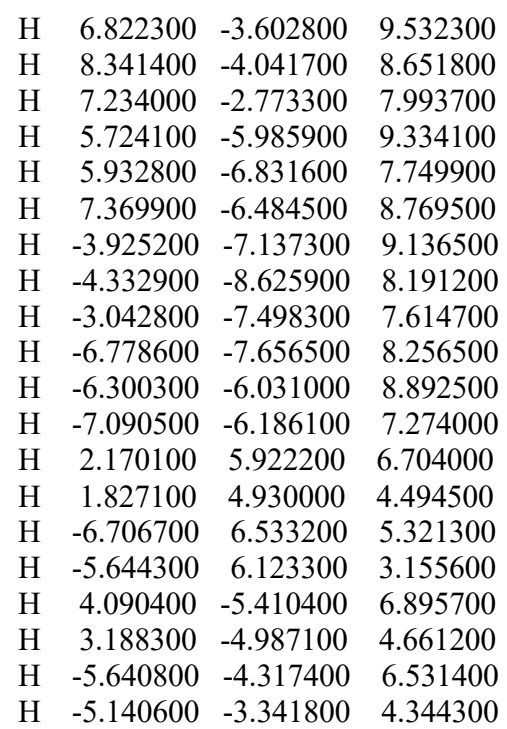

E3

$\begin{array}{lllll}\mathrm{Rh} & 0.000000 & 0.000000 & -0.442700\end{array}$

$\begin{array}{lllll}\mathrm{Rh} & 0.000000 & 0.000000 & -2.824900\end{array}$

$\begin{array}{llll}\text { O } & 1.908600 & -0.776800 & -0.504400\end{array}$

$\begin{array}{llll}\mathrm{O} & 1.848500 & -0.916500 & -2.758400\end{array}$

$\begin{array}{llll}\mathrm{O} & -0.775600 & -1.908600 & -0.504100\end{array}$

$\begin{array}{llll}\mathrm{O} & -0.916500 & -1.849000 & -2.758100\end{array}$

$\begin{array}{lllll}\mathrm{O} & -1.908600 & 0.776900 & -0.504400\end{array}$

$\begin{array}{lllll}\mathrm{O} & -1.848500 & 0.916500 & -2.758500\end{array}$

$\begin{array}{lllll}\mathrm{O} & 0.775600 & 1.908700 & -0.504100\end{array}$

$\begin{array}{llll}\mathrm{O} & 0.916500 & 1.849000 & -2.758000\end{array}$

$\begin{array}{lllll}\mathrm{Cl} & 4.615200 & 0.950600 & -0.322500\end{array}$

$\begin{array}{lllll}\mathrm{Cl} & 0.965100 & -4.610200 & -0.337400\end{array}$

$\begin{array}{llll}\text { Cl } & -4.615100 & -0.950700 & -0.322700\end{array}$

$\begin{array}{llll}\mathrm{Cl} & -0.965000 & 4.610300 & -0.337400\end{array}$

$\begin{array}{lrrr}\mathrm{N} & -8.192700 & 1.923300 & 7.523600\end{array}$

$\begin{array}{llll}\mathrm{N} & 1.855900 & 8.160800 & 7.543700\end{array}$

$\begin{array}{lrrr}\mathrm{N} & 8.192100 & -1.923600 & 7.524000\end{array}$

$\begin{array}{lrrr}\mathrm{N} & -1.855800 & -8.160600 & 7.543700\end{array}$

C $\quad 2.416900 \quad-1.083700 \quad-1.628900$

C $\quad 3.752300 \quad-1.803600 \quad-1.579200$

$\begin{array}{llll}\text { C } & 3.713700 & -3.096800 & -2.398500\end{array}$

C $\quad 4.646100 \quad-2.002300 \quad-2.846200$

C $\quad 4.382600 \quad-1.802600 \quad-0.203600$

$\begin{array}{llll}\text { C } & 4.824900 & -0.629200 & 0.431300\end{array}$

$\begin{array}{llll}\text { C } & 5.437800 & -0.642700 & 1.682200\end{array}$

$\begin{array}{llll}\text { C } & 5.635400 & -1.848000 & 2.370600\end{array}$

$\begin{array}{llll}\text { C } & 5.183000 & -3.026500 & 1.762700\end{array}$

C $\quad 4.572000 \quad-2.992000 \quad 0.510700$

$\begin{array}{llll}\text { C } & 6.296100 & -1.867600 & 3.703400\end{array}$

$\begin{array}{llll}\text { C } & 5.845400 & -2.737000 & 4.712600\end{array}$

C $\quad 6.467000 \quad-2.754700 \quad 5.964300$

$\begin{array}{llll}\text { C } & 7.551400 & -1.904600 & 6.233000\end{array}$

C $\quad 8.003500 \quad-1.036100 \quad 5.227100$

C $\quad 7.382700 \quad-1.017200 \quad 3.975200$

C $\quad 4.316200 \quad-1.267200 \quad-4.104100$

$\begin{array}{llll}\text { C } & 4.314600 & 0.136700 & -4.129800\end{array}$

$\begin{array}{llll}\text { C } & 4.018000 & 0.818600 & -5.313000\end{array}$

$\begin{array}{llll}\text { C } & 3.727200 & 0.106400 & -6.479100\end{array}$

$\begin{array}{llll}\text { C } & 3.753200 & -1.290800 & -6.468500\end{array}$

$\begin{array}{llll}\text { C } & 4.060800 & -1.976100 & -5.289500\end{array}$
C $\quad 6.113000 \quad-2.226600 \quad-2.634500$

C $\quad 6.935900 \quad-1.184700 \quad-2.174400$

$\begin{array}{llll}\text { C } & 8.305300 & -1.393200 & -1.988000\end{array}$

C $\quad 8.870600 \quad-2.636000 \quad-2.281900$

C $\quad 8.069200 \quad-3.668000 \quad-2.775200$

C $\quad 6.698800 \quad-3.463500 \quad-2.961300$

C $\quad-1.081700 \quad-2.417800 \quad-1.628500$

C $\quad-1.797900-3.755100-1.577100$

C $\quad-1.787800 \quad-4.386000 \quad-0.201800$

$\begin{array}{llll}\text { C } & -0.609000 & -4.820500 & 0.428200\end{array}$

C $\quad-0.612000 \quad-5.428000 \quad 1.681900$

C $\quad-1.812500 \quad-5.630000 \quad 2.377100$

C $\quad-2.997100 \quad-5.189700 \quad 1.771900$

C $\quad-2.972700 \quad-4.581100 \quad 0.518600$

C $\quad-1.822900 \quad-6.284200 \quad 3.713400$

C $\quad-2.852600 \quad-7.175000 \quad 4.064400$

C $\quad-2.862700 \quad-7.789700 \quad 5.319500$

C $\quad-1.844700 \quad-7.526500 \quad 6.249500$

C $\quad-0.816000 \quad-6.637000 \quad 5.901900$

C $\quad-0.804500 \quad-6.021800 \quad 4.647100$

C $\quad-2.000900-4.648300 \quad-2.843500$

C $\quad-3.095400 \quad-3.718800 \quad-2.389600$

C $\quad-1.273200 \quad-4.316000 \quad-4.105100$

C $\quad-1.988700 \quad-4.064100 \quad-5.287100$

C $\quad-1.310300 \quad-3.753600 \quad-6.469400$

C $\quad 0.086600 \quad-3.721100 \quad-6.486600$

$\begin{array}{llll}\text { C } & 0.805700 & -4.008800 & -5.323800\end{array}$

C $\quad 0.130700 \quad-4.308800 \quad-4.137600$

$\begin{array}{llll}\text { C } & -2.220900 & -6.115900 & -2.631700\end{array}$

C $\quad-1.174700 \quad-6.936700 \quad-2.177300$

C $\quad-1.379200 \quad-8.306600 \quad-1.990800$

$\begin{array}{llll}\text { C } & -2.622100 & -8.874700 & -2.278800\end{array}$

C $\quad-3.658500 \quad-8.075300 \quad-2.766400$

C $\quad-3.458100 \quad-6.704300 \quad-2.952600$

C $\quad-2.416900 \quad 1.083800 \quad-1.629000$

C $\quad-3.752200 \quad 1.803600 \quad-1.579300$

C $\quad-3.713700 \quad 3.096900 \quad-2.398500$

$\begin{array}{llll}\text { C } & -4.646000 & 2.002300 & -2.846300\end{array}$

$\begin{array}{llll}\text { C } & -4.382600 & 1.802500 & -0.203700\end{array}$

$\begin{array}{llll}\text { C } & -4.824900 & 0.629200 & 0.431100\end{array}$

$\begin{array}{llll}\text { C } & -5.437900 & 0.642600 & 1.682000\end{array}$

$\begin{array}{llll}\text { C } & -5.635500 & 1.847900 & 2.370400\end{array}$

C $\quad-5.183000 \quad 3.026400 \quad 1.762600$

$\begin{array}{llll}\text { C } & -4.572000 & 2.991900 & 0.510600\end{array}$

C $\quad-6.296400 \quad 1.867400 \quad 3.703200$

C $\quad-5.845800 \quad 2.736800 \quad 4.712400$

C $\quad-6.467500 \quad 2.754400 \quad 5.964100$

$\begin{array}{llll}\text { C } & -7.551900 & 1.904400 & 6.232700\end{array}$

$\begin{array}{llll}\text { C } & -8.003800 & 1.035900 & 5.226700\end{array}$

$\begin{array}{llll}\text { C } & -7.382900 & 1.017000 & 3.974800\end{array}$

C $\quad-4.316000 \quad 1.267300 \quad-4.104300$

C $\quad-4.314400 \quad-0.136700 \quad-4.130000$

$\begin{array}{llll}\text { C } & -4.017800 & -0.818600 & -5.313100\end{array}$

C $\quad-3.727000 \quad-0.106300 \quad-6.479300$

C $\quad-3.753000 \quad 1.290900 \quad-6.468600$

C $\quad-4.060700 \quad 1.976200 \quad-5.289600$

C $\quad-6.112900 \quad 2.226600 \quad-2.634700$

C $\quad-6.935900 \quad 1.184700 \quad-2.174600$

C $\quad-8.305200 \quad 1.393100 \quad-1.988300$

C $\quad-8.870600 \quad 2.635900 \quad-2.282200$

$\begin{array}{llll}\text { C } & -8.069100 & 3.667900 & -2.775400\end{array}$

C $\quad-6.698700 \quad 3.463500 \quad-2.961500$ $\begin{array}{llll}\text { C } & 1.081700 & 2.417800 & -1.628400\end{array}$

C $\quad 1.798000 \quad 3.755100 \quad-1.577000$

$\begin{array}{llll}\text { C } & 1.787900 & 4.386000 & -0.201700\end{array}$

C $\quad 0.609000 \quad 4.820500 \quad 0.428300$

$\begin{array}{llll}\text { C } & 0.612000 & 5.428000 & 1.682000\end{array}$

C $\quad 1.812600 \quad 5.630100 \quad 2.377100$

C $\quad 2.997200 \quad 5.189800 \quad 1.772000$

$\begin{array}{llll}\text { C } & 2.972700 & 4.581200 & 0.518600\end{array}$

$\begin{array}{llll}\text { C } & 1.823000 & 6.284300 & 3.713400\end{array}$

C $\quad 2.852700 \quad 7.175100 \quad 4.064400$

$\begin{array}{llll}\text { C } & 2.862800 & 7.789800 & 5.319500\end{array}$

$\begin{array}{llll}\text { C } & 1.844800 & 7.526700 & 6.249500\end{array}$

$\begin{array}{llll}\text { C } & 0.816100 & 6.637100 & 5.901900\end{array}$

$\begin{array}{llll}\text { C } & 0.804600 & 6.021900 & 4.647200\end{array}$

$\begin{array}{llll}\text { C } & 2.001000 & 4.648400 & -2.843500\end{array}$

C $\quad 3.095500 \quad 3.718800 \quad-2.389500$

C $\quad 1.273200 \quad 4.316100 \quad-4.105000$

$\begin{array}{llll}\text { C } & 1.988800 & 4.064200 & -5.287100\end{array}$

C $\quad 1.310400 \quad 3.753600 \quad-6.469300$

C $\quad-0.086500 \quad 3.721200 \quad-6.486500$

$\begin{array}{llll}\text { C } & -0.805600 & 4.008900 & -5.323800\end{array}$

$\begin{array}{llll}\text { C } & -0.130600 & 4.308800 & -4.137500\end{array}$

$\begin{array}{llll}\text { C } & 2.221000 & 6.115900 & -2.631600\end{array}$

$\begin{array}{llll}\text { C } & 1.174900 & 6.936700 & -2.177200\end{array}$

C $\quad 1.379300 \quad 8.306700 \quad-1.990700$

$\begin{array}{llll}\text { C } & 2.622300 & 8.874700 & -2.278700\end{array}$

$\begin{array}{llll}\text { C } & 3.658600 & 8.075300 & -2.766300\end{array}$

$\begin{array}{llll}\text { C } & 3.458200 & 6.704300 & -2.952600\end{array}$

$\begin{array}{llll}\text { C } & 0.639900 & 8.759800 & 8.096600\end{array}$

$\begin{array}{llll}\text { C } & 3.083400 & 8.222200 & 8.339200\end{array}$

$\begin{array}{llll}\text { C } & -8.540400 & 3.189600 & 8.170700\end{array}$

$\begin{array}{llll}\text { C } & -8.513200 & 0.676600 & 8.220900\end{array}$

$\begin{array}{lrrr}\text { C } & 8.539700 & -3.189900 & 8.171100\end{array}$

$\begin{array}{llll}\text { C } & 8.512600 & -0.676900 & 8.221400\end{array}$

$\begin{array}{llll}\text { C } & -0.639800 & -8.759600 & 8.096700\end{array}$

$\begin{array}{llll}\text { C } & -3.083300 & -8.222100 & 8.339200\end{array}$

H $\quad 4.115400 \quad-4.001300-1.953200$

$\mathrm{H} \quad 2.802500 \quad-3.257000 \quad-2.963200$

H $\quad 5.732400 \quad 0.312300 \quad 2.106000$

H $\quad 5.308600 \quad-3.995000 \quad 2.239800$

H $\quad 4.217900 \quad-3.922800 \quad 0.082200$

$\mathrm{H} \quad 4.998100 \quad-3.388900 \quad 4.542100$

$\mathrm{H} \quad 6.090500 \quad-3.419700 \quad 6.731200$

$\mathrm{H} \quad 4.539400 \quad 0.700400 \quad-3.235400$

$\mathrm{H} \quad 4.020800 \quad 1.898800 \quad-5.327200$

H $\quad 3.493900 \quad 0.637200 \quad-7.393100$

H $\quad 3.542600 \quad-1.842800 \quad-7.375400$

H $\quad 4.102700 \quad-3.057700 \quad-5.299100$

$\mathrm{H} \quad 6.523400 \quad-0.208400 \quad-1.971100$

H $\quad 8.929600 \quad-0.587800 \quad-1.623300$

$\mathrm{H} \quad 9.931600 \quad-2.795200 \quad-2.139500$

H $\quad 8.510600 \quad-4.625800 \quad-3.018500$

H $\quad 6.096900 \quad-4.269600 \quad-3.361400$

H $\quad 0.343200 \quad-5.751700 \quad 2.083100$

H $\quad-3.957600 \quad-5.285000 \quad 2.271900$

$\mathrm{H} \quad-3.907100 \quad-4.229600 \quad 0.095900$

H $\quad-3.640000 \quad-7.413600 \quad 3.360600$

$\mathrm{H} \quad-3.656200 \quad-8.485900 \quad 5.559800$

$\mathrm{H} \quad-3.996800 \quad-4.122800 \quad-1.940000$

H $\quad-3.260600 \quad-2.807500 \quad-2.952800$

$\mathrm{H} \quad-3.070100 \quad-4.111000 \quad-5.291600$

H $\quad-1.867600 \quad-3.545500 \quad-7.373600$ 


\begin{tabular}{|c|c|c|c|}
\hline & 0.612000 & -3.485300 & -7.403000 \\
\hline & 1.885900 & -4.006300 & -5.343100 \\
\hline & 0.699400 & -4.531500 & -3.245900 \\
\hline 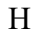 & -0.198500 & -6.521900 & -1.978500 \\
\hline & -0.570500 & -8.929400 & -1.630600 \\
\hline & -2.778300 & -9.936100 & -2.136200 \\
\hline & -4.616500 & -8.518800 & -3.005100 \\
\hline & -4.267500 & -6.104000 & -3.348300 \\
\hline & -4.115400 & 4.001300 & -1.953300 \\
\hline & -2.802500 & 3.257100 & -2.963200 \\
\hline & -5.732500 & -0.312400 & 2.105700 \\
\hline & -5.308800 & 3.994900 & 2.239700 \\
\hline & -4.218000 & 3.922700 & 0.082200 \\
\hline & -4.998500 & 3.388800 & 4.542000 \\
\hline & -6.091100 & 3.419400 & 6.731000 \\
\hline & -4.539300 & -0.700400 & -3.235600 \\
\hline $\mathrm{H}$ & -4.020600 & -1.898700 & -5.327400 \\
\hline & -3.493600 & -0.637000 & -7.393200 \\
\hline & -3.542400 & 1.842900 & -7.375400 \\
\hline & -4.102500 & 3.057800 & -5.299200 \\
\hline & -6.523300 & 0.208400 & -1.971300 \\
\hline $\mathrm{H}$ & -8.929600 & 0.587800 & -1.623600 \\
\hline & -9.931600 & 2.795200 & -2.139700 \\
\hline 1 & -8.510600 & 4.625700 & -3.018700 \\
\hline & -6.096800 & 4.269600 & -3.361500 \\
\hline & -0.343200 & 5.751800 & 2.083200 \\
\hline 11 & 3.957600 & 5.285100 & 2.272000 \\
\hline & 3.907200 & 4.229600 & 0.095900 \\
\hline & 3.640100 & 7.413700 & 3.360600 \\
\hline & 3.656300 & 8.486000 & 5.559800 \\
\hline $\mathrm{H}$ & 3.996800 & 4.122800 & -1.939900 \\
\hline $\mathrm{H}$ & 3.260600 & 2.807500 & -2.952700 \\
\hline H & 3.070200 & 4.111000 & -5.291600 \\
\hline H & 1.867700 & 3.545600 & -7.373600 \\
\hline 日 & -0.611900 & 3.485400 & -7.402900 \\
\hline $\mathrm{H}$ & -1.885800 & 4.006400 & -5.343100 \\
\hline $\mathrm{H}$ & -0.699300 & 4.531600 & -3.245900 \\
\hline $\mathrm{H}$ & 0.198600 & 6.522000 & -1.978500 \\
\hline $\mathrm{H}$ & 0.570700 & 8.929400 & -1.630600 \\
\hline & 2.778400 & 9.936100 & -2.136200 \\
\hline $\mathrm{H}$ & 4.616600 & 8.518800 & -3.005000 \\
\hline $\mathrm{H}$ & 4.267700 & 6.104000 & -3.348200 \\
\hline $\mathrm{H}$ & 0.839200 & 9.813200 & 8.386100 \\
\hline $\mathrm{H}$ & -0.184900 & 8.760700 & 7.352600 \\
\hline & 0.316600 & 8.190200 & 8.993100 \\
\hline $\mathrm{H}$ & 2.895000 & 7.806500 & 9.351600 \\
\hline $\mathrm{H}$ & 3.897800 & 7.628200 & 7.872600 \\
\hline $\mathrm{H}$ & 3.415200 & 9.277400 & 8.434100 \\
\hline $\mathrm{H}$ & -9.616400 & 3.187000 & 8.445500 \\
\hline $\mathrm{H}$ & -7.929800 & 3.320500 & 9.088800 \\
\hline $\mathrm{H}$ & -8.363200 & 4.052000 & 7.493300 \\
\hline $\mathrm{H}$ & -9.615100 & 0.560000 & 8.291500 \\
\hline $\mathrm{H}$ & -8.090300 & -0.203400 & 7.691100 \\
\hline & -8.082400 & 0.698100 & 9.244300 \\
\hline $\mathrm{H}$ & 9.615700 & -3.187400 & 8.446000 \\
\hline $\mathrm{H}$ & 7.929100 & -3.320900 & 9.089100 \\
\hline $\mathrm{H}$ & 8.362600 & -4.052300 & 7.493700 \\
\hline & 9.614400 & -0.560400 & 8.292000 \\
\hline & 8.089700 & 0.203100 & 7.691500 \\
\hline & 8.081700 & -0.698400 & 9.244700 \\
\hline 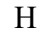 & -0.839100 & -9.813000 & 8.386100 \\
\hline & 0.185000 & -8.760500 & 7.352600 \\
\hline
\end{tabular}

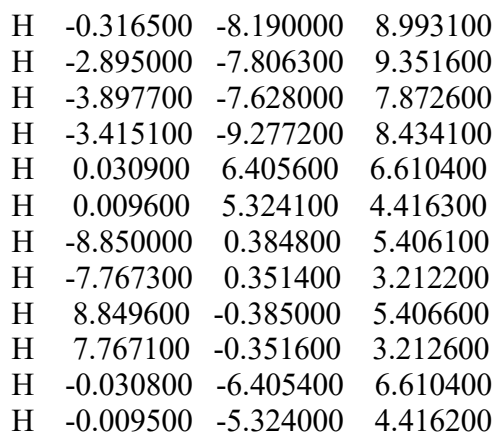

\section{E4}

$\begin{array}{llll}\mathrm{Rh} & -0.003600 & -0.011700 & -0.441100\end{array}$

$\begin{array}{llll}\mathrm{Rh} & -0.019400 & -0.070600 & -2.822400\end{array}$

$\begin{array}{llll}\mathrm{O} & -0.699700 & -1.952000 & -0.449700\end{array}$

$\begin{array}{llll}\mathrm{O} & -0.860900 & -1.952100 & -2.703200\end{array}$

$\begin{array}{llll}\mathrm{O} & -1.943300 & 0.683200 & -0.506700\end{array}$

$\begin{array}{llll}\mathrm{O} & -1.902800 & 0.772900 & -2.763800\end{array}$

$\begin{array}{llll}\mathrm{O} & 0.691400 & 1.925700 & -0.555400\end{array}$

$\begin{array}{lllll}\mathrm{O} & 0.823100 & 1.814500 & -2.807900\end{array}$

$\begin{array}{llll}\mathrm{O} & 1.935800 & -0.709300 & -0.498600\end{array}$

$\begin{array}{llll}\text { O } & 1.864700 & -0.911300 & -2.747600\end{array}$

$\begin{array}{llll}\mathrm{Cl} & 1.144700 & -4.578400 & -0.230100\end{array}$

$\begin{array}{lrrr}\text { Cl } & -4.578600 & -1.143300 & -0.263300\end{array}$

$\begin{array}{llll}\text { Cl } & -1.147900 & 4.561200 & -0.437100\end{array}$

$\begin{array}{lllll}\mathrm{Cl} & 4.568600 & 1.132400 & -0.388100\end{array}$

$\begin{array}{llll}\mathrm{N} & 1.596400 & 8.420800 & 7.323700\end{array}$

$\begin{array}{lrrr}\mathrm{N} & 8.313100 & -1.376600 & 7.507000\end{array}$

$\begin{array}{llll}\mathrm{N} & -1.484300 & -8.077900 & 7.738100\end{array}$

$\begin{array}{llll}\mathrm{N} & -8.217300 & 1.775500 & 7.537300\end{array}$

$\begin{array}{llll}\text { C } & -0.994600 & -2.499500 & -1.559000\end{array}$

C $\quad-1.657600 \quad-3.862100 \quad-1.470100$

$\begin{array}{llll}\text { C } & -2.960600 & -3.896100 & -2.273900\end{array}$

C $\quad-1.833400 \quad-4.792900 \quad-2.713500$

$\begin{array}{llll}\text { C } & -1.614000 & -4.459500 & -0.080600\end{array}$

$\begin{array}{llll}\text { C } & -0.415300 & -4.833500 & 0.550600\end{array}$

$\begin{array}{llll}\text { C } & -0.387100 & -5.413600 & 1.816900\end{array}$

C $\quad-1.574600 \quad-5.647700 \quad 2.524200$

C $\quad-2.779200 \quad-5.266500 \quad 1.918200$

$\begin{array}{llll}\text { C } & -2.785900 & -4.684500 & 0.652100\end{array}$

$\begin{array}{llll}\text { C } & -1.551500 & -6.275100 & 3.873100\end{array}$

C $\quad-0.538200 \quad-5.954800 \quad 4.794200$

$\begin{array}{llll}\text { C } & -0.516800 & -6.545900 & 6.060300\end{array}$

$\begin{array}{llll}\text { C } & -1.507000 & -7.468700 & 6.432200\end{array}$

$\begin{array}{llll}\text { C } & -2.520000 & -7.789500 & 5.514900\end{array}$

$\begin{array}{llll}\text { C } & -2.542800 & -7.198900 & 4.248400\end{array}$

$\begin{array}{llll}\text { C } & -1.127000 & -4.463000 & -3.987800\end{array}$

$\begin{array}{llll}\text { C } & 0.275100 & -4.401700 & -4.030400\end{array}$

$\begin{array}{llll}\text { C } & 0.930700 & -4.104600 & -5.228200\end{array}$

$\begin{array}{llll}\text { C } & 0.193800 & -3.873700 & -6.392400\end{array}$

$\begin{array}{llll}\text { C } & -1.200700 & -3.960100 & -6.364600\end{array}$

$\begin{array}{llll}\text { C } & -1.859200 & -4.268000 & -5.170500\end{array}$

$\begin{array}{llll}\text { C } & -1.994400 & -6.262400 & -2.465200\end{array}$

$\begin{array}{llll}\text { C } & -0.913600 & -7.030100 & -1.999600\end{array}$

$\begin{array}{llll}\text { C } & -1.062100 & -8.402200 & -1.779300\end{array}$

$\begin{array}{llll}\text { C } & -2.283400 & -9.025700 & -2.044400\end{array}$

$\begin{array}{llll}\text { C } & -3.354000 & -8.279600 & -2.542700\end{array}$

$\begin{array}{llll}\text { C } & -3.209500 & -6.906500 & -2.762600\end{array}$

$\begin{array}{llll}\text { C } & -2.470300 & 0.943600 & -1.634400\end{array}$

$\begin{array}{llll}\text { C } & -3.832600 & 1.611500 & -1.591600\end{array}$
C $\quad-4.453200 \quad 1.618800 \quad-0.211600$

$\begin{array}{llll}\text { C } & -4.845200 & 0.444800 & 0.454300\end{array}$

$\begin{array}{llll}\text { C } & -5.450300 & 0.464400 & 1.709000\end{array}$

C $\quad-5.690400 \quad 1.677400 \quad 2.369800$

$\begin{array}{lrrr}\text { C } & -5.287900 & 2.857600 & 1.731000\end{array}$

C $\quad-4.684000 \quad 2.816800 \quad 0.475700$

$\begin{array}{llll}\text { C } & -6.343400 & 1.703200 & 3.706400\end{array}$

C $\quad-7.393900 \quad 0.817600 \quad 4.005600$

$\begin{array}{llll}\text { C } & -8.007200 & 0.841600 & 5.261000\end{array}$

C $\quad-7.583800 \quad 1.751000 \quad 6.243000$

$\begin{array}{llll}\text { C } & -6.535600 & 2.636400 & 5.946800\end{array}$

C $\quad-5.921500 \quad 2.613600 \quad 4.691500$

$\begin{array}{llll}\text { C } & -4.741800 & 1.744700 & -2.856000\end{array}$

C $\quad-3.850200 \quad 2.885300 \quad-2.441500$

C $\quad-4.391700 \quad 0.993100 \quad-4.098700$

$\begin{array}{llll}\text { C } & -4.173200 & 1.682500 & -5.302700\end{array}$

$\begin{array}{llll}\text { C } & -3.846900 & 0.981400 & -6.467300\end{array}$

C $\quad-3.765500 \quad-0.413400 \quad-6.444600$

C $\quad-4.019400 \quad-1.108100 \quad-5.259400$

$\begin{array}{llll}\text { C } & -4.334600 & -0.409900 & -4.090700\end{array}$

$\begin{array}{llll}\text { C } & -6.215000 & 1.916600 & -2.638600\end{array}$

C $\quad-6.992900 \quad 0.854100 \quad-2.148300$

$\begin{array}{llll}\text { C } & -8.368200 & 1.012500 & -1.956600\end{array}$

$\begin{array}{llll}\text { C } & -8.984400 & 2.224500 & -2.275400\end{array}$

C $\quad-8.227800 \quad 3.275700 \quad-2.798500$

C $\quad-6.851500 \quad 3.121300 \quad-2.989800$

$\begin{array}{llll}\text { C } & 0.971900 & 2.417500 & -1.694000\end{array}$

C $\quad 1.636100 \quad 3.782300 \quad-1.680900$

$\begin{array}{llll}\text { C } & 2.927600 & 3.776200 & -2.503700\end{array}$

$\begin{array}{llll}\text { C } & 1.794400 & 4.651500 & -2.970300\end{array}$

$\begin{array}{llll}\text { C } & 1.612500 & 4.446000 & -0.321200\end{array}$

C $\quad 0.423100 \quad 4.850700 \quad 0.308600$

C $\quad 0.413100 \quad 5.489300 \quad 1.546700$

$\begin{array}{llll}\text { C } & 1.610600 & 5.754700 & 2.225600\end{array}$

$\begin{array}{llll}\text { C } & 2.806300 & 5.344500 & 1.620900\end{array}$

$\begin{array}{llll}\text { C } & 2.794900 & 4.704000 & 0.383400\end{array}$

$\begin{array}{llll}\text { C } & 1.606900 & 6.443400 & 3.544400\end{array}$

$\begin{array}{llll}\text { C } & 2.604100 & 7.382200 & 3.862900\end{array}$

C $\quad 2.599800 \quad 8.030100 \quad 5.101200$

C $\quad 1.600000 \quad 7.752600 \quad 6.046700$

C $\quad 0.603900 \quad 6.815200 \quad 5.731500$

C $\quad 0.606700 \quad 6.166800 \quad 4.493600$

$\begin{array}{llll}\text { C } & 1.070100 & 4.260300 & -4.216900\end{array}$

$\begin{array}{llll}\text { C } & -0.332700 & 4.198700 & -4.236700\end{array}$

C $\quad-1.005200 \quad 3.844100 \quad-5.409200$

C $\quad-0.284900 \quad 3.555500 \quad-6.571000$

$\begin{array}{llll}\text { C } & 1.109800 & 3.641500 & -6.567000\end{array}$

$\begin{array}{llll}\text { C } & 1.785300 & 4.006700 & -5.398800\end{array}$

$\begin{array}{llll}\text { C } & 1.958900 & 6.131200 & -2.796100\end{array}$

$\begin{array}{llll}\text { C } & 0.885100 & 6.921400 & -2.352800\end{array}$

$\begin{array}{llll}\text { C } & 1.036700 & 8.302500 & -2.201800\end{array}$

$\begin{array}{llll}\text { C } & 2.254100 & 8.911400 & -2.514600\end{array}$

$\begin{array}{llll}\text { C } & 3.317300 & 8.141300 & -2.991400\end{array}$

$\begin{array}{llll}\text { C } & 3.169600 & 6.759200 & -3.142100\end{array}$

C $\quad 2.447700 \quad-1.025100 \quad-1.619000$

C $\quad 3.811900-1.688300 \quad-1.561400$

C $\quad 4.452000 \quad-1.623600 \quad-0.191800$

$\begin{array}{llll}\text { C } & 4.846300 & -0.415500 & 0.408600\end{array}$

$\begin{array}{llll}\text { C } & 5.465600 & -0.368300 & 1.655600\end{array}$

C $\quad 5.720600 \quad-1.545000 \quad 2.373900$

$\begin{array}{llll}\text { C } & 5.319600 & -2.758300 & 1.799000\end{array}$

$\begin{array}{llll}\text { C } & 4.698600 & -2.784200 & 0.551900\end{array}$ 


\begin{tabular}{|c|c|c|c|}
\hline & 6.389900 & -1.501600 & \\
\hline & 7.324100 & -2.488300 & 4.063600 \\
\hline & 7.954300 & -2.446600 & 5.310400 \\
\hline & 7.663100 & -1.419000 & 6.221400 \\
\hline & 6.730100 & -0.433500 & 5.863200 \\
\hline & 6.099500 & -0.473800 & 4.616800 \\
\hline & 4.703700 & -1.885000 & -2.830100 \\
\hline & 3.820300 & -3.004000 & -2.345000 \\
\hline & 4.334700 & -1.198600 & -4.104600 \\
\hline & 4.101100 & -1.949300 & -5.268500 \\
\hline & 3.756800 & -1.309400 & -6.462800 \\
\hline & 3.672200 & 4700 & -6.510400 \\
\hline 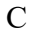 & 3.941100 & 0.839800 & -5.366100 \\
\hline & 4.274500 & 0.202900 & -4.167700 \\
\hline & 6.180000 & -2.043700 & -2.624900 \\
\hline & 6.962600 & 600 & 0200 \\
\hline $\mathrm{C}$ & 8.340800 & 3200 & -2.020300 \\
\hline C & 8.955000 & -2.329200 & -2.285800 \\
\hline- & 8.193100 & -3.406500 & -2.744000 \\
\hline & 6.814000 & -3.263800 & -2.923300 \\
\hline 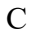 & 8.431 & -2.5 & 8400 \\
\hline $\mathrm{C}$ & 8.870700 & -0.12 & 8.025600 \\
\hline $\mathrm{C}$ & 0.362200 & 6200 & 7.871700 \\
\hline C & 2.826600 & 8.550000 & 8.106700 \\
\hline C & -0.241600 & -8.616900 & 8.293500 \\
\hline $\mathrm{C}$ & -2.702800 & -8.1 & 3900 \\
\hline $\mathrm{C}$ & -8.484800 & 100 & 8.265800 \\
\hline $\mathrm{C}$ & -8.611500 & 3.042300 & 8.156200 \\
\hline $\mathrm{H}$ & -3.842500 & -4.324000 & -1.808300 \\
\hline H & -3.164800 & -3.0 & -2.857400 \\
\hline $\mathrm{H}$ & 0.582 & -5.6 & 8200 \\
\hline $\mathrm{H}$ & -3.732100 & -5.38 & 2.426800 \\
\hline $\mathrm{H}$ & -3.736000 & -4.378900 & 0.228400 \\
\hline $\mathrm{H}$ & 0.226800 & -5.230700 & 4.544300 \\
\hline H & 0.263200 & -6.2 & 6.758400 \\
\hline $\mathrm{H}$ & 0.857700 & -4.580100 & -3.137600 \\
\hline $\mathrm{H}$ & 2.009700 & 0600 & -5.255100 \\
\hline $\mathrm{H}$ & 0.704000 & -3.640000 & -7.318000 \\
\hline$H$ & -1.771200 & -3.796100 & -7.269700 \\
\hline H & -2.937 & -4.3 & -5.166200 \\
\hline $\mathrm{H}$ & 0.046800 & -6.572600 & -1.818200 \\
\hline $\mathrm{H}$ & -0.227000 & -8.983700 & -1.410800 \\
\hline $\mathrm{H}$ & -2.396300 & -10.088800 & -1.875800 \\
\hline $\mathrm{H}$ & -4.295200 & -8.766300 & -2.763600 \\
\hline H & -4.044800 & -6.3 & -3.165900 \\
\hline $\mathrm{H}$ & -5.704600 & -0.490800 & 2.157400 \\
\hline $\mathrm{H}$ & -5.448300 & 3.831700 & 2.185800 \\
\hline $\mathrm{H}$ & -4.369300 & 3.750200 & 0.022700 \\
\hline & -7.756500 & 0.119500 & 3.261600 \\
\hline $\mathrm{H}$ & -8.825800 & 0.162000 & 5.461600 \\
\hline $\mathrm{H}$ & -4.284200 & 3.783700 & -2.014600 \\
\hline $\mathrm{H}$ & -2.949700 & 3.067400 & -3.016700 \\
\hline $\mathrm{H}$ & -4.258000 & 2.761100 & -5.337900 \\
\hline H & -3.664900 & 1.519100 & -7.388800 \\
\hline $\mathrm{H}$ & -3.517600 & -0.956700 & -7.347400 \\
\hline $\mathrm{H}$ & -3.979400 & -2.187600 & -5.247600 \\
\hline $\mathrm{H}$ & -4.530400 & -0.960200 & -3.181200 \\
\hline & -6.540600 & -0.100000 & -1.925400 \\
\hline & -8.957600 & 0.191900 & -1.568600 \\
\hline $\mathrm{H}$ & -10.050000 & 2.345000 & -2.129000 \\
\hline $\mathrm{H}$ & -8.708600 & 4.209100 & -3.060900 \\
\hline$\pi$ & -6.284700 & 3.940900 & -3.412900 \\
\hline
\end{tabular}

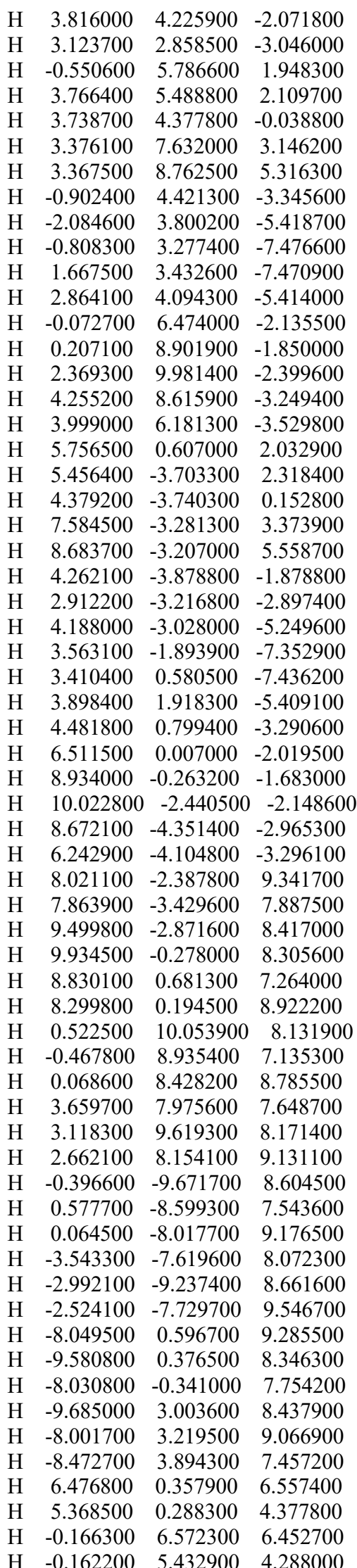

\begin{abstract}
H $\quad-3.283600 \quad-8.512000 \quad 5.774100$
$\mathrm{H} \quad-3.324700 \quad-7.481800 \quad 3.555000$

$\mathrm{H} \quad-6.181100 \quad 3.333800 \quad 6.695100$

$\mathrm{H} \quad-5.101600 \quad 3.294300 \quad 4.499800$
\end{abstract}

\section{E5}

$\begin{array}{llll}\mathrm{Rh} & 0.021700 & -0.007900 & -0.447900\end{array}$

$\begin{array}{llll}\mathrm{Rh} & 0.125000 & -0.039800 & -2.827800\end{array}$

O $\quad 1.137100 \quad 1.725000 \quad-0.484200$

$\begin{array}{llll}\text { O } & 1.363100 & 1.609200 & -2.728900\end{array}$

O $\quad 1.756200 \quad-1.121600 \quad-0.419200$

$\begin{array}{llll}\mathrm{O} & 1.767800 & -1.280600 & -2.672700\end{array}$

$\begin{array}{llll}\mathrm{O} & -1.089900 & -1.741500 & -0.534500\end{array}$

O $\quad-1.120100 \quad-1.686600 \quad-2.792800$

$\begin{array}{llll}\mathrm{O} & -1.707000 & 1.103700 & -0.599100\end{array}$

O $\quad-1.523700 \quad 1.203300 \quad-2.848500$

Cl $\quad-0.073200 \quad 4.705300 \quad-0.403200$

$\begin{array}{lllll}\mathrm{Cl} & 4.725200 & 0.088400 & -0.137800\end{array}$

$\mathrm{Cl} \quad 0.087900 \quad-4.717100 \quad-0.249600$

Cl $\quad-4.688700 \quad-0.106000 \quad-0.545900$

$\begin{array}{llll}\mathrm{N} & -3.735200 & -7.486300 & 7.516600\end{array}$

$\begin{array}{lrrr}\mathrm{N} & -7.958600 & 3.414000 & 7.174000\end{array}$

$\begin{array}{llll}\mathrm{N} & 3.033100 & 7.753300 & 7.580800\end{array}$

N $\quad 7.349200 \quad-3.250100 \quad 7.902800$

C $\quad 1.581400 \quad 2.153100 \quad-1.596200$

$\begin{array}{llll}\text { C } & 2.530900 & 3.334800 & -1.520000\end{array}$

C $\quad 3.831400 \quad 3.047900 \quad-2.275900$

$\begin{array}{llll}\text { C } & 2.948600 & 4.158500 & -2.781000\end{array}$

$\begin{array}{llll}\text { C } & 2.581600 & 3.973600 & -0.149200\end{array}$

$\begin{array}{llll}\text { C } & 1.479600 & 4.627500 & 0.428100\end{array}$

$\begin{array}{llll}\text { C } & 1.544200 & 5.238200 & 1.678600\end{array}$

$\begin{array}{llll}\text { C } & 2.731500 & 5.220800 & 2.423800\end{array}$

C $\quad 3.837200 \quad 4.560600 \quad 1.871500$

C $3.751300 \quad 3.952900 \quad 0.620400$

$\begin{array}{llll}\text { C } & 2.808400 & 5.876200 & 3.757300\end{array}$

C $\quad 3.970900 \quad 6.563100 \quad 4.149500$

C $\quad 4.043900 \quad 7.178100 \quad 5.402200$

$\begin{array}{llll}\text { C } & 2.957200 & 7.119100 & 6.288900\end{array}$

C $\quad 1.795600 \quad 6.433500 \quad 5.900100$

C $\quad 1.721200 \quad 5.818200 \quad 4.647500$

C $\quad 2.223900 \quad 3.951000 \quad-4.070700$

$\begin{array}{llll}\text { C } & 0.846100 & 4.205700 & -4.163300\end{array}$

$\begin{array}{llll}\text { C } & 0.176300 & 4.021600 & -5.375800\end{array}$

C $\quad 0.875900 \quad 3.589100 \quad-6.505100$

$\begin{array}{llll}\text { C } & 2.252300 & 3.359700 & -6.428100\end{array}$

C $\quad 2.927600 \quad 3.553800 \quad-5.219500$

C $\quad 3.429000 \quad 5.562200 \quad-2.567300$

$\begin{array}{llll}\text { C } & 2.536600 & 6.568900 & -2.161700\end{array}$

C $\quad 2.984700 \quad 7.879300 \quad-1.973700$

$\begin{array}{llll}\text { C } & 4.322400 & 8.202800 & -2.211600\end{array}$

$\begin{array}{llll}\text { C } & 5.210800 & 7.218300 & -2.650800\end{array}$

$\begin{array}{llll}\text { C } & 4.766200 & 5.906300 & -2.838500\end{array}$

$\begin{array}{llll}\text { C } & 2.247100 & -1.532300 & -1.518000\end{array}$

C $3.424900-2.483000-1.403600$

C $\quad 3.986900-2.573200 \quad-0.001700$

$\begin{array}{llll}\text { C } & 4.605900 & -1.488100 & 0.642400\end{array}$

$\begin{array}{llll}\text { C } & 5.148400 & -1.589100 & 1.921600\end{array}$

$\begin{array}{llll}\text { C } & 5.093700 & -2.797900 & 2.629500\end{array}$

$\begin{array}{llll}\text { C } & 4.466500 & -3.887300 & 2.010100\end{array}$

C $\quad 3.927000 \quad-3.765000 \quad 0.731100$

C $\quad 5.677100 \quad-2.913900 \quad 3.993200$

$\begin{array}{llll}\text { C } & 6.344900 & -4.087100 & 4.386900\end{array}$ 


\begin{tabular}{|c|c|c|c|}
\hline E & 6.892800 & -4.196400 & 5.667900 \\
\hline $\mathrm{C}$ & 6.784300 & -3.136500 & 6.581800 \\
\hline $\mathrm{C}$ & 6.117600 & -1.964400 & 6.191500 \\
\hline $\mathrm{C}$ & 5.569500 & -1.853500 & 4.910700 \\
\hline & 4.318600 & -2.863600 & -2.628200 \\
\hline & 3.183200 & -3.761200 & -2.211600 \\
\hline & 4.181800 & -2.102300 & -3.906300 \\
\hline 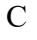 & 3.852500 & -2.773100 & -5.095500 \\
\hline & 3.724700 & -2.063500 & -6.293200 \\
\hline & 3.953300 & -0.685000 & -6.317400 \\
\hline $\mathrm{C}$ & 4.319300 & -0.017600 & -5.145800 \\
\hline $\mathrm{C}$ & 4.437000 & -0.722100 & -3.944700 \\
\hline $\mathrm{C}$ & 5.709100 & -3.349400 & -2.350400 \\
\hline$c$ & 6.689800 & -2.468000 & -1.864400 \\
\hline & 7.988600 & -2.920700 & -1.616400 \\
\hline $\mathrm{C}$ & 8.327600 & -4.251000 & -1.873600 \\
\hline $\mathrm{C}$ & 7.371200 & -5.127000 & -2.391800 \\
\hline $\mathrm{C}$ & 6.070600 & -4.678100 & -2.639700 \\
\hline $\mathrm{C}$ & -1.438300 & -2.198600 & -1.668800 \\
\hline$c$ & -2.396200 & -3.375900 & -1.643600 \\
\hline $\mathrm{C}$ & -3.620900 & -3.110400 & -2.523700 \\
\hline $\mathrm{C}$ & -2.700200 & -4.237000 & -2.912000 \\
\hline $\mathrm{C}$ & -2.575300 & -3.971100 & -0.264000 \\
\hline $\mathrm{C}$ & -1.534900 & -4.611100 & 0.431300 \\
\hline $\mathrm{C}$ & -1.717500 & -5.181400 & 1.689200 \\
\hline $\mathrm{C}$ & -2.967100 & -5.133400 & 2.323200 \\
\hline $\mathrm{C}$ & -4.012700 & -4.484700 & 1.653100 \\
\hline $\mathrm{C}$ & -3.809500 & -3.920000 & 0.395400 \\
\hline $\mathrm{C}$ & -3.166800 & -5.742600 & 3.665700 \\
\hline $\mathrm{C}$ & -2.546900 & -6.960300 & 3.997900 \\
\hline $\mathrm{C}$ & -2.733900 & -7.531300 & 5.259700 \\
\hline $\mathrm{C}$ & -3.543600 & -6.897400 & 6.215200 \\
\hline $\mathrm{C}$ & -4.163700 & -5.681900 & 5.886000 \\
\hline $\mathrm{C}$ & -3.977900 & -5.110000 & 4.624500 \\
\hline $\mathrm{C}$ & -1.859600 & -4.069500 & -4.135500 \\
\hline $\mathrm{C}$ & -0.480700 & -4.331900 & -4.094100 \\
\hline $\mathrm{C}$ & 0.298100 & -4.186700 & -5.245200 \\
\hline $\mathrm{C}$ & -0.292600 & -3.784700 & -6.445800 \\
\hline $\mathrm{C}$ & -1.668500 & -3.547500 & -6.501600 \\
\hline $\mathrm{C}$ & -2.452800 & -3.703300 & -5.354800 \\
\hline $\mathrm{C}$ & -3.204200 & -5.632900 & -2.701000 \\
\hline $\mathrm{C}$ & -2.357100 & -6.627800 & -2.184200 \\
\hline $\mathrm{C}$ & -2.826200 & -7.931300 & -1.998700 \\
\hline $\mathrm{C}$ & -4.137500 & -8.260100 & -2.349600 \\
\hline $\mathrm{C}$ & -4.977200 & -7.288500 & -2.898800 \\
\hline $\mathrm{C}$ & -4.511900 & -5.983300 & -3.084200 \\
\hline $\mathrm{C}$ & -2.101100 & 1.484400 & -1.746700 \\
\hline $\mathrm{C}$ & -3.284200 & 2.435300 & -1.758800 \\
\hline $\mathrm{C}$ & -3.964000 & 2.559400 & -0.412600 \\
\hline $\mathrm{C}$ & -4.634700 & 1.488900 & 0.203400 \\
\hline $\mathrm{C}$ & -5.280800 & 1.619000 & 1.430800 \\
\hline $\mathrm{C}$ & -5.284100 & 2.844200 & 2.112200 \\
\hline $\mathrm{C}$ & -4.608600 & 3.919900 & 1.520600 \\
\hline $\mathrm{C}$ & -3.965400 & 3.768500 & 0.293600 \\
\hline $\mathrm{C}$ & -5.976100 & 2.990500 & 3.421100 \\
\hline $\mathrm{C}$ & -5.943000 & 1.951800 & 4.368700 \\
\hline $\mathrm{C}$ & -6.592800 & 2.091300 & 5.598100 \\
\hline $\mathrm{C}$ & -7.288800 & 3.270800 & 5.905900 \\
\hline $\mathrm{C}$ & -7.323200 & 4.309200 & 4.961900 \\
\hline $\mathrm{C}$ & -6.673500 & 4.171100 & 3.732200 \\
\hline $\mathrm{C}$ & -4.068900 & 2.782800 & -3.065000 \\
\hline$C$ & -2.973700 & 3.693200 & -2.575000 \\
\hline
\end{tabular}

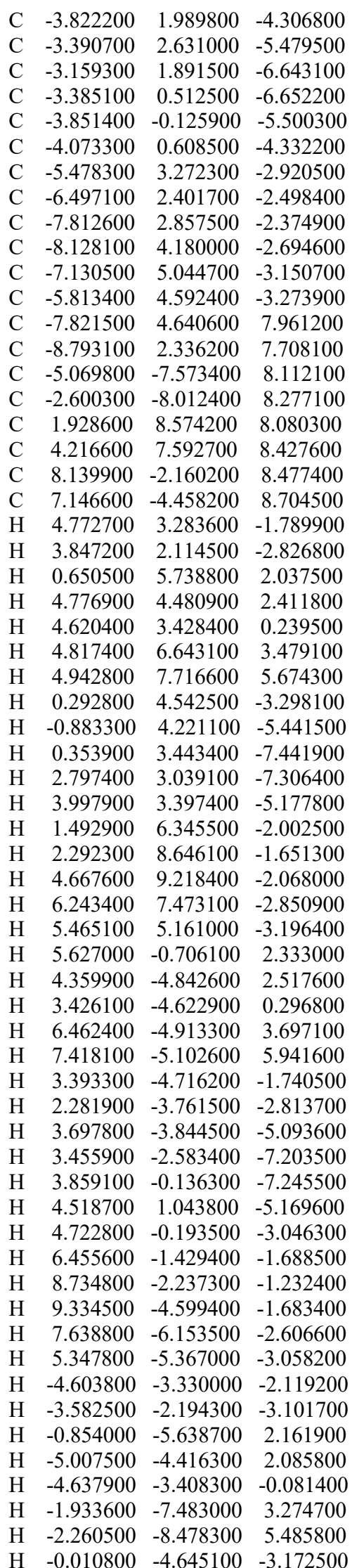

H $\quad 1.357900 \quad-4.392900 \quad-5.208100$

$\mathrm{H} \quad 0.313800 \quad-3.669100 \quad-7.334800$

$\mathrm{H} \quad-2.129000 \quad-3.250500 \quad-7.435000$

$\mathrm{H} \quad-3.521200 \quad-3.541100 \quad-5.415800$

$\mathrm{H} \quad-1.331700 \quad-6.401200 \quad-1.936000$

$\mathrm{H} \quad-2.169800 \quad-8.688700 \quad-1.590400$

$\mathrm{H} \quad-4.498600 \quad-9.270400 \quad-2.207700$

$\mathrm{H} \quad-5.988100 \quad-7.547800 \quad-3.185800$

$\mathrm{H} \quad-5.171700 \quad-5.248400 \quad-3.527700$

$\mathrm{H} \quad-5.791400 \quad 0.744900 \quad 1.822300$

$\mathrm{H} \quad-4.544100 \quad 4.887200 \quad 2.012300$

$\mathrm{H} \quad-3.430100 \quad 4.616800 \quad-0.118000$

$\mathrm{H} \quad-5.396100 \quad 1.039000 \quad 4.169100$

$\mathrm{H} \quad-6.537800 \quad 1.285100 \quad 6.318500$

$\mathrm{H} \quad-3.223700 \quad 4.659200 \quad-2.148200$

H $\quad-2.024000 \quad 3.680400 \quad-3.097400$

$\mathrm{H} \quad-3.236100 \quad 3.702300 \quad-5.491200$

$\mathrm{H} \quad-2.812300 \quad 2.388900 \quad-7.539600$

H $\quad-3.210300 \quad-0.059300 \quad-7.554400$

$\mathrm{H} \quad-4.047700 \quad-1.188200 \quad-5.514300$

$\mathrm{H} \quad-4.436300 \quad 0.102300 \quad-3.449000$

$\mathrm{H} \quad-6.279000 \quad 1.368300 \quad-2.276300$

$\mathrm{H} \quad-8.589100 \quad 2.182500 \quad-2.039300$

$\mathrm{H} \quad-9.147600 \quad 4.531100 \quad-2.600900$

$\begin{array}{llll}\mathrm{H} & -7.378600 & 6.064700 & -3.414100\end{array}$

$\mathrm{H} \quad-5.057300 \quad 5.272000 \quad-3.646200$

$\mathrm{H} \quad-7.486600 \quad 4.390200 \quad 8.990000$

$\begin{array}{llll}\mathrm{H} & -7.069500 & 5.325600 & 7.514900\end{array}$

$\mathrm{H} \quad-8.799700 \quad 5.163100 \quad 8.013200$

$\mathrm{H} \quad-9.801500 \quad 2.730300 \quad 7.955000$

$\begin{array}{llll}\mathrm{H} & -8.918600 & 1.517300 & 6.968100\end{array}$

$\mathrm{H} \quad-8.327600 \quad 1.923400 \quad 8.627600$

$\mathrm{H} \quad-5.279900 \quad-8.622500 \quad 8.409300$

$\mathrm{H} \quad-5.121900 \quad-6.922600 \quad 9.010100$

$\mathrm{H} \quad-5.855200 \quad-7.258900 \quad 7.392200$

$\mathrm{H} \quad-2.694900 \quad-9.114200 \quad 8.376100$

H $\quad-1.635700 \quad-7.776200 \quad 7.779500$

$\mathrm{H} \quad-2.583100 \quad-7.555400 \quad 9.289000$

H $\quad 2.309100 \quad 9.574900 \quad 8.375500$

$\mathrm{H} \quad 1.150000 \quad 8.721300 \quad 7.301900$

$\mathrm{H} \quad 1.467900 \quad 8.084100 \quad 8.963600$

H $\quad 4.924200 \quad 6.852400 \quad 7.997300$

$\mathrm{H} \quad 4.735500 \quad 8.568400 \quad 8.534100$

$\mathrm{H} \quad 3.912300 \quad 7.229800 \quad 9.432000$

H $\quad 9.124300 \quad-2.548600 \quad 8.814000$

H $\quad 8.326300 \quad-1.358800 \quad 7.731200$

H $\quad 7.602600 \quad-1.726200 \quad 9.346600$

$\mathrm{H} \quad 6.730300 \quad-4.184000 \quad 9.696800$

H $\quad 6.431900 \quad-5.153400 \quad 8.215000$

H $\quad 8.116500 \quad-4.979300 \quad 8.847400$

$\begin{array}{llll}\mathrm{H} & -7.868800 & 5.220500 & 5.171100\end{array}$

H $\quad-6.734900 \quad 4.981000 \quad 3.016200$

$\mathrm{H} \quad-4.777700 \quad-5.166700 \quad 6.613800$

H $\quad-4.451800 \quad-4.161000 \quad 4.407500$

H $\quad 0.952500 \quad 6.359800 \quad 6.575200$

$\mathrm{H} \quad 0.820200 \quad 5.278400 \quad 4.384900$

H $\quad 6.006200 \quad-1.141700 \quad 6.886300$

H $\quad 5.042500 \quad-0.945500 \quad 4.646400$

\section{E6}

$\begin{array}{llll}\text { Rh } & -0.004000 & -0.015900 & -0.442600\end{array}$ $\begin{array}{lllll}\mathrm{Rh} & -0.016300 & -0.093800 & -2.823700\end{array}$ 
$\begin{array}{llll}\text { O } & 2.054900 & -0.083300 & -0.512600\end{array}$

O $\quad 2.036500 \quad-0.306700 \quad-2.760500$

$\begin{array}{llll}\mathrm{O} & -0.070100 & -2.076500 & -0.436600\end{array}$

$\begin{array}{llll}\mathrm{O} & -0.231000 & -2.142500 & -2.689100\end{array}$

$\begin{array}{llll}\text { O } & -2.063800 & 0.048700 & -0.495400\end{array}$

$\begin{array}{llll}\mathrm{O} & -2.068100 & 0.123500 & -2.753300\end{array}$

$\begin{array}{llll}\mathrm{O} & 0.062900 & 2.040400 & -0.571400\end{array}$

$\begin{array}{lllll}\mathrm{O} & 0.198600 & 1.959500 & -2.825100\end{array}$

$\begin{array}{lllll}\mathrm{Cl} & 3.984800 & 2.492400 & -0.441900\end{array}$

$\begin{array}{llll}\mathrm{Cl} & 2.495500 & -4.005000 & -0.211200\end{array}$

$\mathrm{Cl} \quad-3.998400 \quad-2.508300 \quad-0.225900$

$\begin{array}{llll}\mathrm{Cl} & -2.496500 & 3.984600 & -0.439000\end{array}$

$\begin{array}{llll}\mathrm{N} & -8.282100 & -0.817200 & 7.609300\end{array}$

$\begin{array}{llll}\mathrm{N} & -0.992700 & 8.551100 & 7.274200\end{array}$

$\begin{array}{llll}\mathrm{N} & 8.341800 & 1.345500 & 7.455200\end{array}$

$\begin{array}{llll}\mathrm{N} & 1.108400 & -8.092500 & 7.787000\end{array}$

C $\quad 2.632800 \quad-0.228900 \quad-1.635900$

$\begin{array}{llll}\text { C } & 4.135900 & -0.434900 & -1.585800\end{array}$

$\begin{array}{llll}\text { C } & 4.547600 & -1.690600 & -2.359500\end{array}$

C $\quad 5.037200 \quad-0.357300 \quad-2.860400$

$\begin{array}{llll}\text { C } & 4.731700 & -0.161100 & -0.222100\end{array}$

C $\quad 4.733100 \quad 1.115500 \quad 0.365800$

$\begin{array}{llll}\text { C } & 5.311000 & 1.365200 & 1.608600\end{array}$

$\begin{array}{llll}\text { C } & 5.921700 & 0.333300 & 2.334900\end{array}$

$\begin{array}{llll}\text { C } & 5.917000 & -0.949900 & 1.772000\end{array}$

$\begin{array}{llll}\text { C } & 5.330600 & -1.179900 & 0.529000\end{array}$

$\begin{array}{llll}\text { C } & 6.546800 & 0.595300 & 3.659300\end{array}$

$\begin{array}{llll}\text { C } & 7.742500 & -0.048200 & 4.024600\end{array}$

$\begin{array}{llll}\text { C } & 8.330200 & 0.198300 & 5.268300\end{array}$

$\begin{array}{llll}\text { C } & 7.735300 & 1.092100 & 6.172600\end{array}$

C $\quad 6.541400 \quad 1.735500 \quad 5.810600$

$\begin{array}{llll}\text { C } & 5.952800 & 1.490200 & 4.567100\end{array}$

C $\quad 4.466100 \quad 0.168100 \quad-4.137000$

$\begin{array}{llll}\text { C } & 3.972400 & 1.480500 & -4.210300\end{array}$

$\begin{array}{llll}\text { C } & 3.450300 & 1.970200 & -5.410500\end{array}$

$\begin{array}{llll}\text { C } & 3.423600 & 1.157900 & -6.546900\end{array}$

$\begin{array}{llll}\text { C } & 3.938600 & -0.139800 & -6.489600\end{array}$

$\begin{array}{llll}\text { C } & 4.471700 & -0.629000 & -5.293500\end{array}$

$\begin{array}{llll}\text { C } & 6.491000 & -0.047800 & -2.666600\end{array}$

$\begin{array}{llll}\text { C } & 6.899900 & 1.232400 & -2.256500\end{array}$

$\begin{array}{llll}\text { C } & 8.256500 & 1.522400 & -2.087100\end{array}$

$\begin{array}{llll}\text { C } & 9.219300 & 0.545100 & -2.348400\end{array}$

$\begin{array}{llll}\text { C } & 8.826800 & -0.719800 & -2.792400\end{array}$

C $\quad 7.470600 \quad-1.013800 \quad-2.961500$

C $\quad-0.185400 \quad-2.696200-1.541000$

$\begin{array}{llll}\text { C } & -0.395400 & -4.196000 & -1.441000\end{array}$

C $\quad-0.165000 \quad-4.741700 \quad-0.048500$

$\begin{array}{llll}\text { C } & 1.093600 & -4.726900 & 0.577100\end{array}$

C $\quad 1.303800 \quad-5.264600 \quad 1.844800$

$\begin{array}{llll}\text { C } & 0.248000 & -5.846900 & 2.560400\end{array}$

$\begin{array}{llll}\text { C } & -1.018600 & -5.853700 & 1.961400\end{array}$

$\begin{array}{llll}\text { C } & -1.208400 & -5.310200 & 0.692200\end{array}$

$\begin{array}{llll}\text { C } & 0.469800 & -6.428100 & 3.911900\end{array}$

$\begin{array}{llll}\text { C } & 1.648400 & -7.139200 & 4.199600\end{array}$

$\begin{array}{llll}\text { C } & 1.857000 & -7.684200 & 5.469400\end{array}$

$\begin{array}{llll}\text { C } & 0.892700 & -7.530100 & 6.477600\end{array}$

C $\quad-0.284900 \quad-6.820800 \quad 6.193100$

C $\quad-0.494900 \quad-6.275400 \quad 4.923500$

C $\quad-0.279900 \quad-5.143900 \quad-2.678500$

C $\quad-1.627300 \quad-4.635700 \quad-2.237400$

C $\quad 0.285500 \quad-4.620400 \quad-3.958300$

C $\quad-0.475700 \quad-4.667200 \quad-5.137700$
C $\quad 0.051500 \quad-4.179200 \quad-6.337000$

C $\quad 1.351900 \quad-3.669000 \quad-6.373200$

C $\quad 2.128800 \quad-3.655300 \quad-5.212200$

$\begin{array}{llll}\text { C } & 1.601000 & -4.131600 & -4.009100\end{array}$

C $\quad 0.021300 \quad-6.589500-2.421800$

C $\quad 1.287800 \quad-6.983400 \quad-1.957600$

C $\quad 1.570400 \quad-8.333000 \quad-1.729200$

C $\quad 0.600200 \quad-9.304500-1.985000$

$\begin{array}{llll}\text { C } & -0.649800 & -8.928400 & -2.482100\end{array}$

$\begin{array}{llll}\text { C } & -0.936500 & -7.579200 & -2.710000\end{array}$

C $\quad-2.653000 \quad 0.121300 \quad-1.619900$

$\begin{array}{llll}\text { C } & -4.155100 & 0.333600 & -1.569000\end{array}$

$\begin{array}{llll}\text { C } & -4.572500 & 1.534200 & -2.423200\end{array}$

C $\quad-5.068900 \quad 0.170700 \quad-2.826500$

C $\quad-4.737900 \quad 0.156100 \quad-0.184000$

$\begin{array}{llll}\text { C } & -4.739600 & -1.077600 & 0.489500\end{array}$

C $\quad-5.309300 \quad-1.239400 \quad 1.750400$

C $\quad-5.909100 \quad-0.157300 \quad 2.409900$

C $\quad-5.900700 \quad 1.085300 \quad 1.762600$

C $\quad-5.325100 \quad 1.226600 \quad 0.501300$

C $\quad-6.523400 \quad-0.327300 \quad 3.754300$

C $\quad-7.241400 \quad-1.494600 \quad 4.069800$

C $\quad-7.817300 \quad-1.654400 \quad 5.333000$

$\begin{array}{llll}\text { C } & -7.687900 & -0.651600 & 6.306700\end{array}$

C $\quad-6.971800 \quad 0.514700 \quad 5.994300$

C $\quad-6.395500 \quad 0.676000 \quad 4.731300$

C $\quad-4.511200 \quad-0.442400 \quad-4.069300$

$\begin{array}{llll}\text { C } & -4.021700 & -1.758500 & -4.056900\end{array}$

$\begin{array}{llll}\text { C } & -3.513000 & -2.331200 & -5.225700\end{array}$

C $\quad-3.494900-1.599000 \quad-6.415300$

C $\quad-4.005700 \quad-0.298500 \quad-6.442300$

$\begin{array}{llll}\text { C } & -4.525800 & 0.273500 & -5.277600\end{array}$

C $\quad-6.521300 \quad-0.121600 \quad-2.597800$

C $\quad-6.927800 \quad-1.369700 \quad-2.096300$

$\begin{array}{llll}\text { C } & -8.283100 & -1.644400 & -1.893800\end{array}$

C $\quad-9.247200 \quad-0.685500 \quad-2.212600$

C $\quad-8.857300 \quad 0.544900 \quad-2.746700$

C $\quad-7.502400 \quad 0.823800 \quad-2.948900$

$\begin{array}{llll}\text { C } & 0.166400 & 2.586700 & -1.715000\end{array}$

$\begin{array}{llll}\text { C } & 0.378800 & 4.089700 & -1.715200\end{array}$

$\begin{array}{llll}\text { C } & 0.167200 & 4.723700 & -0.357800\end{array}$

$\begin{array}{llll}\text { C } & -1.082900 & 4.750600 & 0.284200\end{array}$

C $\quad-1.275000 \quad 5.365900 \quad 1.519200$

$\begin{array}{llll}\text { C } & -0.208700 & 5.988500 & 2.183300\end{array}$

$\begin{array}{llll}\text { C } & 1.049500 & 5.956300 & 1.567700\end{array}$

C $\quad 1.221300 \quad 5.335200 \quad 0.332000$

$\begin{array}{llll}\mathrm{C} & -0.410900 & 6.651300 & 3.499800\end{array}$

C $\quad 0.567500 \quad 6.558300 \quad 4.505500$

C $\quad 0.376100 \quad 7.180800 \quad 5.742000$

$\begin{array}{llll}\text { C } & -0.796100 & 7.909000 & 5.998900\end{array}$

$\begin{array}{llll}\text { C } & -1.774300 & 8.003700 & 4.996600\end{array}$

$\begin{array}{llll}\text { C } & -1.584300 & 7.381400 & 3.759800\end{array}$

$\begin{array}{llll}\text { C } & 0.248500 & 4.955800 & -3.009900\end{array}$

C $\quad 1.600900 \quad 4.474900 \quad-2.553900$

C $\quad-0.333500 \quad 4.351500 \quad-4.245900$

$\begin{array}{llll}\text { C } & 0.413500 & 4.318800 & -5.435000\end{array}$

C $\quad-0.129500 \quad 3.755200 \quad-6.593400$

C $\quad-1.431300 \quad 3.247500 \quad-6.580500$

C $\quad-2.193900 \quad 3.311900 \quad-5.411700$

C $\quad-1.650400 \quad 3.864100 \quad-4.248800$

$\begin{array}{llll}\text { C } & -0.048200 & 6.415600 & -2.843400\end{array}$

C $\quad-1.308000 \quad 6.840800 \quad-2.388900$ $\begin{array}{llll}\text { C } & -1.586400 & 8.202800 & -2.244700\end{array}$

$\begin{array}{llll}\text { C } & -0.619000 & 9.154100 & -2.575700\end{array}$

C $\quad 0.623900 \quad 8.744500 \quad-3.063900$

$\begin{array}{llll}\text { C } & 0.906500 & 7.382900 & -3.207800\end{array}$

$\begin{array}{llll}\text { C } & -2.268200 & 8.437100 & 7.983700\end{array}$

$\begin{array}{llll}\text { C } & 0.078100 & 9.334500 & 7.893200\end{array}$

$\begin{array}{llll}\text { C } & -9.044100 & 0.267700 & 8.230200\end{array}$

$\begin{array}{lrrr}\text { C } & -8.139300 & -2.074600 & 8.345400\end{array}$

$\begin{array}{llll}\text { C } & 8.831100 & 0.243300 & 8.285100\end{array}$

$\begin{array}{llll}\text { C } & 8.484000 & 2.711700 & 7.961700\end{array}$

$\begin{array}{llll}\text { C } & 2.393400 & -7.933000 & 8.470200\end{array}$

$\begin{array}{llll}\text { C } & 0.048300 & -8.837900 & 8.467800\end{array}$

$\mathrm{H} \quad 5.241700 \quad-2.380500 \quad-1.890500$

H $\quad 3.747200 \quad-2.180000 \quad-2.902400$

H $\quad 5.285000 \quad 2.386200 \quad 1.976100$

H $\quad 6.343000 \quad-1.800400 \quad 2.298100$

H $\quad 5.323600 \quad-2.191700 \quad 0.139600$

H $\quad 8.235700 \quad-0.726600 \quad 3.339900$

H $\quad 9.260600 \quad-0.295200 \quad 5.519600$

H $\quad 3.988800 \quad 2.120500 \quad-3.339400$

H $\quad 3.073200 \quad 2.981300 \quad-5.461200$

H $\quad 3.015000 \quad 1.538500 \quad-7.474100$

H $\quad 3.931900 \quad-0.764200 \quad-7.373700$

$\mathrm{H} \quad 4.890700 \quad-1.626800 \quad-5.267300$

H $\quad 6.173000 \quad 2.010200 \quad-2.079400$

$\mathrm{H} \quad 8.561500 \quad 2.508300 \quad-1.761000$

$\mathrm{H} \quad 10.269700 \quad 0.771700 \quad-2.219100$

H $\quad 9.574300 \quad-1.471600 \quad-3.010800$

$\mathrm{H} \quad 7.186700 \quad-1.993900 \quad-3.323600$

H $\quad 2.304500 \quad-5.193800 \quad 2.259200$

H $\quad-1.878900 \quad-6.293400 \quad 2.459300$

$\mathrm{H} \quad-2.207400 \quad-5.317100 \quad 0.270900$

H $\quad 2.401100 \quad-7.292400 \quad 3.436400$

H $\quad 2.764300 \quad-8.242800 \quad 5.661400$

$\mathrm{H} \quad-2.332300 \quad-5.311900 \quad-1.764700$

$\begin{array}{llll}\mathrm{H} & -2.098500 & -3.855700 & -2.824300\end{array}$

$\mathrm{H} \quad-1.474900 \quad-5.083300 \quad-5.126900$

$\mathrm{H} \quad-0.545400 \quad-4.204000 \quad-7.239500$

H $\quad 1.761900 \quad-3.295700 \quad-7.302700$

H $\quad 3.142100 \quad-3.282500 \quad-5.245600$

$\mathrm{H} \quad 2.213800 \quad-4.116800 \quad-3.118900$

H $\quad 2.060700 \quad-6.250800 \quad-1.783400$

$\mathrm{H} \quad 2.545100 \quad-8.626200 \quad-1.361700$

$\mathrm{H} \quad 0.821200 \quad-10.349400 \quad-1.810100$

$\mathrm{H} \quad-1.395600 \quad-9.683100 \quad-2.695800$

$\mathrm{H} \quad-1.904500 \quad-7.308500 \quad-3.112400$

$\mathrm{H} \quad-5.260900 \quad 2.256300 \quad-1.996300$

$\mathrm{H} \quad-3.776500 \quad 1.983100 \quad-3.006100$

$\mathrm{H} \quad-5.249100 \quad-2.223500 \quad 2.204600$

$\mathrm{H} \quad-6.352800 \quad 1.963700 \quad 2.215800$

$\mathrm{H} \quad-5.321000 \quad 2.208700 \quad 0.041900$

$\mathrm{H} \quad-7.375800 \quad-2.276200 \quad 3.332800$

H $\quad-8.380400 \quad-2.554100 \quad 5.546200$

H $\quad-4.031300 \quad-2.337100 \quad-3.144000$

$\mathrm{H} \quad-3.139700 \quad-3.344800 \quad-5.210500$

$\mathrm{H} \quad-3.096700 \quad-2.043700 \quad-7.318100$

H $\quad-4.005700 \quad 0.263700 \quad-7.367200$

$\mathrm{H} \quad-4.941800 \quad 1.272100 \quad-5.316000$

$\mathrm{H} \quad-6.200400 \quad-2.134900-1.873000$

H $\quad-8.586300 \quad-2.604800 \quad-1.497400$

H $\quad-10.296400 \quad-0.900600 \quad-2.057700$

H $\quad-9.605900 \quad 1.281300 \quad-3.009100$ 


$\begin{array}{cccc}\mathrm{H} & -7.220700 & 1.776000 & -3.380500 \\ \mathrm{H} & -2.270100 & 5.322700 & 1.950800 \\ \mathrm{H} & 1.917100 & 6.423900 & 2.025900 \\ \mathrm{H} & 2.214400 & 5.313900 & -0.102300 \\ \mathrm{H} & 1.472400 & 5.985900 & 4.344600 \\ \mathrm{H} & 1.135700 & 7.079800 & 6.507000 \\ \mathrm{H} & 2.312500 & 5.179000 & -2.134900 \\ \mathrm{H} & 2.063800 & 3.657800 & -3.095100 \\ \mathrm{H} & 1.413800 & 4.731600 & -5.463500 \\ \mathrm{H} & 0.456300 & 3.719100 & -7.502800 \\ \mathrm{H} & -1.853600 & 2.815600 & -7.478600 \\ \mathrm{H} & -3.208400 & 2.940700 & -5.408400 \\ \mathrm{H} & -2.252200 & 3.909200 & -3.352100 \\ \mathrm{H} & -2.079200 & 6.122300 & -2.157500 \\ \mathrm{H} & -2.556100 & 8.520700 & -1.884000 \\ \mathrm{H} & -0.836900 & 10.208500 & -2.465900 \\ \mathrm{H} & 1.367300 & 9.482600 & -3.336000 \\ \mathrm{H} & 1.868800 & 7.085100 & -3.604600 \\ \mathrm{H} & -2.091200 & 8.068200 & 9.016000 \\ \mathrm{H} & -2.760100 & 9.431200 & 8.031100 \\ \mathrm{H} & -2.949800 & 7.720800 & 7.477600 \\ \mathrm{H} & -0.296700 & 10.349400 & 8.143500 \\ \mathrm{H} & 0.419500 & 8.832300 & 8.822700 \\ \mathrm{H} & 0.943500 & 9.450200 & 7.206500 \\ \mathrm{H} & -10.048300 & -0.101700 & 8.527200 \\ \mathrm{H} & -8.508500 & 0.632700 & 9.131700 \\ \mathrm{H} & -9.186800 & 1.115600 & 7.527100 \\ \mathrm{H} & -9.130000 & -2.566300 & 8.441900 \\ \mathrm{H} & -7.440800 & -2.767800 & 7.830200 \\ \mathrm{H} & -7.732700 & -1.871600 & 9.358700 \\ \mathrm{H} & 8.382400 & 0.309400 & 9.298700 \\ \mathrm{H} & 8.554500 & -0.741900 & 7.853100 \\ \mathrm{H} & 6.055700 & 2.414800 & 6.499500 \\ \mathrm{H} & -1.034000 & -6.674300 & 6.961000 \\ \mathrm{H} & -1.403100 & -5.715200 & 4.740600 \\ \mathrm{H} & 8.193300 & 0.301900 & 8.371100 \\ \mathrm{H} & 7.843300 & 2.460100 & 7.194000 \\ \mathrm{H} & 9.542800 & 2.900500 & 8.238300 \\ \mathrm{H} & 2.229700 & -7.501600 & 9.480400 \\ \mathrm{H} & 2.888300 & -8.921600 & 8.571800 \\ \mathrm{H} & 3.066200 & -7.248100 & 7.911700 \\ \mathrm{H} & 0.428900 & -9.835100 & 8.774700 \\ \mathrm{H} & -0.281600 & -8.280200 & 9.369400 \\ \mathrm{H} & -0.826300 & -8.997000 & 7.801600 \\ & -2.677900 & 8.575100 & 5.166600 \\ \mathrm{H} & -2.347200 & 7.489600 & 2.999100 \\ \mathrm{H} & -534100 & 1.293000 & 6.736000 \\ & & \\ \mathrm{H} & & \\ \mathrm{H} & & \\ \mathrm{H} & & \end{array}$

\section{E7}

$\begin{array}{llll}\mathrm{Rh} & -0.003200 & 0.003200 & -0.451600\end{array}$

$\begin{array}{llll}\mathrm{Rh} & -0.037700 & 0.006600 & -2.834000\end{array}$

$\begin{array}{llll}\mathrm{O} & -0.124400 & 2.060400 & -0.507900\end{array}$

$\begin{array}{llll}\mathrm{O} & -0.019700 & 2.069800 & -2.764600\end{array}$

$\begin{array}{llll}\text { O } & 2.052700 & 0.122900 & -0.542800\end{array}$

$\begin{array}{llll}\text { O } & 2.026600 & -0.010500 & -2.797800\end{array}$

O $\quad 0.116300 \quad-2.053300 \quad-0.517500$

O $\quad-0.053900 \quad-2.057400 \quad-2.770200$

$\begin{array}{llll}\text { O } & -2.061000 & -0.117600 & -0.482900\end{array}$

$\begin{array}{llll}\text { O } & -2.099900 & 0.023700 & -2.737300\end{array}$
$\mathrm{Cl} \quad-2.852000 \quad 3.748200 \quad-0.273700$

$\begin{array}{llll}\mathrm{Cl} & 3.748100 & 2.848400 & -0.364300\end{array}$

$\mathrm{Cl} \quad 2.852600 \quad-3.736600 \quad-0.372300$

$\mathrm{Cl} \quad-3.740200 \quad-2.853900 \quad-0.278800$

$\begin{array}{llll}\mathrm{N} & 1.873800 & -8.103800 & 7.544200\end{array}$

$\begin{array}{llll}\mathrm{N} & 8.245300 & 1.697100 & 7.457000\end{array}$

$\begin{array}{llll}\mathrm{N} & -1.660500 & 8.175800 & 7.574700\end{array}$

$\begin{array}{llll}\mathrm{N} & -8.086700 & -1.830500 & 7.637900\end{array}$

C $\quad-0.091800 \quad 2.652800 \quad-1.632500$

$\begin{array}{llll}\text { C } & -0.020700 & 4.168100 & -1.581800\end{array}$

C $\quad 1.147000 \quad 4.694200 \quad-2.421100$

C $\quad-0.251100 \quad 5.061700 \quad-2.843300$

$\begin{array}{llll}\text { C } & -0.269100 & 4.733000 & -0.200200\end{array}$

C $\quad-1.505700 \quad 4.620000 \quad 0.458200$

C $\quad-1.735300 \quad 5.172600 \quad 1.716200$

$\begin{array}{llll}\text { C } & -0.721900 & 5.870800 & 2.387800\end{array}$

C $\quad 0.523900 \quad 5.978300 \quad 1.755700$

$\begin{array}{llll}\mathrm{C} & 0.733600 & 5.418100 & 0.497000\end{array}$

$\begin{array}{lrrr}\mathrm{C} & -0.964500 & 6.467100 & 3.729100\end{array}$

C $\quad 0.034200 \quad 6.432300 \quad 4.718400$

$\begin{array}{llll}\text { C } & -0.194600 & 6.992200 & 5.978300\end{array}$

C $\quad-1.425300 \quad 7.598700 \quad 6.275200$

C $\quad-2.423700 \quad 7.635300 \quad 5.289100$

$\begin{array}{llll}\text { C } & -2.196300 & 7.075500 & 4.029000\end{array}$

C $\quad-0.793800 \quad 4.448200 \quad-4.092700$

C $\quad-0.065100 \quad 4.525000 \quad-5.291000$

C $\quad-0.571400 \quad 3.953400 \quad-6.462000$

$\begin{array}{llll}\text { C } & -1.820800 & 3.327600 & -6.451500\end{array}$

C $\quad-2.568400 \quad 3.281500 \quad-5.272100$

C $\quad-2.060300 \quad 3.841500-4.097000$

C $\quad-0.679500 \quad 6.481000 \quad-2.621600$

C $\quad-1.965600 \quad 6.771600 \quad-2.135600$

C $\quad-2.366300 \quad 8.096100 \quad-1.939900$

$\begin{array}{llll}\text { C } & -1.496400 & 9.143900 & -2.250000\end{array}$

$\begin{array}{llll}\text { C } & -0.229200 & 8.868300 & -2.768600\end{array}$

C $\quad 0.175600 \quad 7.544500 \quad-2.964000$

C $\quad 2.627500 \quad 0.075300 \quad-1.676100$

C $\quad 4.143500 \quad 0.002600 \quad-1.648300$

C $\quad 4.729400 \quad 0.264600 \quad-0.278100$

$\begin{array}{llll}\text { C } & 4.623300 & 1.506500 & 0.371400\end{array}$

$\begin{array}{llll}\text { C } & 5.188000 & 1.746000 & 1.622300\end{array}$

$\begin{array}{llll}\text { C } & 5.894200 & 0.738400 & 2.294300\end{array}$

$\begin{array}{llll}\text { C } & 5.999400 & -0.510800 & 1.668500\end{array}$

$\begin{array}{llll}\text { C } & 5.424600 & -0.731500 & 0.418500\end{array}$

$\begin{array}{llll}\text { C } & 6.502600 & 0.988800 & 3.628800\end{array}$

C $\quad 7.752200 \quad 0.434900 \quad 3.959300$

$\begin{array}{llll}\text { C } & 8.322900 & 0.667900 & 5.213600\end{array}$

$\begin{array}{llll}\text { C } & 7.656700 & 1.458300 & 6.163300\end{array}$

C $\quad 6.409200 \quad 2.012300 \quad 5.836000$

$\begin{array}{llll}\text { C } & 5.837500 & 1.780300 & 4.582000\end{array}$

$\begin{array}{llll}\text { C } & 5.017400 & 0.217900 & -2.926300\end{array}$

$\begin{array}{llll}\text { C } & 4.654700 & -1.174900 & -2.483200\end{array}$

$\begin{array}{llll}\text { C } & 4.384800 & 0.748200 & -4.171400\end{array}$

C $\quad 4.439800 \quad 0.005900 \quad-5.362500$

C $\quad 3.850200 \quad 0.500800 \quad-6.529600$

$\begin{array}{llll}\text { C } & 3.228000 & 1.752000 & -6.522700\end{array}$

C $\quad 3.203200 \quad 2.512700 \quad-5.351100$

$\begin{array}{llll}\text { C } & 3.781300 & 2.016100 & -4.179700\end{array}$

$\begin{array}{llll}\text { C } & 6.440800 & 0.646000 & -2.731800\end{array}$

C $\quad 7.497200 \quad-0.214100 \quad-3.083700$

C $\quad 8.824700 \quad 0.190200 \quad-2.913900$

C $\quad 9.110700 \quad 1.461900 \quad-2.412000$ $\begin{array}{llll}\text { C } & 8.069500 & 2.336400 & -2.092900\end{array}$

$\begin{array}{llll}\text { C } & 6.741300 & 1.936000 & -2.262900\end{array}$

C $\quad 0.051500 \quad-2.643100 \quad-1.642200$

C $\quad-0.016700 \quad-4.158800 \quad-1.592000$

$\begin{array}{llll}\text { C } & -1.209100 & -4.684600 & -2.396100\end{array}$

C $\quad 0.175900 \quad-5.050300 \quad-2.861100$

$\begin{array}{llll}\text { C } & 0.274300 & -4.724100 & -0.218900\end{array}$

$\begin{array}{llll}\text { C } & 1.528600 & -4.604100 & 0.403700\end{array}$

$\begin{array}{llll}\text { C } & 1.794900 & -5.147800 & 1.658400\end{array}$

$\begin{array}{llll}\text { C } & 0.803400 & -5.846200 & 2.361700\end{array}$

C $\quad-0.457400 \quad-5.966200 \quad 1.762400$

C $\quad-0.705100 \quad-5.412000 \quad 0.508000$

C $\quad 1.082200 \quad-6.430400 \quad 3.701300$

C $\quad 0.540400 \quad-7.676200 \quad 4.064200$

$\begin{array}{llll}\text { C } & 0.800600 & -8.224100 & 5.323300\end{array}$

$\begin{array}{llll}\text { C } & 1.606700 & -7.538600 & 6.245600\end{array}$

C $\quad 2.148500 \quad-6.294500 \quad 5.886000$

$\begin{array}{llll}\text { C } & 1.889300 & -5.745500 & 4.627300\end{array}$

C $\quad 0.680100 \quad-4.434600 \quad-4.125400$

C $\quad-0.084600 \quad-4.510300 \quad-5.301200$

$\begin{array}{llll}\text { C } & 0.385500 & -3.936200 & -6.486100\end{array}$

C $\quad 1.633900-3.308900-6.512400$

$\begin{array}{llll}\text { C } & 2.416800 & -3.263800 & -5.356100\end{array}$

C $\quad 1.945200 \quad-3.826600 \quad-4.167100$

C $\quad 0.611400 \quad-6.469500 \quad-2.654600$

$\begin{array}{llll}\text { C } & 1.911300 & -6.760100 & -2.206900\end{array}$

$\begin{array}{llll}\text { C } & 2.318500 & -8.084700 & -2.025700\end{array}$

C $\quad 1.440700 \quad-9.132600 \quad-2.312200$

$\begin{array}{llll}\text { C } & 0.158500 & -8.857000 & -2.792800\end{array}$

$\begin{array}{llll}\text { C } & -0.252600 & -7.533100 & -2.973800\end{array}$

C $\quad-2.668100 \quad-0.067500-1.599100$

C $\quad-4.182700 \quad 0.000300 \quad-1.527300$

$\begin{array}{llll}\text { C } & -4.729700 & -0.275400 & -0.143600\end{array}$

$\begin{array}{llll}\text { C } & -4.606300 & -1.524300 & 0.489200\end{array}$

$\begin{array}{llll}\text { C } & -5.144500 & -1.779900 & 1.748400\end{array}$

$\begin{array}{lrrr}\text { C } & -5.837700 & -0.781800 & 2.447500\end{array}$

$\begin{array}{llll}\text { C } & -5.954700 & 0.476000 & 1.841200\end{array}$

$\begin{array}{llll}\text { C } & -5.408900 & 0.711800 & 0.580900\end{array}$

C $\quad-6.419600 \quad-1.052700 \quad 3.789700$

C $\quad-6.377400 \quad-0.073800 \quad 4.798300$

C $\quad-6.923700 \quad-0.329400 \quad 6.059100$

$\begin{array}{llll}\text { C } & -7.523700 & -1.567600 & 6.337500\end{array}$

$\begin{array}{llll}\text { C } & -7.567700 & -2.546100 & 5.332100\end{array}$

C $\quad-7.021600 \quad-2.292000 \quad 4.071100$

$\begin{array}{lrrr}\text { C } & -5.092600 & -0.208400 & -2.780900\end{array}$

C $\quad-4.721600 \quad 1.182400 \quad-2.337900$

$\begin{array}{llll}\text { C } & -4.495100 & -0.726900 & -4.048100\end{array}$

$\begin{array}{llll}\text { C } & -3.885500 & -1.991500 & -4.083700\end{array}$

C $\quad-3.339900 \quad-2.476500 \quad-5.275300$

C $\quad-3.404000 \quad-1.707900 \quad-6.440100$

$\begin{array}{llll}\text { C } & -4.033100 & -0.460300 & -6.419400\end{array}$

$\begin{array}{llll}\text { C } & -4.590100 & 0.023200 & -5.231800\end{array}$

$\begin{array}{llll}\text { C } & -6.508000 & -0.643400 & -2.548300\end{array}$

$\begin{array}{llll}\text { C } & -7.577500 & 0.216200 & -2.860000\end{array}$

$\begin{array}{llll}\text { C } & -8.897900 & -0.194600 & -2.654400\end{array}$

C $\quad-9.164500 \quad-1.472000 \quad-2.156400$

C $\quad-8.111200 \quad-2.345700 \quad-1.877100$

C $\quad-6.790000 \quad-1.939000 \quad-2.083000$

$\begin{array}{llll}\text { C } & -7.818800 & -3.092000 & 8.330900\end{array}$

$\begin{array}{llll}\text { C } & -8.941200 & -0.843100 & 8.299600\end{array}$

$\begin{array}{llll}\text { C } & -2.908600 & 7.918700 & 8.295500\end{array}$

$\begin{array}{llll}\text { C } & -0.657700 & 9.034200 & 8.207800\end{array}$ 


\begin{tabular}{|c|c|c|c|}
\hline & 8.270000 & 3.042500 & \\
\hline & 8.833200 & 0.600500 & 8.228400 \\
\hline 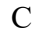 & 0.795400 & -8.682500 & 8.347300 \\
\hline & 3.230400 & -8.112600 & 8.094500 \\
\hline & 1.796500 & 5.447200 & -1.986400 \\
\hline & 1.675600 & 3.942600 & -2.996000 \\
\hline $\mathrm{H}$ & -2.715000 & 5.021300 & 2.158600 \\
\hline $\mathrm{H}$ & 1.350700 & 6.510400 & 2.218900 \\
\hline & 1.717200 & 5.505900 & 0.049500 \\
\hline & 0.985700 & 5.952700 & 4.526200 \\
\hline & 0.583000 & 6.936700 & 6.729600 \\
\hline $\mathrm{H}$ & -3.373600 & 8.113900 & 0400 \\
\hline & -2.977700 & 7.137700 & 3.282100 \\
\hline & 0.892100 & 5.029500 & -5.317000 \\
\hline & 0.001400 & 2600 & 8900 \\
\hline 11 & -2.21 & 9400 & 200 \\
\hline $\mathrm{H}$ & -3.544500 & 2.818500 & -5.270000 \\
\hline & -2.650100 & 3.800400 & -3.192100 \\
\hline & -2.663000 & 5.977000 & 9200 \\
\hline & -3.35 & 8.310200 & -1.5 \\
\hline & -1.8 & 200 & 0300 \\
\hline $\mathrm{H}$ & 0.438500 & 9.680900 & -3.0 \\
\hline & 1.1 & & -3.3 \\
\hline & 5.07 & 2.72 & 000 \\
\hline & 6.50 & -1.3 & 2.1 \\
\hline$\pi$ & 5.5 & 300 & -0. \\
\hline $\mathrm{H}$ & 8.298900 & 2100 & 0300 \\
\hline 11 & 9.294000 & & 5.43 \\
\hline & 5.869500 & 2.60 & 6.5 \\
\hline r & 4.86 & 2.2 & \\
\hline$\pi$ & 5.4 & 700 & -2. \\
\hline $\mathrm{H}$ & 3.89 & 500 & -3.0 \\
\hline П & 4.941100 & -0.95 & 200 \\
\hline $\mathrm{H}$ & 3.882800 & -0.082300 & -7.4 \\
\hline H & 2.77 & 500 & -7.4 \\
\hline $\mathrm{H}$ & 2.74 & 0100 & 300 \\
\hline $\mathrm{H}$ & 3.75 & 100 & 900 \\
\hline $\mathrm{H}$ & 7.295300 & -1.196900 & -3.490600 \\
\hline & 9.632000 & -0.481200 & -3.1 \\
\hline & 10.1 & 4100 & 2200 \\
\hline $\mathrm{H}$ & 8.291600 & 3.328400 & 500 \\
\hline $\mathrm{H}$ & 5.951500 & 2.636900 & -2.0 \\
\hline $\mathrm{H}$ & -1.844300 & -5.438800 & 42900 \\
\hline & -1.755600 & -3.932700 & -2. \\
\hline & 2.79 & -5.0 & 2.0 \\
\hline $\mathrm{H}$ & -1.279400 & -6.466300 & 2.268100 \\
\hline $\mathrm{H}$ & -1.701600 & -5.502200 & 0.090500 \\
\hline & -0.068300 & -8.237700 & 3.366700 \\
\hline & 0.38 & -9.19 & \\
\hline & 2.757800 & -5.739900 & 6.588400 \\
\hline $\mathrm{H}$ & 2.303200 & -4.774200 & 4.387300 \\
\hline $\mathrm{H}$ & -1.041800 & -5.015600 & -5.298900 \\
\hline & -0.214900 & -3.984600 & -7.385300 \\
\hline P & 1.999900 & -2.868600 & -7.430900 \\
\hline $\mathrm{H}$ & 3.391800 & -2.799300 & -5.382800 \\
\hline $\mathrm{H}$ & 2.562200 & -3.786500 & -3.280600 \\
\hline & 2.614300 & -5.965500 & -2.009400 \\
\hline & 3.318300 & -8.298900 & -1.670900 \\
\hline & 1.758200 & -10.158000 & -2.173700 \\
\hline & -0.515800 & -9.669600 & -3.030200 \\
\hline $\mathrm{H}$ & -1.243800 & -7.339500 & -3.364100 \\
\hline & -4.986100 & -2.767800 & 2.169600 \\
\hline
\end{tabular}
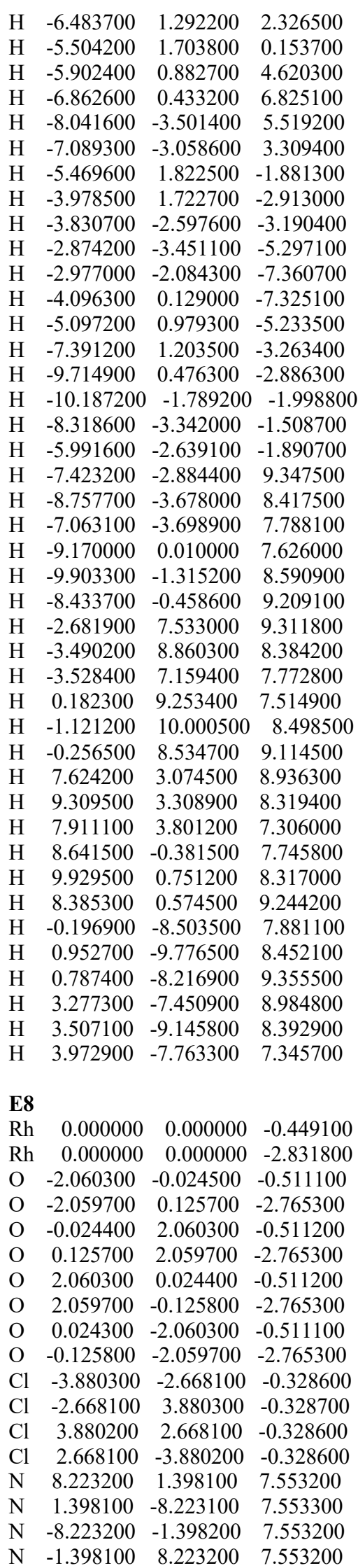

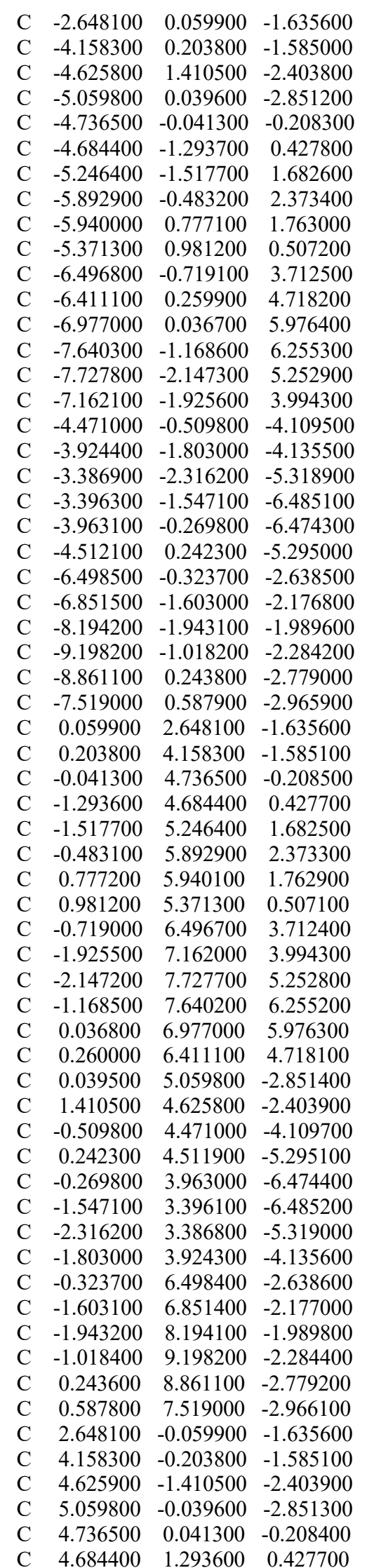




\begin{tabular}{|c|c|c|c|}
\hline C & 5.246400 & 1.517700 & 1.682500 \\
\hline 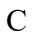 & 5.892900 & 0.483100 & 2.373400 \\
\hline $\mathrm{C}$ & 5.940000 & -0.777300 & 1.763000 \\
\hline C & 5.371200 & -0.981200 & 0.507200 \\
\hline 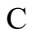 & 6.496700 & 0.719000 & 3.712400 \\
\hline $\mathrm{C}$ & 7.162100 & 1.925500 & 3.994300 \\
\hline $\mathrm{C}$ & 7.727800 & 2.147200 & 5.252800 \\
\hline C & 7.640200 & 1.168500 & 6.255300 \\
\hline $\mathrm{C}$ & 6.977000 & -0.036800 & 5.976300 \\
\hline $\mathrm{C}$ & 6.411100 & -0.259900 & 4.718100 \\
\hline $\mathrm{C}$ & 4.471000 & 0.509700 & -4.109600 \\
\hline $\mathrm{C}$ & 3.924400 & 1.803000 & -4.135600 \\
\hline $\mathrm{C}$ & 3.386800 & 2.316100 & -5.319000 \\
\hline $\mathrm{C}$ & 3.396300 & 1.547000 & -6.485200 \\
\hline $\mathrm{C}$ & 3.963200 & 0.269700 & -6.474400 \\
\hline $\mathrm{C}$ & 4.512100 & -0.242300 & -5.295100 \\
\hline $\mathrm{C}$ & 6.498500 & 0.323700 & -2.638500 \\
\hline $\mathrm{C}$ & 6.851400 & 1.603100 & -2.176900 \\
\hline $\mathrm{C}$ & 8.194000 & 1.943300 & -1.989600 \\
\hline $\mathrm{C}$ & 9.198200 & 1.018400 & -2.284200 \\
\hline $\mathrm{C}$ & 8.861000 & -0.243600 & -2.778900 \\
\hline $\mathrm{C}$ & 7.519100 & -0.587800 & -2.965900 \\
\hline $\mathrm{C}$ & -0.060000 & -2.648100 & -1.635600 \\
\hline $\mathrm{C}$ & -0.203800 & -4.158300 & -1.585100 \\
\hline $\mathrm{C}$ & 0.041300 & -4.736500 & -0.208400 \\
\hline $\mathrm{C}$ & 1.293600 & -4.684400 & 0.427800 \\
\hline $\mathrm{C}$ & 1.517700 & -5.246400 & 1.682600 \\
\hline $\mathrm{C}$ & 0.483100 & -5.892900 & 2.373400 \\
\hline $\mathrm{C}$ & -0.777200 & -5.940100 & 1.762900 \\
\hline $\mathrm{C}$ & -0.981200 & -5.371300 & 0.507100 \\
\hline $\mathrm{C}$ & 0.719000 & -6.496700 & 3.712400 \\
\hline $\mathrm{C}$ & 1.925500 & -7.162000 & 3.994400 \\
\hline $\mathrm{C}$ & 2.147300 & -7.727600 & 5.252900 \\
\hline $\mathrm{C}$ & 1.168600 & -7.640100 & 6.255300 \\
\hline $\mathrm{C}$ & -0.036800 & -6.977000 & 5.976300 \\
\hline $\mathrm{C}$ & -0.259900 & -6.411100 & 4.718100 \\
\hline $\mathrm{C}$ & -0.039500 & -5.059800 & -2.851300 \\
\hline $\mathrm{C}$ & -1.410500 & -4.625900 & -2.403900 \\
\hline $\mathrm{C}$ & 0.509700 & -4.471000 & -4.109600 \\
\hline $\mathrm{C}$ & -0.242400 & -4.512000 & -5.295100 \\
\hline $\mathrm{C}$ & 0.269700 & -3.963000 & -6.474400 \\
\hline $\mathrm{C}$ & 1.546900 & -3.396100 & -6.485200 \\
\hline $\mathrm{C}$ & 2.316100 & -3.386700 & -5.319000 \\
\hline $\mathrm{C}$ & 1.803000 & -3.924300 & -4.135600 \\
\hline $\mathrm{C}$ & 0.323800 & -6.498400 & -2.638600 \\
\hline $\mathrm{C}$ & 1.603300 & -6.851300 & -2.176900 \\
\hline $\mathrm{C}$ & 1.943400 & -8.193900 & -1.989700 \\
\hline $\mathrm{C}$ & 1.018600 & -9.198100 & -2.284300 \\
\hline $\mathrm{C}$ & -0.243400 & -8.861000 & -2.779000 \\
\hline $\mathrm{C}$ & -0.587600 & -7.519100 & -2.965900 \\
\hline $\mathrm{C}$ & 2.668900 & -8.022600 & 8.252100 \\
\hline $\mathrm{C}$ & 0.366500 & -9.030300 & 8.206900 \\
\hline $\mathrm{C}$ & 9.030400 & 0.366500 & 8.206800 \\
\hline $\mathrm{C}$ & 8.022800 & 2.668900 & 8.252000 \\
\hline $\mathrm{C}$ & -9.030400 & -0.366600 & 8.206900 \\
\hline $\mathrm{C}$ & -8.022800 & -2.669000 & 8.252000 \\
\hline $\mathrm{C}$ & -2.668800 & 8.022700 & 8.252000 \\
\hline $\mathrm{C}$ & -0.366400 & 9.030300 & 8.206800 \\
\hline $\mathrm{H}$ & -5.347300 & 2.087600 & -1.958100 \\
\hline $\mathrm{H}$ & -3.848600 & 1.912200 & -2.968500 \\
\hline $\mathrm{H}$ & -5.142800 & -2.511300 & 2.107500 \\
\hline $\mathrm{H}$ & -6.430400 & 1.620900 & 2.241500 \\
\hline
\end{tabular}

\begin{tabular}{|c|c|c|c|}
\hline & -5.410700 & 1.975700 & \\
\hline $\mathrm{H}$ & -5.887200 & 1.190400 & 4.540000 \\
\hline $\mathrm{H}$ & -6.882100 & 0.797900 & 6.740300 \\
\hline & -3.912200 & -2.409700 & -3.241100 \\
\hline & -2.969700 & -3.312700 & -5.333400 \\
\hline & -2.975700 & -1.945600 & -7.399300 \\
\hline & -3.984200 & 0.320500 & -7.381200 \\
\hline & -4.971000 & 1.222500 & -5.304600 \\
\hline & -6.091900 & -2.342100 & -1.972900 \\
\hline & -8.456300 & -2.927300 & -1.623700 \\
\hline & -10.237600 & -1.283600 & -2.141100 \\
\hline & -9.640200 & 0.954300 & -3.022700 \\
\hline & -7.277900 & 1.564200 & -3.367100 \\
\hline & -2.511300 & 5.142800 & 2.107400 \\
\hline & 1.621000 & 6.430500 & 2.241400 \\
\hline & 1.975600 & 5.410800 & 0.077000 \\
\hline & -2.690600 & 7.263600 & 4900 \\
\hline & -3.076700 & 8.250200 & 5.440300 \\
\hline & 2.087600 & 5.347300 & -1.958200 \\
\hline & 1.912300 & 3.848600 & -2.968600 \\
\hline & 1.222500 & 4.970900 & -5.304700 \\
\hline & 0.320500 & 3.984000 & 1300 \\
\hline & -1.945600 & 2.975500 & -7.399400 \\
\hline & -3.312700 & 2.969600 & -5.333500 \\
\hline & -2.409700 & 3.912200 & -3.241200 \\
\hline & -2.342100 & 6.091800 & 3100 \\
\hline & -2.927500 & 8.456200 & -1.623900 \\
\hline & -1.283800 & 10.237600 & -2.141300 \\
\hline & 0.954100 & 9.640200 & -3.023000 \\
\hline & 1.564100 & 7.277900 & -3.367300 \\
\hline & 5.347300 & -2.087600 & 8100 \\
\hline $\mathrm{H}$ & 3.848600 & -1.912300 & -2.968600 \\
\hline $\mathrm{H}$ & 5.142800 & 2.511200 & 2.107500 \\
\hline $\mathrm{H}$ & 6.430300 & -1.621000 & 1500 \\
\hline $\mathrm{H}$ & 5.410700 & -1.975800 & 0.077100 \\
\hline & 7.263600 & 2.690600 & 3.234900 \\
\hline $\mathrm{H}$ & 8.250300 & 3.076700 & 0300 \\
\hline $\mathrm{H}$ & 3.912100 & 2.409700 & -3.241200 \\
\hline $\mathrm{H}$ & 2.969600 & 3.312600 & -5.333500 \\
\hline $\mathrm{H}$ & 2.975700 & 1.945500 & -7.399400 \\
\hline $\mathrm{H}$ & 3.984300 & -0.320600 & -7.381300 \\
\hline $\mathrm{H}$ & 4.971100 & -1.222600 & -5.304600 \\
\hline $\mathrm{H}$ & 6.091800 & 2.342100 & -1.973100 \\
\hline $\mathrm{H}$ & 8.456200 & 2.927600 & -1.623800 \\
\hline & 10.237600 & 1.283900 & -2.141100 \\
\hline $\mathrm{H}$ & 9.640300 & -0.954100 & -3.022600 \\
\hline $\mathrm{H}$ & 7.277900 & -1.564100 & -3.367000 \\
\hline $\mathrm{H}$ & 2.511200 & -5.142700 & 2.107500 \\
\hline $\mathrm{H}$ & -1.620900 & -6.430400 & 2.241400 \\
\hline & -1.975700 & -5.410900 & 0.077000 \\
\hline $\mathrm{H}$ & 2.690700 & -7.263500 & 3.235000 \\
\hline $\mathrm{H}$ & 3.076800 & -8.250100 & 5.440400 \\
\hline $\mathrm{H}$ & -2.087600 & -5.347400 & -1.958100 \\
\hline & -1.912300 & -3.848700 & -2.968500 \\
\hline & -1.222600 & -4.971000 & -5.304600 \\
\hline $\mathrm{H}$ & -0.320700 & -3.984100 & -7.381300 \\
\hline $\mathrm{H}$ & 1.945400 & -2.975400 & -7.399400 \\
\hline & 3.312500 & -2.969400 & -5.333500 \\
\hline & 2.409700 & -3.912100 & -3.241200 \\
\hline & 2.342100 & -6.091700 & -1.973100 \\
\hline & 2.927800 & -8.456000 & -1.623900 \\
\hline & 1.284200 & -10.237500 & -2.141200 \\
\hline
\end{tabular}

$\begin{array}{cccc}\mathrm{H} & -0.953900 & -9.640300 & -3.022700 \\ \mathrm{H} & -1.564000 & -7.278000 & -3.367000 \\ \mathrm{H} & 2.477600 & -7.622400 & 9.270200 \\ \mathrm{H} & 3.206600 & -8.990300 & 8.335600 \\ \mathrm{H} & 3.315100 & -7.295600 & 7.715700 \\ \mathrm{H} & 0.788500 & -10.016400 & 8.494400 \\ \mathrm{H} & 0.004700 & -8.508900 & 9.117800 \\ \mathrm{H} & -0.494500 & -9.212200 & 7.529000 \\ \mathrm{H} & 10.016500 & 0.788400 & 8.494300 \\ \mathrm{H} & 8.509000 & 0.004700 & 9.117800 \\ \mathrm{H} & 9.212200 & -0.494600 & 7.529000 \\ \mathrm{H} & 8.990500 & 3.206500 & 8.335500 \\ \mathrm{H} & 7.295800 & 3.315200 & 7.715600 \\ \mathrm{H} & 7.622600 & 2.477700 & 9.270100 \\ \mathrm{H} & -10.016600 & -0.788500 & 8.494400 \\ \mathrm{H} & -8.509000 & -0.004800 & 9.117800 \\ \mathrm{H} & -9.212200 & 0.494500 & 7.529100 \\ \mathrm{H} & -8.990600 & -3.206700 & 8.335500 \\ \mathrm{H} & -7.295900 & -3.315200 & 7.715600 \\ \mathrm{H} & -7.622700 & -2.477800 & 9.270200 \\ \mathrm{H} & -2.477600 & 7.622600 & 9.270100 \\ \mathrm{H} & -3.206500 & 8.990500 & 8.335500 \\ \mathrm{H} & -3.315100 & 7.295800 & 7.715600 \\ \mathrm{H} & -0.788300 & 10.016600 & 8.494300 \\ \mathrm{H} & -0.004700 & 8.509000 & 9.117700 \\ \mathrm{H} & 0.494600 & 9.212200 & 7.528900 \\ \mathrm{H} & -0.798100 & -6.882000 & 6.740200 \\ \mathrm{H} & -1.190500 & -5.887200 & 4.539900 \\ \mathrm{H} & 6.882000 & -0.798000 & 6.740300 \\ \mathrm{H} & 5.887100 & -1.190400 & 4.539900 \\ \mathrm{H} & -8.250300 & -3.076800 & 5.440300 \\ \mathrm{H} & -7.263600 & -2.690700 & 3.234900 \\ \mathrm{H} & 0.798100 & 6.882000 & 6.740200 \\ \mathrm{H} & 1.190500 & 5.887100 & 4.539800\end{array}$

\section{F1}

$\begin{array}{llll}\mathrm{Rh} & -0.008300 & -0.020800 & -1.176100\end{array}$

$\begin{array}{llll}\mathrm{Rh} & -0.020000 & -0.061400 & -3.557900\end{array}$

$\begin{array}{llll}\mathrm{O} & -1.907500 & 0.773000 & -3.495600\end{array}$

$\begin{array}{lllll}\mathrm{O} & -1.947800 & 0.673300 & -1.239000\end{array}$

$\begin{array}{llll}\mathrm{O} & 0.813700 & 1.827200 & -3.526800\end{array}$

O $\quad 0.686700 \quad 1.916900 \quad-1.273000$

$\begin{array}{llll}\mathrm{O} & -0.852800 & -1.948000 & -3.454500\end{array}$

$\begin{array}{llll}\mathrm{O} & -0.703700 & -1.960600 & -1.200300\end{array}$

$\begin{array}{llll}\mathrm{O} & 1.868300 & -0.893600 & -3.485700\end{array}$

O $\quad 1.930700 \quad-0.716800-1.234300$

$\mathrm{Cl} \quad-4.577500 \quad-1.168900 \quad-1.030300$

$\mathrm{Cl} \quad-1.154900 \quad 4.550900 \quad-1.141000$

Cl $\quad 1.140800 \quad-4.588500 \quad-0.997000$

Cl $\quad 4.562400 \quad 1.130400 \quad-1.114800$

F $\quad 10.285600 \quad-0.383000 \quad 5.148900$

F $\quad \begin{array}{llll}9.504900 & 1.113900 & 3.666400\end{array}$

F $\quad 8.831400 \quad 1.205400 \quad 5.807700$

F $\quad 5.615200 \quad-2.414300 \quad 7.476500$

F $\quad 7.076100 \quad-4.003200 \quad 6.834200$

F $\quad 4.978400 \quad-4.011200 \quad 6.030100$

F $\quad-1.067600 \quad 8.888200 \quad 5.739100$

F $\quad 0.505100 \quad 10.335400 \quad 5.029100$

F $\quad-1.026300 \quad 9.540700 \quad 3.589800$

F $\quad 4.163400 \quad 7.141300 \quad 6.660500$

F $\quad 2.589900 \quad 5.687300 \quad 7.354200$

F $\quad 4.152500 \quad 5.035800 \quad 5.877000$ 


\begin{tabular}{|c|c|c|c|}
\hline F & -5.534500 & 2.668900 & 7.445100 \\
\hline $\mathrm{F}$ & -7.004600 & 4.233700 & 6.765800 \\
\hline $\mathrm{F}$ & -4.916400 & 4.215600 & 5.937200 \\
\hline $\mathrm{F}$ & -10.229600 & 0.555300 & 5.243800 \\
\hline & -9.464600 & -0.991100 & 3.804500 \\
\hline $\mathrm{F}$ & -8.765900 & -1.008400 & 5.939600 \\
\hline $\mathrm{F}$ & -4.107000 & -4.837900 & 6.082300 \\
\hline $\mathrm{F}$ & -4.099500 & -6.913600 & 6.941600 \\
\hline $\mathrm{F}$ & -2.528500 & -5.426900 & 7.568800 \\
\hline & 0.230700 & -9.497400 & 6.238400 \\
\hline $\mathrm{F}$ & 0.042800 & -10.085500 & 4.078100 \\
\hline $\mathrm{F}$ & 1.616700 & -8.604800 & 4.711900 \\
\hline $\mathrm{C}$ & -2.475100 & 0.937900 & -2.365500 \\
\hline $\mathrm{C}$ & -3.838200 & 1.604200 & -2.318000 \\
\hline $\mathrm{C}$ & -4.744000 & 1.753900 & -3.582500 \\
\hline $\mathrm{C}$ & -3.852400 & 2.888700 & -3.151600 \\
\hline $\mathrm{C}$ & -4.459400 & 1.592500 & -0.938400 \\
\hline $\mathrm{C}$ & -4.844300 & 0.407400 & -0.287700 \\
\hline $\mathrm{C}$ & -5.439500 & 0.406500 & 0.971700 \\
\hline $\mathrm{C}$ & -5.677200 & 1.608900 & 1.651800 \\
\hline $\mathrm{C}$ & -5.286800 & 2.800200 & 1.026400 \\
\hline $\mathrm{C}$ & -4.692200 & 2.780000 & -0.233900 \\
\hline $\mathrm{C}$ & -6.311600 & 1.611900 & 2.997300 \\
\hline $\mathrm{C}$ & -7.375800 & 0.739500 & 3.283800 \\
\hline $\mathrm{C}$ & -7.978000 & 0.734100 & 4.553700 \\
\hline $\mathrm{C}$ & -7.504900 & 1.613100 & 5.539400 \\
\hline $\mathrm{C}$ & -6.444200 & 2.491600 & 5.272900 \\
\hline $\mathrm{C}$ & -5.852300 & 2.485300 & 3.998200 \\
\hline $\mathrm{C}$ & -4.391900 & 1.016100 & -4.832800 \\
\hline $\mathrm{C}$ & -4.341000 & -0.387200 & -4.841900 \\
\hline $\mathrm{C}$ & -4.023100 & -1.072400 & -6.017600 \\
\hline $\mathrm{C}$ & -3.760300 & -0.364200 & -7.192900 \\
\hline $\mathrm{C}$ & -3.835800 & 1.031200 & -7.198900 \\
\hline $\mathrm{C}$ & -4.164800 & 1.719300 & -6.027200 \\
\hline $\mathrm{C}$ & -6.217200 & 1.927000 & -3.367300 \\
\hline $\mathrm{C}$ & -6.849700 & 3.135100 & -3.714300 \\
\hline $\mathrm{C}$ & -8.225800 & 3.292600 & -3.524800 \\
\hline $\mathrm{C}$ & -8.986400 & 2.241500 & -3.007600 \\
\hline $\mathrm{C}$ & -8.374100 & 1.026200 & -2.692900 \\
\hline $\mathrm{C}$ & -6.998800 & 0.864600 & -2.882800 \\
\hline $\mathrm{C}$ & 0.965300 & 2.419400 & -2.407500 \\
\hline $\mathrm{C}$ & 1.631800 & 3.782900 & -2.381800 \\
\hline $\mathrm{C}$ & 1.796100 & 4.661200 & -3.663800 \\
\hline $\mathrm{C}$ & 2.925600 & 3.778700 & -3.201100 \\
\hline $\mathrm{C}$ & 1.605200 & 4.433500 & -1.016000 \\
\hline $\mathrm{C}$ & 0.413300 & 4.832800 & -0.386800 \\
\hline $\mathrm{C}$ & 0.398800 & 5.454600 & 0.859500 \\
\hline $\mathrm{C}$ & 1.593900 & 5.706100 & 1.547700 \\
\hline $\mathrm{C}$ & 2.791700 & 5.301800 & 0.944100 \\
\hline $\mathrm{C}$ & 2.785000 & 4.680500 & -0.303400 \\
\hline $\mathrm{C}$ & 1.582500 & 6.369200 & 2.879300 \\
\hline $\mathrm{C}$ & 0.708000 & 7.439900 & 3.133200 \\
\hline $\mathrm{C}$ & 0.689200 & 8.069400 & 4.389700 \\
\hline $\mathrm{C}$ & 1.556900 & 7.616900 & 5.395000 \\
\hline $\mathrm{C}$ & 2.437300 & 6.550100 & 5.161100 \\
\hline $\mathrm{C}$ & 2.444400 & 5.930900 & 3.899400 \\
\hline $\mathrm{C}$ & 1.072000 & 4.282300 & -4.914300 \\
\hline $\mathrm{C}$ & -0.331200 & 4.232500 & -4.937900 \\
\hline $\mathrm{C}$ & -1.003500 & 3.889500 & -6.114000 \\
\hline $\mathrm{C}$ & -0.282600 & 3.600300 & -7.275200 \\
\hline $\mathrm{C}$ & 1.112900 & 3.674200 & -7.267200 \\
\hline$C$ & 1.788300 & 4.028300 & -6.095400 \\
\hline
\end{tabular}

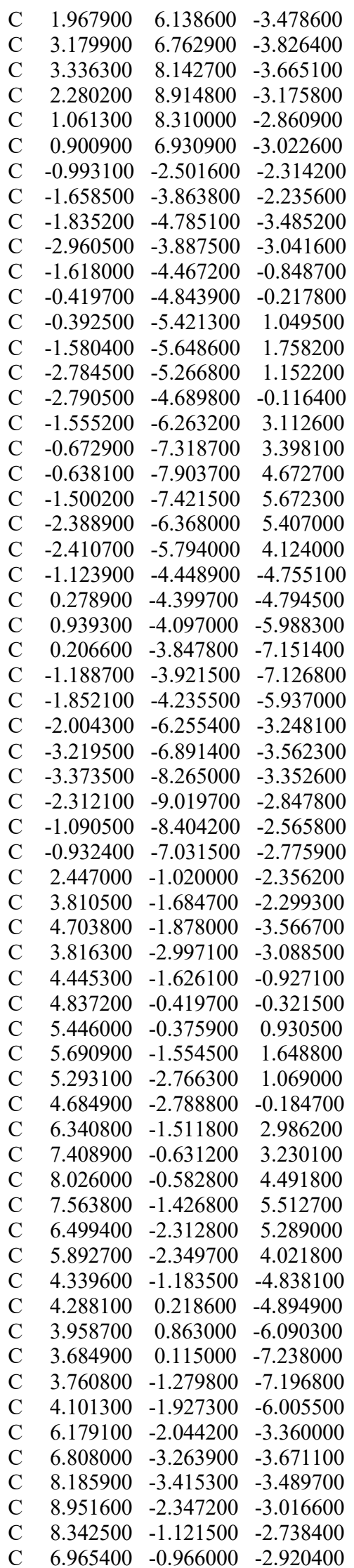

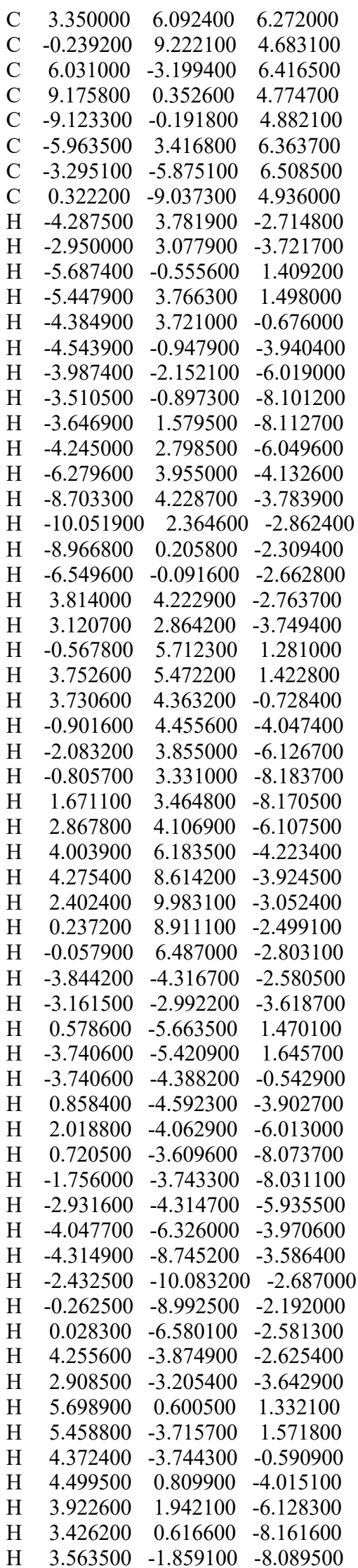



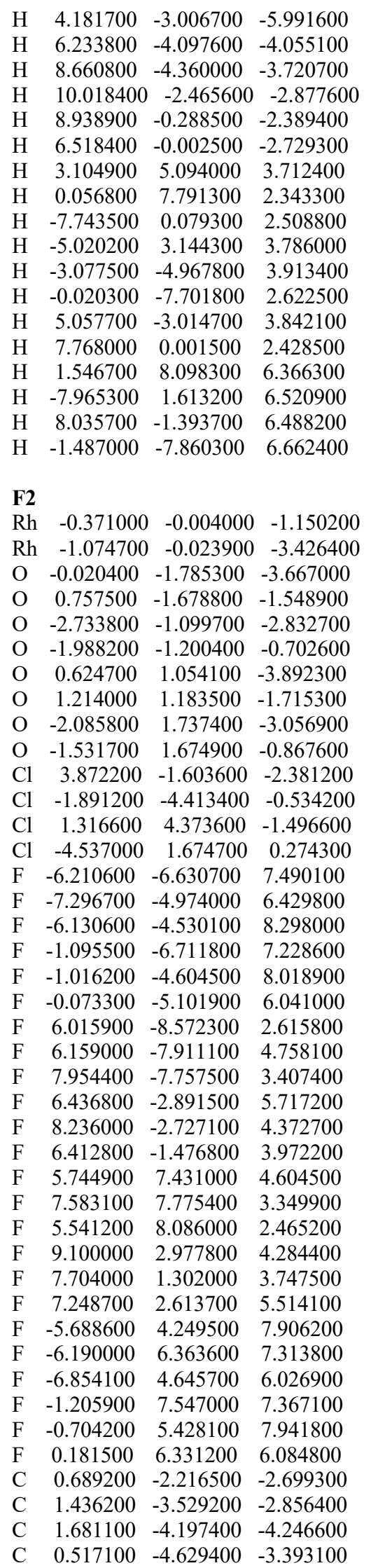

C $\quad 2.364200 \quad-3.817600 \quad-1.696500$

C $\quad 3.483200 \quad-3.018700 \quad-1.405200$

$\begin{array}{llll}\text { C } & 4.329700 & -3.281500 & -0.331300\end{array}$

$\begin{array}{llll}\text { C } & 4.093400 & -4.373300 & 0.515100\end{array}$

$\begin{array}{llll}\text { C } & 2.983200 & -5.183000 & 0.241400\end{array}$

$\begin{array}{llll}\text { C } & 2.144900 & -4.899900 & -0.835700\end{array}$

$\begin{array}{llll}\text { C } & 4.984100 & -4.646100 & 1.674800\end{array}$

$\begin{array}{llll}\text { C } & 5.476900 & -3.587000 & 2.457000\end{array}$

$\begin{array}{llll}\text { C } & 6.317300 & -3.835700 & 3.555400\end{array}$

$\begin{array}{llll}\text { C } & 6.662800 & -5.159200 & 3.866700\end{array}$

C $\quad 6.179800 \quad-6.230100 \quad 3.099500$

$\begin{array}{llll}\text { C } & 5.339500 & -5.965200 & 2.004200\end{array}$

$\begin{array}{llll}\text { C } & 1.391400 & -3.430000 & -5.495300\end{array}$

C $\quad 2.071500 \quad-2.232600 \quad-5.770800$

$\begin{array}{llll}\text { C } & 1.800300 & -1.522600 & -6.943600\end{array}$

C $\quad 0.857500 \quad-2.006600 \quad-7.854000$

C $\quad 0.198200 \quad-3.212600 \quad-7.601600$

C $\quad 0.473600 \quad-3.929800 \quad-6.433600$

C $\quad 2.829500 \quad-5.153100 \quad-4.366300$

C $\quad 2.596200 \quad-6.520500 \quad-4.602100$

$\begin{array}{llll}\text { C } & 3.668000 & -7.409500 & -4.726700\end{array}$

C $\quad 4.980700 \quad-6.940600 \quad-4.638200$

$\begin{array}{llll}\text { C } & 5.223400 & -5.579600 & -4.439600\end{array}$

$\begin{array}{llll}\text { C } & 4.154300 & -4.687900 & -4.316100\end{array}$

C $\quad-2.823800 \quad-1.480800-1.619000$

C $\quad-4.065800 \quad-2.231600 \quad-1.174400$

C $\quad-5.056800 \quad-2.889500 \quad-2.187900$

$\begin{array}{lrrr}\text { C } & -5.338500 & -1.516400 & -1.636400\end{array}$

$\begin{array}{llll}\text { C } & -3.968300 & -2.759700 & 0.240200\end{array}$

$\begin{array}{llll}\text { C } & -3.043700 & -3.747200 & 0.621600\end{array}$

C $\quad-2.976400 \quad-4.245600 \quad 1.920600$

C $\quad-3.842100 \quad-3.770300 \quad 2.915300$

$\begin{array}{llll}\text { C } & -4.762800 & -2.776500 & 2.558000\end{array}$

$\begin{array}{lrrr}\text { C } & -4.812100 & -2.288500 & 1.253600\end{array}$

C $\quad-3.776100 \quad-4.300800 \quad 4.303700$

C $\quad-2.534300 \quad-4.575000 \quad 4.902900$

C $\quad-2.464400 \quad-5.076400 \quad 6.213900$

C $\quad-3.652600 \quad-5.301300 \quad 6.924900$

C $\quad-4.901600 \quad-5.033000 \quad 6.345100$

C $\quad-4.955100 \quad-4.532400 \quad 5.032800$

$\begin{array}{llll}\text { C } & -4.671300 & -3.005700 & -3.626500\end{array}$

$\begin{array}{llll}\text { C } & -3.540000 & -3.747100 & -4.003900\end{array}$

C $\quad-3.190900 \quad-3.861200 \quad-5.352100$

$\begin{array}{llll}\text { C } & -3.970500 & -3.243700 & -6.333300\end{array}$

C $\quad-5.113500 \quad-2.527300 \quad-5.968200$

C $\quad-5.471800 \quad-2.420300 \quad-4.621100$

C $\quad-5.915700 \quad-4.013000 \quad-1.690900$

$\begin{array}{llll}\text { C } & -7.310800 & -3.855500 & -1.597900\end{array}$

C $\quad-8.112500 \quad-4.907700 \quad-1.145700$

$\begin{array}{llll}\text { C } & -7.533800 & -6.131700 & -0.802500\end{array}$

$\begin{array}{llll}\text { C } & -6.153900 & -6.309700 & -0.925000\end{array}$

$\begin{array}{llll}\text { C } & -5.349600 & -5.260400 & -1.378200\end{array}$

C $\quad 1.389400 \quad 1.442000 \quad-2.947800$

C $\quad 2.674200 \quad 2.172200 \quad-3.295900$

C $\quad 2.909600 \quad 2.831200 \quad-4.693000$

C $\quad 3.434900 \quad 1.448800 \quad-4.410900$

C $\quad 3.419600 \quad 2.673100 \quad-2.078100$

C $\quad 2.903700 \quad 3.657900 \quad-1.217900$

C $\quad 3.597900 \quad 4.107700 \quad-0.096700$

$\begin{array}{llll}\text { C } & 4.859600 & 3.585800 & 0.221800\end{array}$

$\begin{array}{llll}\text { C } & 5.389500 & 2.601400 & -0.622600\end{array}$

C $4.678100 \quad 2.162600 \quad-1.737200$ $\begin{array}{llll}\text { C } & 5.597200 & 4.050000 & 1.427800\end{array}$

$\begin{array}{llll}\text { C } & 5.588100 & 5.408300 & 1.789200\end{array}$

$\begin{array}{llll}\text { C } & 6.277600 & 5.852500 & 2.930600\end{array}$

C $\quad 6.981400 \quad 4.922600 \quad 3.710400$

C $\quad 7.004300 \quad 3.563000 \quad 3.365500$

$\begin{array}{llll}\text { C } & 6.308200 & 3.133800 & 2.222500\end{array}$

$\begin{array}{llll}\text { C } & 1.765000 & 2.969900 & -5.643000\end{array}$

C $\quad 0.645800 \quad 3.746000 \quad-5.301100$

C $\quad-0.414600 \quad 3.886200 \quad-6.200500$

$\begin{array}{llll}\text { C } & -0.365800 & 3.256300 & -7.446500\end{array}$

$\begin{array}{llll}\text { C } & 0.754800 & 2.501600 & -7.803700\end{array}$

$\begin{array}{llll}\text { C } & 1.823300 & 2.370000 & -6.911900\end{array}$

$\begin{array}{llll}\text { C } & 3.917100 & 3.937500 & -4.779600\end{array}$

C $\quad 5.101000 \quad 3.764600 \quad-5.519900$

$\begin{array}{llll}\text { C } & 6.035200 & 4.800600 & -5.611700\end{array}$

$\begin{array}{llll}\text { C } & 5.788400 & 6.023800 & -4.984100\end{array}$

$\begin{array}{llll}\text { C } & 4.600200 & 6.217400 & -4.275900\end{array}$

$\begin{array}{llll}\text { C } & 3.663400 & 5.184300 & -4.183400\end{array}$

C $\quad-2.121500 \quad 2.194900-1.866700$

C $\quad-2.816600 \quad 3.519500 \quad-1.610400$

C $\quad-3.815900 \quad 4.152700 \quad-2.631500$

C $\quad-2.371800 \quad 4.579900 \quad-2.621400$

C $\quad-2.908000 \quad 3.871100 \quad-0.141600$

$\begin{array}{llll}\text { C } & -3.667100 & 3.123900 & 0.775700\end{array}$

C $\quad-3.765000 \quad 3.471300 \quad 2.121200$

$\begin{array}{llll}\text { C } & -3.094200 & 4.596200 & 2.620900\end{array}$

$\begin{array}{lrrr}\text { C } & -2.319300 & 5.344800 & 1.725400\end{array}$

$\begin{array}{llll}\text { C } & -2.233900 & 4.980600 & 0.383100\end{array}$

C $\quad-3.195800 \quad 4.970400 \quad 4.057200$

C $\quad-4.423200 \quad 4.866200 \quad 4.734300$

C $\quad-4.526600 \quad 5.216200 \quad 6.091600$

$\begin{array}{llll}\text { C } & -3.386700 & 5.674000 & 6.769200\end{array}$

C $\quad-2.152800 \quad 5.785300 \quad 6.111000$

C $\quad-2.065200 \quad 5.431300 \quad 4.753700$

$\begin{array}{llll}\text { C } & -4.302800 & 3.339900 & -3.786600\end{array}$

$\begin{array}{lrrr}\text { C } & -5.026900 & 2.156400 & -3.570100\end{array}$

$\begin{array}{llll}\text { C } & -5.491100 & 1.407000 & -4.654500\end{array}$

$\begin{array}{llll}\text { C } & -5.241800 & 1.834800 & -5.960900\end{array}$

C $\quad-4.545000 \quad 3.025200 \quad-6.184600$

$\begin{array}{llll}\text { C } & -4.089400 & 3.783500 & -5.102100\end{array}$

$\begin{array}{llll}\text { C } & -4.820200 & 5.133000 & -2.105100\end{array}$

$\begin{array}{llll}\text { C } & -4.761700 & 6.486200 & -2.486400\end{array}$

$\begin{array}{lrrr}\text { C } & -5.705900 & 7.397900 & -2.004800\end{array}$

C $\quad-6.727800 \quad 6.965500 \quad-1.156500$

$\begin{array}{llll}\text { C } & -6.815500 & 5.617900 & -0.800300\end{array}$

C $\quad-5.874300 \quad 4.703400 \quad-1.281200$

$\begin{array}{llll}\text { C } & -6.154700 & -5.292700 & 7.144400\end{array}$

C $\quad-1.142300 \quad-5.374900 \quad 6.877200$

$\begin{array}{llll}\text { C } & -0.951100 & 6.278200 & 6.879100\end{array}$

$\begin{array}{llll}\text { C } & -5.834400 & 5.114900 & 6.837200\end{array}$

C $\quad 6.853500 \quad-2.713800 \quad 4.410400$

$\begin{array}{llll}\text { C } & 6.577900 & -7.637800 & 3.468600\end{array}$

$\begin{array}{llll}\text { C } & 7.771500 & 2.596300 & 4.233800\end{array}$

C $\quad 6.282300 \quad 7.305800 \quad 3.336200$

H $\quad 0.534700 \quad-5.598500 \quad-2.904600$

$\mathrm{H} \quad-0.477000 \quad-4.303100 \quad-3.677400$

H $\quad 5.178200 \quad-2.619800 \quad-0.187400$

H $\quad 2.729400 \quad-6.027800 \quad 0.876700$

H $\quad 1.276200 \quad-5.529000 \quad-0.994900$

H $\quad 2.805100 \quad-1.848500 \quad-5.076500$

H $\quad 2.324900 \quad-0.600500 \quad-7.149300$

H $\quad 0.646700 \quad-1.453200 \quad-8.760000$ 
$\mathrm{H} \quad-0.521600 \quad-3.595100 \quad-8.313600$

H $\quad-0.026300 \quad-4.874000 \quad-6.259400$

$\begin{array}{llll}\mathrm{H} & 1.586000 & -6.899700 & -4.691400\end{array}$

H $\quad 3.480400 \quad-8.461700 \quad-4.897000$

H $\quad 5.809500 \quad-7.629700 \quad-4.736100$

H $\quad 6.240800 \quad-5.213700 \quad-4.389900$

$\mathrm{H} \quad 4.362500 \quad-3.636100 \quad-4.194800$

$\mathrm{H} \quad-6.140200 \quad-1.375700 \quad-0.918600$

$\mathrm{H} \quad-5.177000 \quad-0.660800 \quad-2.282100$

$\mathrm{H} \quad-2.245200 \quad-5.022100 \quad 2.122300$

H $\quad-5.439300 \quad-2.342800 \quad 3.290000$

$\mathrm{H} \quad-5.519400 \quad-1.499900 \quad 1.022500$

$\mathrm{H} \quad-2.928600 \quad-4.231400 \quad-3.255600$

$\mathrm{H} \quad-2.320200 \quad-4.434000 \quad-5.636400$

$\mathrm{H} \quad-3.695600 \quad-3.330400 \quad-7.376500$

H $\quad-5.726100 \quad-2.061300 \quad-6.729100$

$\mathrm{H} \quad-6.372700 \quad-1.884000 \quad-4.352000$

$\mathrm{H} \quad-7.779400 \quad-2.919900 \quad-1.876000$

H $\quad-9.183800 \quad-4.775400 \quad-1.066200$

H $\quad-8.155800 \quad-6.945800 \quad-0.453700$

$\mathrm{H} \quad-5.707900 \quad-7.264100 \quad-0.676700$

$\mathrm{H} \quad-4.289800 \quad-5.427200 \quad-1.495400$

$\mathrm{H} \quad 4.501700 \quad 1.291600 \quad-4.288800$

H $\quad 2.916700 \quad 0.603400 \quad-4.848200$

$\mathrm{H} \quad 3.113400 \quad 4.852000 \quad 0.527400$

H $\quad 6.366600 \quad 2.162500 \quad-0.437900$

H $\quad 5.110200 \quad 1.379700 \quad-2.349800$

H $\quad 0.594900 \quad 4.239600 \quad-4.340700$

$\mathrm{H} \quad-1.270500 \quad 4.489400 \quad-5.934500$

$\mathrm{H} \quad-1.190600 \quad 3.362900 \quad-8.139100$

$\mathrm{H} \quad 0.799200 \quad 2.025200 \quad-8.774500$

$\begin{array}{llll}\mathrm{H} & 2.696200 & 1.803000 & -7.209200\end{array}$

H $\quad 5.301700 \quad 2.829500 \quad-6.027300$

$\mathrm{H} \quad 6.948700 \quad 4.656500 \quad-6.173900$

$\mathrm{H} \quad 6.511800 \quad 6.825500 \quad-5.057100$

H $\quad 4.400800 \quad 7.171300 \quad-3.805200$

$\mathrm{H} \quad 2.738100 \quad 5.363500 \quad-3.658200$

H $\quad-2.101500 \quad 5.563800 \quad-2.251800$

$\mathrm{H} \quad-1.723800 \quad 4.217900 \quad-3.411100$

$\begin{array}{llll}\mathrm{H} & -4.357300 & 2.827500 & 2.764000\end{array}$

$\begin{array}{llll}\mathrm{H} & -1.778500 & 6.230900 & 2.047800\end{array}$

$\mathrm{H} \quad-1.607600 \quad 5.572800 \quad-0.274500$

$\mathrm{H} \quad-5.228200 \quad 1.814100 \quad-2.564800$

H $\quad-6.050200 \quad 0.498900 \quad-4.481700$

H $\quad-5.598900 \quad 1.250200 \quad-6.798800$

H $\quad-4.364800 \quad 3.364800 \quad-7.196300$

$\mathrm{H} \quad-3.573500 \quad 4.716800 \quad-5.287200$

$\mathrm{H} \quad-3.987300 \quad 6.836600 \quad-3.156900$

$\mathrm{H} \quad-5.647900 \quad 8.439300 \quad-2.293800$

$\mathrm{H} \quad-7.458700 \quad 7.672100 \quad-0.785400$

$\begin{array}{llll}\mathrm{H} & -7.618100 & 5.279700 & -0.157900\end{array}$

$\mathrm{H} \quad-5.977000 \quad 3.661400 \quad-1.019900$

H $\quad-5.913000 \quad-4.339200 \quad 4.567100$

H $\quad-1.624000 \quad-4.378200 \quad 4.351300$

H $\quad 5.187400 \quad-2.571700 \quad 2.218200$

$\begin{array}{llll}\mathrm{H} & 4.974300 & -6.780700 & 1.393300\end{array}$

$\begin{array}{llll}\mathrm{H} & 6.299800 & 2.084100 & 1.958800\end{array}$

H $\quad 5.057200 \quad 6.118700 \quad 1.168600$

$\mathrm{H} \quad-1.114700 \quad 5.493800 \quad 4.239800$

$\mathrm{H} \quad-5.299300 \quad 4.529700 \quad 4.195100$

$\mathrm{H} \quad-3.460300 \quad 5.945400 \quad 7.816300$

H $\quad 7.515300 \quad 5.259400 \quad 4.591800$
H $\quad-3.605000 \quad-5.687800 \quad 7.936700$

H $\quad 7.311200 \quad-5.357400 \quad 4.712700$

\section{F3}

$\begin{array}{llll}\text { Rh } & 0.302500 & -0.093900 & -1.168800\end{array}$

$\mathrm{Rh} \quad 0.878800 \quad-0.258400 \quad-3.474600$

$\begin{array}{llll}\mathrm{O} & -0.859500 & 0.770400 & -3.912900\end{array}$

O $\quad-1.463700 \quad 0.798100 \quad-1.736500$

O $\quad 1.886200 \quad 1.533400 \quad-3.280600$

O $\quad 1.223600 \quad 1.748200 \quad-1.131500$

O $\quad-0.160700 \quad-2.042100 \quad-3.539700$

$\begin{array}{llll}\mathrm{O} & -0.595900 & -1.941100 & -1.325200\end{array}$

O $\quad 2.582600 \quad-1.276100 \quad-2.905500$

O $\quad 2.099200 \quad-0.995600 \quad-0.716500$

Cl $\quad-4.224600 \quad-0.801300 \quad-2.176700$

$\mathrm{Cl} \quad-0.297000 \quad 4.553000 \quad-1.515100$

$\mathrm{Cl} \quad 0.724300 \quad-4.727400 \quad-0.490300$

$\begin{array}{llll}\mathrm{Cl} & 4.805900 & 0.599400 & 0.005200\end{array}$

F $\quad-2.057400 \quad 5.690200 \quad 5.012100$

F $\quad-0.939600 \quad 6.063500 \quad 6.925100$

F $\quad-1.841100 \quad 7.786800 \quad 5.789800$

F $\quad 2.765000 \quad 10.006900 \quad 5.478500$

F $\quad 4.453400 \quad 8.817500 \quad 4.593800$

$\begin{array}{llll}\text { F } & 3.662900 & 8.292400 & 6.630100\end{array}$

F $\quad 8.151600 \quad-3.538500 \quad 7.729000$

F $\quad 7.362200 \quad-5.066000 \quad 6.282600$

F $\quad 6.228900 \quad-4.624400 \quad 8.171400$

F $\quad \begin{array}{llll}5.689900 & 0.939500 & 8.085100\end{array}$

F $\quad 3.763400 \quad-0.147000 \quad 8.509100$

F $\quad 3.899200 \quad 1.267700 \quad 6.768700$

F $\quad-1.377500 \quad-8.960300 \quad 4.510300$

F $\quad-1.836800 \quad-8.019400 \quad 6.498000$

F $\quad-3.338700 \quad-9.384200 \quad 5.521500$

F $\quad-6.823300 \quad-5.641000 \quad 5.687600$

F $\quad-5.319300 \quad-4.256100 \quad 6.631900$

F $\quad-6.323900 \quad-3.683900 \quad 4.705200$

F $\quad-6.289900 \quad-0.640700 \quad 4.346700$

F $\quad-8.472900 \quad-0.261600 \quad 4.719300$

F $\quad-6.915500 \quad 0.962100 \quad 5.791200$

F $\quad-10.517600 \quad 4.043400 \quad 2.818500$

F $\quad-9.174500 \quad 5.429900 \quad 1.668700$

F $\quad-8.956400 \quad 5.255600 \quad 3.897900$

C $\quad-1.667200 \quad 1.049000 \quad-2.966500$

C $\quad-2.926800 \quad 1.838700 \quad-3.276200$

C $\quad-3.473700 \quad 2.016000 \quad-4.728300$

C $\quad-2.610800 \quad 3.084200 \quad-4.108100$

C $\quad-3.861800 \quad 1.938100 \quad-2.090600$

C $\quad-4.494300 \quad 0.815600 \quad-1.529400$

$\begin{array}{llll}\text { C } & -5.345100 & 0.908700 & -0.431100\end{array}$

$\begin{array}{llll}\text { C } & -5.608300 & 2.148800 & 0.166500\end{array}$

$\begin{array}{llll}\text { C } & -4.989100 & 3.280900 & -0.379200\end{array}$

$\begin{array}{llll}\text { C } & -4.136100 & 3.165800 & -1.475700\end{array}$

$\begin{array}{llll}\text { C } & -6.500400 & 2.249700 & 1.352500\end{array}$

$\begin{array}{llll}\mathrm{C} & -6.444000 & 1.279900 & 2.368600\end{array}$

C $\quad-7.281700 \quad 1.368200 \quad 3.493400$

C $\quad-8.181300 \quad 2.440000 \quad 3.593900$

C $\quad-8.251400 \quad 3.419100 \quad 2.591300$

C $\quad-7.406300 \quad 3.317200 \quad 1.472700$

C $\quad-2.895500 \quad 1.201500 \quad-5.839300$

C $\quad-2.971000 \quad-0.200500 \quad-5.810100$

C $\quad-2.433600 \quad-0.956600 \quad-6.855400$

C $\quad-1.827100 \quad-0.320200 \quad-7.941300$
C $\quad-1.774600 \quad 1.075400 \quad-7.992300$

$\begin{array}{llll}\text { C } & -2.319100 & 1.834900 & -6.952500\end{array}$

$\begin{array}{llll}\text { C } & -4.931100 & 2.326300 & -4.889300\end{array}$

$\begin{array}{llll}C & -5.341700 & 3.571800 & -5.399300\end{array}$

C $\quad-6.700800 \quad 3.856100 \quad-5.561700$

C $\quad-7.661000 \quad 2.895000 \quad-5.237100$

$\begin{array}{llll}\text { C } & -7.262500 & 1.643000 & -4.763500\end{array}$

$\begin{array}{llll}\text { C } & -5.904500 & 1.354900 & -4.601300\end{array}$

$\begin{array}{llll}\text { C } & 1.824000 & 2.162700 & -2.173000\end{array}$

$\begin{array}{llll}\text { C } & 2.608200 & 3.452600 & -2.015300\end{array}$

$\begin{array}{llll}\text { C } & 3.171100 & 4.246900 & -3.237700\end{array}$

$\begin{array}{llll}\text { C } & 4.053700 & 3.281900 & -2.490700\end{array}$

$\begin{array}{llll}\text { C } & 2.321600 & 4.167500 & -0.712900\end{array}$

$\begin{array}{llll}\text { C } & 1.061800 & 4.708200 & -0.402700\end{array}$

$\begin{array}{llll}\text { C } & 0.811200 & 5.380600 & 0.791200\end{array}$

$\begin{array}{llll}\text { C } & 1.823700 & 5.544400 & 1.746700\end{array}$

C $\quad 3.084000 \quad 5.003300 \quad 1.460500$

$\begin{array}{llll}\text { C } & 3.313700 & 4.330900 & 0.261800\end{array}$

$\begin{array}{llll}\text { C } & 1.561200 & 6.260200 & 3.024200\end{array}$

C $\quad 2.527500 \quad 7.125500 \quad 3.565500$

$\begin{array}{llll}\text { C } & 2.288200 & 7.804100 & 4.772900\end{array}$

C $\quad 1.068300 \quad 7.609000 \quad 5.437700$

C $\quad 0.091300 \quad 6.749300 \quad 4.914000$

$\begin{array}{llll}\text { C } & 0.345500 & 6.077400 & 3.706100\end{array}$

C $\quad 2.735800 \quad 3.879500 \quad-4.618800$

$\begin{array}{llll}\text { C } & 1.383100 & 3.965000 & -4.986200\end{array}$

$\begin{array}{llll}\text { C } & 0.983700 & 3.631500 & -6.283100\end{array}$

$\begin{array}{llll}\text { C } & 1.929700 & 3.216800 & -7.223700\end{array}$

$\begin{array}{llll}\text { C } & 3.281200 & 3.154600 & -6.873800\end{array}$

$\begin{array}{llll}\text { C } & 3.685800 & 3.498300 & -5.580500\end{array}$

C $\quad 3.447300 \quad 5.708100 \quad-3.050200$

$\begin{array}{llll}\text { C } & 4.766400 & 6.195200 & -3.098900\end{array}$

$\begin{array}{llll}\text { C } & 5.022800 & 7.559700 & -2.936100\end{array}$

$\begin{array}{llll}\text { C } & 3.966100 & 8.452800 & -2.744300\end{array}$

$\begin{array}{llll}\text { C } & 2.650600 & 7.984200 & -2.729600\end{array}$

$\begin{array}{llll}\text { C } & 2.390500 & 6.620800 & -2.893500\end{array}$

C $\quad-0.657300 \quad-2.508700 \quad-2.461100$

C $\quad-1.487600 \quad-3.778200 \quad-2.525500$

C $\quad-1.420000 \quad-4.759700 \quad-3.739700$

C $\quad-2.501700 \quad-3.714900 \quad-3.671400$

C $\quad-1.920700 \quad-4.275300-1.163500$

C $\quad-1.013100 \quad-4.727500 \quad-0.189900$

C $\quad-1.425500 \quad-5.180900 \quad 1.061500$

$\begin{array}{llll}\text { C } & -2.785100 & -5.203400 & 1.403200\end{array}$

$\begin{array}{llll}\text { C } & -3.704700 & -4.754100 & 0.446700\end{array}$

$\begin{array}{llll}\text { C } & -3.272100 & -4.301800 & -0.798000\end{array}$

$\begin{array}{llll}\text { C } & -3.229600 & -5.671200 & 2.743800\end{array}$

$\begin{array}{llll}\text { C } & -4.268300 & -5.007400 & 3.419700\end{array}$

C $\quad-4.693000 \quad-5.441000 \quad 4.687300$

C $\quad-4.065300 \quad-6.548000 \quad 5.277700$

C $\quad-3.026800 \quad-7.223900 \quad 4.619600$

C $\quad-2.614700 \quad-6.779500 \quad 3.351400$

C $\quad-0.341800 \quad-4.593200 \quad-4.760200$

$\begin{array}{llll}\text { C } & 1.008400 & -4.720800 & -4.396400\end{array}$

$\begin{array}{llll}\text { C } & 2.012500 & -4.583900 & -5.358700\end{array}$

$\begin{array}{llll}\text { C } & 1.678000 & -4.319200 & -6.689000\end{array}$

$\begin{array}{llll}\text { C } & 0.335900 & -4.212700 & -7.063400\end{array}$

C $\quad-0.671800 \quad-4.363500-6.106300$

C $\quad-1.832500 \quad-6.181900 \quad-3.508600$

C $\quad-2.976000 \quad-6.702300 \quad-4.142100$

C $\quad-3.354300 \quad-8.032500 \quad-3.937200$

C $\quad-2.585500 \quad-8.860600 \quad-3.116100$ 


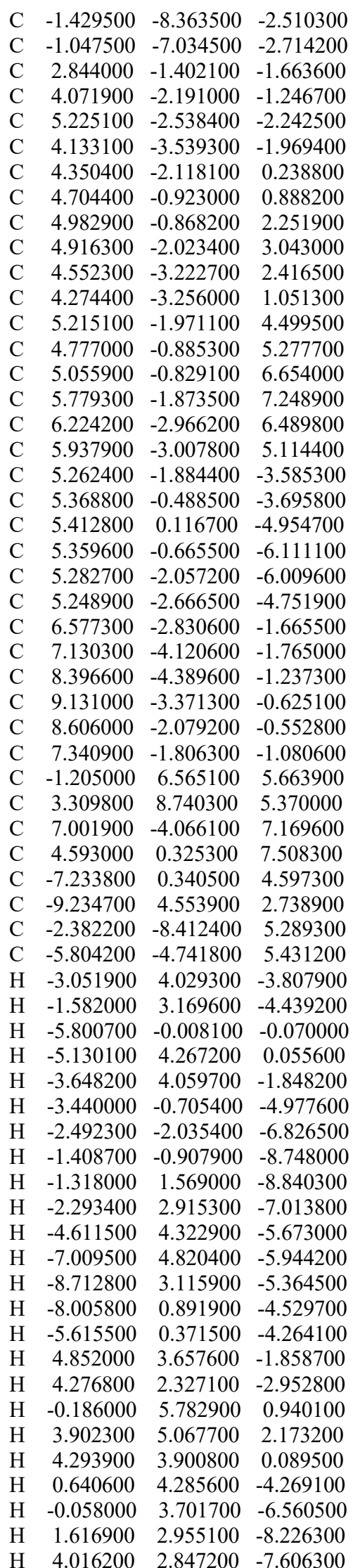

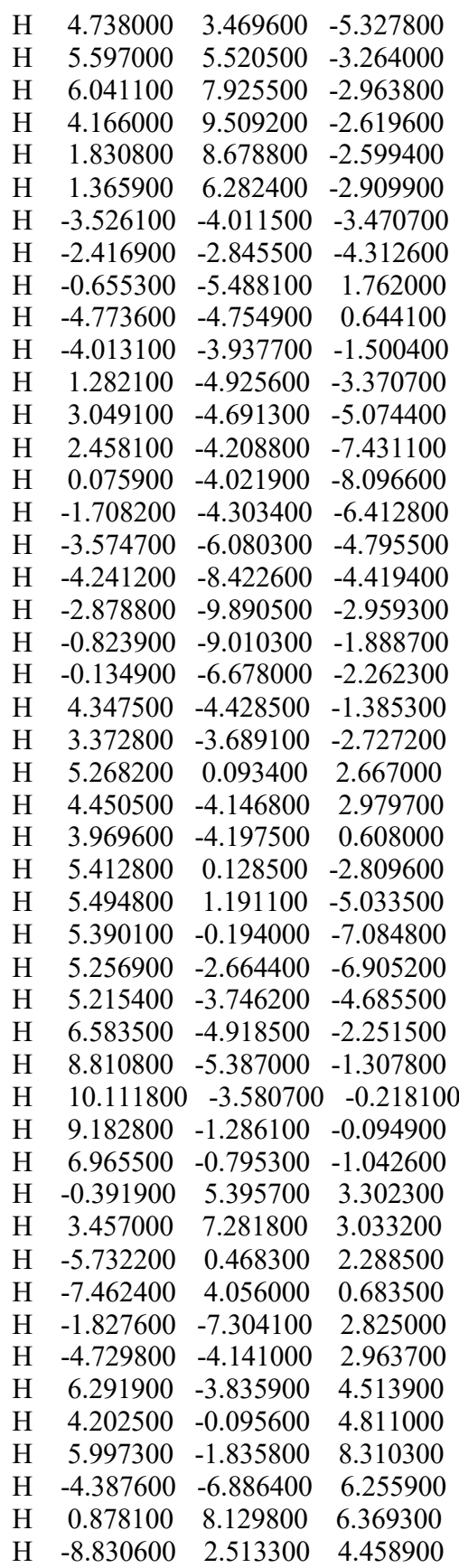

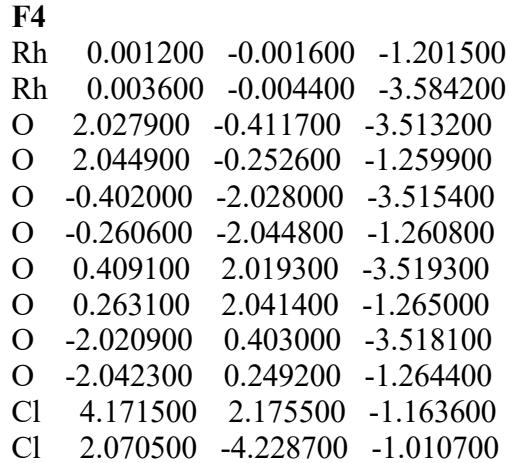

\begin{tabular}{|c|c|c|c|}
\hline & -2.069100 & 4.225700 & \\
\hline & -4.169100 & -2.178300 & -1.165900 \\
\hline $\mathrm{F}$ & 2.837200 & -8.962100 & 3.933400 \\
\hline & 2.676800 & -8.233300 & 6.052500 \\
\hline & 1.458200 & -10.000500 & 5.371600 \\
\hline & -1.610500 & -5.794400 & 7.441200 \\
\hline & -2.825900 & -7.571500 & 6.781000 \\
\hline $\mathrm{F}$ & -3.228200 & -5.543500 & 5.902200 \\
\hline & -4.695500 & -2.744700 & 5.632300 \\
\hline & -5.438000 & -1.429700 & 7.295900 \\
\hline & -6.748200 & -3.071800 & 6.484700 \\
\hline $\mathrm{F}$ & -9.079600 & 2.005000 & 6.209300 \\
\hline $\mathrm{F}$ & -10.386000 & 0.366400 & 5.384600 \\
\hline & -9.827100 & 2.118600 & 4.093800 \\
\hline $\mathrm{F}$ & 3.214200 & 5.557200 & 5.896000 \\
\hline & 1.593000 & 5.811200 & 7.430900 \\
\hline & 2.809600 & 7.587100 & 6.769400 \\
\hline $\mathrm{F}$ & -1.471900 & 10.012100 & 5.345100 \\
\hline $\mathrm{F}$ & -2.847400 & 8.970200 & 3.906100 \\
\hline $\mathrm{F}$ & -2.691600 & 8.246100 & 6.027100 \\
\hline & 5.427200 & 1.449500 & 7.302300 \\
\hline $\mathrm{F}$ & 6.738500 & 3.089600 & 6.488400 \\
\hline $\mathrm{F}$ & 4.687100 & 2.759900 & 5.634000 \\
\hline $\mathrm{F}$ & 9.821500 & -2.106800 & 4.116100 \\
\hline $\mathrm{F}$ & 9.070900 & -1.987500 & 6.230200 \\
\hline F & 10.378300 & -0.351000 & 5.402900 \\
\hline $\mathrm{C}$ & 2.618600 & -0.414000 & -2.383200 \\
\hline $\mathrm{C}$ & 4.099800 & -0.742200 & -2.324200 \\
\hline $\mathrm{C}$ & 5.017500 & -0.724400 & -3.588100 \\
\hline $\mathrm{C}$ & 4.415500 & -2.019200 & -3.106900 \\
\hline $\mathrm{C}$ & 4.696200 & -0.530300 & -0.949900 \\
\hline $\mathrm{C}$ & 4.785500 & 0.737600 & -0.350400 \\
\hline $\mathrm{C}$ & 5.343500 & 0.930300 & 0.910800 \\
\hline $\mathrm{C}$ & 5.844200 & -0.153600 & 1.645000 \\
\hline $\mathrm{C}$ & 5.758200 & -1.427500 & 1.067600 \\
\hline $\mathrm{C}$ & 5.193000 & -1.599900 & -0.194900 \\
\hline $\mathrm{C}$ & 6.434100 & 0.047100 & 2.996000 \\
\hline $\mathrm{C}$ & 5.855700 & 0.958300 & 3.896900 \\
\hline $\mathrm{C}$ & 6.408300 & 1.155400 & 5.174000 \\
\hline $\mathrm{C}$ & 7.549000 & 0.428200 & 5.545400 \\
\hline $\mathrm{C}$ & 8.140000 & -0.487400 & 4.661600 \\
\hline $\mathrm{C}$ & 7.576200 & -0.672500 & 3.387400 \\
\hline $\mathrm{C}$ & 4.505000 & -0.141300 & -4.864700 \\
\hline $\mathrm{C}$ & 4.111500 & 1.205300 & -4.926900 \\
\hline $\mathrm{C}$ & 3.638300 & 1.746100 & -6.125600 \\
\hline C & 3.565300 & 0.951500 & -7.272500 \\
\hline $\mathrm{C}$ & 3.984200 & -0.381000 & -7.226700 \\
\hline $\mathrm{C}$ & 4.466000 & -0.922400 & -6.031200 \\
\hline $\mathrm{C}$ & 6.489400 & -0.537000 & -3.376200 \\
\hline $\mathrm{C}$ & 7.389700 & -1.577600 & -3.670400 \\
\hline $\mathrm{C}$ & 8.763500 & -1.397800 & -3.484000 \\
\hline $\mathrm{C}$ & 9.253500 & -0.173600 & -3.023200 \\
\hline $\mathrm{C}$ & 8.371300 & 0.877200 & -2.762300 \\
\hline $\mathrm{C}$ & 6.997400 & 0.701400 & -2.949000 \\
\hline $\mathrm{C}$ & -0.425300 & -2.616900 & -2.384200 \\
\hline $\mathrm{C}$ & -0.786400 & -4.090300 & -2.332000 \\
\hline $\mathrm{C}$ & -0.728800 & -5.011000 & -3.593500 \\
\hline $\mathrm{C}$ & -2.031600 & -4.383500 & -3.174100 \\
\hline $\mathrm{C}$ & -0.656900 & -4.686300 & -0.947100 \\
\hline 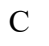 & 0.574100 & -4.806700 & -0.279200 \\
\hline . & 0.681600 & -5.368100 & 0.991500 \\
\hline $\mathrm{C}$ & -0.454400 & -5.837900 & 1.665100 \\
\hline
\end{tabular}




\begin{tabular}{|c|c|c|c|}
\hline C & -1.692000 & -5.716900 & 1.020100 \\
\hline $\mathrm{C}$ & -1.778600 & -5.152400 & -0.250700 \\
\hline $\mathrm{C}$ & -0.345800 & -6.430000 & 3.025300 \\
\hline $\mathrm{C}$ & 0.722400 & -7.285300 & 3.345800 \\
\hline $\mathrm{C}$ & 0.831900 & -7.847700 & 4.629300 \\
\hline $\mathrm{C}$ & -0.140900 & -7.545700 & 5.594200 \\
\hline $\mathrm{C}$ & -1.214700 & -6.694200 & 5.293700 \\
\hline $\mathrm{C}$ & -1.310300 & -6.139800 & 4.005800 \\
\hline $\mathrm{C}$ & -0.074300 & -4.510400 & -4.839500 \\
\hline $\mathrm{C}$ & 1.287600 & -4.168300 & -4.840300 \\
\hline $\mathrm{C}$ & 1.898800 & -3.715800 & -6.012700 \\
\hline $\mathrm{C}$ & 1.158000 & -3.603500 & -7.191800 \\
\hline $\mathrm{C}$ & -0.192000 & -3.964400 & -7.205100 \\
\hline $\mathrm{C}$ & -0.803500 & -4.430000 & -6.037400 \\
\hline $\mathrm{C}$ & -0.581400 & -6.486200 & -3.372700 \\
\hline $\mathrm{C}$ & -1.621900 & -7.365600 & -3.724800 \\
\hline $\mathrm{C}$ & -1.479800 & -8.742700 & -3.53 \\
\hline $\mathrm{C}$ & -0.292700 & -9.257000 & -3.003800 \\
\hline $\mathrm{C}$ & 0.759200 & -8.395800 & -2.683900 \\
\hline $\mathrm{C}$ & 0.621100 & -7.018500 & -2.878100 \\
\hline $\mathrm{C}$ & 0.430000 & 2.610900 & -2.389500 \\
\hline $\mathrm{C}$ & 0.790800 & 4.084500 & -2.340100 \\
\hline $\mathrm{C}$ & 0.735800 & 5.002000 & -3.603900 \\
\hline $\mathrm{C}$ & 2.037800 & 4.375700 & -3.180200 \\
\hline $\mathrm{C}$ & 0.658100 & 4.683800 & -0.956900 \\
\hline $\mathrm{C}$ & -0.574300 & 4.805600 & -0.292000 \\
\hline $\mathrm{C}$ & -0.684800 & 5.369800 & 0.97 \\
\hline $\mathrm{C}$ & 0.449700 & 5.841500 & 1.652100 \\
\hline $\mathrm{C}$ & 1.688800 & 5.719200 & 1.010100 \\
\hline $\mathrm{C}$ & 1.778400 & 5.151700 & -0.259200 \\
\hline $\mathrm{C}$ & 0.338100 & 6.436700 & 3.010700 \\
\hline $\mathrm{C}$ & 1.300500 & 6.148800 & 3.9 \\
\hline $\mathrm{C}$ & 1.201900 & 6.706100 & 5.280500 \\
\hline $\mathrm{C}$ & 0.127200 & 7.558100 & 5.576700 \\
\hline $\mathrm{C}$ & -0.843500 & 7.857800 & 4.608900 \\
\hline $\mathrm{C}$ & -0.731100 & 7.292400 & 3.327000 \\
\hline $\mathrm{C}$ & 0.084100 & 4.498300 & -4.850100 \\
\hline $\mathrm{C}$ & -1.277700 & 4.156000 & -4.853100 \\
\hline $\mathrm{C}$ & -1.886100 & 3.700500 & -6.025700 \\
\hline $\mathrm{C}$ & -1.142800 & 3.585500 & -7.203000 \\
\hline $\mathrm{C}$ & 0.207100 & 3.946700 & -7.214200 \\
\hline $\mathrm{C}$ & 0.816000 & 4.415300 & -6.046300 \\
\hline $\mathrm{C}$ & 0.587800 & 6.477700 & -3.387000 \\
\hline $\mathrm{C}$ & 1.628900 & 7.356500 & -3.738800 \\
\hline $\mathrm{C}$ & 1.486100 & 8.734100 & -3.548400 \\
\hline $\mathrm{C}$ & 0.297900 & 9.249400 & -3.025400 \\
\hline $\mathrm{C}$ & -0.754600 & 8.388800 & -2.705800 \\
\hline $\mathrm{C}$ & -0.615900 & 7.011100 & -2.896400 \\
\hline $\mathrm{C}$ & -2.613800 & 0.408100 & -2.389300 \\
\hline $\mathrm{C}$ & -4.095000 & 0.736500 & -2.333900 \\
\hline $\mathrm{C}$ & -5.010300 & 0.715500 & -3.599500 \\
\hline $\mathrm{C}$ & -4.409300 & 2.011500 & -3.120400 \\
\hline $\mathrm{C}$ & -4.694000 & 0.528200 & -0.960100 \\
\hline $\mathrm{C}$ & -4.784400 & -0.738200 & -0.357500 \\
\hline $\mathrm{C}$ & -5.344600 & -0.927500 & 0.903300 \\
\hline $\mathrm{C}$ & -5.846400 & 0.158400 & 1.633800 \\
\hline $\mathrm{C}$ & -5.759400 & 1.430700 & 1.053200 \\
\hline $\mathrm{C}$ & -5.192100 & 1.599600 & -0.208800 \\
\hline $\mathrm{C}$ & -6.438400 & -0.038700 & 2.984500 \\
\hline $\mathrm{C}$ & -7.581000 & 0.682100 & 3.372300 \\
\hline $\mathrm{C}$ & -8.146700 & 0.500400 & 4.646200 \\
\hline & -7.557100 & -0.412900 & 5.533200 \\
\hline
\end{tabular}

C $\quad-6.415900 \quad-1.141100 \quad 5.165400$

$\begin{array}{llll}\text { C } & -5.861400 & -0.947600 & 3.888700\end{array}$

$\begin{array}{llll}\text { C } & -4.495500 & 0.129100 & -4.873600\end{array}$

C $\quad-4.102100 \quad-1.217700 \quad-4.931700$

C $\quad-3.626600 \quad-1.761600 \quad-6.128000$

C $\quad-3.551200 \quad-0.969900 \quad-7.276800$

$\begin{array}{llll}\text { C } & -3.970100 & 0.362700 & -7.235100\end{array}$

$\begin{array}{llll}\text { C } & -4.454100 & 0.907200 & -6.042000\end{array}$

$\begin{array}{llll}\text { C } & -6.482700 & 0.528700 & -3.389900\end{array}$

C $\quad-7.382300 \quad 1.568600 \quad-3.688500$

C $\quad-8.756500 \quad 1.389400 \quad-3.504300$

$\begin{array}{llll}\text { C } & -9.247500 & 0.166400 & -3.041300\end{array}$

$\begin{array}{llll}\text { C } & -8.365900 & -0.883700 & -2.775900\end{array}$

C $\quad-6.991500 \quad-0.708500 \quad-2.960400$

C $\quad-2.236200 \quad-6.392500 \quad 6.362600$

$\begin{array}{llll}\text { C } & 1.967900 & -8.771500 & 4.993800\end{array}$

C $\quad-5.811300 \quad-2.110500 \quad 6.151400$

$\begin{array}{llll}\text { C } & -9.375100 & 1.260500 & 5.081700\end{array}$

C $\quad 5.802100 \quad 2.127300 \quad 6.156400$

C $\quad 9.368000 \quad-1.246100 \quad 5.101100$

$\begin{array}{llll}\text { C } & -1.980500 & 8.782100 & 4.968900\end{array}$

C $\quad 2.221100 \quad 6.406900 \quad 6.352300$

H $\quad 5.045700 \quad-2.768200 \quad-2.637900$

H $\quad 3.583700 \quad-2.436700 \quad-3.662900$

$\mathrm{H} \quad 5.387400 \quad 1.946700 \quad 1.289200$

H $\quad 6.099900 \quad-2.312800 \quad 1.597700$

$\mathrm{H} \quad 5.116800 \quad-2.604600 \quad-0.595800$

$\mathrm{H} \quad 4.165800 \quad 1.831800 \quad-4.048200$

$\mathrm{H} \quad 3.333800 \quad 2.782100 \quad-6.166800$

H $\quad 3.195400 \quad 1.371600 \quad-8.198900$

H $\quad 3.942000 \quad-0.992500 \quad-8.118700$

$\mathrm{H} \quad 4.810800 \quad-1.948300 \quad-6.013400$

H $\quad 7.030300 \quad-2.527600 \quad-4.045300$

H $\quad 9.449000 \quad-2.206500 \quad-3.701900$

H $\quad 10.317400 \quad-0.035400 \quad-2.880600$

$\mathrm{H} \quad 8.752800 \quad 1.831600 \quad-2.423300$

H $\quad 6.335000 \quad 1.534900 \quad-2.772100$

$\mathrm{H} \quad-2.812700 \quad-4.999800 \quad-2.741000$

$\mathrm{H} \quad-2.406300 \quad-3.544800 \quad-3.749500$

H $\quad 1.668200 \quad-5.403000 \quad 1.443000$

$\mathrm{H} \quad-2.609600 \quad-6.067800 \quad 1.485200$

$\mathrm{H} \quad-2.757100 \quad-5.054400 \quad-0.706800$

$\mathrm{H} \quad \begin{array}{llll}1.873300 & -4.250300 & -3.935400\end{array}$

H $\quad 2.948100 \quad-3.459900 \quad-6.008400$

H $\quad 1.633000 \quad-3.248200 \quad-8.097100$

$\mathrm{H} \quad-0.762700 \quad-3.891000 \quad-8.121800$

H $\quad-1.843500 \quad-4.729200 \quad-6.065500$

$\mathrm{H} \quad-2.542600 \quad-6.987100 \quad-4.150500$

H $\quad-2.288600 \quad-9.411900 \quad-3.793700$

H $\quad-0.183600 \quad-10.323400 \quad-2.855300$

$\mathrm{H} \quad 1.685700 \quad-8.796100 \quad-2.293000$

H $\quad 1.456300 \quad-6.373100 \quad-2.654100$

$\mathrm{H} \quad 2.817900 \quad 4.993200 \quad-2.746900$

H $\quad 2.413900 \quad 3.535800 \quad-3.752700$

$\mathrm{H} \quad-1.672300 \quad 5.405800 \quad 1.426400$

H $\quad 2.605300 \quad 6.071400 \quad 1.476400$

H $\quad 2.757800 \quad 5.052700 \quad-0.712900$

$\mathrm{H} \quad-1.865300 \quad 4.240100 \quad-3.949700$

$\mathrm{H} \quad-2.935500 \quad 3.444400 \quad-6.023100$

H $\quad-1.615800 \quad 3.228000 \quad-8.108500$

$\mathrm{H} \quad 0.779900 \quad 3.871100 \quad-8.129400$

H $\quad 1.856000 \quad 4.714600 \quad-6.072800$
H $\quad 2.550600 \quad 6.977100 \quad-4.161700$

H $\quad 2.295500 \quad 9.402700 \quad-3.811200$

H $\quad 0.188300 \quad 10.316200 \quad-2.879700$

$\mathrm{H} \quad-1.682100 \quad 8.789900 \quad-2.317900$

$\mathrm{H} \quad-1.451500 \quad 6.366100 \quad-2.672700$

$\mathrm{H} \quad-5.040400 \quad 2.761600 \quad-2.654500$

$\mathrm{H} \quad-3.576300 \quad 2.427500 \quad-3.675900$

$\mathrm{H} \quad-5.389200 \quad-1.943000 \quad 1.284300$

H $\quad-6.101900 \quad 2.317400 \quad 1.580400$

$\mathrm{H} \quad-5.115200 \quad 2.603300 \quad-0.612200$

$\mathrm{H} \quad-4.158100 \quad-1.841900 \quad-4.051400$

H $\quad-3.322200 \quad-2.797700 \quad-6.166000$

$\mathrm{H} \quad-3.179600 \quad-1.392400 \quad-8.201400$

$\mathrm{H} \quad-3.926000 \quad 0.971900 \quad-8.128600$

$\mathrm{H} \quad-4.798700 \quad 1.933200 \quad-6.027400$

$\mathrm{H} \quad-7.022200 \quad 2.517600 \quad-4.065200$

$\mathrm{H} \quad-9.441400 \quad 2.197600 \quad-3.725600$

H $\quad-10.311700 \quad 0.028700 \quad-2.900300$

H $\quad-8.748000 \quad-1.837300 \quad-2.435100$

$\mathrm{H} \quad-6.329600 \quad-1.541600 \quad-2.780100$

$\mathrm{H} \quad-2.123200 \quad-5.465800 \quad 3.767300$

H $\quad 1.456000 \quad-7.524800 \quad 2.586600$

H $\quad 4.963800 \quad 1.498400 \quad 3.606200$

$\mathrm{H} \quad 8.033800 \quad-1.361500 \quad 2.689200$

$\mathrm{H} \quad-1.463000 \quad 7.530100 \quad 2.565600$

$\mathrm{H} \quad 2.113900 \quad 5.474500 \quad 3.758800$

$\mathrm{H} \quad-4.969100 \quad-1.488600 \quad 3.600800$

$\mathrm{H} \quad-8.037400 \quad 1.369200 \quad 2.671500$

$\mathrm{H} \quad-7.989300 \quad-0.557600 \quad 6.516900$

$\mathrm{H} \quad 0.045700 \quad 7.991100 \quad 6.567300$

H $\quad-0.061600 \quad-7.976500 \quad 6.586000$

H $\quad 7.979900 \quad 0.575700 \quad 6.529300$

\section{F5}

$\begin{array}{llll}\mathrm{Rh} & -0.153400 & -0.317100 & -1.158900\end{array}$

Rh $\quad-0.436200 \quad-0.936900 \quad-3.441900$

O $\quad-1.454200 \quad-2.610600 \quad-2.791100$

$\begin{array}{llll}\mathrm{O} & -1.066700 & -2.086400 & -0.628000\end{array}$

$\begin{array}{llll}\text { O } & -2.200900 & 0.135000 & -3.443200\end{array}$

$\begin{array}{llll}\text { O } & -1.994700 & 0.604100 & -1.242900\end{array}$

O $\quad 1.346200 \quad-1.974000 \quad-3.310900$

O $\quad 1.674100 \quad-1.265000 \quad-1.191300$

$\begin{array}{llll}\mathrm{O} & 0.597400 & 0.773800 & -3.965400\end{array}$

$\begin{array}{llll}\mathrm{O} & 0.750000 & 1.417200 & -1.805300\end{array}$

$\mathrm{Cl} \quad 0.481200 \quad-4.794800 \quad 0.139300$

Cl $\quad-4.766700 \quad-0.745100 \quad-0.329200$

$\begin{array}{lllll}\mathrm{Cl} & 4.462100 & 0.228800 & -1.813500\end{array}$

Cl $\quad-0.691800 \quad 4.254500 \quad-2.103700$

F $\quad-8.965400 \quad 1.312600 \quad 4.710600$

$\begin{array}{llll}\text { F } & -8.014100 & 1.747100 & 6.698900\end{array}$

F $\quad-9.380000 \quad 3.263200 \quad 5.746000$

F $\quad-4.252400 \quad 5.219000 \quad 6.861400$

F $\quad-5.622300 \quad 6.741100 \quad 5.924500$

F $\quad-3.666000 \quad 6.232600 \quad 4.943900$

F $\quad 5.964400 \quad 7.486400 \quad 4.315500$

F $\quad 4.358400 \quad 6.406800 \quad 5.467300$

F $\quad 5.613500 \quad 5.294600 \quad 3.971800$

F $\quad 0.895000 \quad 9.743400 \quad 3.687300$

F $\quad 2.502500 \quad 10.796500 \quad 2.513100$

F $\quad 0.697000 \quad 9.970900 \quad 1.461000$

F $\quad 7.905900 \quad 2.142200 \quad 5.284400$

$\begin{array}{llll}\text { F } & 6.242500 & 1.273200 & 6.529400\end{array}$ 


\begin{tabular}{|c|c|c|c|}
\hline & 5.777200 & 2.290400 & 4.581100 \\
\hline & 8.970100 & -4.198200 & 4.363500 \\
\hline & 8.503500 & -3.315700 & 6.376100 \\
\hline & 10.169600 & -2.458500 & 5.126800 \\
\hline & 0.342800 & -8.178400 & 6.123400 \\
\hline & 0.682200 & -6.959400 & 7.979800 \\
\hline $\mathrm{F}$ & -1.101100 & -8.327400 & 7.838500 \\
\hline $\mathrm{F}$ & -4.206000 & -4.350300 & 8.725400 \\
\hline $\mathrm{F}$ & -4.057700 & -2.570700 & 7.362200 \\
\hline & -2.423100 & -2.979900 & 8.848500 \\
\hline $\mathrm{C}$ & -1.531700 & -2.841600 & -1.539000 \\
\hline $\mathrm{C}$ & -2.330400 & -4.041100 & -1.062300 \\
\hline $\mathrm{C}$ & -2.727200 & -5.215600 & -2.013900 \\
\hline $\mathrm{C}$ & -3.700200 & -4.098300 & -1.744400 \\
\hline C & -2.220700 & -4.277300 & 0.428300 \\
\hline $\mathrm{C}$ & -1.016400 & -4.638900 & 1.056500 \\
\hline $\mathrm{C}$ & -0.932100 & -4.879700 & 2.426100 \\
\hline $\mathrm{C}$ & -2.064300 & -4.763900 & 3.244600 \\
\hline $\mathrm{C}$ & -3.270900 & -4.389100 & 2.639300 \\
\hline $\mathrm{C}$ & -3.334400 & -4.151900 & 1.267700 \\
\hline $\mathrm{C}$ & -1.978600 & -5.020000 & 4.707500 \\
\hline $\mathrm{C}$ & -1.199800 & -6.081700 & 5.199400 \\
\hline $\mathrm{C}$ & -1.112600 & -6.328800 & 6.580200 \\
\hline $\mathrm{C}$ & -1.814700 & -5.501500 & 7.469300 \\
\hline $\mathrm{C}$ & -2.597700 & -4.437100 & 6.998200 \\
\hline $\mathrm{C}$ & -2.674600 & -4.202500 & 5.614600 \\
\hline $\mathrm{C}$ & -2.112600 & -5.302500 & -3.372700 \\
\hline $\mathrm{C}$ & -0.723300 & -5.446200 & -3.518400 \\
\hline $\mathrm{C}$ & -0.155400 & -5.541200 & -4.791600 \\
\hline $\mathrm{C}$ & -0.967900 & -5.498500 & -5.927300 \\
\hline $\mathrm{C}$ & -2.353700 & -5.381600 & -5.790100 \\
\hline $\mathrm{C}$ & -2.926500 & -5.298700 & -4.517500 \\
\hline $\mathrm{C}$ & -3.026600 & -6.545400 & -1.390200 \\
\hline $\mathrm{C}$ & -4.328100 & -7.077700 & -1.439100 \\
\hline $\mathrm{C}$ & -4.604400 & -8.323500 & -0.868000 \\
\hline $\mathrm{C}$ & -3.582700 & -9.058200 & -0.261900 \\
\hline $\mathrm{C}$ & -2.280200 & -8.554500 & -0.239200 \\
\hline $\mathrm{C}$ & -2.000100 & -7.310200 & -0.810800 \\
\hline $\mathrm{C}$ & -2.614700 & 0.649700 & -2.352000 \\
\hline $\mathrm{C}$ & -3.882900 & 1.483700 & -2.367600 \\
\hline $\mathrm{C}$ & -4.908100 & 1.428600 & -3.545800 \\
\hline $\mathrm{C}$ & -3.856600 & 2.506100 & -3.507000 \\
\hline $\mathrm{C}$ & -4.334800 & 1.906000 & -0.986800 \\
\hline $\mathrm{C}$ & -4.766600 & 0.989100 & -0.012900 \\
\hline $\mathrm{C}$ & -5.200600 & 1.389200 & 1.248800 \\
\hline $\mathrm{C}$ & -5.220000 & 2.745600 & 1.602500 \\
\hline $\mathrm{C}$ & -4.780400 & 3.674200 & 0.650000 \\
\hline $\mathrm{C}$ & -4.348900 & 3.253600 & -0.606500 \\
\hline $\mathrm{C}$ & -5.679800 & 3.176600 & 2.950200 \\
\hline $\mathrm{C}$ & -6.786800 & 2.557500 & 3.556000 \\
\hline $\mathrm{C}$ & -7.224700 & 2.956600 & 4.830400 \\
\hline $\mathrm{C}$ & -6.543400 & 3.986100 & 5.496900 \\
\hline $\mathrm{C}$ & -5.436900 & 4.616900 & 4.909000 \\
\hline C & -5.010600 & 4.206200 & 3.634300 \\
\hline $\mathrm{C}$ & -4.783800 & 0.359300 & -4.581800 \\
\hline $\mathrm{C}$ & -4.882300 & -0.994900 & -4.223800 \\
\hline $\mathrm{C}$ & -4.777200 & -1.990300 & -5.199100 \\
\hline $\mathrm{C}$ & -4.579800 & -1.642600 & -6.537700 \\
\hline $\mathrm{C}$ & -4.508000 & -0.296500 & -6.906000 \\
\hline $\mathrm{C}$ & -4.624000 & 0.702100 & -5.934600 \\
\hline $\mathrm{C}$ & -6.319600 & 1.843400 & -3.259200 \\
\hline$C$ & -6.856700 & 2.999700 & -3.854300 \\
\hline
\end{tabular}

C $\quad-8.177200 \quad 3.379500 \quad-3.596500$

$\begin{array}{llll}\text { C } & -8.979700 & 2.599700 & -2.760500\end{array}$

$\begin{array}{llll}\text { C } & -8.467100 & 1.431100 & -2.192900\end{array}$

$\begin{array}{llll}\text { C } & -7.147900 & 1.047400 & -2.449900\end{array}$

$\begin{array}{llll}\text { C } & 2.030900 & -1.892500 & -2.238300\end{array}$

C $\quad 3.323100 \quad-2.681600 \quad-2.123500$

C $\quad 4.046200 \quad-3.298000 \quad-3.363200$

C $\quad 3.118300 \quad-4.143200 \quad-2.529600$

$\begin{array}{llll}C & 4.106700 & -2.346600 & -0.873200\end{array}$

$\begin{array}{llll}\text { C } & 4.662600 & -1.076000 & -0.646000\end{array}$

$\begin{array}{llll}\text { C } & 5.380600 & -0.769800 & 0.507200\end{array}$

$\begin{array}{llll}\text { C } & 5.579900 & -1.735600 & 1.503100\end{array}$

C $\quad 5.033000 \quad-3.008900 \quad 1.296300$

C $\quad 4.312900 \quad-3.293800 \quad 0.137200$

$\begin{array}{llll}\text { C } & 6.333000 & -1.407900 & 2.743200\end{array}$

$\begin{array}{llll}\text { C } & 6.158800 & -0.163000 & 3.372500\end{array}$

$\begin{array}{llll}\text { C } & 6.865900 & 0.154800 & 4.544600\end{array}$

$\begin{array}{llll}\text { C } & 7.753600 & -0.787600 & 5.084800\end{array}$

$\begin{array}{llll}\text { C } & 7.939900 & -2.036300 & 4.472700\end{array}$

C $\quad 7.224800 \quad-2.339600 \quad 3.301200$

C $\quad 3.606100 \quad-2.917500 \quad-4.739400$

C $\quad 3.667500 \quad-1.580300 \quad-5.164000$

C $\quad 3.259800 \quad-1.231900 \quad-6.454600$

$\begin{array}{llll}\text { C } & 2.799000 & -2.215200 & -7.333700\end{array}$

C $\quad 2.763600 \quad-3.552400 \quad-6.929100$

$\begin{array}{llll}\text { C } & 3.178800 & -3.905400 & -5.641700\end{array}$

C $\quad 5.513900-3.577400-3.244200$

$\begin{array}{llll}\text { C } & 5.990700 & -4.901000 & -3.262600\end{array}$

C $\quad 7.360900 \quad-5.160300 \quad-3.164400$

C $\quad 8.268700 \quad-4.103200 \quad-3.068100$

C $\quad 7.808400 \quad-2.784700 \quad-3.084600$

C $\quad 6.439300 \quad-2.521700 \quad-3.184100$

$\begin{array}{llll}\text { C } & 0.950700 & 1.588000 & -3.049100\end{array}$

C $\quad 1.773800 \quad 2.806500 \quad-3.427000$

$\begin{array}{llll}\text { C } & 1.859100 & 3.335700 & -4.894800\end{array}$

$\begin{array}{llll}\text { C } & 2.929700 & 2.424800 & -4.356200\end{array}$

C $\quad 2.021500 \quad 3.739700 \quad-2.261700$

$\begin{array}{llll}\text { C } & 0.990600 & 4.438000 & -1.609400\end{array}$

$\begin{array}{llll}\text { C } & 1.231000 & 5.291300 & -0.534500\end{array}$

$\begin{array}{llll}\text { C } & 2.533300 & 5.489700 & -0.054400\end{array}$

$\begin{array}{llll}\text { C } & 3.574000 & 4.800500 & -0.690500\end{array}$

$\begin{array}{llll}\text { C } & 3.312500 & 3.948900 & -1.761700\end{array}$

$\begin{array}{llll}\text { C } & 2.793200 & 6.386600 & 1.104100\end{array}$

$\begin{array}{llll}\text { C } & 3.738000 & 6.029100 & 2.081800\end{array}$

$\begin{array}{llll}\text { C } & 3.987100 & 6.868400 & 3.181100\end{array}$

C $\quad 3.277900 \quad 8.073300 \quad 3.296700$

$\begin{array}{llll}\text { C } & 2.330900 & 8.447900 & 2.331800\end{array}$

$\begin{array}{llll}\text { C } & 2.094500 & 7.598900 & 1.236900\end{array}$

C $\quad 0.926000 \quad 2.793200 \quad-5.927600$

C $\quad-0.461400 \quad 2.962500 \quad-5.792900$

C $\quad-1.328900 \quad 2.469200 \quad-6.771300$

$\begin{array}{llll}\text { C } & -0.819600 & 1.805600 & -7.890300\end{array}$

$\begin{array}{llll}\text { C } & 0.561000 & 1.653200 & -8.043200\end{array}$

$\begin{array}{llll}\text { C } & 1.432500 & 2.157500 & -7.073400\end{array}$

$\begin{array}{llll}\text { C } & 2.227800 & 4.774500 & -5.095300\end{array}$

$\begin{array}{llll}\text { C } & 3.441400 & 5.117300 & -5.719200\end{array}$

C $\quad 3.780000 \quad 6.459000 \quad-5.918200$

$\begin{array}{llll}\text { C } & 2.903600 & 7.469600 & -5.515900\end{array}$

$\begin{array}{llll}\text { C } & 1.681000 & 7.139100 & -4.927200\end{array}$

C $\quad 1.338600 \quad 5.798700 \quad-4.728200$

C $\quad-4.730600 \quad 5.716700 \quad 5.662900$

$\begin{array}{llll}\text { C } & -8.411500 & 2.307200 & 5.498400\end{array}$ $\begin{array}{llll}\text { C } & 1.593400 & 9.754300 & 2.493500\end{array}$

$\begin{array}{llll}\text { C } & 4.994800 & 6.502800 & 4.243100\end{array}$

C $\quad-0.284900 \quad-7.464500 \quad 7.129800$

$\begin{array}{llll}\text { C } & -3.333000 & -3.569500 & 7.989700\end{array}$

$\begin{array}{lrrr}\text { C } & 8.906000 & -3.019400 & 5.086500\end{array}$

C $\quad 6.690500 \quad 1.483600 \quad 5.237900$

$\mathrm{H} \quad-4.575500 \quad-4.280200 \quad-1.129100$

$\mathrm{H} \quad-3.859000 \quad-3.358000 \quad-2.519800$

H $\quad 0.041800 \quad-5.135500 \quad 2.831700$

$\mathrm{H} \quad-4.186300 \quad-4.286600 \quad 3.216500$

H $\quad-4.281500 \quad-3.843100 \quad 0.839800$

$\mathrm{H} \quad-0.082600 \quad-5.481700 \quad-2.648600$

$\mathrm{H} \quad 0.913600 \quad-5.655500 \quad-4.897200$

$\mathrm{H} \quad-0.524800 \quad-5.568000 \quad-6.912300$

$\mathrm{H} \quad-2.984800 \quad-5.363500 \quad-6.669100$

$\mathrm{H} \quad-4.002800 \quad-5.233700 \quad-4.422800$

H $\quad-5.129400 \quad-6.530700 \quad-1.919800$

$\mathrm{H} \quad-5.610400 \quad-8.721500 \quad-0.899800$

H $\quad-3.797800 \quad-10.023000 \quad 0.178800$

$\mathrm{H} \quad-1.485100 \quad-9.132200 \quad 0.214100$

$\mathrm{H} \quad-0.982100 \quad-6.952300 \quad-0.810600$

$\mathrm{H} \quad-4.139700 \quad 3.530500 \quad-3.287400$

$\mathrm{H} \quad-3.010900 \quad 2.422300 \quad-4.179300$

$\mathrm{H} \quad-5.497200 \quad 0.612700 \quad 1.947000$

H $\quad-4.775700 \quad 4.740900 \quad 0.858900$

$\mathrm{H} \quad-3.995700 \quad 4.000400 \quad-1.308700$

$\mathrm{H} \quad-5.036600 \quad-1.278100 \quad-3.192100$

H $\quad-4.855600 \quad-3.030600 \quad-4.918000$

$\mathrm{H} \quad-4.494700 \quad-2.415700 \quad-7.290400$

$\mathrm{H} \quad-4.370300 \quad-0.026400 \quad-7.945000$

H $\quad-4.592600 \quad 1.741300 \quad-6.235000$

$\mathrm{H} \quad-6.255400 \quad 3.607300 \quad-4.518800$

$\mathrm{H} \quad-8.579900 \quad 4.276300 \quad-4.049200$

H $\quad-10.002200 \quad 2.894300 \quad-2.562600$

$\mathrm{H} \quad-9.094700 \quad 0.817200 \quad-1.560000$

H $\quad-6.780000 \quad 0.125300 \quad-2.027300$

H $\quad 3.523100 \quad-4.917900-1.886200$

$\mathrm{H} \quad 2.138500 \quad-4.377900 \quad-2.929900$

H $\quad 5.786600 \quad 0.233200 \quad 0.594100$

H $\quad 5.127700 \quad-3.792800 \quad 2.043300$

H $\quad 3.876600 \quad-4.280500 \quad 0.028000$

H $\quad 4.024700 \quad-0.810400 \quad-4.495200$

H $\quad 3.306600 \quad-0.200900 \quad-6.775100$

H $\quad 2.480700 \quad-1.942500 \quad-8.331500$

H $\quad 2.420700 \quad-4.316300 \quad-7.614800$

H $\quad 3.169400 \quad-4.947000 \quad-5.347200$

H $\quad 5.303800 \quad-5.732800 \quad-3.354400$

H $\quad 7.719300 \quad-6.181600 \quad-3.168100$

H $\quad 9.329300 \quad-4.305400 \quad-2.993400$

H $\quad 8.513200 \quad-1.965300 \quad-3.028800$

H $\quad 6.105700 \quad-1.496400 \quad-3.226900$

H $\quad 3.916900 \quad 2.820600 \quad-4.140700$

H $\quad 2.936000 \quad 1.391300 \quad-4.682200$

H $\quad 0.372900 \quad 5.770000 \quad-0.073200$

H $\quad 4.607400 \quad 4.917100 \quad-0.374200$

H $\quad 4.141700 \quad 3.412900 \quad-2.209000$

H $\quad-0.869300 \quad 3.474400 \quad-4.932500$

$\mathrm{H} \quad-2.395000 \quad 2.608300 \quad-6.665800$

$\mathrm{H} \quad-1.494200 \quad 1.420400 \quad-8.644000$

H $\quad 0.956500 \quad 1.151800 \quad-8.917000$

$\mathrm{H} \quad 2.500800 \quad 2.054900 \quad-7.214900$

H $\quad 4.125600 \quad 4.347500 \quad-6.053100$ 


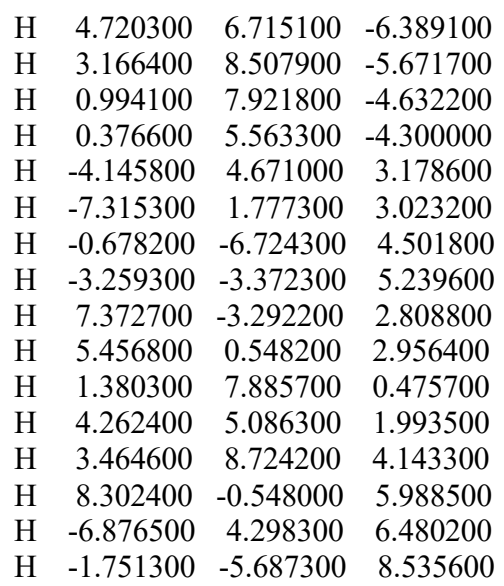

F6

Rh $\quad-0.001800 \quad-0.002000 \quad-1.199500$

$\begin{array}{llll}\text { Rh } & -0.004900 & -0.006300 & -3.581700\end{array}$

O $\quad-0.073200 \quad-2.068400 \quad-3.511700$

$\begin{array}{llll}\mathrm{O} & 0.071200 & -2.060500 & -1.257100\end{array}$

$\begin{array}{llll}\mathrm{O} & -2.068400 & 0.063500 & -3.511000\end{array}$

$\begin{array}{llll}\mathrm{O} & -2.058800 & -0.085400 & -1.256800\end{array}$

$\begin{array}{llll}\mathrm{O} & 2.058800 & -0.076000 & -3.516100\end{array}$

$\begin{array}{llll}\text { O } & 2.054900 & 0.081300 & -1.262500\end{array}$

$\begin{array}{llll}\mathrm{O} & 0.063600 & 2.056200 & -3.519300\end{array}$

$\begin{array}{llll}\text { O } & -0.075000 & 2.056300 & -1.264400\end{array}$

Cl $\quad 2.733500 \quad-3.832700 \quad-1.019900$

$\mathrm{Cl} \quad-3.765300 \quad-2.823500 \quad-1.143300$

Cl $\quad 3.761100 \quad 2.820400 \quad-1.164400$

$\mathrm{Cl} \quad-2.735500 \quad 3.830100 \quad-1.024700$

F $\quad 0.610700 \quad 6.018600 \quad 7.426100$

F $\quad \begin{array}{llll}1.528100 & 7.963800 & 6.758200\end{array}$

F $\quad 2.253600 \quad 6.021500 \quad 5.893300$

F $\quad-4.007700 \quad 7.726300 \quad 6.010400$

F $\quad-3.087600 \quad 9.663400 \quad 5.323000$

F $\quad-4.275500 \quad 8.408700 \quad 3.886700$

F $\quad 9.290600 \quad-0.510000 \quad 6.207600$

F $\quad 10.317000 \quad 1.315000 \quad 5.378300$

F $\quad 10.043700 \quad-0.506200 \quad 4.091000$

F $\quad 6.178600 \quad 4.128800 \quad 6.479800$

F $\quad \begin{array}{llll}5.147100 & 2.300200 & 7.294500\end{array}$

F $\quad 4.203400 \quad 3.477900 \quad 5.630100$

F $\quad 3.108800 \quad-9.650200 \quad 5.340800$

F $\quad 4.031900 \quad-7.711600 \quad 6.019900$

F $\quad 4.291500 \quad-8.399300 \quad 3.896900$

F $\quad-0.580400 \quad-5.999700 \quad 7.449300$

F $\quad-1.501300 \quad-7.946000 \quad 6.789300$

F $\quad-2.229100 \quad-6.005200 \quad 5.922800$

F $\quad-4.196800 \quad-3.456600 \quad 5.654200$

F $\quad-5.136500 \quad-2.271700 \quad 7.316000$

F $\quad-6.171100 \quad-4.102500 \quad 6.510100$

$\begin{array}{llll}\text { F } & -9.280300 & 0.537000 & 6.226000\end{array}$

F $\quad-10.309300 \quad-1.290300 \quad 5.405200$

F $\quad-10.037100 \quad 0.526000 \quad 4.110800$

C $\quad 0.001400 \quad-2.652600 \quad-2.380100$

$\begin{array}{llll}\text { C } & -0.113300 & -4.165300 & -2.326900\end{array}$

$\begin{array}{llll}\text { C } & 0.088700 & -5.064800 & -3.588900\end{array}$

C $\quad-1.297300 \quad-4.658400 \quad-3.163800$

$\begin{array}{llll}\text { C } & 0.118500 & -4.731900 & -0.943200\end{array}$

$\begin{array}{llll}\text { C } & 1.356400 & -4.650400 & -0.282200\end{array}$ $\begin{array}{llll}\text { C } & 1.562300 & -5.189500 & 0.985800\end{array}$

$\begin{array}{llll}\text { C } & 0.522400 & -5.840100 & 1.664300\end{array}$

C $\quad-0.722600 \quad-5.920700 \quad 1.027200$

$\begin{array}{llll}\text { C } & -0.908100 & -5.375600 & -0.241500\end{array}$

C $\quad 0.735000 \quad-6.410800 \quad 3.021300$

$\begin{array}{llll}\text { C } & 1.929700 & -7.083400 & 3.331000\end{array}$

$\begin{array}{llll}\text { C } & 2.138100 & -7.624400 & 4.611400\end{array}$

C $\quad 1.136700 \quad-7.485700 \quad 5.584400$

$\begin{array}{llll}C & -0.062400 & -6.817300 & 5.294900\end{array}$

C $\quad-0.256100 \quad-6.282300 \quad 4.009700$

C $\quad 0.646700 \quad-4.465000 \quad-4.838000$

$\begin{array}{llll}\text { C } & 1.933800 & -3.903500 & -4.845200\end{array}$

C $\quad 2.456800 \quad-3.357800 \quad-6.020500$

C $\quad 1.702500 \quad-3.370300 \quad-7.196300$

$\begin{array}{llll}\text { C } & 0.430500 & -3.948800 & -7.203500\end{array}$

$\begin{array}{llll}\text { C } & -0.090900 & -4.507400 & -6.032800\end{array}$

C $\quad 0.476300 \quad-6.495900 \quad-3.368800$

C $\quad-0.408100 \quad-7.533900 \quad-3.715200$

C $\quad-0.041700 \quad-8.869100 \quad-3.521700$

C $\quad 1.216100 \quad-9.181900 \quad-3.001200$

$\begin{array}{llll}\text { C } & 2.114700 & -8.159900 & -2.687000\end{array}$

C $\quad 1.752400 \quad-6.824000 \quad-2.880700$

C $\quad-2.651500 \quad-0.024100 \quad-2.380300$

$\begin{array}{llll}\text { C } & -4.166100 & 0.060900 & -2.321200\end{array}$

C $\quad-5.069100 \quad-0.111700 \quad-3.584100$

$\begin{array}{llll}\text { C } & -4.684000 & 1.265900 & -3.110600\end{array}$

C $\quad-4.720600 \quad-0.236600 \quad-0.945100$

$\begin{array}{llll}\text { C } & -4.604900 & -1.499000 & -0.338500\end{array}$

$\begin{array}{llll}\text { C } & -5.126200 & -1.772700 & 0.923500\end{array}$

C $\quad-5.795800 \quad-0.779700 \quad 1.651700$

$\begin{array}{llll}\text { C } & -5.914800 & 0.488500 & 1.067600\end{array}$

$\begin{array}{lrrr}\text { C } & -5.383600 & 0.742800 & -0.195700\end{array}$

C $\quad-6.348300 \quad-1.065900 \quad 3.003300$

C $\quad-7.591100 \quad-0.535700 \quad 3.390400$

$\begin{array}{llll}\text { C } & -8.120100 & -0.802600 & 4.665000\end{array}$

C $\quad-7.392400 \quad-1.608600 \quad 5.553400$

C $\quad-6.149800 \quad-2.146600 \quad 5.186200$

C $\quad-5.633500 \quad-1.869600 \quad 3.908800$

C $\quad-4.469400 \quad-0.611600 \quad-4.857900$

C $\quad-3.865300 \quad-1.878200 \quad-4.913300$

C $\quad-3.311600 \quad-2.342600 \quad-6.109500$

C $\quad-3.366200 \quad-1.552600 \quad-7.260500$

$\begin{array}{llll}\text { C } & -3.992800 & -0.304100 & -7.221300\end{array}$

$\begin{array}{llll}\text { C } & -4.555400 & 0.159600 & -6.028500\end{array}$

C $\quad-6.491700 \quad-0.532600 \quad-3.369900$

$\begin{array}{llll}\text { C } & -7.547800 & 0.347300 & -3.670300\end{array}$

$\begin{array}{llll}\text { C } & -8.874700 & -0.050400 & -3.481800\end{array}$

$\begin{array}{llll}\text { C } & -9.161200 & -1.334500 & -3.012700\end{array}$

C $\quad-8.121200 \quad-2.227600 \quad-2.745500$

$\begin{array}{llll}\text { C } & -6.793400 & -1.833900 & -2.934300\end{array}$

$\begin{array}{llll}\text { C } & 2.644800 & 0.016000 & -2.387200\end{array}$

$\begin{array}{llll}\text { C } & 4.159600 & -0.068500 & -2.331600\end{array}$

$\begin{array}{llll}\text { C } & 5.059400 & 0.099300 & -3.597500\end{array}$

C $\quad 4.675800 \quad-1.276500 \quad-3.117600$

C $\quad 4.717300 \quad 0.234600 \quad-0.958100$

$\begin{array}{llll}\text { C } & 4.602500 & 1.499300 & -0.356200\end{array}$

$\begin{array}{llll}\text { C } & 5.126100 & 1.777900 & 0.903800\end{array}$

$\begin{array}{llll}\text { C } & 5.797400 & 0.787900 & 1.634500\end{array}$

$\begin{array}{llll}\text { C } & 5.915800 & -0.482400 & 1.055000\end{array}$

$\begin{array}{llll}\text { C } & 5.382100 & -0.741800 & -0.206300\end{array}$

$\begin{array}{llll}\text { C } & 6.352100 & 1.079300 & 2.984000\end{array}$

C $\quad 7.595800 \quad 0.551300 \quad 3.371000$
C $\quad 8.126900 \quad 0.823300 \quad 4.643700$

$\begin{array}{llll}\text { C } & 7.400000 & 1.632000 & 5.530400\end{array}$

C $\quad 6.156600 \quad 2.167900 \quad 5.163300$

C $\quad 5.638300 \quad 1.885900 \quad 3.887800$

$\begin{array}{llll}\text { C } & 4.456400 & 0.594000 & -4.871700\end{array}$

$\begin{array}{llll}\text { C } & 3.851600 & 1.860100 & -4.930700\end{array}$

$\begin{array}{llll}\text { C } & 3.294800 & 2.319500 & -6.127300\end{array}$

$\begin{array}{llll}\text { C } & 3.346800 & 1.525000 & -7.275400\end{array}$

$\begin{array}{llll}\text { C } & 3.974100 & 0.276900 & -7.232800\end{array}$

$\begin{array}{llll}\text { C } & 4.539900 & -0.181800 & -6.039500\end{array}$

$\begin{array}{llll}\text { C } & 6.482300 & 0.521500 & -3.388400\end{array}$

$\begin{array}{llll}\text { C } & 7.538000 & -0.359400 & -3.687800\end{array}$

$\begin{array}{llll}\text { C } & 8.865300 & 0.039400 & -3.504100\end{array}$

C $\quad 9.152500 \quad 1.325500 \quad-3.041000$

$\begin{array}{llll}\text { C } & 8.112900 & 2.219500 & -2.774900\end{array}$

$\begin{array}{llll}\text { C } & 6.784800 & 1.824600 & -2.958800\end{array}$

C $\quad-0.008000 \quad 2.644400 \quad-2.389700$

C $\quad 0.107300 \quad 4.157300 \quad-2.342200$

$\begin{array}{llll}\text { C } & -0.098500 & 5.052300 & -3.606700\end{array}$

$\begin{array}{llll}\text { C } & 1.288800 & 4.647000 & -3.184500\end{array}$

$\begin{array}{llll}\text { C } & -0.119900 & 4.728700 & -0.959700\end{array}$

C $\quad-1.355600 \quad 4.649500 \quad-0.294200$

$\begin{array}{llll}\text { C } & -1.556900 & 5.192400 & 0.973100\end{array}$

$\begin{array}{llll}\text { C } & -0.514500 & 5.844600 & 1.646000\end{array}$

$\begin{array}{llll}\text { C } & 0.728200 & 5.923400 & 1.004200\end{array}$

$\begin{array}{llll}\text { C } & 0.909100 & 5.374400 & -0.263500\end{array}$

$\begin{array}{llll}\text { C } & -0.722000 & 6.418700 & 3.002300\end{array}$

C $\quad 0.272900 \quad 6.292900 \quad 3.987300$

C $\quad 0.084000 \quad 6.831100 \quad 5.271800$

$\begin{array}{llll}\text { C } & -1.114100 & 7.499900 & 5.564300\end{array}$

$\begin{array}{llll}\text { C } & -2.119200 & 7.636000 & 4.594800\end{array}$

C $\quad-1.915600 \quad 7.091900 \quad 3.314900$

$\begin{array}{llll}\text { C } & -0.660600 & 4.448100 & -4.851900\end{array}$

C $\quad-1.948000 \quad 3.887400 \quad-4.853000$

$\begin{array}{llll}\text { C } & -2.475000 & 3.337600 & -6.024600\end{array}$

C $\quad-1.724300 \quad 3.345200 \quad-7.202800$

C $\quad-0.451900 \quad 3.922900 \quad-7.216000$

$\begin{array}{llll}\text { C } & 0.073400 & 4.485600 & -6.049000\end{array}$

$\begin{array}{llll}\text { C } & -0.485000 & 6.484300 & -3.390600\end{array}$

$\begin{array}{llll}\text { C } & 0.398600 & 7.520800 & -3.743900\end{array}$

$\begin{array}{llll}\text { C } & 0.033200 & 8.856800 & -3.554100\end{array}$

$\begin{array}{llll}\text { C } & -1.222700 & 9.171800 & -3.030300\end{array}$

$\begin{array}{llll}\text { C } & -2.120700 & 8.151300 & -2.709300\end{array}$

$\begin{array}{llll}\text { C } & -1.759300 & 6.814600 & -2.899200\end{array}$

$\begin{array}{lrrr}\text { C } & -5.398400 & -3.005800 & 6.173400\end{array}$

C $\quad-9.453900 \quad-0.247100 \quad 5.100000$

$\begin{array}{llll}\text { C } & -3.390900 & 8.366300 & 4.950800\end{array}$

$\begin{array}{llll}\text { C } & 1.136700 & 6.703100 & 6.345500\end{array}$

$\begin{array}{llll}\text { C } & 3.411000 & -8.354100 & 4.964300\end{array}$

$\begin{array}{llll}\text { C } & -1.110900 & -6.686400 & 6.372200\end{array}$

$\begin{array}{llll}\text { C } & 5.406300 & 3.030200 & 6.148700\end{array}$

$\begin{array}{llll}\text { C } & 9.461700 & 0.270200 & 5.078400\end{array}$

$\mathrm{H} \quad-1.965700 \quad-5.393900 \quad-2.727900$

$\mathrm{H} \quad-1.806300 \quad-3.892700 \quad-3.737700$

H $\quad 2.544100 \quad-5.064300 \quad 1.431800$

$\mathrm{H} \quad-1.567800 \quad-6.417200 \quad 1.497000$

H $\quad-1.892200 \quad-5.437700 \quad-0.691700$

H $\quad 2.528900 \quad-3.887100 \quad-3.942900$

H $\quad 3.449700 \quad-2.932300 \quad-6.021000$

H $\quad 2.108600 \quad-2.942600 \quad-8.103900$

$\mathrm{H} \quad-0.148500 \quad-3.971400 \quad-8.117600$

H $\quad-1.067500 \quad-4.973900 \quad-6.056100$ 
H $\quad-1.380600 \quad-7.311500 \quad-4.136200$

$\mathrm{H} \quad-0.731800 \quad-9.661800 \quad-3.780400$

H $\quad 1.498900 \quad-10.216000 \quad-2.853100$

H $\quad 3.096400 \quad-8.403000 \quad-2.301000$

H $\quad 2.472000 \quad-6.050400 \quad-2.661200$

$\mathrm{H} \quad-5.427200 \quad 1.905600 \quad-2.645200$

$\mathrm{H} \quad-3.930900 \quad 1.809300 \quad-3.669800$

H $\quad-5.006600 \quad-2.781100 \quad 1.307200$

$\mathrm{H} \quad-6.394800 \quad 1.310200 \quad 1.592900$

$\begin{array}{llll}\mathrm{H} & -5.469900 & 1.744600 & -0.601700\end{array}$

$\mathrm{H} \quad-3.818900 \quad-2.500800 \quad-4.031200$

$\mathrm{H} \quad-2.845700 \quad-3.317000 \quad-6.145500$

$\mathrm{H} \quad-2.933700 \quad-1.913100 \quad-8.184900$

H $\quad-4.048600 \quad 0.301700 \quad-8.116500$

$\mathrm{H} \quad-5.059700 \quad 1.117200 \quad-6.015800$

H $\quad-7.346100 \quad 1.340400 \quad-4.051500$

$\mathrm{H} \quad-9.681500 \quad 0.635900 \quad-3.704500$

$\mathrm{H} \quad-10.189000 \quad-1.641400 \quad-2.868400$

H $\quad-8.344000 \quad-3.228900-2.400000$

$\mathrm{H} \quad-6.005500 \quad-2.548500 \quad-2.752300$

H $\quad 5.420300 \quad-1.914200 \quad-2.651400$

H $\quad 3.921400 \quad-1.822300 \quad-3.672800$

$\begin{array}{llll}\mathrm{H} & 5.007000 & 2.787700 & 1.283800\end{array}$

$\mathrm{H} \quad 6.397100 \quad-1.302000 \quad 1.582500$

H $\quad 5.468000 \quad-1.745100 \quad-0.608500$

$\mathrm{H} \quad 3.807200 \quad 2.486100 \quad-4.051000$

H $\quad 2.828300 \quad 3.293500 \quad-6.166000$

H $\quad 2.911900 \quad 1.881500 \quad-8.200100$

H $\quad 4.028000 \quad-0.332400 \quad-8.125700$

H $\quad 5.044500 \quad-1.139200 \quad-6.024200$

H $\quad 7.335600 \quad-1.354000 \quad-4.064500$

H $\quad 9.671600 \quad-0.647600 \quad-3.726000$

H $\quad 10.180500 \quad 1.633300 \quad-2.900400$

H $\quad 8.336300 \quad 3.222200 \quad-2.434000$

H $\quad 5.997100 \quad 2.539700 \quad-2.777900$

H $\quad 1.958700 \quad 5.384000 \quad-2.753300$

H $\quad 1.795800 \quad 3.879200 \quad-3.757200$

$\mathrm{H} \quad-2.537200 \quad 5.068500 \quad 1.422800$

$\mathrm{H} \quad 1.575100 \quad 6.421100 \quad 1.469500$

H $\quad 1.891700 \quad 5.435100 \quad-0.717300$

$\mathrm{H} \quad-2.540300 \quad 3.874700 \quad-3.948800$

H $\quad-3.468200 \quad 2.912800-6.020500$

$\mathrm{H} \quad-2.133400 \quad 2.914500 \quad-8.107600$

H $\quad 0.124300 \quad 3.941700 \quad-8.132000$

H $\quad 1.050200 \quad 4.951300 \quad-6.077100$

H $\quad 1.369500 \quad 7.296600 \quad-4.167400$

H $\quad 0.722600 \quad 9.648300 \quad-3.818000$

H $\quad-1.504700 \quad 10.206600 \quad-2.885000$

$\mathrm{H} \quad-3.100800 \quad 8.396100 \quad-2.320800$

$\mathrm{H} \quad-2.478300 \quad 6.042000 \quad-2.674400$

$\mathrm{H} \quad-4.666500 \quad-2.261800 \quad 3.621400$

$\begin{array}{llll}\mathrm{H} & -8.151600 & 0.068500 & 2.688700\end{array}$

$\begin{array}{llll}\mathrm{H} & 2.686500 & -7.199300 & 2.565700\end{array}$

H $\quad-1.168700 \quad-5.747600 \quad 3.779300$

$\mathrm{H} \quad 4.670600 \quad 2.276500 \quad 3.600700$

$\begin{array}{lrrr}\mathrm{H} & 8.155500 & -0.055000 & 2.670500\end{array}$

$\begin{array}{llll}\mathrm{H} & -2.675400 & 7.205800 & 2.552300\end{array}$

$\mathrm{H} \quad 1.184600 \quad 5.757700 \quad 3.754800$

$\begin{array}{llll}\mathrm{H} & -1.265600 & 7.917300 & 6.553400\end{array}$

$\begin{array}{llll}\mathrm{H} & 7.805000 & 1.845800 & 6.513100\end{array}$

$\mathrm{H} \quad-7.795600 \quad-1.818400 \quad 6.537600$

H $\quad 1.291900 \quad-7.900700 \quad 6.573900$
G1

Rh $\quad 0.243500 \quad-0.415700 \quad-0.492100$

$\begin{array}{llll}\mathrm{Rh} & 1.083900 & -1.840700 & -2.206500\end{array}$

O $\quad-1.419800 \quad-0.088500 \quad-1.663900$

$\begin{array}{llll}\mathrm{O} & -0.557900 & -1.324100 & -3.347400\end{array}$

O $\quad 1.199400 \quad 1.158400 \quad-1.416800$

O $\quad 2.114600 \quad-0.216600 \quad-2.958000$

$\begin{array}{llll}\text { O } & 1.951400 & -0.814200 & 0.591200\end{array}$

O $\quad 2.678300 \quad-2.277100 \quad-0.969200$

$\begin{array}{llll}\mathrm{O} & -0.671800 & -2.062900 & 0.343000\end{array}$

O $\quad 0.006500 \quad-3.384300 \quad-1.359200$

$\mathrm{Cl} \quad-4.190600 \quad-1.697900 \quad-1.417600$

$\mathrm{Cl} \quad-0.181700 \quad 3.162300 \quad-3.520500$

$\begin{array}{llll}\mathrm{Cl} & 4.589400 & 1.025600 & 0.592800\end{array}$

$\mathrm{Cl} \quad 0.565000 \quad-3.855100 \quad 2.705500$

C $\quad-1.463600 \quad-0.588600 \quad-2.832200$

C $\quad-2.642600 \quad-0.166200 \quad-3.689900$

C $\quad-2.175200 \quad 0.368100 \quad-5.046700$

C $\quad-3.007700 \quad-0.886600 \quad-5.027800$

C $\quad-3.708500 \quad 0.577000 \quad-2.914600$

$\begin{array}{llll}\text { C } & -4.469400 & -0.024500 & -1.897200\end{array}$

C $\quad-5.466700 \quad 0.660400 \quad-1.206700$

C $\quad-5.751800 \quad 2.000900 \quad-1.502300$

C $\quad-4.993200 \quad 2.622600 \quad-2.502800$

C $\quad-3.999100 \quad 1.920300 \quad-3.181700$

C $\quad-6.816500 \quad 2.735600 \quad-0.767300$

C $\quad-8.016000 \quad 2.096900 \quad-0.420100$

$\begin{array}{llll}\text { C } & -9.014800 & 2.785900 & 0.271300\end{array}$

$\begin{array}{llll}\text { C } & -8.849300 & 4.138400 & 0.641500\end{array}$

C $\quad-7.635700 \quad 4.771700 \quad 0.285900$

C $\quad-6.638800 \quad 4.079900 \quad-0.407200$

$\begin{array}{llll}\text { C } & -9.976700 & 4.862600 & 1.406200\end{array}$

C $\quad-2.342500 \quad-2.178400 \quad-5.375100$

C $\quad-2.491400 \quad-3.302100 \quad-4.546200$

C $\quad-1.877400 \quad-4.511400 \quad-4.883100$

C $\quad-1.116500 \quad-4.610500 \quad-6.050600$

C $\quad-0.985800 \quad-3.504400 \quad-6.894600$

C $\quad-1.608200 \quad-2.296300 \quad-6.566600$

C $\quad-4.420800 \quad-0.774700 \quad-5.515000$

C $\quad-5.464500 \quad-1.426000 \quad-4.836000$

C $\quad-6.779700 \quad-1.328900 \quad-5.298600$

C $\quad-7.062900 \quad-0.601000 \quad-6.456400$

$\begin{array}{llll}\text { C } & -6.028600 & 0.020000 & -7.160500\end{array}$

C $\quad-4.712000 \quad-0.073700 \quad-6.699800$

$\begin{array}{llll}\text { C } & 1.934500 & 0.929700 & -2.428900\end{array}$

C $\quad 2.729000 \quad 2.113200 \quad-2.950900$

C $\quad 2.305100 \quad 3.431600 \quad-2.341500$

C $\quad 1.034100 \quad 3.993700 \quad-2.551900$

C $\quad 0.660300 \quad 5.220300 \quad-2.007600$

C $\quad 1.552800 \quad 5.953000 \quad-1.212500$

C $\quad 2.820400 \quad 5.402800 \quad-0.980500$

$\begin{array}{llll}\text { C } & 3.174100 & 4.172500 & -1.531200\end{array}$

C $\quad 1.160400 \quad 7.265100 \quad-0.631000$

C $\quad-0.139300 \quad 7.472500 \quad-0.144900$

C $\quad-0.509200 \quad 8.703500 \quad 0.403400$

$\begin{array}{llll}\text { C } & 0.413400 & 9.772500 & 0.482400\end{array}$

$\begin{array}{llll}\text { C } & 1.717500 & 9.549500 & -0.010800\end{array}$

C $\quad 2.084400 \quad 8.317600 \quad-0.556900$

C $\quad 3.460600 \quad 2.090100 \quad-4.331900$

C $\quad 4.217000 \quad 1.771200 \quad-3.069200$

$\begin{array}{llll}\text { C } & 3.190900 & 0.977300 & -5.291200\end{array}$

C $4.243600 \quad 0.165300 \quad-5.744300$ $\begin{array}{llll}\text { C } & 3.994200 & -0.876500 & -6.642500\end{array}$

C $\quad 2.698100 \quad-1.096100 \quad-7.116700$

$\begin{array}{llll}\text { C } & 1.652900 & -0.268000 & -6.700300\end{array}$

$\begin{array}{llll}\text { C } & 1.896600 & 0.767400 & -5.794000\end{array}$

C $\quad 3.751800 \quad 3.406700 \quad-4.986800$

$\begin{array}{llll}\text { C } & 2.709400 & 4.189500 & -5.511400\end{array}$

$\begin{array}{llll}\text { C } & 2.984400 & 5.411600 & -6.131400\end{array}$

C $\quad 4.303700 \quad 5.852800 \quad-6.255000$

$\begin{array}{llll}\text { C } & 5.350900 & 5.065800 & -5.770400\end{array}$

$\begin{array}{llll}\text { C } & 5.079500 & 3.843600 & -5.148400\end{array}$

$\begin{array}{llll}\text { C } & 2.788800 & -1.658300 & 0.140500\end{array}$

$\begin{array}{llll}\text { C } & 3.935000 & -2.015000 & 1.069400\end{array}$

$\begin{array}{llll}\text { C } & 4.046800 & -3.531300 & 1.252100\end{array}$

$\begin{array}{llll}\text { C } & 5.190100 & -2.811700 & 0.586500\end{array}$

$\begin{array}{llll}\text { C } & 4.036600 & -1.101500 & 2.271400\end{array}$

$\begin{array}{llll}\text { C } & 4.343800 & 0.265800 & 2.164600\end{array}$

C $\quad 4.463500 \quad 1.093900 \quad 3.278500$

$\begin{array}{llll}\text { C } & 4.275900 & 0.586300 & 4.571800\end{array}$

$\begin{array}{llll}\text { C } & 3.955100 & -0.771800 & 4.698000\end{array}$

C $\quad 3.836500 \quad-1.582900 \quad 3.571000$

$\begin{array}{llll}\text { C } & 4.407500 & 1.464600 & 5.765600\end{array}$

C $\quad 3.939200 \quad 2.786900 \quad 5.736300$

$\begin{array}{llll}\text { C } & 4.060600 & 3.612800 & 6.857000\end{array}$

$\begin{array}{llll}\text { C } & 4.658400 & 3.140700 & 8.048600\end{array}$

$\begin{array}{llll}\text { C } & 5.125200 & 1.808100 & 8.063400\end{array}$

$\begin{array}{llll}\text { C } & 5.001000 & 0.984800 & 6.942200\end{array}$

$\begin{array}{llll}\text { C } & 4.814300 & 4.016900 & 9.308900\end{array}$

C $\quad 5.406400 \quad-3.032800 \quad-0.875000$

C $\quad 5.566200 \quad-1.942200 \quad-1.745000$

$\begin{array}{llll}\text { C } & 5.780400 & -2.155700 & -3.109300\end{array}$

C $\quad 5.843500 \quad-3.456800 \quad-3.614200$

$\begin{array}{llll}\text { C } & 5.713100 & -4.547800 & -2.750700\end{array}$

$\begin{array}{llll}\text { C } & 5.509900 & -4.337900 & -1.383600\end{array}$

$\begin{array}{llll}\text { C } & 6.454900 & -2.662900 & 1.377200\end{array}$

$\begin{array}{llll}\text { C } & 7.170200 & -1.453500 & 1.359700\end{array}$

C $\quad 8.354900 \quad-1.320800 \quad 2.089200$

$\begin{array}{llll}\text { C } & 8.849700 & -2.400700 & 2.823700\end{array}$

$\begin{array}{llll}\text { C } & 8.166900 & -3.619100 & 2.818900\end{array}$

C $\quad 6.980700 \quad-3.755100 \quad 2.091600$

C $\quad-0.613800 \quad-3.178800 \quad-0.263900$

$\begin{array}{llll}\text { C } & -1.448900 & -4.299700 & 0.328000\end{array}$

$\begin{array}{llll}\text { C } & -2.006900 & -3.971100 & 1.695500\end{array}$

C $\quad-1.192300 \quad-3.778100 \quad 2.824500$

C $\quad-1.716800 \quad-3.505800 \quad 4.086000$

$\begin{array}{llll}\text { C } & -3.101900 & -3.407500 & 4.280200\end{array}$

C $\quad-3.929800 \quad-3.579800 \quad 3.163100$

C $\quad-3.385500 \quad-3.851000 \quad 1.909200$

C $\quad-3.666100 \quad-3.119100 \quad 5.626300$

$\begin{array}{llll}\text { C } & -3.100000 & -3.690400 & 6.775800\end{array}$

C $\quad-3.627200 \quad-3.420400 \quad 8.041600$

C $\quad-4.742200 \quad-2.564900 \quad 8.199100$

$\begin{array}{lrrr}\text { C } & -5.301700 & -1.997300 & 7.033500\end{array}$

$\begin{array}{llll}\text { C } & -4.773400 & -2.270300 & 5.769800\end{array}$

$\begin{array}{lrrr}C & -5.355700 & -2.237200 & 9.576300\end{array}$

C $\quad-1.283200 \quad-5.790800 \quad-0.110500$

C $\quad-2.353800 \quad-4.933500 \quad-0.732300$

C $\quad-0.116900 \quad-6.185200 \quad-0.956600$

$\begin{array}{llll}\text { C } & -0.324500 & -6.787300 & -2.208500\end{array}$

$\begin{array}{llll}\text { C } & 0.765000 & -7.158800 & -3.001700\end{array}$

C $\quad 2.068200 \quad-6.959000 \quad-2.538200$

$\begin{array}{llll}\text { C } & 2.281900 & -6.396000 & -1.277600\end{array}$

$\begin{array}{llll}\text { C } & 1.195200 & -6.012600 & -0.487000\end{array}$ 


\begin{tabular}{|c|c|c|c|}
\hline C & -1.730800 & -6.851900 & 0.848900 \\
\hline & -1.021200 & -7.084300 & 2.039200 \\
\hline & -1.434400 & -8.081600 & 2.927000 \\
\hline & -2.545000 & -8.872200 & 2.624100 \\
\hline & -3.236100 & -8.675100 & 1.426500 \\
\hline & -2.826600 & -7.677400 & 0.536900 \\
\hline & 0.048900 & 11.147900 & 1.078700 \\
\hline $\mathrm{C}$ & -11.263200 & 4.858900 & 0.551900 \\
\hline $\mathrm{C}$ & -9.636800 & 6.336000 & 1.736800 \\
\hline $\mathrm{C}$ & -10.245100 & 4.132900 & 2.740400 \\
\hline & -6.831800 & -2.690000 & 9.603400 \\
\hline $\mathrm{C}$ & -4.627500 & -2.939100 & 10.747900 \\
\hline $\mathrm{C}$ & -5.281700 & -0.714600 & 9.823600 \\
\hline $\mathrm{C}$ & -1.415500 & 11.226300 & 1.573900 \\
\hline C & 0.239600 & 12.241500 & 0.005100 \\
\hline & 0.967500 & 11.446500 & 2.283600 \\
\hline $\mathrm{C}$ & 4.251500 & 5.44 & 9.127300 \\
\hline $\mathrm{C}$ & 4.060200 & 2900 & 10.487200 \\
\hline $\mathrm{C}$ & 6.311400 & 4.144300 & 9.665500 \\
\hline $\mathrm{H}$ & -2.603900 & 1.296200 & -5.410700 \\
\hline H & -1.112000 & 0.275500 & -5.236300 \\
\hline $\mathrm{H}$ & -5.991600 & 4000 & -0.422300 \\
\hline $\mathrm{H}$ & -5.166600 & 7900 & -2.784900 \\
\hline $\mathrm{H}$ & -3.419600 & 2.442800 & -3.934500 \\
\hline $\mathrm{H}$ & -8.191900 & 1.065500 & -0.699200 \\
\hline $\mathrm{H}$ & -9.923500 & 2.251900 & 0.513300 \\
\hline $\mathrm{H}$ & -7.433600 & 2000 & 100 \\
\hline $\mathrm{H}$ & -5.716600 & 4.595500 & -0.644500 \\
\hline $\mathrm{H}$ & -3.078600 & -3.240100 & -3.640900 \\
\hline H & -1.997400 & -5.373300 & -4.242900 \\
\hline $\mathrm{H}$ & -0.638800 & -5.547200 & -6.307000 \\
\hline $\mathrm{H}$ & -0.408800 & 5000 & 6700 \\
\hline $\mathrm{H}$ & -1.520800 & -1.453600 & -7.240200 \\
\hline $\mathrm{H}$ & -5.262000 & -2.018400 & -3.957100 \\
\hline $\mathrm{H}$ & -7.578800 & -1.825400 & -4.763400 \\
\hline $\mathrm{H}$ & -8.081500 & -0.528600 & -6.814900 \\
\hline $\mathrm{H}$ & -6.246200 & 200 & -8.066800 \\
\hline $\mathrm{H}$ & -3.921500 & 0.399400 & -7.268500 \\
\hline $\mathrm{H}$ & -0.334300 & 5.588100 & -2.239100 \\
\hline $\mathrm{H}$ & 3.547200 & 5.903400 & -0.345900 \\
\hline $\mathrm{H}$ & 4.154100 & 3.767400 & -1.305500 \\
\hline $\mathrm{H}$ & -0.870000 & 6.673700 & -0.170000 \\
\hline $\mathrm{H}$ & -1.522900 & 8.795400 & 0.763200 \\
\hline $\mathrm{H}$ & 2.463600 & 10.331700 & 0.020600 \\
\hline $\mathrm{H}$ & 3.090200 & 8.195700 & -0.938900 \\
\hline $\mathrm{H}$ & 4.941200 & 2.479000 & -2.678800 \\
\hline $\mathrm{H}$ & 4.468700 & 0.735900 & -2.869800 \\
\hline $\mathrm{H}$ & 5.255500 & 0.341600 & -5.402800 \\
\hline $\mathrm{H}$ & 4.806900 & -1.508500 & -6.976400 \\
\hline H & 2.505800 & -1.899800 & -7.815700 \\
\hline $\mathrm{H}$ & 0.655400 & -0.423800 & -7.084800 \\
\hline $\mathrm{H}$ & 1.079200 & 1.401800 & -5.481800 \\
\hline $\mathrm{H}$ & 1.686300 & 3.851600 & -5.453100 \\
\hline $\mathrm{H}$ & 2.174400 & 6.012900 & -6.523500 \\
\hline H & 4.515400 & 6.798300 & -6.737500 \\
\hline $\mathrm{H}$ & 6.374200 & 5.400400 & -5.881200 \\
\hline $\mathrm{H}$ & 5.905200 & 3.239100 & -4.795000 \\
\hline $\mathrm{H}$ & 4.165300 & -3.922200 & 2.257500 \\
\hline & 3.380500 & -4.119900 & 0.631900 \\
\hline & 4.724300 & 2.132900 & 3.103700 \\
\hline $\mathrm{H}$ & 3.763900 & -1.220400 & 5.669500 \\
\hline & 3.560700 & -2.622300 & 3.708700 \\
\hline
\end{tabular}

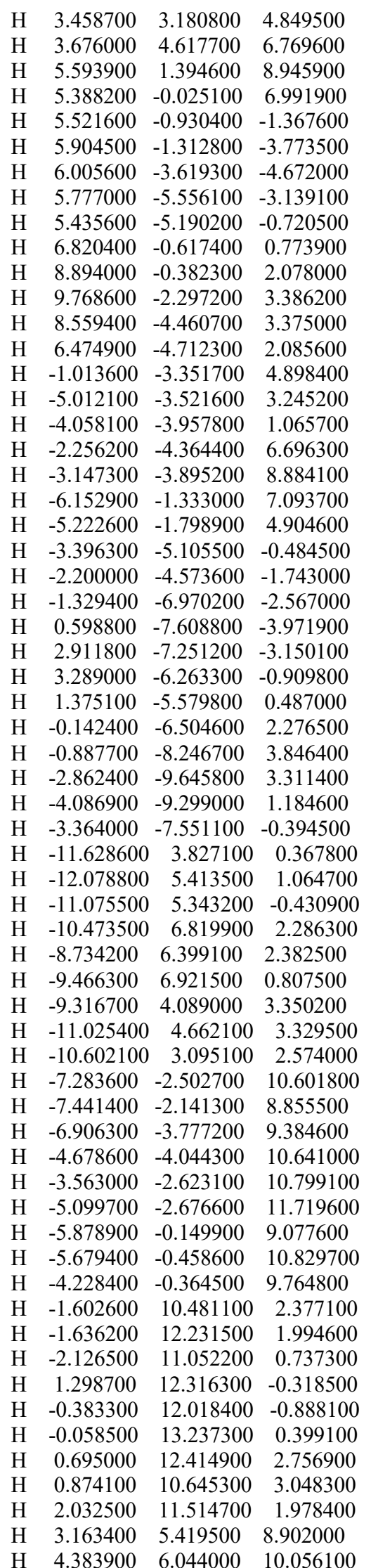

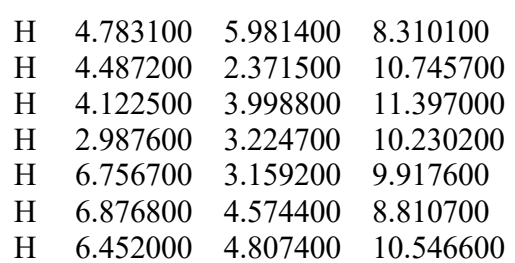

\begin{tabular}{|c|c|c|c|}
\hline G2 & & & \\
\hline $\mathrm{Rh}$ & 0.004100 & -0.001700 & -0.676000 \\
\hline $\mathrm{Rh}$ & 0.017800 & -0.006900 & -3.058000 \\
\hline $\mathrm{O}$ & -1.423300 & 1.484100 & -0.749400 \\
\hline $\mathrm{O}$ & -1.294200 & 1.586000 & -3.002600 \\
\hline $\mathrm{O}$ & 1.489200 & 1.426500 & -0.732300 \\
\hline $\mathrm{O}$ & 1.610300 & 1.305400 & -2.984900 \\
\hline $\mathrm{O}$ & 1.432200 & -1.487800 & -0.726700 \\
\hline $\mathrm{O}$ & 1.329100 & -1.599400 & -2.980800 \\
\hline $\mathrm{O}$ & -1.480200 & -1.430200 & -0.743200 \\
\hline $\mathrm{O}$ & -1.575500 & -1.318800 & -2.997600 \\
\hline $\mathrm{Cl}$ & -4.598800 & 1.007500 & -0.591300 \\
\hline $\mathrm{Cl}$ & 0.995600 & 4.602500 & -0.583800 \\
\hline $\mathrm{Cl}$ & 4.605400 & -1.010800 & -0.533500 \\
\hline $\mathrm{Cl}$ & -0.989300 & -4.605400 & -0.574500 \\
\hline $\mathrm{C}$ & -1.753900 & 1.970800 & -1.876800 \\
\hline $\mathrm{C}$ & -2.681000 & 3.171900 & -1.834800 \\
\hline $\mathrm{C}$ & -2.113000 & 4.337000 & -2.650500 \\
\hline $\mathrm{C}$ & -3.406900 & 3.716500 & -3.107500 \\
\hline $\mathrm{C}$ & -3.267200 & 3.428200 & -0.463500 \\
\hline $\mathrm{C}$ & -4.152500 & 2.535900 & 0.165600 \\
\hline $\mathrm{C}$ & -4.714600 & 2.797200 & 1.413200 \\
\hline $\mathrm{C}$ & -4.409200 & 3.978600 & 2.103600 \\
\hline $\mathrm{C}$ & -3.513500 & 4.871800 & 1.501100 \\
\hline $\mathrm{C}$ & -2.961600 & 4.592000 & 0.252500 \\
\hline $\mathrm{C}$ & -5.012400 & 4.264300 & 3.433300 \\
\hline $\mathrm{C}$ & -4.255200 & 4.870300 & 4.447000 \\
\hline $\mathrm{C}$ & -4.819700 & 5.138300 & 5.697100 \\
\hline $\mathrm{C}$ & -6.167000 & 4.808900 & 5.973900 \\
\hline $\mathrm{C}$ & -6.917000 & 4.200500 & 4.943900 \\
\hline $\mathrm{C}$ & -6.349600 & 3.932800 & 3.696300 \\
\hline $\mathrm{C}$ & -6.832300 & 5.087800 & 7.337700 \\
\hline $\mathrm{C}$ & -3.395600 & 2.909800 & -4.364800 \\
\hline $\mathrm{C}$ & -3.965200 & 1.626600 & -4.393200 \\
\hline $\mathrm{C}$ & -3.963300 & 0.881900 & -5.575600 \\
\hline $\mathrm{C}$ & -3.399700 & 1.413100 & -6.738300 \\
\hline $\mathrm{C}$ & -2.855200 & 2.700100 & -6.725200 \\
\hline $\mathrm{C}$ & -2.865700 & 3.452400 & -5.546900 \\
\hline $\mathrm{C}$ & -4.656800 & 4.518900 & -2.905700 \\
\hline $\mathrm{C}$ & -5.835600 & 3.902000 & -2.453700 \\
\hline $\mathrm{C}$ & -7.003300 & 4.649300 & -2.276700 \\
\hline $\mathrm{C}$ & -7.012600 & 6.014200 & -2.572300 \\
\hline $\mathrm{C}$ & -5.856900 & 6.630800 & -3.057600 \\
\hline $\mathrm{C}$ & -4.686600 & 5.886700 & -3.234000 \\
\hline $\mathrm{C}$ & 1.984300 & 1.762400 & -1.854500 \\
\hline $\mathrm{C}$ & 3.182100 & 2.693100 & -1.797800 \\
\hline $\mathrm{C}$ & 3.418600 & 3.279200 & -0.423000 \\
\hline $\mathrm{C}$ & 2.512600 & 4.157400 & 0.196400 \\
\hline $\mathrm{C}$ & 2.751400 & 4.715100 & 1.450400 \\
\hline $\mathrm{C}$ & 3.924400 & 4.414000 & 2.156700 \\
\hline $\mathrm{C}$ & 4.833300 & 3.528800 & 1.561900 \\
\hline $\mathrm{C}$ & 4.574500 & 2.978800 & 0.308000 \\
\hline $\mathrm{C}$ & 4.187700 & 5.011100 & 3.493800 \\
\hline $\mathrm{C}$ & 3.145900 & 5.184300 & 4.417400 \\
\hline
\end{tabular}




\begin{tabular}{|c|c|c|c|}
\hline C & 3.390600 & 5.745100 & 5.673700 \\
\hline 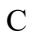 & 4.691800 & 6.153500 & 6.048300 \\
\hline $\mathrm{C}$ & 5.730400 & 5.973100 & 5.108800 \\
\hline $\mathrm{C}$ & 5.482700 & 5.410900 & 3.854500 \\
\hline $\mathrm{C}$ & 3.740800 & 3.421000 & -3.063000 \\
\hline $\mathrm{C}$ & 4.359000 & 2.128500 & -2.598800 \\
\hline $\mathrm{C}$ & 2.950600 & 3.408300 & -4.330700 \\
\hline C & 3.511200 & 2.883300 & -5.506600 \\
\hline $\mathrm{C}$ & 2.774200 & 2.870700 & -6.694400 \\
\hline $\mathrm{C}$ & 1.484600 & 3.408500 & -6.723300 \\
\hline $\mathrm{C}$ & 0.935500 & 3.967500 & -5.566700 \\
\hline $\mathrm{C}$ & 1.665200 & 3.971900 & -4.375000 \\
\hline $\mathrm{C}$ & 4.537300 & 4.673000 & -2.850200 \\
\hline $\mathrm{C}$ & 3.911400 & 5.849800 & -2.405700 \\
\hline $\mathrm{C}$ & 4.653300 & 7.019500 & -2.218600 \\
\hline $\mathrm{C}$ & 6.021900 & 7.032500 & -2.496400 \\
\hline $\mathrm{C}$ & 6.647800 & 5.878700 & -2.974200 \\
\hline $\mathrm{C}$ & 5.909200 & 4.706500 & -3.160800 \\
\hline $\mathrm{C}$ & 1.775700 & -1.979400 & -1.848100 \\
\hline $\mathrm{C}$ & 2.702100 & -3.180400 & -1.790300 \\
\hline $\mathrm{C}$ & 2.143600 & -4.348800 & -2.607700 \\
\hline $\mathrm{C}$ & 3.442700 & -3.730300 & -3.052200 \\
\hline $\mathrm{C}$ & 3.272300 & -3.430900 & -0.411300 \\
\hline $\mathrm{C}$ & 4.150300 & -2.536100 & 0.224500 \\
\hline $\mathrm{C}$ & 4.697800 & -2.792200 & 1.479600 \\
\hline $\mathrm{C}$ & 4.384300 & -3.970700 & 2.171300 \\
\hline $\mathrm{C}$ & 3.495700 & -4.866500 & 1.562100 \\
\hline $\mathrm{C}$ & 2.958400 & -4.591800 & 0.305900 \\
\hline $\mathrm{C}$ & 4.971900 & -4.251000 & 3.509000 \\
\hline $\mathrm{C}$ & 6.306000 & -3.918300 & 3.786300 \\
\hline $\mathrm{C}$ & 6.858900 & -4.181000 & 5.041400 \\
\hline $\mathrm{C}$ & 6.096900 & -4.785100 & 6.065100 \\
\hline $\mathrm{C}$ & 4.752900 & -5.115700 & 5.774100 \\
\hline $\mathrm{C}$ & 4.203000 & -4.852700 & 4.516400 \\
\hline $\mathrm{C}$ & 6.746200 & -5.058500 & 7.437700 \\
\hline $\mathrm{C}$ & 3.446100 & -2.928800 & -4.312900 \\
\hline $\mathrm{C}$ & 4.016200 & -1.645800 & -4.340100 \\
\hline $\mathrm{C}$ & 4.028200 & -0.906000 & -5.525400 \\
\hline $\mathrm{C}$ & 3.478100 & -1.442100 & -6.692400 \\
\hline $\mathrm{C}$ & 2.933100 & -2.728800 & -6.680200 \\
\hline $\mathrm{C}$ & 2.929800 & -3.476300 & -5.498800 \\
\hline $\mathrm{C}$ & 4.690100 & -4.531900 & -2.832600 \\
\hline $\mathrm{C}$ & 5.863600 & -3.913200 & -2.369400 \\
\hline $\mathrm{C}$ & 7.029100 & -4.659800 & -2.175700 \\
\hline $\mathrm{C}$ & 7.041700 & -6.025900 & -2.465600 \\
\hline $\mathrm{C}$ & 5.891800 & -6.644400 & -2.961800 \\
\hline $\mathrm{C}$ & 4.723700 & -5.901100 & -3.154900 \\
\hline $\mathrm{C}$ & -1.962500 & -1.770900 & -1.869500 \\
\hline $\mathrm{C}$ & -3.161100 & -2.701100 & -1.822600 \\
\hline $\mathrm{C}$ & -3.413700 & -3.280900 & -0.447900 \\
\hline $\mathrm{C}$ & -2.515200 & -4.156300 & 0.186000 \\
\hline $\mathrm{C}$ & -2.768500 & -4.708000 & 1.439900 \\
\hline $\mathrm{C}$ & -3.949400 & -4.403200 & 2.131200 \\
\hline $\mathrm{C}$ & -4.851300 & -3.520800 & 1.521700 \\
\hline $\mathrm{C}$ & -4.577900 & -2.976800 & 0.268200 \\
\hline $\mathrm{C}$ & -4.228200 & -4.993600 & 3.468100 \\
\hline $\mathrm{C}$ & -5.527300 & -5.391400 & 3.816000 \\
\hline $\mathrm{C}$ & -5.789400 & -5.947100 & 5.070200 \\
\hline $\mathrm{C}$ & -4.761600 & -6.122900 & 6.022400 \\
\hline $\mathrm{C}$ & -3.456200 & -5.716700 & 5.660700 \\
\hline $\mathrm{C}$ & -3.197100 & -5.162400 & 4.404500 \\
\hline & -5.093600 & -6.739400 & 7.397300 \\
\hline
\end{tabular}

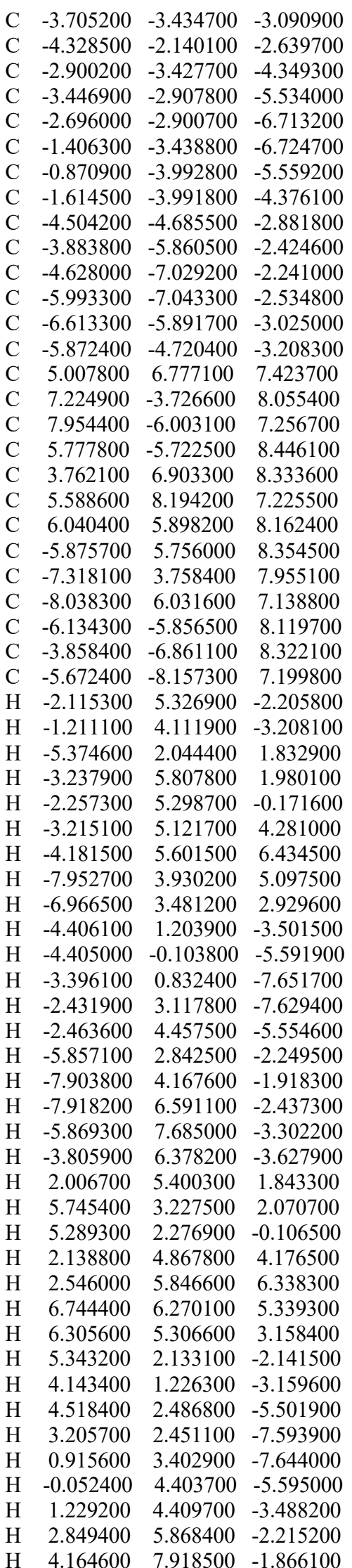

H $\quad 6.594500 \quad 7.939600 \quad-2.353600$

$\mathrm{H} \quad 7.705100 \quad 5.894000 \quad-3.205000$

$\mathrm{H} \quad 6.408000 \quad 3.827300 \quad-3.548700$

$\mathrm{H} \quad 2.140700 \quad-5.336900 \quad-2.158900$

H $\quad 1.248300 \quad-4.126000 \quad-3.176600$

$\mathrm{H} \quad 5.352800 \quad-2.037600 \quad 1.903900$

$\mathrm{H} \quad 3.214500 \quad-5.800500 \quad 2.041700$

$\mathrm{H} \quad 2.259100 \quad-5.300400 \quad-0.123400$

H $\quad 6.931900 \quad-3.469900 \quad 3.024900$

H $\quad 7.892700 \quad-3.910000 \quad 5.206000$

$\mathrm{H} \quad 4.106100 \quad-5.575800 \quad 6.505800$

$\mathrm{H} \quad 3.164900 \quad-5.104900 \quad 4.339300$

$\mathrm{H} \quad 4.446800 \quad-1.219500 \quad-3.445000$

H $\quad 4.470300 \quad 0.079400 \quad-5.540800$

H $\quad 3.485100 \quad-0.865100 \quad-7.608200$

$\mathrm{H} \quad 2.520300 \quad-3.150300 \quad-7.587600$

$\mathrm{H} \quad 2.527700 \quad-4.481300 \quad-5.507000$

$\mathrm{H} \quad 5.882900 \quad-2.852900 \quad-2.169300$

H $\quad 7.925400 \quad-4.176700 \quad-1.808700$

H $\quad 7.945700 \quad-6.602300 \quad-2.317700$

H $\quad 5.906900 \quad-7.699700 \quad-3.201900$

H $\quad 3.847500 \quad-6.394100 \quad-3.557000$

$\mathrm{H} \quad-2.028500 \quad-5.391300 \quad 1.844600$

$\mathrm{H} \quad-5.769100 \quad-3.216900 \quad 2.018500$

H $\quad-5.287700 \quad-2.276800 \quad-0.157900$

$\mathrm{H} \quad-6.342100 \quad-5.290500 \quad 3.110000$

$\mathrm{H} \quad-6.806100 \quad-6.242700 \quad 5.290700$

H $\quad-2.619300 \quad-5.814900 \quad 6.335400$

$\mathrm{H} \quad-2.187100 \quad-4.847200 \quad 4.173500$

$\mathrm{H} \quad-5.317900 \quad-2.142500 \quad-2.193900$

$\mathrm{H} \quad-4.106300 \quad-1.240300 \quad-3.202000$

$\mathrm{H} \quad-4.454000 \quad-2.511100 \quad-5.542800$

$\mathrm{H} \quad-3.117000 \quad-2.484900 \quad-7.619500$

$\mathrm{H} \quad-0.826700 \quad-3.437500 \quad-7.638700$

$\mathrm{H} \quad 0.117100 \quad-4.429400 \quad-5.574100$

$\mathrm{H} \quad-1.188900 \quad-4.425600 \quad-3.482400$

$\mathrm{H} \quad-2.824100 \quad-5.878300 \quad-2.221600$

$\mathrm{H} \quad-4.143600 \quad-7.926600 \quad-1.878700$

$\mathrm{H} \quad-6.567700 \quad-7.949700 \quad-2.394600$

$\mathrm{H} \quad-7.667900 \quad-5.907900 \quad-3.268200$

$\mathrm{H} \quad-6.366600 \quad-3.842900 \quad-3.606000$

H $\quad 7.658500 \quad-3.893400 \quad 9.065400$

H $\quad 8.008800 \quad-3.246100 \quad 7.433400$

H $\quad 6.373900 \quad-3.018200 \quad 8.153000$

H $\quad 8.744300 \quad-5.540700 \quad 6.628600$

H $\quad 8.413100 \quad-6.249500 \quad 8.238900$

H $\quad 7.634200 \quad-6.951300 \quad 6.772800$

H $\quad 4.901000 \quad-5.068500 \quad 8.642900$

H $\quad 5.425400 \quad-6.706600 \quad 8.067800$

H $\quad 6.284400 \quad-5.902200 \quad 9.419400$

$\begin{array}{llll}\mathrm{H} & 3.319500 & 5.904800 & 8.540300\end{array}$

$\mathrm{H} \quad 4.032800 \quad 7.356200 \quad 9.312300$

$\mathrm{H} \quad 2.994100 \quad 7.556500 \quad 7.865700$

$\begin{array}{llll}\mathrm{H} & 6.549400 & 8.168000 & 6.670300\end{array}$

H $\quad 4.874100 \quad 8.828400 \quad 6.657300$

H $\quad 5.784600 \quad 8.680700 \quad 8.205700$

$\begin{array}{llll}\mathrm{H} & 6.252300 & 6.305100 & 9.175000\end{array}$

$\mathrm{H} \quad 5.654700 \quad 4.861900 \quad 8.275900$

H $\quad 7.004800 \quad 5.853000 \quad 7.614700$

$\mathrm{H} \quad-6.393500 \quad 5.939500 \quad 9.321200$

$\mathrm{H} \quad-5.001200 \quad 5.102700 \quad 8.564300$

H $\quad-5.518900 \quad 6.738500 \quad 7.976400$ 


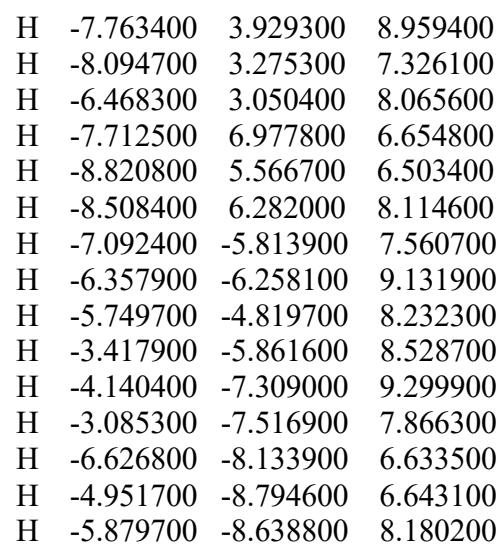

\section{G3}

$\begin{array}{llll}\mathrm{Rh} & -0.060800 & -0.540000 & -0.424700\end{array}$

$\begin{array}{llll}\mathrm{Rh} & -0.268900 & -2.398400 & -1.900900\end{array}$

$\begin{array}{llll}\mathrm{O} & -0.803900 & 0.659200 & -1.927300\end{array}$

$\begin{array}{lllll}\mathrm{O} & -0.874400 & -1.078900 & -3.368700\end{array}$

$\begin{array}{llll}\text { O } & 1.848600 & -0.258100 & -1.147300\end{array}$

$\begin{array}{llll}\text { O } & 1.699400 & -2.099400 & -2.447900\end{array}$

$\begin{array}{llll}\mathrm{O} & 0.671700 & -1.834200 & 1.002000\end{array}$

$\begin{array}{llll}\mathrm{O} & 0.348800 & -3.614400 & -0.350800\end{array}$

$\begin{array}{llll}\mathrm{O} & -1.980700 & -0.917600 & 0.222700\end{array}$

$\begin{array}{llll}\mathrm{O} & -2.226000 & -2.593500 & -1.272100\end{array}$

$\begin{array}{llll}\mathrm{Cl} & -3.894700 & 1.491900 & -2.245800\end{array}$

$\begin{array}{llll}\text { Cl } & 2.635100 & 1.773000 & -3.513600\end{array}$

$\begin{array}{llll}\text { Cl } & 3.794500 & -2.380900 & 1.548400\end{array}$

$\begin{array}{lrrr}\text { Cl } & -2.734500 & -2.659600 & 2.821100\end{array}$

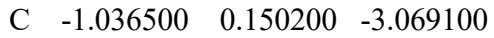

$\begin{array}{llll}\text { C } & -1.426700 & 1.127600 & -4.162900\end{array}$

$\begin{array}{llll}\text { C } & -0.536800 & 0.958300 & -5.397600\end{array}$

$\begin{array}{llll}\text { C } & -2.006800 & 0.661400 & -5.537300\end{array}$

$\begin{array}{llll}\text { C } & -1.737500 & 2.513900 & -3.642100\end{array}$

$\begin{array}{lrrr}\text { C } & -2.827700 & 2.782700 & -2.796700\end{array}$

$\begin{array}{llll}\text { C } & -3.122300 & 4.066300 & -2.342600\end{array}$

$\begin{array}{llll}\text { C } & -2.326800 & 5.158500 & -2.716200\end{array}$

$\begin{array}{llll}\text { C } & -1.227400 & 4.908600 & -3.548400\end{array}$

$\begin{array}{llll}\text { C } & -0.947300 & 3.616600 & -3.989000\end{array}$

$\begin{array}{llll}\text { C } & -2.638600 & 6.532300 & -2.237700\end{array}$

$\begin{array}{llll}\text { C } & -3.100100 & 6.746900 & -0.930100\end{array}$

$\begin{array}{llll}\text { C } & -3.391700 & 8.036700 & -0.478000\end{array}$

$\begin{array}{llll}\text { C } & -3.231200 & 9.158700 & -1.323600\end{array}$

$\begin{array}{llll}\text { C } & -2.766600 & 8.927600 & -2.636800\end{array}$

$\begin{array}{llll}\text { C } & -2.474800 & 7.637500 & -3.085200\end{array}$

C $\quad-3.541100 \quad 10.599600 \quad-0.867400$

$\begin{array}{llll}\text { C } & -2.424100 & -0.761700 & -5.717300\end{array}$

C $\quad-3.443000 \quad-1.311600 \quad-4.922600$

$\begin{array}{llll}\text { C } & -3.837700 & -2.639800 & -5.104100\end{array}$

$\begin{array}{llll}\text { C } & -3.224800 & -3.426300 & -6.082700\end{array}$

$\begin{array}{llll}\text { C } & -2.229100 & -2.878400 & -6.895900\end{array}$

$\begin{array}{llll}\text { C } & -1.839600 & -1.546700 & -6.724600\end{array}$

$\begin{array}{llll}\text { C } & -2.829700 & 1.644200 & -6.314300\end{array}$

$\begin{array}{llll}\text { C } & -4.104700 & 2.023900 & -5.861700\end{array}$

$\begin{array}{llll}\text { C } & -4.874700 & 2.932100 & -6.593600\end{array}$

$\begin{array}{llll}\text { C } & -4.389900 & 3.451700 & -7.796000\end{array}$

$\begin{array}{lllll}\text { C } & -3.139600 & 3.053300 & -8.274400\end{array}$

C $\quad-2.366100 \quad 2.146200 \quad-7.544200$

$\begin{array}{lrrr}\text { C } & 2.325900 & -1.091500 & -1.980700\end{array}$

$\begin{array}{llll}\text { C } & 3.789800 & -0.906900 & -2.337400\end{array}$
C $\quad 4.363700 \quad 0.403700 \quad-1.845100$

$\begin{array}{llll}\text { C } & 3.929200 & 1.652100 & -2.322600\end{array}$

C $\quad 4.481100 \quad 2.853400 \quad-1.883100$

$\begin{array}{llll}\text { C } & 5.507800 & 2.863100 & -0.928600\end{array}$

$\begin{array}{llll}\text { C } & 5.945400 & 1.628800 & -0.430500\end{array}$

$\begin{array}{llll}\text { C } & 5.378000 & 0.438100 & -0.880300\end{array}$

$\begin{array}{llll}\text { C } & 6.103900 & 4.142600 & -0.458300\end{array}$

$\begin{array}{llll}\text { C } & 5.300300 & 5.275000 & -0.256400\end{array}$

$\begin{array}{llll}\text { C } & 5.858100 & 6.477200 & 0.186900\end{array}$

$\begin{array}{llll}\text { C } & 7.244700 & 6.586400 & 0.442700\end{array}$

C $\quad 8.041000 \quad 5.439000 \quad 0.234900$

$\begin{array}{llll}\text { C } & 7.480300 & 4.238600 & -0.206800\end{array}$

C $\quad 4.463000 \quad-1.654500 \quad-3.533500$

C $\quad 4.574600 \quad-2.206400 \quad-2.136800$

$\begin{array}{llll}\text { C } & 3.611800 & -2.390700 & -4.516000\end{array}$

C $\quad 3.806600 \quad-3.765400 \quad-4.727900$

C $\quad 3.012400 \quad-4.456600 \quad-5.647700$

$\begin{array}{llll}\text { C } & 2.039000 & -3.774800 & -6.382900\end{array}$

C $\quad 1.866200 \quad-2.399900 \quad-6.205500$

C $\quad 2.650400 \quad-1.708000 \quad-5.278600$

$\begin{array}{llll}\text { C } & 5.697900 & -1.046800 & -4.127100\end{array}$

C $\quad 5.623000 \quad 0.146500 \quad-4.865300$

$\begin{array}{llll}\text { C } & 6.774300 & 0.705400 & -5.427100\end{array}$

$\begin{array}{llll}\text { C } & 8.007300 & 0.066600 & -5.277700\end{array}$

C $\quad 8.088000 \quad-1.139200 \quad-4.577300$

$\begin{array}{llll}\text { C } & 6.938600 & -1.700400 & -4.012900\end{array}$

$\begin{array}{llll}\text { C } & 0.708100 & -3.080000 & 0.750100\end{array}$

$\begin{array}{llll}\text { C } & 1.107500 & -3.978100 & 1.906900\end{array}$

$\begin{array}{llll}\text { C } & 0.075000 & -5.087700 & 2.126300\end{array}$

$\begin{array}{llll}\text { C } & 1.467200 & -5.486700 & 1.713200\end{array}$

C $\quad 1.657600 \quad-3.215600 \quad 3.092400$

$\begin{array}{llll}\text { C } & 2.858800 & -2.487600 & 3.038800\end{array}$

C $\quad 3.371000 \quad-1.809000 \quad 4.142300$

C $\quad 2.695000 \quad-1.825900 \quad 5.370400$

C $\quad 1.489300 \quad-2.536100 \quad 5.440600$

C $\quad 0.991100 \quad-3.204400 \quad 4.323900$

C $\quad 3.239200 \quad-1.106900 \quad 6.553700$

$\begin{array}{llll}\text { C } & 3.861700 & 0.141700 & 6.408000\end{array}$

$\begin{array}{llll}\text { C } & 4.369900 & 0.817400 & 7.519500\end{array}$

$\begin{array}{llll}\text { C } & 4.274800 & 0.268100 & 8.816800\end{array}$

$\begin{array}{llll}\text { C } & 3.645900 & -0.991400 & 8.951000\end{array}$

$\begin{array}{llll}\text { C } & 3.137100 & -1.664400 & 7.836900\end{array}$

$\begin{array}{llll}\text { C } & 4.848800 & 1.047600 & 10.018300\end{array}$

$\begin{array}{llll}\text { C } & 1.665500 & -6.026500 & 0.334500\end{array}$

$\begin{array}{llll}\text { C } & 2.680100 & -5.516500 & -0.491600\end{array}$

$\begin{array}{llll}\text { C } & 2.869000 & -6.034300 & -1.775800\end{array}$

C $\quad 2.052700 \quad-7.067500 \quad-2.242600$

C $\quad 1.058700 \quad-7.599100 \quad-1.416800$

C $\quad 0.874300 \quad-7.091300 \quad-0.127300$

$\begin{array}{llll}\text { C } & 2.327500 & -6.135900 & 2.754900\end{array}$

$\begin{array}{llll}\text { C } & 3.683600 & -5.789000 & 2.879200\end{array}$

C $\quad 4.486500 \quad-6.403600 \quad 3.844200$

C $\quad 3.950100 \quad-7.387200 \quad 4.678100$

$\begin{array}{llll}\text { C } & 2.613000 & -7.766500 & 4.539100\end{array}$

C $\quad \begin{array}{llll}\text { C } & 1.806600 & -7.152700 & 3.576000\end{array}$

$\begin{array}{llll}\text { C } & -2.654600 & -1.838900 & -0.337700\end{array}$

C $\quad-4.109400 \quad-1.944800 \quad 0.082700$

C $\quad-4.442200 \quad-1.107000 \quad 1.297900$

$\begin{array}{llll}\text { C } & -3.896300 & -1.357800 & 2.568500\end{array}$

C $\quad-4.228700 \quad-0.597900 \quad 3.687700$

C $\quad-5.134800 \quad 0.467300 \quad 3.586900$

$\begin{array}{llll}\text { C } & -5.679300 & 0.741700 & 2.325300\end{array}$ $\begin{array}{lrrr}\text { C } & -5.331300 & -0.028200 & 1.217100\end{array}$

$\begin{array}{llll}\text { C } & -5.497000 & 1.279900 & 4.779300\end{array}$

C $\quad-6.812100 \quad 1.731500 \quad 4.962300$

$\begin{array}{llll}\text { C } & -7.153200 & 2.494800 & 6.081000\end{array}$

$\begin{array}{llll}\text { C } & -6.190500 & 2.835300 & 7.056300\end{array}$

$\begin{array}{llll}\text { C } & -4.867700 & 2.375100 & 6.860400\end{array}$

$\begin{array}{llll}\text { C } & -4.529900 & 1.610400 & 5.740700\end{array}$

$\begin{array}{llll}\text { C } & -6.608500 & 3.681200 & 8.277100\end{array}$

C $\quad-5.003300 \quad-3.171600 \quad-0.289400$

C $\quad-5.038400 \quad-1.925600 \quad-1.134500$

C $\quad-4.371700 \quad-4.396400 \quad-0.866100$

$\begin{array}{llll}\text { C } & -4.774400 & -4.872400 & -2.124800\end{array}$

$\begin{array}{llll}\text { C } & -4.185800 & -6.019000 & -2.666400\end{array}$

C $\quad-3.213000 \quad-6.714400 \quad-1.943000$

$\begin{array}{llll}\text { C } & -2.835500 & -6.268600 & -0.673900\end{array}$

C $\quad-3.413500 \quad-5.116400 \quad-0.134300$

C $\quad-6.200300 \quad-3.446200 \quad 0.570000$

C $\quad-6.043100 \quad-3.912500 \quad 1.886200$

$\begin{array}{llll}\text { C } & -7.160900 & -4.178300 & 2.681900\end{array}$

$\begin{array}{llll}\text { C } & -8.446200 & -4.004100 & 2.164100\end{array}$

C $\quad-8.615100 \quad-3.576400 \quad 0.845200$

$\begin{array}{lrrr}\text { C } & -7.499200 & -3.308300 & 0.046900\end{array}$

$\begin{array}{llll}\text { C } & 7.903500 & 7.893000 & 0.932000\end{array}$

$\begin{array}{llll}\text { C } & 8.964900 & 8.349400 & -0.092400\end{array}$

$\begin{array}{llll}\text { C } & 8.581000 & 7.647700 & 2.297900\end{array}$

$\begin{array}{llll}\text { C } & 6.893700 & 9.052400 & 1.110200\end{array}$

$\begin{array}{llll}\text { C } & -2.263200 & 11.460000 & -0.975600\end{array}$

$\begin{array}{llll}\text { C } & -4.641300 & 11.200000 & -1.769400\end{array}$

$\begin{array}{llll}\text { C } & -4.038300 & 10.679400 & 0.596200\end{array}$

$\begin{array}{llll}\text { C } & 6.362300 & 1.272500 & 9.810500\end{array}$

$\begin{array}{llll}\text { C } & 4.138200 & 2.413800 & 10.132600\end{array}$

$\begin{array}{llll}\text { C } & 4.664500 & 0.308200 & 11.365500\end{array}$

$\begin{array}{lrrr}\text { C } & -5.437200 & 3.966400 & 9.247700\end{array}$

$\begin{array}{llll}\text { C } & -7.160500 & 5.040900 & 7.796500\end{array}$

$\begin{array}{llll}\text { C } & -7.703100 & 2.934700 & 9.070200\end{array}$

$\mathrm{H} \quad-0.118700 \quad 1.847700 \quad-5.857800$

H $\quad 0.153500 \quad 0.123800 \quad-5.356400$

$\mathrm{H} \quad-3.996100 \quad 4.184700 \quad-1.709600$

$\mathrm{H} \quad-0.551500 \quad 5.705500 \quad-3.847700$

$\mathrm{H} \quad-0.071800 \quad 3.460500 \quad-4.609200$

$\mathrm{H} \quad-3.218800 \quad 5.916600 \quad-0.245200$

$\mathrm{H} \quad-3.738200 \quad 8.132800 \quad 0.540000$

$\mathrm{H} \quad-2.628300 \quad 9.747300 \quad-3.328400$

$\mathrm{H} \quad-2.137100 \quad 7.504300 \quad-4.105400$

$\mathrm{H} \quad-3.927100 \quad-0.714000 \quad-4.163200$

$\mathrm{H} \quad-4.623700 \quad-3.056800 \quad-4.491300$

$\mathrm{H} \quad-3.529700 \quad-4.455900 \quad-6.218400$

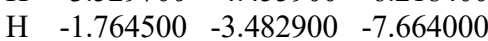

H $\quad-1.085400 \quad-1.125700 \quad-7.376900$

$\mathrm{H} \quad-4.509700 \quad 1.610400 \quad-4.950900$

$\mathrm{H} \quad-5.851500 \quad 3.226800 \quad-6.232300$

$\mathrm{H} \quad-4.988200 \quad 4.153100 \quad-8.362900$

$\mathrm{H} \quad-2.771400 \quad 3.443500 \quad-9.214400$

$\mathrm{H} \quad-1.407200 \quad 1.835500 \quad-7.939700$

H $\quad 4.098500 \quad 3.769700 \quad-2.321500$

$\mathrm{H} \quad 6.714300 \quad 1.567800 \quad 0.335400$

$\begin{array}{llll}\mathrm{H} & 5.724900 & -0.494800 & -0.450400\end{array}$

H $\quad 4.231400 \quad 5.228000 \quad-0.423000$

$\begin{array}{llll}\mathrm{H} & 5.183800 & 7.308700 & 0.326400\end{array}$

H $\quad 9.107600 \quad 5.465100 \quad 0.411000$

H $\quad 8.130700 \quad 3.388300 \quad-0.369300$

H $\quad 5.522900 \quad-2.160300 \quad-1.611000$ 


\begin{tabular}{|c|c|c|c|}
\hline & 3.974400 & -3.070900 & \\
\hline & 4.574300 & -4.298900 & -4.182400 \\
\hline & 3.157000 & -5.518900 & -5.795600 \\
\hline & 1.427500 & -4.309000 & -7.098600 \\
\hline & 1.129600 & -1.868900 & -6.791000 \\
\hline & 2.507200 & -0.644100 & -5.152900 \\
\hline & 4.675100 & 0.639200 & -5.017800 \\
\hline & 6.708900 & 1.630300 & -5.985500 \\
\hline & 8.897700 & 0.499300 & -5.715200 \\
\hline & 9.040800 & -1.642300 & -4.475900 \\
\hline & 7.017800 & -2.644400 & -3.488800 \\
\hline & -0.265600 & -5.282300 & 3.138100 \\
\hline & -0.713700 & -5.135500 & 1.384500 \\
\hline & 4.315800 & -1.289600 & 4.016500 \\
\hline & 0.899000 & -2.560200 & 6.353000 \\
\hline & 0.040700 & -3.718900 & 4.410500 \\
\hline & 3.940000 & 0.60 & 200 \\
\hline & 4.837100 & 1.779200 & 57400 \\
\hline & 3.538600 & -1.477700 & 9.908900 \\
\hline & 2.677800 & -2.634600 & 7.979700 \\
\hline & 3.320100 & -4.71 & -0.142800 \\
\hline & 3.65 & -5.6 & 06000 \\
\hline & 2.198700 & -7.464000 & -3.239100 \\
\hline & 0.435900 & -8.409700 & -1.772500 \\
\hline & 0.118200 & -7.527700 & 0.512400 \\
\hline & 4.124400 & -5.05 & 24100 \\
\hline & 5.527400 & -6.12 & 9600 \\
\hline & 4.573500 & -7.86 & 5.423300 \\
\hline & 2.202100 & -8.540300 & 5.174400 \\
\hline & 0.777600 & -7.472500 & 3.471100 \\
\hline & -3.77 & -0.8 & 3800 \\
\hline$\pi$ & -6.361 & 1.5 & 72100 \\
\hline H & -5.752700 & 0.23 & 0.253000 \\
\hline $\mathrm{H}$ & -7.585700 & 1.479200 & 4.248000 \\
\hline & -8.180900 & 2.816300 & 6.180400 \\
\hline & -4.075500 & 2.59 & 7.559300 \\
\hline $\mathrm{H}$ & -3.501800 & 1.293200 & 8500 \\
\hline H & -5.909200 & -1.278500 & -1.108200 \\
\hline $\mathrm{H}$ & -4.537700 & -1.943400 & -2.095700 \\
\hline$H$ & -5.544700 & -4.356300 & 3200 \\
\hline$\Pi$ & -4.489500 & -6.37 & -3.643200 \\
\hline $\mathrm{H}$ & -2.760900 & -7.604900 & -2.360300 \\
\hline $\mathrm{H}$ & -2.100500 & -6.819700 & -0.105400 \\
\hline $\mathrm{H}$ & -3.113300 & -4.784000 & 0.849300 \\
\hline $\mathrm{H}$ & -5.058700 & -4.082700 & 2.294300 \\
\hline $\mathrm{H}$ & -7.030200 & -4.527500 & 3.698000 \\
\hline $\mathrm{H}$ & -9.311100 & -4.212500 & 2.780600 \\
\hline $\mathrm{H}$ & -9.611200 & -3.457400 & 0.439100 \\
\hline $\mathrm{H}$ & -7.649700 & -2.994800 & -0.978400 \\
\hline & 9.423800 & 9.31 & 0.217900 \\
\hline $\mathrm{H}$ & 9.785200 & 7.607800 & -0.189100 \\
\hline $\mathrm{H}$ & 8.501200 & 8.489400 & -1.092800 \\
\hline $\mathrm{H}$ & 9.026600 & 8.587000 & 2.691500 \\
\hline 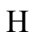 & 7.837600 & 7.277300 & 3.036800 \\
\hline $\mathrm{H}$ & 9.397800 & 6.900200 & 2.220500 \\
\hline $\mathrm{H}$ & 6.397200 & 9.297100 & 0.146200 \\
\hline $\mathrm{H}$ & 6.121400 & 8.792700 & 1.866400 \\
\hline П & 7.408200 & 9.972800 & 1.462700 \\
\hline & -1.910500 & 11.537400 & -2.025300 \\
\hline & -2.451300 & 12.493200 & -0.611300 \\
\hline & -1.446700 & 11.017300 & -0.364900 \\
\hline & -4.912300 & 12.224200 & -1.432600 \\
\hline
\end{tabular}

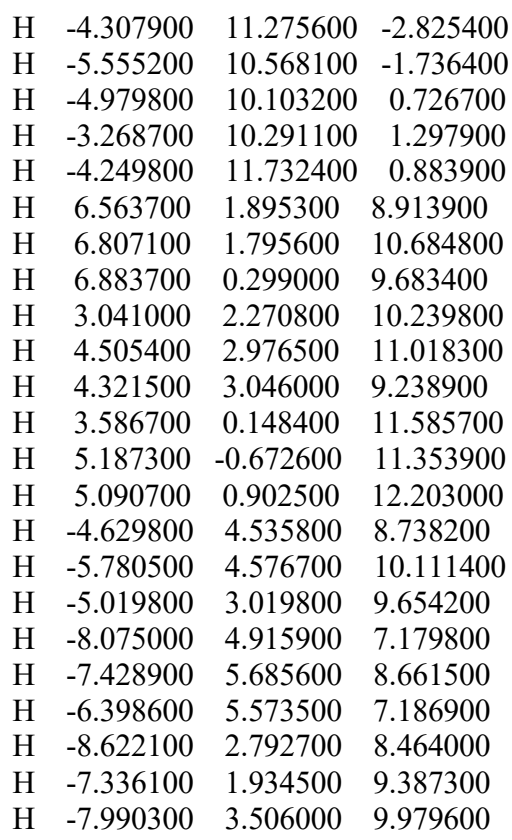

\section{G4}

Rh $\quad-0.102400 \quad-0.391800 \quad-0.556400$

$\begin{array}{llll}\mathrm{Rh} & -0.461700 & -1.751500 & -2.479500\end{array}$

$\begin{array}{llll}\text { O } & -0.203200 & -2.099200 & 0.594100\end{array}$

$\begin{array}{llll}\mathrm{O} & -0.683800 & -3.366700 & -1.213300\end{array}$

$\begin{array}{llll}\text { O } & -2.146400 & -0.172800 & -0.406000\end{array}$

$\begin{array}{llll}\text { O } & -2.478400 & -1.343500 & -2.309300\end{array}$

$\begin{array}{llll}\mathrm{O} & -0.019800 & 1.245200 & -1.805800\end{array}$

$\begin{array}{llll}\mathrm{O} & -0.220000 & -0.060000 & -3.638800\end{array}$

O $\quad 1.922800 \quad-0.679600 \quad-0.806200$

$\begin{array}{llll}\mathrm{O} & 1.575600 & -2.083500 & -2.541700\end{array}$

$\begin{array}{llll}\mathrm{Cl} & 2.340600 & -3.819700 & 1.555300\end{array}$

$\mathrm{Cl} \quad-4.055200 \quad-1.981500 \quad 1.447500$

$\mathrm{Cl} \quad-2.498200 \quad 3.182400 \quad-2.474200$

Cl $\quad 3.881600 \quad 1.330200 \quad-2.380800$

$\begin{array}{llll}\text { C } & -0.483700 & -3.207000 & 0.036100\end{array}$

$\begin{array}{llll}\text { C } & -0.699400 & -4.383500 & 0.970700\end{array}$

$\begin{array}{llll}\text { C } & -2.039900 & -5.070100 & 0.693900\end{array}$

C $\quad-0.778100 \quad-5.858300 \quad 0.459600$

$\begin{array}{llll}\text { C } & -0.279200 & -4.098300 & 2.396000\end{array}$

$\begin{array}{lrrr}\text { C } & 1.055600 & -3.853700 & 2.761900\end{array}$

C $\quad 1.437300 \quad-3.617300 \quad 4.080800$

C $\quad 0.487000 \quad-3.611400 \quad 5.111400$

C $\quad-0.851200 \quad-3.839900 \quad 4.764400$

C $\quad-1.213200 \quad-4.071200 \quad 3.438900$

$\begin{array}{lrrr}\text { C } & 0.888900 & -3.363200 & 6.522300\end{array}$

$\begin{array}{llll}\text { C } & 1.880500 & -2.418900 & 6.828400\end{array}$

$\begin{array}{llll}\text { C } & 2.257400 & -2.182600 & 8.153100\end{array}$

$\begin{array}{llll}\text { C } & 1.652500 & -2.887400 & 9.219500\end{array}$

C $\quad 0.656500 \quad-3.834800 \quad 8.896600$

$\begin{array}{llll}\text { C } & 0.281100 & -4.067700 & 7.571600\end{array}$

$\begin{array}{llll}\text { C } & 2.037000 & -2.659300 & 10.696100\end{array}$

C $\quad-0.395300 \quad-6.172000 \quad-0.950000$

C $\quad 0.906700 \quad-5.915000 \quad-1.409100$

$\begin{array}{llll}\text { C } & 1.262300 & -6.222000 & -2.725200\end{array}$

C $\quad 0.325400 \quad-6.793100 \quad-3.589900$

C $\quad-0.964200 \quad-7.077400 \quad-3.132600$

C $\quad-1.320000 \quad-6.781800 \quad-1.813400$
C $\quad-0.465600 \quad-6.951100 \quad 1.436800$

$\begin{array}{llll}\text { C } & 0.847600 & -7.143000 & 1.898300\end{array}$

$\begin{array}{llll}\text { C } & 1.138300 & -8.169600 & 2.801100\end{array}$

C $\quad 0.126100 \quad-9.029300 \quad 3.233500$

C $\quad-1.176000 \quad-8.871300 \quad 2.753100$

C $\quad-1.470500 \quad-7.844600 \quad 1.851100$

$\begin{array}{llll}\text { C } & -2.892000 & -0.672100 & -1.306900\end{array}$

C $\quad-4.367300 \quad-0.328300 \quad-1.211500$

$\begin{array}{llll}\text { C } & -4.743800 & 0.331400 & 0.097200\end{array}$

C $\quad-4.659500 \quad-0.329100 \quad 1.334900$

$\begin{array}{llll}\text { C } & -5.034700 & 0.276500 & 2.532100\end{array}$

$\begin{array}{llll}\text { C } & -5.516900 & 1.592800 & 2.548700\end{array}$

$\begin{array}{lrrr}\text { C } & -5.595100 & 2.272900 & 1.326100\end{array}$

C $\quad-5.211600 \quad 1.650600 \quad 0.139600$

$\begin{array}{llll}\text { C } & -5.923300 & 2.243500 & 3.823500\end{array}$

C $\quad-5.196100 \quad 2.023200 \quad 5.003000$

$\begin{array}{llll}\text { C } & -5.575000 & 2.635100 & 6.200900\end{array}$

$\begin{array}{llll}\text { C } & -6.699800 & 3.490400 & 6.258100\end{array}$

$\begin{array}{llll}\text { C } & -7.422100 & 3.702800 & 5.063600\end{array}$

C $\quad-7.040000 \quad 3.090900 \quad 3.867800$

C $\quad-5.456900-1.063600 \quad-2.057000$

$\begin{array}{llll}\text { C } & -4.886600 & 0.246600 & -2.532400\end{array}$

C $\quad-5.095200 \quad-2.303300 \quad-2.808200$

C $\quad-5.273000 \quad-2.362200 \quad-4.200100$

C $\quad-4.936300 \quad-3.521200 \quad-4.905600$

C $\quad-4.447800 \quad-4.638500 \quad-4.223000$

C $\quad-4.304900-4.600200 \quad-2.833700$

$\begin{array}{llll}\text { C } & -4.629900 & -3.439500 & -2.126800\end{array}$

C $\quad-6.864600 \quad-1.047800 \quad-1.542500$

C $\quad-7.213200 \quad-1.775600 \quad-0.392200$

$\begin{array}{llll}\text { C } & -8.528900 & -1.768000 & 0.079300\end{array}$

C $\quad-9.514200 \quad-1.053800 \quad-0.606100$

$\begin{array}{llll}\text { C } & -9.187200 & -0.356700 & -1.771600\end{array}$

C $\quad-7.871800 \quad-0.360900 \quad-2.245100$

$\begin{array}{llll}\text { C } & -0.077500 & 1.069500 & -3.063900\end{array}$

$\begin{array}{llll}\text { C } & 0.157500 & 2.303100 & -3.916500\end{array}$

$\begin{array}{llll}\text { C } & 1.248500 & 2.052300 & -4.961400\end{array}$

$\begin{array}{llll}\text { C } & -0.148100 & 2.334600 & -5.448800\end{array}$

C $\quad 0.160600 \quad 3.588400 \quad-3.117900$

$\begin{array}{llll}\text { C } & -0.979100 & 4.076900 & -2.456000\end{array}$

C $\quad-0.973200 \quad 5.274100 \quad-1.743900$

$\begin{array}{llll}\text { C } & 0.191800 & 6.049100 & -1.654700\end{array}$

C $\quad 1.342500 \quad 5.571000-2.295400$

$\begin{array}{llll}\text { C } & 1.316300 & 4.370500 & -3.002500\end{array}$

$\begin{array}{llll}\text { C } & 0.202600 & 7.327600 & -0.894000\end{array}$

C $\quad-0.899200 \quad 8.194300 \quad-0.937500$

C $\quad-0.889900 \quad 9.393600 \quad-0.222100$

$\begin{array}{llll}\text { C } & 0.221600 & 9.768700 & 0.563800\end{array}$

$\begin{array}{llll}\text { C } & 1.326500 & 8.886800 & 0.599900\end{array}$

$\begin{array}{llll}\text { C } & 1.314600 & 7.688000 & -0.118300\end{array}$

$\begin{array}{llll}\text { C } & 0.191100 & 11.101800 & 1.340000\end{array}$

$\begin{array}{llll}\text { C } & -0.917000 & 1.214600 & -6.070300\end{array}$

$\begin{array}{llll}\text { C } & -2.228600 & 0.933900 & -5.654500\end{array}$

C $\quad-2.947700 \quad-0.106600 \quad-6.248800$

$\begin{array}{llll}\text { C } & -2.367000 & -0.869900 & -7.264500\end{array}$

$\begin{array}{llll}\text { C } & -1.072000 & -0.579600 & -7.702500\end{array}$

$\begin{array}{llll}\text { C } & -0.354100 & 0.468000 & -7.118300\end{array}$

C $\quad-0.391600 \quad 3.671000 \quad-6.082700$

$\begin{array}{llll}\text { C } & -1.561400 & 4.394900 & -5.796600\end{array}$

C $\quad-1.791300 \quad 5.635700 \quad-6.397500$

$\begin{array}{llll}\text { C } & -0.867900 & 6.154600 & -7.307800\end{array}$

$\begin{array}{llll}\text { C } & 0.280400 & 5.427000 & -7.628200\end{array}$ 


\begin{tabular}{|c|c|c|c|}
\hline C & 0.514400 & 4.186300 & -7.027700 \\
\hline & 2.329600 & -1.464000 & -1.720800 \\
\hline & 3.820700 & -1.748000 & -1.734400 \\
\hline & 4.609900 & -0.834700 & -0.822100 \\
\hline & 4.713900 & 0.550400 & -1.036500 \\
\hline & 5.462700 & 1.381000 & -0.205700 \\
\hline & 6.151400 & 0.857700 & 0.897600 \\
\hline & 6.049300 & -0.519400 & 1.135500 \\
\hline & 5.292300 & -1.332800 & 0.294600 \\
\hline & 6.955100 & 1.739300 & 1.786800 \\
\hline & 8.177100 & 1.297300 & 2.314500 \\
\hline$c$ & 8.932300 & 2.124000 & 3.149300 \\
\hline C & 8.491400 & 3.422000 & 3.487700 \\
\hline 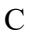 & 7.257100 & 3.856000 & 2.950800 \\
\hline & 6.504400 & 3.026900 & 2.114800 \\
\hline$c$ & 9.355500 & 4.303200 & 4.413500 \\
\hline $\mathrm{C}$ & 4.529400 & -2.458400 & -2.932600 \\
\hline $\mathrm{C}$ & 4.096000 & -3.254400 & -1.729700 \\
\hline C & 3.788400 & -2.659300 & -4.214200 \\
\hline C & 3.611500 & -3.953000 & -4.731800 \\
\hline C & 2.919100 & -4.144000 & -5.931000 \\
\hline C & 2.424200 & -3.043700 & -6.636000 \\
\hline $\mathrm{C}$ & 2.630100 & -1.751400 & -6.146900 \\
\hline $\mathrm{C}$ & 3.312400 & -1.557400 & -4.942800 \\
\hline$c$ & 6.000800 & -2.230100 & -3.104800 \\
\hline C & 6.486300 & -0.9 & -3.513200 \\
\hline $\mathrm{C}$ & 7.857900 & -0.769100 & -3.683600 \\
\hline $\mathrm{C}$ & 8.756200 & -1.817300 & -3.471300 \\
\hline $\mathrm{C}$ & 8.282300 & -3.077900 & -3.100800 \\
\hline $\mathrm{C}$ & 6.911100 & -3.288400 & -2.928100 \\
\hline $\mathrm{C}$ & -7.154800 & 200 & 7.557900 \\
\hline $\mathrm{C}$ & -8.593900 & 3.744200 & 7.901800 \\
\hline $\mathrm{C}$ & -7.121900 & 5.717400 & 7.360900 \\
\hline $\mathrm{C}$ & -6.257100 & 3.849500 & 8.773000 \\
\hline $\mathrm{C}$ & 0.795300 & -2.189800 & 11.485100 \\
\hline $\mathrm{C}$ & 2.560300 & -3.980000 & 11.301900 \\
\hline $\mathrm{C}$ & 3.141000 & -1.589600 & 10.877800 \\
\hline $\mathrm{C}$ & 0.017100 & 12.271700 & 0.347200 \\
\hline $\mathrm{C}$ & -0.991100 & 11.092000 & 2.333400 \\
\hline $\mathrm{C}$ & 1.483700 & 11.362000 & 2.150600 \\
\hline $\mathrm{C}$ & 8.730700 & 5.693000 & 4.685700 \\
\hline $\mathrm{C}$ & 9.537300 & 3.596600 & 5.774500 \\
\hline $\mathrm{C}$ & 10.738400 & 4.530200 & 3.764700 \\
\hline $\mathrm{H}$ & -2.679500 & -5.310900 & 1.536900 \\
\hline $\mathrm{H}$ & -2.578900 & -4.696400 & -0.169100 \\
\hline $\mathrm{H}$ & 2.493400 & -3.458500 & 4.275000 \\
\hline $\mathrm{H}$ & -1.641500 & -3.815200 & 5.510300 \\
\hline $\mathrm{H}$ & -2.262400 & -4.216300 & 3.207100 \\
\hline $\mathrm{H}$ & 2.354900 & -1.844800 & 6.042400 \\
\hline $\mathrm{H}$ & 3.023000 & -1.439800 & 8.320100 \\
\hline $\mathrm{H}$ & 0.162200 & -4.405600 & 9.670700 \\
\hline $\mathrm{H}$ & -0.475100 & -4.815500 & 7.368000 \\
\hline $\mathrm{H}$ & 1.642100 & -5.475100 & -0.750500 \\
\hline $\mathrm{H}$ & 2.266000 & -6.023800 & -3.072200 \\
\hline $\mathrm{H}$ & 0.602300 & -7.026300 & -4.609900 \\
\hline $\mathrm{H}$ & -1.685900 & -7.533700 & -3.797900 \\
\hline $\mathrm{H}$ & -2.313900 & -7.029400 & -1.463100 \\
\hline & 1.649200 & -6.508700 & 1.552400 \\
\hline & 2.150900 & -8.303600 & 3.159200 \\
\hline $\mathrm{H}$ & 0.353000 & -9.825300 & 3.930800 \\
\hline $\mathrm{H}$ & -1.956700 & -9.547900 & 3.075600 \\
\hline 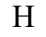 & -2.481700 & -7.748900 & 1.476000 \\
\hline
\end{tabular}

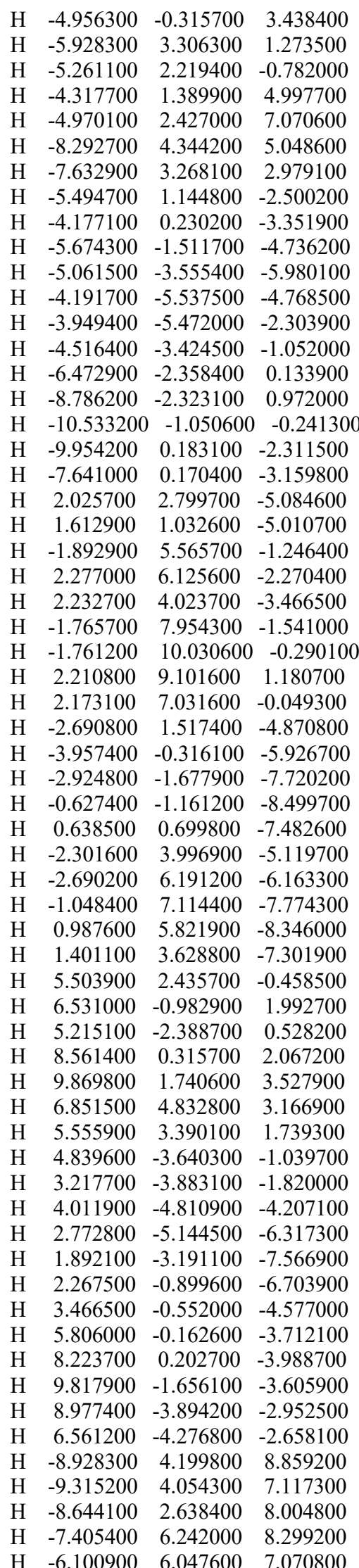

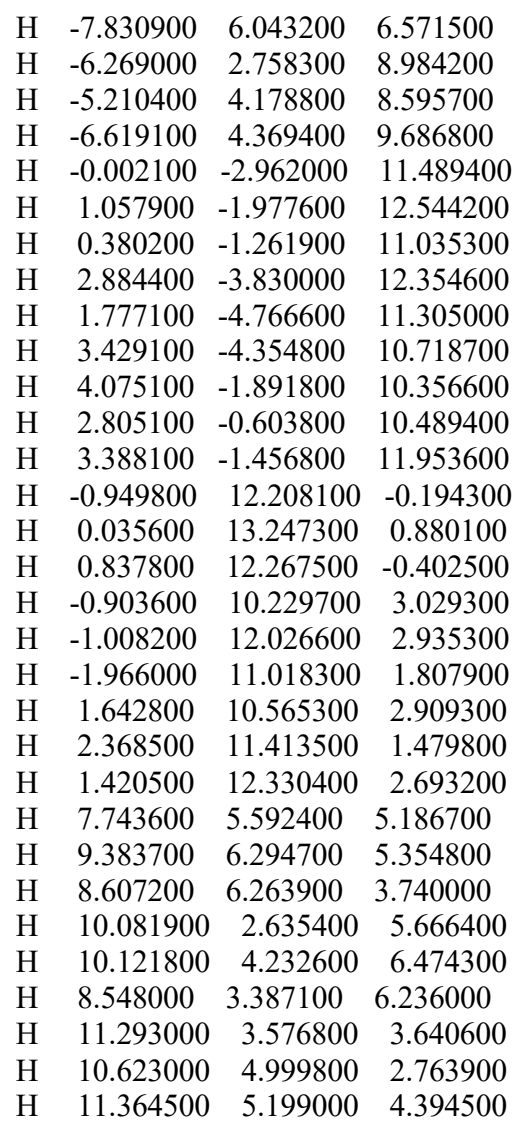

$$
\begin{array}{lccc}
\text { G5 } & & & \\
\text { Rh } & 0.003800 & -0.489800 & -0.486500 \\
\text { Rh } & 0.068000 & -2.137000 & -2.206900 \\
\text { O } & 0.372900 & -1.988200 & 0.880700 \\
\text { O } & 0.303600 & -3.565000 & -0.735800 \\
\text { O } & -2.022700 & -0.832800 & -0.320400 \\
\text { O } & -1.981700 & -2.303600 & -2.034700 \\
\text { O } & -0.361600 & 0.923700 & -1.939400 \\
\text { O } & -0.173700 & -0.614500 & -3.583100 \\
\text { O } & 2.032900 & -0.227100 & -0.745900 \\
\text { O } & 2.114500 & -1.880400 & -2.283300 \\
\text { Cl } & 3.279400 & -2.773900 & 2.013000 \\
\text { Cl } & -3.427100 & -2.753700 & 1.832100 \\
\text { Cl } & -3.276600 & 1.990700 & -2.781100 \\
\text { Cl } & 3.365700 & 2.035200 & -2.608500 \\
\text { C } & 0.428000 & -3.193000 & 0.477900 \\
\text { C } & 0.537000 & -4.250800 & 1.560400 \\
\text { C } & -0.548900 & -5.318200 & 1.398000 \\
\text { C } & 0.888300 & -5.740900 & 1.243900 \\
\text { C } & 0.833300 & -3.681000 & 2.930300 \\
\text { C } & 2.040200 & -3.030700 & 3.240500 \\
\text { C } & 2.322300 & -2.549500 & 4.517100 \\
\text { C } & 1.394100 & -2.692800 & 5.558100 \\
\text { C } & 0.175300 & -3.317600 & 5.262500 \\
\text { C } & -0.087900 & -3.792000 & 3.979000 \\
\text { C } & 1.697000 & -2.193500 & 6.926500 \\
\text { C } & 0.698100 & -1.589800 & 7.704300 \\
\text { C } & 0.981400 & -1.125000 & 8.990500 \\
\text { C } & 2.273800 & -1.248800 & 9.545600 \\
\text { C } & 3.272700 & -1.858900 & 8.751700
\end{array}
$$




\begin{tabular}{|c|c|c|c|}
\hline & 2.986300 & -2.321800 & 7.464700 \\
\hline & 2.544400 & -0.724800 & 10.971300 \\
\hline & 1.372600 & -6.111000 & -0.120100 \\
\hline & 0.675000 & -7.061700 & -0.883200 \\
\hline & 1.127400 & -7.411500 & -2.158900 \\
\hline & 2.294800 & -6.837000 & -2.669000 \\
\hline & 3.015100 & -5.919800 & -1.899800 \\
\hline- & 2.559200 & -5.558800 & -0.629000 \\
\hline$c$ & 1.478500 & -6.565600 & 2.347700 \\
\hline & 2.782600 & -6.315800 & 2.807500 \\
\hline & 3.334400 & -7.093000 & 3.829700 \\
\hline$c$ & 2.599500 & -8.142300 & 4.385900 \\
\hline $\mathrm{C}$ & 1.316300 & -8.423800 & 3.911400 \\
\hline $\mathrm{C}$ & 0.760400 & -7.647300 & 2.890300 \\
\hline $\mathrm{C}$ & -2.584400 & -1.637600 & -1.128800 \\
\hline C & -4.099400 & -1.701200 & -1.060700 \\
\hline $\mathrm{C}$ & -4.678200 & -0.968400 & 9700 \\
\hline $\mathrm{C}$ & -4.452400 & -1.368800 & 1.458100 \\
\hline $\mathrm{C}$ & -5.010900 & -0.699000 & 2.544900 \\
\hline $\mathrm{C}$ & -5.832100 & 0.420800 & 2.351400 \\
\hline C & -6.058300 & 0.842500 & 1.034500 \\
\hline $\mathrm{C}$ & -5.487800 & 0.16 & -0.0 \\
\hline $\mathrm{C}$ & -6.433000 & 1.140900 & 3.506300 \\
\hline $\mathrm{C}$ & -7.737100 & 1.651500 & 3.428000 \\
\hline $\mathrm{C}$ & -8.299500 & 2.336000 & 4.507500 \\
\hline $\mathrm{C}$ & -7.577100 & 2.536200 & 4000 \\
\hline $\mathrm{C}$ & -6.264000 & 2.0 & 100 \\
\hline $\mathrm{C}$ & -5.704500 & 1.329300 & 4.690500 \\
\hline $\mathrm{C}$ & -4.921200 & -2.832300 & -1.760200 \\
\hline $\mathrm{C}$ & -4.719100 & -1.508500 & -2.448100 \\
\hline $\mathrm{C}$ & -4.211100 & -4.027700 & -2.306600 \\
\hline $\mathrm{C}$ & -4.311700 & -4.347100 & -3.670900 \\
\hline $\mathrm{C}$ & -3.652800 & -5.468900 & -4.182800 \\
\hline $\mathrm{C}$ & -2.914300 & -6.295500 & -3.331900 \\
\hline $\mathrm{C}$ & -2.841000 & -6.004900 & -1.967400 \\
\hline $\mathrm{C}$ & -3.486500 & -4.876500 & 4300 \\
\hline $\mathrm{C}$ & -6.294200 & -3.122500 & -1.233600 \\
\hline $\mathrm{C}$ & -7.428500 & -2.866900 & -2.025800 \\
\hline $\mathrm{C}$ & -8.708600 & -3.149200 & -1.539900 \\
\hline $\mathrm{C}$ & -8.868100 & -3.707200 & -0.269400 \\
\hline $\mathrm{C}$ & -7.746800 & -3.997200 & 0.510900 \\
\hline $\mathrm{C}$ & -6.465400 & -3.717900 & 0.027600 \\
\hline $\mathrm{C}$ & -0.357300 & 0.575400 & -3.162300 \\
\hline $\mathrm{C}$ & -0.468800 & 1.704700 & -4.171100 \\
\hline $\mathrm{C}$ & 0.658700 & 1.634500 & -5.204800 \\
\hline $\mathrm{C}$ & -0.756600 & 1.452300 & -5.685900 \\
\hline $\mathrm{C}$ & -0.829500 & 3.031200 & -3.538800 \\
\hline $\mathrm{C}$ & -2.062400 & 3.262600 & -2.905100 \\
\hline $\mathrm{C}$ & -2.388900 & 4.489400 & -2.331400 \\
\hline $\mathrm{C}$ & -1.483100 & 5.559000 & -2.363700 \\
\hline $\mathrm{C}$ & -0.245800 & 5.345600 & -2.985900 \\
\hline $\mathrm{C}$ & 0.063400 & 4.109700 & -3.550400 \\
\hline $\mathrm{C}$ & -1.820800 & 6.868300 & -1.743200 \\
\hline $\mathrm{C}$ & -1.427800 & 8.069300 & -2.351800 \\
\hline $\mathrm{C}$ & -1.736700 & 9.298500 & -1.765400 \\
\hline $\mathrm{C}$ & -2.448200 & 9.370800 & -0.547800 \\
\hline $\mathrm{C}$ & -2.840400 & 8.153500 & 0.055700 \\
\hline $\mathrm{C}$ & -2.531000 & 6.925100 & -0.534500 \\
\hline $\mathrm{C}$ & -2.764600 & 10.749000 & 0.069500 \\
\hline $\mathrm{C}$ & -1.176500 & 0.092500 & -6.140300 \\
\hline $\mathrm{C}$ & -0.417000 & -0.596900 & -7.099800 \\
\hline $\mathrm{C}$ & -0.808900 & -1.868500 & -7.528700 \\
\hline
\end{tabular}

$\begin{array}{llll}\text { C } & -1.976600 & -2.447700 & -7.024900\end{array}$

C $\quad-2.757600 \quad-1.751600 \quad-6.098900$

C $\quad-2.362600 \quad-0.485200 \quad-5.659200$

C $\quad-1.357200 \quad 2.575400 \quad-6.476300$

C $\quad-2.684400 \quad 2.978000 \quad-6.251100$

C $\quad-3.245500 \quad 4.018000 \quad-6.997500$

C $\quad-2.495500 \quad 4.649900 \quad-7.991900$

C $\quad-1.187400 \quad 4.233700 \quad-8.249800$

C $\quad-0.622500 \quad 3.194800 \quad-7.504200$

C $\quad 2.654200 \quad-0.975200 \quad-1.565200$

C $\quad 4.164800 \quad-0.830400 \quad-1.582400$

$\begin{array}{llll}\text { C } & 4.662100 & 0.376000 & -0.816100\end{array}$

C $\quad 4.385100 \quad 1.696200 \quad-1.210800$

$\begin{array}{llll}\text { C } & 4.887000 & 2.801100 & -0.526400\end{array}$

$\begin{array}{llll}\text { C } & 5.697000 & 2.635300 & 0.605800\end{array}$

$\begin{array}{llll}\text { C } & 5.964800 & 1.326600 & 1.028900\end{array}$

C $\quad 5.453000 \quad 0.234600 \quad 0.330700$

$\begin{array}{llll}\text { C } & 6.248600 & 3.812000 & 1.330200\end{array}$

$\begin{array}{llll}\text { C } & 6.330600 & 3.815600 & 2.730700\end{array}$

$\begin{array}{llll}\text { C } & 6.852600 & 4.917800 & 3.413100\end{array}$

$\begin{array}{llll}\text { C } & 7.313500 & 6.056800 & 2.712800\end{array}$

C $\quad 7.224200 \quad 6.039400 \quad 1.303800$

$\begin{array}{llll}\text { C } & 6.700700 & 4.936900 & 0.624900\end{array}$

$\begin{array}{llll}\text { C } & 7.900600 & 7.293900 & 3.423900\end{array}$

C $\quad 5.055800 \quad-1.461800-2.701000$

C $\quad 4.851400 \quad-2.186500-1.396700$

C $\quad 4.413400 \quad-2.023300 \quad-3.927400$

C $\quad 3.655600 \quad-1.199900 \quad-4.775800$

$\begin{array}{llll}\text { C } & 3.067900 & -1.728000 & -5.928400\end{array}$

C $\quad 3.236800 \quad-3.077800 \quad-6.246500$

C $\quad 4.012200 \quad-3.897600 \quad-5.422100$

C $\quad 4.611000 \quad-3.369900 \quad-4.274300$

$\begin{array}{llll}\text { C } & 6.405700 & -0.855400 & -2.940200\end{array}$

C $\quad 7.574700 \quad-1.584800 \quad-2.655400$

$\begin{array}{llll}\text { C } & 8.833300 & -1.023000 & -2.889800\end{array}$

$\begin{array}{llll}\text { C } & 8.937700 & 0.261400 & -3.428800\end{array}$

$\begin{array}{llll}\text { C } & 7.783400 & 0.979900 & -3.748100\end{array}$

$\begin{array}{lrrr}\text { C } & 6.523600 & 0.420600 & -3.516800\end{array}$

$\begin{array}{lrrr}\text { C } & -8.233300 & 3.303000 & 6.870900\end{array}$

$\begin{array}{llll}\text { C } & 1.628000 & -1.461000 & 11.972800\end{array}$

$\begin{array}{llll}\text { C } & 4.007400 & -0.938300 & 11.429200\end{array}$

$\begin{array}{llll}\text { C } & 2.252400 & 0.790500 & 11.027100\end{array}$

$\begin{array}{lrrr}\text { C } & -9.511100 & 2.561500 & 7.320600\end{array}$

$\begin{array}{llll}\text { C } & -8.604300 & 4.728300 & 6.406300\end{array}$

$\begin{array}{llll}\text { C } & -7.309100 & 3.437200 & 8.105200\end{array}$

$\begin{array}{llll}\text { C } & -1.445900 & 11.500900 & 0.353400\end{array}$

$\begin{array}{llll}\text { C } & -3.621300 & 11.571100 & -0.917900\end{array}$

C $\quad-3.546200 \quad 10.652500 \quad 1.401900$

C $\quad 7.049900 \quad 8.536400 \quad 3.081900$

C $\quad 7.927200 \quad 7.152500 \quad 4.965000$

$\begin{array}{llll}\text { C } & 9.352600 & 7.519800 & 2.949100\end{array}$

$\mathrm{H} \quad-1.110600 \quad-5.620700 \quad 2.275800$

$\mathrm{H} \quad-1.154700 \quad-5.223900 \quad 0.504400$

H $\quad 3.272600 \quad-2.046000 \quad 4.664100$

$\mathrm{H} \quad-0.586300 \quad-3.463800 \quad 6.024000$

$\mathrm{H} \quad-1.049400 \quad-4.254300 \quad 3.785600$

$\mathrm{H} \quad-0.302600 \quad-1.459100 \quad 7.312000$

$\mathrm{H} \quad 0.179500 \quad-0.663400 \quad 9.550200$

$\mathrm{H} \quad 4.283100 \quad-1.993000 \quad 9.107500$

H $\quad 3.774200 \quad-2.800200 \quad 6.896300$

$\mathrm{H} \quad-0.215300 \quad-7.533100 \quad-0.487800$

H $\quad 0.577600 \quad-8.133800 \quad-2.748200$
H $\quad 2.648000 \quad-7.111700 \quad-3.654500$

H $\quad 3.930400 \quad-5.494300 \quad-2.284700$

H $\quad 3.127400 \quad-4.849200 \quad-0.044200$

H $\quad 3.377700 \quad-5.529100 \quad 2.369700$

H $\quad 4.335700 \quad-6.886700 \quad 4.184800$

H $\quad 3.028900 \quad-8.745000 \quad 5.175700$

$\mathrm{H} \quad 0.753500 \quad-9.247300 \quad 4.331500$

$\mathrm{H} \quad-0.228500 \quad-7.891400 \quad 2.523500$

H $\quad-4.799700 \quad-1.089100 \quad 3.535600$

$\mathrm{H} \quad-6.657200 \quad 1.723100 \quad 0.816800$

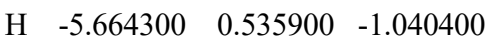

$\mathrm{H} \quad-8.332300 \quad 1.507800 \quad 2.535000$

$\mathrm{H} \quad-9.309000 \quad 2.708600 \quad 4.400200$

H $\quad-5.648100 \quad 2.130600 \quad 6.651200$

$\mathrm{H} \quad-4.688800 \quad 0.963800 \quad 4.775500$

$\mathrm{H} \quad-5.551800 \quad-0.820300 \quad-2.550300$

H $\quad-4.008500 \quad-1.457700 \quad-3.264500$

$\mathrm{H} \quad-4.902400 \quad-3.729600 \quad-4.334900$

$\mathrm{H} \quad-3.722500 \quad-5.702100 \quad-5.237400$

H $\quad-2.409500 \quad-7.167500 \quad-3.727000$

H $\quad-2.291100-6.658300 \quad-1.305700$

H $\quad-3.422100 \quad-4.664500 \quad-0.396200$

$\mathrm{H} \quad-7.323700 \quad-2.450500 \quad-3.019800$

$\mathrm{H} \quad-9.577100 \quad-2.939600 \quad-2.150900$

H $\quad-9.859700 \quad-3.926300 \quad 0.105100$

$\mathrm{H} \quad-7.869600 \quad-4.446600 \quad 1.487900$

$\mathrm{H} \quad-5.610600 \quad-3.977500 \quad 0.632800$

$\mathrm{H} \quad 1.197600 \quad 2.546000 \quad-5.442400$

$\mathrm{H} \quad 1.293600 \quad 0.758800 \quad-5.134400$

$\mathrm{H} \quad-3.370300 \quad 4.584300 \quad-1.877500$

$\mathrm{H} \quad 0.513600 \quad 6.122700 \quad-3.017300$

H $\quad 1.043100 \quad 3.974900 \quad-3.994800$

$\mathrm{H} \quad-0.893600 \quad 8.060600 \quad-3.293600$

H $\quad-1.414900 \quad 10.197200 \quad-2.273700$

$\mathrm{H} \quad-3.383600 \quad 8.126600 \quad 0.988200$

$\mathrm{H} \quad-2.831500 \quad 6.015400 \quad-0.029600$

$\mathrm{H} \quad 0.475600 \quad-0.147800 \quad-7.515900$

$\mathrm{H} \quad-0.211600 \quad-2.401100 \quad-8.257300$

H $\quad-2.282800 \quad-3.430000 \quad-7.360700$

$\mathrm{H} \quad-3.672600 \quad-2.190100 \quad-5.727500$

$\mathrm{H} \quad-2.977500 \quad 0.043300 \quad-4.944400$

$\mathrm{H} \quad-3.290100 \quad 2.481700 \quad-5.508700$

$\mathrm{H} \quad-4.265300 \quad 4.328200 \quad-6.810300$

$\mathrm{H} \quad-2.932100 \quad 5.453500 \quad-8.570800$

$\mathrm{H} \quad-0.612200 \quad 4.712700 \quad-9.031600$

H $\quad 0.386500 \quad 2.874000 \quad-7.730400$

$\mathrm{H} \quad 4.610000 \quad 3.784900 \quad-0.891700$

$\begin{array}{llll}\mathrm{H} & 6.588300 & 1.127600 & 1.896600\end{array}$

H $\quad 5.668500 \quad-0.762000 \quad 0.699500$

$\begin{array}{llll}\mathrm{H} & 5.973000 & 2.969900 & 3.304700\end{array}$

$\mathrm{H} \quad 6.883800 \quad 4.857800 \quad 4.490500$

$\mathrm{H} \quad \begin{array}{llll}7.564500 & 6.881000 & 0.716100\end{array}$

$\begin{array}{llll}\mathrm{H} & 6.668600 & 4.958700 & -0.457200\end{array}$

H $\quad 5.666700 \quad-2.258800 \quad-0.684200$

H $\quad 4.185000 \quad-3.041400 \quad-1.384000$

H $\quad 3.518500 \quad-0.153400 \quad-4.542800$

H $\quad 2.487500 \quad-1.089200 \quad-6.578400$

H $\quad 2.777200 \quad-3.485100 \quad-7.137700$

H $\quad 4.156000 \quad-4.939700 \quad-5.676200$

H $\quad 5.230400 \quad-4.007800 \quad-3.657100$

$\mathrm{H} \quad 7.513900 \quad-2.588300 \quad-2.253500$

H $\quad 9.728100 \quad-1.585900 \quad-2.657900$ 


$\begin{array}{cccc}\mathrm{H} & 9.912700 & 0.694600 & -3.610700 \\ \mathrm{H} & 7.864400 & 1.967700 & -4.182900 \\ \mathrm{H} & 5.644100 & 0.979100 & -3.798600 \\ \mathrm{H} & 1.835200 & -1.130300 & 13.013800 \\ \mathrm{H} & 0.555400 & -1.260900 & 11.768600 \\ \mathrm{H} & 1.795500 & -2.558300 & 11.915000 \\ \mathrm{H} & 4.264800 & -2.019600 & 11.442400 \\ \mathrm{H} & 4.714200 & -0.399800 & 10.761200 \\ \mathrm{H} & 4.159400 & -0.547500 & 12.458900 \\ \mathrm{H} & 1.184900 & 1.009000 & 10.815600 \\ \mathrm{H} & 2.481600 & 1.199500 & 12.035200 \\ \mathrm{H} & 2.873900 & 1.331400 & 10.281000 \\ \mathrm{H} & -9.978700 & 3.073000 & 8.189800 \\ \mathrm{H} & -10.269000 & 2.525000 & 6.510400 \\ \mathrm{H} & -9.267800 & 1.518500 & 7.618300 \\ \mathrm{H} & -9.040400 & 5.315300 & 7.243700 \\ \mathrm{H} & -7.701200 & 5.262000 & 6.038600 \\ \mathrm{H} & -9.354900 & 4.709300 & 5.588700 \\ \mathrm{H} & -7.035600 & 2.437100 & 8.506000 \\ \mathrm{H} & -6.383600 & 3.996500 & 7.848200 \\ \mathrm{H} & -7.819100 & 3.994600 & 8.921000 \\ \mathrm{H} & -0.881600 & 11.703300 & -0.580800 \\ \mathrm{H} & -1.647000 & 12.480600 & 0.838800 \\ \mathrm{H} & -0.797900 & 10.902300 & 1.029800 \\ \mathrm{H} & -4.556200 & 11.023700 & -1.166400 \\ \mathrm{H} & -3.898100 & 12.553300 & -0.476800 \\ \mathrm{H} & -3.074700 & 11.774000 & -1.862400 \\ \mathrm{H} & -4.525100 & 10.147800 & 1.251400 \\ \mathrm{H} & -2.959700 & 10.097900 & 2.166100 \\ \mathrm{H} & -3.753400 & 11.665100 & 1.811800 \\ \mathrm{H} & 7.429100 & 9.433600 & 3.617700 \\ \mathrm{H} & 7.076100 & 8.763100 & 1.995600 \\ \mathrm{H} & 5.991200 & 8.373400 & 3.379000 \\ \mathrm{H} & 8.356700 & 8.062900 & 5.437100 \\ \mathrm{H} & 6.899800 & 7.021600 & 5.368700 \\ \mathrm{H} & 8.556400 & 6.289500 & 5.273000 \\ \mathrm{H} & 9.969300 & 6.616900 & 3.149100 \\ \mathrm{H} & 9.397700 & 7.738900 & 1.861900 \\ \mathrm{H} & 9.811800 & 8.381400 & 3.480700 \\ & & & \end{array}$

$\begin{array}{lccc}\text { G6 } & & & \\ \text { Rh } & -0.221500 & -0.015300 & -0.651800 \\ \text { Rh } & -0.975600 & -0.086200 & -2.910500 \\ \text { O } & 0.549800 & 1.857600 & -1.033200 \\ \text { O } & -0.039300 & 1.729400 & -3.210400 \\ \text { O } & 1.546600 & -0.867700 & -1.279700 \\ \text { O } & 0.776400 & -1.066200 & -3.394300 \\ \text { O } & -1.030500 & -1.892500 & -0.387000 \\ \text { O } & -1.869600 & -1.897900 & -2.484400 \\ \text { O } & -2.028200 & 0.833600 & -0.139300 \\ \text { O } & -2.684800 & 0.898200 & -2.300000 \\ \text { Cl } & -0.957600 & 4.627900 & -0.415500 \\ \text { Cl } & 4.204800 & 0.778700 & -2.041400 \\ \text { Cl } & 0.597400 & -4.652000 & -0.645900 \\ \text { Cl } & -4.578000 & -0.790000 & 0.956400 \\ \text { C } & 0.495100 & 2.322200 & -2.215600 \\ \text { C } & 1.229000 & 3.630600 & -2.447900 \\ \text { C } & 2.192500 & 3.520800 & -3.633000 \\ \text { C } & 1.037700 & 4.481000 & -3.745200 \\ \text { C } & 1.683200 & 4.295500 & -1.166900 \\ \text { C } & 0.785200 & 4.793700 & -0.207200 \\ \text { C } & 1.212700 & 5.428200 & 0.956900\end{array}$

$\begin{array}{llll}\text { C } & 2.579400 & 5.593100 & 1.222700\end{array}$

$\begin{array}{llll}\text { C } & 3.490500 & 5.088900 & 0.285200\end{array}$

$\begin{array}{llll}\text { C } & 3.042700 & 4.456600 & -0.873100\end{array}$

$\begin{array}{llll}\text { C } & 3.039900 & 6.270700 & 2.464500\end{array}$

C $\quad 4.164600 \quad 5.801600 \quad 3.158900$

C $\quad 4.595800 \quad 6.436100 \quad 4.326000$

C $\quad 3.918300 \quad 7.563500 \quad 4.839600$

$\begin{array}{llll}\text { C } & 2.786700 & 8.028000 & 4.129900\end{array}$

C $\quad 2.356900 \quad 7.390000 \quad 2.963200$

C $\quad 4.427100 \quad 8.234900 \quad 6.132200$

C $\quad-0.068500 \quad 4.144500 \quad-4.691200$

C $\quad-1.407100 \quad 4.198100 \quad-4.270600$

$\begin{array}{llll}\text { C } & -2.438200 & 3.895000 & -5.163700\end{array}$

C $\quad-2.142600 \quad 3.542500 \quad-6.482900$

$\begin{array}{llll}\text { C } & -0.814500 & 3.512900 & -6.916600\end{array}$

C $\quad 0.218900 \quad 3.826900 \quad-6.028900$

C $\quad 1.357500 \quad 5.943500 \quad-3.671600$

C $\quad 0.540900 \quad 6.817200 \quad-2.933800$

C $\quad 0.835200 \quad 8.182300 \quad-2.875700$

C $\quad 1.932800 \quad 8.692300 \quad-3.572600$

$\begin{array}{llll}\text { C } & 2.730100 & 7.839400 & -4.339300\end{array}$

C $\quad 2.439600 \quad 6.473200 \quad-4.398400$

C $\quad 1.667200 \quad-1.222200 \quad-2.495000$

C $\quad 2.933100-1.985800 \quad-2.839200$

C $\quad 3.965600 \quad-1.958800 \quad-1.733300$

C $\quad 4.605000 \quad-0.778300 \quad-1.317900$

C $\quad 5.573900-0.765700-0.316900$

$\begin{array}{llll}\text { C } & 5.952900 & -1.951400 & 0.327900\end{array}$

C $\quad 5.316700 \quad-3.137100 \quad-0.063100$

C $\quad 4.347300 \quad-3.128100 \quad-1.064100$

$\begin{array}{llll}\text { C } & 6.989900 & -1.945000 & 1.394800\end{array}$

$\begin{array}{llll}\text { C } & 7.055000 & -0.895000 & 2.323200\end{array}$

C $\quad 8.027300 \quad-0.887800 \quad 3.326800$

C $\quad 8.971400 \quad-1.935400 \quad 3.434000$

C $\quad 8.894000 \quad-2.985300 \quad 2.492900$

C $\quad 7.920000 \quad-2.990100 \quad 1.492000$

$\begin{array}{llll}\text { C } & 3.375000 & -2.258500 & -4.313300\end{array}$

C $\quad 2.608400 \quad-3.304200 \quad-3.547100$

$\begin{array}{llll}\text { C } & 2.680300 & -1.555900 & -5.433700\end{array}$

C $\quad 2.048200 \quad-2.295700 \quad-6.446600$

$\begin{array}{llll}\text { C } & 1.397700 & -1.641000 & -7.496600\end{array}$

$\begin{array}{llll}\text { C } & 1.398600 & -0.244900 & -7.558100\end{array}$

$\begin{array}{llll}\text { C } & 2.058500 & 0.496500 & -6.574900\end{array}$

$\begin{array}{llll}\text { C } & 2.700600 & -0.154400 & -5.518100\end{array}$

$\begin{array}{llll}\text { C } & 4.828000 & -2.526700 & -4.565600\end{array}$

$\begin{array}{llll}\text { C } & 5.777500 & -1.499400 & -4.433400\end{array}$

C $\quad 7.130400 \quad-1.749300 \quad-4.679700$

C $\quad 7.545600 \quad-3.020200 \quad-5.083500$

C $\quad 6.606000 \quad-4.039500 \quad-5.254200$

C $\quad 5.252200 \quad-3.793500 \quad-5.007200$

C $\quad-1.688100 \quad-2.423800 \quad-1.336800$

C $\quad-2.389700 \quad-3.729100-1.008300$

$\begin{array}{llll}\text { C } & -3.873800 & -3.667700 & -1.380500\end{array}$

C $\quad-3.000500-4.653800 \quad-2.110400$

C $\quad-1.970800 \quad-4.314500 \quad 0.322700$

$\begin{array}{llll}\text { C } & -0.667900 & -4.776900 & 0.575400\end{array}$

C $\quad-0.299600 \quad-5.343100 \quad 1.793700$

C $\quad-1.229900 \quad-5.471300 \quad 2.834700$

C $\quad-2.529800 \quad-5.000000 \quad 2.607900$

C $\quad-2.878300 \quad-4.436100 \quad 1.382100$

C $\quad-0.841400 \quad-6.078100 \quad 4.136300$

C $\quad 0.023200 \quad-7.181700 \quad 4.180300$ $\begin{array}{llll}\text { C } & 0.388700 & -7.751100 & 5.401900\end{array}$

$\begin{array}{llll}\text { C } & -0.096300 & -7.236100 & 6.623900\end{array}$

C $\quad-0.967100 \quad-6.123200 \quad 6.567000$

C $\quad-1.332100 \quad-5.556700 \quad 5.342700$

$\begin{array}{llll}\text { C } & 0.333100 & -7.893700 & 7.952000\end{array}$

C $\quad-2.691200 \quad-4.391100 \quad-3.548100$

C $\quad-1.367500 \quad-4.444300 \quad-4.013700$

C $\quad-1.085800 \quad-4.210700 \quad-5.362300$

C $\quad-2.121500 \quad-3.928600 \quad-6.256600$

C $\quad-3.444100 \quad-3.900400 \quad-5.806500$

$\begin{array}{llll}\text { C } & -3.730700 & -4.145100 & -4.460200\end{array}$

$\begin{array}{llll}\text { C } & -3.184400 & -6.103800 & -1.778400\end{array}$

C $\quad-2.072200 \quad-6.950300 \quad-1.633300$

C $\quad-2.246600 \quad-8.304300 \quad-1.334300$

C $\quad-3.533000 \quad-8.831800-1.201100$

C $\quad-4.646800 \quad-8.008300 \quad-1.379900$

C $\quad-4.475900 \quad-6.652900-1.677400$

C $\quad-2.859900 \quad 1.122700 \quad-1.056700$

$\begin{array}{llll}\text { C } & -4.091600 & 1.892500 & -0.615800\end{array}$

$\begin{array}{llll}\text { C } & -4.250100 & 1.955000 & 0.887600\end{array}$

$\begin{array}{llll}\text { C } & -4.493000 & 0.817000 & 1.676000\end{array}$

C $\quad-4.666700 \quad 0.887900 \quad 3.056400$

$\begin{array}{llll}\text { C } & -4.600100 & 2.118600 & 3.724500\end{array}$

$\begin{array}{llll}\text { C } & -4.342200 & 3.262700 & 2.957900\end{array}$

C $\quad-4.170500 \quad 3.170600 \quad 1.578000$

$\begin{array}{llll}\text { C } & -4.787100 & 2.198800 & 5.198400\end{array}$

C $\quad-5.742900 \quad 1.399000 \quad 5.841700$

$\begin{array}{llll}\text { C } & -5.917700 & 1.472800 & 7.225300\end{array}$

$\begin{array}{llll}\text { C } & -5.141400 & 2.348300 & 8.015500\end{array}$

$\begin{array}{llll}\text { C } & -4.180900 & 3.149700 & 7.355700\end{array}$

$\begin{array}{llll}\text { C } & -4.009500 & 3.074500 & 5.970900\end{array}$

$\begin{array}{llll}\text { C } & -5.364700 & 2.398100 & 9.541400\end{array}$

$\begin{array}{llll}\text { C } & -5.336000 & 2.086300 & -1.541300\end{array}$

C $\quad-4.281900 \quad 3.158600 \quad-1.455700$

$\begin{array}{llll}\text { C } & -5.442100 & 1.302500 & -2.808500\end{array}$

C $\quad-5.557700 \quad 1.966000 \quad-4.041200$

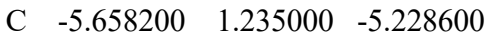

C $\quad-5.673300 \quad-0.161900 \quad-5.191100$

C $\quad-5.597600 \quad-0.827900 \quad-3.965300$

C $\quad-5.485600 \quad-0.100600 \quad-2.777300$

$\begin{array}{llll}\text { C } & -6.653200 & 2.370900 & -0.884900\end{array}$

$\begin{array}{llll}\text { C } & -7.312700 & 1.374900 & -0.144900\end{array}$

$\begin{array}{llll}\text { C } & -8.546000 & 1.639400 & 0.457200\end{array}$

$\begin{array}{llll}\text { C } & -9.144300 & 2.892400 & 0.306500\end{array}$

C $\quad-8.515800 \quad 3.878300 \quad-0.457700$

C $\quad-7.281400 \quad 3.617800 \quad-1.059900$

$\begin{array}{llll}\text { C } & 10.062100 & -1.968400 & 4.524600\end{array}$

C $\quad 11.455500 \quad-1.983400 \quad 3.859000$

$\begin{array}{llll}\text { C } & 10.008500 & -0.747100 & 5.474100\end{array}$

C $\quad 9.891200 \quad-3.238900 \quad 5.385500$

C $\quad 4.394200 \quad 7.213700 \quad 7.290100$

C $\quad 5.876300 \quad 8.725100 \quad 5.921800$

$\begin{array}{llll}\text { C } & 3.575800 & 9.455400 & 6.558100\end{array}$

C $\quad-0.111200 \quad-9.372500 \quad 7.961700$

C $\quad \begin{array}{llll}\text { C } & 1.869300 & -7.818800 & 8.090500\end{array}$

$\begin{array}{llll}\text { C } & -0.284300 & -7.209700 & 9.195700\end{array}$

C $\quad-6.822300 \quad 2.815200 \quad 9.835000$

$\begin{array}{llll}\text { C } & -4.434300 & 3.405900 & 10.258500\end{array}$

$\begin{array}{lrrr}\text { C } & -5.098900 & 1.002500 & 10.146400\end{array}$

H $\quad 3.202100 \quad 3.900700 \quad-3.513500$

H $\quad 2.140200 \quad 2.588900 \quad-4.184100$

H $\quad 0.450600 \quad 5.764500 \quad 1.652900$ 


\begin{tabular}{|c|c|c|c|}
\hline & 4.563500 & 5.188400 & 0.427300 \\
\hline & 3.779000 & 4.061400 & -1.563900 \\
\hline & 4.703200 & 4.929200 & 2.811000 \\
\hline & 5.465000 & 6.034800 & 4.828900 \\
\hline & 2.219200 & 8.886200 & 4.457000 \\
\hline & 1.496100 & 7.787100 & 2.439600 \\
\hline 1 & -1.650300 & 4.471000 & -3.253400 \\
\hline & -3.466300 & 3.940400 & -4.835100 \\
\hline & -2.943000 & 3.304200 & -7.171300 \\
\hline & -0.585900 & 3.254300 & -7.942500 \\
\hline & 1.241900 & 3.824900 & -6.382500 \\
\hline H & -0.329300 & 6.447500 & -2.413600 \\
\hline & 0.206500 & 8.846300 & -2.296500 \\
\hline & 2.158000 & 9.750100 & -3.528700 \\
\hline & 3.570700 & 8.237700 & -4.892500 \\
\hline & 3.058400 & 5.830600 & -5.012000 \\
\hline & 6.030500 & 0.188800 & -0.074600 \\
\hline & 5.539900 & -4.085000 & 0.419900 \\
\hline & 3.858400 & -4.061700 & -1.319000 \\
\hline & 6.339100 & -0.083300 & 2.286800 \\
\hline & 8.019500 & -0.052900 & 4.011200 \\
\hline 1 & 9.591800 & -3.810700 & 2.524500 \\
\hline & 7.907900 & -3.806500 & 0.780800 \\
\hline & 3.110200 & -4.206300 & -3.212300 \\
\hline & 1.561900 & -3.451800 & -3.787800 \\
\hline & 2.062400 & -3.377900 & -6.422700 \\
\hline H & 0.899400 & -2.216300 & -8.266200 \\
\hline$\pi$ & 0.898800 & 0.262000 & -8.373500 \\
\hline & 2.078500 & 1.575100 & -6.635300 \\
\hline & 3.209000 & 0.431500 & -4.765500 \\
\hline त & 5.472700 & -0.503100 & -4.152300 \\
\hline $\mathrm{H}$ & 7.856100 & -0.954500 & -4.565600 \\
\hline $\boldsymbol{B}$ & 8.593200 & -3.211500 & -5.276400 \\
\hline ( & 6.926100 & -5.019700 & -5.583100 \\
\hline 1 & 4.536600 & -4.591100 & -5.162200 \\
\hline $\mathrm{H}$ & -4.601700 & -4.021300 & -0.657500 \\
\hline $\mathrm{H}$ & -4.180200 & -2.769000 & -1.903300 \\
\hline $\mathrm{H}$ & 0.733400 & -5.656200 & 1.908700 \\
\hline 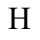 & -3.300600 & -5.073500 & 3.370700 \\
\hline 1 & -3.888600 & -4.064700 & 1.252600 \\
\hline $\mathrm{H}$ & 0.405000 & -7.619500 & 3.266400 \\
\hline $\mathrm{H}$ & 1.054500 & -8.603100 & 5.384500 \\
\hline $\mathrm{H}$ & -1.376000 & -5.671000 & 7.457900 \\
\hline H & -1.986500 & -4.694000 & 5.343200 \\
\hline $\mathrm{H}$ & -0.556800 & -4.663200 & -3.333200 \\
\hline $\mathrm{H}$ & -0.065800 & -4.255600 & -5.715200 \\
\hline $\mathrm{H}$ & -1.899900 & -3.744000 & -7.299900 \\
\hline $\mathrm{H}$ & -4.248100 & -3.696800 & -6.501800 \\
\hline $\mathrm{H}$ & -4.760700 & -4.145600 & -4.127900 \\
\hline $\mathrm{H}$ & -1.071300 & -6.568300 & -1.762700 \\
\hline $\mathrm{H}$ & -1.383800 & -8.946600 & -1.214000 \\
\hline $\mathrm{H}$ & -3.666700 & -9.881100 & -0.972000 \\
\hline $\mathrm{H}$ & -5.643500 & -8.421000 & -1.292900 \\
\hline $\mathrm{H}$ & -5.351200 & -6.034200 & -1.829900 \\
\hline $\mathrm{H}$ & -4.830600 & -0.043500 & 3.589000 \\
\hline $\mathrm{H}$ & -4.283200 & 4.248300 & 3.412500 \\
\hline 11 & -3.952000 & 4.075800 & 1.022700 \\
\hline & -6.371900 & 0.726100 & 5.272300 \\
\hline & -6.669800 & 0.839200 & 7.675200 \\
\hline $\mathrm{H}$ & -3.547200 & 3.840100 & 7.891500 \\
\hline $\mathrm{H}$ & -3.250500 & 3.691500 & 5.506200 \\
\hline & -4.498100 & 4.090200 & -0.942700 \\
\hline
\end{tabular}

\begin{tabular}{|c|c|c|c|c|c|c|c|}
\hline H & -3.593300 & 3.268000 & -2.285600 & $\mathrm{Cl}$ & 2.367500 & & \\
\hline $\mathrm{H}$ & -5.572300 & 3.047500 & -4.080300 & $\mathrm{C}$ & -2.427700 & -1.057200 & \\
\hline & -5.732000 & 1.751600 & -6.176700 & $\mathrm{C}$ & -3.856900 & -1.560500 & -1.234000 \\
\hline & -5.756000 & -0.727900 & -6.109900 & $\mathrm{C}$ & -4.610700 & & -2.496900 \\
\hline & -5.632800 & -1.907300 & -3.934900 & $\mathrm{C}$ & -4.789400 & -0.665100 & -2.054400 \\
\hline & -5.429800 & -0.628800 & -1.835900 & $\mathrm{C}$ & -4.285500 & -2.041200 & 0.135300 \\
\hline & -6.881400 & 0.391100 & -0.042600 & $\mathrm{C}$ & -3.714900 & & \\
\hline & -9.041900 & 0.869400 & 1.033900 & $\mathrm{C}$ & -4.189000 & & 1.962300 \\
\hline & -10.100600 & 3.095100 & 0.771100 & $\mathrm{C}$ & -5.259100 & -3.047300 & 6700 \\
\hline & -8.987700 & 4.843600 & -0.587800 & $\mathrm{C}$ & -5.817800 & -1.900300 & 0000 \\
\hline & -6.818000 & 4.388300 & -1.663100 & $\mathrm{C}$ & -5.327700 & & \\
\hline & 12.259500 & -1.963900 & & $\mathrm{C}$ & -5.812800 & & \\
\hline & 11.605300 & -2.896100 & 3.245100 & $\mathrm{C}$ & -6.800900 & & 200 \\
\hline & 78900 & -1.096000 & 3.201100 & $\mathrm{C}$ & -7.319500 & 66100 & 600 \\
\hline & 10.160700 & 0.199200 & & $\mathrm{C}$ & & & \\
\hline & 9.035700 & -0.704400 & 6.010 & $\mathrm{C}$ & -5.891000 & -3.7 & \\
\hline & 600 & -0.80 & & $\mathrm{C}$ & -5.3 & & \\
\hline & 10.641000 & -3.263200 & & $\mathrm{C}$ & -7.308900 & & 100 \\
\hline & 8.876400 & -3.265100 & 5.838 & $\mathrm{C}$ & -7.442600 & & \\
\hline & 10.028200 & -4.161600 & 4.783800 & $\mathrm{C}$ & -4.300800 & -2.0 & \\
\hline & 4.716400 & 7.687200 & 8.24 & $\mathrm{C}$ & -3.849 & & \\
\hline & 3.3 & 6.81 & & $\mathrm{C}$ & -2.809400 & & \\
\hline & 5.07 & $6.35^{\circ}$ & & $\mathrm{C}$ & -2.107000 & & 500 \\
\hline & 6.25 & 9.251 & 6.82 & $\mathrm{C}$ & -2.441200 & & \\
\hline & 6.56 & 7.88 & 5.71 & $\mathrm{C}$ & -3.49 & -1. & \\
\hline & 5.92 & 9.42 & 5.0 & $\mathrm{C}$ & -4.20 & & \\
\hline & 3.5 & 10.2 & & $\mathrm{C}$ & & & \\
\hline & 2.5 & 9.15 & & $\mathrm{C}$ & -7.076300 & & \\
\hline & $3.9^{7}$ & 9.90 & & $\mathrm{C}$ & -8.14 & & \\
\hline & 0.3 & -9.95 & & $\mathrm{C}$ & -7.91 & & \\
\hline & 0.1 & -9.8 & & $\mathrm{C}$ & -6.6 & & \\
\hline $\mathrm{H}$ & -1.2 & -9.44 & & $\mathrm{C}$ & -5.53 & & \\
\hline & 2.2 & -6.76 & & $\mathrm{C}$ & -1.05 & & \\
\hline & 2.15 & -8.24 & & $\mathrm{C}$ & & & \\
\hline & 2.3 & -8.38 & & $\mathrm{C}$ & -2.0 & & \\
\hline & 0.0 & -6.1 & & $\mathrm{C}$ & -0.6 & & \\
\hline $\mathrm{H}$ & -1.3 & -7.26 & & $\mathrm{C}$ & -2.0 & & \\
\hline & 0.0 & -7.71 & & $\mathrm{C}$ & -3.1 & & \\
\hline & -7.5 & & & $\mathrm{C}$ & & & \\
\hline $\mathrm{H}$ & & & & $\mathrm{C}$ & & & \\
\hline $\mathrm{H}$ & -7.0 & 3.7 & & $\mathrm{C}$ & -1.5 & & \\
\hline $\mathrm{H}$ & -4.6 & & & $\mathrm{C}$ & & & \\
\hline 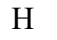 & -3.3 & & & $\mathrm{C}$ & -3.623000 & & \\
\hline & -4.60 & & & $\mathrm{C}$ & -4.63 & & \\
\hline $\mathrm{H}$ & -4.0 & & & $\mathrm{C}$ & & & \\
\hline & -5.212100 & 1.0 & & $\mathrm{C}$ & -4.7 & & \\
\hline & \multirow[t]{3}{*}{-5.809700} & 0.245100 & 9.754900 & $\mathrm{C}$ & -3.716 & & 00 \\
\hline & & & & $\mathrm{C}$ & & & \\
\hline 11 & & & & $\mathrm{C}$ & & & \\
\hline $\mathrm{Rh}$ & & & & $\mathrm{C}$ & & & \\
\hline $\mathrm{Rh}$ & -0.000100 & -0.000500 & & $\mathrm{C}$ & -2.090 & & \\
\hline $\mathrm{O}$ & -1.917600 & -0.760900 & -2.415100 & $\mathrm{C}$ & -2.350300 & 3.848500 & 7300 \\
\hline & -1.864700 & -0.875500 & -0.1 & $\mathrm{C}$ & -3.293000 & 2.808000 & 900 \\
\hline & -0.760600 & & -2.4 & $\mathrm{C}$ & -3.534100 & & \\
\hline D & -0.8 & & -0.1 & $\mathrm{C}$ & -2.84 & & \\
\hline & 0.760300 & -1.918 & -2.41 & $\mathrm{C}$ & -1.922700 & 3.491500 & 300 \\
\hline $\mathrm{O}$ & 0.875500 & -1.864900 & -0.158600 & $\mathrm{C}$ & -1.686700 & 4.203200 & -4.943400 \\
\hline & 1.917500 & 0.759900 & -2.415200 & $\mathrm{C}$ & -3.031100 & 5.758900 & -2.269600 \\
\hline & 1.864700 & & -0.158900 & $\mathrm{C}$ & -2.631600 & & \\
\hline $\mathrm{Cl}$ & -2.367200 & -4.032800 & & $\mathrm{C}$ & -3.499400 & 8.146400 & -2.333200 \\
\hline & -4.033100 & 2.365800 & 0.015100 & $\mathrm{C}$ & -4.778800 & 7.913900 & -1.823000 \\
\hline & 4.032900 & -2.366900 & 0.015200 & $\mathrm{C}$ & -5.199200 & 6.607900 & -1.562800 \\
\hline
\end{tabular}




\begin{tabular}{|c|c|c|c|}
\hline C & -4.336100 & 5.534300 & -1.798400 \\
\hline C & 1.056900 & -2.428000 & -1.284200 \\
\hline $\mathrm{C}$ & 1.560400 & -3.857100 & -1.233800 \\
\hline $\mathrm{C}$ & 2.093100 & -4.611000 & -2.496900 \\
\hline $\mathrm{C}$ & 0.664700 & -4.789800 & -2.053800 \\
\hline C & 2.041600 & -4.285500 & 0.135400 \\
\hline $\mathrm{C}$ & 3.170100 & -3.714600 & 0.752800 \\
\hline $\mathrm{C}$ & 3.673200 & -4.188400 & 1.961800 \\
\hline $\mathrm{C}$ & 3.048800 & -5.258400 & 2.606600 \\
\hline $\mathrm{C}$ & 1.901600 & -5.817300 & 2.040400 \\
\hline $\mathrm{C}$ & 1.413300 & -5.327600 & 0.830700 \\
\hline $\mathrm{C}$ & 3.621800 & -5.811600 & 3.854700 \\
\hline $\mathrm{C}$ & 3.163100 & -5.354700 & 5.111800 \\
\hline $\mathrm{C}$ & 3.716500 & -5.888900 & 6.285800 \\
\hline $\mathrm{C}$ & 4.716500 & -6.868000 & 6.224100 \\
\hline $\mathrm{C}$ & 5.168500 & -7.317900 & 4.978500 \\
\hline $\mathrm{C}$ & 4.631800 & -6.799800 & 3.790100 \\
\hline $\mathrm{C}$ & 2.092200 & -4.299100 & 5.210600 \\
\hline $\mathrm{C}$ & 5.309100 & -7.440100 & 7.478700 \\
\hline $\mathrm{C}$ & 5.138600 & -7.308300 & 2.464000 \\
\hline $\mathrm{C}$ & 2.349200 & -3.850000 & -3.756600 \\
\hline $\mathrm{C}$ & 3.292100 & -2.809700 & -3.782400 \\
\hline $\mathrm{C}$ & 3.533300 & -2.107400 & -4.966300 \\
\hline $\mathrm{C}$ & 2.841800 & -2.441700 & -6.133300 \\
\hline $\mathrm{C}$ & 1.921500 & -3.493300 & -6.122500 \\
\hline $\mathrm{C}$ & 1.685400 & -4.204800 & -4.942500 \\
\hline $\mathrm{C}$ & 3.029500 & -5.760500 & -2.268700 \\
\hline $\mathrm{C}$ & 2.629500 & -7.076600 & -2.564600 \\
\hline $\mathrm{C}$ & 3.496900 & -8.148200 & -2.332300 \\
\hline $\mathrm{C}$ & 4.776400 & -7.916100 & -1.822200 \\
\hline $\mathrm{C}$ & 5.197300 & -6.610200 & -1.562100 \\
\hline $\mathrm{C}$ & 4.334600 & -5.536300 & -1.797600 \\
\hline $\mathrm{C}$ & 2.427700 & 1.056500 & -1.284600 \\
\hline $\mathrm{C}$ & 3.856800 & 1.559900 & -1.234500 \\
\hline $\mathrm{C}$ & 4.610500 & 2.092800 & -2.497700 \\
\hline $\mathrm{C}$ & 4.789200 & 0.664300 & -2.054900 \\
\hline $\mathrm{C}$ & 4.285800 & 2.040900 & 0.134600 \\
\hline $\mathrm{C}$ & 3.715600 & 3.169600 & 0.752100 \\
\hline $\mathrm{C}$ & 4.190500 & 3.673000 & 1.960600 \\
\hline $\mathrm{C}$ & 5.261000 & 3.048500 & 2.604700 \\
\hline $\mathrm{C}$ & 5.819000 & 1.901000 & 2.038500 \\
\hline $\mathrm{C}$ & 5.328200 & 1.412400 & 0.829400 \\
\hline $\mathrm{C}$ & 5.815900 & 3.622200 & 3.851700 \\
\hline $\mathrm{C}$ & 6.806400 & 4.629800 & 3.785200 \\
\hline $\mathrm{C}$ & 7.326000 & 5.167300 & 4.972600 \\
\hline $\mathrm{C}$ & 6.875200 & 4.718400 & 6.219000 \\
\hline $\mathrm{C}$ & 5.893800 & 3.720900 & 6.282700 \\
\hline $\mathrm{C}$ & 5.358100 & 3.166700 & 5.109600 \\
\hline $\mathrm{C}$ & 7.315700 & 5.133400 & 2.458000 \\
\hline $\mathrm{C}$ & 7.448800 & 5.311900 & 7.472500 \\
\hline $\mathrm{C}$ & 4.300000 & 2.098300 & 5.210300 \\
\hline $\mathrm{C}$ & 3.849100 & 2.349100 & -3.757200 \\
\hline $\mathrm{C}$ & 2.808800 & 3.292000 & -3.782600 \\
\hline $\mathrm{C}$ & 2.106100 & 3.533300 & -4.966200 \\
\hline $\mathrm{C}$ & 2.440100 & 2.842000 & -6.133400 \\
\hline $\mathrm{C}$ & 3.491700 & 1.921800 & -6.123100 \\
\hline $\mathrm{C}$ & 4.203500 & 1.685500 & -4.943300 \\
\hline $\mathrm{C}$ & 5.760000 & 3.029100 & -2.269700 \\
\hline $\mathrm{C}$ & 7.076100 & 2.629000 & -2.565600 \\
\hline $\mathrm{C}$ & 8.147700 & 3.496300 & -2.333200 \\
\hline $\mathrm{C}$ & 7.915800 & 4.775900 & -1.823300 \\
\hline C & 6.609900 & 5.196900 & -1.563300 \\
\hline
\end{tabular}

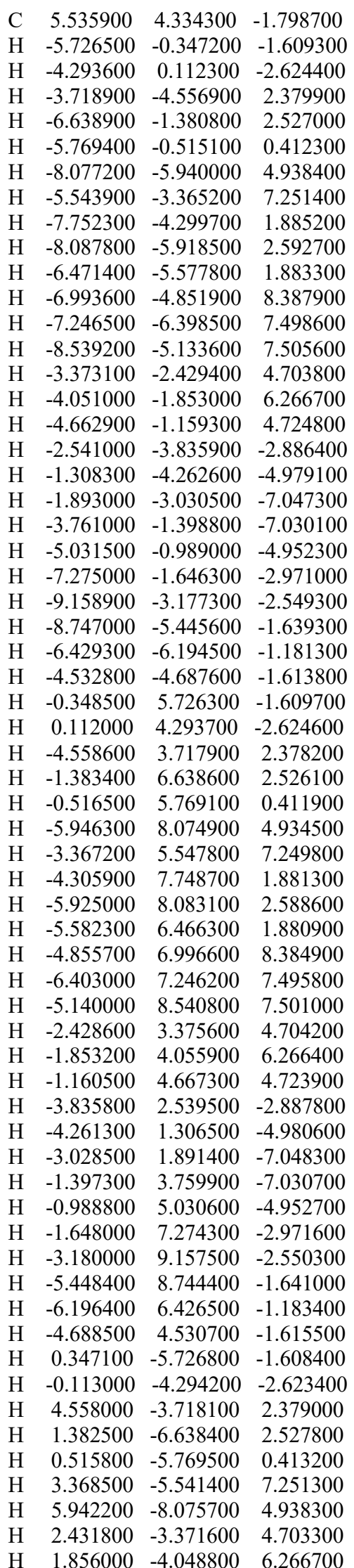

$\begin{array}{lrrr}\mathrm{H} & 1.161700 & -4.661300 & 4.725200 \\ \mathrm{H} & 6.401900 & -7.244000 & 7.498000 \\ \mathrm{H} & 5.136900 & -8.536600 & 7.506000 \\ \mathrm{H} & 4.855600 & -6.990700 & 8.387700 \\ \mathrm{H} & 4.300800 & -7.751900 & 1.885700 \\ \mathrm{H} & 5.919900 & -8.087200 & 2.592600 \\ \mathrm{H} & 5.578900 & -6.471100 & 1.882700 \\ \mathrm{H} & 3.835200 & -2.541200 & -2.887400 \\ \mathrm{H} & 4.260600 & -1.308600 & -4.980300 \\ \mathrm{H} & 3.027600 & -1.893600 & -7.047900 \\ \mathrm{H} & 1.396000 & -3.761700 & -7.029900 \\ \mathrm{H} & 0.987400 & -5.032100 & -4.951700 \\ \mathrm{H} & 1.645700 & -7.275400 & -2.970500 \\ \mathrm{H} & 3.177100 & -9.159100 & -2.549300 \\ \mathrm{H} & 5.445700 & -8.746900 & -1.640200 \\ \mathrm{H} & 6.194600 & -6.429100 & -1.182700 \\ \mathrm{H} & 4.687400 & -4.532800 & -1.614800 \\ \mathrm{H} & 5.726400 & 0.346500 & -1.609800 \\ \mathrm{H} & 4.293400 & -0.113300 & -2.624500 \\ \mathrm{H} & 3.720900 & 4.558100 & 2.377900 \\ \mathrm{H} & 6.640300 & 1.381600 & 2.525400 \\ \mathrm{H} & 5.769600 & 0.514600 & 0.411800 \\ \mathrm{H} & 8.085700 & 5.939300 & 4.930900 \\ \mathrm{H} & 5.545600 & 3.375300 & 7.248800 \\ \mathrm{H} & 7.757500 & 4.293700 & 1.881000 \\ \mathrm{H} & 8.096100 & 5.913300 & 2.585000 \\ \mathrm{H} & 6.479100 & 5.574200 & 1.876100 \\ \mathrm{H} & 8.545000 & 5.137200 & 7.500000 \\ \mathrm{H} & 6.998400 & 4.861000 & 8.382400 \\ \mathrm{H} & 7.255200 & 6.405200 & 7.489900 \\ \mathrm{H} & 3.373100 & 2.439300 & 4.702900 \\ \mathrm{H} & 4.049700 & 1.864100 & 6.266800 \\ \mathrm{H} & 4.659900 & 1.166300 & 4.726100 \\ \mathrm{H} & 2.540400 & 3.834800 & -2.887400 \\ \mathrm{H} & 1.307200 & 4.260600 & -4.979900 \\ \mathrm{H} & 1.891700 & 3.028000 & -7.047900 \\ \mathrm{H} & 3.759900 & 1.396400 & -7.030600 \\ \mathrm{H} & 5.030800 & 0.987400 & -4.952800 \\ \mathrm{H} & 7.274900 & 1.645200 & -2.971300 \\ \mathrm{H} & 9.158700 & 3.176400 & -2.550200 \\ \mathrm{H} & 8.746600 & 5.445100 & -1.641300 \\ \mathrm{H} & 6.428900 & 6.194300 & -1.184000 \\ \mathrm{H} & 4.532400 & 4.687200 & -1.616000\end{array}$

$\begin{array}{lccc}\text { H2 } & & & \\ \mathrm{Rh} & 0.000000 & 0.000000 & -0.097800 \\ \mathrm{Rh} & -0.000200 & -0.000300 & -2.480900 \\ \mathrm{O} & 1.885100 & 0.836600 & -2.415200 \\ \mathrm{O} & 1.828900 & 0.947700 & -0.158700 \\ \mathrm{O} & 0.836700 & -1.885700 & -2.414700 \\ \mathrm{O} & 0.947700 & -1.829000 & -0.158200 \\ \mathrm{O} & -0.837200 & 1.885100 & -2.415100 \\ \mathrm{O} & -0.947700 & 1.829000 & -0.158600 \\ \mathrm{O} & -1.885700 & -0.837300 & -2.414600 \\ \mathrm{O} & -1.829100 & -0.947700 & -0.158100 \\ \mathrm{Cl} & 2.208200 & 4.122200 & 0.017300 \\ \mathrm{Cl} & 4.122200 & -2.208100 & 0.018000 \\ \mathrm{Cl} & -4.122200 & 2.208200 & 0.018100 \\ \mathrm{Cl} & -2.208200 & -4.122200 & 0.018900 \\ \mathrm{C} & 2.383900 & 1.151900 & -1.284500 \\ \mathrm{C} & 3.792400 & 1.710300 & -1.234400 \\ \mathrm{C} & 4.524700 & 2.272700 & -2.497500\end{array}$




\begin{tabular}{|c|c|c|c|}
\hline C & 4.758700 & 0.851900 & -2.055600 \\
\hline 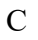 & 4.202800 & 2.206900 & 0.134700 \\
\hline $\mathrm{C}$ & 3.589300 & 3.312400 & 0.753000 \\
\hline C & 4.044900 & 3.833600 & 1.961300 \\
\hline C & 5.139400 & 3.251100 & 2.604500 \\
\hline C & 5.741400 & 2.126300 & 2.037800 \\
\hline $\mathrm{C}$ & 5.269300 & 1.619400 & 0.828800 \\
\hline C & 5.672600 & 3.846200 & 3.851000 \\
\hline C & 5.237700 & 3.370100 & 5.109400 \\
\hline C & 5.752400 & 3.944800 & 6.281900 \\
\hline $\mathrm{C}$ & 6.690800 & 4.982900 & 6.217300 \\
\hline $\mathrm{C}$ & 7.119100 & 5.452100 & 4.970400 \\
\hline $\mathrm{C}$ & 6.619600 & 4.894700 & 3.783500 \\
\hline $\mathrm{C}$ & 4.225700 & 2.258000 & 5.211200 \\
\hline 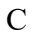 & 7.242000 & 5.598300 & 7.470200 \\
\hline C & 7.104100 & 5.420800 & 2.455800 \\
\hline $\mathrm{C}$ & 3.753600 & 2.500300 & -3.756600 \\
\hline $\mathrm{C}$ & 2.678200 & 3.403000 & -3.781200 \\
\hline $\mathrm{C}$ & 1.966400 & 3.617900 & -4.964500 \\
\hline $\mathrm{C}$ & 2.325900 & 2.940200 & -6.132100 \\
\hline $\mathrm{C}$ & 3.411700 & 2.060500 & -6.122500 \\
\hline C & 4.132500 & 1.851100 & -4.943100 \\
\hline $\mathrm{C}$ & 5.637200 & 3.252500 & -2.269100 \\
\hline $\mathrm{C}$ & 6.967600 & 2.903900 & -2.566100 \\
\hline $\mathrm{C}$ & 8.005200 & 3.811800 & -2.333500 \\
\hline $\mathrm{C}$ & 7.724200 & 5.081000 & -1.822100 \\
\hline $\mathrm{C}$ & 6.403200 & 5.450900 & -1.561000 \\
\hline $\mathrm{C}$ & 5.363100 & 4.547600 & -1.796800 \\
\hline $\mathrm{C}$ & 1.151800 & -2.384200 & -1.283900 \\
\hline $\mathrm{C}$ & 1.710400 & -3.792700 & -1.233500 \\
\hline $\mathrm{C}$ & 2.272900 & -4.525300 & -2.496300 \\
\hline $\mathrm{C}$ & 0.852100 & -4.759100 & -2.054400 \\
\hline $\mathrm{C}$ & 2.207000 & -4.202800 & 0.135800 \\
\hline $\mathrm{C}$ & 3.312400 & -3.589100 & 0.753900 \\
\hline $\mathrm{C}$ & 3.833600 & -4.044500 & 1.962300 \\
\hline $\mathrm{C}$ & 3.251300 & -5.138800 & 2.605800 \\
\hline $\mathrm{C}$ & 2.126400 & -5.740900 & 2.039200 \\
\hline $\mathrm{C}$ & 1.619400 & -5.269100 & 0.830100 \\
\hline $\mathrm{C}$ & 3.846300 & -5.671800 & 3.852400 \\
\hline $\mathrm{C}$ & 4.894700 & -6.618800 & 3.785000 \\
\hline $\mathrm{C}$ & 5.452200 & -7.118100 & 4.971900 \\
\hline $\mathrm{C}$ & 4.983100 & -6.689500 & 6.218700 \\
\hline $\mathrm{C}$ & 3.944900 & -5.751200 & 6.283300 \\
\hline $\mathrm{C}$ & 3.370200 & -5.236700 & 5.110700 \\
\hline $\mathrm{C}$ & 5.420900 & -7.103600 & 2.457400 \\
\hline $\mathrm{C}$ & 5.598500 & -7.240600 & 7.471800 \\
\hline $\mathrm{C}$ & 2.258200 & -4.224600 & 5.212400 \\
\hline $\mathrm{C}$ & 2.500400 & -3.754400 & -3.755600 \\
\hline $\mathrm{C}$ & 3.403100 & -2.679000 & -3.780400 \\
\hline $\mathrm{C}$ & 3.618100 & -1.967600 & -4.963900 \\
\hline $\mathrm{C}$ & 2.940500 & -2.327300 & -6.131500 \\
\hline $\mathrm{C}$ & 2.060700 & -3.413100 & -6.121600 \\
\hline $\mathrm{C}$ & 1.851200 & -4.133600 & -4.942100 \\
\hline $\mathrm{C}$ & 3.252700 & -5.637700 & -2.267700 \\
\hline $\mathrm{C}$ & 2.904100 & -6.968200 & -2.564300 \\
\hline $\mathrm{C}$ & 3.812000 & -8.005600 & -2.331400 \\
\hline $\mathrm{C}$ & 5.081100 & -7.724500 & -1.820100 \\
\hline $\mathrm{C}$ & 5.451100 & -6.403500 & -1.559300 \\
\hline $\mathrm{C}$ & 4.547800 & -5.363500 & -1.795400 \\
\hline $\mathrm{C}$ & -1.152200 & 2.383900 & -1.284400 \\
\hline $\mathrm{C}$ & -1.710600 & 3.792400 & -1.234300 \\
\hline$C$ & -2.273400 & 4.524600 & -2.497200 \\
\hline
\end{tabular}

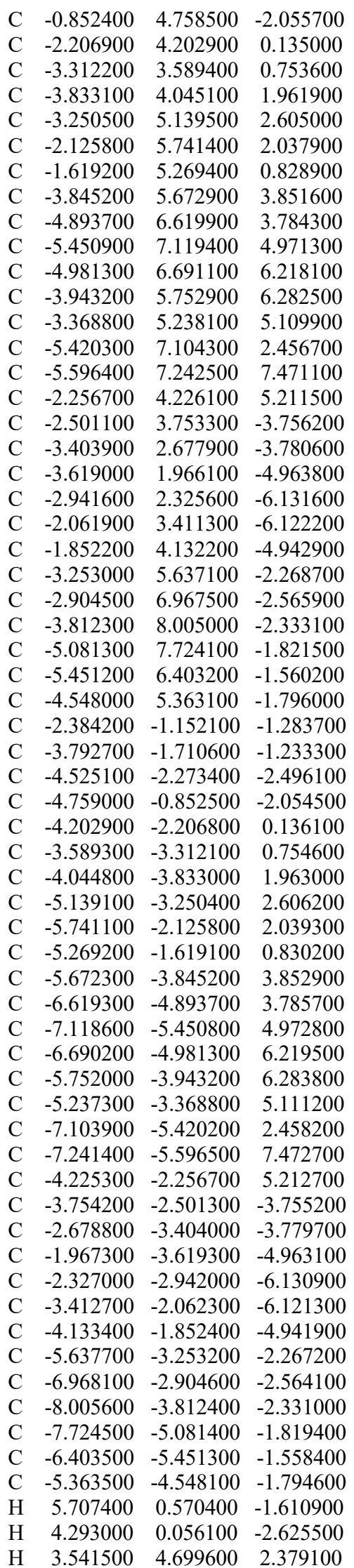

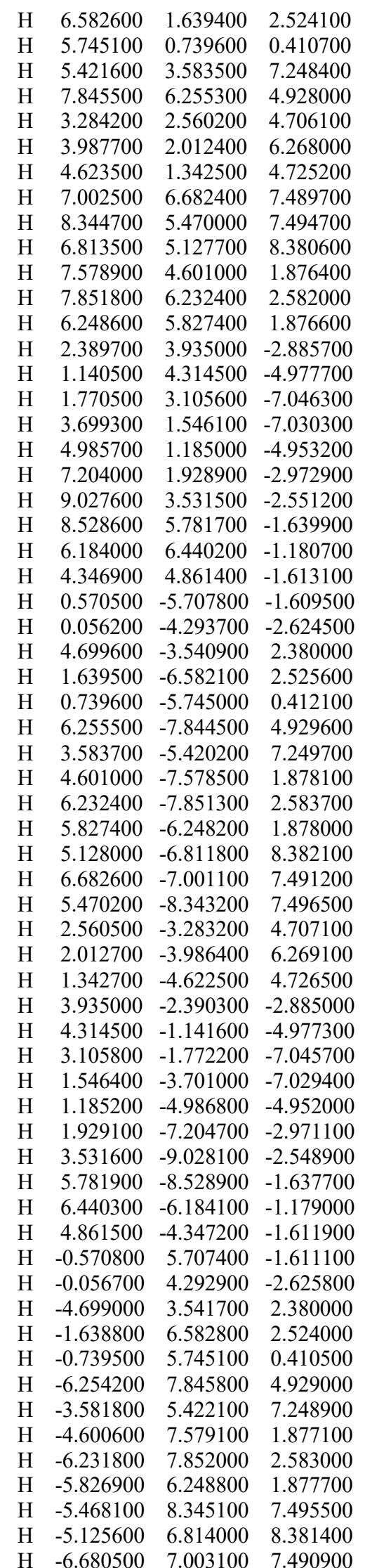




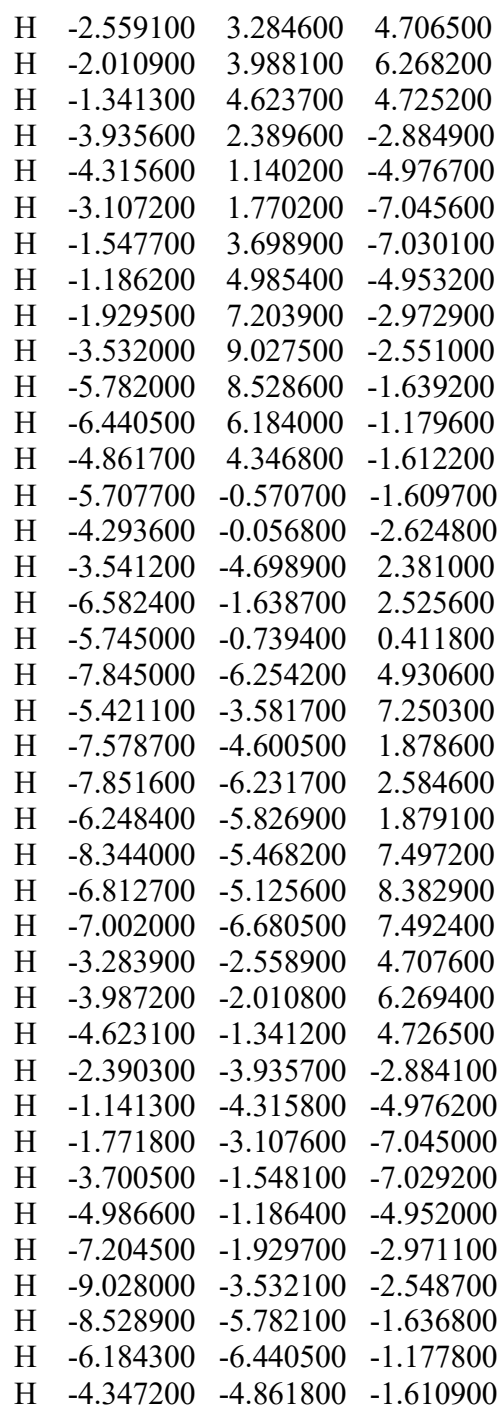

H3

$\begin{array}{llll}\mathrm{Rh} & 0.000000 & 0.000000 & -0.097300\end{array}$

$\begin{array}{llll}\mathrm{Rh} & 0.000000 & -0.000100 & -2.480300\end{array}$

$\begin{array}{llll}\mathrm{O} & -1.377500 & 1.535300 & -2.414400\end{array}$

$\begin{array}{lllll}\text { O } & -1.466300 & 1.446800 & -0.157900\end{array}$

O $\quad 1.535400 \quad 1.377500 \quad-2.414300$

$\begin{array}{llll}\mathrm{O} & 1.446800 & 1.466300 & -0.157900\end{array}$

$\begin{array}{llll}\mathrm{O} & -1.535400 & -1.377700 & -2.414300\end{array}$

$\begin{array}{llll}\mathrm{O} & -1.446900 & -1.466300 & -0.157800\end{array}$

$\begin{array}{llll}\mathrm{O} & 1.377600 & -1.535500 & -2.414200\end{array}$

$\begin{array}{llll}\text { O } & 1.466300 & -1.446800 & -0.157800\end{array}$

$\begin{array}{llll}\mathrm{Cl} & -4.602900 & 0.827400 & 0.017900\end{array}$

$\begin{array}{llll}\mathrm{Cl} & 0.827500 & 4.602900 & 0.018000\end{array}$

$\mathrm{Cl} \quad-0.827700 \quad-4.602900 \quad 0.018400$

$\begin{array}{lllll}\mathrm{Cl} & 4.603000 & -0.827400 & 0.018000\end{array}$

C $\quad-1.831600 \quad 1.911900 \quad-1.283700$

C $\quad-2.797800 \quad 3.079100 \quad-1.233400$

C $\quad-3.559100 \quad 3.601900 \quad-2.496400$

C $\quad-2.280000 \quad 4.263300 \quad-2.054400$

C $\quad-3.397000 \quad 3.315900 \quad 0.135800$

$\begin{array}{llll}\text { C } & -4.259200 & 2.391100 & 0.753800\end{array}$

$\begin{array}{llll}\text { C } & -4.896000 & 2.663500 & 1.961800\end{array}$

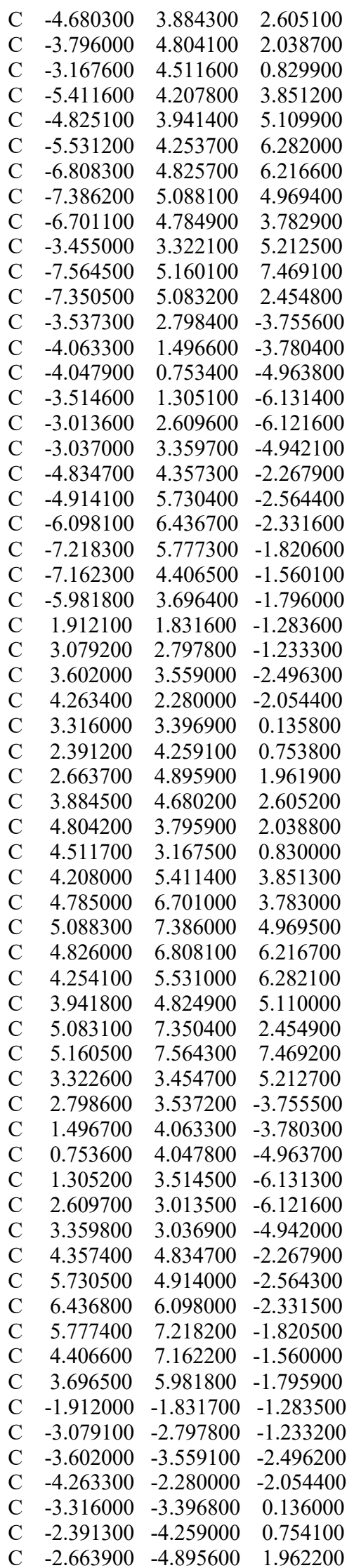

$\begin{array}{llll}\text { C } & -3.884700 & -4.679900 & 2.605400\end{array}$

$\begin{array}{llll}\text { C } & -4.804400 & -3.795600 & 2.038900\end{array}$

C $\quad-4.511900 \quad-3.167300 \quad 0.830100$

$\begin{array}{llll}\text { C } & -4.208400 & -5.410900 & 3.851600\end{array}$

C $\quad-3.942200 \quad-4.824300 \quad 5.110300$

C $\quad-4.254600 \quad-5.530300 \quad 6.282400$

$\begin{array}{llll}\text { C } & -4.826300 & -6.807500 & 6.217100\end{array}$

$\begin{array}{lrrr}\text { C } & -5.088500 & -7.385500 & 4.969900\end{array}$

C $\quad-4.785200 \quad-6.700600 \quad 3.783300$

C $\quad-3.323200 \quad-3.454100 \quad 5.212800$

$\begin{array}{lrrr}\text { C } & -5.160900 & -7.563600 & 7.469600\end{array}$

$\begin{array}{lrrr}\text { C } & -5.083200 & -7.350200 & 2.455300\end{array}$

C $\quad-2.798500 \quad-3.537500 \quad-3.755300$

C $\quad-1.496800 \quad-4.063700 \quad-3.780000$

$\begin{array}{llll}\text { C } & -0.753500 & -4.048400 & -4.963400\end{array}$

C $\quad-1.305000 \quad-3.515100-6.131000$

C $\quad-2.609400 \quad-3.014000 \quad-6.121400$

C $\quad-3.359600 \quad-3.037300 \quad-4.941900$

$\begin{array}{llll}\text { C } & -4.357500 & -4.834700 & -2.267600\end{array}$

C $\quad-5.730600 \quad-4.914000 \quad-2.564200$

C $\quad-6.437000 \quad-6.098000 \quad-2.331300$

C $\quad-5.777700 \quad-7.218200 \quad-1.820100$

C $\quad-4.406900-7.162200 \quad-1.559500$

$\begin{array}{llll}\text { C } & -3.696800 & -5.981800 & -1.795500\end{array}$

C $\quad 1.831700 \quad-1.912100 \quad-1.283500$

C $\quad 2.797900 \quad-3.079100 \quad-1.233100$

C $\quad 3.559100 \quad-3.602100 \quad-2.496100$

$\begin{array}{llll}\text { C } & 2.280000 & -4.263400 & -2.054100\end{array}$

$\begin{array}{llll}\text { C } & 3.397100 & -3.315900 & 0.136000\end{array}$

C $\quad 4.259300 \quad-2.391100 \quad 0.753900$

$\begin{array}{llll}\text { C } & 4.896100 & -2.663500 & 1.962000\end{array}$

$\begin{array}{llll}\text { C } & 4.680500 & -3.884300 & 2.605300\end{array}$

$\begin{array}{llll}\text { C } & 3.796200 & -4.804000 & 2.039000\end{array}$

C $\quad 3.167700 \quad-4.511600 \quad 0.830300$

$\begin{array}{llll}\text { C } & 5.411900 & -4.207700 & 3.851300\end{array}$

C $\quad 4.825700 \quad-3.940900 \quad 5.110100$

$\begin{array}{llll}\text { C } & 5.532000 & -4.253200 & 6.282100\end{array}$

$\begin{array}{llll}\text { C } & 6.808900 & -4.825500 & 6.216600\end{array}$

$\begin{array}{llll}\text { C } & 7.386500 & -5.088300 & 4.969300\end{array}$

$\begin{array}{llll}\text { C } & 6.701300 & -4.785200 & 3.782900\end{array}$

C $\quad 3.455800 \quad-3.321300 \quad 5.212900$

$\begin{array}{llll}\text { C } & 7.565300 & -5.159900 & 7.469000\end{array}$

$\begin{array}{llll}\text { C } & 7.350400 & -5.083800 & 2.454700\end{array}$

$\begin{array}{llll}\text { C } & 3.537300 & -2.798700 & -3.755400\end{array}$

C $\quad 4.063300 \quad-1.496900 \quad-3.780200$

$\begin{array}{llll}\text { C } & 4.047800 & -0.753800 & -4.963700\end{array}$

C $\quad 3.514500 \quad-1.305500 \quad-6.131200$

$\begin{array}{llll}\text { C } & 3.013500 & -2.610000 & -6.121400\end{array}$

C $\quad 3.037000 \quad-3.360000 \quad-4.941800$

C $\quad 4.834700 \quad-4.357500 \quad-2.267600$

$\begin{array}{llll}\text { C } & 4.914000 & -5.730600 & -2.564000\end{array}$

$\begin{array}{lrrr}\text { C } & 6.098100 & -6.436900 & -2.331200\end{array}$

$\begin{array}{llll}\text { C } & 7.218300 & -5.777400 & -1.820200\end{array}$

C $\quad 7.162300 \quad-4.406600 \quad-1.559800$

$\begin{array}{llll}\text { C } & 5.981900 & -3.696600 & -1.795700\end{array}$

$\mathrm{H} \quad-2.305400 \quad 5.252700 \quad-1.609600$

$\begin{array}{llll}\mathrm{H} & -1.379300 & 4.066400 & -2.624400\end{array}$

$\begin{array}{llll}\mathrm{H} & -5.564500 & 1.917200 & 2.379400\end{array}$

$\mathrm{H} \quad-3.592800 \quad 5.754600 \quad 2.525000$

H $\quad-2.477600 \quad 5.235800 \quad 0.412000$

$\begin{array}{llll}\mathrm{H} & -5.086000 & 4.050600 & 7.248800\end{array}$

H $\quad-8.374500 \quad 5.531000 \quad 4.926400$ 


\begin{tabular}{|c|c|c|c|}
\hline H & -3.451500 & 2.333300 & 4.707600 \\
\hline & -3.148500 & 3.171700 & 6.269500 \\
\hline & -2.706700 & 3.983000 & 4.726800 \\
\hline & -6.985100 & 4.897900 & 8.379800 \\
\hline & -8.521700 & 4.597600 & 7.488200 \\
\hline & -7.783000 & 6.248500 & 7.493400 \\
\hline & -6.717000 & 5.787700 & 1.875700 \\
\hline & -8.353300 & 5.543900 & 2.580400 \\
\hline & -7.472700 & 4.143800 & 1.875700 \\
\hline & -4.480000 & 1.057800 & -2.884900 \\
\hline & -4.454900 & -0.247400 & -4.977100 \\
\hline & -3.500300 & 0.725800 & -7.045600 \\
\hline$\pi$ & -2.613400 & 3.042200 & -7.029400 \\
\hline & -2.667300 & 4.376900 & -4.952100 \\
\hline & -4.059700 & 6.256700 & -2.970900 \\
\hline & -6.147200 & 7.495800 & -2.549000 \\
\hline $\mathrm{H}$ & -8.133400 & 6.325800 & -1.638300 \\
\hline $\mathrm{H}$ & -8.035500 & 3.892300 & -1.180000 \\
\hline$\Pi$ & -5.966300 & 2.632900 & -1.612700 \\
\hline 1 & 5.252800 & 2.305300 & -1.609500 \\
\hline$\pi$ & 4.066500 & 1.379200 & -2.624400 \\
\hline $\mathrm{H}$ & 1.917400 & 5.564500 & 2.379500 \\
\hline $\mathrm{H}$ & 5.754700 & 3.592600 & 2.525100 \\
\hline H & 5.235900 & 2.477400 & 0.412100 \\
\hline H & 5.530900 & 8.374400 & 4.926500 \\
\hline H & 4.051100 & 5.085700 & 7.248900 \\
\hline $\mathrm{H}$ & 5.543800 & 3300 & 2.580500 \\
\hline $\mathrm{H}$ & 4.143700 & 7.472600 & 1.875900 \\
\hline $\mathrm{H}$ & 5.787600 & 6.717100 & 1.875700 \\
\hline H & 4.598000 & 8.521400 & 7.488300 \\
\hline $\mathrm{H}$ & 6.248900 & 7.782800 & 7.493300 \\
\hline $\mathrm{H}$ & 4.898500 & 6.984800 & 8.379900 \\
\hline $\mathrm{H}$ & 3.172200 & 3.148200 & 6.269600 \\
\hline $\mathrm{H}$ & 3.983500 & 2.706500 & 4.726900 \\
\hline $\mathrm{H}$ & 2.333700 & 3.451100 & 4.707800 \\
\hline $\mathrm{H}$ & 1.057900 & 4.480000 & -2.884900 \\
\hline $\mathrm{H}$ & -0.247200 & 4.454900 & -4.977000 \\
\hline $\mathrm{H}$ & 0.726000 & 3.500200 & -7.045500 \\
\hline $\mathrm{H}$ & 3.042400 & 2.613200 & -7.029300 \\
\hline $\mathrm{H}$ & 4.377000 & 2.667200 & -4.952000 \\
\hline $\mathrm{H}$ & 6.256800 & 4.059600 & -2.970800 \\
\hline $\mathrm{H}$ & 7.495900 & 6.147100 & -2.548800 \\
\hline $\mathrm{H}$ & 6.325900 & 8.133300 & -1.638200 \\
\hline $\mathrm{H}$ & 3.892400 & 8.035400 & -1.179900 \\
\hline $\mathrm{H}$ & 2.633000 & 5.966200 & -1.612600 \\
\hline $\mathrm{H}$ & -5.252700 & -2.305300 & -1.609600 \\
\hline $\mathrm{H}$ & -4.066400 & -1.379400 & -2.624400 \\
\hline $\mathrm{H}$ & -1.917700 & -5.564100 & 2.379900 \\
\hline $\mathrm{H}$ & -5.755000 & -3.592200 & 2.525200 \\
\hline $\mathrm{H}$ & -5.235900 & -2.477200 & 0.412100 \\
\hline $\mathrm{H}$ & -4.051800 & -5.084900 & 7.249100 \\
\hline $\mathrm{H}$ & -5.531100 & -8.373900 & 4.927000 \\
\hline $\mathrm{H}$ & -2.334300 & -3.450400 & 4.708000 \\
\hline $\mathrm{H}$ & -3.173000 & -3.147300 & 6.269700 \\
\hline $\mathrm{H}$ & -3.984200 & -2.706000 & 4.726900 \\
\hline $\mathrm{H}$ & -4.598300 & -8.520700 & 7.488900 \\
\hline $\mathrm{H}$ & -6.249300 & -7.782100 & 7.493700 \\
\hline $\mathrm{H}$ & -4.898900 & -6.984000 & 8.380300 \\
\hline $\mathrm{H}$ & -5.787700 & -6.716900 & 1.876000 \\
\hline $\mathrm{H}$ & -5.543800 & -8.353100 & 2.581000 \\
\hline $\mathrm{H}$ & -4.143700 & -7.472400 & 1.876400 \\
\hline $\mathrm{H}$ & -1.057900 & -4.480300 & -2.884500 \\
\hline
\end{tabular}

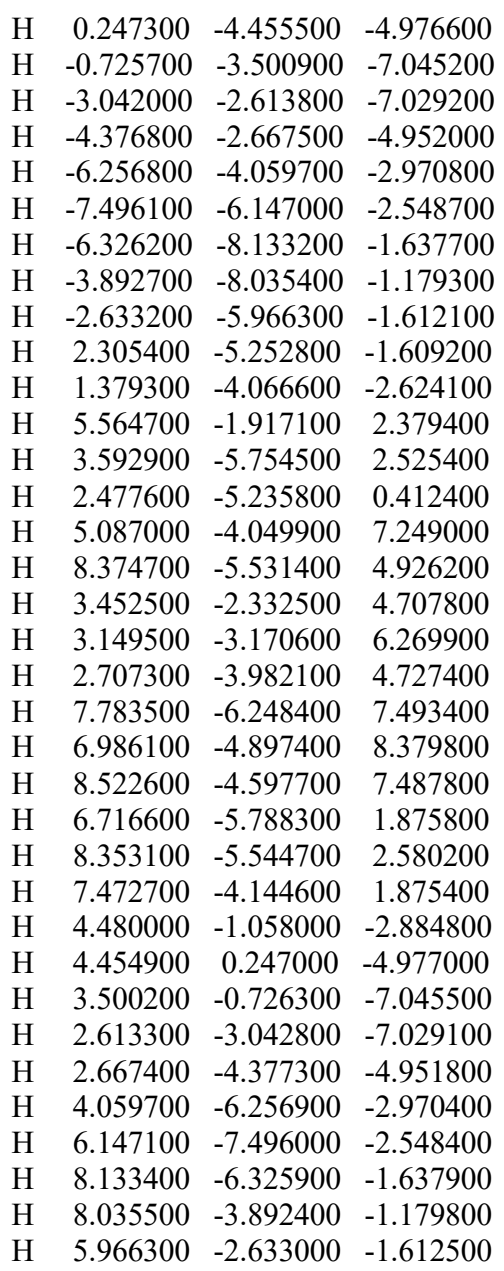

\section{H4}

$\begin{array}{llll}\mathrm{Rh} & -0.000100 & 0.000300 & -0.095400\end{array}$

$\begin{array}{lllll}\mathrm{Rh} & -0.001200 & 0.000900 & -2.478400\end{array}$

$\begin{array}{llll}\mathrm{O} & -0.783100 & 1.909900 & -2.411600\end{array}$

$\begin{array}{lllll}\mathrm{O} & -0.897300 & 1.854700 & -0.155200\end{array}$

$\begin{array}{llll}\text { O } & 1.907800 & 0.782900 & -2.413200\end{array}$

$\begin{array}{llll}\mathrm{O} & 1.854300 & 0.897400 & -0.156800\end{array}$

$\begin{array}{lllll}\mathrm{O} & -1.910100 & -0.781000 & -2.411700\end{array}$

$\begin{array}{llll}\mathrm{O} & -1.854400 & -0.896900 & -0.155400\end{array}$

$\begin{array}{llll}\mathrm{O} & 0.780800 & -1.908000 & -2.413300\end{array}$

$\begin{array}{llll}\text { O } & 0.897100 & -1.853900 & -0.157000\end{array}$

$\begin{array}{llll}\mathrm{Cl} & -4.059900 & 2.324000 & 0.021300\end{array}$

$\begin{array}{llll}\mathrm{Cl} & 2.324600 & 4.059600 & 0.020000\end{array}$

$\begin{array}{lrrr}\mathrm{Cl} & -2.323200 & -4.059800 & 0.018300\end{array}$

$\begin{array}{llll}\mathrm{Cl} & 4.060200 & -2.322900 & 0.015600\end{array}$

$\begin{array}{llll}\text { C } & -1.085000 & 2.416200 & -1.280600\end{array}$

$\begin{array}{llll}\text { C } & -1.603800 & 3.839700 & -1.229900\end{array}$

$\begin{array}{llll}\text { C } & -2.145800 & 4.588000 & -2.492700\end{array}$

$\begin{array}{llll}\text { C } & -0.718900 & 4.782000 & -2.050800\end{array}$

$\begin{array}{llll}\text { C } & -2.089000 & 4.263700 & 0.139200\end{array}$

$\begin{array}{llll}\text { C } & -3.211900 & 3.682100 & 0.756900\end{array}$

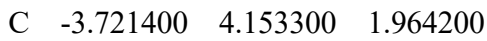

$\begin{array}{llll}\text { C } & -3.109100 & 5.231600 & 2.607100\end{array}$

$\begin{array}{llll}\text { C } & -1.966800 & 5.800800 & 2.041300\end{array}$

$\begin{array}{lrrr}\text { C } & -1.472000 & 5.313500 & 0.833400\end{array}$

$\begin{array}{llll}\text { C } & -3.691000 & 5.783500 & 3.851700\end{array}$ $\begin{array}{lrrr}\text { C } & -4.711800 & 6.760100 & 3.780700\end{array}$

$\begin{array}{lrrr}\text { C } & -5.256900 & 7.277300 & 4.965700\end{array}$

$\begin{array}{llll}\text { C } & -4.802600 & 6.837600 & 6.214200\end{array}$

$\begin{array}{lrrr}\text { C } & -3.791900 & 5.870000 & 6.282300\end{array}$

$\begin{array}{llll}\text { C } & -3.230000 & 5.337100 & 5.111700\end{array}$

$\begin{array}{llll}\text { C } & -5.221200 & 7.257500 & 2.451200\end{array}$

$\begin{array}{lrrr}\text { C } & -5.404400 & 7.408200 & 7.465100\end{array}$

$\begin{array}{lrrr}\text { C } & -2.147600 & 4.293800 & 5.217200\end{array}$

$\begin{array}{llll}\text { C } & -2.394900 & 3.824000 & -3.752100\end{array}$

$\begin{array}{llll}\text { C } & -3.326700 & 2.773800 & -3.777000\end{array}$

$\begin{array}{llll}\text { C } & -3.561400 & 2.068800 & -4.960600\end{array}$

$\begin{array}{llll}\text { C } & -2.874500 & 2.410300 & -6.128200\end{array}$

$\begin{array}{llll}\text { C } & -1.965400 & 3.471600 & -6.118400\end{array}$

$\begin{array}{llll}\text { C } & -1.735900 & 4.185700 & -4.938600\end{array}$

$\begin{array}{llll}\text { C } & -3.094300 & 5.727300 & -2.263800\end{array}$

$\begin{array}{lllll}\text { C } & -2.708400 & 7.047700 & -2.559200\end{array}$

$\begin{array}{llll}\text { C } & -3.587000 & 8.110100 & -2.325800\end{array}$

$\begin{array}{llll}\text { C } & -4.863700 & 7.864100 & -1.815300\end{array}$

$\begin{array}{llll}\text { C } & -5.270600 & 6.553700 & -1.555800\end{array}$

$\begin{array}{llll}\text { C } & -4.396700 & 5.489100 & -1.792300\end{array}$

$\begin{array}{llll}\text { C } & 2.415000 & 1.084800 & -1.282700\end{array}$

$\begin{array}{llll}\text { C } & 3.838700 & 1.603400 & -1.233100\end{array}$

$\begin{array}{llll}\text { C } & 4.585900 & 2.145400 & -2.496400\end{array}$

$\begin{array}{llll}\text { C } & 4.780000 & 0.718400 & -2.055000\end{array}$

$\begin{array}{llll}\text { C } & 4.263900 & 2.088100 & 0.135800\end{array}$

$\begin{array}{llll}\text { C } & 3.683000 & 3.210900 & 0.754300\end{array}$

$\begin{array}{llll}\text { C } & 4.155000 & 3.719700 & 1.961600\end{array}$

$\begin{array}{llll}\text { C } & 5.233400 & 3.106800 & 2.603600\end{array}$

$\begin{array}{llll}\text { C } & 5.802000 & 1.964700 & 2.036900\end{array}$

$\begin{array}{llll}\text { C } & 5.314000 & 1.470600 & 0.829000\end{array}$

$\begin{array}{llll}\text { C } & 5.785900 & 3.687700 & 3.848400\end{array}$

$\begin{array}{llll}\text { C } & 5.340900 & 3.224700 & 5.108300\end{array}$

C $\quad 5.874300 \quad 3.785700 \quad 6.279100$

C $\quad 6.841100 \quad 4.797200 \quad 6.211400$

C $\quad 7.279400 \quad 5.253300 \quad 4.963100$

$\begin{array}{llll}\text { C } & 6.761600 & 4.709200 & 3.777800\end{array}$

C $\quad 4.298500 \quad 2.141500 \quad 5.213400$

$\begin{array}{llll}\text { C } & 7.412300 & 5.397800 & 7.462600\end{array}$

$\begin{array}{llll}\text { C } & 7.257700 & 5.220700 & 2.448600\end{array}$

$\begin{array}{llll}\text { C } & 3.820800 & 2.394700 & -3.755100\end{array}$

$\begin{array}{lrrr}\text { C } & 2.770900 & 3.327000 & -3.779000\end{array}$

C $\quad 2.065000 \quad 3.562200 \quad-4.961900$

$\begin{array}{llll}\text { C } & 2.405100 & 2.875200 & -6.129900\end{array}$

C $\quad 3.466000 \quad 1.965600 \quad-6.121100$

C $\quad 4.181100 \quad 1.735700 \quad-4.942000$

$\begin{array}{llll}\text { C } & 5.725600 & 3.093600 & -2.268400\end{array}$

$\begin{array}{llll}\text { C } & 7.045700 & 2.707500 & -2.565300\end{array}$

$\begin{array}{llll}\text { C } & 8.108400 & 3.585900 & -2.332900\end{array}$

C $\quad 7.863200 \quad 4.862500 \quad-1.821800$

$\begin{array}{llll}\text { C } & 6.553200 & 5.269600 & -1.560800\end{array}$

$\begin{array}{lrrr}\text { C } & 5.488200 & 4.396000 & -1.796400\end{array}$

C $\quad-2.416200 \quad-1.083800 \quad-1.280900$

C $\quad-3.839600 \quad-1.602800 \quad-1.230200$

$\begin{array}{llll}\text { C } & -4.588200 & -2.143800 & -2.493200\end{array}$

$\begin{array}{llll}\text { C } & -4.782200 & -0.717400 & -2.050100\end{array}$

$\begin{array}{llll}\text { C } & -4.263200 & -2.089300 & 0.138600\end{array}$

$\begin{array}{llll}\text { C } & -3.681300 & -3.212700 & 0.755000\end{array}$

$\begin{array}{llll}\text { C } & -4.152200 & -3.723600 & 1.961900\end{array}$

$\begin{array}{llll}\text { C } & -5.230500 & -3.112100 & 2.605600\end{array}$

$\begin{array}{llll}\text { C } & -5.799800 & -1.969300 & 2.041100\end{array}$

$\begin{array}{llll}\text { C } & -5.312900 & -1.473200 & 0.833600\end{array}$

$\begin{array}{lrrr}\text { C } & -5.782100 & -3.695400 & 3.849600\end{array}$ 


\begin{tabular}{|c|c|c|c|}
\hline $\mathrm{C}$ & -6.758700 & -4.716200 & 3.777600 \\
\hline & -7.275600 & -5.262700 & 4.962100 \\
\hline & -6.835700 & -4.809900 & 6.211000 \\
\hline 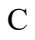 & -5.868100 & -3.799200 & 6.280100 \\
\hline $\mathrm{C}$ & -5.335500 & -3.235900 & 5.110100 \\
\hline$C$ & -7.256300 & -5.224200 & 2.447600 \\
\hline $\mathrm{C}$ & -7.406000 & -5.413200 & 7.461300 \\
\hline $\mathrm{C}$ & -4.292300 & -2.153600 & 5.216700 \\
\hline $\mathrm{C}$ & -3.824600 & -2.391700 & -3.753100 \\
\hline $\mathrm{C}$ & -2.774100 & -3.323300 & -3.779100 \\
\hline $\mathrm{C}$ & -2.069500 & -3.556900 & -4.963100 \\
\hline $\mathrm{C}$ & -2.411600 & -2.869000 & -6.130100 \\
\hline $\mathrm{C}$ & -3.473000 & -1.960200 & -6.119100 \\
\hline $\mathrm{C}$ & -4.186700 & -1.731800 & -4.939000 \\
\hline $\mathrm{C}$ & -5.727300 & -3.092600 & -2.264800 \\
\hline C & -7.047900 & -2.706700 & -2.559400 \\
\hline $\mathrm{C}$ & -8.110000 & -3.585600 & -2.326400 \\
\hline $\mathrm{C}$ & -7.863700 & -4.862700 & -1.817100 \\
\hline $\mathrm{C}$ & -6.553300 & -5.269800 & -1.558500 \\
\hline $\mathrm{C}$ & -5.488800 & -4.395500 & -1.794600 \\
\hline $\mathrm{C}$ & 1.083800 & -2.414900 & -1.282900 \\
\hline $\mathrm{C}$ & 1.602900 & -3.838400 & -1.233200 \\
\hline $\mathrm{C}$ & 2.143600 & -4.586200 & -2.496700 \\
\hline $\mathrm{C}$ & 0.717200 & -4.780500 & -2.053400 \\
\hline $\mathrm{C}$ & 2.089500 & -4.262600 & 0.135400 \\
\hline $\mathrm{C}$ & 3.212900 & -3.681000 & 0.752000 \\
\hline $\mathrm{C}$ & 3.723300 & -4.151900 & 1.959000 \\
\hline $\mathrm{C}$ & 3.111500 & -5.230100 & 2.602600 \\
\hline $\mathrm{C}$ & 1.968900 & -5.799300 & 2.037800 \\
\hline $\mathrm{C}$ & 1.473200 & -5.312400 & 0.830100 \\
\hline $\mathrm{C}$ & 3.694100 & -5.781300 & 3.847100 \\
\hline $\mathrm{C}$ & 4.713700 & -6.759200 & 3.776000 \\
\hline $\mathrm{C}$ & 5.259500 & -7.275700 & 4.961000 \\
\hline $\mathrm{C}$ & 4.807100 & -6.834000 & 6.209500 \\
\hline $\mathrm{C}$ & 3.797600 & -5.865100 & 6.277700 \\
\hline $\mathrm{C}$ & 3.235000 & -5.332900 & 5.107100 \\
\hline $\mathrm{C}$ & 5.221200 & -7.258600 & 2.446500 \\
\hline $\mathrm{C}$ & 5.409600 & -7.404100 & 7.460400 \\
\hline $\mathrm{C}$ & 2.153900 & -4.288200 & 5.212700 \\
\hline $\mathrm{C}$ & 2.391000 & -3.821800 & -3.756200 \\
\hline $\mathrm{C}$ & 3.322800 & -2.771400 & -3.781800 \\
\hline $\mathrm{C}$ & 3.556100 & -2.066100 & -4.965500 \\
\hline $\mathrm{C}$ & 2.868000 & -2.407500 & -6.132500 \\
\hline $\mathrm{C}$ & 1.959000 & -3.468900 & -6.122000 \\
\hline $\mathrm{C}$ & 1.731000 & -4.183300 & -4.942100 \\
\hline $\mathrm{C}$ & 3.092400 & -5.725500 & -2.269200 \\
\hline $\mathrm{C}$ & 2.706400 & -7.045900 & -2.564600 \\
\hline $\mathrm{C}$ & 3.585400 & -8.108100 & -2.332500 \\
\hline $\mathrm{C}$ & 4.862600 & -7.862200 & -1.823400 \\
\hline $\mathrm{C}$ & 5.269700 & -6.551800 & -1.563900 \\
\hline $\mathrm{C}$ & 4.395400 & -5.487200 & -1.799100 \\
\hline $\mathrm{H}$ & -0.410900 & 5.722300 & -1.605800 \\
\hline $\mathrm{H}$ & 0.063500 & 4.294500 & -2.621100 \\
\hline $\mathrm{H}$ & -4.601800 & 3.675100 & 2.381300 \\
\hline $\mathrm{H}$ & -1.456800 & 6.628300 & 2.527500 \\
\hline $\mathrm{H}$ & -0.578600 & 5.763800 & 0.415800 \\
\hline $\mathrm{H}$ & -6.039000 & 8.026300 & 4.920600 \\
\hline $\mathrm{H}$ & -3.442200 & 5.530400 & 7.250000 \\
\hline $\mathrm{H}$ & -4.387200 & 7.707600 & 1.872700 \\
\hline $\mathrm{H}$ & -6.011300 & 8.028400 & 2.574700 \\
\hline $\mathrm{H}$ & -5.651100 & 6.413000 & 1.872500 \\
\hline $\mathrm{H}$ & -5.244500 & 8.506800 & 7.488200 \\
\hline
\end{tabular}

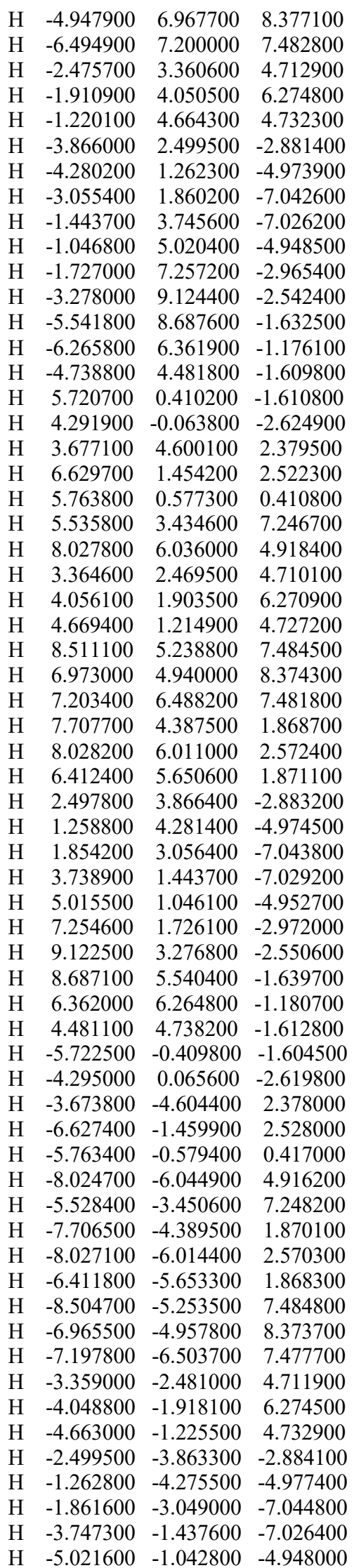

$\begin{array}{lrrr}\mathrm{H} & -7.257700 & -1.724900 & -2.964600 \\ \mathrm{H} & -9.124500 & -3.276600 & -2.542300 \\ \mathrm{H} & -8.687100 & -5.541100 & -1.634600 \\ \mathrm{H} & -6.361100 & -6.265300 & -1.179800 \\ \mathrm{H} & -4.481400 & -4.737700 & -1.612800 \\ \mathrm{H} & 0.409800 & -5.721000 & -1.608300 \\ \mathrm{H} & -0.065900 & -4.292900 & -2.622700 \\ \mathrm{H} & 4.604100 & -3.673600 & 2.375400 \\ \mathrm{H} & 1.459200 & -6.626800 & 2.524500 \\ \mathrm{H} & 0.579400 & -5.762800 & 0.413300 \\ \mathrm{H} & 6.040700 & -8.025800 & 4.915800 \\ \mathrm{H} & 3.449300 & -5.524100 & 7.245500 \\ \mathrm{H} & 4.386000 & -7.708500 & 1.869400 \\ \mathrm{H} & 6.010700 & -8.030000 & 2.569800 \\ \mathrm{H} & 5.651200 & -6.415100 & 1.866400 \\ \mathrm{H} & 5.248500 & -8.502500 & 7.484800 \\ \mathrm{H} & 4.954700 & -6.962000 & 8.372300 \\ \mathrm{H} & 6.500400 & -7.197100 & 7.476600 \\ \mathrm{H} & 2.482700 & -3.355800 & 4.707300 \\ \mathrm{H} & 1.918600 & -4.043600 & 6.270400 \\ \mathrm{H} & 1.225500 & -4.658200 & 4.729100 \\ \mathrm{H} & 3.863000 & -2.497300 & -2.886800 \\ \mathrm{H} & 4.274600 & -1.259400 & -4.979400 \\ \mathrm{H} & 3.047700 & -1.857000 & -7.046900 \\ \mathrm{H} & 1.436400 & -3.742700 & -7.029300 \\ \mathrm{H} & 1.041900 & -5.018200 & -4.951500 \\ \mathrm{H} & 1.724500 & -7.255400 & -2.969700 \\ \mathrm{H} & 3.276300 & -9.122500 & -2.549000 \\ \mathrm{H} & 5.541000 & -8.685700 & -1.641600 \\ \mathrm{H} & 6.265300 & -6.360000 & -1.185300 \\ \mathrm{H} & 4.737600 & -4.480000 & -1.616800\end{array}$

$\begin{array}{lrrr}\text { H5 } & & & \\ \mathrm{Rh} & 0.000400 & -0.000200 & -0.097100 \\ \mathrm{Rh} & 0.001100 & -0.000300 & -2.479900 \\ \mathrm{O} & 1.473200 & -1.445500 & -2.413800 \\ \mathrm{O} & 1.555100 & -1.351600 & -0.157400 \\ \mathrm{O} & -1.444100 & -1.472500 & -2.414600 \\ \mathrm{O} & -1.350900 & -1.554900 & -0.158200 \\ \mathrm{O} & 1.446200 & 1.471800 & -2.413900 \\ \mathrm{O} & 1.351800 & 1.554500 & -0.157500 \\ \mathrm{O} & -1.471000 & 1.444800 & -2.414700 \\ \mathrm{O} & -1.554200 & 1.351300 & -0.158300 \\ \mathrm{Cl} & 4.647300 & -0.533500 & 0.018900 \\ \mathrm{Cl} & -0.533800 & -4.647000 & 0.019400 \\ \mathrm{Cl} & 0.533900 & 4.646900 & 0.018000 \\ \mathrm{Cl} & -4.646500 & 0.533700 & 0.016400 \\ \mathrm{C} & 1.949900 & -1.792400 & -1.282900 \\ \mathrm{C} & 2.988600 & -2.895300 & -1.232200 \\ \mathrm{C} & 3.782100 & -3.368900 & -2.494700 \\ \mathrm{C} & 2.547800 & -4.110600 & -2.052900 \\ \mathrm{C} & 3.601100 & -3.093200 & 0.137300 \\ \mathrm{C} & 4.403100 & -2.115700 & 0.755000 \\ \mathrm{C} & 5.055900 & -2.347100 & 1.963200 \\ \mathrm{C} & 4.917300 & -3.578800 & 2.606900 \\ \mathrm{C} & 4.092600 & -4.552600 & 2.040900 \\ \mathrm{C} & 3.447100 & -4.300700 & 0.832000 \\ \mathrm{C} & 5.667600 & -3.855400 & 3.853000 \\ \mathrm{C} & 6.990600 & -4.351100 & 3.784800 \\ \mathrm{C} & 7.693500 & -4.610200 & 4.971300 \\ \mathrm{C} & 7.100700 & -4.383200 & 6.218500 \\ \mathrm{C} & 5.790500 & -3.891800 & 6.283800\end{array}$




\begin{tabular}{|c|c|c|c|}
\hline 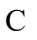 & 5.066000 & -3.625000 & 5.111700 \\
\hline $\mathrm{C}$ & 7.657000 & -4.609300 & 2.456700 \\
\hline $\mathrm{C}$ & 7.876500 & -4.669000 & 7.471100 \\
\hline & 3.660000 & -3.092200 & 5.214300 \\
\hline & 3.709300 & -2.568900 & -3.754300 \\
\hline $\mathrm{C}$ & 4.150700 & -1.236000 & -3.779300 \\
\hline & 4.087800 & -0.495800 & -4.963000 \\
\hline $\mathrm{C}$ & 3.591600 & -1.081000 & -6.130600 \\
\hline & 3.175500 & -2.415000 & -6.120500 \\
\hline & 3.246700 & -3.161600 & -4.940700 \\
\hline $\mathrm{C}$ & 5.103400 & -4.041100 & -2.265600 \\
\hline $\mathrm{C}$ & 5.270400 & -5.406600 & -2.560800 \\
\hline $\mathrm{C}$ & 6.497000 & -6.035700 & -2.327300 \\
\hline & 7.572900 & -5.305500 & -1.816900 \\
\hline & 7.429400 & -3.940900 & -1.557700 \\
\hline $\mathrm{C}$ & 6.206000 & -3.307800 & -1.794300 \\
\hline $\mathrm{C}$ & -1.791500 & -1.949400 & -1.283900 \\
\hline $\mathrm{C}$ & -2.894600 & -2.988000 & -1.233700 \\
\hline $\mathrm{C}$ & -3.367400 & -3.781700 & -2.496500 \\
\hline & -4.109200 & -2.547300 & -2.055300 \\
\hline $\mathrm{C}$ & -3.093400 & -3.600400 & 0.135700 \\
\hline $\mathrm{C}$ & -2.116500 & -4.402600 & 0.754200 \\
\hline $\mathrm{C}$ & -2.349100 & -5.055600 & 1.962000 \\
\hline $\mathrm{C}$ & -3.581400 & -4.917000 & 2.604600 \\
\hline C & -4.554500 & -4.091900 & 2.038100 \\
\hline $\mathrm{C}$ & -4.301400 & -3.446200 & 0.829500 \\
\hline $\mathrm{C}$ & -3.859400 & -5.668000 & 3.850000 \\
\hline $\mathrm{C}$ & -4.350900 & -6.992600 & 3.780600 \\
\hline $\mathrm{C}$ & -4.611600 & -7.695900 & 4.966500 \\
\hline $\mathrm{C}$ & -4.390400 & -7.102200 & 6.214400 \\
\hline $\mathrm{C}$ & -3.903200 & -5.790500 & 6.280900 \\
\hline $\mathrm{C}$ & -3.634800 & -5.065400 & 5.109400 \\
\hline $\mathrm{C}$ & -4.603400 & -7.659900 & 2.451900 \\
\hline $\mathrm{C}$ & -4.677900 & -7.878600 & 7.466300 \\
\hline $\mathrm{C}$ & -3.106400 & -3.657900 & 5.213500 \\
\hline $\mathrm{C}$ & -2.566500 & -3.709100 & -3.755400 \\
\hline $\mathrm{C}$ & -1.233800 & -4.151000 & -3.779600 \\
\hline $\mathrm{C}$ & -0.492700 & -4.088500 & -4.962800 \\
\hline $\mathrm{C}$ & -1.076900 & -3.591900 & -6.130800 \\
\hline $\mathrm{C}$ & -2.410600 & -3.175200 & -6.121600 \\
\hline $\mathrm{C}$ & -3.158100 & -3.246000 & -4.942300 \\
\hline $\mathrm{C}$ & -4.039900 & -5.102900 & -2.267600 \\
\hline $\mathrm{C}$ & -3.307100 & -6.205400 & -1.795500 \\
\hline $\mathrm{C}$ & -3.940500 & -7.428600 & -1.559100 \\
\hline $\mathrm{C}$ & -5.305000 & -7.572000 & -1.819400 \\
\hline $\mathrm{C}$ & -6.034600 & -6.496200 & -2.330600 \\
\hline $\mathrm{C}$ & -5.405200 & -5.269700 & -2.563800 \\
\hline $\mathrm{C}$ & 1.792900 & 1.948900 & -1.283000 \\
\hline $\mathrm{C}$ & 2.895900 & 2.987600 & -1.232300 \\
\hline $\mathrm{C}$ & 3.369800 & 3.780800 & -2.495000 \\
\hline $\mathrm{C}$ & 4.111300 & 2.546600 & -2.052700 \\
\hline $\mathrm{C}$ & 3.093600 & 3.600500 & 0.137000 \\
\hline $\mathrm{C}$ & 2.116000 & 4.402800 & 0.754400 \\
\hline C & 2.347300 & 5.056000 & 1.962400 \\
\hline $\mathrm{C}$ & 3.578900 & 4.917500 & 2.606300 \\
\hline C & 4.552700 & 4.092400 & 2.040800 \\
\hline $\mathrm{C}$ & 4.300900 & 3.446600 & 0.832100 \\
\hline $\mathrm{C}$ & 3.855400 & 5.668300 & 3.852100 \\
\hline $\mathrm{C}$ & 3.628000 & 5.066000 & 5.111100 \\
\hline $\mathrm{C}$ & 3.894900 & 5.790900 & 6.283000 \\
\hline C & 4.383400 & 7.102200 & 6.217100 \\
\hline$C$ & 4.607400 & 7.695600 & 4.969700 \\
\hline
\end{tabular}

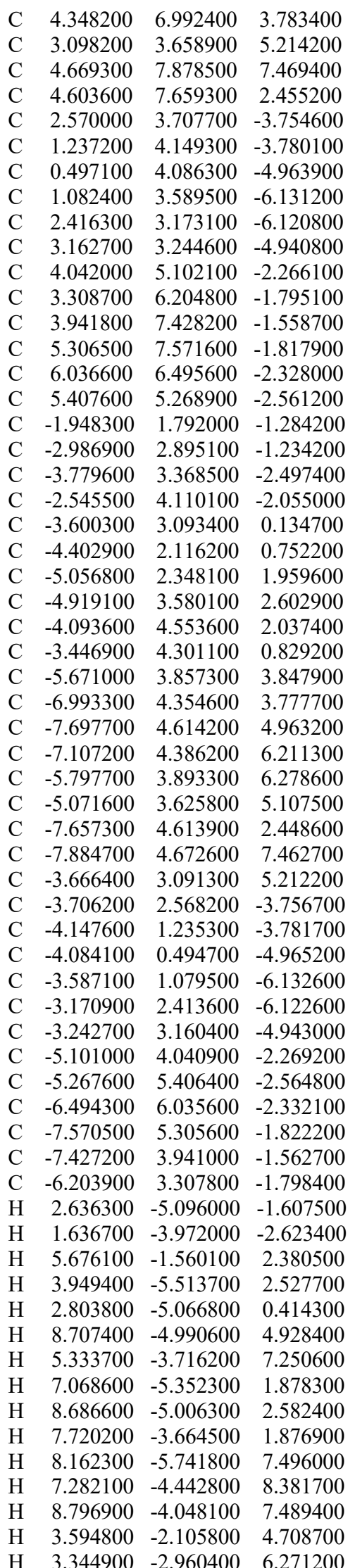

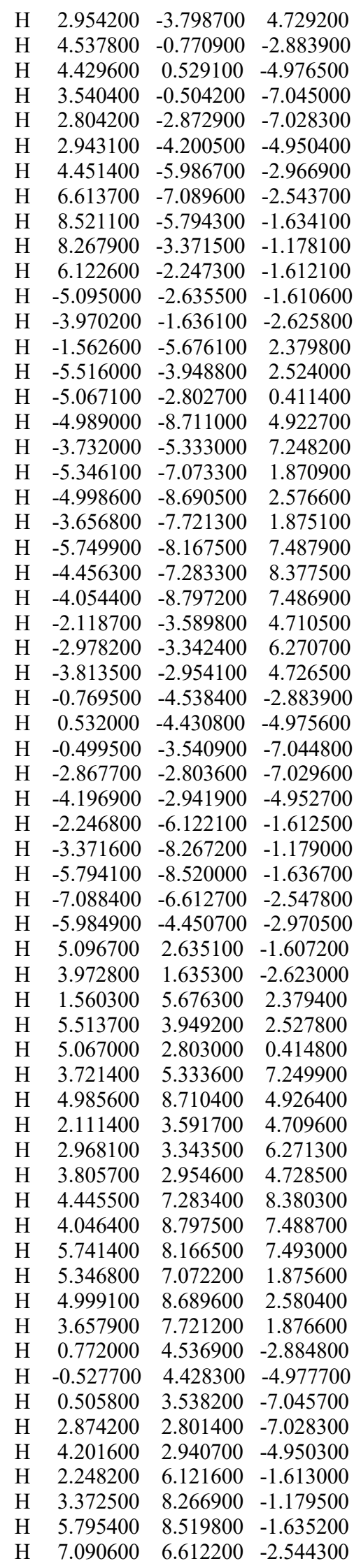




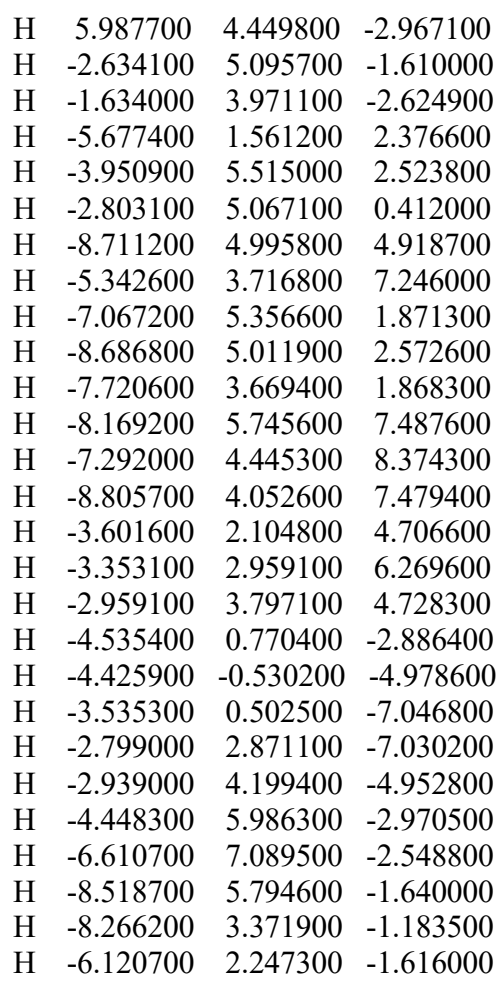

$\begin{array}{lccc}\text { H6 } & & & \\ \mathrm{Rh} & 0.000800 & -0.000500 & -0.096800 \\ \mathrm{Rh} & 0.001600 & -0.001000 & -2.480100 \\ \mathrm{O} & 2.064000 & -0.021700 & -2.413800 \\ \mathrm{O} & 2.058600 & 0.097300 & -0.157100 \\ \mathrm{O} & -0.019200 & -2.063400 & -2.414000 \\ \mathrm{O} & 0.098700 & -2.058400 & -0.157200 \\ \mathrm{O} & 0.022300 & 2.061500 & -2.415000 \\ \mathrm{O} & -0.097100 & 2.057300 & -0.158200 \\ \mathrm{O} & -2.060900 & 0.019700 & -2.415100 \\ \mathrm{O} & -2.057000 & -0.098200 & -0.158300 \\ \mathrm{Cl} & 3.733300 & 2.823400 & 0.019700 \\ \mathrm{Cl} & 2.824600 & -3.733500 & 0.020700 \\ \mathrm{Cl} & -2.823600 & 3.731900 & 0.016600 \\ \mathrm{Cl} & -3.733100 & -2.823300 & 0.019700 \\ \mathrm{C} & 2.648500 & 0.053400 & -1.282800 \\ \mathrm{C} & 4.161300 & -0.029500 & -1.232600 \\ \mathrm{C} & 5.061800 & 0.174200 & -2.495600 \\ \mathrm{C} & 4.677900 & -1.213900 & -2.054200 \\ \mathrm{C} & 4.742000 & 0.248900 & 0.136700 \\ \mathrm{C} & 4.647900 & 1.509300 & 0.755400 \\ \mathrm{C} & 5.279800 & 1.791400 & 1.963900 \\ \mathrm{C} & 6.029500 & 0.803900 & 2.606900 \\ \mathrm{C} & 6.105300 & -0.469300 & 2.039600 \\ \mathrm{C} & 5.464500 & -0.731600 & 0.830400 \\ \mathrm{C} & 6.762300 & 1.120300 & 3.854000 \\ \mathrm{C} & 8.061300 & 1.676100 & 3.787500 \\ \mathrm{C} & 8.747600 & 1.972400 & 4.975000 \\ \mathrm{C} & 8.161600 & 1.724800 & 6.221400 \\ \mathrm{C} & 6.874800 & 1.174700 & 6.285000 \\ \mathrm{C} & 6.167400 & 0.869100 & 5.111900 \\ \mathrm{C} & 8.722200 & 1.952000 & 2.460400 \\ \mathrm{C} & 8.919200 & 2.052100 & 7.475000 \\ \mathrm{C} & 4.782800 & 0.282700 & 5.212700\end{array}$

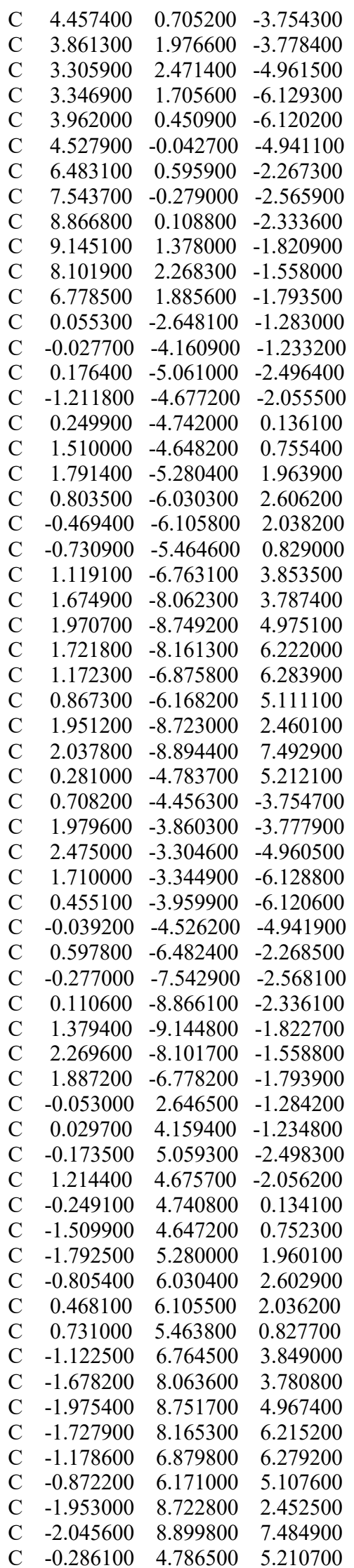

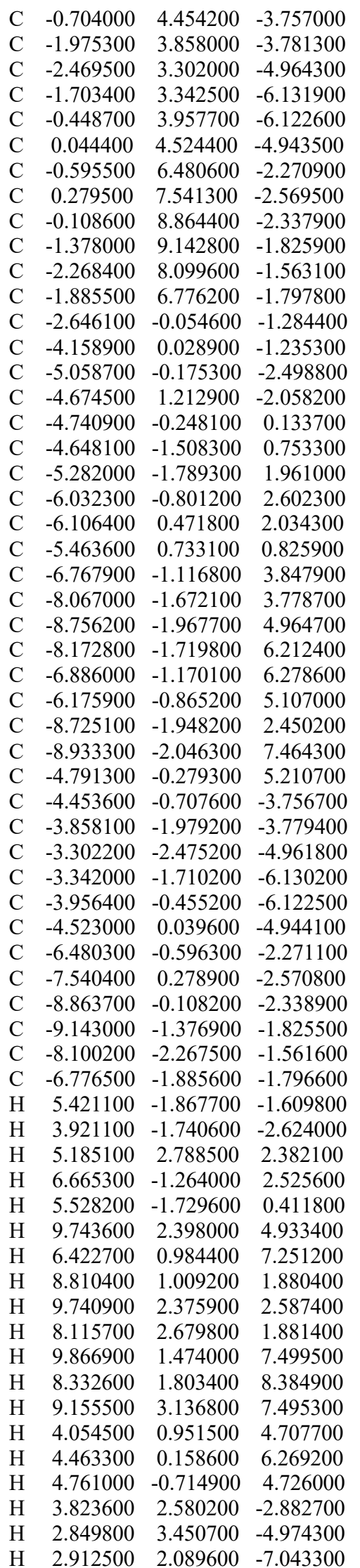




\begin{tabular}{|c|c|c|c|}
\hline & 4.006500 & -0.136600 & \\
\hline H & 5.022100 & -1.005800 & -4.951600 \\
\hline & 7.348500 & -1.262700 & -2.973800 \\
\hline & 9.676800 & -0.574800 & -2.552700 \\
\hline & 10.169600 & 1.675800 & -1.638900 \\
\hline & 8.318800 & 3.257700 & -1.176600 \\
\hline & 5.988100 & 2.596900 & -1.608400 \\
\hline & -1.865900 & -5.420500 & -1.611800 \\
\hline & -1.738300 & -3.920100 & -2.625400 \\
\hline & 2.788300 & -5.185900 & 2.382700 \\
\hline & -1.264400 & -6.665800 & 2.523600 \\
\hline & -1.728700 & -5.528200 & 0.409900 \\
\hline & 2.395900 & -9.744700 & 4.929400 \\
\hline & 0.983100 & -6.427100 & 7.252300 \\
\hline & 1.008600 & -8.810800 & 1.879700 \\
\hline & 2.375000 & -9.741800 & 2.586700 \\
\hline & 2.679200 & -8.1 & 1600 \\
\hline & 2.466900 & -9.898800 & 7.290500 \\
\hline & 1.110500 & -9.022800 & 8.089900 \\
\hline & 2.773500 & -8.311700 & 8.086400 \\
\hline & 0.95 & -4.0 & 7600 \\
\hline & 0.15 & -4.4 & 8700 \\
\hline & -0.716400 & -4.761500 & 4.725000 \\
\hline & 2.582800 & -3.823000 & -2.881900 \\
\hline & 3.45 & -2.8 & 72600 \\
\hline & 2.09 & -2.9 & -7.0 \\
\hline & -0.131900 & -4.0 & 28900 \\
\hline & -1.002300 & -5.020300 & -4.953100 \\
\hline & -1.260400 & -7.3 & -2.976500 \\
\hline & -0.57 & -9.6 & -2.5 \\
\hline & $1.6^{\circ}$ & -10 . & -1 \\
\hline & 3.2 & -8.3 & -1.1 \\
\hline & 2.59 & -5.9 & 08000 \\
\hline & 1.868000 & 5.419200 & 2000 \\
\hline & 1.741400 & 3.9 & 25500 \\
\hline & -2.79 & 5.1 & 2.3 \\
\hline & 1.26 & 6.6 & 2.522200 \\
\hline H & 1.729200 & 5.52 & 0.409600 \\
\hline H & -2.400600 & & 4.920100 \\
\hline & -0.99 & & \\
\hline & -1.009700 & 8.8 & 1.8 \\
\hline$\theta$ & -2.376800 & 9.7 & 2.577500 \\
\hline $\mathrm{H}$ & -2.680400 & 8.115600 & 1.873800 \\
\hline H & -2.474300 & 9.904000 & 7.281000 \\
\hline & -1.1 & & \\
\hline & -2.7818 & 8.3 & $8.0^{\circ}$ \\
\hline${ }_{H}^{H}$ & -0.954700 & 4.057500 & 4.706200 \\
\hline & -0.162900 & 4.468600 & 6.267800 \\
\hline & 0.711900 & 4.7 & \\
\hline & $-2.5^{\circ}$ & & -2.8 \\
\hline & -3.448800 & 2.845600 & -4.977300 \\
\hline $\mathrm{H}$ & -2.086900 & 2.907600 & -7.045800 \\
\hline & 0.139000 & 4.001900 & -7.030400 \\
\hline & 1.007300 & 5.018700 & -4.954000 \\
\hline H & 1.263400 & 7.346000 & -2.976900 \\
\hline $\mathrm{H}$ & 0.575100 & 9.674300 & -2.557000 \\
\hline $\mathrm{H}$ & -1.676000 & 10.167300 & -1.644500 \\
\hline & -3.257900 & 8.316500 & -1.182200 \\
\hline & -2.596700 & 5.985800 & -1.612700 \\
\hline & -5.417700 & 1.867400 & -1.614800 \\
\hline & -3.917000 & 1.738900 & -2.627900 \\
\hline & -5.188500 & -2.786400 & 2.379800 \\
\hline
\end{tabular}

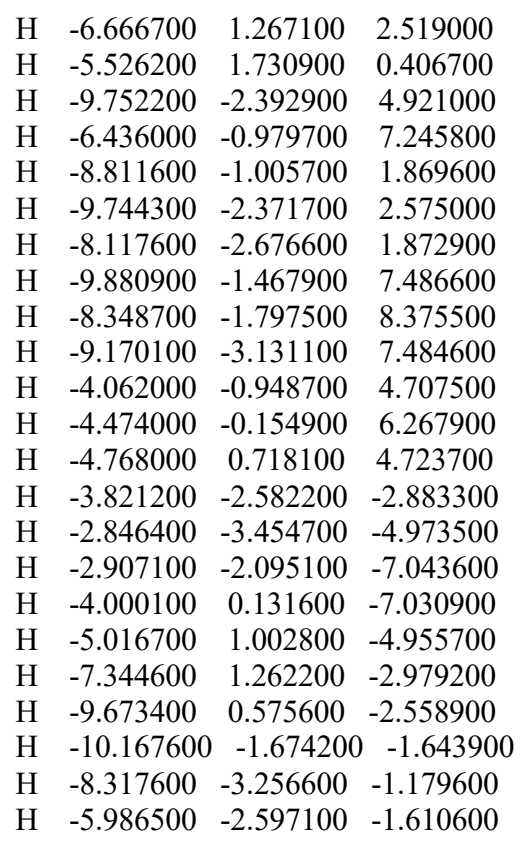

\section{I1}

$\begin{array}{llll}\text { Rh } & 0.000000 & 0.000000 & -0.847500\end{array}$ $\begin{array}{llll}\mathrm{Rh} & 0.000000 & 0.000000 & 1.534600\end{array}$ $\begin{array}{llll}\text { O } & 1.393300 & 1.521600 & 1.468500\end{array}$

$\begin{array}{llll}\mathrm{O} & 1.489600 & 1.424600 & -0.786500\end{array}$

O $\quad 1.521700 \quad-1.393200 \quad 1.468500$

O $\quad 1.424600 \quad-1.489600 \quad-0.786400$

$\begin{array}{llll}\mathrm{O} & -1.393200 & -1.521700 & 1.468500\end{array}$

$\begin{array}{llll}\text { O } & -1.489600 & -1.424600 & -0.786400\end{array}$

$\begin{array}{llll}\mathrm{O} & -1.521600 & 1.393200 & 1.468500\end{array}$

$\begin{array}{llll}\mathrm{O} & -1.424600 & 1.489600 & -0.786400\end{array}$

$\begin{array}{lllll}\mathrm{Cl} & 4.648700 & 0.830300 & -0.983700\end{array}$

Cl $\quad 0.830300 \quad-4.648600 \quad-0.983600$

$\mathrm{Cl} \quad-4.648600 \quad-0.830200 \quad-0.983500$

$\begin{array}{lllll}\mathrm{Cl} & -0.830400 & 4.648600 & -0.983700\end{array}$

$\begin{array}{llll}\mathrm{Br} & 3.234000 & 6.380700 & -3.941700\end{array}$

$\begin{array}{llll}\mathrm{Br} & 6.380700 & -3.234300 & -3.941500\end{array}$

$\mathrm{Br} \quad-3.234400 \quad-6.380500 \quad-3.941700$

$\mathrm{Br} \quad-6.381000 \quad 3.234000 \quad-3.941200$

$\begin{array}{llll}\text { C } & 1.854000 & 1.892500 & 0.338400\end{array}$

$\begin{array}{llll}\text { C } & 2.826800 & 3.056800 & 0.290200\end{array}$

$\begin{array}{llll}\text { C } & 3.586800 & 3.566600 & 1.557500\end{array}$

$\begin{array}{llll}\text { C } & 3.404000 & 3.299500 & -1.088200\end{array}$

C $\quad 4.248200 \quad 2.375000 \quad-1.731200$

C $\quad 4.798700 \quad 2.623900 \quad-2.986900$

$\begin{array}{llll}\text { C } & 4.513100 & 3.818400 & -3.646800\end{array}$

$\begin{array}{llll}\text { C } & 3.665900 & 4.747700 & -3.046500\end{array}$

$\begin{array}{llll}\text { C } & 3.123100 & 4.480500 & -1.790200\end{array}$

$\begin{array}{llll}\text { C } & 2.311700 & 4.237200 & 1.119300\end{array}$

C $\quad 3.560400 \quad 2.754100 \quad 2.810900$

C $\quad 3.062900 \quad 3.309100 \quad 4.001500$

$\begin{array}{llll}\text { C } & 3.039700 & 2.552000 & 5.176500\end{array}$

C $\quad 3.539800 \quad 1.247200 \quad 5.178100$

$\begin{array}{llll}\text { C } & 4.071800 & 0.702300 & 4.006800\end{array}$

$\begin{array}{llll}\text { C } & 4.085600 & 1.451900 & 2.827500\end{array}$

$\begin{array}{llll}\mathrm{C} & 4.862700 & 4.324400 & 1.345900\end{array}$

$\begin{array}{llll}\text { C } & 6.013700 & 3.668300 & 0.877700\end{array}$

C $\quad 7.206100 \quad 4.374100 \quad 0.693900$ $\begin{array}{llll}\text { C } & 7.267700 & 5.735800 & 0.997900\end{array}$

$\begin{array}{llll}\text { C } & 6.140200 & 6.390500 & 1.498700\end{array}$

$\begin{array}{llll}\text { C } & 4.945500 & 5.688100 & 1.682700\end{array}$

$\begin{array}{llll}\text { C } & 1.892500 & -1.853900 & 0.338400\end{array}$

$\begin{array}{llll}\text { C } & 3.056800 & -2.826700 & 0.290300\end{array}$

$\begin{array}{llll}\text { C } & 3.566600 & -3.586700 & 1.557600\end{array}$

$\begin{array}{llll}\text { C } & 3.299500 & -3.404100 & -1.088100\end{array}$

C $\quad 2.375000 \quad-4.248300-1.731100$

$\begin{array}{llll}\text { C } & 2.624000 & -4.798800 & -2.986700\end{array}$

C $\quad 3.818400 \quad-4.513200 \quad-3.646600$

C $\quad 4.747800 \quad-3.666100-3.046400$

C $\quad 4.480500 \quad-3.123100 \quad-1.790000$

$\begin{array}{llll}\text { C } & 4.237200 & -2.311600 & 1.119400\end{array}$

C $\quad 2.754100 \quad-3.560300 \quad 2.811000$

$\begin{array}{llll}\text { C } & 3.309000 & -3.062700 & 4.001600\end{array}$

C $\quad 2.552000 \quad-3.039300 \quad 5.176600$

C $\quad 1.247100 \quad-3.539500 \quad 5.178200$

$\begin{array}{llll}\text { C } & 0.702300 & -4.071600 & 4.006900\end{array}$

$\begin{array}{llll}\text { C } & 1.451900 & -4.085400 & 2.827600\end{array}$

$\begin{array}{llll}\text { C } & 4.324400 & -4.862600 & 1.346100\end{array}$

$\begin{array}{llll}\text { C } & 3.668400 & -6.013600 & 0.877900\end{array}$

C $\quad 4.374100 \quad-7.206000 \quad 0.694300$

$\begin{array}{llll}\text { C } & 5.735800 & -7.267700 & 0.998400\end{array}$

$\begin{array}{llll}\text { C } & 6.390500 & -6.140100 & 1.499100\end{array}$

$\begin{array}{llll}\text { C } & 5.688000 & -4.945400 & 1.683000\end{array}$

$\begin{array}{llll}\text { C } & -1.853900 & -1.892500 & 0.338500\end{array}$

$\begin{array}{llll}\text { C } & -2.826700 & -3.056800 & 0.290200\end{array}$

C $\quad-3.586600 \quad-3.566600 \quad 1.557600$

C $\quad-3.404000 \quad-3.299400-1.088100$

C $\quad-4.248300 \quad-2.374900-1.731000$

C $\quad-4.798800 \quad-2.623800 \quad-2.986700$

C $\quad-4.513300 \quad-3.818200 \quad-3.646600$

C $\quad-3.666100 \quad-4.747600 \quad-3.046400$

C $\quad-3.123200 \quad-4.480400 \quad-1.790100$

C $\quad-2.311600 \quad-4.237200 \quad 1.119400$

C $\quad-3.560300 \quad-2.754100 \quad 2.811000$

C $\quad-3.062700 \quad-3.309100 \quad 4.001600$

C $\quad-3.039300 \quad-2.552100 \quad 5.176600$

C $\quad-3.539500 \quad-1.247300 \quad 5.178300$

C $\quad-4.071500 \quad-0.702400 \quad 4.007000$

C $\quad-4.085400 \quad-1.452000 \quad 2.827600$

$\begin{array}{llll}\text { C } & -4.862600 & -4.324400 & 1.346000\end{array}$

$\begin{array}{llll}\text { C } & -6.013600 & -3.668300 & 0.877900\end{array}$

C $\quad-7.206000 \quad-4.374000 \quad 0.694200$

$\begin{array}{llll}\text { C } & -7.267700 & -5.735700 & 0.998200\end{array}$

$\begin{array}{llll}\text { C } & -6.140100 & -6.390500 & 1.498900\end{array}$

$\begin{array}{llll}\text { C } & -4.945400 & -5.688100 & 1.682800\end{array}$

$\begin{array}{llll}\text { C } & -1.892500 & 1.853900 & 0.338500\end{array}$

$\begin{array}{llll}\text { C } & -3.056800 & 2.826700 & 0.290300\end{array}$

C $\quad-3.566500 \quad 3.586700 \quad 1.557700$

C $\quad-3.299600 \quad 3.404000-1.088100$

C $\quad-2.375100 \quad 4.248200 \quad-1.731100$

$\begin{array}{llll}\text { C } & -2.624200 & 4.798700 & -2.986700\end{array}$

C $\quad-3.818700 \quad 4.513100 \quad-3.646600$

C $\quad-4.748000 \quad 3.665900-3.046200$

$\begin{array}{llll}\text { C } & -4.480600 & 3.123100 & -1.789900\end{array}$

$\begin{array}{llll}\mathrm{C} & -4.237100 & 2.311600 & 1.119600\end{array}$

C $\quad-2.753900 \quad 3.560300 \quad 2.811100$

$\begin{array}{llll}\text { C } & -3.308700 & 3.062700 & 4.001700\end{array}$

C $\quad-2.551500 \quad 3.039500 \quad 5.176700$

C $\quad-1.246800 \quad 3.539700 \quad 5.178100$

$\begin{array}{llll}\text { C } & -0.702100 & 4.071800 & 4.006800\end{array}$ 


\begin{tabular}{|c|c|c|c|}
\hline C & -1.451800 & 4.085600 & 27500 \\
\hline $\mathrm{C}$ & -4.324300 & 4.862600 & 46200 \\
\hline $\mathrm{C}$ & -3.668300 & 6.013600 & 0.877900 \\
\hline $\mathrm{C}$ & -4.374100 & 7.206000 & 0.694300 \\
\hline $\mathrm{C}$ & -5.735700 & 7.267600 & 0.998400 \\
\hline $\mathrm{C}$ & -6.390400 & 6.140100 & 1.499300 \\
\hline $\mathrm{C}$ & -5.687900 & 4.945400 & 1.683100 \\
\hline $\mathrm{H}$ & 5.444800 & 1.879800 & -3.440000 \\
\hline $\mathrm{H}$ & 4.948700 & 4.005600 & -4.624200 \\
\hline $\mathrm{H}$ & 2.446100 & 5.199300 & -1.342700 \\
\hline $\mathrm{H}$ & 2.345100 & 5.229600 & 0.681600 \\
\hline $\mathrm{H}$ & 1.408600 & 4.041200 & 1.685600 \\
\hline $\mathrm{H}$ & 2.697100 & 4.327500 & 4.018600 \\
\hline $\mathrm{H}$ & 2.641600 & 2.980000 & 6.087400 \\
\hline $\mathrm{H}$ & 3.526600 & 0.662900 & 6.089100 \\
\hline $\mathrm{H}$ & 4.480400 & -0.297700 & 4.014400 \\
\hline $\mathrm{H}$ & 4.502100 & 1.018600 & 1.929100 \\
\hline $\mathrm{H}$ & 5.994900 & 2.610200 & 0.666600 \\
\hline $\mathrm{H}$ & 8.085100 & 3.862500 & 0.323600 \\
\hline $\mathrm{H}$ & 8.192400 & 6.280500 & 0.857500 \\
\hline $\mathrm{H}$ & 6.193100 & 7.442000 & 1.749800 \\
\hline $\mathrm{H}$ & 4.087600 & 6.208300 & 2.089300 \\
\hline $\mathrm{H}$ & 1.879800 & -5.444900 & -3.439800 \\
\hline $\mathrm{H}$ & 4.005600 & -4.948900 & -4.624000 \\
\hline $\mathrm{H}$ & 5.199300 & -2.446200 & -1.342600 \\
\hline $\mathrm{H}$ & 5.229600 & -2.345100 & 0.681600 \\
\hline $\mathrm{H}$ & 4.041200 & -1.408600 & 1.685600 \\
\hline $\mathrm{H}$ & 4.327500 & -2.696800 & 4.018700 \\
\hline $\mathrm{H}$ & 2.979900 & -2.641100 & 6.087500 \\
\hline $\mathrm{H}$ & 0.662800 & -3.526300 & 6.089100 \\
\hline $\mathrm{H}$ & -0.297800 & -4.480300 & 4.014500 \\
\hline $\mathrm{H}$ & 1.018700 & -4.502000 & 1.929300 \\
\hline $\mathrm{H}$ & 2.610300 & -5.994900 & 0.666800 \\
\hline $\mathrm{H}$ & 3.862600 & -8.085100 & 0.324000 \\
\hline $\mathrm{H}$ & 6.280500 & -8.192300 & 0.858000 \\
\hline $\mathrm{H}$ & 7.442000 & -6.193000 & 1.750300 \\
\hline $\mathrm{H}$ & 6.208300 & -4.087400 & 2.089600 \\
\hline $\mathrm{H}$ & -5.445000 & -1.879600 & -3.439700 \\
\hline $\mathrm{H}$ & -4.948900 & -4.005400 & -4.624000 \\
\hline $\mathrm{H}$ & -2.446200 & -5.199200 & -1.342700 \\
\hline $\mathrm{H}$ & -2.345100 & -5.229600 & 0.681500 \\
\hline $\mathrm{H}$ & -1.408500 & -4.041300 & 1.685600 \\
\hline $\mathrm{H}$ & -2.696900 & -4.327600 & 4.018600 \\
\hline $\mathrm{H}$ & -2.641200 & -2.980200 & 6.087500 \\
\hline $\mathrm{H}$ & -3.526200 & -0.663000 & 6.089200 \\
\hline $\mathrm{H}$ & -4.480100 & 0.297700 & 4.014600 \\
\hline $\mathrm{H}$ & -4.501900 & -1.018700 & 1.929300 \\
\hline $\mathrm{H}$ & -5.994900 & -2.610200 & 0.666900 \\
\hline $\mathrm{H}$ & -8.085100 & -3.862400 & 0.324000 \\
\hline $\mathrm{H}$ & -8.192300 & -6.280400 & 0.857700 \\
\hline $\mathrm{H}$ & -6.193000 & -7.442000 & 1.749900 \\
\hline $\mathrm{H}$ & -4.087400 & -6.208400 & 2.089300 \\
\hline $\mathrm{H}$ & -1.880100 & 5.444800 & -3.439900 \\
\hline $\mathrm{H}$ & -4.006000 & 4.948700 & -4.624000 \\
\hline $\mathrm{H}$ & -5.199400 & 2.446000 & -1.342400 \\
\hline $\mathrm{H}$ & -5.229500 & 2.345000 & 0.681900 \\
\hline $\mathrm{H}$ & -4.041100 & 1.408600 & 1.685800 \\
\hline $\mathrm{H}$ & -4.327200 & 2.696900 & 4.018800 \\
\hline $\mathrm{H}$ & -2.979400 & 2.641300 & 6.087600 \\
\hline $\mathrm{H}$ & -0.662300 & 3.526500 & 6.089000 \\
\hline $\mathrm{H}$ & 0.298000 & 4.480500 & 4.014300 \\
\hline & -1.018600 & 4.502100 & 1.929200 \\
\hline
\end{tabular}

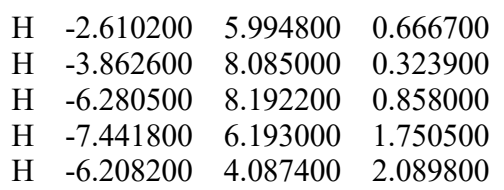

\section{I'1}

$\begin{array}{llll}\text { Rh } & 0.243800 & 0.045500 & -1.007900\end{array}$

$\begin{array}{llll}\mathrm{Rh} & -0.012000 & -0.244400 & 1.344600\end{array}$

$\begin{array}{llll}\mathrm{O} & -1.488900 & -1.637800 & 0.957200\end{array}$

$\begin{array}{llll}\mathrm{O} & -1.281400 & -1.317600 & -1.269300\end{array}$

$\begin{array}{llll}\mathrm{O} & -1.488100 & 1.200000 & 1.299600\end{array}$

$\begin{array}{llll}\mathrm{O} & -1.238400 & 1.489900 & -0.927300\end{array}$

$\begin{array}{llll}\mathrm{O} & 1.404200 & 1.225500 & 1.609800\end{array}$

$\begin{array}{llll}\mathrm{O} & 1.695100 & 1.457500 & -0.618400\end{array}$

O $\quad 1.486700 \quad-1.646400 \quad 1.266300$

O $\quad 1.673100 \quad-1.431700 \quad-0.974700$

$\mathrm{Cl} \quad-1.736800 \quad-4.969900 \quad 0.498800$

Cl $\quad-0.979200 \quad 4.693700 \quad-0.900000$

Cl $\quad 4.900600 \quad 0.983200 \quad-0.509100$

Cl $\quad 1.207200 \quad-4.596800 \quad-1.561200$

$\mathrm{Br} \quad-6.798200 \quad-2.531900 \quad 3.652900$

$\begin{array}{llll}\mathrm{Br} & -6.132600 & 2.852200 & -4.309100\end{array}$

$\begin{array}{llll}\mathrm{Br} & 3.584400 & 6.819000 & -2.910900\end{array}$

$\mathrm{Br} \quad 6.940100 \quad-2.673400 \quad-3.784600$

C $\quad-1.881200 \quad-1.780500 \quad-0.247300$

C $\quad-3.255900-2.403600 \quad-0.423000$

C $\quad-3.859800 \quad-2.720800 \quad-1.823900$

C $\quad-3.749600 \quad-3.113200 \quad 0.818900$

C $\quad-3.136000 \quad-4.280800 \quad 1.307600$

C $\quad-3.592800 \quad-4.932100 \quad 2.452000$

C $\quad-4.687700 \quad-4.425000 \quad 3.150200$

C $\quad-5.310500 \quad-3.262100 \quad 2.699900$

C $\quad-4.838400 \quad-2.623000 \quad 1.553800$

C $\quad-4.177100 \quad-1.411600 \quad-1.146800$

C $\quad-2.976600 \quad-2.697100 \quad-3.029300$

C $\quad-1.911700 \quad-3.605400-3.148100$

C $\quad-1.091000 \quad-3.585900 \quad-4.279800$

C $\quad-1.336000 \quad-2.670500 \quad-5.306500$

C $\quad-2.407200 \quad-1.778600 \quad-5.206900$

C $\quad-3.230200 \quad-1.795000 \quad-4.076900$

$\begin{array}{llll}\text { C } & -4.968100 & -3.727200 & -1.895000\end{array}$

C $\quad-6.256600 \quad-3.335600 \quad-2.302600$

$\begin{array}{llll}\text { C } & -7.288500 & -4.275100 & -2.384700\end{array}$

$\begin{array}{llll}\text { C } & -7.040600 & -5.615700 & -2.081300\end{array}$

$\begin{array}{llll}\text { C } & -5.757000 & -6.020900 & -1.708700\end{array}$

C $\quad-4.722600 \quad-5.084400 \quad-1.626700$

C $\quad-1.814000 \quad 1.721000 \quad 0.183500$

$\begin{array}{llll}\text { C } & -3.067400 & 2.576400 & 0.122800\end{array}$

$\begin{array}{llll}\text { C } & -3.720500 & 3.173700 & 1.408900\end{array}$

$\begin{array}{llll}\text { C } & -3.291400 & 3.211000 & -1.232100\end{array}$

$\begin{array}{llll}\text { C } & -2.420400 & 4.176800 & -1.770600\end{array}$

C $\quad-2.642900 \quad 4.758000 \quad-3.017500$

C $\quad-3.751000 \quad 4.379900 \quad-3.774600$

C $\quad-4.623600 \quad 3.413300 \quad-3.278500$

$\begin{array}{llll}\text { C } & -4.386300 & 2.843900 & -2.027800\end{array}$

$\begin{array}{llll}\text { C } & -4.225000 & 1.872700 & 0.842400\end{array}$

C $\quad-2.956600 \quad 3.147300 \quad 2.693500$

$\begin{array}{llll}\text { C } & -1.763300 & 3.877100 & 2.824200\end{array}$

C $\quad-1.063900 \quad 3.873600 \quad 4.034200$

C $\quad-1.545400 \quad 3.138900 \quad 5.120200$

C $\quad-2.738800 \quad 2.421100 \quad 5.003600$
C $\quad-3.449100 \quad 2.432700 \quad 3.799100$

C $\quad-4.613600 \quad 4.366900 \quad 1.249700$

$\begin{array}{llll}\text { C } & -4.089700 & 5.608800 & 0.852500\end{array}$

C $\quad-4.926200 \quad 6.720800 \quad 0.720500$

$\begin{array}{llll}\text { C } & -6.289000 & 6.609800 & 1.005100\end{array}$

C $\quad-6.815000 \quad 5.389000 \quad 1.433900$

C $\quad-5.981500 \quad 4.274500 \quad 1.565900$

$\begin{array}{llll}\text { C } & 1.941600 & 1.769700 & 0.589900\end{array}$

$\begin{array}{llll}\text { C } & 2.849700 & 2.967000 & 0.798300\end{array}$

$\begin{array}{llll}\text { C } & 3.415500 & 3.343700 & 2.205100\end{array}$

C $\quad 3.566500 \quad 3.405000 \quad-0.460000$

$\begin{array}{llll}\text { C } & 4.518300 & 2.595600 & -1.107700\end{array}$

$\begin{array}{llll}\text { C } & 5.194500 & 3.018300 & -2.250300\end{array}$

$\begin{array}{llll}\text { C } & 4.931900 & 4.278000 & -2.786900\end{array}$

$\begin{array}{llll}\text { C } & 3.982800 & 5.098200 & -2.179900\end{array}$

C $\quad 3.314000 \quad 4.657000 \quad-1.038500$

$\begin{array}{llll}\text { C } & 2.163000 & 4.005000 & 1.692200\end{array}$

$\begin{array}{llll}\text { C } & 3.296000 & 2.367100 & 3.330200\end{array}$

$\begin{array}{llll}\text { C } & 2.580300 & 2.706200 & 4.490700\end{array}$

$\begin{array}{llll}\text { C } & 2.466200 & 1.790800 & 5.541300\end{array}$

$\begin{array}{llll}\text { C } & 3.087200 & 0.542100 & 5.452200\end{array}$

C $\quad 3.829400 \quad 0.211200 \quad 4.315900$

$\begin{array}{llll}\text { C } & 3.936000 & 1.118800 & 3.258700\end{array}$

$\begin{array}{llll}\text { C } & 4.653600 & 4.186500 & 2.254100\end{array}$

$\begin{array}{llll}\text { C } & 4.607700 & 5.483700 & 2.796900\end{array}$

$\begin{array}{llll}\text { C } & 5.764600 & 6.266200 & 2.857500\end{array}$

$\begin{array}{llll}\text { C } & 6.980200 & 5.755600 & 2.396300\end{array}$

$\begin{array}{llll}\text { C } & 7.043100 & 4.456900 & 1.886400\end{array}$

$\begin{array}{llll}\text { C } & 5.888700 & 3.671300 & 1.825600\end{array}$

$\begin{array}{llll}\text { C } & 2.010600 & -1.940900 & 0.142300\end{array}$

$\begin{array}{llll}\text { C } & 3.210100 & -2.868800 & 0.114700\end{array}$

$\begin{array}{llll}\text { C } & 3.620600 & -3.745500 & 1.340900\end{array}$

$\begin{array}{llll}\text { C } & 3.615700 & -3.277400 & -1.285900\end{array}$

C $\quad 2.791600 \quad-4.068500 \quad-2.107900$

$\begin{array}{llll}\text { C } & 3.179700 & -4.456100 & -3.389200\end{array}$

C $\quad 4.417000 \quad-4.056100 \quad-3.891700$

C $\quad 5.250300 \quad-3.258900 \quad-3.109000$

C $\quad 4.844400 \quad-2.877900-1.830300$

$\begin{array}{llll}\text { C } & 4.280300 & -2.410400 & 1.111000\end{array}$

$\begin{array}{llll}\text { C } & 2.694300 & -3.875800 & 2.506700\end{array}$

C $\quad 3.123900 \quad-3.506600 \quad 3.792000$

$\begin{array}{llll}\text { C } & 2.259900 & -3.621800 & 4.884700\end{array}$

$\begin{array}{llll}\text { C } & 0.969700 & -4.128300 & 4.706300\end{array}$

C $\quad 0.546200 \quad-4.530100 \quad 3.436800$

$\begin{array}{llll}\text { C } & 1.407600 & -4.413600 & 2.341500\end{array}$

$\begin{array}{llll}\text { C } & 4.443500 & -4.969100 & 1.073500\end{array}$

C $\quad 3.877100 \quad-6.082500 \quad 0.430400$

$\begin{array}{llll}\text { C } & 4.641600 & -7.228200 & 0.193200\end{array}$

$\begin{array}{llll}\text { C } & 5.971500 & -7.282600 & 0.616400\end{array}$

$\begin{array}{llll}\text { C } & 6.534500 & -6.195800 & 1.289400\end{array}$

$\begin{array}{llll}\text { C } & 5.773000 & -5.047800 & 1.527400\end{array}$

$\mathrm{H} \quad-3.085300 \quad-5.829500 \quad 2.789400$

$\mathrm{H} \quad-5.038200 \quad-4.939600 \quad 4.040300$

$\mathrm{H} \quad-5.316600 \quad-1.707300 \quad 1.223400$

H $\quad-5.170800 \quad-1.246700 \quad-0.741800$

$\mathrm{H} \quad-3.699900 \quad-0.508400 \quad-1.514000$

$\mathrm{H} \quad-1.714000 \quad-4.321900 \quad-2.363400$

H $\quad-0.266000-4.281600 \quad-4.361900$

$\mathrm{H} \quad-0.699600 \quad-2.656500 \quad-6.181800$

H $\quad-2.601500 \quad-1.075700 \quad-6.006700$

H $\quad-4.061600-1.103400-4.016700$

H $\quad-6.463300 \quad-2.303900 \quad-2.558200$ 
$\begin{array}{rrrr}\mathrm{H} & -8.279700 & -3.964200 & -2.688700 \\ \mathrm{H} & -7.840000 & -6.342400 & -2.146700 \\ \mathrm{H} & -5.560900 & -7.062800 & -1.491300 \\ \mathrm{H} & -3.731600 & -5.422500 & -1.366900 \\ \mathrm{H} & -1.943500 & 5.499000 & -3.389400 \\ \mathrm{H} & -3.915600 & 4.838400 & -4.745500 \\ \mathrm{H} & -5.060800 & 2.079100 & -1.658500 \\ \mathrm{H} & -5.193800 & 1.847600 & 0.353000 \\ \mathrm{H} & -3.964700 & 0.948600 & 1.348100 \\ \mathrm{H} & -1.375300 & 4.448400 & 1.992400 \\ \mathrm{H} & -0.151800 & 4.446200 & 4.131100 \\ \mathrm{H} & -0.998600 & 3.133100 & 6.054200 \\ \mathrm{H} & -3.117000 & 1.860700 & 5.848700 \\ \mathrm{H} & -4.379600 & 1.883300 & 3.727300 \\ \mathrm{H} & -3.035100 & 5.722900 & 0.656100 \\ \mathrm{H} & -4.515700 & 7.671300 & 0.405100 \\ \mathrm{H} & -6.935100 & 7.472300 & 0.904600 \\ \mathrm{H} & -7.868300 & 5.307200 & 1.669100 \\ \mathrm{H} & -6.403400 & 3.340600 & 1.915700 \\ \mathrm{H} & 5.920100 & 2.357700 & -2.712600 \\ \mathrm{H} & 5.465700 & 4.601100 & -3.676100 \\ \mathrm{H} & 2.560800 & 5.293100 & -0.587000 \\ \mathrm{H} & 2.190100 & 5.047000 & 1.389700 \\ \mathrm{H} & 1.209100 & 3.695900 & 2.103800 \\ \mathrm{H} & 2.112300 & 3.677500 & 4.581100 \\ \mathrm{H} & 1.901800 & 2.052500 & 6.427000 \\ \mathrm{H} & 3.002300 & -0.164800 & 6.267500 \\ \mathrm{H} & 4.327600 & -0.746100 & 4.256200 \\ \mathrm{H} & 4.512500 & 0.847600 & 2.385400 \\ \mathrm{H} & 3.677600 & 5.889700 & 3.174400 \\ \mathrm{H} & 5.719400 & 7.267100 & 3.266800 \\ \mathrm{H} & 6.221000 & -4.223000 & 2.067000 \\ \mathrm{H} & 7.875400 & 6.362000 & 2.445000 \\ \mathrm{H} & 7.988500 & 4.055300 & 1.545200 \\ \mathrm{H} & 5.963300 & 2.660500 & 1.454700 \\ \mathrm{H} & 2.508400 & -5.066400 & -3.984000 \\ \mathrm{H} & 4.713000 & -4.365200 & -4.890200 \\ \mathrm{H} & 5.488800 & -2.236300 & -1.240000 \\ \mathrm{H} & 5.311000 & -2.365200 & 0.774400 \\ \mathrm{H} & 3.994200 & -1.580300 & 1.747300 \\ \mathrm{H} & 4.127600 & -3.131800 & 3.945900 \\ \mathrm{H} & 2.592200 & -3.322500 & 5.870300 \\ & 0.301600 & -4.217900 & 5.553000 \\ \mathrm{H} & -1.070700 & -4.733000 & 3.302900 \\ & & & \\ \mathrm{H} & & \end{array}$

\section{J1}

$\begin{array}{llll}\mathrm{Rh} & 0.000000 & 0.000000 & -0.872500\end{array}$ $\begin{array}{llll}\mathrm{Rh} & 0.000000 & 0.000000 & 1.509700\end{array}$

O $\quad 1.916200 \quad-0.764700 \quad 1.443400$

$\begin{array}{llll}\mathrm{O} & 1.859300 & -0.888800 & -0.811700\end{array}$

$\begin{array}{llll}\mathrm{O} & -0.764700 & -1.916200 & 1.443400\end{array}$

O $\quad-0.888800 \quad-1.859300 \quad-0.811700$

$\begin{array}{llll}\mathrm{O} & -1.916200 & 0.764700 & 1.443400\end{array}$

$\begin{array}{llll}\mathrm{O} & -1.859300 & 0.888800 & -0.811600\end{array}$

$\begin{array}{llll}\text { O } & 0.764700 & 1.916200 & 1.443400\end{array}$

$\begin{array}{llll}\mathrm{O} & 0.888800 & 1.859300 & -0.811600\end{array}$

Cl $\quad 2.434300 \quad-4.053900 \quad-1.006500$
Cl $\quad-4.054000 \quad-2.434300 \quad-1.006400$

$\mathrm{Cl} \quad-2.434300 \quad 4.054000 \quad-1.006300$

$\mathrm{Cl} \quad 4.054000 \quad 2.434300 \quad-1.006400$

F $\quad 0.504900 \quad 6.320900 \quad-5.027600$

F $\quad-0.131200 \quad 7.273700 \quad-3.094100$

F $\quad 1.799600 \quad 7.892100 \quad-4.064000$

F $\quad-6.320800 \quad 0.505000 \quad-5.027700$

F $\quad-7.892100 \quad 1.799700 \quad-4.064000$

F $\quad-7.273800 \quad-0.131100 \quad-3.094200$

F $\quad-0.505000 \quad-6.320700 \quad-5.027800$

F $\quad-1.799600 \quad-7.892000 \quad-4.064300$

F $\quad 0.131200 \quad-7.273600 \quad-3.094400$

F $\quad 7.892000 \quad-1.799500 \quad-4.064200$

F $\quad 6.320700 \quad-0.504800 \quad-5.027800$

F $\quad 7.273700 \quad 0.131200 \quad-3.094300$

C $\quad 2.426900 \quad-1.061900 \quad 0.313000$

C $\quad 3.862200 \quad-1.553600 \quad 0.263800$

$\begin{array}{llll}\text { C } & 4.609900 & -2.082000 & 1.530800\end{array}$

$\begin{array}{lrrr}\text { C } & 4.292900 & -2.005700 & -1.116000\end{array}$

C $\quad 3.730900 \quad-3.125200 \quad-1.754200$

C $\quad 4.161600 \quad-3.545400 \quad-3.009300$

$\begin{array}{llll}\text { C } & 5.171000 & -2.846800 & -3.667700\end{array}$

$\begin{array}{llll}\text { C } & 5.746300 & -1.713200 & -3.081600\end{array}$

$\begin{array}{llll}\text { C } & 5.290700 & -1.314000 & -1.818100\end{array}$

$\begin{array}{llll}\text { C } & 4.778800 & -0.650700 & 1.094900\end{array}$

$\begin{array}{llll}\text { C } & 3.841500 & -2.350400 & 2.783700\end{array}$

$\begin{array}{llll}\text { C } & 4.179300 & -1.686700 & 3.974700\end{array}$

C $\quad 3.463800 \quad-1.937700 \quad 5.149100$

$\begin{array}{llll}\text { C } & 2.426200 & -2.873900 & 5.149700\end{array}$

C $\quad 2.110100 \quad-3.565900 \quad 3.978000$

C $\quad 2.815200 \quad-3.308700 \quad 2.799200$

$\begin{array}{llll}\text { C } & 5.775300 & -3.000500 & 1.318300\end{array}$

$\begin{array}{llll}\text { C } & 5.576900 & -4.309400 & 0.847600\end{array}$

$\begin{array}{llll}\text { C } & 6.664100 & -5.167900 & 0.662200\end{array}$

$\begin{array}{llll}\text { C } & 7.957000 & -4.736600 & 0.967100\end{array}$

$\begin{array}{lrrr}\text { C } & 8.162700 & -3.450100 & 1.470700\end{array}$

$\begin{array}{llll}\text { C } & 7.077700 & -2.588300 & 1.656300\end{array}$

C $\quad-1.061900 \quad-2.426900 \quad 0.313000$

C $\quad-1.553700 \quad-3.862200 \quad 0.263800$

$\begin{array}{llll}\text { C } & -2.082000 & -4.609900 & 1.530800\end{array}$

$\begin{array}{llll}\text { C } & -2.005800 & -4.293000 & -1.116000\end{array}$

$\begin{array}{llll}\text { C } & -3.125400 & -3.730800 & -1.754200\end{array}$

C $\quad-3.545400 \quad-4.161500 \quad-3.009300$

C $\quad-2.846900 \quad-5.171000 \quad-3.667700$

C $\quad-1.713300 \quad-5.746200 \quad-3.081600$

$\begin{array}{llll}\text { C } & -1.314000 & -5.290600 & -1.818100\end{array}$

$\begin{array}{llll}\text { C } & -0.650700 & -4.778800 & 1.094900\end{array}$

C $\quad-2.350300 \quad-3.841600 \quad 2.783700$

C $\quad-1.686600 \quad-4.179400 \quad 3.974700$

C $\quad-1.937600 \quad-3.463800 \quad 5.149200$

C $\quad-2.873800 \quad-2.426400 \quad 5.149700$

C $\quad-3.565800 \quad-2.110200 \quad 3.978000$

$\begin{array}{llll}\text { C } & -3.308700 & -2.815300 & 2.799200\end{array}$

C $\quad-3.000300 \quad-5.775400 \quad 1.318400$

C $\quad-4.309400 \quad-5.576900 \quad 0.847700$

C $\quad-5.167900 \quad-6.664100 \quad 0.662200$

C $\quad-4.736500 \quad-7.956900 \quad 0.967200$

C $\quad-3.450100 \quad-8.162700 \quad 1.470700$

$\begin{array}{llll}\text { C } & -2.588200 & -7.077600 & 1.656300\end{array}$

$\begin{array}{llll}\text { C } & -2.426900 & 1.061900 & 0.313100\end{array}$

$\begin{array}{llll}\text { C } & -3.862200 & 1.553700 & 0.263900\end{array}$

C $\quad-4.609900 \quad 2.082000 \quad 1.530900$ $\begin{array}{llll}\text { C } & -4.293000 & 2.005800 & -1.115900\end{array}$

$\begin{array}{llll}\text { C } & -3.730900 & 3.125300 & -1.754100\end{array}$

C $\quad-4.161700 \quad 3.545500 \quad-3.009100$

$\begin{array}{lrrr}C & -5.171100 & 2.846900 & -3.667500\end{array}$

C $\quad-5.746300 \quad 1.713300 \quad-3.081500$

C $\quad-5.290700 \quad 1.314000 \quad-1.817900$

$\begin{array}{llll}\text { C } & -4.778800 & 0.650600 & 1.095000\end{array}$

$\begin{array}{lrrr}\text { C } & -3.841500 & 2.350300 & 2.783800\end{array}$

C $\quad-4.179300 \quad 1.686600 \quad 3.974800$

$\begin{array}{llll}\text { C } & -3.463700 & 1.937500 & 5.149300\end{array}$

C $\quad-2.426200 \quad 2.873700 \quad 5.149800$

C $\quad-2.110100 \quad 3.565700 \quad 3.978100$

$\begin{array}{llll}\text { C } & -2.815200 & 3.308600 & 2.799300\end{array}$

C $\quad-5.775300 \quad 3.000400 \quad 1.318600$

$\begin{array}{llll}\text { C } & -5.576900 & 4.309400 & 0.847800\end{array}$

$\begin{array}{llll}\text { C } & -6.664100 & 5.167900 & 0.662500\end{array}$

C $\quad-7.956900 \quad 4.736500 \quad 0.967400$

$\begin{array}{llll}\text { C } & -8.162600 & 3.450000 & 1.470900\end{array}$

$\begin{array}{llll}\text { C } & -7.077600 & 2.588200 & 1.656500\end{array}$

$\begin{array}{llll}\text { C } & 1.061900 & 2.426900 & 0.313100\end{array}$

$\begin{array}{llll}\text { C } & 1.553700 & 3.862100 & 0.263900\end{array}$

$\begin{array}{llll}\text { C } & 2.082000 & 4.609800 & 1.530900\end{array}$

$\begin{array}{llll}\text { C } & 2.005800 & 4.293000 & -1.115900\end{array}$

$\begin{array}{llll}\text { C } & 3.125300 & 3.730900 & -1.754100\end{array}$

C $\quad 3.545400 \quad 4.161600 \quad-3.009200$

$\begin{array}{llll}\text { C } & 2.846900 & 5.171100 & -3.667600\end{array}$

C $\quad 1.713200 \quad 5.746300 \quad-3.081500$

C $\quad 1.313900 \quad 5.290700 \quad-1.817900$

$\begin{array}{llll}\text { C } & 0.650700 & 4.778800 & 1.095000\end{array}$

$\begin{array}{llll}\text { C } & 2.350300 & 3.841500 & 2.783800\end{array}$

C $\quad 1.686800 \quad 4.179200 \quad 3.974800$

$\begin{array}{llll}\text { C } & 1.937700 & 3.463600 & 5.149300\end{array}$

$\begin{array}{llll}\text { C } & 2.873900 & 2.426100 & 5.149800\end{array}$

C $\quad 3.565800 \quad 2.110000 \quad 3.978100$

$\begin{array}{llll}\text { C } & 3.308700 & 2.815200 & 2.799300\end{array}$

C $\quad 3.000400 \quad 5.775300 \quad 1.318500$

$\begin{array}{llll}\text { C } & 4.309400 & 5.576900 & 0.847800\end{array}$

$\begin{array}{llll}\text { C } & 5.167900 & 6.664000 & 0.662400\end{array}$

C $\quad 4.736500 \quad 7.956900 \quad 0.967400$

$\begin{array}{llll}\text { C } & 3.450100 & 8.162600 & 1.470900\end{array}$

$\begin{array}{llll}\text { C } & 2.588300 & 7.077500 & 1.656500\end{array}$

C $\quad-0.957300 \quad-6.822400 \quad-3.821000$

$\begin{array}{llll}\text { C } & -6.822500 & 0.957300 & -3.820800\end{array}$

$\begin{array}{llll}\text { C } & 6.822400 & -0.957200 & -3.821000\end{array}$

C $\quad 0.957300 \quad 6.822500 \quad-3.820800$

$\mathrm{H} \quad 3.702800 \quad-4.415700 \quad-3.466000$

$\mathrm{H} \quad 5.492600 \quad-3.197000 \quad-4.645700$

H $\quad 5.694000 \quad-0.426400 \quad-1.344100$

H $\quad 5.718300 \quad-0.325200 \quad 0.660000$

H $\quad 4.271300 \quad 0.121200 \quad 1.661700$

H $\quad 4.997800 \quad-0.978800 \quad 3.992600$

$\mathrm{H} \quad 3.719100 \quad-1.412500 \quad 6.060400$

H $\quad 1.875700 \quad-3.072100 \quad 6.060200$

$\mathrm{H} \quad 1.324000 \quad-4.307000 \quad 3.984700$

H $\quad 2.561300 \quad-3.852900 \quad 1.900500$

$\mathrm{H} \quad 4.582800 \quad-4.671700 \quad 0.635700$

H $\quad 6.502900 \quad-6.171200 \quad 0.289700$

H $\quad 8.797600 \quad-5.403200 \quad 0.825300$

H $\quad 9.162700 \quad-3.121900 \quad 1.722500$

H $\quad 7.254700 \quad-1.601500 \quad 2.065000$

$\mathrm{H} \quad-4.415800 \quad-3.702700 \quad-3.465900$

H $\quad-3.197100 \quad-5.492500 \quad-4.645700$ 
$\begin{array}{rrrr}\text { H } & -0.426400 & -5.694000 & -1.344200 \\ \mathrm{H} & -0.325100 & -5.718200 & 0.659900 \\ \mathrm{H} & 0.121200 & -4.271400 & 1.661600 \\ \mathrm{H} & -0.978800 & -4.997900 & 3.992500 \\ \mathrm{H} & -1.412400 & -3.719300 & 6.060300 \\ \mathrm{H} & -3.072000 & -1.875800 & 6.060300 \\ \mathrm{H} & -4.306800 & -1.324100 & 3.984800 \\ \mathrm{H} & -3.852900 & -2.561300 & 1.900600 \\ \mathrm{H} & -4.671700 & -4.582800 & 0.635800 \\ \mathrm{H} & -6.171300 & -6.502800 & 0.289800 \\ \mathrm{H} & -5.403200 & -8.797600 & 0.825400 \\ \mathrm{H} & -3.121900 & -9.162800 & 1.722500 \\ \mathrm{H} & -1.601400 & -7.254800 & 2.065000 \\ \mathrm{H} & -3.702800 & 4.415800 & -3.465800 \\ \mathrm{H} & -5.492700 & 3.197100 & -4.645500 \\ \mathrm{H} & -5.694000 & 0.426400 & -1.344000 \\ \mathrm{H} & -5.718200 & 0.325100 & 0.660100 \\ \mathrm{H} & -4.271300 & -0.121300 & 1.661700 \\ \mathrm{H} & -4.997700 & 0.978700 & 3.992700 \\ \mathrm{H} & -3.719100 & 1.412300 & 6.060500 \\ \mathrm{H} & -1.875600 & 3.071900 & 6.060300 \\ \mathrm{H} & -1.323800 & 4.306800 & 3.984900 \\ \mathrm{H} & -2.561300 & 3.852900 & 1.900700 \\ \mathrm{H} & -4.582800 & 4.671700 & 0.635900 \\ \mathrm{H} & -6.502900 & 6.171200 & 0.290100 \\ \mathrm{H} & -8.797600 & 5.403200 & 0.825600 \\ \mathrm{H} & -9.162700 & 3.121900 & 1.722800 \\ \mathrm{H} & -7.254700 & 1.601400 & 2.065200 \\ \mathrm{H} & 4.415800 & 3.702800 & -3.465900 \\ \mathrm{H} & 3.197100 & 5.492800 & -4.645500 \\ \mathrm{H} & 0.426400 & 5.694000 & -1.343900 \\ \mathrm{H} & 0.325100 & 5.718200 & 0.660100 \\ \mathrm{H} & -0.121200 & 4.271300 & 1.661800 \\ \mathrm{H} & 0.978800 & 4.997800 & 3.992700 \\ \mathrm{H} & 1.412500 & 3.719000 & 6.060500 \\ \mathrm{H} & 3.072000 & 1.875600 & 6.060300 \\ \mathrm{H} & 4.306900 & 1.323900 & 3.984800 \\ \mathrm{H} & 3.852900 & 2.561200 & 1.900700 \\ \mathrm{H} & 4.671700 & 4.582800 & 0.635900 \\ \mathrm{H} & 6.171300 & 6.502800 & 0.290000 \\ \mathrm{H} & 5.403200 & 8.797600 & 0.825600 \\ \mathrm{H} & 3.121900 & 9.162700 & 1.722800 \\ \mathrm{H} & 1.601500 & 7.254700 & 2.065200\end{array}$

$\begin{array}{lccc}\text { J"1 } & & & \\ \mathrm{Rh} & 0.234700 & 0.047400 & -1.017900 \\ \mathrm{Rh} & 0.003200 & -0.208300 & 1.341300 \\ \mathrm{O} & -1.499800 & -1.583400 & 0.989000 \\ \mathrm{O} & -1.311200 & -1.297400 & -1.243900 \\ \mathrm{O} & -1.453700 & 1.255500 & 1.297900 \\ \mathrm{O} & -1.231100 & 1.507500 & -0.936500 \\ \mathrm{O} & 1.444300 & 1.242800 & 1.568000 \\ \mathrm{O} & 1.709900 & 1.443600 & -0.666400 \\ \mathrm{O} & 1.478900 & -1.633600 & 1.262600 \\ \mathrm{O} & 1.644600 & -1.447100 & -0.982600 \\ \mathrm{Cl} & -1.816000 & -4.925100 & 0.559000 \\ \mathrm{Cl} & -1.011700 & 4.715500 & -1.030900 \\ \mathrm{Cl} & 4.913800 & 0.910500 & -0.570700 \\ \mathrm{Cl} & 1.127300 & -4.607500 & -1.543900 \\ \mathrm{~F} & -6.115900 & -1.188600 & 3.946000 \\ \mathrm{~F} & -6.811900 & -3.193900 & 4.686600 \\ \mathrm{~F} & -7.577100 & -2.396600 & 2.728900\end{array}$

C $\quad-1.908200 \quad-1.734600 \quad-0.209200$

C $\quad-3.296700 \quad-2.332500 \quad-0.360800$

C $\quad-3.927800 \quad-2.647600 \quad-1.749800$

C $\quad-3.783300 \quad-3.022500 \quad 0.894600$

C $\quad-3.186200 \quad-4.197000 \quad 1.382200$

C $\quad-3.641100 \quad-4.821400 \quad 2.540600$

C $\quad-4.710500 \quad-4.283400 \quad 3.254700$

C $\quad-5.325800 \quad-3.105000 \quad 2.813900$

$\begin{array}{llll}\text { C } & -4.845400 & -2.500900 & 1.644900\end{array}$

C $\quad-4.208800 \quad-1.327500 \quad-1.078000$

C $\quad-3.063700 \quad-2.648400 \quad-2.969100$

C $\quad-2.016400 \quad-3.575600 \quad-3.097700$

C $\quad-1.212500 \quad-3.577300-4.241400$

$\begin{array}{llll}\text { C } & -1.457300 & -2.664400 & -5.270500\end{array}$

$\begin{array}{llll}\text { C } & -2.511400 & -1.753400 & -5.160900\end{array}$

C $\quad-3.317500-1.748400 \quad-4.018600$

C $\quad-5.056900 \quad-3.631900 \quad-1.796400$

C $\quad-6.343800 \quad-3.217300 \quad-2.185900$

C $\quad-7.395700 \quad-4.136300 \quad-2.243900$

$\begin{array}{llll}\text { C } & -7.169700 & -5.479500 & -1.934500\end{array}$

C $\quad-5.888500 \quad-5.907800 \quad-1.580100$

C $\quad-4.834300 \quad-4.991800 \quad-1.522100$

$\begin{array}{llll}\text { C } & -1.794300 & 1.756300 & 0.176700\end{array}$

$\begin{array}{llll}C & -3.051000 & 2.606700 & 0.116100\end{array}$

C $\quad-3.673400 \quad 3.247700 \quad 1.396100$

C $\quad-3.306600 \quad 3.184900 \quad-1.258900$

C $\quad-2.459100 \quad 4.138000 \quad-1.850400$

C $\quad-2.711800 \quad 4.650800 \quad-3.119700$

C $\quad-3.818000 \quad 4.209000 \quad-3.842600$

C $\quad-4.676300 \quad 3.242700 \quad-3.304700$

$\begin{array}{llll}\text { C } & -4.401400 & 2.752000 & -2.021500\end{array}$

$\begin{array}{llll}\text { C } & -4.189400 & 1.927400 & 0.888200\end{array}$

$\begin{array}{llll}\text { C } & -2.879100 & 3.264600 & 2.662400\end{array}$

$\begin{array}{llll}\text { C } & -1.681200 & 3.994700 & 2.739300\end{array}$

$\begin{array}{llll}\text { C } & -0.953900 & 4.032000 & 3.932300\end{array}$

C $\quad-1.411500 \quad 3.337300 \quad 5.054400$

$\begin{array}{llll}\text { C } & -2.608800 & 2.619400 & 4.990900\end{array}$

$\begin{array}{llll}\text { C } & -3.347000 & 2.590800 & 3.803700\end{array}$

$\begin{array}{llll}\text { C } & -4.573300 & 4.433200 & 1.218100\end{array}$

$\begin{array}{llll}\text { C } & -4.061500 & 5.663200 & 0.771800\end{array}$

$\begin{array}{llll}\text { C } & -4.903800 & 6.768600 & 0.622100\end{array}$

C $\quad-6.260200 \quad 6.663400 \quad 0.937800$

C $\quad-6.773400 \quad 5.455600 \quad 1.416000$

$\begin{array}{llll}\text { C } & -5.934000 & 4.347800 & 1.566000\end{array}$

$\begin{array}{llll}\text { C } & 1.976900 & 1.766100 & 0.534800\end{array}$

$\begin{array}{llll}\text { C } & 2.906400 & 2.951200 & 0.716900\end{array}$

$\begin{array}{llll}\text { C } & 3.488200 & 3.339200 & 2.114000\end{array}$

C $3.620600 \quad 3.355400-0.554700$

C $\quad 4.552700 \quad 2.518200 \quad-1.192200$

$\begin{array}{llll}\text { C } & 5.223300 & 2.914200 & -2.345600\end{array}$

C $\quad 4.973500 \quad 4.168200 \quad-2.898600$

$\begin{array}{llll}\text { C } & 4.039400 & 5.029100 & -2.310200\end{array}$

C $\quad 3.377900 \quad 4.601100-1.151400$
C $\quad 2.242300 \quad 4.012500 \quad 1.600500$

C $\quad 3.362000 \quad 2.380700 \quad 3.254100$

$\begin{array}{llll}\text { C } & 2.653200 & 2.744200 & 4.411600\end{array}$

C $\quad 2.530800 \quad 1.844700 \quad 5.474800$

C $\quad 3.135500 \quad 0.587000 \quad 5.401000$

$\begin{array}{llll}\text { C } & 3.870000 & 0.231100 & 4.267100\end{array}$

C $\quad 3.985700 \quad 1.123300 \quad 3.197800$

$\begin{array}{llll}\text { C } & 4.739000 & 4.164100 & 2.141600\end{array}$

$\begin{array}{llll}\text { C } & 4.716600 & 5.468800 & 2.667300\end{array}$

$\begin{array}{llll}\text { C } & 5.885000 & 6.235300 & 2.707000\end{array}$

C $\quad 7.089100 \quad 5.701200 \quad 2.242000$

$\begin{array}{llll}\text { C } & 7.128700 & 4.395000 & 1.749200\end{array}$

C $\quad 5.962600 \quad 3.625300 \quad 1.709200$

C $\quad 1.986400 \quad-1.948300 \quad 0.136600$

$\begin{array}{llll}\text { C } & 3.171400 & -2.894100 & 0.107200\end{array}$

$\begin{array}{llll}\text { C } & 3.577900 & -3.766200 & 1.337900\end{array}$

C $\quad 3.557200 \quad-3.320200 \quad-1.293800$

C $\quad 2.715200 \quad-4.103900-2.102000$

C $\quad 3.093500 \quad-4.500600-3.381900$

C $\quad 4.331000 \quad-4.120300 \quad-3.898600$

$\begin{array}{llll}\text { C } & 5.194000 & -3.324500 & -3.135200\end{array}$

C $\quad 4.784700 \quad-2.940000 \quad-1.851800$

$\begin{array}{llll}\text { C } & 4.256300 & -2.443700 & 1.091300\end{array}$

$\begin{array}{llll}\text { C } & 2.658100 & -3.872000 & 2.511300\end{array}$

C $\quad 3.100900 \quad-3.493500 \quad 3.789500$

$\begin{array}{llll}\text { C } & 2.242700 & -3.585200 & 4.889000\end{array}$

$\begin{array}{llll}\text { C } & 0.945000 & -4.076800 & 4.724800\end{array}$

C $\quad 0.507700 \quad-4.487000 \quad 3.462700$

$\begin{array}{llll}\text { C } & 1.363000 & -4.394000 & 2.360400\end{array}$

$\begin{array}{llll}\text { C } & 4.379600 & -5.004600 & 1.074200\end{array}$

$\begin{array}{llll}\text { C } & 3.791400 & -6.113800 & 0.443400\end{array}$

$\begin{array}{llll}\text { C } & 4.536900 & -7.272600 & 0.208400\end{array}$

$\begin{array}{llll}\text { C } & 5.869100 & -7.344000 & 0.621600\end{array}$

$\begin{array}{llll}\text { C } & 6.453600 & -6.261200 & 1.282500\end{array}$

$\begin{array}{llll}\text { C } & 5.711300 & -5.100200 & 1.518200\end{array}$

C $\quad 6.534600 \quad-2.865000 \quad-3.652000$

C $\quad 3.768600 \quad 6.374900 \quad-2.936400$

$\begin{array}{llll}\text { C } & -5.849100 & 2.750600 & -4.116300\end{array}$

C $\quad-6.472500 \quad-2.467600 \quad 3.558200$

$\mathrm{H} \quad-3.151500 \quad-5.727300 \quad 2.881700$

$\mathrm{H} \quad-5.034500 \quad-4.803400 \quad 4.152200$

$\mathrm{H} \quad-5.297900 \quad-1.576000 \quad 1.301100$

H $\quad-5.192900 \quad-1.139400 \quad-0.659700$

$\mathrm{H} \quad-3.719500 \quad-0.437100 \quad-1.460000$

$\mathrm{H} \quad-1.818600 \quad-4.289900 \quad-2.310800$

$\mathrm{H} \quad-0.400500 \quad-4.287300 \quad-4.330900$

$\mathrm{H} \quad-0.833800 \quad-2.666700 \quad-6.155200$

$\mathrm{H} \quad-2.705400 \quad-1.052100 \quad-5.962100$

H $\quad-4.135400 \quad-1.041600 \quad-3.950400$

$\mathrm{H} \quad-6.534000 \quad-2.183400 \quad-2.445700$

$\mathrm{H} \quad-8.385300 \quad-3.807600 \quad-2.533800$

H $\quad-7.984600 \quad-6.190300 \quad-1.981300$

$\mathrm{H} \quad-5.709700 \quad-6.951900 \quad-1.358100$

H $\quad-3.846000 \quad-5.347800 \quad-1.276000$

H $\quad-2.035600 \quad 5.387200 \quad-3.540200$

$\mathrm{H} \quad-3.990600 \quad 4.623300 \quad-4.832900$

H $\quad-5.032900 \quad 1.990000 \quad-1.578200$

$\begin{array}{llll}\mathrm{H} & -5.170200 & 1.885600 & 0.424300\end{array}$

$\begin{array}{llll}\mathrm{H} & -3.915900 & 1.021400 & 1.418900\end{array}$

H $\quad-1.311100 \quad 4.534600 \quad 1.878800$

H $\quad-0.038100 \quad 4.604600 \quad 3.988000$

H $\quad-0.842900 \quad 3.362600 \quad 5.975100$ 
$\begin{array}{rrrr}\mathrm{H} & -2.968300 & 2.090000 & 5.863800 \\ \mathrm{H} & -4.280100 & 2.041800 & 3.773100 \\ \mathrm{H} & -3.011400 & 5.774100 & 0.551900 \\ \mathrm{H} & -4.502500 & 7.709700 & 0.268900 \\ \mathrm{H} & -6.910700 & 7.520800 & 0.823700 \\ \mathrm{H} & -7.821300 & 5.379000 & 1.675700 \\ \mathrm{H} & -6.346000 & 3.424800 & 1.954400 \\ \mathrm{H} & 5.936100 & 2.238600 & -2.806100 \\ \mathrm{H} & 5.513100 & 4.456400 & -3.797600 \\ \mathrm{H} & 2.629800 & 5.229600 & -0.681400 \\ \mathrm{H} & 2.283000 & 5.050100 & 1.284900 \\ \mathrm{H} & 1.286700 & 3.723200 & 2.022000 \\ \mathrm{H} & 2.195800 & 3.721700 & 4.489500 \\ \mathrm{H} & 1.971800 & 2.125200 & 6.358200 \\ \mathrm{H} & 3.043500 & -0.107800 & 6.225700 \\ \mathrm{H} & 4.354500 & -0.733900 & 4.218400 \\ \mathrm{H} & 4.555600 & 0.832600 & 2.326400 \\ \mathrm{H} & 3.795900 & 5.893300 & 3.047500 \\ \mathrm{H} & 5.857900 & 7.242100 & 3.103200 \\ \mathrm{H} & 7.993100 & 6.295200 & 2.274500 \\ \mathrm{H} & 8.065000 & 3.975300 & 1.405000 \\ \mathrm{H} & 6.019400 & 2.608800 & 1.351100 \\ \mathrm{H} & 2.413000 & -5.105900 & -3.971400 \\ \mathrm{H} & 4.587500 & -4.454900 & -4.900300 \\ \mathrm{H} & 5.431300 & -2.302000 & -1.258200 \\ \mathrm{H} & 5.285200 & -2.417900 & 0.747200 \\ \mathrm{H} & 3.988000 & -1.603500 & 1.722200 \\ \mathrm{H} & 4.109900 & -3.128900 & 3.932500 \\ \mathrm{H} & 2.585300 & -3.278900 & 5.868900 \\ \mathrm{H} & 0.281400 & -4.148200 & 5.576800 \\ \mathrm{H} & -0.493700 & -4.878100 & 3.340000 \\ \mathrm{H} & 1.015000 & -4.716200 & 1.389600 \\ \mathrm{H} & 2.754600 & -6.089200 & 0.144700 \\ \mathrm{H} & 4.078400 & -8.118700 & -0.286800 \\ \mathrm{H} & 6.444400 & -8.242800 & 0.441100 \\ \mathrm{H} & 7.481000 & -6.323100 & 1.617200 \\ \mathrm{H} & 6.176000 & -4.278400 & 2.048300\end{array}$

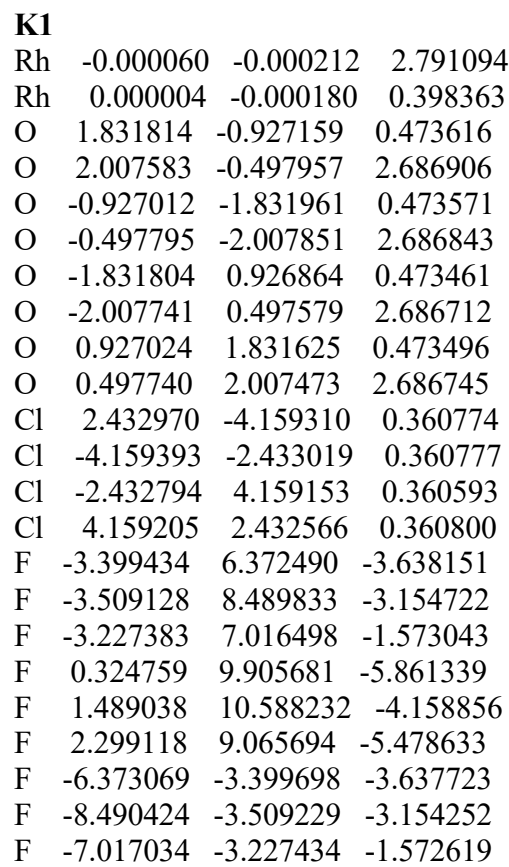

$\begin{array}{llll}F & -9.065637 & 2.298487 & -5.479194\end{array}$

F $\quad-9.906714 \quad 0.324351 \quad-5.860645$

F $\quad-10.588193 \quad 1.489849 \quad-4.158562$

F $\quad-0.323687 \quad-9.905646 \quad-5.861557$

F $\quad-2.297725 \quad-9.064036 \quad-5.480764$

F $\quad-1.490048 \quad-10.586983-4.160000$

F $\quad 3.509403 \quad-8.490148 \quad-3.154063$

F $\quad 3.400183 \quad-6.372943 \quad-3.638203$

F $\quad 3.227672 \quad-7.016250 \quad-1.572901$

F $\quad 9.065212 \quad-2.298091 \quad-5.479492$

F $\quad 9.906485 \quad-0.323987 \quad-5.860671$

F $\quad \begin{array}{llll}10.587826 & -1.489786 & -4.158724\end{array}$

F $\quad 7.016650 \quad 3.227681 \quad-1.572665$

$\begin{array}{llll}\text { F } & 8.490108 & 3.509479 & -3.154233\end{array}$

F $\quad 6.372792 \quad 3.399886 \quad-3.637822$

$\begin{array}{llll}\text { C } & 3.793210 & -1.623118 & 1.575805\end{array}$

$\begin{array}{llll}\text { C } & 4.321990 & -2.417512 & 2.821694\end{array}$

$\begin{array}{llll}\text { C } & 4.840136 & -1.047965 & 2.511630\end{array}$

$\begin{array}{llll}\text { C } & 2.442417 & -0.973930 & 1.591616\end{array}$

$\begin{array}{llll}\text { C } & 4.217901 & -2.072541 & 0.209717\end{array}$

$\begin{array}{llll}\text { C } & 3.689421 & -3.208328 & -0.420894\end{array}$

$\begin{array}{llll}\text { C } & 4.120073 & -3.605340 & -1.684577\end{array}$

$\begin{array}{llll}\text { C } & 5.067443 & -2.841477 & -2.361476\end{array}$

$\begin{array}{llll}\text { C } & 5.587465 & -1.675478 & -1.783815\end{array}$

$\begin{array}{llll}\text { C } & 5.155424 & -1.322790 & -0.499264\end{array}$

$\begin{array}{llll}\text { C } & 5.172378 & -3.629300 & 2.511665\end{array}$

$\begin{array}{llll}\text { C } & 4.688602 & -4.909445 & 2.820554\end{array}$

$\begin{array}{llll}\text { C } & 5.423557 & -6.051579 & 2.507329\end{array}$

$\begin{array}{llll}\text { C } & 6.665764 & -5.939731 & 1.880936\end{array}$

$\begin{array}{llll}\text { C } & 7.165493 & -4.672345 & 1.580227\end{array}$

C $\quad 6.427975 \quad-3.531162 \quad 1.895552$

$\begin{array}{llll}\text { C } & 3.467095 & -2.553914 & 4.054576\end{array}$

$\begin{array}{llll}\text { C } & 4.019096 & -2.199241 & 5.291486\end{array}$

$\begin{array}{llll}\text { C } & 3.267991 & -2.304243 & 6.463415\end{array}$

$\begin{array}{llll}\text { C } & 1.950383 & -2.765243 & 6.407773\end{array}$

$\begin{array}{llll}\text { C } & 1.398765 & -3.134469 & 5.179083\end{array}$

$\begin{array}{llll}\text { C } & 2.158362 & -3.040778 & 4.013442\end{array}$

$\begin{array}{llll}\text { C } & 6.556932 & -0.817395 & -2.501245\end{array}$

C $\quad 7.519803 \quad-1.366118 \quad-3.359834$

C $\quad 8.425721 \quad-0.542957 \quad-4.026726$

$\begin{array}{llll}\text { C } & 8.397715 & 0.840549 & -3.853254\end{array}$

$\begin{array}{llll}\text { C } & 7.442124 & 1.388558 & -2.999694\end{array}$

$\begin{array}{llll}\text { C } & 6.528991 & 0.574199 & -2.332048\end{array}$

$\begin{array}{llll}\text { C } & -1.623166 & -3.793329 & 1.575693\end{array}$

$\begin{array}{llll}\text { C } & -2.417646 & -4.322040 & 2.821559\end{array}$

C $\quad-1.048165 \quad-4.840369 \quad 2.511483$

$\begin{array}{llll}\text { C } & -0.973822 & -2.442600 & 1.591542\end{array}$

$\begin{array}{llll}\text { C } & -2.072573 & -4.217883 & 0.209558\end{array}$

C $\quad-3.208336 \quad-3.689335 \quad-0.421024$

$\begin{array}{llll}\text { C } & -3.605291 & -4.119814 & -1.684792\end{array}$

$\begin{array}{llll}\text { C } & -2.841398 & -5.067087 & -2.361783\end{array}$

$\begin{array}{llll}\text { C } & -1.675422 & -5.587189 & -1.784134\end{array}$

C $\quad-1.322800 \quad-5.155321 \quad-0.499511$

C $\quad-3.629542 \quad-5.172256 \quad 2.511504$

C $\quad-4.909602 \quad-4.688566 \quad 2.820910$

$\begin{array}{llll}\text { C } & -6.051843 & -5.423361 & 2.507694\end{array}$

$\begin{array}{llll}\text { C } & -5.940195 & -6.665323 & 1.880765\end{array}$

$\begin{array}{llll}\text { C } & -4.672910 & -7.164959 & 1.579538\end{array}$

$\begin{array}{llll}\text { C } & -3.531614 & -6.427612 & 1.894862\end{array}$

$\begin{array}{llll}\text { C } & -2.553894 & -3.467149 & 4.054462\end{array}$

$\begin{array}{llll}\text { C } & -2.199277 & -4.019224 & 5.291357\end{array}$

C $\quad-2.304143 \quad-3.268125 \quad 6.463301$ $\begin{array}{llll}\text { C } & -2.764959 & -1.950445 & 6.407682\end{array}$

$\begin{array}{llll}\text { C } & -3.134137 & -1.398758 & 5.179016\end{array}$

$\begin{array}{llll}\text { C } & -3.040589 & -2.158355 & 4.013367\end{array}$

$\begin{array}{llll}\text { C } & -0.817329 & -6.556574 & -2.501653\end{array}$

C $\quad-1.366008 \quad-7.519236 \quad-3.360511$

$\begin{array}{llll}\text { C } & -0.542830 & -8.425070 & -4.027484\end{array}$

$\begin{array}{llll}\text { C } & 0.840654 & -8.397197 & -3.853841\end{array}$

$\begin{array}{llll}\text { C } & 1.388633 & -7.441821 & -3.000019\end{array}$

$\begin{array}{llll}\text { C } & 0.574257 & -6.528761 & -2.332289\end{array}$

$\begin{array}{llll}\text { C } & -3.793175 & 1.622988 & 1.575622\end{array}$

$\begin{array}{llll}\text { C } & -4.321913 & 2.417424 & 2.821524\end{array}$

$\begin{array}{llll}\text { C } & -4.840200 & 1.047943 & 2.511418\end{array}$

$\begin{array}{llll}\text { C } & -2.442461 & 0.973643 & 1.591421\end{array}$

$\begin{array}{llll}\text { C } & -4.217854 & 2.072489 & 0.209550\end{array}$

$\begin{array}{llll}\text { C } & -3.689350 & 3.208282 & -0.421039\end{array}$

$\begin{array}{llll}\text { C } & -4.120063 & 3.605386 & -1.684667\end{array}$

C $\quad-5.067524 \quad 2.841624 \quad-2.361559$

$\begin{array}{llll}\text { C } & -5.587558 & 1.675610 & -1.783919\end{array}$

$\begin{array}{llll}\text { C } & -5.155465 & 1.322839 & -0.499410\end{array}$

C $\quad-5.172152 \quad 3.629302 \quad 2.511462$

$\begin{array}{llll}\text { C } & -4.688221 & 4.909398 & 2.820364\end{array}$

$\begin{array}{llll}\text { C } & -5.423037 & 6.051619 & 2.507119\end{array}$

$\begin{array}{llll}\text { C } & -6.665245 & 5.939921 & 1.880702\end{array}$

$\begin{array}{llll}\text { C } & -7.165119 & 4.672603 & 1.579980\end{array}$

$\begin{array}{llll}\text { C } & -6.427743 & 3.531326 & 1.895315\end{array}$

$\begin{array}{llll}\text { C } & -3.467115 & 2.553706 & 4.054483\end{array}$

$\begin{array}{llll}\text { C } & -4.019356 & 2.199231 & 5.291353\end{array}$

$\begin{array}{llll}\text { C } & -3.268371 & 2.304119 & 6.463365\end{array}$

$\begin{array}{llll}\text { C } & -1.950649 & 2.764801 & 6.407866\end{array}$

$\begin{array}{llll}\text { C } & -1.398784 & 3.133828 & 5.179227\end{array}$

$\begin{array}{llll}\text { C } & -2.158271 & 3.040271 & 4.013497\end{array}$

$\begin{array}{llll}\text { C } & -6.557106 & 0.817594 & -2.501310\end{array}$

C $\quad-6.529186-0.574001 \quad-2.332116$

$\begin{array}{llll}\text { C } & -7.442398 & -1.388331 & -2.999701\end{array}$

$\begin{array}{llll}\text { C } & -8.398010 & -0.840299 & -3.853202\end{array}$

$\begin{array}{llll}\text { C } & -8.425994 & 0.543213 & -4.026670\end{array}$

$\begin{array}{llll}\text { C } & -7.520016 & 1.366347 & -3.359841\end{array}$

$\begin{array}{llll}\text { C } & 1.623053 & 3.793015 & 1.575694\end{array}$

$\begin{array}{llll}\text { C } & 2.417349 & 4.321812 & 2.821619\end{array}$

$\begin{array}{llll}\text { C } & 1.047847 & 4.839989 & 2.511435\end{array}$

$\begin{array}{llll}\text { C } & 0.973786 & 2.442251 & 1.591476\end{array}$

$\begin{array}{llll}\text { C } & 2.072601 & 4.217682 & 0.209639\end{array}$

$\begin{array}{llll}\text { C } & 3.208410 & 3.689156 & -0.420889\end{array}$

$\begin{array}{llll}\text { C } & 3.605561 & 4.119829 & -1.684522\end{array}$

$\begin{array}{llll}\text { C } & 2.841814 & 5.067269 & -2.361461\end{array}$

C $\quad 1.675774 \quad 5.587305 \quad-1.783900$

$\begin{array}{llll}\text { C } & 1.322961 & 5.155258 & -0.499382\end{array}$

$\begin{array}{llll}\text { C } & 3.629176 & 5.172151 & 2.511644\end{array}$

$\begin{array}{llll}\text { C } & 4.909292 & 4.688217 & 2.820408\end{array}$

$\begin{array}{llll}\text { C } & 6.051482 & 5.423117 & 2.507234\end{array}$

C $\quad 5.939710 \quad 6.665435 \quad 1.881033$

$\begin{array}{llll}\text { C } & 4.672354 & 7.165308 & 1.580449\end{array}$

$\begin{array}{llll}\text { C } & 3.531116 & 6.427847 & 1.895715\end{array}$

C $\quad 2.553657 \quad 3.466974 \quad 4.054556$

$\begin{array}{llll}\text { C } & 2.199069 & 4.019112 & 5.291422\end{array}$

$\begin{array}{llll}\text { C } & 2.303969 & 3.268063 & 6.463408\end{array}$

$\begin{array}{llll}\text { C } & 2.764780 & 1.950393 & 6.407848\end{array}$

$\begin{array}{llll}\text { C } & 3.133912 & 1.398626 & 5.179202\end{array}$

$\begin{array}{llll}\text { C } & 3.040324 & 2.158162 & 4.013512\end{array}$

$\begin{array}{llll}\text { C } & 0.817759 & 6.556786 & -2.501381\end{array}$

$\begin{array}{llll}\text { C } & 1.366526 & 7.519707 & -3.359895\end{array}$

$\begin{array}{llll}\text { C } & 0.543398 & 8.425608 & -4.026821\end{array}$ 


\begin{tabular}{|c|c|c|c|}
\hline $\mathrm{C}$ & -0.840134 & 8.397551 & -3.853465 \\
\hline $\mathrm{C}$ & -1.388184 & 7.441936 & -2.999976 \\
\hline $\mathrm{C}$ & -0.573841 & 6.528797 & -2.332296 \\
\hline $\mathrm{C}$ & -9.493535 & 1.162745 & -4.885647 \\
\hline & -7.341952 & -2.879019 & -2.838336 \\
\hline $\mathrm{C}$ & -1.162336 & -9.492345 & -4.886828 \\
\hline $\mathrm{C}$ & 2.879315 & -7.341500 & -2.838544 \\
\hline $\mathrm{C}$ & 7.341641 & 2.879248 & -2.838379 \\
\hline $\mathrm{C}$ & 9.493213 & -1.162468 & -4.885779 \\
\hline C & -2.878874 & 7.341408 & -2.838740 \\
\hline $\mathrm{C}$ & 1.162871 & 9.493196 & -4.885778 \\
\hline $\mathrm{H}$ & 5.844537 & -0.920807 & 2.128337 \\
\hline $\mathrm{H}$ & 4.504277 & -0.248832 & 3.159294 \\
\hline $\mathrm{H}$ & 3.711248 & -4.494080 & -2.145812 \\
\hline$H$ & 5.371731 & -3.142402 & -3.358252 \\
\hline $\mathrm{H}$ & 5.570163 & -0.442555 & -0.022243 \\
\hline $\mathrm{H}$ & 3.726360 & -5.011686 & 3.307593 \\
\hline $\mathrm{H}$ & 5.022012 & -7.030080 & 2.753154 \\
\hline $\mathrm{H}$ & 7.238950 & -6.828239 & 1.634660 \\
\hline H & 8.133628 & -4.566103 & 1.100225 \\
\hline $\mathrm{H}$ & 6.843805 & -2.563194 & 1.644815 \\
\hline $\mathrm{H}$ & 5.039038 & -1.827201 & 5.330892 \\
\hline $\mathrm{H}$ & 3.708095 & -2.018308 & 7.414081 \\
\hline $\mathrm{H}$ & 1.355594 & -2.835360 & 7.313672 \\
\hline $\mathrm{H}$ & 0.369092 & -3.470140 & 5.127531 \\
\hline $\mathrm{H}$ & 1.716474 & -3.298682 & 3.059587 \\
\hline $\mathrm{H}$ & 7.573875 & -2.439837 & -3.496789 \\
\hline $\mathrm{H}$ & 5.780710 & 1.024774 & -1.690217 \\
\hline $\mathrm{H}$ & -0.921171 & -5.844782 & 2.128148 \\
\hline $\mathrm{H}$ & -0.248974 & -4.504648 & 3.159146 \\
\hline $\mathrm{H}$ & -4.494021 & -3.710936 & -2.146005 \\
\hline $\mathrm{H}$ & -3.142277 & -5.371236 & -3.358616 \\
\hline $\mathrm{H}$ & -0.442586 & -5.570114 & -0.022485 \\
\hline $\mathrm{H}$ & -5.011696 & -3.726508 & 3.308335 \\
\hline $\mathrm{H}$ & -7.030273 & -5.021885 & 2.753929 \\
\hline $\mathrm{H}$ & -6.828793 & -7.238383 & 1.634502 \\
\hline $\mathrm{H}$ & -4.566810 & -8.132908 & 1.099117 \\
\hline $\mathrm{H}$ & -2.563734 & -6.843380 & 1.643696 \\
\hline $\mathrm{H}$ & -1.827382 & -5.039212 & 5.330742 \\
\hline $\mathrm{H}$ & -2.018258 & -3.708274 & 7.413956 \\
\hline $\mathrm{H}$ & -2.834977 & -1.355659 & 7.313603 \\
\hline $\mathrm{H}$ & -3.469662 & -0.369034 & 5.127488 \\
\hline $\mathrm{H}$ & -3.298459 & -1.716419 & 3.059523 \\
\hline $\mathrm{H}$ & -2.439712 & -7.573207 & -3.497602 \\
\hline $\mathrm{H}$ & 1.024811 & -5.780635 & -1.690267 \\
\hline $\mathrm{H}$ & -5.844602 & 0.920892 & 2.128081 \\
\hline $\mathrm{H}$ & -4.504442 & 0.248760 & 3.159074 \\
\hline $\mathrm{H}$ & -3.711221 & 4.494148 & -2.145863 \\
\hline $\mathrm{H}$ & -5.371893 & 3.142633 & -3.358283 \\
\hline $\mathrm{H}$ & -5.570215 & 0.442590 & -0.022421 \\
\hline $\mathrm{H}$ & -3.725978 & 5.011504 & 3.307425 \\
\hline $\mathrm{H}$ & -5.021379 & 7.030080 & 2.752943 \\
\hline $\mathrm{H}$ & -7.238313 & 6.828496 & 1.634417 \\
\hline $\mathrm{H}$ & -8.133262 & 4.566476 & 1.099962 \\
\hline $\mathrm{H}$ & -6.843682 & 2.563402 & 1.644562 \\
\hline $\mathrm{H}$ & -5.039389 & 1.827433 & 5.330647 \\
\hline $\mathrm{H}$ & -3.708662 & 2.018339 & 7.413998 \\
\hline H & -1.355948 & 2.834812 & 7.313839 \\
\hline $\mathrm{H}$ & -0.369026 & 3.469253 & 5.127778 \\
\hline $\mathrm{H}$ & -1.716202 & 3.298028 & 3.059692 \\
\hline $\mathrm{H}$ & -5.780893 & -1.024585 & -1.690307 \\
\hline 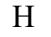 & -7.574039 & 2.440073 & -3.496786 \\
\hline
\end{tabular}

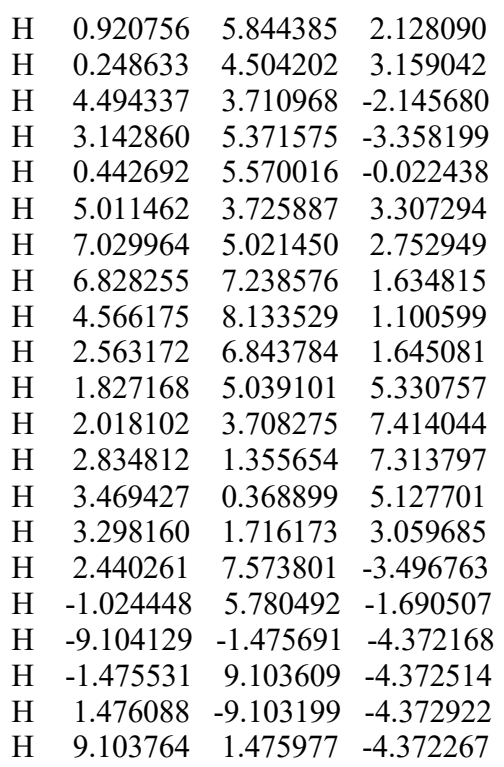

K2

$\begin{array}{llll}\text { Rh } & 0.000094 & -0.000131 & 2.796375\end{array}$ $\begin{array}{lllll}\text { Rh } & 0.000060 & -0.000157 & 0.403674\end{array}$ $\begin{array}{llll}\mathrm{O} & -0.707536 & -1.927338 & 0.478959\end{array}$

$\begin{array}{llll}\mathrm{O} & -0.260284 & -2.052098 & 2.692141\end{array}$

$\begin{array}{llll}\text { O } & -1.927309 & 0.707182 & 0.478913\end{array}$

$\begin{array}{llll}\mathrm{O} & -2.051961 & 0.260011 & 2.692103\end{array}$

$\begin{array}{llll}\mathrm{O} & 0.707686 & 1.927009 & 0.478710\end{array}$

$\begin{array}{llll}\text { O } & 0.260465 & 2.051925 & 2.691868\end{array}$

$\begin{array}{llll}\mathrm{O} & 1.927442 & -0.707459 & 0.479006\end{array}$

$\begin{array}{llll}\text { O } & 2.052045 & -0.260277 & 2.692207\end{array}$

$\begin{array}{llll}\mathrm{Cl} & -3.849148 & -2.901817 & 0.368632\end{array}$

$\begin{array}{llll}\mathrm{Cl} & -2.900930 & 3.847469 & 0.366406\end{array}$

$\begin{array}{llll}\mathrm{Cl} & 3.849086 & 2.901763 & 0.367826\end{array}$

$\mathrm{Cl} \quad 2.901007 \quad-3.847566 \quad 0.366653$

$\begin{array}{llll}\text { F } & 5.936407 & 4.117682 & -3.632248\end{array}$

F $\quad 8.026631 \quad 4.472667 \quad-3.148657$

F $\quad 6.595847 \quad 4.021804 \quad-1.567065$

F $\quad 9.879690 \quad 0.829269 \quad-5.853769$

F $\quad 10.691623 \quad-0.248840 \quad-4.151313$

F $\quad 9.273700 \quad-1.229192 \quad-5.472119$

$\begin{array}{llll}\text { F } & -4.124324 & 5.937292 & -3.626560\end{array}$

$\begin{array}{llll}\text { F } & -4.470838 & 8.028728 & -3.141813\end{array}$

F $\quad-4.018014 \quad 6.596711 \quad-1.561971$

F $\quad 0.232979 \quad 9.003579 \quad-6.209664$

F $\quad-0.825463 \quad 10.520254 \quad-5.070078$

F $\quad 1.234358 \quad 10.014266 \quad-4.569059$

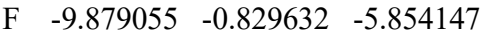

$\begin{array}{llll}\text { F } & -9.273785 & 1.228917 & -5.471847\end{array}$

$\begin{array}{llll}F & -10.691691 & 0.247783 & -4.151601\end{array}$

F $\quad-8.026895 \quad-4.472939-3.147737$

F $\quad-5.936793 \quad-4.118226-3.632076$

F $\quad-6.595544 \quad-4.021961 \quad-1.566688$

$\begin{array}{llll}\text { F } & -0.233672 & -9.001869 & -6.210644\end{array}$

F $\quad 0.824716 \quad-10.519031 \quad-5.071659$

F $\quad-1.235034 \quad-10.013060 \quad-4.570327$

F $\quad 4.017723-6.596606-1.562372$

F $\quad 4.470291 \quad-8.028848 \quad-3.142101$

$\begin{array}{llll}\text { F } & 4.124200 & -5.937377 & -3.627015\end{array}$

C $\quad-1.170270 \quad-3.956448 \quad 1.581016$ $\begin{array}{llll}\text { C } & -1.897040 & -4.574515 & 2.827086\end{array}$

$\begin{array}{llll}\text { C } & -0.476575 & -4.929528 & 2.516203\end{array}$

$\begin{array}{llll}\text { C } & -0.682706 & -2.539289 & 1.596921\end{array}$

$\begin{array}{llll}\text { C } & -1.567954 & -4.430166 & 0.215009\end{array}$

$\begin{array}{llll}\text { C } & -2.758680 & -4.038587 & -0.414119\end{array}$

$\begin{array}{llll}\text { C } & -3.104297 & -4.513149 & -1.677174\end{array}$

$\begin{array}{llll}\text { C } & -2.235300 & -5.364181 & -2.355218\end{array}$

$\begin{array}{llll}\text { C } & -1.015190 & -5.743069 & -1.779454\end{array}$

$\begin{array}{llll}\text { C } & -0.713941 & -5.272710 & -0.495275\end{array}$

$\begin{array}{llll}\text { C } & -3.001566 & -5.560367 & 2.517471\end{array}$

$\begin{array}{llll}\text { C } & -4.329029 & -5.230081 & 2.828903\end{array}$

$\begin{array}{llll}\text { C } & -5.377876 & -6.093141 & 2.516171\end{array}$

$\begin{array}{llll}\text { C } & -5.122531 & -7.312862 & 1.887663\end{array}$

$\begin{array}{llll}\text { C } & -3.805869 & -7.660508 & 1.584382\end{array}$

$\begin{array}{llll}\text { C } & -2.758265 & -6.795045 & 1.899272\end{array}$

$\begin{array}{llll}\text { C } & -2.131607 & -3.741599 & 4.060236\end{array}$

$\begin{array}{llll}\text { C } & -1.714798 & -4.248871 & 5.296916\end{array}$

$\begin{array}{llll}\text { C } & -1.906256 & -3.515382 & 6.469074\end{array}$

$\begin{array}{llll}\text { C } & -2.517553 & -2.260395 & 6.413880\end{array}$

C $\quad-2.948793 \quad-1.755216 \quad 5.185419$

$\begin{array}{llll}\text { C } & -2.767591 & -2.498465 & 4.019567\end{array}$

C $\quad-0.049906 \quad-6.603364-2.499740$

$\begin{array}{llll}\text { C } & -0.483010 & -7.627600 & -3.353507\end{array}$

$\begin{array}{llll}\text { C } & 0.439848 & -8.434012 & -4.017665\end{array}$

$\begin{array}{llll}\text { C } & 1.810714 & -8.243443 & -3.846032\end{array}$

$\begin{array}{llll}\text { C } & 2.243943 & -7.226742 & -2.997090\end{array}$

$\begin{array}{llll}\text { C } & 1.328918 & -6.411434 & -2.333177\end{array}$

$\begin{array}{llll}\text { C } & -3.956434 & 1.169850 & 1.581285\end{array}$

$\begin{array}{llll}\text { C } & -4.573947 & 1.897045 & 2.827429\end{array}$

$\begin{array}{llll}\text { C } & -4.929118 & 0.476544 & 2.517164\end{array}$

$\begin{array}{llll}\text { C } & -2.539238 & 0.682330 & 1.596886\end{array}$

$\begin{array}{llll}\text { C } & -4.430862 & 1.567089 & 0.215400\end{array}$

$\begin{array}{llll}\text { C } & -4.038542 & 2.756925 & -0.414974\end{array}$

$\begin{array}{llll}\text { C } & -4.513265 & 3.101628 & -1.678217\end{array}$

$\begin{array}{llll}\text { C } & -5.365621 & 2.232936 & -2.355013\end{array}$

$\begin{array}{llll}\text { C } & -5.745952 & 1.014043 & -1.777664\end{array}$

$\begin{array}{llll}\text { C } & -5.275101 & 0.713585 & -0.493467\end{array}$

$\begin{array}{llll}\text { C } & -5.559724 & 3.001598 & 2.517767\end{array}$

$\begin{array}{lrrr}\text { C } & -5.228349 & 4.329328 & 2.826941\end{array}$

$\begin{array}{llll}\text { C } & -6.091365 & 5.378142 & 2.513999\end{array}$

$\begin{array}{llll}\text { C } & -7.312126 & 5.122496 & 1.887618\end{array}$

$\begin{array}{llll}\text { C } & -7.660836 & 3.805600 & 1.586662\end{array}$

$\begin{array}{llll}\text { C } & -6.795401 & 2.758019 & 1.901694\end{array}$

C $\quad-3.740588 \quad 2.131858 \quad 4.060246$

$\begin{array}{llll}\text { C } & -4.247364 & 1.715113 & 5.297152\end{array}$

$\begin{array}{llll}\text { C } & -3.513429 & 1.906676 & 6.469013\end{array}$

$\begin{array}{llll}\text { C } & -2.258473 & 2.517997 & 6.413287\end{array}$

$\begin{array}{llll}\text { C } & -1.753781 & 2.949136 & 5.184599\end{array}$

$\begin{array}{llll}\text { C } & -2.497473 & 2.767823 & 4.019048\end{array}$

$\begin{array}{llll}\text { C } & -6.609293 & 0.049174 & -2.494966\end{array}$

$\begin{array}{llll}\text { C } & -7.629661 & 0.482339 & -3.353252\end{array}$

$\begin{array}{llll}\text { C } & -8.433899 & -0.440463 & -4.020046\end{array}$

$\begin{array}{llll}\text { C } & -8.245237 & -1.811358 & -3.846757\end{array}$

$\begin{array}{llll}\text { C } & -7.232239 & -2.244672 & -2.993463\end{array}$

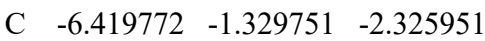

$\begin{array}{llll}\text { C } & 1.170301 & 3.956251 & 1.580664\end{array}$

$\begin{array}{llll}\text { C } & 1.897076 & 4.574433 & 2.826673\end{array}$

$\begin{array}{llll}\text { C } & 0.476562 & 4.929256 & 2.515897\end{array}$

$\begin{array}{llll}\text { C } & 0.682836 & 2.539059 & 1.596629\end{array}$

$\begin{array}{llll}\text { C } & 1.567853 & 4.430097 & 0.214659\end{array}$

$\begin{array}{llll}\text { C } & 2.758534 & 4.038644 & -0.414633\end{array}$

$\begin{array}{llll}\text { C } & 3.104062 & 4.513452 & -1.677629\end{array}$ 


\begin{tabular}{|c|c|c|c|}
\hline & 2.235016 & 5.364615 & -2.355456 \\
\hline $\mathrm{C}$ & 1.014950 & 5.743374 & -1.779530 \\
\hline $\mathrm{C}$ & 0.713789 & 5.272782 & -0.495418 \\
\hline $\mathrm{C}$ & 3.001456 & 5.560406 & 2.516949 \\
\hline $\mathrm{C}$ & 4.329067 & 5.229911 & 2.827547 \\
\hline$C$ & 5.377778 & 6.093065 & 2.514658 \\
\hline $\mathrm{C}$ & 5.122141 & 7.313104 & 1.886866 \\
\hline $\mathrm{C}$ & 3.805345 & 7.660949 & 1.584446 \\
\hline $\mathrm{C}$ & 2.757875 & 6.795376 & 1.899467 \\
\hline $\mathrm{C}$ & 2.131861 & 3.741586 & 4.059834 \\
\hline $\mathrm{C}$ & 1.715229 & 4.248936 & 5.296545 \\
\hline $\mathrm{C}$ & 1.906797 & 3.515500 & 6.468718 \\
\hline $\mathrm{C}$ & 2.518021 & 2.260483 & 6.413512 \\
\hline $\mathrm{C}$ & 2.949089 & 1.755218 & 5.185022 \\
\hline $\mathrm{C}$ & 2.767784 & 2.498424 & 4.019154 \\
\hline $\mathrm{C}$ & 0.049578 & 6.603787 & -2.499564 \\
\hline $\mathrm{C}$ & -1.329209 & 6.411653 & -2.333067 \\
\hline $\mathrm{C}$ & -2.244342 & 7.227077 & -2.996695 \\
\hline $\mathrm{C}$ & -1.811225 & 8.244119 & -3.845273 \\
\hline $\mathrm{C}$ & -0.440372 & 8.434894 & -4.016851 \\
\hline $\mathrm{C}$ & 0.482583 & 7.628355 & -3.352979 \\
\hline $\mathrm{C}$ & 3.956615 & -1.169927 & 1.581354 \\
\hline $\mathrm{C}$ & 4.574198 & -1.897062 & 2.827501 \\
\hline $\mathrm{C}$ & 4.929267 & -0.476525 & 2.517195 \\
\hline $\mathrm{C}$ & 2.539369 & -0.682560 & 1.596983 \\
\hline $\mathrm{C}$ & 4.430994 & -1.567245 & 0.215468 \\
\hline $\mathrm{C}$ & 4.038639 & -2.757098 & -0.414828 \\
\hline $\mathrm{C}$ & 4.513311 & -3.101869 & -1.678076 \\
\hline $\mathrm{C}$ & 5.365646 & -2.233197 & -2.354930 \\
\hline $\mathrm{C}$ & 5.746001 & -1.014279 & -1.777660 \\
\hline $\mathrm{C}$ & 5.275191 & -0.713748 & -0.493475 \\
\hline $\mathrm{C}$ & 5.560076 & -3.001534 & 2.517812 \\
\hline $\mathrm{C}$ & 5.228826 & -4.329272 & 2.827029 \\
\hline $\mathrm{C}$ & 6.091913 & -5.378037 & 2.514071 \\
\hline & 7.312614 & -5.122310 & 1.887621 \\
\hline & 7.661206 & -3.805392 & 1.586606 \\
\hline $\mathrm{C}$ & 6.795698 & -2.757877 & 1.901665 \\
\hline $\mathrm{C}$ & 3.740866 & -2.131942 & 4.060315 \\
\hline $\mathrm{C}$ & 4.247582 & -1.715071 & 5.297199 \\
\hline $\mathrm{C}$ & 3.513661 & -1.906654 & 6.469070 \\
\hline $\mathrm{C}$ & 2.258794 & -2.518129 & 6.413365 \\
\hline & 1.754157 & -2.949402 & 5.184693 \\
\hline $\mathrm{C}$ & 2.497831 & -2.768077 & 4.019133 \\
\hline $\mathrm{C}$ & 6.609331 & -0.049464 & -2.495047 \\
\hline & 7.629751 & -0.482696 & -3.353222 \\
\hline $\mathrm{C}$ & 8.434011 & 0.440059 & -4.020069 \\
\hline $\mathrm{C}$ & 8.245298 & 1.810977 & -3.846937 \\
\hline $\mathrm{C}$ & 7.232229 & 2.244341 & -2.993771 \\
\hline $\mathrm{C}$ & 6.419741 & 1.329479 & -2.326202 \\
\hline $\mathrm{C}$ & 0.049624 & 9.497094 & -4.962024 \\
\hline $\mathrm{C}$ & -3.713500 & 6.958966 & -2.828926 \\
\hline $\mathrm{C}$ & -9.566388 & 0.050803 & -4.878781 \\
\hline $\mathrm{C}$ & -6.959250 & -3.713608 & -2.832279 \\
\hline $\mathrm{C}$ & 3.713161 & -6.958905 & -2.829298 \\
\hline & -0.050277 & -9.495840 & -4.963183 \\
\hline $\mathrm{C}$ & 6.959154 & 3.713284 & -2.832735 \\
\hline & 9.566541 & -0.051315 & -4.878694 \\
\hline $\mathrm{H}$ & -0.233492 & -5.912191 & 2.132554 \\
\hline & 0.278301 & -4.502982 & 3.163601 \\
\hline $\mathrm{H}$ & -4.035649 & -4.211675 & -2.137017 \\
\hline $\mathrm{H}$ & -2.499729 & -5.702315 & -3.351454 \\
\hline & 0.209692 & -5.580989 & -0.019598 \\
\hline
\end{tabular}

\begin{tabular}{|c|c|c|c|}
\hline $\mathrm{H}$ & -4.542352 & -4.287050 & 3.317530 \\
\hline $\mathrm{H}$ & -6.396228 & -5.809112 & 2.764015 \\
\hline $\mathrm{H}$ & -5.938300 & -7.985668 & 1.641739 \\
\hline $\mathrm{H}$ & -3.587888 & -8.608889 & 1.102691 \\
\hline $\mathrm{H}$ & -1.748728 & -7.094487 & 1.646449 \\
\hline $\mathrm{H}$ & -1.226544 & -5.218569 & 5.335958 \\
\hline $\mathrm{H}$ & -1.570794 & -3.919414 & 7.419565 \\
\hline $\mathrm{H}$ & -2.656217 & -1.678040 & 7.319969 \\
\hline & -3.402139 & -0.771637 & 5.134183 \\
\hline & -3.075475 & -2.089408 & 3.065898 \\
\hline $\mathrm{H}$ & -1.542871 & -7.816778 & -3.477216 \\
\hline $\mathrm{H}$ & 1.689190 & -5.615275 & -1.691905 \\
\hline $\mathrm{H}$ & -5.911958 & 0.233379 & 2.134014 \\
\hline $\mathrm{H}$ & -4.502354 & -0.278158 & 3.164613 \\
\hline $\mathrm{H}$ & -4.211117 & 4.032222 & -2.139164 \\
\hline $\mathrm{H}$ & -5.703474 & 2.496845 & -3.351464 \\
\hline $\mathrm{H}$ & -5.584348 & -0.209090 & -0.016578 \\
\hline $\mathrm{H}$ & -4.284551 & 4.542851 & 3.313977 \\
\hline $\mathrm{H}$ & -5.806516 & 6.396708 & 2.760028 \\
\hline $\mathrm{H}$ & -7.984906 & 5.938258 & 1.641561 \\
\hline $\mathrm{H}$ & -8.610044 & 3.587406 & 1.106679 \\
\hline $\mathrm{H}$ & -7.095679 & 1.748256 & 1.650764 \\
\hline $\mathrm{H}$ & -5.217025 & 1.226826 & 5.336619 \\
\hline $\mathrm{H}$ & -3.917087 & 1.571271 & 7.419686 \\
\hline $\mathrm{H}$ & -1.675746 & 2.656744 & 7.319118 \\
\hline $\mathrm{H}$ & -0.770204 & 3.402416 & 5.132952 \\
\hline $\mathrm{H}$ & -2.088782 & 3.075587 & 3.065168 \\
\hline $\mathrm{H}$ & -7.808214 & 1.542507 & -3.490042 \\
\hline $\mathrm{H}$ & -5.624050 & -1.690374 & -1.684339 \\
\hline $\mathrm{H}$ & 0.233329 & 5.911865 & 2.132216 \\
\hline $\mathrm{H}$ & -0.278233 & 4.502660 & 3.163360 \\
\hline $\mathrm{H}$ & 4.035377 & 4.212077 & -2.137597 \\
\hline $\mathrm{H}$ & 2.499373 & 5.702967 & -3.351631 \\
\hline $\mathrm{H}$ & -0.209824 & 5.580979 & -0.019644 \\
\hline $\mathrm{H}$ & 4.542582 & 4.286643 & 3.315651 \\
\hline $\mathrm{H}$ & 6.396258 & 5.808891 & 2.761825 \\
\hline $\mathrm{H}$ & 5.937807 & 7.985996 & 1.640830 \\
\hline $\mathrm{H}$ & 3.587158 & 8.609569 & 1.103317 \\
\hline $\mathrm{H}$ & 1.748202 & 7.094956 & 1.647339 \\
\hline $\mathrm{H}$ & 1.226985 & 5.218645 & 5.335603 \\
\hline $\mathrm{H}$ & 1.571474 & 3.919609 & 7.419228 \\
\hline $\mathrm{H}$ & 2.656784 & 1.678158 & 7.319604 \\
\hline $\mathrm{H}$ & 3.402339 & 0.771597 & 5.133778 \\
\hline $\mathrm{H}$ & 3.075563 & 2.089323 & 3.065472 \\
\hline $\mathrm{H}$ & -1.689391 & 5.615252 & -1.692029 \\
\hline $\mathrm{H}$ & 1.542421 & 7.817683 & -3.476619 \\
\hline $\mathrm{H}$ & 5.912097 & -0.233284 & 2.134056 \\
\hline $\mathrm{H}$ & 4.502445 & 0.278147 & 3.164637 \\
\hline $\mathrm{H}$ & 4.211157 & -4.032479 & -2.138970 \\
\hline $\mathrm{H}$ & 5.703466 & -2.497151 & -3.351381 \\
\hline $\mathrm{H}$ & 5.584446 & 0.208956 & -0.016644 \\
\hline $\mathrm{H}$ & 4.285052 & -4.542881 & 3.314093 \\
\hline $\mathrm{H}$ & 5.807142 & -6.396618 & 2.760141 \\
\hline $\mathrm{H}$ & 7.985450 & -5.938018 & 1.641548 \\
\hline $\mathrm{H}$ & 8.610364 & -3.587131 & 1.106570 \\
\hline $\mathrm{H}$ & 7.095890 & -1.748097 & 1.650695 \\
\hline $\mathrm{H}$ & 5.217183 & -1.226638 & 5.336632 \\
\hline П & 3.917290 & -1.571150 & 7.419717 \\
\hline $\mathrm{H}$ & 1.676063 & -2.656889 & 7.319195 \\
\hline $\mathrm{H}$ & 0.770638 & -3.402806 & 5.133079 \\
\hline $\mathrm{H}$ & 2.089201 & -3.075938 & 3.065272 \\
\hline & 7.808336 & -1.542869 & -3.489893 \\
\hline
\end{tabular}

H $\quad 5.623958 \quad 1.690134 \quad-1.684674$

$\mathrm{H} \quad-2.524593 \quad 8.881731 \quad-4.351368$

$\mathrm{H} \quad 8.872861 \quad 2.524062 \quad-4.365904$

$\mathrm{H} \quad-8.872785 \quad-2.524481 \quad-4.365691$

H $\quad 2.524018 \quad-8.880965 \quad-4.352338$

\section{K'1}

$\begin{array}{llll}\text { Rh } & 0.000009 & -0.000213 & -0.168650\end{array}$

$\begin{array}{llll}\mathrm{Rh} & 0.000090 & -0.000130 & -2.556730\end{array}$

$\begin{array}{llll}\mathrm{O} & -1.453638 & 1.485396 & -2.446250\end{array}$

$\begin{array}{lllll}\mathrm{O} & -1.735126 & 1.084990 & -0.239338\end{array}$

$\begin{array}{llll}\mathrm{O} & 1.485581 & 1.453568 & -2.446095\end{array}$

$\begin{array}{llll}\mathrm{O} & 1.085281 & 1.734930 & -0.239139\end{array}$

$\begin{array}{llll}\mathrm{O} & 1.453792 & -1.485657 & -2.446190\end{array}$

O $\quad 1.735131 \quad-1.085473 \quad-0.239221$

$\begin{array}{llll}\text { O } & -1.485473 & -1.453863 & -2.446405\end{array}$

$\begin{array}{llll}\text { O } & -1.085242 & -1.735317 & -0.239460\end{array}$

$\begin{array}{llll}\mathrm{Cl} & -2.308738 & 4.801481 & 0.476287\end{array}$

$\begin{array}{llll}\mathrm{Cl} & 4.801765 & 2.308877 & 0.476671\end{array}$

$\begin{array}{lllll}\mathrm{Cl} & 2.309070 & -4.802098 & 0.476403\end{array}$

$\begin{array}{llll}\mathrm{Cl} & -4.802010 & -2.308883 & 0.475850\end{array}$

F $\quad-7.568440 \quad-2.499346 \quad 5.735555$

F $\quad-5.785499 \quad-1.302704 \quad 6.105624$

$\begin{array}{llll}\text { F } & -5.648791 & -3.138937 & 4.950815\end{array}$

F $\quad-8.357827 \quad-1.935061 \quad-0.279310$

$\begin{array}{llll}\text { F } & -9.708962 & -2.469669 & 1.343371\end{array}$

$\begin{array}{llll}\text { F } & -9.741546 & -0.459207 & 0.504393\end{array}$

F $\quad-3.140788 \quad 5.651106 \quad 4.949711$

$\begin{array}{llll}\text { F } & -0.459015 & 9.741229 & 0.503564\end{array}$

$\begin{array}{llll}\text { F } & -2.469396 & 9.709149 & 1.342746\end{array}$

$\begin{array}{llll}\text { F } & -1.935209 & 8.357723 & -0.279815\end{array}$

$\begin{array}{llll}\text { F } & -2.497983 & 7.569074 & 5.735878\end{array}$

$\begin{array}{llll}\text { F } & -1.304209 & 5.783917 & 6.104467\end{array}$

$\begin{array}{llll}\text { F } & 5.779655 & 1.305565 & 6.103220\end{array}$

F $\quad 5.653883 \quad 3.143022 \quad 4.948974$

F $\quad \begin{array}{llll}7.568703 & 2.494559 & 5.738067\end{array}$

F $\quad 8.357564 \quad 1.935502 \quad-0.279200$

$\begin{array}{llll}\text { F } & 9.708909 & 2.469519 & 1.343500\end{array}$

F $\quad 9.741162 \quad 0.459273 \quad 0.504008$

F $\quad 0.459159 \quad-9.741352 \quad 0.505357$

F $\quad 2.469545 \quad-9.708612 \quad 1.344515$

F $\quad \begin{array}{llll}1.935100 & -8.357811 & -0.278495\end{array}$

$\begin{array}{llll}\text { F } & 2.492275 & -7.567918 & 5.739896\end{array}$

F $\quad 3.144576 \quad-5.655380 \quad 4.948500$

$\begin{array}{llll}\text { F } & 1.306614 & -5.776158 & 6.102533\end{array}$

$\begin{array}{llll}\text { C } & -3.334325 & 2.436030 & -1.301959\end{array}$

$\begin{array}{llll}\text { C } & -4.356047 & 2.365740 & -2.502499\end{array}$

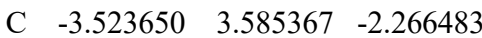

$\begin{array}{llll}\text { C } & -2.080270 & 1.614356 & -1.348090\end{array}$

$\begin{array}{llll}\text { C } & -3.858571 & 2.556870 & 0.098300\end{array}$

$\begin{array}{llll}\text { C } & -3.441898 & 3.553926 & 0.988039\end{array}$

$\begin{array}{llll}\text { C } & -3.864020 & 3.572464 & 2.319070\end{array}$

$\begin{array}{llll}\text { C } & -4.716296 & 2.574746 & 2.783840\end{array}$

$\begin{array}{llll}\text { C } & -5.150444 & 1.557597 & 1.921341\end{array}$

$\begin{array}{llll}\text { C } & -4.703691 & 1.565707 & 0.598106\end{array}$

$\begin{array}{llll}\text { C } & -5.819864 & 2.337369 & -2.128103\end{array}$

$\begin{array}{llll}\text { C } & -6.557634 & 1.157230 & -2.314134\end{array}$

$\begin{array}{llll}\text { C } & -7.883173 & 1.062079 & -1.892945\end{array}$

$\begin{array}{llll}\text { C } & -8.507178 & 2.153448 & -1.284731\end{array}$

C $\quad-7.793014 \quad 3.340511-1.117191$

$\begin{array}{llll}\text { C } & -6.464530 & 3.430574 & -1.534871\end{array}$

$\begin{array}{llll}\text { C } & -4.018098 & 1.561465 & -3.731476\end{array}$ 


\begin{tabular}{|c|c|c|c|}
\hline C & -4.031538 & 2.205351 & -4.974980 \\
\hline $\mathrm{C}$ & -3.731300 & 1.504704 & -6.144186 \\
\hline $\mathrm{C}$ & -3.410364 & 0.146675 & -6.079928 \\
\hline $\mathrm{C}$ & -3.406626 & -0.504707 & -4.844918 \\
\hline $\mathrm{C}$ & -3.721044 & 0.197340 & -3.681757 \\
\hline $\mathrm{C}$ & -6.051020 & 0.468232 & 2.357385 \\
\hline $\mathrm{C}$ & -5.867466 & -0.199961 & 3.575243 \\
\hline $\mathrm{C}$ & -6.682732 & -1.278934 & 3.912630 \\
\hline $\mathrm{C}$ & -7.693842 & -1.713855 & 3.052116 \\
\hline $\mathrm{C}$ & -7.883966 & -1.038829 & 1.850430 \\
\hline $\mathrm{C}$ & -7.077852 & 0.045306 & 1.506786 \\
\hline $\mathrm{C}$ & 2.436300 & 3.334181 & -1.301769 \\
\hline $\mathrm{C}$ & 2.366038 & 4.355856 & -2.502354 \\
\hline $\mathrm{C}$ & 3.585639 & 3.523389 & -2.266309 \\
\hline $\mathrm{C}$ & 1.614617 & 2.080121 & -1.347896 \\
\hline $\mathrm{C}$ & 2.557034 & 3.858452 & 0.098488 \\
\hline $\mathrm{C}$ & 3.554057 & 3.441917 & 0.988306 \\
\hline $\mathrm{C}$ & 3.572458 & 3.864140 & 2.319319 \\
\hline $\mathrm{C}$ & 2.574673 & 4.716398 & 2.783940 \\
\hline $\mathrm{C}$ & 1.557568 & 5.150443 & 1.921328 \\
\hline $\mathrm{C}$ & 1.565782 & 4.703558 & 0.598147 \\
\hline $\mathrm{C}$ & 2.337771 & 5.819687 & -2.127987 \\
\hline $\mathrm{C}$ & 3.430852 & 6.464186 & -1.534327 \\
\hline $\mathrm{C}$ & 3.340837 & 7.792667 & -1.116636 \\
\hline $\mathrm{C}$ & 2.153918 & 8.506994 & -1.284528 \\
\hline $\mathrm{C}$ & 1.062671 & 7.883156 & -1.893122 \\
\hline $\mathrm{C}$ & 1.157794 & 6.557625 & -2.314375 \\
\hline $\mathrm{C}$ & 1.561744 & 4.017910 & -3.731316 \\
\hline $\mathrm{C}$ & 0.197610 & 3.720937 & -3.681603 \\
\hline $\mathrm{C}$ & -0.504421 & 3.406475 & -4.844760 \\
\hline $\mathrm{C}$ & 0.146985 & 3.410115 & -6.0 \\
\hline $\mathrm{C}$ & 1.505025 & 3.731002 & -6.144012 \\
\hline $\mathrm{C}$ & 2.205655 & 4.031267 & -4.974803 \\
\hline $\mathrm{C}$ & 0.468170 & 6.051059 & 2.357180 \\
\hline $\mathrm{C}$ & -0.200186 & 5.867572 & 3.574981 \\
\hline $\mathrm{C}$ & -1.279230 & 6.682817 & 3.912140 \\
\hline $\mathrm{C}$ & -1.714041 & 7.693865 & 3.051488 \\
\hline $\mathrm{C}$ & -1.038843 & 7.883951 & 1.849903 \\
\hline $\mathrm{C}$ & 0.045356 & 7.077826 & 1.506460 \\
\hline $\mathrm{C}$ & 3.334330 & -2.436506 & -1.301890 \\
\hline $\mathrm{C}$ & 4.356122 & -2.366103 & -2.502421 \\
\hline $\mathrm{C}$ & 3.523751 & -3.585771 & -2.266447 \\
\hline $\mathrm{C}$ & 2.080305 & -1.614795 & -1.347999 \\
\hline $\mathrm{C}$ & 3.858621 & -2.557325 & 0.098361 \\
\hline $\mathrm{C}$ & 3.442102 & -3.554420 & 0.988122 \\
\hline $\mathrm{C}$ & 3.864312 & -3.572900 & 2.319131 \\
\hline $\mathrm{C}$ & 4.716498 & -2.575102 & 2.783851 \\
\hline $\mathrm{C}$ & 5.150514 & -1.557918 & 1.921320 \\
\hline $\mathrm{C}$ & 4.703690 & -1.566074 & 0.598105 \\
\hline $\mathrm{C}$ & 5.819913 & -2.337629 & -2.127997 \\
\hline $\mathrm{C}$ & 6.464576 & -3.430706 & -1.534499 \\
\hline $\mathrm{C}$ & 7.793056 & -3.340555 & -1.116808 \\
\hline $\mathrm{C}$ & 8.507208 & -2.153506 & -1.284558 \\
\hline $\mathrm{C}$ & 7.883204 & -1.062262 & -1.892984 \\
\hline $\mathrm{C}$ & 6.557674 & -1.157523 & -2.314226 \\
\hline $\mathrm{C}$ & 4.018104 & -1.561818 & -3.731374 \\
\hline $\mathrm{C}$ & 4.031542 & -2.205709 & -4.974880 \\
\hline $\mathrm{C}$ & 3.731184 & -1.505100 & -6.144074 \\
\hline $\mathrm{C}$ & 3.410112 & -0.147096 & -6.079793 \\
\hline $\mathrm{C}$ & 3.406398 & 0.504284 & -4.844792 \\
\hline $\mathrm{C}$ & 3.720965 & -0.197711 & -3.681643 \\
\hline & 6.050963 & -0.468428 & 2.357307 \\
\hline
\end{tabular}

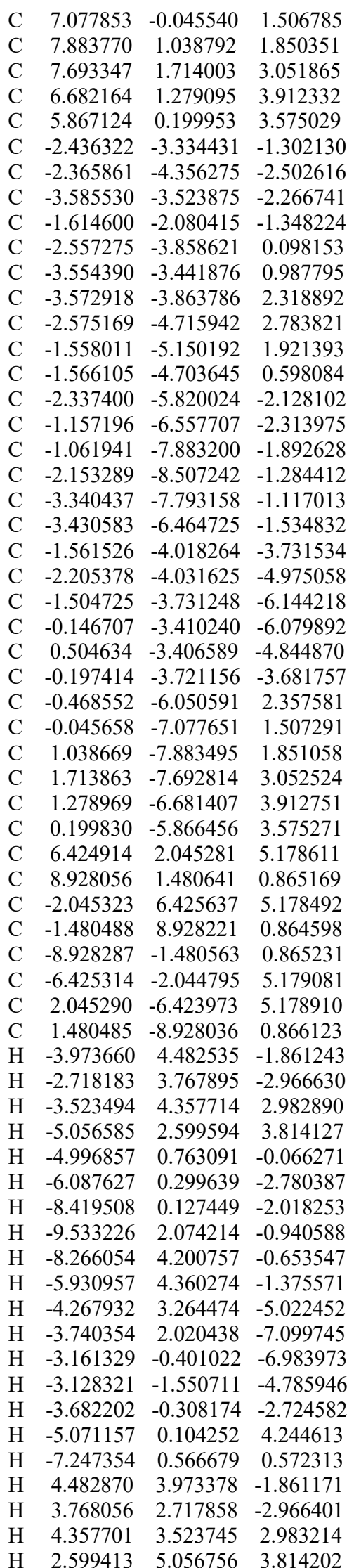

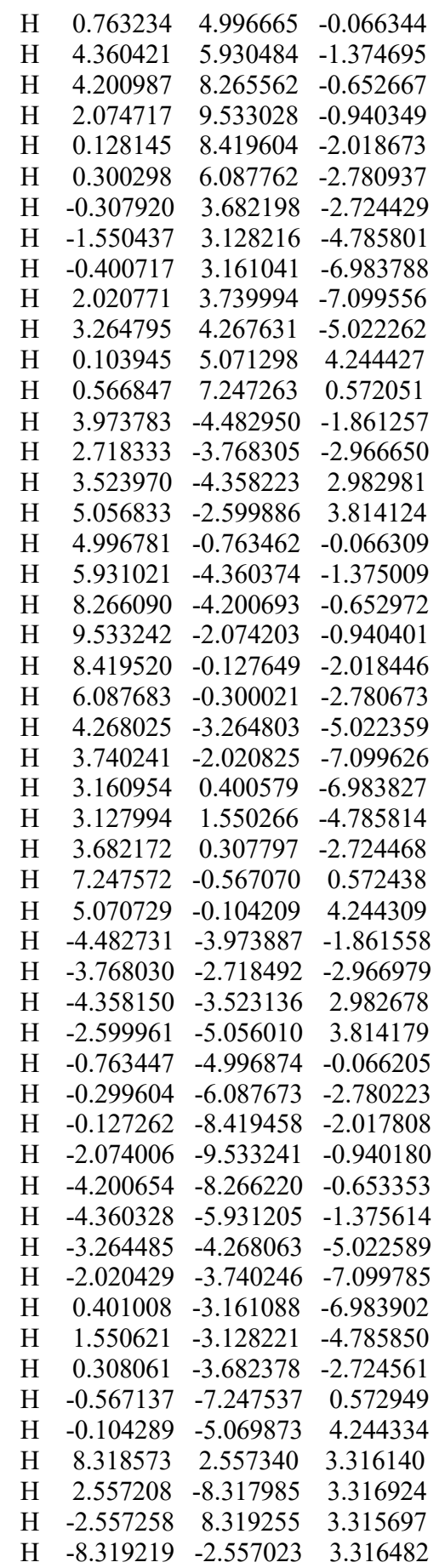
$\begin{array}{lccc}\text { K'2 } & & & \\ \mathrm{Rh} & 0.058028 & -0.542818 & -2.832475 \\ \mathrm{Rh} & 0.039248 & -0.196579 & -0.469246 \\ \mathrm{O} & 0.451839 & 1.782744 & -0.824147 \\ \mathrm{O} & 0.963178 & 1.315092 & -2.977634 \\ \mathrm{O} & -1.977857 & 0.155215 & -0.619353 \\ \mathrm{O} & -1.812624 & 0.364551 & -2.863598 \\ \mathrm{O} & -0.359586 & -2.191757 & -0.267225 \\ \mathrm{O} & -0.853642 & -2.372352 & -2.466502 \\ \mathrm{O} & 2.047939 & -0.601529 & -0.461317\end{array}$ 
$\begin{array}{llll}\text { O } & 1.914133 & -1.429660 & -2.562123\end{array}$

$\begin{array}{lllll}\mathrm{Cl} & -1.591019 & 4.307817 & -1.068307\end{array}$

$\begin{array}{llll}\mathrm{Cl} & -4.638252 & -2.075575 & -0.602589\end{array}$

$\begin{array}{llll}\mathrm{Cl} & 1.476610 & -4.944452 & 0.464880\end{array}$

$\begin{array}{lllll}\mathrm{Cl} & 4.654234 & 1.428630 & -0.658460\end{array}$

F $\quad 4.046802 \quad 9.024746 \quad 4.132997$

$\begin{array}{llll}\text { F } & 6.078705 & 8.446721 & 4.669211\end{array}$

F $\quad 5.692948 \quad 9.450031 \quad 2.781540$

F $\quad 8.171570 \quad 4.565935 \quad 2.503012$

F $\quad 7.027360 \quad 3.389584 \quad 1.068146$

$\begin{array}{llll}\text { F } & 6.733486 & 3.089655 & 3.197087\end{array}$

F $\quad-7.339081 \quad 5.606570 \quad 5.888825$

$\begin{array}{llll}\mathrm{F} & -8.750353 & 5.127419 & 4.308118\end{array}$

F $\quad-7.945134 \quad 3.540163 \quad 5.552516$

$\begin{array}{llll}\text { F } & -3.344203 & 6.629690 & 1.177593\end{array}$

F $\quad-2.475774 \quad 6.333216 \quad 3.144152$

F $\quad-4.117630 \quad 7.736101 \quad 2.889011$

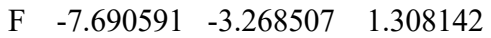

$\begin{array}{llll}\text { F } & -7.530789 & -5.370049 & 1.835271\end{array}$

$\begin{array}{llll}\text { F } & -8.604348 & -3.996412 & 3.143032\end{array}$

$\begin{array}{llll}\mathrm{F} & -3.923885 & -1.736414 & 6.669297\end{array}$

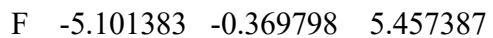

$\begin{array}{lllll}\text { F } & -6.100870 & -1.818825 & 6.729600\end{array}$

F $\quad 3.854326 \quad-7.457988 \quad 4.720108$

F $\quad 2.387589 \quad-5.851910 \quad 4.741487$

$\begin{array}{llll}\text { F } & 3.043633 & -6.689579 & 2.849918\end{array}$

F $\quad 8.818208 \quad-5.235452 \quad 5.131843$

F $\quad 7.558384 \quad-5.115721 \quad 6.896852$

F $\quad 8.374581 \quad-3.299218 \quad 6.011253$

C $\quad 1.236510 \quad 3.544598 \quad-2.174888$

$\begin{array}{llll}\text { C } & 0.985769 & 4.274043 & -3.541540\end{array}$

$\begin{array}{llll}\text { C } & 2.360587 & 3.828106 & -3.153601\end{array}$

$\begin{array}{llll}\text { C } & 0.849592 & 2.108690 & -1.990678\end{array}$

$\begin{array}{llll}\text { C } & 1.158212 & 4.346548 & -0.910594\end{array}$

$\begin{array}{llll}\text { C } & -0.054622 & 4.757877 & -0.338805\end{array}$

$\begin{array}{llll}\text { C } & -0.088116 & 5.510965 & 0.832017\end{array}$

$\begin{array}{llll}\text { C } & 1.101674 & 5.840972 & 1.475179\end{array}$

$\begin{array}{llll}\text { C } & 2.332429 & 5.416833 & 0.956504\end{array}$

$\begin{array}{llll}\text { C } & 2.331613 & 4.681834 & -0.235688\end{array}$

C $\quad 0.616449 \quad 5.737383 \quad-3.444589$

$\begin{array}{llll}\text { C } & -0.668097 & 6.152905 & -3.826062\end{array}$

$\begin{array}{llll}\text { C } & -1.058008 & 7.486001 & -3.708569\end{array}$

$\begin{array}{llll}\text { C } & -0.168348 & 8.438634 & -3.209667\end{array}$

$\begin{array}{llll}\text { C } & 1.116541 & 8.041929 & -2.838033\end{array}$

$\begin{array}{llll}\text { C } & 1.504248 & 6.707403 & -2.957832\end{array}$

C $\quad 0.328382 \quad 3.535440 \quad-4.677338$

$\begin{array}{llll}\text { C } & 0.962727 & 3.519733 & -5.925272\end{array}$

C $\quad 0.391483 \quad 2.845388 \quad-7.005972$

$\begin{array}{llll}\text { C } & -0.825246 & 2.177527 & -6.846410\end{array}$

$\begin{array}{llll}\text { C } & -1.469514 & 2.201711 & -5.607823\end{array}$

$\begin{array}{llll}\text { C } & -0.899796 & 2.885229 & -4.534894\end{array}$

$\begin{array}{llll}\text { C } & 3.608811 & 5.718607 & 1.642468\end{array}$

$\begin{array}{llll}\text { C } & 3.795005 & 6.925535 & 2.331498\end{array}$

$\begin{array}{llll}\text { C } & 5.002691 & 7.196982 & 2.971775\end{array}$

$\begin{array}{llll}\text { C } & 6.052201 & 6.278891 & 2.938766\end{array}$

$\begin{array}{llll}\text { C } & 5.870391 & 5.079352 & 2.253545\end{array}$

$\begin{array}{llll}\text { C } & 4.664404 & 4.796542 & 1.613944\end{array}$

$\begin{array}{llll}\text { C } & -3.936931 & 0.694393 & -1.806855\end{array}$

C $\quad-4.823175 \quad 0.341039 \quad-3.048243$

$\begin{array}{llll}\text { C } & -4.370649 & 1.750267 & -2.805976\end{array}$

$\begin{array}{lllll}\text { C } & -2.469487 & 0.372267 & -1.776082\end{array}$

$\begin{array}{llll}\text { C } & -4.544603 & 0.662501 & -0.435746\end{array}$ $\begin{array}{llll}\text { C } & -4.871673 & -0.537001 & 0.210592\end{array}$

$\begin{array}{llll}\text { C } & -5.358977 & -0.556757 & 1.513850\end{array}$

$\begin{array}{llll}\text { C } & -5.510556 & 0.638754 & 2.209168\end{array}$

$\begin{array}{llll}\text { C } & -5.191669 & 1.861723 & 1.600079\end{array}$

$\begin{array}{llll}\text { C } & -4.718385 & 1.846190 & 0.280654\end{array}$

$\begin{array}{llll}\text { C } & -6.262318 & -0.029527 & -2.758315\end{array}$

$\begin{array}{llll}\text { C } & -6.749882 & -1.283560 & -3.156667\end{array}$

$\begin{array}{llll}\text { C } & -8.057740 & -1.673033 & -2.872095\end{array}$

$\begin{array}{llll}\text { C } & -8.914423 & -0.814296 & -2.182379\end{array}$

$\begin{array}{llll}\text { C } & -8.447867 & 0.440656 & -1.790192\end{array}$

$\begin{array}{llll}\text { C } & -7.139678 & 0.828663 & -2.078690\end{array}$

$\begin{array}{llll}\text { C } & -4.222162 & -0.387506 & -4.222612\end{array}$

$\begin{array}{llll}\text { C } & -4.433375 & 0.122652 & -5.508695\end{array}$

$\begin{array}{llll}\text { C } & -3.907875 & -0.526565 & -6.627812\end{array}$

C $\quad-3.162051 \quad-1.697048 \quad-6.469694$

$\begin{array}{llll}\text { C } & -2.960685 & -2.220960 & -5.190179\end{array}$

C $\quad-3.499153 \quad-1.574942 \quad-4.078128$

$\begin{array}{llll}\text { C } & -5.339390 & 3.142368 & 2.328227\end{array}$

$\begin{array}{llll}\text { C } & -6.353005 & 3.313813 & 3.282639\end{array}$

$\begin{array}{llll}\text { C } & -6.491413 & 4.521164 & 3.963785\end{array}$

$\begin{array}{llll}\text { C } & -5.632352 & 5.589190 & 3.706807\end{array}$

$\begin{array}{llll}\text { C } & -4.626199 & 5.423831 & 2.757469\end{array}$

$\begin{array}{llll}\text { C } & -4.472984 & 4.215917 & 2.077538\end{array}$

$\begin{array}{llll}\text { C } & -1.203567 & -4.250357 & -1.021792\end{array}$

$\begin{array}{llll}\text { C } & -0.935423 & -5.380001 & -2.068955\end{array}$

C $\quad-2.308588-4.802006 \quad-1.900073$

$\begin{array}{llll}\text { C } & -0.766449 & -2.838856 & -1.287655\end{array}$

$\begin{array}{llll}\text { C } & -1.236369 & -4.537110 & 0.450807\end{array}$

$\begin{array}{llll}\text { C } & -0.101722 & -4.804807 & 1.225785\end{array}$

$\begin{array}{llll}\text { C } & -0.177527 & -4.903858 & 2.615121\end{array}$

$\begin{array}{llll}\text { C } & -1.389894 & -4.664491 & 3.260398\end{array}$

$\begin{array}{llll}\text { C } & -2.537892 & -4.353970 & 2.518369\end{array}$

$\begin{array}{llll}\text { C } & -2.439544 & -4.332649 & 1.123841\end{array}$

C $\quad-0.630315-6.753953 \quad-1.518802$

$\begin{array}{llll}\text { C } & 0.554057 & -7.407553 & -1.890943\end{array}$

$\begin{array}{llll}\text { C } & 0.877408 & -8.661953 & -1.375508\end{array}$

$\begin{array}{llll}\text { C } & 0.021124 & -9.295776 & -0.474461\end{array}$

$\begin{array}{llll}\text { C } & -1.164423 & -8.661475 & -0.101619\end{array}$

C $\quad-1.487267 \quad-7.407966 \quad-0.620532$

C $\quad-0.225972 \quad-5.032658 \quad-3.351978$

$\begin{array}{llll}\text { C } & -0.844221 & -5.348877 & -4.567488\end{array}$

$\begin{array}{llll}\text { C } & -0.229614 & -5.039459 & -5.781694\end{array}$

$\begin{array}{llll}\text { C } & 1.015498 & -4.406210 & -5.790072\end{array}$

C $\quad 1.643806 \quad-4.101311-4.581044$

C $\quad 1.030994 \quad-4.422420 \quad-3.370129$

$\begin{array}{llll}\text { C } & -3.816878 & -3.946883 & 3.143628\end{array}$

$\begin{array}{llll}\text { C } & -5.040112 & -4.304294 & 2.561290\end{array}$

$\begin{array}{llll}\text { C } & -6.241611 & -3.822475 & 3.079084\end{array}$

C $\quad-6.253762 \quad-2.983682 \quad 4.191409$

$\begin{array}{llll}\text { C } & -5.037964 & -2.633038 & 4.779678\end{array}$

$\begin{array}{llll}\text { C } & -3.831631 & -3.106388 & 4.266684\end{array}$

$\begin{array}{llll}\text { C } & 3.994948 & -1.534139 & -1.394295\end{array}$

$\begin{array}{llll}\text { C } & 4.925701 & -1.474628 & -2.656612\end{array}$

$\begin{array}{llll}\text { C } & 4.429463 & -2.786088 & -2.131019\end{array}$

C $\quad 2.547783 \quad-1.157886 \quad-1.494295$

$\begin{array}{llll}\text { C } & 4.584205 & -1.250926 & -0.044743\end{array}$

$\begin{array}{llll}\text { C } & 4.941272 & 0.037219 & 0.378270\end{array}$

$\begin{array}{llll}\text { C } & 5.506106 & 0.261238 & 1.631373\end{array}$

$\begin{array}{llll}\text { C } & 5.683918 & -0.806508 & 2.506710\end{array}$

$\begin{array}{llll}\text { C } & 5.292608 & -2.101742 & 2.141022\end{array}$

$\begin{array}{llll}\text { C } & 4.757259 & -2.297328 & 0.861866\end{array}$

$\begin{array}{llll}\text { C } & 6.358360 & -1.060995 & -2.404416\end{array}$ $\begin{array}{llll}\text { C } & 6.849098 & 0.121851 & -2.977537\end{array}$

$\begin{array}{llll}\text { C } & 8.150895 & 0.555557 & -2.731654\end{array}$

$\begin{array}{llll}\text { C } & 8.997105 & -0.187529 & -1.907549\end{array}$

$\begin{array}{llll}\text { C } & 8.526694 & -1.372142 & -1.340076\end{array}$

$\begin{array}{llll}\text { C } & 7.224405 & -1.804581 & -1.589353\end{array}$

C $\quad 4.363185 \quad-1.020674 \quad-3.978651$

$\begin{array}{llll}\text { C } & 4.534419 & -1.843399 & -5.098269\end{array}$

$\begin{array}{llll}\text { C } & 4.023020 & -1.469485 & -6.342162\end{array}$

$\begin{array}{llll}\text { C } & 3.332476 & -0.262650 & -6.477807\end{array}$

$\begin{array}{llll}\text { C } & 3.172258 & 0.570431 & -5.368372\end{array}$

$\begin{array}{llll}\text { C } & 3.695334 & 0.197244 & -4.130891\end{array}$

$\begin{array}{llll}\text { C } & 5.424515 & -3.238279 & 3.080563\end{array}$

$\begin{array}{llll}\text { C } & 6.517573 & -3.327007 & 3.955059\end{array}$

$\begin{array}{llll}\text { C } & 6.628229 & -4.392835 & 4.844969\end{array}$

C $\quad 5.656786 \quad-5.393372 \quad 4.885906$

$\begin{array}{llll}\text { C } & 4.571659 & -5.309655 & 4.016732\end{array}$

$\begin{array}{llll}\text { C } & 4.451744 & -4.246488 & 3.121170\end{array}$

$\begin{array}{llll}\text { C } & -5.038018 & -1.647975 & 5.915049\end{array}$

$\begin{array}{llll}\text { C } & -7.520732 & -4.121490 & 2.349369\end{array}$

$\begin{array}{llll}\text { C } & -7.628024 & 4.697742 & 4.932364\end{array}$

$\begin{array}{llll}\text { C } & -3.650508 & 6.535633 & 2.493812\end{array}$

$\begin{array}{llll}\text { C } & 6.956348 & 4.040810 & 2.251533\end{array}$

$\begin{array}{llll}\text { C } & 5.203408 & 8.527332 & 3.643469\end{array}$

$\begin{array}{llll}\text { C } & 3.474095 & -6.334442 & 4.080088\end{array}$

$\begin{array}{llll}\text { C } & 7.842503 & -4.507031 & 5.724549\end{array}$

H $\quad 3.107508 \quad 4.557516 \quad-2.867649$

H $\quad 2.753178 \quad 2.967618 \quad-3.679204$

$\begin{array}{llll}\mathrm{H} & -1.035862 & 5.824871 & 1.246322\end{array}$

$\mathrm{H} \quad 1.060613 \quad 6.401098 \quad 2.403333$

H $\quad 3.274920 \quad 4.368887 \quad-0.667730$

$\begin{array}{llll}\mathrm{H} & -1.369288 & 5.425008 & -4.215865\end{array}$

$\mathrm{H} \quad-2.060262 \quad 7.778285 \quad-4.007230$

H $\quad-0.470642 \quad 9.477141 \quad-3.116109$

H $\quad 1.824463 \quad 8.770760 \quad-2.455086$

$\begin{array}{llll}\mathrm{H} & 2.508019 & 6.433717 & -2.657417\end{array}$

H $\quad 1.914799 \quad 4.029386 \quad-6.043932$

$\mathrm{H} \quad 0.899474 \quad 2.835824 \quad-7.965740$

$\mathrm{H} \quad-1.269157 \quad 1.639055 \quad-7.678546$

H $\quad-2.401486 \quad 1.665971 \quad-5.472412$

$\begin{array}{llll}\mathrm{H} & -1.385607 & 2.872271 & -3.567268\end{array}$

H $\quad 3.000968 \quad 7.662567 \quad 2.355611$

H $\quad 4.538603 \quad 3.847983 \quad 1.104683$

H $\quad-5.084574 \quad 2.496067 \quad-2.478105$

$\mathrm{H} \quad-3.592714 \quad 2.127826 \quad-3.457543$

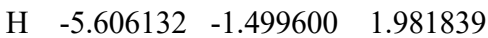

$\begin{array}{llll}\mathrm{H} & -5.839381 & 0.604532 & 3.241523\end{array}$

H $\quad-4.478396 \quad 2.780127 \quad-0.215484$

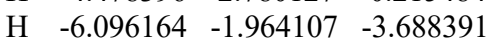

$\mathrm{H} \quad-8.404480-2.652033 \quad-3.189152$

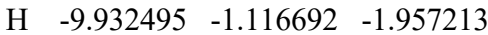

$\begin{array}{llll}\mathrm{H} & -9.101622 & 1.125482 & -1.258565\end{array}$

$\mathrm{H} \quad-6.811754 \quad 1.807457 \quad-1.752121$

$\mathrm{H} \quad-5.003800 \quad 1.039333 \quad-5.629811$

H $\quad-4.074150 \quad-0.113917 \quad-7.618410$

H $\quad-2.742188 \quad-2.198782 \quad-7.336486$

$\mathrm{H} \quad-2.371504 \quad-3.122143 \quad-5.054780$

H $\quad-3.317541 \quad-1.970641 \quad-3.088172$

$\begin{array}{llll}\mathrm{H} & -7.047182 & 2.506681 & 3.484396\end{array}$

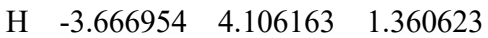

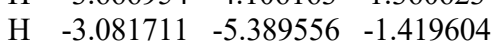

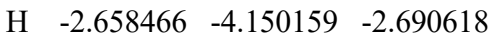

H $\quad 0.707476 \quad-5.142602 \quad 3.190753$ 


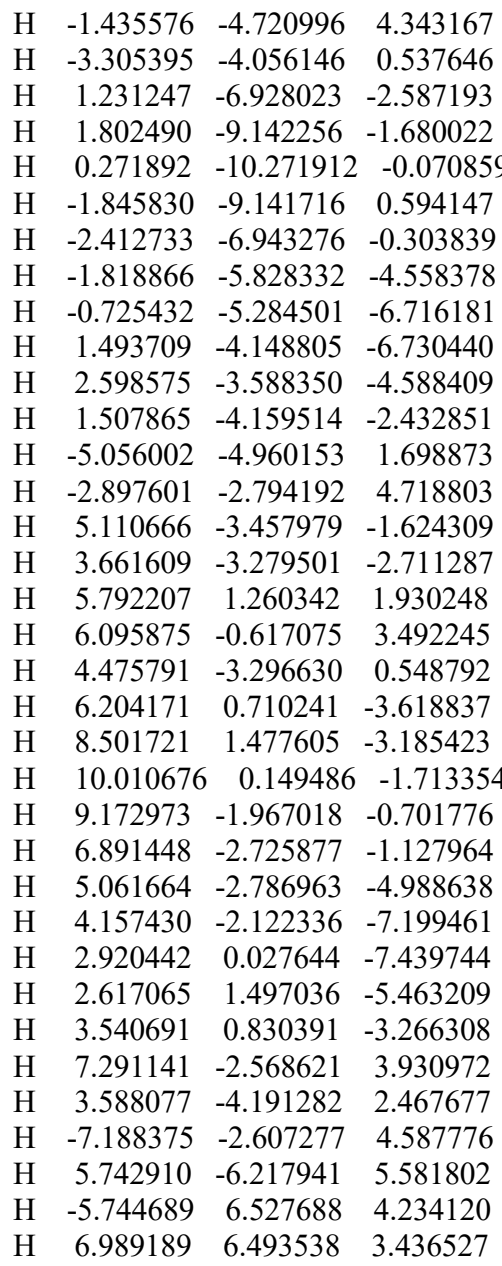

\section{K'3}

$\begin{array}{llll}\mathrm{Rh} & 0.072280 & -0.064500 & 2.893730\end{array}$

$\begin{array}{llll}\mathrm{Rh} & 0.065380 & -0.087720 & 0.505450\end{array}$

$\begin{array}{llll}\mathrm{O} & 1.008590 & 1.734700 & 0.566290\end{array}$

$\begin{array}{llll}\mathrm{O} & 0.504080 & 1.964900 & 2.757110\end{array}$

$\begin{array}{llll}\text { O } & 1.893100 & -1.000730 & 0.595190\end{array}$

$\begin{array}{llll}\mathrm{O} & 2.101860 & -0.488720 & 2.788040\end{array}$

$\begin{array}{llll}\mathrm{O} & -0.848720 & -1.920090 & 0.592510\end{array}$

$\begin{array}{llll}\mathrm{O} & -0.341240 & -2.094570 & 2.790050\end{array}$

$\begin{array}{llll}\mathrm{O} & -1.773990 & 0.820790 & 0.569160\end{array}$

$\begin{array}{lrrr}\mathrm{O} & -1.952610 & 0.358240 & 2.776250\end{array}$

$\begin{array}{llll}\mathrm{Cl} & 4.380310 & 2.486990 & 0.824230\end{array}$

$\begin{array}{llll}\mathrm{Cl} & 3.005270 & -4.190950 & 0.312590\end{array}$

$\begin{array}{llll}\text { Cl } & -4.056830 & -2.738500 & 0.529460\end{array}$

$\begin{array}{llll}\text { Cl } & -2.530800 & 3.984140 & 0.394020\end{array}$

F $\quad 4.448070 \quad 1.368060 \quad-6.389160$

F $\quad 5.863510 \quad 3.022540 \quad-6.483290$

F $\quad 3.984150 \quad 3.257550 \quad-5.420560$

F $\quad 8.706780 \quad 4.080320 \quad-2.649190$

F $\quad 8.990470 \quad 2.538720 \quad-1.134980$

F $\quad \begin{array}{llll}7.381280 & 3.983390 & -0.927190\end{array}$

F $\quad-3.135040 \quad 5.480330 \quad-4.074520$

$\begin{array}{llll}\text { F } & -3.044430 & 6.541400 & -2.183660\end{array}$

F $\quad-3.256090 \quad 7.651400 \quad-4.047620$

F $\quad \begin{array}{llll}1.764830 & 9.598890 & -5.143500\end{array}$

$\begin{array}{llll}\text { F } & 2.660720 & 7.880590 & -6.124020\end{array}$
F $\quad 0.704670 \quad 8.603730 \quad-6.757580$

F $\quad-6.226570 \quad-3.794320 \quad-3.466940$

F $\quad-8.338480 \quad-3.956230 \quad-2.973820$

F $\quad-6.868510 \quad-3.607110 \quad-1.402650$

F $\quad-8.943940 \quad 0.749030 \quad-6.097700$

F $\quad-9.828090 \quad 1.886620 \quad-4.472690$

F $\quad-10.536520 \quad-0.127730 \quad-4.908770$

F $\quad 3.628540 \quad-5.918840 \quad-3.734490$

F $\quad 3.664040 \quad-6.753150 \quad-1.731290$

F $\quad 3.974210 \quad-8.048140 \quad-3.455040$

F $\quad-0.916180 \quad-8.947040 \quad-6.147260$

F $\quad-1.851190 \quad-9.933690 \quad-4.453640$

F $\quad 0.164560 \quad-10.491070 \quad-5.066940$

$\begin{array}{llll}\text { C } & 1.657000 & 3.733690 & 1.625330\end{array}$

$\begin{array}{llll}\text { C } & 2.346980 & 4.350460 & 2.888360\end{array}$

$\begin{array}{llll}\text { C } & 0.990820 & 4.818090 & 2.450760\end{array}$

C $\quad 1.015570 \quad 2.375520 \quad 1.669050$

$\begin{array}{llll}\text { C } & 2.197240 & 4.052760 & 0.262770\end{array}$

$\begin{array}{llll}\text { C } & 3.404210 & 3.518750 & -0.208000\end{array}$

C $\quad 3.854500 \quad 3.757760 \quad-1.502740$

$\begin{array}{llll}\text { C } & 3.084310 & 4.529750 & -2.366840\end{array}$

C $\quad 1.867800 \quad 5.080360 \quad-1.936500$

$\begin{array}{llll}\text { C } & 1.451850 & 4.832430 & -0.620960\end{array}$

$\begin{array}{llll}\mathrm{C} & 3.549560 & 5.232770 & 2.628210\end{array}$

$\begin{array}{llll}\text { C } & 4.790420 & 4.898300 & 3.191570\end{array}$

$\begin{array}{llll}\text { C } & 5.927170 & 5.665040 & 2.940450\end{array}$

C $\quad 5.850470 \quad 6.790920 \quad 2.119710$

C $\quad 4.621060 \quad 7.143420 \quad 1.562360$

$\begin{array}{llll}\text { C } & 3.484900 & 6.375730 & 1.817380\end{array}$

C $\quad 2.426630 \quad 3.558230 \quad 4.167880$

$\begin{array}{llll}\text { C } & 2.021800 & 4.167630 & 5.361060\end{array}$

$\begin{array}{llll}\text { C } & 2.089890 & 3.476710 & 6.572680\end{array}$

$\begin{array}{llll}\text { C } & 2.564600 & 2.163270 & 6.601590\end{array}$

C $\quad 2.985250 \quad 1.553920 \quad 5.416720$

$\begin{array}{llll}\text { C } & 2.927440 & 2.254070 & 4.212410\end{array}$

C $\quad 1.031850 \quad 5.897590 \quad-2.844990$

C $\quad 1.619200 \quad 6.706880 \quad-3.828790$

$\begin{array}{llll}\text { C } & 0.830000 & 7.477620 & -4.679580\end{array}$

$\begin{array}{llll}C & -0.560050 & 7.469460 & -4.567620\end{array}$

$\begin{array}{llll}\text { C } & -1.147010 & 6.669190 & -3.589910\end{array}$

C $\quad-0.366280 \quad 5.886360 \quad-2.739880$

$\begin{array}{llll}\text { C } & 3.941800 & -1.496830 & 1.632660\end{array}$

$\begin{array}{llll}\text { C } & 4.551600 & -2.279990 & 2.840690\end{array}$

$\begin{array}{llll}\text { C } & 4.956330 & -0.863200 & 2.563320\end{array}$

$\begin{array}{llll}\text { C } & 2.539820 & -0.962990 & 1.693370\end{array}$

C $\quad 4.343780 \quad-1.797960 \quad 0.218600$

$\begin{array}{llll}\text { C } & 3.942530 & -2.937830 & -0.488000\end{array}$

$\begin{array}{llll}\text { C } & 4.215050 & -3.082790 & -1.848340\end{array}$

$\begin{array}{llll}\text { C } & 4.837640 & -2.046890 & -2.543220\end{array}$

$\begin{array}{llll}\text { C } & 5.216770 & -0.873920 & -1.875840\end{array}$

$\begin{array}{llll}\text { C } & 4.990930 & -0.793270 & -0.498420\end{array}$

$\begin{array}{llll}\text { C } & 5.500990 & -3.407100 & 2.506340\end{array}$

$\begin{array}{llll}\text { C } & 5.250430 & -4.698520 & 2.994040\end{array}$

$\begin{array}{llll}\text { C } & 6.091150 & -5.764470 & 2.676650\end{array}$

$\begin{array}{llll}\text { C } & 7.206780 & -5.564120 & 1.863000\end{array}$

$\begin{array}{llll}\text { C } & 7.473330 & -4.283460 & 1.377460\end{array}$

$\begin{array}{llll}\text { C } & 6.632790 & -3.218040 & 1.698600\end{array}$

C $\quad 3.708120 \quad-2.497460 \quad 4.070060$

$\begin{array}{llll}\text { C } & 4.202520 & -2.069610 & 5.307880\end{array}$

C $\quad 3.453780 \quad-2.242360 \quad 6.472920$

C $\quad 2.196230 \quad-2.847160 \quad 6.408840$

C $\quad 1.703950 \quad-3.289230 \quad 5.179330$ $\begin{array}{llll}\text { C } & 2.460210 & -3.124250 & 4.019240\end{array}$

$\begin{array}{llll}\text { C } & 5.746250 & 0.318090 & -2.576770\end{array}$

$\begin{array}{llll}\text { C } & 5.217250 & 0.718360 & -3.812900\end{array}$

$\begin{array}{llll}\text { C } & 5.638180 & 1.904610 & -4.411190\end{array}$

$\begin{array}{llll}\text { C } & 6.592270 & 2.717540 & -3.797810\end{array}$

$\begin{array}{llll}\text { C } & 7.122470 & 2.319410 & -2.572590\end{array}$

$\begin{array}{llll}\text { C } & 6.710720 & 1.132230 & -1.968430\end{array}$

$\begin{array}{llll}\text { C } & -1.409810 & -3.924230 & 1.688370\end{array}$

$\begin{array}{llll}\text { C } & -2.162020 & -4.490240 & 2.944350\end{array}$

$\begin{array}{llll}\text { C } & -0.774790 & -4.945640 & 2.611590\end{array}$

$\begin{array}{llll}\text { C } & -0.828780 & -2.542970 & 1.705200\end{array}$

$\begin{array}{llll}\text { C } & -1.847130 & -4.361970 & 0.322380\end{array}$

C $\quad-3.030130 \quad-3.913560 \quad-0.281900$

C $\quad-3.416750 \quad-4.358640 \quad-1.543620$

$\begin{array}{llll}\text { C } & -2.594130 & -5.235010 & -2.245860\end{array}$

$\begin{array}{llll}\text { C } & -1.378340 & -5.666500 & -1.697950\end{array}$

C $\quad-1.034810 \quad-5.224890 \quad-0.414180$

$\begin{array}{llll}\text { C } & -3.336680 & -5.399070 & 2.659420\end{array}$

$\begin{array}{llll}\text { C } & -4.624610 & -5.012670 & 3.059820\end{array}$

$\begin{array}{llll}\text { C } & -5.735530 & -5.805530 & 2.776210\end{array}$

$\begin{array}{llll}\text { C } & -5.584260 & -7.009380 & 2.086790\end{array}$

$\begin{array}{llll}\text { C } & -4.308000 & -7.412170 & 1.692390\end{array}$

$\begin{array}{llll}\text { C } & -3.198110 & -6.617830 & 1.979220\end{array}$

$\begin{array}{llll}\mathrm{C} & -2.313630 & -3.632080 & 4.173610\end{array}$

$\begin{array}{llll}\text { C } & -1.893900 & -4.146360 & 5.406140\end{array}$

$\begin{array}{llll}\text { C } & -2.003920 & -3.384690 & 6.570750\end{array}$

$\begin{array}{llll}\text { C } & -2.537790 & -2.094980 & 6.512470\end{array}$

$\begin{array}{llll}\text { C } & -2.973690 & -1.582070 & 5.288790\end{array}$

C $\quad-2.872430 \quad-2.352070 \quad 4.130700$

C $\quad-0.467520 \quad-6.554890 \quad-2.454930$

C $\quad 0.922390 \quad-6.398830 \quad-2.361070$

$\begin{array}{llll}\text { C } & 1.778230 & -7.235120 & -3.078120\end{array}$

C $\quad 1.273210 \quad-8.239350 \quad-3.901110$

$\begin{array}{llll}\text { C } & -0.109300 & -8.397800 & -3.994970\end{array}$

$\begin{array}{llll}\text { C } & -0.972640 & -7.568200 & -3.282440\end{array}$

$\begin{array}{llll}\text { C } & -3.779730 & 1.404880 & 1.655400\end{array}$

$\begin{array}{llll}\text { C } & -4.343070 & 2.188550 & 2.893000\end{array}$

$\begin{array}{llll}\text { C } & -4.794870 & 0.791930 & 2.601660\end{array}$

$\begin{array}{llll}\text { C } & -2.398430 & 0.824580 & 1.680610\end{array}$

$\begin{array}{llll}\text { C } & -4.225220 & 1.815970 & 0.284130\end{array}$

$\begin{array}{llll}\text { C } & -3.745460 & 2.962760 & -0.365310\end{array}$

C $\quad-4.191250 \quad 3.319220-1.635490$

$\begin{array}{llll}\text { C } & -5.109180 & 2.508730 & -2.297850\end{array}$

$\begin{array}{llll}\text { C } & -5.583850 & 1.334160 & -1.698880\end{array}$

$\begin{array}{llll}\text { C } & -5.133360 & 1.017870 & -0.411020\end{array}$

C $\quad-5.252290 \quad 3.353620 \quad 2.572080$

$\begin{array}{llll}\text { C } & -4.833420 & 4.659500 & 2.867810\end{array}$

C $\quad-5.626130 \quad 5.760000 \quad 2.546050$

$\begin{array}{llll}\text { C } & -6.861980 & 5.579770 & 1.923090\end{array}$

$\begin{array}{llll}\text { C } & -7.296760 & 4.285980 & 1.634030\end{array}$

C $\quad-6.501940 \quad 3.186620 \quad 1.958390$

C $\quad-3.482780 \quad 2.381600 \quad 4.113820$

C $\quad-3.991580 \quad 2.001520 \quad 5.361490$

$\begin{array}{llll}\text { C } & -3.227770 & 2.157830 & 6.519550\end{array}$

$\begin{array}{llll}\text { C } & -1.941810 & 2.697940 & 6.438700\end{array}$

C $\quad-1.434620 \quad 3.092140 \quad 5.198900$

C $\quad-2.205720 \quad 2.943820 \quad 4.047210$

C $\quad-6.527550 \quad 0.433850 \quad-2.398600$

$\begin{array}{llll}\text { C } & -6.452800 & -0.953790 & -2.213790\end{array}$

C $\quad-7.349780 \quad-1.804240 \quad-2.858420$

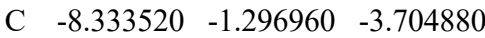

$\begin{array}{llll}\text { C } & -8.407620 & 0.082800 & -3.894640\end{array}$ 


\begin{tabular}{|c|c|c|c|}
\hline $\mathrm{C}$ & -7.518920 & 0.941660 & -3.250370 \\
\hline $\mathrm{C}$ & -0.677140 & -9.446240 & -4.911280 \\
\hline $\mathrm{C}$ & 3.260580 & -7.000870 & -2.996340 \\
\hline $\mathrm{C}$ & 4.988660 & 2.379830 & -5.680710 \\
\hline $\mathrm{C}$ & 8.059060 & 3.228450 & -1.827870 \\
\hline $\mathrm{C}$ & -2.643460 & 6.595960 & -3.476360 \\
\hline $\mathrm{C}$ & 1.489390 & 8.386340 & -5.679920 \\
\hline $\mathrm{C}$ & -9.432730 & 0.647750 & -4.838870 \\
\hline $\mathrm{C}$ & -7.207470 & -3.288570 & -2.672650 \\
\hline $\mathrm{H}$ & 0.877540 & 5.798150 & 2.003490 \\
\hline $\mathrm{H}$ & 0.150570 & 4.499440 & 3.054620 \\
\hline $\mathrm{H}$ & 4.792700 & 3.333990 & -1.833210 \\
\hline $\mathrm{H}$ & 3.420790 & 4.663130 & -3.388650 \\
\hline $\mathrm{H}$ & 0.521650 & 5.258860 & -0.261950 \\
\hline $\mathrm{H}$ & 4.868050 & 4.024320 & 3.826720 \\
\hline $\mathrm{H}$ & 6.874560 & 5.377720 & 3.386600 \\
\hline $\mathrm{H}$ & 6.735120 & 7.387860 & 1.920480 \\
\hline $\mathrm{H}$ & 4.540000 & 8.020000 & 0.926720 \\
\hline $\mathrm{H}$ & 2.550140 & 6.676710 & 1.361470 \\
\hline $\mathrm{H}$ & 1.641360 & 5.184980 & 5.335820 \\
\hline $\mathrm{H}$ & 1.764930 & 3.960840 & 8760 \\
\hline $\mathrm{H}$ & 2.608230 & 1.618520 & 7.540040 \\
\hline $\mathrm{H}$ & 3.342050 & 0.529140 & 5.425550 \\
\hline $\mathrm{H}$ & 3.229740 & 1.768180 & 3.294730 \\
\hline $\mathrm{H}$ & 2.698000 & 6.747800 & -3.921350 \\
\hline $\mathrm{H}$ & -0.849480 & 5.254580 & -2.002940 \\
\hline $\mathrm{H}$ & 5.941120 & -0.656380 & 2.161860 \\
\hline $\mathrm{H}$ & 4.574920 & -0.110520 & 3.241750 \\
\hline $\mathrm{H}$ & 3.922720 & -3.987140 & -2.366360 \\
\hline $\mathrm{H}$ & 5.025580 & -2.158070 & -3.606220 \\
\hline $\mathrm{H}$ & 5.240640 & 0.119620 & 0.024970 \\
\hline $\mathrm{H}$ & 4.386880 & -4.871880 & 3.624520 \\
\hline $\mathrm{H}$ & 5.870240 & -6.753470 & 3.066880 \\
\hline $\mathrm{H}$ & 7.861730 & -6.393180 & 1.613380 \\
\hline $\mathrm{H}$ & 8.339990 & -4.107200 & 0.747500 \\
\hline $\mathrm{H}$ & 6.867700 & -2.239460 & 1.298720 \\
\hline $\mathrm{H}$ & 5.174530 & -1.586860 & 5.353150 \\
\hline $\mathrm{H}$ & 3.847870 & -1.898280 & 7.424480 \\
\hline $\mathrm{H}$ & 1.601100 & -2.972120 & 7.308530 \\
\hline $\mathrm{H}$ & 0.718210 & -3.736850 & 5.127220 \\
\hline $\mathrm{H}$ & 2.063960 & -3.442710 & 3.061820 \\
\hline $\mathrm{H}$ & 4.450570 & 0.119820 & -4.290620 \\
\hline $\mathrm{H}$ & 7.141610 & 0.840180 & -1.018070 \\
\hline $\mathrm{H}$ & -0.612040 & -5.943120 & 2.223300 \\
\hline $\mathrm{H}$ & 0.019850 & -4.575610 & 3.245100 \\
\hline $\mathrm{H}$ & -4.343190 & -4.015430 & -1.983300 \\
\hline $\mathrm{H}$ & -2.888740 & -5.551830 & -3.240690 \\
\hline $\mathrm{H}$ & -0.112680 & -5.570730 & 0.039650 \\
\hline $\mathrm{H}$ & -4.758510 & -4.080410 & 3.594980 \\
\hline $\mathrm{H}$ & -6.720830 & -5.478590 & 3.094720 \\
\hline $\mathrm{H}$ & -6.448410 & -7.627380 & 1.863330 \\
\hline $\mathrm{H}$ & -4.170110 & -8.348960 & 1.160960 \\
\hline $\mathrm{H}$ & -2.223520 & -6.959050 & 1.653260 \\
\hline $\mathrm{H}$ & -1.468020 & -5.144970 & 5.447970 \\
\hline $\mathrm{H}$ & -1.666610 & -3.794950 & 7.517940 \\
\hline $\mathrm{H}$ & -2.613370 & -1.492170 & 7.412630 \\
\hline $\mathrm{H}$ & -3.367350 & -0.573170 & 5.234200 \\
\hline $\mathrm{H}$ & -3.181140 & -1.938450 & 3.178920 \\
\hline $\mathrm{H}$ & 1.337470 & -5.613290 & -1.739250 \\
\hline $\mathrm{H}$ & -2.042490 & -7.727950 & -3.348350 \\
\hline $\mathrm{H}$ & -5.793250 & 0.612110 & 2.223980 \\
\hline 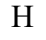 & -4.417900 & 0.017970 & 3.257180 \\
\hline
\end{tabular}
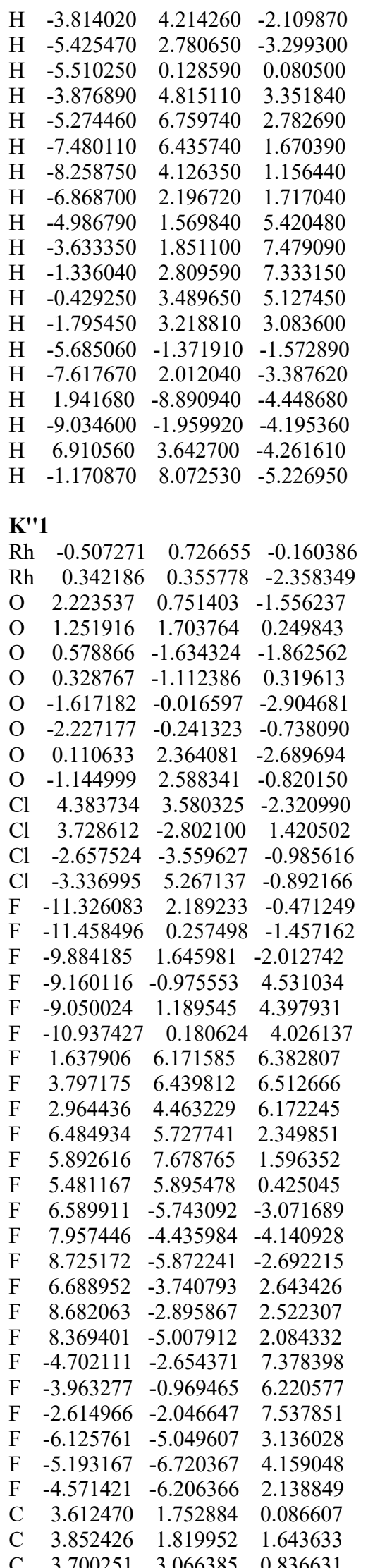

$\begin{array}{llll}\text { C } & 2.266222 & 1.373602 & -0.442088\end{array}$

$\begin{array}{llll}\text { C } & 4.731781 & 1.317836 & -0.804088\end{array}$

$\begin{array}{llll}\text { C } & 5.156658 & 2.049800 & -1.918487\end{array}$

$\begin{array}{llll}\text { C } & 6.160231 & 1.573981 & -2.764385\end{array}$

$\begin{array}{llll}\text { C } & 6.743269 & 0.334366 & -2.513262\end{array}$

$\begin{array}{llll}\text { C } & 6.321143 & -0.436618 & -1.420728\end{array}$

$\begin{array}{llll}\text { C } & 5.321866 & 0.073056 & -0.589781\end{array}$

$\begin{array}{llll}\text { C } & 5.218425 & 1.404056 & 2.138596\end{array}$

$\begin{array}{llll}\text { C } & 5.358592 & 0.180068 & 2.811674\end{array}$

$\begin{array}{llll}\text { C } & 6.609805 & -0.277938 & 3.220157\end{array}$

$\begin{array}{llll}\text { C } & 7.751438 & 0.489415 & 2.975943\end{array}$

$\begin{array}{llll}\text { C } & 7.620064 & 1.724538 & 2.340308\end{array}$

$\begin{array}{llll}\text { C } & 6.366000 & 2.178835 & 1.927621\end{array}$

$\begin{array}{llll}\text { C } & 2.739357 & 1.482675 & 2.603352\end{array}$

$\begin{array}{llll}\text { C } & 2.025186 & 0.283989 & 2.541111\end{array}$

$\begin{array}{llll}\text { C } & 0.999912 & 0.020105 & 3.448509\end{array}$

$\begin{array}{llll}\text { C } & 0.697259 & 0.943777 & 4.452005\end{array}$

$\begin{array}{llll}\text { C } & 1.430447 & 2.130089 & 4.543362\end{array}$

$\begin{array}{llll}\text { C } & 2.443821 & 2.395754 & 3.621220\end{array}$

$\begin{array}{llll}\text { C } & 6.869603 & -1.778589 & -1.133441\end{array}$

$\begin{array}{llll}\text { C } & 7.126237 & -2.152742 & 0.190096\end{array}$

$\begin{array}{llll}\text { C } & 7.602499 & -3.429005 & 0.487423\end{array}$

$\begin{array}{llll}\text { C } & 7.839423 & -4.352586 & -0.526129\end{array}$

$\begin{array}{llll}\text { C } & 7.577649 & -3.982068 & -1.847256\end{array}$

$\begin{array}{llll}\text { C } & 7.097704 & -2.710846 & -2.154565\end{array}$

$\begin{array}{llll}\text { C } & 1.070534 & -3.297508 & -0.213521\end{array}$

C $2.001488-4.170608-1.114238$

C $\quad 0.519061-4.393656-1.114750$

$\begin{array}{llll}\text { C } & 0.647846 & -1.908895 & -0.624860\end{array}$

$\begin{array}{llll}\text { C } & 1.056853 & -3.461554 & 1.277977\end{array}$

$\begin{array}{llll}\text { C } & 2.169634 & -3.241170 & 2.099193\end{array}$

$\begin{array}{llll}\text { C } & 2.076051 & -3.327318 & 3.488449\end{array}$

$\begin{array}{llll}\text { C } & 0.843894 & -3.572455 & 4.084072\end{array}$

$\begin{array}{llll}\text { C } & -0.303953 & -3.742988 & 3.295105\end{array}$

$\begin{array}{llll}\text { C } & -0.166789 & -3.715151 & 1.903736\end{array}$

$\begin{array}{llll}\text { C } & 2.858136 & -5.232710 & -0.453120\end{array}$

$\begin{array}{llll}\text { C } & 4.191645 & -5.407173 & -0.852818\end{array}$

$\begin{array}{llll}\text { C } & 4.991969 & -6.393842 & -0.278455\end{array}$

$\begin{array}{llll}\text { C } & 4.486409 & -7.221620 & 0.723076\end{array}$

$\begin{array}{llll}\text { C } & 3.160278 & -7.063924 & 1.127148\end{array}$

$\begin{array}{llll}\text { C } & 2.354329 & -6.091174 & 0.537682\end{array}$

$\begin{array}{llll}\text { C } & 2.620572 & -3.558795 & -2.343088\end{array}$

$\begin{array}{llll}\text { C } & 2.522177 & -4.246390 & -3.558358\end{array}$

$\begin{array}{llll}\text { C } & 3.123775 & -3.742688 & -4.712497\end{array}$

$\begin{array}{llll}\text { C } & 3.821059 & -2.532896 & -4.663660\end{array}$

$\begin{array}{llll}\text { C } & 3.924390 & -1.843769 & -3.453129\end{array}$

$\begin{array}{llll}\text { C } & 3.343145 & -2.364177 & -2.296896\end{array}$

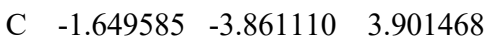

$\begin{array}{llll}\text { C } & -2.623611 & -4.708100 & 3.350595\end{array}$

$\begin{array}{llll}\text { C } & -3.905977 & -4.752338 & 3.891522\end{array}$

$\begin{array}{llll}\text { C } & -4.249474 & -3.960191 & 4.988818\end{array}$

C $\quad-3.281941 \quad-3.126949 \quad 5.542716$

$\begin{array}{llll}\text { C } & -1.993693 & -3.076124 & 5.008790\end{array}$

C $\quad-3.718300 \quad-1.016600 \quad-2.393800$

$\begin{array}{llll}\text { C } & -3.862990 & -1.723126 & -3.789072\end{array}$

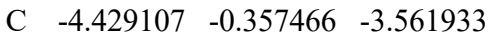

$\begin{array}{llll}\text { C } & -2.419620 & -0.387319 & -1.991731\end{array}$

C $\quad-4.522631 \quad-1.560850 \quad-1.251264$

C $\quad-4.141460 \quad-2.722607 \quad-0.565103$

$\begin{array}{llll}\text { C } & -4.903239 & -3.248717 & 0.472822\end{array}$

$\begin{array}{llll}\text { C } & -6.068343 & -2.600426 & 0.867361\end{array}$

$\begin{array}{llll}\text { C } & -6.474165 & -1.421936 & 0.225924\end{array}$ 


\begin{tabular}{|c|c|c|c|}
\hline C & -5.689410 & -0.925091 & \\
\hline & -4.792178 & -2.918069 & -3.813182 \\
\hline 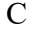 & -6.181066 & -2.791122 & -3.669635 \\
\hline 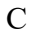 & -7.008552 & -3.913827 & -3.644101 \\
\hline & -6.464967 & -5.192433 & -3.769458 \\
\hline c & -5.085719 & -5.333102 & -3.930066 \\
\hline $\mathrm{C}$ & -4.261653 & -4.209075 & -3.954976 \\
\hline $\mathrm{C}$ & -2.678121 & -1.828275 & -4.715601 \\
\hline $\mathrm{C}$ & -1.491438 & -2.469927 & -4.345050 \\
\hline 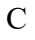 & -0.417773 & -2.549454 & -5.229706 \\
\hline $\mathrm{C}$ & -0.520900 & -1.992464 & -6.507311 \\
\hline $\mathrm{C}$ & -1.705535 & -1.360254 & -6.891658 \\
\hline $\mathrm{C}$ & -2.776518 & -1.281369 & -5.999378 \\
\hline $\mathrm{C}$ & -7.710230 & -0.719772 & 0.638753 \\
\hline C & -8.530118 & -0.092424 & -0.309337 \\
\hline $\mathrm{C}$ & -9.693201 & 0.567406 & 0.084361 \\
\hline $\mathrm{C}$ & -10.068046 & 0.614115 & 1.425971 \\
\hline $\mathrm{C}$ & -9.255991 & -0.010720 & 2.371772 \\
\hline $\mathrm{C}$ & -8.089785 & -0.669727 & 1.987619 \\
\hline 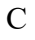 & -0.433800 & 4.554446 & -1.949643 \\
\hline $\mathrm{C}$ & -0.825513 & 5.2 & -3.31 \\
\hline $\mathrm{C}$ & 0.614240 & 5.063093 & -2.917573 \\
\hline $\mathrm{C}$ & -0.512980 & 3.056879 & -1.824019 \\
\hline $\mathrm{C}$ & -0.601612 & 5.241755 & -0.627880 \\
\hline $\mathrm{C}$ & -1.830161 & 5.590079 & -0.055852 \\
\hline $\mathrm{C}$ & -1.897419 & 6.1 & 1.2 \\
\hline $\mathrm{C}$ & -0.733778 & 6.38 & 1.939990 \\
\hline $\mathrm{C}$ & 0.513522 & 5.999831 & 1.424236 \\
\hline $\mathrm{C}$ & 0.549561 & 5.451579 & 0.136659 \\
\hline $\mathrm{C}$ & -1.438273 & 6.595075 & -3.291399 \\
\hline $\mathrm{C}$ & -0.900243 & 7.6 & 1939 \\
\hline $\mathrm{C}$ & -1.485601 & 8.89 & -2.491997 \\
\hline $\mathrm{C}$ & -2.618325 & 9.164912 & -3.262065 \\
\hline $\mathrm{C}$ & -3.152324 & 8.150425 & -4.056010 \\
\hline $\mathrm{C}$ & -2.569371 & 6.88 & -4.069906 \\
\hline $\mathrm{C}$ & -1.352059 & 4.279680 & -4.373395 \\
\hline $\mathrm{C}$ & -0.626444 & 3000 & -5.549349 \\
\hline $\mathrm{C}$ & -1.111186 & 3.213431 & -6.541563 \\
\hline $\mathrm{C}$ & -2.342354 & 2.575960 & -6.376149 \\
\hline $\mathrm{C}$ & -3.083299 & 2.801150 & -5.213509 \\
\hline $\mathrm{C}$ & -2.586252 & 3.637175 & -4.213727 \\
\hline $\mathrm{C}$ & 1.753430 & 6.103578 & 2.227944 \\
\hline $\mathrm{C}$ & 1.710873 & 5.978602 & 3.624423 \\
\hline $\mathrm{C}$ & 2.883266 & 5.998116 & 4.376358 \\
\hline $\mathrm{C}$ & 4.128312 & 6.113854 & 3.758998 \\
\hline $\mathrm{C}$ & 4.176186 & 6.239307 & 2.371831 \\
\hline $\mathrm{C}$ & 3.005809 & 6.255626 & 1.615032 \\
\hline $\mathrm{C}$ & -9.603665 & 0.091409 & 3.831166 \\
\hline $\mathrm{C}$ & -10.588758 & 1.170008 & -0.962388 \\
\hline $\mathrm{C}$ & -4.942540 & -5.684685 & 3.331179 \\
\hline $\mathrm{C}$ & -3.642111 & -2.204266 & 6.674221 \\
\hline $\mathrm{C}$ & 7.722690 & -5.002464 & -2.939527 \\
\hline $\mathrm{C}$ & 7.841369 & -3.777665 & 1.929540 \\
\hline $\mathrm{C}$ & 5.507628 & 6.387977 & 1.688340 \\
\hline $\mathrm{C}$ & 2.817997 & 5.777839 & 5.861718 \\
\hline $\mathrm{H}$ & 4.553787 & 3.692417 & 0.620681 \\
\hline $\mathrm{H}$ & 2.764986 & 3.596811 & 0.955003 \\
\hline $\mathrm{H}$ & 6.475379 & 2.168847 & -3.613666 \\
\hline $\mathrm{H}$ & 7.529924 & -0.028817 & -3.166394 \\
\hline $\mathrm{H}$ & 4.962752 & -0.522177 & 0.238884 \\
\hline $\mathrm{H}$ & 4.482431 & -0.427124 & 3.002437 \\
\hline$\pi$ & 6.693862 & -1.243266 & 3.708373 \\
\hline
\end{tabular}

\begin{tabular}{|c|c|c|c|}
\hline & 8.728517 & & \\
\hline & 8.495721 & 2.340943 & 60999 \\
\hline & 6.297259 & 3.145902 & 1.444306 \\
\hline & 2.230045 & -0.426074 & 1.752073 \\
\hline & 0.438888 & -0.902898 & 3.360840 \\
\hline & -0.099397 & 0.737850 & 5.161185 \\
\hline & 1.221617 & 2.847135 & 5.330188 \\
\hline & 3.011281 & 3.317078 & 3.690321 \\
\hline & 6.971771 & -1.436675 & 0.988546 \\
\hline & 6.871911 & -2.453394 & -3.182275 \\
\hline & 0.122836 & -5.285473 & -0.643618 \\
\hline $\mathrm{H}$ & -0.020601 & -4.052682 & -1.988892 \\
\hline H & 2.960944 & -3.176837 & 4.095399 \\
\hline & 0.775733 & -3.622785 & 5.165562 \\
\hline & -1.043581 & -3.823154 & 1.274842 \\
\hline & 4.609999 & -4.775347 & -1.624880 \\
\hline $\mathrm{H}$ & 6.009 & -6.5 & 9449 \\
\hline & 5.112128 & -7.985353 & 1.174508 \\
\hline & 2.743194 & -7.704593 & 1.898306 \\
\hline & 1.329574 & -6.003882 & 0.875595 \\
\hline & 1.970731 & -5.181537 & -3.59 \\
\hline $\mathrm{H}$ & 3.04 & 392 & 7551 \\
\hline 11 & 4.280907 & -2.130918 & -5.561462 \\
\hline П & 4.457273 & -0.901258 & -3.404708 \\
\hline & 3.423358 & -1.818420 & 5360 \\
\hline H & -2.375762 & 722 & 3967 \\
\hline $\mathrm{H}$ & -1.261 & -2.402037 & 407 \\
\hline $\mathrm{H}$ & -5.498882 & -0.208957 & 9034 \\
\hline $\mathrm{H}$ & -3.889898 & 0.461252 & -4.019943 \\
\hline H & -4.594668 & -4.169787 & 7169 \\
\hline H & -6.66 & -3.03 & 890 \\
\hline $\mathrm{H}$ & -5.980163 & -0.0 & -1.315053 \\
\hline $\mathrm{H}$ & -6.636462 & -1.813505 & -3.571302 \\
\hline $\mathrm{H}$ & -8.080367 & -3.784037 & -3.528365 \\
\hline $\mathrm{H}$ & -7.107999 & -6.066894 & -3.748351 \\
\hline $\mathrm{H}$ & -4.646221 & -6.320341 & -4.03 \\
\hline $\mathrm{H}$ & -3.194331 & -4.336340 & 35871 \\
\hline $\mathrm{H}$ & -1.390187 & -2.859146 & -3.340599 \\
\hline $\mathrm{H}$ & 0.501976 & -3.030562 & -4.915054 \\
\hline H & 0.31 & -2.05 & 6556 \\
\hline $\mathrm{H}$ & -1.796141 & -0.924729 & -7.8 \\
\hline $\mathrm{H}$ & -3.692969 & -0.779121 & -6.295331 \\
\hline $\mathrm{H}$ & -8.269552 & -0.132908 & -1.360554 \\
\hline $\mathrm{H}$ & -7.464765 & -1.133007 & 2.742004 \\
\hline & 1.160759 & 522 & -2.6 \\
\hline $\mathrm{H}$ & 1.200574 & 4.304705 & -3.419843 \\
\hline $\mathrm{H}$ & -2.859470 & 6.469118 & 1.613293 \\
\hline $\mathrm{H}$ & -0.803419 & 6.854443 & 2.915273 \\
\hline $\mathrm{H}$ & 1.492905 & 5.128658 & -0.288910 \\
\hline H & -0.024265 & 7.46 & -1.89 \\
\hline $\mathrm{H}$ & -1.049383 & 9.675820 & -1.872938 \\
\hline $\mathrm{H}$ & -3.073312 & 10.150456 & -3.247088 \\
\hline $\mathrm{H}$ & -4.027842 & 8.340524 & -4.669684 \\
\hline $\mathrm{H}$ & -3.002255 & 6.111154 & -4.693044 \\
\hline $\mathrm{H}$ & 0.328505 & 4.566941 & -5.683217 \\
\hline $\mathrm{H}$ & -0.529909 & 3.050396 & -7.444095 \\
\hline $\mathrm{H}$ & -2.722947 & 1.911897 & -7.145565 \\
\hline $\mathrm{H}$ & -4.052904 & 2.330407 & -5.088348 \\
\hline & -3.155016 & 3.796939 & -3.303954 \\
\hline $\mathrm{H}$ & 0.762992 & 5.832580 & 4.127300 \\
\hline $\mathrm{H}$ & 3.073838 & 6.370302 & 0.540139 \\
\hline & & & \\
\hline
\end{tabular}

H $\quad 5.038950 \quad 6.098458 \quad 4.344650$

H $\quad-5.250148 \quad-3.991439 \quad 5.402213$

H $\quad 8.220893 \quad-5.338373 \quad-0.294158$

\section{K"2}

$\begin{array}{llll}\text { Rh } & -0.000036 & 1.189934 & 0.000304\end{array}$

$\begin{array}{llll}\mathrm{Rh} & -0.000043 & -1.189640 & -0.000102\end{array}$

O $\quad 1.655322 \quad-1.053201-1.243102$

$\begin{array}{llll}\text { O } & 1.169133 & 1.106565 & -1.697282\end{array}$

$\begin{array}{llll}\mathrm{O} & 1.168958 & -1.106270 & 1.697579\end{array}$

$\begin{array}{llll}\mathrm{O} & 1.655220 & 1.053490 & 1.243468\end{array}$

$\begin{array}{llll}\text { O } & -1.655502 & -1.053509 & 1.242797\end{array}$

$\begin{array}{llll}\mathrm{O} & -1.169216 & 1.106042 & 1.697876\end{array}$

O

$\begin{array}{lllll}\text { O } & -1.655397 & 1.053807 & -1.242741\end{array}$

Cl $\quad 1.545784 \quad-1.973555 \quad-4.670034$

$\begin{array}{llll}\mathrm{Cl} & 1.546238 & 1.973582 & 4.670730\end{array}$

$\begin{array}{lllll}\mathrm{Cl} & -1.545661 & -1.974947 & 4.669775\end{array}$

Cl $-1.545498 \quad 1.975408 \quad-4.669438$

F $\quad-6.414640 \quad-6.456544 \quad-1.161794$

F $\quad-6.677400 \quad-4.837219 \quad-2.585512$

F $\quad-8.401538 \quad-5.985122 \quad-1.926280$

F $\quad-9.840724 \quad-0.749584 \quad 1.348131$

$\begin{array}{lllll}\text { F } & -10.577879 & -1.752893 & -0.442093\end{array}$

F $\quad-10.916527 \quad-2.628629 \quad 1.515491$

$\begin{array}{llll}\text { F } & -6.678399 & 4.837175 & 2.585618\end{array}$

F $\quad-6.414889 \quad 6.456349 \quad 1.161856$

F $\quad-8.402121 \quad 5.985344 \quad 1.925743$

F $\quad-10.578060 \quad 1.752592 \quad 0.441921$

$\begin{array}{llll}\text { F } & -9.840724 & 0.749256 & -1.348200\end{array}$

F $\quad-10.916681 \quad 2.628210 \quad-1.515737$

F $\quad 9.841132 \quad 0.749279 \quad 1.348124$

F $\quad 10.577994 \quad 1.752815 \quad-0.442086$

F $\quad 10.916808 \quad 2.628399 \quad 1.515545$

F $\quad 8.401151 \quad 5.985645 \quad-1.924874$

$\begin{array}{llll}\text { F } & 6.677428 & 4.837451 & -2.584663\end{array}$

F $\quad 6.414027 \quad 6.456241 \quad-1.160467$

$\begin{array}{llll}\text { F } & 9.841240 & -0.749705 & -1.348781\end{array}$

F $\quad 10.578157 \quad-1.752939 \quad 0.441583$

F $\quad 10.916763 \quad-2.628935 \quad-1.515894$

F $\quad 6.677363 \quad-4.837140 \quad 2.584860$

F $\quad 8.401170 \quad-5.985351 \quad 1.925293$

F $\quad 6.414085 \quad-6.456160 \quad 1.160888$

$\begin{array}{llll}\text { C } & 2.985038 & 0.068529 & -2.844544\end{array}$

$\begin{array}{llll}\text { C } & 3.866634 & 1.363476 & -3.015535\end{array}$

$\begin{array}{llll}\text { C } & 2.835626 & 0.994627 & -4.035989\end{array}$

$\begin{array}{llll}\text { C } & 1.853990 & 0.047364 & -1.858317\end{array}$

$\begin{array}{llll}\text { C } & 3.620992 & -1.279153 & -3.010478\end{array}$

$\begin{array}{llll}\text { C } & 3.042768 & -2.285044 & -3.794879\end{array}$

C $\quad 3.591273 \quad-3.565631 \quad-3.870050$

C $4.731593 \quad-3.867998-3.130286$

$\begin{array}{llll}\text { C } & 5.326118 & -2.891169 & -2.321910\end{array}$

$\begin{array}{llll}\text { C } & 4.764395 & -1.614005 & -2.280344\end{array}$

$\begin{array}{llll}\text { C } & 5.332981 & 1.146083 & -3.311457\end{array}$

$\begin{array}{llll}\text { C } & 5.775443 & 0.558400 & -4.504182\end{array}$

$\begin{array}{llll}\text { C } & 7.127159 & 0.286562 & -4.714496\end{array}$

$\begin{array}{llll}\text { C } & 8.066863 & 0.596948 & -3.731446\end{array}$

$\begin{array}{llll}\text { C } & 7.642100 & 1.200930 & -2.546576\end{array}$

$\begin{array}{llll}\text { C } & 6.292464 & 1.483108 & -2.342987\end{array}$

$\begin{array}{llll}\text { C } & 3.583744 & 2.582017 & -2.174714\end{array}$

$\begin{array}{llll}\text { C } & 3.484676 & 3.824219 & -2.813842\end{array}$

$\begin{array}{llll}\text { C } & 3.194686 & 4.977535 & -2.085292\end{array}$ 


\begin{tabular}{|c|c|c|c|}
\hline C & 2.977012 & 4.895856 & -0.706495 \\
\hline $\mathrm{C}$ & 3.093568 & 3.663095 & -0.061380 \\
\hline $\mathrm{C}$ & 3.426789 & 2.521431 & -0.789527 \\
\hline $\mathrm{C}$ & 6.483402 & -3.201471 & -1.449153 \\
\hline 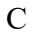 & 7.656263 & -2.438240 & -1.511590 \\
\hline C & 8.717235 & -2.716935 & -0.650207 \\
\hline $\mathrm{C}$ & 8.614244 & -3.726757 & 0.307924 \\
\hline C & 7.435962 & -4.465675 & 0.384843 \\
\hline $\mathrm{C}$ & 6.386632 & -4.225687 & -0.500780 \\
\hline $\mathrm{C}$ & 2.985003 & -0.068411 & 2.844763 \\
\hline $\mathrm{C}$ & 3.866477 & -1.363470 & 3.015551 \\
\hline $\mathrm{C}$ & 2.835616 & -0.994606 & 4.036143 \\
\hline $\mathrm{C}$ & 1.853871 & -0.047103 & 1.858633 \\
\hline $\mathrm{C}$ & 3.621109 & 1.279172 & 3.010750 \\
\hline $\mathrm{C}$ & 3.043114 & 2.285029 & 3.795374 \\
\hline $\mathrm{C}$ & 3.591788 & 3.565537 & 3.870639 \\
\hline $\mathrm{C}$ & 4.732038 & 3.867862 & 3.130760 \\
\hline $\mathrm{C}$ & 5.326333 & 2.891079 & 2.322162 \\
\hline $\mathrm{C}$ & 4.764447 & 1.613982 & 2.280501 \\
\hline $\mathrm{C}$ & 5.332880 & -1.146223 & 3.311330 \\
\hline $\mathrm{C}$ & 5.775488 & -0.558581 & 4.504019 \\
\hline $\mathrm{C}$ & 7.127252 & -0.286831 & 4.714208 \\
\hline $\mathrm{C}$ & 8.066839 & -0.597253 & 3.731052 \\
\hline $\mathrm{C}$ & 7.641921 & -1.201182 & 2.546219 \\
\hline $\mathrm{C}$ & 6.292247 & -1.483281 & 2.342756 \\
\hline $\mathrm{C}$ & 3.583357 & -2.581902 & 2.174652 \\
\hline $\mathrm{C}$ & 3.483925 & -3.824115 & 2.813691 \\
\hline $\mathrm{C}$ & 3.193686 & -4.977305 & 2.085051 \\
\hline $\mathrm{C}$ & 2.976140 & -4.895490 & 0.706243 \\
\hline $\mathrm{C}$ & 3.093043 & -3.662715 & 0.061220 \\
\hline $\mathrm{C}$ & 3.426493 & -2.521184 & 0.789460 \\
\hline $\mathrm{C}$ & 6.483519 & 3.201360 & 1.449291 \\
\hline $\mathrm{C}$ & 7.656329 & 2.438020 & 1.511495 \\
\hline $\mathrm{C}$ & 8.717226 & 2.716734 & 0.650028 \\
\hline $\mathrm{C}$ & 8.614207 & 3.726684 & -0.307963 \\
\hline $\mathrm{C}$ & 7.435980 & 4.465691 & -0.384670 \\
\hline $\mathrm{C}$ & 6.386709 & 4.225676 & 0.501028 \\
\hline $\mathrm{C}$ & -2.985065 & 0.067595 & 2.844836 \\
\hline $\mathrm{C}$ & -3.866736 & 1.362441 & 3.016169 \\
\hline $\mathrm{C}$ & -2.835676 & 0.993390 & 4.036510 \\
\hline $\mathrm{C}$ & -1.854094 & 0.046800 & 1.858504 \\
\hline $\mathrm{C}$ & -3.620927 & -1.280167 & 3.010420 \\
\hline $\mathrm{C}$ & -3.042630 & -2.286259 & 3.794509 \\
\hline $\mathrm{C}$ & -3.591082 & -3.566886 & 3.869314 \\
\hline $\mathrm{C}$ & -4.731418 & -3.869079 & 3.129501 \\
\hline $\mathrm{C}$ & -5.326033 & -2.892035 & 2.321446 \\
\hline $\mathrm{C}$ & -4.764372 & -1.614836 & 2.280268 \\
\hline $\mathrm{C}$ & -5.333058 & 1.144864 & 3.312031 \\
\hline $\mathrm{C}$ & -5.775435 & 0.556075 & 4.504247 \\
\hline $\mathrm{C}$ & -7.127130 & 0.283992 & 4.714389 \\
\hline $\mathrm{C}$ & -8.066898 & 0.595257 & 3.731682 \\
\hline $\mathrm{C}$ & -7.642233 & 1.200338 & 2.547350 \\
\hline $\mathrm{C}$ & -6.292619 & 1.482740 & 2.343932 \\
\hline $\mathrm{C}$ & -3.583852 & 2.581174 & 2.175625 \\
\hline $\mathrm{C}$ & -3.427090 & 2.520910 & 0.790404 \\
\hline $\mathrm{C}$ & -3.093768 & 3.662699 & 0.062512 \\
\hline $\mathrm{C}$ & -2.976939 & 4.895281 & 0.707921 \\
\hline $\mathrm{C}$ & -3.194422 & 4.976652 & 2.086766 \\
\hline $\mathrm{C}$ & -3.484504 & 3.823196 & 2.815059 \\
\hline $\mathrm{C}$ & -6.483322 & -3.202064 & 1.448635 \\
\hline $\mathrm{C}$ & -7.656038 & -2.438603 & 1.511091 \\
\hline & -8.717059 & -2.717049 & 0.649686 \\
\hline
\end{tabular}

$\begin{array}{llll}\text { C } & -8.614235 & -3.726820 & -0.308515\end{array}$

$\begin{array}{llll}\text { C } & -7.436080 & -4.465913 & -0.385509\end{array}$

$\begin{array}{llll}\text { C } & -6.386707 & -4.226201 & 0.500155\end{array}$

$\begin{array}{llll}\text { C } & -2.984904 & -0.067376 & -2.844783\end{array}$

C $\quad-3.866383 \quad-1.362351 \quad-3.016253$

C $\quad-2.835333 \quad-0.993073 \quad-4.036511$

$\begin{array}{llll}\text { C } & -1.853964 & -0.046514 & -1.858427\end{array}$

$\begin{array}{llll}\text { C } & -3.620891 & 1.280325 & -3.010350\end{array}$

$\begin{array}{llll}\text { C } & -3.042619 & 2.286502 & -3.794351\end{array}$

$\begin{array}{llll}\text { C } & -3.591187 & 3.567077 & -3.869160\end{array}$

C $\quad-4.731600 \quad 3.869143 \quad-3.129431$

$\begin{array}{llll}\text { C } & -5.326176 & 2.892033 & -2.321425\end{array}$

C $\quad-4.764406 \quad 1.614866 \quad-2.280261$

$\begin{array}{llll}\text { C } & -5.332720 & -1.144998 & -3.312181\end{array}$

$\begin{array}{llll}\text { C } & -6.292310 & -1.483322 & -2.344277\end{array}$

$\begin{array}{llll}\text { C } & -7.641985 & -1.201280 & -2.547818\end{array}$

$\begin{array}{llll}\text { C } & -8.066660 & -0.596077 & -3.732093\end{array}$

$\begin{array}{llll}\text { C } & -7.126849 & -0.284347 & -4.714607\end{array}$

$\begin{array}{llll}\text { C } & -5.775104 & -0.556098 & -4.504351\end{array}$

C $\quad-3.583346-2.581112 \quad-2.175801$

C $\quad-3.483505-3.823023 \quad-2.815375$

$\begin{array}{llll}\text { C } & -3.193287 & -4.976487 & -2.087153\end{array}$

$\begin{array}{llll}\text { C } & -2.976170 & -4.895241 & -0.708246\end{array}$

$\begin{array}{llll}\text { C } & -3.093481 & -3.662772 & -0.062719\end{array}$

$\begin{array}{llll}\text { C } & -3.426919 & -2.520970 & -0.790534\end{array}$

$\begin{array}{llll}\text { C } & -6.483517 & 3.201982 & -1.448649\end{array}$

$\begin{array}{llll}\text { C } & -7.656191 & 2.438459 & -1.511172\end{array}$

$\begin{array}{llll}\text { C } & -8.717259 & 2.716857 & -0.649813\end{array}$

$\begin{array}{llll}\text { C } & -8.614554 & 3.726659 & 0.308369\end{array}$

$\begin{array}{llll}\text { C } & -7.436449 & 4.465834 & 0.385399\end{array}$

$\begin{array}{llll}\text { C } & -6.387001 & 4.226139 & -0.500180\end{array}$

$\begin{array}{llll}\text { C } & -7.236259 & -5.442384 & -1.509637\end{array}$

$\begin{array}{llll}\text { C } & -10.008118 & -1.957366 & 0.769432\end{array}$

$\begin{array}{llll}\text { C } & 10.008373 & 1.957129 & 0.769522\end{array}$

$\begin{array}{llll}\text { C } & 7.235981 & 5.442430 & -1.508525\end{array}$

$\begin{array}{llll}\text { C } & 7.235991 & -5.442257 & 1.508838\end{array}$

C $\quad 10.008429 \quad-1.957456 \quad-0.769942$

$\begin{array}{llll}\text { C } & -7.236799 & 5.442358 & 1.509507\end{array}$

$\begin{array}{llll}\text { C } & -10.008261 & 1.957059 & -0.769577\end{array}$

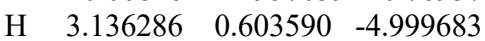

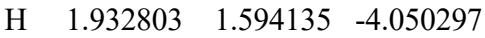

H $3.119566-4.318474 \quad-4.490705$

H $\quad 5.155321 \quad-4.865798 \quad-3.177549$

H $\quad 5.206861 \quad-0.864598 \quad-1.636375$

$\begin{array}{llll}\mathrm{H} & 5.066977 & 0.286770 & -5.277330\end{array}$

H $\quad 7.441331 \quad-0.174545 \quad-5.645881$

H $\quad 9.117443 \quad 0.369317 \quad-3.880473$

H $\quad 8.364648 \quad 1.441133 \quad-1.778278$

H $\quad 5.979352 \quad 1.955144 \quad-1.419038$

H $3.625487 \quad 3.880900 \quad-3.889394$

$\begin{array}{llll}\mathrm{H} & 3.124737 & 5.934410 & -2.593539\end{array}$

H $\quad 2.726293 \quad 5.787487 \quad-0.139320$

H $\quad 2.926200 \quad 3.580528 \quad 1.005260$

H $\quad 3.504666 \quad 1.572846 \quad-0.274982$

H $\quad 7.737294 \quad-1.635840 \quad-2.235582$

$\mathrm{H} \quad 5.471540 \quad-4.797604 \quad-0.419575$

$\begin{array}{llll}\mathrm{H} & 3.136416 & -0.603685 & 4.999838\end{array}$

H $\quad 1.932738 \quad-1.594025 \quad 4.050501$

$\mathrm{H} \quad 3.120255 \quad 4.318357 \quad 4.491477$

H $\quad 5.155890 \quad 4.865610 \quad 3.178105$

$\begin{array}{llll}\mathrm{H} & 5.206741 & 0.864614 & 1.636386\end{array}$

$\begin{array}{llll}\mathrm{H} & 5.067124 & -0.286899 & 5.277236\end{array}$
H $\quad 7.441540 \quad 0.174241 \quad 5.645565$

H $\quad 9.117448 \quad-0.369684 \quad 3.879984$

H $\quad 8.364373 \quad-1.441405 \quad 1.777834$

H $\quad 5.979020 \quad-1.955281 \quad 1.418816$

H $\quad 3.624625 \quad-3.880902 \quad 3.889257$

H $\quad 3.123444 \quad-5.934198 \quad 2.593232$

H $\quad 2.725238 \quad-5.787019 \quad 0.138992$

$\begin{array}{llll}\mathrm{H} & 2.925741 & -3.580037 & -1.005427\end{array}$

H $\quad 3.504595 \quad-1.572561 \quad 0.274982$

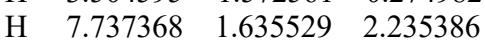

$\begin{array}{llll}\mathrm{H} & 5.471651 & 4.797670 & 0.419982\end{array}$

$\mathrm{H} \quad-3.136302 \quad 0.602116 \quad 5.000118$

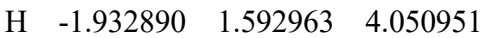

$\begin{array}{llll}\mathrm{H} & -3.119331 & -4.319895 & 4.489737\end{array}$

$\mathrm{H} \quad-5.155095 \quad-4.866911 \quad 3.176507$

$\begin{array}{llll}\mathrm{H} & -5.206932 & -0.865231 & 1.636600\end{array}$

$\begin{array}{llll}\mathrm{H} & -5.066918 & 0.283674 & 5.277073\end{array}$

$\mathrm{H} \quad-7.441220 \quad-0.178012 \quad 5.645356$

$\begin{array}{llll}\mathrm{H} & -9.117463 & 0.367437 & 3.880570\end{array}$

$\begin{array}{llll}\mathrm{H} & -8.364839 & 1.441245 & 1.779322\end{array}$

$\begin{array}{llll}\mathrm{H} & -5.979580 & 1.955627 & 1.420388\end{array}$

$\begin{array}{llll}\mathrm{H} & -3.505141 & 1.572452 & 0.275630\end{array}$

H $\quad-2.926500 \quad 3.580366 \quad-1.004154$

$\begin{array}{llll}\mathrm{H} & -2.726141 & 5.787013 & 0.140939\end{array}$

$\begin{array}{llll}\mathrm{H} & -3.124241 & 5.933390 & 2.595257\end{array}$

$\mathrm{H} \quad-3.625140 \quad 3.879624 \quad 3.890656$

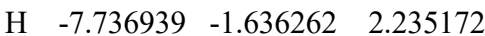

H $\quad-5.471721 \quad-4.798285 \quad 0.418892$

H $\quad-3.135967-0.601783 \quad-5.000100$

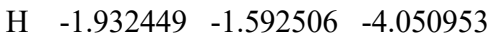

$\begin{array}{lllll}\mathrm{H} & -3.119428 & 4.320169 & -4.489497\end{array}$

$\mathrm{H} \quad-5.155344 \quad 4.866948 \quad-3.176409$

H $\quad-5.206946 \quad 0.865201 \quad-1.636638$

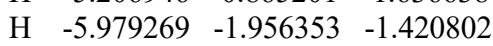

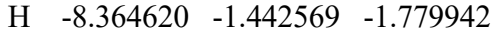

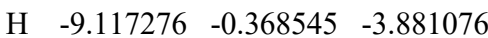

H $\quad-7.440948 \quad 0.177733 \quad-5.645535$

$\mathrm{H} \quad-5.066571 \quad-0.283378 \quad-5.277047$

H $\quad-3.623848 \quad-3.879347 \quad-3.891006$

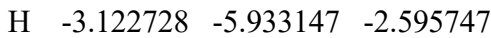

H $\quad-2.725278 \quad-5.786975 \quad-0.141320$

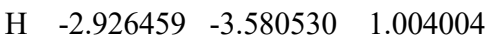

H $\quad-3.505318-1.572591 \quad-0.275668$

$\mathrm{H} \quad-7.737000 \quad 1.636089 \quad-2.235222$

H $\quad-5.472057 \quad 4.798286 \quad-0.418896$

H $\quad-9.429000 \quad-3.918424 \quad-0.995812$

H $\quad-9.429378 \quad 3.918242 \quad 0.995604$

H $\quad 9.428861 \quad 3.918481 \quad-0.995339$

H $\quad 9.428951 \quad-3.918543 \quad 0.995241$

\section{K"3}

$\begin{array}{llll}\mathrm{Rh} & 1.459180 & -0.580312 & 0.610505\end{array}$

$\begin{array}{lllll}\mathrm{Rh} & 1.007556 & 0.191727 & -1.603887\end{array}$

$\begin{array}{llll}\text { O } & 2.186330 & 1.867175 & -1.174835\end{array}$

$\begin{array}{llll}\text { O } & 2.284551 & 1.259334 & 1.000904\end{array}$

$\begin{array}{llll}\text { O } & 2.527611 & -1.065629 & -2.231373\end{array}$

$\begin{array}{llll}\text { O } & 3.232755 & -1.339014 & -0.097912\end{array}$

$\begin{array}{llll}\text { O } & -0.262590 & -1.437229 & -1.771639\end{array}$

$\begin{array}{llll}\text { O } & 0.604484 & -2.382266 & 0.089125\end{array}$

$\begin{array}{llll}\text { O } & -0.514196 & 1.339454 & -0.835169\end{array}$

$\begin{array}{llll}\text { O } & -0.414811 & 0.192880 & 1.107245\end{array}$

$\mathrm{Cl} \quad 3.815715 \quad 4.712508 \quad-2.282743$ 
$\begin{array}{llll}\mathrm{Cl} & 3.087801 & -5.042038 & -0.694833\end{array}$

$\begin{array}{lllll}\mathrm{Cl} & 0.339940 & -4.336235 & -3.786136\end{array}$

$\begin{array}{llll}\mathrm{Cl} & -0.848030 & 2.938182 & 3.187868\end{array}$

$\begin{array}{llll}\text { F } & -2.136471 & 6.311273 & 4.222875\end{array}$

$\begin{array}{llll}\text { F } & -4.229235 & 6.859082 & 3.957907\end{array}$

F $\quad-3.620741 \quad 4.779965 \quad 3.790900$

$\begin{array}{llll}\text { F } & -5.608751 & 5.093635 & -1.274450\end{array}$

F $\quad-4.827865 \quad 6.825323 \quad-2.332047$

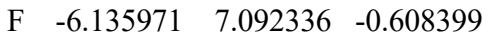

$\begin{array}{llll}\text { F } & -6.711715 & 1.904768 & -4.165415\end{array}$

F $\quad-6.717522 \quad 0.739012 \quad-5.998763$

F $\quad-8.552122 \quad 0.968310 \quad-4.843391$

F $\quad-8.450904 \quad-3.621711 \quad-0.953121$

F $\quad-9.221162-1.595481 \quad-0.730856$

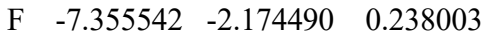

$\begin{array}{llll}\text { F } & 8.706337 & -1.964037 & 5.691051\end{array}$

F $\quad 8.650885 \quad 0.114231 \quad 5.061748$

F $\quad \begin{array}{llll}10.562905 & -0.884710 & 5.320312\end{array}$

$\begin{array}{llll}\text { F } & 11.651058 & -2.017489 & -0.538694\end{array}$

F $\quad 10.744845 \quad-0.045267 \quad-0.596155$

F $\quad 12.318611 \quad-0.474054 \quad 0.845311$

F $\quad-9.270426-2.955103 \quad 2.135603$

$\begin{array}{llll}\text { F } & -8.930734 & -0.801501 & 2.123452\end{array}$

F $\quad-9.540983 \quad-1.801292 \quad 3.953286$

F $\quad-3.901838 \quad-5.282214 \quad 3.977301$

F $\quad-5.957833 \quad-5.998183 \quad 3.885059$

F $\quad-5.159071 \quad-5.206122 \quad 5.746456$

$\begin{array}{llll}\text { C } & 2.685188 & 3.538568 & 0.464804\end{array}$

$\begin{array}{llll}\text { C } & 4.080798 & 3.876019 & 1.074087\end{array}$

$\begin{array}{llll}\text { C } & 2.866918 & 3.755104 & 1.950175\end{array}$

$\begin{array}{llll}\text { C } & 2.385288 & 2.110324 & 0.058940\end{array}$

$\begin{array}{llll}\text { C } & 1.894692 & 4.547702 & -0.318866\end{array}$

$\begin{array}{llll}\text { C } & 2.283070 & 5.117670 & -1.538048\end{array}$

$\begin{array}{llll}\text { C } & 1.471921 & 6.041128 & -2.200206\end{array}$

$\begin{array}{llll}\text { C } & 0.240671 & 6.399550 & -1.662333\end{array}$

$\begin{array}{llll}\text { C } & -0.192720 & 5.837772 & -0.453305\end{array}$

$\begin{array}{llll}\text { C } & 0.648230 & 4.922492 & 0.191795\end{array}$

$\begin{array}{llll}\text { C } & 4.662049 & 5.253997 & 0.840364\end{array}$

$\begin{array}{llll}\text { C } & 5.997472 & 5.401026 & 0.434844\end{array}$

$\begin{array}{llll}\text { C } & 6.548139 & 6.661476 & 0.204871\end{array}$

$\begin{array}{llll}\text { C } & 5.776598 & 7.810952 & 0.374221\end{array}$

$\begin{array}{llll}\text { C } & 4.450570 & 7.682072 & 0.788965\end{array}$

$\begin{array}{llll}\text { C } & 3.903649 & 6.421779 & 1.025464\end{array}$

C $\quad 5.094196 \quad 2.763976 \quad 1.115186$

$\begin{array}{llll}\text { C } & 5.650190 & 2.367551 & 2.336382\end{array}$

$\begin{array}{llll}\text { C } & 6.686889 & 1.433044 & 2.375180\end{array}$

$\begin{array}{llll}\text { C } & 7.182226 & 0.893476 & 1.186645\end{array}$

$\begin{array}{llll}\text { C } & 6.617440 & 1.265542 & -0.034796\end{array}$

$\begin{array}{llll}\text { C } & 5.575164 & 2.192718 & -0.069581\end{array}$

$\begin{array}{llll}\text { C } & -1.531278 & 6.119799 & 0.111953\end{array}$

$\begin{array}{llll}\text { C } & -2.645915 & 6.245143 & -0.728832\end{array}$

$\begin{array}{llll}\text { C } & -3.928555 & 6.346912 & -0.192961\end{array}$

$\begin{array}{llll}\text { C } & -4.130653 & 6.325487 & 1.185842\end{array}$

$\begin{array}{llll}\text { C } & -3.021954 & 6.217105 & 2.024779\end{array}$

$\begin{array}{llll}\text { C } & -1.734598 & 6.131704 & 1.499285\end{array}$

$\begin{array}{llll}\text { C } & 4.414698 & -2.463331 & -1.800200\end{array}$

C $\quad 5.246673 \quad-2.106559 \quad-3.083686$

$\begin{array}{llll}\text { C } & 4.228934 & -3.201912 & -3.109856\end{array}$

C $3.311422-1.549350-1.355990$

C $\quad 5.081251 \quad-3.152298 \quad-0.647601$

$\begin{array}{llll}\text { C } & 4.552354 & -4.305671 & -0.054192\end{array}$

$\begin{array}{llll}\text { C } & 5.133154 & -4.884581 & 1.074874\end{array}$ $\begin{array}{llll}\text { C } & 6.270887 & -4.309848 & 1.634109\end{array}$

$\begin{array}{llll}\text { C } & 6.831220 & -3.160709 & 1.061258\end{array}$

$\begin{array}{llll}\text { C } & 6.219144 & -2.596998 & -0.061078\end{array}$

$\begin{array}{llll}\text { C } & 6.723714 & -2.427876 & -3.048677\end{array}$

C $\quad 7.661713 \quad-1.382832-3.056769$

$\begin{array}{llll}\text { C } & 9.027497 & -1.638597 & -2.941064\end{array}$

$\begin{array}{llll}\text { C } & 9.490009 & -2.951623 & -2.832561\end{array}$

C $\quad 8.569938-4.001108 \quad-2.845716$

$\begin{array}{llll}\text { C } & 7.203452 & -3.741119 & -2.951660\end{array}$

$\begin{array}{llll}\text { C } & 4.913470 & -0.856830 & -3.853445\end{array}$

C $\quad 4.703142 \quad-0.958096 \quad-5.232983$

$\begin{array}{llll}\text { C } & 4.443797 & 0.179538 & -5.998032\end{array}$

$\begin{array}{llll}\text { C } & 4.395518 & 1.434794 & -5.387069\end{array}$

$\begin{array}{llll}\text { C } & 4.607207 & 1.542132 & -4.010929\end{array}$

$\begin{array}{llll}\text { C } & 4.865220 & 0.402674 & -3.250508\end{array}$

$\begin{array}{llll}\text { C } & 8.044204 & -2.513616 & 1.608107\end{array}$

$\begin{array}{llll}\text { C } & 9.077150 & -2.137014 & 0.741892\end{array}$

$\begin{array}{llll}\text { C } & 10.183341 & -1.436891 & 1.223818\end{array}$

$\begin{array}{llll}\text { C } & 10.282021 & -1.102176 & 2.572380\end{array}$

$\begin{array}{llll}\text { C } & 9.259635 & -1.493480 & 3.438702\end{array}$

$\begin{array}{llll}\text { C } & 8.152644 & -2.198453 & 2.968100\end{array}$

$\begin{array}{llll}\text { C } & -1.067234 & -3.566942 & -1.126818\end{array}$

C $\quad-1.700102-4.310308 \quad 0.114305$

$\begin{array}{llll}\text { C } & -0.570013 & -4.917798 & -0.654214\end{array}$

$\begin{array}{llll}\text { C } & -0.172946 & -2.381289 & -0.914559\end{array}$

$\begin{array}{llll}\text { C } & -1.858601 & -3.466720 & -2.393854\end{array}$

C $-1.324742 \quad-3.778176 \quad-3.650203$

C $\quad-2.065721-3.612580 \quad-4.821563$

C $\quad-3.362296-3.107266 \quad-4.752002$

C $\quad-3.916915 \quad-2.762856 \quad-3.512787$

C $\quad-3.152209-2.950081 \quad-2.359506$

$\begin{array}{llll}\text { C } & -3.106377 & -4.846265 & -0.040389\end{array}$

$\begin{array}{llll}\text { C } & -3.432968 & -5.840586 & -0.973099\end{array}$

$\begin{array}{llll}\text { C } & -4.750063 & -6.270519 & -1.134812\end{array}$

$\begin{array}{llll}\text { C } & -5.772776 & -5.716159 & -0.363601\end{array}$

$\begin{array}{llll}\text { C } & -5.460141 & -4.737848 & 0.581614\end{array}$

$\begin{array}{llll}\text { C } & -4.142112 & -4.311791 & 0.742904\end{array}$

C $\quad-1.387220 \quad-3.840005 \quad 1.511238$

C $\quad-1.578459 \quad-2.519684 \quad 1.919846$

C $\quad-1.261368 \quad-2.124318 \quad 3.219058$

$\begin{array}{llll}\text { C } & -0.796122 & -3.062855 & 4.142351\end{array}$

C $\quad-0.640639 \quad-4.396826 \quad 3.753606$

$\begin{array}{llll}\text { C } & -0.926181 & -4.778033 & 2.442822\end{array}$

$\begin{array}{llll}\text { C } & -5.250103 & -2.142846 & -3.366768\end{array}$

$\begin{array}{llll}\text { C } & -6.081322 & -2.538606 & -2.314778\end{array}$

$\begin{array}{llll}\text { C } & -7.292745 & -1.888189 & -2.086043\end{array}$

$\begin{array}{llll}\text { C } & -7.701224 & -0.834892 & -2.899700\end{array}$

C $\quad-6.869113 \quad-0.437753 \quad-3.948996$

$\begin{array}{llll}\text { C } & -5.660523 & -1.087004 & -4.191248\end{array}$

$\begin{array}{llll}\text { C } & -2.270631 & 1.623126 & 0.745703\end{array}$

$\begin{array}{llll}\text { C } & -3.390699 & 2.032666 & -0.285415\end{array}$

$\begin{array}{llll}\text { C } & -2.463906 & 3.053843 & 0.286361\end{array}$

$\begin{array}{llll}\text { C } & -0.980846 & 0.998746 & 0.298702\end{array}$

$\begin{array}{llll}\text { C } & -2.694578 & 1.205091 & 2.119405\end{array}$

C $\quad-2.130948 \quad 1.734956 \quad 3.286109$

$\begin{array}{llll}\text { C } & -2.529633 & 1.309050 & 4.553266\end{array}$

$\begin{array}{llll}\text { C } & -3.502212 & 0.318088 & 4.670333\end{array}$

$\begin{array}{llll}\text { C } & -4.075707 & -0.243246 & 3.523750\end{array}$

$\begin{array}{llll}\text { C } & -3.663027 & 0.208675 & 2.270770\end{array}$

$\begin{array}{llll}\text { C } & -4.821298 & 2.015560 & 0.208480\end{array}$

$\begin{array}{llll}\text { C } & -5.246813 & 2.806608 & 1.286478\end{array}$

$\begin{array}{llll}\text { C } & -6.559643 & 2.747974 & 1.749295\end{array}$ $\begin{array}{llll}\text { C } & -7.481900 & 1.895700 & 1.141590\end{array}$

$\begin{array}{llll}\text { C } & -7.074670 & 1.109252 & 0.063538\end{array}$

$\begin{array}{llll}\text { C } & -5.761568 & 1.171443 & -0.402702\end{array}$

$\begin{array}{llll}\text { C } & -3.239927 & 1.714043 & -1.749866\end{array}$

$\begin{array}{llll}\text { C } & -3.575881 & 2.713695 & -2.673313\end{array}$

$\begin{array}{llll}\text { C } & -3.436964 & 2.492296 & -4.042436\end{array}$

$\begin{array}{llll}\text { C } & -2.933997 & 1.272387 & -4.504344\end{array}$

$\begin{array}{llll}\text { C } & -2.614282 & 0.267826 & -3.589855\end{array}$

$\begin{array}{llll}\text { C } & -2.797043 & 0.478482 & -2.222768\end{array}$

$\begin{array}{llll}\text { C } & -5.080367 & -1.335701 & 3.601479\end{array}$

$\begin{array}{llll}\text { C } & -6.401425 & -1.117489 & 3.190037\end{array}$

$\begin{array}{llll}\text { C } & -7.324426 & -2.161674 & 3.223858\end{array}$

$\begin{array}{llll}\text { C } & -6.939080 & -3.440167 & 3.630114\end{array}$

$\begin{array}{llll}\text { C } & -5.617485 & -3.659940 & 4.011314\end{array}$

$\begin{array}{llll}\text { C } & -4.695260 & -2.612356 & 4.021309\end{array}$

$\begin{array}{llll}\text { C } & -7.216637 & 0.788471 & -4.744657\end{array}$

$\begin{array}{llll}\text { C } & -8.094945 & -2.320167 & -0.892559\end{array}$

$\begin{array}{llll}\text { C } & 11.231599 & -0.994550 & 0.241815\end{array}$

$\begin{array}{llll}\text { C } & 9.301021 & -1.065362 & 4.878601\end{array}$

$\begin{array}{llll}\text { C } & -3.245120 & 6.056384 & 3.501755\end{array}$

$\begin{array}{llll}\text { C } & -5.123634 & 6.351485 & -1.104557\end{array}$

$\begin{array}{llll}\text { C } & -5.161498 & -5.037455 & 4.402945\end{array}$

C $\quad-8.761792 \quad-1.924413 \quad 2.851106$

H $\quad 2.455481 \quad 4.659947 \quad 2.380834$

$\begin{array}{llll}\mathrm{H} & 2.780525 & 2.872767 & 2.570372\end{array}$

$\mathrm{H} \quad 1.811867 \quad 6.478802 \quad-3.131430$

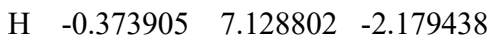

$\begin{array}{llll}\mathrm{H} & 0.319889 & 4.451195 & 1.110570\end{array}$

$\mathrm{H} \quad 6.614361 \quad 4.523143 \quad 0.291266$

H $\quad 7.584537 \quad 6.740570 \quad-0.109621$

$\begin{array}{llll}\mathrm{H} & 6.202581 & 8.792630 & 0.191409\end{array}$

$\begin{array}{llll}\mathrm{H} & 3.834823 & 8.564640 & 0.933966\end{array}$

H $\quad 2.870263 \quad 6.365088 \quad 1.342934$

$\begin{array}{llll}\mathrm{H} & 5.278717 & 2.808060 & 3.256888\end{array}$

$\begin{array}{llll}\mathrm{H} & 7.121260 & 1.136362 & 3.324378\end{array}$

H $\quad 8.009546 \quad 0.196982 \quad 1.208354$

H $\quad \begin{array}{llll}7.003465 & 0.847047 & -0.959250\end{array}$

H $\quad 5.146158 \quad 2.503136-1.015511$

H $\quad-2.518106 \quad 6.209273 \quad-1.804124$

$\begin{array}{llll}\mathrm{H} & -0.890463 & 6.046916 & 2.171727\end{array}$

H $\quad 4.546843 \quad-4.236489-3.133344$

H $\quad 3.305499 \quad-2.997547 \quad-3.638888$

H $\quad 4.696091 \quad-5.777224 \quad 1.506939$

H $\quad 6.733686 \quad-4.767917 \quad 2.502214$

H $\quad 6.624483 \quad-1.687445 \quad-0.484139$

H $\quad \begin{array}{llll}7.319983 & -0.359035 & -3.152318\end{array}$

H $\quad 9.728436 \quad-0.811151-2.918325$

H $\quad 10.551065 \quad-3.150516 \quad-2.727675$

$\mathrm{H} \quad 8.913118 \quad-5.028094 \quad-2.767138$

H $\quad 6.514270 \quad-4.577008 \quad-2.939724$

$\mathrm{H} \quad 4.741171 \quad-1.935478 \quad-5.705224$

H $\quad 4.280061 \quad 0.085751 \quad-7.067374$

H $\quad 4.193333 \quad 2.323180 \quad-5.977696$

H $\quad 4.555914 \quad 2.509926 \quad-3.527921$

H $\quad 5.000053 \quad 0.487676 \quad-2.178542$

H $\quad 9.010376 \quad-2.386075 \quad-0.310710$

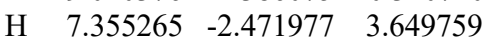

H $\quad-0.747965 \quad-5.759549 \quad-1.312027$

H $\quad 0.396838 \quad-4.916470 \quad-0.166873$

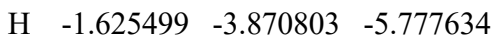

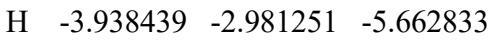

H $\quad-3.559823 \quad-2.655683 \quad-1.401240$ 
H $\quad-2.664879 \quad-6.277668 \quad-1.599947$

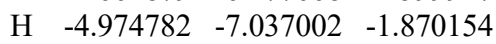

H $\quad-6.801154 \quad-6.034402 \quad-0.500595$

$\begin{array}{llll}\mathrm{H} & -6.244122 & -4.288754 & 1.178446\end{array}$

H $\quad-3.918706-3.551307 \quad 1.480142$

H $\quad-1.927881 \quad-1.779737 \quad 1.211962$

H $\quad-1.372795 \quad-1.084857 \quad 3.499795$

H $\quad-0.556833 \quad-2.757834 \quad 5.156792$

$\mathrm{H} \quad-0.288101 \quad-5.136020 \quad 4.466576$

$\begin{array}{llll}\mathrm{H} & -0.787703 & -5.810042 & 2.133636\end{array}$

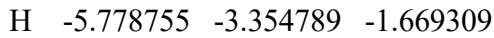

H $\quad-5.013122 \quad-0.739328 \quad-4.986170$

$\begin{array}{llll}\mathrm{H} & -2.829653 & 3.760641 & 1.014198\end{array}$

H $\quad-1.692028 \quad 3.426533 \quad-0.371941$

$\begin{array}{llll}\mathrm{H} & -2.075023 & 1.743914 & 5.435653\end{array}$

H $\quad-3.811440 \quad-0.019752 \quad 5.653877$

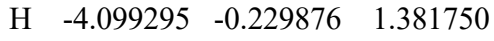

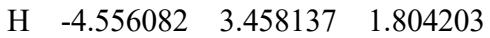

$\begin{array}{llll}\mathrm{H} & -6.856613 & 3.369179 & 2.588862\end{array}$

$\begin{array}{llll}\mathrm{H} & -8.502325 & 1.836927 & 1.505418\end{array}$

$\begin{array}{llll}\mathrm{H} & -7.786003 & 0.445885 & -0.409295\end{array}$

$\begin{array}{llll}\mathrm{H} & -5.462627 & 0.557402 & -1.245128\end{array}$

H $\quad-3.959165 \quad 3.661033 \quad-2.310659$

$\mathrm{H} \quad-3.709750 \quad 3.272960 \quad-4.746013$

H $\quad-2.800695 \quad 1.103818 \quad-5.568983$

$\mathrm{H} \quad-2.222661 \quad-0.682013 \quad-3.932225$

$\mathrm{H} \quad-2.540930-0.311304 \quad-1.528667$

H $\quad-6.699116 \quad-0.136363 \quad 2.838610$

H $\quad-3.663945 \quad-2.797185 \quad 4.298302$

H $\quad-8.637263 \quad-0.323404 \quad-2.715860$

$\mathrm{H} \quad-7.648854 \quad-4.258155 \quad 3.617094$

H $\quad 11.135832 \quad-0.549571 \quad 2.942130$

$\begin{array}{llll}\mathrm{H} & -5.131269 & 6.381500 & 1.596822\end{array}$

$\begin{array}{lccc}\text { K"4 } & & \\ \text { Rh } & -0.425846 & -1.902692 & -1.112827 \\ \text { Rh } & 0.426329 & -1.902643 & 1.112727 \\ \text { O } & -1.167427 & -0.670177 & 1.621000 \\ \text { O } & -1.542433 & -0.244638 & -0.564045 \\ \text { O } & -0.744454 & -3.587554 & 1.448231 \\ \text { O } & -1.918613 & -3.120501 & -0.429353 \\ \text { O } & 1.919263 & -3.120223 & 0.429245 \\ \text { O } & 0.745225 & -3.587351 & -1.448397 \\ \text { O } & 1.542486 & -0.244266 & 0.563989 \\ \text { O } & 1.167628 & -0.669897 & -1.621067 \\ \text { Cl } & -5.111695 & -0.246365 & -0.183756 \\ \text { Cl } & -2.255686 & -6.740154 & -1.260681 \\ \text { Cl } & 2.256983 & -6.739891 & 1.260336 \\ \text { Cl } & 5.111753 & -0.245159 & 0.183541 \\ \text { F } & 7.955354 & 0.698093 & 3.290946 \\ \text { F } & 9.990286 & -0.056199 & 3.266521 \\ \text { F } & 8.461328 & -1.079375 & 4.434428 \\ \text { F } & 9.739673 & -2.698997 & -2.123108 \\ \text { F } & 8.338904 & -1.086447 & -2.497278 \\ \text { F } & 10.254238 & -0.659758 & -1.552548 \\ F & 2.302251 & 9.055973 & 1.986557 \\ \text { F } & 1.505411 & 7.978823 & 0.277225 \\ \text { F } & 0.164890 & 8.706240 & 1.834371 \\ \text { F } & 0.335310 & 6.547892 & 6.356330 \\ \text { F } & 1.113888 & 4.515464 & 6.486959 \\ \text { F } & -0.779904 & 4.895627 & 5.491670 \\ \text { F } & -1.507361 & 7.978534 & -0.276936\end{array}$

F $\quad-0.166965 \quad 8.706357 \quad-1.833995$

$\begin{array}{llll}\text { F } & -2.304409 & 9.055514 & -1.986277\end{array}$

$\begin{array}{llll}\text { F } & 0.779027 & 4.896260 & -5.491421\end{array}$

F $\quad-0.336813 \quad 6.548085 \quad-6.356118$

F $\quad-1.114619 \quad 4.515373 \quad-6.486728$

F $\quad-8.338592 \quad-1.087927 \quad 2.497392$

F $\quad-10.254045 \quad-0.661646 \quad 1.552693$

F $\quad-9.739005 \quad-2.700788 \quad 2.123200$

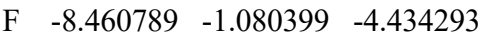

$\begin{array}{llll}\text { F } & -7.955566 & 0.697083 & -3.290493\end{array}$

$\begin{array}{llll}\text { F } & -9.990239 & -0.057925 & -3.266429\end{array}$

$\begin{array}{llll}\text { C } & -2.613019 & 1.137449 & 1.032532\end{array}$

$\begin{array}{llll}\text { C } & -2.329169 & 2.038552 & 2.294662\end{array}$

$\begin{array}{llll}\text { C } & -3.419018 & 1.013320 & 2.311254\end{array}$

$\begin{array}{llll}\text { C } & -1.704309 & -0.007999 & 0.681069\end{array}$

$\begin{array}{llll}\text { C } & -3.239428 & 1.766225 & -0.176687\end{array}$

C $\quad-4.355121 \quad 1.212152-0.816626$

$\begin{array}{llll}\text { C } & -4.881251 & 1.760116 & -1.987139\end{array}$

$\begin{array}{llll}\text { C } & -4.275279 & 2.881213 & -2.549461\end{array}$

$\begin{array}{llll}\text { C } & -3.154467 & 3.458665 & -1.939339\end{array}$

$\begin{array}{llll}\text { C } & -2.658066 & 2.892819 & -0.763855\end{array}$

$\begin{array}{llll}\text { C } & -2.681527 & 3.503398 & 2.173945\end{array}$

$\begin{array}{llll}\text { C } & -1.655552 & 4.461660 & 2.153037\end{array}$

$\begin{array}{llll}\text { C } & -1.943976 & 5.811023 & 1.957720\end{array}$

$\begin{array}{llll}\mathrm{C} & -3.264235 & 6.236211 & 1.799007\end{array}$

$\begin{array}{llll}\text { C } & -4.294305 & 5.296999 & 1.845471\end{array}$

$\begin{array}{llll}\text { C } & -4.003428 & 3.945662 & 2.030785\end{array}$

$\begin{array}{llll}\text { C } & -1.133444 & 1.770593 & 3.173406\end{array}$

$\begin{array}{llll}\text { C } & 0.146602 & 1.545718 & 2.666759\end{array}$

$\begin{array}{llll}\text { C } & 1.202173 & 1.221550 & 3.518744\end{array}$

$\begin{array}{llll}\text { C } & 1.000576 & 1.180700 & 4.899815\end{array}$

$\begin{array}{llll}\text { C } & -0.261945 & 1.475744 & 5.422524\end{array}$

$\begin{array}{llll}\text { C } & -1.322651 & 1.758487 & 4.561978\end{array}$

$\begin{array}{llll}\text { C } & -2.445805 & 4.614849 & -2.539744\end{array}$

C $\quad-1.920698 \quad 4.522046 \quad-3.833324$

C $\quad-1.178843 \quad 5.574102 \quad-4.367975$

C $\quad-0.992299 \quad 6.751520 \quad-3.647076$

C $\quad-1.532701 \quad 6.851507 \quad-2.364194$

$\begin{array}{llll}\text { C } & -2.237269 & 5.787148 & -1.801545\end{array}$

$\begin{array}{llll}\text { C } & -2.816788 & -4.715794 & 1.040592\end{array}$

$\begin{array}{llll}\text { C } & -3.275690 & -4.830966 & 2.545208\end{array}$

$\begin{array}{llll}\text { C } & -2.461122 & -5.907906 & 1.901687\end{array}$

C $\quad-1.738722 \quad-3.743498 \quad 0.672400$

$\begin{array}{llll}\text { C } & -3.835600 & -4.845910 & -0.051537\end{array}$

$\begin{array}{llll}\text { C } & -3.676090 & -5.704917 & -1.144505\end{array}$

$\begin{array}{llll}\text { C } & -4.590325 & -5.725364 & -2.199405\end{array}$

$\begin{array}{llll}\text { C } & -5.680187 & -4.859566 & -2.183841\end{array}$

$\begin{array}{llll}\text { C } & -5.860232 & -3.971271 & -1.113843\end{array}$

$\begin{array}{llll}\text { C } & -4.930903 & -3.982187 & -0.071459\end{array}$

$\begin{array}{llll}\text { C } & -4.759232 & -5.014521 & 2.774222\end{array}$

$\begin{array}{llll}\text { C } & -5.504099 & -3.978165 & 3.360001\end{array}$

$\begin{array}{llll}\text { C } & -6.889466 & -4.069398 & 3.489767\end{array}$

$\begin{array}{llll}\text { C } & -7.563254 & -5.209874 & 3.048135\end{array}$

$\begin{array}{llll}\text { C } & -6.831857 & -6.259349 & 2.490251\end{array}$

C $\quad-5.446520 \quad-6.161859 \quad 2.355491$

$\begin{array}{llll}\text { C } & -2.585405 & -4.000849 & 3.595843\end{array}$

C $-2.004196 \quad-4.645896 \quad 4.693500$

C $\quad-1.371185 \quad-3.910795 \quad 5.697110$

C $\quad-1.310071 \quad-2.517613 \quad 5.609463$

C $\quad-1.892257 \quad-1.867333 \quad 4.519800$

$\begin{array}{llll}\text { C } & -2.534085 & -2.606390 & 3.527288\end{array}$

C $\quad-6.977272 \quad-3.003759-1.057740$
C $\quad-7.368737 \quad-2.268330 \quad-2.184534$

$\begin{array}{llll}\text { C } & -8.359740 & -1.295042 & -2.077630\end{array}$

$\begin{array}{llll}\text { C } & -8.988590 & -1.039942 & -0.856429\end{array}$

$\begin{array}{llll}\text { C } & -8.612006 & -1.784498 & 0.257474\end{array}$

$\begin{array}{llll}\text { C } & -7.618196 & -2.757792 & 0.161363\end{array}$

$\begin{array}{llll}\text { C } & 2.817771 & -4.715231 & -1.040797\end{array}$

$\begin{array}{llll}\text { C } & 3.276785 & -4.830169 & -2.545396\end{array}$

$\begin{array}{llll}\text { C } & 2.462389 & -5.907329 & -1.902037\end{array}$

$\begin{array}{llll}\text { C } & 1.739515 & -3.743167 & -0.672569\end{array}$

$\begin{array}{llll}\text { C } & 3.836579 & -4.845255 & 0.051357\end{array}$

$\begin{array}{llll}\text { C } & 3.677208 & -5.704398 & 1.144246\end{array}$

$\begin{array}{llll}\text { C } & 4.591429 & -5.724771 & 2.199163\end{array}$

$\begin{array}{llll}\text { C } & 5.681139 & -4.858769 & 2.183692\end{array}$

$\begin{array}{llll}\text { C } & 5.861036 & -3.970344 & 1.113769\end{array}$

$\begin{array}{llll}\text { C } & 4.931720 & -3.981328 & 0.071385\end{array}$

$\begin{array}{llll}\text { C } & 4.760385 & -5.013374 & -2.774320\end{array}$

$\begin{array}{llll}\text { C } & 5.447894 & -6.160626 & -2.355722\end{array}$

$\begin{array}{llll}\text { C } & 6.833255 & -6.257786 & -2.490383\end{array}$

$\begin{array}{llll}\text { C } & 7.564465 & -5.208061 & -3.048033\end{array}$

$\begin{array}{llll}\text { C } & 6.890469 & -4.067667 & -3.489545\end{array}$

$\begin{array}{llll}\text { C } & 5.505069 & -3.976760 & -3.359875\end{array}$

$\begin{array}{llll}\text { C } & 2.586409 & -4.000066 & -3.595987\end{array}$

$\begin{array}{llll}\text { C } & 2.005411 & -4.645109 & -4.693755\end{array}$

$\begin{array}{llll}\text { C } & 1.372346 & -3.910020 & -5.697342\end{array}$

$\begin{array}{llll}\text { C } & 1.310974 & -2.516861 & -5.609573\end{array}$

$\begin{array}{llll}\text { C } & 1.892947 & -1.866592 & -4.519793\end{array}$

$\begin{array}{llll}\text { C } & 2.534829 & -2.605624 & -3.527300\end{array}$

$\begin{array}{llll}\text { C } & 6.977906 & -3.002635 & 1.057757\end{array}$

C $\quad 7.369236-2.267204 \quad 2.184591$

$\begin{array}{llll}\text { C } & 8.360078 & -1.293733 & 2.077753\end{array}$

$\begin{array}{llll}\text { C } & 8.988897 & -1.038443 & 0.856564\end{array}$

$\begin{array}{llll}\text { C } & 8.612440 & -1.783016 & -0.257372\end{array}$

$\begin{array}{llll}\text { C } & 7.618801 & -2.756493 & -0.161327\end{array}$

$\begin{array}{llll}\text { C } & 2.612719 & 1.138107 & -1.032579\end{array}$

$\begin{array}{llll}\text { C } & 2.328610 & 2.039174 & -2.294697\end{array}$

$\begin{array}{llll}\text { C } & 3.418694 & 1.014206 & -2.311331\end{array}$

$\begin{array}{llll}\text { C } & 1.704326 & -0.007595 & -0.681129\end{array}$

$\begin{array}{llll}\text { C } & 3.239015 & 1.766991 & 0.176637\end{array}$

$\begin{array}{llll}\text { C } & 4.354852 & 1.213144 & 0.816519\end{array}$

$\begin{array}{llll}\text { C } & 4.880890 & 1.761170 & 1.987045\end{array}$

$\begin{array}{llll}\text { C } & 4.274677 & 2.882103 & 2.549431\end{array}$

$\begin{array}{llll}\text { C } & 3.153703 & 3.459321 & 1.939376\end{array}$

$\begin{array}{llll}\text { C } & 2.657399 & 2.893408 & 0.763884\end{array}$

$\begin{array}{llll}\text { C } & 2.680644 & 3.504098 & -2.173997\end{array}$

$\begin{array}{llll}\text { C } & 1.654456 & 4.462137 & -2.153173\end{array}$

$\begin{array}{llll}\text { C } & 1.942598 & 5.811574 & -1.957897\end{array}$

$\begin{array}{llll}\text { C } & 3.262748 & 6.237056 & -1.799142\end{array}$

$\begin{array}{llll}\text { C } & 4.293025 & 5.298057 & -1.845519\end{array}$

$\begin{array}{llll}\text { C } & 4.002440 & 3.946655 & -2.030789\end{array}$

$\begin{array}{llll}\text { C } & 1.132918 & 1.770943 & -3.173403\end{array}$

$\begin{array}{llll}\text { C } & 1.322089 & 1.758839 & -4.561976\end{array}$

$\begin{array}{llll}\text { C } & 0.261420 & 1.475832 & -5.422492\end{array}$

$\begin{array}{llll}\text { C } & -1.001011 & 1.180496 & -4.899731\end{array}$

$\begin{array}{llll}\text { C } & -1.202589 & 1.221366 & -3.518662\end{array}$

$\begin{array}{llll}\text { C } & -0.147067 & 1.545808 & -2.666722\end{array}$

$\begin{array}{llll}\text { C } & 2.444799 & 4.615297 & 2.539867\end{array}$

$\begin{array}{llll}\text { C } & 2.235948 & 5.787579 & 1.801729\end{array}$

$\begin{array}{llll}\text { C } & 1.531153 & 6.851760 & 2.364448\end{array}$

$\begin{array}{llll}\text { C } & 0.990838 & 6.751597 & 3.647354\end{array}$

$\begin{array}{llll}\text { C } & 1.177689 & 5.574185 & 4.368197\end{array}$

$\begin{array}{llll}\text { C } & 1.919767 & 4.522320 & 3.833475\end{array}$

C $\quad 9.244369-1.551176 \quad-1.601132$ 


\begin{tabular}{|c|c|c|c|}
\hline E & 8.696852 & -0.442582 & 3.268757 \\
\hline $\mathrm{C}$ & -8.696671 & -0.443852 & -3.268569 \\
\hline $\mathrm{C}$ & -9.243970 & -1.552839 & 1.601245 \\
\hline $\mathrm{C}$ & -1.378589 & 8.142765 & -1.610182 \\
\hline & -0.470654 & 5.385535 & -5.679531 \\
\hline $\mathrm{C}$ & 0.469592 & 5.385382 & 5.679758 \\
\hline & 1.376663 & 8.142992 & 1.610469 \\
\hline $\mathrm{H}$ & -4.456461 & 1.318491 & 2.266129 \\
\hline $\mathrm{H}$ & -3.239446 & 0.124144 & 2.901112 \\
\hline & -5.752184 & 1.309397 & -2.448208 \\
\hline $\mathrm{H}$ & -4.676948 & 3.308919 & -3.462323 \\
\hline $\mathrm{H}$ & -1.778432 & 3.323249 & -0.301892 \\
\hline $\mathrm{H}$ & -0.625502 & 4.147841 & 2.278329 \\
\hline $\mathrm{H}$ & -1.140654 & 6.533509 & 1.919693 \\
\hline & -3.481855 & 7.286847 & 1.636530 \\
\hline $\mathrm{H}$ & -5.327409 & 5.611122 & 1.732328 \\
\hline $\mathrm{H}$ & -4.823153 & 3.237753 & 2.043463 \\
\hline $\mathrm{H}$ & 0.321075 & 1.567343 & 1.600106 \\
\hline $\mathrm{H}$ & 2.176329 & 1.007360 & 3.095341 \\
\hline 7 & 1.822807 & 0.934536 & 5.565144 \\
\hline $\mathrm{H}$ & -0.423795 & 1.471272 & 6.495909 \\
\hline $\mathrm{H}$ & -2.311243 & 1.958897 & 4.964902 \\
\hline $\mathrm{H}$ & -2.045924 & 3.608749 & -4.399620 \\
\hline $\mathrm{H}$ & -2.631870 & 5.865350 & -0.795314 \\
\hline $\mathrm{H}$ & -2.918863 & -6.852144 & 1.634987 \\
\hline $\mathrm{H}$ & -1.423434 & -5.978000 & 2.207177 \\
\hline $\mathrm{H}$ & -4.441475 & -6.409084 & -3.027030 \\
\hline $\mathrm{H}$ & -6.395942 & -4.885690 & -2.999047 \\
\hline $\mathrm{H}$ & -5.038735 & -3.278337 & 0.743559 \\
\hline $\mathrm{H}$ & -4.998677 & -3.084220 & 3.704567 \\
\hline $\mathrm{H}$ & -7.443585 & -3.238454 & 3.913704 \\
\hline $\mathrm{H}$ & -8.643230 & -5.274116 & 3.132402 \\
\hline $\mathrm{H}$ & -7.338996 & -7.156793 & 2.149470 \\
\hline $\mathrm{H}$ & -4.910420 & -6.985518 & 1.898950 \\
\hline $\mathrm{H}$ & -2.041474 & -5.729696 & 4.754622 \\
\hline $\mathrm{H}$ & -0.922393 & -4.425523 & 6.541469 \\
\hline $\mathrm{H}$ & -0.807926 & -1.940434 & 6.380255 \\
\hline $\mathrm{H}$ & -1.820879 & -0.789589 & 4.432581 \\
\hline $\mathrm{H}$ & -2.951648 & -2.099026 & 2.665745 \\
\hline $\mathrm{H}$ & -6.874940 & -2.432666 & -3.135262 \\
\hline $\mathrm{H}$ & -7.345930 & -3.335929 & 1.036363 \\
\hline $\mathrm{H}$ & 2.920284 & -6.851502 & -1.635407 \\
\hline $\mathrm{H}$ & 1.424732 & -5.977575 & -2.207599 \\
\hline $\mathrm{H}$ & 4.442699 & -6.408591 & 3.026725 \\
\hline $\mathrm{H}$ & 6.396890 & -4.884835 & 2.998899 \\
\hline $\mathrm{H}$ & 5.039435 & -3.277398 & -0.743578 \\
\hline $\mathrm{H}$ & 4.911936 & -6.984467 & -1.899349 \\
\hline $\mathrm{H}$ & 7.340565 & -7.155172 & -2.149699 \\
\hline $\mathrm{H}$ & 8.644467 & -5.272049 & -3.132227 \\
\hline $\mathrm{H}$ & 7.444443 & -3.236530 & -3.913290 \\
\hline $\mathrm{H}$ & 4.999477 & -3.082865 & -3.704341 \\
\hline $\mathrm{H}$ & 2.042903 & -5.728899 & -4.754992 \\
\hline $\mathrm{H}$ & 0.923710 & -4.424752 & -6.541790 \\
\hline $\mathrm{H}$ & 0.808788 & -1.939690 & -6.380344 \\
\hline $\mathrm{H}$ & 1.821378 & -0.788865 & -4.432473 \\
\hline $\mathrm{H}$ & 2.952230 & -2.098269 & -2.665663 \\
\hline $\mathrm{H}$ & 6.875465 & -2.431678 & 3.135313 \\
\hline $\mathrm{H}$ & 7.346666 & -3.334635 & -1.036364 \\
\hline $\mathrm{H}$ & 4.456073 & 1.319615 & -2.266246 \\
\hline $\mathrm{H}$ & 3.239308 & 0.124981 & -2.901194 \\
\hline $\mathrm{H}$ & 5.751929 & 1.310619 & 2.448072 \\
\hline & 4.676267 & 3.309848 & 3.462314 \\
\hline
\end{tabular}

$\begin{array}{crrr}\mathrm{H} & 1.777654 & 3.323667 & 0.301979 \\ \mathrm{H} & 0.624483 & 4.148105 & -2.278487 \\ \mathrm{H} & 1.139118 & 6.533887 & -1.919941 \\ \mathrm{H} & 3.480149 & 7.287750 & -1.636704 \\ \mathrm{H} & 5.326061 & 5.612415 & -1.732333 \\ \mathrm{H} & 4.822320 & 3.238923 & -2.043409 \\ \mathrm{H} & 2.310627 & 1.959459 & -4.964944 \\ \mathrm{H} & 0.423251 & 1.471354 & -6.495874 \\ \mathrm{H} & -1.823205 & 0.934120 & -5.565039 \\ \mathrm{H} & -2.176667 & 1.006956 & -3.095213 \\ \mathrm{H} & -0.321516 & 1.567443 & -1.600066 \\ \mathrm{H} & 2.630487 & 5.865907 & 0.795482 \\ \mathrm{H} & 2.045227 & 3.609038 & 4.399718 \\ \mathrm{H} & 9.758044 & -0.280756 & 0.779744 \\ \mathrm{H} & 0.416812 & 7.568026 & 4.068144 \\ \mathrm{H} & -9.757878 & -0.282388 & -0.779568 \\ \mathrm{H} & -0.418450 & 7.568111 & -4.067813\end{array}$

\section{K"5}

$\begin{array}{llll}\mathrm{Rh} & -0.967925 & -1.238765 & -1.610066\end{array}$

$\begin{array}{llll}\mathrm{Rh} & -1.265036 & -0.453302 & 0.629738\end{array}$

$\begin{array}{llll}\text { O } & 0.245472 & -1.791672 & 1.138330\end{array}$

$\begin{array}{llll}\mathrm{O} & 0.143749 & -2.808756 & -0.876340\end{array}$

$\begin{array}{llll}\mathrm{O} & 0.141244 & 1.001035 & 0.276666\end{array}$

O $\quad 0.731734 \quad-0.024502 \quad-1.645385$

$\begin{array}{lllll}\mathrm{O} & -2.622281 & 0.931338 & -0.093684\end{array}$

$\begin{array}{llll}\mathrm{O} & -2.053372 & 0.407075 & -2.218576\end{array}$

$\begin{array}{llll}\text { O } & -2.751657 & -1.853749 & 0.831414\end{array}$

$\begin{array}{llll}\text { O } & -2.684966 & -2.370162 & -1.370040\end{array}$

$\mathrm{Cl} \quad 0.039683 \quad-4.838155 \quad 2.988128$

$\begin{array}{llll}\mathrm{Cl} & 0.959769 & 2.897198 & -3.558365\end{array}$

$\begin{array}{llll}\mathrm{Cl} & -5.392598 & 2.894195 & 0.293815\end{array}$

$\begin{array}{llll}\mathrm{Cl} & -3.766378 & -4.092002 & 2.879283\end{array}$

$\begin{array}{llll}\text { F } & 7.668929 & -0.761659 & 0.864915\end{array}$

F $\quad \begin{array}{llll}9.065232 & 0.230259 & 2.214957\end{array}$

$\begin{array}{llll}\text { F } & 8.760160 & -1.922343 & 2.340948\end{array}$

$\begin{array}{llll}\text { F } & 6.909898 & 2.371417 & 6.093151\end{array}$

F $\quad 4.892608 \quad 1.785036 \quad 6.676416$

F $\quad 5.206012 \quad 2.995400 \quad 4.899106$

F $\quad 6.344609 \quad-3.969780 \quad-5.174248$

F $\quad 8.259879 \quad-4.424595 \quad-4.236123$

F $\quad 6.407456-4.709698 \quad-3.134505$

F $\quad 9.247554 \quad 0.800111-0.766964$

F $\quad 9.923225 \quad-1.270456 \quad-0.633604$

F $\quad 10.456347 \quad-0.061987 \quad-2.353945$

$\begin{array}{llll}\text { F } & 2.835615 & 5.910682 & -2.569304\end{array}$

F $\quad 3.666773 \quad 7.746151 \quad-1.742907$

F $\quad 1.751775 \quad 7.778172 \quad-2.782080$

F $\quad \begin{array}{llll}1.803850 & 6.188395 & 4.092442\end{array}$

F $\quad 3.712370 \quad 6.253713 \quad 3.044969$

F $\quad 2.602396 \quad 8.087102 \quad 3.389750$

F $\quad-10.458956 \quad 2.736408 \quad-1.377079$

$\begin{array}{llll}\text { F } & -8.495868 & 3.303472 & -0.635821\end{array}$

$\begin{array}{llll}\text { F } & -8.687424 & 1.663084 & -2.051424\end{array}$

F $\quad-12.581866 \quad 1.405202 \quad 2.823964$

F $\quad-12.721115 \quad-0.691383 \quad 2.272920$

F $\quad-11.663998 \quad-0.119561 \quad 4.082057$

$\begin{array}{llll}\text { C } & 1.748940 & -3.571582 & 0.717798\end{array}$

$\begin{array}{llll}\text { C } & 2.840076 & -4.061099 & -0.319298\end{array}$

$\begin{array}{llll}\text { C } & 1.764905 & -4.979209 & 0.160722\end{array}$

$\begin{array}{llll}\text { C } & 0.631348 & -2.663398 & 0.287947\end{array}$

$\begin{array}{llll}\text { C } & 2.167891 & -3.329329 & 2.135002\end{array}$ $\begin{array}{llll}\text { C } & 1.469723 & -3.840656 & 3.235731\end{array}$

C $\quad 1.853144 \quad-3.548423 \quad 4.545733$

$\begin{array}{llll}\text { C } & 2.952405 & -2.723385 & 4.775428\end{array}$

C $3.665156-2.186185 \quad 3.696246$

$\begin{array}{llll}\text { C } & 3.252119 & -2.494878 & 2.398532\end{array}$

C $\quad 4.258705 \quad-4.228391 \quad 0.177630$

$\begin{array}{llll}\text { C } & 4.592283 & -5.121549 & 1.206637\end{array}$

$\begin{array}{llll}\text { C } & 5.903400 & -5.230235 & 1.668070\end{array}$

$\begin{array}{llll}\text { C } & 6.915520 & -4.450741 & 1.105005\end{array}$

$\begin{array}{llll}\text { C } & 6.601613 & -3.569009 & 0.070293\end{array}$

$\begin{array}{llll}\text { C } & 5.288328 & -3.459202 & -0.386701\end{array}$

$\begin{array}{llll}\text { C } & 2.752915 & -3.646757 & -1.765258\end{array}$

$\begin{array}{llll}\text { C } & 2.821545 & -4.643515 & -2.747432\end{array}$

C $\quad 2.748580 \quad-4.313766 \quad-4.100402$

$\begin{array}{llll}\text { C } & 2.583091 & -2.979409 & -4.485638\end{array}$

$\begin{array}{llll}\text { C } & 2.525705 & -1.981587 & -3.510906\end{array}$

$\begin{array}{llll}\text { C } & 2.639859 & -2.315260 & -2.161406\end{array}$

$\begin{array}{llll}\text { C } & 4.815131 & -1.272205 & 3.857587\end{array}$

$\begin{array}{llll}\text { C } & 5.935213 & -1.436024 & 3.037937\end{array}$

$\begin{array}{llll}\text { C } & 6.992735 & -0.529273 & 3.093720\end{array}$

$\begin{array}{llll}\text { C } & 6.953349 & 0.559635 & 3.959723\end{array}$

$\begin{array}{llll}\text { C } & 5.832457 & 0.723870 & 4.777986\end{array}$

$\begin{array}{llll}\text { C } & 4.778214 & -0.185801 & 4.741729\end{array}$

$\begin{array}{llll}\text { C } & 2.071832 & 1.778440 & -0.879027\end{array}$

$\begin{array}{llll}\text { C } & 2.816732 & 2.398333 & 0.360904\end{array}$

$\begin{array}{llll}\text { C } & 1.814598 & 3.197347 & -0.409466\end{array}$

$\begin{array}{llll}\text { C } & 0.904261 & 0.844287 & -0.728073\end{array}$

$\begin{array}{llll}\text { C } & 2.867920 & 1.536799 & -2.124260\end{array}$

$\begin{array}{llll}\text { C } & 2.459274 & 1.988283 & -3.384715\end{array}$

$\begin{array}{llll}\text { C } & 3.194423 & 1.710308 & -4.537397\end{array}$

C $4.359501 \quad 0.952665 \quad-4.441929$

$\begin{array}{llll}\text { C } & 4.792333 & 0.479019 & -3.197520\end{array}$

$\begin{array}{llll}\text { C } & 4.041236 & 0.779863 & -2.061612\end{array}$

$\begin{array}{llll}\text { C } & 4.284832 & 2.723802 & 0.186624\end{array}$

$\begin{array}{llll}\text { C } & 4.732540 & 3.686659 & -0.729234\end{array}$

$\begin{array}{llll}\text { C } & 6.092912 & 3.936722 & -0.903579\end{array}$

C $\quad 7.037782 \quad 3.233198 \quad-0.157133$

$\begin{array}{llll}\text { C } & 6.604683 & 2.286500 & 0.772324\end{array}$

$\begin{array}{llll}\text { C } & 5.244197 & 2.035004 & 0.945592\end{array}$

$\begin{array}{llll}\text { C } & 2.445227 & 2.025015 & 1.773176\end{array}$

$\begin{array}{llll}\text { C } & 2.431079 & 3.060168 & 2.717738\end{array}$

$\begin{array}{llll}\text { C } & 2.064183 & 2.819544 & 4.039419\end{array}$

$\begin{array}{llll}\text { C } & 1.689093 & 1.530797 & 4.430585\end{array}$

$\begin{array}{llll}\text { C } & 1.730363 & 0.488913 & 3.502551\end{array}$

$\begin{array}{llll}\text { C } & 2.133843 & 0.730045 & 2.187991\end{array}$

$\begin{array}{llll}\text { C } & 5.994895 & -0.382262 & -3.068615\end{array}$

$\begin{array}{llll}\text { C } & 6.009640 & -1.648387 & -3.661086\end{array}$

$\begin{array}{llll}\text { C } & 7.112336 & -2.487412 & -3.497271\end{array}$

$\begin{array}{llll}\text { C } & 8.227563 & -2.062297 & -2.779887\end{array}$

$\begin{array}{llll}\text { C } & 8.217315 & -0.791190 & -2.200811\end{array}$

$\begin{array}{llll}\text { C } & 7.103943 & 0.038761 & -2.322300\end{array}$

$\begin{array}{llll}\text { C } & -3.029022 & 2.539070 & -1.797014\end{array}$

$\begin{array}{llll}\text { C } & -4.095227 & 2.632957 & -2.931804\end{array}$

$\begin{array}{llll}\text { C } & -2.647377 & 2.903321 & -3.216220\end{array}$

$\begin{array}{llll}\text { C } & -2.554065 & 1.181211 & -1.342538\end{array}$

$\begin{array}{llll}\text { C } & -2.920494 & 3.568107 & -0.712179\end{array}$

$\begin{array}{llll}\text { C } & -3.880461 & 3.782611 & 0.284636\end{array}$

$\begin{array}{llll}\text { C } & -3.667451 & 4.706211 & 1.308677\end{array}$

$\begin{array}{llll}C & -2.471404 & 5.414426 & 1.368850\end{array}$

$\begin{array}{llll}\text { C } & -1.480130 & 5.214347 & 0.397884\end{array}$

$\begin{array}{llll}\text { C } & -1.732133 & 4.297922 & -0.629813\end{array}$

$\begin{array}{llll}\text { C } & -5.050950 & 3.805190 & -2.928996\end{array}$ 


\begin{tabular}{|c|c|c|c|}
\hline C & -6.419951 & 3.596734 & \\
\hline & -7.320231 & 4.661808 & -3.153960 \\
\hline $\mathrm{C}$ & -6.872344 & 5.966079 & -2.951958 \\
\hline 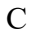 & -5.510427 & 6.191034 & -2.742833 \\
\hline 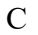 & -4.611434 & 5.126116 & -2.736630 \\
\hline - & -4.673634 & 1.330867 & -3.416825 \\
\hline $\mathrm{C}$ & -5.397871 & 0.501122 & -2.552568 \\
\hline $\mathrm{C}$ & -5.987927 & -0.669326 & -3.026697 \\
\hline $\mathrm{C}$ & -5.845313 & -1.036659 & -4.366546 \\
\hline $\mathrm{C}$ & -5.115378 & -0.218743 & -5.231705 \\
\hline c & -4.540288 & 0.962188 & -4.758658 \\
\hline $\mathrm{C}$ & -0.165810 & 5.890191 & 0.468300 \\
\hline $\mathrm{C}$ & 0.479604 & 6.069845 & 1.701859 \\
\hline $\mathrm{C}$ & 1.766547 & 6.596025 & 1.754390 \\
\hline $\mathrm{C}$ & 2.447745 & 6.944693 & 0.585246 \\
\hline C & 1.801125 & 6.785762 & -0.636186 \\
\hline $\mathrm{C}$ & 0.502596 & 6.277896 & 7907 \\
\hline $\mathrm{C}$ & -4.516286 & -3.155141 & -0.046278 \\
\hline $\mathrm{C}$ & -4.614418 & -4.660065 & -0.489195 \\
\hline $\mathrm{C}$ & -5.202840 & -3.568638 & -1.328338 \\
\hline C & -3.211389 & -2.420801 & -0.211974 \\
\hline $\mathrm{C}$ & -5.328526 & -2.662153 & 5577 \\
\hline $\mathrm{C}$ & -5.083003 & -3.017084 & 2.450056 \\
\hline $\mathrm{C}$ & -5.876968 & -2.531049 & 3.487732 \\
\hline $\mathrm{C}$ & -6.938908 & -1.677559 & 3.209464 \\
\hline $\mathrm{C}$ & -7.215943 & -1.294976 & 508 \\
\hline $\mathrm{C}$ & -6.39 & -1.79 & 347 \\
\hline $\mathrm{C}$ & -5.558264 & -5.551149 & 0.296508 \\
\hline $\mathrm{C}$ & -5.120548 & -6.778364 & 0.818532 \\
\hline $\mathrm{C}$ & -5.974584 & -7.600377 & 1.553891 \\
\hline $\mathrm{C}$ & -7.295332 & -7.219880 & 7532 \\
\hline $\mathrm{C}$ & -7.752120 & -6.010245 & 3713 \\
\hline $\mathrm{C}$ & -6.898230 & -5.193305 & 0.524746 \\
\hline $\mathrm{C}$ & -3.363775 & -5.333358 & -0.984759 \\
\hline $\mathrm{C}$ & -2.323142 & -5.652218 & -0.103602 \\
\hline $\mathrm{C}$ & -1.235355 & -6.407832 & -0.536748 \\
\hline $\mathrm{C}$ & -1.142680 & -6.808257 & 1792 \\
\hline $\mathrm{C}$ & -2.158143 & -6.462118 & -2.765660 \\
\hline $\mathrm{C}$ & -3.268766 & -5.743479 & -2.318669 \\
\hline $\mathrm{C}$ & -8.360154 & -0.413371 & 1.566674 \\
\hline $\mathrm{C}$ & -8.305811 & 0.470325 & 0.481312 \\
\hline $\mathrm{C}$ & -9.397464 & 1.278925 & 0.167019 \\
\hline $\mathrm{C}$ & -10.562445 & 1.236100 & 0.927837 \\
\hline $\mathrm{C}$ & -10.620240 & 0.360113 & 2.012123 \\
\hline $\mathrm{C}$ & -9.538104 & -0.457622 & 2.328269 \\
\hline $\mathrm{C}$ & 2.517045 & 7.070777 & -1.925441 \\
\hline $\mathrm{C}$ & 2.464410 & 6.781390 & 3.073600 \\
\hline $\mathrm{C}$ & 7.036805 & -3.895496 & -4.015908 \\
\hline $\mathrm{C}$ & 9.452630 & -0.324273 & -1.481783 \\
\hline $\mathrm{C}$ & 5.711737 & 1.964051 & 5.617950 \\
\hline $\mathrm{C}$ & 8.133124 & -0.743121 & 2.141099 \\
\hline $\mathrm{C}$ & -11.894987 & 0.242382 & 2.801346 \\
\hline $\mathrm{C}$ & -9.268636 & 2.246084 & -0.975181 \\
\hline $\mathrm{H}$ & 1.995565 & -5.780902 & 0.851762 \\
\hline $\mathrm{H}$ & 0.984070 & -5.201431 & -0.553091 \\
\hline $\mathrm{H}$ & 1.293163 & -3.963916 & 5.375397 \\
\hline $\mathrm{H}$ & 3.256320 & -2.505814 & 5.794161 \\
\hline $\mathrm{H}$ & 3.776467 & -2.057385 & 1.558855 \\
\hline $\mathrm{H}$ & 3.827541 & -5.730277 & 1.674020 \\
\hline $\mathrm{H}$ & 6.131028 & -5.924529 & 2.471117 \\
\hline $\mathrm{H}$ & 7.934114 & -4.521523 & 1.472087 \\
\hline $\mathrm{H}$ & 7.375842 & -2.953783 & -0.370070 \\
\hline
\end{tabular}

H $\quad 5.064082 \quad-2.772011-1.192835$

H $\quad 2.925760 \quad-5.681513 \quad-2.444841$

H $\quad 2.807311 \quad-5.095809 \quad-4.851312$

$\mathrm{H} \quad 2.503567 \quad-2.720531 \quad-5.537337$

H $\quad 2.391362 \quad-0.944493 \quad-3.791305$

$\mathrm{H} \quad 2.588011 \quad-1.534177 \quad-1.416054$

H $\quad 5.981786 \quad-2.274057 \quad 2.352603$

H $\quad 3.905957 \quad-0.022418 \quad 5.360898$

H $\quad 2.134559 \quad 3.999968 \quad-1.056884$

H $\quad 0.859211 \quad 3.351306 \quad 0.072945$

$\mathrm{H} \quad 2.851052 \quad 2.077145 \quad-5.497623$

H $\quad 4.930729 \quad 0.728283 \quad-5.336691$

H $4.364109 \quad 0.401411-1.099591$

H $4.030809 \quad 4.246836 \quad-1.331697$

H $\quad 6.409518 \quad 4.684747 \quad-1.624087$

H $\quad 8.098546 \quad 3.416836 \quad-0.294285$

$\begin{array}{llll}\mathrm{H} & 7.335432 & 1.748425 & 1.358767\end{array}$

H $\quad 4.922992 \quad 1.296523 \quad 1.672381$

$\begin{array}{llll}\mathrm{H} & 2.725538 & 4.054556 & 2.405716\end{array}$

H $\quad 2.061533 \quad 3.637767 \quad 4.751843$

$\mathrm{H} \quad 1.380421 \quad 1.338167 \quad 5.453912$

H $\quad 1.456842 \quad-0.517907 \quad 3.795815$

H $\quad 2.157575 \quad-0.092920 \quad 1.486620$

H $\quad 5.143943 \quad-1.992333 \quad-4.213901$

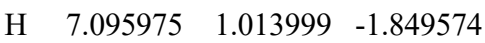

$\mathrm{H} \quad-2.348368 \quad 3.926570 \quad-3.408959$

H $\quad-2.091406 \quad 2.150597 \quad-3.759201$

H $\quad-4.436568 \quad 4.864154 \quad 2.055623$

$\mathrm{H} \quad-2.318830 \quad 6.134380 \quad 2.165897$

H $\quad-0.969148 \quad 4.103433 \quad-1.375607$

$\mathrm{H} \quad-6.793651 \quad 2.593224 \quad-3.288723$

$\mathrm{H} \quad-8.375477 \quad 4.462763 \quad-3.311970$

H $\quad-7.571678 \quad 6.796505 \quad-2.957505$

$\mathrm{H} \quad-5.140781 \quad 7.200043 \quad-2.586435$

$\mathrm{H} \quad-3.564167 \quad 5.341960 \quad-2.567612$

H $\quad-5.489161 \quad 0.779595 \quad-1.508633$

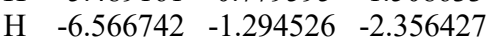

H $\quad-6.301676 \quad-1.951148 \quad-4.732403$

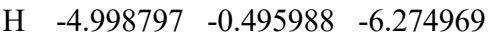

$\mathrm{H} \quad-3.981298 \quad 1.603877 \quad-5.433123$

$\begin{array}{llll}\mathrm{H} & -0.005415 & 5.752062 & 2.616390\end{array}$

H $\quad 0.017429 \quad 6.165618-1.660904$

H $\quad-6.280750 \quad-3.484923 \quad-1.382232$

$\mathrm{H} \quad-4.682553 \quad-3.300712 \quad-2.238478$

H $\quad-5.659052 \quad-2.819876 \quad 4.509237$

$\begin{array}{llll}\mathrm{H} & -7.537065 & -1.294730 & 4.029038\end{array}$

H $\quad-6.603156-1.529131 \quad-0.158093$

$\begin{array}{llll}\mathrm{H} & -4.102364 & -7.102565 & 0.650357\end{array}$

H $\quad-5.599988 \quad-8.542318 \quad 1.943330$

H $\quad-7.959938 \quad-7.857100 \quad 2.362686$

$\begin{array}{llll}\mathrm{H} & -8.779177 & -5.696731 & 1.424685\end{array}$

H $\quad-7.296000 \quad-4.263845 \quad 0.140138$

$\mathrm{H} \quad-2.381057 \quad-5.327948 \quad 0.928379$

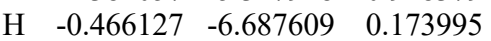

H $\quad-0.288965 \quad-7.387165 \quad-2.210604$

$\mathrm{H} \quad-2.096400 \quad-6.767377 \quad-3.805829$

H $\quad-4.072018-5.503740 \quad-3.008573$

H $\quad-7.406345 \quad 0.537836 \quad-0.118524$

$\mathrm{H} \quad-9.618509-1.146591 \quad 3.160475$

$\begin{array}{llll}\mathrm{H} & 3.462108 & 7.321372 & 0.625718\end{array}$

H $\quad-11.404896 \quad 1.869884 \quad 0.682894$

H $\quad 9.085350 \quad-2.713842 \quad-2.663894$
H $\quad 7.767732 \quad 1.272180 \quad 3.990743$

K"6

$\begin{array}{llll}\mathrm{Rh} & -0.760263 & 0.076190 & 0.991368\end{array}$

$\begin{array}{llll}\mathrm{Rh} & -0.982554 & -1.456469 & -0.819174\end{array}$

O $\quad 0.771924 \quad-0.577920 \quad-1.552522$

$\begin{array}{llll}\text { O } & 0.505531 & 1.198592 & -0.184009\end{array}$

$\begin{array}{llll}\mathrm{O} & 0.088885 & -2.815966 & 0.317930\end{array}$

$\begin{array}{llll}\text { O } & 0.779868 & -1.127657 & 1.654251\end{array}$

$\begin{array}{llll}\text { O } & -2.730233 & -2.111415 & 0.091455\end{array}$

$\begin{array}{llll}\text { O } & -2.048612 & -1.143523 & 2.019605\end{array}$

$\begin{array}{llll}\text { O } & -2.016981 & -0.001220 & -1.831525\end{array}$

$\begin{array}{llll}\text { O } & -2.276362 & 1.163757 & 0.090344\end{array}$

Cl $\quad 0.855555 \quad 1.338969 \quad-4.536290$

$\mathrm{Cl} \quad 0.413316 \quad-3.269405 \quad 4.580894$

$\begin{array}{llll}\text { Cl } & -3.547830 & -5.368235 & 1.520050\end{array}$

$\begin{array}{lllll}\mathrm{Cl} & -5.536744 & 2.776808 & -0.113914\end{array}$

F $\quad 9.107100 \quad-1.836764 \quad 1.797706$

F $\quad 7.848234 \quad-1.135371 \quad 0.173004$

$\begin{array}{llll}\text { F } & 9.510604 & 0.093060 & 0.870654\end{array}$

$\begin{array}{llll}\text { F } & 6.156934 & 4.004547 & 2.810209\end{array}$

F $\quad 8.066867 \quad 3.748083 \quad 3.814405$

F $\quad 6.207410 \quad 3.580351 \quad 4.940454$

F $\quad 9.833440 \quad-2.477403 \quad-1.187620$

F $\quad 10.184709 \quad-2.122941 \quad-3.298102$

$\begin{array}{llll}\text { F } & 9.308887 & -0.554107 & -2.075318\end{array}$

F $\quad 5.429484-6.225706 \quad-3.897778$

F $\quad 7.421898 \quad-6.530765 \quad-3.065642$

F $\quad 5.731756 \quad-6.160471-1.749601$

$\begin{array}{llll}\text { F } & 1.642635 & 9.066953 & 2.476380\end{array}$

F $\quad 3.015925 \quad 7.421461 \quad 2.822078$

F $\quad 0.976955 \quad 7.316304 \quad 3.581463$

$\begin{array}{llll}\text { F } & 3.766985 & 5.570944 & -1.853650\end{array}$

F $\quad 3.288561 \quad 7.600146 \quad-2.458729$

F $\quad 2.180761 \quad 5.921555 \quad-3.299516$

F $\quad-13.200514 \quad-2.095269 \quad-0.198948$

$\begin{array}{llll}\text { F } & -12.232247 & -3.979136 & 0.314469\end{array}$

$\begin{array}{llll}\text { F } & -12.275169 & -2.349857 & 1.750954\end{array}$

$\begin{array}{llll}F & -9.144005 & 0.956752 & -2.971020\end{array}$

$\begin{array}{llll}\text { F } & -8.409719 & 1.751836 & -1.086868\end{array}$

F $\quad-10.544982 \quad 1.765368 \quad-1.515883$

$\begin{array}{llll}\text { C } & 2.274084 & 1.246947 & -1.776144\end{array}$

$\begin{array}{llll}\text { C } & 3.227207 & 2.198230 & -0.957873\end{array}$

$\begin{array}{llll}\text { C } & 2.181164 & 2.758810 & -1.869096\end{array}$

$\begin{array}{llll}\text { C } & 1.104387 & 0.573396 & -1.116193\end{array}$

C $\quad 2.860662 \quad 0.437597 \quad-2.892293$

$\begin{array}{llll}\text { C } & 2.294704 & 0.391271 & -4.171380\end{array}$

$\begin{array}{llll}\text { C } & 2.817794 & -0.420577 & -5.178185\end{array}$

$\begin{array}{llll}\text { C } & 3.924138 & -1.221090 & -4.906461\end{array}$

$\begin{array}{llll}\text { C } & 4.509739 & -1.207505 & -3.634392\end{array}$

$\begin{array}{llll}\text { C } & 3.970212 & -0.378881 & -2.650527\end{array}$

$\begin{array}{llll}\text { C } & 4.674832 & 2.245329 & -1.395237\end{array}$

$\begin{array}{llll}\text { C } & 5.050864 & 2.658748 & -2.681753\end{array}$

$\begin{array}{llll}\text { C } & 6.383073 & 2.627236 & -3.089045\end{array}$

$\begin{array}{llll}\text { C } & 7.375585 & 2.189907 & -2.211347\end{array}$

$\begin{array}{llll}\text { C } & 7.019107 & 1.798522 & -0.920518\end{array}$

$\begin{array}{llll}\text { C } & 5.685071 & 1.827754 & -0.514329\end{array}$

$\begin{array}{llll}\text { C } & 3.026184 & 2.388613 & 0.523629\end{array}$

$\begin{array}{llll}\text { C } & 3.077979 & 3.693894 & 1.029033\end{array}$

$\begin{array}{llll}\text { C } & 2.881397 & 3.941374 & 2.386739\end{array}$

$\begin{array}{llll}\text { C } & 2.606164 & 2.880669 & 3.253862\end{array}$

$\begin{array}{llll}\text { C } & 2.572236 & 1.575307 & 2.759657\end{array}$ 


\begin{tabular}{|c|c|c|c|}
\hline C & 2.812117 & 1.329317 & 1.407545 \\
\hline & 5.650558 & -2.098600 & -3.304619 \\
\hline & 6.889349 & -1.570288 & -2.916334 \\
\hline & 7.947154 & -2.426606 & -2.614735 \\
\hline & 7.777822 & -3.812713 & -2.650096 \\
\hline & 6.537349 & -4.333239 & -3.009159 \\
\hline & 5.484548 & -3.486013 & -3.355779 \\
\hline & 1.888519 & -3.196958 & 1.847547 \\
\hline & 2.741816 & -4.177450 & 0.953760 \\
\hline & 1.669775 & -4.693895 & 1.863975 \\
\hline & 0.837167 & -2.328537 & 1.221679 \\
\hline & 2.539782 & -2.520601 & 3.015654 \\
\hline & 1.957341 & -2.474470 & 4.287829 \\
\hline - & 2.540670 & -1.759768 & 5.335636 \\
\hline & 3.721891 & -1.054650 & 5.116334 \\
\hline & 4.317842 & -1.060833 & 3.848654 \\
\hline$c$ & 3.712247 & -1.792628 & 2.824806 \\
\hline $\mathrm{C}$ & 4.201190 & -4.341632 & 1.312712 \\
\hline $\mathrm{C}$ & 4.619480 & -4.885440 & 2.535145 \\
\hline $\mathcal{C}$ & 5.973473 & -4.962145 & 2.861074 \\
\hline & 6.940981 & -4.499701 & 1.967346 \\
\hline $\mathrm{C}$ & 6.539931 & 2313 & 8864 \\
\hline $\mathrm{C}$ & 5.185451 & -3.896189 & 0.416095 \\
\hline $\mathrm{C}$ & 2.471507 & -4.278367 & -0.525837 \\
\hline $\mathrm{C}$ & 2.352989 & -5.554125 & -1.092904 \\
\hline C & 2.097602 & -5.705337 & 5450 \\
\hline $\mathrm{C}$ & 1.933860 & -4.5 & 4743 \\
\hline $\mathrm{C}$ & 2.066951 & -3.303260 & -2.707889 \\
\hline $\mathrm{C}$ & 2.365858 & -3.160422 & -1.352963 \\
\hline $\mathrm{C}$ & 5.523225 & -0.275155 & 3.513569 \\
\hline $\mathrm{C}$ & 5.665910 & 1.06 & 435 \\
\hline $\mathrm{C}$ & 6.746807 & 4742 & 6500 \\
\hline $\mathrm{C}$ & 7.715495 & 1.253599 & 2.620417 \\
\hline $\mathrm{C}$ & 7.576591 & -0.078306 & 2.240563 \\
\hline $\mathrm{C}$ & 6.495126 & -0.838024 & 2.682200 \\
\hline $\mathrm{C}$ & -4.093506 & -2.327664 & 2.021794 \\
\hline $\mathrm{C}$ & -4.706875 & -1.487620 & 3.219657 \\
\hline $\mathrm{C}$ & -3.913118 & -2.725584 & 3.478769 \\
\hline $\mathrm{C}$ & -2.872631 & -1.822324 & 1.327562 \\
\hline $\mathrm{C}$ & -5.045190 & -3.099126 & 1.160333 \\
\hline $\mathrm{C}$ & -4.952552 & -4.478773 & 0.944464 \\
\hline $\mathrm{C}$ & -5.955373 & -5.182444 & 0.274857 \\
\hline $\mathrm{C}$ & -7.092072 & -4.509508 & -0.166598 \\
\hline $\mathrm{C}$ & -7.198189 & -3.121226 & -0.002722 \\
\hline $\mathrm{C}$ & -6.144259 & -2.439395 & 0.609262 \\
\hline $\mathrm{C}$ & -6.216694 & -1.521673 & 3.346098 \\
\hline $\mathrm{C}$ & -6.970440 & -0.388498 & 2.997286 \\
\hline $\mathrm{C}$ & -8.361896 & -0.441819 & 2.927683 \\
\hline $\mathrm{C}$ & -9.038654 & -1.623913 & 3.232052 \\
\hline $\mathrm{C}$ & -8.304959 & -2.739539 & 3.635985 \\
\hline $\mathrm{C}$ & -6.911927 & -2.686716 & 3.696380 \\
\hline $\mathrm{C}$ & -4.086970 & -0.165749 & 3.591207 \\
\hline $\mathrm{C}$ & -3.636432 & 0.027913 & 4.901302 \\
\hline $\mathrm{C}$ & -3.071064 & 1.244861 & 5.286466 \\
\hline $\mathrm{C}$ & -2.952913 & 2.284949 & 4.360965 \\
\hline $\mathrm{C}$ & -3.409843 & 2.101422 & 3.053544 \\
\hline $\mathrm{C}$ & -3.978763 & 0.885983 & 2.675886 \\
\hline $\mathrm{C}$ & -8.431080 & -2.367647 & -0.322116 \\
\hline $\mathrm{C}$ & -9.680777 & -2.882874 & 0.046268 \\
\hline $\mathrm{C}$ & -10.833594 & -2.115943 & -0.104983 \\
\hline $\mathrm{C}$ & -10.772414 & -0.829420 & -0.641535 \\
\hline & -9.532700 & -0.325029 & -1.025586 \\
\hline
\end{tabular}

$\begin{array}{llll}\text { C } & -8.371072 & -1.079935 & -0.865899\end{array}$

$\begin{array}{llll}\text { C } & -3.012594 & 2.163323 & -1.920132\end{array}$

$\begin{array}{llll}\text { C } & -4.002494 & 1.933109 & -3.100569\end{array}$

$\begin{array}{llll}\text { C } & -2.567886 & 2.276836 & -3.368368\end{array}$

$\begin{array}{llll}\text { C } & -2.413361 & 1.008822 & -1.168048\end{array}$

$\begin{array}{llll}\text { C } & -3.042654 & 3.400144 & -1.074562\end{array}$

$\begin{array}{llll}\text { C } & -4.089839 & 3.761274 & -0.221352\end{array}$

$\begin{array}{llll}\text { C } & -3.989547 & 4.884334 & 0.602309\end{array}$

$\begin{array}{llll}\text { C } & -2.809246 & 5.621522 & 0.632928\end{array}$

$\begin{array}{llll}\text { C } & -1.713135 & 5.248699 & -0.162123\end{array}$

C $\quad-1.870866 \quad 4.156333 \quad-1.020444$

$\begin{array}{llll}\text { C } & -5.058666 & 2.985048 & -3.352778\end{array}$

$\begin{array}{llll}\text { C } & -6.402525 & 2.608322 & -3.483793\end{array}$

$\begin{array}{llll}\text { C } & -7.399241 & 3.558532 & -3.702318\end{array}$

$\begin{array}{llll}\text { C } & -7.074143 & 4.911482 & -3.796427\end{array}$

$\begin{array}{llll}\text { C } & -5.739667 & 5.301216 & -3.673031\end{array}$

$\begin{array}{llll}\text { C } & -4.744065 & 4.349094 & -3.458264\end{array}$

$\begin{array}{llll}\text { C } & -4.423317 & 0.520289 & -3.408356\end{array}$

$\begin{array}{llll}\text { C } & -4.948407 & -0.336557 & -2.435907\end{array}$

C $\quad-5.321283 \quad-1.639597 \quad-2.770353$

$\begin{array}{llll}\text { C } & -5.193796 & -2.092645 & -4.084914\end{array}$

$\begin{array}{llll}\text { C } & -4.686440 & -1.236804 & -5.064987\end{array}$

$\begin{array}{llll}\text { C } & -4.301967 & 0.060060 & -4.724170\end{array}$

$\begin{array}{llll}\text { C } & -0.399351 & 5.925498 & -0.065727\end{array}$

$\begin{array}{llll}\text { C } & 0.484456 & 5.940290 & -1.156358\end{array}$

$\begin{array}{llll}\text { C } & 1.756213 & 6.491984 & -1.033870\end{array}$

$\begin{array}{llll}\text { C } & 2.169571 & 7.077024 & 0.162961\end{array}$

$\begin{array}{llll}\text { C } & 1.289999 & 7.079168 & 1.243883\end{array}$

$\begin{array}{llll}\text { C } & 0.025075 & 6.503512 & 1.138931\end{array}$

$\begin{array}{llll}\text { C } & -9.416867 & 1.039504 & -1.642032\end{array}$

C $\quad-12.136542 \quad-2.637395 \quad 0.429874$

$\begin{array}{llll}\text { C } & 6.798449 & 3.284872 & 3.762544\end{array}$

$\begin{array}{llll}\text { C } & 8.525835 & -0.733391 & 1.280282\end{array}$

$\begin{array}{llll}\text { C } & 6.284910 & -5.812805 & -2.936181\end{array}$

C $\quad 9.311362 \quad-1.884821 \quad-2.289280$

$\begin{array}{llll}\text { C } & 1.724057 & 7.718984 & 2.533622\end{array}$

$\begin{array}{llll}\text { C } & 2.738512 & 6.402962 & -2.168338\end{array}$

H $\quad 2.462129 \quad 3.229558 \quad-2.801294$

$\mathrm{H} \quad 1.315261 \quad 3.193021 \quad-1.385154$

H $\quad 2.355652 \quad-0.429942 \quad-6.158389$

H $\quad 4.330839 \quad-1.859615 \quad-5.683689$

H $\quad 4.409787 \quad-0.378275 \quad-1.660536$

$\begin{array}{llll}\mathrm{H} & 4.304454 & 2.990927 & -3.392071\end{array}$

H $\quad 6.642262 \quad 2.944753 \quad-4.094413$

H $\quad 8.413561 \quad 2.153074 \quad-2.525932$

$\begin{array}{llll}\mathrm{H} & 7.787148 & 1.469812 & -0.234324\end{array}$

H $\quad 5.425639 \quad 1.519441 \quad 0.492436$

$\begin{array}{llll}\mathrm{H} & 3.273814 & 4.513763 & 0.348182\end{array}$

$\begin{array}{llll}\mathrm{H} & 2.936509 & 4.956967 & 2.762148\end{array}$

H $\quad 2.429434 \quad 3.069342 \quad 4.308807$

$\begin{array}{llll}\mathrm{H} & 2.360483 & 0.743359 & 3.420255\end{array}$

$\mathrm{H} \quad 2.776723 \quad 0.310587 \quad 1.042041$

$\mathrm{H} \quad 7.024704 \quad-0.496122 \quad-2.864937$

H $\quad 4.522572 \quad-3.901541 \quad-3.629312$

$\begin{array}{llll}\mathrm{H} & 1.935014 & -5.215057 & 2.775684\end{array}$

$\mathrm{H} \quad 0.760647 \quad-5.043132 \quad 1.388193$

H $\quad 2.066540 \quad-1.751142 \quad 6.310160$

H $\quad 4.176198 \quad-0.501704 \quad 5.932026$

H $\quad 4.143642 \quad-1.770971 \quad 1.832651$

H $\quad 3.891344 \quad-5.238263 \quad 3.255933$

H $\quad 6.269588 \quad-5.381123 \quad 3.817914$

H $\quad 7.994263 \quad-4.542711 \quad 2.225173$
H $\quad 7.280070 \quad-3.604488 \quad 0.039300$

H $\quad 4.890953 \quad-3.483891 \quad-0.540960$

H $\quad 2.455319 \quad-6.429330 \quad-0.457785$

H $\quad 2.012865-6.700140 \quad-2.882082$

H $\quad 1.710923 \quad-4.689566 \quad-4.321583$

$\mathrm{H} \quad 1.939888-2.417708 \quad-3.317457$

H $\quad 2.466157 \quad-2.166471 \quad-0.938754$

$\mathrm{H} \quad 4.906938 \quad 1.527002 \quad 4.524921$

H $\quad 6.402385 \quad-1.871908 \quad 2.371174$

H $\quad-4.408518 \quad-3.632782 \quad 3.799724$

$\begin{array}{llll}\mathrm{H} & -2.913852 & -2.591471 & 3.876463\end{array}$

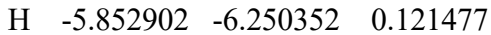

$\begin{array}{llll}\mathrm{H} & -7.889178 & -5.065482 & -0.649261\end{array}$

$\begin{array}{llll}\mathrm{H} & -6.229502 & -1.373317 & 0.764124\end{array}$

$\begin{array}{llll}\mathrm{H} & -6.463103 & 0.534357 & 2.741824\end{array}$

$\begin{array}{llll}\mathrm{H} & -8.915086 & 0.439306 & 2.617117\end{array}$

H $\quad-10.118087 \quad-1.680658 \quad 3.138696$

$\mathrm{H} \quad-8.814600 \quad-3.663620 \quad 3.891605$

$\begin{array}{llll}\mathrm{H} & -6.373303 & -3.581402 & 3.984609\end{array}$

$\mathrm{H} \quad-3.719608 \quad-0.783783 \quad 5.618319$

H $\quad-2.722736 \quad 1.379319 \quad 6.306292$

H $\quad-2.509611 \quad 3.231395 \quad 4.656443$

$\begin{array}{llll}\mathrm{H} & -3.312127 & 2.894795 & 2.321519\end{array}$

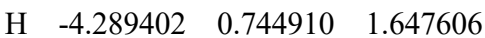

H $\quad-9.743716 \quad-3.864739 \quad 0.500470$

$\mathrm{H} \quad-7.418120 \quad-0.661957 \quad-1.166707$

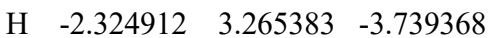

$\mathrm{H} \quad-1.928780 \quad 1.478762 \quad-3.723421$

$\mathrm{H} \quad-4.826146 \quad 5.163662 \quad 1.232050$

H $\quad-2.748545 \quad 6.489574 \quad 1.280279$

H $\quad-1.039524 \quad 3.822157 \quad-1.630342$

H $\quad-6.677831 \quad 1.565223 \quad-3.403386$

$\mathrm{H} \quad-8.429704 \quad 3.232204 \quad-3.790456$

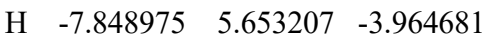

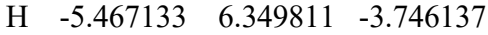

$\mathrm{H} \quad-3.720718 \quad 4.689792 \quad-3.359600$

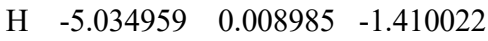

$\mathrm{H} \quad-5.699239 \quad-2.305570 \quad-2.003796$

H $\quad-5.486719-3.106179 \quad-4.341281$

H $\quad-4.584282-1.579266 \quad-6.090315$

$\mathrm{H} \quad-3.895526 \quad 0.724231 \quad-5.481385$

H $\quad 0.182569 \quad 5.521800 \quad-2.107763$

$\begin{array}{llll}\mathrm{H} & -0.616467 & 6.474835 & 2.010674\end{array}$

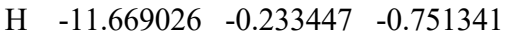

H $\quad 3.162559 \quad 7.500720 \quad 0.258345$

$\begin{array}{llll}\mathrm{H} & 8.548217 & 1.847413 & 2.265914\end{array}$

H $\quad 8.596639 \quad-4.474305 \quad-2.394151$

\section{L1}

$\begin{array}{llll}\mathrm{Rh} & 0.000101 & -0.000406 & -1.668094\end{array}$

$\begin{array}{llll}\mathrm{Rh} & 0.000201 & -0.000206 & 0.721906\end{array}$

$\begin{array}{llll}\text { O } & -1.708452 & 1.140065 & 0.643406\end{array}$

$\begin{array}{llll}\mathrm{O} & -1.407136 & 1.511052 & -1.567094\end{array}$

$\begin{array}{llll}\text { O } & -1.140970 & -1.708159 & 0.643706\end{array}$

$\begin{array}{llll}\text { O } & -1.511658 & -1.407243 & -1.566894\end{array}$

$\begin{array}{llll}\text { O } & 1.708853 & -1.140578 & 0.643406\end{array}$

$\begin{array}{llll}\text { O } & 1.407138 & -1.511965 & -1.566994\end{array}$

$\begin{array}{llll}\mathrm{O} & 1.141272 & 1.707746 & 0.643306\end{array}$

$\begin{array}{llll}\text { O } & 1.511759 & 1.406431 & -1.567294\end{array}$

Cl $\quad-4.658606 \quad-0.166312 \quad 0.517406$

$\mathrm{Cl} \quad 0.152506 \quad-4.663013 \quad 0.529306$

$\begin{array}{llll}\text { Cl } & 4.658708 & 0.166499 & 0.516506\end{array}$ 


\begin{tabular}{|c|c|c|c|}
\hline & -0.153505 & 4.662200 & \\
\hline . & -3.307597 & 2.453932 & -0.481594 \\
\hline & -4.197492 & 2.571069 & -1.767894 \\
\hline & -3.296345 & 3.693531 & -1.359494 \\
\hline & -2.052731 & 1.635979 & -0.478294 \\
\hline$C$ & -4.008795 & 2.493961 & 0.843106 \\
\hline & -4.662942 & 1.371888 & 1.374806 \\
\hline$c$ & -5.321940 & 1.427615 & 2.599806 \\
\hline $\mathrm{C}$ & -5.331790 & 2.616216 & 3.325006 \\
\hline & -4.672043 & 3.755088 & 2.841206 \\
\hline & -4.016947 & 3.662561 & 1.605206 \\
\hline $\mathrm{C}$ & -5.684787 & 2.681031 & -1.512194 \\
\hline $\mathrm{C}$ & -6.510533 & 1.577265 & -1.772694 \\
\hline $\mathrm{C}$ & -7.878131 & 1.625122 & -1.508994 \\
\hline & -8.454183 & 2.784346 & -0.985894 \\
\hline $\mathrm{C}$ & -7.647237 & 3.895012 & -0.738394 \\
\hline $\mathrm{C}$ & -6.277939 & 3.843255 & -1.001794 \\
\hline $\mathrm{C}$ & -3.850923 & 1.819254 & -3.026494 \\
\hline $\mathrm{C}$ & -3.818494 & 2.521553 & -4.237094 \\
\hline$c$ & -3.520321 & 1.865240 & -5.433194 \\
\hline $\mathrm{C}$ & -3.247279 & 0.495229 & -5.428494 \\
\hline $\mathrm{C}$ & -3.290508 & -0.213769 & -4.225594 \\
\hline $\mathrm{C}$ & -3.602881 & 0.444244 & -3.037094 \\
\hline $\mathrm{C}$ & -4.664290 & 5.024688 & 3.604206 \\
\hline $\mathrm{C}$ & -4.594490 & 5.021985 & 5.007006 \\
\hline $\mathrm{C}$ & -4.584140 & 6.218385 & 2506 \\
\hline $\mathrm{C}$ & -4.641589 & 7.440887 & 5.049506 \\
\hline $\mathrm{C}$ & -4.711888 & 7.456990 & 3.654706 \\
\hline $\mathrm{C}$ & -4.724638 & 6.260791 & 2.939206 \\
\hline $\mathrm{C}$ & -2.460137 & -3.303904 & -0.479894 \\
\hline $\mathrm{C}$ & -2.576274 & -4.196299 & -1.764394 \\
\hline $\mathrm{C}$ & -3.697336 & -3.291352 & -1.360894 \\
\hline $\mathrm{C}$ & -1.638185 & -2.051638 & -0.477694 \\
\hline $\mathrm{C}$ & -2.505866 & -4.001302 & 0.846606 \\
\hline $\mathrm{C}$ & -1.387793 & -4.658549 & 1.382806 \\
\hline $\mathrm{C}$ & -1.449221 & -5.313346 & 2.609906 \\
\hline $\mathrm{C}$ & -2.639321 & -5.315396 & 3.332606 \\
\hline $\mathrm{C}$ & -3.774093 & -4.651849 & 2.844306 \\
\hline $\mathrm{C}$ & -3.676066 & -4.001653 & 1.606206 \\
\hline $\mathrm{C}$ & -2.691036 & -5.682794 & -1.506194 \\
\hline $\mathrm{C}$ & -1.589771 & -6.512540 & -1.764794 \\
\hline $\mathrm{C}$ & -1.642028 & -7.879638 & -1.498794 \\
\hline $\mathrm{C}$ & -2.803251 & -8.450990 & -0.975094 \\
\hline $\mathrm{C}$ & -3.911418 & -7.640043 & -0.729394 \\
\hline $\mathrm{C}$ & -3.855261 & -6.271446 & -0.995094 \\
\hline $\mathrm{C}$ & -1.820060 & -3.853731 & -3.021494 \\
\hline $\mathrm{C}$ & -2.519158 & -3.819901 & -4.233894 \\
\hline $\mathrm{C}$ & -1.858846 & -3.524729 & -5.428494 \\
\hline $\mathrm{C}$ & -0.487935 & -3.255986 & -5.420394 \\
\hline $\mathrm{C}$ & 0.217663 & -3.300515 & -4.215694 \\
\hline $\mathrm{C}$ & -0.444350 & -3.609888 & -3.028594 \\
\hline $\mathrm{C}$ & -5.044992 & -4.634896 & 3.605006 \\
\hline $\mathrm{C}$ & -6.280295 & -4.690545 & 2.938106 \\
\hline $\mathrm{C}$ & -7.477594 & -4.668795 & 3.651506 \\
\hline $\mathrm{C}$ & -7.463591 & -4.593995 & 5.046106 \\
\hline $\mathrm{C}$ & -6.241988 & -4.541246 & 5.721106 \\
\hline $\mathrm{C}$ & -5.044389 & -4.560596 & 5.007706 \\
\hline $\mathrm{C}$ & 3.308099 & -2.454344 & -0.481794 \\
\hline $\mathrm{C}$ & 4.197694 & -2.571481 & -1.768194 \\
\hline $\mathrm{C}$ & 3.296847 & -3.694044 & -1.359394 \\
\hline $\mathrm{C}$ & 2.053033 & -1.636592 & -0.478394 \\
\hline$C$ & 4.009597 & -2.493974 & 0.842806 \\
\hline
\end{tabular}

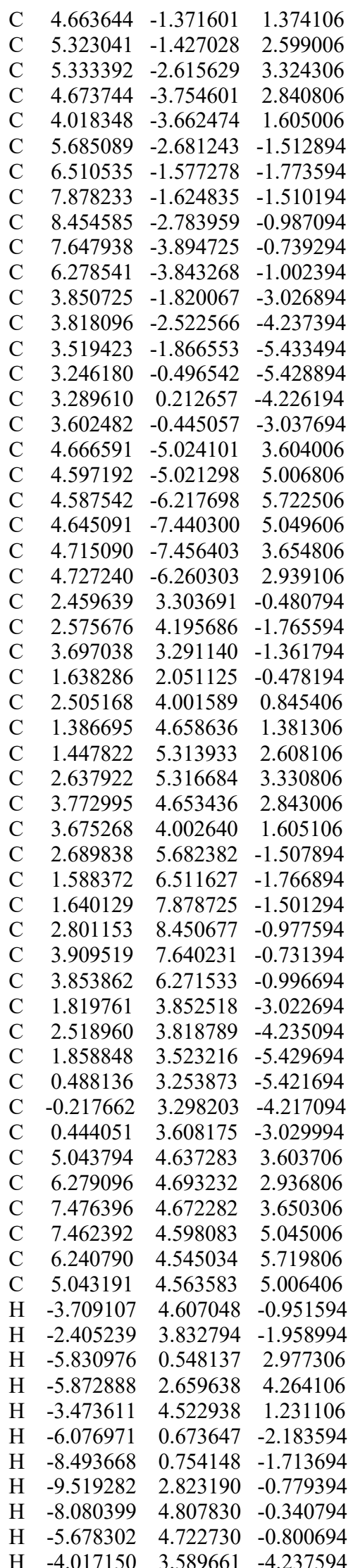

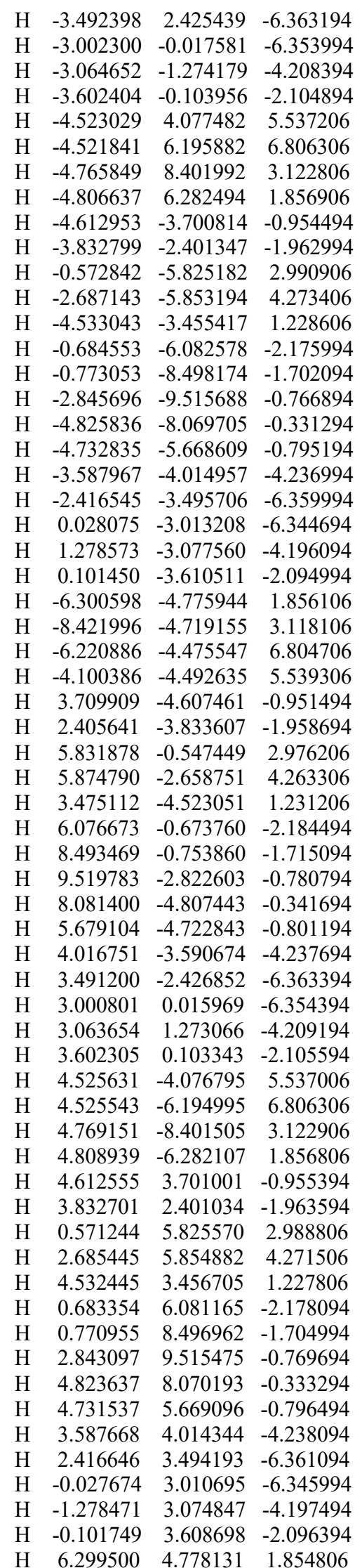




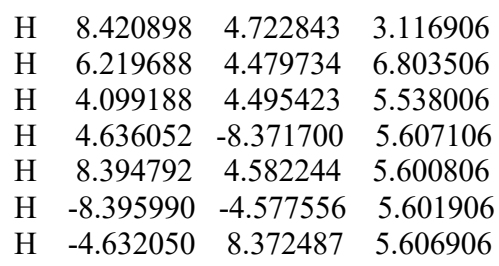

L2

$\begin{array}{llll}\text { Rh } & -0.000198 & -0.000901 & -1.728603\end{array}$

$\begin{array}{llll}\mathrm{Rh} & -0.000198 & -0.000401 & 0.662697\end{array}$

$\begin{array}{llll}\text { O } & 1.922605 & -0.712691 & 0.593297\end{array}$

$\begin{array}{llll}\text { O } & 1.718607 & -1.156592 & -1.614303\end{array}$

$\begin{array}{llll}\mathrm{O} & 0.723492 & 1.919103 & 0.583897\end{array}$

$\begin{array}{llll}\text { O } & 1.163793 & 1.708005 & -1.623903\end{array}$

$\begin{array}{llll}\mathrm{O} & -1.922902 & 0.711990 & 0.592697\end{array}$

$\begin{array}{lllll}\mathrm{O} & -1.719004 & 1.155091 & -1.615003\end{array}$

$\begin{array}{llll}\mathrm{O} & -0.723989 & -1.919904 & 0.584797\end{array}$

$\begin{array}{llll}\text { O } & -1.164490 & -1.709506 & -1.623003\end{array}$

$\begin{array}{llll}\mathrm{Cl} & 4.532396 & 1.213422 & 0.484597\end{array}$

$\begin{array}{llll}\text { Cl } & -1.022722 & 4.680694 & 0.636397\end{array}$

$\begin{array}{llll}\text { Cl } & -4.534092 & -1.212123 & 0.486497\end{array}$

$\begin{array}{llll}\text { Cl } & 1.021225 & -4.681895 & 0.638997\end{array}$

$\begin{array}{llll}\text { C } & 3.782310 & -1.636482 & -0.515303\end{array}$

$\begin{array}{llll}\text { C } & 4.687509 & -1.546677 & -1.793303\end{array}$

$\begin{array}{llll}\text { C } & 4.066016 & -2.846180 & -1.387703\end{array}$

$\begin{array}{llll}\text { C } & 2.373607 & -1.125289 & -0.524903\end{array}$

$\begin{array}{llll}\text { C } & 4.457509 & -1.520479 & 0.819097\end{array}$

$\begin{array}{llll}\text { C } & 4.848803 & -0.284077 & 1.354797\end{array}$

$\begin{array}{llll}\text { C } & 5.480402 & -0.194073 & 2.592097\end{array}$

$\begin{array}{llll}\text { C } & 5.727108 & -1.350972 & 3.326297\end{array}$

$\begin{array}{llll}\text { C } & 5.333214 & -2.605374 & 2.838797\end{array}$

$\begin{array}{llll}\text { C } & 4.698215 & -2.658277 & 1.590097\end{array}$

$\begin{array}{llll}\text { C } & 6.157708 & -1.308470 & -1.526403\end{array}$

$\begin{array}{llll}\text { C } & 6.715002 & -0.053867 & -1.815703\end{array}$

$\begin{array}{llll}\text { C } & 8.054100 & 0.218439 & -1.541903\end{array}$

$\begin{array}{llll}\text { C } & 8.871105 & -0.763357 & -0.978403\end{array}$

$\begin{array}{llll}\text { C } & 8.334012 & -2.020659 & -0.700803\end{array}$

$\begin{array}{llll}\text { C } & 6.992713 & -2.289666 & -0.975203\end{array}$

C $\quad 4.183606-0.899580 \quad-3.057103$

$\begin{array}{llll}\text { C } & 4.319009 & -1.595679 & -4.264203\end{array}$

$\begin{array}{llll}\text { C } & 3.879407 & -1.032781 & -5.464103\end{array}$

$\begin{array}{llll}\text { C } & 3.296000 & 0.236516 & -5.467203\end{array}$

$\begin{array}{llll}\text { C } & 3.170497 & 0.942315 & -4.268203\end{array}$

$\begin{array}{llll}\text { C } & 3.623000 & 0.380117 & -3.075403\end{array}$

$\begin{array}{llll}\text { C } & 5.578921 & -3.844473 & 3.612397\end{array}$

$\begin{array}{llll}\text { C } & 5.914126 & -5.042571 & 2.959897\end{array}$

C $\quad 6.141632 \quad-6.210570 \quad 3.685997$

$\begin{array}{llll}\text { C } & 6.041132 & -6.202971 & 5.078997\end{array}$

$\begin{array}{llll}\text { C } & 5.710726 & -5.017672 & 5.739597\end{array}$

$\begin{array}{llll}\text { C } & 5.481021 & -3.849873 & 5.013597\end{array}$

$\begin{array}{llll}\text { C } & 1.720683 & 3.746808 & -0.518403\end{array}$

$\begin{array}{llll}\text { C } & 1.592178 & 4.678407 & -1.773403\end{array}$

$\begin{array}{llll}\text { C } & 2.892182 & 4.011414 & -1.445803\end{array}$

$\begin{array}{llll}\text { C } & 1.155490 & 2.358405 & -0.531303\end{array}$

$\begin{array}{llll}\text { C } & 1.706680 & 4.373408 & 0.843497\end{array}$

$\begin{array}{llll}\text { C } & 0.529778 & 4.833302 & 1.451597\end{array}$

$\begin{array}{llll}\text { C } & 0.544875 & 5.395202 & 2.725697\end{array}$

$\begin{array}{llll}\text { C } & 1.743574 & 5.480108 & 3.429897\end{array}$

$\begin{array}{llll}\text { C } & 2.938277 & 5.003914 & 2.870397\end{array}$

$\begin{array}{llll}\text { C } & 2.889379 & 4.468114 & 1.576297\end{array}$

$\begin{array}{llll}\text { C } & 1.412271 & 6.151406 & -1.480003\end{array}$ $\begin{array}{llll}\text { C } & 0.203468 & 6.780200 & -1.814303\end{array}$

$\begin{array}{llll}\text { C } & -0.012839 & 8.126099 & -1.523203\end{array}$

$\begin{array}{llll}\text { C } & 0.979458 & 8.878704 & -0.893203\end{array}$

$\begin{array}{lllll}\text { C } & 2.190961 & 8.269410 & -0.565103\end{array}$

$\begin{array}{llll}\text { C } & 2.404767 & 6.922611 & -0.858403\end{array}$

$\begin{array}{llll}\text { C } & 0.871181 & 4.197904 & -3.005603\end{array}$

$\begin{array}{llll}\text { C } & 1.521480 & 4.290107 & -4.241903\end{array}$

$\begin{array}{llll}\text { C } & 0.890382 & 3.864304 & -5.412303\end{array}$

$\begin{array}{llll}\text { C } & -0.402315 & 3.337498 & -5.355603\end{array}$

$\begin{array}{llll}\text { C } & -1.061115 & 3.254694 & -4.127003\end{array}$

$\begin{array}{llll}\text { C } & -0.431017 & 3.693497 & -2.963503\end{array}$

$\begin{array}{llll}\text { C } & 4.216677 & 5.040420 & 3.617997\end{array}$

$\begin{array}{llll}\text { C } & 4.511271 & 6.091922 & 4.500897\end{array}$

$\begin{array}{llll}\text { C } & 5.715171 & 6.116328 & 5.203897\end{array}$

$\begin{array}{llll}\mathrm{C} & 6.649076 & 5.091532 & 5.036297\end{array}$

$\begin{array}{llll}\text { C } & 6.368782 & 4.041031 & 4.159297\end{array}$

$\begin{array}{llll}\text { C } & 5.164382 & 4.014425 & 3.458697\end{array}$

$\begin{array}{llll}\text { C } & -3.782307 & 1.636381 & -0.515703\end{array}$

$\begin{array}{llll}\text { C } & -4.688206 & 1.545876 & -1.793103\end{array}$

$\begin{array}{lllll}\text { C } & -4.065913 & 2.845479 & -1.388903\end{array}$

$\begin{array}{llll}\text { C } & -2.373804 & 1.124488 & -0.525503\end{array}$

$\begin{array}{llll}\text { C } & -4.456706 & 1.521777 & 0.819297\end{array}$

$\begin{array}{llll}\text { C } & -4.848500 & 0.286076 & 1.356097\end{array}$

$\begin{array}{llll}\text { C } & -5.478999 & 0.197372 & 2.594097\end{array}$

$\begin{array}{llll}\text { C } & -5.723805 & 1.354971 & 3.327897\end{array}$

$\begin{array}{llll}\text { C } & -5.329111 & 2.608773 & 2.839297\end{array}$

$\begin{array}{llll}\text { C } & -4.695512 & 2.660276 & 1.589797\end{array}$

$\begin{array}{llll}\text { C } & -6.158405 & 1.308469 & -1.525303\end{array}$

$\begin{array}{llll}\text { C } & -6.716499 & 0.054266 & -1.814603\end{array}$

$\begin{array}{llll}\text { C } & -8.055597 & -0.217340 & -1.540003\end{array}$

$\begin{array}{llll}\text { C } & -8.871702 & 0.764856 & -0.975703\end{array}$

$\begin{array}{llll}\text { C } & -8.333608 & 2.021658 & -0.698203\end{array}$

$\begin{array}{llll}\text { C } & -6.992410 & 2.290065 & -0.973303\end{array}$

$\begin{array}{llll}\text { C } & -4.184903 & 0.897579 & -3.056503\end{array}$

$\begin{array}{lllll}\text { C } & -4.320306 & 1.592778 & -4.264103\end{array}$

$\begin{array}{llll}\text { C } & -3.881104 & 1.028880 & -5.463703\end{array}$

$\begin{array}{llll}\text { C } & -3.298297 & -0.240817 & -5.465903\end{array}$

$\begin{array}{llll}\text { C } & -3.172894 & -0.945716 & -4.266403\end{array}$

$\begin{array}{llll}\text { C } & -3.624897 & -0.382318 & -3.074003\end{array}$

$\begin{array}{llll}\text { C } & -5.572517 & 3.848472 & 3.612597\end{array}$

$\begin{array}{llll}\text { C } & -5.907023 & 5.046770 & 2.959897\end{array}$

$\begin{array}{llll}\text { C } & -6.132129 & 6.215269 & 3.685697\end{array}$

$\begin{array}{llll}\text { C } & -6.030029 & 6.208270 & 5.078697\end{array}$

$\begin{array}{llll}\text { C } & -5.700323 & 5.022771 & 5.739497\end{array}$

$\begin{array}{llll}\text { C } & -5.472917 & 3.854472 & 5.013697\end{array}$

$\begin{array}{llll}\text { C } & -1.721780 & -3.747809 & -0.516603\end{array}$

$\begin{array}{llll}\text { C } & -1.593175 & -4.680008 & -1.771103\end{array}$

$\begin{array}{llll}\text { C } & -2.893079 & -4.012715 & -1.444003\end{array}$

$\begin{array}{llll}\text { C } & -1.156187 & -2.359506 & -0.530203\end{array}$

$\begin{array}{llll}\text { C } & -1.708077 & -4.373609 & 0.845697\end{array}$

$\begin{array}{llll}\text { C } & -0.531475 & -4.833703 & 1.453997\end{array}$

$\begin{array}{llll}\text { C } & -0.546772 & -5.394903 & 2.728497\end{array}$

$\begin{array}{llll}\text { C } & -1.745671 & -5.479109 & 3.432597\end{array}$

$\begin{array}{llll}\text { C } & -2.940174 & -5.002815 & 2.872697\end{array}$

$\begin{array}{llll}\text { C } & -2.890976 & -4.467615 & 1.578397\end{array}$

$\begin{array}{llll}\text { C } & -1.413568 & -6.152907 & -1.477003\end{array}$

$\begin{array}{llll}\text { C } & -0.204865 & -6.782202 & -1.811003\end{array}$

C $\quad 0.011042-8.128000 \quad-1.519303$

$\begin{array}{llll}\text { C } & -0.981355 & -8.880105 & -0.889003\end{array}$

$\begin{array}{llll}\text { C } & -2.192658 & -8.270411 & -0.561203\end{array}$

$\begin{array}{llll}\text { C } & -2.406164 & -6.923612 & -0.855103\end{array}$

$\begin{array}{llll}\text { C } & -0.871878 & -4.200105 & -3.003403\end{array}$
C $\quad-1.521977-4.292608 \quad-4.239703$

$\begin{array}{llll}\text { C } & -0.890679 & -3.867305 & -5.410203\end{array}$

$\begin{array}{llll}\text { C } & 0.402018 & -3.340499 & -5.353403\end{array}$

$\begin{array}{llll}\text { C } & 1.060718 & -3.257395 & -4.124803\end{array}$

$\begin{array}{llll}\text { C } & 0.430420 & -3.695798 & -2.961203\end{array}$

$\begin{array}{llll}\text { C } & -4.218574 & -5.038721 & 3.620297\end{array}$

$\begin{array}{llll}\text { C } & -5.166079 & -4.012626 & 3.460397\end{array}$

$\begin{array}{llll}\text { C } & -6.370579 & -4.038632 & 4.160997\end{array}$

$\begin{array}{llll}\text { C } & -6.651073 & -5.088733 & 5.038497\end{array}$

$\begin{array}{llll}\text { C } & -5.717268 & -6.113629 & 5.206597\end{array}$

$\begin{array}{llll}\text { C } & -4.513368 & -6.089723 & 4.503597\end{array}$

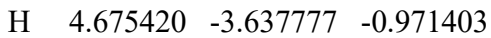

$\begin{array}{llll}\mathrm{H} & 3.236917 & -3.190985 & -1.992603\end{array}$

$\begin{array}{llll}\mathrm{H} & 5.783798 & 0.773528 & 2.974897\end{array}$

$\begin{array}{llll}\mathrm{H} & 6.245908 & -1.275370 & 4.275897\end{array}$

$\begin{array}{llll}\mathrm{H} & 4.356719 & -3.615379 & 1.212097\end{array}$

$\begin{array}{lllll}\mathrm{H} & 6.093098 & 0.715530 & -2.257003\end{array}$

$\mathrm{H} \quad 8.458096 \quad 1.200141 \quad-1.770003$

$\begin{array}{llll}\mathrm{H} & 9.914304 & -0.552952 & -0.763703\end{array}$

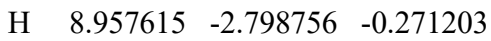

$\begin{array}{llll}\mathrm{H} & 6.606018 & -3.275768 & -0.748703\end{array}$

$\begin{array}{llll}\mathrm{H} & 4.761114 & -2.588077 & -4.258803\end{array}$

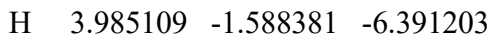

$\begin{array}{llll}\mathrm{H} & 2.941598 & 0.673614 & -6.396103\end{array}$

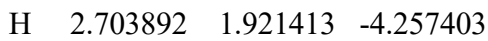

$\begin{array}{llll}\mathrm{H} & 3.492297 & 0.917017 & -2.145903\end{array}$

$\begin{array}{llll}\mathrm{H} & 6.020426 & -5.051871 & 1.879497\end{array}$

$\begin{array}{llll}\mathrm{H} & 6.406137 & -7.125069 & 3.163797\end{array}$

H $\quad \begin{array}{llll}6.622626 & -5.003573 & 6.821797\end{array}$

$\begin{array}{llll}\mathrm{H} & 5.199216 & -2.939675 & 5.533597\end{array}$

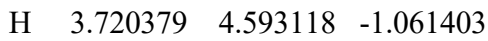

H $\quad 3.181186 \quad 3.187115 \quad-2.085603$

$\begin{array}{llll}\mathrm{H} & -0.380027 & 5.743698 & 3.170797\end{array}$

$\begin{array}{llll}\mathrm{H} & 1.738272 & 5.888508 & 4.434897\end{array}$

$\begin{array}{llll}\mathrm{H} & 3.804981 & 4.116118 & 1.116297\end{array}$

$\begin{array}{llll}\mathrm{H} & -0.575529 & 6.209397 & -2.304903\end{array}$

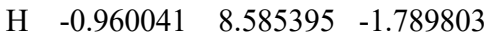

$\mathrm{H} \quad 0.812753 \quad 9.927003 \quad-0.664903$

$\begin{array}{lllll}\mathrm{H} & 2.976958 & 8.841114 & -0.080903\end{array}$

$\begin{array}{llll}\mathrm{H} & 3.355170 & 6.480816 & -0.586303\end{array}$

$\mathrm{H} \quad 2.531978 \quad 4.686912 \quad-4.282403$

H $\quad 1.410882 \quad 3.936006 \quad-6.362803$

$\begin{array}{llll}\mathrm{H} & -0.893713 & 2.991495 & -6.260203\end{array}$

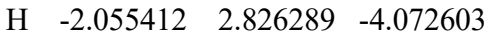

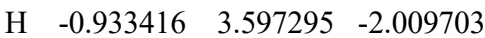

$\begin{array}{llll}\mathrm{H} & 3.803667 & 6.906518 & 4.619197\end{array}$

$\begin{array}{llll}\text { H } & 5.927567 & 6.941729 & 5.876697\end{array}$

$\begin{array}{llll}\mathrm{H} & 7.086386 & 3.236834 & 4.025997\end{array}$

$\begin{array}{llll}\mathrm{H} & 4.945586 & 3.182324 & 2.797097\end{array}$

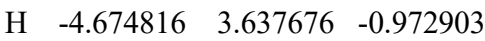

$\begin{array}{lllll}\mathrm{H} & -3.237014 & 3.189484 & -1.994503\end{array}$

$\begin{array}{llll}\mathrm{H} & -5.782795 & -0.769729 & 2.977797\end{array}$

$\begin{array}{llll}\mathrm{H} & -6.241605 & 1.280369 & 4.278097\end{array}$

$\begin{array}{llll}\mathrm{H} & -4.353316 & 3.616778 & 1.210897\end{array}$

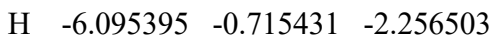

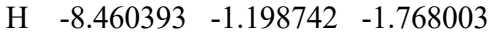

$\begin{array}{llll}\mathrm{H} & -9.914901 & 0.554951 & -0.760403\end{array}$

$\begin{array}{llll}\mathrm{H} & -8.956512 & 2.800155 & -0.267903\end{array}$

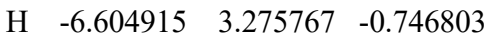

$\begin{array}{llll}\mathrm{H} & -4.761911 & 2.585376 & -4.259303\end{array}$

$\begin{array}{lllll}\mathrm{H} & -3.986806 & 1.583780 & -6.391203\end{array}$

$\begin{array}{llll}\mathrm{H} & -2.944295 & -0.678715 & -6.394603\end{array}$ 
$\begin{array}{rrrr}\mathrm{H} & -2.706689 & -1.925014 & -4.255003 \\ \mathrm{H} & -3.494294 & -0.918618 & -2.144103 \\ \mathrm{H} & -6.014523 & 5.055570 & 1.879697 \\ \mathrm{H} & -6.396234 & 7.129868 & 3.163497 \\ \mathrm{H} & -5.611023 & 5.009172 & 6.821497 \\ \mathrm{H} & -5.191613 & 2.944074 & 5.533797 \\ \mathrm{H} & -3.721476 & -4.594019 & -1.059503 \\ \mathrm{H} & -3.181983 & -3.188716 & -2.084303 \\ \mathrm{H} & 0.377930 & -5.743499 & 3.173797 \\ \mathrm{H} & -1.740569 & -5.887009 & 4.437697 \\ \mathrm{H} & -3.806378 & -4.115619 & 1.118097 \\ \mathrm{H} & 0.574232 & -6.211698 & -2.301803 \\ \mathrm{H} & 0.958244 & -8.587496 & -1.785703 \\ \mathrm{H} & -0.814949 & -9.928305 & -0.660303 \\ \mathrm{H} & -2.978855 & -8.841615 & -0.076803 \\ \mathrm{H} & -3.356566 & -6.481517 & -0.583203 \\ \mathrm{H} & -2.532575 & -4.689313 & -4.280203 \\ \mathrm{H} & -1.411079 & -3.939107 & -6.360703 \\ \mathrm{H} & 0.893616 & -2.994696 & -6.258003 \\ \mathrm{H} & 2.054916 & -2.829090 & -4.070303 \\ \mathrm{H} & 0.932619 & -3.599396 & -2.007403 \\ \mathrm{H} & -4.947083 & -3.180825 & 2.798397 \\ \mathrm{H} & -7.087982 & -3.234436 & 4.027197 \\ \mathrm{H} & -5.929864 & -6.938630 & 5.879797 \\ \mathrm{H} & -3.805964 & -6.904419 & 4.622297 \\ \mathrm{H} & -6.205934 & 7.118169 & 5.643997 \\ \mathrm{H} & -7.588773 & -5.108638 & 5.585097 \\ \mathrm{H} & 7.586776 & 5.111937 & 5.582997 \\ \mathrm{H} & 6.218837 & -7.112470 & 5.644597\end{array}$

\section{L3}

$\begin{array}{llll}\mathrm{Rh} & 0.024098 & -0.155301 & -1.699302\end{array}$

$\begin{array}{llll}\mathrm{Rh} & 0.003299 & -0.078301 & 0.690598\end{array}$

$\begin{array}{llll}\mathrm{O} & -1.812579 & 0.869440 & 0.568498\end{array}$

$\begin{array}{lllll}\mathrm{O} & -1.530771 & 1.214134 & -1.648202\end{array}$

$\begin{array}{llll}\text { O } & -0.961141 & -1.891179 & 0.660398\end{array}$

$\begin{array}{lllll}\mathrm{O} & -1.353037 & -1.694870 & -1.557802\end{array}$

$\begin{array}{llll}\text { O } & 1.830278 & -1.017542 & 0.666398\end{array}$

$\begin{array}{lllll}\text { O } & 1.587767 & -1.506036 & -1.527602\end{array}$

$\begin{array}{llll}\mathrm{O} & 0.948440 & 1.741478 & 0.564398\end{array}$

$\begin{array}{llll}\text { O } & 1.383833 & 1.403468 & -1.628702\end{array}$

$\begin{array}{llll}\mathrm{Cl} & -4.704813 & -0.613995 & 0.533998\end{array}$

$\begin{array}{llll}\text { Cl } & 0.342892 & -4.874408 & 0.870298\end{array}$

$\begin{array}{lllll}\mathrm{Cl} & 4.566917 & 0.708297 & 0.405198\end{array}$

$\begin{array}{llll}\mathrm{Cl} & -0.635696 & 4.556014 & 0.342998\end{array}$

C $\quad-3.504753 \quad 2.029978 \quad-0.585302$

$\begin{array}{llll}\text { C } & -4.416453 & 2.016699 & -1.861102\end{array}$

$\begin{array}{llll}\text { C } & -3.606526 & 3.226680 & -1.514102\end{array}$

$\begin{array}{llll}\text { C } & -2.189369 & 1.312449 & -0.564902\end{array}$

$\begin{array}{llll}\text { C } & -4.179352 & 2.080993 & 0.753198\end{array}$

$\begin{array}{llll}\text { C } & -4.759577 & 0.948506 & 1.343598\end{array}$

$\begin{array}{llll}\text { C } & -5.375276 & 1.013220 & 2.590498\end{array}$

$\begin{array}{llll}\text { C } & -5.411649 & 2.222521 & 3.279898\end{array}$

$\begin{array}{llll}\text { C } & -4.822723 & 3.373808 & 2.737298\end{array}$

$\begin{array}{llll}\text { C } & -4.213925 & 3.272194 & 1.478698\end{array}$

$\begin{array}{llll}\text { C } & -5.905453 & 2.013332 & -1.592802\end{array}$

$\begin{array}{llll}\text { C } & -6.650580 & 0.855149 & -1.860702\end{array}$

$\begin{array}{llll}\text { C } & -8.015581 & 0.798380 & -1.585302\end{array}$

$\begin{array}{llll}\text { C } & -8.670456 & 1.904494 & -1.040902\end{array}$

$\begin{array}{llll}\text { C } & -7.944830 & 3.067878 & -0.783702\end{array}$

$\begin{array}{llll}\text { C } & -6.578329 & 3.121247 & -1.059802\end{array}$

C $\quad-4.013571 \quad 1.245090 \quad-3.090802$ $\begin{array}{llll}\text { C } & -4.049856 & 1.896690 & -4.329402\end{array}$

$\begin{array}{llll}\mathrm{C} & -3.702171 & 1.220583 & -5.500402\end{array}$

C $\quad-3.309501 \quad-0.118926 \quad-5.442602$

$\begin{array}{llll}\text { C } & -3.282716 & -0.778427 & -4.211602\end{array}$

C $\quad-3.644701 \quad-0.101819 \quad-3.047502$

$\begin{array}{llll}\text { C } & -4.833394 & 4.663508 & 3.465698\end{array}$

$\begin{array}{llll}\text { C } & -4.960666 & 5.878711 & 2.772098\end{array}$

$\begin{array}{llll}\text { C } & -4.960939 & 7.093511 & 3.455698\end{array}$

$\begin{array}{llll}\text { C } & -4.836939 & 7.117408 & 4.846698\end{array}$

$\begin{array}{llll}\text { C } & -4.712466 & 5.916005 & 5.547998\end{array}$

$\begin{array}{llll}\text { C } & -4.709593 & 4.701105 & 4.864398\end{array}$

$\begin{array}{llll}\text { C } & -2.205180 & -3.593151 & -0.392402\end{array}$

C $-2.180302-4.582952-1.609202$

C $\quad-3.380783 \quad-3.728825-1.341502$

C $\quad-1.445351 \quad-2.301768 \quad-0.444302$

$\begin{array}{llll}\text { C } & -2.313192 & -4.157949 & 0.991998\end{array}$

C $\quad-1.231306 \quad-4.761373 \quad 1.648698$

$\begin{array}{llll}\text { C } & -1.357517 & -5.262670 & 2.941798\end{array}$

C $\quad-2.571114 \quad-5.142843 \quad 3.614598$

$\begin{array}{llll}\text { C } & -3.670200 & -4.520118 & 3.004798\end{array}$

$\begin{array}{llll}\text { C } & -3.513190 & -4.048422 & 1.694398\end{array}$

$\begin{array}{llll}\text { C } & -2.216335 & -6.054651 & -1.262002\end{array}$

$\begin{array}{llll}\text { C } & -1.109853 & -6.863676 & -1.561602\end{array}$

$\begin{array}{llll}\text { C } & -1.093084 & -8.215676 & -1.222602\end{array}$

C $\quad-2.187497 \quad-8.793852 \quad-0.577802$

$\begin{array}{llll}\text { C } & -3.299179 & -8.004026 & -0.283202\end{array}$

C $\quad-3.313448-6.651726 \quad-0.624602$

$\begin{array}{llll}\text { C } & -1.370094 & -4.251270 & -2.835102\end{array}$

$\begin{array}{llll}\text { C } & -1.999895 & -4.287056 & -4.084902\end{array}$

$\begin{array}{llll}\text { C } & -1.289889 & -3.989272 & -5.249602\end{array}$

$\begin{array}{llll}\text { C } & 0.063319 & -3.650402 & -5.173502\end{array}$

C $\quad 0.700520 \quad-3.626916 \quad-3.931202$

$\begin{array}{llll}\text { C } & -0.010087 & -3.936500 & -2.772702\end{array}$

$\begin{array}{llll}\text { C } & -4.957597 & -4.344189 & 3.716598\end{array}$

$\begin{array}{llll}\text { C } & -5.418018 & -5.306079 & 4.630398\end{array}$

$\begin{array}{llll}\text { C } & -6.628814 & -5.131952 & 5.299498\end{array}$

$\begin{array}{llll}\text { C } & -7.404789 & -3.994434 & 5.066198\end{array}$

$\begin{array}{llll}\text { C } & -6.959167 & -3.031544 & 4.157698\end{array}$

$\begin{array}{llll}\text { C } & -5.747271 & -3.203171 & 3.491098\end{array}$

C $3.601253-2.134582-0.412502$

C $\quad 4.489351-2.210502 \quad-1.703502$

C $3.755024-3.417485-1.211002$

$\begin{array}{llll}\text { C } & 2.243567 & -1.500751 & -0.438202\end{array}$

$\begin{array}{llll}\text { C } & 4.315656 & -1.992698 & 0.898898\end{array}$

$\begin{array}{llll}\text { C } & 4.805784 & -0.757509 & 1.350498\end{array}$

$\begin{array}{llll}\text { C } & 5.484087 & -0.641524 & 2.560698\end{array}$

$\begin{array}{llll}\text { C } & 5.682661 & -1.770329 & 3.351098\end{array}$

$\begin{array}{llll}\text { C } & 5.194033 & -3.021018 & 2.947198\end{array}$

$\begin{array}{llll}\text { C } & 4.511131 & -3.099902 & 1.725298\end{array}$

$\begin{array}{llll}\text { C } & 5.979354 & -2.098935 & -1.462802\end{array}$

$\begin{array}{llll}\text { C } & 6.638981 & -0.902750 & -1.782402\end{array}$

$\begin{array}{llll}\text { C } & 8.001084 & -0.745081 & -1.533702\end{array}$

$\begin{array}{llll}\text { C } & 8.738461 & -1.786797 & -0.967502\end{array}$

C $\quad 8.098534 \quad-2.988083 \quad-0.662102$

C $\quad 6.734031 \quad-3.141952 \quad-0.910402$

$\begin{array}{llll}\text { C } & 4.039265 & -1.595792 & -3.003202\end{array}$

C $\quad 4.098948 \quad-2.373293 \quad-4.165802$

C $\quad 3.709960 \quad-1.843384 \quad-5.397902$

$\begin{array}{llll}\text { C } & 3.254989 & -0.524974 & -5.478302\end{array}$

$\begin{array}{llll}\text { C } & 3.207107 & 0.261227 & -4.324402\end{array}$

$\begin{array}{llll}\text { C } & 3.608195 & -0.270082 & -3.099602\end{array}$

$\begin{array}{llll}\text { C } & 5.395006 & -4.230722 & 3.778098\end{array}$ $\begin{array}{llll}\text { C } & 5.638978 & -5.478328 & 3.179898\end{array}$

$\begin{array}{llll}\text { C } & 5.826352 & -6.618532 & 3.959598\end{array}$

$\begin{array}{llll}\text { C } & 5.776554 & -6.533331 & 5.352698\end{array}$

$\begin{array}{llll}\text { C } & 5.537382 & -5.298225 & 5.959498\end{array}$

$\begin{array}{llll}\text { C } & 5.347808 & -4.158121 & 5.179998\end{array}$

$\begin{array}{llll}\text { C } & 2.107878 & 3.428552 & -0.602002\end{array}$

$\begin{array}{llll}\text { C } & 2.136698 & 4.288051 & -1.913802\end{array}$

$\begin{array}{llll}\text { C } & 3.346081 & 3.523324 & -1.476502\end{array}$

$\begin{array}{llll}\text { C } & 1.424348 & 2.096267 & -0.562802\end{array}$

$\begin{array}{llll}\text { C } & 2.069695 & 4.165153 & 0.703498\end{array}$

$\begin{array}{llll}\text { C } & 0.886808 & 4.723779 & 1.211498\end{array}$

$\begin{array}{llll}\text { C } & 0.869023 & 5.411380 & 2.421898\end{array}$

C $\quad 2.044126 \quad 5.545053 \quad 3.156898$

$\begin{array}{llll}\text { C } & 3.243513 & 4.982026 & 2.697598\end{array}$

$\begin{array}{llll}\text { C } & 3.224798 & 4.297127 & 1.474598\end{array}$

$\begin{array}{llll}\text { C } & 2.086131 & 5.785752 & -1.703902\end{array}$

$\begin{array}{llll}\text { C } & 0.911647 & 6.485379 & -2.018802\end{array}$

$\begin{array}{llll}\text { C } & 0.811278 & 7.857681 & -1.797602\end{array}$

$\begin{array}{llll}\text { C } & 1.889394 & 8.565257 & -1.262702\end{array}$

$\begin{array}{llll}\text { C } & 3.068979 & 7.884630 & -0.960202\end{array}$

$\begin{array}{llll}\text { C } & 3.165748 & 6.510528 & -1.181702\end{array}$

$\begin{array}{llll}\text { C } & 1.433887 & 3.823667 & -3.162802\end{array}$

$\begin{array}{llll}\text { C } & 2.141687 & 3.829451 & -4.370502\end{array}$

$\begin{array}{llll}\text { C } & 1.525578 & 3.429865 & -5.558402\end{array}$

$\begin{array}{llll}\text { C } & 0.191969 & 3.013695 & -5.547902\end{array}$

$\begin{array}{llll}\text { C } & -0.522731 & 3.015111 & -4.347702\end{array}$

$\begin{array}{llll}\text { C } & 0.093378 & 3.430097 & -3.168102\end{array}$

$\begin{array}{llll}\text { C } & 4.499416 & 5.101698 & 3.473698\end{array}$

$\begin{array}{llll}\text { C } & 5.734119 & 5.255070 & 2.821098\end{array}$

$\begin{array}{llll}\text { C } & 6.917722 & 5.361144 & 3.549798\end{array}$

$\begin{array}{llll}\text { C } & 6.890021 & 5.318944 & 4.945598\end{array}$

$\begin{array}{llll}\text { C } & 5.668718 & 5.169272 & 5.606298\end{array}$

C $\quad 4.485115 \quad 5.060698 \quad 4.877698$

H $\quad-4.090106 \quad 4.118991 \quad-1.137702$

$\mathrm{H} \quad-2.738822 \quad 3.414561 \quad-2.134102$

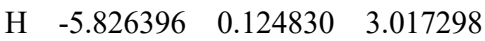

$\begin{array}{llll}\mathrm{H} & -5.916348 & 2.270332 & 4.238798\end{array}$

$\mathrm{H} \quad-3.723106 \quad 4.142483 \quad 1.058198$

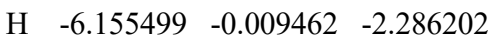

$\mathrm{H} \quad-8.566801 \quad-0.112908 \quad-1.797102$

$\mathrm{H} \quad-9.733657 \quad 1.862018 \quad-0.825102$

H $\quad-8.440010 \quad 3.940489-0.368502$

$\mathrm{H} \quad-6.043708 \quad 4.039035 \quad-0.847902$

$\mathrm{H} \quad-4.341833 \quad 2.942297 \quad-4.371302$

H $\quad-3.729160 \quad 1.741883 \quad-6.452702$

H $\quad-3.025713 \quad-0.645633 \quad-6.349002$

$\mathrm{H} \quad-2.960840 \quad-1.812734 \quad-4.154702$

$\begin{array}{llll}\mathrm{H} & -3.592012 & -0.608620 & -2.093502\end{array}$

$\begin{array}{llll}\mathrm{H} & -5.083467 & 5.869014 & 1.693598\end{array}$

$\begin{array}{llll}\mathrm{H} & -5.066818 & 8.021513 & 2.902198\end{array}$

$\begin{array}{llll}\mathrm{H} & -4.607665 & 5.924603 & 6.628698\end{array}$

$\mathrm{H} \quad-4.584914 \quad 3.774202 \quad 5.415398$

H $\quad-4.289893 \quad-4.175204 \quad-0.959002$

$\begin{array}{llll}\mathrm{H} & -3.538564 & -2.898321 & -2.017602\end{array}$

H $\quad-0.504628 \quad-5.725389 \quad 3.424998$

$\mathrm{H} \quad-2.648423 \quad-5.507441 \quad 4.633298$

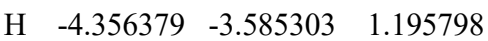

H $\quad-0.253443-6.429595 \quad-2.063002$

H $\quad-0.221197 \quad-8.816496 \quad-1.463602$

H $\quad-2.176020 \quad-9.846552 \quad-0.312402$

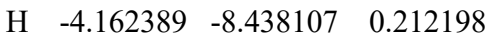

H $\quad-4.191035 \quad-6.067606 \quad-0.377002$ 

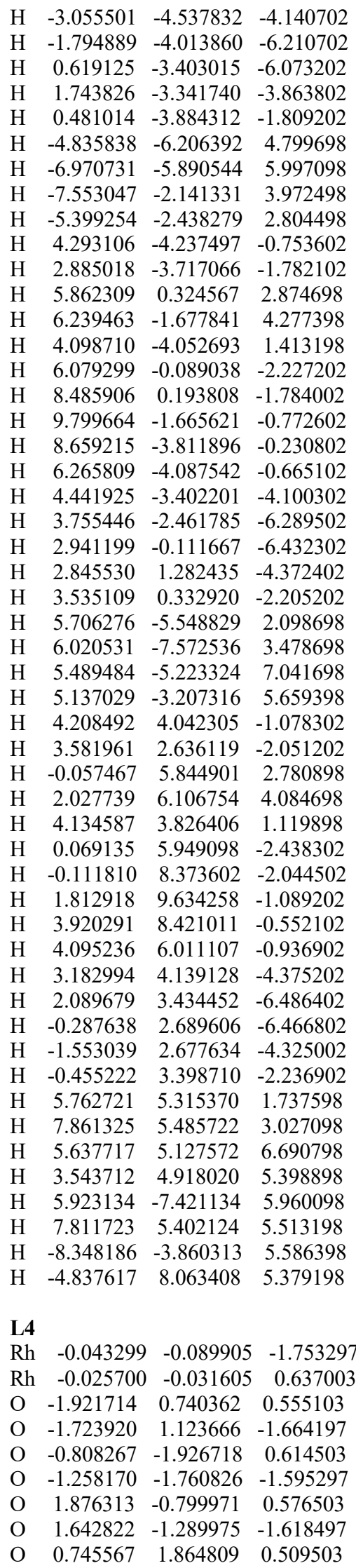

O $\quad 1.165671 \quad 1.593016 \quad-1.695597$

$\begin{array}{llll}\mathrm{Cl} & -4.761184 & -0.930088 & 0.654703\end{array}$

Cl $\quad 0.817583 \quad-4.774090 \quad 0.751803$

$\begin{array}{llll}\mathrm{Cl} & 4.521081 & 1.069975 & 0.392203\end{array}$

$\begin{array}{llll}\mathrm{Cl} & -0.955583 & 4.668679 & 0.484103\end{array}$

$\begin{array}{llll}\text { C } & -3.735532 & 1.759630 & -0.541097\end{array}$

$\begin{array}{llll}\text { C } & -4.690829 & 1.641113 & -1.778197\end{array}$

$\begin{array}{llll}\text { C } & -3.985852 & 2.927626 & -1.475797\end{array}$

$\begin{array}{llll}\text { C } & -2.362121 & 1.158054 & -0.565497\end{array}$

$\begin{array}{llll}\text { C } & -4.331232 & 1.782220 & 0.835103\end{array}$

$\begin{array}{llll}\text { C } & -4.823612 & 0.631011 & 1.465803\end{array}$

$\begin{array}{llll}\text { C } & -5.342413 & 0.677002 & 2.757203\end{array}$

$\begin{array}{llll}\text { C } & -5.350134 & 1.882202 & 3.455403\end{array}$

$\begin{array}{llll}\text { C } & -4.838454 & 3.051311 & 2.873103\end{array}$

$\begin{array}{llll}\text { C } & -4.347453 & 2.971819 & 1.562803\end{array}$

$\begin{array}{llll}\text { C } & -6.163527 & 1.502988 & -1.461297\end{array}$

$\begin{array}{llll}\text { C } & -6.838006 & 0.322376 & -1.807397\end{array}$

$\begin{array}{llll}\text { C } & -8.185203 & 0.144752 & -1.496797\end{array}$

$\begin{array}{llll}\text { C } & -8.893521 & 1.148340 & -0.834397\end{array}$

$\begin{array}{llll}\text { C } & -8.238542 & 2.332051 & -0.493197\end{array}$

$\begin{array}{llll}\text { C } & -6.890745 & 2.507375 & -0.806397\end{array}$

$\begin{array}{llll}\text { C } & -4.247316 & 0.896921 & -3.010697\end{array}$

$\begin{array}{llll}\text { C } & -4.346428 & 1.537319 & -4.251697\end{array}$

$\begin{array}{llll}\text { C } & -3.953716 & 0.886626 & -5.422797\end{array}$

C $\quad-3.454093 \quad-0.416665 \quad-5.362397$

C $\quad-3.365382 \quad-1.066163 \quad-4.129197$

$\begin{array}{llll}\text { C } & -3.770993 & -0.415770 & -2.964497\end{array}$

$\begin{array}{llll}\text { C } & -4.792177 & 4.333112 & 3.614403\end{array}$

C $\quad-5.804283 \quad 4.681494 \quad 4.523403$

$\begin{array}{llll}\text { C } & -5.752204 & 5.888095 & 5.220203\end{array}$

$\begin{array}{llll}\text { C } & -4.688519 & 6.770813 & 5.020003\end{array}$

$\begin{array}{llll}\text { C } & -3.676514 & 6.436431 & 4.117203\end{array}$

$\begin{array}{llll}\text { C } & -3.726092 & 5.229030 & 3.422703\end{array}$

$\begin{array}{llll}\text { C } & -1.882435 & -3.745537 & -0.424097\end{array}$

$\begin{array}{llll}\text { C } & -1.789618 & -4.721936 & -1.647897\end{array}$

$\begin{array}{llll}\text { C } & -3.065330 & -4.000858 & -1.338097\end{array}$

C $\quad-1.265759 \quad-2.380326 \quad-0.485097$

$\begin{array}{llll}\text { C } & -1.890025 & -4.319537 & 0.960803\end{array}$

$\begin{array}{llll}\text { C } & -0.734716 & -4.816017 & 1.580403\end{array}$

$\begin{array}{llll}\text { C } & -0.769907 & -5.325118 & 2.876203\end{array}$

$\begin{array}{llll}\text { C } & -1.966007 & -5.316739 & 3.589803\end{array}$

$\begin{array}{llll}\text { C } & -3.137516 & -4.797559 & 3.019203\end{array}$

$\begin{array}{llll}\text { C } & -3.070325 & -4.317858 & 1.704003\end{array}$

$\begin{array}{llll}\text { C } & -1.660392 & -6.190833 & -1.311197\end{array}$

$\begin{array}{llll}\text { C } & -0.493380 & -6.883213 & -1.668197\end{array}$

$\begin{array}{llll}\text { C } & -0.324556 & -8.227710 & -1.341197\end{array}$

$\begin{array}{llll}\text { C } & -1.323244 & -8.915527 & -0.650597\end{array}$

$\begin{array}{llll}\text { C } & -2.493556 & -8.242748 & -0.297897\end{array}$

C $\quad-2.660380 \quad-6.897851 \quad-0.627797$

$\begin{array}{llll}\text { C } & -1.056525 & -4.300523 & -2.894597\end{array}$

$\begin{array}{llll}\text { C } & -1.716023 & -4.395434 & -4.125797\end{array}$

$\begin{array}{llll}\text { C } & -1.076230 & -4.018223 & -5.307997\end{array}$

$\begin{array}{llll}\text { C } & 0.235662 & -3.539600 & -5.268497\end{array}$

$\begin{array}{llll}\text { C } & 0.904060 & -3.456188 & -4.045197\end{array}$

C $\quad 0.264367 \quad-3.845300 \quad-2.869297$

$\begin{array}{llll}\text { C } & -4.408818 & -4.733582 & 3.777203\end{array}$

$\begin{array}{llll}\text { C } & -4.754300 & -5.735188 & 4.698903\end{array}$

$\begin{array}{llll}\text { C } & -5.950601 & -5.665709 & 5.412003\end{array}$

C $\quad-6.825720 \quad-4.595124 \quad 5.215803$

$\begin{array}{llll}\text { C } & -6.494138 & -3.593518 & 4.300303\end{array}$

$\begin{array}{llll}\text { C } & -5.297236 & -3.660497 & 3.589403\end{array}$

$\begin{array}{llll}\text { C } & 3.705031 & -1.790139 & -0.526697\end{array}$ $\begin{array}{llll}\text { C } & 4.596630 & -1.754424 & -1.817297\end{array}$

$\begin{array}{llll}\text { C } & 3.951553 & -3.028435 & -1.369997\end{array}$

$\begin{array}{llll}\text { C } & 2.308021 & -1.248264 & -0.535597\end{array}$

$\begin{array}{llll}\text { C } & 4.399328 & -1.653327 & 0.796003\end{array}$

$\begin{array}{llll}\text { C } & 4.821007 & -0.411520 & 1.295003\end{array}$

$\begin{array}{llll}\text { C } & 5.472305 & -0.302808 & 2.520703\end{array}$

$\begin{array}{llll}\text { C } & 5.708925 & -1.445604 & 3.279903\end{array}$

$\begin{array}{llll}\text { C } & 5.285447 & -2.704111 & 2.829403\end{array}$

$\begin{array}{llll}\text { C } & 4.630948 & -2.775923 & 1.591703\end{array}$

$\begin{array}{llll}\text { C } & 6.074826 & -1.542498 & -1.572797\end{array}$

$\begin{array}{llll}\text { C } & 6.654805 & -0.305987 & -1.893697\end{array}$

$\begin{array}{llll}\text { C } & 8.002700 & -0.057164 & -1.641297\end{array}$

$\begin{array}{llll}\text { C } & 8.805618 & -1.045050 & -1.068297\end{array}$

$\begin{array}{llll}\text { C } & 8.245540 & -2.284859 & -0.759597\end{array}$

$\begin{array}{llll}\text { C } & 6.895644 & -2.530383 & -1.012397\end{array}$

$\begin{array}{llll}\text { C } & 4.092119 & -1.128632 & -3.091497\end{array}$

$\begin{array}{llll}\text { C } & 4.189532 & -1.860831 & -4.280697\end{array}$

$\begin{array}{llll}\text { C } & 3.746723 & -1.318838 & -5.488997\end{array}$

$\begin{array}{llll}\text { C } & 3.198900 & -0.034048 & -5.518697\end{array}$

$\begin{array}{llll}\text { C } & 3.111787 & 0.707550 & -4.337997\end{array}$

$\begin{array}{llll}\text { C } & 3.566896 & 0.165358 & -3.136997\end{array}$

$\begin{array}{llll}\text { C } & 5.521668 & -3.928207 & 3.629403\end{array}$

$\begin{array}{llll}\text { C } & 5.826790 & -5.147302 & 3.001503\end{array}$

$\begin{array}{llll}\text { C } & 6.045810 & -6.301098 & 3.752603\end{array}$

$\begin{array}{llll}\text { C } & 5.966609 & -6.257999 & 5.146303\end{array}$

$\begin{array}{llll}\text { C } & 5.666288 & -5.051405 & 5.782503\end{array}$

$\begin{array}{llll}\text { C } & 5.445068 & -3.897909 & 5.031603\end{array}$

$\begin{array}{llll}\text { C } & 1.769235 & 3.646827 & -0.638297\end{array}$

$\begin{array}{llll}\text { C } & 1.664019 & 4.552025 & -1.914097\end{array}$

$\begin{array}{llll}\text { C } & 2.949131 & 3.866448 & -1.566697\end{array}$

$\begin{array}{llll}\text { C } & 1.177960 & 2.269116 & -0.618797\end{array}$

$\begin{array}{llll}\text { C } & 1.764124 & 4.302327 & 0.710603\end{array}$

C $\quad 0.595715 \quad 4.798506 \quad 1.306403$

$\begin{array}{llll}\text { C } & 0.616705 & 5.378307 & 2.572303\end{array}$

$\begin{array}{llll}\text { C } & 1.814004 & 5.447128 & 3.280803\end{array}$

$\begin{array}{llll}\text { C } & 3.000313 & 4.937048 & 2.733403\end{array}$

$\begin{array}{llll}\text { C } & 2.945322 & 4.382648 & 1.447403\end{array}$

$\begin{array}{llll}\text { C } & 1.512393 & 6.034422 & -1.653397\end{array}$

$\begin{array}{llll}\text { C } & 0.318782 & 6.680301 & -2.008997\end{array}$

$\begin{array}{llll}\text { C } & 0.127858 & 8.035998 & -1.747297\end{array}$

$\begin{array}{llll}\text { C } & 1.131045 & 8.781516 & -1.126197\end{array}$

$\begin{array}{llll}\text { C } & 2.327756 & 8.154937 & -0.777297\end{array}$

$\begin{array}{llll}\text { C } & 2.516280 & 6.798340 & -1.041097\end{array}$

$\begin{array}{llll}\text { C } & 0.939528 & 4.060212 & -3.139897\end{array}$

$\begin{array}{llll}\text { C } & 1.596727 & 4.118224 & -4.374697\end{array}$

$\begin{array}{llll}\text { C } & 0.962535 & 3.684013 & -5.540197\end{array}$

$\begin{array}{llll}\text { C } & -0.339956 & 3.182290 & -5.480297\end{array}$

$\begin{array}{llll}\text { C } & -1.005456 & 3.132878 & -4.253297\end{array}$

$\begin{array}{llll}\text { C } & -0.371763 & 3.580489 & -3.094897\end{array}$

$\begin{array}{llll}\text { C } & 4.276012 & 4.956571 & 3.486203\end{array}$

$\begin{array}{llll}\text { C } & 5.200131 & 3.906287 & 3.348103\end{array}$

$\begin{array}{llll}\text { C } & 6.402031 & 3.916608 & 4.053403\end{array}$

$\begin{array}{llll}\text { C } & 6.703212 & 4.975113 & 4.913703\end{array}$

$\begin{array}{llll}\text { C } & 5.792694 & 6.024098 & 5.060203\end{array}$

$\begin{array}{llll}\text { C } & 4.591294 & 6.015676 & 4.352503\end{array}$

$\mathrm{H} \quad-4.541067 \quad 3.773316 \quad-1.090197$

$\begin{array}{llll}\mathrm{H} & -3.165357 & 3.190840 & -2.131097\end{array}$

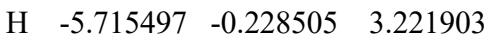

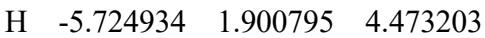

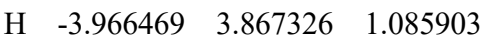

H $\quad-6.301892 \quad-0.465415 \quad-2.322597$

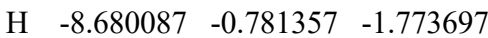




\begin{tabular}{|c|c|c|c|}
\hline & -9.942718 & 1.011721 & -0.590897 \\
\hline & -8.775156 & 3.126442 & 0.016703 \\
\hline & -6.413061 & 3.436783 & -0.522297 \\
\hline & -4.722845 & 2.555413 & -4.295397 \\
\hline & -4.030125 & 1.399525 & -6.376897 \\
\hline & -3.134684 & -0.923259 & -6.268397 \\
\hline & -2.958264 & -2.069656 & -4.072297 \\
\hline & -3.671385 & -0.911569 & -2.007897 \\
\hline & -6.648471 & 4.014179 & 4.667103 \\
\hline & -6.548008 & 6.142781 & 5.913603 \\
\hline & -2.842626 & 7.113746 & 3.958203 \\
\hline & -2.921988 & 4.968444 & 2.741703 \\
\hline & -3.909621 & -4.543073 & -0.931497 \\
\hline & -3.331545 & -3.188663 & -2.002097 \\
\hline & 0.138400 & -5.704802 & 3.330003 \\
\hline & -1.975101 & -5.684639 & 4.610203 \\
\hline$\pi$ & -3.968632 & -3.933374 & 1.235203 \\
\hline & 0.290711 & -6.363299 & -2.205197 \\
\hline & 0.591153 & -8.736494 & -1.627497 \\
\hline & -1.193426 & -9.962425 & -0.394197 \\
\hline & -3.284247 & -8.763162 & 0.234103 \\
\hline $\mathrm{H}$ & -3.579288 & -6.406267 & -0.333997 \\
\hline & -2.740517 & -4.755952 & -4.153197 \\
\hline & -1.604229 & -4.090532 & -6.254197 \\
\hline & 0.734956 & -3.231191 & -6.182397 \\
\hline & 1.913953 & -3.06 & -4.005397 \\
\hline $\mathrm{H}$ & 0.775365 & -3.747991 & -1.919897 \\
\hline 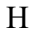 & -4.093285 & -6.584576 & 4.840203 \\
\hline ה & -6.203187 & -6.453613 & 6.115103 \\
\hline & -7.165252 & -2.754130 & 4.144003 \\
\hline 1 & -5.038650 & -2.864693 & 2.898303 \\
\hline $\mathrm{H}$ & 4.548466 & -3.822324 & -0.940097 \\
\hline त & 3.107159 & -3.370450 & -1.954897 \\
\hline & 5.799288 & 0.668098 & 2.874503 \\
\hline & 6.243223 & -1.356095 & 4.219703 \\
\hline $\mathrm{H}$ & 4.267265 & -3.735729 & 1.242403 \\
\hline $\mathrm{H}$ & 6.044091 & 0.467702 & -2.342897 \\
\hline $\mathrm{H}$ & 8.424583 & 0.911044 & -1.893697 \\
\hline$\theta$ & 9.855714 & -0.852831 & -0.870297 \\
\hline & 8.857853 & -3.067649 & -0.322197 \\
\hline $\mathrm{H}$ & 6.490961 & -3.503490 & -0.762397 \\
\hline $\mathrm{H}$ & 4.604250 & -2.864623 & -4.254397 \\
\hline $\mathrm{H}$ & 3.822533 & -1.902237 & -6.401797 \\
\hline & 2.842493 & 0.387446 & -6.453997 \\
\hline & 2.673270 & 1.699543 & -4.347097 \\
\hline $\mathrm{H}$ & 3.464387 & 0.730557 & -2.220797 \\
\hline $\mathrm{H}$ & 5.916590 & -5.184500 & 1.920303 \\
\hline $\mathrm{H}$ & 6.287126 & -7.232394 & 3.249103 \\
\hline & 5.594987 & -5.009606 & 6.865203 \\
\hline H & 5.186552 & -2.970713 & 5.533203 \\
\hline $\mathrm{H}$ & 3.788021 & 4.439462 & -1.192397 \\
\hline $\mathrm{H}$ & 3.223046 & 3.022152 & -2.186997 \\
\hline $\mathrm{H}$ & -0.301602 & 5.752590 & 3.009903 \\
\hline & 1.813196 & 5.869728 & 4.279803 \\
\hline $\mathrm{H}$ & 3.854829 & 4.002463 & 0.997703 \\
\hline $\mathrm{H}$ & -0.468508 & 6.115088 & -2.493097 \\
\hline $\mathrm{H}$ & -0.808250 & 8.508482 & -2.029897 \\
\hline & 0.984027 & 9.837413 & -0.920897 \\
\hline & 3.122046 & 8.720751 & -0.299697 \\
\hline & 3.455788 & 6.343256 & -0.753297 \\
\hline $\mathrm{H}$ & 2.614620 & 4.495342 & -4.417597 \\
\hline $\mathrm{H}$ & 1.488134 & 3.729222 & -6.489397 \\
\hline
\end{tabular}

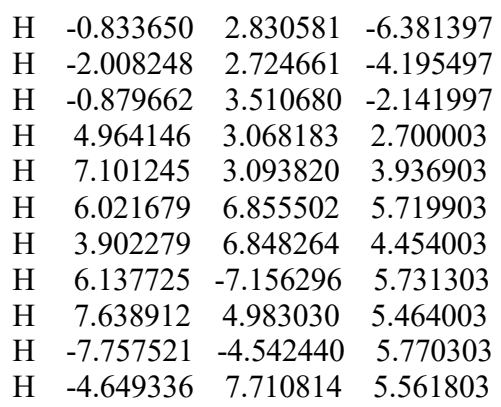

\section{L5}

$\begin{array}{llll}\mathrm{Rh} & -0.042803 & -0.134999 & -1.725803\end{array}$ $\begin{array}{llll}\mathrm{Rh} & -0.032502 & -0.057600 & 0.662897\end{array}$ $\begin{array}{lllll}\text { O } & -1.117236 & -1.798378 & 0.646897\end{array}$

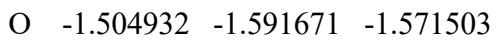

$\begin{array}{lllll}\text { O } & 1.723678 & -1.123934 & 0.619497\end{array}$

$\begin{array}{llll}\text { O } & 1.431869 & -1.581428 & -1.575503\end{array}$

$\begin{array}{llll}\text { O } & 1.038632 & 1.687979 & 0.529297\end{array}$

$\begin{array}{llll}\mathrm{O} & 1.412526 & 1.337072 & -1.673103\end{array}$

$\begin{array}{llll}\mathrm{O} & -1.778381 & 1.013635 & 0.554097\end{array}$

$\begin{array}{llll}\text { O } & -1.508175 & 1.326529 & -1.669003\end{array}$

$\begin{array}{llll}\mathrm{Cl} & 0.037004 & -4.857001 & 0.837397\end{array}$

$\begin{array}{lllll}\mathrm{Cl} & 4.601007 & 0.387810 & 0.351997\end{array}$

$\begin{array}{llll}\mathrm{Cl} & -0.274409 & 4.672205 & 0.403897\end{array}$

$\begin{array}{llll}\mathrm{Cl} & -4.828805 & -0.206606 & 0.629897\end{array}$

$\begin{array}{llll}\text { C } & -2.454768 & -3.435252 & -0.388603\end{array}$

$\begin{array}{llll}\text { C } & -2.504687 & -4.429751 & -1.600203\end{array}$

$\begin{array}{llll}\text { C } & -3.652169 & -3.510129 & -1.316603\end{array}$

C $\quad-1.626643 \quad-2.187268 \quad-0.454603$

$\begin{array}{llll}\text { C } & -2.570378 & -3.985850 & 1.001097\end{array}$

$\begin{array}{llll}\text { C } & -1.514691 & -4.649771 & 1.641797\end{array}$

$\begin{array}{llll}\text { C } & -1.646701 & -5.135468 & 2.940297\end{array}$

$\begin{array}{llll}\text { C } & -2.836897 & -4.935745 & 3.635497\end{array}$

C $\quad-3.905584-4.246624 \quad 3.043497$

$\begin{array}{llll}\text { C } & -3.746375 & -3.795027 & 1.726297\end{array}$

$\begin{array}{llll}\text { C } & -2.615116 & -5.895749 & -1.244803\end{array}$

$\begin{array}{llll}\text { C } & -1.561733 & -6.766270 & -1.563003\end{array}$

C $\quad-1.613559 \quad-8.116069-1.218903$

$\begin{array}{llll}\text { C } & -2.725069 & -8.630247 & -0.549603\end{array}$

$\begin{array}{llll}\text { C } & -3.784453 & -7.778426 & -0.235403\end{array}$

C $\quad-3.730326 \quad-6.428527 \quad-0.582203$

$\begin{array}{llll}\text { C } & -1.699482 & -4.148067 & -2.841803\end{array}$

$\begin{array}{llll}\text { C } & -2.351382 & -4.156254 & -4.080703\end{array}$

$\begin{array}{llll}\text { C } & -1.646477 & -3.901768 & -5.258703\end{array}$

$\begin{array}{llll}\text { C } & -0.276272 & -3.634295 & -5.207203\end{array}$

C $\quad 0.382528 \quad-3.638708 \quad-3.975803$

$\begin{array}{llll}\text { C } & -0.324077 & -3.904894 & -2.804103\end{array}$

$\begin{array}{llll}\text { C } & -5.162478 & -3.980299 & 3.781097\end{array}$

$\begin{array}{llll}\text { C } & -5.673597 & -4.909489 & 4.701597\end{array}$

$\begin{array}{llll}\text { C } & -6.855391 & -4.650066 & 5.394697\end{array}$

C $\quad-7.550168 \quad-3.457753 \quad 5.179597$

C $\quad-7.052850 \quad-2.526262 \quad 4.265197$

$\begin{array}{llll}\text { C } & -5.870055 & -2.783385 & 3.574197\end{array}$

$\begin{array}{llll}\text { C } & 3.402253 & -2.359167 & -0.478103\end{array}$

C $\quad 4.278851 \quad-2.479284 \quad-1.774103$

C $\quad 3.465328 \quad-3.639368 \quad-1.292403$

$\begin{array}{llll}\text { C } & 2.093268 & -1.629941 & -0.490403\end{array}$

$\begin{array}{llll}\text { C } & 4.125355 & -2.283781 & 0.833797\end{array}$

$\begin{array}{llll}\text { C } & 4.715878 & -1.096092 & 1.291997\end{array}$

C $\quad 5.397079 \quad-1.042106 \quad 2.504797$
C $\quad 5.495157 \quad-2.186508 \quad 3.291597$

$\begin{array}{llll}\text { C } & 4.902033 & -3.389496 & 2.882197\end{array}$

C $\quad 4.221133 \quad-3.406283 \quad 1.656797$

C $\quad 5.774151-2.471513 \quad-1.540503$

$\begin{array}{llll}\text { C } & 6.518873 & -1.335328 & -1.890803\end{array}$

C $\quad 7.890075-1.271955-1.649403$

C $\quad 8.551354-2.349867 \quad-1.058003$

C $\quad 7.825831 \quad-3.492453 \quad-0.720103$

C $\quad 6.453030 \quad-3.552326 \quad-0.962103$

C $3.861564-1.819476 \quad-3.062803$

$\begin{array}{llll}\text { C } & 3.853549 & -2.585376 & -4.234503\end{array}$

$\begin{array}{llll}\text { C } & 3.489660 & -2.014468 & -5.456003\end{array}$

$\begin{array}{llll}\text { C } & 3.128286 & -0.666461 & -5.516503\end{array}$

$\begin{array}{llll}\text { C } & 3.148202 & 0.107438 & -4.353503\end{array}$

$\begin{array}{llll}\text { C } & 3.524190 & -0.465669 & -3.139503\end{array}$

$\begin{array}{llll}\text { C } & 4.991509 & -4.613398 & 3.711897\end{array}$

$\begin{array}{llll}\text { C } & 5.123985 & -5.877400 & 3.113097\end{array}$

$\begin{array}{llll}\text { C } & 5.204862 & -7.030602 & 3.891997\end{array}$

$\begin{array}{llll}\text { C } & 5.157864 & -6.942601 & 5.285097\end{array}$

$\begin{array}{llll}\text { C } & 5.028888 & -5.691599 & 5.892497\end{array}$

C $\quad 4.945711 \quad-4.538297 \quad 5.113797$

$\begin{array}{llll}\text { C } & 2.315564 & 3.286355 & -0.635603\end{array}$

$\begin{array}{llll}\text { C } & 2.374081 & 4.168953 & -1.930903\end{array}$

C $\quad 3.531964 \quad 3.303031 \quad-1.544103$

$\begin{array}{llll}\text { C } & 1.525539 & 2.013470 & -0.602003\end{array}$

$\begin{array}{llll}\text { C } & 2.378977 & 3.984753 & 0.690197\end{array}$

$\begin{array}{llll}\text { C } & 1.272990 & 4.646575 & 1.243997\end{array}$

$\begin{array}{llll}\text { C } & 1.350903 & 5.283473 & 2.479597\end{array}$

$\begin{array}{llll}\text { C } & 2.544302 & 5.255450 & 3.196397\end{array}$

$\begin{array}{llll}\text { C } & 3.666289 & 4.581528 & 2.692597\end{array}$

$\begin{array}{llll}\text { C } & 3.553777 & 3.957730 & 1.442397\end{array}$

$\begin{array}{llll}\text { C } & 2.446710 & 5.661852 & -1.696003\end{array}$

$\begin{array}{llll}\text { C } & 1.345326 & 6.465973 & -2.025603\end{array}$

$\begin{array}{llll}\text { C } & 1.358653 & 7.838673 & -1.784203\end{array}$

$\begin{array}{llll}\text { C } & 2.479565 & 8.441951 & -1.211503\end{array}$

$\begin{array}{llll}\text { C } & 3.587149 & 7.656530 & -0.891103\end{array}$

$\begin{array}{llll}\text { C } & 3.570722 & 6.282930 & -1.134003\end{array}$

C $\quad 1.603873 \quad 3.777568 \quad-3.165103$

$\begin{array}{llll}\text { C } & 2.280173 & 3.733955 & -4.390003\end{array}$

$\begin{array}{llll}\text { C } & 1.605566 & 3.393968 & -5.564303\end{array}$

$\begin{array}{llll}\text { C } & 0.242860 & 3.088595 & -5.523003\end{array}$

$\begin{array}{llll}\text { C } & -0.440139 & 3.140408 & -4.305803\end{array}$

$\begin{array}{llll}\text { C } & 0.235868 & 3.494395 & -3.138903\end{array}$

$\begin{array}{llll}\text { C } & 4.935688 & 4.517103 & 3.453297\end{array}$

$\begin{array}{llll}\text { C } & 6.173388 & 4.543379 & 2.788897\end{array}$

C $\quad 7.368187 \quad 4.470856 \quad 3.503297$

C $\quad 7.349485 \quad 4.373656 \quad 4.896397$

$\begin{array}{llll}\text { C } & 6.125685 & 4.349280 & 5.568897\end{array}$

$\begin{array}{llll}\text { C } & 4.930586 & 4.419203 & 4.854597\end{array}$

$\begin{array}{llll}\text { C } & -3.402456 & 2.286666 & -0.576903\end{array}$

$\begin{array}{llll}\text { C } & -4.349255 & 2.304485 & -1.826403\end{array}$

$\begin{array}{llll}\text { C } & -3.454733 & 3.467867 & -1.527603\end{array}$

$\begin{array}{llll}\text { C } & -2.140172 & 1.477642 & -0.576203\end{array}$

$\begin{array}{llll}\text { C } & -4.009753 & 2.416378 & 0.788097\end{array}$

$\begin{array}{llll}\text { C } & -4.677474 & 1.358591 & 1.421197\end{array}$

$\begin{array}{llll}\text { C } & -5.210571 & 1.498402 & 2.699997\end{array}$

$\begin{array}{llll}\text { C } & -5.058148 & 2.702499 & 3.383297\end{array}$

$\begin{array}{llll}\text { C } & -4.370327 & 3.776085 & 2.798597\end{array}$

$\begin{array}{llll}\text { C } & -3.868130 & 3.606475 & 1.501197\end{array}$

C $\quad-5.828654 \quad 2.401214 \quad-1.525603$

$\begin{array}{llll}\text { C } & -6.670075 & 1.324130 & -1.842703\end{array}$

$\begin{array}{llll}\text { C } & -8.031374 & 1.361757 & -1.545203\end{array}$ 


\begin{tabular}{|c|c|c|c|}
\hline $\mathrm{C}$ & -8.586052 & 2.482768 & -0.925803 \\
\hline $\mathrm{C}$ & -7.763331 & 3.565552 & -0.614403 \\
\hline $\mathrm{C}$ & -6.401632 & 3.525325 & -0.914103 \\
\hline $\mathrm{C}$ & -4.016671 & 1.487078 & -3.047503 \\
\hline & -4.008159 & 2.121878 & -4.295203 \\
\hline $\mathrm{C}$ & -3.714073 & 1.405772 & -5.457103 \\
\hline $\mathrm{C}$ & -3.422300 & 0.041667 & -5.380603 \\
\hline $\mathrm{C}$ & -3.441512 & -0.600633 & -4.140403 \\
\hline $\mathrm{C}$ & -3.748198 & 0.117273 & -2.985103 \\
\hline c & -4.156102 & 5.049481 & 3.524597 \\
\hline $\mathrm{C}$ & -2.967687 & 5.779258 & 3.348097 \\
\hline $\mathrm{C}$ & -2.759964 & 6.977854 & 4.028297 \\
\hline $\mathrm{C}$ & -3.733754 & 7.469373 & 4.900897 \\
\hline $\mathrm{C}$ & -4.918168 & 6.752696 & 5.085497 \\
\hline c & -5.127892 & 5.554900 & 4.403297 \\
\hline $\mathrm{H}$ & -4.577877 & -3.903911 & -0.916203 \\
\hline $\mathrm{H}$ & -3.776053 & -2.674826 & -1.993703 \\
\hline $\mathrm{H}$ & -0.814711 & -5.647184 & 3.410397 \\
\hline $\mathrm{H}$ & -2.917404 & -5.288843 & 4.657997 \\
\hline $\mathrm{H}$ & -4.568265 & -3.281511 & 1.241197 \\
\hline $\mathrm{H}$ & -0.692725 & -6.382287 & 33403 \\
\hline $\mathrm{H}$ & -0.781672 & -8.765385 & -1.475103 \\
\hline $\mathrm{H}$ & -2.767090 & -9.681046 & -0.280203 \\
\hline $\mathrm{H}$ & -4.660060 & -8.161909 & 0.279497 \\
\hline $\mathrm{H}$ & -4.568314 & -5.795411 & -0.318703 \\
\hline $\mathrm{H}$ & -3.419486 & -4.351533 & 17603 \\
\hline $\mathrm{H}$ & -2.168377 & -3.904258 & -6.211103 \\
\hline $\mathrm{H}$ & 0.276133 & -3.421106 & -6.117703 \\
\hline $\mathrm{H}$ & 1.440433 & -3.408728 & -3.926903 \\
\hline $\mathrm{H}$ & 0.185224 & -3.874204 & -1.849403 \\
\hline $\mathrm{H}$ & -5.155015 & -5.850399 & 4.857297 \\
\hline $\mathrm{H}$ & -7.238306 & -5.384259 & 6.097097 \\
\hline $\mathrm{H}$ & -7.582732 & -1.593752 & 4.094297 \\
\hline $\mathrm{H}$ & -5.480040 & -2.042493 & 2.883797 \\
\hline $\mathrm{H}$ & 3.948111 & -4.499877 & -0.847603 \\
\hline $\mathrm{H}$ & 2.575624 & -3.872151 & -1.864203 \\
\hline $\mathrm{H}$ & 5.854197 & -0.112415 & 2.824097 \\
\hline $\mathrm{H}$ & 6.053658 & -2.144919 & 4.220597 \\
\hline $\mathrm{H}$ & 3.728915 & -4.318773 & 1.339197 \\
\hline $\mathrm{H}$ & 6.018990 & -0.493418 & -2.354203 \\
\hline $\mathrm{H}$ & 8.441192 & -0.377765 & -1.924703 \\
\hline $\mathrm{H}$ & 9.619254 & -2.301988 & -0.868403 \\
\hline $\mathrm{H}$ & 8.325815 & -4.343763 & -0.268003 \\
\hline $\mathrm{H}$ & 5.918512 & -4.453816 & -0.689003 \\
\hline $\mathrm{H}$ & 4.123328 & -3.636681 & -4.184503 \\
\hline $\mathrm{H}$ & 3.481448 & -2.623868 & -6.354903 \\
\hline $\mathrm{H}$ & 2.833995 & -0.220956 & -6.462303 \\
\hline $\mathrm{H}$ & 2.857222 & 1.151744 & -4.386003 \\
\hline $\mathrm{H}$ & 3.504602 & 0.129831 & -2.237503 \\
\hline $\mathrm{H}$ & 5.188083 & -5.953102 & 2.032097 \\
\hline $\mathrm{H}$ & 5.313743 & -7.997804 & 3.410597 \\
\hline $\mathrm{H}$ & 4.983690 & -5.613598 & 6.974597 \\
\hline $\mathrm{H}$ & 4.819430 & -3.572994 & 5.593897 \\
\hline $\mathrm{H}$ & 4.442973 & 3.746113 & -1.162603 \\
\hline $\mathrm{H}$ & 3.682347 & 2.413328 & -2.143003 \\
\hline $\mathrm{H}$ & 0.484813 & 5.801090 & 2.875697 \\
\hline $\mathrm{H}$ & 2.603612 & 5.775349 & 4.146497 \\
\hline $\mathrm{H}$ & 4.399266 & 3.403714 & 1.051297 \\
\hline $\mathrm{H}$ & 0.469717 & 6.011591 & -2.473303 \\
\hline $\mathrm{H}$ & 0.490064 & 8.436090 & -2.044603 \\
\hline $\mathrm{H}$ & 2.491586 & 9.510951 & -1.022003 \\
\hline 1 & 4.470658 & 8.110312 & -0.452303 \\
\hline
\end{tabular}
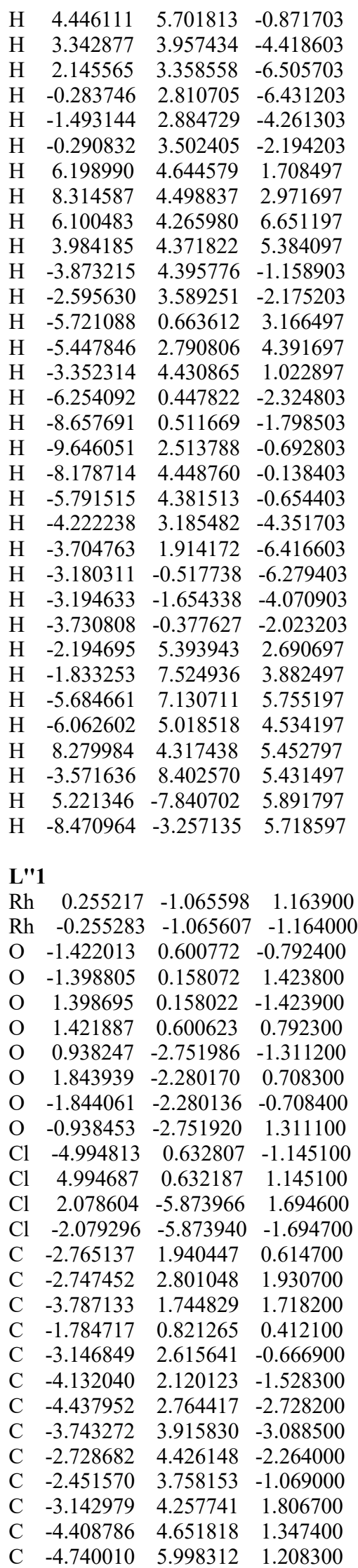

$\begin{array}{llll}\text { C } & -3.811728 & 6.988129 & 1.533300\end{array}$

$\begin{array}{llll}\text { C } & -2.551521 & 6.612351 & 1.999100\end{array}$

$\begin{array}{llll}\text { C } & -2.219897 & 5.263457 & 2.134100\end{array}$

$\begin{array}{llll}\text { C } & -1.705848 & 2.551066 & 2.989100\end{array}$

$\begin{array}{llll}\text { C } & -0.341747 & 2.479891 & 2.701400\end{array}$

C $\quad 0.586557 \quad 2.250408 \quad 3.716800$

C $\quad 0.161760 \quad 2.126400 \quad 5.041200$

C $\quad-1.198342 \quad 2.233276 \quad 5.344200$

$\begin{array}{llll}\text { C } & -2.124346 & 2.441859 & 4.321500\end{array}$

$\begin{array}{llll}\text { C } & -1.956503 & 5.632462 & -2.635500\end{array}$

$\begin{array}{llll}\text { C } & -1.530507 & 5.836370 & -3.957500\end{array}$

$\begin{array}{llll}\text { C } & -0.815828 & 6.982982 & -4.302800\end{array}$

$\begin{array}{llll}\text { C } & -0.520445 & 7.945288 & -3.334200\end{array}$

$\begin{array}{llll}\text { C } & -0.927141 & 7.743480 & -2.012500\end{array}$

$\begin{array}{llll}\text { C } & -1.635221 & 6.594868 & -1.663900\end{array}$

$\begin{array}{llll}\text { C } & 2.765263 & 1.940247 & -0.614700\end{array}$

$\begin{array}{llll}\text { C } & 2.747648 & 2.800947 & -1.930700\end{array}$

$\begin{array}{llll}\text { C } & 3.787267 & 1.744565 & -1.718200\end{array}$

$\begin{array}{llll}\text { C } & 1.784683 & 0.821229 & -0.412200\end{array}$

$\begin{array}{llll}\text { C } & 3.147051 & 2.615354 & 0.666900\end{array}$

$\begin{array}{llll}\text { C } & 4.132060 & 2.119671 & 1.528300\end{array}$

$\begin{array}{llll}\text { C } & 4.438048 & 2.763777 & 2.728300\end{array}$

$\begin{array}{llll}\text { C } & 3.743528 & 3.915264 & 3.088600\end{array}$

$\begin{array}{llll}\text { C } & 2.729018 & 4.425846 & 2.264000\end{array}$

$\begin{array}{llll}\text { C } & 2.451830 & 3.757941 & 1.069000\end{array}$

$\begin{array}{llll}\text { C } & 3.143421 & 4.257554 & -1.806600\end{array}$

$\begin{array}{llll}\text { C } & 2.220503 & 5.263437 & -2.134200\end{array}$

$\begin{array}{llll}\text { C } & 2.552279 & 6.612243 & -1.999300\end{array}$

C $\quad 3.812572 \quad 6.987766 \quad-1.533300$

$\begin{array}{llll}\text { C } & 4.740490 & 5.997882 & -1.208100\end{array}$

C $\quad 4.409114 \quad 4.651376 \quad-1.347100$

$\begin{array}{llll}\text { C } & 1.706052 & 2.551028 & -2.989100\end{array}$

$\begin{array}{llll}\text { C } & 2.124554 & 2.441735 & -4.321400\end{array}$

$\begin{array}{llll}\text { C } & 1.198458 & 2.233319 & -5.344100\end{array}$

$\begin{array}{llll}\text { C } & -0.161640 & 2.126394 & -5.041200\end{array}$

$\begin{array}{llll}\text { C } & -0.586443 & 2.250587 & -3.716800\end{array}$

$\begin{array}{llll}\text { C } & 0.341853 & 2.480103 & -2.701400\end{array}$

$\begin{array}{llll}\text { C } & 1.957097 & 5.632332 & 2.635600\end{array}$

C $\quad 1.530993 \quad 5.836225 \quad 3.957500$

C $\quad 0.816472 \quad 6.982912 \quad 4.302800$

$\begin{array}{llll}\text { C } & 0.521455 & 7.945307 & 3.334200\end{array}$

$\begin{array}{llll}\text { C } & 0.928159 & 7.743514 & 2.012600\end{array}$

$\begin{array}{llll}\text { C } & 1.636079 & 6.594827 & 1.664000\end{array}$

C $\quad 2.895669-3.925251 \quad-0.605200$

$\begin{array}{llll}\text { C } & 3.504872 & -4.130940 & -2.042700\end{array}$

$\begin{array}{llll}\text { C } & 2.561790 & -5.132957 & -1.456700\end{array}$

$\begin{array}{llll}\text { C } & 1.810450 & -2.913770 & -0.400800\end{array}$

$\begin{array}{llll}\text { C } & 3.798171 & -4.037135 & 0.585300\end{array}$

$\begin{array}{llll}\text { C } & 3.531686 & -4.874939 & 1.672400\end{array}$

$\begin{array}{llll}\text { C } & 4.368986 & -4.908124 & 2.788200\end{array}$

$\begin{array}{llll}\text { C } & 5.481471 & -4.074004 & 2.837800\end{array}$

$\begin{array}{llll}\text { C } & 5.763055 & -3.189699 & 1.783300\end{array}$

$\begin{array}{llll}\text { C } & 4.907955 & -3.193315 & 0.677700\end{array}$

C $4.985278-4.436813 \quad-2.103200$

$\begin{array}{llll}\text { C } & 5.860062 & -3.538798 & -2.734000\end{array}$

$\begin{array}{llll}\text { C } & 7.237366 & -3.761473 & -2.745700\end{array}$

$\begin{array}{llll}\text { C } & 7.773586 & -4.893363 & -2.130400\end{array}$

$\begin{array}{llll}\text { C } & 6.913702 & -5.802579 & -1.512700\end{array}$

$\begin{array}{llll}\text { C } & 5.538098 & -5.577203 & -1.502000\end{array}$

$\begin{array}{llll}\text { C } & 2.995457 & -3.286849 & -3.179800\end{array}$

C $\quad 2.486668-3.912358-4.323000$

$\begin{array}{llll}\text { C } & 2.020155 & -3.155167 & -5.399000\end{array}$ 


\begin{tabular}{|c|c|c|c|}
\hline & 2.058830 & -1.759766 & -5.339900 \\
\hline $\mathrm{C}$ & 2.573318 & -1.129757 & -4.204900 \\
\hline $\mathrm{C}$ & 3.045032 & -1.891048 & -3.137700 \\
\hline $\mathrm{C}$ & 6.916139 & -2.261579 & 1.827200 \\
\hline & 7.308628 & -1.655971 & 3.031700 \\
\hline $\mathrm{C}$ & 8.373412 & -0.756852 & 3.062700 \\
\hline $\mathrm{C}$ & 9.065406 & -0.446040 & 1.889800 \\
\hline C & 8.687217 & -1.046347 & 0.686900 \\
\hline & 7.624033 & -1.947366 & 0.654600 \\
\hline & -2.896031 & -3.924955 & 0.605100 \\
\hline $\mathrm{C}$ & -3.505128 & -4.130666 & 2.042700 \\
\hline $\mathrm{C}$ & -2.562210 & -5.132749 & 1.456600 \\
\hline $\mathrm{C}$ & -1.810650 & -2.913735 & 0.400700 \\
\hline & -3.798529 & -4.036771 & -0.585300 \\
\hline & -3.532214 & -4.874666 & -1.672400 \\
\hline $\mathrm{C}$ & -4.369614 & -4.907781 & -2.788200 \\
\hline $\mathrm{C}$ & -5.482029 & -4.073501 & -2.837700 \\
\hline $\mathrm{C}$ & -5.763345 & -3.189106 & -1.783200 \\
\hline $\mathrm{C}$ & -4.908245 & -3.192891 & -0.677700 \\
\hline & -4.985522 & -4.436393 & 2.103200 \\
\hline $\mathrm{C}$ & -5.538502 & -5.576802 & 1.502000 \\
\hline $\mathrm{C}$ & -6.914198 & -5.802027 & 1.512700 \\
\hline $\mathrm{C}$ & -7.773914 & -4.892743 & 2.130400 \\
\hline $\mathrm{C}$ & -7.237634 & -3.760833 & 2.745600 \\
\hline C & -5.860338 & -3.538308 & 2.734000 \\
\hline $\mathrm{C}$ & -2.995643 & -3.286657 & 3.179700 \\
\hline $\mathrm{C}$ & -3.045068 & -1.890858 & 3.137600 \\
\hline $\mathrm{C}$ & -2.573282 & -1.129649 & 4.204900 \\
\hline $\mathrm{C}$ & -2.058870 & -1.759740 & 5.339900 \\
\hline $\mathrm{C}$ & -2.020345 & -3.155139 & 5.399000 \\
\hline $\mathrm{C}$ & -2.486932 & -3.912248 & 4.322900 \\
\hline $\mathrm{C}$ & -6.916361 & -2.260927 & -1.827100 \\
\hline $\mathrm{C}$ & -7.624167 & -1.946540 & -0.654400 \\
\hline $\mathrm{C}$ & -8.687283 & -1.045459 & -0.686700 \\
\hline $\mathrm{C}$ & -9.065494 & -0.445066 & -1.889600 \\
\hline $\mathrm{C}$ & -8.373588 & -0.756053 & -3.062600 \\
\hline $\mathrm{C}$ & -7.308972 & -1.655334 & -3.031600 \\
\hline $\mathrm{H}$ & -4.810138 & 2.025811 & 1.503800 \\
\hline $\mathrm{H}$ & -3.682617 & 0.836631 & 2.297300 \\
\hline $\mathrm{H}$ & -5.214845 & 2.364503 & -3.369800 \\
\hline $\mathrm{H}$ & -3.995782 & 4.423725 & -4.013400 \\
\hline $\mathrm{H}$ & -1.662276 & 4.129167 & -0.426600 \\
\hline $\mathrm{H}$ & -5.148472 & 3.910305 & 1.071600 \\
\hline $\mathrm{H}$ & -5.726415 & 6.270694 & 0.845500 \\
\hline $\mathrm{H}$ & -4.066847 & 8.037724 & 1.425300 \\
\hline $\mathrm{H}$ & -1.815134 & 7.363764 & 2.257700 \\
\hline $\mathrm{H}$ & -1.234092 & 4.996675 & 2.497600 \\
\hline $\mathrm{H}$ & 0.003352 & 2.562197 & 1.678700 \\
\hline $\mathrm{H}$ & 1.637259 & 2.168627 & 3.467000 \\
\hline $\mathrm{H}$ & 0.885163 & 1.951013 & 5.831900 \\
\hline $\mathrm{H}$ & -1.538641 & 2.146269 & 6.371700 \\
\hline $\mathrm{H}$ & -3.183647 & 2.513340 & 4.552200 \\
\hline $\mathrm{H}$ & -1.737193 & 5.078966 & -4.706900 \\
\hline $\mathrm{H}$ & -0.486230 & 7.123688 & -5.327700 \\
\hline $\mathrm{H}$ & -0.698955 & 8.484485 & -1.252200 \\
\hline $\mathrm{H}$ & -1.968918 & 6.456262 & -0.641100 \\
\hline $\mathrm{H}$ & 4.810262 & 2.025484 & -1.503800 \\
\hline $\mathrm{H}$ & 3.682683 & 0.836363 & -2.297300 \\
\hline $\mathrm{H}$ & 5.214855 & 2.363791 & 3.369900 \\
\hline $\mathrm{H}$ & 3.996018 & 4.423169 & 4.013500 \\
\hline $\mathrm{H}$ & 1.662724 & 4.129027 & 0.426600 \\
\hline $\mathrm{H}$ & 1.234708 & 4.996819 & -2.497900 \\
\hline
\end{tabular}

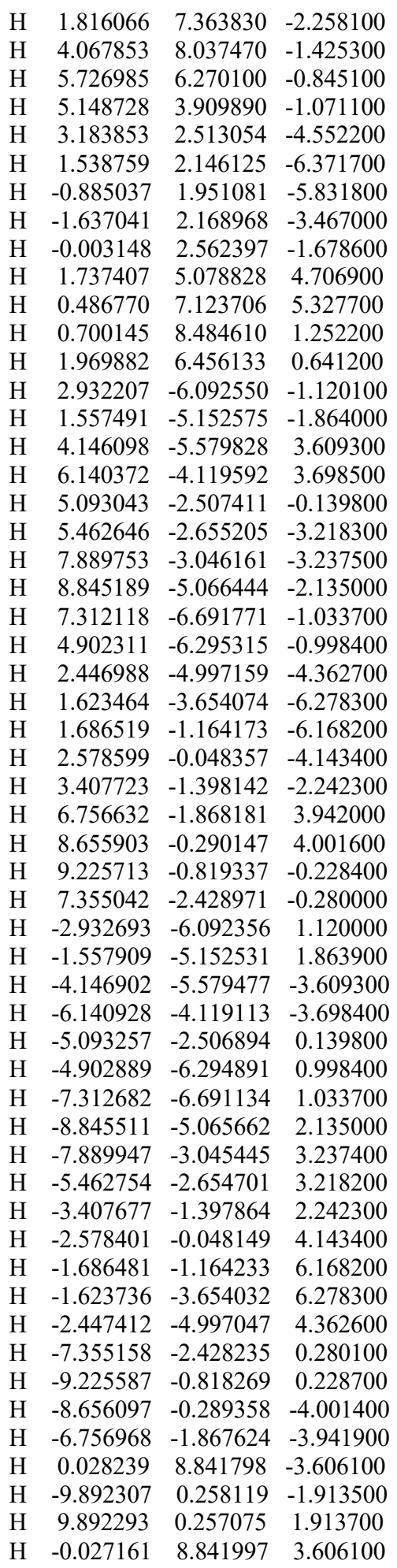

\section{L"2}

$\begin{array}{llll}\text { Rh } & 0.279301 & -0.104100 & 1.252605\end{array}$ $\begin{array}{llll}\mathrm{Rh} & 0.033001 & -0.772600 & -1.017595\end{array}$ O $\quad 1.866001 \quad 0.148300 \quad-1.338495$ $\begin{array}{llll}\mathrm{O} & 1.673501 & 1.265200 & 0.618705\end{array}$ O $\quad 0.983401 \quad-2.517800 \quad-0.444395$ $\begin{array}{llll}\text { O } & 1.861501 & -1.467800 & 1.349105\end{array}$

$\begin{array}{llll}\text { O } & -1.748999 & -1.629900 & -0.453395\end{array}$

$\begin{array}{llll}\mathrm{O} & -1.114799 & -1.558400 & 1.717705\end{array}$

$\begin{array}{llll}\text { O } & -0.890799 & 1.031400 & -1.349495\end{array}$

$\begin{array}{llll}\text { O } & -1.255099 & 1.246400 & 0.872705\end{array}$

$\begin{array}{llll}\mathrm{Cl} & 2.326402 & 4.592600 & -0.528395\end{array}$

$\mathrm{Cl} \quad 3.200401 \quad-4.263000 \quad 3.383705$

Cl $\quad-2.181799 \quad-5.149700 \quad-0.568295$

$\begin{array}{lllll}\mathrm{Cl} & -0.927698 & 4.750700 & 1.049305\end{array}$

$\begin{array}{llll}\text { C } & 3.548501 & 1.727000 & -0.732095\end{array}$

$\begin{array}{llll}\text { C } & 4.008401 & 2.082900 & -2.180795\end{array}$

$\begin{array}{llll}\text { C } & 4.589901 & 0.902200 & -1.469095\end{array}$

$\begin{array}{llll}\text { C } & 2.257001 & 1.006800 & -0.486595\end{array}$

C $3.961802 \quad 2.594400 \quad 0.418405$

C $3.498302 \quad 3.905400 \quad 0.587605$

C $3.912802 \quad 4.693300 \quad 1.659305$

C $\quad 4.764702 \quad 4.153700 \quad 2.618505$

$\begin{array}{llll}\text { C } & 5.188102 & 2.820600 & 2.525505\end{array}$

$\begin{array}{llll}\text { C } & 4.790201 & 2.069300 & 1.412905\end{array}$

C $\quad 4.880002 \quad 3.310000 \quad-2.342995$

$\begin{array}{llll}\text { C } & 4.482702 & 4.341300 & -3.206395\end{array}$

C $\quad 5.262702 \quad 5.485800 \quad-3.369395$

$\begin{array}{llll}\text { C } & 6.463302 & 5.625700 & -2.672295\end{array}$

$\begin{array}{llll}\text { C } & 6.874302 & 4.604700 & -1.814295\end{array}$

C $\quad 6.094202 \quad 3.460400 \quad-1.654195$

$\begin{array}{llll}\text { C } & 3.071401 & 1.814800 & -3.329595\end{array}$

$\begin{array}{llll}\text { C } & 1.760502 & 2.303000 & -3.350695\end{array}$

$\begin{array}{llll}\text { C } & 0.927501 & 2.051600 & -4.440095\end{array}$

$\begin{array}{llll}\text { C } & 1.400701 & 1.320400 & -5.531995\end{array}$

$\begin{array}{llll}\text { C } & 2.714101 & 0.844000 & -5.526695\end{array}$

$\begin{array}{llll}\text { C } & 3.541001 & 1.088100 & -4.428795\end{array}$

$\begin{array}{llll}\text { C } & 6.016502 & 2.208500 & 3.589205\end{array}$

$\begin{array}{llll}C & 5.679501 & 0.950500 & 4.111305\end{array}$

$\begin{array}{llll}\text { C } & 6.461801 & 0.362600 & 5.104805\end{array}$

$\begin{array}{llll}\text { C } & 7.599001 & 1.017300 & 5.582605\end{array}$

C $\quad 7.942202 \quad 2.271100 \quad 5.069305$

C $7.152602 \quad 2.865700 \quad 4.085705$

$\begin{array}{llll}\text { C } & 3.069001 & -3.280500 & 0.391905\end{array}$

$\begin{array}{llll}\text { C } & 3.406001 & -4.032600 & -0.963195\end{array}$

$\begin{array}{llll}\text { C } & 2.754101 & -4.749900 & 0.174905\end{array}$

$\begin{array}{llll}\text { C } & 1.888401 & -2.359500 & 0.435405\end{array}$

$\begin{array}{llll}\text { C } & 4.219801 & -2.854100 & 1.253305\end{array}$

$\begin{array}{llll}\text { C } & 4.405901 & -3.263700 & 2.576805\end{array}$

$\begin{array}{llll}\text { C } & 5.535201 & -2.882100 & 3.304305\end{array}$

$\begin{array}{llll}\text { C } & 6.491001 & -2.061300 & 2.716005\end{array}$

C $\quad 6.320601 \quad-1.586100 \quad 1.405505$

$\begin{array}{llll}\text { C } & 5.177701 & -1.991700 & 0.708705\end{array}$

$\begin{array}{llll}\text { C } & 4.876101 & -4.273600 & -1.240495\end{array}$

$\begin{array}{llll}\text { C } & 5.483001 & -3.708500 & -2.372995\end{array}$

$\begin{array}{llll}\text { C } & 6.848601 & -3.863300 & -2.615195\end{array}$

C $\quad 7.643901-4.592300 \quad-1.731095$

$\begin{array}{llll}\text { C } & 7.051701 & -5.174500 & -0.609695\end{array}$

$\begin{array}{llll}\text { C } & 5.687101 & -5.020200 & -0.371895\end{array}$

C $\quad 2.569801 \quad-3.779500 \quad-2.192795$

C $\quad 2.524101 \quad-2.524800 \quad-2.811095$

$\begin{array}{llll}\text { C } & 1.724601 & -2.306800 & -3.931295\end{array}$

$\begin{array}{llll}\text { C } & 0.966901 & -3.354700 & -4.463195\end{array}$

C $\quad 1.027201 \quad-4.618100 \quad-3.871695$

$\begin{array}{llll}\text { C } & 1.827301 & -4.827100 & -2.745795\end{array}$

$\begin{array}{llll}\text { C } & 7.296601 & -0.668700 & 0.770405\end{array}$

$\begin{array}{llll}\text { C } & 7.538401 & -0.725800 & -0.613395\end{array}$

$\begin{array}{llll}\text { C } & 8.382501 & 0.198600 & -1.227195\end{array}$ 


\begin{tabular}{|c|c|c|c|}
\hline E & 9.005301 & 1.194899 & -0.470495 \\
\hline $\mathrm{C}$ & 8.796401 & 1.241100 & 0.909605 \\
\hline & 7.958301 & 0.313100 & 1.526005 \\
\hline & -3.119499 & -2.713400 & 1.126805 \\
\hline & -3.861299 & -2.478399 & 2.491505 \\
\hline & -2.968299 & -3.664200 & 2.299005 \\
\hline $\mathrm{C}$ & -1.905999 & -1.902500 & 0.784205 \\
\hline $\mathrm{C}$ & -3.923299 & -3.081299 & -0.082195 \\
\hline $\mathrm{C}$ & -3.593899 & -4.155000 & -0.916695 \\
\hline & -4.337499 & -4.444599 & -2.060895 \\
\hline $\mathrm{C}$ & -5.424299 & -3.641199 & -2.394695 \\
\hline $\mathrm{C}$ & -5.767699 & -2.536199 & -1.600295 \\
\hline $\mathrm{C}$ & -5.002999 & -2.279199 & -0.459595 \\
\hline $\mathrm{C}$ & -5.362199 & -2.681799 & 2.504405 \\
\hline & -5.951899 & -3.903299 & 2.143105 \\
\hline $\mathrm{C}$ & -7.336499 & -4.058999 & 2.121905 \\
\hline $\mathrm{C}$ & -8.170299 & -2.995099 & 2.469105 \\
\hline $\mathrm{C}$ & -7.599899 & -1.777399 & 2.839805 \\
\hline $\mathrm{C}$ & -6.212899 & -1.622399 & 2.857005 \\
\hline C & -3.384599 & -1.380600 & 3.403705 \\
\hline $\mathrm{C}$ & -3.289299 & -0.055800 & 2.971105 \\
\hline $\mathrm{C}$ & -2.870499 & 0.947800 & 3.843105 \\
\hline $\mathrm{C}$ & -2.562899 & 0.637800 & 5.169905 \\
\hline $\mathrm{C}$ & -2.671899 & -0.682200 & 5.616105 \\
\hline $\mathrm{C}$ & -3.079699 & -1.684600 & 4.734905 \\
\hline $\mathrm{C}$ & -6.911099 & 62099 & -1.944695 \\
\hline $\mathrm{C}$ & -7.774099 & -1.193699 & -0.939795 \\
\hline $\mathrm{C}$ & -8.876599 & -0.406199 & -1.265495 \\
\hline $\mathrm{C}$ & -9.131399 & -0.066399 & -2.596395 \\
\hline $\mathrm{C}$ & -8.263899 & -0.505399 & -3.599895 \\
\hline $\mathrm{C}$ & -7.162399 & -1.297699 & -3.276995 \\
\hline $\mathrm{C}$ & -2.479398 & 2.633100 & -0.610995 \\
\hline $\mathrm{C}$ & -3.308198 & 2.613700 & -1.949695 \\
\hline $\mathrm{C}$ & -2.147498 & 3.543400 & -1.780495 \\
\hline $\mathrm{C}$ & -1.473799 & 1.553300 & -0.346495 \\
\hline $\mathrm{C}$ & -3.126898 & 3.165900 & 0.629005 \\
\hline $\mathrm{C}$ & -2.527398 & 4.132300 & 1.444705 \\
\hline $\mathrm{C}$ & -3.155798 & 4.602900 & 2.598105 \\
\hline $\mathrm{C}$ & -4.399998 & 4.093301 & 2.958905 \\
\hline $\mathrm{C}$ & -5.018598 & 3.099601 & 2.184505 \\
\hline $\mathrm{C}$ & -4.361798 & 2.653801 & 1.034605 \\
\hline $\mathrm{C}$ & -4.711698 & 3.182501 & -1.903995 \\
\hline $\mathrm{C}$ & -4.968598 & 4.501401 & -1.497395 \\
\hline $\mathrm{C}$ & -6.268798 & 4.998001 & -1.432095 \\
\hline $\mathrm{C}$ & -7.350598 & 4.187401 & -1.779195 \\
\hline $\mathrm{C}$ & -7.112098 & 2.877001 & -2.192695 \\
\hline $\mathrm{C}$ & -5.809098 & 2.380101 & -2.253595 \\
\hline $\mathrm{C}$ & -3.147099 & 1.454400 & -2.897095 \\
\hline $\mathrm{C}$ & -3.381599 & 0.137100 & -2.494195 \\
\hline $\mathrm{C}$ & -3.221199 & -0.919300 & -3.389295 \\
\hline $\mathrm{C}$ & -2.845599 & -0.665600 & -4.711095 \\
\hline $\mathrm{C}$ & -2.637399 & 0.650900 & -5.130695 \\
\hline $\mathrm{C}$ & -2.790499 & 1.703400 & -4.226695 \\
\hline $\mathrm{C}$ & -6.337598 & 2.541101 & 2.556505 \\
\hline $\mathrm{C}$ & -7.309898 & 2.301401 & 1.571105 \\
\hline $\mathrm{C}$ & -8.570099 & 1.822001 & 1.923405 \\
\hline $\mathrm{C}$ & -8.877699 & 1.565701 & 3.261905 \\
\hline $\mathrm{C}$ & -7.908199 & 1.775101 & 4.246005 \\
\hline $\mathrm{C}$ & -6.647798 & 2.259501 & 3.896505 \\
\hline $\mathrm{H}$ & 5.610801 & 0.937900 & -1.115795 \\
\hline $\mathrm{H}$ & 4.261501 & -0.072400 & -1.804695 \\
\hline $\mathrm{H}$ & 3.549302 & 5.710200 & 1.752105 \\
\hline
\end{tabular}

\begin{tabular}{|c|c|c|c|}
\hline & 5.066202 & 4.758000 & 3.467905 \\
\hline $\mathrm{H}$ & 5.137301 & 1.047500 & 1.308805 \\
\hline $\mathrm{H}$ & 3.552502 & 4.248300 & -3.753695 \\
\hline $\mathrm{H}$ & 4.928302 & 6.268600 & -4.043695 \\
\hline 1 & 7.071902 & 6.516000 & -2.798395 \\
\hline $\mathrm{H}$ & 7.808602 & 4.690400 & -1.267395 \\
\hline & 6.448702 & 2.694200 & -0.976795 \\
\hline & 1.383502 & 2.854500 & -2.497395 \\
\hline & -0.095598 & 2.411400 & -4.431795 \\
\hline & 0.749201 & 1.120700 & -6.377395 \\
\hline & 3.091301 & 0.273800 & -6.370395 \\
\hline $\mathrm{H}$ & 4.558301 & 0.707200 & -4.417895 \\
\hline & 4.802501 & 0.433200 & 3.735505 \\
\hline & 6.184601 & -0.609100 & 5.500605 \\
\hline & 8.828302 & 2.783400 & 5.431505 \\
\hline & 7.430202 & 3.832200 & 3.676005 \\
\hline $\mathrm{H}$ & 3.314501 & -5.471200 & 0.754905 \\
\hline $\mathrm{H}$ & 1.702301 & -4.987100 & 0.062805 \\
\hline & 5.662201 & -3.233300 & 4.321905 \\
\hline & 7.375401 & -1.789600 & 3.280505 \\
\hline $\mathrm{H}$ & 5.022401 & -1.646300 & -0.304795 \\
\hline $\mathrm{H}$ & 4.886301 & -3.141500 & -3.076095 \\
\hline $\mathrm{H}$ & 7.287801 & -3.409400 & -3.498595 \\
\hline & 8.707401 & -4.708700 & -1.914595 \\
\hline & 7.651701 & -5.751600 & 0.087205 \\
\hline 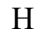 & 5.268701 & -5.473300 & 0.518305 \\
\hline $\mathrm{H}$ & 3.060801 & -1.692500 & -2.374995 \\
\hline $\mathrm{H}$ & 1.689001 & -1.319100 & -4.378195 \\
\hline & 0.339201 & -3.187400 & -5.333595 \\
\hline & 0.450001 & -5.441200 & -4.282395 \\
\hline $\mathrm{H}$ & 1.860101 & -5.808000 & -2.280895 \\
\hline $\mathrm{H}$ & 7.070301 & -1.502800 & -1.208395 \\
\hline $\mathrm{H}$ & 8.551801 & 0.141500 & -2.298095 \\
\hline & 9.273101 & 2.009399 & 1.510605 \\
\hline H & 7.784201 & 0.379200 & 2.592405 \\
\hline $\mathrm{H}$ & -3.398999 & -4.652200 & 2.197805 \\
\hline $\mathrm{H}$ & -2.005799 & -3.636000 & 2.796105 \\
\hline $\mathrm{H}$ & -4.064899 & -5.290599 & -2.681195 \\
\hline $\mathrm{H}$ & -6.012999 & -3.879699 & -3.274195 \\
\hline $\mathrm{H}$ & -5.245899 & -1.422999 & 0.157905 \\
\hline $\mathrm{H}$ & -5.336699 & -4.745499 & 1.850905 \\
\hline $\mathrm{H}$ & -7.761299 & -5.015299 & 1.832105 \\
\hline $\mathrm{H}$ & -9.249299 & -3.113799 & 2.451205 \\
\hline $\mathrm{H}$ & -8.226999 & -0.936699 & 3.112505 \\
\hline $\mathrm{H}$ & -5.795299 & -0.665899 & 3.147605 \\
\hline $\mathrm{H}$ & -3.504299 & 0.192600 & 1.938705 \\
\hline $\mathrm{H}$ & -2.777599 & 1.963700 & 3.480905 \\
\hline $\mathrm{H}$ & -2.239599 & 1.419200 & 5.851305 \\
\hline $\mathrm{H}$ & -2.437399 & -0.931300 & 6.646805 \\
\hline $\mathrm{H}$ & -3.158099 & -2.712600 & 5.077105 \\
\hline $\mathrm{H}$ & -7.599699 & -1.476899 & 0.092905 \\
\hline $\mathrm{H}$ & -9.545399 & -0.070699 & -0.479295 \\
\hline $\mathrm{H}$ & -8.443299 & -0.228199 & -4.634195 \\
\hline $\mathrm{H}$ & -6.479399 & -1.621699 & -4.056095 \\
\hline $\mathrm{H}$ & -2.320798 & 4.604100 & -1.652695 \\
\hline $\mathrm{H}$ & -1.238298 & 3.293400 & -2.313895 \\
\hline $\mathrm{H}$ & -2.672998 & 5.361500 & 3.203405 \\
\hline $\mathrm{H}$ & -4.896398 & 4.476601 & 3.844205 \\
\hline & -4.821399 & 1.882501 & 0.428305 \\
\hline $\mathrm{H}$ & -4.156098 & 5.154301 & -1.203295 \\
\hline $\mathrm{H}$ & -6.433198 & 6.021201 & -1.107895 \\
\hline & -8.364598 & 4.571801 & -1.727495 \\
\hline
\end{tabular}

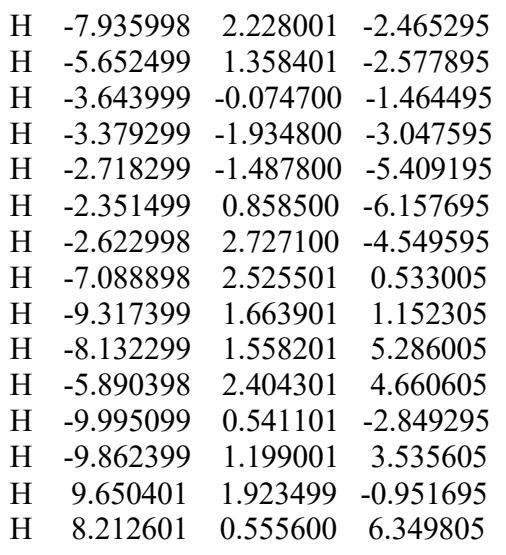

L"3

$\begin{array}{llll}\mathrm{Rh} & 0.274002 & -1.076896 & 1.028997\end{array}$

$\begin{array}{llll}\text { Rh } & 0.215601 & -0.030697 & -1.109003\end{array}$

$\begin{array}{llll}\text { O } & -1.538098 & -1.070799 & -1.440103\end{array}$

$\begin{array}{llll}\text { O } & -1.038896 & -2.476498 & 0.260597\end{array}$

$\begin{array}{llll}\mathrm{O} & -0.812401 & 1.506602 & -0.222003\end{array}$

$\begin{array}{llll}\text { O } & -1.311899 & 0.171502 & 1.533097\end{array}$

$\begin{array}{llll}\mathrm{O} & 1.933600 & 0.963306 & -0.522803\end{array}$

$\begin{array}{llll}\text { O } & 1.543000 & 0.417505 & 1.635697\end{array}$

$\begin{array}{llll}\mathrm{O} & 1.291703 & -1.649095 & -1.806003\end{array}$

$\begin{array}{llll}\text { O } & 1.898903 & -2.149395 & 0.312897\end{array}$

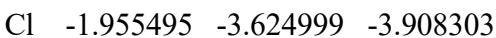

$\begin{array}{llll}\mathrm{Cl} & -1.050002 & 2.621102 & 4.074597\end{array}$

$\begin{array}{llll}\mathrm{Cl} & 5.714799 & 1.125710 & -0.250403\end{array}$

$\begin{array}{llll}\mathrm{Cl} & 2.589608 & -5.687094 & -0.164703\end{array}$

$\begin{array}{llll}\text { C } & -2.979696 & -2.899700 & -1.059903\end{array}$

$\begin{array}{llll}\text { C } & -3.792495 & -3.621601 & 0.077197\end{array}$

$\begin{array}{llll}\text { C } & -2.875394 & -4.393600 & -0.818403\end{array}$

$\begin{array}{llll}\text { C } & -1.764397 & -2.093499 & -0.711003\end{array}$

C $\quad-3.721096-2.346601 \quad-2.237403$

C $\quad-3.349196 \quad-2.604401 \quad-3.560903$

$\begin{array}{llll}\text { C } & -4.036497 & -2.039202 & -4.635503\end{array}$

C $\quad-5.109898 \quad-1.187503 \quad-4.391603$

$\begin{array}{llll}\text { C } & -5.496398 & -0.881103 & -3.077103\end{array}$

$\begin{array}{llll}\text { C } & -4.786297 & -1.467503 & -2.026403\end{array}$

$\begin{array}{llll}\text { C } & -5.289695 & -3.743803 & -0.119503\end{array}$

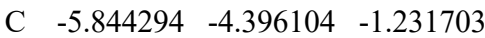

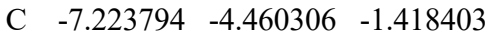

$\begin{array}{llll}\text { C } & -8.088295 & -3.877207 & -0.490903\end{array}$

$\begin{array}{llll}\text { C } & -7.553795 & -3.234106 & 0.625497\end{array}$

$\begin{array}{llll}\text { C } & -6.171795 & -3.168804 & 0.808897\end{array}$

$\begin{array}{llll}\text { C } & -3.377595 & -3.426401 & 1.510497\end{array}$

$\begin{array}{llll}\text { C } & -3.316497 & -2.155701 & 2.087497\end{array}$

$\begin{array}{llll}\text { C } & -2.955697 & -1.998300 & 3.424497\end{array}$

$\begin{array}{llll}\text { C } & -2.671495 & -3.119200 & 4.207997\end{array}$

$\begin{array}{llll}\text { C } & -2.746194 & -4.395800 & 3.643797\end{array}$

$\begin{array}{llll}\text { C } & -3.097394 & -4.545801 & 2.301397\end{array}$

$\begin{array}{llll}\text { C } & -6.627799 & 0.030795 & -2.797603\end{array}$

$\begin{array}{llll}\text { C } & -7.538599 & -0.262806 & -1.769003\end{array}$

$\begin{array}{llll}\text { C } & -8.630600 & 0.570193 & -1.533003\end{array}$

$\begin{array}{llll}\text { C } & -8.826801 & 1.712793 & -2.312403\end{array}$

$\begin{array}{llll}\text { C } & -7.911302 & 2.027894 & -3.319703\end{array}$

$\begin{array}{llll}\text { C } & -6.820401 & 1.193095 & -3.561203\end{array}$

$\begin{array}{llll}\text { C } & -2.457602 & 2.235000 & 1.324197\end{array}$

$\begin{array}{llll}\text { C } & -3.191403 & 3.160099 & 0.283297\end{array}$

$\begin{array}{llll}\text { C } & -2.062504 & 3.685901 & 1.113797\end{array}$ 


\begin{tabular}{|c|c|c|c|}
\hline $\mathrm{C}$ & -1.460501 & 1.229001 & 0.836897 \\
\hline $\mathrm{C}$ & -3.187301 & 1.798099 & 2.555197 \\
\hline $\mathrm{C}$ & -2.654301 & 1.929300 & 3.842397 \\
\hline $\mathrm{C}$ & -3.353201 & 1.495399 & 4.968897 \\
\hline $\mathrm{C}$ & -4.604700 & 0.905698 & 4.814397 \\
\hline $\mathrm{C}$ & -5.160800 & 0.729497 & 3.537897 \\
\hline $\mathrm{C}$ & -4.432801 & 1.177098 & 2.432297 \\
\hline $\mathrm{C}$ & -4.605603 & 3.588798 & 0.617297 \\
\hline $\mathrm{C}$ & -4.913804 & 4.268497 & 1.806897 \\
\hline $\mathrm{C}$ & -6.224205 & 4.623596 & 2.119297 \\
\hline $\mathrm{C}$ & -7.265604 & 4.313894 & 1.243297 \\
\hline $\mathrm{C}$ & -6.975604 & 3.647695 & 0.052997 \\
\hline $\mathrm{C}$ & -5.662503 & 3.288496 & -0.256103 \\
\hline $\mathrm{C}$ & -2.932403 & 2.942300 & -1.184403 \\
\hline $\mathrm{C}$ & -2.391304 & 3.982100 & -1.947303 \\
\hline $\mathrm{C}$ & -2.132904 & 3.807201 & -3.307703 \\
\hline $\mathrm{C}$ & -2.423002 & 2.585700 & -3.920903 \\
\hline $\mathrm{C}$ & -2.978901 & 1.547900 & -3.168403 \\
\hline $\mathrm{C}$ & -3.236701 & 1.730099 & -1.810803 \\
\hline $\mathrm{C}$ & -6.490799 & 0.106095 & 3.356497 \\
\hline $\mathrm{C}$ & -6.873598 & -1.007405 & 4.121197 \\
\hline $\mathrm{C}$ & -8.145797 & -1.561407 & 3.980397 \\
\hline $\mathrm{C}$ & -9.055198 & -1.010008 & 3.074197 \\
\hline $\mathrm{C}$ & -8.675399 & 0.085293 & 2.294497 \\
\hline $\mathrm{C}$ & -7.402700 & 0.637194 & 2.428997 \\
\hline $\mathrm{C}$ & 3.086998 & 2.169807 & 1.145197 \\
\hline $\mathrm{C}$ & 3.861598 & 2.073708 & 2.496397 \\
\hline $\mathrm{C}$ & 2.641397 & 2.938206 & 2.380797 \\
\hline $\mathrm{C}$ & 2.133100 & 1.087006 & 0.730497 \\
\hline $\mathrm{C}$ & 3.634097 & 2.916008 & -0.035303 \\
\hline $\mathrm{C}$ & 4.777498 & 2.528909 & -0.743403 \\
\hline $\mathrm{C}$ & 5.195297 & 3.220509 & -1.879903 \\
\hline $\mathrm{C}$ & 4.443296 & 4.291209 & -2.353003 \\
\hline $\mathrm{C}$ & 3.265395 & 4.683907 & -1.697403 \\
\hline $\mathrm{C}$ & 2.899396 & 3.994207 & -0.535503 \\
\hline $\mathrm{C}$ & 5.215498 & 2.746209 & 2.575497 \\
\hline $\mathrm{C}$ & 5.409896 & 4.079410 & 2.180197 \\
\hline $\mathrm{C}$ & 6.667695 & 4.676711 & 2.250997 \\
\hline $\mathrm{C}$ & 7.763596 & 3.958613 & 2.730697 \\
\hline $\mathrm{C}$ & 7.583498 & 2.635912 & 3.134797 \\
\hline $\mathrm{C}$ & 6.326998 & 2.036911 & 3.055497 \\
\hline $\mathrm{C}$ & 3.732500 & 0.812108 & 3.310097 \\
\hline $\mathrm{C}$ & 4.108701 & -0.434592 & 2.800997 \\
\hline $\mathrm{C}$ & 3.975603 & -1.585592 & 3.576397 \\
\hline $\mathrm{C}$ & 3.480503 & -1.500393 & 4.880197 \\
\hline $\mathrm{C}$ & 3.123001 & -0.255493 & 5.403697 \\
\hline $\mathrm{C}$ & 3.249900 & 0.892507 & 4.620297 \\
\hline $\mathrm{C}$ & 2.413494 & 5.774806 & -2.225803 \\
\hline $\mathrm{C}$ & 2.983393 & 6.916807 & -2.811003 \\
\hline $\mathrm{C}$ & 2.177791 & 7.938806 & -3.311603 \\
\hline $\mathrm{C}$ & 0.786991 & 7.838604 & -3.235003 \\
\hline $\mathrm{C}$ & 0.208493 & 6.706203 & -2.657103 \\
\hline $\mathrm{C}$ & 1.012394 & 5.681704 & -2.159403 \\
\hline $\mathrm{C}$ & 3.269304 & -2.923493 & -1.457603 \\
\hline $\mathrm{C}$ & 3.936504 & -2.461192 & -2.814403 \\
\hline $\mathrm{C}$ & 3.093205 & -3.694993 & -2.751403 \\
\hline $\mathrm{C}$ & 2.065903 & -2.188194 & -0.952203 \\
\hline $\mathrm{C}$ & 4.150805 & -3.434592 & -0.359903 \\
\hline $\mathrm{C}$ & 3.944106 & -4.655592 & 0.289397 \\
\hline $\mathrm{C}$ & 4.773107 & -5.077191 & 1.329997 \\
\hline C & 5.824106 & -4.264490 & 1.744997 \\
\hline $\mathrm{C}$ & 6.048304 & -3.018290 & 1.137597 \\
\hline
\end{tabular}

$\begin{array}{llll}\text { C } & 5.196904 & -2.630291 & 0.099197\end{array}$

$\begin{array}{llll}\text { C } & 5.442504 & -2.594390 & -2.908403\end{array}$

C $\quad 6.093905 \quad-3.828789 \quad-2.762503$

C $7.484206-3.921288-2.799903$

$\begin{array}{llll}\text { C } & 8.263304 & -2.779087 & -2.990603\end{array}$

C $\quad 7.630703 \quad-1.545788-3.149403$

$\begin{array}{llll}\text { C } & 6.238903 & -1.456289 & -3.111003\end{array}$

$\begin{array}{llll}\text { C } & 3.373602 & -1.269893 & -3.546303\end{array}$

$\begin{array}{llll}\text { C } & 3.362601 & 0.003007 & -2.968603\end{array}$

$\begin{array}{llll}\text { C } & 2.836900 & 1.095207 & -3.656603\end{array}$

$\begin{array}{llll}\text { C } & 2.330900 & 0.928306 & -4.948603\end{array}$

$\begin{array}{llll}\text { C } & 2.359001 & -0.335894 & -5.543603\end{array}$

C $\quad 2.879403 \quad-1.427293 \quad-4.844903$

$\begin{array}{llll}\text { C } & 7.143603 & -2.122788 & 1.573697\end{array}$

$\begin{array}{llll}\text { C } & 7.872802 & -1.383987 & 0.626897\end{array}$

$\begin{array}{llll}\text { C } & 8.901401 & -0.533586 & 1.029097\end{array}$

$\begin{array}{llll}\text { C } & 9.222801 & -0.410286 & 2.382297\end{array}$

$\begin{array}{llll}\text { C } & 8.500602 & -1.136087 & 3.332697\end{array}$

$\begin{array}{llll}\text { C } & 7.469103 & -1.984788 & 2.932397\end{array}$

H $\quad-3.286893 \quad-5.050101 \quad-1.574303$

$\mathrm{H} \quad-1.939293 \quad-4.728199 \quad-0.387103$

H $\quad-3.731896 \quad-2.267301 \quad-5.650403$

$\mathrm{H} \quad-5.656298 \quad-0.765604 \quad-5.228503$

$\mathrm{H} \quad-5.063098 \quad-1.234103 \quad-1.005403$

H $\quad-5.204093 \quad-4.844903 \quad-1.981203$

H $\quad-7.620493 \quad-4.967506 \quad-2.292703$

H $\quad-9.163194 \quad-3.923808 \quad-0.636303$

$\begin{array}{llll}\mathrm{H} & -8.204996 & -2.773307 & 1.359197\end{array}$

$\mathrm{H} \quad-5.783196 \quad-2.663104 \quad 1.684397$

$\mathrm{H} \quad-3.510198 \quad-1.278101 \quad 1.482497$

H $\quad-2.888098 \quad-1.002900 \quad 3.844497$

$\mathrm{H} \quad-2.393096 \quad-2.999000 \quad 5.250897$

$\mathrm{H} \quad-2.529793 \quad-5.272700 \quad 4.246897$

$\mathrm{H} \quad-3.149193 \quad-5.536501 \quad 1.858997$

H $\quad-7.409998 \quad-1.163706 \quad-1.178403$

$\begin{array}{llll}\mathrm{H} & -9.336500 & 0.316792 & -0.748703\end{array}$

$\mathrm{H} \quad-8.045303 \quad 2.925094 \quad-3.916103$

$\mathrm{H} \quad-6.100901 \quad 1.449896 \quad-4.332303$

$\mathrm{H} \quad-2.266604 \quad 4.379200 \quad 1.919397$

$\begin{array}{llll}\mathrm{H} & -1.113404 & 3.839402 & 0.613997\end{array}$

$\mathrm{H} \quad-2.919801 \quad 1.621300 \quad 5.954397$

H $\quad-5.156400 \quad 0.589397 \quad 5.693297$

H $\quad-4.842700 \quad 1.042897 \quad 1.438397$

$\begin{array}{llll}\mathrm{H} & -4.135105 & 4.510498 & 2.519597\end{array}$

H $\quad-6.428305 \quad 5.142795 \quad 3.050697$

$\begin{array}{llll}\mathrm{H} & -8.287805 & 4.588093 & 1.485297\end{array}$

H $\quad-7.767403 \quad 3.395494 \quad-0.642403$

$\mathrm{H} \quad-5.467203 \quad 2.771097 \quad-1.187603$

H $\quad-2.158905 \quad 4.927001 \quad-1.464303$

$\mathrm{H} \quad-1.699205 \quad 4.618501 \quad-3.884303$

$\mathrm{H} \quad-2.218802 \quad 2.443901 \quad-4.978003$

$\mathrm{H} \quad-3.201700 \quad 0.593899 \quad-3.630603$

$\begin{array}{llll}\mathrm{H} & -3.634700 & 0.906599 & -1.228703\end{array}$

H $\quad-6.163197 \quad-1.447904 \quad 4.814097$

H $\quad-8.425896 \quad-2.425107 \quad 4.575597$

$\begin{array}{llll}\mathrm{H} & -9.377100 & 0.523292 & 1.591797\end{array}$

$\begin{array}{llll}\mathrm{H} & -7.126401 & 1.505695 & 1.840397\end{array}$

$\begin{array}{llll}\mathrm{H} & 2.756896 & 4.015306 & 2.368997\end{array}$

$\begin{array}{llll}\mathrm{H} & 1.734998 & 2.577605 & 2.852197\end{array}$

H $\quad 6.086797 \quad 2.898911-2.405603$

$\mathrm{H} \quad 4.752695 \quad 4.796209 \quad-3.261803$

H $2.006496 \quad 4.294106 \quad 0.003197$ $\begin{array}{llll}\text { H } & 4.585295 & 4.666609 & 1.796097\end{array}$

$\begin{array}{llll}\mathrm{H} & 6.785994 & 5.708111 & 1.932597\end{array}$

$\begin{array}{llll}\mathrm{H} & 8.742795 & 4.424114 & 2.789597\end{array}$

$\begin{array}{llll}\mathrm{H} & 8.421898 & 2.057713 & 3.507497\end{array}$

$\mathrm{H} \quad 6.217200 \quad 1.003811 \quad 3.360897$

H $\quad 4.481201-0.507191 \quad 1.786397$

H $\quad 4.252204 \quad-2.547092 \quad 3.158297$

$\mathrm{H} \quad 3.377504 \quad-2.397093 \quad 5.483997$

H $\quad 2.742901 \quad-0.178294 \quad 6.418097$

H $\quad 2.960799 \quad 1.859307 \quad 5.021997$

H $\quad 4.063892 \quad 7.013508 \quad-2.852803$

H $\quad 2.636990 \quad 8.817606 \quad-3.753903$

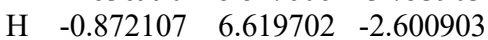

H $\quad 0.550495 \quad 4.792304 \quad-1.742203$

$\mathrm{H} \quad 3.557306 \quad-4.670993 \quad-2.813103$

H $\quad 2.107605 \quad-3.634894 \quad-3.198103$

$\begin{array}{llll}\mathrm{H} & 4.595108 & -6.034591 & 1.805997\end{array}$

H $\quad 6.480006 \quad-4.606789 \quad 2.538597$

H $\quad 5.339903 \quad-1.667290 \quad-0.373603$

H $\quad 5.523907 \quad-4.734490 \quad-2.594803$

H $\quad 7.957407 \quad-4.890787 \quad-2.677603$

H $\quad 9.346104 \quad-2.849986 \quad-3.015703$

H $\quad 8.218302 \quad-0.645387 \quad-3.301503$

H $\quad 5.770701 \quad-0.488390 \quad-3.235303$

H $\quad 3.717201 \quad 0.133908 \quad-1.955403$

H $\quad 2.816598 \quad 2.067207 \quad-3.176403$

H $\quad 1.921299 \quad 1.776805 \quad-5.488903$

H $\quad 1.974501-0.473494 \quad-6.549903$

H $\quad 2.889804 \quad-2.411293 \quad-5.304903$

H $\quad \begin{array}{llll}7.650503 & -1.498788 & -0.428503\end{array}$

$\begin{array}{llll}\mathrm{H} & 9.458101 & 0.025015 & 0.282797\end{array}$

$\mathrm{H} \quad 8.735502 \quad-1.035786 \quad 4.387997$

H $\quad 6.893404 \quad-2.522889 \quad 3.678097$

$\mathrm{H} \quad-9.683102 \quad 2.355792 \quad-2.132903$

H $\quad-10.049197 \quad-1.434709 \quad 2.971997$

H $\quad 0.159390 \quad 8.634803 \quad-3.623003$

H $\quad 10.029201 \quad 0.246015 \quad 2.693997$
L"4
$\begin{array}{llll}\text { Rh } & 0.258103 & -0.195499 & -1.055698\end{array}$
$\begin{array}{llll}\mathrm{Rh} & 0.327103 & -1.096299 & 1.146102\end{array}$
$\begin{array}{llll}\text { O } & 1.978804 & -2.186998 & 0.497302\end{array}$
$\begin{array}{llll}\text { O } & 1.334804 & -1.855698 & -1.642198\end{array}$
$\begin{array}{llll}\mathrm{O} & 1.580202 & 0.447802 & 1.662302\end{array}$
$\begin{array}{llll}\text { O } & 1.965102 & 0.844302 & -0.529698\end{array}$
$\begin{array}{llll}\text { O } & -1.265898 & 0.183399 & 1.557202\end{array}$
$\begin{array}{llll}\text { O } & -0.762599 & 1.399700 & -0.281498\end{array}$
$\begin{array}{llll}\text { O } & -0.990795 & -2.542501 & 0.465202\end{array}$
$\begin{array}{llll}\text { O } & -1.491596 & -1.249201 & -1.322698\end{array}$
$\begin{array}{llll}\mathrm{Cl} & 2.970908 & -5.685297 & 0.279502\end{array}$
$\begin{array}{llll}\mathrm{Cl} & 5.670701 & 1.392506 & -0.425098\end{array}$
$\begin{array}{llll}\mathrm{Cl} & -1.015900 & 2.785999 & 3.954702\end{array}$

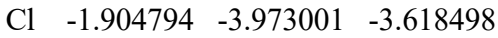
$\begin{array}{llll}\text { C } & 3.385505 & -2.983697 & -1.242998\end{array}$
$\begin{array}{llll}\text { C } & 3.980305 & -2.588396 & -2.656998\end{array}$
$\begin{array}{llll}\text { C } & 3.224506 & -3.865097 & -2.467398\end{array}$
$\begin{array}{llll}\text { C } & 2.144505 & -2.294298 & -0.763898\end{array}$
$\begin{array}{llll}\text { C } & 4.337706 & -3.340196 & -0.143898\end{array}$
$\begin{array}{llll}\text { C } & 4.257607 & -4.524196 & 0.595602\end{array}$
$\begin{array}{llll}\text { C } & 5.167107 & -4.809595 & 1.615202\end{array}$
$\begin{array}{llll}\text { C } & 6.173206 & -3.895894 & 1.915402\end{array}$
$\begin{array}{llll}\text { C } & 6.270005 & -2.681694 & 1.216302\end{array}$ 


\begin{tabular}{|c|c|c|c|}
\hline & 5.338705 & -2.429395 & 0.205002 \\
\hline $\mathrm{C}$ & 5.488405 & -2.638695 & -2.797098 \\
\hline $\mathrm{C}$ & 6.221406 & -3.811794 & -2.558098 \\
\hline $\mathrm{C}$ & 7.612306 & -3.826393 & -2.644498 \\
\hline $\mathrm{C}$ & 8.311705 & -2.666192 & -2.979998 \\
\hline$C$ & 7.598104 & -1.494793 & -3.233198 \\
\hline $\mathrm{C}$ & 6.205504 & -1.482894 & -3.144498 \\
\hline $\mathrm{C}$ & 3.316404 & -1.494197 & -3.453698 \\
\hline $\mathrm{C}$ & 3.280003 & -0.176197 & -2.988098 \\
\hline $\mathrm{C}$ & 2.644802 & 0.823503 & -3.722998 \\
\hline $\mathrm{C}$ & 2.053602 & 0.518402 & -4.951998 \\
\hline $\mathrm{C}$ & 2.111403 & -0.789798 & -5.439798 \\
\hline $\mathrm{C}$ & 2.741704 & -1.788197 & -4.694098 \\
\hline $\mathrm{C}$ & 7.317304 & -1.682793 & 1.528002 \\
\hline $\mathrm{C}$ & 7.938203 & -0.963192 & 0.493502 \\
\hline $\mathrm{C}$ & 8.919402 & -0.014591 & 0.777802 \\
\hline $\mathrm{C}$ & 9.300902 & 0.227409 & 2.099002 \\
\hline $\mathrm{C}$ & 8.687603 & -0.480092 & 3.135702 \\
\hline $\mathrm{C}$ & 7.703404 & -1.426893 & 2.853302 \\
\hline $\mathrm{C}$ & 3.007300 & 2.261003 & 1.035902 \\
\hline $\mathrm{C}$ & 3.827600 & 2.313504 & 2.359502 \\
\hline $\mathrm{C}$ & 2.537200 & 3.067803 & 2.236502 \\
\hline $\mathrm{C}$ & 2.138101 & 1.080702 & 0.710902 \\
\hline $\mathrm{C}$ & 3.433800 & 2.980103 & -0.209598 \\
\hline $\mathrm{C}$ & 4.571500 & 2.657205 & -0.956398 \\
\hline $\mathrm{C}$ & 4.843299 & 3.292105 & -2.168198 \\
\hline $\mathrm{C}$ & 3.941799 & 4.219604 & -2.681598 \\
\hline $\mathrm{C}$ & 2.755298 & 4.524003 & -1.993698 \\
\hline $\mathrm{C}$ & 2.546699 & 3.912803 & -0.752498 \\
\hline $\mathrm{C}$ & 5.125500 & 3.090505 & 2.349202 \\
\hline $\mathrm{C}$ & 5.202198 & 4.406205 & 1.865602 \\
\hline $\mathrm{C}$ & 6.411498 & 5.100106 & 1.854402 \\
\hline $\mathrm{C}$ & 7.574398 & 4.499007 & 2.337702 \\
\hline $\mathrm{C}$ & 7.510800 & 3.194907 & 2.828302 \\
\hline $\mathrm{C}$ & 6.302800 & 2.498806 & 2.831002 \\
\hline $\mathrm{C}$ & 3.819701 & 1.098404 & 3.249902 \\
\hline $\mathrm{C}$ & 3.388101 & 1.228003 & 4.574102 \\
\hline $\mathrm{C}$ & 3.370002 & 0.124103 & 5.427602 \\
\hline $\mathrm{C}$ & 3.785004 & -1.125396 & 4.960302 \\
\hline $\mathrm{C}$ & 4.228804 & -1.258396 & 3.642502 \\
\hline $\mathrm{C}$ & 4.254703 & -0.150096 & 2.796602 \\
\hline $\mathrm{C}$ & 1.717497 & 5.407002 & -2.575998 \\
\hline $\mathrm{C}$ & 1.496997 & 5.429802 & -3.963198 \\
\hline $\mathrm{C}$ & 0.491497 & 6.223601 & -4.513698 \\
\hline $\mathrm{C}$ & -0.320704 & 7.003700 & -3.687498 \\
\hline $\mathrm{C}$ & -0.111704 & 6.990000 & -2.306198 \\
\hline $\mathrm{C}$ & 0.899197 & 6.203001 & -1.756798 \\
\hline $\mathrm{C}$ & -2.396300 & 2.240398 & 1.215702 \\
\hline $\mathrm{C}$ & -3.108200 & 3.116397 & 0.116602 \\
\hline $\mathrm{C}$ & -1.978401 & 3.671598 & 0.925602 \\
\hline $\mathrm{C}$ & -1.409699 & 1.195299 & 0.794802 \\
\hline $\mathrm{C}$ & -3.141899 & 1.885297 & 2.463202 \\
\hline $\mathrm{C}$ & -2.621799 & 2.090698 & 3.746002 \\
\hline $\mathrm{C}$ & -3.335699 & 1.731497 & 4.889302 \\
\hline $\mathrm{C}$ & -4.589999 & 1.142796 & 4.757202 \\
\hline $\mathrm{C}$ & -5.134598 & 0.894496 & 3.487802 \\
\hline $\mathrm{C}$ & -4.391899 & 1.268396 & 2.364802 \\
\hline $\mathrm{C}$ & -4.518201 & 3.581796 & 0.416902 \\
\hline $\mathrm{C}$ & -4.823402 & 4.330096 & 1.565502 \\
\hline $\mathrm{C}$ & -6.130302 & 4.719195 & 1.850702 \\
\hline $\mathrm{C}$ & -7.171602 & 4.375394 & 0.987402 \\
\hline & -6.884501 & 3.641294 & -0.162898 \\
\hline
\end{tabular}

$\begin{array}{llll}\text { C } & -5.574901 & 3.248395 & -0.444698\end{array}$

C $\quad-2.837900 \quad 2.813098 \quad-1.334298$

$\begin{array}{llll}\text { C } & -2.244201 & 3.788598 & -2.141798\end{array}$

$\begin{array}{llll}\text { C } & -1.968401 & 3.528298 & -3.485098\end{array}$

C $\quad-2.293200 \quad 2.285898 \quad-4.035898$

$\begin{array}{llll}\text { C } & -2.901599 & 1.312098 & -3.239198\end{array}$

$\begin{array}{llll}\text { C } & -3.176299 & 1.578997 & -1.898998\end{array}$

$\begin{array}{llll}\text { C } & -6.469598 & 0.274294 & 3.333402\end{array}$

$\begin{array}{llll}\text { C } & -6.867097 & -0.789706 & 4.158902\end{array}$

$\begin{array}{llll}\text { C } & -8.144996 & -1.336707 & 4.045302\end{array}$

$\begin{array}{llll}\text { C } & -9.045397 & -0.827708 & 3.105902\end{array}$

$\begin{array}{llll}\text { C } & -8.650898 & 0.217092 & 2.266502\end{array}$

$\begin{array}{llll}\text { C } & -7.372298 & 0.761493 & 2.373502\end{array}$

$\begin{array}{llll}\text { C } & -2.932395 & -3.047902 & -0.829198\end{array}$

$\begin{array}{llll}\text { C } & -3.756494 & -3.686303 & 0.348302\end{array}$

$\begin{array}{llll}\text { C } & -2.838393 & -4.522202 & -0.487098\end{array}$

C $\quad-1.716495-2.223401-0.528998$

$\begin{array}{llll}\text { C } & -3.663495 & -2.568303 & -2.044898\end{array}$

C $\quad-3.287495 \quad-2.915203 \quad-3.346398$

C $\quad-3.959895 \quad-2.410103 \quad-4.459598$

C $\quad-5.022096-1.529204 \quad-4.277998$

$\begin{array}{llll}\text { C } & -5.412296 & -1.134405 & -2.988398\end{array}$

$\begin{array}{llll}\text { C } & -4.717196 & -1.662504 & -1.897598\end{array}$

$\begin{array}{llll}\text { C } & -5.252994 & -3.810105 & 0.149002\end{array}$

C $\quad-6.136395 \quad-3.161205 \quad 1.026002$

$\begin{array}{llll}\text { C } & -7.517395 & -3.225507 & 0.835402\end{array}$

$\begin{array}{llll}\text { C } & -8.049594 & -3.941807 & -0.236798\end{array}$

$\begin{array}{llll}\text { C } & -7.183793 & -4.599406 & -1.111798\end{array}$

$\begin{array}{llll}\text { C } & -5.805293 & -4.535605 & -0.917998\end{array}$

$\begin{array}{llll}\text { C } & -3.349194 & -3.395603 & 1.767302\end{array}$

C $\quad-3.278996 \quad-2.088003 \quad 2.253802$

$\begin{array}{llll}\text { C } & -2.925496 & -1.840402 & 3.579102\end{array}$

$\begin{array}{llll}\text { C } & -2.658395 & -2.906102 & 4.441602\end{array}$

$\begin{array}{llll}\text { C } & -2.742294 & -4.218502 & 3.968102\end{array}$

$\begin{array}{llll}\text { C } & -3.085593 & -4.459303 & 2.636902\end{array}$

$\begin{array}{llll}\text { C } & -6.531297 & -0.189606 & -2.775398\end{array}$

$\begin{array}{llll}\text { C } & -7.451897 & -0.403007 & -1.735998\end{array}$

$\begin{array}{llll}\text { C } & -8.531098 & 0.460692 & -1.559298\end{array}$

$\begin{array}{llll}\text { C } & -8.704699 & 1.554792 & -2.410198\end{array}$

$\begin{array}{llll}\text { C } & -7.779399 & 1.790693 & -3.430098\end{array}$

$\begin{array}{llll}\text { C } & -6.701398 & 0.924794 & -3.612198\end{array}$

H $\quad 3.752407 \quad-4.809996 \quad-2.472198$

H $\quad 2.223306 \quad-3.906498 \quad-2.880098$

H $\quad 5.087408 \quad-5.741895 \quad 2.162202$

H $\quad 6.893706 \quad-4.133493 \quad 2.691102$

H $\quad 5.385604 \quad-1.494495 \quad-0.339198$

H $\quad 5.716607 \quad-4.727994 \quad-2.277298$

H $\quad 8.148607 \quad-4.749492 \quad-2.446598$

H $\quad 9.395305 \quad-2.676091 \quad-3.043598$

H $\quad 8.122203 \quad-0.581692 \quad-3.499098$

$\begin{array}{llll}\mathrm{H} & 5.675203 & -0.561394 & -3.345998\end{array}$

H $3.697802 \quad 0.061604 \quad-2.018898$

$\mathrm{H} \quad 2.605501 \quad 1.832003 \quad-3.326698$

H $\quad 1.555901 \quad 1.294402 \quad-5.526398$

H $\quad \begin{array}{llll}1.663503 & -1.034698 & -6.398498\end{array}$

$\begin{array}{llll}\mathrm{H} & 2.772105 & -2.807097 & -5.069198\end{array}$

H $\quad 7.670504 \quad-1.168393 \quad-0.537298$

$\mathrm{H} \quad 9.392102 \quad 0.528009 \quad-0.035098$

$\begin{array}{llll}\mathrm{H} & 8.969803 & -0.288991 & 4.166602\end{array}$

H $\quad 7.210304 \quad-1.950893 \quad 3.665202$

$\begin{array}{llll}\mathrm{H} & 2.562599 & 4.147603 & 2.151402\end{array}$

H $\quad 1.679600 \quad 2.666402 \quad 2.762402$
H $\quad 5.745500 \quad 3.041906 \quad-2.714398$

H $\quad 4.161998 \quad 4.702804 \quad-3.627398$

$\begin{array}{llll}\mathrm{H} & 1.628899 & 4.107602 & -0.208198\end{array}$

H $\quad 4.322098 \quad 4.902804 \quad 1.476202$

$\mathrm{H} \quad 6.439697 \quad 6.115106 \quad 1.469302$

$\begin{array}{llll}\mathrm{H} & 8.515598 & 5.040308 & 2.332702\end{array}$

H $\quad 8.403300 \quad 2.707208 \quad 3.205202$

$\mathrm{H} \quad 6.282501 \quad 1.481506 \quad 3.201702$

H $\quad 3.053300 \quad 2.197503 \quad 4.931602$

$\begin{array}{llll}\mathrm{H} & 3.028702 & 0.238503 & 6.452202\end{array}$

H $\quad 3.765604 \quad-1.988896 \quad 5.618602$

H $\quad 4.549005 \quad-2.224395 \quad 3.268902$

H $\quad 4.590303 \quad-0.259595 \quad 1.772102$

$\begin{array}{lllll}\text { H } & 2.096898 & 4.799202 & -4.611698\end{array}$

H $\quad 0.333297 \quad 6.221401 \quad-5.587898$

$\begin{array}{llll}\mathrm{H} & -0.731005 & 7.599900 & -1.655598\end{array}$

$\begin{array}{llll}\mathrm{H} & 1.065897 & 6.218901 & -0.684498\end{array}$

$\mathrm{H} \quad-2.176702 \quad 4.412798 \quad 1.688902$

$\begin{array}{llll}\mathrm{H} & -1.025201 & 3.783499 & 0.422802\end{array}$

H $\quad-2.911399 \quad 1.913998 \quad 5.869802$

H $\quad-5.153598 \quad 0.884596 \quad 5.647502$

H $\quad-4.793199 \quad 1.078296 \quad 1.376302$

$\begin{array}{llll}\mathrm{H} & -4.045202 & 4.600897 & 2.268302\end{array}$

$\mathrm{H} \quad-6.331702 \quad 5.291494 \quad 2.751102$

$\begin{array}{llll}\mathrm{H} & -8.191302 & 4.675693 & 1.208302\end{array}$

$\mathrm{H} \quad-7.676101 \quad 3.361893 \quad-0.848098$

$\mathrm{H} \quad-5.382400 \quad 2.678395 \quad-1.345398$

$\begin{array}{llll}\mathrm{H} & -1.972702 & 4.749098 & -1.714698\end{array}$

$\mathrm{H} \quad-1.493202 \quad 4.292999 \quad-4.090798$

$\begin{array}{llll}\mathrm{H} & -2.074699 & 2.077298 & -5.079298\end{array}$

$\begin{array}{lllll}\mathrm{H} & -3.152398 & 0.342497 & -3.652798\end{array}$

$\begin{array}{lllll}\mathrm{H} & -3.615098 & 0.803997 & -1.280198\end{array}$

H $\quad-6.163896 \quad-1.196805 \quad 4.878802$

H $\quad-8.436596 \quad-2.162008 \quad 4.687602$

$\begin{array}{llll}\mathrm{H} & -9.346198 & 0.622692 & 1.538402\end{array}$

$\begin{array}{llll}\mathrm{H} & -7.084099 & 1.591894 & 1.737202\end{array}$

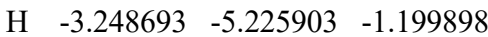

H $\quad-1.906993 \quad-4.831901 \quad-0.027898$

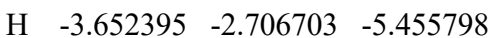

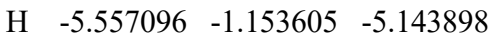

$\mathrm{H} \quad-4.995396-1.359504 \quad-0.895398$

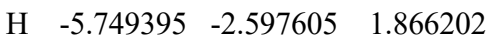

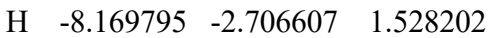

H $\quad-9.123594 \quad-3.987908 \quad-0.388198$

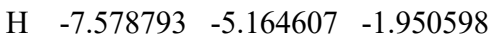

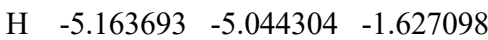

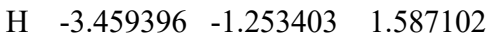

$\mathrm{H} \quad-2.849197 \quad-0.818302 \quad 3.927602$

$\mathrm{H} \quad-2.385695 \quad-2.715502 \quad 5.475502$

$\mathrm{H} \quad-2.538893 \quad-5.052802 \quad 4.633002$

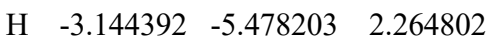

H $\quad-7.340796 \quad-1.266706 \quad-1.088898$

H $\quad-9.244598 \quad 0.268692 \quad-0.764698$

$\begin{array}{llll}\mathrm{H} & -7.895900 & 2.650493 & -4.082798\end{array}$

$\begin{array}{llll}\mathrm{H} & -5.973999 & 1.121095 & -4.393598\end{array}$

H $\quad-10.043896 \quad-1.246209 \quad 3.024602$

H $\quad-9.551400 \quad 2.221291 \quad-2.276698$

H $\quad 10.069602 \quad 0.961410 \quad 2.319202$

H $\quad-1.108305 \quad 7.615999-4.115098$

L"5

$\begin{array}{llll}\mathrm{Rh} & 0.040997 & 0.867506 & 0.775194\end{array}$ 
Rh $\quad 0.275196 \quad-0.069495 \quad-1.398906$

$\begin{array}{llll}\text { O } & 1.748797 & 1.372904 & -1.715806\end{array}$

$\begin{array}{llll}\mathrm{O} & 0.843999 & 2.583005 & -0.042506\end{array}$

$\begin{array}{llll}\text { O } & 1.776795 & -1.249996 & -0.637006\end{array}$

$\begin{array}{llll}\mathrm{O} & 1.935196 & 0.106604 & 1.166494\end{array}$

$\begin{array}{llll}\text { O } & -1.183705 & -1.437893 & -0.809406\end{array}$

$\begin{array}{llll}\text { O } & -0.761405 & -0.936093 & 1.353894\end{array}$

$\begin{array}{llll}\mathrm{O} & -1.214603 & 1.234507 & -2.003306\end{array}$

$\begin{array}{llll}\mathrm{O} & -1.793902 & 1.551608 & 0.161394\end{array}$

$\begin{array}{llll}\mathrm{Cl} & 2.910300 & 3.658103 & -4.218806\end{array}$

$\begin{array}{lllll}\mathrm{Cl} & 1.800992 & -3.981996 & 1.235694\end{array}$

$\mathrm{Cl} \quad-0.981209 \quad-4.992293 \quad-0.612706$

$\begin{array}{llll}\text { Cl } & -2.220899 & 5.117008 & -0.209406\end{array}$

C $\quad 2.829699 \quad 3.389203 \quad-1.070506$

$\begin{array}{llll}\text { C } & 3.100301 & 4.399503 & 0.114094\end{array}$

$\begin{array}{llll}\text { C } & 2.385501 & 4.838403 & -1.127806\end{array}$

C $\quad 1.728598 \quad 2.379404 \quad-0.931606$

$\begin{array}{llll}\text { C } & 4.008099 & 2.864302 & -1.831206\end{array}$

$\begin{array}{llll}\text { C } & 4.155799 & 2.917502 & -3.219206\end{array}$

C $\quad 5.276098 \quad 2.371500 \quad-3.849806$

$\begin{array}{llll}\text { C } & 6.270098 & 1.759299 & -3.090906\end{array}$

C $\quad 6.153298 \quad 1.675800 \quad-1.693706$

$\begin{array}{llll}\text { C } & 5.011598 & 2.221901 & -1.103206\end{array}$

$\begin{array}{llll}\text { C } & 4.537601 & 4.838501 & 0.315194\end{array}$

$\begin{array}{llll}\text { C } & 5.166801 & 4.659901 & 1.557194\end{array}$

$\begin{array}{llll}\text { C } & 6.504801 & 5.009499 & 1.748594\end{array}$

$\begin{array}{llll}\text { C } & 7.249502 & 5.551598 & 0.701794\end{array}$

$\begin{array}{llll}\text { C } & 6.633802 & 5.750199 & -0.534906\end{array}$

$\begin{array}{llll}\text { C } & 5.297702 & 5.402300 & -0.722406\end{array}$

$\begin{array}{llll}\text { C } & 2.298800 & 4.272603 & 1.384894\end{array}$

$\begin{array}{llll}\text { C } & 2.411099 & 3.146103 & 2.209694\end{array}$

$\begin{array}{llll}\text { C } & 1.632299 & 3.022404 & 3.357994\end{array}$

$\begin{array}{llll}\text { C } & 0.738100 & 4.039105 & 3.709394\end{array}$

$\begin{array}{llll}\text { C } & 0.643901 & 5.181105 & 2.912194\end{array}$

$\begin{array}{llll}\text { C } & 1.424801 & 5.296104 & 1.759394\end{array}$

$\begin{array}{llll}\text { C } & 7.188697 & 1.049699 & -0.839306\end{array}$

$\begin{array}{llll}\text { C } & 7.440298 & 1.556298 & 0.448694\end{array}$

$\begin{array}{llll}\text { C } & 8.364997 & 0.940797 & 1.289294\end{array}$

$\begin{array}{llll}\text { C } & 9.072496 & -0.182803 & 0.854094\end{array}$

$\begin{array}{llll}\text { C } & 8.848995 & -0.679103 & -0.431706\end{array}$

$\begin{array}{llll}\text { C } & 7.912896 & -0.072702 & -1.270806\end{array}$

$\begin{array}{llll}\text { C } & 3.625695 & -1.539598 & 0.794794\end{array}$

$\begin{array}{llll}\text { C } & 4.221895 & -1.483098 & 2.233094\end{array}$

$\begin{array}{llll}\text { C } & 4.746696 & -0.579999 & 1.165994\end{array}$

$\begin{array}{llll}\text { C } & 2.339895 & -0.853397 & 0.437694\end{array}$

$\begin{array}{llll}\text { C } & 3.913693 & -2.757198 & -0.031606\end{array}$

$\begin{array}{llll}\text { C } & 3.127592 & -3.914197 & 0.083694\end{array}$

$\begin{array}{llll}\text { C } & 3.350191 & -5.036398 & -0.708706\end{array}$

$\begin{array}{llll}\text { C } & 4.387491 & -5.025299 & -1.636706\end{array}$

$\begin{array}{llll}\text { C } & 5.208892 & -3.898299 & -1.771906\end{array}$

$\begin{array}{llll}\text { C } & 4.950393 & -2.779199 & -0.966406\end{array}$

$\begin{array}{llll}\text { C } & 5.070393 & -2.674099 & 2.627194\end{array}$

$\begin{array}{llll}\text { C } & 6.423893 & -2.767101 & 2.278394\end{array}$

$\begin{array}{llll}\text { C } & 7.177992 & -3.887601 & 2.631494\end{array}$

C $\quad 6.592791 \quad-4.933301 \quad 3.345494$

$\begin{array}{llll}\text { C } & 5.249291 & -4.841000 & 3.716694\end{array}$

$\begin{array}{llll}\text { C } & 4.499492 & -3.719599 & 3.366694\end{array}$

$\begin{array}{llll}\text { C } & 3.458595 & -0.887198 & 3.387294\end{array}$

$\begin{array}{llll}\text { C } & 4.107496 & 0.048002 & 4.201894\end{array}$

$\begin{array}{llll}\text { C } & 3.478197 & 0.561602 & 5.336994\end{array}$

$\begin{array}{llll}\text { C } & 2.184696 & 0.148904 & 5.666594\end{array}$

C $\quad 1.526495 \quad-0.775196 \quad 4.851194$ $\begin{array}{llll}\text { C } & 2.161995 & -1.292496 & 3.722794\end{array}$

$\begin{array}{llll}\text { C } & 6.331392 & -3.886101 & -2.737806\end{array}$

C $\quad 6.176592 \quad-4.390400 \quad-4.038206$

C $\quad 7.240292-4.364102 \quad-4.940006$

$\begin{array}{llll}\text { C } & 8.475092 & -3.835203 & -4.556906\end{array}$

C $\quad 8.642193 \quad-3.339903 \quad-3.261406$

$\begin{array}{llll}\text { C } & 7.579693 & -3.369202 & -2.359506\end{array}$

$\begin{array}{llll}\text { C } & -2.361007 & -2.624292 & 0.880194\end{array}$

$\begin{array}{llll}\text { C } & -3.107306 & -2.439691 & 2.257994\end{array}$

$\begin{array}{llll}\text { C } & -1.959907 & -3.391692 & 2.128994\end{array}$

$\begin{array}{llll}\text { C } & -1.368405 & -1.587293 & 0.443794\end{array}$

$\begin{array}{llll}\text { C } & -3.097507 & -3.285991 & -0.244706\end{array}$

$\begin{array}{llll}\text { C } & -2.584208 & -4.367292 & -0.970906\end{array}$

C $\quad-3.309209 \quad-4.964191 \quad-2.002806$

C $\quad-4.569608 \quad-4.474290 \quad-2.329306$

$\begin{array}{llll}\text { C } & -5.109707 & -3.375989 & -1.643006\end{array}$

C $\quad-4.354607 \quad-2.800290 \quad-0.617606$

$\begin{array}{llll}\text { C } & -4.508807 & -3.006590 & 2.371694\end{array}$

$\begin{array}{llll}\text { C } & -5.582506 & -2.174789 & 2.726794\end{array}$

$\begin{array}{llll}\text { C } & -6.882707 & -2.674887 & 2.816994\end{array}$

$\begin{array}{llll}\text { C } & -7.143508 & -4.018787 & 2.552094\end{array}$

$\begin{array}{llll}\text { C } & -6.085909 & -4.858888 & 2.200494\end{array}$

$\begin{array}{llll}\text { C } & -4.788308 & -4.358889 & 2.115894\end{array}$

$\begin{array}{llll}\text { C } & -2.893105 & -1.171191 & 3.041394\end{array}$

C $\quad-2.400605 \quad-1.241992 \quad 4.348594$

$\begin{array}{llll}\text { C } & -2.205904 & -0.079992 & 5.097894\end{array}$

$\begin{array}{llll}\text { C } & -2.503503 & 1.167808 & 4.543894\end{array}$

$\begin{array}{llll}\text { C } & -3.010803 & 1.243709 & 3.244094\end{array}$

$\begin{array}{llll}\text { C } & -3.214204 & 0.079209 & 2.505694\end{array}$

$\begin{array}{llll}\text { C } & -6.456907 & -2.859588 & -1.972406\end{array}$

$\begin{array}{llll}\text { C } & -6.882407 & -2.761587 & -3.306906\end{array}$

$\begin{array}{llll}\text { C } & -8.176606 & -2.339386 & -3.609706\end{array}$

$\begin{array}{llll}\text { C } & -9.065606 & -2.010185 & -2.583206\end{array}$

C $\quad-8.642406 \quad-2.080986 \quad-1.253806$

C $\quad-7.347506 \quad-2.496287 \quad-0.948706$

$\begin{array}{llll}\text { C } & -3.206701 & 2.452609 & -1.489506\end{array}$

C $\quad-4.013402 \quad 2.047310 \quad-2.773506$

C $\quad-3.109301 \quad 3.240109 \quad-2.781506$

C $\quad-1.982602 \quad 1.682008 \quad-1.095006$

$\begin{array}{llll}\text { C } & -3.949501 & 2.981510 & -0.300906\end{array}$

$\begin{array}{llll}\text { C } & -3.586800 & 4.160309 & 0.359594\end{array}$

$\begin{array}{llll}\text { C } & -4.257599 & 4.591710 & 1.504394\end{array}$

$\begin{array}{llll}\text { C } & -5.303500 & 3.828711 & 2.014894\end{array}$

$\begin{array}{llll}\text { C } & -5.681101 & 2.626911 & 1.395494\end{array}$

$\begin{array}{llll}\text { C } & -4.990702 & 2.228411 & 0.248194\end{array}$

$\begin{array}{llll}\text { C } & -5.511902 & 2.257511 & -2.731406\end{array}$

$\begin{array}{llll}\text { C } & -6.378703 & 1.162612 & -2.870306\end{array}$

$\begin{array}{llll}\text { C } & -7.762303 & 1.326814 & -2.788106\end{array}$

$\begin{array}{llll}\text { C } & -8.312101 & 2.589314 & -2.565006\end{array}$

$\begin{array}{llll}\text { C } & -7.462000 & 3.688113 & -2.432906\end{array}$

C $\quad-6.081000 \quad 3.522612-2.518706$

$\begin{array}{llll}\text { C } & -3.581003 & 0.836709 & -3.555506\end{array}$

$\begin{array}{llll}\text { C } & -3.436504 & -0.415391 & -2.953406\end{array}$

$\begin{array}{llll}\text { C } & -3.053905 & -1.525391 & -3.704906\end{array}$

C $\quad-2.832505 \quad-1.397191 \quad-5.077906$

$\begin{array}{llll}\text { C } & -2.991104 & -0.151791 & -5.692106\end{array}$

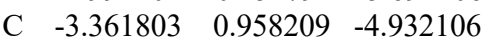

$\begin{array}{llll}\text { C } & -6.782502 & 1.797613 & 1.933394\end{array}$

$\begin{array}{llll}\text { C } & -6.915602 & 1.591513 & 3.315794\end{array}$

$\begin{array}{llll}\text { C } & -7.977803 & 0.842914 & 3.822294\end{array}$

$\begin{array}{llll}\text { C } & -8.924304 & 0.291715 & 2.954694\end{array}$

$\begin{array}{llll}\text { C } & -8.788003 & 0.475215 & 1.576494\end{array}$ $\begin{array}{llll}\text { C } & -7.723403 & 1.216713 & 1.067194\end{array}$

H $\quad 2.884302 \quad 5.491103 \quad-1.832706$

H $\quad 1.316201 \quad 4.998704 \quad-1.048106$

H $\quad 5.368999 \quad 2.435100 \quad-4.927906$

H $\quad \begin{array}{llll}7.150397 & 1.366499 & -3.589006\end{array}$

H $\quad 4.892998 \quad 2.168201 \quad-0.029806$

H $\quad 4.609700 \quad 4.244201 \quad 2.386794$

$\begin{array}{llll}\mathrm{H} & 6.961301 & 4.853999 & 2.721494\end{array}$

H $\quad 8.291602 \quad 5.818597 \quad 0.846994$

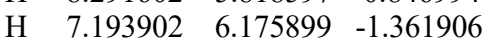

H $\quad 4.863402 \quad 5.555001 \quad-1.702606$

$\begin{array}{llll}\mathrm{H} & 3.061598 & 2.328103 & 1.921494\end{array}$

H $\quad 1.717498 \quad 2.131004 \quad 3.968694$

H $\quad 0.125200 \quad 3.941606 \quad 4.600694$

H $\quad-0.039998 \quad 5.980606 \quad 3.181594$

$\begin{array}{llll}\mathrm{H} & 1.335402 & 6.177904 & 1.131794\end{array}$

$\begin{array}{llll}\mathrm{H} & 6.918799 & 2.444699 & 0.787794\end{array}$

$\begin{array}{llll}\mathrm{H} & 8.539997 & 1.345197 & 2.281494\end{array}$

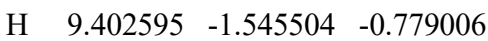

H $\quad 7.723896-0.492702 \quad-2.253206$

H $\quad 5.724295 \quad-0.743100 \quad 0.735894$

H $\quad 4.453997 \quad 0.459001 \quad 1.231394$

H $\quad 2.721990 \quad-5.911797 \quad-0.591106$

H $\quad 4.575890 \quad-5.907699 \quad-2.239306$

H $\quad 5.559394 \quad-1.890400 \quad-1.089606$

$\begin{array}{llll}\mathrm{H} & 6.908594 & -1.963601 & 1.736494\end{array}$

H $\quad 8.225392 \quad-3.936502 \quad 2.348594$

H $\quad 7.177690 \quad-5.806701 \quad 3.617494$

$\mathrm{H} \quad 4.783190 \quad-5.642799 \quad 4.281594$

H $\quad 3.460892 \quad-3.653798 \quad 3.667394$

$\begin{array}{llll}\mathrm{H} & 5.112696 & 0.369101 & 3.943294\end{array}$

$\begin{array}{llll}\mathrm{H} & 3.994397 & 1.286902 & 5.958694\end{array}$

H $\quad 1.690297 \quad 0.551204 \quad 6.545694$

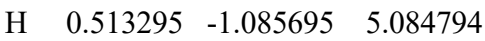

$\mathrm{H} \quad 1.639494 \quad-1.992096 \quad 3.082894$

H $\quad 5.212391 \quad-4.781699 \quad-4.348206$

H $\quad 7.102191 \quad-4.748901 \quad-5.945906$

H $\quad 9.603593 \quad-2.940604-2.951606$

H $\quad 7.713893 \quad-3.010302 \quad-1.345406$

H $\quad-2.143208-4.458192 \quad 2.142294$

$\mathrm{H} \quad-1.019407 \quad-3.087993 \quad 2.572894$

$\mathrm{H} \quad-2.888410 \quad-5.807791 \quad-2.537706$

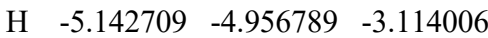

$\mathrm{H} \quad-4.756006-1.950089-0.078906$

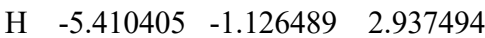

$\begin{array}{llll}\mathrm{H} & -7.687406 & -2.001687 & 3.088994\end{array}$

H $\quad-8.155608 \quad-4.406186 \quad 2.616994$

$\begin{array}{llll}\mathrm{H} & -6.267210 & -5.908488 & 1.989694\end{array}$

$\begin{array}{llll}\mathrm{H} & -3.997409 & -5.038190 & 1.822894\end{array}$

$\mathrm{H} \quad-2.157206 \quad-2.211192 \quad 4.774694$

H $\quad-1.819004 \quad-0.148992 \quad 6.110494$

$\begin{array}{llll}\mathrm{H} & -2.342802 & 2.073908 & 5.120794\end{array}$

$\mathrm{H} \quad-3.239602 \quad 2.203909 \quad 2.797594$

$\begin{array}{llll}\mathrm{H} & -3.578804 & 0.153009 & 1.487994\end{array}$

H $\quad-6.189207 \quad-3.003788 \quad-4.106406$

$\mathrm{H} \quad-8.490506-2.265886 \quad-4.646406$

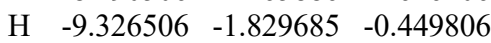

$\begin{array}{llll}\mathrm{H} & -7.037406 & -2.578487 & 0.087394\end{array}$

H $\quad-3.530900 \quad 4.236909-2.790306$

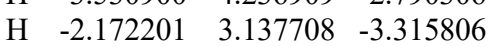

$\begin{array}{llll}\mathrm{H} & -3.959698 & 5.514910 & 1.987894\end{array}$

H $\quad-5.835400 \quad 4.173812 \quad 2.895294$ 

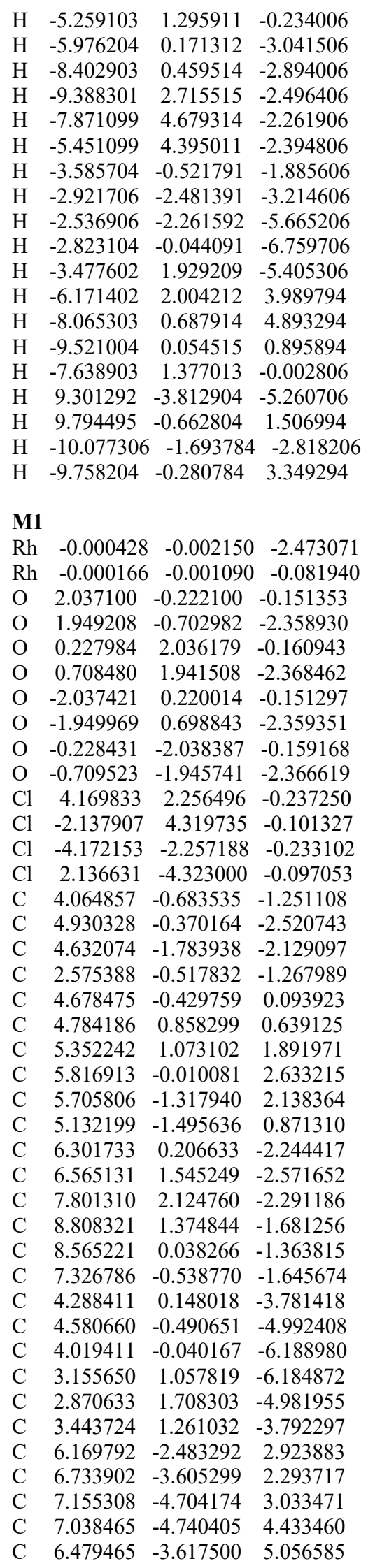

\begin{tabular}{|c|c|c|c|c|c|c|c|}
\hline C & 6.053001 & -2.512343 & 4.319805 & $\mathrm{C}$ & 0.660625 & -4.812142 & 0.728470 \\
\hline $\mathrm{C}$ & 0.749772 & 4.053600 & -1.256143 & $\mathrm{C}$ & 0.773443 & -5.333199 & 2.015068 \\
\hline C & 0.396865 & 4.930612 & -2.506556 & $\mathrm{C}$ & -0.375058 & -5.665166 & 2.730118 \\
\hline $\mathrm{C}$ & 1.821044 & 4.601130 & -2.180069 & $\mathrm{C}$ & -1.647540 & -5.480979 & 2.168737 \\
\hline $\mathrm{C}$ & 0.540723 & 2.568992 & -1.275343 & $\mathrm{C}$ & -1.720279 & -4.986203 & 0.859263 \\
\hline C & 0.588592 & 4.643889 & 0.112594 & $\mathrm{C}$ & 0.136055 & -6.318730 & -2.204178 \\
\hline $\mathrm{C}$ & -0.662150 & 4.810695 & 0.723569 & $\mathrm{C}$ & 1.447690 & -6.649855 & -2.576370 \\
\hline $\mathrm{C}$ & -0.775293 & 5.333460 & 2.009451 & $\mathrm{C}$ & 1.984976 & -7.902713 & -2.285008 \\
\hline $\mathrm{C}$ & 0.373021 & 5.667086 & 2.724050 & $\mathrm{C}$ & 1.219831 & -8.859019 & -1.615959 \\
\hline $\mathrm{C}$ & 1.645606 & 5.482822 & 2.162950 & $\mathrm{C}$ & -0.089567 & -8.547426 & -1.248578 \\
\hline $\mathrm{C}$ & 1.718649 & 4.986219 & 0.854185 & $\mathrm{C}$ & -0.624910 & -7.293494 & -1.542631 \\
\hline $\mathrm{C}$ & -0.137203 & 6.314492 & -2.210323 & $\mathrm{C}$ & 0.182287 & 5451 & 5920 \\
\hline $\mathrm{C}$ & -1.449385 & 6.644619 & -2.581500 & $\mathrm{C}$ & -0.430577 & -4.540688 & 70400 \\
\hline $\mathrm{C}$ & -1.986845 & 7.897609 & -2.291122 & $\mathrm{C}$ & 0.075720 & -3.974983 & -6.142152 \\
\hline $\mathrm{C}$ & -1.221326 & 8.855080 & -1.624141 & $\mathrm{C}$ & 1.204411 & -3.153442 & \\
\hline $\mathrm{C}$ & 0.088604 & 8.544493 & -1.257853 & $\mathrm{C}$ & 1.828062 & -2.915387 & 1908 \\
\hline $\mathrm{C}$ & 0.624125 & 7.29 & -1.5 & $\mathrm{C}$ & 1.325260 & 829 & 875 \\
\hline $\mathrm{C}$ & -0.183182 & 4.289922 & -3.740291 & $\mathrm{C}$ & -2.884739 & -5.764849 & 2.930106 \\
\hline $\mathrm{C}$ & 0.429646 & 4.534347 & -4.974951 & $\mathrm{C}$ & -4.038079 & -4.991231 & 2.736187 \\
\hline $\mathrm{C}$ & -0.076575 & 3.967702 & -6.146290 & $\mathrm{C}$ & -5.206692 & -5.244872 & 3.453078 \\
\hline $\mathrm{C}$ & -1.205138 & 3.146004 & -6.092157 & $\mathrm{C}$ & -5.275656 & -6.27 & 240 \\
\hline $\mathrm{C}$ & -1.82 & 2.9 & -4.8 & $\mathrm{C}$ & -4.116703 & -7.0 & 6978 \\
\hline $\mathrm{C}$ & -1.326008 & 3.487113 & -3.700646 & $\mathrm{C}$ & -2.948610 & -6.803436 & 3.873174 \\
\hline $\mathrm{C}$ & 2.882635 & 5.768589 & 2.92 & $\mathrm{C}$ & -6.541186 & 3812 & 5.207765 \\
\hline $\mathrm{C}$ & 2.945925 & 6.80 & 3.86 & $\mathrm{C}$ & -6.227 & -6.4 & 591 \\
\hline $\mathrm{C}$ & 4.113887 & 7.05 & 4.5 & $\mathrm{C}$ & -7.69 & -5.6 & 211 \\
\hline $\mathrm{C}$ & 5.273284 & 6.28 & & $\mathrm{C}$ & -6.9 & -8. & \\
\hline $\mathrm{C}$ & 5.204895 & 5.250817 & 3.44 & $\mathrm{C}$ & -7.503283 & & 5.203521 \\
\hline $\mathrm{C}$ & 4.036421 & 4.995300 & 2.73 & $\mathrm{C}$ & -7.2915 & 231 & 420 \\
\hline $\mathrm{C}$ & -4.065280 & 0.68 & -1.2 & $\mathrm{C}$ & -9.009 & & \\
\hline $\mathrm{C}$ & -4.93 & 0.3 & -2.5 & $\mathrm{C}$ & -6.7 & & 552 \\
\hline $\mathrm{C}$ & -4.6 & 1.78 & -2.1 & $\mathrm{C}$ & & & 008 \\
\hline $\mathrm{C}$ & -2.575898 & 0.514999 & -1.26 & $\mathrm{C}$ & 6.225582 & & 091 \\
\hline $\mathrm{C}$ & -4.678403 & 0.429878 & & $\mathrm{C}$ & & & \\
\hline $\mathrm{C}$ & -4.784772 & -0.8 & & $\mathrm{C}$ & 7.69 & & \\
\hline $\mathrm{C}$ & -5.3 & -1.0 & & $\mathrm{C}$ & 7.5 & -5 & 5.2 \\
\hline $\mathrm{C}$ & -5.81 & 0.0 & 2.6 & $\mathrm{C}$ & 9.019291 & -6. & 625 \\
\hline $\mathrm{C}$ & -5.703399 & 1.321724 & 2.13 & $\mathrm{C}$ & 7.302673 & -5.8 & 6.7 \\
\hline $\mathrm{C}$ & -5.130657 & & & $\mathrm{C}$ & & & 690 \\
\hline $\mathrm{C}$ & -6.3 & & & $\mathrm{H}$ & & -2 & 303 \\
\hline $\mathrm{C}$ & $-6.5 t$ & -1.5 & -2.5 & $\mathrm{H}$ & 761 & -2.3 & -2. \\
\hline $\mathrm{C}$ & -7.803636 & -2.126474 & -2.287 & $\mathrm{H}$ & & & 665 \\
\hline $\mathrm{C}$ & -8.809819 & -1.375661 & -1.676888 & $\mathrm{H}$ & 6.282036 & 0.168451 & 768 \\
\hline $\mathrm{C}$ & -8.565708 & -0.039067 & -1.360279 & $\mathrm{H}$ & 5.006774 & -2.500702 & 0.484239 \\
\hline $\mathrm{C}$ & -7.32 & & & $\mathrm{H}$ & & & \\
\hline $\mathrm{C}$ & -4.289912 & -0.153415 & -3.7 & $\mathrm{H}$ & 7.975688 & 4822 & -2.550225 \\
\hline $\mathrm{C}$ & -4.582090 & 0.483878 & -4.991644 & $\mathrm{H}$ & 9.771597 & 1.824683 & 1090 \\
\hline $\mathrm{C}$ & -4.021276 & 0.031653 & -6.187750 & $\mathrm{H}$ & 9.339896 & -0.562390 & -0.89 \\
\hline $\mathrm{C}$ & -3.158027 & -1.066728 & & $\mathrm{H}$ & & -1.57 & -1.3849 \\
\hline $\mathrm{C}$ & -2.873073 & -1.715836 & -4.97 & $\mathrm{H}$ & 5.2428 & -1.352009 & -4.992621 \\
\hline $\mathrm{C}$ & -3.445735 & -1.266824 & -3.789554 & $\mathrm{H}$ & 4.250414 & -0.550849 & -7.119126 \\
\hline $\mathrm{C}$ & -6.165639 & 2.488584 & 2.923131 & $\mathrm{H}$ & 2.707201 & 1.404824 & -7.111108 \\
\hline $\mathrm{C}$ & -6.729179 & 3.610271 & 2.291877 & $\mathrm{H}$ & 2.187139 & 2.550314 & -4.965990 \\
\hline $\mathrm{C}$ & -7.148829 & 4.710617 & 3.030452 & $\mathrm{H}$ & 3.193055 & 1.747814 & -2.859615 \\
\hline $\mathrm{C}$ & -7.030713 & 4.748703 & 4.430282 & $\mathrm{H}$ & 6.864241 & -3.607293 & 1.215836 \\
\hline $\mathrm{C}$ & -6.472314 & 3.626109 & 5.054498 & $\mathrm{H}$ & 7.591157 & -5.547298 & 2.506760 \\
\hline $\mathrm{C}$ & -6.047611 & 2.519478 & 4.318899 & $\mathrm{H}$ & 6.358779 & -3.594970 & 6.132934 \\
\hline $\mathrm{C}$ & -0.750890 & -4.056780 & -1.252208 & $\mathrm{H}$ & 5.598739 & -1.673406 & 4.838042 \\
\hline $\mathrm{C}$ & -0.397874 & -4.935079 & -2.501685 & $\mathrm{H}$ & 2.480170 & 5.367244 & -1.791591 \\
\hline $\mathrm{C}$ & -1.822085 & -4.605203 & -2.175662 & $\mathrm{H}$ & 2.304421 & 3.876156 & -2.822797 \\
\hline $\mathrm{C}$ & -0.541594 & -2.572212 & -1.272968 & $\mathrm{H}$ & -1.755380 & 5.454339 & 2.456589 \\
\hline C & -0.590006 & -4.645504 & 0.117235 & $\mathrm{H}$ & 0.274269 & 6.037703 & 3.738777 \\
\hline
\end{tabular}




\begin{tabular}{|c|c|c|c|}
\hline & 2.690044 & 4.859418 & \\
\hline & -2.055880 & 5.912441 & -3.100539 \\
\hline & -3.006872 & 8.123430 & -2.587490 \\
\hline & -1.638275 & 9.831305 & -1.396399 \\
\hline & 0.701307 & 9.278956 & -0.743818 \\
\hline & 1.642402 & 7.082054 & -1.247301 \\
\hline & 1.314947 & 5.162831 & -5.013452 \\
\hline & 0.414466 & 4.161145 & -7.095396 \\
\hline & -1.596187 & 2.691077 & -6.997471 \\
\hline & -2.691158 & 2.254040 & -4.813207 \\
\hline & -1.793517 & 3.274775 & -2.747832 \\
\hline & 2.080161 & 7.444386 & 4.024177 \\
\hline & 4.121690 & 7.875187 & 5.291282 \\
\hline & 6.068239 & 4.622052 & 3.264454 \\
\hline & 4.016926 & 4.168602 & 2.028454 \\
\hline & -5.410017 & 2.408983 & -1.714930 \\
\hline$H$ & -3.913112 & 2.306462 & 5275 \\
\hline & -5.434001 & -2.076677 & 2.288601 \\
\hline & -6.279707 & -0.162182 & 3.599736 \\
\hline 11 & -5.004651 & 2.501626 & 0.482538 \\
\hline$\Pi$ & -5.796530 & -2.141697 & -3.044841 \\
\hline $\mathrm{H}$ & -7.978789 & -3.166564 & 45489 \\
\hline 11 & -9.773217 & -1.824821 & -1.455894 \\
\hline П & -9.339726 & 0.562280 & -0.893016 \\
\hline$\pi$ & -7.169810 & 1.576575 & -1.383066 \\
\hline $\mathrm{H}$ & -5.243832 & 1.345549 & 2800 \\
\hline $\mathrm{H}$ & -4.252225 & 0.541282 & 18485 \\
\hline H & -2.709887 & -1.415093 & -7.108322 \\
\hline $\mathrm{H}$ & -2.189907 & -2.558089 & -4.961910 \\
\hline $\mathrm{H}$ & -3.195114 & -1.752561 & -2.856296 \\
\hline $\mathrm{H}$ & -6.860461 & 0883 & 4108 \\
\hline $\mathrm{H}$ & -7.584292 & 5.553427 & 2.502933 \\
\hline $\mathrm{H}$ & -6.350700 & 3.604976 & 6.130769 \\
\hline $\mathrm{H}$ & -5.593737 & 1.680816 & 4.837922 \\
\hline $\mathrm{H}$ & -2.481210 & -5.370965 & -1.786480 \\
\hline $\mathrm{H}$ & -2.305415 & -3.880885 & -2.819154 \\
\hline $\mathrm{H}$ & 1.753467 & -5.453997 & 2.462401 \\
\hline $\mathrm{H}$ & -0.276527 & -6.034411 & 3.745370 \\
\hline $\mathrm{H}$ & -2.691588 & -4.859508 & 0.396203 \\
\hline $\mathrm{H}$ & 2.053923 & -5.918592 & -3.096991 \\
\hline $\mathrm{H}$ & 3.004575 & -8.129309 & -2.582240 \\
\hline $\mathrm{H}$ & 1.636633 & -9.835125 & -1.387462 \\
\hline $\mathrm{H}$ & -0.701981 & -9.280976 & -0.732904 \\
\hline $\mathrm{H}$ & -1.642744 & -7.084343 & -1.238081 \\
\hline $\mathrm{H}$ & -1.315978 & -5.169064 & -5.008439 \\
\hline $\mathrm{H}$ & -0.415360 & -4.169050 & -7.091116 \\
\hline $\mathrm{H}$ & 1.595530 & -2.699218 & -6.994271 \\
\hline $\mathrm{H}$ & 2.690580 & -2.260779 & -4.810374 \\
\hline $\mathrm{H}$ & 1.792834 & -3.279896 & -2.744208 \\
\hline $\mathrm{H}$ & -4.018126 & -4.165733 & 2.032033 \\
\hline $\mathrm{H}$ & -6.069687 & -4.615943 & 3.268780 \\
\hline $\mathrm{H}$ & -4.124953 & -7.866730 & 5.301112 \\
\hline $\mathrm{H}$ & -2.083218 & -7.439259 & 4.033229 \\
\hline $\mathrm{H}$ & -7.120709 & -6.663891 & 7.308353 \\
\hline $\mathrm{H}$ & -5.436781 & -7.128928 & 7.028305 \\
\hline $\mathrm{H}$ & -5.904189 & -5.423617 & 6.948800 \\
\hline $\mathrm{H}$ & -7.441772 & -4.588877 & 5.076062 \\
\hline $\mathrm{H}$ & -7.984726 & -5.706687 & 3.809505 \\
\hline $\mathrm{H}$ & -8.574836 & -5.888222 & 5.465479 \\
\hline $\mathrm{H}$ & -6.227940 & -8.759253 & 5.178680 \\
\hline $\mathrm{H}$ & -7.898835 & -8.266811 & 5.488198 \\
\hline & -7.228300 & -8.154223 & 3.849579 \\
\hline
\end{tabular}

$\begin{array}{crrr}\mathrm{H} & -7.643485 & 6.746390 & 7.225421 \\ \mathrm{H} & -7.849701 & 4.993762 & 7.127738 \\ \mathrm{H} & -6.234238 & 5.710802 & 6.970257 \\ \mathrm{H} & -9.362332 & 7.090646 & 5.483156 \\ \mathrm{H} & -9.218231 & 6.357418 & 3.879548 \\ \mathrm{H} & -9.592255 & 5.343154 & 5.281240 \\ \mathrm{H} & -5.640985 & 7.090300 & 4.893447 \\ \mathrm{H} & -6.860630 & 7.400636 & 3.648781 \\ \mathrm{H} & -7.041816 & 8.116679 & 5.256540 \\ \mathrm{H} & 7.118266 & 6.677672 & 7.300418 \\ \mathrm{H} & 5.434072 & 7.141269 & 7.019637 \\ \mathrm{H} & 5.902413 & 5.436081 & 6.943122 \\ \mathrm{H} & 7.895361 & 8.277831 & 5.477383 \\ \mathrm{H} & 7.224829 & 8.161933 & 3.839005 \\ \mathrm{H} & 6.224171 & 8.768750 & 5.167054 \\ \mathrm{H} & 7.440387 & 4.598897 & 5.071760 \\ \mathrm{H} & 7.982677 & 5.714799 & 3.803238 \\ \mathrm{H} & 8.572725 & 5.899565 & 5.458863 \\ \mathrm{H} & 9.373284 & -7.078691 & 5.487394 \\ \mathrm{H} & 9.226889 & -6.347982 & 3.882853 \\ \mathrm{H} & 9.601434 & -5.331298 & 5.282640 \\ \mathrm{H} & 7.655957 & -6.733413 & 7.230910 \\ \mathrm{H} & 7.860462 & -4.980737 & 7.130423 \\ \mathrm{H} & 6.245495 & -5.699506 & 6.975675 \\ \mathrm{H} & 5.651333 & -7.082563 & 4.901526 \\ \mathrm{H} & 6.869960 & -7.393613 & 3.656032 \\ \mathrm{H} & 7.053486 & -8.107152 & 5.264632\end{array}$

\section{M2}

$\begin{array}{llll}\mathrm{Rh} & -0.000135 & -0.000036 & -2.533547\end{array}$ $\begin{array}{lllll}\mathrm{Rh} & -0.000090 & 0.000020 & -0.144957\end{array}$ $\begin{array}{llll}\text { O } & 1.180632 & 1.673994 & -0.221798\end{array}$

$\begin{array}{llll}\text { O } & 1.561314 & 1.359421 & -2.428633\end{array}$

$\begin{array}{llll}\mathrm{O} & -1.674079 & 1.180705 & -0.221855\end{array}$

$\begin{array}{lllll}\mathrm{O} & -1.359644 & 1.561389 & -2.428710\end{array}$

$\begin{array}{llll}\text { O } & -1.180799 & -1.673956 & -0.221816\end{array}$

O $\quad-1.561561 \quad-1.359510 \quad-2.428661$

$\begin{array}{llll}\text { O } & 1.673887 & -1.180703 & -0.221769\end{array}$

$\begin{array}{llll}\text { O } & 1.359269 & -1.561499 & -2.428585\end{array}$

$\begin{array}{lllll}\mathrm{Cl} & 0.238448 & 4.865218 & -0.188705\end{array}$

$\begin{array}{lllll}\mathrm{Cl} & -4.865051 & 0.238183 & -0.188433\end{array}$

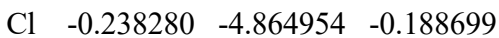

$\begin{array}{lllll}\mathrm{Cl} & 4.865163 & -0.238487 & -0.188981\end{array}$

$\begin{array}{llll}\text { C } & 2.616799 & 3.187908 & -1.304433\end{array}$

$\begin{array}{llll}\text { C } & 2.745809 & 4.129791 & -2.550044\end{array}$

C $3.830468 \quad 3.152463 \quad-2.212549$

$\begin{array}{llll}\text { C } & 1.715662 & 1.989135 & -1.334983\end{array}$

$\begin{array}{llll}\text { C } & 2.746179 & 3.766141 & 0.073400\end{array}$

$\begin{array}{llll}\text { C } & 1.735865 & 4.529551 & 0.673910\end{array}$

$\begin{array}{llll}\text { C } & 1.864982 & 5.007739 & 1.975507\end{array}$

C $3.002883 \quad 4.695113 \quad 2.715775$

$\begin{array}{llll}\text { C } & 4.022411 & 3.903738 & 2.165110\end{array}$

$\begin{array}{llll}\text { C } & 3.872405 & 3.469878 & 0.840534\end{array}$

$\begin{array}{llll}\text { C } & 2.946075 & 5.599223 & -2.252648\end{array}$

$\begin{array}{llll}\text { C } & 1.987482 & 6.530313 & -2.680288\end{array}$

$\begin{array}{llll}\text { C } & 2.124019 & 7.888165 & -2.395641\end{array}$

$\begin{array}{llll}\text { C } & 3.227378 & 8.349775 & -1.676663\end{array}$

C $\quad 4.193006 \quad 7.436632 \quad-1.251892$

$\begin{array}{llll}\text { C } & 4.054639 & 6.078925 & -1.539560\end{array}$

$\begin{array}{llll}\text { C } & 1.942541 & 3.847895 & -3.793213\end{array}$

$\begin{array}{llll}\text { C } & 2.611184 & 3.765984 & -5.020297\end{array}$

$\begin{array}{llll}\text { C } & 1.908614 & 3.507430 & -6.198783\end{array}$ $\begin{array}{llll}\text { C } & 0.523984 & 3.326196 & -6.159619\end{array}$

$\begin{array}{llll}\text { C } & -0.151281 & 3.421199 & -4.940658\end{array}$

$\begin{array}{llll}\text { C } & 0.554095 & 3.692404 & -3.769022\end{array}$

$\begin{array}{llll}\text { C } & 5.208185 & 3.501286 & 2.954509\end{array}$

$\begin{array}{llll}\text { C } & 5.766219 & 4.344403 & 3.929102\end{array}$

C $\quad 6.874136 \quad 3.945135 \quad 4.669104$

C $\quad 7.480224 \quad 2.692048 \quad 4.475562$

$\begin{array}{llll}\text { C } & 6.920381 & 1.856809 & 3.500368\end{array}$

$\begin{array}{llll}\text { C } & 5.808026 & 2.249324 & 2.756985\end{array}$

$\begin{array}{llll}\text { C } & -3.188153 & 2.616745 & -1.304377\end{array}$

$\begin{array}{llll}\text { C } & -4.130174 & 2.745682 & -2.549880\end{array}$

$\begin{array}{llll}\text { C } & -3.152901 & 3.830419 & -2.212490\end{array}$

$\begin{array}{llll}\text { C } & -1.989316 & 1.715697 & -1.335025\end{array}$

$\begin{array}{llll}\text { C } & -3.766256 & 2.746062 & 0.073537\end{array}$

$\begin{array}{llll}\text { C } & -4.529451 & 1.735643 & 0.674138\end{array}$

$\begin{array}{llll}\text { C } & -5.007424 & 1.864658 & 1.975817\end{array}$

$\begin{array}{llll}\text { C } & -4.694788 & 3.002562 & 2.716084\end{array}$

$\begin{array}{llll}\text { C } & -3.903608 & 4.022180 & 2.165322\end{array}$

$\begin{array}{llll}\text { C } & -3.469957 & 3.872277 & 0.840668\end{array}$

$\begin{array}{llll}\text { C } & -5.599598 & 2.945836 & -2.252350\end{array}$

$\begin{array}{llll}\text { C } & -6.530669 & 1.987210 & -2.679929\end{array}$

$\begin{array}{llll}\text { C } & -7.888494 & 2.123654 & -2.395168\end{array}$

$\begin{array}{llll}\text { C } & -8.350110 & 3.226962 & -1.676130\end{array}$

$\begin{array}{llll}\text { C } & -7.436999 & 4.192640 & -1.251407\end{array}$

$\begin{array}{llll}\text { C } & -6.079308 & 4.054367 & -1.539189\end{array}$

$\begin{array}{llll}\text { C } & -3.848332 & 1.942386 & -3.793042\end{array}$

$\begin{array}{llll}C & -3.766606 & 2.610966 & -5.020168\end{array}$

$\begin{array}{llll}\text { C } & -3.508117 & 1.908333 & -6.198652\end{array}$

$\begin{array}{llll}\text { C } & -3.326764 & 0.523721 & -6.159407\end{array}$

C $\quad-3.421572 \quad-0.151473 \quad-4.940395$

$\begin{array}{llll}\text { C } & -3.692708 & 0.553953 & -3.768776\end{array}$

$\begin{array}{llll}\text { C } & -3.501079 & 5.207918 & 2.954738\end{array}$

$\begin{array}{llll}\text { C } & -2.249175 & 5.807845 & 2.757032\end{array}$

$\begin{array}{llll}\text { C } & -1.856567 & 6.920135 & 3.500448\end{array}$

$\begin{array}{llll}\text { C } & -2.691647 & 7.479836 & 4.475875\end{array}$

$\begin{array}{llll}\text { C } & -3.944666 & 6.873662 & 4.669601\end{array}$

$\begin{array}{llll}\text { C } & -4.344036 & 5.765808 & 3.929563\end{array}$

C $\quad-2.616949 \quad-3.187966 \quad-1.304281$

C $\quad-2.746144 \quad-4.129888-2.549831$

$\begin{array}{llll}\text { C } & -3.830761 & -3.152558 & -2.212216\end{array}$

$\begin{array}{llll}\text { C } & -1.715843 & -1.989184 & -1.334971\end{array}$

$\begin{array}{llll}\text { C } & -2.746124 & -3.766150 & 0.073606\end{array}$

$\begin{array}{llll}\text { C } & -1.735626 & -4.529362 & 0.674067\end{array}$

$\begin{array}{llll}\text { C } & -1.864463 & -5.007342 & 1.975760\end{array}$

$\begin{array}{llll}\text { C } & -3.002248 & -4.694685 & 2.716191\end{array}$

C $\quad-4.021954 \quad-3.903508 \quad 2.165576$

$\begin{array}{llll}\text { C } & -3.872239 & -3.469872 & 0.840892\end{array}$

$\begin{array}{llll}\text { C } & -2.946332 & -5.599309 & -2.252326\end{array}$

$\begin{array}{llll}\text { C } & -1.987721 & -6.530388 & -2.679927\end{array}$

$\begin{array}{llll}\text { C } & -2.124184 & -7.888213 & -2.395184\end{array}$

$\begin{array}{llll}\text { C } & -3.227507 & -8.349830 & -1.676151\end{array}$

$\begin{array}{llll}\text { C } & -4.193160 & -7.436700 & -1.251422\end{array}$

$\begin{array}{llll}\text { C } & -4.054867 & -6.079011 & -1.539175\end{array}$

$\begin{array}{llll}\text { C } & -1.943034 & -3.848026 & -3.793111\end{array}$

$\begin{array}{llll}\text { C } & -2.611810 & -3.766294 & -5.020138\end{array}$

C $\quad-1.909350 \quad-3.507773 \quad-6.198723$

$\begin{array}{llll}\text { C } & -0.524740 & -3.326403 & -6.159686\end{array}$

$\begin{array}{llll}\text { C } & 0.150632 & -3.421214 & -4.940777\end{array}$

$\begin{array}{llll}\text { C } & -0.554600 & -3.692380 & -3.769053\end{array}$

$\begin{array}{llll}C & -5.207556 & -3.500944 & 2.955177\end{array}$

$\begin{array}{llll}\text { C } & -5.807533 & -2.249064 & 2.757511\end{array}$

$\begin{array}{llll}\text { C } & -6.919660 & -1.856407 & 3.501139\end{array}$ 


\begin{tabular}{|c|c|c|c|}
\hline C & -7.479147 & -2.691407 & 4.476755 \\
\hline $\mathrm{C}$ & -6.872941 & -3.944416 & 4.670423 \\
\hline $\mathrm{C}$ & -5.765242 & -4.343834 & 3.930174 \\
\hline $\mathrm{C}$ & 3.187763 & -2.616945 & -1.304368 \\
\hline C & 4.129553 & -2.746071 & -2.550034 \\
\hline $\mathrm{C}$ & 3.152255 & -3.830706 & -2.212365 \\
\hline $\mathrm{C}$ & 1.989010 & -1.715787 & -1.334929 \\
\hline $\mathrm{C}$ & 3.766088 & -2.746191 & 0.073449 \\
\hline $\mathrm{C}$ & 4.529495 & -1.735802 & 0.673828 \\
\hline $\mathrm{C}$ & 5.007680 & -1.864742 & 1.975429 \\
\hline $\mathrm{C}$ & 4.695079 & -3.002557 & 2.715853 \\
\hline $\mathrm{C}$ & 3.903728 & -4.022169 & 2.165305 \\
\hline $\mathrm{C}$ & 3.469850 & -3.872338 & 0.840718 \\
\hline $\mathrm{C}$ & 5.599008 & -2.946284 & -2.252724 \\
\hline $\mathrm{C}$ & 6.530040 & -1.987677 & -2.680445 \\
\hline $\mathrm{C}$ & 7.887909 & -2.124159 & -2.395877 \\
\hline $\mathrm{C}$ & 8.349595 & -3.227489 & -1.676905 \\
\hline $\mathrm{C}$ & 7.436511 & -4.193130 & -1.252057 \\
\hline $\mathrm{C}$ & 6.078787 & -4.054818 & -1.539636 \\
\hline $\mathrm{C}$ & 3.847544 & -1.942922 & -3.793253 \\
\hline $\mathrm{C}$ & 3.765613 & -2.611683 & -5.020277 \\
\hline $\mathrm{C}$ & 3.506945 & -1.909218 & -6.198823 \\
\hline $\mathrm{C}$ & 3.325606 & -0.524606 & -6.159758 \\
\hline $\mathrm{C}$ & 3.420614 & 0.150766 & -4.940860 \\
\hline $\mathrm{C}$ & 3.691943 & -0.554491 & -3.769179 \\
\hline $\mathrm{C}$ & 3.501274 & -5.207832 & 2.954869 \\
\hline $\mathrm{C}$ & 2.249355 & -5.807770 & 2.757384 \\
\hline $\mathrm{C}$ & 1.856837 & -6.920003 & 3.500939 \\
\hline $\mathrm{C}$ & 2.692039 & -7.479636 & 4.476291 \\
\hline $\mathrm{C}$ & 3.945087 & -6.873460 & 4.669796 \\
\hline $\mathrm{C}$ & 4.344365 & -5.765664 & 3.929613 \\
\hline $\mathrm{C}$ & 8.700630 & 2.293659 & 5.314822 \\
\hline $\mathrm{C}$ & 8.316859 & 2.308633 & 6.810808 \\
\hline $\mathrm{C}$ & 9.216169 & 0.888819 & 4.961885 \\
\hline $\mathrm{C}$ & 9.840907 & 3.305824 & 5.067902 \\
\hline $\mathrm{C}$ & 2.293639 & -8.699885 & 5.315778 \\
\hline $\mathrm{C}$ & 2.308459 & -8.315787 & 6.811679 \\
\hline $\mathrm{C}$ & 0.888858 & -9.215571 & 4.962817 \\
\hline $\mathrm{C}$ & 3.305882 & -9.840158 & 5.069193 \\
\hline $\mathrm{C}$ & -8.699258 & -2.292825 & 5.316355 \\
\hline $\mathrm{C}$ & -8.314904 & -2.307288 & 6.812189 \\
\hline $\mathrm{C}$ & -9.215027 & -0.888133 & 4.963152 \\
\hline $\mathrm{C}$ & -9.839561 & -3.305154 & 5.070215 \\
\hline $\mathrm{C}$ & -2.293133 & 8.700145 & 5.315229 \\
\hline $\mathrm{C}$ & -2.307705 & 8.316146 & 6.811158 \\
\hline $\mathrm{C}$ & -0.888414 & 9.215833 & 4.962010 \\
\hline $\mathrm{C}$ & -3.305435 & 9.840391 & 5.068733 \\
\hline $\mathrm{H}$ & 4.771693 & 3.506168 & -1.810404 \\
\hline $\mathrm{H}$ & 3.913788 & 2.285056 & -2.854927 \\
\hline $\mathrm{H}$ & 1.068182 & 5.595136 & 2.417277 \\
\hline $\mathrm{H}$ & 3.075653 & 5.039004 & 3.742008 \\
\hline $\mathrm{H}$ & 4.656299 & 2.874963 & 0.386330 \\
\hline $\mathrm{H}$ & 1.125188 & 6.187880 & -3.239356 \\
\hline $\mathrm{H}$ & 1.364241 & 8.584793 & -2.737232 \\
\hline $\mathrm{H}$ & 3.335337 & 9.406758 & -1.453415 \\
\hline $\mathrm{H}$ & 5.061068 & 7.777847 & -0.695912 \\
\hline $\mathrm{H}$ & 4.820349 & 5.398535 & -1.188647 \\
\hline $\mathrm{H}$ & 3.689762 & 3.893455 & -5.047126 \\
\hline $\mathrm{H}$ & 2.442849 & 3.439322 & -7.141794 \\
\hline $\mathrm{H}$ & -0.026386 & 3.111340 & -7.070883 \\
\hline $\mathrm{H}$ & -1.222806 & 3.260188 & -4.900175 \\
\hline $\mathrm{H}$ & 0.030735 & 3.734328 & -2.822576 \\
\hline
\end{tabular}

\begin{tabular}{|c|c|c|c|}
\hline 1 & 5.343497 & 5.330894 & 4.093688 \\
\hline $\mathrm{H}$ & 7.278234 & 4.630615 & 5.407399 \\
\hline $\mathrm{H}$ & 7.345251 & 0.878057 & 3.311232 \\
\hline & 5.389920 & 1.561315 & 2.029495 \\
\hline & -3.506630 & 4.771624 & -1.810308 \\
\hline & -2.285552 & 3.913812 & -2.854940 \\
\hline & -5.594651 & 1.067767 & 2.417672 \\
\hline & -5.038490 & 3.075246 & 3.742381 \\
\hline & -2.875188 & 4.656250 & 0.386386 \\
\hline & -6.188227 & 1.124956 & -3.239058 \\
\hline & -8.585114 & 1.363840 & -2.736722 \\
\hline & -9.407086 & 3.334859 & -1.452793 \\
\hline & -7.778222 & 5.060661 & -0.695379 \\
\hline & -5.398936 & 4.820096 & -1.188310 \\
\hline & -3.894180 & 3.689535 & -5.047063 \\
\hline & -3.440154 & 2.442515 & -7.141698 \\
\hline $\mathrm{H}$ & -3.111962 & -0.026683 & -7.070672 \\
\hline & -3.260461 & -1.222983 & -4.899859 \\
\hline & -3.734480 & 0.030659 & -2.822291 \\
\hline & -1.561289 & 5.389844 & 2.029368 \\
\hline$\Pi$ & -0.877884 & 7.345083 & 3.311173 \\
\hline $\mathrm{H}$ & -4.630012 & 7.277641 & 5.408077 \\
\hline & -5.330464 & 5.343017 & 4.094295 \\
\hline & -4.771933 & -3.506227 & -1.809921 \\
\hline & -3.914167 & -2.285159 & -2.854607 \\
\hline $\mathrm{H}$ & -1.067502 & -5.594545 & 2.417509 \\
\hline $\mathrm{H}$ & -3.074779 & -5.038387 & 3.742499 \\
\hline 1 & -4.656268 & -2.875079 & 0.386736 \\
\hline & -1.125462 & -6.187947 & -3.239059 \\
\hline H & -1.364385 & -8.584843 & -2.736743 \\
\hline $\mathrm{H}$ & -3.335418 & -9.406802 & -1.452830 \\
\hline $\mathrm{H}$ & -5.061195 & -7.777925 & -0.695392 \\
\hline $\mathrm{H}$ & -4.820593 & -5.398627 & -1.188297 \\
\hline & -3.690371 & -3.893878 & -5.046869 \\
\hline $\mathrm{H}$ & -2.443677 & -3.439803 & -7.141692 \\
\hline $\mathrm{H}$ & 0.025518 & -3.111571 & -7.071023 \\
\hline $\mathrm{H}$ & 1.222161 & -3.260092 & -4.900389 \\
\hline $\mathrm{H}$ & -0.031163 & -3.734149 & -2.822649 \\
\hline 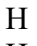 & -5.389689 & -1.561231 & 2.029721 \\
\hline $\mathrm{H}$ & -7.344651 & -0.877732 & 3.311884 \\
\hline $\mathrm{H}$ & -7.276764 & -4.629716 & 5.409028 \\
\hline $\mathrm{H}$ & -5.342421 & -5.330255 & 4.094879 \\
\hline $\mathrm{H}$ & 3.505990 & -4.771893 & -1.810156 \\
\hline 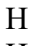 & 2.284795 & -3.914080 & -2.854664 \\
\hline $\mathrm{H}$ & 5.595054 & -1.067872 & 2.417104 \\
\hline $\mathrm{H}$ & 5.038950 & -3.075177 & 3.742085 \\
\hline $\mathrm{H}$ & 2.874932 & -4.656293 & 0.386609 \\
\hline H & 6.187542 & -1.125408 & -3.239527 \\
\hline $\mathrm{H}$ & 8.584493 & -1.364366 & -2.737532 \\
\hline $\mathrm{H}$ & 9.406604 & -3.335408 & -1.453722 \\
\hline $\mathrm{H}$ & 7.777792 & -5.061165 & -0.696076 \\
\hline $\mathrm{H}$ & 5.398447 & -4.820534 & -1.188674 \\
\hline & 3.893165 & -3.690251 & -5.047032 \\
\hline $\mathrm{H}$ & 3.438831 & -2.443547 & -7.141777 \\
\hline $\mathrm{H}$ & 3.110659 & 0.025669 & -7.071053 \\
\hline $\mathrm{H}$ & 3.259524 & 1.222288 & -4.900456 \\
\hline & 3.733865 & -0.031059 & -2.822774 \\
\hline & 1.561370 & -5.389836 & 2.029769 \\
\hline $\mathrm{H}$ & 0.878121 & -7.344952 & 3.311823 \\
\hline $\mathrm{H}$ & 4.630540 & -7.277400 & 5.408200 \\
\hline $\mathrm{H}$ & 5.330824 & -5.342876 & 4.094172 \\
\hline 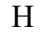 & 9.179737 & 2.031070 & 7.425729 \\
\hline
\end{tabular}

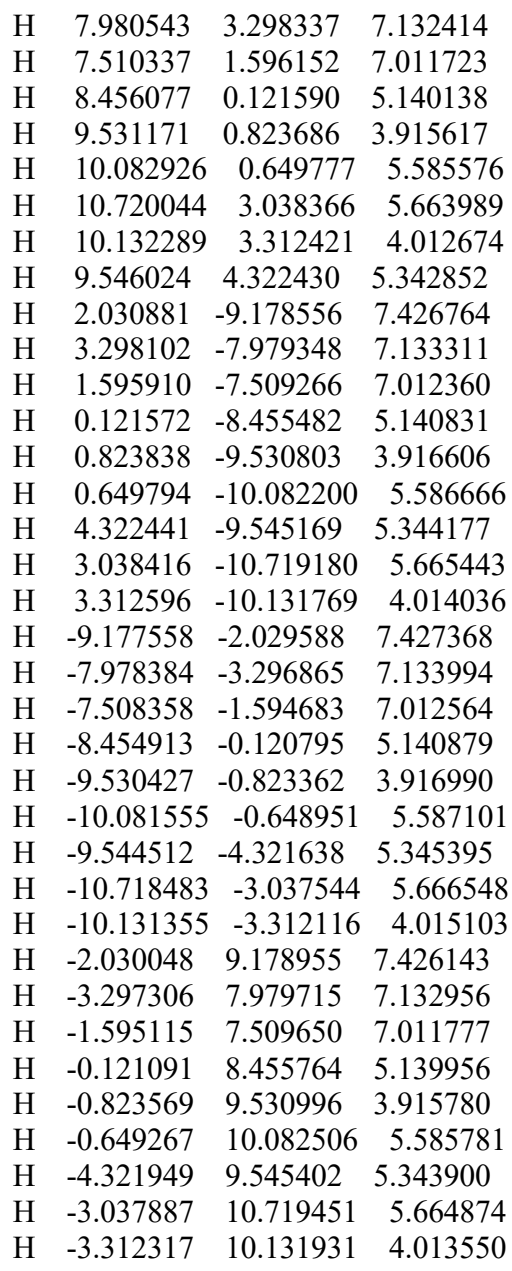

$$
\begin{array}{lccc}
\text { M'1 } & & & \\
\mathrm{Rh} & 0.045009 & -0.463531 & -2.554519 \\
\mathrm{Rh} & 0.047989 & -0.122106 & -0.192105 \\
\mathrm{O} & -0.292329 & -2.126129 & 0.014564 \\
\mathrm{O} & -0.825671 & -2.311985 & -2.174839 \\
\mathrm{O} & 2.065373 & -0.460248 & -0.215877 \\
\mathrm{O} & 1.926415 & -1.306136 & -2.308706 \\
\mathrm{O} & 0.386382 & 1.864726 & -0.546455 \\
\mathrm{O} & 0.906721 & 1.420081 & -2.701707 \\
\mathrm{O} & -1.972363 & 0.179555 & -0.314542 \\
\mathrm{O} & -1.845189 & 0.405888 & -2.559275 \\
\mathrm{Cl} & 1.577535 & -4.885335 & 0.696250 \\
\mathrm{Cl} & 4.687592 & 1.638218 & -0.495442 \\
\mathrm{Cl} & -1.736021 & 4.401703 & -0.841864 \\
\mathrm{Cl} & -4.649125 & -2.041586 & -0.227995 \\
\mathrm{C} & -1.138518 & -4.191303 & -0.716977 \\
\mathrm{C} & -0.880862 & -5.318593 & -1.768121 \\
\mathrm{C} & -2.255258 & -4.748952 & -1.574727 \\
\mathrm{C} & -0.713833 & -2.777230 & -0.997066 \\
\mathrm{C} & -1.136714 & -4.465090 & 0.759052 \\
\mathrm{C} & 0.022823 & -4.711705 & 1.503342 \\
\mathrm{C} & -0.005707 & -4.757556 & 2.896420 \\
\mathrm{C} & -1.198254 & -4.504176 & 3.572763 \\
\mathrm{C} & -2.375878 & -4.226175 & 2.862553 \\
\mathrm{C} & -2.320436 & -4.246883 & 1.464330 \\
\mathrm{C} & -0.564821 & -6.694145 & -1.228610
\end{array}
$$




\begin{tabular}{|c|c|c|c|}
\hline $\mathrm{C}$ & 0.590095 & -7.365590 & -1.657385 \\
\hline C & 0.920174 & -8.624543 & -1.157048 \\
\hline $\mathrm{C}$ & 0.100771 & -9.245314 & -0.213815 \\
\hline $\mathrm{C}$ & -1.055407 & -8.593057 & 0.216282 \\
\hline $\mathrm{C}$ & -1.385608 & -7.335550 & -0.287843 \\
\hline $\mathrm{C}$ & -0.188372 & -4.962304 & -3.058154 \\
\hline $\mathrm{C}$ & -0.822016 & -5.263006 & -4.269472 \\
\hline $\mathrm{C}$ & -0.220137 & -4.943677 & -5.487658 \\
\hline $\mathrm{C}$ & 1.028561 & -4.317796 & -5.504472 \\
\hline $\mathrm{C}$ & 1.673153 & -4.029823 & -4.299732 \\
\hline $\mathrm{C}$ & 1.072105 & -4.358628 & -3.085203 \\
\hline $\mathrm{C}$ & -3.633394 & -3.816131 & 3.529064 \\
\hline $\mathrm{C}$ & -4.884050 & -4.228541 & 3.040413 \\
\hline $\mathrm{C}$ & -6.063296 & -3.755065 & 3.605222 \\
\hline $\mathrm{C}$ & -6.054940 & -2.841847 & 4.674436 \\
\hline $\mathrm{C}$ & -4.804498 & -2.450123 & 5.169830 \\
\hline $\mathrm{C}$ & -3.617507 & -2.928199 & 4.612166 \\
\hline $\mathrm{C}$ & 4.027776 & -1.339002 & -1.161811 \\
\hline $\mathrm{C}$ & 4.939705 & -1.268971 & -2.433696 \\
\hline $\mathrm{C}$ & 4.488472 & -2.588864 & -1.884028 \\
\hline $\mathrm{C}$ & 2.567229 & -1.008278 & -1.252152 \\
\hline $\mathrm{C}$ & 4.592361 & -1.022219 & 1119 \\
\hline $\mathrm{C}$ & 4.915848 & 0.278422 & 0.598594 \\
\hline $\mathrm{C}$ & 5.380041 & 0.540454 & 1.885464 \\
\hline $\mathrm{C}$ & 5.486224 & -0.499752 & 2.805736 \\
\hline $\mathrm{C}$ & 5.130652 & -1.810404 & 2.451905 \\
\hline $\mathrm{C}$ & 4.707318 & -2.042212 & 938 \\
\hline $\mathrm{C}$ & 6.367310 & -0.821425 & -2.213971 \\
\hline $\mathrm{C}$ & 6.855975 & 0.305179 & -2.892707 \\
\hline $\mathrm{C}$ & 8.157918 & 0.760979 & -2.690261 \\
\hline $\mathrm{C}$ & 9.006680 & 0.098174 & -1.803043 \\
\hline $\mathrm{C}$ & 8.537376 & -1.028309 & 6565 \\
\hline $\mathrm{C}$ & 7.235477 & -1.483686 & -1.332807 \\
\hline $\mathrm{C}$ & 4.339550 & -0.843220 & -3.748774 \\
\hline $\mathrm{C}$ & 4.491462 & -1.676868 & -4.862676 \\
\hline $\mathrm{C}$ & 3.941196 & -1.324621 & -6.096496 \\
\hline $\mathrm{C}$ & 3.231666 & -0.128332 & -6.227774 \\
\hline $\mathrm{C}$ & 3.090921 & 0.716567 & -5.124419 \\
\hline $\mathrm{C}$ & 3.651993 & 0.364408 & -3.897750 \\
\hline $\mathrm{C}$ & 5.156598 & -2.916165 & 3.435715 \\
\hline $\mathrm{C}$ & 6.125628 & -2.979937 & 4.449942 \\
\hline $\mathrm{C}$ & 6.124956 & -4.020508 & 5.373049 \\
\hline $\mathrm{C}$ & 5.163698 & -5.044995 & 5.331999 \\
\hline $\mathrm{C}$ & 4.201169 & -4.976236 & 4.316635 \\
\hline $\mathrm{C}$ & 4.194233 & -3.934800 & 3.389638 \\
\hline $\mathrm{C}$ & 1.115867 & 3.657176 & -1.879307 \\
\hline $\mathrm{C}$ & 0.853300 & 4.391593 & -3.238112 \\
\hline $\mathrm{C}$ & 2.239997 & 3.984217 & -2.842598 \\
\hline $\mathrm{C}$ & 0.772267 & 2.206915 & -1.712325 \\
\hline $\mathrm{C}$ & 1.005432 & 4.420101 & -0.592510 \\
\hline $\mathrm{C}$ & -0.222871 & 4.803588 & -0.037000 \\
\hline $\mathrm{C}$ & -0.288487 & 5.473265 & 1.182306 \\
\hline $\mathrm{C}$ & 0.881295 & 5.734856 & 1.891510 \\
\hline $\mathrm{C}$ & 2.128559 & 5.331729 & 1.390866 \\
\hline $\mathrm{C}$ & 2.158742 & 4.694592 & 0.142399 \\
\hline $\mathrm{C}$ & 0.445882 & 5.844768 & -3.141311 \\
\hline $\mathrm{C}$ & -0.802043 & 6.250360 & -3.638081 \\
\hline $\mathrm{C}$ & -1.222287 & 7.575775 & -3.536587 \\
\hline $\mathrm{C}$ & -0.401219 & 8.531231 & -2.936452 \\
\hline $\mathrm{C}$ & 0.845875 & 8.144206 & -2.444780 \\
\hline $\mathrm{C}$ & 1.264654 & 6.818021 & -2.549598 \\
\hline & 0.226160 & 3.639091 & -4.382430 \\
\hline
\end{tabular}

$\begin{array}{llll}\text { C } & 0.864983 & 3.649839 & -5.627850\end{array}$

$\begin{array}{llll}\text { C } & 0.318278 & 2.964445 & -6.714510\end{array}$

$\begin{array}{llll}\text { C } & -0.877789 & 2.258444 & -6.563516\end{array}$

$\begin{array}{llll}\text { C } & -1.527464 & 2.255777 & -5.327220\end{array}$

$\begin{array}{llll}\text { C } & -0.982696 & 2.951367 & -4.249164\end{array}$

$\begin{array}{llll}\text { C } & 3.377814 & 5.534058 & 2.158739\end{array}$

$\begin{array}{llll}\text { C } & 3.561950 & 6.653137 & 2.986926\end{array}$

$\begin{array}{llll}\text { C } & 4.737816 & 6.819953 & 3.710856\end{array}$

$\begin{array}{llll}\text { C } & 5.785412 & 5.885428 & 3.645498\end{array}$

$\begin{array}{llll}\text { C } & 5.596713 & 4.773257 & 2.815182\end{array}$

$\begin{array}{llll}\text { C } & 4.419414 & 4.598010 & 2.089221\end{array}$

$\begin{array}{llll}\text { C } & -3.953170 & 0.721577 & -1.455169\end{array}$

$\begin{array}{llll}\text { C } & -4.864669 & 0.361796 & -2.672583\end{array}$

C $\quad-4.414859 \quad 1.775255 \quad-2.442613$

$\begin{array}{llll}\text { C } & -2.484031 & 0.403340 & -1.460836\end{array}$

$\begin{array}{llll}\text { C } & -4.503201 & 0.695160 & -0.059475\end{array}$

$\begin{array}{llll}\text { C } & -4.801523 & -0.500410 & 0.606233\end{array}$

$\begin{array}{llll}\text { C } & -5.174960 & -0.517305 & 1.946889\end{array}$

$\begin{array}{llll}\text { C } & -5.236791 & 0.680831 & 2.653863\end{array}$

$\begin{array}{llll}\text { C } & -4.936869 & 1.902588 & 2.030112\end{array}$

$\begin{array}{llll}\text { C } & -4.587568 & 1.879776 & 0.672323\end{array}$

$\begin{array}{llll}\text { C } & -6.296890 & -0.011597 & -2.356728\end{array}$

$\begin{array}{llll}\text { C } & -6.813089 & -1.236060 & -2.807504\end{array}$

$\begin{array}{llll}\text { C } & -8.119338 & -1.622228 & -2.510308\end{array}$

C $\quad-8.945320 \quad-0.790556 \quad-1.753399$

$\begin{array}{llll}\text { C } & -8.448858 & 0.433713 & -1.304618\end{array}$

$\begin{array}{llll}\text { C } & -7.143056 & 0.819302 & -1.606514\end{array}$

$\begin{array}{llll}\text { C } & -4.279627 & -0.364201 & -3.856588\end{array}$

$\begin{array}{llll}\text { C } & -4.502674 & 0.149378 & -5.139167\end{array}$

$\begin{array}{llll}\text { C } & -3.991206 & -0.498865 & -6.265360\end{array}$

C $\quad-3.247216-1.671815 \quad-6.117667$

$\begin{array}{llll}\text { C } & -3.033137 & -2.198623 & -4.841363\end{array}$

$\begin{array}{llll}\text { C } & -3.557514 & -1.553639 & -3.722059\end{array}$

$\begin{array}{llll}\text { C } & -4.940094 & 3.175941 & 2.784628\end{array}$

$\begin{array}{llll}\text { C } & -4.021284 & 4.191875 & 2.484802\end{array}$

$\begin{array}{llll}\text { C } & -4.011504 & 5.388305 & 3.200712\end{array}$

C $\quad-4.911942 \quad 5.622963 \quad 4.247531$

$\begin{array}{llll}\text { C } & -5.829835 & 4.600669 & 4.543075\end{array}$

$\begin{array}{llll}\text { C } & -5.847427 & 3.405723 & 3.831856\end{array}$

$\begin{array}{llll}\text { C } & 5.201061 & -6.172752 & 6.370859\end{array}$

$\begin{array}{llll}\text { C } & 6.548279 & -6.920367 & 6.262243\end{array}$

$\begin{array}{llll}\text { C } & 5.063019 & -5.567797 & 7.785196\end{array}$

C $\quad 4.065884 \quad-7.189503 \quad 6.167135$

$\begin{array}{llll}\text { C } & -7.380351 & -2.315915 & 5.240068\end{array}$

$\begin{array}{llll}\text { C } & -7.166725 & -1.281767 & 6.357288\end{array}$

$\begin{array}{llll}\text { C } & -8.187884 & -3.499526 & 5.816794\end{array}$

$\begin{array}{llll}\text { C } & -8.192923 & -1.644854 & 4.110267\end{array}$

$\begin{array}{llll}\text { C } & -4.926029 & 6.923958 & 5.059673\end{array}$

$\begin{array}{llll}\text { C } & -4.672689 & 6.597768 & 6.547931\end{array}$

C $\quad-3.847150 \quad 7.914257 \quad 4.591303$

$\begin{array}{llll}\text { C } & -6.305642 & 7.602358 & 4.911191\end{array}$

$\begin{array}{llll}\text { C } & 7.064014 & 6.109187 & 4.462459\end{array}$

$\begin{array}{llll}\text { C } & 8.087595 & 4.979453 & 4.262640\end{array}$

$\begin{array}{llll}\text { C } & 7.718470 & 7.439776 & 4.030212\end{array}$

$\begin{array}{llll}\text { C } & 6.703834 & 6.178489 & 5.962768\end{array}$

$\mathrm{H} \quad-3.014018 \quad-5.343713 \quad-1.080346$

H $\quad-2.624974 \quad-4.098489 \quad-2.357305$

H $\quad 0.905463 \quad-4.960058 \quad 3.448004$

$\mathrm{H} \quad-1.208444 \quad-4.520154 \quad 4.657784$

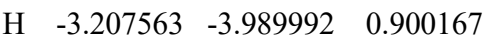

H $\quad 1.239373 \quad-6.896794 \quad-2.386652$

H $\quad 1.821906 \quad-9.118409 \quad-1.506779$
H $\quad 0.356793 \quad-10.224852 \quad 0.178173$

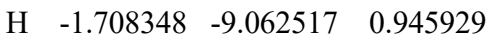

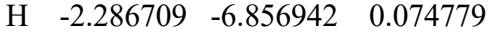

$\mathrm{H} \quad-1.798576 \quad-5.738448 \quad-4.253942$

$\mathrm{H} \quad-0.728407 \quad-5.176068 \quad-6.418742$

H $\quad 1.497191 \quad-4.053663 \quad-6.447824$

H $\quad 2.631973 \quad-3.524433 \quad-4.312338$

$\mathrm{H} \quad 1.561872 \quad-4.107605 \quad-2.151211$

$\mathrm{H} \quad-4.930882 \quad-4.928612 \quad 2.212040$

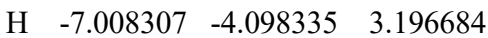

H $\quad-4.739604 \quad-1.746162 \quad 5.990532$

H $\quad-2.667010 \quad-2.572754 \quad 4.998625$

H $\quad 5.198508 \quad-3.230109-1.376309$

$\begin{array}{llll}\mathrm{H} & 3.728155 & -3.114052 & -2.446837\end{array}$

$\mathrm{H} \quad 5.625528 \quad 1.555487 \quad 2.175676$

H $\quad 5.808641 \quad-0.278660 \quad 3.817671$

H $\quad 4.446754 \quad-3.049959 \quad 0.831663$

H $\quad 6.209560 \quad 0.832403 \quad-3.583639$

$\begin{array}{llll}\mathrm{H} & 8.506649 & 1.637390 & -3.228372\end{array}$

H $\quad 10.020556 \quad 0.452022 \quad-1.643185$

H $\quad 9.184588-1.560269-0.435749$

H $\quad 6.903954-2.356630 \quad-0.784808$

H $\quad 5.034233 \quad-2.612034-4.756732$

H $\quad 4.060606 \quad-1.986204 \quad-6.949385$

H $\quad 2.790166 \quad 0.144881 \quad-7.181680$

$\mathrm{H} \quad 2.522407 \quad 1.635615 \quad-5.213892$

H $\quad 3.512140 \quad 1.005487 \quad-3.036490$

$\mathrm{H} \quad 6.900154 \quad-2.220788 \quad 4.503147$

$\begin{array}{lllll}\mathrm{H} & 6.896477 & -4.036138 & 6.136529\end{array}$

H $\quad 3.434347 \quad-5.737983 \quad 4.238509$

H $\quad 3.414459 \quad-3.906307 \quad 2.635922$

H $\quad 2.958807 \quad 4.736210 \quad-2.541699$

H $\quad 2.664728 \quad 3.142399 \quad-3.374309$

$\begin{array}{llll}\mathrm{H} & -1.250911 & 5.765018 & 1.585884\end{array}$

$\mathrm{H} \quad 0.814238 \quad 6.220987 \quad 2.858927$

H $\quad 3.112432 \quad 4.393826 \quad-0.275981$

H $\quad-1.451246 \quad 5.520577 \quad-4.106383$

$\mathrm{H} \quad-2.194766 \quad 7.859406 \quad-3.927865$

$\begin{array}{lllll}\mathrm{H} & -0.726873 & 9.563774 & -2.855778\end{array}$

H $\quad 1.500607 \quad 8.874592 \quad-1.979019$

$\mathrm{H} \quad 2.235984 \quad 6.552614 \quad-2.151595$

H $\quad 1.801702 \quad 4.188615 \quad-5.740046$

H $\quad 0.829809 \quad 2.975745 \quad-7.672416$

H $\quad-1.301605 \quad 1.712235 \quad-7.401120$

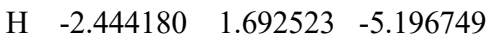

H $\quad-1.472475 \quad 2.919947 \quad-3.284266$

H $\quad 2.787253 \quad 7.411441 \quad 3.047712$

H $\quad 4.841868 \quad 7.702911 \quad 4.333669$

H $\quad \begin{array}{llll}6.371105 & 4.020136 & 2.728654\end{array}$

H $\quad 4.303270 \quad 3.709124 \quad 1.478092$

$\mathrm{H} \quad-5.127575 \quad 2.514148 \quad-2.096362$

H $\quad-3.656340 \quad 2.158082 \quad-3.113996$

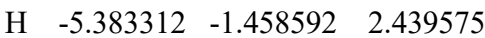

$\begin{array}{llll}\mathrm{H} & -5.479726 & 0.654697 & 3.710874\end{array}$

$\begin{array}{llll}\mathrm{H} & -4.353822 & 2.809814 & 0.165686\end{array}$

$\mathrm{H} \quad-6.183592 \quad-1.896248 \quad-3.391482$

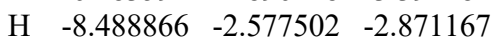

$\mathrm{H} \quad-9.962234-1.090101 \quad-1.519105$

$\begin{array}{llll}\mathrm{H} & -9.077703 & 1.096838 & -0.718144\end{array}$

$\begin{array}{llll}\mathrm{H} & -6.790992 & 1.772824 & -1.233779\end{array}$

$\mathrm{H} \quad-5.071449 \quad 1.068132 \quad-5.251990$

H $\quad-4.166662 \quad-0.083522 \quad-7.253264$ 


$\begin{array}{rrrr}\mathrm{H} & -2.838064 & -2.172998 & -6.989907 \\ \mathrm{H} & -2.443436 & -3.100724 & -4.714918 \\ \mathrm{H} & -3.367056 & -1.951484 & -2.734209 \\ \mathrm{H} & -3.288320 & 4.040128 & 1.698746 \\ \mathrm{H} & -3.279783 & 6.142057 & 2.935130 \\ \mathrm{H} & -6.552996 & 4.739873 & 5.340531 \\ \mathrm{H} & -6.587446 & 2.650006 & 4.077481 \\ \mathrm{H} & 7.396432 & -6.253303 & 6.440326 \\ \mathrm{H} & 6.594310 & -7.727138 & 7.001684 \\ \mathrm{H} & 6.668327 & -7.361116 & 5.267357 \\ \mathrm{H} & 4.113057 & -5.033436 & 7.887822 \\ \mathrm{H} & 5.092983 & -6.360521 & 8.540447 \\ \mathrm{H} & 5.871096 & -4.864538 & 8.005187 \\ \mathrm{H} & 3.080931 & -6.720322 & 6.255996 \\ \mathrm{H} & 4.127695 & -7.675565 & 5.188346 \\ \mathrm{H} & 4.132766 & -7.970287 & 6.930870 \\ \mathrm{H} & -8.137133 & -0.930315 & 6.720612 \\ \mathrm{H} & -6.627761 & -1.708317 & 7.208993 \\ \mathrm{H} & -6.609487 & -0.409399 & 6.000579 \\ \mathrm{H} & -9.142286 & -3.146018 & 6.221527 \\ \mathrm{H} & -8.404762 & -4.249775 & 5.051016 \\ \mathrm{H} & -7.634598 & -3.990467 & 6.623828 \\ \mathrm{H} & -7.648599 & -0.795672 & 3.686105 \\ \mathrm{H} & -8.411575 & -2.342251 & 3.296991 \\ \mathrm{H} & -9.147440 & -1.277086 & 4.501461 \\ \mathrm{H} & -4.684951 & 7.516479 & 7.144206 \\ \mathrm{H} & -5.436373 & 5.926445 & 6.950629 \\ \mathrm{H} & -3.697691 & 6.117607 & 6.679386 \\ \mathrm{H} & -2.840644 & 7.496998 & 4.696606 \\ \mathrm{H} & -3.990668 & 8.205458 & 3.545965 \\ \mathrm{H} & -3.895821 & 8.822796 & 5.199210 \\ \mathrm{H} & -7.113492 & 6.958992 & 5.271116 \\ \mathrm{H} & -6.334262 & 8.532368 & 5.489116 \\ \mathrm{H} & -6.508302 & 7.844652 & 3.863006 \\ \mathrm{H} & 8.403784 & 4.898947 & 3.217806 \\ \mathrm{H} & 8.978413 & 5.182294 & 4.864859 \\ \mathrm{H} & 7.687609 & 4.009956 & 4.576140 \\ \mathrm{H} & 7.049584 & 8.290154 & 4.189839 \\ \mathrm{H} & 8.631706 & 7.617674 & 4.608206 \\ \mathrm{H} & 7.985084 & 7.414377 & 2.968736 \\ \mathrm{H} & 6.007274 & 6.994593 & 6.174317 \\ \mathrm{H} & 6.239681 & 5.243976 & 6.294006 \\ \mathrm{H} & 7.606156 & 6.343378 & 6.561419\end{array}$

$\begin{array}{lccc}\text { M'2 } & & & \\ \mathrm{Rh} & 0.617785 & 0.153553 & -2.492279 \\ \mathrm{Rh} & 0.213975 & 0.120379 & -0.140919 \\ \mathrm{O} & 2.235548 & -0.033462 & 0.113224 \\ \mathrm{O} & 2.521828 & -0.551871 & -2.069002 \\ \mathrm{O} & 0.373775 & 2.162201 & -0.155908 \\ \mathrm{O} & 1.284088 & 2.097598 & -2.225772 \\ \mathrm{O} & -1.793579 & 0.255463 & -0.551439 \\ \mathrm{O} & -1.338475 & 0.824164 & -2.692265 \\ \mathrm{O} & 0.116001 & -1.921035 & -0.280782 \\ \mathrm{O} & -0.068390 & -1.806404 & -2.530416 \\ \mathrm{Cl} & 4.891021 & 1.991554 & 0.888775 \\ \mathrm{Cl} & -1.862583 & 4.648555 & -0.404965 \\ \mathrm{Cl} & -3.740107 & -2.320819 & -1.262394 \\ \mathrm{Cl} & 2.471928 & -4.579410 & -0.081625 \\ \mathrm{C} & 4.372176 & -0.745057 & -0.555655 \\ \mathrm{C} & 5.511743 & -0.410808 & -1.570667 \\ \mathrm{C} & 5.024765 & -1.819761 & -1.400045\end{array}$

C $\quad 2.943139 \quad-0.410604 \quad-0.878077$

$\begin{array}{llll}\text { C } & 4.599220 & -0.739031 & 0.927986\end{array}$

$\begin{array}{llll}\text { C } & 4.771691 & 0.426020 & 1.683932\end{array}$

$\begin{array}{llll}\text { C } & 4.786636 & 0.390687 & 3.077492\end{array}$

$\begin{array}{llll}\text { C } & 4.577669 & -0.817652 & 3.740223\end{array}$

$\begin{array}{llll}\text { C } & 4.372085 & -2.002789 & 3.017513\end{array}$

$\begin{array}{llll}\text { C } & 4.418146 & -1.937217 & 1.620233\end{array}$

$\begin{array}{llll}\text { C } & 6.851060 & -0.015529 & -0.993904\end{array}$

$\begin{array}{llll}\text { C } & 7.468424 & 1.173509 & -1.410453\end{array}$

$\begin{array}{llll}\text { C } & 8.693936 & 1.574105 & -0.879480\end{array}$

$\begin{array}{llll}\text { C } & 9.333953 & 0.793361 & 0.083520\end{array}$

$\begin{array}{llll}\text { C } & 8.735264 & -0.395502 & 0.502363\end{array}$

C $\quad 7.511594 \quad-0.796494 \quad-0.032763$

$\begin{array}{llll}\text { C } & 5.150072 & 0.264427 & -2.868546\end{array}$

$\begin{array}{llll}\text { C } & 5.539201 & -0.338092 & -4.070499\end{array}$

$\begin{array}{llll}\text { C } & 5.218333 & 0.244533 & -5.297435\end{array}$

$\begin{array}{llll}\text { C } & 4.502687 & 1.443582 & -5.332608\end{array}$

$\begin{array}{llll}\text { C } & 4.127224 & 2.059276 & -4.136842\end{array}$

$\begin{array}{llll}\text { C } & 4.457278 & 1.477834 & -2.912841\end{array}$

$\begin{array}{llll}\text { C } & 4.022579 & -3.283381 & 3.674319\end{array}$

$\begin{array}{llll}\text { C } & 4.517859 & -4.506785 & 3.194224\end{array}$

C $\quad 4.115360 \quad-5.711755 \quad 3.759859$

$\begin{array}{llll}\text { C } & 3.194440 & -5.757771 & 4.821508\end{array}$

C $\quad 2.715714-4.533205 \quad 5.305035$

C $\quad 3.122218 \quad-3.320401 \quad 4.746603$

$\begin{array}{llll}\text { C } & 1.157228 & 4.184220 & -1.065097\end{array}$

$\begin{array}{llll}\text { C } & 1.039564 & 5.096409 & -2.333644\end{array}$

$\begin{array}{llll}\text { C } & 2.380793 & 4.724957 & -1.776796\end{array}$

$\begin{array}{llll}\text { C } & 0.917481 & 2.707540 & -1.172195\end{array}$

$\begin{array}{llll}\text { C } & 0.798883 & 4.723985 & 0.286941\end{array}$

$\begin{array}{llll}\text { C } & -0.520809 & 4.969746 & 0.687290\end{array}$

$\begin{array}{llll}\text { C } & -0.814496 & 5.432039 & 1.968103\end{array}$

$\begin{array}{llll}\text { C } & 0.213923 & 5.614589 & 2.889472\end{array}$

$\begin{array}{llll}\text { C } & 1.545018 & 5.335796 & 2.543698\end{array}$

$\begin{array}{llll}\text { C } & 1.806633 & 4.911178 & 1.233743\end{array}$

$\begin{array}{llll}\text { C } & 0.504451 & 6.493299 & -2.113709\end{array}$

C $\quad-0.642754 \quad 6.915987 \quad-2.802174$

C $\quad-1.175299 \quad 8.189126 \quad-2.603824$

C $\quad-0.571202 \quad 9.074452 \quad-1.710464$

$\begin{array}{llll}\text { C } & 0.573860 & 8.670582 & -1.023017\end{array}$

$\begin{array}{llll}\text { C } & 1.106133 & 7.397577 & -1.225184\end{array}$

$\begin{array}{llll}\text { C } & 0.659251 & 4.472562 & -3.651283\end{array}$

$\begin{array}{llll}\text { C } & 1.493049 & 4.667696 & -4.758269\end{array}$

$\begin{array}{llll}\text { C } & 1.182842 & 4.095772 & -5.993578\end{array}$

C $\quad 0.027787 \quad 3.322290-6.133272$

$\begin{array}{llll}\text { C } & -0.817790 & 3.138787 & -5.036995\end{array}$

$\begin{array}{llll}\text { C } & -0.507266 & 3.718902 & -3.808309\end{array}$

$\begin{array}{llll}\text { C } & 2.644231 & 5.446295 & 3.528939\end{array}$

$\begin{array}{llll}\text { C } & 2.645314 & 6.433401 & 4.527638\end{array}$

$\begin{array}{llll}\text { C } & 3.682830 & 6.513195 & 5.450792\end{array}$

$\begin{array}{llll}\text { C } & 4.766508 & 5.618577 & 5.424845\end{array}$

$\begin{array}{lllll}\text { C } & 4.759554 & 4.637168 & 4.425418\end{array}$

$\begin{array}{llll}\text { C } & 3.721339 & 4.549478 & 3.498977\end{array}$

C $\quad-3.615522 \quad 0.689868 \quad-1.973637$

$\begin{array}{llll}\text { C } & -4.232927 & 0.465179 & -3.395496\end{array}$

$\begin{array}{llll}\text { C } & -4.062469 & 1.848260 & -2.846885\end{array}$

$\begin{array}{llll}\text { C } & -2.139077 & 0.575152 & -1.736268\end{array}$

$\begin{array}{llll}\text { C } & -4.441302 & 0.294719 & -0.784254\end{array}$

$\begin{array}{llll}\text { C } & -4.561571 & -1.042114 & -0.376114\end{array}$

$\begin{array}{llll}\text { C } & -5.309184 & -1.402823 & 0.741179\end{array}$

$\begin{array}{llll}\text { C } & -5.959937 & -0.415521 & 1.475554\end{array}$

C $\quad-5.855265 \quad 0.936068 \quad 1.115058$ $\begin{array}{llll}\text { C } & -5.086866 & 1.261142 & -0.012551\end{array}$

$\begin{array}{llll}\text { C } & -5.610491 & -0.162578 & -3.410231\end{array}$

C $\quad-5.763330-1.476783 \quad-3.877066$

$\begin{array}{llll}\text { C } & -7.007926 & -2.104079 & -3.867040\end{array}$

$\begin{array}{llll}\text { C } & -8.133743 & -1.427297 & -3.394791\end{array}$

$\begin{array}{llll}\text { C } & -7.999095 & -0.114979 & -2.941041\end{array}$

$\begin{array}{llll}\text { C } & -6.751793 & 0.509913 & -2.952127\end{array}$

$\begin{array}{llll}\text { C } & -3.371452 & 0.067199 & -4.566198\end{array}$

$\begin{array}{llll}\text { C } & -3.499683 & 0.785463 & -5.760904\end{array}$

$\begin{array}{llll}\text { C } & -2.743928 & 0.442456 & -6.883955\end{array}$

$\begin{array}{llll}\text { C } & -1.849455 & -0.628567 & -6.822192\end{array}$

C $\quad-1.726441-1.359036 \quad-5.637495$

$\begin{array}{llll}\text { C } & -2.489777 & -1.016718 & -4.521998\end{array}$

$\begin{array}{llll}\text { C } & -6.528019 & 1.993444 & 1.902183\end{array}$

$\begin{array}{llll}\text { C } & -7.054693 & 3.138212 & 1.280847\end{array}$

$\begin{array}{llll}\text { C } & -7.679076 & 4.132504 & 2.025303\end{array}$

$\begin{array}{llll}\text { C } & -7.811798 & 4.036502 & 3.421024\end{array}$

$\begin{array}{llll}\text { C } & -7.288049 & 2.891542 & 4.034698\end{array}$

$\begin{array}{llll}\text { C } & -6.658911 & 1.891108 & 3.293622\end{array}$

$\begin{array}{llll}\text { C } & -0.266517 & -3.932323 & -1.434212\end{array}$

$\begin{array}{llll}\text { C } & 0.188879 & -4.814926 & -2.638524\end{array}$

C $\quad-1.255763-4.450555 \quad-2.461012$

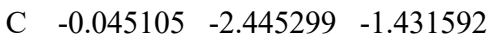

$\begin{array}{llll}\text { C } & -0.273052 & -4.473083 & -0.034099\end{array}$

$\begin{array}{llll}\text { C } & 0.897301 & -4.737374 & 0.686853\end{array}$

$\begin{array}{llll}\text { C } & 0.860130 & -5.073355 & 2.038045\end{array}$

$\begin{array}{llll}C & -0.363516 & -5.117146 & 2.701393\end{array}$

$\begin{array}{llll}\text { C } & -1.559991 & -4.826005 & 2.025147\end{array}$

$\begin{array}{llll}\text { C } & -1.483039 & -4.531256 & 0.657620\end{array}$

$\begin{array}{llll}\text { C } & 0.626796 & -6.228788 & -2.324052\end{array}$

$\begin{array}{llll}\text { C } & 1.866969 & -6.695773 & -2.785019\end{array}$

$\begin{array}{llll}\text { C } & 2.305900 & -7.986733 & -2.493646\end{array}$

$\begin{array}{llll}\text { C } & 1.513319 & -8.845867 & -1.731963\end{array}$

$\begin{array}{llll}\text { C } & 0.274330 & -8.398175 & -1.272260\end{array}$

$\begin{array}{llll}\text { C } & -0.164545 & -7.108163 & -1.568721\end{array}$

$\begin{array}{llll}\text { C } & 0.922160 & -4.173538 & -3.787820\end{array}$

$\begin{array}{lllll}\text { C } & 0.453867 & -4.381069 & -5.089772\end{array}$

$\begin{array}{llll}\text { C } & 1.120267 & -3.824318 & -6.183284\end{array}$

$\begin{array}{llll}\text { C } & 2.264354 & -3.048429 & -5.982428\end{array}$

$\begin{array}{llll}\text { C } & 2.743531 & -2.846043 & -4.685758\end{array}$

$\begin{array}{llll}\text { C } & 2.080942 & -3.414898 & -3.598807\end{array}$

$\begin{array}{llll}\text { C } & -2.861465 & -4.737412 & 2.725957\end{array}$

$\begin{array}{llll}\text { C } & -4.071580 & -4.995324 & 2.058320\end{array}$

$\begin{array}{llll}\text { C } & -5.294049 & -4.828255 & 2.699532\end{array}$

$\begin{array}{llll}\text { C } & -5.374705 & -4.406702 & 4.038125\end{array}$

$\begin{array}{llll}\text { C } & -4.166289 & -4.169172 & 4.704338\end{array}$

$\begin{array}{llll}\text { C } & -2.936996 & -4.326381 & 4.063618\end{array}$

$\begin{array}{llll}\text { C } & -8.506568 & 5.159246 & 4.201708\end{array}$

$\begin{array}{llll}\text { C } & -8.551656 & 4.877087 & 5.712316\end{array}$

$\begin{array}{llll}\text { C } & -9.956979 & 5.311398 & 3.692835\end{array}$

$\begin{array}{llll}\text { C } & -7.743047 & 6.483054 & 3.977412\end{array}$

$\begin{array}{llll}\text { C } & 5.888866 & 5.744387 & 6.462573\end{array}$

$\begin{array}{llll}\text { C } & 5.293787 & 5.583833 & 7.878705\end{array}$

$\begin{array}{llll}\text { C } & 6.544910 & 7.137020 & 6.337042\end{array}$

$\begin{array}{llll}\text { C } & 6.979285 & 4.677236 & 6.272386\end{array}$

$\begin{array}{llll}\text { C } & 2.754115 & -7.111507 & 5.393006\end{array}$

$\begin{array}{llll}\text { C } & 1.711450 & -6.958845 & 6.512283\end{array}$

$\begin{array}{llll}\text { C } & 3.987162 & -7.841248 & 5.969987\end{array}$

$\begin{array}{llll}\text { C } & 2.133448 & -7.968761 & 4.267504\end{array}$

C $\quad-6.744714 \quad-4.206170 \quad 4.697356$

$\begin{array}{llll}\text { C } & -6.626774 & -3.833529 & 6.184330\end{array}$

$\begin{array}{llll}\text { C } & -7.569801 & -5.506873 & 4.588741\end{array}$ 
$\begin{array}{llll}\text { C } & -7.486497 & -3.063886 & 3.968182\end{array}$

H $\quad 5.651654 \quad-2.541483 \quad-0.890078$

$\mathrm{H} \quad 4.422668 \quad-2.225555 \quad-2.202535$

$\mathrm{H} \quad 4.933063 \quad 1.306590 \quad 3.638923$

H $4.573137 \quad-0.835458 \quad 4.825233$

$\mathrm{H} \quad 4.216202 \quad-2.831813 \quad 1.044216$

H $\quad 6.983310 \quad 1.793596 \quad-2.154355$

H $\quad 9.146461 \quad 2.500470 \quad-1.220637$

$\begin{array}{llll}\mathrm{H} & 10.287395 & 1.104310 & 0.499387\end{array}$

H $\quad 9.220741 \quad-1.018924 \quad 1.247216$

H $\quad 7.073998 \quad-1.721648 \quad 0.321070$

H $\quad 6.085942 \quad-1.276314 \quad-4.040791$

H $\quad 5.519956 \quad-0.240086 \quad-6.221213$

H $\quad 4.238035 \quad 1.896421 \quad-6.283434$

H $\quad 3.555461 \quad 2.979965 \quad-4.165307$

H $4.140569 \quad 1.944210 \quad-1.986695$

H $\quad 5.228374 \quad-4.511789 \quad 2.373543$

H $\quad 4.522910 \quad-6.634259 \quad 3.358979$

H $\quad 2.000923 \quad-4.510181 \quad 6.118588$

$\begin{array}{llll}\mathrm{H} & 2.703287 & -2.393075 & 5.125706\end{array}$

$\mathrm{H} \quad 2.973154 \quad 5.471613 \quad-1.262498$

H $\quad 2.955078 \quad 4.000482 \quad-2.338412$

$\begin{array}{llll}\mathrm{H} & -1.844388 & 5.620027 & 2.249277\end{array}$

$\mathrm{H} \quad-0.029489 \quad 5.937061 \quad 3.896301$

H $\quad 2.829956 \quad 4.712608 \quad 0.934698$

$\mathrm{H} \quad-1.124752 \quad 6.240800 \quad-3.498567$

$\mathrm{H} \quad-2.065422 \quad 8.486718 \quad-3.150110$

$\mathrm{H} \quad-0.984640 \quad 10.066072 \quad-1.554020$

$\begin{array}{llll}\text { H } & 1.060549 & 9.346929 & -0.326747\end{array}$

H $\quad 1.991252 \quad 7.116914-0.668296$

H $\quad 2.395699 \quad 5.261776 \quad-4.645538$

H $\quad 1.844774 \quad 4.249000 \quad-6.840805$

$\begin{array}{llll}\mathrm{H} & -0.213608 & 2.862592 & -7.087166\end{array}$

$\mathrm{H} \quad-1.703311 \quad 2.521802 \quad-5.134524$

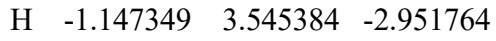

$\begin{array}{llll}\mathrm{H} & 1.838835 & 7.159341 & 4.568107\end{array}$

$\begin{array}{llll}\mathrm{H} & 3.648883 & 7.296206 & 6.201888\end{array}$

$\begin{array}{llll}\mathrm{H} & 5.568197 & 3.918810 & 4.358924\end{array}$

H $\quad 3.742616 \quad 3.757644 \quad 2.758066$

H $\quad-4.923944 \quad 2.421130 \quad-2.528319$

$\mathrm{H} \quad-3.265150 \quad 2.440660 \quad-3.278440$

$\mathrm{H} \quad-5.379611-2.444441 \quad 1.034083$

$\mathrm{H} \quad-6.569044 \quad-0.704359 \quad 2.324330$

$\begin{array}{llll}\mathrm{H} & -4.962878 & 2.303043 & -0.286686\end{array}$

H $\quad-4.898742 \quad-2.012818 \quad-4.249375$

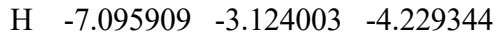

H $\quad-9.104018 \quad-1.914363 \quad-3.385507$

$\begin{array}{llll}\mathrm{H} & -8.865486 & 0.429625 & -2.577944\end{array}$

$\mathrm{H} \quad-6.682264 \quad 1.528932 \quad-2.592048$

H $\quad-4.189568 \quad 1.623664 \quad-5.805199$

$\begin{array}{llll}\mathrm{H} & -2.850028 & 1.014473 & -7.800940\end{array}$

$\begin{array}{lllll}\mathrm{H} & -1.253763 & -0.894177 & -7.690576\end{array}$

H $\quad-1.029367-2.187835 \quad-5.575528$

$\begin{array}{llll}\mathrm{H} & -2.363764 & -1.565433 & -3.598778\end{array}$

$\begin{array}{llll}\mathrm{H} & -6.995067 & 3.238855 & 0.201417\end{array}$

$\begin{array}{llll}\mathrm{H} & -8.078041 & 4.996971 & 1.503991\end{array}$

$\begin{array}{llll}\mathrm{H} & -7.355956 & 2.769347 & 5.108974\end{array}$

H $\quad-6.242121 \quad 1.032004 \quad 3.810492$

H $\quad-1.961742 \quad-5.208262 \quad-2.142289$

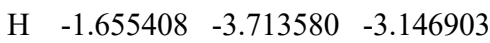

$\begin{array}{llll}\mathrm{H} & 1.781066 & -5.276868 & 2.569702\end{array}$

H $\quad-0.378538 \quad-5.373419 \quad 3.755539$
H $\quad-2.382209-4.254854 \quad 0.121435$

H $\quad 2.498155 \quad-6.040380 \quad-3.372362$

$\mathrm{H} \quad 3.271835 \quad-8.318152 \quad-2.863031$

$\mathrm{H} \quad 1.854154 \quad-9.850725 \quad-1.502163$

$\mathrm{H} \quad-0.358854 \quad-9.053312 \quad-0.681503$

$\mathrm{H} \quad-1.128030 \quad-6.793978 \quad-1.187505$

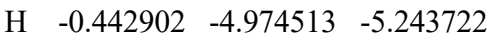

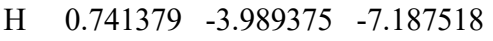

H $\quad 2.779383 \quad-2.604378 \quad-6.829089$

H $\quad 3.621535 \quad-2.230079 \quad-4.521400$

H $\quad 2.439321 \quad-3.237179 \quad-2.592876$

H $\quad-4.057162 \quad-5.340791 \quad 1.029608$

$\begin{array}{llll}\mathrm{H} & -6.202708 & -5.035821 & 2.143191\end{array}$

$\mathrm{H} \quad-4.168326 \quad-3.838894 \quad 5.736061$

H $\quad-2.026833 \quad-4.091074 \quad 4.606303$

$\begin{array}{llll}\mathrm{H} & -9.055093 & 5.703057 & 6.223937\end{array}$

H $\quad-9.105196 \quad 3.959866 \quad 5.936802$

$\mathrm{H} \quad-7.547253 \quad 4.785181 \quad 6.137570$

H $\quad-10.466581 \quad 6.114084 \quad 4.236666$

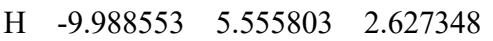

$\begin{array}{llll}\mathrm{H} & -10.520388 & 4.384939 & 3.843630\end{array}$

H $\quad-6.710862 \quad 6.400728 \quad 4.332802$

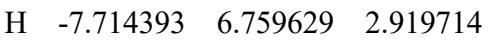

$\mathrm{H} \quad-8.229364 \quad 7.297888 \quad 4.524464$

H $\quad 6.082139 \quad 5.676857 \quad 8.633431$

$\mathrm{H} \quad 4.537301 \quad 6.345300 \quad 8.088296$

$\begin{array}{llll}\mathrm{H} & 4.824724 & 4.601317 & 7.993411\end{array}$

H $\quad 7.347004 \quad 7.245563 \quad 7.075013$

$\mathrm{H} \quad 6.976301 \quad 7.274350 \quad 5.340282$

H $\quad 5.822771 \quad 7.940794 \quad 6.505890$

H $\quad 6.577280 \quad 3.664329 \quad 6.375234$

$\mathrm{H} \quad 7.459011 \quad 4.758342 \quad 5.291884$

H $\quad 7.754541 \quad 4.806481 \quad 7.033762$

H $\quad \begin{array}{llll}1.423200 & -7.947846 & 6.881063\end{array}$

H $\quad 2.105842 \quad-6.389912 \quad 7.360049$

H $\quad 0.804687 \quad-6.459454 \quad 6.155893$

H $\quad 3.694455 \quad-8.813829 \quad 6.379875$

H $\quad 4.747739 \quad-8.014938 \quad 5.203423$

$\begin{array}{llll}\mathrm{H} & 4.444777 & -7.254918 & 6.773268\end{array}$

H $\quad 1.248203 \quad-7.482527 \quad 3.846349$

H $\quad 2.840632 \quad-8.142150 \quad 3.451665$

$\mathrm{H} \quad 1.830863 \quad-8.944750 \quad 4.661619$

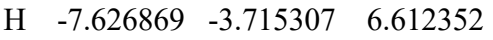

H $\quad-6.108073 \quad-4.610136 \quad 6.755363$

H $\quad-6.092767 \quad-2.888659 \quad 6.325131$

H $\quad-8.549284 \quad-5.371672 \quad 5.059493$

H $\quad-7.737786 \quad-5.798135 \quad 3.548372$

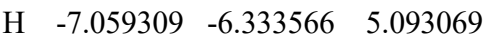

$\mathrm{H} \quad-6.928973 \quad-2.126404 \quad 4.062003$

H $\quad-7.613129 \quad-3.279337 \quad 2.903203$

Н $\quad-8.480239-2.914036 \quad 4.403715$

\section{M"1}

$\begin{array}{llll}\mathrm{Rh} & -0.003600 & 1.185832 & -0.048481\end{array}$

$\begin{array}{llll}\mathrm{Rh} & -0.001599 & -1.190731 & 0.065916\end{array}$

$\begin{array}{llll}\mathrm{O} & -1.686342 & -1.000188 & 1.265388\end{array}$

$\begin{array}{llll}\text { O } & -1.170092 & 1.168138 & 1.644491\end{array}$

$\begin{array}{llll}\mathrm{O} & -1.167247 & -1.177039 & -1.627267\end{array}$

$\begin{array}{lllll}\mathrm{O} & -1.684733 & 0.991951 & -1.252204\end{array}$

O $\quad 1.682379-1.112455 \quad-1.144280$

$\begin{array}{llll}\text { O } & 1.166995 & 1.010760 & -1.729398\end{array}$

O $\quad 1.169751 \quad-1.010562 \quad 1.747080$ $\begin{array}{llll}\text { O } & 1.680166 & 1.113699 & 1.161521\end{array}$

Cl $\quad-1.793850 \quad-1.999852 \quad 4.637980$

$\begin{array}{llll}\mathrm{Cl} & -1.774199 & 1.987173 & -4.629003\end{array}$

Cl $\quad 1.773656 \quad-2.436098 \quad-4.401948$

$\begin{array}{llll}\mathrm{Cl} & 1.789293 & 2.436146 & 4.420337\end{array}$

$\begin{array}{llll}\text { C } & -3.002348 & 0.210123 & 2.817293\end{array}$

$\begin{array}{llll}\text { C } & -3.763201 & 1.574695 & 2.999371\end{array}$

$\begin{array}{llll}\text { C } & -2.755263 & 1.119069 & 4.007923\end{array}$

C $\quad-1.873763 \quad 0.125653 \quad 1.834042$

$\begin{array}{llll}\text { C } & -3.759190 & -1.073357 & 2.957408\end{array}$

$\begin{array}{llll}\text { C } & -3.310449 & -2.135403 & 3.750638\end{array}$

$\begin{array}{llll}\text { C } & -4.021414 & -3.332426 & 3.837540\end{array}$

$\begin{array}{llll}\text { C } & -5.201495 & -3.483548 & 3.114180\end{array}$

$\begin{array}{llll}\text { C } & -5.673107 & -2.450468 & 2.289508\end{array}$

$\begin{array}{llll}\text { C } & -4.932627 & -1.266856 & 2.224106\end{array}$

$\begin{array}{llll}\text { C } & -5.234636 & 1.514197 & 3.355613\end{array}$

$\begin{array}{llll}\text { C } & -5.702224 & 0.831214 & 4.490069\end{array}$

$\begin{array}{llll}\text { C } & -7.061855 & 0.772142 & 4.789734\end{array}$

$\begin{array}{llll}\text { C } & -7.994173 & 1.402589 & 3.963650\end{array}$

$\begin{array}{llll}\text { C } & -7.545210 & 2.087373 & 2.835095\end{array}$

$\begin{array}{llll}\text { C } & -6.184057 & 2.141384 & 2.534360\end{array}$

$\begin{array}{llll}\text { C } & -3.398622 & 2.733781 & 2.110372\end{array}$

$\begin{array}{llll}\text { C } & -2.985419 & 3.939674 & 2.685269\end{array}$

$\begin{array}{llll}\text { C } & -2.672184 & 5.037661 & 1.882156\end{array}$

$\begin{array}{llll}\text { C } & -2.773151 & 4.938195 & 0.491822\end{array}$

$\begin{array}{llll}\text { C } & -3.194625 & 3.738711 & -0.087675\end{array}$

$\begin{array}{llll}\text { C } & -3.512601 & 2.648644 & 0.720177\end{array}$

$\begin{array}{llll}\text { C } & -6.934335 & -2.585863 & 1.528636\end{array}$

C $\quad-7.851476-1.524146 \quad 1.474430$

$\begin{array}{llll}\text { C } & -9.065470 & -1.668049 & 0.811435\end{array}$

$\begin{array}{llll}\text { C } & -9.415792 & -2.861420 & 0.159001\end{array}$

$\begin{array}{llll}\text { C } & -8.482535 & -3.907672 & 0.196619\end{array}$

$\begin{array}{llll}\text { C } & -7.266778 & -3.776006 & 0.868926\end{array}$

$\begin{array}{llll}\text { C } & -2.992900 & -0.218402 & -2.810070\end{array}$

$\begin{array}{llll}\text { C } & -3.754407 & -1.582238 & -2.994457\end{array}$

$\begin{array}{llll}\text { C } & -2.741621 & -1.128342 & -3.998933\end{array}$

$\begin{array}{llll}\text { C } & -1.869427 & -0.134307 & -1.821053\end{array}$

$\begin{array}{llll}\text { C } & -3.747099 & 1.066162 & -2.954077\end{array}$

$\begin{array}{llll}\text { C } & -3.293103 & 2.126859 & -3.746089\end{array}$

$\begin{array}{llll}\text { C } & -4.000233 & 3.325990 & -3.834860\end{array}$

$\begin{array}{llll}\text { C } & -5.181928 & 3.480442 & -3.114853\end{array}$

$\begin{array}{llll}\text { C } & -5.659059 & 2.448496 & -2.291954\end{array}$

C $\quad-4.922266 \quad 1.262746 \quad-2.224385$

$\begin{array}{llll}\text { C } & -5.224291 & -1.519811 & -3.356342\end{array}$

$\begin{array}{llll}\text { C } & -6.177889 & -2.145325 & -2.538699\end{array}$

$\begin{array}{llll}\text { C } & -7.537849 & -2.088695 & -2.844395\end{array}$

$\begin{array}{llll}\text { C } & -7.981398 & -1.403031 & -3.974549\end{array}$

$\begin{array}{llll}\text { C } & -7.044840 & -0.774402 & -4.797250\end{array}$

$\begin{array}{llll}\text { C } & -5.686440 & -0.835961 & -4.492549\end{array}$

C $\quad-3.393929-2.740288-2.102555$

$\begin{array}{llll}\text { C } & -2.974689 & -3.945927 & -2.673500\end{array}$

$\begin{array}{llll}\text { C } & -2.663675 & -5.042128 & -1.867040\end{array}$

$\begin{array}{llll}\text { C } & -2.772996 & -4.941052 & -0.477461\end{array}$

$\begin{array}{llll}\text { C } & -3.200687 & -3.741837 & 0.098024\end{array}$

$\begin{array}{llll}\text { C } & -3.516349 & -2.653668 & -0.713174\end{array}$

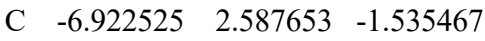

$\begin{array}{llll}\text { C } & -7.845100 & 1.530299 & -1.488262\end{array}$

$\begin{array}{llll}\text { C } & -9.061608 & 1.678751 & -0.830928\end{array}$

$\begin{array}{llll}\text { C } & -9.409196 & 2.872382 & -0.177422\end{array}$

$\begin{array}{llll}\text { C } & -8.470335 & 3.913804 & -0.207364\end{array}$

$\begin{array}{llll}\text { C } & -7.251910 & 3.777634 & -0.874016\end{array}$

$\begin{array}{llll}\text { C } & 2.992785 & -0.060873 & -2.811209\end{array}$ 


\begin{tabular}{|c|c|c|c|}
\hline & 3.753852 & 1.278081 & -3.130219 \\
\hline & 2.741543 & 0.726194 & -4.084890 \\
\hline & 1.868304 & -0.046735 & -1.819871 \\
\hline & 3.747557 & -1.353017 & -2.828369 \\
\hline & 3.294650 & -2.486379 & -3.513204 \\
\hline & 4.004884 & -3.686683 & -3.487391 \\
\hline & 5.188375 & -3.767874 & -2.758347 \\
\hline & 5.663983 & -2.660472 & -2.039256 \\
\hline & 4.924126 & -1.475520 & -2.084974 \\
\hline & 5.224044 & 1.182216 & -3.483790 \\
\hline & 6.175974 & 1.888207 & -2.732343 \\
\hline 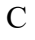 & 7.536102 & 1.805160 & -3.030996 \\
\hline C & 7.981568 & 1.011821 & -4.087501 \\
\hline 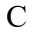 & 7.046788 & 0.301944 & -4.843464 \\
\hline & 5.688136 & 0.389948 & -4.546371 \\
\hline $\mathrm{C}$ & 3.392017 & 2.517913 & 6868 \\
\hline $\mathrm{C}$ & 2.969932 & 3.660664 & 3171 \\
\hline $\mathrm{C}$ & 2.656961 & 4.830118 & -2.348020 \\
\hline- & 2.767679 & 4.866215 & -0.955370 \\
\hline & 3.198524 & 3.730240 & -0.265280 \\
\hline $\mathrm{C}$ & 3.515547 & 2.5682 & 863 \\
\hline $\mathrm{C}$ & 6.929154 & -2.722823 & -1.275472 \\
\hline $\mathrm{C}$ & 7.846279 & -1.660934 & -1.328056 \\
\hline $\mathcal{C}$ & 9.064586 & -1.741793 & -0.662398 \\
\hline 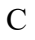 & 9.419424 & -2.868048 & 8052 \\
\hline $\mathrm{C}$ & 8.485 & 2468 & 636 \\
\hline $\mathrm{C}$ & 7.265873 & -3.844792 & -0.507380 \\
\hline $\mathrm{C}$ & 2.999939 & 0.061735 & 2.821133 \\
\hline C & 3.763793 & -1.276986 & 3.135640 \\
\hline $\mathrm{C}$ & 2.755728 & -0.726418 & 510 \\
\hline $\mathrm{C}$ & 1.870467 & 0.04 & 249 \\
\hline $\mathrm{C}$ & 3.754676 & 1.354056 & 482 \\
\hline $\mathrm{C}$ & 3.305656 & 2.486843 & 3.523803 \\
\hline $\mathrm{C}$ & 4.016292 & 3.686876 & 3.495954 \\
\hline $\mathrm{C}$ & 5.196240 & 3.768432 & 257 \\
\hline $\mathrm{C}$ & 5.667812 & 2.661693 & 3466 \\
\hline $\mathrm{C}$ & 4.927654 & 1.477038 & 5473 \\
\hline $\mathrm{C}$ & 5.235627 & -1.180216 & 3.482240 \\
\hline $\mathrm{C}$ & 6.184431 & -1.885563 & 2.726197 \\
\hline C & 7.545921 & -1.801881 & 416 \\
\hline $\mathrm{C}$ & 7.995934 & -1.008294 & 4.072826 \\
\hline $\mathrm{C}$ & 7.064343 & -0.299093 & 4.833346 \\
\hline $\mathrm{C}$ & 5.704331 & -0.387933 & 4.542800 \\
\hline $\mathrm{C}$ & 3.399592 & -2.517409 & 2.364315 \\
\hline $\mathrm{C}$ & 3.516323 & -2.568409 & 0.972739 \\
\hline $\mathrm{C}$ & 3.196911 & -3.731157 & 0.274419 \\
\hline $\mathrm{C}$ & 2.771176 & -4.867360 & 0.967270 \\
\hline $\mathrm{C}$ & 2.667888 & -4.830778 & 2.360478 \\
\hline $\mathrm{C}$ & 2.982789 & -3.660512 & 3.053354 \\
\hline $\mathrm{C}$ & 6.929101 & 2.724675 & 1.268337 \\
\hline $\mathrm{C}$ & 7.846375 & 1.662665 & 1.314912 \\
\hline $\mathrm{C}$ & 9.061607 & 1.744575 & 0.643733 \\
\hline $\mathrm{C}$ & 9.413047 & 2.872064 & -0.116460 \\
\hline $\mathrm{C}$ & 8.479131 & 3.916501 & -0.179288 \\
\hline $\mathrm{C}$ & 7.262186 & 3.847740 & 0.500252 \\
\hline $\mathrm{C}$ & -10.790082 & -3.004117 & $7 \quad-0.510314$ \\
\hline $\mathrm{C}$ & -10.858483 & -4.230850 & -1.436770 \\
\hline $\mathrm{C}$ & -11.853183 & -3.160649 & 0.600380 \\
\hline & -11.126204 & -1.751785 & -1.348148 \\
\hline & 10.789552 & 2.952794 & -0.791437 \\
\hline $\mathrm{C}$ & 11.126375 & 1.630675 & -1.513700 \\
\hline & 10.863043 & 4.092326 & -1.822696 \\
\hline
\end{tabular}

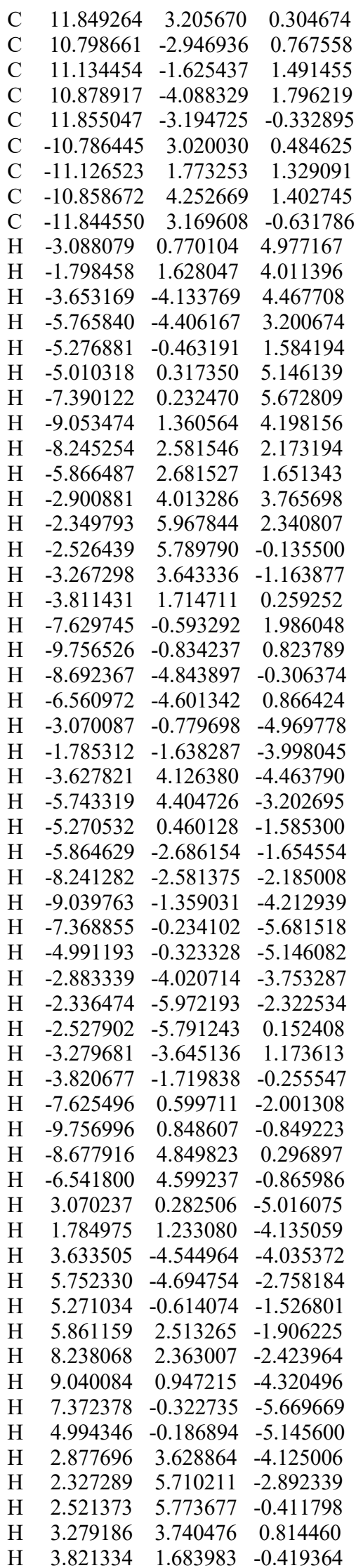

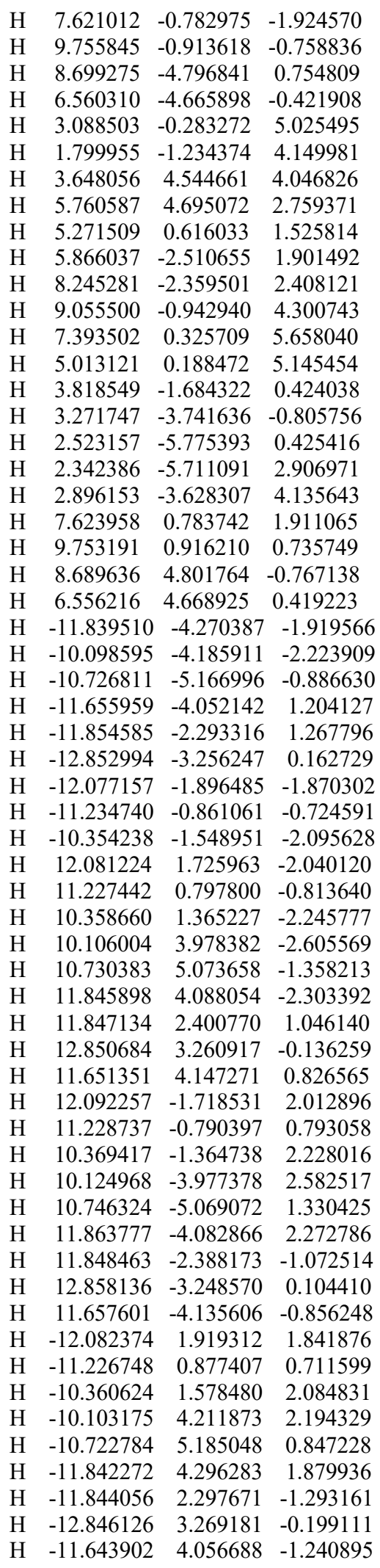

M"2

$\begin{array}{lllll}\mathrm{Rh} & 0.494375 & 0.393931 & -1.155732\end{array}$ 
$\begin{array}{llll}\mathrm{Rh} & 0.483212 & -0.397520 & 1.098290\end{array}$

$\begin{array}{llll}\text { O } & -1.165831 & 0.829089 & 1.378631\end{array}$

$\begin{array}{lllll}\mathrm{O} & -0.762433 & 1.902034 & -0.568658\end{array}$

$\begin{array}{llll}\mathrm{O} & -0.754467 & -1.911008 & 0.485688\end{array}$

$\begin{array}{lllll}\text { O } & -1.153140 & -0.835068 & -1.459489\end{array}$

$\begin{array}{llll}\text { O } & 2.122319 & -1.560746 & 0.638927\end{array}$

$\begin{array}{llll}\text { O } & 1.856813 & -1.115719 & -1.563300\end{array}$

$\begin{array}{llll}\mathrm{O} & 1.825954 & 1.113905 & 1.537346\end{array}$

$\begin{array}{llll}\text { O } & 2.118602 & 1.564596 & -0.659866\end{array}$

$\begin{array}{llll}\mathrm{Cl} & -1.290985 & 3.616373 & 3.431100\end{array}$

$\begin{array}{llll}\text { Cl } & -1.356709 & -3.602895 & -3.531235\end{array}$

$\begin{array}{lllll}\mathrm{Cl} & 3.008065 & -3.458577 & -3.707652\end{array}$

$\begin{array}{llll}\mathrm{Cl} & 2.953139 & 3.445635 & 3.703844\end{array}$

$\begin{array}{llll}\text { C } & -2.574915 & 2.636620 & 0.782376\end{array}$

$\begin{array}{llll}\text { C } & -3.375127 & 3.268521 & -0.418375\end{array}$

$\begin{array}{llll}\text { C } & -2.379961 & 4.077700 & 0.346472\end{array}$

$\begin{array}{llll}\text { C } & -1.420938 & 1.720907 & 0.504328\end{array}$

$\begin{array}{llll}\text { C } & -3.294101 & 2.327841 & 2.058767\end{array}$

$\begin{array}{llll}\text { C } & -2.812764 & 2.735788 & 3.309114\end{array}$

$\begin{array}{llll}\text { C } & -3.498844 & 2.437268 & 4.485997\end{array}$

$\begin{array}{llll}\text { C } & -4.688015 & 1.715471 & 4.423999\end{array}$

$\begin{array}{llll}\text { C } & -5.191315 & 1.265398 & 3.193460\end{array}$

$\begin{array}{llll}\text { C } & -4.469743 & 1.574181 & 2.035294\end{array}$

$\begin{array}{llll}\text { C } & -4.853981 & 3.526194 & -0.219055\end{array}$

$\begin{array}{llll}\text { C } & -5.336057 & 4.385581 & 0.780468\end{array}$

$\begin{array}{llll}\text { C } & -6.703328 & 4.589622 & 0.960784\end{array}$

$\begin{array}{llll}\text { C } & -7.627333 & 3.939379 & 0.141085\end{array}$

$\begin{array}{llll}\text { C } & -7.162920 & 3.085371 & -0.858847\end{array}$

$\begin{array}{llll}\text { C } & -5.794613 & 2.883138 & -1.037812\end{array}$

$\begin{array}{llll}\text { C } & -3.010222 & 2.883947 & -1.827820\end{array}$

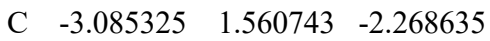

$\begin{array}{llll}\text { C } & -2.770566 & 1.229710 & -3.585087\end{array}$

C $\quad-2.386298 \quad 2.228621 \quad-4.483086$

$\begin{array}{llll}\text { C } & -2.318600 & 3.556976 & -4.054054\end{array}$

$\begin{array}{llll}\text { C } & -2.631078 & 3.880723 & -2.732800\end{array}$

$\begin{array}{llll}\text { C } & -6.467735 & 0.522221 & 3.105861\end{array}$

$\begin{array}{llll}\text { C } & -6.856576 & -0.393723 & 4.096124\end{array}$

$\begin{array}{llll}\text { C } & -8.088609 & -1.038699 & 4.029245\end{array}$

$\begin{array}{llll}\text { C } & -8.986603 & -0.807064 & 2.973352\end{array}$

$\begin{array}{llll}\text { C } & -8.582264 & 0.096930 & 1.981108\end{array}$

$\begin{array}{llll}\text { C } & -7.350402 & 0.745063 & 2.039576\end{array}$

$\begin{array}{llll}\text { C } & -2.580542 & -2.629775 & -0.850398\end{array}$

$\begin{array}{llll}\text { C } & -3.361418 & -3.264745 & 0.362848\end{array}$

$\begin{array}{llll}\text { C } & -2.383705 & -4.073978 & -0.424265\end{array}$

$\begin{array}{llll}\text { C } & -1.415693 & -1.723718 & -0.584155\end{array}$

$\begin{array}{llll}\text { C } & -3.324311 & -2.313289 & -2.110578\end{array}$

$\begin{array}{llll}\text { C } & -2.873716 & -2.720610 & -3.372557\end{array}$

C $\quad-3.589943 \quad-2.423999 \quad-4.531911$

C $\quad-4.779555-1.706045 \quad-4.440196$

$\begin{array}{llll}\text { C } & -5.253037 & -1.256776 & -3.197627\end{array}$

$\begin{array}{llll}\text { C } & -4.500660 & -1.562130 & -2.058223\end{array}$

$\begin{array}{llll}\text { C } & -4.844546 & -3.520542 & 0.191431\end{array}$

$\begin{array}{llll}\text { C } & -5.347655 & -4.372796 & -0.803954\end{array}$

$\begin{array}{llll}\text { C } & -6.718260 & -4.578573 & -0.954877\end{array}$

$\begin{array}{llll}\text { C } & -7.624985 & -3.937003 & -0.109450\end{array}$

$\begin{array}{llll}\text { C } & -7.139852 & -3.089126 & 0.885781\end{array}$

$\begin{array}{llll}\text { C } & -5.768234 & -2.885408 & 1.035490\end{array}$

$\begin{array}{llll}\text { C } & -2.970666 & -2.884900 & 1.766773\end{array}$

$\begin{array}{llll}\text { C } & -2.567118 & -3.882345 & 2.660198\end{array}$

C $\quad-2.227395 \quad-3.560658 \quad 3.975330$

$\begin{array}{llll}\text { C } & -2.292229 & -2.233952 & 4.409853\end{array}$

C $\quad-2.701778 \quad-1.234405 \quad 3.523553$ $\begin{array}{llll}\text { C } & -3.044046 & -1.563495 & 2.213645\end{array}$

$\begin{array}{llll}\text { C } & -6.531657 & -0.521969 & -3.076715\end{array}$

$\begin{array}{llll}\text { C } & -6.958603 & 0.383780 & -4.060650\end{array}$

$\begin{array}{llll}\text { C } & -8.193771 & 1.018499 & -3.960348\end{array}$

$\begin{array}{llll}\text { C } & -9.056998 & 0.786658 & -2.875900\end{array}$

$\begin{array}{llll}\text { C } & -8.614715 & -0.106989 & -1.890560\end{array}$

$\begin{array}{llll}\text { C } & -7.379747 & -0.744923 & -1.982688\end{array}$

$\begin{array}{llll}\text { C } & 3.815321 & -2.269040 & -0.884580\end{array}$

$\begin{array}{llll}\text { C } & 4.102053 & -3.719759 & -0.381020\end{array}$

$\begin{array}{llll}\text { C } & 4.690962 & -2.534048 & 0.317925\end{array}$

C $\quad 2.487269-1.624140 \quad-0.579623$

$\begin{array}{llll}\text { C } & 4.428675 & -1.709995 & -2.132816\end{array}$

$\begin{array}{llll}\text { C } & 4.108105 & -2.116017 & -3.433007\end{array}$

C $\quad 4.624429-1.452630 \quad-4.546545$

$\begin{array}{llll}\text { C } & 5.450810 & -0.343981 & -4.374956\end{array}$

$\begin{array}{llll}\text { C } & 5.788523 & 0.098460 & -3.087885\end{array}$

$\begin{array}{llll}\text { C } & 5.280003 & -0.611742 & -1.995326\end{array}$

$\begin{array}{llll}\text { C } & 5.029490 & -4.586403 & -1.201933\end{array}$

$\begin{array}{llll}\text { C } & 4.705270 & -5.925177 & -1.467564\end{array}$

C $\quad 5.566472 \quad-6.747191 \quad-2.195306$

$\begin{array}{llll}\text { C } & 6.774420 & -6.249846 & -2.682365\end{array}$

$\begin{array}{llll}\text { C } & 7.112145 & -4.920356 & -2.425331\end{array}$

$\begin{array}{llll}\text { C } & 6.256327 & -4.103173 & -1.688914\end{array}$

$\begin{array}{llll}\text { C } & 2.980782 & -4.441938 & 0.314304\end{array}$

$\begin{array}{llll}\text { C } & 1.835470 & -4.841349 & -0.384449\end{array}$

$\begin{array}{llll}\text { C } & 0.838461 & -5.577102 & 0.256513\end{array}$

$\begin{array}{llll}\text { C } & 0.957979 & -5.893010 & 1.612167\end{array}$

$\begin{array}{llll}\text { C } & 2.093452 & -5.487457 & 2.317278\end{array}$

$\begin{array}{llll}\text { C } & 3.103824 & -4.776575 & 1.667148\end{array}$

$\begin{array}{llll}\text { C } & 6.573273 & 1.328704 & -2.841627\end{array}$

$\begin{array}{llll}C & 7.543865 & 1.383522 & -1.833369\end{array}$

$\begin{array}{llll}\text { C } & 8.163295 & 2.588327 & -1.496600\end{array}$

$\begin{array}{llll}\text { C } & 7.836709 & 3.784501 & -2.149335\end{array}$

$\begin{array}{llll}\text { C } & 6.890972 & 3.712220 & -3.186513\end{array}$

$\begin{array}{llll}\text { C } & 6.273998 & 2.514054 & -3.530894\end{array}$

$\begin{array}{llll}\text { C } & 3.793676 & 2.268313 & 0.885332\end{array}$

$\begin{array}{llll}\text { C } & 4.085112 & 3.721288 & 0.391949\end{array}$

$\begin{array}{llll}\text { C } & 4.680331 & 2.539325 & -0.308102\end{array}$

$\begin{array}{llll}\text { C } & 2.468837 & 1.625112 & 0.562870\end{array}$

$\begin{array}{llll}\text { C } & 4.394353 & 1.705340 & 2.138034\end{array}$

$\begin{array}{llll}\text { C } & 4.060205 & 2.107514 & 3.436088\end{array}$

$\begin{array}{llll}\text { C } & 4.569375 & 1.444463 & 4.553093\end{array}$

$\begin{array}{llll}\text { C } & 5.402826 & 0.340385 & 4.387024\end{array}$

$\begin{array}{llll}\text { C } & 5.753896 & -0.098609 & 3.102364\end{array}$

C $\quad 5.250632 \quad 0.610165 \quad 2.006341$

$\begin{array}{llll}\text { C } & 5.006228 & 4.584062 & 1.224411\end{array}$

$\begin{array}{llll}\text { C } & 6.229863 & 4.099280 & 1.717914\end{array}$

$\begin{array}{llll}\text { C } & 7.080296 & 4.913866 & 2.463429\end{array}$

$\begin{array}{llll}\text { C } & 6.740350 & 6.242195 & 2.723536\end{array}$

$\begin{array}{llll}\text { C } & 5.535382 & 6.740996 & 2.230597\end{array}$

$\begin{array}{llll}\text { C } & 4.679493 & 5.921558 & 1.493772\end{array}$

$\begin{array}{llll}\text { C } & 2.968706 & 4.447429 & -0.306683\end{array}$

C $\quad 3.102241 \quad 4.794478-1.655371$

$\begin{array}{llll}\text { C } & 2.096995 & 5.512001 & -2.306212\end{array}$

C $\quad 0.956750 \quad 5.912058 \quad-1.605542$

$\begin{array}{llll}\text { C } & 0.826469 & 5.582922 & -0.253984\end{array}$

$\begin{array}{llll}\text { C } & 1.817933 & 4.839920 & 0.387112\end{array}$

$\begin{array}{llll}\text { C } & 6.549133 & -1.323520 & 2.862852\end{array}$

$\begin{array}{llll}\text { C } & 6.249609 & -2.511845 & 3.546872\end{array}$

$\begin{array}{llll}\text { C } & 6.878237 & -3.705404 & 3.207812\end{array}$

$\begin{array}{llll}\text { C } & 7.836397 & -3.769997 & 2.181571\end{array}$

$\begin{array}{llll}\text { C } & 8.163127 & -2.570669 & 1.534765\end{array}$ $\begin{array}{llll}\text { C } & 7.532104 & -1.370377 & 1.866298\end{array}$

$\begin{array}{llll}\text { C } & -10.361853 & -1.489045 & 2.954658\end{array}$

$\begin{array}{llll}\text { C } & -11.243194 & -0.827961 & 4.038225\end{array}$

$\begin{array}{llll}\text { C } & -11.059386 & -1.339871 & 1.591074\end{array}$

C $\quad-10.233730 \quad-2.996720 \quad 3.261186$

$\begin{array}{llll}\text { C } & 8.469092 & -5.118494 & 1.816212\end{array}$

$\begin{array}{llll}\text { C } & 9.455620 & -4.998195 & 0.643610\end{array}$

$\begin{array}{llll}\text { C } & 9.229211 & -5.670048 & 3.042067\end{array}$

$\begin{array}{llll}\text { C } & 7.355051 & -6.110079 & 1.411510\end{array}$

$\begin{array}{llll}\text { C } & 8.456677 & 5.137299 & -1.778116\end{array}$

$\begin{array}{llll}\text { C } & 7.332221 & 6.120798 & -1.382988\end{array}$

$\begin{array}{llll}\text { C } & 9.433315 & 5.023389 & -0.596571\end{array}$

$\begin{array}{llll}\text { C } & 9.224247 & 5.694675 & -2.996669\end{array}$

C $\quad-10.436666 \quad 1.457558 \quad-2.818741$

$\begin{array}{llll}\text { C } & -10.331062 & 2.963788 & -3.140527\end{array}$

$\begin{array}{llll}\text { C } & -11.346282 & 0.780726 & -3.868595\end{array}$

C $\quad-11.089111 \quad 1.313357 \quad-1.432502$

$\begin{array}{llll}\mathrm{H} & -2.714306 & 4.849969 & 1.027726\end{array}$

$\begin{array}{llll}\mathrm{H} & -1.442280 & 4.283499 & -0.151845\end{array}$

H $\quad-3.107609 \quad 2.775150 \quad 5.438692$

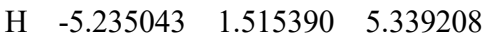

$\mathrm{H} \quad-4.833366 \quad 1.225097 \quad 1.076830$

H $\quad-4.648426 \quad 4.894038 \quad 1.445306$

$\begin{array}{llll}\mathrm{H} & -7.043545 & 5.257992 & 1.745935\end{array}$

$\begin{array}{llll}\mathrm{H} & -8.692988 & 4.092145 & 0.282493\end{array}$

$\mathrm{H} \quad-7.856667 \quad 2.558620 \quad-1.500471$

$\begin{array}{llll}\mathrm{H} & -5.464379 & 2.216477 & -1.824454\end{array}$

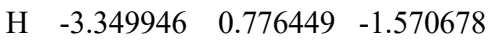

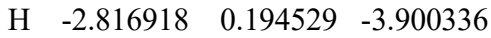

$\mathrm{H} \quad-2.141444 \quad 1.973964 \quad-5.510125$

H $\quad-2.022430 \quad 4.339759 \quad-4.746201$

$\mathrm{H} \quad-2.571391 \quad 4.911816 \quad-2.396384$

H $\quad-6.182299 \quad-0.613443 \quad 4.918390$

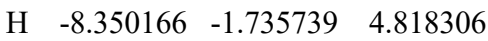

$\begin{array}{llll}\mathrm{H} & -9.234969 & 0.319894 & 1.146877\end{array}$

$\begin{array}{llll}\mathrm{H} & -7.093527 & 1.459378 & 1.265887\end{array}$

H $\quad-2.734716-4.842121 \quad-1.101739$

$\mathrm{H} \quad-1.437187 \quad-4.285883 \quad 0.054365$

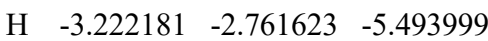

$\mathrm{H} \quad-5.350566-1.509263 \quad-5.341339$

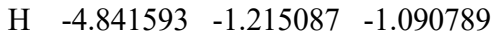

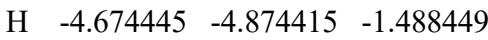

H $\quad-7.074611 \quad-5.241535 \quad-1.737443$

$\mathrm{H} \quad-8.693234-4.091194 \quad-0.227917$

$\mathrm{H} \quad-7.820000 \quad-2.567868 \quad 1.546296$

$\mathrm{H} \quad-5.421993 \quad-2.224196 \quad 1.819743$

H $\quad-2.508589-4.912028 \quad 2.319408$

H $\quad-1.911789-4.343910 \quad 4.658299$

H $\quad-2.025771 \quad-1.981105 \quad 5.431964$

H $\quad-2.745917 \quad-0.199955 \quad 3.841957$

$\begin{array}{llll}\mathrm{H} & -3.326500 & -0.778138 & 1.523858\end{array}$

$\begin{array}{llll}\mathrm{H} & -6.312087 & 0.603702 & -4.904846\end{array}$

H $\quad-8.485592 \quad 1.707722 \quad-4.745628$

$\begin{array}{llll}\mathrm{H} & -9.239176 & -0.329225 & -1.034820\end{array}$

H $\quad-7.093743 \quad-1.451873 \quad-1.212744$

H $\quad 5.753536 \quad-2.361768 \quad 0.221832$

$\begin{array}{llll}\mathrm{H} & 4.272778 & -2.259054 & 1.275708\end{array}$

H $\quad 4.368154 \quad-1.797530 \quad-5.541747$

H $\quad 5.839095 \quad 0.173406 \quad-5.246300$

$\begin{array}{llll}\mathrm{H} & 5.507834 & -0.267664 & -0.994416\end{array}$

H $\quad 3.774418-6.334735 \quad-1.096715$

H $\quad \begin{array}{llll}5.287557 & -7.780567 & -2.378410\end{array}$ 
H $\quad 7.445025 \quad-6.887738 \quad-3.249611$

H $\quad 8.049877 \quad-4.513840 \quad-2.791949$

H $\quad 6.560674 \quad-3.082109 \quad-1.501686$

H $\quad 1.734693 \quad-4.583005-1.433285$

H $\quad-0.027321 \quad-5.910734 \quad-0.305558$

$\begin{array}{llll}\mathrm{H} & 0.175285 & -6.455304 & 2.112037\end{array}$

H $\quad 2.197287 \quad-5.731987 \quad 3.370175$

H $3.993809 \quad-4.472934 \quad 2.210511$

H $\quad 7.809730 \quad 0.476965 \quad-1.297052$

H $\quad 8.899539 \quad 2.584900 \quad-0.702046$

H $\quad 6.612712 \quad 4.612261 \quad-3.725434$

H $\quad 5.515563 \quad 2.503690 \quad-4.307998$

H $\quad 5.742093 \quad 2.367267 \quad-0.203921$

H $\quad 4.270448 \quad 2.268749 \quad-1.270760$

$\mathrm{H} \quad 4.302635 \quad 1.786518 \quad 5.546524$

H $\quad 5.786519 \quad-0.176119 \quad 5.260907$

$\begin{array}{llll}\mathrm{H} & 5.486329 & 0.268002 & 1.006630\end{array}$

$\begin{array}{llll}\mathrm{H} & 6.536193 & 3.079165 & 1.528617\end{array}$

$\begin{array}{llll}\mathrm{H} & 8.015765 & 4.506278 & 2.834640\end{array}$

$\begin{array}{llll}\mathrm{H} & 7.406909 & 6.878067 & 3.297792\end{array}$

$\mathrm{H} \quad 5.254745 \quad 7.773485 \quad 2.416057$

H $\quad 3.751078 \quad 6.332251 \quad 1.118123$

H $3.996481 \quad 4.495798 \quad-2.194592$

$\mathrm{H} \quad 2.208695 \quad 5.766564 \quad-3.355914$

H $\quad 0.178475 \quad 6.480092 \quad-2.105886$

$\begin{array}{llll}\mathrm{H} & -0.043415 & 5.911117 & 0.304848\end{array}$

$\mathrm{H} \quad 1.708847 \quad 4.571228 \quad 1.432473$

H $\quad 5.482041 \quad-2.507641 \quad 4.315020$

$\begin{array}{llll}\mathrm{H} & 6.599537 & -4.608227 & 3.741865\end{array}$

H $\quad 8.908588 \quad-2.561153 \quad 0.748879$

H $\quad 7.798168 \quad-0.461194 \quad 1.334526$

$\begin{array}{llll}\mathrm{H} & -12.237777 & -1.286990 & 4.055490\end{array}$

H $\quad-10.798187 \quad-0.941614 \quad 5.031448$

H $\quad-11.362419 \quad 0.242292 \quad 3.841395$

$\mathrm{H} \quad-11.279118 \quad-0.294889 \quad 1.354506$

$\begin{array}{llll}\mathrm{H} & -10.449676 & -1.755647 & 0.781571\end{array}$

$\begin{array}{llll}\mathrm{H} & -12.011191 & -1.879253 & 1.604416\end{array}$

H $\quad-11.224794 \quad-3.461672 \quad 3.248617$

$\begin{array}{llll}\mathrm{H} & -9.613967 & -3.503814 & 2.515722\end{array}$

$\mathrm{H} \quad-9.796650 \quad-3.182408 \quad 4.245520$

H $\quad 9.872489 \quad-5.983957 \quad 0.415284$

H $\quad 8.961180 \quad-4.629989 \quad-0.259535$

$\begin{array}{llll}\mathrm{H} & 10.290859 & -4.331718 & 0.881550\end{array}$

H $\quad 10.027801 \quad-4.985338 \quad 3.345749$

H $\quad 8.564045 \quad-5.813765 \quad 3.898469$

H $\quad 9.681556 \quad-6.638251 \quad 2.801906$

$\begin{array}{llll}\mathrm{H} & 6.800280 & -5.741331 & 0.545939\end{array}$

$\begin{array}{llll}\mathrm{H} & 7.792870 & -7.079794 & 1.151162\end{array}$

$\begin{array}{llll}\mathrm{H} & 6.643030 & -6.273646 & 2.225637\end{array}$

H $\quad 7.760869 \quad 7.093324 \quad-1.117900$

H $\quad 6.626453 \quad 6.279886 \quad-2.203391$

$\begin{array}{llll}\mathrm{H} & 6.772461 & 5.747437 & -0.522556\end{array}$

$\begin{array}{llll}\mathrm{H} & 8.932755 & 4.653223 & 0.302404\end{array}$

H $\quad 10.274364 \quad 4.361418 \quad-0.826523$

H $\quad \begin{array}{llll}9.842552 & 6.011637 & -0.365279\end{array}$

$\mathrm{H} \quad 8.566123 \quad 5.834406 \quad-3.859101$

H $\quad 9.667814 \quad 6.665778 \quad-2.751888$

H $\quad 10.030270 \quad 5.015615 \quad-3.293371$

H $\quad-11.324964 \quad 3.420925 \quad-3.098749$

H $\quad-9.691299 \quad 3.481510 \quad-2.419655$

H $\quad-9.928237 \quad 3.145438 \quad-4.140148$

H $\quad-10.933756 \quad 0.890128 \quad-4.876198$
H $\quad-11.450204 \quad-0.288857 \quad-3.659810$

$\mathrm{H} \quad-12.344740 \quad 1.231454 \quad-3.857888$

$\mathrm{H} \quad-10.457550 \quad 1.740451 \quad-0.645991$

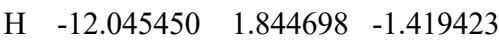

H $\quad-11.292334 \quad 0.268496-1.181163$

\section{N1}

$\begin{array}{llll}\mathrm{Rh} & -0.000494 & -0.000184 & -2.094232\end{array}$

$\begin{array}{llll}\mathrm{Rh} & -0.000018 & -0.000098 & 0.297218\end{array}$

$\begin{array}{llll}\mathrm{O} & -1.526799 & 1.366325 & 0.228398\end{array}$

$\begin{array}{llll}\text { O } & -1.193669 & 1.691979 & -1.984022\end{array}$

$\begin{array}{llll}\mathrm{O} & -1.366491 & -1.526821 & 0.228508\end{array}$

$\begin{array}{llll}\text { O } & -1.692629 & -1.193382 & -1.983792\end{array}$

$\begin{array}{llll}\text { O } & 1.526722 & -1.366492 & 0.227908\end{array}$

$\begin{array}{llll}\text { O } & 1.192728 & -1.692364 & -1.984362\end{array}$

$\begin{array}{llll}\text { O } & 1.366448 & 1.526613 & 0.227838\end{array}$

$\begin{array}{llll}\text { O } & 1.691701 & 1.192991 & -1.984582\end{array}$

$\begin{array}{llll}\mathrm{Cl} & -4.749705 & 0.711257 & 0.307328\end{array}$

$\begin{array}{llll}\text { Cl } & -0.711335 & -4.749909 & 0.306508\end{array}$

$\begin{array}{llll}\mathrm{Cl} & 4.749905 & -0.711170 & 0.305118\end{array}$

$\begin{array}{llll}\mathrm{Cl} & 0.711352 & 4.749509 & 0.306138\end{array}$

$\begin{array}{llll}\text { C } & -2.900409 & 2.933344 & -0.864152\end{array}$

C $\quad-3.833174 \quad 3.138793 \quad-2.108022$

$\begin{array}{llll}\text { C } & -2.754688 & 4.127354 & -1.787692\end{array}$

$\begin{array}{llll}\text { C } & -1.796431 & 1.918837 & -0.888012\end{array}$

$\begin{array}{llll}\text { C } & -3.468669 & 3.139126 & 0.508368\end{array}$

$\begin{array}{llll}\text { C } & -4.311151 & 2.206007 & 1.128378\end{array}$

$\begin{array}{llll}\text { C } & -4.798183 & 2.412297 & 2.416428\end{array}$

$\begin{array}{llll}\text { C } & -4.423673 & 3.553575 & 3.121948\end{array}$

$\begin{array}{llll}\text { C } & -3.557179 & 4.497354 & 2.550878\end{array}$

$\begin{array}{llll}\text { C } & -3.107553 & 4.268028 & 1.243288\end{array}$

$\begin{array}{llll}\text { C } & -5.274750 & 3.475564 & -1.798512\end{array}$

$\begin{array}{llll}\text { C } & -6.286973 & 2.568593 & -2.146782\end{array}$

$\begin{array}{llll}\text { C } & -7.622277 & 2.827233 & -1.841632\end{array}$

$\begin{array}{llll}\text { C } & -7.978660 & 4.004497 & -1.182132\end{array}$

$\begin{array}{llll}\text { C } & -6.983879 & 4.920594 & -0.839112\end{array}$

$\begin{array}{llll}\text { C } & -5.648663 & 4.659591 & -1.146932\end{array}$

$\begin{array}{llll}\text { C } & -3.639805 & 2.304723 & -3.347722\end{array}$

$\begin{array}{llll}\text { C } & -3.509450 & 2.956882 & -4.579712\end{array}$

$\begin{array}{llll}\text { C } & -3.326734 & 2.227741 & -5.756172\end{array}$

$\begin{array}{llll}\text { C } & -3.270006 & 0.832593 & -5.710182\end{array}$

$\begin{array}{llll}\text { C } & -3.412916 & 0.175215 & -4.486242\end{array}$

$\begin{array}{llll}\text { C } & -3.609439 & 0.908260 & -3.316582\end{array}$

$\begin{array}{llll}\text { C } & -3.104094 & 5.693342 & 3.298978\end{array}$

$\begin{array}{llll}\text { C } & -3.959461 & 6.352007 & 4.194888\end{array}$

$\begin{array}{llll}\text { C } & -3.538452 & 7.485469 & 4.895878\end{array}$

$\begin{array}{llll}\text { C } & -2.238686 & 7.962704 & 4.683928\end{array}$

$\begin{array}{llll}\text { C } & -1.360836 & 7.325411 & 3.800228\end{array}$

$\begin{array}{llll}\text { C } & -1.805712 & 6.191405 & 3.114348\end{array}$

$\begin{array}{llll}\text { C } & -2.933731 & -2.900291 & -0.863882\end{array}$

$\begin{array}{llll}\text { C } & -3.139523 & -3.832733 & -2.107902\end{array}$

$\begin{array}{llll}\text { C } & -4.127997 & -2.754324 & -1.787072\end{array}$

$\begin{array}{llll}\text { C } & -1.919250 & -1.796299 & -0.887822\end{array}$

$\begin{array}{llll}\text { C } & -3.139097 & -3.468808 & 0.508588\end{array}$

$\begin{array}{llll}\text { C } & -2.205817 & -4.311470 & 1.128118\end{array}$

$\begin{array}{llll}\text { C } & -2.411717 & -4.798779 & 2.416128\end{array}$

$\begin{array}{llll}\text { C } & -3.552741 & -4.424346 & 3.122098\end{array}$

$\begin{array}{llll}\text { C } & -4.496626 & -3.557644 & 2.551548\end{array}$

$\begin{array}{llll}\text { C } & -4.267728 & -3.107754 & 1.243978\end{array}$

$\begin{array}{llll}\text { C } & -3.476311 & -5.274388 & -1.798702\end{array}$

$\begin{array}{llll}\text { C } & -2.569602 & -6.286609 & -2.147652\end{array}$

$\begin{array}{llll}\text { C } & -2.828275 & -7.621976 & -1.842792\end{array}$ $\begin{array}{llll}\text { C } & -4.005306 & -7.978432 & -1.182922\end{array}$

$\begin{array}{llll}\text { C } & -4.921163 & -6.983650 & -0.839242\end{array}$

$\begin{array}{llll}\text { C } & -4.660127 & -5.648371 & -1.146762\end{array}$

$\begin{array}{llll}\text { C } & -2.305707 & -3.639120 & -3.347732\end{array}$

$\begin{array}{llll}\text { C } & -2.958111 & -3.508317 & -4.579542\end{array}$

$\begin{array}{llll}\text { C } & -2.229191 & -3.325397 & -5.756112\end{array}$

C $\quad-0.834013 \quad-3.268964-5.710402$

$\begin{array}{llll}\text { C } & -0.176395 & -3.412343 & -4.486642\end{array}$

$\begin{array}{llll}\text { C } & -0.909227 & -3.609010 & -3.316882\end{array}$

$\begin{array}{llll}\text { C } & -5.692312 & -3.104621 & 3.300158\end{array}$

$\begin{array}{llll}\text { C } & -6.350838 & -3.960197 & 4.195988\end{array}$

$\begin{array}{llll}\text { C } & -7.484010 & -3.539244 & 4.897478\end{array}$

$\begin{array}{llll}\text { C } & -7.961094 & -2.239326 & 4.686128\end{array}$

$\begin{array}{llll}\text { C } & -7.323930 & -1.361265 & 3.802548\end{array}$

$\begin{array}{llll}\text { C } & -6.190208 & -1.806104 & 3.116138\end{array}$

$\begin{array}{llll}\text { C } & 2.900036 & -2.933467 & -0.865072\end{array}$

$\begin{array}{llll}\text { C } & 3.832151 & -3.138984 & -2.109412\end{array}$

$\begin{array}{llll}\text { C } & 2.753939 & -4.127616 & -1.788372\end{array}$

$\begin{array}{llll}\text { C } & 1.795922 & -1.919097 & -0.888572\end{array}$

$\begin{array}{llll}\text { C } & 3.468939 & -3.138995 & 0.507218\end{array}$

$\begin{array}{llll}\text { C } & 4.311742 & -2.205765 & 1.126648\end{array}$

$\begin{array}{llll}\text { C } & 4.799437 & -2.411859 & 2.414478\end{array}$

$\begin{array}{llll}\text { C } & 4.425263 & -3.553010 & 3.120378\end{array}$

$\begin{array}{llll}\text { C } & 3.558433 & -4.496852 & 2.549918\end{array}$

$\begin{array}{llll}\text { C } & 3.108142 & -4.267745 & 1.242528\end{array}$

$\begin{array}{llll}\text { C } & 5.273923 & -3.475578 & -1.800622\end{array}$

$\begin{array}{llll}\text { C } & 6.285910 & -2.568723 & -2.149872\end{array}$

$\begin{array}{llll}\text { C } & 7.621404 & -2.827198 & -1.845422\end{array}$

$\begin{array}{llll}\text { C } & 7.978238 & -4.004164 & -1.185642\end{array}$

$\begin{array}{llll}\text { C } & 6.983690 & -4.920167 & -0.841662\end{array}$

$\begin{array}{llll}\text { C } & 5.648287 & -4.659337 & -1.148792\end{array}$

$\begin{array}{llll}\text { C } & 3.638030 & -2.305054 & -3.349072\end{array}$

$\begin{array}{llll}\text { C } & 3.506923 & -2.957323 & -4.580922\end{array}$

$\begin{array}{llll}\text { C } & 3.323528 & -2.228289 & -5.757342\end{array}$

$\begin{array}{llll}\text { C } & 3.266902 & -0.833130 & -5.711452\end{array}$

$\begin{array}{llll}\text { C } & 3.410560 & -0.175634 & -4.487662\end{array}$

$\begin{array}{llll}\text { C } & 3.607711 & -0.908577 & -3.318052\end{array}$

$\begin{array}{llll}\text { C } & 3.105730 & -5.692690 & 3.298468\end{array}$

$\begin{array}{llll}\text { C } & 1.807132 & -6.190527 & 3.114938\end{array}$

$\begin{array}{llll}\text { C } & 1.362625 & -7.324407 & 3.801298\end{array}$

$\begin{array}{llll}\text { C } & 2.241090 & -7.961790 & 4.684298\end{array}$

$\begin{array}{llll}\text { C } & 3.541121 & -7.484765 & 4.895148\end{array}$

$\begin{array}{llll}\text { C } & 3.961742 & -6.351436 & 4.193738\end{array}$

$\begin{array}{llll}\text { C } & 2.933241 & 2.900006 & -0.865332\end{array}$

$\begin{array}{llll}\text { C } & 3.138428 & 3.832436 & -2.109482\end{array}$

$\begin{array}{llll}\text { C } & 4.127061 & 2.754057 & -1.789082\end{array}$

$\begin{array}{lllll}\text { C } & 1.918753 & 1.796001 & -0.888742\end{array}$

$\begin{array}{llll}\text { C } & 3.139288 & 3.468590 & 0.507018\end{array}$

$\begin{array}{llll}\text { C } & 2.206244 & 4.311168 & 1.127028\end{array}$

$\begin{array}{llll}\text { C } & 2.412780 & 4.798519 & 2.414918\end{array}$

$\begin{array}{llll}\text { C } & 3.554233 & 4.424220 & 3.120288\end{array}$

$\begin{array}{llll}\text { C } & 4.497941 & 3.557651 & 2.549228\end{array}$

$\begin{array}{llll}\text { C } & 4.268368 & 3.107707 & 1.241788\end{array}$

$\begin{array}{llll}\text { C } & 3.475239 & 5.274088 & -1.800422\end{array}$

$\begin{array}{llll}\text { C } & 2.568161 & 6.286215 & -2.148712\end{array}$

$\begin{array}{llll}\text { C } & 2.826862 & 7.621590 & -1.843942\end{array}$

$\begin{array}{llll}\text { C } & 4.004276 & 7.978166 & -1.184812\end{array}$

$\begin{array}{lllll}\text { C } & 4.920489 & 6.983485 & -0.841802\end{array}$

$\begin{array}{llll}\text { C } & 4.659436 & 5.648191 & -1.149252\end{array}$

$\begin{array}{llll}\text { C } & 2.304023 & 3.638733 & -3.348902\end{array}$

$\begin{array}{llll}\text { C } & 2.955848 & 3.507938 & -4.581022\end{array}$

$\begin{array}{llll}\text { C } & 2.226378 & 3.324898 & -5.757232\end{array}$ 


\begin{tabular}{|c|c|c|c|}
\hline $\mathrm{C}$ & 0.831236 & 3.268319 & -5.710842 \\
\hline & 0.174189 & 3.411678 & -4.486782 \\
\hline & 0.907568 & 3.608474 & -3.317382 \\
\hline & 5.694118 & 3.104835 & 3.297168 \\
\hline . & 6.352952 & 3.960496 & 4.192678 \\
\hline & 7.486605 & 3.539746 & 4.893518 \\
\hline & 7.963850 & 2.239951 & 4.681828 \\
\hline & 7.326397 & 1.361811 & 3.798538 \\
\hline $\mathrm{C}$ & 6.192206 & 1.806440 & 3.112788 \\
\hline$c$ & 4.464360 & -8.177417 & 5.868078 \\
\hline & -0.047324 & -7.829991 & 3.611028 \\
\hline & -8.176419 & -4.462020 & 5.871018 \\
\hline$C$ & -7.829605 & 0.048572 & 3.611718 \\
\hline $\mathrm{C}$ & 0.048837 & 7.831307 & 3.608738 \\
\hline $\mathrm{C}$ & -4.461007 & 8.178009 & 5.869528 \\
\hline$C$ & 7.832302 & -0.047891 & 3.607318 \\
\hline & 8.179329 & 4.462620 & 5.866738 \\
\hline $\mathrm{H}$ & -3.011107 & 5.102900 & -1.394362 \\
\hline $\mathrm{H}$ & -1.887941 & 4.119118 & -2.436032 \\
\hline $\mathrm{H}$ & -5.447183 & 1.674317 & 2.872248 \\
\hline $\mathrm{H}$ & -4.782282 & 3.689202 & 4.136738 \\
\hline $\mathrm{H}$ & -2.455232 & 4.995699 & 0.774788 \\
\hline $\mathrm{H}$ & -6.025790 & 1.650672 & -2.659332 \\
\hline $\mathrm{H}$ & -8.383954 & 2.104992 & -2.119932 \\
\hline $\mathrm{H}$ & -9.017973 & 4.207349 & -0.942512 \\
\hline $\mathrm{H}$ & -7.243254 & 5.844792 & -0.331522 \\
\hline $\mathrm{H}$ & -4.902000 & 5.390062 & -0.861542 \\
\hline $\mathrm{H}$ & -3.538673 & 4.042419 & -4.611862 \\
\hline $\mathrm{H}$ & -3.220099 & 2.748734 & -6.703022 \\
\hline $\mathrm{H}$ & -3.113625 & 0.260327 & -6.619862 \\
\hline $\mathrm{H}$ & -3.345792 & -0.906003 & -4.440082 \\
\hline $\mathrm{H}$ & -3.688006 & 0.395748 & -2.366702 \\
\hline $\mathrm{H}$ & -4.977121 & 5.995099 & 4.324568 \\
\hline $\mathrm{H}$ & -1.126203 & 5.680294 & 2.439178 \\
\hline $\mathrm{H}$ & -5.103446 & -3.010850 & -1.393562 \\
\hline $\mathrm{H}$ & -4.119906 & -1.887414 & -2.435192 \\
\hline $\mathrm{H}$ & -1.673635 & -5.447936 & 2.871548 \\
\hline $\mathrm{H}$ & -3.688065 & -4.783185 & 4.136848 \\
\hline $\mathrm{H}$ & -4.995502 & -2.455276 & 0.775868 \\
\hline $\mathrm{H}$ & -1.651880 & -6.025394 & -2.660512 \\
\hline $\mathrm{H}$ & -2.106233 & -8.383652 & -2.121612 \\
\hline $\mathrm{H}$ & -4.208175 & -9.017793 & -0.943532 \\
\hline $\mathrm{H}$ & -5.845181 & -7.243064 & -0.331352 \\
\hline $\mathrm{H}$ & -5.390396 & -4.901719 & -0.860842 \\
\hline $\mathrm{H}$ & -4.043663 & -3.537325 & -4.611472 \\
\hline $\mathrm{H}$ & -2.750361 & -3.218399 & -6.702812 \\
\hline $\mathrm{H}$ & -0.261923 & -3.112460 & -6.620172 \\
\hline $\mathrm{H}$ & 0.904851 & -3.345474 & -4.440712 \\
\hline $\mathrm{H}$ & -0.396542 & -3.687962 & -2.367122 \\
\hline $\mathrm{H}$ & -5.994036 & -4.977953 & 4.325208 \\
\hline $\mathrm{H}$ & -5.679205 & -1.126451 & 2.441028 \\
\hline $\mathrm{H}$ & 3.010672 & -5.103094 & -1.395062 \\
\hline $\mathrm{H}$ & 1.886828 & -4.119525 & -2.436232 \\
\hline $\mathrm{H}$ & 5.448678 & -1.673813 & 2.869848 \\
\hline $\mathrm{H}$ & 4.784417 & -3.688498 & 4.134988 \\
\hline $\mathrm{H}$ & 2.455535 & -4.995474 & 0.774498 \\
\hline $\mathrm{H}$ & 6.024400 & -1.651030 & -2.662652 \\
\hline $\mathrm{H}$ & 8.382895 & -2.105036 & -2.124482 \\
\hline $\mathrm{H}$ & 9.017699 & -4.206886 & -0.946572 \\
\hline П & 7.243395 & -5.844146 & -0.333852 \\
\hline $\mathrm{H}$ & 4.901829 & -5.389724 & -0.862642 \\
\hline & 3.536105 & -4.042862 & -4.612992 \\
\hline
\end{tabular}

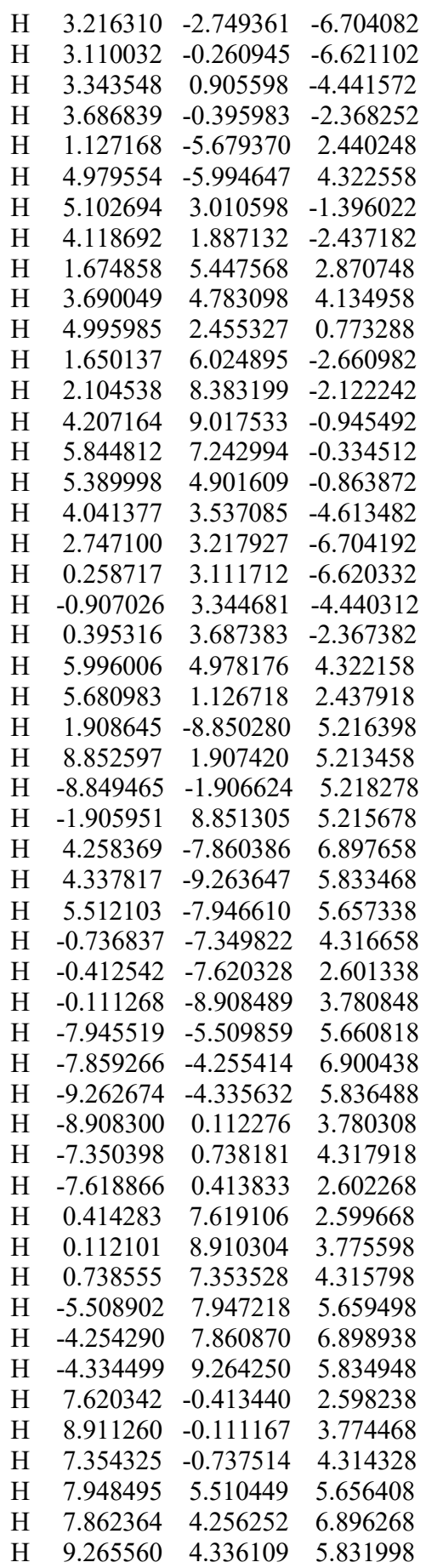

$$
\begin{array}{lccc}
\text { N'1 } & & & \\
\text { Rh } & -0.000357 & -0.002588 & -2.137463 \\
\text { Rh } & 0.000106 & -0.000424 & 0.247683 \\
\text { O } & 1.981683 & -0.542056 & 0.159273 \\
\text { O } & 1.753463 & -1.106760 & -2.016908 \\
\text { O } & 0.598465 & 1.956515 & 0.159632 \\
\text { O } & 1.121481 & 1.742064 & -2.029291 \\
\text { O } & -1.981589 & 0.540994 & 0.159072 \\
\text { O } & -1.754147 & 1.101991 & -2.018128 \\
\text { O } & -0.598155 & -1.957582 & 0.163263
\end{array}
$$

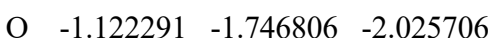

$\begin{array}{lllll}\text { Cl } & 4.801228 & 1.459965 & -0.468524\end{array}$

$\begin{array}{llll}\mathrm{Cl} & -0.843925 & 4.976679 & 0.474517\end{array}$

$\begin{array}{llll}\mathrm{Cl} & -4.804330 & -1.460024 & -0.462217\end{array}$

$\begin{array}{llll}\mathrm{Cl} & 0.841783 & -4.978395 & 0.483907\end{array}$

$\begin{array}{llll}\text { C } & 3.864545 & -1.437824 & -0.931158\end{array}$

$\begin{array}{llll}\text { C } & 4.727666 & -1.469704 & -2.234864\end{array}$

$\begin{array}{llll}\text { C } & 4.153435 & -2.733813 & -1.665165\end{array}$

$\begin{array}{llll}\text { C } & 2.430763 & -0.988564 & -0.947914\end{array}$

$\begin{array}{llll}\text { C } & 4.540145 & -1.138563 & 0.374878\end{array}$

$\begin{array}{llll}\text { C } & 4.981835 & 0.152529 & 0.693465\end{array}$

$\begin{array}{llll}\text { C } & 5.543776 & 0.452480 & 1.929812\end{array}$

$\begin{array}{llll}\text { C } & 5.664399 & -0.552281 & 2.884326\end{array}$

$\begin{array}{llll}\text { C } & 5.219383 & -1.856516 & 2.618554\end{array}$

$\begin{array}{llll}\text { C } & 4.664487 & -2.121951 & 1.357219\end{array}$

$\begin{array}{llll}\text { C } & 6.208515 & -1.199218 & -2.060813\end{array}$

$\begin{array}{llll}\text { C } & 6.798286 & -0.114939 & -2.728304\end{array}$

$\begin{array}{llll}\text { C } & 8.151461 & 0.180788 & -2.570823\end{array}$

$\begin{array}{llll}\text { C } & 8.952409 & -0.604567 & -1.740875\end{array}$

$\begin{array}{llll}\text { C } & 8.382522 & -1.691212 & -1.076891\end{array}$

$\begin{array}{llll}\text { C } & 7.028888 & -1.985866 & -1.238505\end{array}$

$\begin{array}{llll}\text { C } & 4.170379 & -0.971011 & -3.544437\end{array}$

$\begin{array}{llll}\text { C } & 4.328257 & -1.771375 & -4.681794\end{array}$

$\begin{array}{llll}\text { C } & 3.859428 & -1.345054 & -5.926163\end{array}$

$\begin{array}{llll}\text { C } & 3.227759 & -0.105224 & -6.046481\end{array}$

$\begin{array}{llll}\text { C } & 3.081651 & 0.707631 & -4.919186\end{array}$

$\begin{array}{llll}\text { C } & 3.558812 & 0.279456 & -3.680551\end{array}$

$\begin{array}{llll}\text { C } & 5.311720 & -2.917511 & 3.648279\end{array}$

$\begin{array}{llll}\text { C } & 5.568495 & -4.247823 & 3.290801\end{array}$

$\begin{array}{llll}\text { C } & 5.652679 & -5.255301 & 4.258406\end{array}$

$\begin{array}{llll}\text { C } & 5.484271 & -4.909978 & 5.603072\end{array}$

$\begin{array}{llll}\text { C } & 5.226019 & -3.588905 & 5.993101\end{array}$

$\begin{array}{llll}\text { C } & 5.141978 & -2.604494 & 5.006839\end{array}$

$\begin{array}{llll}\text { C } & 1.721203 & 3.751582 & -0.864702\end{array}$

$\begin{array}{llll}\text { C } & 1.581128 & 4.741297 & -2.068996\end{array}$

$\begin{array}{llll}\text { C } & 2.870151 & 4.020180 & -1.813511\end{array}$

$\begin{array}{llll}\text { C } & 1.095180 & 2.386393 & -0.932477\end{array}$

$\begin{array}{llll}\text { C } & 1.804458 & 4.241180 & 0.552073\end{array}$

$\begin{array}{llll}\text { C } & 0.708631 & 4.738073 & 1.268332\end{array}$

$\begin{array}{llll}\text { C } & 0.791876 & 4.996807 & 2.635645\end{array}$

$\begin{array}{llll}\text { C } & 1.970768 & 4.713975 & 3.323961\end{array}$

$\begin{array}{llll}\text { C } & 3.084115 & 4.200781 & 2.644032\end{array}$

$\begin{array}{llll}\text { C } & 2.982743 & 4.006748 & 1.262296\end{array}$

$\begin{array}{llll}\text { C } & 1.472159 & 6.210736 & -1.734912\end{array}$

$\begin{array}{llll}\text { C } & 0.399873 & 6.965915 & -2.233342\end{array}$

$\begin{array}{llll}\text { C } & 0.264347 & 8.318639 & -1.923139\end{array}$

C $\quad 1.200634 \quad 8.951830 \quad-1.105356$

$\begin{array}{llll}\text { C } & 2.276428 & 8.215410 & -0.607759\end{array}$

$\begin{array}{llll}\text { C } & 2.412024 & 6.863610 & -0.921946\end{array}$

$\begin{array}{llll}\text { C } & 0.797804 & 4.295478 & -3.275492\end{array}$

$\begin{array}{llll}\text { C } & 1.425427 & 4.274145 & -4.526193\end{array}$

$\begin{array}{llll}\text { C } & 0.731997 & 3.858059 & -5.664168\end{array}$

$\begin{array}{llll}\text { C } & -0.603197 & 3.460135 & -5.559837\end{array}$

$\begin{array}{llll}\text { C } & -1.238800 & 3.495294 & -4.317451\end{array}$

$\begin{array}{llll}\text { C } & -0.544180 & 3.916045 & -3.184891\end{array}$

$\begin{array}{llll}\text { C } & 4.316474 & 3.765128 & 3.343887\end{array}$

$\begin{array}{llll}\text { C } & 4.228484 & 2.970166 & 4.493325\end{array}$

$\begin{array}{llll}\text { C } & 5.377175 & 2.456177 & 5.106595\end{array}$

$\begin{array}{llll}\text { C } & 6.625410 & 2.763338 & 4.555350\end{array}$

$\begin{array}{llll}\text { C } & 6.745923 & 3.565930 & 3.411969\end{array}$

$\begin{array}{llll}\text { C } & 5.582706 & 4.059073 & 2.815092\end{array}$

$\begin{array}{llll}\text { C } & -3.864597 & 1.435754 & -0.931985\end{array}$ 


\begin{tabular}{|c|c|c|c|}
\hline C & -4.728557 & 1.464840 & \\
\hline 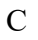 & -4.153398 & 2.730081 & -1.668932 \\
\hline $\mathrm{C}$ & -2.431005 & 0.985831 & -0.948647 \\
\hline $\mathrm{C}$ & -4.539276 & 1.140014 & 0.375301 \\
\hline $\mathrm{C}$ & -4.982110 & -0.149860 & 0.697165 \\
\hline$C$ & -5.543118 & -0.446435 & 1.934750 \\
\hline $\mathrm{C}$ & -5.661532 & 0.560541 & 2.887208 \\
\hline $\mathrm{C}$ & -5.215248 & 1.863670 & 2.618139 \\
\hline $\mathrm{C}$ & -4.661393 & 2.125687 & 1.355641 \\
\hline $\mathrm{C}$ & -6.209483 & 1.195712 & -2.059807 \\
\hline $\mathrm{C}$ & -6.800691 & 0.111419 & -2.726023 \\
\hline $\mathrm{C}$ & -8.154049 & -0.182908 & -2.567466 \\
\hline $\mathrm{C}$ & -8.953746 & 0.603841 & -1.737650 \\
\hline $\mathrm{C}$ & -8.382412 & 1.690466 & -1.074859 \\
\hline $\mathrm{C}$ & -7.028620 & 1.983747 & -1.237583 \\
\hline $\mathrm{C}$ & -4.172136 & 0.962607 & -3.543784 \\
\hline $\mathrm{C}$ & -4.330536 & 1.759980 & -4.683180 \\
\hline $\mathrm{C}$ & -3.862304 & 1.330358 & -5.926634 \\
\hline $\mathrm{C}$ & -3.230676 & 0.090209 & -6.043957 \\
\hline $\mathrm{C}$ & -3.084098 & -0.719686 & -4.914588 \\
\hline $\mathrm{C}$ & -3.560704 & -0.288234 & -3.676880 \\
\hline $\mathrm{C}$ & -5.305182 & 2.927091 & 3.645575 \\
\hline $\mathrm{C}$ & -5.134357 & 2.617027 & 5.004640 \\
\hline $\mathrm{C}$ & -5.216082 & 3.603880 & 5.988708 \\
\hline $\mathrm{C}$ & -5.473155 & 4.924311 & 5.595913 \\
\hline $\mathrm{C}$ & -5.642618 & 5.266719 & 0595 \\
\hline $\mathrm{C}$ & -5.560716 & 4.256928 & 3.285269 \\
\hline $\mathrm{C}$ & -1.722659 & -3.753817 & -0.857049 \\
\hline $\mathrm{C}$ & -1.583572 & -4.746061 & -2.059349 \\
\hline $\mathrm{C}$ & -2.871977 & -4.023377 & -1.805157 \\
\hline $\mathrm{C}$ & -1.095716 & -2.389183 & -0.927744 \\
\hline $\mathrm{C}$ & -1.805943 & -4.240482 & 0.560733 \\
\hline $\mathrm{C}$ & -0.710360 & -4.736937 & 1.277641 \\
\hline $\mathrm{C}$ & -0.793470 & -4.993068 & 2.645455 \\
\hline $\mathrm{C}$ & -1.971978 & -4.708050 & 3.333510 \\
\hline $\mathrm{C}$ & -3.085083 & -4.195259 & 2.652902 \\
\hline $\mathrm{C}$ & -2.983878 & -4.003800 & 1.270800 \\
\hline $\mathrm{C}$ & -1.475942 & -6.214968 & -1.722459 \\
\hline $\mathrm{C}$ & -0.404842 & -6.972304 & -2.220157 \\
\hline $\mathrm{C}$ & -0.270659 & -8.324613 & -1.907516 \\
\hline $\mathrm{C}$ & -1.207154 & -8.955207 & -1.087977 \\
\hline $\mathrm{C}$ & -2.281782 & -8.216622 & -0.591067 \\
\hline $\mathrm{C}$ & -2.416034 & -6.865264 & -0.907684 \\
\hline $\mathrm{C}$ & -0.799857 & -4.303340 & -3.266727 \\
\hline $\mathrm{C}$ & -1.427464 & -4.283931 & -4.517465 \\
\hline $\mathrm{C}$ & -0.733638 & -3.870798 & -5.656274 \\
\hline $\mathrm{C}$ & 0.601933 & -3.473988 & -5.552761 \\
\hline $\mathrm{C}$ & 1.237528 & -3.507297 & -4.310319 \\
\hline $\mathrm{C}$ & 0.542515 & -3.925070 & -3.176894 \\
\hline $\mathrm{C}$ & -4.316996 & -3.757643 & 3.352308 \\
\hline $\mathrm{C}$ & -5.583534 & -4.051724 & 2.824388 \\
\hline $\mathrm{C}$ & -6.746309 & -3.557078 & 3.420922 \\
\hline $\mathrm{C}$ & -6.625004 & -2.752797 & 4.562998 \\
\hline $\mathrm{C}$ & -5.376409 & -2.445359 & 5.113308 \\
\hline $\mathrm{C}$ & -4.228204 & -2.960914 & 4.500487 \\
\hline $\mathrm{C}$ & -5.893864 & 6.698167 & 3.842493 \\
\hline $\mathrm{C}$ & -5.010363 & 3.262260 & 7.444651 \\
\hline $\mathrm{C}$ & 5.264910 & 1.584739 & 6.334184 \\
\hline $\mathrm{C}$ & 8.101782 & 3.846724 & 2.811670 \\
\hline $\mathrm{C}$ & 5.021835 & -3.244133 & 7.448513 \\
\hline $\mathrm{C}$ & 5.905272 & -6.687333 & 3.853223 \\
\hline $\mathrm{C}$ & -5.263297 & -1.571959 & 6.339426 \\
\hline
\end{tabular}

$\begin{array}{llll}\text { C } & -8.102473 & -3.838012 & 2.821366\end{array}$

H $\quad 4.803745 \quad-3.455961 \quad-1.186911$

H $\quad 3.313799 \quad-3.165572 \quad-2.195065$

$\begin{array}{llll}\mathrm{H} & 5.876298 & 1.459347 & 2.147725\end{array}$

$\mathrm{H} \quad 6.120240 \quad-0.314713 \quad 3.838944$

H $\quad 4.281513 \quad-3.113774 \quad 1.140349$

$\begin{array}{llll}\mathrm{H} & 6.189934 & 0.506270 & -3.374160\end{array}$

H $\quad 8.577741 \quad 1.028864 \quad-3.098414$

$\mathrm{H} \quad 10.006290 \quad-0.375516 \quad-1.615851$

H $\quad 8.990315 \quad-2.317188 \quad-0.430469$

H $\quad 6.621329 \quad-2.832322 \quad-0.700510$

H $\quad 4.815996 \quad-2.737701 \quad-4.587071$

$\begin{array}{llll}\mathrm{H} & 3.983858 & -1.982139 & -6.796755\end{array}$

H $\quad 2.855786 \quad 0.226919 \quad-7.011185$

$\mathrm{H} \quad 2.588043 \quad 1.671413 \quad-4.998725$

H $\quad 3.416206 \quad 0.903361 \quad-2.809217$

H $\quad 5.732372 \quad-4.500156 \quad 2.247008$

H $\quad 4.920561 \quad-1.581659 \quad 5.296755$

$\mathrm{H} \quad 3.720285 \quad 4.568355 \quad-1.425621$

H $\quad 3.118520 \quad 3.218378 \quad-2.497658$

$\mathrm{H} \quad-0.070282 \quad 5.391850 \quad 3.160839$

H $\quad 2.022052 \quad 4.892887 \quad 4.393132$

H $\quad 3.820499 \quad 3.572954 \quad 0.732534$

$\mathrm{H} \quad-0.337533 \quad 6.490511 \quad-2.868296$

H $\quad-0.577319 \quad 8.876234 \quad-2.323177$

H $1.096192 \quad 10.004674 \quad-0.861814$

H $\quad 3.018191 \quad 8.691824 \quad 0.026256$

H $\quad 3.256088 \quad 6.322949 \quad-0.512474$

H $\quad 2.467255 \quad 4.571527 \quad-4.603311$

H $\quad 1.235316 \quad 3.838951 \quad-6.626327$

H $\quad-1.146728 \quad 3.120259 \quad-6.436328$

$\mathrm{H} \quad-2.267092 \quad 3.166536 \quad-4.232510$

H $\quad-1.029535 \quad 3.911378-2.215378$

H $\quad 3.249722 \quad 2.713070 \quad 4.888774$

H $\quad 5.657682 \quad 4.679222 \quad 1.926372$

H $\quad-4.803211 \quad 3.453602 \quad-1.192056$

H $\quad-3.313951 \quad 3.160262 \quad-2.200403$

$\mathrm{H} \quad-5.876624 \quad-1.452426 \quad 2.155209$

H $\quad-6.116660 \quad 0.325604 \quad 3.842822$

$\mathrm{H} \quad-4.277440 \quad 3.116561 \quad 1.136191$

$\mathrm{H} \quad-6.193333 \quad-0.510890 \quad-3.371760$

H $\quad-8.581446 \quad-1.031008 \quad-3.094119$

H $\quad-10.007766 \quad 0.375882 \quad-1.611797$

$\mathrm{H} \quad-8.989203 \quad 2.317495 \quad-0.428528$

H $\quad-6.619936 \quad 2.830214 \quad-0.700442$

$\mathrm{H} \quad-4.818153 \quad 2.726584 \quad-4.590753$

H $\quad-3.987096 \quad 1.965145 \quad-6.798844$

H $\quad-2.859122 \quad-0.244472 \quad-7.007945$

H $\quad-2.590475 \quad-1.683639-4.991790$

H $\quad-3.417765 \quad-0.909844 \quad-2.803982$

$\begin{array}{llll}\mathrm{H} & -4.913919 & 1.594592 & 5.296727\end{array}$

$\begin{array}{llll}\mathrm{H} & -5.725450 & 4.507017 & 2.241088\end{array}$

$\mathrm{H} \quad-3.722485-4.570170 \quad-1.416116$

H $\quad-3.119820 \quad-3.222737 \quad-2.490865$

$\mathrm{H} \quad 0.068497 \quad-5.387850 \quad 3.171166$

$\mathrm{H} \quad-2.023167 \quad-4.884996 \quad 4.403025$

$\begin{array}{llll}\mathrm{H} & -3.821440 & -3.570358 & 0.740428\end{array}$

$\mathrm{H} \quad 0.332705 \quad-6.498951 \quad-2.856474$

$\mathrm{H} \quad 0.570107 \quad-8.883912 \quad-2.307066$

H $\quad-1.103770 \quad-10.007716 \quad-0.842547$

H $\quad-3.023692 \quad-8.691011 \quad 0.044299$

H $\quad-3.259210 \quad-6.322876 \quad-0.498668$
H $\quad-2.469575 \quad-4.580484 \quad-4.593960$

H $\quad-1.236960 \quad-3.853106 \quad-6.618471$

H $\quad 1.145797 \quad-3.136388-6.429923$

H $\quad 2.266178 \quad-3.179485 \quad-4.226096$

H $\quad 1.027909-3.919063 \quad-2.207400$

$\mathrm{H} \quad-5.659127 \quad-4.673214 \quad 1.936653$

H $\quad-3.249178 \quad-2.703723 \quad 4.895212$

$\mathrm{H} \quad-5.544627 \quad 5.700330 \quad 6.354763$

H $\quad-7.523037 \quad-2.350906 \quad 5.026312$

$\mathrm{H} \quad \begin{array}{llll}7.523796 & 2.362612 & 5.018987\end{array}$

$\begin{array}{llll}\mathrm{H} & 5.557461 & -5.684148 & 6.363643\end{array}$

$\mathrm{H} \quad-6.525102 \quad 6.753604 \quad 2.950848$

$\mathrm{H} \quad-6.379180 \quad 7.262550 \quad 4.643599$

$\begin{array}{llll}\mathrm{H} & -4.952342 & 7.208879 & 3.606129\end{array}$

H $\quad-5.787157 \quad 3.711971 \quad 8.071553$

$\begin{array}{llll}\mathrm{H} & -5.025092 & 2.181002 & 7.605321\end{array}$

H $\quad-4.046799 \quad 3.639968 \quad 7.806495$

$\mathrm{H} \quad 6.148580 \quad 0.952309 \quad 6.456577$

$\begin{array}{llll}\mathrm{H} & 5.166120 & 2.191442 & 7.242392\end{array}$

$\begin{array}{llll}\mathrm{H} & 4.384013 & 0.937682 & 6.280695\end{array}$

H $\quad 8.854715 \quad 4.017156 \quad 3.586928$

$\begin{array}{llll}\mathrm{H} & 8.443343 & 2.996284 & 2.209117\end{array}$

H $\quad 8.075013 \quad 4.722898 \quad 2.158564$

H $\quad 5.031064-2.162346 \quad 7.605960$

$\begin{array}{llll}\mathrm{H} & 4.061277 & -3.626127 & 7.813803\end{array}$

H $\quad 5.802615 \quad-3.687696 \quad 8.074874$

H $\quad 4.964712-7.197900 \quad 3.612803$

H $\quad 6.540782 \quad-6.744082 \quad 2.964678$

$\mathrm{H} \quad 6.386522 \quad-7.250955 \quad 4.657298$

H $\quad-4.381635 \quad-0.926035 \quad 6.284957$

H $\quad-6.146231 \quad-0.938248 \quad 6.460579$

$\mathrm{H} \quad-5.165418-2.177200 \quad 7.248705$

$\mathrm{H} \quad-8.442356 \quad-2.989468 \quad 2.215186$

H $\quad-8.077003 \quad-4.716882 \quad 2.171825$

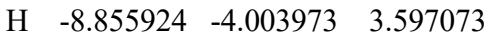

$\begin{array}{lccc}\text { N'2 } & & & \\ \mathrm{Rh} & 0.288548 & -0.222881 & -2.158301 \\ \mathrm{Rh} & 0.128004 & 0.005230 & 0.210925 \\ \mathrm{O} & -1.101946 & -1.628283 & 0.193901 \\ \mathrm{O} & -1.386970 & -1.453104 & -2.040575 \\ \mathrm{O} & 1.742953 & -1.233850 & 0.359073 \\ \mathrm{O} & 1.511162 & -1.864723 & -1.800524 \\ \mathrm{O} & 1.387880 & 1.613412 & 0.075562 \\ \mathrm{O} & 1.942220 & 1.021468 & -2.036141 \\ \mathrm{O} & -1.501484 & 1.205607 & -0.084348 \\ \mathrm{O} & -0.976929 & 1.427202 & -2.271166 \\ \mathrm{Cl} & -0.764470 & -5.090987 & 0.618945 \\ \mathrm{Cl} & 5.135363 & -1.022694 & 0.891581 \\ \mathrm{Cl} & 0.807898 & 4.864444 & -0.194838 \\ \mathrm{Cl} & -4.756867 & 0.227960 & -0.723048 \\ \mathrm{C} & -2.678291 & -3.058689 & -0.803296 \\ \mathrm{C} & -2.854633 & -4.100694 & -1.954074 \\ \mathrm{C} & -3.835143 & -2.978867 & -1.777659 \\ \mathrm{C} & -1.640808 & -1.973668 & -0.908645 \\ \mathrm{C} & -2.927797 & -3.400910 & 0.637676 \\ \mathrm{C} & -2.075468 & -4.211704 & 1.396870 \\ \mathrm{C} & -2.189299 & -4.296501 & 2.783283 \\ \mathrm{C} & -3.149357 & -3.528189 & 3.440889 \\ \mathrm{C} & -4.021437 & -2.702123 & 2.716415 \\ \mathrm{C} & -3.909907 & -2.682630 & 1.321626 \\ \mathrm{C} & -3.258793 & -5.500872 & -1.554137\end{array}$




\begin{tabular}{|c|c|c|c|}
\hline t & -2.528794 & -6.603215 & \\
\hline 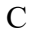 & -2.862137 & -7.903349 & -1.645909 \\
\hline $\mathrm{C}$ & -3.937889 & -8.134575 & -0.788121 \\
\hline $\mathrm{C}$ & -4.679765 & -7.048806 & -0.321214 \\
\hline & -4.347334 & -5.749632 & -0.703504 \\
\hline $\mathrm{C}$ & -1.943615 & -4.014911 & -3.151361 \\
\hline $\mathrm{C}$ & -2.505684 & -3.890479 & -4.426707 \\
\hline $\mathrm{C}$ & -1.692299 & -3.807928 & -5.558414 \\
\hline $\mathrm{C}$ & -0.302404 & -3.846894 & -5.423056 \\
\hline & 0.264727 & -3.983711 & -4.154006 \\
\hline $\mathrm{C}$ & -0.552456 & -4.077528 & -3.028212 \\
\hline $\mathrm{C}$ & -4.973333 & -1.773624 & 3.373261 \\
\hline $\mathrm{C}$ & -6.283847 & -1.632140 & 2.891628 \\
\hline $\mathrm{C}$ & -7.156288 & -0.688563 & 3.440372 \\
\hline C & -6.694446 & 0.125379 & 4.484454 \\
\hline $\mathrm{C}$ & -5.394073 & 0.006878 & 410 \\
\hline $\mathrm{C}$ & -4.544441 & -0.954681 & 4.425171 \\
\hline $\mathrm{C}$ & 3.067733 & -3.020631 & -0.385258 \\
\hline $\mathrm{C}$ & 4.123217 & -3.389644 & -1.475799 \\
\hline $\mathrm{C}$ & 2.956935 & -4.292549 & -1.199540 \\
\hline $\mathrm{C}$ & 2.038870 & -1.957737 & -0.648027 \\
\hline $\mathrm{C}$ & 3.366885 & -3.117754 & 1.082427 \\
\hline $\mathrm{C}$ & 4.224720 & -2.249381 & 1.766175 \\
\hline $\mathrm{C}$ & 4.328987 & -2.280969 & 3.155990 \\
\hline $\mathrm{C}$ & 3.524093 & -3.149501 & 3.890397 \\
\hline $\mathrm{C}$ & 2.616418 & -4.003330 & 3.244399 \\
\hline $\mathrm{C}$ & 2.585554 & -3.987271 & 1.845121 \\
\hline $\mathrm{C}$ & 5.498726 & -3.788889 & -0.995176 \\
\hline $\mathrm{C}$ & 6.634303 & -3.151287 & -1.517024 \\
\hline $\mathrm{C}$ & 7.914378 & -3.481253 & -1.073491 \\
\hline $\mathrm{C}$ & 8.090892 & -4.460288 & -0.095318 \\
\hline $\mathrm{C}$ & 6.971204 & -5.109041 & 0.426894 \\
\hline $\mathrm{C}$ & 5.692105 & -4.780371 & -0.020655 \\
\hline $\mathrm{C}$ & 4.092749 & -2.647736 & -2.787208 \\
\hline $\mathrm{C}$ & 3.988548 & -3.382088 & -3.974267 \\
\hline $\mathrm{C}$ & 3.942246 & -2.736713 & -5.210799 \\
\hline $\mathrm{C}$ & 4.002948 & -1.342473 & -5.270765 \\
\hline $\mathrm{C}$ & 4.125962 & -0.604853 & -4.091453 \\
\hline C & 4.179940 & -1.254350 & -2.858463 \\
\hline $\mathrm{C}$ & 1.641989 & -4.836475 & 3.988301 \\
\hline $\mathrm{C}$ & 1.033007 & -4.344928 & 5.154865 \\
\hline $\mathrm{C}$ & 0.046333 & -5.076457 & 5.819018 \\
\hline $\mathrm{C}$ & -0.334232 & -6.321001 & 5.296266 \\
\hline C & 0.253273 & -6.837064 & 4.136988 \\
\hline $\mathrm{C}$ & 1.247311 & -6.087380 & 3.498240 \\
\hline $\mathrm{C}$ & 3.066755 & 2.859100 & -1.000882 \\
\hline $\mathrm{C}$ & 3.364102 & 3.659693 & -2.315984 \\
\hline $\mathrm{C}$ & 4.326980 & 2.629097 & -1.810447 \\
\hline $\mathrm{C}$ & 2.055231 & 1.752546 & -1.001947 \\
\hline $\mathrm{C}$ & 3.167912 & 3.547459 & 0.327087 \\
\hline $\mathrm{C}$ & 2.215142 & 4.467376 & 0.784932 \\
\hline $\mathrm{C}$ & 2.329488 & 5.065221 & 2.037747 \\
\hline $\mathrm{C}$ & 3.389770 & 4.721560 & 2.873149 \\
\hline $\mathrm{C}$ & 4.342871 & 3.774329 & 2.469827 \\
\hline $\mathrm{C}$ & 4.212187 & 3.215967 & 1.191126 \\
\hline $\mathrm{C}$ & 3.685368 & 5.127537 & -2.146884 \\
\hline $\mathrm{C}$ & 2.866947 & 6.093097 & -2.752285 \\
\hline $\mathrm{C}$ & 3.117582 & 7.455035 & -2.590722 \\
\hline $\mathrm{C}$ & 4.197531 & 7.886263 & -1.819502 \\
\hline $\mathrm{C}$ & 5.024884 & 6.937617 & -1.217536 \\
\hline $\mathrm{C}$ & 4.773304 & 5.575886 & -1.382812 \\
\hline$C$ & 2.605760 & 3.318111 & -3.571999 \\
\hline
\end{tabular}

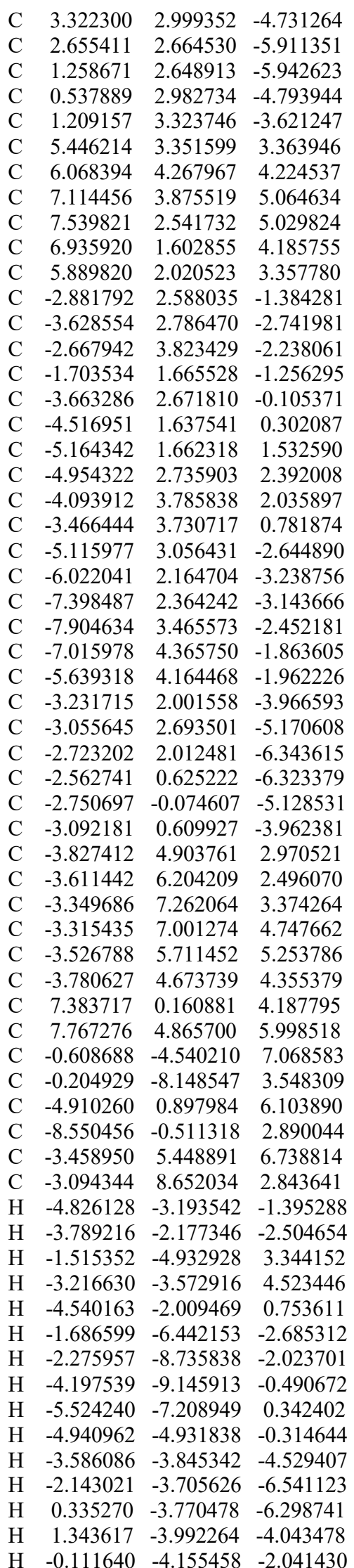

H $\quad-6.623238 \quad-2.267612 \quad 2.078510$

H $\quad-3.521564 \quad-1.039319 \quad 4.781929$

$\mathrm{H} \quad 3.121386 \quad-5.229984 \quad-0.681816$

H $\quad 2.171799 \quad-4.314247 \quad-1.943479$

H $\quad 5.016089-1.611631 \quad 3.659815$

$\mathrm{H} \quad 3.600838 \quad-3.155855 \quad 4.972705$

$\mathrm{H} \quad 1.864370 \quad-4.606181 \quad 1.325672$

H $\quad 6.514992 \quad-2.385557 \quad-2.273753$

H $\quad 8.774089-2.968585 \quad-1.494668$

H $\quad 9.086582-4.717321 \quad 0.252845$

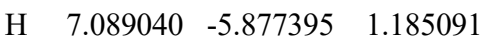

H $\quad 4.846363 \quad-5.299105 \quad 0.413292$

$\mathrm{H} \quad 3.931424 \quad-4.465766 \quad-3.924328$

H $\quad 3.852200 \quad-3.320360 \quad-6.122087$

H $\quad 3.953976 \quad-0.832091 \quad-6.228028$

H $\quad 4.152573 \quad 0.477914 \quad-4.137670$

H $\quad 4.246852 \quad-0.677494 \quad-1.942722$

H $\quad 1.306810 \quad-3.361798 \quad 5.526366$

$\begin{array}{llll}\mathrm{H} & 1.716280 & -6.483752 & 2.602761\end{array}$

H $\quad 5.268681 \quad 2.939499 \quad-1.375284$

H $\quad 4.368913 \quad 1.696842 \quad-2.357475$

$\mathrm{H} \quad 1.579535 \quad 5.776338 \quad 2.364899$

H $\quad 3.452279 \quad 5.167298 \quad 3.860208$

$\mathrm{H} \quad 4.949658 \quad 2.498257 \quad 0.849254$

H $\quad 2.024275 \quad 5.775253 \quad-3.353981$

H $\quad 2.464852 \quad 8.178753 \quad-3.069753$

H $\quad 4.393937 \quad 8.946371 \quad-1.691770$

H $\quad 5.872987 \quad 7.253768 \quad-0.617646$

$\begin{array}{llll}\mathrm{H} & 5.432749 & 4.868851 & -0.895517\end{array}$

H $\quad 4.408385 \quad 3.003027 \quad-4.703021$

H $\quad 3.225721 \quad 2.411088 \quad-6.800166$

$\mathrm{H} \quad 0.732405 \quad 2.377495 \quad-6.853093$

$\mathrm{H} \quad-0.544991 \quad 2.953633 \quad-4.805512$

H $\quad 0.646642 \quad 3.546206 \quad-2.722868$

H $\quad 5.754188 \quad 5.307771 \quad 4.217550$

$\begin{array}{llll}\mathrm{H} & 5.404260 & 1.295231 & 2.712077\end{array}$

H $\quad-3.037883 \quad 4.770126 \quad-1.863846$

$\mathrm{H} \quad-1.712831 \quad 3.881792 \quad-2.745965$

$\begin{array}{llll}\mathrm{H} & -5.811893 & 0.845067 & 1.824028\end{array}$

$\begin{array}{llll}\mathrm{H} & -5.472566 & 2.752530 & 3.344247\end{array}$

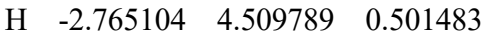

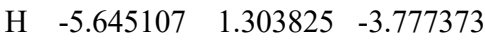

$\begin{array}{llll}\mathrm{H} & -8.074748 & 1.654651 & -3.611058\end{array}$

$\mathrm{H} \quad-8.976242 \quad 3.622456 \quad-2.376071$

$\mathrm{H} \quad-7.390660 \quad 5.231480 \quad-1.325785$

H $\quad-4.981027 \quad 4.880298 \quad-1.486231$

H $\quad-3.172383 \quad 3.773583 \quad-5.183860$

H $\quad-2.583167 \quad 2.565728 \quad-7.267491$

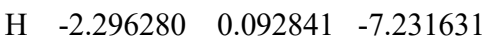

H $\quad-2.622964 \quad-1.152133 \quad-5.098731$

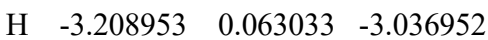

$\begin{array}{llll}\mathrm{H} & -3.673476 & 6.402797 & 1.429852\end{array}$

H $\quad-3.921949 \quad 3.666439 \quad 4.735558$

$\begin{array}{llll}\mathrm{H} & 8.360873 & 2.229325 & 5.671282\end{array}$

$\begin{array}{llll}\mathrm{H} & -3.121839 & 7.818398 & 5.439016\end{array}$

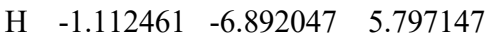

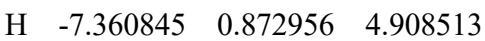

H $\quad 7.182955 \quad-0.319529 \quad 3.226251$

$\begin{array}{llll}\mathrm{H} & 8.453797 & 0.075814 & 4.398286\end{array}$

H $\quad 6.857005 \quad-0.413425 \quad 4.960345$

$\begin{array}{llll}\mathrm{H} & 8.844642 & 4.690997 & 6.075385\end{array}$

H $\quad 7.608962 \quad 5.894318 \quad 5.663316$ 


$\begin{array}{rrrr}\mathrm{H} & 7.353027 & 4.782214 & 7.010670 \\ \mathrm{H} & -1.696888 & -4.651928 & 7.024669 \\ \mathrm{H} & -0.265181 & -5.081892 & 7.957846 \\ \mathrm{H} & -0.379698 & -3.481533 & 7.215050 \\ \mathrm{H} & -0.766833 & -8.740395 & 4.275478 \\ \mathrm{H} & -0.857230 & -7.974227 & 2.683923 \\ \mathrm{H} & 0.641898 & -8.746461 & 3.197601 \\ \mathrm{H} & -3.861534 & 1.176600 & 5.962191 \\ \mathrm{H} & -5.503139 & 1.814723 & 6.167873 \\ \mathrm{H} & -4.982245 & 0.391171 & 7.073746 \\ \mathrm{H} & -8.593331 & 0.346722 & 2.208104 \\ \mathrm{H} & -8.873340 & -1.392962 & 2.330277 \\ \mathrm{H} & -9.274689 & -0.325563 & 3.688833 \\ \mathrm{H} & -3.825732 & 4.448367 & 6.982929 \\ \mathrm{H} & -2.429081 & 5.525760 & 7.106813 \\ \mathrm{H} & -4.054735 & 6.177463 & 7.298320 \\ \mathrm{H} & -2.038922 & 8.780369 & 2.573891 \\ \mathrm{H} & -3.684428 & 8.849919 & 1.943981 \\ \mathrm{H} & -3.336491 & 9.414777 & 3.588791\end{array}$

$\begin{array}{lccc}\text { N'3 } & & & \\ \mathrm{Rh} & 0.048067 & -0.276894 & -2.122346 \\ \mathrm{Rh} & 0.039126 & -0.068063 & 0.256797 \\ \mathrm{O} & 0.565337 & 1.897434 & 0.021900 \\ \mathrm{O} & 1.050489 & 1.539153 & -2.157441 \\ \mathrm{O} & -1.949148 & 0.420460 & 0.129741 \\ \mathrm{O} & -1.768935 & 0.731925 & -2.101013 \\ \mathrm{O} & -0.474048 & -2.044443 & 0.346370 \\ \mathrm{O} & -0.960206 & -2.074587 & -1.861458 \\ \mathrm{O} & 2.019029 & -0.584480 & 0.240171 \\ \mathrm{O} & 1.857603 & -1.269961 & -1.908995 \\ \mathrm{Cl} & -1.357494 & 4.525745 & -0.074104 \\ \mathrm{Cl} & -4.700909 & -1.621677 & 0.078058 \\ \mathrm{Cl} & 1.213857 & -4.955794 & 0.907179 \\ \mathrm{Cl} & 4.707667 & 1.367826 & 0.136078 \\ \mathrm{C} & 1.436035 & 3.699995 & -1.212657 \\ \mathrm{C} & 1.223759 & 4.528492 & -2.527373 \\ \mathrm{C} & 2.576183 & 3.996017 & -2.167515 \\ \mathrm{C} & 0.978876 & 2.275004 & -1.123000 \\ \mathrm{C} & 1.389575 & 4.410855 & 0.107263 \\ \mathrm{C} & 0.192391 & 4.828436 & 0.705466 \\ \mathrm{C} & 0.185339 & 5.464815 & 1.943858 \\ \mathrm{C} & 1.383833 & 5.672259 & 2.622383 \\ \mathrm{C} & 2.601451 & 5.243205 & 2.073999 \\ \mathrm{C} & 2.571841 & 4.626219 & 0.815734 \\ \mathrm{C} & 0.919935 & 5.997356 & -2.336339 \\ \mathrm{C} & -0.331970 & 6.500594 & -2.720922 \\ \mathrm{C} & -0.661000 & 7.840742 & -2.523612 \\ \mathrm{C} & 0.258169 & 8.712877 & -1.938451 \\ \mathrm{C} & 1.510778 & 8.228553 & -1.560311 \\ \mathrm{C} & 1.837761 & 6.887412 & -1.760408 \\ \mathrm{C} & 0.543057 & 3.897073 & -3.713404 \\ \mathrm{C} & 1.183814 & 3.941537 & -4.957401 \\ \mathrm{C} & 0.592925 & 3.366621 & -6.084064 \\ \mathrm{C} & -0.649767 & 2.737543 & -5.975041 \\ \mathrm{C} & -1.300085 & 2.701734 & -4.739800 \\ \mathrm{C} & -0.711013 & 3.288535 & -3.620898 \\ \mathrm{C} & 3.884466 & 5.415226 & 2.795081 \\ \mathrm{C} & 4.115846 & 6.543836 & 3.595578 \\ \mathrm{C} & 5.327986 & 6.716500 & 4.269801 \\ \mathrm{C} & 6.321485 & 5.739795 & 4.127053 \\ \mathrm{C} & 6.119827 & 4.601508 & 3.338568\end{array}$

\begin{tabular}{|c|c|c|c|c|c|c|c|}
\hline & 4.896664 & 450691 & 2.678817 & $\mathrm{C}$ & 4.894098 & -0.079550 & 1.120975 \\
\hline & -3.867406 & 1.148168 & 1.022416 & $\mathrm{C}$ & 5.391347 & 0.066260 & 2.413483 \\
\hline & -4.773966 & 0.900668 & -2.274808 & $\mathrm{C}$ & 5.484335 & -1.046075 & 3.246786 \\
\hline & -4.236686 & 2.268673 & -1.975019 & $\mathrm{C}$ & 5.076992 & -2.312299 & .800883 \\
\hline & -2.424789 & 0.728557 & -1.012354 & $\mathrm{C}$ & 4.609350 & -2.425274 & 1.484546 \\
\hline & -4.473784 & 1.099423 & 0.348906 & $\mathrm{C}$ & 6.313063 & -1.079994 & -1.731568 \\
\hline & -4.856220 & -0.104844 & 0.954205 & $\mathrm{C}$ & 6.857648 & 0.093939 & -2.273737 \\
\hline & -5.336825 & -0.148088 & 2.259712 & $\mathrm{C}$ & 8.176115 & 0.464465 & -2.013245 \\
\hline & -5.434311 & 1.033650 & 2.990354 & $\mathrm{C}$ & 8.985692 & -0.334064 & -1.204401 \\
\hline & -5.055473 & 2.262914 & 2.427782 & $\mathrm{C}$ & 8.460726 & -1.509171 & -0.665642 \\
\hline & -4.584649 & 2.264925 & 1.106684 & $\mathrm{C}$ & 7.141933 & 8396 & -0.929482 \\
\hline & -6.231506 & 0.60 & -1.996026 & $\mathrm{C}$ & 20634 & 7483 & 8891 \\
\hline & -6.790670 & -0.60 & -2.440639 & $\mathrm{C}$ & 48251 & 9874 & 9255 \\
\hline & -8.119710 & -0.927843 & -2.170726 & $\mathrm{C}$ & 7059 & 1538 & -5.679004 \\
\hline & -8.926249 & -0.046801 & -1.449305 & $\mathrm{C}$ & & 0275 & 49257 \\
\hline & -8.387502 & 1.162720 & -1.0095 & $\mathrm{C}$ & 263 & 278 & 528 \\
\hline & -7.05 & & -1.28 & $\mathrm{C}$ & 208 & & \\
\hline C & -4.217795 & 0.191181 & -3.482631 & $\mathrm{C}$ & 957 & 919 & 851 \\
\hline C & -4.390333 & 0.775213 & -4.742854 & $\mathrm{C}$ & 38255 & 72044 & 25342 \\
\hline & -3.902662 & 0.148763 & -5.891666 & $\mathrm{C}$ & 3941 & -4.7 & \\
\hline & -3.233540 & -1.07 & -5.7896 & $\mathrm{C}$ & 773 & -5 & 762 \\
\hline $\mathrm{C}$ & -3.07 & -1.6 & -4.5 & $\mathrm{C}$ & & & 427 \\
\hline $\mathrm{C}$ & -3.571852 & -1.0 & -3.395080 & $\mathrm{C}$ & 2807 & 829 & 042 \\
\hline $\mathrm{C}$ & -5.130517 & 3.524797 & 3.201257 & $\mathrm{C}$ & 1551 & & 6151 \\
\hline C & -6.155686 & 3.7 & 4.13 & $\mathrm{C}$ & 504 & -3 . & 493 \\
\hline C & -6.242603 & & $4.8 \mathrm{c}$ & $\mathrm{C}$ & & & \\
\hline $\mathrm{C}$ & -5.28 & & 4.6 & $\mathrm{C}$ & & & \\
\hline $\mathrm{C}$ & -4.250578 & 5.7 & 3.716 & $\mathrm{C}$ & 140 & & \\
\hline C & -4.183010 & 4.5 & 3.00 & $\mathrm{C}$ & & & \\
\hline $\mathrm{C}$ & -1.422748 & -4.0 & -0.5 & $\mathrm{C}$ & & -6.6 & \\
\hline $\mathrm{C}$ & -1.21 & & -1.6 & $\mathrm{C}$ & & & \\
\hline $\mathrm{C}$ & -2.55 & -4.4 & -1.4 & $\mathrm{H}$ & & & 027 \\
\hline $\mathrm{C}$ & -0.907276 & -2.6 & -0.7 & $\mathrm{H}$ & & & 971 \\
\hline C & -1.470833 & -4.3 & & $\mathrm{H}$ & 950 & & \\
\hline $\mathrm{C}$ & -0.350023 & & 1.6 & $\mathrm{H}$ & & & \\
\hline C & -0.42 & -4.8 & 3.0 & $\mathrm{H}$ & & & \\
\hline $\mathrm{C}$ & -1.6 & -4 & 3.73 & $\mathrm{H}$ & & & 726 \\
\hline $\mathrm{C}$ & -2.747223 & -4.1 & 3.02 & $\mathrm{H}$ & 940 & & 758 \\
\hline $\mathrm{C}$ & -2.654784 & & & $\mathrm{H}$ & & & 933 \\
\hline $\mathrm{C}$ & -0.9 & & & $\mathrm{H}$ & & & 389 \\
\hline $\mathrm{C}$ & 0.1 & -7. & -1.6 & $\mathrm{H}$ & & & 980 \\
\hline $\mathrm{C}$ & 0.376419 & -8.5 & -1.17 & $\mathrm{H}$ & & & 165 \\
\hline $\mathrm{C}$ & -0.501538 & -9.1 & -0.284261 & $\mathrm{H}$ & & & 516 \\
\hline $\mathrm{C}$ & -1.629751 & -8.45 & & $\mathrm{H}$ & & 5608 & 134 \\
\hline $\mathrm{C}$ & -1.87 & & & $\mathrm{H}$ & & & 583 \\
\hline $\mathrm{C}$ & -0.48 & -4.7 & -2.8 & $\mathrm{H}$ & 449 & & 624 \\
\hline $\mathrm{C}$ & -1.111594 & -4.90 & -4.12 & $\mathrm{H}$ & 301 & & 514 \\
\hline $\mathrm{C}$ & -0.472938 & -4.561598 & -5.317643 & $\mathrm{H}$ & 5177 & 3091 & 1749 \\
\hline C & 0.809554 & & & $\mathrm{H}$ & & & 2265 \\
\hline $\mathrm{C}$ & 1.45 & & & $\mathrm{H}$ & & & 805 \\
\hline $\mathrm{C}$ & 0.812308 & -4.17 & -2.8 & $\mathrm{H}$ & & -1.0 & 866 \\
\hline $\mathrm{C}$ & -3.988969 & -3.708219 & 3.681806 & $\mathrm{H}$ & -5.781299 & 0.989245 & 4.017380 \\
\hline $\mathrm{C}$ & -5.244486 & -3.965178 & 3.111502 & $\mathrm{H}$ & -4.295073 & 3.203097 & 0.646397 \\
\hline$C$ & -6.414548 & & & $\mathrm{H}$ & & -1.301964 & 8082 \\
\hline $\mathrm{C}$ & -6.313859 & -2.6 & 4.82 & $\mathrm{H}$ & & -1.8 & -2.524777 \\
\hline $\mathrm{C}$ & -5.075724 & $-2.3 \xi$ & $5.41\}$ & $\mathrm{H}$ & 1067 & -0.2 & -1.236335 \\
\hline $\mathrm{C}$ & -3.921307 & -2.921460 & 4.840549 & $\mathrm{H}$ & -9.000959 & 1.863956 & -0.451833 \\
\hline & 3.933364 & -1.523978 & -0.746982 & $\mathrm{H}$ & -6.673181 & 2.427964 & -0.918777 \\
\hline & 4.867056 & & -2.003288 & $\mathrm{H}$ & & 1.731652 & -4.819760 \\
\hline $\mathrm{C}$ & 4.325544 & -2.749830 & -1.546907 & $\mathrm{H}$ & -4.038053 & 0.618686 & -6.861258 \\
\hline & 2.499673 & -1.091478 & -0.826561 & $\mathrm{H}$ & -2.842320 & -1.558151 & -6.679136 \\
\hline & 4.512979 & -1.332412 & 0.622818 & $\mathrm{H}$ & -2.537836 & -2.610313 & -4.444439 \\
\hline
\end{tabular}


H $\quad-3.419284-1.498668 \quad-2.425000$

$\begin{array}{llll}\mathrm{H} & -6.914237 & 2.967042 & 4.278634\end{array}$

$\begin{array}{llll}\mathrm{H} & -3.371931 & 4.387879 & 2.297105\end{array}$

H $\quad-3.359475 \quad-5.018387 \quad-0.981433$

$\begin{array}{lllll}\mathrm{H} & -2.868496 & -3.731248 & -2.179000\end{array}$

H $\quad 0.457198 \quad-5.178616 \quad 3.629163$

$\begin{array}{llll}\mathrm{H} & -1.651620 & -4.670497 & 4.817814\end{array}$

H $\quad-3.506652 \quad-3.765368 \quad 1.059620$

H $\quad 0.825542 \quad-6.752205 \quad-2.292629$

H $\quad \begin{array}{llll}1.257566 & -9.049653 & -1.527739\end{array}$

H $\quad-0.311990 \quad-10.160811 \quad 0.057089$

$\begin{array}{llll}\mathrm{H} & -2.327297 & -8.924845 & 0.841890\end{array}$

$\begin{array}{llll}\mathrm{H} & -2.756059 & -6.644020 & 0.084971\end{array}$

H $\quad-2.114296 \quad-5.325014 \quad-4.148060$

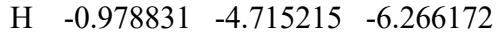

H $\quad \begin{array}{llll}1.307571 & -3.723495 & -6.206887\end{array}$

H $\quad 2.435858 \quad-3.372059-4.032142$

H $\quad 1.300728 \quad-4.005465 \quad-1.918798$

$\begin{array}{llll}\mathrm{H} & -5.310860 & -4.585584 & 2.222655\end{array}$

H $\quad-2.952186 \quad-2.693148 \quad 5.275089$

H $4.983228 \quad-3.469071 \quad-1.074920$

H $\quad 3.542855 \quad-3.186432 \quad-2.152932$

$\begin{array}{llll}\mathrm{H} & 5.679222 & 1.046515 & 2.773429\end{array}$

H $\quad 5.840908 \quad-0.917508 \quad 4.263255$

H $\quad 4.308199 \quad-3.397902 \quad 1.111699$

$\begin{array}{llll}\mathrm{H} & 6.242144 & 0.725287 & -2.902921\end{array}$

H $\quad 8.568714 \quad 1.380667 \quad-2.444160$

H $\quad 10.012408 \quad-0.046770 \quad-0.999384$

H $\quad 9.077092 \quad-2.146050 \quad-0.038299$

H $\quad 6.766300 \quad-2.793384 \quad-0.488998$

$\mathrm{H} \quad 4.932782 \quad-2.630549 \quad-4.401112$

$\begin{array}{llll}\mathrm{H} & 4.046974 & -1.816099 & -6.568770\end{array}$

H $\quad 2.907110 \quad 0.396334 \quad-6.692514$

H $\quad 2.681070 \quad 1.777656 \quad-4.641485$

$\begin{array}{llll}\mathrm{H} & 3.584897 & 0.959526 & -2.489364\end{array}$

H $\quad 6.938804 \quad-2.940116 \quad 4.683992$

H $\quad 3.288488 \quad-4.339709 \quad 2.894425$

$\begin{array}{llll}\mathrm{H} & -7.217513 & -2.243924 & 5.266710\end{array}$

H $\quad 5.185416 \quad-6.632946 \quad 5.978657$

H $\quad-5.350144 \quad 6.851784 \quad 5.193812$

H $\quad 7.272971 \quad 5.871451 \quad 4.637330$

$\mathrm{H} \quad-4.812100 \quad-0.443054 \quad 6.322365$

$\begin{array}{llll}\mathrm{H} & -5.903310 & -1.506257 & 7.214009\end{array}$

H $\quad-4.150142 \quad-1.771075 \quad 7.275977$

H $\quad-7.947677 \quad-2.921589 \quad 2.247441$

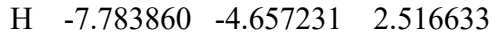

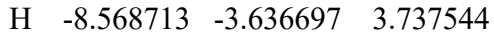

$\begin{array}{llll}\text { H } & -8.159737 & 4.418618 & 5.732873\end{array}$

$\begin{array}{lllll}\mathrm{H} & -6.963359 & 4.987497 & 6.901668\end{array}$

H $\quad-7.748966 \quad 6.140955 \quad 5.825434$

$\begin{array}{llll}\mathrm{H} & -3.580759 & 7.796518 & 3.810482\end{array}$

$\begin{array}{llll}\mathrm{H} & -2.308579 & 6.609526 & 4.099736\end{array}$

$\begin{array}{llll}\mathrm{H} & -2.904922 & 6.873668 & 2.457660\end{array}$

$\begin{array}{llll}\mathrm{H} & 7.134426 & 3.025007 & 2.259295\end{array}$

H $\quad 8.189698 \quad 3.971953 \quad 3.322494$

$\mathrm{H} \quad 7.082228 \quad 2.784936 \quad 4.008885$

H $4.904866 \quad 8.754365 \quad 4.858815$

$\begin{array}{llll}\mathrm{H} & 5.359350 & 7.694282 & 6.195454\end{array}$

$\begin{array}{llll}\mathrm{H} & 6.596657 & 8.270486 & 5.079424\end{array}$

H $\quad 2.552183 \quad-6.632791 \quad 3.338908$

H $\quad 3.383273 \quad-7.635331 \quad 4.542585$

H $\quad 2.219590 \quad-6.417968 \quad 5.061028$
H $\quad 8.165314 \quad-4.388031 \quad 6.200924$

H $\quad 6.955427 \quad-4.617770 \quad 7.465954$

H $\quad 7.566095 \quad-6.013750 \quad 6.578930$

\section{N'4}

$\begin{array}{llll}\text { Rh } & 0.174142 & -0.371622 & -2.150987\end{array}$

$\begin{array}{llll}\mathrm{Rh} & 0.102466 & -0.058076 & 0.213991\end{array}$

$\begin{array}{llll}\mathrm{O} & -1.281151 & 1.422885 & -0.094081\end{array}$

$\begin{array}{lllll}\mathrm{O} & -0.772210 & 1.475691 & -2.295867\end{array}$

$\begin{array}{llll}\mathrm{O} & -1.395233 & -1.452819 & 0.258948\end{array}$

$\begin{array}{llll}\mathrm{O} & -1.680711 & -1.298540 & -1.977108\end{array}$

$\begin{array}{llll}\text { O } & 1.474775 & -1.559812 & 0.366330\end{array}$

$\begin{array}{llll}\text { O } & 1.096926 & -2.193628 & -1.771814\end{array}$

$\begin{array}{llll}\text { O } & 1.622703 & 1.300083 & 0.014691\end{array}$

$\begin{array}{llll}\text { O } & 2.020919 & 0.568448 & -2.088554\end{array}$

$\begin{array}{lllll}\mathrm{Cl} & -4.652940 & 1.264095 & -0.341074\end{array}$

$\begin{array}{llll}\mathrm{Cl} & -1.747332 & -4.912674 & 0.818420\end{array}$

$\begin{array}{llll}\mathrm{Cl} & 4.856967 & -1.985061 & 0.863878\end{array}$

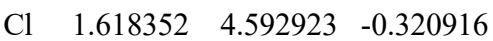

$\begin{array}{llll}\text { C } & -2.352848 & 3.046632 & -1.413087\end{array}$

C $\quad-3.151758 \quad 3.278670 \quad-2.738544$

$\begin{array}{llll}\text { C } & -1.961726 & 4.127063 & -2.401717\end{array}$

$\begin{array}{llll}\text { C } & -1.402857 & 1.891390 & -1.273632\end{array}$

$\begin{array}{llll}\text { C } & -2.970113 & 3.420820 & -0.097699\end{array}$

$\begin{array}{llll}\text { C } & -3.986362 & 2.660525 & 0.496091\end{array}$

$\begin{array}{llll}\text { C } & -4.473890 & 2.955987 & 1.765741\end{array}$

$\begin{array}{llll}\text { C } & -3.940158 & 4.032568 & 2.469749\end{array}$

$\begin{array}{llll}\text { C } & -2.921370 & 4.822941 & 1.914579\end{array}$

$\begin{array}{llll}\text { C } & -2.460338 & 4.495111 & 0.631171\end{array}$

$\begin{array}{llll}\text { C } & -4.541113 & 3.858164 & -2.586981\end{array}$

C $\quad-5.648975 \quad 3.137695 \quad-3.058620$

$\begin{array}{llll}\text { C } & -6.945387 & 3.627827 & -2.908731\end{array}$

$\begin{array}{llll}\text { C } & -7.166602 & 4.855428 & -2.283196\end{array}$

C $\quad-6.074301 \quad 5.586975 \quad-1.815915$

C $\quad-4.778175 \quad 5.095206 \quad-1.969366$

C $\quad-3.003239 \quad 2.313631 \quad-3.886329$

C $\quad-2.720574 \quad 2.821522 \quad-5.159597$

C $\quad-2.583548 \quad 1.965131 \quad-6.254069$

C $\quad-2.727620 \quad 0.586133 \quad-6.083495$

$\begin{array}{llll}\text { C } & -3.024140 & 0.073286 & -4.818168\end{array}$

$\begin{array}{llll}\text { C } & -3.171767 & 0.934825 & -3.732072\end{array}$

$\begin{array}{llll}\text { C } & -2.330952 & 5.960495 & 2.658746\end{array}$

C $\quad-3.119533 \quad 6.737965 \quad 3.521547$

$\begin{array}{llll}\text { C } & -2.578686 & 7.819445 & 4.220782\end{array}$

$\begin{array}{llll}\text { C } & -1.223837 & 8.126072 & 4.039577\end{array}$

$\begin{array}{llll}\text { C } & -0.411103 & 7.369828 & 3.188895\end{array}$

$\begin{array}{llll}\text { C } & -0.975719 & 6.287859 & 2.505511\end{array}$

$\begin{array}{llll}\text { C } & -3.232003 & -2.586182 & -0.670611\end{array}$

C $\quad-3.635666-3.611680 \quad-1.774951$

$\begin{array}{llll}\text { C } & -4.368177 & -2.309194 & -1.635186\end{array}$

$\begin{array}{llll}\text { C } & -2.006976 & -1.726152 & -0.825546\end{array}$

$\begin{array}{llll}\text { C } & -3.526497 & -2.822965 & 0.782303\end{array}$

$\begin{array}{llll}\text { C } & -2.859784 & -3.772396 & 1.565184\end{array}$

$\begin{array}{llll}\text { C } & -3.003528 & -3.804591 & 2.951337\end{array}$

$\begin{array}{llll}\text { C } & -3.794740 & -2.845774 & 3.582830\end{array}$

$\begin{array}{llll}\text { C } & -4.465179 & -1.865246 & 2.835030\end{array}$

$\begin{array}{llll}\text { C } & -4.337153 & -1.899447 & 1.441922\end{array}$

$\begin{array}{llll}\text { C } & -4.309473 & -4.885005 & -1.316392\end{array}$

$\begin{array}{llll}\text { C } & -3.818622 & -6.128906 & -1.740892\end{array}$

$\begin{array}{llll}\text { C } & -4.404480 & -7.319743 & -1.313321\end{array}$

C $\quad-5.499254 \quad-7.296288-0.448746$

C $\quad-6.003970 \quad-6.066313 \quad-0.024989$
C $\quad-5.419294 \quad-4.876405 \quad-0.457230$

$\begin{array}{llll}\text { C } & -2.749830 & -3.762944 & -2.983999\end{array}$

C $\quad-3.313483-3.627551 \quad-4.257585$

$\begin{array}{llll}\text { C } & -2.527457 & -3.765685 & -5.402954\end{array}$

$\begin{array}{llll}\text { C } & -1.163639 & -4.042635 & -5.283035\end{array}$

$\begin{array}{llll}\text { C } & -0.597796 & -4.193664 & -4.014915\end{array}$

$\begin{array}{llll}\text { C } & -1.389151 & -4.063732 & -2.874131\end{array}$

$\begin{array}{llll}\text { C } & -5.209299 & -0.746310 & 3.460855\end{array}$

$\begin{array}{llll}\text { C } & -6.383841 & -0.253053 & 2.874394\end{array}$

$\begin{array}{llll}\text { C } & -7.043574 & 0.861784 & 3.400480\end{array}$

$\begin{array}{llll}\text { C } & -6.509517 & 1.482783 & 4.536275\end{array}$

$\begin{array}{llll}\text { C } & -5.338849 & 1.013592 & 5.144883\end{array}$

$\begin{array}{llll}\text { C } & -4.703177 & -0.107250 & 4.602065\end{array}$

$\begin{array}{llll}\text { C } & 2.428087 & -3.583500 & -0.334674\end{array}$

$\begin{array}{llll}\text { C } & 3.380275 & -4.175442 & -1.421346\end{array}$

$\begin{array}{llll}\text { C } & 2.067207 & -4.832439 & -1.111078\end{array}$

$\begin{array}{llll}\text { C } & 1.614890 & -2.353072 & -0.621948\end{array}$

$\begin{array}{llll}\text { C } & 2.721218 & -3.693922 & 1.133401\end{array}$

$\begin{array}{llll}\text { C } & 3.738894 & -2.987014 & 1.783130\end{array}$

$\begin{array}{llll}\text { C } & 3.853255 & -2.995346 & 3.172421\end{array}$

$\begin{array}{llll}\text { C } & 2.905415 & -3.669085 & 3.939693\end{array}$

C $\quad 1.842765 \quad-4.351800 \quad 3.327655$

$\begin{array}{llll}\text { C } & 1.796930 & -4.373793 & 1.928837\end{array}$

$\begin{array}{llll}\text { C } & 4.659623 & -4.819538 & -0.940151\end{array}$

C $\quad 5.889653 \quad-4.433004 \quad-1.493251$

C $7.087196-4.995009-1.052252$

$\begin{array}{llll}\text { C } & 7.084196 & -5.960270 & -0.044987\end{array}$

$\begin{array}{llll}\text { C } & 5.867606 & -6.360459 & 0.509126\end{array}$

$\begin{array}{llll}\text { C } & 4.670776 & -5.800286 & 0.064004\end{array}$

$\begin{array}{llll}\text { C } & 3.475859 & -3.474024 & -2.751791\end{array}$

$\begin{array}{llll}\text { C } & 3.199490 & -4.198698 & -3.916821\end{array}$

$\begin{array}{llll}\text { C } & 3.264710 & -3.587858 & -5.169907\end{array}$

$\begin{array}{llll}\text { C } & 3.614775 & -2.239367 & -5.269383\end{array}$

$\begin{array}{llll}\text { C } & 3.911978 & -1.515785 & -4.112597\end{array}$

$\begin{array}{llll}\text { C } & 3.849614 & -2.131067 & -2.862357\end{array}$

$\begin{array}{llll}\text { C } & 0.737122 & -4.957690 & 4.107132\end{array}$

$\begin{array}{llll}\text { C } & 0.237934 & -4.310971 & 5.247874\end{array}$

$\begin{array}{llll}\text { C } & -0.859693 & -4.821318 & 5.946598\end{array}$

$\begin{array}{llll}\text { C } & -1.453995 & -6.005423 & 5.491451\end{array}$

$\begin{array}{llll}\text { C } & -0.979252 & -6.673020 & 4.356115\end{array}$

$\begin{array}{llll}\text { C } & 0.121382 & -6.141172 & 3.677848\end{array}$

$\begin{array}{llll}\text { C } & 3.472461 & 2.201746 & -1.120225\end{array}$

$\begin{array}{llll}\text { C } & 3.879193 & 2.905341 & -2.461181\end{array}$

C $\quad 4.655413 \quad 1.733276 \quad-1.943022$

$\begin{array}{llll}\text { C } & 2.282207 & 1.292000 & -1.076439\end{array}$

$\begin{array}{llll}\text { C } & 3.720939 & 2.889385 & 0.188735\end{array}$

$\begin{array}{llll}\text { C } & 2.954543 & 3.970953 & 0.642781\end{array}$

$\begin{array}{llll}\text { C } & 3.197543 & 4.561669 & 1.880186\end{array}$

$\begin{array}{llll}\text { C } & 4.197046 & 4.052131 & 2.705333\end{array}$

$\begin{array}{llll}\text { C } & 4.960140 & 2.944419 & 2.307022\end{array}$

$\begin{array}{llll}\text { C } & 4.707809 & 2.394968 & 1.042787\end{array}$

$\begin{array}{llll}\text { C } & 4.455192 & 4.297561 & -2.336081\end{array}$

$\begin{array}{llll}\text { C } & 3.804918 & 5.377679 & -2.951841\end{array}$

$\begin{array}{llll}\text { C } & 4.292413 & 6.678062 & -2.828802\end{array}$

$\begin{array}{lllll}\text { C } & 5.446725 & 6.930734 & -2.086634\end{array}$

$\begin{array}{llll}\text { C } & 6.108773 & 5.865489 & -1.475234\end{array}$

$\begin{array}{llll}\text { C } & 5.620431 & 4.565382 & -1.601984\end{array}$

$\begin{array}{llll}\text { C } & 3.050400 & 2.670228 & -3.697171\end{array}$

$\begin{array}{llll}\text { C } & 3.680855 & 2.197495 & -4.853884\end{array}$

$\begin{array}{llll}\text { C } & 2.947128 & 1.953847 & -6.016285\end{array}$

$\begin{array}{llll}\text { C } & 1.569226 & 2.184563 & -6.032198\end{array}$

$\begin{array}{llll}\text { C } & 0.936639 & 2.671459 & -4.886205\end{array}$ 


\begin{tabular}{|c|c|c|c|}
\hline $\mathrm{C}$ & 1.675959 & 2.920782 & -3.731044 \\
\hline $\mathrm{C}$ & 5.987401 & 2.349313 & 3.193611 \\
\hline $\mathrm{C}$ & 6.779289 & 3.157027 & 4.022510 \\
\hline $\mathrm{C}$ & 7.752747 & 2.600768 & 4.857918 \\
\hline $\mathrm{C}$ & 7.930091 & 1.212027 & 4.850525 \\
\hline $\mathrm{C}$ & 7.152014 & 0.378908 & 4.038076 \\
\hline $\mathrm{C}$ & 6.184420 & 0.960042 & 3.213673 \\
\hline $\mathrm{C}$ & -1.665283 & -7.913974 & 3.840681 \\
\hline $\mathrm{C}$ & -1.426619 & -4.079902 & 7.132785 \\
\hline $\mathrm{C}$ & -8.278181 & 1.410748 & 2.727997 \\
\hline $\mathrm{C}$ & -4.747368 & 1.734914 & 6.331360 \\
\hline $\mathrm{C}$ & 1.054224 & 7.696238 & 3.029390 \\
\hline $\mathrm{C}$ & -3.431964 & 8.639037 & 5.158166 \\
\hline $\mathrm{C}$ & 7.332262 & -1.119779 & 4.071646 \\
\hline & 8.587768 & 3.478248 & 5.758767 \\
\hline $\mathrm{H}$ & -2.101554 & 5.159259 & -2.104858 \\
\hline $\mathrm{H}$ & -1.059548 & 3.939821 & -2.970302 \\
\hline $\mathrm{H}$ & -5.240944 & 2.334303 & 2.211471 \\
\hline $\mathrm{H}$ & -4.302934 & 4.236051 & 3.471842 \\
\hline $\mathrm{H}$ & -1.676712 & 5.092847 & 0.178951 \\
\hline $\mathrm{H}$ & -5.494297 & 2.182066 & -3.544608 \\
\hline $\mathrm{H}$ & -7.783011 & 3.045933 & -3.281677 \\
\hline $\mathrm{H}$ & -8.175483 & 5.238608 & -2.164613 \\
\hline $\mathrm{H}$ & -6.226061 & 6.546661 & -1.331053 \\
\hline $\mathrm{H}$ & -3.955804 & 5.687265 & -1.588126 \\
\hline $\mathrm{H}$ & -2.596661 & 3.893133 & -5.288681 \\
\hline $\mathrm{H}$ & -2.356822 & 2.374116 & -7.234177 \\
\hline $\mathrm{H}$ & -2.610415 & -0.084717 & -6.929474 \\
\hline $\mathrm{H}$ & -3.121229 & -0.997144 & -4.671644 \\
\hline $\mathrm{H}$ & -3.372481 & 0.530499 & -2.749017 \\
\hline $\mathrm{H}$ & -4.177547 & 6.515809 & 3.625268 \\
\hline $\mathrm{H}$ & -0.346313 & 5.684735 & 1.857451 \\
\hline $\mathrm{H}$ & -5.374938 & -2.305980 & -1.234353 \\
\hline $\mathrm{H}$ & -4.176815 & -1.560655 & -2.393670 \\
\hline $\mathrm{H}$ & -2.479802 & -4.554260 & 3.532391 \\
\hline $\mathrm{H}$ & -3.887774 & -2.864020 & 4.664143 \\
\hline $\mathrm{H}$ & -4.805188 & -1.122006 & 0.851952 \\
\hline $\mathrm{H}$ & -2.965327 & -6.166043 & -2.406906 \\
\hline $\mathrm{H}$ & -4.000223 & -8.267187 & -1.657232 \\
\hline $\mathrm{H}$ & -5.955445 & -8.222408 & -0.112740 \\
\hline $\mathrm{H}$ & -6.858714 & -6.027425 & 0.643645 \\
\hline $\mathrm{H}$ & -5.832997 & -3.941452 & -0.100706 \\
\hline $\mathrm{H}$ & -4.372012 & -3.401042 & -4.348322 \\
\hline $\mathrm{H}$ & -2.977677 & -3.650036 & -6.384340 \\
\hline $\mathrm{H}$ & -0.544949 & -4.140377 & -6.170100 \\
\hline $\mathrm{H}$ & 0.464559 & -4.390162 & -3.919221 \\
\hline $\mathrm{H}$ & -0.944662 & -4.157300 & -1.890227 \\
\hline $\mathrm{H}$ & -6.793246 & -0.749946 & 1.999782 \\
\hline $\mathrm{H}$ & -3.780932 & -0.466831 & 5.049889 \\
\hline $\mathrm{H}$ & 2.057927 & -5.770205 & -0.568508 \\
\hline $\mathrm{H}$ & 1.282068 & -4.723763 & -1.847155 \\
\hline $\mathrm{H}$ & 4.662798 & -2.455946 & 3.649549 \\
\hline $\mathrm{H}$ & 2.991975 & -3.656745 & 5.021222 \\
\hline $\mathrm{H}$ & 0.963354 & -4.858570 & 1.434978 \\
\hline $\mathrm{H}$ & 5.911065 & -3.681825 & -2.273241 \\
\hline $\mathrm{H}$ & 8.023753 & -4.674034 & -1.498515 \\
\hline $\mathrm{H}$ & 8.015630 & -6.397642 & 0.301145 \\
\hline$\Pi$ & 5.844641 & -7.114147 & 1.290507 \\
\hline $\mathrm{H}$ & 3.746980 & -6.130009 & 0.522800 \\
\hline $\mathrm{H}$ & 2.920502 & -5.245508 & -3.836647 \\
\hline $\mathrm{H}$ & 3.037831 & -4.161745 & -6.063403 \\
\hline $\mathrm{H}$ & 3.657142 & -1.753985 & -6.239810 \\
\hline
\end{tabular}
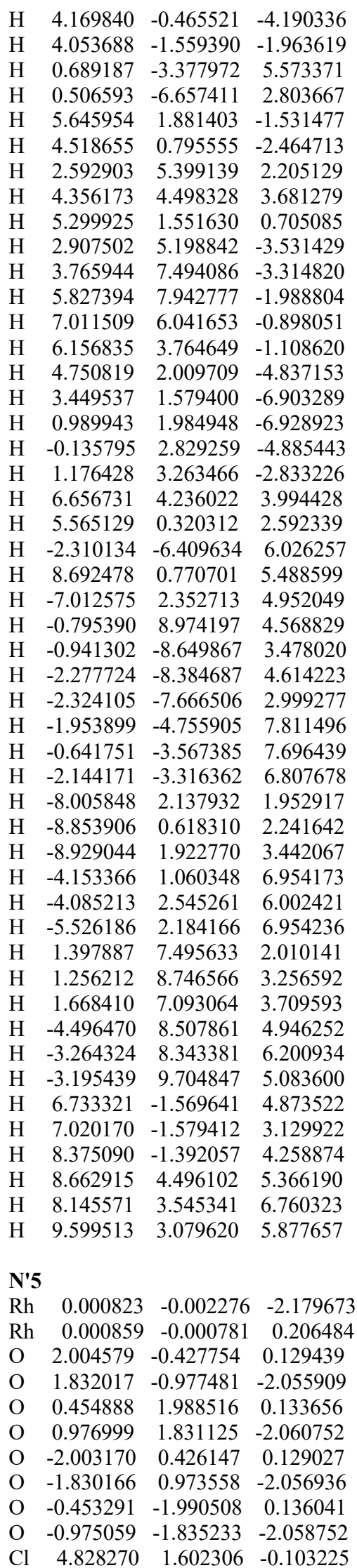

$\begin{array}{llll}\mathrm{Cl} & -1.197884 & 4.912137 & 0.514272\end{array}$

$\begin{array}{llll}\mathrm{Cl} & -4.828637 & -1.600914 & -0.099225\end{array}$

$\mathrm{Cl} \quad 1.196806 \quad-4.915095 \quad 0.521334$

$\begin{array}{llll}\text { C } & 3.934072 & -1.250629 & -0.936249\end{array}$

$\begin{array}{llll}\text { C } & 4.835127 & -1.116144 & -2.209231\end{array}$

$\begin{array}{llll}\text { C } & 4.317913 & -2.455108 & -1.772548\end{array}$

$\begin{array}{llll}\text { C } & 2.485939 & -0.850095 & -0.973707\end{array}$

$\begin{array}{llll}\text { C } & 4.523352 & -1.071445 & 0.431409\end{array}$

$\begin{array}{llll}\text { C } & 4.921350 & 0.177699 & 0.923728\end{array}$

$\begin{array}{llll}\text { C } & 5.367073 & 0.339958 & 2.232569\end{array}$

$\begin{array}{llll}\text { C } & 5.408572 & -0.763282 & 3.081452\end{array}$

$\begin{array}{llll}\text { C } & 5.011116 & -2.033482 & 2.634000\end{array}$

$\begin{array}{llll}\text { C } & 4.581378 & -2.157404 & 1.305188\end{array}$

$\begin{array}{llll}\text { C } & 6.292023 & -0.780226 & -1.975869\end{array}$

$\begin{array}{llll}\text { C } & 6.857895 & 0.340608 & -2.602492\end{array}$

$\begin{array}{llll}\text { C } & 8.188550 & 0.694925 & -2.384479\end{array}$

$\begin{array}{llll}\text { C } & 8.990162 & -0.065902 & -1.532760\end{array}$

$\begin{array}{llll}\text { C } & 8.444647 & -1.188949 & -0.910004\end{array}$

$\begin{array}{llll}\text { C } & 7.114285 & -1.543257 & -1.132648\end{array}$

$\begin{array}{llll}\text { C } & 4.254057 & -0.532956 & -3.471717\end{array}$

C $\quad 4.381311 \quad-1.248028-4.667725$

$\begin{array}{llll}\text { C } & 3.858964 & -0.741819 & -5.859690\end{array}$

$\begin{array}{llll}\text { C } & 3.203821 & 0.491939 & -5.866216\end{array}$

$\begin{array}{llll}\text { C } & 3.088487 & 1.219982 & -4.679125\end{array}$

$\begin{array}{llll}\text { C } & 3.620994 & 0.713257 & -3.494475\end{array}$

$\begin{array}{llll}\text { C } & 5.020498 & -3.211201 & 3.533465\end{array}$

C $\quad 6.006862 \quad-3.352290 \quad 4.521140$

$\begin{array}{llll}\text { C } & 6.030763 & -4.464146 & 5.367769\end{array}$

$\begin{array}{llll}\text { C } & 5.050037 & -5.450704 & 5.208178\end{array}$

$\begin{array}{llll}\text { C } & 4.051127 & -5.337828 & 4.234534\end{array}$

$\begin{array}{llll}\text { C } & 4.045726 & -4.212611 & 3.405032\end{array}$

$\begin{array}{llll}\text { C } & 1.429549 & 3.869311 & -0.877881\end{array}$

$\begin{array}{llll}\text { C } & 1.213164 & 4.864340 & -2.063668\end{array}$

$\begin{array}{llll}\text { C } & 2.554233 & 4.236995 & -1.823412\end{array}$

$\begin{array}{llll}\text { C } & 0.908438 & 2.462351 & -0.959364\end{array}$

$\begin{array}{llll}\text { C } & 1.491858 & 4.339610 & 0.546574\end{array}$

$\begin{array}{llll}\text { C } & 0.380050 & 4.766806 & 1.281790\end{array}$

$\begin{array}{llll}\text { C } & 0.472250 & 5.024073 & 2.648794\end{array}$

$\begin{array}{llll}\text { C } & 1.675596 & 4.804136 & 3.316872\end{array}$

$\begin{array}{llll}\text { C } & 2.803268 & 4.342481 & 2.621649\end{array}$

$\begin{array}{llll}\text { C } & 2.687728 & 4.152984 & 1.240084\end{array}$

$\begin{array}{llll}\text { C } & 0.993641 & 6.315236 & -1.704320\end{array}$

$\begin{array}{llll}\text { C } & -0.139224 & 6.990353 & -2.183154\end{array}$

$\begin{array}{llll}\text { C } & -0.380777 & 8.321957 & -1.847351\end{array}$

$\begin{array}{llll}\text { C } & 0.507357 & 9.013167 & -1.022716\end{array}$

$\begin{array}{llll}\text { C } & 1.642217 & 8.356833 & -0.544669\end{array}$

$\begin{array}{llll}\text { C } & 1.883879 & 7.026179 & -0.884596\end{array}$

$\begin{array}{llll}\text { C } & 0.466985 & 4.385611 & -3.281518\end{array}$

$\begin{array}{llll}\text { C } & 1.086083 & 4.481040 & -4.533296\end{array}$

$\begin{array}{llll}\text { C } & 0.435625 & 4.038606 & -5.686179\end{array}$

$\begin{array}{llll}\text { C } & -0.847692 & 3.494335 & -5.595692\end{array}$

C $\quad-1.476833 \quad 3.411981 \quad-4.352087$

$\begin{array}{llll}\text { C } & -0.826807 & 3.862994 & -3.204033\end{array}$

$\begin{array}{llll}\text { C } & 4.064101 & 3.964148 & 3.303054\end{array}$

$\begin{array}{llll}\text { C } & 4.031079 & 3.302378 & 4.540687\end{array}$

$\begin{array}{llll}\text { C } & 5.204156 & 2.853954 & 5.152674\end{array}$

$\begin{array}{llll}\text { C } & 6.427392 & 3.076910 & 4.505527\end{array}$

$\begin{array}{llll}\text { C } & 6.493292 & 3.736086 & 3.273261\end{array}$

$\begin{array}{llll}\text { C } & 5.303468 & 4.179797 & 2.685980\end{array}$

$\begin{array}{llll}\text { C } & -3.931958 & 1.249695 & -0.937540\end{array}$

$\begin{array}{llll}\text { C } & -4.833164 & 1.113577 & -2.210257\end{array}$

$\begin{array}{llll}\text { C } & -4.314983 & 2.452949 & -1.775978\end{array}$ 


\begin{tabular}{|c|c|c|c|}
\hline $\mathrm{C}$ & -2.484161 & 0.847827 & -0.974525 \\
\hline $\mathrm{C}$ & -4.521609 & 1.073557 & 0.430359 \\
\hline $\mathrm{C}$ & -4.921076 & -0.174250 & 0.924877 \\
\hline $\mathrm{C}$ & -5.368146 & -0.333444 & 2.233645 \\
\hline & -5.409709 & 0.771621 & 3.080145 \\
\hline $\mathrm{C}$ & -5.010902 & 2.040598 & 2.630421 \\
\hline $\mathrm{C}$ & -4.579579 & 2.161377 & 1.301823 \\
\hline $\mathrm{C}$ & -6.290305 & 0.779117 & -1.976455 \\
\hline $\mathrm{C}$ & -6.856912 & -0.342337 & -2.601309 \\
\hline c & -8.187894 & -0.695249 & -2.383020 \\
\hline $\mathrm{C}$ & -8.989079 & 0.067622 & -1.532729 \\
\hline $\mathrm{C}$ & -8.442795 & 1.191219 & -0.911638 \\
\hline $\mathrm{C}$ & -7.112128 & 1.544162 & -1.134607 \\
\hline $\mathrm{C}$ & -4.252369 & 0.527618 & -3.471572 \\
\hline c & -4.379459 & 1.240222 & -4.669067 \\
\hline $\mathrm{C}$ & -3.857146 & 0.731479 & -5.859973 \\
\hline $\mathrm{C}$ & -3.202100 & -0.502344 & -5.863907 \\
\hline $\mathrm{C}$ & -3.086984 & -1.227945 & -4.675315 \\
\hline $\mathrm{C}$ & -3.619476 & -0.718719 & -3.491733 \\
\hline $\mathrm{C}$ & -5.020932 & 3.220387 & 3.527171 \\
\hline $\mathrm{C}$ & -4.045694 & 4.221169 & 97581 \\
\hline $\mathrm{C}$ & -4.051935 & 5.348500 & 4.224212 \\
\hline $\mathrm{C}$ & -5.052136 & 5.464117 & 5.196196 \\
\hline $\mathrm{C}$ & -6.033322 & 4.478204 & 5.356969 \\
\hline $\mathrm{C}$ & -6.008571 & 3.364214 & 4.513165 \\
\hline $\mathrm{C}$ & -1.428918 & -3.871754 & -0.873903 \\
\hline $\mathrm{C}$ & -1.212764 & -4.868207 & -2.058582 \\
\hline $\mathrm{C}$ & -2.553422 & -4.239552 & -1.819637 \\
\hline $\mathrm{C}$ & -0.906849 & -2.465269 & -0.956584 \\
\hline $\mathrm{C}$ & -1.492534 & -4.340549 & 0.550982 \\
\hline $\mathrm{C}$ & -0.381662 & -4.768169 & 1.287368 \\
\hline $\mathrm{C}$ & -0.475250 & -5.024670 & 2.654436 \\
\hline $\mathrm{C}$ & -1.678997 & -4.803471 & 3.321368 \\
\hline $\mathrm{C}$ & -2.805656 & -4.341017 & 2.625028 \\
\hline $\mathrm{C}$ & -2.688712 & -4.152253 & 1.243471 \\
\hline $\mathrm{C}$ & -0.994653 & -6.318905 & -1.697522 \\
\hline $\mathrm{C}$ & 0.138365 & -6.995330 & -2.174150 \\
\hline $\mathrm{C}$ & 0.378524 & -8.326758 & -1.836636 \\
\hline $\mathrm{C}$ & -0.511248 & -9.016518 & -1.012571 \\
\hline $\mathrm{C}$ & -1.646274 & -8.358890 & -0.536707 \\
\hline $\mathrm{C}$ & -1.886504 & -7.028394 & -0.878287 \\
\hline $\mathrm{C}$ & -0.465598 & -4.391458 & -3.276631 \\
\hline $\mathrm{C}$ & -1.084713 & -4.486434 & -4.528440 \\
\hline $\mathrm{C}$ & -0.433199 & -4.046087 & -5.681513 \\
\hline $\mathrm{C}$ & 0.851312 & -3.504616 & -5.591239 \\
\hline $\mathrm{C}$ & 1.480507 & -3.422875 & -4.347626 \\
\hline $\mathrm{C}$ & 0.829294 & -3.871502 & -3.199295 \\
\hline $\mathrm{C}$ & -4.066747 & -3.961254 & 3.305172 \\
\hline $\mathrm{C}$ & -5.305654 & -4.174237 & 2.686309 \\
\hline $\mathrm{C}$ & -6.495526 & -3.728969 & 3.272363 \\
\hline $\mathrm{C}$ & -6.430179 & -3.071173 & 4.505361 \\
\hline $\mathrm{C}$ & -5.207405 & -2.850976 & 5.154392 \\
\hline $\mathrm{C}$ & -4.034302 & -3.300750 & 4.543542 \\
\hline $\mathrm{C}$ & -7.090332 & 4.612619 & 6.426232 \\
\hline $\mathrm{C}$ & -2.983877 & 6.406592 & 4.086490 \\
\hline $\mathrm{C}$ & 5.160388 & 2.140824 & 6.482132 \\
\hline $\mathrm{C}$ & 7.810621 & 3.920957 & 2.560675 \\
\hline $\mathrm{C}$ & 2.983501 & -6.396524 & 4.098062 \\
\hline $\mathrm{C}$ & 7.086343 & -4.595569 & 6.438827 \\
\hline $\mathrm{C}$ & -5.164281 & -2.138998 & 6.484480 \\
\hline $\mathrm{C}$ & -7.812079 & -3.910729 & 2.557555 \\
\hline П & 4.999927 & -3.174968 & -1.336496 \\
\hline
\end{tabular}

H $\quad 3.523466 \quad-2.887682 \quad-2.366202$

$\mathrm{H} \quad 5.653820 \quad 1.320840 \quad 2.591521$

H $\quad 5.723393 \quad-0.622940 \quad 4.110216$

H $\quad 4.277278 \quad-3.1293120 .931859$

$\begin{array}{llll}\mathrm{H} & 6.248391 & 0.945842 & -3.262244\end{array}$

$\mathrm{H} \quad 8.596309 \quad 1.570148 \quad-2.881524$

H $\quad 10.025996 \quad 0.209488 \quad-1.359739$

H $\quad 9.053820 \quad-1.797033 \quad-0.247987$

H $\quad 6.724637 \quad-2.416261 \quad-0.624672$

H $\quad 4.884011 \quad-2.211190 \quad-4.660245$

H $3.958729 \quad-1.312724 \quad-6.778077$

$\begin{array}{llll}\mathrm{H} & 2.786977 & 0.884615 & -6.788972\end{array}$

H $\quad 2.566098 \quad 2.171142 \quad-4.670873$

H $\quad 3.502968 \quad 1.265580 \quad-2.571522$

H $\quad 6.785548 \quad-2.600453 \quad 4.611370$

H $\quad 3.262388 \quad-4.110059 \quad 2.659804$

H $3.362122 \quad 4.839950 \quad-1.426565$

H $\quad 2.860744 \quad 3.467926-2.520956$

$\begin{array}{llll}\mathrm{H} & -0.399553 & 5.370035 & 3.190510\end{array}$

$\begin{array}{llll}\mathrm{H} & 1.732152 & 4.990617 & 4.384349\end{array}$

H $\quad 3.533386 \quad 3.759247 \quad 0.692792$

$\mathrm{H} \quad-0.840630 \quad 6.467733 \quad-2.821977$

H $\quad-1.267320 \quad 8.817282 \quad-2.232051$

H $\quad 0.320348 \quad 10.049533 \quad-0.758792$

$\begin{array}{llll}\mathrm{H} & 2.347418 & 8.879837 & 0.094308\end{array}$

$\begin{array}{llll}\mathrm{H} & 2.771013 & 6.547401 & -0.489167\end{array}$

H $\quad 2.089266 \quad 4.892415 \quad-4.599166$

H $\quad 0.932857 \quad 4.111412 \quad-6.648844$

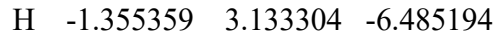

$\begin{array}{llll}\mathrm{H} & -2.462382 & 2.968079 & -4.279800\end{array}$

$\begin{array}{llll}\mathrm{H} & -1.305716 & 3.770187 & -2.235623\end{array}$

$\mathrm{H} \quad 3.073770 \quad 3.100993 \quad 5.012612$

H $\quad 5.343455 \quad 4.699202 \quad 1.733136$

H $\quad-4.996573 \quad 3.173904 \quad-1.341112$

$\begin{array}{llll}\mathrm{H} & -3.520390 & 2.884110 & -2.370460\end{array}$

H $\quad-5.656046 \quad-1.313351 \quad 2.594356$

$\begin{array}{llll}\mathrm{H} & -5.725821 & 0.633729 & 4.108841\end{array}$

$\begin{array}{llll}\mathrm{H} & -4.274507 & 3.132272 & 0.926659\end{array}$

$\begin{array}{llll}\mathrm{H} & -6.247732 & -0.949117 & -3.259933\end{array}$

$\begin{array}{lllll}\mathrm{H} & -8.596223 & -1.570964 & -2.878718\end{array}$

$\mathrm{H} \quad-10.025180 \quad-0.206649 \quad-1.359508$

$\begin{array}{llll}\mathrm{H} & -9.051640 & 1.800841 & -0.250729\end{array}$

$\begin{array}{lllll}\mathrm{H} & -6.721938 & 2.417704 & -0.627969\end{array}$

H $\quad-4.881976 \quad 2.203495 \quad-4.663578$

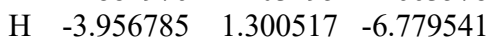

H $\quad-2.785183 \quad-0.896946 \quad-6.785806$

H $\quad-2.564496 \quad-2.179031-4.665008$

H $\quad-3.501611 \quad-1.269288 \quad-2.567711$

$\mathrm{H} \quad-3.261414 \quad 4.116520 \quad 2.653648$

H $\quad-6.787649 \quad 2.612888 \quad 4.604206$

H $\quad-3.361996-4.841450 \quad-1.422581$

H $\quad-2.859010 \quad-3.471008 \quad-2.518129$

$\begin{array}{llll}\mathrm{H} & 0.395815 & -5.371053 & 3.197065\end{array}$

H $\quad-1.736597 \quad-4.989527 \quad 4.388862$

$\mathrm{H} \quad-3.533437 \quad-3.757847 \quad 0.695236$

H $\quad 0.841009 \quad-6.473913 \quad-2.812590$

H $\quad \begin{array}{llll}1.265252 & -8.823100 & -2.219617\end{array}$

H $\quad-0.325359-10.052763 \quad-0.747372$

$\begin{array}{llll}\mathrm{H} & -2.352718 & -8.880739 & 0.101829\end{array}$

H $\quad-2.773789 \quad-6.548589 \quad-0.484466$

H $\quad-2.088708 \quad-4.895851 \quad-4.594179$

$\begin{array}{llll}\mathrm{H} & -0.930478 & -4.118427 & -6.644186\end{array}$
H $\quad 1.359916 \quad-3.145347 \quad-6.480924$

H $\quad 2.467178 \quad-2.981420 \quad-4.275602$

$\mathrm{H} \quad 1.308238 \quad-3.779135 \quad-2.230837$

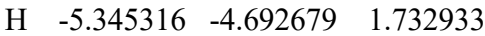

H $\quad-3.077316 \quad-3.101394 \quad 5.016955$

$\mathrm{H} \quad-5.070485 \quad 6.341810 \quad 5.838395$

H $\quad-7.348103 \quad-2.713432 \quad 4.965804$

$\begin{array}{llll}\mathrm{H} & 7.345290 & 2.720307 & 4.966875\end{array}$

H $\quad 5.067725 \quad-6.326740 \quad 5.852667$

H $\quad-6.714338 \quad 4.262721 \quad 7.395402$

$\begin{array}{llll}\mathrm{H} & -7.396586 & 5.654922 & 6.555748\end{array}$

H $\quad-7.977865 \quad 4.020673 \quad 6.186438$

$\mathrm{H} \quad-2.598974 \quad 6.451623 \quad 3.063821$

$\begin{array}{llll}\mathrm{H} & -3.365811 & 7.396200 & 4.353333\end{array}$

H $\quad-2.135920 \quad 6.198515 \quad 4.751068$

$\begin{array}{llll}\mathrm{H} & 4.155393 & 1.771700 & 6.702622\end{array}$

$\begin{array}{llll}\mathrm{H} & 5.849403 & 1.290622 & 6.499303\end{array}$

H $\quad 5.454179 \quad 2.811239 \quad 7.298717$

$\begin{array}{llll}\mathrm{H} & 8.653908 & 3.849810 & 3.252657\end{array}$

$\begin{array}{llll}\mathrm{H} & 7.946027 & 3.149285 & 1.792941\end{array}$

H $\quad 7.858798 \quad 4.890526 \quad 2.056098$

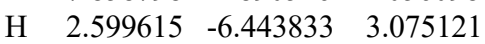

H $\quad 3.365462 \quad-7.385452 \quad 4.367396$

H $\quad 2.134855 \quad-6.187243 \quad 4.761376$

$\begin{array}{llll}\mathrm{H} & 7.974140 & -4.004173 & 6.198664\end{array}$

$\begin{array}{llll}\mathrm{H} & 6.708997 & -4.243113 & 7.406545\end{array}$

H $\quad \begin{array}{llll}7.392537 & -5.637483 & 6.571554\end{array}$

H $\quad-5.848072 \quad-1.284529 \quad 6.499559$

$\mathrm{H} \quad-5.465419 \quad-2.808041 \quad 7.299507$

H $\quad-4.157880 \quad-1.776189 \quad 6.709022$

$\begin{array}{llll}\mathrm{H} & -7.943323 & -3.140010 & 1.788130\end{array}$

$\mathrm{H} \quad-7.862515 \quad-4.881038 \quad 2.054605$

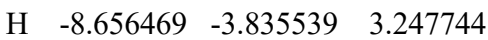

\section{N"1}

$\begin{array}{llll}\text { Rh } & 0.318806 & -0.169248 & 1.179066\end{array}$

$\begin{array}{lllll}\mathrm{Rh} & 0.320343 & 0.161887 & -1.187936\end{array}$

$\begin{array}{llll}\mathrm{O} & -1.304511 & -1.141394 & -1.221636\end{array}$

$\begin{array}{llll}\text { O } & -0.932832 & -1.774309 & 0.913803\end{array}$

$\begin{array}{lllll}\mathrm{O} & -0.941894 & 1.760360 & -0.926843\end{array}$

$\begin{array}{llll}\mathrm{O} & -1.309325 & 1.129313 & 1.209798\end{array}$

$\begin{array}{llll}\text { O } & 1.935993 & 1.424962 & -0.936359\end{array}$

$\begin{array}{llll}\mathrm{O} & 1.675905 & 1.383392 & 1.309414\end{array}$

O $\quad 1.688029-1.379651 \quad-1.317552$

$\begin{array}{llll}\text { O } & 1.940114 & -1.426224 & 0.928942\end{array}$

Cl $\quad-1.278641 \quad-4.387050 \quad-2.466501$

$\begin{array}{llll}\mathrm{Cl} & -1.268185 & 4.375155 & 2.453902\end{array}$

$\begin{array}{llll}\mathrm{Cl} & 2.837001 & 3.944287 & 3.109415\end{array}$

Cl $2.859159 \quad-3.944491 \quad-3.115573$

$\begin{array}{llll}\text { C } & -2.739853 & -2.773815 & -0.271348\end{array}$

$\begin{array}{llll}\text { C } & -3.633979 & -3.055919 & 0.997484\end{array}$

$\begin{array}{llll}\text { C } & -2.638272 & -4.062601 & 0.521291\end{array}$

C $\quad-1.575464-1.828793 \quad-0.181575$

$\begin{array}{llll}\text { C } & -3.365912 & -2.802217 & -1.632208\end{array}$

$\begin{array}{llll}\text { C } & -2.781696 & -3.494129 & -2.701668\end{array}$

$\begin{array}{llll}\text { C } & -3.335614 & -3.467432 & -3.981012\end{array}$

$\begin{array}{llll}\text { C } & -4.491763 & -2.725435 & -4.212190\end{array}$

C $\quad-5.096023 \quad-2.007062 \quad-3.171745$

$\begin{array}{llll}\text { C } & -4.520123 & -2.063670 & -1.899751\end{array}$

$\begin{array}{llll}\text { C } & -5.112769 & -3.287595 & 0.779314\end{array}$

$\begin{array}{llll}\text { C } & -5.595782 & -4.375424 & 0.037621\end{array}$

$\begin{array}{llll}\text { C } & -6.962357 & -4.555742 & -0.171851\end{array}$ 


\begin{tabular}{|c|c|c|c|}
\hline $\mathrm{C}$ & -7.880965 & -3.649998 & 0.360799 \\
\hline C & -7.414963 & -2.564992 & 1.104531 \\
\hline $\mathrm{C}$ & -6.047588 & -2.387076 & 1.315270 \\
\hline $\mathrm{C}$ & -3.317713 & -2.389103 & 2.311623 \\
\hline $\mathrm{C}$ & -3.174281 & -3.197250 & 3.446667 \\
\hline C & -2.892660 & -2.632785 & 4.690755 \\
\hline $\mathrm{C}$ & -2.734369 & -1.248465 & 4.808323 \\
\hline $\mathrm{C}$ & -2.881693 & -0.438399 & 3.681059 \\
\hline $\mathrm{C}$ & -3.193973 & -1.006544 & 2.446653 \\
\hline $\mathrm{C}$ & -6.292683 & -1.165211 & -3.397485 \\
\hline $\mathrm{C}$ & -7.391761 & -1.257327 & -2.530168 \\
\hline $\mathrm{C}$ & -8.523887 & -0.463823 & -2.722935 \\
\hline $\mathrm{C}$ & -8.528143 & 0.455925 & -3.782179 \\
\hline $\mathrm{C}$ & -7.435720 & 0.584950 & -4.645038 \\
\hline $\mathrm{C}$ & -6.325618 & -0.246261 & -4.451407 \\
\hline $\mathrm{C}$ & -2.741896 & 2.765554 & 0.263911 \\
\hline $\mathrm{C}$ & -3.640025 & 3.050563 & -1.001303 \\
\hline $\mathrm{C}$ & -2.638597 & 4.053768 & -0.529160 \\
\hline $\mathrm{C}$ & -1.580889 & 1.816999 & 0.170235 \\
\hline $\mathrm{C}$ & -3.362443 & 2.795322 & 1.627206 \\
\hline $\mathrm{C}$ & -2.772480 & 3.485707 & 2.694471 \\
\hline $\mathrm{C}$ & -3.321528 & 3.459950 & 3.975966 \\
\hline $\mathrm{C}$ & -4.478413 & 2.720535 & 4.211453 \\
\hline $\mathrm{C}$ & -5.088116 & 2.003329 & 3.173315 \\
\hline $\mathrm{C}$ & -4.516927 & 2.058827 & 1.899171 \\
\hline $\mathrm{C}$ & -5.116871 & 3.287540 & -0.776865 \\
\hline $\mathrm{C}$ & -5.593024 & 4.374103 & -0.028802 \\
\hline $\mathrm{C}$ & -6.958086 & 4.558406 & 0.186757 \\
\hline $\mathrm{C}$ & -7.881982 & 3.657959 & -0.345722 \\
\hline $\mathrm{C}$ & -7.422759 & 2.574137 & -1.095335 \\
\hline $\mathrm{C}$ & -6.056846 & 2.392199 & -1.312316 \\
\hline $\mathrm{C}$ & -3.330090 & 2.382593 & -2.316375 \\
\hline $\mathrm{C}$ & -3.190269 & 3.190431 & -3.452137 \\
\hline $\mathrm{C}$ & -2.908824 & 2.625973 & -4.696226 \\
\hline $\mathrm{C}$ & -2.746745 & 1.242005 & -4.813016 \\
\hline $\mathrm{C}$ & -2.891357 & 0.432247 & -3.685245 \\
\hline $\mathrm{C}$ & -3.204649 & 1.000171 & -2.451009 \\
\hline $\mathrm{C}$ & -6.286588 & 1.165365 & 3.403111 \\
\hline $\mathrm{C}$ & -6.322581 & 0.252062 & 4.461814 \\
\hline $\mathrm{C}$ & -7.435809 & -0.573854 & 4.660359 \\
\hline $\mathrm{C}$ & -8.527586 & -0.446096 & 3.796627 \\
\hline $\mathrm{C}$ & -8.520088 & 0.467642 & 2.732151 \\
\hline $\mathrm{C}$ & -7.385357 & 1.256246 & 2.535137 \\
\hline $\mathrm{C}$ & 3.624083 & 2.416718 & 0.431622 \\
\hline $\mathrm{C}$ & 3.861237 & 3.790912 & -0.271311 \\
\hline $\mathrm{C}$ & 4.453195 & 2.532386 & -0.825787 \\
\hline $\mathrm{C}$ & 2.301792 & 1.712066 & 0.249757 \\
\hline $\mathrm{C}$ & 4.281992 & 2.052701 & 1.729858 \\
\hline $\mathrm{C}$ & 3.971365 & 2.609692 & 2.976387 \\
\hline $\mathrm{C}$ & 4.529295 & 2.107784 & 4.153151 \\
\hline $\mathrm{C}$ & 5.392256 & 1.014058 & 4.107526 \\
\hline $\mathrm{C}$ & 5.730045 & 0.430963 & 2.879033 \\
\hline $\mathrm{C}$ & 5.177426 & 0.982607 & 1.718322 \\
\hline $\mathrm{C}$ & 4.797931 & 4.773929 & 0.394507 \\
\hline $\mathrm{C}$ & 4.426095 & 6.115966 & 0.562932 \\
\hline $\mathrm{C}$ & 5.288299 & 7.034788 & 1.162271 \\
\hline $\mathrm{C}$ & 6.547871 & 6.635354 & 1.608355 \\
\hline $\mathrm{C}$ & 6.935478 & 5.304976 & 1.441558 \\
\hline $\mathrm{C}$ & 6.074424 & 4.389605 & 0.839657 \\
\hline $\mathrm{C}$ & 2.687182 & 4.392319 & -0.993011 \\
\hline $\mathrm{C}$ & 1.565549 & 4.838479 & -0.283615 \\
\hline $\mathrm{C}$ & 0.510966 & 5.463553 & -0.947405 \\
\hline
\end{tabular}

$\begin{array}{llll}\text { C } & 0.549763 & 5.621581 & -2.335133\end{array}$

$\begin{array}{llll}\text { C } & 1.657829 & 5.162687 & -3.051414\end{array}$

$\begin{array}{llll}\text { C } & 2.724458 & 4.560934 & -2.380818\end{array}$

$\begin{array}{llll}\text { C } & 6.556233 & -0.792634 & 2.754495\end{array}$

$\begin{array}{llll}\text { C } & 6.296729 & -1.909715 & 3.562277\end{array}$

$\begin{array}{llll}\text { C } & 6.929939 & -3.131697 & 3.322478\end{array}$

$\begin{array}{llll}\text { C } & 7.857673 & -3.215821 & 2.274483\end{array}$

$\begin{array}{llll}\text { C } & 8.164432 & -2.109313 & 1.476871\end{array}$

$\begin{array}{llll}\text { C } & 7.505963 & -0.900156 & 1.731484\end{array}$

$\begin{array}{llll}\text { C } & 3.632939 & -2.415387 & -0.434699\end{array}$

$\begin{array}{llll}\text { C } & 3.865445 & -3.790312 & 0.268460\end{array}$

$\begin{array}{llll}\text { C } & 4.455873 & -2.532631 & 0.826665\end{array}$

$\begin{array}{llll}\text { C } & 2.310069 & -1.710676 & -0.256415\end{array}$

C $\quad 4.295308-2.050145 \quad-1.730281$

$\begin{array}{llll}\text { C } & 3.989759 & -2.607057 & -2.978093\end{array}$

$\begin{array}{llll}\text { C } & 4.549939 & -2.103127 & -4.152937\end{array}$

C $\quad 5.409761 \quad-1.007071 \quad-4.104170$

$\begin{array}{llll}\text { C } & 5.742616 & -0.424100 & -2.874287\end{array}$

$\begin{array}{llll}\text { C } & 5.188346 & -0.978064 & -1.715455\end{array}$

C $4.806324 \quad-4.773326 \quad-0.391557$

$\begin{array}{llll}\text { C } & 4.439468 & -6.117478 & -0.553903\end{array}$

$\begin{array}{llll}\text { C } & 5.306314 & -7.036577 & -1.146146\end{array}$

$\begin{array}{llll}\text { C } & 6.565590 & -6.635277 & -1.591239\end{array}$

$\begin{array}{llll}\text { C } & 6.948291 & -5.302685 & -1.430483\end{array}$

$\begin{array}{llll}\text { C } & 6.082761 & -4.387047 & -0.835470\end{array}$

$\begin{array}{llll}\text { C } & 2.686204 & -4.391042 & 0.982264\end{array}$

$\begin{array}{llll}\text { C } & 1.571707 & -4.841516 & 0.264338\end{array}$

$\begin{array}{llll}\text { C } & 0.511778 & -5.465049 & 0.920898\end{array}$

$\begin{array}{llll}\text { C } & 0.537852 & -5.617352 & 2.309602\end{array}$

$\begin{array}{llll}\text { C } & 1.638637 & -5.154103 & 3.034193\end{array}$

$\begin{array}{llll}\text { C } & 2.710681 & -4.553752 & 2.370984\end{array}$

$\begin{array}{llll}\text { C } & 6.565517 & 0.801307 & -2.745977\end{array}$

$\begin{array}{llll}\text { C } & 6.304702 & 1.919896 & -3.551201\end{array}$

$\begin{array}{llll}\text { C } & 6.934583 & 3.142807 & -3.306879\end{array}$

$\begin{array}{llll}\text { C } & 7.860356 & 3.226427 & -2.257130\end{array}$

$\begin{array}{llll}\text { C } & 8.168336 & 2.118432 & -1.462012\end{array}$

$\begin{array}{llll}\text { C } & 7.513145 & 0.908558 & -1.721021\end{array}$

$\begin{array}{llll}\text { C } & 9.148339 & -2.219449 & 0.337435\end{array}$

$\begin{array}{llll}\text { C } & 6.575835 & -4.358042 & 4.127109\end{array}$

$\begin{array}{llll}\text { C } & -7.432661 & -1.591481 & 5.774995\end{array}$

$\begin{array}{llll}\text { C } & -9.718490 & 0.608661 & 1.825199\end{array}$

$\begin{array}{llll}\text { C } & -7.428707 & 1.609336 & -5.753442\end{array}$

$\begin{array}{llll}\text { C } & -9.722704 & -0.605869 & -1.816704\end{array}$

$\begin{array}{llll}\text { C } & 9.148905 & 2.227731 & -0.319698\end{array}$

C $\quad 6.578961 \quad 4.370591 \quad-4.108593$

$\begin{array}{llll}\mathrm{H} & -2.966738 & -4.967422 & 0.024816\end{array}$

$\mathrm{H} \quad-1.745962 \quad-4.170889 \quad 1.123022$

H $\quad-2.861086-4.017498 \quad-4.785483$

H $\quad-4.925654 \quad-2.703338 \quad-5.206567$

$\mathrm{H} \quad-4.968783 \quad-1.495120 \quad-1.095068$

$\begin{array}{llll}\mathrm{H} & -4.907448 & -5.089231 & -0.398568\end{array}$

$\begin{array}{lllll}\mathrm{H} & -7.306449 & -5.406881 & -0.751569\end{array}$

H $\quad-8.945648 \quad-3.787625 \quad 0.199031$

H $\quad-8.109389-1.853484 \quad 1.533790$

H $\quad-5.711456-1.542962 \quad 1.906617$

H $\quad-3.276572 \quad-4.274257 \quad 3.348096$

H $\quad-2.785068 \quad-3.271507 \quad 5.562469$

H $\quad-2.498355 \quad-0.805457 \quad 5.771480$

$\mathrm{H} \quad-2.752217 \quad 0.633725 \quad 3.754285$

$\mathrm{H} \quad-3.290130 \quad-0.368102 \quad 1.578730$

H $\quad-7.367300 \quad-1.971252 \quad-1.713264$

H $\quad-5.456678 \quad-0.141980 \quad-5.094000$
H $\quad-2.961620 \quad 4.960088 \quad-0.031894$

H $\quad-1.748057 \quad 4.158359 \quad-1.134257$

$\mathrm{H} \quad-2.842855 \quad 4.008963 \quad 4.778700$

H $\quad-4.908789 \quad 2.699766 \quad 5.207365$

$\mathrm{H} \quad-4.970205 \quad 1.491262 \quad 1.096346$

H $\quad-4.900524 \quad 5.083377 \quad 0.408235$

H $\quad-7.296828 \quad 5.408345 \quad 0.771354$

$\begin{array}{lllll}\mathrm{H} & -8.945520 & 3.798592 & -0.179075\end{array}$

$\mathrm{H} \quad-8.121353 \quad 1.866403 \quad-1.524018$

$\mathrm{H} \quad-5.725562 \quad 1.548891 \quad-1.907566$

$\begin{array}{llll}\mathrm{H} & -3.293971 & 4.267333 & -3.353807\end{array}$

H $\quad-2.803645 \quad 3.264437 \quad-5.568424$

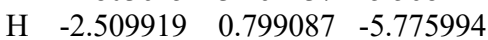

$\begin{array}{llll}\mathrm{H} & -2.758733 & -0.639532 & -3.757769\end{array}$

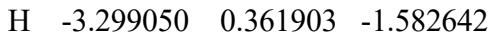

$\begin{array}{llll}\mathrm{H} & -5.453995 & 0.148338 & 5.105068\end{array}$

$\begin{array}{llll}\mathrm{H} & -7.358747 & 1.965843 & 1.714521\end{array}$

H $\quad 5.520974 \quad 2.390204 \quad-0.747116$

H $\quad 4.007243 \quad 2.118354 \quad-1.718667$

$\begin{array}{llll}\mathrm{H} & 4.276103 & 2.566780 & 5.101987\end{array}$

H $\quad 5.808799 \quad 0.621419 \quad 5.029504$

$\begin{array}{llll}\mathrm{H} & 5.408601 & 0.532408 & 0.763492\end{array}$

$\begin{array}{llll}\mathrm{H} & 3.454693 & 6.450094 & 0.221069\end{array}$

$\begin{array}{llll}\mathrm{H} & 4.970206 & 8.066742 & 1.277373\end{array}$

$\begin{array}{llll}\mathrm{H} & 7.219275 & 7.349621 & 2.074938\end{array}$

$\begin{array}{llll}\mathrm{H} & 7.914281 & 4.973453 & 1.775331\end{array}$

$\begin{array}{llll}\mathrm{H} & 6.411650 & 3.368333 & 0.727789\end{array}$

$\begin{array}{llll}\mathrm{H} & 1.527862 & 4.700747 & 0.791120\end{array}$

$\mathrm{H} \quad-0.338563 \quad 5.824967 \quad-0.378102$

$\begin{array}{llll}\mathrm{H} & -0.276356 & 6.098979 & -2.853317\end{array}$

$\begin{array}{llll}\mathrm{H} & 1.696012 & 5.280392 & -4.130197\end{array}$

H $\quad 3.589397 \quad 4.210764 \quad-2.936145$

H $\quad 5.545158 \quad-1.839334 \quad 4.343562$

H $\quad 7.728083 \quad-0.029040 \quad 1.124256$

$\mathrm{H} \quad 5.524265 \quad-2.392328 \quad 0.753432$

H $\quad 4.006076 \quad-2.117968 \quad 1.717352$

H $\quad 4.300708 \quad-2.562289 \quad-5.102753$

$\mathrm{H} \quad 5.827884 \quad-0.612762 \quad-5.024726$

H $\quad 5.416602 \quad-0.528424 \quad-0.759590$

H $\quad 3.468506 \quad-6.453272 \quad-0.212465$

$\mathrm{H} \quad 4.991964 \quad-8.070208 \quad-1.256351$

H $\quad 7.240611 \quad-7.349689 \quad-2.052339$

$\mathrm{H} \quad 7.926822 \quad-4.969644 \quad-1.763502$

$\mathrm{H} \quad 6.416440 \quad-3.364117 \quad-0.727779$

H $\quad 1.543690 \quad-4.708295 \quad-0.811287$

H $\quad-0.332276 \quad-5.829195 \quad 0.345188$

H $\quad-0.292609 \quad-6.093344 \quad 2.822132$

H $\quad 1.666971 \quad-5.267252 \quad 4.113768$

H $\quad 3.569967 \quad-4.200144 \quad 2.932850$

H $\quad 5.554565 \quad 1.850081 \quad-4.333909$

H $\quad 7.736572 \quad 0.036496 \quad-1.115625$

H $\quad 8.343787 \quad-4.166798 \quad 2.071501$

H $\quad 8.344145 \quad 4.177914 \quad-2.051058$

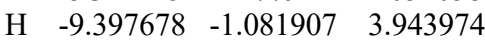

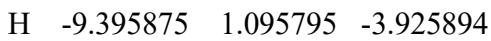

H $\quad 8.631516 \quad-2.226968 \quad-0.630092$

$\begin{array}{llll}\mathrm{H} & 9.840993 & -1.371995 & 0.324456\end{array}$

$\begin{array}{llll}\mathrm{H} & 9.733655 & -3.139837 & 0.403588\end{array}$

H $\quad 6.056057 \quad-4.093608 \quad 5.051613$

$\begin{array}{llll}\mathrm{H} & 5.916108 & -5.019423 & 3.552237\end{array}$

H $\quad 7.466870 \quad-4.938169 \quad 4.385971$

H $\quad-6.551704 \quad-2.239268 \quad 5.707940$ 


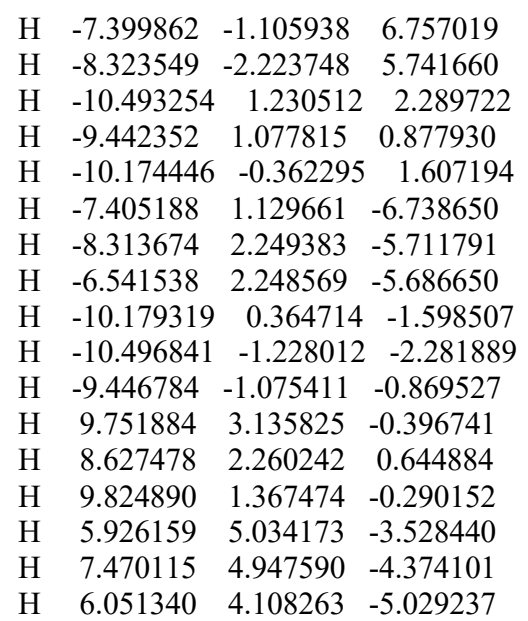

N"2

$\begin{array}{llll}\text { Rh } & 0.331352 & -1.298133 & 1.144375\end{array}$

$\begin{array}{llll}\mathrm{Rh} & -0.329282 & -1.298684 & -1.143969\end{array}$

$\begin{array}{llll}\text { O } & 1.304486 & -0.070036 & -1.517352\end{array}$

$\begin{array}{llll}\mathrm{O} & 1.475002 & 0.365254 & 0.692230\end{array}$

$\begin{array}{llll}\mathrm{O} & 0.860141 & -2.980917 & -1.373523\end{array}$

$\begin{array}{llll}\text { O } & 1.887872 & -2.509525 & 0.586851\end{array}$

$\begin{array}{llll}\mathrm{O} & -1.884310 & -2.511912 & -0.586324\end{array}$

$\begin{array}{llll}\mathrm{O} & -0.855979 & -2.981839 & 1.374076\end{array}$

$\begin{array}{llll}\text { O } & -1.475011 & 0.363308 & -0.692062\end{array}$

$\begin{array}{llll}\mathrm{O} & -1.303924 & -0.071401 & 1.517581\end{array}$

$\begin{array}{llll}\mathrm{Cl} & 5.035098 & 0.315745 & 0.664667\end{array}$

$\begin{array}{llll}\mathrm{Cl} & 2.179985 & -6.113819 & 1.503247\end{array}$

$\begin{array}{llll}\mathrm{Cl} & -2.170911 & -6.116767 & -1.502237\end{array}$

$\begin{array}{lllll}\text { Cl } & -5.034532 & 0.308747 & -0.665609\end{array}$

$\begin{array}{llll}\text { C } & 2.690994 & 1.739649 & -0.796121\end{array}$

$\begin{array}{llll}\text { C } & 2.521668 & 2.638188 & -2.077890\end{array}$

$\begin{array}{llll}\text { C } & 3.617961 & 1.621968 & -1.989420\end{array}$

$\begin{array}{llll}\text { C } & 1.752483 & 0.595288 & -0.533283\end{array}$

$\begin{array}{llll}\text { C } & 3.201557 & 2.362150 & 0.469120\end{array}$

$\begin{array}{llll}\text { C } & 4.241588 & 1.790265 & 1.213368\end{array}$

$\begin{array}{llll}\text { C } & 4.657211 & 2.337009 & 2.427640\end{array}$

C $\quad 4.020782 \quad 3.472051 \quad 2.923620$

$\begin{array}{llll}\text { C } & 2.968720 & 4.065700 & 2.212526\end{array}$

C $\quad 2.580723 \quad 3.496263 \quad 0.997027$

$\begin{array}{llll}\text { C } & 2.841491 & 4.110295 & -1.940756\end{array}$

$\begin{array}{llll}\text { C } & 1.820415 & 5.060711 & -2.102329\end{array}$

$\begin{array}{llll}\text { C } & 2.079460 & 6.422126 & -1.943782\end{array}$

$\begin{array}{llll}\text { C } & 3.364331 & 6.866006 & -1.627964\end{array}$

$\begin{array}{llll}\text { C } & 4.389179 & 5.931501 & -1.472664\end{array}$

$\begin{array}{llll}\text { C } & 4.128748 & 4.570745 & -1.629300\end{array}$

$\begin{array}{llll}\text { C } & 1.423277 & 2.355604 & -3.070951\end{array}$

$\begin{array}{llll}\text { C } & 0.091258 & 2.172188 & -2.699775\end{array}$

$\begin{array}{llll}\text { C } & -0.878725 & 1.876987 & -3.657260\end{array}$

$\begin{array}{llll}\text { C } & -0.534432 & 1.812822 & -5.008627\end{array}$

$\begin{array}{llll}\text { C } & 0.787936 & 2.047801 & -5.396928\end{array}$

$\begin{array}{llll}\text { C } & 1.759858 & 2.310155 & -4.430668\end{array}$

$\begin{array}{llll}\text { C } & 2.247687 & 5.249774 & 2.732808\end{array}$

C $\quad 1.754472 \quad 5.264089 \quad 4.041282$

$\begin{array}{llll}\text { C } & 1.032470 & 6.361844 & 4.525285\end{array}$

$\begin{array}{llll}\text { C } & 0.847447 & 7.464624 & 3.686460\end{array}$

$\begin{array}{llll}\text { C } & 1.343684 & 7.481229 & 2.374311\end{array}$

$\begin{array}{llll}\text { C } & 2.025251 & 6.358435 & 1.901930\end{array}$

$\begin{array}{llll}\text { C } & 2.875563 & -4.130822 & -0.803688\end{array}$
C $\quad 3.410064 \quad-4.295241 \quad-2.277160$

$\begin{array}{llll}\text { C } & 2.521775 & -5.328663 & -1.659433\end{array}$

$\begin{array}{llll}\text { C } & 1.790717 & -3.137639 & -0.521972\end{array}$

$\begin{array}{llll}\text { C } & 3.837652 & -4.254794 & 0.337674\end{array}$

$\begin{array}{llll}\text { C } & 3.626105 & -5.106699 & 1.426358\end{array}$

$\begin{array}{llll}\text { C } & 4.513305 & -5.146585 & 2.502661\end{array}$

$\begin{array}{llll}\text { C } & 5.624090 & -4.307868 & 2.509752\end{array}$

$\begin{array}{llll}\text { C } & 5.854174 & -3.414172 & 1.451246\end{array}$

$\begin{array}{llll}\text { C } & 4.947591 & -3.409288 & 0.387406\end{array}$

$\begin{array}{llll}\text { C } & 4.891161 & -4.563417 & -2.430019\end{array}$

$\begin{array}{llll}\text { C } & 5.702516 & -3.640740 & -3.108482\end{array}$

$\begin{array}{llll}\text { C } & 7.081100 & -3.830214 & -3.210846\end{array}$

$\begin{array}{llll}\text { C } & 7.682714 & -4.951806 & -2.638995\end{array}$

$\begin{array}{llll}\text { C } & 6.886124 & -5.884542 & -1.972999\end{array}$

$\begin{array}{llll}\text { C } & 5.508873 & -5.693199 & -1.873047\end{array}$

$\begin{array}{llll}\text { C } & 2.815230 & -3.448048 & -3.370480\end{array}$

C $\quad 2.254108 \quad-4.073252 \quad-4.489330$

$\begin{array}{llll}\text { C } & 1.705782 & -3.316485 & -5.526328\end{array}$

$\begin{array}{llll}\text { C } & 1.712985 & -1.921227 & -5.451786\end{array}$

$\begin{array}{llll}\text { C } & 2.278968 & -1.291094 & -4.341811\end{array}$

$\begin{array}{llll}\text { C } & 2.834361 & -2.051901 & -3.314716\end{array}$

$\begin{array}{llll}\text { C } & 7.003886 & -2.481518 & 1.441569\end{array}$

C $\quad 7.444372 \quad-1.866815 \quad 2.621564$

$\begin{array}{llll}\text { C } & 8.499337 & -0.949208 & 2.606922\end{array}$

$\begin{array}{llll}\text { C } & 9.112341 & -0.649462 & 1.384271\end{array}$

$\begin{array}{llll}\text { C } & 8.696752 & -1.251012 & 0.190493\end{array}$

$\begin{array}{llll}\text { C } & 7.645150 & -2.169801 & 0.233404\end{array}$

C $\quad-2.869852 \quad-4.134484 \quad 0.804257$

$\begin{array}{llll}\text { C } & -3.404361 & -4.299365 & 2.277658\end{array}$

$\begin{array}{llll}\text { C } & -2.514583 & -5.331703 & 1.660246\end{array}$

$\begin{array}{llll}\text { C } & -1.786327 & -3.139857 & 0.522515\end{array}$

$\begin{array}{llll}\text { C } & -3.831560 & -4.259993 & -0.337268\end{array}$

$\begin{array}{llll}\text { C } & -3.618512 & -5.111727 & -1.425802\end{array}$

$\begin{array}{llll}\text { C } & -4.505357 & -5.153001 & -2.502333\end{array}$

$\begin{array}{llll}\text { C } & -5.617311 & -4.315828 & -2.509843\end{array}$

$\begin{array}{llll}\text { C } & -5.848928 & -3.422325 & -1.451504\end{array}$

C $\quad-4.942662 \quad-3.416058 \quad-0.387401$

$\begin{array}{llll}\text { C } & -4.885116 & -4.569449 & 2.430335\end{array}$

$\begin{array}{llll}\text { C } & -5.501195 & -5.700287 & 1.873711\end{array}$

$\begin{array}{llll}\text { C } & -6.878217 & -5.893434 & 1.973447\end{array}$

$\begin{array}{llll}\text { C } & -7.676186 & -4.961480 & 2.638872\end{array}$

$\begin{array}{llll}\text { C } & -7.076200 & -3.838823 & 3.210369\end{array}$

$\begin{array}{llll}\text { C } & -5.697845 & -3.647567 & 3.108231\end{array}$

C $\quad-2.810817 \quad-3.451198 \quad 3.370933$

$\begin{array}{llll}\text { C } & -2.249202 & -4.075504 & 4.490053\end{array}$

$\begin{array}{llll}\text { C } & -1.702086 & -3.317862 & 5.527046\end{array}$

C $\quad-1.710979-1.922620 \quad 5.452223$

$\begin{array}{llll}\text { C } & -2.277437 & -1.293404 & 4.341967\end{array}$

C $\quad-2.831628 \quad-2.055096 \quad 3.314879$

$\begin{array}{llll}\text { C } & -6.999860 & -2.491169 & -1.442336\end{array}$

$\begin{array}{llll}\text { C } & -7.440714 & -1.877172 & -2.622565\end{array}$

$\begin{array}{llll}\text { C } & -8.496841 & -0.960889 & -2.608394\end{array}$

$\begin{array}{llll}\text { C } & -9.110657 & -0.661786 & -1.386001\end{array}$

C $\quad-8.694738 \quad-1.262666 \quad-0.192005$

$\begin{array}{llll}\text { C } & -7.641953 & -2.180126 & -0.234424\end{array}$

$\begin{array}{llll}\text { C } & -2.693091 & 1.736163 & 0.796013\end{array}$

$\begin{array}{llll}\text { C } & -2.525633 & 2.634973 & 2.077788\end{array}$

$\begin{array}{llll}\text { C } & -3.620148 & 1.616844 & 1.989080\end{array}$

$\begin{array}{llll}\text { C } & -1.752808 & 0.593175 & 0.533409\end{array}$

$\begin{array}{llll}\text { C } & -3.204217 & 2.357952 & -0.469359\end{array}$

$\begin{array}{llll}\text { C } & -4.243085 & 1.784487 & -1.214009\end{array}$

C $\quad-4.659140 \quad 2.330653 \quad-2.428388$ $\begin{array}{llll}\text { C } & -4.024335 & 3.466721 & -2.924057\end{array}$

$\begin{array}{llll}\text { C } & -2.973509 & 4.062042 & -2.212511\end{array}$

$\begin{array}{llll}\text { C } & -2.585037 & 3.493149 & -0.996915\end{array}$

$\begin{array}{llll}\text { C } & -2.848101 & 4.106506 & 1.940488\end{array}$

$\begin{array}{llll}\text { C } & -1.828794 & 5.058790 & 2.102186\end{array}$

$\begin{array}{llll}\text { C } & -2.090333 & 6.419724 & 1.943542\end{array}$

$\begin{array}{llll}\text { C } & -3.375967 & 6.861224 & 1.627537\end{array}$

$\begin{array}{llll}\text { C } & -4.399074 & 5.924840 & 1.472084\end{array}$

$\begin{array}{llll}\text { C } & -4.136139 & 4.564575 & 1.628797\end{array}$

$\begin{array}{llll}\text { C } & -1.426939 & 2.354378 & 3.071081\end{array}$

$\begin{array}{llll}\text { C } & -1.763654 & 2.308747 & 4.430756\end{array}$

$\begin{array}{llll}\text { C } & -0.791480 & 2.048120 & 5.397239\end{array}$

$\begin{array}{llll}\text { C } & 0.531304 & 1.815070 & 5.009192\end{array}$

$\begin{array}{llll}\text { C } & 0.875701 & 1.879445 & 3.657860\end{array}$

$\begin{array}{llll}\text { C } & -0.094582 & 2.172924 & 2.700156\end{array}$

$\begin{array}{llll}\text { C } & -2.254397 & 5.247438 & -2.732498\end{array}$

$\begin{array}{llll}\text { C } & -2.035710 & 6.357206 & -1.902028\end{array}$

$\begin{array}{llll}\text { C } & -1.356618 & 7.481481 & -2.374386\end{array}$

$\begin{array}{llll}\text { C } & -0.858899 & 7.465346 & -3.686017\end{array}$

$\begin{array}{llll}\text { C } & -1.040016 & 6.361537 & -4.524275\end{array}$

$\begin{array}{llll}\text { C } & -1.759708 & 5.262173 & -4.040367\end{array}$

$\begin{array}{llll}\text { C } & -9.348361 & -0.910674 & 1.122310\end{array}$

$\begin{array}{llll}\text { C } & -8.973971 & -0.316814 & -3.887616\end{array}$

$\begin{array}{llll}\text { C } & 8.976123 & -0.304353 & 3.885878\end{array}$

$\begin{array}{llll}\text { C } & 9.349444 & -0.898334 & -1.124104\end{array}$

$\begin{array}{llll}\text { C } & 1.160674 & 8.693340 & 1.493706\end{array}$

$\begin{array}{llll}\text { C } & 0.452571 & 6.328054 & 5.918319\end{array}$

$\begin{array}{llll}\text { C } & -0.458568 & 6.328358 & -5.916683\end{array}$

$\begin{array}{llll}\text { C } & -1.177450 & 8.694749 & -1.494574\end{array}$

H $\quad 4.643135 \quad 1.937421 \quad-1.842339$

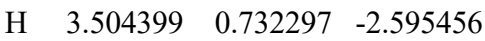

$\begin{array}{llll}\mathrm{H} & 5.468638 & 1.871529 & 2.975785\end{array}$

H $\quad 4.344328 \quad 3.901122 \quad 3.866440$

$\begin{array}{llll}\mathrm{H} & 1.754678 & 3.935210 & 0.451377\end{array}$

H $\quad 0.815993 \quad 4.741011-2.356605$

H $\quad 1.270452 \quad 7.129656 \quad-2.076236$

H $\quad 3.563806 \quad 7.926117 \quad-1.505313$

H $\quad 5.395623 \quad 6.258489 \quad-1.229583$

H $\quad 4.942545 \quad 3.869439 \quad-1.489641$

H $\quad-0.193740 \quad 2.212293 \quad-1.657329$

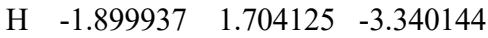

$\begin{array}{lllll}\mathrm{H} & -1.290818 & 1.588384 & -5.754882\end{array}$

$\mathrm{H} \quad 1.065166 \quad 2.010573 \quad-6.446191$

H $\quad 2.792853 \quad 2.469125 \quad-4.727249$

H $\quad 1.887993 \quad 4.389680 \quad 4.670074$

$\begin{array}{llll}\mathrm{H} & 2.410070 & 6.349183 & 0.887198\end{array}$

H $\quad 2.931586-6.284746-1.359950$

H $\quad 1.498093 \quad-5.365169-2.013792$

H $\quad 4.330443 \quad-5.827138 \quad 3.326346$

H $\quad 6.321665 \quad-4.357049 \quad 3.339336$

H $\quad 5.092830 \quad-2.714234 \quad-0.430017$

H $\quad 5.254201 \quad-2.764100 \quad-3.559680$

H $\quad 7.682818 \quad-3.097132 \quad-3.739757$

$\begin{array}{llll}\mathrm{H} & 8.755608 & -5.098828 & -2.713634\end{array}$

H $\quad 7.335748 \quad-6.765953 \quad-1.526062$

H $\quad 4.924123 \quad-6.429104 \quad-1.334392$

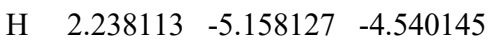

H $\quad 1.269644 \quad-3.815639 \quad-6.386588$

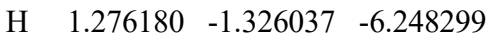

H $\quad 2.259285 \quad-0.210493 \quad-4.266491$

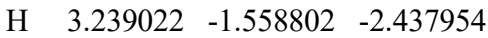

H $\quad 6.936963 \quad-2.079933 \quad 3.558176$ 


\begin{tabular}{|c|c|c|c|}
\hline & 7.332475 & -2.662413 & -0.681811 \\
\hline & -2.923065 & -6.288380 & 1.360844 \\
\hline & -1.490914 & -5.366777 & 2.014800 \\
\hline & -4.321319 & -5.833387 & -3.325896 \\
\hline & -6.314575 & -4.366067 & -3.339622 \\
\hline & -5.089104 & -2.721093 & 0.429883 \\
\hline & -4.915334 & -6.435642 & 1.335501 \\
\hline & -7.326576 & -6.775633 & 1.526789 \\
\hline & -8.748896 & -5.109889 & 2.713345 \\
\hline & -7.679009 & -3.106325 & 3.738836 \\
\hline & -5.250793 & -2.770137 & 3.559132 \\
\hline & -2.231908 & -5.160349 & 4.541083 \\
\hline & -1.265554 & -3.816298 & 6.387511 \\
\hline & -1.275114 & -1.326740 & 6.248734 \\
\hline & -2.259090 & -0.212795 & 4.266444 \\
\hline & -3.236659 & -1.562665 & 2.437915 \\
\hline & -6.932700 & -2.089754 & -3.558959 \\
\hline & -7.328947 & -2.672214 & 0.680963 \\
\hline & -4.645844 & 1.930491 & 1.841779 \\
\hline & -3.505172 & 0.727345 & 2.595089 \\
\hline & -5.469623 & 1.863913 & -2.976864 \\
\hline & -4.348177 & 3.895350 & -3.866972 \\
\hline & -1.759931 & 3.933412 & -0.450904 \\
\hline & -0.823817 & 4.740930 & 2.356624 \\
\hline & -1.282678 & 7.128764 & 2.076009 \\
\hline & -3.577400 & 7.920972 & 1.504884 \\
\hline & -5.406076 & 6.249961 & 1.228848 \\
\hline & -4.948635 & 3.861783 & 1.489032 \\
\hline & -2.796935 & 2.466217 & 4.727145 \\
\hline & -1.068809 & 2.010709 & 6.446456 \\
\hline & 1.287922 & 1.591978 & 5604 \\
\hline & 1.897235 & 1.708098 & 3.340950 \\
\hline & 0.190513 & 2.213199 & 1.657725 \\
\hline & -2.421728 & 6.347607 & -0.887758 \\
\hline & -1.890339 & 4.387053 & -4.668781 \\
\hline $\mathrm{H}$ & -9.928442 & 0.055135 & -1.364113 \\
\hline H & -0.305430 & 8.326229 & -4.054351 \\
\hline 1 & 9.929213 & 0.068488 & 1.362008 \\
\hline & 0.291991 & 8.324220 & 4.054804 \\
\hline & -10.416905 & -0.711283 & 30.99879 \\
\hline $\mathrm{H}$ & -9.228959 & -1.716585 & 1.851724 \\
\hline $\mathrm{H}$ & -8.896925 & -0.009047 & 1.554175 \\
\hline $\mathrm{H}$ & -8.171411 & -0.261102 & -4.628503 \\
\hline & -9.793414 & -0.891845 & -4.336093 \\
\hline$H$ & -9.347965 & 0.695462 & -3.708906 \\
\hline $\mathrm{H}$ & 9.347676 & 0.708810 & 3.707077 \\
\hline $\mathrm{H}$ & 8.174084 & -0.250625 & 4.627479 \\
\hline & 9.797267 & -0.877622 & 4.333489 \\
\hline & 8.896300 & 0.002339 & -1.556181 \\
\hline 1 & 10.417661 & -0.697025 & -1.00094 \\
\hline $\mathrm{H}$ & 9.231264 & -1.704713 & -1.853211 \\
\hline $\mathrm{H}$ & 1.887016 & 9.475673 & 1.744795 \\
\hline & 1.299299 & 8.439283 & 0.440230 \\
\hline & 0.164481 & 9.131272 & 1.612559 \\
\hline $\mathrm{H}$ & -0.169572 & 7.205085 & 6.114120 \\
\hline $\mathrm{H}$ & -0.163478 & 5.433225 & 6.059942 \\
\hline & 1.242027 & 6.297693 & 6.678022 \\
\hline & 0.161846 & 7.206667 & -6.112267 \\
\hline & 0.159613 & 5.434828 & -6.057220 \\
\hline & -1.247155 & 6.295869 & -6.677201 \\
\hline & -1.325476 & 8.443193 & -0.441780 \\
\hline & -0.178971 & 9.129519 & -1.605958 \\
\hline
\end{tabular}

H $\quad-1.899179 \quad 9.478815 \quad-1.753390$

N"3

$\begin{array}{llll}\text { Rh } & -0.762044 & -1.026187 & 0.931394\end{array}$

$\begin{array}{llll}\mathrm{Rh} & -0.547585 & -0.501054 & -1.386345\end{array}$

$\begin{array}{llll}\mathrm{O} & -1.992560 & 0.941827 & -1.084509\end{array}$

$\begin{array}{llll}\mathrm{O} & -2.534934 & 0.017083 & 0.908941\end{array}$

$\begin{array}{llll}\mathrm{O} & 0.896180 & 0.872340 & -0.824103\end{array}$

$\begin{array}{llll}\mathrm{O} & 0.263580 & 0.724962 & 1.337210\end{array}$

O $\quad 0.878237 \quad-1.988596 \quad-1.524895$

O $\quad 1.052652 \quad-2.021115 \quad 0.726958$

$\begin{array}{llll}\mathrm{O} & -2.048566 & -1.869724 & -1.750569\end{array}$

$\begin{array}{llll}\mathrm{O} & -1.914952 & -2.647571 & 0.370038\end{array}$

$\begin{array}{llll}\mathrm{Cl} & -5.582046 & 0.763690 & -2.325936\end{array}$

$\begin{array}{lllll}\mathrm{Cl} & 0.334717 & 4.386528 & -0.985713\end{array}$

$\begin{array}{llll}\text { Cl } & 1.539713 & -5.451308 & 0.996410\end{array}$

$\begin{array}{llll}\mathrm{Cl} & -3.293804 & -5.450287 & 1.404961\end{array}$

$\begin{array}{llll}\text { C } & -3.861562 & 1.803846 & 0.079723\end{array}$

$\begin{array}{llll}\text { C } & -4.274876 & 2.355398 & 1.494009\end{array}$

$\begin{array}{llll}\text { C } & -5.071277 & 1.286678 & 0.828479\end{array}$

C $\quad-2.710813 \quad 0.856289 \quad-0.031796$

$\begin{array}{llll}\text { C } & -4.019004 & 2.685273 & -1.118844\end{array}$

$\begin{array}{llll}\text { C } & -4.757331 & 2.322217 & -2.250651\end{array}$

$\begin{array}{llll}\text { C } & -4.822413 & 3.148218 & -3.373404\end{array}$

$\begin{array}{llll}\text { C } & -4.118037 & 4.348527 & -3.387036\end{array}$

$\begin{array}{llll}\text { C } & -3.336832 & 4.733174 & -2.285528\end{array}$

$\begin{array}{llll}\text { C } & -3.311005 & 3.887831 & -1.172086\end{array}$

$\begin{array}{llll}\text { C } & -4.817467 & 3.764679 & 1.539633\end{array}$

$\begin{array}{llll}\text { C } & -4.009343 & 4.805738 & 2.022489\end{array}$

$\begin{array}{llll}\text { C } & -4.470821 & 6.120841 & 2.047733\end{array}$

$\begin{array}{llll}\text { C } & -5.757956 & 6.423284 & 1.596777\end{array}$

$\begin{array}{llll}\text { C } & -6.577877 & 5.393914 & 1.134569\end{array}$

$\begin{array}{llll}\text { C } & -6.112996 & 4.077705 & 1.108141\end{array}$

$\begin{array}{llll}\text { C } & -3.483293 & 1.969931 & 2.715401\end{array}$

$\begin{array}{llll}\text { C } & -4.165655 & 1.411119 & 3.802445\end{array}$

$\begin{array}{llll}\text { C } & -3.480246 & 1.047298 & 4.962029\end{array}$

$\begin{array}{llll}\text { C } & -2.098203 & 1.238068 & 5.044578\end{array}$

$\begin{array}{llll}\text { C } & -1.412903 & 1.801818 & 3.966685\end{array}$

$\begin{array}{llll}\text { C } & -2.104571 & 2.173587 & 2.814348\end{array}$

$\begin{array}{llll}\text { C } & -2.527994 & 5.973342 & -2.287117\end{array}$

$\begin{array}{llll}\text { C } & -2.375801 & 6.716983 & -1.109046\end{array}$

$\begin{array}{llll}\text { C } & -1.562261 & 7.853588 & -1.072168\end{array}$

$\begin{array}{llll}\text { C } & -0.905944 & 8.247194 & -2.242790\end{array}$

$\begin{array}{llll}\text { C } & -1.048505 & 7.531167 & -3.438950\end{array}$

C $\quad-1.862471 \quad 6.395603 \quad-3.447459$

$\begin{array}{llll}\text { C } & 1.922413 & 2.334061 & 0.722891\end{array}$

$\begin{array}{llll}\text { C } & 2.693337 & 2.354632 & 2.094071\end{array}$

$\begin{array}{llll}\text { C } & 1.544667 & 3.281476 & 1.844451\end{array}$

$\begin{array}{llll}\text { C } & 0.958180 & 1.226786 & 0.399514\end{array}$

$\begin{array}{llll}\text { C } & 2.593433 & 2.885961 & -0.499650\end{array}$

$\begin{array}{llll}\text { C } & 1.957500 & 3.803249 & -1.346337\end{array}$

$\begin{array}{llll}\text { C } & 2.561643 & 4.257144 & -2.518883\end{array}$

$\begin{array}{llll}\text { C } & 3.822098 & 3.780996 & -2.870008\end{array}$

C $\quad 4.484476 \quad 2.852856 \quad-2.054541$

C $\quad 3.855634 \quad 2.425995 \quad-0.882050$

$\begin{array}{llll}\text { C } & 4.109175 & 2.886491 & 2.084678\end{array}$

$\begin{array}{llll}\text { C } & 5.176914 & 2.021650 & 2.373502\end{array}$

$\begin{array}{llll}\text { C } & 6.495364 & 2.474928 & 2.329848\end{array}$

$\begin{array}{llll}\text { C } & 6.776628 & 3.803053 & 2.006055\end{array}$

$\begin{array}{llll}\text { C } & 5.723067 & 4.674523 & 1.726677\end{array}$

$\begin{array}{llll}\text { C } & 4.405657 & 4.219613 & 1.767437\end{array}$

$\begin{array}{llll}\text { C } & 2.467276 & 1.267071 & 3.113036\end{array}$ $\begin{array}{llll}\text { C } & 2.506967 & -0.091061 & 2.797857\end{array}$

$\begin{array}{llll}\text { C } & 2.251700 & -1.054307 & 3.773541\end{array}$

$\begin{array}{llll}\text { C } & 2.001862 & -0.668981 & 5.091744\end{array}$

$\begin{array}{llll}\text { C } & 2.009324 & 0.687984 & 5.428784\end{array}$

$\begin{array}{llll}\text { C } & 2.234201 & 1.647892 & 4.441609\end{array}$

$\begin{array}{llll}\text { C } & 5.805460 & 2.297418 & -2.427491\end{array}$

$\begin{array}{llll}\text { C } & 6.015518 & 1.762380 & -3.702385\end{array}$

$\begin{array}{llll}\text { C } & 7.250979 & 1.203482 & -4.052446\end{array}$

$\begin{array}{llll}\text { C } & 8.285276 & 1.222910 & -3.112388\end{array}$

$\begin{array}{llll}\text { C } & 8.102952 & 1.760989 & -1.829492\end{array}$

$\begin{array}{llll}\text { C } & 6.851453 & 2.278864 & -1.492382\end{array}$

$\begin{array}{llll}\text { C } & 2.772729 & -3.049791 & -0.532551\end{array}$

$\begin{array}{llll}\text { C } & 3.751452 & -2.803624 & -1.744689\end{array}$

$\begin{array}{llll}\text { C } & 2.911958 & -4.037989 & -1.674826\end{array}$

$\begin{array}{llll}\text { C } & 1.474554 & -2.297968 & -0.445467\end{array}$

$\begin{array}{llll}\text { C } & 3.344117 & -3.382886 & 0.813199\end{array}$

$\begin{array}{llll}\text { C } & 2.855829 & -4.440375 & 1.591875\end{array}$

$\begin{array}{llll}\text { C } & 3.353789 & -4.704369 & 2.867290\end{array}$

$\begin{array}{llll}\text { C } & 4.354377 & -3.891672 & 3.393789\end{array}$

$\begin{array}{llll}\text { C } & 4.857665 & -2.813836 & 2.653226\end{array}$

$\begin{array}{llll}\text { C } & 4.341618 & -2.582442 & 1.375432\end{array}$

$\begin{array}{llll}\text { C } & 5.238422 & -2.886218 & -1.475797\end{array}$

$\begin{array}{llll}\text { C } & 6.047995 & -1.753892 & -1.661178\end{array}$

$\begin{array}{llll}\text { C } & 7.416574 & -1.799902 & -1.393949\end{array}$

$\begin{array}{llll}\text { C } & 8.010097 & -2.979177 & -0.942274\end{array}$

$\begin{array}{llll}\text { C } & 7.217593 & -4.113479 & -0.759153\end{array}$

$\begin{array}{llll}\text { C } & 5.849907 & -4.065383 & -1.024645\end{array}$

$\begin{array}{llll}\text { C } & 3.386055 & -1.809262 & -2.816417\end{array}$

$\begin{array}{llll}\text { C } & 3.080782 & -0.478022 & -2.531721\end{array}$

$\begin{array}{llll}\text { C } & 2.731398 & 0.404916 & -3.552565\end{array}$

$\begin{array}{llll}\text { C } & 2.727106 & -0.026022 & -4.880658\end{array}$

$\begin{array}{llll}\text { C } & 3.067349 & -1.348808 & -5.179669\end{array}$

$\begin{array}{llll}\text { C } & 3.387526 & -2.234663 & -4.150524\end{array}$

$\begin{array}{llll}\text { C } & 5.892315 & -1.910981 & 3.206956\end{array}$

$\begin{array}{llll}\text { C } & 7.033249 & -1.601199 & 2.451226\end{array}$

$\begin{array}{llll}\text { C } & 8.022859 & -0.760241 & 2.962884\end{array}$

$\begin{array}{llll}\text { C } & 7.835784 & -0.196464 & 4.233923\end{array}$

$\begin{array}{llll}\text { C } & 6.692946 & -0.463784 & 4.992961\end{array}$

$\begin{array}{llll}\text { C } & 5.733223 & -1.341794 & 4.474422\end{array}$

$\begin{array}{llll}\text { C } & -3.725485 & -3.380880 & -0.982958\end{array}$

C $\quad-3.687572 \quad-4.593004 \quad-1.961731$

$\begin{array}{llll}\text { C } & -4.343089 & -3.297675 & -2.356310\end{array}$

$\begin{array}{llll}\text { C } & -2.455677 & -2.587982 & -0.782065\end{array}$

$\begin{array}{llll}\text { C } & -4.612729 & -3.336025 & 0.227482\end{array}$

$\begin{array}{llll}\text { C } & -4.495654 & -4.171528 & 1.345483\end{array}$

$\begin{array}{llll}\text { C } & -5.325373 & -4.010953 & 2.456136\end{array}$

$\begin{array}{llll}\text { C } & -6.271976 & -2.990510 & 2.478661\end{array}$

$\begin{array}{llll}\text { C } & -6.407563 & -2.119385 & 1.387860\end{array}$

$\begin{array}{llll}\text { C } & -5.572585 & -2.320232 & 0.280748\end{array}$

C $\quad-4.562941-5.788710 \quad-1.667203$

$\begin{array}{llll}\text { C } & -4.043452 & -7.088843 & -1.758791\end{array}$

$\begin{array}{llll}\text { C } & -4.833976 & -8.203834 & -1.480349\end{array}$

$\begin{array}{llll}\text { C } & -6.167224 & -8.047480 & -1.102093\end{array}$

$\begin{array}{llll}\text { C } & -6.701727 & -6.761046 & -1.018021\end{array}$

$\begin{array}{llll}\text { C } & -5.912624 & -5.647969 & -1.303284\end{array}$

C $\quad-2.348032 \quad-4.901901 \quad-2.575970$

$\begin{array}{llll}\text { C } & -2.167495 & -4.804344 & -3.959392\end{array}$

$\begin{array}{llll}\text { C } & -0.942187 & -5.134302 & -4.541340\end{array}$

C $\quad 0.112203 \quad-5.582566 \quad-3.742931$

$\begin{array}{llll}\text { C } & -0.063379 & -5.688483 & -2.361040\end{array}$

$\begin{array}{llll}\text { C } & -1.282061 & -5.340227 & -1.779965\end{array}$

$\begin{array}{llll}\text { C } & -7.331051 & -0.961035 & 1.405105\end{array}$ 


\begin{tabular}{|c|c|c|c|}
\hline $\mathrm{C}$ & -7.465685 & -0.183251 & 2.567279 \\
\hline $\mathrm{C}$ & -8.185159 & 1.012428 & 2.555158 \\
\hline $\mathrm{C}$ & -8.811102 & 1.408008 & 1.363473 \\
\hline $\mathrm{C}$ & -8.722834 & 0.638430 & 0.199978 \\
\hline $\mathrm{C}$ & -7.978130 & -0.547055 & 0.235764 \\
\hline $\mathrm{C}$ & 6.468744 & 0.193573 & 6.332815 \\
\hline $\mathrm{C}$ & 9.275608 & -0.478483 & 2.169753 \\
\hline $\mathrm{C}$ & 7.438420 & 0.577156 & -5.412743 \\
\hline $\mathrm{C}$ & 9.240284 & 1.794600 & -0.838044 \\
\hline $\mathrm{C}$ & -0.343262 & 7.986218 & -4.693712 \\
\hline $\mathrm{C}$ & -1.377247 & 8.615709 & 0.217506 \\
\hline $\mathrm{C}$ & -9.378838 & 1.083965 & -1.083389 \\
\hline $\mathrm{C}$ & -8.226654 & 1.905773 & 3.769964 \\
\hline $\mathrm{H}$ & -6.043506 & 1.504294 & 0.411663 \\
\hline $\mathrm{H}$ & -4.966656 & 0.289584 & 1.229833 \\
\hline $\mathrm{H}$ & -5.415047 & 2.846917 & -4.229605 \\
\hline $\mathrm{H}$ & -4.182553 & 3008 & -4.257523 \\
\hline $\mathrm{H}$ & -2.696321 & 4.150788 & -0.320665 \\
\hline $\mathrm{H}$ & -3.011016 & 4.583973 & 2.382771 \\
\hline $\mathrm{H}$ & -3.824130 & 6.908736 & 2.422438 \\
\hline $\mathrm{H}$ & -6.118771 & 7.446941 & 1.614595 \\
\hline $\mathrm{H}$ & -7.586170 & 386 & 4496 \\
\hline $\mathrm{H}$ & -6.774695 & 3.297059 & 0.752929 \\
\hline $\mathrm{H}$ & -5.236034 & 1.249607 & 3.726741 \\
\hline $\mathrm{H}$ & -4.022751 & 0.610318 & 5.795313 \\
\hline $\mathrm{H}$ & -1.555536 & 0.944722 & 5.938376 \\
\hline $\mathrm{H}$ & -0.33 & 297 & 4.016941 \\
\hline $\mathrm{H}$ & -1.560563 & 2.571121 & 1.964593 \\
\hline $\mathrm{H}$ & -2.909196 & 6.414557 & -0.213258 \\
\hline $\mathrm{H}$ & -1.952841 & 5.811296 & -4.358714 \\
\hline $\mathrm{H}$ & 1.721833 & 4.335176 & 1.670909 \\
\hline $\mathrm{H}$ & 0.621884 & 188 & 4523 \\
\hline $\mathrm{H}$ & 2.042770 & 4.971877 & -3.147778 \\
\hline $\mathrm{H}$ & 4.295378 & 4.132707 & -3.780997 \\
\hline $\mathrm{H}$ & 4.352356 & 1.695285 & -0.255813 \\
\hline $\mathrm{H}$ & 4.982218 & 0.987969 & 2.636833 \\
\hline $\mathrm{H}$ & 7.296654 & 1.782948 & 2.557119 \\
\hline $\mathrm{H}$ & 7.803562 & 4.153817 & 1.973207 \\
\hline $\mathrm{H}$ & 5.922805 & 5.711993 & 1.476278 \\
\hline $\mathrm{H}$ & 3.610615 & 4.917300 & 1.533264 \\
\hline $\mathrm{H}$ & 2.691435 & -0.408481 & 1.780445 \\
\hline $\mathrm{H}$ & 2.251710 & -2.101196 & 3.496496 \\
\hline $\mathrm{H}$ & 1.807948 & -1.419372 & 5.852456 \\
\hline $\mathrm{H}$ & 1.825837 & 0.998620 & 6.453005 \\
\hline $\mathrm{H}$ & 2.218381 & 2.704101 & 4.695712 \\
\hline $\mathrm{H}$ & 5.193164 & 1.736241 & -4.410705 \\
\hline $\mathrm{H}$ & 6.688990 & 2.695174 & -0.503348 \\
\hline $\mathrm{H}$ & 3.363658 & -4.994617 & -1.442873 \\
\hline $\mathrm{H}$ & 2.071760 & -4.084832 & -2.354937 \\
\hline $\mathrm{H}$ & 2.955835 & -5.534219 & 3.439889 \\
\hline $\mathrm{H}$ & 4.746519 & -4.095223 & 4.384862 \\
\hline $\mathrm{H}$ & 4.712667 & -1.740386 & 0.804656 \\
\hline $\mathrm{H}$ & 5.613638 & -0.828427 & -2.021271 \\
\hline $\mathrm{H}$ & 8.011890 & -0.908523 & -1.549683 \\
\hline $\mathrm{H}$ & 9.075476 & -3.013725 & -0.736173 \\
\hline $\mathrm{H}$ & 7.661614 & -5.040600 & -0.409541 \\
\hline $\mathrm{H}$ & 5.260709 & -4.959238 & -0.859309 \\
\hline $\mathrm{H}$ & 3.064162 & -0.135032 & -1.505726 \\
\hline $\mathrm{H}$ & 2.463245 & 1.424199 & -3.304409 \\
\hline & 2.461939 & 0.663079 & -5.677180 \\
\hline $\mathrm{H}$ & 3.072517 & -1.692370 & -6.209894 \\
\hline & 3.631945 & -3.268304 & -4.378494 \\
\hline
\end{tabular}

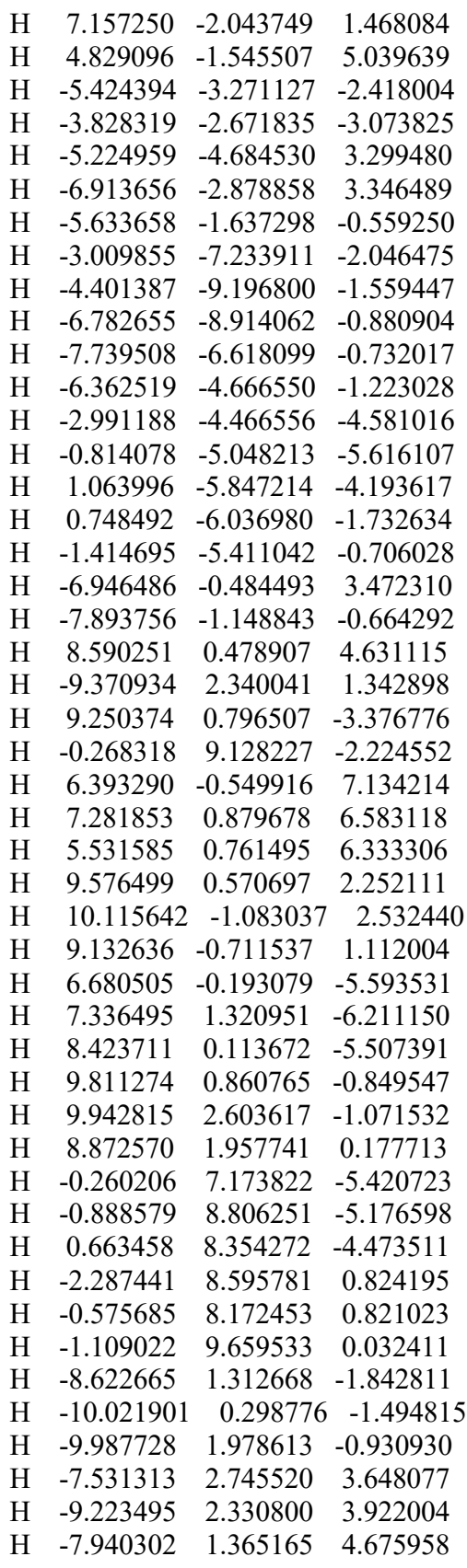

$\begin{array}{lrrr}\text { N"4 } & & & \\ \mathrm{Rh} & -0.000106 & -0.000406 & -1.189259 \\ \mathrm{Rh} & -0.000090 & 0.000637 & 1.189103 \\ \mathrm{O} & 1.668177 & -1.225357 & 1.056031 \\ \mathrm{O} & 1.167852 & -1.692868 & -1.098985 \\ \mathrm{O} & 1.167772 & 1.693171 & 1.098775 \\ \mathrm{O} & 1.668107 & 1.225646 & -1.056232 \\ \mathrm{O} & -1.668258 & 1.226657 & 1.055094 \\ \mathrm{O} & -1.167997 & 1.692182 & -1.100362 \\ \mathrm{O} & -1.167859 & -1.692032 & 1.100230 \\ \mathrm{O} & -1.668199 & -1.226523 & -1.055216 \\ \mathrm{Cl} & 1.593127 & -4.577937 & 2.114528 \\ \mathrm{Cl} & 1.593217 & 4.578042 & -2.114913 \\ \mathrm{Cl} & -1.593443 & 4.580435 & 2.110138\end{array}$
Cl $\quad-1.593134 \quad-4.580046 \quad-2.110586$

$\begin{array}{llll}\text { C } & 2.971384 & -2.852222 & -0.065366\end{array}$

$\begin{array}{llll}\text { C } & 3.790306 & -3.067336 & -1.391459\end{array}$

$\begin{array}{llll}\text { C } & 2.760249 & -4.062388 & -0.955041\end{array}$

$\begin{array}{llll}\text { C } & 1.855989 & -1.849388 & -0.040742\end{array}$

$\begin{array}{llll}\text { C } & 3.660481 & -2.983795 & 1.257235\end{array}$

$\begin{array}{llll}\text { C } & 3.120663 & -3.720499 & 2.318534\end{array}$

$\begin{array}{llll}\text { C } & 3.739755 & -3.766513 & 3.567677\end{array}$

C $\quad 4.921055 \quad-3.057137 \quad 3.775053$

$\begin{array}{llll}\text { C } & 5.485343 & -2.300440 & 2.739398\end{array}$

$\begin{array}{llll}\text { C } & 4.839802 & -2.277035 & 1.501250\end{array}$

$\begin{array}{llll}\text { C } & 5.261210 & -3.404849 & -1.261594\end{array}$

$\begin{array}{llll}\text { C } & 5.707426 & -4.566738 & -0.614801\end{array}$

$\begin{array}{llll}\text { C } & 7.068029 & -4.842724 & -0.483089\end{array}$

$\begin{array}{llll}\text { C } & 8.019335 & -3.960231 & -0.996952\end{array}$

$\begin{array}{llll}\text { C } & 7.591279 & -2.800211 & -1.643937\end{array}$

C $\quad 6.229596 \quad-2.528453 \quad-1.778543$

$\begin{array}{llll}\text { C } & 3.468935 & -2.226743 & -2.599481\end{array}$

C $3.173413-2.866158 \quad-3.808635$

$\begin{array}{llll}\text { C } & 2.893837 & -2.122846 & -4.956006\end{array}$

$\begin{array}{llll}\text { C } & 2.900473 & -0.726143 & -4.901373\end{array}$

$\begin{array}{llll}\text { C } & 3.200564 & -0.082684 & -3.698546\end{array}$

$\begin{array}{llll}\text { C } & 3.498690 & -0.830869 & -2.560799\end{array}$

$\begin{array}{llll}\text { C } & 6.736420 & -1.527953 & 2.919575\end{array}$

$\begin{array}{llll}\text { C } & 7.794889 & -1.715261 & 2.023532\end{array}$

$\begin{array}{llll}\text { C } & 8.993936 & -1.010192 & 2.170294\end{array}$

$\begin{array}{llll}\text { C } & 9.100514 & -0.082315 & 3.212217\end{array}$

$\begin{array}{llll}\text { C } & 8.042154 & 0.148687 & 4.103301\end{array}$

$\begin{array}{llll}\text { C } & 6.868078 & -0.596453 & 3.958669\end{array}$

$\begin{array}{llll}\text { C } & 2.971360 & 2.852469 & 0.065159\end{array}$

$\begin{array}{llll}\text { C } & 3.790278 & 3.067556 & 1.391276\end{array}$

$\begin{array}{llll}\text { C } & 2.760314 & 4.062673 & 0.954791\end{array}$

$\begin{array}{llll}\text { C } & 1.855935 & 1.849676 & 0.040546\end{array}$

$\begin{array}{llll}\text { C } & 3.660503 & 2.983948 & -1.257417\end{array}$

C $\quad 3.120751 \quad 3.720584 \quad-2.318793$

$\begin{array}{llll}\text { C } & 3.739899 & 3.766490 & -3.567916\end{array}$

C $4.921199 \quad 3.057076 \quad-3.775188$

$\begin{array}{llll}\text { C } & 5.485421 & 2.300438 & -2.739448\end{array}$

$\begin{array}{llll}\text { C } & 4.839812 & 2.277129 & -1.501338\end{array}$

$\begin{array}{llll}\text { C } & 5.261214 & 3.404905 & 1.261452\end{array}$

$\begin{array}{llll}\text { C } & 5.707577 & 4.566708 & 0.614603\end{array}$

$\begin{array}{llll}\text { C } & 7.068225 & 4.842535 & 0.482930\end{array}$

$\begin{array}{llll}\text { C } & 8.019419 & 3.959962 & 0.996887\end{array}$

$\begin{array}{llll}\text { C } & 7.591205 & 2.800029 & 1.643926\end{array}$

$\begin{array}{llll}\text { C } & 6.229488 & 2.528430 & 1.778482\end{array}$

$\begin{array}{llll}\text { C } & 3.468779 & 2.227046 & 2.599324\end{array}$

$\begin{array}{llll}\text { C } & 3.173241 & 2.866536 & 3.808432\end{array}$

$\begin{array}{llll}\text { C } & 2.893577 & 2.123279 & 4.955835\end{array}$

$\begin{array}{llll}\text { C } & 2.900151 & 0.726576 & 4.901252\end{array}$

$\begin{array}{llll}\text { C } & 3.200249 & 0.083042 & 3.698471\end{array}$

$\begin{array}{llll}\text { C } & 3.498464 & 0.831173 & 2.560710\end{array}$

$\begin{array}{llll}\text { C } & 6.736484 & 1.527906 & -2.919447\end{array}$

$\begin{array}{llll}\text { C } & 7.794765 & 1.715099 & -2.023159\end{array}$

$\begin{array}{llll}\text { C } & 8.993768 & 1.009893 & -2.169619\end{array}$

$\begin{array}{llll}\text { C } & 9.100520 & 0.082061 & -3.211540\end{array}$

$\begin{array}{llll}\text { C } & 8.042360 & -0.148815 & -4.102898\end{array}$

$\begin{array}{llll}\text { C } & 6.868316 & 0.596424 & -3.958532\end{array}$

$\begin{array}{llll}\text { C } & -2.971615 & 2.852376 & -0.067810\end{array}$

$\begin{array}{llll}\text { C } & -3.790615 & 3.066139 & -1.394089\end{array}$

$\begin{array}{llll}\text { C } & -2.760643 & 4.061703 & -0.958656\end{array}$

$\begin{array}{llll}\text { C } & -1.856138 & 1.849657 & -0.042264\end{array}$

$\begin{array}{llll}\text { C } & -3.660655 & 2.985186 & 1.254690\end{array}$ 


\begin{tabular}{|c|c|c|c|}
\hline & -3.120842 & 3.723022 & \\
\hline & -3.739793 & 3.770185 & 3.564361 \\
\hline & -4.920966 & 3.060829 & 3.772565 \\
\hline 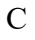 & -5.485258 & 2.303025 & 2.737719 \\
\hline & -4.839857 & 2.278495 & 1.499526 \\
\hline & -5.261548 & 3.403560 & -1.264494 \\
\hline 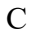 & -5.707903 & 4.566079 & -0.618926 \\
\hline $\mathrm{C}$ & -7.068555 & 4.841972 & -0.487418 \\
\hline $\mathrm{C}$ & -8.019742 & 3.958754 & -1.000255 \\
\hline & -7.591538 & 2.798115 & -1.646022 \\
\hline 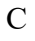 & -6.229816 & 2.526444 & -1.780436 \\
\hline $\mathrm{C}$ & -3.469192 & 2.224478 & -2.601358 \\
\hline $\mathrm{C}$ & -3.498643 & 0.828639 & -2.561380 \\
\hline $\mathrm{C}$ & -3.200510 & 0.079459 & -3.698474 \\
\hline & -2.900745 & 0.721876 & -4.901939 \\
\hline $\mathrm{C}$ & -2.894424 & 2.118525 & 7876 \\
\hline $\mathrm{C}$ & -3.173987 & 2.862839 & 11146 \\
\hline $\mathrm{C}$ & -6.736150 & 1.530466 & 2.918822 \\
\hline $\mathrm{C}$ & -7.794751 & 1.716573 & 2.022704 \\
\hline $\mathcal{C}$ & -8.993577 & 1.011269 & 2.170261 \\
\hline $\mathrm{C}$ & -9.099790 & 0.08 & 110 \\
\hline $\mathrm{C}$ & -8.041278 & -0.145394 & 327 \\
\hline $\mathrm{C}$ & -6.867444 & 0.599948 & 3.958859 \\
\hline $\mathrm{C}$ & -2.971381 & -2.852390 & 0.067675 \\
\hline 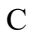 & -3.790302 & -3.066295 & 3959 \\
\hline $\mathrm{C}$ & -2.760283 & -4.0 & 424 \\
\hline $\mathrm{C}$ & -1.855998 & -1.849563 & 138 \\
\hline $\mathrm{C}$ & -3.660491 & -2.985136 & -1.254800 \\
\hline $\mathrm{C}$ & -3.120644 & -3.722773 & -2.315426 \\
\hline $\mathrm{C}$ & -3.739664 & -3.76 & 4564 \\
\hline $\mathrm{C}$ & -4.920921 & -3.06 & 2613 \\
\hline $\mathrm{C}$ & -5.485249 & -2.303001 & 7642 \\
\hline $\mathrm{C}$ & -4.839779 & -2.278557 & -1.499480 \\
\hline $\mathrm{C}$ & -5.261228 & -3.403794 & 1.264363 \\
\hline $\mathrm{C}$ & -6.229540 & -2.526758 & 374 \\
\hline $\mathrm{C}$ & -7.591248 & -2.798470 & 1.645910 \\
\hline $\mathrm{C}$ & -8.019394 & -3.959082 & 1.000048 \\
\hline $\mathrm{C}$ & -7.068162 & -4.842220 & 0.487164 \\
\hline $\mathrm{C}$ & -5.707524 & -4.566286 & 0.618721 \\
\hline $\mathrm{C}$ & -3.468895 & -2.224718 & 2.60 \\
\hline $\mathrm{C}$ & -3.173619 & -2.863163 & 3.811006 \\
\hline $\mathrm{C}$ & -2.894072 & -2.118915 & 4.957794 \\
\hline $\mathrm{C}$ & -2.900471 & -0.722266 & 4.901967 \\
\hline $\mathrm{C}$ & -3.200306 & -0.079765 & 3.698567 \\
\hline C & -3.498423 & -0.828871 & 2.561416 \\
\hline $\mathrm{C}$ & -6.736246 & -1.530567 & -2.918543 \\
\hline $\mathrm{C}$ & -7.794765 & -1.716929 & -2.022370 \\
\hline $\mathrm{C}$ & -8.993669 & -1.011708 & -2.169710 \\
\hline $\mathrm{C}$ & -9.100046 & -0.084684 & -3.212390 \\
\hline C & -8.041628 & 0.145375 & -4.103662 \\
\hline $\mathrm{C}$ & -6.867713 & -0.599886 & -3.958409 \\
\hline $\mathrm{C}$ & -8.144178 & -1.219392 & 5.158493 \\
\hline $\mathrm{C}$ & -10.149332 & 1.284627 & 1.239538 \\
\hline $\mathrm{C}$ & 10.149098 & 1.284374 & -1.238704 \\
\hline $\mathrm{C}$ & 8.145785 & -1.223789 & -5.156022 \\
\hline $\mathrm{C}$ & 8.145438 & 1.223666 & 5.156436 \\
\hline $\mathrm{C}$ & 10.149576 & -1.284943 & 1.239846 \\
\hline $\mathrm{C}$ & -8.144709 & 1.219547 & -5.157630 \\
\hline $\mathrm{C}$ & -10.149305 & -1.285327 & -1.238933 \\
\hline $\mathrm{H}$ & 3.068221 & -5.021886 & -0.559282 \\
\hline $\mathrm{H}$ & 1.829817 & -4.076875 & -1.510673 \\
\hline $\mathrm{H}$ & 3.296462 & -4.348473 & 4.367519 \\
\hline
\end{tabular}

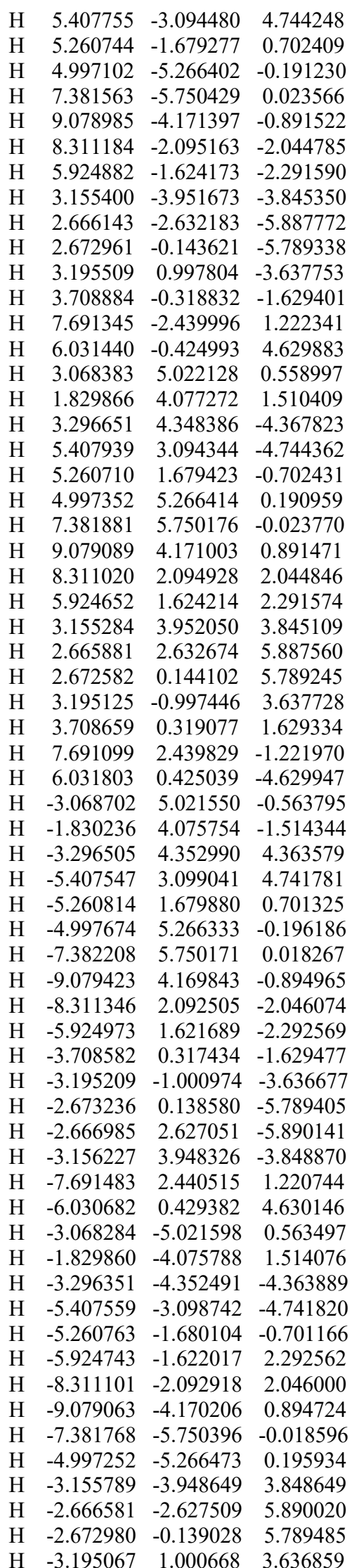

$\begin{array}{llcc}\mathrm{H} & -3.708404 & -0.317597 & 1.629561 \\ \mathrm{H} & -7.691354 & -2.440985 & -1.220538 \\ \mathrm{H} & -6.031014 & -0.429106 & -4.629724 \\ \mathrm{H} & -10.023531 & -0.477728 & 3.332005 \\ \mathrm{H} & -10.023845 & 0.477385 & -3.331121 \\ \mathrm{H} & 10.024420 & -0.479992 & -3.329609 \\ \mathrm{H} & 10.024434 & 0.479647 & 3.330481 \\ \mathrm{H} & -7.856219 & -2.192344 & 4.741083 \\ \mathrm{H} & -7.480379 & -1.016003 & 6.003238 \\ \mathrm{H} & -9.166400 & -1.316141 & 5.535817 \\ \mathrm{H} & -9.806376 & 1.385546 & 0.205814 \\ \mathrm{H} & -10.894789 & 0.486710 & 1.278872 \\ \mathrm{H} & -10.651285 & 2.222529 & 1.506012 \\ \mathrm{H} & 9.805897 & 1.384675 & -0.205000 \\ \mathrm{H} & 10.895467 & 0.487321 & -1.278120 \\ \mathrm{H} & 10.649983 & 2.222933 & -1.504881 \\ \mathrm{H} & 7.484296 & -1.019887 & -6.002443 \\ \mathrm{H} & 9.168693 & -1.322631 & -5.530892 \\ \mathrm{H} & 7.855108 & -2.195908 & -4.738547 \\ \mathrm{H} & 7.483208 & 1.020179 & 6.002381 \\ \mathrm{H} & 9.168131 & 1.321921 & 5.532053 \\ \mathrm{H} & 7.855636 & 2.195930 & 4.738705 \\ \mathrm{H} & 10.895436 & -0.487371 & 1.278492 \\ \mathrm{H} & 10.651061 & -2.222857 & 1.507202 \\ \mathrm{H} & 9.806626 & -1.386652 & 0.206199 \\ \mathrm{H} & -9.166967 & 1.316253 & -5.534861 \\ \mathrm{H} & -7.856827 & 2.192454 & -4.740074 \\ \mathrm{H} & -7.480958 & 1.016373 & -6.002449 \\ \mathrm{H} & -9.806260 & -1.386248 & -0.205237 \\ \mathrm{H} & -10.894897 & -0.487538 & -1.278167 \\ \mathrm{H} & -10.651121 & -2.223304 & -1.505427\end{array}$

\begin{tabular}{|c|c|c|c|}
\hline \multicolumn{4}{|c|}{ N"5 } \\
\hline $\mathrm{Rh}$ & -0.719944 & -0.398358 & -0.889542 \\
\hline Rh & -0.452274 & -1.017983 & 1.394681 \\
\hline $\mathrm{O}$ & 1.264659 & -2.002250 & 0.795016 \\
\hline $\mathrm{O}$ & 0.594296 & -1.917539 & -1.361823 \\
\hline $\mathrm{O}$ & 0.621895 & 0.735913 & 1.641931 \\
\hline $\mathrm{O}$ & 0.845315 & 0.926930 & -0.598653 \\
\hline $\mathrm{O}$ & -2.219881 & 0.030464 & 1.758580 \\
\hline $\mathrm{O}$ & -2.043019 & 1.033644 & -0.260303 \\
\hline $\mathrm{O}$ & -1.526230 & -2.726105 & 0.943565 \\
\hline $\mathrm{O}$ & -2.252164 & -1.766691 & -0.972708 \\
\hline $\mathrm{Cl}$ & 1.516779 & -5.470500 & 0.975438 \\
\hline $\mathrm{Cl}$ & 0.509817 & 4.566877 & -0.541197 \\
\hline $\mathrm{Cl}$ & -5.846372 & 0.937643 & -0.764261 \\
\hline $\mathrm{Cl}$ & -2.116716 & -4.989963 & -2.828286 \\
\hline $\mathrm{C}$ & 2.553545 & -3.159150 & -0.816962 \\
\hline $\mathrm{C}$ & 3.238510 & -2.993828 & -2.222241 \\
\hline $\mathrm{C}$ & 2.307962 & -4.135656 & -1.953013 \\
\hline $\mathrm{C}$ & 1.387404 & -2.292133 & -0.442886 \\
\hline $\mathrm{C}$ & 3.388629 & -3.553183 & 0.362974 \\
\hline $\mathrm{C}$ & 3.008736 & -4.570313 & 1.247197 \\
\hline $\mathrm{C}$ & 3.763296 & -4.877254 & 2.379287 \\
\hline $\mathrm{C}$ & 4.921783 & -4.151549 & 2.648383 \\
\hline $\mathrm{C}$ & 5.329486 & -3.121472 & 1.791099 \\
\hline $\mathrm{C}$ & 4.552314 & -2.840530 & 0.665912 \\
\hline $\mathrm{C}$ & 4.728586 & -3.253985 & -2.304683 \\
\hline $\mathrm{C}$ & 5.286364 & -4.501607 & -1.987086 \\
\hline $\mathrm{C}$ & 6.663249 & -4.712513 & -2.040279 \\
\hline $\mathrm{C}$ & 7.519391 & -3.677060 & -2.418412 \\
\hline$C^{2}$ & 6.979195 & -2.431798 & -2.740524 \\
\hline
\end{tabular}




\begin{tabular}{|c|c|c|c|}
\hline & 5.601054 & -2.221974 & -2.687038 \\
\hline $\mathrm{C}$ & 2.758916 & -1.924218 & -3.168856 \\
\hline $\mathrm{C}$ & 2.440057 & -2.290724 & -4.481741 \\
\hline $\mathrm{C}$ & 2.014910 & -1.334512 & -5.404523 \\
\hline $\mathrm{C}$ & 1.893558 & 0.002706 & -5.016571 \\
\hline $\mathrm{C}$ & 2.215256 & 0.374924 & -3.709773 \\
\hline $\mathrm{C}$ & 2.663164 & -0.580833 & -2.798838 \\
\hline $\mathrm{C}$ & 6.552455 & -2.324698 & 2.059536 \\
\hline $\mathrm{C}$ & 7.599161 & -2.316974 & 1.128188 \\
\hline $\mathrm{C}$ & 8.770337 & -1.593098 & 1.373895 \\
\hline $\mathrm{C}$ & 8.853102 & -0.830288 & 2.546433 \\
\hline $\mathrm{C}$ & 7.805099 & -0.792524 & 3.474337 \\
\hline $\mathrm{C}$ & 6.665343 & -1.567501 & 3.231354 \\
\hline $\mathrm{C}$ & 2.206227 & 2.294352 & 0.766634 \\
\hline $\mathrm{C}$ & 3.234387 & 2.206318 & 1.957523 \\
\hline $\mathrm{C}$ & 2.116772 & 3.202095 & 1.976306 \\
\hline $\mathrm{C}$ & 1.148963 & 1.240348 & 0.600310 \\
\hline $\mathrm{C}$ & 2.667836 & 2.855375 & -0.543770 \\
\hline $\mathrm{C}$ & 1.977568 & 3.868990 & -1.219212 \\
\hline $\mathrm{C}$ & 2.400603 & 4.331797 & -2.466227 \\
\hline $\mathrm{C}$ & 3.526668 & 3.771017 & -3.063339 \\
\hline $\mathrm{C}$ & 4.232259 & 2.740214 & -2.425020 \\
\hline $\mathrm{C}$ & 3.782177 & 2.303615 & -1.176437 \\
\hline $\mathrm{C}$ & 4.652062 & 2.659499 & 1.686086 \\
\hline $\mathrm{C}$ & 4.963092 & 3.976982 & 1.321160 \\
\hline $\mathrm{C}$ & 6.275403 & 4.354889 & 1.038349 \\
\hline $\mathrm{C}$ & 7.310605 & 3.422230 & 1.117639 \\
\hline $\mathrm{C}$ & 7.017494 & 2.108388 & 1.486677 \\
\hline $\mathrm{C}$ & 5.703918 & 1.733026 & 1.769936 \\
\hline $\mathrm{C}$ & 3.149246 & 1.073863 & 2.947213 \\
\hline $\mathrm{C}$ & 3.115818 & -0.265088 & 2.556745 \\
\hline $\mathrm{C}$ & 3.022165 & -1.280379 & 3.507903 \\
\hline $\mathrm{C}$ & 3.009018 & -0.967154 & 4.868773 \\
\hline $\mathrm{C}$ & 3.087131 & 0.369185 & 5.271649 \\
\hline $\mathrm{C}$ & 3.151067 & 1.381863 & 4.313769 \\
\hline $\mathrm{C}$ & 5.408415 & 2.088760 & -3.040940 \\
\hline $\mathrm{C}$ & 6.519946 & 1.772396 & -2.249778 \\
\hline $\mathrm{C}$ & 7.632000 & 1.126961 & -2.795743 \\
\hline $\mathrm{C}$ & 7.611596 & 0.789541 & -4.154250 \\
\hline $\mathrm{C}$ & 6.506607 & 1.079516 & -4.965546 \\
\hline $\mathrm{C}$ & 5.412056 & 1.738058 & -4.397734 \\
\hline $\mathrm{C}$ & -3.631006 & 1.896288 & 1.244194 \\
\hline $\mathrm{C}$ & -3.707275 & 2.503071 & 2.696861 \\
\hline $\mathrm{C}$ & -4.706154 & 1.492466 & 2.228675 \\
\hline $\mathrm{C}$ & -2.554199 & 0.908588 & 0.904010 \\
\hline $\mathrm{C}$ & -3.972552 & 2.776591 & 0.079971 \\
\hline $\mathrm{C}$ & -4.936447 & 2.442758 & -0.877255 \\
\hline $\mathrm{C}$ & -5.172485 & 3.259959 & -1.984746 \\
\hline $\mathrm{C}$ & -4.423751 & 4.419936 & -2.158547 \\
\hline $\mathrm{C}$ & -3.427095 & 4.773532 & -1.234198 \\
\hline $\mathrm{C}$ & -3.225539 & 3.936911 & -0.133382 \\
\hline $\mathrm{C}$ & -4.083526 & 3.963759 & 2.793152 \\
\hline $\mathrm{C}$ & -5.328609 & 4.447876 & 2.367998 \\
\hline $\mathrm{C}$ & -5.621943 & 5.810549 & 2.407628 \\
\hline $\mathrm{C}$ & -4.677187 & 6.722941 & 2.879524 \\
\hline $\mathrm{C}$ & -3.438250 & 6.253801 & 3.319177 \\
\hline $\mathrm{C}$ & -3.147198 & 4.890241 & 3.277622 \\
\hline $\mathrm{C}$ & -2.733862 & 2.047970 & 3.751898 \\
\hline $\mathrm{C}$ & -1.350525 & 2.099715 & 3.567024 \\
\hline $\mathrm{C}$ & -0.482677 & 1.659659 & 4.565572 \\
\hline $\mathrm{C}$ & -0.990744 & 1.186698 & 5.777143 \\
\hline & -2.372076 & 1.158844 & 5.984165 \\
\hline
\end{tabular}

\begin{tabular}{|c|c|c|c|c|c|c|c|}
\hline E & -3.236217 & 1.586417 & 4.974632 & $\mathrm{H}$ & 1.847172 & 5.123162 & -2.959439 \\
\hline & -2.580638 & 5.976073 & -1.404626 & $\mathrm{H}$ & 3.861299 & 4.139202 & -4.027715 \\
\hline & -2.193455 & 6.725899 & -0.284719 & $\mathrm{H}$ & 4.304170 & 1.494142 & -0.681799 \\
\hline & -1.338598 & 7.823984 & -0.412027 & $\mathrm{H}$ & 4.180685 & 4.721638 & \\
\hline & -0.881478 & 8.174937 & -1.687142 & $\mathrm{H}$ & 6.485648 & 5.381785 & 0.755086 \\
\hline & -1.262265 & 7.453535 & -2.825649 & $\Pi$ & 8.331888 & 3.714802 & 0.893948 \\
\hline & -2.113000 & 6.354974 & -2.670427 & $\mathrm{H}$ & 7.805063 & 1.365930 & 6513 \\
\hline & -3.334934 & -3.728227 & -0.250588 & $\mathrm{H}$ & 5.501997 & 0.708737 & 9269 \\
\hline & -4.088393 & -4.292554 & 1.013457 & $\mathrm{H}$ & 3.117495 & -0.524806 & 6366 \\
\hline $\mathrm{C}$ & -3.040912 & -5.101315 & 0.315005 & $\mathrm{H}$ & 2.958918 & -2.309837 & 3.179099 \\
\hline & -2.292849 & -2.668451 & -0.069663 & $\mathrm{H}$ & 2.942170 & 58001 & 0047 \\
\hline & -4.085126 & -3.568675 & -1.5 & $\mathrm{H}$ & 3.086203 & 357 & \\
\hline $\mathrm{C}$ & -3.631637 & -4.088858 & -2.75 & $\mathrm{H}$ & 3.193086 & 2299 & 592 \\
\hline $\mathrm{C}$ & -4.324738 & -3.866731 & -3.94 & $\mathrm{H}$ & 6.524394 & 6616 & 938 \\
\hline $\mathrm{C}$ & -5.482249 & -3.093743 & -3.931340 & $\mathrm{H}$ & & & 699 \\
\hline & -5.951518 & -2.52 & -2.7 & $\mathrm{H}$ & 3884 & 058 & 982 \\
\hline $\mathrm{C}$ & -5.234043 & -2.7 & -1.5 & $\mathrm{H}$ & 575 & & \\
\hline $\mathrm{C}$ & -5.551950 & -4.629819 & 0.83 & $\mathrm{H}$ & 4617 & 283 & 529 \\
\hline $\mathrm{C}$ & -5.989901 & -5.581782 & -0.101224 & $\mathrm{H}$ & -4.622340 & 484 & 2408 \\
\hline $\mathrm{C}$ & -7.347949 & -5.829109 & -0.295566 & $\mathrm{H}$ & -2.446458 & 7007 & 310 \\
\hline $\mathrm{C}$ & -8.305846 & -5.133501 & 0.4 & $\mathrm{H}$ & -6.0 & & 12 \\
\hline $\mathrm{C}$ & -7.885633 & -4.1 & & $\mathrm{H}$ & 638 & 584 & 800 \\
\hline $\mathrm{C}$ & -6.525659 & -3.9 & $1.5^{\circ}$ & $\mathrm{H}$ & 4320 & 043 & 176 \\
\hline $\mathrm{C}$ & -3.738142 & -3.7 & 2.37 & $\mathrm{H}$ & 3213 & & 779 \\
\hline $\mathrm{C}$ & -3.311781 & -4.6 & 3.37 & $\mathrm{H}$ & 0492 & & 26 \\
\hline $\mathrm{C}$ & -2.990598 & -4.1 & & $\mathrm{H}$ & -0.5 & & \\
\hline $\mathrm{C}$ & -3.092513 & -2.8 & & $\mathrm{H}$ & & & \\
\hline $\mathrm{C}$ & -3.525497 & -1.9 & 3.9 & $\mathrm{H}$ & 876 & & 534 \\
\hline $\mathrm{C}$ & -3.854746 & -2.3 & 2.68 & $\mathrm{H}$ & 502 & & \\
\hline $\mathrm{C}$ & -7.156290 & -1.6 & -2.6 & $\mathrm{H}$ & & & 05 \\
\hline $\mathrm{C}$ & -7.441 & -0.7 & -3.7 & $\mathrm{H}$ & & 60 & 204 \\
\hline $\mathrm{C}$ & -8.549 & 0.0 & -3.6 & $\mathrm{H}$ & 886 & & 774 \\
\hline C & -9.375841 & 0.02 & -2.5 & $\mathrm{H}$ & 094 & & 176 \\
\hline $\mathrm{C}$ & -9.118890 & -0.8 & & $\mathrm{H}$ & & & 756 \\
\hline $\mathrm{C}$ & -8.009543 & -1.7 & -1.5 & $\mathrm{H}$ & -3 & & 458 \\
\hline $\mathrm{C}$ & -0.772074 & & -4.1 & $\mathrm{H}$ & -6 . & & 899 \\
\hline $\mathrm{C}$ & -0.894304 & 8.5 & & $\mathrm{H}$ & 005 & & 824 \\
\hline $\mathrm{C}$ & 8.814638 & 0.80 & -1.9 & $\mathrm{H}$ & 073 & & .771 \\
\hline $\mathrm{C}$ & 6.487783 & & & $\mathrm{H}$ & & & \\
\hline $\mathrm{C}$ & 7.8695 & & & $\mathrm{H}$ & & & 97 \\
\hline $\mathrm{C}$ & 9.937 & $-1.6^{\circ}$ & 0.4 & & -8 & & 274 \\
\hline $\mathrm{C}$ & -10.002658 & -0.899556 & $5-0$. & $\mathrm{H}$ & 20 & & 452 \\
\hline $\mathrm{C}$ & -8.855084 & 1.037138 & -4.79 & $\mathrm{H}$ & 288 & & 795 \\
\hline $\mathrm{H}$ & 2.699491 & -5.1 & -1.8 & $\mathrm{H}$ & 257 & & 438 \\
\hline $\mathrm{H}$ & 1.326 & & & $\mathrm{H}$ & & & \\
\hline $\mathrm{H}$ & 3.441 & -5.6 & & $\mathrm{H}$ & 062 & & 751 \\
\hline $\mathrm{H}$ & 5.512303 & -4.38 & & $\mathrm{H}$ & 3644 & 299 & 9746 \\
\hline $\mathrm{H}$ & 4.854127 & -2.035006 & 0.00 & $\mathrm{H}$ & 2026 & 141 & 1616 \\
\hline $\mathrm{H}$ & 4.652631 & & & $\mathrm{H}$ & 5569 & & 2647 \\
\hline $\mathrm{H}$ & 7.06 & & & & & & -1.7 \\
\hline $\mathrm{H}$ & 8.592 & -3.8 & -2.4 & $\mathrm{H}$ & 5814 & 36807 & $7 \quad-2.47049$ \\
\hline $\mathrm{H}$ & 7.623944 & -1.615671 & -3.04 & $\mathrm{H}$ & 8.466972 & 0.276396 & -4.587791 \\
\hline $\mathrm{H}$ & 5.206780 & -1.24 & -2.9 & $\mathrm{H}$ & 9.751744 & -0.247872 & 2.738148 \\
\hline $\mathrm{H}$ & 2.520830 & -3.3 & & $\mathrm{H}$ & & & \\
\hline $\mathrm{H}$ & 1.771802 & -1.6 & & $\mathrm{H}$ & -0.8 & & -4.8 \\
\hline $\mathrm{H}$ & 1.551098 & 0.749041 & -5.727362 & $\mathrm{H}$ & 5015 & & -4.613045 \\
\hline $\mathrm{H}$ & 2.117914 & 1.405940 & -3.393738 & $\mathrm{H}$ & -1.635512 & 8.522393 & 1.611363 \\
\hline $\mathrm{H}$ & 2.894043 & -0.279967 & -1.784051 & $\mathrm{H}$ & 0.047380 & 8.186322 & 1.201682 \\
\hline $\mathrm{H}$ & 7.507538 & -2.905676 & & $\mathrm{H}$ & -0.725829 & & \\
\hline $\mathrm{H}$ & 5.839303 & -1.550995 & 3.936066 & & 8.488046 & 0.320135 & -0.99 \\
\hline$\Pi$ & 2.323357 & 4.252223 & 1.812519 & $\mathrm{H}$ & 9.519708 & 0.134814 & -2.419423 \\
\hline & 1.302219 & 3.006838 & 2.662102 & $\mathrm{H}$ & 9.358498 & 1.710436 & -1.632031 \\
\hline
\end{tabular}




$\begin{array}{clcc}\mathrm{H} & 6.703281 & 1.545829 & -7.068753 \\ \mathrm{H} & 7.233653 & -0.083652 & -6.637291 \\ \mathrm{H} & 5.504840 & 0.301916 & -6.712686 \\ \mathrm{H} & 7.272928 & -0.278292 & 5.506621 \\ \mathrm{H} & 8.897677 & 0.252363 & 5.024580 \\ \mathrm{H} & 7.470239 & 1.104191 & 4.427015 \\ \mathrm{H} & 10.460938 & -0.716416 & 0.342370 \\ \mathrm{H} & 10.667818 & -2.416664 & 0.763027 \\ \mathrm{H} & 9.612155 & -1.970509 & -0.580330 \\ \mathrm{H} & -11.049151 & -0.708187 & -0.516616 \\ \mathrm{H} & -9.941385 & -1.866767 & 0.244966 \\ \mathrm{H} & -9.698295 & -0.132088 & 0.460661 \\ \mathrm{H} & -7.948110 & 1.310241 & -5.338267 \\ \mathrm{H} & -9.547857 & 0.585692 & -5.512346 \\ \mathrm{H} & -9.323321 & 1.954225 & -4.422386\end{array}$

$\begin{array}{lrrr}\text { O1 } & & & \\ \mathrm{Rh} & 0.344347 & -0.295649 & -0.531840 \\ \mathrm{Rh} & 0.651166 & -1.226000 & 1.641793 \\ \mathrm{O} & -0.762043 & -1.973172 & -1.005270 \\ \mathrm{O} & 0.091022 & -3.033596 & 0.800794 \\ \mathrm{O} & 1.485584 & 1.296014 & 0.110768 \\ \mathrm{O} & 1.227007 & 0.664510 & 2.266280 \\ \mathrm{O} & 2.073057 & -1.268969 & -1.073692 \\ \mathrm{O} & 2.604700 & -1.616116 & 1.096405 \\ \mathrm{O} & -1.348477 & 0.645083 & 0.177405 \\ \mathrm{O} & -1.328053 & -0.682556 & 2.005734 \\ \mathrm{Cl} & -0.142619 & -5.001659 & -3.476911 \\ \mathrm{Cl} & 4.943847 & 2.131179 & 0.140453 \\ \mathrm{Cl} & 3.248089 & -4.264464 & -2.595479 \\ \mathrm{Cl} & -1.255707 & 3.448540 & 1.983354 \\ \mathrm{C} & -1.358543 & -4.236303 & -0.658124 \\ \mathrm{C} & -1.908376 & -5.206764 & 0.455453 \\ \mathrm{C} & -3.450646 & -3.467965 & -1.761010 \\ \mathrm{C} & -4.285568 & -3.222842 & -2.850444 \\ \mathrm{C} & -2.188796 & -4.059218 & -1.893136 \\ \mathrm{C} & -3.220560 & -5.896537 & 0.153512 \\ \mathrm{C} & -2.590025 & -4.180369 & -4.295952 \\ \mathrm{C} & -1.725590 & -4.866310 & 1.910251 \\ \mathrm{C} & -1.968855 & -3.362048 & 3.797924 \\ \mathrm{C} & -0.622689 & -2.994531 & -0.251388 \\ \mathrm{C} & -3.329640 & -6.919784 & -0.797129 \\ \mathrm{C} & -1.140151 & -5.811470 & 2.760004 \\ \mathrm{C} & -0.692324 & -5.559540 & -0.336116 \\ \mathrm{C} & -0.959893 & -5.533663 & 4.116628 \\ \mathrm{C} & -1.767622 & -4.384088 & -3.186261 \\ \mathrm{C} & -4.383212 & -5.502089 & 0.833984 \\ \mathrm{C} & -5.705705 & -7.123886 & -0.379223 \\ \mathrm{C} & -3.846194 & -3.606007 & -4.125149 \\ \mathrm{C} & -2.153510 & -3.649620 & 2.447237 \\ \mathrm{C} & -1.369126 & -4.303652 & 4.637469 \\ \mathrm{C} & -4.557434 & -7.527899 & -1.060982 \\ \mathrm{C} & -5.611998 & -6.103373 & 0.569083 \\ \mathrm{C} & 2.340941 & 2.726606 & 1.779709 \\ \mathrm{C} & 3.224946 & 2.784145 & 3.069654 \\ \mathrm{C} & 1.720853 & 4.538159 & 0.186528 \\ \mathrm{C} & 1.860329 & 5.271836 & -0.993606 \\ \mathrm{C} & 2.660918 & 3.592233 & 0.598124 \\ \mathrm{C} & 4.414528 & 3.718208 & 3.045578 \\ \mathrm{C} & 3.997798 & 4.183370 & -1.352337 \\ \mathrm{C} & 3.425862 & 1.530566 & 3.878777 \\ \mathrm{C} & 3.338021 & 0.413537 & 6.032862 \\ & & & \end{array}$

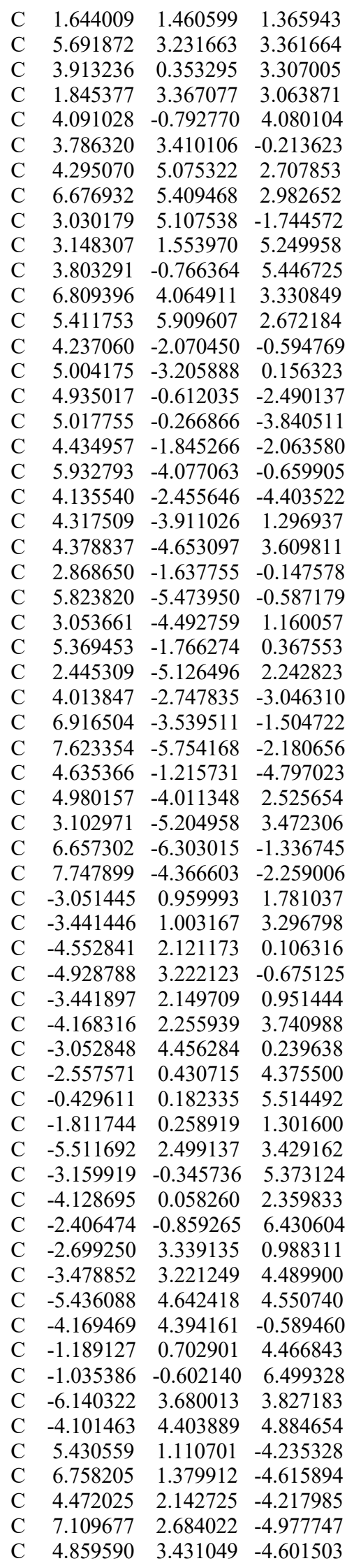

\begin{tabular}{|c|c|c|c|}
\hline$C$ & 6.174294 & 3.723797 & -4.976332 \\
\hline & 0.723121 & 6.118567 & -1.454663 \\
\hline & -0.419792 & 5.484499 & -1.984745 \\
\hline & 0.765687 & 7.519982 & -1.335034 \\
\hline $\mathrm{C}$ & -1.503277 & 6.271737 & -2.387338 \\
\hline $\mathrm{C}$ & -0.342112 & 8.270453 & -1.741954 \\
\hline $\mathrm{C}$ & -1.488488 & 7.664209 & -2.265697 \\
\hline $\mathrm{C}$ & -6.094621 & 3.131255 & -1.599126 \\
\hline$C$ & -7.401234 & 3.009023 & -1.086503 \\
\hline $\mathrm{C}$ & -5.884705 & 3.148644 & -2.995927 \\
\hline$C$ & -8.474896 & 2.902139 & -1.977077 \\
\hline $\mathrm{C}$ & -6.982605 & 3.012388 & -3.849123 \\
\hline $\mathrm{C}$ & -8.286479 & 2.885429 & -3.360391 \\
\hline $\mathrm{C}$ & -5.591103 & -2.535155 & -2.639701 \\
\hline $\mathrm{C}$ & -6.794925 & -3.261944 & -2.668639 \\
\hline $\mathrm{C}$ & -5.603669 & -1.148744 & -2.382711 \\
\hline $\mathrm{C}$ & -7.996488 & -2.590136 & -2.415982 \\
\hline $\mathrm{C}$ & -6.824928 & -0.510301 & -2.147838 \\
\hline $\mathrm{C}$ & -8.032457 & -1.219024 & -2.150974 \\
\hline $\mathrm{C}$ & -7.666775 & 2.978587 & 0.399971 \\
\hline $\mathrm{C}$ & -4.503950 & 3.310419 & -3.590196 \\
\hline $\mathrm{C}$ & -9.449913 & 2.711194 & -4.304781 \\
\hline $\mathrm{C}$ & 1.982231 & 8.205448 & -0.759640 \\
\hline $\mathrm{C}$ & -0.489959 & 3.980777 & -2.123443 \\
\hline $\mathrm{C}$ & -2.690572 & 8.486013 & -2.662139 \\
\hline $\mathrm{C}$ & 7.790589 & 0.277926 & -4.624615 \\
\hline $\mathrm{C}$ & 3.049669 & 1.876343 & -3.782824 \\
\hline $\mathrm{C}$ & 6.578182 & 5.131778 & -5.340065 \\
\hline $\mathrm{C}$ & -4.319029 & -0.352395 & -2.371011 \\
\hline $\mathrm{C}$ & -6.798719 & -4.744159 & -2.954556 \\
\hline $\mathrm{C}$ & -9.341760 & -0.514279 & -1.892175 \\
\hline $\mathrm{H}$ & -3.786262 & -3.189181 & -0.768329 \\
\hline $\mathrm{H}$ & -2.235205 & -4.451919 & -5.283663 \\
\hline $\mathrm{H}$ & -2.273874 & -2.399089 & 4.187710 \\
\hline $\mathrm{H}$ & -2.457193 & -7.256404 & -1.344032 \\
\hline $\mathrm{H}$ & -0.815374 & -6.764516 & 2.351755 \\
\hline $\mathrm{H}$ & -0.496216 & -6.274261 & 4.761662 \\
\hline $\mathrm{H}$ & -4.324693 & -4.720521 & 1.582653 \\
\hline $\mathrm{H}$ & -6.661161 & -7.595898 & -0.585618 \\
\hline $\mathrm{H}$ & -2.580134 & -2.895615 & 1.795773 \\
\hline $\mathrm{H}$ & -1.222708 & -4.075564 & 5.689198 \\
\hline $\mathrm{H}$ & -4.612554 & -8.321198 & -1.800305 \\
\hline $\mathrm{H}$ & -6.496277 & -5.774861 & 1.106703 \\
\hline $\mathrm{H}$ & -4.479684 & -3.432469 & -4.989164 \\
\hline $\mathrm{H}$ & 0.259840 & -5.487701 & 0.174430 \\
\hline $\mathrm{H}$ & -0.739206 & -6.331975 & -1.091151 \\
\hline $\mathrm{H}$ & 0.821119 & 4.661474 & 0.777281 \\
\hline $\mathrm{H}$ & 4.881557 & 4.023833 & -1.957440 \\
\hline $\mathrm{H}$ & 3.114269 & 0.444551 & 7.095089 \\
\hline $\mathrm{H}$ & 5.813477 & 2.189107 & 3.629139 \\
\hline $\mathrm{H}$ & 4.110424 & 0.327854 & 2.242938 \\
\hline $\mathrm{H}$ & 4.432650 & -1.710551 & 3.614975 \\
\hline $\mathrm{H}$ & 3.331047 & 5.497219 & 2.452710 \\
\hline $\mathrm{H}$ & 7.546096 & 6.059393 & 2.956241 \\
\hline $\mathrm{H}$ & 2.772344 & 2.467692 & 5.701342 \\
\hline $\mathrm{H}$ & 3.940259 & -1.659544 & 6.049084 \\
\hline $\mathrm{H}$ & 7.785505 & 3.658341 & 2578550 \\
\hline$H$ & 5.287523 & 6.954099 & 2.402673 \\
\hline $\mathrm{H}$ & 3.171167 & 5.672529 & -2.660494 \\
\hline $\mathrm{H}$ & 1.717547 & 4.442830 & 3.071361 \\
\hline $\mathrm{H}$ & 1.090814 & 2.813413 & 3.608775 \\
\hline $\mathrm{H}$ & 5.221273 & 0.117024 & -1.740174 \\
\hline
\end{tabular}




\begin{tabular}{|c|c|c|c|}
\hline & 3.813711 & -3.181911 & -5.141133 \\
\hline $\mathrm{H}$ & 4.901991 & -4.713964 & 4.559463 \\
\hline & 5.074690 & -5.916506 & 0.058029 \\
\hline & 2.528700 & -4.412791 & 0.215281 \\
\hline & 1.448468 & -5.539291 & 2.138427 \\
\hline & 7.038055 & -2.467557 & -1.598452 \\
\hline & 8.272069 & -6.397885 & -2.766834 \\
\hline & 5.968019 & -3.572063 & 2.632770 \\
\hline & 2.622717 & -5.692953 & 4.315217 \\
\hline & 6.547355 & -7.380684 & -1.260260 \\
\hline & 8.496117 & -3.920305 & -2.907236 \\
\hline & 4.700950 & -0.973094 & -5.8 \\
\hline & 6.293532 & -1.386699 & 51517 \\
\hline & 5.082795 & -1.325787 & 1.312631 \\
\hline & -5.133982 & 1.208236 & 0.040982 \\
\hline $\mathrm{H}$ & -2.451262 & 5.355779 & 0.288252 \\
\hline & 0.634964 & 0.388 & \\
\hline & -6.085662 & 1.769659 & 1901 \\
\hline & -4.224858 & -0.552317 & 5.313838 \\
\hline & -2.888303 & -1.464963 & 7.192196 \\
\hline & -2.446058 & 3.043436 & 4.7 \\
\hline & -5.923127 & 5.562609 & \\
\hline & -0.705107 & 1.287210 & 3.696838 \\
\hline & -0.443808 & -1.006547 & 7.315278 \\
\hline & -7.183353 & 1470 & 2700 \\
\hline & -3.542164 & 476 & 5.4 \\
\hline 11 & -4.446466 & 5.262377 & -1. \\
\hline & -3.859712 & -0.987177 & 2.452732 \\
\hline П & -5.153038 & 0.232004 & 2.056003 \\
\hline & 8.136639 & 1885 & -5.2 \\
\hline & 4.116048 & 1083 & -4.6 \\
\hline П & -2.379673 & 415 & -2.8 \\
\hline П & -0.310150 & 9.353039 & 3239 \\
\hline $\mathrm{H}$ & -9.482032 & 2.818815 & -1.577149 \\
\hline $\mathrm{H}$ & -6.814550 & 3.005299 & -4.9 \\
\hline & -8.925822 & -3.155138 & -2.4 \\
\hline H & -6.836002 & 9092 & -1.9 \\
\hline H & -7.504684 & 4690 & 0120 \\
\hline $\mathrm{H}$ & -7.006503 & 3.654818 & \\
\hline H & -8.703234 & 3.253059 & 0.6 \\
\hline & -4.198541 & 4.363144 & -3.6 \\
\hline $\mathrm{H}$ & -3.745020 & 2.768702 & -3.020230 \\
\hline $\mathrm{H}$ & -4.483798 & 2.950267 & -4.621731 \\
\hline H & -10.404483 & 32.860947 & -3.79350 \\
\hline $\mathrm{H}$ & -9.394709 & 3.414067 & -5.142087 \\
\hline H & -9.457617 & 1.701685 & -4.733623 \\
\hline $\mathrm{H}$ & 2.250249 & 7.785113 & 0.215515 \\
\hline $\mathrm{H}$ & 2.858452 & 8.080253 & -1.405099 \\
\hline $\mathrm{H}$ & 1.805983 & 9.276702 & -0.635558 \\
\hline $\mathrm{H}$ & 0.415584 & 3.577625 & -2.587338 \\
\hline $\mathrm{H}$ & -0.590156 & 3.490969 & -1.149897 \\
\hline $\mathrm{H}$ & -1.350817 & 3.689293 & -2.730004 \\
\hline $\mathrm{H}$ & -2.398376 & 9.480425 & -3.011743 \\
\hline $\mathrm{H}$ & -3.263201 & 7.996827 & -3.455491 \\
\hline $\mathrm{H}$ & -3.368416 & 8.626680 & -1.811062 \\
\hline $\mathrm{H}$ & 7.869657 & -0.199099 & -3.641559 \\
\hline $\mathrm{H}$ & 7.526437 & -0.513540 & -5.334266 \\
\hline $\mathrm{H}$ & 8.775268 & 0.664733 & -4.897768 \\
\hline & 2.618447 & 1.024016 & -4.317358 \\
\hline $\mathrm{H}$ & 2.999836 & 1.640968 & -2.714112 \\
\hline $\mathrm{H}$ & 2.420140 & 2.751884 & -3.958077 \\
\hline & 6.914101 & 5.685471 & -4.454644 \\
\hline
\end{tabular}

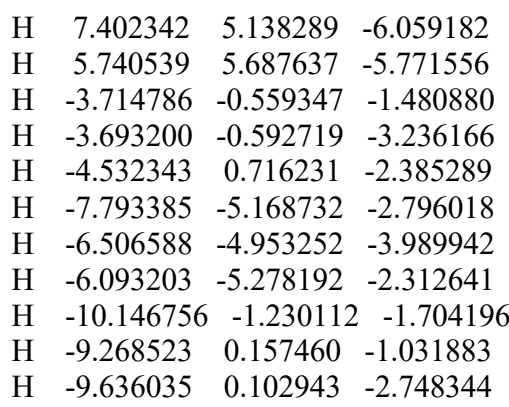

\section{O'1}

$\begin{array}{llll}\mathrm{Rh} & 0.000945 & -0.000149 & -2.316799\end{array}$ $\begin{array}{llll}\mathrm{Rh} & 0.000448 & -0.000087 & 0.068304\end{array}$ $\begin{array}{lllll}\text { O } & -1.669345 & -1.180784 & -0.024969\end{array}$ $\begin{array}{lllll}\mathrm{O} & -1.973519 & -0.623007 & -2.196324\end{array}$

$\begin{array}{llll}\text { O } & 1.181198 & -1.669797 & -0.024667\end{array}$

$\begin{array}{llll}\text { O } & 0.624015 & -1.974328 & -2.196000\end{array}$

$\begin{array}{llll}\mathrm{O} & 1.670150 & 1.180617 & -0.024523\end{array}$

$\begin{array}{lllll}\mathrm{O} & 1.975161 & 0.622817 & -2.195672\end{array}$

$\begin{array}{lllll}\text { O } & -1.180300 & 1.669665 & -0.024925\end{array}$

$\begin{array}{llll}\mathrm{O} & -0.621719 & 1.974389 & -2.195897\end{array}$

$\begin{array}{lllll}\mathrm{Cl} & -2.553888 & -4.564129 & -0.002624\end{array}$

$\begin{array}{llll}\text { Cl } & 4.564835 & -2.554596 & -0.000943\end{array}$

$\begin{array}{lllll}\mathrm{Cl} & 2.553549 & 4.564212 & -0.001767\end{array}$

$\begin{array}{lllll}\mathrm{Cl} & -4.563807 & 2.553061 & -0.003551\end{array}$

$\begin{array}{llll}\text { C } & -3.687526 & -1.828201 & -1.035883\end{array}$

$\begin{array}{llll}\text { C } & -4.279892 & -2.580132 & -2.269351\end{array}$

$\begin{array}{llll}\text { C } & -4.772275 & -1.212152 & -1.897995\end{array}$

$\begin{array}{llll}\text { C } & -2.340381 & -1.171264 & -1.110075\end{array}$

$\begin{array}{llll}\text { C } & -4.004689 & -2.255898 & 0.364949\end{array}$

$\begin{array}{llll}\text { C } & -3.545877 & -3.449594 & 0.933205\end{array}$

$\begin{array}{llll}\text { C } & -3.787846 & -3.761458 & 2.268592\end{array}$

C $\quad-4.428252-2.830518 \quad 3.084222$

$\begin{array}{llll}\text { C } & -4.833480 & -1.592348 & 2.569866\end{array}$

$\begin{array}{llll}\text { C } & -4.646856 & -1.345258 & 1.208059\end{array}$

$\begin{array}{llll}\text { C } & -5.161286 & -3.775828 & -1.986428\end{array}$

$\begin{array}{llll}\text { C } & -4.888603 & -5.006649 & -2.601950\end{array}$

$\begin{array}{llll}\text { C } & -5.672697 & -6.130896 & -2.345637\end{array}$

$\begin{array}{llll}\text { C } & -6.751759 & -6.052006 & -1.464812\end{array}$

$\begin{array}{llll}\text { C } & -7.039131 & -4.832895 & -0.849690\end{array}$

C $\quad-6.256421 \quad-3.708956 \quad-1.110802$

$\begin{array}{llll}\text { C } & -3.445550 & -2.687745 & -3.519093\end{array}$

$\begin{array}{llll}\text { C } & -3.988048 & -2.262649 & -4.736777\end{array}$

$\begin{array}{llll}\text { C } & -3.242368 & -2.340102 & -5.914510\end{array}$

$\begin{array}{llll}\text { C } & -1.941534 & -2.848615 & -5.883976\end{array}$

$\begin{array}{llll}\text { C } & -1.400781 & -3.292134 & -4.674909\end{array}$

$\begin{array}{llll}\text { C } & -2.153186 & -3.220346 & -3.503376\end{array}$

$\begin{array}{llll}\text { C } & -5.357070 & -0.512846 & 3.454949\end{array}$

$\begin{array}{llll}\text { C } & -6.694405 & -0.522024 & 3.892507\end{array}$

$\begin{array}{llll}\text { C } & -7.145778 & 0.511814 & 4.718779\end{array}$

$\begin{array}{llll}\text { C } & -6.300613 & 1.549287 & 5.125324\end{array}$

$\begin{array}{llll}\text { C } & -4.973399 & 1.532551 & 4.686047\end{array}$

$\begin{array}{llll}\text { C } & -4.486611 & 0.524244 & 3.847071\end{array}$

$\begin{array}{llll}\text { C } & 1.829035 & -3.688090 & -1.034937\end{array}$

$\begin{array}{llll}\text { C } & 2.581284 & -4.280623 & -2.268013\end{array}$

C $\quad 1.213214 \quad-4.773083-1.897032$

$\begin{array}{llll}\text { C } & 1.171994 & -2.341059 & -1.109560\end{array}$

$\begin{array}{llll}\text { C } & 2.256208 & -4.004942 & 0.366161\end{array}$

$\begin{array}{llll}\text { C } & 3.449659 & -3.545992 & 0.934698\end{array}$

$\begin{array}{llll}\text { C } & 3.760917 & -3.787560 & 2.270376\end{array}$ $\begin{array}{llll}\text { C } & 2.829463 & -4.427422 & 3.085823\end{array}$

$\begin{array}{llll}\text { C } & 1.591422 & -4.832612 & 2.571180\end{array}$

$\begin{array}{llll}\text { C } & 1.344992 & -4.646748 & 1.209188\end{array}$

$\begin{array}{llll}\text { C } & 3.776907 & -5.161929 & -1.984475\end{array}$

$\begin{array}{llll}\text { C } & 5.007957 & -4.889518 & -2.599866\end{array}$

$\begin{array}{llll}\text { C } & 6.132135 & -5.673472 & -2.342964\end{array}$

$\begin{array}{llll}\text { C } & 6.053015 & -6.752327 & -1.461919\end{array}$

$\begin{array}{llll}\text { C } & 4.833675 & -7.039425 & -0.846953\end{array}$

$\begin{array}{llll}\text { C } & 3.709806 & -6.256845 & -1.108557\end{array}$

$\begin{array}{llll}\text { C } & 2.689253 & -3.446508 & -3.517739\end{array}$

$\begin{array}{llll}\text { C } & 2.264179 & -3.988992 & -4.735534\end{array}$

$\begin{array}{llll}\text { C } & 2.341962 & -3.243361 & -5.913326\end{array}$

$\begin{array}{llll}\text { C } & 2.850691 & -1.942608 & -5.882714\end{array}$

$\begin{array}{llll}\text { C } & 3.294190 & -1.401853 & -4.673637\end{array}$

$\begin{array}{llll}\text { C } & 3.222166 & -2.154160 & -3.502057\end{array}$

$\begin{array}{llll}\text { C } & 0.511510 & -5.355533 & 3.456179\end{array}$

$\begin{array}{llll}\text { C } & -0.525667 & -4.484653 & 3.847192\end{array}$

$\begin{array}{llll}\text { C } & -1.534281 & -4.970649 & 4.686167\end{array}$

$\begin{array}{llll}\text { C } & -1.551238 & -6.297477 & 5.126699\end{array}$

$\begin{array}{llll}\text { C } & -0.513676 & -7.143035 & 4.721212\end{array}$

$\begin{array}{llll}\text { C } & 0.520468 & -6.692480 & 3.894878\end{array}$

$\begin{array}{llll}\text { C } & 3.688437 & 1.828607 & -1.034710\end{array}$

$\begin{array}{llll}\text { C } & 4.281064 & 2.580712 & -2.267844\end{array}$

$\begin{array}{llll}\text { C } & 4.773832 & 1.212927 & -1.896300\end{array}$

$\begin{array}{llll}\text { C } & 2.341598 & 1.171242 & -1.109320\end{array}$

$\begin{array}{llll}\text { C } & 4.004879 & 2.256349 & 0.366361\end{array}$

$\begin{array}{llll}\text { C } & 3.545310 & 3.449798 & 0.934507\end{array}$

$\begin{array}{llll}\text { C } & 3.786271 & 3.761456 & 2.270222\end{array}$

$\begin{array}{llll}\text { C } & 4.426195 & 2.830442 & 3.086094\end{array}$

$\begin{array}{llll}\text { C } & 4.832052 & 1.592429 & 2.571849\end{array}$

$\begin{array}{llll}\text { C } & 4.646706 & 1.345613 & 1.209752\end{array}$

$\begin{array}{llll}\text { C } & 5.161871 & 3.776689 & -1.984566\end{array}$

$\begin{array}{llll}\text { C } & 4.888928 & 5.007499 & -2.600146\end{array}$

$\begin{array}{llll}\text { C } & 5.672387 & 6.132083 & -2.343457\end{array}$

$\begin{array}{llll}\text { C } & 6.751277 & 6.053593 & -1.462280\end{array}$

$\begin{array}{llll}\text { C } & 7.038908 & 4.834494 & -0.847101\end{array}$

$\begin{array}{llll}\text { C } & 6.256695 & 3.710286 & -1.108490\end{array}$

$\begin{array}{llll}\text { C } & 3.447203 & 2.687880 & -3.518006\end{array}$

$\begin{array}{llll}\text { C } & 3.990679 & 2.263083 & -4.735424\end{array}$

$\begin{array}{llll}\text { C } & 3.245385 & 2.339962 & -5.913495\end{array}$

$\begin{array}{llll}\text { C } & 1.944234 & 2.847415 & -5.883630\end{array}$

$\begin{array}{llll}\text { C } & 1.402489 & 3.290655 & -4.674794\end{array}$

$\begin{array}{llll}\text { C } & 2.154426 & 3.219639 & -3.502923\end{array}$

$\begin{array}{llll}\text { C } & 5.354930 & 0.512788 & 3.457168\end{array}$

$\begin{array}{llll}\text { C } & 4.484273 & -0.524491 & 3.848047\end{array}$

$\begin{array}{llll}\text { C } & 4.970175 & -1.532876 & 4.687473\end{array}$

$\begin{array}{llll}\text { C } & 6.296804 & -1.549227 & 5.128498\end{array}$

$\begin{array}{llll}\text { C } & 7.142234 & -0.511499 & 4.723141\end{array}$

$\begin{array}{llll}\text { C } & 6.691713 & 0.522261 & 3.896411\end{array}$

$\begin{array}{lllll}\text { C } & -1.827968 & 3.687806 & -1.035657\end{array}$

$\begin{array}{llll}\text { C } & -2.579720 & 4.280175 & -2.269285\end{array}$

$\begin{array}{llll}\text { C } & -1.211932 & 4.772897 & -1.897365\end{array}$

$\begin{array}{llll}\text { C } & -1.170517 & 2.340922 & -1.109835\end{array}$

$\begin{array}{llll}\text { C } & -2.256141 & 4.004596 & 0.365148\end{array}$

$\begin{array}{llll}\text { C } & -3.449834 & 3.545168 & 0.932912\end{array}$

$\begin{array}{llll}\text { C } & -3.762078 & 3.786752 & 2.268337\end{array}$

$\begin{array}{llll}\text { C } & -2.831507 & 4.427031 & 3.084293\end{array}$

$\begin{array}{llll}\text { C } & -1.593295 & 4.832737 & 2.570435\end{array}$

$\begin{array}{llll}\text { C } & -1.345854 & 4.646724 & 1.208624\end{array}$

$\begin{array}{llll}\text { C } & -3.775774 & 5.161073 & -1.986522\end{array}$

$\begin{array}{llll}\text { C } & -5.006353 & 4.887860 & -2.602233\end{array}$

$\begin{array}{llll}\text { C } & -6.131008 & 5.671461 & -2.346135\end{array}$ 


\begin{tabular}{|c|c|c|c|}
\hline C & -6.052751 & 6.750559 & -1.465277 \\
\hline$C$ & -4.833847 & 7.038368 & -0.849941 \\
\hline $\mathrm{C}$ & -3.709509 & 6.256151 & -1.110838 \\
\hline $\mathrm{C}$ & -2.686473 & 3.446217 & -3.519262 \\
\hline 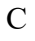 & -2.261713 & 3.989577 & -4.736767 \\
\hline $\mathrm{C}$ & -2.338261 & 3.244233 & -5.914778 \\
\hline $\mathrm{C}$ & -2.845459 & 1.942910 & -5.884792 \\
\hline $\mathrm{C}$ & -3.288625 & 1.401204 & -4.675969 \\
\hline $\mathrm{C}$ & -3.217881 & 2.153331 & -3.504146 \\
\hline $\mathrm{C}$ & -0.513993 & 5.356094 & 3.456039 \\
\hline $\mathrm{C}$ & 0.523274 & 4.485670 & 3.847727 \\
\hline $\mathrm{C}$ & 1.531363 & 4.972046 & 4.687055 \\
\hline $\mathrm{C}$ & 1.547618 & 6.299001 & 5.127324 \\
\hline $\mathrm{C}$ & 0.509941 & 7.144133 & 4.721210 \\
\hline C & -0.523619 & 6.693077 & 3.894542 \\
\hline $\mathrm{C}$ & 7.630041 & 1.627283 & 3.473344 \\
\hline $\mathrm{C}$ & 3.048269 & -0.561216 & 3.377062 \\
\hline $\mathrm{C}$ & 6.808342 & -2.672354 & 5.997182 \\
\hline $\mathrm{C}$ & 1.625325 & -7.631026 & 3.471735 \\
\hline $\mathrm{C}$ & -0.561808 & -3.048537 & 3.376720 \\
\hline $\mathrm{C}$ & -2.674703 & -6.808963 & 5.994947 \\
\hline $\mathrm{C}$ & -7.632462 & -1.626846 & 3.468174 \\
\hline $\mathrm{C}$ & -3.050038 & 0.560553 & 3.377854 \\
\hline $\mathrm{C}$ & -6.812964 & 2.672497 & 5.993442 \\
\hline $\mathrm{C}$ & -1.628587 & 7.631141 & 3.470583 \\
\hline $\mathrm{C}$ & 0.560099 & 3.049397 & 3.377497 \\
\hline $\mathrm{C}$ & 2.670502 & 6.811086 & 5.995978 \\
\hline $\mathrm{H}$ & -5.757362 & -1.102115 & -1.460620 \\
\hline $\mathrm{H}$ & -4.460506 & -0.393217 & -2.533114 \\
\hline $\mathrm{H}$ & -3.425490 & -4.696330 & 2.678802 \\
\hline $\mathrm{H}$ & -4.574361 & -3.051221 & 4.137021 \\
\hline $\mathrm{H}$ & -4.954287 & -0.388552 & 0.796925 \\
\hline $\mathrm{H}$ & -4.051617 & -5.086843 & -3.284664 \\
\hline $\mathrm{H}$ & -5.435570 & -7.070285 & -2.836123 \\
\hline $\mathrm{H}$ & -7.362308 & -6.926643 & -1.262490 \\
\hline $\mathrm{H}$ & -7.877100 & -4.750404 & -0.163972 \\
\hline $\mathrm{H}$ & -6.504976 & -2.782512 & -0.608219 \\
\hline $\mathrm{H}$ & -4.996199 & -1.858178 & -4.756876 \\
\hline $\mathrm{H}$ & -3.673856 & -1.997920 & -6.850478 \\
\hline $\mathrm{H}$ & -1.352370 & -2.900464 & -6.794845 \\
\hline $\mathrm{H}$ & -0.385374 & -3.671585 & -4.643791 \\
\hline $\mathrm{H}$ & -1.722500 & -3.539399 & -2.561684 \\
\hline $\mathrm{H}$ & -8.180654 & 0.505996 & 5.052877 \\
\hline $\mathrm{H}$ & -4.296775 & 2.320590 & 5.007178 \\
\hline $\mathrm{H}$ & 1.103157 & -5.758024 & -1.459462 \\
\hline $\mathrm{H}$ & 0.394535 & -4.461325 & -2.532493 \\
\hline $\mathrm{H}$ & 4.695621 & -3.425172 & 2.680838 \\
\hline $\mathrm{H}$ & 3.049692 & -4.573092 & 4.138880 \\
\hline $\mathrm{H}$ & 0.388513 & -4.954079 & 0.797702 \\
\hline $\mathrm{H}$ & 5.088299 & -4.052711 & -3.282764 \\
\hline $\mathrm{H}$ & 7.071625 & -5.436578 & -2.833438 \\
\hline $\mathrm{H}$ & 6.927663 & -7.362819 & -1.259218 \\
\hline $\mathrm{H}$ & 4.751130 & -7.877185 & -0.160954 \\
\hline $\mathrm{H}$ & 2.783258 & -6.505192 & -0.606106 \\
\hline $\mathrm{H}$ & 1.859712 & -4.997105 & -4.755532 \\
\hline $\mathrm{H}$ & 1.999762 & -3.674836 & -6.849278 \\
\hline $\mathrm{H}$ & 2.902833 & -1.353466 & -6.793546 \\
\hline $\mathrm{H}$ & 3.673986 & -0.386585 & -4.642510 \\
\hline $\mathrm{H}$ & 3.541142 & -1.723534 & -2.560285 \\
\hline $\mathrm{H}$ & -2.322417 & -4.293690 & 5.006561 \\
\hline $\mathrm{H}$ & -0.508047 & -8.177641 & 5.056229 \\
\hline$\pi$ & 5.758681 & 1.103201 & -1.458474 \\
\hline
\end{tabular}

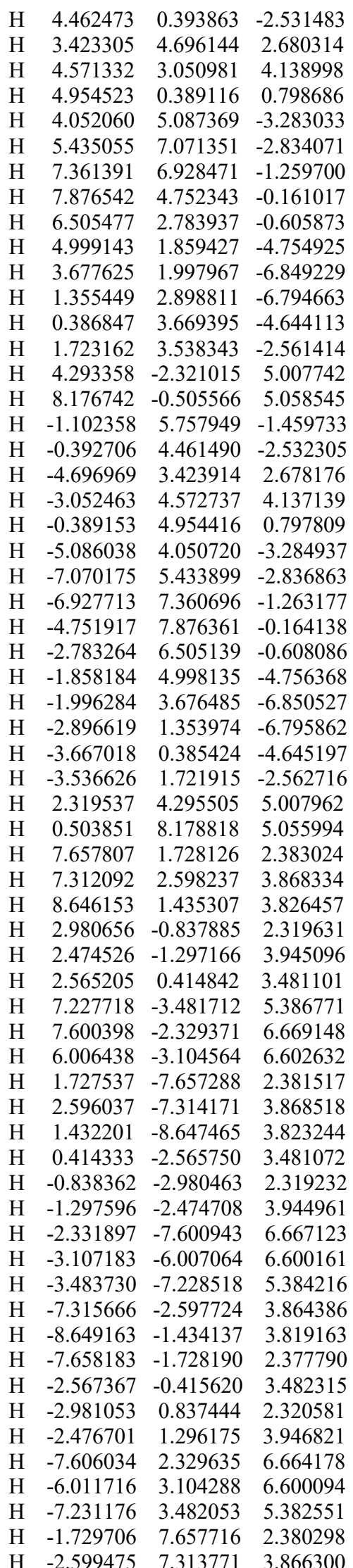

$\begin{array}{cccc}\mathrm{H} & -1.436305 & 8.647575 & 3.822475 \\ \mathrm{H} & -0.416083 & 2.566415 & 3.481546 \\ \mathrm{H} & 0.837092 & 2.981188 & 2.320183 \\ \mathrm{H} & 1.295770 & 2.475886 & 3.946158 \\ \mathrm{H} & 2.327300 & 7.603529 & 6.667305 \\ \mathrm{H} & 3.102449 & 6.009565 & 6.602167 \\ \mathrm{H} & 3.480067 & 7.229958 & 5.385508\end{array}$

O'2

$\begin{array}{llll}\text { Rh } & -0.000127 & 0.000550 & 0.074704\end{array}$

$\begin{array}{lllll}\mathrm{Rh} & 0.000284 & 0.000618 & -2.309503\end{array}$

$\begin{array}{lllll}\text { O } & 0.438120 & 1.998156 & -0.019398\end{array}$

$\begin{array}{llll}\text { O } & 1.038314 & 1.790458 & -2.190926\end{array}$

$\begin{array}{llll}\text { O } & -0.438522 & -1.997012 & -0.019695\end{array}$

$\begin{array}{llll}\text { O } & -1.037626 & -1.789186 & -2.191578\end{array}$

$\begin{array}{llll}\text { O } & 1.997515 & -0.437929 & -0.019267\end{array}$

$\begin{array}{llll}\mathrm{O} & 1.790084 & -1.037442 & -2.190973\end{array}$

$\begin{array}{llll}\text { O } & -1.997676 & 0.438805 & -0.019826\end{array}$

$\begin{array}{llll}\mathrm{O} & -1.789551 & 1.038389 & -2.191532\end{array}$

Cl $\quad-1.200062 \quad 5.095040 \quad-0.002366$

$\begin{array}{llll}\mathrm{Cl} & 1.200107 & -5.094752 & -0.004127\end{array}$

$\begin{array}{llll}\text { Cl } & 5.095299 & 1.199424 & -0.001919\end{array}$

$\begin{array}{llll}\text { Cl } & -5.094291 & -1.200918 & -0.004773\end{array}$

$\begin{array}{llll}\text { C } & 1.484798 & 3.839527 & -1.033670\end{array}$

$\begin{array}{llll}\text { C } & 1.412252 & 4.791574 & -2.268996\end{array}$

$\begin{array}{llll}\text { C } & 2.517277 & 4.134976 & 1.209782\end{array}$

$\begin{array}{llll}\text { C } & 2.487797 & 4.444460 & 2.571228\end{array}$

$\begin{array}{llll}\text { C } & 1.428699 & 4.371673 & 0.366165\end{array}$

$\begin{array}{llll}\text { C } & 1.251857 & 6.269061 & -1.989669\end{array}$

$\begin{array}{llll}\text { C } & 0.252010 & 5.338118 & 2.268344\end{array}$

C $\quad 0.724100 \quad 4.304351 \quad-3.517161$

$\begin{array}{llll}\text { C } & -1.192011 & 3.362878 & -4.667741\end{array}$

$\begin{array}{llll}\text { C } & 0.938048 & 2.443875 & -1.105215\end{array}$

C $\quad 0.219934 \quad 6.989595 \quad-2.609622$

$\begin{array}{llll}\text { C } & 1.407010 & 4.362638 & -4.737108\end{array}$

$\begin{array}{llll}\text { C } & 2.700620 & 4.118648 & -1.896104\end{array}$

$\begin{array}{llll}\text { C } & 0.802412 & 3.915739 & -5.913548\end{array}$

$\begin{array}{llll}\text { C } & 0.284197 & 4.942943 & 0.933346\end{array}$

$\begin{array}{llll}\text { C } & 2.101170 & 6.962835 & -1.113483\end{array}$

$\begin{array}{llll}\text { C } & 0.881609 & 9.022708 & -1.475867\end{array}$

$\begin{array}{llll}\text { C } & 1.352664 & 5.084571 & 3.084678\end{array}$

$\begin{array}{llll}\text { C } & -0.586312 & 3.818422 & -3.497594\end{array}$

$\begin{array}{llll}\text { C } & -0.498170 & 3.406766 & -5.879373\end{array}$

$\begin{array}{llll}\text { C } & 0.035865 & 8.348540 & -2.357131\end{array}$

$\begin{array}{llll}\text { C } & 1.916426 & 8.320772 & -0.856294\end{array}$

$\begin{array}{llll}\text { C } & -1.484539 & -3.838401 & -1.034729\end{array}$

C $\quad-1.412098-4.790223-2.270110$

$\begin{array}{llll}\text { C } & -2.516835 & -4.134048 & 1.208833\end{array}$

$\begin{array}{llll}\text { C } & -2.487224 & -4.444042 & 2.570196\end{array}$

$\begin{array}{llll}\text { C } & -1.428618 & -4.370926 & 0.364945\end{array}$

$\begin{array}{llll}\text { C } & -1.252148 & -6.267864 & -1.991037\end{array}$

$\begin{array}{llll}\text { C } & -0.251995 & -5.338884 & 2.266576\end{array}$

C $\quad-0.723750 \quad-4.303027 \quad-3.518252$

$\begin{array}{llll}\text { C } & -0.801295 & -3.915594 & -5.914779\end{array}$

$\begin{array}{llll}\text { C } & -0.937834 & -2.442809 & -1.105884\end{array}$

$\begin{array}{llll}\text { C } & -0.220006 & -6.988347 & -2.610687\end{array}$

$\begin{array}{llll}\text { C } & 0.586497 & -3.816630 & -3.498519\end{array}$

$\begin{array}{llll}\text { C } & -2.700407 & -4.117141 & -1.897299\end{array}$

$\begin{array}{llll}\text { C } & 1.192447 & -3.361360 & -4.668578\end{array}$

$\begin{array}{llll}\text { C } & -0.284185 & -4.942897 & 0.931866\end{array}$

$\begin{array}{llll}\text { C } & -2.102045 & -6.961615 & -1.115419\end{array}$

$\begin{array}{llll}\text { C } & -0.882804 & -9.021589 & -1.477737\end{array}$ 


\begin{tabular}{|c|c|c|c|}
\hline C & -1.352456 & -5.085162 & \\
\hline C & -1.406174 & -4.362227 & -4.738411 \\
\hline $\mathrm{C}$ & 0.499054 & -3.406172 & -5.880454 \\
\hline $\mathrm{C}$ & -0.036355 & -8.347423 & -2.358377 \\
\hline $\mathrm{C}$ & -1.917740 & -8.319690 & -0.858607 \\
\hline $\mathrm{C}$ & 3.838845 & -1.484791 & -1.033425 \\
\hline $\mathrm{C}$ & 4.791210 & -1.412367 & -2.268425 \\
\hline $\mathrm{C}$ & 4.133409 & -2.517528 & 1.210075 \\
\hline $\mathrm{C}$ & 4.442846 & -2.488138 & 2.571532 \\
\hline $\mathrm{C}$ & 4.370711 & -1.429125 & 0.366476 \\
\hline $\mathrm{C}$ & 6.268724 & -1.252751 & -1.988818 \\
\hline $\mathrm{C}$ & 5.337954 & -0.253016 & 2.268673 \\
\hline $\mathrm{C}$ & 4.304564 & -0.723864 & -3.516712 \\
\hline $\mathrm{C}$ & 3.917377 & -0.801307 & -5.913288 \\
\hline $\mathrm{C}$ & 2.443397 & -0.937713 & -1.105155 \\
\hline $\mathrm{C}$ & 6.961927 & -2.102582 & -1.112754 \\
\hline $\mathrm{C}$ & 3.818589 & 0.586582 & -3.496969 \\
\hline $\mathrm{C}$ & 4.117666 & -2.700554 & -1.895996 \\
\hline $\mathrm{C}$ & 3.363759 & 1.192636 & -4.667136 \\
\hline $\mathrm{C}$ & 4.942639 & -0.284911 & 0.933811 \\
\hline $\mathrm{C}$ & 6.989670 & -0.220849 & -2.608311 \\
\hline $\mathrm{C}$ & 9.022389 & -0.883840 & -1.474448 \\
\hline $\mathrm{C}$ & 5.083764 & -1.353526 & 3.084924 \\
\hline $\mathrm{C}$ & 4.363551 & -1.406279 & -4.736841 \\
\hline $\mathrm{C}$ & 3.408432 & 0.499279 & -5.879007 \\
\hline $\mathrm{C}$ & 8.319928 & -1.91 & -0.85 \\
\hline $\mathrm{C}$ & 8.348687 & -0.037478 & -2.355468 \\
\hline $\mathrm{C}$ & -3.839194 & 1.484516 & -1.034976 \\
\hline $\mathrm{C}$ & -4.790688 & 1.411851 & -2.270681 \\
\hline $\mathrm{C}$ & -4.135809 & 2.516434 & 1.208532 \\
\hline $\mathrm{C}$ & -4.445950 & 2.486718 & 2.569882 \\
\hline $\mathrm{C}$ & -4.371888 & 1.428076 & 0.364629 \\
\hline $\mathrm{C}$ & -6.268194 & 1.251034 & -1.991987 \\
\hline $\mathrm{C}$ & -5.339042 & 0.250778 & 2.266144 \\
\hline $\mathrm{C}$ & -4.302730 & 0.723986 & -3.518799 \\
\hline $\mathrm{C}$ & -3.913772 & 0.802415 & -5.915032 \\
\hline $\mathrm{C}$ & -2.443309 & 0.938257 & -1.105944 \\
\hline $\mathrm{C}$ & -6.988044 & 0.218704 & -2.612096 \\
\hline $\mathrm{C}$ & -3.816267 & -0.586302 & -3.499240 \\
\hline $\mathrm{C}$ & -4.118217 & 2.700336 & -1.897410 \\
\hline $\mathrm{C}$ & -3.360212 & -1.191682 & -4.669176 \\
\hline $\mathrm{C}$ & -4.943192 & 0.283260 & 0.931299 \\
\hline $\mathrm{C}$ & -6.962701 & 2.100281 & -1.116250 \\
\hline $\mathrm{C}$ & -9.021871 & 0.880053 & -1.479356 \\
\hline $\mathrm{C}$ & -5.086143 & 1.351252 & 3.082694 \\
\hline $\mathrm{C}$ & -4.361215 & 1.406803 & -4.738676 \\
\hline $\mathrm{C}$ & -3.404239 & -0.497807 & -5.880811 \\
\hline $\mathrm{C}$ & -8.347040 & 0.034254 & -2.360038 \\
\hline $\mathrm{C}$ & -8.320604 & 1.915178 & -0.859689 \\
\hline $\mathrm{C}$ & 4.000914 & -3.602924 & 3.457429 \\
\hline $\mathrm{C}$ & 2.647188 & -3.664937 & 3.845384 \\
\hline $\mathrm{C}$ & 4.910588 & -4.584759 & 3.891815 \\
\hline $\mathrm{C}$ & 2.231760 & -4.708531 & 4.679692 \\
\hline $\mathrm{C}$ & 4.452323 & -5.619630 & 4.712727 \\
\hline $\mathrm{C}$ & 3.116951 & -5.695894 & 5.121916 \\
\hline $\mathrm{C}$ & -3.601838 & -4.002352 & 3.456461 \\
\hline $\mathrm{C}$ & -3.664277 & -2.648567 & 3.844037 \\
\hline $\mathrm{C}$ & -4.583138 & -4.912359 & 3.891520 \\
\hline $\mathrm{C}$ & -4.707597 & -2.233323 & 4.678753 \\
\hline $\mathrm{C}$ & -5.617700 & -4.454337 & 4.712786 \\
\hline $\mathrm{C}$ & -5.694425 & -3.118970 & 5.121715 \\
\hline & -4.004803 & 3.601895 & 3.455588 \\
\hline
\end{tabular}

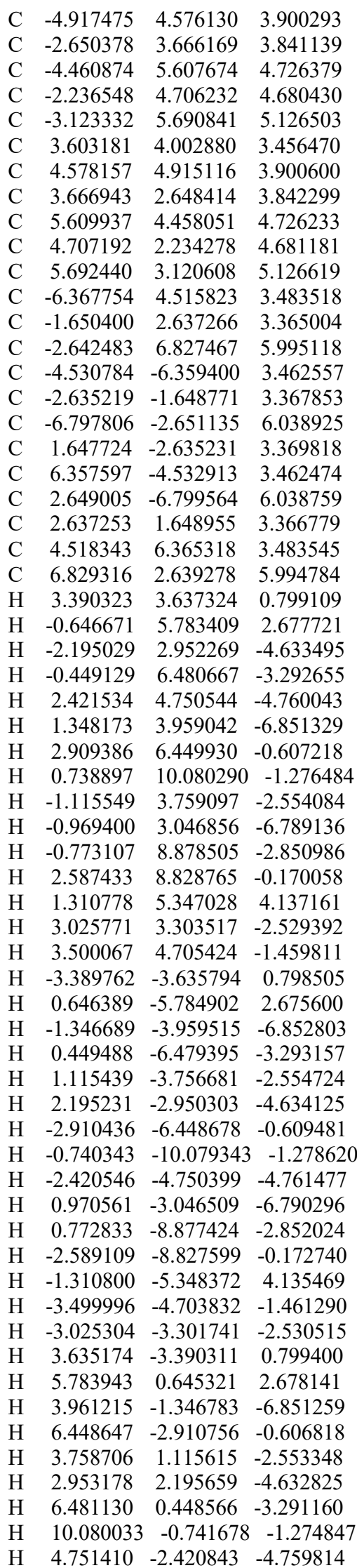

H $\quad 3.049095 \quad 0.970820 \quad-6.788839$

H $\quad 8.827429 \quad-2.589934 \quad-0.169167$

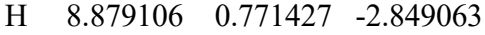

H $\quad 5.346468 \quad-1.312032 \quad 4.137477$

H $\quad 4.704075 \quad-3.500367 \quad-1.459752$

$\mathrm{H} \quad 3.302512 \quad-3.025345 \quad-2.529549$

$\begin{array}{llll}\mathrm{H} & -3.638261 & 3.389803 & 0.798204\end{array}$

$\mathrm{H} \quad-5.784450 \quad-0.648058 \quad 2.675078$

$\mathrm{H} \quad-3.957185 \quad 1.348290 \quad-6.852865$

H $\quad-6.478619 \quad-0.450320 \quad-3.294648$

$\mathrm{H} \quad-3.756878-1.115446 \quad-2.555657$

$\mathrm{H} \quad-2.949115-2.194554 \quad-4.634886$

H $\quad-6.450248 \quad 2.908798 \quad-0.609984$

H $\quad-10.079554 \quad 0.736941 \quad-1.280357$

H $\quad-4.749447 \quad 2.421229 \quad-4.761703$

$\begin{array}{llll}\mathrm{H} & -3.044017 & -0.968902 & -6.790583\end{array}$

H $\quad-8.876577 \quad-0.775097 \quad-2.854044$

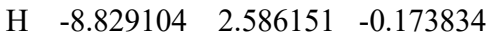

$\begin{array}{llll}\mathrm{H} & -5.349106 & 1.309202 & 4.135047\end{array}$

H $\quad-3.302865 \quad 3.025791 \quad-2.530361$

H $\quad-4.705390 \quad 3.499640 \quad-1.461254$

H $\quad 1.191313 \quad-4.748194 \quad 4.992503$

H $\quad 5.153760 \quad-6.384147 \quad 5.038568$

H $\quad-4.747616 \quad-1.192898 \quad 4.991268$

$\begin{array}{llll}\mathrm{H} & -6.381766 & -5.156017 & 5.039300\end{array}$

$\mathrm{H} \quad-5.166216 \quad 6.362615 \quad 5.065825$

$\mathrm{H} \quad-1.197137 \quad 4.743713 \quad 4.996717$

$\begin{array}{llll}\mathrm{H} & 6.365328 & 5.163009 & 5.065203\end{array}$

$\begin{array}{llll}\mathrm{H} & 4.744208 & 1.194825 & 4.997662\end{array}$

$\begin{array}{llll}\mathrm{H} & -6.465611 & 4.468041 & 2.393551\end{array}$

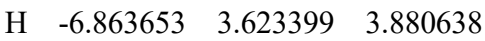

$\mathrm{H} \quad-6.914483 \quad 5.392466 \quad 3.839222$

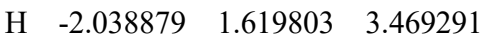

H $\quad-1.405074 \quad 2.776499 \quad 2.306776$

H $\quad-0.718547 \quad 2.714722 \quad 3.929964$

H $\quad-3.431473 \quad 7.179474 \quad 6.666193$

H $\quad-1.782498 \quad 6.529131 \quad 6.601652$

H $\quad-2.329025 \quad 7.683338 \quad 5.384642$

$\begin{array}{llll}\mathrm{H} & -4.485479 & -6.448913 & 2.371800\end{array}$

$\begin{array}{llll}\mathrm{H} & -3.639850 & -6.862443 & 3.854024\end{array}$

$\mathrm{H} \quad-5.409315 \quad-6.904835 \quad 3.815707$

H $\quad-1.617945 \quad-2.037962 \quad 3.471896$

$\begin{array}{llll}\mathrm{H} & -2.774565 & -1.403081 & 2.309687\end{array}$

$\mathrm{H} \quad-2.711909-0.717128 \quad 3.933208$

H $\quad-7.724649 \quad-3.204921 \quad 5.862553$

$\begin{array}{llll}\mathrm{H} & -6.526035 & -2.799977 & 7.091270\end{array}$

H $\quad-7.003083 \quad-1.585425 \quad 5.903165$

H $\quad 2.037263 \quad-1.618193 \quad 3.474533$

H $\quad 1.402076-2.773886 \quad 2.311539$

H $\quad 0.716024 \quad-2.712043 \quad 3.935062$

$\begin{array}{llll}\mathrm{H} & 6.446786 & -4.487242 & 2.371713\end{array}$

H $\quad 6.860893 \quad-3.642226 \quad 3.854073$

$\begin{array}{llll}\mathrm{H} & 6.902791 & -5.411736 & 3.815167\end{array}$

H $\quad 1.582868 \quad-7.003474 \quad 5.904096$

H $\quad 3.201353 \quad-7.726984 \quad 5.860834$

H $\quad 2.799492 \quad-6.528861 \quad 7.091103$

$\begin{array}{llll}\mathrm{H} & 1.620106 & 2.037890 & 3.471637\end{array}$

$\begin{array}{llll}\mathrm{H} & 2.775909 & 1.403588 & 2.308437\end{array}$

$\begin{array}{llll}\mathrm{H} & 2.714732 & 0.717037 & 3.931629\end{array}$

$\begin{array}{llll}\mathrm{H} & 4.470872 & 6.463046 & 2.393596\end{array}$

$\begin{array}{llll}\mathrm{H} & 3.625941 & 6.861517 & 3.880372\end{array}$

$\begin{array}{llll}\mathrm{H} & 5.395067 & 6.911868 & 3.839494\end{array}$ 
$\begin{array}{llll}\mathrm{H} & 7.684578 & 2.325025 & 5.383993\end{array}$

$\begin{array}{llll}\mathrm{H} & 7.182131 & 3.428217 & 6.665359\end{array}$

$\begin{array}{llll}\mathrm{H} & 6.530745 & 1.779681 & 6.601794\end{array}$

P1

$\begin{array}{llll}\mathrm{Rh} & -0.000100 & 0.000100 & -1.117800\end{array}$

$\begin{array}{llll}\mathrm{Rh} & -0.000100 & 0.000100 & 1.263300\end{array}$

$\begin{array}{llll}\mathrm{O} & -1.553700 & -1.359500 & -1.048000\end{array}$

$\begin{array}{llll}\mathrm{O} & -1.442200 & -1.476500 & 1.201600\end{array}$

$\begin{array}{llll}\mathrm{O} & -1.425600 & 1.502400 & -1.058700\end{array}$

$\begin{array}{llll}\mathrm{O} & -1.465300 & 1.458100 & 1.198800\end{array}$

$\begin{array}{llll}\mathrm{O} & 1.553400 & 1.359800 & -1.048000\end{array}$

$\begin{array}{llll}\text { O } & 1.442200 & 1.476600 & 1.201600\end{array}$

O $\quad 1.425400 \quad-1.502200 \quad-1.058900$

$\begin{array}{llll}\text { O } & 1.465000 & -1.458100 & 1.198500\end{array}$

$\begin{array}{llll}\text { C } & -4.044400 & -4.248900 & -1.740900\end{array}$

C $\quad-3.204700 \quad-2.663100 \quad 0.080400$

$\begin{array}{llll}\text { C } & -4.174700 & -2.668700 & 1.320400\end{array}$

$\begin{array}{llll}\text { C } & -4.709300 & -2.114700 & -3.387500\end{array}$

C $\quad-3.780400 \quad-2.947700 \quad-1.296400$

$\begin{array}{llll}\text { C } & -4.623600 & -4.485900 & -2.989900\end{array}$

C $\quad-5.615700 \quad-2.958100 \quad 1.038200$

$\begin{array}{llll}\text { C } & -1.985400 & -1.761900 & 0.080600\end{array}$

C $\quad-3.961100 \quad-1.718300 \quad 2.453600$

C $\quad-3.758600 \quad-2.206700 \quad 3.755600$

C $\quad-3.172200 \quad-3.770900 \quad 1.131600$

$\begin{array}{llll}\text { C } & -6.391300 & -2.039900 & 0.308900\end{array}$

C $\quad-4.131400 \quad-1.881900-2.142300$

$\begin{array}{llll}\text { C } & -6.225300 & -4.117000 & 1.548500\end{array}$

$\begin{array}{llll}\text { C } & -4.957000 & -3.419400 & -3.821400\end{array}$

C $\quad-8.332600 \quad-3.465800 \quad 0.546200$

$\begin{array}{llll}\text { C } & -3.607600 & -1.319500 & 4.825300\end{array}$

$\begin{array}{llll}\text { C } & -3.898600 & 0.550700 & 3.318000\end{array}$

$\begin{array}{llll}\text { C } & -4.040600 & -0.333200 & 2.244400\end{array}$

$\begin{array}{llll}\text { C } & -7.576600 & -4.371700 & 1.295200\end{array}$

$\begin{array}{llll}\text { C } & -7.741000 & -2.298400 & 0.057000\end{array}$

$\begin{array}{llll}\text { C } & -3.676800 & 0.058700 & 4.606400\end{array}$

$\begin{array}{llll}\text { C } & -4.210700 & 2.033400 & -2.015900\end{array}$

C $\quad-3.038900 \quad 2.872500 \quad 0.081100$

C $\quad-2.765200 \quad 4.406000 \quad 0.107400$

C $\quad-6.625500 \quad 1.737300 \quad-0.657900$

$\begin{array}{llll}\text { C } & -4.265000 & 2.342300 & -0.646000\end{array}$

C $\quad-5.348800 \quad 1.600600 \quad-2.695300$

$\begin{array}{llll}\text { C } & -3.763300 & 5.302300 & -0.558700\end{array}$

C $\quad-1.878600 \quad 1.879700 \quad 0.070200$

C $\quad-1.361700 \quad 4.909300 \quad 0.045300$

$\begin{array}{llll}\text { C } & -0.871400 & 5.742200 & 1.064700\end{array}$

$\begin{array}{llll}\text { C } & -3.201500 & 3.667100 & 1.354400\end{array}$

$\begin{array}{llll}\text { C } & -4.529700 & 6.203500 & 0.201400\end{array}$

C $\quad-5.488300 \quad 2.181900 \quad 0.019800$

$\begin{array}{llll}\text { C } & -3.896800 & 5.302700 & -1.957900\end{array}$

$\begin{array}{llll}\text { C } & -6.562400 & 1.448900 & -2.020100\end{array}$

C $\quad-5.595500 \quad 7.022000 \quad-1.813400$

$\begin{array}{llll}\text { C } & 0.385400 & 6.340000 & 0.941700\end{array}$

C $\quad 0.679100 \quad 5.284600 \quad-1.216900$

$\begin{array}{llll}\text { C } & -0.574600 & 4.679900 & -1.095000\end{array}$

$\begin{array}{llll}\text { C } & -4.816700 & 6.151600 & -2.579800\end{array}$

C $\quad-5.447700 \quad 7.052100 \quad-0.424500$

$\begin{array}{llll}\text { C } & 1.152900 & 6.123800 & -0.204700\end{array}$

$\begin{array}{llll}\text { C } & 4.131000 & 1.882400 & -2.142700\end{array}$

$\begin{array}{llll}\text { C } & 3.204600 & 2.663200 & 0.080300\end{array}$

$\begin{array}{llll}\text { C } & 4.174900 & 2.668400 & 1.320300\end{array}$ $\begin{array}{llll}\text { C } & 4.623600 & 4.486500 & -2.989700\end{array}$

$\begin{array}{llll}\text { C } & 3.780400 & 2.948000 & -1.296500\end{array}$

$\begin{array}{llll}\text { C } & 4.708800 & 2.115300 & -3.387800\end{array}$

$\begin{array}{lllr}\text { C } & 5.615900 & 2.957600 & 1.037800\end{array}$

$\begin{array}{llll}\text { C } & 1.985300 & 1.762000 & 0.080600\end{array}$

$\begin{array}{llll}\text { C } & 3.961300 & 1.718000 & 2.453500\end{array}$

$\begin{array}{llll}\text { C } & 3.759100 & 2.206500 & 3.755600\end{array}$

$\begin{array}{llll}\text { C } & 3.172500 & 3.770900 & 1.131700\end{array}$

$\begin{array}{llll}\text { C } & 6.225800 & 4.116300 & 1.548100\end{array}$

$\begin{array}{lllr}\text { C } & 4.044300 & 4.249200 & -1.740700\end{array}$

$\begin{array}{llll}\text { C } & 6.391200 & 2.039200 & 0.308400\end{array}$

$\begin{array}{llll}\text { C } & 4.956800 & 3.420100 & -3.821400\end{array}$

C $\quad 8.332800 \quad 3.464700 \quad 0.545500$

$\begin{array}{llll}\text { C } & 3.608100 & 1.319300 & 4.825300\end{array}$

$\begin{array}{llll}\text { C } & 3.898300 & -0.550900 & 3.317800\end{array}$

$\begin{array}{llll}\text { C } & 4.040400 & 0.332900 & 2.244200\end{array}$

C $\quad 7.740900 \quad 2.297500 \quad 0.056300$

$\begin{array}{llll}\text { C } & 7.577100 & 4.370800 & 1.294700\end{array}$

$\begin{array}{llll}\text { C } & 3.676900 & -0.058900 & 4.606300\end{array}$

$\begin{array}{llll}\text { C } & 4.211000 & -2.033200 & -2.015600\end{array}$

$\begin{array}{llll}\text { C } & 3.038600 & -2.872500 & 0.081000\end{array}$

$\begin{array}{llll}\text { C } & 2.765000 & -4.406000 & 0.107100\end{array}$

$\begin{array}{llll}\text { C } & 6.625500 & -1.737500 & -0.656900\end{array}$

$\begin{array}{llll}\text { C } & 4.264900 & -2.342200 & -0.645600\end{array}$

$\begin{array}{llll}\text { C } & 5.349300 & -1.600300 & -2.694600\end{array}$

$\begin{array}{llll}\text { C } & 3.763400 & -5.302300 & -0.558800\end{array}$

$\begin{array}{llll}\text { C } & 1.878400 & -1.879600 & 0.070000\end{array}$

$\begin{array}{llll}\text { C } & 1.361600 & -4.909400 & 0.044500\end{array}$

C $\quad 0.871000 \quad-5.742500 \quad 1.063600$

$\begin{array}{llll}\text { C } & 3.200900 & -3.667200 & 1.354300\end{array}$

C $\quad 4.529600 \quad-6.203400 \quad 0.201400$

$\begin{array}{llll}\text { C } & 5.488100 & -2.182100 & 0.020500\end{array}$

C $\quad 3.897200 \quad-5.302600 \quad-1.958000$

C $\quad 6.562700 \quad-1.448800 \quad-2.019000$

$\begin{array}{llll}\text { C } & 5.595900 & -7.021700 & -1.813200\end{array}$

C $\quad-0.385800 \quad-6.340200 \quad 0.940300$

C $\quad-0.678900 \quad-5.284400 \quad-1.218200$

C $\quad 0.574700 \quad-4.679700 \quad-1.096000$

C $\quad 4.817400 \quad-6.151300 \quad-2.579700$

C $\quad 5.447800 \quad-7.051900-0.424300$

C $\quad-1.153000 \quad-6.123800 \quad-0.206200$

H $\quad-3.802600 \quad-5.100000-1.115400$

$\mathrm{H} \quad-4.973400 \quad-1.269700 \quad-4.017500$

$\mathrm{H} \quad-4.811300 \quad-5.508600 \quad-3.306800$

$\mathrm{H} \quad-3.722100 \quad-3.272900 \quad 3.941900$

$\mathrm{H} \quad-3.512900 \quad-4.757700 \quad 0.838300$

H $\quad-2.296700 \quad-3.783500 \quad 1.773700$

H $\quad-5.950600 \quad-1.126000 \quad-0.066400$

$\mathrm{H} \quad-5.656200 \quad-4.821600 \quad 2.141700$

$\mathrm{H} \quad-9.378600 \quad-3.664500 \quad 0.351900$

$\mathrm{H} \quad-3.445500 \quad-1.700600 \quad 5.825200$

$\mathrm{H} \quad-3.964800 \quad 1.616500 \quad 3.154400$

$\mathrm{H} \quad-4.205400 \quad 0.055800 \quad 1.250100$

H $\quad-8.039000 \quad-5.270000 \quad 1.683400$

$\mathrm{H} \quad-8.328800 \quad-1.591200 \quad-0.513900$

$\mathrm{H} \quad-3.565500 \quad 0.744900 \quad 5.435900$

$\mathrm{H} \quad-3.271200 \quad 2.140900 \quad-2.546200$

$\mathrm{H} \quad-7.558900 \quad 1.613300 \quad-0.115000$

$\mathrm{H} \quad-5.286300 \quad 1.385600 \quad-3.759000$

$\mathrm{H} \quad-1.474700 \quad 5.952100 \quad 1.939200$

$\mathrm{H} \quad-4.203200 \quad 3.828000 \quad 1.740000$

H $\quad-2.467000 \quad 3.479400 \quad 2.130600$
$\mathrm{H} \quad-4.416300 \quad 6.251100 \quad 1.277100$

$\mathrm{H} \quad-3.286700 \quad 4.650600 \quad-2.568000$

H $\quad-6.305900 \quad 7.680300 \quad-2.296500$

H $\quad 0.754900 \quad 6.989000 \quad 1.725000$

$\mathrm{H} \quad 1.272500 \quad 5.119700 \quad-2.106300$

$\mathrm{H} \quad-0.944800 \quad 4.062600 \quad-1.903300$

$\mathrm{H} \quad-4.921200 \quad 6.138900 \quad-3.657000$

$\mathrm{H} \quad-6.041400 \quad 7.736700 \quad 0.167300$

H $\quad 2.112600 \quad 6.611700 \quad-0.308400$

H $\quad 3.955700 \quad 0.862100 \quad-1.821800$

H $\quad 4.811400 \quad 5.509200 \quad-3.306300$

H $\quad 4.972800 \quad 1.270400 \quad-4.018000$

$\mathrm{H} \quad 3.722900 \quad 3.272700 \quad 3.941800$

H $\quad 3.513300 \quad 4.757600 \quad 0.838400$

H $\quad 2.297100 \quad 3.783600 \quad 1.773900$

H $\quad 5.656900 \quad 4.821100 \quad 2.141500$

H $\quad 5.950200 \quad 1.125500 \quad-0.066800$

$\begin{array}{llll}\mathrm{H} & 9.378800 & 3.663300 & 0.351000\end{array}$

$\mathrm{H} \quad 3.446300 \quad 1.700400 \quad 5.825200$

H $\quad 3.964200 \quad-1.616800 \quad 3.154100$

H $\quad 4.204800 \quad-0.056000 \quad 1.249900$

$\mathrm{H} \quad 8.328500 \quad 1.590200 \quad-0.514700$

$\begin{array}{llll}\mathrm{H} & 8.039800 & 5.269000 & 1.682800\end{array}$

H $\quad 3.565600 \quad-0.745100 \quad 5.435800$

H $\quad 3.271500 \quad-2.140500 \quad-2.546100$

$\mathrm{H} \quad 7.558800 \quad-1.613700 \quad-0.113800$

H $\quad 5.287000 \quad-1.385000 \quad-3.758200$

$\mathrm{H} \quad 1.474200 \quad-5.952500 \quad 1.938300$

H $\quad 4.202500 \quad-3.828100 \quad 1.740300$

H $\quad 2.466100 \quad-3.479600 \quad 2.130300$

H $\quad 4.415900 \quad-6.251100 \quad 1.277100$

H $\quad 3.287300 \quad-4.650300 \quad-2.568200$

H $\quad 6.306500 \quad-7.679900 \quad-2.296100$

H $\quad-0.755400 \quad-6.989400 \quad 1.723400$

$\mathrm{H} \quad-1.272200 \quad-5.119300 \quad-2.107700$

H $\quad 0.945100 \quad-4.062300 \quad-1.904000$

$\mathrm{H} \quad 4.922100 \quad-6.138500 \quad-3.656800$

H $\quad 6.041400 \quad-7.736600 \quad 0.167600$

$\mathrm{H} \quad-2.112700 \quad-6.611700 \quad-0.310300$

$\mathrm{H} \quad 5.556800 \quad-2.392900 \quad 1.082700$

$\mathrm{H} \quad-3.956200 \quad-0.861700 \quad-1.821300$

H $\quad 3.802700 \quad 5.100400-1.115000$

$\mathrm{H} \quad-5.557300 \quad 2.392300 \quad 1.082100$

$\mathrm{H} \quad-5.408500 \quad-3.599600 \quad-4.793500$

$\mathrm{H} \quad-7.446800 \quad 1.106700 \quad-2.550300$

H $\quad 7.447300 \quad-1.106500 \quad-2.549000$

H $\quad 5.408200 \quad 3.600500 \quad-4.793500$

P2

$\begin{array}{llll}\text { Rh } & -0.033400 & -0.210100 & -1.342600\end{array}$

$\begin{array}{llll}\mathrm{Rh} & -0.244800 & 0.005700 & 1.017400\end{array}$

$\begin{array}{llll}\text { O } & 1.099900 & -1.916800 & -1.025800\end{array}$

$\begin{array}{llll}\mathrm{O} & 1.063000 & -1.582800 & 1.209000\end{array}$

O $\quad-1.800000 \quad-1.280800 \quad-1.321500$

$\begin{array}{llll}\mathrm{O} & -1.824700 & -1.314300 & 0.934500\end{array}$

$\begin{array}{llll}\mathrm{O} & -1.087500 & 1.544300 & -1.522500\end{array}$

$\begin{array}{llll}\mathrm{O} & -1.545400 & 1.585400 & 0.686500\end{array}$

$\begin{array}{llll}\mathrm{O} & 1.693800 & 0.895500 & -1.239200\end{array}$

$\begin{array}{llll}\text { O } & 1.338000 & 1.350600 & 0.944200\end{array}$

$\begin{array}{llll}\text { C } & 4.989100 & -2.974200 & 0.664100\end{array}$

$\begin{array}{llll}\text { C } & 2.477700 & -3.311800 & 0.366200\end{array}$

$\begin{array}{llll}\text { C } & 2.045200 & -4.804800 & 0.342300\end{array}$ 


\begin{tabular}{|c|c|c|c|}
\hline & 5.279700 & -2.177700 & -1.983300 \\
\hline $\mathrm{C}$ & 3.851900 & -2.914300 & -0.154000 \\
\hline $\mathrm{C}$ & 6.251600 & -2.639400 & 0.170100 \\
\hline $\mathrm{C}$ & 3.044500 & -5.805300 & -0.148800 \\
\hline & 1.455400 & -2.196900 & 0.163900 \\
\hline $\mathrm{C}$ & 0.627200 & -5.168700 & 0.047800 \\
\hline $\mathrm{C}$ & -0.109400 & -5.920300 & 0.978700 \\
\hline $\mathrm{C}$ & 2.342500 & -4.093600 & 1.648800 \\
\hline $\mathrm{C}$ & 3.548900 & -6.790500 & 0.717800 \\
\hline & 4.017600 & -2.504500 & -1.488100 \\
\hline $\mathrm{C}$ & 3.442900 & -5.809500 & -1.496600 \\
\hline $\mathrm{C}$ & 6.404100 & -2.241800 & -1.157100 \\
\hline $\mathrm{C}$ & 4.881800 & -7.712300 & -1.082100 \\
\hline $\mathrm{C}$ & -1.383600 & -6.392100 & 0.652900 \\
\hline & -1.208700 & -5.366000 & -1.532200 \\
\hline $\mathrm{C}$ & 0.068100 & -4.895900 & -1.212000 \\
\hline $\mathrm{C}$ & 4.365200 & -6.753400 & -1.957100 \\
\hline $\mathrm{C}$ & 4.469900 & -7.734000 & 0.252900 \\
\hline $\mathrm{C}$ & -1.926800 & -6.126700 & -0.606000 \\
\hline $\mathrm{C}$ & -4.513200 & -3.807800 & -2.181600 \\
\hline $\mathrm{C}$ & -3.621600 & -2.382900 & -0.255900 \\
\hline $\mathrm{C}$ & -4.597400 & -2.398300 & 0.973800 \\
\hline $\mathrm{C}$ & -5.017000 & -1.535300 & -3.702800 \\
\hline $\mathrm{C}$ & -4.186300 & -2.554900 & -1.652400 \\
\hline $\mathrm{C}$ & -5.082900 & -3.929500 & -3.451900 \\
\hline $\mathrm{C}$ & -6.047100 & -2.645200 & 0.690400 \\
\hline $\mathrm{C}$ & -2.332000 & -1.586000 & -0.206600 \\
\hline $\mathrm{C}$ & -4.352500 & -1.479500 & 2.126000 \\
\hline $\mathrm{C}$ & -4.440000 & -0.089000 & 1.951700 \\
\hline $\mathrm{C}$ & -3.643100 & -3.537200 & 0.746300 \\
\hline $\mathrm{C}$ & -6.685700 & -3.787100 & 1.205000 \\
\hline $\mathrm{C}$ & -4.451000 & -1.418600 & -2.436500 \\
\hline $\mathrm{C}$ & -6.801700 & -1.709500 & -0.038700 \\
\hline $\mathrm{C}$ & -5.336800 & -2.794500 & -4.218500 \\
\hline $\mathrm{C}$ & -8.777400 & -3.088000 & 0.204200 \\
\hline $\mathrm{C}$ & -4.259900 & 0.768200 & 3.040400 \\
\hline $\mathrm{C}$ & -3.903200 & -1.135200 & 4.490100 \\
\hline $\mathrm{C}$ & -4.099100 & -1.998000 & 3.407500 \\
\hline $\mathrm{C}$ & -8.158000 & -1.936700 & -0.288200 \\
\hline $\mathrm{C}$ & -8.043000 & -4.009700 & 0.954600 \\
\hline $\mathrm{C}$ & -3.982300 & 0.247500 & 4.306300 \\
\hline $\mathrm{C}$ & -0.890100 & 4.247200 & -2.627000 \\
\hline $\mathrm{C}$ & -2.301500 & 3.408000 & -0.705900 \\
\hline $\mathrm{C}$ & -2.527400 & 4.425200 & 0.465600 \\
\hline $\mathrm{C}$ & -3.116600 & 4.646900 & -4.245800 \\
\hline $\mathrm{C}$ & -2.163200 & 3.931100 & -2.123900 \\
\hline $\mathrm{C}$ & -0.732600 & 4.759700 & -3.911500 \\
\hline $\mathrm{C}$ & -2.598700 & 5.875100 & 0.100700 \\
\hline $\mathrm{C}$ & -1.583200 & 2.092000 & -0.484300 \\
\hline $\mathrm{C}$ & -1.941500 & 4.169500 & 1.816800 \\
\hline $\mathrm{C}$ & -2.774900 & 4.135500 & 2.946900 \\
\hline $\mathrm{C}$ & -3.644800 & 3.524100 & 0.009700 \\
\hline $\mathrm{C}$ & -3.796700 & 6.594200 & 0.257600 \\
\hline $\mathrm{C}$ & -3.270000 & 4.130200 & -2.955900 \\
\hline $\mathrm{C}$ & -1.447200 & 6.550900 & -0.338400 \\
\hline $\mathrm{C}$ & -1.848800 & 4.967100 & -4.727200 \\
\hline $\mathrm{C}$ & -2.708400 & 8.608500 & -0.532700 \\
\hline $\mathrm{C}$ & -2.235600 & 3.902100 & 4.215000 \\
\hline $\mathrm{C}$ & -0.017400 & 3.799300 & 3.254400 \\
\hline $\mathrm{C}$ & -0.554000 & 4.031200 & 1.982900 \\
\hline 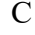 & -1.506200 & 7.909000 & -0.663400 \\
\hline & -3.851100 & 7.953000 & -0.067000 \\
\hline
\end{tabular}

\begin{tabular}{|c|c|c|c|}
\hline & -0.858200 & 3.727600 & 4.367700 \\
\hline $\mathrm{C}$ & 3.795700 & 3.815100 & -2.131900 \\
\hline $\mathrm{C}$ & 3.253400 & 2.317400 & -0.151400 \\
\hline $\mathrm{C}$ & 4.200300 & 2.330400 & 1.101400 \\
\hline $\mathrm{C}$ & 4.889400 & 1.689900 & -3.551700 \\
\hline $\mathrm{C}$ & 3.818900 & 2.545300 & -1.541700 \\
\hline $\mathrm{C}$ & 4.320500 & 4.026600 & -3.409700 \\
\hline $\mathrm{C}$ & 5.664500 & 2.499500 & 0.843300 \\
\hline $\mathrm{C}$ & 2.007000 & 1.456100 & -0.139000 \\
\hline $\mathrm{C}$ & 3.880800 & 1.467300 & 2.280100 \\
\hline $\mathrm{C}$ & 3.692000 & 2.045800 & 3.546800 \\
\hline $\mathrm{C}$ & 3.277300 & 3.485300 & 0.825900 \\
\hline $\mathrm{C}$ & 6.403400 & 1.454600 & 0.261900 \\
\hline $\mathrm{C}$ & 4.362200 & 1.482000 & -2.279300 \\
\hline & 6.332600 & 3.670300 & 1.242100 \\
\hline $\mathrm{C}$ & 4.875200 & 2.965300 & -4.122800 \\
\hline $\mathrm{C}$ & 8.425000 & 2.777200 & 0.410000 \\
\hline $\mathrm{C}$ & 3.401400 & 1.241700 & 4.652600 \\
\hline $\mathrm{C}$ & 3.522600 & -0.729400 & 3.254300 \\
\hline & 3.811500 & 0.071900 & 2.145700 \\
\hline $\mathrm{C}$ & 7.705500 & 3.810100 & 1.016800 \\
\hline C & 7.775000 & 1.598000 & 0.037700 \\
\hline $\mathrm{C}$ & 3.313900 & -0.145000 & 4.505800 \\
\hline $\mathrm{H}$ & 4.896800 & -3.272800 & 1.702600 \\
\hline H & 5.383500 & -1.876800 & -3.022800 \\
\hline $\mathrm{H}$ & 7.114200 & -2.687600 & 0.829400 \\
\hline $\mathrm{H}$ & 0.315300 & -6.165900 & 1.944200 \\
\hline $\mathrm{H}$ & 3.238500 & -4.355000 & 2.202600 \\
\hline $\mathrm{H}$ & 1.512800 & -3.814000 & 2.290600 \\
\hline $\mathrm{H}$ & 3.229400 & -6.828800 & 1.751700 \\
\hline $\mathrm{H}$ & 3.039200 & -5.084200 & -2.190500 \\
\hline $\mathrm{H}$ & 5.594400 & -8.443700 & -1.440500 \\
\hline $\mathrm{H}$ & -1.942900 & -6.978800 & 1.370000 \\
\hline $\mathrm{H}$ & -1.632500 & -5.159500 & -2.506500 \\
\hline $\mathrm{H}$ & 0.631800 & -4.349400 & -1.956600 \\
\hline $\mathrm{H}$ & 4.675300 & -6.744600 & -2.993900 \\
\hline $\mathrm{H}$ & 4.861400 & -8.484800 & 0.927000 \\
\hline $\mathrm{H}$ & -2.904200 & -6.512900 & -0.862500 \\
\hline $\mathrm{H}$ & -4.328900 & -4.707300 & -1.605800 \\
\hline $\mathrm{H}$ & -5.209500 & -0.640300 & -4.288500 \\
\hline $\mathrm{H}$ & -5.324100 & -4.916600 & -3.837700 \\
\hline $\mathrm{H}$ & -4.650400 & 0.327600 & 0.975100 \\
\hline $\mathrm{H}$ & -4.032700 & -4.492600 & 0.411300 \\
\hline $\mathrm{H}$ & -2.770000 & -3.619400 & 1.386000 \\
\hline $\mathrm{H}$ & -6.133700 & -4.504300 & 1.799500 \\
\hline $\mathrm{H}$ & -6.341600 & -0.805400 & -0.414500 \\
\hline $\mathrm{H}$ & -9.828200 & -3.261600 & 0.011700 \\
\hline $\mathrm{H}$ & -4.346800 & 1.835500 & 2.905500 \\
\hline $\mathrm{H}$ & -3.699600 & -1.539000 & 5.473400 \\
\hline $\mathrm{H}$ & -4.054300 & -3.068100 & 3.567700 \\
\hline $\mathrm{H}$ & -8.730200 & -1.217200 & -0.859500 \\
\hline $\mathrm{H}$ & -8.526600 & -4.895300 & 1.346000 \\
\hline $\mathrm{H}$ & -3.839000 & 0.915200 & 5.146100 \\
\hline $\mathrm{H}$ & -0.014000 & 4.079800 & -2.007200 \\
\hline $\mathrm{H}$ & -3.992600 & 4.793300 & -4.872400 \\
\hline $\mathrm{H}$ & 0.263800 & 4.993800 & -4.276800 \\
\hline $\mathrm{H}$ & -3.842800 & 4.283500 & 2.843700 \\
\hline$\Pi$ & -4.478500 & 3.936100 & -0.549400 \\
\hline $\mathrm{H}$ & -3.915800 & 2.687900 & 0.644100 \\
\hline 11 & -4.687100 & 6.105300 & 0.632300 \\
\hline 21 & -0.504000 & 6.027800 & -0.426200 \\
\hline & -2.752100 & 9.660700 & -0.782700 \\
\hline
\end{tabular}

$\begin{array}{rrrr}\mathrm{H} & -2.885500 & 3.859100 & 5.079300 \\ \mathrm{H} & 1.049100 & 3.676500 & 3.382200 \\ \mathrm{H} & 0.103100 & 4.087900 & 1.125100 \\ \mathrm{H} & -0.618000 & 8.420400 & -1.010900 \\ \mathrm{H} & -4.778500 & 8.499200 & 0.047000 \\ \mathrm{H} & -0.441100 & 3.546300 & 5.349800 \\ \mathrm{H} & 3.356700 & 4.652500 & -1.597700 \\ \mathrm{H} & 5.311900 & 0.852100 & -4.100600 \\ \mathrm{H} & 4.292900 & 5.022800 & -3.843800 \\ \mathrm{H} & 3.765400 & 3.118600 & 3.675900 \\ \mathrm{H} & 3.685200 & 4.422200 & 0.461600 \\ \mathrm{H} & 2.408300 & 3.602100 & 1.459700 \\ \mathrm{H} & 5.919900 & 0.527300 & -0.012800 \\ \mathrm{H} & 5.793800 & 4.473100 & 1.729300 \\ \mathrm{H} & 9.487900 & 2.886700 & 0.237700 \\ \mathrm{H} & 3.246400 & 1.693600 & 5.623800 \\ \mathrm{H} & 3.456300 & -1.802700 & 3.144700 \\ \mathrm{H} & 3.962600 & -0.387500 & 1.179200 \\ \mathrm{H} & 8.212800 & 4.717500 & 1.317700 \\ \mathrm{H} & 8.334700 & 0.791900 & -0.418900 \\ \mathrm{H} & 3.088200 & -0.766800 & 5.362400 \\ \mathrm{H} & 4.364100 & 0.483400 & -1.855800 \\ \mathrm{H} & 3.150500 & -2.450100 & -2.136500 \\ \mathrm{H} & -4.265500 & 3.873100 & -2.607600 \\ \mathrm{H} & -4.206600 & -0.434500 & -2.049100 \\ \mathrm{H} & 7.385800 & -1.981800 & -1.543600 \\ \mathrm{H} & 5.288700 & 3.127000 & -5.114800 \\ \mathrm{H} & -5.779300 & -2.886400 & -5.206800 \\ \mathrm{H} & -1.728000 & 5.369600 & -5.729400\end{array}$

\section{Q1}

$\begin{array}{llll}\mathrm{Rh} & 0.000100 & 0.000000 & 0.672700\end{array}$

$\begin{array}{llll}\mathrm{Rh} & -0.000100 & 0.000200 & -1.708800\end{array}$

$\begin{array}{llll}\mathrm{O} & 1.701100 & -1.171800 & 0.600900\end{array}$

$\begin{array}{llll}\mathrm{O} & 1.590400 & -1.313900 & -1.647300\end{array}$

$\begin{array}{llll}\mathrm{O} & 1.239200 & 1.657700 & 0.614100\end{array}$

$\begin{array}{llll}\text { O } & 1.300100 & 1.608200 & -1.642800\end{array}$

$\begin{array}{llll}\mathrm{O} & -1.700900 & 1.172000 & 0.601300\end{array}$

$\begin{array}{llll}\mathrm{O} & -1.590500 & 1.314400 & -1.646900\end{array}$

$\begin{array}{llll}\text { O } & -1.239000 & -1.657600 & 0.614100\end{array}$

O $\quad-1.300200 \quad-1.607800 \quad-1.642900$

$\begin{array}{llll}\text { O } & 6.220600 & -2.047000 & 5.376900\end{array}$

$\begin{array}{llll}\text { O } & 6.360800 & -4.164700 & 5.026800\end{array}$

$\begin{array}{llll}\text { O } & 8.654200 & 1.665200 & 1.698700\end{array}$

O $\quad 7.511100 \quad 1.347900 \quad 3.492800$

$\begin{array}{llll}\mathrm{O} & -6.218000 & 2.047100 & 5.379000\end{array}$

$\begin{array}{llll}\text { O } & -6.357000 & 4.165000 & 5.029700\end{array}$

$\begin{array}{llll}\text { O } & -8.656800 & -1.669100 & 1.693700\end{array}$

$\begin{array}{llll}\mathrm{O} & -7.514800 & -1.347200 & 3.487700\end{array}$

$\mathrm{N} \quad 6.004500 \quad-3.032100 \quad 4.640800$

$\mathrm{N} \quad \begin{array}{llll}7.555200 & 1.682400 & 2.290700\end{array}$

$\mathrm{N} \quad-6.001600 \quad 3.032200 \quad 4.643100$

$\mathrm{N} \quad-7.557900 \quad-1.683700 \quad 2.286100$

$\begin{array}{llll}\text { C } & 4.494700 & -3.785600 & 1.284500\end{array}$

C $\quad 3.472100 \quad-2.304400 \quad-0.531900$

C $\quad 4.432800 \quad-2.210600 \quad-1.776000$

C $\quad 4.950600 \quad-1.595000 \quad 2.918500$

C $\quad 4.083500 \quad-2.525100 \quad 0.840200$

$\begin{array}{lrrr}\text { C } & 5.117300 & -3.957700 & 2.523100\end{array}$

C $\quad 5.897300 \quad-2.353000 \quad-1.501000$

C $\quad 2.166400 \quad-1.534000 \quad-0.528000$

$\begin{array}{llll}\text { C } & 4.118000 & -1.283900 & -2.905100\end{array}$ 


\begin{tabular}{|c|c|c|c|}
\hline $\mathrm{C}$ & 4.054200 & 0.101200 & -2.690600 \\
\hline & 3.549100 & -3.409700 & -1.583600 \\
\hline $\mathrm{C}$ & 6.579900 & -1.363000 & -0.772400 \\
\hline $\mathrm{C}$ & 4.329300 & -1.431100 & 1.683800 \\
\hline $\mathrm{C}$ & 6.618000 & -3.444200 & -2.015600 \\
\hline $\mathrm{C}$ & 5.356600 & -2.863600 & 3.363800 \\
\hline $\mathrm{C}$ & 8.652800 & -2.588200 & -1.017600 \\
\hline $\mathrm{C}$ & 3.816100 & 0.969400 & -3.759900 \\
\hline $\mathrm{C}$ & 3.714000 & -0.915600 & -5.273500 \\
\hline $\mathrm{C}$ & 3.961000 & -1.786200 & -4.208000 \\
\hline $\mathrm{C}$ & 7.988800 & -3.563400 & -1.766700 \\
\hline $\mathrm{C}$ & 7.949300 & -1.486600 & -0.524500 \\
\hline $\mathrm{C}$ & 3.640700 & 0.461500 & -5.049400 \\
\hline $\mathrm{C}$ & 3.948300 & 2.457700 & 1.575700 \\
\hline $\mathrm{C}$ & 2.703800 & 3.188100 & -0.519300 \\
\hline $\mathrm{C}$ & 2.271600 & 4.683900 & -0.541900 \\
\hline $\mathrm{C}$ & 6.379600 & 2.387000 & 0.229300 \\
\hline $\mathrm{C}$ & 3.973500 & 2.781900 & 0.210500 \\
\hline $\mathrm{C}$ & 5.115800 & 2.115400 & 2.255600 \\
\hline $\mathrm{C}$ & 3.168400 & 5.676700 & 0.131100 \\
\hline $\mathrm{C}$ & 1.656300 & 2.077000 & -0.513600 \\
\hline $\mathrm{C}$ & 0.822700 & 5.036400 & -0.482700 \\
\hline $\mathrm{C}$ & 0.060800 & 4.721300 & 0.654400 \\
\hline $\mathrm{C}$ & 2.787700 & 3.998600 & -1.789600 \\
\hline $\mathrm{C}$ & 3.299200 & 5.683200 & 1.530600 \\
\hline $\mathrm{C}$ & 5.209500 & 2.741400 & -0.445500 \\
\hline $\mathrm{C}$ & 3.837400 & 7200 & -0.622700 \\
\hline $\mathrm{C}$ & 6.353300 & 2.065500 & 1.593000 \\
\hline $\mathrm{C}$ & 4.808800 & 7.571600 & 1.398600 \\
\hline $\mathrm{C}$ & -1.251200 & 5.187100 & 0.772700 \\
\hline $\mathrm{C}$ & -1.065100 & 6.273300 & -1.382600 \\
\hline $\mathrm{C}$ & 0.249200 & 5.814400 & -1.502100 \\
\hline $\mathrm{C}$ & 4.660600 & 7.593800 & 0.009500 \\
\hline $\mathrm{C}$ & 4.124400 & 6.620200 & 2.158700 \\
\hline $\mathrm{C}$ & -1.809000 & 5.972700 & -0.239700 \\
\hline $\mathrm{C}$ & -4.329100 & 1.431200 & 1.684600 \\
\hline $\mathrm{C}$ & -3.472200 & 2.304500 & -0.531000 \\
\hline $\mathrm{C}$ & -4.433300 & 2.210300 & -1.774800 \\
\hline $\mathrm{C}$ & -5.114900 & 3.958000 & 2.525300 \\
\hline $\mathrm{C}$ & -4.083000 & 2.525300 & 0.841300 \\
\hline $\mathrm{C}$ & -4.949600 & 1.595000 & 2.919800 \\
\hline $\mathrm{C}$ & -5.897800 & 2.352600 & -1.499300 \\
\hline $\mathrm{C}$ & -2.166300 & 1.534300 & -0.527400 \\
\hline $\mathrm{C}$ & -4.118700 & 1.283500 & -2.903800 \\
\hline $\mathrm{C}$ & -4.054600 & -0.101500 & -2.689100 \\
\hline $\mathrm{C}$ & -3.549700 & 3.409600 & -1.582900 \\
\hline $\mathrm{C}$ & -6.618700 & 3.443800 & -2.013700 \\
\hline C & -4.493000 & 3.785900 & 1.286300 \\
\hline $\mathrm{C}$ & -6.580000 & 1.362600 & -0.770500 \\
\hline $\mathrm{C}$ & -5.354500 & 2.863800 & 3.365700 \\
\hline $\mathrm{C}$ & -8.653100 & 2.587600 & -1.015100 \\
\hline $\mathrm{C}$ & -3.816700 & -0.969900 & -3.758300 \\
\hline $\mathrm{C}$ & -3.715500 & 0.914800 & -5.272300 \\
\hline $\mathrm{C}$ & -3.962400 & 1.785600 & -4.206900 \\
\hline $\mathrm{C}$ & -7.949300 & 1.486000 & -0.522200 \\
\hline $\mathrm{C}$ & -7.989400 & 3.562900 & -1.764400 \\
\hline $\mathrm{C}$ & -3.641900 & -0.462200 & -5.047900 \\
\hline $\mathrm{C}$ & -5.209500 & -2.743300 & -0.447500 \\
\hline $\mathrm{C}$ & -2.703400 & -3.188100 & -0.519300 \\
\hline $\mathrm{C}$ & -2.270900 & -4.683800 & -0.540600 \\
\hline $\mathrm{C}$ & -5.118100 & -2.113900 & 2.252900 \\
\hline & -3.973900 & -2.781700 & 0.209300 \\
\hline
\end{tabular}

$\begin{array}{llll}\text { C } & -6.380400 & -2.389400 & 0.226200\end{array}$

$\begin{array}{llll}\text { C } & -3.168000 & -5.676300 & 0.132600\end{array}$

C $\quad-1.656200 \quad-2.076800 \quad-0.513600$

C $\quad-0.822000 \quad-5.036100 \quad-0.480300$

C $\quad-0.247900 \quad-5.814800 \quad-1.498800$

C $\quad-2.786300 \quad-3.999500 \quad-1.789200$

C $\quad-3.300100 \quad-5.681500 \quad 1.531900$

$\begin{array}{llll}\text { C } & -3.949800 & -2.455800 & 1.574000\end{array}$

C $\quad-3.836100 \quad-6.657600 \quad-0.621000$

C $\quad-6.355200 \quad-2.066200 \quad 1.589500$

$\begin{array}{llll}\text { C } & -4.809000 & -7.570500 & 1.400300\end{array}$

$\begin{array}{llll}\text { C } & 1.066300 & -6.273400 & -1.378300\end{array}$

$\begin{array}{llll}\text { C } & 1.251300 & -5.185600 & 0.776200\end{array}$

C $\quad-0.060700 \quad-4.720100 \quad 0.657000$

$\begin{array}{llll}\text { C } & -4.659600 & -7.593800 & 0.011400\end{array}$

$\begin{array}{llll}\text { C } & -4.125600 & -6.618200 & 2.160200\end{array}$

C $\quad 1.809700 \quad-5.971900-0.235200$

H $\quad 4.339500 \quad-4.661900 \quad 0.666000$

$\mathrm{H} \quad 5.118200 \quad-0.705700 \quad 3.520500$

H $\quad 5.409000 \quad-4.965600 \quad 2.808500$

$\mathrm{H} \quad 4.181900 \quad 0.501500 \quad-1.695400$

H $\quad 3.990700 \quad-4.356100 \quad-1.291700$

H $\quad 2.676700 \quad-3.512000 \quad-2.221700$

H $\quad 6.051400 \quad-0.498500 \quad-0.393800$

H $\quad 6.120200 \quad-4.201300 \quad-2.608400$

H $\quad 9.713900 \quad-2.682200 \quad-0.826300$

$\mathrm{H} \quad 3.771600 \quad 2.035800 \quad-3.591900$

H $\quad 3.587500 \quad-1.307900 \quad-6.274300$

$\mathrm{H} \quad 4.034000 \quad-2.849900 \quad-4.398100$

H $\quad 8.537200 \quad-4.410500 \quad-2.157900$

H $\quad 8.465500 \quad-0.725900 \quad 0.046400$

$\mathrm{H} \quad 3.454900 \quad 1.135400 \quad-5.875600$

$\mathrm{H} \quad 3.004000 \quad 2.475000 \quad 2.108100$

H $\quad 7.305700 \quad 2.358700 \quad-0.339700$

$\mathrm{H} \quad 5.036000 \quad 1.889800 \quad 3.316400$

$\mathrm{H} \quad 0.492400 \quad 4.145500 \quad 1.462800$

H $\quad 3.769000 \quad 4.264300 \quad-2.170100$

$\mathrm{H} \quad 2.080100 \quad 3.737500 \quad-2.569700$

H $\quad 2.760100 \quad 4.967200 \quad 2.135900$

H $\quad 3.721300 \quad 6.698300 \quad-1.698400$

$\mathrm{H} \quad 5.445700 \quad 8.297900 \quad 1.886600$

$\mathrm{H} \quad-1.826800 \quad 4.956600 \quad 1.659300$

H $\quad-1.499500 \quad 6.880500 \quad-2.166000$

H $\quad 0.829400 \quad 6.089600 \quad-2.374200$

$\mathrm{H} \quad 5.180200 \quad 8.340000 \quad-0.577400$

$\mathrm{H} \quad 4.228300 \quad 6.612400 \quad 3.236000$

$\mathrm{H} \quad-2.816400 \quad 6.353400 \quad-0.138800$

$\mathrm{H} \quad-4.041600 \quad 0.434900 \quad 1.370400$

$\begin{array}{llll}\mathrm{H} & -5.405800 & 4.966000 & 2.811200\end{array}$

$\mathrm{H} \quad-5.117400 \quad 0.705700 \quad 3.521700$

$\mathrm{H} \quad-4.181800 \quad-0.501600 \quad-1.693700$

$\mathrm{H} \quad-3.991200 \quad 4.356000 \quad-1.291000$

$\mathrm{H} \quad-2.677500 \quad 3.511900 \quad-2.221300$

H $\quad-6.121100 \quad 4.200900 \quad-2.606600$

H $\quad-6.051400 \quad 0.498100 \quad-0.392200$

$\mathrm{H} \quad-9.714100 \quad 2.681500 \quad-0.823500$

H $\quad-3.771700 \quad-2.036200 \quad-3.590100$

$\mathrm{H} \quad-3.589600 \quad 1.307000 \quad-6.273200$

$\mathrm{H} \quad-4.035700 \quad 2.849200 \quad-4.397200$

H $\quad-8.465400 \quad 0.725300 \quad 0.048800$

H $\quad-8.538000 \quad 4.409900 \quad-2.155500$

H $\quad-3.456200 \quad-1.136200 \quad-5.874100$
H $\quad-5.267300 \quad-2.973200 \quad-1.506500$

$\mathrm{H} \quad-5.039100 \quad-1.886900 \quad 3.313400$

$\mathrm{H} \quad-7.306200 \quad-2.362900 \quad-0.343400$

$\mathrm{H} \quad-0.827600 \quad-6.090600 \quad-2.371000$

$\mathrm{H} \quad-3.767200 \quad-4.265600 \quad-2.170300$

$\mathrm{H} \quad-2.078100 \quad-3.738800 \quad-2.568900$

$\mathrm{H} \quad-2.761700 \quad-4.964800 \quad 2.137000$

$\mathrm{H} \quad-3.719200 \quad-6.699600 \quad-1.696600$

$\mathrm{H} \quad-5.446200 \quad-8.296500 \quad 1.888400$

H $\quad 1.501200 \quad-6.881200 \quad-2.161000$

$\mathrm{H} \quad 1.826500 \quad-4.954400 \quad 1.662900$

$\mathrm{H} \quad-0.492800 \quad-4.143800 \quad 1.464800$

$\mathrm{H} \quad-5.178600 \quad-8.340700 \quad-0.575300$

$\mathrm{H} \quad-4.230400 \quad-6.609500 \quad 3.237400$

H $\quad 2.817000 \quad-6.352600 \quad-0.133500$

$\mathrm{H} \quad-3.005800 \quad-2.471400 \quad 2.106900$

$\mathrm{H} \quad 4.041100 \quad-0.434800 \quad 1.370000$

$\mathrm{H} \quad-4.337600 \quad 4.662300 \quad 0.668000$

$\begin{array}{llll}\mathrm{H} & 5.268100 & 2.970000 & -1.504700\end{array}$

\section{R1}

$\begin{array}{llll}\mathrm{Rh} & -0.000100 & -0.000100 & 0.506600\end{array}$

$\begin{array}{lllll}\mathrm{Rh} & -0.000100 & 0.000000 & -1.874500\end{array}$

$\begin{array}{llll}\text { O } & 1.752000 & -1.106300 & 0.423600\end{array}$

O $\quad 1.608900 \quad-1.281700 \quad-1.820700$

$\begin{array}{llll}\mathrm{O} & 1.173400 & 1.697600 & 0.456000\end{array}$

$\begin{array}{llll}\text { O } & 1.279300 & 1.629200 & -1.798900\end{array}$

$\begin{array}{llll}\text { O } & -1.752100 & 1.106200 & 0.423900\end{array}$

$\begin{array}{llll}\mathrm{O} & -1.609100 & 1.281700 & -1.820500\end{array}$

$\begin{array}{llll}\text { O } & -1.173400 & -1.697600 & 0.455800\end{array}$

O $\quad-1.279500 \quad-1.629200 \quad-1.799100$

F $\quad 5.473200 \quad-3.755000 \quad 5.247500$

F $\quad 7.452300 \quad-3.491100 \quad 4.204100$

F $\quad 6.401100 \quad-1.713100 \quad 5.091000$

F $\quad \begin{array}{llll}7.951900 & 0.647200 & 1.974200\end{array}$

F $\quad 7.143900 \quad 1.661600 \quad 3.808700$

F $\quad 8.364000 \quad 2.833400 \quad 2.328900$

F $\quad-8.364200 \quad-2.834900 \quad 2.328000$

F $\quad-7.144700 \quad-1.662300 \quad 3.807700$

F $\quad-7.953000 \quad-0.648600 \quad 1.972900$

F $\quad-6.401200 \quad 1.713900 \quad 5.091300$

F $\quad-7.451800 \quad 3.492300 \quad 4.204500$

F $\quad-5.472700 \quad 3.755400 \quad 5.248000$

$\begin{array}{llll}\text { C } & 4.569600 & -3.683800 & 1.085600\end{array}$

C $\quad 3.524900 \quad-2.222600 \quad-0.731600$

C $\quad 4.455400 \quad-2.128000 \quad-1.997800$

$\begin{array}{llll}\text { C } & 5.092200 & -1.462400 & 2.665600\end{array}$

C $\quad 4.166400 \quad-2.425600 \quad 0.627300$

$\begin{array}{llll}\text { C } & 5.222700 & -3.834000 & 2.310700\end{array}$

$\begin{array}{llll}\text { C } & 5.928000 & -2.264300 & -1.764900\end{array}$

$\begin{array}{llll}\text { C } & 2.206900 & -1.474100 & -0.707900\end{array}$

C $\quad 4.105800 \quad-1.208900-3.122900$

$\begin{array}{llll}\text { C } & 3.915500 & -1.719100 & -4.418300\end{array}$

C $\quad 3.588600 \quad-3.334600 \quad-1.777300$

$\begin{array}{llll}\text { C } & 6.635600 & -3.356700 & -2.296200\end{array}$

$\begin{array}{llll}\text { C } & 4.441800 & -1.317700 & 1.443200\end{array}$

$\begin{array}{llll}\text { C } & 6.632100 & -1.263900 & -1.072100\end{array}$

$\begin{array}{llll}\text { C } & 5.497700 & -2.726700 & 3.121500\end{array}$

$\begin{array}{llll}\text { C } & 8.702000 & -2.479500 & -1.387200\end{array}$

C $\quad 3.638700 \quad-0.855300 \quad-5.482000$

$\begin{array}{llll}\text { C } & 3.778500 & 1.038900 & -3.982900\end{array}$

$\begin{array}{llll}\text { C } & 4.044100 & 0.177600 & -2.914500\end{array}$ 


\begin{tabular}{|c|c|c|c|}
\hline r & 8.011700 & -1.376100 & -0.879600 \\
\hline $\mathrm{C}$ & 8.015100 & -3.466700 & -2.098400 \\
\hline $\mathrm{C}$ & 3.569900 & 0.523000 & -5.264300 \\
\hline $\mathrm{C}$ & 5.147200 & 2.754300 & -0.447600 \\
\hline $\mathrm{C}$ & 2.652100 & 3.225300 & -0.652800 \\
\hline $\mathrm{C}$ & 2.215500 & 4.718700 & -0.729500 \\
\hline $\mathrm{C}$ & 4.920800 & 2.276900 & 2.281300 \\
\hline $\mathrm{C}$ & 3.884000 & 2.841600 & 0.149200 \\
\hline $\mathrm{C}$ & 6.278200 & 2.426400 & 0.303000 \\
\hline & 3.080500 & 5.734700 & -0.048200 \\
\hline $\mathrm{C}$ & 1.609900 & 2.109900 & -0.666600 \\
\hline $\mathrm{C}$ & 0.762000 & 5.057600 & -0.726300 \\
\hline $\mathrm{C}$ & 0.212100 & 5.803900 & -1.781500 \\
\hline & 2.785500 & 4.005500 & -1.937400 \\
\hline & 3.782400 & 6.691900 & -0.802500 \\
\hline $\mathrm{C}$ & 3.791100 & 2.586400 & 1.526300 \\
\hline $\mathrm{C}$ & 3.146700 & 5.789100 & 1.354900 \\
\hline $\mathrm{C}$ & 6.186500 & 2.186600 & 1.680000 \\
\hline $\mathrm{C}$ & 4.660600 & 7.674400 & 1.229400 \\
\hline $\mathrm{C}$ & -1.111800 & 6.247000 & -1.714700 \\
\hline $\mathrm{C}$ & -1.352700 & 5.209900 & 0.459100 \\
\hline $\mathrm{C}$ & -0.032300 & 4.759000 & 0.393100 \\
\hline $\mathrm{C}$ & 3.942200 & 6.747800 & 1.988600 \\
\hline $\mathrm{C}$ & 4.575600 & 7.650400 & -0.164800 \\
\hline C & -1.888500 & 5.962700 & -0.589100 \\
\hline $\mathrm{C}$ & -4.442100 & 1.318100 & 1.443400 \\
\hline $\mathrm{C}$ & -3.525000 & 2.222800 & -0.731300 \\
\hline $\mathrm{C}$ & -4.455500 & 2.128200 & -1.997400 \\
\hline $\mathrm{C}$ & -5.222100 & 3.834600 & 2.311100 \\
\hline $\mathrm{C}$ & -4.166300 & 2.425900 & 0.627600 \\
\hline $\mathrm{C}$ & -5.092400 & 1.462900 & 2.665800 \\
\hline $\mathrm{C}$ & -5.928100 & 2.264800 & -1.764600 \\
\hline $\mathrm{C}$ & -2.207000 & 1.474100 & -0.707600 \\
\hline $\mathrm{C}$ & -4.106200 & 1.208900 & -3.122400 \\
\hline $\mathrm{C}$ & -3.915800 & 1.718900 & -4.417900 \\
\hline $\mathrm{C}$ & -3.588500 & 3.334600 & -1.777100 \\
\hline $\mathrm{C}$ & -6.632400 & 1.264500 & -1.071700 \\
\hline $\mathrm{C}$ & -4.569100 & 3.684200 & 1.086000 \\
\hline $\mathrm{C}$ & -6.635400 & 3.357300 & -2.295900 \\
\hline $\mathrm{C}$ & -5.497400 & 2.727300 & 3.121900 \\
\hline $\mathrm{C}$ & -8.702000 & 2.480500 & -1.386800 \\
\hline $\mathrm{C}$ & -3.639100 & 0.854800 & -5.481400 \\
\hline $\mathrm{C}$ & -3.779100 & -1.039100 & -3.982100 \\
\hline $\mathrm{C}$ & -4.044600 & -0.177600 & -2.913800 \\
\hline $\mathrm{C}$ & -8.015000 & 3.467600 & -2.098100 \\
\hline $\mathrm{C}$ & -8.012000 & 1.377100 & -0.879200 \\
\hline $\mathrm{C}$ & -3.570400 & -0.523500 & -5.263500 \\
\hline $\mathrm{C}$ & -3.791300 & -2.586400 & 1.525900 \\
\hline $\mathrm{C}$ & -2.651900 & -3.225600 & -0.652900 \\
\hline $\mathrm{C}$ & -2.215100 & -4.718900 & -0.729200 \\
\hline $\mathrm{C}$ & -6.278400 & -2.427400 & 0.302300 \\
\hline $\mathrm{C}$ & -3.884000 & -2.842000 & 0.148800 \\
\hline $\mathrm{C}$ & -4.921200 & -2.277100 & 2.280600 \\
\hline $\mathrm{C}$ & -3.080000 & -5.734900 & -0.047800 \\
\hline $\mathrm{C}$ & -1.609900 & -2.110000 & -0.666700 \\
\hline $\mathrm{C}$ & -0.761600 & -5.057700 & -0.725800 \\
\hline $\mathrm{C}$ & -0.211500 & -5.804100 & -1.780800 \\
\hline $\mathrm{C}$ & -2.785100 & -4.006100 & -1.937300 \\
\hline $\mathrm{C}$ & -3.781700 & -6.692400 & -0.801900 \\
\hline $\mathrm{C}$ & -5.147200 & -2.755100 & -0.448100 \\
\hline $\mathrm{C}$ & -3.146300 & -5.788900 & 1.355300 \\
\hline C & -6.186900 & -2.187300 & 1.679300 \\
\hline
\end{tabular}

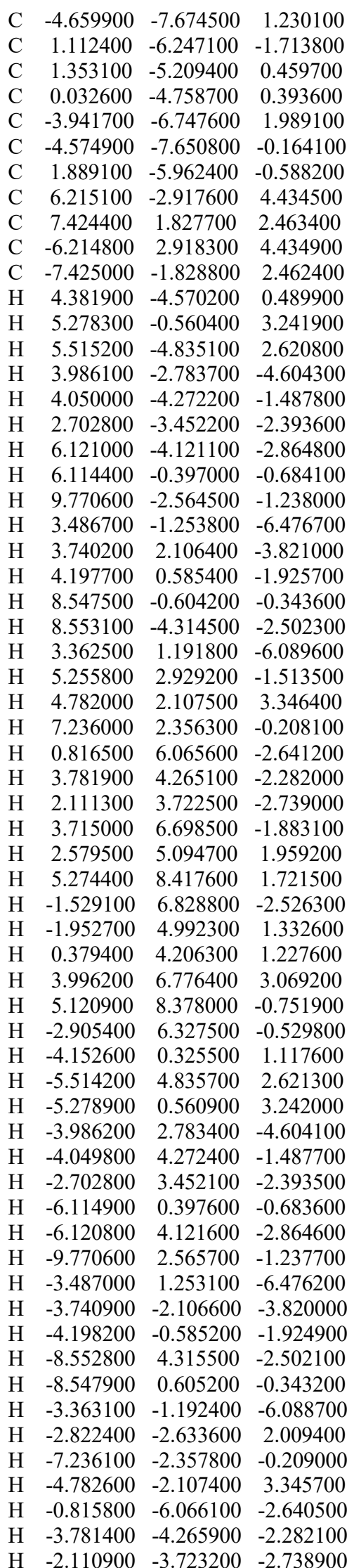

$\begin{array}{rrrr}\mathrm{H} & -3.714200 & -6.699100 & -1.882500 \\ \mathrm{H} & -2.579200 & -5.094300 & 1.959500 \\ \mathrm{H} & -5.273700 & -8.417700 & 1.722400 \\ \mathrm{H} & 1.529800 & -6.829000 & -2.525300 \\ \mathrm{H} & 1.953000 & -4.991500 & 1.333200 \\ \mathrm{H} & -0.379200 & -4.205800 & 1.227900 \\ \mathrm{H} & -3.995800 & -6.776000 & 3.069700 \\ \mathrm{H} & -5.120100 & -8.378600 & -0.751100 \\ \mathrm{H} & 2.906000 & -6.327100 & -0.528700 \\ \mathrm{H} & -5.255700 & -2.930300 & -1.514000 \\ \mathrm{H} & 4.152000 & -0.325200 & 1.117500 \\ \mathrm{H} & -4.381000 & 4.570700 & 0.490300 \\ \mathrm{H} & 2.822000 & 2.634000 & 2.009700\end{array}$

\section{R2}

$\begin{array}{llll}\mathrm{Rh} & 0.003200 & 0.027900 & 0.580900\end{array}$

$\begin{array}{lllll}\mathrm{Rh} & -0.136600 & 0.054700 & -1.796600\end{array}$

O $\quad 1.725400 \quad-1.116200 \quad 0.395600$

$\begin{array}{llll}\text { O } & 1.471300 & -1.226500 & -1.840800\end{array}$

$\begin{array}{llll}\mathrm{O} & 1.212800 & 1.702100 & 0.469600\end{array}$

$\begin{array}{llll}\mathrm{O} & 1.140000 & 1.682500 & -1.787800\end{array}$

$\begin{array}{llll}\mathrm{O} & -1.727400 & 1.163400 & 0.615100\end{array}$

$\begin{array}{llll}\mathrm{O} & -1.742800 & 1.330200 & -1.634300\end{array}$

$\begin{array}{llll}\mathrm{O} & -1.183100 & -1.665600 & 0.574200\end{array}$

$\begin{array}{llll}\text { O } & -1.350700 & -1.611600 & -1.678300\end{array}$

F $\quad-7.060200 \quad 3.659800 \quad 4.819500$

F $\quad-4.995800 \quad 3.985400 \quad 5.660300$

F $\quad \begin{array}{rrrr}-5.899800 & 1.927300 & 5.658900\end{array}$

F $\quad-8.285300 \quad-1.601700 \quad 1.609400$

F $\quad-8.639000 \quad-1.687900-0.610000$

F $\quad-8.735500 \quad-3.570100 \quad 0.622800$

$\begin{array}{llll}\text { F } & 8.009200 & 0.492500 & 1.501600\end{array}$

F $\quad 7.383800 \quad 1.530500 \quad 3.393800$

$\begin{array}{llll}\text { F } & 8.531800 & 2.659900 & 1.825800\end{array}$

F $\quad 6.797000 \quad-1.661500 \quad 4.664600$

F $\quad 6.141200 \quad-3.808800 \quad 4.780800$

F $\quad 7.953700 \quad-3.245900 \quad 3.567000$

C $\quad 4.797600 \quad-3.637800 \quad 0.744500$

C $\quad 3.423300 \quad-2.228500 \quad-0.882900$

C $\quad 4.240200 \quad-2.181500 \quad-2.229800$

$\begin{array}{llll}\text { C } & 5.222100 & -1.453300 & 2.397900\end{array}$

$\begin{array}{llll}\text { C } & 4.195700 & -2.419200 & 0.408800\end{array}$

$\begin{array}{lrrr}\text { C } & 5.593700 & -3.767200 & 1.883700\end{array}$

$\begin{array}{llll}\text { C } & 5.722900 & -2.358400 & -2.122000\end{array}$

C $\quad 2.122800 \quad-1.456500 \quad-0.765700$

C $\quad 3.826300 \quad-1.253400 \quad-3.325600$

C $\quad 3.500400 \quad-1.758100 \quad-4.595500$

C $\quad 3.357200 \quad-3.356400 \quad-1.915500$

C $\quad 6.508900 \quad-1.386100 \quad-1.478100$

$\begin{array}{llll}\text { C } & 4.435400 & -1.326200 & 1.255700\end{array}$

$\begin{array}{llll}\text { C } & 6.352200 & -3.467400 & -2.712700\end{array}$

$\begin{array}{lrrr}\text { C } & 5.818800 & -2.678000 & 2.733000\end{array}$

$\begin{array}{llll}\text { C } & 8.509200 & -2.661200 & -1.961000\end{array}$

C $\quad 3.153900 \quad-0.886000 \quad-5.631800$

$\begin{array}{llll}\text { C } & 3.490000 & 1.004100 & -4.158000\end{array}$

$\begin{array}{llll}\text { C } & 3.828800 & 0.135000 & -3.117300\end{array}$

$\begin{array}{llll}\text { C } & 7.739100 & -3.620500 & -2.623900\end{array}$

$\begin{array}{llll}\text { C } & 7.894800 & -1.542500 & -1.392800\end{array}$

$\begin{array}{llll}\text { C } & 3.147700 & 0.494200 & -5.412900\end{array}$

C $\quad 3.903100 \quad 2.585500 \quad 1.372500$

$\begin{array}{llll}\text { C } & 2.622400 & 3.233700 & -0.725500\end{array}$

$\begin{array}{llll}\text { C } & 2.198700 & 4.731500 & -0.777900\end{array}$ 


\begin{tabular}{|c|c|c|c|}
\hline & 6.282500 & 2.332400 & -0.036000 \\
\hline $\mathrm{C}$ & 3.899100 & 2.828600 & -0.009800 \\
\hline $\mathrm{C}$ & 5.076300 & 2.240800 & 2.041200 \\
\hline & 3.113500 & 5.738100 & -0.150100 \\
\hline & 1.566200 & 2.133000 & -0.675500 \\
\hline $\mathrm{C}$ & 0.751200 & 5.084300 & -0.688400 \\
\hline $\mathrm{C}$ & 0.020300 & 4.788700 & 0.474300 \\
\hline $\mathrm{C}$ & 2.687200 & 4.011600 & -2.017200 \\
\hline & 3.262300 & 5.792700 & 1.246500 \\
\hline & 5.109500 & 2.696800 & -0.700000 \\
\hline $\mathrm{C}$ & 3.780500 & 6.686800 & -0.945700 \\
\hline $\mathrm{C}$ & 6.287700 & 2.100200 & 1.345100 \\
\hline $\mathrm{C}$ & 4.786900 & 7.661200 & 1.029900 \\
\hline $\mathrm{C}$ & -1.292600 & 5.244400 & 0.614600 \\
\hline & -1.170700 & 6.281500 & -1.569200 \\
\hline $\mathrm{C}$ & 0.145700 & 5.833600 & -1.710500 \\
\hline $\mathrm{C}$ & 4.620200 & 7.637000 & -0.356900 \\
\hline $\mathrm{C}$ & 4.104000 & 6.743100 & 1.831200 \\
\hline $\mathrm{C}$ & -1.884200 & 5.999000 & -0.402000 \\
\hline & -4.283400 & 1.441800 & 1.854500 \\
\hline $\mathrm{C}$ & -3.568400 & 2.292600 & -0.417200 \\
\hline $\mathrm{C}$ & -4.593000 & 2.181600 & -1.608800 \\
\hline $\mathrm{C}$ & -5.023700 & 3.970400 & 2.714200 \\
\hline $\mathrm{C}$ & -4.097700 & 2.527400 & 0.984100 \\
\hline C & -4.822700 & 1.613000 & 3.126800 \\
\hline $\mathrm{C}$ & -6.042000 & 2.355900 & -1.277500 \\
\hline $\mathrm{C}$ & -2.258400 & 1.530900 & -0.483100 \\
\hline $\mathrm{C}$ & -4.335100 & 1.230800 & -2.732200 \\
\hline $\mathrm{C}$ & -4.244100 & -0.149100 & -2.490900 \\
\hline $\mathrm{C}$ & -3.698100 & 3.381800 & -1.480300 \\
\hline $\mathrm{C}$ & -6.705900 & 1.417200 & -0.469200 \\
\hline $\mathrm{C}$ & -4.480700 & 3.792400 & 1.439900 \\
\hline $\mathrm{C}$ & -6.768200 & 3.433700 & -1.813400 \\
\hline $\mathrm{C}$ & -5.204600 & 2.886200 & 3.580400 \\
\hline $\mathrm{C}$ & -8.764800 & 2.675400 & -0.672300 \\
\hline $\mathrm{C}$ & -4.006400 & -1.033800 & -3.547600 \\
\hline $\mathrm{C}$ & -3.997400 & 0.819000 & -5.103800 \\
\hline $\mathrm{C}$ & -4.229200 & 1.707400 & -4.049800 \\
\hline $\mathrm{C}$ & -8.122200 & 3.594600 & -1.505200 \\
\hline $\mathrm{C}$ & -8.057800 & 1.586300 & -0.157200 \\
\hline $\mathrm{C}$ & -3.881000 & -0.550200 & -4.852100 \\
\hline $\mathrm{C}$ & -4.881500 & -3.600400 & -1.167800 \\
\hline $\mathrm{C}$ & -2.411900 & -3.413500 & -0.549500 \\
\hline $\mathrm{C}$ & -1.813000 & -4.767100 & -0.083700 \\
\hline $\mathrm{C}$ & -5.619800 & -2.108500 & 1.060300 \\
\hline $\mathrm{C}$ & -3.880500 & -3.113300 & -0.320400 \\
\hline $\mathrm{C}$ & -6.228600 & -3.349000 & -0.906400 \\
\hline $\mathrm{C}$ & -2.752800 & -5.781100 & 0.491500 \\
\hline $\mathrm{C}$ & -1.574300 & -2.138400 & -0.541100 \\
\hline $\mathrm{C}$ & -0.443700 & -4.798900 & 0.513300 \\
\hline $\mathrm{C}$ & -0.209700 & -4.221300 & 1.773000 \\
\hline $\mathrm{C}$ & -1.963800 & -4.440200 & -1.559800 \\
\hline $\mathrm{C}$ & -2.927500 & -7.022800 & -0.143600 \\
\hline $\mathrm{C}$ & -4.274100 & -2.361000 & 0.797000 \\
\hline $\mathrm{C}$ & -3.426500 & -5.529400 & 1.699600 \\
\hline $\mathrm{C}$ & -6.622300 & -2.601500 & 0.210800 \\
\hline $\mathrm{C}$ & -4.480200 & -7.706400 & 1.584800 \\
\hline $\mathrm{C}$ & 1.035100 & -4.358000 & 2.391900 \\
\hline $\mathrm{C}$ & 1.826900 & -5.664400 & 0.515100 \\
\hline $\mathrm{C}$ & 0.585000 & -5.524300 & -0.110800 \\
\hline $\mathrm{C}$ & -4.293300 & -6.485100 & 2.236900 \\
\hline$C$ & -3.793800 & -7.977000 & 0.398300 \\
\hline
\end{tabular}

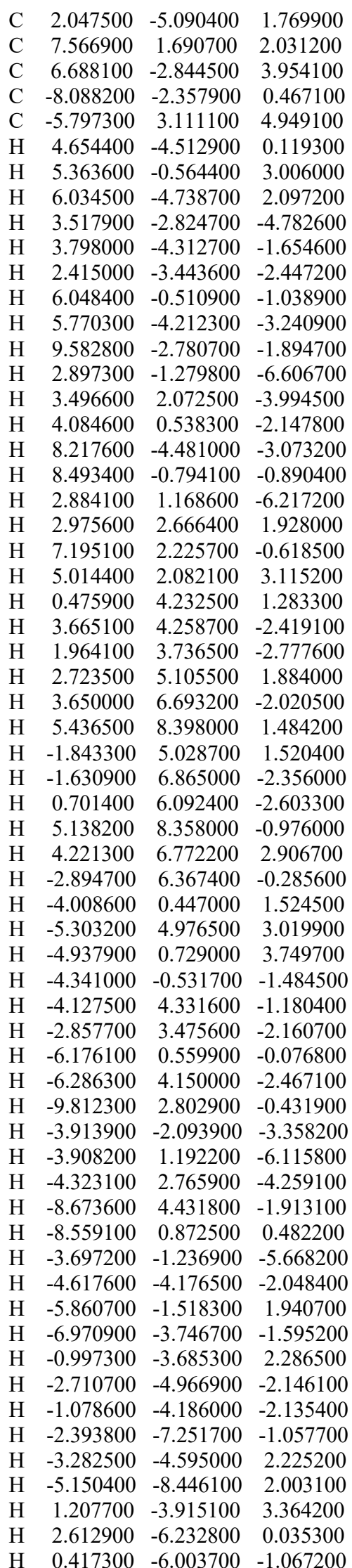

$\mathrm{H} \quad-4.816800 \quad-6.280400 \quad 3.161800$

$\mathrm{H} \quad-3.930600 \quad-8.927900 \quad-0.100200$

$\mathrm{H} \quad 2.999800 \quad-5.220800 \quad 2.265900$

$\mathrm{H} \quad-3.514000 \quad-1.984000 \quad 1.474400$

$\mathrm{H} \quad 4.011600 \quad-0.357900 \quad 1.019800$

$\mathrm{H} \quad-4.361300 \quad 4.663100 \quad 0.804700$

H $\quad 5.142100 \quad 2.863200 \quad-1.772200$

\begin{tabular}{|c|c|c|c|}
\hline$?$ & & & \\
\hline $\mathrm{Rh}$ & 0.005300 & 0.000700 & 0.653100 \\
\hline $\mathrm{Rh}$ & -0.019300 & 0.023500 & -1.729000 \\
\hline $\mathrm{O}$ & -1.726000 & 1.136200 & 0.614700 \\
\hline $\mathrm{O}$ & -1.645500 & 1.278500 & -1.632900 \\
\hline $\mathrm{O}$ & -1.178300 & -1.693500 & 0.582400 \\
\hline $\mathrm{O}$ & -1.214600 & -1.656700 & -1.677100 \\
\hline $\mathrm{O}$ & 1.733100 & -1.140100 & 0.554400 \\
\hline $\mathrm{O}$ & 1.606100 & -1.235300 & -1.693300 \\
\hline $\mathrm{O}$ & 1.190400 & 1.697500 & 0.593100 \\
\hline $\mathrm{O}$ & 1.183500 & 1.697300 & -1.666800 \\
\hline $\mathrm{F}$ & -7.627200 & 3.238400 & 4.347600 \\
\hline $\mathrm{F}$ & -5.707400 & 3.799500 & 5.384200 \\
\hline $\mathrm{F}$ & -6.367000 & 1.651400 & 5.320600 \\
\hline $\mathrm{F}$ & 5.778000 & -4.112900 & 5.133400 \\
\hline $\mathrm{F}$ & 6.422200 & -1.959700 & 5.183000 \\
\hline $\mathrm{F}$ & 7.677100 & -3.478700 & 4.100800 \\
\hline $\mathrm{F}$ & 8.305900 & 1.387700 & 1.132800 \\
\hline $\mathrm{F}$ & 8.755000 & 3.387500 & 0.209600 \\
\hline $\mathrm{F}$ & 8.525200 & 1.568600 & -1.098000 \\
\hline $\mathrm{F}$ & -8.369700 & -1.497500 & 0.872400 \\
\hline $\mathrm{F}$ & -8.762500 & -3.430100 & -0.204400 \\
\hline $\mathrm{F}$ & -8.447700 & -1.531900 & -1.375300 \\
\hline $\mathrm{C}$ & -4.748500 & 3.659600 & 1.242200 \\
\hline $\mathrm{C}$ & -3.513500 & 2.271400 & -0.510300 \\
\hline $\mathrm{C}$ & -4.429200 & 2.254300 & -1.792300 \\
\hline $\mathrm{C}$ & -5.015100 & 1.458400 & 2.910500 \\
\hline $\mathrm{C}$ & -4.175800 & 2.447000 & 0.842500 \\
\hline $\mathrm{C}$ & -5.437700 & 3.775600 & 2.450700 \\
\hline $\mathrm{C}$ & -5.895800 & 2.468300 & -1.583700 \\
\hline $\mathrm{C}$ & -2.211500 & 1.491900 & -0.508300 \\
\hline $\mathrm{C}$ & -4.109100 & 1.331900 & -2.923600 \\
\hline $\mathrm{C}$ & -3.859100 & 1.847300 & -4.206400 \\
\hline $\mathrm{C}$ & -3.508400 & 3.413800 & -1.529300 \\
\hline $\mathrm{C}$ & -6.658000 & 1.531900 & -0.863900 \\
\hline $\mathrm{C}$ & -4.334600 & 1.346800 & 1.700200 \\
\hline $\mathrm{C}$ & -6.536300 & 3.584500 & -2.148700 \\
\hline $\mathrm{C}$ & -5.581400 & 2.679700 & 3.308900 \\
\hline $\mathrm{C}$ & -8.650500 & 2.862500 & -1.214200 \\
\hline $\mathrm{C}$ & -3.566900 & 0.984800 & -5.267000 \\
\hline $\mathrm{C}$ & -3.801000 & -0.917400 & -3.789200 \\
\hline $\mathrm{C}$ & -4.099200 & -0.058900 & -2.726600 \\
\hline $\mathrm{C}$ & -7.907000 & 3.782300 & -1.957900 \\
\hline $\mathrm{C}$ & -8.026800 & 1.737200 & -0.670000 \\
\hline $\mathrm{C}$ & -3.533100 & -0.396200 & -5.057300 \\
\hline $\mathrm{C}$ & -4.314900 & -2.363900 & 0.529000 \\
\hline $\mathrm{C}$ & -2.335300 & -3.450700 & -0.601000 \\
\hline $\mathrm{C}$ & -1.789800 & -4.788300 & -0.039400 \\
\hline $\mathrm{C}$ & -6.081600 & -3.293200 & -1.397300 \\
\hline $\mathrm{C}$ & -3.814800 & -3.127800 & -0.536200 \\
\hline $\mathrm{C}$ & -5.673700 & -2.070900 & 0.632900 \\
\hline $\mathrm{C}$ & -2.783000 & -5.781200 & 0.479000 \\
\hline $\mathrm{C}$ & -1.501100 & -2.175900 & -0.550900 \\
\hline $\mathrm{C}$ & -0.478600 & -4.802600 & 0.677700 \\
\hline
\end{tabular}




\begin{tabular}{|c|c|c|c|}
\hline C & 0.607000 & -5.527700 & 0.158000 \\
\hline $\mathrm{C}$ & -1.802400 & -4.509100 & -1.533700 \\
\hline $\mathrm{C}$ & -3.566200 & -5.488300 & 1.609100 \\
\hline $\mathrm{C}$ & -4.721000 & -3.585500 & -1.498100 \\
\hline $\mathrm{C}$ & -2.901000 & -7.044100 & -0.126800 \\
\hline $\mathrm{C}$ & -6.581900 & -2.533600 & -0.331800 \\
\hline $\mathrm{C}$ & -4.609900 & -7.668800 & 1.471500 \\
\hline $\mathrm{C}$ & 1.797900 & -5.628100 & 0.882700 \\
\hline $\mathrm{C}$ & 0.838900 & -4.289600 & 2.655900 \\
\hline $\mathrm{C}$ & -0.355900 & -4.193100 & 1.938200 \\
\hline $\mathrm{C}$ & -3.816500 & -7.979600 & 0.364200 \\
\hline $\mathrm{C}$ & -4.481800 & -6.425500 & 2.095700 \\
\hline $\mathrm{C}$ & 1.910900 & -5.014800 & 2.133300 \\
\hline $\mathrm{C}$ & 4.751800 & -3.742700 & 1.022000 \\
\hline $\mathrm{C}$ & 3.489400 & -2.264600 & -0.634400 \\
\hline $\mathrm{C}$ & 4.394300 & -2.183300 & -1.923400 \\
\hline $\mathrm{C}$ & 5.030900 & -1.639600 & 2.809500 \\
\hline $\mathrm{C}$ & 4.166600 & -2.512200 & 0.700100 \\
\hline $\mathrm{C}$ & 5.461800 & -3.922700 & 2.210300 \\
\hline $\mathrm{C}$ & 5.862600 & -2.405100 & -1.736900 \\
\hline $\mathrm{C}$ & 2.192800 & -1.477300 & -0.584900 \\
\hline $\mathrm{C}$ & 4.063800 & -1.204900 & -3.003600 \\
\hline $\mathrm{C}$ & 3.801500 & -1.654700 & -4.308400 \\
\hline $\mathrm{C}$ & 3.474400 & -3.353300 & -1.710000 \\
\hline $\mathrm{C}$ & 6.500100 & -3.491300 & -2.360500 \\
\hline $\mathrm{C}$ & 4.332300 & -1.462300 & 7500 \\
\hline $\mathrm{C}$ & 6.628500 & -1.506100 & -0.974100 \\
\hline $\mathrm{C}$ & 5.611300 & -2.877100 & 3.128200 \\
\hline $\mathrm{C}$ & 8.618500 & -2.818500 & -1.398900 \\
\hline $\mathrm{C}$ & 3.498200 & -0.739700 & -5.320900 \\
\hline $\mathrm{C}$ & 3.745800 & 1.085100 & -3.750700 \\
\hline $\mathrm{C}$ & 4.055300 & 0.173800 & -2.736300 \\
\hline $\mathrm{C}$ & 7.997700 & -1.722300 & -0.795400 \\
\hline $\mathrm{C}$ & 7.871500 & -3.698700 & -2.185900 \\
\hline $\mathrm{C}$ & 3.465300 & 0.628800 & -5.040900 \\
\hline $\mathrm{C}$ & 4.798300 & 3.589200 & -1.345600 \\
\hline $\mathrm{C}$ & 2.366700 & 3.447300 & -0.582600 \\
\hline $\mathrm{C}$ & 1.825200 & 4.806700 & -0.067600 \\
\hline $\mathrm{C}$ & 5.631300 & 2.010900 & 0.787300 \\
\hline $\mathrm{C}$ & 3.838700 & 3.106500 & -0.448900 \\
\hline $\mathrm{C}$ & 6.151100 & 3.292500 & -1.178400 \\
\hline $\mathrm{C}$ & 2.818200 & 5.789300 & 0.470600 \\
\hline $\mathrm{C}$ & 1.503200 & 2.191400 & -0.538300 \\
\hline $\mathrm{C}$ & 0.489500 & 4.860200 & 0.600000 \\
\hline $\mathrm{C}$ & 0.306800 & 4.270400 & 1.862400 \\
\hline $\mathrm{C}$ & 1.888000 & 4.497600 & -1.553600 \\
\hline $\mathrm{C}$ & 2.985500 & 7.038000 & -0.152400 \\
\hline $\mathrm{C}$ & 4.280400 & 2.312700 & 0.620500 \\
\hline $\mathrm{C}$ & 3.553800 & 5.499800 & 1.633200 \\
\hline $\mathrm{C}$ & 6.591900 & 2.498900 & -0.111600 \\
\hline $\mathrm{C}$ & 4.648400 & 7.655400 & 1.496600 \\
\hline $\mathrm{C}$ & -0.903600 & 4.421700 & 2.543500 \\
\hline $\mathrm{C}$ & -1.763700 & 5.762900 & 0.722200 \\
\hline $\mathrm{C}$ & -0.555700 & 5.610700 & 0.035500 \\
\hline $\mathrm{C}$ & 4.471000 & 6.426400 & 2.136900 \\
\hline $\mathrm{C}$ & 3.902700 & 7.962700 & 0.355600 \\
\hline $\mathrm{C}$ & -1.932100 & 5.178900 & 1.980200 \\
\hline $\mathrm{C}$ & 8.062400 & 2.202500 & 0.040700 \\
\hline $\mathrm{C}$ & 6.381100 & -3.103800 & 4.404900 \\
\hline $\mathrm{C}$ & -8.058700 & -2.240600 & -0.253200 \\
\hline $\mathrm{C}$ & -6.329400 & 2.838000 & 4.608900 \\
\hline & -4.663600 & 4.540700 & 0.614600 \\
\hline
\end{tabular}

\begin{tabular}{|c|c|c|c|}
\hline $\mathrm{H}$ & -5.092900 & 0.566800 & 3.528400 \\
\hline $\mathrm{H}$ & -5.858600 & 4.744000 & 2.712800 \\
\hline $\mathrm{H}$ & -3.887500 & 2.915400 & -4.383200 \\
\hline $\mathrm{H}$ & -3.917700 & 4.371200 & -1.223600 \\
\hline & -2.605700 & 3.498400 & -2.125600 \\
\hline $\mathrm{H}$ & -6.190700 & 0.650300 & -0.445700 \\
\hline $\mathrm{H}$ & -5.974700 & 4.301900 & -2.733900 \\
\hline H & -9.711100 & 3.018300 & -1.065300 \\
\hline & -3.366900 & 1.387100 & -6.251600 \\
\hline & -3.773800 & -1.986500 & -3.632100 \\
\hline & -4.305200 & -0.472000 & -1.748500 \\
\hline $\mathrm{H}$ & -8.393100 & 4.648400 & -2.387900 \\
\hline & -8.605900 & 1.023700 & -0.099600 \\
\hline & -3.302900 & -1.062700 & -5.878400 \\
\hline & -3.628100 & -2.008100 & 1.291200 \\
\hline 11 & -6.748700 & -3.666600 & -2.171300 \\
\hline $\mathrm{H}$ & -5.998300 & -1.469000 & 1.477600 \\
\hline $\mathrm{H}$ & 0.524500 & -6.032800 & -0.796200 \\
\hline & -2.498200 & -5.051400 & -2.167100 \\
\hline & -0.866800 & -4.279000 & -2.035400 \\
\hline $\mathrm{H}$ & -3.468900 & -4.536900 & 2.114200 \\
\hline $\mathrm{H}$ & -2.284700 & -7.303900 & -0.978500 \\
\hline $\mathrm{H}$ & -5.317900 & -8.394300 & 1.850800 \\
\hline & 2.628300 & -6.194100 & 0.481100 \\
\hline & 0.927100 & -3.817900 & 3.625900 \\
\hline $\mathrm{H}$ & -1.189400 & -3.656300 & 2.372400 \\
\hline $\mathrm{H}$ & -3.908300 & -8.947300 & -0.111800 \\
\hline $\mathrm{H}$ & -5.088600 & -6.189600 & 2.960300 \\
\hline 1 & 2.825600 & -5.108800 & 2.702800 \\
\hline H & 4.664000 & -4.587000 & 0.346500 \\
\hline $\mathrm{H}$ & 5.112700 & -0.784400 & 3.476500 \\
\hline $\mathrm{H}$ & 5.893000 & -4.901500 & 2.409400 \\
\hline $\mathrm{H}$ & 3.828600 & -2.712400 & -4.539500 \\
\hline 4 & 3.884200 & -4.325600 & -1.456600 \\
\hline $\mathrm{H}$ & 2.566400 & -3.406200 & -2.301900 \\
\hline $\mathrm{H}$ & 5.935300 & -4.178600 & -2.978000 \\
\hline $\mathrm{H}$ & 6.163400 & -0.646800 & -0.509200 \\
\hline $\mathrm{H}$ & 9.679400 & -2.982500 & -1.261700 \\
\hline $\mathrm{H}$ & 3.288600 & -1.091600 & -6.322600 \\
\hline $\mathrm{H}$ & 3.718400 & 2.144800 & -3.539000 \\
\hline $\mathrm{H}$ & 4.271100 & 0.536200 & -1.740600 \\
\hline $\mathrm{H}$ & 8.578900 & -1.040000 & -0.190000 \\
\hline $\mathrm{H}$ & 8.355400 & -4.542100 & -2.661300 \\
\hline $\mathrm{H}$ & 3.226200 & 1.336000 & -5.824500 \\
\hline $\mathrm{H}$ & 4.497000 & 4.196300 & -2.192800 \\
\hline $\mathrm{H}$ & 5.909000 & 1.385000 & 1.631200 \\
\hline $\mathrm{H}$ & 6.859700 & 3.689100 & -1.902500 \\
\hline $\mathrm{H}$ & 1.108400 & 3.713100 & 2.329700 \\
\hline $\mathrm{H}$ & 2.614200 & 5.015800 & -2.172500 \\
\hline $\mathrm{H}$ & 0.967500 & 4.271800 & -2.083800 \\
\hline $\mathrm{H}$ & 2.406700 & 7.294900 & -1.030900 \\
\hline $\mathrm{H}$ & 3.419200 & 4.558400 & 2.148800 \\
\hline $\mathrm{H}$ & 5.357900 & 8.372400 & 1.888700 \\
\hline H & -1.036500 & 3.970100 & 3.518100 \\
\hline $\mathrm{H}$ & -2.563000 & 6.348700 & 0.287100 \\
\hline $\mathrm{H}$ & -0.426800 & 6.098500 & -0.922600 \\
\hline $\mathrm{H}$ & 5.041300 & 6.193200 & 3.026700 \\
\hline & 4.033100 & 8.919300 & -0.133600 \\
\hline & -2.856100 & 5.320900 & 2.523900 \\
\hline $\mathrm{H}$ & 3.554500 & 1.938900 & 1.335700 \\
\hline $\mathrm{H}$ & -3.922600 & 0.387600 & 1.413900 \\
\hline 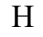 & 3.913600 & -0.490300 & 1.392000 \\
\hline
\end{tabular}

Н $\quad-4.369400 \quad-4.165300 \quad-2.345100$

S1

$\begin{array}{llll}\mathrm{Rh} & -0.000100 & 0.000000 & 0.469800\end{array}$

$\begin{array}{llll}\mathrm{Rh} & -0.000100 & 0.000000 & -1.911700\end{array}$

O $\quad 1.721900 \quad-1.140900 \quad 0.398100$

O $\quad 1.612300 \quad-1.287500 \quad-1.849800$

$\begin{array}{llll}\text { O } & 1.208600 & 1.680300 & 0.411100\end{array}$

$\begin{array}{llll}\text { O } & 1.271900 & 1.630300 & -1.845700\end{array}$

$\begin{array}{llll}\text { O } & -1.722000 & 1.140900 & 0.398200\end{array}$

$\begin{array}{llll}\mathrm{O} & -1.612500 & 1.287400 & -1.849800\end{array}$

O $\quad-1.208700 \quad-1.680400 \quad 0.411200$

$\begin{array}{llll}\mathrm{O} & -1.272200 & -1.630400 & -1.845600\end{array}$

$\mathrm{Br} \quad 6.258400 \quad-2.970400 \quad 4.837700$

$\begin{array}{llll}\mathrm{Br} & 7.894600 & 1.687400 & 2.304200\end{array}$

$\mathrm{Br} \quad-6.257200 \quad 2.971800 \quad 4.838300$

$\mathrm{Br} \quad-7.895300 \quad-1.687900 \quad 2.303300$

$\begin{array}{llll}\text { C } & 4.557400 & -3.707900 & 1.081300\end{array}$

$\begin{array}{llll}\text { C } & 3.512700 & -2.242400 & -0.734500\end{array}$

C $\quad 4.471100 \quad-2.131400 \quad-1.979100$

C $\quad 4.978600 \quad-1.501500 \quad 2.713200$

C $\quad 4.128500 \quad-2.451200 \quad 0.638200$

$\begin{array}{lrrr}\text { C } & 5.182400 & -3.867300 & 2.320800\end{array}$

$\begin{array}{llll}\text { C } & 5.938100 & -2.244900 & -1.703800\end{array}$

C $\quad 2.192900-1.496100 \quad-0.730600$

C $\quad 4.138500 \quad-1.213100 \quad-3.110000$

$\begin{array}{llll}\text { C } & 3.994100 & -1.720600 & -4.412400\end{array}$

$\begin{array}{llll}\text { C } & 3.610200 & -3.346700 & -1.785500\end{array}$

$\begin{array}{llll}\text { C } & 6.680000 & -3.323400 & -2.214900\end{array}$

$\begin{array}{llll}\text { C } & 4.355500 & -1.347800 & 1.477600\end{array}$

C $\quad 6.601200 \quad-1.239300 \quad-0.978700$

$\begin{array}{llll}\text { C } & 5.395500 & -2.763600 & 3.144000\end{array}$

C $\quad 8.697900 \quad-2.423800 \quad-1.220700$

$\begin{array}{llll}\text { C } & 3.730600 & -0.857000 & -5.479600\end{array}$

$\begin{array}{llll}\text { C } & 3.790800 & 1.032000 & -3.968900\end{array}$

$\begin{array}{llll}\text { C } & 4.045500 & 0.170600 & -2.897800\end{array}$

$\begin{array}{llll}\text { C } & 7.972900 & -1.334500 & -0.731200\end{array}$

$\begin{array}{llll}\text { C } & 8.052900 & -3.414700 & -1.966000\end{array}$

$\begin{array}{llll}\text { C } & 3.628200 & 0.518600 & -5.257800\end{array}$

$\begin{array}{llll}\text { C } & 5.157900 & 2.824600 & -0.654800\end{array}$

C $\quad 2.646700 \quad 3.236100 \quad-0.722900$

$\begin{array}{llll}\text { C } & 2.188700 & 4.724000 & -0.748600\end{array}$

$\begin{array}{llll}\text { C } & 5.072800 & 2.213200 & 2.056200\end{array}$

C $\quad 3.923400 \quad 2.852500 \quad 0.008000$

$\begin{array}{llll}\text { C } & 6.332900 & 2.487800 & 0.021100\end{array}$

$\begin{array}{llll}\text { C } & 3.068000 & 5.733900 & -0.077600\end{array}$

$\begin{array}{llll}\text { C } & 1.619000 & 2.106400 & -0.716600\end{array}$

$\begin{array}{llll}\text { C } & 0.733900 & 5.051500 & -0.690500\end{array}$

C $\quad 0.147600 \quad 5.818200 \quad-1.711100$

$\begin{array}{llll}\text { C } & 2.716800 & 4.045400 & -1.994900\end{array}$

C $\quad 3.722000 \quad 6.722600 \quad-0.833800$

$\begin{array}{llll}\text { C } & 3.899200 & 2.536900 & 1.376900\end{array}$

$\begin{array}{llll}\text { C } & 3.196100 & 5.747800 & 1.322000\end{array}$

$\begin{array}{llll}\text { C } & 6.295300 & 2.179500 & 1.380300\end{array}$

$\begin{array}{llll}\text { C } & 4.674700 & 7.660200 & 1.185700\end{array}$

C $\quad-1.174400 \quad 6.254600 \quad-1.592800$

C $\quad-1.342700 \quad 5.168200 \quad 0.563800$

$\begin{array}{llll}\text { C } & -0.023000 & 4.724800 & 0.446700\end{array}$

$\begin{array}{llll}\text { C } & 4.004700 & 6.700500 & 1.948200\end{array}$

$\begin{array}{llll}\text { C } & 4.528600 & 7.674900 & -0.203600\end{array}$

$\begin{array}{llll}\text { C } & -1.913600 & 5.942800 & -0.449700\end{array}$

$\begin{array}{llll}\text { C } & -4.355400 & 1.348400 & 1.478000\end{array}$ 


\begin{tabular}{|c|c|c|c|}
\hline C & -3.512900 & 2.242400 & -0.734400 \\
\hline C & -4.471400 & 2.131200 & -1.978800 \\
\hline $\mathrm{C}$ & -5.181600 & 3.868100 & 2.321000 \\
\hline $\mathrm{C}$ & -4.128400 & 2.451600 & 0.638400 \\
\hline $\mathrm{C}$ & -4.978100 & 1.502300 & 2.713700 \\
\hline $\mathrm{C}$ & -5.938400 & 2.244700 & -1.703300 \\
\hline $\mathrm{C}$ & -2.193100 & 1.496100 & -0.730500 \\
\hline $\mathrm{C}$ & -4.139000 & 1.212700 & -3.109500 \\
\hline $\mathrm{C}$ & -3.994800 & 1.719900 & -4.412000 \\
\hline $\mathrm{C}$ & -3.610500 & 3.346500 & -1.785600 \\
\hline $\mathrm{C}$ & -6.601400 & 1.239200 & -0.978000 \\
\hline $\mathrm{C}$ & -4.556900 & 3.708400 & 1.081400 \\
\hline $\mathrm{C}$ & -6.680400 & 3.323100 & -2.214500 \\
\hline $\mathrm{C}$ & -5.394700 & 2.764600 & 3.144500 \\
\hline $\mathrm{C}$ & -8.698100 & 2.423600 & -1.220000 \\
\hline $\mathrm{C}$ & -3.731400 & 0.856100 & -5.479200 \\
\hline $\mathrm{C}$ & -3.791400 & -1.032700 & -3.968000 \\
\hline $\mathrm{C}$ & -4.045900 & -0.171100 & -2.897100 \\
\hline $\mathrm{C}$ & -8.053300 & 3.414400 & -1.965500 \\
\hline $\mathrm{C}$ & -7.973100 & 1.334400 & -0.730300 \\
\hline $\mathrm{C}$ & -3.629000 & -0.519500 & -5.257100 \\
\hline $\mathrm{C}$ & -3.899600 & -2.536400 & 1.376700 \\
\hline $\mathrm{C}$ & -2.646600 & -3.236400 & -0.722600 \\
\hline $\mathrm{C}$ & -2.188400 & -4.724200 & -0.747800 \\
\hline $\mathrm{C}$ & -6.333100 & -2.488700 & 0.020700 \\
\hline $\mathrm{C}$ & -3.923600 & -2.852800 & 0.00 \\
\hline $\mathrm{C}$ & -5.073300 & -2.212800 & 2.055700 \\
\hline $\mathrm{C}$ & -3.067700 & -5.734000 & -0.076600 \\
\hline $\mathrm{C}$ & -1.619100 & -2.106600 & -0.716500 \\
\hline $\mathrm{C}$ & -0.733600 & -5.051500 & -0.689400 \\
\hline $\mathrm{C}$ & -0.147100 & -5.818600 & -1.709700 \\
\hline $\mathrm{C}$ & -2.716500 & -4.046100 & -1.994400 \\
\hline $\mathrm{C}$ & -3.721400 & -6.723100 & -0.832600 \\
\hline $\mathrm{C}$ & -5.158000 & -2.825500 & -0.655000 \\
\hline $\mathrm{C}$ & -3.196100 & -5.747400 & 1.323000 \\
\hline $\mathrm{C}$ & -6.295800 & -2.179800 & 1.379700 \\
\hline $\mathrm{C}$ & -4.674300 & -7.660100 & 1.187100 \\
\hline $\mathrm{C}$ & 1.174900 & -6.254900 & -1.591000 \\
\hline $\mathrm{C}$ & 1.342900 & -5.167700 & 0.565200 \\
\hline $\mathrm{C}$ & 0.023100 & -4.724400 & 0.447700 \\
\hline $\mathrm{C}$ & -4.004600 & -6.700000 & 1.949300 \\
\hline $\mathrm{C}$ & -4.527900 & -7.675300 & -0.202200 \\
\hline $\mathrm{C}$ & 1.913900 & -5.942700 & -0.448000 \\
\hline $\mathrm{H}$ & 4.413500 & -4.584900 & 0.461300 \\
\hline $\mathrm{H}$ & 5.143700 & -0.624300 & 3.332700 \\
\hline $\mathrm{H}$ & 5.498900 & -4.859900 & 2.630700 \\
\hline $\mathrm{H}$ & 4.089600 & -2.782800 & -4.600700 \\
\hline $\mathrm{H}$ & 4.069300 & -4.284600 & -1.493400 \\
\hline $\mathrm{H}$ & 2.739800 & -3.465600 & -2.423500 \\
\hline $\mathrm{H}$ & 6.197100 & -4.092200 & -2.804900 \\
\hline $\mathrm{H}$ & 6.055700 & -0.384300 & -0.603000 \\
\hline $\mathrm{H}$ & 9.760700 & -2.496000 & -1.029700 \\
\hline $\mathrm{H}$ & 3.613900 & -1.253500 & -6.479900 \\
\hline $\mathrm{H}$ & 3.723400 & 2.097500 & -3.802600 \\
\hline $\mathrm{H}$ & 4.162800 & 0.574800 & -1.903000 \\
\hline $\mathrm{H}$ & 8.474200 & -0.561300 & -0.163600 \\
\hline $\mathrm{H}$ & 8.617900 & -4.252100 & -2.354500 \\
\hline $\mathrm{H}$ & 3.429500 & 1.187100 & -6.085400 \\
\hline $\mathrm{H}$ & 5.207100 & 3.048000 & -1.715600 \\
\hline $\mathrm{H}$ & 5.023800 & 1.986000 & 3.118100 \\
\hline $\mathrm{H}$ & 7.271200 & 2.459300 & -0.526600 \\
\hline $\mathrm{H}$ & 0.723400 & 6.102200 & -2.583300 \\
\hline
\end{tabular}
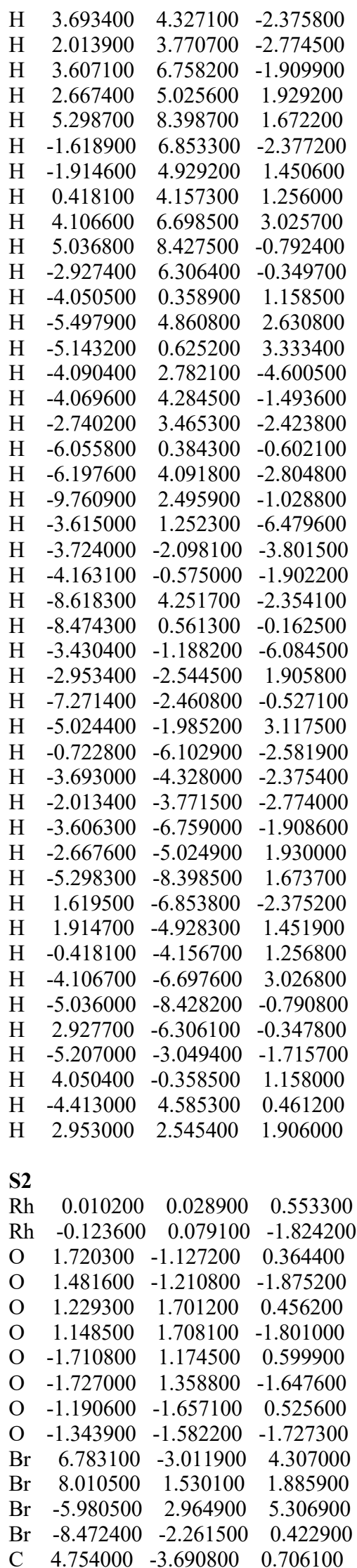

C $\quad 3.425800 \quad-2.226300 \quad-0.911400$

C $\quad 4.273100 \quad-2.132400 \quad-2.237800$

$\begin{array}{llll}\text { C } & 5.162900 & -1.550300 & 2.423700\end{array}$

$\begin{array}{llll}\text { C } & 4.171400 & -2.455800 & 0.391600\end{array}$

$\begin{array}{llll}\text { C } & 5.523700 & -3.860400 & 1.859300\end{array}$

C $\quad 5.754800 \quad-2.290400 \quad-2.094100$

$\begin{array}{llll}\text { C } & 2.124000 & -1.456400 & -0.798000\end{array}$

$\begin{array}{llll}\text { C } & 3.873500 & -1.182000 & -3.319700\end{array}$

C $\quad 3.593000 \quad-1.657800 \quad-4.611500$

C $\quad 3.394000 \quad-3.323100 \quad-1.977500$

C $\quad 6.506000 \quad-1.322600 \quad-1.403500$

$\begin{array}{llll}\text { C } & 4.401500 & -1.383200 & 1.269500\end{array}$

$\begin{array}{llll}\text { C } & 6.417900 & -3.376500 & -2.689600\end{array}$

$\begin{array}{lrrr}\text { C } & 5.728900 & -2.790400 & 2.727500\end{array}$

$\begin{array}{llll}\text { C } & 8.538200 & -2.559300 & -1.849100\end{array}$

$\begin{array}{llll}\text { C } & 3.258700 & -0.764300 & -5.633600\end{array}$

$\begin{array}{llll}\text { C } & 3.515800 & 1.090500 & -4.100600\end{array}$

$\begin{array}{llll}\text { C } & 3.843900 & 0.199600 & -3.074800\end{array}$

$\begin{array}{llll}\text { C } & 7.803500 & -3.512500 & -2.559900\end{array}$

C $\quad 7.890000 \quad-1.463000 \quad-1.274600$

$\begin{array}{llll}\text { C } & 3.218800 & 0.608900 & -5.377900\end{array}$

$\begin{array}{llll}\text { C } & 3.987200 & 2.517100 & 1.267200\end{array}$

$\begin{array}{llll}\text { C } & 2.606000 & 3.273400 & -0.730100\end{array}$

$\begin{array}{llll}\text { C } & 2.159600 & 4.763900 & -0.693200\end{array}$

C $\quad 6.314600 \quad 2.425200 \quad-0.261800$

$\begin{array}{llll}\text { C } & 3.922200 & 2.856200 & -0.094600\end{array}$

$\begin{array}{llll}\text { C } & 5.196700 & 2.149000 & 1.854500\end{array}$

$\begin{array}{llll}\text { C } & 3.085800 & 5.751400 & -0.053300\end{array}$

$\begin{array}{llll}\text { C } & 1.570600 & 2.152500 & -0.685000\end{array}$

$\begin{array}{llll}\text { C } & 0.713000 & 5.098400 & -0.539800\end{array}$

$\begin{array}{llll}\text { C } & 0.072300 & 5.893600 & -1.504400\end{array}$

$\begin{array}{llll}\text { C } & 2.605700 & 4.111100 & -1.985300\end{array}$

$\begin{array}{llll}\text { C } & 3.702400 & 6.752500 & -0.824500\end{array}$

$\begin{array}{llll}\text { C } & 5.104700 & 2.808100 & -0.845000\end{array}$

$\begin{array}{llll}\text { C } & 3.296500 & 5.732000 & 1.336200\end{array}$

$\begin{array}{llll}\text { C } & 6.365600 & 2.092000 & 1.090900\end{array}$

$\begin{array}{llll}\text { C } & 4.780700 & 7.636500 & 1.155700\end{array}$

$\begin{array}{lrrr}\text { C } & -1.239800 & 6.328300 & -1.299900\end{array}$

$\begin{array}{llll}\text { C } & -1.290100 & 5.182900 & 0.832000\end{array}$

$\begin{array}{llll}\text { C } & 0.020600 & 4.742800 & 0.629600\end{array}$

$\begin{array}{llll}\text { C } & 4.148700 & 6.664300 & 1.934700\end{array}$

$\begin{array}{llll}\text { C } & 4.552900 & 7.684200 & -0.221800\end{array}$

$\begin{array}{llll}\text { C } & -1.914800 & 5.985400 & -0.126400\end{array}$

$\begin{array}{llll}\text { C } & -4.266700 & 1.386700 & 1.826400\end{array}$

C $\quad-3.556500 \quad 2.305300 \quad-0.420000$

C $\quad-4.569800 \quad 2.225700 \quad-1.623300$

$\begin{array}{llll}\text { C } & -5.070500 & 3.888700 & 2.735400\end{array}$

$\begin{array}{llll}\text { C } & -4.100000 & 2.498600 & 0.983300\end{array}$

C $\quad-4.816200 \quad 1.522500 \quad 3.098600$

C $\quad-6.021500 \quad 2.390200 \quad-1.299200$

C $\quad-2.243800 \quad 1.548600 \quad-0.495000$

$\begin{array}{llll}\text { C } & -4.303000 & 1.301400 & -2.766700\end{array}$

$\begin{array}{llll}\text { C } & -4.182900 & 1.808800 & -4.071500\end{array}$

C $\quad-3.676000 \quad 3.422000 \quad-1.456200$

C $\quad-6.745400 \quad 3.479700 \quad-1.814100$

$\begin{array}{llll}\text { C } & -4.516200 & 3.746600 & 1.460500\end{array}$

$\begin{array}{llll}\text { C } & -6.690000 & 1.430100 & -0.520600\end{array}$

$\begin{array}{llll}\text { C } & -5.221500 & 2.777400 & 3.562000\end{array}$

$\begin{array}{llll}\text { C } & -8.750700 & 2.687000 & -0.711900\end{array}$

$\begin{array}{llll}\text { C } & -3.942800 & 0.944900 & -5.143800\end{array}$

$\begin{array}{llll}\text { C } & -3.971400 & -0.944300 & -3.632000\end{array}$

$\begin{array}{llll}\text { C } & -4.217500 & -0.084200 & -2.557200\end{array}$ 


\begin{tabular}{|c|c|c|c|}
\hline C & -8.045400 & 1.586900 & -0.218000 \\
\hline C & -8.102600 & 3.629200 & -1.514600 \\
\hline $\mathrm{C}$ & -3.832100 & -0.430300 & -4.923400 \\
\hline $\mathrm{C}$ & -4.296000 & -2.360400 & 0.716900 \\
\hline $\mathrm{C}$ & -2.419800 & -3.390300 & -0.625700 \\
\hline $\mathrm{C}$ & -1.833200 & -4.750800 & -0.165400 \\
\hline $\mathrm{C}$ & -6.229700 & -3.300400 & -1.051600 \\
\hline $\mathrm{C}$ & -3.891000 & -3.086700 & -0.415300 \\
\hline $\mathrm{C}$ & -5.645800 & -2.112400 & 0.962400 \\
\hline $\mathrm{C}$ & -2.784600 & -5.767800 & 0.385400 \\
\hline $\mathrm{C}$ & -1.577000 & -2.118500 & -0.596400 \\
\hline $\mathrm{C}$ & -0.472700 & -4.794700 & 0.450100 \\
\hline $\mathrm{C}$ & 0.560700 & -5.520100 & -0.166100 \\
\hline $\mathrm{C}$ & -1.961400 & -4.408500 & -1.640100 \\
\hline $\mathrm{C}$ & -2.957300 & -7.000500 & -0.267800 \\
\hline $\mathrm{C}$ & -4.877500 & -3.548000 & -1.295600 \\
\hline $\mathrm{C}$ & -3.470400 & -5.529300 & 1.589200 \\
\hline $\mathrm{C}$ & -6.620100 & -2.584100 & 0.079400 \\
\hline $\mathrm{C}$ & -4.532000 & -7.699900 & 1.434100 \\
\hline $\mathrm{C}$ & 1.792500 & -5.673500 & 0.476400 \\
\hline $\mathrm{C}$ & 0.981000 & -4.380400 & 2.354100 \\
\hline $\mathrm{C}$ & -0.253700 & -4.230100 & 1.718400 \\
\hline $\mathrm{C}$ & -4.347100 & -6.488200 & 2.104300 \\
\hline $\mathrm{C}$ & -3.833700 & -7.957900 & 0.251800 \\
\hline $\mathrm{C}$ & 1.997800 & -5.113300 & 1.740000 \\
\hline $\mathrm{H}$ & 4.613300 & -4.545900 & 0.053700 \\
\hline $\mathrm{H}$ & 5.320300 & -0.697100 & 3.077700 \\
\hline $\mathrm{H}$ & 5.954500 & -4.836000 & 2.069000 \\
\hline $\mathrm{H}$ & 3.636200 & -2.718400 & -4.826400 \\
\hline $\mathrm{H}$ & 3.836200 & -4.283400 & -1.735100 \\
\hline $\mathrm{H}$ & 2.465600 & -3.401600 & -2.534600 \\
\hline $\mathrm{H}$ & 6.019000 & -0.464900 & -0.958900 \\
\hline $\mathrm{H}$ & 5.863300 & -4.117200 & -3.252000 \\
\hline $\mathrm{H}$ & 9.610600 & -2.666600 & -1.749700 \\
\hline $\mathrm{H}$ & 3.037200 & -1.136100 & -6.625600 \\
\hline $\mathrm{H}$ & 3.493900 & 2.153800 & -3.907500 \\
\hline $\mathrm{H}$ & 4.063900 & 0.579500 & -2.087600 \\
\hline $\mathrm{H}$ & 8.308400 & -4.355700 & -3.013100 \\
\hline $\mathrm{H}$ & 8.460000 & -0.720000 & -0.731800 \\
\hline $\mathrm{H}$ & 2.963500 & 1.300000 & -6.170700 \\
\hline $\mathrm{H}$ & 3.081500 & 2.539300 & 1.863000 \\
\hline $\mathrm{H}$ & 7.209800 & 2.380100 & -0.876500 \\
\hline $\mathrm{H}$ & 5.217700 & 1.903400 & 2.913200 \\
\hline $\mathrm{H}$ & 0.598400 & 6.199400 & -2.400300 \\
\hline $\mathrm{H}$ & 3.560600 & 4.394000 & -2.416900 \\
\hline $\mathrm{H}$ & 1.855200 & 3.860300 & -2.727700 \\
\hline $\mathrm{H}$ & 3.524300 & 6.813700 & -1.890700 \\
\hline $\mathrm{H}$ & 2.798000 & 5.000200 & 1.957200 \\
\hline $\mathrm{H}$ & 5.438600 & 8.359100 & 1.620900 \\
\hline $\mathrm{H}$ & -1.726100 & 6.948500 & -2.041600 \\
\hline $\mathrm{H}$ & -1.813100 & 4.918700 & 1.741600 \\
\hline $\mathrm{H}$ & 0.504900 & 4.153100 & 1.397100 \\
\hline $\mathrm{H}$ & 4.313900 & 6.636800 & 3.004000 \\
\hline $\mathrm{H}$ & 5.031800 & 8.446600 & -0.822500 \\
\hline $\mathrm{H}$ & -2.920800 & 6.346300 & 0.039900 \\
\hline $\mathrm{H}$ & -3.968100 & 0.406700 & 1.474400 \\
\hline $\mathrm{H}$ & -5.379500 & 4.875100 & 3.071400 \\
\hline $\mathrm{H}$ & -4.927800 & 0.641500 & 3.726000 \\
\hline $\mathrm{H}$ & -4.272200 & 2.872100 & -4.256500 \\
\hline $\mathrm{H}$ & -4.106000 & 4.365500 & -1.137600 \\
\hline $\mathrm{H}$ & -2.828800 & 3.531400 & -2.125800 \\
\hline & -6.259200 & 4.213400 & -2.444800 \\
\hline
\end{tabular}

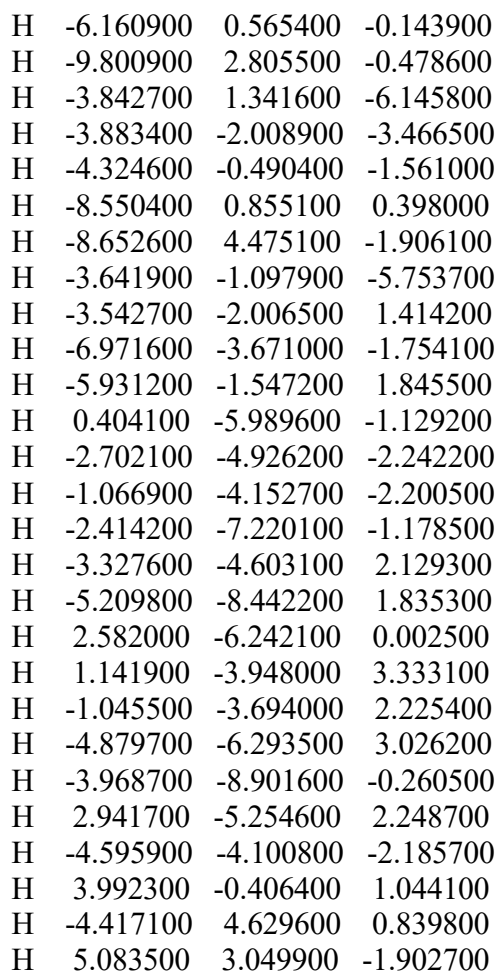

T1

$\begin{array}{llll}\text { Rh } & -0.015500 & 0.012000 & 0.273000\end{array}$ Rh $\quad-0.091200 \quad-0.156400 \quad-2.101200$

$\begin{array}{llll}\text { O } & 1.756000 & -1.068300 & 0.216000\end{array}$

O $\quad 1.580300 \quad-1.347400 \quad-2.012100$

$\begin{array}{llll}\mathrm{O} & 1.124100 & 1.716900 & 0.066500\end{array}$

$\begin{array}{llll}\mathrm{O} & 1.115500 & 1.530000 & -2.183700\end{array}$

$\begin{array}{llll}\mathrm{O} & -1.784800 & 1.081700 & 0.167600\end{array}$

$\begin{array}{llll}\mathrm{O} & -1.760400 & 1.048500 & -2.087300\end{array}$

$\begin{array}{llll}\mathrm{O} & -1.143000 & -1.712100 & 0.384300\end{array}$

$\begin{array}{llll}\text { O } & -1.255600 & -1.855600 & -1.867000\end{array}$

C $\quad 5.052700 \quad-3.272000 \quad 0.820000$

C $\quad 3.561500 \quad-2.153900-0.932300$

C $4.394600 \quad-2.191300 \quad-2.269900$

$\begin{array}{llll}\text { C } & 5.205300 & -0.935700 & 2.287600\end{array}$

$\begin{array}{llll}\text { C } & 4.320200 & -2.161800 & 0.380700\end{array}$

$\begin{array}{llll}\text { C } & 5.868100 & -3.211700 & 1.952100\end{array}$

C $\quad 5.883400 \quad-2.293700 \quad-2.144400$

C $\quad 2.211600 \quad-1.460000-0.906900$

$\begin{array}{llll}\text { C } & 3.950400 & -1.395300 & -3.453900\end{array}$

$\begin{array}{llll}\text { C } & 3.905400 & 0.006800 & -3.388800\end{array}$

$\begin{array}{llll}\text { C } & 3.573000 & -3.377400 & -1.852600\end{array}$

$\begin{array}{llll}\text { C } & 6.560200 & -3.420300 & -2.643300\end{array}$

$\begin{array}{llll}\text { C } & 4.416800 & -0.988800 & 1.143000\end{array}$

$\begin{array}{llll}\text { C } & 6.627500 & -1.235400 & -1.593100\end{array}$

$\begin{array}{llll}\text { C } & 5.968300 & -2.035500 & 2.708200\end{array}$

C $\quad 8.682200 \quad-2.454900 \quad-1.989600$

$\begin{array}{llll}\text { C } & 3.548900 & 0.752300 & -4.516100\end{array}$

$\begin{array}{llll}\text { C } & 3.282100 & -1.289000 & -5.788200\end{array}$

C $\quad 3.650200 \quad-2.038100 \quad-4.666800$

C $\quad 8.021000 \quad-1.319300 \quad-1.516000$

C $\quad 7.952600 \quad-3.503300 \quad-2.555100$

$\begin{array}{llll}\text { C } & 3.231300 & 0.105300 & -5.712800\end{array}$

$\begin{array}{llll}\text { C } & 4.996000 & 2.754800 & -0.848600\end{array}$ $\begin{array}{llll}\text { C } & 2.513600 & 3.210400 & -1.187900\end{array}$

$\begin{array}{llll}\text { C } & 2.042200 & 4.669800 & -1.467900\end{array}$

$\begin{array}{llll}\text { C } & 4.694200 & 2.677500 & 1.908800\end{array}$

$\begin{array}{llll}\text { C } & 3.722600 & 2.957700 & -0.305800\end{array}$

$\begin{array}{llll}\text { C } & 6.099300 & 2.496800 & -0.030900\end{array}$

$\begin{array}{llll}\text { C } & 2.856100 & 5.790000 & -0.894800\end{array}$

C $\quad 1.495200 \quad 2.076300 \quad-1.097200$

C $\quad 0.579100 \quad 4.955200 \quad-1.543200$

$\begin{array}{llll}\text { C } & -0.238100 & 4.757900 & -0.417800\end{array}$

$\begin{array}{llll}\text { C } & 2.671900 & 3.827900 & -2.556200\end{array}$

$\begin{array}{llll}\text { C } & 2.858700 & 6.035000 & 0.489500\end{array}$

$\begin{array}{llll}\text { C } & 3.592900 & 2.914200 & 1.090800\end{array}$

$\begin{array}{llll}\text { C } & 3.570600 & 6.656000 & -1.742100\end{array}$

$\begin{array}{llll}\text { C } & 5.969400 & 2.448000 & 1.364500\end{array}$

$\begin{array}{llll}\text { C } & 4.338900 & 7.924600 & 0.172300\end{array}$

$\begin{array}{llll}\text { C } & -1.576500 & 5.156000 & -0.441700\end{array}$

C $\quad-1.308900 \quad 5.933500 \quad-2.718300$

$\begin{array}{llll}\text { C } & 0.034000 & 5.545400 & -2.695100\end{array}$

C $\quad 4.315600 \quad 7.711500-1.208000$

$\begin{array}{llll}\text { C } & 3.606200 & 7.090300 & 1.019700\end{array}$

$\begin{array}{lrrr}\text { C } & -2.109600 & 5.751100 & -1.587900\end{array}$

C $\quad-4.715600 \quad 3.644600 \quad 0.668300$

$\begin{array}{llll}\text { C } & -3.661200 & 2.012600 & -0.991500\end{array}$

$\begin{array}{llll}\text { C } & -4.667700 & 1.702900 & -2.164700\end{array}$

$\begin{array}{llll}\text { C } & -4.884000 & 1.678400 & 2.610500\end{array}$

$\begin{array}{llll}\text { C } & -4.222100 & 2.369700 & 0.371700\end{array}$

$\begin{array}{llll}\text { C } & -5.299200 & 3.931800 & 1.904200\end{array}$

C $\quad-6.126400 \quad 1.789500 \quad-1.841000$

$\begin{array}{llll}\text { C } & -2.309700 & 1.324900 & -0.967400\end{array}$

$\begin{array}{llll}\text { C } & -4.336600 & 0.667600 & -3.189900\end{array}$

C $\quad-4.151200 \quad-0.670800 \quad-2.813700$

C $\quad-3.860900 \quad 2.968800 \quad-2.165200$

$\begin{array}{llll}\text { C } & -6.935400 & 2.761100 & -2.455300\end{array}$

$\begin{array}{llll}\text { C } & -4.324400 & 1.389500 & 1.370000\end{array}$

C $\quad-6.717300 \quad 0.854200 \quad-0.973100$

$\begin{array}{llll}\text { C } & -5.397300 & 2.952900 & 2.902000\end{array}$

$\begin{array}{llll}\text { C } & -8.877900 & 1.899400 & -1.293900\end{array}$

C $\quad-3.877800 \quad-1.643000 \quad-3.780700$

C $\quad-3.999500 \quad 0.040600 \quad-5.514100$

C $\quad-4.275700 \quad 1.014800 \quad-4.550200$

$\begin{array}{llll}\text { C } & -8.086900 & 0.913400 & -0.699900\end{array}$

$\begin{array}{llll}\text { C } & -8.303300 & 2.820500 & -2.173200\end{array}$

C $\quad-3.798000 \quad-1.287200 \quad-5.129100$

$\begin{array}{llll}\text { C } & -3.892600 & -2.536200 & 1.277300\end{array}$

C $\quad-2.437700 \quad-3.489300 \quad-0.576300$

C $\quad-1.834600-4.900200-0.322700$

C $\quad-6.226900 \quad-3.079300 \quad-0.124100$

$\begin{array}{llll}\text { C } & -3.793900 & -3.131800 & 0.010700\end{array}$

$\begin{array}{llll}\text { C } & -5.132400 & -2.225600 & 1.832300\end{array}$

$\begin{array}{llll}\text { C } & -2.662500 & -5.875900 & 0.454600\end{array}$

C $\quad-1.531100 \quad-2.267200 \quad-0.693900$

C $\quad-0.362900-5.057600 \quad-0.122500$

$\begin{array}{llll}\text { C } & 0.272100 & -4.476000 & 0.988100\end{array}$

$\begin{array}{llll}\text { C } & -2.329900 & -4.492200 & -1.697200\end{array}$

$\begin{array}{llll}\text { C } & -2.917000 & -5.663000 & 1.820400\end{array}$

C $\quad-4.984600 \quad-3.401000 \quad-0.673800$

$\begin{array}{llll}\text { C } & -3.142700 & -7.047300 & -0.156600\end{array}$

$\begin{array}{llll}\text { C } & -6.328400 & -2.483600 & 1.141400\end{array}$

$\begin{array}{llll}\text { C } & -4.177500 & -7.727600 & 1.924800\end{array}$

$\begin{array}{llll}\text { C } & 1.618700 & -4.744200 & 1.249900\end{array}$

C $\quad 1.713100 \quad-6.183900-0.693400$

$\begin{array}{llll}\text { C } & 0.369300 & -5.912200 & -0.963200\end{array}$ 


\begin{tabular}{|c|c|c|c|}
\hline C & -3.904000 & -7.963600 & 0.575200 \\
\hline $\mathrm{C}$ & -3.680100 & -6.580400 & 2.548000 \\
\hline & 2.332800 & -5.611200 & 0.419500 \\
\hline $\mathrm{C}$ & 7.118100 & 2.088200 & 2.238900 \\
\hline $\mathrm{C}$ & 7.290800 & 2.707800 & 3.490700 \\
\hline . & 8.017200 & 1.077600 & 1.851400 \\
\hline $\mathrm{C}$ & 8.340400 & 2.324200 & 4.331600 \\
\hline $\mathrm{C}$ & 9.064900 & 0.698100 & 2.694800 \\
\hline $\mathrm{C}$ & 9.226400 & 1.320400 & 3.934100 \\
\hline $\mathrm{C}$ & 6.892400 & -1.926000 & 3.869900 \\
\hline $\mathrm{C}$ & 6.543500 & -1.153200 & 4.994400 \\
\hline C & 8.150300 & -2.558000 & 3.844200 \\
\hline $\mathrm{C}$ & 7.433000 & -1.016600 & 100 \\
\hline $\mathrm{C}$ & 9.036000 & -2.417100 & 4.916700 \\
\hline $\mathrm{C}$ & 8.678200 & -1.647000 & 6.025100 \\
\hline $\mathrm{C}$ & -7.650500 & -2.120100 & 1700 \\
\hline $\mathrm{C}$ & -8.776000 & -2.940 & 800 \\
\hline $\mathrm{C}$ & -7.801000 & -0.941600 & 2.477600 \\
\hline $\mathrm{C}$ & -10.019300 & -2.585600 & 2.046600 \\
\hline $\mathrm{C}$ & -9.046200 & -0.590500 & 3.006000 \\
\hline $\mathrm{C}$ & -10.154600 & -1.411600 & 2.790800 \\
\hline $\mathrm{C}$ & -6.042100 & 3.241800 & 100 \\
\hline $\mathrm{C}$ & -5.524600 & 2.695100 & 800 \\
\hline $\mathrm{C}$ & -7.188200 & 4.056600 & 4.279100 \\
\hline $\mathrm{C}$ & -6.139800 & 2.959900 & \\
\hline $\mathrm{C}$ & -7.799900 & 7100 & 100 \\
\hline $\mathrm{C}$ & -7.27 & 600 & 300 \\
\hline $\mathrm{H}$ & 5.03 & -4.200500 & 200 \\
\hline $\mathrm{H}$ & 5.239 & 0.010800 & 7100 \\
\hline $\mathrm{H}$ & 6.411600 & -4.111700 & 0800 \\
\hline $\mathrm{H}$ & 4.14 & 0.5 & -2.4 \\
\hline $\mathrm{H}$ & 4.06 & -4.2 & 3900 \\
\hline $\mathrm{H}$ & 2.6 & -3.57 & 8700 \\
\hline $\mathrm{H}$ & 6.010700 & -4.232800 & -3.102100 \\
\hline $\mathrm{H}$ & 6.129800 & -0.347000 & 6200 \\
\hline $\mathrm{H}$ & 9.760900 & -2.5 & -1.9 \\
\hline $\mathrm{H}$ & 3.5 & $1.8 ?$ & -4.4 \\
\hline $\mathrm{H}$ & 600 & -1.789000 & 500 \\
\hline $\mathrm{H}$ & 3.705400 & -3.116800 & 4200 \\
\hline $\mathrm{H}$ & 8.591700 & -0.501100 & 8700 \\
\hline $\mathrm{H}$ & $8.46^{\circ}$ & -4.37 & -2.9 \\
\hline $\mathrm{H}$ & 2.95 & 0.684600 & 3800 \\
\hline $\mathrm{H}$ & 5.134600 & 2.777700 & -1.925300 \\
\hline $\mathrm{H}$ & 4.525400 & 2.640800 & 2.982800 \\
\hline $\mathrm{H}$ & 7.062200 & 2.338000 & -0.511100 \\
\hline $\mathrm{H}$ & 0.16 & 4.32 & \\
\hline $\mathrm{H}$ & 3.675300 & 4.067500 & -2.895700 \\
\hline $\mathrm{H}$ & 2.031700 & 3.429000 & -3.335500 \\
\hline $\mathrm{H}$ & 2.277700 & 5.416100 & 1.158700 \\
\hline $\mathrm{H}$ & 3.550100 & 6.516900 & -2.815600 \\
\hline $\mathrm{H}$ & 4.915200 & 8.742800 & 0.5 \\
\hline $\mathrm{H}$ & -2.193400 & 5.019400 & 0.435800 \\
\hline $\mathrm{H}$ & -1.723600 & 6.394000 & -3.605500 \\
\hline $\mathrm{H}$ & 0.654500 & 5.727000 & -3.563900 \\
\hline $\mathrm{H}$ & 4.871100 & 8.367800 & -1.865300 \\
\hline $\mathrm{H}$ & 3.611200 & 7.265700 & 2.087700 \\
\hline $\mathrm{H}$ & -3.142800 & 6.072400 & -1.600100 \\
\hline $\mathrm{H}$ & -4.653500 & 4.441300 & -0.065500 \\
\hline & -4.937500 & 0.872100 & 3.338400 \\
\hline & -5.654000 & 4.946200 & 2.072300 \\
\hline $\mathrm{H}$ & -4.207200 & -0.951400 & -1.771800 \\
\hline & -4.354900 & 3.913400 & -1.967500 \\
\hline
\end{tabular}
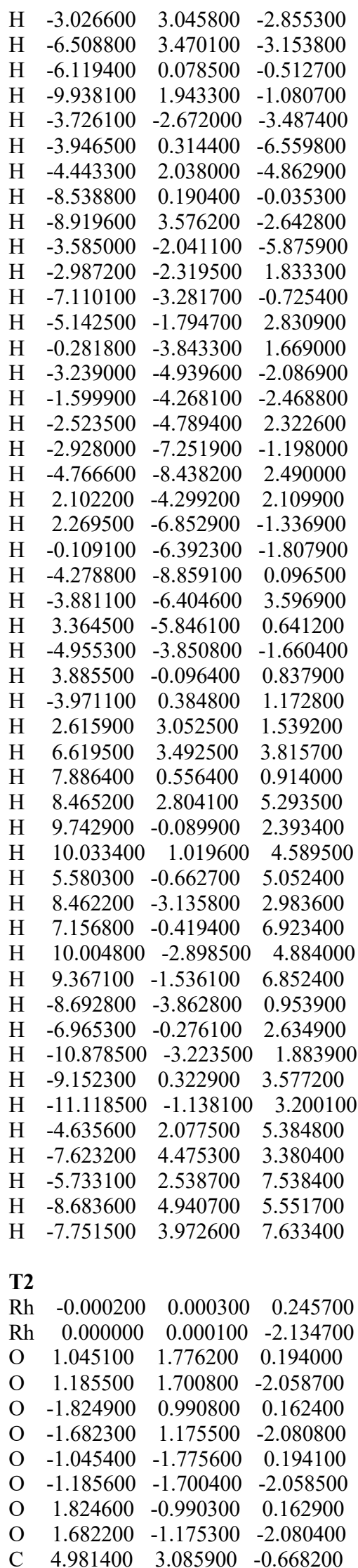

\begin{tabular}{|c|c|c|c|}
\hline $\mathrm{C}$ & 2.459000 & 3.370400 & -0.901400 \\
\hline $\mathrm{C}$ & 1.951900 & 4.842000 & -0.959200 \\
\hline $\mathrm{C}$ & 4.752500 & 2.464000 & 2.024100 \\
\hline & 3.705200 & 3.041800 & -0.097000 \\
\hline $\mathrm{C}$ & 6.125000 & 2.823700 & 0.089500 \\
\hline & 2.771800 & 5.886900 & -0.265900 \\
\hline & 1.474800 & 2.204900 & -0.925300 \\
\hline & 0.484900 & 5.115300 & -0.946700 \\
\hline $\mathrm{C}$ & -0.100400 & 5.853200 & -1.988600 \\
\hline & 2.551700 & 4.170800 & -2.177100 \\
\hline & 3.417500 & 6.892000 & -1.007600 \\
\hline $\mathrm{C}$ & 3.613100 & 2.719800 & 1.265600 \\
\hline $\mathrm{C}$ & 2.852400 & 5.914800 & 1.137200 \\
\hline $\mathrm{C}$ & 6.036800 & 2.507100 & 1.453900 \\
\hline $\mathrm{C}$ & 4.271000 & 7.874100 & 1.035100 \\
\hline $\mathrm{C}$ & -1.439300 & 6.245600 & -1.907100 \\
\hline $\mathrm{C}$ & -1.626400 & 5.170300 & 0.253500 \\
\hline $\mathrm{C}$ & -0.290100 & 4.771200 & 0.173300 \\
\hline $\mathrm{C}$ & 3.608000 & 6.897800 & 1.782400 \\
\hline $\mathrm{C}$ & 4.170600 & 7.874800 & -0.358400 \\
\hline $\mathrm{C}$ & -2.196800 & 5.918000 & -0.780100 \\
\hline $\mathrm{C}$ & -4.539900 & 1.120000 & 1.141400 \\
\hline $\mathrm{C}$ & -3.622900 & 2.070300 & -1.003300 \\
\hline $\mathrm{C}$ & -4.528400 & 1.973200 & -2.286800 \\
\hline $\mathrm{C}$ & -5.493700 & 3.585700 & 1.986400 \\
\hline $\mathrm{C}$ & -4.295100 & 2.245400 & 0.340900 \\
\hline $\mathrm{C}$ & -5.247200 & 1.224600 & 2.334000 \\
\hline $\mathrm{C}$ & -6.004600 & 2.155700 & -2.108300 \\
\hline $\mathrm{C}$ & -2.289600 & 1.345800 & -0.969300 \\
\hline $\mathrm{C}$ & -4.164500 & 1.017000 & -3.374700 \\
\hline $\mathrm{C}$ & -4.137700 & -0.363700 & -3.121100 \\
\hline $\mathrm{C}$ & -3.657900 & 3.176500 & -2.057700 \\
\hline $\mathrm{C}$ & -6.658900 & 3.241800 & -2.717000 \\
\hline $\mathrm{C}$ & -4.781100 & 3.477000 & 0.789500 \\
\hline $\mathrm{C}$ & -6.764000 & 1.216000 & -1.388600 \\
\hline $\mathrm{C}$ & -5.747600 & 2.459200 & 2.781100 \\
\hline $\mathrm{C}$ & -8.778400 & 2.490300 & -1.822400 \\
\hline $\mathrm{C}$ & -3.841400 & -1.262100 & -4.149900 \\
\hline $\mathrm{C}$ & -3.604800 & 0.582600 & -5.698800 \\
\hline $\mathrm{C}$ & -3.910000 & 1.484200 & -4.674800 \\
\hline $\mathrm{C}$ & -8.143400 & 1.391600 & -1.239400 \\
\hline $\mathrm{C}$ & -8.037700 & 3.411400 & -2.565600 \\
\hline $\mathrm{C}$ & -3.569400 & -0.789500 & -5.436200 \\
\hline $\mathrm{C}$ & -3.612800 & -2.719600 & 1.266400 \\
\hline $\mathrm{C}$ & -2.459600 & -3.369500 & -0.901300 \\
\hline $\mathrm{C}$ & -1.953100 & -4.841300 & -0.959900 \\
\hline $\mathrm{C}$ & -6.125100 & -2.822800 & 0.091100 \\
\hline $\mathrm{C}$ & -3.705500 & -3.041000 & -0.096300 \\
\hline $\mathrm{C}$ & -4.751900 & -2.464000 & 2.025500 \\
\hline $\mathrm{C}$ & -2.773100 & -5.886300 & -0.266900 \\
\hline $\mathrm{C}$ & -1.475100 & -2.204300 & -0.925100 \\
\hline $\mathrm{C}$ & -0.486100 & -5.115000 & -0.947800 \\
\hline $\mathrm{C}$ & 0.289100 & -4.771600 & 0.172200 \\
\hline $\mathrm{C}$ & -2.552900 & -4.169400 & -2.177300 \\
\hline $\mathrm{C}$ & -2.853300 & -5.914800 & 1.136200 \\
\hline $\mathrm{C}$ & -4.981900 & -3.084700 & -0.667100 \\
\hline $\mathrm{C}$ & -3.419300 & -6.890800 & -1.008900 \\
\hline $\mathrm{C}$ & -6.036400 & -2.506900 & 1.455700 \\
\hline $\mathrm{C}$ & -4.272600 & -7.873600 & 1.033400 \\
\hline & 1.625300 & -5.171100 & 0.252100 \\
\hline & 1.437600 & -6.245500 & -1.908900 \\
\hline & 0.098800 & -5.852700 & -1.990100 \\
\hline
\end{tabular}




\begin{tabular}{|c|c|c|c|}
\hline $\mathrm{C}$ & -4.172600 & -7.873700 & -0.360000 \\
\hline $\mathrm{C}$ & -3.609100 & -6.897800 & 1.781100 \\
\hline $\mathrm{C}$ & 2.195300 & -5.918600 & -0.781900 \\
\hline & 4.539900 & -1.120200 & 1.142000 \\
\hline & 3.622300 & -2.070400 & -1.002500 \\
\hline $\mathrm{C}$ & 4.528100 & -1.973500 & -2.285900 \\
\hline $\mathrm{C}$ & 5.493100 & -3.586100 & 1.987000 \\
\hline $\mathrm{C}$ & 4.294400 & -2.245600 & 0.341800 \\
\hline $\mathrm{C}$ & 5.247900 & -1.224800 & 2.334200 \\
\hline & 6.004300 & -2.156600 & -2.107100 \\
\hline $\mathrm{C}$ & 2.289300 & -1.345500 & -0.968700 \\
\hline $\mathrm{C}$ & 4.164700 & -1.017000 & -3.373600 \\
\hline $\mathrm{C}$ & 4.138200 & 0.363600 & -3.119700 \\
\hline $\mathrm{C}$ & 3.657200 & -3.176600 & -2.057000 \\
\hline & 6.763800 & -1.217100 & -1.387300 \\
\hline $\mathrm{C}$ & 4.779900 & -3.477400 & 0.790500 \\
\hline $\mathrm{C}$ & 6.658200 & -3.243000 & -2.715600 \\
\hline $\mathrm{C}$ & 5.748100 & -2.459500 & 2.781100 \\
\hline $\mathrm{C}$ & 8.777800 & -2.492400 & -1.820400 \\
\hline $\mathrm{C}$ & 3.842200 & 1.262400 & -4.148300 \\
\hline $\mathrm{C}$ & 3.605500 & -0.581900 & -5.697800 \\
\hline $\mathrm{C}$ & 3.910400 & -1.483700 & -4.673900 \\
\hline $\mathrm{C}$ & 8.036900 & -3.413200 & -2.563900 \\
\hline $\mathrm{C}$ & 8.143100 & -1.393400 & -1.237700 \\
\hline $\mathrm{C}$ & 3.570300 & 0.790200 & -5.434800 \\
\hline $\mathrm{C}$ & -6.535800 & 2.557600 & 4.039500 \\
\hline $\mathrm{C}$ & -7.435000 & 1.535900 & 4.399300 \\
\hline $\mathrm{C}$ & -6.396900 & 3.670800 & 4.889800 \\
\hline $\mathrm{C}$ & -8.175900 & 1.626000 & 5.580900 \\
\hline $\mathrm{C}$ & -7.140100 & 3.756100 & 6.071400 \\
\hline $\mathrm{C}$ & -8.028500 & 2.735000 & 6.416600 \\
\hline $\mathrm{C}$ & 7.250300 & 2.201500 & 2.262800 \\
\hline $\mathrm{C}$ & 8.341900 & 1.524400 & 1.686900 \\
\hline $\mathrm{C}$ & 7.322600 & 2.569700 & 3.620600 \\
\hline $\mathrm{C}$ & 9.473500 & 1.221300 & 2.449600 \\
\hline $\mathrm{C}$ & 8.456500 & 2.263600 & 4.379500 \\
\hline $\mathrm{C}$ & 9.530900 & 1.590200 & 3.794900 \\
\hline $\mathrm{C}$ & 6.537500 & -2.557900 & 4.038700 \\
\hline $\mathrm{C}$ & 6.398800 & -3.670800 & 4.889500 \\
\hline $\mathrm{C}$ & 7.437900 & -1.536700 & 4.397000 \\
\hline $\mathrm{C}$ & 7.143000 & -3.756100 & 6.070400 \\
\hline $\mathrm{C}$ & 8.179900 & -1.626800 & 5.578000 \\
\hline $\mathrm{C}$ & 8.032600 & -2.735400 & 6.414300 \\
\hline $\mathrm{C}$ & -7.249600 & -2.201600 & 2.265200 \\
\hline $\mathrm{C}$ & -7.321400 & -2.571100 & 3.622800 \\
\hline $\mathrm{C}$ & -8.341200 & -1.523900 & 1.690400 \\
\hline $\mathrm{C}$ & -8.455000 & -2.265300 & 4.382400 \\
\hline $\mathrm{C}$ & -9.472500 & -1.221100 & 2.453700 \\
\hline $\mathrm{C}$ & -9.529400 & -1.591100 & 3.798700 \\
\hline $\mathrm{H}$ & 5.096200 & 3.318200 & -1.722200 \\
\hline $\mathrm{H}$ & 4.606000 & 2.207800 & 3.070900 \\
\hline $\mathrm{H}$ & 7.085600 & 2.892000 & -0.415300 \\
\hline $\mathrm{H}$ & 0.488000 & 6.150000 & -2.848000 \\
\hline $\mathrm{H}$ & 3.532000 & 4.484500 & -2.522600 \\
\hline $\mathrm{H}$ & 1.890800 & 3.862200 & -2.980500 \\
\hline $\mathrm{H}$ & 3.337900 & 6.916200 & -2.087100 \\
\hline $\mathrm{H}$ & 2.328600 & 5.179300 & 1.732500 \\
\hline $\mathrm{H}$ & 4.854000 & 8.636000 & 1.536100 \\
\hline $\mathrm{H}$ & -1.883800 & 6.822200 & -2.707900 \\
\hline $\mathrm{H}$ & -2.212800 & 4.917400 & 1.126900 \\
\hline $\mathrm{H}$ & 0.148500 & 4.225400 & 0.998500 \\
\hline $\mathrm{H}$ & 3.674300 & 6.906100 & 2.862600 \\
\hline
\end{tabular}

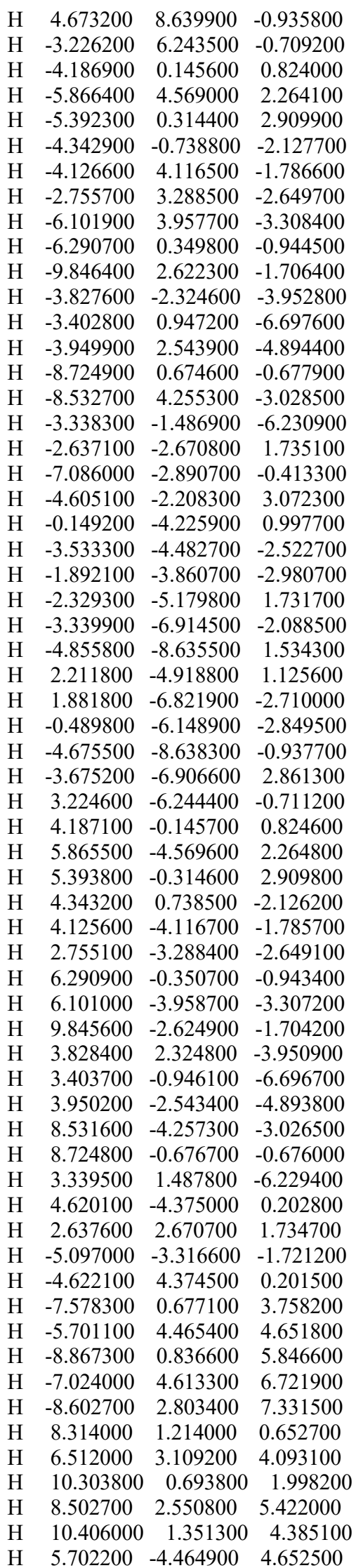

$\begin{array}{cccc}\mathrm{H} & 7.581100 & -0.678400 & 3.755400 \\ \mathrm{H} & 7.027000 & -4.612900 & 6.721400 \\ \mathrm{H} & 8.872100 & -0.837800 & 5.842600 \\ \mathrm{H} & 8.607600 & -2.803800 & 7.328600 \\ \mathrm{H} & -6.510700 & -3.111100 & 4.094400 \\ \mathrm{H} & -8.313600 & -1.212500 & 0.656400 \\ \mathrm{H} & -8.500800 & -2.553300 & 5.424700 \\ \mathrm{H} & -10.302800 & -0.693000 & 2.003100 \\ \mathrm{H} & -10.404200 & -1.352500 & 4.389500\end{array}$

T3

$\begin{array}{llll}\text { Rh } & -0.008900 & 0.006800 & 0.256300\end{array}$

$\begin{array}{llll}\mathrm{Rh} & -0.079600 & -0.150900 & -2.118700\end{array}$

O $\quad 1.130400 \quad 1.526000 \quad-2.199400$

$\begin{array}{llll}\mathrm{O} & 1.127700 & 1.717600 & 0.050800\end{array}$

$\begin{array}{llll}\text { O } & 1.593000 & -1.343200 & -2.023000\end{array}$

$\begin{array}{llll}\mathrm{O} & 1.762100 & -1.068100 & 0.207500\end{array}$

O $\quad-1.255700 \quad-1.841500 \quad-1.901100$

$\begin{array}{llll}\mathrm{O} & -1.137400 & -1.724100 & 0.351300\end{array}$

$\begin{array}{llll}\mathrm{O} & -1.746400 & 1.062700 & -2.098700\end{array}$

$\begin{array}{llll}\mathrm{O} & -1.775700 & 1.078100 & 0.156000\end{array}$

C $\quad 3.690400 \quad 2.798100 \quad 1.007700$

C $\quad 2.517700 \quad 3.210200 \quad-1.209000$

$\begin{array}{llll}\text { C } & 2.037000 & 4.677800 & -1.420800\end{array}$

$\begin{array}{llll}\text { C } & 6.175600 & 2.629100 & -0.219100\end{array}$

$\begin{array}{llll}\text { C } & 3.764500 & 2.937600 & -0.386700\end{array}$

$\begin{array}{llll}\text { C } & 4.836200 & 2.593300 & 1.772400\end{array}$

$\begin{array}{llll}\text { C } & 2.876700 & 5.776300 & -0.843700\end{array}$

$\begin{array}{llll}\text { C } & 1.503300 & 2.075000 & -1.112200\end{array}$

C $\quad 0.573600 \quad 4.970200 \quad-1.429400$

C $\quad-0.196800 \quad 4.755900 \quad-0.274600$

$\begin{array}{llll}\text { C } & 2.619700 & 3.872500 & -2.561900\end{array}$

$\begin{array}{llll}C & 2.958300 & 5.956000 & 0.548200\end{array}$

C $\quad 5.026600 \quad 2.845000 \quad-0.982900$

$\begin{array}{llll}\text { C } & 3.540200 & 6.682900 & -1.689400\end{array}$

C $\quad 6.105900 \quad 2.501900 \quad 1.176200$

$\begin{array}{llll}\text { C } & 4.412700 & 7.866200 & 0.235100\end{array}$

$\begin{array}{llll}\text { C } & -1.530800 & 5.167300 & -0.232800\end{array}$

C $\quad-1.352600 \quad 5.990500 \quad-2.502100$

C $\quad-0.014300 \quad 5.589600 \quad-2.544400$

C $\quad 4.311600 \quad 7.716700 \quad-1.150200$

$\begin{array}{llll}\text { C } & 3.732100 & 6.989600 & 1.083300\end{array}$

$\begin{array}{lrrr}\text { C } & -2.105800 & 5.792400 & -1.342400\end{array}$

$\begin{array}{llll}\text { C } & 4.866500 & -3.422100 & 0.891500\end{array}$

C $\quad 3.542000 \quad-2.205700 \quad-0.924600$

C $\quad 4.396900 \quad-2.255000 \quad-2.244000$

$\begin{array}{llll}\text { C } & 5.169300 & -1.083000 & 2.337900\end{array}$

$\begin{array}{llll}\text { C } & 4.264200 & -2.257000 & 0.406200\end{array}$

$\begin{array}{lrrr}\text { C } & 5.600900 & -3.426400 & 2.079000\end{array}$

C $\quad 5.876100 \quad-2.437300 \quad-2.097700$

$\begin{array}{llll}\text { C } & 2.213700 & -1.470500 & -0.913100\end{array}$

C $\quad 4.008600 \quad-1.405100 \quad-3.409200$

$\begin{array}{llll}\text { C } & 4.015500 & -0.005400 & -3.295700\end{array}$

C $\quad 3.518300 \quad-3.413900-1.864700$

C $\quad 6.665500 \quad-1.442900-1.492700$

$\begin{array}{llll}\text { C } & 4.444000 & -1.081600 & 1.150500\end{array}$

$\begin{array}{llll}\text { C } & 6.500800 & -3.581400 & -2.624900\end{array}$

$\begin{array}{lrrr}\text { C } & 5.764600 & -2.255700 & 2.832500\end{array}$

$\begin{array}{llll}\text { C } & 8.656400 & -2.766800 & -1.883900\end{array}$

$\begin{array}{llll}\text { C } & 3.697300 & 0.791500 & -4.398900\end{array}$

$\begin{array}{llll}\text { C } & 3.371100 & -1.193900 & -5.744400\end{array}$

$\begin{array}{llll}\text { C } & 3.698000 & -1.994600 & -4.645900\end{array}$ 


\begin{tabular}{|c|c|c|c|}
\hline $\mathrm{C}$ & 7.883500 & -3.747700 & -2.508900 \\
\hline $\mathrm{C}$ & 8.048900 & -1.613800 & -1.381600 \\
\hline & 3.369800 & 0.198200 & -5.620600 \\
\hline $\mathrm{C}$ & -5.018100 & -3.337300 & -0.717800 \\
\hline & -2.472700 & -3.463000 & -0.632900 \\
\hline & -1.911300 & -4.895800 & -0.408300 \\
\hline $\mathrm{C}$ & -5.139000 & -2.169400 & 1.792900 \\
\hline $\mathrm{C}$ & -3.820500 & -3.085100 & -0.038300 \\
\hline $\mathrm{C}$ & -6.253700 & -3.004400 & -0.160500 \\
\hline & -2.766500 & -5.859500 & 0.354300 \\
\hline $\mathrm{C}$ & -1.537800 & -2.260500 & -0.732200 \\
\hline $\mathrm{C}$ & -0.445400 & -5.107800 & -0.221600 \\
\hline $\mathrm{C}$ & 0.217300 & -4.569100 & 0.893900 \\
\hline $\mathrm{C}$ & -2.396000 & -4.447400 & -1.773200 \\
\hline & -3.011600 & -5.661400 & 1.724000 \\
\hline $\mathrm{C}$ & -3.905500 & -2.494100 & 1.231500 \\
\hline $\mathrm{C}$ & -3.284000 & -7.004900 & -0.275300 \\
\hline $\mathrm{C}$ & -6.340800 & -2.411700 & 1.106900 \\
\hline $\mathrm{C}$ & -4.334700 & -7.687800 & 1.797400 \\
\hline$C$ & 1.550200 & -4.904100 & 1.149900 \\
\hline $\mathrm{C}$ & 1.577000 & -6.316500 & -0.815600 \\
\hline $\mathrm{C}$ & 0.246600 & -5.980600 & -1.077700 \\
\hline $\mathrm{C}$ & -4.071600 & -7.909500 & 0.443200 \\
\hline $\mathrm{C}$ & -3.801000 & -6.567000 & 2.438200 \\
\hline $\mathrm{C}$ & 2.222300 & -5.792000 & 0.306000 \\
\hline $\mathrm{C}$ & -4.651900 & 3.694000 & 3500 \\
\hline $\mathrm{C}$ & -3.634600 & 2.049400 & -0.997200 \\
\hline $\mathrm{C}$ & -4.643000 & 1.768300 & -2.174600 \\
\hline $\mathrm{C}$ & -4.824200 & 1.736600 & 2.622200 \\
\hline $\mathrm{C}$ & -4.186800 & 2.410300 & 0.369700 \\
\hline $\mathrm{C}$ & -5.194800 & 3.998900 & 1.923200 \\
\hline $\mathrm{C}$ & -6.100200 & 1.877000 & -1.852000 \\
\hline $\mathrm{C}$ & -2.294200 & 1.338700 & -0.977900 \\
\hline $\mathrm{C}$ & -4.328100 & 0.737200 & -3.209000 \\
\hline $\mathrm{C}$ & -4.262300 & 1.095600 & -4.566200 \\
\hline $\mathrm{C}$ & -3.813900 & 3.019900 & -2.163100 \\
\hline $\mathrm{C}$ & -6.892500 & 2.864800 & -2.461900 \\
\hline $\mathrm{C}$ & -4.298600 & 1.432600 & 1.369700 \\
\hline $\mathrm{C}$ & -6.706900 & 0.946200 & -0.990300 \\
\hline $\mathrm{C}$ & -5.288900 & 3.026700 & 2.928000 \\
\hline $\mathrm{C}$ & -8.850700 & 2.026000 & -1.309900 \\
\hline $\mathrm{C}$ & -4.000600 & 0.126300 & -5.538800 \\
\hline $\mathrm{C}$ & -3.903000 & -1.574400 & -3.820600 \\
\hline $\mathrm{C}$ & -4.162100 & -0.607100 & -2.844800 \\
\hline $\mathrm{C}$ & -8.076100 & 1.024600 & -0.719900 \\
\hline $\mathrm{C}$ & -8.260000 & 2.943500 & -2.182400 \\
\hline $\mathrm{C}$ & -3.818300 & -1.207700 & -5.165800 \\
\hline $\mathrm{C}$ & 6.552900 & -2.248800 & 4.095800 \\
\hline $\mathrm{C}$ & 7.348300 & -1.138100 & 4.436300 \\
\hline $\mathrm{C}$ & 6.517200 & -3.351100 & 4.971300 \\
\hline $\mathrm{C}$ & 8.091400 & -1.132400 & 5.620000 \\
\hline $\mathrm{C}$ & 7.261500 & -3.340000 & 6.155300 \\
\hline $\mathrm{C}$ & 8.048200 & -2.232400 & 6.478900 \\
\hline $\mathrm{C}$ & 7.325100 & 2.256500 & 1.995000 \\
\hline $\mathrm{C}$ & 8.359900 & 1.436400 & 1.507600 \\
\hline $\mathrm{C}$ & 7.459400 & 2.829000 & 3.274600 \\
\hline $\mathrm{C}$ & 9.499300 & 1.195100 & 2.280800 \\
\hline $\mathrm{C}$ & 8.600800 & 2.584000 & 4.044600 \\
\hline $\mathrm{C}$ & 9.619700 & 1.768200 & 3.548400 \\
\hline $\mathrm{C}$ & -5.866100 & 3.345500 & 4.262600 \\
\hline $\mathrm{C}$ & -6.665300 & 2.405200 & 4.940900 \\
\hline$C$ & -5.624200 & 4.593500 & 4.868100 \\
\hline
\end{tabular}

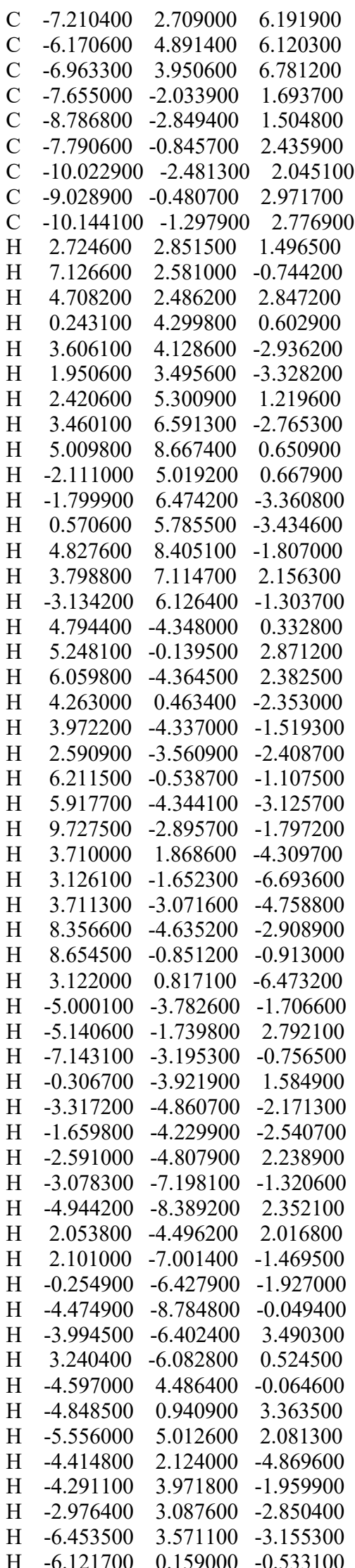

$\begin{array}{llll}\mathrm{H} & -9.910600 & 2.084700 & -1.098900\end{array}$

$\mathrm{H} \quad-3.943800 \quad 0.408600 \quad-6.582100$

$\mathrm{H} \quad-3.766700 \quad-2.608000 \quad-3.536800$

$\mathrm{H} \quad-4.222000 \quad-0.895900 \quad-1.805500$

$\begin{array}{llll}\mathrm{H} & -8.540600 & 0.304600 & -0.060700\end{array}$

$\mathrm{H} \quad-8.863600 \quad 3.711300 \quad-2.648700$

H $\quad-3.616600 \quad-1.957900 \quad-5.919400$

$\mathrm{H} \quad-3.970300 \quad 0.420400 \quad 1.168400$

$\mathrm{H} \quad 5.124200 \quad 2.932700 \quad-2.060500$

$\mathrm{H} \quad-2.994900 \quad-2.292400 \quad 1.784400$

H $\quad 4.017600 \quad-0.149000 \quad 0.801300$

H $\quad 7.409500 \quad-0.282000 \quad 3.780000$

H $\quad 5.900500 \quad-4.213200 \quad 4.751600$

$\mathrm{H} \quad 8.703300 \quad-0.275000 \quad 5.869500$

H $\quad 7.225000 \quad-4.190100 \quad 6.824300$

H $\quad 8.623700 \quad-2.226200 \quad 7.395500$

H $\quad 8.278900 \quad 0.966100 \quad 0.538500$

$\mathrm{H} \quad 6.691600 \quad 3.479000 \quad 3.674100$

H $\quad \begin{array}{llll}10.285700 & 0.556700 & 1.899500\end{array}$

$\mathrm{H} \quad 8.695700 \quad 3.028700 \quad 5.026800$

$\mathrm{H} \quad 10.500500 \quad 1.577000 \quad 4.147500$

$\mathrm{H} \quad-6.884200 \quad 1.442800 \quad 4.498500$

$\mathrm{H} \quad-4.998400 \quad 5.331900 \quad 4.383300$

$\mathrm{H} \quad-7.827700 \quad 1.981700 \quad 6.703200$

$\mathrm{H} \quad-5.976100 \quad 5.851700 \quad 6.580100$

$\mathrm{H} \quad-7.385800 \quad 4.183600 \quad 7.750000$

$\mathrm{H} \quad-8.713600 \quad-3.778300 \quad 0.953800$

H $\quad-6.947900 \quad-0.184300 \quad 2.574100$

H $\quad-10.887400 \quad-3.115600 \quad 1.897600$

H $\quad-9.125400 \quad 0.440300 \quad 3.531900$

H $\quad-11.102400 \quad-1.013700 \quad 3.191900$
T4
$\begin{array}{llll}\text { Rh } & -0.006600 & 0.017800 & 0.405500\end{array}$
$\begin{array}{lllll}\mathrm{Rh} & -0.170400 & 0.093000 & -1.969800\end{array}$
$\begin{array}{llll}\text { O } & 1.705000 & -1.119900 & 0.181300\end{array}$
$\begin{array}{llll}\text { O } & 1.493900 & -1.119800 & -2.060400\end{array}$
$\begin{array}{llll}\mathrm{O} & 1.189700 & 1.707800 & 0.316700\end{array}$
$\begin{array}{llll}\text { O } & 1.017300 & 1.785300 & -1.934200\end{array}$
$\begin{array}{llll}\mathrm{O} & -1.740700 & 1.135600 & 0.494200\end{array}$
$\begin{array}{llll}\mathrm{O} & -1.834300 & 1.289600 & -1.752700\end{array}$
$\begin{array}{llll}\mathrm{O} & -1.171900 & -1.685800 & 0.371500\end{array}$
$\begin{array}{llll}\text { O } & -1.299900 & -1.633700 & -1.884100\end{array}$
$\begin{array}{llll}\text { C } & 4.982300 & -3.498100 & 0.393300\end{array}$
C $\quad 3.471100 \quad-2.093400 \quad-1.114700$
C $\quad 4.318700 \quad-1.917600 \quad-2.435800$
$\begin{array}{llll}\text { C } & 5.046700 & -1.522300 & 2.325100\end{array}$
$\begin{array}{llll}\text { C } & 4.222600 & -2.338600 & 0.182800\end{array}$
C $\quad 5.783300 \quad-3.655800 \quad 1.525200$
$\begin{array}{llll}\text { C } & 5.805900 & -2.013100 & -2.294700\end{array}$
$\begin{array}{llll}\text { C } & 2.135800 & -1.384100 & -0.987800\end{array}$
$\begin{array}{llll}\text { C } & 3.880000 & -0.951800 & -3.488400\end{array}$
$\begin{array}{llll}\text { C } & 3.781700 & 0.417200 & -3.197100\end{array}$
$\begin{array}{llll}\text { C } & 3.492100 & -3.153000 & -2.219400\end{array}$
C $\quad 6.517400 \quad-3.038800 \quad-2.939500$
$\begin{array}{llll}\text { C } & 4.283700 & -1.348100 & 1.174600\end{array}$
$\begin{array}{llll}\text { C } & 6.512300 & -1.045900 & -1.557100\end{array}$
$\begin{array}{llll}\text { C } & 5.833700 & -2.667500 & 2.516900\end{array}$
$\begin{array}{llll}\text { C } & 8.599200 & -2.163900 & -2.062600\end{array}$
C $\quad 3.403000 \quad 1.324400 \quad-4.191000$
$\begin{array}{llll}\text { C } & 3.245000 & -0.487200 & -5.787900\end{array}$
$\begin{array}{llll}\text { C } & 3.625000 & -1.396900 & -4.796400\end{array}$ 


\begin{tabular}{|c|c|c|c|}
\hline & 7.902500 & -1.126500 & -1.438400 \\
\hline $\mathrm{C}$ & 7.907800 & -3.116900 & -2.815400 \\
\hline $\mathrm{C}$ & 3.131400 & 0.872200 & -5.484600 \\
\hline & 4.034900 & 2.539800 & 0.903800 \\
\hline $\mathrm{C}$ & 2.417300 & 3.402600 & -0.866000 \\
\hline $\mathrm{C}$ & 1.885500 & 4.850000 & -0.662100 \\
\hline & 6.208800 & 2.825600 & -0.799100 \\
\hline C & 3.813500 & 3.029000 & -0.392300 \\
\hline & 5.316700 & 2.206700 & 1.339100 \\
\hline & 2.806600 & 5.840600 & -0.021300 \\
\hline $\mathrm{C}$ & 1.458100 & 2.216200 & -0.820200 \\
\hline $\mathrm{C}$ & 0.440500 & 5.078200 & -0.361600 \\
\hline $\mathrm{C}$ & -0.325500 & 5.902800 & -1.202300 \\
\hline S & 2.248400 & 4.333900 & -2.040800 \\
\hline & 3.161600 & 5.708200 & 1.332100 \\
\hline $\mathrm{C}$ & 4.926700 & 3.170700 & -1.229000 \\
\hline $\mathrm{C}$ & 3.277600 & 6.948500 & -0.747400 \\
\hline $\mathrm{C}$ & 6.433200 & 2.333800 & 0.495000 \\
\hline $\mathrm{C}$ & 4.498100 & 7.722500 & 1.196000 \\
\hline$c$ & -1.637900 & 6.235300 & -0.857600 \\
\hline $\mathrm{C}$ & -1.443000 & 4.921700 & 1.166100 \\
\hline $\mathrm{C}$ & -0.128200 & 4.589500 & 0.826700 \\
\hline $\mathrm{C}$ & 4.126500 & 7.879600 & -0.141600 \\
\hline $\mathrm{C}$ & 4.012000 & 6.640200 & 1.933500 \\
\hline $\mathrm{C}$ & -2.190600 & 5.757100 & 0.332100 \\
\hline $\mathrm{C}$ & -4.784900 & 3.439100 & 1.442000 \\
\hline $\mathrm{C}$ & -3.668500 & 2.165100 & -0.473500 \\
\hline $\mathrm{C}$ & -4.688000 & 2.110900 & -1.673200 \\
\hline $\mathrm{C}$ & -4.841000 & 1.165000 & 3.022000 \\
\hline $\mathrm{C}$ & -4.216900 & 2.265500 & 0.937400 \\
\hline $\mathrm{C}$ & -5.369000 & 3.483100 & 2.709700 \\
\hline $\mathrm{C}$ & -6.142200 & 2.221700 & -1.335800 \\
\hline $\mathrm{C}$ & -2.325800 & 1.467500 & -0.587700 \\
\hline $\mathrm{C}$ & -4.405500 & 1.246800 & -2.858600 \\
\hline $\mathrm{C}$ & -4.281300 & -0.144200 & -2.712400 \\
\hline $\mathrm{C}$ & -3.828300 & 3.325000 & -1.457700 \\
\hline $\mathrm{C}$ & -6.904900 & 3.295600 & -1.826200 \\
\hline $\mathrm{C}$ & -4.264200 & 1.125800 & 1.756100 \\
\hline $\mathrm{C}$ & -6.771600 & 1.232100 & -0.562000 \\
\hline $\mathrm{C}$ & -5.408800 & 2.346900 & 3.528200 \\
\hline $\mathrm{C}$ & -8.870600 & 2.428900 & -0.707400 \\
\hline $\mathrm{C}$ & -4.021000 & -0.947700 & -3.826900 \\
\hline $\mathrm{C}$ & -4.057000 & 1.007500 & -5.252000 \\
\hline $\mathrm{C}$ & -4.310500 & 1.815500 & -4.139900 \\
\hline $\mathrm{C}$ & -8.125000 & 1.347000 & -0.234200 \\
\hline $\mathrm{C}$ & -8.262600 & 3.399000 & -1.508400 \\
\hline $\mathrm{C}$ & -3.907400 & -0.372500 & -5.094800 \\
\hline $\mathrm{C}$ & -4.672900 & -3.695700 & -1.605000 \\
\hline $\mathrm{C}$ & -2.267500 & -3.501600 & -0.776900 \\
\hline $\mathrm{C}$ & -1.611800 & -4.805100 & -0.256300 \\
\hline $\mathrm{C}$ & -5.611700 & -2.290800 & 0.599900 \\
\hline $\mathrm{C}$ & -3.756800 & -3.258800 & -0.643700 \\
\hline $\mathrm{C}$ & -6.035000 & -3.419500 & -1.478900 \\
\hline $\mathrm{C}$ & -2.512800 & -5.863500 & 0.299300 \\
\hline $\mathrm{C}$ & -1.510700 & -2.177700 & -0.752900 \\
\hline $\mathrm{C}$ & -0.268100 & -4.737700 & 0.396000 \\
\hline $\mathrm{C}$ & 0.838700 & -5.377400 & -0.187500 \\
\hline $\mathrm{C}$ & -1.718200 & -4.517500 & -1.745800 \\
\hline $\mathrm{C}$ & -3.255800 & -5.630700 & 1.469700 \\
\hline $\mathrm{C}$ & -4.251600 & -2.559800 & 0.466900 \\
\hline $\mathrm{C}$ & -2.580200 & -7.126500 & -0.314000 \\
\hline & -6.529900 & -2.702800 & -0.379900 \\
\hline
\end{tabular}

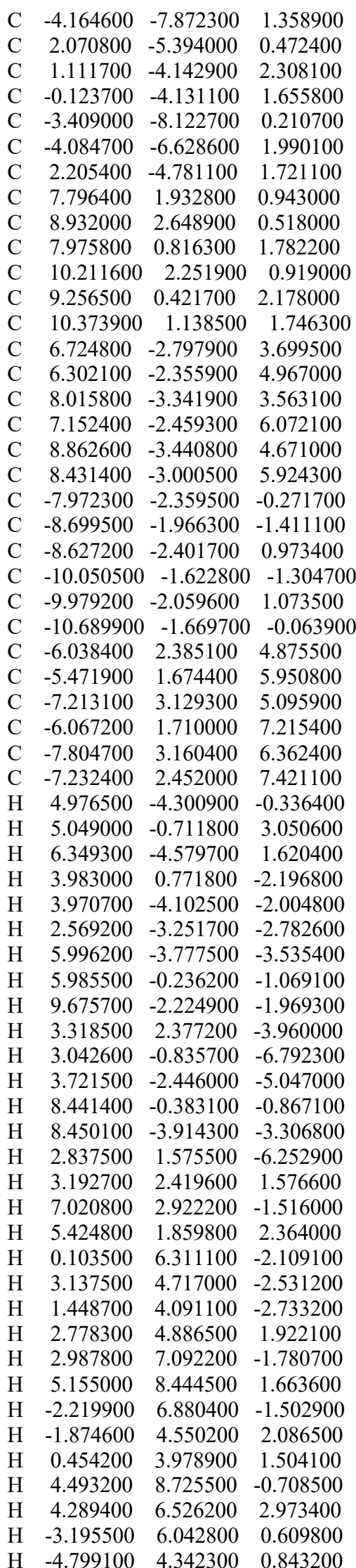

$\begin{array}{llll}\mathrm{H} & -4.860300 & 0.241600 & 3.597000\end{array}$

$\mathrm{H} \quad-5.774800 \quad 4.434900 \quad 3.045000$

$\mathrm{H} \quad-4.368500 \quad-0.598200 \quad-1.734800$

$\mathrm{H} \quad-4.278700 \quad 4.243300 \quad-1.095500$

$\mathrm{H} \quad-2.988000 \quad 3.486900 \quad-2.125700$

$\mathrm{H} \quad-6.448000 \quad 4.053100 \quad-2.450700$

$\mathrm{H} \quad-6.210800 \quad 0.381400 \quad-0.202200$

$\mathrm{H} \quad-9.920200 \quad 2.512900 \quad-0.457200$

$\mathrm{H} \quad-3.902900 \quad-2.015700 \quad-3.711200$

$\mathrm{H} \quad-3.976700 \quad 1.451500 \quad-6.235800$

$\mathrm{H} \quad-4.430100 \quad 2.883200 \quad-4.276100$

$\mathrm{H} \quad-8.596200 \quad 0.597300 \quad 0.386300$

$\mathrm{H} \quad-8.843100 \quad 4.232500 \quad-1.882000$

$\mathrm{H} \quad-3.706800 \quad-0.996700 \quad-5.955900$

$\mathrm{H} \quad-4.325600 \quad-4.235200 \quad-2.480200$

$\mathrm{H} \quad-5.933800 \quad-1.716200 \quad 1.465000$

$\mathrm{H} \quad-6.700400 \quad-3.782700 \quad-2.258800$

$\mathrm{H} \quad 0.743500 \quad-5.879100 \quad-1.142300$

$\mathrm{H} \quad-2.412600 \quad-5.097800 \quad-2.346500$

$\mathrm{H} \quad-0.826300 \quad-4.226600 \quad-2.293200$

$\mathrm{H} \quad-3.193000 \quad-4.680100 \quad 1.981300$

$\mathrm{H} \quad-1.991500 \quad-7.339700 \quad-1.197600$

H $\quad-4.805400 \quad-8.644600 \quad 1.764100$

$\mathrm{H} \quad 2.918400 \quad-5.892900 \quad 0.021000$

H $\quad 1.216900 \quad-3.671500 \quad 3.276600$

$\mathrm{H} \quad-0.969600 \quad-3.659300 \quad 2.138600$

$\mathrm{H} \quad-3.462700 \quad-9.090300 \quad-0.271200$

H $\quad-4.661200 \quad-6.439200 \quad 2.886300$

$\mathrm{H} \quad 3.154800 \quad-4.807100 \quad 2.238900$

$\mathrm{H} \quad-3.558000 \quad-2.222300 \quad 1.231300$

H $\quad 3.742400 \quad-0.421500 \quad 1.045100$

$\mathrm{H} \quad-3.852300 \quad 0.192800 \quad 1.392300$

H $\quad 4.800700 \quad 3.530900 \quad-2.244300$

$\mathrm{H} \quad 8.831700 \quad 3.523700 \quad-0.111500$

$\mathrm{H} \quad 7.131200 \quad 0.232300 \quad 2.111700$

$\begin{array}{llll}\mathrm{H} & 11.078400 & 2.809700 & 0.588800\end{array}$

$\mathrm{H} \quad 9.381900 \quad-0.444300 \quad 2.815500$

$\mathrm{H} \quad 11.365400 \quad 0.831700 \quad 2.053200$

H $\quad 5.310500 \quad-1.944100 \quad 5.106200$

H $\quad 8.378500 \quad-3.668900 \quad 2.596700$

$\mathrm{H} \quad 6.818600 \quad-2.120400 \quad 7.044300$

H $\quad 9.856200 \quad-3.854600 \quad 4.556400$

H $\quad 9.088700 \quad-3.077800 \quad 6.780700$

$\mathrm{H} \quad-8.217600 \quad-1.899400 \quad-2.378400$

$\mathrm{H} \quad-8.098100 \quad-2.711300 \quad 1.865800$

$\mathrm{H} \quad-10.600100 \quad-1.313000 \quad-2.184100$

$\mathrm{H} \quad-10.476100 \quad-2.096600 \quad 2.034300$

H $\quad-11.735400 \quad-1.401700 \quad 0.016600$

$\mathrm{H} \quad-4.559700 \quad 1.106400 \quad 5.818900$

$\mathrm{H} \quad-7.684900 \quad 3.671000 \quad 4.285800$

$\mathrm{H} \quad-5.622600 \quad 1.164200 \quad 8.037500$

$\mathrm{H} \quad-8.710200 \quad 3.731600 \quad 6.521900$

H $\quad-7.691900 \quad 2.477700 \quad 8.400700$

\section{T5}

$\begin{array}{llll}\mathrm{Rh} & -0.001400 & 0.003100 & 0.517900\end{array}$

$\begin{array}{llll}\mathrm{Rh} & -0.002500 & 0.002500 & -1.864700\end{array}$

O $\quad 1.684200 \quad-1.175200 \quad-1.798400$

$\begin{array}{llll}\text { O } & 1.766300 & -1.071300 & 0.451500\end{array}$

$\begin{array}{llll}\text { O } & -1.111600 & -1.737200 & -1.805300\end{array}$

O $\quad-1.113900 \quad-1.737200 \quad 0.455200$

$\begin{array}{llll}\text { O } & -1.689700 & 1.179600 & -1.798200\end{array}$ 


\begin{tabular}{|c|c|c|c|}
\hline $\mathrm{O}$ & -1.769200 & 1.076800 & 0.451700 \\
\hline & 1.107400 & 1.741800 & -1.806900 \\
\hline & 1.112000 & 1.742600 & 0.453600 \\
\hline C & 4.388200 & -1.182400 & 1.510000 \\
\hline $\mathrm{C}$ & 3.580400 & -2.137200 & -0.691600 \\
\hline$C$ & 4.490100 & -2.098900 & -1.977300 \\
\hline $\mathrm{C}$ & 5.597800 & -3.566400 & 2.246500 \\
\hline $\mathrm{C}$ & 4.256600 & -2.289600 & 0.657300 \\
\hline $\mathrm{C}$ & 5.100500 & -1.265800 & 2.702900 \\
\hline $\mathrm{C}$ & 5.963300 & -2.268900 & -1.772700 \\
\hline C & 2.258200 & -1.392700 & -0.679000 \\
\hline $\mathrm{C}$ & 4.139600 & -1.194500 & -3.113900 \\
\hline $\mathrm{C}$ & 3.905800 & -1.726400 & -4.393000 \\
\hline $\mathrm{C}$ & 3.602900 & -3.282900 & -1.706100 \\
\hline $\mathrm{C}$ & 6.695700 & -1.317800 & -1.041800 \\
\hline $\mathrm{C}$ & 4.878100 & -3.479100 & 1.053100 \\
\hline $\mathrm{C}$ & 6.637700 & -3.361600 & -2.343900 \\
\hline $\mathrm{C}$ & 5.725700 & -2.460600 & 3.096800 \\
\hline $\mathrm{C}$ & 8.725500 & -2.590100 & -1.388500 \\
\hline $\mathrm{C}$ & 3.588900 & -0.880900 & -5.460100 \\
\hline $\mathrm{C}$ & 3.765300 & 1.038000 & -3.996200 \\
\hline $\mathrm{C}$ & 4.088300 & 0.196600 & -2.927000 \\
\hline $\mathrm{C}$ & 8.012800 & -3.521800 & -2.147800 \\
\hline $\mathrm{C}$ & 8.066800 & -1.489700 & -0.835700 \\
\hline $\mathrm{C}$ & 3.514100 & 0.499800 & -5.260600 \\
\hline $\mathrm{C}$ & -4.521700 & -3.727400 & -1.709500 \\
\hline $\mathrm{C}$ & -2.177100 & -3.558200 & -0.716100 \\
\hline $\mathrm{C}$ & -1.596900 & -4.861400 & -0.113400 \\
\hline $\mathrm{C}$ & -5.579300 & -2.212900 & 0.365000 \\
\hline $\mathrm{C}$ & -3.665500 & -3.273600 & -0.701900 \\
\hline $\mathrm{C}$ & -5.882300 & -3.417400 & -1.691800 \\
\hline $\mathrm{C}$ & -2.565000 & -5.877900 & 0.406100 \\
\hline $\mathrm{C}$ & -1.394600 & -2.250500 & -0.675400 \\
\hline $\mathrm{C}$ & -0.300300 & -4.811800 & 0.629300 \\
\hline $\mathrm{C}$ & 0.823900 & -5.500600 & 0.143000 \\
\hline $\mathrm{C}$ & -1.588300 & -4.616100 & -1.614200 \\
\hline $\mathrm{C}$ & -3.384400 & -5.587800 & 1.510900 \\
\hline $\mathrm{C}$ & -4.220400 & -2.518300 & 0.341500 \\
\hline $\mathrm{C}$ & -2.623400 & -7.157700 & -0.172200 \\
\hline $\mathrm{C}$ & -6.436400 & -2.645700 & -0.660200 \\
\hline $\mathrm{C}$ & -4.345800 & -7.807400 & 1.401500 \\
\hline $\mathrm{C}$ & 2.006700 & -5.532400 & 0.887300 \\
\hline $\mathrm{C}$ & 0.962000 & -4.199400 & 2.615500 \\
\hline $\mathrm{C}$ & -0.224600 & -4.171700 & 1.878400 \\
\hline $\mathrm{C}$ & -3.516100 & -8.114400 & 0.320100 \\
\hline $\mathrm{C}$ & -4.276900 & -6.546400 & 1.998800 \\
\hline $\mathrm{C}$ & 2.073700 & -4.886200 & 2.124500 \\
\hline $\mathrm{C}$ & -4.391400 & 1.180000 & 1.510700 \\
\hline $\mathrm{C}$ & -3.586100 & 2.139200 & -0.689200 \\
\hline $\mathrm{C}$ & -4.498400 & 2.099800 & -1.972900 \\
\hline $\mathrm{C}$ & -5.592800 & 3.564700 & 2.258000 \\
\hline $\mathrm{C}$ & -4.259600 & 2.289600 & 0.661200 \\
\hline $\mathrm{C}$ & -5.099900 & 1.261300 & 2.705900 \\
\hline $\mathrm{C}$ & -5.971500 & 2.267200 & -1.765200 \\
\hline $\mathrm{C}$ & -2.262800 & 1.397000 & -0.678400 \\
\hline $\mathrm{C}$ & -4.148500 & 1.196900 & -3.110800 \\
\hline $\mathrm{C}$ & -4.093100 & -0.194300 & -2.924900 \\
\hline $\mathrm{C}$ & -3.612700 & 3.285400 & -1.702900 \\
\hline $\mathrm{C}$ & -6.648900 & 3.359300 & -2.333900 \\
\hline $\mathrm{C}$ & -4.877100 & 3.479300 & 1.062000 \\
\hline $\mathrm{C}$ & -6.700900 & 1.313800 & -1.034300 \\
\hline & -5.720600 & 2.456700 & 3.105300 \\
\hline
\end{tabular}

C $\quad-8.733600 \quad 2.582900 \quad-1.375600$

C $\quad-3.770600 \quad-1.034100 \quad-3.995400$

C $\quad-3.603100 \quad 0.886000 \quad-5.458600$

$\begin{array}{llll}\text { C } & -3.919400 & 1.730100 & -4.390200\end{array}$

C $\quad-8.071900 \quad 1.483000 \quad-0.825400$

C $\quad-8.023900 \quad 3.516800 \quad-2.135000$

C $\quad-3.524200 \quad-0.494600 \quad-5.260200$

C $\quad 4.525400 \quad 3.728600 \quad-1.707800$

$\begin{array}{llll}\text { C } & 2.178600 & 3.560500 & -0.719100\end{array}$

$\begin{array}{llll}\text { C } & 1.601000 & 4.864800 & -0.116500\end{array}$

$\begin{array}{llll}\text { C } & 5.576700 & 2.206600 & 0.364300\end{array}$

$\begin{array}{llll}\text { C } & 3.666400 & 3.272700 & -0.703500\end{array}$

C $\quad 5.885600 \quad 3.417000 \quad-1.688000$

$\begin{array}{llll}\text { C } & 2.571200 & 5.879000 & 0.403400\end{array}$

$\begin{array}{llll}\text { C } & 1.392700 & 2.254800 & -0.677600\end{array}$

$\begin{array}{llll}\text { C } & 0.304200 & 4.817400 & 0.626000\end{array}$

C $\quad-0.819500 \quad 5.506000 \quad 0.138200$

$\begin{array}{llll}\text { C } & 1.592300 & 4.619600 & -1.617400\end{array}$

$\begin{array}{llll}\text { C } & 2.631800 & 7.159100 & -0.174100\end{array}$

$\begin{array}{llll}\text { C } & 4.218200 & 2.513600 & 0.338700\end{array}$

$\begin{array}{llll}\text { C } & 3.390600 & 5.586500 & 1.507500\end{array}$

C $\quad 6.436600 \quad 2.641500 \quad-0.657600$

$\begin{array}{llll}\text { C } & 4.356200 & 7.804400 & 1.399300\end{array}$

$\begin{array}{llll}\mathrm{C} & -2.002700 & 5.539100 & 0.881800\end{array}$

C $\quad-0.959200 \quad 4.208500 \quad 2.612500$

$\begin{array}{llll}\text { C } & 0.227700 & 4.179100 & 1.875900\end{array}$

$\begin{array}{llll}\text { C } & 4.285200 & 6.543100 & 1.995700\end{array}$

C $\quad 3.526500 \quad 8.113700 \quad 0.318500$

$\begin{array}{llll}\text { C } & -2.070500 & 4.894800 & 2.119900\end{array}$

C $\quad-7.875300 \quad-2.271000 \quad-0.666600$

$\begin{array}{llll}\text { C } & -8.613500 & -2.242000 & 0.531600\end{array}$

$\begin{array}{llll}\text { C } & -8.516900 & -1.922600 & -1.870300\end{array}$

$\begin{array}{llll}\text { C } & -9.963000 & -1.876500 & 0.522700\end{array}$

C $\quad-9.866100 \quad-1.556100 \quad-1.873000$

C $\quad-10.588700 \quad-1.533800 \quad-0.678100$

C $\quad 6.502300 \quad-2.544400 \quad 4.362300$

C $\quad 7.309200 \quad-1.468400 \quad 4.778600$

$\begin{array}{llll}\text { C } & 6.444700 & -3.701400 & 5.162300\end{array}$

C $\quad 8.040100 \quad-1.550300 \quad 5.967600$

$\begin{array}{llll}\text { C } & 7.176800 & -3.777100 & 6.351100\end{array}$

$\begin{array}{llll}\text { C } & 7.973800 & -2.703100 & 6.753300\end{array}$

$\begin{array}{llll}\text { C } & 7.875300 & 2.265200 & -0.661800\end{array}$

C $\quad 8.519900 \quad 1.921500 \quad-1.865300$

$\begin{array}{llll}\text { C } & 8.610200 & 2.230100 & 0.538300\end{array}$

C $\quad 9.868700 \quad 1.553700 \quad-1.865900$

C $\quad 9.959400 \quad 1.863300 \quad 0.531400$

$\begin{array}{llll}\text { C } & 10.588000 & 1.525300 & -0.669100\end{array}$

$\begin{array}{llll}\text { C } & -6.492200 & 2.538800 & 4.374000\end{array}$

C $\quad-6.428400 \quad 3.693100 \quad 5.177200$

$\begin{array}{llll}\text { C } & -7.300400 & 1.463600 & 4.790000\end{array}$

$\begin{array}{llll}\text { C } & -7.155900 & 3.767300 & 6.369000\end{array}$

$\begin{array}{llll}\text { C } & -8.026600 & 1.543900 & 5.982100\end{array}$

$\begin{array}{llll}\text { C } & -7.954200 & 2.694200 & 6.771000\end{array}$

$\mathrm{H} \quad 3.932500 \quad-0.240800 \quad 1.232200$

$\mathrm{H} \quad 6.070700 \quad-4.517100 \quad 2.481600$

$\mathrm{H} \quad 5.144900 \quad-0.376500 \quad 3.328200$

H $\quad 3.966500 \quad-2.794400 \quad-4.562100$

$\mathrm{H} \quad 4.038600 \quad-4.228000 \quad-1.398700$

$\mathrm{H} \quad 2.700800 \quad-3.393900 \quad-2.299500$

H $\quad 6.201700 \quad-0.454200 \quad-0.618900$

H $\quad 6.098700 \quad-4.090200 \quad-2.936500$

H $\quad 9.788700 \quad-2.718400 \quad-1.232000$
H $\quad 3.401900 \quad-1.296200 \quad-6.441800$

H $\quad 3.706400 \quad 2.106900-3.847800$

$\mathrm{H} \quad 4.281200 \quad 0.622500 \quad-1.951900$

H $\quad 8.525600 \quad-4.369700 \quad-2.582900$

H $\quad 8.618800 \quad-0.770400 \quad-0.246700$

H $\quad 3.265200 \quad 1.153200 \quad-6.086800$

$\mathrm{H} \quad-4.127000 \quad-4.308300 \quad-2.536600$

$\mathrm{H} \quad-5.947100 \quad-1.595000 \quad 1.180400$

$\mathrm{H} \quad-6.499100 \quad-3.798400 \quad-2.502600$

H $\quad 0.779300 \quad-6.028700 \quad-0.801200$

$\mathrm{H} \quad-2.253200 \quad-5.196200 \quad-2.247500$

$\mathrm{H} \quad-0.651400 \quad-4.365000 \quad-2.103300$

$\mathrm{H} \quad-3.332400 \quad-4.622600 \quad 1.995900$

H $\quad-1.978400 \quad-7.414300 \quad-1.003400$

$\mathrm{H} \quad-5.036100 \quad-8.549300 \quad 1.781700$

$\begin{array}{llll}\mathrm{H} & 2.867300 & -6.069600 & 0.510600\end{array}$

H $\quad 1.014200 \quad-3.702900 \quad 3.575700$

$\mathrm{H} \quad-1.087300 \quad-3.662100 \quad 2.287500$

$\begin{array}{llll}\mathrm{H} & -3.562100 & -9.095400 & -0.134900\end{array}$

$\mathrm{H} \quad-4.911700 \quad-6.313000 \quad 2.843800$

H $\quad 2.983900 \quad-4.924900 \quad 2.707900$

$\mathrm{H} \quad-3.939000 \quad 0.238100 \quad 1.228300$

$\mathrm{H} \quad-6.062600 \quad 4.515900 \quad 2.497700$

$\mathrm{H} \quad-5.144700 \quad 0.370300 \quad 3.328700$

H $\quad-4.282300 \quad-0.621300 \quad-1.949400$

$\mathrm{H} \quad-4.049600 \quad 4.229500 \quad-1.394100$

$\mathrm{H} \quad-2.712000 \quad 3.398400 \quad-2.298200$

H $\quad-6.112300 \quad 4.089600 \quad-2.926600$

$\mathrm{H} \quad-6.204600 \quad 0.450400 \quad-0.613600$

$\mathrm{H} \quad-9.796700 \quad 2.709200-1.217100$

H $\quad-3.708500 \quad-2.102900 \quad-3.847800$

$\mathrm{H} \quad-3.419700 \quad 1.302400 \quad-6.440600$

$\mathrm{H} \quad-3.983400 \quad 2.798000 \quad-4.558600$

$\mathrm{H} \quad-8.621500 \quad 0.761900 \quad-0.236400$

$\mathrm{H} \quad-8.539000 \quad 4.364400 \quad-2.568200$

H $\quad-3.275700 \quad-1.146900 \quad-6.087300$

H $\quad 4.133400 \quad 4.312700 \quad-2.534100$

H $\quad 5.941800 \quad 1.585700 \quad 1.178700$

$\mathrm{H} \quad 6.504600 \quad 3.800100 \quad-2.496200$

$\mathrm{H} \quad-0.774400 \quad 6.032700 \quad-0.806800$

$\mathrm{H} \quad 2.258600 \quad 5.198300 \quad-2.250400$

$\mathrm{H} \quad 0.655100 \quad 4.370500 \quad-2.106900$

$\begin{array}{llll}\mathrm{H} & 1.987000 & 7.417400 & -1.004900\end{array}$

H $\quad 3.337100 \quad 4.621000 \quad 1.991800$

H $\quad 5.048100 \quad 8.544700 \quad 1.779700$

$\mathrm{H} \quad-2.863100 \quad 6.075700 \quad 0.503900$

H $\quad-1.012100 \quad 3.713400 \quad 3.573400$

$\mathrm{H} \quad 1.089900 \quad 3.669500 \quad 2.286100$

$\begin{array}{llll}\mathrm{H} & 4.920000 & 6.307900 & 2.840200\end{array}$

H $\quad 3.574200 \quad 9.094900 \quad-0.135800$

$\mathrm{H} \quad-2.980900 \quad 4.934700 \quad 2.702900$

H $\quad 3.570600 \quad 2.159300 \quad 1.135100$

H $\quad 4.817200 \quad-4.364500 \quad 0.429200$

$\begin{array}{llll}\mathrm{H} & -4.815400 & 4.366500 & 0.440600\end{array}$

H $\quad-3.574900 \quad-2.165400 \quad 1.140200$

$\mathrm{H} \quad-8.152100 \quad-2.515900 \quad 1.472000$

H $\quad-7.970100 \quad-1.909600 \quad-2.804700$

H $\quad-10.524200 \quad-1.859300 \quad 1.448100$

H $\quad-10.349700 \quad-1.282000 \quad-2.801700$

H $\quad-11.632600 \quad-1.247900 \quad-0.682100$

H $\quad 7.390000 \quad-0.572300 \quad 4.176100$

H $\quad 5.820200 \quad-4.539100 \quad 4.878500$ 


$\begin{array}{cccc}\mathrm{H} & 8.661400 & -0.720200 & 6.278200 \\ \mathrm{H} & 7.123500 & -4.668600 & 6.962600 \\ \mathrm{H} & 8.540200 & -2.764100 & 7.673600 \\ \mathrm{H} & 7.975600 & 1.913300 & -2.801200 \\ \mathrm{H} & 8.146600 & 2.500300 & 1.478600 \\ \mathrm{H} & 10.354500 & 1.283300 & -2.794500 \\ \mathrm{H} & 10.518000 & 1.841400 & 1.458200 \\ \mathrm{H} & 11.631600 & 1.238400 & -0.671600 \\ \mathrm{H} & -5.802700 & 4.530000 & 4.893600 \\ \mathrm{H} & -7.385900 & 0.569600 & 4.185200 \\ \mathrm{H} & -7.097700 & 4.656700 & 6.983000 \\ \mathrm{H} & -8.649000 & 0.714600 & 6.292500 \\ \mathrm{H} & -8.516900 & 2.754000 & 7.693600\end{array}$

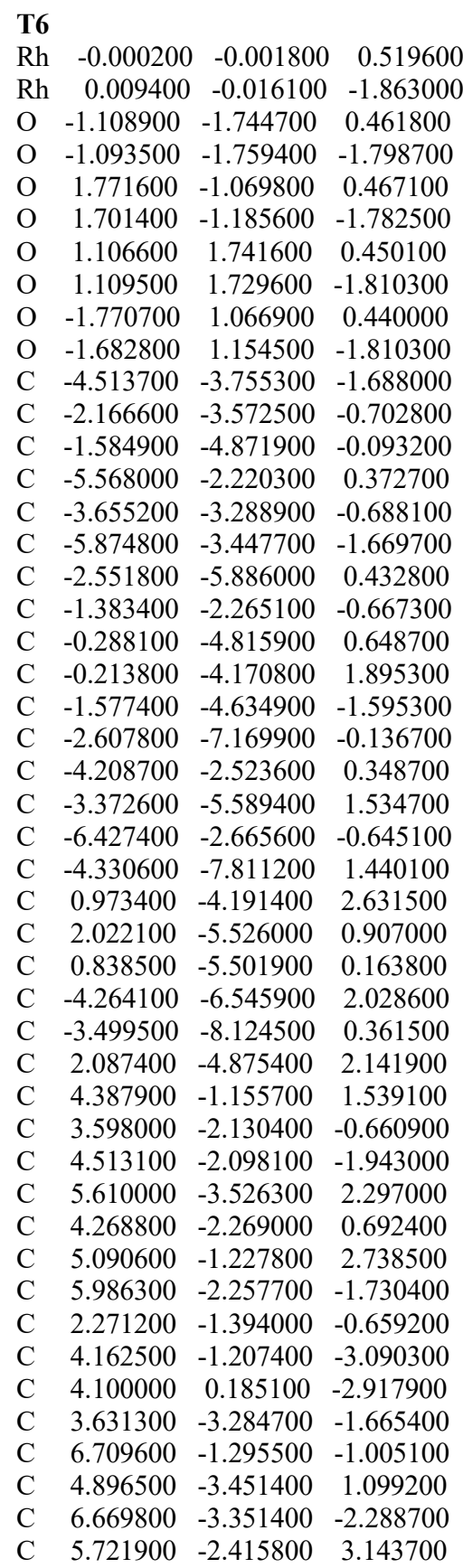

C $\quad 8.748800 \quad-2.558200 \quad-1.331700$

$\begin{array}{llll}\text { C } & 3.777400 & 1.013200 & -3.997600\end{array}$

$\begin{array}{llll}\text { C } & 3.624200 & -0.921600 & -5.443000\end{array}$

C $\quad 3.940500 \quad-1.753900 \quad-4.365500$

C $\quad 8.045000 \quad-3.501300 \quad-2.085200$

$\begin{array}{llll}\text { C } & 8.080900 & -1.456800 & -0.791900\end{array}$

$\begin{array}{llll}\text { C } & 3.538100 & 0.460500 & -5.258000\end{array}$

$\begin{array}{llll}\text { C } & 4.495700 & 3.736400 & -1.740700\end{array}$

$\begin{array}{llll}\text { C } & 2.159600 & 3.563700 & -0.728900\end{array}$

$\begin{array}{llll}\text { C } & 1.574300 & 4.864500 & -0.126400\end{array}$

$\begin{array}{llll}\text { C } & 5.575600 & 2.242500 & 0.337500\end{array}$

$\begin{array}{llll}\text { C } & 3.649600 & 3.287500 & -0.722600\end{array}$

C $\quad 5.857300 \quad 3.430600 \quad-1.732100$

$\begin{array}{llll}\text { C } & 2.538200 & 5.888300 & 0.386300\end{array}$

$\begin{array}{llll}\text { C } & 1.386200 & 2.250900 & -0.682600\end{array}$

$\begin{array}{llll}\text { C } & 0.281000 & 4.808700 & 0.621700\end{array}$

C $\quad 0.213700 \quad 4.170300 \quad 1.872100$

$\begin{array}{llll}\text { C } & 1.560100 & 4.615800 & -1.626800\end{array}$

C $\quad 3.363700 \quad 5.606300 \quad 1.488600$

$\begin{array}{llll}\text { C } & 4.215400 & 2.542700 & 0.322500\end{array}$

$\begin{array}{llll}\text { C } & 2.586400 & 7.166900 & -0.195600\end{array}$

$\begin{array}{llll}\text { C } & 6.422200 & 2.669100 & -0.698900\end{array}$

$\begin{array}{llll}\text { C } & 4.311000 & 7.831500 & 1.369400\end{array}$

$\begin{array}{llll}\text { C } & -0.971000 & 4.190200 & 2.612500\end{array}$

C $\quad-2.029900 \quad 5.514100 \quad 0.886000$

$\begin{array}{llll}\text { C } & -0.849200 & 5.489600 & 0.138200\end{array}$

$\begin{array}{llll}\text { C } & 3.475200 & 8.130400 & 0.290500\end{array}$

C $\quad 4.252200 \quad 6.571700 \quad 1.970500$

$\begin{array}{llll}\text { C } & -2.089200 & 4.868100 & 2.123700\end{array}$

$\begin{array}{llll}\text { C } & -4.891000 & 3.472000 & 1.014300\end{array}$

C $\quad-3.584700 \quad 2.119300 \quad-0.715800$

$\begin{array}{llll}\text { C } & -4.491100 & 2.068500 & -2.003500\end{array}$

$\begin{array}{llll}\text { C } & -5.107700 & 1.273300 & 2.684200\end{array}$

$\begin{array}{llll}\text { C } & -4.264500 & 2.281200 & 0.630400\end{array}$

$\begin{array}{llll}\text { C } & -5.612400 & 3.567800 & 2.205900\end{array}$

C $\quad-5.965200 \quad 2.236300 \quad-1.803800$

C $\quad-2.260500 \quad 1.378600 \quad-0.694100$

C $\quad-4.135300 \quad 1.156300 \quad-3.132100$

$\begin{array}{llll}\text { C } & -3.899500 & 1.678900 & -4.414700\end{array}$

C $\quad-3.607600 \quad 3.257000 \quad-1.739300$

C $\quad-6.641100 \quad 3.322300 \quad-2.385800$

$\begin{array}{llll}\text { C } & -4.393800 & 1.181300 & 1.492900\end{array}$

$\begin{array}{llll}\text { C } & -6.696800 & 1.289500 & -1.066600\end{array}$

$\begin{array}{llll}\text { C } & -5.737100 & 2.469700 & 3.066500\end{array}$

C $\quad-8.729100 \quad 2.553600 \quad-1.428500$

$\begin{array}{llll}\text { C } & -3.577400 & 0.825900 & -5.474300\end{array}$

C $\quad-3.752600 \quad-1.082000 \quad-3.995900$

C $\quad-4.080800 \quad-0.233200 \quad-2.934300$

C $\quad-8.068800 \quad 1.459700 \quad-0.864900$

C $\quad-8.017100 \quad 3.480600 \quad-2.194200$

C $\quad-3.499500 \quad-0.553100 \quad-5.263800$

C $\quad 6.487200 \quad-2.488100 \quad 4.417200$

C $\quad 6.025600 \quad-1.822100 \quad 5.568400$

$\begin{array}{llll}\text { C } & 7.687000 & -3.221100 & 4.490400\end{array}$

C $\quad 6.748100 \quad-1.890200 \quad 6.763500$

$\begin{array}{lrrr}\text { C } & 8.406000 & -3.284800 & 5.688000\end{array}$

C $\quad 7.937000 \quad-2.620400 \quad 6.823300$

C $\quad-7.867100 \quad-2.293200 \quad-0.651500$

$\begin{array}{llll}\text { C } & -8.513700 & -1.962300 & -1.857500\end{array}$

C $\quad-8.601000 \quad-2.249100 \quad 0.548900$

C $\quad-9.863600 \quad-1.598500 \quad-1.860300$

C $\quad-9.951300 \quad-1.886400 \quad 0.539900$
C $\quad-10.582000 \quad-1.561400 \quad-0.663200$

C $\quad-6.514200 \quad 2.562900 \quad 4.331000$

C $\quad-7.318000 \quad 1.488400 \quad 4.757100$

C $\quad-6.460200 \quad 3.727400 \quad 5.120100$

$\begin{array}{llll}\text { C } & -8.049400 & 1.579200 & 5.945300\end{array}$

C $\quad-7.192800 \quad 3.812100 \quad 6.308000$

$\begin{array}{llll}\text { C } & -7.986600 & 2.739500 & 6.720100\end{array}$

C $\quad 7.861500 \quad 2.297100 \quad-0.715900$

$\begin{array}{llll}\text { C } & 8.613200 & 2.288300 & 0.474000\end{array}$

$\begin{array}{llll}\text { C } & 8.489600 & 1.930900 & -1.921400\end{array}$

C $\quad 9.963400 \quad 1.925500 \quad 0.455100$

$\begin{array}{llll}\text { C } & 9.839300 & 1.566600 & -1.933900\end{array}$

$\begin{array}{llll}\text { C } & 10.575800 & 1.564700 & -0.747300\end{array}$

H $\quad-4.120800 \quad-4.344700 \quad-2.510100$

H $\quad-5.934300 \quad-1.594300 \quad 1.182600$

$\mathrm{H} \quad-6.493100 \quad-3.839100 \quad-2.474300$

H $\quad-1.078000 \quad-3.662700 \quad 2.303200$

$\mathrm{H} \quad-2.242100 \quad-5.219300 \quad-2.224800$

H $\quad-0.641300 \quad-4.385600 \quad-2.086700$

H $\quad-1.961900 \quad-7.431200 \quad-0.965700$

H $\quad-3.322700 \quad-4.620600 \quad 2.012900$

$\begin{array}{llll}\mathrm{H} & -5.020100 & -8.551400 & 1.824900\end{array}$

H $\quad 1.024600 \quad-3.691200 \quad 3.589900$

H $\quad 2.884800 \quad-6.060600 \quad 0.531300$

H $\quad 0.795300 \quad-6.033400 \quad-0.778500$

$\mathrm{H} \quad-4.900000 \quad-6.307600 \quad 2.871400$

H $\quad-3.543700 \quad-9.108600 \quad-0.086600$

H $\quad 2.998000 \quad-4.908700 \quad 2.724900$

$\mathrm{H} \quad 3.932400 \quad-0.217200 \quad 1.250100$

H $\quad 6.055100 \quad-4.483300 \quad 2.559400$

H $\quad 5.157000 \quad-0.323400 \quad 3.339600$

$\mathrm{H} \quad 4.283600 \quad 0.622200 \quad-1.945900$

H $\quad 4.070800 \quad-4.224900-1.348400$

H $\quad 2.732400 \quad-3.405700 \quad-2.261800$

H $\quad 6.208400 \quad-0.431200 \quad-0.592200$

H $\quad 6.137800 \quad-4.088600 \quad-2.876900$

$\mathrm{H} \quad 9.812100 \quad-2.678500 \quad-1.169700$

$\mathrm{H} \quad 3.709800 \quad 2.083000 \quad-3.860500$

H $\quad 3.446400 \quad-1.348100 \quad-6.421700$

$\mathrm{H} \quad 4.010200 \quad-2.823000 \quad-4.523500$

H $\quad 8.564800 \quad-4.350100 \quad-2.510300$

H $\quad 8.626100 \quad-0.728400 \quad-0.207700$

H $\quad 3.289700 \quad 1.103700 \quad-6.092300$

H $\quad 4.091900 \quad 4.308900 \quad-2.569400$

H $\quad 5.952900 \quad 1.633300 \quad 1.155200$

$\begin{array}{llll}\mathrm{H} & 6.466400 & 3.805900 & -2.551400\end{array}$

H $\quad 1.081200 \quad 3.667300 \quad 2.279400$

$\mathrm{H} \quad 2.218700 \quad 5.198300-2.264500$

H $\quad 0.622400 \quad 4.358200 \quad-2.110900$

H $\quad 3.319300 \quad 4.642200 \quad 1.976700$

$\mathrm{H} \quad 1.936600 \quad 7.417300 \quad-1.025000$

$\begin{array}{llll}\mathrm{H} & 4.998200 & 8.578600 & 1.744900\end{array}$

$\mathrm{H} \quad-1.017100 \quad 3.694200 \quad 3.573300$

H $\quad-2.895100 \quad 6.045300 \quad 0.511400$

H $\quad-0.811200 \quad 6.016700 \quad-0.806800$

H $\quad 3.513400 \quad 9.110400 \quad-0.167300$

H $\quad 4.891700 \quad 6.344600 \quad 2.813600$

$\mathrm{H} \quad-2.998300 \quad 4.900000 \quad 2.709200$

H $\quad-4.832900 \quad 4.351700 \quad 0.382100$

$\begin{array}{llll}\mathrm{H} & -5.149800 & 0.389600 & 3.317600\end{array}$

$\mathrm{H} \quad-6.088900 \quad 4.519000 \quad 2.431700$

H $\quad-3.962600 \quad 2.745300 \quad-4.592400$ 


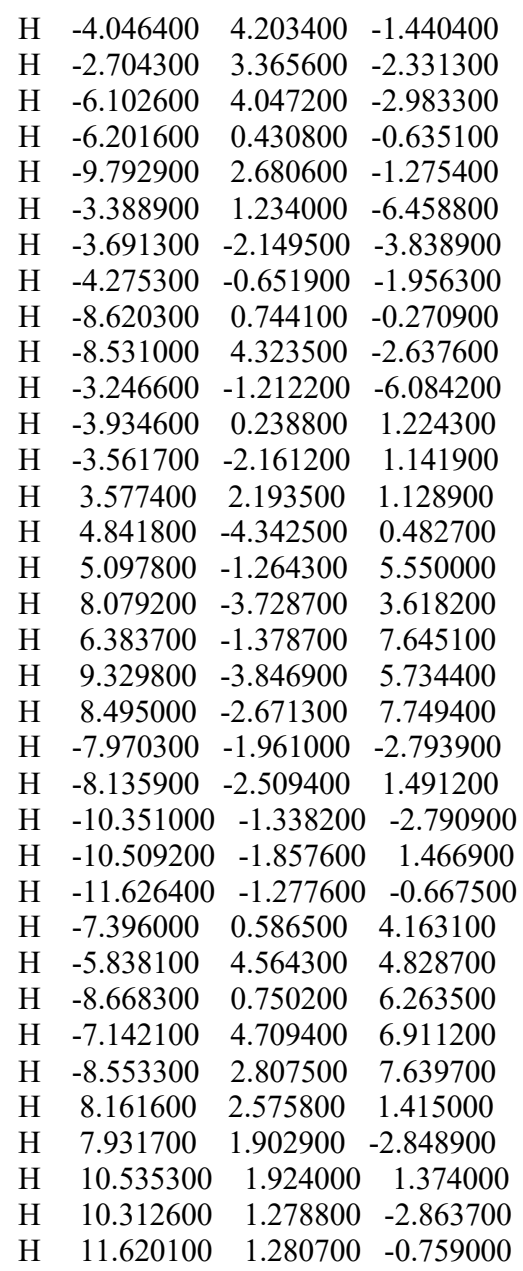

T7

$\begin{array}{llll}\mathrm{Rh} & 0.000100 & 0.000000 & 0.521000\end{array}$

$\begin{array}{lllll}\mathrm{Rh} & 0.000000 & 0.000300 & -1.861700\end{array}$

$\begin{array}{llll}\mathrm{O} & -1.773100 & 1.063900 & 0.455900\end{array}$

$\begin{array}{lllll}\mathrm{O} & -1.698900 & 1.161500 & -1.794400\end{array}$

$\begin{array}{llll}\mathrm{O} & -1.103000 & -1.746100 & 0.456800\end{array}$

$\begin{array}{llll}\mathrm{O} & -1.092100 & -1.750000 & -1.803700\end{array}$

$\begin{array}{lrrr}\mathrm{O} & 1.773200 & -1.063800 & 0.455500\end{array}$

$\begin{array}{llll}\text { O } & 1.698800 & -1.160800 & -1.794800\end{array}$

$\begin{array}{llll}\mathrm{O} & 1.103100 & 1.746200 & 0.457200\end{array}$

$\begin{array}{llll}\text { O } & 1.092100 & 1.750600 & -1.803300\end{array}$

$\begin{array}{llll}\text { C } & -4.904600 & 3.443100 & 1.064200\end{array}$

$\begin{array}{llll}\text { C } & -3.598700 & 2.112800 & -0.683200\end{array}$

$\begin{array}{llll}\text { C } & -4.510300 & 2.072700 & -1.967300\end{array}$

$\begin{array}{llll}\text { C } & -5.090700 & 1.233800 & 2.723500\end{array}$

$\begin{array}{llll}\text { C } & -4.272400 & 2.259400 & 0.668000\end{array}$

$\begin{array}{llll}\text { C } & -5.618200 & 3.526300 & 2.261400\end{array}$

$\begin{array}{llll}\text { C } & -5.984100 & 2.231600 & -1.759000\end{array}$

$\begin{array}{llll}\text { C } & -2.271100 & 1.378000 & -0.673900\end{array}$

$\begin{array}{llll}\text { C } & -4.156000 & 1.176500 & -3.109200\end{array}$

$\begin{array}{llll}\text { C } & -4.092900 & -0.215100 & -2.929500\end{array}$

$\begin{array}{llll}\text { C } & -3.630500 & 3.261700 & -1.694100\end{array}$

$\begin{array}{llll}\text { C } & -6.667900 & 3.320700 & -2.325700\end{array}$

$\begin{array}{llll}\text { C } & -4.388400 & 1.153100 & 1.524300\end{array}$

$\begin{array}{llll}\text { C } & -6.707700 & 1.273100 & -1.029000\end{array}$

$\begin{array}{llll}\text { C } & -5.725300 & 2.423400 & 3.118500\end{array}$ $\begin{array}{llll}\text { C } & -8.747900 & 2.530700 & -1.368400\end{array}$

$\begin{array}{llll}\text { C } & -3.767100 & -1.048500 & -4.004000\end{array}$

C $\quad-3.611500 \quad 0.878900 \quad-5.459000$

C $\quad-3.931200 \quad 1.716500 \quad-4.386600$

C $\quad-8.079700 \quad 1.433800 \quad-0.820000$

C $\quad-8.043700 \quad 3.470000 \quad-2.126300$

C $\quad-3.524900 \quad-0.502200 \quad-5.266700$

C $\quad-4.206400 \quad-2.553200 \quad 0.325400$

C $\quad-2.146600 \quad-3.577700 \quad-0.715300$

$\begin{array}{llll}\text { C } & -1.559900 & -4.873700 & -0.103900\end{array}$

C $\quad-5.842200 \quad-3.456200 \quad-1.727600$

C $\quad-3.637000 \quad-3.303700 \quad-0.713600$

C $\quad-5.567100 \quad-2.254800 \quad 0.335300$

C $\quad-2.522700 \quad-5.896100 \quad 0.413500$

C $\quad-1.375100 \quad-2.263600 \quad-0.674000$

C $\quad-0.268100 \quad-4.810300 \quad 0.646100$

$\begin{array}{llll}\text { C } & 0.865400 & -5.489100 & 0.167500\end{array}$

C $\quad-1.543800 \quad-4.634100 \quad-1.605700$

C $\quad-2.566900-7.178600 \quad-0.160000$

C $\quad-4.480100 \quad-3.760100 \quad-1.731000$

C $\quad-3.351300 \quad-5.608600 \quad 1.512100$

C $\quad-6.410600 \quad-2.689100 \quad-0.700500$

$\begin{array}{llll}\text { C } & -4.293500 & -7.836600 & 1.405600\end{array}$

$\begin{array}{llll}\text { C } & 2.045300 & -5.505100 & 0.916800\end{array}$

C $\quad 0.978800 \quad-4.176900 \quad 2.635400$

C $\quad-0.205000 \quad-4.165200 \quad 1.893300$

$\begin{array}{llll}\text { C } & -4.238700 & -6.572700 & 1.998400\end{array}$

$\begin{array}{llll}\text { C } & -3.454600 & -8.140800 & 0.330600\end{array}$

$\begin{array}{lrrr}\text { C } & 2.100200 & -4.852800 & 2.151400\end{array}$

$\begin{array}{llll}\text { C } & 4.904600 & -3.443700 & 1.062800\end{array}$

C $\quad 3.598600 \quad-2.112600 \quad-0.684000$

C $\quad 4.510200 \quad-2.072000-1.968100$

$\begin{array}{lrrr}\text { C } & 5.090700 & -1.235200 & 2.723100\end{array}$

C $\quad 4.272400 \quad-2.259700 \quad 0.667100$

$\begin{array}{llll}\text { C } & 5.618200 & -3.527400 & 2.260000\end{array}$

$\begin{array}{llll}\text { C } & 5.984100 & -2.231100 & -1.759900\end{array}$

C $\quad 2.271100 \quad-1.377700 \quad-0.674400$

C $\quad 4.156000 \quad-1.175400 \quad-3.109600$

$\begin{array}{llll}\text { C } & 4.092900 & 0.216200 & -2.929400\end{array}$

C $\quad 3.630300 \quad-3.261100-1.695400$

$\begin{array}{llll}\text { C } & 6.667700 & -3.320000 & -2.327000\end{array}$

$\begin{array}{lrrr}\text { C } & 4.388400 & -1.153900 & 1.524000\end{array}$

C $\quad 6.707700 \quad-1.273000 \quad-1.029500$

$\begin{array}{llll}\text { C } & 5.725300 & -2.425000 & 3.117500\end{array}$

C $\quad 8.747800 \quad-2.530600 \quad-1.369400$

$\begin{array}{llll}\text { C } & 3.767200 & 1.050000 & -4.003700\end{array}$

$\begin{array}{llll}\text { C } & 3.611500 & -0.876900 & -5.459300\end{array}$

C $\quad 3.931200 \quad-1.714900 \quad-4.387300$

C $\quad 8.079700 \quad-1.433800 \quad-0.820500$

C $\quad 8.043600 \quad-3.469500 \quad-2.127700$

C $\quad 3.525000 \quad 0.504100 \quad-5.266500$

C $\quad 4.480400 \quad 3.760700 \quad-1.729800$

$\begin{array}{llll}\text { C } & 2.146700 & 3.578100 & -0.714600\end{array}$

C $\quad 1.560000 \quad 4.874000 \quad-0.103100$

$\begin{array}{llll}\text { C } & 5.567000 & 2.254600 & 0.336200\end{array}$

$\begin{array}{llll}\text { C } & 3.637100 & 3.304000 & -0.712700\end{array}$

C $\quad 5.842500 \quad 3.456700 \quad-1.726200$

C $\quad 2.522800 \quad 5.896300 \quad 0.414500$

$\begin{array}{llll}\text { C } & 1.375200 & 2.264000 & -0.673500\end{array}$

C $\quad 0.268100 \quad 4.810500 \quad 0.646700$

$\begin{array}{llll}\text { C } & -0.865400 & 5.489400 & 0.168100\end{array}$

C $\quad 1.544100 \quad 4.634600 \quad-1.605000$
C $\quad 3.351300 \quad 5.608700 \quad 1.513100$

$\begin{array}{llll}\text { C } & 4.206300 & 2.553200 & 0.326200\end{array}$

$\begin{array}{llll}\text { C } & 2.566900 & 7.178900 & -0.159000\end{array}$

$\begin{array}{llll}\text { C } & 6.410700 & 2.689200 & -0.699300\end{array}$

$\begin{array}{lrrr}\text { C } & 4.293400 & 7.836800 & 1.406900\end{array}$

C $\quad-2.045300 \quad 5.505300 \quad 0.917400$

$\begin{array}{llll}\text { C } & -0.978900 & 4.177000 & 2.635900\end{array}$

C $\quad 0.204900 \quad 4.165300 \quad 1.893800$

$\begin{array}{llll}\text { C } & 3.454600 & 8.141100 & 0.331800\end{array}$

C $\quad 4.238700 \quad 6.572800 \quad 1.999500$

$\begin{array}{lrrr}\text { C } & -2.100300 & 4.853000 & 2.151900\end{array}$

$\begin{array}{llll}\text { C } & -7.850500 & -2.319600 & -0.723000\end{array}$

C $\quad-8.476400 \quad-1.961800 \quad-1.932100$

$\begin{array}{lrrr}\text { C } & -8.604900 & -2.305000 & 0.465100\end{array}$

$\begin{array}{llll}\text { C } & -9.826800 & -1.600100 & -1.949900\end{array}$

$\begin{array}{llll}\text { C } & -9.955800 & -1.944900 & 0.441000\end{array}$

C $\quad-10.566000 \quad-1.592500 \quad-0.765000$

$\begin{array}{llll}\text { C } & -6.488500 & 2.505300 & 4.392700\end{array}$

C $\quad-6.022200 \quad 1.852200 \quad 5.549400$

C $\quad-7.690600 \quad 3.234900 \quad 4.461200$

$\begin{array}{llll}\text { C } & -6.742600 & 1.929400 & 6.745300\end{array}$

C $\quad-8.407400 \quad 3.307700 \quad 5.659500$

$\begin{array}{llll}\text { C } & -7.934000 & 2.656100 & 6.800300\end{array}$

$\begin{array}{llll}\text { C } & 7.850500 & 2.319600 & -0.721700\end{array}$

C $\quad 8.476700 \quad 1.962200 \quad-1.930800$

$\begin{array}{llll}\text { C } & 8.604700 & 2.304600 & 0.466500\end{array}$

$\begin{array}{llll}\text { C } & 9.827000 & 1.600400 & -1.948500\end{array}$

C $\quad 9.955600 \quad 1.944300 \quad 0.442500$

$\begin{array}{llll}\text { C } & 10.566000 & 1.592400 & -0.763500\end{array}$

$\begin{array}{llll}\text { C } & 6.488400 & -2.507500 & 4.391700\end{array}$

C $\quad 6.022100 \quad-1.854900 \quad 5.548800$

$\begin{array}{llll}\text { C } & 7.690600 & -3.237100 & 4.459900\end{array}$

$\begin{array}{llll}\text { C } & 6.742500 & -1.932700 & 6.744600\end{array}$

$\begin{array}{llll}\text { C } & 8.407400 & -3.310500 & 5.658200\end{array}$

$\begin{array}{llll}\text { C } & 7.933900 & -2.659400 & 6.799300\end{array}$

H $\quad-4.853900 \quad 4.328700 \quad 0.439600$

$\mathrm{H} \quad-5.153700 \quad 0.334700 \quad 3.332800$

$\mathrm{H} \quad-6.066600 \quad 4.484100 \quad 2.515200$

$\mathrm{H} \quad-4.278800 \quad-0.647300 \quad-1.955700$

$\mathrm{H} \quad-4.071200 \quad 4.203300 \quad-1.383100$

$\mathrm{H} \quad-2.730200 \quad 3.380100 \quad-2.289000$

H $\quad-6.135500 \quad 4.055000 \quad-2.917300$

$\mathrm{H} \quad-6.206100 \quad 0.412400 \quad-0.609200$

$\mathrm{H} \quad-9.811700 \quad 2.650500 \quad-1.209600$

$\mathrm{H} \quad-3.699100 \quad-2.117500 \quad-3.861300$

$\mathrm{H} \quad-3.431500 \quad 1.300500 \quad-6.439400$

$\mathrm{H} \quad-4.001300 \quad 2.784800 \quad-4.550300$

$\mathrm{H} \quad-8.625100 \quad 0.708400 \quad-0.232300$

$\mathrm{H} \quad-8.563800 \quad 4.315300 \quad-2.558000$

H $\quad-3.274000 \quad-1.149400 \quad-6.097000$

$\mathrm{H} \quad-3.570800 \quad-2.198000 \quad 1.131100$

$\mathrm{H} \quad-6.448800 \quad-3.837500 \quad-2.545900$

H $\quad-5.947100 \quad-1.640900 \quad 1.148100$

H $\quad 0.830800 \quad-6.021000 \quad-0.775000$

H $\quad-2.200500 \quad-5.221900 \quad-2.240600$

$\mathrm{H} \quad-0.606000 \quad-4.377800 \quad-2.090300$

H $\quad-1.914700 \quad-7.433000 \quad-0.986300$

H $\quad-3.310200 \quad-4.641200 \quad 1.993800$

$\mathrm{H} \quad-4.979900 \quad-8.582700 \quad 1.784500$

H $\quad 2.913300 \quad-6.034300 \quad 0.545900$

$\mathrm{H} \quad 1.021600 \quad-3.675900 \quad 3.593800$

H $\quad-1.074900 \quad-3.663100 \quad 2.296600$ 
H $\quad-4.880600 \quad-6.341400 \quad 2.838600$

H $\quad-3.489600 \quad-9.123900 \quad-0.120800$

$\begin{array}{llll}\mathrm{H} & 3.008500 & -4.878500 & 2.738400\end{array}$

H $\quad 4.853900 \quad-4.328900 \quad 0.437800$

$\mathrm{H} \quad 5.153600 \quad-0.336400 \quad 3.332900$

$\begin{array}{llll}\mathrm{H} & 6.066600 & -4.485400 & 2.513300\end{array}$

H $\quad 4.278800 \quad 0.648000 \quad-1.955500$

H $\quad 4.071000 \quad-4.202800 \quad-1.384700$

$\mathrm{H} \quad 2.730100 \quad-3.379200 \quad-2.290300$

H $\quad 6.135300 \quad-4.054000 \quad-2.919000$

H $\quad 6.206100 \quad-0.412400 \quad-0.609200$

H $\quad 9.811600 \quad-2.650500 \quad-1.210600$

H $\quad 3.699200 \quad 2.119000 \quad-3.860600$

H $\quad 3.431500 \quad-1.298200 \quad-6.439900$

$\mathrm{H} \quad 4.001200 \quad-2.783100 \quad-4.551300$

$\mathrm{H} \quad 8.625200 \quad-0.708800 \quad-0.232500$

H $\quad 8.563600 \quad-4.314600 \quad-2.559800$

H $\quad 3.274100 \quad 1.151700 \quad-6.096600$

$\mathrm{H} \quad 4.074100 \quad 4.338100 \quad-2.553800$

$\begin{array}{llll}\mathrm{H} & 5.946800 & 1.640400 & 1.148900\end{array}$

$\mathrm{H} \quad 6.449200 \quad 3.838300 \quad-2.544300$

H $\quad-0.830700 \quad 6.021400 \quad-0.774300$

H $\quad 2.200800 \quad 5.222400 \quad-2.239700$

$\mathrm{H} \quad 0.606300 \quad 4.378300 \quad-2.089600$

$\mathrm{H} \quad 3.310100 \quad 4.641300 \quad 1.994700$

$\mathrm{H} \quad 1.914800 \quad 7.433400 \quad-0.985200$

$\mathrm{H} \quad 4.979800 \quad 8.582800 \quad 1.785900$

$\mathrm{H} \quad-2.913200 \quad 6.034600 \quad 0.546500$

$\mathrm{H} \quad-1.021800 \quad 3.675900 \quad 3.594200$

$\mathrm{H} \quad 1.074700 \quad 3.663200 \quad 2.297200$

$\mathrm{H} \quad 3.489700 \quad 9.124200 \quad-0.119500$

$\begin{array}{llll}\mathrm{H} & 4.880500 & 6.341400 & 2.839700\end{array}$

$\mathrm{H} \quad-3.008600 \quad 4.878700 \quad 2.738800$

$\mathrm{H} \quad 3.570500 \quad 2.197800 \quad 1.131700$

$\begin{array}{llll}\mathrm{H} & -3.929600 & 0.213700 & 1.244100\end{array}$

$\mathrm{H} \quad 3.929600 \quad-0.214300 \quad 1.244200$

$\mathrm{H} \quad-4.073600 \quad-4.337200 \quad-2.555100$

$\mathrm{H} \quad-7.916500 \quad-1.938400 \quad-2.858500$

$\mathrm{H} \quad-8.154900 \quad-2.586000 \quad 1.408800$

$\mathrm{H} \quad-10.298500 \quad-1.318700 \quad-2.882500$

$\mathrm{H} \quad-10.529800 \quad-1.939000 \quad 1.358500$

H $\quad-11.610800 \quad-1.310500 \quad-0.780800$

$\mathrm{H} \quad-5.092500 \quad 1.297500 \quad 5.534600$

$\mathrm{H} \quad-8.086200 \quad 3.732700 \quad 3.584900$

$\begin{array}{llll}\mathrm{H} & -6.374800 & 1.427800 & 7.631100\end{array}$

H $\quad-9.333100 \quad 3.867100 \quad 5.702200$

$\begin{array}{llll}\mathrm{H} & -8.490300 & 2.714100 & 7.726900\end{array}$

H $\quad 7.916900 \quad 1.939100 \quad-2.857300$

$\mathrm{H} \quad 8.154600 \quad 2.585200 \quad 1.410300$

H $\quad 10.298800 \quad 1.319400 \quad-2.881100$

$\begin{array}{llll}\mathrm{H} & 10.529500 & 1.938100 & 1.360100\end{array}$

$\begin{array}{lllll}\mathrm{H} & 11.610800 & 1.310300 & -0.779200\end{array}$

$\begin{array}{llll}\mathrm{H} & 5.092500 & -1.300300 & 5.534200\end{array}$

H $\quad 8.086200 \quad-3.734400 \quad 3.583300$

H $\quad 6.374600 \quad-1.431600 \quad 7.630600$

$\mathrm{H} \quad 9.333000 \quad-3.869900 \quad 5.700700$

H $\quad 8.490200 \quad-2.717900 \quad 7.725900$

U1

$\begin{array}{llll}\mathrm{Rh} & -0.571412 & -0.664875 & 0.988422\end{array}$

$\begin{array}{llll}\mathrm{Rh} & 0.138114 & 0.030069 & -1.186197\end{array}$

O $\quad-1.671054 \quad 1.011945 \quad-1.340395$
$\begin{array}{llll}\mathrm{O} & -2.484447 & -0.158533 & 0.409690\end{array}$

$\begin{array}{lllll}\text { O } & 0.926386 & 1.696471 & -0.282876\end{array}$

$\begin{array}{llll}\mathrm{O} & -0.247632 & 1.287629 & 1.606350\end{array}$

$\begin{array}{llll}\text { O } & 1.936362 & -0.930066 & -0.824908\end{array}$

$\begin{array}{llll}\text { O } & 1.404323 & -1.119038 & 1.365956\end{array}$

$\begin{array}{llll}\mathrm{O} & -0.812994 & -1.663127 & -1.936627\end{array}$

$\begin{array}{llll}\mathrm{O} & -0.942137 & -2.502126 & 0.153432\end{array}$

F $\quad-7.492114 \quad-1.476359 \quad 0.394815$

F $\quad-8.005376 \quad-3.383569-0.506206$

F $\quad-7.006537 \quad-1.856329-1.692585$

F $\quad-3.554598 \quad-5.422956 \quad 3.234110$

F $\quad-5.049700 \quad-3.992796 \quad 3.923475$

F $\quad-2.984687 \quad-3.366473 \quad 3.616369$

F $\quad 4.251883 \quad-2.258270 \quad-4.553784$

F $\quad 5.218838-4.011723 \quad-3.712726$

F $\quad 6.410575 \quad-2.486587 \quad-4.707138$

F $\quad 9.082600 \quad-0.339681 \quad-1.126943$

F $\quad 8.027246 \quad 0.754946 \quad 0.430897$

$\begin{array}{llll}\text { F } & 7.962991 & 1.444603 & -1.634245\end{array}$

F $\quad 6.359372 \quad 3.994676 \quad-1.548547$

F $\quad 5.177672 \quad 2.186755 \quad-1.822471$

$\begin{array}{llll}\text { F } & 6.009310 & 2.646825 & 0.130735\end{array}$

F $\quad \begin{array}{llll}1.788093 & 7.978011 & -0.451094\end{array}$

$\begin{array}{llll}\text { F } & 3.440132 & 7.817819 & -1.864381\end{array}$

$\begin{array}{lllll}\text { F } & 1.471583 & 7.060887 & -2.390969\end{array}$

F $\quad-1.354153 \quad 6.047750 \quad-0.598574$

F $\quad-1.546553 \quad 6.759027 \quad-2.649283$

F $\quad-0.366577 \quad 4.980003 \quad-2.210556$

F $\quad-6.987082 \quad 5.098238 \quad-3.258405$

F $\quad-6.447781 \quad 3.498716 \quad-4.622927$

$\begin{array}{llll}\text { F } & -5.628087 & 5.498146 & -4.907785\end{array}$

$\begin{array}{llll}\text { C } & -2.766000 & 3.598378 & -1.317533\end{array}$

$\begin{array}{llll}\text { C } & -3.873035 & 1.485866 & -0.597529\end{array}$

$\begin{array}{llll}\text { C } & -4.810387 & 1.553592 & 0.658453\end{array}$

C $\quad-4.800220 \quad 4.224520 \quad-3.088119$

$\begin{array}{llll}\text { C } & -3.830833 & 2.701717 & -1.469910\end{array}$

$\begin{array}{llll}\text { C } & -2.708281 & 4.772860 & -2.060914\end{array}$

$\begin{array}{llll}\text { C } & -5.650773 & 2.801753 & 0.769902\end{array}$

C $\quad-2.584623 \quad 0.728854 \quad-0.495885$

$\begin{array}{llll}\text { C } & -4.360920 & 1.008174 & 1.987681\end{array}$

$\begin{array}{llll}\text { C } & -5.067453 & -0.045380 & 2.576270\end{array}$

$\begin{array}{llll}\text { C } & -5.117817 & 0.619420 & -0.461962\end{array}$

$\begin{array}{llll}\text { C } & -7.028267 & 2.748217 & 0.535500\end{array}$

$\begin{array}{llll}\text { C } & -4.845858 & 3.027299 & -2.369249\end{array}$

$\begin{array}{llll}\text { C } & -5.070588 & 4.026712 & 1.126163\end{array}$

$\begin{array}{llll}\text { C } & -3.730393 & 5.105650 & -2.948646\end{array}$

$\begin{array}{llll}\text { C } & -7.222990 & 5.119582 & 0.970746\end{array}$

$\begin{array}{llll}\text { C } & -4.661196 & -0.571922 & 3.803519\end{array}$

$\begin{array}{llll}\text { C } & -2.847198 & 1.022348 & 3.881997\end{array}$

$\begin{array}{llll}\text { C } & -3.260991 & 1.548878 & 2.660508\end{array}$

$\begin{array}{llll}\text { C } & -5.848816 & 5.178253 & 1.220613\end{array}$

$\begin{array}{llll}\text { C } & -7.811544 & 3.900378 & 0.634417\end{array}$

$\begin{array}{llll}\text { C } & -3.545082 & -0.043815 & 4.455905\end{array}$

$\begin{array}{llll}\text { C } & 1.796185 & 5.204727 & 0.054095\end{array}$

$\begin{array}{llll}\text { C } & 1.118112 & 3.235556 & 1.494334\end{array}$

$\begin{array}{llll}\text { C } & 1.407857 & 3.322973 & 3.033255\end{array}$

$\begin{array}{llll}\text { C } & 4.183732 & 3.945997 & -0.603626\end{array}$

$\begin{array}{llll}\text { C } & 2.072607 & 3.947505 & 0.583384\end{array}$

$\begin{array}{llll}\text { C } & 2.714401 & 5.831604 & -0.793783\end{array}$

$\begin{array}{llll}\text { C } & 2.656829 & 4.092037 & 3.388418\end{array}$

$\begin{array}{llll}\text { C } & 0.553836 & 1.981242 & 0.906010\end{array}$

C $\quad 1.130243 \quad 2.174455 \quad 3.968133$ $\begin{array}{llll}\text { C } & 1.608096 & 0.881064 & 3.743381\end{array}$

$\begin{array}{llll}\text { C } & 0.239394 & 4.038641 & 2.435262\end{array}$

C $\quad 3.880982 \quad 3.410295 \quad 3.457081$

$\begin{array}{llll}\text { C } & 3.276196 & 3.321670 & 0.244978\end{array}$

$\begin{array}{llll}\text { C } & 2.641008 & 5.468825 & 3.628489\end{array}$

$\begin{array}{llll}\text { C } & 3.913891 & 5.210309 & -1.130290\end{array}$

$\begin{array}{llll}\text { C } & 5.039079 & 5.472456 & 3.958959\end{array}$

$\begin{array}{llll}\text { C } & 1.331248 & -0.145899 & 4.645410\end{array}$

$\begin{array}{llll}\text { C } & 0.146970 & 1.414738 & 6.056124\end{array}$

$\begin{array}{llll}\text { C } & 0.405503 & 2.433861 & 5.137789\end{array}$

$\begin{array}{llll}\text { C } & 3.824047 & 6.155433 & 3.913002\end{array}$

$\begin{array}{llll}\text { C } & 5.062849 & 4.093334 & 3.732305\end{array}$

$\begin{array}{llll}\text { C } & 0.600839 & 0.117495 & 5.806440\end{array}$

$\begin{array}{llll}\text { C } & 5.736713 & -0.650863 & -0.137082\end{array}$

$\begin{array}{llll}\text { C } & 3.597188 & -1.699763 & 0.692381\end{array}$

$\begin{array}{llll}\text { C } & 3.713958 & -3.017124 & 1.546374\end{array}$

$\begin{array}{llll}\text { C } & 5.511373 & -1.958133 & -2.575245\end{array}$

$\begin{array}{llll}\text { C } & 4.629438 & -1.472106 & -0.371399\end{array}$

$\begin{array}{llll}\text { C } & 6.730837 & -0.509181 & -1.103554\end{array}$

$\begin{array}{llll}\text { C } & 4.849902 & -3.935705 & 1.167637\end{array}$

$\begin{array}{llll}\text { C } & 2.212241 & -1.215022 & 0.386831\end{array}$

$\begin{array}{llll}\text { C } & 2.455225 & -3.718255 & 1.989390\end{array}$

$\begin{array}{llll}\text { C } & 1.551609 & -4.268207 & 1.075045\end{array}$

$\begin{array}{llll}\text { C } & 4.024346 & -1.685053 & 2.146362\end{array}$

$\begin{array}{llll}\text { C } & 6.109279 & -3.833989 & 1.767844\end{array}$

$\begin{array}{llll}\text { C } & 4.521816 & -2.120321 & -1.604434\end{array}$

$\begin{array}{llll}\text { C } & 4.658547 & -4.908234 & 0.175524\end{array}$

$\begin{array}{llll}\text { C } & 6.627082 & -1.162362 & -2.330682\end{array}$

$\begin{array}{llll}\text { C } & 6.961896 & -5.618896 & 0.372191\end{array}$

$\begin{array}{llll}\text { C } & 0.414605 & -4.938856 & 1.522669\end{array}$

$\begin{array}{llll}\text { C } & 1.046156 & -4.506738 & 3.807384\end{array}$

$\begin{array}{llll}\text { C } & 2.196085 & -3.855402 & 3.359292\end{array}$

$\begin{array}{llll}\text { C } & 5.703629 & -5.737466 & -0.223833\end{array}$

$\begin{array}{llll}\text { C } & 7.157906 & -4.668717 & 1.374104\end{array}$

$\begin{array}{llll}\text { C } & 0.147049 & -5.052824 & 2.886878\end{array}$

$\begin{array}{llll}\text { C } & -3.234225 & -3.895919 & 0.771442\end{array}$

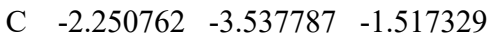

C $\quad-1.741351 \quad-4.849326 \quad-2.205392$

$\begin{array}{llll}\text { C } & -5.715829 & -2.973711 & -0.047291\end{array}$

$\begin{array}{llll}\text { C } & -3.418838 & -3.548609 & -0.569556\end{array}$

$\begin{array}{llll}\text { C } & -4.276748 & -3.788507 & 1.686825\end{array}$

$\begin{array}{llll}\text { C } & -2.548355 & -6.099946 & -1.958775\end{array}$

$\begin{array}{llll}\text { C } & -1.239723 & -2.502368 & -1.083647\end{array}$

$\begin{array}{llll}\text { C } & -0.251635 & -5.054782 & -2.362710\end{array}$

$\begin{array}{llll}\text { C } & 0.377092 & -6.171871 & -1.788841\end{array}$

$\begin{array}{llll}\text { C } & -2.443199 & -3.765882 & -2.985661\end{array}$

$\begin{array}{llll}\text { C } & -2.648334 & -6.689223 & -0.691435\end{array}$

$\begin{array}{llll}\text { C } & -4.674856 & -3.088360 & -0.972018\end{array}$

$\begin{array}{llll}\text { C } & -3.187565 & -6.722922 & -3.037592\end{array}$

$\begin{array}{llll}\text { C } & -5.529489 & -3.329019 & 1.289274\end{array}$

$\begin{array}{llll}\text { C } & -4.016582 & -8.471250 & -1.586455\end{array}$

$\begin{array}{llll}\text { C } & 1.749808 & -6.378677 & -1.926165\end{array}$

$\begin{array}{llll}\text { C } & 1.917633 & -4.369266 & -3.237813\end{array}$

$\begin{array}{llll}\text { C } & 0.546605 & -4.160279 & -3.094046\end{array}$

$\begin{array}{llll}\text { C } & -3.916010 & -7.899051 & -2.855832\end{array}$

$\begin{array}{llll}\text { C } & -3.378352 & -7.862855 & -0.504177\end{array}$

$\begin{array}{llll}\text { C } & 2.529697 & -5.479105 & -2.653549\end{array}$

$\begin{array}{llll}\text { C } & 5.437571 & 3.204337 & -0.964963\end{array}$

$\begin{array}{llll}\text { C } & 2.360649 & 7.171372 & -1.374235\end{array}$

$\begin{array}{llll}\text { C } & -5.959720 & 4.580624 & -3.974528\end{array}$

$\begin{array}{llll}\text { C } & -1.500492 & 5.649095 & -1.890311\end{array}$

$\begin{array}{llll}\text { C } & -7.051263 & -2.428915 & -0.470492\end{array}$ 


\begin{tabular}{|c|c|c|c|}
\hline E & -3.978940 & -4.140573 & 3.115685 \\
\hline $\mathrm{C}$ & 5.360198 & -2.673543 & -3.885942 \\
\hline $\mathrm{C}$ & 7.944070 & 0.340698 & -0.849013 \\
\hline $\mathrm{H}$ & -1.968259 & 3.372798 & -0.621149 \\
\hline & -5.922404 & -0.466126 & 2.058352 \\
\hline $\mathrm{H}$ & -5.958956 & 0.820923 & -1.113482 \\
\hline $\mathrm{H}$ & -4.912723 & -0.429325 & -0.296953 \\
\hline $\mathrm{H}$ & -7.489645 & 1.800145 & 0.276392 \\
\hline $\mathrm{H}$ & -4.003152 & 4.083221 & 1.309978 \\
\hline & -7.828528 & 6.018035 & 1.038968 \\
\hline $\mathrm{H}$ & -5.201975 & -1.406135 & 4.238109 \\
\hline $\mathrm{H}$ & -1.970399 & 1.428119 & 4.373969 \\
\hline $\mathrm{H}$ & -2.683115 & 2.339607 & 2.196108 \\
\hline $\mathrm{H}$ & -5.382512 & 6.123081 & 1.482843 \\
\hline & -8.879238 & 3.843328 & 0.445716 \\
\hline $\mathrm{H}$ & -3.216721 & -0.461175 & 5.403192 \\
\hline $\mathrm{H}$ & 0.862008 & 5.699016 & 0.285817 \\
\hline $\mathrm{H}$ & 2.149375 & 0.657583 & 2.833641 \\
\hline $\mathrm{H}$ & 0.259671 & 5.116302 & 2.328534 \\
\hline 1 & -0.738355 & 3.620678 & 2.642621 \\
\hline $\mathrm{H}$ & 3.906830 & 2.338781 & 8198 \\
\hline $\mathrm{H}$ & 1.704037 & 6.014168 & 3.596790 \\
\hline $\mathrm{H}$ & 5.959356 & 6.007187 & 4.172519 \\
\hline $\mathrm{H}$ & 1.667614 & -1.155604 & 4.431665 \\
\hline $\mathrm{H}$ & -0.419058 & 1.631337 & 6.957036 \\
\hline $\mathrm{H}$ & 0.036742 & 3.438759 & 2444 \\
\hline $\mathrm{H}$ & 3.792131 & 7.225046 & 4.096816 \\
\hline $\mathrm{H}$ & 6.002463 & 3.550428 & 3.766996 \\
\hline $\mathrm{H}$ & 0.385780 & -0.681399 & 6.509497 \\
\hline $\mathrm{H}$ & 5.833707 & -0.122831 & 0.802520 \\
\hline $\mathrm{H}$ & 1.705882 & -4.148739 & 0.008698 \\
\hline $\mathrm{H}$ & 5.055158 & -1.426355 & 2.350545 \\
\hline $\mathrm{H}$ & 3.310298 & -1.281702 & 2.850119 \\
\hline $\mathrm{H}$ & 6.279164 & -3.104997 & 2.552445 \\
\hline $\mathrm{H}$ & 3.689449 & -5.015938 & -0.293949 \\
\hline $\mathrm{H}$ & 7.778382 & -6.263326 & 0.061474 \\
\hline $\mathrm{H}$ & -0.264617 & -5.359094 & 0.791508 \\
\hline $\mathrm{H}$ & 0.855113 & -4.591064 & 4.872981 \\
\hline $\mathrm{H}$ & 2.897911 & -3.440632 & 4.076895 \\
\hline $\mathrm{H}$ & 5.534955 & -6.476225 & -1.001818 \\
\hline $\mathrm{H}$ & 8.127344 & -4.573388 & 1.853704 \\
\hline $\mathrm{H}$ & -0.756296 & -5.549178 & 3.227800 \\
\hline $\mathrm{H}$ & -2.256960 & -4.204820 & 1.111891 \\
\hline $\mathrm{H}$ & -0.209964 & -6.897774 & -1.240335 \\
\hline $\mathrm{H}$ & -3.447595 & -3.993014 & -3.324018 \\
\hline $\mathrm{H}$ & -1.876239 & -3.138861 & -3.661126 \\
\hline $\mathrm{H}$ & -2.160648 & -6.230603 & 0.159401 \\
\hline $\mathrm{H}$ & -3.110958 & -6.282218 & -4.026830 \\
\hline $\mathrm{H}$ & -4.587427 & -9.383182 & -1.441586 \\
\hline $\mathrm{H}$ & 2.206480 & -7.249619 & -1.466165 \\
\hline $\mathrm{H}$ & 2.500663 & -3.669968 & -3.825450 \\
\hline $\mathrm{H}$ & 0.108321 & -3.289915 & -3.562564 \\
\hline $\mathrm{H}$ & -4.405148 & -8.366087 & -3.705176 \\
\hline $\mathrm{H}$ & -3.448944 & -8.297824 & 0.487947 \\
\hline $\mathrm{H}$ & 3.595666 & -5.636930 & -2.771972 \\
\hline $\mathrm{H}$ & -4.835708 & -2.778111 & -1.996943 \\
\hline $\mathrm{H}$ & -5.683568 & 2.357444 & -2.516652 \\
\hline $\mathrm{H}$ & 3.668793 & -2.757154 & -1.802301 \\
\hline $\mathrm{H}$ & 3.502658 & 2.340454 & 0.644811 \\
\hline $\mathrm{H}$ & -3.690300 & 6.023385 & -3.520447 \\
\hline $\mathrm{H}$ & -6.333232 & -3.228095 & 2.007807 \\
\hline & 7.403019 & -1.060767 & -3.079111 \\
\hline
\end{tabular}

\begin{tabular}{|c|c|c|c|c|c|c|c|}
\hline & 4.626754 & 5.699583 & -1.781091 & $\mathrm{C}$ & -2.145689 & 4.643134 & -2.103060 \\
\hline & & & & $\mathrm{C}$ & -3.305448 & 3.109793 & -0.629125 \\
\hline $\mathrm{U} 2$ & & & & $\mathrm{C}$ & -4.244913 & 3.694590 & -2.778906 \\
\hline h & -0.149210 & -0.365029 & $9 \quad 1.803446$ & $\mathrm{C}$ & -4.122483 & 4.453300 & 1.788666 \\
\hline & -0.009234 & -0.180118 & -0.573802 & $\mathrm{C}$ & -2.211881 & 1.260247 & 0.626226 \\
\hline & -1.377273 & -1.711118 & -0.689049 & $\mathrm{C}$ & -3.072991 & 2.713830 & 3.272272 \\
\hline & -1.061011 & -2.208413 & 1.492152 & $\mathrm{C}$ & -1.688870 & 2.794199 & 3.446947 \\
\hline & -1.559532 & 1.154542 & -0.464763 & $\mathrm{C}$ & -4.595686 & 1.945672 & 1.331086 \\
\hline & -2.013073 & 0.555304 & 1.666102 & $\mathrm{C}$ & -3.134849 & 5.442892 & 1.903363 \\
\hline & 1.301935 & 1.373022 & -0.280593 & $\mathrm{C}$ & -2.219279 & 3.946545 & -0.900325 \\
\hline & 0.712402 & 1.515632 & 1.897362 & $\mathrm{C}$ & -5.436011 & 4.846560 & 1.516366 \\
\hline & 1.583268 & -1.482101 & -0.521900 & $\mathrm{C}$ & -3.159229 & 4541 & \\
\hline & 1.745704 & -1.192193 & 1.716367 & $\mathrm{C}$ & -4.762487 & 0185 & \\
\hline & 7.564345 & -2.947932 & -3.695211 & $\mathrm{C}$ & -1.113114 & 2.566300 & 5880 \\
\hline & 6.340512 & -1.280426 & -4.344051 & $\mathrm{C}$ & & 2.214759 & \\
\hline & 7.355986 & -1.19 & -2.42 & $\mathrm{C}$ & -3.879133 & 2.42 & \\
\hline & 3.323 & -6.59 & -2.7 & $\mathrm{C}$ & & & \\
\hline & 1.680320 & -5.293746 & -3.32 & $\mathrm{C}$ & & 7857 & \\
\hline & 3.373694 & -5.558058 & -4.669937 & $\mathrm{C}$ & -1.923175 & 2.277585 & 5.796798 \\
\hline & -5.902461 & -6.077050 & -3.30 & $\mathrm{C}$ & & & \\
\hline & -5.326168 & -4.95 & -5.07 & C & & & \\
\hline & -3.838 & -6.10 & -3.9 & $\mathrm{C}$ & & & \\
\hline & -6.522146 & -0.47 & -3.60 & $\mathrm{C}$ & & 593 & \\
\hline & -5.991253 & 0.15 & -1.58 & $\mathrm{C}$ & & & -0.2 \\
\hline & -4.561577 & 0.37 & -3.21 & C & & 2.7 & \\
\hline & -1.11 & 6.38 & -3.3 & & & & \\
\hline & -0.42 & 6.05 & -1.2 & $\mathrm{C}$ & & & \\
\hline & -5.695 & 4.67 & -4.3 & $\mathrm{C}$ & & & \\
\hline & -6.39 & 2.87 & -3.3 & $\mathrm{C}$ & & & \\
\hline & -4.82 & 2.74 & -4.8 & C & & & \\
\hline & 2.14 & 6.77 & -2.5 & & & & \\
\hline & 2.39 & 5.53 & $-4.2^{\prime}$ & $\mathrm{C}$ & & & \\
\hline & 4.09 & 6.61 & -3.4 & $\mathrm{C}$ & & & \\
\hline & 4.864 & 0.58 & -2.9 & C & & & \\
\hline & 6.34 & 2.09 & -3.42 & & & & \\
\hline & 6.33 & $1.1 \mathrm{~S}$ & -1.4 & 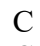 & & & \\
\hline & 0.08 & 4.63 & -2.85 & $\mathrm{C}$ & & & \\
\hline & -3.81 & -4.27 & -1.8 & $\mathrm{C}$ & & & \\
\hline 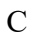 & -2.783623 & -3.33 & & & & & \\
\hline & -3.57 & -3.7 & & C & & & \\
\hline & -4.96 & -1.8 & -2.4 & C & & & \\
\hline $\mathrm{C}$ & -3.58 & -3.21 & -0.9 & $\mathrm{C}$ & & -2.2 & \\
\hline $\mathrm{C}$ & -4.628286 & -4.11 & -2.97 & $\mathrm{C}$ & & -2. & \\
\hline $\mathrm{C}$ & -5.04 & -4.069408 & & C & & & \\
\hline & -1.65 & -2.3 & & 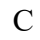 & & & \\
\hline $\mathrm{C}$ & -3.31 & -3.18 & 2.9 & $\mathrm{C}$ & & -2.8 & -0 . \\
\hline $\mathrm{C}$ & -3.381695 & -1.808463 & 3.14 & $\mathrm{C}$ & & -2.6 & -2 \\
\hline $\mathrm{C}$ & -2.565552 & -4.689174 & 0.937976 & $\mathrm{C}$ & 4.397748 & -4.7 & \\
\hline & -5.961920 & -3.011915 & 1.276038 & $\mathrm{C}$ & & -1.6 & \\
\hline $\mathrm{C}$ & -4.17 & -1.9 & -1.2 & C & & & \\
\hline $\mathrm{C}$ & -5.51 & -5.37 & 1.14 & $\mathrm{C}$ & & -3.9 & \\
\hline $\mathrm{C}$ & -5.207201 & -2.884586 & -3.269340 & $\mathrm{C}$ & 4.460931 & -2.149426 & 1.670163 \\
\hline & -7.771229 & -4.561703 & 0.859312 & $\mathrm{C}$ & 5.779534 & -4.814339 & 1.118222 \\
\hline & -3.164821 & -1.293183 & & $\mathrm{C}$ & 3.253756 & 5464 & \\
\hline $\mathrm{C}$ & -2.861421 & -3.53 & $5.2^{\prime}$ & $\mathrm{C}$ & & -5.8 & \\
\hline $\mathrm{C}$ & -3.068250 & -4.044321 & 3.98 & $\mathrm{C}$ & 4.844640 & -3.683920 & -3.286937 \\
\hline & -6.866537 & -5.621272 & 0.917351 & $\mathrm{C}$ & 5.564393 & -7.095835 & 0.338133 \\
\hline & -7.313480 & -3.253609 & 1.043151 & $\mathrm{C}$ & 0.504566 & -4.050462 & 3.722963 \\
\hline & -2.901524 & -2.155991 & 5.490254 & & 2.313597 & -3.640663 & \\
\hline & -4.320812 & 2.993035 & -1.574427 & $C$ & 3.191298 & -3.493915 & 4.192697 \\
\hline & -3.294392 & 2.296273 & 0.631627 & $C$ & 4.185697 & -7.027005 & 0.551807 \\
\hline & -3.737307 & 3.001300 & 1.951901 & $\mathrm{C}$ & 6.359113 & -5.988182 & 0.632574 \\
\hline
\end{tabular}




\begin{tabular}{|c|c|c|c|}
\hline $\mathrm{C}$ & 0.963436 & -3.912774 & 5.034024 \\
\hline & -0.907214 & 5.439394 & -2.399378 \\
\hline & -5.294385 & 3.494732 & -3.835547 \\
\hline $\mathrm{C}$ & -5.517784 & -0.448159 & -2.708895 \\
\hline$c$ & -4.921278 & -5.310065 & -3.840150 \\
\hline & 3.026732 & -5.423882 & -3.372772 \\
\hline & 6.686368 & -2.012002 & -3.259412 \\
\hline & 2.988384 & 5.913883 & -3.119125 \\
\hline & 5.542690 & 1.654486 & -2.439575 \\
\hline $\mathrm{H}$ & -3.368693 & -5.246472 & -1.656678 \\
\hline H & -3.542313 & -1.126714 & 2.321030 \\
\hline & -2.892402 & -5.571510 & 0.401314 \\
\hline $\mathrm{H}$ & -1.622739 & -4.803641 & 1.459917 \\
\hline $\mathrm{H}$ & -5.615949 & -1.993710 & 1.418753 \\
\hline $\mathrm{H}$ & -4.816538 & -6.207826 & 1.192100 \\
\hline $\mathrm{H}$ & -8.824166 & -4.751030 & 0.675029 \\
\hline $\mathrm{H}$ & -3.195384 & -0.220731 & 4.579567 \\
\hline $\mathrm{H}$ & -2.665219 & -4.213945 & 6.095697 \\
\hline $\mathrm{H}$ & -3.030677 & -5.116334 & 3.815304 \\
\hline $\mathrm{H}$ & -7.211985 & -6.641969 & 0.784404 \\
\hline $\mathrm{H}$ & -8.009390 & -2.421247 & 1.001190 \\
\hline $\mathrm{H}$ & -2.732792 & -1.755081 & 6.485338 \\
\hline $\mathrm{H}$ & -5.158262 & 2.334861 & -1.392711 \\
\hline $\mathrm{H}$ & -1.047902 & 2.979817 & 2.595260 \\
\hline $\mathrm{H}$ & -5.514790 & 2.220026 & 0.827882 \\
\hline $\mathrm{H}$ & -4.621539 & 0.984695 & 1.831491 \\
\hline $\mathrm{H}$ & -2.114193 & 5.156699 & 2.134179 \\
\hline $\mathrm{H}$ & -6.221048 & 4.102052 & 1.442643 \\
\hline $\mathrm{H}$ & -5.008780 & 8.218062 & 1.298632 \\
\hline $\mathrm{H}$ & -0.034593 & 2.605332 & 4.803428 \\
\hline $\mathrm{H}$ & -3.947711 & 1.990828 & 6.486992 \\
\hline $\mathrm{H}$ & -4.956302 & 2.367943 & 4.253227 \\
\hline $\mathrm{H}$ & -6.781463 & 6.480796 & 1.132465 \\
\hline $\mathrm{H}$ & -2.667679 & 7.537774 & 1.805824 \\
\hline $\mathrm{H}$ & -1.476516 & 2.101160 & 6.770738 \\
\hline $\mathrm{H}$ & 4.192553 & 1.620163 & -0.147979 \\
\hline $\mathrm{H}$ & 2.460795 & 4.102379 & 4.855181 \\
\hline $\mathrm{H}$ & 2.031370 & 5.217261 & 1.595718 \\
\hline $\mathrm{H}$ & 0.953271 & 4.057029 & 2.501857 \\
\hline $\mathrm{H}$ & 3.903127 & 5.977306 & 2.112254 \\
\hline $\mathrm{H}$ & 5.325219 & 1.924500 & 2.233197 \\
\hline $\mathrm{H}$ & 8.085850 & 5.154857 & 1.608583 \\
\hline $\mathrm{H}$ & 2.249345 & 2.690998 & 6.882619 \\
\hline $\mathrm{H}$ & 3.030250 & -0.810359 & 4.507092 \\
\hline $\mathrm{H}$ & 3.251966 & 0.604618 & 2.503553 \\
\hline $\mathrm{H}$ & 7.628672 & 2.719121 & 1.854409 \\
\hline $\mathrm{H}$ & 6.204351 & 6.775534 & 1.755152 \\
\hline $\mathrm{H}$ & 2.526600 & 0.222271 & 6.714037 \\
\hline $\mathrm{H}$ & 5.546834 & -1.412132 & -0.854491 \\
\hline $\mathrm{H}$ & 1.004078 & -3.976424 & 1.639313 \\
\hline $\mathrm{H}$ & 5.499162 & -2.133363 & 1.362565 \\
\hline $\mathrm{H}$ & 4.195094 & -1.396456 & 2.400395 \\
\hline $\mathrm{H}$ & 6.415856 & -3.964388 & 1.336587 \\
\hline $\mathrm{H}$ & 2.540934 & -5.817601 & 1.213354 \\
\hline $\mathrm{H}$ & 6.013802 & -8.004749 & -0.049529 \\
\hline $\mathrm{H}$ & -0.546704 & -4.231119 & 3.535765 \\
\hline $\mathrm{H}$ & 2.682617 & -3.534297 & 6.283896 \\
\hline $\mathrm{H}$ & 4.238960 & -3.269821 & 4.372255 \\
\hline $\mathrm{H}$ & 3.555594 & -7.882824 & 0.330413 \\
\hline & 7.433584 & -6.032112 & 0.482696 \\
\hline $\mathrm{H}$ & 0.269629 & -4.010122 & 5.863532 \\
\hline & 2.381694 & -4.303161 & -1.031039 \\
\hline
\end{tabular}

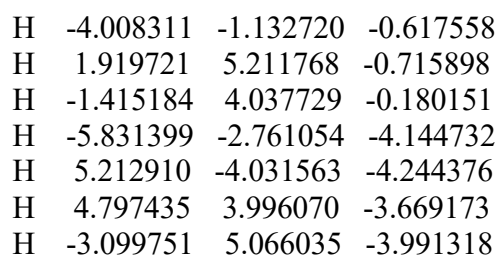

U3

$\begin{array}{lllll}\mathrm{Rh} & 0.153337 & 0.063167 & -1.144889\end{array}$

$\begin{array}{llll}\mathrm{Rh} & -0.534063 & -0.675197 & 1.021744\end{array}$

$\begin{array}{llll}\text { O } & 1.450509 & -1.104551 & 1.384712\end{array}$

$\begin{array}{llll}\text { O } & 1.964344 & -0.881132 & -0.807536\end{array}$

$\begin{array}{llll}\mathrm{O} & -0.233797 & 1.270108 & 1.670238\end{array}$

$\begin{array}{llll}\mathrm{O} & 0.927806 & 1.718853 & -0.218137\end{array}$

$\begin{array}{llll}\text { O } & -2.453362 & -0.187703 & 0.457319\end{array}$

$\begin{array}{llll}\mathrm{O} & -1.668173 & 1.029467 & -1.274855\end{array}$

$\begin{array}{llll}\mathrm{O} & -0.883518 & -2.504812 & 0.158112\end{array}$

$\begin{array}{llll}\text { O } & -0.783315 & -1.627955 & -1.917924\end{array}$

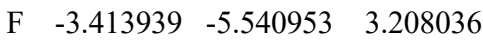

F $\quad-2.871415 \quad-3.479992 \quad 3.606444$

F $\quad-4.923710 \quad-4.140516 \quad 3.926074$

$\begin{array}{llll}\text { F } & -7.419979 & -1.601495 & 0.428959\end{array}$

F $\quad-7.942156-3.530585 \quad-0.418434$

F $\quad-6.985750 \quad-2.021018 \quad-1.662042$

$\begin{array}{llll}\text { F } & 5.988475 & 2.858601 & 0.150492\end{array}$

F $\quad 6.270738 \quad 4.223902 \quad-1.527682$

$\begin{array}{llll}\text { F } & 5.189937 & 2.355580 & -1.806647\end{array}$

$\begin{array}{llll}\text { F } & 2.989218 & 7.963748 & -1.666260\end{array}$

$\begin{array}{llll}\text { F } & 1.695893 & 6.758510 & -2.923160\end{array}$

F $\quad 0.927783 \quad 7.590399 \quad-1.066527$

$\begin{array}{llll}\text { F } & -0.616566 & 5.229972 & -2.219838\end{array}$

$\begin{array}{llll}\text { F } & -1.615819 & 6.009684 & -0.455445\end{array}$

$\begin{array}{llll}\text { F } & -1.987907 & 6.910460 & -2.405423\end{array}$

F $\quad-5.803572 \quad 5.290722 \quad-4.909453$

$\begin{array}{llll}\text { F } & -7.177690 & 4.784537 & -3.302439\end{array}$

$\begin{array}{lllll}\text { F } & -6.481803 & 3.235097 & -4.653670\end{array}$

$\begin{array}{llll}\text { F } & 7.956986 & 1.653128 & -1.606168\end{array}$

$\begin{array}{llll}\text { F } & 7.998721 & 0.970533 & 0.462152\end{array}$

F $\quad 9.095454 \quad-0.111974 \quad-1.075181$

F $\quad 6.473724 \quad-2.243820 \quad-4.725720$

$\begin{array}{llll}\text { F } & 5.317807 & -3.812452 & -3.756735\end{array}$

F $\quad 4.310348 \quad-2.068008 \quad-4.568274$

$\begin{array}{llll}\text { C } & 4.581100 & -1.979029 & -1.615626\end{array}$

$\begin{array}{llll}\text { C } & 3.651738 & -1.628301 & 0.690422\end{array}$

$\begin{array}{llll}\text { C } & 3.803039 & -2.963422 & 1.511192\end{array}$

$\begin{array}{llll}\text { C } & 6.746986 & -0.318471 & -1.088787\end{array}$

$\begin{array}{llll}\text { C } & 4.673968 & -1.351116 & -0.371132\end{array}$

$\begin{array}{llll}\text { C } & 5.563651 & -1.772363 & -2.585046\end{array}$

$\begin{array}{llll}\text { C } & 4.956332 & -3.846686 & 1.102010\end{array}$

$\begin{array}{llll}\text { C } & 2.254101 & -1.171634 & 0.399805\end{array}$

$\begin{array}{llll}\text { C } & 2.560856 & -3.700957 & 1.941235\end{array}$

$\begin{array}{llll}\text { C } & 1.660636 & -4.239257 & 1.016683\end{array}$

$\begin{array}{llll}\text { C } & 4.084903 & -1.639036 & 2.142142\end{array}$

$\begin{array}{llll}\text { C } & 6.225085 & -3.715344 & 1.676451\end{array}$

$\begin{array}{llll}\text { C } & 5.759171 & -0.504515 & -0.123436\end{array}$

$\begin{array}{llll}\text { C } & 4.772450 & -4.812742 & 0.102108\end{array}$

$\begin{array}{llll}\text { C } & 6.657892 & -0.951240 & -2.327871\end{array}$

$\begin{array}{llll}\text { C } & 7.099561 & -5.456652 & 0.239748\end{array}$

$\begin{array}{llll}\text { C } & 0.538420 & -4.942423 & 1.451549\end{array}$

$\begin{array}{llll}\text { C } & 1.177958 & -4.566637 & 3.744047\end{array}$

$\begin{array}{llll}\text { C } & 2.313117 & -3.882001 & 3.308165\end{array}$ $\begin{array}{llll}\text { C } & 5.832620 & -5.605440 & -0.330371\end{array}$

$\begin{array}{llll}\text { C } & 7.288990 & -4.513533 & 1.249565\end{array}$

$\begin{array}{llll}\text { C } & 0.282426 & -5.101394 & 2.813434\end{array}$

$\begin{array}{llll}\text { C } & 3.256658 & 3.429413 & 0.322557\end{array}$

$\begin{array}{llll}\text { C } & 1.101119 & 3.242996 & 1.568744\end{array}$

$\begin{array}{llll}\text { C } & 1.412201 & 3.326788 & 3.101178\end{array}$

$\begin{array}{llll}\text { C } & 2.462559 & 5.786650 & -0.909879\end{array}$

$\begin{array}{llll}\text { C } & 2.011295 & 3.978927 & 0.631926\end{array}$

$\begin{array}{llll}\text { C } & 4.096544 & 4.056391 & -0.593623\end{array}$

$\begin{array}{llll}\text { C } & 2.655770 & 4.111495 & 3.441767\end{array}$

$\begin{array}{llll}\text { C } & 0.553580 & 1.984051 & 0.974913\end{array}$

$\begin{array}{llll}\text { C } & 1.159476 & 2.164473 & 4.026245\end{array}$

$\begin{array}{llll}\text { C } & 0.431896 & 2.397073 & 5.199737\end{array}$

$\begin{array}{llll}\text { C } & 0.221882 & 4.025926 & 2.524517\end{array}$

$\begin{array}{llll}\text { C } & 2.638450 & 5.497798 & 3.621531\end{array}$

$\begin{array}{llll}\text { C } & 1.617834 & 5.162086 & 0.009129\end{array}$

$\begin{array}{llll}\text { C } & 3.880298 & 3.435189 & 3.549885\end{array}$

$\begin{array}{llll}\text { C } & 3.709203 & 5.241684 & -1.217320\end{array}$

$\begin{array}{llll}\text { C } & 5.034376 & 5.518454 & 3.967451\end{array}$

$\begin{array}{llll}\text { C } & 0.187683 & 1.361646 & 6.103516\end{array}$

$\begin{array}{llll}\text { C } & 1.395285 & -0.161803 & 4.672063\end{array}$

$\begin{array}{llll}\text { C } & 1.655334 & 0.881017 & 3.783175\end{array}$

$\begin{array}{llll}\text { C } & 5.060008 & 4.130853 & 3.802563\end{array}$

$\begin{array}{llll}\text { C } & 3.819033 & 6.197230 & 3.883162\end{array}$

$\begin{array}{llll}\text { C } & 0.661019 & 0.074869 & 5.836338\end{array}$

$\begin{array}{llll}\text { C } & -4.918979 & 2.874731 & -2.350594\end{array}$

$\begin{array}{llll}\text { C } & -3.894801 & 1.407848 & -0.547214\end{array}$

$\begin{array}{llll}\text { C } & -4.833328 & 1.437991 & 0.709133\end{array}$

$\begin{array}{llll}\text { C } & -2.919872 & 4.767356 & -1.983336\end{array}$

$\begin{array}{llll}\text { C } & -3.906921 & 2.619538 & -1.425937\end{array}$

$\begin{array}{llll}\text { C } & -4.938049 & 4.072860 & -3.069418\end{array}$

$\begin{array}{llll}\text { C } & -5.734861 & 2.642817 & 0.819231\end{array}$

$\begin{array}{llll}\text { C } & -2.577329 & 0.704422 & -0.440773\end{array}$

$\begin{array}{llll}\text { C } & -4.355040 & 0.917714 & 2.038777\end{array}$

$\begin{array}{llll}\text { C } & -3.293219 & 1.524813 & 2.716599\end{array}$

$\begin{array}{llll}\text { C } & -5.100504 & 0.488623 & -0.408852\end{array}$

$\begin{array}{llll}\text { C } & -5.218443 & 3.899448 & 1.162781\end{array}$

$\begin{array}{llll}\text { C } & -2.907647 & 3.585067 & -1.249990\end{array}$

$\begin{array}{llll}\text { C } & -7.109990 & 2.513129 & 0.599969\end{array}$

$\begin{array}{llll}\text { C } & -3.940318 & 5.028976 & -2.897007\end{array}$

$\begin{array}{llll}\text { C } & -7.428786 & 4.872791 & 1.025067\end{array}$

$\begin{array}{llll}\text { C } & -2.853043 & 1.023798 & 3.939777\end{array}$

$\begin{array}{llll}\text { C } & -4.560757 & -0.683234 & 3.849900\end{array}$

$\begin{array}{llll}\text { C } & -4.995879 & -0.179352 & 2.623131\end{array}$

$\begin{array}{llll}\text { C } & -7.953720 & 3.621344 & 0.701652\end{array}$

$\begin{array}{llll}\text { C } & -6.057330 & 5.007603 & 1.259299\end{array}$

$\begin{array}{llll}\text { C } & -3.483019 & -0.086666 & 4.508149\end{array}$

$\begin{array}{llll}\text { C } & -3.135366 & -3.966592 & 0.761749\end{array}$

$\begin{array}{llll}\text { C } & -2.174982 & -3.542206 & -1.525675\end{array}$

$\begin{array}{llll}\text { C } & -1.638224 & -4.826305 & -2.244965\end{array}$

$\begin{array}{llll}\text { C } & -5.645070 & -3.095865 & -0.027431\end{array}$

C $\quad-3.336946-3.601650 \quad-0.572105$

$\begin{array}{llll}\text { C } & -4.174627 & -3.900395 & 1.684943\end{array}$

$\begin{array}{llll}\text { C } & -2.414532 & -6.100213 & -2.019490\end{array}$

C $-1.186832-2.490922 \quad-1.077386$

$\begin{array}{llll}\text { C } & -0.145141 & -4.993828 & -2.416387\end{array}$

$\begin{array}{llll}\text { C } & 0.628801 & -4.063177 & -3.128731\end{array}$

$\begin{array}{llll}\text { C } & -2.370912 & -3.743919 & -2.997561\end{array}$

$\begin{array}{llll}\text { C } & -2.492075 & -6.717583 & -0.764041\end{array}$

$\begin{array}{llll}\text { C } & -4.607205 & -3.167804 & -0.959671\end{array}$

$\begin{array}{llll}\text { C } & -3.047384 & -6.715046 & -3.106660\end{array}$

$\begin{array}{llll}\text { C } & -5.441381 & -3.468064 & 1.302073\end{array}$ 


\begin{tabular}{|c|c|c|c|}
\hline C & -3.826455 & -8.511173 & -1.686528 \\
\hline$C$ & 2.003428 & -4.237386 & -3.284765 \\
\hline & 1.887875 & -6.283659 & -2.025596 \\
\hline 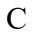 & 0.511779 & -6.111156 & -1.875448 \\
\hline 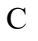 & -3.747935 & -7.910822 & -2.944403 \\
\hline 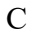 & -3.194076 & -7.911060 & -0.596225 \\
\hline $\mathrm{C}$ & 2.643462 & -5.348069 & -2.732901 \\
\hline C & 2.014751 & 7.024541 & -1.634754 \\
\hline C & 5.391525 & 3.384220 & -0.946114 \\
\hline C & 7.942126 & 0.551767 & -0.817733 \\
\hline $\mathrm{C}$ & 5.428332 & -2.468737 & -3.907674 \\
\hline $\mathrm{C}$ & -6.994487 & -2.569271 & -0.428721 \\
\hline $\mathrm{C}$ & -3.858414 & -4.264214 & 3.106874 \\
\hline $\mathrm{C}$ & -1.792953 & 5.738047 & -1.774802 \\
\hline C & -6.094370 & 4.347175 & -3.988687 \\
\hline $\mathrm{H}$ & 3.745398 & -2.635248 & -1.823519 \\
\hline $\mathrm{H}$ & 1.805197 & -4.085113 & -0.046673 \\
\hline $\mathrm{H}$ & 5.109868 & -1.359906 & 2.348794 \\
\hline $\mathrm{H}$ & 3.364459 & -1.271022 & 2.858618 \\
\hline$\pi$ & 6.391284 & -2.991753 & 2.466699 \\
\hline $\mathrm{H}$ & 3.796984 & -4.943528 & -0.347885 \\
\hline $\mathrm{H}$ & 7.927809 & -6.072454 & -0.096821 \\
\hline $\mathrm{H}$ & -0.139001 & -5.351646 & 0.712691 \\
\hline $\mathrm{H}$ & 0.995393 & -4.685334 & 4.807857 \\
\hline H & 3.011887 & -3.474997 & 4.033168 \\
\hline $\mathrm{H}$ & 5.668703 & -6.339380 & -1.113884 \\
\hline $\mathrm{H}$ & 8.265340 & -4.395238 & 1.709630 \\
\hline $\mathrm{H}$ & -0.609868 & -5.623318 & 3.145308 \\
\hline H & 3.569105 & 2.504038 & 0.791172 \\
\hline $\mathrm{H}$ & 0.048505 & 3.393855 & 5.398156 \\
\hline $\mathrm{H}$ & 0.222493 & 5.103874 & 2.417071 \\
\hline $\mathrm{H}$ & -0.744932 & 3.590372 & 2.746588 \\
\hline $\mathrm{H}$ & 1.702451 & 6.041888 & 3.560385 \\
\hline $\mathrm{H}$ & 3.908504 & 2.357186 & 3.430558 \\
\hline $\mathrm{H}$ & 5.952791 & 6.062940 & 4.163615 \\
\hline $\mathrm{H}$ & -0.381412 & 1.557444 & 7.007270 \\
\hline $\mathrm{H}$ & 1.746742 & -1.163551 & 4.445237 \\
\hline $\mathrm{H}$ & 2.197097 & 0.677328 & 2.868818 \\
\hline$H$ & 5.999321 & 3.590402 & 3.869041 \\
\hline $\mathrm{H}$ & 3.784823 & 7.273799 & 4.019899 \\
\hline $\mathrm{H}$ & 0.458011 & -0.736605 & 6.528494 \\
\hline $\mathrm{H}$ & -5.704503 & 2.148332 & -2.517008 \\
\hline $\mathrm{H}$ & -2.767107 & 2.354450 & 2.257867 \\
\hline $\mathrm{H}$ & -5.950933 & 0.654829 & -1.058214 \\
\hline $\mathrm{H}$ & -4.851437 & -0.550192 & -0.243307 \\
\hline $\mathrm{H}$ & -4.154046 & 4.015590 & 1.335503 \\
\hline $\mathrm{H}$ & -7.521307 & 1.539297 & 0.352216 \\
\hline $\mathrm{H}$ & -8.081552 & 5.737372 & 1.095489 \\
\hline $\mathrm{H}$ & -2.007068 & 1.483735 & 4.438384 \\
\hline $\mathrm{H}$ & -5.050118 & -1.550672 & 4.280416 \\
\hline $\mathrm{H}$ & -5.822766 & -0.649831 & 2.102677 \\
\hline $\mathrm{H}$ & -9.018764 & 3.505130 & 0.525425 \\
\hline $\mathrm{H}$ & -5.640388 & 5.977869 & 1.511511 \\
\hline $\mathrm{H}$ & -3.132859 & -0.483625 & 5.456409 \\
\hline $\mathrm{H}$ & -2.148417 & -4.255494 & 1.091588 \\
\hline $\mathrm{H}$ & 0.168507 & -3.191030 & -3.572079 \\
\hline $\mathrm{H}$ & -3.371646 & -3.987917 & -3.335033 \\
\hline $\mathrm{H}$ & -1.823197 & -3.089159 & -3.662506 \\
\hline $\mathrm{H}$ & -2.008456 & -6.265579 & 0.092636 \\
\hline $\mathrm{H}$ & -2.987871 & -6.252489 & -4.087059 \\
\hline $\mathrm{H}$ & -4.375647 & -9.438545 & -1.556772 \\
\hline П & 2.567754 & -3.509944 & -3.856510 \\
\hline
\end{tabular}

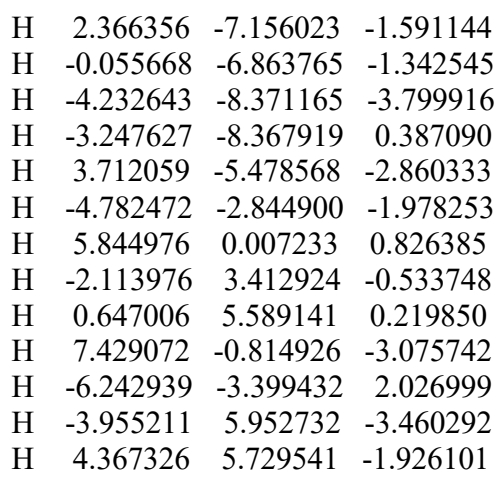

U4

$\begin{array}{llll}\mathrm{Rh} & -0.435915 & -0.778451 & 1.956470\end{array}$

$\begin{array}{llll}\mathrm{Rh} & -0.330639 & -0.494827 & -0.421951\end{array}$

$\begin{array}{llll}\mathrm{O} & -1.386774 & 1.246056 & -0.199287\end{array}$

$\begin{array}{llll}\text { O } & -1.753250 & 0.825144 & 1.990249\end{array}$

$\begin{array}{llll}\text { O } & 1.419041 & 0.569637 & -0.217205\end{array}$

$\begin{array}{llll}\mathrm{O} & 1.149985 & 0.573969 & 2.030008\end{array}$

$\begin{array}{llll}\text { O } & 0.786098 & -2.216567 & -0.496904\end{array}$

$\begin{array}{llll}\mathrm{O} & 0.827389 & -2.398083 & 1.758668\end{array}$

$\begin{array}{llll}\mathrm{O} & -2.116710 & -1.514659 & -0.492209\end{array}$

$\begin{array}{llll}\text { O } & -2.018769 & -2.075013 & 1.694068\end{array}$

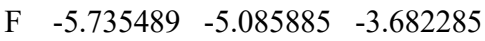

$\begin{array}{lllll}\text { F } & -6.704274 & -3.599270 & -4.948760\end{array}$

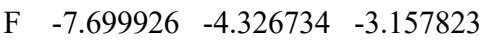

$\begin{array}{llll}\text { F } & -5.892649 & 1.071805 & -4.107303\end{array}$

$\begin{array}{llll}\text { F } & -4.034716 & 1.333277 & -2.997106\end{array}$

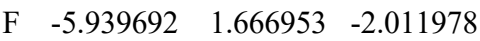

$\begin{array}{llll}\text { F } & -5.921780 & 4.579170 & -2.374241\end{array}$

$\begin{array}{llll}\text { F } & -4.807934 & 5.940313 & -3.661187\end{array}$

$\begin{array}{llll}\text { F } & -4.827896 & 3.813906 & -4.088896\end{array}$

$\begin{array}{lllll}\text { F } & 0.105439 & 6.102440 & -3.426585\end{array}$

$\begin{array}{llll}\text { F } & 0.973704 & 4.270119 & -2.627615\end{array}$

F $\quad 0.762245 \quad 6.012121 \quad-1.348365$

F $\quad 3.177158 \quad 5.972302 \quad-2.939305$

F $\quad 3.412762 \quad 6.439233 \quad-0.825650$

$\begin{array}{llll}\text { F } & 5.156023 & 6.302993 & -2.121978\end{array}$

$\begin{array}{llll}\text { F } & 5.845007 & 1.182301 & -4.030886\end{array}$

F $\quad 7.360101 \quad 2.069507 \quad-2.760314$

F $\quad \begin{array}{llll}6.431357 & 0.190926 & -2.180896\end{array}$

$\begin{array}{llll}\text { F } & 6.986784 & -2.651412 & 1.832709\end{array}$

F $\quad 8.122012 \quad-2.662657 \quad-0.027150$

F $\quad 7.431849 \quad-4.532512 \quad 0.848869$

$\begin{array}{llll}\text { F } & 3.873449 & -0.671977 & -3.389109\end{array}$

F $\quad 3.660785 \quad-2.637580 \quad-4.282271$

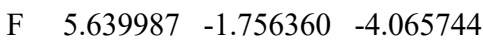

$\begin{array}{llll}\text { C } & -3.638728 & 3.835094 & -1.022646\end{array}$

$\begin{array}{llll}\text { C } & -2.531115 & 2.878551 & 1.047062\end{array}$

$\begin{array}{llll}\text { C } & -2.462798 & 3.727555 & 2.364738\end{array}$

$\begin{array}{llll}\text { C } & -1.197223 & 4.829774 & -1.902880\end{array}$

$\begin{array}{llll}\text { C } & -2.497917 & 3.637342 & -0.246785\end{array}$

$\begin{array}{llll}\text { C } & -3.556873 & 4.539471 & -2.225858\end{array}$

$\begin{array}{llll}\text { C } & -2.290346 & 5.209619 & 2.138244\end{array}$

$\begin{array}{llll}\text { C } & -1.844851 & 1.550837 & 0.953216\end{array}$

$\begin{array}{llll}\text { C } & -1.778039 & 3.226252 & 3.610256\end{array}$

$\begin{array}{llll}\text { C } & -0.464989 & 2.749460 & 3.622283\end{array}$

$\begin{array}{llll}\text { C } & -3.730475 & 3.044224 & 1.958993\end{array}$

$\begin{array}{llll}\text { C } & -0.993610 & 5.744226 & 2.101657\end{array}$

$\begin{array}{llll}\text { C } & -1.275944 & 4.134009 & -0.700781\end{array}$ $\begin{array}{llll}\text { C } & -3.373808 & 6.064755 & 1.917350\end{array}$

$\begin{array}{llll}\text { C } & -2.337222 & 5.045970 & -2.674829\end{array}$

$\begin{array}{llll}\text { C } & -1.873560 & 7.935501 & 1.584044\end{array}$

$\begin{array}{lrrr}\text { C } & 0.116718 & 2.298842 & 4.807359\end{array}$

$\begin{array}{llll}\text { C } & -1.901312 & 2.856001 & 6.009614\end{array}$

$\begin{array}{llll}\text { C } & -2.485771 & 3.286567 & 4.817575\end{array}$

$\begin{array}{llll}\text { C } & -3.167723 & 7.418959 & 1.643324\end{array}$

$\begin{array}{llll}\text { C } & -0.784532 & 7.091410 & 1.817936\end{array}$

$\begin{array}{llll}\text { C } & -0.599219 & 2.350702 & 6.005420\end{array}$

$\begin{array}{llll}\text { C } & 3.360618 & 3.698140 & -0.498206\end{array}$

$\begin{array}{llll}\text { C } & 2.967287 & 1.783542 & 1.088186\end{array}$

$\begin{array}{llll}\text { C } & 4.025005 & 1.456668 & 2.217254\end{array}$

$\begin{array}{llll}\text { C } & 5.135231 & 2.167653 & -1.991789\end{array}$

$\begin{array}{llll}\text { C } & 3.533408 & 2.348617 & -0.186386\end{array}$

$\begin{array}{llll}\text { C } & 4.085138 & 4.281733 & -1.540961\end{array}$

$\begin{array}{llll}\text { C } & 5.464473 & 1.646128 & 1.798326\end{array}$

$\begin{array}{llll}\text { C } & 1.759953 & 0.910605 & 0.964723\end{array}$

$\begin{array}{llll}\text { C } & 3.786881 & 0.300078 & 3.149656\end{array}$

$\begin{array}{llll}\text { C } & 3.624564 & -1.010254 & 2.690391\end{array}$

$\begin{array}{llll}\text { C } & 3.107723 & 2.629518 & 2.340356\end{array}$

$\begin{array}{llll}\text { C } & 6.238369 & 0.523267 & 1.468460\end{array}$

$\begin{array}{llll}\text { C } & 4.389958 & 1.572792 & -0.978302\end{array}$

$\begin{array}{llll}\text { C } & 6.028087 & 2.915120 & 1.613901\end{array}$

$\begin{array}{llll}\text { C } & 4.992727 & 3.526441 & -2.276511\end{array}$

$\begin{array}{llll}\text { C } & 8.039671 & 1.935211 & 0.689940\end{array}$

$\begin{array}{llll}\text { C } & 3.447952 & -2.060407 & 3.590123\end{array}$

$\begin{array}{llll}\text { C } & 3.595742 & -0.507385 & 5.432576\end{array}$

$\begin{array}{llll}\text { C } & 3.774075 & 0.540562 & 4.528842\end{array}$

$\begin{array}{llll}\text { C } & 7.300619 & 3.059167 & 1.061145\end{array}$

$\begin{array}{llll}\text { C } & 7.507747 & 0.663970 & 0.911142\end{array}$

$\begin{array}{llll}\text { C } & 3.432437 & -1.813351 & 4.963925\end{array}$

$\begin{array}{llll}\text { C } & 3.383096 & -2.968022 & -1.494778\end{array}$

$\begin{array}{llll}\text { C } & 2.116825 & -3.919771 & 0.452806\end{array}$

$\begin{array}{llll}\text { C } & 1.534319 & -5.315731 & 0.137229\end{array}$

$\begin{array}{llll}\text { C } & 5.800191 & -3.140242 & -0.148800\end{array}$

$\begin{array}{llll}\text { C } & 3.403224 & -3.497195 & -0.198793\end{array}$

C $\quad 4.554487 \quad-2.523981 \quad-2.098520$

$\begin{array}{llll}\text { C } & 2.346877 & -6.182750 & -0.793203\end{array}$

$\begin{array}{llll}\text { C } & 1.155726 & -2.765573 & 0.589588\end{array}$

$\begin{array}{llll}\text { C } & 0.044124 & -5.500639 & -0.007890\end{array}$

$\begin{array}{llll}\text { C } & -0.604415 & -6.439173 & 0.805461\end{array}$

$\begin{array}{llll}\text { C } & 2.094164 & -4.996384 & 1.500591\end{array}$

$\begin{array}{llll}\text { C } & 3.194949 & -7.180840 & -0.303928\end{array}$

$\begin{array}{llll}\text { C } & 4.625320 & -3.582565 & 0.467403\end{array}$

$\begin{array}{llll}\text { C } & 2.256373 & -5.996449 & -2.180172\end{array}$

$\begin{array}{llll}\text { C } & 5.778342 & -2.608349 & -1.434173\end{array}$

$\begin{array}{llll}\text { C } & 3.863765 & -7.764121 & -2.555348\end{array}$

$\begin{array}{llll}\text { C } & -1.953748 & -6.736518 & 0.613385\end{array}$

$\begin{array}{llll}\text { C } & -2.043313 & -5.140438 & -1.196713\end{array}$

$\begin{array}{llll}\text { C } & -0.692721 & -4.846411 & -1.004979\end{array}$

$\begin{array}{llll}\text { C } & 3.011157 & -6.775458 & -3.054301\end{array}$

$\begin{array}{llll}\text { C } & 3.948929 & -7.967579 & -1.178185\end{array}$

$\begin{array}{llll}\text { C } & -2.673172 & -6.095923 & -0.397912\end{array}$

$\begin{array}{llll}\text { C } & -5.248125 & -3.213083 & -1.643371\end{array}$

$\begin{array}{llll}\text { C } & -4.016870 & -2.530753 & 0.471076\end{array}$

$\begin{array}{llll}\text { C } & -4.936885 & -2.537348 & 1.745475\end{array}$

$\begin{array}{llll}\text { C } & -5.330500 & -0.568903 & -2.487401\end{array}$

$\begin{array}{llll}\text { C } & -4.679255 & -2.228241 & -0.839603\end{array}$

$\begin{array}{llll}\text { C } & -5.858607 & -2.876169 & -2.855694\end{array}$

$\begin{array}{llll}\text { C } & -6.398783 & -2.272702 & 1.476156\end{array}$

$\begin{array}{llll}\text { C } & -2.614994 & -2.010154 & 0.571894\end{array}$

$\begin{array}{llll}\text { C } & -4.463386 & -1.992981 & 3.066190\end{array}$ 


\begin{tabular}{|c|c|c|c|}
\hline C & -4.121677 & -0.648345 & 3.226963 \\
\hline $\mathrm{C}$ & -4.340598 & -3.802886 & 1.233478 \\
\hline $\mathrm{C}$ & -6.850907 & -0.964256 & 1.253223 \\
\hline $\mathrm{C}$ & -4.736195 & -0.898210 & -1.274423 \\
\hline & -7.328261 & -3.316902 & 1.459228 \\
\hline $\mathrm{C}$ & -5.904303 & -1.554873 & -3.291087 \\
\hline $\mathrm{C}$ & -9.117468 & -1.761734 & 0.975570 \\
\hline $\mathrm{C}$ & -3.708649 & -0.160052 & 4.464353 \\
\hline $\mathrm{C}$ & -4.010719 & -2.356010 & 5.423626 \\
\hline c & -4.412367 & -2.841492 & 4.177609 \\
\hline $\mathrm{C}$ & -8.679735 & -3.064844 & 1.210969 \\
\hline $\mathrm{C}$ & -8.196813 & -0.710197 & 0.999988 \\
\hline $\mathrm{C}$ & -3.653342 & -1.013256 & 5.569086 \\
\hline $\mathrm{C}$ & 6.184700 & 1.396506 & -2.740639 \\
\hline C & 3.950594 & 5.746987 & -1.850539 \\
\hline $\mathrm{C}$ & 0.155069 & 5.312346 & -2.337101 \\
\hline $\mathrm{C}$ & -4.780177 & 4.718329 & -3.080186 \\
\hline $\mathrm{C}$ & -5.306864 & 0.871909 & -2.912314 \\
\hline $\mathrm{C}$ & -6.497209 & -3.967087 & -3.666905 \\
\hline $\mathrm{C}$ & 7.087349 & -3.247014 & 0.616835 \\
\hline $\mathrm{C}$ & 4.446308 & -1.902324 & -3.461817 \\
\hline $\mathrm{H}$ & -4.591286 & 3.433061 & -0.702563 \\
\hline $\mathrm{H}$ & 0.089190 & 2.675433 & 2.696310 \\
\hline $\mathrm{H}$ & -4.560910 & 3.620923 & 1.569868 \\
\hline $\mathrm{H}$ & -4.018309 & 2.180610 & 2.544918 \\
\hline $\mathrm{H}$ & -0.142917 & 5.096710 & 2.287105 \\
\hline $\mathrm{H}$ & -4.386843 & 5.679822 & 1.956012 \\
\hline $\mathrm{H}$ & -1.713465 & 8.985855 & 1.361529 \\
\hline $\mathrm{H}$ & 1.120564 & 1.885486 & 4.792846 \\
\hline $\mathrm{H}$ & -2.465721 & 2.905171 & 6.935844 \\
\hline $\mathrm{H}$ & -3.504319 & 3.664137 & 4.817296 \\
\hline $\mathrm{H}$ & -4.022033 & 8.067290 & 1.473953 \\
\hline $\mathrm{H}$ & 0.227847 & 7.481307 & 1.776037 \\
\hline $\mathrm{H}$ & -0.146427 & 1.998998 & 6.927419 \\
\hline $\mathrm{H}$ & 2.701431 & 4.313804 & 0.102010 \\
\hline $\mathrm{H}$ & 3.610388 & -1.207657 & 1.624145 \\
\hline $\mathrm{H}$ & 3.489780 & 3.634123 & 2.211279 \\
\hline $\mathrm{H}$ & 2.312927 & 2.543620 & 3.067786 \\
\hline $\mathrm{H}$ & 5.842055 & -0.470632 & 1.629487 \\
\hline $\mathrm{H}$ & 5.468997 & 3.805841 & 1.874422 \\
\hline $\mathrm{H}$ & 9.021492 & 2.048876 & 0.241316 \\
\hline $\mathrm{H}$ & 3.297810 & -3.067357 & 3.218410 \\
\hline $\mathrm{H}$ & 3.585924 & -0.304720 & 6.499241 \\
\hline $\mathrm{H}$ & 3.903559 & 1.555835 & 4.892297 \\
\hline $\mathrm{H}$ & 7.708777 & 4.054282 & 0.912858 \\
\hline $\mathrm{H}$ & 8.070675 & -0.222602 & 0.640134 \\
\hline $\mathrm{H}$ & 3.292570 & -2.631628 & 5.663650 \\
\hline $\mathrm{H}$ & 2.442133 & -2.887916 & -2.020618 \\
\hline $\mathrm{H}$ & -0.040060 & -6.949029 & 1.580630 \\
\hline $\mathrm{H}$ & 3.036802 & -5.444055 & 1.792041 \\
\hline $\mathrm{H}$ & 1.387519 & -4.865494 & 2.311570 \\
\hline $\mathrm{H}$ & 3.265042 & -7.351824 & 0.765106 \\
\hline $\mathrm{H}$ & 1.593458 & -5.235931 & -2.577946 \\
\hline $\mathrm{H}$ & 4.453606 & -8.370834 & -3.235434 \\
\hline $\mathrm{H}$ & -2.439848 & -7.472949 & 1.245955 \\
\hline $\mathrm{H}$ & -2.601463 & -4.623352 & -1.970991 \\
\hline $\mathrm{H}$ & -0.215185 & -4.096981 & -1.623227 \\
\hline $\mathrm{H}$ & 2.936037 & -6.608811 & -4.124534 \\
\hline $\mathrm{H}$ & 4.601387 & -8.738560 & -0.779660 \\
\hline $\mathrm{H}$ & -3.718931 & -6.339196 & -0.557690 \\
\hline $\mathrm{H}$ & -5.210774 & -4.252544 & -1.342237 \\
\hline 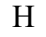 & -4.117860 & 0.006204 & 2.365420 \\
\hline
\end{tabular}
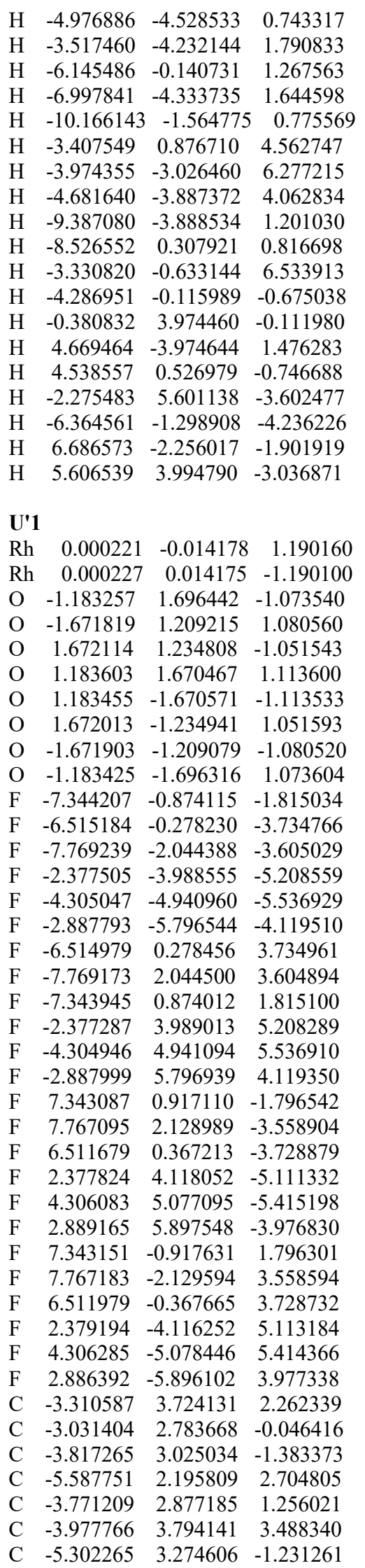

$\begin{array}{llll}\text { C } & -1.880531 & 1.825936 & -0.017521\end{array}$

$\begin{array}{llll}\text { C } & -3.450945 & 2.237386 & -2.614365\end{array}$

$\begin{array}{llll}\text { C } & -3.149055 & 2.919793 & -3.797363\end{array}$

$\begin{array}{llll}\text { C } & -2.835044 & 4.037585 & -0.884591\end{array}$

$\begin{array}{llll}\text { C } & -5.815334 & 4.432814 & -0.633036\end{array}$

$\begin{array}{llll}\text { C } & -4.906779 & 2.096244 & 1.495399\end{array}$

$\begin{array}{llll}\text { C } & -6.208510 & 2.280570 & -1.630402\end{array}$

$\begin{array}{llll}\text { C } & -5.127639 & 3.042874 & 3.714494\end{array}$

$\begin{array}{llll}\text { C } & -8.071697 & 3.564886 & -0.779559\end{array}$

$\begin{array}{lllll}\text { C } & -2.836054 & 2.216127 & -4.961787\end{array}$

$\begin{array}{llll}\text { C } & -3.131764 & 0.131467 & -3.777335\end{array}$

$\begin{array}{llll}\text { C } & -3.448356 & 0.839949 & -2.620097\end{array}$

$\begin{array}{llll}\text { C } & -7.575350 & 2.415920 & -1.397508\end{array}$

$\begin{array}{llll}\text { C } & -7.185185 & 4.577115 & -0.409899\end{array}$

$\begin{array}{llll}\text { C } & -2.823984 & 0.818672 & -4.953719\end{array}$

$\begin{array}{llll}\text { C } & 3.310739 & 3.778973 & -2.172366\end{array}$

$\begin{array}{llll}\text { C } & 3.031547 & 2.782084 & 0.112580\end{array}$

$\begin{array}{llll}\text { C } & 3.817810 & 2.991190 & 1.454722\end{array}$

$\begin{array}{llll}\text { C } & 5.586761 & 2.260479 & -2.653041\end{array}$

$\begin{array}{llll}\text { C } & 3.771033 & 2.907114 & -1.187392\end{array}$

$\begin{array}{llll}\text { C } & 3.977751 & 3.878892 & -3.396375\end{array}$

$\begin{array}{llll}\text { C } & 5.302789 & 3.244068 & 1.308188\end{array}$

$\begin{array}{llll}\text { C } & 1.880795 & 1.825215 & 0.060918\end{array}$

$\begin{array}{llll}\text { C } & 3.451687 & 2.174425 & 2.666645\end{array}$

$\begin{array}{llll}\text { C } & 3.448145 & 0.777263 & 2.638885\end{array}$

$\begin{array}{llll}\text { C } & 2.835544 & 4.015507 & 0.980665\end{array}$

$\begin{array}{llll}\text { C } & 6.208968 & 2.240357 & 1.682507\end{array}$

$\begin{array}{llll}\text { C } & 4.906059 & 2.131638 & -1.446242\end{array}$

$\begin{array}{llll}\text { C } & 5.815880 & 4.416506 & 0.738374\end{array}$

$\begin{array}{llll}\text { C } & 5.127007 & 3.132579 & -3.641363\end{array}$

$\begin{array}{llll}\text { C } & 8.072126 & 3.544805 & 0.862809\end{array}$

$\begin{array}{llll}\text { C } & 3.131825 & 0.041442 & 3.779025\end{array}$

$\begin{array}{llll}\text { C } & 2.838200 & 2.097317 & 5.013270\end{array}$

$\begin{array}{llll}\text { C } & 3.150941 & 2.828475 & 3.865847\end{array}$

$\begin{array}{llll}\text { C } & 7.185699 & 4.565968 & 0.518383\end{array}$

$\begin{array}{llll}\text { C } & 7.575766 & 2.381112 & 1.452554\end{array}$

$\begin{array}{llll}\text { C } & 2.825238 & 0.700463 & 4.971734\end{array}$

$\begin{array}{llll}\text { C } & 4.906039 & -2.131976 & 1.446137\end{array}$

$\begin{array}{llll}\text { C } & 3.031390 & -2.782251 & -0.112567\end{array}$

$\begin{array}{llll}\text { C } & 3.817588 & -2.991360 & -1.454746\end{array}$

$\begin{array}{llll}\text { C } & 3.977476 & -3.878890 & 3.396450\end{array}$

$\begin{array}{llll}\text { C } & 3.770904 & -2.907299 & 1.187384\end{array}$

$\begin{array}{llll}\text { C } & 5.586736 & -2.260790 & 2.652953\end{array}$

$\begin{array}{llll}\text { C } & 5.302579 & -3.244272 & -1.308286\end{array}$

$\begin{array}{llll}\text { C } & 1.880683 & -1.825319 & -0.060886\end{array}$

$\begin{array}{llll}\text { C } & 3.451460 & -2.174497 & -2.666608\end{array}$

$\begin{array}{llll}\text { C } & 3.150527 & -2.828453 & -3.865818\end{array}$

$\begin{array}{llll}\text { C } & 2.835304 & -4.015650 & -0.980675\end{array}$

$\begin{array}{llll}\text { C } & 5.815688 & -4.416667 & -0.738412\end{array}$

$\begin{array}{llll}\text { C } & 3.310489 & -3.779008 & 2.172452\end{array}$

$\begin{array}{llll}\text { C } & 6.208760 & -2.240604 & -1.682740\end{array}$

$\begin{array}{llll}\text { C } & 5.126861 & -3.132709 & 3.641349\end{array}$

$\begin{array}{llll}\text { C } & 8.071932 & -3.545024 & -0.863037\end{array}$

$\begin{array}{llll}\text { C } & 2.837747 & -2.097192 & -5.013158\end{array}$

$\begin{array}{llll}\text { C } & 3.131723 & -0.041423 & -3.778824\end{array}$

$\begin{array}{llll}\text { C } & 3.448092 & -0.777346 & -2.638768\end{array}$

$\begin{array}{llll}\text { C } & 7.575552 & -2.381363 & -1.452844\end{array}$

$\begin{array}{llll}\text { C } & 7.185509 & -4.566144 & -0.518489\end{array}$

$\begin{array}{llll}\text { C } & 2.824930 & -0.700339 & -4.971540\end{array}$

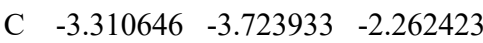

$\begin{array}{llll}\text { C } & -3.031509 & -2.783577 & 0.046383\end{array}$

$\begin{array}{llll}\text { C } & -3.817377 & -3.025018 & 1.383317\end{array}$ 


\begin{tabular}{|c|c|c|c|}
\hline C & -5.587871 & -2.195674 & \\
\hline C & -3.771313 & -2.877046 & -1.256063 \\
\hline $\mathrm{C}$ & -3.977826 & -3.793909 & -3.488422 \\
\hline $\mathrm{C}$ & -5.302367 & -3.274645 & 1.231178 \\
\hline 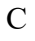 & -1.880682 & -1.825789 & 0.017560 \\
\hline C & -3.451077 & -2.237400 & 2.614328 \\
\hline $\mathrm{C}$ & -3.149317 & -2.919832 & 3.797345 \\
\hline $\mathrm{C}$ & -2.835128 & -4.037526 & 0.884501 \\
\hline $\mathrm{C}$ & -5.815358 & -4.432887 & 0.632953 \\
\hline $\mathrm{C}$ & -4.906903 & -2.096141 & -1.495405 \\
\hline $\mathrm{C}$ & -6.208672 & -2.280667 & 1.630312 \\
\hline $\mathrm{C}$ & -5.127724 & -3.042670 & -3.714559 \\
\hline $\mathrm{C}$ & -8.071775 & -3.565099 & 0.779473 \\
\hline $\mathrm{C}$ & -2.836348 & -2.216200 & 4.961795 \\
\hline $\mathrm{C}$ & -3.131808 & -0.131504 & 3.777314 \\
\hline $\mathrm{C}$ & -3.448347 & -0.839963 & 2.620049 \\
\hline $\mathrm{C}$ & -7.575512 & -2.416092 & 1.397414 \\
\hline $\mathrm{C}$ & -7.185207 & -4.577274 & 0.409823 \\
\hline $\mathrm{C}$ & -2.824189 & -0.818744 & 4.953725 \\
\hline $\mathrm{C}$ & 6.803398 & 1.424177 & -2.928333 \\
\hline $\mathrm{C}$ & 3.396331 & 4.747632 & -4.474393 \\
\hline $\mathrm{C}$ & -6.805208 & 1.354054 & 2.959022 \\
\hline $\mathrm{C}$ & -3.395657 & 4.635086 & 4.587797 \\
\hline $\mathrm{C}$ & -6.805365 & -1.353962 & -2.958998 \\
\hline $\mathrm{C}$ & -3.395685 & -4.634774 & -4.587929 \\
\hline $\mathrm{C}$ & 3.395937 & -4.747192 & 4.474752 \\
\hline $\mathrm{C}$ & 6.803509 & -1.424644 & 2.928137 \\
\hline $\mathrm{H}$ & -2.428964 & 4.332289 & 2.091114 \\
\hline $\mathrm{H}$ & -3.152342 & 4.006053 & -3.801930 \\
\hline $\mathrm{H}$ & -3.183105 & 4.970426 & -0.457684 \\
\hline $\mathrm{H}$ & -1.894584 & 4.102582 & -1.418854 \\
\hline $\mathrm{H}$ & -5.151967 & 5.228950 & -0.316556 \\
\hline $\mathrm{H}$ & -5.843295 & 1.391291 & -2.123748 \\
\hline $\mathrm{H}$ & -9.135699 & 3.673303 & -0.593459 \\
\hline $\mathrm{H}$ & -2.600979 & 2.758144 & -5.872956 \\
\hline $\mathrm{H}$ & -3.114146 & -0.952189 & -3.753755 \\
\hline $\mathrm{H}$ & -3.648720 & 0.298651 & -1.704293 \\
\hline $\mathrm{H}$ & -8.244066 & 1.616037 & -1.698232 \\
\hline $\mathrm{H}$ & -7.556502 & 5.482539 & 0.060497 \\
\hline $\mathrm{H}$ & -2.577302 & 0.270007 & -5.857813 \\
\hline $\mathrm{H}$ & 2.429646 & 4.383416 & -1.985910 \\
\hline $\mathrm{H}$ & 3.647514 & 0.257945 & 1.710236 \\
\hline $\mathrm{H}$ & 3.183546 & 4.958259 & 0.576072 \\
\hline $\mathrm{H}$ & 1.895268 & 4.067759 & 1.516660 \\
\hline $\mathrm{H}$ & 5.843768 & 1.339342 & 2.154093 \\
\hline $\mathrm{H}$ & 5.152535 & 5.220293 & 0.441831 \\
\hline $\mathrm{H}$ & 9.136078 & 3.657565 & 0.679088 \\
\hline $\mathrm{H}$ & 3.113568 & -1.041324 & 3.729493 \\
\hline $\mathrm{H}$ & 2.604041 & 2.617486 & 5.937320 \\
\hline $\mathrm{H}$ & 3.154947 & 3.914318 & 3.896448 \\
\hline $\mathrm{H}$ & 7.557049 & 5.482596 & 0.070232 \\
\hline $\mathrm{H}$ & 8.244420 & 1.573981 & 1.733385 \\
\hline $\mathrm{H}$ & 2.578763 & 0.130443 & 5.862582 \\
\hline $\mathrm{H}$ & 5.277518 & -1.450887 & 0.692830 \\
\hline $\mathrm{H}$ & 3.154401 & -3.914284 & -3.896480 \\
\hline $\mathrm{H}$ & 3.183292 & -4.958433 & -0.576119 \\
\hline $\mathrm{H}$ & 1.895000 & -4.067852 & -1.516620 \\
\hline $\mathrm{H}$ & 5.152352 & -5.220422 & -0.441748 \\
\hline $\mathrm{H}$ & 5.843540 & -1.339609 & -2.154356 \\
\hline $\mathrm{H}$ & 9.135899 & -3.657774 & -0.679362 \\
\hline $\mathrm{H}$ & 2.603437 & -2.617281 & -5.937214 \\
\hline $\mathrm{H}$ & 3.113600 & 1.041342 & -3.729209 \\
\hline
\end{tabular}
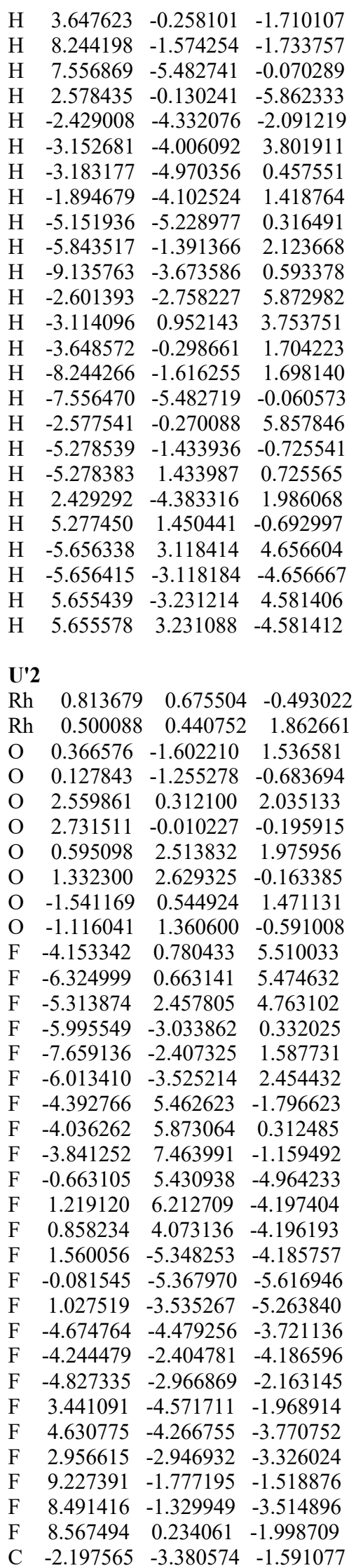

$\begin{array}{llll}\text { C } & -0.403259 & -3.392210 & 0.144908\end{array}$

$\begin{array}{llll}\text { C } & -1.082204 & -4.241580 & 1.268085\end{array}$

$\begin{array}{llll}\text { C } & -0.446609 & -4.249087 & -3.563492\end{array}$

$\begin{array}{llll}\text { C } & -0.861883 & -3.635569 & -1.263197\end{array}$

$\begin{array}{llll}\text { C } & -2.654497 & -3.581816 & -2.888071\end{array}$

$\begin{array}{llll}\text { C } & -2.121877 & -5.234020 & 0.787033\end{array}$

$\begin{array}{llll}\text { C } & 0.053052 & -1.980067 & 0.364135\end{array}$

$\begin{array}{llll}\text { C } & -1.416143 & -3.647427 & 2.610709\end{array}$

$\begin{array}{llll}\text { C } & -1.125742 & -4.397332 & 3.756726\end{array}$

$\begin{array}{llll}\text { C } & 0.355613 & -4.464070 & 0.909122\end{array}$

$\begin{array}{llll}\text { C } & -3.474219 & -4.999823 & 1.074525\end{array}$

$\begin{array}{llll}\text { C } & 0.012718 & -4.072224 & -2.254199\end{array}$

$\begin{array}{llll}\text { C } & -1.796584 & -6.355650 & 0.012475\end{array}$

$\begin{array}{llll}\text { C } & -1.780251 & -4.013505 & -3.888490\end{array}$

$\begin{array}{llll}\text { C } & -4.135444 & -6.941525 & -0.205867\end{array}$

$\begin{array}{llll}\text { C } & -1.485592 & -3.932371 & 5.022773\end{array}$

$\begin{array}{llll}\text { C } & -2.442962 & -1.957097 & 4.014340\end{array}$

$\begin{array}{llll}\text { C } & -2.092966 & -2.431580 & 2.749745\end{array}$

$\begin{array}{llll}\text { C } & -2.791784 & -7.199523 & -0.480396\end{array}$

$\begin{array}{llll}\text { C } & -4.471418 & -5.838633 & 0.580007\end{array}$

$\begin{array}{llll}\text { C } & -2.139817 & -2.705452 & 5.154151\end{array}$

$\begin{array}{llll}\text { C } & 6.400227 & -0.850949 & -0.588529\end{array}$

$\begin{array}{llll}\text { C } & 4.561497 & -0.646930 & 1.156656\end{array}$

$\begin{array}{llll}\text { C } & 4.937471 & -1.387947 & 2.489978\end{array}$

$\begin{array}{llll}\text { C } & 4.889851 & -2.703063 & -2.002088\end{array}$

$\begin{array}{llll}\text { C } & 5.142352 & -1.207791 & -0.106908\end{array}$

$\begin{array}{llll}\text { C } & 6.902932 & -1.428863 & -1.758902\end{array}$

$\begin{array}{llll}\text { C } & 5.875110 & -2.559174 & 2.326835\end{array}$

$\begin{array}{llll}\text { C } & 3.186457 & -0.069327 & 0.995740\end{array}$

$\begin{array}{llll}\text { C } & 3.930928 & -1.570340 & 3.593664\end{array}$

$\begin{array}{llll}\text { C } & 2.747462 & -2.285976 & 3.399001\end{array}$

$\begin{array}{llll}\text { C } & 5.453250 & -0.017972 & 2.214444\end{array}$

$\begin{array}{llll}\text { C } & 7.234117 & -2.442997 & 2.633294\end{array}$

$\begin{array}{llll}\text { C } & 4.398250 & -2.152799 & -0.823916\end{array}$

$\begin{array}{llll}\text { C } & 5.388945 & -3.793341 & 1.872734\end{array}$

$\begin{array}{llll}\text { C } & 6.152687 & -2.352982 & -2.480657\end{array}$

$\begin{array}{llll}\text { C } & 7.605941 & -4.750686 & 2.008922\end{array}$

$\begin{array}{llll}\text { C } & 1.836779 & -2.454068 & 4.439031\end{array}$

C $\quad 3.301030 \quad-1.220325 \quad 5.911800$

$\begin{array}{llll}\text { C } & 4.204215 & -1.045348 & 4.861491\end{array}$

$\begin{array}{llll}\text { C } & 6.246412 & -4.878873 & 1.710006\end{array}$

$\begin{array}{llll}\text { C } & 8.095867 & -3.531246 & 2.475901\end{array}$

$\begin{array}{llll}\text { C } & 2.111873 & -1.922756 & 5.701413\end{array}$

$\begin{array}{llll}\text { C } & 0.491320 & 5.056531 & -1.528470\end{array}$

$\begin{array}{llll}\text { C } & 0.825093 & 4.651928 & 0.928893\end{array}$

$\begin{array}{llll}\text { C } & 2.042364 & 5.488051 & 1.387767\end{array}$

$\begin{array}{llll}\text { C } & -2.155036 & 5.797907 & -1.128419\end{array}$

$\begin{array}{llll}\text { C } & -0.014452 & 5.112753 & -0.227635\end{array}$

$\begin{array}{llll}\text { C } & -0.322130 & 5.369056 & -2.616428\end{array}$

$\begin{array}{llll}\text { C } & 2.306969 & 6.760811 & 0.619777\end{array}$

C $\quad 0.941805 \quad 3.149690 \quad 0.931912$

$\begin{array}{llll}\text { C } & 3.277948 & 4.755879 & 1.845856\end{array}$

$\begin{array}{llll}\text { C } & 3.791794 & 5.007514 & 3.123980\end{array}$

$\begin{array}{llll}\text { C } & 0.802711 & 5.380689 & 2.240483\end{array}$

$\begin{array}{llll}\text { C } & 1.696051 & 7.971298 & 0.965118\end{array}$

$\begin{array}{llll}\text { C } & -1.344800 & 5.492038 & -0.033814\end{array}$

$\begin{array}{llll}\text { C } & 3.165192 & 6.739938 & -0.490354\end{array}$

$\begin{array}{llll}\text { C } & -1.649138 & 5.744417 & -2.427510\end{array}$

$\begin{array}{llll}\text { C } & 2.755395 & 9.087559 & -0.903432\end{array}$

$\begin{array}{llll}\text { C } & 4.970597 & 4.394783 & 3.550126\end{array}$

$\begin{array}{llll}\text { C } & 5.148981 & 3.266862 & 1.422477\end{array}$

$\begin{array}{llll}\text { C } & 3.966106 & 3.874168 & 1.000817\end{array}$ 


\begin{tabular}{|c|c|c|c|}
\hline & 3.382208 & 7.887941 & -1.249319 \\
\hline $\mathrm{C}$ & 1.917721 & 9.125673 & 0.210846 \\
\hline $\mathrm{C}$ & 5.655751 & 3.525988 & 2.697450 \\
\hline $\mathrm{C}$ & -4.417735 & 1.178157 & 2.430914 \\
\hline $\mathrm{C}$ & -3.373365 & 1.260265 & 0.145458 \\
\hline $\mathrm{C}$ & -3.954719 & 1.498368 & -1.288544 \\
\hline $\mathrm{C}$ & -5.608975 & -1.269385 & 1.868324 \\
\hline $\mathrm{C}$ & -4.230530 & 0.596648 & 1.179175 \\
\hline $\mathrm{C}$ & -5.180933 & 0.525819 & 3.403126 \\
\hline $\mathrm{C}$ & -5.384603 & 1.047720 & -1.512703 \\
\hline $\mathrm{C}$ & -1.905370 & 1.025613 & 0.345338 \\
\hline $\mathrm{C}$ & -3.049300 & 1.326333 & -2.479732 \\
\hline $\mathrm{C}$ & -2.452053 & 0.093310 & -2.764784 \\
\hline$C$ & -3.699880 & 2.654178 & -0.372017 \\
\hline & -6.455693 & 1.551578 & -0.758573 \\
\hline $\mathrm{C}$ & -4.818974 & -0.644033 & 0.909564 \\
\hline $\mathrm{C}$ & -5.662378 & 0.050478 & -2.458277 \\
\hline $\mathrm{C}$ & -5.790024 & -0.695107 & 3.127952 \\
\hline $\mathrm{C}$ & -8.002813 & 0.038583 & -1.844253 \\
\hline & -1.623480 & -0.057359 & -3.874174 \\
\hline $\mathrm{C}$ & -1.974716 & 2.267425 & -4.439494 \\
\hline $\mathrm{C}$ & -2.813871 & 2.407809 & -3.331981 \\
\hline $\mathrm{C}$ & -6.951883 & -0.456530 & -2.615504 \\
\hline $\mathrm{C}$ & -7.747516 & 1.053937 & -0.920814 \\
\hline C & -1.377363 & 1.034681 & -4.712653 \\
\hline $\mathrm{C}$ & 3.993387 & -3.627126 & -2.773352 \\
\hline $\mathrm{C}$ & 8.293307 & -1.074472 & -2.204062 \\
\hline $\mathrm{C}$ & 0.515686 & -4.630481 & -4.653378 \\
\hline $\mathrm{C}$ & -4.099349 & -3.359105 & -3.231280 \\
\hline $\mathrm{C}$ & -6.315971 & -2.555341 & 1.552050 \\
\hline $\mathrm{C}$ & -5.255549 & 1.108892 & 4.784201 \\
\hline $\mathrm{C}$ & -3.601660 & 6.153951 & -0.935734 \\
\hline $\mathrm{C}$ & 0.266398 & 5.274858 & -3.994962 \\
\hline $\mathrm{H}$ & -2.885555 & -3.055077 & -0.822384 \\
\hline $\mathrm{H}$ & -0.612447 & -5.348946 & 3.651732 \\
\hline $\mathrm{H}$ & 0.653993 & -5.350978 & 0.363779 \\
\hline $\mathrm{H}$ & 1.088464 & -4.104105 & 1.620647 \\
\hline $\mathrm{H}$ & -3.750999 & -4.154314 & 1.690227 \\
\hline $\mathrm{H}$ & -0.764557 & -6.578243 & -0.230010 \\
\hline $\mathrm{H}$ & -4.909828 & -7.595143 & -0.595228 \\
\hline $\mathrm{H}$ & -1.249205 & -4.523371 & 5.902386 \\
\hline $\mathrm{H}$ & -2.952038 & -1.005777 & 4.111558 \\
\hline $\mathrm{H}$ & -2.326354 & -1.834374 & 1.877440 \\
\hline $\mathrm{H}$ & -2.512566 & -8.059151 & -1.082049 \\
\hline $\mathrm{H}$ & -5.508384 & -5.620860 & 0.809748 \\
\hline $\mathrm{H}$ & -2.416639 & -2.335813 & 6.136872 \\
\hline $\mathrm{H}$ & 6.998543 & -0.115189 & -0.065466 \\
\hline $\mathrm{H}$ & 2.502712 & -2.656641 & 2.411799 \\
\hline $\mathrm{H}$ & 6.499194 & 0.122083 & 1.969536 \\
\hline $\mathrm{H}$ & 5.006798 & 0.797719 & 2.767968 \\
\hline $\mathrm{H}$ & 7.623773 & -1.498788 & 2.999621 \\
\hline $\mathrm{H}$ & 4.336445 & -3.901847 & 1.634471 \\
\hline $\mathrm{H}$ & 8.275406 & -5.595403 & 1.879258 \\
\hline $\mathrm{H}$ & 0.908981 & -2.982776 & 4.258933 \\
\hline $\mathrm{H}$ & 3.525007 & -0.805443 & 6.890168 \\
\hline $\mathrm{H}$ & 5.123981 & -0.490311 & 5.021719 \\
\hline $\mathrm{H}$ & 5.855028 & -5.823666 & 1.345197 \\
\hline $\mathrm{H}$ & 9.148836 & -3.422417 & 2.717385 \\
\hline $\mathrm{H}$ & 1.401491 & -2.054296 & 6.512329 \\
\hline $\mathrm{H}$ & 1.512072 & 4.738635 & -1.690911 \\
\hline $\mathrm{H}$ & 3.262928 & 5.689087 & 3.783583 \\
\hline $\mathrm{H}$ & 0.142823 & 6.235738 & 2.322865 \\
\hline
\end{tabular}

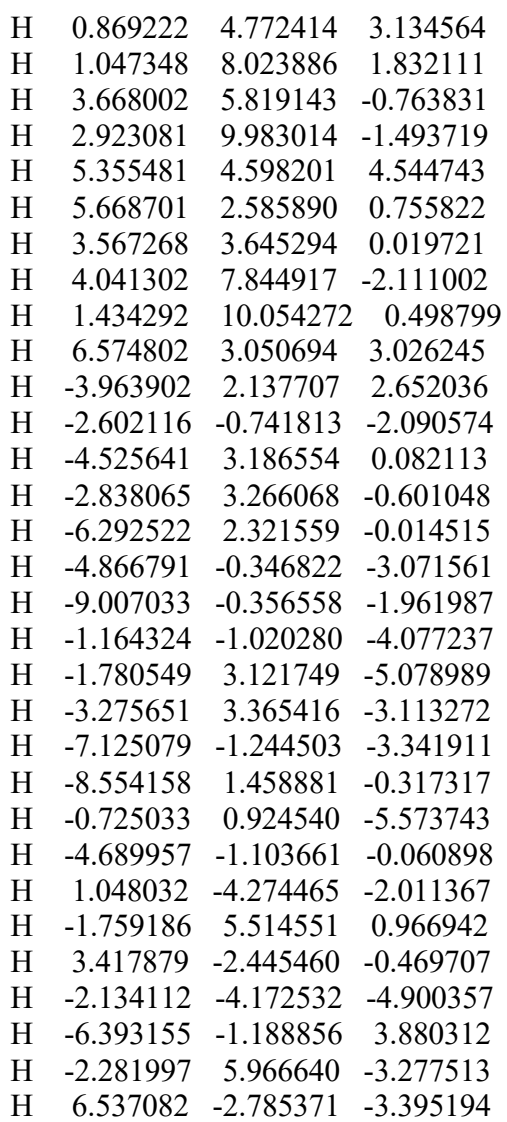

\section{U'3}

$\begin{array}{llll}\mathrm{Rh} & -0.132637 & -1.209234 & 0.951461\end{array}$

$\begin{array}{llll}\mathrm{Rh} & -0.457501 & -0.130798 & -1.150215\end{array}$

$\begin{array}{llll}\mathrm{O} & -1.781235 & 1.117994 & -0.204870\end{array}$

$\begin{array}{llll}\mathrm{O} & -1.909754 & -0.293556 & 1.554383\end{array}$

$\begin{array}{llll}\mathrm{O} & 1.102619 & 1.132825 & -0.610091\end{array}$

$\begin{array}{llll}\mathrm{O} & 0.905190 & 0.476680 & 1.544193\end{array}$

$\begin{array}{llll}\mathrm{O} & 0.884203 & -1.486103 & -1.938850\end{array}$

$\begin{array}{llll}\text { O } & 1.599678 & -1.991494 & 0.146179\end{array}$

$\begin{array}{llll}\text { O } & -2.005305 & -1.460929 & -1.466112\end{array}$

$\begin{array}{llll}\mathrm{O} & -1.206444 & -2.794338 & 0.171778\end{array}$

F $\quad 3.013846 \quad-6.074464 \quad 2.544773$

F $\quad 5.015618 \quad-6.874162 \quad 2.253671$

F $\quad 3.480587 \quad-7.186233 \quad 0.738330$

$\begin{array}{llll}\text { F } & 8.016768 & -3.043401 & 1.528099\end{array}$

F $\quad 7.296408 \quad-1.278824 \quad 0.468346$

$\begin{array}{llll}\text { F } & 6.663363 & -1.662102 & 2.512788\end{array}$

$\begin{array}{llll}\text { F } & 1.729080 & 6.851658 & -0.941057\end{array}$

F $\quad \begin{array}{llll}1.564182 & 6.111590 & -2.979633\end{array}$

F $\quad 3.360226 \quad 7.154244 \quad-2.351415$

F $\quad \begin{array}{llll}6.590160 & 1.832296 & -1.903719\end{array}$

$\begin{array}{llll}\text { F } & 5.552858 & 2.160027 & -3.785377\end{array}$

F $\quad 6.759047 \quad 3.748460 \quad-2.929760$

F $\quad-6.876853 \quad 3.760356 \quad-1.532598$

F $\quad-6.215606 \quad 5.797887 \quad-1.932150$

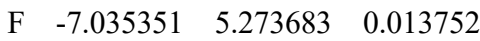

$\begin{array}{llll}\text { F } & -0.543884 & 5.060847 & -1.518675\end{array}$

F $\quad-1.568404 \quad 6.933083 \quad-1.086142$

F $\quad-0.504776 \quad 5.872190 \quad 0.495530$

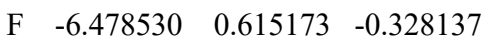

$\begin{array}{llll}\text { F } & -7.449120 & 0.869476 & -2.263783\end{array}$

$\begin{array}{lllll}\text { F } & -5.339614 & 1.351855 & -2.026658\end{array}$

$\begin{array}{llll}\text { F } & -7.686715 & -3.036014 & -5.125358\end{array}$

F $\quad-6.129291 \quad-4.557659 \quad-5.025138$

$\begin{array}{llll}\text { F } & -7.803777 & -4.624403 & -3.645557\end{array}$

$\begin{array}{llll}\text { C } & -2.384560 & 3.772567 & 0.499978\end{array}$

$\begin{array}{llll}\text { C } & -3.249497 & 1.687868 & 1.550071\end{array}$

$\begin{array}{llll}\text { C } & -3.370462 & 1.817749 & 3.104660\end{array}$

$\begin{array}{llll}\text { C } & -4.872707 & 4.470251 & -0.497606\end{array}$

$\begin{array}{llll}\text { C } & -3.478635 & 2.938857 & 0.755998\end{array}$

$\begin{array}{llll}\text { C } & -2.532232 & 4.924540 & -0.263200\end{array}$

$\begin{array}{llll}\text { C } & -3.726135 & 3.201345 & 3.594142\end{array}$

$\begin{array}{llll}\text { C } & -2.238125 & 0.766580 & 0.934726\end{array}$

$\begin{array}{llll}\text { C } & -2.442896 & 1.092910 & 4.041824\end{array}$

$\begin{array}{llll}\text { C } & -2.990943 & 0.411721 & 5.135084\end{array}$

$\begin{array}{llll}\text { C } & -4.384743 & 1.043932 & 2.328069\end{array}$

$\begin{array}{llll}\text { C } & -2.708081 & 4.147976 & 3.778655\end{array}$

$\begin{array}{llll}\text { C } & -4.724982 & 3.301346 & 0.256077\end{array}$

$\begin{array}{llll}\text { C } & -5.045523 & 3.569434 & 3.871319\end{array}$

$\begin{array}{llll}\text { C } & -3.781320 & 5.289520 & -0.769629\end{array}$

$\begin{array}{llll}\text { C } & -4.330205 & 5.802174 & 4.471552\end{array}$

$\begin{array}{lrrr}\text { C } & -2.162953 & -0.218453 & 6.066381\end{array}$

$\begin{array}{llll}\text { C } & -0.223476 & 0.497183 & 4.817078\end{array}$

$\begin{array}{llll}\text { C } & -1.053854 & 1.142718 & 3.902835\end{array}$

C $\quad \begin{array}{rlll}\text { C.347050 } & 4.862073 & 4.307752\end{array}$

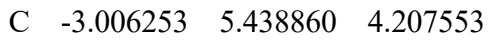

$\begin{array}{llll}\text { C } & -0.775620 & -0.183558 & 5.904567\end{array}$

$\begin{array}{llll}\text { C } & 2.475957 & 4.209834 & -0.491472\end{array}$

$\begin{array}{llll}\text { C } & 2.477719 & 2.179589 & 1.006307\end{array}$

$\begin{array}{llll}\text { C } & 3.448320 & 1.896229 & 2.209282\end{array}$

$\begin{array}{llll}\text { C } & 4.701630 & 3.263416 & -1.869619\end{array}$

$\begin{array}{lllll}\text { C } & 3.028168 & 2.976897 & -0.142443\end{array}$

$\begin{array}{llll}\text { C } & 3.035615 & 4.961079 & -1.529121\end{array}$

$\begin{array}{llll}\text { C } & 4.860842 & 2.407035 & 2.029538\end{array}$

$\begin{array}{llll}\text { C } & 1.423740 & 1.186612 & 0.623374\end{array}$

$\begin{array}{llll}\text { C } & 3.379070 & 0.599695 & 2.973382\end{array}$

$\begin{array}{llll}\text { C } & 3.384424 & 0.647912 & 4.372554\end{array}$

$\begin{array}{llll}\text { C } & 2.314768 & 2.861875 & 2.355751\end{array}$

$\begin{array}{llll}\text { C } & 5.881753 & 1.486797 & 1.748930\end{array}$

$\begin{array}{lllll}\text { C } & 4.132039 & 2.499552 & -0.855393\end{array}$

$\begin{array}{llll}\text { C } & 5.184716 & 3.768867 & 2.053209\end{array}$

$\begin{array}{llll}\text { C } & 4.157782 & 4.500319 & -2.214915\end{array}$

$\begin{array}{llll}\text { C } & 7.479533 & 3.275494 & 1.455021\end{array}$

$\begin{array}{llll}\text { C } & 3.370108 & -0.528381 & 5.124338\end{array}$

$\begin{array}{llll}\text { C } & 3.355174 & -1.823526 & 3.085942\end{array}$

$\begin{array}{llll}\text { C } & 3.384269 & -0.645532 & 2.340737\end{array}$

$\begin{array}{llll}\text { C } & 6.481441 & 4.199180 & 1.768373\end{array}$

$\begin{array}{llll}\text { C } & 7.173946 & 1.913208 & 1.452716\end{array}$

$\begin{array}{llll}\text { C } & 3.346515 & -1.768622 & 4.481308\end{array}$

$\begin{array}{llll}\text { C } & 4.929078 & -2.517307 & -0.159040\end{array}$

$\begin{array}{llll}\text { C } & 2.926748 & -2.680680 & -1.684538\end{array}$

$\begin{array}{llll}\text { C } & 3.548812 & -2.197060 & -3.040805\end{array}$

$\begin{array}{llll}\text { C } & 4.373269 & -5.004331 & 0.945616\end{array}$

C $\quad 3.832152 \quad-3.246476 \quad-0.629596$

$\begin{array}{llll}\text { C } & 5.759606 & -3.049119 & 0.822671\end{array}$

$\begin{array}{llll}\text { C } & 5.055401 & -2.302888 & -3.142758\end{array}$

$\begin{array}{llll}\text { C } & 1.715918 & -1.998331 & -1.126990\end{array}$

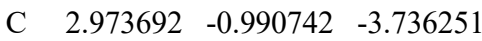

$\begin{array}{llll}\text { C } & 2.850769 & 0.241511 & -3.088902\end{array}$

$\begin{array}{llll}\text { C } & 2.717839 & -3.440291 & -2.986191\end{array}$

$\begin{array}{llll}\text { C } & 5.826164 & -1.130626 & -3.131610\end{array}$

$\begin{array}{lllll}\text { C } & 3.559581 & -4.492791 & -0.068661\end{array}$ 


\begin{tabular}{|c|c|c|c|}
\hline & 5.725881 & -3.532793 & -3.168915 \\
\hline $\mathrm{C}$ & 5.486989 & -4.295702 & 1.388105 \\
\hline $\mathrm{C}$ & 7.873859 & -2.415962 & -3.102289 \\
\hline & 2.352208 & 1.355355 & -3.760801 \\
\hline $\mathrm{C}$ & 2.105828 & 0.024535 & -5.761835 \\
\hline $\mathrm{C}$ & 2.602723 & -1.088596 & -5.081152 \\
\hline $\mathrm{C}$ & 7.119873 & -3.589220 & -3.149847 \\
\hline $\mathrm{C}$ & 7.218012 & -1.183900 & -3.098734 \\
\hline & 1.977410 & 1.249937 & -5.102195 \\
\hline & -4.832729 & -1.492927 & -1.362702 \\
\hline $\mathrm{C}$ & -3.273358 & -3.368678 & -0.860398 \\
\hline $\mathrm{C}$ & -3.701247 & -4.288174 & 0.340803 \\
\hline $\mathrm{C}$ & -6.182404 & -2.943795 & -3.296171 \\
\hline & -4.390645 & -2.785237 & -1.670803 \\
\hline & -5.921736 & -0.933479 & -2.022487 \\
\hline $\mathrm{C}$ & -5.187083 & -4.530642 & 0.460530 \\
\hline $\mathrm{C}$ & -2.074567 & -2.482791 & -0.704326 \\
\hline $\mathrm{C}$ & -2.991352 & -4.216793 & 1.667151 \\
\hline $\mathrm{C}$ & -3.021973 & -3.057942 & 2.448987 \\
\hline & -2.995775 & -4.864689 & -0.840961 \\
\hline $\mathrm{C}$ & -6.035067 & -3.527809 & 0.951278 \\
\hline $\mathrm{C}$ & -5.074580 & -3.501580 & -2.652108 \\
\hline $\mathrm{C}$ & -5.742370 & -5.759484 & 0.091274 \\
\hline $\mathrm{C}$ & -6.615990 & -1.655889 & -2.992154 \\
\hline C & -7.955876 & -4.969693 & 0.663915 \\
\hline $\mathrm{C}$ & -2.353053 & -3.003773 & 3.669418 \\
\hline $\mathrm{C}$ & -1.645663 & -5.295093 & 3.375805 \\
\hline $\mathrm{C}$ & -2.309896 & -5.339424 & 2.147945 \\
\hline $\mathrm{C}$ & -7.118194 & -5.979533 & 0.191138 \\
\hline $\mathrm{C}$ & -7.407976 & -3.742278 & 1.047457 \\
\hline $\mathrm{C}$ & -1.659872 & -4.123965 & 4.136959 \\
\hline $\mathrm{C}$ & 5.901459 & 2.754123 & -2.615786 \\
\hline $\mathrm{C}$ & 2.420744 & 6.269284 & -1.943873 \\
\hline $\mathrm{C}$ & -6.244335 & 4.829423 & -0.992738 \\
\hline $\mathrm{C}$ & -1.297544 & 5.710005 & -0.593654 \\
\hline $\mathrm{C}$ & -6.948964 & -3.784188 & -4.277648 \\
\hline $\mathrm{C}$ & -6.308049 & 0.473826 & -1.670972 \\
\hline $\mathrm{C}$ & 3.980279 & -6.288645 & 1.616306 \\
\hline $\mathrm{C}$ & 6.934951 & -2.261312 & 1.326044 \\
\hline $\mathrm{H}$ & -1.406396 & 3.505603 & 0.880802 \\
\hline $\mathrm{H}$ & -4.070208 & 0.375120 & 5.252726 \\
\hline $\mathrm{H}$ & -5.379411 & 1.447109 & 2.185575 \\
\hline $\mathrm{H}$ & -4.341538 & -0.035262 & 2.412361 \\
\hline $\mathrm{H}$ & -1.677808 & 3.870734 & 3.580839 \\
\hline $\mathrm{H}$ & -5.845416 & 2.846642 & 3.750730 \\
\hline $\mathrm{H}$ & -4.564350 & 6.808480 & 4.804765 \\
\hline $\mathrm{H}$ & -2.601932 & -0.745060 & 6.908470 \\
\hline $\mathrm{H}$ & 0.850337 & 0.515875 & 4.671066 \\
\hline $\mathrm{H}$ & -0.615146 & 1.640916 & 3.047813 \\
\hline $\mathrm{H}$ & -6.377796 & 5.130741 & 4.518087 \\
\hline $\mathrm{H}$ & -2.206610 & 6.162399 & 4.333846 \\
\hline $\mathrm{H}$ & -0.128571 & -0.685641 & 6.617620 \\
\hline $\mathrm{H}$ & 1.623144 & 4.594822 & 0.053880 \\
\hline $\mathrm{H}$ & 3.393157 & 1.612945 & 4.871378 \\
\hline $\mathrm{H}$ & 2.505774 & 3.927733 & 2.390231 \\
\hline $\mathrm{H}$ & 1.477231 & 2.532059 & 2.958133 \\
\hline $\mathrm{H}$ & 5.660917 & 0.428546 & 1.755389 \\
\hline $\mathrm{H}$ & 4.426589 & 4.510494 & 2.275797 \\
\hline $\mathrm{H}$ & 8.484349 & 3.613518 & 1.221384 \\
\hline $\mathrm{H}$ & 3.369630 & -0.475322 & 6.208898 \\
\hline $\mathrm{H}$ & 3.336993 & -2.779456 & 2.574668 \\
\hline $\mathrm{H}$ & 3.373180 & -0.703497 & 1.260555 \\
\hline
\end{tabular}

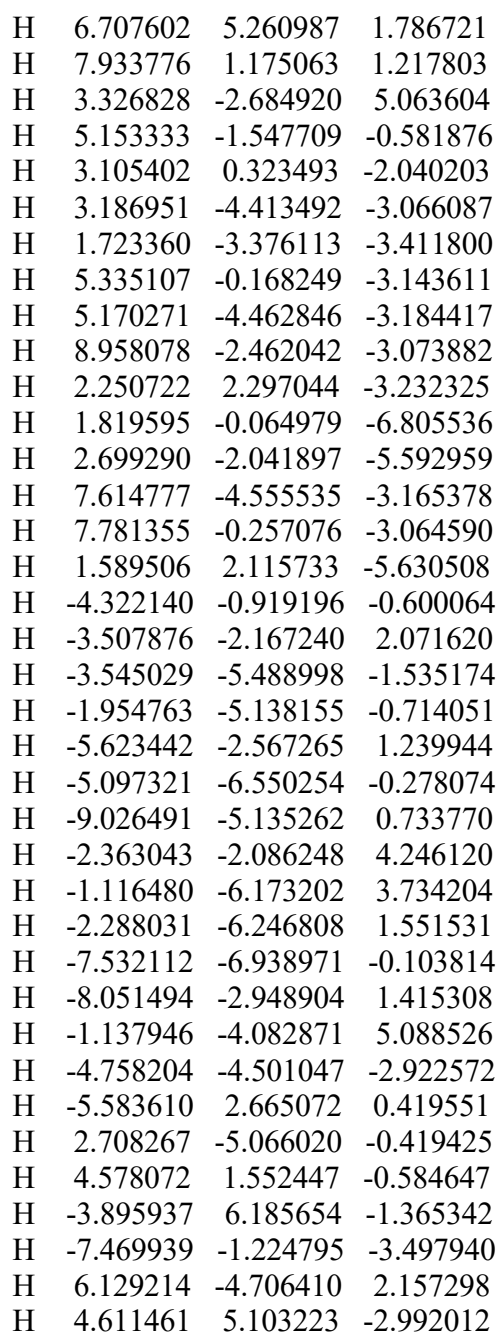

$$
\begin{array}{lrrr}
\text { U'4 } & & & \\
\text { Rh } & -0.454220 & -0.492590 & -1.078460 \\
\text { Rh } & -0.042750 & -0.996670 & 1.215410 \\
\text { O } & 1.785040 & -1.733570 & 0.601570 \\
\text { O } & 1.054230 & -1.824160 & -1.536800 \\
\text { O } & 0.775430 & 0.893680 & 1.381730 \\
\text { O } & 0.933170 & 1.041380 & -0.868620 \\
\text { O } & -1.927470 & -0.185030 & 1.601990 \\
\text { O } & -1.935270 & 0.775480 & -0.442710 \\
\text { O } & -0.904810 & -2.838720 & 0.843910 \\
\text { O } & -1.825740 & -2.036180 & -1.055260 \\
\text { F } & -6.536400 & -0.303800 & -0.395110 \\
\text { F } & -7.493540 & -0.631950 & -2.326330 \\
\text { F } & -5.461710 & 0.143580 & -2.230800 \\
\text { F } & -7.207320 & -5.100920 & -4.182410 \\
\text { F } & -5.482540 & -6.357690 & -3.738960 \\
\text { F } & -7.164180 & -6.297500 & -2.367810 \\
\text { F } & 2.488340 & 6.707140 & -4.009480 \\
\text { F } & 0.841800 & 5.337110 & -4.355100 \\
\text { F } & 0.878500 & 6.552160 & -2.551490 \\
\text { F } & 6.321030 & 2.070150 & -2.347160 \\
F & 6.278750 & 3.694790 & -3.800360 \\
\text { F } & 5.286810 & 1.815630 & -4.241780 \\
\text { F } & -7.650840 & 4.202560 & -1.159660
\end{array}
$$

F $\quad-7.284490 \quad 2.397920 \quad-2.306620$

F $\quad-6.863890 \quad 4.348290 \quad-3.182740$

F $\quad-2.401140 \quad 6.200200 \quad-2.677910$

F $\quad-1.248230 \quad 5.678550-0.901060$

F $\quad-1.152870 \quad 4.415920 \quad-2.665590$

F $\quad 3.633250 \quad-4.936950 \quad 3.883200$

F $\quad 4.263510 \quad-6.377580 \quad 2.385030$

F $\quad 5.721600 \quad-5.534370 \quad 3.768130$

F $\quad 7.348730 \quad-0.284020 \quad 0.689340$

F $\quad 8.254050 \quad-1.647830 \quad 2.130240$

F $\quad 6.726440 \quad-0.246550 \quad 2.772280$

$\begin{array}{llll}\text { C } & 5.158200 & -1.911370 & 0.396670\end{array}$

$\begin{array}{llll}\text { C } & 3.219310 & -2.670550 & -1.027120\end{array}$

C $\quad 3.804920 \quad-2.449700 \quad-2.465400$

$\begin{array}{llll}\text { C } & 4.883870 & -4.116630 & 2.062810\end{array}$

$\begin{array}{llll}\text { C } & 4.165660 & -2.858000 & 0.122680\end{array}$

$\begin{array}{llll}\text { C } & 6.027880 & -2.092500 & 1.467760\end{array}$

$\begin{array}{llll}\text { C } & 5.315030 & -2.394320 & -2.554190\end{array}$

$\begin{array}{llll}\text { C } & 1.925440 & -2.026590 & -0.634970\end{array}$

C $\quad 3.103250 \quad-1.520220-3.421080$

$\begin{array}{llll}\text { C } & 2.821510 & -0.194040 & -3.082750\end{array}$

C $\quad 3.127300 \quad-3.735580-2.109760$

$\begin{array}{llll}\text { C } & 6.127880 & -3.505630 & -2.295040\end{array}$

$\begin{array}{llll}\text { C } & 4.033950 & -3.960670 & 0.964720\end{array}$

$\begin{array}{llll}\text { C } & 5.940010 & -1.168540 & -2.828480\end{array}$

$\begin{array}{llll}\text { C } & 5.896070 & -3.194350 & 2.314250\end{array}$

C $\quad 8.125550 \quad-2.154310 \quad-2.515890$

$\begin{array}{llll}\text { C } & 2.206090 & 0.660950 & -3.994300\end{array}$

$\begin{array}{llll}\text { C } & 2.158980 & -1.124600 & -5.620810\end{array}$

C $\quad 2.772410 \quad-1.977050 \quad-4.700850$

C $\quad 7.327390 \quad-1.044480 \quad-2.797630$

C $\quad 7.518030 \quad-3.387630 \quad-2.276920$

$\begin{array}{llll}\text { C } & 1.872240 & 0.197180 & -5.268820\end{array}$

$\begin{array}{llll}\text { C } & 1.926960 & 4.201320 & -1.496270\end{array}$

$\begin{array}{llll}\text { C } & 2.142930 & 2.598100 & 0.439790\end{array}$

C $\quad 3.117710 \quad 2.726280 \quad 1.665660$

$\begin{array}{llll}\text { C } & 4.274840 & 3.230660 & -2.633720\end{array}$

C $\quad 2.615690 \quad 3.161490-0.870730$

$\begin{array}{llll}\text { C } & 2.412240 & 4.747670 & -2.688040\end{array}$

C $\quad 4.462320 \quad 3.346200 \quad 1.355600$

C $\quad 1.222490 \quad 1.423210 \quad 0.313740$

$\begin{array}{llll}\text { C } & 3.189400 & 1.648520 & 2.715870\end{array}$

C $\quad 3.162900 \quad 2.026300 \quad 4.063680$

C $\quad 1.874470 \quad 3.555480 \quad 1.590640$

$\begin{array}{llll}\text { C } & 4.621130 & 4.704040 & 1.053820\end{array}$

$\begin{array}{llll}\text { C } & 3.781640 & 2.665410 & -1.461610\end{array}$

$\begin{array}{llll}\text { C } & 5.590530 & 2.515190 & 1.289480\end{array}$

$\begin{array}{llll}\text { C } & 3.593930 & 4.276250 & -3.256600\end{array}$

$\begin{array}{llll}\text { C } & 6.968940 & 4.362870 & 0.565060\end{array}$

$\begin{array}{llll}\text { C } & 3.274890 & 1.068020 & 5.072750\end{array}$

$\begin{array}{llll}\text { C } & 3.452410 & -0.664270 & 3.399320\end{array}$

$\begin{array}{llll}\text { C } & 3.354730 & 0.298980 & 2.396110\end{array}$

$\begin{array}{llll}\text { C } & 6.827920 & 3.011810 & 0.887530\end{array}$

C $\quad 5.862360 \quad 5.207640 \quad 0.661940$

C $\quad 3.411280 \quad-0.282630 \quad 4.742010$

$\begin{array}{llll}\text { C } & -2.864070 & 3.429000 & -0.380750\end{array}$

$\begin{array}{llll}\mathrm{C} & -3.493840 & 1.563050 & 1.141840\end{array}$

$\begin{array}{llll}\text { C } & -3.658740 & 2.041170 & 2.622550\end{array}$

$\begin{array}{llll}\text { C } & -5.398780 & 3.567440 & -1.489300\end{array}$

$\begin{array}{llll}\text { C } & -3.855560 & 2.554090 & 0.076480\end{array}$

$\begin{array}{llll}\text { C } & -3.133800 & 4.341900 & -1.393250\end{array}$

$\begin{array}{llll}\text { C } & -4.186200 & 3.448080 & 2.773820\end{array}$ 


\begin{tabular}{|c|c|c|c|}
\hline C & -2.368330 & 0.651110 & \\
\hline $\mathrm{C}$ & -2.669020 & 1.675500 & 3.695250 \\
\hline $\mathrm{C}$ & -1.293500 & 1.857940 & 3.534280 \\
\hline $\mathrm{C}$ & -4.558590 & 0.989390 & 2.061560 \\
\hline $\mathrm{C}$ & -3.292100 & 4.527070 & 2.719130 \\
\hline C & -5.126770 & 2.635920 & -0.482100 \\
\hline $\mathrm{C}$ & -5.545200 & 3.709600 & 2.968360 \\
\hline $\mathrm{C}$ & -4.407920 & 4.424310 & -1.958690 \\
\hline $\mathrm{C}$ & -5.113110 & 6.090400 & 3.015430 \\
\hline $\mathrm{C}$ & -0.409220 & 1.551560 & 4.566900 \\
\hline $\mathrm{C}$ & -2.272410 & 0.923150 & 5.969790 \\
\hline $\mathrm{C}$ & -3.152160 & 1.210990 & 4.924240 \\
\hline $\mathrm{C}$ & -6.007170 & 5.022760 & 3.088790 \\
\hline $\mathrm{C}$ & -3.750400 & 5.837160 & 2.832600 \\
\hline $\mathrm{C}$ & -0.896390 & 1.085680 & 5.790230 \\
\hline $\mathrm{C}$ & -4.607120 & -4.652300 & -1.693650 \\
\hline $\mathrm{C}$ & -2.868970 & -3.884620 & -0.001990 \\
\hline $\mathrm{C}$ & -3.206760 & -4.538550 & 1.387110 \\
\hline $\mathrm{C}$ & -5.766460 & -2.129420 & -1.680920 \\
\hline $\mathrm{C}$ & -4.032340 & -3.647680 & -0.916320 \\
\hline $\mathrm{C}$ & -5.761010 & -4.399760 & -2.440610 \\
\hline $\mathrm{C}$ & -4.655200 & -4.922060 & 1.576270 \\
\hline $\mathrm{C}$ & -1.787190 & -2.849690 & -0.072360 \\
\hline $\mathrm{C}$ & -2.534930 & -4.071430 & 2.651530 \\
\hline $\mathrm{C}$ & -2.717210 & -2.772970 & 3.137260 \\
\hline $\mathrm{C}$ & -2.416020 & -5.289300 & 0.368430 \\
\hline $\mathrm{C}$ & -5.625360 & -3.940650 & 1.823650 \\
\hline $\mathrm{C}$ & -4.631140 & -2.382040 & -0.918370 \\
\hline $\mathrm{C}$ & -5.053410 & -6.260890 & 1.515440 \\
\hline $\mathrm{C}$ & -6.350960 & -3.138380 & -2.445430 \\
\hline $\mathrm{C}$ & -7.355260 & -5.629280 & 1.907690 \\
\hline $\mathrm{C}$ & -2.081870 & -2.352580 & 4.303220 \\
\hline $\mathrm{C}$ & -1.102350 & -4.546880 & 4.552800 \\
\hline $\mathrm{C}$ & -1.734000 & -4.958960 & 3.377210 \\
\hline $\mathrm{C}$ & -6.394820 & -6.614430 & 1.679440 \\
\hline $\mathrm{C}$ & -6.964490 & -4.289320 & 1.982670 \\
\hline $\mathrm{C}$ & -1.269380 & -3.239510 & 5.015370 \\
\hline $\mathrm{C}$ & 5.540400 & 2.707330 & -3.250350 \\
\hline $\mathrm{C}$ & 1.653610 & 5.837010 & -3.394670 \\
\hline $\mathrm{C}$ & 4.635450 & -5.244940 & 3.021440 \\
\hline $\mathrm{C}$ & 7.090670 & -1.072110 & 1.758900 \\
\hline $\mathrm{C}$ & -6.324800 & -0.736080 & -1.668150 \\
\hline $\mathrm{C}$ & -6.402830 & -5.533820 & -3.188390 \\
\hline $\mathrm{C}$ & -6.794100 & 3.632190 & -2.041000 \\
\hline $\mathrm{C}$ & -1.995610 & 5.169680 & -1.912800 \\
\hline $\mathrm{H}$ & 5.272520 & -1.048920 & -0.245450 \\
\hline $\mathrm{H}$ & 3.044430 & 0.163460 & -2.085840 \\
\hline $\mathrm{H}$ & 3.710570 & -4.636360 & -1.961550 \\
\hline $\mathrm{H}$ & 2.140550 & -3.893960 & -2.528660 \\
\hline $\mathrm{H}$ & 5.687630 & -4.472980 & -2.084460 \\
\hline $\mathrm{H}$ & 5.337940 & -0.302590 & -3.063200 \\
\hline $\mathrm{H}$ & 9.206790 & -2.061430 & -2.488080 \\
\hline $\mathrm{H}$ & 1.982520 & 1.681440 & -3.702820 \\
\hline $\mathrm{H}$ & 1.905480 & -1.492030 & -6.610760 \\
\hline $\mathrm{H}$ & 2.992060 & -3.005440 & -4.973280 \\
\hline $\mathrm{H}$ & 7.775340 & -0.074900 & -2.989440 \\
\hline $\mathrm{H}$ & 8.124930 & -4.263430 & -2.068140 \\
\hline $\mathrm{H}$ & 1.393610 & 0.860290 & -5.983230 \\
\hline $\mathrm{H}$ & 1.024290 & 4.598870 & -1.049000 \\
\hline $\mathrm{H}$ & 3.047250 & 3.075720 & 4.319660 \\
\hline $\mathrm{H}$ & 1.936210 & 4.614240 & 1.369410 \\
\hline $\mathrm{H}$ & 1.071140 & 3.279190 & 2.262530 \\
\hline
\end{tabular}
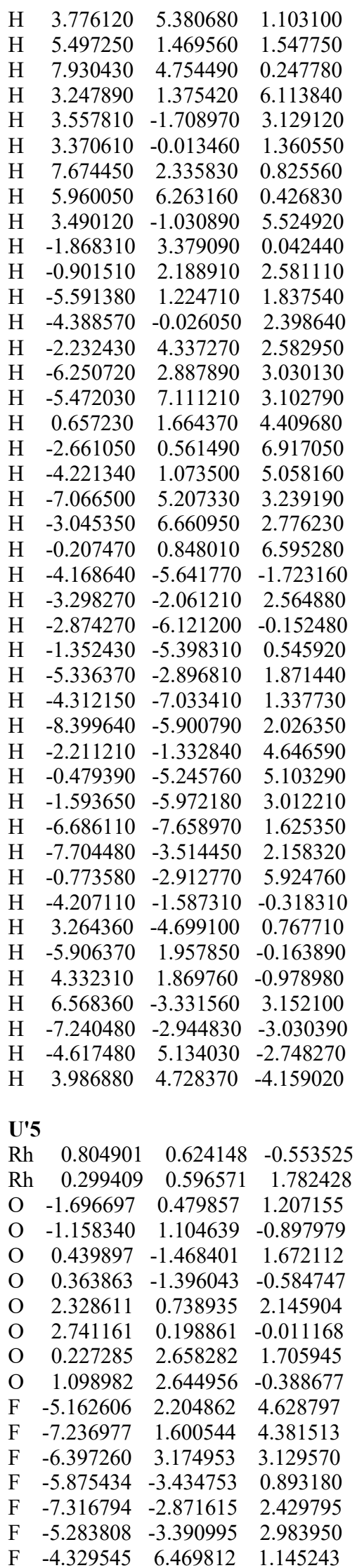

$\begin{array}{llll}\text { F } & -5.021645 & 4.550985 & 0.397467\end{array}$

F $\quad-5.280626 \quad 6.338085 \quad-0.811305$

F $\quad-2.811592 \quad 4.348860 \quad-4.582155$

F $\quad-1.234811 \quad 5.843090 \quad-4.643201$

F $\quad-0.730185 \quad 3.757076 \quad-4.312348$

F $\quad-3.162269 \quad-5.796530 \quad-4.074029$

F $\quad-3.199385 \quad-3.631805 \quad-4.224808$

F $\quad-3.942086 \quad-4.600055 \quad-2.426437$

F $\quad 1.638656 \quad-6.493242 \quad-4.550206$

$\begin{array}{llll}\text { F } & 2.348628 & -4.444649 & -4.631778\end{array}$

F $\quad 3.008822 \quad-5.755703 \quad-3.026576$

$\begin{array}{llll}\text { F } & 4.587238 & -4.196082 & -1.275637\end{array}$

F $\quad 3.732731 \quad-2.895608 \quad-2.790075$

F $\quad 5.715489 \quad-3.744442 \quad-3.087685$

$\begin{array}{llll}\text { F } & 8.543490 & 0.207708 & -3.153740\end{array}$

$\begin{array}{llll}\text { F } & 7.578502 & 1.969974 & -2.310218\end{array}$

F $\quad 9.065153 \quad 0.893541 \quad-1.153529$

$\begin{array}{llll}\text { C } & -4.846109 & 1.351142 & 1.660818\end{array}$

$\begin{array}{llll}\text { C } & -3.446292 & 0.829085 & -0.354344\end{array}$

$\begin{array}{llll}\text { C } & -3.885234 & 0.563440 & -1.839549\end{array}$

$\begin{array}{llll}\text { C } & -5.620168 & -1.308295 & 1.907735\end{array}$

$\begin{array}{llll}\text { C } & -4.366093 & 0.409946 & 0.752200\end{array}$

$\begin{array}{llll}\text { C } & -5.696810 & 0.958149 & 2.697683\end{array}$

$\begin{array}{llll}\text { C } & -5.192810 & -0.179776 & -1.998870\end{array}$

$\begin{array}{llll}\text { C } & -1.996688 & 0.798617 & 0.006935\end{array}$

$\begin{array}{llll}\text { C } & -2.843522 & 0.227392 & -2.875050\end{array}$

$\begin{array}{llll}\text { C } & -2.005624 & -0.884825 & -2.748851\end{array}$

$\begin{array}{llll}\text { C } & -3.875425 & 1.951289 & -1.285489\end{array}$

$\begin{array}{llll}\text { C } & -5.177674 & -1.516706 & -2.423700\end{array}$

$\begin{array}{llll}\text { C } & -4.747144 & -0.928195 & 0.893001\end{array}$

$\begin{array}{llll}\text { C } & -6.426445 & 0.390657 & -1.659022\end{array}$

$\begin{array}{llll}\text { C } & -6.098652 & -0.369080 & 2.823467\end{array}$

$\begin{array}{llll}\text { C } & -7.566486 & -1.700517 & -2.097788\end{array}$

$\begin{array}{llll}\text { C } & -1.060002 & -1.180193 & -3.727976\end{array}$

$\begin{array}{llll}\text { C } & -1.773731 & 0.753073 & -4.988327\end{array}$

$\begin{array}{llll}\text { C } & -2.721812 & 1.041012 & -4.005316\end{array}$

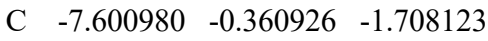

$\begin{array}{llll}\text { C } & -6.346805 & -2.273577 & -2.461602\end{array}$

$\begin{array}{llll}\text { C } & -0.940508 & -0.359895 & -4.853032\end{array}$

$\begin{array}{llll}\text { C } & 0.958038 & -4.463866 & -1.670850\end{array}$

$\begin{array}{llll}\text { C } & 0.038057 & -3.485210 & 0.466106\end{array}$

$\begin{array}{llll}\text { C } & -0.740027 & -4.221144 & 1.614497\end{array}$

$\begin{array}{llll}\text { C } & -1.596746 & -4.595285 & -2.771229\end{array}$

$\begin{array}{llll}\text { C } & -0.134597 & -4.009962 & -0.931175\end{array}$

$\begin{array}{llll}\text { C } & 0.768505 & -4.978200 & -2.956729\end{array}$

$\begin{array}{llll}\text { C } & -1.636936 & -5.354020 & 1.165378\end{array}$

$\begin{array}{llll}\text { C } & 0.293790 & -2.009956 & 0.531531\end{array}$

$\begin{array}{llll}\text { C } & -1.295662 & -3.454768 & 2.786398\end{array}$

$\begin{array}{llll}\text { C } & -1.072818 & -3.950150 & 4.076640\end{array}$

$\begin{array}{llll}\text { C } & 0.746497 & -4.350644 & 1.495437\end{array}$

$\begin{array}{llll}\text { C } & -3.026887 & -5.163652 & 1.179741\end{array}$

$\begin{array}{llll}\text { C } & -1.409968 & -4.057790 & -1.501460\end{array}$

$\begin{array}{llll}\text { C } & -1.139582 & -6.561358 & 0.659734\end{array}$

$\begin{array}{llll}\text { C } & -0.508544 & -5.060818 & -3.509128\end{array}$

$\begin{array}{llll}\text { C } & -3.381558 & -7.314715 & 0.136762\end{array}$

$\begin{array}{llll}\text { C } & -1.609139 & -3.300473 & 5.189920\end{array}$

$\begin{array}{llll}\text { C } & -2.617872 & -1.656426 & 3.736631\end{array}$

$\begin{array}{llll}\text { C } & -2.087160 & -2.314766 & 2.628509\end{array}$

$\begin{array}{llll}\text { C } & -2.003089 & -7.532176 & 0.150446\end{array}$

$\begin{array}{llll}\text { C } & -3.890649 & -6.125046 & 0.660716\end{array}$

$\begin{array}{llll}\text { C } & -2.377702 & -2.145778 & 5.022354\end{array}$

$\begin{array}{llll}\text { C } & 4.882304 & -1.582939 & -0.299800\end{array}$ 


\begin{tabular}{|c|c|c|c|}
\hline C & 4.535026 & 0.084135 & 1.514252 \\
\hline $\mathrm{C}$ & 4.935813 & -0.481583 & 2.916943 \\
\hline $\mathrm{C}$ & 6.949650 & -0.123808 & -1.426683 \\
\hline $\mathrm{C}$ & 5.296854 & -0.398337 & 0.319768 \\
\hline $\mathrm{C}$ & 5.478445 & -2.015224 & -1.477271 \\
\hline $\mathrm{C}$ & 6.089155 & -1.456174 & 2.903394 \\
\hline $\mathrm{C}$ & 3.096094 & 0.364592 & 1.203643 \\
\hline $\mathrm{C}$ & 3.885557 & -0.800011 & 3.946281 \\
\hline $\mathrm{C}$ & 3.979486 & -0.224051 & 5.217938 \\
\hline & 5.197912 & 0.947158 & 2.570710 \\
\hline & 5.850679 & -2.813635 & 2.643038 \\
\hline $\mathrm{C}$ & 6.343355 & 0.320020 & -0.245758 \\
\hline $\mathrm{C}$ & 7.401929 & -1.041519 & 3.147701 \\
\hline $\mathrm{C}$ & 6.521397 & -1.287594 & -2.057025 \\
\hline & 8.211395 & -3.301325 & 2.839719 \\
\hline $\mathrm{C}$ & 3.033517 & -0.517946 & 6.201946 \\
\hline $\mathrm{C}$ & 1.883913 & -1.972821 & 4.654015 \\
\hline $\mathrm{C}$ & 2.837268 & -1.684816 & 3.680270 \\
\hline $\mathrm{C}$ & 8.456948 & -1.956790 & 3.116462 \\
\hline$C$ & 6.901001 & -3.727606 & 2.605658 \\
\hline $\mathrm{C}$ & 1.979781 & -1.391082 & 5.920146 \\
\hline $\mathrm{C}$ & -1.959880 & 5.333072 & 0.209855 \\
\hline $\mathrm{C}$ & 0.455527 & 4.714728 & 0.540684 \\
\hline $\mathrm{C}$ & 1.692831 & 5.625127 & 0.570087 \\
\hline $\mathrm{C}$ & -1.767852 & 4.917094 & -2.532529 \\
\hline $\mathrm{C}$ & -0.727117 & 4.987754 & -0.346276 \\
\hline $\mathrm{C}$ & -3.086606 & 5.470346 & -0.603963 \\
\hline $\mathrm{C}$ & 1.669227 & 6.854982 & -0.305867 \\
\hline $\mathrm{C}$ & 0.635456 & 3.223895 & 0.643873 \\
\hline $\mathrm{C}$ & 3.058735 & 4.998854 & 0.737212 \\
\hline $\mathrm{C}$ & 3.642174 & 4.218479 & -0.272432 \\
\hline $\mathrm{C}$ & 0.738564 & 5.563987 & 1.742770 \\
\hline $\mathrm{C}$ & 1.362375 & 8.112231 & 0.225513 \\
\hline $\mathrm{C}$ & -0.643005 & 4.765448 & -1.724605 \\
\hline $\mathrm{C}$ & 1.971546 & 6.762222 & -1.672421 \\
\hline $\mathrm{C}$ & -2.998102 & 5.276651 & -1.980903 \\
\hline $\mathrm{C}$ & 1.623847 & 9.139779 & -1.949677 \\
\hline $\mathrm{C}$ & 4.938756 & 3.723481 & -0.131658 \\
\hline $\mathrm{C}$ & 5.095140 & 4.757983 & 2.044616 \\
\hline $\mathrm{C}$ & 3.800309 & 5.258560 & 1.896507 \\
\hline $\mathrm{C}$ & 1.944158 & 7.891179 & -2.488750 \\
\hline $\mathrm{C}$ & 1.338939 & 9.247001 & -0.588573 \\
\hline $\mathrm{C}$ & 5.670535 & 3.995920 & 1.026517 \\
\hline $\mathrm{C}$ & -2.973212 & -4.660511 & -3.369287 \\
\hline $\mathrm{C}$ & 1.943544 & -5.419505 & -3.784703 \\
\hline $\mathrm{C}$ & -6.027367 & -2.747287 & 2.049615 \\
\hline $\mathrm{C}$ & -6.131633 & 1.981883 & 3.707046 \\
\hline $\mathrm{C}$ & -1.637512 & 4.714475 & -4.016027 \\
\hline $\mathrm{C}$ & -4.425408 & 5.721143 & 0.028224 \\
\hline $\mathrm{C}$ & 8.037111 & 0.725430 & -2.017210 \\
\hline $\mathrm{C}$ & 4.893500 & -3.216782 & -2.161124 \\
\hline $\mathrm{H}$ & -4.574440 & 2.394772 & 1.548662 \\
\hline $\mathrm{H}$ & -2.054969 & -1.494880 & -1.855544 \\
\hline $\mathrm{H}$ & -4.799508 & 2.460611 & -1.044569 \\
\hline $\mathrm{H}$ & -3.080092 & 2.598397 & -1.629942 \\
\hline $\mathrm{H}$ & -4.245454 & -1.973034 & -2.724429 \\
\hline $\mathrm{H}$ & -6.482840 & 1.422962 & -1.334597 \\
\hline $\mathrm{H}$ & -8.479219 & -2.287724 & -2.122761 \\
\hline $\mathrm{H}$ & -0.410906 & -2.040242 & -3.603579 \\
\hline $\mathrm{H}$ & -1.679668 & 1.406107 & -5.850302 \\
\hline $\mathrm{H}$ & -3.352328 & 1.918447 & -4.103376 \\
\hline $\mathrm{H}$ & -8.543502 & 0.103523 & -1.434367 \\
\hline
\end{tabular}
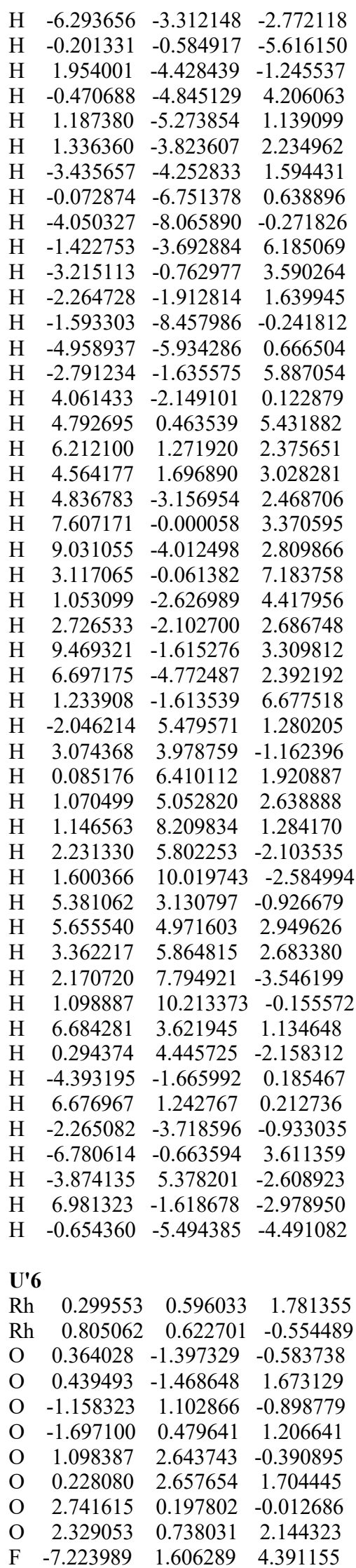

$\begin{array}{llll}\text { F } & -6.395812 & 3.179139 & 3.129586\end{array}$

$\begin{array}{llll}\mathrm{F} & -5.149494 & 2.216248 & 4.623726\end{array}$

$\begin{array}{llll}\text { F } & -5.880730 & -3.430793 & 0.897636\end{array}$

$\begin{array}{llll}\mathrm{F} & -7.319760 & -2.864443 & 2.435283\end{array}$

$\begin{array}{llll}\text { F } & -5.286893 & -3.386169 & 2.987747\end{array}$

F $\quad-5.019130 \quad 4.551610 \quad 0.397902$

$\begin{array}{llll}\text { F } & -4.328107 & 6.472774 & 1.140643\end{array}$

$\begin{array}{lllll}\text { F } & -5.280244 & 6.335605 & -0.815034\end{array}$

F $\quad-1.234466 \quad 5.841012 \quad-4.646142$

$\begin{array}{llll}\text { F } & -0.728338 & 3.755486 & -4.314407\end{array}$

F $\quad-2.810164 \quad 4.345742 \quad-4.584299$

F $\quad 2.347244 \quad-4.445768 \quad-4.630604$

F $\quad 1.637012 \quad-6.494222 \quad-4.548346$

$\begin{array}{llll}\text { F } & 3.007483 & -5.756458 & -3.025111\end{array}$

F $\quad-3.943279 \quad-4.596881 \quad-2.426186$

$\begin{array}{llll}\text { F } & -3.199117 & -3.631982 & -4.225780\end{array}$

$\begin{array}{llll}\text { F } & -3.164117 & -5.796470 & -4.071825\end{array}$

$\begin{array}{llll}\text { F } & 3.733077 & -2.896611 & -2.790785\end{array}$

F $\quad 4.586869-4.197339 \quad-1.276174$

F $\quad 5.715516 \quad-3.746346 \quad-3.088136$

$\begin{array}{llll}\text { F } & 7.580418 & 1.967911 & -2.309294\end{array}$

F $\quad 8.544578 \quad 0.205696 \quad-3.153865$

F $\quad 9.066800 \quad 0.890309 \quad-1.153368$

$\begin{array}{llll}\text { C } & 0.956675 & -4.464541 & -1.669220\end{array}$

$\begin{array}{llll}\text { C } & 0.036551 & -3.485786 & 0.467648\end{array}$

$\begin{array}{llll}\text { C } & -0.742791 & -4.220576 & 1.615951\end{array}$

$\begin{array}{llll}\text { C } & -1.597862 & -4.594857 & -2.770281\end{array}$

$\begin{array}{llll}\text { C } & -0.136033 & -4.010439 & -0.929697\end{array}$

$\begin{array}{llll}\text { C } & 0.767262 & -4.978630 & -2.955217\end{array}$

$\begin{array}{llll}\text { C } & -1.641446 & -5.352013 & 1.166726\end{array}$

$\begin{array}{llll}\text { C } & 0.293370 & -2.010725 & 0.532754\end{array}$

$\begin{array}{llll}\text { C } & -1.297469 & -3.453240 & 2.787675\end{array}$

$\begin{array}{llll}\text { C } & -2.087857 & -2.312533 & 2.629371\end{array}$

$\begin{array}{llll}\text { C } & 0.743602 & -4.352034 & 1.497249\end{array}$

$\begin{array}{llll}\text { C } & -3.031165 & -5.159711 & 1.181564\end{array}$

$\begin{array}{llll}\text { C } & -1.411242 & -4.057730 & -1.500341\end{array}$

$\begin{array}{llll}\text { C } & -1.145904 & -6.559759 & 0.660272\end{array}$

$\begin{array}{llll}\text { C } & -0.509682 & -5.060697 & -3.507960\end{array}$

$\begin{array}{llll}\text { C } & -3.389030 & -7.309640 & 0.137319\end{array}$

$\begin{array}{llll}\text { C } & -2.617795 & -1.653226 & 3.737285\end{array}$

C $\quad-1.610606 \quad-3.297700 \quad 5.191147$

$\begin{array}{llll}\text { C } & -1.075050 & -3.948380 & 4.078069\end{array}$

$\begin{array}{llll}\text { C } & -2.010893 & -7.529070 & 0.150578\end{array}$

$\begin{array}{llll}\text { C } & -3.896357 & -6.119606 & 0.662172\end{array}$

C $\quad-2.377941 \quad-2.142250 \quad 5.023189$

$\begin{array}{llll}\text { C } & -4.843099 & 1.353982 & 1.661000\end{array}$

$\begin{array}{llll}\text { C } & -3.446453 & 0.828881 & -0.355344\end{array}$

$\begin{array}{llll}\text { C } & -3.885534 & 0.562460 & -1.840351\end{array}$

$\begin{array}{llll}\text { C } & -5.621470 & -1.303902 & 1.910287\end{array}$

$\begin{array}{llll}\text { C } & -4.366315 & 0.411614 & 0.751897\end{array}$

$\begin{array}{llll}\text { C } & -5.692684 & 0.962914 & 2.699520\end{array}$

$\begin{array}{llll}\text { C } & -5.193446 & -0.180177 & -1.999393\end{array}$

$\begin{array}{llll}\text { C } & -1.996854 & 0.797778 & 0.006195\end{array}$

$\begin{array}{llll}\text { C } & -2.844066 & 0.225583 & -2.875890\end{array}$

$\begin{array}{llll}\text { C } & -2.722865 & 1.038287 & -4.006895\end{array}$

$\begin{array}{llll}\text { C } & -3.875019 & 1.950706 & -1.287248\end{array}$

$\begin{array}{llll}\text { C } & -5.178953 & -1.517352 & -2.423481\end{array}$

$\begin{array}{llll}\text { C } & -4.749715 & -0.925742 & 0.893772\end{array}$

$\begin{array}{llll}\text { C } & -6.426833 & 0.391161 & -1.660188\end{array}$

$\begin{array}{llll}\text { C } & -6.096626 & -0.363534 & 2.826590\end{array}$

$\begin{array}{llll}\text { C } & -7.567987 & -1.699566 & -2.098161\end{array}$

$\begin{array}{llll}\text { C } & -1.774918 & 0.749803 & -4.989880\end{array}$ 


\begin{tabular}{|c|c|c|c|}
\hline C & -1.060375 & -1.182217 & \\
\hline $\mathrm{C}$ & -2.005875 & -0.886320 & -2.749022 \\
\hline $\mathrm{C}$ & -7.601788 & -0.359774 & -1.709140 \\
\hline $\mathrm{C}$ & -6.348530 & -2.273538 & -2.461319 \\
\hline C & -0.941339 & -0.362802 & -4.853838 \\
\hline $\mathrm{C}$ & -0.641856 & 4.764223 & -1.727025 \\
\hline $\mathrm{C}$ & 0.456201 & 4.713819 & 0.538693 \\
\hline C & 1.693894 & 5.623624 & 0.569463 \\
\hline $\mathrm{C}$ & -3.085410 & 5.470073 & -0.606837 \\
\hline $\mathrm{C}$ & -0.725998 & 4.987180 & -0.348773 \\
\hline $\mathrm{C}$ & -1.766657 & 4.915605 & -2.535019 \\
\hline $\mathrm{C}$ & 1.671868 & 6.853999 & -0.305786 \\
\hline $\mathrm{C}$ & 0.635709 & 3.222928 & 0.641942 \\
\hline $\mathrm{C}$ & 3.059373 & 4.996583 & 0.737285 \\
\hline $\mathrm{C}$ & 3.643328 & 4.216744 & -0.272511 \\
\hline $\mathrm{C}$ & 0.738605 & 5.562596 & 1.741279 \\
\hline $\mathrm{C}$ & 1.366578 & 8.111255 & 0.226481 \\
\hline $\mathrm{C}$ & -1.958708 & 5.333105 & 0.207078 \\
\hline $\mathrm{C}$ & 1.974521 & 6.761841 & -1.672298 \\
\hline $\mathrm{C}$ & -2.996915 & 5.275573 & -1.983640 \\
\hline $\mathrm{C}$ & 1.630024 & 9.140064 & -1.947861 \\
\hline $\mathrm{C}$ & 4.939761 & 3.721505 & -0.131248 \\
\hline $\mathrm{C}$ & 5.094845 & 4.754326 & 2.045907 \\
\hline $\mathrm{C}$ & 3.800186 & 5.255224 & 1.897308 \\
\hline $\mathrm{C}$ & 1.948913 & 7.891466 & -2.487766 \\
\hline $\mathrm{C}$ & 1.344852 & 9.246663 & -0.586752 \\
\hline $\mathrm{C}$ & 5.670840 & 3.993044 & 1.027578 \\
\hline $\mathrm{C}$ & 6.344363 & 0.317931 & -0.245968 \\
\hline $\mathrm{C}$ & 4.535257 & 0.082746 & 1.513309 \\
\hline $\mathrm{C}$ & 4.935285 & -0.482569 & 2.916392 \\
\hline $\mathrm{C}$ & 5.478994 & -2.016935 & -1.477833 \\
\hline $\mathrm{C}$ & 5.297408 & -0.400044 & 0.319198 \\
\hline $\mathrm{C}$ & 6.950838 & -0.126032 & -1.426732 \\
\hline $\mathrm{C}$ & 6.088552 & -1.457266 & 2.904121 \\
\hline $\mathrm{C}$ & 3.096494 & 0.363485 & 1.202171 \\
\hline $\mathrm{C}$ & 3.884333 & -0.800499 & 3.945151 \\
\hline $\mathrm{C}$ & 3.976962 & -0.223898 & 5.216608 \\
\hline $\mathrm{C}$ & 5.197979 & 0.945923 & 2.569748 \\
\hline $\mathrm{C}$ & 5.850638 & -2.814641 & 2.642787 \\
\hline $\mathrm{C}$ & 4.882581 & -1.584425 & -0.300576 \\
\hline $\mathrm{C}$ & 7.400833 & -1.042671 & 3.151236 \\
\hline $\mathrm{C}$ & 6.522393 & -1.289694 & -2.057230 \\
\hline $\mathrm{C}$ & 8.211009 & -3.302300 & 2.843890 \\
\hline $\mathrm{C}$ & 3.030061 & -0.517502 & 6.199816 \\
\hline $\mathrm{C}$ & 1.882140 & -1.973226 & 4.651413 \\
\hline $\mathrm{C}$ & 2.836448 & -1.685567 & 3.678510 \\
\hline $\mathrm{C}$ & 8.455951 & -1.957881 & 3.121718 \\
\hline $\mathrm{C}$ & 6.901087 & -3.728546 & 2.607088 \\
\hline $\mathrm{C}$ & 1.976715 & -1.390899 & 5.917367 \\
\hline $\mathrm{C}$ & -6.030558 & -2.742181 & 2.053677 \\
\hline $\mathrm{C}$ & -6.123778 & 1.988287 & 3.708758 \\
\hline $\mathrm{C}$ & -2.974182 & -4.659585 & -3.368698 \\
\hline $\mathrm{C}$ & 1.942168 & -5.420327 & -3.783161 \\
\hline C & 4.893650 & -3.218259 & -2.161725 \\
\hline $\mathrm{C}$ & 8.038567 & 0.723037 & -2.016985 \\
\hline $\mathrm{C}$ & -4.424064 & 5.721358 & 0.025468 \\
\hline $\mathrm{C}$ & -1.636293 & 4.712366 & -4.018425 \\
\hline $\mathrm{H}$ & 1.952593 & -4.429446 & -1.243727 \\
\hline $\mathrm{H}$ & -2.265277 & -1.910897 & 1.640643 \\
\hline $\mathrm{H}$ & 1.183357 & -5.275854 & 1.141013 \\
\hline $\mathrm{H}$ & 1.334018 & -3.825851 & 2.236942 \\
\hline $\mathrm{H}$ & -3.438633 & -4.248599 & 1.596971 \\
\hline
\end{tabular}
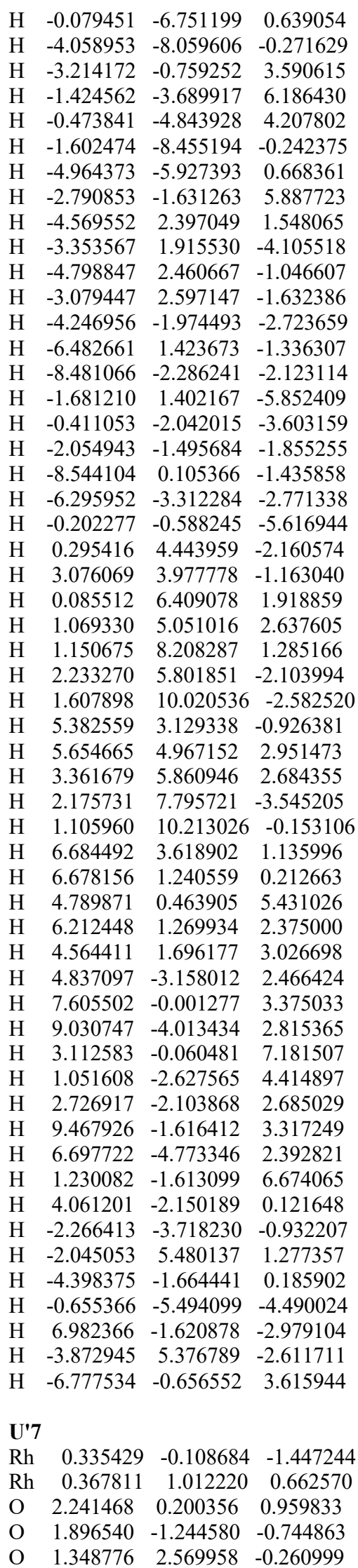

$\begin{array}{llll}\text { O } & 1.676340 & 1.305955 & -2.116009\end{array}$

$\begin{array}{llll}\text { O } & -1.512097 & 1.735813 & 0.143091\end{array}$

$\begin{array}{llll}\mathrm{O} & -1.197283 & 1.135550 & -2.014511\end{array}$

$\begin{array}{llll}\text { O } & -0.490012 & -0.684060 & 1.464926\end{array}$

$\begin{array}{lllll}\mathrm{O} & -1.009507 & -1.441731 & -0.598951\end{array}$

$\begin{array}{llll}\text { F } & -4.640304 & 6.682898 & 2.527646\end{array}$

$\begin{array}{llll}\text { F } & -2.570352 & 6.106174 & 2.171159\end{array}$

$\begin{array}{llll}\text { F } & -3.745138 & 6.950225 & 0.556247\end{array}$

$\begin{array}{llll}\text { F } & -7.058619 & 0.934235 & 1.636489\end{array}$

$\begin{array}{llll}\text { F } & -5.962118 & 1.387258 & 3.456686\end{array}$

$\begin{array}{llll}\text { F } & -7.539012 & 2.721898 & 2.790518\end{array}$

F $\quad-6.644823 \quad-2.549266 \quad-0.844519$

F $\quad-6.821313-4.485837 \quad-1.830954$

F $\quad-5.922327 \quad-2.810278-2.877515$

F $\quad-1.528736 \quad-6.397450 \quad-2.777120$

$\begin{array}{llll}\text { F } & -1.265897 & -7.109560 & -0.738458\end{array}$

$\begin{array}{llll}\text { F } & -3.085497 & -7.581500 & -1.837569\end{array}$

$\begin{array}{llll}\text { F } & 0.804947 & -5.319676 & -1.754846\end{array}$

$\begin{array}{llll}\text { F } & 2.017706 & -7.097107 & -1.419125\end{array}$

$\begin{array}{llll}\text { F } & 1.158733 & -6.017039 & 0.270672\end{array}$

F $\quad 7.004405 \quad-3.581608 \quad-2.813721$

F $\quad 7.392913-5.179261 \quad-1.396860$

F $\quad 6.301533 \quad-5.602634 \quad-3.228492$

F $\quad 4.070085 \quad 3.302092 \quad 3.193166$

F $\quad 5.777554 \quad 4.592767 \quad 2.827005$

$\begin{array}{llll}\text { F } & 6.095599 & 2.532235 & 3.440179\end{array}$

$\begin{array}{llll}\text { F } & 7.690399 & 1.189572 & -2.526601\end{array}$

F $\quad 8.740910 \quad 1.377940 \quad-0.626499$

F $\quad 7.349542 \quad-0.259218 \quad-0.941738$

C $\quad 5.071882 \quad-3.178862 \quad-0.823101$

$\begin{array}{llll}\text { C } & 3.730698 & -1.637822 & 0.689287\end{array}$

$\begin{array}{llll}\text { C } & 4.155703 & -1.663704 & 2.195226\end{array}$

$\begin{array}{llll}\text { C } & 2.959079 & -4.988153 & -0.864456\end{array}$

$\begin{array}{llll}\text { C } & 3.914828 & -2.893475 & -0.102526\end{array}$

$\begin{array}{llll}\text { C } & 5.175618 & -4.375219 & -1.542001\end{array}$

$\begin{array}{llll}\text { C } & 4.770279 & -2.956827 & 2.672767\end{array}$

$\begin{array}{llll}\text { C } & 2.535788 & -0.833886 & 0.280926\end{array}$

$\begin{array}{llll}\text { C } & 3.318778 & -0.963712 & 3.232165\end{array}$

$\begin{array}{llll}\text { C } & 1.997376 & -1.344038 & 3.486736\end{array}$

$\begin{array}{llll}\text { C } & 4.917459 & -0.845422 & 1.207983\end{array}$

$\begin{array}{llll}\text { C } & 6.153680 & -3.076352 & 2.837548\end{array}$

$\begin{array}{llll}\text { C } & 2.863590 & -3.816526 & -0.124419\end{array}$

$\begin{array}{llll}\text { C } & 3.958168 & -4.058368 & 2.977580\end{array}$

$\begin{array}{llll}\text { C } & 4.122564 & -5.286048 & -1.575648\end{array}$

$\begin{array}{llll}\text { C } & 5.903112 & -5.368284 & 3.570716\end{array}$

$\begin{array}{llll}\text { C } & 1.240817 & -0.679777 & 4.448772\end{array}$

$\begin{array}{llll}\text { C } & 3.124687 & 0.749403 & 4.939687\end{array}$

$\begin{array}{llll}\text { C } & 3.878541 & 0.080708 & 3.973817\end{array}$

$\begin{array}{llll}\text { C } & 4.518367 & -5.255374 & 3.417911\end{array}$

$\begin{array}{llll}\text { C } & 6.718285 & -4.273588 & 3.283774\end{array}$

$\begin{array}{llll}\text { C } & 1.801339 & 0.372286 & 5.177917\end{array}$

$\begin{array}{llll}\text { C } & 4.236930 & 3.218404 & 0.423269\end{array}$

$\begin{array}{llll}\text { C } & 3.045850 & 3.210842 & -1.787652\end{array}$

$\begin{array}{llll}\text { C } & 2.716929 & 4.630796 & -2.278491\end{array}$

$\begin{array}{llll}\text { C } & 6.433105 & 1.900983 & -0.648523\end{array}$

$\begin{array}{llll}\text { C } & 4.250595 & 2.909151 & -0.941131\end{array}$

$\begin{array}{llll}\text { C } & 5.329461 & 2.909511 & 1.229571\end{array}$

$\begin{array}{llll}\text { C } & 3.707586 & 5.736520 & -1.999401\end{array}$

$\begin{array}{llll}\text { C } & 1.912714 & 2.307657 & -1.370271\end{array}$

$\begin{array}{llll}\text { C } & 1.271838 & 5.070588 & -2.262400\end{array}$

C $\quad 0.672622 \quad 5.485916 \quad-1.063681$

$\begin{array}{llll}\text { C } & 3.154523 & 3.552160 & -3.243775\end{array}$ 


\begin{tabular}{|c|c|c|c|}
\hline C & 4.415296 & 6.314609 & -3.060263 \\
\hline & 5.352394 & 2.233267 & -1.468902 \\
\hline & 3.911058 & 6.240088 & -0.707322 \\
\hline 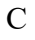 & 6.441011 & 2.254154 & 0.699687 \\
\hline & 5.516880 & 7.844253 & -1.544710 \\
\hline C & -0.637812 & 5.957617 & -1.045418 \\
\hline $\mathrm{C}$ & -0.802779 & 5.579347 & -3.423752 \\
\hline$c$ & 0.517763 & 5.121056 & -3.439954 \\
\hline$c$ & 4.811043 & 7.280551 & -0.479782 \\
\hline & 5.312553 & 7.360355 & -2.837654 \\
\hline $\mathrm{C}$ & -1.381833 & 6.006460 & -2.228417 \\
\hline $\mathrm{C}$ & -3.617519 & 4.153058 & 0.123558 \\
\hline $\mathrm{C}$ & -3.209928 & 2.246200 & -1.428305 \\
\hline $\mathrm{C}$ & -4.050453 & 1.654983 & -2.613437 \\
\hline $\mathrm{C}$ & -5.517802 & 2.733043 & 1.559843 \\
\hline C & -3.926171 & 2.851336 & -0.258407 \\
\hline $\mathrm{C}$ & -4.244399 & 4.738955 & 1.224289 \\
\hline $\mathrm{C}$ & -5.550624 & 1.677541 & -2.415611 \\
\hline $\mathrm{C}$ & -1.878474 & 1.659883 & -1.078105 \\
\hline $\mathrm{C}$ & -3.550746 & 0.446570 & -3.360971 \\
\hline $\mathrm{C}$ & -3.229995 & -0.745703 & -2.707357 \\
\hline $\mathrm{C}$ & -3.299001 & 2.937202 & -2.781049 \\
\hline $\mathrm{C}$ & -6.229201 & 0.471014 & -2.187143 \\
\hline $\mathrm{C}$ & -4.872049 & 2.134938 & 0.480546 \\
\hline $\mathrm{C}$ & -6.287957 & 2.867888 & -2.371250 \\
\hline $\mathrm{C}$ & -5.208869 & 4.040117 & 1.945188 \\
\hline $\mathrm{C}$ & -8.305396 & 1.651050 & -1.810256 \\
\hline $\mathrm{C}$ & -2.803609 & -1.861267 & -3.424963 \\
\hline $\mathrm{C}$ & -3.032460 & -0.614196 & -5.481095 \\
\hline $\mathrm{C}$ & -3.453980 & 0.501345 & -4.755322 \\
\hline $\mathrm{C}$ & -7.650771 & 2.855275 & -2.072049 \\
\hline $\mathrm{C}$ & -7.587217 & 0.455954 & -1.876224 \\
\hline $\mathrm{C}$ & -2.703667 & -1.798901 & -4.816624 \\
\hline $\mathrm{C}$ & -2.149088 & -4.530896 & -0.183069 \\
\hline $\mathrm{C}$ & -2.047385 & -2.470149 & 1.263555 \\
\hline $\mathrm{C}$ & -2.809886 & -2.161958 & 2.603996 \\
\hline $\mathrm{C}$ & -4.661723 & -3.811855 & -1.140770 \\
\hline $\mathrm{C}$ & -2.736511 & -3.341112 & 0.251367 \\
\hline $\mathrm{C}$ & -2.818678 & -5.352408 & -1.094065 \\
\hline $\mathrm{C}$ & -4.215688 & -2.712993 & 2.694885 \\
\hline $\mathrm{C}$ & -1.116943 & -1.455318 & 0.672797 \\
\hline $\mathrm{C}$ & -2.646963 & -0.833986 & 3.296906 \\
\hline $\mathrm{C}$ & -2.373321 & -0.824892 & 4.669604 \\
\hline $\mathrm{C}$ & -1.633889 & -3.087490 & 2.589836 \\
\hline $\mathrm{C}$ & -5.299656 & -1.825709 & 2.608059 \\
\hline $\mathrm{C}$ & -3.988238 & -2.977686 & -0.252984 \\
\hline $\mathrm{C}$ & -4.488277 & -4.083590 & 2.783250 \\
\hline $\mathrm{C}$ & -4.083019 & -5.006103 & -1.568032 \\
\hline $\mathrm{C}$ & -6.868443 & -3.664216 & 2.622779 \\
\hline $\mathrm{C}$ & -2.254847 & 0.380970 & 5.363005 \\
\hline $\mathrm{C}$ & -2.700607 & 1.590979 & 3.321206 \\
\hline $\mathrm{C}$ & -2.827721 & 0.383319 & 2.636964 \\
\hline $\mathrm{C}$ & -5.801363 & -4.554831 & 2.747465 \\
\hline $\mathrm{C}$ & -6.610538 & -2.293597 & 2.559861 \\
\hline $\mathrm{C}$ & -2.410970 & 1.593929 & 4.687347 \\
\hline $\mathrm{C}$ & 7.556693 & 1.064780 & -1.191630 \\
\hline $\mathrm{C}$ & 5.317116 & 3.330479 & 2.671999 \\
\hline $\mathrm{C}$ & 1.745250 & -5.867667 & -0.943153 \\
\hline $\mathrm{C}$ & 6.463553 & -4.685903 & -2.250410 \\
\hline $\mathrm{C}$ & -6.011954 & -3.417816 & -1.667320 \\
\hline $\mathrm{C}$ & -2.172351 & -6.609998 & -1.605711 \\
\hline$C$ & -6.521936 & 1.948364 & 2.355372 \\
\hline
\end{tabular}

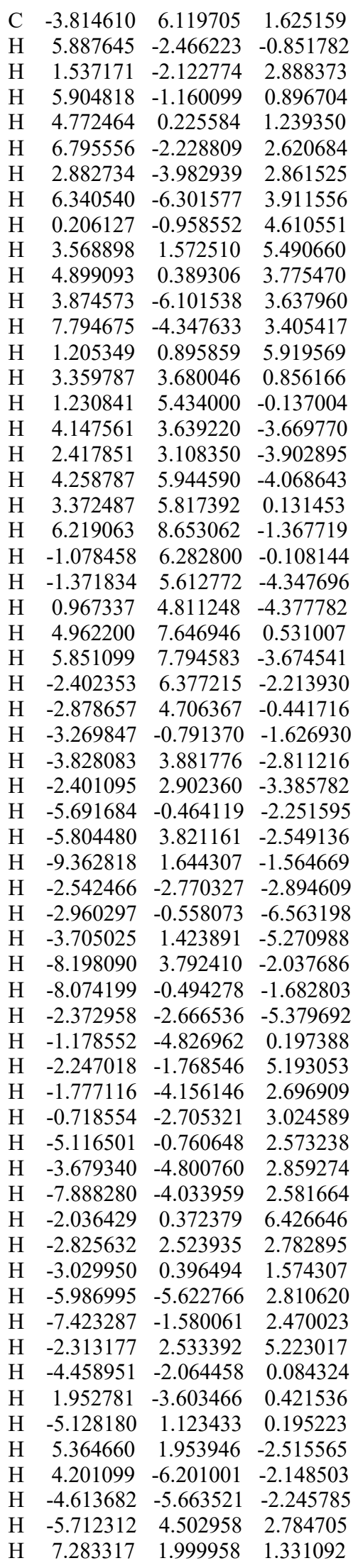

\begin{tabular}{|c|c|c|c|}
\hline \multicolumn{4}{|c|}{ U'8 } \\
\hline $\mathrm{Rh}$ & -0.430138 & -0.963318 & -1.109735 \\
\hline $\mathrm{Rh}$ & 0.437248 & -0.960722 & 1.111441 \\
\hline $\mathrm{O}$ & -1.157472 & 0.266554 & 1.612507 \\
\hline $\mathrm{O}$ & -1.542754 & 0.701654 & -0.572117 \\
\hline $\mathrm{O}$ & -0.742051 & -2.630910 & 1.448994 \\
\hline $\mathrm{O}$ & -1.931170 & -2.183618 & -0.420329 \\
\hline $\mathrm{O}$ & 1.943974 & -2.173691 & 0.421852 \\
\hline $\mathrm{O}$ & 0.757702 & -2.627756 & -1.447838 \\
\hline $\mathrm{O}$ & 1.540125 & 0.711356 & 0.572131 \\
\hline $\mathrm{O}$ & 1.157685 & 0.272225 & -1.612119 \\
\hline $\mathrm{F}$ & 1.389838 & 6.362183 & 1.387314 \\
\hline $\mathrm{F}$ & 3.090829 & 6.826604 & 2.670191 \\
\hline $\mathrm{F}$ & 1.527657 & 5.496425 & 3.375543 \\
\hline $\mathrm{F}$ & 6.932513 & 3.898851 & 2.795970 \\
\hline $\mathrm{F}$ & 6.074110 & 1.996613 & 3.391367 \\
\hline $\mathrm{F}$ & 7.046212 & 2.186491 & 1.455443 \\
\hline $\mathrm{F}$ & 5.557038 & -0.310932 & 1.929846 \\
\hline $\mathrm{F}$ & 6.494178 & -0.204688 & -0.026852 \\
\hline $\mathrm{F}$ & 7.603227 & -1.010014 & 1.669442 \\
\hline $\mathrm{F}$ & 7.312921 & -5.787338 & 2.528770 \\
\hline $\mathrm{F}$ & 7.269685 & -6.548204 & 0.493074 \\
\hline $\mathrm{F}$ & 5.591586 & -6.920337 & 1.818812 \\
\hline $\mathrm{F}$ & -6.498222 & -0.243262 & 0.025947 \\
\hline $\mathrm{F}$ & -5.556965 & -0.342688 & -1.929148 \\
\hline $\mathrm{F}$ & -7.598539 & -1.056521 & -1.672265 \\
\hline $\mathrm{F}$ & -7.273757 & -5.830600 & -2.530699 \\
\hline $\mathrm{F}$ & -7.220041 & -6.596860 & -0.497245 \\
\hline $\mathrm{F}$ & -5.540406 & -6.948758 & -1.826491 \\
\hline $\mathrm{F}$ & -7.061748 & 2.144412 & -1.452627 \\
\hline $\mathrm{F}$ & -6.958355 & 3.857190 & -2.793418 \\
\hline $\mathrm{F}$ & -6.092002 & 1.959070 & -3.390189 \\
\hline $\mathrm{F}$ & -3.132619 & 6.803536 & -2.676276 \\
\hline $\mathrm{F}$ & -1.561926 & 5.481775 & -3.380750 \\
\hline $\mathrm{F}$ & -1.428575 & 6.350432 & -1.393452 \\
\hline $\mathrm{C}$ & -2.473591 & 3.896313 & -0.690152 \\
\hline $\mathrm{C}$ & -2.591934 & 2.079129 & 1.039095 \\
\hline $\mathrm{C}$ & -2.356155 & 2.911267 & 2.350136 \\
\hline $\mathrm{C}$ & -4.953467 & 3.195327 & -1.726553 \\
\hline $\mathrm{C}$ & -3.167585 & 2.815883 & -0.137413 \\
\hline $\mathrm{C}$ & -3.034105 & 4.640680 & -1.724039 \\
\hline $\mathrm{C}$ & -2.682936 & 4.384565 & 2.241139 \\
\hline $\mathrm{C}$ & -1.693208 & 0.936505 & 0.675318 \\
\hline $\mathrm{C}$ & -1.181857 & 2.633424 & 3.251809 \\
\hline $\mathrm{C}$ & 0.122843 & 2.506422 & 2.770271 \\
\hline $\mathrm{C}$ & -3.453427 & 1.896477 & 2.279316 \\
\hline $\mathrm{C}$ & -3.992799 & 4.867177 & 2.134851 \\
\hline $\mathrm{C}$ & -4.408874 & 2.463939 & -0.667122 \\
\hline $\mathrm{C}$ & -1.627876 & 5.305590 & 2.164521 \\
\hline $\mathrm{C}$ & -4.277860 & 4.295004 & -2.252020 \\
\hline C & -3.177836 & 7.124963 & 1.809574 \\
\hline $\mathrm{C}$ & 1.188778 & 2.299693 & 3.644513 \\
\hline $\mathrm{C}$ & -0.335195 & 2.389192 & 5.515263 \\
\hline $\mathrm{C}$ & -1.400048 & 2.585382 & 4.634032 \\
\hline $\mathrm{C}$ & -1.868300 & 6.658616 & 1.939049 \\
\hline $\mathrm{C}$ & -4.238062 & 6.224285 & 1.920714 \\
\hline $\mathrm{C}$ & 0.962877 & 2.237196 & 5.021000 \\
\hline $\mathrm{C}$ & -4.660621 & -4.825065 & -0.226097 \\
\hline $\mathrm{C}$ & -2.860829 & -3.680433 & 1.159148 \\
\hline $\mathrm{C}$ & -3.095575 & -3.888010 & 2.696330 \\
\hline${ }^{2}$ & -5.840713 & -2.370202 & -0.764060 \\
\hline & -4.082490 & -3.667254 & 0.291398 \\
\hline
\end{tabular}

U'8

$\begin{array}{llll}\mathrm{Rh} & 0.437248 & -0.960722 & 1.111441\end{array}$

$\begin{array}{llll}\text { O } & -1.157472 & 0.266554 & 1.612507\end{array}$

$\begin{array}{lllll}\mathrm{O} & -1.542754 & 0.701654 & -0.572117\end{array}$

$\begin{array}{llll}\mathrm{O} & -0.742051 & -2.630910 & 1.448994\end{array}$

$\begin{array}{llll}\text { O } & -1.931170 & -2.183618 & -0.420329\end{array}$

$\begin{array}{llll}\text { O } & 1.943974 & -2.173691 & 0.421852\end{array}$

$\begin{array}{lllll}\text { O } & 0.757702 & -2.627756 & -1.447838\end{array}$

$\begin{array}{llll}\mathrm{O} & 1.540125 & 0.711356 & 0.572131\end{array}$

$\begin{array}{llll}\mathrm{O} & 1.157685 & 0.272225 & -1.612119\end{array}$

$\begin{array}{llll}\text { F } & 1.389838 & 6.362183 & 1.387314\end{array}$

$\begin{array}{llll}\text { F } & 3.090829 & 6.826604 & 2.670191\end{array}$

F $\quad 1.527657 \quad 5.496425 \quad 3.375543$

$\begin{array}{llll}F & 6.932513 & 3.898851 & 2.795970\end{array}$

$\begin{array}{llll}\mathrm{F} & 6.074110 & 1.996613 & 3.391367\end{array}$

$\begin{array}{llll}\text { F } & 7.046212 & 2.186491 & 1.455443\end{array}$

$\begin{array}{llll}\text { F } & 5.557038 & -0.310932 & 1.929846\end{array}$

F $\quad 6.494178 \quad-0.204688 \quad-0.026852$

$\begin{array}{llll}\text { F } & 7.603227 & -1.010014 & 1.669442\end{array}$

$\begin{array}{llll}\text { F } & 7.312921 & -5.787338 & 2.528770\end{array}$

F $\quad 7.269685 \quad-6.548204 \quad 0.493074$

$\begin{array}{llll}\text { F } & 5.591586 & -6.920337 & 1.818812\end{array}$

$\begin{array}{llll}\text { F } & -6.498222 & -0.243262 & 0.025947\end{array}$

$\begin{array}{llll}\text { F } & -5.556965 & -0.342688 & -1.929148\end{array}$

$\begin{array}{llll}\text { F } & -7.598539 & -1.056521 & -1.672265\end{array}$

$\begin{array}{llll}\text { F } & -7.273757 & -5.830600 & -2.530699\end{array}$

F $\quad-7.220041 \quad-6.596860 \quad-0.497245$

F $\quad-5.540406 \quad-6.948758 \quad-1.826491$

$\begin{array}{llll}\text { F } & -7.061748 & 2.144412 & -1.452627\end{array}$

$\begin{array}{llll}\text { F } & -6.958355 & 3.857190 & -2.793418\end{array}$

$\begin{array}{llll}\text { F } & -6.092002 & 1.959070 & -3.390189\end{array}$

$\begin{array}{llll}\text { F } & -3.132619 & 6.803536 & -2.676276\end{array}$

$\begin{array}{llll}\text { F } & -1.561926 & 5.481775 & -3.380750\end{array}$

$\begin{array}{lllll}\text { F } & -1.428575 & 6.350432 & -1.393452\end{array}$

$\begin{array}{lllll}\text { C } & -2.473591 & 3.896313 & -0.690152\end{array}$

$\begin{array}{llll}\text { C } & -2.591934 & 2.079129 & 1.039095\end{array}$

$\begin{array}{llll}\text { C } & -2.356155 & 2.911267 & 2.350136\end{array}$

$\begin{array}{llll}\text { C } & -4.953467 & 3.195327 & -1.726553\end{array}$

$\begin{array}{llll}\text { C } & -3.167585 & 2.815883 & -0.137413\end{array}$

$\begin{array}{llll}\text { C } & -3.034105 & 4.640680 & -1.724039\end{array}$

$\begin{array}{llll}\text { C } & -2.682936 & 4.384565 & 2.241139\end{array}$

$\begin{array}{llll}\text { C } & -1.693208 & 0.936505 & 0.675318\end{array}$

$\begin{array}{llll}\text { C } & -1.181857 & 2.633424 & 3.251809\end{array}$

$\begin{array}{llll}\text { C } & 0.122843 & 2.506422 & 2.770271\end{array}$

$\begin{array}{llll}\text { C } & -3.453427 & 1.896477 & 2.279316\end{array}$

$\begin{array}{llll}\text { C } & -3.992799 & 4.867177 & 2.134851\end{array}$

$\begin{array}{llll}\text { C } & -4.408874 & 2.463939 & -0.667122\end{array}$

$\begin{array}{llll}\text { C } & -1.627876 & 5.305590 & 2.164521\end{array}$

$\begin{array}{llll}\text { C } & -4.277860 & 4.295004 & -2.252020\end{array}$

$\begin{array}{llll}\text { C } & -3.177836 & 7.124963 & 1.809574\end{array}$

$\begin{array}{llll}\text { C } & 1.188778 & 2.299693 & 3.644513\end{array}$

$\begin{array}{llll}\text { C } & -0.335195 & 2.389192 & 5.515263\end{array}$

$\begin{array}{llll}\text { C } & -1.400048 & 2.585382 & 4.634032\end{array}$

$\begin{array}{llll}\text { C } & -1.868300 & 6.658616 & 1.939049\end{array}$

$\begin{array}{llll}\text { C } & -4.238062 & 6.224285 & 1.920714\end{array}$

$\begin{array}{llll}\text { C } & 0.962877 & 2.237196 & 5.021000\end{array}$

$\begin{array}{llll}\text { C } & -4.660621 & -4.825065 & -0.226097\end{array}$

$\begin{array}{llll}\text { C } & -2.860829 & -3.680433 & 1.159148\end{array}$

$\begin{array}{llll}\text { C } & -5.840713 & -2.370202 & -0.764060\end{array}$

$\begin{array}{llll}\text { C } & -4.082490 & -3.667254 & 0.291398\end{array}$ 


\begin{tabular}{|c|c|c|c|}
\hline C & -5.827727 & -4.753795 & -0.992969 \\
\hline $\mathrm{C}$ & -4.534349 & -4.101944 & 3.101180 \\
\hline $\mathrm{C}$ & -1.762500 & -2.767062 & 0.703471 \\
\hline $\mathrm{C}$ & -2.297086 & -3.119474 & 3.714515 \\
\hline $\mathrm{C}$ & -2.304148 & -1.722609 & 3.748580 \\
\hline C & -2.423490 & -4.943823 & 1.883922 \\
\hline $\mathrm{C}$ & -5.024010 & -5.380123 & 3.385198 \\
\hline $\mathrm{C}$ & -4.694595 & -2.438186 & 0.020127 \\
\hline $\mathrm{C}$ & -5.403229 & -3.006957 & 3.210989 \\
\hline $\mathrm{C}$ & -6.425939 & -3.528481 & -1.274753 \\
\hline $\mathrm{C}$ & -7.218996 & -4.472945 & 3.846593 \\
\hline $\mathrm{C}$ & -1.571839 & -1.029363 & 4.708803 \\
\hline $\mathrm{C}$ & -0.835343 & -3.127653 & 5.652217 \\
\hline $\mathrm{C}$ & -1.563181 & -3.816819 & 4.679873 \\
\hline $\mathrm{C}$ & -6.735097 & -3.189870 & 3.575073 \\
\hline $\mathrm{C}$ & -6.358079 & -5.566304 & 3.755854 \\
\hline $\mathrm{C}$ & -0.834712 & -1.730615 & 5.666196 \\
\hline $\mathrm{C}$ & 4.690828 & -4.800041 & 0.227338 \\
\hline $\mathrm{C}$ & 2.881201 & -3.667631 & -1.155575 \\
\hline $\mathrm{C}$ & 3.117563 & -3.877109 & -2.692197 \\
\hline $\mathrm{C}$ & 5.853496 & -2.336426 & 0.764095 \\
\hline $\mathrm{C}$ & 4.103074 & -3.646357 & -0.288480 \\
\hline $\mathrm{C}$ & 5.858868 & -4.720236 & 0.991929 \\
\hline $\mathrm{C}$ & 4.557035 & -4.088839 & -3.095978 \\
\hline $\mathrm{C}$ & 1.778471 & -2.758571 & -0.701676 \\
\hline $\mathrm{C}$ & 2.318239 & -3.109797 & -3.710438 \\
\hline $\mathrm{C}$ & 2.324793 & -1.712891 & -3.743373 \\
\hline $\mathrm{C}$ & 2.448280 & -4.933674 & -1.878428 \\
\hline $\mathrm{C}$ & 5.424645 & -2.992855 & -3.205900 \\
\hline $\mathrm{C}$ & 4.706598 & -2.412836 & -0.018154 \\
\hline $\mathrm{C}$ & 5.048622 & -5.366600 & -3.378722 \\
\hline $\mathrm{C}$ & 6.448268 & -3.490512 & 1.273265 \\
\hline $\mathrm{C}$ & 7.242832 & -4.457018 & -3.838873 \\
\hline $\mathrm{C}$ & 1.591429 & -1.019190 & -4.702368 \\
\hline $\mathrm{C}$ & 0.854921 & -3.117132 & -5.646746 \\
\hline $\mathrm{C}$ & 1.583862 & -3.806832 & -4.675589 \\
\hline $\mathrm{C}$ & 6.383224 & -5.551400 & -3.748067 \\
\hline $\mathrm{C}$ & 6.757052 & -3.174382 & -3.568754 \\
\hline $\mathrm{C}$ & 0.853717 & -1.720051 & -5.659596 \\
\hline $\mathrm{C}$ & 4.392986 & 2.492010 & 0.666535 \\
\hline $\mathrm{C}$ & 2.579517 & 2.095151 & -1.040370 \\
\hline $\mathrm{C}$ & 2.337970 & 2.924246 & -2.352287 \\
\hline $\mathrm{C}$ & 3.005334 & 4.662215 & 1.720226 \\
\hline $\mathrm{C}$ & 3.150330 & 2.836746 & 0.135360 \\
\hline $\mathrm{C}$ & 4.932465 & 3.227210 & 1.725916 \\
\hline $\mathrm{C}$ & 2.654961 & 4.399831 & -2.245246 \\
\hline $\mathrm{C}$ & 1.688821 & 0.946515 & -0.675394 \\
\hline $\mathrm{C}$ & 1.165322 & 2.637631 & -3.253371 \\
\hline $\mathrm{C}$ & 1.383335 & 2.589973 & -4.635641 \\
\hline $\mathrm{C}$ & 3.442057 & 1.916963 & -2.280512 \\
\hline $\mathrm{C}$ & 1.593782 & 5.313832 & -2.169072 \\
\hline $\mathrm{C}$ & 2.449856 & 3.913752 & 0.686553 \\
\hline $\mathrm{C}$ & 3.961636 & 4.891378 & -2.140711 \\
\hline $\mathrm{C}$ & 4.250469 & 4.323753 & 2.249713 \\
\hline $\mathrm{C}$ & 3.131756 & 7.144118 & -1.818170 \\
\hline $\mathrm{C}$ & 0.319618 & 2.385143 & -5.516283 \\
\hline $\mathrm{C}$ & -1.202986 & 2.286505 & -3.644874 \\
\hline $\mathrm{C}$ & -0.138230 & 2.501776 & -2.771239 \\
\hline $\mathrm{C}$ & 4.197941 & 6.250432 & -1.928826 \\
\hline $\mathrm{C}$ & 1.825271 & 6.668748 & -1.945743 \\
\hline $\mathrm{C}$ & -0.977147 & 2.224261 & -5.021380 \\
\hline & -6.387468 & -1.011074 & -1.088167 \\
\hline
\end{tabular}

C $\quad-6.464674 \quad-6.028812 \quad-1.468067$

$\begin{array}{llll}\text { C } & -6.266911 & 2.788345 & -2.334907\end{array}$

$\begin{array}{llll}\text { C } & -2.293851 & 5.818960 & -2.289983\end{array}$

$\begin{array}{llll}\text { C } & 2.257935 & 5.836739 & 2.284624\end{array}$

C $\quad 6.246893 \quad 2.826752 \quad 2.336392$

$\begin{array}{llll}\text { C } & 6.390828 & -0.973311 & 1.087446\end{array}$

$\begin{array}{llll}\text { C } & 6.507319 & -5.990406 & 1.464381\end{array}$

$\mathrm{H} \quad-1.518005 \quad 4.188680 \quad-0.276745$

H $\quad 0.311986 \quad 2.536492 \quad 1.705922$

H $\quad-4.487526 \quad 2.212061 \quad 2.209810$

H $\quad-3.295539 \quad 0.980935 \quad 2.835523$

$\mathrm{H} \quad-4.835688 \quad 4.189223 \quad 2.199527$

$\begin{array}{llll}\mathrm{H} & -0.610193 & 4.958642 & 2.275854\end{array}$

$\begin{array}{llll}\mathrm{H} & -3.370811 & 8.178094 & 1.630171\end{array}$

$\mathrm{H} \quad 2.191394 \quad 2.190455 \quad 3.244662$

$\begin{array}{llll}\mathrm{H} & -0.520447 & 2.349063 & 6.584401\end{array}$

$\begin{array}{llll}\mathrm{H} & -2.410408 & 2.697935 & 5.017073\end{array}$

$\mathrm{H} \quad-1.027140 \quad 7.339792 \quad 1.862049$

$\begin{array}{llll}\mathrm{H} & -5.262221 & 6.574653 & 1.835362\end{array}$

$\begin{array}{llll}\mathrm{H} & 1.791661 & 2.078476 & 5.704250\end{array}$

H $\quad-4.206993 \quad-5.791675 \quad-0.045004$

H $\quad-2.831559 \quad-1.170600 \quad 2.980385$

$\mathrm{H} \quad-2.955672 \quad-5.859868 \quad 1.658554$

$\mathrm{H} \quad-1.353366 \quad-5.058311 \quad 2.010030$

H $\quad-4.361456 \quad-6.237009 \quad 3.320765$

$\mathrm{H} \quad-5.037615 \quad-2.007427 \quad 3.002221$

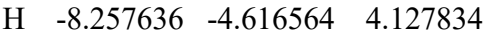

H $\quad-1.558437 \quad 0.053935 \quad 4.696043$

$\mathrm{H} \quad-0.267664 \quad-3.681648 \quad 6.394022$

H $-1.556305 \quad-4.902851 \quad 4.662529$

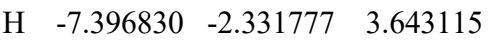

$\mathrm{H} \quad-6.721183 \quad-6.566499 \quad 3.971767$

H $\quad-0.262312 \quad-1.189673 \quad 6.413979$

H $\quad 4.244324 \quad-5.769965 \quad 0.046392$

$\mathrm{H} \quad 2.853008 \quad-1.161650 \quad-2.975011$

H $\quad 2.983207 \quad-5.847762 \quad-1.651524$

H $\quad 1.378508 \quad-5.051562 \quad-2.004380$

$\mathrm{H} \quad 5.057841 \quad-1.993613 \quad-2.997966$

H $\quad 4.387110 \quad-6.224285 \quad-3.314237$

H $\quad 8.281906 \quad-4.599535 \quad-4.119065$

$\mathrm{H} \quad 1.576842 \quad 0.064078 \quad-4.688546$

H $\quad 0.286744 \quad-3.670703 \quad-6.388479$

H $\quad 1.577283 \quad-4.892886 \quad-4.658904$

$\mathrm{H} \quad 6.747757 \quad-6.551312 \quad-3.962891$

H $\quad 7.417754 \quad-2.315493 \quad-3.636819$

H $\quad 0.280301 \quad-1.178712 \quad-6.406289$

$\mathrm{H} \quad 4.950015 \quad 1.665555 \quad 0.243811$

$\mathrm{H} \quad 2.392704 \quad 2.709526 \quad-5.019183$

H $\quad 4.474009 \quad 2.239588 \quad-2.211509$

H $3.290246 \quad 0.999663 \quad-2.835515$

$\begin{array}{llll}\mathrm{H} & 0.578379 & 4.959843 & -2.279037\end{array}$

H $\quad 4.808981 \quad 4.218978 \quad-2.205123$

H $\quad 3.317783 \quad 8.198802 \quad-1.640576$

H $\quad 0.504754 \quad 2.345323 \quad-6.585463$

H $\quad-2.204645 \quad 2.170327 \quad-3.244553$

$\mathrm{H} \quad-0.327022 \quad 2.531307 \quad-1.706845$

H $\quad 5.219793 \quad 6.607812 \quad-1.844895$

$\mathrm{H} \quad 0.979644 \quad 7.344387 \quad-1.869068$

$\mathrm{H} \quad-1.805014 \quad 2.058855 \quad-5.704164$

$\mathrm{H} \quad 1.492801 \quad 4.200024 \quad 0.272280$

$\mathrm{H} \quad-4.961044 \quad 1.634923 \quad-0.243054$

H $\quad 4.268040 \quad-1.503366 \quad-0.409473$
H $\quad-4.263517 \quad-1.525472 \quad 0.412112$

H $\quad-4.721755 \quad 4.883783 \quad-3.045229$

H $\quad 4.690325 \quad 4.915540 \quad 3.042948$

H $\quad 7.344161 \quad-3.430733 \quad 1.877357$

H $\quad-7.321164 \quad-3.475209 \quad-1.880424$

\section{U'9}

$\begin{array}{llll}\text { Rh } & 0.433187 & -0.962334 & 1.110834\end{array}$

Rh $\quad-0.433228 \quad-0.962541 \quad-1.110736$

$\begin{array}{llll}\text { O } & 1.158520 & 0.269096 & -1.611181\end{array}$

$\begin{array}{llll}\mathrm{O} & 1.541708 & 0.705802 & 0.573451\end{array}$

$\begin{array}{llll}\text { O } & 0.750577 & -2.630189 & -1.446842\end{array}$

$\begin{array}{llll}\text { O } & 1.937697 & -2.179129 & 0.422949\end{array}$

$\begin{array}{llll}\text { O } & -1.937478 & -2.179497 & -0.422592\end{array}$

$\begin{array}{llll}\text { O } & -0.750297 & -2.630177 & 1.447275\end{array}$

$\begin{array}{llll}\text { O } & -1.542011 & 0.705564 & -0.573527\end{array}$

$\begin{array}{llll}\mathrm{O} & -1.158748 & 0.269080 & 1.611133\end{array}$

$\begin{array}{llll}\text { F } & -6.083327 & 1.977489 & -3.393692\end{array}$

$\begin{array}{llll}\text { F } & -6.945243 & 3.877934 & -2.797713\end{array}$

$\begin{array}{llll}\text { F } & -7.054088 & 2.165472 & -1.456940\end{array}$

$\begin{array}{llll}\text { F } & -1.409622 & 6.356077 & -1.392073\end{array}$

$\begin{array}{llll}\text { F } & -3.110478 & 6.813595 & -2.677595\end{array}$

$\begin{array}{llll}\text { F } & -1.542323 & 5.487329 & -3.379298\end{array}$

$\begin{array}{llll}\text { F } & 1.541806 & 5.487598 & 3.379219\end{array}$

$\begin{array}{llll}\text { F } & 3.109663 & 6.813952 & 2.677043\end{array}$

$\begin{array}{llll}\text { F } & 1.408690 & 6.355991 & 1.391856\end{array}$

$\begin{array}{llll}\text { F } & 6.083069 & 1.978267 & 3.393687\end{array}$

F $\quad 6.944679 \quad 3.878782 \quad 2.797516$

$\begin{array}{llll}\text { F } & 7.053801 & 2.166214 & 1.456921\end{array}$

F $\quad-6.496488-0.222920 \quad 0.023489$

F $\quad-5.558175 \quad-0.328035 \quad-1.932706$

F $\quad-7.602179 \quad-1.033574 \quad-1.672442$

F $\quad-7.296381 \quad-5.811128-2.525580$

$\begin{array}{lllll}\text { F } & -7.254009 & -6.567798 & -0.488347\end{array}$

$\begin{array}{llll}\text { F } & -5.573239 & -6.938557 & -1.811088\end{array}$

F $\quad 7.298098 \quad-5.810489 \quad 2.524676$

F $\quad 7.255337 \quad-6.566639 \quad 0.487255$

F $\quad 5.575008 \quad-6.938111 \quad 1.810365$

F $\quad 6.497148 \quad-0.222295 \quad-0.023133$

$\begin{array}{llll}\text { F } & 7.601852 & -1.032491 & 1.673669\end{array}$

F $\quad 5.557621 \quad-0.327050 \quad 1.932482$

$\begin{array}{llll}\text { C } & 4.400891 & 2.477881 & 0.669546\end{array}$

$\begin{array}{llll}\text { C } & 2.586520 & 2.086738 & -1.037800\end{array}$

$\begin{array}{llll}\text { C } & 2.348688 & 2.917344 & -2.349470\end{array}$

$\begin{array}{llll}\text { C } & 3.018750 & 4.650887 & 1.724515\end{array}$

C $3.159032 \quad 2.825931 \quad 0.138653$

$\begin{array}{llll}\text { C } & 4.942413 & 3.211268 & 1.729145\end{array}$

$\begin{array}{llll}\text { C } & 2.670519 & 4.391803 & -2.241230\end{array}$

$\begin{array}{llll}\text { C } & 1.691634 & 0.941056 & -0.673958\end{array}$

$\begin{array}{llll}\text { C } & 1.175732 & 2.635247 & -3.251581\end{array}$

C $\quad 1.394552 \quad 2.587936 \quad-4.633732$

$\begin{array}{llll}\text { C } & 3.449290 & 1.906279 & -2.277458\end{array}$

$\begin{array}{llll}\text { C } & 1.612365 & 5.309356 & -2.165624\end{array}$

$\begin{array}{llll}\text { C } & 2.461359 & 3.904466 & 0.690398\end{array}$

$\begin{array}{llll}\text { C } & 3.978731 & 4.878880 & -2.135050\end{array}$

$\begin{array}{llll}\text { C } & 4.263079 & 4.309116 & 2.253708\end{array}$

C $\quad 3.156123 \quad 7.134205 \quad-1.811855$

$\begin{array}{llll}\text { C } & 0.330703 & 2.387911 & -5.515312\end{array}$

$\begin{array}{llll}\text { C } & -1.193544 & 2.292970 & -3.645084\end{array}$

$\begin{array}{llll}\text { C } & -0.128655 & 2.503605 & -2.770489\end{array}$

$\begin{array}{llll}\text { C } & 4.219393 & 6.236982 & -1.921971\end{array}$

C $\quad 1.848183 \quad 6.663360-1.941203$ 


\begin{tabular}{|c|c|c|c|}
\hline C & -0.966992 & 2.231285 & -5.021487 \\
\hline 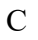 & 4.701321 & -2.424393 & -0.016459 \\
\hline & 2.872429 & -3.673260 & -1.156214 \\
\hline C & 3.107388 & -3.881067 & -2.693419 \\
\hline C & 5.847994 & -4.736680 & 0.988834 \\
\hline 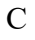 & 4.094658 & -3.655795 & -0.289334 \\
\hline C & 5.848846 & -2.352553 & 0.765395 \\
\hline C & 4.546427 & -4.092530 & -3.098618 \\
\hline $\mathrm{C}$ & 1.771227 & -2.763093 & -0.700962 \\
\hline$C$ & 2.307069 & -3.114586 & -3.711728 \\
\hline $\mathrm{C}$ & 2.312377 & -1.717746 & -3.746970 \\
\hline C & 2.438043 & -4.938086 & -1.880331 \\
\hline $\mathrm{C}$ & 5.037028 & -5.369749 & -3.385385 \\
\hline $\mathrm{C}$ & 4.679595 & -4.811930 & 0.224405 \\
\hline $\mathrm{C}$ & 5.414391 & -2.996613 & -3.206489 \\
\hline C & 6.440847 & -3.509062 & 1.272139 \\
\hline $\mathrm{C}$ & 7.231062 & -4.459881 & -3.845842 \\
\hline $\mathrm{C}$ & 1.577787 & -1.026143 & -4.706616 \\
\hline $\mathrm{C}$ & 0.842799 & -3.126110 & -5.647481 \\
\hline $\mathrm{C}$ & 1.572817 & -3.813613 & -4.675601 \\
\hline $\mathrm{C}$ & 6.746239 & -3.177754 & -3.571554 \\
\hline $\mathrm{C}$ & 6.371088 & -5.554140 & -3.756932 \\
\hline $\mathrm{C}$ & 0.840244 & -1.729068 & -5.662451 \\
\hline $\mathrm{C}$ & -4.678654 & -4.812517 & -0.22445 \\
\hline $\mathrm{C}$ & -2.871991 & -3.673626 & 1.156650 \\
\hline $\mathrm{C}$ & -3.107052 & -3.881409 & 2.693824 \\
\hline $\mathrm{C}$ & -5.848504 & -2.353312 & -0.765002 \\
\hline $\mathrm{C}$ & -4.094142 & -3.656362 & 0.289652 \\
\hline $\mathrm{C}$ & -5.846946 & -4.737397 & -0.989077 \\
\hline $\mathrm{C}$ & -4.546075 & -4.093136 & 3.098953 \\
\hline $\mathrm{C}$ & -1.770912 & -2.763313 & 0.701376 \\
\hline $\mathrm{C}$ & -2.306912 & -3.114690 & 3.712091 \\
\hline $\mathrm{C}$ & -1.572621 & -3.813453 & 4.676116 \\
\hline $\mathrm{C}$ & -2.437463 & -4.938360 & 1.880827 \\
\hline $\mathrm{C}$ & -5.036545 & -5.370478 & 3.385399 \\
\hline $\mathrm{C}$ & -4.701103 & -2.425043 & 0.017020 \\
\hline $\mathrm{C}$ & -5.414175 & -2.997353 & 3.207030 \\
\hline $\mathrm{C}$ & -6.440081 & -3.509875 & -1.272169 \\
\hline $\mathrm{C}$ & -7.230714 & -4.460975 & 3.845965 \\
\hline $\mathrm{C}$ & -0.842744 & -3.125675 & 5.647932 \\
\hline $\mathrm{C}$ & -1.577953 & -1.025973 & 4.706644 \\
\hline $\mathrm{C}$ & -2.312387 & -1.717839 & 3.747071 \\
\hline $\mathrm{C}$ & -6.746027 & -3.178732 & 3.571989 \\
\hline $\mathrm{C}$ & -6.370603 & -5.555096 & 3.756850 \\
\hline $\mathrm{C}$ & -0.840376 & -1.728637 & 5.662650 \\
\hline $\mathrm{C}$ & -4.401221 & 2.477495 & -0.669701 \\
\hline $\mathrm{C}$ & -2.586827 & 2.086608 & 1.037660 \\
\hline $\mathrm{C}$ & -2.349040 & 2.917252 & 2.349288 \\
\hline $\mathrm{C}$ & -3.019387 & 4.650689 & -1.724692 \\
\hline $\mathrm{C}$ & -3.159407 & 2.825735 & -0.138808 \\
\hline $\mathrm{C}$ & -4.942866 & 3.210828 & -1.729266 \\
\hline $\mathrm{C}$ & -2.670980 & 4.391693 & 2.241066 \\
\hline $\mathrm{C}$ & -1.691917 & 0.940929 & 0.673855 \\
\hline $\mathrm{C}$ & -1.176051 & 2.635269 & 3.251399 \\
\hline $\mathrm{C}$ & -1.394839 & 2.588060 & 4.633553 \\
\hline $\mathrm{C}$ & -3.449601 & 1.906127 & 2.277322 \\
\hline $\mathrm{C}$ & -3.979227 & 4.878697 & 2.135025 \\
\hline $\mathrm{C}$ & -2.461876 & 3.904340 & -0.690571 \\
\hline $\mathrm{C}$ & -1.612882 & 5.309301 & 2.165341 \\
\hline $\mathrm{C}$ & -4.263704 & 4.308781 & -2.253834 \\
\hline $\mathrm{C}$ & -3.156784 & 7.134072 & 1.811810 \\
\hline $\mathrm{C}$ & -0.330964 & 2.388135 & 5.515125 \\
\hline
\end{tabular}

\begin{tabular}{|c|c|c|c|}
\hline C & 1.193247 & 2.293106 & 3.644863 \\
\hline $\mathrm{C}$ & 0.128333 & 2.503651 & 2.770278 \\
\hline $\mathrm{C}$ & -1.848802 & 6.663297 & 1.940991 \\
\hline C & -4.219987 & 6.236795 & 1.922019 \\
\hline C & 0.966732 & 2.231511 & 5.021278 \\
\hline & 6.493129 & -6.009402 & 1.459002 \\
\hline & 6.390176 & -0.991442 & 1.090473 \\
\hline C & 2.274288 & 5.826975 & 2.289507 \\
\hline $\mathrm{C}$ & 6.256511 & 2.808035 & 2.338557 \\
\hline $\mathrm{C}$ & -2.275018 & 5.826802 & -2.289758 \\
\hline C & -6.256905 & 2.807341 & -2.338645 \\
\hline $\mathrm{C}$ & -6.390148 & -0.992303 & -1.090008 \\
\hline $\mathrm{C}$ & -6.491664 & -6.010145 & -1.459744 \\
\hline $\mathrm{H}$ & 4.955826 & 1.650289 & 0.246303 \\
\hline H & 2.404633 & 2.704140 & -5.016440 \\
\hline $\mathrm{H}$ & 4.482317 & 2.225319 & -2.207596 \\
\hline $\mathrm{H}$ & 3.294694 & 0.989868 & -2.833156 \\
\hline $\mathrm{H}$ & 0.595903 & 4.958921 & -2.277039 \\
\hline $\mathrm{H}$ & 4.823895 & 4.203705 & -2.199079 \\
\hline $\mathrm{H}$ & 3.345535 & 8.188126 & -1.633354 \\
\hline $\mathrm{H}$ & 0.516454 & 2.348434 & -6.584400 \\
\hline $\mathrm{H}$ & -2.195874 & 2.180031 & -3.245537 \\
\hline $\mathrm{H}$ & -0.318254 & 2.532984 & -1.706216 \\
\hline $\mathrm{H}$ & 5.242361 & 6.590866 & -1.836723 \\
\hline $\mathrm{H}$ & 1.004737 & 7.341783 & -1.865037 \\
\hline $\mathrm{H}$ & -1.794986 & 2.069576 & -5.705000 \\
\hline $\mathrm{H}$ & 4.265100 & -1.512843 & -0.405386 \\
\hline $\mathrm{H}$ & 2.840236 & -1.164430 & -2.980035 \\
\hline $\mathrm{H}$ & 2.972395 & -5.852855 & -1.654880 \\
\hline $\mathrm{H}$ & 1.368121 & -5.055073 & -2.005873 \\
\hline $\mathrm{H}$ & 4.375119 & -6.227257 & -3.322431 \\
\hline $\mathrm{H}$ & 5.048232 & -1.997802 & -2.995242 \\
\hline $\mathrm{H}$ & 8.269707 & -4.602089 & -4.127805 \\
\hline $\mathrm{H}$ & 1.562725 & 0.057154 & -4.694453 \\
\hline $\mathrm{H}$ & 0.274853 & -3.681398 & -6.388121 \\
\hline $\mathrm{H}$ & 1.567258 & -4.899641 & -4.657388 \\
\hline $\mathrm{H}$ & 7.407281 & -2.319005 & -3.638101 \\
\hline $\mathrm{H}$ & 6.734921 & -6.553610 & -3.974957 \\
\hline $\mathrm{H}$ & 0.266050 & -1.189441 & -6.409806 \\
\hline $\mathrm{H}$ & -4.229403 & -5.780889 & -0.042141 \\
\hline $\mathrm{H}$ & -1.566909 & -4.899485 & 4.658099 \\
\hline $\mathrm{H}$ & -2.971695 & -5.853192 & 1.655327 \\
\hline $\mathrm{H}$ & -1.367540 & -5.055214 & 2.006472 \\
\hline $\mathrm{H}$ & -4.374549 & -6.227890 & 3.322304 \\
\hline $\mathrm{H}$ & -5.048150 & -1.998443 & 2.996054 \\
\hline $\mathrm{H}$ & -8.269354 & -4.603368 & 4.127838 \\
\hline $\mathrm{H}$ & -0.274774 & -3.680758 & 6.388694 \\
\hline $\mathrm{H}$ & -1.563007 & 0.057331 & 4.694285 \\
\hline $\mathrm{H}$ & -2.840273 & -1.164735 & 2.979994 \\
\hline $\mathrm{H}$ & -7.407166 & -2.320060 & 3.638707 \\
\hline $\mathrm{H}$ & -6.734332 & -6.554667 & 3.974634 \\
\hline $\mathrm{H}$ & -0.266302 & -1.188804 & 6.409941 \\
\hline $\mathrm{H}$ & -4.956027 & 1.649816 & -0.246455 \\
\hline $\mathrm{H}$ & -2.404911 & 2.704246 & 5.016278 \\
\hline $\mathrm{H}$ & -4.482633 & 2.225107 & 2.207445 \\
\hline $\mathrm{H}$ & -3.294948 & 0.989727 & 2.833041 \\
\hline $\mathrm{H}$ & -4.824338 & 4.203472 & 2.199129 \\
\hline $\mathrm{H}$ & -0.596376 & 4.958916 & 2.276626 \\
\hline $\mathrm{H}$ & -3.346263 & 8.188002 & 1.633368 \\
\hline $\mathrm{H}$ & -0.516685 & 2.348731 & 6.584217 \\
\hline $\mathrm{H}$ & 2.195572 & 2.180175 & 3.245300 \\
\hline$\pi$ & 0.317891 & 2.532972 & 1.705999 \\
\hline
\end{tabular}

$\begin{array}{rrrc}\mathrm{H} & -1.005406 & 7.341774 & 1.864742 \\ \mathrm{H} & -5.242985 & 6.590611 & 1.836891 \\ \mathrm{H} & 1.794752 & 2.069880 & 5.704788 \\ \mathrm{H} & -1.505711 & 4.193654 & -0.276377 \\ \mathrm{H} & 1.505169 & 4.193672 & 0.276175 \\ \mathrm{H} & -4.265175 & -1.513483 & 0.406245 \\ \mathrm{H} & 4.230589 & -5.780378 & 0.041920 \\ \mathrm{H} & 4.704410 & 4.899514 & 3.047142 \\ \mathrm{H} & -4.705125 & 4.899133 & -3.047255 \\ \mathrm{H} & -7.336322 & -3.453665 & -1.876083 \\ \mathrm{H} & 7.337177 & -3.452761 & 1.875921\end{array}$

\section{U'10}

$\begin{array}{llll}\mathrm{Rh} & -0.326046 & 0.110411 & -1.461963\end{array}$ $\begin{array}{llll}\mathrm{Rh} & -0.378660 & -1.020535 & 0.641930\end{array}$

$\begin{array}{llll}\mathrm{O} & 0.502752 & 0.658595 & 1.455088\end{array}$

$\begin{array}{llll}\text { O } & 1.037425 & 1.417502 & -0.604548\end{array}$

$\begin{array}{llll}\mathrm{O} & -2.241382 & -0.184837 & 0.940825\end{array}$

$\begin{array}{llll}\text { O } & -1.869679 & 1.266964 & -0.752413\end{array}$

O $\quad-1.382683 \quad-2.557313 \quad-0.295550$

$\begin{array}{llll}\mathrm{O} & -1.696034 & -1.272705 & -2.138285\end{array}$

$\begin{array}{llll}\text { O } & 1.491042 & -1.764922 & 0.125912\end{array}$

O $\quad 1.190723 \quad-1.157268 \quad-2.031384$

F $\quad 7.064339 \quad-1.029150 \quad 1.618589$

F $\quad 5.974196 \quad-1.451880 \quad 3.450422$

$\begin{array}{llll}\text { F } & 7.515113 & -2.823084 & 2.774744\end{array}$

$\begin{array}{llll}\text { F } & 2.473625 & -5.953377 & 2.449359\end{array}$

F $\quad 4.508352 \quad-6.718179 \quad 2.555396$

F $\quad 3.352797 \quad-6.919795 \quad 0.716691$

F $\quad-4.003021 \quad-3.291535 \quad 3.203532$

F $\quad-6.021920 \quad-2.526846 \quad 3.513662$

$\begin{array}{llll}\mathrm{F} & -5.718771 & -4.579225 & 2.867088\end{array}$

F $\quad-7.374412 \quad 0.305430 \quad-0.875197$

$\begin{array}{lllll}\text { F } & -8.769712 & -1.301677 & -0.440649\end{array}$

F $\quad-7.811355 \quad-1.185996-2.394545$

F $\quad-6.988326 \quad 3.667388 \quad-2.776342$

F $\quad-6.233759 \quad 5.659453 \quad-3.237948$

F $\quad-7.317672 \quad 5.303285 \quad-1.388026$

$\begin{array}{llll}\text { F } & -0.739310 & 5.326314 & -1.752373\end{array}$

$\begin{array}{llll}\mathrm{F} & -1.932990 & 7.117937 & -1.423001\end{array}$

$\begin{array}{llll}\text { F } & 5.965927 & 2.747999 & -2.872322\end{array}$

$\begin{array}{llll}\text { F } & 6.879434 & 4.409961 & -1.816893\end{array}$

$\begin{array}{llll}\text { F } & 6.683602 & 2.470706 & -0.839651\end{array}$

F $\quad 3.177830 \quad 7.542588 \quad-1.810328$

$\begin{array}{llll}\text { F } & 1.350548 & 7.083916 & -0.718553\end{array}$

F $\quad 1.611265 \quad 6.380176 \quad-2.760496$

$\begin{array}{llll}\text { F } & -1.090557 & 6.031229 & 0.270899\end{array}$

$\begin{array}{llll}\text { C } & 2.206735 & 4.493345 & -0.173699\end{array}$

$\begin{array}{llll}\text { C } & 2.081954 & 2.427304 & 1.263619\end{array}$

$\begin{array}{llll}\text { C } & 2.839364 & 2.106297 & 2.603699\end{array}$

C $\quad 4.712842 \quad 3.753329-1.132158$

$\begin{array}{llll}\text { C } & 2.781463 & 3.295428 & 0.255770\end{array}$

$\begin{array}{llll}\text { C } & 2.885670 & 5.312393 & -1.079943\end{array}$

$\begin{array}{llll}\text { C } & 4.251338 & 2.640308 & 2.697494\end{array}$

$\begin{array}{llll}\text { C } & 1.141162 & 1.425272 & 0.667544\end{array}$

$\begin{array}{llll}\text { C } & 2.659929 & 0.779245 & 3.294708\end{array}$

$\begin{array}{llll}\text { C } & 2.819307 & -0.440320 & 2.633358\end{array}$

$\begin{array}{llll}\text { C } & 1.674141 & 3.045363 & 2.591368\end{array}$

$\begin{array}{llll}\text { C } & 5.324223 & 1.740519 & 2.603039\end{array}$

$\begin{array}{llll}\text { C } & 4.029969 & 2.921704 & -0.249091\end{array}$

$\begin{array}{llll}\text { C } & 4.540299 & 4.006691 & 2.796579\end{array}$

$\begin{array}{llll}\text { C } & 4.146878 & 4.955412 & -1.554266\end{array}$ 


\begin{tabular}{|c|c|c|c|}
\hline E & 6.915181 & 3.559528 & 2.632788 \\
\hline $\mathrm{C}$ & 2.673222 & -1.646223 & 3.317310 \\
\hline $\mathrm{C}$ & 2.255056 & -0.430426 & 5.361642 \\
\hline & 2.390729 & 0.773638 & 4.668418 \\
\hline & 5.859096 & 4.462147 & 2.764473 \\
\hline $\mathrm{C}$ & 6.640693 & 2.192627 & 2.558654 \\
\hline & 2.388198 & -1.645216 & 4.684410 \\
\hline $\mathrm{C}$ & -5.032580 & 3.235509 & -0.823846 \\
\hline $\mathrm{C}$ & -3.704092 & 1.674518 & 0.679093 \\
\hline & -4.125751 & 1.702524 & 2.186236 \\
\hline & -2.899108 & 5.020491 & -0.866667 \\
\hline $\mathrm{C}$ & -3.875956 & 2.934285 & -0.108708 \\
\hline $\mathrm{C}$ & -5.124924 & 4.435084 & -1.538589 \\
\hline $\mathrm{C}$ & -4.723547 & 3.002167 & 2.667161 \\
\hline & -2.518777 & 0.858030 & 0.268089 \\
\hline $\mathrm{C}$ & -3.296326 & 0.990827 & 3.221094 \\
\hline $\mathrm{C}$ & -3.870650 & -0.043850 & 3.965212 \\
\hline $\mathrm{C}$ & -4.898591 & 0.894832 & 1.199005 \\
\hline $\mathrm{C}$ & -6.105338 & 3.139975 & 2.831264 \\
\hline C & -2.814553 & 3.845262 & -0.130817 \\
\hline $\mathrm{C}$ & -3.896804 & 4.091816 & 2.975309 \\
\hline $\mathrm{C}$ & -4.061212 & 5.333812 & -1.573060 \\
\hline $\mathrm{C}$ & -5.824249 & 5.426484 & 3.570358 \\
\hline $\mathrm{C}$ & -3.125774 & -0.722558 & 4.930971 \\
\hline $\mathrm{C}$ & -1.220911 & 0.676470 & 4.434338 \\
\hline $\mathrm{C}$ & -1.968803 & 1.351061 & 3.472671 \\
\hline $\mathrm{C}$ & -4.441058 & 5.295218 & 3.418220 \\
\hline $\mathrm{C}$ & -6.653964 & 4.343628 & 3.280150 \\
\hline $\mathrm{C}$ & -1.796391 & -0.365469 & 5.166304 \\
\hline $\mathrm{C}$ & -5.411226 & -2.177825 & -1.411312 \\
\hline $\mathrm{C}$ & -3.113096 & -3.144191 & -1.804761 \\
\hline $\mathrm{C}$ & -2.807775 & -4.552985 & -2.345035 \\
\hline $\mathrm{C}$ & -5.314218 & -2.877024 & 1.278677 \\
\hline $\mathrm{C}$ & -4.292344 & -2.852583 & -0.919561 \\
\hline $\mathrm{C}$ & -6.472020 & -1.858052 & -0.560191 \\
\hline $\mathrm{C}$ & -3.793117 & -5.662809 & -2.068127 \\
\hline $\mathrm{C}$ & -1.952854 & -2.271921 & -1.396150 \\
\hline $\mathrm{C}$ & -1.362664 & -4.992592 & -2.384449 \\
\hline C & -0.747947 & -5.502512 & -1.230872 \\
\hline $\mathrm{C}$ & -3.269426 & -3.445745 & -3.265193 \\
\hline $\mathrm{C}$ & -4.497326 & -6.238136 & -3.133057 \\
\hline $\mathrm{C}$ & -4.241815 & -3.173510 & 0.441595 \\
\hline $\mathrm{C}$ & -3.994785 & -6.173954 & -0.778908 \\
\hline C & -6.442785 & -2.222225 & 0.784500 \\
\hline $\mathrm{C}$ & -5.589551 & -7.783619 & -1.627105 \\
\hline $\mathrm{C}$ & 0.568434 & -5.957135 & -1.263557 \\
\hline $\mathrm{C}$ & 0.705083 & -5.381380 & -3.603278 \\
\hline $\mathrm{C}$ & -0.619902 & -4.937920 & -3.569124 \\
\hline C & -4.888373 & -7.221760 & -0.558354 \\
\hline $\mathrm{C}$ & -5.388166 & -7.290440 & -2.917275 \\
\hline $\mathrm{C}$ & 1.301759 & -5.896690 & -2.452509 \\
\hline $\mathrm{C}$ & 3.530604 & -4.177300 & 0.158042 \\
\hline $\mathrm{C}$ & 3.193089 & -2.287526 & -1.435060 \\
\hline $\mathrm{C}$ & 4.046524 & -1.701118 & -2.614246 \\
\hline $\mathrm{C}$ & 5.482551 & -2.793636 & 1.563757 \\
\hline $\mathrm{C}$ & 3.891133 & -2.897401 & -0.256021 \\
\hline $\mathrm{C}$ & 4.131364 & -4.756199 & 1.276674 \\
\hline $\mathrm{C}$ & 5.546170 & -1.736625 & -2.413711 \\
\hline $\mathrm{C}$ & 1.863592 & -1.689714 & -1.093628 \\
\hline $\mathrm{C}$ & 3.558666 & -0.487978 & -3.361899 \\
\hline $\mathrm{C}$ & 3.462064 & -0.540703 & -4.756289 \\
\hline$C$ & 3.286297 & -2.976665 & -2.788311 \\
\hline
\end{tabular}

$\begin{array}{lrrrrrrr}\mathrm{C} & 6.270280 & -2.934441 & -2.351507 & \mathrm{H} & 5.776350 & -3.884944 & -2.514736 \\ \mathrm{C} & 4.859961 & -2.198435 & 0.469195 & \mathrm{H} & 5.711801 & 0.405537 & -2.280023 \\ \mathrm{C} & 6.238390 & -0.534727 & -2.201992 & \mathrm{H} & 9.357991 & -1.734468 & -1.559005 \\ \mathrm{C} & 5.122869 & -4.076673 & 1.980659 & \mathrm{H} & 2.979059 & 0.524923 & -6.563426 \\ \mathrm{C} & 8.300851 & -1.732779 & -1.805875 & \mathrm{H} & 2.581138 & 2.738020 & -2.893040 \\ \mathrm{C} & 3.051355 & 0.579419 & -5.481259 & \mathrm{H} & 3.289293 & 0.750727 & -1.626974 \\ \mathrm{C} & 2.833646 & 1.826901 & -3.424096 & \mathrm{H} & 8.093802 & 0.416401 & -1.708922 \\ \mathrm{C} & 3.249036 & 0.706657 & -2.707397 & \mathrm{H} & 8.169258 & -3.875846 & -2.002576 \\ \mathrm{C} & 7.596283 & -0.530806 & -1.889820 & \mathrm{H} & 2.411453 & 2.637886 & -5.378329 \\ \mathrm{C} & 7.632811 & -2.933089 & -2.051201 & \mathrm{H} & 5.150323 & -1.202370 & 0.163728 \\ \mathrm{C} & 2.733618 & 1.766638 & -4.815873 & \mathrm{H} & 4.491029 & 2.002033 & 0.084028 \\ \mathrm{C} & -1.675508 & 5.886415 & -0.944148 & \mathrm{H} & -3.351765 & -3.635207 & 0.846910 \\ \mathrm{C} & -6.411331 & 4.766712 & -2.240293 & \mathrm{H} & -1.904757 & 3.620185 & 0.411940 \\ \mathrm{C} & 6.059881 & 3.348880 & -1.658889 & \mathrm{H} & 4.685127 & 5.610674 & -2.228104 \\ \mathrm{C} & 2.253851 & 6.579512 & -1.586135 & \mathrm{H} & 5.604799 & -4.536255 & 2.834695 \\ \mathrm{C} & 6.511698 & -2.028379 & 2.345765 & \mathrm{H} & -7.269613 & -1.978245 & 1.439766 \\ \mathrm{C} & 3.629086 & -6.088230 & 1.751206 & \mathrm{H} & -4.130893 & 6.251334 & -2.143034\end{array}$

\section{U'11}

$\begin{array}{llll}\mathrm{Rh} & 0.367718 & 1.012331 & 0.664062\end{array}$ $\begin{array}{lllll}\mathrm{Rh} & 0.335781 & -0.108543 & -1.445770\end{array}$

$\begin{array}{llll}\text { O } & 1.677533 & 1.305778 & -2.114423\end{array}$

O $\quad 1.348354 \quad 2.570153 \quad-0.260122$

$\begin{array}{llll}\text { O } & 1.895471 & -1.245757 & -0.743448\end{array}$

$\begin{array}{llll}\text { O } & 2.241079 & 0.199458 & 0.960926\end{array}$

$\begin{array}{llll}\mathrm{O} & -1.009806 & -1.440964 & -0.597740\end{array}$

$\begin{array}{llll}\text { O } & -0.490043 & -0.683704 & 1.466328\end{array}$

$\begin{array}{llll}\text { O } & -1.196458 & 1.135269 & -2.013706\end{array}$

$\begin{array}{llll}\mathrm{O} & -1.512530 & 1.735642 & 0.143758\end{array}$

F $\quad 4.069656 \quad 3.298818 \quad 3.194576$

$\begin{array}{llll}\text { F } & 5.777003 & 4.590015 & 2.829811\end{array}$

$\begin{array}{llll}\text { F } & 6.095239 & 2.529001 & 3.441353\end{array}$

F $\quad 8.740663 \quad 1.376612 \quad-0.625141$

$\begin{array}{llll}\text { F } & 7.349033 & -0.259750 & -0.943203\end{array}$

F $\quad \begin{array}{llll}7.691054 & 1.190992 & -2.526017\end{array}$

F $\quad \begin{array}{llll}1.157099 & -6.012999 & 0.272011\end{array}$

F $\quad 0.808326 \quad-5.328697 \quad-1.758890$

$\begin{array}{llll}\text { F } & 2.022478 & -7.102244 & -1.408543\end{array}$

F $\quad 6.301027 \quad-5.610226 \quad-3.225948$

$\begin{array}{llll}\text { F } & 7.400838 & -5.146539 & -1.408937\end{array}$

$\begin{array}{llll}\text { F } & 6.981826 & -3.574914 & -2.845817\end{array}$

$\begin{array}{llll}\text { F } & -3.755277 & 6.950141 & 0.553689\end{array}$

$\begin{array}{llll}\text { F } & -2.576584 & 6.108939 & 2.167248\end{array}$

$\begin{array}{llll}\text { F } & -4.647410 & 6.681003 & 2.526200\end{array}$

$\begin{array}{llll}\text { F } & -7.055247 & 0.927058 & 1.634849\end{array}$

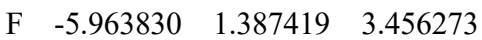

F $\quad-7.543085 \quad 2.716018 \quad 2.783698$

$\begin{array}{llll}\text { F } & -6.643159 & -2.544272 & -0.842815\end{array}$

F $\quad-6.823259 \quad-4.482294 \quad-1.825742$

F $\quad-1.267183 \quad-7.109310 \quad-0.740583$

F $\quad-1.530844 \quad-6.395389-2.778561$

$\begin{array}{lllll}\text { F } & -3.087404 & -7.579884 & -1.839268\end{array}$

$\begin{array}{llll}\text { F } & -5.923126 & -2.809773 & -2.876109\end{array}$

C $\quad 5.352362 \quad 2.233200 \quad-1.467942$

C $\quad 3.045895 \quad 3.211487 \quad-1.785913$

$\begin{array}{llll}\text { C } & 2.717334 & 4.632067 & -2.274954\end{array}$

$\begin{array}{llll}\text { C } & 5.329422 & 2.907926 & 1.230952\end{array}$

$\begin{array}{llll}\text { C } & 4.250676 & 2.908942 & -0.939781\end{array}$

$\begin{array}{llll}\text { C } & 6.433041 & 1.900409 & -0.647703\end{array}$

$\begin{array}{llll}\text { C } & 3.708397 & 5.737040 & -1.994218\end{array}$

$\begin{array}{llll}\text { C } & 1.912980 & 2.307854 & -1.368990\end{array}$

$\begin{array}{llll}\text { C } & 1.272451 & 5.072552 & -2.258284\end{array}$ 


\begin{tabular}{|c|c|c|c|}
\hline & 0.519586 & 5.128344 & -3.436361 \\
\hline $\mathrm{C}$ & 3.154318 & 3.554451 & -3.241680 \\
\hline $\mathrm{C}$ & 4.419028 & 6.314316 & -3.053552 \\
\hline $\mathrm{C}$ & 4.236968 & 3.217421 & 0.424766 \\
\hline $\mathrm{C}$ & 3.909476 & 6.240628 & -0.701756 \\
\hline $\mathrm{C}$ & 6.440950 & 2.252856 & 0.700707 \\
\hline $\mathrm{C}$ & 5.518825 & 7.843015 & -1.535733 \\
\hline $\mathrm{C}$ & -0.800611 & 5.587646 & -3.419601 \\
\hline $\mathrm{C}$ & -0.637626 & 5.956263 & -1.039595 \\
\hline & 0.672404 & 5.483461 & -1.058477 \\
\hline $\mathrm{C}$ & 4.810000 & 7.280181 & -0.472327 \\
\hline $\mathrm{C}$ & 5.316857 & 7.359183 & -2.829059 \\
\hline $\mathrm{C}$ & -1.380443 & 6.010508 & -2.223111 \\
\hline $\mathrm{C}$ & 5.069684 & -3.176189 & -0.828173 \\
\hline & 3.730574 & -1.638479 & 0.689369 \\
\hline $\mathrm{C}$ & 4.156315 & -1.665321 & 2.194934 \\
\hline $\mathrm{C}$ & 2.959471 & -4.988736 & -0.864365 \\
\hline $\mathrm{C}$ & 3.914623 & -2.893348 & -0.103688 \\
\hline $\mathrm{C}$ & 5.172846 & -4.371813 & -1.548660 \\
\hline $\mathrm{C}$ & 4.771468 & -2.958508 & 2.671543 \\
\hline $\mathrm{C}$ & 2.535203 & -0.834791 & 0.281960 \\
\hline $\mathrm{C}$ & 3.319598 & -0.966300 & 3.232698 \\
\hline $\mathrm{C}$ & 3.879361 & 0.077642 & 3.975002 \\
\hline $\mathrm{C}$ & 4.917418 & -0.846130 & 1.207883 \\
\hline $\mathrm{C}$ & 6.154961 & -3.077711 & 2.835798 \\
\hline $\mathrm{C}$ & 2.864585 & -3.817953 & -0.123150 \\
\hline $\mathrm{C}$ & 3.959757 & -4.060336 & 2.976317 \\
\hline $\mathrm{C}$ & 4.121234 & -5.284169 & -1.579663 \\
\hline $\mathrm{C}$ & 5.905209 & -5.369928 & 3.568409 \\
\hline $\mathrm{C}$ & 3.125755 & 0.745115 & 4.941914 \\
\hline $\mathrm{C}$ & 1.242219 & -0.684444 & 4.450806 \\
\hline $\mathrm{C}$ & 1.998474 & -1.347389 & 3.487628 \\
\hline $\mathrm{C}$ & 4.520389 & -5.257325 & 3.416112 \\
\hline $\mathrm{C}$ & 6.720015 & -4.274939 & 3.281480 \\
\hline $\mathrm{C}$ & 1.802714 & 0.367209 & 5.180560 \\
\hline $\mathrm{C}$ & -3.987786 & -2.976800 & -0.251061 \\
\hline $\mathrm{C}$ & -2.046576 & -2.470453 & 1.264778 \\
\hline $\mathrm{C}$ & -2.808870 & -2.162688 & 2.605450 \\
\hline $\mathrm{C}$ & -2.819651 & -5.351457 & -1.094072 \\
\hline $\mathrm{C}$ & -2.735982 & -3.341041 & 0.252518 \\
\hline $\mathrm{C}$ & -4.662029 & -3.810061 & -1.139027 \\
\hline $\mathrm{C}$ & -4.214129 & -2.715057 & 2.697137 \\
\hline $\mathrm{C}$ & -1.116821 & -1.454964 & 0.674051 \\
\hline $\mathrm{C}$ & -2.646788 & -0.834265 & 3.297633 \\
\hline $\mathrm{C}$ & -2.372528 & -0.824105 & 4.670174 \\
\hline $\mathrm{C}$ & -1.632210 & -3.087426 & 2.590927 \\
\hline $\mathrm{C}$ & -4.485306 & -4.085985 & 2.784939 \\
\hline $\mathrm{C}$ & -2.149203 & -4.530755 & -0.182945 \\
\hline $\mathrm{C}$ & -5.299087 & -1.828841 & 2.611906 \\
\hline $\mathrm{C}$ & -4.084062 & -5.004352 & -1.567233 \\
\hline $\mathrm{C}$ & -6.866071 & -3.668911 & 2.627501 \\
\hline $\mathrm{C}$ & -2.254529 & 0.382290 & 5.362756 \\
\hline $\mathrm{C}$ & -2.702009 & 1.590686 & 3.320369 \\
\hline $\mathrm{C}$ & -2.828715 & 0.382522 & 2.636989 \\
\hline $\mathrm{C}$ & -6.609569 & -2.298032 & 2.564888 \\
\hline $\mathrm{C}$ & -5.797942 & -4.558536 & 2.750455 \\
\hline $\mathrm{C}$ & -2.411749 & 1.594725 & 4.686390 \\
\hline $\mathrm{C}$ & -4.872542 & 2.133074 & 0.478111 \\
\hline $\mathrm{C}$ & -3.209102 & 2.246303 & -1.429262 \\
\hline $\mathrm{C}$ & -4.048038 & 1.655736 & -2.615807 \\
\hline $\mathrm{C}$ & -4.248750 & 4.737883 & 1.222433 \\
\hline & -3.927067 & 2.850775 & -0.260141 \\
\hline
\end{tabular}

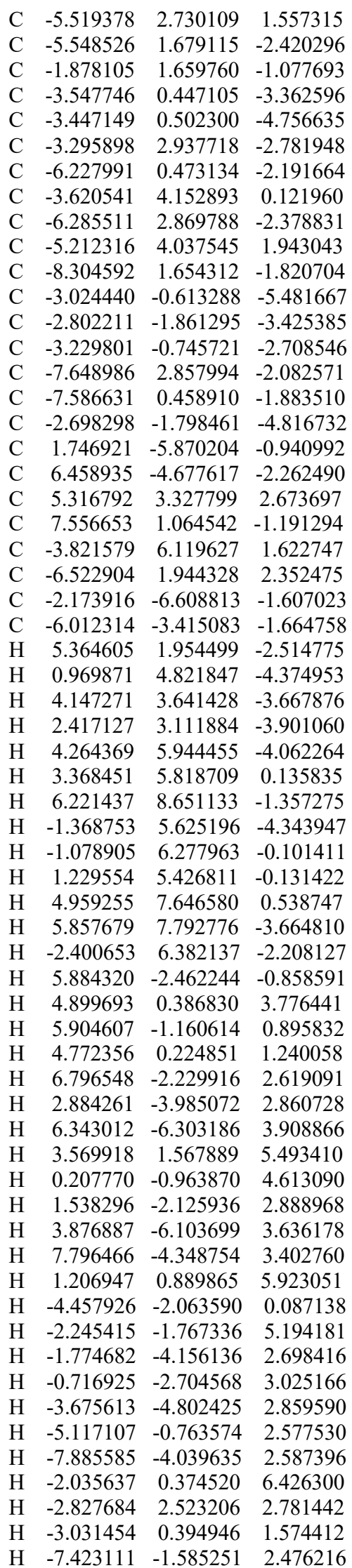

$\begin{array}{crrr}\mathrm{H} & -5.982426 & -5.626682 & 2.813247 \\ \mathrm{H} & -2.314270 & 2.534593 & 5.221394 \\ \mathrm{H} & -5.127373 & 1.121359 & 0.192392 \\ \mathrm{H} & -3.696023 & 1.425238 & -5.272653 \\ \mathrm{H} & -3.824567 & 3.882495 & -2.812802 \\ \mathrm{H} & -2.397055 & 2.902647 & -3.385296 \\ \mathrm{H} & -5.690694 & -0.462265 & -2.253913 \\ \mathrm{H} & -5.801364 & 3.822665 & -2.556969 \\ \mathrm{H} & -9.362550 & 1.648220 & -1.577435 \\ \mathrm{H} & -2.949228 & -0.556798 & -6.563540 \\ \mathrm{H} & -2.543154 & -2.770737 & -2.894655 \\ \mathrm{H} & -3.272752 & -0.791842 & -1.628256 \\ \mathrm{H} & -8.196075 & 3.795347 & -2.050585 \\ \mathrm{H} & -8.074317 & -0.490929 & -1.689852 \\ \mathrm{H} & -2.366611 & -2.666118 & -5.379211 \\ \mathrm{H} & -2.882154 & 4.707250 & -0.442902 \\ \mathrm{H} & 3.359862 & 3.678973 & 0.857833 \\ \mathrm{H} & -1.178490 & -4.827377 & 0.196694 \\ \mathrm{H} & 1.955332 & -3.606657 & 0.426038 \\ \mathrm{H} & 7.283215 & 1.998211 & 1.331972 \\ \mathrm{H} & -5.716661 & 4.499476 & 2.782532 \\ \mathrm{H} & -4.615286 & -5.661138 & -2.245177 \\ \mathrm{H} & 4.199541 & -6.198638 & -2.153286\end{array}$

\section{U'12}

$\begin{array}{llll}\mathrm{Rh} & -0.195874 & 0.754888 & 2.141220\end{array}$ $\begin{array}{lllll}\mathrm{Rh} & -0.168790 & 0.531497 & -0.244968\end{array}$

$\begin{array}{llll}\text { O } & 1.491679 & -0.626477 & -0.089272\end{array}$

$\begin{array}{llll}\text { O } & 1.632331 & -0.297023 & 2.139382\end{array}$

$\begin{array}{llll}\text { O } & -1.311815 & -1.176862 & -0.013751\end{array}$

$\begin{array}{llll}\text { O } & -1.131151 & -1.076450 & 2.240329\end{array}$

$\begin{array}{llll}\mathrm{O} & -1.855785 & 1.697578 & -0.319349\end{array}$

$\begin{array}{llll}\text { O } & -2.007277 & 1.742278 & 1.935803\end{array}$

$\begin{array}{llll}\text { O } & 1.069856 & 2.174481 & -0.284399\end{array}$

O $\quad 0.763813 \quad 2.575138 \quad 1.918682$

$\begin{array}{llll}\mathrm{F} & -8.906188 & 2.498514 & 0.653098\end{array}$

F $\quad-8.784244 \quad 3.207059 \quad-1.395300$

$\begin{array}{llll}F & -9.490561 & 1.190602 & -0.990157\end{array}$

F $\quad-5.725313 \quad-0.808660 \quad-3.817945$

F $\quad-5.600812 \quad-2.110639-2.087961$

F $\quad-3.827866-1.048682 \quad-2.773703$

F $\quad-2.434519 \quad-5.007359-4.560746$

F $\quad-3.984941 \quad-3.884023 \quad-3.524207$

F $\quad-2.132824 \quad-2.904195 \quad-4.096073$

F $\quad-0.033868 \quad-7.808930 \quad 0.090524$

F $\quad \begin{array}{llll}1.617967 & -6.478712 & -0.360733\end{array}$

F $\quad 0.633248 \quad-7.572025 \quad-1.967329$

$\begin{array}{llll}\text { F } & 3.967913 & 6.173714 & -4.743694\end{array}$

F $\quad 3.585149 \quad 7.367525 \quad-2.961206$

F $\quad 1.951796 \quad 6.320588 \quad-3.932609$

F $\quad 4.156465 \quad 0.563691 \quad-3.086260$

$\begin{array}{llll}\text { F } & 5.744428 & 1.766737 & -3.963943\end{array}$

$\begin{array}{llll}\text { F } & 5.896455 & 1.091575 & -1.896104\end{array}$

$\begin{array}{llll}\text { F } & 6.538640 & -1.039984 & -3.822181\end{array}$

F $\quad 7.485357-1.311123 \quad-1.880538$

$\begin{array}{llll}F & 7.266927 & -2.997109 & -3.241411\end{array}$

F $\quad 0.902082 \quad-3.428045 \quad-3.103205$

$\begin{array}{llll}\text { F } & 2.021836 & -5.268968 & -2.821228\end{array}$

F $\quad 2.441994-4.000908 \quad-4.533705$

C $\quad 4.966819 \quad-1.768320 \quad-0.900713$

$\begin{array}{llll}\text { C } & 3.322269 & -1.593159 & 1.018615\end{array}$

$\begin{array}{llll}\text { C } & 3.709057 & -2.500403 & 2.228160\end{array}$ 


\begin{tabular}{|c|c|c|c|}
\hline E & 3.149358 & -3.223933 & -2.409540 \\
\hline $\mathrm{C}$ & 3.717995 & -2.067218 & -0.353928 \\
\hline $\mathrm{C}$ & 5.311158 & -2.216348 & -2.175486 \\
\hline $\mathrm{C}$ & 4.447975 & -3.770145 & 1.878236 \\
\hline $\mathrm{C}$ & 2.052327 & -0.787826 & 1.044550 \\
\hline $\mathrm{C}$ & 2.832327 & -2.654562 & 3.442035 \\
\hline $\mathrm{C}$ & 3.341925 & -2.257024 & 4.684329 \\
\hline $\mathrm{C}$ & 4.405312 & -1.200582 & 2.012428 \\
\hline & 3.771401 & -4.847541 & 1.292098 \\
\hline & 2.815944 & -2.816661 & -1.119104 \\
\hline $\mathrm{C}$ & 5.810042 & -3.902386 & 2.164197 \\
\hline $\mathrm{C}$ & 4.404119 & -2.939834 & -2.945091 \\
\hline $\mathrm{C}$ & 5.809385 & -6.148742 & 1.266058 \\
\hline$C$ & 2.622170 & -2.479460 & 5.858600 \\
\hline & 0.879759 & -3.540059 & 4.570041 \\
\hline $\mathrm{C}$ & 1.589998 & -3.295636 & 3.394148 \\
\hline $\mathrm{C}$ & 6.488583 & -5.084995 & 1.860708 \\
\hline $\mathrm{C}$ & 4.446282 & -6.026570 & 0.982940 \\
\hline $\mathrm{C}$ & 1.387770 & -3.128005 & 5.804192 \\
\hline C & -0.828305 & -4.975973 & 0.138356 \\
\hline $\mathrm{C}$ & -1.999165 & -3.056508 & 1.251769 \\
\hline $\mathrm{C}$ & -3.334155 & -3.275213 & 2.035903 \\
\hline $\mathrm{C}$ & -2.036701 & -4.427976 & -2.295516 \\
\hline $\mathrm{C}$ & -1.723386 & -3.910281 & 0.048999 \\
\hline $\mathrm{C}$ & -0.548657 & -5.767120 & -0.978083 \\
\hline $\mathrm{C}$ & -4.277925 & -4.280901 & 1.429507 \\
\hline $\mathrm{C}$ & -1.444663 & -1.664928 & 1.157543 \\
\hline $\mathrm{C}$ & -4.008138 & -2.118859 & 2.725796 \\
\hline $\mathrm{C}$ & -4.438507 & -0.975746 & 2.046354 \\
\hline $\mathrm{C}$ & -2.028614 & -3.720744 & 2.608771 \\
\hline $\mathrm{C}$ & -5.197833 & -3.857564 & 0.460624 \\
\hline $\mathrm{C}$ & -2.329656 & -3.642005 & -1.183003 \\
\hline $\mathrm{C}$ & -4.245206 & -5.633981 & 1.779932 \\
\hline $\mathrm{C}$ & -1.149462 & -5.500459 & -2.202816 \\
\hline $\mathrm{C}$ & -5.999772 & -6.120940 & 0.184106 \\
\hline $\mathrm{C}$ & -5.111016 & 0.038472 & 2.729546 \\
\hline $\mathrm{C}$ & -4.927978 & -1.213033 & 4.784843 \\
\hline $\mathrm{C}$ & -4.260660 & -2.227241 & 4.099598 \\
\hline $\mathrm{C}$ & -5.102426 & -6.548464 & 1.163020 \\
\hline $\mathrm{C}$ & -6.044846 & -4.768546 & -0.165173 \\
\hline $\mathrm{C}$ & -5.357216 & -0.075054 & 4.098094 \\
\hline $\mathrm{C}$ & -6.190206 & 2.267998 & 0.035538 \\
\hline $\mathrm{C}$ & -3.775061 & 2.691761 & 0.635353 \\
\hline $\mathrm{C}$ & -3.718396 & 4.234836 & 0.422385 \\
\hline $\mathrm{C}$ & -5.519860 & 0.216943 & -1.694749 \\
\hline $\mathrm{C}$ & -4.839793 & 1.919877 & -0.097528 \\
\hline $\mathrm{C}$ & -7.178409 & 1.621800 & -0.705032 \\
\hline $\mathrm{C}$ & -4.791491 & 4.846592 & -0.444336 \\
\hline $\mathrm{C}$ & -2.442967 & 1.995388 & 0.768787 \\
\hline $\mathrm{C}$ & -2.397964 & 4.945022 & 0.276865 \\
\hline $\mathrm{C}$ & -2.017074 & 5.899066 & 1.227684 \\
\hline $\mathrm{C}$ & -4.121577 & 3.655276 & 1.746000 \\
\hline $\mathrm{C}$ & -4.872667 & 4.546466 & -1.810219 \\
\hline $\mathrm{C}$ & -4.522995 & 0.871343 & -0.974271 \\
\hline $\mathrm{C}$ & -5.720744 & 5.731257 & 0.111970 \\
\hline $\mathrm{C}$ & -6.858131 & 0.589233 & -1.580520 \\
\hline $\mathrm{C}$ & -6.814254 & 5.970953 & -2.031763 \\
\hline $\mathrm{C}$ & -0.903571 & 6.711789 & 1.009623 \\
\hline $\mathrm{C}$ & -0.510524 & 5.602046 & -1.101670 \\
\hline $\mathrm{C}$ & -1.621336 & 4.789385 & -0.880135 \\
\hline $\mathrm{C}$ & -6.728373 & 6.290687 & -0.676278 \\
\hline & -5.878835 & 5.101246 & -2.598245 \\
\hline
\end{tabular}

$\begin{array}{llll}\text { C } & -0.161053 & 6.577257 & -0.164377\end{array}$

$\begin{array}{llll}\text { C } & 3.771297 & 2.793444 & -1.089404\end{array}$

$\begin{array}{llll}\text { C } & 2.400191 & 3.839576 & 0.725971\end{array}$

$\begin{array}{llll}\text { C } & 3.293928 & 4.153579 & 1.979070\end{array}$

$\begin{array}{llll}\text { C } & 3.501922 & 5.017602 & -2.724623\end{array}$

$\begin{array}{llll}\text { C } & 3.058707 & 3.902854 & -0.620030\end{array}$

$\begin{array}{llll}\text { C } & 4.336107 & 2.801422 & -2.360604\end{array}$

$\begin{array}{llll}\text { C } & 4.738821 & 4.445743 & 1.654458\end{array}$

$\begin{array}{llll}\text { C } & 1.327718 & 2.795735 & 0.800684\end{array}$

C $\quad 3.104619 \quad 3.452469 \quad 3.297454$

$\begin{array}{llll}\text { C } & 2.912557 & 4.226713 & 4.447598\end{array}$

$\begin{array}{llll}\text { C } & 2.234208 & 5.106824 & 1.541797\end{array}$

$\begin{array}{llll}\text { C } & 5.648747 & 3.383857 & 1.543718\end{array}$

$\begin{array}{llll}\text { C } & 2.943165 & 5.017388 & -1.443575\end{array}$

$\begin{array}{llll}\text { C } & 5.200234 & 5.748677 & 1.445974\end{array}$

$\begin{array}{llll}\text { C } & 4.201687 & 3.912183 & -3.196489\end{array}$

C $\quad 7.429592 \quad 4.923165 \quad 0.990450$

$\begin{array}{llll}\text { C } & 2.770559 & 3.620038 & 5.696809\end{array}$

C $3.003093 \quad 1.449697 \quad 4.663325$

C $\quad 3.160012 \quad 2.063422 \quad 3.421960$

$\begin{array}{llll}\text { C } & 6.536897 & 5.987125 & 1.116500\end{array}$

$\begin{array}{llll}\text { C } & 6.980099 & 3.617684 & 1.208702\end{array}$

$\begin{array}{llll}\text { C } & 2.812046 & 2.227971 & 5.807577\end{array}$

$\begin{array}{llll}\text { C } & -2.647324 & -4.063277 & -3.618886\end{array}$

$\begin{array}{llll}\text { C } & 0.411976 & -6.908590 & -0.817132\end{array}$

$\begin{array}{llll}\text { C } & 2.134746 & -3.978925 & -3.219981\end{array}$

$\begin{array}{llll}\text { C } & 6.650961 & -1.886742 & -2.769603\end{array}$

$\begin{array}{llll}\text { C } & 3.264033 & 6.216321 & -3.595225\end{array}$

$\begin{array}{llll}\text { C } & 5.039001 & 1.562223 & -2.834972\end{array}$

$\begin{array}{llll}\text { C } & -5.163536 & -0.929911 & -2.597411\end{array}$

$\begin{array}{llll}\text { C } & -8.591879 & 2.122367 & -0.607119\end{array}$

H $\quad 5.677793 \quad-1.168988 \quad-0.349732$

H $\quad 4.314821 \quad-1.777307 \quad 4.729563$

H $\quad 5.430310 \quad-1.203208 \quad 1.664406$

$\mathrm{H} \quad 4.161622 \quad-0.391867 \quad 2.687001$

H $\quad 2.716898 \quad-4.762305 \quad 1.058807$

$\begin{array}{llll}\mathrm{H} & 6.343351 & -3.078889 & 2.628617\end{array}$

$\mathrm{H} \quad 6.336883 \quad-7.066046 \quad 1.023230$

$\mathrm{H} \quad 3.031077 \quad-2.157958 \quad 6.811624$

$\mathrm{H} \quad-0.068913 \quad-4.061904 \quad 4.528827$

H $\quad 1.175419 \quad-3.599048 \quad 2.438411$

H $\quad \begin{array}{llll}7.546780 & -5.171530 & 2.088118\end{array}$

$\begin{array}{llll}\mathrm{H} & 3.906068 & -6.842392 & 0.514714\end{array}$

H $\quad 0.827966 \quad-3.318676 \quad 6.714488$

H $\quad-0.341641 \quad-5.195887 \quad 1.081612$

$\begin{array}{llll}\mathrm{H} & -4.233840 & -0.868647 & 0.986772\end{array}$

H $\quad-1.794403 \quad-4.777300 \quad 2.649361$

H $\quad-1.641671 \quad-3.130418 \quad 3.427273$

$\mathrm{H} \quad-5.241762 \quad-2.812659 \quad 0.181200$

$\mathrm{H} \quad-3.552378 \quad-5.981121 \quad 2.538878$

$\begin{array}{llll}\mathrm{H} & -6.658646 & -6.833752 & -0.302064\end{array}$

$\begin{array}{llll}\mathrm{H} & -5.443437 & 0.917124 & 2.192573\end{array}$

H $\quad-5.113468 \quad-1.311782 \quad 5.850123$

H $\quad-3.928213 \quad-3.114257 \quad 4.630555$

H $\quad-5.064710 \quad-7.595438 \quad 1.448338$

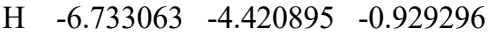

$\begin{array}{llll}\mathrm{H} & -5.879604 & 0.717820 & 4.624505\end{array}$

$\begin{array}{llll}\mathrm{H} & -6.492871 & 3.062168 & 0.702833\end{array}$

$\begin{array}{llll}\mathrm{H} & -2.614217 & 6.024608 & 2.125645\end{array}$

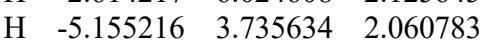

$\begin{array}{llll}\mathrm{H} & -3.405488 & 3.700272 & 2.557906\end{array}$

H $\quad-4.167689 \quad 3.847895 \quad-2.248483$
H $\quad-5.661520 \quad 5.972946 \quad 1.168934$

$\begin{array}{llll}\mathrm{H} & -7.604787 & 6.394671 & -2.643209\end{array}$

H $\quad-0.627706 \quad 7.459489 \quad 1.746819$

H $\quad 0.072093 \quad 5.488636-2.010440$

H $\quad-1.895088 \quad 4.037911-1.611311$

H $\quad-7.448421 \quad 6.968955 \quad-0.228797$

$\mathrm{H} \quad-5.940677 \quad 4.845114 \quad-3.651430$

$\begin{array}{llll}\mathrm{H} & 0.683108 & 7.234973 & -0.347518\end{array}$

H $\quad 3.870787 \quad 1.913852 \quad-0.464476$

$\begin{array}{llll}\mathrm{H} & 2.869248 & 5.308502 & 4.360015\end{array}$

$\begin{array}{llll}\mathrm{H} & 2.508559 & 6.038712 & 1.064445\end{array}$

$\mathrm{H} \quad 1.339621 \quad 5.160056 \quad 2.149515$

$\begin{array}{llll}\mathrm{H} & 5.310678 & 2.367643 & 1.716222\end{array}$

$\begin{array}{llll}\mathrm{H} & 4.518244 & 6.586483 & 1.543272\end{array}$

$\mathrm{H} \quad 8.466666 \quad 5.107369 \quad 0.727717$

$\begin{array}{llll}\mathrm{H} & 2.622452 & 4.233639 & 6.580434\end{array}$

$\begin{array}{llll}\mathrm{H} & 3.007753 & 0.367205 & 4.732161\end{array}$

$\mathrm{H} \quad 3.263517 \quad 1.455265 \quad 2.534685$

$\begin{array}{llll}\mathrm{H} & 6.876780 & 7.006149 & 0.958486\end{array}$

$\begin{array}{llll}\mathrm{H} & 7.666042 & 2.781305 & 1.114814\end{array}$

H $\quad 2.691445 \quad 1.752818 \quad 6.776534$

H $\quad 2.401699 \quad 5.888500 \quad-1.099868$

H $\quad 1.836421 \quad-3.052065 \quad-0.721598$

$\begin{array}{llll}\mathrm{H} & -3.495817 & 0.568016 & -1.106817\end{array}$

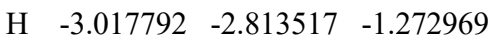

$\mathrm{H} \quad 4.660876 \quad-3.265170 \quad-3.945692$

H $\quad 4.633415 \quad 3.912069-4.188592$

H $\quad-7.629032 \quad 0.090184 \quad-2.154742$

H $\quad-0.921306 \quad-6.103620 \quad-3.070713$

\section{U'13}

$\begin{array}{llll}\mathrm{Rh} & -0.123515 & 0.014154 & -0.994269\end{array}$

$\begin{array}{llll}\mathrm{Rh} & -1.032371 & 0.325516 & 1.195206\end{array}$

$\begin{array}{llll}\text { O } & -0.932689 & -1.712374 & 1.480088\end{array}$

$\begin{array}{llll}\mathrm{O} & -0.480838 & -2.004152 & -0.711817\end{array}$

$\begin{array}{llll}\text { O } & 0.932341 & 0.498816 & 1.857177\end{array}$

$\begin{array}{llll}\text { O } & 1.726034 & -0.212069 & -0.138140\end{array}$

$\begin{array}{llll}\mathrm{O} & -1.096464 & 2.330161 & 0.759188\end{array}$

$\begin{array}{llll}\text { O } & 0.213413 & 2.052808 & -1.059745\end{array}$

$\begin{array}{llll}\mathrm{O} & -2.898258 & 0.204284 & 0.343697\end{array}$

$\begin{array}{lllll}\text { O } & -2.009467 & 0.365130 & -1.732960\end{array}$

$\begin{array}{llll}\text { F } & -7.225111 & -0.817581 & 2.979895\end{array}$

F $\quad-8.449108 \quad-0.944488 \quad 1.190172$

$\begin{array}{llll}\text { F } & -8.857589 & 0.587067 & 2.687997\end{array}$

$\begin{array}{llll}\mathrm{F} & -6.750822 & 5.099101 & 1.704652\end{array}$

F $\quad-5.142323 \quad 4.350326 \quad 2.952199$

$\begin{array}{llll}F & -4.709536 & 5.073776 & 0.944599\end{array}$

F $\quad 0.076499 \quad 7.232456 \quad-4.477972$

$\begin{array}{llll}\text { F } & 2.187308 & 7.344135 & -5.009735\end{array}$

F $\quad 1.418693 \quad 8.500834 \quad-3.337449$

F $\quad 4.997746 \quad 3.989630 \quad-0.753225$

F $\quad 5.373195 \quad 4.446966 \quad-2.850912$

F $\quad 4.380003 \quad 2.588954 \quad-2.294371$

F $\quad \begin{array}{llll}7.231496 & 2.390137 & -2.049795\end{array}$

$\begin{array}{llll}\text { F } & 7.440532 & 2.733883 & 0.088011\end{array}$

$\begin{array}{llll}\text { F } & 8.654887 & 1.222603 & -0.905330\end{array}$

$\begin{array}{llll}\text { F } & 6.487489 & -2.823612 & -2.676272\end{array}$

$\begin{array}{llll}\text { F } & 4.315709 & -2.889522 & -2.549136\end{array}$

F $\quad 5.523048 \quad-3.783063 \quad-0.978115$

$\begin{array}{llll}\text { F } & 3.625459 & -6.793437 & 1.845729\end{array}$

F $\quad 4.752380 \quad-4.999102 \quad 1.378220$

$\begin{array}{llll}\text { F } & 4.900708 & -6.736302 & 0.079680\end{array}$ 


\begin{tabular}{|c|c|c|c|}
\hline & 0.844381 & -5.295729 & -4.140381 \\
\hline $\mathrm{F}$ & 1.934348 & -3.429248 & -3.952389 \\
\hline $\mathrm{F}$ & 3.024606 & -5.306733 & -4.122127 \\
\hline $\mathrm{C}$ & 0.784326 & -4.383523 & -1.411939 \\
\hline $\mathrm{C}$ & -0.524601 & -3.924859 & 0.686402 \\
\hline $\mathrm{C}$ & -1.796686 & -4.847199 & 0.840309 \\
\hline $\mathrm{C}$ & 2.884202 & -5.491569 & 0.016552 \\
\hline $\mathrm{C}$ & 0.688212 & -4.464307 & -0.017862 \\
\hline $\mathrm{C}$ & 1.906726 & -4.863532 & -2.076104 \\
\hline $\mathrm{C}$ & -1.638746 & -6.270066 & 0.356995 \\
\hline $\mathrm{C}$ & -0.680052 & -2.436923 & 0.466788 \\
\hline $\mathrm{C}$ & -3.194096 & -4.279755 & 0.726440 \\
\hline $\mathrm{C}$ & -4.136392 & -4.883777 & -0.123289 \\
\hline $\mathrm{C}$ & -0.937338 & -4.476901 & 2.018445 \\
\hline $\mathrm{C}$ & -1.714763 & -7.313713 & 1.287470 \\
\hline $\mathrm{C}$ & 1.754803 & -5.017548 & 0.691096 \\
\hline $\mathrm{C}$ & -1.462917 & -6.593395 & -0.994728 \\
\hline $\mathrm{C}$ & 2.969041 & -5.425997 & -1.370934 \\
\hline $\mathrm{C}$ & -1.419366 & -8.953060 & -0.465725 \\
\hline $\mathrm{C}$ & -5.446482 & -4.411571 & -0.202968 \\
\hline $\mathrm{C}$ & -4.931260 & -2.716347 & 1.421588 \\
\hline $\mathrm{C}$ & -3.620757 & -3.185771 & 1.498495 \\
\hline $\mathrm{C}$ & -1.349631 & -7.921759 & -1.403994 \\
\hline $\mathrm{C}$ & -1.606808 & -8.644697 & 0.882643 \\
\hline $\mathrm{C}$ & -5.855070 & -3.326016 & 0.571872 \\
\hline $\mathrm{C}$ & 4.414483 & -1.193898 & -0.151604 \\
\hline $\mathrm{C}$ & 3.283507 & 0.233703 & 1.582502 \\
\hline $\mathrm{C}$ & 3.576735 & -0.255261 & 3.039235 \\
\hline $\mathrm{C}$ & 6.366726 & 0.732192 & -0.594478 \\
\hline $\mathrm{C}$ & 4.356876 & 0.011664 & 0.556293 \\
\hline $\mathrm{C}$ & 5.434209 & -1.422919 & -1.075347 \\
\hline $\mathrm{C}$ & 4.924445 & -0.901607 & 3.250407 \\
\hline $\mathrm{C}$ & 1.874807 & 0.163169 & 1.073012 \\
\hline $\mathrm{C}$ & 2.489196 & -0.850848 & 3.891650 \\
\hline $\mathrm{C}$ & 2.184857 & -0.239631 & 5.113679 \\
\hline $\mathrm{C}$ & 3.525842 & 1.202545 & 2.727579 \\
\hline $\mathrm{C}$ & 5.974388 & -0.201363 & 3.851924 \\
\hline $\mathrm{C}$ & 5.337286 & 0.976310 & 0.313506 \\
\hline $\mathrm{C}$ & 5.137440 & -2.229470 & 2.855853 \\
\hline $\mathrm{C}$ & 6.424649 & -0.469296 & -1.297178 \\
\hline $\mathrm{C}$ & 7.424879 & -2.125732 & 3.632948 \\
\hline $\mathrm{C}$ & 1.238834 & -0.796289 & 5.974459 \\
\hline $\mathrm{C}$ & 0.904514 & -2.607206 & 4.413943 \\
\hline $\mathrm{C}$ & 1.837673 & -2.039422 & 3.547084 \\
\hline $\mathrm{C}$ & 6.377967 & -2.835904 & 3.038878 \\
\hline $\mathrm{C}$ & 7.217741 & -0.808856 & 4.043620 \\
\hline $\mathrm{C}$ & 0.597770 & -1.987302 & 5.627151 \\
\hline $\mathrm{C}$ & 0.608810 & 5.725892 & -2.172891 \\
\hline $\mathrm{C}$ & -0.164034 & 4.257179 & -0.252609 \\
\hline $\mathrm{C}$ & -0.314153 & 5.092178 & 1.068943 \\
\hline $\mathrm{C}$ & 3.134824 & 4.580062 & -2.077395 \\
\hline $\mathrm{C}$ & 0.859882 & 4.716249 & -1.244024 \\
\hline $\mathrm{C}$ & 1.616934 & 6.166131 & -3.034234 \\
\hline $\mathrm{C}$ & 0.552135 & 6.324141 & 1.169335 \\
\hline $\mathrm{C}$ & -0.360026 & 2.773697 & -0.176265 \\
\hline $\mathrm{C}$ & -0.549357 & 4.402734 & 2.388820 \\
\hline $\mathrm{C}$ & -1.796913 & 4.501081 & 3.013213 \\
\hline $\mathrm{C}$ & -1.400771 & 5.091626 & 0.046314 \\
\hline $\mathrm{C}$ & -0.030226 & 7.595155 & 1.125437 \\
\hline $\mathrm{C}$ & 2.138958 & 4.148007 & -1.207144 \\
\hline$C$ & 1.939710 & 6.223833 & 1.341512 \\
\hline$C$ & 2.887838 & 5.598882 & -2.996549 \\
\hline
\end{tabular}

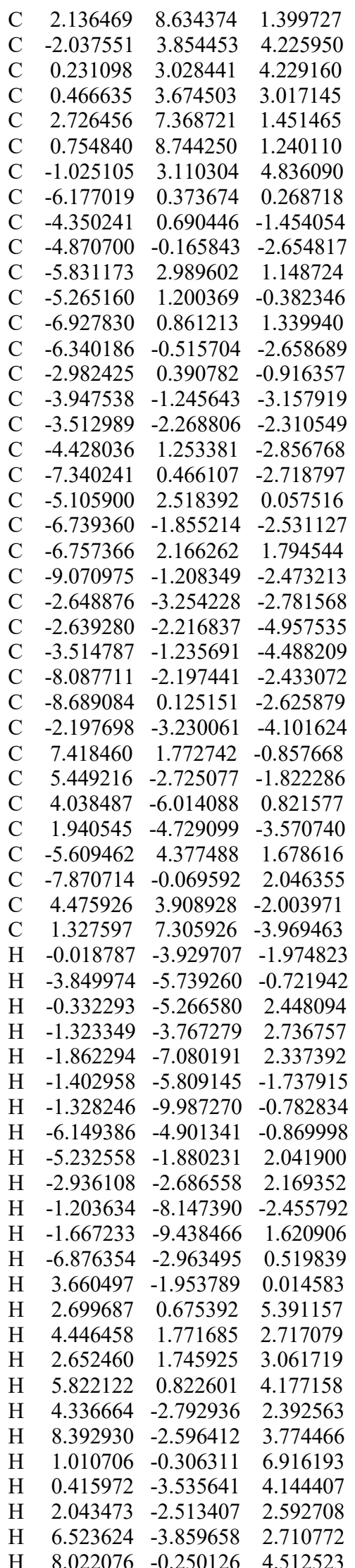

$\begin{array}{cccc}\mathrm{H} & -0.131711 & -2.431885 & 6.297128 \\ \mathrm{H} & -0.373362 & 6.176717 & -2.239517 \\ \mathrm{H} & -2.593451 & 5.061300 & 2.536860 \\ \mathrm{H} & -1.559195 & 5.984908 & -0.545332 \\ \mathrm{H} & -2.299713 & 4.531895 & 0.269665 \\ \mathrm{H} & -1.105025 & 7.685949 & 1.002818 \\ \mathrm{H} & 2.413171 & 5.249070 & 1.368955 \\ \mathrm{H} & 2.749977 & 9.526258 & 1.481600 \\ \mathrm{H} & -3.018143 & 3.928296 & 4.685931 \\ \mathrm{H} & 1.018812 & 2.444445 & 4.694127 \\ \mathrm{H} & 1.432861 & 3.577018 & 2.534602 \\ \mathrm{H} & 3.801283 & 7.272379 & 1.571107 \\ \mathrm{H} & 0.285655 & 9.722667 & 1.202226 \\ \mathrm{H} & -1.209749 & 2.601809 & 5.777590 \\ \mathrm{H} & -6.300677 & -0.648592 & -0.058603 \\ \mathrm{H} & -3.829582 & -2.282949 & -1.273708 \\ \mathrm{H} & -5.168451 & 2.024328 & -3.031946 \\ \mathrm{H} & -3.481431 & 1.402872 & -3.361280 \\ \mathrm{H} & -7.077929 & 1.512485 & -2.819289 \\ \mathrm{H} & -5.991484 & -2.636209 & -2.492510 \\ \mathrm{H} & -10.120675 & -1.472745 & -2.392521 \\ \mathrm{H} & -2.327296 & -4.033318 & -2.103907 \\ \mathrm{H} & -2.300641 & -2.188827 & -5.988857 \\ \mathrm{H} & -3.851252 & -0.444922 & -5.152309 \\ \mathrm{H} & -8.366033 & -3.241309 & -2.323066 \\ \mathrm{H} & -9.441186 & 0.906991 & -2.668728 \\ \mathrm{H} & -1.501256 & -3.984683 & -4.454548 \\ \mathrm{H} & -4.393827 & 3.165286 & -0.441243 \\ \mathrm{H} & 1.716598 & -5.081958 & 1.772049 \\ \mathrm{H} & 2.356069 & 3.359134 & -0.496917 \\ \mathrm{H} & 5.303954 & 1.927950 & 0.825299 \\ \mathrm{H} & 3.846313 & -5.787664 & -1.888530 \\ \mathrm{H} & -7.334731 & 2.535681 & 2.633793 \\ \mathrm{H} & 3.665314 & 5.936097 & -3.669180 \\ \mathrm{H} & 7.224958 & -0.662198 & -2.000264\end{array}$

\section{V1}

$\begin{array}{llll}\mathrm{Rh} & -0.432470 & -0.733339 & 2.001531\end{array}$

$\begin{array}{llll}\text { Rh } & -0.301641 & -0.509297 & -0.380686\end{array}$

$\begin{array}{llll}\text { O } & 0.912880 & -2.169056 & -0.405912\end{array}$

$\begin{array}{llll}\mathrm{O} & 0.913414 & -2.290394 & 1.855615\end{array}$

$\begin{array}{llll}\text { O } & -2.053768 & -1.596617 & -0.457210\end{array}$

$\begin{array}{llll}\text { O } & -1.963647 & -2.089763 & 1.745494\end{array}$

$\begin{array}{llll}\mathrm{O} & -1.421262 & 1.201515 & -0.222388\end{array}$

$\begin{array}{llll}\text { O } & -1.803754 & 0.827635 & 1.972431\end{array}$

$\begin{array}{llll}\text { O } & 1.395876 & 0.627820 & -0.160238\end{array}$

$\begin{array}{llll}\text { O } & 1.102763 & 0.671078 & 2.082201\end{array}$

$\begin{array}{llll}\mathrm{Br} & 7.529916 & -2.486416 & 0.711632\end{array}$

$\begin{array}{llll}\mathrm{Br} & 4.537728 & -2.743662 & -4.137141\end{array}$

$\begin{array}{llll}\mathrm{Br} & 4.021175 & 6.029013 & -2.170841\end{array}$

$\begin{array}{llll}\mathrm{Br} & 5.776960 & 0.715429 & -3.199809\end{array}$

$\begin{array}{llll}\mathrm{Br} & -5.067453 & 4.655257 & -3.459953\end{array}$

$\begin{array}{lllll}\mathrm{Br} & 0.479830 & 5.555797 & -2.424526\end{array}$

$\begin{array}{llll}\mathrm{Br} & -5.531869 & 1.055161 & -2.985696\end{array}$

$\begin{array}{llll}\mathrm{Br} & -6.665018 & -4.490752 & -3.737040\end{array}$

$\begin{array}{llll}\text { C } & 3.480754 & -3.209619 & -1.529573\end{array}$

$\begin{array}{llll}\text { C } & 2.316025 & -3.767873 & 0.623600\end{array}$

$\begin{array}{llll}\text { C } & 1.822895 & -5.230537 & 0.482936\end{array}$

$\begin{array}{llll}\text { C } & 5.881137 & -2.775314 & -0.210821\end{array}$

$\begin{array}{llll}\text { C } & 3.545088 & -3.360243 & -0.138539\end{array}$

$\begin{array}{llll}\text { C } & 4.626720 & -2.862954 & -2.233171\end{array}$

$\begin{array}{llll}\text { C } & 2.647969 & -6.122044 & -0.415698\end{array}$ 


\begin{tabular}{|c|c|c|c|}
\hline $\mathrm{C}$ & 1.291225 & -2.666055 & 0.702887 \\
\hline & 0.345534 & -5.523945 & 0.437549 \\
\hline $\mathrm{C}$ & -0.175912 & -6.493440 & 1.303983 \\
\hline $\mathrm{C}$ & 2.417070 & -4.728169 & 1.774664 \\
\hline $\mathrm{C}$ & 2.289512 & -6.267678 & -1.764559 \\
\hline C & 4.755310 & -3.143555 & 0.524200 \\
\hline $\mathrm{C}$ & 3.788817 & -6.788238 & 0.044473 \\
\hline $\mathrm{C}$ & 5.845674 & -2.631736 & -1.594345 \\
\hline $\mathrm{C}$ & 4.210121 & -7.682069 & -2.165323 \\
\hline $\mathrm{C}$ & -1.507120 & -6.896576 & 1.201508 \\
\hline $\mathrm{C}$ & -1.831949 & -5.347898 & -0.623061 \\
\hline $\mathrm{C}$ & -0.499604 & -4.945421 & -0.519353 \\
\hline $\mathrm{C}$ & 4.564396 & -7.561725 & -0.822012 \\
\hline $\mathrm{C}$ & 3.064351 & -7.033542 & -2.632674 \\
\hline $\mathrm{C}$ & -2.334936 & -6.333683 & 0.227221 \\
\hline C & -4.737082 & -1.044658 & -1.226068 \\
\hline $\mathrm{C}$ & -3.939554 & -2.63 & \\
\hline $\mathrm{C}$ & -4.857389 & -2.640090 & 05707 \\
\hline $\mathrm{C}$ & -5.877392 & -3.076716 & -2.718281 \\
\hline $\mathrm{C}$ & -4.626754 & -2.371098 & -0.782021 \\
\hline $\mathrm{C}$ & -5.410910 & -0.765043 & -2.405207 \\
\hline $\mathrm{C}$ & -6.328779 & -2.42 & \\
\hline $\mathrm{C}$ & -2.551699 & -2.070998 & 6871 \\
\hline $\mathrm{C}$ & -4.397747 & -2.058118 & 3.115361 \\
\hline $\mathrm{C}$ & -4.307926 & -2.886871 & 4.239245 \\
\hline $\mathrm{C}$ & -4.223931 & -3.895938 & 1.318284 \\
\hline $\mathrm{C}$ & -7.223586 & -3.50 & 1.5 \\
\hline $\mathrm{C}$ & -5.199247 & -3.392391 & -1.540535 \\
\hline $\mathrm{C}$ & -6.826266 & -1.139451 & 1.301532 \\
\hline $\mathrm{C}$ & -6.000425 & -1.769034 & -3.175510 \\
\hline $\mathrm{C}$ & -9.067941 & -2.015786 & 1.0 \\
\hline $\mathrm{C}$ & -3.918746 & -2.36 & 5465 \\
\hline $\mathrm{C}$ & -3.708409 & -0.176124 & 4.481679 \\
\hline $\mathrm{C}$ & -4.108109 & -0.699021 & 3.2542 \\
\hline $\mathrm{C}$ & -8.182384 & -0.934308 & 1.058541 \\
\hline $\mathrm{C}$ & -8.585051 & -3.299403 & 1.315871 \\
\hline $\mathrm{C}$ & -3.613777 & -1.009529 & 5.598806 \\
\hline $\mathrm{C}$ & -1.306351 & 4.142370 & -0.718236 \\
\hline $\mathrm{C}$ & -2.602611 & 2.848120 & 0.9750 \\
\hline $\mathrm{C}$ & -2.620248 & 3.707851 & 2.286140 \\
\hline $\mathrm{C}$ & -3.528845 & 4.454958 & -2.339779 \\
\hline $\mathrm{C}$ & -2.535256 & 3.603014 & -0.321543 \\
\hline $\mathrm{C}$ & -1.215538 & 4.832744 & -1.917974 \\
\hline $\mathrm{C}$ & -2.504721 & 5.196810 & 2.061920 \\
\hline $\mathrm{C}$ & -1.893005 & 1.528825 & 0.918018 \\
\hline $\mathrm{C}$ & -1.961047 & 3.248861 & 3.561071 \\
\hline $\mathrm{C}$ & -2.697863 & 3.326230 & 4.749601 \\
\hline $\mathrm{C}$ & -3.844947 & 2.975771 & 1.836205 \\
\hline $\mathrm{C}$ & -1.236532 & 5.795301 & 2.108902 \\
\hline $\mathrm{C}$ & -3.654103 & 3.756008 & -1.139590 \\
\hline $\mathrm{C}$ & -3.613057 & 5.999994 & 1.777605 \\
\hline $\mathrm{C}$ & -2.320709 & 5.008582 & -2.752679 \\
\hline $\mathrm{C}$ & -2.191151 & 7.946403 & 1.554113 \\
\hline $\mathrm{C}$ & -2.130460 & 2.942265 & 5.965868 \\
\hline $\mathrm{C}$ & -0.072522 & 2.399627 & 4.825452 \\
\hline $\mathrm{C}$ & -0.638606 & 2.803160 & 3.616358 \\
\hline $\mathrm{C}$ & -3.458400 & 7.365118 & 1.525199 \\
\hline $\mathrm{C}$ & -1.078473 & 7.154735 & 1.851507 \\
\hline $\mathrm{C}$ & -0.817167 & 2.468079 & 6.005214 \\
\hline $\mathrm{C}$ & 4.156646 & 1.498705 & -0.981773 \\
\hline $\mathrm{C}$ & 2.881838 & 1.934934 & 1.130986 \\
\hline & 3.895785 & 1.740958 & 2.315171 \\
\hline
\end{tabular}

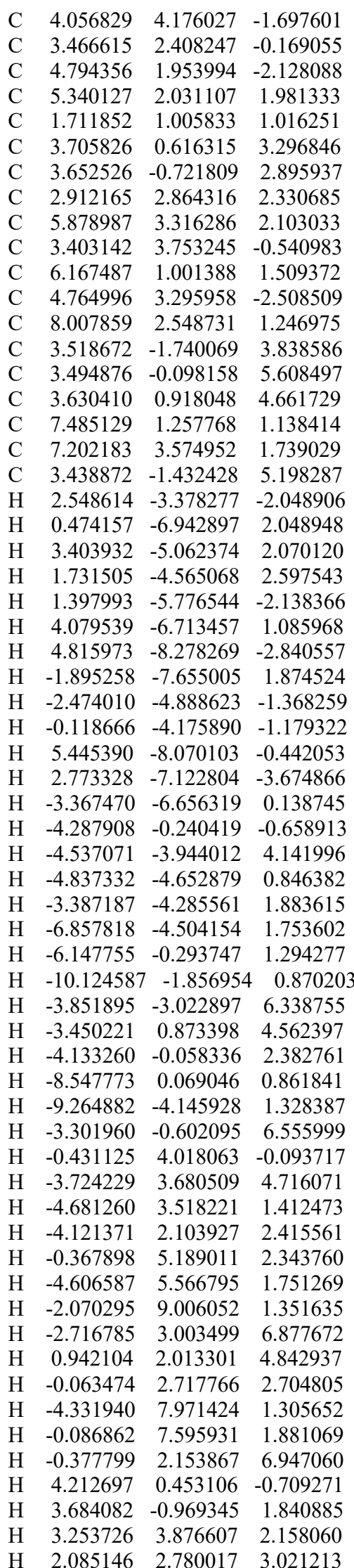

$\begin{array}{rrrr}\mathrm{H} & 5.269450 & 4.126658 & 2.486503 \\ \mathrm{H} & 5.781721 & -0.007958 & 1.424122 \\ \mathrm{H} & 9.034125 & 2.749872 & 0.955522 \\ \mathrm{H} & 3.454016 & -2.771866 & 3.511418 \\ \mathrm{H} & 3.434395 & 0.151651 & 6.663431 \\ \mathrm{H} & 3.676659 & 1.955536 & 4.979805 \\ \mathrm{H} & 8.097740 & 0.445365 & 0.762294 \\ \mathrm{H} & 7.598943 & 4.580506 & 1.840975 \\ \mathrm{H} & 3.332237 & -2.225706 & 5.931774 \\ \mathrm{H} & 2.864724 & 4.468795 & 0.066811 \\ \mathrm{H} & 4.825000 & -3.260691 & 1.597672 \\ \mathrm{H} & -4.606680 & 3.326403 & -0.857858 \\ \mathrm{H} & -5.128909 & -4.424821 & -1.225530 \\ \mathrm{H} & 6.729409 & -2.357168 & -2.153554 \\ \mathrm{H} & 5.280037 & 3.638901 & -3.395800 \\ \mathrm{H} & -2.239883 & 5.551355 & -3.685039 \\ \mathrm{H} & -6.529162 & -1.539362 & -4.090911\end{array}$

\section{V2}

$\begin{array}{llll}\text { Rh } & -0.503283 & 0.179422 & 1.110276\end{array}$ $\begin{array}{llll}\mathrm{Rh} & -0.748935 & -0.402523 & -1.190607\end{array}$

$\begin{array}{llll}\mathrm{O} & -0.001151 & -2.255067 & -0.691300\end{array}$

$\begin{array}{llll}\text { O } & 0.758547 & -1.451131 & 1.282141\end{array}$

$\begin{array}{llll}\mathrm{O} & -2.626440 & -1.123297 & -0.702492\end{array}$

$\begin{array}{llll}\text { O } & -2.110655 & -1.039896 & 1.502144\end{array}$

$\begin{array}{llll}\text { O } & -1.415499 & 1.533492 & -1.524144\end{array}$

$\begin{array}{llll}\mathrm{O} & -1.680561 & 1.824223 & 0.698957\end{array}$

$\begin{array}{llll}\text { O } & 1.178030 & 0.333899 & -1.458972\end{array}$

$\begin{array}{llll}\mathrm{O} & 1.070185 & 1.371374 & 0.546945\end{array}$

$\begin{array}{llll}\mathrm{Br} & 6.079256 & -2.019628 & 2.979917\end{array}$

$\begin{array}{llll}\mathrm{Br} & 2.004078 & -4.905532 & 5.726674\end{array}$

$\begin{array}{llll}\mathrm{Br} & 6.202359 & -1.675967 & -3.351244\end{array}$

$\begin{array}{llll}\mathrm{Br} & 3.654385 & 2.630602 & -6.076277\end{array}$

$\mathrm{Br} \quad-0.103281 \quad 4.841130 \quad 4.261776$

$\begin{array}{llll}\mathrm{Br} & 2.401205 & 6.981225 & -0.391753\end{array}$

$\begin{array}{lllll}\mathrm{Br} & -4.627207 & -6.608548 & -2.050119\end{array}$

$\mathrm{Br} \quad-7.385201 \quad-1.796816 \quad-3.382832$

$\begin{array}{llll}\text { C } & 1.898150 & -4.136272 & 2.971543\end{array}$

$\begin{array}{llll}\text { C } & 1.562226 & -3.549092 & 0.546589\end{array}$

$\begin{array}{llll}\text { C } & 2.157724 & -4.297251 & -0.697186\end{array}$

$\begin{array}{llll}\text { C } & 4.369182 & -2.872372 & 2.986287\end{array}$

$\begin{array}{llll}\text { C } & 2.376136 & -3.533190 & 1.807171\end{array}$

$\begin{array}{llll}\text { C } & 2.669365 & -4.087987 & 4.131428\end{array}$

$\begin{array}{llll}\text { C } & 3.532280 & -4.894318 & -0.487424\end{array}$

$\begin{array}{llll}\text { C } & 0.713989 & -2.330857 & 0.358374\end{array}$

$\begin{array}{llll}\text { C } & 1.932398 & -3.757932 & -2.085182\end{array}$

$\begin{array}{llll}\text { C } & 1.402673 & -4.604424 & -3.064994\end{array}$

$\begin{array}{llll}\text { C } & 0.983207 & -4.859149 & 0.040196\end{array}$

$\begin{array}{llll}\text { C } & 3.747948 & -6.028615 & 0.304914\end{array}$

$\begin{array}{llll}\text { C } & 3.618680 & -2.889807 & 1.818063\end{array}$

C $4.648942 \quad-4.258926-1.051180$

$\begin{array}{llll}\text { C } & 3.914778 & -3.465218 & 4.164379\end{array}$

$\begin{array}{llll}\text { C } & 6.143787 & -5.838839 & 0.006065\end{array}$

$\begin{array}{llll}\text { C } & 1.222054 & -4.150602 & -4.372712\end{array}$

$\begin{array}{llll}\text { C } & 2.104306 & -1.992430 & -3.741159\end{array}$

$\begin{array}{llll}\text { C } & 2.287147 & -2.453055 & -2.438784\end{array}$

$\begin{array}{llll}\text { C } & 5.939981 & -4.718696 & -0.802040\end{array}$

$\begin{array}{llll}\text { C } & 5.039999 & -6.496005 & 0.550295\end{array}$

C $\quad \begin{array}{llll}\text { C } & 1.571851 & -2.841397 & -4.714100\end{array}$

$\begin{array}{llll}\text { C } & -4.449264 & -4.153535 & -0.588674\end{array}$

$\begin{array}{llll}\text { C } & -4.116740 & -2.120697 & 0.847035\end{array}$

$\begin{array}{llll}\text { C } & -5.059854 & -1.543412 & 1.964015\end{array}$ 


\begin{tabular}{|c|c|c|c|}
\hline C & -6.173588 & -2.760648 & \\
\hline $\mathrm{C}$ & -4.737516 & -2.812318 & -0.328675 \\
\hline $\mathrm{C}$ & -5.031527 & -4.773722 & -1.692574 \\
\hline $\mathrm{C}$ & -6.544158 & -1.699713 & 1.721377 \\
\hline $\mathrm{C}$ & -2.859993 & -1.370272 & 0.528050 \\
\hline $\mathrm{C}$ & -4.659045 & -0.255028 & 2.632414 \\
\hline $\mathrm{C}$ & -4.488231 & 0.923747 & 1.900474 \\
\hline $\mathrm{C}$ & -4.208910 & -2.750135 & 2.220024 \\
\hline $\mathrm{C}$ & -7.164451 & -2.956522 & 1.668616 \\
\hline $\mathrm{C}$ & -5.600449 & -2.109054 & -1.174318 \\
\hline $\mathrm{C}$ & -7.334589 & -0.566437 & 1.476410 \\
\hline $\mathrm{C}$ & -5.905037 & -4.099379 & -2.542332 \\
\hline $\mathrm{C}$ & -9.287010 & -1.944240 & 1.093671 \\
\hline $\mathrm{C}$ & -4.155262 & 2.113020 & 2.546315 \\
\hline $\mathrm{C}$ & -4.147162 & 0.965820 & 4.668212 \\
\hline $\mathrm{C}$ & -4.492495 & -0.221721 & 4.021790 \\
\hline $\mathrm{C}$ & -8.687366 & -0.686208 & 1.160002 \\
\hline $\mathrm{C}$ & -8.518134 & -3.078583 & 1.357347 \\
\hline $\mathrm{C}$ & -3.977379 & 2.140470 & 3.929480 \\
\hline $\mathrm{C}$ & 0.081000 & 5.309480 & -0.393392 \\
\hline $\mathrm{C}$ & -1.858584 & 3.731530 & -0.690411 \\
\hline $\mathrm{C}$ & -3.296419 & 4.263480 & -1.014034 \\
\hline $\mathrm{C}$ & -0.020876 & 5.044730 & 2.363224 \\
\hline $\mathrm{C}$ & -0.912114 & 4.513501 & 0.184520 \\
\hline $\mathrm{C}$ & 1.007805 & 5.956987 & 0.422977 \\
\hline $\mathrm{C}$ & -3.640128 & 5.628183 & -0.472373 \\
\hline $\mathrm{C}$ & -1.659484 & 2.246436 & -0.499397 \\
\hline $\mathrm{C}$ & -4.448691 & 3.287569 & -1.104146 \\
\hline $\mathrm{C}$ & -5.602812 & 3.473048 & -0.325198 \\
\hline $\mathrm{C}$ & -2.209278 & 4.229177 & -2.059650 \\
\hline $\mathrm{C}$ & -3.898958 & 6.676493 & -1.363161 \\
\hline $\mathrm{C}$ & -0.956844 & 4.383696 & 1.580668 \\
\hline $\mathrm{C}$ & -3.749167 & 5.874640 & 0.902491 \\
\hline $\mathrm{C}$ & 0.980525 & 5.842858 & 1.809326 \\
\hline $\mathrm{C}$ & -4.345159 & 8.179674 & 0.478369 \\
\hline $\mathrm{C}$ & -6.686376 & 2.599450 & -0.418188 \\
\hline $\mathrm{C}$ & -5.514850 & 1.328689 & -2.089218 \\
\hline $\mathrm{C}$ & -4.428031 & 2.196353 & -1.989256 \\
\hline $\mathrm{C}$ & -4.096275 & 7.139656 & 1.376102 \\
\hline $\mathrm{C}$ & -4.248608 & 7.943453 & -0.894083 \\
\hline $\mathrm{C}$ & -6.651617 & 1.521933 & -1.303563 \\
\hline $\mathrm{C}$ & 3.381945 & 2.064416 & -3.284030 \\
\hline $\mathrm{C}$ & 2.959943 & 1.764699 & -0.825453 \\
\hline $\mathrm{C}$ & 3.876695 & 2.207537 & 0.365259 \\
\hline $\mathrm{C}$ & 5.048339 & -0.152990 & -3.330102 \\
\hline $\mathrm{C}$ & 3.614351 & 1.346563 & -2.108693 \\
\hline $\mathrm{C}$ & 3.988195 & 1.651585 & -4.468321 \\
\hline $\mathrm{C}$ & 5.363906 & 2.109015 & 0.107112 \\
\hline C & 1.646825 & 1.102632 & -0.554393 \\
\hline $\mathrm{C}$ & 3.476574 & 1.899185 & 1.784942 \\
\hline $\mathrm{C}$ & 3.258829 & 0.591334 & 2.230134 \\
\hline $\mathrm{C}$ & 3.061732 & 3.214891 & -0.379298 \\
\hline $\mathrm{C}$ & 6.048873 & 3.035287 & -0.688026 \\
\hline C & 4.448088 & 0.223622 & -2.135151 \\
\hline $\mathrm{C}$ & 6.084492 & 1.026916 & 0.634894 \\
\hline $\mathrm{C}$ & 4.834725 & 0.546139 & -4.517171 \\
\hline $\mathrm{C}$ & 8.104576 & 1.774555 & -0.464798 \\
\hline $\mathrm{C}$ & 2.921589 & 0.338532 & 3.558252 \\
\hline $\mathrm{C}$ & 3.014317 & 2.706470 & 4.027086 \\
\hline $\mathrm{C}$ & 3.354668 & 2.953327 & 2.696221 \\
\hline $\mathrm{C}$ & 7.436374 & 0.855238 & 0.346849 \\
\hline & 7.405584 & 2.869666 & -0.972712 \\
\hline
\end{tabular}

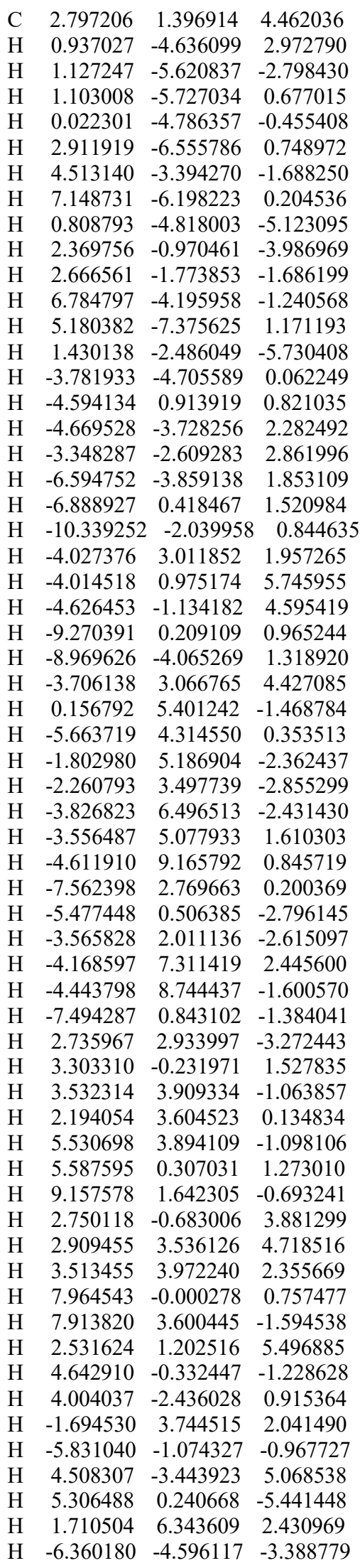

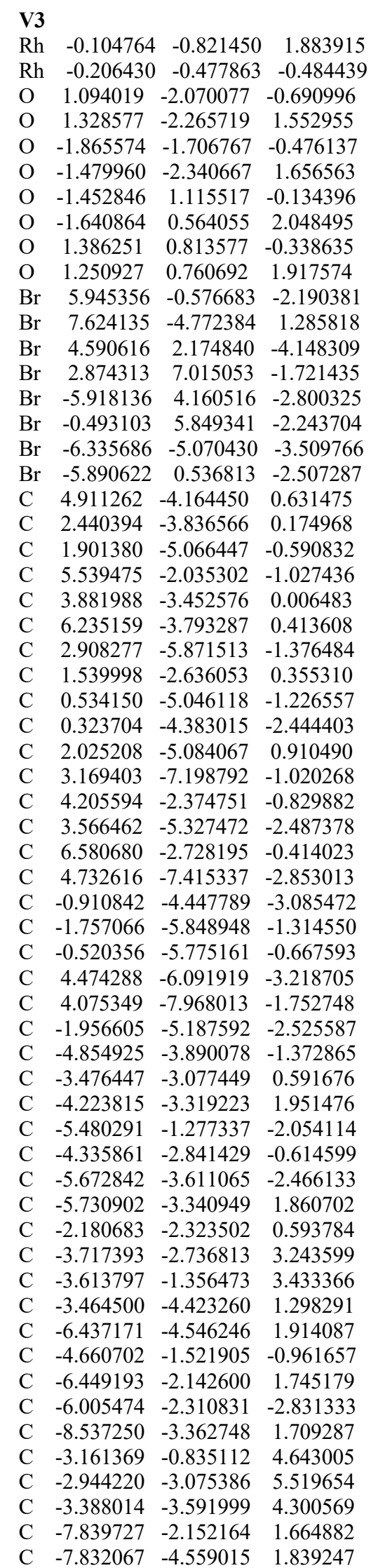




\begin{tabular}{|c|c|c|c|}
\hline $\mathrm{C}$ & -2.825386 & -1.694104 & \\
\hline $\mathrm{C}$ & -1.846401 & 4.063979 & -0.486742 \\
\hline $\mathrm{C}$ & -2.765489 & 2.507745 & 1.239075 \\
\hline $\mathrm{C}$ & -2.777579 & 3.292151 & 2.597268 \\
\hline & -4.253638 & 4.125921 & -1.856665 \\
\hline $\mathrm{C}$ & -2.934348 & 3.323304 & -0.010926 \\
\hline $\mathrm{C}$ & -1.989376 & 4.829120 & -1.635622 \\
\hline $\mathrm{C}$ & -2.882343 & 4.791250 & 2.453464 \\
\hline $\mathrm{C}$ & -1.889700 & 1.307353 & 1.049890 \\
\hline $\mathrm{C}$ & -1.951823 & 2.864277 & 3.783062 \\
\hline $\mathrm{C}$ & -0.581107 & 2.607030 & 3.707464 \\
\hline $\mathrm{C}$ & -3.927390 & 2.417129 & 2.210418 \\
\hline $\mathrm{C}$ & -4.108621 & 5.446825 & 2.309513 \\
\hline $\mathrm{C}$ & -4.144662 & 3.348863 & -0.704208 \\
\hline C & -1.705403 & 5.554608 & 2.426702 \\
\hline $\mathrm{C}$ & -3.191457 & 4.881536 & -2.343104 \\
\hline $\mathrm{C}$ & -2.982752 & 7.577107 & 2.074832 \\
\hline $\mathrm{C}$ & 0.132053 & 2.218033 & 4.841526 \\
\hline $\mathrm{C}$ & -1.876907 & 2.400157 & 6.167761 \\
\hline $\mathrm{C}$ & -2.590049 & 2.769310 & 5.026299 \\
\hline $\mathrm{C}$ & -1.752625 & 6.932843 & 2.232822 \\
\hline $\mathrm{C}$ & -4.159428 & 6.830031 & 2.121317 \\
\hline $\mathrm{C}$ & -0.513079 & 2.113168 & 6.075748 \\
\hline $\mathrm{C}$ & 2.770523 & 4.437485 & -0.475900 \\
\hline $\mathrm{C}$ & 2.723150 & 2.328400 & 0.900554 \\
\hline $\mathrm{C}$ & 3.926345 & 2.147295 & 1.895475 \\
\hline $\mathrm{C}$ & 3.856104 & 3.091669 & -2.636846 \\
\hline $\mathrm{C}$ & 3.001466 & 3.061466 & -0.383016 \\
\hline $\mathrm{C}$ & 3.119480 & 5.120470 & -1.638960 \\
\hline $\mathrm{C}$ & 5.251763 & 2.677816 & 1.410586 \\
\hline $\mathrm{C}$ & 1.714974 & 1.219342 & 0.825175 \\
\hline $\mathrm{C}$ & 4.032803 & 0.905352 & 2.739886 \\
\hline $\mathrm{C}$ & 4.129442 & -0.374487 & 2.186034 \\
\hline $\mathrm{C}$ & 2.819922 & 3.098096 & 2.204858 \\
\hline $\mathrm{C}$ & 5.690351 & 3.967662 & 1.724646 \\
\hline $\mathrm{C}$ & 3.549201 & 2.382606 & -1.481809 \\
\hline $\mathrm{C}$ & 6.066713 & 1.866819 & 0.609168 \\
\hline $\mathrm{C}$ & 3.661779 & 4.468913 & -2.739943 \\
\hline $\mathrm{C}$ & 7.704690 & 3.634544 & 0.423570 \\
\hline $\mathrm{C}$ & 4.278454 & -1.491290 & 3.007832 \\
\hline $\mathrm{C}$ & 4.236611 & -0.066759 & 4.956920 \\
\hline $\mathrm{C}$ & 4.091028 & 1.048612 & 4.131795 \\
\hline $\mathrm{C}$ & 7.278087 & 2.340373 & 0.112907 \\
\hline $\mathrm{C}$ & 6.909641 & 4.443366 & 1.235771 \\
\hline $\mathrm{C}$ & 4.332076 & -1.342084 & 4.394803 \\
\hline $\mathrm{H}$ & 4.692909 & -4.996707 & 1.285728 \\
\hline $\mathrm{H}$ & 1.128087 & -3.805879 & -2.884484 \\
\hline $\mathrm{H}$ & 2.785097 & -5.713042 & 1.358402 \\
\hline $\mathrm{H}$ & 1.117034 & -5.002525 & 1.497307 \\
\hline $\mathrm{H}$ & 2.659963 & -7.630454 & -0.163948 \\
\hline $\mathrm{H}$ & 3.385737 & -4.296033 & -2.768499 \\
\hline $\mathrm{H}$ & 5.442582 & -8.009453 & -3.420146 \\
\hline $\mathrm{H}$ & -1.057126 & -3.925399 & -4.026171 \\
\hline $\mathrm{H}$ & -2.556800 & -6.436124 & -0.874297 \\
\hline $\mathrm{H}$ & -0.369211 & -6.305798 & 0.267148 \\
\hline $\mathrm{H}$ & 4.984401 & -5.651985 & -4.070267 \\
\hline $\mathrm{H}$ & 4.268605 & -8.996064 & -1.461543 \\
\hline $\mathrm{H}$ & -2.914621 & -5.246890 & -3.031678 \\
\hline $\mathrm{H}$ & -4.622771 & -4.916519 & -1.128639 \\
\hline $\mathrm{H}$ & -3.825747 & -0.687577 & 2.608740 \\
\hline $\mathrm{H}$ & -4.008769 & -5.237101 & 0.836493 \\
\hline$H$ & -2.514804 & -4.705634 & 1.736257 \\
\hline
\end{tabular}
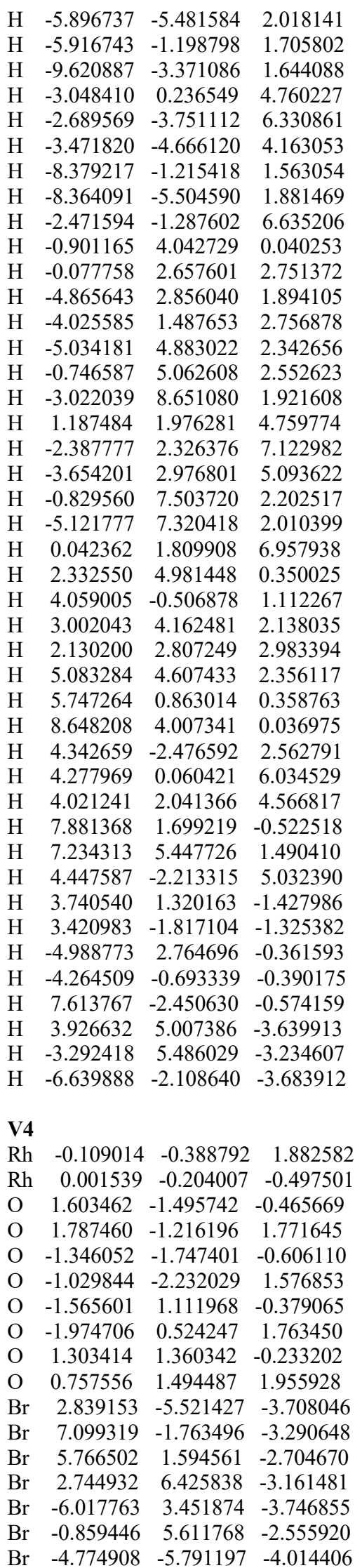

$\begin{array}{llll}\mathrm{Br} & -5.635479 & -0.270640 & -2.873777\end{array}$

$\begin{array}{llll}\text { C } & 5.082927 & -2.186026 & -1.297740\end{array}$

$\begin{array}{llll}\text { C } & 3.470511 & -2.446594 & 0.625857\end{array}$

$\begin{array}{llll}\text { C } & 3.760869 & -3.475879 & 1.776259\end{array}$

$\begin{array}{llll}\text { C } & 3.753998 & -4.154579 & -2.733064\end{array}$

$\begin{array}{llll}\text { C } & 3.957552 & -2.804719 & -0.748611\end{array}$

$\begin{array}{llll}\text { C } & 5.531379 & -2.578637 & -2.558181\end{array}$

$\begin{array}{llll}\text { C } & 4.441573 & -4.749922 & 1.338427\end{array}$

$\begin{array}{llll}\text { C } & 2.193728 & -1.664894 & 0.652084\end{array}$

$\begin{array}{llll}\text { C } & 2.773260 & -3.655333 & 2.899414\end{array}$

$\begin{array}{llll}\text { C } & 1.425479 & -3.954059 & 2.679689\end{array}$

$\begin{array}{llll}\text { C } & 4.494622 & -2.174612 & 1.710490\end{array}$

$\begin{array}{llll}\text { C } & 3.663071 & -5.883714 & 1.060718\end{array}$

$\begin{array}{llll}\text { C } & 3.283421 & -3.790871 & -1.478775\end{array}$

$\begin{array}{llll}\text { C } & 5.826577 & -4.832930 & 1.160075\end{array}$

$\begin{array}{llll}\text { C } & 4.885444 & -3.564799 & -3.297540\end{array}$

$\begin{array}{llll}\text { C } & 5.632275 & -7.123734 & 0.402431\end{array}$

$\begin{array}{llll}\text { C } & 0.548018 & -4.099703 & 3.752863\end{array}$

$\begin{array}{llll}\text { C } & 2.360096 & -3.690885 & 5.294350\end{array}$

$\begin{array}{llll}\text { C } & 3.233724 & -3.537032 & 4.216811\end{array}$

$\begin{array}{llll}\text { C } & 6.418028 & -6.009380 & 0.695212\end{array}$

$\begin{array}{llll}\text { C } & 4.249686 & -7.056965 & 0.591932\end{array}$

C $\quad 1.010058 \quad-3.966051 \quad 5.063080$

$\begin{array}{llll}\text { C } & -3.718477 & -4.398525 & -1.750332\end{array}$

$\begin{array}{llll}\text { C } & -2.737498 & -3.386317 & 0.361308\end{array}$

$\begin{array}{llll}\text { C } & -3.533673 & -3.819744 & 1.638455\end{array}$

$\begin{array}{llll}\text { C } & -4.876188 & -1.965814 & -2.408920\end{array}$

C $\quad-3.524402-3.297522 \quad-0.916401$

C $\quad-4.504550 \quad-4.264569 \quad-2.894854$

$\begin{array}{llll}\text { C } & -4.993400 & -4.128915 & 1.404212\end{array}$

$\begin{array}{llll}\text { C } & -1.624403 & -2.384795 & 0.463454\end{array}$

C $\quad-3.281332 \quad-3.212233 \quad 2.993133$

C $\quad-3.366408-1.836102 \quad 3.220184$

$\begin{array}{llll}\text { C } & -2.508865 & -4.727183 & 1.041643\end{array}$

$\begin{array}{llll}\text { C } & -5.451233 & -5.444198 & 1.287214\end{array}$

$\begin{array}{llll}\text { C } & -4.115589 & -2.070147 & -1.253742\end{array}$

$\begin{array}{llll}\text { C } & -5.920915 & -3.080879 & 1.320164\end{array}$

$\begin{array}{llll}\text { C } & -5.095872 & -3.057342 & -3.250667\end{array}$

$\begin{array}{llll}\text { C } & -7.718912 & -4.660566 & 0.973250\end{array}$

$\begin{array}{llll}\text { C } & -3.154204 & -1.310155 & 4.493190\end{array}$

C $\quad-2.817935 \quad-3.541843 \quad 5.354688$

$\begin{array}{llll}\text { C } & -3.020144 & -4.061745 & 4.074265\end{array}$

C $\quad-7.271121 \quad-3.342364 \quad 1.100899$

$\begin{array}{llll}\text { C } & -6.806004 & -5.710121 & 1.072741\end{array}$

$\begin{array}{llll}\text { C } & -2.876996 & -2.162580 & 5.565557\end{array}$

$\begin{array}{llll}\text { C } & -2.339173 & 3.842944 & -0.878378\end{array}$

$\begin{array}{llll}\text { C } & -3.318512 & 2.216285 & 0.739907\end{array}$

$\begin{array}{llll}\text { C } & -3.723363 & 2.937272 & 2.067231\end{array}$

$\begin{array}{llll}\text { C } & -4.516308 & 3.632772 & -2.574875\end{array}$

$\begin{array}{llll}\text { C } & -3.412068 & 3.011887 & -0.529392\end{array}$

$\begin{array}{llll}\text { C } & -2.372592 & 4.541404 & -2.076078\end{array}$

$\begin{array}{llll}\text { C } & -4.162521 & 4.372729 & 1.899707\end{array}$

$\begin{array}{llll}\text { C } & -2.205783 & 1.212575 & 0.719293\end{array}$

$\begin{array}{llll}\text { C } & -2.988830 & 2.695163 & 3.358899\end{array}$

$\begin{array}{llll}\text { C } & -1.601709 & 2.823235 & 3.463039\end{array}$

$\begin{array}{llll}\text { C } & -4.576130 & 1.845413 & 1.506859\end{array}$

$\begin{array}{llll}\text { C } & -5.504623 & 4.720401 & 1.721590\end{array}$

$\begin{array}{llll}\text { C } & -4.508762 & 2.909904 & -1.382462\end{array}$

$\begin{array}{llll}\text { C } & -3.200475 & 5.393134 & 1.923125\end{array}$

$\begin{array}{llll}\text { C } & -3.460136 & 4.457989 & -2.948580\end{array}$

$\begin{array}{llll}\text { C } & -4.912552 & 7.061496 & 1.558255\end{array}$

$\begin{array}{llll}\text { C } & -0.956529 & 2.626926 & 4.682824\end{array}$ 


\begin{tabular}{|c|c|c|c|}
\hline C & -3.090675 & 2.218026 & 5.737865 \\
\hline $\mathrm{C}$ & -3.729253 & 2.398177 & 4.508996 \\
\hline $\mathrm{C}$ & -3.569297 & 6.724573 & 1.748394 \\
\hline $\mathrm{C}$ & -5.878504 & 6.055844 & 1.551720 \\
\hline $\mathrm{C}$ & -1.700503 & 2.325070 & 5.826018 \\
\hline $\mathrm{C}$ & 2.601800 & 4.589597 & -0.971340 \\
\hline $\mathrm{C}$ & 2.282219 & 3.085555 & 1.037788 \\
\hline $\mathrm{C}$ & 3.083417 & 3.352565 & 2.356347 \\
\hline $\mathrm{C}$ & 4.502669 & 2.846138 & -2.001252 \\
\hline $\mathrm{C}$ & 2.949757 & 3.442561 & -0.258271 \\
\hline $\mathrm{C}$ & 3.226660 & 4.848451 & -2.191182 \\
\hline $\mathrm{C}$ & 4.471984 & 3.908818 & 2.152592 \\
\hline $\mathrm{C}$ & 1.382988 & 1.893152 & 0.922514 \\
\hline $\mathrm{C}$ & 2.976098 & 2.430838 & 3.543190 \\
\hline $\mathrm{C}$ & 3.159852 & 1.049409 & 3.445032 \\
\hline $\mathrm{C}$ & 1.891396 & 4.185609 & 2.006740 \\
\hline $\mathrm{C}$ & 4.717443 & 5.280776 & 2.043113 \\
\hline $\mathrm{C}$ & 3.910143 & 2.567231 & -0.778690 \\
\hline $\mathrm{C}$ & 5.554782 & 3.022633 & 2.050468 \\
\hline $\mathrm{C}$ & 4.182349 & 3.992438 & -2.731072 \\
\hline $\mathrm{C}$ & 7.077694 & 4.867726 & 1.699175 \\
\hline $\mathrm{C}$ & 3.059665 & 0.235209 & 4.572672 \\
\hline $\mathrm{C}$ & 2.642335 & 2.183819 & 5.937074 \\
\hline $\mathrm{C}$ & 2.726967 & 2.991805 & 4.801755 \\
\hline $\mathrm{C}$ & 6.844419 & 3.494995 & 1.820070 \\
\hline $\mathrm{C}$ & 6.010913 & 5.757957 & 1.817772 \\
\hline $\mathrm{C}$ & 2.800825 & 0.800505 & 5.823431 \\
\hline $\mathrm{H}$ & 5.607541 & -1.411820 & -0.752150 \\
\hline $\mathrm{H}$ & 1.041393 & -4.017154 & 1.667255 \\
\hline $\mathrm{H}$ & 5.533064 & -2.154989 & 1.404648 \\
\hline $\mathrm{H}$ & 4.227857 & -1.430724 & 2.449066 \\
\hline $\mathrm{H}$ & 2.589477 & -5.844877 & 1.209106 \\
\hline $\mathrm{H}$ & 6.456760 & -3.979726 & 1.383375 \\
\hline $\mathrm{H}$ & 6.091231 & -8.036436 & 0.035323 \\
\hline $\mathrm{H}$ & -0.503462 & -4.282270 & 3.569158 \\
\hline $\mathrm{H}$ & 2.732043 & -3.587846 & 6.309370 \\
\hline $\mathrm{H}$ & 4.281313 & -3.310814 & 4.394127 \\
\hline $\mathrm{H}$ & 7.494946 & -6.050974 & 0.563220 \\
\hline $\mathrm{H}$ & 3.626588 & -7.918767 & 0.373098 \\
\hline $\mathrm{H}$ & 0.318893 & -4.068997 & 5.894105 \\
\hline $\mathrm{H}$ & -3.267253 & -5.354681 & -1.521593 \\
\hline $\mathrm{H}$ & -3.536016 & -1.162374 & 2.390673 \\
\hline $\mathrm{H}$ & -2.825607 & -5.619439 & 0.515857 \\
\hline $\mathrm{H}$ & -1.568057 & -4.826396 & 1.569938 \\
\hline $\mathrm{H}$ & -4.749762 & -6.268236 & 1.366219 \\
\hline $\mathrm{H}$ & -5.581091 & -2.055417 & 1.419109 \\
\hline $\mathrm{H}$ & -8.770854 & -4.865505 & 0.800113 \\
\hline $\mathrm{H}$ & -3.196526 & -0.236931 & 4.642529 \\
\hline $\mathrm{H}$ & -2.610670 & -4.213553 & 6.182474 \\
\hline $\mathrm{H}$ & -2.967702 & -5.134126 & 3.908470 \\
\hline $\mathrm{H}$ & -7.973898 & -2.518099 & 1.026047 \\
\hline $\mathrm{H}$ & -7.144124 & -6.738012 & 0.983074 \\
\hline $\mathrm{H}$ & -2.711379 & -1.753429 & 6.557801 \\
\hline $\mathrm{H}$ & -1.474602 & 3.916853 & -0.231642 \\
\hline $\mathrm{H}$ & -1.012713 & 3.020569 & 2.577110 \\
\hline $\mathrm{H}$ & -5.525759 & 2.085501 & 1.044787 \\
\hline $\mathrm{H}$ & -4.551614 & 0.894438 & 2.025492 \\
\hline $\mathrm{H}$ & -6.268324 & 3.950355 & 1.719806 \\
\hline $\mathrm{H}$ & -2.156902 & 5.141080 & 2.079196 \\
\hline $\mathrm{H}$ & -5.201720 & 8.098839 & 1.421106 \\
\hline $\mathrm{H}$ & 0.124563 & 2.694980 & 4.734973 \\
\hline $\mathrm{H}$ & -3.677783 & 1.984534 & 6.621016 \\
\hline
\end{tabular}
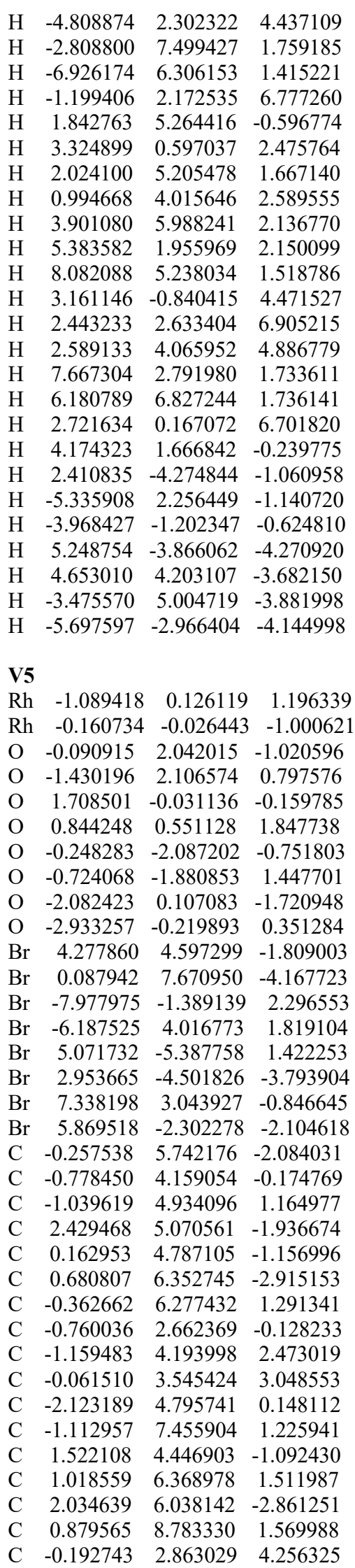

C $\quad \begin{array}{llll}-2.521170 & 3.493554 & 4.356408\end{array}$

C $\quad-2.385851 \quad 4.173236 \quad 3.145031$

$\begin{array}{llll}\text { C } & 1.636191 & 7.610791 & 1.645246\end{array}$

$\begin{array}{llll}\text { C } & -0.497862 & 8.701999 & 1.364498\end{array}$

$\begin{array}{llll}\text { C } & -1.425251 & 2.830950 & 4.914216\end{array}$

$\begin{array}{llll}\text { C } & 5.130329 & 1.636361 & 0.313601\end{array}$

$\begin{array}{llll}\text { C } & 3.208691 & 0.581928 & 1.561200\end{array}$

$\begin{array}{llll}\text { C } & 3.573210 & 0.076962 & 2.997569\end{array}$

$\begin{array}{llll}\text { C } & 5.569996 & -0.698955 & -1.108310\end{array}$

$\begin{array}{llll}\text { C } & 4.298440 & 0.534643 & 0.527529\end{array}$

$\begin{array}{llll}\text { C } & 6.182812 & 1.540231 & -0.594737\end{array}$

$\begin{array}{llll}\text { C } & 4.989861 & -0.413355 & 3.175801\end{array}$

C $\quad 1.816642 \quad 0.346320 \quad 1.054778$

$\begin{array}{llll}\text { C } & 2.571657 & -0.673559 & 3.832587\end{array}$

$\begin{array}{llll}\text { C } & 2.049007 & -1.908990 & 3.438932\end{array}$

$\begin{array}{llll}\text { C } & 3.341347 & 1.528385 & 2.741537\end{array}$

$\begin{array}{llll}\text { C } & 5.965977 & 0.395776 & 3.764924\end{array}$

$\begin{array}{llll}\text { C } & 4.521927 & -0.645267 & -0.198576\end{array}$

$\begin{array}{llll}\text { C } & 5.346202 & -1.704152 & 2.759595\end{array}$

$\begin{array}{llll}\text { C } & 6.426677 & 0.381427 & -1.321387\end{array}$

$\begin{array}{llll}\text { C } & 7.623778 & -1.344968 & 3.489161\end{array}$

$\begin{array}{llll}\text { C } & 1.196508 & -2.615741 & 4.285933\end{array}$

$\begin{array}{llll}\text { C } & 1.353753 & -0.853257 & 5.927304\end{array}$

$\begin{array}{llll}\text { C } & 2.219162 & -0.155554 & 5.084481\end{array}$

$\begin{array}{llll}\text { C } & 6.651846 & -2.164474 & 2.908571\end{array}$

$\begin{array}{llll}\text { C } & 7.275165 & -0.065789 & 3.922174\end{array}$

$\begin{array}{llll}\text { C } & 0.842657 & -2.090921 & 5.530579\end{array}$

$\begin{array}{llll}\text { C } & 1.472811 & -4.286019 & -1.365136\end{array}$

$\begin{array}{llll}\text { C } & -0.052307 & -4.012377 & 0.630606\end{array}$

$\begin{array}{llll}\text { C } & -1.198570 & -5.091097 & 0.684032\end{array}$

$\begin{array}{llll}\text { C } & 3.598867 & -4.975540 & 0.275428\end{array}$

$\begin{array}{llll}\text { C } & 1.279961 & -4.358487 & 0.021508\end{array}$

$\begin{array}{llll}\text { C } & 2.717225 & -4.589246 & -1.899857\end{array}$

$\begin{array}{llll}\text { C } & -0.835963 & -6.458266 & 0.157387\end{array}$

$\begin{array}{llll}\text { C } & -0.388460 & -2.553480 & 0.422544\end{array}$

$\begin{array}{llll}\text { C } & -2.653171 & -4.700853 & 0.530500\end{array}$

$\begin{array}{llll}\text { C } & -3.491891 & -5.413907 & -0.342381\end{array}$

C $\quad-0.460986-4.655755 \quad 1.922292$

$\begin{array}{llll}\text { C } & -0.770490 & -7.541335 & 1.041700\end{array}$

$\begin{array}{llll}\text { C } & 2.357571 & -4.697862 & 0.844964\end{array}$

C $\quad-0.601874-6.692211-1.203922$

$\begin{array}{llll}\text { C } & 3.802370 & -4.946992 & -1.099581\end{array}$

$\begin{array}{llll}\text { C } & -0.223626 & -9.041583 & -0.774959\end{array}$

$\begin{array}{llll}\text { C } & -4.851788 & -5.119091 & -0.441854\end{array}$

$\begin{array}{llll}\text { C } & -4.595310 & -3.390370 & 1.207963\end{array}$

C $\quad-3.234742 \quad-3.678963 \quad 1.301502$

$\begin{array}{llll}\text { C } & -0.295090 & -7.970688 & -1.668139\end{array}$

$\begin{array}{llll}\text { C } & -0.466525 & -8.823607 & 0.582060\end{array}$

$\begin{array}{llll}\text { C } & -5.415145 & -4.108830 & 0.337459\end{array}$

$\begin{array}{llll}\text { C } & -5.434477 & 1.867728 & 0.089889\end{array}$

$\begin{array}{llll}\text { C } & -4.441372 & 0.163687 & -1.429364\end{array}$

$\begin{array}{llll}\text { C } & -4.877646 & -0.704706 & -2.653089\end{array}$

$\begin{array}{llll}\text { C } & -6.946691 & -0.047856 & 1.403752\end{array}$

$\begin{array}{llll}\text { C } & -5.404661 & 0.538950 & -0.344079\end{array}$

$\begin{array}{llll}\text { C } & -6.207908 & 2.207647 & 1.195377\end{array}$

$\begin{array}{llll}\text { C } & -6.299895 & -1.211591 & -2.662855\end{array}$

$\begin{array}{llll}\text { C } & -3.046935 & -0.002075 & -0.901500\end{array}$

$\begin{array}{llll}\text { C } & -3.856124 & -1.658972 & -3.215739\end{array}$

$\begin{array}{llll}\text { C } & -3.518676 & -1.586856 & -4.571773\end{array}$

$\begin{array}{llll}\text { C } & -4.590725 & 0.760021 & -2.811583\end{array}$

$\begin{array}{llll}\text { C } & -7.402854 & -0.345303 & -2.674700\end{array}$

$\begin{array}{llll}\text { C } & -6.170187 & -0.424338 & 0.314357\end{array}$ 


\begin{tabular}{|c|c|c|c|}
\hline C & -6.545932 & -2.591729 & -2.601097 \\
\hline & -6.981354 & 1.265095 & 1.871941 \\
\hline & -8.935172 & -2.213384 & -2.524963 \\
\hline 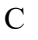 & -2.574517 & -2.458789 & -5.115888 \\
\hline & -2.306062 & -3.501285 & -2.956804 \\
\hline c & -3.248542 & -2.629787 & -2.414530 \\
\hline C & -7.847465 & -3.087313 & -2.527000 \\
\hline $\mathrm{C}$ & -8.704922 & -0.839201 & -2.605873 \\
\hline $\mathrm{C}$ & -1.960757 & -3.418648 & -4.306054 \\
\hline & -1.300577 & 6.017570 & -2.162893 \\
\hline $\mathrm{H}$ & 0.887800 & 3.530858 & 2.524935 \\
\hline $\mathrm{H}$ & -2.415695 & 5.666932 & -0.424915 \\
\hline $\mathrm{H}$ & -2.927295 & 4.102960 & 0.364996 \\
\hline $\mathrm{H}$ & -2.185494 & 7.399425 & 1.068951 \\
\hline & 1.617537 & 5.466990 & 1.563091 \\
\hline $\mathrm{H}$ & 1.361119 & 9.751078 & 1.67 \\
\hline $\mathrm{H}$ & 0.658661 & 2.338292 & 4.677864 \\
\hline $\mathrm{H}$ & -3.483189 & 3.479524 & 4.860227 \\
\hline $\mathrm{H}$ & -3.246821 & 4.666792 & 2.705727 \\
\hline 1 & 2.709184 & 7.663259 & 1.802724 \\
\hline $\mathrm{H}$ & -1.096165 & 9.606433 & 912 \\
\hline $\mathrm{H}$ & -1.528064 & 2.296619 & 3897 \\
\hline $\mathrm{H}$ & 4.966517 & 2.564302 & 0.843884 \\
\hline त & 2.290160 & -2.311451 & 2.461050 \\
\hline 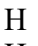 & 4.184819 & 2.206442 & 2.747277 \\
\hline $\mathrm{H}$ & 2.412574 & 1.947320 & 3.10 \\
\hline $\mathrm{H}$ & 5.704671 & 1.391470 & 4.107887 \\
\hline $\mathrm{H}$ & 4.604990 & -2.353576 & 2.308012 \\
\hline H & 8.642492 & -1.702432 & 3.603671 \\
\hline $\mathrm{H}$ & 0.804390 & -3.576103 & 3.974492 \\
\hline $\mathrm{H}$ & 1.086665 & -0.43 & 193 \\
\hline $\mathrm{H}$ & 2.632235 & 0.798310 & 5.399087 \\
\hline $\mathrm{H}$ & 6.906808 & -3.161600 & 2.564223 \\
\hline $\mathrm{H}$ & 8.019686 & 0.577033 & 4.382006 \\
\hline $\mathrm{H}$ & 0.176101 & -2.643391 & 6.185654 \\
\hline $\mathrm{H}$ & 0.661373 & -3.981003 & -2.009478 \\
\hline $\mathrm{H}$ & -3.084408 & -6.217258 & -0.942920 \\
\hline $\mathrm{H}$ & 0.220118 & -5.374846 & 2.361633 \\
\hline $\mathrm{H}$ & -0.980258 & -4.034885 & 2.639021 \\
\hline $\mathrm{H}$ & -0.962313 & -7.379081 & 2.097815 \\
\hline $\mathrm{H}$ & -0.653352 & -5.872019 & -1.908673 \\
\hline $\mathrm{H}$ & 0.018665 & -10.036977 & -1.134112 \\
\hline $\mathrm{H}$ & -5.471468 & -5.689452 & -1.127162 \\
\hline $\mathrm{H}$ & -5.014681 & -2.605272 & 1.828102 \\
\hline $\mathrm{H}$ & -2.634698 & -3.096195 & 1.985820 \\
\hline $\mathrm{H}$ & -0.109323 & -8.127259 & -2.726173 \\
\hline $\mathrm{H}$ & -0.419056 & -9.650026 & 1.284589 \\
\hline $\mathrm{H}$ & -6.474892 & -3.885244 & 0.269170 \\
\hline $\mathrm{H}$ & -4.838537 & 2.617709 & -0.416548 \\
\hline $\mathrm{H}$ & -3.990462 & -0.835489 & -5.198339 \\
\hline $\mathrm{H}$ & -5.410785 & 1.452786 & -2.957137 \\
\hline $\mathrm{H}$ & -3.670617 & 1.023609 & -3.318429 \\
\hline $\mathrm{H}$ & -7.258470 & 0.727588 & -2.719195 \\
\hline $\mathrm{H}$ & -5.713901 & -3.283803 & -2.597856 \\
\hline $\mathrm{H}$ & -9.948873 & -2.597110 & -2.465271 \\
\hline $\mathrm{H}$ & -2.317050 & -2.387758 & -6.168398 \\
\hline $\mathrm{H}$ & -1.842114 & -4.238119 & -2.314692 \\
\hline$\Pi$ & -3.494580 & -2.692854 & -1.360589 \\
\hline $\mathrm{H}$ & -8.007276 & -4.159956 & -2.470425 \\
\hline $\mathrm{H}$ & -9.539933 & -0.145279 & -2.612198 \\
\hline $\mathrm{H}$ & -1.220078 & -4.094713 & -4.722689 \\
\hline & -6.149485 & -1.450806 & -0.019224 \\
\hline
\end{tabular}
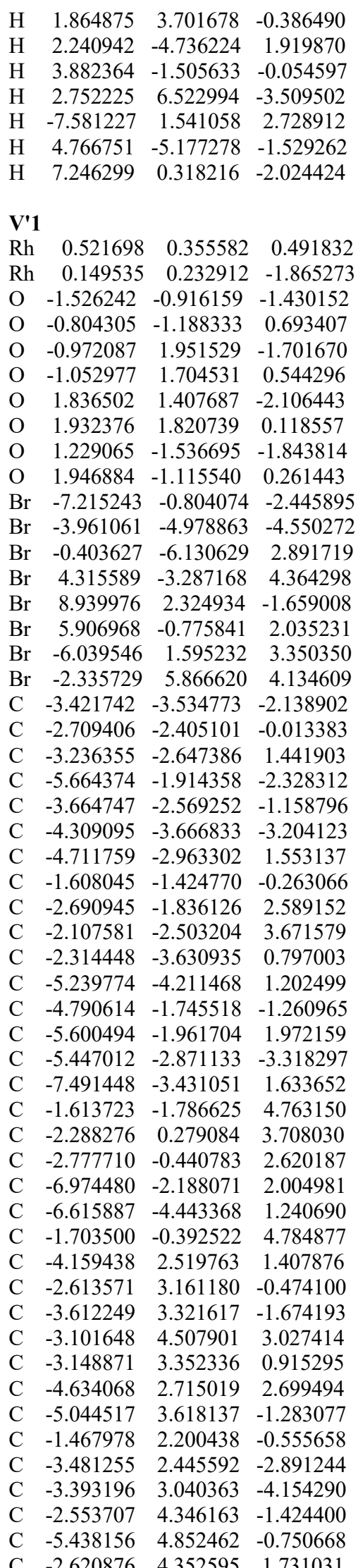

$\begin{array}{llll}\text { C } & -6.011972 & 2.608207 & -1.393771\end{array}$

$\begin{array}{llll}\text { C } & -4.118612 & 3.704477 & 3.537416\end{array}$

$\begin{array}{llll}\text { C } & -7.692710 & 4.039077 & -0.405077\end{array}$

$\begin{array}{llll}\text { C } & -3.317118 & 2.253367 & -5.304872\end{array}$

$\begin{array}{llll}\text { C } & -3.420536 & 0.260081 & -3.944052\end{array}$

$\begin{array}{llll}\text { C } & -3.501479 & 1.051476 & -2.800014\end{array}$

$\begin{array}{llll}\text { C } & -7.317816 & 2.811080 & -0.953029\end{array}$

$\begin{array}{llll}\text { C } & -6.747371 & 5.061164 & -0.315067\end{array}$

$\begin{array}{llll}\text { C } & -3.329551 & 0.859600 & -5.202345\end{array}$

$\begin{array}{llll}\text { C } & 4.819307 & 1.135400 & 0.215394\end{array}$

$\begin{array}{llll}\text { C } & 3.601110 & 2.770340 & -1.282980\end{array}$

$\begin{array}{llll}\text { C } & 3.474142 & 4.310737 & -1.149951\end{array}$

$\begin{array}{llll}\text { C } & 7.262865 & 1.826190 & -0.890999\end{array}$

$\begin{array}{llll}\text { C } & 4.859962 & 2.096482 & -0.806064\end{array}$

$\begin{array}{llll}\text { C } & 5.999842 & 0.538227 & 0.648805\end{array}$

$\begin{array}{llll}\text { C } & 4.666936 & 5.057045 & -0.606247\end{array}$

$\begin{array}{llll}\text { C } & 2.351091 & 1.947011 & -1.076740\end{array}$

$\begin{array}{llll}\text { C } & 2.166676 & 4.942986 & -0.746951\end{array}$

$\begin{array}{llll}\text { C } & 1.403057 & 5.646359 & -1.685220\end{array}$

$\begin{array}{llll}\text { C } & 3.600727 & 3.663168 & -2.499551\end{array}$

$\begin{array}{llll}\text { C } & 5.300243 & 6.022151 & -1.395899\end{array}$

$\begin{array}{llll}\text { C } & 6.105025 & 2.437659 & -1.356312\end{array}$

$\begin{array}{llll}\text { C } & 5.137969 & 4.825853 & 0.692473\end{array}$

$\begin{array}{llll}\text { C } & 7.242466 & 0.862365 & 0.114235\end{array}$

$\begin{array}{llll}\text { C } & 6.857501 & 6.500189 & 0.391814\end{array}$

$\begin{array}{llll}\text { C } & 0.274388 & 6.366866 & -1.288171\end{array}$

$\begin{array}{llll}\text { C } & 0.644304 & 5.675931 & 0.996432\end{array}$

$\begin{array}{llll}\text { C } & 1.765879 & 4.952827 & 0.596875\end{array}$

$\begin{array}{llll}\text { C } & 6.227048 & 5.540751 & 1.188133\end{array}$

$\begin{array}{llll}\text { C } & 6.390243 & 6.740670 & -0.901316\end{array}$

$\begin{array}{llll}\text { C } & -0.100000 & 6.394239 & 0.055196\end{array}$

$\begin{array}{llll}\text { C } & 3.212825 & -3.381482 & 1.729036\end{array}$

$\begin{array}{llll}\text { C } & 2.388970 & -3.247854 & -0.651244\end{array}$

$\begin{array}{llll}\text { C } & 3.583906 & -3.649572 & -1.562842\end{array}$

$\begin{array}{llll}\text { C } & 1.086369 & -5.027771 & 2.419427\end{array}$

$\begin{array}{llll}\text { C } & 2.299549 & -3.781955 & 0.749998\end{array}$

$\begin{array}{llll}\text { C } & 3.047730 & -3.825547 & 3.034721\end{array}$

$\begin{array}{llll}\text { C } & 4.656521 & -4.482161 & -0.908128\end{array}$

$\begin{array}{llll}\text { C } & 1.819895 & -1.863207 & -0.767080\end{array}$

$\begin{array}{llll}\text { C } & 4.093502 & -2.660969 & -2.578164\end{array}$

$\begin{array}{llll}\text { C } & 4.240810 & -3.082582 & -3.905619\end{array}$

$\begin{array}{llll}\text { C } & 2.212163 & -4.209727 & -1.800573\end{array}$

$\begin{array}{llll}\text { C } & 4.550322 & -5.870786 & -0.779738\end{array}$

$\begin{array}{llll}\text { C } & 1.235303 & -4.619334 & 1.095416\end{array}$

$\begin{array}{llll}\text { C } & 5.781743 & -3.842170 & -0.366448\end{array}$

C $\quad 1.985258 \quad-4.647183 \quad 3.412169$

$\begin{array}{llll}\text { C } & 6.637781 & -5.953350 & 0.444035\end{array}$

C $\quad 4.748242 \quad-2.217281 \quad-4.873985$

$\begin{array}{llll}\text { C } & 4.974398 & -0.489477 & -3.202057\end{array}$

$\begin{array}{llll}\text { C } & 4.467013 & -1.358410 & -2.234590\end{array}$

$\begin{array}{llll}\text { C } & 6.758937 & -4.567720 & 0.310346\end{array}$

$\begin{array}{llll}\text { C } & 5.533768 & -6.601742 & -0.109494\end{array}$

C $\quad 5.116913 \quad-0.915873-4.523036$

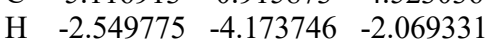

$\begin{array}{llll}\mathrm{H} & -2.023010 & -3.585709 & 3.648900\end{array}$

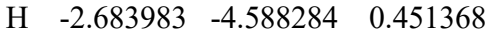

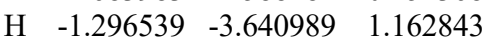

$\begin{array}{llll}\mathrm{H} & -4.584530 & -5.015782 & 0.889669\end{array}$

$\mathrm{H} \quad-5.220530 \quad-0.993186 \quad 2.271532$

$\begin{array}{llll}\mathrm{H} & -8.561950 & -3.609931 & 1.655776\end{array}$

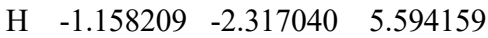

$\begin{array}{llll}\mathrm{H} & -2.357314 & 1.361743 & 3.708166\end{array}$ 


\begin{tabular}{|c|c|c|c|}
\hline H & -3.184947 & 0.092169 & 1.769774 \\
\hline & -7.638947 & -1.388863 & 2.319999 \\
\hline & -7.000803 & -5.419326 & 0.960798 \\
\hline H & -1.320277 & 0.166313 & 5.633441 \\
\hline & -4.589922 & 1.751785 & 0.780176 \\
\hline & -3.380025 & 4.123709 & -4.233203 \\
\hline & -2.812354 & 5.314617 & -1.016021 \\
\hline & -1.710749 & 4.355786 & -2.103915 \\
\hline & -4.725284 & 5.662796 & -0.656425 \\
\hline & -5.745816 & 1.650894 & -1.823200 \\
\hline & -8.708663 & 4.199279 & -0.057765 \\
\hline & -3.248412 & 2.727702 & -6.279352 \\
\hline & -3.419535 & -0.819646 & -3.846057 \\
\hline & -3.534442 & 0.576208 & -1.828570 \\
\hline & -8.038754 & 2.003530 & -1.037525 \\
\hline & -7.024816 & 6.026211 & 0.098095 \\
\hline & -3.268643 & 0.246237 & -6.096414 \\
\hline & 3.879005 & 0.848911 & 0.663526 \\
\hline & 1.707775 & 5.652534 & -2.726782 \\
\hline & 4.520383 & 3.792264 & -3.057160 \\
\hline & 2.714277 & 3.600118 & -3.119736 \\
\hline & 4.938649 & 6.207132 & -2.403069 \\
\hline 11 & 4.669478 & 4.062945 & 1.305214 \\
\hline H & 7.709334 & 7.052996 & 0.775703 \\
\hline & -0.300856 & 6.917334 & -2.026116 \\
\hline & 0.354239 & 5.687766 & 2.043058 \\
\hline & 2.342823 & 4.403611 & 1.331711 \\
\hline I & 6.588967 & 5.342623 & 2.192375 \\
\hline 1 & 6.874795 & 7.484001 & -1.527060 \\
\hline 7 & -0.963761 & 6.972835 & 0.368788 \\
\hline & 4.030142 & -2.724335 & 1.472845 \\
\hline H & 3.951050 & -4.093882 & -4.175729 \\
\hline $\mathrm{H}$ & 2.002427 & -5.240144 & -1.539943 \\
\hline $\mathrm{H}$ & 1.678468 & -3.837005 & -2.666193 \\
\hline $\mathrm{H}$ & 3.699780 & -6.392675 & -1.203773 \\
\hline & 5.886267 & -2.767373 & -0.462241 \\
\hline $\mathrm{H}$ & 7.398158 & -6.521037 & 0.971355 \\
\hline $\mathrm{H}$ & 4.854169 & -2.556931 & -5.899886 \\
\hline $\mathrm{H}$ & 5.256342 & 0.517880 & -2.921249 \\
\hline $\mathrm{H}$ & 4.347169 & -1.014347 & -1.213821 \\
\hline & 7.612125 & -4.048875 & 0.736617 \\
\hline $\mathrm{H}$ & 5.433256 & -7.679275 & -0.021242 \\
\hline $\mathrm{H}$ & 5.511774 & -0.238640 & -5.274195 \\
\hline $\mathrm{H}$ & 0.524943 & -4.935914 & 0.341891 \\
\hline & -4.992727 & -1.000151 & -0.504216 \\
\hline & 6.185792 & 3.182068 & -2.134474 \\
\hline $\mathrm{H}$ & -1.835720 & 4.991185 & 1.354398 \\
\hline $\mathrm{H}$ & -6.134252 & -2.989754 & -4.145217 \\
\hline & 1.864299 & -4.975073 & 4.436001 \\
\hline & 8.150461 & 0.389294 & 0.462936 \\
\hline & -4.493908 & 3.840623 & 4.54284 \\
\hline
\end{tabular}

$\begin{array}{lrrr}\mathbf{V}^{\prime} \mathbf{2} & & & \\ \mathrm{Rh} & 0.207375 & 0.690333 & 1.742565 \\ \mathrm{Rh} & 0.689867 & 0.757279 & -0.597460 \\ \mathrm{O} & 2.681869 & 0.668671 & -0.080069 \\ \mathrm{O} & 2.193257 & 1.142338 & 2.075928 \\ \mathrm{O} & 0.595734 & -1.311098 & -0.585801 \\ \mathrm{O} & 0.657028 & -1.327890 & 1.672755 \\ \mathrm{O} & -1.320759 & 0.896448 & -0.940551 \\ \mathrm{O} & -1.760836 & 0.271515 & 1.187210 \\ \mathrm{O} & 0.623617 & 2.802527 & -0.471515\end{array}$

$\begin{array}{llll}\mathrm{O} & -0.173871 & 2.714218 & 1.650289\end{array}$

$\mathrm{Br} \quad 5.386223 \quad-2.925604 \quad-2.212566$

$\begin{array}{llll}\mathrm{Br} & 8.688721 & 1.717376 & -1.772439\end{array}$

$\begin{array}{llll}\mathrm{Br} & -5.627734 & 4.621375 & 0.237160\end{array}$

$\begin{array}{llll}\mathrm{Br} & -2.421843 & 4.594362 & -4.468970\end{array}$

$\begin{array}{llll}\mathrm{Br} & -5.475334 & -3.878023 & 2.292508\end{array}$

$\begin{array}{llll}\mathrm{Br} & -6.840373 & 1.474928 & 3.704937\end{array}$

$\begin{array}{llll}\mathrm{Br} & 3.182384 & -5.447021 & -3.746005\end{array}$

$\mathrm{Br} \quad-2.491276 \quad-4.970964 \quad-3.569450$

$\begin{array}{llll}\text { C } & 6.420526 & 1.143967 & -0.128083\end{array}$

$\begin{array}{llll}\text { C } & 4.469830 & 0.818157 & 1.461682\end{array}$

$\begin{array}{llll}\text { C } & 4.912144 & 0.366902 & 2.898282\end{array}$

$\begin{array}{llll}\text { C } & 5.844156 & -1.276745 & -1.355311\end{array}$

$\begin{array}{llll}\text { C } & 5.338706 & 0.385747 & 0.319127\end{array}$

$\begin{array}{llll}\text { C } & 7.204163 & 0.667469 & -1.179534\end{array}$

$\begin{array}{llll}\text { C } & 6.201004 & -0.416902 & 2.958021\end{array}$

$\begin{array}{llll}\text { C } & 3.009027 & 0.883005 & 1.134246\end{array}$

$\begin{array}{llll}\text { C } & 3.899055 & -0.071662 & 3.921055\end{array}$

$\begin{array}{llll}\text { C } & 3.037489 & -1.146915 & 3.688716\end{array}$

$\begin{array}{llll}\text { C } & 4.973168 & 1.803362 & 2.502653\end{array}$

$\begin{array}{llll}\text { C } & 7.401520 & 0.191208 & 3.336898\end{array}$

$\begin{array}{llll}\text { C } & 5.057162 & -0.839911 & -0.301045\end{array}$

$\begin{array}{llll}\text { C } & 6.206821 & -1.784295 & 2.647874\end{array}$

$\begin{array}{llll}\text { C } & 6.937611 & -0.540992 & -1.815375\end{array}$

$\begin{array}{llll}\text { C } & 8.586510 & -1.900543 & 3.064396\end{array}$

$\begin{array}{llll}\text { C } & 2.117689 & -1.544148 & 4.656109\end{array}$

$\begin{array}{llll}\text { C } & 2.932274 & 0.184619 & 6.133142\end{array}$

$\begin{array}{llll}\text { C } & 3.844445 & 0.585605 & 5.154925\end{array}$

$\begin{array}{llll}\text { C } & 7.388876 & -2.519523 & 2.694985\end{array}$

$\begin{array}{llll}\text { C } & 8.587852 & -0.544562 & 3.390525\end{array}$

$\begin{array}{llll}\text { C } & 2.062436 & -0.879843 & 5.883627\end{array}$

$\begin{array}{llll}\text { C } & 1.695331 & -4.357991 & -1.549528\end{array}$

$\begin{array}{llll}C & 0.596786 & -3.405416 & 0.510487\end{array}$

$\begin{array}{llll}\text { C } & -0.080344 & -4.231080 & 1.660775\end{array}$

C $\quad-0.780771 \quad-4.715444 \quad-2.757040$

$\begin{array}{llll}\text { C } & 0.531263 & -3.984164 & -0.874342\end{array}$

$\begin{array}{llll}\text { C } & 1.593212 & -4.910184 & -2.825326\end{array}$

$\begin{array}{llll}\text { C } & -0.788045 & -5.491798 & 1.216972\end{array}$

$\begin{array}{llll}\text { C } & 0.612827 & -1.906847 & 0.542306\end{array}$

$\begin{array}{llll}\text { C } & -0.760742 & -3.550270 & 2.819572\end{array}$

$\begin{array}{llll}\text { C } & -1.713344 & -2.543460 & 2.648922\end{array}$

$\begin{array}{llll}\text { C } & 1.410338 & -4.133253 & 1.566702\end{array}$

$\begin{array}{llll}\text { C } & -0.102934 & -6.639420 & 0.801642\end{array}$

$\begin{array}{llll}\text { C } & -0.713797 & -4.156204 & -1.488112\end{array}$

$\begin{array}{llll}\text { C } & -2.189972 & -5.496201 & 1.157102\end{array}$

$\begin{array}{llll}\text { C } & 0.364192 & -5.104514 & -3.451782\end{array}$

$\begin{array}{llll}\text { C } & -2.189226 & -7.729495 & 0.230901\end{array}$

$\begin{array}{llll}\text { C } & -2.348435 & -1.970185 & 3.749752\end{array}$

C $\quad-1.118437 \quad-3.438913 \quad 5.219847$

$\begin{array}{llll}\text { C } & -0.478288 & -4.001972 & 4.114544\end{array}$

C $\quad-2.884193 \quad-6.597636 \quad 0.662553$

$\begin{array}{llll}\text { C } & -0.796465 & -7.747500 & 0.311909\end{array}$

$\begin{array}{llll}\text { C } & -2.051694 & -2.414811 & 5.039815\end{array}$

$\begin{array}{llll}\text { C } & -5.105266 & 0.735708 & 1.555437\end{array}$

$\begin{array}{llll}\text { C } & -3.548788 & 0.350905 & -0.375133\end{array}$

$\begin{array}{llll}\text { C } & -3.954565 & -0.003665 & -1.851322\end{array}$

$\begin{array}{llll}\text { C } & -5.326267 & -1.995808 & 1.998098\end{array}$

$\begin{array}{llll}\text { C } & -4.413037 & -0.160318 & 0.737617\end{array}$

$\begin{array}{llll}\text { C } & -5.896571 & 0.241196 & 2.589437\end{array}$

C $\quad-5.153410 \quad-0.915070 \quad-1.981800$

$\begin{array}{llll}\text { C } & -2.106543 & 0.512749 & -0.018335\end{array}$

C $\quad-2.894254 \quad-0.210333 \quad-2.902382$ $\begin{array}{llll}\text { C } & -2.912911 & 0.596087 & -4.045433\end{array}$

$\begin{array}{llll}\text { C } & -4.122338 & 1.385299 & -1.328019\end{array}$

$\begin{array}{llll}\text { C } & -6.457414 & -0.478966 & -1.716418\end{array}$

$\begin{array}{llll}\text { C } & -4.521466 & -1.536341 & 0.963398\end{array}$

$\begin{array}{llll}\text { C } & -4.961045 & -2.263780 & -2.317254\end{array}$

$\begin{array}{llll}\text { C } & -6.027846 & -1.124661 & 2.831005\end{array}$

$\begin{array}{llll}\text { C } & -7.324814 & -2.712414 & -2.067446\end{array}$

$\begin{array}{llll}\text { C } & -1.958326 & 0.428238 & -5.049716\end{array}$

C $\quad-0.950733-1.365397 \quad-3.784253$

$\begin{array}{llll}\text { C } & -1.906071 & -1.192357 & -2.784957\end{array}$

C $\quad-6.031593 \quad-3.154774 \quad-2.352898$

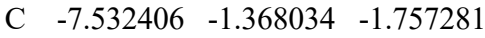

$\begin{array}{llll}\text { C } & -0.973946 & -0.554805 & -4.922097\end{array}$

$\begin{array}{llll}\text { C } & -1.409370 & 4.780420 & -1.801825\end{array}$

$\begin{array}{llll}\text { C } & -0.290816 & 4.767108 & 0.462906\end{array}$

$\begin{array}{llll}\text { C } & 0.782939 & 5.866019 & 0.487909\end{array}$

$\begin{array}{llll}\text { C } & -3.914680 & 4.757584 & -0.600931\end{array}$

C $\quad-1.514986 \quad 4.846613 \quad-0.408101$

$\begin{array}{llll}\text { C } & -2.568809 & 4.725994 & -2.568396\end{array}$

$\begin{array}{llll}\text { C } & 0.575793 & 7.052115 & -0.423383\end{array}$

$\begin{array}{llll}\text { C } & 0.112346 & 3.320488 & 0.570732\end{array}$

$\begin{array}{llll}\text { C } & 2.227569 & 5.469316 & 0.691574\end{array}$

$\begin{array}{llll}\text { C } & 2.918587 & 4.662295 & -0.223501\end{array}$

$\begin{array}{llll}\text { C } & -0.153160 & 5.659420 & 1.659392\end{array}$

$\begin{array}{llll}\text { C } & -0.131698 & 8.185092 & -0.007764\end{array}$

$\begin{array}{llll}\text { C } & -2.776153 & 4.828130 & 0.196612\end{array}$

$\begin{array}{llll}\text { C } & 1.094613 & 7.030514 & -1.727119\end{array}$

$\begin{array}{llll}\text { C } & -3.838868 & 4.723013 & -1.991196\end{array}$

$\begin{array}{llll}\text { C } & 0.169643 & 9.220240 & -2.174996\end{array}$

$\begin{array}{llll}\text { C } & 4.268274 & 4.363907 & -0.031752\end{array}$

$\begin{array}{llll}\text { C } & 4.271681 & 5.678437 & 1.992563\end{array}$

$\begin{array}{llll}\text { C } & 2.920928 & 5.971411 & 1.800839\end{array}$

$\begin{array}{llll}\text { C } & 0.890111 & 8.099729 & -2.596576\end{array}$

$\begin{array}{llll}\text { C } & -0.335006 & 9.260606 & -0.875762\end{array}$

$\begin{array}{llll}\text { C } & 4.950593 & 4.875882 & 1.073108\end{array}$

$\begin{array}{llll}\mathrm{H} & 6.657258 & 2.095378 & 0.328917\end{array}$

H $\quad 3.040231 \quad-1.635667 \quad 2.721791$

H $\quad 5.937046 \quad 2.263252 \quad 2.326610$

H $\quad 4.224243 \quad 2.470163 \quad 2.911547$

H $\quad \begin{array}{llll}7.412741 & 1.244655 & 3.596537\end{array}$

H $\quad 5.282513 \quad-2.273767 \quad 2.361193$

H $\quad 9.508657 \quad-2.472402 \quad 3.099288$

$\mathrm{H} \quad 1.426097 \quad-2.351092 \quad 4.444544$

$\begin{array}{llll}\mathrm{H} & 2.898815 & 0.705886 & 7.085323\end{array}$

H $\quad 4.513478 \quad 1.420243 \quad 5.343534$

$\mathrm{H} \quad 7.376556 \quad-3.574869 \quad 2.440060$

H $\quad 9.510743 \quad-0.055171 \quad 3.686726$

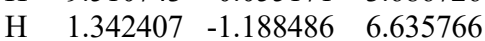

$\begin{array}{llll}\mathrm{H} & 2.666160 & -4.220869 & -1.088741\end{array}$

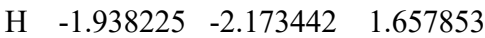

$\begin{array}{llll}\mathrm{H} & 1.994817 & -4.982821 & 1.234879\end{array}$

H $\quad 1.897390 \quad-3.510165 \quad 2.306122$

H $\quad 0.979336-6.677722 \quad 0.842782$

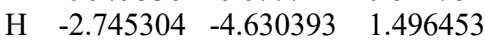

H $\quad-2.726459 \quad-8.588810 \quad-0.158198$

$\mathrm{H} \quad-3.072315-1.178198 \quad 3.592532$

$\begin{array}{llll}\mathrm{H} & -0.885221 & -3.796335 & 6.218297\end{array}$

H $\quad 0.251309 \quad-4.794877 \quad 4.253525$

H $\quad-3.968676 \quad-6.566321 \quad 0.614876$

H $\quad-0.242989-8.624830 \quad-0.008871$

$\mathrm{H} \quad-2.547242 \quad-1.971049 \quad 5.898016$

$\begin{array}{llll}\mathrm{H} & -5.036118 & 1.802859 & 1.379765\end{array}$ 


$\begin{array}{crcc}\mathrm{H} & -3.666349 & 1.372352 & -4.139770 \\ \mathrm{H} & -5.104131 & 1.777145 & -1.093032 \\ \mathrm{H} & -3.419133 & 2.119597 & -1.695665 \\ \mathrm{H} & -6.647404 & 0.557432 & -1.463542 \\ \mathrm{H} & -3.968035 & -2.626027 & -2.551354 \\ \mathrm{H} & -8.160464 & -3.404944 & -2.091416 \\ \mathrm{H} & -1.981609 & 1.068579 & -5.926382 \\ \mathrm{H} & -0.185797 & -2.125217 & -3.666778 \\ \mathrm{H} & -1.847920 & -1.790453 & -1.884369 \\ \mathrm{H} & -5.849964 & -4.195917 & -2.603003 \\ \mathrm{H} & -8.533416 & -1.005707 & -1.543517 \\ \mathrm{H} & -0.229480 & -0.687044 & -5.701545 \\ \mathrm{H} & -0.437333 & 4.753904 & -2.273401 \\ \mathrm{H} & 2.393396 & 4.242450 & -1.071425 \\ \mathrm{H} & -0.946351 & 6.376256 & 1.835207 \\ \mathrm{H} & 0.266724 & 5.221276 & 2.557308 \\ \mathrm{H} & -0.522271 & 8.239051 & 1.002224 \\ \mathrm{H} & 1.664291 & 6.171702 & -2.064620 \\ \mathrm{H} & 0.008640 & 10.053789 & -2.851584 \\ \mathrm{H} & 4.783555 & 3.727433 & -0.744762 \\ \mathrm{H} & 4.792899 & 6.078121 & 2.857103 \\ \mathrm{H} & 2.396055 & 6.599179 & 2.514750 \\ \mathrm{H} & 1.293209 & 8.057353 & -3.603845 \\ \mathrm{H} & -0.886494 & 10.130161 & -0.531139 \\ \mathrm{H} & 6.002845 & 4.651408 & 1.218851 \\ \mathrm{H} & -2.861884 & 4.841531 & 1.276101 \\ \mathrm{H} & 4.214902 & -1.434375 & 0.026770 \\ \mathrm{H} & -4.002936 & -2.236222 & 0.322240 \\ \mathrm{H} & -1.619151 & -3.887705 & -0.961796 \\ \mathrm{H} & 7.548991 & -0.892920 & -2.635541 \\ \mathrm{H} & -4.730614 & 4.660988 & -2.600478 \\ \mathrm{H} & -6.651278 & -1.495540 & 3.633530 \\ \mathrm{H} & 0.300267 & -5.544314 & -4.438042 \\ & & & \end{array}$

$\begin{array}{lccc}\text { V'3 } & & & \\ \mathrm{Rh} & 0.295791 & -0.037660 & -1.469235 \\ \mathrm{Rh} & 0.309099 & 1.060151 & 0.652344 \\ \mathrm{O} & -0.489799 & -0.660734 & 1.447590 \\ \mathrm{O} & -0.991023 & -1.425519 & -0.619573 \\ \mathrm{O} & 2.194412 & 0.287896 & 0.930490 \\ \mathrm{O} & 1.900264 & -1.142534 & -0.794603 \\ \mathrm{O} & 1.255874 & 2.648502 & -0.259080 \\ \mathrm{O} & 1.591995 & 1.418839 & -2.134761 \\ \mathrm{O} & -1.600130 & 1.739863 & 0.167065 \\ \mathrm{O} & -1.276203 & 1.175994 & -1.998951 \\ \mathrm{Br} & -6.117884 & -3.465342 & -1.933930 \\ \mathrm{Br} & -1.752267 & -7.105869 & -1.620548 \\ \mathrm{Br} & -6.714076 & 1.284493 & 2.656043 \\ \mathrm{Br} & -4.447799 & 6.432231 & 1.695267 \\ \mathrm{Br} & 5.193982 & 3.886965 & 3.036874 \\ \mathrm{Br} & 7.720978 & 1.044139 & -1.200175 \\ \mathrm{Br} & 7.111356 & -4.575582 & -2.228943 \\ \mathrm{Br} & 1.759238 & -6.128106 & -0.965931 \\ \mathrm{C} & -1.940083 & -4.642981 & -0.162880 \\ \mathrm{C} & -1.907261 & -2.555674 & 1.247659 \\ \mathrm{C} & -2.658118 & -2.334952 & 2.607472 \\ \mathrm{C} & -4.412210 & -3.947218 & -1.219784 \\ \mathrm{C} & -2.558558 & -3.455381 & 0.236571 \\ \mathrm{C} & -2.580721 & -5.465478 & -1.087819 \\ \mathrm{C} & -4.007186 & -3.009481 & 2.713753 \\ \mathrm{C} & -1.072537 & -1.463651 & 0.653473 \\ \mathrm{C} & -2.595709 & -1.012999 & 3.327831\end{array}$

\begin{tabular}{|c|c|c|c|}
\hline $\mathrm{C}$ & -2.874089 & 0.203148 & 2.699909 \\
\hline $\mathrm{C}$ & -1.409037 & -3.157704 & 2.550673 \\
\hline $\mathrm{C}$ & -4.151782 & -4.393624 & 2.862659 \\
\hline $\mathrm{C}$ & -3.799351 & -3.102112 & -0.304217 \\
\hline 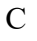 & -5.167046 & -2.228713 & 2.595310 \\
\hline $\mathrm{C}$ & -3.822769 & -5.142239 & -1.629663 \\
\hline $\mathrm{C}$ & -6.561611 & -4.200233 & 2.717034 \\
\hline $\mathrm{C}$ & -2.836893 & 1.398920 & 3.415925 \\
\hline $\mathrm{C}$ & -2.279293 & 0.176425 & 5.422328 \\
\hline C & -2.309742 & -1.016386 & 4.698247 \\
\hline $\mathrm{C}$ & -6.430027 & -2.815830 & 2.588447 \\
\hline $\mathrm{C}$ & -5.416684 & -4.984051 & 2.863192 \\
\hline $\mathrm{C}$ & -2.537735 & 1.390025 & 4.779986 \\
\hline $\mathrm{C}$ & 5.241308 & -2.995370 & -0.751485 \\
\hline C & 3.766794 & -1.464395 & 0.631794 \\
\hline $\mathrm{C}$ & 4.214455 & -1.424235 & 2.132793 \\
\hline $\mathrm{C}$ & 3.204333 & -4.877751 & -0.861921 \\
\hline $\mathrm{C}$ & 4.019842 & -2.726746 & -0.132572 \\
\hline $\mathrm{C}$ & 5.424892 & -4.215888 & -1.401312 \\
\hline C & 4.943833 & -2.646041 & 2.636157 \\
\hline $\mathrm{C}$ & 2.527303 & -0.723178 & 0.234417 \\
\hline $\mathrm{C}$ & 3.339228 & -0.764566 & 3.166463 \\
\hline $\mathrm{C}$ & 2.113283 & -1.324545 & 3.541226 \\
\hline $\mathrm{C}$ & 4.906063 & -0.581117 & 1.115684 \\
\hline $\mathrm{C}$ & 6.311540 & -2.578292 & 2.921678 \\
\hline $\mathrm{C}$ & 2.995873 & -3.683247 & -0.189960 \\
\hline $\mathrm{C}$ & 4.267921 & -3.853541 & 2.858768 \\
\hline $\mathrm{C}$ & 4.421882 & -5.177887 & -1.474400 \\
\hline $\mathrm{C}$ & 6.316004 & -4.895158 & 3.615841 \\
\hline $\mathrm{C}$ & 1.306305 & -0.698351 & 4.488191 \\
\hline $\mathrm{C}$ & 2.951316 & 1.052213 & 4.728233 \\
\hline $\mathrm{C}$ & 3.757306 & 0.419697 & 3.780697 \\
\hline $\mathrm{C}$ & 4.948132 & -4.970421 & 3.340143 \\
\hline $\mathrm{C}$ & 6.995112 & -3.694154 & 3.409003 \\
\hline $\mathrm{C}$ & 1.718559 & 0.497865 & 5.080808 \\
\hline $\mathrm{C}$ & 5.212974 & 2.405529 & -1.409465 \\
\hline $\mathrm{C}$ & 2.917575 & 3.354318 & -1.795141 \\
\hline $\mathrm{C}$ & 2.539548 & 4.740163 & -2.344334 \\
\hline $\mathrm{C}$ & 5.209675 & 3.288610 & 1.222013 \\
\hline $\mathrm{C}$ & 4.122322 & 3.132405 & -0.922629 \\
\hline $\mathrm{C}$ & 6.284028 & 2.130355 & -0.561930 \\
\hline $\mathrm{C}$ & 3.459272 & 5.901749 & -2.049689 \\
\hline $\mathrm{C}$ & 1.814947 & 2.414700 & -1.376840 \\
\hline $\mathrm{C}$ & 1.074477 & 5.101662 & -2.408655 \\
\hline $\mathrm{C}$ & 0.421114 & 5.202321 & -3.642511 \\
\hline $\mathrm{C}$ & 3.056836 & 3.653600 & -3.258481 \\
\hline $\mathrm{C}$ & 4.304020 & 6.403699 & -3.046349 \\
\hline $\mathrm{C}$ & 4.112628 & 3.557053 & 0.412068 \\
\hline C & 3.463541 & 6.526444 & -0.794234 \\
\hline $\mathrm{C}$ & 6.316640 & 2.579141 & 0.755908 \\
\hline $\mathrm{C}$ & 5.150478 & 8.093633 & -1.536432 \\
\hline $\mathrm{C}$ & -0.917108 & 5.597012 & -3.712260 \\
\hline $\mathrm{C}$ & -0.972789 & 5.814160 & -1.309255 \\
\hline C & 0.356554 & 5.401365 & -1.241757 \\
\hline $\mathrm{C}$ & 4.303654 & 7.608386 & -0.537043 \\
\hline C & 5.143965 & 7.490337 & -2.794121 \\
\hline $\mathrm{C}$ & -1.615695 & 5.913236 & -2.545967 \\
\hline $\mathrm{C}$ & -3.962767 & 4.098996 & 0.119778 \\
\hline $\mathrm{C}$ & -3.321295 & 2.211111 & -1.396809 \\
\hline $\mathrm{C}$ & -4.138444 & 1.600465 & -2.589967 \\
\hline C & -5.588575 & 2.437047 & 1.628032 \\
\hline$C$ & -4.083540 & 2.751838 & -0.223300 \\
\hline
\end{tabular}

\begin{tabular}{|c|c|c|c|}
\hline & & 589707 & \\
\hline & -5.637754 & 562431 & \\
\hline & -1.970097 & 1.666772 & \\
\hline & -3.601814 & 0.424565 & -3.363328 \\
\hline & -3.519330 & 0.513264 & -4.757060 \\
\hline & -3.439611 & 13815 & -2.739600 \\
\hline & -6.259667 & & \\
\hline & -4.898961 & & 0.542069 \\
\hline & -6.431368 & 2.715710 & -2.417631 \\
\hline & -5.481207 & 3.778196 & 5854 \\
\hline & -8.3 & & -1.8 \\
\hline & & & \\
\hline & -2.785907 & -1.85 & 7720 \\
\hline & -3.238254 & -0.772018 & 9975 \\
\hline & -7.797277 & 2.65 & -2.1 \\
\hline & -7.6 & & \\
\hline & -2.7 & & \\
\hline & -0.97 & -4.92 & 0830 \\
\hline & -3.08 & 0.22 & \\
\hline & -1.4 & -4.2 & \\
\hline & -0.5 & & \\
\hline $\mathrm{H}$ & -3.2 & & \\
\hline & -5.08 & & \\
\hline & -7.5 & -4.6 & \\
\hline & -3.0 & & \\
\hline H & -2.0 & & \\
\hline I & -2.1 & & \\
\hline & -7.30 & & \\
\hline & -5.5 & -6.0 & \\
\hline & -2.5 & & \\
\hline F & & & \\
\hline I & & & \\
\hline & 5.91 & & \\
\hline & 4.69 & & \\
\hline & & & \\
\hline $\mathrm{H}$ & 3.2 & & \\
\hline $\mathrm{H}$ & & & \\
\hline & 0.34 & -1.1 & \\
\hline & & & \\
\hline & 4.7 & & \\
\hline $\mathrm{H}$ & 4.41 & -5.9 & \\
\hline F & & -3.6 & \\
\hline & 1.081238 & & \\
\hline & 5.213894 & & \\
\hline & & & \\
\hline $\mathrm{H}$ & 4.06 & & \\
\hline & 2.35 & & -3.9 \\
\hline & 4.301007 & & -4.0 \\
\hline & 2.80 & & \\
\hline & 5.80 & & \\
\hline $\mathrm{H}$ & -1.407963 & & -4.6780 \\
\hline $\mathrm{H}$ & -1.502567 & & -0.394760 \\
\hline & 0.833274 & 5.297597 & -0.2 \\
\hline & $4.2977^{\prime}$ & & \\
\hline $\mathrm{H}$ & & & \\
\hline $\mathrm{H}$ & -2.650843 & 6.236400 & -2.599767 \\
\hline & -3.331497 & 4.749574 & -0.469625 \\
\hline & -3.803279 & & \\
\hline & -4.006461 & & -2.757890 \\
\hline $\mathrm{H}$ & -2.541805 & 2.924552 & -3.345297 \\
\hline & -5.677237 & -0.574416 & -2.097305 \\
\hline & -5.991595 & 3.679222 & -2.646151 \\
\hline
\end{tabular}

$\begin{array}{llll}\text { C } & -5.637754 & 1.562431 & -2.389264\end{array}$

$\begin{array}{llll}\text { C } & -1.970097 & 1.666772 & -1.052831\end{array}$

$\begin{array}{llll}\text { C } & -3.601814 & 0.424565 & -3.363328\end{array}$

$\begin{array}{llll}\text { C } & -3.519330 & 0.513264 & -4.757060\end{array}$

$\begin{array}{llll}\text { C } & -3.439611 & 2.913815 & -2.739600\end{array}$

$\begin{array}{llll}\text { C } & -6.259667 & 0.338102 & -2.101001\end{array}$

$\begin{array}{llll}\text { C } & -4.898961 & 1.912050 & 0.542069\end{array}$

$\begin{array}{llll}\text { C } & -6.431368 & 2.715710 & -2.417631\end{array}$

$\begin{array}{llll}\text { C } & -5.481207 & 3.778196 & 1.995854\end{array}$

$\begin{array}{llll}\text { C } & -8.398353 & 1.432821 & -1.825297\end{array}$

$\begin{array}{llll}\text { C } & -3.072290 & -0.572250 & -5.512512\end{array}$

$\begin{array}{llll}\text { C } & -2.785907 & -1.857469 & -3.487720\end{array}$

$\begin{array}{llll}\text { C } & -3.238254 & -0.772018 & -2.739975\end{array}$

$\begin{array}{llll}\text { C } & -7.797277 & 2.652498 & -2.137686\end{array}$

$\begin{array}{llll}\text { C } & -7.621202 & 0.272853 & -1.814024\end{array}$

$\begin{array}{llll}\text { C } & -2.702737 & -1.761548 & -4.878717\end{array}$

$\mathrm{H} \quad-0.974282 \quad-4.922607 \quad 0.240830$

$\begin{array}{llll}\mathrm{H} & -3.081372 & 0.229428 & 1.638356\end{array}$

$\begin{array}{llll}\mathrm{H} & -1.457183 & -4.235915 & 2.643735\end{array}$

H $\quad-0.518779 \quad-2.701874 \quad 2.965765$

$\mathrm{H} \quad-3.279625 \quad-5.028237 \quad 2.967164$

$\begin{array}{llll}\mathrm{H} & -5.083888 & -1.152709 & 2.503181\end{array}$

$\begin{array}{llll}\mathrm{H} & -7.544147 & -4.661767 & 2.708992\end{array}$

$\begin{array}{llll}\mathrm{H} & -3.040621 & 2.332311 & 2.903058\end{array}$

$\begin{array}{llll}\mathrm{H} & -2.051218 & 0.157678 & 6.483807\end{array}$

$\begin{array}{llll}\mathrm{H} & -2.106805 & -1.960627 & 5.196001\end{array}$

$\begin{array}{llll}\mathrm{H} & -7.309287 & -2.187621 & 2.480578\end{array}$

$\begin{array}{llll}\mathrm{H} & -5.503504 & -6.060522 & 2.975612\end{array}$

$\begin{array}{llll}\mathrm{H} & -2.510123 & 2.320411 & 5.339146\end{array}$

H $\quad 6.042427 \quad-2.268002 \quad-0.738001$

$\begin{array}{llll}\mathrm{H} & 1.767186 & -2.231654 & 3.057708\end{array}$

$\begin{array}{llll}\mathrm{H} & 5.912312 & -0.840007 & 0.809881\end{array}$

$\begin{array}{llll}\mathrm{H} & 4.691450 & 0.478312 & 1.115834\end{array}$

H $\quad 6.844711 \quad-1.646101 \quad 2.763416$

$\begin{array}{llll}\text { H } & 3.209509 & -3.928279 & 2.637422\end{array}$

$\mathrm{H} \quad 6.846612 \quad-5.766263 \quad 3.987721$

$\mathrm{H} \quad 0.343652 \quad-1.124694 \quad 4.746533$

$\begin{array}{llll}\mathrm{H} & 3.285806 & 1.980902 & 5.180853\end{array}$

$\begin{array}{llll}\mathrm{H} & 4.704788 & 0.866016 & 3.497550\end{array}$

$\begin{array}{llll}\mathrm{H} & 4.411167 & -5.901396 & 3.494688\end{array}$

$\begin{array}{llll}\mathrm{H} & 8.057091 & -3.623333 & 3.623817\end{array}$

$\begin{array}{llll}\mathrm{H} & 1.081238 & 0.990510 & 5.809008\end{array}$

$\mathrm{H} \quad 5.213894 \quad 2.040180 \quad-2.428708$

$\begin{array}{llll}\mathrm{H} & 0.965767 & 4.978015 & -4.554244\end{array}$

$\begin{array}{lllll}\mathrm{H} & 4.060653 & 3.759326 & -3.653080\end{array}$

H $\quad 2.357108 \quad 3.164412 \quad-3.925534$

$\begin{array}{llll}\mathrm{H} & 4.301007 & 5.946222 & -4.030209\end{array}$

$\begin{array}{llll}\mathrm{H} & 2.808895 & 6.168742 & -0.008857\end{array}$

$\begin{array}{llll}\mathrm{H} & 5.806382 & 8.935189 & -1.336076\end{array}$

$\begin{array}{llll}\mathrm{H} & -1.407963 & 5.668248 & -4.678013\end{array}$

$\begin{array}{lllll}\mathrm{H} & -1.502567 & 6.063851 & -0.394760\end{array}$

$\begin{array}{llll}\mathrm{H} & 0.833274 & 5.297597 & -0.274998\end{array}$

$\begin{array}{llll}\mathrm{H} & 4.297770 & 8.070460 & 0.445437\end{array}$

$\begin{array}{lllll}\mathrm{H} & 5.790807 & 7.863673 & -3.582281\end{array}$

$\begin{array}{lllll}\mathrm{H} & -2.650843 & 6.236400 & -2.599767\end{array}$

$\begin{array}{llll}\mathrm{H} & -3.331497 & 4.749574 & -0.469625\end{array}$

$\begin{array}{llll}\mathrm{H} & -3.803279 & 1.439134 & -5.249165\end{array}$

H $\quad-4.006461 \quad 3.836172 \quad-2.757890$

$\begin{array}{lrrr}\mathrm{H} & -5.677237 & -0.574416 & -2.097305 \\ \mathrm{H} & -5.991595 & 3.679222 & -2.646151\end{array}$ 


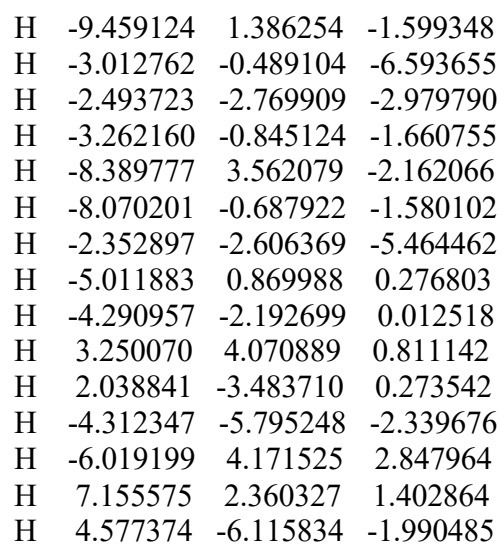

\section{V'4}

$\begin{array}{llll}\mathrm{Rh} & -0.000074 & 0.000753 & 1.930372\end{array}$

$\begin{array}{lllll}\mathrm{Rh} & 0.000148 & 0.000751 & -0.466988\end{array}$

$\begin{array}{llll}\mathrm{O} & -1.653542 & -1.223731 & -0.388972\end{array}$

$\begin{array}{llll}\mathrm{O} & -1.642233 & -1.237940 & 1.863922\end{array}$

$\begin{array}{llll}\text { O } & -1.303732 & 1.603540 & -0.401293\end{array}$

$\begin{array}{llll}\mathrm{O} & -1.163598 & 1.703823 & 1.854893\end{array}$

$\begin{array}{llll}\mathrm{O} & 1.654036 & 1.225007 & -0.388594\end{array}$

$\begin{array}{llll}\mathrm{O} & 1.642073 & 1.239533 & 1.864293\end{array}$

$\begin{array}{llll}\text { O } & 1.303809 & -1.602129 & -0.401413\end{array}$

$\begin{array}{llll}\mathrm{O} & 1.163333 & -1.702464 & 1.854752\end{array}$

$\begin{array}{lllll}\mathrm{Br} & -6.535884 & -5.645088 & -2.087627\end{array}$

$\begin{array}{llll}\mathrm{Br} & -4.341100 & -0.902707 & -4.358480\end{array}$

$\begin{array}{llll}\mathrm{Br} & 7.308281 & -4.058893 & 2.801969\end{array}$

$\begin{array}{llll}\mathrm{Br} & 6.400915 & -1.062482 & -1.949142\end{array}$

$\begin{array}{llll}\mathrm{Br} & 4.341109 & 0.902672 & -4.357847\end{array}$

$\begin{array}{llll}\mathrm{Br} & 6.536799 & 5.644898 & -2.087570\end{array}$

$\begin{array}{lllll}\mathrm{Br} & -6.400743 & 1.062040 & -1.949285\end{array}$

$\begin{array}{llll}\mathrm{Br} & -7.309169 & 4.056946 & 2.802566\end{array}$

$\begin{array}{llll}\text { C } & -4.018983 & -1.726763 & -1.641125\end{array}$

$\begin{array}{llll}\text { C } & -3.453072 & -2.297202 & 0.763785\end{array}$

$\begin{array}{llll}\text { C } & -4.385752 & -2.087888 & 2.017868\end{array}$

$\begin{array}{llll}\text { C } & -5.496436 & -4.047166 & -1.925779\end{array}$

$\begin{array}{llll}\text { C } & -4.116162 & -2.606435 & -0.552363\end{array}$

$\begin{array}{llll}\text { C } & -4.605163 & -2.060073 & -2.857208\end{array}$

$\begin{array}{llll}\text { C } & -5.865678 & -2.207999 & 1.757302\end{array}$

$\begin{array}{llll}\text { C } & -2.161123 & -1.527420 & 0.738646\end{array}$

$\begin{array}{llll}\text { C } & -4.076759 & -1.073060 & 3.088328\end{array}$

$\begin{array}{llll}\text { C } & -4.183943 & 0.301293 & 2.852899\end{array}$

$\begin{array}{llll}\text { C } & -3.537948 & -3.304877 & 1.908784\end{array}$

$\begin{array}{llll}\text { C } & -6.609959 & -3.236328 & 2.342200\end{array}$

$\begin{array}{llll}\text { C } & -4.883137 & -3.769522 & -0.710555\end{array}$

$\begin{array}{llll}\text { C } & -6.519285 & -1.268590 & 0.951729\end{array}$

$\begin{array}{llll}\text { C } & -5.359721 & -3.216034 & -3.033953\end{array}$

$\begin{array}{llll}\text { C } & -8.628139 & -2.394242 & 1.305066\end{array}$

$\begin{array}{llll}\text { C } & -3.951174 & 1.212160 & 3.881646\end{array}$

$\begin{array}{llll}\text { C } & -3.529004 & -0.607947 & 5.408466\end{array}$

$\begin{array}{llll}\text { C } & -3.759057 & -1.517947 & 4.376267\end{array}$

$\begin{array}{llll}\text { C } & -7.889369 & -1.360962 & 0.721741\end{array}$

$\begin{array}{llll}\text { C } & -7.985461 & -3.329935 & 2.117385\end{array}$

$\begin{array}{llll}\text { C } & -3.620744 & 0.763532 & 5.162269\end{array}$

$\begin{array}{llll}\text { C } & -4.357689 & 2.305055 & -0.384269\end{array}$

$\begin{array}{llll}\text { C } & -2.392306 & 3.394138 & 0.740278\end{array}$

$\begin{array}{llll}\text { C } & -1.834777 & 4.667327 & 0.065951\end{array}$

$\begin{array}{llll}\text { C } & -6.117013 & 3.361704 & 1.483450\end{array}$

$\begin{array}{llll}\text { C } & -3.864557 & 3.105642 & 0.657636\end{array}$ $\begin{array}{llll}\text { C } & -5.726679 & 2.078310 & -0.481955\end{array}$

$\begin{array}{llll}\text { C } & -2.836613 & 5.611851 & -0.554078\end{array}$

$\begin{array}{llll}\text { C } & -1.546373 & 2.140598 & 0.725520\end{array}$

$\begin{array}{llll}\text { C } & -0.514662 & 4.625163 & -0.661189\end{array}$

$\begin{array}{llll}\text { C } & 0.631391 & 5.189859 & -0.090976\end{array}$

$\begin{array}{llll}\text { C } & -1.859235 & 4.532958 & 1.567253\end{array}$

$\begin{array}{llll}\text { C } & -3.007340 & 6.893269 & -0.018604\end{array}$

$\begin{array}{llll}\text { C } & -4.756890 & 3.640873 & 1.590247\end{array}$

$\begin{array}{llll}\text { C } & -3.577380 & 5.251468 & -1.687682\end{array}$

$\begin{array}{llll}\mathrm{C} & -6.631846 & 2.587141 & 0.449197\end{array}$

$\begin{array}{llll}\text { C } & -4.643906 & 7.422993 & -1.718688\end{array}$

$\begin{array}{llll}\text { C } & 1.833975 & 5.228946 & -0.800296\end{array}$

C $\quad 0.767633 \quad 4.133601 \quad-2.664995$

C $\quad-0.431525 \quad 4.096300 \quad-1.957206$

$\begin{array}{llll}\text { C } & -4.476615 & 6.147774 & -2.263789\end{array}$

$\begin{array}{llll}\text { C } & -3.903778 & 7.794672 & -0.595313\end{array}$

$\begin{array}{llll}\text { C } & 1.906029 & 4.703004 & -2.089387\end{array}$

$\begin{array}{llll}\text { C } & 4.884096 & 3.769572 & -0.710112\end{array}$

$\begin{array}{llll}\text { C } & 3.453906 & 2.297504 & 0.764419\end{array}$

$\begin{array}{llll}\text { C } & 4.386633 & 2.087291 & 2.018376\end{array}$

$\begin{array}{llll}\text { C } & 4.605582 & 2.060047 & -2.856649\end{array}$

$\begin{array}{llll}\text { C } & 4.117016 & 2.606569 & -0.551753\end{array}$

$\begin{array}{llll}\text { C } & 5.497179 & 4.047111 & -1.925477\end{array}$

$\begin{array}{llll}\text { C } & 5.866564 & 2.206474 & 1.757481\end{array}$

$\begin{array}{llll}\text { C } & 2.161442 & 1.528575 & 0.739128\end{array}$

$\begin{array}{llll}\text { C } & 4.077112 & 1.072495 & 3.088696\end{array}$

$\begin{array}{llll}\text { C } & 3.759462 & 1.517355 & 4.376667\end{array}$

$\begin{array}{llll}\text { C } & 3.539607 & 3.304852 & 1.909620\end{array}$

$\begin{array}{llll}\text { C } & 6.519393 & 1.266517 & 0.951895\end{array}$

$\begin{array}{llll}\text { C } & 4.019608 & 1.726822 & -1.640442\end{array}$

$\begin{array}{llll}\text { C } & 6.611641 & 3.234435 & 2.341993\end{array}$

$\begin{array}{llll}\text { C } & 5.360162 & 3.215960 & -3.033593\end{array}$

$\begin{array}{llll}\text { C } & 8.629024 & 2.390938 & 1.304447\end{array}$

$\begin{array}{llll}\text { C } & 3.528847 & 0.607319 & 5.408699\end{array}$

$\begin{array}{llll}\text { C } & 3.950416 & -1.212776 & 3.881709\end{array}$

$\begin{array}{llll}\text { C } & 4.183740 & -0.301862 & 2.853126\end{array}$

$\begin{array}{llll}\text { C } & 7.987149 & 3.327157 & 2.116779\end{array}$

$\begin{array}{llll}\text { C } & 7.889458 & 1.357996 & 0.721512\end{array}$

$\begin{array}{llll}\text { C } & 3.619991 & -0.764172 & 5.162326\end{array}$

$\begin{array}{llll}\text { C } & 4.357371 & -2.304946 & -0.384277\end{array}$

$\begin{array}{llll}\text { C } & 2.391540 & -3.393191 & 0.740226\end{array}$

$\begin{array}{llll}\text { C } & 1.833806 & -4.666158 & 0.065724\end{array}$

$\begin{array}{llll}\text { C } & 6.116325 & -3.362752 & 1.483144\end{array}$

$\begin{array}{llll}\text { C } & 3.863932 & -3.105380 & 0.657588\end{array}$

$\begin{array}{llll}\text { C } & 5.726461 & -2.078821 & -0.482031\end{array}$

$\begin{array}{llll}\text { C } & 2.835664 & -5.610730 & -0.554237\end{array}$

$\begin{array}{llll}\text { C } & 1.546094 & -2.139332 & 0.725415\end{array}$

$\begin{array}{llll}\text { C } & 0.513908 & -4.623902 & -0.661756\end{array}$

$\begin{array}{llll}\text { C } & -0.631643 & -5.190954 & -0.092851\end{array}$

$\begin{array}{llll}\text { C } & 1.857921 & -4.531907 & 1.567042\end{array}$

$\begin{array}{llll}\text { C } & 3.007249 & -6.891796 & -0.018192\end{array}$

$\begin{array}{llll}\text { C } & 4.756062 & -3.641193 & 1.590048\end{array}$

$\begin{array}{llll}\text { C } & 3.575729 & -5.250568 & -1.688374\end{array}$

$\begin{array}{llll}\text { C } & 6.631453 & -2.588295 & 0.448957\end{array}$

C $\quad 4.643326 \quad-7.421578 \quad-1.718738$

$\begin{array}{llll}\text { C } & -1.833911 & -5.230498 & -0.802670\end{array}$

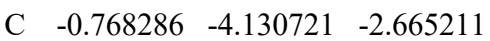

C $\quad 0.430556 \quad-4.092964 \quad-1.956890$

$\begin{array}{llll}\text { C } & 4.475150 & -6.146708 & -2.264426\end{array}$

$\begin{array}{llll}\text { C } & 3.903871 & -7.793053 & -0.594863\end{array}$

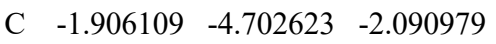

H $\quad-3.462802 \quad-0.808282 \quad-1.553410$
$\begin{array}{llll}\mathrm{H} & -4.426631 & 0.661739 & 1.860097\end{array}$

$\begin{array}{llll}\mathrm{H} & -3.998578 & -4.263777 & 1.709574\end{array}$

$\mathrm{H} \quad-2.655064 \quad-3.353232 \quad 2.533893$

H $\quad-6.110892 \quad-3.967678 \quad 2.971035$

H $\quad-5.946545 \quad-0.482319 \quad 0.477249$

H $\quad-9.695664 \quad-2.472001 \quad 1.123296$

H $\quad-4.023165 \quad 2.274485 \quad 3.682100$

$\mathrm{H} \quad-3.283211-0.969910 \quad 6.402455$

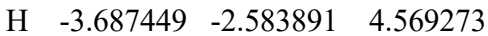

$\begin{array}{llll}\mathrm{H} & -8.373006 & -0.634504 & 0.075949\end{array}$

$\mathrm{H} \quad-8.551979 \quad-4.136234 \quad 2.573359$

H $\quad-3.443255 \quad 1.476717 \quad 5.961503$

$\mathrm{H} \quad-3.675778 \quad 1.883631 \quad-1.111697$

H $\quad 0.580474 \quad 5.615084 \quad 0.906303$

$\mathrm{H} \quad-2.554822 \quad 5.151214 \quad 2.121473$

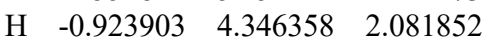

$\begin{array}{llll}\mathrm{H} & -2.432750 & 7.186017 & 0.854909\end{array}$

$\mathrm{H} \quad-3.465485 \quad 4.261232 \quad-2.113552$

$\begin{array}{llll}\mathrm{H} & -5.346509 & 8.119803 & -2.165334\end{array}$

H $\quad 2.712952 \quad 5.673263 \quad-0.345369$

H $\quad 0.815949 \quad 3.714319 \quad-3.665642$

H $\quad-1.306840 \quad 3.643777 \quad-2.407906$

$\mathrm{H} \quad-5.050210 \quad 5.847642 \quad-3.135474$

H $\quad-4.024159 \quad 8.784466 \quad-0.165349$

H $\quad 2.842276 \quad 4.724773 \quad-2.636101$

H $\quad 5.020480 \quad 4.466055 \quad 0.102676$

$\mathrm{H} \quad 3.688316 \quad 2.583308 \quad 4.569811$

$\mathrm{H} \quad 4.000883 \quad 4.263481 \quad 1.710629$

$\mathrm{H} \quad 2.656837 \quad 3.353650 \quad 2.534861$

H $\quad 5.946036 \quad 0.480491 \quad 0.477765$

$\begin{array}{llll}\mathrm{H} & 6.113211 & 3.966202 & 2.970848\end{array}$

$\begin{array}{llll}\mathrm{H} & 9.696546 & 2.468011 & 1.122377\end{array}$

H $\quad 3.283081 \quad 0.969267 \quad 6.402710$

H $\quad 4.021971-2.275098 \quad 3.682025$

H $\quad 4.426432-0.662295 \quad 1.860317$

H $\quad 8.554278 \quad 4.133185 \quad 2.572454$

$\begin{array}{llll}\mathrm{H} & 8.372454 & 0.631145 & 0.075675\end{array}$

H $\quad 3.442057 \quad-1.477379 \quad 5.961453$

H $\quad 3.675607 \quad-1.883108 \quad-1.111599$

H $\quad-0.580500 \quad-5.617631 \quad 0.903811$

H $\quad 2.553114 \quad-5.150327 \quad 2.121552$

H $\quad 0.922414 \quad-4.345058 \quad 2.081243$

$\begin{array}{llll}\mathrm{H} & 2.433200 & -7.184415 & 0.855706\end{array}$

H $\quad 3.463090 \quad-4.260625 \quad-2.114723$

H $\quad 5.346072 \quad-8.118262 \quad-2.165342$

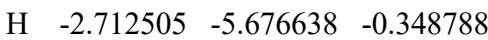

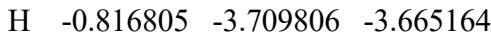

H $\quad 1.305409-3.638375 \quad-2.406419$

$\mathrm{H} \quad 5.048204 \quad-5.846745 \quad-3.136522$

H $\quad 4.024919 \quad-8.782573 \quad-0.164457$

$\mathrm{H} \quad-2.842103 \quad-4.724766 \quad-2.638106$

H $\quad 4.400610 \quad-4.260296 \quad 2.401960$

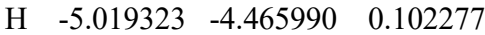

$\begin{array}{llll}\mathrm{H} & 3.463351 & 0.808402 & -1.552587\end{array}$

$\begin{array}{llll}\mathrm{H} & -4.401680 & 4.260024 & 2.402224\end{array}$

$\mathrm{H} \quad-5.815662-3.457279 \quad-3.984598$

H $\quad 7.691536 \quad-2.389590 \quad 0.368016$

H $\quad 5.815899 \quad 3.457155 \quad-3.984339$

$\begin{array}{llll}\mathrm{H} & -7.691815 & 2.387832 & 0.368338\end{array}$

V'5

$\begin{array}{llll}\mathrm{Rh} & 0.437839 & -0.958747 & 1.108921\end{array}$ 
$\begin{array}{llll}\text { Rh } & -0.437937 & -0.958641 & -1.108992\end{array}$

$\begin{array}{llll}\text { O } & 0.750387 & -2.620068 & -1.452133\end{array}$

$\begin{array}{llll}\text { O } & 1.943376 & -2.173027 & 0.414799\end{array}$

$\begin{array}{llll}\text { O } & -1.943750 & -2.172571 & -0.414814\end{array}$

$\begin{array}{llll}\text { O } & -0.750835 & -2.619900 & 1.452085\end{array}$

$\begin{array}{llll}\mathrm{O} & -1.550774 & 0.704605 & -0.564599\end{array}$

$\begin{array}{llll}\mathrm{O} & -1.149979 & 0.274273 & 1.618442\end{array}$

$\begin{array}{llll}\text { O } & 1.150110 & 0.274050 & -1.618460\end{array}$

$\begin{array}{llll}\mathrm{O} & 1.550977 & 0.704308 & 0.564575\end{array}$

$\begin{array}{llll}\mathrm{Br} & 6.701263 & -0.686021 & 1.063235\end{array}$

$\begin{array}{llll}\mathrm{Br} & 6.631451 & -6.369739 & 1.574482\end{array}$

$\begin{array}{llll}\mathrm{Br} & 2.078359 & 5.954824 & 2.596972\end{array}$

$\begin{array}{llll}\mathrm{Br} & 6.791409 & 2.781024 & 2.252495\end{array}$

$\begin{array}{lllll}\mathrm{Br} & -2.077184 & 5.955699 & -2.596388\end{array}$

$\begin{array}{lllll}\mathrm{Br} & -6.790443 & 2.782049 & -2.253129\end{array}$

$\begin{array}{llll}\mathrm{Br} & -6.632697 & -6.368590 & -1.574471\end{array}$

$\begin{array}{llll}\mathrm{Br} & -6.701362 & -0.684857 & -1.063143\end{array}$

$\begin{array}{llll}\text { C } & 4.676551 & -4.817771 & 0.177642\end{array}$

$\begin{array}{llll}\text { C } & 2.877995 & -3.652854 & -1.180581\end{array}$

$\begin{array}{llll}\text { C } & 3.098233 & -3.851318 & -2.721394\end{array}$

$\begin{array}{llll}\text { C } & 5.901839 & -2.383000 & 0.685376\end{array}$

$\begin{array}{llll}\text { C } & 4.109750 & -3.645415 & -0.323904\end{array}$

$\begin{array}{llll}\text { C } & 5.859360 & -4.749629 & 0.913570\end{array}$

$\begin{array}{llll}\text { C } & 4.531936 & -4.061964 & -3.145843\end{array}$

$\begin{array}{llll}\text { C } & 1.776196 & -2.750494 & -0.711956\end{array}$

C $\quad 2.288716 \quad-3.081203 \quad-3.730080$

$\begin{array}{llll}\text { C } & 2.294000 & -1.684354 & -3.761845\end{array}$

$\begin{array}{llll}\text { C } & 2.436890 & -4.913691 & -1.909016\end{array}$

$\begin{array}{llll}\text { C } & 5.016824 & -5.337618 & -3.449017\end{array}$

$\begin{array}{llll}\text { C } & 4.736779 & -2.417504 & -0.066055\end{array}$

$\begin{array}{llll}\text { C } & 5.398588 & -2.965982 & -3.262137\end{array}$

$\begin{array}{llll}\text { C } & 6.495213 & -3.542747 & 1.185279\end{array}$

C $\quad 7.203162 \quad-4.426123 \quad-3.941535$

$\begin{array}{llll}\text { C } & 1.553322 & -0.989719 & -4.714841\end{array}$

$\begin{array}{llll}\text { C } & 0.811703 & -3.087111 & -5.656279\end{array}$

$\begin{array}{llll}\text { C } & 1.547882 & -3.777381 & -4.691057\end{array}$

$\begin{array}{llll}\text { C } & 6.723928 & -3.145525 & -3.651054\end{array}$

$\begin{array}{llll}\text { C } & 6.344027 & -5.520454 & -3.844814\end{array}$

C $\quad 0.809528 \quad-1.689984 \quad-5.667791$

$\begin{array}{llll}\text { C } & -4.677392 & -4.817017 & -0.177751\end{array}$

$\begin{array}{llll}\text { C } & -2.878569 & -3.652435 & 1.180425\end{array}$

$\begin{array}{llll}\text { C } & -3.098869 & -3.851030 & 2.721212\end{array}$

$\begin{array}{llll}\text { C } & -5.902255 & -2.381998 & -0.685360\end{array}$

$\begin{array}{llll}\text { C } & -4.110328 & -3.644783 & 0.323764\end{array}$

$\begin{array}{llll}\text { C } & -5.860239 & -4.748629 & -0.913588\end{array}$

$\begin{array}{llll}\text { C } & -4.532594 & -4.061705 & 3.145581\end{array}$

$\begin{array}{llll}\text { C } & -1.776665 & -2.750144 & 0.711893\end{array}$

$\begin{array}{llll}\text { C } & -2.289419 & -3.080985 & 3.729995\end{array}$

$\begin{array}{llll}\text { C } & -2.294707 & -1.684144 & 3.761828\end{array}$

$\begin{array}{llll}\text { C } & -2.437534 & -4.913368 & 1.908774\end{array}$

$\begin{array}{llll}\text { C } & -5.017475 & -5.337361 & 3.448748\end{array}$

$\begin{array}{llll}\text { C } & -4.737151 & -2.416744 & 0.065991\end{array}$

$\begin{array}{llll}\text { C } & -5.399281 & -2.965736 & 3.261786\end{array}$

$\begin{array}{llll}\text { C } & -6.495882 & -3.541621 & -1.185239\end{array}$

C $\quad-7.203860 \quad-4.425905 \quad 3.941102$

$\begin{array}{llll}\text { C } & -1.554065 & -0.989548 & 4.714875\end{array}$

$\begin{array}{llll}\text { C } & -0.812501 & -3.086987 & 5.656268\end{array}$

$\begin{array}{llll}\text { C } & -1.548642 & -3.777207 & 4.690981\end{array}$

$\begin{array}{llll}\text { C } & -6.724639 & -3.145293 & 3.650626\end{array}$

C $\quad-6.344698 \quad-5.520225 \quad 3.844460$

$\begin{array}{llll}\text { C } & -0.810310 & -1.689854 & 5.667837\end{array}$

$\begin{array}{llll}\text { C } & -4.482129 & 2.475414 & -0.578644\end{array}$ $\begin{array}{llll}\text { C } & -2.603637 & 2.072433 & 1.053055\end{array}$

$\begin{array}{llll}\text { C } & -2.353690 & 2.922207 & 2.349080\end{array}$

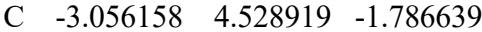

$\begin{array}{llll}\text { C } & -3.202534 & 2.796640 & -0.120118\end{array}$

$\begin{array}{llll}\text { C } & -5.025945 & 3.197342 & -1.639505\end{array}$

$\begin{array}{llll}\text { C } & -2.676655 & 4.393444 & 2.218015\end{array}$

$\begin{array}{llll}\text { C } & -1.697565 & 0.937518 & 0.683511\end{array}$

$\begin{array}{llll}\mathrm{C} & -1.181356 & 2.659533 & 3.258143\end{array}$

$\begin{array}{llll}\text { C } & 0.125920 & 2.516003 & 2.789187\end{array}$

$\begin{array}{llll}\text { C } & -3.450196 & 1.905308 & 2.304435\end{array}$

$\begin{array}{llll}\text { C } & -1.620764 & 5.302880 & 2.056890\end{array}$

$\begin{array}{llll}\text { C } & -2.481165 & 3.823675 & -0.738000\end{array}$

$\begin{array}{llll}\text { C } & -3.986692 & 4.883661 & 2.185263\end{array}$

$\begin{array}{llll}\text { C } & -4.334564 & 4.235398 & -2.259297\end{array}$

$\begin{array}{llll}\text { C } & -3.177986 & 7.130072 & 1.776310\end{array}$

$\begin{array}{llll}\text { C } & 1.184143 & 2.326408 & 3.677351\end{array}$

$\begin{array}{llll}\text { C } & -0.353099 & 2.464342 & 5.533960\end{array}$

$\begin{array}{llll}\mathrm{C} & -1.409937 & 2.642815 & 4.639906\end{array}$

$\begin{array}{llll}\text { C } & -4.235493 & 6.239645 & 1.964693\end{array}$

$\begin{array}{llll}\text { C } & -1.865636 & 6.654770 & 1.830080\end{array}$

$\begin{array}{llll}\mathrm{C} & 0.948316 & 2.298231 & 5.053211\end{array}$

$\begin{array}{llll}\text { C } & 4.482796 & 2.474689 & 0.578365\end{array}$

$\begin{array}{llll}\text { C } & 2.603993 & 2.072019 & -1.053064\end{array}$

$\begin{array}{llll}\text { C } & 2.354050 & 2.921966 & -2.348987\end{array}$

$\begin{array}{llll}\text { C } & 3.057106 & 4.528085 & 1.786892\end{array}$

$\begin{array}{llll}\text { C } & 3.203148 & 2.796033 & 0.120094\end{array}$

$\begin{array}{llll}\text { C } & 5.026819 & 3.196451 & 1.639243\end{array}$

$\begin{array}{llll}\text { C } & 2.677278 & 4.393149 & -2.217796\end{array}$

$\begin{array}{llll}\text { C } & 1.697807 & 0.937205 & -0.683529\end{array}$

$\begin{array}{llll}\text { C } & 1.181573 & 2.659610 & -3.257951\end{array}$

C $\quad-0.125693 \quad 2.516360 \quad-2.788882$

$\begin{array}{llll}\text { C } & 3.450404 & 1.904895 & -2.304559\end{array}$

$\begin{array}{llll}\text { C } & 3.987394 & 4.883152 & -2.185225\end{array}$

$\begin{array}{llll}\text { C } & 2.481929 & 3.823028 & 0.738227\end{array}$

$\begin{array}{llll}\text { C } & 1.621556 & 5.302713 & -2.056412\end{array}$

$\begin{array}{llll}\text { C } & 4.335576 & 4.234435 & 2.259309\end{array}$

$\begin{array}{llll}\text { C } & 3.179096 & 7.129641 & -1.775901\end{array}$

$\begin{array}{llll}\text { C } & -1.184033 & 2.326995 & -3.676958\end{array}$

$\begin{array}{llll}\text { C } & 0.353076 & 2.464641 & -5.533687\end{array}$

$\begin{array}{llll}\text { C } & 1.410022 & 2.642878 & -4.639734\end{array}$

$\begin{array}{llll}\text { C } & 1.866659 & 6.654544 & -1.829498\end{array}$

$\begin{array}{llll}\text { C } & 4.236439 & 6.239079 & -1.964562\end{array}$

$\begin{array}{llll}\text { C } & -0.948327 & 2.298782 & -5.052832\end{array}$

H $\quad 4.211377 \quad-5.778372 \quad 0.001679$

H $\quad 2.827444 \quad-1.134122 \quad-2.996813$

$\mathrm{H} \quad 2.973980 \quad-5.829618 \quad-1.695822$

$\mathrm{H} \quad 1.365870 \quad-5.030190 \quad-2.025126$

H $\quad 4.355480 \quad-6.195102-3.380306$

H $\quad 5.035789 \quad-1.968498 \quad-3.039767$

H $\quad 8.236654 \quad-4.567099 \quad-4.242550$

$\begin{array}{llll}\mathrm{H} & 1.539028 & 0.093730 & -4.700312\end{array}$

H $\quad 0.238817 \quad-3.640291 \quad-6.394703$

$\mathrm{H} \quad 1.542280 \quad-4.863454 \quad-4.675892$

H $\quad 7.384174 \quad-2.286671 \quad-3.724090$

H $\quad 6.703287 \quad-6.518729-4.075702$

H $\quad 0.230963 \quad-1.148342 \quad-6.410297$

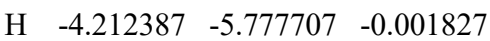

H $\quad-2.828118 \quad-1.133876 \quad 2.996790$

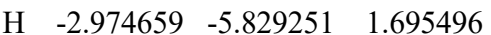

$\begin{array}{llll}\mathrm{H} & -1.366524 & -5.029918 & 2.024900\end{array}$

H $\quad-4.356101 \quad-6.194836 \quad 3.380090$

$\begin{array}{llll}\mathrm{H} & -5.036484 & -1.968250 & 3.039403\end{array}$
H $\quad-8.237360 \quad-4.566890 \quad 4.242058$

$\begin{array}{llll}\mathrm{H} & -1.539727 & 0.093902 & 4.700374\end{array}$

$\mathrm{H} \quad-0.239652 \quad-3.640197 \quad 6.394699$

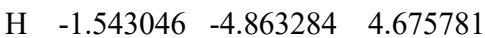

$\mathrm{H} \quad-7.384914 \quad-2.286457 \quad 3.723599$

$\mathrm{H} \quad-6.703947 \quad-6.518490 \quad 4.075351$

$\mathrm{H} \quad-0.231768 \quad-1.148246 \quad 6.410382$

$\mathrm{H} \quad-5.047167 \quad 1.676775 \quad-0.114074$

H $\quad 0.323584 \quad 2.516644 \quad 1.726361$

$\begin{array}{llll}\mathrm{H} & -4.485491 & 2.217649 & 2.239668\end{array}$

$\mathrm{H} \quad-3.284532 \quad 0.999766 \quad 2.874362$

$\begin{array}{llll}\mathrm{H} & -0.597727 & 4.950789 & 2.105828\end{array}$

$\begin{array}{llll}\mathrm{H} & -4.827013 & 4.212384 & 2.318604\end{array}$

$\begin{array}{llll}\mathrm{H} & -3.373930 & 8.182457 & 1.595917\end{array}$

$\mathrm{H} \quad 2.189274 \quad 2.205148 \quad 3.288030$

$\begin{array}{llll}\mathrm{H} & -0.546875 & 2.449751 & 6.602229\end{array}$

$\begin{array}{llll}\mathrm{H} & -2.422894 & 2.767133 & 5.012258\end{array}$

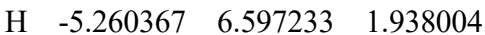

$\begin{array}{llll}\mathrm{H} & -1.028270 & 7.332838 & 1.694182\end{array}$

$\begin{array}{llll}\mathrm{H} & 1.771789 & 2.153651 & 5.745931\end{array}$

$\mathrm{H} \quad 5.047730 \quad 1.676097 \quad 0.113593$

$\mathrm{H} \quad-0.323267 \quad 2.517049 \quad-1.726036$

H $\quad 4.485750 \quad 2.217073 \quad-2.239861$

H $\quad 3.284543 \quad 0.999437 \quad-2.874567$

H $\quad 4.827579 \quad 4.211759 \quad-2.318782$

H $\quad 0.598457 \quad 4.950795 \quad-2.105249$

H $\quad 3.375230 \quad 8.181977 \quad-1.595431$

$\mathrm{H} \quad-2.189147 \quad 2.205915 \quad-3.287537$

$\mathrm{H} \quad 0.546751 \quad 2.450032 \quad-6.601988$

H $\quad 2.422985 \quad 2.766980 \quad-5.012167$

H $\quad 1.029424 \quad 7.332736 \quad-1.693398$

H $\quad 5.261369 \quad 6.596510 \quad-1.938009$

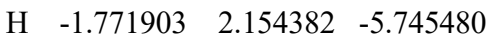

H $\quad 1.498185 \quad 4.088725 \quad 0.377482$

H $\quad 4.307761 \quad-1.497074 \quad-0.438524$

H $\quad-1.497458 \quad 4.089258 \quad-0.377078$

$\begin{array}{llll}\mathrm{H} & -4.307924 & -1.496414 & 0.438451\end{array}$

H $\quad 7.408517 \quad-3.504285 \quad 1.763734$

$\mathrm{H} \quad 4.775914 \quad 4.794857 \quad 3.073123$

H $\quad-4.774740 \quad 4.795956-3.073112$

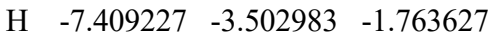

\section{V'6}

$\begin{array}{llll}\mathrm{Rh} & -0.004319 & 0.037745 & -1.829882\end{array}$

$\begin{array}{lllll}\mathrm{Rh} & 0.354183 & 0.417314 & 0.499811\end{array}$

$\begin{array}{llll}\mathrm{O} & 1.484208 & 2.059189 & -0.023831\end{array}$

$\begin{array}{llll}\text { O } & 1.503266 & 1.413996 & -2.194329\end{array}$

$\begin{array}{llll}\text { O } & -1.409826 & 1.518906 & 0.457395\end{array}$

$\begin{array}{llll}\mathrm{O} & -1.342281 & 1.595834 & -1.801620\end{array}$

$\begin{array}{llll}\mathrm{O} & -0.688394 & -1.314818 & 0.858297\end{array}$

$\begin{array}{llll}\text { O } & -1.500823 & -1.290698 & -1.249451\end{array}$

$\begin{array}{llll}\text { O } & 2.030395 & -0.768630 & 0.339712\end{array}$

$\begin{array}{llll}\text { O } & 1.347052 & -1.525284 & -1.683111\end{array}$

$\begin{array}{llll}\mathrm{Br} & 5.361936 & 0.542777 & 2.785147\end{array}$

$\begin{array}{lllll}\mathrm{Br} & 8.189680 & 1.186558 & -2.113876\end{array}$

$\begin{array}{llll}\mathrm{Br} & 0.930088 & -4.874949 & 3.850587\end{array}$

$\begin{array}{llll}\mathrm{Br} & 6.283320 & -2.920408 & 3.846942\end{array}$

$\begin{array}{llll}\mathrm{Br} & -3.462012 & -5.992679 & -3.822585\end{array}$

$\mathrm{Br} \quad-7.111628 \quad-1.896132 \quad-2.288651$

$\begin{array}{llll}\mathrm{Br} & -6.339452 & 1.326297 & 3.368636\end{array}$

$\begin{array}{llll}\mathrm{Br} & -2.930392 & 5.895773 & 3.633757\end{array}$

C $\quad 5.554433 \quad 2.167603 \quad-1.599492$ 


\begin{tabular}{|c|c|c|c|}
\hline & 3.151333 & 2.963674 & \\
\hline $\mathrm{C}$ & 2.961648 & 4.493965 & -1.432123 \\
\hline $\mathrm{C}$ & 5.447475 & 1.191677 & 0.993251 \\
\hline $\mathrm{C}$ & 4.383147 & 2.329372 & -0.852297 \\
\hline $\mathrm{C}$ & 6.637848 & 1.493113 & -1.041235 \\
\hline $\mathrm{C}$ & 4.099915 & 5.329286 & -0.900931 \\
\hline $\mathrm{C}$ & 1.934994 & 2.096383 & -1.213950 \\
\hline $\mathrm{C}$ & 1.596755 & 5.067117 & -1.142050 \\
\hline $\mathrm{C}$ & 0.778511 & 5.537213 & -2.175207 \\
\hline $\mathrm{C}$ & 3.197129 & 3.753448 & -2.724472 \\
\hline $\mathrm{C}$ & 4.731248 & 6.251554 & -1.743194 \\
\hline $\mathrm{C}$ & 4.340598 & 1.838971 & 0.461385 \\
\hline $\mathrm{C}$ & 4.515537 & 5.238116 & 0.433851 \\
\hline $\mathrm{C}$ & 6.614083 & 0.992243 & 0.257168 \\
\hline & 6.172203 & 6.962394 & 0.064255 \\
\hline $\mathrm{C}$ & -0.447943 & 6.144772 & -1.894054 \\
\hline $\mathrm{C}$ & -0.060154 & 5.822144 & 0.466970 \\
\hline $\mathrm{C}$ & 1.157343 & 5.208162 & 0.182661 \\
\hline $\mathrm{C}$ & 5.545793 & 6.046960 & 0.912887 \\
\hline $\mathrm{C}$ & 5.760690 & 7.064054 & -1.265918 \\
\hline $\mathrm{C}$ & -0.866468 & 6.297208 & -0.572401 \\
\hline $\mathrm{C}$ & -4.575834 & 2.183912 & 1.290850 \\
\hline $\mathrm{C}$ & -3.096023 & 2.735564 & -0.675653 \\
\hline $\mathrm{C}$ & -4.106280 & 2.672021 & -1.872950 \\
\hline $\mathrm{C}$ & -3.622797 & 4.387288 & 2.682509 \\
\hline $\mathrm{C}$ & -3.631455 & 3.026363 & 0.695925 \\
\hline $\mathrm{C}$ & -5.036727 & 2.474540 & 2.569573 \\
\hline $\mathrm{C}$ & -5.562382 & 2.854591 & -1.503122 \\
\hline $\mathrm{C}$ & -1.863522 & 1.885592 & -0.677171 \\
\hline $\mathrm{C}$ & -3.886245 & 1.700682 & -3.002526 \\
\hline $\mathrm{C}$ & -3.888320 & 2.178759 & -4.317293 \\
\hline $\mathrm{C}$ & -3.157642 & 3.819686 & -1.739974 \\
\hline $\mathrm{C}$ & -6.089156 & 4.089163 & -1.104037 \\
\hline $\mathrm{C}$ & -3.154245 & 4.134254 & 1.397382 \\
\hline $\mathrm{C}$ & -6.414108 & 1.739655 & -1.499873 \\
\hline $\mathrm{C}$ & -4.575290 & 3.574214 & 3.292838 \\
\hline $\mathrm{C}$ & -8.245855 & 3.079054 & -0.663650 \\
\hline $\mathrm{C}$ & -3.736175 & 1.300860 & -5.391883 \\
\hline $\mathrm{C}$ & -3.581680 & -0.552176 & -3.850071 \\
\hline $\mathrm{C}$ & -3.739151 & 0.328842 & -2.781897 \\
\hline $\mathrm{C}$ & -7.737138 & 1.846340 & -1.077374 \\
\hline $\mathrm{C}$ & -7.416722 & 4.200854 & -0.688006 \\
\hline $\mathrm{C}$ & -3.581141 & -0.068590 & -5.160405 \\
\hline $\mathrm{C}$ & -4.626635 & -2.446521 & -0.989322 \\
\hline $\mathrm{C}$ & -2.509430 & -2.739345 & 0.336040 \\
\hline $\mathrm{C}$ & -3.054567 & -2.798047 & 1.807824 \\
\hline $\mathrm{C}$ & -3.945011 & -4.551690 & -2.662407 \\
\hline $\mathrm{C}$ & -3.430084 & -3.140163 & -0.779624 \\
\hline $\mathrm{C}$ & -5.467602 & -2.833950 & -2.025037 \\
\hline $\mathrm{C}$ & -4.507580 & -3.194953 & 1.947474 \\
\hline $\mathrm{C}$ & -1.497052 & -1.704578 & -0.042715 \\
\hline $\mathrm{C}$ & -2.594883 & -1.781226 & 2.820501 \\
\hline $\mathrm{C}$ & -2.753842 & -0.407641 & 2.616290 \\
\hline $\mathrm{C}$ & -2.049545 & -3.796393 & 1.327786 \\
\hline $\mathrm{C}$ & -4.958181 & -4.498258 & 1.703272 \\
\hline $\mathrm{C}$ & -3.086779 & -4.199139 & -1.623434 \\
\hline $\mathrm{C}$ & -5.459140 & -2.216115 & 2.272015 \\
\hline $\mathrm{C}$ & -5.149948 & -3.887402 & -2.880945 \\
\hline $\mathrm{C}$ & -7.256804 & -3.818660 & 2.048989 \\
\hline $\mathrm{C}$ & -2.336579 & 0.509545 & 3.578540 \\
\hline$C$ & -1.606464 & -1.312097 & 4.986900 \\
\hline & -2.022689 & -2.225598 & 4.017020 \\
\hline
\end{tabular}

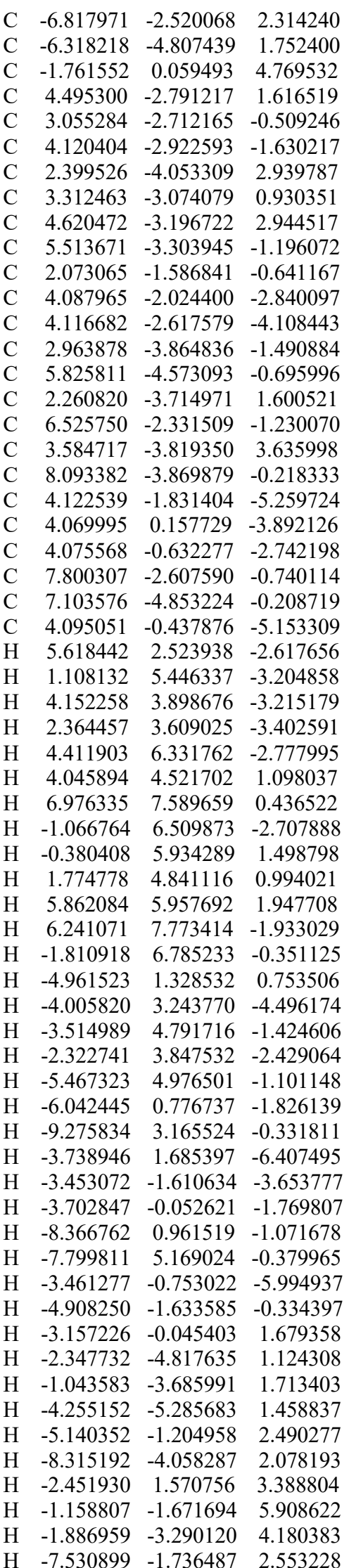

$\begin{array}{rrrr}\mathrm{H} & -6.641680 & -5.824910 & 1.554693 \\ \mathrm{H} & -1.435827 & 0.771760 & 5.521813 \\ \mathrm{H} & 5.312616 & -2.283165 & 1.127121 \\ \mathrm{H} & 4.129946 & -3.700764 & -4.187439 \\ \mathrm{H} & 3.116543 & -4.853329 & -1.073997 \\ \mathrm{H} & 2.191583 & -3.796577 & -2.247645 \\ \mathrm{H} & 5.069807 & -5.349220 & -0.665785 \\ \mathrm{H} & 6.312412 & -1.348781 & -1.631903 \\ \mathrm{H} & 9.084113 & -4.086800 & 0.168594 \\ \mathrm{H} & 4.143936 & -2.304027 & -6.237056 \\ \mathrm{H} & 4.027013 & 1.236144 & -3.801763 \\ \mathrm{H} & 4.049949 & -0.165231 & -1.765211 \\ \mathrm{H} & 8.561278 & -1.833542 & -0.771532 \\ \mathrm{H} & 7.321691 & -5.842194 & 0.182567 \\ \mathrm{H} & 4.091244 & 0.178216 & -6.047315 \\ \mathrm{H} & 1.339583 & -3.931731 & 1.072894 \\ \mathrm{H} & 3.441445 & 1.951503 & 1.048813 \\ \mathrm{H} & -2.161245 & -4.740617 & -1.469021 \\ \mathrm{H} & -2.416355 & 4.779277 & 0.942143 \\ \mathrm{H} & 7.459371 & 0.461460 & 0.673692 \\ \mathrm{H} & 3.697042 & -4.114231 & 4.670756 \\ \mathrm{H} & -5.812674 & -4.176758 & -3.685448 \\ \mathrm{H} & -4.939551 & 3.783936 & 4.289626\end{array}$

$V^{\prime 7}$

$\begin{array}{llll}\mathrm{Rh} & -0.050795 & -1.178701 & 1.159288\end{array}$ $\begin{array}{llll}\mathrm{Rh} & -0.478736 & -0.208873 & -0.976183\end{array}$

$\begin{array}{llll}\text { O } & -1.785482 & 1.062783 & -0.036663\end{array}$

$\begin{array}{llll}\mathrm{O} & -1.788939 & -0.238124 & 1.811220\end{array}$

$\begin{array}{llll}\text { O } & 1.069926 & 1.113708 & -0.569036\end{array}$

$\begin{array}{llll}\text { O } & 1.002739 & 0.538377 & 1.616229\end{array}$

$\begin{array}{llll}\mathrm{O} & 0.852648 & -1.577544 & -1.757427\end{array}$

$\begin{array}{llll}\text { O } & 1.655400 & -1.997786 & 0.315157\end{array}$

$\begin{array}{llll}\text { O } & -2.005044 & -1.571400 & -1.181407\end{array}$

$\begin{array}{llll}\text { O } & -1.173570 & -2.800546 & 0.523253\end{array}$

$\begin{array}{lllll}\mathrm{Br} & -1.460717 & 6.029660 & -0.810882\end{array}$

$\mathrm{Br} \quad-6.841297 \quad 4.175031 \quad-1.352223$

$\mathrm{Br} \quad-6.518311 \quad-4.520724 \quad-4.630942$

$\begin{array}{lllll}\mathrm{Br} & -6.576391 & 0.449095 & -1.832775\end{array}$

$\begin{array}{llll}\mathrm{Br} & 4.545047 & -6.760167 & 1.602319\end{array}$

$\begin{array}{llll}\mathrm{Br} & 7.244624 & -1.745081 & 1.459666\end{array}$

$\begin{array}{lllll}\mathrm{Br} & 1.942299 & 6.673584 & -2.158881\end{array}$

$\mathrm{Br} \quad 5.818429 \quad 2.640445 \quad-3.211980$

$\begin{array}{llll}\text { C } & -4.832260 & 2.961299 & 0.279396\end{array}$

$\begin{array}{llll}\text { C } & -3.203169 & 1.688455 & 1.738368\end{array}$

C $\quad-3.232799 \quad 1.966974 \quad 3.278423$

$\begin{array}{llll}\text { C } & -2.860878 & 4.811044 & -0.348047\end{array}$

$\begin{array}{llll}\text { C } & -3.561370 & 2.833480 & 0.836773\end{array}$

$\begin{array}{llll}\text { C } & -5.095478 & 4.024641 & -0.583558\end{array}$

$\begin{array}{llll}\text { C } & -3.598477 & 3.382004 & 3.657311\end{array}$

$\begin{array}{llll}\text { C } & -2.182897 & 0.766241 & 1.139906\end{array}$

C $\quad-2.233386 \quad 1.345369 \quad 4.216522$

$\begin{array}{llll}\text { C } & -0.857341 & 1.391045 & 3.984144\end{array}$

$\begin{array}{llll}\text { C } & -4.259253 & 1.083656 & 2.645410\end{array}$

$\begin{array}{llll}\text { C } & -4.922327 & 3.779082 & 3.866314\end{array}$

$\begin{array}{llll}\text { C } & -2.570977 & 3.772925 & 0.524288\end{array}$

$\begin{array}{llll}\text { C } & -2.580169 & 4.337016 & 3.794340\end{array}$

$\begin{array}{llll}\text { C } & -4.126795 & 4.966382 & -0.916804\end{array}$

$\begin{array}{llll}\text { C } & -4.208963 & 6.050628 & 4.302305\end{array}$

$\begin{array}{llll}\text { C } & 0.037351 & 0.821983 & 4.888985\end{array}$

$\begin{array}{llll}\text { C } & -1.809641 & 0.196942 & 6.314480\end{array}$

$\begin{array}{llll}\text { C } & -2.701936 & 0.751840 & 5.395094\end{array}$ 


\begin{tabular}{|c|c|c|c|}
\hline $\mathrm{C}$ & -2.880998 & 5.660092 & 4.107746 \\
\hline C & -5.226745 & 5.104419 & 4.186642 \\
\hline $\mathrm{C}$ & -0.436411 & 0.223421 & 6.058453 \\
\hline C & 2.288040 & 4.287730 & -0.603890 \\
\hline C & 2.453457 & 2.299475 & 0.938180 \\
\hline . & 3.501219 & 2.119502 & 2.092575 \\
\hline $\mathrm{C}$ & 4.373972 & 3.312818 & -2.157218 \\
\hline $\mathrm{C}$ & 2.896250 & 3.074780 & -0.270588 \\
\hline $\mathrm{C}$ & 2.745885 & 4.995355 & -1.714290 \\
\hline $\mathrm{C}$ & 4.872406 & 2.691907 & 1.812465 \\
\hline C & 1.440463 & 1.235074 & 0.645333 \\
\hline $\mathrm{C}$ & 3.541304 & 0.860682 & 2.919303 \\
\hline $\mathrm{C}$ & 3.573597 & -0.414554 & 2.350579 \\
\hline $\mathrm{C}$ & 2.327605 & 3.032039 & 2.263712 \\
\hline $\mathrm{C}$ & 5.132532 & 4.067115 & 1.804335 \\
\hline $\mathrm{C}$ & 3.939999 & 2.580828 & -1.060262 \\
\hline $\mathrm{C}$ & 5.917328 & 1.815523 & 1.483597 \\
\hline $\mathrm{C}$ & 3.792763 & 4.530725 & -2.507246 \\
\hline $\mathrm{C}$ & 7.415001 & 3.673336 & 1.095895 \\
\hline $\mathrm{C}$ & 3.650282 & -1.550938 & 3.154757 \\
\hline $\mathrm{C}$ & 3.708464 & -0.151116 & 5.121615 \\
\hline $\mathrm{C}$ & 3.621334 & 0.982629 & 4.311947 \\
\hline $\mathrm{C}$ & 7.172410 & 2.298242 & 1.121134 \\
\hline $\mathrm{C}$ & 6.391440 & 4.553698 & 1.448057 \\
\hline $\mathrm{C}$ & 3.716219 & -1.423226 & 4.543786 \\
\hline $\mathrm{C}$ & 4.926300 & -2.401989 & -0.075122 \\
\hline $\mathrm{C}$ & 2.918608 & -2.730116 & -1.549721 \\
\hline $\mathrm{C}$ & 3.471070 & -2.284272 & -2.949571 \\
\hline $\mathrm{C}$ & 4.709833 & -4.984615 & 0.912200 \\
\hline $\mathrm{C}$ & 3.902204 & -3.236788 & -0.534835 \\
\hline $\mathrm{C}$ & 5.834627 & -2.885366 & 0.857905 \\
\hline $\mathrm{C}$ & 4.974573 & -2.357542 & -3.104621 \\
\hline $\mathrm{C}$ & 1.722983 & -2.048421 & -0.960247 \\
\hline $\mathrm{C}$ & 2.844147 & -1.129214 & -3.686150 \\
\hline $\mathrm{C}$ & 2.439947 & -1.312887 & -5.012842 \\
\hline $\mathrm{C}$ & 2.673223 & -3.542293 & -2.811424 \\
\hline $\mathrm{C}$ & 5.722692 & -1.170511 & -3.089866 \\
\hline $\mathrm{C}$ & 3.792675 & -4.536765 & -0.036050 \\
\hline $\mathrm{C}$ & 5.664678 & -3.572082 & -3.200720 \\
\hline $\mathrm{C}$ & 5.749109 & -4.178616 & 1.371448 \\
\hline $\mathrm{C}$ & 7.792594 & -2.415541 & -3.207293 \\
\hline $\mathrm{C}$ & 1.899804 & -0.253225 & -5.743682 \\
\hline $\mathrm{C}$ & 2.164652 & 1.195894 & -3.829743 \\
\hline $\mathrm{C}$ & 2.707925 & 0.135316 & -3.107340 \\
\hline $\mathrm{C}$ & 7.059166 & -3.601613 & -3.250518 \\
\hline $\mathrm{C}$ & 7.114800 & -1.197153 & -3.132023 \\
\hline $\mathrm{C}$ & 1.759201 & 1.005184 & -5.152650 \\
\hline $\mathrm{C}$ & -4.841979 & -1.747010 & -1.254595 \\
\hline $\mathrm{C}$ & -3.214881 & -3.486354 & -0.499874 \\
\hline $\mathrm{C}$ & -3.684642 & -4.296141 & 0.759534 \\
\hline $\mathrm{C}$ & -5.834565 & -3.378914 & -3.257481 \\
\hline $\mathrm{C}$ & -4.288893 & -3.023545 & -1.441410 \\
\hline $\mathrm{C}$ & -5.868779 & -1.312793 & -2.080066 \\
\hline $\mathrm{C}$ & -5.168969 & -4.561160 & 0.839917 \\
\hline $\mathrm{C}$ & -2.045502 & -2.556915 & -0.371252 \\
\hline $\mathrm{C}$ & -3.044455 & -4.085089 & 2.105988 \\
\hline $\mathrm{C}$ & -3.098456 & -2.848132 & 2.753688 \\
\hline $\mathrm{C}$ & -2.907572 & -4.966344 & -0.323248 \\
\hline $\mathrm{C}$ & -6.048811 & -3.543768 & 1.235213 \\
\hline $\mathrm{C}$ & -4.789314 & -3.839029 & -2.457069 \\
\hline $\mathrm{C}$ & -5.689957 & -5.825198 & 0.547894 \\
\hline $\mathrm{C}$ & -6.394517 & -2.116671 & -3.092464 \\
\hline
\end{tabular}

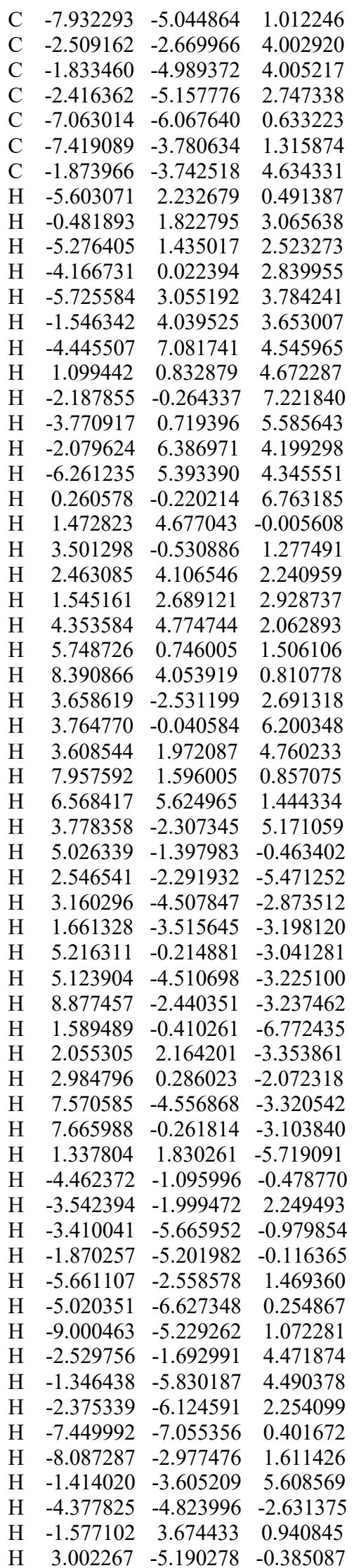

H $\quad 4.426860 \quad 1.651797 \quad-0.798201$

$\mathrm{H} \quad-4.342506 \quad 5.780343 \quad-1.596020$

$\mathrm{H} \quad-7.200801 \quad-1.770389-3.725072$

H $\quad 6.461184 \quad-4.541665 \quad 2.100369$

H $\quad 4.144083 \quad 5.095931-3.360080$

\section{V'8}

$\begin{array}{llll}\text { Rh } & -0.000883 & 0.000123 & -1.152617\end{array}$

$\begin{array}{llll}\mathrm{Rh} & -0.000911 & -0.000254 & 1.242961\end{array}$

$\begin{array}{llll}\text { O } & 1.594297 & 1.324360 & 1.148540\end{array}$

$\begin{array}{llll}\text { O } & 1.760641 & 1.046257 & -1.089384\end{array}$

$\begin{array}{llll}\mathrm{O} & -1.463780 & 1.447595 & 1.171623\end{array}$

$\begin{array}{llll}\text { O } & -1.180899 & 1.686994 & -1.062669\end{array}$

$\begin{array}{llll}\mathrm{O} & -1.595825 & -1.325209 & 1.148922\end{array}$

$\begin{array}{llll}\mathrm{O} & -1.762525 & -1.045808 & -1.088830\end{array}$

$\begin{array}{llll}\text { O } & 1.461968 & -1.448253 & 1.172088\end{array}$

$\begin{array}{llll}\text { O } & 1.179263 & -1.686702 & -1.062265\end{array}$

$\begin{array}{llll}\mathrm{Br} & 4.447230 & 4.430283 & 4.760986\end{array}$

$\begin{array}{llll}\mathrm{Br} & 5.718389 & -1.076234 & 3.956320\end{array}$

$\begin{array}{llll}\mathrm{Br} & 6.366917 & -0.858198 & -3.496255\end{array}$

$\begin{array}{llll}\mathrm{Br} & 2.814952 & -5.079157 & -4.953362\end{array}$

$\begin{array}{llll}\mathrm{Br} & -5.714127 & 1.069125 & 3.962798\end{array}$

$\begin{array}{llll}\mathrm{Br} & -4.450272 & -4.440825 & 4.755920\end{array}$

$\begin{array}{llll}\mathrm{Br} & -2.813313 & 5.091621 & -4.947764\end{array}$

$\mathrm{Br} \quad-6.361665 \quad 0.863065 \quad-3.503608$

$\begin{array}{llll}\text { C } & 4.687353 & 0.686074 & 1.955634\end{array}$

$\begin{array}{llll}\text { C } & 3.627919 & 1.934675 & 0.078158\end{array}$

$\begin{array}{llll}\text { C } & 4.050565 & 3.022085 & -0.968258\end{array}$

$\begin{array}{llll}\text { C } & 4.558141 & 2.899101 & 3.621588\end{array}$

$\begin{array}{llll}\text { C } & 4.199125 & 1.899439 & 1.462605\end{array}$

$\begin{array}{llll}\text { C } & 5.091890 & 0.600890 & 3.284025\end{array}$

$\begin{array}{llll}\text { C } & 5.084194 & 4.040995 & -0.538923\end{array}$

$\begin{array}{llll}C & 2.216426 & 1.412543 & 0.039220\end{array}$

$\begin{array}{llll}\text { C } & 2.953525 & 3.531960 & -1.867362\end{array}$

C $\quad 1.913762 \quad 4.309848 \quad-1.342895$

$\begin{array}{llll}\text { C } & 4.474980 & 1.591019 & -1.129287\end{array}$

$\begin{array}{llll}\text { C } & 4.847343 & 5.414098 & -0.704450\end{array}$

$\begin{array}{llll}\text { C } & 4.146762 & 3.016844 & 2.298748\end{array}$

$\begin{array}{llll}\text { C } & 6.303895 & 3.655646 & 0.043701\end{array}$

$\begin{array}{llll}\text { C } & 5.035705 & 1.697475 & 4.142938\end{array}$

$\begin{array}{llll}\text { C } & 6.981445 & 5.965179 & 0.298340\end{array}$

$\begin{array}{llll}\text { C } & 0.879155 & 4.765013 & -2.159123\end{array}$

$\begin{array}{llll}\text { C } & 1.910997 & 3.683028 & -4.052816\end{array}$

$\begin{array}{llll}\text { C } & 2.949644 & 3.236346 & -3.232720\end{array}$

$\begin{array}{llll}\text { C } & 7.237581 & 4.602380 & 0.458779\end{array}$

$\begin{array}{llll}\text { C } & 5.782175 & 6.364055 & -0.289340\end{array}$

$\begin{array}{llll}\text { C } & 0.868369 & 4.443310 & -3.518497\end{array}$

$\begin{array}{llll}\text { C } & -2.969978 & 3.892850 & -2.357918\end{array}$

$\begin{array}{llll}\text { C } & -2.889199 & 2.934495 & -0.020105\end{array}$

$\begin{array}{llll}\text { C } & -2.855048 & 4.187793 & 0.909901\end{array}$

$\begin{array}{llll}\text { C } & -4.997465 & 2.120236 & -3.039493\end{array}$

$\begin{array}{llll}\text { C } & -3.483788 & 3.019857 & -1.394998\end{array}$

$\begin{array}{llll}\text { C } & -3.497717 & 3.871208 & -3.643380\end{array}$

$\begin{array}{llll}\text { C } & -3.347686 & 5.476266 & 0.298325\end{array}$

$\begin{array}{llll}\text { C } & -1.757389 & 1.954453 & 0.043407\end{array}$

$\begin{array}{llll}\text { C } & -1.694752 & 4.341087 & 1.857797\end{array}$

$\begin{array}{llll}\text { C } & -1.946318 & 4.589911 & 3.211732\end{array}$

$\begin{array}{llll}\text { C } & -3.798062 & 3.053456 & 1.183079\end{array}$

$\begin{array}{llll}\text { C } & -4.691569 & 5.677495 & -0.041782\end{array}$

$\begin{array}{llll}\text { C } & -4.508815 & 2.133974 & -1.736133\end{array}$

$\begin{array}{llll}\text { C } & -2.427076 & 6.491315 & -0.006744\end{array}$

$\begin{array}{llll}\text { C } & -4.512002 & 2.988206 & -4.014819\end{array}$ 


\begin{tabular}{|c|c|c|c|}
\hline $\mathrm{C}$ & -4.172892 & 7.838430 & -1.002144 \\
\hline C & -0.891677 & 4.760443 & 4.110052 \\
\hline $\mathrm{C}$ & 0.683865 & 4.440876 & 2.311960 \\
\hline $\mathrm{C}$ & -0.370718 & 4.271458 & 1.416808 \\
\hline $\mathrm{C}$ & -2.832925 & 7.656831 & -0.655179 \\
\hline $\mathrm{C}$ & -5.100562 & 6.845564 & -0.685680 \\
\hline $\mathrm{C}$ & 0.430066 & 4.685343 & 3.662147 \\
\hline $\mathrm{C}$ & -4.148664 & -3.022810 & 2.296483 \\
\hline $\mathrm{C}$ & -3.629138 & -1.936176 & 0.077909 \\
\hline $\mathrm{C}$ & -4.051393 & -3.021230 & -0.971107 \\
\hline $\mathrm{C}$ & -5.090410 & -0.607616 & 3.286921 \\
\hline $\mathrm{C}$ & -4.199991 & -1.903708 & 1.462586 \\
\hline $\mathrm{C}$ & -4.559522 & -2.907156 & 3.619715 \\
\hline $\mathrm{C}$ & -5.084561 & -4.041749 & -0.544376 \\
\hline $\mathrm{C}$ & -2.217975 & -1.413059 & 0.039555 \\
\hline $\mathrm{C}$ & -2.954339 & -3.528076 & -1.872005 \\
\hline $\mathrm{C}$ & -1.914165 & -4.307299 & -1.350326 \\
\hline $\mathrm{C}$ & -4.476712 & -1.590026 & -1.128538 \\
\hline $\mathrm{C}$ & -4.847549 & -5.414256 & -0.714422 \\
\hline $\mathrm{C}$ & -4.686623 & -0.690720 & 1.958210 \\
\hline $\mathrm{C}$ & -6.304131 & -3.658473 & 0.039898 \\
\hline $\mathrm{C}$ & -5.03 & -1.70 & 4.143658 \\
\hline $\mathrm{C}$ & -6.981213 & -5.968935 & 0.287332 \\
\hline $\mathrm{C}$ & -0.879221 & -4.758877 & -2.168131 \\
\hline $\mathrm{C}$ & -1.911626 & -3.670779 & -4.057973 \\
\hline $\mathrm{C}$ & -2.950562 & -3.227663 & -3.236315 \\
\hline $\mathrm{C}$ & -7.237509 & 6695 & 0.4 \\
\hline $\mathrm{C}$ & -5.782075 & -6.365712 & -0.302056 \\
\hline $\mathrm{C}$ & -0.868504 & -4.432251 & -3.526326 \\
\hline $\mathrm{C}$ & 2.970446 & -3.886418 & -2.360688 \\
\hline $\mathrm{C}$ & 2.888346 & -2.933681 & -0.020744 \\
\hline $\mathrm{C}$ & 2.853618 & -4.189148 & 0.906271 \\
\hline $\mathrm{C}$ & 5.000504 & -2.114494 & -3.036268 \\
\hline $\mathrm{C}$ & 3.484167 & -3.016061 & -1.395307 \\
\hline $\mathrm{C}$ & 3.499428 & -3.862450 & -3.645570 \\
\hline $\mathrm{C}$ & 3.347339 & -5.475856 & 0.291848 \\
\hline $\mathrm{C}$ & 1.755891 & -1.954389 & 0.043616 \\
\hline $\mathrm{C}$ & 1.692387 & -4.345088 & 1.852557 \\
\hline $\mathrm{C}$ & 0.368777 & -4.275435 & 1.410263 \\
\hline $\mathrm{C}$ & 3.795916 & -3.055059 & 1.183076 \\
\hline $\mathrm{C}$ & 4.691482 & -5.675399 & -0.048251 \\
\hline $\mathrm{C}$ & 4.510437 & -2.130473 & -1.733444 \\
\hline C & 2.427453 & -6.490587 & -0.016385 \\
\hline $\mathrm{C}$ & 4.515083 & -2.979747 & -4.014003 \\
\hline $\mathrm{C}$ & 4.174475 & -7.834063 & -1.014581 \\
\hline $\mathrm{C}$ & -0.686650 & -4.447313 & 2.303962 \\
\hline $\mathrm{C}$ & 0.887146 & -4.769427 & 4.103108 \\
\hline $\mathrm{C}$ & 1.942644 & -4.596464 & 3.206267 \\
\hline $\mathrm{C}$ & 2.834246 & -7.654137 & -0.667750 \\
\hline $\mathrm{C}$ & 5.101433 & -6.841502 & -0.695062 \\
\hline $\mathrm{C}$ & -0.434161 & -4.694259 & 3.653930 \\
\hline $\mathrm{H}$ & 4.724702 & -0.185168 & 1.313964 \\
\hline $\mathrm{H}$ & 1.917282 & 4.558849 & -0.287571 \\
\hline $\mathrm{H}$ & 5.513227 & 1.335130 & -0.957727 \\
\hline $\mathrm{H}$ & 3.983255 & 1.006093 & -1.895664 \\
\hline $\mathrm{H}$ & 3.928726 & 5.750450 & -1.167357 \\
\hline $\mathrm{H}$ & 6.534315 & 2.608976 & 0.198891 \\
\hline $\mathrm{H}$ & 7.708083 & 6.703091 & 0.623624 \\
\hline $\mathrm{H}$ & 0.083195 & 5.369781 & -1.734614 \\
\hline $\mathrm{H}$ & 1.915871 & 3.435254 & -5.110029 \\
\hline $\mathrm{H}$ & 3.752445 & 2.637387 & -3.651748 \\
\hline & 8.167555 & 4.270268 & 0.909997 \\
\hline
\end{tabular}
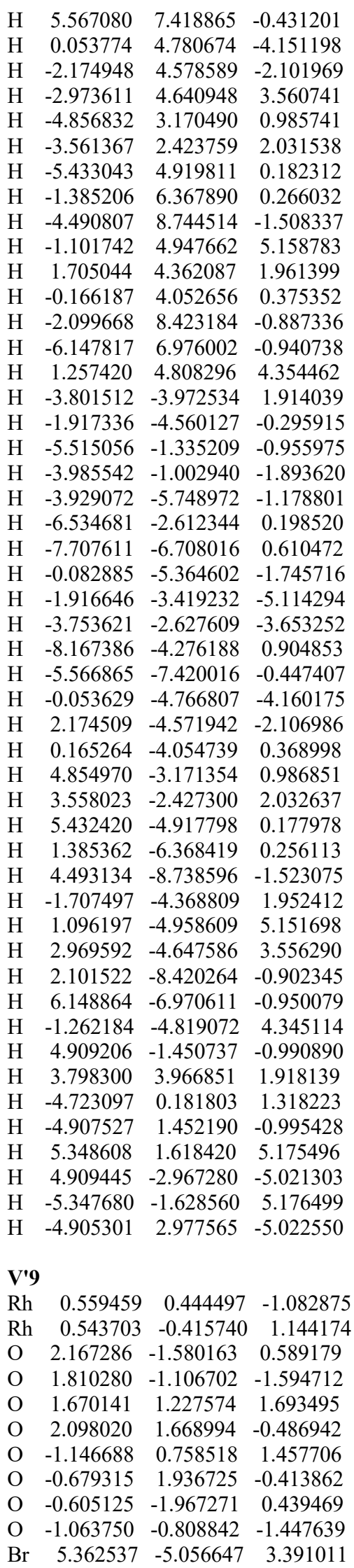

$\begin{array}{llll}\mathrm{Br} & 0.239227 & -6.747879 & 1.528039\end{array}$

$\begin{array}{llll}\mathrm{Br} & -6.335179 & 0.018284 & -3.298804\end{array}$

$\begin{array}{lllll}\mathrm{Br} & -2.868854 & -3.406775 & -6.252837\end{array}$

$\begin{array}{llll}\mathrm{Br} & -6.498164 & -0.175064 & 3.118113\end{array}$

$\begin{array}{llll}\mathrm{Br} & -3.160771 & 3.239238 & 6.227473\end{array}$

$\begin{array}{lllll}\mathrm{Br} & 0.119048 & 6.907000 & -1.328770\end{array}$

$\begin{array}{llll}\mathrm{Br} & 5.146132 & 5.155709 & -3.383006\end{array}$

$\begin{array}{llll}\text { C } & 1.928361 & -4.852766 & 0.211284\end{array}$

$\begin{array}{llll}\text { C } & 3.086457 & -3.040772 & -1.039658\end{array}$

$\begin{array}{llll}\text { C } & 4.297740 & -2.886053 & -2.020676\end{array}$

$\begin{array}{llll}\text { C } & 3.959382 & -4.987648 & 2.094178\end{array}$

$\begin{array}{llll}\text { C } & 3.068205 & -4.056977 & 0.062390\end{array}$

$\begin{array}{llll}\text { C } & 1.820587 & -5.693369 & 1.314906\end{array}$

$\begin{array}{llll}\text { C } & 5.473192 & -3.822833 & -1.842375\end{array}$

$\begin{array}{llll}\text { C } & 2.309655 & -1.806382 & -0.657598\end{array}$

$\begin{array}{llll}\text { C } & 4.630378 & -1.474297 & -2.432042\end{array}$

$\begin{array}{llll}\text { C } & 5.190437 & -0.581912 & -1.508934\end{array}$

$\begin{array}{llll}\text { C } & 2.988201 & -3.455935 & -2.488685\end{array}$

$\begin{array}{llll}\text { C } & 5.298508 & -5.213100 & -1.727527\end{array}$

$\begin{array}{llll}\text { C } & 4.097819 & -4.137965 & 1.002355\end{array}$

$\begin{array}{llll}\text { C } & 6.784115 & -3.325765 & -1.783082\end{array}$

$\begin{array}{llll}\text { C } & 2.825254 & -5.777573 & 2.277716\end{array}$

$\begin{array}{llll}\text { C } & 7.681299 & -5.553675 & -1.473461\end{array}$

$\begin{array}{llll}\text { C } & 5.486188 & 0.731337 & -1.872072\end{array}$

$\begin{array}{llll}\text { C } & 4.675575 & 0.288067 & -4.100346\end{array}$

C $\quad 4.389766-1.029344 \quad-3.734608$

$\begin{array}{llll}\text { C } & 7.873575 & -4.178972 & -1.599073\end{array}$

$\begin{array}{llll}\text { C } & 6.384521 & -6.065655 & -1.542925\end{array}$

$\begin{array}{llll}\text { C } & 5.219924 & 1.175170 & -3.169378\end{array}$

$\begin{array}{llll}\text { C } & 1.826869 & 4.980676 & -0.084464\end{array}$

$\begin{array}{llll}\text { C } & 2.978588 & 3.141828 & 1.123034\end{array}$

$\begin{array}{llll}\text { C } & 4.214779 & 3.001692 & 2.063226\end{array}$

C $\quad 3.779506 \quad 5.075320 \quad-2.046664$

$\begin{array}{llll}\text { C } & 2.947667 & 4.149976 & 0.009910\end{array}$

$\begin{array}{llll}\text { C } & 1.691584 & 5.827813 & -1.179777\end{array}$

$\begin{array}{llll}\text { C } & 5.416631 & 3.843745 & 1.715796\end{array}$

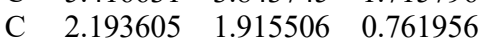

$\begin{array}{llll}\text { C } & 4.541947 & 1.638319 & 2.614906\end{array}$

$\begin{array}{llll}\text { C } & 4.615065 & 0.508425 & 1.797707\end{array}$

$\begin{array}{llll}\text { C } & 2.941028 & 3.628701 & 2.552827\end{array}$

$\begin{array}{llll}\text { C } & 6.575829 & 3.218198 & 1.229566\end{array}$

$\begin{array}{llll}\text { C } & 3.931238 & 4.194975 & -0.981070\end{array}$

$\begin{array}{llll}\text { C } & 5.400974 & 5.242181 & 1.792598\end{array}$

$\begin{array}{llll}\text { C } & 2.662681 & 5.900537 & -2.176409\end{array}$

$\begin{array}{llll}\text { C } & 7.637810 & 5.362643 & 0.874066\end{array}$

$\begin{array}{llll}\text { C } & 4.931386 & -0.737835 & 2.336244\end{array}$

$\begin{array}{llll}\text { C } & 5.131841 & 0.258428 & 4.523694\end{array}$

$\begin{array}{llll}\text { C } & 4.808394 & 1.503570 & 3.982790\end{array}$

$\begin{array}{llll}\text { C } & 6.499571 & 5.994424 & 1.375999\end{array}$

$\begin{array}{llll}\text { C } & 7.672233 & 3.968564 & 0.806937\end{array}$

$\begin{array}{llll}\text { C } & 5.191397 & -0.869927 & 3.700463\end{array}$

$\begin{array}{llll}\text { C } & -2.925121 & 2.842292 & 3.402623\end{array}$

$\begin{array}{llll}\text { C } & -2.494267 & 2.639425 & 0.934711\end{array}$

$\begin{array}{llll}\text { C } & -3.254245 & 3.348039 & -0.241245\end{array}$

$\begin{array}{llll}\text { C } & -5.038086 & 1.051632 & 3.240305\end{array}$

C $\quad-3.270264 \quad 2.286261 \quad 2.169105$

$\begin{array}{llll}\text { C } & -3.646341 & 2.483968 & 4.539563\end{array}$

$\begin{array}{llll}\text { C } & -4.736516 & 3.554088 & -0.018959\end{array}$

$\begin{array}{llll}\text { C } & -1.362194 & 1.708993 & 0.634148\end{array}$

$\begin{array}{llll}\text { C } & -2.880189 & 3.046005 & -1.669558\end{array}$

$\begin{array}{llll}\text { C } & -2.922678 & 1.748438 & -2.189110\end{array}$

$\begin{array}{llll}\text { C } & -2.264949 & 4.102248 & 0.589992\end{array}$ 


\begin{tabular}{|c|c|c|c|}
\hline C & -5.236668 & 4.503950 & 0.880225 \\
\hline $\mathrm{C}$ & -4.331360 & 1.377913 & 2.089909 \\
\hline $\mathrm{C}$ & -5.653467 & 2.727115 & -0.685434 \\
\hline $\mathrm{C}$ & -4.715012 & 1.592109 & 4.484392 \\
\hline $\mathrm{C}$ & -7.505716 & 3.759258 & 0.478057 \\
\hline C & -2.595329 & 1.502571 & -3.520793 \\
\hline $\mathrm{C}$ & -2.182799 & 3.860062 & -3.847164 \\
\hline $\mathrm{C}$ & -2.511386 & 4.100059 & -2.511822 \\
\hline $\mathrm{C}$ & -7.020679 & 2.821356 & -0.435430 \\
\hline $\mathrm{C}$ & -6.606556 & 4.605478 & 1.127414 \\
\hline $\mathrm{C}$ & -2.224372 & 2.559416 & -4.355955 \\
\hline $\mathrm{C}$ & -2.724088 & -2.964969 & -3.428472 \\
\hline $\mathrm{C}$ & -2.373712 & -2.719453 & -0.950527 \\
\hline $\mathrm{C}$ & -3.167980 & -3.424013 & 0.205466 \\
\hline $\mathrm{C}$ & -4.858590 & -1.193303 & -3.358535 \\
\hline $\mathrm{C}$ & -3.112601 & -2.394046 & -2.215114 \\
\hline $\mathrm{C}$ & -3.413248 & -2.630587 & -4.592460 \\
\hline $\mathrm{C}$ & -4.637129 & -3.656185 & -0.070847 \\
\hline $\mathrm{C}$ & -1.271311 & -1.761322 & -0.625222 \\
\hline $\mathrm{C}$ & -2.855234 & -3.097409 & 1.642628 \\
\hline $\mathrm{C}$ & -2.926578 & -1.792626 & 2.139469 \\
\hline $\mathrm{C}$ & -2.135666 & -4.172837 & -0.576694 \\
\hline $\mathrm{C}$ & -5.591459 & -2.840180 & 0.555185 \\
\hline $\mathrm{C}$ & -4.184516 & -1.495464 & -2.182459 \\
\hline $\mathrm{C}$ & -5.088034 & -4.619904 & -0.981403 \\
\hline $\mathrm{C}$ & -4.491477 & -1.748700 & 3978 \\
\hline $\mathrm{C}$ & -7.382072 & -3.909104 & -0.669710 \\
\hline $\mathrm{C}$ & -2.658729 & -1.524701 & 3.480400 \\
\hline $\mathrm{C}$ & -2.252379 & -3.875070 & 3.861354 \\
\hline $\mathrm{C}$ & -2.520704 & -4.136810 & 2.516858 \\
\hline $\mathrm{C}$ & -6.445855 & -4.744974 & -1.279005 \\
\hline $\mathrm{C}$ & -6.946628 & -2.957880 & 0.254869 \\
\hline $\mathrm{C}$ & -2.320744 & -2.566742 & 4.347268 \\
\hline $\mathrm{H}$ & 1.129121 & -4.793651 & -0.517482 \\
\hline $\mathrm{H}$ & 5.402409 & -0.920999 & -0.501464 \\
\hline $\mathrm{H}$ & 2.933379 & -4.516655 & -2.701198 \\
\hline $\mathrm{H}$ & 2.364817 & -2.829782 & -3.113993 \\
\hline $\mathrm{H}$ & 4.307925 & -5.649062 & -1.762733 \\
\hline $\mathrm{H}$ & 6.963249 & -2.263742 & -1.888425 \\
\hline $\mathrm{H}$ & 8.527510 & -6.217738 & -1.327642 \\
\hline $\mathrm{H}$ & 5.922819 & 1.406498 & -1.142439 \\
\hline $\mathrm{H}$ & 4.473009 & 0.620806 & -5.113928 \\
\hline $\mathrm{H}$ & 3.962927 & -1.712217 & -4.462470 \\
\hline $\mathrm{H}$ & 8.874787 & -3.760855 & -1.557163 \\
\hline $\mathrm{H}$ & 6.212877 & -7.133880 & -1.452475 \\
\hline $\mathrm{H}$ & 5.432463 & 2.201846 & -3.449425 \\
\hline $\mathrm{H}$ & 1.060108 & 4.943661 & 0.680160 \\
\hline $\mathrm{H}$ & 4.392119 & 0.597429 & 0.741881 \\
\hline $\mathrm{H}$ & 2.888760 & 4.701035 & 2.699588 \\
\hline $\mathrm{H}$ & 2.351191 & 3.040474 & 3.245447 \\
\hline $\mathrm{H}$ & 6.618148 & 2.135890 & 1.181891 \\
\hline $\mathrm{H}$ & 4.525355 & 5.760415 & 2.165446 \\
\hline $\mathrm{H}$ & 8.489601 & 5.949012 & 0.544101 \\
\hline $\mathrm{H}$ & 4.948487 & -1.608929 & 1.694077 \\
\hline $\mathrm{H}$ & 5.331184 & 0.168048 & 5.587242 \\
\hline $\mathrm{H}$ & 4.753558 & 2.378842 & 4.623708 \\
\hline $\mathrm{H}$ & 6.461402 & 7.077429 & 1.442473 \\
\hline $\mathrm{H}$ & 8.553603 & 3.461344 & 0.426499 \\
\hline $\mathrm{H}$ & 5.430242 & -1.845057 & 4.114034 \\
\hline $\mathrm{H}$ & -2.103688 & 3.545047 & 3.473221 \\
\hline $\mathrm{H}$ & -3.162355 & 0.915017 & -1.540712 \\
\hline $\mathrm{H}$ & -2.603614 & 4.834045 & 1.313097 \\
\hline
\end{tabular}
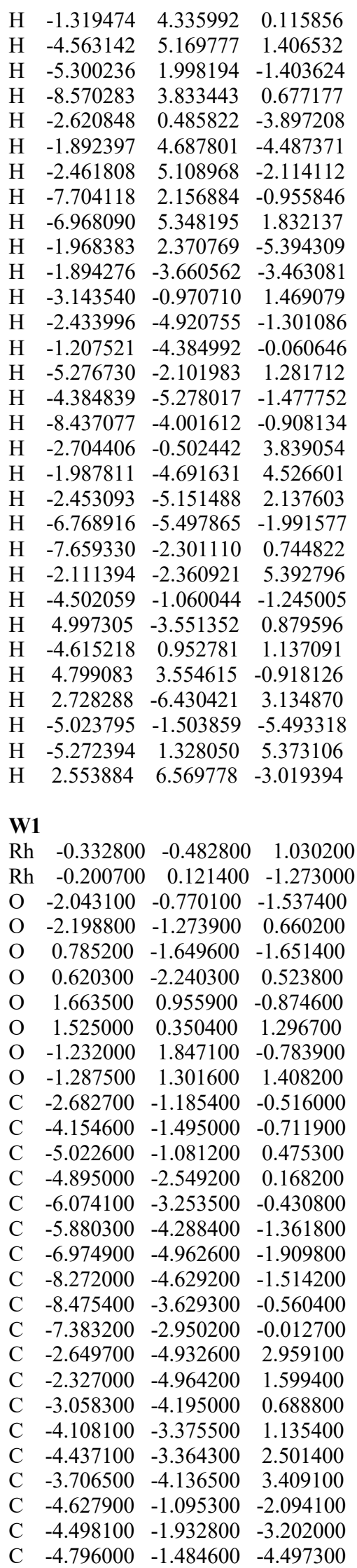

$\begin{array}{lrrr}\text { C } & -5.178800 & -0.148900 & -4.681200\end{array}$

C $\quad-5.305800 \quad 0.719600 \quad-3.587600$

$\begin{array}{llll}\text { C } & -5.059400 & 0.218300 & -2.301900\end{array}$

C $\quad-5.609100 \quad 2.163200 \quad-3.763400$

C $\quad-6.498300 \quad 2.839600 \quad-2.918000$

C $\quad-6.700400 \quad 4.216400 \quad-3.016800$

$\begin{array}{llll}\text { C } & -6.014900 & 4.997100 & -3.970700\end{array}$

$\begin{array}{llll}\text { C } & -5.142400 & 4.299800 & -4.832500\end{array}$

$\begin{array}{llll}\text { C } & -4.947300 & 2.923800 & -4.736100\end{array}$

C $\quad-6.161500 \quad 6.525500 \quad-4.088400$

C $\quad-7.182500 \quad 7.106500 \quad-3.081900$

$\begin{array}{llll}\text { C } & -4.796500 & 7.194400 & -3.818600\end{array}$

$\begin{array}{llll}\text { C } & -6.640900 & 6.891100 & -5.509600\end{array}$

$\begin{array}{llll}\text { C } & -4.664600 & -2.423400 & -5.638600\end{array}$

C $\quad-5.158100 \quad-3.731700 \quad-5.552500$

C $\quad-4.984700 \quad-4.648800 \quad-6.588300$

$\begin{array}{llll}\text { C } & -4.297400 & -4.303900 & -7.770600\end{array}$

$\begin{array}{llll}\text { C } & -3.828700 & -2.975600 & -7.854100\end{array}$

C $\quad-4.010200 \quad-2.058800 \quad-6.821200$

C $\quad-4.025900 \quad-5.293000 \quad-8.918800$

$\begin{array}{llll}\text { C } & -4.659900 & -4.759700 & -10.221200\end{array}$

C $\quad-4.612300 \quad-6.698300 \quad-8.646500$

C $\quad-2.501900 \quad-5.445800 \quad-9.112500$

$\begin{array}{llll}\text { C } & 1.064200 & -2.399500 & -0.658400\end{array}$

C $\quad 2.061500 \quad-3.524700 \quad-0.868600$

$\begin{array}{llll}\text { C } & 2.919300 & -3.393500 & -2.118000\end{array}$

$\begin{array}{llll}\text { C } & 1.916900 & -4.516400 & -2.064300\end{array}$

$\begin{array}{llll}\text { C } & 2.429100 & -5.909400 & -1.864300\end{array}$

$\begin{array}{llll}\text { C } & 1.770300 & -6.792000 & -0.990600\end{array}$

$\begin{array}{llll}\text { C } & 2.245200 & -8.093800 & -0.809600\end{array}$

C $\quad 3.366900 \quad-8.534200 \quad-1.515900$

$\begin{array}{llll}\text { C } & 4.008800 & -7.676400 & -2.412500\end{array}$

$\begin{array}{llll}\text { C } & 3.538300 & -6.372400 & -2.594300\end{array}$

C $\quad-1.537200 \quad-4.405400 \quad-4.615800$

$\begin{array}{llll}\text { C } & -0.268600 & -4.170400 & -5.149900\end{array}$

C $\quad 0.854700 \quad-4.187700 \quad-4.318000$

C $\quad 0.711000 \quad-4.420500 \quad-2.939700$

C $\quad-0.568300 \quad-4.652700 \quad-2.410600$

C $\quad-1.686900-4.650000 \quad-3.248300$

$\begin{array}{llll}\text { C } & 2.725000 & -3.925600 & 0.430600\end{array}$

$\begin{array}{llll}\text { C } & 2.024000 & -4.601200 & 1.432900\end{array}$

$\begin{array}{llll}\text { C } & 2.599000 & -4.850800 & 2.685800\end{array}$

$\begin{array}{llll}\text { C } & 3.903100 & -4.392400 & 2.939600\end{array}$

$\begin{array}{llll}\text { C } & 4.624900 & -3.707200 & 1.953900\end{array}$

$\begin{array}{llll}\text { C } & 4.021000 & -3.492500 & 0.704500\end{array}$

C $\quad 5.972700 \quad-3.127600 \quad 2.179600$

$\begin{array}{llll}\text { C } & 6.944600 & -3.175400 & 1.170500\end{array}$

$\begin{array}{llll}\text { C } & 8.145600 & -2.483000 & 1.287600\end{array}$

$\begin{array}{llll}\text { C } & 8.448400 & -1.699500 & 2.420400\end{array}$

C $\quad 7.493700 \quad-1.711300 \quad 3.459100$

$\begin{array}{llll}\text { C } & 6.290300 & -2.410100 & 3.339800\end{array}$

$\begin{array}{llll}\text { C } & 9.739100 & -0.862100 & 2.449000\end{array}$

$\begin{array}{llll}\text { C } & 9.885100 & -0.040600 & 3.750800\end{array}$

$\begin{array}{llll}\text { C } & 9.734300 & 0.125400 & 1.260800\end{array}$

$\begin{array}{llll}\text { C } & 10.963100 & -1.795400 & 2.335800\end{array}$

$\begin{array}{llll}\text { C } & 1.769800 & -5.125900 & 5.051200\end{array}$

C $\quad \begin{array}{llll}\text { C } & 1.790800 & -5.548700 & 3.716500\end{array}$

C $\quad 0.965100 \quad-6.627500 \quad 3.372700$

$\begin{array}{llll}\text { C } & 0.130800 & -7.238900 & 4.306500\end{array}$

C $\quad 0.070800 \quad-6.795700 \quad 5.643900$

$\begin{array}{llll}\text { C } & 0.930800 & -5.730500 & 5.985000\end{array}$

$\begin{array}{llll}\text { C } & -0.881400 & -7.390800 & 6.697300\end{array}$ 


\begin{tabular}{|c|c|c|c|}
\hline C & -1.832700 & -6.288100 & 7.209800 \\
\hline 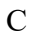 & -0.059900 & -7.951700 & 7.877400 \\
\hline C & -1.750200 & -8.540100 & 6.133900 \\
\hline 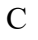 & 2.157600 & 0.820800 & 0.291900 \\
\hline 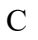 & 3.618800 & 1.171800 & 0.500700 \\
\hline & 4.266000 & 0.382600 & 1.638500 \\
\hline$C$ & 4.074200 & 1.859400 & 1.838700 \\
\hline $\mathrm{C}$ & 5.288800 & 2.736500 & 1.777800 \\
\hline $\mathrm{C}$ & 6.467300 & 2.373000 & 2.454300 \\
\hline $\mathrm{C}$ & 7.596000 & 3.196500 & 2.396600 \\
\hline C & 7.555600 & 4.392200 & 1.675800 \\
\hline $\mathrm{C}$ & 6.380800 & 4.776200 & 1.025600 \\
\hline $\mathrm{C}$ & 5.248200 & 3.960400 & 1.086700 \\
\hline $\mathrm{C}$ & 1.204900 & 3.341700 & 4.669600 \\
\hline $\mathrm{C}$ & 2.102600 & 2.340000 & 5.046100 \\
\hline C & 3.022200 & 1.839800 & 4.119700 \\
\hline $\mathrm{C}$ & 3.033400 & 2.326500 & 2.802100 \\
\hline $\mathrm{C}$ & 2.120600 & 3.327200 & 2.428600 \\
\hline $\mathrm{C}$ & 1.216000 & 3.837400 & 3.363000 \\
\hline $\mathrm{C}$ & 4.407900 & 1.461700 & -0.759500 \\
\hline C & 4.052300 & 2.543300 & -1.577400 \\
\hline $\mathrm{C}$ & 4.845400 & 2.927000 & -2.664900 \\
\hline $\mathrm{C}$ & 6.012500 & 2.201300 & -2.945700 \\
\hline $\mathrm{C}$ & 6.387300 & 1.110700 & -2.152900 \\
\hline $\mathrm{C}$ & 5.566200 & 0.746000 & -1.072500 \\
\hline $\mathrm{C}$ & 7.665800 & 0.389800 & -2.377200 \\
\hline $\mathrm{C}$ & 8.850600 & 1.081500 & -2.66 \\
\hline $\mathrm{C}$ & 10.066500 & 0.412900 & -2.784300 \\
\hline $\mathrm{C}$ & 10.172900 & -0.985600 & -2.631900 \\
\hline $\mathrm{C}$ & 8.967900 & -1.675600 & -2.384400 \\
\hline $\mathrm{C}$ & 7.752900 & -1.002800 & -2.257300 \\
\hline $\mathrm{C}$ & 11.547700 & -1.672900 & -2.719000 \\
\hline $\mathrm{C}$ & 11.465500 & -3.200000 & -2.486100 \\
\hline $\mathrm{C}$ & 12.152200 & -1.436000 & -4.118700 \\
\hline $\mathrm{C}$ & 12.489000 & -1.083400 & -1.646000 \\
\hline $\mathrm{C}$ & 4.471700 & 4.116600 & -3.472800 \\
\hline $\mathrm{C}$ & 4.407100 & 4.073200 & -4.870000 \\
\hline $\mathrm{C}$ & 4.018500 & 5.191200 & -5.606000 \\
\hline $\mathrm{C}$ & 3.685200 & 6.416800 & -4.991500 \\
\hline $\mathrm{C}$ & 3.789500 & 6.456800 & -3.585600 \\
\hline $\mathrm{C}$ & 4.167400 & 5.333800 & -2.851100 \\
\hline $\mathrm{C}$ & 3.217600 & 7.609700 & -5.845400 \\
\hline $\mathrm{C}$ & 1.941400 & 7.217500 & -6.620900 \\
\hline $\mathrm{C}$ & 4.328800 & 7.995600 & -6.844600 \\
\hline $\mathrm{C}$ & 2.888300 & 8.859100 & -4.994800 \\
\hline $\mathrm{C}$ & -1.640000 & 2.015000 & 0.413300 \\
\hline $\mathrm{C}$ & -2.701000 & 3.067700 & 0.675800 \\
\hline $\mathrm{C}$ & -3.684400 & 3.233800 & -0.481100 \\
\hline $\mathrm{C}$ & -2.766900 & 4.382700 & -0.158500 \\
\hline $\mathrm{C}$ & -3.355800 & 5.596600 & 0.492700 \\
\hline $\mathrm{C}$ & -2.617500 & 6.316400 & 1.448200 \\
\hline $\mathrm{C}$ & -3.155100 & 7.461400 & 2.042600 \\
\hline $\mathrm{C}$ & -4.422500 & 7.914400 & 1.669900 \\
\hline $\mathrm{C}$ & -5.149200 & 7.229700 & 0.693100 \\
\hline $\mathrm{C}$ & -4.616600 & 6.082000 & 0.099100 \\
\hline $\mathrm{C}$ & 0.365700 & 5.239400 & -2.974600 \\
\hline $\mathrm{C}$ & 0.673100 & 5.070500 & -1.621300 \\
\hline $\mathrm{C}$ & -0.341800 & 4.792400 & -0.701800 \\
\hline $\mathrm{C}$ & -1.673600 & 4.675000 & -1.134000 \\
\hline $\mathrm{C}$ & -1.976600 & 4.867600 & -2.492300 \\
\hline $\mathrm{C}$ & -0.958700 & 5.143200 & -3.408800 \\
\hline & -3.273900 & 2.957000 & 2.073800 \\
\hline
\end{tabular}

\begin{tabular}{|c|c|c|c|c|c|c|c|}
\hline & -4.382500 & 2.134300 & 293600 & $\mathrm{H}$ & -5.716000 & -6.649300 & -8.524400 \\
\hline & -4.836300 & 1.841500 & 3.587900 & $\mathrm{H}$ & -2.031500 & -4.486100 & -9.412800 \\
\hline & -4.197400 & 2.450800 & 4.676700 & $\mathrm{H}$ & -2.278200 & -6.190500 & -9.907000 \\
\hline & -3.108000 & .311800 & 4.484300 & $\mathrm{H}$ & -2.024300 & -5.786900 & -8.168100 \\
\hline & -2.644400 & 3.529900 & 3.178900 & $\mathrm{H}$ & 2.711300 & -2.539600 & -2.753600 \\
\hline & -2.418100 & 3.962600 & 5.624600 & $\mathrm{H}$ & 3.975000 & -3.625700 & -2.014700 \\
\hline & -1.950700 & 5.280300 & & $\mathrm{H}$ & 0.886100 & -6.477900 & 4000 \\
\hline & -1.244500 & 5.873600 & & $\mathrm{H}$ & 1.738900 & -8.763200 & -0.126100 \\
\hline & -0.963700 & 5.188000 & 7.772100 & $\mathrm{H}$ & 3.732200 & -9.543400 & -1.376500 \\
\hline & -1.461500 & 3.871900 & 7.867700 & $\mathrm{H}$ & 4.869200 & -8.023100 & -2.970000 \\
\hline & -2.169400 & 3.280200 & 6.82 & $\mathrm{H}$ & 4.039100 & -5.726000 & 04000 \\
\hline & -0.137900 & & & $\mathrm{H}$ & 0000 & & 4800 \\
\hline & 1.253500 & 6.2 & & $\mathrm{H}$ & 4400 & -3. & 1300 \\
\hline & 0.072300 & 4.96 & 12100 & $\mathrm{H}$ & 6100 & 1600 & 3500 \\
\hline & -0.862600 & 7.15 & & $\mathrm{H}$ & & & 5100 \\
\hline & -5.788200 & & & $\mathrm{H}$ & 000 & -4 & 400 \\
\hline $\mathrm{C}$ & -5.91 & & & $\mathrm{H}$ & & & \\
\hline $\mathrm{C}$ & -7.049700 & & & $\mathrm{H}$ & 300 & & 100 \\
\hline $\mathrm{C}$ & -8.004300 & -0.21 & & $\mathrm{H}$ & & & 100 \\
\hline & -7.870700 & -1.2 & 4.0270 & $\mathrm{H}$ & & -3.7 & 400 \\
\hline & -6.73 & -1. & & $\mathrm{H}$ & & -2.5 & \\
\hline $\mathrm{C}$ & -8.86 & & & $\mathrm{H}$ & & & \\
\hline $\mathrm{C}$ & -8.131900 & -3.746500 & & $\mathrm{H}$ & & & \\
\hline $\mathrm{C}$ & -10.050300 & -2.307800 & 74500 & $\mathrm{H}$ & & & \\
\hline $\mathrm{C}$ & -9.441600 & -2.442700 & 5.59 & $\mathrm{H}$ & 9000 & 2400 & 900 \\
\hline $\mathrm{H}$ & -4.5 & & & $\mathrm{H}$ & & -0.7 & \\
\hline $\mathrm{H}$ & -5.9 & & & $\mathrm{H}$ & & & \\
\hline $\mathrm{H}$ & -4.88 & & & $\mathrm{H}$ & 5100 & 0 & 3200 \\
\hline $\mathrm{H}$ & -6.8 & -5.7 & & $\mathrm{H}$ & & & \\
\hline $\mathrm{H}$ & -9.11 & & & 11 & & & 000 \\
\hline $\mathrm{H}$ & -9.4 & & & & & & \\
\hline $\mathrm{H}$ & -7.5 & & & $\mathrm{H}$ & & 300 & 7900 \\
\hline $\mathrm{H}$ & -2.08 & & & $\mathrm{H}$ & & -4.2 & \\
\hline $\mathrm{H}$ & -1.5 & & & $\mathrm{H}$ & & & \\
\hline $\mathrm{H}$ & -2.80 & -4.2 & -0.3 & $\mathrm{H}$ & & -8 & \\
\hline $\mathrm{H}$ & -5.2 & & & $\mathrm{H}$ & & & \\
\hline $\mathrm{H}$ & $-3.9 c$ & -4.1 & & $\mathrm{H}$ & & & 300 \\
\hline $\mathrm{H}$ & -4.10 & -2 & -3.0 & $\mathrm{H}$ & & & \\
\hline $\mathrm{H}$ & -5.40 & & & $\mathrm{H}$ & & & \\
\hline $\mathrm{H}$ & -5.1 & & & $\mathrm{H}$ & & & \\
\hline $\mathrm{H}$ & -7.0 & & & $\mathrm{H}$ & -0 . & & \\
\hline $\mathrm{H}$ & -7.4 & & & $\mathrm{H}$ & & & 000 \\
\hline $\mathrm{H}$ & -4.56 & & & $\mathrm{H}$ & 300 & & \\
\hline $\mathrm{H}$ & -4.234400 & & & $\mathrm{H}$ & 5600 & & \\
\hline $\mathrm{H}$ & & & & 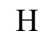 & & & \\
\hline $\mathrm{H}$ & -7.26 & & & & & & \\
\hline $\mathrm{H}$ & -6.86 & & & $\mathrm{H}$ & & -0. & \\
\hline $\mathrm{H}$ & -4.429000 & & & $\mathrm{H}$ & & & \\
\hline $\mathrm{H}$ & -4.88 & & & $\mathrm{H}$ & & & \\
\hline $\mathrm{H}$ & & & & & & & \\
\hline $\mathrm{H}$ & -5.89 & & & $\mathrm{H}$ & & & \\
\hline $\mathrm{H}$ & -6.801700 & & & $\mathrm{H}$ & & 3100 & 2800 \\
\hline $\mathrm{H}$ & -7.599500 & 6.377000 & & $\mathrm{H}$ & 300 & 1600 & 5.395200 \\
\hline & -5.693500 & 100 & & & & & \\
\hline $\mathrm{H}$ & -5.406400 & & & & & & \\
\hline $\mathrm{H}$ & -3.287500 & -2.623300 & & $\mathrm{H}$ & & & \\
\hline $\mathrm{H}$ & -3.600100 & -1.057400 & -6.920600 & $\mathrm{H}$ & 0.537900 & 4.629900 & 3.077800 \\
\hline & -5.751900 & -4.603900 & -10.083800 & $\mathrm{H}$ & 3.148200 & 3.100300 & -1.358100 \\
\hline & -4.511500 & & & $\mathrm{H}$ & & & \\
\hline & -4.206100 & -3.794700 & -10.529900 & & & -0.079000 & -0.443100 \\
\hline & -4.162500 & -7.144500 & -7.733100 & $\mathrm{H}$ & 8.830500 & 2.164400 & -2.749000 \\
\hline & -4.399000 & -7.385300 & -9.494200 & $\mathrm{H}$ & 10.941600 & 1.025700 & -2.985900 \\
\hline
\end{tabular}




\begin{tabular}{|c|c|c|c|}
\hline $\mathrm{H}$ & 8.917000 & -2.755900 & -2.282400 \\
\hline $\mathrm{H}$ & 6.850900 & -1.578200 & -2.068500 \\
\hline $\mathrm{H}$ & 11.049800 & -3.423600 & -1.479300 \\
\hline $\mathrm{H}$ & 12.475000 & -3.662200 & -2.543300 \\
\hline $\mathrm{H}$ & 10.832000 & -3.685200 & -3.259700 \\
\hline $\mathrm{H}$ & 11.469300 & -1.823600 & -4.905500 \\
\hline $\mathrm{H}$ & 13.130500 & -1.954600 & -4.218000 \\
\hline $\mathrm{H}$ & 12.324900 & -0.355700 & -4.308500 \\
\hline $\mathrm{H}$ & 12.673600 & -0.001900 & -1.814200 \\
\hline $\mathrm{H}$ & 13.475300 & -1.595800 & -1.662600 \\
\hline $\mathrm{H}$ & 12.048900 & -1.208100 & -0.633700 \\
\hline $\mathrm{H}$ & 4.631700 & 3.144700 & -5.388400 \\
\hline $\mathrm{H}$ & 3.970000 & 5.071200 & -6.685500 \\
\hline $\mathrm{H}$ & 3.576600 & 7.347300 & -3.001700 \\
\hline $\mathrm{H}$ & 4.239200 & 5.410400 & -1.769700 \\
\hline $\mathrm{H}$ & 2.134200 & 6.382700 & -7.326800 \\
\hline $\mathrm{H}$ & 1.562200 & 8.076700 & -7.215600 \\
\hline $\mathrm{H}$ & 1.142000 & 6.900900 & -5.916300 \\
\hline $\mathrm{H}$ & 5.267600 & 8.241600 & -6.302600 \\
\hline $\mathrm{H}$ & 4.028700 & 8.882200 & -7.444300 \\
\hline $\mathrm{H}$ & 4.542400 & 7.169500 & -7.554800 \\
\hline $\mathrm{H}$ & 2.069300 & 8.642500 & -4.274900 \\
\hline $\mathrm{H}$ & 2.552500 & 9.698800 & -5.641400 \\
\hline $\mathrm{H}$ & 3.784800 & 9.204700 & -4.436000 \\
\hline $\mathrm{H}$ & -3.486100 & 2.653700 & -1.375100 \\
\hline $\mathrm{H}$ & -4.735000 & 3.295400 & -0.214100 \\
\hline $\mathrm{H}$ & -1.621500 & 6.000700 & 1.726200 \\
\hline $\mathrm{H}$ & -2.584400 & 8.002600 & 2.786200 \\
\hline $\mathrm{H}$ & -4.836100 & 8.803200 & 2.128500 \\
\hline $\mathrm{H}$ & -6.123800 & 7.591600 & 0.392300 \\
\hline $\mathrm{H}$ & -5.186700 & 5.578000 & -0.670100 \\
\hline $\mathrm{H}$ & 1.150300 & 5.446500 & -3.688100 \\
\hline $\mathrm{H}$ & 1.696300 & 5.155900 & -1.282300 \\
\hline $\mathrm{H}$ & -0.090000 & 4.656400 & 0.342200 \\
\hline $\mathrm{H}$ & -2.998800 & 4.795700 & -2.840300 \\
\hline $\mathrm{H}$ & -1.196600 & 5.281700 & -4.455600 \\
\hline $\mathrm{H}$ & -4.856900 & 1.649200 & 1.447200 \\
\hline $\mathrm{H}$ & -4.570200 & 2.273600 & 5.681700 \\
\hline $\mathrm{H}$ & -1.743000 & 4.111400 & 3.038200 \\
\hline $\mathrm{H}$ & -2.146900 & 5.854000 & 4.627200 \\
\hline $\mathrm{H}$ & -0.911900 & 6.896700 & 6.414700 \\
\hline $\mathrm{H}$ & -1.309100 & 3.245600 & 8.741600 \\
\hline $\mathrm{H}$ & -2.499000 & 2.250300 & 6.928200 \\
\hline $\mathrm{H}$ & 1.771000 & 5.336400 & 7.937400 \\
\hline $\mathrm{H}$ & 1.886100 & 6.698300 & 9.113300 \\
\hline $\mathrm{H}$ & 1.177200 & 6.976600 & 7.491500 \\
\hline $\mathrm{H}$ & -0.902100 & 4.685800 & 10.569200 \\
\hline $\mathrm{H}$ & 0.669900 & 5.487500 & 10.889400 \\
\hline $\mathrm{H}$ & 0.622800 & 4.038500 & 9.834200 \\
\hline $\mathrm{H}$ & -0.954600 & 7.891700 & 8.526900 \\
\hline $\mathrm{H}$ & -0.303800 & 7.637400 & 10.176100 \\
\hline $\mathrm{H}$ & -1.883600 & 6.904000 & 9.712100 \\
\hline $\mathrm{H}$ & -4.911800 & -0.187400 & 5.390100 \\
\hline $\mathrm{H}$ & -7.198300 & 1.579800 & 2.220400 \\
\hline $\mathrm{H}$ & -8.844400 & -0.143500 & 2.394700 \\
\hline $\mathrm{H}$ & -6.549800 & -1.939600 & 5.620400 \\
\hline $\mathrm{H}$ & -7.687800 & -3.736300 & 2.859800 \\
\hline $\mathrm{H}$ & -8.835500 & -4.604600 & 3.942000 \\
\hline $\mathrm{H}$ & -7.316600 & -3.926100 & 4.610000 \\
\hline $\mathrm{H}$ & -10.628300 & -1.375500 & 3.353600 \\
\hline $\mathrm{H}$ & -10.747000 & -3.165000 & 3.299700 \\
\hline $\mathrm{H}$ & -9.694400 & -2.318900 & 2.122800 \\
\hline
\end{tabular}

H $\quad-8.650400 \quad-2.628300 \quad 6.348300$

H $\quad-10.197800 \quad-3.250800 \quad 5.697300$

H $\quad-9.931000 \quad-1.473200 \quad 5.829200$

\section{W2}

$\begin{array}{llll}\mathrm{Rh} & -0.238400 & 0.229700 & -1.259600\end{array}$

$\begin{array}{llll}\mathrm{Rh} & -0.367200 & -0.652400 & 0.952000\end{array}$

$\begin{array}{llll}\mathrm{O} & -1.364200 & 1.050600 & 1.532100\end{array}$

$\begin{array}{llll}\mathrm{O} & -1.322800 & 1.857000 & -0.578100\end{array}$

$\begin{array}{llll}\mathrm{O} & 1.474600 & 0.184100 & 1.321200\end{array}$

O $\quad 1.591900 \quad 1.068400 \quad-0.753800$

$\begin{array}{llll}\mathrm{O} & 0.629100 & -2.315100 & 0.254700\end{array}$

O $\quad 0.810300 \quad-1.456200 \quad-1.827300$

O $\quad-2.216500 \quad-1.439800 \quad 0.473900$

$\begin{array}{llll}\mathrm{O} & -2.052300 & -0.671300 & -1.645300\end{array}$

C $\quad-1.736500 \quad 1.868300 \quad 0.628800$

C $\quad-2.827300 \quad 2.850400 \quad 1.014800$

C $\quad-3.804700 \quad 3.145200-0.120600$

C $\quad-2.920600 \quad 4.262900 \quad 0.363200$

C $\quad-3.547700 \quad 5.362600 \quad 1.164300$

$\begin{array}{llll}C & -2.844000 & 5.955900 & 2.226800\end{array}$

C $\quad-3.418800 \quad 6.995000 \quad 2.963700$

$\begin{array}{llll}\text { C } & -4.689300 & 7.470000 & 2.630600\end{array}$

$\begin{array}{lrrr}\text { C } & -5.381900 & 6.914200 & 1.552600\end{array}$

C $\quad-4.812100 \quad 5.872000 \quad 0.815700$

C $\quad 0.219400 \quad 5.573700 \quad-2.261600$

C $\quad 0.513700 \quad 5.237100 \quad-0.937000$

C $\quad-0.503200 \quad 4.807200 \quad-0.080900$

$\begin{array}{llll}\text { C } & -1.824700 & 4.709900 & -0.547700\end{array}$

C $\quad-2.116000 \quad 5.072600 \quad-1.873400$

C $\quad-1.095200 \quad 5.496500 \quad-2.727900$

C $\quad-3.401400 \quad 2.544300 \quad 2.382300$

C $\quad-2.767900 \quad 2.958300 \quad 3.554200$

C $\quad-3.223700 \quad 2.556500 \quad 4.817800$

C $\quad-4.311400 \quad 1.674500 \quad 4.891500$

C $\quad-4.954200 \quad 1.222900 \quad 3.730600$

$\begin{array}{llll}\text { C } & -4.506800 & 1.695900 & 2.488100\end{array}$

C $\quad-6.025800 \quad 0.195700 \quad 3.782500$

C $\quad-7.159700 \quad 0.263400 \quad 2.962400$

$\begin{array}{llll}\text { C } & -8.106900 & -0.760400 & 2.934900\end{array}$

C $\quad-7.968100 \quad-1.917200 \quad 3.730100$

C $\quad-6.838200 \quad-1.958900 \quad 4.573500$

C $\quad-5.896100 \quad-0.933400 \quad 4.601900$

C $\quad-8.953700 \quad-3.099800 \quad 3.698200$

C $\quad-10.135000 \quad-2.865700 \quad 2.727600$

$\begin{array}{llll}\text { C } & -8.211400 & -4.374900 & 3.242900\end{array}$

C $\quad-9.539200 \quad-3.322400 \quad 5.109100$

C $\quad-2.525400 \quad 3.036700 \quad 6.035000$

C $\quad-2.053100 \quad 4.353000 \quad 6.124500$

C $\quad-1.336700 \quad 4.800800 \quad 7.233300$

C $\quad-1.051200 \quad 3.948900 \quad 8.320400$

$\begin{array}{llll}\text { C } & -1.555600 & 2.634300 & 8.229800\end{array}$

$\begin{array}{llll}\text { C } & -2.273400 & 2.190300 & 7.122000\end{array}$

C $\quad-0.217900 \quad 4.375100 \quad 9.542600$

C $\quad 1.039900 \quad 3.485100 \quad 9.639700$

$\begin{array}{lrrr}\text { C } & -1.062300 & 4.215400 & 10.824900\end{array}$

$\begin{array}{llll}\text { C } & 0.248600 & 5.847800 & 9.462000\end{array}$

$\begin{array}{llll}\text { C } & 2.092400 & 0.797200 & 0.386100\end{array}$

C $\quad 3.545100 \quad 1.155900 \quad 0.631100$

$\begin{array}{llll}\mathrm{C} & 4.235100 & 0.213700 & 1.617600\end{array}$

$\begin{array}{llll}\text { C } & 4.009900 & 1.633700 & 2.053900\end{array}$

$\begin{array}{llll}\text { C } & 5.198500 & 2.546300 & 2.110700\end{array}$ $\begin{array}{llll}\text { C } & 5.102600 & 3.867000 & 1.637600\end{array}$

C $\quad 6.211000 \quad 4.716500 \quad 1.681000$

$\begin{array}{llll}\text { C } & 7.417300 & 4.265500 & 2.220900\end{array}$

$\begin{array}{llll}\text { C } & 7.513700 & 2.967500 & 2.727500\end{array}$

$\begin{array}{llll}\text { C } & 6.409300 & 2.111100 & 2.679900\end{array}$

C $\quad 1.153500 \quad 2.540700 \quad 5.131500$

C $\quad 2.096900 \quad 1.530000 \quad 5.330700$

$\begin{array}{llll}\text { C } & 3.014100 & 1.220000 & 4.322500\end{array}$

$\begin{array}{llll}\text { C } & 2.978400 & 1.908300 & 3.098500\end{array}$

C $\quad 2.021600 \quad 2.919000 \quad 2.903300$

C $\quad 1.118300 \quad 3.237300 \quad 3.920700$

$\begin{array}{llll}\text { C } & 4.302100 & 1.663400 & -0.579800\end{array}$

$\begin{array}{llll}\text { C } & 5.460500 & 1.028400 & -1.034900\end{array}$

$\begin{array}{llll}\text { C } & 6.245400 & 1.578900 & -2.062800\end{array}$

$\begin{array}{llll}\text { C } & 5.834000 & 2.778500 & -2.654700\end{array}$

$\begin{array}{llll}\text { C } & 4.668500 & 3.430500 & -2.226800\end{array}$

C $\quad 3.911000 \quad 2.859200 \quad-1.198200$

C $\quad 4.264800 \quad 4.733100 \quad-2.817200$

C $\quad 4.155500 \quad 4.916900-4.200200$

$\begin{array}{llll}\text { C } & 3.747800 & 6.138800 & -4.732400\end{array}$

$\begin{array}{llll}\text { C } & 3.438400 & 7.248000 & -3.916900\end{array}$

C $\quad 3.584600 \quad 7.058600 \quad-2.527000$

$\begin{array}{llll}\text { C } & 3.982900 & 5.832100 & -1.996900\end{array}$

$\begin{array}{llll}\text { C } & 2.950800 & 8.563500 & -4.551200\end{array}$

$\begin{array}{llll}\text { C } & 2.654800 & 9.658700 & -3.499500\end{array}$

$\begin{array}{llll}\text { C } & 1.649200 & 8.302500 & -5.339500\end{array}$

$\begin{array}{llll}\text { C } & 4.032600 & 9.106200 & -5.509100\end{array}$

$\begin{array}{llll}\text { C } & 8.682500 & 1.682300 & -2.675300\end{array}$

$\begin{array}{llll}\text { C } & 7.523700 & 0.928300 & -2.446400\end{array}$

C $\quad 7.638000 \quad-0.463600 \quad-2.550100$

$\begin{array}{llll}\text { C } & 8.854400 & -1.080300 & -2.842500\end{array}$

$\begin{array}{llll}\text { C } & 10.033800 & -0.332800 & -3.042200\end{array}$

$\begin{array}{llll}\text { C } & 9.899300 & 1.069400 & -2.964700\end{array}$

C $\quad 11.409900 \quad-0.965900 \quad-3.317400$

C $\quad 12.409400 \quad-0.527300 \quad-2.224800$

C $\quad 11.921300 \quad-0.501200 \quad-4.696900$

C $\quad 11.363600 \quad-2.512100 \quad-3.320300$

C $\quad 1.101200 \quad-2.311600 \quad-0.927800$

C $\quad 2.141400 \quad-3.368200-1.252200$

$\begin{array}{llll}\text { C } & 2.970400 & -3.098700 & -2.498600\end{array}$

$\begin{array}{llll}\text { C } & 2.005300 & -4.253800 & -2.531000\end{array}$

$\begin{array}{llll}\text { C } & 2.562100 & -5.640800 & -2.460600\end{array}$

$\begin{array}{llll}\text { C } & 3.656800 & -6.010600 & -3.261700\end{array}$

$\begin{array}{llll}\text { C } & 4.167000 & -7.310800 & -3.202200\end{array}$

$\begin{array}{llll}\text { C } & 3.579600 & -8.256000 & -2.357400\end{array}$

C $\quad 2.473500 \quad-7.905600 \quad-1.579400$

C $\quad 1.959400 \quad-6.607200 \quad-1.637300$

$\begin{array}{lrrr}\text { C } & -1.458800 & -4.008400 & -5.061300\end{array}$

C $\quad-0.198400 \quad-3.692500 \quad-5.571500$

C $\quad 0.926300 \quad-3.755500 \quad-4.744100$

C $\quad 0.792500 \quad-4.115700 \quad-3.391900$

C $\quad-0.478500 \quad-4.431300 \quad-2.887100$

$\begin{array}{llll}\text { C } & -1.599100 & -4.380700 & -3.722300\end{array}$

C $\quad 2.855400 \quad-3.849800 \quad-0.004200$

$\begin{array}{llll}\text { C } & 4.165700 & -3.434500 & 0.232700\end{array}$

C $\quad 4.839700 \quad-3.741000 \quad 1.425600$

$\begin{array}{llll}\text { C } & 4.169100 & -4.495200 & 2.394800\end{array}$

$\begin{array}{llll}\text { C } & 2.845200 & -4.918100 & 2.190200\end{array}$

C $\quad 2.203300 \quad-4.591500 \quad 0.988000$

C $\quad 2.110400 \quad-5.648900 \quad 3.253900$

$\begin{array}{llll}\text { C } & 2.706800 & -6.674800 & 3.997300\end{array}$

$\begin{array}{llll}\text { C } & 2.005400 & -7.337900 & 5.002500\end{array}$ 


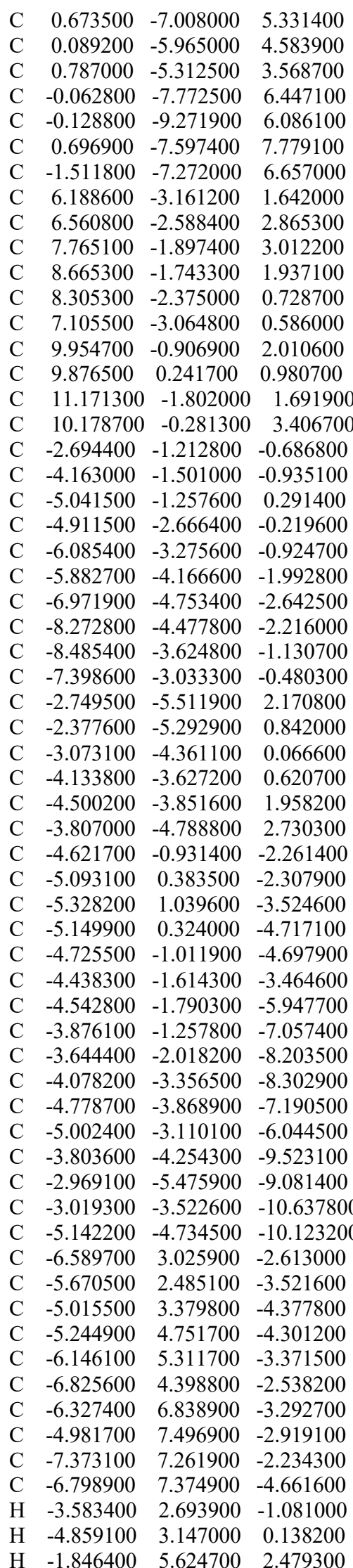

\begin{tabular}{|c|c|c|c|}
\hline & -2.874600 & 7.438000 & 3.787700 \\
\hline $\mathrm{H}$ & -5.131600 & 8.277200 & 3.199900 \\
\hline $\mathrm{H}$ & -6.359100 & 7.294400 & 1.284700 \\
\hline $\mathrm{H}$ & -5.356500 & 5.468000 & -0.027700 \\
\hline $\mathrm{H}$ & 1.006700 & 5.896300 & -2.927500 \\
\hline & 1.527900 & 5.310500 & -0.570500 \\
\hline $\mathrm{H}$ & -0.260400 & 4.541100 & 0.940200 \\
\hline $\mathrm{H}$ & -3.131200 & 5.019100 & -2.244800 \\
\hline $\mathrm{H}$ & -1.323400 & 5.765700 & -3.751100 \\
\hline & -1.867300 & 3.555000 & 3.490400 \\
\hline & -4.678200 & 1.355800 & 5.863100 \\
\hline $\mathrm{H}$ & -4.982500 & 1.331600 & 1.584000 \\
\hline $\mathrm{H}$ & -7.312400 & 1.137900 & 2.335100 \\
\hline $\mathrm{H}$ & -8.945500 & -0.604400 & 2.262700 \\
\hline $\mathrm{H}$ & -6.647900 & -2.812500 & 5.219200 \\
\hline & -5.021700 & -1.034300 & 5.238800 \\
\hline $\mathrm{H}$ & -10.721900 & -1.971000 & 3.028200 \\
\hline $\mathrm{H}$ & -10.825400 & -3.737100 & 2.729500 \\
\hline $\mathrm{H}$ & -9.772300 & -2.730200 & 1.686800 \\
\hline & -7.762600 & -4.225000 & 2.237900 \\
\hline $\mathrm{H}$ & -8.909600 & -5.237900 & 3.186600 \\
\hline $\mathrm{H}$ & -7.398600 & -4.646900 & 3.948700 \\
\hline $\mathrm{H}$ & -8.750600 & -3.602800 & 5.838200 \\
\hline $\mathrm{H}$ & -10.289800 & -4.142400 & 5.099300 \\
\hline $\mathrm{H}$ & -10.037200 & -2.397000 & 5.471400 \\
\hline $\mathrm{H}$ & -2.254700 & 5.047800 & 5.314000 \\
\hline $\mathrm{H}$ & -1.017400 & 5.838100 & 7.195800 \\
\hline $\mathrm{H}$ & -1.381800 & 1.908600 & 9.020400 \\
\hline $\mathrm{H}$ & -2.605300 & 1.156300 & 7.086200 \\
\hline $\mathrm{H}$ & 0.773000 & 2.419900 & 9.802800 \\
\hline $\mathrm{H}$ & 1.682700 & 3.802400 & 10.489300 \\
\hline $\mathrm{H}$ & 1.636300 & 3.555200 & 8.704000 \\
\hline $\mathrm{H}$ & -1.993400 & 4.818200 & 10.752000 \\
\hline $\mathrm{H}$ & -0.493100 & 4.557500 & 11.716400 \\
\hline $\mathrm{H}$ & -1.345300 & 3.155900 & 10.997000 \\
\hline $\mathrm{H}$ & 0.891500 & 6.010900 & 8.570000 \\
\hline $\mathrm{H}$ & 0.845400 & 6.121800 & 10.359200 \\
\hline $\mathrm{H}$ & -0.622600 & 6.536500 & 9.416000 \\
\hline $\mathrm{H}$ & 3.605500 & -0.562800 & 2.036700 \\
\hline $\mathrm{H}$ & 5.240800 & -0.118400 & 1.385300 \\
\hline $\mathrm{H}$ & 4.172200 & 4.239300 & 1.231500 \\
\hline $\mathrm{H}$ & 6.132500 & 5.726600 & 1.300000 \\
\hline $\mathrm{H}$ & 8.274600 & 4.925200 & 2.256200 \\
\hline $\mathrm{H}$ & 8.445400 & 2.626800 & 3.158700 \\
\hline $\mathrm{H}$ & 6.500800 & 1.110900 & 3.084700 \\
\hline $\mathrm{H}$ & 0.460300 & 2.790700 & 5.920900 \\
\hline $\mathrm{H}$ & 2.127600 & 0.998100 & 6.272800 \\
\hline $\mathrm{H}$ & 3.758500 & 0.453300 & 4.498800 \\
\hline $\mathrm{H}$ & 1.985400 & 3.468600 & 1.971200 \\
\hline $\mathrm{H}$ & 0.404100 & 4.036100 & 3.775700 \\
\hline $\mathrm{H}$ & 5.802500 & 0.116700 & -0.557500 \\
\hline $\mathrm{H}$ & 6.433400 & 3.223600 & -3.444000 \\
\hline $\mathrm{H}$ & 3.006300 & 3.356200 & -0.866500 \\
\hline $\mathrm{H}$ & 4.361600 & 4.085500 & -4.869200 \\
\hline $\mathrm{H}$ & 3.665500 & 6.195600 & -5.815000 \\
\hline $\mathrm{H}$ & 3.392000 & 7.841800 & -1.799800 \\
\hline $\mathrm{H}$ & 4.088100 & 5.732000 & -0.920100 \\
\hline & 1.856800 & 9.328900 & -2.799300 \\
\hline & 2.304300 & 10.592400 & -3.990800 \\
\hline 11 & 3.569900 & 9.908300 & -2.920100 \\
\hline & 1.815100 & 7.591500 & -6.175800 \\
\hline $\mathrm{H}$ & 1.257400 & 9.246500 & -5.776700 \\
\hline
\end{tabular}

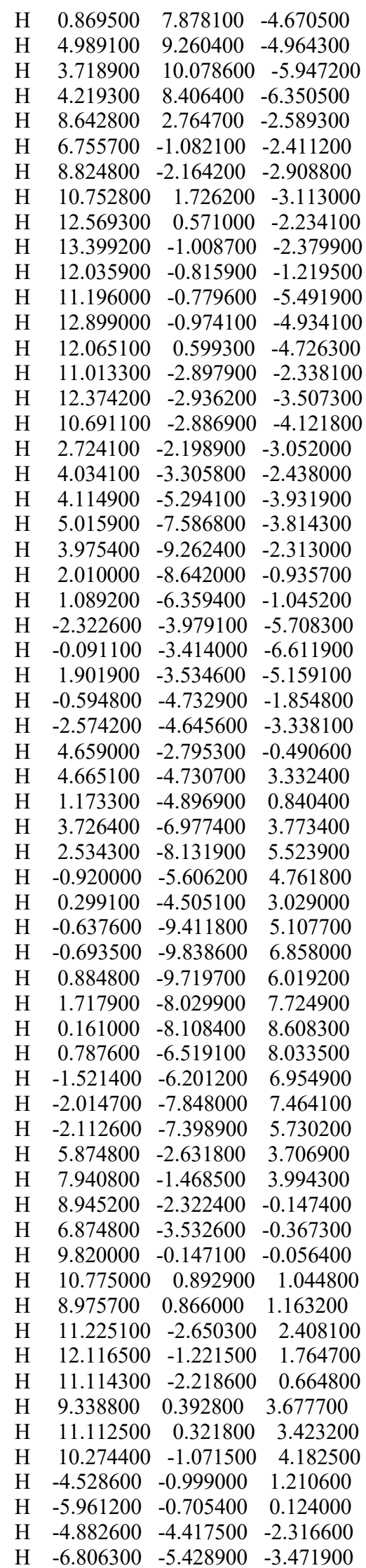




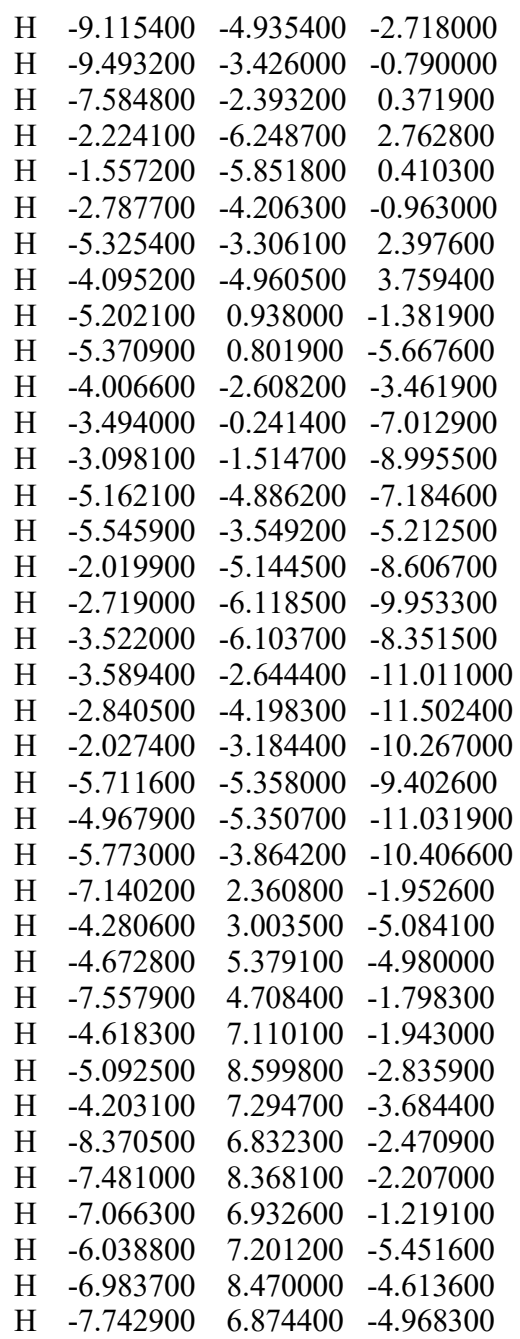

$\begin{array}{lrrr}\text { W3 } & & & \\ \mathrm{Rh} & -0.354800 & -0.496200 & 1.020500 \\ \mathrm{Rh} & -0.217500 & 0.142600 & -1.272900 \\ \mathrm{O} & 1.632400 & 0.991200 & -0.854700 \\ \mathrm{O} & 1.502400 & 0.338700 & 1.303300 \\ \mathrm{O} & -1.275100 & 1.847200 & -0.758200 \\ \mathrm{O} & -1.309300 & 1.279200 & 1.428300 \\ \mathrm{O} & -2.044700 & -0.764000 & -1.567200 \\ \mathrm{O} & -2.221400 & -1.282600 & 0.625600 \\ \mathrm{O} & 0.792400 & -1.612500 & -1.668900 \\ \mathrm{O} & 0.601900 & -2.244000 & 0.492600 \\ \mathrm{C} & 2.129800 & 0.834800 & 0.307600 \\ \mathrm{C} & 3.589000 & 1.194000 & 0.518200 \\ \mathrm{C} & 4.240300 & 0.408300 & 1.655500 \\ \mathrm{C} & 4.039100 & 1.883600 & 1.856500 \\ \mathrm{C} & 5.248700 & 2.767800 & 1.797100 \\ \mathrm{C} & 5.202200 & 3.991600 & 1.106100 \\ \mathrm{C} & 6.330400 & 4.813700 & 1.046500 \\ \mathrm{C} & 7.506500 & 4.436000 & 1.698000 \\ \mathrm{C} & 7.552700 & 3.240400 & 2.418600 \\ \mathrm{C} & 6.428500 & 2.410700 & 2.474900 \\ \mathrm{C} & 1.168700 & 3.366700 & 4.684200 \\ \mathrm{C} & 2.070800 & 2.370200 & 5.064200 \\ \mathrm{C} & 2.988600 & 1.866900 & 4.137900\end{array}$

$\begin{array}{llll}\text { C } & 2.994100 & 2.345600 & 2.817400\end{array}$

$\begin{array}{llll}\text { C } & 2.075100 & 3.339100 & 2.439900\end{array}$

C $\quad 1.171800 \quad 3.852000 \quad 3.373900$

$\begin{array}{llll}\text { C } & 4.374400 & 1.493500 & -0.742000\end{array}$

C $\quad 5.540200 \quad 0.790600 \quad-1.056700$

C $\quad 6.355100 \quad 1.164400 \quad-2.138800$

$\begin{array}{llll}\text { C } & 5.966200 & 2.249800 & -2.931900\end{array}$

C $\quad 4.791200 \quad 2.962100 \quad-2.649700$

C $\quad 4.005700 \quad 2.571100 \quad-1.559500$

C $\quad 4.397900 \quad 4.142700 \quad-3.461200$

C $\quad 4.060900 \quad 5.353200 \quad-2.843500$

$\begin{array}{llll}\text { C } & 3.660400 & 6.465700 & -3.582000\end{array}$

$\begin{array}{llll}\text { C } & 3.566400 & 6.421700 & -4.988400\end{array}$

C $\quad 3.933500 \quad 5.203800 \quad-5.599100$

C $\quad 4.343400 \quad 4.096200 \quad-4.858900$

C $\quad 3.077600 \quad 7.602600 \quad-5.847200$

C $\quad 2.710100 \quad 8.843800 \quad-5.000100$

$\begin{array}{llll}\text { C } & 4.188400 & 8.015100 & -6.836200\end{array}$

C $\quad \begin{array}{llll}\text { C } & 1.818500 & 7.179700 & -6.634500\end{array}$

$\begin{array}{llll}\text { C } & 7.640700 & 0.457400 & -2.366700\end{array}$

$\begin{array}{llll}\text { C } & 7.741800 & -0.934800 & -2.253000\end{array}$

C $\quad 8.963200 \quad-1.594900 \quad-2.385100$

C $\quad 10.160900 \quad-0.891800 \quad-2.631200$

$\begin{array}{llll}\text { C } & 10.040400 & 0.506300 & -2.777100\end{array}$

C $\quad 8.818000 \quad 1.162200 \quad-2.650100$

C $\quad 11.542300 \quad-1.565000 \quad-2.723200$

C $\quad 12.478900 \quad-0.971200 \quad-1.648400$

C $\quad 12.142900 \quad-1.315400 \quad-4.122400$

$\begin{array}{llll}\text { C } & 11.475600 & -3.093900 & -2.497400\end{array}$

C $\quad-1.675600 \quad 1.999600 \quad 0.443600$

C $\quad-2.745900 \quad 3.038100 \quad 0.727800$

C $\quad-3.750800 \quad 3.187200 \quad-0.413100$

C $\quad-2.846500 \quad 4.351200 \quad-0.105600$

C $\quad-3.446600 \quad 5.558300 \quad 0.548800$

$\begin{array}{llll}\text { C } & -2.701100 & 6.304200 & 1.478400\end{array}$

C $\quad-3.247900 \quad 7.444800 \quad 2.072800$

$\begin{array}{llll}\text { C } & -4.532400 & 7.868400 & 1.724600\end{array}$

$\begin{array}{llll}\text { C } & -5.266600 & 7.159400 & 0.771300\end{array}$

C $\quad-4.724600 \quad 6.016000 \quad 0.177200$

C $\quad 0.233700 \quad 5.249800 \quad-2.966700$

C $\quad-1.095800 \quad 5.138400 \quad-3.381400$

C $\quad-2.096600 \quad 4.849200 \quad-2.450300$

C $\quad-1.771600 \quad 4.659200 \quad-1.097000$

C $\quad-0.434900 \quad 4.792500 \quad-0.684300$

C $\quad 0.563100 \quad 5.082600 \quad-1.618300$

$\begin{array}{llll}\text { C } & -3.289400 & 2.913300 & 2.136500\end{array}$

$\begin{array}{llll}\text { C } & -2.666600 & 3.524100 & 3.224700\end{array}$

C $\quad-3.090700 \quad 3.291500 \quad 4.541100$

C $\quad-4.127400 \quad 2.374700 \quad 4.760200$

C $\quad-4.755100 \quad 1.722600 \quad 3.689300$

$\begin{array}{llll}\text { C } & -4.349300 & 2.034300 & 2.383600\end{array}$

C $\quad-5.763800 \quad 0.657700 \quad 3.926400$

C $\quad-6.927100 \quad 0.537700 \quad 3.155000$

C $\quad-7.818100 \quad-0.516500 \quad 3.351000$

C $\quad-7.597900 \quad-1.515700 \quad 4.322900$

C $\quad-6.432200 \quad-1.375100 \quad 5.103900$

C $\quad-5.545500 \quad-0.317300 \quad 4.908900$

C $\quad-8.599400 \quad-2.674000 \quad 4.488400$

C $\quad-8.159700 \quad-3.691800 \quad 5.567200$

C $\quad-8.739300 \quad-3.435800 \quad 3.152900$

C $\quad-9.973700 \quad-2.106600 \quad 4.900500$

C $\quad-2.113300 \quad 3.319600 \quad 6.862500$
C $\quad-2.411800 \quad 3.981400 \quad 5.664600$

C $\quad-2.003700 \quad 5.317300 \quad 5.551700$

C $\quad-1.305300 \quad 5.959200 \quad 6.573100$

$\begin{array}{llll}\text { C } & -0.975100 & 5.294400 & 7.772200\end{array}$

$\begin{array}{llll}\text { C } & -1.414100 & 3.958100 & 7.883700\end{array}$

C $\quad-0.165200 \quad 5.940200 \quad 8.911100$

$\begin{array}{lrrr}\text { C } & -1.002100 & 5.934000 & 10.208100\end{array}$

$\begin{array}{llll}\text { C } & 0.227900 & 7.405100 & 8.607000\end{array}$

C $\quad 1.135100 \quad 5.138000 \quad 9.133300$

C $\quad-2.692800 \quad-1.188900 \quad-0.555400$

$\begin{array}{llll}\text { C } & -4.160100 & -1.504900 & -0.772900\end{array}$

C $\quad-5.046600 \quad-1.117500 \quad 0.409100$

$\begin{array}{llll}\text { C } & -4.905100 & -2.579100 & 0.077100\end{array}$

$\begin{array}{llll}\text { C } & -6.067200 & -3.282400 & -0.556200\end{array}$

C $\quad-5.847000 \quad-4.314900-1.484200$

$\begin{array}{llll}\text { C } & -6.925400 & -4.988300 & -2.064400\end{array}$

C $\quad-8.233400 \quad-4.655300 \quad-1.705700$

C $\quad-8.464000 \quad-3.655200 \quad-0.758300$

$\begin{array}{llll}\text { C } & -7.388000 & -2.976400 & -0.179500\end{array}$

$\begin{array}{llll}\text { C } & -2.668200 & -4.978400 & 2.860600\end{array}$

C $\quad-3.747000 \quad-4.210100 \quad 3.307500$

C $\quad-4.477200 \quad-3.434900 \quad 2.401800$

C $\quad-4.125200 \quad-3.415200 \quad 1.041500$

C $\quad-3.054900 \quad-4.209000 \quad 0.597500$

C $\quad-2.324400 \quad-4.981100 \quad 1.505900$

C $\quad-4.621300 \quad-1.089600 \quad-2.154500$

$\begin{array}{llll}\text { C } & -5.065600 & 0.221600 & -2.348300\end{array}$

$\begin{array}{llll}\text { C } & -5.307200 & 0.737800 & -3.629000\end{array}$

C $\quad-5.160500 \quad-0.114200 \quad-4.733000$

C $\quad-4.765000 \quad-1.448300 \quad-4.563700$

C $\quad-4.474000 \quad-1.911400 \quad-3.272000$

C $\quad-4.614800 \quad-2.370000 \quad-5.716500$

C $\quad-3.948700 \quad-1.985100 \quad-6.885900$

C $\quad-3.749900 \quad-2.886000 \quad-7.929500$

C $\quad-4.212300 \quad-4.217800 \quad-7.870500$

C $\quad-4.911900-4.583100 \quad-6.701600$

C $\quad-5.102300 \quad-3.682000 \quad-5.654900$

C $\quad-3.921600 \quad-5.189000 \quad-9.029100$

C $\quad-2.394500 \quad-5.331100 \quad-9.206400$

C $\quad-4.503500 \quad-6.601200 \quad-8.783900$

$\begin{array}{llll}\text { C } & -4.542700 & -4.640600 & -10.331500\end{array}$

$\begin{array}{llll}\text { C } & -5.628500 & 2.179700 & -3.784900\end{array}$

$\begin{array}{llll}\text { C } & -4.976000 & 2.963900 & -4.744800\end{array}$

C $\quad-5.186200 \quad 4.339300 \quad-4.817300$

C $\quad-6.065300 \quad 5.012100 \quad-3.942600$

C $\quad-6.743400 \quad 4.207200 \quad-3.003700$

C $\quad-6.526200 \quad 2.831300 \quad-2.929000$

C $\quad-6.222700 \quad 6.541500 \quad-4.027800$

C $\quad-6.697200 \quad 6.935200 \quad-5.443200$

C $\quad-4.864000 \quad 7.213400 \quad-3.735400$

$\begin{array}{llll}\text { C } & -7.253200 & 7.092900 & -3.014400\end{array}$

$\begin{array}{llll}\text { C } & 1.063600 & -2.378200 & -0.685800\end{array}$

$\begin{array}{llll}\text { C } & 2.072100 & -3.492300 & -0.900900\end{array}$

$\begin{array}{llll}\text { C } & 2.936400 & -3.342700 & -2.143700\end{array}$

C $\quad 1.942300 \quad-4.473400 \quad-2.107300$

C $\quad 2.463300 \quad-5.864300 \quad-1.916700$

C $\quad 1.808200 \quad-6.757800 \quad-1.051400$

$\begin{array}{llll}\text { C } & 2.291400 & -8.057800 & -0.879100\end{array}$

$\begin{array}{llll}\text { C } & 3.417900 & -8.485200 & -1.585700\end{array}$

C $\quad 4.056500 \quad-7.616100 \quad-2.473800$

C $\quad 3.577800 \quad-6.313900 \quad-2.646900$

$\begin{array}{llll}\text { C } & -1.493000 & -4.360700 & -4.683900\end{array}$ 


\begin{tabular}{|c|c|c|c|}
\hline C & -1.651100 & -4.622600 & -3.320700 \\
\hline 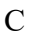 & -0.538900 & -4.626500 & -2.474400 \\
\hline & 0.742400 & -4.377600 & -2.990700 \\
\hline 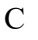 & 0.894600 & -4.127600 & -4.365200 \\
\hline 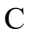 & -0.222300 & -4.109400 & -5.205500 \\
\hline & 2.733300 & -3.899700 & 0.398200 \\
\hline & 4.027300 & -3.462500 & 0.675800 \\
\hline$c$ & 4.633100 & -3.686900 & 1.922400 \\
\hline 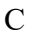 & 3.916200 & -4.387100 & 2.900400 \\
\hline & 2.612600 & -4.847200 & 2.645300 \\
\hline C & 2.035100 & -4.586700 & 1.395300 \\
\hline C & 1.815900 & -5.561100 & 3.674300 \\
\hline $\mathrm{C}$ & 0.961300 & -6.615200 & 3.323400 \\
\hline C & 0.158800 & -7.241900 & 4.272600 \\
\hline C & 0.158100 & -6.853300 & 5.628800 \\
\hline C & 1.039400 & -5.808300 & 5.975500 \\
\hline $\mathrm{C}$ & 1.844900 & -5.184100 & 5.022800 \\
\hline $\mathrm{C}$ & -0.781700 & -7.547400 & 6.631700 \\
\hline $\mathrm{C}$ & -0.641200 & -6.986800 & 8.066600 \\
\hline C & -0.461400 & -9.056700 & 6.679900 \\
\hline $\mathrm{C}$ & -2.246400 & -7.346300 & 6.186700 \\
\hline $\mathrm{C}$ & 6.287400 & -2.390600 & 3.321300 \\
\hline $\mathrm{C}$ & 5.977000 & -3.100600 & 2.154500 \\
\hline $\mathrm{C}$ & 6.950600 & -3.132600 & 1.146600 \\
\hline C & 8.145900 & -2.431600 & 1.271200 \\
\hline $\mathrm{C}$ & 8.440500 & -1.654600 & 2.410600 \\
\hline $\mathrm{C}$ & 7.484800 & -1.682900 & 3.448000 \\
\hline $\mathrm{C}$ & 9.723600 & -0.805800 & 2.447300 \\
\hline $\mathrm{C}$ & 9.860600 & 0.007100 & 3.755500 \\
\hline $\mathrm{C}$ & 9.711200 & 0.190900 & 1.266800 \\
\hline $\mathrm{C}$ & 10.956100 & -1.727000 & 2.328400 \\
\hline $\mathrm{H}$ & 3.585800 & -0.280000 & 2.177600 \\
\hline $\mathrm{H}$ & 5.243500 & 0.025800 & 1.502800 \\
\hline $\mathrm{H}$ & 4.294900 & 4.309400 & 0.611100 \\
\hline $\mathrm{H}$ & 6.290500 & 5.746600 & 0.499200 \\
\hline $\mathrm{H}$ & 8.379100 & 5.074800 & 1.653200 \\
\hline $\mathrm{H}$ & 8.461000 & 2.957600 & 2.934000 \\
\hline $\mathrm{H}$ & 6.480200 & 1.491000 & 3.044400 \\
\hline $\mathrm{H}$ & 0.479700 & 3.771800 & 5.410200 \\
\hline $\mathrm{H}$ & 2.071500 & 2.002000 & 6.082000 \\
\hline $\mathrm{H}$ & 3.704700 & 1.117600 & 4.452400 \\
\hline $\mathrm{H}$ & 2.072600 & 3.729700 & 1.430100 \\
\hline $\mathrm{H}$ & 0.489800 & 4.640100 & 3.085700 \\
\hline $\mathrm{H}$ & 5.865100 & -0.031000 & -0.427900 \\
\hline $\mathrm{H}$ & 6.589800 & 2.558400 & -3.766600 \\
\hline $\mathrm{H}$ & 3.095800 & 3.118200 & -1.339000 \\
\hline $\mathrm{H}$ & 4.124500 & 5.433200 & -1.761800 \\
\hline $\mathrm{H}$ & 3.422300 & 7.351500 & -3.000600 \\
\hline $\mathrm{H}$ & 3.895600 & 5.081500 & -6.678800 \\
\hline $\mathrm{H}$ & 4.592700 & 3.172600 & -5.374600 \\
\hline $\mathrm{H}$ & 1.890800 & 8.607000 & -4.286900 \\
\hline $\mathrm{H}$ & 2.358900 & 9.674600 & -5.650000 \\
\hline $\mathrm{H}$ & 3.593000 & 9.211900 & -4.434000 \\
\hline $\mathrm{H}$ & 5.115700 & 8.284300 & -6.285600 \\
\hline $\mathrm{H}$ & 3.872300 & 8.893800 & -7.439400 \\
\hline $\mathrm{H}$ & 4.428800 & 7.194000 & -7.543600 \\
\hline $\mathrm{H}$ & 2.037900 & 6.349600 & -7.338100 \\
\hline П & 1.424600 & 8.029300 & -7.233300 \\
\hline $\mathrm{H}$ & 1.020200 & 6.844400 & -5.937400 \\
\hline $\mathrm{H}$ & 6.845800 & -1.519900 & -2.065200 \\
\hline $\mathrm{H}$ & 8.923200 & -2.676100 & -2.288100 \\
\hline & 10.909000 & 1.128700 & -2.977200 \\
\hline
\end{tabular}

H $\quad 8.786800 \quad 2.245100 \quad-2.732800$

H $\quad 12.652400 \quad 0.112900 \quad-1.811700$

$\mathrm{H} \quad 13.470300 \quad-1.473600 \quad-1.668500$

H $\quad 12.041300 \quad-1.105100 \quad-0.636200$

H $\quad 11.463000 \quad-1.706100 \quad-4.910200$

H $\quad 13.126100 \quad-1.823900 \quad-4.225200$

$\mathrm{H} \quad 12.304700 \quad-0.232600 \quad-4.307200$

H $\quad 11.063500 \quad-3.326400 \quad-1.491200$

$\mathrm{H} \quad 12.489600 \quad-3.545800 \quad-2.558000$

H $\quad 10.846000 \quad-3.581700 \quad-3.272500$

$\mathrm{H} \quad-3.557100 \quad 2.610600 \quad-1.310300$

$\mathrm{H} \quad-4.797600 \quad 3.229800 \quad-0.126900$

$\mathrm{H} \quad-1.692000 \quad 6.013500 \quad 1.735000$

$\mathrm{H} \quad-2.671300 \quad 8.005900 \quad 2.796700$

$\mathrm{H} \quad-4.953100 \quad 8.754000 \quad 2.182900$

H $\quad-6.254200 \quad 7.499400 \quad 0.488000$

$\mathrm{H} \quad-5.300800 \quad 5.495300 \quad-0.576200$

$\mathrm{H} \quad 1.005700 \quad 5.467200 \quad-3.691100$

H $\quad-1.351000 \quad 5.275200 \quad-4.424400$

$\mathrm{H} \quad-3.122900 \quad 4.764900 \quad-2.782700$

$\mathrm{H} \quad-0.165900 \quad 4.658100 \quad 0.355700$

$\mathrm{H} \quad 1.590200 \quad 5.178000 \quad-1.294500$

H $\quad-1.797700 \quad 4.147700 \quad 3.062700$

$\mathrm{H} \quad-4.468400 \quad 2.183300 \quad 5.773700$

$\begin{array}{llll}\mathrm{H} & -4.815300 & 1.521400 & 1.548900\end{array}$

$\mathrm{H} \quad-7.149200 \quad 1.286600 \quad 2.399100$

$\mathrm{H} \quad-8.700500 \quad-0.534700 \quad 2.716100$

H $\quad-6.151500 \quad-2.081400 \quad 5.879400$

$\mathrm{H} \quad-4.642400 \quad-0.271600 \quad 5.511300$

$\mathrm{H} \quad-7.177700 \quad-4.143100 \quad 5.306200$

H $\quad-8.897900 \quad-4.518700 \quad 5.653400$

$\mathrm{H} \quad-8.086400 \quad-3.206000 \quad 6.564200$

$\mathrm{H} \quad-9.180900 \quad-2.792400 \quad 2.364200$

H $\quad-9.405900 \quad-4.317600 \quad 3.269000$

H $\quad-7.746400 \quad-3.793300 \quad 2.803900$

$\mathrm{H} \quad-9.885900 \quad-1.529500 \quad 5.846500$

H $\quad-10.707200 \quad-2.926900 \quad 5.057800$

H $\quad-10.385200 \quad-1.433500 \quad 4.119200$

H $\quad-2.393100 \quad 2.276900 \quad 6.984400$

$\mathrm{H} \quad-2.242400 \quad 5.872800 \quad 4.649200$

$\mathrm{H} \quad-1.037400 \quad 6.993400 \quad 6.377900$

$\mathrm{H} \quad-1.202400 \quad 3.368000 \quad 8.771900$

$\begin{array}{llll}\mathrm{H} & -1.961800 & 6.472600 & 10.051300\end{array}$

$\mathrm{H} \quad-0.451500 \quad 6.433800 \quad 11.034500$

$\mathrm{H} \quad-1.232600 \quad 4.899800 \quad 10.539100$

$\mathrm{H} \quad 0.862100 \quad 7.463600 \quad 7.696000$

$\mathrm{H} \quad 0.810100 \quad 7.839800 \quad 9.448500$

$\mathrm{H} \quad-0.676400 \quad 8.035600 \quad 8.464300$

$\mathrm{H} \quad 0.921200 \quad 4.097700 \quad 9.456500$

$\mathrm{H} \quad 1.760200 \quad 5.610900 \quad 9.921600$

$\mathrm{H} \quad 1.728500 \quad 5.095700 \quad 8.194100$

H $\quad-4.541600 \quad-0.747300 \quad 1.293800$

$\mathrm{H} \quad-5.964400 \quad-0.590700 \quad 0.166200$

H $\quad-4.841700 \quad-4.606100 \quad-1.753600$

H $\quad-6.746200 \quad-5.774000 \quad-2.786900$

$\mathrm{H} \quad-9.067700 \quad-5.179900 \quad-2.153000$

H $\quad-9.477100 \quad-3.408700 \quad-0.468000$

H $\quad-7.588900 \quad-2.217200 \quad 0.564200$

$\mathrm{H} \quad-2.102300 \quad-5.574100 \quad 3.563200$

H $\quad-4.017300 \quad-4.214500 \quad 4.355700$

$\mathrm{H} \quad-5.311400 \quad-2.845000 \quad 2.759000$

H $\quad-2.780700 \quad-4.214800 \quad-0.447600$
H $\quad-1.493000 \quad-5.581600 \quad 1.159900$

$\mathrm{H} \quad-5.150500 \quad 0.881300 \quad-1.491400$

$\mathrm{H} \quad-5.385600 \quad 0.255300 \quad-5.729800$

$\mathrm{H} \quad-4.071100 \quad-2.910200 \quad-3.159400$

$\mathrm{H} \quad-3.543200 \quad-0.980100 \quad-6.966200$

$\mathrm{H} \quad-3.200200 \quad-2.518500 \quad-8.792400$

$\mathrm{H} \quad-5.330300 \quad-5.573500 \quad-6.548300$

$\mathrm{H} \quad-5.646400 \quad-4.005800 \quad-4.771900$

$\mathrm{H} \quad-1.925800 \quad-4.364700 \quad-9.487300$

$\mathrm{H} \quad-2.157200 \quad-6.063200 \quad-10.008600$

$\mathrm{H} \quad-1.926600 \quad-5.683000 \quad-8.261200$

H $\quad-4.062400 \quad-7.057900 \quad-7.871500$

H $\quad-4.276400 \quad-7.275000 \quad-9.638600$

$\mathrm{H} \quad-5.608900 \quad-6.559700 \quad-8.674500$

H $\quad-5.637100 \quad-4.492600 \quad-10.205200$

H $\quad-4.380300 \quad-5.348000 \quad-11.173500$

H $\quad-4.090400 \quad-3.669100 \quad-10.621000$

$\mathrm{H} \quad-4.257200 \quad 2.501000 \quad-5.415400$

$\mathrm{H} \quad-4.615600 \quad 4.879500 \quad-5.568500$

$\mathrm{H} \quad-7.459700 \quad 4.607200 \quad-2.292200$

$\mathrm{H} \quad-7.074600 \quad 2.251700 \quad-2.190900$

$\mathrm{H} \quad-5.945500 \quad 6.667900 \quad-6.214900$

$\mathrm{H} \quad-6.866700 \quad 8.031800 \quad-5.511300$

$\mathrm{H} \quad-7.650200 \quad 6.418500 \quad-5.689100$

H $\quad-4.502000 \quad 6.931600 \quad-2.723400$

$\mathrm{H} \quad-4.955800 \quad 8.320500 \quad-3.775300$

$\mathrm{H} \quad-4.093700 \quad 6.913100 \quad-4.476500$

$\mathrm{H} \quad-8.258200 \quad 6.653900 \quad-3.194900$

$\mathrm{H} \quad-7.345900 \quad 8.196800 \quad-3.107700$

$\mathrm{H} \quad-6.941600 \quad 6.872800 \quad-1.971500$

H $\quad 2.726100 \quad-2.484100 \quad-2.772100$

$\mathrm{H} \quad 3.993100 \quad-3.568400 \quad-2.036800$

$\mathrm{H} \quad 0.920800 \quad-6.453400 \quad-0.514400$

H $\quad \begin{array}{llll}1.788000 & -8.735800 & -0.202000\end{array}$

H $\quad 3.789700 \quad-9.492900-1.453100$

$\mathrm{H} \quad 4.920900 \quad-7.952600 \quad-3.031500$

H $\quad 4.076200 \quad-5.658400 \quad-3.349900$

$\mathrm{H} \quad-2.350700 \quad-4.366900 \quad-5.339500$

$\mathrm{H} \quad-2.632600 \quad-4.839300 \quad-2.922900$

$\mathrm{H} \quad-0.670100 \quad-4.841600 \quad-1.422400$

$\mathrm{H} \quad 1.877900 \quad-3.958700 \quad-4.786400$

$\mathrm{H} \quad-0.101500 \quad-3.916400 \quad-6.263800$

H $\quad 4.559100 \quad-2.871700 \quad-0.062000$

$\mathrm{H} \quad 4.382400 \quad-4.599200 \quad 3.858800$

$\mathrm{H} \quad 1.000400 \quad-4.862500 \quad 1.227000$

$\mathrm{H} \quad 0.928400 \quad-6.959000 \quad 2.293300$

$\mathrm{H} \quad-0.479200 \quad-8.046800 \quad 3.916000$

H $\quad 1.128700 \quad-5.423300 \quad 6.986900$

H $\quad 2.480400 \quad-4.359200 \quad 5.333500$

H $\quad 0.393100 \quad-7.130400 \quad 8.447500$

H $\quad-1.331000 \quad-7.510600 \quad 8.763600$

$\mathrm{H} \quad-0.894700 \quad-5.905000 \quad 8.095300$

$\mathrm{H} \quad-0.644200 \quad-9.543500 \quad 5.699100$

$\mathrm{H} \quad-1.099300 \quad-9.571000 \quad 7.431100$

H $\quad 0.603200 \quad-9.217300 \quad 6.956600$

$\mathrm{H} \quad-2.483800 \quad-6.262600 \quad 6.114700$

H $\quad-2.946700 \quad-7.809400 \quad 6.915300$

$\mathrm{H} \quad-2.438700 \quad-7.811200 \quad 5.196900$

$\mathrm{H} \quad 5.557400 \quad-2.337800 \quad 4.124700$

H $\quad 6.766200 \quad-3.709400 \quad 0.244300$

H $\quad 8.834700 \quad-2.486800 \quad 0.433200$

H $\quad 7.610600 \quad-1.139600 \quad 4.379900$ 

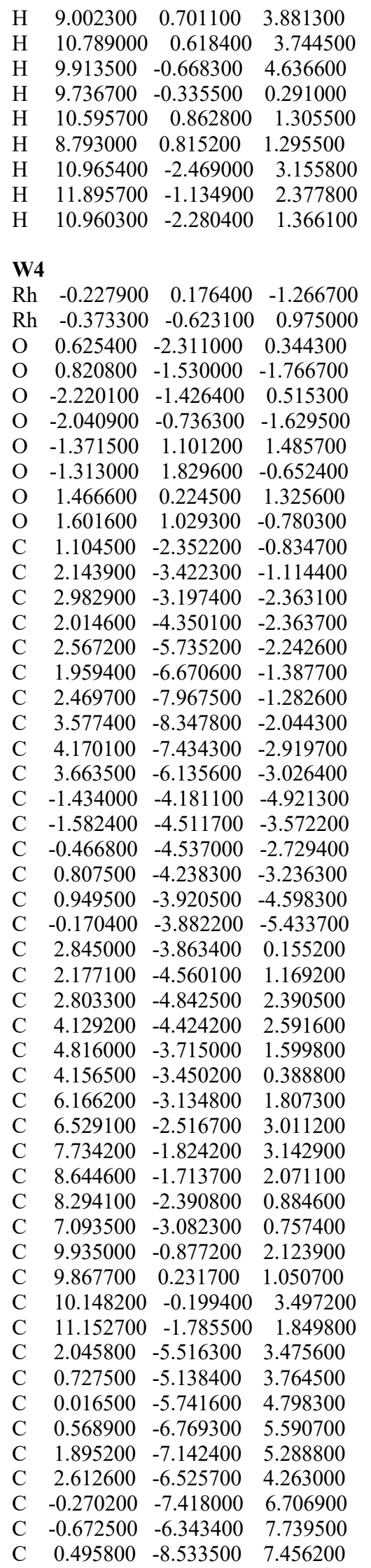

\begin{tabular}{|c|c|c|c|c|c|c|c|}
\hline C & 1.542200 & -8.044800 & 6.096000 & $\mathrm{C}$ & -4.344900 & 1.858000 & 4.797500 \\
\hline $\mathrm{C}$ & -2.690100 & -1.241500 & -0.656000 & $\mathrm{C}$ & -4.979300 & 365300 & 3.648800 \\
\hline $\mathrm{C}$ & -4.157600 & -1.536600 & -0.903200 & $\mathrm{C}$ & -4.520000 & 1.790300 & 2.393400 \\
\hline $\mathrm{C}$ & -5.043700 & -1.247300 & 0.307800 & $\mathrm{C}$ & -6.054300 & 0.343500 & 3.729900 \\
\hline $\mathrm{C}$ & -4.912800 & -2.674000 & -0.150800 & $\mathrm{C}$ & -5.934000 & -0.754700 & 4.591700 \\
\hline $\mathrm{C}$ & -6.083000 & -3.306700 & -0.841600 & $\mathrm{C}$ & -6.878600 & -1.778400 & 4.593800 \\
\hline $\mathrm{C}$ & -5.874300 & -4.238500 & -1.873200 & $\mathrm{C}$ & -8.001600 & -1.765400 & 3.740400 \\
\hline $\mathrm{C}$ & -6.960000 & -4.846400 & -2.509300 & $\mathrm{C}$ & -8.131000 & -0.638700 & 2.901700 \\
\hline $\mathrm{C}$ & -8.263500 & -4.551100 & -2.104500 & $\mathrm{C}$ & -7.181400 & 0.383300 & 2.898700 \\
\hline $\mathrm{C}$ & -8.482400 & -3.656800 & -1.054200 & $\mathrm{C}$ & -8.990100 & -2.946000 & 3.744900 \\
\hline $\mathrm{C}$ & -7.399100 & -3.044100 & -0.417700 & $\mathrm{C}$ & -9.587900 & -3.113700 & 5.158200 \\
\hline $\mathrm{C}$ & -2.772600 & -5.437200 & 2.353500 & $\mathrm{C}$ & -10.162800 & -2.745700 & 2.756300 \\
\hline $\mathrm{C}$ & -3.832500 & -4.692900 & 2.879800 & $\mathrm{C}$ & -8.247700 & -4.239200 & 4300 \\
\hline $\mathrm{C}$ & -4.518300 & -3.781900 & 2.070500 & $\mathrm{C}$ & -2.563400 & 3.256000 & 4900 \\
\hline $\mathrm{C}$ & -4.142200 & -3.605200 & 0.728600 & $\mathrm{C}$ & & & 5.943400 \\
\hline $\mathrm{C}$ & -3.078800 & -4.359700 & 0.208200 & $\mathrm{C}$ & -1.368700 & 5.044600 & 5100 \\
\hline $\mathrm{C}$ & -2.39 & -5.26 & 1.020600 & $\mathrm{C}$ & 000 & & 400 \\
\hline $\mathrm{C}$ & -4.605400 & -1.015500 & -2.252800 & $\mathrm{C}$ & -1.630300 & 2.929200 & 8.136200 \\
\hline $\mathrm{C}$ & -4.415300 & -1.744000 & -3.427800 & $\mathrm{C}$ & -2.337200 & 2.455300 & 1400 \\
\hline $\mathrm{C}$ & -4.685700 & -1.185700 & -4.685300 & $\mathrm{C}$ & -0.280100 & 4.794800 & 9.346100 \\
\hline $\mathrm{C}$ & -5.102100 & 0.15 & -4.7589 & $\mathrm{C}$ & & 2000 & 600 \\
\hline $\mathrm{C}$ & -5.29 & 0.9 & -3.5 & $\mathrm{C}$ & 300 & 500 & 7000 \\
\hline $\mathrm{C}$ & -5.069900 & 0.298900 & -2.352800 & $\mathrm{C}$ & 400 & 6.035400 & 9.935600 \\
\hline $\mathrm{C}$ & -5.627000 & 6200 & -3.649300 & $\mathrm{C}$ & & 0.799800 & 2600 \\
\hline $\mathrm{C}$ & -6.551900 & 2.93 & -2.769400 & $\mathrm{C}$ & 00 & 1.1 & 000 \\
\hline $\mathrm{C}$ & -6.7 & 4.30 & -2.748 & $\mathrm{C}$ & & & 300 \\
\hline $\mathrm{C}$ & -6.0 & 5.18 & -3.60 & $\mathrm{C}$ & & & 800 \\
\hline $\mathrm{C}$ & -5.18 & 4.590700 & -4.510300 & $\mathrm{C}$ & 300 & 2.599700 & 100 \\
\hline $\mathrm{C}$ & -4.962400 & 3.216200 & -4.533300 & $\mathrm{C}$ & & & 000 \\
\hline $\mathrm{C}$ & -6.273700 & 6.71 & -3.590700 & $\mathrm{C}$ & 00 & & 200 \\
\hline $\mathrm{C}$ & -7.3 & 7.18 & -2.5 & $\mathrm{C}$ & & & 00 \\
\hline $\mathrm{C}$ & -6.73 & 7.2 & -4.98 & $\mathrm{C}$ & 000 & 4.748500 & 100 \\
\hline $\mathrm{C}$ & -4.92 & 7.38 & -3.231000 & $\mathrm{C}$ & 500 & 3.902400 & 800 \\
\hline $\mathrm{C}$ & $-3.77^{\prime}$ & -1.535300 & -7.0093 & $\mathrm{C}$ & & & 400 \\
\hline $\mathrm{C}$ & -4.49 & -2.01 & -5.9 & $\mathrm{C}$ & & & 400 \\
\hline $\mathrm{C}$ & -4.9 & -3.3 & -5.9 & $\mathrm{C}$ & & & 700 \\
\hline $\mathrm{C}$ & -4.749200 & -4.13 & -7.0745 & $\mathrm{C}$ & 200 & 00 & 700 \\
\hline $\mathrm{C}$ & -4.000800 & -3.68 & -8.179900 & $\mathrm{C}$ & 000 & 3.0 & 500 \\
\hline $\mathrm{C}$ & -3.53 & -2.350500 & & $\mathrm{C}$ & 1500 & 71000 & 200 \\
\hline $\mathrm{C}$ & -3.6 & & & $\mathrm{C}$ & & & 300 \\
\hline $\mathrm{C}$ & -2.12 & -4.69 & 20500 & $\mathrm{C}$ & 500 & 4900 & 3200 \\
\hline $\mathrm{C}$ & -4.224400 & -3.905000 & -10.678800 & $\mathrm{C}$ & 300 & 3.325600 & 5800 \\
\hline $\mathrm{C}$ & -4.253500 & -5.982700 & -9.292400 & $\mathrm{C}$ & 5.866100 & 2.654700 & -2.709700 \\
\hline $\mathrm{C}$ & -1.735000 & 1.886200 & 0.550400 & $\mathrm{C}$ & 6.268600 & 1.476400 & 0500 \\
\hline $\mathrm{C}$ & & & & $\mathrm{C}$ & & & 700 \\
\hline $\mathrm{C}$ & -3.792900 & 3.141600 & -0.260300 & $\mathrm{C}$ & 7.547800 & 0.807900 & 8800 \\
\hline $\mathrm{C}$ & -2.908400 & 4.273100 & 0.189200 & $\mathrm{C}$ & 8.711000 & 1.549000 & 6500 \\
\hline $\mathrm{C}$ & -3.537500 & 5.403400 & 0.944600 & $\mathrm{C}$ & 9.928000 & 0.921600 & -2.92 \\
\hline $\mathrm{C}$ & -4.796800 & & & $\mathrm{C}$ & 10.058300 & -0.482900 & -2.945100 \\
\hline $\mathrm{C}$ & -5.368500 & 6.97 & & $\mathrm{C}$ & 74700 & -1.21 & -2.72 \\
\hline $\mathrm{C}$ & -4.682800 & 7.567000 & 2.323700 & $\mathrm{C}$ & 7.658100 & -0.587300 & -2.468500 \\
\hline $\mathrm{C}$ & -3.417200 & 7.099400 & 2.684800 & $\mathrm{C}$ & 11.434400 & -1.130400 & -3.184200 \\
\hline $\mathrm{C}$ & -2.840500 & 6.032000 & 1.991000 & $\mathrm{C}$ & 11.959200 & -0.720000 & -4.575800 \\
\hline $\mathrm{C}$ & 0.256500 & & -2.457200 & $\mathrm{C}$ & 11.382800 & -2.675400 & -3.129000 \\
\hline $\mathrm{C}$ & 0.539300 & 5.188700 & -1.118700 & $\mathrm{C}$ & 12.426200 & -0.653900 & -2.100400 \\
\hline $\mathrm{C}$ & -0.485700 & 4.793600 & -0.255500 & $\mathrm{C}$ & 4.206700 & 4.740200 & -4.346400 \\
\hline $\mathrm{C}$ & -1.803800 & 4.682900 & -0.728900 & $\mathrm{C}$ & 4.304300 & 4.606700 & -2.956800 \\
\hline $\mathrm{C}$ & -2.083500 & 4.997000 & -2.069400 & $\mathrm{C}$ & 4.018500 & 5.735500 & -2.179400 \\
\hline $\mathrm{C}$ & -1.054600 & & -2.930800 & $\mathrm{C}$ & & & -2.757200 \\
\hline $\mathrm{C}$ & -3.410500 & 2.630300 & 2.265600 & $\mathrm{C}$ & 3.492900 & 7.081500 & -4.154200 \\
\hline $\mathrm{C}$ & -2.784400 & 3.084300 & 3.426700 & $\mathrm{C}$ & 3.806300 & 5.942700 & -4.926100 \\
\hline $\mathrm{C}$ & -3.252600 & 2.732200 & 4.700500 & $\mathrm{C}$ & 3.013100 & 8.373900 & -4.840000 \\
\hline
\end{tabular}


$\begin{array}{llll}\text { C } & 4.103400 & 8.879200 & -5.808500\end{array}$

$\begin{array}{llll}\text { C } & 2.711200 & 9.507300 & -3.831400\end{array}$

C $\quad 1.717100 \quad 8.086600 \quad-5.628400$

H $\quad 2.743600 \quad-2.315800 \quad-2.947900$

H $\quad 4.045400 \quad-3.405700 \quad-2.287900$

$\begin{array}{llll}\mathrm{H} & 1.088100 & -6.399800 & -0.807300\end{array}$

H $\quad 2.002200 \quad-8.679500 \quad-0.615000$

H $\quad 3.970300 \quad-9.353000 \quad-1.963400$

H $\quad 5.020200 \quad-7.733800 \quad-3.518900$

H $\quad 4.125800 \quad-5.444100 \quad-3.719600$

H $\quad-2.294100 \quad-4.171300 \quad-5.573600$

$\mathrm{H} \quad-2.559800 \quad-4.764000 \quad-3.185400$

$\mathrm{H} \quad-0.589500 \quad-4.806100 \quad-1.688800$

H $\quad 1.927700 \quad-3.713300 \quad-5.014200$

$\mathrm{H} \quad-0.057000 \quad-3.636100 \quad-6.481800$

H $\quad 1.145700 \quad-4.861700 \quad 1.023800$

$\mathrm{H} \quad 4.613100 \quad-4.625600 \quad 3.543400$

$\mathrm{H} \quad 4.662100 \quad-2.843300 \quad-0.353500$

H $\quad 5.835100 \quad-2.525400 \quad 3.847400$

H $\quad 7.902200 \quad-1.358400 \quad 4.109400$

H $\quad 8.942200 \quad-2.373500 \quad 0.013100$

H $\quad 6.870200 \quad-3.585600 \quad-0.179500$

H $\quad 9.818600 \quad-0.196100 \quad 0.028600$

$\begin{array}{llll}\mathrm{H} & 10.766900 & 0.883300 & 1.097300\end{array}$

$\begin{array}{llll}\mathrm{H} & 8.966600 & 0.864100 & 1.201900\end{array}$

$\begin{array}{llll}\mathrm{H} & 9.307400 & 0.486200 & 3.735000\end{array}$

$\mathrm{H} \quad 11.083100 \quad 0.402200 \quad 3.499000$

H $\quad 10.235400 \quad-0.959700 \quad 4.303300$

H $\quad 11.198500 \quad-2.606400 \quad 2.597700$

H $\quad 12.098300 \quad-1.204100 \quad 1.909300$

H $\quad 11.104100 \quad-2.240100 \quad 0.838500$

H $\quad 0.259900 \quad-4.342800 \quad 3.190700$

$\begin{array}{llll}\mathrm{H} & -0.991800 & -5.375400 & 4.974600\end{array}$

H $\quad 2.425100 \quad-7.923200 \quad 5.826400$

H $\quad 3.627500 \quad-6.856500 \quad 4.058000$

H $\quad 0.232200 \quad-5.852300 \quad 8.158900$

H $\quad-1.244400 \quad-6.797300 \quad 8.577800$

$\begin{array}{llll}\mathrm{H} & -1.314300 & -5.560600 & 7.283600\end{array}$

$\mathrm{H} \quad 0.786400 \quad-9.349800 \quad 6.759900$

H $\quad-0.138900 \quad-8.981500 \quad 8.251600$

$\begin{array}{llll}\mathrm{H} & 1.407900 & -8.127600 & 7.944800\end{array}$

$\begin{array}{llll}\mathrm{H} & -2.191700 & -7.275300 & 5.628100\end{array}$

$\mathrm{H} \quad-2.143300 \quad-8.557700 \quad 6.878000$

$\mathrm{H} \quad-1.270300 \quad-8.791000 \quad 5.318300$

H $\quad-4.536300 \quad-0.956200 \quad 1.220400$

H $\quad-5.961100 \quad-0.699900 \quad 0.114300$

H $\quad-4.872400 \quad-4.504500 \quad-2.178900$

H $\quad-6.789700 \quad-5.553400 \quad-3.310900$

$\mathrm{H} \quad-9.103300 \quad-5.025000 \quad-2.595800$

$\mathrm{H} \quad-9.492200 \quad-3.442300 \quad-0.729800$

H $\quad-7.590400 \quad-2.371500 \quad 0.407900$

H $\quad-2.253000 \quad-6.154000 \quad 2.974600$

H $\quad-4.128300 \quad-4.828100 \quad 3.912200$

H $\quad-5.345600 \quad-3.220000 \quad 2.484700$

$\mathrm{H} \quad-2.785700 \quad-4.241700 \quad-0.824200$

$\mathrm{H} \quad-1.568300 \quad-5.840300 \quad 0.614600$

H $\quad-3.990100 \quad-2.739500 \quad-3.384600$

$\mathrm{H} \quad-5.308900 \quad 0.594100 \quad-5.729100$

$\mathrm{H} \quad-5.184700 \quad 0.888100 \quad-1.449200$

H $\quad-7.109800 \quad 2.295000 \quad-2.089000$

H $\quad-7.521700 \quad 4.647900 \quad-2.026600$

H $\quad-4.609200 \quad 5.190600 \quad-5.207700$
H $\quad-4.222900 \quad 2.811700 \quad-5.218900$

$\begin{array}{llll}\mathrm{H} & -8.323600 & 6.746300 & -2.786400\end{array}$

$\mathrm{H} \quad-7.432600 \quad 8.289100 \quad-2.573700$

$\mathrm{H} \quad-7.029600 \quad 6.891400 \quad-1.528400$

$\mathrm{H} \quad-5.966200 \quad 6.996600 \quad-5.759200$

$\mathrm{H} \quad-6.914900 \quad 8.298500 \quad-4.978000$

$\mathrm{H} \quad-7.675100 \quad 6.692100 \quad-5.278300$

H $\quad-4.575600 \quad 7.036100 \quad-2.237700$

$\mathrm{H} \quad-5.038200 \quad 8.491400 \quad-3.191200$

$\mathrm{H} \quad-4.145000 \quad 7.152600 \quad-3.981200$

H $\quad-3.369800 \quad-0.528100 \quad-6.991700$

$\mathrm{H} \quad-5.559200 \quad-3.713600 \quad-5.134300$

H $\quad-5.174200 \quad-5.133900 \quad-7.018900$

H $\quad-2.950500 \quad-1.917000 \quad-8.919800$

$\mathrm{H} \quad-1.646400 \quad-3.707500 \quad-9.702300$

$\mathrm{H} \quad-1.855800 \quad-5.357800 \quad-10.367800$

$\mathrm{H} \quad-1.701300 \quad-5.119900 \quad-8.587200$

$\mathrm{H} \quad-5.323200 \quad-3.762800 \quad-10.588500$

H $\quad-4.026200 \quad-4.543800-11.566800$

H $\quad-3.759300 \quad-2.915400-10.870300$

$\mathrm{H} \quad-3.852300 \quad-6.512600 \quad-8.401300$

H $\quad-3.990000 \quad-6.587000 \quad-10.187700$

H $\quad-5.362500 \quad-5.945700 \quad-9.227600$

$\mathrm{H} \quad-3.566400 \quad 2.654900 \quad-1.202000$

H $\quad-4.849200 \quad 3.156600 \quad-0.009600$

$\mathrm{H} \quad-5.335500 \quad 5.472500 \quad-0.266100$

$\begin{array}{llll}\mathrm{H} & -6.341700 & 7.349300 & 0.970300\end{array}$

$\mathrm{H} \quad-5.126600 \quad 8.396100 \quad 2.859400$

$\mathrm{H} \quad-2.878300 \quad 7.569900 \quad 3.497000$

$\mathrm{H} \quad-1.846400 \quad 5.706400 \quad 2.264000$

H $\quad 1.050000 \quad 5.772600 \quad-3.128200$

$\mathrm{H} \quad 1.550800 \quad 5.272900 \quad-0.747100$

$\mathrm{H} \quad-0.251900 \quad 4.564900 \quad 0.776700$

H $\quad-3.095800 \quad 4.932500 \quad-2.446700$

$\mathrm{H} \quad-1.273900 \quad 5.617900 \quad-3.965100$

$\mathrm{H} \quad-1.880400 \quad 3.674000 \quad 3.347900$

$\mathrm{H} \quad-4.722100 \quad 1.577600 \quad 5.776900$

$\mathrm{H} \quad-4.989700 \quad 1.394600 \quad 1.499300$

$\mathrm{H} \quad-5.065000 \quad-0.833900 \quad 5.238900$

H $\quad-6.695600 \quad-2.607800 \quad 5.272400$

$\mathrm{H} \quad-8.963900 \quad-0.505800 \quad 2.217500$

H $\quad-7.326800 \quad 1.234200 \quad 2.238100$

$\mathrm{H} \quad-8.806100 \quad-3.368100 \quad 5.904000$

$\mathrm{H} \quad-10.340600 \quad-3.931700 \quad 5.173100$

$\mathrm{H} \quad-10.086500 \quad-2.174100 \quad 5.481100$

$\mathrm{H} \quad-10.749800 \quad-1.839000 \quad 3.018200$

H $\quad-10.855500 \quad-3.614800 \quad 2.785100$

$\mathrm{H} \quad-9.791000 \quad-2.650200 \quad 1.714200$

$\mathrm{H} \quad-7.789700 \quad-4.128300 \quad 2.338400$

$\mathrm{H} \quad-8.947900 \quad-5.101900 \quad 3.314200$

$\mathrm{H} \quad-7.441900 \quad-4.486700 \quad 4.066900$

$\mathrm{H} \quad-2.253400 \quad 5.232600 \quad 5.102200$

$\mathrm{H} \quad-1.017900 \quad 6.072300 \quad 6.992500$

$\mathrm{H} \quad-1.496300 \quad 2.217300 \quad 8.945200$

$\mathrm{H} \quad-2.683500 \quad 1.425400 \quad 7.033500$

$\mathrm{H} \quad 1.625500 \quad 4.317800 \quad 8.369000$

$\mathrm{H} \quad 1.757200 \quad 5.555600 \quad 9.673400$

$\mathrm{H} \quad 1.065700 \quad 6.004800 \quad 8.082500$

H $\quad-1.079300 \quad 3.464000 \quad 10.908600$

$\mathrm{H} \quad 0.504600 \quad 4.200600 \quad 11.315700$

$\begin{array}{llll}\mathrm{H} & 0.439500 & 2.863300 & 10.123000\end{array}$

H $\quad-1.056100 \quad 6.855600 \quad 9.191100$
H $\quad-0.422900 \quad 6.428200 \quad 10.811000$

$\mathrm{H} \quad-2.012500 \quad 5.774600 \quad 10.268000$

H $\quad 3.588500 \quad-0.504000 \quad 2.083400$

H $\quad 5.230300 \quad-0.090000 \quad 1.428600$

$\mathrm{H} \quad 6.482400 \quad 1.196100 \quad 3.091100$

H $\quad 8.432700 \quad 2.706000 \quad 3.123500$

H $\quad 8.278200 \quad 4.970200 \quad 2.135600$

$\mathrm{H} \quad 6.146600 \quad 5.744100 \quad 1.134400$

$\mathrm{H} \quad 4.180600 \quad 4.263000 \quad 1.106100$

$\mathrm{H} \quad 0.424600 \quad 2.997900 \quad 5.816400$

$\mathrm{H} \quad 2.082400 \quad 1.213200 \quad 6.245900$

$\begin{array}{llll}\mathrm{H} & 3.726000 & 0.600000 & 4.506600\end{array}$

$\mathrm{H} \quad 1.985500 \quad 3.529300 \quad 1.858000$

$\mathrm{H} \quad 0.391300 \quad 4.166300 \quad 3.627600$

H $\quad 3.025800 \quad 3.306600 \quad-0.967400$

H $\quad 6.473500 \quad 3.068600 \quad-3.509800$

H $\quad 5.808400 \quad 0.072200 \quad-0.516100$

$\begin{array}{llll}\mathrm{H} & 8.674400 & 2.633900 & -2.621900\end{array}$

H $\quad 10.785000 \quad 1.569600 \quad-3.087900$

H $\quad 8.841900 \quad-2.304100 \quad-2.752300$

H $\quad 6.772600 \quad-1.197400 \quad-2.313600$

H $\quad 11.239700 \quad-1.025900 \quad-5.366000$

$\mathrm{H} \quad 12.937100 \quad-1.204700 \quad-4.786600$

H $\quad 12.107200 \quad 0.378200 \quad-4.645600$

H $\quad 11.022800 \quad-3.022600 \quad-2.135900$

H $\quad 12.393300 \quad-3.109600 \quad-3.291200$

$\mathrm{H} \quad 10.715700 \quad-3.078200 \quad-3.921500$

H $\quad 12.589800 \quad 0.442700 \quad-2.149800$

$\mathrm{H} \quad 13.415700 \quad-1.143800 \quad-2.228900$

H $\quad 12.043400 \quad-0.903400 \quad-1.088200$

H $\quad 4.416300 \quad 3.884500 \quad-4.982900$

$\mathrm{H} \quad 4.114600 \quad 5.674500 \quad-1.098900$

H $\quad 3.430900 \quad 7.752300 \quad-2.060600$

H $\quad 3.732800 \quad 5.960100 \quad-6.010700$

H $\quad 5.056000 \quad 9.051400 \quad-5.262200$

H $\quad 3.795300 \quad 9.835500 \quad-6.284200$

H $\quad 4.295200 \quad 8.148800 \quad-6.622200$

H $\quad 1.907000 \quad 9.204900 \quad-3.125900$

H $\quad 2.366600 \quad 10.423100 \quad-4.359200$

H $\quad 3.622300 \quad 9.776100 \quad-3.254200$

$\mathrm{H} \quad 1.888100 \quad 7.345300 \quad-6.436900$

H $\quad 1.330600 \quad 9.014800 \quad-6.102700$

H $\quad 0.931400 \quad 7.688100 \quad-4.950400$

\section{W5}

$\begin{array}{llll}\mathrm{Rh} & -0.214200 & 0.128600 & -1.264700\end{array}$

$\begin{array}{llll}\mathrm{Rh} & -0.360100 & -0.449900 & 1.044000\end{array}$

$\begin{array}{llll}\mathrm{O} & -1.255300 & 1.360500 & 1.418400\end{array}$

$\begin{array}{llll}\mathrm{O} & -1.247800 & 1.862300 & -0.784700\end{array}$

$\begin{array}{llll}\mathrm{O} & 1.525500 & 0.334500 & 1.298100\end{array}$

$\begin{array}{llll}\text { O } & 1.648100 & 0.955400 & -0.869600\end{array}$

$\begin{array}{llll}\text { O } & 0.535800 & -2.238500 & 0.556500\end{array}$

O $\quad 0.766000 \quad-1.655500 \quad-1.614600$

O $\quad-2.253800 \quad-1.183000 \quad 0.667400$

O $\quad-2.052600 \quad-0.755400 \quad-1.542600$

C $\quad-1.625500 \quad 2.057200 \quad 0.418300$

$\begin{array}{llll}\text { C } & -2.672800 & 3.121100 & 0.691800\end{array}$

C $\quad-3.695200 \quad 3.262500 \quad-0.434200$

$\begin{array}{llll}\text { C } & -2.765400 & 4.416800 & -0.167700\end{array}$

C $\quad-3.326500 \quad 5.647800 \quad 0.477000$

$\begin{array}{llll}\text { C } & -2.536800 & 6.407100 & 1.358200\end{array}$

C $\quad-3.046200 \quad 7.570000 \quad 1.942700$ 


\begin{tabular}{|c|c|c|c|}
\hline C & -4.338100 & 8.001000 & 1.633500 \\
\hline $\mathrm{C}$ & -5.118400 & 7.275500 & 0.730400 \\
\hline $\mathrm{C}$ & -4.614200 & 6.109600 & 0.147400 \\
\hline $\mathrm{C}$ & 0.262400 & 5.191400 & -3.119600 \\
\hline $\mathrm{C}$ & -1.079700 & 5.107300 & -3.498800 \\
\hline C & -2.063100 & 4.859200 & -2.537800 \\
\hline $\mathrm{C}$ & -1.708200 & 4.682800 & -1.190000 \\
\hline $\mathrm{C}$ & -0.358600 & 4.789000 & -0.813400 \\
\hline $\mathrm{C}$ & 0.622000 & 5.037800 & -1.777300 \\
\hline $\mathrm{C}$ & -3.200500 & 3.041900 & 2.110300 \\
\hline $\mathrm{C}$ & -2.558600 & 3.672100 & 3.176000 \\
\hline $\mathrm{C}$ & -2.977900 & 3.485200 & 4.501500 \\
\hline $\mathrm{C}$ & -4.031200 & 2.595800 & 4.753800 \\
\hline $\mathrm{C}$ & -4.679400 & 1.926400 & 3.706200 \\
\hline $\mathrm{C}$ & -4.275600 & 2.192000 & 2.390000 \\
\hline $\mathrm{C}$ & -5.712000 & 0.892700 & 3.975600 \\
\hline $\mathrm{C}$ & -6.875000 & 0.775700 & 3.203100 \\
\hline $\mathrm{C}$ & -7.788200 & -0.254000 & 3.423800 \\
\hline $\mathrm{C}$ & -7.592000 & -1.230700 & 4.423100 \\
\hline $\mathrm{C}$ & -6.428700 & -1.090600 & 5.207700 \\
\hline $\mathrm{C}$ & -5.519200 & -0.057000 & 4.987700 \\
\hline $\mathrm{C}$ & -8.613600 & -2.368000 & 4.609400 \\
\hline $\mathrm{C}$ & -8.196800 & -3.367600 & 5.714000 \\
\hline $\mathrm{C}$ & -9.980400 & -1.768400 & 5.000100 \\
\hline $\mathrm{C}$ & -8.758900 & -3.158300 & 3.290900 \\
\hline $\mathrm{C}$ & -2.280600 & 4.196300 & 5.600300 \\
\hline $\mathrm{C}$ & -1.842400 & 5.518200 & 5.442800 \\
\hline $\mathrm{C}$ & -1.130600 & 6.167700 & 6.447500 \\
\hline $\mathrm{C}$ & -0.815500 & 5.538200 & 7.670200 \\
\hline $\mathrm{C}$ & -1.282900 & 4.216900 & 7.827600 \\
\hline $\mathrm{C}$ & -1.995600 & 3.568600 & 6.819500 \\
\hline $\mathrm{C}$ & 0.011200 & 6.284100 & 8.733400 \\
\hline $\mathrm{C}$ & 0.261200 & 5.432200 & 10.000200 \\
\hline $\mathrm{C}$ & -0.735800 & 7.564500 & 9.163700 \\
\hline $\mathrm{C}$ & 1.384900 & 6.664200 & 8.140200 \\
\hline $\mathrm{C}$ & 2.153400 & 0.804600 & 0.290000 \\
\hline $\mathrm{C}$ & 3.621300 & 1.139700 & 0.480500 \\
\hline $\mathrm{C}$ & 4.265800 & 0.377800 & 1.636800 \\
\hline $\mathrm{C}$ & 4.094300 & 1.862300 & 1.793000 \\
\hline $\mathrm{C}$ & 5.319300 & 2.721600 & 1.695300 \\
\hline $\mathrm{C}$ & 6.499400 & 2.362800 & 2.371600 \\
\hline $\mathrm{C}$ & 7.639300 & 3.167200 & 2.275800 \\
\hline $\mathrm{C}$ & 7.608500 & 4.339800 & 1.517500 \\
\hline $\mathrm{C}$ & 6.432700 & 4.719800 & 0.866700 \\
\hline $\mathrm{C}$ & 5.289400 & 3.922800 & 0.964800 \\
\hline $\mathrm{C}$ & 1.274300 & 3.488100 & 4.592600 \\
\hline $\mathrm{C}$ & 1.278200 & 3.933600 & 3.268200 \\
\hline $\mathrm{C}$ & 2.163700 & 3.373700 & 2.344200 \\
\hline $\mathrm{C}$ & 3.065100 & 2.373600 & 2.745800 \\
\hline $\mathrm{C}$ & 3.059100 & 1.935100 & 4.080100 \\
\hline $\mathrm{C}$ & 2.158200 & 2.484800 & 4.996600 \\
\hline $\mathrm{C}$ & 4.402700 & 1.385600 & -0.794200 \\
\hline $\mathrm{C}$ & 5.556800 & 0.656400 & -1.091800 \\
\hline $\mathrm{C}$ & 6.366000 & 0.977100 & -2.195100 \\
\hline $\mathrm{C}$ & 5.981900 & 2.035800 & -3.026200 \\
\hline $\mathrm{C}$ & 4.820800 & 2.775900 & -2.759200 \\
\hline $\mathrm{C}$ & 4.041700 & 2.438600 & -1.646700 \\
\hline $\mathrm{C}$ & 4.434700 & 3.928600 & -3.613200 \\
\hline $\mathrm{C}$ & 4.114600 & 5.165700 & -3.040900 \\
\hline $\mathrm{C}$ & 3.720900 & 6.252900 & -3.819600 \\
\hline $\mathrm{C}$ & 3.617500 & 6.155600 & -5.222700 \\
\hline $\mathrm{C}$ & 3.968200 & 4.911200 & -5.787900 \\
\hline
\end{tabular}

$\begin{array}{llll}\text { C } & 4.370700 & 3.828700 & -5.007700\end{array}$

$\begin{array}{llll}\text { C } & 3.137500 & 7.308300 & -6.123900\end{array}$

$\begin{array}{llll}\text { C } & 2.784900 & 8.583900 & -5.322900\end{array}$

C $\quad 1.871800 \quad 6.869100 \quad-6.891600$

$\begin{array}{llll}\text { C } & 4.249300 & 7.672100 & -7.130600\end{array}$

C $\quad 7.731200 \quad-1.140700 \quad-2.221400$

$\begin{array}{llll}\text { C } & 7.637600 & 0.243300 & -2.416000\end{array}$

$\begin{array}{llll}\text { C } & 8.810400 & 0.916200 & -2.783500\end{array}$

$\begin{array}{llll}\text { C } & 10.025200 & 0.247500 & -2.931800\end{array}$

$\begin{array}{llll}\text { C } & 10.134200 & -1.142600 & -2.721600\end{array}$

C $\quad 8.942300 \quad-1.811900 \quad-2.371800$

C $\quad 11.456100 \quad-1.923400 \quad-2.835000$

C $\quad 12.646500 \quad-1.024100 \quad-3.243700$

C $\quad 11.313200 \quad-3.031500 \quad-3.900400$

C $\quad 11.791900 \quad-2.561200 \quad-1.470400$

C $\quad \begin{array}{llll}\text { C } & 1.006100 & -2.408500 & -0.613800\end{array}$

C $\quad \begin{array}{llll}\text { C } & 1.986900 & -3.554000 & -0.792600\end{array}$

$\begin{array}{llll}\text { C } & 2.847500 & -3.473200 & -2.043500\end{array}$

C $\quad 1.826600 \quad-4.577700 \quad-1.958500$

C $\quad 2.316100 \quad-5.972400 \quad-1.717500$

$\begin{array}{llll}\text { C } & 3.416300 & -6.475100 & -2.434600\end{array}$

C $\quad 3.865400 \quad-7.780800 \quad-2.214700$

$\begin{array}{llll}\text { C } & 3.210900 & -8.600900 & -1.292300\end{array}$

C $\quad 2.097800 \quad-8.121100 \quad-0.598000$

C $\quad 1.644300 \quad-6.817500 \quad-0.817000$

C $\quad-1.622600 \quad-4.499900 \quad-4.516800$

C $\quad-0.350700 \quad-4.295500 \quad-5.055600$

$\begin{array}{llll}\text { C } & 0.771100 & -4.299700 & -4.221700\end{array}$

$\begin{array}{llll}\text { C } & 0.622900 & -4.488000 & -2.837100\end{array}$

$\begin{array}{llll}\text { C } & -0.659900 & -4.687300 & -2.303100\end{array}$

C $\quad-1.777200 \quad-4.698500 \quad-3.142500$

$\begin{array}{llll}\text { C } & 2.640700 & -3.928800 & 0.520200\end{array}$

C $\quad 1.924900 \quad-4.559000 \quad 1.542300$

$\begin{array}{llll}\text { C } & 2.493900 & -4.783900 & 2.802800\end{array}$

$\begin{array}{llll}\text { C } & 3.807900 & -4.345500 & 3.041800\end{array}$

C $\quad 4.542800 \quad-3.702300 \quad 2.038400$

C $\quad 3.945200 \quad-3.513300 \quad 0.782000$

$\begin{array}{llll}\text { C } & 5.898200 & -3.137400 & 2.255200\end{array}$

C $\quad 6.879900 \quad-3.234500 \quad 1.259500$

$\begin{array}{llll}\text { C } & 8.091700 & -2.559400 & 1.368900\end{array}$

C $\quad 8.394300 \quad-1.743000 \quad 2.478500$

C $\quad 7.427200 \quad-1.703200 \quad 3.504800$

C $\quad 6.214300 \quad-2.386200 \quad 3.394400$

$\begin{array}{llll}\text { C } & 9.701600 & -0.931600 & 2.499100\end{array}$

$\begin{array}{llll}\text { C } & 9.836500 & -0.054400 & 3.765500\end{array}$

$\begin{array}{llll}\text { C } & 10.906000 & -1.895100 & 2.458200\end{array}$

$\begin{array}{llll}\text { C } & 9.745700 & 0.001200 & 1.267600\end{array}$

C $\quad 1.678500 \quad-5.434700 \quad 3.858800$

$\begin{array}{lrrr}\text { C } & 0.792500 & -6.475900 & 3.549400\end{array}$

$\begin{array}{llll}\text { C } & -0.024800 & -7.043100 & 4.523000\end{array}$

C $\quad-0.009500 \quad-6.605000 \quad 5.863900$

$\begin{array}{llll}\text { C } & 0.902000 & -5.573000 & 6.169100\end{array}$

C $\quad 1.721700 \quad-5.008000 \quad 5.192100$

C $\quad-0.963200 \quad-7.236000 \quad 6.895100$

C $\quad-0.800000 \quad-6.628300 \quad 8.308200$

$\begin{array}{llll}\text { C } & -0.684600 & -8.750900 & 6.996500\end{array}$

C $\quad-2.424000 \quad-7.010400 \quad 6.449100$

C $\quad-2.716300 \quad-1.125300 \quad-0.519500$

$\begin{array}{llll}\text { C } & -4.189900 & -1.414300 & -0.736300\end{array}$

C $\quad-5.071400 \quad-0.945200 \quad 0.419600$

C $\quad-4.967500 \quad-2.425100 \quad 0.161000$

C $\quad-6.149000 \quad-3.127700 \quad-0.436700$
C $\quad-5.958300 \quad-4.219400 \quad-1.301500$

C $\quad-7.055300 \quad-4.894500-1.843700$

C $\quad-8.353400 \quad-4.504000 \quad-1.507800$

C $\quad-8.555600 \quad-3.445000 \quad-0.620000$

C $\quad-7.460900-2.764700 \quad-0.079300$

C $\quad-2.796300 \quad-4.740500 \quad 3.065400$

C $\quad-3.853900 \quad-3.921700 \quad 3.471400$

C $\quad-4.563000 \quad-3.174300 \quad 2.526500$

C $\quad-4.211400 \quad-3.233400 \quad 1.167500$

C $\quad-3.163100 \quad-4.077700 \quad 0.765600$

$\begin{array}{llll}\text { C } & -2.453300 & -4.821700 & 1.713000\end{array}$

C $\quad-4.626700 \quad-1.052500 \quad-2.141000$

C $\quad-4.524500 \quad-1.943300 \quad-3.209200$

C $\quad-4.788200 \quad-1.539900 \quad-4.526800$

C $\quad-5.101100 \quad-0.196400 \quad-4.772600$

$\begin{array}{llll}\text { C } & -5.192800 & 0.727000 & -3.720800\end{array}$

$\begin{array}{llll}\text { C } & -4.990600 & 0.270900 & -2.411000\end{array}$

C $\quad-5.412200 \quad 2.174400 \quad-3.975200$

$\begin{array}{llll}\text { C } & -6.263400 & 2.951400 & -3.178600\end{array}$

C $\quad-6.388200 \quad 4.324800 \quad-3.380600$

$\begin{array}{llll}\text { C } & -5.664800 & 5.005100 & -4.383000\end{array}$

C $\quad-4.827200 \quad 4.209300 \quad-5.191900$

C $\quad-4.709500 \quad 2.834300 \quad-4.991900$

C $\quad-5.812900 \quad 6.529100 \quad-4.549100$

C $\quad-5.447400 \quad 7.236600 \quad-3.225900$

$\begin{array}{llll}\text { C } & -7.272700 & 6.862300 & -4.920800\end{array}$

$\begin{array}{llll}\text { C } & -4.895200 & 7.098900 & -5.656400\end{array}$

C $\quad-5.227900 \quad-3.820400 \quad-5.477000$

C $\quad-4.696500 \quad-2.532500 \quad-5.625400$

C $\quad-4.046900 \quad-2.240100 \quad-6.830600$

C $\quad-3.904000 \quad-3.197900 \quad-7.834200$

C $\quad-4.412300 \quad-4.505200 \quad-7.686400$

C $\quad-5.090300 \quad-4.777100 \quad-6.479300$

$\begin{array}{llll}\text { C } & -4.241500 & -5.611900 & -8.743000\end{array}$

C $\quad-5.629800 \quad-6.102800 \quad-9.205900$

$\begin{array}{llll}\text { C } & -3.457400 & -6.790600 & -8.127200\end{array}$

C $\quad-3.467900 \quad-5.129400 \quad-9.992700$

$\mathrm{H} \quad-3.524100 \quad 2.665900 \quad-1.322800$

H $\quad-4.735800 \quad 3.323900 \quad-0.129900$

$\mathrm{H} \quad-1.523200 \quad 6.107400 \quad 1.586500$

$\mathrm{H} \quad-2.435200 \quad 8.141800 \quad 2.629200$

$\mathrm{H} \quad-4.729800 \quad 8.903900 \quad 2.083800$

$\begin{array}{llll}\mathrm{H} & -6.112700 & 7.619800 & 0.476800\end{array}$

H $\quad-5.228300 \quad 5.574200 \quad-0.564400$

H $\quad 1.021000 \quad 5.377100 \quad-3.866600$

$\mathrm{H} \quad-1.358300 \quad 5.233300 \quad-4.537200$

$\mathrm{H} \quad-3.099300 \quad 4.795700 \quad-2.842600$

$\begin{array}{llll}\mathrm{H} & -0.066300 & 4.664800 & 0.221600\end{array}$

H $\quad 1.659200 \quad 5.111400 \quad-1.481100$

$\mathrm{H} \quad-1.681800 \quad 4.277600 \quad 2.989400$

$\mathrm{H} \quad-4.369800 \quad 2.441900 \quad 5.774400$

H $\quad-4.759100 \quad 1.667000 \quad 1.573400$

$\mathrm{H} \quad-7.078500 \quad 1.507400 \quad 2.425500$

$\begin{array}{llll}\mathrm{H} & -8.667900 & -0.271800 & 2.785000\end{array}$

H $\quad-6.166600 \quad-1.780100 \quad 6.004500$

$\mathrm{H} \quad-4.618200 \quad-0.012900 \quad 5.593300$

$\mathrm{H} \quad-7.220500 \quad-3.840200 \quad 5.469600$

$\mathrm{H} \quad-8.948600 \quad-4.180500 \quad 5.814400$

$\mathrm{H} \quad-8.121700 \quad-2.860300 \quad 6.700000$

H $\quad-9.888300 \quad-1.171300 \quad 5.933200$

H $\quad-10.728600 \quad-2.572500 \quad 5.171600$

H $\quad-10.375900 \quad-1.106500 \quad 4.201100$ 
H $\quad-9.185500 \quad-2.526400 \quad 2.484900$

$\mathrm{H} \quad-9.440700 \quad-4.026100 \quad 3.423400$

H $\quad-7.770400 \quad-3.540000 \quad 2.955300$

$\mathrm{H} \quad-2.066900 \quad 6.051300 \quad 4.523400$

$\mathrm{H} \quad-0.822900 \quad 7.190300 \quad 6.243700$

$\mathrm{H} \quad-1.102000 \quad 3.630000 \quad 8.723100$

$\mathrm{H} \quad-2.299700 \quad 2.536900 \quad 6.973300$

$\mathrm{H} \quad-0.699400 \quad 5.151800 \quad 10.484000$

$\begin{array}{llll}\mathrm{H} & 0.858600 & 6.000800 & 10.745900\end{array}$

$\mathrm{H} \quad 0.828400 \quad 4.509400 \quad 9.750800$

$\mathrm{H} \quad-0.857700 \quad 8.269700 \quad 8.315100$

$\begin{array}{llll}\mathrm{H} & -0.175800 & 8.097600 & 9.962400\end{array}$

H $\quad-1.745100 \quad 7.310300 \quad 9.554200$

$\begin{array}{llll}\mathrm{H} & 1.917600 & 5.755100 & 7.785300\end{array}$

$\begin{array}{llll}\mathrm{H} & 2.018600 & 7.166000 & 8.903300\end{array}$

H $\quad 1.278900 \quad 7.361900 \quad 7.283200$

$\begin{array}{llll}\mathrm{H} & 3.602000 & -0.281000 & 2.184600\end{array}$

H $\quad 5.260200 \quad-0.028900 \quad 1.490000$

$\begin{array}{llll}\mathrm{H} & 6.539300 & 1.461100 & 2.969900\end{array}$

$\begin{array}{llll}\mathrm{H} & 8.547800 & 2.882900 & 2.789800\end{array}$

$\mathrm{H} \quad 8.493000 \quad 4.958900 \quad 1.442400$

H $\quad 6.405300 \quad 5.634900 \quad 0.289300$

H $\quad 4.383700 \quad 4.241600 \quad 0.467500$

$\mathrm{H} \quad 0.599100 \quad 3.929200 \quad 5.310600$

$\mathrm{H} \quad 0.610900 \quad 4.727000 \quad 2.960600$

$\mathrm{H} \quad 2.161600 \quad 3.733500 \quad 1.323000$

$\mathrm{H} \quad 3.762000 \quad 1.181100 \quad 4.412600$

H $\quad 2.158500 \quad 2.147200 \quad 6.025000$

H $\quad 5.876100 \quad-0.145200 \quad-0.435100$

$\mathrm{H} \quad 6.598300 \quad 2.301600 \quad-3.880500$

H $\quad 3.142000 \quad 3.006800 \quad-1.438300$

$\mathrm{H} \quad 4.186700 \quad 5.286900 \quad-1.963500$

H $\quad 3.495800 \quad 7.163000 \quad-3.271600$

H $\quad 3.923000 \quad 4.747900 \quad-6.861800$

$\mathrm{H} \quad 4.606800 \quad 2.883100 \quad-5.488800$

$\mathrm{H} \quad 1.966500 \quad 8.381700 \quad-4.598100$

$\begin{array}{llll}\mathrm{H} & 2.438700 & 9.392800 & -6.002400\end{array}$

$\begin{array}{llll}\mathrm{H} & 3.673500 & 8.965000 & -4.774600\end{array}$

$\begin{array}{llll}\mathrm{H} & 2.080400 & 6.011000 & -7.564100\end{array}$

H $\quad 1.485100 \quad 7.699800 \quad-7.521100$

H $\quad 1.071800 \quad 6.568900 \quad-6.180700$

$\mathrm{H} \quad 5.180800 \quad 7.952800 \quad-6.593000$

H $\quad 3.940000 \quad 8.530300 \quad-7.765900$

$\begin{array}{llll}\mathrm{H} & 4.479600 & 6.822200 & -7.806800\end{array}$

H $\quad 6.841600 \quad-1.706300 \quad-1.959200$

H $\quad 8.784900 \quad 1.993600 \quad-2.922200$

$\mathrm{H} \quad 10.871900 \quad 0.871400 \quad-3.202400$

H $\quad 8.927600 \quad-2.886800 \quad-2.207200$

$\mathrm{H} \quad 12.464100 \quad-0.553500 \quad-4.234100$

$\mathrm{H} \quad 13.581900-1.620100 \quad-3.321700$

$\mathrm{H} \quad 12.820000 \quad-0.228500 \quad-2.487200$

H $\quad 10.545700 \quad-3.779300 \quad-3.610900$

$\mathrm{H} \quad 12.273200 \quad-3.575900 \quad-4.034000$

$\mathrm{H} \quad 11.021800 \quad-2.591800 \quad-4.878800$

$\mathrm{H} \quad 11.886900 \quad-1.774800 \quad-0.691000$

$\mathrm{H} \quad 12.752400 \quad-3.118300 \quad-1.521700$

H $\quad 11.005300 \quad-3.276600 \quad-1.150500$

H $\quad 2.654200 \quad-2.634700 \quad-2.703700$

H $\quad 3.899200 \quad-3.719900 \quad-1.933200$

H $\quad 3.926500 \quad-5.858400 \quad-3.163800$

H $\quad 4.719000 \quad-8.158100 \quad-2.762700$

H $\quad 3.559600 \quad-9.611400 \quad-1.123500$
H $\quad 1.581700 \quad-8.761400 \quad 0.105700$

H $\quad 0.766500 \quad-6.472800 \quad-0.288800$

$\mathrm{H} \quad-2.484200 \quad-4.519700 \quad-5.167100$

$\mathrm{H} \quad-0.232700 \quad-4.151100 \quad-6.121900$

H $\quad 1.754900 \quad-4.169600 \quad-4.655300$

$\mathrm{H} \quad-0.789000 \quad-4.854200 \quad-1.242000$

$\mathrm{H} \quad-2.760000 \quad-4.879000 \quad-2.730100$

H $\quad 0.883800 \quad-4.814600 \quad 1.382900$

H $\quad 4.267900 \quad-4.529500 \quad 4.009000$

H $\quad 4.492200 \quad-2.965500 \quad 0.022300$

H $\quad 6.689800 \quad-3.843900 \quad 0.380000$

H $\quad 8.788900 \quad-2.668800 \quad 0.543000$

H $\quad 7.556900 \quad-1.124500 \quad 4.414600$

H $\quad 5.477800 \quad-2.282400 \quad 4.186900$

$\mathrm{H} \quad 8.994100 \quad 0.665800 \quad 3.838700$

H $\quad 10.780600 \quad 0.531900 \quad 3.740300$

H $\quad 9.856900 \quad-0.683200 \quad 4.681800$

H $\quad 10.880500 \quad-2.583100 \quad 3.330700$

$\mathrm{H} \quad 11.862900 \quad-1.329800 \quad 2.485100$

$\mathrm{H} \quad 10.904700 \quad-2.509300 \quad 1.534200$

H $\quad 9.782200 \quad-0.575400 \quad 0.320500$

$\mathrm{H} \quad 10.646800 \quad 0.651200 \quad 1.294900$

H $\quad 8.844600 \quad 0.650600 \quad 1.240400$

H $\quad 0.746600 \quad-6.857200 \quad 2.533100$

H $\quad-0.686900 \quad-7.841900 \quad 4.198100$

$\mathrm{H} \quad 1.005500 \quad-5.153000 \quad 7.165300$

H $\quad 2.381200 \quad-4.190300 \quad 5.470400$

H $\quad 0.231900 \quad-6.786600 \quad 8.689800$

$\mathrm{H} \quad-1.500500 \quad-7.107400 \quad 9.026500$

$\mathrm{H} \quad-1.023600 \quad-5.539500 \quad 8.298900$

$\mathrm{H} \quad-0.886100 \quad-9.267500 \quad 6.034800$

$\mathrm{H} \quad-1.332400 \quad-9.219800 \quad 7.768600$

H $\quad 0.376600 \quad-8.931000 \quad 7.274100$

H $\quad-2.632400 \quad-5.923800 \quad 6.341400$

$\mathrm{H} \quad-3.133000 \quad-7.429300 \quad 7.195800$

$\mathrm{H} \quad-2.633500 \quad-7.503300 \quad 5.476500$

$\mathrm{H} \quad-4.560600 \quad-0.546100 \quad 1.288100$

$\mathrm{H} \quad-5.972500 \quad-0.405700 \quad 0.143300$

H $\quad-4.961800 \quad-4.556300 \quad-1.549800$

$\mathrm{H} \quad-6.898200 \quad-5.726200 \quad-2.518300$

$\mathrm{H} \quad-9.202000 \quad-5.030100 \quad-1.925300$

$\mathrm{H} \quad-9.561400 \quad-3.154500 \quad-0.345200$

$\mathrm{H} \quad-7.641200 \quad-1.960900 \quad 0.621900$

$\mathrm{H} \quad-2.245700 \quad-5.314200 \quad 3.797900$

$\mathrm{H} \quad-4.123800 \quad-3.865000 \quad 4.518200$

H $\quad-5.380000 \quad-2.543900 \quad 2.851900$

H $\quad-2.888600 \quad-4.143900 \quad-0.277500$

$\mathrm{H} \quad-1.638100 \quad-5.460800 \quad 1.398800$

$\mathrm{H} \quad-4.177900 \quad-2.954500 \quad-3.038500$

$\mathrm{H} \quad-5.303800 \quad 0.128800 \quad-5.789200$

$\mathrm{H} \quad-5.038300 \quad 0.981200 \quad-1.592300$

H $\quad-6.848800 \quad 2.475000 \quad-2.396400$

$\mathrm{H} \quad-7.074700 \quad 4.854400 \quad-2.724700$

$\mathrm{H} \quad-4.219500 \quad 4.617200 \quad-5.994000$

$\mathrm{H} \quad-4.023600 \quad 2.269400 \quad-5.617300$

$\mathrm{H} \quad-6.154700 \quad 6.969200 \quad-2.414000$

H $\quad-5.486700 \quad 8.341100 \quad-3.344900$

$\mathrm{H} \quad-4.420400 \quad 6.958000 \quad-2.906000$

$\mathrm{H} \quad-7.562500 \quad 6.338500 \quad-5.857400$

H $\quad-7.399300 \quad 7.955600 \quad-5.077600$

$\mathrm{H} \quad-7.975300 \quad 6.554200 \quad-4.118000$

$\mathrm{H} \quad-3.826900 \quad 6.893800 \quad-5.426600$
$\begin{array}{llll}\mathrm{H} & -5.016100 & 8.200900 & -5.740500\end{array}$

$\mathrm{H} \quad-5.148000 \quad 6.660300 \quad-6.645800$

$\mathrm{H} \quad-5.761400 \quad-4.077200 \quad-4.566100$

$\mathrm{H} \quad-3.607800 \quad-1.256700 \quad-6.975100$

$\mathrm{H} \quad-3.364800 \quad-2.871000 \quad-8.718300$

$\mathrm{H} \quad-5.526400 \quad-5.753700 \quad-6.284600$

H $\quad-6.200600 \quad-6.558500 \quad-8.369900$

$\mathrm{H} \quad-5.530200 \quad-6.873700 \quad-10.000600$

$\mathrm{H} \quad-6.223300 \quad-5.255600 \quad-9.613000$

$\mathrm{H} \quad-2.471300 \quad-6.443300 \quad-7.748900$

$\mathrm{H} \quad-3.281900 \quad-7.585700 \quad-8.884000$

H $\quad-4.011500 \quad-7.250500 \quad-7.282100$

H $\quad-4.004700 \quad-4.293800 \quad-10.491700$

$\mathrm{H} \quad-3.364700 \quad-5.951600 \quad-10.734000$

H $\quad-2.443700 \quad-4.795000 \quad-9.719400$

\section{W6}

$\begin{array}{llll}\mathrm{Rh} & -0.319800 & -0.367300 & 1.094300\end{array}$

$\begin{array}{llll}\mathrm{Rh} & -0.174600 & 0.081100 & -1.243600\end{array}$

$\begin{array}{llll}\mathrm{O} & -1.930600 & -0.974500 & -1.484700\end{array}$

$\begin{array}{llll}\mathrm{O} & -2.105700 & -1.332100 & 0.740000\end{array}$

$\begin{array}{llll}\text { O } & 0.960900 & -1.622400 & -1.472500\end{array}$

$\begin{array}{llll}\mathrm{O} & 0.791900 & -2.069200 & 0.736400\end{array}$

$\begin{array}{llll}\mathrm{O} & 1.603300 & 1.088800 & -0.864100\end{array}$

$\begin{array}{llll}\mathrm{O} & 1.458300 & 0.630600 & 1.343000\end{array}$

$\begin{array}{llll}\text { O } & -1.350200 & 1.746300 & -0.906000\end{array}$

$\begin{array}{llll}\text { O } & -1.424400 & 1.357300 & 1.318600\end{array}$

C $\quad-2.562600 \quad-1.367000 \quad-0.449800$

C $\quad-3.998700 \quad-1.809100 \quad-0.655300$

$\begin{array}{llll}\text { C } & -4.930300 & -1.386000 & 0.479200\end{array}$

$\begin{array}{llll}\text { C } & -4.676000 & -2.856500 & 0.281500\end{array}$

C $\quad-5.776900 \quad-3.696100 \quad-0.291300$

$\begin{array}{llll}\text { C } & -7.117900 & -3.471800 & 0.072000\end{array}$

$\begin{array}{llll}\text { C } & -8.135600 & -4.275800 & -0.449400\end{array}$

C $\quad-7.824800 \quad-5.321700 \quad-1.321300$

C $\quad-6.494000 \quad-5.575400 \quad-1.660500$

C $\quad-5.473500 \quad-4.776000 \quad-1.138300$

C $\quad-2.325200 \quad-4.844700 \quad 3.287000$

C $\quad-1.961900 \quad-4.944000 \quad 1.940800$

C $\quad-2.726900 \quad-4.303700 \quad 0.960700$

C $\quad-3.852300 \quad-3.545200 \quad 1.323200$

C $\quad-4.220100 \quad-3.465900 \quad 2.677000$

C $\quad-3.455600 \quad-4.109700 \quad 3.654200$

C $\quad-4.462700 \quad-1.547100 \quad-2.073100$

C $\quad-4.237600 \quad-2.448200 \quad-3.113700$

C $\quad-4.531000 \quad-2.117300 \quad-4.444900$

C $\quad-5.008600 \quad-0.831800 \quad-4.734200$

$\begin{array}{llll}\text { C } & -5.232400 & 0.099600 & -3.710100\end{array}$

$\begin{array}{llll}\text { C } & -4.986300 & -0.288800 & -2.385800\end{array}$

$\begin{array}{llll}\text { C } & -5.637900 & 1.499400 & -3.998700\end{array}$

$\begin{array}{llll}\text { C } & -5.006900 & 2.236300 & -5.009400\end{array}$

C $\quad-5.302000 \quad 3.582500 \quad-5.212900$

$\begin{array}{llll}\text { C } & -6.249300 & 4.271100 & -4.426300\end{array}$

C $\quad-6.901400 \quad 3.511100 \quad-3.432900$

C $\quad-6.599400 \quad 2.164700 \quad-3.227000$

C $\quad-6.508000 \quad 5.770700 \quad-4.662100$

C $\quad-6.973600 \quad 5.992500 \quad-6.117200$

$\begin{array}{llll}\text { C } & -7.598000 & 6.343000 & -3.725500\end{array}$

$\begin{array}{llll}\text { C } & -5.205800 & 6.561700 & -4.413100\end{array}$

C $\quad-4.294100 \quad-3.120900 \quad-5.511600$

C $\quad-3.636900 \quad-2.789500 \quad-6.702400$

$\begin{array}{llll}\text { C } & -3.354000 & -3.759500 & -7.661100\end{array}$ 


\begin{tabular}{|c|c|c|c|}
\hline C & -3.718300 & -5.112100 & -7.491500 \\
\hline $\mathrm{C}$ & -4.409900 & -5.426700 & -6.303300 \\
\hline $\mathrm{C}$ & -4.685100 & -4.455400 & -5.341500 \\
\hline C & -3.336600 & -6.155000 & -8.557800 \\
\hline 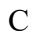 & -3.973200 & -5.768700 & -9.909800 \\
\hline & -3.817400 & -7.580400 & -8.197000 \\
\hline C & -1.800300 & -6.197200 & -8.704800 \\
\hline $\mathrm{C}$ & 1.278600 & -2.271800 & -0.422100 \\
\hline $\mathrm{C}$ & 2.374300 & -3.316200 & -0.534100 \\
\hline $\mathrm{C}$ & 3.248100 & -3.192900 & -1.773400 \\
\hline C & 2.344200 & -4.392700 & -1.664000 \\
\hline $\mathrm{C}$ & 2.968800 & -5.719800 & -1.361500 \\
\hline $\mathrm{C}$ & 4.131700 & -6.130800 & -2.037100 \\
\hline $\mathrm{C}$ & 4.708100 & -7.373600 & -1.758300 \\
\hline $\mathrm{C}$ & 4.120400 & -8.223600 & -0.818100 \\
\hline C & 2.947700 & -7.837600 & -0.164700 \\
\hline $\mathrm{C}$ & 2.367000 & -6.597200 & -0.442500 \\
\hline $\mathrm{C}$ & -1.044600 & -4.746800 & -4.280100 \\
\hline $\mathrm{C}$ & 0.212600 & -4.441400 & -4.805700 \\
\hline $\mathrm{C}$ & 1.313000 & -4.307700 & -3.954000 \\
\hline $\mathrm{C}$ & 1.156000 & -4.459400 & -2.565900 \\
\hline $\mathrm{C}$ & -0.112000 & -4.763500 & -2.045700 \\
\hline $\mathrm{C}$ & -1.206500 & -4.911100 & -2.902100 \\
\hline $\mathrm{C}$ & 3.034300 & -3.580400 & 0.801000 \\
\hline $\mathrm{C}$ & 2.356900 & -4.240700 & 1.829700 \\
\hline $\mathrm{C}$ & 2.921600 & -4.381700 & 3.103700 \\
\hline $\mathrm{C}$ & 4.190400 & -3.83 & 9600 \\
\hline $\mathrm{C}$ & 4.888300 & -3.158600 & 2.337600 \\
\hline $\mathrm{C}$ & 4.295100 & -3.050300 & 1.069500 \\
\hline $\mathrm{C}$ & 6.203700 & -2.506300 & 2.556000 \\
\hline $\mathrm{C}$ & 6.474600 & -1.740200 & 3.697000 \\
\hline $\mathrm{C}$ & 7.664100 & -1.022300 & 3.815400 \\
\hline $\mathrm{C}$ & 8.652100 & -1.031600 & 2.807300 \\
\hline $\mathrm{C}$ & 8.387700 & -1.841600 & 1.684200 \\
\hline $\mathrm{C}$ & 7.197400 & -2.556300 & 1.568700 \\
\hline $\mathrm{C}$ & 9.914900 & -0.162800 & 2.947700 \\
\hline $\mathrm{C}$ & 9.503800 & 1.321100 & 3.040100 \\
\hline $\mathrm{C}$ & 10.878000 & -0.311900 & 1.746100 \\
\hline $\mathrm{C}$ & 10.682500 & -0.566700 & 4.224200 \\
\hline $\mathrm{C}$ & 2.067500 & -4.579100 & 5.468000 \\
\hline $\mathrm{C}$ & 2.141000 & -5.072900 & 4.159800 \\
\hline $\mathrm{C}$ & 1.395300 & -6.222300 & 3.865500 \\
\hline $\mathrm{C}$ & 0.586700 & -6.836000 & 4.820100 \\
\hline $\mathrm{C}$ & 0.472800 & -6.324400 & 6.129300 \\
\hline $\mathrm{C}$ & 1.253500 & -5.186200 & 6.422200 \\
\hline $\mathrm{C}$ & -0.456600 & -6.923300 & 7.200700 \\
\hline $\mathrm{C}$ & 0.378800 & -7.361400 & 8.422300 \\
\hline $\mathrm{C}$ & -1.236500 & -8.158100 & 6.690500 \\
\hline $\mathrm{C}$ & -1.488500 & -5.860800 & 7.636400 \\
\hline $\mathrm{C}$ & 2.077000 & 1.074800 & 0.318200 \\
\hline $\mathrm{C}$ & 3.501100 & 1.557100 & 0.524200 \\
\hline $\mathrm{C}$ & 4.177700 & 0.949400 & 1.750800 \\
\hline $\mathrm{C}$ & 3.855400 & 2.417500 & 1.787700 \\
\hline $\mathrm{C}$ & 4.992500 & 3.385900 & 1.665200 \\
\hline $\mathrm{C}$ & 4.874600 & 4.523300 & 0.847000 \\
\hline $\mathrm{C}$ & 5.928800 & 5.434700 & 0.746000 \\
\hline $\mathrm{C}$ & 7.098300 & 5.236100 & 1.483100 \\
\hline $\mathrm{C}$ & 7.211500 & 4.130000 & 2.328500 \\
\hline $\mathrm{C}$ & 6.163200 & 3.209400 & 2.424400 \\
\hline $\mathrm{C}$ & 0.785000 & 3.926100 & 4.384500 \\
\hline C & 1.746700 & 3.043600 & 4.881600 \\
\hline & 2.733500 & 2.533300 & 4.033200 \\
\hline
\end{tabular}

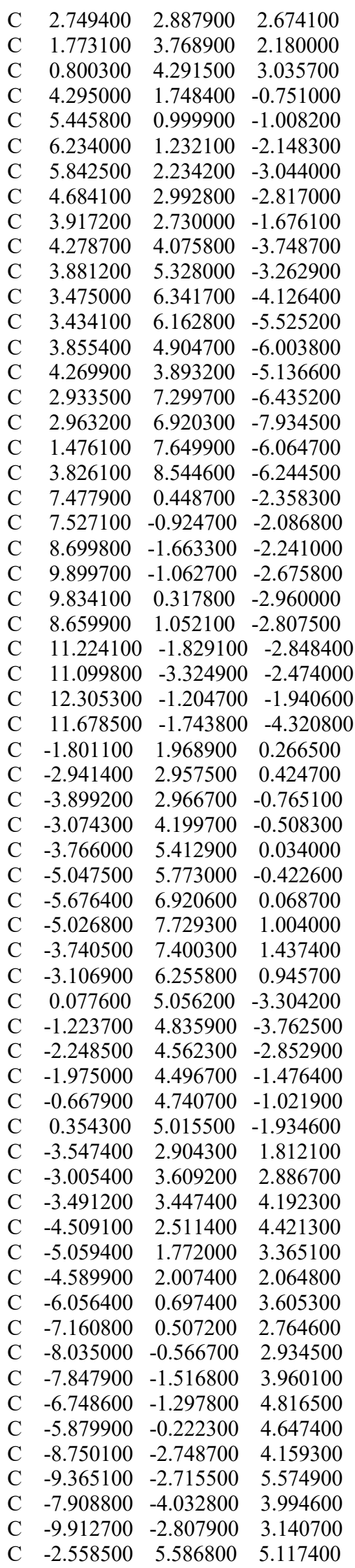

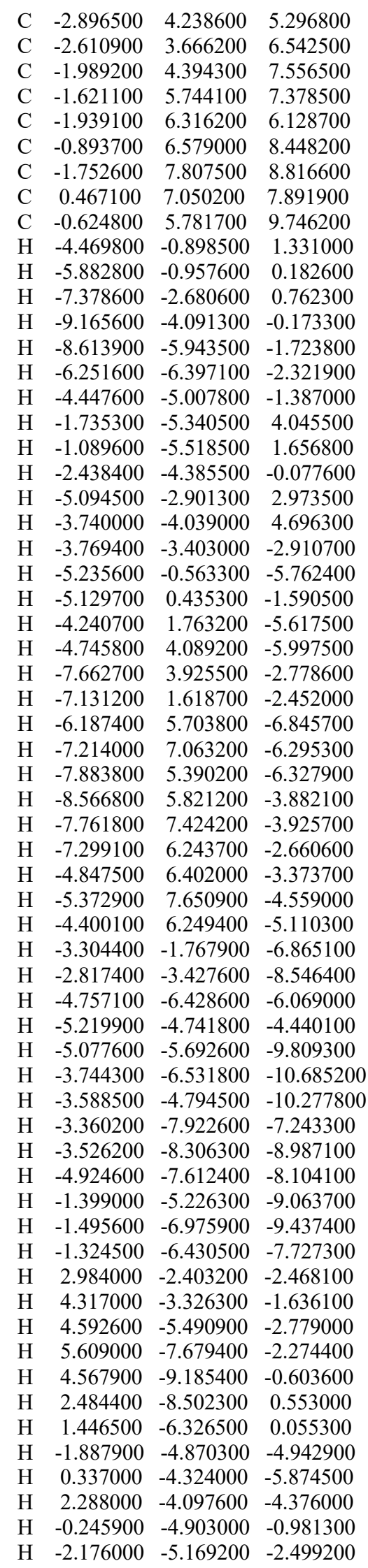




\begin{tabular}{|c|c|c|c|}
\hline & 1.345600 & -4.592900 & 1.661600 \\
\hline & 4.647800 & -3.952500 & 4.327800 \\
\hline & 4.808500 & -2.482100 & 0.301300 \\
\hline & 5.726900 & -1.662400 & 4.481900 \\
\hline & 7.785600 & -0.426900 & 4.716900 \\
\hline & 9.074500 & -1.941100 & 0.849500 \\
\hline & 7.039000 & -3.169800 & 0.686000 \\
\hline & 8.924300 & 1.614700 & 2.138000 \\
\hline & 10.399500 & 1.975800 & 3.108600 \\
\hline & 8.877200 & 1.515200 & 3.935900 \\
\hline & 11.222700 & -1.363800 & 1.644800 \\
\hline & 11.779200 & 0.325100 & 1.880900 \\
\hline & 10.384500 & 0.004200 & 0.801000 \\
\hline & 10.079500 & -0.382300 & 5.137700 \\
\hline & 11.621800 & 0.020000 & 4.321000 \\
\hline & 10.946200 & -1.646000 & 4.191100 \\
\hline & 2.619400 & -3.681900 & 5200 \\
\hline & 1.451100 & -6.652900 & 2.869400 \\
\hline & 0.051700 & -7.718300 & 4.481600 \\
\hline & 1.231100 & -4.726300 & 7.407100 \\
\hline & 1.154200 & -8.096800 & 8.116500 \\
\hline & -0.268100 & -7.835000 & 2400 \\
\hline & 0.886600 & -6.497100 & 8.899200 \\
\hline & -1.880800 & -7.889000 & 5.825400 \\
\hline & -1.897700 & -8.563400 & 7.487200 \\
\hline & -0.539200 & -8.969500 & 9100 \\
\hline & -0.996000 & -4.983500 & 5700 \\
\hline & -2.198500 & -6.282800 & 8.380500 \\
\hline & -2.072000 & -5.505200 & 6.759400 \\
\hline & 3.567700 & 0.267900 & 2.332600 \\
\hline & 5.212900 & 0.6 & 1.65 \\
\hline & 3.966500 & 4.70 & 0900 \\
\hline & 5.834700 & 6.300300 & 0.103100 \\
\hline & 7.911900 & 5.946000 & 1.408900 \\
\hline & 8.110400 & 3.989800 & 2.914200 \\
\hline & 6.263600 & 2.363700 & 3.093 \\
\hline & 0.039000 & 4.33 & 5.048800 \\
\hline & 1.738100 & 2.769200 & 5.928600 \\
\hline & 3.492200 & 1.874200 & 4.437000 \\
\hline & 1.778100 & 4.064700 & 1.138800 \\
\hline & 0.071200 & 4.994000 & 2.656000 \\
\hline $\mathrm{H}$ & 5.769500 & 0.245200 & -0.299600 \\
\hline & 6.446800 & 2.434600 & -3.924500 \\
\hline & 3.006800 & 3.294700 & -1.507700 \\
\hline & 3.903400 & 5.518600 & -2.193700 \\
\hline & 3.179400 & 7.282400 & -3.668500 \\
\hline$\theta$ & 3.869500 & 4.649900 & -7.059300 \\
\hline & 4.561200 & 2.931100 & -5.549800 \\
\hline & 3.998600 & 6.682600 & -8.261600 \\
\hline & 2.598400 & 7.762900 & -8.561400 \\
\hline $\mathrm{H}$ & 2.306600 & 6.046100 & -8.134700 \\
\hline $\mathrm{H}$ & 0.824000 & 6.756100 & -6.171900 \\
\hline & 1.082600 & 8.450300 & -6.728200 \\
\hline & 1.397900 & 8.014000 & -5.018800 \\
\hline & 3.766100 & 8.934000 & -5.206600 \\
\hline $\mathrm{H}$ & 3.510300 & 9.364100 & -6.926100 \\
\hline $\mathrm{H}$ & 4.887200 & 8.296800 & -6.464000 \\
\hline & 6.623500 & -1.435900 & -1.766500 \\
\hline & 8.618200 & -2.722700 & -2.015000 \\
\hline & 10.709500 & 0.864300 & -3.301800 \\
\hline & 8.672800 & 2.119300 & -3.011700 \\
\hline & 10.792400 & -3.441900 & -1.412000 \\
\hline
\end{tabular}

H $\quad 12.074100 \quad-3.845200 \quad-2.600900$

$\mathrm{H} \quad 10.360300 \quad-3.836500 \quad-3.127300$

$\mathrm{H} \quad 12.509100 \quad-0.148700 \quad-2.214800$

$\mathrm{H} \quad 13.262700 \quad-1.762800 \quad-2.027500$

$\mathrm{H} \quad 11.981300 \quad-1.233800 \quad-0.878800$

$\mathrm{H} \quad 10.893700 \quad-2.154900 \quad-4.992100$

H $\quad 12.613200 \quad-2.324600 \quad-4.478900$

$\mathrm{H} \quad 11.881500 \quad-0.695000 \quad-4.623000$

$\mathrm{H} \quad-3.634800 \quad 2.338200 \quad-1.607900$

$\mathrm{H} \quad-4.958800 \quad 2.971400 \quad-0.528400$

$\mathrm{H} \quad-5.559500 \quad 5.171500 \quad-1.161900$

$\mathrm{H} \quad-6.666000 \quad 7.186000 \quad-0.279900$

$\mathrm{H} \quad-5.514900 \quad 8.617800 \quad 1.383000$

$\mathrm{H} \quad-3.230000 \quad 8.037200 \quad 2.148200$

$\begin{array}{llll}\mathrm{H} & -2.098700 & 6.036400 & 1.268300\end{array}$

H $\quad 0.868600 \quad 5.259600 \quad-4.011600$

$\mathrm{H} \quad-1.438200 \quad 4.875600 \quad-4.822700$

$\mathrm{H} \quad-3.252300 \quad 4.392100 \quad-3.219900$

$\mathrm{H} \quad-0.440400 \quad 4.704200 \quad 0.035400$

H $\quad 1.359600 \quad 5.197100 \quad-1.578900$

$\mathrm{H} \quad-2.152600 \quad 4.255700 \quad 2.727900$

H $\quad-4.897100 \quad 2.376400 \quad 5.427000$

$\mathrm{H} \quad-4.994200 \quad 1.422900 \quad 1.245100$

H $\quad-7.348900 \quad 1.216900 \quad 1.962900$

H $\quad-8.857100 \quad-0.617800 \quad 2.226600$

$\mathrm{H} \quad-6.523600 \quad-1.981400 \quad 5.631300$

$\mathrm{H} \quad-5.024500 \quad-0.122500 \quad 5.309700$

$\mathrm{H} \quad-8.583800 \quad-2.781200 \quad 6.360700$

H $\quad-10.058600 \quad-3.571500 \quad 5.723400$

H $\quad-9.935200 \quad-1.773400 \quad 5.726700$

H $\quad-7.438500 \quad-4.062400 \quad 2.988600$

H $\quad-8.544700 \quad-4.937300 \quad 4.107900$

$\mathrm{H} \quad-7.102800 \quad-4.091900 \quad 4.755900$

H $\quad-10.566600 \quad-1.914200 \quad 3.235500$

H $\quad-10.543700 \quad-3.706500 \quad 3.314600$

H $\quad-9.527100 \quad-2.868100 \quad 2.101100$

$\mathrm{H} \quad-2.787900 \quad 6.075500 \quad 4.174800$

$\mathrm{H} \quad-2.841100 \quad 2.618200 \quad 6.714000$

$\mathrm{H} \quad-1.796800 \quad 3.846500 \quad 8.474100$

$\mathrm{H} \quad-1.705000 \quad 7.354600 \quad 5.907500$

$\mathrm{H} \quad-1.897600 \quad 8.479500 \quad 7.945000$

$\mathrm{H} \quad-1.264900 \quad 8.402700 \quad 9.618900$

$\mathrm{H} \quad-2.752700 \quad 7.485200 \quad 9.179200$

$\mathrm{H} \quad 1.080900 \quad 6.176900 \quad 7.580900$

$\mathrm{H} \quad 1.032200 \quad 7.619000 \quad 8.661900$

$\begin{array}{llll}\mathrm{H} & 0.338700 & 7.715700 & 7.012600\end{array}$

$\begin{array}{llll}\mathrm{H} & -1.577800 & 5.439200 & 10.204400\end{array}$

$\mathrm{H} \quad-0.100800 \quad 6.414000 \quad 10.495700$

$\mathrm{H} \quad 0.020800 \quad 4.900100 \quad 9.542400$

\section{W7}

$\begin{array}{llll}\mathrm{Rh} & 0.057200 & -0.134700 & 1.280300\end{array}$

$\begin{array}{llll}\mathrm{Rh} & 0.306300 & -0.445900 & -1.070000\end{array}$

$\begin{array}{llll}\mathrm{O} & -0.768000 & -2.191200 & -0.854400\end{array}$

$\begin{array}{llll}\text { O } & -1.095200 & -1.846200 & 1.354600\end{array}$

$\begin{array}{lrrr}\text { O } & 2.099700 & -1.389700 & -0.698200\end{array}$

$\begin{array}{llll}\text { O } & 1.794200 & -1.218200 & 1.534800\end{array}$

$\begin{array}{llll}\mathrm{O} & 1.375300 & 1.324800 & -1.170000\end{array}$

$\begin{array}{llll}\mathrm{O} & 1.246000 & 1.546800 & 1.072300\end{array}$

$\begin{array}{lrrrr}\mathrm{O} & -1.500400 & 0.495900 & -1.309600\end{array}$

$\begin{array}{lllll}\mathrm{O} & -1.704600 & 0.901500 & 0.902300\end{array}$

$\begin{array}{lrrr}\text { C } & -1.344000 & -2.439200 & 0.253600\end{array}$ $\begin{array}{lrrr}\text { C } & -2.464400 & -3.463900 & 0.227600\end{array}$

C $\quad-3.406100 \quad-3.416200 \quad 1.421100$

$\begin{array}{llll}\text { C } & -2.519200 & -4.624000 & 1.269500\end{array}$

C $\quad-3.147000 \quad-5.910200 \quad 0.829300$

$\begin{array}{llll}\text { C } & -4.353300 & -6.348000 & 1.404200\end{array}$

$\begin{array}{llll}\text { C } & -4.932400 & -7.553800 & 0.997400\end{array}$

C $\quad-4.305000 \quad-8.340700 \quad 0.028300$

C $\quad-3.090600 \quad-7.929500 \quad-0.526000$

$\begin{array}{lrrr}\text { C } & -2.507200 & -6.726200 & -0.120000\end{array}$

$\begin{array}{llll}\text { C } & 0.697300 & -5.243600 & 4.048700\end{array}$

$\begin{array}{llll}\text { C } & -0.583900 & -4.949600 & 4.519700\end{array}$

$\begin{array}{llll}\text { C } & -1.627800 & -4.730300 & 3.616500\end{array}$

$\begin{array}{llll}\text { C } & -1.389500 & -4.784000 & 2.232600\end{array}$

$\begin{array}{llll}\text { C } & -0.098300 & -5.078400 & 1.767800\end{array}$

$\begin{array}{llll}\text { C } & 0.939600 & -5.311500 & 2.674300\end{array}$

C $\quad-3.061200 \quad-3.597600 \quad-1.157500$

$\begin{array}{llll}\text { C } & -2.362000 & -4.202100 & -2.205700\end{array}$

C $\quad-2.866300 \quad-4.208900 \quad-3.513100$

C $\quad-4.093700 \quad-3.575100 \quad-3.771600$

$\begin{array}{llll}\text { C } & -4.809500 & -2.952500 & -2.741300\end{array}$

C $\quad-4.280000 \quad-2.983000 \quad-1.441100$

$\begin{array}{llll}\text { C } & -6.067300 & -2.195700 & -2.962300\end{array}$

C $\quad-6.221800 \quad-1.305700 \quad-4.032800$

$\begin{array}{llll}\text { C } & -7.330800 & -0.462000 & -4.126800\end{array}$

$\begin{array}{llll}\text { C } & -8.351200 & -0.470200 & -3.152400\end{array}$

$\begin{array}{llll}\text { C } & -8.217000 & -1.420300 & -2.118800\end{array}$

$\begin{array}{llll}\text { C } & -7.109100 & -2.256900 & -2.026300\end{array}$

$\begin{array}{llll}\text { C } & -9.540700 & 0.506300 & -3.145400\end{array}$

$\begin{array}{llll}\text { C } & -9.501200 & 1.498900 & -4.330400\end{array}$

$\begin{array}{llll}\text { C } & -10.861000 & -0.287900 & -3.233600\end{array}$

$\begin{array}{llll}\text { C } & -9.521700 & 1.327200 & -1.836600\end{array}$

$\begin{array}{llll}\text { C } & -2.067800 & -4.846000 & -4.590000\end{array}$

$\begin{array}{llll}\text { C } & -1.366400 & -6.037300 & -4.359800\end{array}$

$\begin{array}{llll}\text { C } & -0.554400 & -6.598800 & -5.341100\end{array}$

$\begin{array}{llll}\text { C } & -0.389800 & -6.003400 & -6.609500\end{array}$

$\begin{array}{llll}\text { C } & -1.121800 & -4.820100 & -6.839800\end{array}$

$\begin{array}{llll}\text { C } & -1.938400 & -4.261900 & -5.856200\end{array}$

$\begin{array}{llll}\text { C } & 0.563200 & -6.633600 & -7.641600\end{array}$

$\begin{array}{llll}\text { C } & 0.098800 & -8.070000 & -7.964100\end{array}$

$\begin{array}{llll}\text { C } & 0.993600 & -6.673100 & -7.061500\end{array}$

$\begin{array}{llll}\text { C } & 0.612100 & -5.841700 & -8.969500\end{array}$

$\begin{array}{llll}\text { C } & 2.478300 & -1.542300 & 0.509900\end{array}$

$\begin{array}{llll}\text { C } & 3.884300 & -2.054600 & 0.759500\end{array}$

C $\quad 4.886800 \quad-1.592000 \quad-0.294900$

$\begin{array}{llll}\text { C } & 4.565500 & -3.060600 & -0.219700\end{array}$

$\begin{array}{llll}\text { C } & 5.609800 & -3.982800 & 0.332400\end{array}$

$\begin{array}{llll}\text { C } & 5.235700 & -5.105900 & 1.090200\end{array}$

$\begin{array}{llll}\text { C } & 6.205700 & -5.981600 & 1.585700\end{array}$

$\begin{array}{llll}\text { C } & 7.556200 & -5.760700 & 1.307100\end{array}$

C $\quad 7.937400 \quad-4.669700 \quad 0.522700$

$\begin{array}{llll}\text { C } & 6.970400 & -3.789200 & 0.029600\end{array}$

C $\quad 2.247000 \quad-4.720200 \quad-3.442300$

$\begin{array}{llll}\text { C } & 3.394400 & -3.972100 & -3.719100\end{array}$

C $4.149500 \quad-3.436100 \quad-2.672100$

C $\quad 3.756900 \quad-3.638100 \quad-1.338400$

$\begin{array}{llll}\text { C } & 2.611300 & -4.404600 & -1.069100\end{array}$

$\begin{array}{llll}\text { C } & 1.857200 & -4.939500 & -2.118100\end{array}$

$\begin{array}{llll}\text { C } & 4.281500 & -1.910500 & 2.213300\end{array}$

C $\quad 3.982500 \quad-2.876400 \quad 3.173900$

C $\quad 4.225500 \quad-2.651800 \quad 4.537400$

$\begin{array}{llll}\text { C } & 4.721300 & -1.404300 & 4.940600\end{array}$

$\begin{array}{llll}\text { C } & 5.012800 & -0.407100 & 3.998800\end{array}$ 


\begin{tabular}{|c|c|c|c|}
\hline C & 4.821100 & -0.693200 & 2.640200 \\
\hline $\mathrm{C}$ & 5.432300 & 0.958600 & 4.404600 \\
\hline $\mathrm{C}$ & 6.429600 & 1.660300 & 3.714900 \\
\hline C & 6.741100 & 2.984300 & 4.023600 \\
\hline 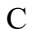 & 6.062500 & 3.684600 & 5.042500 \\
\hline C & 5.081900 & 2.957100 & 5.749300 \\
\hline $\mathrm{C}$ & 4.777800 & 1.632400 & 5.443900 \\
\hline $\mathrm{C}$ & 6.318100 & 5.165800 & 5.376400 \\
\hline $\mathrm{C}$ & 7.454600 & 5.781300 & 4.526700 \\
\hline $\mathrm{C}$ & 6.715000 & 5.302000 & 6.862300 \\
\hline $\mathrm{C}$ & 5.033000 & 5.978500 & 5.110100 \\
\hline $\mathrm{C}$ & 3.247700 & -3.462300 & 6.713900 \\
\hline $\mathrm{C}$ & 3.924300 & -3.724600 & 5.516700 \\
\hline $\mathrm{C}$ & 4.278800 & -5.054800 & 5.255900 \\
\hline $\mathrm{C}$ & 3.946700 & -6.076400 & 6.141800 \\
\hline $\mathrm{C}$ & 3.237500 & -5.830700 & 7.336500 \\
\hline $\mathrm{C}$ & 2.908700 & -4.484600 & 7.600000 \\
\hline $\mathrm{C}$ & 2.844600 & -7.001000 & 8.256400 \\
\hline $\mathrm{C}$ & 2.068100 & -6.538500 & 9.511800 \\
\hline $\mathrm{C}$ & 4.117000 & -7.735100 & 8.730600 \\
\hline $\mathrm{C}$ & 1.943100 & -7.983100 & 7.477700 \\
\hline $\mathrm{C}$ & 1.727300 & 1.859400 & -0.068400 \\
\hline $\mathrm{C}$ & 2.885800 & 2.846700 & -0.112700 \\
\hline $\mathrm{C}$ & 3.830200 & 2.655600 & 1.080900 \\
\hline $\mathrm{C}$ & 3.059300 & 3.944800 & 0.984500 \\
\hline $\mathrm{C}$ & 3.808300 & 5.200400 & 0.641400 \\
\hline $\mathrm{C}$ & 3.155200 & 6.248800 & -0.032100 \\
\hline $\mathrm{C}$ & 3.833700 & 7.433200 & -0.331200 \\
\hline $\mathrm{C}$ & 5.161000 & 7.599200 & 0.067900 \\
\hline $\mathrm{C}$ & 5.806900 & 6.586500 & 0.779600 \\
\hline $\mathrm{C}$ & 5.132900 & 5.398600 & 1.078100 \\
\hline $\mathrm{C}$ & -0.104900 & 4.546200 & 3.833000 \\
\hline $\mathrm{C}$ & 1.183600 & 4.234200 & 4.274900 \\
\hline $\mathrm{C}$ & 2.212800 & 4.041000 & 3.349200 \\
\hline $\mathrm{C}$ & 1.955900 & 4.148300 & 1.972400 \\
\hline $\mathrm{C}$ & 0.662100 & 4.481400 & 1.537400 \\
\hline $\mathrm{C}$ & -0.363000 & 4.675900 & 2.465500 \\
\hline $\mathrm{C}$ & 3.538800 & 2.933700 & -1.481400 \\
\hline $\mathrm{C}$ & 3.298700 & 3.955100 & -2.401300 \\
\hline $\mathrm{C}$ & 3.901000 & 3.968800 & -3.671700 \\
\hline $\mathrm{C}$ & 4.698400 & 2.875300 & -4.038600 \\
\hline $\mathrm{C}$ & 4.954900 & 1.830100 & -3.141600 \\
\hline $\mathrm{C}$ & 4.397600 & 1.894000 & -1.859800 \\
\hline $\mathrm{C}$ & 5.772900 & 0.655700 & -3.547900 \\
\hline $\mathrm{C}$ & 6.839900 & 0.194400 & -2.766800 \\
\hline $\mathrm{C}$ & 7.580700 & -0.924600 & -3.144400 \\
\hline $\mathrm{C}$ & 7.289900 & -1.656800 & -4.314800 \\
\hline $\mathrm{C}$ & 6.216500 & -1.178800 & -5.095500 \\
\hline $\mathrm{C}$ & 5.485200 & -0.050600 & -4.722300 \\
\hline $\mathrm{C}$ & 8.132800 & -2.891000 & -4.688300 \\
\hline $\mathrm{C}$ & 7.616300 & -3.600400 & -5.962100 \\
\hline $\mathrm{C}$ & 9.589800 & -2.452700 & -4.943000 \\
\hline $\mathrm{C}$ & 8.103200 & -3.917800 & -3.534600 \\
\hline $\mathrm{C}$ & 3.724300 & 5.128100 & -4.586700 \\
\hline $\mathrm{C}$ & 2.594500 & 5.956900 & -4.537000 \\
\hline $\mathrm{C}$ & 2.440600 & 7.050800 & -5.386800 \\
\hline $\mathrm{C}$ & 3.418600 & 7.388400 & -6.344200 \\
\hline $\mathrm{C}$ & 4.557000 & 6.557000 & -6.380900 \\
\hline $\mathrm{C}$ & 4.706900 & 5.465600 & -5.529300 \\
\hline $\mathrm{C}$ & 3.297800 & 8.584100 & -7.306400 \\
\hline $\mathrm{C}$ & 4.470000 & 9.559100 & -7.065600 \\
\hline C & 1.979400 & 9.370700 & -7.116400 \\
\hline
\end{tabular}

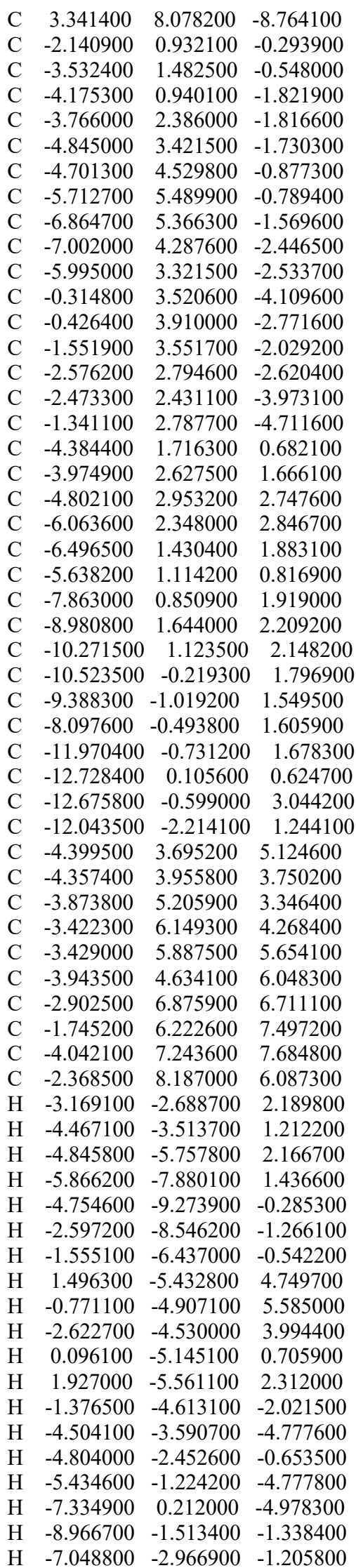

H $\quad-8.573600 \quad 2.109400 \quad-4.303600$

$\mathrm{H} \quad-10.365200 \quad 2.197200 \quad-4.288700$

$\mathrm{H} \quad-9.552800 \quad 0.958600 \quad-5.300300$

$\mathrm{H} \quad-10.879700 \quad-0.905500 \quad-4.157500$

$\mathrm{H} \quad-11.733400 \quad 0.400700 \quad-3.254400$

H $\quad-10.989300 \quad-0.963500 \quad-2.362100$

$\mathrm{H} \quad-9.673600 \quad 0.679500 \quad-0.949300$

H $\quad-10.331500 \quad 2.088600 \quad-1.834200$

$\mathrm{H} \quad-8.547900 \quad 1.848700 \quad-1.719200$

$\mathrm{H} \quad-1.461500 \quad-6.538800 \quad-3.400700$

$\mathrm{H} \quad-0.039600 \quad-7.519800 \quad-5.079100$

$\mathrm{H} \quad-1.078900 \quad-4.273100 \quad-7.776900$

$\mathrm{H} \quad-2.452800 \quad-3.328700 \quad-6.069200$

$\mathrm{H} \quad 0.141900 \quad-8.723200 \quad-7.067600$

$\mathrm{H} \quad 0.747600 \quad-8.528500 \quad-8.741600$

$\mathrm{H} \quad-0.946800 \quad-8.062600 \quad-8.341100$

$\mathrm{H} \quad 2.331600 \quad-5.648200 \quad-6.794700$

$\mathrm{H} \quad 2.707600 \quad-7.094400 \quad-7.802100$

H $\quad 2.046000 \quad-7.304700 \quad-6.149900$

$\mathrm{H} \quad-0.392300 \quad-5.803700 \quad-9.444100$

$\mathrm{H} \quad 1.306200 \quad-6.325000 \quad-9.691100$

H $\quad 0.975600 \quad-4.805200 \quad-8.799100$

H $\quad 4.489600 \quad-1.030200 \quad-1.131400$

$\mathrm{H} \quad 5.838700 \quad-1.226300 \quad 0.078800$

H $\quad 4.194300 \quad-5.313300 \quad 1.288700$

H $\quad 5.908700 \quad-6.837200 \quad 2.178400$

H $\quad 8.306000 \quad-6.441900 \quad 1.688000$

H $\quad 8.982400 \quad-4.508800 \quad 0.291500$

$\mathrm{H} \quad 7.285800 \quad-2.961100 \quad-0.590900$

$\mathrm{H} \quad 1.662900 \quad-5.131300 \quad-4.253600$

H $\quad 3.698600 \quad-3.806300 \quad-4.744800$

$\mathrm{H} \quad 5.032800 \quad-2.855000 \quad-2.898700$

H $\quad 2.299600 \quad-4.574600 \quad-0.048600$

$\mathrm{H} \quad 0.972300 \quad-5.524800 \quad-1.904500$

$\mathrm{H} \quad 3.496800 \quad-3.798600 \quad 2.881200$

H $\quad 4.909600 \quad-1.217900 \quad 5.994400$

$\mathrm{H} \quad 5.021900 \quad 0.080800 \quad 1.906600$

$\mathrm{H} \quad 6.981000 \quad 1.161500 \quad 2.922000$

H $\quad 7.529800 \quad 3.431000 \quad 3.425400$

$\mathrm{H} \quad 4.502700 \quad 3.417900 \quad 6.545500$

$\mathrm{H} \quad 3.984200 \quad 1.130700 \quad 5.990800$

H $\quad 8.410900 \quad 5.240600 \quad 4.695800$

$\begin{array}{llll}\mathrm{H} & 7.616900 & 6.847100 & 4.797900\end{array}$

$\mathrm{H} \quad 7.203900 \quad 5.748100 \quad 3.445700$

$\mathrm{H} \quad 5.892500 \quad 4.982900 \quad 7.535800$

$\begin{array}{llll}\mathrm{H} & 6.955400 & 6.358600 & 7.110700\end{array}$

H $\quad 7.609100 \quad 4.679300 \quad 7.082200$

$\mathrm{H} \quad 4.725800 \quad 5.880200 \quad 4.046600$

H $\quad 5.197000 \quad 7.056400 \quad 5.326300$

$\mathrm{H} \quad 4.193000 \quad 5.631200 \quad 5.747900$

$\mathrm{H} \quad 2.943400 \quad-2.444500 \quad 6.942800$

H $\quad 4.827100 \quad-5.294100 \quad 4.349000$

H $\quad 4.253400 \quad-7.081800 \quad 5.864100$

H $\quad 2.365800 \quad-4.172800 \quad 8.487200$

H $\quad 2.684200 \quad-5.845400 \quad 10.124600$

$\mathrm{H} \quad 1.802500 \quad-7.407000 \quad 10.153100$

H $\quad \begin{array}{llll}1.120900 & -6.031400 & 9.226600\end{array}$

H $\quad 4.670200 \quad-8.183400 \quad 7.879000$

$\mathrm{H} \quad 3.858500 \quad-8.559100 \quad 9.430700$

H $\quad 4.796800 \quad-7.030000 \quad 9.256200$

H $\quad 1.042400 \quad-7.457900 \quad 7.091700$

H $\quad 1.608800 \quad-8.816000 \quad 8.133600$ 


\begin{tabular}{|c|c|c|c|}
\hline & 2.479900 & -8.431800 & 6.615600 \\
\hline & 3.527000 & 1.932100 & 1.829100 \\
\hline & 4.889300 & 2.634000 & 0.842400 \\
\hline & 2.119800 & 6.151100 & -0.330100 \\
\hline & 3.327000 & 8.225800 & -0.866500 \\
\hline & 5.684400 & 8.518300 & -0.161400 \\
\hline & 6.829000 & 6.725300 & 1.106800 \\
\hline & 5.645800 & 4.644800 & 1.659800 \\
\hline & -0.901200 & 4.688300 & 4.550300 \\
\hline & 1.385300 & 4.141600 & 5.334400 \\
\hline & 3.207000 & 3.800700 & 3.701600 \\
\hline & 0.445500 & 4.576200 & 0.480900 \\
\hline & -1.355300 & 4.927600 & 2.120600 \\
\hline & 2.646900 & 4.774600 & -2.136400 \\
\hline & 5.134800 & 2.831900 & -5.031700 \\
\hline & 4.590300 & 1.090500 & -1.157700 \\
\hline & 7.109300 & 0.72 & 9800 \\
\hline & 8.403800 & -1.207800 & -2.493000 \\
\hline & 5.894700 & -1.659700 & -6.014300 \\
\hline & 4.653900 & 0.269300 & -5.344800 \\
\hline & 6.566800 & -3.941000 & -5.824600 \\
\hline & 8.234000 & -4.495500 & 3200 \\
\hline & 7.669600 & -2.924300 & -6.842600 \\
\hline & 9.629400 & -1.693900 & -5.754400 \\
\hline & 10.215200 & -3.320800 & -5.245000 \\
\hline & 10.044400 & -2.011000 & -4.031200 \\
\hline & 500 & -3.51 & -2.618800 \\
\hline & 8.657500 & -4.840100 & -3.813500 \\
\hline & 7.057600 & -4.202900 & -3.291500 \\
\hline & 1.79 & 5.747000 & 0900 \\
\hline & 1.5 & 7.6 & 60000 \\
\hline 11 & 5.371200 & 6.746 & 76000 \\
\hline 7 & 5.623300 & 4.885900 & -5.585100 \\
\hline H & 4.477000 & 9.901500 & -6.008100 \\
\hline & 4.380800 & 10.452100 & 721700 \\
\hline & 5.4 & 9.08 & 3800 \\
\hline त & 1.099300 & 8.718800 & -7.305900 \\
\hline H & 1.923700 & 10.224000 & -7.827000 \\
\hline$\theta$ & 1.911600 & 9.786600 & -6.087900 \\
\hline & 4.31 & 7.5 & 600 \\
\hline H & 3.215100 & 8.921200 & -9.477700 \\
\hline $\mathrm{H}$ & 2.526500 & 7.343600 & -8.942900 \\
\hline $\mathrm{H}$ & -3.576700 & 0.231900 & -2.382800 \\
\hline $\mathrm{H}$ & -5.230100 & 0.690900 & -1.791900 \\
\hline H & -3.809700 & 4.650100 & -0.277300 \\
\hline $\mathrm{H}$ & -5.601100 & 6.332100 & -0.118800 \\
\hline $\mathrm{H}$ & -7.646800 & 6.111300 & -1.501600 \\
\hline $\mathrm{H}$ & -7.890000 & 4.200900 & -3.058700 \\
\hline & -6.114900 & 2.495400 & -3.223500 \\
\hline H & 0.563200 & 3.786700 & -4.682200 \\
\hline $\mathrm{H}$ & 0.355700 & 4.487200 & -2.303000 \\
\hline $\mathrm{H}$ & -1.616100 & 3.846100 & -0.989200 \\
\hline $\mathrm{H}$ & -3.262000 & 1.861900 & -4.449600 \\
\hline $\mathrm{H}$ & -1.258100 & 2.493700 & -5.749900 \\
\hline $\mathrm{H}$ & -2.997800 & 3.091100 & 1.587500 \\
\hline $\mathrm{H}$ & -6.717000 & 2.610000 & 3.674400 \\
\hline $\mathrm{H}$ & -5.992200 & 0.422800 & 0.060200 \\
\hline $\mathrm{H}$ & -8.844200 & 2.695800 & 2.445000 \\
\hline $\mathrm{H}$ & -11.085100 & 1.810900 & 2.365800 \\
\hline $\mathrm{H}$ & -9.453000 & -2.075700 & 1.305300 \\
\hline $\mathrm{H}$ & -7.254100 & -1.148900 & 1.407000 \\
\hline $\mathrm{H}$ & -12.805500 & 1.170600 & 0.927600 \\
\hline
\end{tabular}

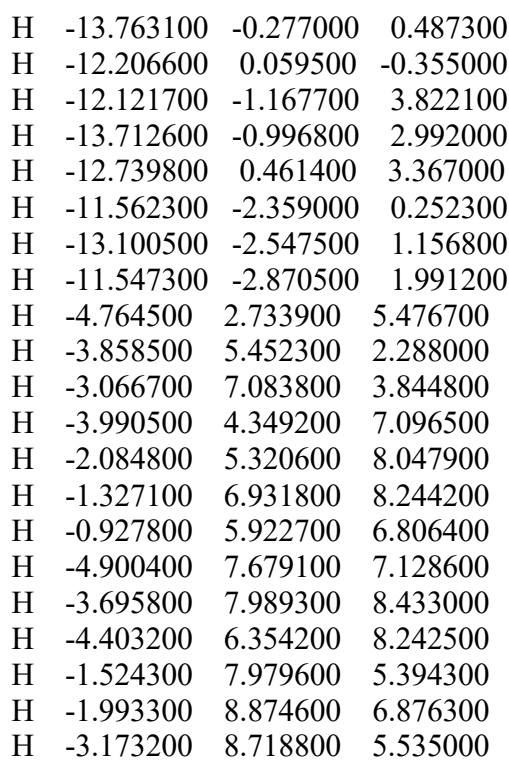

\section{W'1}

$\begin{array}{llll}\text { Rh } & -0.374101 & -0.739402 & -1.067599\end{array}$

$\begin{array}{llll}\mathrm{Rh} & -0.346102 & 0.764298 & 0.778401\end{array}$

$\begin{array}{llll}\text { O } & -1.323603 & 2.135197 & -0.418299\end{array}$

$\begin{array}{llll}\text { O } & -1.571502 & 0.608897 & -2.065399\end{array}$

$\begin{array}{llll}\mathrm{O} & 1.545197 & 1.354899 & 0.203601\end{array}$

$\begin{array}{llll}\text { O } & 1.395098 & 0.161899 & -1.700299\end{array}$

$\begin{array}{llll}\text { O } & 0.627099 & -0.690901 & 1.863001\end{array}$

$\begin{array}{lllll}\mathrm{O} & 0.801400 & -2.020901 & 0.045701\end{array}$

$\begin{array}{llll}\mathrm{O} & -2.216502 & 0.045897 & 1.278901\end{array}$

$\begin{array}{llll}\text { O } & -2.110501 & -1.545503 & -0.322399\end{array}$

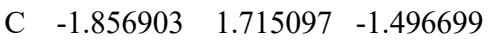

$\begin{array}{llll}\text { C } & -2.987804 & 2.550096 & -2.066199\end{array}$

$\begin{array}{llll}\text { C } & -4.141103 & 1.712995 & -2.621799\end{array}$

C $\quad-3.338904 \quad 2.546796 \quad-3.584299$

$\begin{array}{llll}\text { C } & -3.974104 & 3.774895 & -4.159199\end{array}$

$\begin{array}{llll}\text { C } & -5.332904 & 3.780494 & -4.525799\end{array}$

$\begin{array}{llll}\text { C } & -5.907805 & 4.923994 & -5.087999\end{array}$

$\begin{array}{llll}\text { C } & -5.129806 & 6.062894 & -5.309099\end{array}$

$\begin{array}{llll}\text { C } & -3.771406 & 6.054195 & -4.984399\end{array}$

C $\quad-3.192205 \quad 4.911596 \quad-4.425799$

$\begin{array}{llll}\text { C } & -0.871502 & 0.402998 & -6.380199\end{array}$

$\begin{array}{llll}\text { C } & -0.300603 & 1.373298 & -5.551499\end{array}$

$\begin{array}{llll}\text { C } & -1.098603 & 2.075497 & -4.643899\end{array}$

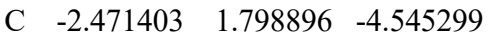

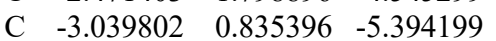

$\begin{array}{llll}\text { C } & -2.241702 & 0.137097 & -6.304199\end{array}$

C $\quad-3.360604 \quad 3.683496 \quad-1.130299$

$\begin{array}{llll}\text { C } & -4.256004 & 3.412495 & -0.089999\end{array}$

$\begin{array}{llll}\text { C } & -4.488405 & 4.329195 & 0.943301\end{array}$

$\begin{array}{llll}\text { C } & -3.851306 & 5.576995 & 0.885501\end{array}$

C $\quad-2.989106 \quad 5.896196 \quad-0.172199$

C $\quad-2.733705 \quad 4.929196 \quad-1.157099$

$\begin{array}{llll}\text { C } & -2.318407 & 7.216997 & -0.253299\end{array}$

$\begin{array}{llll}\text { C } & -1.710607 & 7.802597 & 0.863601\end{array}$

$\begin{array}{llll}\text { C } & -1.022608 & 9.012097 & 0.770501\end{array}$

$\begin{array}{llll}\text { C } & -0.910509 & 9.706498 & -0.451999\end{array}$

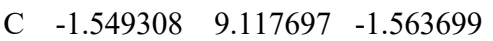

$\begin{array}{llll}\text { C } & -2.234007 & 7.908897 & -1.468699\end{array}$

C $\quad-0.132910 \quad 11.025298 \quad-0.616299$ $\begin{array}{lrrr}\text { C } & -1.089010 & 12.129297 & -1.116099\end{array}$

$\begin{array}{llll}\text { C } & 0.502890 & 11.511899 & 0.707301\end{array}$

$\begin{array}{llll}\text { C } & 1.003790 & 10.825799 & -1.641699\end{array}$

C $\quad-5.335905 \quad 3.936694 \quad 2.099301$

$\begin{array}{llll}\text { C } & -4.906605 & 4.159795 & 3.413901\end{array}$

$\begin{array}{llll}\text { C } & -5.648104 & 3.722194 & 4.511401\end{array}$

C $\quad-6.872604 \quad 3.040193 \quad 4.352801$

$\begin{array}{llll}\text { C } & -7.306304 & 2.844493 & 3.024501\end{array}$

$\begin{array}{llll}\text { C } & -6.558704 & 3.274093 & 1.929501\end{array}$

C $\quad-7.723803 \quad 2.529693 \quad 5.530701$

$\begin{array}{llll}\text { C } & -7.954002 & 1.008592 & 5.391701\end{array}$

$\begin{array}{llll}\text { C } & -9.085804 & 3.254292 & 5.526101\end{array}$

$\begin{array}{llll}\text { C } & -7.053004 & 2.780193 & 6.901901\end{array}$

$\begin{array}{llll}\text { C } & 2.050698 & 0.860000 & -0.857599\end{array}$

$\begin{array}{llll}\text { C } & 3.523498 & 1.143901 & -1.121199\end{array}$

$\begin{array}{llll}\text { C } & 4.210397 & 1.997701 & -0.056799\end{array}$

C $\quad 3.876896 \quad 2.665201 \quad-1.361199$

$\begin{array}{llll}\text { C } & 4.978196 & 2.997202 & -2.314399\end{array}$

$\begin{array}{llll}\text { C } & 4.896196 & 2.602202 & -3.660099\end{array}$

$\begin{array}{llll}\text { C } & 5.911996 & 2.946002 & -4.556899\end{array}$

$\begin{array}{llll}\text { C } & 7.008596 & 3.694703 & -4.119999\end{array}$

$\begin{array}{llll}\text { C } & 7.090595 & 4.101903 & -2.785599\end{array}$

$\begin{array}{llll}\text { C } & 6.075896 & 3.762303 & -1.885999\end{array}$

$\begin{array}{llll}\text { C } & 0.941794 & 5.801899 & -1.458799\end{array}$

$\begin{array}{llll}\text { C } & 0.910495 & 4.789799 & -2.420299\end{array}$

$\begin{array}{llll}\text { C } & 1.827096 & 3.734900 & -2.361299\end{array}$

C $\quad 2.792996 \quad 3.697100 \quad-1.342099$

$\begin{array}{llll}\text { C } & 2.820995 & 4.726300 & -0.383299\end{array}$

$\begin{array}{llll}\text { C } & 1.889094 & 5.765800 & -0.435299\end{array}$

$\begin{array}{llll}\text { C } & 4.359198 & -0.006499 & -1.696199\end{array}$

$\begin{array}{llll}\text { C } & 3.795799 & -1.236299 & -2.073799\end{array}$

C $\quad 4.571200 \quad-2.328498 \quad-2.500999$

$\begin{array}{llll}\text { C } & 5.959900 & -2.177297 & -2.591699\end{array}$

$\begin{array}{llll}\text { C } & 6.562899 & -0.972897 & -2.216999\end{array}$

$\begin{array}{llll}\text { C } & 5.763898 & 0.077802 & -1.748899\end{array}$

$\begin{array}{llll}\text { C } & 8.035099 & -0.792296 & -2.297899\end{array}$

$\begin{array}{llll}\text { C } & 8.589898 & 0.357704 & -2.872699\end{array}$

$\begin{array}{llll}\text { C } & 9.968398 & 0.547805 & -2.925999\end{array}$

$\begin{array}{llll}\text { C } & 10.874599 & -0.394094 & -2.394899\end{array}$

$\begin{array}{llll}\text { C } & 10.305799 & -1.557594 & -1.835299\end{array}$

$\begin{array}{llll}\text { C } & 8.924300 & -1.750195 & -1.795099\end{array}$

C $\quad 12.388398 \quad-0.115493 \quad-2.429599$

$\begin{array}{llll}\text { C } & 13.219599 & -1.257592 & -1.799399\end{array}$

$\begin{array}{llll}\text { C } & 12.690197 & 1.178107 & -1.642799\end{array}$

$\begin{array}{llll}\text { C } & 12.848698 & 0.053408 & -3.893199\end{array}$

C $\quad 2.588701 \quad-3.701200 \quad-3.266699$

$\begin{array}{llll}\text { C } & 3.919101 & -3.625599 & -2.827499\end{array}$

$\begin{array}{llll}\text { C } & 4.604602 & -4.841998 & -2.690999\end{array}$

$\begin{array}{llll}\text { C } & 3.998003 & -6.059699 & -2.988399\end{array}$

C $\quad 2.656803 \quad-6.146300 \quad-3.414699$

C $\quad 1.970702 \quad-4.920700 \quad-3.541999$

$\begin{array}{llll}\text { C } & 2.032604 & -7.514800 & -3.745699\end{array}$

$\begin{array}{llll}\text { C } & 2.178404 & -8.467100 & -2.537499\end{array}$

$\begin{array}{llll}\text { C } & 2.761904 & -8.122200 & -4.961299\end{array}$

$\begin{array}{llll}\text { C } & 0.527904 & -7.412801 & -4.090699\end{array}$

$\begin{array}{llll}\text { C } & 1.124500 & -1.679401 & 1.233001\end{array}$

$\begin{array}{llll}\text { C } & 2.238000 & -2.450800 & 1.919101\end{array}$

$\begin{array}{llll}\text { C } & 3.060901 & -3.323300 & 0.983901\end{array}$

$\begin{array}{llll}\text { C } & 2.186401 & -4.009300 & 1.998601\end{array}$

C $\quad 2.858902 \quad-4.670900 \quad 3.162701$

C $\quad 2.331602 \quad-4.550100 \quad 4.460501$

$\begin{array}{llll}\text { C } & 2.958302 & -5.178100 & 5.540301\end{array}$ 


\begin{tabular}{|c|c|c|c|}
\hline C & 4.102003 & -5.952399 & 5.332501 \\
\hline & 4.613903 & -6.108998 & 4.042301 \\
\hline & 3.991402 & -5.480199 & 2.959901 \\
\hline$\tau$ & -1.373797 & -6.068203 & 0.768101 \\
\hline$\tau$ & -1.422998 & -5.158303 & 1.824301 \\
\hline & -0.271898 & -4.465102 & 2.202201 \\
\hline & 0.941702 & -4.688501 & 1.530901 \\
\hline$C$ & 0.982502 & -5.605401 & 0.466401 \\
\hline $\mathrm{C}$ & -0.178197 & -6.284202 & 0.082001 \\
\hline 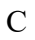 & 2.931300 & -1.604900 & 2.962401 \\
\hline & 4.153299 & -0.998199 & 2.670901 \\
\hline $\mathrm{C}$ & 4.744598 & -0.079298 & 3.554101 \\
\hline $\mathrm{C}$ & 4.095198 & 0.205801 & 4.763001 \\
\hline $\mathrm{C}$ & 2.868299 & -0.397000 & 5.082801 \\
\hline $\mathrm{C}$ & 2.297499 & -1.288600 & 4.166801 \\
\hline C & 2.133298 & -0.081600 & 6.332901 \\
\hline $\mathrm{C}$ & 1.465999 & -1.084501 & 7.048901 \\
\hline $\mathrm{C}$ & 0.699699 & -0.794301 & 8.176401 \\
\hline $\mathrm{C}$ & 0.551798 & 0.526699 & 8.647901 \\
\hline $\mathrm{C}$ & 1.253297 & 1.522799 & 7.936501 \\
\hline $\mathrm{C}$ & 2.024997 & 1.228900 & 6.814301 \\
\hline $\mathrm{C}$ & -0.334602 & 0.910098 & 7001 \\
\hline $\mathrm{C}$ & -1.027701 & -0.313503 & 10.49130 \\
\hline $\mathrm{C}$ & 0.528897 & 1.590799 & 10.930201 \\
\hline $\mathrm{C}$ & -1.437003 & 1.884897 & 9.379401 \\
\hline $\mathrm{C}$ & 5.993198 & 0.610803 & 3.144401 \\
\hline $\mathrm{C}$ & 6.179897 & 1303 & 801 \\
\hline $\mathrm{C}$ & 7.293596 & 2.659603 & 2.872301 \\
\hline $\mathrm{C}$ & 8.289297 & 1.997704 & 2.123901 \\
\hline $\mathrm{C}$ & 8.112498 & 0.609104 & 1.943501 \\
\hline $\mathrm{C}$ & 6.998098 & -0.063397 & 2.437401 \\
\hline $\mathrm{C}$ & 9.500396 & 2.708805 & 1.494201 \\
\hline $\mathrm{C}$ & 9.495995 & 4.234005 & 1.749801 \\
\hline $\mathrm{C}$ & 9.486197 & 2.486105 & -0.032199 \\
\hline $\mathrm{C}$ & 10.803097 & 2.128706 & 2.083701 \\
\hline $\mathrm{C}$ & -2.712901 & -0.920404 & 0.610801 \\
\hline $\mathrm{C}$ & -4.155001 & -1.310405 & 0.874501 \\
\hline $\mathrm{C}$ & -5.029102 & -0.149205 & 1.339901 \\
\hline $\mathrm{C}$ & -4.742801 & -1.255705 & 2.318801 \\
\hline $\mathrm{C}$ & -5.853900 & -2.200606 & 2.653201 \\
\hline $\mathrm{C}$ & -7.156200 & -1.719707 & 2.879101 \\
\hline $\mathrm{C}$ & -8.186700 & -2.602208 & 3.215201 \\
\hline $\mathrm{C}$ & -7.924499 & -3.968408 & 3.343901 \\
\hline $\mathrm{C}$ & -6.627998 & -4.451707 & 3.151201 \\
\hline $\mathrm{C}$ & -5.594299 & -3.571206 & 2.820301 \\
\hline $\mathrm{C}$ & -2.135601 & -0.327003 & 5.613101 \\
\hline $\mathrm{C}$ & -1.892801 & -1.459303 & 4.830301 \\
\hline $\mathrm{C}$ & -2.739300 & -1.765304 & 3.760101 \\
\hline $\mathrm{C}$ & -3.832101 & -0.935205 & 3.461601 \\
\hline $\mathrm{C}$ & -4.077702 & 0.191595 & 4.264401 \\
\hline $\mathrm{C}$ & -3.229202 & 0.496196 & 5.332301 \\
\hline $\mathrm{C}$ & -4.698100 & -2.205105 & -0.222699 \\
\hline $\mathrm{C}$ & -5.338000 & -1.607006 & -1.312399 \\
\hline $\mathrm{C}$ & -5.659900 & -2.326206 & -2.472799 \\
\hline $\mathrm{C}$ & -5.372099 & -3.697706 & -2.504899 \\
\hline $\mathrm{C}$ & -4.767599 & -4.332205 & -1.412199 \\
\hline $\mathrm{C}$ & -4.423099 & -3.574105 & -0.283899 \\
\hline $\mathrm{C}$ & -4.418897 & -5.776405 & -1.460899 \\
\hline $\mathrm{C}$ & -4.853897 & -6.669305 & -0.474699 \\
\hline $\mathrm{C}$ & -4.440896 & -8.000905 & -0.480499 \\
\hline $\mathrm{C}$ & -3.556495 & -8.506804 & -1.457299 \\
\hline $\mathrm{C}$ & -3.163596 & -7.606904 & -2.469199 \\
\hline
\end{tabular}

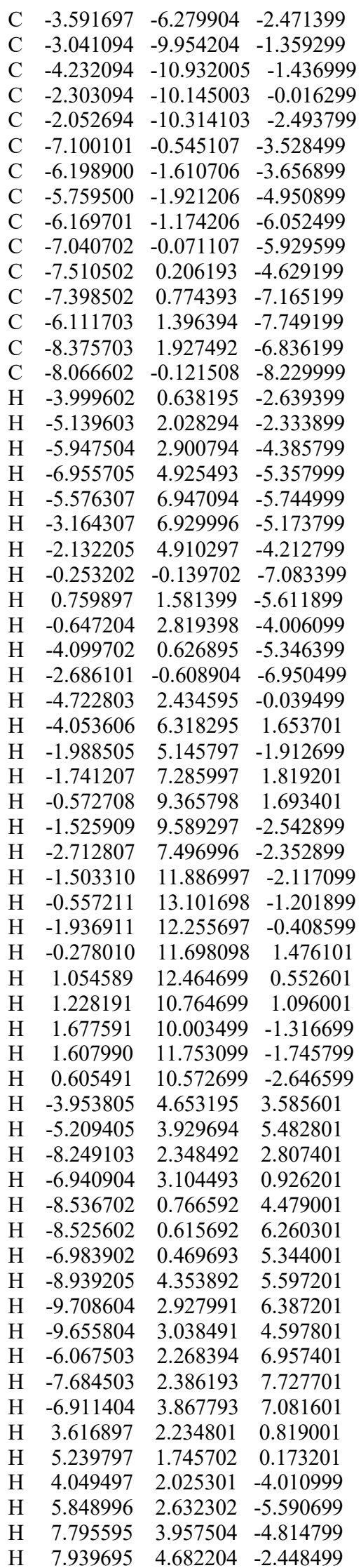

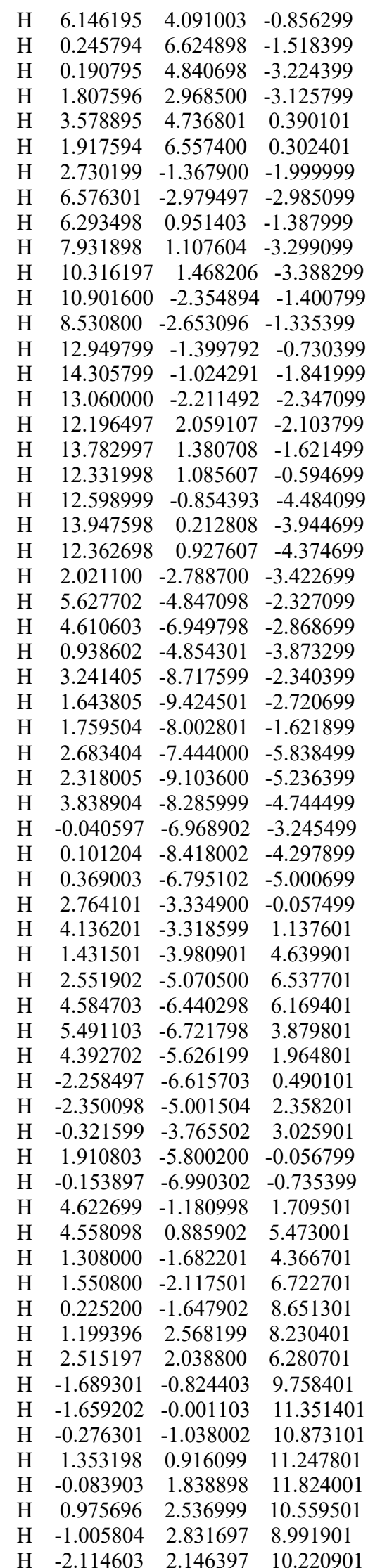




\begin{tabular}{|c|c|c|c|}
\hline & -2.045603 & & \\
\hline & 5.417296 & 2.543702 & 3.900701 \\
\hline & 7.325096 & 3.724304 & 3.083901 \\
\hline & 8.838398 & 0.013405 & 1.396001 \\
\hline & 6.909599 & -1.132897 & 2.268701 \\
\hline & 8.583995 & 4.703004 & 1.320601 \\
\hline & 10.377595 & 4.715706 & 1.273601 \\
\hline & 9.543195 & 4.453005 & 2.838401 \\
\hline & 9.618897 & 1.413705 & -0.278999 \\
\hline & 10.309396 & 3.047506 & -0.524299 \\
\hline & 8.521696 & 2.825804 & -0.464799 \\
\hline & 5097 & 2.250106 & 8401 \\
\hline & 0896 & 19707 & 1.663901 \\
\hline & 10.908498 & 1.048206 & 1.850401 \\
\hline & -4.535802 & 0.804295 & 1.487301 \\
\hline & -6.0 & -0.0 & 601 \\
\hline & -7.3 & -0.6 & 301 \\
\hline & -9.188000 & -2.225708 & 9801 \\
\hline & -8.723298 & -4.650708 & 3.604101 \\
\hline & -6.421598 & -5.5 & 7301 \\
\hline & -4.5 & -3.9 & 2.7 \\
\hline & -1.4 & -0.0 & 501 \\
\hline & -1.0 & -2.1 & 4301 \\
\hline & -2.539800 & -2.6 & 6901 \\
\hline & -4.919402 & 0.8 & 201 \\
\hline & -3.4 & 1.3 & 501 \\
\hline & -5.5 & -0.5 & 799 \\
\hline & -5.6 & -4.2 & 999 \\
\hline & -3.80 & -4.0 & 401 \\
\hline & -5.51 & -6.3 & 301 \\
\hline & -4.8 & -8.6 & 001 \\
\hline & -2.5 & -7.8 & 199 \\
\hline & -3.2 & -5.6 & 399 \\
\hline & -4.9 & 38605 & 37199 \\
\hline & 9093 & -11. & 05599 \\
\hline & -4.7 & -10 . & 32899 \\
\hline & -1.46 & -9.41 & 0.07 \\
\hline & -1.881694 & 70903 & 59701 \\
\hline & -2.984794 & 00004 & 0.847701 \\
\hline & -2.5 & -10 . & 8599 \\
\hline & -1.65 & -11.3 & $\begin{array}{l}3.391299 \\
\end{array}$ \\
\hline & -1.157795 & -9.655903 & 9299 \\
\hline & -7.496301 & -0.296007 & 7699 \\
\hline & -5.046200 & -2.727505 & -5.0 \\
\hline & -5.7 & -1.4 & -7.0 \\
\hline & -8.20 & 1.012192 & -4.412499 \\
\hline $\mathrm{H}$ & -5.606003 & 2.027894 & -6.987299 \\
\hline & -6.346503 & 2.033694 & -8.629099 \\
\hline & -5.396702 & 0.614894 & -8.081999 \\
\hline & -9.332303 & 1.531191 & -6.432099 \\
\hline $\mathrm{H}$ & -8.613703 & 2.515292 & -7.749399 \\
\hline $\mathrm{H}$ & -7.929504 & 2.626292 & -6.097099 \\
\hline & -7.374201 & -0.909807 & -8.592399 \\
\hline & -8.376702 & 0.479492 & -9.112299 \\
\hline & -8.969001 & -0.615208 & -7.808699 \\
\hline
\end{tabular}

\section{W'2}

$\begin{array}{llll}\text { Rh } & 0.001300 & -0.001798 & -1.194298\end{array}$ $\begin{array}{llll}\mathrm{Rh} & -0.004100 & -0.002997 & 1.189702\end{array}$ $\begin{array}{llll}\text { O } & 1.368447 & -1.528545 & 1.132702\end{array}$ $\begin{array}{llll}\text { O } & 1.539752 & -1.381351 & -1.116698\end{array}$ $\begin{array}{llll}\text { O } & -1.602245 & -1.313042 & 1.110502\end{array}$

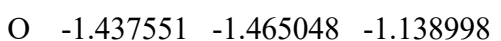

$\begin{array}{lllll}\mathrm{O} & -1.376247 & 1.522850 & 1.128102\end{array}$

$\begin{array}{llll}\mathrm{O} & -1.537252 & 1.378056 & -1.122298\end{array}$

$\begin{array}{llll}\text { O } & 1.594345 & 1.307047 & 1.118902\end{array}$

$\begin{array}{llll}\text { O } & 1.440051 & 1.461153 & -1.131298\end{array}$

$\begin{array}{llll}\text { C } & 1.936738 & -1.791965 & 0.024002\end{array}$

$\begin{array}{llll}\text { C } & 3.233711 & -2.573510 & 0.103802\end{array}$

$\begin{array}{llll}\text { C } & 4.227723 & -2.224544 & -0.998298\end{array}$

$\begin{array}{llll}\text { C } & 3.626575 & -3.605623 & -0.993298\end{array}$

$\begin{array}{llll}\text { C } & 4.503936 & -4.751154 & -0.591398\end{array}$

C $\quad 5.839833 \quad-4.816800 \quad-1.028298$

$\begin{array}{llll}\text { C } & 6.647696 & -5.900228 & -0.672098\end{array}$

$\begin{array}{llll}\text { C } & 6.127060 & -6.935010 & 0.108602\end{array}$

$\begin{array}{llll}\text { C } & 4.793061 & -6.895064 & 0.521102\end{array}$

$\begin{array}{llll}\text { C } & 3.980699 & -5.815835 & 0.162502\end{array}$

C $\quad 0.809041 \quad-4.602626 \quad-4.083798$

$\begin{array}{llll}\text { C } & 2.057159 & -4.071769 & -4.419198\end{array}$

$\begin{array}{llll}\text { C } & 2.970171 & -3.741500 & -3.413998\end{array}$

C $\quad 2.634564 \quad-3.930389 \quad-2.062798$

$\begin{array}{llll}\text { C } & 1.380245 & -4.473245 & -1.733998\end{array}$

$\begin{array}{llll}\text { C } & 0.472034 & -4.806614 & -2.743098\end{array}$

$\begin{array}{llll}\text { C } & 3.729408 & -2.652727 & 1.531302\end{array}$

$\begin{array}{llll}\text { C } & 3.192877 & -3.566008 & 2.439902\end{array}$

$\begin{array}{llll}\text { C } & 3.515978 & -3.519819 & 3.803402\end{array}$

C $4.364214 \quad-2.498949 \quad 4.258602$

$\begin{array}{llll}\text { C } & 4.895646 & -1.550167 & 3.373802\end{array}$

C $\quad 4.594143-1.664857 \quad 2.008402$

$\begin{array}{llll}\text { C } & 5.668987 & -0.376894 & 3.855802\end{array}$

$\begin{array}{llll}\text { C } & 5.262912 & 0.329520 & 4.996002\end{array}$

C $\quad 5.904752 \quad 1.502198 \quad 5.387302$

$\begin{array}{llll}\text { C } & 6.990571 & 2.040860 & 4.665802\end{array}$

C $\quad 7.412545 \quad 1.304646 \quad 3.539002$

$\begin{array}{llll}\text { C } & 6.767805 & 0.130268 & 3.149302\end{array}$

C $\quad 7.635717 \quad 3.365738 \quad 5.112602$

C $\quad 6.578456 \quad 4.490875 \quad 5.082202$

C $\quad 8.181711 \quad 3.213919 \quad 6.548402$

C $\quad 8.810532 \quad 3.794297 \quad 4.201902$

C $\quad 2.910944 \quad-4.504898 \quad 4.733402$

$\begin{array}{llll}\text { C } & 2.704298 & -5.835691 & 4.345602\end{array}$

C $\quad 2.074766 \quad-6.754969 \quad 5.183202$

$\begin{array}{llll}\text { C } & 1.616079 & -6.391454 & 6.466302\end{array}$

$\begin{array}{llll}\text { C } & 1.854625 & -5.056562 & 6.856002\end{array}$

$\begin{array}{llll}\text { C } & 2.482457 & -4.139884 & 6.016202\end{array}$

$\begin{array}{llll}\text { C } & 0.867146 & -7.351028 & 7.408902\end{array}$

$\begin{array}{llll}\text { C } & 0.700597 & -8.766922 & 6.808702\end{array}$

$\begin{array}{llll}\text { C } & -0.542435 & -6.790379 & 7.696202\end{array}$

$\begin{array}{llll}\text { C } & 1.647341 & -7.487055 & 8.733802\end{array}$

C $\quad-2.016959 \quad-1.704328 \quad-0.030598$

C $\quad-3.347384 \quad-2.427382 \quad-0.111098$

$\begin{array}{llll}\text { C } & -4.324670 & -2.035848 & 0.991602\end{array}$

$\begin{array}{llll}\text { C } & -3.785319 & -3.442167 & 0.985002\end{array}$

$\begin{array}{llll}\text { C } & -4.712957 & -4.547134 & 0.582502\end{array}$

$\begin{array}{llll}\text { C } & -6.049858 & -4.554188 & 1.021202\end{array}$

$\begin{array}{llll}\text { C } & -6.905394 & -5.600258 & 0.664502\end{array}$

C $\quad-6.432130 \quad-6.655875 \quad-0.118398$

C $\quad-5.098231 \quad-6.674521 \quad-0.532698$

C $\quad-4.238395 \quad-5.632751 \quad-0.173598$

C $\quad-1.013458-4.566962 \quad 4.073002$

C $\quad-2.236238 \quad-3.980620 \quad 4.409602$

C $\quad-3.134125 \quad-3.609089 \quad 3.405102$

C $\quad-2.808232 \quad-3.811900 \quad 2.053702$

C $\quad-1.579753 \quad-4.410343 \quad 1.723602$ $\begin{array}{llll}\text { C } & -0.686766 & -4.784874 & 2.731902\end{array}$

$\begin{array}{llll}\text { C } & -3.846386 & -2.482864 & -1.538598\end{array}$

$\begin{array}{llll}\text { C } & -3.350618 & -3.417682 & -2.448498\end{array}$

$\begin{array}{llll}\text { C } & -3.671616 & -3.355570 & -3.811798\end{array}$

$\begin{array}{llll}\text { C } & -4.474279 & -2.297743 & -4.265598\end{array}$

C $\quad-4.963246-1.327626 \quad-3.379398$

$\begin{array}{llll}\text { C } & -4.666850 & -1.457236 & -2.014198\end{array}$

$\begin{array}{llll}\text { C } & -5.684404 & -0.120801 & -3.859698\end{array}$

C $\quad-6.759085 \quad 0.433736 \quad-3.151698$

$\begin{array}{llll}\text { C } & -7.351343 & 1.635957 & -3.539498\end{array}$

$\begin{array}{llll}\text { C } & -6.898318 & 2.353941 & -4.665998\end{array}$

$\begin{array}{llll}\text { C } & -5.838139 & 1.768405 & -5.389198\end{array}$

$\begin{array}{llll}\text { C } & -5.248480 & 0.568084 & -4.999598\end{array}$

$\begin{array}{llll}\text { C } & -7.484471 & 3.706762 & -5.110698\end{array}$

$\begin{array}{llll}\text { C } & -8.638055 & 4.186002 & -4.198298\end{array}$

$\begin{array}{llll}\text { C } & -8.037976 & 3.580881 & -6.546098\end{array}$

$\begin{array}{llll}\text { C } & -6.378134 & 4.783623 & -5.080098\end{array}$

$\begin{array}{llll}\text { C } & -2.665739 & -4.017805 & -6.025198\end{array}$

$\begin{array}{llll}\text { C } & -3.110651 & -4.365190 & -4.743098\end{array}$

$\begin{array}{llll}\text { C } & -2.963797 & -5.704395 & -4.357098\end{array}$

$\begin{array}{llll}\text { C } & -2.375430 & -6.649715 & -5.195698\end{array}$

$\begin{array}{llll}\text { C } & -1.900518 & -6.305232 & -6.478098\end{array}$

$\begin{array}{llll}\text { C } & -2.079072 & -4.960426 & -6.865998\end{array}$

$\begin{array}{llll}\text { C } & -1.194852 & -7.295956 & -7.421798\end{array}$

$\begin{array}{llll}\text { C } & -1.091902 & -8.718760 & -6.823598\end{array}$

$\begin{array}{llll}\text { C } & 0.238465 & -6.798506 & -7.707898\end{array}$

$\begin{array}{llll}\text { C } & -1.979756 & -7.395029 & -8.747198\end{array}$

$\begin{array}{llll}\text { C } & -1.939238 & 1.787770 & 0.017102\end{array}$

$\begin{array}{llll}\text { C } & -3.236111 & 2.569914 & 0.091602\end{array}$

$\begin{array}{llll}\text { C } & -4.225123 & 2.222349 & -1.015298\end{array}$

C $\quad-3.623375 \quad 3.603128 \quad-1.006398$

$\begin{array}{llll}\text { C } & -4.502335 & 4.748658 & -0.607998\end{array}$

$\begin{array}{llll}\text { C } & -3.982799 & 5.812340 & 0.149702\end{array}$

$\begin{array}{llll}\text { C } & -4.796561 & 6.891669 & 0.505002\end{array}$

$\begin{array}{llll}\text { C } & -6.128260 & 6.932515 & 0.085302\end{array}$

$\begin{array}{llll}\text { C } & -6.645096 & 5.898833 & -0.699198\end{array}$

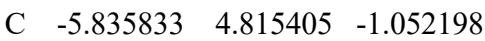

$\begin{array}{llll}\text { C } & -0.791340 & 4.601930 & -4.083298\end{array}$

$\begin{array}{llll}\text { C } & -0.461033 & 4.806318 & -2.740898\end{array}$

$\begin{array}{llll}\text { C } & -1.373845 & 4.472150 & -1.736098\end{array}$

C $\quad-2.626264 \quad 3.928493 \quad-2.071098$

$\begin{array}{llll}\text { C } & -2.955170 & 3.739405 & -3.423798\end{array}$

$\begin{array}{llll}\text { C } & -2.037459 & 4.069973 & -4.424598\end{array}$

$\begin{array}{llll}\text { C } & -3.738508 & 2.647932 & 1.516902\end{array}$

$\begin{array}{llll}\text { C } & -3.206277 & 3.560213 & 2.428902\end{array}$

$\begin{array}{llll}\text { C } & -3.536078 & 3.512725 & 3.790802\end{array}$

$\begin{array}{llll}\text { C } & -4.386514 & 2.491454 & 4.240902\end{array}$

$\begin{array}{llll}\text { C } & -4.913746 & 1.543573 & 3.352602\end{array}$

$\begin{array}{llll}\text { C } & -4.605642 & 1.659562 & 1.988802\end{array}$

$\begin{array}{llll}\text { C } & -5.689587 & 0.369899 & 3.829702\end{array}$

$\begin{array}{llll}\text { C } & -6.785005 & -0.136363 & 3.117402\end{array}$

$\begin{array}{llll}\text { C } & -7.431745 & -1.310940 & 3.502802\end{array}$

$\begin{array}{llll}\text { C } & -7.015471 & -2.048255 & 4.631002\end{array}$

C $\quad-5.933152 \quad-1.510492 \quad 5.358302$

$\begin{array}{llll}\text { C } & -5.289212 & -0.337614 & 4.971202\end{array}$

$\begin{array}{llll}\text { C } & -7.663117 & -3.373532 & 5.073502\end{array}$

$\begin{array}{llll}\text { C } & -8.216011 & -3.222913 & 6.506702\end{array}$

$\begin{array}{llll}\text { C } & -8.833531 & -3.800892 & 4.156702\end{array}$

$\begin{array}{llll}\text { C } & -6.605956 & -4.498769 & 5.047202\end{array}$

$\begin{array}{llll}\text { C } & -2.935444 & 4.496904 & 4.724602\end{array}$

$\begin{array}{llll}\text { C } & -2.727298 & 5.828197 & 4.339102\end{array}$

$\begin{array}{llll}\text { C } & -2.101766 & 6.746675 & 5.180502\end{array}$ 


\begin{tabular}{|c|c|c|c|}
\hline $\mathrm{C}$ & -1.648779 & 6.381860 & 6.465302 \\
\hline $\mathrm{C}$ & -1.888925 & 5.046568 & 6.852502 \\
\hline $\mathrm{C}$ & -2.512757 & 4.130589 & 6.009002 \\
\hline $\mathrm{C}$ & -0.904246 & 7.340434 & 7.412202 \\
\hline $\mathrm{C}$ & -1.690341 & 7.474961 & 8.733802 \\
\hline C & -0.735097 & 8.757028 & 6.814302 \\
\hline $\mathrm{C}$ & 0.504135 & 6.779685 & 7.705302 \\
\hline $\mathrm{C}$ & 2.014559 & 1.699133 & -0.019998 \\
\hline $\mathrm{C}$ & 3.345484 & 2.421687 & -0.093598 \\
\hline $\mathrm{C}$ & 4.317070 & 2.030153 & 1.014202 \\
\hline $\mathrm{C}$ & 3.777919 & 3.436472 & 1.004902 \\
\hline $\mathrm{C}$ & 4.707557 & 4.541339 & 0.607102 \\
\hline $\mathrm{C}$ & 6.042058 & 4.548793 & 1.053202 \\
\hline $\mathrm{C}$ & 6.899394 & 5.594763 & 0.700702 \\
\hline C & 6.430330 & 6.649980 & -0.085298 \\
\hline $\mathrm{C}$ & 5.098731 & 6.668226 & -0.506998 \\
\hline $\mathrm{C}$ & 4.237095 & 5.626556 & -0.152198 \\
\hline $\mathrm{C}$ & 0.990558 & 4.561668 & 4.078702 \\
\hline $\mathrm{C}$ & 2.211338 & 3.974926 & 4.421502 \\
\hline $\mathrm{C}$ & 3.114125 & 3.603095 & 3.421702 \\
\hline $\mathrm{C}$ & 2.795332 & 3.806206 & 2.068602 \\
\hline $\mathrm{C}$ & 1.568753 & 4.405148 & 1.732202 \\
\hline $\mathrm{C}$ & 0.670866 & 4.779979 & 2.736002 \\
\hline $\mathrm{C}$ & 3.852186 & 2.477769 & -1.518298 \\
\hline $\mathrm{C}$ & 3.359818 & 3.411986 & -2.430598 \\
\hline $\mathrm{C}$ & 3.690816 & 3.353075 & -3.791798 \\
\hline $\mathrm{C}$ & 4.499380 & 2.297747 & -4.240998 \\
\hline $\mathrm{C}$ & 4.983346 & 1.326930 & -3.352898 \\
\hline $\mathrm{C}$ & 4.677651 & 1.454240 & -1.989498 \\
\hline $\mathrm{C}$ & 5.708204 & 0.121205 & -3.830098 \\
\hline $\mathrm{C}$ & 6.778585 & -0.434132 & -3.116198 \\
\hline $\mathrm{C}$ & 7.373144 & -1.635953 & -3.501598 \\
\hline $\mathrm{C}$ & 6.926719 & -2.352837 & -4.631498 \\
\hline $\mathrm{C}$ & 5.871039 & -1.766501 & -5.360498 \\
\hline $\mathrm{C}$ & 5.279181 & -0.566580 & -4.973298 \\
\hline $\mathrm{C}$ & 7.515272 & -3.705458 & -5.073898 \\
\hline $\mathrm{C}$ & 8.662855 & -4.185998 & -4.154698 \\
\hline $\mathrm{C}$ & 8.077876 & -3.578277 & -6.505598 \\
\hline $\mathrm{C}$ & 6.408435 & -4.782020 & -5.051498 \\
\hline $\mathrm{C}$ & 2.966297 & 5.698300 & -4.331998 \\
\hline $\mathrm{C}$ & 3.134451 & 4.363494 & -4.725098 \\
\hline $\mathrm{C}$ & 2.714739 & 4.021208 & -6.017098 \\
\hline $\mathrm{C}$ & 2.131072 & 4.956929 & -6.870498 \\
\hline $\mathrm{C}$ & 1.931118 & 6.295636 & -6.474098 \\
\hline $\mathrm{C}$ & 2.380330 & 6.633720 & -5.180198 \\
\hline $\mathrm{C}$ & 1.241855 & 7.353459 & -7.354898 \\
\hline $\mathrm{C}$ & -0.025827 & 7.868203 & -6.639398 \\
\hline $\mathrm{C}$ & 0.814635 & 6.795574 & -8.732898 \\
\hline $\mathrm{C}$ & 2.209096 & 8.531226 & -7.599898 \\
\hline $\mathrm{H}$ & 3.892248 & -1.518932 & -1.749698 \\
\hline $\mathrm{H}$ & 5.261228 & -2.091680 & -0.692198 \\
\hline $\mathrm{H}$ & 6.256960 & -4.033214 & -1.647198 \\
\hline $\mathrm{H}$ & 7.676594 & -5.939663 & -1.005498 \\
\hline $\mathrm{H}$ & 6.753631 & -7.773431 & 0.383602 \\
\hline $\mathrm{H}$ & 4.384933 & -7.705449 & 1.111302 \\
\hline $\mathrm{H}$ & 2.942199 & -5.819699 & 0.462402 \\
\hline $\mathrm{H}$ & 0.107032 & -4.861001 & -4.863298 \\
\hline $\mathrm{H}$ & 2.319364 & -3.920778 & -5.458498 \\
\hline $\mathrm{H}$ & 3.936985 & -3.338234 & -3.686398 \\
\hline $\mathrm{H}$ & 1.106040 & -4.635036 & -0.698898 \\
\hline $\mathrm{H}$ & -0.489281 & -5.230181 & -2.485798 \\
\hline $\mathrm{H}$ & 2.449352 & -4.277782 & 2.101902 \\
\hline
\end{tabular}

H $\quad 4.626615 \quad-2.450758 \quad 5.311702$

H $\quad 4.969768 \quad-0.914170 \quad 1.320502$

H $\quad 4.401900 \quad-0.016050 \quad 5.561102$

$\begin{array}{llll}\mathrm{H} & 5.510169 & 2.001712 & 6.268502\end{array}$

$\mathrm{H} \quad 8.250456 \quad 1.606917 \quad 2.917702$

H $\quad 7.134086 \quad-0.404845 \quad 2.277002$

H $\quad 6.156759 \quad 4.600289 \quad 4.060702$

$\begin{array}{llll}\mathrm{H} & 7.029589 & 5.462959 & 5.377302\end{array}$

H $\quad 5.742349 \quad 4.284004 \quad 5.782602$

H $\quad 7.367204 \quad 3.007747 \quad 7.274002$

H $\quad 8.692444 \quad 4.145301 \quad 6.876402$

H $\quad 8.913083 \quad 2.378294 \quad 6.597602$

$\mathrm{H} \quad 9.618205 \quad 3.030969 \quad 4.213302$

H $\quad 9.250365 \quad 4.753582 \quad 4.551502$

H $\quad 8.467337 \quad 3.946109 \quad 3.156402$

H $\quad 3.049787 \quad-6.166503 \quad 3.370002$

H $\quad \begin{array}{llll}1.964732 & -7.756666 & 4.778402\end{array}$

$\mathrm{H} \quad 1.533038 \quad-4.687051 \quad 7.826502$

$\begin{array}{llll}\mathrm{H} & 2.603192 & -3.113588 & 6.351702\end{array}$

H $\quad 0.113198 \quad-8.730302 \quad 5.865602$

H $\quad 0.158374 \quad-9.432903 \quad 7.514802$

H $\quad 1.690781 \quad-9.229056 \quad 6.605202$

H $\quad-0.492701 \quad-5.811181 \quad 8.216902$

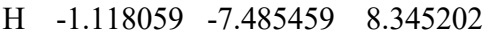

H $\quad-1.104730 \quad-6.651859 \quad 6.747202$

H $\quad 2.679928 \quad-7.849290 \quad 8.538902$

H $\quad \begin{array}{llll}1.145716 & -8.209437 & 9.413802\end{array}$

$\mathrm{H} \quad 1.713274 \quad-6.516757 \quad 9.269102$

$\mathrm{H} \quad-3.958146 \quad-1.346461 \quad 1.743502$

$\mathrm{H} \quad-5.351364 \quad-1.857112 \quad 0.686102$

H $\quad-6.431030 \quad-3.753875 \quad 1.641802$

$\mathrm{H} \quad-7.934694 \quad-5.594523 \quad 0.999302$

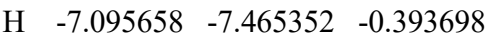

H $\quad-4.727160 \quad-7.501234 \quad-1.124498$

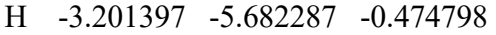

H $\quad-0.323268 \quad-4.857086 \quad 4.851902$

H $\quad-2.490732 \quad-3.818711 \quad 5.449102$

$\mathrm{H} \quad-4.081509 \quad-3.162756 \quad 3.678502$

H $\quad-1.313759 \quad-4.583452 \quad 0.688202$

$\begin{array}{llll}\mathrm{H} & 0.254418 & -5.251006 & 2.473702\end{array}$

$\mathrm{H} \quad-2.639044 \quad-4.161806 \quad-2.111598$

H $\quad-4.734477 \quad-2.236734 \quad-5.318598$

H $\quad-5.008824 \quad-0.691624 \quad-1.325298$

$\mathrm{H} \quad-7.147903 \quad-0.085550 \quad-2.279498$

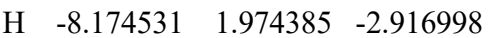

$\mathrm{H} \quad-5.422622 \quad 2.250890 \quad-6.270298$

H $\quad-4.404093 \quad 0.185255 \quad-5.565898$

H $\quad-9.478980 \quad 3.459231 \quad-4.209698$

H $\quad-9.035321 \quad 5.164215 \quad-4.546398$

H $\quad-8.287450 \quad 4.321189 \quad-3.152998$

H $\quad-7.234084 \quad 3.339653 \quad-7.272698$

H $\quad-8.507143 \quad 4.534497 \quad-6.872498$

$\mathrm{H} \quad-8.805804 \quad 2.778707 \quad-6.595398$

H $\quad-5.950931 \quad 4.873008 \quad-4.058898$

$\mathrm{H} \quad-6.785900 \quad 5.775237 \quad-5.373498$

$\mathrm{H} \quad-5.552743 \quad 4.540795 \quad-5.781698$

H $\quad-2.740403 \quad-2.986703 \quad-6.359198$

H $\quad-3.324008 \quad-6.020782 \quad-3.382098$

H $\quad-2.310365 \quad-7.655918 \quad-4.792298$

H $\quad-1.740759 \quad-4.604237 \quad-7.835898$

H $\quad-0.503901 \quad-8.709780 \quad-5.880298$

H $\quad-0.579726 \quad-9.407278 \quad-7.530498$

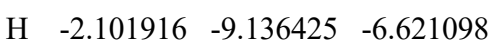

H $\quad 0.232799 \quad-5.817306 \quad-8.227198$

H $\quad 0.782740 \quad-7.517625 \quad-8.357798$

H $\quad 0.806169-6.686726 \quad-6.758598$

H $\quad-3.027667 \quad-7.711093 \quad-8.553098$

$\mathrm{H} \quad-1.510782 \quad-8.138245 \quad-9.428098$

H $\quad-2.002022-6.422028 \quad-9.281098$

$\begin{array}{llll}\mathrm{H} & -3.886547 & 1.517237 & -1.765798\end{array}$

$\begin{array}{llll}\mathrm{H} & -5.260127 & 2.089785 & -0.714198\end{array}$

H $\quad-2.945898 \quad 5.815604 \quad 0.455202$

$\begin{array}{llll}\mathrm{H} & -4.391333 & 7.701254 & 1.098102\end{array}$

H $\quad-6.756031 \quad 7.771036 \quad 0.357702$

$\mathrm{H} \quad-7.672194 \quad 5.938968-1.038198$

$\mathrm{H} \quad-6.249860 \quad 4.032519 \quad-1.674098$

H $\quad-0.085632 \quad 4.860305 \quad-4.859598$

$\begin{array}{llll}\mathrm{H} & 0.498581 & 5.230685 & -2.478998\end{array}$

H $\quad-1.104839 \quad 4.634041 \quad-0.699698$

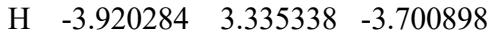

$\begin{array}{llll}\mathrm{H} & -2.294564 & 3.918382 & -5.464998\end{array}$

$\mathrm{H} \quad-2.461152 \quad 4.272288 \quad 2.095302$

$\mathrm{H} \quad-4.654015 \quad 2.442264 \quad 5.292702$

$\begin{array}{llll}\mathrm{H} & -4.977968 & 0.909675 & 1.298402\end{array}$

H $\quad-7.146786 \quad 0.399650 \quad 2.243802$

$\begin{array}{llll}\mathrm{H} & -8.266756 & -1.612511 & 2.877102\end{array}$

H $\quad-5.542969 \quad-2.011006 \quad 6.241002$

H $\quad-4.430800 \quad 0.007256 \quad 5.540902$

$\begin{array}{llll}\mathrm{H} & -7.405004 & -3.017641 & 7.236402\end{array}$

$\begin{array}{llll}\mathrm{H} & -8.728544 & -4.154395 & 6.831302\end{array}$

$\mathrm{H} \quad-8.947483 \quad-2.387188 \quad 6.553102$

H $\quad-9.641205 \quad-3.037364 \quad 4.164802$

$\begin{array}{llll}\mathrm{H} & -9.275365 & -4.760476 & 4.503302\end{array}$

$\mathrm{H} \quad-8.485337 \quad-3.951904 \quad 3.112702$

H $\quad-6.179359-4.607384 \quad 4.027502$

H $\quad-7.058689 \quad-5.471053 \quad 5.339102$

H $\quad-5.773149 \quad-4.292798 \quad 5.751802$

$\begin{array}{llll}\mathrm{H} & -3.068287 & 6.159909 & 3.362302\end{array}$

$\begin{array}{llll}\mathrm{H} & -1.990032 & 7.748871 & 4.777402\end{array}$

H $\quad-1.571638 \quad 4.675957 \quad 7.824102$

$\begin{array}{llll}\mathrm{H} & -2.634892 & 3.103994 & 6.342802\end{array}$

$\begin{array}{llll}\mathrm{H} & -2.722128 & 7.837397 & 8.534602\end{array}$

$\mathrm{H} \quad-1.191916 \quad 8.196744 \quad 9.416802$

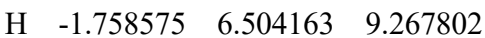

H $\quad-0.143498 \quad 8.721507 \quad 5.873902$

H $\quad-0.196174 \quad 9.422309 \quad 7.523502$

$\begin{array}{llll}\mathrm{H} & -1.724481 & 9.219262 & 6.606902\end{array}$

$\begin{array}{llll}\mathrm{H} & 0.452201 & 5.799987 & 8.224802\end{array}$

H $\quad 1.076759 \quad 7.474165 \quad 8.357602$

$\begin{array}{llll}\mathrm{H} & 1.070730 & 6.642165 & 6.758702\end{array}$

$\begin{array}{llll}\mathrm{H} & 3.946647 & 1.340666 & 1.764102\end{array}$

$\mathrm{H} \quad 5.345364 \quad 1.851517 \quad 0.714002$

$\begin{array}{llll}\mathrm{H} & 6.419830 & 3.748680 & 1.676102\end{array}$

$\begin{array}{llll}\mathrm{H} & 7.926794 & 5.589328 & 1.041102\end{array}$

H $\quad 7.095258 \quad 7.459457 \quad-0.357398$

H $\quad 4.730860 \quad 7.494639-1.101298$

$\begin{array}{lllll}\mathrm{H} & 3.201797 & 5.675692 & -0.459298\end{array}$

H $\quad 0.296468 \quad 4.852092 \quad 4.854102$

$\begin{array}{llll}\mathrm{H} & 2.460332 & 3.812717 & 5.462302\end{array}$

H $\quad 4.060109 \quad 3.156362 \quad 3.699802$

$\begin{array}{llll}\mathrm{H} & 1.308259 & 4.578557 & 0.695502\end{array}$

$\begin{array}{llll}\mathrm{H} & -0.268718 & 5.246612 & 2.473102\end{array}$

H $\quad 2.643744 \quad 4.153311 \quad-2.097198$

H $\quad 4.768178 \quad 2.238837 \quad-5.291898$ 

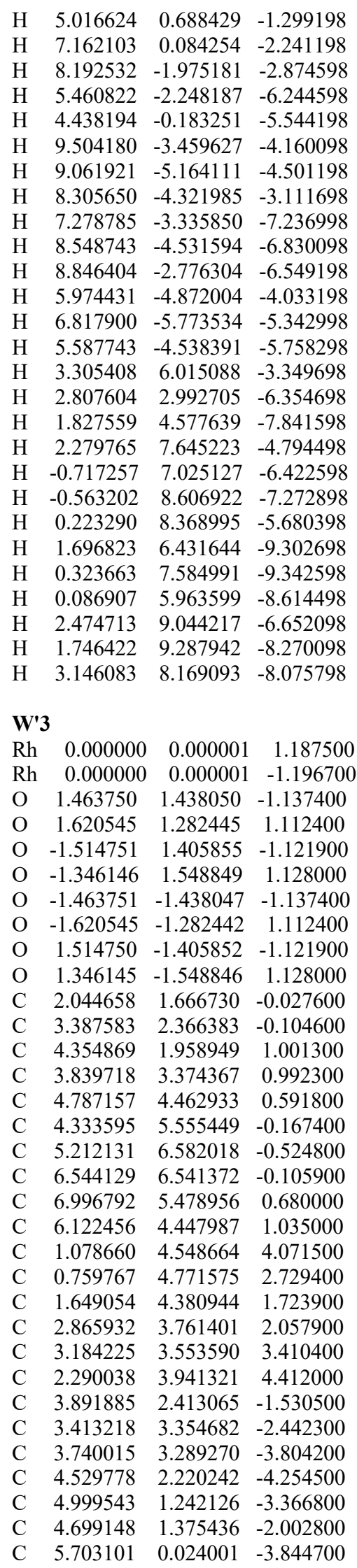

\begin{tabular}{|c|c|c|c|c|c|c|c|}
\hline & 5.260577 & -0.656983 & -4.986700 & $\mathrm{C}$ & -3.387583 & -2.366380 & -0.104600 \\
\hline & 5.831434 & -1.867003 & -5.374200 & $\mathrm{C}$ & -4.354869 & -1.958946 & 1.001300 \\
\hline & 6.878313 & -2.470640 & -4.646600 & $\mathrm{C}$ & -3.839719 & -3.374364 & 0.992300 \\
\hline & 7.338738 & -1.760756 & -3.518000 & $\mathrm{C}$ & -4.787157 & -4.462931 & 0.591800 \\
\hline & 6.765281 & -0.548836 & -3.132300 & $\mathrm{C}$ & -4.333595 & -5.555446 & -0.167400 \\
\hline & 7.443165 & -3.833260 & -5.089100 & $\mathrm{C}$ & -5.212131 & -6.582016 & -0.524800 \\
\hline & 8.583948 & -4.332700 & -4.171500 & $\mathrm{C}$ & -6.544130 & -6.541369 & -0.105900 \\
\hline & 6.318228 & -4.890920 & -5.064300 & $\mathrm{C}$ & -6.996792 & -5.478953 & 9900 \\
\hline & 8.005569 & -3.716480 & -6.521800 & $\mathrm{C}$ & -6.122456 & -4.447984 & 35000 \\
\hline & 3.198451 & 4.308189 & -4.737000 & $\mathrm{C}$ & -1.078760 & -4.548661 & 71600 \\
\hline & 3.054098 & 5.645994 & -4.344600 & $\mathrm{C}$ & -0.759768 & -4.771572 & 9400 \\
\hline & 2.481931 & 6.590614 & & $\mathrm{C}$ & 054 & & 900 \\
\hline & 2.023620 & 6.259230 & -6.484400 & $\mathrm{C}$ & 5932 & -3.761398 & 7900 \\
\hline & 2.199672 & 4.917024 & -6.880200 & $\mathrm{C}$ & -3.184225 & -3.553587 & 9400 \\
\hline & 2.769539 & 3.972204 & & $\mathrm{C}$ & -2.290039 & -3.941318 & \\
\hline & 1.350257 & 7.327954 & & $\mathrm{C}$ & -3.891885 & -2.413062 & 500 \\
\hline C & 0.093 & 4998 & & $\mathrm{C}$ & 218 & 54679 & \\
\hline & 0.909838 & 6.776269 & & $\mathrm{C}$ & 0016 & -3.289267 & 200 \\
\hline & 2.336798 & 8.488819 & -7.612900 & $\mathrm{C}$ & 29778 & -2.220240 & 4500 \\
\hline & -1.907036 & 1.822768 & & $\mathrm{C}$ & -4.999544 & -1.242123 & 800 \\
\hline & -3.190408 & 2.626613 & & $\mathrm{C}$ & 148 & 75434 & 800 \\
\hline $\mathrm{C}$ & -4.18 & $2.2 \mathrm{~S}$ & & $\mathrm{C}$ & 101 & 24098 & 700 \\
\hline C & -3.563171 & 3.665827 & & $\mathrm{C}$ & & 56986 & 700 \\
\hline 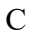 & -4.421431 & 4.826157 & -0.6 & $\mathrm{C}$ & & 1.867006 & 200 \\
\hline & -3.881294 & 5.881538 & & $\mathrm{C}$ & -6. & 2.470643 & -4 \\
\hline & -4.675555 & 6.97 & & $\mathrm{C}$ & & 1.760759 & 000 \\
\hline $\mathrm{C}$ & -6.00 & 7.0 & & $\mathrm{C}$ & & 48739 & \\
\hline $\mathrm{C}$ & -6.544889 & 6.0 & & $\mathrm{C}$ & -7. & 33263 & 200 \\
\hline C & -5.755128 & 4.915003 & & $\mathrm{C}$ & -8.5 & 4.332603 & 500 \\
\hline & -0.723238 & 4.614727 & & $\mathrm{C}$ & & 4.890923 & \\
\hline $\mathrm{C}$ & -0.38 & 4.8 & & $\mathrm{C}$ & & 16482 & \\
\hline $\mathrm{C}$ & -1.30 & & & $\mathrm{C}$ & & 08186 & 000 \\
\hline $\mathrm{C}$ & -2.563661 & 3.973691 & & $\mathrm{C}$ & 098 & 45991 & 600 \\
\hline $\mathrm{C}$ & -2.899867 & 3.790403 & & $\mathrm{C}$ & -2. & 90611 & \\
\hline & -1.979556 & 4.10 & & $\mathrm{C}$ & -2.0 & 59228 & 400 \\
\hline E & -3.687 & 2.71 & & $\mathrm{C}$ & & 17021 & 200 \\
\hline $\mathrm{C}$ & -3.13 & 3.6 & & $\mathrm{C}$ & -2 & 72201 & 500 \\
\hline $\mathrm{C}$ & -3.465 & 3.576423 & & $\mathrm{C}$ & -1. & 27951 & 1300 \\
\hline $\mathrm{C}$ & -4.331610 & 2.569753 & & $\mathrm{C}$ & & 64995 & 5000 \\
\hline & -4.87 & & & $\mathrm{C}$ & & 76267 & 700 \\
\hline $\mathrm{C}$ & -4.57 & 1.7 & & $\mathrm{C}$ & -2 & 8817 & 900 \\
\hline $\mathrm{C}$ & -5.67 & 0.47 & & $\mathrm{C}$ & & 22766 & 200 \\
\hline $\mathrm{C}$ & -6.777101 & -0.017661 & & $\mathrm{C}$ & & -2.626611 & \\
\hline $\mathrm{C}$ & -7.442642 & -1.180937 & & $\mathrm{C}$ & & -2.295346 & 800 \\
\hline & -7.03 & & & $\mathrm{C}$ & & & \\
\hline $\mathrm{C}$ & -5.94 & -1.4 & & $\mathrm{C}$ & & 26154 & \\
\hline $\mathrm{C}$ & -5.279909 & -0.2 & & $\mathrm{C}$ & & 535 & 600 \\
\hline $\mathrm{C}$ & -7.704714 & -3.238628 & & $\mathrm{C}$ & & -6.974563 & 100 \\
\hline & -8.251208 & -3.078009 & & $\mathrm{C}$ & & -7.037509 & \\
\hline $\mathrm{C}$ & -6.66 & & & $\mathrm{C}$ & & 2128 & \\
\hline $\mathrm{C}$ & -8.884628 & -3.646487 & & $\mathrm{C}$ & & -4.915001 & \\
\hline $\mathrm{C}$ & -2.426453 & 4.178387 & & $\mathrm{C}$ & & -4.614724 & -4.087300 \\
\hline $\mathrm{C}$ & -2.845940 & 4.550901 & 4.726500 & $\mathrm{C}$ & 5431 & -4.813412 & 5900 \\
\hline & -2.616494 & 5.878293 & & $\mathrm{C}$ & & -4.495444 & \\
\hline & -1.973562 & 6.786671 & & $\mathrm{C}$ & & -3.973689 & -2.0 \\
\hline $\mathrm{C}$ & -1.523775 & 6.415155 & & $\mathrm{C}$ & 2.899867 & -3.790400 & -3.421300 \\
\hline & -1.785322 & 5.084264 & 6.851600 & $\mathrm{C}$ & 1.979556 & -4.104868 & -4.424900 \\
\hline & -0.761042 & 7.361628 & 7.407500 & $\mathrm{C}$ & 3.687905 & -2.713828 & 1.521900 \\
\hline & 0.638438 & 6.777479 & & $\mathrm{C}$ & & -3.617609 & \\
\hline & -1.541537 & 7.510156 & 8.730900 & $\mathrm{C}$ & 3.465274 & -3.576420 & 3.794800 \\
\hline & -0.569592 & 8.774821 & 6.808300 & $\mathrm{C}$ & 4.331610 & -2.569751 & 4.247700 \\
\hline & -2.044659 & -1.666727 & -0.027600 & $\mathrm{C}$ & 4.876943 & -1.630470 & 3.361300 \\
\hline
\end{tabular}




\begin{tabular}{|c|c|c|c|}
\hline $\mathrm{C}$ & 4.570339 & -1.740459 & 1.996600 \\
\hline & 5.671283 & -0.470298 & 3.841000 \\
\hline $\mathrm{C}$ & 6.777100 & 0.017664 & 3.131800 \\
\hline $\mathrm{C}$ & 7.442641 & 1.180940 & 3.519500 \\
\hline $\mathrm{C}$ & 7.035867 & 1.924754 & 4.646800 \\
\hline $\mathrm{C}$ & 5.942549 & 1.405193 & 5.370900 \\
\hline $\mathrm{C}$ & 5.279808 & 0.243516 & 4.981600 \\
\hline $\mathrm{C}$ & 7.704714 & 3.238631 & 5.091600 \\
\hline $\mathrm{C}$ & 8.251208 & 3.078012 & 6.526200 \\
\hline $\mathrm{C}$ & 6.666854 & 4.381767 & 5.063100 \\
\hline $\mathrm{C}$ & 8.884628 & 3.646490 & 4.178100 \\
\hline $\mathrm{C}$ & 2.426453 & -4.178384 & 6.010200 \\
\hline $\mathrm{C}$ & 2.845940 & -4.550898 & 4.726600 \\
\hline $\mathrm{C}$ & 2.616393 & -5.878290 & 4.339700 \\
\hline $\mathrm{C}$ & 1.973562 & -6.786668 & 5.179000 \\
\hline $\mathrm{C}$ & 1.523775 & -6.415152 & 6.463000 \\
\hline $\mathrm{C}$ & 1.785321 & -5.084261 & 6.851600 \\
\hline $\mathrm{C}$ & 0.761041 & -7.361625 & 7.407500 \\
\hline $\mathrm{C}$ & -0.638438 & -6.777476 & 7.697600 \\
\hline $\mathrm{C}$ & 1.541536 & -7.510153 & 8.730900 \\
\hline $\mathrm{C}$ & 0.569492 & -8.774819 & 6.808300 \\
\hline $\mathrm{H}$ & 3.974345 & 1.276362 & 1.752500 \\
\hline $\mathrm{H}$ & 5.379262 & 1.762713 & 0.699000 \\
\hline $\mathrm{H}$ & 3.298697 & 5.621986 & -0.472400 \\
\hline $\mathrm{H}$ & 4.857160 & 7.414131 & -1.119000 \\
\hline $\mathrm{H}$ & 7.222157 & 7.339148 & -0.380000 \\
\hline $\mathrm{H}$ & 8.024691 & 5220 & 200 \\
\hline $\mathrm{H}$ & 6.487928 & 3.641974 & 1.657800 \\
\hline $\mathrm{H}$ & 0.391370 & 4.851388 & 4.848200 \\
\hline $\mathrm{H}$ & -0.172316 & 5.254007 & 2.468200 \\
\hline $\mathrm{H}$ & 1.389260 & 4.558253 & $0.68^{\circ}$ \\
\hline $\mathrm{H}$ & 4.122908 & 3.090957 & 6800 \\
\hline $\mathrm{H}$ & 2.538532 & 3.775512 & 5.452400 \\
\hline $\mathrm{H}$ & 2.710444 & 4.108206 & -2.107800 \\
\hline $\mathrm{H}$ & 4.795076 & 2.156133 & -5.306000 \\
\hline $\mathrm{H}$ & 5.026721 & 0.604625 & -1.312800 \\
\hline $\mathrm{H}$ & 4.425091 & -0.259854 & -5.556400 \\
\hline $\mathrm{H}$ & 5.411718 & -2.342089 & -6.257300 \\
\hline $\mathrm{H}$ & 8.153326 & -2.113285 & -2.892100 \\
\hline $\mathrm{H}$ & 7.158999 & -0.036350 & -2.258300 \\
\hline $\mathrm{H}$ & 9.437373 & -3.620730 & -4.178700 \\
\hline $\mathrm{H}$ & 8.965713 & -5.317613 & -4.518000 \\
\hline $\mathrm{H}$ & 8.226143 & -4.461987 & -3.127800 \\
\hline $\mathrm{H}$ & 5.884225 & -4.972805 & -4.045400 \\
\hline $\mathrm{H}$ & 6.710393 & -5.889334 & -5.355600 \\
\hline $\mathrm{H}$ & 5.500837 & -4.633892 & -5.770200 \\
\hline $\mathrm{H}$ & 7.209478 & -3.460852 & -7.252100 \\
\hline $\mathrm{H}$ & 8.459636 & -4.677896 & -6.846500 \\
\hline $\mathrm{H}$ & 8.787597 & -2.927707 & -6.567100 \\
\hline $\mathrm{H}$ & 3.401109 & 5.957682 & -3.363400 \\
\hline $\mathrm{H}$ & 2.399667 & 7.604017 & -4.806900 \\
\hline $\mathrm{H}$ & 1.887159 & 4.542135 & -7.850200 \\
\hline $\mathrm{H}$ & 2.844003 & 2.942002 & -6.364500 \\
\hline $\mathrm{H}$ & -0.611753 & 7.034023 & -6.426700 \\
\hline $\mathrm{H}$ & -0.432798 & 8.612317 & -7.278700 \\
\hline $\mathrm{H}$ & 0.353493 & 8.362089 & -5.688000 \\
\hline $\mathrm{H}$ & 1.784224 & 6.396939 & -9.312600 \\
\hline $\mathrm{H}$ & 0.430866 & 7.573586 & -9.349800 \\
\hline $\mathrm{H}$ & 0.168409 & 5.956996 & -8.619700 \\
\hline $\mathrm{H}$ & 2.613716 & 8.998010 & -6.666200 \\
\hline $\mathrm{H}$ & 1.885425 & 9.252835 & -8.282500 \\
\hline & 3.266285 & 8.110287 & -8.090900 \\
\hline
\end{tabular}

\begin{tabular}{|c|c|c|c|}
\hline 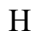 & -3.863345 & 1.584337 & -1.759800 \\
\hline $\mathrm{H}$ & -5.224324 & 2.180185 & -0.704900 \\
\hline $\mathrm{H}$ & -2.843394 & 5.867401 & 0.455500 \\
\hline $\mathrm{H}$ & -4.254727 & 7.777651 & 1.102100 \\
\hline & -6.620523 & 7.886634 & 0.370100 \\
\hline & -7.572387 & 6.069267 & -1.021100 \\
\hline $\mathrm{H}$ & -6.184555 & 4.138819 & -1.660700 \\
\hline & -0.015330 & 4.860602 & -4.865700 \\
\hline & 0.582383 & 5.220881 & -2.486900 \\
\hline & -1.026137 & 4.652637 & -0.702900 \\
\hline $\mathrm{H}$ & -3.872881 & 3.403437 & -3.695600 \\
\hline $\mathrm{H}$ & -2.242261 & 3.957780 & -5.464600 \\
\hline $\mathrm{H}$ & -2.382049 & 4.316885 & 2.096100 \\
\hline $\mathrm{H}$ & -4.597311 & 2.525663 & 5.300100 \\
\hline & -4.957065 & 0.996475 & 1.307600 \\
\hline $\mathrm{H}$ & -7.132182 & 0.524152 & 2.258900 \\
\hline $\mathrm{H}$ & -8.284352 & -1.468508 & 2.896100 \\
\hline $\mathrm{H}$ & -5.558467 & -1.911903 & 6.252700 \\
\hline П & -4.414197 & 0.086956 & 5.548800 \\
\hline & -8.968278 & -2.229984 & 6.574100 \\
\hline $\mathrm{H}$ & -7.434901 & -2.886138 & 7.253600 \\
\hline $\mathrm{H}$ & -8.778541 & -4.000590 & 6.852600 \\
\hline $\mathrm{H}$ & -6.244958 & -4.498079 & 4.042400 \\
\hline $\mathrm{H}$ & -7.135388 & -5.346048 & 5.356700 \\
\hline $\mathrm{H}$ & -5.828847 & -4.189594 & 5.765400 \\
\hline $\mathrm{H}$ & -9.679201 & -2.869359 & 4.188000 \\
\hline $\mathrm{H}$ & -9.341762 & -4.598271 & 4.526300 \\
\hline $\mathrm{H}$ & -8.541834 & -3.803799 & 3.133300 \\
\hline $\mathrm{H}$ & -2.564989 & 3.154091 & 6.344900 \\
\hline $\mathrm{H}$ & -2.954182 & 6.215105 & 3.363500 \\
\hline $\mathrm{H}$ & -1.846027 & 7.786566 & 4.775000 \\
\hline $\mathrm{H}$ & -1.472035 & 4.709053 & 7.822700 \\
\hline $\mathrm{H}$ & 0.571303 & 5.799081 & 8.217900 \\
\hline $\mathrm{H}$ & 1.224262 & 7.462658 & 8.348100 \\
\hline $\mathrm{H}$ & 1.200333 & 6.629959 & 6.749800 \\
\hline $\mathrm{H}$ & -2.567623 & 7.889692 & 8.533900 \\
\hline $\mathrm{H}$ & -1.029511 & 8.223838 & 9.412200 \\
\hline $\mathrm{H}$ & -1.624871 & 6.540958 & 9.265600 \\
\hline $\mathrm{H}$ & 0.019106 & 8.728801 & 5.866500 \\
\hline $\mathrm{H}$ & -0.017769 & 9.431402 & 7.515800 \\
\hline $\mathrm{H}$ & -1.551475 & 9.253456 & 6.602900 \\
\hline $\mathrm{H}$ & -3.974445 & -1.276359 & 1.752500 \\
\hline $\mathrm{H}$ & -5.379262 & -1.762710 & 0.699000 \\
\hline $\mathrm{H}$ & -3.298698 & -5.621983 & -0.472400 \\
\hline $\mathrm{H}$ & -4.857160 & -7.414128 & -1.119000 \\
\hline $\mathrm{H}$ & -7.222158 & -7.339145 & -0.380000 \\
\hline $\mathrm{H}$ & -8.024692 & -5.456217 & 1.018200 \\
\hline $\mathrm{H}$ & -6.487928 & -3.641971 & 1.657800 \\
\hline $\mathrm{H}$ & -0.391470 & -4.851285 & 4.848200 \\
\hline $\mathrm{H}$ & 0.172315 & -5.254005 & 2.468200 \\
\hline $\mathrm{H}$ & -1.389260 & -4.558250 & 0.687700 \\
\hline $\mathrm{H}$ & -4.122909 & -3.090954 & 3.686800 \\
\hline $\mathrm{H}$ & -2.538533 & -3.775509 & 5.452400 \\
\hline $\mathrm{H}$ & -2.710444 & -4.108203 & -2.107800 \\
\hline $\mathrm{H}$ & -4.795076 & -2.156130 & -5.306000 \\
\hline $\mathrm{H}$ & -5.026721 & -0.604622 & -1.312800 \\
\hline $\mathrm{H}$ & -4.425091 & 0.259857 & -5.556400 \\
\hline & -5.411718 & 2.342091 & -6.257300 \\
\hline $\mathrm{H}$ & -8.153326 & 2.113288 & -2.892200 \\
\hline $\mathrm{H}$ & -7.158999 & 0.036353 & -2.258300 \\
\hline$\Pi$ & -9.437373 & 3.620733 & -4.178700 \\
\hline & -8.965713 & 5.317616 & -4.518000 \\
\hline
\end{tabular}

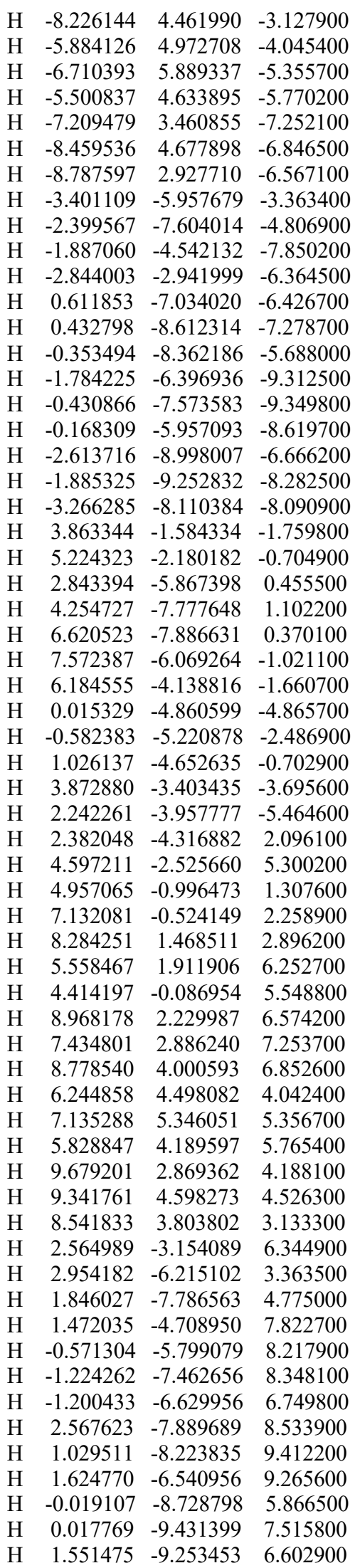




\begin{tabular}{|c|c|c|c|}
\hline & & & \\
\hline $\mathrm{Rh}$ & 0.035900 & 0.234900 & 1.116400 \\
\hline $\mathrm{Rh}$ & 0.102800 & 0.240600 & -1.268100 \\
\hline $\mathrm{O}$ & 1.276900 & 1.929500 & -1.167200 \\
\hline $\mathrm{O}$ & -1.022900 & -1.475700 & -1.240700 \\
\hline $\mathrm{O}$ & 1.234100 & 1.914700 & 1.088800 \\
\hline $\mathrm{O}$ & -1.098700 & -1.476100 & 1.014600 \\
\hline $\mathrm{O}$ & 1.807500 & -0.899500 & -1.161700 \\
\hline $\mathrm{O}$ & -1.649300 & 1.313300 & -1.248100 \\
\hline $\mathrm{O}$ & -1.698800 & 1.342300 & 1.008000 \\
\hline $\mathrm{O}$ & 1.722700 & -0.937800 & 1.094100 \\
\hline $\mathrm{O}$ & 4.926200 & -2.810800 & 2.544400 \\
\hline $\mathrm{O}$ & 1.051600 & -3.869300 & 0.300700 \\
\hline $\mathrm{O}$ & 3.382000 & 5.017200 & 2.462300 \\
\hline $\mathrm{O}$ & 4.134700 & 0.919500 & 0.508700 \\
\hline $\mathrm{O}$ & -1.817000 & 4.409600 & 0.265400 \\
\hline $\mathrm{O}$ & -5.389500 & 2.293300 & 2.251400 \\
\hline $\mathrm{O}$ & -4.036800 & -1.028800 & 0.425900 \\
\hline $\mathrm{O}$ & -2.579300 & -4.977700 & 2.276200 \\
\hline $\mathrm{N}$ & 3.155600 & -3.227700 & 1.083300 \\
\hline $\mathrm{N}$ & 3.651500 & 3.111200 & 1.146100 \\
\hline $\mathrm{N}$ & -3.703200 & 3.191900 & 0.910100 \\
\hline $\mathrm{N}$ & -3.200700 & -3.128000 & 0.997400 \\
\hline $\mathrm{C}$ & -2.204100 & 1.563900 & -0.140800 \\
\hline $\mathrm{C}$ & 2.226800 & -1.266700 & -0.029200 \\
\hline $\mathrm{C}$ & 1.653300 & 2.342200 & -0.035000 \\
\hline $\mathrm{C}$ & -1.380500 & -1.958300 & -0.130200 \\
\hline $\mathrm{C}$ & 3.477400 & -2.162600 & 0.126900 \\
\hline $\mathrm{C}$ & 3.893300 & -3.393100 & 2.273900 \\
\hline $\mathrm{C}$ & 1.929900 & -3.920400 & 1.140600 \\
\hline $\mathrm{C}$ & 3.134200 & -4.380800 & 3.091000 \\
\hline $\mathrm{C}$ & 1.946300 & -4.679200 & 2.420700 \\
\hline $\mathrm{C}$ & 3.428200 & -4.937600 & 4.327200 \\
\hline $\mathrm{C}$ & 0.993400 & -5.530000 & 2.963700 \\
\hline $\mathrm{C}$ & 2.482900 & -5.813100 & 4.878800 \\
\hline $\mathrm{C}$ & 1.284400 & -6.099200 & 4.211400 \\
\hline $\mathrm{C}$ & 2.687500 & 3.491800 & 0.108000 \\
\hline $\mathrm{C}$ & 3.850600 & 3.909700 & 2.295500 \\
\hline $\mathrm{C}$ & 4.221500 & 1.835200 & 1.304500 \\
\hline $\mathrm{C}$ & 4.710800 & 3.10 & 3.206500 \\
\hline $\mathrm{C}$ & 4.916500 & 1.854200 & 2.620000 \\
\hline $\mathrm{C}$ & 5.239200 & 3.417200 & 4.450500 \\
\hline $\mathrm{C}$ & 5.643800 & 0.857800 & 3.256700 \\
\hline $\mathrm{C}$ & 5.988400 & 2.425300 & 5.098000 \\
\hline $\mathrm{C}$ & 6.182500 & 1.166500 & 4.513500 \\
\hline $\mathrm{C}$ & -3.654200 & 2.097700 & -0.061800 \\
\hline $\mathrm{C}$ & -2.744400 & 4.220000 & 1.025500 \\
\hline $\mathrm{C}$ & -4.549500 & 3.146200 & 2.034900 \\
\hline $\mathrm{C}$ & -3.105000 & 4.970600 & 2.262100 \\
\hline $\mathrm{C}$ & -4.188100 & 4.329700 & 2.865100 \\
\hline $\mathrm{C}$ & -2.528600 & 6.103000 & 2.818700 \\
\hline $\mathrm{C}$ & -4.741300 & 4.797400 & 4.048500 \\
\hline $\mathrm{C}$ & -3.082000 & 6.586200 & 4.012100 \\
\hline $\mathrm{C}$ & -4.171100 & 5.944400 & 4.616700 \\
\hline $\mathrm{C}$ & -2.179700 & -3.280800 & -0.043000 \\
\hline $\mathrm{C}$ & -3.977700 & -1.970500 & 1.192500 \\
\hline $\mathrm{C}$ & -3.246000 & -3.971800 & 2.126100 \\
\hline $\mathrm{C}$ & -4.660200 & -2.147100 & 2.501200 \\
\hline $\mathrm{C}$ & -4.238300 & -3.358000 & 3.053900 \\
\hline $\mathrm{C}$ & -5.548500 & -1.308500 & 3.159900 \\
\hline $\mathrm{C}$ & -4.704000 & -3.790700 & 4.287000 \\
\hline $\mathrm{C}$ & -6.025700 & -1.740800 & 4.405200 \\
\hline
\end{tabular}

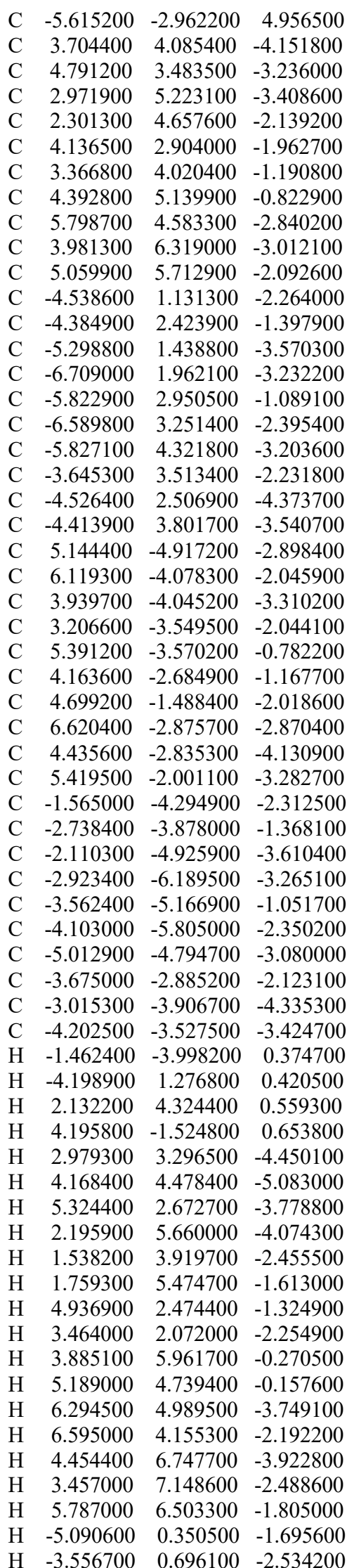

H $\quad-5.384800 \quad 0.510100 \quad-4.175800$

$\mathrm{H} \quad-7.275600 \quad 1.191000 \quad-2.665000$

H $\quad-7.273300 \quad 2.166600 \quad-4.168600$

$\mathrm{H} \quad-5.778200 \quad 3.883100 \quad-0.484900$

$\mathrm{H} \quad-6.388800 \quad 2.199300 \quad-0.494100$

$\mathrm{H} \quad-7.606700 \quad 3.628000 \quad-2.150000$

$\mathrm{H} \quad-6.379300 \quad 4.556400 \quad-4.140100$

$\mathrm{H} \quad-5.756400 \quad 5.263700 \quad-2.616200$

$\mathrm{H} \quad-2.613300 \quad 3.197700 \quad-2.487000$

$\mathrm{H} \quad-3.568700 \quad 4.457400 \quad-1.651900$

$\mathrm{H} \quad-3.511600 \quad 2.130300 \quad-4.630000$

$\mathrm{H} \quad-5.053700 \quad 2.716000 \quad-5.330300$

$\mathrm{H} \quad-3.868000 \quad 4.572100 \quad-4.127700$

$\mathrm{H} \quad 4.795300 \quad-5.800200 \quad-2.319100$

$\mathrm{H} \quad 5.663900 \quad-5.299900 \quad-3.804300$

H $\quad 6.985300 \quad-4.708100 \quad-1.747000$

H $\quad 3.241100 \quad-4.649800 \quad-3.928800$

$\mathrm{H} \quad 2.852600 \quad-4.433400 \quad-1.472600$

H $\quad 2.309200 \quad-2.976200 \quad-2.354000$

$\mathrm{H} \quad 6.103600 \quad-2.989400 \quad-0.154800$

$\mathrm{H} \quad 5.067900 \quad-4.450100 \quad-0.183700$

H $\quad 3.882300 \quad-0.812500 \quad-2.340000$

H $\quad 5.405000 \quad-0.874200 \quad-1.416300$

$\mathrm{H} \quad 7.159400 \quad-3.232700 \quad-3.775400$

H $\quad 7.340100 \quad-2.276200 \quad-2.270500$

$\mathrm{H} \quad 4.938800 \quad-3.187000 \quad-5.058300$

$\mathrm{H} \quad 3.572000 \quad-2.206400 \quad-4.440500$

H $\quad 5.781000 \quad-1.135500 \quad-3.879700$

$\mathrm{H} \quad-0.899600 \quad-5.024500 \quad-1.799100$

$\mathrm{H} \quad-0.932900 \quad-3.429700 \quad-2.592700$

H $\quad-1.261200 \quad-5.203300 \quad-4.272500$

$\mathrm{H} \quad-2.272800 \quad-6.933700 \quad-2.755100$

$\mathrm{H} \quad-3.302900-6.663800 \quad-4.196800$

H $\quad-4.425100 \quad-4.929400 \quad-0.391200$

$\mathrm{H} \quad-2.929100 \quad-5.906200 \quad-0.512600$

$\mathrm{H} \quad-4.688500 \quad-6.716300 \quad-2.099600$

$\mathrm{H} \quad-5.418800 \quad-5.250800 \quad-4.009700$

$\mathrm{H} \quad-5.879900 \quad-4.528900 \quad-2.435900$

$\mathrm{H} \quad-3.147700 \quad-1.943600 \quad-2.379000$

$\mathrm{H} \quad-4.545300 \quad-2.615800 \quad-1.488800$

$\mathrm{H} \quad-2.431100 \quad-2.996900 \quad-4.596600$

$\mathrm{H} \quad-3.391600 \quad-4.343400 \quad-5.286500$

$\mathrm{H} \quad-4.858100 \quad-2.802500 \quad-3.954700$

$\mathrm{H} \quad 5.070500 \quad 4.390500 \quad 4.900500$

$\begin{array}{llll}\mathrm{H} & 6.419700 & 2.631400 & 6.073600\end{array}$

$\begin{array}{llll}\mathrm{H} & 6.757000 & 0.414500 & 5.046800\end{array}$

$\mathrm{H} \quad 5.769600 \quad-0.124100 \quad 2.809800$

H $\quad 0.053800 \quad-5.724300 \quad 2.455200$

H $\quad 0.564800 \quad-6.769600 \quad 4.672500$

H $\quad 2.676700 \quad-6.271000 \quad 5.844800$

$\mathrm{H} \quad 4.352100 \quad-4.696300 \quad 4.843600$

$\mathrm{H} \quad-5.838100 \quad-0.351600 \quad 2.735100$

$\mathrm{H} \quad-6.722300 \quad-1.115700 \quad 4.956500$

H $\quad-6.004200 \quad-3.266600 \quad 5.924100$

H $\quad-4.368600 \quad-4.731000 \quad 4.713300$

$\mathrm{H} \quad-1.681200 \quad 6.588300 \quad 2.344600$

$\mathrm{H} \quad-5.581600 \quad 4.289100 \quad 4.511100$

$\mathrm{H} \quad-4.576700 \quad 6.342700 \quad 5.542500$

$\begin{array}{llll}\mathrm{H} & -2.659300 & 7.471400 & 4.478900\end{array}$

X2

$\begin{array}{llll}\mathrm{Rh} & -0.027300 & 0.028200 & 0.891500\end{array}$ 
$\begin{array}{llll}\mathrm{Rh} & 0.303100 & 0.366800 & -1.449400\end{array}$

$\begin{array}{llll}\mathrm{O} & -0.900600 & 2.021100 & -1.348500\end{array}$

$\begin{array}{lrrr}\text { O } & 1.529900 & -1.275500 & -1.444100\end{array}$

$\begin{array}{llll}\text { O } & -1.252900 & 1.689500 & 0.863900\end{array}$

$\begin{array}{llll}\text { O } & 1.226800 & -1.597400 & 0.770100\end{array}$

$\begin{array}{llll}\text { O } & 1.943800 & 1.526600 & -0.979700\end{array}$

$\begin{array}{llll}\mathrm{O} & -1.368800 & -0.805900 & -1.764900\end{array}$

$\begin{array}{llll}\text { O } & -1.633900 & -1.164900 & 0.449300\end{array}$

$\begin{array}{llll}\mathrm{O} & 1.606400 & 1.237500 & 1.232300\end{array}$

$\begin{array}{llll}\text { O } & 4.303900 & 3.405700 & 3.271700\end{array}$

$\begin{array}{llll}\text { O } & 4.160200 & -0.440800 & 0.758200\end{array}$

$\begin{array}{llll}\mathrm{O} & -3.397000 & 2.602800 & 2.503500\end{array}$

$\begin{array}{llll}\text { O } & -4.561200 & 4.001100 & -1.756600\end{array}$

$\begin{array}{llll}\mathrm{O} & -3.544700 & -4.828000 & -0.178300\end{array}$

$\begin{array}{llll}\text { O } & -4.336400 & -0.551900 & 1.385800\end{array}$

$\begin{array}{llll}\text { O } & 3.633000 & -4.573000 & 2.041100\end{array}$

$\begin{array}{llll}\mathrm{O} & 0.002900 & -4.088700 & -0.740600\end{array}$

$\begin{array}{llll}\mathrm{N} & 4.227900 & 1.691500 & 1.694100\end{array}$

$\begin{array}{llll}\mathrm{N} & -3.593300 & 3.420600 & 0.290300\end{array}$

$\begin{array}{llll}\mathrm{N} & -3.857000 & -2.554200 & 0.241600\end{array}$

N $\quad 2.055700 \quad-4.193400 \quad 0.363800$

C $\quad-1.987000 \quad-1.275100 \quad-0.763000$

$\begin{array}{llll}\text { C } & 2.268700 & 1.665300 & 0.233300\end{array}$

$\begin{array}{llll}\text { C } & -1.395800 & 2.330100 & -0.215300\end{array}$

$\begin{array}{llll}\text { C } & 1.745100 & -1.884200 & -0.359600\end{array}$

$\begin{array}{llll}\text { C } & 3.536000 & 2.456500 & 0.654000\end{array}$

$\begin{array}{llll}\text { C } & 4.479500 & 2.242900 & 2.972200\end{array}$

$\begin{array}{llll}\text { C } & 4.400000 & 0.296600 & 1.695100\end{array}$

C $\quad 4.964700 \quad 1.112400 \quad 3.812000$

$\begin{array}{llll}\text { C } & 4.900100 & -0.056300 & 3.050400\end{array}$

$\begin{array}{llll}\text { C } & 5.389700 & 1.094000 & 5.132500\end{array}$

$\begin{array}{llll}\text { C } & 5.241600 & -1.294000 & 3.577900\end{array}$

C $\quad 5.751800 \quad-0.147300 \quad 5.673200$

$\begin{array}{lrrr}\text { C } & 5.674500 & -1.320800 & 4.910900\end{array}$

$\begin{array}{llll}\text { C } & -2.236800 & 3.617700 & -0.240400\end{array}$

$\begin{array}{llll}\text { C } & -4.048300 & 2.851700 & 1.516700\end{array}$

$\begin{array}{llll}\text { C } & -4.667800 & 3.570100 & -0.625000\end{array}$

$\begin{array}{llll}\text { C } & -5.516800 & 2.648900 & 1.334900\end{array}$

C $\quad-5.886600 \quad 3.088100 \quad 0.067700$

$\begin{array}{llll}\text { C } & -6.444200 & 2.102900 & 2.210300\end{array}$

$\begin{array}{llll}\text { C } & -7.196100 & 2.999500 & -0.385800\end{array}$

$\begin{array}{llll}\text { C } & -7.769000 & 2.007900 & 1.767000\end{array}$

$\begin{array}{llll}\text { C } & -8.140000 & 2.448200 & 0.488100\end{array}$

$\begin{array}{llll}\text { C } & -3.253300 & -2.123200 & -1.023000\end{array}$

C $\quad-3.897100 \quad-3.927300 \quad 0.560900$

C $\quad-4.279200 \quad-1.760100 \quad 1.336800$

$\begin{array}{llll}\text { C } & -4.440200 & -4.021700 & 1.941800\end{array}$

C $\quad-4.656700 \quad-2.727200 \quad 2.408600$

C $\quad-4.718200 \quad-5.133700 \quad 2.724800$

C $\quad-5.154300 \quad-2.487600 \quad 3.681400$

$\begin{array}{llll}\text { C } & -5.222600 & -4.904600 & 4.011300\end{array}$

$\begin{array}{lrrr}\text { C } & -5.435200 & -3.601700 & 4.482900\end{array}$

$\begin{array}{lrrr}\text { C } & 2.740300 & -3.064500 & -0.271200\end{array}$

C $\quad 2.559400 \quad-4.814800 \quad 1.523200$

C $\quad 0.720700 \quad-4.572600 \quad 0.111500$

$\begin{array}{llll}\text { C } & 1.510700 & -5.784700 & 1.950100\end{array}$

C $\quad 0.406900 \quad-5.634300 \quad 1.108300$

$\begin{array}{llll}\text { C } & 1.509900 & -6.703700 & 2.989300\end{array}$

$\begin{array}{llll}\text { C } & -0.746100 & -6.389700 & 1.271100\end{array}$

$\begin{array}{llll}\text { C } & 0.356400 & -7.481800 & 3.158400\end{array}$

$\begin{array}{llll}\text { C } & -0.751600 & -7.325900 & 2.314800\end{array}$

C $\quad 0.928700 \quad 6.190500 \quad 1.458600$ $\begin{array}{lrrr}\text { C } & -0.353700 & 5.992700 & 2.294000\end{array}$

$\begin{array}{llll}\text { C } & 0.553000 & 6.363600 & -0.027400\end{array}$

C $\quad-0.184500 \quad 5.100400 \quad-0.516200$

$\begin{array}{llll}\text { C } & -1.100500 & 4.728700 & 1.811800\end{array}$

C $\quad-1.490400 \quad 4.867700 \quad 0.309900$

$\begin{array}{llll}\text { C } & -2.391200 & 6.130200 & 0.153600\end{array}$

$\begin{array}{llll}\text { C } & -1.270400 & 7.223800 & 2.130600\end{array}$

C $\quad-0.365800 \quad 7.591700 \quad-0.192000$

$\begin{array}{llll}\mathrm{C} & -1.647100 & 7.392100 & 0.643400\end{array}$

$\begin{array}{llll}\text { C } & 5.892200 & 5.223600 & -1.830600\end{array}$

C $\quad 6.655200 \quad 4.223500 \quad-0.939300$

$\begin{array}{llll}\text { C } & 4.691200 & 4.512600 & -2.485700\end{array}$

C $\quad 5.191500 \quad 3.331500 \quad-3.345000$

C $\quad 7.164200 \quad 3.049000 \quad-1.801100$

$\begin{array}{llll}\text { C } & 5.964200 & 2.333600 & -2.456200\end{array}$

$\begin{array}{llll}\text { C } & 5.029100 & 1.785600 & -1.356000\end{array}$

C $\quad 5.710800 \quad 3.685500 \quad 0.157700$

$\begin{array}{llll}\text { C } & 3.745900 & 3.983500 & -1.387400\end{array}$

$\begin{array}{llll}\text { C } & 4.484900 & 2.952800 & -0.475600\end{array}$

C $\quad 4.198300 \quad-3.105600 \quad-4.442000$

$\begin{array}{llll}\text { C } & 5.176300 & -2.669900 & -3.330000\end{array}$

C $\quad 3.372900 \quad-4.318300 \quad-3.960900$

C $\quad 4.322900 \quad-5.483700 \quad-3.614300$

$\begin{array}{llll}\text { C } & 6.122200 & -3.837100 & -2.983700\end{array}$

C $\quad 5.292300 \quad-5.042300 \quad-2.498100$

C $\quad 4.484100 \quad-4.641800 \quad-1.244500$

$\begin{array}{llll}\text { C } & 4.377800 & -2.266700 & -2.073400\end{array}$

C $\quad 2.557700 \quad-3.927900 \quad-2.708100$

C $3.510400 \quad-3.463100 \quad-1.564500$

C $\quad-5.657300 \quad-2.247200 \quad-1.918600$

C $\quad-4.279700 \quad-1.518800-2.040000$

$\begin{array}{llll}\text { C } & -6.667100 & -1.692100 & -2.946000\end{array}$

C $\quad-6.874800 \quad-0.183100 \quad-2.698500$

C $\quad-4.519000 \quad 0.009600 \quad-1.821000$

$\begin{array}{llll}\text { C } & -5.528700 & 0.553600 & -2.855700\end{array}$

C $\quad-4.981000 \quad 0.332900 \quad-4.280300$

C $\quad-3.766400 \quad-1.731700 \quad-3.501400$

C $\quad-6.124100 \quad-1.912100 \quad-4.371700$

C $\quad-4.778200 \quad-1.176400 \quad-4.526100$

H $\quad 3.477400 \quad-2.729800 \quad 0.466600$

$\mathrm{H} \quad-2.877300 \quad-3.066800 \quad-1.442500$

$\mathrm{H} \quad-2.423100 \quad 3.801900 \quad-1.302900$

$\mathrm{H} \quad 3.161000 \quad 3.338900 \quad 1.188800$

$\mathrm{H} \quad 1.602200 \quad 5.314100 \quad 1.581800$

$\mathrm{H} \quad 1.482000 \quad 7.086200 \quad 1.817100$

$\mathrm{H} \quad-0.081200 \quad 5.875100 \quad 3.365500$

$\mathrm{H} \quad 1.476200 \quad 6.510800 \quad-0.628700$

$\mathrm{H} \quad 0.502000 \quad 4.230800 \quad-0.419200$

H $\quad-0.424900 \quad 5.209900 \quad-1.597200$

$\mathrm{H} \quad-2.006300 \quad 4.606500 \quad 2.437500$

$\mathrm{H} \quad-0.449100 \quad 3.843300 \quad 1.972300$

$\mathrm{H} \quad-2.684400 \quad 6.265100 \quad-0.911400$

$\mathrm{H} \quad-3.328900 \quad 6.013600 \quad 0.740600$

$\mathrm{H} \quad-0.750100 \quad 8.136200 \quad 2.496500$

$\mathrm{H} \quad-2.189700 \quad 7.098500 \quad 2.744000$

$\mathrm{H} \quad 0.165700 \quad 8.510500 \quad 0.140500$

H $\quad-0.628000 \quad 7.729800 \quad-1.264000$

$\mathrm{H} \quad-2.306400 \quad 8.279600 \quad 0.526900$

$\mathrm{H} \quad 6.569100 \quad 5.627700 \quad-2.615200$

H $\quad 5.539100 \quad 6.084400 \quad-1.221100$

H $\quad 7.520200 \quad 4.736400 \quad-0.465200$

$\mathrm{H} \quad 4.142800 \quad 5.233400 \quad-3.130600$
H $\quad 5.852800 \quad 3.706900 \quad-4.156700$

H $\quad 4.329800 \quad 2.820900 \quad-3.829000$

$\mathrm{H} \quad 7.736000 \quad 2.333500 \quad-1.170000$

H $\quad 7.856900 \quad 3.425100 \quad-2.585700$

$\mathrm{H} \quad 6.332900 \quad 1.490600 \quad-3.080800$

H $\quad 4.206200 \quad 1.220900-1.839000$

H $\quad 5.602100 \quad 1.066400 \quad-0.733700$

$\mathrm{H} \quad 5.367600 \quad 4.530500 \quad 0.795400$

$\begin{array}{llll}\mathrm{H} & 6.287500 & 2.993700 & 0.810300\end{array}$

H $\quad 3.375900 \quad 4.836700 \quad-0.776900$

H $\quad 2.861000 \quad 3.536900-1.882100$

H $\quad 4.764700 \quad-3.373000 \quad-5.361100$

$\mathrm{H} \quad 3.520200 \quad-2.263700 \quad-4.703700$

H $\quad 5.774800 \quad-1.801500 \quad-3.682500$

H $\quad 2.678900 \quad-4.636500 \quad-4.769100$

H $\quad 4.896200 \quad-5.788400 \quad-4.517400$

H $\quad 3.735700 \quad-6.367600 \quad-3.281000$

$\mathrm{H} \quad 6.839000 \quad-3.525000 \quad-2.192500$

H $\quad 6.718800 \quad-4.121400 \quad-3.878400$

H $\quad 5.974300 \quad-5.883400 \quad-2.246500$

H $\quad 3.919200 \quad-5.531900 \quad-0.890600$

H $\quad 5.186000 \quad-4.352200 \quad-0.430900$

H $\quad 3.755300 \quad-1.386400 \quad-2.328900$

$\mathrm{H} \quad 5.086500 \quad-1.941500 \quad-1.280200$

H $\quad 1.838700 \quad-3.131700 \quad-2.987500$

$\mathrm{H} \quad 1.964100 \quad-4.808600 \quad-2.382400$

$\mathrm{H} \quad-6.087100 \quad-2.109900 \quad-0.902300$

$\mathrm{H} \quad-5.528300 \quad-3.340900 \quad-2.078100$

$\mathrm{H} \quad-7.638100 \quad-2.222000 \quad-2.834600$

H $\quad-7.282800 \quad-0.016100 \quad-1.677000$

H $\quad-7.616400 \quad 0.222500 \quad-3.421300$

$\mathrm{H} \quad-3.571600 \quad 0.581900 \quad-1.913900$

$\mathrm{H} \quad-4.923700 \quad 0.204500 \quad-0.810500$

$\mathrm{H} \quad-5.684400 \quad 1.640200 \quad-2.688800$

$\mathrm{H} \quad-5.691800 \quad 0.747400 \quad-5.028700$

H $\quad-4.016400 \quad 0.872700 \quad-4.404500$

$\mathrm{H} \quad-3.605000 \quad-2.814700 \quad-3.699500$

$\mathrm{H} \quad-2.794400 \quad-1.224700 \quad-3.665600$

H $\quad-5.988400 \quad-2.999200 \quad-4.564000$

H $\quad-6.853900 \quad-1.531300 \quad-5.119700$

$\mathrm{H} \quad-4.387700 \quad-1.333900 \quad-5.555200$

H $\quad 2.371000 \quad-6.810600 \quad 3.641500$

$\mathrm{H} \quad 0.318200 \quad-8.216200 \quad 3.958100$

$\mathrm{H} \quad-1.631400 \quad-7.943000 \quad 2.475100$

$\mathrm{H} \quad-1.605800 \quad-6.246200 \quad 0.622900$

$\mathrm{H} \quad-4.550500 \quad-6.138200 \quad 2.348900$

$\mathrm{H} \quad-5.452900 \quad-5.748200 \quad 4.655800$

$\mathrm{H} \quad-5.824100 \quad-3.456300 \quad 5.486800$

$\mathrm{H} \quad-5.312700 \quad-1.473600 \quad 4.034900$

H $\quad-6.140600 \quad 1.755500 \quad 3.192300$

$\mathrm{H} \quad-8.524300 \quad 1.581000 \quad 2.421000$

H $\quad-9.175200 \quad 2.355600 \quad 0.171900$

$\mathrm{H} \quad-7.465900 \quad 3.337300 \quad-1.381500$

$\begin{array}{llll}\mathrm{H} & 5.155300 & -2.202300 & 2.988400\end{array}$

H $\quad 5.949900 \quad-2.268900 \quad 5.363600$

H $\quad 6.090200 \quad-0.203800 \quad 6.703900$

$\begin{array}{llll}\mathrm{H} & 5.431300 & 2.006400 & 5.719100\end{array}$

$\begin{array}{lccc}\text { X3 } & & & \\ \mathrm{Rh} & -0.129600 & 0.305800 & 0.953800 \\ \mathrm{Rh} & 0.290900 & 0.234800 & -1.394600 \\ \mathrm{O} & 1.974400 & 1.379600 & -1.075100\end{array}$ 


\begin{tabular}{|c|c|c|c|}
\hline D & -1.359300 & -0.968800 & -1.579400 \\
\hline 0 & 1.592700 & 1.442900 & 1.147300 \\
\hline ก & -1.767800 & -0.896000 & 0.638600 \\
\hline $\mathrm{O}$ & 1.416400 & -1.454700 & -1.071400 \\
\hline O & -0.847100 & 1.933400 & -1.595500 \\
\hline $\mathrm{O}$ & -1.243400 & 2.009100 & 0.633200 \\
\hline O & 1.013000 & -1.393900 & 1.147900 \\
\hline $\mathrm{O}$ & 3.315500 & -4.109400 & 2.989100 \\
\hline $\mathrm{O}$ & -0.447400 & -3.975400 & 0.351300 \\
\hline & 4.467300 & 3.637600 & 2.893900 \\
\hline $\mathrm{O}$ & 4.016700 & -0.520100 & 0.980000 \\
\hline $\mathrm{O}$ & -4.518600 & 4.164600 & -2.081600 \\
\hline $\mathrm{O}$ & -2.920900 & 3.622000 & 2.234700 \\
\hline & -4.489300 & 0.167800 & -0.509200 \\
\hline & -4.255800 & -3.921200 & 1.593000 \\
\hline $\mathrm{N}$ & 1.655500 & -4.001500 & 1.352700 \\
\hline $\mathrm{N}$ & 4.247200 & 1.717100 & 1.591600 \\
\hline $\mathrm{N}$ & -3.332400 & 3.920200 & -0.080500 \\
\hline $\mathrm{N}$ & -4.207000 & -2.034800 & 0.218500 \\
\hline & -1.326600 & 2.464500 & -0.541800 \\
\hline & 1.528400 & -1.907700 & 0.101800 \\
\hline & 2.300800 & 1.660800 & 0.112500 \\
\hline $\mathrm{C}$ & -1.985200 & -1.325800 & -0.541400 \\
\hline$C$ & 2.391700 & -3.154500 & 0.409700 \\
\hline & 2.180500 & -4.338900 & 2.617100 \\
\hline $\mathrm{C}$ & 0.275400 & -4.265200 & 5500 \\
\hline $\mathrm{C}$ & 1.065800 & -4.992100 & 3.360000 \\
\hline $\mathrm{C}$ & -0.082100 & -4.928300 & 2.567500 \\
\hline $\mathrm{C}$ & 1.039600 & -5.555900 & 4.627200 \\
\hline C & -1.307400 & -5.406900 & 3.010100 \\
\hline $\mathrm{C}$ & -0.187400 & -6.059400 & 5.080900 \\
\hline $\mathrm{C}$ & -1.340700 & -5.981300 & 4.288600 \\
\hline $\mathrm{C}$ & 3.640500 & 2.378100 & 0.433500 \\
\hline $\mathrm{C}$ & 4.540500 & 2.431800 & 2.776500 \\
\hline $\mathrm{C}$ & 4.304400 & 0.328500 & 1.802800 \\
\hline $\mathrm{C}$ & 4.922000 & 1.404800 & 3.784900 \\
\hline $\mathrm{C}$ & 4.763600 & 0.144100 & 3.205700 \\
\hline $\mathrm{C}$ & 5.337500 & 1.551400 & 5.100300 \\
\hline $\mathrm{C}$ & 4.997800 & -1.024000 & 3.918500 \\
\hline $\mathrm{C}$ & 5.591900 & 0.380400 & 5.827300 \\
\hline $\mathrm{C}$ & 5.420400 & -0.884000 & 5.247900 \\
\hline $\mathrm{C}$ & -2.050200 & 3.800100 & -0.791100 \\
\hline $\mathrm{C}$ & -4.502800 & 4.064700 & -0.871800 \\
\hline $\mathrm{C}$ & -3.664300 & 3.792700 & 1.295700 \\
\hline $\mathrm{C}$ & -5.650700 & 4.087100 & 0.070200 \\
\hline $\mathrm{C}$ & -5.151300 & 3.929300 & 1.358900 \\
\hline $\mathrm{C}$ & -7.009100 & 4.241600 & -0.173600 \\
\hline $\mathrm{C}$ & -5.987300 & 3.925700 & 2.466900 \\
\hline $\mathrm{C}$ & -7.864000 & 4.238500 & 0.936200 \\
\hline $\mathrm{C}$ & -7.360200 & 4.085800 & 2.236800 \\
\hline $\mathrm{C}$ & -3.054500 & -2.442200 & -0.587700 \\
\hline $\mathrm{C}$ & -4.796800 & -0.753900 & 0.219100 \\
\hline $\mathrm{C}$ & -4.673400 & -2.819200 & 1.291100 \\
\hline $\mathrm{C}$ & -5.826300 & -0.786000 & 1.297600 \\
\hline $\mathrm{C}$ & -5.750300 & -2.021700 & 1.941600 \\
\hline $\mathrm{C}$ & -6.740700 & 0.181300 & 1.687800 \\
\hline $\mathrm{C}$ & -6.584500 & -2.340900 & 3.003100 \\
\hline $\mathrm{C}$ & -7.591500 & -0.132800 & 2.756200 \\
\hline $\mathrm{C}$ & -7.514900 & -1.372800 & 3.404100 \\
\hline $\mathrm{C}$ & -3.143800 & -3.190500 & -4.941500 \\
\hline $\mathrm{C}$ & -2.611500 & -4.308300 & -4.019600 \\
\hline$C$ & -4.389000 & -2.539600 & -4.302100 \\
\hline
\end{tabular}

$\begin{array}{lrrr}\text { C } & -5.480600 & -3.610300 & -4.094600\end{array}$

C $\quad-3.705000 \quad-5.374900 \quad-3.811600$

C $\quad-4.942900 \quad-4.720000 \quad-3.167300$

$\begin{array}{llll}\text { C } & -4.552500 & -4.106600 & -1.804800\end{array}$

C $\quad-2.217700 \quad-3.704100 \quad-2.656100$

$\begin{array}{llll}\text { C } & -4.009800 & -1.919400 & -2.938800\end{array}$

C $\quad-3.448900 \quad-3.014800 \quad-1.982100$

$\begin{array}{llll}\mathrm{C} & -0.579900 & 5.229300 & 0.799900\end{array}$

$\begin{array}{llll}\text { C } & 0.331800 & 6.474600 & 0.883000\end{array}$

$\begin{array}{lrrr}\text { C } & -1.124000 & 5.047400 & -0.648500\end{array}$

C $\quad-1.912000 \quad 6.335500 \quad-1.039200$

$\begin{array}{llll}\text { C } & -0.477100 & 7.730600 & 0.494700\end{array}$

$\begin{array}{lrrr}\text { C } & -1.001500 & 7.579800 & -0.948900\end{array}$

C $\quad 0.189800 \quad 7.417700 \quad-1.915000$

$\begin{array}{llll}\text { C } & 1.523400 & 6.312300 & -0.081700\end{array}$

$\begin{array}{llll}\text { C } & 0.096600 & 4.918400 & -1.615700\end{array}$

C $\quad 1.000600 \quad 6.164900 \quad-1.525900$

$\begin{array}{llll}\text { C } & 5.073200 & 1.261100 & -1.419100\end{array}$

C $\quad 4.642800 \quad 2.597900 \quad-0.739600$

$\begin{array}{llll}\text { C } & 6.066400 & 1.537000 & -2.569100\end{array}$

$\begin{array}{llll}\text { C } & 5.405100 & 2.456300 & -3.617700\end{array}$

C $\quad 4.020200 \quad 3.543300 \quad-1.816300$

$\begin{array}{llll}\text { C } & 5.021600 & 3.799600 & -2.961100\end{array}$

$\begin{array}{llll}\text { C } & 6.283600 & 4.481100 & -2.395600\end{array}$

C $\quad 5.932400 \quad 3.295000 \quad-0.199000$

$\begin{array}{llll}\text { C } & 7.328100 & 2.222800 & -2.004300\end{array}$

$\begin{array}{llll}\text { C } & 6.934500 & 3.560800 & -1.344100\end{array}$

$\begin{array}{llll}\text { C } & 3.289600 & -6.456400 & -2.388300\end{array}$

C $\quad 4.405200 \quad-5.966400 \quad-1.441500$

C $\quad 2.511500 \quad-5.242700 \quad-2.938500$

C $\quad 1.874300 \quad-4.466000 \quad-1.765400$

$\begin{array}{llll}\text { C } & 3.777500 & -5.179200 & -0.270300\end{array}$

$\begin{array}{llll}\text { C } & 2.979500 & -3.943800 & -0.796600\end{array}$

C $\quad 3.980900 \quad-3.047500 \quad-1.595100$

C $\quad 5.373400 \quad-5.051200 \quad-2.217200$

$\begin{array}{llll}\text { C } & 3.476800 & -4.318400 & -3.710700\end{array}$

C $\quad 4.599800 \quad-3.836400 \quad-2.767300$

$\mathrm{H} \quad-2.592700 \quad-3.255900 \quad-0.015100$

$\mathrm{H} \quad-2.359200 \quad 3.752100 \quad-1.839900$

H $\quad 3.357100 \quad 3.364700 \quad 0.822200$

$\begin{array}{llll}\mathrm{H} & 3.230500 & -2.755500 & 0.991700\end{array}$

$\mathrm{H} \quad-3.405500 \quad-3.612800 \quad-5.936600$

$\mathrm{H} \quad-2.354900 \quad-2.423400 \quad-5.104100$

$\mathrm{H} \quad-1.719700 \quad-4.780400 \quad-4.486700$

$\mathrm{H} \quad-4.776400 \quad-1.743900 \quad-4.975100$

$\mathrm{H} \quad-5.778400 \quad-4.044400 \quad-5.074300$

$\mathrm{H} \quad-6.388100 \quad-3.147500 \quad-3.648000$

$\mathrm{H} \quad-3.322700 \quad-6.190500 \quad-3.159000$

H $\quad-3.980700 \quad-5.832200 \quad-4.787300$

$\mathrm{H} \quad-5.731000 \quad-5.489300 \quad-3.015400$

$\mathrm{H} \quad-5.465400 \quad-3.671300 \quad-1.342300$

$\mathrm{H} \quad-4.191700 \quad-4.912500 \quad-1.127600$

$\mathrm{H} \quad-1.386000 \quad-2.992600 \quad-2.825400$

H $\quad-1.821200 \quad-4.510100-1.999700$

H $\quad-3.269300 \quad-1.111600 \quad-3.109100$

$\mathrm{H} \quad-4.915300 \quad-1.450100 \quad-2.499600$

$\mathrm{H} \quad-1.413700 \quad 5.378000 \quad 1.512300$

$\mathrm{H} \quad-0.007400 \quad 4.332200 \quad 1.121300$

H $\quad 0.711300 \quad 6.586400 \quad 1.922000$

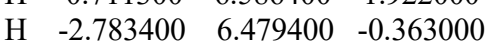

$\mathrm{H} \quad-2.310100 \quad 6.244400 \quad-2.074300$

$\begin{array}{llll}\mathrm{H} & 0.164900 & 8.635500 & 0.573300\end{array}$
$\begin{array}{llll}\mathrm{H} & -1.329000 & 7.867400 & 1.196600\end{array}$

H $\quad-1.583100 \quad 8.485300 \quad-1.227800$

$\mathrm{H} \quad-0.177900 \quad 7.323500 \quad-2.960500$

$\mathrm{H} \quad 0.839800 \quad 8.319200 \quad-1.871800$

$\mathrm{H} \quad 2.117400 \quad 5.418400 \quad 0.204600$

H $\quad 2.196200 \quad 7.194800 \quad-0.009000$

$\mathrm{H} \quad 0.709000 \quad 4.027100 \quad-1.359400$

$\mathrm{H} \quad-0.252800 \quad 4.789700 \quad-2.664200$

H $\quad 1.860100 \quad 6.050700 \quad-2.221700$

H $\quad 4.200900 \quad 0.710500 \quad-1.826400$

H $\quad 5.569900 \quad 0.595600 \quad-0.682600$

$\mathrm{H} \quad 6.352600 \quad 0.575500 \quad-3.048700$

$\mathrm{H} \quad 4.499500 \quad 1.965000 \quad-4.036700$

H $\quad 6.106700 \quad 2.634100 \quad-4.462200$

H $\quad 3.740500 \quad 4.514500 \quad-1.354500$

H $\quad 3.096900 \quad 3.118500-2.256300$

H $\quad 4.553300 \quad 4.462700 \quad-3.720900$

H $\quad 7.003700 \quad 4.689800 \quad-3.217200$

H $\quad 6.015200 \quad 5.456800 \quad-1.933800$

H $\quad 6.431600 \quad 2.659200 \quad 0.564800$

H $\quad 5.672100 \quad 4.258200 \quad 0.293700$

H $\quad 7.820300 \quad 1.560600 \quad-1.258300$

H $\quad 8.060900 \quad 2.402900 \quad-2.821400$

H $\quad 7.843500 \quad 4.052700 \quad-0.934600$

H $\quad 2.598900 \quad-7.136400 \quad-1.842800$

$\mathrm{H} \quad 3.731100 \quad-7.037200 \quad-3.227900$

H $\quad 4.963200 \quad-6.841600 \quad-1.043100$

$\mathrm{H} \quad 1.711300 \quad-5.597300 \quad-3.624400$

$\mathrm{H} \quad 1.180800 \quad-5.147500 \quad-1.229400$

H $\quad 1.267100 \quad-3.634300 \quad-2.177300$

H $\quad 4.580800 \quad-4.850600 \quad 0.426200$

$\mathrm{H} \quad 3.110500 \quad-5.864800 \quad 0.297000$

H $\quad 3.488500 \quad-2.147100 \quad-2.011600$

H $\quad 4.792600 \quad-2.686900-0.924500$

H $\quad 5.841700 \quad-5.614600 \quad-3.054100$

H $\quad 6.193100 \quad-4.709500 \quad-1.547500$

H $\quad 3.915900 \quad-4.865200 \quad-4.574000$

$\mathrm{H} \quad 2.921700 \quad-3.445200 \quad-4.119100$

H $\quad 5.297900 \quad-3.178000 \quad-3.329100$

$\mathrm{H} \quad-7.384500 \quad 4.362200 \quad-1.184900$

$\mathrm{H} \quad-8.933500 \quad 4.361500 \quad 0.790100$

$\mathrm{H} \quad-8.047800 \quad 4.096900 \quad 3.078100$

$\mathrm{H} \quad-5.583300 \quad 3.805900 \quad 3.467300$

$\mathrm{H} \quad 4.838300 \quad-2.001000 \quad 3.471800$

$\mathrm{H} \quad 5.613400 \quad-1.772500 \quad 5.842100$

$\begin{array}{llll}\mathrm{H} & 5.919500 & 0.452600 & 6.860600\end{array}$

$\mathrm{H} \quad 5.452800 \quad 2.535500 \quad 5.543700$

$\mathrm{H} \quad-6.513900 \quad-3.304200 \quad 3.498600$

H $\quad-8.187500 \quad-1.585000 \quad 4.230400$

$\mathrm{H} \quad-8.321800 \quad 0.598900 \quad 3.090100$

$\mathrm{H} \quad-6.792100 \quad 1.140300 \quad 1.183100$

H $\quad 1.934900 \quad-5.597800 \quad 5.239700$

$\mathrm{H} \quad-0.249000 \quad-6.510200 \quad 6.067400$

H $\quad-2.279100 \quad-6.368000 \quad 4.675500$

H $\quad-2.201200 \quad-5.315300 \quad 2.399900$

$\begin{array}{lccc}\text { X4 } & & & \\ \text { Rh } & 0.116600 & 0.247200 & 0.811000 \\ \text { Rh } & 0.283600 & -0.358900 & -1.492600 \\ \text { O } & 0.314700 & -1.757500 & 1.279800 \\ \text { O } & -0.088900 & 2.204900 & 0.210300 \\ \text { O } & 0.476800 & -2.320000 & -0.898100\end{array}$


$\begin{array}{llll}\text { O } & 0.061900 & 1.625000 & -1.972700\end{array}$

$\begin{array}{llll}\text { O } & 2.171900 & 0.468900 & 0.822300\end{array}$

$\begin{array}{llll}\mathrm{O} & -1.913700 & -0.031700 & 0.684900\end{array}$

$\begin{array}{llll}\mathrm{O} & -1.756400 & -0.595900 & -1.493900\end{array}$

O $\quad 2.329100 \quad-0.123200 \quad-1.351700$

$\begin{array}{llll}\text { O } & 5.851100 & 2.671200 & 0.095500\end{array}$

$\begin{array}{llll}\text { O } & 3.973800 & -0.565300 & 2.813300\end{array}$

$\begin{array}{llll}\text { O } & 0.857500 & -5.105900 & 3.502500\end{array}$

$\begin{array}{llll}\mathrm{O} & -2.370000 & -3.232000 & 0.817100\end{array}$

$\begin{array}{llll}\text { O } & -3.701200 & 2.204000 & -0.488000\end{array}$

$\begin{array}{llll}\text { O } & -5.546800 & -1.190500 & 2.016500\end{array}$

$\begin{array}{llll}\mathrm{O} & -0.057700 & 4.697400 & 1.584400\end{array}$

$\begin{array}{llll}\mathrm{O} & -2.433900 & 5.264100 & -2.353600\end{array}$

$\begin{array}{llll}\mathrm{N} & 4.800000 & 0.862300 & 1.126500\end{array}$

$\mathrm{N} \quad-0.557800 \quad-4.259000 \quad 1.854800$

$\begin{array}{llll}\mathrm{N} & -4.591700 & 0.260600 & 0.457700\end{array}$

$\mathrm{N} \quad-0.968000 \quad 4.762600 \quad-0.601900$

C $\quad-2.406100 \quad-0.420500 \quad-0.424300$

$\begin{array}{llll}\text { C } & 2.824600 & 0.181000 & -0.222700\end{array}$

$\begin{array}{llll}\text { C } & 0.400000 & -2.601500 & 0.331700\end{array}$

$\begin{array}{llll}\text { C } & -0.040900 & 2.467700 & -1.025500\end{array}$

$\begin{array}{llll}\text { C } & 4.364400 & 0.280200 & -0.145000\end{array}$

$\begin{array}{llll}\text { C } & 5.496600 & 2.092800 & 1.104200\end{array}$

$\begin{array}{llll}\text { C } & 4.534100 & 0.446100 & 2.456700\end{array}$

$\begin{array}{llll}\text { C } & 5.694600 & 2.489100 & 2.520800\end{array}$

C $\quad 5.117100 \quad 1.510600 \quad 3.326700$

$\begin{array}{llll}\text { C } & 6.319800 & 3.604900 & 3.060000\end{array}$

$\begin{array}{llll}\text { C } & 5.142700 & 1.603800 & 4.710900\end{array}$

C $\quad 6.348600 \quad 3.711200 \quad 4.456200$

$\begin{array}{llll}\text { C } & 5.769600 & 2.725900 & 5.268300\end{array}$

C $\quad 0.468100 \quad-4.071700 \quad 0.827300$

C $\quad-0.235100 \quad-4.707500 \quad 3.157200$

$\begin{array}{llll}\text { C } & -1.866700 & -3.755200 & 1.794000\end{array}$

C $\quad-1.487200 \quad-4.570200 \quad 3.951900$

$\begin{array}{llll}\text { C } & -2.460400 & -3.986100 & 3.138100\end{array}$

$\begin{array}{llll}\text { C } & -1.749500 & -4.898500 & 5.273800\end{array}$

C $\quad-3.731600 \quad-3.691400 \quad 3.611800$

C $\quad-3.033500 \quad-4.619300 \quad 5.761800$

C $\quad-4.004800 \quad-4.023100 \quad 4.946400$

$\begin{array}{llll}\text { C } & -3.909400 & -0.769700 & -0.328800\end{array}$

$\begin{array}{llll}\text { C } & -4.393000 & 1.652500 & 0.343900\end{array}$

$\begin{array}{llll}\text { C } & -5.320800 & -0.063400 & 1.617800\end{array}$

$\begin{array}{llll}\text { C } & -5.178400 & 2.262800 & 1.455800\end{array}$

$\begin{array}{llll}\text { C } & -5.732900 & 1.234600 & 2.218800\end{array}$

C $\quad-5.380800 \quad 3.596300 \quad 1.780000$

C $\quad-6.512300 \quad 1.495900 \quad 3.336400$

$\begin{array}{llll}\text { C } & -6.169600 & 3.871800 & 2.905200\end{array}$

$\begin{array}{llll}\text { C } & -6.726400 & 2.839500 & 3.671500\end{array}$

$\begin{array}{llll}\text { C } & -0.122000 & 3.936900 & -1.474600\end{array}$

$\begin{array}{llll}\text { C } & -0.899500 & 5.043800 & 0.787300\end{array}$

$\begin{array}{llll}\text { C } & -2.122700 & 5.349100 & -1.183500\end{array}$

C $\quad-2.093500 \quad 5.895900 \quad 1.078100$

$\begin{array}{llll}\text { C } & -2.817700 & 6.082700 & -0.095100\end{array}$

$\begin{array}{llll}\text { C } & -2.491000 & 6.472600 & 2.276400\end{array}$

$\begin{array}{llll}\text { C } & -3.971700 & 6.855000 & -0.127100\end{array}$

$\begin{array}{llll}\text { C } & -3.653800 & 7.254100 & 2.259900\end{array}$

$\begin{array}{llll}\text { C } & -4.383100 & 7.443100 & 1.076400\end{array}$

C $\quad 1.742200 \quad-5.096200 \quad-1.142500$

$\begin{array}{llll}\text { C } & 1.771600 & -6.230900 & -2.186500\end{array}$

C $\quad 0.464400 \quad-5.197600 \quad-0.250900$

C $\quad-0.800800 \quad-5.152400 \quad-1.162700$

C $\quad 0.515500 \quad-6.138300 \quad-3.078900$
C $\quad-0.750800 \quad-6.287800 \quad-2.208800$

C $\quad-0.721500 \quad-7.650900 \quad-1.486100$

$\begin{array}{llll}\text { C } & 1.796900 & -7.592300 & -1.463800\end{array}$

C $\quad 0.496900 \quad-6.593200 \quad 0.451000$

C $\quad 0.533300 \quad-7.732200 \quad-0.591800$

C $\quad 6.791700 \quad-3.420200 \quad 0.110100$

$\begin{array}{llll}\text { C } & 7.424900 & -2.169900 & -0.534800\end{array}$

C $\quad 5.327100 \quad-3.555600 \quad-0.356200$

$\begin{array}{llll}\text { C } & 4.527000 & -2.306400 & 0.077800\end{array}$

$\begin{array}{llll}\text { C } & 6.634200 & -0.916200 & -0.105400\end{array}$

$\begin{array}{llll}\text { C } & 5.140400 & -1.017700 & -0.552900\end{array}$

$\begin{array}{llll}\text { C } & 5.128000 & -1.184000 & -2.108100\end{array}$

$\begin{array}{llll}\text { C } & 7.385600 & -2.303000 & -2.069800\end{array}$

C $\quad 5.287200 \quad-3.688700 \quad-1.892800$

C $\quad 5.922000 \quad-2.437500 \quad-2.534200$

$\begin{array}{llll}\text { C } & 1.095600 & 6.093900 & -2.118200\end{array}$

$\begin{array}{llll}\text { C } & 2.450200 & 6.728800 & -2.498700\end{array}$

$\begin{array}{llll}\text { C } & 1.264600 & 4.573000 & -1.810900\end{array}$

C $\quad 1.863200 \quad 3.892800 \quad-3.084200$

$\begin{array}{llll}\text { C } & 3.008500 & 6.034700 & -3.756900\end{array}$

$\begin{array}{llll}\text { C } & 3.213200 & 4.534400 & -3.466800\end{array}$

$\begin{array}{llll}\text { C } & 4.206600 & 4.361300 & -2.300200\end{array}$

C $\quad 3.444100 \quad 6.558300 \quad-1.330400$

$\begin{array}{llll}\text { C } & 2.295400 & 4.417600 & -0.651000\end{array}$

C $\quad 3.647600 \quad 5.056000 \quad-1.041000$

$\begin{array}{llll}\text { C } & -4.580800 & 0.005100 & -2.707700\end{array}$

C $\quad-4.656100 \quad-1.134200 \quad-1.646600$

$\begin{array}{lrrr}\text { C } & -5.337800 & -0.404800 & -3.990700\end{array}$

$\begin{array}{llll}\text { C } & -4.714400 & -1.691100 & -4.573700\end{array}$

$\begin{array}{llll}\text { C } & -4.067900 & -2.447100 & -2.259500\end{array}$

C $\quad-4.828500 \quad-2.835700 \quad-3.544100$

$\begin{array}{llll}\text { C } & -6.312000 & -3.085400 & -3.207100\end{array}$

C $\quad-6.164300-1.402000-1.337200$

C $\quad-6.822100 \quad-0.658900 \quad-3.653600$

C $\quad-6.926700 \quad-1.799600 \quad-2.619900$

$\mathrm{H} \quad-0.698800 \quad 3.915200 \quad-2.405700$

$\mathrm{H} \quad-3.920700 \quad-1.656900 \quad 0.315800$

$\mathrm{H} \quad 1.412100 \quad-4.142800 \quad 1.384500$

$\mathrm{H} \quad 4.639900 \quad 1.060700 \quad-0.866200$

$\mathrm{H} \quad 1.791700 \quad-4.132200 \quad-1.684800$

$\begin{array}{llll}\mathrm{H} & 2.652600 & -5.158000 & -0.508400\end{array}$

H $\quad 2.682300 \quad-6.130700 \quad-2.816700$

$\mathrm{H} \quad-0.887200 \quad-4.182800 \quad-1.694000$

$\mathrm{H} \quad-1.720500 \quad-5.278700 \quad-0.553900$

$\mathrm{H} \quad 0.540600 \quad-6.937100 \quad-3.852300$

$\mathrm{H} \quad 0.497700 \quad-5.161800 \quad-3.611200$

H $\quad-1.652800 \quad-6.233000 \quad-2.856600$

$\mathrm{H} \quad-1.638000 \quad-7.774500 \quad-0.868200$

$\mathrm{H} \quad-0.711300 \quad-8.476200 \quad-2.231700$

H $\quad 2.707700 \quad-7.670200 \quad-0.829900$

$\mathrm{H} \quad 1.839200 \quad-8.417400 \quad-2.208400$

H $\quad 1.388100 \quad-6.671300 \quad 1.112800$

$\mathrm{H} \quad-0.401900 \quad-6.731700 \quad 1.091100$

H $\quad 0.552000 \quad-8.713100 \quad-0.068800$

$\mathrm{H} \quad 6.832300 \quad-3.339000 \quad 1.218700$

$\mathrm{H} \quad 7.367000 \quad-4.328000 \quad-0.176600$

H $\quad 8.480600 \quad-2.075000 \quad-0.199300$

$\mathrm{H} \quad 4.880500 \quad-4.461600 \quad 0.106600$

$\mathrm{H} \quad 4.543700 \quad-2.250000 \quad 1.182300$

H $\quad 3.467300 \quad-2.432800 \quad-0.233100$

H $\quad 7.106200 \quad-0.010700 \quad-0.547700$

H $\quad 6.708600 \quad-0.821100 \quad 0.999900$
H $\quad 4.094000 \quad-1.288000 \quad-2.493600$

H $\quad 5.570800 \quad-0.287500 \quad-2.596400$

H $\quad 7.969400 \quad-3.194000 \quad-2.389500$

$\mathrm{H} \quad 7.854600 \quad-1.411600 \quad-2.541600$

H $\quad 5.839000 \quad-4.600800 \quad-2.209700$

$\mathrm{H} \quad 4.237600 \quad-3.800700 \quad-2.240400$

$\mathrm{H} \quad 5.893900 \quad-2.535400 \quad-3.641300$

$\mathrm{H} \quad 0.371500 \quad 6.239300 \quad-2.950400$

$\mathrm{H} \quad 0.691700 \quad 6.630400 \quad-1.231700$

H $\quad 2.306400 \quad 7.811500-2.707000$

H $\quad 1.158100 \quad 3.983100 \quad-3.940200$

H $\quad 2.034000 \quad 2.809500 \quad-2.908700$

H $\quad 3.974900 \quad 6.500200 \quad-4.050900$

H $\quad 2.304500 \quad 6.165400 \quad-4.608100$

H $\quad 3.617600 \quad 4.034400 \quad-4.373800$

H $\quad 4.367500 \quad 3.279800 \quad-2.100600$

H $\quad 5.192400 \quad 4.799400 \quad-2.570700$

H $\quad 3.056700 \quad 7.071900 \quad-0.423000$

$\begin{array}{llll}\mathrm{H} & 4.417500 & 7.030700 & -1.587800\end{array}$

$\begin{array}{llll}\mathrm{H} & 1.933600 & 4.920100 & 0.264700\end{array}$

H $\quad 2.457400 \quad 3.344900 \quad-0.406600$

$\mathrm{H} \quad 4.366800 \quad 4.939300 \quad-0.201800$

H $\quad-3.530600 \quad 0.242200 \quad-2.974300$

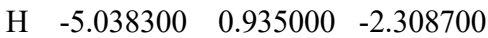

H $\quad-5.265000 \quad 0.413400 \quad-4.740100$

$\mathrm{H} \quad-3.646500 \quad-1.514000 \quad-4.829300$

$\mathrm{H} \quad-5.237600 \quad-1.973700 \quad-5.513700$

H $\quad-4.138000 \quad-3.279600 \quad-1.524300$

$\mathrm{H} \quad-2.996300 \quad-2.340000 \quad-2.517300$

$\mathrm{H} \quad-4.386000 \quad-3.762600 \quad-3.970300$

H $\quad-6.864600 \quad-3.387500 \quad-4.123900$

$\mathrm{H} \quad-6.401600 \quad-3.918800 \quad-2.475900$

$\mathrm{H} \quad-6.645600 \quad-0.494300 \quad-0.911400$

H $\quad-6.264300 \quad-2.213500 \quad-0.582300$

$\mathrm{H} \quad-7.284300 \quad 0.267500 \quad-3.246700$

H $\quad-7.380600 \quad-0.929500 \quad-4.576600$

$\mathrm{H} \quad-7.996100 \quad-1.979700 \quad-2.374900$

$\mathrm{H} \quad-4.945800 \quad 4.391000 \quad 1.183500$

$\mathrm{H} \quad-6.349500 \quad 4.904600 \quad 3.189700$

$\mathrm{H} \quad-7.332000 \quad 3.086300 \quad 4.539000$

$\mathrm{H} \quad-6.935400 \quad 0.686800 \quad 3.923500$

H $\quad 4.690800 \quad 0.834300 \quad 5.328900$

$\begin{array}{llll}\mathrm{H} & 5.808300 & 2.837000 & 6.348300\end{array}$

$\mathrm{H} \quad 6.826300 \quad 4.569900 \quad 4.919300$

$\mathrm{H} \quad 6.763200 \quad 4.360500 \quad 2.419200$

$\mathrm{H} \quad-1.915000 \quad 6.320900 \quad 3.183900$

$\mathrm{H} \quad-3.994900 \quad 7.727600 \quad 3.176400$

H $\quad-5.277400 \quad 8.059900 \quad 1.094100$

$\mathrm{H} \quad-4.524000 \quad 6.995100 \quad-1.051100$

$\mathrm{H} \quad-4.470000 \quad-3.205900 \quad 2.980900$

$\mathrm{H} \quad-4.986900 \quad-3.809200 \quad 5.358200$

$\mathrm{H} \quad-3.279000 \quad-4.862100 \quad 6.791900$

$\mathrm{H} \quad-0.985400 \quad-5.348000 \quad 5.900200$

\section{Y1}

$\begin{array}{llll}\text { Rh } & 0.000100 & 0.000000 & 0.214900\end{array}$

$\begin{array}{llll}\text { Rh } & 0.000100 & 0.000000 & -2.170900\end{array}$

O $\quad 1.122100 \quad 1.711000 \quad-2.113600$

O $\quad-1.122000 \quad-1.710900 \quad-2.113600$

$\begin{array}{llll}\mathrm{O} & 1.210000 & 1.672200 & 0.142200\end{array}$

$\begin{array}{llll}\text { O } & -1.209800 & -1.672200 & 0.142300\end{array}$

$\begin{array}{llll}\text { O } & 1.679100 & -1.178600 & -2.104900\end{array}$ 


\begin{tabular}{|c|c|c|c|}
\hline ) & -1.679000 & 1.178600 & -2.104700 \\
\hline J & -1.675000 & 1.199700 & 0.151600 \\
\hline 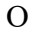 & 1.675200 & -1.199900 & 0.151400 \\
\hline U & 0.920700 & -4.074500 & -0.268000 \\
\hline $\mathrm{O}$ & 5.106100 & -3.062900 & 1.321600 \\
\hline $\mathrm{O}$ & 4.113200 & 1.136800 & -1.124500 \\
\hline $\mathrm{O}$ & 3.399200 & 4.932200 & 1.358600 \\
\hline $\mathrm{O}$ & -0.921000 & 4.074500 & -0.268600 \\
\hline $\mathrm{O}$ & -5.105800 & 3.062700 & 1.322400 \\
\hline $\mathrm{O}$ & -4.113000 & -1.136700 & -1.123900 \\
\hline $\mathrm{O}$ & -3.398700 & -4.932400 & 1.358700 \\
\hline $\mathrm{Cl}$ & -0.365400 & -5.999800 & 1500 \\
\hline $\mathrm{Cl}$ & 5.452700 & -4.751600 & 3.962400 \\
\hline $\mathrm{Cl}$ & 0.677100 & -7.325900 & 4.526500 \\
\hline $\mathrm{Cl}$ & 3.563800 & -6.702600 & 5.576000 \\
\hline $\mathrm{Cl}$ & 6.772100 & -0.131300 & \\
\hline $\mathrm{Cl}$ & 5.738200 & 5.030900 & 5800 \\
\hline $\mathrm{Cl}$ & 8.213500 & 3.168300 & 4.080500 \\
\hline $\mathrm{Cl}$ & 0.365200 & 6.000800 & 1.860000 \\
\hline $\mathrm{Cl}$ & -5.452100 & 4.752300 & 3.962600 \\
\hline $\mathrm{Cl}$ & -0.676900 & 7.327700 & 4.524700 \\
\hline $\mathrm{Cl}$ & -3.563100 & 6.704200 & 5.575100 \\
\hline $\mathrm{Cl}$ & -6.772000 & 0.131100 & -0.012300 \\
\hline $\mathrm{Cl}$ & -5.737300 & -5.031200 & \\
\hline $\mathrm{Cl}$ & -8.727400 & -0.608800 & 8500 \\
\hline $\mathrm{Cl}$ & -8.212400 & -3.168800 & 1600 \\
\hline $\mathrm{Cl}$ & 8.728000 & 0.608400 & 2.347200 \\
\hline $\mathrm{N}$ & 3.117400 & -3.462000 & 0.187100 \\
\hline $\mathrm{N}$ & 3.554800 & 3.183200 & -0.167200 \\
\hline $\mathrm{N}$ & -3.117500 & 3.461800 & 0.187200 \\
\hline $\mathrm{N}$ & -3.554500 & -3.183200 & -0.1 \\
\hline $\mathrm{C}$ & -2.121900 & 1.557200 & -0.986100 \\
\hline $\mathrm{C}$ & 2.122000 & -1.557400 & -0.986300 \\
\hline $\mathrm{C}$ & 1.484000 & 2.173300 & -0.996800 \\
\hline $\mathrm{C}$ & -1.483900 & -2.173200 & -0.996900 \\
\hline $\mathrm{C}$ & 3.352700 & -2.494600 & -0.891700 \\
\hline $\mathrm{C}$ & 3.853400 & -3.163300 & -2.208000 \\
\hline $\mathrm{C}$ & 4.388200 & -2.079300 & -3.198800 \\
\hline $\mathrm{C}$ & 5.041600 & -4.126900 & -1.889100 \\
\hline $\mathrm{C}$ & 2.739600 & -3.996700 & -2.913800 \\
\hline $\mathrm{C}$ & 1.906000 & -4.120800 & 0.437800 \\
\hline $\mathrm{C}$ & 4.030700 & -3.615200 & 1.246600 \\
\hline $\mathrm{C}$ & 4.919900 & -2.737300 & -4.489300 \\
\hline $\mathrm{C}$ & 5.581400 & -4.780900 & -3.179700 \\
\hline $\mathrm{C}$ & 3.285200 & -4.639100 & -4.207700 \\
\hline $\mathrm{C}$ & 2.101800 & -4.854000 & 1.729100 \\
\hline $\mathrm{C}$ & 3.383500 & -4.565400 & 2.204600 \\
\hline $\mathrm{C}$ & 6.084200 & -3.685500 & -4.140400 \\
\hline $\mathrm{C}$ & 3.783800 & -3.535400 & -5.164700 \\
\hline $\mathrm{C}$ & 4.453200 & -5.585000 & -3.859000 \\
\hline $\mathrm{C}$ & 1.239700 & -5.692100 & 2.424400 \\
\hline $\mathrm{C}$ & 3.861600 & -5.114900 & 3.385800 \\
\hline $\mathrm{C}$ & 1.710400 & -6.266700 & 3.627600 \\
\hline $\mathrm{C}$ & 3.008200 & -5.984500 & 4.101400 \\
\hline $\mathrm{C}$ & 2.311600 & 3.478500 & -0.893900 \\
\hline $\mathrm{C}$ & 2.550100 & 4.286500 & -2.204600 \\
\hline $\mathrm{C}$ & 1.186100 & 4.703100 & -2.842400 \\
\hline $\mathrm{C}$ & 3.328700 & 5.599100 & -1.872200 \\
\hline $\mathrm{C}$ & 3.385100 & 3.490500 & -3.254700 \\
\hline $\mathrm{C}$ & 4.326000 & 2.021500 & -0.322200 \\
\hline $\mathrm{C}$ & 3.958200 & 3.938500 & 0.949100 \\
\hline & 1.413700 & 5.539200 & -4.118200 \\
\hline
\end{tabular}

$\begin{array}{llll}\text { C } & 3.550800 & 6.444200 & -3.145600\end{array}$

$\begin{array}{llll}\text { C } & 3.595300 & 4.338900 & -4.528600\end{array}$

$\begin{array}{llll}\text { C } & 5.410200 & 2.114700 & 0.707100\end{array}$

C $\quad 5.184600 \quad 3.259500 \quad 1.475400$

C $\quad 2.187500 \quad 6.823000 \quad-3.758000$

$\begin{array}{llll}\text { C } & 2.225800 & 4.710900 & -5.136600\end{array}$

$\begin{array}{llll}\text { C } & 4.366000 & 5.626100 & -4.168400\end{array}$

$\begin{array}{llll}\text { C } & 6.491000 & 1.277900 & 0.949600\end{array}$

$\begin{array}{llll}\text { C } & 6.027500 & 3.610400 & 2.520600\end{array}$

C $\quad 7.363700 \quad 1.618100 \quad 2.008500$

$\begin{array}{llll}\text { C } & 7.133000 & 2.771200 & 2.787000\end{array}$

$\begin{array}{llll}\text { C } & -3.352900 & 2.494100 & -0.891300\end{array}$

C $\quad-3.854100 \quad 3.162300 \quad-2.207600$

C $\quad-4.388800 \quad 2.078000 \quad-3.198100$

C $\quad-5.042600 \quad 4.125600 \quad-1.888600$

$\begin{array}{llll}\text { C } & -2.740800 & 3.996100 & -2.913800\end{array}$

C $\quad-1.906100 \quad 4.120800 \quad 0.437500$

$\begin{array}{llll}\text { C } & -4.030600 & 3.615200 & 1.246900\end{array}$

$\begin{array}{llll}\text { C } & -4.921000 & 2.735600 & -4.488600\end{array}$

C $\quad-5.583000 \quad 4.779100 \quad-3.179100$

$\begin{array}{llll}\text { C } & -3.287000 & 4.638000 & -4.207700\end{array}$

$\begin{array}{llll}\text { C } & -2.101700 & 4.854400 & 1.728500\end{array}$

$\begin{array}{llll}\text { C } & -3.383300 & 4.565800 & 2.204400\end{array}$

$\begin{array}{llll}\text { C } & -6.085600 & 3.683300 & -4.139500\end{array}$

C $\quad-3.785400 \quad 3.534000-5.164400$

C $\quad-4.455200 \quad 5.583600 \quad-3.858900$

$\begin{array}{llll}\text { C } & -1.239700 & 5.692900 & 2.423300\end{array}$

C $\quad-3.861200 \quad 5.115600 \quad 3.385500$

C $\quad-1.710200 \quad 6.267900 \quad 3.626400$

$\begin{array}{llll}\text { C } & -3.007800 & 5.985600 & 4.100600\end{array}$

C $\quad-2.311400 \quad-3.478400 \quad-0.893900$

$\begin{array}{llll}\text { C } & -2.550200 & -4.286200 & -2.204700\end{array}$

C $\quad-1.186300 \quad-4.702800 \quad-2.842800$

C $\quad-3.328800 \quad-5.598900 \quad-1.872300$

C $\quad-3.385300 \quad-3.490100 \quad-3.254600$

C $\quad-4.325800 \quad-2.021500-0.321700$

C $\quad-3.957700 \quad-3.938600 \quad 0.949300$

C $\quad-1.414100 \quad-5.538700 \quad-4.118600$

C $\quad-3.551100 \quad-6.443800 \quad-3.145800$

C $\quad-3.595800 \quad-4.338400 \quad-4.528500$

$\begin{array}{llll}\text { C } & -5.409800 & -2.114800 & 0.707700\end{array}$

C $\quad-5.184000 \quad-3.259700 \quad 1.476000$

C $\quad-2.187900 \quad-6.822600 \quad-3.758400$

C $\quad-2.226400 \quad-4.710300 \quad-5.136800$

$\begin{array}{llll}\text { C } & -4.366500 & -5.625600 & -4.168400\end{array}$

$\begin{array}{llll}\text { C } & -6.490600 & -1.278200 & 0.950500\end{array}$

$\begin{array}{lrrr}\text { C } & -6.026700 & -3.610700 & 2.521300\end{array}$

C $\quad-7.363100 \quad-1.618500 \quad 2.009500$

C $\quad-7.132200 \quad-2.771500 \quad 2.787900$

H $\quad 3.600500 \quad-1.355700 \quad-3.485100$

H $\quad 5.203900 \quad-1.492100 \quad-2.720900$

H $\quad 5.863800 \quad-3.571100 \quad-1.385800$

$\mathrm{H} \quad 4.717800 \quad-4.935500 \quad-1.197700$

H $\quad 2.377300 \quad-4.805300 \quad-2.245000$

$\mathrm{H} \quad \begin{array}{llll}1.865900 & -3.366500 & -3.174200\end{array}$

$\mathrm{H} \quad 5.282300 \quad-1.949100 \quad-5.184800$

H $\quad 6.421200 \quad-5.463800 \quad-2.926000$

H $\quad 2.475100 \quad-5.218800 \quad-4.701900$

H $\quad 6.488700 \quad-4.148300 \quad-5.067300$

H $\quad 6.912200 \quad-3.113800 \quad-3.666100$

H $\quad 2.945000 \quad-2.854300 \quad-5.429100$

H $\quad 4.151200 \quad-3.991200 \quad-6.110300$
H $\quad 4.836300 \quad-6.072300 \quad-4.782500$

$\mathrm{H} \quad 4.099700 \quad-6.392700 \quad-3.180800$

$\mathrm{H} \quad 0.575500 \quad 3.820500 \quad-3.115600$

H $\quad 0.589400 \quad 5.298900-2.117100$

H $\quad 2.767300 \quad 6.202100 \quad-1.124100$

H $\quad 4.321500 \quad 5.364500 \quad-1.430000$

$\mathrm{H} \quad 4.379200 \quad 3.223800 \quad-2.836400$

$\mathrm{H} \quad 2.885800 \quad 2.543300 \quad-3.541000$

H $\quad 0.431100 \quad 5.811800 \quad-4.561900$

H $\quad 4.108200 \quad 7.369800 \quad-2.883800$

H $\quad 4.181800 \quad 3.753500 \quad-5.269900$

H $\quad 2.338200 \quad 7.444100 \quad-4.668200$

$\mathrm{H} \quad 1.600900 \quad 7.431100 \quad-3.034500$

H $\quad 1.667000 \quad 3.788300 \quad-5.408400$

H $\quad 2.372200 \quad 5.296900 \quad-6.070500$

H $\quad 4.544200 \quad 6.231400 \quad-5.084400$

H $\quad 5.361000 \quad 5.368100 \quad-3.743300$

$\mathrm{H} \quad-3.600800 \quad 1.354600 \quad-3.484500$

$\begin{array}{llll}\mathrm{H} & -5.204100 & 1.490600 & -2.719900\end{array}$

$\mathrm{H} \quad-5.864500 \quad 3.569500 \quad-1.385000$

$\mathrm{H} \quad-4.719000 \quad 4.934400 \quad-1.197400$

$\begin{array}{llll}\mathrm{H} & -2.378700 & 4.805000 & -2.245200\end{array}$

H $\quad-1.866900 \quad 3.366200 \quad-3.174300$

$\mathrm{H} \quad-5.283200 \quad 1.947100 \quad-5.183800$

H $\quad-6.423000 \quad 5.461700 \quad-2.925400$

$\mathrm{H} \quad-2.477200 \quad 5.218000 \quad-4.702200$

$\mathrm{H} \quad-6.490500 \quad 4.145900 \quad-5.066500$

H $\quad-6.913200 \quad 3.111400-3.665000$

$\mathrm{H} \quad-2.946300 \quad 2.853200 \quad-5.428800$

$\mathrm{H} \quad-4.153200 \quad 3.989500 \quad-6.110000$

$\mathrm{H} \quad-4.838800 \quad 6.070500 \quad-4.782400$

$\mathrm{H} \quad-4.101900 \quad 6.391500 \quad-3.180900$

$\mathrm{H} \quad-0.575800 \quad-3.820200 \quad-3.116000$

$\mathrm{H} \quad-0.589500 \quad-5.298700 \quad-2.117600$

$\mathrm{H} \quad-2.767200 \quad-6.202000 \quad-1.124300$

$\mathrm{H} \quad-4.321500 \quad-5.364300 \quad-1.429900$

$\mathrm{H} \quad-4.379400 \quad-3.223500 \quad-2.836100$

$\mathrm{H} \quad-2.886000 \quad-2.542900 \quad-3.540800$

H $\quad-0.431600 \quad-5.811400 \quad-4.562400$

$\mathrm{H} \quad-4.108500 \quad-7.369500 \quad-2.884000$

$\mathrm{H} \quad-4.182300 \quad-3.752900 \quad-5.269600$

$\mathrm{H} \quad-2.338800 \quad-7.443600 \quad-4.668700$

$\mathrm{H} \quad-1.601200 \quad-7.430800 \quad-3.035100$

$\mathrm{H} \quad-1.667600 \quad-3.787700 \quad-5.408600$

$\mathrm{H} \quad-2.372900 \quad-5.296300 \quad-6.070700$

$\mathrm{H} \quad-4.544800 \quad-6.230800 \quad-5.084300$

$\mathrm{H} \quad-5.361400 \quad-5.367600 \quad-3.743100$

$\mathrm{H} \quad-1.730600 \quad-4.108500 \quad-0.211900$

$\begin{array}{llll}\mathrm{H} & -4.160900 & 1.856900 & -0.509600\end{array}$

$\mathrm{H} \quad 1.730900 \quad 4.108500 \quad-0.211700$

$\mathrm{H} \quad 4.161100 \quad-1.857600 \quad-0.510300$

\section{Y2}

$\begin{array}{llll}\mathrm{Rh} & -0.252100 & -0.344100 & -0.090100\end{array}$

$\begin{array}{llll}\mathrm{Rh} & -0.811800 & -0.976400 & -2.324300\end{array}$

$\begin{array}{llll}\mathrm{O} & -2.115300 & 0.604400 & -2.361600\end{array}$

$\begin{array}{llll}\mathrm{O} & 0.480400 & -2.564900 & -2.154700\end{array}$

$\begin{array}{lrrr}\mathrm{O} & -1.595000 & 1.210300 & -0.251300\end{array}$

$\begin{array}{llll}\text { O } & 1.031900 & -1.963300 & -0.044600\end{array}$

$\begin{array}{llll}\mathrm{O} & 0.775000 & 0.166200 & -2.967200\end{array}$

$\begin{array}{llll}\mathrm{O} & -2.327700 & -2.130000 & -1.553800\end{array}$

$\begin{array}{llll}\mathrm{O} & -1.792700 & -1.557400 & 0.561000\end{array}$ 
$\begin{array}{llll}\text { O } & 1.285600 & 0.795900 & -0.860800\end{array}$

O $\quad 3.658000 \quad 4.055500 \quad-2.311700$

$\begin{array}{llll}\text { O } & 3.935900 & 0.030400 & -0.083400\end{array}$

$\begin{array}{llll}\mathrm{O} & -4.270500 & 4.221200 & 0.825200\end{array}$

O $\quad-0.843800 \quad 3.582700 \quad-2.163900$

$\begin{array}{llll}\text { O } & -4.471500 & -0.184600 & 0.548200\end{array}$

$\begin{array}{llll}\text { O } & -3.926500 & -4.038300 & 2.979200\end{array}$

O $\quad 1.597400 \quad-3.892400 \quad 2.068900$

O $\quad 4.816200 \quad-3.642600 \quad-1.221900$

$\begin{array}{llll}\mathrm{Cl} & 5.196300 & 6.125500 & -0.507700\end{array}$

$\begin{array}{llll}\mathrm{Cl} & 5.435300 & 0.632100 & 2.585800\end{array}$

$\begin{array}{llll}\mathrm{Cl} & 6.636400 & 5.940800 & 2.296000\end{array}$

$\begin{array}{llll}\text { Cl } & 6.749600 & 3.211000 & 3.830400\end{array}$

$\begin{array}{llll}\mathrm{Cl} & -3.642300 & 6.931900 & 2.292600\end{array}$

$\begin{array}{llll}\mathrm{Cl} & 1.126500 & 5.997500 & -1.731000\end{array}$

$\begin{array}{llll}\mathrm{Cl} & 1.093300 & 8.552800 & 0.125300\end{array}$

$\begin{array}{llll}\mathrm{Cl} & -5.480700 & 1.981100 & 2.589700\end{array}$

$\begin{array}{llll}\text { Cl } & -4.877800 & -3.349100 & 5.909800\end{array}$

$\mathrm{Cl} \quad-6.227800 \quad 1.930100 \quad 5.657400$

$\begin{array}{llll}\mathrm{Cl} & -5.921400 & -0.716100 & 7.310000\end{array}$

Cl $\quad 3.097900 \quad-3.483800 \quad 4.765100$

$\begin{array}{llll}\mathrm{Cl} & 7.525800 & -3.151500 & 0.280700\end{array}$

$\begin{array}{llll}\mathrm{Cl} & 6.116800 & -3.008400 & 5.548600\end{array}$

Cl $\quad 8.319100 \quad-2.842300 \quad 3.324100$

$\begin{array}{llll}\mathrm{Cl} & -1.272600 & 9.013300 & 2.129300\end{array}$

N $\quad 3.623000 \quad 1.884800 \quad-1.484700$

$\mathrm{N} \quad-2.769000 \quad 3.671700 \quad-0.864800$

$\mathrm{N} \quad-4.210600 \quad-2.307500 \quad 1.456900$

N $\quad 2.953800 \quad-3.846500 \quad 0.154900$

C $\quad-2.511400 \quad-2.151100 \quad-0.303800$

$\begin{array}{llll}\text { C } & 1.526100 & 0.707100 & -2.102600\end{array}$

C $\quad-2.266300 \quad 1.316300 \quad-1.329800$

C $\quad 1.092100 \quad-2.724300 \quad-1.052600$

$\begin{array}{llll}\text { C } & 2.832200 & 1.366900 & -2.606900\end{array}$

$\begin{array}{llll}\text { C } & 3.946100 & 3.254400 & -1.447700\end{array}$

$\begin{array}{llll}\text { C } & 4.065700 & 1.206700 & -0.331400\end{array}$

C $\quad 4.688300 \quad 3.472100 \quad-0.170600$

$\begin{array}{llll}\text { C } \quad 4.746100 & 2.252500 & 0.505900\end{array}$

$\begin{array}{llll}\text { C } & 5.261900 & 4.624700 & 0.350300\end{array}$

$\begin{array}{llll}\text { C } & 5.372900 & 2.141300 & 1.739600\end{array}$

C $\quad 5.906300 \quad 4.531600 \quad 1.603700$

$\begin{array}{lrrr}\text { C } & 5.958800 & 3.302300 & 2.292800\end{array}$

$\begin{array}{llll}\text { C } & -3.380600 & 2.391600 & -1.244900\end{array}$

C $\quad-3.264100 \quad 4.455000 \quad 0.192000$

$\begin{array}{llll}\text { C } & -1.526400 & 4.133200 & -1.327200\end{array}$

C $\quad-2.303100 \quad 5.593300 \quad 0.336900$

$\begin{array}{llll}\text { C } & -1.254700 & 5.394200 & -0.564500\end{array}$

$\begin{array}{llll}\text { C } & -2.336800 & 6.694400 & 1.181300\end{array}$

$\begin{array}{llll}\text { C } & -0.189900 & 6.281300 & -0.648700\end{array}$

$\begin{array}{llll}\text { C } & -1.270900 & 7.618800 & 1.102300\end{array}$

$\begin{array}{llll}\text { C } & -0.208000 & 7.412700 & 0.199100\end{array}$

$\begin{array}{llll}\text { C } & -3.636900 & -3.021500 & 0.314800\end{array}$

$\begin{array}{llll}\text { C } & -4.524900 & -0.942900 & 1.494100\end{array}$

$\begin{array}{llll}\text { C } & -4.253500 & -2.898400 & 2.735400\end{array}$

C $\quad-4.913300 \quad-0.658200 \quad 2.912500$

C $\quad-4.765200 \quad-1.834600 \quad 3.652600$

$\begin{array}{llll}\text { C } & -5.353000 & 0.520300 & 3.501500\end{array}$

C $\quad-5.063400 \quad-1.884900 \quad 5.007000$

$\begin{array}{llll}\text { C } & -5.667400 & 0.488200 & 4.879800\end{array}$

C $\quad-5.527400 \quad-0.701200 \quad 5.624000$

$\begin{array}{llll}\text { C } & 2.034500 & -3.940900 & -0.982100\end{array}$

C $\quad 2.672900 \quad-3.765100 \quad 1.535600$
C $\quad 4.322400 \quad-3.641200 \quad-0.114600$

C $\quad 4.001100 \quad-3.522300 \quad 2.195000$

C $\quad 4.984100 \quad-3.451200 \quad 1.208000$

C $\quad 4.317600 \quad-3.389600 \quad 3.540500$

$\begin{array}{llll}\text { C } & 6.320500 & -3.240800 & 1.519600\end{array}$

C $\quad 5.672300 \quad-3.176500 \quad 3.882400$

$\begin{array}{lrrr}\text { C } & 6.664300 & -3.102100 & 2.881900\end{array}$

$\begin{array}{llll}\text { C } & -5.338700 & 1.683600 & -5.143100\end{array}$

C $\quad-4.631400 \quad 3.040200 \quad-4.934200$

C $\quad-6.103900 \quad 1.294200 \quad-3.860100$

C $\quad-5.108400 \quad 1.167100 \quad-2.689000$

C $\quad-3.622000 \quad 2.926800 \quad-3.769700$

C $\quad-4.354400 \quad 2.515800 \quad-2.455800$

C $\quad-5.432900 \quad 3.604000 \quad-2.150300$

C $\quad-5.680900 \quad 4.121600 \quad-4.603300$

$\begin{array}{llll}\text { C } & -7.149200 & 2.377600 & -3.529700\end{array}$

C $\quad-6.436500 \quad 3.727900 \quad-3.317100$

$\begin{array}{llll}\text { C } & -4.076700 & -4.637000 & -1.623100\end{array}$

C $\quad-4.718700 \quad-3.595600 \quad-0.651700$

C $\quad-5.143400 \quad-5.237600 \quad-2.561700$

C $\quad-6.214900 \quad-5.962000 \quad-1.723100$

C $\quad-5.818100 \quad-4.344500 \quad 0.168900$

C $\quad-6.884100 \quad-4.954300 \quad-0.767700$

C $\quad-7.550800 \quad-3.830400 \quad-1.588100$

C $\quad-5.423300 \quad-2.483200-1.487100$

C $\quad-5.801100 \quad-4.107000-3.381700$

C $\quad-6.480400 \quad-3.101900 \quad-2.427300$

C $\quad 6.040400 \quad-1.167700 \quad-4.332900$

$\begin{array}{llll}\text { C } & 5.941700 & 0.303000 & -4.789800\end{array}$

C $\quad 4.624200 \quad-1.770700 \quad-4.231100$

C $\quad 3.799000 \quad-0.975200 \quad-3.196200$

$\begin{array}{llll}\text { C } & 5.115100 & 1.106200 & -3.762600\end{array}$

$\begin{array}{llll}\text { C } & 3.672200 & 0.520000 & -3.624500\end{array}$

$\begin{array}{llll}\text { C } & 3.011800 & 0.574000 & -5.041100\end{array}$

$\begin{array}{llll}\text { C } & 5.255800 & 0.370800 & -6.168300\end{array}$

$\begin{array}{llll}\text { C } & 3.932800 & -1.701300 & -5.608300\end{array}$

C $\quad 3.840200 \quad-0.230500 \quad-6.065300$

C $\quad 1.038800 \quad-5.605400 \quad-2.649900$

C $\quad 1.324600 \quad-5.328600 \quad-1.137500$

C $\quad 0.368200 \quad-6.982400 \quad-2.841400$

$\begin{array}{llll}\text { C } & 1.301300 & -8.087900 & -2.309100\end{array}$

C $\quad 2.244600 \quad-6.477800 \quad-0.615800$

C $\quad 1.570100 \quad-7.852600 \quad-0.809600$

C $\quad 0.236600 \quad-7.884400 \quad-0.033200$

C $\quad-0.034500 \quad-5.399200 \quad-0.370200$

$\begin{array}{llll}\text { C } & -0.966500 & -7.014400 & -2.069400\end{array}$

C $\quad-0.698600 \quad-6.779700 \quad-0.568000$

H $\quad 2.715800 \quad-3.822000 \quad-1.834200$

H $\quad-3.118400 \quad-3.872000 \quad 0.778700$

$\mathrm{H} \quad-3.969400 \quad 2.089400 \quad-0.371000$

$\mathrm{H} \quad 2.516400 \quad 2.284400 \quad-3.121500$

$\mathrm{H} \quad-4.590600 \quad 0.899200 \quad-5.392900$

$\mathrm{H} \quad-6.045200 \quad 1.752100 \quad-5.999400$

$\mathrm{H} \quad-4.092100 \quad 3.322600 \quad-5.864600$

$\mathrm{H} \quad-6.617700 \quad 0.320400 \quad-4.016100$

$\mathrm{H} \quad-4.409300 \quad 0.343300 \quad-2.928100$

$\mathrm{H} \quad-5.658000 \quad 0.869200 \quad-1.768700$

$\mathrm{H} \quad-3.115100 \quad 3.907000 \quad-3.638500$

$\mathrm{H} \quad-2.842200 \quad 2.190100 \quad-4.049000$

H $\quad-5.983700 \quad 3.350000 \quad-1.217300$

$\mathrm{H} \quad-4.956000 \quad 4.596100 \quad-1.994800$

H $\quad-6.397000 \quad 4.227700 \quad-5.447700$
H $\quad-5.182600 \quad 5.106500 \quad-4.465900$

$\mathrm{H} \quad-7.884900 \quad 2.461900 \quad-4.359600$

H $\quad-7.713100 \quad 2.096000 \quad-2.613300$

$\mathrm{H} \quad-7.189900 \quad 4.509400 \quad-3.076800$

$\mathrm{H} \quad-3.598300 \quad-5.454900 \quad-1.040900$

$\mathrm{H} \quad-3.284800 \quad-4.184500 \quad-2.251000$

$\mathrm{H} \quad-4.661700 \quad-5.964100 \quad-3.252100$

$\mathrm{H} \quad-5.749200 \quad-6.787500 \quad-1.140900$

H $\quad-6.978000 \quad-6.417100 \quad-2.392100$

$\mathrm{H} \quad-6.327400 \quad-3.648500 \quad 0.871100$

H $\quad-5.358800 \quad-5.153000 \quad 0.779900$

H $\quad-7.655500 \quad-5.476700 \quad-0.160900$

H $\quad-8.330900 \quad-4.258100 \quad-2.255700$

H $\quad-8.056100 \quad-3.110300 \quad-0.907400$

H $\quad-4.695200 \quad-1.917500 \quad-2.100800$

H $\quad-5.929500 \quad-1.757100 \quad-0.817500$

$\mathrm{H} \quad-5.034100 \quad-3.587300 \quad-3.997500$

H $\quad-6.553500 \quad-4.534100-4.080600$

H $\quad-6.962800 \quad-2.295400 \quad-3.021700$

H $\quad 6.551100 \quad-1.227300 \quad-3.346400$

H $\quad 6.650400 \quad-1.750800 \quad-5.057400$

H $\quad 6.962900 \quad 0.736400 \quad-4.863800$

H $\quad 4.696900 \quad-2.832500 \quad-3.910000$

H $\quad 4.299100 \quad-1.059200 \quad-2.212900$

H $\quad 2.800400 \quad-1.448600 \quad-3.104400$

$\mathrm{H} \quad 5.067700 \quad 2.172100 \quad-4.078400$

$\mathrm{H} \quad 5.649100 \quad 1.073200 \quad-2.787800$

H $\quad 1.985500 \quad 0.156600 \quad-5.027900$

$\mathrm{H} \quad 2.928100 \quad 1.628400 \quad-5.387000$

H $\quad 5.854000 \quad-0.191000 \quad-6.919000$

H $\quad 5.197300 \quad 1.426600 \quad-6.513100$

$\mathrm{H} \quad 4.508500 \quad-2.294500 \quad-6.352300$

$\mathrm{H} \quad 2.915100 \quad-2.146000 \quad-5.545500$

H $\quad 3.346400 \quad-0.183200 \quad-7.060300$

$\mathrm{H} \quad 1.985800 \quad-5.578300 \quad-3.234000$

$\mathrm{H} \quad 0.370500 \quad-4.829700 \quad-3.077500$

H $\quad 0.173600 \quad-7.152700 \quad-3.922800$

H $\quad 2.259800 \quad-8.081900 \quad-2.873400$

H $\quad 0.833600 \quad-9.085700 \quad-2.459700$

H $\quad 2.461300 \quad-6.348100 \quad 0.466500$

$\mathrm{H} \quad 3.219200 \quad-6.462200 \quad-1.152700$

H $\quad 2.240900 \quad-8.651600 \quad-0.425100$

$\mathrm{H} \quad-0.246500 \quad-8.879500 \quad-0.148900$

$\mathrm{H} \quad 0.424100 \quad-7.731400 \quad 1.052400$

$\mathrm{H} \quad-0.735700 \quad-4.616800 \quad-0.732400$

$\mathrm{H} \quad 0.113300 \quad-5.231800 \quad 0.713200$

$\mathrm{H} \quad-1.649000 \quad-6.231000 \quad-2.464200$

$\mathrm{H} \quad-1.468800 \quad-7.995600 \quad-2.217200$

H $\quad-1.660800 \quad-6.810200 \quad-0.011700$ 


\subsection{Carboxylate Ligands, Rotational Transition States, and SMART Probe}

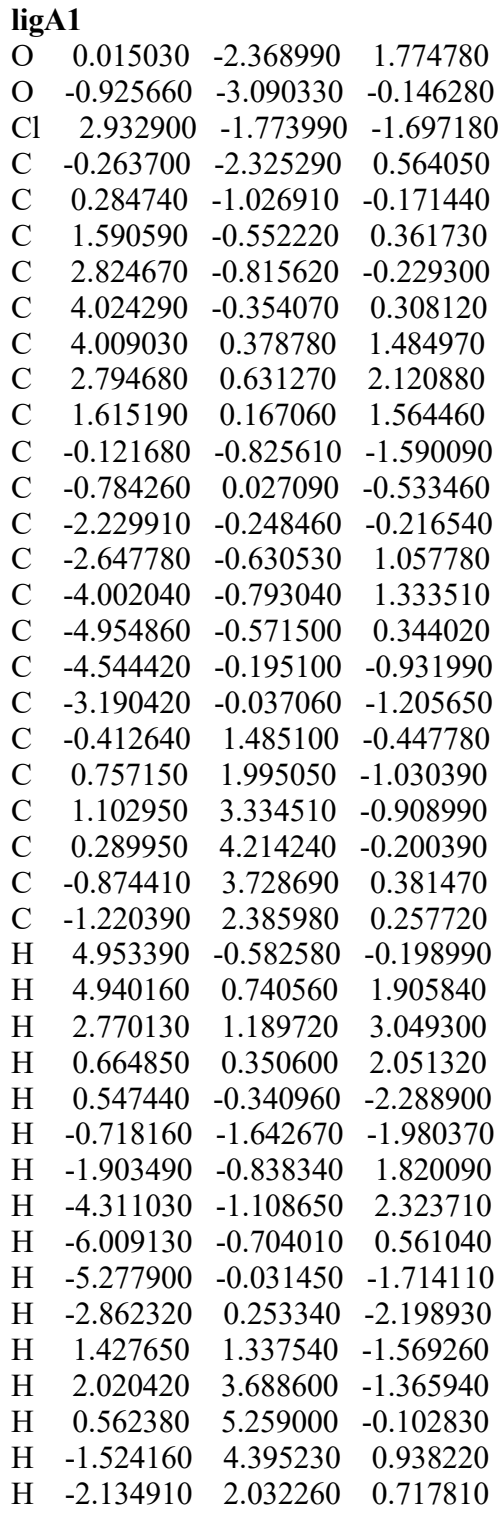

\section{ligA_TSrot}

$\begin{array}{llll}\text { O } & -0.313839 & -2.591770 & -1.352410\end{array}$

$\begin{array}{llll}\text { O } & 0.377351 & -3.162320 & 0.716580\end{array}$

$\mathrm{Cl} \quad-2.131791 \quad 1.996939-0.242420$

C $\quad-0.103759-2.406710-0.140340$

C $\quad-0.538670 \quad-0.970970 \quad 0.362960$

C $\quad-2.018290 \quad-0.762541 \quad 0.105900$

C $\quad-2.794050 \quad 0.378869-0.121490$

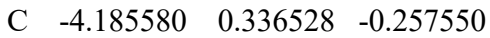

C $\quad-4.870320-0.857832-0.151520$

$\begin{array}{llll}\text { C } & -4.143120 & -2.017112 & 0.102620\end{array}$

C $\quad-2.767980-1.957061 \quad 0.223100$

C $\quad-0.001620 \quad-0.559240 \quad 1.711020$

C $\quad 0.682570 \quad-0.041130 \quad 0.479620$

$\begin{array}{llll}\text { C } & 2.038670 & -0.644549 & 0.152010\end{array}$

$\begin{array}{llll}\text { C } & 2.350730 & -1.137079 & -1.115090\end{array}$ $\begin{array}{llll}\text { C } & 3.638360 & -1.582179 & -1.396000\end{array}$

C $\quad 4.631500 \quad-1.534918-0.423080$

$\begin{array}{llll}\text { C } & 4.325990 & -1.044468 & 0.843040\end{array}$

$\begin{array}{llll}\text { C } & 3.038380 & -0.603209 & 1.123290\end{array}$

$\begin{array}{llll}\text { C } & 0.776219 & 1.436670 & 0.205310\end{array}$

$\begin{array}{llll}\text { C } & 0.807319 & 2.365050 & 1.242330\end{array}$

$\begin{array}{llll}\text { C } & 0.946798 & 3.725830 & 0.978600\end{array}$

$\begin{array}{llll}\text { C } & 1.076408 & 4.173940 & -0.330310\end{array}$

$\begin{array}{llll}\text { C } & 1.082878 & 3.247330 & -1.373030\end{array}$

$\begin{array}{llll}\text { C } & 0.943369 & 1.894340 & -1.105080\end{array}$

$\mathrm{H} \quad-4.710501 \quad 1.264508 \quad-0.445820$

H $\quad-5.948290 \quad-0.880652 \quad-0.261780$

$\mathrm{H} \quad-4.643749 \quad-2.973302 \quad 0.200640$

$\mathrm{H} \quad-2.226969-2.873331 \quad 0.422380$

$\mathrm{H} \quad-0.606700 \quad 0.149520 \quad 2.267830$

H $\quad 0.479720 \quad-1.352890 \quad 2.272190$

H $\quad 1.565880 \quad-1.225709 \quad-1.857290$

H $\quad 3.859990 \quad-1.984089-2.378430$

H $\quad 5.632190 \quad-1.889208 \quad-0.644970$

H $\quad 5.088400 \quad-1.011698 \quad 1.613860$

$\mathrm{H} \quad 2.795110 \quad-0.223809 \quad 2.110770$

$\mathrm{H} \quad 0.700819 \quad 2.020080 \quad 2.264670$

$\mathrm{H} \quad 0.951898 \quad 4.435320 \quad 1.798990$

H $\quad 1.179078 \quad 5.232960 \quad-0.538950$

H $\quad 1.189388 \quad 3.584990 \quad-2.398020$

H $\quad 0.931279 \quad 1.174690 \quad-1.916170$

\section{ligA_TSalt}

O $\quad 0.312810 \quad-2.526701 \quad-0.735310$

O $\quad-0.232860 \quad-3.067751 \quad 1.386890$

$\begin{array}{llll}\mathrm{Cl} & -2.654720 & -2.495121 & -0.924750\end{array}$

$\begin{array}{llll}\text { C } & -0.073290 & -2.303411 & 0.423970\end{array}$

$\begin{array}{llll}\text { C } & -0.385900 & -0.786931 & 0.733700\end{array}$

$\begin{array}{llll}\text { C } & -1.770550 & -0.245551 & 0.465030\end{array}$

$\begin{array}{llll}\text { C } & -2.796120 & -0.894391 & -0.250480\end{array}$

$\begin{array}{llll}\text { C } & -4.045140 & -0.301591 & -0.457440\end{array}$

$\begin{array}{llll}\text { C } & -4.338020 & 0.954049 & 0.042530\end{array}$

C $\quad-3.362600 \quad 1.616559 \quad 0.777620$

$\begin{array}{llll}\text { C } & -2.129750 & 1.019089 & 0.976200\end{array}$

C $\quad 0.330290 \quad-0.253711 \quad 1.950280$

$\begin{array}{llll}\text { C } & 0.826470 & 0.150549 & 0.591200\end{array}$

$\begin{array}{llll}\text { C } & 2.177030 & -0.366981 & 0.150650\end{array}$

$\begin{array}{llll}\text { C } & 2.426350 & -0.707431 & -1.181560\end{array}$

$\begin{array}{llll}\text { C } & 3.701330 & -1.074021 & -1.586910\end{array}$

$\begin{array}{llll}\text { C } & 4.752780 & -1.106851 & -0.671430\end{array}$

$\begin{array}{llll}\text { C } & 4.514370 & -0.773651 & 0.655070\end{array}$

$\begin{array}{llll}\text { C } & 3.232020 & -0.405071 & 1.058900\end{array}$

$\begin{array}{llll}\text { C } & 0.647790 & 1.563629 & 0.102600\end{array}$

$\begin{array}{llll}\text { C } & 1.094830 & 2.644469 & 0.861740\end{array}$

$\begin{array}{llll}\text { C } & 0.958500 & 3.948589 & 0.394890\end{array}$

$\begin{array}{llll}\text { C } & 0.377230 & 4.186819 & -0.847020\end{array}$

C $\quad-0.060940 \quad 3.112479 \quad-1.617440$

C $\quad 0.074370 \quad 1.812209 \quad-1.145750$

$\mathrm{H} \quad-4.784460 \quad-0.859251 \quad-1.018270$

$\mathrm{H} \quad-5.311330 \quad 1.397949 \quad-0.131460$

H $\quad-3.552450 \quad 2.598729 \quad 1.194960$

$\begin{array}{llll}\mathrm{H} & -1.401180 & 1.578169 & 1.544730\end{array}$

$\mathrm{H} \quad-0.151400 \quad 0.485259 \quad 2.579830$

H $\quad 0.892300 \quad-1.009871 \quad 2.485140$
H $\quad 1.598740 \quad-0.733161 \quad-1.877670$

H $\quad 3.873700 \quad-1.356251 \quad-2.619500$

H $\quad 5.746060 \quad-1.402801 \quad-0.990860$

$\begin{array}{llll}\mathrm{H} & 5.320570 & -0.804211 & 1.379930\end{array}$

H $\quad 3.045170 \quad-0.149301 \quad 2.096520$

$\begin{array}{llll}\mathrm{H} & 1.547800 & 2.453019 & 1.829680\end{array}$

$\mathrm{H} \quad 1.306740 \quad 4.778579 \quad 1.000080$

$\mathrm{H} \quad 0.265210 \quad 5.201769 \quad-1.211550$

$\mathrm{H} \quad-0.520620 \quad 3.289079 \quad-2.583420$

$\mathrm{H} \quad-0.290430 \quad 0.971859 \quad-1.728090$

\section{ligA2}

$\begin{array}{llll}\mathrm{O} & -1.262610 & -2.973620 & -0.873390\end{array}$

$\begin{array}{llll}\mathrm{O} & 0.178230 & -2.919960 & 0.863230\end{array}$

$\begin{array}{llll}\mathrm{Cl} & 1.017260 & -0.040820 & 2.141070\end{array}$

$\begin{array}{llll}\text { C } & -0.348500 & -2.506700 & -0.180480\end{array}$

$\begin{array}{llll}\text { C } & 0.250610 & -1.127920 & -0.705830\end{array}$

$\begin{array}{llll}\text { C } & 1.686500 & -0.940990 & -0.357050\end{array}$

$\begin{array}{llll}\text { C } & 2.140320 & -0.495220 & 0.887440\end{array}$

$\begin{array}{llll}\text { C } & 3.494790 & -0.367010 & 1.172670\end{array}$

C $\quad 4.441650 \quad-0.701720 \quad 0.213110$

$\begin{array}{llll}\text { C } & 4.022460 & -1.167800 & -1.028620\end{array}$

$\begin{array}{llll}\text { C } & 2.664580 & -1.281270 & -1.296580\end{array}$

$\begin{array}{llll}\text { C } & -0.264080 & -0.648770 & -2.024460\end{array}$

$\begin{array}{llll}\text { C } & -0.699170 & 0.077980 & -0.771210\end{array}$

$\begin{array}{llll}\text { C } & -2.124090 & -0.103180 & -0.320240\end{array}$

$\begin{array}{llll}\text { C } & -2.436390 & -0.685780 & 0.906970\end{array}$

$\begin{array}{llll}\text { C } & -3.759620 & -0.764790 & 1.329100\end{array}$

$\begin{array}{llll}\text { C } & -4.785050 & -0.260740 & 0.534490\end{array}$

$\begin{array}{llll}\text { C } & -4.480260 & 0.316120 & -0.695330\end{array}$

C $\quad-3.156870 \quad 0.391400 \quad-1.115850$

$\begin{array}{llll}\text { C } & -0.155710 & 1.464380 & -0.527660\end{array}$

$\begin{array}{llll}\text { C } & 1.031190 & 1.920430 & -1.117620\end{array}$

C $\quad 1.531710 \quad 3.190130 \quad-0.852340$

$\begin{array}{llll}\text { C } & 0.857000 & 4.051760 & 0.005430\end{array}$

C $\quad-0.327430 \quad 3.620640 \quad 0.591910$

C $\quad-0.824030 \quad 2.349030 \quad 0.329580$

H $\quad 3.794120 \quad-0.006500 \quad 2.148820$

H $\quad 5.496900 \quad-0.602800 \quad 0.440170$

$\mathrm{H} \quad 4.749110 \quad-1.444190 \quad-1.784200$

$\mathrm{H} \quad 2.328100 \quad-1.649250 \quad-2.260210$

$\mathrm{H} \quad 0.393480 \quad-0.120070 \quad-2.705160$

$\mathrm{H} \quad-1.000910 \quad-1.309280 \quad-2.466260$

$\mathrm{H} \quad-1.638920 \quad-1.103850 \quad 1.512490$

$\mathrm{H} \quad-3.989910 \quad-1.236040 \quad 2.278010$

$\mathrm{H} \quad-5.815910 \quad-0.328930 \quad 0.864540$

$\mathrm{H} \quad-5.272850 \quad 0.701030 \quad-1.327980$

$\mathrm{H} \quad-2.911050 \quad 0.838800 \quad-2.074140$

$\mathrm{H} \quad 1.594880 \quad 1.275430 \quad-1.778660$

H $\quad 2.459910 \quad 3.501610-1.318850$

$\mathrm{H} \quad 1.249230 \quad 5.040630 \quad 0.214320$

$\mathrm{H} \quad-0.870220 \quad 4.273760 \quad 1.266560$

H $\quad-1.741860 \quad 2.032250 \quad 0.808980$

\section{ligB1}

$\begin{array}{llll}\text { O } & 2.266630 & -3.006150 & -0.200250\end{array}$

O $\quad 0.871080 \quad-2.267870 \quad-1.814720$

$\mathrm{Cl} \quad-1.344820 \quad-2.336860 \quad 2.202810$

$\begin{array}{llll}\mathrm{Br} & -5.060450 & 0.354350 & -0.844010\end{array}$ 


$\begin{array}{rrrr}\text { C } & -0.932430 & -0.024510 & -1.051940 \\ \mathrm{C} & 0.847360 & -1.142240 & 0.279750 \\ \mathrm{C} & 1.826960 & 0.022060 & 0.542570 \\ \mathrm{C} & -2.967180 & -0.939350 & 0.591300 \\ \mathrm{C} & -0.584630 & -0.812020 & 0.053810 \\ \mathrm{C} & -2.240720 & 0.326610 & -1.336310 \\ \mathrm{C} & 1.256510 & 1.411410 & 0.670330 \\ \mathrm{C} & 1.386370 & -2.286930 & -0.683420 \\ \mathrm{C} & 3.203920 & -0.013960 & -0.065410 \\ \mathrm{C} & 3.409590 & -0.248160 & -1.424470 \\ \mathrm{C} & 1.503690 & -0.997020 & 1.609080 \\ \mathrm{C} & 0.192190 & 1.708520 & 1.533740 \\ \mathrm{C} & -1.636770 & -1.274820 & 0.840450 \\ \mathrm{C} & 1.752070 & 2.457140 & -0.118550 \\ \mathrm{C} & -3.246750 & -0.131710 & -0.494710 \\ \mathrm{C} & 0.146570 & 4.008990 & 0.801720 \\ \mathrm{C} & 4.692520 & -0.190740 & -1.960580 \\ \mathrm{C} & 5.585790 & 0.336130 & 0.209330 \\ \mathrm{C} & 4.303470 & 0.274810 & 0.742990 \\ \mathrm{C} & 1.204680 & 3.735330 & -0.056150 \\ \mathrm{C} & -0.354360 & 2.983750 & 1.598330 \\ \mathrm{C} & 5.783300 & 0.105680 & -1.149410 \\ \mathrm{H} & -0.129080 & 0.313090 & -1.695230 \\ \mathrm{H} & -3.752540 & -1.312740 & 1.233870 \\ \mathrm{H} & -2.477980 & 0.944510 & -2.192010 \\ \mathrm{H} & 2.564770 & -0.513400 & -2.052250 \\ \mathrm{H} & 0.935810 & -0.673520 & 2.471330 \\ \mathrm{H} & 2.264620 & -1.748350 & 1.788370 \\ \mathrm{H} & -0.239290 & 0.932410 & 2.153440 \\ \mathrm{H} & 2.578190 & 2.268190 & -0.793170 \\ \mathrm{H} & -0.283780 & 5.002770 & 0.848320 \\ \mathrm{H} & 4.839480 & -0.393240 & -3.015510 \\ \mathrm{H} & 6.430370 & 0.556950 & 0.852960 \\ \mathrm{H} & 4.141820 & 0.450520 & 1.802040 \\ \mathrm{H} & 1.611980 & 4.519020 & -0.685590 \\ \mathrm{H} & -1.183590 & 3.172410 & 2.271030 \\ \mathrm{H} & 6.782260 & 0.144190 & -1.569660\end{array}$

\section{ligB TSrot}

O $\quad \begin{array}{lll}1.155719 & -3.144720 & 0.752280\end{array}$

$\begin{array}{lllll}\text { O } & 0.455449 & -2.515670 & -1.297140\end{array}$

$\begin{array}{llll}\mathrm{Cl} & -0.846110 & 2.236851 & -0.166190\end{array}$

$\begin{array}{llll}\mathrm{Br} & -5.724670 & -0.189148 & -0.123980\end{array}$

$\begin{array}{llll}\text { C } & -1.858771 & -1.629429 & 0.332520\end{array}$

$\begin{array}{llll}\text { C } & 0.459599 & -0.872760 & 0.410170\end{array}$

$\begin{array}{llll}\text { C } & 1.770620 & -0.070960 & 0.486560\end{array}$

$\begin{array}{llll}\text { C } & -3.051360 & 0.799501 & -0.120820\end{array}$

$\begin{array}{llll}\text { C } & -0.996290 & -0.516989 & 0.191270\end{array}$

C $\quad-3.236351 \quad-1.562608 \quad 0.250460$

$\begin{array}{llll}\text { C } & 2.002950 & 1.388700 & 0.198300\end{array}$

$\begin{array}{llll}\text { C } & 0.728089 & -2.348490 & -0.095120\end{array}$

$\begin{array}{llll}\text { C } & 3.046899 & -0.811680 & 0.122890\end{array}$

$\begin{array}{llll}\text { C } & 3.267779 & -1.336491 & -1.150390\end{array}$

$\begin{array}{llll}\text { C } & 1.073360 & -0.511600 & 1.740270\end{array}$

$\begin{array}{llll}\text { C } & 2.157560 & 2.313820 & 1.227130\end{array}$

$\begin{array}{llll}\text { C } & -1.658810 & 0.696221 & -0.020790\end{array}$

$\begin{array}{llll}\text { C } & 2.173950 & 1.821070 & -1.120070\end{array}$

C $\quad-3.826560 \quad-0.330018 \quad 0.011990$

$\begin{array}{llll}\text { C } & 2.553841 & 4.080000 & -0.366980\end{array}$

$\begin{array}{llll}\text { C } & 4.493069 & -1.913921 & -1.466770\end{array}$

$\begin{array}{llll}\text { C } & 5.299019 & -1.446481 & 0.748700\end{array}$

$\begin{array}{llll}\text { C } & 4.073459 & -0.872451 & 1.064480\end{array}$
}

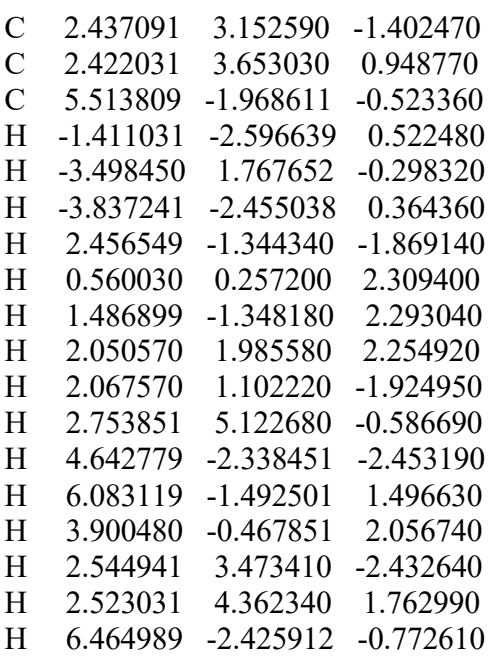

\section{ligB_TSalt}

$\begin{array}{lrrrrrrr}\mathrm{O} & 1.440500 & -3.125720 & 1.384919 & \mathrm{C} & -0.363240 & 4.109930 & -0.027370 \\ \mathrm{O} & 1.805170 & -2.476500 & -0.746161 & \mathrm{C} & -4.643560 & -0.968800 & 1.471850 \\ \mathrm{Cl} & -1.099020 & -3.064071 & -0.865291 & \mathrm{C} & -5.525780 & 0.109190 & -0.489130 \\ \mathrm{Br} & -5.257840 & 0.371128 & -0.100521 & \mathrm{C} & -4.232530 & 0.260170 & -0.977210 \\ \mathrm{C} & -1.273910 & 0.471649 & 1.052209 & \mathrm{C} & -1.480910 & 3.605010 & 0.627330 \\ \mathrm{C} & 0.802870 & -0.924620 & 0.755879 & \mathrm{C} & 0.298200 & 3.301320 & -0.944840 \\ \mathrm{C} & 1.785940 & 0.248910 & 0.593379 & \mathrm{C} & -5.734300 & -0.504570 & 0.742900 \\ \mathrm{C} & -2.902520 & -1.218351 & -0.357261 & \mathrm{H} & 1.288300 & -1.463220 & -2.443400 \\ \mathrm{C} & -0.668840 & -0.686161 & 0.522919 & \mathrm{H} & 2.903920 & 0.217080 & 1.902200 \\ \mathrm{C} & -2.606521 & 0.809159 & 0.888609 & \mathrm{H} & 3.721500 & -1.117690 & -2.097420 \\ \mathrm{C} & 1.296579 & 1.593030 & 0.122749 & \mathrm{H} & -2.502340 & -1.201170 & 1.537330 \\ \mathrm{C} & 1.414840 & -2.342430 & 0.425069 & \mathrm{H} & -0.757860 & -0.056390 & -2.767680 \\ \mathrm{C} & 3.203320 & 0.029740 & 0.115259 & \mathrm{H} & -2.065660 & -1.324880 & -2.459570 \\ \mathrm{C} & 3.484960 & -0.257770 & -1.222831 & \mathrm{H} & 0.400900 & 1.409030 & -1.912390 \\ \mathrm{C} & 1.420490 & -0.256210 & 1.959769 & \mathrm{H} & -2.790140 & 1.937510 & 0.905510 \\ \mathrm{C} & 1.507259 & 2.739450 & 0.887869 & \mathrm{H} & -0.011690 & 5.115270 & 0.174690 \\ \mathrm{C} & -1.551520 & -1.533401 & -0.176421 & \mathrm{H} & -4.798230 & -1.467880 & 2.421810 \\ \mathrm{C} & 0.660979 & 1.718720 & -1.113881 & \mathrm{H} & -6.369440 & 0.464110 & -1.071110 \\ \mathrm{C} & -3.415910 & -0.050481 & 0.165329 & \mathrm{H} & -4.061650 & 0.736820 & -1.937690 \\ \mathrm{C} & 0.435329 & 4.098419 & -0.790971 & \mathrm{H} & -2.011870 & 4.216520 & 1.348630 \\ \mathrm{C} & 4.798300 & -0.344320 & -1.661331 & \mathrm{H} & 1.174310 & 3.671550 & -1.465750 \\ \mathrm{C} & 5.585200 & 0.138311 & 0.558219 & \mathrm{H} & -6.741070 & -0.631940 & 1.125370\end{array}$

$\begin{array}{llll}\mathrm{C} & 4.264610 & 0.225110 & 0.995659\end{array}$

$\begin{array}{llll}\mathrm{C} & 0.232559 & 2.960449 & -1.567811\end{array}$

$\begin{array}{llll}\text { C } & 1.077159 & 3.984760 & 0.438669\end{array}$

$\begin{array}{lrrr}\text { C } & 5.855190 & -0.144319 & -0.774041\end{array}$

$\begin{array}{llll}\mathrm{H} & -0.669561 & 1.169259 & 1.612799\end{array}$

$\mathrm{H} \quad-3.531740 \quad-1.906141 \quad-0.904831$

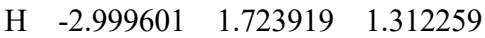

$\mathrm{H} \quad 2.664930 \quad-0.466970 \quad-1.896581$

$\begin{array}{llll}\mathrm{H} & 0.810170 & 0.361700 & 2.608159\end{array}$

$\begin{array}{llll}\mathrm{H} & 2.142170 & -0.879220 & 2.474279\end{array}$

$\begin{array}{llll}\mathrm{H} & 2.008599 & 2.645400 & 1.846259\end{array}$

$\begin{array}{llll}\mathrm{H} & 0.481699 & 0.823529 & -1.701231\end{array}$

$\begin{array}{llll}\mathrm{H} & 0.094018 & 5.066029 & -1.140991\end{array}$

$\begin{array}{llll}\mathrm{H} & 5.000820 & -0.589459 & -2.697861\end{array}$

$\begin{array}{llll}\mathrm{H} & 6.397530 & 0.286441 & 1.261309\end{array}$

$\begin{array}{llll}\mathrm{H} & 4.053850 & 0.441690 & 2.037649\end{array}$

$\begin{array}{llll}\mathrm{H} & -0.272231 & 3.038999 & -2.524071\end{array}$

$\mathrm{H} \quad 1.243319 \quad 4.866160 \quad 1.048399$

H $\quad 6.880250 \quad-0.221699 \quad-1.119551$

\section{ligB2}

$\begin{array}{llll}\mathrm{O} & -2.135440 & -3.015410 & -0.866530\end{array}$

$\begin{array}{llll}\mathrm{O} & -0.598950 & -2.881800 & 0.782640\end{array}$

$\begin{array}{llll}\mathrm{Cl} & 0.131670 & 0.013050 & 2.045280\end{array}$

$\begin{array}{llll}\mathrm{Br} & 5.314880 & -0.131900 & 0.206840\end{array}$

$\begin{array}{llll}\text { C } & 1.650820 & -1.085640 & -1.493350\end{array}$

$\begin{array}{llll}\text { C } & -0.730470 & -1.081810 & -0.771180\end{array}$

$\begin{array}{llll}\text { C } & -1.748520 & 0.068030 & -0.772740\end{array}$

$\begin{array}{llll}\text { C } & 2.560140 & -0.149270 & 0.944660\end{array}$

$\begin{array}{llll}\text { C } & 0.707710 & -0.813870 & -0.499440\end{array}$

$\begin{array}{llll}\text { C } & 3.014440 & -0.897650 & -1.308310\end{array}$

$\begin{array}{llll}\text { C } & -1.267610 & 1.479950 & -0.542450\end{array}$

$\begin{array}{llll}\text { C } & -1.212870 & -2.498410 & -0.224170\end{array}$

$\begin{array}{llll}\text { C } & -3.134820 & -0.194720 & -0.247540\end{array}$

$\begin{array}{llll}\text { C } & -3.350500 & -0.813960 & 0.982280\end{array}$

C $\quad-1.344100 \quad-0.624970 \quad-2.054780$

C $\quad-0.149850 \quad 2.010250 \quad-1.200940$

$\begin{array}{llll}\text { C } & 1.202260 & -0.357430 & 0.726060\end{array}$

$\begin{array}{llll}\text { C } & -1.924610 & 2.312370 & 0.373570\end{array}$

$\begin{array}{llll}\text { C } & 3.449580 & -0.420560 & -0.081670\end{array}$

$\begin{array}{llll}\text { C } & -0.363240 & 4.109930 & -0.027370\end{array}$

$\begin{array}{llll}\text { C } & -4.643560 & -0.968800 & 1.471850\end{array}$

$\begin{array}{llll}\text { C } & -5.525780 & 0.109190 & -0.489130\end{array}$

$\begin{array}{llll}\text { C } & -4.232530 & 0.260170 & -0.977210\end{array}$

$\begin{array}{llll}\text { C } & -1.480910 & 3.605010 & 0.627330\end{array}$

$\begin{array}{llll}\text { C } & 0.298200 & 3.301320 & -0.944840\end{array}$

$\begin{array}{lrrr}\text { C } & -5.734300 & -0.504570 & 0.742900\end{array}$

H $\quad 1.288300 \quad-1.463220 \quad-2.443400$

H $\quad 2.903920 \quad 0.217080 \quad 1.902200$

$\mathrm{H} \quad 3.721500 \quad-1.117690 \quad-2.097420$

$\mathrm{H} \quad-2.502340 \quad-1.201170 \quad 1.537330$

$\mathrm{H} \quad-0.757860 \quad-0.056390 \quad-2.767680$

$\mathrm{H} \quad-2.065660 \quad-1.324880 \quad-2.459570$

H $\quad 0.400900 \quad 1.409030 \quad-1.912390$

$\mathrm{H} \quad-2.790140 \quad 1.937510 \quad 0.905510$

$\mathrm{H} \quad-0.011690 \quad 5.115270 \quad 0.174690$

$\mathrm{H} \quad-4.798230 \quad-1.467880 \quad 2.421810$

$\mathrm{H} \quad-6.369440 \quad 0.464110 \quad-1.071110$

$\mathrm{H} \quad-4.061650 \quad 0.736820 \quad-1.937690$

H $\quad 1.174310 \quad 3.671550 \quad-1.465750$

H $\quad-6.741070 \quad-0.631940 \quad 1.125370$

\section{$\operatorname{ligC1}$}

$\begin{array}{llll}\mathrm{O} & 1.646480 & -3.059480 & 0.013160\end{array}$

O $\quad 0.458660 \quad-2.341630 \quad-1.766930$

$\begin{array}{llll}\mathrm{O} & -4.856090 & 0.564840 & -1.448180\end{array}$

$\begin{array}{llll}\mathrm{Cl} & -2.137800 & -2.019920 & 2.023360\end{array}$

$\begin{array}{llll}\text { C } & -1.277520 & 0.050840 & -1.303870\end{array}$

$\begin{array}{llll}\text { C } & 0.304490 & -1.094240 & 0.250450\end{array}$

$\begin{array}{llll}\text { C } & 1.329330 & 0.024260 & 0.546170\end{array}$

$\begin{array}{llll}\text { C } & -3.508760 & -0.637560 & 0.180570\end{array}$

$\begin{array}{llll}\text { C } & -1.078470 & -0.691100 & -0.126280\end{array}$

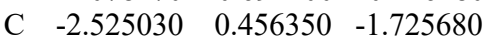

$\begin{array}{llll}\text { C } & 0.844390 & 1.450840 & 0.549850\end{array}$

$\begin{array}{llll}\text { C } & 0.860430 & -2.319150 & -0.589760\end{array}$

$\begin{array}{llll}\text { C } & 2.750930 & -0.130550 & 0.075270\end{array}$

C $\quad 3.063200 \quad-0.447660 \quad-1.246340$

$\begin{array}{llll}\text { C } & 0.842440 & -0.909950 & 1.627810\end{array}$

$\begin{array}{llll}\mathrm{C} & -0.281140 & 1.858150 & 1.281170\end{array}$

$\begin{array}{lrrr}\text { C } & -2.224860 & -1.032760 & 0.572940\end{array}$

$\begin{array}{llll}\text { C } & 1.491030 & 2.423770 & -0.222900\end{array}$ 


$\begin{array}{rrrr}\mathrm{C} & -3.652670 & 0.117490 & -0.973220 \\ \mathrm{C} & -0.088070 & 4.119980 & 0.456990 \\ \mathrm{C} & 4.390360 & -0.498440 & -1.661740 \\ \mathrm{C} & 5.116540 & 0.083380 & 0.555920 \\ \mathrm{C} & 3.789760 & 0.129910 & 0.969060 \\ \mathrm{C} & 1.031650 & 3.736830 & -0.270810 \\ \mathrm{C} & -0.740010 & 3.168180 & 1.235320 \\ \mathrm{C} & 5.420700 & -0.228750 & -0.766340 \\ \mathrm{C} & -6.003470 & 0.225960 & -0.709220 \\ \mathrm{H} & -0.398620 & 0.300050 & -1.885980 \\ \mathrm{H} & -4.351890 & -0.941390 & 0.783910 \\ \mathrm{H} & -2.657340 & 1.033630 & -2.632160 \\ \mathrm{H} & 2.262270 & -0.693170 & -1.936670 \\ \mathrm{H} & 0.216950 & -0.502510 & 2.411110 \\ \mathrm{H} & 1.533660 & -1.692260 & 1.920780 \\ \mathrm{H} & -0.828630 & 1.141670 & 1.880320 \\ \mathrm{H} & 2.367510 & 2.149880 & -0.797380 \\ \mathrm{H} & -0.449720 & 5.141190 & 0.417770 \\ \mathrm{H} & 4.618570 & -0.764550 & -2.687760 \\ \mathrm{H} & 5.912250 & 0.284030 & 1.265210 \\ \mathrm{H} & 3.544780 & 0.369620 & 1.999190 \\ \mathrm{H} & 1.556710 & 4.461170 & -0.884030 \\ \mathrm{H} & -1.619610 & 3.442330 & 1.807320 \\ \mathrm{H} & 6.454040 & -0.274370 & -1.092350 \\ \mathrm{H} & -5.957590 & 0.632240 & 0.307350 \\ \mathrm{H} & -6.848160 & 0.666610 & -1.236220 \\ \mathrm{H} & -6.136350 & -0.860040 & -0.654310\end{array}$

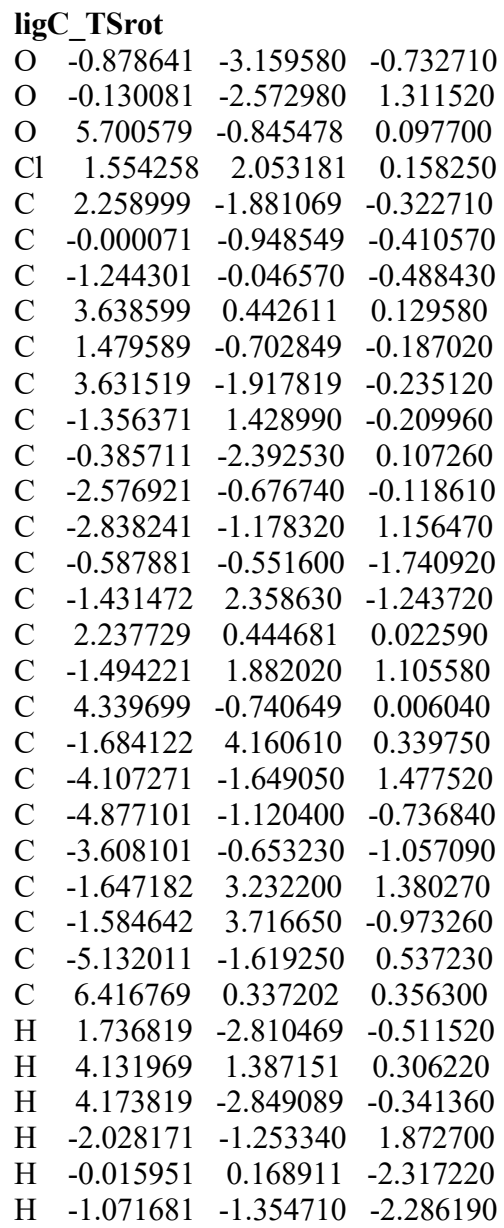

$\mathrm{H} \quad-1.348832 \quad 2.017030 \quad-2.269370$

$\mathrm{H} \quad-1.448591 \quad 1.161170 \quad 1.914420$

$\mathrm{H} \quad-1.797412 \quad 5.217560 \quad 0.553380$

$\mathrm{H} \quad-4.289361 \quad-2.057360 \quad 2.465420$

H $\quad-5.664461 \quad-1.101281 \quad-1.482640$

$\mathrm{H} \quad-3.404031 \quad-0.267170 \quad-2.050870$

$\mathrm{H} \quad-1.730552 \quad 3.566380 \quad 2.408550$

H $\quad-1.624322 \quad 4.427350 \quad-1.791680$

H $\quad-6.117941 \quad-1.993501 \quad 0.790270$

H $\quad 6.114819 \quad 0.785052 \quad 1.309300$

$\begin{array}{llll}\mathrm{H} & 7.467009 & 0.054502 & 0.406050\end{array}$

H $\quad 6.275909 \quad 1.072222 \quad-0.443890$

\section{ligC_TSalt}

O $\quad 0.676430 \quad-3.123850 \quad 1.392489$

$\begin{array}{llll}\text { O } & 1.071370 & -2.512110 & -0.743721\end{array}$

$\begin{array}{llll}\text { O } & -5.167929 & 0.923851 & 0.124919\end{array}$

$\mathrm{Cl} \quad-1.885770 \quad-2.807770 \quad-0.822701$

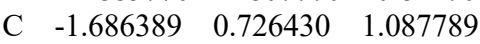

$\begin{array}{llll}\text { C } & 0.246420 & -0.869650 & 0.769019\end{array}$

$\begin{array}{llll}\text { C } & 1.338521 & 0.197940 & 0.589399\end{array}$

$\begin{array}{llll}\text { C } & -3.495030 & -0.794940 & -0.285851\end{array}$

$\begin{array}{llll}\text { C } & -1.200180 & -0.488140 & 0.553019\end{array}$

$\begin{array}{llll}\text { C } & -2.979329 & 1.184350 & 0.945959\end{array}$

C $\quad 0.979551 \quad 1.581540 \quad 0.116469$

$\begin{array}{llll}\text { C } & 0.711670 & -2.339340 & 0.433069\end{array}$

$\begin{array}{llll}\text { C } & 2.723371 & -0.159560 & 0.099559\end{array}$

C $\quad 2.963750 \quad-0.470790 \quad-1.241491$

$\begin{array}{llll}\text { C } & 0.940291 & -0.264770 & 1.963489\end{array}$

$\begin{array}{llll}\text { C } & 1.321291 & 2.706740 & 0.865729\end{array}$

C $\quad-2.172630 \quad-1.236060 \quad-0.125611$

$\begin{array}{llll}\text { C } & 0.337441 & 1.761920 & -1.110081\end{array}$

$\begin{array}{llll}\text { C } & -3.902319 & 0.417530 & 0.239949\end{array}$

$\begin{array}{llll}\text { C } & 0.367231 & 4.155170 & -0.809271\end{array}$

$\begin{array}{llll}\text { C } & 4.257830 & -0.687511 & -1.692131\end{array}$

$\begin{array}{llll}\text { C } & 5.108641 & -0.292161 & 0.520789\end{array}$

$\begin{array}{llll}\text { C } & 3.807211 & -0.074341 & 0.970139\end{array}$

$\begin{array}{llll}\text { C } & 0.032721 & 3.037500 & -1.569921\end{array}$

$\begin{array}{llll}\text { C } & 1.015231 & 3.986060 & 0.410739\end{array}$

$\begin{array}{llll}\text { C } & 5.337510 & -0.596951 & -0.814311\end{array}$

$\begin{array}{llll}\text { C } & -6.103509 & 0.152041 & -0.589321\end{array}$

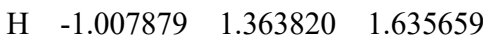

$\mathrm{H} \quad-4.174150 \quad-1.440920 \quad-0.822931$

H $\quad-3.288439 \quad 2.131900 \quad 1.369529$

H $\quad 2.120940 \quad-0.594080 \quad-1.908291$

$\begin{array}{llll}\mathrm{H} & 0.402081 & 0.413540 & 2.615459\end{array}$

H $\quad 1.604610 \quad-0.952760 \quad 2.472549$

$\begin{array}{llll}\mathrm{H} & 1.826851 & 2.569180 & 1.816649\end{array}$

H $\quad 0.054711 \quad 0.884290 \quad-1.682991$

$\mathrm{H} \quad 0.122671 \quad 5.150080 \quad-1.164031$

H $\quad 4.425450 \quad-0.949071 \quad-2.730961$

H $\quad \begin{array}{llll}5.937811 & -0.228341 & 1.217039\end{array}$

H $\quad 3.628331 \quad 0.159009 \quad 2.014449$

$\mathrm{H} \quad-0.478369 \quad 3.159800 \quad-2.518311$

$\begin{array}{llll}\mathrm{H} & 1.282681 & 4.850880 & 1.008209\end{array}$

H $\quad 6.346600 \quad-0.775841 \quad-1.169271$

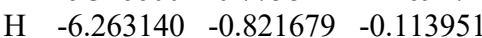

H $\quad-7.034849 \quad 0.715811 \quad-0.580451$

H $\quad-5.782559 \quad-0.004699 \quad-1.624741$

$\operatorname{ligC2}$

O $\quad-0.208540 \quad-2.909110 \quad 0.791120$
O $\quad-1.778360 \quad-2.988290 \quad-0.828510$

O $\quad 5.313500 \quad-0.426180 \quad-0.112230$

$\begin{array}{llll}\mathrm{Cl} & 0.684750 & -0.028950 & 2.003540\end{array}$

$\begin{array}{llll}\text { C } & 2.085240 & -1.200860 & -1.555990\end{array}$

C $\quad-0.286140 \quad-1.119610 \quad-0.778750$

$\begin{array}{llll}\text { C } & -1.259130 & 0.071390 & -0.771400\end{array}$

$\begin{array}{llll}\text { C } & 3.080610 & -0.269030 & 0.844640\end{array}$

$\begin{array}{llll}\text { C } & 1.167660 & -0.896380 & -0.542860\end{array}$

$\begin{array}{llll}\text { C } & 3.451320 & -1.044550 & -1.403080\end{array}$

$\begin{array}{llll}\text { C } & -0.720190 & 1.465390 & -0.565440\end{array}$

C $\quad-0.820050 \quad-2.504740 \quad-0.209580$

$\begin{array}{llll}\text { C } & -2.643070 & -0.131620 & -0.214620\end{array}$

$\begin{array}{llll}\text { C } & -2.854570 & -0.722760 & 1.029930\end{array}$

$\begin{array}{llll}\text { C } & -0.908920 & -0.649010 & -2.053730\end{array}$

$\begin{array}{llll}\text { C } & 0.404170 & 1.946090 & -1.250880\end{array}$

$\begin{array}{lrrr}\text { C } & 1.709540 & -0.444710 & 0.656210\end{array}$

$\begin{array}{lrrr}\text { C } & -1.327050 & 2.332830 & 0.353070\end{array}$

$\begin{array}{llll}\text { C } & 3.953960 & -0.568150 & -0.193160\end{array}$

$\begin{array}{llll}\text { C } & 0.292000 & 4.066240 & -0.098930\end{array}$

$\begin{array}{llll}\text { C } & -4.141250 & -0.821570 & 1.549670\end{array}$

$\begin{array}{llll}\text { C } & -5.026360 & 0.256780 & -0.409710\end{array}$

$\begin{array}{llll}\text { C } & -3.739380 & 0.351690 & -0.927860\end{array}$

$\begin{array}{llll}\text { C } & -0.830670 & 3.610810 & 0.583010\end{array}$

$\begin{array}{llll}\text { C } & 0.904600 & 3.222410 & -1.018910\end{array}$

$\begin{array}{llll}\text { C } & -5.230220 & -0.328880 & 0.836670\end{array}$

$\begin{array}{llll}\text { C } & 5.837810 & 0.045750 & 1.105510\end{array}$

H $\quad 1.691300 \quad-1.576940 \quad-2.494290$

H $\quad 3.425930 \quad 0.096830 \quad 1.800810$

$\mathrm{H} \quad 4.144930 \quad-1.286380 \quad-2.199000$

$\mathrm{H} \quad-2.008600 \quad-1.132120 \quad 1.572370$

H $\quad-0.314210-0.111210-2.783390$

$\mathrm{H} \quad-1.666760 \quad-1.322030 \quad-2.437200$

H $\quad 0.919420 \quad 1.315990 \quad-1.963700$

$\mathrm{H} \quad-2.195500 \quad 1.997060 \quad 0.905920$

$\mathrm{H} \quad 0.683850 \quad 5.060520 \quad 0.083460$

$\mathrm{H} \quad-4.293310 \quad-1.299580 \quad 2.510910$

$\mathrm{H} \quad-5.869100 \quad 0.633190 \quad-0.979550$

$\mathrm{H} \quad-3.572110 \quad 0.805810 \quad-1.899830$

$\mathrm{H} \quad-1.324710 \quad 4.249990 \quad 1.306690$

H $\quad 1.783400 \quad 3.553270 \quad-1.561600$

$\mathrm{H} \quad-6.232400 \quad-0.412540 \quad 1.242730$

H $\quad 6.918090 \quad 0.088190 \quad 0.976550$

$\mathrm{H} \quad 5.460040 \quad 1.046720 \quad 1.340820$

H $\quad 5.596780 \quad-0.631040 \quad 1.932160$

\section{ligD1}

$\begin{array}{llll}\text { O } & -2.201430 & -2.001430 & 2.130890\end{array}$

$\begin{array}{llll}\mathrm{O} & -3.835850 & -2.815840 & 0.803470\end{array}$

$\begin{array}{llll}\text { O } & 7.474290 & 0.650690 & 0.274900\end{array}$

$\begin{array}{lllll}\mathrm{Cl} & -0.477610 & -2.862380 & -2.045820\end{array}$

$\begin{array}{llll}\text { C } & -2.845170 & -2.128930 & 1.074990\end{array}$

C $\quad-2.326700 \quad-1.197860 \quad-0.105330$

$\begin{array}{llll}\text { C } & -3.230120 & 0.008290 & -0.444560\end{array}$

C $\quad-3.122220 \quad-1.184620 \quad-1.364680$

$\begin{array}{llll}\text { C } & -0.855810 & -0.980450 & -0.085090\end{array}$

$\begin{array}{llll}\text { C } & 0.058250 & -1.649780 & -0.895000\end{array}$

$\begin{array}{llll}\text { C } & 1.426700 & -1.401570 & -0.843420\end{array}$

$\begin{array}{llll}\text { C } & 1.935950 & -0.468520 & 0.055010\end{array}$

$\begin{array}{llll}\text { C } & 1.039810 & 0.188410 & 0.906890\end{array}$

C $\quad-0.315170 \quad-0.072620 \quad 0.836060$

$\begin{array}{llll}\text { C } & 3.389620 & -0.185280 & 0.110630\end{array}$

$\begin{array}{llll}\text { C } & 3.853740 & 1.122580 & 0.283620\end{array}$ 


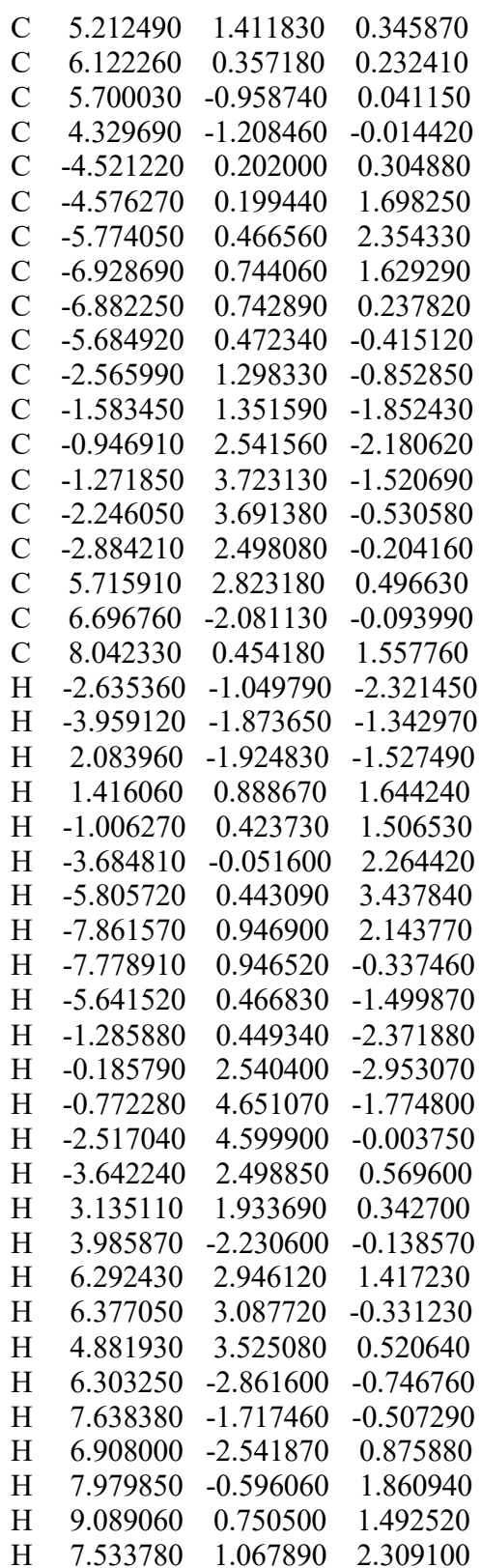

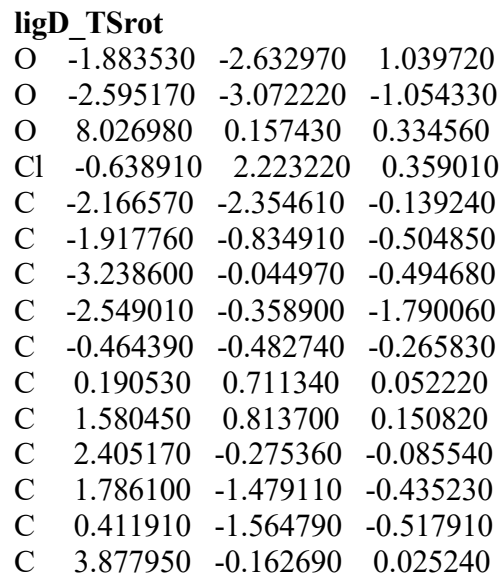

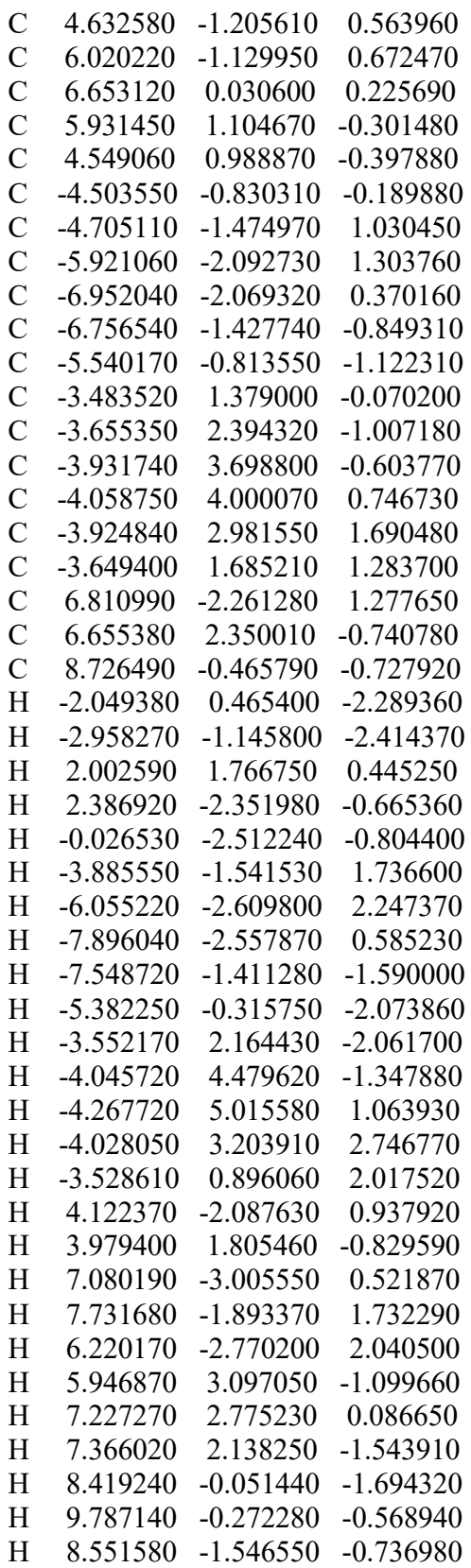

\section{ligD_TSalt}

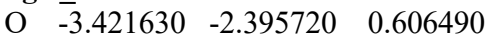

$\begin{array}{llll}\mathrm{O} & -3.232830 & -2.930610 & -1.578340\end{array}$

$\begin{array}{llll}\mathrm{O} & 7.618230 & 0.171410 & 0.172740\end{array}$

$\mathrm{Cl} \quad-0.588580 \quad-3.299360 \quad 0.533220$

C $\quad-3.071690 \quad-2.224100 \quad-0.572760$

$\begin{array}{llll}\text { C } & -2.317930 & -0.863420 & -0.845060\end{array}$

$\begin{array}{llll}\text { C } & -3.149830 & 0.397050 & -0.544230\end{array}$

$\begin{array}{llll}\text { C } & -2.919320 & -0.044880 & -1.961130\end{array}$

C $\quad-0.818780 \quad-0.803940 \quad-0.685350$

$\begin{array}{llll}\text { C } & -0.000760 & -1.778840 & -0.080950\end{array}$

$\begin{array}{llll}\text { C } & 1.379980 & -1.615860 & 0.044840\end{array}$

$\begin{array}{llll}\text { C } & 2.034730 & -0.486130 & -0.428470\end{array}$

$\begin{array}{llll}\text { C } & 1.255410 & 0.480940 & -1.063950\end{array}$

$\begin{array}{llll}\text { C } & -0.111130 & 0.309710 & -1.182460\end{array}$ $\begin{array}{llll}\text { C } & 3.497860 & -0.321060 & -0.266370\end{array}$

$\begin{array}{llll}\text { C } & 4.270070 & 0.255580 & -1.279750\end{array}$

$\begin{array}{llll}\text { C } & 5.642640 & 0.430410 & -1.141550\end{array}$

$\begin{array}{llll}\text { C } & 6.249060 & 0.014320 & 0.046650\end{array}$

$\begin{array}{llll}\text { C } & 5.517600 & -0.583460 & 1.073340\end{array}$

$\begin{array}{llll}\text { C } & 4.143510 & -0.738760 & 0.897840\end{array}$

$\begin{array}{llll}\text { C } & -4.554970 & 0.303310 & 0.005280\end{array}$

$\begin{array}{llll}\text { C } & -4.793850 & -0.037260 & 1.339310\end{array}$

C $\quad-6.082540 \quad-0.009260 \quad 1.851950$

$\begin{array}{llll}\text { C } & -7.157590 & 0.360020 & 1.044200\end{array}$

$\begin{array}{llll}\text { C } & -6.930830 & 0.696350 & -0.283550\end{array}$

$\begin{array}{llll}\text { C } & -5.634510 & 0.667840 & -0.795380\end{array}$

$\begin{array}{llll}\text { C } & -2.483940 & 1.640210 & -0.016160\end{array}$

C $\quad-2.608670 \quad 2.857740 \quad-0.684160$

$\begin{array}{llll}\text { C } & -2.016510 & 4.011770 & -0.179100\end{array}$

$\begin{array}{llll}\text { C } & -1.295420 & 3.961780 & 1.010140\end{array}$

C $\quad-1.176130 \quad 2.752410 \quad 1.690580$

$\begin{array}{llll}\text { C } & -1.766700 & 1.602270 & 1.181000\end{array}$

$\begin{array}{llll}\text { C } & 6.482090 & 1.018720 & -2.244800\end{array}$

$\begin{array}{llll}\text { C } & 6.192440 & -1.044440 & 2.339660\end{array}$

$\begin{array}{llll}\text { C } & 7.977030 & 1.338600 & 0.890790\end{array}$

$\mathrm{H} \quad-2.278560 \quad 0.547660 \quad-2.603640$

$\mathrm{H} \quad-3.734770 \quad-0.545070 \quad-2.469610$

$\mathrm{H} \quad 1.944670 \quad-2.420150 \quad 0.500430$

H $\quad 1.711780 \quad 1.390750 \quad-1.438150$

$\mathrm{H} \quad-0.660160 \quad 1.105940 \quad-1.663010$

$\mathrm{H} \quad-3.966540 \quad-0.375650 \quad 1.948560$

H $\quad-6.254170 \quad-0.297200 \quad 2.883070$

$\mathrm{H} \quad-8.164480 \quad 0.371710 \quad 1.446960$

$\mathrm{H} \quad-7.758730 \quad 0.975820 \quad-0.925980$

$\mathrm{H} \quad-5.458170 \quad 0.926320 \quad-1.834170$

$\mathrm{H} \quad-3.171960 \quad 2.891500 \quad-1.611680$

$\mathrm{H} \quad-2.118170 \quad 4.949950 \quad-0.713750$

$\mathrm{H} \quad-0.828380 \quad 4.857710 \quad 1.403390$

$\mathrm{H} \quad-0.610460 \quad 2.702700 \quad 2.614200$

$\mathrm{H} \quad-1.654330 \quad 0.650510 \quad 1.690850$

H $\quad 3.790740 \quad 0.548320 \quad-2.208220$

$\mathrm{H} \quad 3.558280 \quad-1.182750 \quad 1.696990$

H $\quad 6.974760 \quad 1.940530 \quad-1.924620$

H $\quad 7.269560 \quad 0.323520 \quad-2.543030$

$\mathrm{H} \quad 5.864830 \quad 1.246270 \quad-3.114310$

H $\quad 5.679030 \quad-1.916500 \quad 2.746980$

H $\quad 7.234690 \quad-1.305260 \quad 2.151770$

$\mathrm{H} \quad 6.171420 \quad-0.264100 \quad 3.106610$

$\mathrm{H} \quad 7.608560 \quad 1.297780 \quad 1.921030$

$\mathrm{H} \quad 9.065700 \quad 1.387650 \quad 0.897380$

H $\quad 7.572700 \quad 2.234810 \quad 0.407780$

\section{ligD2}

$\begin{array}{llll}\text { O } & -3.707080 & -3.060660 & -0.394990\end{array}$

$\begin{array}{llll}\text { O } & -2.127540 & -2.775980 & 1.192580\end{array}$

$\begin{array}{llll}\text { O } & 7.640930 & 0.130390 & 0.446240\end{array}$

$\begin{array}{llll}\mathrm{Cl} & -1.210260 & 0.246030 & 1.989240\end{array}$

$\begin{array}{llll}\text { C } & -2.741280 & -2.509040 & 0.148800\end{array}$

C $\quad-2.192680 \quad-1.221620 \quad-0.611500$

$\begin{array}{llll}\text { C } & -3.147600 & -0.029010 & -0.773810\end{array}$

$\begin{array}{llll}\text { C } & -2.809210 & -0.929450 & -1.941120\end{array}$

$\begin{array}{llll}\text { C } & -0.736690 & -0.994080 & -0.404910\end{array}$

$\begin{array}{llll}\text { C } & -0.188950 & -0.370910 & 0.719770\end{array}$

$\begin{array}{llll}\text { C } & 1.179660 & -0.192560 & 0.868940\end{array}$

$\begin{array}{llll}\text { C } & 2.067570 & -0.652180 & -0.103630\end{array}$

$\begin{array}{llll}\text { C } & 1.542280 & -1.305190 & -1.221090\end{array}$ 


\begin{tabular}{|c|c|c|c|}
\hline & 0.171500 & -1.465120 & -1.356990 \\
\hline & 3.527400 & -0.443150 & 0.045850 \\
\hline & 4.031650 & 0.753040 & 0.557410 \\
\hline C & 5.400820 & 0.973750 & 0.697720 \\
\hline & 6.274750 & -0.040260 & 0.304580 \\
\hline$C$ & 5.808960 & -1.258700 & -0.196560 \\
\hline & 4.435770 & -1.441710 & -0.319030 \\
\hline $\mathrm{C}$ & -4.533850 & -0.130920 & -0.195590 \\
\hline $\mathrm{C}$ & -4.753670 & -0.537510 & 1.119380 \\
\hline $\mathrm{C}$ & -6.041600 & -0.542290 & 1.645550 \\
\hline $\mathrm{C}$ & -7.122960 & -0.138190 & 0.868330 \\
\hline$C$ & -6.910760 & 0.262540 & -0.447910 \\
\hline $\mathrm{C}$ & -5.622760 & 0.263480 & -0.972060 \\
\hline $\mathrm{C}$ & -2.587780 & 1.372440 & -0.768980 \\
\hline $\mathrm{C}$ & -1.453650 & 1.731640 & -1.510280 \\
\hline $\mathrm{C}$ & -0.932700 & 3.019810 & -1.461070 \\
\hline $\mathrm{C}$ & -1.534660 & 3.996620 & -0.675620 \\
\hline $\mathrm{C}$ & -2.667370 & 3.662000 & 0.057810 \\
\hline $\mathrm{C}$ & -3.183850 & 2.372240 & 0.011440 \\
\hline $\mathrm{C}$ & 5.921860 & 2.273430 & 1.255210 \\
\hline $\mathrm{C}$ & 6.788370 & -2.345680 & -0.553210 \\
\hline $\mathrm{C}$ & 8.226490 & 0.830470 & -0.636820 \\
\hline $\mathrm{H}$ & -2.208020 & -0.508940 & -2.739220 \\
\hline $\mathrm{H}$ & -3.576700 & -1.639590 & -2.225930 \\
\hline $\mathrm{H}$ & 1.550470 & 0.288370 & 1.766050 \\
\hline $\mathrm{H}$ & 2.208600 & -1.661330 & -1.999290 \\
\hline $\mathrm{H}$ & -0.231040 & -1.963950 & -2.232300 \\
\hline $\mathrm{H}$ & -3.914170 & -0.878960 & 1.716080 \\
\hline $\mathrm{H}$ & -6.200550 & -0.877510 & 2.664300 \\
\hline $\mathrm{H}$ & -8.126020 & -0.147950 & 1.280610 \\
\hline $\mathrm{H}$ & -7.747860 & 0.568020 & -1.066360 \\
\hline $\mathrm{H}$ & -5.449080 & 0.573550 & -1.998040 \\
\hline $\mathrm{H}$ & -0.946550 & 0.997380 & -2.122270 \\
\hline $\mathrm{H}$ & -0.046500 & 3.255220 & -2.040310 \\
\hline $\mathrm{H}$ & -1.126880 & 5.000340 & -0.635520 \\
\hline $\mathrm{H}$ & -3.153170 & 4.406260 & 0.679340 \\
\hline $\mathrm{H}$ & -4.059260 & 2.133290 & 0.602340 \\
\hline $\mathrm{H}$ & 3.339720 & 1.540510 & 0.839690 \\
\hline $\mathrm{H}$ & 4.058490 & -2.394120 & -0.677030 \\
\hline $\mathrm{H}$ & 6.882590 & 2.126440 & 1.750290 \\
\hline $\mathrm{H}$ & 6.059080 & 3.017620 & 0.464640 \\
\hline $\mathrm{H}$ & 5.214410 & 2.688060 & 1.974620 \\
\hline $\mathrm{H}$ & 7.457410 & -2.032940 & -1.359240 \\
\hline $\mathrm{H}$ & 7.416840 & -2.594980 & 0.304180 \\
\hline & 6.261120 & -3.243580 & -0.876500 \\
\hline & 7.830150 & 1.848550 & -0.710130 \\
\hline $\mathrm{H}$ & 9.298950 & 0.872570 & -0.447690 \\
\hline & 8.041740 & 0.311780 & -1.583810 \\
\hline
\end{tabular}

\begin{tabular}{lrrr}
\multicolumn{4}{l}{ ligE1 } \\
$\mathrm{O}$ & 2.132560 & -2.026290 & -2.095900 \\
$\mathrm{O}$ & 3.753810 & -2.794640 & -0.725700 \\
$\mathrm{Cl}$ & 0.363020 & -2.835020 & 2.064890 \\
$\mathrm{~N}$ & -7.690220 & 0.530350 & -0.350240 \\
$\mathrm{C}$ & 2.760030 & -2.124340 & -1.027100 \\
$\mathrm{C}$ & 2.212370 & -1.174820 & 0.124030 \\
$\mathrm{C}$ & 0.738750 & -0.977550 & 0.080500 \\
$\mathrm{C}$ & -0.175840 & -1.646160 & 0.890160 \\
$\mathrm{C}$ & -1.546670 & -1.415450 & 0.821120 \\
$\mathrm{C}$ & -2.059500 & -0.504330 & -0.098070 \\
$\mathrm{C}$ & -1.161640 & 0.148420 & -0.951770 \\
$\mathrm{C}$ & 0.196120 & -0.092180 & -0.860960
\end{tabular} $\begin{array}{llll}\text { C } & -3.514990 & -0.241760 & -0.170170\end{array}$

$\begin{array}{llll}\text { C } & -4.010940 & 1.045170 & -0.389010\end{array}$

$\begin{array}{llll}\text { C } & -5.372400 & 1.303360 & -0.465170\end{array}$

$\begin{array}{llll}\text { C } & -6.314770 & 0.272180 & -0.311790\end{array}$

$\begin{array}{llll}\text { C } & -5.818330 & -1.026030 & -0.104980\end{array}$

$\begin{array}{llll}\text { C } & -4.453250 & -1.266640 & -0.033750\end{array}$

$\begin{array}{llll}\text { C } & 3.096560 & 0.049410 & 0.449620\end{array}$

$\begin{array}{lrrr}\text { C } & 2.988200 & -1.123890 & 1.394690\end{array}$

$\begin{array}{llll}\text { C } & 4.396910 & 0.240440 & -0.284420\end{array}$

$\begin{array}{llll}\text { C } & 5.546780 & 0.538580 & 0.446800\end{array}$

$\begin{array}{llll}\text { C } & 6.750890 & 0.808390 & -0.193890\end{array}$

$\begin{array}{llll}\mathrm{C} & 6.818270 & 0.780650 & -1.584210\end{array}$

$\begin{array}{llll}\text { C } & 5.677650 & 0.475170 & -2.320200\end{array}$

$\begin{array}{llll}\text { C } & 4.472970 & 0.208990 & -1.676450\end{array}$

$\begin{array}{llll}\text { C } & 2.413850 & 1.341090 & 0.819790\end{array}$

$\begin{array}{llll}\text { C } & 1.411780 & 1.405060 & 1.799250\end{array}$

C $\quad 0.759040 \quad 2.595420 \quad 2.091990$

$\begin{array}{llll}\text { C } & 1.087010 & 3.767060 & 1.416020\end{array}$

$\begin{array}{llll}\text { C } & 2.080720 & 3.724940 & 0.445910\end{array}$

$\begin{array}{llll}\text { C } & 2.734720 & 2.531160 & 0.154620\end{array}$

$\begin{array}{llll}\text { C } & -8.573210 & -0.600740 & -0.557520\end{array}$

$\begin{array}{llll}\text { C } & -8.114890 & 1.780960 & -0.948730\end{array}$

$\mathrm{H} \quad-2.204260 \quad-1.935700 \quad 1.507300$

$\mathrm{H} \quad-1.538170 \quad 0.829770 \quad-1.706580$

$\mathrm{H} \quad 0.887870 \quad 0.402790 \quad-1.531900$

H $\quad-6.493190 \quad-1.865380-0.009910$

$\mathrm{H} \quad-4.106620 \quad-2.285470 \quad 0.101410$

H $\quad 2.484120 \quad-0.973050 \quad 2.340210$

$\mathrm{H} \quad 3.833580 \quad-1.802760 \quad 1.402380$

$\mathrm{H} \quad 5.486960 \quad 0.555580 \quad 1.530660$

H $\quad 7.636590 \quad 1.033950 \quad 0.390170$

$\mathrm{H} \quad 7.756600 \quad 0.982720 \quad-2.089040$

H $\quad 5.725930 \quad 0.429070 \quad-3.402380$

H $\quad 3.592620 \quad-0.063560 \quad-2.250030$

$\mathrm{H} \quad 1.111420 \quad 0.510210 \quad 2.329690$

$\mathrm{H} \quad-0.017580 \quad 2.602050 \quad 2.848810$

$\mathrm{H} \quad 0.574680 \quad 4.695270 \quad 1.642320$

$\mathrm{H} \quad 2.354410 \quad 4.625590 \quad-0.092930$

$\mathrm{H} \quad 3.507360 \quad 2.523940 \quad-0.604490$

$\mathrm{H} \quad-8.354370 \quad-1.145300 \quad-1.488220$

$\mathrm{H} \quad-8.500390 \quad-1.302700 \quad 0.275100$

$\mathrm{H} \quad-9.601770 \quad-0.243690 \quad-0.594340$

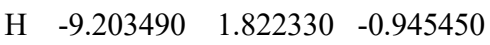

$\mathrm{H} \quad-7.754170 \quad 2.629590 \quad-0.364740$

$\mathrm{H} \quad-7.759470 \quad 1.898060 \quad-1.983340$

$\mathrm{H} \quad-3.312960 \quad 1.869700 \quad-0.486830$

H $\quad-5.693510 \quad 2.321870 \quad-0.634280$

\section{ligE_TSrot}

$\begin{array}{llll}\mathrm{O} & -1.764019 & -2.588080 & 1.153880\end{array}$

O $\quad-2.492759-3.109080 \quad-0.915310$

$\begin{array}{llll}\mathrm{Cl} & -0.466080 & 2.217580 & 0.262860\end{array}$

$\begin{array}{llll}\mathrm{N} & 8.251170 & 0.067392 & 0.287050\end{array}$

C $\quad-2.049919 \quad-2.357520 \quad-0.034700$

$\begin{array}{llll}\text { C } & -1.785439 & -0.858440 & -0.467350\end{array}$

$\begin{array}{llll}\text { C } & -0.326169 & -0.513100 & -0.252720\end{array}$

$\begin{array}{llll}\text { C } & 0.344690 & 0.684880 & 0.011010\end{array}$

$\begin{array}{llll}\text { C } & 1.736510 & 0.775581 & 0.094810\end{array}$

$\begin{array}{llll}\text { C } & 2.548551 & -0.330469 & -0.105050\end{array}$

C $\quad 1.912311-1.540109 \quad-0.400200$

$\begin{array}{llll}\text { C } & 0.536421 & -1.614170 & -0.466470\end{array}$

$\begin{array}{llll}\text { C } & 4.022040 & -0.227119 & -0.012750\end{array}$ $\begin{array}{llll}\text { C } & 4.789031 & -1.255209 & 0.539660\end{array}$

$\begin{array}{llll}\text { C } & 6.171581 & -1.173958 & 0.625870\end{array}$

$\begin{array}{llll}\text { C } & 6.860560 & -0.037858 & 0.168240\end{array}$

$\begin{array}{llll}\text { C } & 6.093010 & 0.992582 & -0.400640\end{array}$

$\begin{array}{llll}\text { C } & 4.710970 & 0.893131 & -0.481670\end{array}$

$\begin{array}{llll}\text { C } & -3.096970 & -0.052950 & -0.482210\end{array}$

$\begin{array}{llll}\text { C } & -2.419999 & -0.430240 & -1.767470\end{array}$

$\begin{array}{llll}\text { C } & -4.369629 & -0.808951 & -0.137670\end{array}$

$\begin{array}{llll}\text { C } & -5.412039 & -0.816631 & -1.063830\end{array}$

$\begin{array}{llll}\text { C } & -6.634239 & -1.403791 & -0.759230\end{array}$

$\begin{array}{llll}\text { C } & -6.829929 & -1.993321 & 0.486230\end{array}$

$\begin{array}{llll}\text { C } & -5.793409 & -1.992071 & 1.413950\end{array}$

$\begin{array}{llll}\text { C } & -4.571589 & -1.401251 & 1.108900\end{array}$

$\begin{array}{llll}\text { C } & -3.322770 & 1.390490 & -0.117190\end{array}$

$\begin{array}{llll}\text { C } & -3.487610 & 2.367650 & -1.095120\end{array}$

$\begin{array}{llll}\text { C } & -3.747440 & 3.690949 & -0.745480\end{array}$

$\begin{array}{llll}\text { C } & -3.864660 & 4.049989 & 0.591730\end{array}$

$\begin{array}{llll}\text { C } & -3.737480 & 3.070419 & 1.576780\end{array}$

$\begin{array}{llll}\mathrm{C} & -3.478600 & 1.755160 & 1.223430\end{array}$

$\begin{array}{llll}\text { C } & 8.913480 & 1.076262 & -0.515760\end{array}$

$\begin{array}{llll}\text { C } & 8.994861 & -1.158638 & 0.502750\end{array}$

H $\quad 2.171260 \quad 1.735031 \quad 0.347410$

H $\quad 2.500811 \quad-2.428579 \quad-0.600870$

$\mathrm{H} \quad 0.085361 \quad-2.567860 \quad-0.709590$

H $\quad 6.568130 \quad 1.879362 \quad-0.796760$

$\mathrm{H} \quad 4.154640 \quad 1.698841 \quad-0.948350$

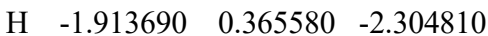

H $\quad-2.843979-1.237350 \quad-2.355090$

$\mathrm{H} \quad-5.253759-0.359561 \quad-2.035560$

H $\quad-7.430819 \quad-1.407171 \quad-1.495370$

$\begin{array}{llll}\mathrm{H} & -7.778439 & -2.461121 & 0.726260\end{array}$

$\mathrm{H} \quad-5.928049 \quad-2.469131 \quad 2.378360$

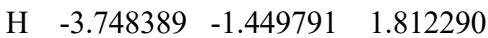

$\begin{array}{llll}\mathrm{H} & -3.392010 & 2.092580 & -2.139490\end{array}$

$\mathrm{H} \quad-3.856180 \quad 4.441259 \quad-1.521140$

$\mathrm{H} \quad-4.060881 \quad 5.080229 \quad 0.866840$

H $\quad-3.833030 \quad 3.337939 \quad 2.623310$

$\mathrm{H} \quad-3.363310 \quad 0.995950 \quad 1.989040$

H $\quad 8.727190 \quad 0.951892 \quad-1.593140$

H $\quad 8.584760 \quad 2.075542 \quad-0.224560$

H $\quad 9.986770 \quad 1.020912 \quad-0.337620$

H $\quad 10.058751 \quad-0.926058 \quad 0.530850$

H $\quad 8.732721 \quad-1.604638 \quad 1.463800$

H $\quad 8.816711-1.906198 \quad-0.284510$

H $\quad 4.289921 \quad-2.136239 \quad 0.928020$

$\begin{array}{llll}\mathrm{H} & 6.708161 & -2.002518 & 1.067040\end{array}$

\section{ligE_TSalt}

$\begin{array}{llll}\mathrm{O} & -3.275930 & -2.378220 & 0.709919\end{array}$

$\begin{array}{llll}\mathrm{O} & -3.081530 & -2.998550 & -1.451541\end{array}$

$\begin{array}{llll}\mathrm{Cl} & -0.435010 & -3.260639 & 0.678079\end{array}$

$\begin{array}{llll}\mathrm{N} & 7.831100 & 0.193021 & 0.184729\end{array}$

C $\quad-2.925740 \quad-2.251080 \quad-0.474981$

$\begin{array}{llll}\text { C } & -2.181990 & -0.896720 & -0.800691\end{array}$

$\begin{array}{llll}\text { C } & -0.683460 & -0.817580 & -0.638591\end{array}$

C $\quad 0.141770 \quad-1.7609890 .004029$

$\begin{array}{llll}\text { C } & 1.521580 & -1.583819 & 0.121829\end{array}$

$\begin{array}{llll}\text { C } & 2.169300 & -0.468389 & -0.393931\end{array}$

$\begin{array}{llll}\text { C } & 1.381300 & 0.468511 & -1.063761\end{array}$

$\begin{array}{llll}\text { C } & 0.016200 & 0.281881 & -1.176921\end{array}$

$\begin{array}{llll}\text { C } & 3.630760 & -0.292229 & -0.241551\end{array}$

$\begin{array}{llll}\text { C } & 4.408760 & 0.267691 & -1.256931\end{array}$ 


$\begin{array}{rrrr}\text { C } & 5.778260 & 0.445201 & -1.119751 \\ \mathrm{C} & 6.445180 & 0.052801 & 0.053409 \\ \mathrm{C} & 5.663130 & -0.498799 & 1.082519 \\ \mathrm{C} & 4.294180 & -0.666519 & 0.928879 \\ \mathrm{C} & -3.027190 & 0.366670 & -0.555621 \\ \mathrm{C} & -2.785960 & -0.131120 & -1.952261 \\ \mathrm{C} & -4.433730 & 0.281810 & -0.008211 \\ \mathrm{C} & -5.513760 & 0.602090 & -0.827011 \\ \mathrm{C} & -6.812430 & 0.637950 & -0.321541 \\ \mathrm{C} & -7.041240 & 0.353770 & 1.018009 \\ \mathrm{C} & -5.965700 & 0.029180 & 1.844159 \\ \mathrm{C} & -4.674690 & -0.006350 & 1.337799 \\ \mathrm{C} & -2.376740 & 1.638060 & -0.078041 \\ \mathrm{C} & -2.513220 & 2.824830 & -0.797251 \\ \mathrm{C} & -1.935840 & 4.005870 & -0.340001 \\ \mathrm{C} & -1.217820 & 4.014430 & 0.852099 \\ \mathrm{C} & -1.086820 & 2.836320 & 1.583349 \\ \mathrm{C} & -1.662770 & 1.658900 & 1.121419 \\ \mathrm{C} & 8.385930 & 0.108641 & 1.521329 \\ \mathrm{C} & 8.496530 & 1.107991 & -0.721931 \\ \mathrm{H} & 2.091970 & -2.366689 & 0.606839 \\ \mathrm{H} & 1.829720 & 1.368061 & -1.471101 \\ \mathrm{H} & -0.538920 & 1.055301 & -1.687131 \\ \mathrm{H} & 6.114560 & -0.794159 & 2.019489 \\ \mathrm{H} & 3.720410 & -1.075429 & 1.753439 \\ \mathrm{H} & -2.147340 & 0.440770 & -2.615341 \\ \mathrm{H} & -3.594170 & -0.659590 & -2.443411 \\ \mathrm{H} & -5.335770 & 0.819680 & -1.874861 \\ \mathrm{H} & -7.640570 & 0.882120 & -0.977941 \\ \mathrm{H} & -8.049870 & 0.371270 & 1.416229 \\ \mathrm{H} & -6.138610 & -0.218020 & 2.885599 \\ \mathrm{H} & -3.846450 & -0.310980 & 1.963419 \\ \mathrm{H} & -3.073820 & 2.812570 & -1.726931 \\ \mathrm{H} & -2.046300 & 4.919380 & -0.914201 \\ \mathrm{H} & -0.761720 & 4.931370 & 1.207979 \\ \mathrm{H} & -0.522830 & 2.832141 & 2.509319 \\ \mathrm{H} & -1.540740 & 0.730740 & 1.670989 \\ \mathrm{H} & 7.957180 & 0.854621 & 2.207159 \\ \mathrm{H} & 8.217420 & -0.882969 & 1.945189 \\ \mathrm{H} & 9.463120 & 0.261481 & 1.466859 \\ \mathrm{H} & 9.556230 & 1.144611 & -0.472221 \\ \mathrm{H} & 8.415850 & 0.756711 & -1.752261 \\ \mathrm{H} & 8.085980 & 2.127511 & -0.671901 \\ \mathrm{H} & 3.933750 & 0.558041 & -2.187711 \\ \mathrm{H} & 6.325370 & 0.881301 & -1.944151\end{array}$

\footnotetext{
ligE2

$\begin{array}{llll}\mathrm{O} & -1.914530 & -2.812650 & 1.061350\end{array}$

O $\quad-3.541520 \quad-3.054470 \quad-0.484580$

$\begin{array}{llll}\mathrm{Cl} & -1.005310 & 0.191090 & 1.957770\end{array}$

$\begin{array}{llll}\mathrm{N} & 7.862170 & 0.240190 & 0.120940\end{array}$

$\begin{array}{llll}\text { C } & -2.567100 & -2.509800 & 0.051360\end{array}$

C $\quad-2.066460 \quad-1.179570 \quad-0.666730$

C $\quad-0.606780 \quad-0.938710 \quad-0.504020$

$\begin{array}{llll}\text { C } & -0.024320 & -0.359740 & 0.626530\end{array}$

$\begin{array}{llll}\text { C } & 1.347380 & -0.175560 & 0.736060\end{array}$

$\begin{array}{llll}\text { C } & 2.205190 & -0.580970 & -0.286700\end{array}$

C $\quad 1.644490 \quad-1.185160 \quad-1.414740$

C $\quad 0.271280 \quad-1.354850 \quad-1.508590$

$\begin{array}{llll}\text { C } & 3.666950 & -0.372590 & -0.173020\end{array}$

$\begin{array}{llll}\text { C } & 4.197920 & 0.790160 & 0.389150\end{array}$

$\begin{array}{llll}\text { C } & 5.566250 & 0.990970 & 0.503690\end{array}$
}

$\begin{array}{llll}\text { C } & 6.480580 & 0.028570 & 0.042620\end{array}$

C $\quad 5.949260 \quad-1.148240 \quad-0.511750$

$\begin{array}{llll}\text { C } & 4.577640 & -1.333660 & -0.616030\end{array}$

$\begin{array}{llll}\text { C } & -3.046320 & 0.001800 & -0.736790\end{array}$

C $\quad-2.737320 \quad-0.836480-1.957360$

C $\quad-4.406690 \quad-0.154420 \quad-0.111170$

C $\quad-4.564730 \quad-0.621750 \quad 1.192530$

$\begin{array}{llll}\text { C } & -5.829860 & -0.678850 & 1.768380\end{array}$

$\begin{array}{llll}\text { C } & -6.950100 & -0.267000 & 1.052650\end{array}$

$\begin{array}{llll}\text { C } & -6.799640 & 0.194570 & -0.252120\end{array}$

C $\quad-5.534100 \quad 0.247920 \quad-0.826140$

$\begin{array}{llll}\mathrm{C} & -2.511170 & 1.411690 & -0.686810\end{array}$

C $\quad-1.407520 \quad 1.824210 \quad-1.446240$

C $\quad-0.907710 \quad 3.118460-1.355840$

$\begin{array}{llll}\text { C } & -1.501460 & 4.048570 & -0.509620\end{array}$

C $\quad-2.604430 \quad 3.660990 \quad 0.242740$

C $\quad-3.099550 \quad 2.365020 \quad 0.155240$

$\begin{array}{lrrr}\text { C } & 8.721490 & -0.918280 & -0.025410\end{array}$

$\begin{array}{llll}\text { C } & 8.326690 & 1.258370 & 1.043230\end{array}$

$\mathrm{H} \quad 1.745550 \quad 0.266150 \quad 1.641660$

$\mathrm{H} \quad 2.285140 \quad-1.497270 \quad-2.232510$

H $\quad-0.157700 \quad-1.818050 \quad-2.390960$

H $\quad 6.601800 \quad-1.938730 \quad-0.855690$

H $\quad 4.204000 \quad-2.265980 \quad-1.025330$

$\mathrm{H} \quad-2.173130 \quad-0.367350 \quad-2.755430$

H $\quad-3.503060 \quad-1.546180 \quad-2.247900$

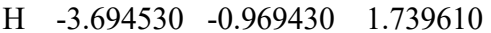

$\mathrm{H} \quad-5.940450 \quad-1.060720 \quad 2.777090$

$\mathrm{H} \quad-7.935380 \quad-0.317590 \quad 1.503100$

$\mathrm{H} \quad-7.667410 \quad 0.506600 \quad-0.823240$

$\mathrm{H} \quad-5.408640 \quad 0.605280 \quad-1.843730$

$\mathrm{H} \quad-0.907120 \quad 1.126810 \quad-2.105110$

$\mathrm{H} \quad-0.043910 \quad 3.395230 \quad-1.950400$

H $\quad-1.109820 \quad 5.056910 \quad-0.437250$

$\mathrm{H} \quad-3.083090 \quad 4.368040 \quad 0.911520$

$\mathrm{H} \quad-3.951440 \quad 2.084130 \quad 0.761840$

H $\quad 8.508760 \quad-1.701650 \quad 0.717380$

H $\quad 8.614570 \quad-1.352310 \quad-1.021140$

H $\quad 9.758940 \quad-0.604420 \quad 0.083990$

$\mathrm{H} \quad 9.415800 \quad 1.277740 \quad 1.028530$

$\mathrm{H} \quad 7.978340 \quad 2.245700 \quad 0.734780$

$\mathrm{H} \quad 7.989850 \quad 1.080500 \quad 2.075290$

$\mathrm{H} \quad 3.522790 \quad 1.566330 \quad 0.733240$

H $\quad 5.914680 \quad 1.913480 \quad 0.947150$

\section{ligF1}

$\begin{array}{llll}\mathrm{O} & -2.641010 & -2.540640 & 1.670970\end{array}$

$\begin{array}{llll}\text { O } & -4.265090 & -3.163560 & 0.230670\end{array}$

$\mathrm{Cl} \quad-1.844250 \quad 0.591100 \quad 1.868600$

F $\quad 7.327920 \quad-1.796060 \quad-0.811000$

$\begin{array}{llll}\text { F } & 6.353870 & -2.905290 & 0.757400\end{array}$

F $\quad 5.687940 \quad-3.121220 \quad-1.278700$

F $\quad 5.260980 \quad 3.301790 \quad-0.483230$

F $\quad 4.407780 \quad 3.228410 \quad 1.492140$

F $\quad 6.442710 \quad 2.581850 \quad 1.167780$

C $\quad-3.302270 \quad-2.494640 \quad 0.623240$

C $\quad-2.824100 \quad-1.357460 \quad-0.388770$

C $\quad-3.820200 \quad-0.245700 \quad-0.747610$

$\begin{array}{llll}\text { C } & -3.481890 & -1.344830 & -1.730120\end{array}$

C $\quad-1.374720 \quad-1.054690 \quad-0.267060$

$\begin{array}{llll}\text { C } & -0.828510 & -0.220510 & 0.714540\end{array}$

$\begin{array}{llll}\text { C } & 0.535980 & 0.014710 & 0.798900\end{array}$ $\begin{array}{llll}\text { C } & 1.417390 & -0.601370 & -0.090010\end{array}$

$\begin{array}{llll}\text { C } & 0.896670 & -1.462180 & -1.058780\end{array}$

C $\quad-0.470820 \quad-1.672740 \quad-1.136010$

$\begin{array}{llll}\text { C } & 2.869800 & -0.339120 & -0.006750\end{array}$

$\begin{array}{llll}\text { C } & 3.799030 & -1.356620 & -0.245620\end{array}$

$\begin{array}{llll}\text { C } & 5.162280 & -1.099800 & -0.181790\end{array}$

$\begin{array}{llll}\text { C } & 5.643840 & 0.164780 & 0.131320\end{array}$

$\begin{array}{llll}\text { C } & 4.721430 & 1.173770 & 0.375930\end{array}$

$\begin{array}{llll}\text { C } & 3.355140 & 0.934880 & 0.304740\end{array}$

$\begin{array}{lrrr}\text { C } & -5.188370 & -0.269230 & -0.119870\end{array}$

C $\quad-5.365920 \quad-0.436150 \quad 1.252300$

$\begin{array}{llll}\text { C } & -6.640230 & -0.372540 & 1.807010\end{array}$

$\begin{array}{llll}\text { C } & -7.749710 & -0.138360 & 1.000410\end{array}$

$\begin{array}{llll}\text { C } & -7.579580 & 0.022510 & -0.371930\end{array}$

$\begin{array}{llll}\text { C } & -6.305150 & -0.044010 & -0.923930\end{array}$

$\begin{array}{llll}\text { C } & -3.300350 & 1.144110 & -1.025340\end{array}$

C $\quad-2.204130 \quad 1.383120 \quad-1.865230$

$\begin{array}{llll}\text { C } & -1.717230 & 2.668640 & -2.074810\end{array}$

C $\quad-2.317000 \quad 3.760620-1.457380$

C $\quad-3.412920 \quad 3.544110 \quad-0.629720$

$\begin{array}{llll}\text { C } & -3.895500 & 2.257850 & -0.418000\end{array}$

$\begin{array}{llll}\text { C } & 5.207030 & 2.568860 & 0.643730\end{array}$

$\begin{array}{llll}\text { C } & 6.131310 & -2.228730 & -0.382530\end{array}$

$\mathrm{H} \quad-2.916410 \quad-1.071350 \quad-2.613630$

H $\quad-4.232610 \quad-2.117460 \quad-1.848070$

$\mathrm{H} \quad 0.906580 \quad 0.660520 \quad 1.586080$

$\mathrm{H} \quad 1.557820 \quad-1.940490 \quad-1.773270$

$\mathrm{H} \quad-0.874430 \quad-2.332890 \quad-1.896030$

$\mathrm{H} \quad-4.504340 \quad-0.646300 \quad 1.877400$

$\mathrm{H} \quad-6.766050 \quad-0.520980 \quad 2.873540$

H $\quad-8.741970 \quad-0.094680 \quad 1.435740$

$\mathrm{H} \quad-8.438750 \quad 0.193810 \quad-1.011300$

H $\quad-6.164410 \quad 0.079830 \quad-1.993410$

H $\quad-1.701390 \quad 0.559200 \quad-2.354680$

$\mathrm{H} \quad-0.861030 \quad 2.812340 \quad-2.724770$

$\mathrm{H} \quad-1.935940 \quad 4.762510-1.618780$

H $\quad-3.896020 \quad 4.380420 \quad-0.136580$

$\mathrm{H} \quad-4.742640 \quad 2.113480 \quad 0.240910$

$\begin{array}{llll}\mathrm{H} & 2.656920 & 1.746700 & 0.474270\end{array}$

H $\quad 3.449700 \quad-2.358880 \quad-0.465040$

H $\quad 6.707390 \quad 0.358300 \quad 0.183310$

\section{ligF_TSrot}

$\begin{array}{llll}\mathrm{O} & -3.316769 & -2.906170 & -1.447970\end{array}$

$\begin{array}{llll}\text { O } & -2.556209 & -2.750150 & 0.669870\end{array}$

$\begin{array}{lllll}\mathrm{Cl} & -1.320950 & 2.146930 & 0.627950\end{array}$

F $\quad 7.038510 \quad 2.548142 \quad 0.135890$

F $\quad 6.282340 \quad 2.427622 \quad-1.876480$

$\begin{array}{llll}\text { F } & 5.144110 & 3.456472 & -0.364920\end{array}$

F $\quad 6.349781 \quad-3.185028-0.393280$

F $\quad 7.305801 \quad-2.131878 \quad 1.224850$

F $\quad 5.472781 \quad-3.219278 \quad 1.572620$

C $\quad-2.869069-2.318560 \quad-0.454030$

$\begin{array}{llll}\text { C } & -2.626359 & -0.762490 & -0.616170\end{array}$

$\begin{array}{llll}\text { C } & -3.943119 & 0.020870 & -0.480130\end{array}$

$\begin{array}{llll}\text { C } & -3.275409 & -0.115860 & -1.816450\end{array}$

$\begin{array}{llll}\text { C } & -1.169549 & -0.448690 & -0.360620\end{array}$

$\begin{array}{llll}\text { C } & -0.505379 & 0.691881 & 0.105550\end{array}$

$\begin{array}{llll}\text { C } & 0.885871 & 0.775841 & 0.191080\end{array}$

$\begin{array}{llll}\text { C } & 1.697141 & -0.277359 & -0.201990\end{array}$

$\begin{array}{llll}\text { C } & 1.071051 & -1.423759 & -0.700010\end{array}$

$\begin{array}{llll}\text { C } & -0.303789 & -1.490489 & -0.773020\end{array}$ 


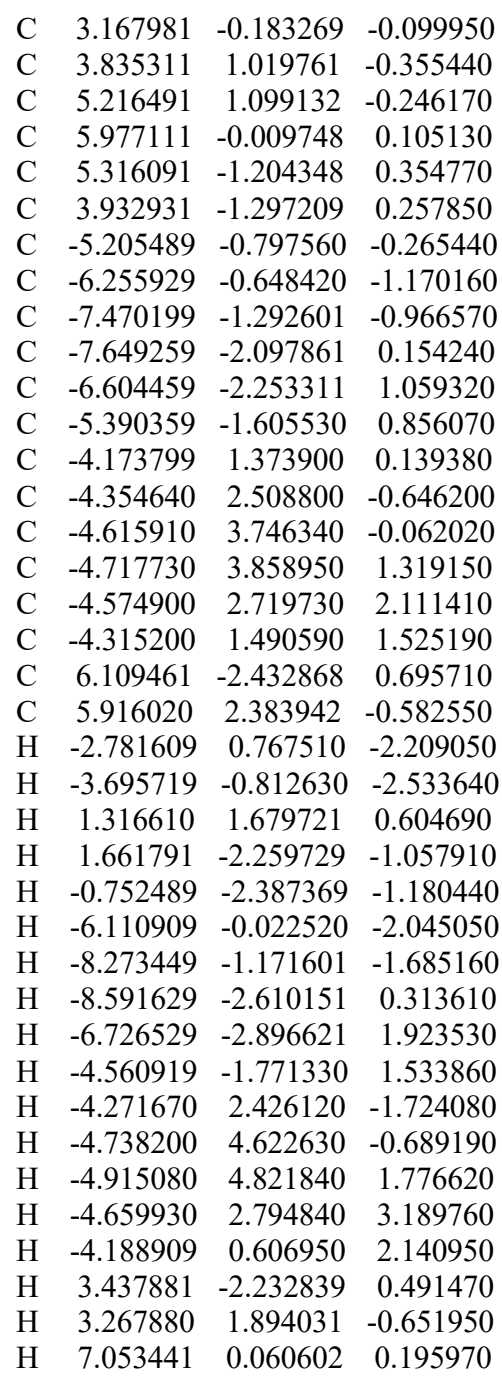

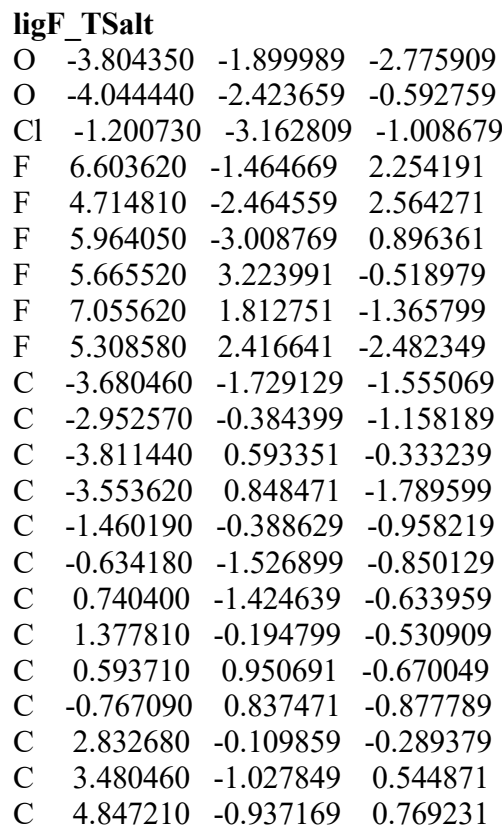

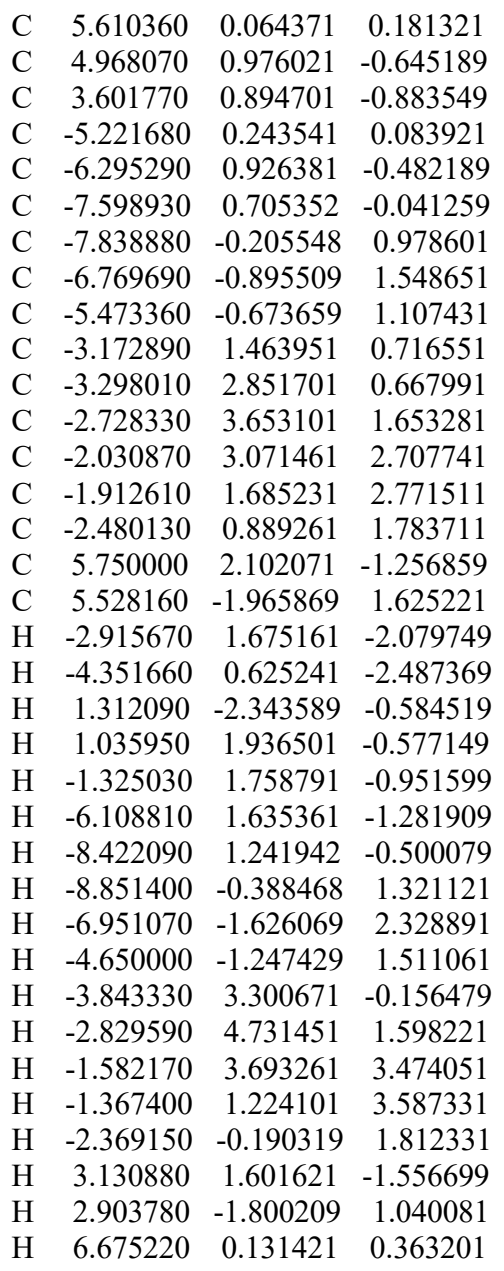

\section{$\operatorname{ligF2}$}

$\begin{array}{llll}\text { O } & 4.520130 & -2.798720 & -0.970610\end{array}$

O $\quad 2.888690 \quad-1.912830 \quad-2.256540$

Cl $\quad 1.087890 \quad-3.059170 \quad 1.831460$

$\begin{array}{llll}\text { F } & -7.199460 & -1.645840 & 0.452660\end{array}$

F $\quad-6.347330 \quad-2.477930 \quad-1.341190$

F $\quad-5.607340 \quad-3.099310 \quad 0.583560$

F $\quad-4.114370 \quad 3.575000 \quad-1.166170$

F $\quad-6.174080 \quad 2.940050 \quad-1.028670$

F $\quad-5.056350 \quad 3.376210 \quad 0.759900$

C $\quad 3.523560 \quad-2.109610 \quad-1.206140$

$\begin{array}{llll}\text { C } & 2.976450 & -1.274580 & 0.034150\end{array}$

$\begin{array}{llll}\text { C } & 3.854060 & -0.085630 & 0.483470\end{array}$

$\begin{array}{llll}\text { C } & 3.747270 & -1.346510 & 1.306920\end{array}$

$\begin{array}{llll}\text { C } & 1.506910 & -1.064080 & -0.003680\end{array}$

$\begin{array}{llll}\text { C } & 0.577700 & -1.779560 & 0.748910\end{array}$

$\begin{array}{llll}\text { C } & -0.788000 & -1.521290 & 0.688350\end{array}$

C $\quad-1.272700 \quad-0.529250 \quad-0.158960$

C $\quad-0.363300 \quad 0.172200 \quad-0.960050$

C $\quad 0.987140 \quad-0.103720-0.884250$

C $\quad-2.717710 \quad-0.222340 \quad-0.204860$

C $\quad-3.678560 \quad-1.234420-0.110810$

$\begin{array}{llll}\text { C } & -5.032640 & -0.928700 & -0.144330\end{array}$

C $\quad-5.473030 \quad 0.381490 \quad-0.283570$

C $\quad-4.519140 \quad 1.385700 \quad-0.383900$

C $\quad-3.161320 \quad 1.096890 \quad-0.340380$ $\begin{array}{llll}\text { C } & 5.158900 & 0.188600 & -0.215550\end{array}$

$\begin{array}{llll}\text { C } & 6.290900 & 0.450000 & 0.556810\end{array}$

$\begin{array}{llll}\text { C } & 7.499930 & 0.788640 & -0.040060\end{array}$

$\begin{array}{llll}\text { C } & 7.590290 & 0.868610 & -1.427030\end{array}$

$\begin{array}{llll}\text { C } & 6.467630 & 0.601980 & -2.204190\end{array}$

$\begin{array}{llll}\text { C } & 5.257600 & 0.266450 & -1.604090\end{array}$

C $\quad 3.147940 \quad 1.157500 \quad 0.962130$

C $\quad 2.170850 \quad 1.125520 \quad 1.967100$

$\begin{array}{llll}\text { C } & 1.483590 & 2.271710 & 2.346360\end{array}$

C $\quad 1.751870 \quad 3.492040 \quad 1.733500$

C $\quad 2.722820 \quad 3.544790 \quad 0.740790$

$\begin{array}{llll}\text { C } & 3.411960 & 2.395710 & 0.363970\end{array}$

$\begin{array}{llll}\text { C } & -4.963920 & 2.817410 & -0.460400\end{array}$

C $\quad-6.043410 \quad-2.038110 \quad-0.107380$

$\mathrm{H} \quad 3.242750 \quad-1.296470 \quad 2.262380$

H $\quad 4.595630 \quad-2.019030 \quad 1.246760$

H $\quad-1.457400 \quad-2.079560 \quad 1.332060$

$\mathrm{H} \quad-0.721610 \quad 0.916700 \quad-1.662220$

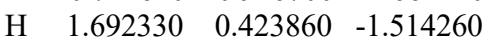

$\begin{array}{llll}\mathrm{H} & 6.213490 & 0.383070 & 1.637620\end{array}$

H $\quad 8.371500 \quad 0.984150 \quad 0.575050$

H $\quad 8.532640 \quad 1.124850 \quad-1.898420$

H $\quad 6.534010 \quad 0.640960 \quad-3.285600$

H $\quad 4.392380 \quad 0.026640 \quad-2.214110$

$\begin{array}{llll}\mathrm{H} & 1.917880 & 0.190790 & 2.451910\end{array}$

$\mathrm{H} \quad 0.728940 \quad 2.206200 \quad 3.122360$

H $\quad 1.212480 \quad 4.385540 \quad 2.026210$

H $\quad 2.950460 \quad 4.485480 \quad 0.251500$

$\mathrm{H} \quad 4.166220 \quad 2.460700 \quad-0.410940$

$\mathrm{H} \quad-2.436100 \quad 1.900780 \quad-0.393600$

$\mathrm{H} \quad-3.361520 \quad-2.267530 \quad-0.028630$

$\mathrm{H} \quad-6.530120 \quad 0.612800 \quad-0.313490$

\section{ligG1}

$\begin{array}{llll}\mathrm{O} & 2.391710 & -2.102440 & -1.995920\end{array}$

O $4.022060-2.808820 \quad-0.603100$

$\begin{array}{llll}\mathrm{Cl} & 0.655880 & -2.732700 & 2.205430\end{array}$

C $\quad 3.026460 \quad-2.153220 \quad-0.928150$

C $\quad 2.485720 \quad-1.152940 \quad 0.183850$

$\begin{array}{llll}\text { C } & 3.269480 & -1.043830 & 1.445680\end{array}$

$\begin{array}{llll}\text { C } & 3.370190 & 0.085260 & 0.447310\end{array}$

$\begin{array}{llll}\text { C } & 1.011850 & -0.963520 & 0.138940\end{array}$

$\begin{array}{llll}\text { C } & 0.104970 & -1.596440 & 0.986200\end{array}$

$\begin{array}{llll}\text { C } & -1.268150 & -1.387630 & 0.903490\end{array}$

C $\quad-1.789960 \quad-0.535220 \quad-0.065820$

$\begin{array}{llll}\text { C } & -0.900270 & 0.083500 & -0.952270\end{array}$

C $\quad 0.460550 \quad-0.133310 \quad-0.846540$

$\begin{array}{llll}\text { C } & -3.249760 & -0.301770 & -0.156770\end{array}$

$\begin{array}{llll}\text { C } & -4.040680 & -0.183760 & 0.985840\end{array}$

$\begin{array}{llll}\text { C } & -5.413420 & 0.033210 & 0.897130\end{array}$

$\begin{array}{llll}\text { C } & -6.052550 & 0.146150 & -0.337560\end{array}$

$\begin{array}{llll}\text { C } & -5.254140 & 0.029230 & -1.481950\end{array}$

$\begin{array}{llll}\text { C } & -3.887910 & -0.190740 & -1.397690\end{array}$

$\begin{array}{llll}\text { C } & -7.557160 & 0.385600 & -0.481970\end{array}$

$\begin{array}{llll}\text { C } & -8.187710 & -0.779220 & -1.264020\end{array}$

$\begin{array}{llll}\text { C } & -8.259510 & 0.486400 & 0.875730\end{array}$

$\begin{array}{llll}\text { C } & -7.795610 & 1.699230 & -1.245620\end{array}$

$\begin{array}{llll}\text { C } & 4.666170 & 0.243640 & -0.302250\end{array}$

$\begin{array}{llll}\text { C } & 4.735120 & 0.147320 & -1.691580\end{array}$

C $\quad 5.936210 \quad 0.384220 \quad-2.353230$

$\begin{array}{llll}\text { C } & 7.080130 & 0.724940 & -1.638160\end{array}$

$\begin{array}{llll}\text { C } & 7.019690 & 0.817980 & -0.250380\end{array}$ 


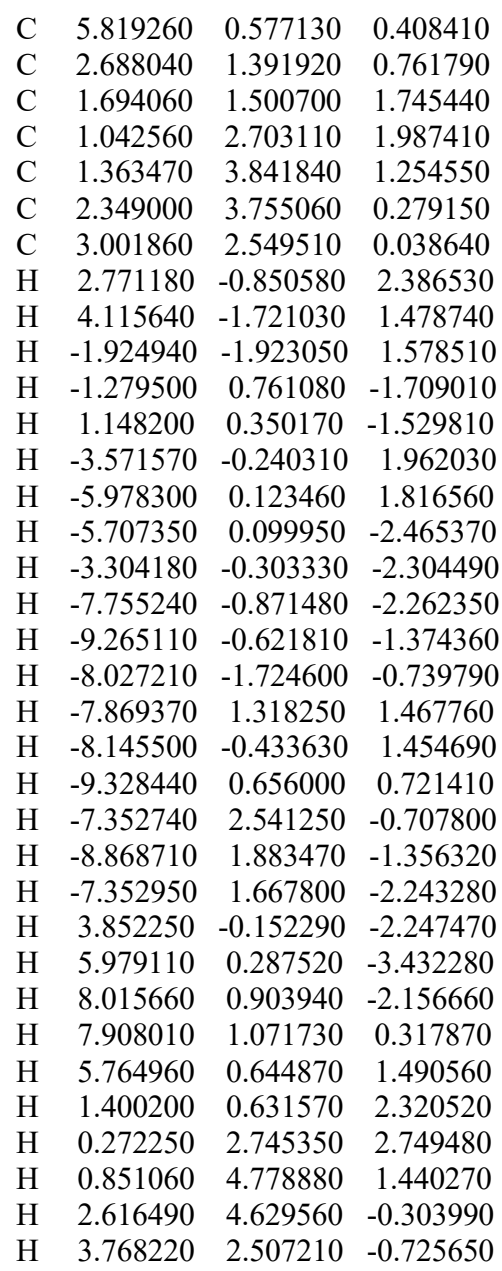

\section{ligG TSrot}

$\begin{array}{llll}\text { O } & -2.047361 & -2.469309 & 1.385060\end{array}$

$\begin{array}{llll}\text { O } & -2.797201 & -3.180449 & -0.619220\end{array}$

$\begin{array}{llll}\mathrm{Cl} & -0.738750 & 2.221210 & 0.033350\end{array}$

$\begin{array}{llll}\text { C } & -2.343841 & -2.351499 & 0.182790\end{array}$

$\begin{array}{llll}\text { C } & -2.077441 & -0.900919 & -0.391750\end{array}$

$\begin{array}{llll}\text { C } & -2.721880 & -0.593999 & -1.721070\end{array}$

$\begin{array}{llll}\text { C } & -3.385220 & -0.093709 & -0.471250\end{array}$

$\begin{array}{llll}\text { C } & -0.614810 & -0.545940 & -0.224960\end{array}$

$\begin{array}{llll}\text { C } & 0.062820 & 0.667660 & -0.069420\end{array}$

$\begin{array}{llll}\text { C } & 1.455360 & 0.758210 & -0.007370\end{array}$

$\begin{array}{llll}\text { C } & 2.259740 & -0.365880 & -0.116730\end{array}$

$\begin{array}{llll}\text { C } & 1.617609 & -1.593580 & -0.302910\end{array}$

$\begin{array}{llll}\text { C } & 0.240699 & -1.666150 & -0.349650\end{array}$

$\begin{array}{llll}\text { C } & 3.734560 & -0.259941 & -0.044450\end{array}$

$\begin{array}{llll}\text { C } & 4.358450 & 0.633899 & 0.825730\end{array}$

$\begin{array}{llll}\text { C } & 5.745940 & 0.733579 & 0.888720\end{array}$

$\begin{array}{llll}\text { C } & 6.568600 & -0.059671 & 0.088830\end{array}$

$\begin{array}{llll}\text { C } & 5.938049 & -0.958151 & -0.780690\end{array}$

$\begin{array}{llll}\text { C } & 4.556919 & -1.057471 & -0.848990\end{array}$

$\begin{array}{llll}\text { C } & 8.096650 & 0.014529 & 0.128110\end{array}$

$\begin{array}{llll}\text { C } & 8.625510 & 0.407068 & -1.261900\end{array}$

$\begin{array}{llll}\text { C } & 8.599370 & 1.047808 & 1.141220\end{array}$

$\begin{array}{llll}\text { C } & 8.666139 & -1.359572 & 0.519710\end{array}$

$\begin{array}{llll}\text { C } & -4.657751 & -0.807509 & -0.045690\end{array}$

$\begin{array}{llll}\text { C } & -4.851391 & -1.277219 & 1.253310\end{array}$

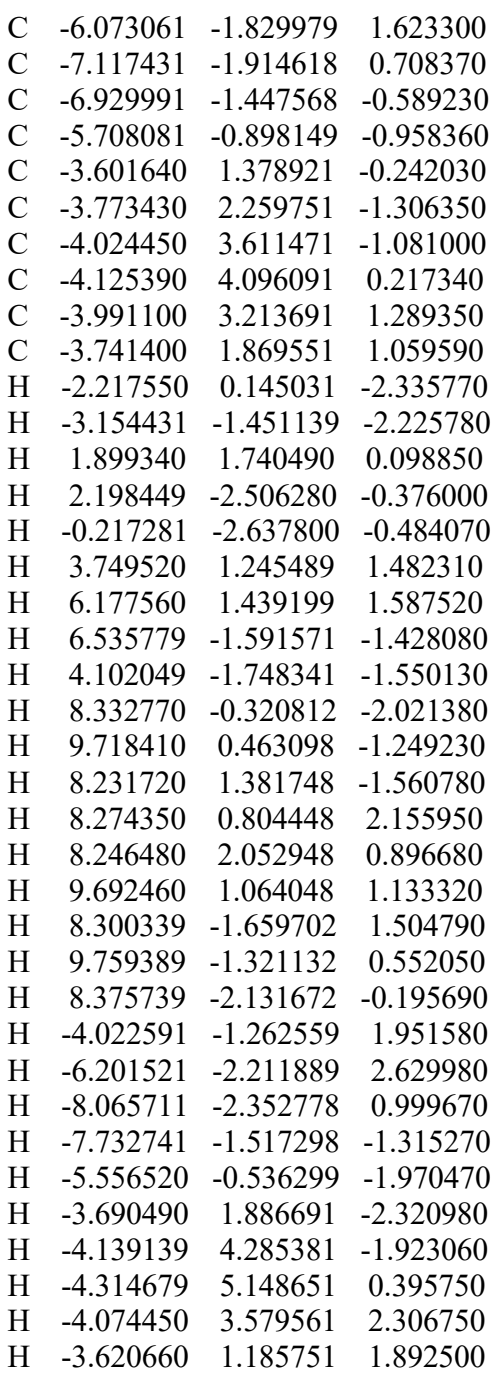

\section{ligG_TSalt}

$\begin{array}{llll}\text { O } & -3.536330 & -2.341529 & 0.851510\end{array}$

$\begin{array}{lllll}\text { O } & -3.374130 & -3.081089 & -1.274980\end{array}$

$\begin{array}{llll}\mathrm{Cl} & -0.703670 & -3.232819 & 0.844240\end{array}$

$\begin{array}{llll}\text { C } & -3.202610 & -2.281709 & -0.343350\end{array}$

$\begin{array}{llll}\text { C } & -2.458770 & -0.950439 & -0.752030\end{array}$

$\begin{array}{llll}\text { C } & -3.066640 & -0.249859 & -1.942680\end{array}$

$\begin{array}{llll}\text { C } & -3.300420 & 0.327321 & -0.575950\end{array}$

$\begin{array}{llll}\text { C } & -0.960480 & -0.862419 & -0.599210\end{array}$

$\begin{array}{llll}\text { C } & -0.131900 & -1.772499 & 0.086250\end{array}$

$\begin{array}{llll}\text { C } & 1.246540 & -1.581559 & 0.199180\end{array}$

$\begin{array}{llll}\text { C } & 1.888440 & -0.490089 & -0.371060\end{array}$

$\begin{array}{llll}\text { C } & 1.098770 & 0.408781 & -1.089000\end{array}$

$\begin{array}{llll}\text { C } & -0.264700 & 0.210331 & -1.194210\end{array}$

$\begin{array}{llll}\text { C } & 3.349170 & -0.297039 & -0.228410\end{array}$

$\begin{array}{llll}\text { C } & 4.234570 & -1.374409 & -0.252440\end{array}$

$\begin{array}{llll}\text { C } & 5.607280 & -1.185369 & -0.114080\end{array}$

$\begin{array}{llll}\text { C } & 6.153110 & 0.087981 & 0.049030\end{array}$

$\begin{array}{llll}\text { C } & 5.260540 & 1.166751 & 0.069280\end{array}$

$\begin{array}{llll}\text { C } & 3.892870 & 0.982391 & -0.063750\end{array}$

$\begin{array}{llll}\text { C } & 7.654320 & 0.340981 & 0.204750\end{array}$

$\begin{array}{llll}\text { C } & 7.919810 & 1.023971 & 1.557200\end{array}$

$\begin{array}{llll}\text { C } & 8.467460 & -0.956339 & 0.155620\end{array}$ $\begin{array}{llll}\text { C } & 8.139930 & 1.256691 & -0.931630\end{array}$

$\begin{array}{lllll}\text { C } & -4.705060 & 0.277031 & -0.019120\end{array}$

$\begin{array}{llll}\mathrm{C} & -4.942890 & 0.058181 & 1.340380\end{array}$

$\begin{array}{llll}\text { C } & -6.232230 & 0.125741 & 1.848040\end{array}$

$\begin{array}{llll}\text { C } & -7.308690 & 0.414201 & 1.009940\end{array}$

$\begin{array}{llll}\text { C } & -7.082820 & 0.629791 & -0.342890\end{array}$

$\begin{array}{llll}\text { C } & -5.786140 & 0.561821 & -0.849810\end{array}$

$\begin{array}{llll}\text { C } & -2.643270 & 1.620401 & -0.171190\end{array}$

$\begin{array}{llll}\text { C } & -2.766651 & 2.764231 & -0.958790\end{array}$

$\begin{array}{llll}\text { C } & -2.179021 & 3.964531 & -0.569510\end{array}$

$\begin{array}{llll}\text { C } & -1.464871 & 4.036301 & 0.622890\end{array}$

$\begin{array}{llll}\text { C } & -1.347921 & 2.901981 & 1.422620\end{array}$

$\begin{array}{llll}\text { C } & -1.933280 & 1.704961 & 1.028040\end{array}$

$\begin{array}{lllll}\mathrm{H} & -2.430300 & 0.280441 & -2.641460\end{array}$

$\begin{array}{llll}\mathrm{H} & -3.878510 & -0.804229 & -2.397920\end{array}$

$\begin{array}{llll}\mathrm{H} & 1.811610 & -2.307009 & 0.771650\end{array}$

$\mathrm{H} \quad 1.552880 \quad 1.257941 \quad-1.587700$

$\begin{array}{llll}\mathrm{H} & -0.819700 & 0.942541 & -1.761770\end{array}$

H $\quad 3.846900 \quad-2.375839-0.403280$

$\begin{array}{llll}\mathrm{H} & 6.249620 & -2.056529 & -0.145540\end{array}$

$\begin{array}{llll}\mathrm{H} & 5.636790 & 2.175761 & 0.203060\end{array}$

$\begin{array}{llll}\mathrm{H} & 3.227680 & 1.837681 & -0.016820\end{array}$

$\begin{array}{llll}\mathrm{H} & 7.399360 & 1.981051 & 1.631320\end{array}$

$\begin{array}{llll}\mathrm{H} & 8.990890 & 1.209601 & 1.683750\end{array}$

$\begin{array}{llll}\mathrm{H} & 7.578000 & 0.390991 & 2.379890\end{array}$

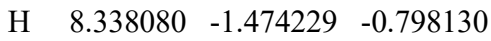

H $\quad 8.182560 \quad-1.638219 \quad 0.960850$

H $\quad 9.529710 \quad-0.725209 \quad 0.271070$

$\begin{array}{llll}\mathrm{H} & 7.958860 & 0.790761 & -1.903490\end{array}$

$\begin{array}{llll}\mathrm{H} & 9.213100 & 1.446801 & -0.832350\end{array}$

H $\quad 7.623220 \quad 2.218591 \quad-0.918650$

$\mathrm{H} \quad-4.114610 \quad-0.219569 \quad 1.978250$

$\begin{array}{llll}\mathrm{H} & -6.402910 & -0.068099 & 2.901070\end{array}$

$\begin{array}{llll}\mathrm{H} & -8.315930 & 0.456871 & 1.409730\end{array}$

$\begin{array}{llll}\mathrm{H} & -7.911830 & 0.845271 & -1.008140\end{array}$

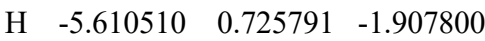

$\mathrm{H} \quad-3.324100 \quad 2.702821 \quad-1.888410$

$\mathrm{H} \quad-2.279151 \quad 4.843801 \quad-1.196580$

$\begin{array}{llll}\mathrm{H} & -1.002231 & 4.968621 & 0.926660\end{array}$

$\begin{array}{llll}\mathrm{H} & -0.788701 & 2.947161 & 2.350440\end{array}$

$\begin{array}{llll}\mathrm{H} & -1.821310 & 0.809461 & 1.631370\end{array}$

\section{ligG2}

$\begin{array}{llll}\text { O } & -2.195760 & -2.934490 & 0.627660\end{array}$

$\begin{array}{llll}\text { O } & -3.832400 & -2.934000 & -0.927150\end{array}$

$\mathrm{Cl} \quad-1.282370 \quad-0.114310 \quad 1.963510$

C $\quad-2.852140 \quad-2.481310 \quad-0.321730$

C $\quad-2.346980 \quad-1.060920-0.837130$

C $\quad-3.018230 \quad-0.527200 \quad-2.060900$

$\begin{array}{llll}\text { C } & -3.319800 & 0.123370 & -0.729200\end{array}$

$\begin{array}{llll}\text { C } & -0.886500 & -0.853440 & -0.642260\end{array}$

$\begin{array}{llll}\text { C } & -0.302000 & -0.468620 & 0.567600\end{array}$

$\begin{array}{llll}\text { C } & 1.070580 & -0.313860 & 0.705100\end{array}$

$\begin{array}{llll}\text { C } & 1.925750 & -0.555780 & -0.370200\end{array}$

C $\quad 1.363290 \quad-0.962290 \quad-1.582520$

C $\quad-0.010690 \quad-1.106300 \quad-1.701900$

$\begin{array}{llll}\text { C } & 3.389860 & -0.388820 & -0.220750\end{array}$

$\begin{array}{llll}\text { C } & 4.165870 & 0.154020 & -1.244600\end{array}$

$\begin{array}{llll}\text { C } & 5.542790 & 0.309380 & -1.105290\end{array}$

$\begin{array}{llll}\text { C } & 6.201360 & -0.067590 & 0.065190\end{array}$

$\begin{array}{llll}\text { C } & 5.418090 & -0.611550 & 1.090580\end{array}$

$\begin{array}{llll}\text { C } & 4.047720 & -0.771350 & 0.954140\end{array}$ 

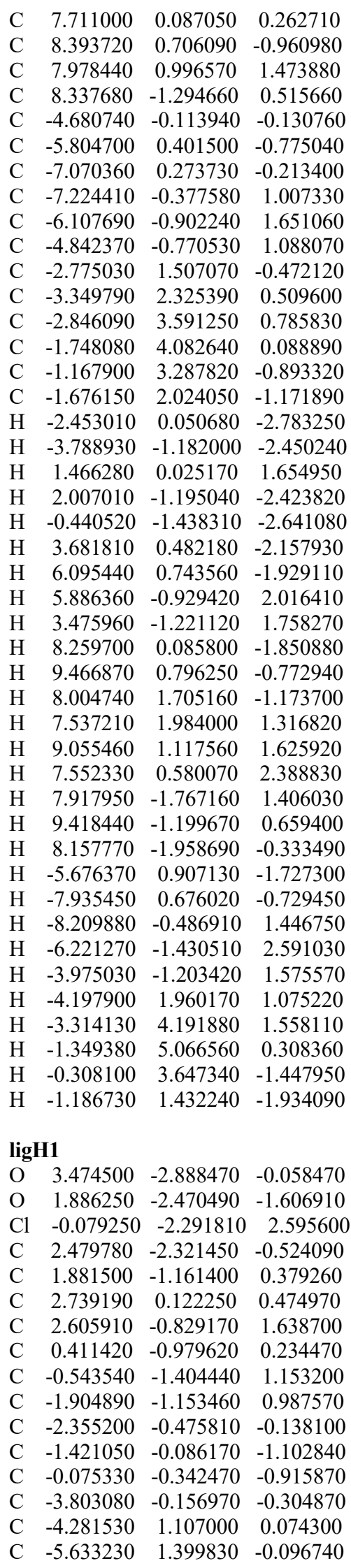

C $\quad-6.520060 \quad 0.468820 \quad-0.634050$

C $\quad-6.024660 \quad-0.776980 \quad-1.007320$

C $\quad-4.677540 \quad-1.106250 \quad-0.850010$

$\begin{array}{llll}\text { C } & -3.342850 & 2.129170 & 0.664460\end{array}$

$\begin{array}{llll}\text { C } & -7.984260 & 0.799160 & -0.781410\end{array}$

C $\quad-4.165960 \quad-2.461870 \quad-1.267200$

$\begin{array}{llll}\text { C } & 4.068020 & 0.190440 & -0.229260\end{array}$

C $\quad 4.218490 \quad-0.157900 \quad-1.571100$

$\begin{array}{llll}\text { C } & 5.448030 & -0.000830 & -2.203830\end{array}$

$\begin{array}{llll}\text { C } & 6.539580 & 0.509260 & -1.508110\end{array}$

$\begin{array}{llll}\text { C } & 6.397960 & 0.854410 & -0.166760\end{array}$

$\begin{array}{llll}\text { C } & 5.169340 & 0.693640 & 0.463720\end{array}$

$\begin{array}{llll}\text { C } & 2.012090 & 1.441750 & 0.526180\end{array}$

$\begin{array}{llll}\text { C } & 1.002120 & 1.708140 & 1.462180\end{array}$

$\begin{array}{llll}\text { C } & 0.297390 & 2.905100 & 1.446560\end{array}$

C $\quad 0.577580 \quad 3.879230 \quad 0.492770$

$\begin{array}{llll}\text { C } & 1.580750 & 3.636510 & -0.437680\end{array}$

$\begin{array}{llll}\text { C } & 2.288960 & 2.438560 & -0.417560\end{array}$

$\begin{array}{llll}\mathrm{H} & 2.065120 & -0.497940 & 2.514870\end{array}$

H $\quad 3.458720 \quad-1.474580 \quad 1.816730$

$\mathrm{H} \quad-2.605280 \quad-1.484650 \quad 1.745950$

$\mathrm{H} \quad-1.760210 \quad 0.427360 \quad-1.996570$

H $\quad 0.651710 \quad-0.042000 \quad-1.660530$

$\mathrm{H} \quad-6.002490 \quad 2.379280 \quad 0.195900$

H $\quad-6.700200 \quad-1.511840 \quad-1.436650$

$\mathrm{H} \quad-2.850540 \quad 1.731730 \quad 1.555790$

$\mathrm{H} \quad-3.880200 \quad 3.039520 \quad 0.935330$

$\mathrm{H} \quad-2.542850 \quad 2.387100 \quad-0.034110$

$\mathrm{H} \quad-8.124220 \quad 1.834230 \quad-1.100250$

$\mathrm{H} \quad-8.509500 \quad 0.672810 \quad 0.169880$

$\mathrm{H} \quad-8.462790 \quad 0.147650 \quad-1.514710$

$\mathrm{H} \quad-3.803850 \quad-3.026430 \quad-0.404460$

$\mathrm{H} \quad-3.319480 \quad-2.367630 \quad-1.951090$

$\mathrm{H} \quad-4.951920 \quad-3.040160 \quad-1.755100$

$\mathrm{H} \quad 3.377660 \quad-0.587490 \quad-2.106670$

H $\quad 5.554350 \quad-0.292800 \quad-3.242580$

H $\quad 7.497380 \quad 0.625430 \quad-2.003310$

$\mathrm{H} \quad 7.244970 \quad 1.242630 \quad 0.388470$

H $\quad 5.052000 \quad 0.958050 \quad 1.510140$

$\mathrm{H} \quad 0.736770 \quad 0.964770 \quad 2.203470$

$\mathrm{H} \quad-0.482260 \quad 3.072170 \quad 2.181880$

H $\quad 0.022570 \quad 4.810420 \quad 0.476890$

$\mathrm{H} \quad 1.818620 \quad 4.381940 \quad-1.188720$

H $\quad 3.067570 \quad 2.271800 \quad-1.152130$

\section{ligH_TSrot}

O $\quad-2.157910 \quad-3.033280 \quad-1.114381$

$\begin{array}{llll}\mathrm{O} & -1.405450 & -2.637180 & 0.974039\end{array}$

$\begin{array}{lllll}\mathrm{Cl} & -0.129570 & 2.220390 & 0.357779\end{array}$

C $\quad-1.706740 \quad-2.335590 \quad-0.194891$

C $\quad-1.451220 \quad-0.811970 \quad-0.537951$

$\begin{array}{llll}\text { C } & -2.764670 & -0.011720 & -0.488071\end{array}$

C $\quad-2.100790 \quad-0.305900 \quad-1.801731$

$\begin{array}{llll}\text { C } & 0.009590 & -0.477080 & -0.314721\end{array}$

$\begin{array}{llll}\text { C } & 0.678700 & 0.702540 & 0.024779\end{array}$

$\begin{array}{llll}\text { C } & 2.072260 & 0.786820 & 0.114979\end{array}$

$\begin{array}{llll}\text { C } & 2.878370 & -0.305970 & -0.151861\end{array}$

$\begin{array}{llll}\text { C } & 2.248100 & -1.493070 & -0.529391\end{array}$

$\begin{array}{llll}\text { C } & 0.870970 & -1.561900 & -0.603701\end{array}$

$\begin{array}{llll}\text { C } & 4.362150 & -0.212100 & -0.036031\end{array}$

$\begin{array}{llll}\text { C } & 5.007520 & -0.740670 & 1.092399\end{array}$

$\begin{array}{llll}\text { C } & 6.395800 & -0.650760 & 1.184939\end{array}$ $\begin{array}{llll}\text { C } & 7.158270 & -0.051271 & 0.185579\end{array}$

$\begin{array}{llll}\text { C } & 6.499940 & 0.461300 & -0.928861\end{array}$

$\begin{array}{llll}\text { C } & 5.112510 & 0.392620 & -1.054681\end{array}$

$\begin{array}{llll}\text { C } & 4.209290 & -1.392790 & 2.193009\end{array}$

$\begin{array}{llll}\text { C } & 8.655690 & 0.065209 & 0.321689\end{array}$

C $\quad 4.430730 \quad 0.951840 \quad-2.278441$

$\begin{array}{llll}\text { C } & -4.031250 & -0.792039 & -0.177091\end{array}$

$\begin{array}{llll}\text { C } & -4.218290 & -1.458449 & 1.033869\end{array}$

$\begin{array}{llll}\text { C } & -5.434820 & -2.071239 & 1.315609\end{array}$

$\begin{array}{llll}\text { C } & -6.480830 & -2.021379 & 0.399929\end{array}$

$\begin{array}{llll}\text { C } & -6.299940 & -1.358079 & -0.810131\end{array}$

$\begin{array}{llll}\text { C } & -5.082980 & -0.748849 & -1.091591\end{array}$

$\begin{array}{llll}\text { C } & -2.988380 & 1.405120 & -0.029721\end{array}$

$\begin{array}{llll}\text { C } & -3.168410 & 2.441560 & -0.941691\end{array}$

C $\quad-3.424380 \quad 3.739691 \quad-0.505651$

$\begin{array}{llll}\text { C } & -3.522630 & 4.013431 & 0.853119\end{array}$

$\begin{array}{llll}\text { C } & -3.380530 & 2.973991 & 1.772469\end{array}$

$\begin{array}{llll}\text { C } & -3.125450 & 1.684080 & 1.333249\end{array}$

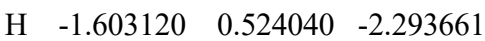

H $\quad-2.529080 \quad-1.075980 \quad-2.434201$

H $\quad 2.515820 \quad 1.732520 \quad 0.404659$

H $\quad 2.843300 \quad-2.370150 \quad-0.761771$

H $\quad 0.418840 \quad-2.499330 \quad-0.901991$

H $\quad 6.892950 \quad-1.059911 \quad 2.060399$

H $\quad 7.079510 \quad 0.922599 \quad-1.724041$

H $\quad 3.404850 \quad-0.736670 \quad 2.532749$

H $\quad 4.849030 \quad-1.637210 \quad 3.042239$

H $\quad 3.730470 \quad-2.310600 \quad 1.843649$

H $\quad 9.067140 \quad-0.777421 \quad 0.880749$

$\begin{array}{llll}\mathrm{H} & 8.927540 & 0.980999 & 0.854989\end{array}$

H $\quad 9.138840 \quad 0.095779 \quad-0.656751$

H $\quad 3.823090 \quad 1.824450 \quad-2.026191$

H $\quad 3.752890 \quad 0.216280 \quad-2.717231$

H $\quad 5.165420 \quad 1.248260 \quad-3.028701$

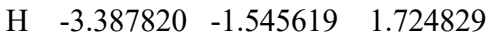

$\mathrm{H} \quad-5.557800 \quad-2.605339 \quad 2.251219$

H $\quad-7.425300 \quad-2.506199 \quad 0.621299$

H $\quad-7.104000 \quad-1.320899 \quad-1.537141$

H $\quad-4.936600 \quad-0.234239 \quad-2.036021$

$\mathrm{H} \quad-3.087410 \quad 2.233120 \quad-2.002591$

$\mathrm{H} \quad-3.545020 \quad 4.537151 \quad-1.230831$

$\begin{array}{llll}\mathrm{H} & -3.715569 & 5.023891 & 1.195599\end{array}$

$\mathrm{H} \quad-3.461620 \quad 3.174831 \quad 2.834999$

$\begin{array}{llll}\mathrm{H} & -2.998270 & 0.878440 & 2.047839\end{array}$

\section{ligH_TSalt}

$\begin{array}{llll}\text { O } & \mathbf{2} .761489 & -3.253349 & 0.905160\end{array}$

$\begin{array}{llll}\text { O } & 2.855649 & -2.261239 & -1.120270\end{array}$

Cl $0.024259-3.110009-1.184020$

$\begin{array}{llll}\text { C } & 2.561409 & -2.347039 & 0.083730\end{array}$

$\begin{array}{llll}\text { C } & 1.837849 & -1.074179 & 0.671720\end{array}$

$\begin{array}{llll}\text { C } & 2.690409 & 0.208941 & 0.637820\end{array}$

$\begin{array}{llll}\text { C } & 2.468599 & -0.528899 & 1.928150\end{array}$

$\begin{array}{llll}\text { C } & 0.341029 & -0.938979 & 0.535360\end{array}$

$\begin{array}{llll}\text { C } & -0.501821 & -1.715179 & -0.283420\end{array}$

$\begin{array}{llll}\text { C } & -1.865951 & -1.439109 & -0.415120\end{array}$

$\begin{array}{llll}\text { C } & -2.474141 & -0.403579 & 0.277910\end{array}$

$\begin{array}{llll}\text { C } & -1.681261 & 0.335261 & 1.153090\end{array}$

$\begin{array}{llll}\text { C } & -0.331011 & 0.059481 & 1.269500\end{array}$

$\begin{array}{llll}\text { C } & -3.917761 & -0.088088 & 0.083870\end{array}$

$\begin{array}{llll}\text { C } & -4.906691 & -0.907148 & 0.647480\end{array}$

$\begin{array}{llll}\text { C } & -6.249421 & -0.581478 & 0.455810\end{array}$ 


\begin{tabular}{|c|c|c|c|}
\hline C & -6.632761 & 0.532482 & -0.285790 \\
\hline$C$ & -5.635671 & 1.334682 & -0.835550 \\
\hline$r$ & -4.283401 & 1.043412 & -0.662330 \\
\hline C & -4.527091 & -2.118318 & 1.462380 \\
\hline $\mathrm{C}$ & -8.089861 & 0.846302 & -0.515210 \\
\hline & -3.228250 & 1.928252 & -1.278940 \\
\hline $\mathrm{C}$ & 4.087689 & 0.217601 & 0.061350 \\
\hline $\mathrm{C}$ & 4.309379 & 0.154231 & -1.316870 \\
\hline $\mathrm{C}$ & 5.593469 & 0.276200 & -1.828280 \\
\hline C & 6.680149 & 0.464980 & -0.975390 \\
\hline $\mathrm{C}$ & 6.470289 & 0.525310 & 0.395900 \\
\hline $\mathrm{C}$ & 5.179309 & 0.402800 & 0.906620 \\
\hline $\mathrm{C}$ & 2.033530 & 1.542951 & 0.397540 \\
\hline $\mathrm{C}$ & 2.158400 & 2.582231 & 1.318100 \\
\hline $\mathrm{C}$ & 1.557200 & 3.816371 & 1.086210 \\
\hline $\mathrm{C}$ & 0.828670 & 4.028761 & -0.080260 \\
\hline $\mathrm{C}$ & 0.713550 & 3.001581 & -1.014410 \\
\hline $\mathrm{C}$ & 1.312030 & 1.770251 & -0.776560 \\
\hline $\mathrm{H}$ & 1.850489 & -0.081509 & 2.697680 \\
\hline $\mathrm{H}$ & 3.282689 & -1.140329 & 2.298710 \\
\hline $\mathrm{H}$ & -2.450771 & -2.057899 & -1.086020 \\
\hline $\mathrm{H}$ & -2.119661 & 1.138221 & 1.736820 \\
\hline $\mathrm{H}$ & 0.234979 & 0.680071 & 1.948040 \\
\hline $\mathrm{H}$ & -7.013581 & -1.212948 & 0.901040 \\
\hline $\mathrm{H}$ & -5.916430 & 2.209182 & -1.416490 \\
\hline $\mathrm{H}$ & -4.091631 & -2.897848 & 0.832460 \\
\hline $\mathrm{H}$ & -5.400891 & -2.533368 & 1.967000 \\
\hline $\mathrm{H}$ & -3.772511 & -1.865018 & 2.210130 \\
\hline $\mathrm{H}$ & -8.707741 & 0.466212 & 0.300500 \\
\hline $\mathrm{H}$ & -8.444761 & 0.386822 & -1.442470 \\
\hline $\mathrm{H}$ & -8.253180 & 1.922752 & -0.596040 \\
\hline $\mathrm{H}$ & -2.504841 & 1.334711 & -1.843100 \\
\hline $\mathrm{H}$ & -2.658010 & 2.461672 & -0.514140 \\
\hline $\mathrm{H}$ & -3.682380 & 2.661842 & -1.947010 \\
\hline $\mathrm{H}$ & 3.472939 & -0.048879 & -1.972040 \\
\hline $\mathrm{H}$ & 5.751579 & 0.203170 & -2.898440 \\
\hline $\mathrm{H}$ & 7.683009 & 0.551020 & -1.379190 \\
\hline $\mathrm{H}$ & 7.307469 & 0.661830 & 1.071730 \\
\hline $\mathrm{H}$ & 5.015939 & 0.445240 & 1.978370 \\
\hline $\mathrm{H}$ & 2.724870 & 2.411081 & 2.228360 \\
\hline $\mathrm{H}$ & 1.656850 & 4.611851 & 1.816710 \\
\hline $\mathrm{H}$ & 0.354660 & 4.987031 & -0.260890 \\
\hline $\mathrm{H}$ & 0.144390 & 3.156661 & -1.924450 \\
\hline & 1.199799 & 0.954771 & -1.484520 \\
\hline
\end{tabular}

\section{ligH2}

$\begin{array}{llll}\mathrm{O} & -1.605480 & -2.974570 & 0.619280\end{array}$

$\begin{array}{llll}\mathrm{O} & -3.231350 & -2.937140 & -0.946230\end{array}$

$\begin{array}{llll}\mathrm{Cl} & -0.743500 & -0.165660 & 2.009440\end{array}$

C $\quad-2.249820 \quad-2.501980 \quad-0.329340$

$\begin{array}{llll}\text { C } & -1.723140 & -1.085380 & -0.826660\end{array}$

$\begin{array}{llll}\text { C } & -2.685310 & 0.110920 & -0.744920\end{array}$

$\begin{array}{llll}\text { C } & -2.357730 & -0.544590 & -2.067370\end{array}$

C $\quad-0.267990 \quad-0.877830 \quad-0.591320$

$\begin{array}{llll}\text { C } & 0.276800 & -0.483970 & 0.633550\end{array}$

$\begin{array}{llll}\text { C } & 1.642560 & -0.292910 & 0.802880\end{array}$

$\begin{array}{llll}\text { C } & 2.523820 & -0.513570 & -0.252510\end{array}$

C $\quad 2.006330 \quad-0.942590 \quad-1.474170$

C $\quad 0.637440 \quad-1.117340 \quad-1.627960$

$\begin{array}{llll}\text { C } & 3.986100 & -0.279840 & -0.072010\end{array}$

$\begin{array}{llll}\text { C } & 4.848410 & -1.361420 & 0.157170\end{array}$

$\begin{array}{lrrr}\text { C } & 6.212200 & -1.118970 & 0.322140\end{array}$

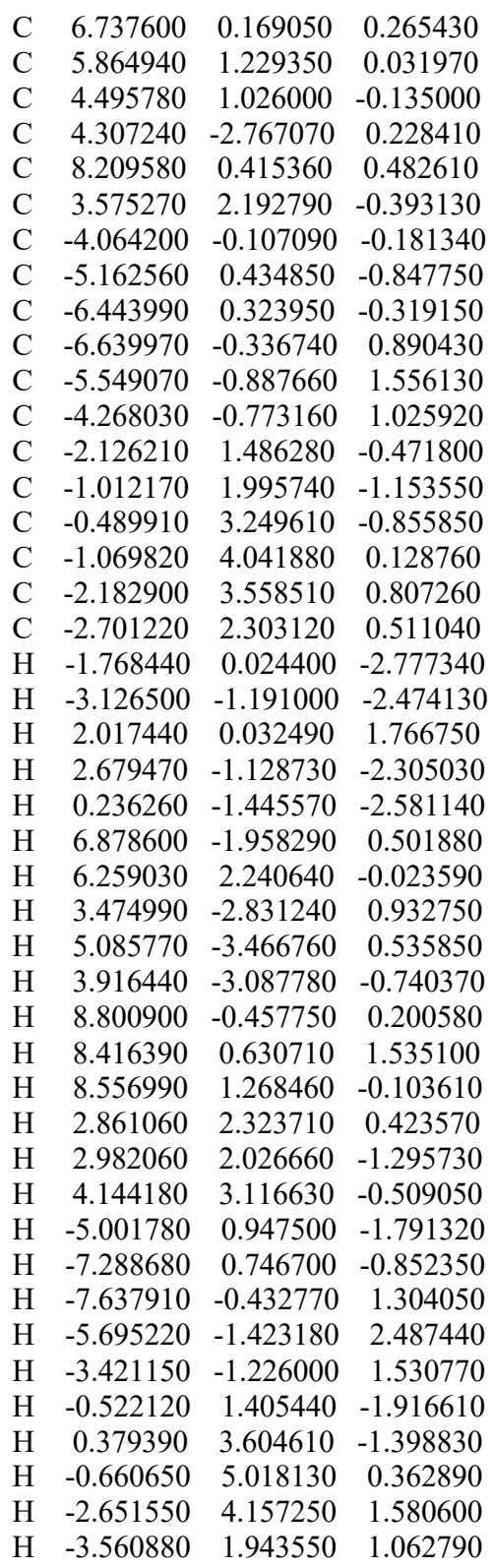

\section{ligI1}

$\begin{array}{llll}\text { O } & -2.243300 & 2.601320 & -1.355100\end{array}$

$\begin{array}{llll}\mathrm{O} & -0.545460 & 1.543780 & -2.403610\end{array}$

$\begin{array}{llll}\mathrm{Cl} & 1.023640 & 3.558270 & 1.376300\end{array}$

$\begin{array}{llll}\mathrm{Br} & 3.493370 & -1.609540 & -1.476660\end{array}$

C $\quad-1.221730 \quad 1.911040 \quad-1.427870$

$\begin{array}{llll}\text { C } & -0.697280 & 1.337090 & -0.039430\end{array}$

$\begin{array}{llll}\text { C } & -1.559400 & 0.224500 & 0.593230\end{array}$

$\begin{array}{llll}\text { C } & 0.781500 & 1.193000 & 0.011440\end{array}$

$\begin{array}{llll}\text { C } & 1.643190 & 2.107150 & 0.614280\end{array}$

$\begin{array}{llll}\text { C } & 3.024070 & 1.927360 & 0.629850\end{array}$

$\begin{array}{llll}\text { C } & 3.586330 & 0.818540 & 0.017830\end{array}$

$\begin{array}{llll}\text { C } & 2.738110 & -0.083550 & -0.614820\end{array}$

$\begin{array}{llll}\text { C } & 1.369940 & 0.093740 & -0.630510\end{array}$

C $\quad-1.516440 \quad 1.618450 \quad 1.172530$

$\begin{array}{llll}\text { C } & -2.825620 & -0.211180 & -0.094710\end{array}$

$\begin{array}{llll}\text { C } & -2.849890 & -0.571610 & -1.441430\end{array}$ $\begin{array}{llll}\text { C } & -4.022130 & -1.049280 & -2.019370\end{array}$

$\begin{array}{llll}\text { C } & -5.181070 & -1.177380 & -1.260280\end{array}$

$\begin{array}{llll}\text { C } & -5.165190 & -0.814590 & 0.083750\end{array}$

$\begin{array}{llll}\text { C } & -3.993420 & -0.334750 & 0.657940\end{array}$

$\begin{array}{llll}\text { C } & -0.854830 & -0.886620 & 1.329670\end{array}$

$\begin{array}{llll}\text { C } & 0.102630 & -0.637270 & 2.323480\end{array}$

$\begin{array}{llll}\text { C } & 0.778440 & -1.673640 & 2.954590\end{array}$

$\begin{array}{llll}\text { C } & 0.519270 & -2.997780 & 2.614130\end{array}$

$\begin{array}{llll}\text { C } & -0.429110 & -3.264700 & 1.634920\end{array}$

C $\quad-1.107140 \quad-2.225130 \quad 1.004910$

$\mathrm{H} \quad 3.650510 \quad 2.663040 \quad 1.118130$

$\mathrm{H} \quad 4.657080 \quad 0.663430 \quad 0.024090$

$\mathrm{H} \quad 0.721160 \quad-0.610180 \quad-1.134990$

H $\quad-1.043740 \quad 1.758050 \quad 2.135680$

$\mathrm{H} \quad-2.377320 \quad 2.243530 \quad 0.963120$

$\mathrm{H} \quad-1.956260 \quad-0.440380 \quad-2.043480$

H $\quad-4.030690 \quad-1.309050 \quad-3.071920$

$\mathrm{H} \quad-6.094160 \quad-1.545360 \quad-1.715130$

$\begin{array}{llll}\mathrm{H} & -6.065980 & -0.900000 & 0.681780\end{array}$

$\mathrm{H} \quad-3.973740 \quad-0.047500 \quad 1.704700$

H $\quad 0.349630 \quad 0.380400 \quad 2.599210$

H $\quad 1.519300 \quad-1.440880 \quad 3.711420$

H $\quad 1.052440 \quad-3.805930 \quad 3.101420$

$\mathrm{H} \quad-0.646070 \quad-4.288780 \quad 1.351750$

H $\quad-1.843220 \quad-2.458160 \quad 0.245250$

\section{ligI_TSrot}

$\begin{array}{llll}\mathrm{O} & -0.132479 & -2.838331 & 0.643999\end{array}$

$\begin{array}{lllll}\text { O } & -0.460839 & -1.989421 & -1.420061\end{array}$

$\begin{array}{llll}\mathrm{Cl} & -0.003181 & 2.907419 & -0.232561\end{array}$

$\begin{array}{llll}\mathrm{Br} & -5.185380 & -0.810933 & 0.096399\end{array}$

$\begin{array}{llll}\text { C } & -0.206549 & -1.938561 & -0.203691\end{array}$

$\begin{array}{llll}\text { C } & 0.048680 & -0.466951 & 0.320999\end{array}$

$\begin{array}{llll}\text { C } & 1.556080 & -0.196731 & 0.474309\end{array}$

$\begin{array}{llll}\text { C } & -1.164900 & 0.394878 & 0.041039\end{array}$

$\begin{array}{llll}\text { C } & -1.327650 & 1.767768 & -0.173581\end{array}$

$\begin{array}{llll}\text { C } & -2.581871 & 2.361988 & -0.342061\end{array}$

$\begin{array}{llll}\text { C } & -3.742110 & 1.617838 & -0.279851\end{array}$

$\begin{array}{llll}\text { C } & -3.609150 & 0.257768 & -0.034101\end{array}$

$\begin{array}{llll}\text { C } & -2.375690 & -0.336312 & 0.116939\end{array}$

$\begin{array}{llll}\text { C } & 0.683680 & -0.355351 & 1.684969\end{array}$

$\begin{array}{llll}\text { C } & 2.492581 & -1.350380 & 0.154309\end{array}$

C $\quad 2.573751 \quad-1.913190 \quad-1.119231$

$\begin{array}{llll}\text { C } & 3.519311 & -2.896230 & -1.391331\end{array}$

$\begin{array}{llll}\text { C } & 4.399431 & -3.323960 & -0.402651\end{array}$

$\begin{array}{llll}\text { C } & 4.323301 & -2.765490 & 0.869739\end{array}$

$\begin{array}{llll}\text { C } & 3.376021 & -1.785600 & 1.141269\end{array}$

$\begin{array}{llll}\text { C } & 2.318540 & 1.077960 & 0.226239\end{array}$

$\begin{array}{llll}\text { C } & 2.752280 & 1.877730 & 1.279959\end{array}$

$\begin{array}{llll}\text { C } & 3.501169 & 3.027910 & 1.041279\end{array}$

C $\quad 3.841769 \quad 3.382300 \quad-0.258501$

$\begin{array}{llll}\text { C } & 3.442629 & 2.566560 & -1.317261\end{array}$

$\begin{array}{llll}\text { C } & 2.697690 & 1.422850 & -1.074321\end{array}$

$\mathrm{H} \quad-2.626931 \quad 3.428628 \quad-0.520621$

H $\quad-4.713051 \quad 2.075897 \quad-0.412031$

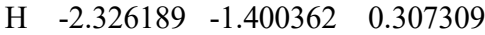

$\begin{array}{llll}\mathrm{H} & 0.457190 & 0.547049 & 2.244409\end{array}$

H $\quad 0.735341 \quad-1.286151 \quad 2.239429$

H $\quad \begin{array}{llll}1.853831 & -1.621711 & -1.875181\end{array}$

H $\quad 3.554861 \quad-3.341680 \quad-2.379241$

H $\quad 5.130281 \quad-4.095740 \quad-0.617621$

H $\quad 4.996611 \quad-3.097860 \quad 1.652359$ 
H $\quad 3.310691 \quad-1.350960 \quad 2.133739$

H $\quad 2.483920 \quad 1.607480 \quad 2.295119$

H $\quad 3.816769 \quad 3.647260 \quad 1.873809$

H $\quad 4.419819 \quad 4.279870 \quad-0.447511$

H $\quad 3.708659 \quad 2.829680 \quad-2.335011$

H $\quad 2.373150 \quad 0.796390 \quad-1.897791$

\section{ligI_TSalt}

$\begin{array}{llll}\mathrm{O} & 1.866766 & 2.862741 & -1.651231\end{array}$

$\begin{array}{llll}\text { O } & 2.382577 & 2.286672 & 0.469819\end{array}$

$\begin{array}{llll}\mathrm{Cl} & -0.176475 & 3.733328 & 0.904779\end{array}$

$\begin{array}{llll}\mathrm{Br} & -4.206999 & -0.995847 & -0.595771\end{array}$

$\begin{array}{llll}\text { C } & 1.780777 & 2.193351 & -0.612081\end{array}$

$\begin{array}{llll}\text { C } & 0.744008 & 1.003459 & -0.687781\end{array}$

$\begin{array}{llll}\text { C } & 1.360350 & -0.397590 & -0.518141\end{array}$

$\begin{array}{llll}\text { C } & -0.675552 & 1.239848 & -0.236041\end{array}$

$\begin{array}{llll}\text { C } & -1.154153 & 2.362587 & 0.468849\end{array}$

$\begin{array}{llll}\text { C } & -2.491413 & 2.472715 & 0.858459\end{array}$

$\begin{array}{llll}\text { C } & -3.421312 & 1.491394 & 0.567609\end{array}$

$\begin{array}{llll}\text { C } & -2.973781 & 0.393235 & -0.148191\end{array}$

$\begin{array}{llll}\text { C } & -1.657461 & 0.274246 & -0.543071\end{array}$

$\begin{array}{llll}\text { C } & 0.941630 & 0.092330 & -1.874451\end{array}$

$\begin{array}{llll}\text { C } & 2.838391 & -0.580868 & -0.259341\end{array}$

$\begin{array}{llll}\text { C } & 3.400800 & -0.298197 & 0.988179\end{array}$

$\begin{array}{llll}\text { C } & 4.736491 & -0.578825 & 1.236469\end{array}$

$\begin{array}{llll}\text { C } & 5.536011 & -1.147984 & 0.245919\end{array}$

$\begin{array}{llll}\text { C } & 4.986842 & -1.429445 & -0.997771\end{array}$

$\begin{array}{llll}\text { C } & 3.644351 & -1.146807 & -1.243991\end{array}$

$\begin{array}{llll}\text { C } & 0.585842 & -1.492501 & 0.167259\end{array}$

$\begin{array}{llll}\text { C } & 0.329433 & -2.700131 & -0.480341\end{array}$

$\begin{array}{llll}\text { C } & -0.368155 & -3.718822 & 0.162269\end{array}$

$\begin{array}{llll}\text { C } & -0.812476 & -3.542333 & 1.468759\end{array}$

$\begin{array}{llll}\text { C } & -0.550067 & -2.343992 & 2.127759\end{array}$

C $\quad 0.144852 \quad-1.328521 \quad 1.481659$

$\begin{array}{llll}\mathrm{H} & -2.793305 & 3.360615 & 1.398979\end{array}$

$\begin{array}{llll}\mathrm{H} & -4.453712 & 1.583603 & 0.877049\end{array}$

H $\quad-1.382239-0.613853 \quad-1.090191$

H $\quad 0.083760 \quad-0.358081 \quad-2.360321$

H $\quad 1.719149 \quad 0.429551 \quad-2.549231$

$\begin{array}{llll}\mathrm{H} & 2.793620 & 0.192712 & 1.736819\end{array}$

$\begin{array}{llll}\mathrm{H} & 5.163590 & -0.333945 & 2.202449\end{array}$

$\begin{array}{llll}\mathrm{H} & 6.581812 & -1.357733 & 0.441969\end{array}$

H $\quad 5.599312 \quad-1.863354 \quad-1.780551$

H $\quad 3.216662 \quad-1.363337 \quad-2.217221$

H $\quad 0.675044-2.831881-1.501021$

$\mathrm{H} \quad-0.568854 \quad-4.648342 \quad-0.358931$

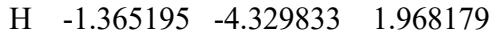

H $\quad-0.900007 \quad-2.195113 \quad 3.143039$

H $\quad 0.328220 \quad-0.381141 \quad 1.979099$

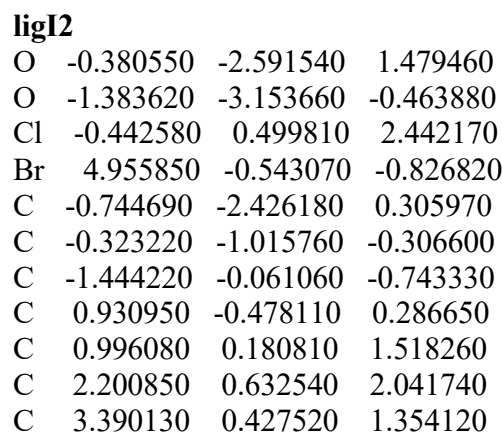

$\begin{array}{llll}\text { C } & 3.335680 & -0.240050 & 0.140300\end{array}$

$\begin{array}{llll}\text { C } & 2.137380 & -0.688470 & -0.388420\end{array}$

C $\quad-0.611570 \quad-0.811950-1.758320$

$\begin{array}{llll}\text { C } & -2.874410 & -0.494060 & -0.559070\end{array}$

C $\quad-3.776850 \quad-0.309560 \quad-1.605900$

C $\quad-5.123210 \quad-0.620380-1.450270$

C $\quad-5.582370-1.124130 \quad-0.236400$

$\begin{array}{llll}\text { C } & -4.685050 & -1.319400 & 0.809190\end{array}$

$\begin{array}{llll}\text { C } & -3.338950 & -1.005200 & 0.651460\end{array}$

C $\quad-1.237500 \quad 1.428300 \quad-0.612000$

C $\quad-0.063190 \quad 2.064730 \quad-1.036300$

$\begin{array}{llll}\text { C } & 0.120340 & 3.433170 & -0.872050\end{array}$

C $\quad-0.869440 \quad 4.213500 \quad-0.285080$

$\begin{array}{llll}\text { C } & -2.045790 & 3.601340 & 0.132440\end{array}$

C $\quad-2.225780 \quad 2.232490 \quad-0.028760$

$\mathrm{H} \quad 2.202980 \quad 1.146860 \quad 2.994350$

H $\quad 4.332020 \quad 0.776500 \quad 1.755950$

$\mathrm{H} \quad 2.116340 \quad-1.214620 \quad-1.335170$

$\mathrm{H} \quad 0.068350 \quad-0.234570 \quad-2.374320$

H $\quad-1.092890 \quad-1.663710 \quad-2.224340$

$\mathrm{H} \quad-3.410880 \quad 0.082850 \quad-2.549830$

$\mathrm{H} \quad-5.811730 \quad-0.475560 \quad-2.275730$

H $\quad-6.629980 \quad-1.375090 \quad-0.111340$

$\mathrm{H} \quad-5.029900 \quad-1.732320 \quad 1.750470$

H $\quad-2.634570 \quad-1.183950 \quad 1.457150$

$\mathrm{H} \quad 0.736630 \quad 1.491830 \quad-1.486900$

$\mathrm{H} \quad 1.047580 \quad 3.887000 \quad-1.204080$

$\mathrm{H} \quad-0.725220 \quad 5.279990 \quad-0.154630$

$\mathrm{H} \quad-2.831310 \quad 4.189300 \quad 0.594400$

$\begin{array}{llll}\mathrm{H} & -3.146000 & 1.777060 & 0.315370\end{array}$

\section{ligJ1}

$\begin{array}{llll}\mathrm{O} & -2.235310 & 2.641520 & -1.276790\end{array}$

$\begin{array}{llll}\mathrm{O} & -0.551950 & 1.605980 & -2.370440\end{array}$

$\begin{array}{llll}\mathrm{Cl} & 1.104560 & 3.505040 & 1.418880\end{array}$

F $\quad 4.092020 \quad-2.049490 \quad-0.535750$

F $\quad 2.402960-2.141200-1.876630$

F $\quad 4.131880 \quad-0.955260 \quad-2.385200$

$\begin{array}{llll}\text { C } & -1.216460 & 1.951260 & -1.377980\end{array}$

$\begin{array}{llll}\text { C } & -0.675980 & 1.343310 & -0.012870\end{array}$

$\begin{array}{llll}\text { C } & -1.528710 & 0.219540 & 0.613500\end{array}$

$\begin{array}{llll}\text { C } & 0.801070 & 1.180060 & 0.007540\end{array}$

$\begin{array}{llll}\text { C } & 1.686950 & 2.062390 & 0.616160\end{array}$

$\begin{array}{llll}\text { C } & 3.068840 & 1.859590 & 0.614260\end{array}$

$\begin{array}{llll}\text { C } & 3.595760 & 0.754020 & -0.022840\end{array}$

$\begin{array}{llll}\text { C } & 2.729980 & -0.129110 & -0.672170\end{array}$

$\begin{array}{llll}\text { C } & 1.366450 & 0.087110 & -0.667660\end{array}$

$\begin{array}{lrrr}\text { C } & -1.468580 & 1.599430 & 1.222470\end{array}$

$\begin{array}{llll}\text { C } & -2.813320 & -0.200170 & -0.050010\end{array}$

C $\quad-3.952250 \quad-0.366060 \quad 0.738190$

C $\quad-5.140610 \quad-0.831120 \quad 0.186620$

$\begin{array}{llll}\text { C } & -5.202610 & -1.136300 & -1.170360\end{array}$

$\begin{array}{llll}\text { C } & -4.072760 & -0.966660 & -1.964160\end{array}$

C $\quad-2.883540 \quad-0.503790 \quad-1.409030$

C $\quad-0.798160 \quad-0.911140 \quad 1.292830$

$\begin{array}{llll}\text { C } & 0.156350 & -0.692430 & 2.295860\end{array}$

C $\quad 0.874890 \quad-1.742720 \quad 2.853020$

C $\quad 0.661580 \quad-3.049720 \quad 2.426140$

$\begin{array}{llll}\text { C } & -0.287270 & -3.286870 & 1.439260\end{array}$

C $\quad-1.008450 \quad-2.233880 \quad 0.884640$

$\begin{array}{llll}\text { C } & 3.320910 & -1.314170 & -1.367340\end{array}$

H $\quad 3.710060 \quad 2.574880 \quad 1.112840$
H $\quad 4.664890 \quad 0.576800 \quad-0.024540$

H $\quad 0.696590 \quad-0.591710 \quad-1.179600$

H $\quad-0.975010 \quad 1.721440 \quad 2.177390$

$\mathrm{H} \quad-2.332860 \quad 2.229540 \quad 1.044590$

$\mathrm{H} \quad-3.896650 \quad-0.123780 \quad 1.795010$

$\mathrm{H} \quad-6.018280 \quad-0.950170 \quad 0.812580$

$\mathrm{H} \quad-6.128840 \quad-1.492730 \quad-1.607440$

$\mathrm{H} \quad-4.117260 \quad-1.182520 \quad-3.025650$

$\mathrm{H} \quad-2.012840 \quad-0.342380 \quad-2.036900$

H $\quad 0.369660 \quad 0.313640 \quad 2.635060$

$\mathrm{H} \quad 1.614170 \quad-1.533720 \quad 3.618270$

$\mathrm{H} \quad 1.230540 \quad-3.867710 \quad 2.852590$

$\mathrm{H} \quad-0.468270 \quad-4.297180 \quad 1.089560$

H $\quad-1.741660 \quad-2.441850 \quad 0.114800$

\section{ligJ TSrot}

O $-0.106182 \quad-2.852043 \quad 0.662278$

$\begin{array}{llll}\mathrm{O} & -0.492550 & -2.011988 & -1.395603\end{array}$

Cl $\quad-0.097995 \quad 2.888555-0.222253$

$\begin{array}{llll}\text { F } & -4.668499 & -1.923320 & 0.439124\end{array}$

F $\quad-5.781543 \quad-0.148683 \quad 0.956810$

F $\quad-5.560457-0.710628 \quad-1.106336$

C $\quad-0.215192 \quad-1.955472 \quad-0.184184$

$\begin{array}{llll}\text { C } & 0.019415 & -0.478010 & 0.333624\end{array}$

$\begin{array}{llll}\text { C } & 1.522562 & -0.181745 & 0.477668\end{array}$

$\begin{array}{llll}\text { C } & -1.209910 & 0.359584 & 0.054158\end{array}$

$\begin{array}{llll}\text { C } & -1.400107 & 1.725902 & -0.164688\end{array}$

$\begin{array}{llll}\text { C } & -2.666158 & 2.302355 & -0.339499\end{array}$

$\begin{array}{llll}\text { C } & -3.805540 & 1.535819 & -0.273346\end{array}$

$\begin{array}{llll}\text { C } & -3.660049 & 0.172142 & -0.019051\end{array}$

$\begin{array}{llll}\text { C } & -2.410622 & -0.389953 & 0.132848\end{array}$

$\begin{array}{llll}\text { C } & 0.660305 & -0.350977 & 1.693901\end{array}$

$\begin{array}{llll}\text { C } & 2.476614 & -1.319766 & 0.154157\end{array}$

$\begin{array}{llll}\text { C } & 2.554929 & -1.887456 & -1.117305\end{array}$

$\begin{array}{llll}\text { C } & 3.514953 & -2.854993 & -1.394077\end{array}$

$\begin{array}{llll}\text { C } & 4.412353 & -3.261842 & -0.412134\end{array}$

$\begin{array}{llll}\text { C } & 4.339131 & -2.698201 & 0.858156\end{array}$

$\begin{array}{llll}\text { C } & 3.377386 & -1.733890 & 1.134425\end{array}$

$\begin{array}{llll}\text { C } & 2.260132 & 1.105654 & 0.220228\end{array}$

$\begin{array}{llll}\text { C } & 2.685223 & 1.917545 & 1.268165\end{array}$

$\begin{array}{llll}\text { C } & 3.410050 & 3.081115 & 1.020236\end{array}$

$\begin{array}{llll}\text { C } & 3.735113 & 3.437060 & -0.283100\end{array}$

$\begin{array}{llll}\text { C } & 3.345397 & 2.609471 & -1.336154\end{array}$

$\begin{array}{llll}\text { C } & 2.624327 & 1.452518 & -1.084066\end{array}$

$\begin{array}{llll}\text { C } & -4.898614 & -0.662769 & 0.068904\end{array}$

H $\quad-2.724855 \quad 3.367439 \quad-0.521403$

$\mathrm{H} \quad-4.785345 \quad 1.978937 \quad-0.405444$

H $\quad-2.339691 \quad-1.451760 \quad 0.331506$

$\begin{array}{llll}\mathrm{H} & 0.422535 & 0.549406 & 2.251891\end{array}$

$\mathrm{H} \quad 0.730605 \quad-1.279188 \quad 2.250646$

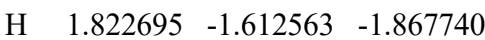

H $\quad 3.548512 \quad-3.304912 \quad-2.380001$

H $\quad 5.154648 \quad-4.021573 \quad-0.630630$

$\begin{array}{llll}\mathrm{H} & 5.026094 & -3.014540 & 1.635508\end{array}$

H $\quad 3.314284 \quad-1.295301 \quad 2.125313$

$\begin{array}{llll}\mathrm{H} & 2.429180 & 1.645914 & 2.286124\end{array}$

H $3.719066 \quad 3.709611 \quad 1.848343$

H $\quad 4.294406 \quad 4.344898 \quad-0.479250$

H $\quad 3.600220 \quad 2.873446 \quad-2.356529$

H $\quad 2.307734 \quad 0.816115 \quad-1.903017$ 


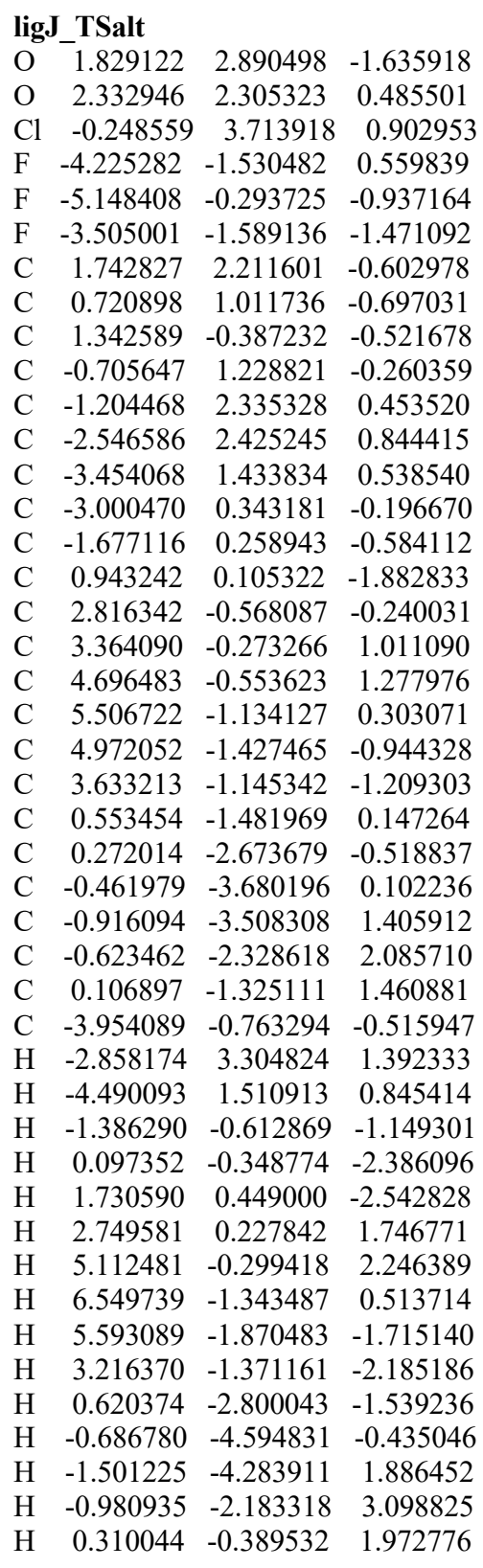

ligJ2

$\begin{array}{llll}\text { O } & -1.375670 & -3.144870 & -0.487340\end{array}$

$\begin{array}{llll}\mathrm{O} & -0.332750 & -2.612240 & 1.443580\end{array}$

$\begin{array}{llll}\mathrm{Cl} & -0.373870 & 0.441020 & 2.446300\end{array}$

F $\quad 5.220310 \quad-1.672800 \quad-0.345200$

F $\quad 4.454620 \quad-0.537020 \quad-2.005820$

F $\quad 5.579300 \quad 0.446370 \quad-0.445750$

C $\quad-0.716850 \quad-2.431670 \quad 0.278460$

C $\quad-0.292990 \quad-1.018820 \quad-0.326240$

$\begin{array}{llll}\text { C } & -1.409780 & -0.049500 & -0.741080\end{array}$

$\begin{array}{llll}\text { C } & 0.970530 & -0.495800 & 0.258410\end{array}$

$\begin{array}{llll}\text { C } & 1.052700 & 0.137850 & 1.503180\end{array}$

$\begin{array}{llll}\text { C } & 2.262900 & 0.582560 & 2.024750\end{array}$

$\begin{array}{llll}\text { C } & 3.438180 & 0.395620 & 1.314350\end{array}$

$\begin{array}{llll}\text { C } & 3.382570 & -0.243370 & 0.080450\end{array}$

$\begin{array}{llll}\text { C } & 2.169470 & -0.685660 & -0.430420\end{array}$
C $\quad-0.595880 \quad-0.798030 \quad-1.772550$

$\begin{array}{llll}\text { C } & -2.842950 & -0.468270 & -0.547370\end{array}$

$\begin{array}{llll}\text { C } & -3.301070 & -0.995830 & 0.658460\end{array}$

$\begin{array}{llll}\text { C } & -4.649260 & -1.296400 & 0.824930\end{array}$

C $\quad-5.554980 \quad-1.071060 \quad-0.207220$

C $\quad-5.102430 \quad-0.550640-1.416520$

C $\quad-3.754100 \quad-0.253420 \quad-1.580870$

C $\quad-1.184490 \quad 1.435740 \quad-0.592920$

$\begin{array}{llll}\text { C } & -0.014290 & 2.067180 & -1.035330\end{array}$

$\begin{array}{llll}\text { C } & 0.187640 & 3.430870 & -0.853530\end{array}$

C $\quad-0.779480 \quad 4.211180 \quad-0.230010$

$\begin{array}{llll}\text { C } & -1.951760 & 3.604120 & 0.206160\end{array}$

$\begin{array}{llll}\text { C } & -2.149940 & 2.240050 & 0.027230\end{array}$

C $\quad 4.646300 \quad-0.499140 \quad-0.676240$

$\begin{array}{llll}\mathrm{H} & 2.271210 & 1.079020 & 2.986370\end{array}$

H $\quad 4.382400 \quad 0.748470 \quad 1.709960$

H $\quad 2.134370 \quad-1.186140 \quad-1.391740$

H $\quad 0.082280 \quad-0.222640 \quad-2.392210$

H $\quad-1.091060 \quad-1.640850 \quad-2.240260$

H $\quad-2.591130 \quad-1.197740 \quad 1.453670$

$\mathrm{H} \quad-4.989260 \quad-1.722260 \quad 1.762180$

H $\quad-6.604220 \quad-1.311470 \quad-0.075490$

$\mathrm{H} \quad-5.797660 \quad-0.382300 \quad-2.231780$

$\mathrm{H} \quad-3.393310 \quad 0.151860 \quad-2.521340$

$\mathrm{H} \quad 0.767650 \quad 1.494320 \quad-1.516330$

$\mathrm{H} \quad 1.110740 \quad 3.880980 \quad-1.201700$

$\mathrm{H} \quad-0.621170 \quad 5.273900 \quad-0.086150$

$\mathrm{H} \quad-2.719680 \quad 4.192340 \quad 0.696460$

H $\quad-3.066310 \quad 1.787900 \quad 0.385810$

\section{ligK1}

$\begin{array}{llll}\mathrm{O} & -2.286170 & 1.635810 & -2.469390\end{array}$

$\begin{array}{llll}O & -4.459060 & 1.705310 & -1.852440\end{array}$

$\mathrm{Cl} \quad-2.731600 \quad 4.103280 \quad 1.131650$

F $\quad 4.105520 \quad-3.570590 \quad-0.091750$

F $\quad 1.960890 \quad-3.412130-0.278210$

F $\quad 2.898580 \quad-3.120560 \quad 1.637050$

$\begin{array}{llll}\text { F } & 5.903900 & 2.362580 & -0.677080\end{array}$

F $\quad 6.819680 \quad 0.408150 \quad-0.598540$

F $\quad 5.838250 \quad 1.068730 \quad-2.397250$

$\begin{array}{llll}\text { C } & -2.802920 & 1.274680 & -0.182530\end{array}$

$\begin{array}{llll}\text { C } & -3.133520 & -0.140260 & 0.336760\end{array}$

$\begin{array}{llll}\text { C } & -3.871850 & 1.081990 & 0.835560\end{array}$

$\begin{array}{llll}\text { C } & -3.242570 & 1.589950 & -1.676490\end{array}$

$\begin{array}{llll}\text { C } & -1.488710 & 1.866510 & 0.174400\end{array}$

$\begin{array}{llll}\text { C } & -1.334740 & 3.113800 & 0.776860\end{array}$

$\begin{array}{llll}\text { C } & -0.082330 & 3.625170 & 1.110250\end{array}$

$\begin{array}{llll}\mathrm{C} & 1.056100 & 2.881170 & 0.848560\end{array}$

$\begin{array}{llll}\text { C } & 0.942330 & 1.633300 & 0.226170\end{array}$

$\begin{array}{llll}\text { C } & -0.318110 & 1.167520 & -0.125100\end{array}$

$\begin{array}{llll}\text { C } & -2.146790 & -0.825390 & 1.249140\end{array}$

$\begin{array}{llll}\text { C } & -1.613770 & -0.199270 & 2.385520\end{array}$

C $\quad-0.657160 \quad-0.823290 \quad 3.177310$

C $\quad-0.201660 \quad-2.101090 \quad 2.863730$

C $\quad-0.723410 \quad-2.742210 \quad 1.746150$

C $\quad-1.680650 \quad-2.113920 \quad 0.952760$

C $\quad-3.885240 \quad-1.089730 \quad-0.558040$

C $\quad-4.981500 \quad-1.779730 \quad-0.041400$

C $\quad-5.651340 \quad-2.728840 \quad-0.805440$

C $\quad-5.228390 \quad-2.997850-2.104160$

C $\quad-4.140090 \quad-2.307720 \quad-2.628760$

C $\quad-3.469530-1.360640 \quad-1.861040$ $\begin{array}{llll}\text { C } & 2.128870 & 0.779200 & 0.004320\end{array}$

$\begin{array}{llll}\text { C } & 3.355040 & 1.317080 & -0.396630\end{array}$

$\begin{array}{llll}\text { C } & 4.461640 & 0.496340 & -0.567490\end{array}$

$\begin{array}{llll}\text { C } & 4.380840 & -0.875630 & -0.356530\end{array}$

$\begin{array}{llll}\text { C } & 3.160830 & -1.407820 & 0.035940\end{array}$

$\begin{array}{llll}\text { C } & 2.045580 & -0.599000 & 0.220550\end{array}$

$\begin{array}{llll}\text { C } & 3.031540 & -2.876580 & 0.321160\end{array}$

$\begin{array}{llll}\text { C } & 5.752400 & 1.084680 & -1.055430\end{array}$

$\mathrm{H} \quad-3.710650 \quad 1.396660 \quad 1.859250$

$\mathrm{H} \quad-4.870900 \quad 1.224300 \quad 0.438530$

$\begin{array}{llll}\mathrm{H} & -0.015740 & 4.594240 & 1.588310\end{array}$

H $\quad 2.026430 \quad 3.255120 \quad 1.156440$

$\begin{array}{llll}\mathrm{H} & -0.426460 & 0.220630 & -0.642040\end{array}$

$\begin{array}{llll}\mathrm{H} & -1.923620 & 0.804850 & 2.647530\end{array}$

$\mathrm{H} \quad-0.260320 \quad-0.300830 \quad 4.040670$

$\mathrm{H} \quad 0.552270 \quad-2.584290 \quad 3.474080$

$\mathrm{H} \quad-0.380280 \quad-3.735570 \quad 1.479360$

$\mathrm{H} \quad-2.069700 \quad-2.633790 \quad 0.085870$

$\begin{array}{llll}\mathrm{H} & -5.307330 & -1.562310 & 0.971110\end{array}$

H $\quad-6.505170 \quad-3.253470 \quad-0.390770$

$\mathrm{H} \quad-5.751620 \quad-3.731900 \quad-2.706890$

$\mathrm{H} \quad-3.816850 \quad-2.495660 \quad-3.646330$

$\mathrm{H} \quad-2.641840 \quad-0.798820 \quad-2.282770$

H $\quad 3.436930 \quad 2.380700 \quad-0.588330$

H $\quad 1.109740 \quad-1.034830 \quad 0.559940$

H $\quad 5.247170 \quad-1.509840 \quad-0.491840$

\section{ligK TSrot}

$\begin{array}{llll}\text { O } & -1.013241 & 1.744980 & -1.060449\end{array}$

$\begin{array}{llll}\text { O } & -1.259441 & 2.326700 & 1.102921\end{array}$

Cl $\quad-2.566191 \quad-3.047530 \quad-0.745869$

$\begin{array}{llll}\text { F } & 5.408989 & 3.344850 & 0.221121\end{array}$

$\begin{array}{llll}\text { F } & 3.424589 & 3.486150 & -0.619239\end{array}$

$\begin{array}{llll}\text { F } & 5.090149 & 2.916350 & -1.863899\end{array}$

F $\quad 6.397529 \quad-2.841920 \quad 0.081371$

F $\quad 7.582769-1.078930 \quad 0.469081$

$\begin{array}{llll}\text { F } & 6.359509 & -1.927020 & 2.028061\end{array}$

$\begin{array}{llll}\text { C } & -1.919571 & 0.120580 & 0.420111\end{array}$

$\begin{array}{llll}\text { C } & -3.448031 & 0.146350 & 0.588931\end{array}$

$\begin{array}{llll}\text { C } & -2.554761 & -0.063290 & 1.776071\end{array}$

$\begin{array}{llll}\text { C } & -1.340281 & 1.568350 & 0.128421\end{array}$

$\begin{array}{llll}\text { C } & -0.922141 & -0.916500 & -0.050439\end{array}$

C $\quad-1.041281-2.220340 \quad-0.540389$

$\begin{array}{llll}\text { C } & 0.069599 & -2.989990 & -0.907279\end{array}$

$\begin{array}{llll}\text { C } & 1.349719 & -2.493450 & -0.792359\end{array}$

$\begin{array}{llll}\text { C } & 1.526499 & -1.207360 & -0.272669\end{array}$

$\begin{array}{llll}\text { C } & 0.410479 & -0.475480 & 0.097041\end{array}$

$\begin{array}{llll}\text { C } & -4.453021 & -0.900440 & 0.184891\end{array}$

$\begin{array}{llll}\text { C } & -5.000511 & -1.778740 & 1.115981\end{array}$

$\begin{array}{llll}\text { C } & -5.963491 & -2.711670 & 0.737011\end{array}$

$\begin{array}{llll}\text { C } & -6.405551 & -2.763840 & -0.579069\end{array}$

C $\quad-5.890611-1.861810 \quad-1.510179$

$\begin{array}{llll}\text { C } & -4.932601 & -0.935630 & -1.127979\end{array}$

$\begin{array}{llll}\text { C } & -4.122591 & 1.501550 & 0.468291\end{array}$

$\begin{array}{llll}\text { C } & -4.924801 & 1.944270 & 1.519151\end{array}$

$\begin{array}{llll}\text { C } & -5.641571 & 3.130500 & 1.417871\end{array}$

$\begin{array}{llll}\mathrm{C} & -5.565461 & 3.890870 & 0.254581\end{array}$

C $\quad-4.764971 \quad 3.456000 \quad-0.796529$

$\begin{array}{llll}\text { C } & -4.049861 & 2.267200 & -0.694789\end{array}$

$\begin{array}{llll}\text { C } & 2.871479 & -0.602800 & -0.154479\end{array}$

$\begin{array}{llll}\text { C } & 3.979989 & -1.372580 & 0.193811\end{array}$

$\begin{array}{llll}\text { C } & 5.243379 & -0.795730 & 0.283801\end{array}$ 


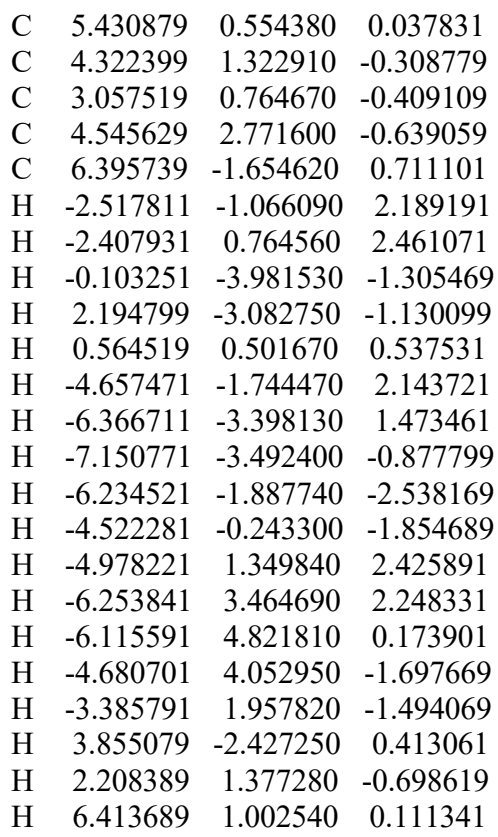

$\begin{array}{lrrr}\text { ligK_TSalt } & & \\ \text { O } & 4.860950 & 1.288749 & -0.079940 \\ \text { O } & 4.592930 & 1.220559 & -2.320680 \\ \text { Cl } & 3.224970 & 3.762739 & -0.575950 \\ \text { F } & -3.595381 & -3.110380 & -1.506060 \\ \text { F } & -3.206731 & -1.921070 & -3.261490 \\ \text { F } & -5.195311 & -2.001399 & -2.423430 \\ \text { F } & -5.182150 & 2.208351 & 1.994660 \\ \text { F } & -6.216320 & 0.329841 & 1.738380 \\ \text { F } & -4.460320 & 0.438651 & 2.983860 \\ \text { C } & 2.782860 & 0.519279 & -0.964980 \\ \text { C } & 2.638689 & -0.800951 & -0.169490 \\ \text { C } & 2.542149 & -0.791171 & -1.669050 \\ \text { C } & 4.249360 & 1.076279 & -1.138790 \\ \text { C } & 1.641970 & 1.500109 & -0.889290 \\ \text { C } & 1.729440 & 2.882489 & -0.631270 \\ \text { C } & 0.588340 & 3.659890 & -0.405180 \\ \text { C } & -0.676900 & 3.103840 & -0.397380 \\ \text { C } & -0.812230 & 1.749480 & -0.701580 \\ \text { C } & 0.329240 & 1.014250 & -0.991990 \\ \text { C } & 1.391419 & -1.050261 & 0.643870 \\ \text { C } & 0.520199 & -2.091830 & 0.324680 \\ \text { C } & -0.675341 & -2.264550 & 1.019390 \\ \text { C } & -1.006791 & -1.402630 & 2.060290 \\ \text { C } & -0.124310 & -0.382800 & 2.412170 \\ \text { C } & 1.064950 & -0.212501 & 1.712840 \\ \text { C } & 3.827319 & -1.479151 & 0.467780 \\ \text { C } & 4.244709 & -2.719301 & -0.009920 \\ \text { C } & 5.266709 & -3.424391 & 0.622780 \\ \text { C } & 5.880529 & -2.890882 & 1.748460 \\ \text { C } & 5.470899 & -1.648581 & 2.230560 \\ \text { C } & 4.454299 & -0.947801 & 1.597410 \\ \text { C } & -2.098470 & 1.031010 & -0.579140 \\ \text { C } & -2.974660 & 1.326960 & 0.464700 \\ \text { C } & -4.131140 & 0.577470 & 0.654180 \\ \text { C } & -4.444160 & -0.483769 & -0.180180 \\ \text { C } & -3.576301 & -0.770690 & -1.229860 \\ \text { C } & -2.424460 & -0.028950 & -1.434910\end{array}$
C $\quad-3.888341-1.944720 \quad-2.110830$

$\begin{array}{llll}\text { C } & -5.000190 & 0.887501 & 1.836740\end{array}$

$\mathrm{H} \quad 1.586579 \quad-0.991961 \quad-2.140960$

H $\quad 3.399989-1.141841-2.230300$

$\mathrm{H} \quad 0.723341 \quad 4.717160 \quad-0.216130$

$\mathrm{H} \quad-1.543540 \quad 3.719850 \quad-0.184030$

$\mathrm{H} \quad 0.187630 \quad-0.033650 \quad-1.201150$

H $\quad 0.769909-2.751011-0.501200$

$\mathrm{H} \quad-1.352441 \quad-3.064200 \quad 0.739000$

$\mathrm{H} \quad-1.944871-1.520500 \quad 2.591410$

$\mathrm{H} \quad-0.374140 \quad 0.293870 \quad 3.222070$

$\begin{array}{llll}\mathrm{H} & 1.729970 & 0.611339 & 1.952760\end{array}$

H $\quad 3.766239 \quad-3.133971 \quad-0.891210$

$\mathrm{H} \quad 5.582339 \quad-4.385281 \quad 0.231500$

H $\quad 6.681629 \quad-3.430612 \quad 2.241340$

H $\quad 5.962749 \quad-1.213892 \quad 3.093440$

$\begin{array}{llll}\mathrm{H} & 4.176930 & 0.042219 & 1.933270\end{array}$

$\mathrm{H} \quad-2.728790 \quad 2.121520 \quad 1.160390$

$\mathrm{H} \quad-1.769280 \quad-0.269720 \quad-2.263420$

H $\quad-5.342581 \quad-1.068039 \quad-0.026150$

\section{$\operatorname{ligK2}$}

$\begin{array}{llll}\text { O } & 2.976680 & 3.120840 & 0.412520\end{array}$

$\begin{array}{llll}\text { O } & 2.374430 & 1.950130 & 2.247300\end{array}$

$\mathrm{Cl} \quad 2.872670 \quad-1.257330 \quad 2.278200$

F $\quad-6.093540 \quad 3.142730 \quad-0.031120$

F $\quad-4.944070 \quad 3.073070 \quad-1.849060$

F $\quad-4.035450 \quad 3.796230 \quad-0.032660$

F $\quad-7.197660 \quad-1.582900 \quad-0.324110$

F $\quad-5.663380-2.684850-1.362570$

F $\quad-5.851720 \quad-2.866530 \quad 0.773090$

$\begin{array}{llll}\text { C } & 2.132140 & 0.901870 & 0.122440\end{array}$

$\begin{array}{llll}\text { C } & 3.220920 & 0.244610 & -0.736740\end{array}$

$\begin{array}{llll}\text { C } & 2.160090 & 1.127690 & -1.354540\end{array}$

$\begin{array}{llll}\text { C } & 2.542910 & 2.143670 & 1.033890\end{array}$

$\begin{array}{llll}\text { C } & 1.054190 & 0.079150 & 0.733410\end{array}$

$\begin{array}{llll}\text { C } & 1.269390 & -0.886500 & 1.722360\end{array}$

$\begin{array}{lrrr}\text { C } & 0.218110 & -1.602100 & 2.283700\end{array}$

C $\quad-1.085250 \quad-1.360210 \quad 1.878660$

$\begin{array}{llll}\text { C } & -1.342730 & -0.387100 & 0.910600\end{array}$

$\begin{array}{llll}\text { C } & -0.269020 & 0.311770 & 0.359660\end{array}$

C $\quad 3.152540 \quad-1.240410-0.998700$

$\begin{array}{llll}\text { C } & 4.287620 & -2.043450 & -0.825380\end{array}$

C $\quad 4.241790 \quad-3.417890 \quad-1.027570$

$\begin{array}{llll}\text { C } & 3.057370 & -4.036840 & -1.410910\end{array}$

$\begin{array}{llll}\text { C } & 1.921880 & -3.255790 & -1.594490\end{array}$

C $\quad 1.970610 \quad-1.880500 \quad-1.395540$

$\begin{array}{llll}\text { C } & 4.623800 & 0.785960 & -0.664470\end{array}$

$\begin{array}{llll}\text { C } & 5.262770 & 1.012710 & 0.553080\end{array}$

$\begin{array}{llll}\text { C } & 6.587370 & 1.437070 & 0.581300\end{array}$

C $\quad 7.289510 \quad 1.636290 \quad-0.603480$

$\begin{array}{llll}\text { C } & 6.654690 & 1.418030 & -1.823010\end{array}$

$\begin{array}{llll}\text { C } & 5.329930 & 0.996230 & -1.848230\end{array}$

C $\quad-2.727430 \quad-0.099950 \quad 0.477210$

C $\quad-3.663180 \quad-1.125030 \quad 0.334440$

$\begin{array}{llll}\text { C } & -4.967650 & -0.846280 & -0.061140\end{array}$

C $\quad-5.372270 \quad 0.450340 \quad-0.334630$

C $\quad-4.438390 \quad 1.473310 \quad-0.195950$

$\begin{array}{llll}\text { C } & -3.137150 & 1.212520 & 0.204260\end{array}$

C $\quad-4.869580 \quad 2.875010 \quad-0.520100$

$\begin{array}{lrrr}\text { C } & -5.922600 & -1.989700 & -0.241160\end{array}$

H $\quad 1.424430 \quad 0.654480 \quad-1.994900$
H $\quad 2.480610 \quad 2.125570 \quad-1.630050$

$\mathrm{H} \quad 0.431360 \quad-2.339120 \quad 3.047360$

$\mathrm{H} \quad-1.901520 \quad-1.901360 \quad 2.344080$

$\mathrm{H} \quad-0.445690 \quad 1.056790 \quad-0.410060$

H $\quad 5.219060 \quad-1.586000 \quad-0.515860$

H $\quad 5.139880 \quad-4.006880-0.877360$

$\mathrm{H} \quad 3.018860 \quad-5.109320 \quad-1.563800$

$\mathrm{H} \quad 0.986370 \quad-3.715790 \quad-1.893300$

$\mathrm{H} \quad 1.061910 \quad-1.310290 \quad-1.537760$

$\mathrm{H} \quad 4.707500 \quad 0.884450 \quad 1.476340$

H $\quad 7.067000 \quad 1.626200 \quad 1.535000$

H $\quad 8.320320 \quad 1.972080 \quad-0.577550$

H $\quad 7.188870 \quad 1.581680 \quad-2.752620$

H $\quad 4.827740 \quad 0.824760 \quad-2.795480$

$\mathrm{H} \quad-3.366390 \quad-2.151140 \quad 0.520930$

$\begin{array}{llll}\mathrm{H} & -2.436950 & 2.029000 & 0.336530\end{array}$

H $\quad-6.389790 \quad 0.665000 \quad-0.635770$

\section{ligL1}

$\begin{array}{llll}\text { O } & -3.034590 & 1.992410 & -1.558080\end{array}$

$\begin{array}{llll}\mathrm{O} & -1.067610 & 1.409690 & -2.503530\end{array}$

$\mathrm{Cl} \quad-0.361130 \quad 4.060450 \quad 0.912250$

$\begin{array}{llll}\text { C } & -1.250110 & 1.266400 & -0.140460\end{array}$

$\begin{array}{llll}\text { C } & -1.802660 & -0.007970 & 0.531330\end{array}$

$\begin{array}{llll}\text { C } & -2.153840 & 1.373430 & 1.037960\end{array}$

C $\quad-1.858620 \quad 1.612410 \quad-1.566820$

$\begin{array}{llll}\text { C } & 0.204010 & 1.555560 & -0.032800\end{array}$

$\begin{array}{llll}\text { C } & 0.716340 & 2.765070 & 0.433510\end{array}$

$\begin{array}{llll}\text { C } & 2.085360 & 2.999560 & 0.528930\end{array}$

$\begin{array}{llll}\text { C } & 2.979480 & 2.008160 & 0.157260\end{array}$

$\begin{array}{llll}\text { C } & 2.506790 & 0.786730 & -0.334350\end{array}$

$\begin{array}{llll}\text { C } & 1.133900 & 0.597560 & -0.442880\end{array}$

$\begin{array}{llll}\text { C } & -0.871830 & -0.868450 & 1.347870\end{array}$

$\begin{array}{llll}\text { C } & -0.017840 & -0.334520 & 2.324940\end{array}$

C $\quad 0.866060 \quad-1.137580 \quad 3.034600$

$\begin{array}{llll}\text { C } & 0.925410 & -2.508110 & 2.796840\end{array}$

C $\quad 0.084380 \quad-3.057240 \quad 1.836610$

$\begin{array}{llll}\text { C } & -0.799140 & -2.250090 & 1.124160\end{array}$

C $\quad-2.882260 \quad-0.788780 \quad-0.169830$

$\begin{array}{llll}\text { C } & -2.740260 & -1.216340 & -1.489560\end{array}$

C $\quad-3.717840 \quad-2.012190 \quad-2.078370$

C $\quad-4.844780 \quad-2.394100 \quad-1.356800$

$\begin{array}{llll}\text { C } & -4.995680 & -1.965460 & -0.040940\end{array}$

$\begin{array}{llll}\text { C } & -4.018650 & -1.167470 & 0.544080\end{array}$

$\begin{array}{llll}\text { C } & 3.439030 & -0.311300 & -0.681800\end{array}$

$\begin{array}{llll}\text { C } & 3.133100 & -1.627760 & -0.318500\end{array}$

$\begin{array}{llll}\text { C } & 4.003420 & -2.666860 & -0.625500\end{array}$

C $\quad 5.195330 \quad-2.410220-1.297590$

$\begin{array}{llll}\text { C } & 5.509230 & -1.104170 & -1.661900\end{array}$

$\begin{array}{llll}\text { C } & 4.637900 & -0.064370 & -1.356830\end{array}$

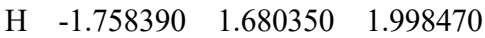

H $\quad-3.145480 \quad 1.732350 \quad 0.785430$

$\mathrm{H} \quad 2.434930 \quad 3.951840 \quad 0.907690$

H $\quad 4.045230 \quad 2.171500 \quad 0.274250$

H $\quad 0.745840 \quad-0.328660 \quad-0.851760$

$\mathrm{H} \quad-0.016410 \quad 0.730560 \quad 2.520650$

H $\quad 1.519350 \quad-0.684210 \quad 3.771700$

H $\quad 1.619340 \quad-3.133030 \quad 3.347260$

$\mathrm{H} \quad 0.111630 \quad-4.122310 \quad 1.633030$

H $\quad-1.443500 \quad-2.703050 \quad 0.380890$

$\mathrm{H} \quad-1.878370 \quad-0.889570 \quad-2.063140$

$\mathrm{H} \quad-3.603260 \quad-2.322460 \quad-3.110860$ 
H $\quad-5.607280 \quad-3.010330-1.820430$

$\mathrm{H} \quad-5.876210 \quad-2.247210 \quad 0.526230$

$\mathrm{H} \quad-4.130500 \quad-0.826520 \quad 1.568700$

H $\quad 2.216300 \quad-1.827120 \quad 0.228060$

H $\quad 3.751250 \quad-3.679670 \quad-0.331700$

$\mathrm{H} \quad 6.429680 \quad-0.895690 \quad-2.195790$

H $\quad 4.872190 \quad 0.948640 \quad-1.665460$

H $\quad 5.873080 \quad-3.221490 \quad-1.537540$

\section{ligL_TSrot}

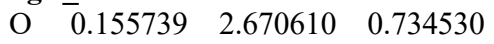

$\begin{array}{llll}\text { O } & 0.466149 & 1.819210 & -1.328770\end{array}$

$\begin{array}{llll}\mathrm{Cl} & -0.671300 & -3.005931 & -0.320000\end{array}$

$\begin{array}{llll}\text { C } & -0.306090 & 0.342350 & 0.365500\end{array}$

$\begin{array}{llll}\text { C } & -1.836460 & 0.264749 & 0.503770\end{array}$

$\begin{array}{llll}\text { C } & -0.962070 & 0.296729 & 1.722970\end{array}$

$\begin{array}{llll}\text { C } & 0.151449 & 1.778520 & -0.124630\end{array}$

$\begin{array}{llll}\text { C } & 0.785850 & -0.665840 & 0.068460\end{array}$

$\begin{array}{llll}\text { C } & 0.781210 & -2.035140 & -0.213390\end{array}$

$\begin{array}{llll}\text { C } & 1.958340 & -2.760410 & -0.427620\end{array}$

$\begin{array}{llll}\text { C } & 3.192980 & -2.149820 & -0.360670\end{array}$

$\begin{array}{llll}\text { C } & 3.259340 & -0.788940 & -0.047900\end{array}$

$\begin{array}{llll}\text { C } & 2.075450 & -0.101730 & 0.172250\end{array}$

$\begin{array}{llll}\text { C } & -2.747050 & -0.904121 & 0.234050\end{array}$

$\begin{array}{llll}\text { C } & -3.265140 & -1.674551 & 1.271560\end{array}$

$\begin{array}{llll}\text { C } & -4.146920 & -2.721491 & 1.011930\end{array}$

$\begin{array}{llll}\text { C } & -4.537190 & -2.998891 & -0.292370\end{array}$

$\begin{array}{llll}\text { C } & -4.052190 & -2.208321 & -1.334380\end{array}$

$\begin{array}{llll}\text { C } & -3.174850 & -1.167991 & -1.070580\end{array}$

$\begin{array}{llll}\text { C } & -2.617171 & 1.529599 & 0.189690\end{array}$

$\begin{array}{llll}\text { C } & -2.590811 & 2.128909 & -1.069350\end{array}$

$\begin{array}{llll}\text { C } & -3.401471 & 3.226579 & -1.339670\end{array}$

$\begin{array}{llll}\text { C } & -4.252311 & 3.734689 & -0.363520\end{array}$

$\begin{array}{llll}\text { C } & -4.282431 & 3.140719 & 0.894870\end{array}$

$\begin{array}{llll}\text { C } & -3.470051 & 2.045959 & 1.164400\end{array}$

$\begin{array}{llll}\text { C } & 4.554010 & -0.069239 & 0.009810\end{array}$

$\begin{array}{llll}\text { C } & 4.647209 & 1.247021 & -0.457830\end{array}$

$\begin{array}{llll}\text { C } & 5.857919 & 1.927791 & -0.412870\end{array}$

$\begin{array}{llll}\text { C } & 6.997169 & 1.307671 & 0.092820\end{array}$

$\begin{array}{llll}\text { C } & 6.915340 & -0.001179 & 0.558470\end{array}$

$\begin{array}{llll}\text { C } & 5.703700 & -0.681959 & 0.518140\end{array}$

$\mathrm{H} \quad-0.855470 \quad-0.633261 \quad 2.272230$

$\begin{array}{llll}\mathrm{H} & -0.902551 & 1.220149 & 2.288630\end{array}$

H $\quad 1.875280 \quad-3.812860 \quad-0.667440$

H $\quad 4.092640 \quad-2.715329 \quad-0.575970$

$\begin{array}{llll}\mathrm{H} & 2.140449 & 0.942260 & 0.452010\end{array}$

H $\quad-2.960660 \quad-1.464421 \quad 2.290590$

$\mathrm{H} \quad-4.527030 \quad-3.320831 \quad 1.832090$

$\mathrm{H} \quad-5.218650 \quad-3.816782 \quad-0.497800$

$\mathrm{H} \quad-4.355220 \quad-2.411281 \quad-2.355580$

$\mathrm{H} \quad-2.785850 \quad-0.562591 \quad-1.881780$

H $\quad-1.889951 \quad 1.768729-1.813940$

$\mathrm{H} \quad-3.353521 \quad 3.696539 \quad-2.315660$

H $\quad-4.877521 \quad 4.594739 \quad-0.576700$

$\begin{array}{llll}\mathrm{H} & -4.933481 & 3.534098 & 1.668040\end{array}$

$\begin{array}{llll}\mathrm{H} & -3.487301 & 1.582129 & 2.145650\end{array}$

$\begin{array}{llll}\mathrm{H} & 3.765069 & 1.726400 & -0.870620\end{array}$

H $\quad 5.910779 \quad 2.946161 \quad-0.780410$

H $\quad 7.794300 \quad-0.489759 \quad 0.964260$

$\begin{array}{llll}\mathrm{H} & 5.638800 & -1.692809 & 0.906150\end{array}$

H $\quad 7.940319 \quad 1.841031 \quad 0.126700$

\section{ligL_TSalt}

$\begin{array}{llll}\mathrm{O} & -2.667270 & 2.434581 & 1.855349\end{array}$

$\begin{array}{llll}\mathrm{O} & -3.118500 & 1.889251 & -0.287981\end{array}$

$\mathrm{Cl} \quad-0.928440 \quad 3.873341 \quad-0.682951$

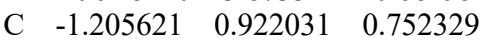

$\begin{array}{llll}\text { C } & -1.504931 & -0.566839 & 0.486719\end{array}$

$\begin{array}{llll}\text { C } & -1.176911 & -0.087739 & 1.872399\end{array}$

$\begin{array}{llll}\text { C } & -2.473800 & 1.861821 & 0.773149\end{array}$

$\begin{array}{llll}\text { C } & 0.119150 & 1.484300 & 0.295899\end{array}$

C $\quad 0.325980 \quad 2.709710 \quad-0.367101$

$\begin{array}{llll}\text { C } & 1.593920 & 3.100100 & -0.807391\end{array}$

$\begin{array}{llll}\text { C } & 2.705310 & 2.303839 & -0.610351\end{array}$

$\begin{array}{llll}\text { C } & 2.559320 & 1.097969 & 0.076179\end{array}$

$\begin{array}{llll}\text { C } & 1.291949 & 0.739540 & 0.519319\end{array}$

$\begin{array}{llll}\text { C } & -0.512121 & -1.410290 & -0.270271\end{array}$

$\begin{array}{llll}\text { C } & 0.064168 & -2.537990 & 0.312769\end{array}$

C $\quad 0.999298 \quad-3.300670 \quad-0.381301$

$\begin{array}{llll}\text { C } & 1.360128 & -2.949430 & -1.678151\end{array}$

C $\quad 0.771388-1.838490 \quad-2.277551$

C $\quad-0.157741-1.077090 \quad-1.578931$

$\begin{array}{llll}\text { C } & -2.908541 & -1.058609 & 0.220149\end{array}$

$\begin{array}{llll}\text { C } & -3.550941 & -0.810579 & -0.995651\end{array}$

C $\quad-4.793781 \quad-1.367788 \quad-1.260141$

$\begin{array}{llll}\text { C } & -5.419072 & -2.183088 & -0.317571\end{array}$

$\begin{array}{llll}\text { C } & -4.790512 & -2.431818 & 0.895259\end{array}$

$\begin{array}{llll}\text { C } & -3.541532 & -1.871419 & 1.157529\end{array}$

$\begin{array}{llll}\text { C } & 3.710419 & 0.192559 & 0.305819\end{array}$

$\begin{array}{llll}\text { C } & 3.845389 & -0.514171 & 1.505779\end{array}$

$\begin{array}{llll}\text { C } & 4.912049 & -1.381512 & 1.707249\end{array}$

$\begin{array}{llll}\text { C } & 5.870649 & -1.558142 & 0.713739\end{array}$

$\begin{array}{llll}\text { C } & 5.750059 & -0.858852 & -0.483251\end{array}$

$\begin{array}{llll}\text { C } & 4.680299 & 0.005619 & -0.684611\end{array}$

$\mathrm{H} \quad-0.232311 \quad-0.372960 \quad 2.321529$

$\begin{array}{llll}\mathrm{H} & -1.996121 & 0.022871 & 2.572709\end{array}$

H $\quad 1.686441 \quad 4.057260 \quad-1.304721$

H $\quad 3.679800 \quad 2.632819 \quad-0.953681$

H $\quad 1.210749 \quad-0.214240 \quad 1.018309$

H $\quad-0.212012 \quad-2.802120 \quad 1.329029$

H $\quad 1.453538 \quad-4.163130 \quad 0.093889$

H $\quad 2.100228 \quad-3.531781 \quad-2.214991$

H $\quad 1.051429-1.553630 \quad-3.285571$

$\mathrm{H} \quad-0.590081 \quad-0.187100 \quad-2.025521$

$\mathrm{H} \quad-3.090481 \quad-0.132679 \quad-1.701571$

H $\quad-5.288861 \quad-1.148428 \quad-2.199471$

H $\quad-6.394622 \quad-2.608988 \quad-0.525131$

$\mathrm{H} \quad-5.269612 \quad-3.056138 \quad 1.641549$

H $\quad-3.051882 \quad-2.062449 \quad 2.106739$

H $\quad 3.111689-0.367521 \quad 2.290499$

H $\quad 4.998038 \quad-1.917032 \quad 2.645979$

$\begin{array}{llll}\mathrm{H} & 6.484639 & -0.996722 & -1.268771\end{array}$

$\begin{array}{llll}\mathrm{H} & 4.573129 & 0.522249 & -1.631941\end{array}$

H $\quad 6.702108 \quad-2.235562 \quad 0.870249$

\section{ligL2}

$\begin{array}{llll}\text { O } & -0.704190 & -2.386340 & 1.803840\end{array}$

$\begin{array}{llll}\mathrm{O} & -1.496790 & -3.199780 & -0.147040\end{array}$

$\begin{array}{llll}\text { Cl } & -0.985570 & 0.804290 & 2.416560\end{array}$

C $\quad-0.563940 \quad-0.996770 \quad-0.126140$

$\begin{array}{llll}\text { C } & -1.687410 & -0.159030 & -0.753970\end{array}$

C $\quad-0.732940 \quad-0.960660 \quad-1.610560$

C $\quad-0.968420 \quad-2.359190 \quad 0.592470$

$\begin{array}{llll}\text { C } & 0.610250 & -0.334030 & 0.505780\end{array}$ $\begin{array}{llll}\text { C } & 0.539990 & 0.463910 & 1.651070\end{array}$

$\begin{array}{llll}\text { C } & 1.676680 & 1.036300 & 2.209580\end{array}$

$\begin{array}{llll}\text { C } & 2.922150 & 0.810000 & 1.642690\end{array}$

$\begin{array}{llll}\text { C } & 3.039460 & -0.001730 & 0.512210\end{array}$

$\begin{array}{llll}\text { C } & 1.879620 & -0.554150 & -0.030690\end{array}$

C $\quad-1.566840 \quad 1.345130 \quad-0.771430$

$\begin{array}{llll}\text { C } & -0.388280 & 2.000090 & -1.154730\end{array}$

C $\quad-0.288760 \quad 3.386650-1.130810$

$\begin{array}{llll}\text { C } & -1.368060 & 4.166510 & -0.730340\end{array}$

$\begin{array}{llll}\text { C } & -2.548400 & 3.534710 & -0.355510\end{array}$

C $\quad-2.645000 \quad 2.148150 \quad-0.375950$

$\begin{array}{llll}\text { C } & -3.104570 & -0.653910 & -0.639840\end{array}$

$\begin{array}{llll}\text { C } & -3.647320 & -1.050010 & 0.581320\end{array}$

$\begin{array}{llll}\text { C } & -4.984070 & -1.426100 & 0.665430\end{array}$

$\begin{array}{llll}\text { C } & -5.794660 & -1.407640 & -0.465700\end{array}$

$\begin{array}{llll}\text { C } & -5.256780 & -1.019280 & -1.689580\end{array}$

C $\quad-3.919620 \quad-0.646310 \quad-1.771160$

$\begin{array}{llll}\text { C } & 4.363370 & -0.277430 & -0.095120\end{array}$

C $\quad 4.673570-1.552050-0.582080$

C $\quad 5.913550 \quad-1.814250 \quad-1.152460$

$\begin{array}{llll}\text { C } & 6.871360 & -0.808550 & -1.244840\end{array}$

$\begin{array}{llll}\text { C } & 6.575920 & 0.462840 & -0.762270\end{array}$

$\begin{array}{llll}\text { C } & 5.334100 & 0.725180 & -0.195190\end{array}$

$\mathrm{H} \quad-0.030110 \quad-0.410300 \quad-2.225590$

H $\quad-1.128990 \quad-1.882020 \quad-2.021180$

$\mathrm{H} \quad 1.575740 \quad 1.648540 \quad 3.096850$

$\mathrm{H} \quad 3.807400 \quad 1.236340 \quad 2.101380$

H $\quad 1.945400 \quad-1.170210 \quad-0.922020$

H $\quad 0.479390 \quad 1.429480 \quad-1.459040$

H $\quad 0.643650 \quad 3.855930-1.425000$

$\mathrm{H} \quad-1.289080 \quad 5.247530 \quad-0.709130$

$\mathrm{H} \quad-3.402880 \quad 4.121810 \quad-0.037050$

$\begin{array}{llll}\mathrm{H} & -3.571180 & 1.679260 & -0.067800\end{array}$

$\mathrm{H} \quad-3.008060 \quad-1.090650 \quad 1.457140$

$\mathrm{H} \quad-5.388870 \quad-1.748680 \quad 1.618110$

$\mathrm{H} \quad-6.834950 \quad-1.706450 \quad-0.397220$

$\mathrm{H} \quad-5.876530 \quad-1.012810 \quad-2.579730$

$\mathrm{H} \quad-3.492260 \quad-0.343330 \quad-2.722190$

H $\quad 3.939920 \quad-2.344900 \quad-0.488840$

$\mathrm{H} \quad 6.135180 \quad-2.810610-1.517680$

$\mathrm{H} \quad 7.311210 \quad 1.256240 \quad-0.836890$

H $\quad 5.099450 \quad 1.724080 \quad 0.155870$

H $\quad 7.838560 \quad-1.013610 \quad-1.688910$

\section{ligM1}

$\begin{array}{llll}\text { O } & -4.293360 & 0.729590 & -1.978560\end{array}$

$\begin{array}{llll}\mathrm{O} & -2.148260 & 0.832550 & -2.678840\end{array}$

$\mathrm{Cl} \quad-2.874640 \quad 3.897080 \quad 0.367820$

C $\quad-2.556920 \quad 0.878290 \quad-0.340490$

$\begin{array}{llll}\text { C } & -2.687640 & -0.434280 & 0.460640\end{array}$

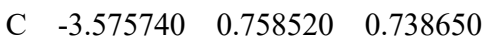

$\begin{array}{llll}\text { C } & -3.068180 & 0.820090 & -1.843470\end{array}$

$\begin{array}{llll}\text { C } & -1.331370 & 1.700920 & -0.163670\end{array}$

$\begin{array}{llll}\text { C } & -1.351970 & 3.055410 & 0.164440\end{array}$

C $\quad-0.182590 \quad 3.792290 \quad 0.332210$

$\begin{array}{llll}\text { C } & 1.047810 & 3.173310 & 0.177970\end{array}$

C $\quad 1.112360 \quad 1.819960-0.170100$

$\begin{array}{llll}\text { C } & -0.075400 & 1.122970 & -0.359680\end{array}$

$\begin{array}{llll}\text { C } & -1.617820 & -0.794020 & 1.460450\end{array}$

$\begin{array}{llll}\text { C } & -1.142240 & 0.120010 & 2.413010\end{array}$

C $\quad-0.124120 \quad-0.215150 \quad 3.296730$

$\begin{array}{llll}\text { C } & 0.454070 & -1.481310 & 3.264430\end{array}$ 


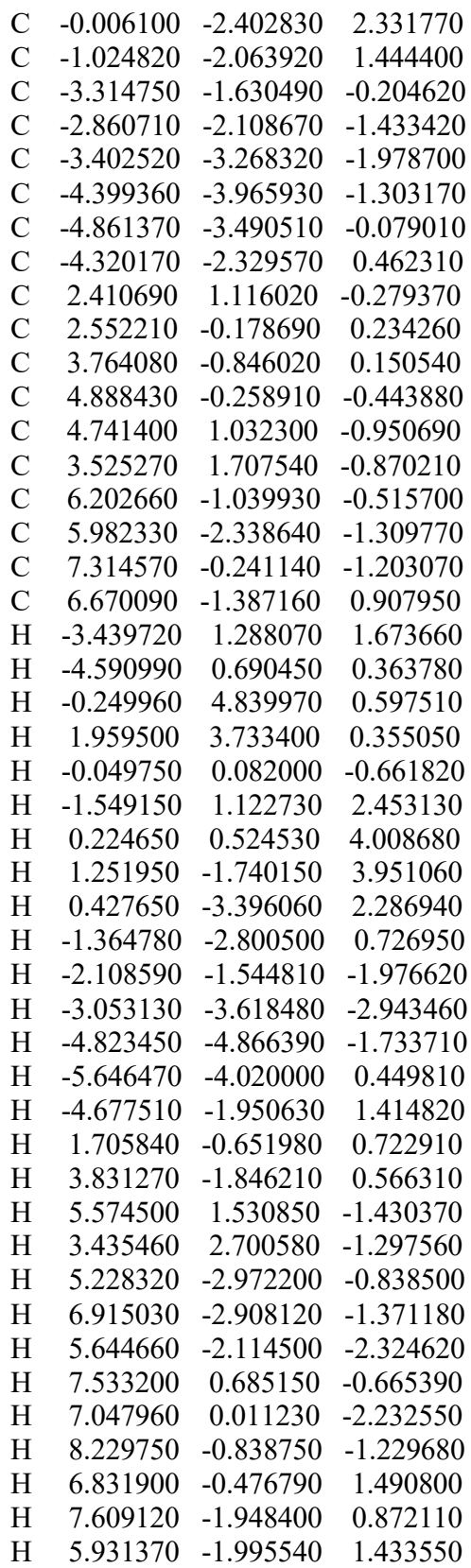

ligM_TSrot
$\begin{array}{llll}\text { O } & -0.585990 & 2.484810 & 0.697759 \\ \text { O } & -0.426080 & 1.582780 & -1.361101 \\ \text { Cl } & -2.250020 & -3.015031 & -0.305881 \\ \text { C } & -1.389960 & 0.247540 & 0.352619 \\ \text { C } & -2.913590 & 0.397109 & 0.506249 \\ \text { C } & -2.030800 & 0.308919 & 1.716629 \\ \text { C } & -0.730850 & 1.597080 & -0.153871 \\ \text { C } & -0.461280 & -0.912390 & 0.053679 \\ \text { C } & -0.669680 & -2.267400 & -0.219491 \\ \text { C } & 0.386310 & -3.158610 & -0.439381 \\ \text { C } & 1.697830 & -2.735720 & -0.386391 \\ \text { C } & 1.966460 & -1.398210 & -0.081001 \\ \text { C } & 0.898010 & -0.543710 & 0.144409 \\ \text { C } & -3.988740 & -0.627101 & 0.254149\end{array}$
C $\quad-4.600370 \quad-1.310121 \quad 1.301849$

$\begin{array}{llll}\text { C } & -5.627850 & -2.218981 & 1.058369\end{array}$

$\begin{array}{llll}\text { C } & -6.070090 & -2.442061 & -0.239771\end{array}$

C $\quad-5.488250 \quad-1.734521 \quad-1.291741$

C $\quad-4.466050 \quad-0.831561 \quad-1.044021$

$\begin{array}{llll}\text { C } & -3.502550 & 1.760879 & 0.188219\end{array}$

$\begin{array}{llll}\text { C } & -3.400690 & 2.339999 & -1.076361\end{array}$

$\begin{array}{llll}\text { C } & -4.043590 & 3.542809 & -1.349941\end{array}$

$\begin{array}{llll}\text { C } & -4.800830 & 4.178269 & -0.371301\end{array}$

$\begin{array}{llll}\text { C } & -4.905550 & 3.605179 & 0.892719\end{array}$

$\begin{array}{llll}\text { C } & -4.260620 & 2.404819 & 1.165409\end{array}$

$\begin{array}{llll}\text { C } & 3.352170 & -0.875990 & -0.033401\end{array}$

$\begin{array}{llll}\text { C } & 3.638780 & 0.415580 & -0.493241\end{array}$

$\begin{array}{llll}\text { C } & 4.933270 & 0.909210 & -0.452671\end{array}$

$\begin{array}{llll}\text { C } & 5.999860 & 0.146020 & 0.039439\end{array}$

$\begin{array}{llll}\text { C } & 5.708120 & -1.140410 & 0.492579\end{array}$

C $\quad 4.408570 \quad-1.641260 \quad 0.457839$

$\begin{array}{llll}\text { C } & 7.409890 & 0.740450 & 0.063029\end{array}$

$\begin{array}{llll}\text { C } & 7.415300 & 2.008660 & 0.933229\end{array}$

$\begin{array}{llll}\text { C } & 8.441340 & -0.237410 & 0.634819\end{array}$

$\begin{array}{llll}\text { C } & 7.835510 & 1.104410 & -1.369541\end{array}$

$\mathrm{H} \quad-2.056030 \quad-0.622271 \quad 2.273539$

$\mathrm{H} \quad-1.829710 \quad 1.218020 \quad 2.272879$

H $\quad 0.147390 \quad-4.188580 \quad-0.672131$

H $\quad 2.502780 \quad-3.428170 \quad-0.605681$

$\mathrm{H} \quad 1.117590 \quad 0.480700 \quad 0.417819$

$\mathrm{H} \quad-4.255970 \quad-1.142801 \quad 2.316069$

$\begin{array}{llll}\mathrm{H} & -6.080690 & -2.753611 & 1.886289\end{array}$

$\mathrm{H} \quad-6.865660 \quad-3.153001 \quad-0.432621$

H $\quad-5.830010-1.895331 \quad-2.308201$

$\mathrm{H} \quad-4.003270 \quad-0.292461 \quad-1.863181$

$\mathrm{H} \quad-2.767690 \quad 1.874849 \quad-1.823531$

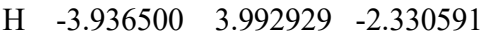

$\mathrm{H} \quad-5.294690 \quad 5.119339 \quad-0.586991$

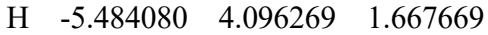

$\mathrm{H} \quad-4.336280 \quad 1.956259 \quad 2.150999$

H $\quad 2.837290 \quad 1.025990 \quad-0.897681$

$\mathrm{H} \quad 5.111510 \quad 1.914260 \quad-0.820301$

H $\quad 6.491760 \quad-1.771990 \quad 0.892459$

H $\quad 4.211560 \quad-2.636160 \quad 0.842999$

$\mathrm{H} \quad 6.724700 \quad 2.761090 \quad 0.547699$

H $\quad 8.417890 \quad 2.447250 \quad 0.957439$

$\begin{array}{llll}\mathrm{H} & 7.114850 & 1.771990 & 1.956989\end{array}$

H $\quad 8.499810 \quad-1.151690 \quad 0.038399$

H $\quad 8.203850 \quad-0.511590 \quad 1.665949$

$\begin{array}{llll}\mathrm{H} & 9.429330 & 0.230920 & 0.630609\end{array}$

$\mathrm{H} \quad 7.836370 \quad 0.216140 \quad-2.006451$

H $\quad 8.843010 \quad 1.532250 \quad-1.368291$

H $\quad 7.157360 \quad 1.835340 \quad-1.814121$

\section{ligM TSalt}

$\begin{array}{llll}\mathrm{O} & \overline{4} .112501 & 1.778598 & -2.040171\end{array}$

$\begin{array}{llll}\text { O } & 4.511951 & 1.204127 & 0.105759\end{array}$

$\begin{array}{llll}\text { Cl } & 2.913592 & 3.709548 & 0.475719\end{array}$

$\begin{array}{llll}\text { C } & 2.367041 & 0.730198 & -0.821711\end{array}$

$\begin{array}{llll}\text { C } & 2.271030 & -0.771302 & -0.475211\end{array}$

$\begin{array}{llll}\text { C } & 2.039840 & -0.292012 & -1.880651\end{array}$

$\begin{array}{llll}\text { C } & 3.831621 & 1.308588 & -0.927941\end{array}$

$\begin{array}{llll}\text { C } & 1.252011 & 1.637629 & -0.361541\end{array}$

$\begin{array}{llll}\text { C } & 1.392242 & 2.891889 & 0.263149\end{array}$

$\begin{array}{llll}\text { C } & 0.286322 & 3.596469 & 0.747719\end{array}$

$\begin{array}{llll}\text { C } & -0.996048 & 3.091620 & 0.644039\end{array}$ $\begin{array}{llll}\text { C } & -1.190619 & 1.872100 & -0.004721\end{array}$

$\begin{array}{llll}\text { C } & -0.079039 & 1.209949 & -0.510771\end{array}$

$\begin{array}{llll}\text { C } & 1.105960 & -1.279461 & 0.335789\end{array}$

$\begin{array}{llll}\text { C } & 0.214649 & -2.211531 & -0.194121\end{array}$

$\begin{array}{llll}\text { C } & -0.894311 & -2.633440 & 0.534309\end{array}$

C $\quad-1.116081 \quad-2.137480 \quad 1.814959$

$\begin{array}{llll}\text { C } & -0.212370 & -1.231851 & 2.365919\end{array}$

$\begin{array}{llll}\text { C } & 0.889900 & -0.809301 & 1.632569\end{array}$

$\begin{array}{llll}\text { C } & 3.498800 & -1.607052 & -0.201071\end{array}$

$\begin{array}{llll}\text { C } & 4.232410 & -1.466643 & 0.979809\end{array}$

$\begin{array}{llll}\text { C } & 5.286199 & -2.325533 & 1.258159\end{array}$

$\begin{array}{llll}\text { C } & 5.627839 & -3.339903 & 0.364709\end{array}$

$\begin{array}{llll}\text { C } & 4.907629 & -3.484113 & -0.813851\end{array}$

$\begin{array}{llll}\text { C } & 3.849189 & -2.620642 & -1.090091\end{array}$

$\begin{array}{llll}\text { C } & -2.525869 & 1.237661 & -0.101151\end{array}$

$\begin{array}{llll}\text { C } & -2.911709 & 0.517111 & -1.237331\end{array}$

$\begin{array}{llll}\text { C } & -4.136930 & -0.127729 & -1.292611\end{array}$

$\begin{array}{llll}\text { C } & -5.039340 & -0.085218 & -0.222581\end{array}$

$\begin{array}{llll}\text { C } & -4.657629 & 0.645431 & 0.902209\end{array}$

$\begin{array}{llll}\text { C } & -3.425569 & 1.292221 & 0.962279\end{array}$

$\begin{array}{llll}\text { C } & -6.368380 & -0.836978 & -0.320531\end{array}$

$\begin{array}{llll}\text { C } & -6.088601 & -2.337158 & -0.514611\end{array}$

$\begin{array}{llll}\text { C } & -7.222690 & -0.667737 & 0.939679\end{array}$

$\begin{array}{llll}\text { C } & -7.172390 & -0.309207 & -1.520681\end{array}$

H $\quad 1.040950 \quad-0.340171 \quad-2.299541$

$\mathrm{H} \quad 2.837840 \quad-0.438722 \quad-2.598711$

H $\quad 0.460972 \quad 4.558899 \quad 1.211759$

H $\quad-1.837178 \quad 3.653640 \quad 1.034569$

$\mathrm{H} \quad-0.264510 \quad 0.257889 \quad-0.982981$

H $\quad 0.381049-2.583031-1.200671$

H $\quad-1.592061 \quad-3.339210 \quad 0.096899$

$\mathrm{H} \quad-1.990081 \quad-2.447540 \quad 2.376429$

H $\quad-0.379830 \quad-0.835760 \quad 3.361329$

H $\quad 1.570700 \quad-0.067611 \quad 2.038589$

H $\quad 4.002940 \quad-0.643332 \quad 1.642709$

H $\quad 5.859549 \quad-2.190483 \quad 2.168449$

H $\quad 6.458058 \quad-4.002824 \quad 0.582559$

H $\quad 5.168878 \quad-4.262413 \quad-1.522551$

H $\quad 3.288399 \quad-2.730012 \quad-2.012591$

$\mathrm{H} \quad-2.241710 \quad 0.470090 \quad-2.088731$

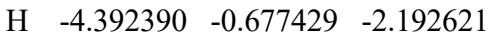

$\begin{array}{llll}\mathrm{H} & -5.311389 & 0.706622 & 1.763349\end{array}$

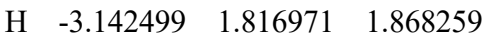

H $\quad-5.520311 \quad-2.522098 \quad-1.428481$

H $\quad-7.028911 \quad-2.893397 \quad-0.580181$

$\mathrm{H} \quad-5.509721 \quad-2.730208 \quad 0.325019$

$\begin{array}{llll}\mathrm{H} & -7.467780 & 0.382343 & 1.118979\end{array}$

H $\quad-6.713620 \quad-1.059868 \quad 1.823779$

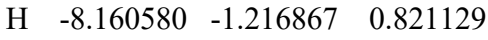

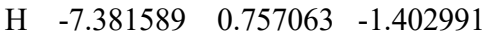

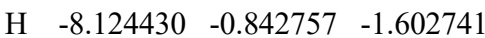

H $\quad-6.628050 \quad-0.443348 \quad-2.457601$

\section{$\operatorname{ligM2}$}

$\begin{array}{llll}\mathrm{O} & -2.380880 & -3.228510 & 0.195700\end{array}$

$\begin{array}{llll}\mathrm{O} & -1.879740 & -2.151630 & 2.115220\end{array}$

$\begin{array}{llll}\mathrm{Cl} & -2.458020 & 1.047190 & 2.320650\end{array}$

$\begin{array}{llll}\text { C } & -1.606200 & -0.969060 & 0.065080\end{array}$

$\begin{array}{llll}\text { C } & -2.699220 & -0.297080 & -0.779120\end{array}$

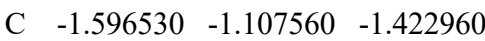

$\begin{array}{llll}\text { C } & -2.000510 & -2.274430 & 0.887080\end{array}$

$\begin{array}{llll}\text { C } & -0.566170 & -0.146170 & 0.742230\end{array}$ 


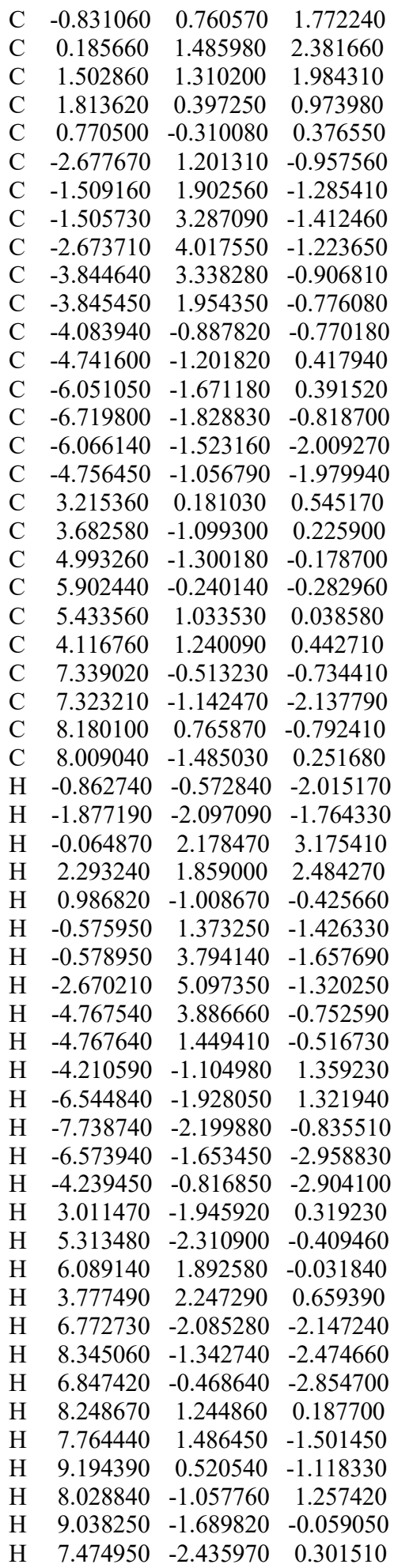

ligN1
$\begin{array}{lccc}\mathrm{O} & -1.487400 & 1.427380 & -2.510350 \\ \mathrm{O} & -3.561220 & 1.726090 & -1.666060 \\ \mathrm{Cl} & -1.327120 & 4.106180 & 0.928960 \\ \mathrm{C} & -1.770580 & 1.223490 & -0.161920 \\ \mathrm{C} & -2.182140 & -0.124090 & 0.467670\end{array}$ $\begin{array}{llll}\text { C } & -2.740730 & 1.190690 & 0.966860\end{array}$

$\begin{array}{llll}\text { C } & -2.345840 & 1.507090 & -1.615490\end{array}$

$\begin{array}{llll}\text { C } & -0.376890 & 1.704400 & 0.027800\end{array}$

$\begin{array}{llll}\mathrm{C} & -0.059060 & 2.968930 & 0.520800\end{array}$

$\begin{array}{llll}\text { C } & 1.258490 & 3.381380 & 0.700020\end{array}$

$\begin{array}{llll}\text { C } & 2.297310 & 2.516250 & 0.393250\end{array}$

$\begin{array}{llll}\text { C } & 2.021540 & 1.244630 & -0.119810\end{array}$

$\begin{array}{llll}\text { C } & 0.694790 & 0.880210 & -0.319650\end{array}$

$\begin{array}{llll}\mathrm{C} & -1.196010 & -0.869760 & 1.331250\end{array}$

$\begin{array}{llll}\text { C } & -0.465940 & -0.242510 & 2.352720\end{array}$

C $\quad 0.465400 \quad-0.938520 \quad 3.113160$

$\begin{array}{llll}\text { C } & 0.698360 & -2.292080 & 2.884190\end{array}$

$\begin{array}{llll}\text { C } & -0.016440 & -2.932640 & 1.879300\end{array}$

C $\quad-0.946810 \quad-2.232210 \quad 1.115200$

$\begin{array}{llll}\text { C } & -3.108420 & -1.027590 & -0.302070\end{array}$

$\begin{array}{llll}\text { C } & -4.229310 & -1.556890 & 0.336320\end{array}$

C $\quad-5.058030 \quad-2.464820 \quad-0.313830$

C $\quad-4.771960 \quad-2.853430 \quad-1.619610$

$\begin{array}{llll}\text { C } & -3.660040 & -2.322110 & -2.266130\end{array}$

$\begin{array}{llll}\text { C } & -2.830820 & -1.416380 & -1.612190\end{array}$

$\begin{array}{llll}\text { C } & 3.104580 & 0.267660 & -0.381480\end{array}$

$\begin{array}{llll}\text { C } & 4.316660 & 0.651990 & -0.955210\end{array}$

C $\quad 5.336960 \quad-0.273620 \quad-1.171650$

$\begin{array}{llll}\text { C } & 5.130800 & -1.600320 & -0.798960\end{array}$

$\begin{array}{llll}\text { C } & 3.926300 & -2.013250 & -0.225800\end{array}$

$\begin{array}{llll}\text { C } & 2.923610 & -1.071630 & -0.020160\end{array}$

$\begin{array}{llll}\text { C } & 3.703140 & -3.454880 & 0.155490\end{array}$

$\begin{array}{llll}\text { C } & 6.625050 & 0.154540 & -1.829670\end{array}$

$\mathrm{H} \quad-2.438780 \quad 1.530450 \quad 1.950080$

$\mathrm{H} \quad-3.757050 \quad 1.419160 \quad 0.665400$

$\mathrm{H} \quad 1.455070 \quad 4.370190 \quad 1.095290$

$\mathrm{H} \quad 3.322520 \quad 2.816280 \quad 0.580460$

$\mathrm{H} \quad 0.459100 \quad-0.088090 \quad-0.746510$

$\mathrm{H} \quad-0.601080 \quad 0.814520 \quad 2.545480$

$\mathrm{H} \quad 1.018830 \quad-0.413800 \quad 3.883840$

$\mathrm{H} \quad 1.428100 \quad-2.833060 \quad 3.476170$

$\mathrm{H} \quad 0.146600 \quad-3.986660 \quad 1.680550$

$\mathrm{H} \quad-1.490030 \quad-2.755580 \quad 0.338250$

$\mathrm{H} \quad-4.447770 \quad-1.246220 \quad 1.353340$

H $\quad-5.929240 \quad-2.863440 \quad 0.194610$

$\mathrm{H} \quad-5.419530 \quad-3.554960 \quad-2.133990$

$\mathrm{H} \quad-3.442780 \quad-2.601160 \quad-3.291010$

H $\quad-1.984000 \quad-0.974200 \quad-2.127990$

$\mathrm{H} \quad 4.457300 \quad 1.685720 \quad-1.257130$

$\mathrm{H} \quad 1.994390 \quad-1.371080 \quad 0.456930$

$\mathrm{H} \quad 5.923640 \quad-2.327390 \quad-0.955650$

H $\quad 3.339390 \quad-4.027740 \quad-0.702500$

$\mathrm{H} \quad 4.627930 \quad-3.922160 \quad 0.500790$

$\mathrm{H} \quad 2.954120 \quad-3.532440 \quad 0.945580$

H $\quad 7.418280 \quad-0.574770 \quad-1.655840$

$\mathrm{H} \quad 6.492240 \quad 0.254250 \quad-2.910700$

H $\quad 6.958860 \quad 1.122130 \quad-1.448980$

\section{ligN_TSrot}

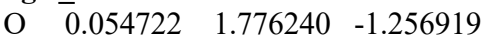
$\begin{array}{llll}\mathrm{O} & -0.198378 & 2.551170 & 0.843761\end{array}$

Cl $\quad-1.289930 \quad-3.034799 \quad-0.441679$

$\begin{array}{llll}\text { C } & -0.769609 & 0.263950 & 0.381181\end{array}$

$\begin{array}{llll}\text { C } & -2.300889 & 0.249321 & 0.530141\end{array}$

$\begin{array}{llll}\text { C } & -1.416959 & 0.190351 & 1.741471\end{array}$

$\begin{array}{llll}\text { C } & -0.251118 & 1.697550 & -0.052369\end{array}$

$\begin{array}{llll}\text { C } & 0.273010 & -0.779370 & 0.031291\end{array}$ $\begin{array}{llll}\text { C } & 0.205300 & -2.133960 & -0.307909\end{array}$

$\begin{array}{llll}\text { C } & 1.347560 & -2.900310 & -0.563839\end{array}$

$\begin{array}{llll}\text { C } & 2.608710 & -2.347541 & -0.485009\end{array}$

$\begin{array}{llll}\text { C } & 2.737910 & -1.005101 & -0.117499\end{array}$

$\begin{array}{llll}\text { C } & 1.586971 & -0.278031 & 0.145791\end{array}$

$\begin{array}{llll}\text { C } & -3.263960 & -0.865838 & 0.218611\end{array}$

$\begin{array}{llll}\text { C } & -3.806460 & -1.658838 & 1.226171\end{array}$

$\begin{array}{llll}\text { C } & -4.733780 & -2.655248 & 0.929281\end{array}$

C $\quad-5.145290 \quad-2.858168 \quad-0.382199$

$\begin{array}{llll}\text { C } & -4.635310 & -2.043238 & -1.393019\end{array}$

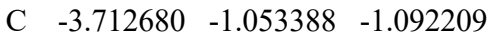

$\begin{array}{llll}\text { C } & -3.026599 & 1.559811 & 0.277251\end{array}$

$\begin{array}{llll}\text { C } & -3.849878 & 2.070332 & 1.280201\end{array}$

$\begin{array}{llll}\text { C } & -4.615488 & 3.209772 & 1.064461\end{array}$

$\begin{array}{llll}\text { C } & -4.567728 & 3.855402 & -0.167669\end{array}$

$\begin{array}{llll}\text { C } & -3.745998 & 3.353462 & -1.171579\end{array}$

$\begin{array}{llll}\text { C } & -2.982188 & 2.211211 & -0.955129\end{array}$

$\begin{array}{llll}\text { C } & 4.061381 & -0.340302 & -0.048969\end{array}$

$\begin{array}{llll}\text { C } & 5.192010 & -1.019392 & 0.418351\end{array}$

$\begin{array}{llll}\text { C } & 6.432941 & -0.394243 & 0.479521\end{array}$

$\begin{array}{llll}\text { C } & 6.538231 & 0.933237 & 0.058971\end{array}$

$\begin{array}{llll}\text { C } & 5.429751 & 1.633128 & -0.412189\end{array}$

$\begin{array}{llll}\text { C } & 4.195601 & 0.985858 & -0.463079\end{array}$

$\begin{array}{llll}\text { C } & 5.551352 & 3.057748 & -0.890399\end{array}$

$\begin{array}{llll}\text { C } & 7.637130 & -1.117203 & 1.030241\end{array}$

$\mathrm{H} \quad-1.348390 \quad-0.765769 \quad 2.250411$

H $\quad-1.311779 \quad 1.085481 \quad 2.344741$

H $\quad 1.216099 \quad-3.937270 \quad-0.845939$

$\mathrm{H} \quad 3.480830 \quad-2.942111 \quad-0.732819$

$\begin{array}{llll}\mathrm{H} & 1.700881 & 0.748839 & 0.469241\end{array}$

H $\quad-3.485090 \quad-1.507468 \quad 2.250421$

H $\quad-5.132441 \quad-3.273838 \quad 1.726001$

H $\quad-5.862191 \quad-3.636967 \quad-0.616909$

H $\quad-4.954500 \quad-2.187858 \quad-2.419209$

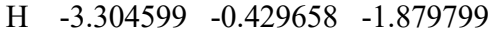

$\begin{array}{llll}\mathrm{H} & -3.881229 & 1.565732 & 2.240781\end{array}$

$\begin{array}{llll}\mathrm{H} & -5.243978 & 3.597172 & 1.859021\end{array}$

$\begin{array}{llll}\mathrm{H} & -5.156527 & 4.749862 & -0.338849\end{array}$

H $\quad-3.683928 \quad 3.862392 \quad-2.127029$

$\begin{array}{llll}\mathrm{H} & -2.302628 & 1.853341 & -1.720249\end{array}$

$\begin{array}{llll}\mathrm{H} & 5.091390 & -2.043412 & 0.765241\end{array}$

H $\quad 3.325501 \quad 1.512559 \quad-0.846049$

$\begin{array}{llll}\mathrm{H} & 7.504031 & 1.430377 & 0.103691\end{array}$

H $\quad 6.486522 \quad 3.509307 \quad-0.554279$

H $\quad 5.527082 \quad 3.100528 \quad-1.982739$

H $\quad 4.719932 \quad 3.661738 \quad-0.522649$

H $\quad 8.536300 \quad-0.876564 \quad 0.458681$

H $\quad 7.820060 \quad-0.831253 \quad 2.070151$

H $\quad 7.493170 \quad-2.198633 \quad 1.002801$

\section{ligN_TSalt}

$\begin{array}{llll}\mathrm{O} & -3.721120 & 1.666510 & -0.185600\end{array}$

$\begin{array}{llll}\mathrm{O} & -3.300040 & 2.141770 & 1.980280\end{array}$

Cl $\quad-1.730690 \quad 3.892530 \quad-0.444600$

$\begin{array}{llll}\text { C } & -1.708990 & 0.841170 & 0.793910\end{array}$

$\begin{array}{llll}\text { C } & -1.846880 & -0.650040 & 0.421910\end{array}$

$\begin{array}{llll}\text { C } & -1.588380 & -0.235470 & 1.842970\end{array}$

$\begin{array}{llll}\text { C } & -3.064550 & 1.646920 & 0.868200\end{array}$

$\begin{array}{llll}\text { C } & -0.447010 & 1.567160 & 0.394680\end{array}$

$\begin{array}{llll}\text { C } & -0.363890 & 2.846170 & -0.188610\end{array}$

$\begin{array}{llll}\text { C } & 0.858790 & 3.386850 & -0.596960\end{array}$

$\begin{array}{llll}\text { C } & 2.041900 & 2.686740 & -0.458580\end{array}$ 


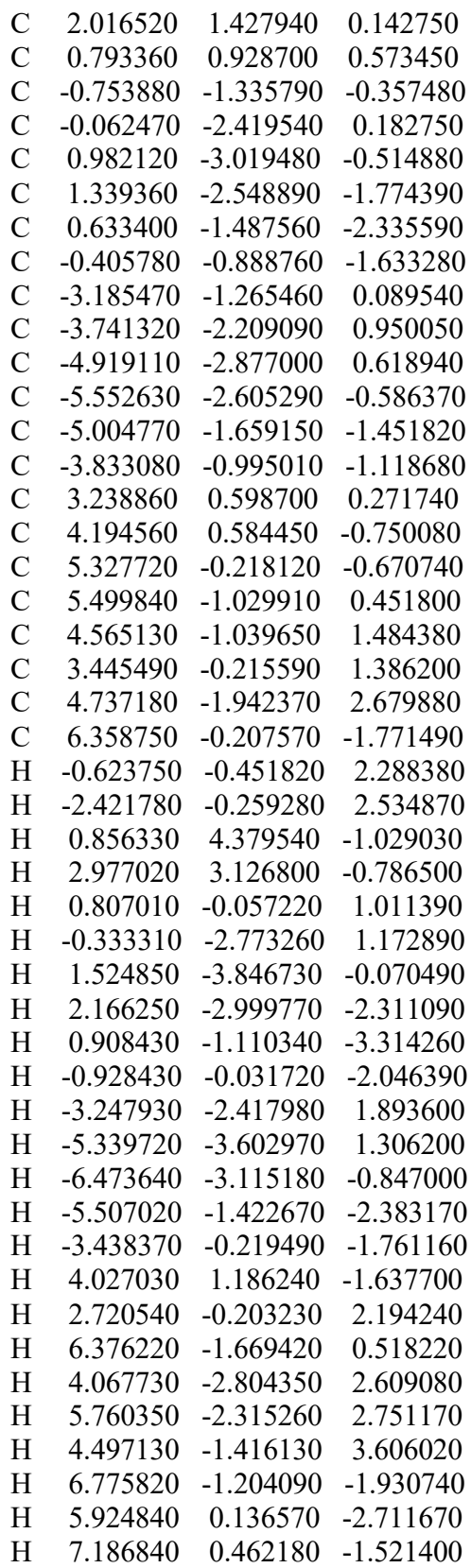

ligN2
$\begin{array}{lrrr}\mathrm{O} & -1.889160 & -3.192810 & 0.078750 \\ \mathrm{O} & -1.207840 & -2.224140 & 2.000410 \\ \mathrm{Cl} & -1.586870 & 0.994560 & 2.373950 \\ \mathrm{C} & -1.012080 & -0.968740 & -0.014880 \\ \mathrm{C} & -2.125390 & -0.205310 & -0.747080 \\ \mathrm{C} & -1.116230 & -1.039770 & -1.503950 \\ \mathrm{C} & -1.416070 & -2.288290 & 0.779500 \\ \mathrm{C} & 0.117760 & -0.232940 & 0.617560 \\ \mathrm{C} & -0.021360 & 0.642100 & 1.698370 \\ \mathrm{C} & 1.075740 & 1.283850 & 2.260390 \\ \mathrm{C} & 2.349800 & 1.051530 & 1.763660 \\ \mathrm{C} & 2.536130 & 0.164140 & 0.701270 \\ \mathrm{C} & 1.414170 & -0.456170 & 0.151890 \\ \mathrm{C} & -2.037880 & 1.296530 & -0.865230\end{array}$ $\begin{array}{llll}\text { C } & -0.859150 & 1.953240 & -1.244910\end{array}$

$\begin{array}{llll}\text { C } & -0.792190 & 3.340220 & -1.314810\end{array}$

$\begin{array}{llll}\text { C } & -1.904960 & 4.118200 & -1.014920\end{array}$

$\begin{array}{llll}\text { C } & -3.085750 & 3.483980 & -0.645540\end{array}$

$\begin{array}{llll}\text { C } & -3.149950 & 2.097380 & -0.572290\end{array}$

C $\quad-3.534870 \quad-0.726890 \quad-0.659550$

$\begin{array}{llll}\text { C } & -4.120610 & -1.058730 & 0.560900\end{array}$

$\begin{array}{llll}\text { C } & -5.451270 & -1.461510 & 0.613320\end{array}$

C $\quad-6.212820 \quad-1.534340 \quad-0.549190$

C $\quad-5.631520 \quad-1.210870 \quad-1.772150$

$\begin{array}{llll}\text { C } & -4.300630 & -0.810980 & -1.821810\end{array}$

$\begin{array}{llll}\text { C } & 3.892030 & -0.120230 & 0.172690\end{array}$

$\begin{array}{llll}\text { C } & 4.840990 & 0.902270 & 0.041990\end{array}$

$\begin{array}{llll}\text { C } & 6.114410 & 0.646610 & -0.451810\end{array}$

$\begin{array}{llll}\text { C } & 6.441750 & -0.659680 & -0.826210\end{array}$

$\begin{array}{llll}\text { C } & 5.520940 & -1.696120 & -0.708360\end{array}$

C $\quad 4.249600 \quad-1.413430 \quad-0.206240$

$\begin{array}{llll}\text { C } & 5.880590 & -3.108570 & -1.095010\end{array}$

C $\quad 7.132540 \quad 1.751130 \quad-0.588010$

H $\quad-0.399150 \quad-0.515960 \quad-2.125650$

$\mathrm{H} \quad-1.473380 \quad-1.996950 \quad-1.865350$

H $\quad 0.921460 \quad 1.955440 \quad 3.095560$

H $\quad 3.203940 \quad 1.533620 \quad 2.226060$

H $\quad 1.533990 \quad-1.131750 \quad-0.689190$

$\mathrm{H} \quad 0.033390 \quad 1.385360 \quad-1.472750$

$\mathrm{H} \quad 0.141170 \quad 3.811560 \quad-1.602640$

$\mathrm{H} \quad-1.851540 \quad 5.199760 \quad-1.066700$

$\mathrm{H} \quad-3.966190 \quad 4.069740 \quad-0.404810$

$\mathrm{H} \quad-4.077740 \quad 1.627550 \quad-0.270510$

$\mathrm{H} \quad-3.518440 \quad-1.029060 \quad 1.463060$

$\mathrm{H} \quad-5.889500 \quad-1.733090 \quad 1.567100$

$\mathrm{H} \quad-7.248320 \quad-1.853630 \quad-0.504760$

$\mathrm{H} \quad-6.212310 \quad-1.275970 \quad-2.685920$

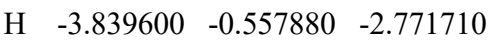

$\mathrm{H} \quad 4.563080 \quad 1.917300 \quad 0.307860$

H $\quad 3.529880 \quad-2.217500 \quad-0.086810$

H $\quad 7.433860 \quad-0.867920 \quad-1.218650$

$\mathrm{H} \quad 5.160420 \quad-3.511960 \quad-1.810430$

H $\quad 6.873670 \quad-3.154490 \quad-1.544840$

H $\quad 5.872270 \quad-3.763240 \quad-0.219860$

$\mathrm{H} \quad 6.682860 \quad 2.725880 \quad-0.394030$

H $\quad 7.954590 \quad 1.612100 \quad 0.119640$

H $\quad 7.562050 \quad 1.767450 \quad-1.592590$

\section{lig01}

$\begin{array}{llll}\text { O } & -1.225140 & -2.706950 & 1.345160\end{array}$

$\begin{array}{llll}\mathrm{O} & -1.991730 & -3.137030 & -0.733730\end{array}$

$\begin{array}{llll}\mathrm{Cl} & -1.683740 & 0.301070 & 2.525400\end{array}$

$\begin{array}{llll}\text { C } & -1.085360 & -0.970300 & -0.281330\end{array}$

C $\quad-2.196380 \quad-0.047160 \quad-0.804660$

C $\quad-1.189510 \quad-0.673200 \quad-1.742730$

$\begin{array}{llll}\text { C } & -1.480820 & -2.448270 & 0.159310\end{array}$

$\begin{array}{llll}\text { C } & 0.037100 & -0.403810 & 0.516020\end{array}$

$\begin{array}{llll}\text { C } & -0.112270 & 0.158160 & 1.786550\end{array}$

$\begin{array}{llll}\text { C } & 0.974370 & 0.646860 & 2.500810\end{array}$

$\begin{array}{llll}\text { C } & 2.252030 & 0.571210 & 1.961860\end{array}$

$\begin{array}{llll}\text { C } & 2.445060 & -0.000700 & 0.705000\end{array}$

C $\quad \begin{array}{llll}\text { C } & 1.336590 & -0.475850 & 0.009260\end{array}$

$\begin{array}{llll}\text { C } & -2.098380 & 1.436470 & -0.545070\end{array}$

$\begin{array}{llll}\text { C } & -0.920410 & 2.162880 & -0.768040\end{array}$

C $\quad-0.841090 \quad 3.522350 \quad-0.488200$

$\begin{array}{llll}\text { C } & -1.942480 & 4.205040 & 0.016240\end{array}$ $\begin{array}{lrrr}\text { C } & -3.122910 & 3.503730 & 0.234170\end{array}$

$\begin{array}{llll}C & -3.198470 & 2.143310 & -0.041690\end{array}$

$\begin{array}{llll}\text { C } & -3.609180 & -0.564860 & -0.847930\end{array}$

$\begin{array}{llll}\text { C } & -4.378810 & -0.340510 & -1.988830\end{array}$

C $\quad-5.712320 \quad-0.731760 \quad-2.038310$

C $\quad-6.292470 \quad-1.357200 \quad-0.938160$

$\begin{array}{llll}\text { C } & -5.527220 & -1.592310 & 0.200220\end{array}$

$\begin{array}{llll}\text { C } & -4.193930 & -1.198080 & 0.247320\end{array}$

$\begin{array}{llll}\text { C } & 3.810820 & -0.108440 & 0.114480\end{array}$

$\begin{array}{llll}\text { C } & 4.501330 & -1.328950 & 0.174010\end{array}$

$\begin{array}{llll}\text { C } & 5.775030 & -1.413610 & -0.386180\end{array}$

$\begin{array}{llll}\text { C } & 6.377410 & -0.320190 & -1.004680\end{array}$

$\begin{array}{llll}\text { C } & 5.671880 & 0.878410 & -1.061250\end{array}$

$\begin{array}{llll}\text { C } & 4.396180 & 1.001360 & -0.510190\end{array}$

$\begin{array}{llll}\text { C } & 3.871510 & -2.529790 & 0.833330\end{array}$

$\begin{array}{llll}\text { C } & 3.646930 & 2.307350 & -0.604980\end{array}$

C $\quad 7.768560 \quad-0.429790 \quad-1.576740$

$\mathrm{H} \quad-0.470570 \quad-0.016830 \quad-2.219310$

$\begin{array}{llll}\mathrm{H} & -1.550190 & -1.511970 & -2.326340\end{array}$

H $\quad 0.809590 \quad 1.081980 \quad 3.478580$

H $\quad 3.102210 \quad 0.948990 \quad 2.520480$

H $\quad 1.475470 \quad-0.925140 \quad-0.970150$

H $\quad-0.037460 \quad 1.664790 \quad-1.146330$

$\mathrm{H} \quad 0.092240 \quad 4.046510 \quad-0.663860$

H $\quad-1.880370 \quad 5.264500 \quad 0.237390$

$\mathrm{H} \quad-3.994270 \quad 4.014290 \quad 0.629460$

$\mathrm{H} \quad-4.125390 \quad 1.616580 \quad 0.148490$

$\mathrm{H} \quad-3.918880 \quad 0.147100 \quad-2.843030$

$\mathrm{H} \quad-6.296270-0.554420 \quad-2.935000$

$\mathrm{H} \quad-7.330110 \quad-1.670620 \quad-0.973370$

H $\quad-5.964640 \quad-2.098660 \quad 1.053300$

$\mathrm{H} \quad-3.589590 \quad-1.405590 \quad 1.124140$

H $\quad 6.308440 \quad-2.359360 \quad-0.340460$

H $\quad 6.120250 \quad 1.737890 \quad-1.552300$

$\mathrm{H} \quad 3.542380 \quad-2.290820 \quad 1.847160$

H $\quad 2.980030 \quad-2.857330 \quad 0.293140$

$\mathrm{H} \quad 4.576090 \quad-3.361430 \quad 0.877280$

$\mathrm{H} \quad 4.210860 \quad 3.036690 \quad-1.188480$

H $\quad 2.670570 \quad 2.160660 \quad-1.073890$

H $\quad 3.455160 \quad 2.726550 \quad 0.385660$

$\mathrm{H} \quad 8.522640 \quad-0.294350 \quad-0.795720$

H $\quad 7.932600 \quad-1.410920 \quad-2.026990$

H $\quad 7.940970 \quad 0.330400 \quad-2.340810$

\section{ligO_TSrot}

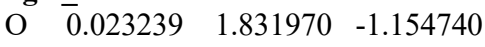

$\begin{array}{llll}\text { O } & -0.291982 & 2.558240 & 0.954420\end{array}$

Cl $\quad-1.396411 \quad-2.986800 \quad-0.487680$

$\begin{array}{llll}\text { C } & -0.850521 & 0.283330 & 0.424010\end{array}$

$\begin{array}{llll}\text { C } & -2.384471 & 0.279650 & 0.553960\end{array}$

$\begin{array}{llll}\text { C } & -1.516101 & 0.175890 & 1.773200\end{array}$

$\begin{array}{llll}\text { C } & -0.317881 & 1.724690 & 0.040700\end{array}$

$\begin{array}{llll}\text { C } & 0.184629 & -0.757230 & 0.046580\end{array}$

$\begin{array}{llll}\text { C } & 0.105449 & -2.096320 & -0.347800\end{array}$

$\begin{array}{llll}\text { C } & 1.238449 & -2.856879 & -0.654750\end{array}$

$\begin{array}{llll}\text { C } & 2.504189 & -2.312709 & -0.569410\end{array}$

$\begin{array}{llll}\text { C } & 2.644659 & -0.993139 & -0.135990\end{array}$

$\begin{array}{llll}\text { C } & 1.504609 & -0.271189 & 0.175160\end{array}$

$\begin{array}{llll}\text { C } & -3.354721 & -0.815840 & 0.197880\end{array}$

C $\quad-3.919291 \quad-1.630140 \quad 1.175840$

$\begin{array}{llll}\text { C } & -4.853031 & -2.607660 & 0.839100\end{array}$

$\begin{array}{llll}\text { C } & -5.249031 & -2.769520 & -0.482880\end{array}$ 


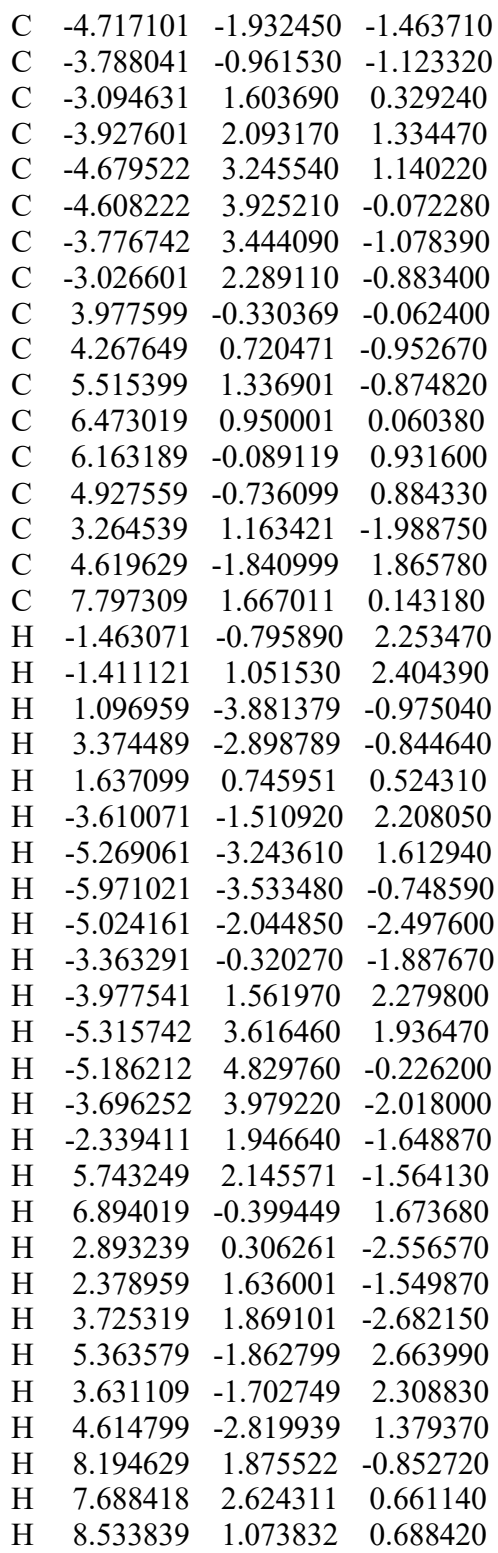

$\begin{array}{llll}\text { C } & 0.099560 & -1.997740 & -2.378860\end{array}$

C $\quad-0.763660-1.204050 \quad-1.633080$

C $\quad-3.311150 \quad-1.173540 \quad 0.394990$

C $\quad-3.808320 \quad-1.975430 \quad 1.420230$

C $\quad-5.039700 \quad-2.616160 \quad 1.298750$

C $\quad-5.788560 \quad-2.460370 \quad 0.139610$

C $\quad-5.300080 \quad-1.657230 \quad-0.889850$

$\begin{array}{llll}\text { C } & -4.073410 & -1.019810 & -0.765850\end{array}$

$\begin{array}{llll}\text { C } & 3.270180 & 0.486000 & 0.111870\end{array}$

$\begin{array}{llll}\text { C } & 3.803200 & 0.419130 & 1.408730\end{array}$

$\begin{array}{llll}\text { C } & 4.938980 & -0.356360 & 1.638260\end{array}$

$\begin{array}{llll}\text { C } & 5.553240 & -1.074690 & 0.615640\end{array}$

$\begin{array}{llll}\text { C } & 5.010990 & -0.994200 & -0.663710\end{array}$

C $\quad 3.879700 \quad-0.224390 \quad-0.933040$

$\begin{array}{llll}\text { C } & 3.164960 & 1.179720 & 2.544760\end{array}$

$\begin{array}{llll}\text { C } & 3.315360 & -0.170440 & -2.330470\end{array}$

C $\quad 6.752920 \quad-1.944610 \quad 0.895340$

$\mathrm{H} \quad-0.530840 \quad-0.226920 \quad 2.239800$

$\begin{array}{llll}\mathrm{H} & -2.292930 & 0.093840 & 2.593310\end{array}$

$\mathrm{H} \quad 0.947250 \quad 4.156050 \quad-1.577760$

$\mathrm{H} \quad 3.047990 \quad 2.882140 \quad-1.216260$

$\mathrm{H} \quad 0.816920 \quad-0.092570 \quad 0.835220$

$\mathrm{H} \quad-0.407350 \quad-2.687490 \quad 1.385090$

H $\quad 1.161530 \quad-4.086990 \quad 0.070510$

H $\quad 1.489830 \quad-3.642470 \quad-2.347140$

$\mathrm{H} \quad 0.241050 \quad-1.794100 \quad-3.434880$

$\mathrm{H} \quad-1.284650 \quad-0.369570 \quad-2.092120$

$\mathrm{H} \quad-3.224560 \quad-2.093790 \quad 2.327140$

$\mathrm{H} \quad-5.411560 \quad-3.230130 \quad 2.111750$

$\mathrm{H} \quad-6.751570 \quad-2.949310 \quad 0.040950$

$\mathrm{H} \quad-5.889620 \quad-1.510510 \quad-1.787910$

$\mathrm{H} \quad-3.720750 \quad-0.350590 \quad-1.538760$

H $\quad 5.353350 \quad-0.399480 \quad 2.642050$

$\mathrm{H} \quad 5.474450 \quad-1.550300 \quad-1.474310$

$\mathrm{H} \quad 2.959000 \quad 2.213260 \quad 2.258830$

H $\quad 2.205190 \quad 0.739130 \quad 2.826450$

$\mathrm{H} \quad 3.813510 \quad 1.176910 \quad 3.422220$

$\mathrm{H} \quad 3.744140 \quad-0.960950 \quad-2.949120$

$\mathrm{H} \quad 2.230390 \quad-0.288000 \quad-2.312480$

$\mathrm{H} \quad 3.528770 \quad 0.791100 \quad-2.805580$

$\mathrm{H} \quad 7.380670 \quad-2.047490 \quad 0.008170$

$\mathrm{H} \quad 7.362340 \quad-1.528790 \quad 1.700230$

H $\quad 6.441860 \quad-2.948210 \quad 1.200210$

\section{ligO2}

$\begin{array}{llll}\mathrm{O} & -0.889860 & 0.076930 & -2.761830\end{array}$

$\begin{array}{llll}\mathrm{O} & -3.096810 & 0.534450 & -2.666980\end{array}$

Cl $\quad-1.409570 \quad 4.107440 \quad-0.391490$

$\begin{array}{llll}\text { C } & -1.769470 & 0.953670 & -0.722890\end{array}$

$\begin{array}{llll}\text { C } & -2.356160 & 0.001840 & 0.344280\end{array}$

$\begin{array}{llll}\text { C } & -3.000830 & 1.329440 & 0.026000\end{array}$

$\begin{array}{llll}\text { C } & -1.943040 & 0.481400 & -2.231510\end{array}$

C $\quad-0.431250 \quad 1.541460 \quad-0.450210$

C $\quad-0.141560 \quad 2.894660 \quad-0.305490$

C $\quad 1.158120 \quad 3.356310 \quad-0.104110$

$\begin{array}{llll}\text { C } & 2.211280 & 2.455570 & -0.076630\end{array}$

$\begin{array}{llll}\text { C } & 1.965340 & 1.089920 & -0.246500\end{array}$

C $\quad 0.657750 \quad 0.663360 \quad-0.412650$

$\begin{array}{llll}\text { C } & -1.593870 & -0.145290 & 1.636170\end{array}$

$\begin{array}{llll}\text { C } & -1.206430 & 0.959970 & 2.407980\end{array}$

C $\quad-0.462260 \quad 0.806030 \quad 3.570970$

C $\quad-0.076530 \quad-0.460130 \quad 4.002010$
C $\quad-0.447780 \quad-1.566720 \quad 3.248180$

$\begin{array}{llll}\text { C } & -1.197480 & -1.410850 & 2.085630\end{array}$

$\begin{array}{llll}\text { C } & -3.074720 & -1.249860 & -0.083450\end{array}$

$\begin{array}{llll}\text { C } & -2.516740 & -2.153990 & -0.986560\end{array}$

C $\quad-3.177430 \quad-3.339530 \quad-1.293450$

C $\quad-4.399340 \quad-3.639550 \quad-0.699620$

$\begin{array}{llll}\text { C } & -4.964690 & -2.739710 & 0.199800\end{array}$

$\begin{array}{llll}\text { C } & -4.303760 & -1.554460 & 0.502030\end{array}$

$\begin{array}{llll}\text { C } & 3.069830 & 0.092460 & -0.313010\end{array}$

$\begin{array}{llll}\text { C } & 3.745260 & -0.304550 & 0.847700\end{array}$

$\begin{array}{llll}\text { C } & 4.759310 & -1.258240 & 0.749600\end{array}$

$\begin{array}{llll}\text { C } & 5.108070 & -1.825520 & -0.472550\end{array}$

C $\quad 4.410450 \quad-1.428450-1.611820$

$\begin{array}{llll}\text { C } & 3.390530 & -0.479530 & -1.556090\end{array}$

$\begin{array}{llll}\text { C } & 3.347950 & 0.255880 & 2.190650\end{array}$

$\begin{array}{llll}\text { C } & 2.648940 & -0.070520 & -2.804920\end{array}$

$\begin{array}{llll}\text { C } & 6.224100 & -2.835700 & -0.569230\end{array}$

$\mathrm{H} \quad-2.974170 \quad 2.107770 \quad 0.776190$

$\begin{array}{llll}\mathrm{H} & -3.896290 & 1.280100 & -0.583220\end{array}$

$\begin{array}{llll}\mathrm{H} & 1.330360 & 4.419520 & 0.008030\end{array}$

$\mathrm{H} \quad 3.227950 \quad 2.811420 \quad 0.054060$

H $\quad 0.451140 \quad-0.392450 \quad-0.547040$

$\mathrm{H} \quad-1.467280 \quad 1.962850 \quad 2.093500$

$\mathrm{H} \quad-0.175320 \quad 1.685240 \quad 4.137350$

H $\quad 0.510460 \quad-0.579110 \quad 4.905720$

$\mathrm{H} \quad-0.152980 \quad-2.562430 \quad 3.560700$

$\mathrm{H} \quad-1.477630 \quad-2.288560 \quad 1.515990$

H $\quad-1.579770 \quad-1.907810 \quad-1.475860$

$\mathrm{H} \quad-2.739100 \quad-4.024510 \quad-2.010450$

$\mathrm{H} \quad-4.913960 \quad-4.562120 \quad-0.944620$

$\mathrm{H} \quad-5.921840 \quad-2.958690 \quad 0.660530$

$\mathrm{H} \quad-4.739980 \quad-0.846620 \quad 1.200080$

H $\quad 5.275960 \quad-1.574680 \quad 1.651840$

H $\quad 4.661170 \quad-1.874010 \quad-2.570880$

$\mathrm{H} \quad 3.554960 \quad 1.327250 \quad 2.253630$

H $\quad 2.274470 \quad 0.131260 \quad 2.358470$

H $\quad 3.888890 \quad-0.247100 \quad 2.994260$

$\mathrm{H} \quad 1.577520 \quad-0.290370 \quad-2.748650$

H $\quad 2.723350 \quad 1.009880 \quad-2.957240$

H $\quad 3.066280 \quad-0.573920 \quad-3.678530$

$\mathrm{H} \quad 7.154620 \quad-2.359800 \quad-0.893120$

H $\quad 6.411060 \quad-3.309320 \quad 0.396390$

H $\quad 5.984640 \quad-3.617520 \quad-1.293120$

\section{ligP1}

$\begin{array}{llll}\text { O } & 1.212920 & -3.056000 & -0.653450\end{array}$

$\begin{array}{llll}\mathrm{O} & 0.095090 & -2.727910 & 1.279030\end{array}$

C $\quad 0.411850 \quad-2.487770 \quad 0.100280$

$\begin{array}{llll}\text { C } & -0.298610 & -1.210160 & -0.507410\end{array}$

$\begin{array}{llll}\text { C } & 0.162510 & -0.735480 & -1.843050\end{array}$

$\begin{array}{llll}\text { C } & 0.576270 & 0.053570 & -0.619000\end{array}$

$\begin{array}{llll}\text { C } & -0.063310 & 1.388460 & -0.339410\end{array}$

$\begin{array}{llll}\text { C } & -1.219550 & 1.824750 & -1.000450\end{array}$

$\begin{array}{llll}\text { C } & -1.818360 & 3.039120 & -0.688590\end{array}$

$\begin{array}{llll}\text { C } & -1.279440 & 3.862160 & 0.295040\end{array}$

$\begin{array}{llll}\text { C } & -0.131060 & 3.448150 & 0.959490\end{array}$

C $\quad 0.467350 \quad 2.232060 \quad 0.645220$

$\begin{array}{llll}\text { C } & 4.739150 & 0.069090 & 0.484160\end{array}$

$\begin{array}{llll}\text { C } & 3.801710 & -0.500280 & 1.340040\end{array}$

$\begin{array}{llll}\text { C } & 2.454940 & -0.537200 & 0.989910\end{array}$

$\begin{array}{llll}\text { C } & 2.030480 & -0.008030 & -0.228470\end{array}$

$\begin{array}{llll}\text { C } & 2.977960 & 0.555220 & -1.084360\end{array}$ 
$\begin{array}{rrrr}\mathrm{C} & 4.322930 & 0.596740 & -0.735300 \\ \mathrm{C} & -1.728670 & -1.053460 & -0.114270 \\ \mathrm{C} & -2.069320 & -0.677580 & 1.188530 \\ \mathrm{C} & -4.418090 & -0.756910 & 0.634450 \\ \mathrm{C} & -2.755510 & -1.300630 & -1.024350 \\ \mathrm{C} & -4.091750 & -1.153600 & -0.657360 \\ \mathrm{C} & -3.398710 & -0.524350 & 1.557180 \\ \mathrm{H} & 0.937640 & -1.351990 & -2.283490 \\ \mathrm{H} & -0.542020 & -0.282270 & -2.530860 \\ \mathrm{H} & -1.681490 & 1.201750 & -1.754490 \\ \mathrm{H} & -2.718530 & 3.337130 & -1.214870 \\ \mathrm{H} & -1.749290 & 4.807760 & 0.540790 \\ \mathrm{H} & 0.306950 & 4.072420 & 1.730790 \\ \mathrm{H} & 1.363070 & 1.931380 & 1.174940 \\ \mathrm{H} & 5.788090 & 0.091380 & 0.758900 \\ \mathrm{H} & 4.119150 & -0.931930 & 2.282710 \\ \mathrm{H} & 1.729740 & -1.009260 & 1.645130 \\ \mathrm{H} & 2.646090 & 0.962450 & -2.034550 \\ \mathrm{H} & 5.045910 & 1.034740 & -1.415050 \\ \mathrm{H} & -1.270470 & -0.516300 & 1.902210 \\ \mathrm{H} & -5.456200 & -0.634220 & 0.923460 \\ \mathrm{H} & -2.499480 & -1.612620 & -2.032020 \\ \mathrm{H} & -3.644320 & -0.222810 & 2.569640 \\ \mathrm{H} & -4.875630 & -1.349470 & -1.381400\end{array}$

\begin{tabular}{lrrr}
\multicolumn{4}{l}{ ligP_TSrot } \\
O & -0.515761 & -3.098739 & 0.728420 \\
O & 0.436369 & -2.652780 & -1.266240 \\
C & 0.118310 & -2.411290 & -0.086640 \\
C & 0.584350 & -0.994100 & 0.420170 \\
C & 0.069630 & -0.564720 & 1.771120 \\
C & -0.536339 & 0.039811 & 0.536160 \\
C & -0.337519 & 1.500191 & 0.226410 \\
C & -0.074488 & 2.444521 & 1.216960 \\
C & 0.101652 & 3.789370 & 0.895590 \\
C & 0.006853 & 4.212290 & -0.424490 \\
C & -0.280808 & 3.279751 & -1.421010 \\
C & -0.458478 & 1.942611 & -1.096270 \\
C & -4.614660 & -0.911497 & -0.486290 \\
C & -3.589620 & -1.237948 & -1.368120 \\
C & -2.263650 & -0.966558 & -1.044010 \\
C & -1.948299 & -0.366789 & 0.174990 \\
C & -2.981019 & -0.047478 & 1.057220 \\
C & -4.305589 & -0.316348 & 0.733900 \\
C & 2.023930 & -0.697310 & 0.104010 \\
C & 2.915290 & -1.771511 & 0.263870 \\
C & 4.806710 & -0.385562 & -0.300980 \\
C & 2.567741 & 0.532379 & -0.275000 \\
C & 3.941281 & 0.684089 & -0.474710 \\
C & 4.279530 & -1.621611 & 0.071280 \\
H & -0.511380 & -1.320509 & 2.287260 \\
H & 0.728481 & 0.053890 & 2.370190 \\
H & 0.005642 & 2.122530 & 2.248560 \\
H & 0.314953 & 4.505760 & 1.681500 \\
H & 0.150073 & 5.256840 & -0.676950 \\
H & -0.360638 & 3.597281 & -2.454770 \\
H & -0.669769 & 1.214331 & -1.871940 \\
H & -5.645820 & -1.130787 & -0.740890 \\
H & -3.818560 & -1.723548 & -2.310230 \\
H & -1.461830 & -1.266729 & -1.711070 \\
H & -2.732799 & 0.412272 & 2.009130 \\
H & -5.095309 & -0.067567 & 1.434660
\end{tabular}

$\begin{array}{rrrr}\mathrm{H} & 2.512299 & -2.741681 & 0.532000 \\ \mathrm{H} & 5.872891 & -0.264972 & -0.458800 \\ \mathrm{H} & 1.936701 & 1.394060 & -0.429220 \\ \mathrm{H} & 4.934860 & -2.476102 & 0.201880 \\ \mathrm{H} & 4.326491 & 1.653339 & -0.773720\end{array}$

\section{ligQ1}

O $\quad 0.581180 \quad-2.705200 \quad 1.190320$

$\begin{array}{llll}\mathrm{O} & 1.874980 & -3.083090 & -0.621620\end{array}$

$\begin{array}{llll}\mathrm{O} & -5.898310 & -0.404980 & -0.439840\end{array}$

$\begin{array}{llll}\mathrm{O} & -5.414970 & 0.078900 & 1.594200\end{array}$

$\begin{array}{llll}\mathrm{N} & -5.101290 & -0.262550 & 0.469770\end{array}$

C $\quad 1.030350 \quad-2.485750 \quad 0.052640$

$\begin{array}{llll}\text { C } & 0.440510 & -1.172910 & -0.623580\end{array}$

$\begin{array}{llll}\text { C } & 1.034620 & -0.724970 & -1.914790\end{array}$

$\begin{array}{llll}\text { C } & 1.372580 & 0.051180 & -0.660510\end{array}$

$\begin{array}{llll}\text { C } & 0.752250 & 1.404770 & -0.424030\end{array}$

$\begin{array}{llll}\mathrm{C} & -0.283050 & 1.912370 & -1.218930\end{array}$

C $\quad-0.881940 \quad 3.134120 \quad-0.934870$

$\begin{array}{llll}\text { C } & -0.461070 & 3.890610 & 0.153640\end{array}$

C $\quad 0.570420 \quad 3.406130 \quad 0.949520$

$\begin{array}{llll}\text { C } & 1.168690 & 2.183610 & 0.662820\end{array}$

$\begin{array}{llll}\text { C } & 5.416410 & -0.084380 & 0.810430\end{array}$

$\begin{array}{llll}\text { C } & 4.388420 & -0.628410 & 1.573430\end{array}$

$\begin{array}{llll}\text { C } & 3.078090 & -0.616830 & 1.104030\end{array}$

$\begin{array}{llll}\text { C } & 2.782480 & -0.063070 & -0.140980\end{array}$

$\begin{array}{llll}\text { C } & 3.819860 & 0.475650 & -0.903000\end{array}$

$\begin{array}{llll}\text { C } & 5.128730 & 0.467530 & -0.435020\end{array}$

C $\quad-1.000320 \quad-0.948010 \quad-0.344020$

C $\quad-1.417290 \quad-0.569790 \quad 0.939420$

$\begin{array}{llll}\text { C } & -3.684120 & -0.514810 & 0.193130\end{array}$

C $\quad-1.965080-1.131530 \quad-1.337750$

C $\quad-2.753670 \quad-0.343910 \quad 1.214600$

$\begin{array}{llll}\text { C } & -3.311840 & -0.916190 & -1.082140\end{array}$

H $\quad 1.818060 \quad-1.377260 \quad-2.282790$

$\mathrm{H} \quad 0.413910 \quad-0.250270 \quad-2.665550$

$\mathrm{H} \quad-0.649100 \quad 1.343880 \quad-2.063720$

H $\quad-1.687350 \quad 3.491100 \quad-1.567050$

$\mathrm{H} \quad-0.931930 \quad 4.840650 \quad 0.378250$

$\mathrm{H} \quad 0.914800 \quad 3.979800 \quad 1.802910$

$\mathrm{H} \quad 1.972300 \quad 1.825500 \quad 1.294880$

$\mathrm{H} \quad 6.436550 \quad-0.099780 \quad 1.177830$

$\mathrm{H} \quad 4.605540 \quad-1.077330 \quad 2.536090$

H $\quad 2.282840 \quad-1.067950 \quad 1.688900$

$\mathrm{H} \quad 3.588520 \quad 0.902960 \quad-1.873890$

H $\quad 5.923760 \quad 0.886200 \quad-1.042370$

$\mathrm{H} \quad-0.668310 \quad-0.461810 \quad 1.713080$

$\mathrm{H} \quad-1.648950 \quad-1.447590 \quad-2.325550$

H $\quad-3.087870 \quad-0.038180 \quad 2.196360$

H $\quad-4.066890 \quad-1.049620 \quad-1.844620$

\section{ligQ_TSrot}

$\begin{array}{llll}\mathrm{O} & -0.207110 & -2.644670 & -1.212779\end{array}$

$\begin{array}{llll}\text { O } & -1.290111 & -3.094780 & 0.714891\end{array}$

$\begin{array}{llll}\mathrm{O} & 6.166830 & -1.054253 & -0.009869\end{array}$

$\begin{array}{llll}\text { O } & 5.841941 & 1.005747 & -0.517029\end{array}$

N $\quad 5.437821 \quad-0.101102 \quad-0.210719$

C $\quad-0.613950 \quad-2.406010 \quad-0.060759$

C $\quad-0.202360 \quad-0.976580 \quad 0.466261$

$\begin{array}{llll}\text { C } & -0.772729 & -0.563610 & 1.802411\end{array}$

$\begin{array}{llll}\text { C } & -1.343769 & 0.036690 & 0.550741\end{array}$ $\begin{array}{llll}\text { C } & -1.154299 & 1.501990 & 0.256681\end{array}$

$\begin{array}{llll}\text { C } & -0.933728 & 2.442280 & 1.261111\end{array}$

C $\quad-0.756098 \quad 3.789950 \quad 0.952401$

$\begin{array}{llll}\text { C } & -0.807037 & 4.218330 & -0.368199\end{array}$

$\begin{array}{llll}\text { C } & -1.054178 & 3.289600 & -1.378919\end{array}$

C $\quad-1.233958 \quad 1.949550 \quad-1.067229$

$\begin{array}{llll}\text { C } & -5.354310 & -0.988998 & -0.643849\end{array}$

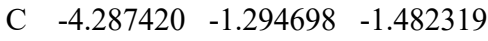

$\begin{array}{llll}\text { C } & -2.982010 & -0.998669 & -1.101999\end{array}$

$\begin{array}{llll}\text { C } & -2.730929 & -0.395249 & 0.129551\end{array}$

$\begin{array}{llll}\text { C } & -3.804809 & -0.095838 & 0.968041\end{array}$

$\begin{array}{llll}\text { C } & -5.108899 & -0.389618 & 0.588501\end{array}$

$\begin{array}{llll}\text { C } & 1.237500 & -0.655651 & 0.210881\end{array}$

$\begin{array}{llll}\text { C } & 1.772351 & 0.590639 & -0.137899\end{array}$

$\begin{array}{llll}\text { C } & 3.992891 & -0.296302 & -0.071479\end{array}$

$\begin{array}{llll}\text { C } & 2.139750 & -1.721511 & 0.396181\end{array}$

$\begin{array}{llll}\text { C } & 3.142761 & 0.776079 & -0.280789\end{array}$

$\begin{array}{llll}\text { C } & 3.504220 & -1.555202 & 0.265711\end{array}$

$\mathrm{H} \quad-1.352180 \quad-1.340240 \quad 2.288651$

$\begin{array}{llll}\mathrm{H} & -0.150189 & 0.062930 & 2.431041\end{array}$

$\begin{array}{llll}\mathrm{H} & -0.888578 & 2.116990 & 2.293771\end{array}$

$\begin{array}{llll}\mathrm{H} & -0.575937 & 4.503830 & 1.748531\end{array}$

$\begin{array}{lllll}\mathrm{H} & -0.661507 & 5.264780 & -0.610439\end{array}$

$\mathrm{H} \quad-1.100878 \quad 3.612160 \quad-2.412969$

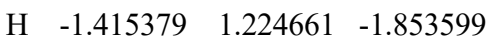

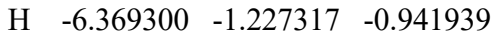

$\mathrm{H} \quad-4.467130 \quad-1.782628 \quad-2.433679$

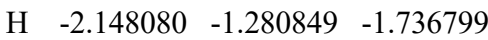

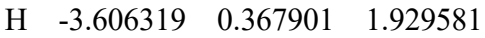

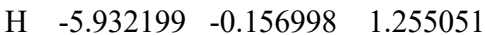

$\begin{array}{llll}\mathrm{H} & 1.131751 & 1.440619 & -0.310929\end{array}$

$\begin{array}{llll}\mathrm{H} & 1.742870 & -2.699981 & 0.637581\end{array}$

H $\quad 3.550972 \quad 1.739898 \quad-0.552889$

H $\quad 4.191000 \quad-2.377452 \quad 0.412221$

\section{ligR1}

$\begin{array}{llll}\text { O } & -0.838120 & -2.717600 & -1.138490\end{array}$

$\begin{array}{llll}\mathrm{O} & -2.157650 & -3.083580 & 0.656480\end{array}$

F $\quad 5.378790 \quad-1.073940 \quad-1.272720$

F $\quad 5.076100 \quad 1.015740 \quad-0.873470$

$\begin{array}{llll}\text { F } & 5.648860 & -0.313570 & 0.727420\end{array}$

C $\quad-1.304960 \quad-2.485610 \quad-0.009430$

$\begin{array}{llll}\text { C } & -0.734980 & -1.164750 & 0.657260\end{array}$

$\begin{array}{llll}\text { C } & -1.356480 & -0.701470 & 1.930390\end{array}$

$\begin{array}{llll}\text { C } & -1.675240 & 0.054610 & 0.659000\end{array}$

$\begin{array}{llll}\text { C } & -1.062530 & 1.410110 & 0.416530\end{array}$

$\begin{array}{llll}\text { C } & -1.476190 & 2.179320 & -0.678440\end{array}$

$\begin{array}{llll}\text { C } & -0.884830 & 3.404450 & -0.968590\end{array}$

$\begin{array}{llll}\text { C } & 0.137390 & 3.901570 & -0.168560\end{array}$

C $\quad 0.555860 \quad 3.154650 \quad 0.927520$

$\begin{array}{llll}\text { C } & -0.036570 & 1.930810 & 1.215450\end{array}$

C $\quad-5.691720 \quad-0.132190 \quad-0.881480$

$\begin{array}{llll}\text { C } & -4.645720 & -0.676580 & -1.619440\end{array}$

$\begin{array}{llll}\text { C } & -3.343990 & -0.648520 & -1.127230\end{array}$

$\begin{array}{llll}\text { C } & -3.074830 & -0.077610 & 0.116160\end{array}$

$\begin{array}{llll}\text { C } & -4.130300 & 0.461110 & 0.852990\end{array}$

$\begin{array}{llll}\text { C } & -5.430650 & 0.436690 & 0.362230\end{array}$

$\begin{array}{llll}\text { C } & 0.713600 & -0.933250 & 0.407020\end{array}$

$\begin{array}{llll}\text { C } & 1.158340 & -0.564650 & -0.869580\end{array}$

$\begin{array}{llll}\text { C } & 3.427220 & -0.477900 & -0.075290\end{array}$

$\begin{array}{llll}\text { C } & 1.655520 & -1.096000 & 1.420200\end{array}$

$\begin{array}{llll}\text { C } & 2.500170 & -0.331200 & -1.109690\end{array}$ 


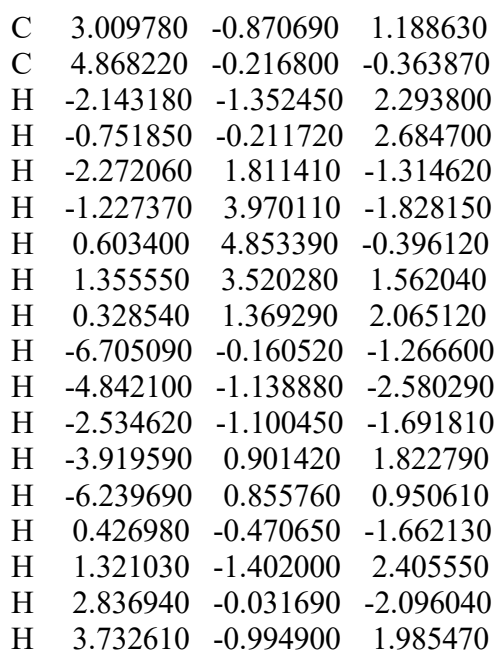

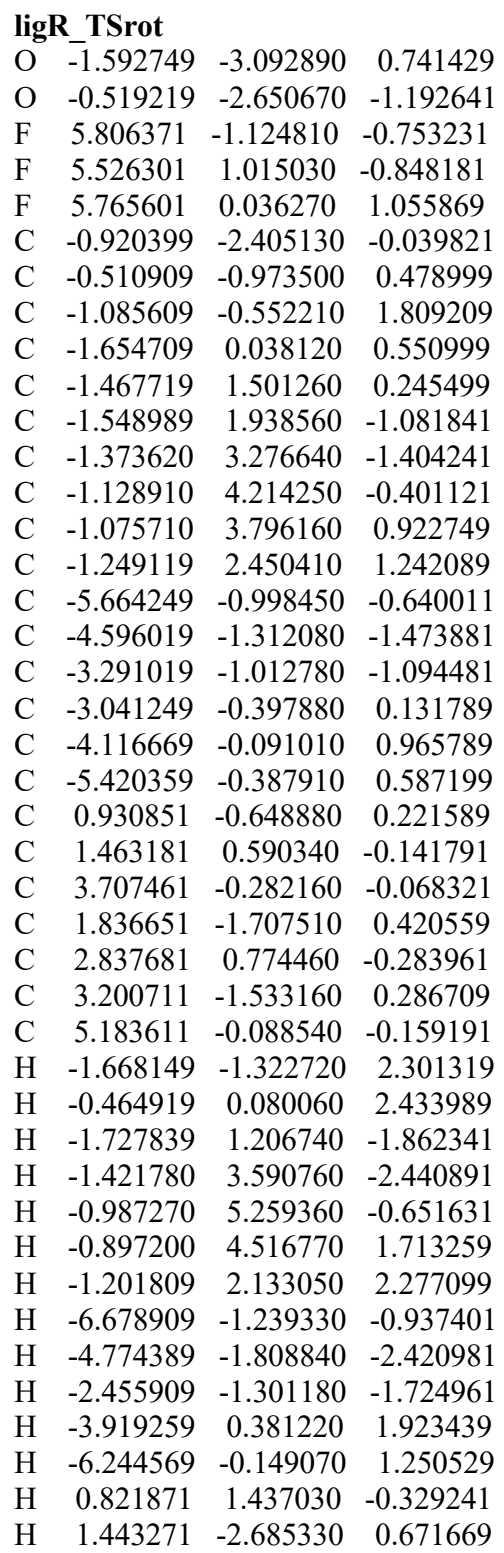

H $\quad 3.223251 \quad 1.744850 \quad-0.572711$ H $\quad 3.875611 \quad-2.367350 \quad 0.441349$

\section{ligS1}

$\begin{array}{llll}\text { O } & 2.199230 & -3.082200 & 0.653820\end{array}$

O $\quad 0.890890 \quad-2.709610 \quad-1.147450$

Br $\quad-5.229690 \quad-0.146810 \quad-0.407340$

C $\quad 1.351070 \quad-2.479290 \quad-0.014780$

$\begin{array}{llll}\text { C } & 0.781520 & -1.159330 & 0.649070\end{array}$

$\begin{array}{llll}\text { C } & 1.727070 & 0.057030 & 0.651750\end{array}$

$\begin{array}{llll}\text { C } & 1.403000 & -0.697090 & 1.922870\end{array}$

$\begin{array}{llll}\text { C } & -0.668210 & -0.923900 & 0.400120\end{array}$

C $\quad-1.610460 \quad-1.080960 \quad 1.413940$

C $\quad-2.967640 \quad-0.859290 \quad 1.187090$

$\begin{array}{llll}\text { C } & -3.372650 & -0.477110 & -0.080790\end{array}$

C $\quad-2.463160-0.329870 \quad-1.121170$

$\begin{array}{llll}\text { C } & -1.117440 & -0.561630 & -0.873420\end{array}$

$\begin{array}{llll}\mathrm{C} & 1.123250 & 1.416100 & 0.409480\end{array}$

$\begin{array}{llll}\mathrm{C} & 0.091660 & 1.937650 & 1.200880\end{array}$

C $\quad-0.489680 \quad 3.167400 \quad 0.915950$

$\begin{array}{llll}\text { C } & -0.054870 & 3.920010 & -0.169940\end{array}$

C $\quad 0.972330 \quad 3.421930 \quad-0.962940$

$\begin{array}{llll}\text { C } & 1.552830 & 2.190880 & -0.675600\end{array}$

$\begin{array}{llll}\text { C } & 3.127450 & -0.082370 & 0.112570\end{array}$

$\begin{array}{llll}\text { C } & 4.184490 & 0.447550 & 0.853610\end{array}$

$\begin{array}{llll}\text { C } & 5.486000 & 0.416700 & 0.366220\end{array}$

$\begin{array}{llll}C & 5.746920 & -0.150240 & -0.878410\end{array}$

$\begin{array}{llll}\text { C } & 4.699440 & -0.686190 & -1.620530\end{array}$

C $\quad 3.396590 \quad-0.651490 \quad-1.131730$

$\mathrm{H} \quad 2.186610 \quad-1.350530 \quad 2.288550$

H $\quad 0.798970 \quad-0.203610 \quad 2.675330$

$\mathrm{H} \quad-1.278320 \quad-1.383550 \quad 2.401570$

$\mathrm{H} \quad-3.692950 \quad-0.981270 \quad 1.981510$

$\mathrm{H} \quad-2.803810 \quad-0.039070 \quad-2.106570$

$\mathrm{H} \quad-0.390500 \quad-0.471340 \quad-1.671020$

H $\quad-0.286520 \quad 1.372000 \quad 2.041930$

$\mathrm{H} \quad-1.293780 \quad 3.533220 \quad 1.544820$

$\mathrm{H} \quad-0.511910 \quad 4.876830 \quad-0.394930$

$\mathrm{H} \quad 1.327830 \quad 3.991660 \quad-1.814580$

$\mathrm{H} \quad 2.353160 \quad 1.822960-1.306120$

H $\quad 3.973890 \quad 0.886120 \quad 1.824230$

H $\quad 6.296060 \quad 0.829030 \quad 0.958010$

H $\quad 6.761140 \quad-0.183800 \quad-1.260940$

H $\quad 4.895570 \quad-1.147320 \quad-2.582030$

$\mathrm{H} \quad 2.585850 \quad-1.097390 \quad-1.699130$

\section{ligS_TSrot}

O $1.661139 \quad-3.090330 \quad 0.748591$

O $\quad 0.566189 \quad-2.653180 \quad-1.174039$

$\begin{array}{lllll}\mathrm{Br} & -5.532070 & -0.043077 & -0.201269\end{array}$

$\begin{array}{llll}\text { C } & 0.977969 & -2.404350 & -0.025639\end{array}$

$\begin{array}{llll}\text { C } & 0.570599 & -0.973960 & 0.497891\end{array}$

$\begin{array}{llll}\text { C } & 1.713640 & 0.039680 & 0.554941\end{array}$

C $\quad 1.161330 \quad-0.552720 \quad 1.820231$

$\begin{array}{llll}\text { C } & -0.874861 & -0.649849 & 0.251141\end{array}$

$\begin{array}{llll}\text { C } & -1.778831 & -1.703499 & 0.465951\end{array}$

$\begin{array}{llll}\text { C } & -3.148851 & -1.538038 & 0.340211\end{array}$

$\begin{array}{llll}\text { C } & -3.642660 & -0.290568 & -0.020749\end{array}$

$\begin{array}{llll}\text { C } & -2.790130 & 0.770582 & -0.256019\end{array}$

$\begin{array}{llll}\text { C } & -1.412980 & 0.584051 & -0.118759\end{array}$

$\begin{array}{llll}\text { C } & 1.519000 & 1.502430 & 0.252641\end{array}$

$\begin{array}{llll}\text { C } & 1.302771 & 2.449930 & 1.251461\end{array}$ $\begin{array}{llll}\text { C } & 1.122341 & 3.795380 & 0.934871\end{array}$

$\begin{array}{llll}\text { C } & 1.165921 & 4.215490 & -0.388719\end{array}$

$\begin{array}{llll}\text { C } & 1.407891 & 3.279860 & -1.394379\end{array}$

$\begin{array}{llll}\text { C } & 1.590350 & 1.942100 & -1.074639\end{array}$

$\begin{array}{llll}\text { C } & 3.096080 & -0.392230 & 0.118471\end{array}$

$\begin{array}{llll}\text { C } & 4.181510 & -0.080921 & 0.937821\end{array}$

$\begin{array}{llll}\text { C } & 5.481140 & -0.374161 & 0.542661\end{array}$

$\begin{array}{llll}\text { C } & 5.711019 & -0.985581 & -0.686849\end{array}$

C $\quad 4.632879 \quad-1.303911 \quad-1.506099$

C $3.331989-1.008291-1.110059$

$\begin{array}{llll}\mathrm{H} & 1.753509 & -1.320260 & 2.305301\end{array}$

$\begin{array}{llll}\mathrm{H} & 0.546880 & 0.077910 & 2.452771\end{array}$

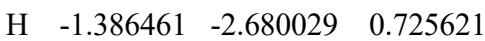

$\mathrm{H} \quad-3.823401 \quad-2.367588 \quad 0.509871$

H $\quad-3.182550 \quad 1.736762 \quad-0.547419$

$\begin{array}{llll}\mathrm{H} & -0.776980 & 1.432941 & -0.316639\end{array}$

$\begin{array}{llll}\mathrm{H} & 1.263050 & 2.131290 & 2.286351\end{array}$

$\mathrm{H} \quad 0.946001 \quad 4.514300 \quad 1.727451$

$\mathrm{H} \quad 1.018892 \quad 5.260410 \quad-0.637029$

$\mathrm{H} \quad 1.448571 \quad 3.595320 \quad-2.430979$

$\mathrm{H} \quad 1.767160 \quad 1.211970 \quad-1.857179$

$\begin{array}{llll}\mathrm{H} & 3.994970 & 0.391809 & 1.897411\end{array}$

H $\quad 6.313190 \quad-0.131802 \quad 1.194881$

H $\quad 6.722489-1.223612 \quad-0.997209$

H $\quad 4.800399 \quad-1.801641 \quad-2.454699$

H $\quad 2.489429 \quad-1.300550 \quad-1.728789$

\section{ligT1}

$\begin{array}{llll}\mathrm{O} & -1.307790 & -2.647470 & -1.339970\end{array}$

$\begin{array}{llll}\text { O } & -2.669670 & -3.107680 & 0.399880\end{array}$

$\begin{array}{llll}\text { C } & -1.787180 & -2.484770 & -0.203730\end{array}$

$\begin{array}{llll}\text { C } & -1.193970 & -1.236140 & 0.568480\end{array}$

$\begin{array}{llll}\text { C } & -2.102520 & 0.006670 & 0.640420\end{array}$

$\begin{array}{llll}\text { C } & -1.834040 & -0.850570 & 1.858480\end{array}$

$\begin{array}{llll}\text { C } & -3.492760 & -0.045790 & 0.061240\end{array}$

$\begin{array}{llll}\text { C } & -3.749750 & -0.516240 & -1.226090\end{array}$

$\begin{array}{lrrr}\text { C } & -5.040800 & -0.471560 & -1.744720\end{array}$

$\begin{array}{llll}\text { C } & -6.088110 & 0.046700 & -0.989820\end{array}$

$\begin{array}{llll}\text { C } & -5.839410 & 0.515240 & 0.297580\end{array}$

$\begin{array}{llll}\text { C } & -4.549960 & 0.466570 & 0.814570\end{array}$

C $\quad-1.452020 \quad 1.360120 \quad 0.517240$

$\begin{array}{llll}\text { C } & -0.419800 & 1.782810 & 1.365120\end{array}$

$\begin{array}{llll}\text { C } & 0.205570 & 3.011320 & 1.189600\end{array}$

$\begin{array}{llll}\text { C } & -0.184240 & 3.862420 & 0.160650\end{array}$

C $\quad-1.211280 \quad 3.463330-0.686810$

$\begin{array}{llll}\text { C } & -1.835740 & 2.232960 & -0.509050\end{array}$

$\begin{array}{llll}\text { C } & 0.267540 & -1.024800 & 0.372310\end{array}$

$\begin{array}{llll}\text { C } & 1.181110 & -1.288630 & 1.391080\end{array}$

$\begin{array}{llll}\text { C } & 2.543430 & -1.070690 & 1.211380\end{array}$

$\begin{array}{llll}\text { C } & 3.030370 & -0.577360 & -0.000150\end{array}$

$\begin{array}{llll}\text { C } & 2.114660 & -0.335040 & -1.032210\end{array}$

$\begin{array}{llll}\text { C } & 0.760310 & -0.564130 & -0.852650\end{array}$

$\begin{array}{llll}\text { C } & 4.475580 & -0.311470 & -0.188570\end{array}$

$\begin{array}{llll}\text { C } & 4.911400 & 0.816550 & -0.893800\end{array}$

$\begin{array}{llll}\text { C } & 6.266390 & 1.070030 & -1.071850\end{array}$

$\begin{array}{llll}\text { C } & 7.218590 & 0.202860 & -0.543930\end{array}$

$\begin{array}{llll}\text { C } & 6.799690 & -0.921710 & 0.161010\end{array}$

$\begin{array}{llll}\text { C } & 5.444150 & -1.176480 & 0.333790\end{array}$

$\mathrm{H} \quad-2.646630 \quad-1.503410 \quad 2.155630$

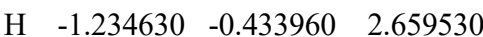

H $\quad-2.940090 \quad-0.948730 \quad-1.805350$

H $\quad-5.228410 \quad-0.857160 \quad-2.740590$ 

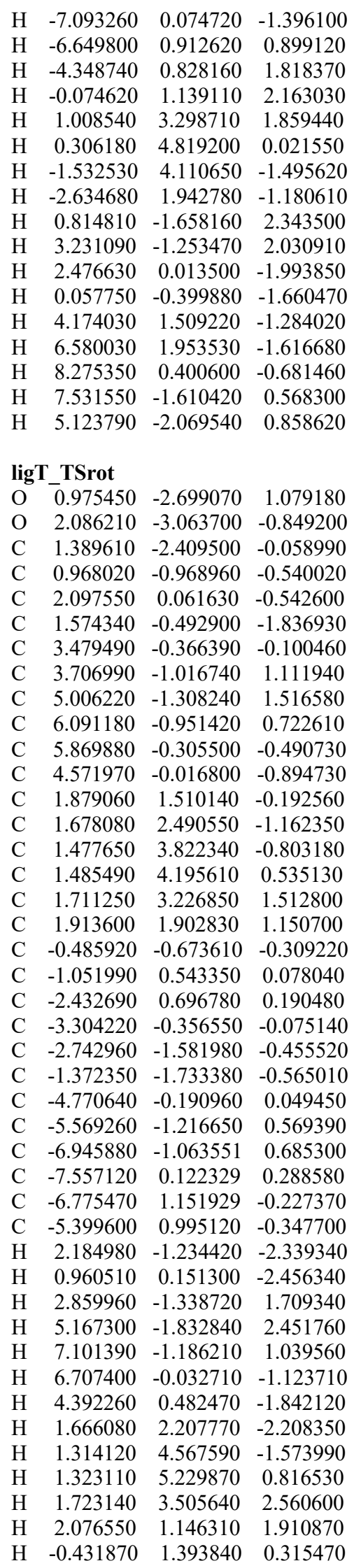

$\begin{array}{rrrr}\mathrm{H} & -2.830040 & 1.650040 & 0.524380 \\ \mathrm{H} & -3.391940 & -2.420610 & -0.685980 \\ \mathrm{H} & -0.960990 & -2.694590 & -0.851350 \\ \mathrm{H} & -5.096070 & -2.132060 & 0.906410 \\ \mathrm{H} & -7.542430 & -1.869861 & 1.097310 \\ \mathrm{H} & -8.630330 & 0.242849 & 0.380610 \\ \mathrm{H} & -7.240500 & 2.077759 & -0.547530 \\ \mathrm{H} & -4.799570 & 1.790780 & -0.774980\end{array}$

\section{$\operatorname{lig} \mathbf{U} 1$}

$\begin{array}{llll}\mathrm{O} & -2.356210 & -2.103560 & 2.417680\end{array}$

$\begin{array}{llll}\mathrm{O} & -1.162100 & -0.249360 & 2.902470\end{array}$

F $\quad 1.265200 \quad 3.328310 \quad 1.195160$

F $\quad 3.106110 \quad 3.111740 \quad 0.088690$

F $\quad 3.068960 \quad 2.706350 \quad 2.200010$

F $\quad \begin{array}{llll}5.093820 & -1.435030 & -0.258340\end{array}$

F $\quad 3.690090 \quad-2.314530 \quad-1.638850$

$\begin{array}{llll}\text { F } & 3.884300 & -3.117710 & 0.346240\end{array}$

C $\quad-1.554060 \quad-1.192330 \quad 2.190800$

$\begin{array}{llll}\text { C } & -0.951690 & -1.176430 & 0.728530\end{array}$

$\begin{array}{llll}\text { C } & -1.896030 & -0.742100 & -0.408710\end{array}$

$\begin{array}{llll}\text { C } & -1.453380 & -2.177710 & -0.258200\end{array}$

$\begin{array}{llll}\text { C } & 0.452250 & -0.693720 & 0.639430\end{array}$

$\begin{array}{llll}\text { C } & 1.492730 & -1.528550 & 0.260320\end{array}$

C $\quad 2.793360 \quad-1.034820 \quad 0.130550$

$\begin{array}{llll}\text { C } & 3.078070 & 0.295010 & 0.375590\end{array}$

$\begin{array}{llll}\text { C } & 2.032920 & 1.128530 & 0.778820\end{array}$

$\begin{array}{llll}\text { C } & 0.743960 & 0.649520 & 0.923980\end{array}$

$\begin{array}{llll}\text { C } & -1.288170 & 0.132810 & -1.475120\end{array}$

C $\quad-1.622220 \quad 1.492020 \quad-1.517140$

$\begin{array}{llll}\text { C } & -1.001430 & 2.360750 & -2.406850\end{array}$

$\begin{array}{llll}\text { C } & -0.032430 & 1.891790 & -3.288070\end{array}$

$\begin{array}{llll}\text { C } & 0.298150 & 0.541660 & -3.271050\end{array}$

$\begin{array}{llll}\text { C } & -0.324070 & -0.325150 & -2.378030\end{array}$

$\begin{array}{llll}\text { C } & -3.341510 & -0.423770 & -0.124940\end{array}$

$\begin{array}{llll}\text { C } & -4.308580 & -0.896100 & -1.013710\end{array}$

$\begin{array}{llll}\text { C } & -5.650830 & -0.577860 & -0.844760\end{array}$

$\begin{array}{llll}\text { C } & -6.043990 & 0.223420 & 0.223840\end{array}$

$\begin{array}{llll}\text { C } & -5.086740 & 0.694950 & 1.115790\end{array}$

$\begin{array}{lrrr}\text { C } & -3.742510 & 0.376650 & 0.943910\end{array}$

$\begin{array}{llll}\text { C } & 3.863020 & -1.965690 & -0.346330\end{array}$

$\begin{array}{llll}\text { C } & 2.353400 & 2.563470 & 1.065890\end{array}$

$\begin{array}{llll}\mathrm{H} & -2.196540 & -2.851040 & 0.152500\end{array}$

$\mathrm{H} \quad-0.781120 \quad-2.601740 \quad-0.994070$

H $\quad 1.291860 \quad-2.574540 \quad 0.053710$

$\begin{array}{llll}\mathrm{H} & -0.059880 & 1.295860 & 1.250750\end{array}$

$\begin{array}{llll}\mathrm{H} & -2.371190 & 1.872960 & -0.832390\end{array}$

$\begin{array}{llll}\mathrm{H} & -1.271240 & 3.410880 & -2.403290\end{array}$

$\begin{array}{llll}\mathrm{H} & 0.460320 & 2.569540 & -3.975440\end{array}$

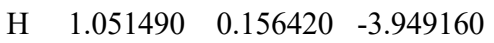

$\begin{array}{llll}\mathrm{H} & -0.032600 & -1.367660 & -2.380490\end{array}$

$\mathrm{H} \quad-3.995000 \quad-1.521810 \quad-1.843800$

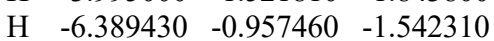

$\begin{array}{llll}\mathrm{H} & -7.090580 & 0.469690 & 0.364850\end{array}$

$\begin{array}{llll}\mathrm{H} & -5.385250 & 1.304990 & 1.960810\end{array}$

$\begin{array}{llll}\mathrm{H} & -3.003860 & 0.724530 & 1.659140\end{array}$

$\begin{array}{llll}\mathrm{H} & 4.081870 & 0.682440 & 0.254640\end{array}$

\section{ligU_TSrot}

$\begin{array}{llll}\mathrm{O} & 1.651801 & -3.261420 & 0.449849\end{array}$

$\begin{array}{llll}\mathrm{O} & 0.526151 & -2.570550 & -1.379621\end{array}$

$\begin{array}{llll}\text { F } & -2.443400 & 3.300020 & 0.677189\end{array}$ $\begin{array}{llll}\text { F } & -4.044120 & 2.735229 & -0.652701\end{array}$

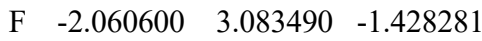

$\begin{array}{llll}\text { F } & -5.068379 & -1.862521 & 1.001089\end{array}$

$\begin{array}{llll}\text { F } & -3.632699 & -3.410761 & 0.555039\end{array}$

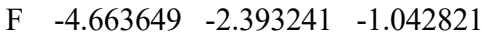

$\begin{array}{llll}\text { C } & 1.003251 & -2.459800 & -0.234571\end{array}$

$\begin{array}{llll}\text { C } & 0.742680 & -1.049020 & 0.421689\end{array}$

$\begin{array}{llll}\text { C } & 1.983830 & -0.171490 & 0.591709\end{array}$

$\begin{array}{llll}\text { C } & 1.349000 & -0.822580 & 1.785849\end{array}$

$\begin{array}{llll}\text { C } & -0.649900 & -0.546460 & 0.185659\end{array}$

$\begin{array}{llll}\text { C } & -1.668999 & -1.519000 & 0.242949\end{array}$

$\begin{array}{llll}\text { C } & -2.999220 & -1.173330 & 0.105209\end{array}$

$\begin{array}{llll}\text { C } & -3.388450 & 0.149190 & -0.114621\end{array}$

$\begin{array}{llll}\text { C } & -2.394460 & 1.102430 & -0.186391\end{array}$

$\begin{array}{llll}\text { C } & -1.043800 & 0.769170 & -0.038921\end{array}$

$\begin{array}{llll}\text { C } & 1.930350 & 1.325190 & 0.429469\end{array}$

$\begin{array}{llll}\text { C } & 2.050520 & 1.877850 & -0.850891\end{array}$

$\begin{array}{llll}\text { C } & 1.956740 & 3.248350 & -1.046841\end{array}$

$\begin{array}{llll}\text { C } & 1.760670 & 4.100630 & 0.039199\end{array}$

$\begin{array}{llll}\text { C } & 1.679460 & 3.565980 & 1.318839\end{array}$

$\begin{array}{llll}\text { C } & 1.770180 & 2.188550 & 1.511449\end{array}$

$\begin{array}{llll}\text { C } & 3.318810 & -0.698470 & 0.116699\end{array}$

$\begin{array}{llll}\text { C } & 4.422390 & -0.577250 & 0.960679\end{array}$

$\begin{array}{llll}\text { C } & 5.688390 & -0.964540 & 0.537809\end{array}$

$\begin{array}{llll}\text { C } & 5.864711 & -1.478320 & -0.743951\end{array}$

C $\quad 4.767081 \quad-1.605990 \quad-1.588811$

C $\quad 3.500440 \quad-1.217040 \quad-1.164671$

$\begin{array}{llll}\text { C } & -4.074499 & -2.215251 & 0.155889\end{array}$

$\begin{array}{llll}\text { C } & -2.736850 & 2.545030 & -0.399531\end{array}$

H $\quad 1.845731 \quad-1.695560 \quad 2.193409$

$\mathrm{H} \quad 0.794460 \quad-0.195850 \quad 2.474799$

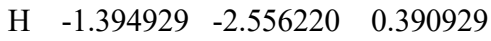

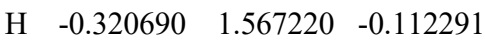

$\begin{array}{llll}\mathrm{H} & 2.185870 & 1.212770 & -1.697071\end{array}$

$\begin{array}{llll}\mathrm{H} & 2.023450 & 3.654030 & -2.049891\end{array}$

$\begin{array}{llll}\mathrm{H} & 1.673460 & 5.169980 & -0.113771\end{array}$

$\begin{array}{llll}\mathrm{H} & 1.536250 & 4.219210 & 2.172289\end{array}$

$\begin{array}{llll}\mathrm{H} & 1.695520 & 1.780510 & 2.512439\end{array}$

H $\quad 4.277330 \quad-0.179140 \quad 1.960429$

$\begin{array}{llll}\mathrm{H} & 6.535320 & -0.870830 & 1.208739\end{array}$

$\mathrm{H} \quad 6.849021 \quad-1.788870 \quad-1.076371$

$\mathrm{H} \quad 4.891131 \quad-2.026980 \quad-2.580151$

H $\quad 2.638511 \quad-1.361140 \quad-1.808661$

H $\quad-4.432390 \quad 0.413719 \quad-0.225651$

\section{ligV1}

$\begin{array}{llll}\mathrm{O} & 1.178160 & -0.289880 & -2.886360\end{array}$

$\begin{array}{llll}\text { O } & 2.305210 & -2.183310 & -2.395500\end{array}$

$\begin{array}{llll}\mathrm{Br} & -4.173940 & -2.181450 & 0.451370\end{array}$

$\begin{array}{llll}\mathrm{Br} & -2.379680 & 2.964290 & -1.108380\end{array}$

$\begin{array}{llll}\text { C } & 1.543050 & -1.237540 & -2.168220\end{array}$

$\begin{array}{llll}\text { C } & 0.966520 & -1.180550 & -0.693850\end{array}$

$\begin{array}{llll}\text { C } & 1.947610 & -0.749760 & 0.412820\end{array}$

$\begin{array}{llll}\text { C } & 1.469720 & -2.178080 & 0.295010\end{array}$

$\begin{array}{llll}\text { C } & -0.432230 & -0.678950 & -0.591710\end{array}$

$\begin{array}{llll}\text { C } & -0.711350 & 0.662040 & -0.882740\end{array}$

$\begin{array}{llll}\text { C } & -2.005130 & 1.127260 & -0.748580\end{array}$

$\begin{array}{llll}\text { C } & -3.060720 & 0.311180 & -0.351030\end{array}$

$\begin{array}{llll}\text { C } & -2.760230 & -1.015820 & -0.094670\end{array}$

C $\quad-1.472920 \quad-1.522750 \quad-0.208120$

$\begin{array}{llll}\text { C } & 1.399290 & 0.145360 & 1.494530\end{array}$

$\begin{array}{llll}\text { C } & 0.404460 & -0.263920 & 2.388670\end{array}$ 


$\begin{array}{rrrr}\mathrm{C} & -0.141610 & 0.617790 & 3.315410 \\ \mathrm{C} & 0.296010 & 1.935840 & 3.377560 \\ \mathrm{C} & 1.292070 & 2.356700 & 2.502980 \\ \mathrm{C} & 1.836890 & 1.472970 & 1.578570 \\ \mathrm{C} & 3.392560 & -0.473850 & 0.084150 \\ \mathrm{C} & 4.373160 & -0.969690 & 0.944860 \\ \mathrm{C} & 5.717810 & -0.687870 & 0.734800 \\ \mathrm{C} & 6.100020 & 0.099870 & -0.347790 \\ \mathrm{C} & 5.129150 & 0.594190 & -1.212370 \\ \mathrm{C} & 3.782580 & 0.312710 & -0.999000 \\ \mathrm{H} & 2.187250 & -2.874570 & -0.122690 \\ \mathrm{H} & 0.808010 & -2.573290 & 1.056100 \\ \mathrm{H} & 0.091160 & 1.306690 & -1.213830 \\ \mathrm{H} & -1.274890 & -2.566390 & 0.001580 \\ \mathrm{H} & 0.029260 & -1.278490 & 2.358160 \\ \mathrm{H} & -0.919250 & 0.269920 & 3.986300 \\ \mathrm{H} & -0.135790 & 2.625420 & 4.093660 \\ \mathrm{H} & 1.645720 & 3.381290 & 2.533380 \\ \mathrm{H} & 2.610930 & 1.818060 & 0.903160 \\ \mathrm{H} & 4.067980 & -1.584580 & 1.786080 \\ \mathrm{H} & 6.466690 & -1.085460 & 1.411130 \\ \mathrm{H} & 7.148130 & 0.317640 & -0.521090 \\ \mathrm{H} & 5.418310 & 1.193490 & -2.068330 \\ \mathrm{H} & 3.031540 & 0.677480 & -1.692540 \\ \mathrm{H} & -4.065200 & 0.696120 & -0.248370\end{array}$

$\begin{array}{lccc}\text { ligV_TSrot } & & \\ \mathrm{O} & 0.601470 & -2.517319 & -1.476460 \\ \mathrm{O} & 1.626530 & -3.300079 & 0.374870 \\ \mathrm{Br} & -4.321050 & -2.454479 & 0.201920 \\ \mathrm{Br} & -2.781030 & 2.957511 & -0.472120 \\ \mathrm{C} & 1.030090 & -2.457669 & -0.309460 \\ \mathrm{C} & 0.780100 & -1.062119 & 0.383500 \\ \mathrm{C} & 2.030220 & -0.204148 & 0.581520 \\ \mathrm{C} & 1.378770 & -0.876239 & 1.755900 \\ \mathrm{C} & -0.609450 & -0.541219 & 0.160010 \\ \mathrm{C} & -0.976590 & 0.789511 & -0.054720 \\ \mathrm{C} & -2.319850 & 1.124261 & -0.182710 \\ \mathrm{C} & -3.340020 & 0.194551 & -0.109530 \\ \mathrm{C} & -2.953340 & -1.125799 & 0.098960 \\ \mathrm{C} & -1.632570 & -1.504039 & 0.226300 \\ \mathrm{C} & 2.008520 & 1.297182 & 0.458030 \\ \mathrm{C} & 1.863870 & 2.135951 & 1.561320 \\ \mathrm{C} & 1.826199 & 3.520731 & 1.406530 \\ \mathrm{C} & 1.944049 & 4.087541 & 0.143830 \\ \mathrm{C} & 2.118780 & 3.258662 & -0.963770 \\ \mathrm{C} & 2.159780 & 1.880792 & -0.805300 \\ \mathrm{C} & 3.362730 & -0.740598 & 0.108160 \\ \mathrm{C} & 4.456700 & -0.662908 & 0.970070 \\ \mathrm{C} & 5.721950 & -1.059078 & 0.553500 \\ \mathrm{C} & 5.907790 & -1.538798 & -0.740180 \\ \mathrm{C} & 4.820010 & -1.623438 & -1.602900 \\ \mathrm{C} & 3.553880 & -1.225178 & -1.185210 \\ \mathrm{H} & 1.862290 & -1.763779 & 2.147730 \\ \mathrm{H} & 0.825570 & -0.259229 & 2.454750 \\ \mathrm{H} & -0.243830 & 1.575541 & -0.131590 \\ \mathrm{H} & -1.372570 & -2.545819 & 0.363620 \\ \mathrm{H} & 1.764240 & 1.703041 & 2.549620 \\ \mathrm{H} & 1.698539 & 4.154821 & 2.276840 \\ \mathrm{H} & 1.901749 & 5.163521 & 0.020440 \\ \mathrm{H} & 2.213659 & 3.689042 & -1.954290 \\ \mathrm{H} & 2.282060 & 1.234752 & -1.668060\end{array}$

H $\quad 4.304180 \quad-0.291298 \quad 1.978880$

H $\quad 6.560800 \quad-0.999138 \quad 1.238380$

$\mathrm{H} \quad 6.891420 \quad-1.856298 \quad-1.068180$

H $\quad 4.950860 \quad-2.017648 \quad-2.604390$

H $\quad 2.698620 \quad-1.336358-1.844220$

H $\quad-4.378510 \quad 0.474221 \quad-0.212900$

\section{ligW1}

$\begin{array}{llll}\mathrm{O} & -2.095800 & -1.535860 & -2.798240\end{array}$

$\begin{array}{llll}\mathrm{O} & -1.623930 & -3.740920 & -2.865090\end{array}$

C $\quad-1.610280 \quad-2.603710 \quad-2.378710$

C $\quad-0.909400 \quad-2.482560 \quad-0.966630$

$\begin{array}{llll}\text { C } & -0.452890 & -3.736520 & -0.299780\end{array}$

C $\quad-1.687490 \quad-3.049680 \quad 0.236930$

C $\quad-1.620800 \quad-2.260690 \quad 1.518860$

C $\quad-2.773080 \quad-1.627310 \quad 2.001010$

C $\quad-2.734960 \quad-0.815720 \quad 3.128980$

C $\quad-1.541320 \quad-0.618890 \quad 3.815120$

C $\quad-0.390100 \quad-1.249580 \quad 3.356760$

$\begin{array}{llll}\text { C } & -0.430970 & -2.059340 & 2.226820\end{array}$

C $\quad-5.480000 \quad-5.053870 \quad-0.152150$

C $\quad-5.246940 \quad-3.882230 \quad-0.864450$

$\begin{array}{llll}\text { C } & -4.020380 & -3.229870 & -0.772100\end{array}$

C $\quad-3.007530 \quad-3.748500 \quad 0.033920$

$\begin{array}{llll}\text { C } & -3.247890 & -4.927510 & 0.741700\end{array}$

$\begin{array}{llll}\text { C } & -4.472510 & -5.578780 & 0.653120\end{array}$

C $\quad-0.159370 \quad-1.212370 \quad-0.751700$

$\begin{array}{llll}\text { C } & 1.226700 & -1.188780 & -0.641450\end{array}$

$\begin{array}{llll}\text { C } & 1.917780 & 0.005600 & -0.406930\end{array}$

$\begin{array}{llll}\text { C } & 1.197040 & 1.192890 & -0.294260\end{array}$

C $\quad-0.195800 \quad 1.193900-0.423610$

$\begin{array}{llll}\text { C } & -0.858880 & -0.008700 & -0.649520\end{array}$

$\begin{array}{llll}\text { C } & -0.963810 & 2.459110 & -0.345460\end{array}$

C $\quad-2.060170 \quad 2.677470 \quad-1.188240$

C $\quad-2.784060 \quad 3.857650 \quad-1.118520$

C $\quad-2.453350 \quad 4.872960 \quad-0.211880$

C $\quad-1.360230 \quad 4.649220 \quad 0.625110$

$\begin{array}{llll}\text { C } & -0.630430 & 3.464470 & 0.560230\end{array}$

C $\quad-3.286380 \quad 6.156370 \quad-0.173350$

C $\quad-3.229570 \quad 6.844930-1.547570$

$\begin{array}{llll}\text { C } & -4.746700 & 5.808360 & 0.161340\end{array}$

$\begin{array}{llll}\text { C } & -2.776790 & 7.146170 & 0.879070\end{array}$

$\begin{array}{llll}\text { C } & 3.395590 & 0.006400 & -0.287500\end{array}$

$\begin{array}{llll}\text { C } & 4.188890 & -0.799730 & -1.112490\end{array}$

$\begin{array}{llll}\text { C } & 5.570550 & -0.799990 & -0.996690\end{array}$

$\begin{array}{llll}\text { C } & 6.230550 & 0.001780 & -0.057280\end{array}$

$\begin{array}{llll}\text { C } & 5.437000 & 0.804120 & 0.762730\end{array}$

$\begin{array}{llll}\text { C } & 4.048650 & 0.804830 & 0.651140\end{array}$

$\begin{array}{llll}\text { C } & 7.758050 & -0.031880 & 0.030960\end{array}$

$\begin{array}{llll}\text { C } & 8.295480 & 0.905860 & 1.116620\end{array}$

$\begin{array}{llll}\text { C } & 8.357670 & 0.400770 & -1.317820\end{array}$

C $\quad 8.221070 \quad-1.461630 \quad 0.358250$

$\mathrm{H} \quad-0.637620 \quad-4.631320 \quad-0.882570$

H $\quad 0.449150 \quad-3.734920 \quad 0.300060$

H $\quad-3.711310 \quad-1.766750 \quad 1.476910$

$\mathrm{H} \quad-3.643770 \quad-0.330320 \quad 3.467210$

$\mathrm{H} \quad-1.508040 \quad 0.018810 \quad 4.691080$

$\mathrm{H} \quad 0.552120 \quad-1.107570 \quad 3.874690$

$\mathrm{H} \quad 0.487960 \quad-2.518690 \quad 1.887160$

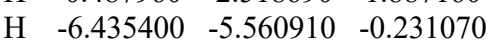

H $\quad-6.019010 \quad-3.475450 \quad-1.507910$

H $\quad-3.833660 \quad-2.330910 \quad-1.351060$
$\mathrm{H} \quad-2.457110 \quad-5.331760 \quad 1.366360$

$\mathrm{H} \quad-4.639630 \quad-6.496130 \quad 1.207210$

$\mathrm{H} \quad 1.777880 \quad-2.121170 \quad-0.712470$

$\mathrm{H} \quad 1.725470 \quad 2.131950 \quad-0.164760$

$\mathrm{H} \quad-1.937650 \quad-0.035710 \quad-0.740960$

$\mathrm{H} \quad-2.328950 \quad 1.915380 \quad-1.912590$

H $\quad-3.623230 \quad 3.988760 \quad-1.793820$

$\mathrm{H} \quad-1.065720 \quad 5.394600 \quad 1.353450$

H $\quad 0.196340 \quad 3.308600 \quad 1.244980$

H $\quad-2.198630 \quad 7.099890 \quad-1.805910$

$\mathrm{H} \quad-3.822670 \quad 7.764810 \quad-1.535420$

$\mathrm{H} \quad-3.622590 \quad 6.198650 \quad-2.334840$

$\mathrm{H} \quad-5.178090 \quad 5.136700 \quad-0.583430$

$\begin{array}{llll}\mathrm{H} & -5.355590 & 6.717410 & 0.192690\end{array}$

$\mathrm{H} \quad-4.809360 \quad 5.314480 \quad 1.134240$

$\begin{array}{llll}\mathrm{H} & -2.820360 & 6.719080 & 1.884260\end{array}$

$\begin{array}{llll}\mathrm{H} & -3.400780 & 8.043940 & 0.867770\end{array}$

$\mathrm{H} \quad-1.746420 \quad 7.449960 \quad 0.676740$

H $\quad 3.711370 \quad-1.415620-1.866520$

H $\quad 6.145390 \quad-1.435040 \quad-1.662900$

$\mathrm{H} \quad 5.891460 \quad 1.438110 \quad 1.513930$

$\mathrm{H} \quad 3.459110 \quad 1.420570 \quad 1.321740$

H $\quad 8.018000 \quad 1.944890 \quad 0.921340$

$\begin{array}{llll}\mathrm{H} & 9.386970 & 0.848230 & 1.140510\end{array}$

H $\quad \begin{array}{llll}7.922530 & 0.628310 & 2.105850\end{array}$

$\mathrm{H} \quad 8.043020 \quad-0.262270 \quad-2.126220$

H $\quad 9.450920 \quad 0.382380 \quad-1.269830$

H $\quad 8.038500 \quad 1.414760 \quad-1.571570$

$\mathrm{H} \quad 7.803350 \quad-1.790750 \quad 1.313020$

H $\quad 9.312810 \quad-1.500300 \quad 0.424880$

H $\quad 7.903430 \quad-2.170450 \quad-0.409090$

\section{ligW_TSrot}

$\begin{array}{llll}\mathrm{O} & -0.586058 & -3.108890 & 1.718960\end{array}$

$\begin{array}{llll}\text { O } & -0.553458 & -4.658110 & 0.081610\end{array}$

C $\quad-0.314238-3.552050 \quad 0.587280$

C $\quad 0.476731 \quad-2.535790 \quad-0.327200$

C $\quad 0.863372 \quad-3.039180 \quad-1.693160$

$\begin{array}{llll}\text { C } & 1.928612 & -2.911209 & -0.639380\end{array}$

$\begin{array}{llll}\text { C } & 3.000291 & -1.858799 & -0.766770\end{array}$

$\begin{array}{llll}\text { C } & 3.750071 & -1.509918 & 0.364380\end{array}$

$\begin{array}{llll}\text { C } & 4.688271 & -0.488268 & 0.314810\end{array}$

$\begin{array}{llll}\text { C } & 4.914390 & 0.201272 & -0.875770\end{array}$

$\begin{array}{llll}\text { C } & 4.209891 & -0.166138 & -2.015270\end{array}$

$\begin{array}{llll}\text { C } & 3.269241 & -1.193129 & -1.961740\end{array}$

$\begin{array}{llll}\text { C } & 3.739063 & -6.468928 & 0.982020\end{array}$

$\begin{array}{llll}\text { C } & 2.988813 & -5.640039 & 1.809820\end{array}$

$\begin{array}{llll}\text { C } & 2.372442 & -4.501919 & 1.298940\end{array}$

$\begin{array}{llll}\text { C } & 2.498072 & -4.181979 & -0.052420\end{array}$

$\begin{array}{llll}\text { C } & 3.248022 & -5.020289 & -0.877720\end{array}$

C $3.864883 \quad-6.157138 \quad-0.369040$

$\begin{array}{llll}\text { C } & -0.043259 & -1.131360 & -0.210510\end{array}$

$\begin{array}{llll}\text { C } & -1.437669 & -1.000050 & -0.246490\end{array}$

$\begin{array}{llll}\text { C } & -2.066730 & 0.236599 & -0.153790\end{array}$

$\begin{array}{llll}\text { C } & -1.287290 & 1.393150 & -0.043440\end{array}$

$\begin{array}{llll}\text { C } & 0.099890 & 1.296300 & -0.010270\end{array}$

$\begin{array}{llll}\text { C } & 0.706330 & 0.035020 & -0.081930\end{array}$

$\begin{array}{llll}\text { C } & 0.949840 & 2.506421 & 0.110370\end{array}$

$\begin{array}{llll}\text { C } & 2.147200 & 2.479321 & 0.836210\end{array}$

$\begin{array}{llll}\text { C } & 2.955189 & 3.601551 & 0.924510\end{array}$

$\begin{array}{llll}\text { C } & 2.613159 & 4.807091 & 0.299510\end{array}$

$\begin{array}{llll}\text { C } & 1.417259 & 4.833281 & -0.417520\end{array}$ 


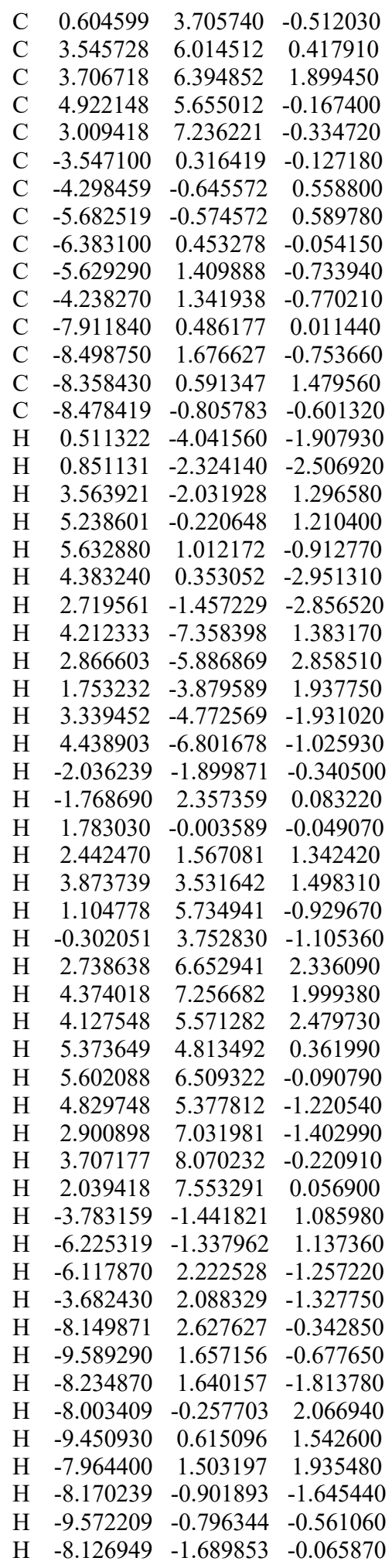

\section{ligX1}

$\begin{array}{llll}\text { O } & 1.055180 & 2.988080 & -0.838350\end{array}$

O $\quad-1.056670 \quad 2.322220 \quad-1.285230$

$\begin{array}{llll}\mathrm{O} & -1.813620 & 1.080250 & 2.307930\end{array}$

O $\quad-0.932470 \quad-0.783200 \quad-1.783930$

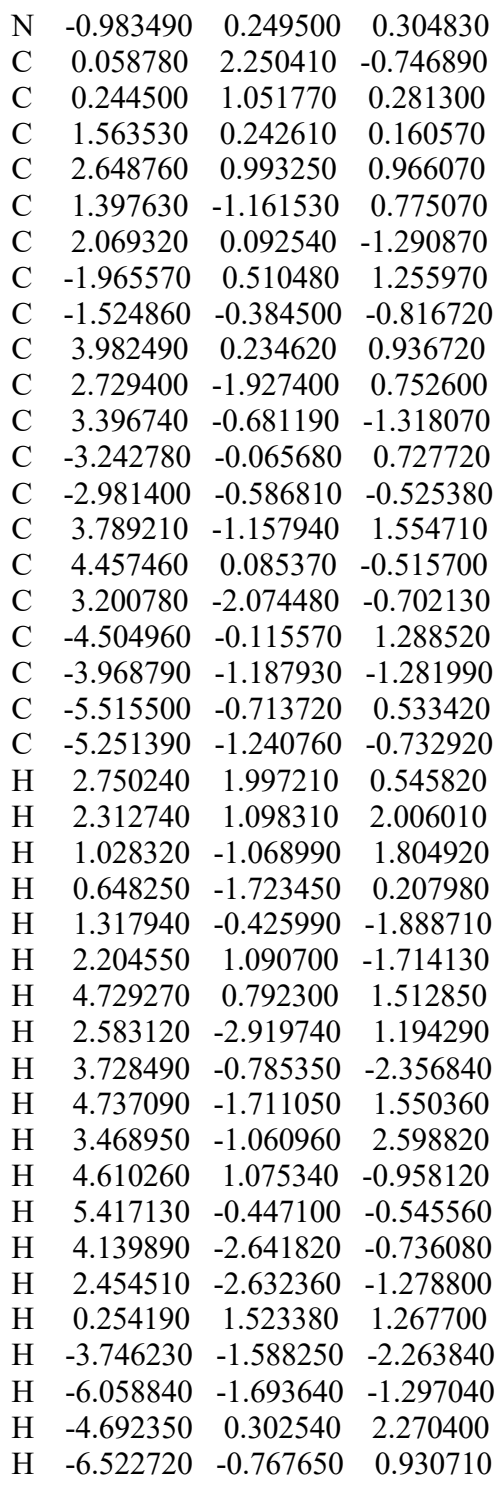

\section{ligX_TSrot}

$\begin{array}{lllll}\text { O } & -0.633149 & 3.360940 & -1.201300\end{array}$

$\begin{array}{llll}\text { O } & -0.508139 & 2.616799 & 0.927390\end{array}$

$\begin{array}{llll}\mathrm{O} & 2.148281 & 2.401668 & 0.338710\end{array}$

$\begin{array}{llll}\text { O } & 1.028109 & -1.466681 & -1.777930\end{array}$

$\begin{array}{llll}\mathrm{N} & 1.036380 & 0.502419 & -0.524010\end{array}$

$\begin{array}{llll}\text { C } & -0.475929 & 2.513889 & -0.311460\end{array}$

$\begin{array}{llll}\text { C } & -0.319160 & 1.024949 & -0.856430\end{array}$

$\begin{array}{llll}\text { C } & -1.497290 & 0.144680 & -0.305710\end{array}$

$\begin{array}{llll}\text { C } & -1.855570 & -1.047520 & -1.227710\end{array}$

$\begin{array}{llll}\text { C } & -1.193190 & -0.396590 & 1.103410\end{array}$

$\begin{array}{llll}\text { C } & -2.772610 & 1.029230 & -0.236810\end{array}$

$\begin{array}{lrrr}\text { C } & 2.083550 & 1.234278 & 0.103090\end{array}$

$\begin{array}{llll}\text { C } & 1.537560 & -0.708571 & -0.987600\end{array}$

$\begin{array}{llll}\text { C } & -3.052351 & -1.841570 & -0.678070\end{array}$

$\begin{array}{llll}\text { C } & -2.395871 & -1.181130 & 1.650920\end{array}$

$\begin{array}{llll}\text { C } & -3.969390 & 0.244701 & 0.316590\end{array}$

$\begin{array}{llll}\text { C } & 3.204760 & 0.256758 & 0.307980\end{array}$

$\begin{array}{llll}\text { C } & 2.887250 & -0.901042 & -0.368900\end{array}$

$\begin{array}{llll}\text { C } & -2.706661 & -2.366240 & 0.724410\end{array}$
C $\quad-4.291290 \quad-0.944889-0.593100$

C $\quad-3.624220 \quad-0.261839 \quad 1.725330$

$\begin{array}{llll}\text { C } & 4.413290 & 0.409598 & 0.962000\end{array}$

$\begin{array}{llll}\text { C } & 3.755419 & -1.976192 & -0.426400\end{array}$

C $\quad 5.292750 \quad-0.673703 \quad 0.934280$

C $\quad 4.969459 \quad-1.848943 \quad 0.249900$

$\mathrm{H} \quad-2.088360 \quad-0.665260 \quad-2.229570$

$\mathrm{H} \quad-1.010521 \quad-1.721980-1.335400$

H $\quad-0.322660 \quad-1.064401 \quad 1.061180$

$\mathrm{H} \quad-0.940010 \quad 0.447970 \quad 1.749930$

H $\quad-2.587619 \quad 1.889930 \quad 0.405190$

$\mathrm{H} \quad-2.996230 \quad 1.410440 \quad-1.240450$

H $\quad-3.251541 \quad-2.688169-1.345120$

H $\quad-2.156021 \quad-1.552530 \quad 2.653540$

H $\quad-4.834510 \quad 0.914421 \quad 0.371990$

H $\quad-3.544821-2.950909 \quad 1.124960$

H $\quad-1.837291 \quad-3.031660 \quad 0.667210$

H $\quad-4.562500 \quad-0.586929-1.593000$

H $\quad-5.146171 \quad-1.510429 \quad-0.199860$

H $\quad-4.476580 \quad-0.806809 \quad 2.151440$

$\mathrm{H} \quad-3.409250 \quad 0.589451 \quad 2.379570$

$\mathrm{H} \quad-0.385250 \quad 1.060489 \quad-1.946670$

H $3.493499 \quad-2.872692 \quad-0.975730$

H $\quad 5.677719 \quad-2.669603 \quad 0.242260$

H $4.653390 \quad 1.335008 \quad 1.471840$

H $\quad 6.246210 \quad-0.604213 \quad 1.445500$

\section{ligY1}

$\begin{array}{llll}\text { O } & 2.639500 & -0.949670 & 2.949350\end{array}$

$\begin{array}{llll}\mathrm{O} & 0.500680 & -0.283980 & 2.646630\end{array}$

O $\quad-0.257460 \quad-2.724790 \quad-0.091180$

$\begin{array}{llll}\mathrm{O} & 0.525760 & 1.763370 & 0.267350\end{array}$

$\mathrm{Cl} \quad-3.331550 \quad-3.016830 \quad-0.503040$

$\begin{array}{llll}\mathrm{Cl} & -2.262340 & 3.123220 & 0.221790\end{array}$

$\mathrm{Cl} \quad-5.199060 \quad 2.063990 \quad-0.133220$

Cl $\quad-5.730410 \quad-0.986790 \quad-0.499710$

$\mathrm{N} \quad 0.525130 \quad-0.551590 \quad 0.074370$

$\begin{array}{llll}\text { C } & 1.627230 & -0.669410 & 2.290030\end{array}$

$\begin{array}{llll}\text { C } & 1.798780 & -0.898840 & 0.724580\end{array}$

$\begin{array}{llll}\text { C } & 3.073300 & -0.301130 & 0.074050\end{array}$

$\begin{array}{llll}\text { C } & 2.854360 & -0.059910 & -1.432590\end{array}$

$\begin{array}{llll}\text { C } & 3.549540 & 1.015610 & 0.726760\end{array}$

C $\quad 4.204120 \quad-1.343320 \quad 0.234970$

C $\quad-0.444750 \quad-1.538960 \quad-0.046270$

$\begin{array}{llll}\text { C } & -0.048160 & 0.714750 & 0.183450\end{array}$

$\begin{array}{llll}\text { C } & 4.150120 & 0.427070 & -2.098920\end{array}$

$\begin{array}{llll}\text { C } & 4.840240 & 1.509550 & 0.054860\end{array}$

$\begin{array}{lrrr}\text { C } & 5.501490 & -0.856300 & -0.423500\end{array}$

$\begin{array}{llll}\text { C } & -1.772800 & -0.833560 & -0.116470\end{array}$

$\begin{array}{llll}\text { C } & -1.536330 & 0.519630 & 0.043880\end{array}$

$\begin{array}{llll}\text { C } & 4.590820 & 1.744330 & -1.442330\end{array}$

$\begin{array}{llll}\text { C } & 5.255380 & -0.624320 & -1.921560\end{array}$

$\begin{array}{llll}\text { C } & 5.946840 & 0.460250 & 0.228960\end{array}$

C $\quad-3.048970 \quad-1.329720 \quad-0.291620$

$\begin{array}{llll}\text { C } & -2.566210 & 1.438360 & 0.034480\end{array}$

$\begin{array}{llll}\text { C } & -4.113330 & -0.415090 & -0.292770\end{array}$

C $\quad-3.874120 \quad 0.955530 \quad-0.129610$

H $\quad 2.509730 \quad-0.988430 \quad-1.906230$

$\mathrm{H} \quad 2.070360 \quad 0.691230 \quad-1.573120$

H $\quad 2.768930 \quad 1.772850 \quad 0.640820$

$\mathrm{H} \quad 3.723310 \quad 0.831550 \quad 1.789630$

H $\quad 4.344670 \quad-1.531760 \quad 1.302750$ 


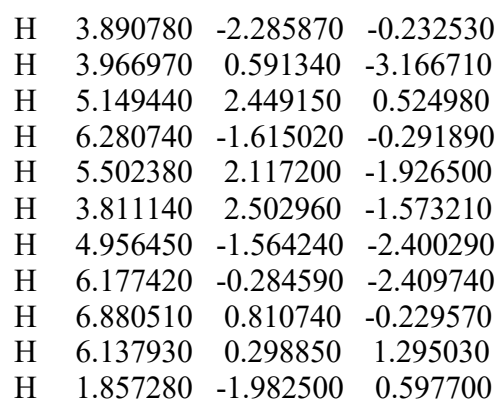

\section{ligY TSrot}

$\begin{array}{llll}O & -2.691815 & 3.019705 & -1.929602\end{array}$

$\begin{array}{llll}\mathrm{O} & -2.160473 & 2.830770 & 0.258358\end{array}$

$\begin{array}{lllll}\mathrm{O} & 0.393004 & 2.788396 & -0.667274\end{array}$

$\begin{array}{llll}\mathrm{O} & -0.370966 & -1.540332 & -1.695089\end{array}$

$\begin{array}{lllll}\mathrm{Cl} & 2.215835 & -3.079069 & -0.937446\end{array}$

$\begin{array}{llll}\mathrm{Cl} & 4.995308 & -2.192598 & 0.230330\end{array}$

$\begin{array}{llll}\text { Cl } & 5.472373 & 0.776469 & 1.054111\end{array}$

$\begin{array}{lllll}\text { Cl } & 3.178187 & 2.899158 & 0.709957\end{array}$

$\begin{array}{llll}\mathrm{N} & -0.525013 & 0.628875 & -0.870557\end{array}$

$\begin{array}{llll}\text { C } & -2.282645 & 2.440563 & -0.915765\end{array}$

$\begin{array}{llll}\text { C } & -1.967757 & 0.895138 & -1.137749\end{array}$

$\begin{array}{llll}\text { C } & -2.939023 & 0.039692 & -0.249536\end{array}$

$\begin{array}{llll}\text { C } & -3.192499 & -1.381883 & -0.809084\end{array}$

$\begin{array}{llll}\text { C } & -2.429358 & -0.087862 & 1.198368\end{array}$

$\begin{array}{llll}\text { C } & -4.319330 & 0.751340 & -0.231961\end{array}$

$\begin{array}{lllll}\text { C } & 0.455993 & 1.604794 & -0.570525\end{array}$

$\begin{array}{llll}\text { C } & 0.089135 & -0.586877 & -1.123210\end{array}$

$\begin{array}{llll}\text { C } & -4.204213 & -2.151699 & 0.056237\end{array}$

$\begin{array}{llll}\text { C } & -3.446972 & -0.850295 & 2.061991\end{array}$

$\begin{array}{llll}\text { C } & -5.330065 & -0.010773 & 0.634714\end{array}$

$\begin{array}{llll}\text { C } & 1.710608 & 0.829794 & -0.236656\end{array}$

$\begin{array}{llll}\text { C } & 1.499612 & -0.484591 & -0.601663\end{array}$

$\begin{array}{llll}\text { C } & -3.660131 & -2.259400 & 1.489409\end{array}$

$\begin{array}{llll}\text { C } & -5.549766 & -1.420501 & 0.077185\end{array}$

$\begin{array}{llll}\text { C } & -4.787212 & -0.099799 & 2.068972\end{array}$

$\begin{array}{llll}\text { C } & 2.925293 & 1.256958 & 0.262294\end{array}$

$\begin{array}{llll}\text { C } & 2.489874 & -1.439702 & -0.480424\end{array}$

$\begin{array}{llll}\text { C } & 3.942425 & 0.300735 & 0.410566\end{array}$

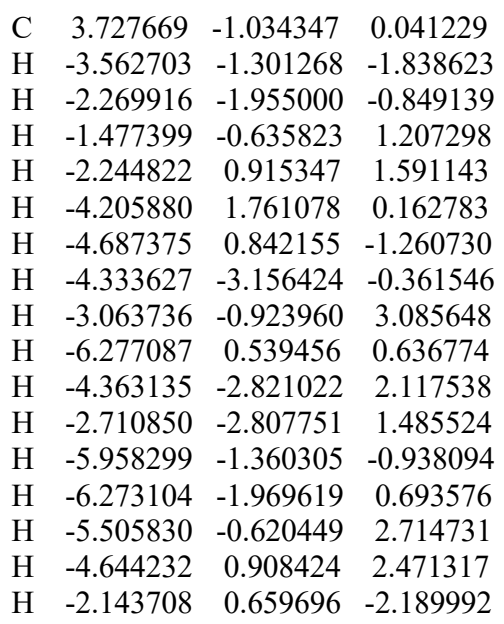

\section{probeA}

$\begin{array}{lll}\text { S } & 2.99781005 & 7.52077791\end{array}$

7.69384669

Si $\quad 3.03493367 \quad 5.37070859$

7.58277162

H $3.33933782 \quad 5.01608725$

6.18904216

H 4.016162124 .71623345

8.46012732

Si $\quad-0.23550355 \quad 6.62320489$

2.92785568

H $\quad-0.84597240 \quad 7.96012617$

2.89837255

H $\quad 0.10042137 \quad 6.20465139$ 1.55932822

Si $\quad 1.71226164 \quad 6.73584504$

4.26071034

H $\quad 2.22349598 \quad 5.36853474$

4.43392116

H $\quad 2.72795147 \quad 7.56517053$

3.59626064

Si $\quad 1.25095954 \quad 7.70641956$

6.36969651
H $\quad 0.88928924 \quad 9.11996141$

6.19082778

H $\quad 0.13100706 \quad 6.98605997$ 6.99234289

Si $\quad-2.16068622 \quad 1.39449517$

4.93706640

H $\quad-3.57482642 \quad 1.29617978$

4.54791145

H $\quad-1.59644082 \quad 0.03713174$

4.92749313

Si $\quad-0.90471051 \quad 2.85486480$

3.59010375

$\mathrm{H} \quad-0.93588802 \quad 2.44582470$

2.17850389

H $\quad 0.48161783 \quad 2.82518368$

4.07807176

Si $\quad-1.74251167 \quad 5.05043032$ 3.82791459

H $\quad-3.03847712 \quad 5.11852020$

3.13745292

H $\quad-1.92153239 \quad 5.37442359$

5.25054593

Si $\quad 0.84863738 \quad 4.76187852$ 8.09558084

H $\quad-0.04655974 \quad 5.75876922$ 7.49082383

$\mathrm{H} \quad 0.60748103 \quad 4.71178667$

9.54479940

Si $\quad-1.88414607 \quad 2.47118468$ 6.95986247

H $\quad-2.40949237 \quad 1.78200987$

8.14727534

H $\quad-2.55638591 \quad 3.77356934$

6.84677936

Si $\quad 0.41943687 \quad 2.77946796$ 6.99949212

H $1.12652764 \quad 1.61499044$ 7.55168300

H $\quad 0.85737522 \quad 3.00219661$

5.61403121 


\section{NMR Spectra for Characterization}

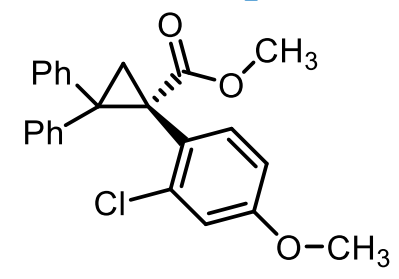

${ }^{1} \mathrm{H} \mathrm{NMR}\left(600 \mathrm{MHz}, \mathrm{CDCl}_{3}\right)$

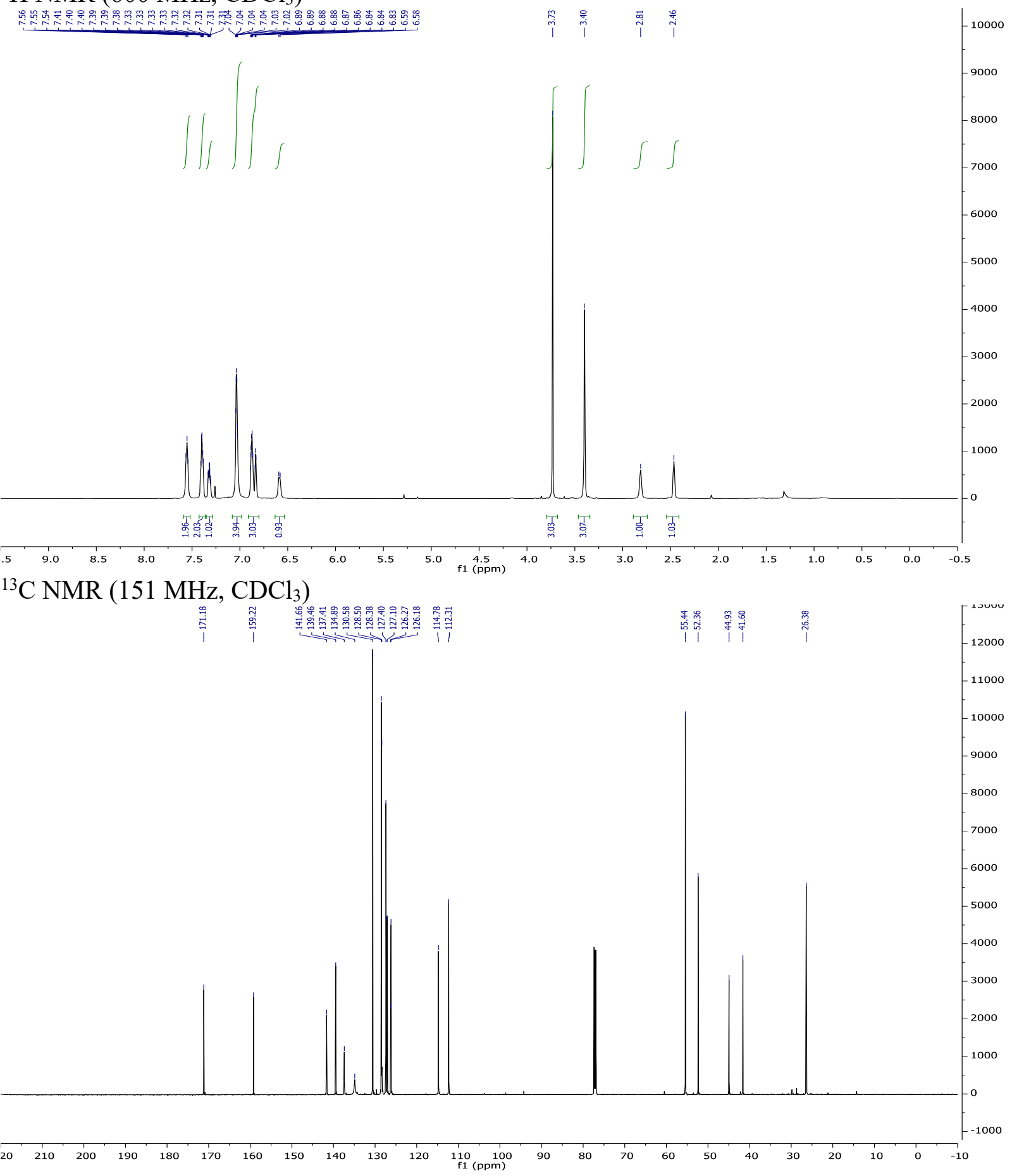




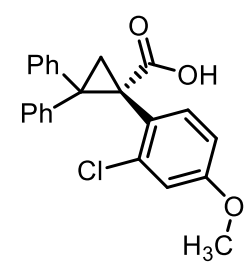

${ }^{1} \mathrm{H} \mathrm{NMR}\left(600 \mathrm{MHz}, \mathrm{CDCl}_{3}\right)$

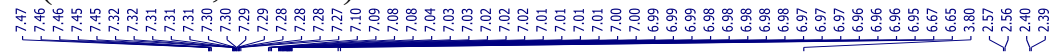

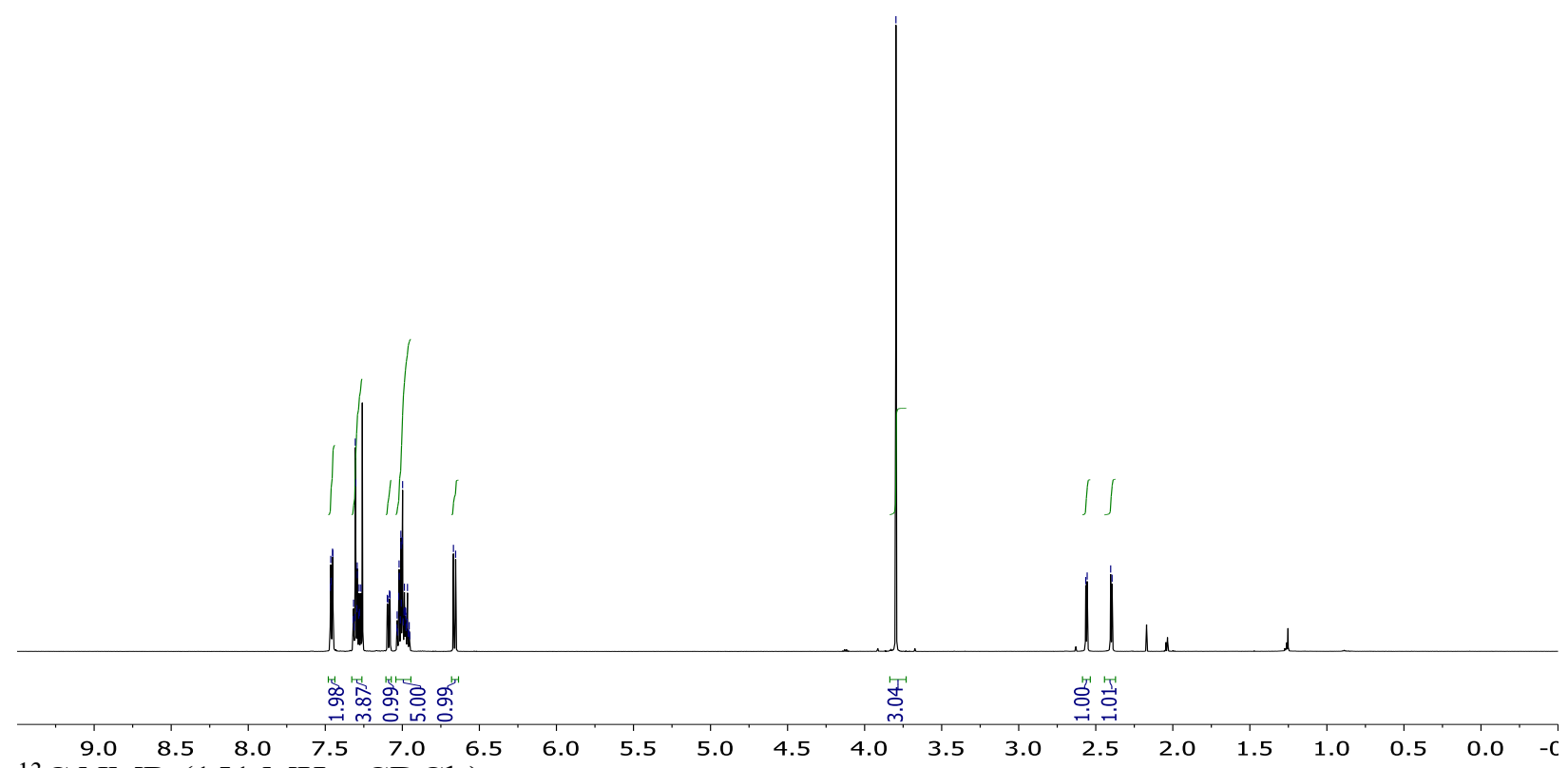
${ }^{13} \mathrm{C}$ NMR $\left(151 \mathrm{MHz}, \mathrm{CDCl}_{3}\right)$

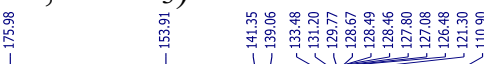

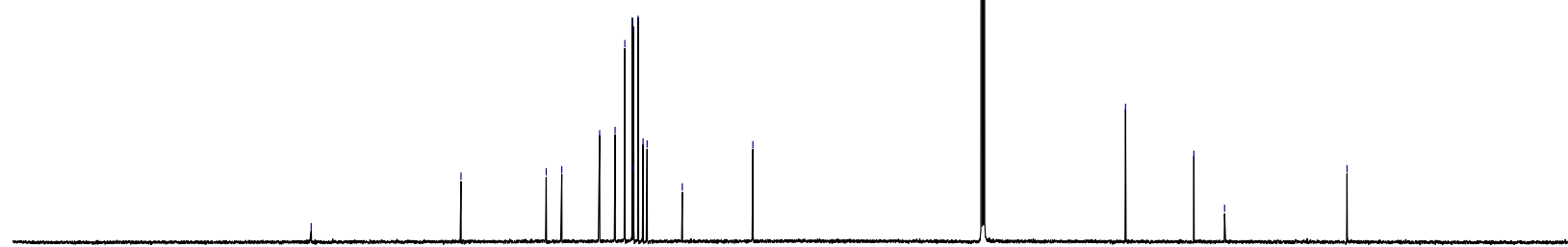

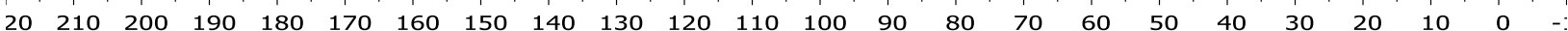




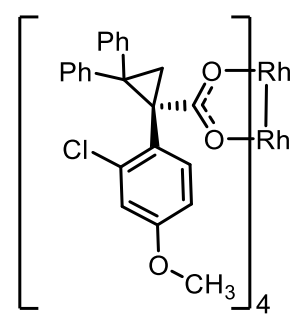

${ }^{1} \mathrm{H}$ NMR (600 MHz, $\left.\mathrm{CDCl}_{3}\right)$

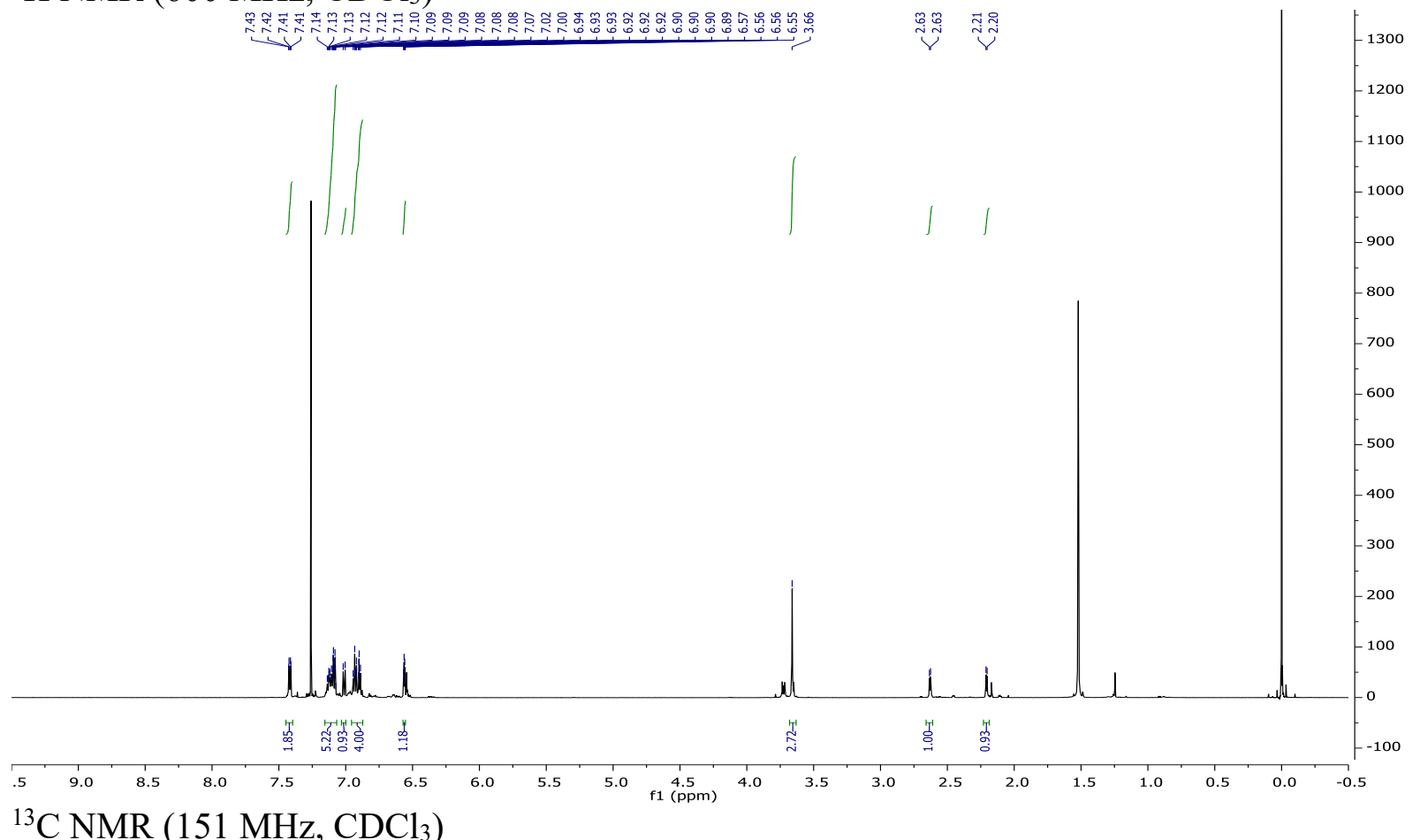
${ }^{13} \mathrm{C} \mathrm{NMR}\left(151 \mathrm{MHz}, \mathrm{CDCl}_{3}\right)$

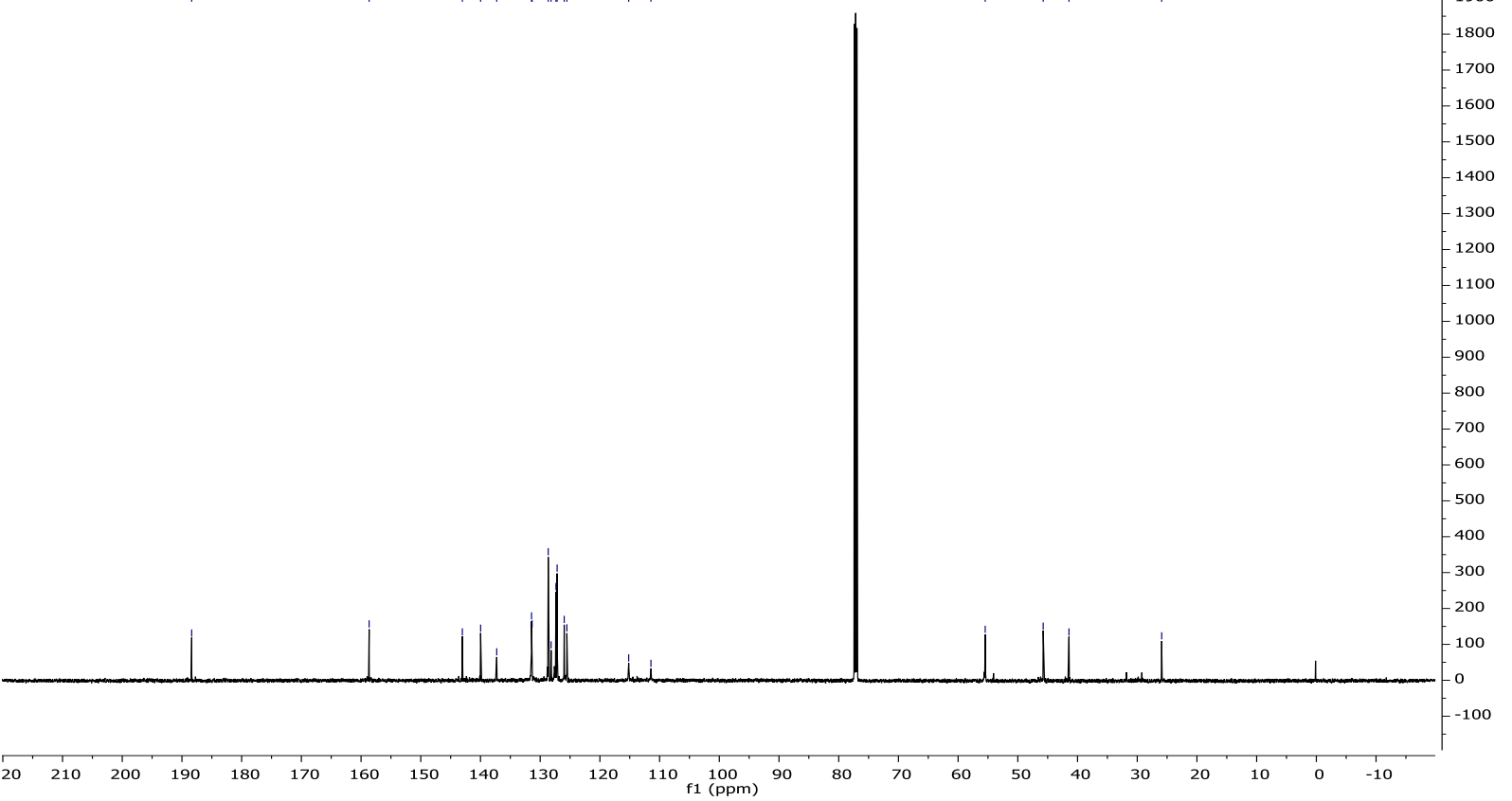




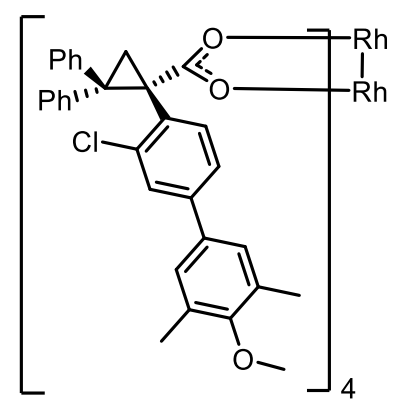

${ }^{1} \mathrm{H} \mathrm{NMR}\left(600 \mathrm{MHz}, \mathrm{CDCl}_{3}\right)$

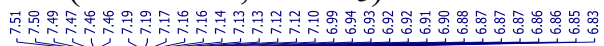
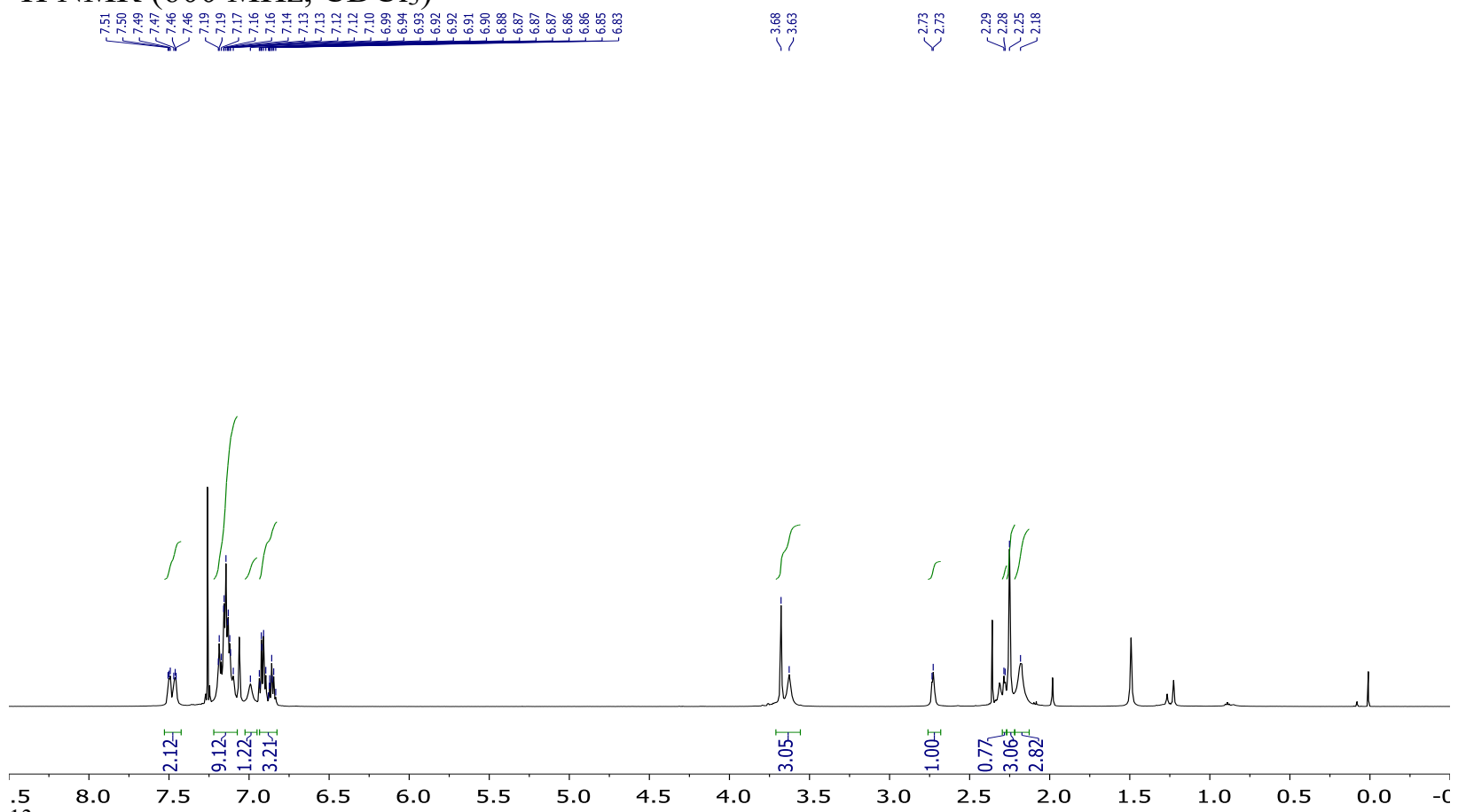

${ }^{13} \mathrm{C}$ NMR (151 MHz, $\left.\mathrm{CDCl}_{3}\right)$

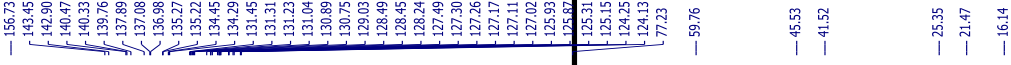

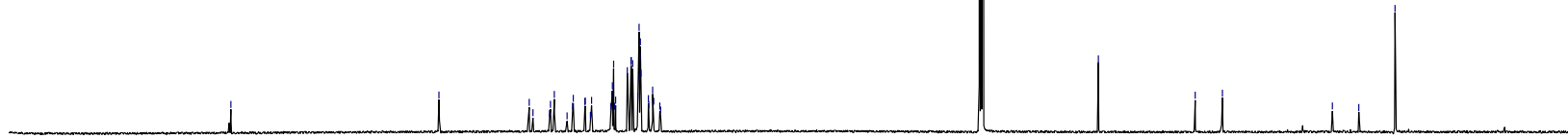

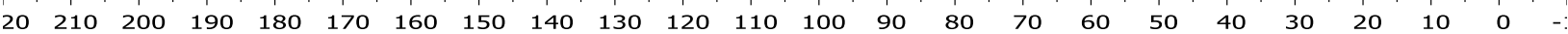




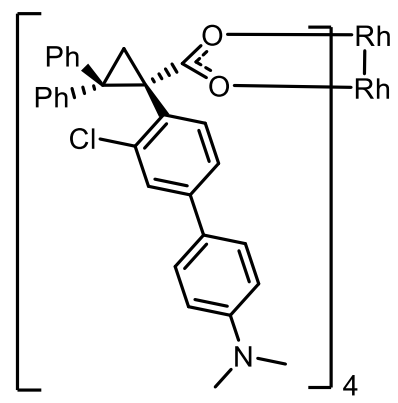

${ }^{1} \mathrm{H} \mathrm{NMR}\left(600 \mathrm{MHz}, \mathrm{CDCl}_{3}\right)$

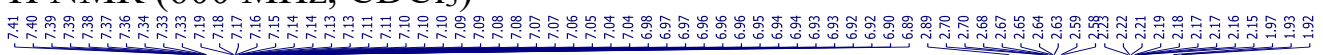

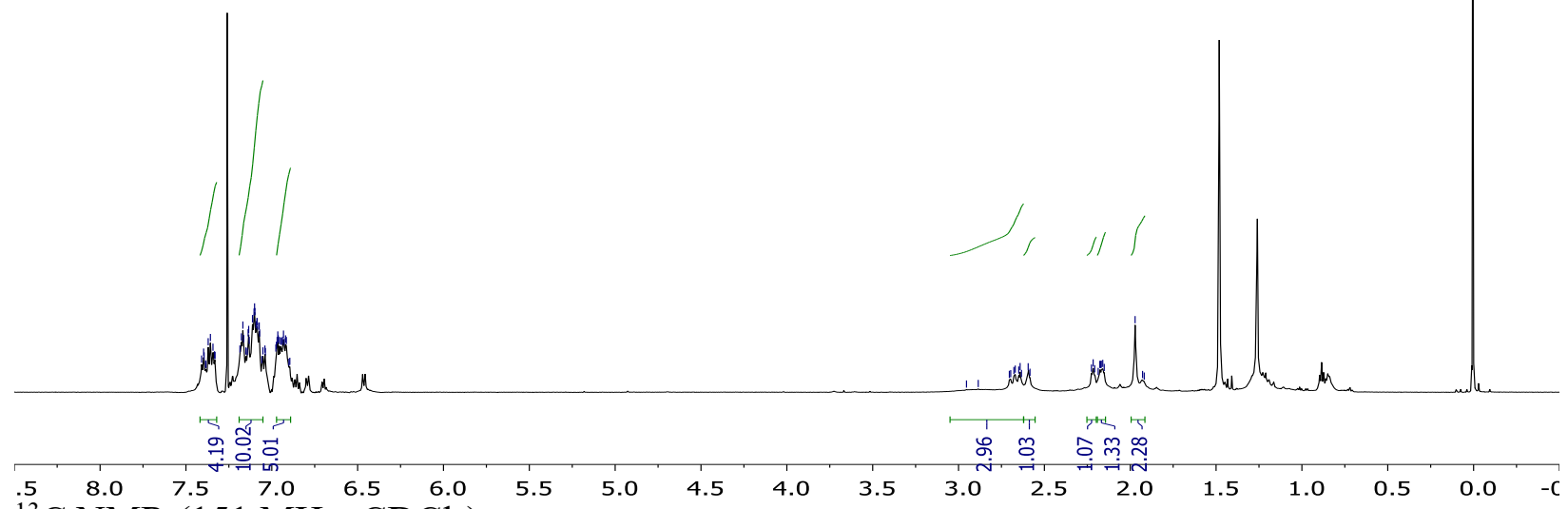

${ }^{13} \mathrm{C}$ NMR (151 MHz, $\left.\mathrm{CDCl}_{3}\right)$

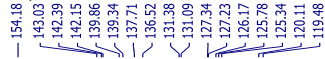
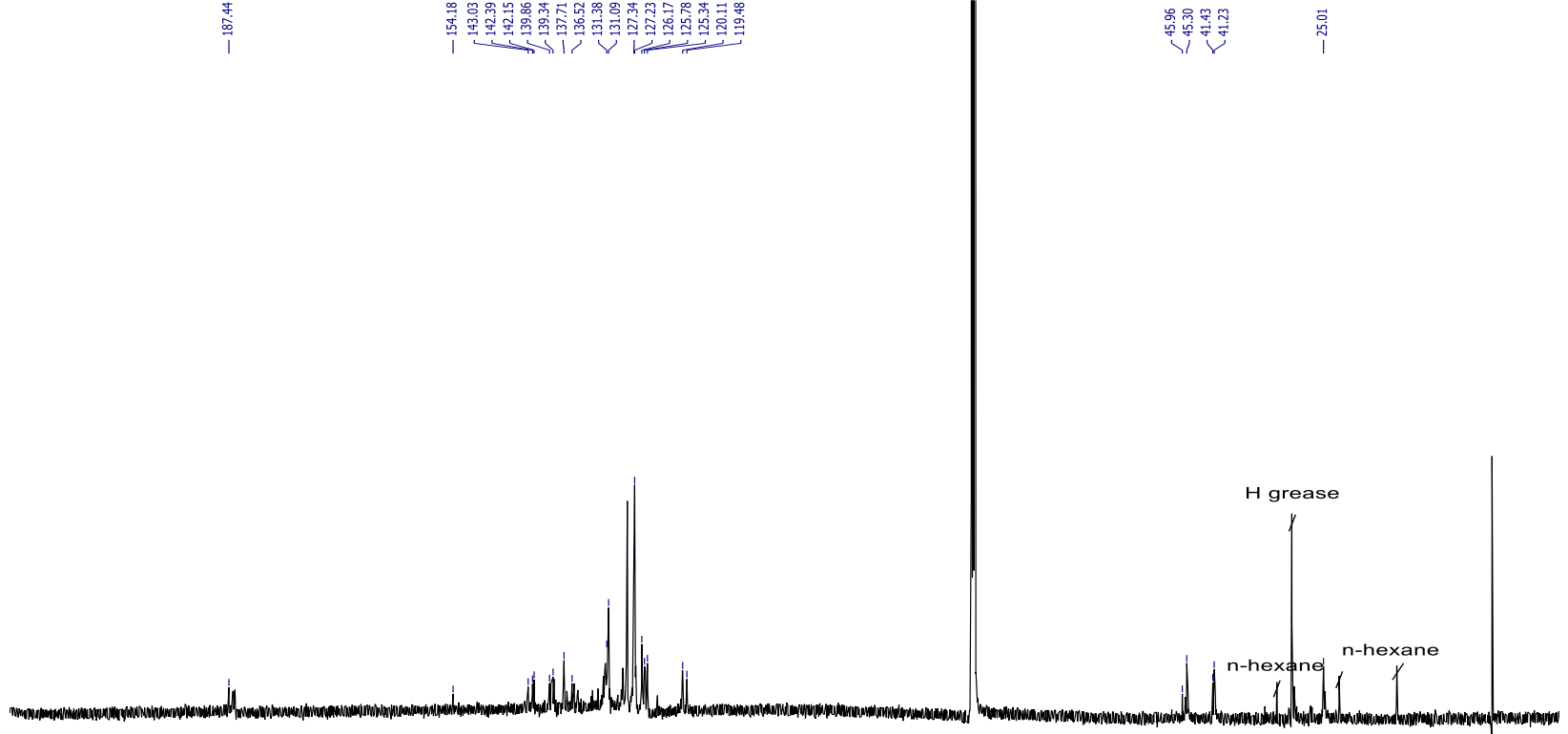

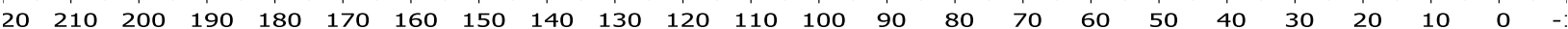




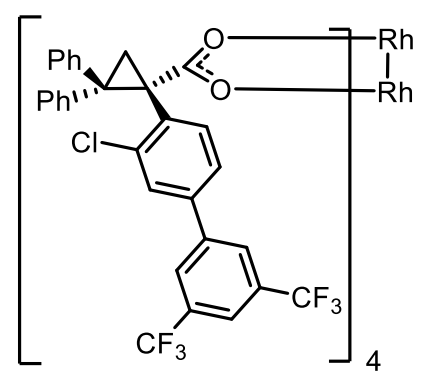

${ }^{1} \mathrm{H} \mathrm{NMR}\left(600 \mathrm{MHz}, \mathrm{CDCl}_{3}\right)$

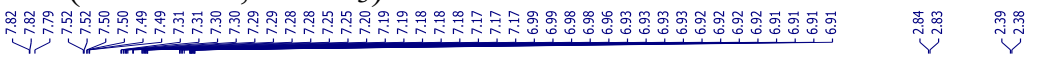

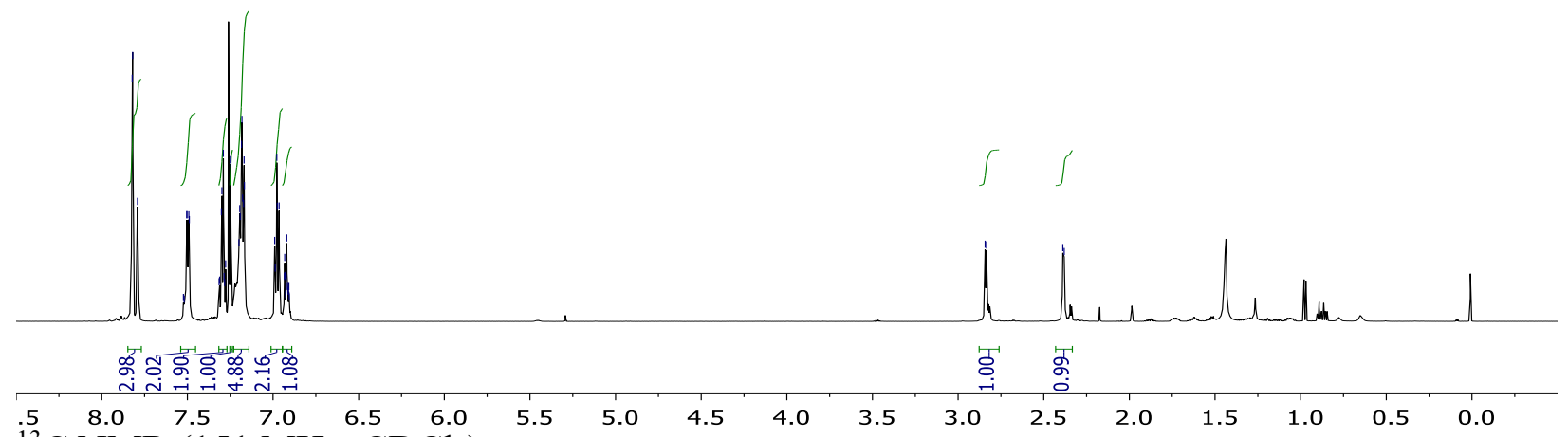
${ }^{.5} \mathrm{C}$ NMR $\left(151^{8.0} \mathrm{MHz}^{7.0} \mathrm{CDCl}_{3}\right)$

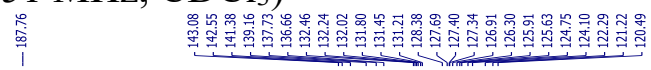

$\begin{array}{llllllllllllllllllllllll}20 & 210 & 200 & 190 & 180 & 170 & 160 & 150 & 140 & 130 & 120 & 110 & 100 & 90 & 80 & 70 & 60 & 50 & 40 & 30 & 20 & 10 & 0 & -:\end{array}$ 


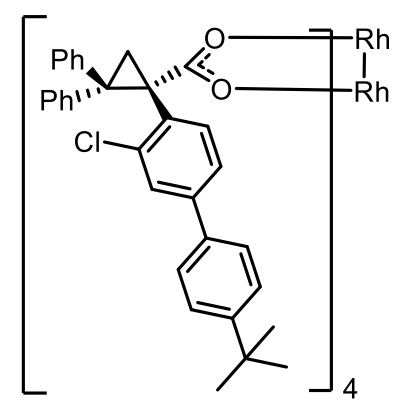

${ }^{1} \mathrm{H}$ NMR $\left(600 \mathrm{MHz}, \mathrm{CDCl}_{3}\right)$

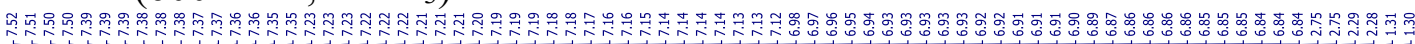

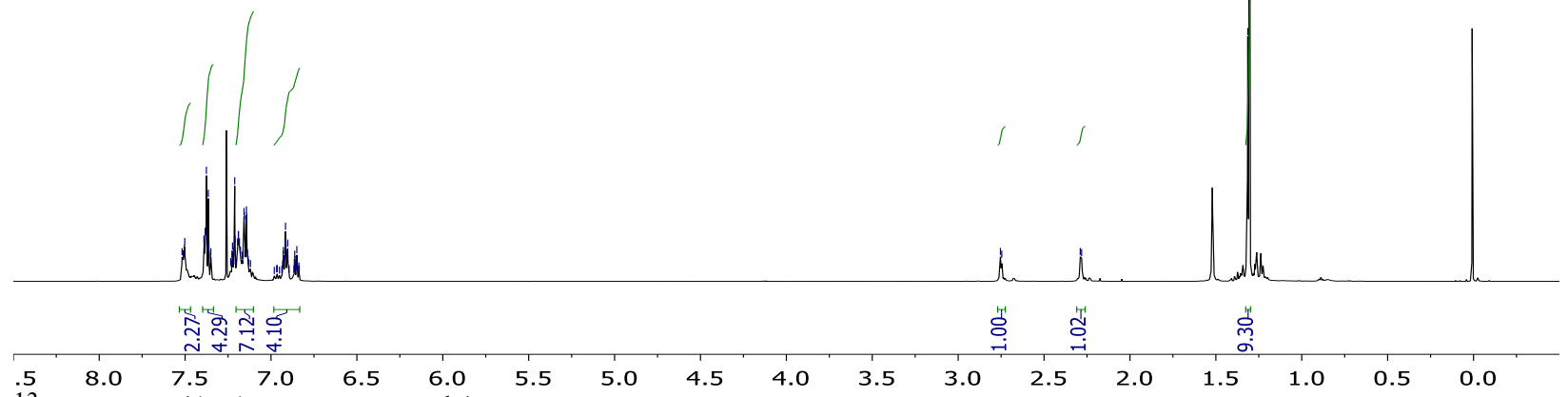
${ }^{13} \mathrm{C}$ NMR $\left(151 \mathrm{MHz}, \mathrm{CDCl}_{3}\right)$

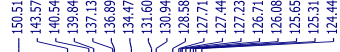

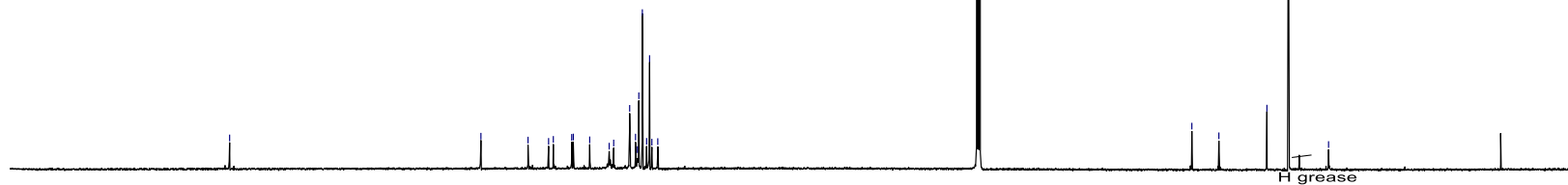

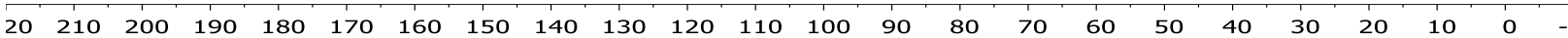




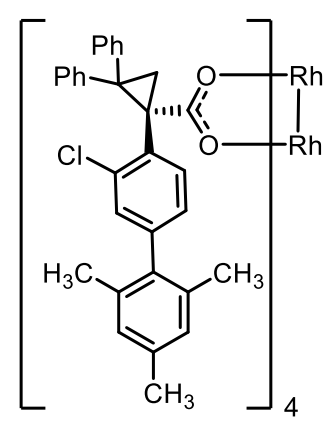

${ }^{1} \mathrm{H} \mathrm{NMR}\left(600 \mathrm{MHz}, \mathrm{CDCl}_{3}\right)$

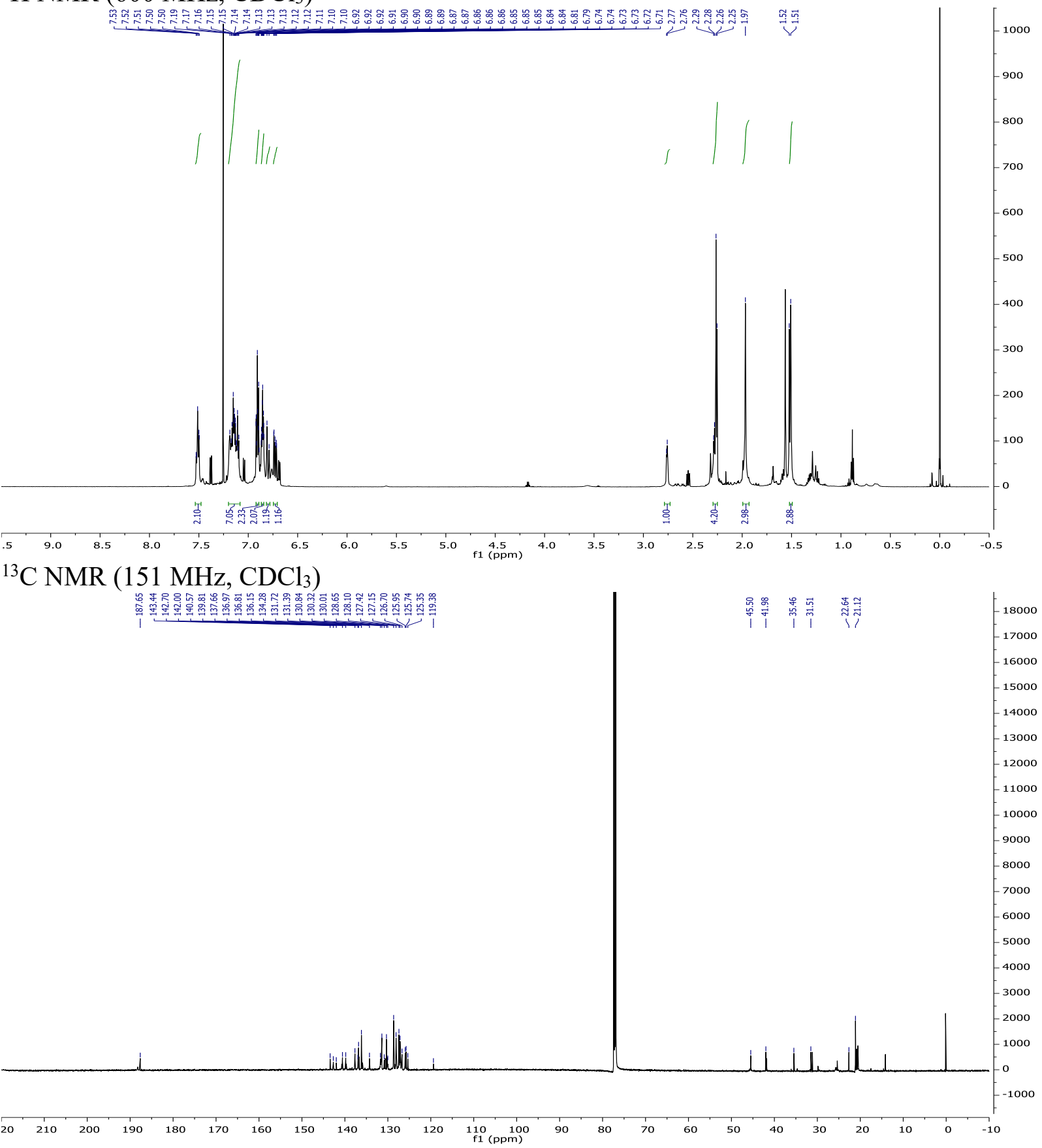




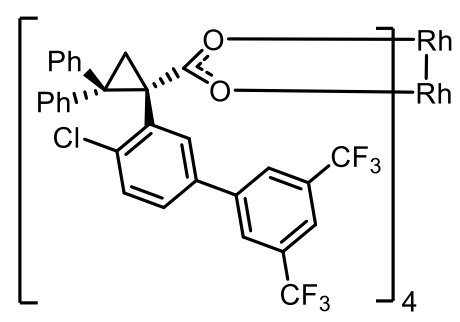

${ }^{1} \mathrm{H} \mathrm{NMR}\left(600 \mathrm{MHz}, \mathrm{CDCl}_{3}\right)$

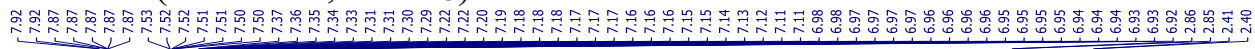

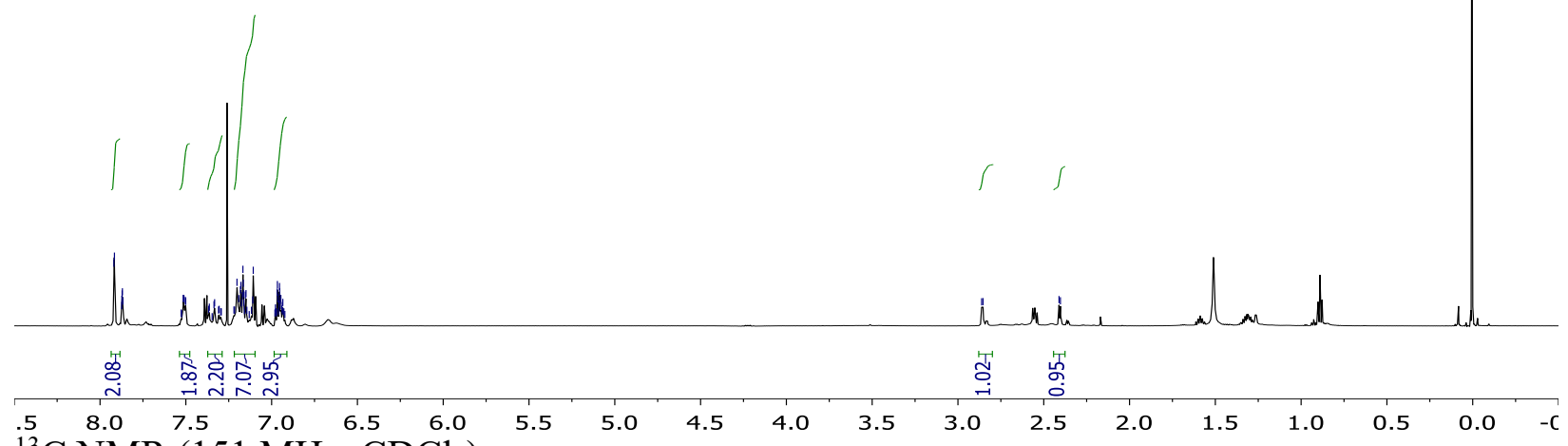

${ }^{13} \mathrm{C}$ NMR $\left(151 \mathrm{MHz}, \mathrm{CDCl}_{3}\right)$

畜

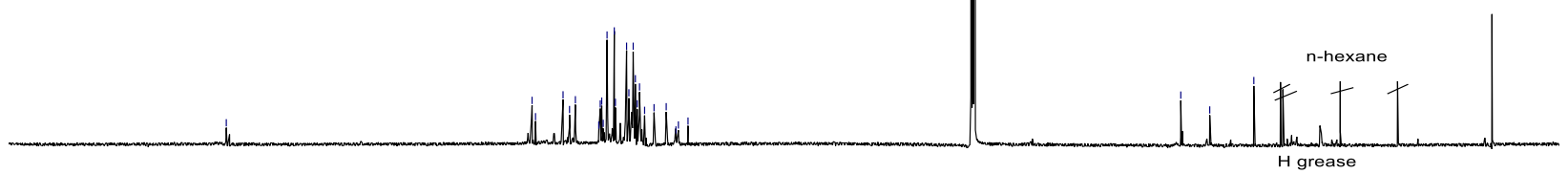

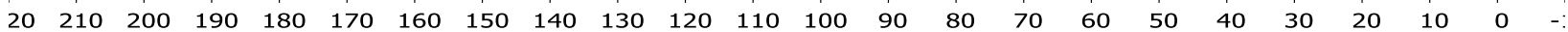




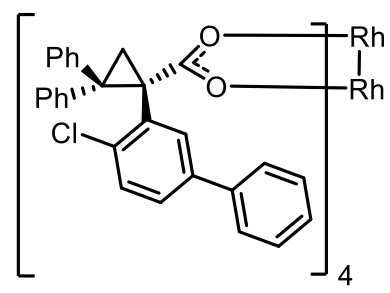

${ }^{1} \mathrm{H} \mathrm{NMR}\left(600 \mathrm{MHz}, \mathrm{CDCl}_{3}\right)$

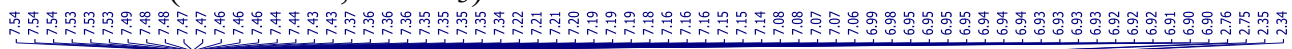

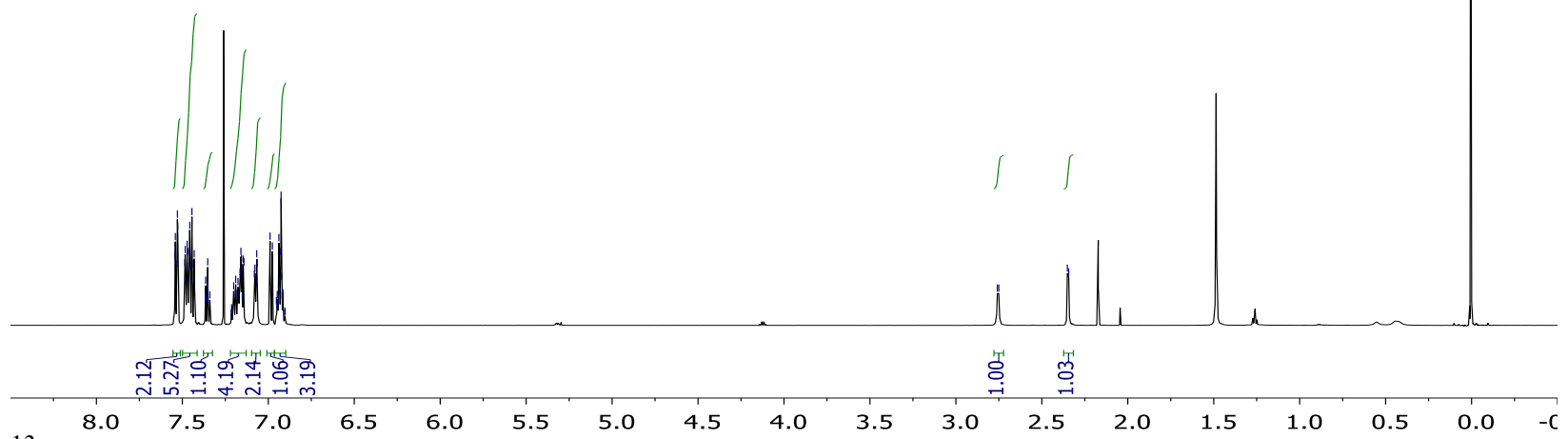
${ }^{13} \mathrm{C} \mathrm{NMR}\left(151 \mathrm{MHz}, \mathrm{CDCl}_{3}\right)$
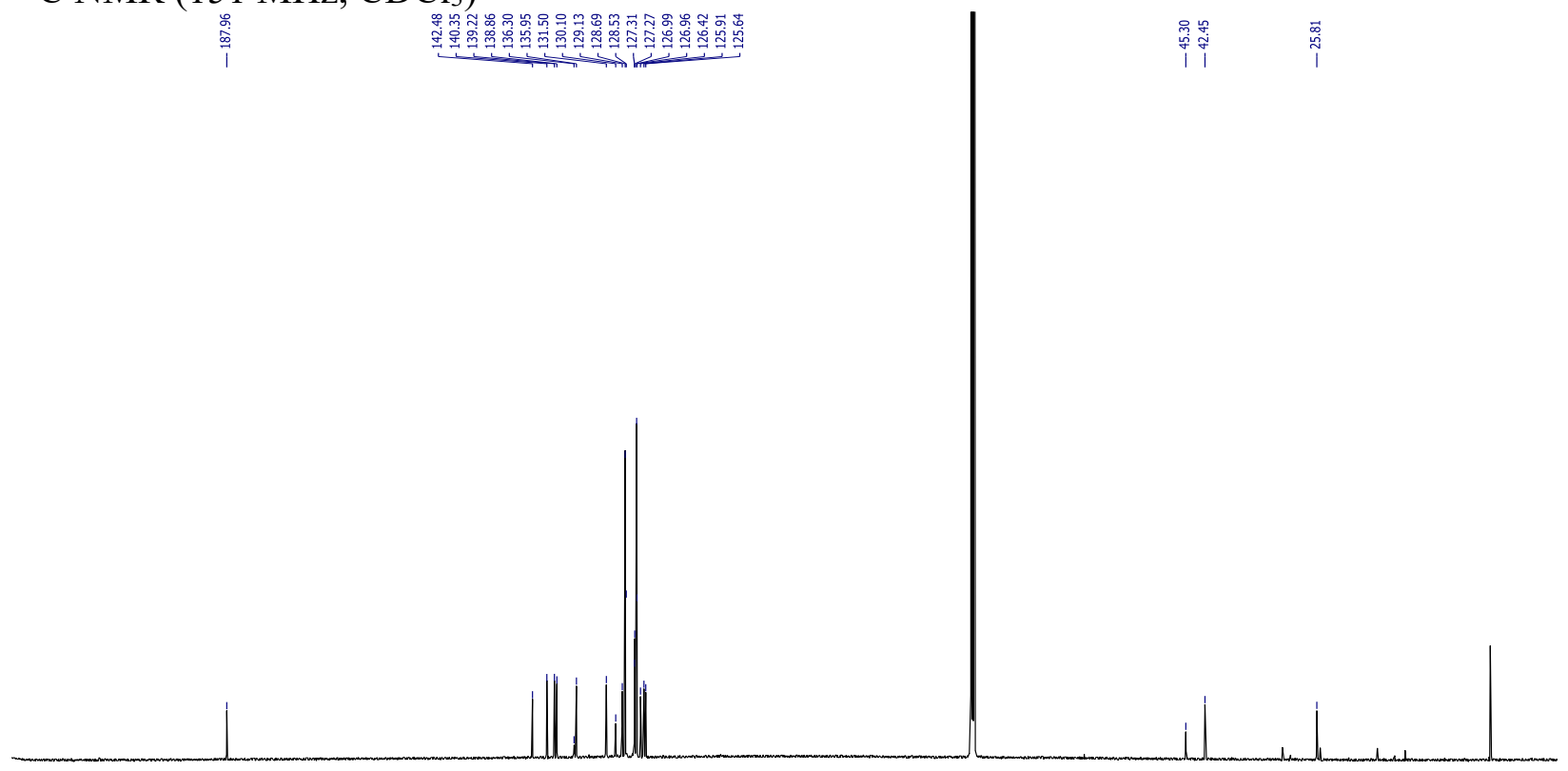

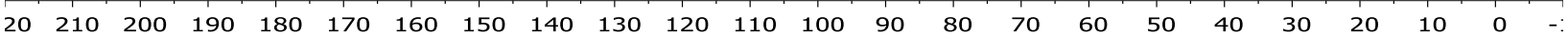




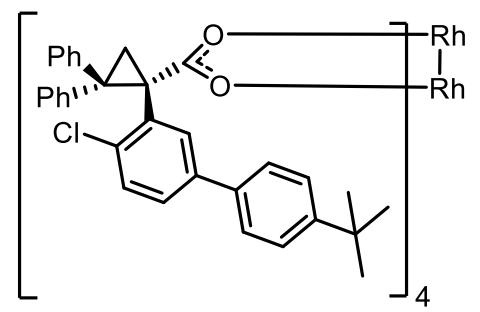

${ }^{1} \mathrm{H} \mathrm{NMR}\left(600 \mathrm{MHz}, \mathrm{CDCl}_{3}\right)$

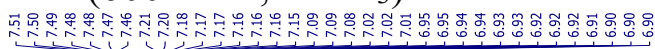
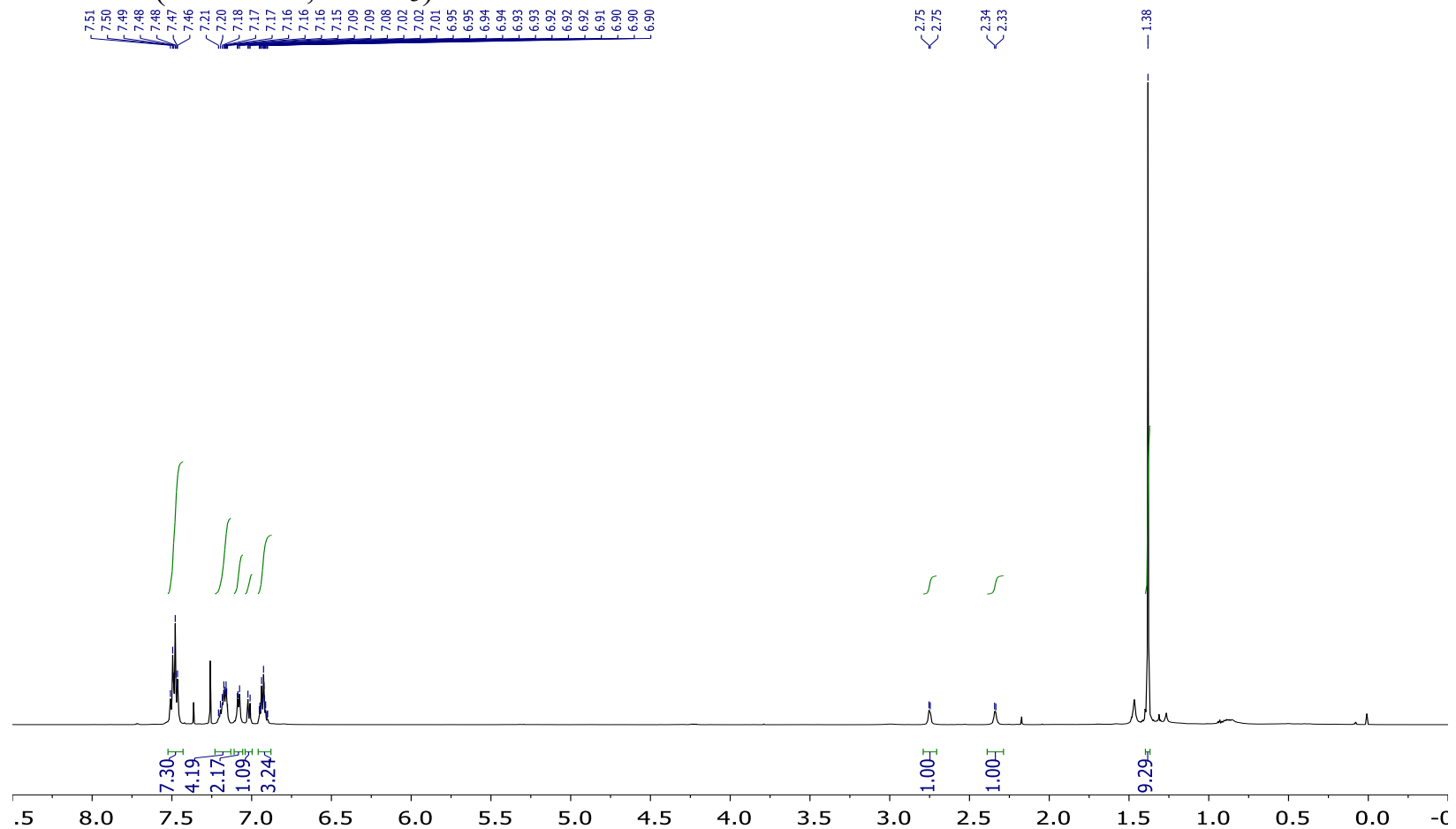
${ }^{13} \mathrm{C}$ NMR $\left(151 \mathrm{MHz}, \mathrm{CDCl}_{3}\right)$
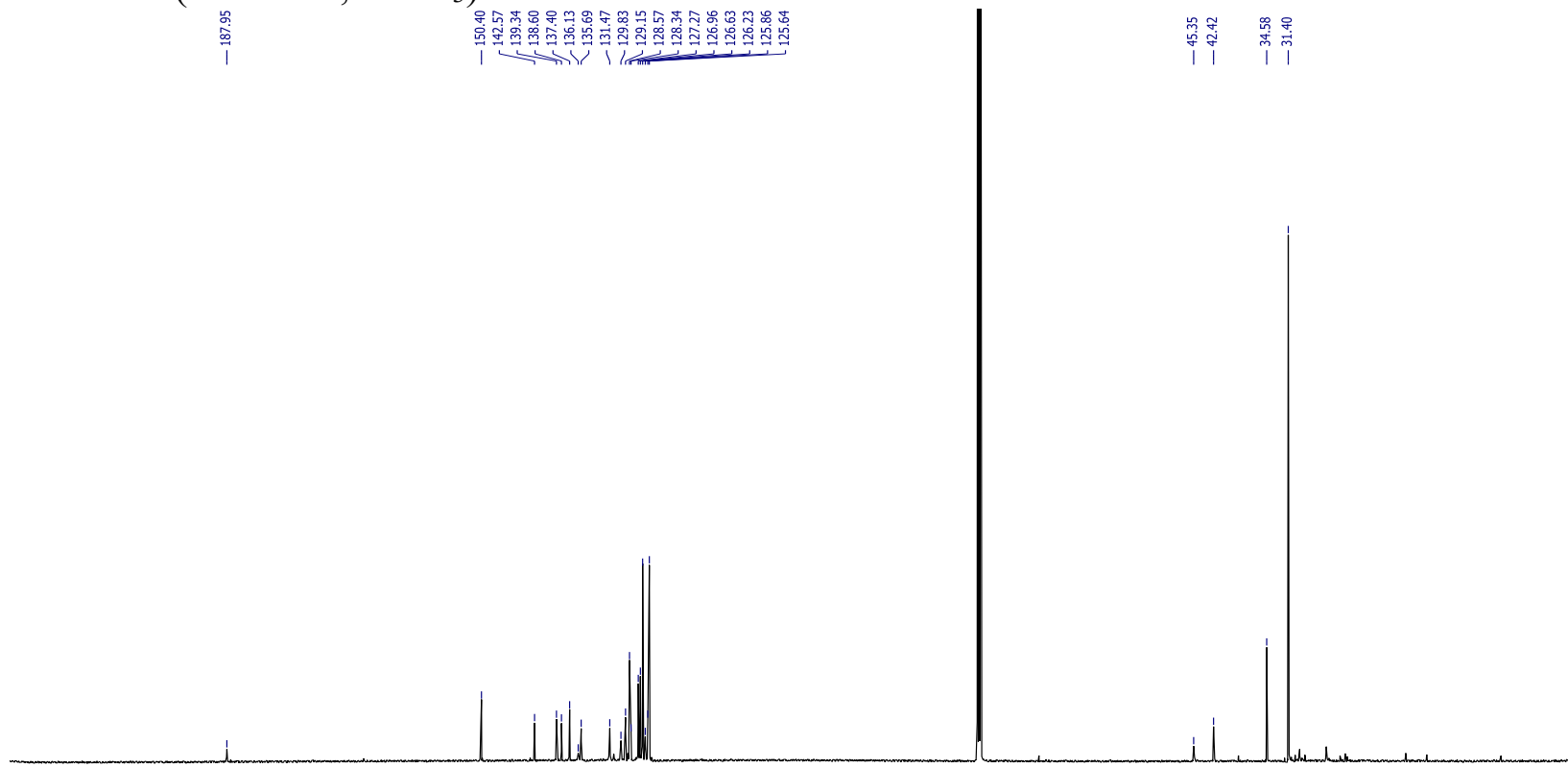

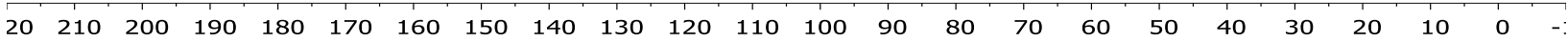




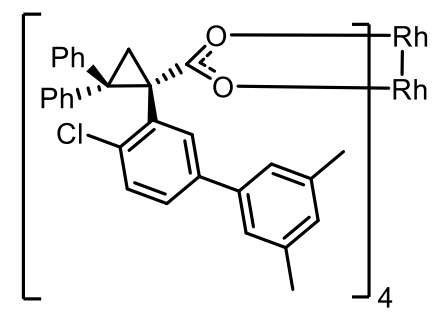

${ }^{1} \mathrm{H}$ NMR $\left(600 \mathrm{MHz}, \mathrm{CDCl}_{3}\right)$

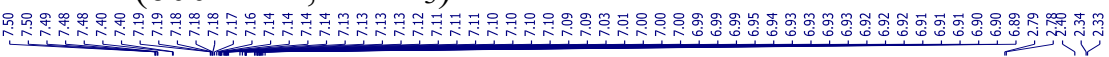

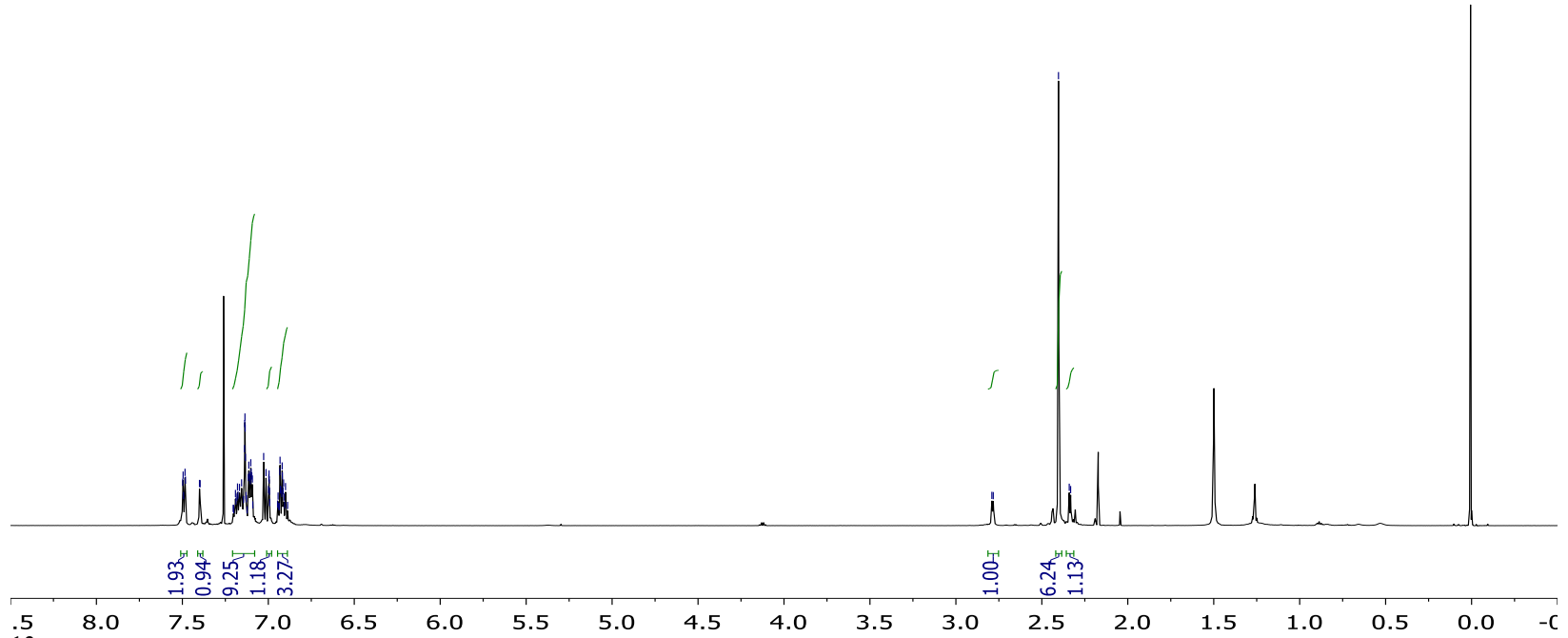

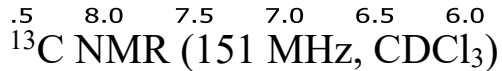
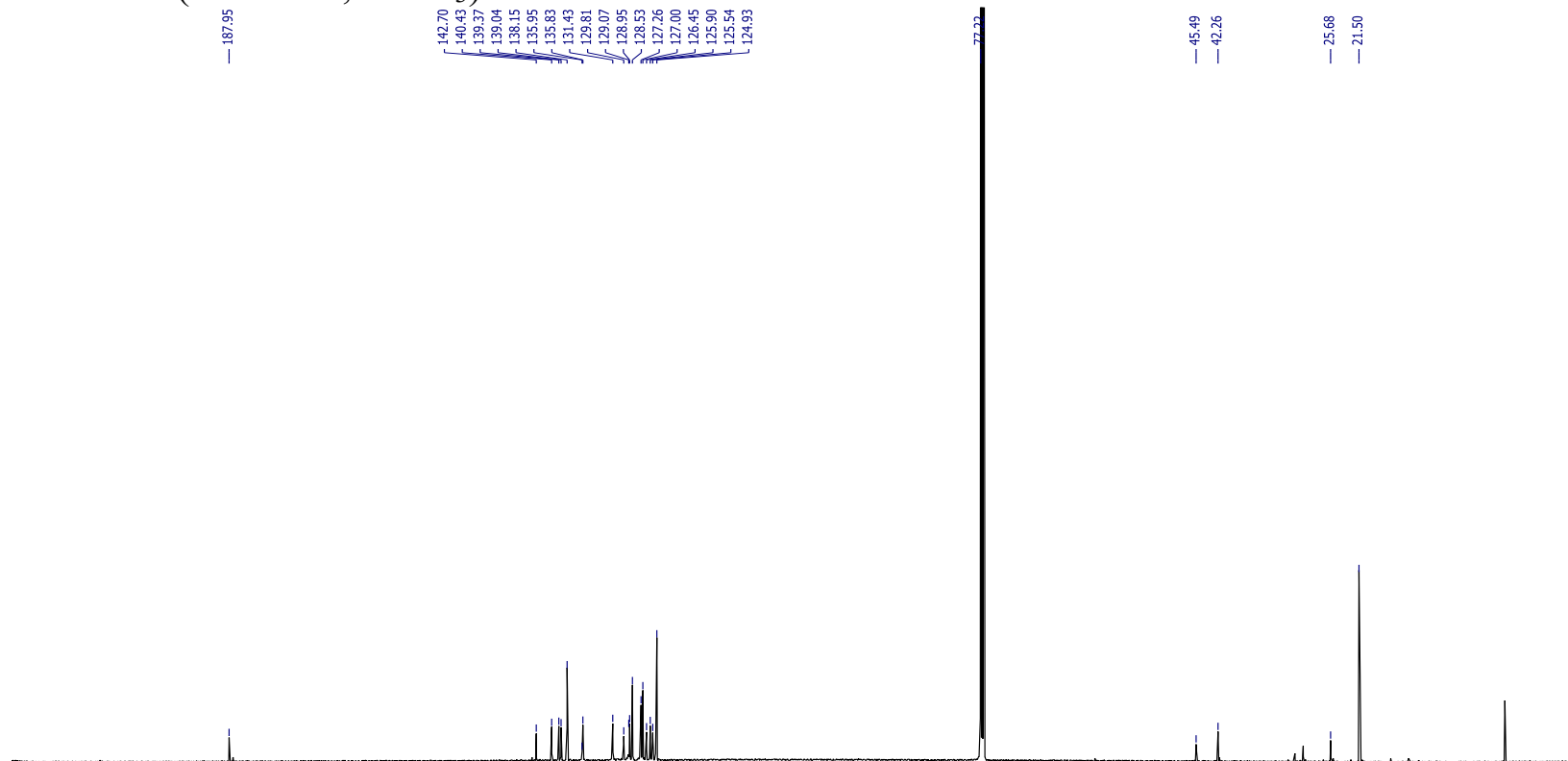

$\begin{array}{llllllllllllllllllllll}20 & 210 & 200 & 190 & 180 & 170 & 160 & 150 & 140 & 130 & 120 & 110 & 100 & 90 & 80 & 70 & 60 & 50 & 40 & 30 & 20 & 10\end{array}$ 


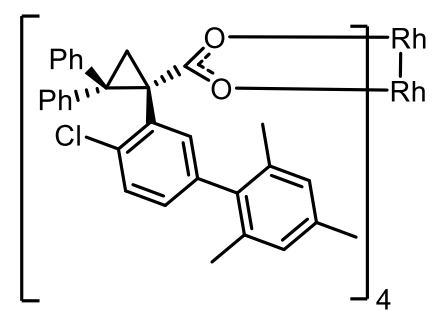

${ }^{1} \mathrm{H}$ NMR (600 MHz, $\mathrm{CDCl}_{3}$ )

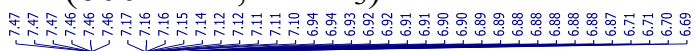

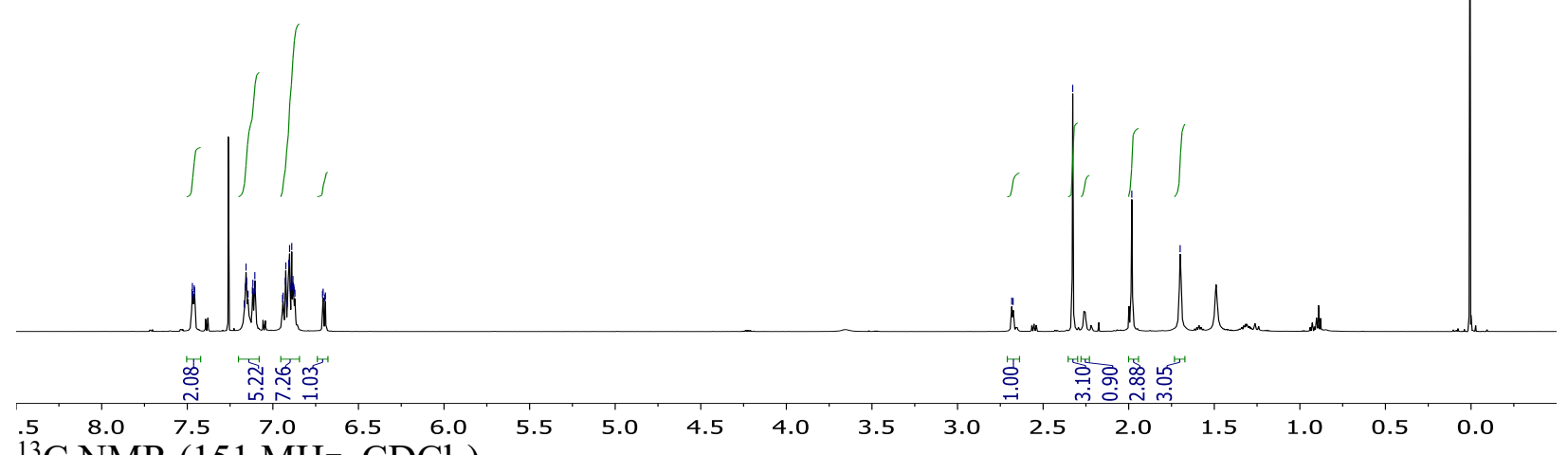

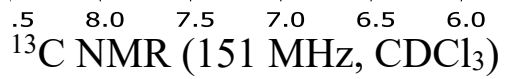

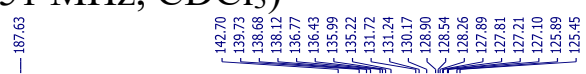

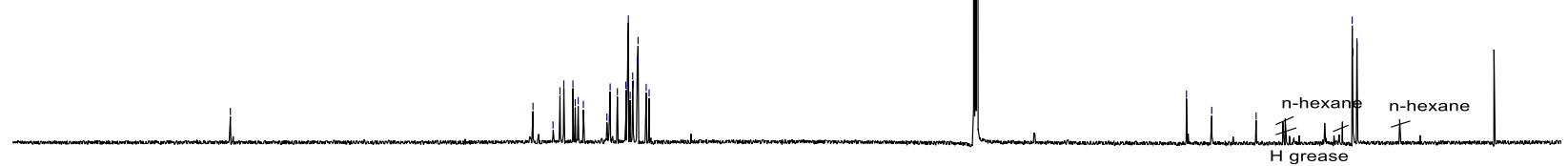

$\begin{array}{lllllllllllllllllllllll}20 & 210 & 200 & 190 & 180 & 170 & 160 & 150 & 140 & 130 & 120 & 110 & 100 & 90 & 80 & 70 & 60 & 50 & 40 & 30 & 20 & 10 & 0\end{array}$ 


\section{HPLC Spectra for Enantioselectivity Determination}

The regioisomers and diastereomers of the $\mathrm{C}-\mathrm{H}$ insertion products are inseparable by flash column chromatography, which causes additional difficulty for enantiomer separation on our available chiral HPLC columns. Hence, for better separation, the isolated products were reduced to the corresponding alcohols, without influence on the chiral centers, and used for enantiomeric excess (ee) determination of the $\mathrm{C}-\mathrm{H}$ functionalization products.
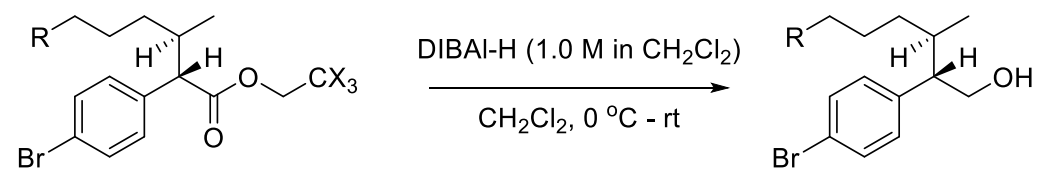

A flask with a solution of ester $(0.2 \mathrm{mmol}, 1.0$ equiv. $)$ in $\mathrm{CH}_{2} \mathrm{Cl}_{2}(1 \mathrm{~mL})$ was flushed with argon and cooled to $0{ }^{\circ} \mathrm{C}$ via ice bath. Under an argon atmosphere, a solution of $1 \mathrm{M}$ diisobutylaluminum hydride (DIBAl-H) in $\mathrm{CH}_{2} \mathrm{Cl}_{2}(0.4 \mathrm{~mL}, 0.4 \mathrm{mmol}, 2.0$ equiv.) was added dropwise into the flask at $0{ }^{\circ} \mathrm{C}$, and the mixture was stirred for $4 \mathrm{~h}$ at room temperature. The reaction was quenched by adding $2 \mathrm{~mL}$ of $2 \mathrm{M}$ aqueous $\mathrm{HCl}$ solution dropwise. The resulting solution was extracted with diethyl ether $(3 \times 3 \mathrm{~mL})$ and dried over $\mathrm{Na}_{2} \mathrm{SO}_{4}$. The crude product was concentrated and confirmed by ${ }^{1} \mathrm{H}$ NMR spectroscopy, and then the crude alcohol product was used for HPLC directly.

HPLC (reduced alcohol): OJH column, 5\% $i$-propanol in hexane, $0.5 \mathrm{~mL} \mathrm{~min}^{-1}, 1 \mathrm{mg} \mathrm{mL}^{-1}, 120$ min, UV $230 \mathrm{~nm}$. 
<smiles>CCCCCc1ccc(Br)cc1</smiles>
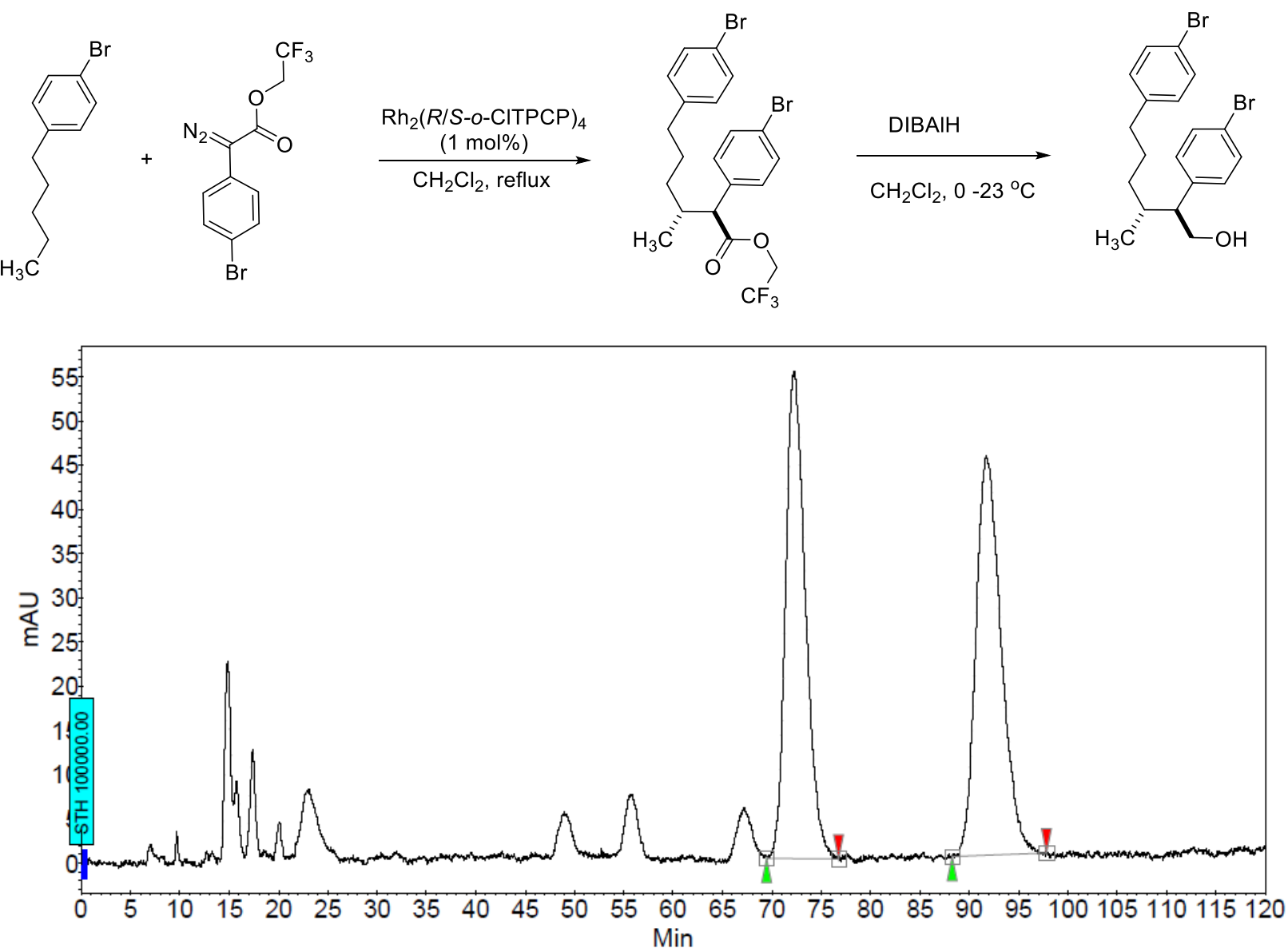

\section{Peak results :}

\begin{tabular}{|c|l|r|r|r|r|r|}
\hline Index & Name & $\begin{array}{r}\text { Time } \\
\text { [Min] }\end{array}$ & $\begin{array}{r}\text { Quantity } \\
\text { [\% Area] }\end{array}$ & $\begin{array}{r}\text { Height } \\
\text { [mAU] }\end{array}$ & \multicolumn{2}{|c|}{$\begin{array}{r}\text { [mAU.Min] } \\
\text { [mArea \% } \\
\text { [\%] }\end{array}$} \\
\hline \hline 1 & UNKNOWN & 72.29 & 48.98 & 55.1 & 128.2 & 48.984 \\
\hline 2 & UNKNOWN & 91.76 & 51.02 & 45.2 & 133.5 & 51.016 \\
\hline & & & & & & \\
\hline Total & & & 100.00 & 100.3 & 261.7 & 100.000 \\
\hline
\end{tabular}

$\rightarrow$ racemic 

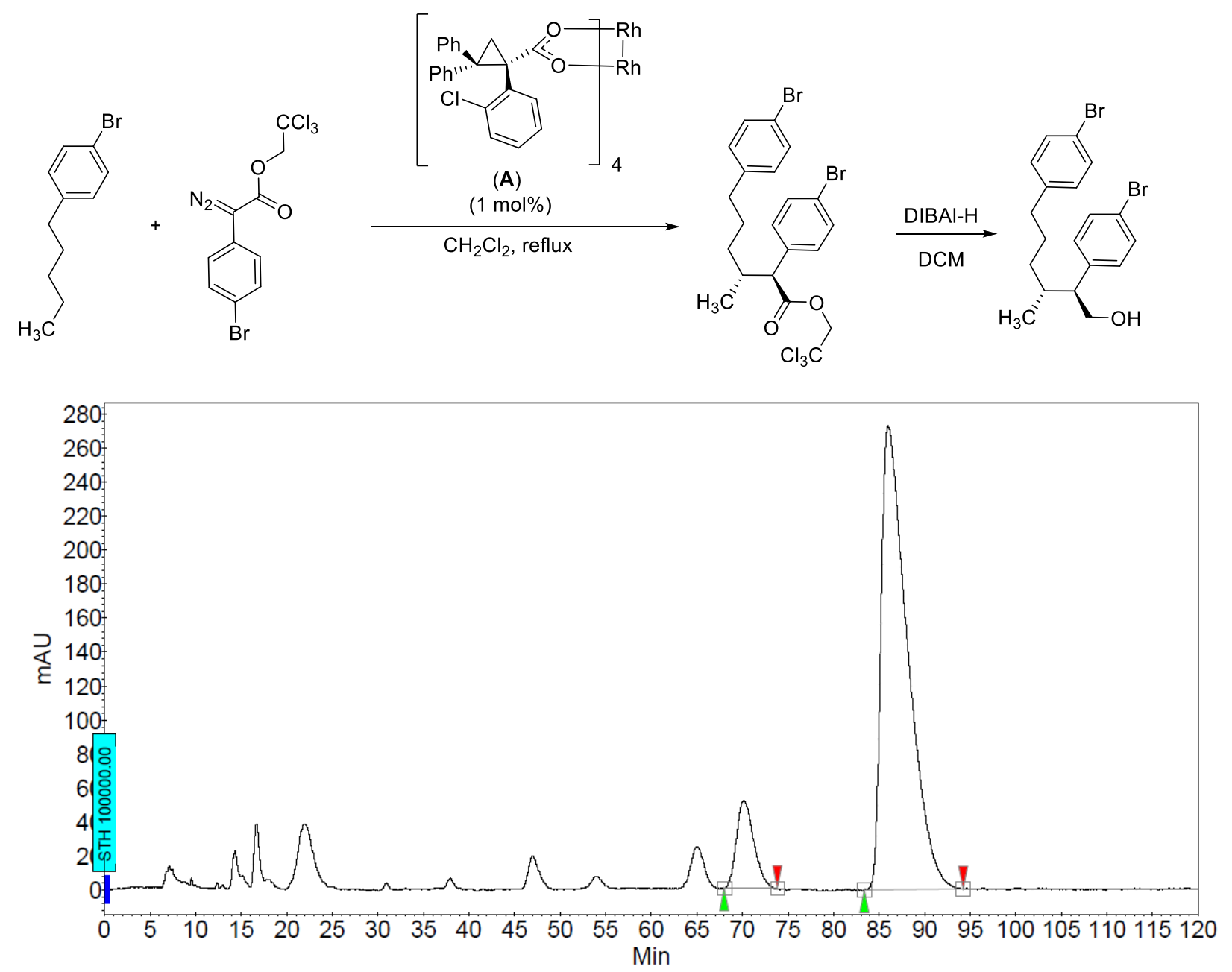

\section{Peak results :}

\begin{tabular}{|c|l|r|r|r|r|r|}
\hline Index & Name & $\begin{array}{r}\text { Time } \\
\text { [Min] }\end{array}$ & $\begin{array}{r}\text { Quantity } \\
\text { [\% Area] }\end{array}$ & $\begin{array}{r}\text { Height } \\
\text { [mAU] }\end{array}$ & $\begin{array}{r}\text { Area } \\
\text { [mAU.Min] }\end{array}$ & $\begin{array}{r}\text { Area \% } \\
{[\%]}\end{array}$ \\
\hline \hline 1 & UNKNOWN & 70.14 & 11.08 & 51.9 & 114.1 & 11.077 \\
\hline 2 & UNKNOWN & 85.99 & 88.92 & 273.1 & 915.7 & 88.923 \\
\hline & & & & & & \\
\hline Total & & & 100.00 & 325.0 & 1029.8 & 100.000 \\
\hline
\end{tabular}

$\rightarrow 78 \%$ ee 

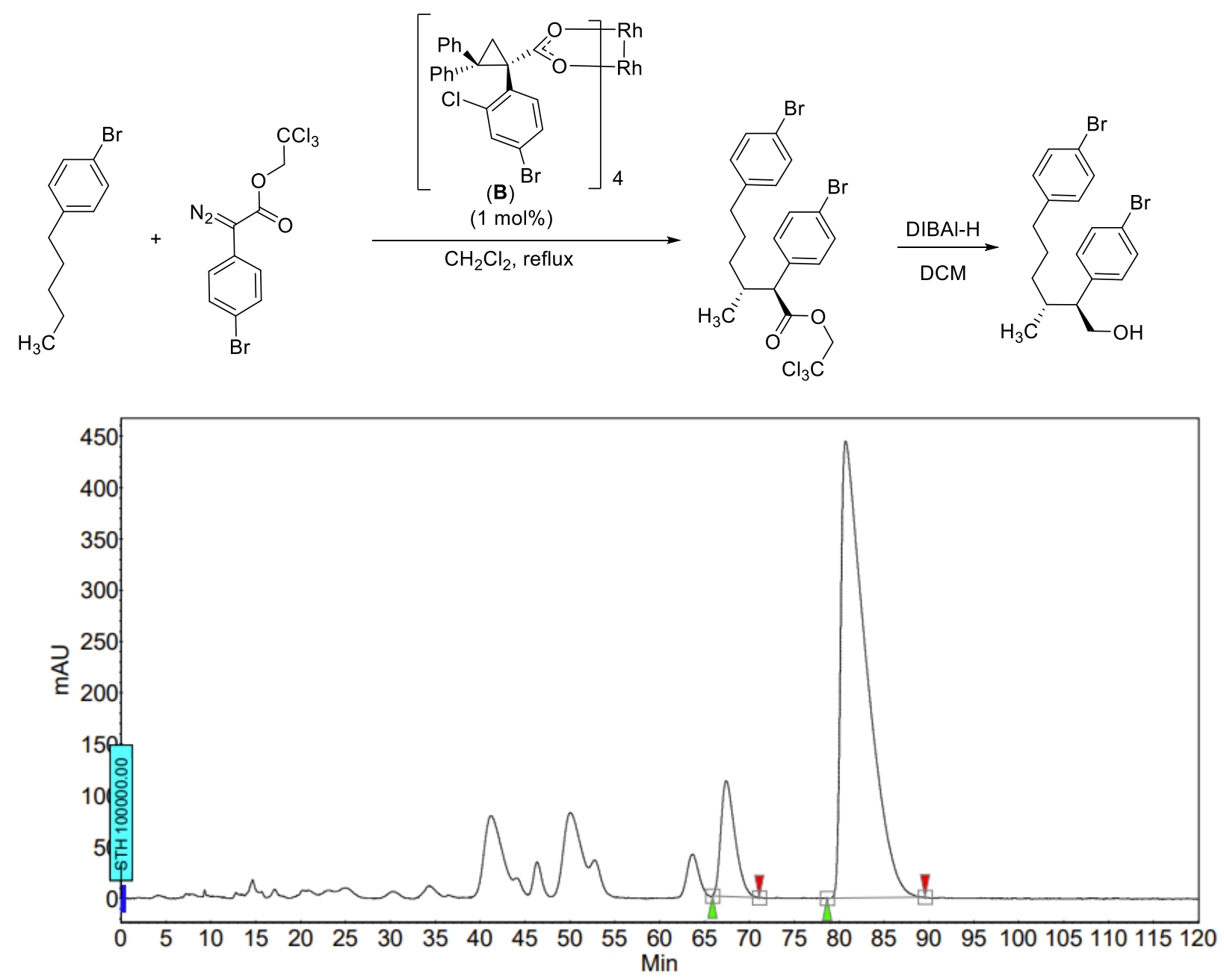

\section{Peak results :}

\begin{tabular}{|c|l|r|r|r|r|r|}
\hline Index & Name & $\begin{array}{r}\text { Time } \\
\text { [Min] }\end{array}$ & $\begin{array}{r}\text { Quantity } \\
\text { [\% Area] }\end{array}$ & $\begin{array}{r}\text { Height } \\
\text { [mAU] }\end{array}$ & $\begin{array}{r}\text { Area } \\
\text { [mAU.Min] }\end{array}$ & $\begin{array}{r}\text { Area \% } \\
{[\%]}\end{array}$ \\
\hline \hline 1 & UNKNOWN & 67.43 & 12.46 & 112.6 & 205.9 & 12.464 \\
\hline 2 & UNKNOWN & 80.72 & 87.54 & 445.0 & 1445.7 & 87.536 \\
\hline & & & & & & \\
\hline Total & & & 100.00 & 557.7 & 1651.6 & 100.000 \\
\hline
\end{tabular}

$\rightarrow 75 \%$ ee 

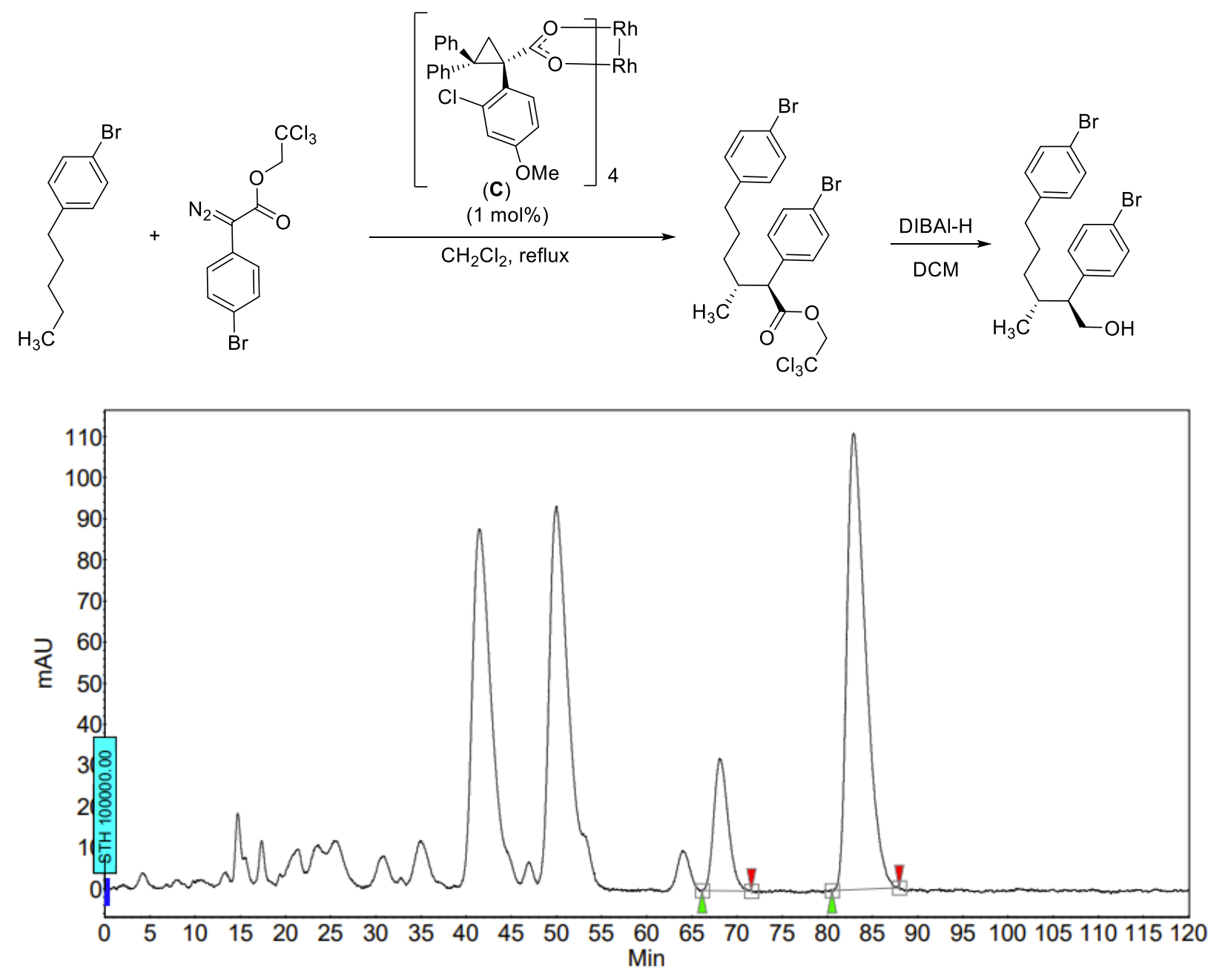

Peak results :

\begin{tabular}{|c|l|r|r|r|r|r|}
\hline Index & Name & $\begin{array}{r}\text { Time } \\
\text { [Min] }\end{array}$ & $\begin{array}{r}\text { Quantity } \\
\text { [\% Area] }\end{array}$ & $\begin{array}{r}\text { Height } \\
\text { [mAU] }\end{array}$ & $\begin{array}{r}\text { Area } \\
\text { [mAU.Min] }\end{array}$ & $\begin{array}{r}\text { Area \% } \\
\text { [\%] }\end{array}$ \\
\hline \hline 1 & UNKNOWN & 68.05 & 18.07 & 32.1 & 59.1 & 18.070 \\
\hline 2 & UNKNOWN & 82.90 & 81.93 & 111.1 & 267.9 & 81.930 \\
\hline & & & & & & \\
\hline Total & & & 100.00 & 143.2 & 326.9 & 100.000 \\
\hline
\end{tabular}

$\rightarrow 64 \%$ ee 

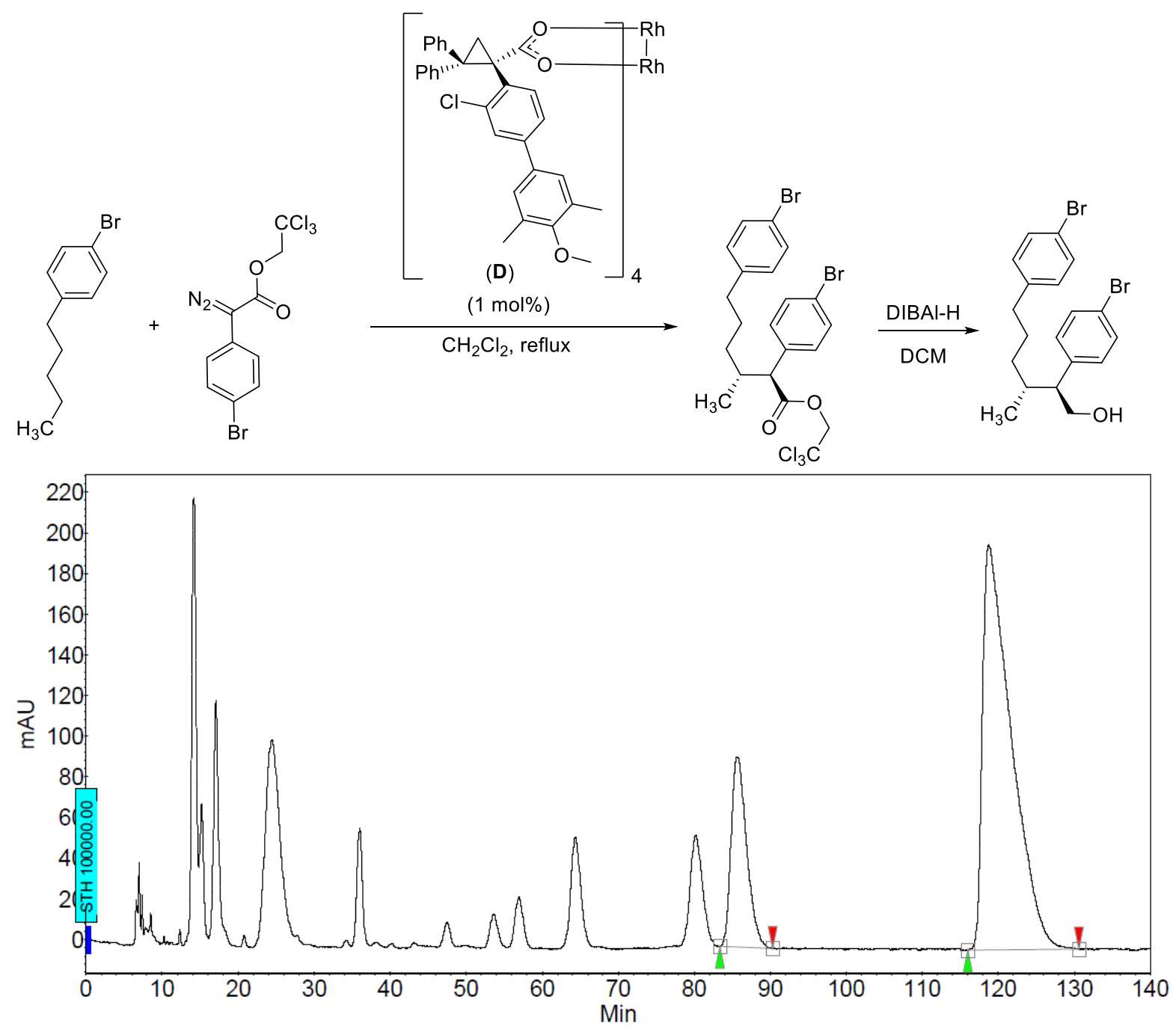

\section{Peak results :}

\begin{tabular}{|c|l|r|r|r|r|r|}
\hline Index & Name & $\begin{array}{r}\text { Time } \\
{[\text { Min] }}\end{array}$ & $\begin{array}{r}\text { Quantity } \\
{[\% \text { Area] }}\end{array}$ & $\begin{array}{r}\text { Height } \\
{[\mathrm{mAU}]}\end{array}$ & $\begin{array}{r}\text { Area } \\
\text { [mAU.Min] }\end{array}$ & $\begin{array}{r}\text { Area \% } \\
{[\%]}\end{array}$ \\
\hline \hline 1 & UNKNOWN & 85.67 & 20.29 & 93.6 & 222.3 & 20.289 \\
\hline 2 & UNKNOWN & 118.78 & 79.71 & 199.3 & 873.6 & 79.711 \\
\hline & & & & & & \\
\hline Total & & & 100.00 & 292.9 & 1095.9 & 100.000 \\
\hline
\end{tabular}

$\rightarrow 59 \%$ ee 

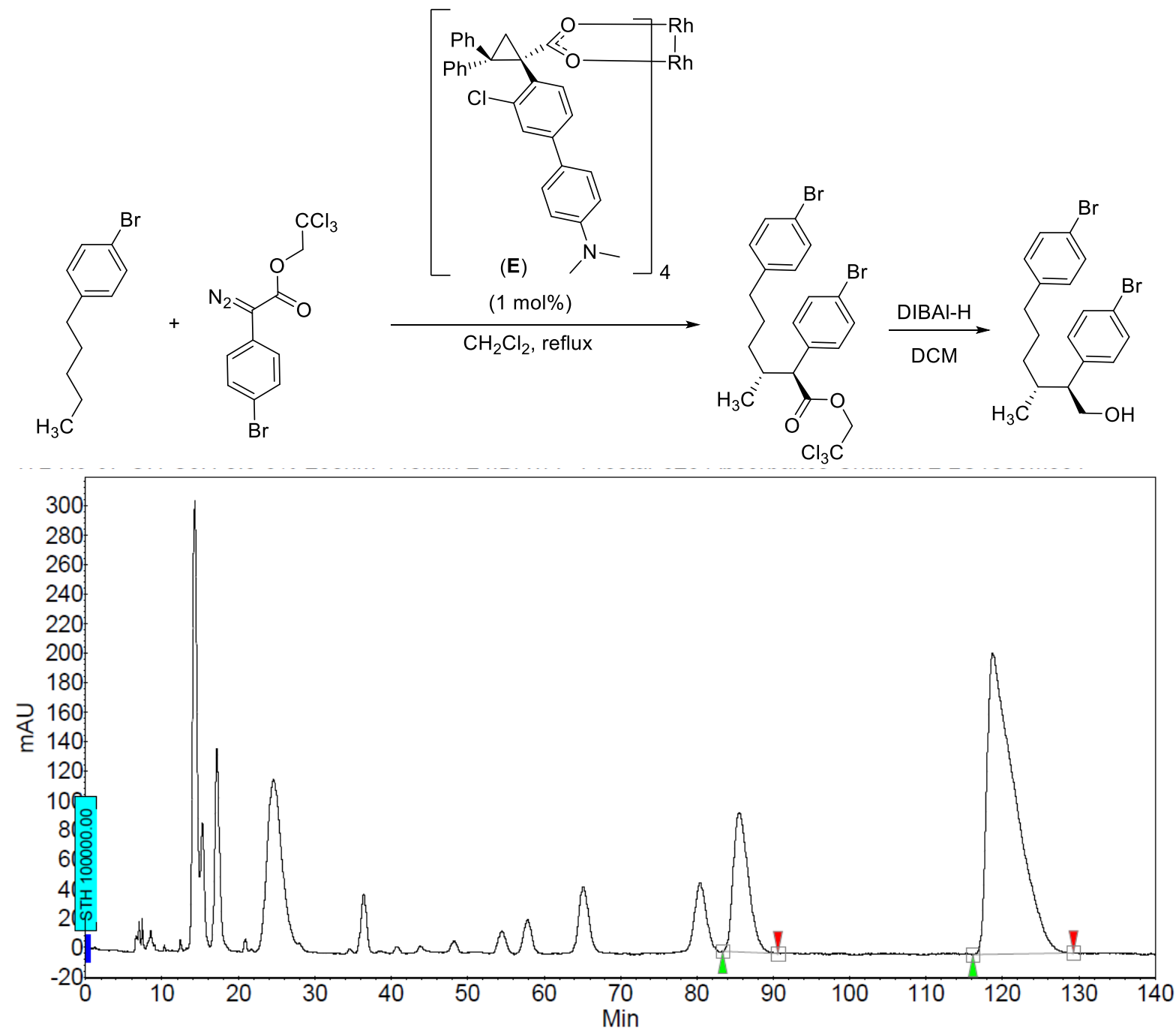

Peak results :

\begin{tabular}{|c|l|r|r|r|r|r|}
\hline Index & Name & $\begin{array}{r}\text { Time } \\
{[\text { Min] }}\end{array}$ & $\begin{array}{r}\text { Quantity } \\
\text { [\% Area] }\end{array}$ & $\begin{array}{r}\text { Height } \\
{[\mathrm{mAU}]}\end{array}$ & $\begin{array}{r}\text { Area } \\
\text { [mAU.Min] }\end{array}$ & $\begin{array}{r}\text { Area \% } \\
{[\%]}\end{array}$ \\
\hline \hline 1 & UNKNOWN & 85.55 & 20.05 & 94.5 & 217.5 & 20.046 \\
\hline 2 & UNKNOWN & 118.71 & 79.95 & 204.3 & 867.6 & 79.954 \\
\hline & & & & & & \\
\hline Total & & & 100.00 & 298.8 & 1085.1 & 100.000 \\
\hline
\end{tabular}

$\rightarrow 60 \%$ ee 


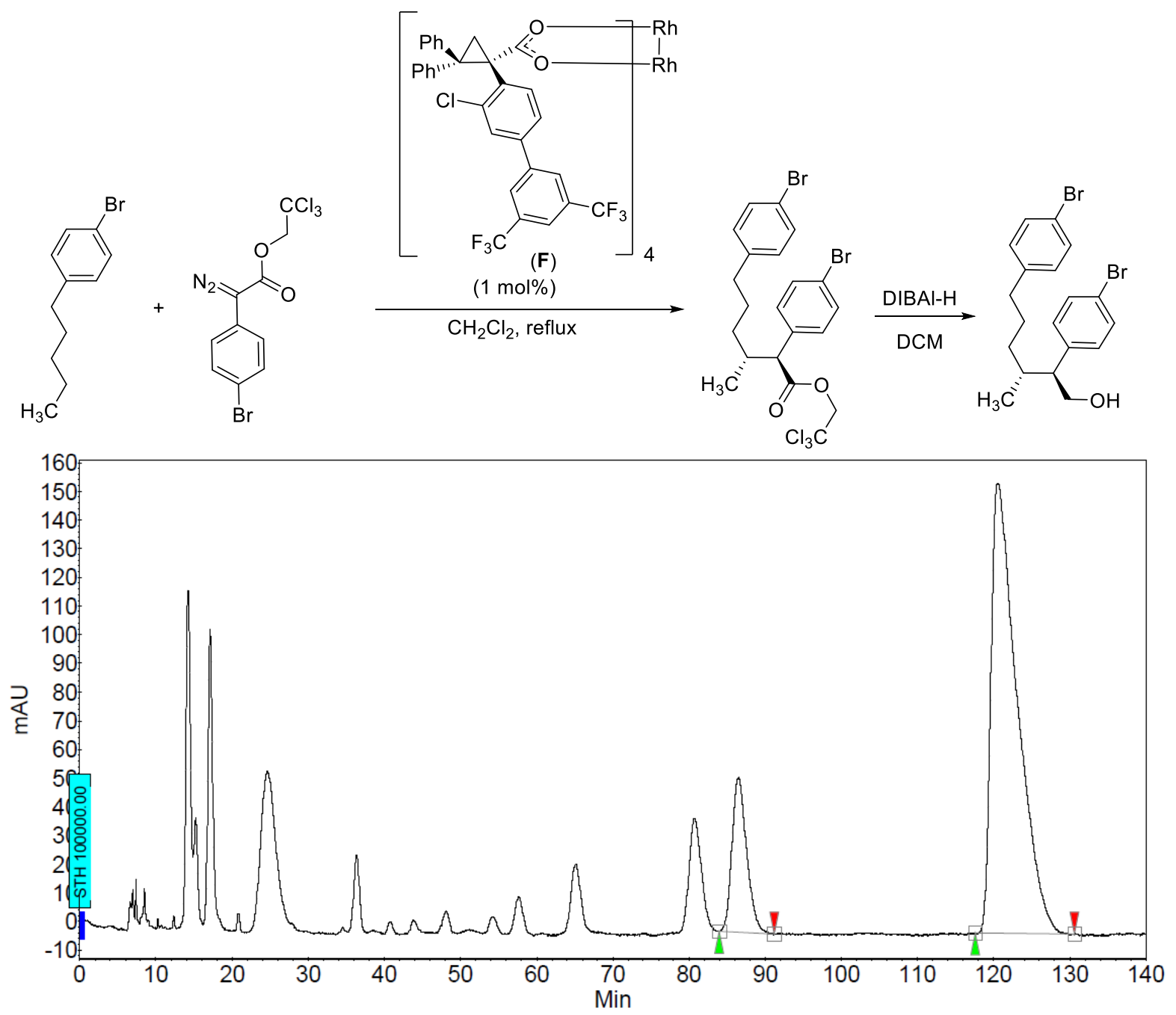

Peak results :

\begin{tabular}{|c|l|r|r|r|r|r|}
\hline Index & Name & $\begin{array}{r}\text { Time } \\
\text { [Min] }\end{array}$ & $\begin{array}{r}\text { Quantity } \\
\text { [\% Area] }\end{array}$ & $\begin{array}{r}\text { Height } \\
\text { [mAU] }\end{array}$ & $\begin{array}{r}\text { Area } \\
\text { [mAU.Min] }\end{array}$ & $\begin{array}{r}\text { Area \% } \\
{[\%]}\end{array}$ \\
\hline \hline 1 & UNKNOWN & 86.54 & 16.25 & 54.1 & 121.5 & 16.254 \\
\hline 2 & UNKNOWN & 120.54 & 83.75 & 157.1 & 626.0 & 83.746 \\
\hline & & & & & & \\
\hline Total & & & 100.00 & 211.1 & 747.5 & 100.000 \\
\hline
\end{tabular}

$\rightarrow 67 \%$ ee 

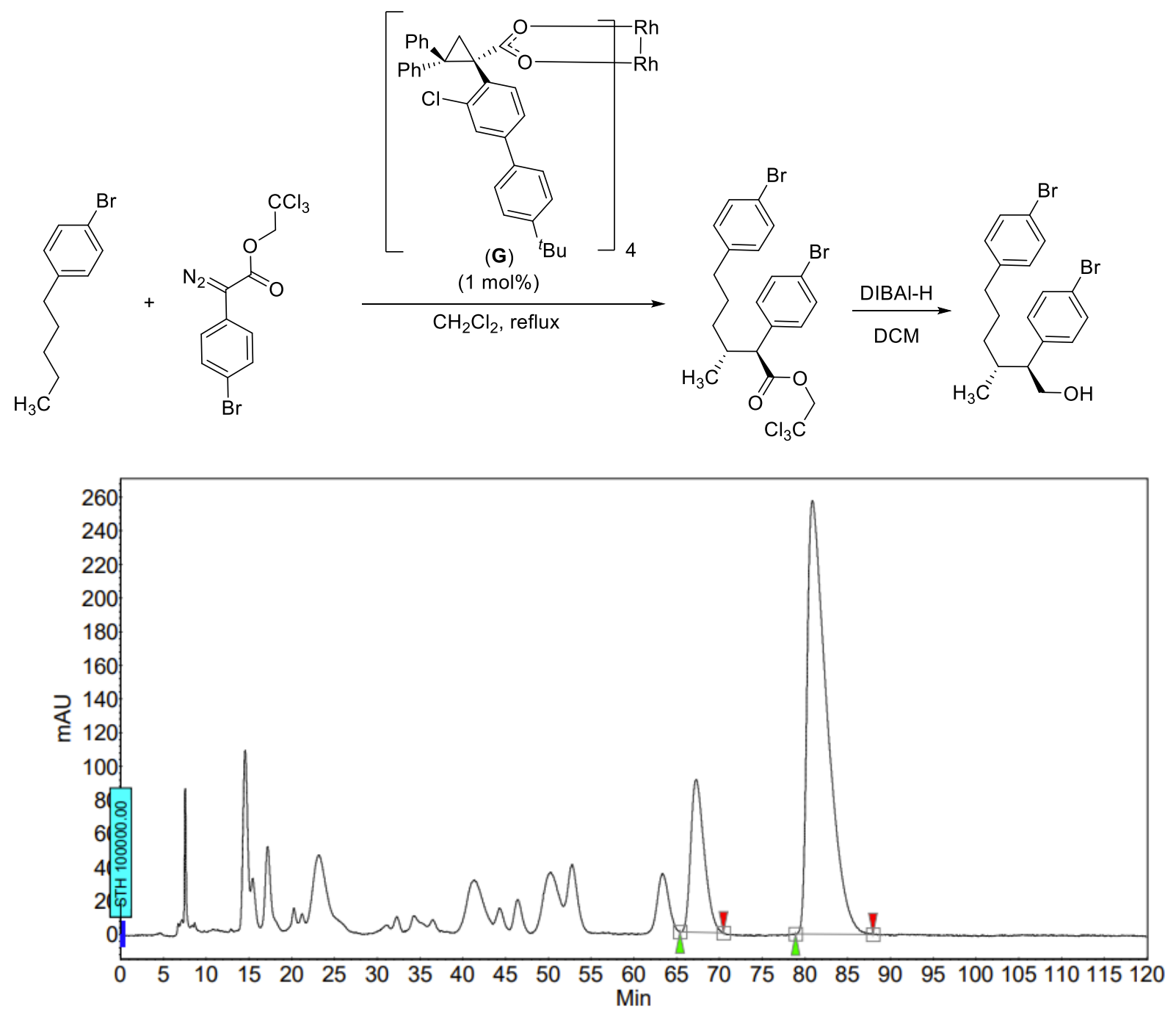

\section{Peak results :}

\begin{tabular}{|c|l|r|r|r|r|r|}
\hline Index & Name & $\begin{array}{r}\text { Time } \\
\text { [Min] }\end{array}$ & $\begin{array}{r}\text { Quantity } \\
\text { [\% Area] }\end{array}$ & $\begin{array}{r}\text { Height } \\
\text { [mAU] }\end{array}$ & $\begin{array}{r}\text { Area } \\
\text { [mAU.Min] }\end{array}$ & $\begin{array}{r}\text { Area \% } \\
{[\%]}\end{array}$ \\
\hline \hline 1 & UNKNOWN & 67.30 & 19.29 & 90.8 & 165.4 & 19.295 \\
\hline 2 & UNKNOWN & 80.88 & 80.71 & 257.9 & 691.7 & 80.705 \\
\hline & & & & & & \\
\hline Total & & & 100.00 & 348.6 & 857.1 & 100.000 \\
\hline
\end{tabular}

$\rightarrow 61 \%$ ee 

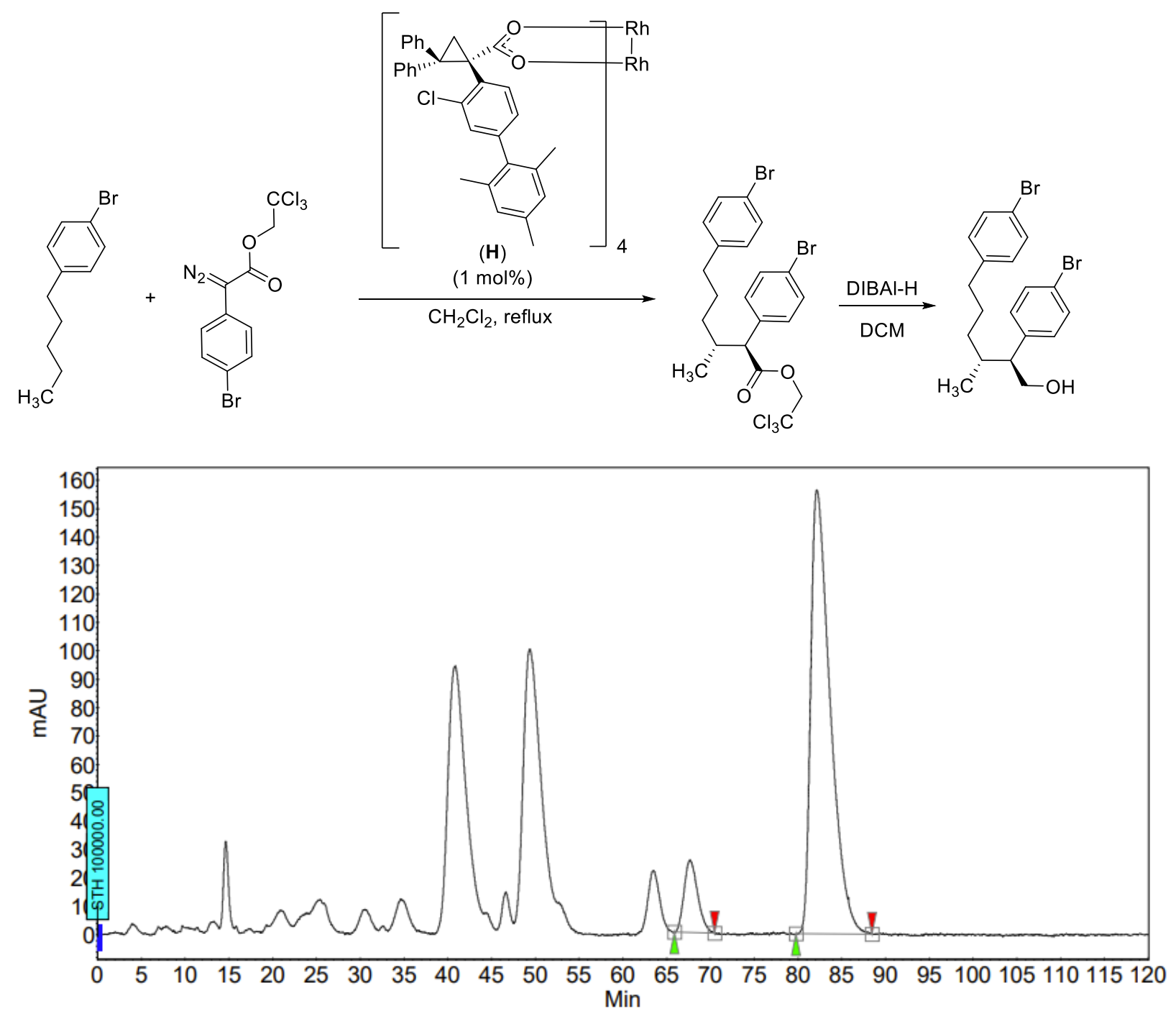

\section{Peak results :}

\begin{tabular}{|c|l|r|r|r|r|r|}
\hline Index & Name & $\begin{array}{r}\text { Time } \\
\text { [Min] }\end{array}$ & $\begin{array}{r}\text { Quantity } \\
\text { [\% Area] }\end{array}$ & $\begin{array}{r}\text { Height } \\
\text { [mAU] }\end{array}$ & $\begin{array}{r}\text { Area } \\
\text { [mAU.Min] }\end{array}$ & $\begin{array}{r}\text { Area \% } \\
{[\%]}\end{array}$ \\
\hline \hline 1 & UNKNOWN & 67.66 & 10.24 & 25.5 & 46.0 & 10.242 \\
\hline 2 & UNKNOWN & 82.15 & 89.76 & 156.3 & 402.9 & 89.758 \\
\hline & & & & & & \\
\hline Total & & & 100.00 & 181.8 & 448.8 & 100.000 \\
\hline
\end{tabular}

$\rightarrow 79 \%$ ee 

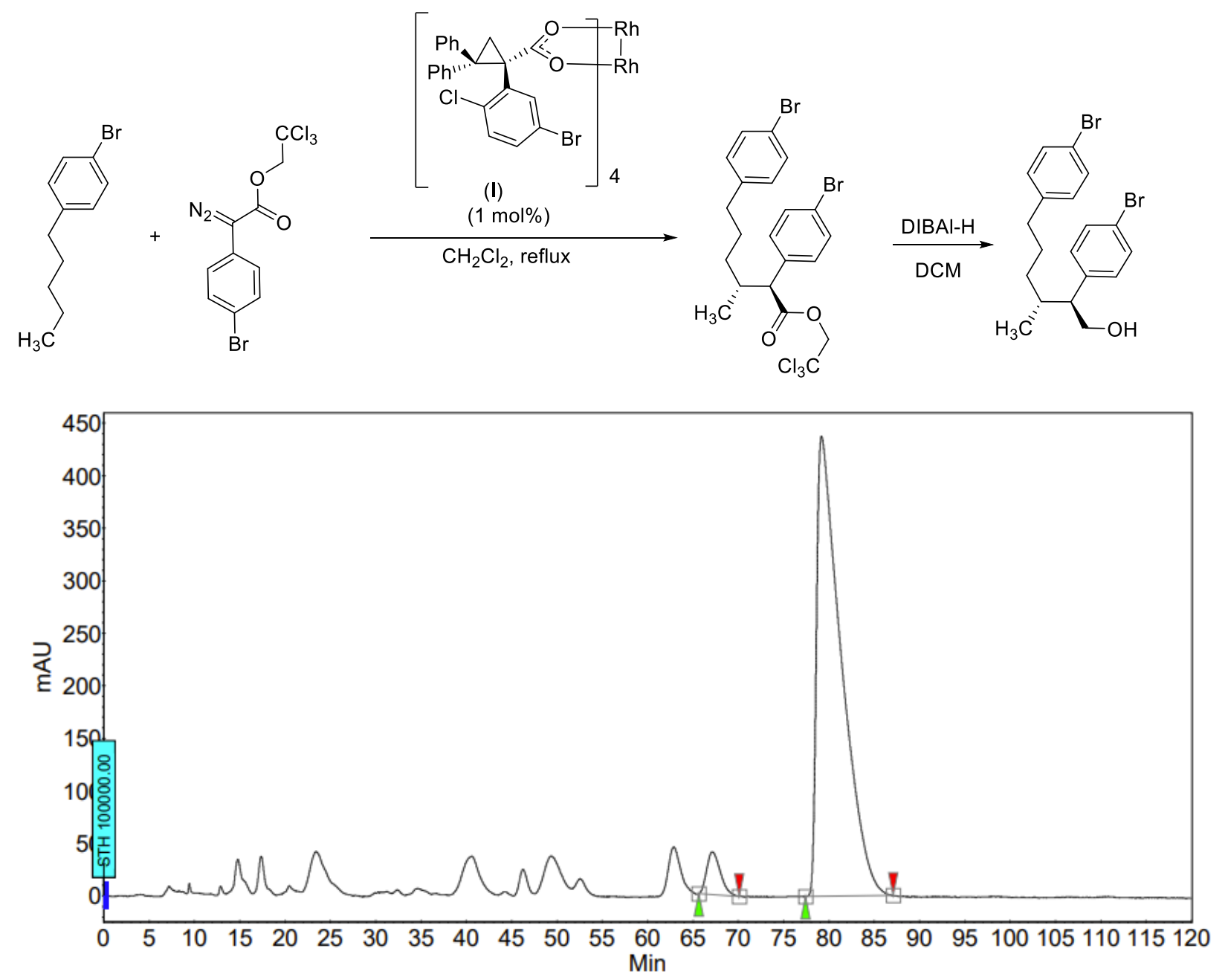

Peak results :

\begin{tabular}{|c|l|r|r|r|r|r|}
\hline Index & Name & $\begin{array}{r}\text { Time } \\
\text { [Min] }\end{array}$ & $\begin{array}{r}\text { Quantity } \\
\text { [\% Area] }\end{array}$ & $\begin{array}{r}\text { Height } \\
\text { [mAU] }\end{array}$ & $\begin{array}{r}\text { Area } \\
\text { [mAU.Min] }\end{array}$ & $\begin{array}{r}\text { Area \% } \\
{[\%]}\end{array}$ \\
\hline \hline 1 & UNKNOWN & 67.14 & 4.92 & 40.8 & 71.2 & 4.923 \\
\hline 2 & UNKNOWN & 79.16 & 95.08 & 438.3 & 1374.3 & 95.077 \\
\hline & & & & & & \\
\hline Total & & & 100.00 & 479.1 & 1445.5 & 100.000 \\
\hline
\end{tabular}

$\rightarrow 90 \%$ ee 

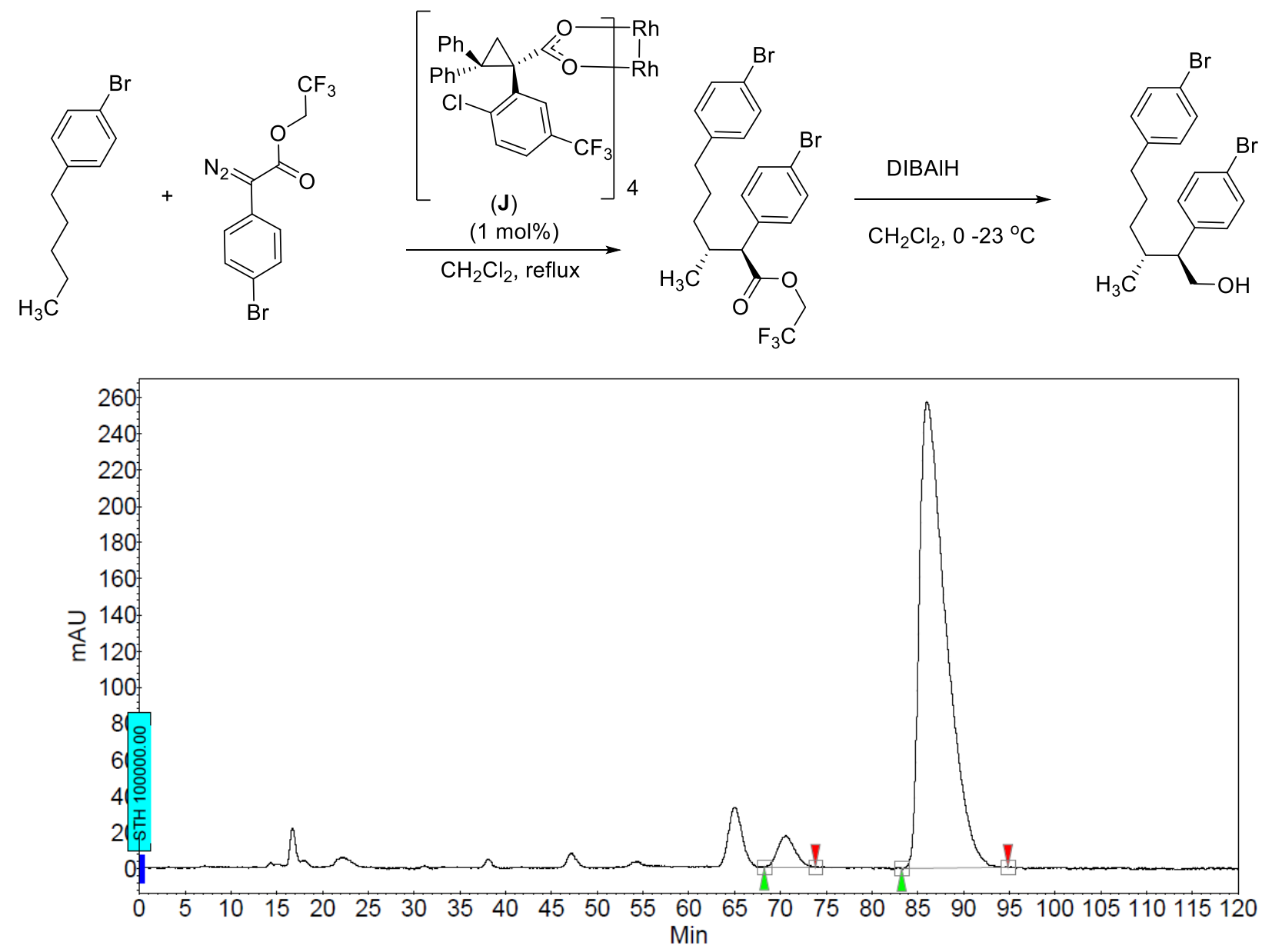

Peak results :

\begin{tabular}{|c|l|r|r|r|r|r|}
\hline Index & Name & $\begin{array}{r}\text { Time } \\
\text { [Min] }\end{array}$ & $\begin{array}{r}\text { Quantity } \\
\text { [\% Area] }\end{array}$ & $\begin{array}{r}\text { Height } \\
\text { [mAU] }\end{array}$ & $\begin{array}{r}\text { Area } \\
\text { [mAU.Min] }\end{array}$ & $\begin{array}{r}\text { Area \% } \\
{[\%]}\end{array}$ \\
\hline \hline 1 & UNKNOWN & 70.59 & 4.25 & 17.7 & 38.5 & 4.255 \\
\hline 2 & UNKNOWN & 86.03 & 95.75 & 257.3 & 867.0 & 95.745 \\
\hline & & & & & & \\
\hline Total & & & 100.00 & 275.0 & 905.5 & 100.000 \\
\hline
\end{tabular}

$\rightarrow 91 \%$ ee 


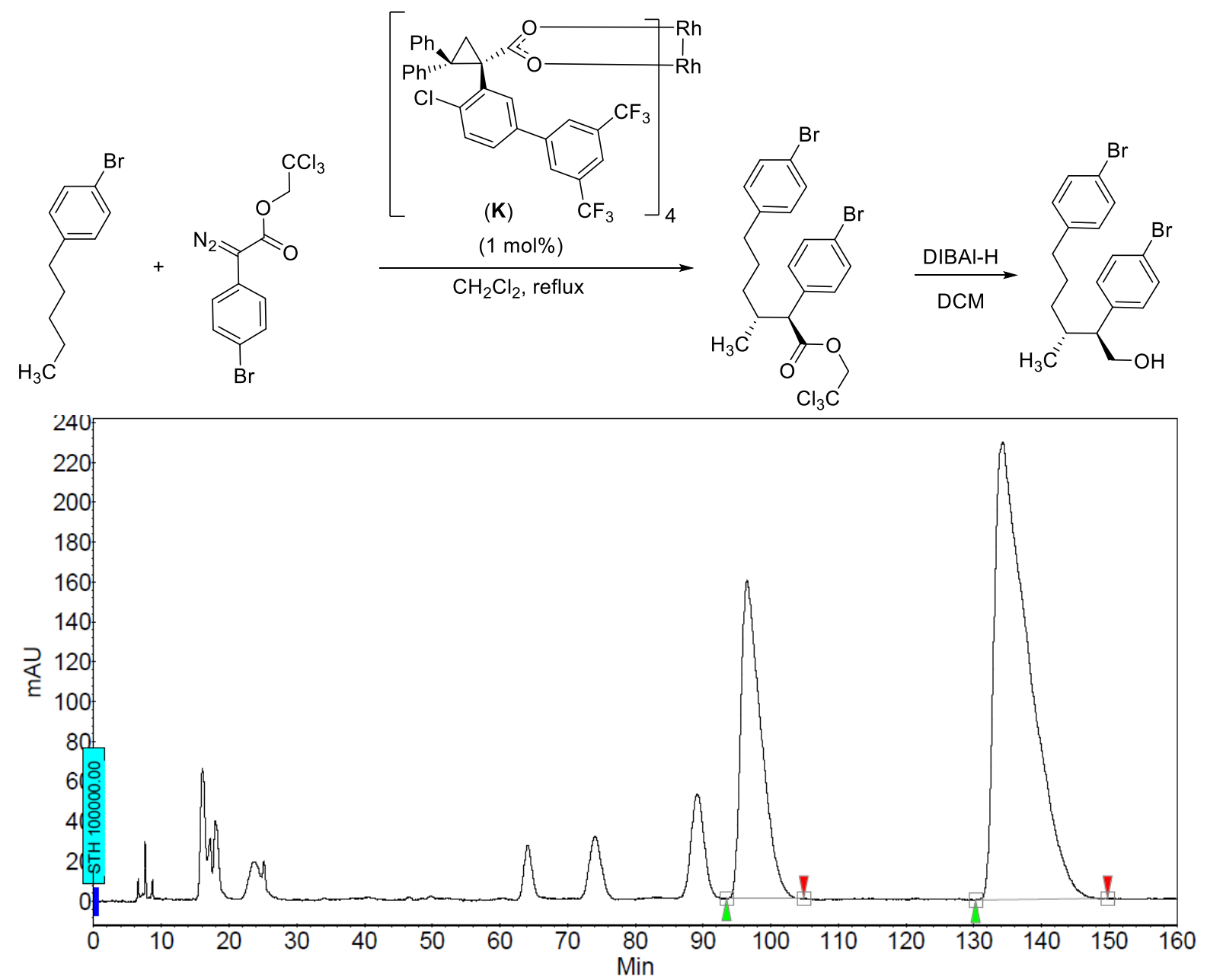

\section{Peak results :}

\begin{tabular}{|c|l|r|r|r|r|r|}
\hline Index & Name & $\begin{array}{r}\text { Time } \\
\text { [Min] }\end{array}$ & $\begin{array}{r}\text { Quantity } \\
\text { [\% Area] }\end{array}$ & $\begin{array}{r}\text { Height } \\
\text { [mAU] }\end{array}$ & $\begin{array}{r}\text { Area } \\
\text { [mAU.Min] }\end{array}$ & $\begin{array}{r}\text { Area \% } \\
{[\%]}\end{array}$ \\
\hline \hline 1 & UNKNOWN & 96.53 & 28.83 & 159.8 & 549.7 & 28.826 \\
\hline 2 & UNKNOWN & 134.25 & 71.17 & 229.5 & 1357.3 & 71.174 \\
\hline & & & & & & \\
\hline Total & & & 100.00 & 389.2 & 1907.0 & 100.000 \\
\hline
\end{tabular}

$\rightarrow 42 \%$ ee 

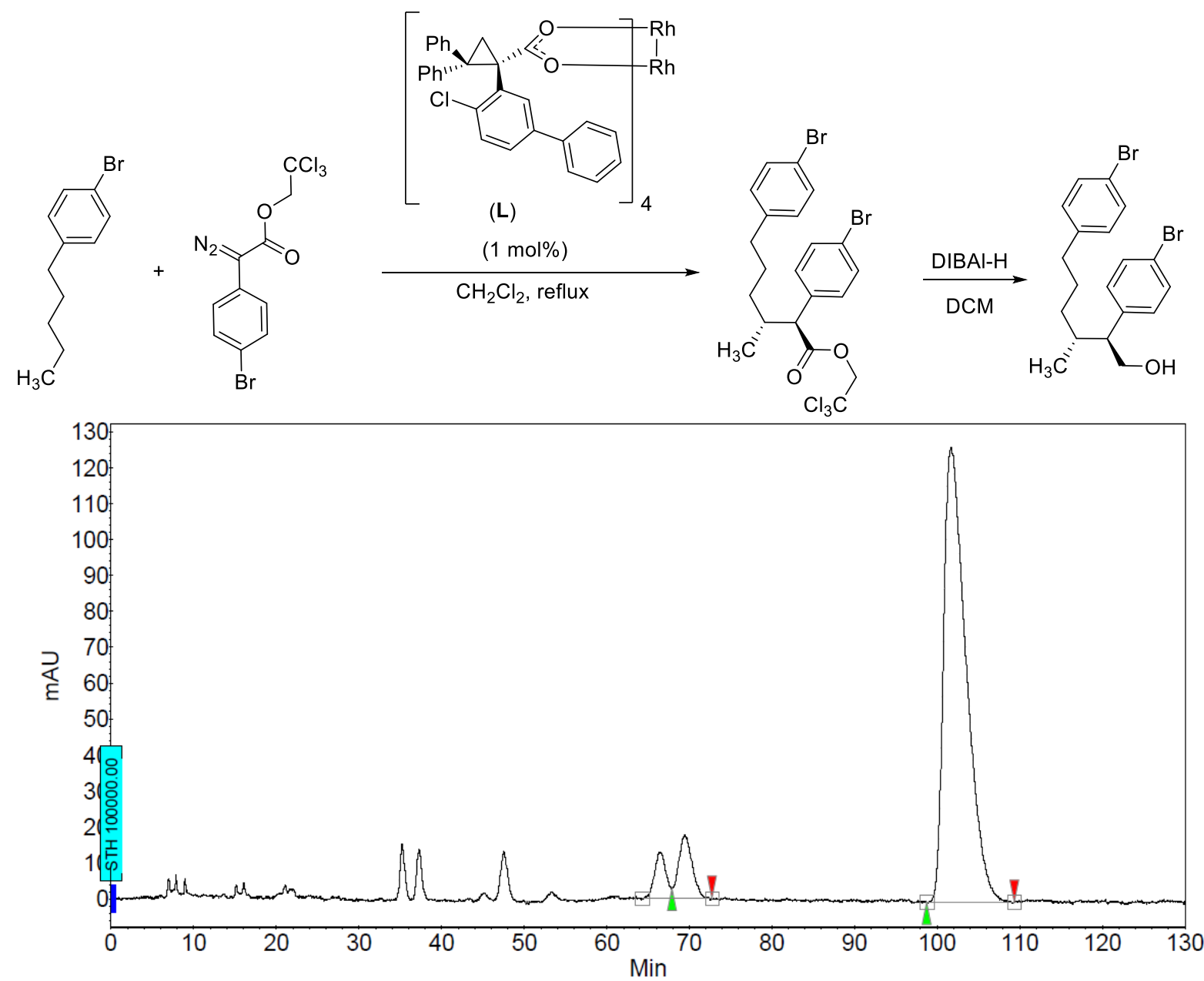

Peak results :

\begin{tabular}{|c|l|r|r|r|r|r|}
\hline Index & Name & $\begin{array}{r}\text { Time } \\
\text { [Min] }\end{array}$ & $\begin{array}{r}\text { Quantity } \\
\text { [\% Area] }\end{array}$ & $\begin{array}{r}\text { Height } \\
\text { [mAU] }\end{array}$ & $\begin{array}{r}\text { Area } \\
\text { [mAU.Min] }\end{array}$ & $\begin{array}{r}\text { Area \% } \\
{[\%]}\end{array}$ \\
\hline \hline 2 & UNKNOWN & 69.47 & 7.69 & 17.6 & 33.2 & 7.688 \\
\hline 1 & UNKNOWN & 101.67 & 92.31 & 126.7 & 398.1 & 92.312 \\
\hline & & & & & & \\
\hline Total & & & 100.00 & 144.3 & 431.3 & 100.000 \\
\hline
\end{tabular}

$\rightarrow 84 \%$ ee 

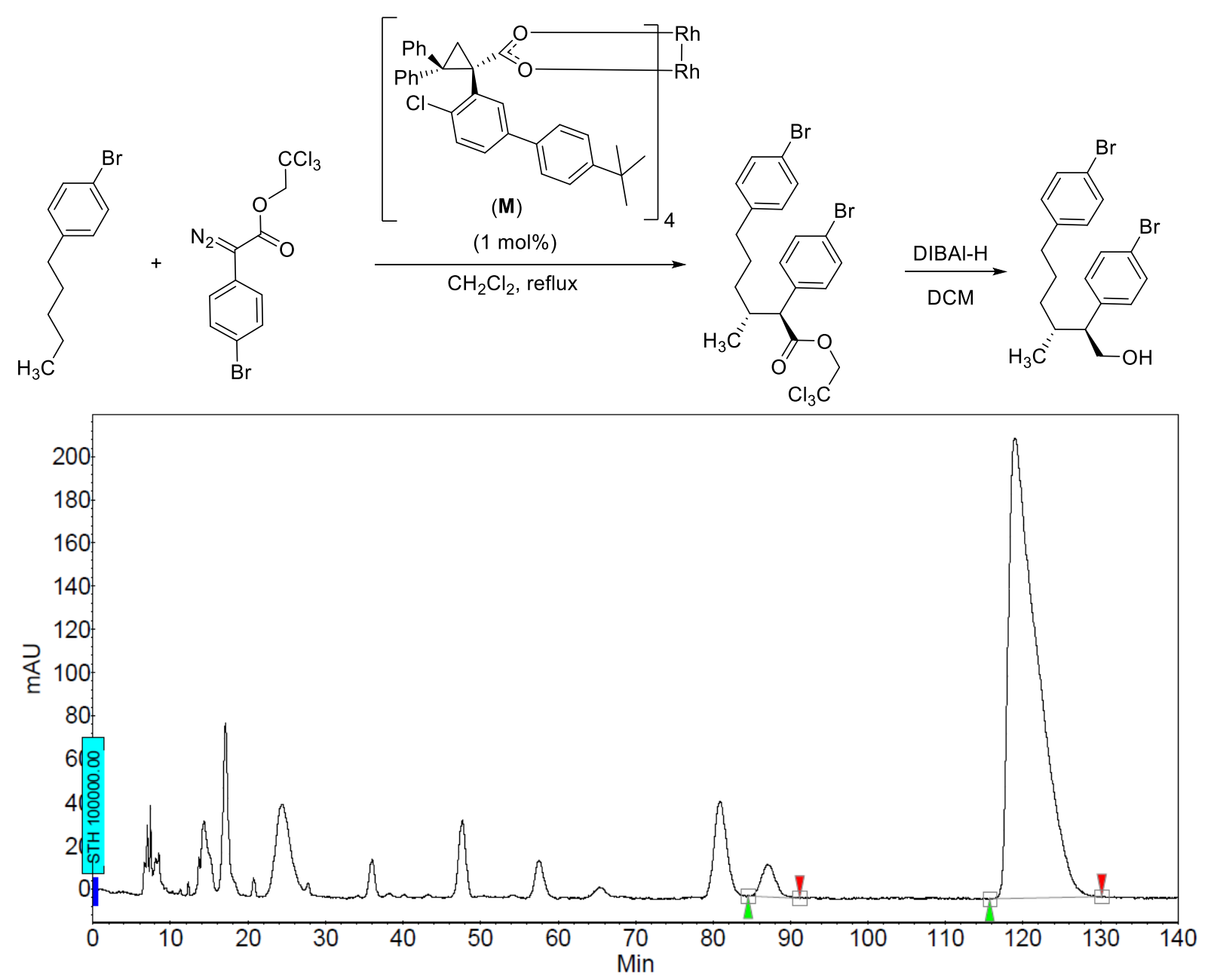

Peak results :

\begin{tabular}{|c|l|r|r|r|r|r|}
\hline Index & Name & $\begin{array}{r}\text { Time } \\
\text { [Min] }\end{array}$ & $\begin{array}{r}\text { Quantity } \\
\text { [\% Area] }\end{array}$ & $\begin{array}{r}\text { Height } \\
\text { [mAU] }\end{array}$ & $\begin{array}{r}\text { Area } \\
\text { [mAU.Min] }\end{array}$ & $\begin{array}{r}\text { Area \% } \\
{[\%]}\end{array}$ \\
\hline \hline 1 & UNKNOWN & 87.12 & 3.44 & 14.9 & 32.7 & 3.441 \\
\hline 2 & UNKNOWN & 118.94 & 96.56 & 212.8 & 917.2 & 96.559 \\
\hline & & & & & & \\
\hline Total & & & 100.00 & 227.8 & 949.9 & 100.000 \\
\hline
\end{tabular}

$\rightarrow 93 \%$ ee 

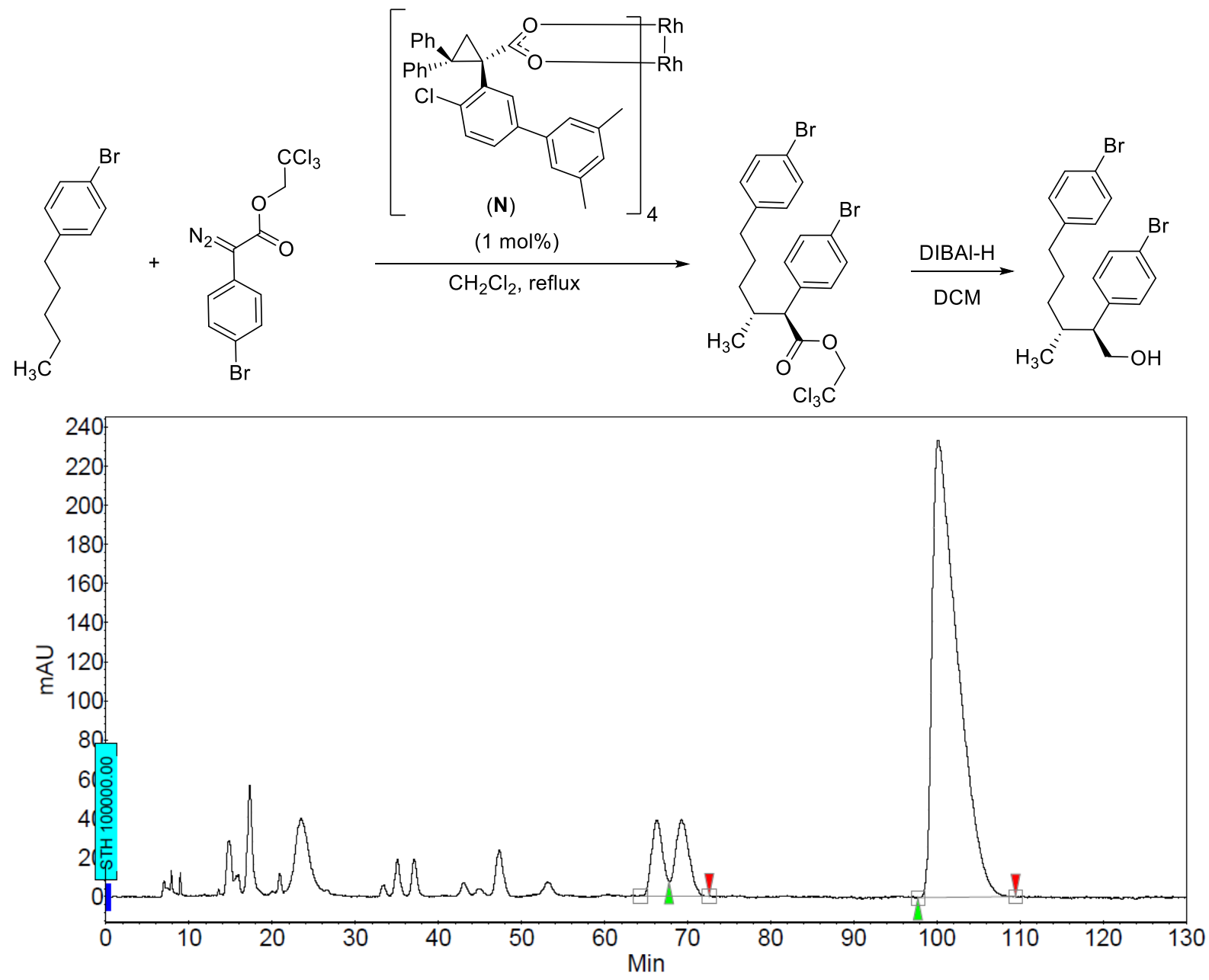

\section{Peak results :}

\begin{tabular}{|c|l|r|r|r|r|r|}
\hline Index & Name & $\begin{array}{r}\text { Time } \\
\text { [Min] }\end{array}$ & $\begin{array}{r}\text { Quantity } \\
\text { [\% Area] }\end{array}$ & $\begin{array}{r}\text { Height } \\
\text { [mAU] }\end{array}$ & $\begin{array}{r}\text { Area } \\
\text { [mAU.Min] }\end{array}$ & $\begin{array}{r}\text { Area \% } \\
{[\%]}\end{array}$ \\
\hline \hline 2 & UNKNOWN & 69.31 & 8.23 & 39.0 & 74.3 & 8.227 \\
\hline 1 & UNKNOWN & 100.20 & 91.77 & 233.8 & 828.6 & 91.773 \\
\hline & & & & & & \\
\hline Total & & & 100.00 & 272.9 & 902.8 & 100.000 \\
\hline
\end{tabular}

$\rightarrow 84 \%$ ee 

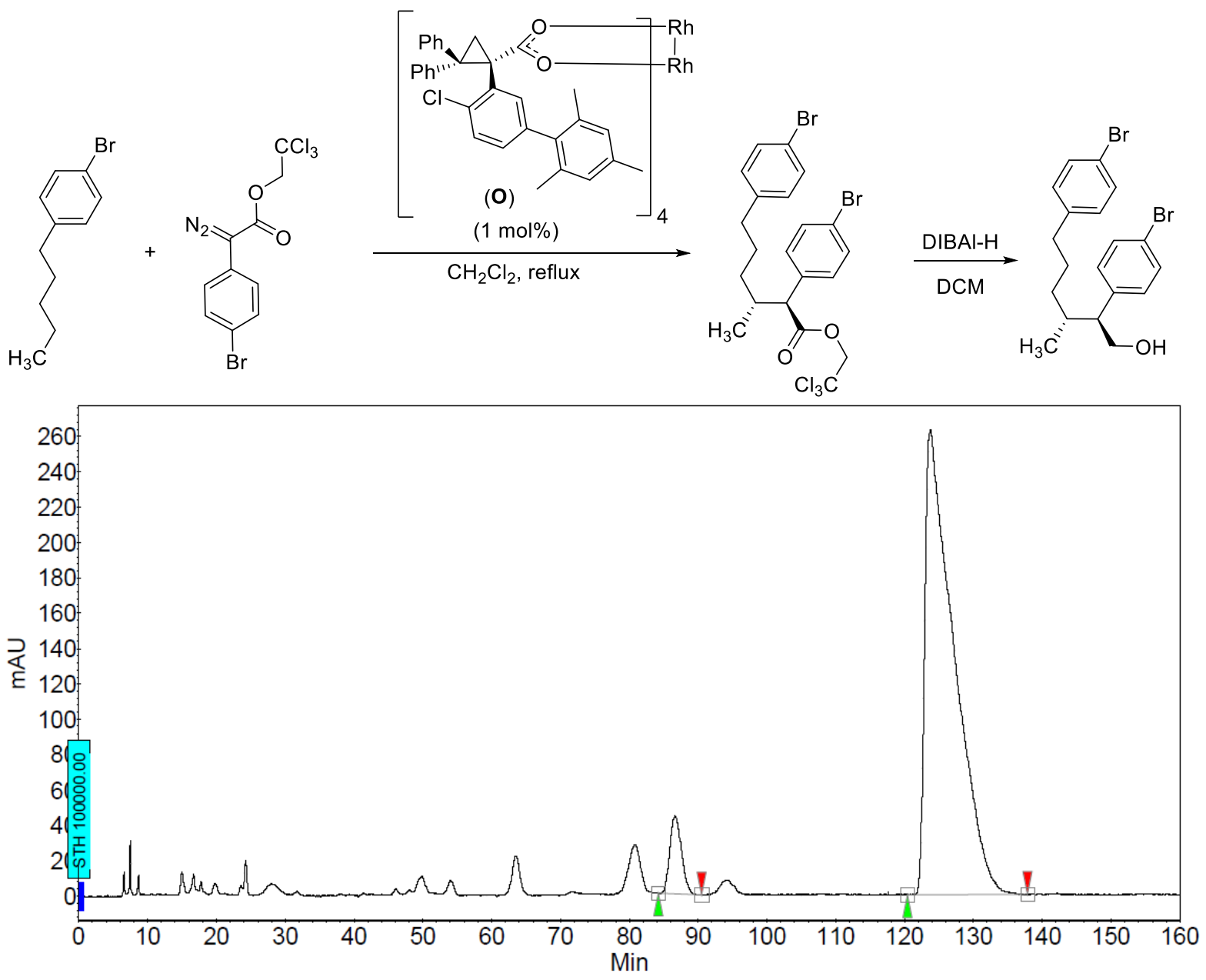

\section{Peak results :}

\begin{tabular}{|c|l|r|r|r|r|r|}
\hline Index & Name & $\begin{array}{r}\text { Time } \\
\text { [Min] }\end{array}$ & $\begin{array}{r}\text { Quantity } \\
\text { [\% Area] }\end{array}$ & $\begin{array}{r}\text { Height } \\
\text { [mAU] }\end{array}$ & $\begin{array}{r}\text { Area } \\
\text { [mAU.Min] }\end{array}$ & $\begin{array}{r}\text { Area \% } \\
{[\%]}\end{array}$ \\
\hline \hline 1 & UNKNOWN & 86.63 & 6.35 & 44.0 & 86.8 & 6.348 \\
\hline 2 & UNKNOWN & 123.73 & 93.65 & 263.0 & 1279.8 & 93.652 \\
\hline & & & & & & \\
\hline Total & & & 100.00 & 307.0 & 1366.6 & 100.000 \\
\hline
\end{tabular}

$\rightarrow 87 \%$ ee 


\section{X-Ray Crystallographic Data for Four Dirhodium Catalysts}

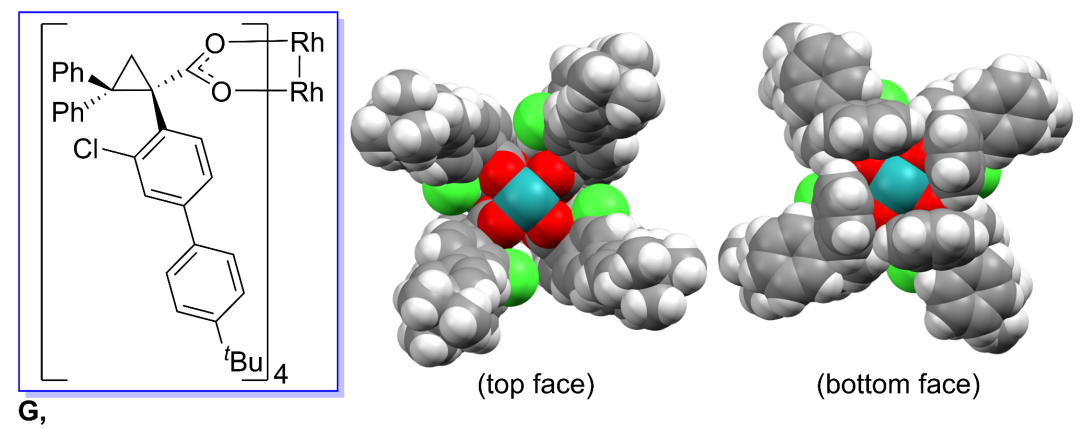

$\mathrm{Rh}_{2}\left(\mathrm{~S}-2-\mathrm{Cl}-4-\left(p-{ }^{t} \mathrm{BuC}_{6} \mathrm{H}_{4}\right) \mathrm{TPCP}\right)_{4}$

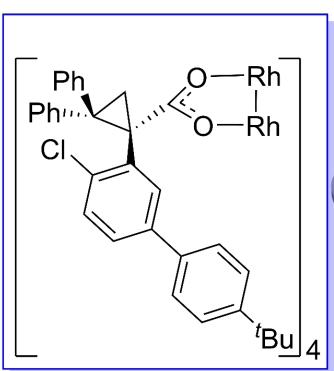

M,

$\mathrm{Rh}_{2}\left(\mathrm{~S}-2-\mathrm{Cl}-5-\left(\mathrm{p}^{\mathrm{t}} \mathrm{BuC}_{6} \mathrm{H}_{4}\right) \mathrm{TPCP}\right)_{4}$

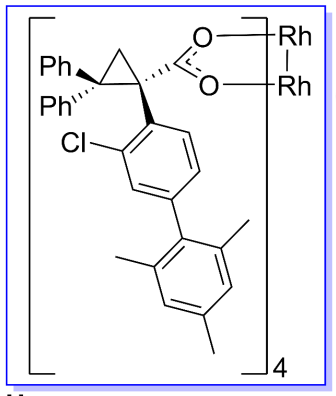

H,

$\mathrm{Rh}_{2}(\mathrm{~S}-2-\mathrm{Cl}-4-\mathrm{MesTPCP})_{4}$

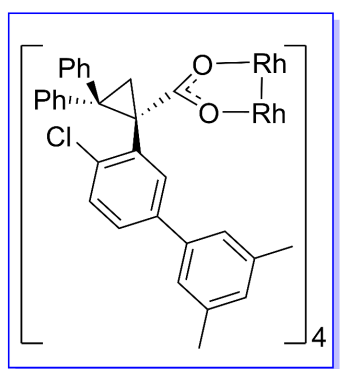

N,

,

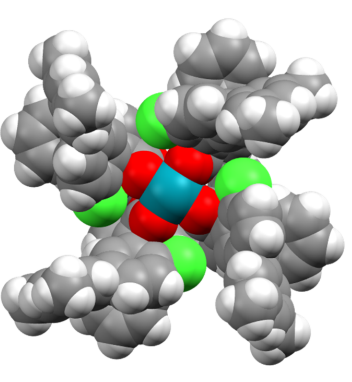

(top face)

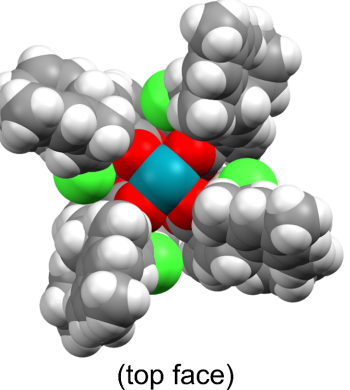

(top face)

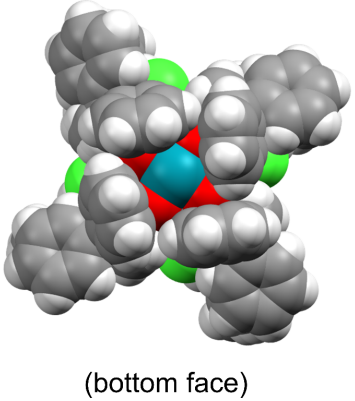

(bottom face)

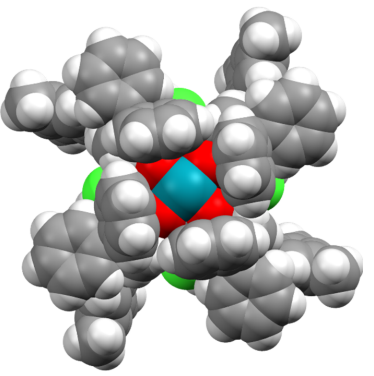

(bottom face)

Figure S44. Space-filling diagrams of four dirhodium catalysts $(\mathbf{G}, \mathbf{M}, \mathbf{H}, \mathbf{N})$ based on their X-ray crystal structures. Note that all subsequent X-ray figures and data tables in this SI section are numbered re-starting at "1" for each individual section (e.g., 13.1, 13.2, 13.3, and 13.4). 


\title{
13.1. X-Ray Crystallographic Data for $\mathrm{Rh}_{2}\left(S-2-\mathrm{Cl}-4-\left(p-{ }^{t} \mathrm{BuC}_{6} \mathrm{H}_{4}\right) \mathrm{TPCP}\right)_{4}$
}

\section{A Commensurately Modulated Structure}

\author{
$R_{1}=7.35 \%$
}

\section{Crystal Data and Experimental}

Note on the modulation. This is a commensurate structure with weak satellite reflections. The diffraction quality was good but there are satellites between the $a^{*}$-axis and the $b^{*}$ axis $(1 / 21 / 2$ 0). This gives a two-fold supercell (with $59.6 \AA$, $59.6 \AA, 14.37 \AA$ ). Because the satellites were weak, and the number of parameters very large a supercell refinement was not attempted (yet). The structure was reasonable without the satellites but with large residual densities close to the rhodium atoms (and exactly at the midpoint of the $\mathrm{Rh}-\mathrm{Rh}$ bonds).

Experimental. Single violet block-shaped crystals of 2cl4tbuph_twin slow evaporation in 50 percent dichloromethane in acetonitrile. A suitable crystal with dimensions $0.51 \times 0.26 \times 0.22 \mathrm{~mm}^{3}$ was selected and mounted on a Bruker APEX-II CCD diffractometer. The crystal was kept at a steady $T=100(2) \mathrm{K}$ during data collection. The structure was solved with the XT (Sheldrick, 2015) solution program using dual methods and by using Olex2 1.3-alpha (Dolomanov et al., 2009) as the graphical interface. The model was refined with ShelXL 2018/3 (Sheldrick, 2015) using full matrix least squares minimisation on $\boldsymbol{F}^{2}$.

Crystal Data. $\mathrm{C}_{132} \mathrm{H}_{118} \mathrm{Cl}_{4} \mathrm{~N}_{2} \mathrm{O}_{8} \mathrm{Rh}_{2}, M_{r}=2207.90$, tetragonal, P4 (No. 75), $\mathrm{a}=29.6743(2) \AA, \quad \mathrm{b}=29.6743(2) \AA, \quad \mathrm{c}=$ 14.37139(16) $\AA, \alpha=\beta=\gamma=90^{\circ}, \quad V=12654.9(2) \AA^{3}, \quad T=$ $100(2) \mathrm{K}, Z=4, Z^{\prime}=1, \mu\left(\mathrm{MoK}_{\alpha}\right)=0.398,119931$ reflections measured, 23980 unique $\left(R_{\text {int }}=0.0632\right)$ which were used in all calculations. The final $w R_{2}$ was 0.2027 (all data) and $R_{1}$ was $0.0735(\mathrm{I} \geq 2 \sigma(\mathrm{I}))$.

\begin{tabular}{|c|c|}
\hline Compound & 2cl4tbuph_twin \\
\hline Formula & $\mathrm{C}_{132} \mathrm{H}_{118} \mathrm{Cl}_{4} \mathrm{~N}_{2} \mathrm{O}_{8} \mathrm{Rh}_{2}$ \\
\hline$D_{\text {calc. }} / \mathrm{g} \mathrm{cm}^{-3}$ & 1.159 \\
\hline$\mu / \mathrm{mm}^{-1}$ & 0.398 \\
\hline Formula Weight & 2207.90 \\
\hline Colour & violet \\
\hline Shape & block-shaped \\
\hline Size $/ \mathrm{mm}^{3}$ & $0.51 \times 0.26 \times 0.22$ \\
\hline$T / \mathrm{K}$ & $100(2)$ \\
\hline Crystal System & tetragonal \\
\hline Flack Parameter & $0.144(10)$ \\
\hline Space Group & $P 4$ \\
\hline$a / \AA$ & $29.6743(2)$ \\
\hline$b / \AA$ & $29.6743(2)$ \\
\hline$c / \AA ̊$ & $14.37139(16)$ \\
\hline$\alpha /^{\circ}$ & 90 \\
\hline$\beta /^{\circ}$ & 90 \\
\hline$\left.\gamma\right|^{\circ}$ & 90 \\
\hline $\mathrm{V} / \AA^{3}$ & $12654.9(2)$ \\
\hline$Z$ & 4 \\
\hline$Z^{\prime}$ & 1 \\
\hline Wavelength/Å & 0.71073 \\
\hline Radiation type & $\mathrm{MoK}_{\alpha}$ \\
\hline$\Theta_{\min } /^{\circ}$ & 1.372 \\
\hline$\left.\Theta_{\max }\right|^{\circ}$ & 25.681 \\
\hline Measured Refl's. & 119931 \\
\hline Indep't Refl's & 23980 \\
\hline Refl's I $\geq 2 \sigma(I)$ & 21073 \\
\hline$R_{\text {int }}$ & 0.0632 \\
\hline Parameters & 1313 \\
\hline Restraints & 1369 \\
\hline Largest Peak & 7.733 \\
\hline Deepest Hole & -3.010 \\
\hline GooF & 1.064 \\
\hline$w R_{2}$ (all data) & 0.2027 \\
\hline$w R_{2}$ & 0.1933 \\
\hline$R_{1}$ (all data) & 0.0820 \\
\hline$R_{1}$ & 0.0735 \\
\hline
\end{tabular}




\section{Structure Quality Indicators}

\section{Reflections:}

Refinement:

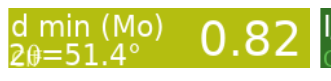

$\mathrm{I} / \sigma(\mathrm{I})$
Rint

Rint

-3.0 Goof 1.064

Full $50.5^{\circ}$

99.6

lack 44(10)

A violet block-shaped-shaped crystal with dimensions $0.51 \times 0.26 \times 0.22 \mathrm{~mm}^{3}$ was mounted. Data were collected using a Bruker APEX-II CCD diffractometer equipped with an Oxford Cryosystems lowtemperature device operating at $T=100(2) \mathrm{K}$.

Data were measured using $\phi$ and $\omega$ scans using $\mathrm{MoK}_{\alpha}$ radiation. The diffraction pattern was indexed and the total number of runs and images was based on the strategy calculation from the program APEX2 v2014.11 (Bruker, 2014). The maximum resolution that was achieved was $\Theta=25.681^{\circ}(0.83 \AA)$.

The unit cell was refined using SAINT v8.34A (Bruker, 2013) on 9227 reflections, 8\% of the observed reflections. There were groups of weaker satellite reflections that bisect the stronger in the ab-plane. Each reflection $\mathbf{a}$ or $\mathbf{b}$ has a weaker partner. This is therefore a modulated structure and the weaker reflections can be described using the modulation vector, q. This structure, based on the intense reflections is already very detailed (with 3 unique dirhodium groups) and therefore a refinement in the supercell or with modulation vectors was not attempted. However, the effects of the modulation can be discerned by groups of residual density peaks that break the symmetry along the c-direction. Thus the entire dirhodium molecules appear to be shifted relative to each other in the c-direction (following the pattern of satellite reflections.

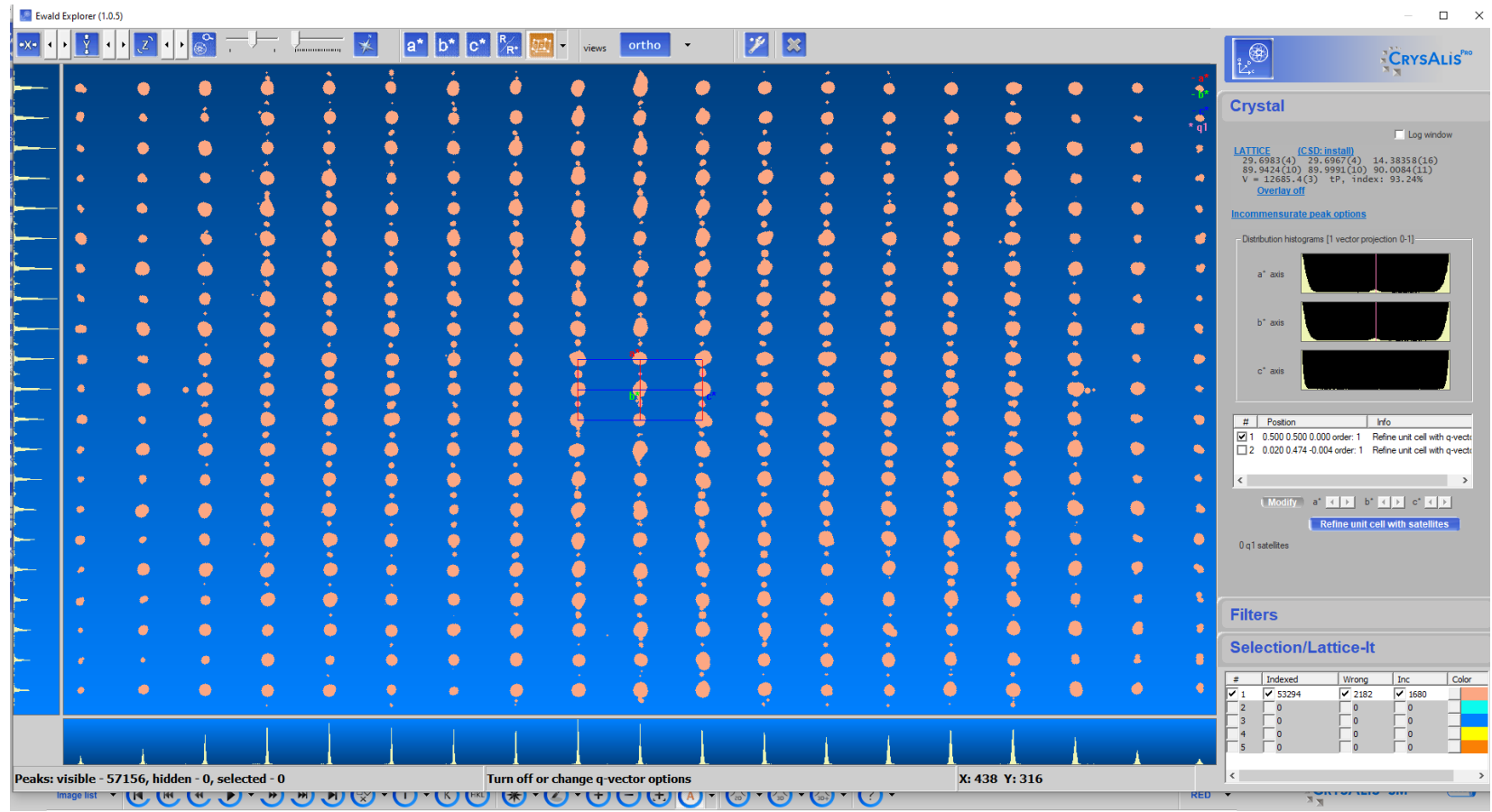

Data reduction, scaling and absorption corrections were performed using SAINT v8.34A (Bruker, 2013). The final completeness is $99.60 \%$ out to $25.681^{\circ}$ in $\Theta$. SADABS-2014/5 (Bruker, 2014/5) was used for 
absorption correction. $w R_{2}$ (int) was 0.1399 before and 0.0789 after correction. The Ratio of minimum to maximum transmission is 0.8794 . The $\lambda / 2$ correction factor is Not present. The absorption coefficient $\mu$ of this material is $0.398 \mathrm{~mm}^{-1}$ at this wavelength $(\lambda=0.71073 \AA)$ and the minimum and maximum transmissions are 0.656 and 0.746 .

The structure was solved and the space group P4 (\# 75) determined by the XT (Sheldrick, 2015) structure solution program using using dual methods and refined by full matrix least squares minimisation on $\boldsymbol{F}^{2}$ using version 2018/3 of ShelXL 2018/3 (Sheldrick, 2015). All non-hydrogen atoms were refined anisotropically. Hydrogen atom positions were calculated geometrically and refined using the riding model.

\section{Data Plots: Diffraction Data}
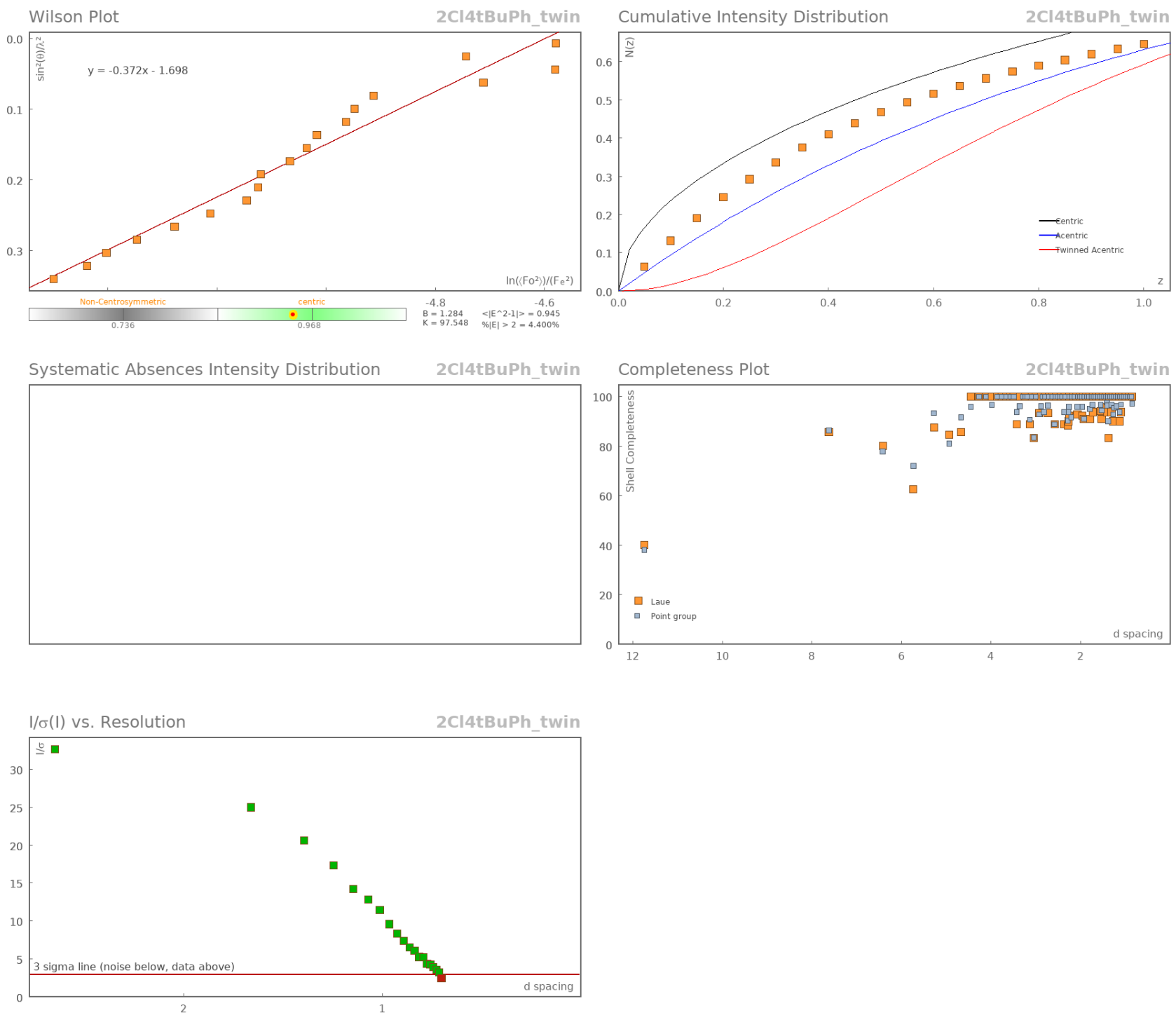


\section{Data Plots: Refinement and Data}
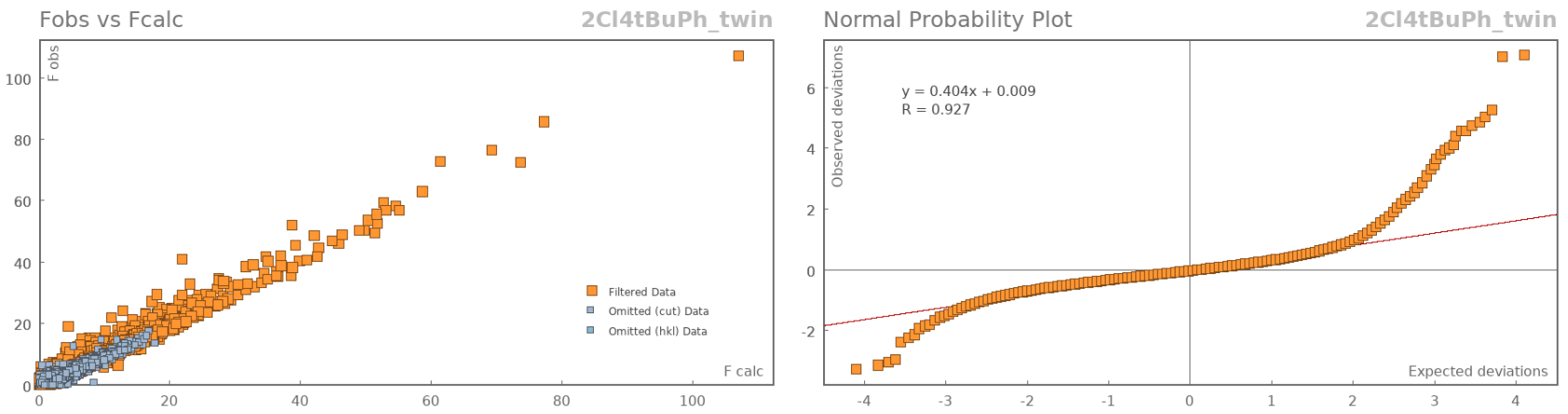

Table 1: Fractional Atomic Coordinates $\left(\times 10^{4}\right)$ and Equivalent Isotropic Displacement Parameters $\left(\AA^{2} \times 10^{3}\right)$ for 2cl4tbuph_twin. $U_{e q}$ is defined as $1 / 3$ of the trace of the orthogonalised $U_{i j}$.

\begin{tabular}{|c|c|c|c|c|}
\hline Atom & $\mathbf{x}$ & $\mathbf{y}$ & $\mathbf{Z}$ & $\boldsymbol{U}_{e q}$ \\
\hline$\overline{\mathrm{Rh} 1}$ & 5000 & 0 & 5811.1 & $5.47(18)$ \\
\hline Rh2 & 5000 & 0 & 4144.79 & $7.38(19)$ \\
\hline $\mathrm{Cl} 1$ & $6244.0(7)$ & $888.8(7)$ & $4144.3(17)$ & $25.2(5)$ \\
\hline $\mathrm{Cl} 2$ & $4025.5(8)$ & $1282.4(7)$ & 4158.1(16) & $27.7(5)$ \\
\hline 01 & $5225.8(17)$ & $652.7(18)$ & $4189(4)$ & $12.6(10)$ \\
\hline 02 & 5093.1(17) & $681.3(17)$ & $5754(4)$ & $9.6(9)$ \\
\hline 04 & $4354.3(18)$ & 236.1(19) & $4196(4)$ & $15.5(11)$ \\
\hline 05 & $4316.8(17)$ & $87.7(17)$ & $5746(4)$ & $9.9(9)$ \\
\hline N1 & 5000 & 0 & $7365(9)$ & $28.4(10)$ \\
\hline N2 & 5000 & 0 & 2461(9) & $28.4(10)$ \\
\hline C1 & $5224(2)$ & $845(2)$ & $4996(6)$ & $11.5(12)$ \\
\hline $\mathrm{C} 2$ & $5388(3)$ & $1331(3)$ & 4987(7) & $18.0(12)$ \\
\hline $\mathrm{C} 3$ & $5125(3)$ & $1647(3)$ & $5606(6)$ & $15.6(13)$ \\
\hline $\mathrm{C} 4$ & $5592(3)$ & $1543(3)$ & $5892(7)$ & $16.5(12)$ \\
\hline $\mathrm{C} 5$ & $5556(3)$ & $1506(3)$ & $4089(6)$ & $19.4(14)$ \\
\hline C6 & $5946(3)$ & $1339(3)$ & $3657(6)$ & $21.7(16)$ \\
\hline C7 & $6115(3)$ & $1514(3)$ & $2826(6)$ & 25.6(19) \\
\hline $\mathrm{C} 8$ & $5891(3)$ & $1875(3)$ & $2373(7)$ & $28.8(19)$ \\
\hline C9 & $5488(4)$ & $2023(4)$ & $2780(8)$ & $36(2)$ \\
\hline C10 & $5332(3)$ & $1846(3)$ & $3601(7)$ & $28.5(19)$ \\
\hline C11 & $6068(3)$ & 2051(3) & $1505(7)$ & $32.4(19)$ \\
\hline C12 & $5797(4)$ & $2120(4)$ & $756(8)$ & $47(3)$ \\
\hline C13 & $5981(4)$ & $2280(4)$ & $-89(8)$ & $46(3)$ \\
\hline C14 & $6425(4)$ & $2364(3)$ & $-213(7)$ & $32.5(18)$ \\
\hline C15 & $6709(4)$ & $2323(4)$ & $599(7)$ & $37(2)$ \\
\hline C16 & $6545(3)$ & $2165(3)$ & $1414(8)$ & $35(2)$ \\
\hline C17 & $6610(4)$ & $2513(4)$ & $-1169(8)$ & $35.7(18)$ \\
\hline C18 & $6405(4)$ & $2942(4)$ & $-1432(9)$ & $48(3)$ \\
\hline C19 & $7127(4)$ & $2565(4)$ & $-1152(9)$ & $49(3)$ \\
\hline $\mathrm{C} 20$ & $6518(5)$ & $2140(4)$ & $-1868(9)$ & $47(3)$ \\
\hline C21 & $5672(3)$ & $1272(3)$ & $6776(6)$ & $15.9(14)$ \\
\hline C22 & $5915(3)$ & $874(3)$ & $6796(7)$ & 21.1(17) \\
\hline C23 & $5982(3)$ & $646(3)$ & $7633(6)$ & $23.2(16)$ \\
\hline
\end{tabular}




\begin{tabular}{|c|c|c|c|c|}
\hline Atom & $\mathbf{x}$ & $\mathbf{y}$ & $\mathbf{z}$ & $U_{e q}$ \\
\hline$\overline{\mathrm{C} 24}$ & $5809(3)$ & $814(3)$ & $8451(7)$ & $25.4(17)$ \\
\hline $\mathrm{C} 25$ & $5583(3)$ & $1219(3)$ & $8457(7)$ & $26.5(17)$ \\
\hline C26 & $5515(3)$ & $1452(3)$ & $7607(6)$ & $23.4(17)$ \\
\hline C27 & $5952(3)$ & $1903(3)$ & $5747(7)$ & $20.3(15)$ \\
\hline $\mathrm{C} 28$ & 6391(3) & 1796(3) & $5555(7)$ & $25.4(18)$ \\
\hline C29 & $6720(3)$ & $2127(3)$ & $5433(8)$ & $32(2)$ \\
\hline C30 & $6609(4)$ & $2574(4)$ & $5538(10)$ & $46(3)$ \\
\hline C31 & $6161(4)$ & $2686(4)$ & $5776(11)$ & $50(3)$ \\
\hline C32 & $5836(4)$ & $2360(3)$ & $5872(10)$ & $42(3)$ \\
\hline C33 & $4157(2)$ & $224(2)$ & $4997(6)$ & $11.6(12)$ \\
\hline C34 & $3673(2)$ & $387(3)$ & $4975(6)$ & $15.4(12)$ \\
\hline C35 & $3506(3)$ & $523(3)$ & $4051(6)$ & $19.2(14)$ \\
\hline C36 & $3636(3)$ & $927(3)$ & $3616(6)$ & $17.4(15)$ \\
\hline C37 & $3485(3)$ & $1052(3)$ & $2739(6)$ & 23.1(17) \\
\hline C38 & $3186(3)$ & $763(3)$ & $2250(6)$ & $22.9(15)$ \\
\hline C39 & $3054(3)$ & $360(3)$ & $2672(6)$ & $22.7(16)$ \\
\hline C40 & $3215(3)$ & $242(3)$ & $3540(7)$ & $25.8(18)$ \\
\hline C41 & $3003(3)$ & $890(3)$ & $1325(7)$ & $27.4(17)$ \\
\hline C42 & $2553(4)$ & $825(4)$ & 1134(7) & $36(2)$ \\
\hline C43 & $2369(3)$ & $969(4)$ & $304(7)$ & $32(2)$ \\
\hline C44 & $2629(3)$ & $1180(4)$ & $-350(6)$ & $29.7(18)$ \\
\hline C45 & $3081(3)$ & $1221(3)$ & $-182(7)$ & $30.0(18)$ \\
\hline C46 & $3257(4)$ & $1070(4)$ & $648(7)$ & $32.7(19)$ \\
\hline C47 & $2399(4)$ & $1372(3)$ & $-1283(7)$ & $32.6(17)$ \\
\hline C48 & $2746(4)$ & $1617(4)$ & $-1860(8)$ & $42(2)$ \\
\hline C49 & $2212(5)$ & $983(4)$ & $-1872(8)$ & $48(3)$ \\
\hline C50 & $2014(4)$ & $1672(4)$ & $-995(8)$ & $42(2)$ \\
\hline C51 & $3456(3)$ & $610(3)$ & $5871(6)$ & $17.2(12)$ \\
\hline C52 & $3348(3)$ & $138(3)$ & $5621(6)$ & $16.6(13)$ \\
\hline C53 & $3728(3)$ & $684(3)$ & $6743(6)$ & $18.8(14)$ \\
\hline C54 & $3549(3)$ & $528(3)$ & $7584(6)$ & $23.2(17)$ \\
\hline C55 & $3776(3)$ & 586(3) & $8429(6)$ & $26.0(17)$ \\
\hline C56 & $4182(3)$ & $807(3)$ & $8425(7)$ & $25.7(17)$ \\
\hline C57 & $4362(3)$ & $967(3)$ & $7606(6)$ & $22.7(16)$ \\
\hline C58 & $4123(3)$ & $906(3)$ & $6768(7)$ & $19.9(15)$ \\
\hline C59 & $3114(3)$ & $976(3)$ & $5668(6)$ & $23.8(15)$ \\
\hline C60 & $3224(3)$ & $1419(3)$ & $5780(8)$ & $33(2)$ \\
\hline C61 & $2930(4)$ & $1780(4)$ & $5602(8)$ & $40(2)$ \\
\hline C62 & $2497(4)$ & $1671(4)$ & 5291(9) & $47(3)$ \\
\hline C63 & $2390(4)$ & $1242(5)$ & $5169(13)$ & $66(4)$ \\
\hline C64 & $2689(3)$ & $880(4)$ & $5351(10)$ & $48(3)$ \\
\hline C65 & 5000 & 0 & $8161(10)$ & $53.3(17)$ \\
\hline C66 & 5000 & 0 & $9086(12)$ & $75(4)$ \\
\hline C67 & 5000 & 0 & $1665(10)$ & $53.3(17)$ \\
\hline C68 & 5000 & 0 & $740(12)$ & $75(4)$ \\
\hline Rh3 & 5000 & 5000 & 8264.1(11) & $20.4(3)$ \\
\hline Rh4 & 5000 & 5000 & $6605.5(13)$ & $24.7(4)$ \\
\hline $\mathrm{Cl} 3$ & $3887.6(8)$ & $3917.7(9)$ & $6722(2)$ & $33.1(5)$ \\
\hline 06 & $4877(2)$ & $4320(2)$ & $6656(5)$ & $24.4(13)$ \\
\hline 07 & $4973(2)$ & $4321(2)$ & $8198(5)$ & $26.5(13)$ \\
\hline N3 & 5000 & 5000 & $5167(7)$ & $28.4(10)$ \\
\hline N4 & 5000 & 5000 & $9741(10)$ & $28.4(10)$ \\
\hline COAA & $4680(4)$ & $2516(4)$ & $8393(9)$ & $40(2)$ \\
\hline
\end{tabular}




\begin{tabular}{|c|c|c|c|c|}
\hline Atom & $\mathbf{x}$ & $\mathbf{y}$ & $\mathbf{z}$ & $U_{e q}$ \\
\hline$\overline{C 69}$ & $4894(3)$ & $4123(3)$ & $7423(7)$ & $25.3(15)$ \\
\hline C70 & $4830(3)$ & $3634(3)$ & $7400(7)$ & $26.8(14)$ \\
\hline C71 & $4683(3)$ & $3444(3)$ & $6489(7)$ & $24.8(15)$ \\
\hline C72 & $4253(3)$ & $3545(3)$ & $6131(7)$ & $28.9(18)$ \\
\hline C73 & $4106(4)$ & $3378(4)$ & $5287(8)$ & $36(2)$ \\
\hline C74 & $4377(3)$ & $3093(3)$ & $4768(7)$ & $31.3(17)$ \\
\hline C75 & $4802(3)$ & $3012(3)$ & $5063(7)$ & $31.2(18)$ \\
\hline C76 & $4949(4)$ & $3192(3)$ & $5934(7)$ & 32.3(19) \\
\hline C77 & $4215(4)$ & $2902(4)$ & $3860(8)$ & $36.7(19)$ \\
\hline C78 & $4479(4)$ & $2914(3)$ & $3043(8)$ & $32.5(18)$ \\
\hline C79 & $4331(4)$ & $2761(4)$ & $2225(9)$ & $45(2)$ \\
\hline C80 & $3878(4)$ & 2598(4) & 2129(8) & $37(2)$ \\
\hline C81 & $3627(4)$ & $2566(4)$ & $2938(8)$ & $40(2)$ \\
\hline C82 & $3777(4)$ & $2725(4)$ & $3763(8)$ & $39(2)$ \\
\hline C83 & $3717(5)$ & $2437(4)$ & $1167(8)$ & $47(2)$ \\
\hline C84 & $4019(5)$ & $2065(4)$ & $780(9)$ & $53(3)$ \\
\hline C85 & $3237(5)$ & $2263(4)$ & 1195(9) & $51(3)$ \\
\hline C86 & $3744(5)$ & $2821(4)$ & 483(9) & $53(3)$ \\
\hline C87 & $4715(3)$ & $3363(3)$ & $8297(7)$ & $28.1(14)$ \\
\hline C88 & $5197(3)$ & $3380(3)$ & $7948(7)$ & $26.6(15)$ \\
\hline C89 & $4618(3)$ & $3593(3)$ & $9199(7)$ & $30.1(16)$ \\
\hline C90 & $4855(4)$ & $3507(4)$ & $9981(8)$ & $38(2)$ \\
\hline C91 & $4745(5)$ & $3706(5)$ & $10845(9)$ & $56(3)$ \\
\hline C92 & $4354(4)$ & $3981(4)$ & $10885(10)$ & $50(2)$ \\
\hline C93 & $4117(5)$ & $4044(4)$ & $10119(10)$ & $52(3)$ \\
\hline C94 & $4220(4)$ & $3861(3)$ & $9271(8)$ & $39(2)$ \\
\hline C95 & $4470(3)$ & 2914(3) & $8183(7)$ & $29.1(16)$ \\
\hline C96 & $4020(3)$ & $2889(3)$ & $7926(7)$ & $30.8(19)$ \\
\hline C97 & $3801(4)$ & $2482(4)$ & $7865(9)$ & $39(2)$ \\
\hline C98 & $4006(4)$ & $2085(4)$ & $8028(8)$ & $37(2)$ \\
\hline C99 & $4474(4)$ & $2099(4)$ & $8353(10)$ & $47(3)$ \\
\hline C100 & 5000 & 5000 & 4371(9) & $53.3(17)$ \\
\hline C102 & 5000 & 5000 & 10538(11) & $53.3(17)$ \\
\hline Rh4B & 0 & 0 & $5563.7(9)$ & $7.0(2)$ \\
\hline Rh3B & 0 & 0 & $7223.5(9)$ & $6.4(2)$ \\
\hline $\mathrm{Cl} 3 \mathrm{~B}$ & $1187.9(9)$ & $-1135.6(7)$ & $5555.7(16)$ & $30.6(5)$ \\
\hline 07B & $687.6(17)$ & $-9.5(18)$ & $7163(4)$ & $12.6(10)$ \\
\hline O6B & $673.3(18)$ & $-162.4(18)$ & $5621(4)$ & 14.1(10) \\
\hline N4B & 0 & 0 & $8725(10)$ & $28.4(10)$ \\
\hline N3B & 0 & 0 & $4093(10)$ & $28.4(10)$ \\
\hline C70B & $1368(2)$ & $-190(2)$ & $6410(6)$ & $10.4(11)$ \\
\hline C69B & $867(2)$ & $-124(2)$ & $6411(5)$ & $7.5(11)$ \\
\hline C71B & $1572(2)$ & $-301(3)$ & $5474(5)$ & $9.9(12)$ \\
\hline C72B & $1524(3)$ & $-725(3)$ & $5048(6)$ & $18.6(15)$ \\
\hline C73B & $1715(3)$ & $-814(3)$ & $4186(6)$ & $23.9(17)$ \\
\hline C74B & 1951(3) & $-485(3)$ & $3719(6)$ & $22.0(15)$ \\
\hline C75B & 1988(3) & $-54(3)$ & $4112(6)$ & $22.1(16)$ \\
\hline C76B & $1795(3)$ & $24(3)$ & $4974(6)$ & $15.8(14)$ \\
\hline C77B & $2173(3)$ & $-575(3)$ & $2804(6)$ & $22.3(16)$ \\
\hline C78B & 2082(3) & $-311(4)$ & $2025(6)$ & $28.0(19)$ \\
\hline C79B & 2311(3) & $-376(4)$ & $1182(6)$ & $33(2)$ \\
\hline C80B & $2610(3)$ & $-719(4)$ & $1053(6)$ & $30.3(18)$ \\
\hline C81B & $2693(5)$ & $-989(5)$ & $1833(8)$ & $54(4)$ \\
\hline
\end{tabular}




\begin{tabular}{lcrcc}
\hline Atom & $\mathbf{x}$ & $\mathbf{y}$ & $\mathbf{z}$ & \multicolumn{1}{c}{$\boldsymbol{U}_{\text {eq }}$} \\
\hline C82B & $2484(4)$ & $-933(5)$ & $2685(8)$ & $50(3)$ \\
C83B & $2853(3)$ & $-818(4)$ & $136(7)$ & $38(2)$ \\
C84B & $2913(5)$ & $-364(5)$ & $-416(10)$ & $61(3)$ \\
C86B & $2547(5)$ & $-1125(6)$ & $-433(10)$ & $66(4)$ \\
C85B & $3312(4)$ & $-1021(5)$ & $255(9)$ & $53(3)$ \\
C87B & $1615(2)$ & $-373(2)$ & $7288(6)$ & $11.6(11)$ \\
C88B & $1636(3)$ & $120(3)$ & $7055(5)$ & $14.4(13)$ \\
C89B & $1358(3)$ & $-505(3)$ & $8160(5)$ & $12.8(13)$ \\
C90B & $1483(3)$ & $-322(3)$ & $9015(6)$ & $20.7(16)$ \\
C91B & $1269(3)$ & $-456(4)$ & $9826(6)$ & $28.8(19)$ \\
C92B & $923(3)$ & $-762(3)$ & $9800(6)$ & $26.3(18)$ \\
C93B & $784(3)$ & $-936(3)$ & $8949(6)$ & $22.9(16)$ \\
C94B & $1000(3)$ & $-814(3)$ & $8140(6)$ & $18.2(15)$ \\
C95B & $2033(3)$ & $-661(3)$ & $7154(6)$ & $15.6(13)$ \\
C1A & $2449(3)$ & $-476(4)$ & $7016(8)$ & $33(2)$ \\
C99B & $2823(4)$ & $-761(4)$ & $6909(9)$ & $46(3)$ \\
C98B & $2788(4)$ & $-1219(4)$ & $6940(7)$ & $39(2)$ \\
C97B & $2367(4)$ & $-1408(4)$ & $7032(9)$ & $43(3)$ \\
C96B & $1984(3)$ & $-1130(3)$ & $7148(8)$ & $27.2(18)$ \\
C1B & 0 & 0 & $9522(11)$ & $53.3(17)$ \\
C103 & 0 & 0 & $10548(11)$ & $53.3(17)$ \\
C1C & 0 & 0 & $3298(11)$ & $53.3(17)$ \\
C101 & 0 & 0 & $2271(11)$ & $53.3(17)$ \\
C1D & 5000 & 5000 & $3370(11)$ & $108(10)$ \\
C1E & 5000 & 5000 & $11539(13)$ & $108(10)$ \\
& & & &
\end{tabular}

Table 2: Anisotropic Displacement Parameters $\left(\times 10^{4}\right)$ for 2cl4tbuph_twin. The anisotropic displacement factor exponent takes the form: $-2 \pi^{2}\left[h^{2} a^{* 2} \times U_{11}+\ldots+2 h k a^{*} \times b^{*} \times U_{12}\right]$

\begin{tabular}{lcccccc}
\hline Atom & $\boldsymbol{U}_{\mathbf{1 1}}$ & $\boldsymbol{U}_{\mathbf{2 2}}$ & $\boldsymbol{U}_{\mathbf{3 3}}$ & $\boldsymbol{U}_{\mathbf{2 3}}$ & $\boldsymbol{U}_{\mathbf{1 3}}$ & $\boldsymbol{U}_{\mathbf{1 2}}$ \\
\hline $\mathrm{Rh} 1$ & $6.3(4)$ & $7.3(4)$ & $2.8(4)$ & 0 & 0 & $-0.6(3)$ \\
$\mathrm{Rh} 2$ & $7.6(4)$ & $11.5(4)$ & $3.1(4)$ & 0 & 0 & $-1.5(3)$ \\
$\mathrm{Cl} 1$ & $21.4(10)$ & $21.2(10)$ & $33.2(11)$ & $4.8(9)$ & $6.9(9)$ & $5.8(8)$ \\
$\mathrm{Cl} 2$ & $34.9(12)$ & $23.6(11)$ & $24.6(11)$ & $5.6(9)$ & $-5.8(10)$ & $-11.4(9)$ \\
01 & $12(2)$ & $16(2)$ & $9.8(18)$ & $1.5(18)$ & $4(2)$ & $0.6(19)$ \\
O2 & $10(2)$ & $13(2)$ & $6.0(18)$ & $-1.6(17)$ & $-3.7(18)$ & $0.6(17)$ \\
O4 & $10(2)$ & $23(3)$ & $13.2(19)$ & $6(2)$ & $0.7(18)$ & $-1(2)$ \\
O5 & $11(2)$ & $9(2)$ & $9.5(18)$ & $-3.5(19)$ & $4.2(17)$ & $0.4(17)$ \\
$\mathrm{N} 1$ & $32(3)$ & $6(2)$ & $47.8(17)$ & 0 & 0 & $2(3)$ \\
$\mathrm{N} 2$ & $32(3)$ & $6(2)$ & $47.8(17)$ & 0 & 0 & $2(3)$ \\
$\mathrm{C} 1$ & $12(3)$ & $13(2)$ & $9.8(18)$ & $1.7(17)$ & $0(2)$ & $2(2)$ \\
$\mathrm{C} 2$ & $20(3)$ & $14(2)$ & $20(2)$ & $0.2(19)$ & $4(2)$ & $-2(2)$ \\
$\mathrm{C} 3$ & $15(3)$ & $8(3)$ & $24(3)$ & $2(3)$ & $6(2)$ & $-4(2)$ \\
$\mathrm{C} 4$ & $16(2)$ & $10(3)$ & $24(2)$ & $-4(2)$ & $4(2)$ & $1(2)$ \\
$\mathrm{C} 5$ & $24(3)$ & $11(3)$ & $23(3)$ & $2(2)$ & $8(2)$ & $-1(2)$ \\
$\mathrm{C} 6$ & $22(3)$ & $16(4)$ & $27(3)$ & $7(3)$ & $9(3)$ & $-1(3)$ \\
$\mathrm{C} 7$ & $26(4)$ & $25(4)$ & $25(4)$ & $11(3)$ & $10(3)$ & $8(3)$ \\
$\mathrm{C} 8$ & $26(4)$ & $27(4)$ & $33(4)$ & $14(3)$ & $8(3)$ & $7(3)$ \\
$\mathrm{C} 9$ & $30(4)$ & $37(5)$ & $41(4)$ & $22(4)$ & $13(3)$ & $16(4)$ \\
$\mathrm{C} 10$ & $35(4)$ & $21(4)$ & $30(4)$ & $8(3)$ & $11(3)$ & $9(3)$ \\
$\mathrm{C} 11$ & $33(3)$ & $31(5)$ & $33(3)$ & $15(3)$ & $7(3)$ & $3(3)$
\end{tabular}




\begin{tabular}{|c|c|c|c|c|c|c|}
\hline Atom & $U_{11}$ & $U_{22}$ & $U_{33}$ & $U_{23}$ & $U_{13}$ & $U_{12}$ \\
\hline$\overline{\mathrm{C} 12}$ & $33(4)$ & $71(8)$ & $37(4)$ & $24(4)$ & $2(3)$ & $-13(5)$ \\
\hline C13 & $40(3)$ & $67(7)$ & $32(4)$ & $20(5)$ & $1(3)$ & $-9(4)$ \\
\hline C14 & $39(3)$ & $25(5)$ & $33(3)$ & $6(3)$ & $8(3)$ & $-2(3)$ \\
\hline C15 & $35(4)$ & $40(6)$ & $37(4)$ & $12(4)$ & $7(3)$ & $-3(4)$ \\
\hline C16 & $31(3)$ & $36(5)$ & $37(4)$ & $13(4)$ & $5(3)$ & $0(4)$ \\
\hline C17 & $40(4)$ & $34(4)$ & $33(3)$ & $5(3)$ & $8(3)$ & $-3(3)$ \\
\hline C18 & $63(6)$ & $42(4)$ & $37(5)$ & $11(4)$ & $20(5)$ & $11(5)$ \\
\hline C19 & $41(4)$ & $63(7)$ & $44(6)$ & $18(6)$ & $7(4)$ & $-7(4)$ \\
\hline $\mathrm{C} 20$ & $60(7)$ & $45(5)$ & $37(4)$ & $-4(4)$ & $7(5)$ & $-2(5)$ \\
\hline $\mathrm{C} 21$ & $12(3)$ & $12(3)$ & $24(2)$ & $-1(2)$ & $4(2)$ & $-5(2)$ \\
\hline C22 & $26(4)$ & $14(3)$ & $24(3)$ & $-4(2)$ & $-2(3)$ & $2(3)$ \\
\hline C23 & $24(4)$ & $17(4)$ & $29(3)$ & $1(2)$ & $-7(3)$ & $-8(3)$ \\
\hline C24 & $24(4)$ & $24(3)$ & $28(3)$ & $1(3)$ & $-6(3)$ & $-10(3)$ \\
\hline C25 & $29(5)$ & $25(4)$ & $25(3)$ & $0(3)$ & $1(3)$ & $-8(3)$ \\
\hline C26 & $25(5)$ & $21(4)$ & $24(3)$ & $-3(2)$ & $6(3)$ & $1(3)$ \\
\hline C27 & $17(2)$ & $14(3)$ & $30(4)$ & $4(3)$ & $-2(3)$ & $-2(2)$ \\
\hline C28 & $17(3)$ & $22(3)$ & $37(5)$ & $4(3)$ & $1(3)$ & $0(2)$ \\
\hline C29 & $19(3)$ & $28(3)$ & $48(6)$ & $8(4)$ & $0(4)$ & $-5(3)$ \\
\hline C30 & $28(4)$ & $27(3)$ & $83(9)$ & $8(4)$ & $0(5)$ & $-5(3)$ \\
\hline C31 & $30(4)$ & $16(3)$ & $103(10)$ & $2(5)$ & $4(5)$ & $-6(3)$ \\
\hline C32 & $26(4)$ & $14(3)$ & $85(8)$ & $-1(4)$ & $5(5)$ & $-2(2)$ \\
\hline C33 & $9(2)$ & $13(3)$ & $12.6(19)$ & $1(2)$ & $1.0(17)$ & $-4(2)$ \\
\hline C34 & $10(2)$ & $21(3)$ & $16(2)$ & $4(2)$ & $1.9(18)$ & $-3(2)$ \\
\hline C35 & $15(3)$ & $25(3)$ & $18(2)$ & $6(2)$ & $-3(2)$ & $-2(2)$ \\
\hline C36 & $16(3)$ & $22(3)$ & $14(3)$ & $2(2)$ & $1(2)$ & $0(3)$ \\
\hline C37 & $23(4)$ & $29(4)$ & $17(3)$ & $8(3)$ & $-2(3)$ & $-3(3)$ \\
\hline C38 & $14(3)$ & $34(3)$ & $20(3)$ & $1(2)$ & $0(2)$ & $1(3)$ \\
\hline C39 & $16(4)$ & $33(4)$ & $19(3)$ & $-1(3)$ & $-2(3)$ & $-3(3)$ \\
\hline C40 & $22(4)$ & $31(4)$ & $25(3)$ & $5(3)$ & $-6(3)$ & $-9(3)$ \\
\hline C41 & $26(3)$ & $32(5)$ & $23(3)$ & $2(3)$ & $-5(2)$ & $6(3)$ \\
\hline C42 & $29(3)$ & $48(6)$ & $31(4)$ & $8(4)$ & $-8(3)$ & $1(4)$ \\
\hline C43 & $25(3)$ & $42(5)$ & $29(3)$ & $7(3)$ & $-4(3)$ & $6(4)$ \\
\hline C44 & $33(3)$ & $36(5)$ & $20(3)$ & $-3(3)$ & $-1(2)$ & $1(3)$ \\
\hline C45 & $34(3)$ & $29(5)$ & $27(3)$ & $2(3)$ & $-2(3)$ & $-3(3)$ \\
\hline C46 & $33(4)$ & $38(5)$ & $27(3)$ & $3(3)$ & $0(3)$ & $5(4)$ \\
\hline C47 & $39(4)$ & $32(4)$ & $26(3)$ & $2(3)$ & $-7(3)$ & $-4(3)$ \\
\hline C48 & $44(5)$ & $48(6)$ & $32(5)$ & $16(4)$ & $-8(4)$ & $-5(4)$ \\
\hline C49 & $58(7)$ & $49(5)$ & $38(5)$ & $-8(4)$ & $-16(5)$ & $-11(4)$ \\
\hline C50 & $38(5)$ & $50(5)$ & $38(5)$ & $3(4)$ & $-12(4)$ & $5(4)$ \\
\hline C51 & $15(3)$ & $20(3)$ & $17(2)$ & $6(2)$ & $7(2)$ & $0(2)$ \\
\hline C52 & $9(3)$ & $20(3)$ & $20(3)$ & $8(2)$ & $3(3)$ & $-1(2)$ \\
\hline C53 & $22(3)$ & $18(4)$ & $16(2)$ & $3(2)$ & $6(2)$ & $2(3)$ \\
\hline C54 & $27(4)$ & $27(4)$ & $16(2)$ & $6(3)$ & $8(2)$ & $7(3)$ \\
\hline C55 & $38(4)$ & $24(4)$ & $16(3)$ & $5(3)$ & $4(3)$ & $8(3)$ \\
\hline C56 & $39(4)$ & $13(4)$ & $25(3)$ & $-7(3)$ & $1(3)$ & $10(3)$ \\
\hline C57 & $31(4)$ & $11(4)$ & $27(3)$ & $-6(3)$ & $-2(2)$ & $4(3)$ \\
\hline C58 & $22(3)$ & $17(4)$ & $21(3)$ & $-5(3)$ & $6(2)$ & $3(3)$ \\
\hline C59 & $21(3)$ & $25(3)$ & $25(4)$ & $7(3)$ & $8(3)$ & $7(2)$ \\
\hline C60 & $23(4)$ & $25(3)$ & $50(6)$ & $7(3)$ & $3(4)$ & $10(2)$ \\
\hline C61 & $40(4)$ & $35(4)$ & $46(6)$ & $9(4)$ & $4(4)$ & $22(3)$ \\
\hline C62 & $36(4)$ & $47(4)$ & 58(7) & $23(5)$ & $1(4)$ & $22(3)$ \\
\hline C63 & $27(4)$ & $51(4)$ & $120(12)$ & $15(5)$ & $-3(6)$ & $17(3)$ \\
\hline C64 & $19(3)$ & $39(4)$ & $86(8)$ & $9(5)$ & $-4(4)$ & $7(3)$ \\
\hline
\end{tabular}




\begin{tabular}{|c|c|c|c|c|c|c|}
\hline Atom & $U_{11}$ & $U_{22}$ & $U_{33}$ & $U_{23}$ & $U_{13}$ & $U_{12}$ \\
\hline C65 & $54.8(19)$ & $54.5(19)$ & $50.4(17)$ & 0 & 0 & $-0.1(10)$ \\
\hline C66 & $93(6)$ & $80(6)$ & $53(2)$ & 0 & 0 & $1(5)$ \\
\hline C67 & $54.8(19)$ & $54.5(19)$ & $50.4(17)$ & 0 & 0 & $-0.1(10)$ \\
\hline C68 & $93(6)$ & $80(6)$ & $53(2)$ & 0 & 0 & $1(5)$ \\
\hline Rh3 & $14.9(4)$ & $14.9(4)$ & $31.3(8)$ & 0 & 0 & 0 \\
\hline $\mathrm{Rh} 4$ & $13.1(4)$ & $13.1(4)$ & $47.8(10)$ & 0 & 0 & 0 \\
\hline $\mathrm{Cl} 3$ & $26.6(12)$ & $35.0(14)$ & $37.7(12)$ & $-1.5(11)$ & $-2.8(10)$ & $6.2(10)$ \\
\hline 06 & $21(3)$ & $19(3)$ & $34(3)$ & $-4(2)$ & $4(2)$ & $5(2)$ \\
\hline 07 & $17(3)$ & $24(3)$ & $39(3)$ & $-7(2)$ & $-7(2)$ & $-1(2)$ \\
\hline N3 & $32(3)$ & $6(2)$ & $47.8(17)$ & 0 & 0 & $2(3)$ \\
\hline N4 & $32(3)$ & $6(2)$ & $47.8(17)$ & 0 & 0 & $2(3)$ \\
\hline COAA & $28(4)$ & $30(3)$ & $62(7)$ & $-1(4)$ & $-1(4)$ & $-1(3)$ \\
\hline C69 & $19(4)$ & $23(3)$ & $34(3)$ & $-3.3(18)$ & $-4(3)$ & $5(2)$ \\
\hline C70 & $25(4)$ & $25(2)$ & $31(3)$ & $-1.6(19)$ & $-4(2)$ & $0(2)$ \\
\hline C71 & $27(3)$ & $18(4)$ & $29(3)$ & $2(3)$ & $-5(2)$ & $3(3)$ \\
\hline C72 & $29(3)$ & $20(4)$ & $38(4)$ & $3(3)$ & $-9(3)$ & $-2(3)$ \\
\hline C73 & $31(4)$ & $37(5)$ & $40(4)$ & $-3(3)$ & $-13(3)$ & $8(3)$ \\
\hline C74 & $35(3)$ & $21(4)$ & $38(3)$ & $5(3)$ & $-3(3)$ & $-1(3)$ \\
\hline C75 & $36(3)$ & $24(4)$ & $33(4)$ & $-3(3)$ & $0(3)$ & $8(3)$ \\
\hline C76 & $34(4)$ & $26(4)$ & $37(4)$ & $-4(3)$ & $-2(3)$ & $4(3)$ \\
\hline C77 & $37(4)$ & $36(5)$ & $38(3)$ & $3(3)$ & $-3(3)$ & $-1(3)$ \\
\hline C78 & $31(4)$ & $27(5)$ & $40(3)$ & $9(3)$ & $-3(3)$ & $-2(3)$ \\
\hline C79 & $52(4)$ & $42(6)$ & $39(4)$ & $7(4)$ & $-2(3)$ & $-18(4)$ \\
\hline C80 & $52(4)$ & $28(5)$ & $31(3)$ & $1(3)$ & $-2(3)$ & $-14(4)$ \\
\hline C81 & $50(5)$ & $36(6)$ & $34(3)$ & $4(3)$ & $-3(3)$ & $-12(4)$ \\
\hline C82 & $35(4)$ & $48(6)$ & $34(4)$ & $-5(4)$ & $1(3)$ & $-3(4)$ \\
\hline C83 & $64(5)$ & $38(5)$ & $37(4)$ & $-3(3)$ & $-9(3)$ & $-12(4)$ \\
\hline C84 & $70(6)$ & $49(5)$ & $39(6)$ & $-13(4)$ & $-13(5)$ & $-7(5)$ \\
\hline C85 & $64(5)$ & $37(6)$ & $52(7)$ & $-4(5)$ & $-16(4)$ & $-13(4)$ \\
\hline C86 & $72(8)$ & $50(5)$ & $38(5)$ & $4(4)$ & $-14(5)$ & $-15(5)$ \\
\hline C87 & $27(3)$ & $28(3)$ & $30(3)$ & $0(2)$ & $-6(3)$ & $1(2)$ \\
\hline C88 & $25(3)$ & $22(4)$ & $33(4)$ & $-3(3)$ & $-7(3)$ & $0(2)$ \\
\hline C89 & $37(4)$ & $19(4)$ & $34(3)$ & $-2(3)$ & $-1(3)$ & $-7(3)$ \\
\hline C90 & $42(5)$ & $38(5)$ & $34(3)$ & $-3(3)$ & $-4(3)$ & $-14(4)$ \\
\hline C91 & $57(6)$ & $70(7)$ & $41(4)$ & $-22(5)$ & $-7(4)$ & $-16(5)$ \\
\hline C92 & $58(6)$ & $43(6)$ & $49(4)$ & $-11(5)$ & $12(4)$ & $-24(4)$ \\
\hline C93 & $62(6)$ & $40(6)$ & $53(4)$ & $-18(4)$ & $10(4)$ & $0(5)$ \\
\hline C94 & $48(5)$ & $26(5)$ & $43(4)$ & $-2(4)$ & $8(4)$ & $3(4)$ \\
\hline C95 & $27(3)$ & $28(3)$ & $32(4)$ & $-7(3)$ & $3(3)$ & $-1(2)$ \\
\hline C96 & $28(3)$ & $28(3)$ & $37(5)$ & $-2(4)$ & $1(3)$ & $-2(3)$ \\
\hline C97 & $31(4)$ & $30(3)$ & $55(6)$ & $-6(4)$ & $3(4)$ & $-6(2)$ \\
\hline C98 & $33(4)$ & $28(3)$ & $52(6)$ & $-5(4)$ & $11(4)$ & $-7(3)$ \\
\hline C99 & $32(4)$ & $30(3)$ & $79(8)$ & $-6(5)$ & $7(4)$ & $0(3)$ \\
\hline C100 & $54.8(19)$ & $54.5(19)$ & $50.4(17)$ & 0 & 0 & $-0.1(10)$ \\
\hline C102 & $54.8(19)$ & $54.5(19)$ & $50.4(17)$ & 0 & 0 & $-0.1(10)$ \\
\hline Rh4B & $6.7(3)$ & $6.7(3)$ & $7.7(5)$ & 0 & 0 & 0 \\
\hline Rh3B & 7.4(3) & $7.4(3)$ & $4.3(5)$ & 0 & 0 & 0 \\
\hline $\mathrm{Cl} 3 \mathrm{~B}$ & $48.6(15)$ & $18.5(11)$ & $24.6(11)$ & $-3.4(9)$ & $12.1(10)$ & $-12.6(10)$ \\
\hline 07B & $13(2)$ & $15(3)$ & $9.7(17)$ & $-2(2)$ & $-1.1(18)$ & $2.3(19)$ \\
\hline O6B & $14.9(19)$ & $17(2)$ & $10.9(16)$ & $-2.3(16)$ & $0.5(15)$ & $0.7(17)$ \\
\hline N4B & $32(3)$ & $6(2)$ & $47.8(17)$ & 0 & 0 & $2(3)$ \\
\hline N3B & $32(3)$ & $6(2)$ & $47.8(17)$ & 0 & 0 & $2(3)$ \\
\hline C70B & $11(2)$ & $10(3)$ & $10(2)$ & $1(2)$ & $2.1(16)$ & $-2(2)$ \\
\hline
\end{tabular}




\begin{tabular}{|c|c|c|c|c|c|c|}
\hline Atom & $U_{11}$ & $U_{22}$ & $U_{33}$ & $U_{23}$ & $U_{13}$ & $U_{12}$ \\
\hline$\overline{\mathrm{C} 69 \mathrm{~B}}$ & $10(2)$ & $2(3)$ & $9.7(18)$ & $1(2)$ & $2.1(15)$ & $-1(2)$ \\
\hline C71B & $4(3)$ & $13(3)$ & $13(2)$ & $0.3(19)$ & $1(2)$ & $1(2)$ \\
\hline C72B & $23(4)$ & $13(3)$ & $20(3)$ & $-3(2)$ & $4(3)$ & $1(2)$ \\
\hline C73B & $28(4)$ & $26(3)$ & $18(3)$ & $-7(2)$ & $5(3)$ & $-4(3)$ \\
\hline C74B & $20(4)$ & $28(3)$ & $18(3)$ & $-3(2)$ & $2(3)$ & $-2(3)$ \\
\hline C75B & $23(4)$ & $28(3)$ & $16(3)$ & $0(2)$ & $6(3)$ & $-5(3)$ \\
\hline C76B & $16(3)$ & $20(3)$ & $11(2)$ & $2(2)$ & $0(2)$ & $-6(3)$ \\
\hline C77B & $16(4)$ & $31(4)$ & $20(3)$ & $-5(2)$ & $2(2)$ & $-5(3)$ \\
\hline C78B & $29(5)$ & $33(5)$ & $22(3)$ & $-2(3)$ & $6(3)$ & $-1(3)$ \\
\hline C79B & $27(4)$ & $53(5)$ & $18(3)$ & $-5(3)$ & $3(3)$ & $1(3)$ \\
\hline C80B & $19(4)$ & $52(5)$ & $20(3)$ & $-9(3)$ & $1(3)$ & $-3(3)$ \\
\hline C81B & $48(7)$ & $83(8)$ & $30(4)$ & $5(4)$ & $17(4)$ & $34(6)$ \\
\hline C82B & $57(7)$ & $60(6)$ & $34(4)$ & $11(4)$ & $24(4)$ & $29(5)$ \\
\hline C83B & $27(4)$ & $63(5)$ & $26(3)$ & $-7(3)$ & $9(3)$ & $-4(3)$ \\
\hline C84B & $63(8)$ & $72(6)$ & $47(6)$ & $8(5)$ & $32(6)$ & $6(5)$ \\
\hline C86B & $57(6)$ & $96(8)$ & $45(6)$ & $-38(6)$ & $6(5)$ & $-15(6)$ \\
\hline C85B & $35(4)$ & $82(8)$ & $41(6)$ & $-5(5)$ & $18(4)$ & $9(4)$ \\
\hline C87B & $10(2)$ & $13(2)$ & $13(2)$ & $1(2)$ & $0.7(19)$ & $2(2)$ \\
\hline C88B & $15(3)$ & $12(2)$ & $17(3)$ & $-1(2)$ & $-5(3)$ & $-1(2)$ \\
\hline C89B & $10(3)$ & $16(3)$ & $13(2)$ & $3(2)$ & $2(2)$ & $6(2)$ \\
\hline C90B & $22(4)$ & $27(4)$ & $13(2)$ & $-1(2)$ & $2(2)$ & $-3(3)$ \\
\hline C91B & $29(4)$ & $43(5)$ & $15(3)$ & $4(3)$ & $4(3)$ & $1(3)$ \\
\hline C92B & $25(4)$ & $39(4)$ & $15(3)$ & $9(3)$ & $7(3)$ & $5(3)$ \\
\hline C93B & $22(4)$ & $29(4)$ & $18(3)$ & $8(3)$ & $6(2)$ & $3(3)$ \\
\hline C94B & $20(3)$ & $19(4)$ & $15(3)$ & $0(3)$ & $4(2)$ & $-2(3)$ \\
\hline C95B & $14(2)$ & $20(3)$ & $13(3)$ & $4(3)$ & $6(2)$ & $6.3(19)$ \\
\hline C1A & $13(3)$ & $31(4)$ & $56(7)$ & $23(4)$ & $8(3)$ & $6(2)$ \\
\hline C99B & $19(3)$ & $46(4)$ & $72(8)$ & $30(4)$ & $17(4)$ & $18(3)$ \\
\hline C98B & $27(3)$ & $46(4)$ & $46(6)$ & $29(4)$ & $13(4)$ & $22(3)$ \\
\hline C97B & $30(4)$ & $34(4)$ & $64(7)$ & $19(5)$ & $15(4)$ & $22(3)$ \\
\hline C96B & $18(3)$ & $20(3)$ & $43(5)$ & $3(3)$ & $-2(4)$ & $8(2)$ \\
\hline C1B & $54.8(19)$ & $54.5(19)$ & $50.4(17)$ & 0 & 0 & $-0.1(10)$ \\
\hline C103 & $54.8(19)$ & $54.5(19)$ & $50.4(17)$ & 0 & 0 & $-0.1(10)$ \\
\hline C1C & $54.8(19)$ & $54.5(19)$ & $50.4(17)$ & 0 & 0 & $-0.1(10)$ \\
\hline C101 & 54.8(19) & 54.5(19) & $50.4(17)$ & 0 & 0 & $-0.1(10)$ \\
\hline C1D & 137(16) & 137(16) & $50(2)$ & 0 & 0 & 0 \\
\hline C1E & $137(16)$ & $137(16)$ & $50(2)$ & 0 & 0 & 0 \\
\hline
\end{tabular}

Table 3: Bond Lengths in Å for 2cl4tbuph_twin.

\begin{tabular}{lll}
\hline Atom & Atom & Length/Å \\
\hline $\mathrm{Rh} 1$ & $\mathrm{Rh} 2$ & $2.39472(4)$ \\
$\mathrm{Rh} 1$ & $\mathrm{O} 2$ & $2.042(5)$ \\
$\mathrm{Rh} 1$ & $\mathrm{O} 2^{1}$ & $2.042(5)$ \\
$\mathrm{Rh} 1$ & $\mathrm{O}^{1}$ & $2.046(5)$ \\
$\mathrm{Rh} 1$ & $\mathrm{O} 5$ & $2.046(5)$ \\
$\mathrm{Rh} 1$ & $\mathrm{~N} 1$ & $2.233(13)$ \\
$\mathrm{Rh} 2$ & $\mathrm{O} 11$ & $2.050(5)$ \\
$\mathrm{Rh} 2$ & $\mathrm{O} 1$ & $2.051(5)$ \\
$\mathrm{Rh} 2$ & $\mathrm{O} 4$ & $2.042(5)$ \\
$\mathrm{Rh} 2$ & $\mathrm{O} 41$ & $2.042(5)$
\end{tabular}

\begin{tabular}{lll}
\hline Atom & Atom & Length/Å \\
\hline Rh2 & N2 & $2.420(13)$ \\
Cl1 & C6 & $1.747(9)$ \\
Cl2 & C36 & $1.748(9)$ \\
O1 & C1 & $1.293(10)$ \\
O2 & C1 & $1.254(10)$ \\
O4 & C33 & $1.292(10)$ \\
O5 & C33 & $1.243(10)$ \\
N1 & C65 & $1.144(10)$ \\
N2 & C67 & $1.144(10)$ \\
C1 & C2 & $1.523(10)$
\end{tabular}




\begin{tabular}{|c|c|c|}
\hline Atom & Atom & Length/Å \\
\hline$\overline{\mathrm{C} 2}$ & C3 & $1.511(11)$ \\
\hline $\mathrm{C} 2$ & C4 & $1.565(13)$ \\
\hline $\mathrm{C} 2$ & $\mathrm{C} 5$ & $1.479(12)$ \\
\hline C3 & $\mathrm{C} 4$ & $1.478(12)$ \\
\hline $\mathrm{C} 4$ & $\mathrm{C} 21$ & $1.522(13)$ \\
\hline $\mathrm{C} 4$ & $\mathrm{C} 27$ & $1.526(11)$ \\
\hline C5 & C6 & $1.404(12)$ \\
\hline C5 & C10 & $1.399(13)$ \\
\hline C6 & C7 & $1.396(12)$ \\
\hline C7 & C8 & $1.416(13)$ \\
\hline C8 & C9 & $1.403(13)$ \\
\hline C8 & $\mathrm{C} 11$ & $1.450(13)$ \\
\hline C9 & $\mathrm{C} 10$ & $1.372(13)$ \\
\hline C11 & C12 & $1.359(16)$ \\
\hline C11 & C16 & $1.462(14)$ \\
\hline C12 & C13 & $1.413(15)$ \\
\hline C13 & C14 & $1.352(15)$ \\
\hline C14 & $\mathrm{C} 15$ & $1.445(15)$ \\
\hline C14 & C17 & $1.546(14)$ \\
\hline C15 & C16 & $1.352(15)$ \\
\hline $\mathrm{C} 17$ & C18 & $1.460(15)$ \\
\hline C17 & C19 & $1.542(15)$ \\
\hline C17 & $\mathrm{C} 20$ & $1.519(16)$ \\
\hline C21 & $\mathrm{C} 22$ & $1.385(12)$ \\
\hline C21 & $\mathrm{C} 26$ & $1.390(12)$ \\
\hline $\mathrm{C} 22$ & $\mathrm{C} 23$ & $1.393(13)$ \\
\hline $\mathrm{C} 23$ & $\mathrm{C} 24$ & $1.377(14)$ \\
\hline $\mathrm{C} 24$ & $\mathrm{C} 25$ & $1.377(14)$ \\
\hline $\mathrm{C} 25$ & $\mathrm{C} 26$ & $1.419(13)$ \\
\hline $\mathrm{C} 27$ & $\mathrm{C} 28$ & $1.370(12)$ \\
\hline $\mathrm{C} 27$ & C32 & $1.411(13)$ \\
\hline $\mathrm{C} 28$ & $\mathrm{C} 29$ & $1.396(13)$ \\
\hline C29 & C30 & $1.375(15)$ \\
\hline C30 & C31 & $1.412(16)$ \\
\hline C31 & C32 & $1.373(14)$ \\
\hline C33 & C34 & $1.516(10)$ \\
\hline C34 & C35 & $1.473(12)$ \\
\hline C34 & C51 & $1.586(12)$ \\
\hline C34 & C52 & $1.530(11)$ \\
\hline C35 & C36 & $1.407(12)$ \\
\hline C35 & $\mathrm{C} 40$ & $1.407(12)$ \\
\hline C36 & C37 & $1.388(12)$ \\
\hline C37 & C38 & $1.419(13)$ \\
\hline C38 & C39 & $1.399(13)$ \\
\hline C38 & $\mathrm{C} 41$ & $1.485(13)$ \\
\hline C39 & $\mathrm{C} 40$ & $1.380(13)$ \\
\hline C41 & $\mathrm{C} 42$ & $1.378(14)$ \\
\hline C41 & C46 & $1.342(14)$ \\
\hline $\mathrm{C} 42$ & $\mathrm{C} 43$ & $1.380(14)$ \\
\hline C43 & $\mathrm{C} 44$ & $1.368(14)$ \\
\hline C44 & $\mathrm{C} 45$ & $1.369(14)$ \\
\hline C44 & $\mathrm{C} 47$ & $1.609(13)$ \\
\hline C45 & $\mathrm{C} 46$ & $1.377(14)$ \\
\hline
\end{tabular}

\begin{tabular}{|c|c|c|}
\hline Atom & Atom & Length/Å \\
\hline$\overline{\mathrm{C} 47}$ & C48 & $1.508(15)$ \\
\hline C47 & C49 & $1.536(15)$ \\
\hline C47 & C50 & $1.506(15)$ \\
\hline C51 & C52 & $1.482(12)$ \\
\hline C51 & C53 & $1.506(13)$ \\
\hline C51 & C59 & $1.514(12)$ \\
\hline C53 & C54 & $1.400(12)$ \\
\hline C53 & C58 & $1.346(13)$ \\
\hline C54 & C55 & $1.400(13)$ \\
\hline C55 & C56 & $1.371(15)$ \\
\hline C56 & C57 & $1.376(13)$ \\
\hline C57 & C58 & $1.409(13)$ \\
\hline C59 & C60 & $1.366(14)$ \\
\hline C59 & C64 & $1.369(15)$ \\
\hline C60 & C61 & $1.403(13)$ \\
\hline C61 & C62 & $1.400(17)$ \\
\hline C62 & C63 & $1.322(18)$ \\
\hline C63 & C64 & $1.420(16)$ \\
\hline C65 & C66 & $1.328(17)$ \\
\hline C67 & C68 & $1.329(17)$ \\
\hline Rh3 & $\mathrm{Rh} 4$ & $2.384(2)$ \\
\hline Rh3 & $07^{2}$ & $2.020(6)$ \\
\hline Rh3 & $07^{3}$ & $2.020(6)$ \\
\hline Rh3 & $07^{4}$ & $2.020(6)$ \\
\hline Rh3 & 07 & $2.020(6)$ \\
\hline Rh3 & N4 & $2.123(15)$ \\
\hline Rh4 & $06^{4}$ & $2.051(6)$ \\
\hline Rh4 & $06^{3}$ & $2.051(6)$ \\
\hline Rh4 & 06 & $2.051(6)$ \\
\hline Rh4 & $06^{2}$ & $2.051(6)$ \\
\hline Rh4 & N3 & $2.067(10)$ \\
\hline $\mathrm{Cl} 3$ & C72 & $1.766(11)$ \\
\hline 06 & C69 & $1.248(12)$ \\
\hline 07 & C69 & $1.281(12)$ \\
\hline N3 & C100 & $1.144(10)$ \\
\hline N4 & C102 & $1.144(10)$ \\
\hline COAA & C95 & $1.369(15)$ \\
\hline COAA & C99 & $1.380(15)$ \\
\hline C69 & C70 & $1.463(13)$ \\
\hline C70 & C71 & $1.492(14)$ \\
\hline C70 & C87 & $1.559(14)$ \\
\hline C70 & C88 & $1.541(13)$ \\
\hline C71 & C72 & $1.408(13)$ \\
\hline C71 & C76 & $1.349(14)$ \\
\hline C72 & C73 & $1.381(14)$ \\
\hline C73 & C74 & $1.385(15)$ \\
\hline C74 & C75 & $1.351(14)$ \\
\hline C74 & C77 & $1.501(15)$ \\
\hline C75 & C76 & $1.430(15)$ \\
\hline C77 & C78 & $1.413(16)$ \\
\hline C77 & C82 & $1.409(15)$ \\
\hline C78 & C79 & $1.336(16)$ \\
\hline C79 & C80 & $1.434(16)$ \\
\hline
\end{tabular}




\begin{tabular}{lll}
\hline Atom & Atom & Length/A \\
\hline C80 & C81 & $1.384(17)$ \\
C80 & C83 & $1.539(16)$ \\
C81 & C82 & $1.352(16)$ \\
C83 & C84 & $1.527(18)$ \\
C83 & C85 & $1.515(18)$ \\
C83 & C86 & $1.508(17)$ \\
C87 & C88 & $1.519(14)$ \\
C87 & C89 & $1.493(14)$ \\
C87 & C95 & $1.526(14)$ \\
C89 & C90 & $1.350(15)$ \\
C89 & C94 & $1.429(15)$ \\
C90 & C91 & $1.413(17)$ \\
C91 & C92 & $1.421(19)$ \\
C92 & C93 & $1.321(19)$ \\
C93 & C94 & $1.369(17)$ \\
C95 & C96 & $1.389(14)$ \\
C96 & C97 & $1.371(14)$ \\
C97 & C98 & $1.347(16)$ \\
C98 & C99 & $1.465(16)$ \\
C100 & C1D & $1.439(13)$ \\
C102 & C1E & $1.439(13)$ \\
Rh4B & Rh3B & $2.3853(14)$ \\
Rh4B & O6B 5 & $2.057(5)$ \\
Rh4B & O6B & $2.057(5)$ \\
Rh4B & O6B 6 & $2.057(5)$ \\
Rh4B & O6B 7 & $2.057(5)$ \\
Rh4B & N3B & $2.113(15)$ \\
Rh3B & O7B 7 & $2.042(5)$ \\
Rh3B & O7B & $2.042(5)$ \\
Rh3B & O7B 5 & $2.042(5)$ \\
Rh3B & O7B 6 & $2.042(5)$ \\
Rh3B & N4B & $2.158(15)$ \\
Cl3B & C72B & $1.735(8)$ \\
O7B & C69B & $1.252(10)$ \\
O6B & C69B & $1.278(10)$ \\
N4B & C1B & $1.144(10)$ \\
N3B & C1C & $1.144(10)$ \\
C70B & C69B & $1.500(10)$ \\
C70B & C71B & $1.511(10)$ \\
& &
\end{tabular}

\begin{tabular}{|c|c|c|}
\hline Atom & Atom & Length/Å \\
\hline$\overline{\mathrm{C} 70 \mathrm{~B}}$ & C87B & $1.556(11)$ \\
\hline C70B & C88B & $1.529(11)$ \\
\hline C71B & C72B & $1.406(11)$ \\
\hline C71B & C76B & $1.373(11)$ \\
\hline C72B & C73B & $1.389(12)$ \\
\hline C73B & C74B & $1.377(13)$ \\
\hline C74B & C75B & $1.401(12)$ \\
\hline C74B & C77B & $1.494(12)$ \\
\hline C75B & C76B & $1.384(12)$ \\
\hline C77B & C78B & $1.394(13)$ \\
\hline C77B & C82B & $1.417(15)$ \\
\hline C78B & C79B & $1.402(13)$ \\
\hline C79B & C80B & $1.361(15)$ \\
\hline C80B & C81B & $1.400(16)$ \\
\hline C80B & C83B & $1.531(13)$ \\
\hline C81B & C82B & $1.382(15)$ \\
\hline C83B & C84B & $1.574(18)$ \\
\hline C83B & C86B & $1.524(16)$ \\
\hline C83B & C85B & $1.500(15)$ \\
\hline C87B & C88B & $1.503(11)$ \\
\hline C87B & C89B & $1.518(11)$ \\
\hline C87B & C95B & $1.519(10)$ \\
\hline C89B & C90B & $1.394(11)$ \\
\hline C89B & C94B & $1.402(12)$ \\
\hline С90B & C91B & $1.386(12)$ \\
\hline C91B & C92B & $1.371(14)$ \\
\hline C92B & C93B & $1.391(13)$ \\
\hline C93B & C94B & $1.376(11)$ \\
\hline C95B & $\mathrm{C} 1 \mathrm{~A}$ & $1.364(13)$ \\
\hline C95B & C96B & $1.399(12)$ \\
\hline C1A & C99B & $1.404(14)$ \\
\hline C99B & C98B & $1.364(17)$ \\
\hline C98B & C97B & $1.375(17)$ \\
\hline C97B & C96B & $1.413(13)$ \\
\hline C1B & C103 & $1.475(9)$ \\
\hline C1C & C101 & $1.475(9)$ \\
\hline
\end{tabular}

Table 4: Bond Angles in ${ }^{\circ}$ for 2cl4tbuph_twin.

\begin{tabular}{llll}
\hline Atom & Atom & Atom & \multicolumn{1}{c}{ Angle $^{\circ}$} \\
\hline $\mathrm{O}^{1}$ & $\mathrm{Rh} 1$ & $\mathrm{Rh} 2$ & $87.69(15)$ \\
$\mathrm{O} 2$ & $\mathrm{Rh} 1$ & $\mathrm{Rh} 2$ & $87.69(15)$ \\
$\mathrm{O} 2$ & $\mathrm{Rh} 1$ & $\mathrm{O}^{1}$ & $175.4(3)$ \\
$\mathrm{O} 2$ & $\mathrm{Rh} 1$ & $\mathrm{O}^{1}$ & $89.43(18)$ \\
$\mathrm{O} 2$ & $\mathrm{Rh} 1$ & $\mathrm{O} 5$ & $90.36(18)$ \\
$\mathrm{O}^{1}$ & $\mathrm{Rh} 1$ & $\mathrm{O} 5$ & $89.43(18)$ \\
$\mathrm{O}^{1}$ & $\mathrm{Rh} 1$ & $\mathrm{O} 5^{1}$ & $90.36(18)$ \\
$\mathrm{O}^{1}$ & $\mathrm{Rh} 1$ & $\mathrm{~N} 1$ & $92.31(15)$ \\
$\mathrm{O} 2$ & $\mathrm{Rh} 1$ & $\mathrm{~N} 1$ & $92.31(15)$
\end{tabular}

\begin{tabular}{llll}
\hline Atom & Atom & Atom & \multicolumn{1}{c}{ Angle $^{\circ}$} \\
\hline O51 & Rh1 & Rh2 & $87.37(15)$ \\
O5 & Rh1 & Rh2 & $87.37(15)$ \\
O5 & Rh1 & O51 & $174.7(3)$ \\
O5 & Rh1 & N1 & $92.63(15)$ \\
O51 & Rh1 & N1 & $92.63(15)$ \\
N1 & Rh1 & Rh2 & 180.0 \\
Rh1 & Rh2 & N2 & 180.0 \\
O1 & Rh2 & Rh1 & $88.23(15)$ \\
011 & Rh2 & Rh1 & $88.24(15)$
\end{tabular}




\begin{tabular}{|c|c|c|c|}
\hline Atom & Atom & Atom & Angle ${ }^{\circ}$ \\
\hline$\overline{011}$ & Rh2 & 01 & $176.5(3)$ \\
\hline 01 & $\mathrm{Rh} 2$ & N2 & $91.77(15)$ \\
\hline $01^{1}$ & $\mathrm{Rh} 2$ & $\mathrm{~N} 2$ & $91.76(15)$ \\
\hline 04 & $\mathrm{Rh} 2$ & Rh1 & $87.94(16)$ \\
\hline 041 & $\mathrm{Rh} 2$ & Rh1 & $87.94(16)$ \\
\hline 04 & $\mathrm{Rh} 2$ & $01^{1}$ & $90.9(2)$ \\
\hline 04 & $\mathrm{Rh} 2$ & 01 & $88.9(2)$ \\
\hline 041 & Rh2 & 01 & $90.9(2)$ \\
\hline 041 & Rh2 & $01^{1}$ & $88.9(2)$ \\
\hline 04 & Rh2 & $04^{1}$ & $175.9(3)$ \\
\hline $04^{1}$ & Rh2 & $\mathrm{N} 2$ & $92.06(16)$ \\
\hline 04 & $\mathrm{Rh} 2$ & N2 & $92.06(16)$ \\
\hline C1 & 01 & $\mathrm{Rh} 2$ & $116.3(5)$ \\
\hline C1 & 02 & $\mathrm{Rh} 1$ & $117.4(5)$ \\
\hline C33 & 04 & $\mathrm{Rh} 2$ & $116.6(5)$ \\
\hline C33 & 05 & Rh1 & $117.3(5)$ \\
\hline C65 & N1 & Rh1 & 180.0 \\
\hline C67 & N2 & $\mathrm{Rh} 2$ & 180.0 \\
\hline 01 & $\mathrm{C} 1$ & $\mathrm{C} 2$ & 114.1(7) \\
\hline 02 & C1 & 01 & $127.6(7)$ \\
\hline 02 & C1 & $\mathrm{C} 2$ & $118.2(7)$ \\
\hline $\mathrm{C} 1$ & $\mathrm{C} 2$ & $\mathrm{C} 4$ & $119.8(7)$ \\
\hline C3 & $\mathrm{C} 2$ & $\mathrm{C} 1$ & $114.8(7)$ \\
\hline C3 & $\mathrm{C} 2$ & $\mathrm{C} 4$ & $57.4(5)$ \\
\hline C5 & $\mathrm{C} 2$ & $\mathrm{C} 1$ & $116.6(7)$ \\
\hline C5 & $\mathrm{C} 2$ & C3 & $118.0(7)$ \\
\hline $\mathrm{C} 5$ & $\mathrm{C} 2$ & C4 & $117.0(7)$ \\
\hline $\mathrm{C} 4$ & $\mathrm{C} 3$ & $\mathrm{C} 2$ & $63.1(6)$ \\
\hline C3 & $\mathrm{C} 4$ & $\mathrm{C} 2$ & $59.5(5)$ \\
\hline C3 & $\mathrm{C} 4$ & $\mathrm{C} 21$ & 119.3(7) \\
\hline C3 & $\mathrm{C} 4$ & $\mathrm{C} 27$ & 118.1(7) \\
\hline C21 & $\mathrm{C} 4$ & $\mathrm{C} 2$ & $122.8(7)$ \\
\hline C21 & C4 & $\mathrm{C} 27$ & $112.0(7)$ \\
\hline $\mathrm{C} 27$ & $\mathrm{C} 4$ & $\mathrm{C} 2$ & $116.0(7)$ \\
\hline C6 & $\mathrm{C} 5$ & $\mathrm{C} 2$ & $122.7(8)$ \\
\hline C10 & $\mathrm{C} 5$ & $\mathrm{C} 2$ & $122.1(8)$ \\
\hline C10 & $\mathrm{C} 5$ & C6 & $115.3(8)$ \\
\hline C5 & C6 & $\mathrm{Cl} 1$ & $120.7(7)$ \\
\hline C7 & C6 & Cl1 & $116.6(7)$ \\
\hline C7 & C6 & $\mathrm{C} 5$ & $122.8(8)$ \\
\hline C6 & C7 & C8 & $120.5(8)$ \\
\hline C7 & C8 & C11 & 119.9(8) \\
\hline C9 & C8 & C7 & 116.4(8) \\
\hline C9 & C8 & C11 & 123.6(9) \\
\hline C10 & C9 & C8 & $121.8(9)$ \\
\hline C9 & C10 & C5 & $123.1(9)$ \\
\hline C8 & C11 & C16 & $120.7(9)$ \\
\hline C12 & C11 & $\mathrm{C} 8$ & $121.5(10)$ \\
\hline C12 & C11 & C16 & 117.8(9) \\
\hline C11 & $\mathrm{C} 12$ & C13 & $120.2(10)$ \\
\hline C14 & $\mathrm{C} 13$ & C12 & $123.4(11)$ \\
\hline C13 & C14 & C15 & $116.5(9)$ \\
\hline C13 & C14 & C17 & 121.1(10) \\
\hline
\end{tabular}

\begin{tabular}{|c|c|c|c|}
\hline Atom & Atom & Atom & Angle ${ }^{\circ}$ \\
\hline C15 & C14 & C17 & $122.3(9)$ \\
\hline C16 & C15 & C14 & $121.2(10)$ \\
\hline C15 & C16 & C11 & $120.4(10)$ \\
\hline C18 & C17 & C14 & 109.3(9) \\
\hline C18 & C17 & C19 & $109.4(10)$ \\
\hline C18 & C17 & $\mathrm{C} 20$ & $112.8(11)$ \\
\hline C19 & C17 & C14 & $111.6(9)$ \\
\hline $\mathrm{C} 20$ & C17 & C14 & $108.4(9)$ \\
\hline $\mathrm{C} 20$ & C17 & C19 & $105.3(10)$ \\
\hline $\mathrm{C} 22$ & $\mathrm{C} 21$ & C4 & 123.3(8) \\
\hline $\mathrm{C} 22$ & $\mathrm{C} 21$ & $\mathrm{C} 26$ & $119.0(8)$ \\
\hline $\mathrm{C} 26$ & $\mathrm{C} 21$ & $\mathrm{C} 4$ & $117.5(7)$ \\
\hline C21 & $\mathrm{C} 22$ & C23 & $120.4(9)$ \\
\hline C24 & $\mathrm{C} 23$ & C22 & $120.6(9)$ \\
\hline $\mathrm{C} 23$ & $\mathrm{C} 24$ & $\mathrm{C} 25$ & $120.2(9)$ \\
\hline C24 & $\mathrm{C} 25$ & C26 & 119.3(9) \\
\hline C21 & C26 & $\mathrm{C} 25$ & 120.3(8) \\
\hline C28 & $\mathrm{C} 27$ & $\mathrm{C} 4$ & $122.1(8)$ \\
\hline $\mathrm{C} 28$ & $\mathrm{C} 27$ & C32 & 118.7(8) \\
\hline C32 & $\mathrm{C} 27$ & C4 & 119.1(8) \\
\hline $\mathrm{C} 27$ & $\mathrm{C} 28$ & C29 & $121.9(9)$ \\
\hline C30 & C29 & $\mathrm{C} 28$ & $119.8(10)$ \\
\hline $\mathrm{C} 29$ & C30 & C31 & $118.7(10)$ \\
\hline C32 & C31 & C30 & $121.3(10)$ \\
\hline C31 & C32 & $\mathrm{C} 27$ & $119.6(10)$ \\
\hline 04 & C33 & C34 & $113.6(7)$ \\
\hline 05 & C33 & 04 & $127.5(7)$ \\
\hline 05 & C33 & C34 & $118.8(7)$ \\
\hline C33 & C34 & C51 & 120.1(7) \\
\hline C33 & C34 & C52 & 115.5(7) \\
\hline C35 & C34 & C33 & $115.2(7)$ \\
\hline C35 & C34 & C51 & $118.7(7)$ \\
\hline C35 & C34 & C52 & $117.8(7)$ \\
\hline C52 & C34 & C51 & $56.8(5)$ \\
\hline C36 & C35 & C34 & $122.7(8)$ \\
\hline $\mathrm{C} 40$ & C35 & C34 & 121.1(8) \\
\hline $\mathrm{C} 40$ & C35 & C36 & $116.2(8)$ \\
\hline C35 & C36 & $\mathrm{Cl} 2$ & $119.9(6)$ \\
\hline C37 & C36 & $\mathrm{Cl} 2$ & $117.2(7)$ \\
\hline C37 & C36 & C35 & $122.8(8)$ \\
\hline C36 & C37 & C38 & 119.4(8) \\
\hline C37 & C38 & C41 & 121.3(8) \\
\hline C39 & C38 & C37 & 118.6(8) \\
\hline C39 & C38 & C41 & 120.1(8) \\
\hline C40 & C39 & C38 & $120.7(8)$ \\
\hline C39 & $\mathrm{C} 40$ & C35 & $122.4(9)$ \\
\hline C42 & C41 & C38 & $119.9(9)$ \\
\hline C46 & C41 & C38 & $123.0(9)$ \\
\hline C46 & C41 & C42 & 117.1(9) \\
\hline C41 & $\mathrm{C} 42$ & $\mathrm{C} 43$ & $120.8(10)$ \\
\hline C44 & C43 & $\mathrm{C} 42$ & $120.9(10)$ \\
\hline C43 & C44 & C47 & $119.6(9)$ \\
\hline C45 & C44 & C43 & 118.3(9) \\
\hline
\end{tabular}




\begin{tabular}{|c|c|c|c|}
\hline Atom & Atom & Atom & Angle $/^{\circ}$ \\
\hline$\overline{C 45}$ & C44 & C47 & 122.1(9) \\
\hline C44 & C45 & C46 & $119.6(10)$ \\
\hline C41 & C46 & C45 & $123.1(10)$ \\
\hline C48 & C47 & C44 & 109.8(8) \\
\hline C48 & $\mathrm{C} 47$ & C49 & 107.7(9) \\
\hline C49 & C47 & C44 & $110.2(9)$ \\
\hline $\mathrm{C} 50$ & $\mathrm{C} 47$ & C44 & $107.7(8)$ \\
\hline C50 & C47 & C48 & $112.6(9)$ \\
\hline C50 & C47 & C49 & $108.7(9)$ \\
\hline C52 & C51 & C34 & $59.7(5)$ \\
\hline C52 & C51 & C53 & $117.1(7)$ \\
\hline C52 & C51 & C59 & 119.1(8) \\
\hline C53 & C51 & C34 & $121.3(7)$ \\
\hline C53 & C51 & C59 & $114.5(8)$ \\
\hline C59 & C51 & C34 & $114.5(7)$ \\
\hline C51 & C52 & C34 & $63.5(6)$ \\
\hline C54 & C53 & C51 & $117.9(8)$ \\
\hline C58 & C53 & C51 & $124.0(8)$ \\
\hline C58 & C53 & C54 & 118.1(9) \\
\hline C55 & C54 & C53 & 121.7(9) \\
\hline C56 & C55 & C54 & 118.6(9) \\
\hline C55 & C56 & C57 & $120.6(9)$ \\
\hline C56 & C57 & C58 & 119.5(9) \\
\hline C53 & C58 & C57 & $121.6(9)$ \\
\hline C60 & C59 & C51 & $120.5(8)$ \\
\hline C60 & C59 & C64 & $117.3(9)$ \\
\hline C64 & C59 & C51 & $122.2(9)$ \\
\hline C59 & C60 & C61 & $124.4(10)$ \\
\hline C62 & C61 & C60 & $116.9(11)$ \\
\hline C63 & C62 & C61 & $119.0(10)$ \\
\hline C62 & C63 & C64 & $123.6(12)$ \\
\hline C59 & C64 & C63 & $118.7(11)$ \\
\hline N1 & C65 & C66 & 180.0 \\
\hline N2 & C67 & C68 & 180.0 \\
\hline 072 & Rh3 & Rh4 & $87.3(2)$ \\
\hline 073 & Rh3 & Rh4 & $87.3(2)$ \\
\hline 074 & Rh3 & Rh4 & $87.3(2)$ \\
\hline 07 & Rh3 & Rh4 & $87.3(2)$ \\
\hline 07 & Rh3 & $07^{3}$ & $174.6(4)$ \\
\hline 07 & Rh3 & $07^{4}$ & $89.88(2)$ \\
\hline 074 & Rh3 & 073 & $89.87(2)$ \\
\hline 07 & Rh3 & 072 & $89.87(2)$ \\
\hline 074 & Rh3 & $07^{2}$ & $174.6(4)$ \\
\hline $07^{3}$ & Rh3 & $07^{2}$ & $89.87(2)$ \\
\hline $07^{2}$ & Rh3 & N4 & $92.7(2)$ \\
\hline $07^{3}$ & Rh3 & $\mathrm{N} 4$ & $92.7(2)$ \\
\hline 07 & Rh3 & $\mathrm{N} 4$ & $92.7(2)$ \\
\hline $07^{4}$ & Rh3 & N4 & $92.7(2)$ \\
\hline N4 & Rh3 & Rh4 & 180.0 \\
\hline $06^{4}$ & Rh4 & Rh3 & $87.96(19)$ \\
\hline 06 & $\mathrm{Rh} 4$ & Rh3 & $87.96(19)$ \\
\hline $06^{3}$ & Rh4 & Rh3 & $87.96(19)$ \\
\hline $06^{2}$ & Rh4 & Rh3 & $87.96(19)$ \\
\hline
\end{tabular}

\begin{tabular}{|c|c|c|c|}
\hline Atom & Atom & Atom & Angle/ ${ }^{\circ}$ \\
\hline $06^{3}$ & Rh4 & $06^{4}$ & $89.927(15)$ \\
\hline $06^{3}$ & Rh4 & $06^{2}$ & $89.927(17)$ \\
\hline 06 & $\mathrm{Rh} 4$ & $06^{3}$ & $175.9(4)$ \\
\hline 06 & Rh4 & $06^{2}$ & $89.928(15)$ \\
\hline 06 & Rh4 & $06^{4}$ & $89.927(16)$ \\
\hline $06^{2}$ & Rh4 & $06^{4}$ & $175.9(4)$ \\
\hline $06^{2}$ & Rh4 & N3 & $92.04(19)$ \\
\hline 06 & Rh4 & N3 & $92.04(19)$ \\
\hline $06^{4}$ & Rh4 & N3 & $92.04(19)$ \\
\hline $06^{3}$ & Rh4 & N3 & 92.04(19) \\
\hline N3 & $\mathrm{Rh} 4$ & Rh3 & 180.0 \\
\hline C69 & 06 & Rh4 & $119.0(6)$ \\
\hline C69 & 07 & Rh3 & $120.3(6)$ \\
\hline C100 & N3 & $\mathrm{Rh} 4$ & 180.0 \\
\hline C102 & N4 & $\mathrm{Rh} 3$ & 180.0 \\
\hline C95 & COAA & C99 & $124.2(11)$ \\
\hline 06 & C69 & 07 & 124.1(8) \\
\hline 06 & C69 & $\mathrm{C} 70$ & $116.1(8)$ \\
\hline 07 & C69 & C70 & $119.8(9)$ \\
\hline C69 & $\mathrm{C} 70$ & C71 & $115.7(8)$ \\
\hline C69 & C70 & C87 & $121.5(8)$ \\
\hline C69 & C70 & C88 & 112.5(8) \\
\hline C71 & C70 & C87 & $117.7(8)$ \\
\hline C71 & C70 & C88 & $118.0(8)$ \\
\hline C88 & C70 & C87 & $58.7(6)$ \\
\hline C72 & C71 & C70 & $120.4(9)$ \\
\hline C76 & $\mathrm{C} 71$ & C70 & 123.8(9) \\
\hline C76 & C71 & C72 & $115.6(9)$ \\
\hline C71 & C72 & $\mathrm{Cl} 3$ & $120.9(7)$ \\
\hline C73 & C72 & $\mathrm{Cl} 3$ & $117.0(8)$ \\
\hline C73 & C72 & C71 & $122.0(10)$ \\
\hline C72 & C73 & C74 & $120.6(10)$ \\
\hline C73 & C74 & C77 & $120.8(9)$ \\
\hline C75 & C74 & C73 & $118.8(10)$ \\
\hline C75 & C74 & C77 & $120.2(10)$ \\
\hline C74 & $\mathrm{C} 75$ & C76 & $119.5(9)$ \\
\hline C71 & C76 & C75 & $123.1(10)$ \\
\hline C78 & C77 & C74 & $122.4(9)$ \\
\hline C82 & C77 & C74 & $121.5(10)$ \\
\hline C82 & C77 & C78 & $116.0(10)$ \\
\hline C79 & C78 & C77 & $122.7(10)$ \\
\hline C78 & C79 & C80 & $120.6(11)$ \\
\hline C79 & $\mathrm{C} 80$ & C83 & $118.9(11)$ \\
\hline C81 & C80 & C79 & $116.5(11)$ \\
\hline C81 & C80 & C83 & $124.4(10)$ \\
\hline C82 & C81 & C80 & $122.4(11)$ \\
\hline C81 & C82 & C77 & $121.4(11)$ \\
\hline C84 & C83 & C80 & $111.6(11)$ \\
\hline C85 & C83 & $\mathrm{C} 80$ & $112.0(10)$ \\
\hline C85 & C83 & C84 & $108.4(10)$ \\
\hline C86 & C83 & C80 & $109.5(9)$ \\
\hline C86 & C83 & C84 & 106.1(11) \\
\hline C86 & C83 & C85 & $108.9(11)$ \\
\hline
\end{tabular}




\begin{tabular}{|c|c|c|c|}
\hline Atom & Atom & Atom & Angle $/^{\circ}$ \\
\hline$\overline{\mathrm{C} 88}$ & C87 & $\mathrm{C} 70$ & $60.1(6)$ \\
\hline C89 & C87 & $\mathrm{C} 70$ & $121.6(8)$ \\
\hline C89 & C87 & C88 & $116.9(8)$ \\
\hline C89 & C87 & C95 & 113.7(8) \\
\hline C95 & C87 & $\mathrm{C} 70$ & $117.9(8)$ \\
\hline C95 & C87 & C88 & $116.2(8)$ \\
\hline C87 & C88 & $\mathrm{C} 70$ & $61.3(6)$ \\
\hline $\mathrm{C} 90$ & C89 & C87 & $122.5(9)$ \\
\hline $\mathrm{C} 90$ & C89 & C94 & $118.4(10)$ \\
\hline C94 & C89 & C87 & $118.4(9)$ \\
\hline C89 & C90 & C91 & $122.2(11)$ \\
\hline C92 & C91 & C90 & $117.7(12)$ \\
\hline C93 & C92 & C91 & $118.9(12)$ \\
\hline C92 & C93 & C94 & $124.5(13)$ \\
\hline C93 & C94 & C89 & $118.1(12)$ \\
\hline COAA & C95 & C87 & $120.8(9)$ \\
\hline COAA & C95 & C96 & $116.8(9)$ \\
\hline C96 & C95 & C87 & $122.2(9)$ \\
\hline C97 & C96 & C95 & $121.2(10)$ \\
\hline C98 & C97 & C96 & 123.1(11) \\
\hline C97 & C98 & C99 & $117.2(10)$ \\
\hline COAA & C99 & C98 & $117.3(10)$ \\
\hline N3 & C100 & C1D & 180.0 \\
\hline N4 & C102 & C1E & $180.000(17)$ \\
\hline O6B & Rh4B & Rh3B & $87.71(15)$ \\
\hline $06 B^{5}$ & Rh4B & Rh3B & $87.71(15)$ \\
\hline $06 \mathrm{~B}^{6}$ & Rh4B & Rh3B & $87.71(15)$ \\
\hline $06 B^{7}$ & Rh4B & Rh3B & 87.71(15) \\
\hline $06 \mathrm{~B}^{7}$ & Rh4B & $06 \mathrm{~B}^{5}$ & $89.908(13)$ \\
\hline $06 B^{7}$ & Rh4B & $06 B^{6}$ & $175.4(3)$ \\
\hline $06 B^{6}$ & Rh4B & $06 B^{5}$ & $89.908(13)$ \\
\hline $06 B^{7}$ & Rh4B & 06B & $89.906(13)$ \\
\hline $06 B^{6}$ & Rh4B & O6B & $89.911(13)$ \\
\hline $06 B^{5}$ & Rh4B & O6B & $175.4(3)$ \\
\hline O6B & Rh4B & N3B & $92.29(15)$ \\
\hline $06 \mathrm{~B}^{6}$ & Rh4B & N3B & $92.29(15)$ \\
\hline $06 \mathrm{~B}^{7}$ & Rh4B & N3B & $92.29(15)$ \\
\hline $06 B^{5}$ & Rh4B & N3B & $92.29(15)$ \\
\hline N3B & Rh4B & Rh3B & 180.0 \\
\hline $07 B^{5}$ & Rh3B & Rh4B & $87.57(16)$ \\
\hline $07 B^{7}$ & Rh3B & Rh4B & $87.57(16)$ \\
\hline 07B & Rh3B & Rh4B & $87.57(16)$ \\
\hline $0^{2} B^{6}$ & Rh3B & Rh4B & $87.57(16)$ \\
\hline $0^{7} B^{6}$ & Rh3B & O7B7 & $175.1(3)$ \\
\hline 07B & Rh3B & $07 B^{5}$ & 175.1(3) \\
\hline O7B & Rh3B & $0^{6} B^{6}$ & $89.894(14)$ \\
\hline $\mathrm{O}^{\mathrm{B}} \mathrm{B}^{5}$ & Rh3B & O7B & $89.897(14)$ \\
\hline $07 B^{6}$ & Rh3B & $07 B^{5}$ & $89.897(15)$ \\
\hline O7B & Rh3B & $07 B^{7}$ & $89.900(15)$ \\
\hline $07 B^{6}$ & Rh3B & N4B & $92.43(16)$ \\
\hline $07 B^{7}$ & Rh3B & N4B & $92.43(16)$ \\
\hline O7B & Rh3B & N4B & $92.43(16)$ \\
\hline $07 B^{5}$ & Rh3B & N4B & $92.43(16)$ \\
\hline
\end{tabular}

\begin{tabular}{|c|c|c|c|}
\hline Atom & Atom & Atom & Angle ${ }^{\circ}$ \\
\hline$\overline{\mathrm{N} 4 \mathrm{~B}}$ & Rh3B & Rh4B & 180.0 \\
\hline C69B & 07B & Rh3B & $117.8(5)$ \\
\hline C69B & O6B & Rh4B & $116.9(5)$ \\
\hline C1B & N4B & Rh3B & 180.0 \\
\hline $\mathrm{C} 1 \mathrm{C}$ & N3B & Rh4B & 180.0 \\
\hline C69B & C70B & C71B & $115.2(6)$ \\
\hline C69B & C70B & C87B & $120.7(6)$ \\
\hline C69B & C70B & C88B & $115.8(6)$ \\
\hline C71B & C70B & C87B & $117.3(6)$ \\
\hline C71B & C70B & C88B & $117.6(6)$ \\
\hline C88B & C70B & C87B & $58.3(5)$ \\
\hline 07B & C69B & O6B & $126.8(7)$ \\
\hline O7B & C69B & C70B & $117.3(7)$ \\
\hline O6B & C69B & C70B & $115.7(6)$ \\
\hline C72B & C71B & C70B & $122.8(7)$ \\
\hline C76B & C71B & C70B & $120.4(7)$ \\
\hline C76B & C71B & C72B & 116.7(7) \\
\hline C71B & C72B & $\mathrm{Cl} 3 \mathrm{~B}$ & $120.2(6)$ \\
\hline C73B & C72B & $\mathrm{Cl} 3 \mathrm{~B}$ & 118.4(6) \\
\hline C73B & C72B & C71B & $121.2(8)$ \\
\hline C74B & C73B & C72B & $120.4(8)$ \\
\hline C73B & C74B & C75B & 119.4(8) \\
\hline C73B & C74B & C77B & 121.7(8) \\
\hline C75B & C74B & C77B & $118.9(8)$ \\
\hline C76B & C75B & C74B & $118.8(8)$ \\
\hline C71B & C76B & C75B & $123.4(8)$ \\
\hline C78B & C77B & C74B & $121.4(8)$ \\
\hline C78B & C77B & C82B & $116.8(9)$ \\
\hline C82B & C77B & C74B & 121.8(9) \\
\hline C77B & C78B & C79B & $121.5(10)$ \\
\hline C80B & C79B & C78B & $122.4(10)$ \\
\hline C79B & C80B & C81B & $115.7(9)$ \\
\hline C79B & C80B & C83B & $124.6(10)$ \\
\hline C81B & C80B & C83B & $119.7(10)$ \\
\hline C82B & C81B & C80B & 124.1(11) \\
\hline C81B & C82B & C77B & $119.3(11)$ \\
\hline C80B & C83B & C84B & 108.8(10) \\
\hline C86B & C83B & C80B & $107.3(9)$ \\
\hline C86B & C83B & C84B & $107.9(11)$ \\
\hline C85B & C83B & C80B & $114.0(9)$ \\
\hline C85B & C83B & C84B & $107.4(10)$ \\
\hline C85B & C83B & C86B & 111.3(11) \\
\hline C88B & C87B & C70B & $59.9(5)$ \\
\hline C88B & C87B & C89B & $117.2(7)$ \\
\hline C88B & C87B & C95B & 119.1(7) \\
\hline C89B & C87B & C70B & $121.5(6)$ \\
\hline C89B & C87B & C95B & $111.7(6)$ \\
\hline C95B & C87B & C70B & 118.5(6) \\
\hline C87B & C88B & C70B & $61.7(5)$ \\
\hline C90B & C89B & C87B & 119.5(7) \\
\hline C94B & C89B & C87B & $122.2(7)$ \\
\hline C94B & C89B & C90B & $118.3(7)$ \\
\hline C91B & C90B & C89B & $120.5(8)$ \\
\hline
\end{tabular}




\begin{tabular}{llll}
\hline Atom & Atom & Atom & Angle $^{\circ}$ \\
\hline C92B & C91B & C90B & $120.7(9)$ \\
C91B & C92B & C93B & $119.4(8)$ \\
C94B & C93B & C92B & $120.5(8)$ \\
C93B & C94B & C89B & $120.4(8)$ \\
C1A & C95B & C87B & $122.0(8)$ \\
C1A & C95B & C96B & $119.5(8)$ \\
C96B & C95B & C87B & $118.4(7)$ \\
C95B & C1A & C99B & $119.3(10)$ \\
C98B & C99B & C1A & $122.4(11)$
\end{tabular}

\begin{tabular}{|c|c|c|c|}
\hline Atom & Atom & Atom & Angle/ ${ }^{\circ}$ \\
\hline$\overline{\mathrm{C} 99 \mathrm{~B}}$ & C98B & C97B & $118.6(10)$ \\
\hline C98B & C97B & C96B & $120.2(11)$ \\
\hline C95B & C96B & C97B & $119.9(9)$ \\
\hline N4B & C1B & C103 & 180.0 \\
\hline N3B & C1C & C101 & 180.0 \\
\hline
\end{tabular}

Table 5: Torsion Angles in ${ }^{\circ}$ for 2cl4tbuph_twin.

\begin{tabular}{|c|c|c|c|c|}
\hline Atom & Atom & Atom & Atom & Angle $/^{\circ}$ \\
\hline Rh1 & 02 & $\mathrm{C} 1$ & 01 & $14.8(10)$ \\
\hline Rh1 & 02 & $\mathrm{C} 1$ & $\mathrm{C} 2$ & $-167.6(5)$ \\
\hline Rh1 & 05 & C33 & 04 & 14.7(10) \\
\hline Rh1 & 05 & C33 & C34 & $-167.2(5)$ \\
\hline Rh2 & 01 & C1 & 02 & $-1.5(10)$ \\
\hline Rh2 & 01 & $\mathrm{C} 1$ & $\mathrm{C} 2$ & $-179.1(5)$ \\
\hline Rh2 & 04 & C33 & 05 & $0.5(10)$ \\
\hline Rh2 & 04 & C33 & C34 & $-177.7(5)$ \\
\hline Cl1 & C6 & C7 & C8 & $179.0(8)$ \\
\hline $\mathrm{Cl} 2$ & C36 & C37 & C38 & $176.8(7)$ \\
\hline 01 & C1 & $\mathrm{C} 2$ & C3 & 139.7(7) \\
\hline 01 & C1 & $\mathrm{C} 2$ & $\mathrm{C} 4$ & $-155.1(7)$ \\
\hline 01 & $\mathrm{C} 1$ & $\mathrm{C} 2$ & $\mathrm{C} 5$ & $-4.3(10)$ \\
\hline 02 & $\mathrm{C} 1$ & $\mathrm{C} 2$ & C3 & $-38.1(10)$ \\
\hline 02 & C1 & $\mathrm{C} 2$ & C4 & 27.1(10) \\
\hline 02 & $\mathrm{C} 1$ & $\mathrm{C} 2$ & $\mathrm{C} 5$ & $177.8(7)$ \\
\hline 04 & C33 & C34 & C35 & 1.1(10) \\
\hline 04 & C33 & C34 & C51 & $-151.4(7)$ \\
\hline 04 & C33 & C34 & C52 & $143.8(7)$ \\
\hline 05 & C33 & C34 & C35 & $-177.3(7)$ \\
\hline 05 & C33 & C34 & C51 & $30.3(10)$ \\
\hline 05 & C33 & C34 & C52 & $-34.6(10)$ \\
\hline C1 & $\mathrm{C} 2$ & C3 & C4 & $110.8(8)$ \\
\hline C1 & $\mathrm{C} 2$ & $\mathrm{C} 4$ & C3 & $-101.9(8)$ \\
\hline $\mathrm{C} 1$ & $\mathrm{C} 2$ & $\mathrm{C} 4$ & $\mathrm{C} 21$ & $5.3(11)$ \\
\hline $\mathrm{C} 1$ & C2 & $\mathrm{C} 4$ & C27 & $149.4(7)$ \\
\hline $\mathrm{C} 1$ & $\mathrm{C} 2$ & $\mathrm{C} 5$ & C6 & $-65.7(11)$ \\
\hline $\mathrm{C} 1$ & $\mathrm{C} 2$ & $\mathrm{C} 5$ & C10 & $113.2(10)$ \\
\hline $\mathrm{C} 2$ & C3 & $\mathrm{C} 4$ & C21 & $-113.0(8)$ \\
\hline C2 & C3 & $\mathrm{C} 4$ & C27 & 105.2(9) \\
\hline C2 & C4 & $\mathrm{C} 21$ & C22 & 53.1(12) \\
\hline $\mathrm{C} 2$ & $\mathrm{C} 4$ & $\mathrm{C} 21$ & $\mathrm{C} 26$ & $-131.1(9)$ \\
\hline $\mathrm{C} 2$ & $\mathrm{C} 4$ & $\mathrm{C} 27$ & $\mathrm{C} 28$ & $-80.5(11)$ \\
\hline C2 & C4 & $\mathrm{C} 27$ & C32 & $103.7(11)$ \\
\hline $\mathrm{C} 2$ & $\mathrm{C} 5$ & C6 & Cl1 & $3.0(13)$ \\
\hline $\mathrm{C} 2$ & $\mathrm{C} 5$ & $\mathrm{C} 6$ & $\mathrm{C} 7$ & $-177.6(9)$ \\
\hline C2 & $\mathrm{C} 5$ & C10 & C9 & 178.1(10) \\
\hline C3 & C2 & $\mathrm{C} 4$ & C21 & 107.3(9) \\
\hline C3 & C2 & $\mathrm{C} 4$ & $\mathrm{C} 27$ & $-108.7(8)$ \\
\hline
\end{tabular}




\begin{tabular}{|c|c|c|c|c|}
\hline Atom & Atom & Atom & Atom & Angle ${ }^{\circ}$ \\
\hline$\overline{\mathrm{C} 3}$ & $\mathrm{C} 2$ & C5 & C6 & $151.4(8)$ \\
\hline C3 & $\mathrm{C} 2$ & $\mathrm{C} 5$ & C10 & $-29.7(13)$ \\
\hline C3 & C4 & $\mathrm{C} 21$ & C22 & 123.7(9) \\
\hline C3 & $\mathrm{C} 4$ & $\mathrm{C} 21$ & C26 & $-60.4(11)$ \\
\hline C3 & C4 & $\mathrm{C} 27$ & C28 & $-148.1(9)$ \\
\hline C3 & $\mathrm{C} 4$ & $\mathrm{C} 27$ & C32 & $36.0(13)$ \\
\hline C4 & $\mathrm{C} 2$ & $\mathrm{C} 5$ & C6 & $85.9(10)$ \\
\hline $\mathrm{C} 4$ & $\mathrm{C} 2$ & $\mathrm{C} 5$ & C10 & $-95.2(10)$ \\
\hline $\mathrm{C} 4$ & $\mathrm{C} 21$ & $\mathrm{C} 22$ & $\mathrm{C} 23$ & $179.0(8)$ \\
\hline $\mathrm{C} 4$ & $\mathrm{C} 21$ & $\mathrm{C} 26$ & $\mathrm{C} 25$ & $-179.4(8)$ \\
\hline $\mathrm{C} 4$ & $\mathrm{C} 27$ & $\mathrm{C} 28$ & $\mathrm{C} 29$ & $-179.3(9)$ \\
\hline C4 & $\mathrm{C} 27$ & C32 & C31 & $177.7(12)$ \\
\hline C5 & $\mathrm{C} 2$ & C3 & C4 & $-105.7(8)$ \\
\hline C5 & $\mathrm{C} 2$ & $\mathrm{C} 4$ & $\mathrm{C} 3$ & $107.5(8)$ \\
\hline C5 & C2 & C4 & C21 & $-145.3(8)$ \\
\hline C5 & $\mathrm{C} 2$ & $\mathrm{C} 4$ & $\mathrm{C} 27$ & $-1.2(10)$ \\
\hline C5 & C6 & $\mathrm{C} 7$ & $\mathrm{C} 8$ & $-0.5(15)$ \\
\hline C6 & C5 & C10 & C9 & $-3.0(15)$ \\
\hline C6 & $\mathrm{C} 7$ & C8 & C9 & $-3.0(15)$ \\
\hline C6 & C7 & C8 & C11 & $-179.4(10)$ \\
\hline C7 & C8 & C9 & C10 & $3.5(17)$ \\
\hline C7 & $\mathrm{C} 8$ & C11 & $\mathrm{C} 12$ & $132.0(12)$ \\
\hline $\mathrm{C} 7$ & $\mathrm{C} 8$ & C11 & C16 & $-48.7(15)$ \\
\hline C8 & C9 & C10 & C5 & $-0.5(18)$ \\
\hline C8 & C11 & C12 & C13 & $-178.2(11)$ \\
\hline C8 & C11 & C16 & C15 & 179.1(10) \\
\hline C9 & C8 & C11 & $\mathrm{C} 12$ & $-44.0(17)$ \\
\hline C9 & C8 & C11 & C16 & $135.2(12)$ \\
\hline C10 & C5 & $\mathrm{C} 6$ & $\mathrm{Cl} 1$ & $-176.0(7)$ \\
\hline C10 & $\mathrm{C} 5$ & $\mathrm{C} 6$ & $\mathrm{C} 7$ & $3.4(14)$ \\
\hline C11 & $\mathrm{C} 8$ & $\mathrm{C} 9$ & C10 & $179.7(11)$ \\
\hline C11 & C12 & C13 & C14 & $1(2)$ \\
\hline C12 & C11 & C16 & C15 & $-1.6(17)$ \\
\hline C12 & C13 & C14 & C15 & $-5.8(18)$ \\
\hline C12 & C13 & C14 & C17 & $176.5(12)$ \\
\hline C13 & C14 & C15 & C16 & $6.5(16)$ \\
\hline $\mathrm{C} 13$ & C14 & C17 & C18 & $61.7(14)$ \\
\hline C13 & C14 & C17 & C19 & $-177.2(11)$ \\
\hline C13 & C14 & C17 & $\mathrm{C} 20$ & $-61.7(14)$ \\
\hline C14 & C15 & C16 & C11 & $-2.9(17)$ \\
\hline C15 & C14 & C17 & C18 & $-116.0(12)$ \\
\hline C15 & C14 & C17 & C19 & $5.2(14)$ \\
\hline C15 & C14 & C17 & $\mathrm{C} 20$ & $120.7(11)$ \\
\hline C16 & C11 & C12 & C13 & $2.5(19)$ \\
\hline $\mathrm{C} 17$ & C14 & C15 & C16 & $-175.8(10)$ \\
\hline C21 & $\mathrm{C} 4$ & $\mathrm{C} 27$ & C28 & $67.4(11)$ \\
\hline C21 & C4 & $\mathrm{C} 27$ & C32 & $-108.5(11)$ \\
\hline $\mathrm{C} 21$ & $\mathrm{C} 22$ & $\mathrm{C} 23$ & $\mathrm{C} 24$ & $-0.1(14)$ \\
\hline $\mathrm{C} 22$ & $\mathrm{C} 21$ & $\mathrm{C} 26$ & $\mathrm{C} 25$ & $-3.4(13)$ \\
\hline $\mathrm{C} 22$ & $\mathrm{C} 23$ & $\mathrm{C} 24$ & $\mathrm{C} 25$ & $-2.8(14)$ \\
\hline C23 & $\mathrm{C} 24$ & $\mathrm{C} 25$ & $\mathrm{C} 26$ & $2.6(14)$ \\
\hline C24 & $\mathrm{C} 25$ & C26 & $\mathrm{C} 21$ & $0.5(14)$ \\
\hline C26 & $\mathrm{C} 21$ & $\mathrm{C} 22$ & $\mathrm{C} 23$ & $3.1(13)$ \\
\hline
\end{tabular}




\begin{tabular}{|c|c|c|c|c|}
\hline Atom & Atom & Atom & Atom & Angle/ ${ }^{\circ}$ \\
\hline$\overline{\mathrm{C} 27}$ & $\mathrm{C} 4$ & $\mathrm{C} 21$ & $\mathrm{C} 22$ & $-92.3(10)$ \\
\hline $\mathrm{C} 27$ & $\mathrm{C} 4$ & $\mathrm{C} 21$ & $\mathrm{C} 26$ & $83.6(10)$ \\
\hline C27 & $\mathrm{C} 28$ & C29 & C30 & $2.3(17)$ \\
\hline C28 & $\mathrm{C} 27$ & C32 & C31 & $1.7(19)$ \\
\hline C28 & $\mathrm{C} 29$ & C30 & C31 & $0.5(19)$ \\
\hline $\mathrm{C} 29$ & C30 & C31 & C32 & $-2(2)$ \\
\hline C30 & C31 & C32 & C27 & $1(2)$ \\
\hline C32 & $\mathrm{C} 27$ & $\mathrm{C} 28$ & C29 & $-3.4(16)$ \\
\hline C33 & C34 & C35 & C36 & $-74.1(10)$ \\
\hline C33 & C34 & C35 & $\mathrm{C} 40$ & $103.9(9)$ \\
\hline C33 & C34 & C51 & C52 & $-102.4(8)$ \\
\hline C33 & C34 & C51 & C53 & $2.7(11)$ \\
\hline C33 & C34 & C51 & C59 & $146.9(7)$ \\
\hline C33 & C34 & C52 & C51 & $110.6(8)$ \\
\hline C34 & C35 & C36 & $\mathrm{Cl} 2$ & $2.2(12)$ \\
\hline C34 & C35 & C36 & C37 & $178.9(8)$ \\
\hline C34 & C35 & $\mathrm{C} 40$ & C39 & $-179.8(8)$ \\
\hline C34 & C51 & C53 & C54 & $-128.9(8)$ \\
\hline C34 & C51 & C53 & C58 & $53.5(12)$ \\
\hline C34 & C51 & C59 & $\mathrm{C} 60$ & $-104.1(10)$ \\
\hline C34 & C51 & C59 & C64 & $74.7(12)$ \\
\hline C35 & C34 & C51 & C52 & $106.1(8)$ \\
\hline C35 & C34 & C51 & C53 & $-148.7(8)$ \\
\hline C35 & C34 & C51 & C59 & $-4.5(10)$ \\
\hline C35 & C34 & C52 & C51 & $-107.7(8)$ \\
\hline C35 & C36 & C37 & C38 & $-0.1(14)$ \\
\hline C36 & C35 & C40 & C39 & $-1.6(14)$ \\
\hline C36 & C37 & C38 & C39 & $0.1(13)$ \\
\hline C36 & C37 & C38 & C41 & $177.8(8)$ \\
\hline C37 & C38 & C39 & C40 & $-0.9(13)$ \\
\hline C37 & C38 & C41 & C42 & $-135.6(10)$ \\
\hline C37 & C38 & C41 & C46 & $44.2(14)$ \\
\hline C38 & C39 & C40 & C35 & $1.7(15)$ \\
\hline C38 & C41 & C42 & C43 & $175.4(10)$ \\
\hline C38 & C41 & C46 & C45 & $-174.6(10)$ \\
\hline C39 & C38 & $\mathrm{C} 41$ & $\mathrm{C} 42$ & $42.0(14)$ \\
\hline C39 & C38 & C41 & C46 & $-138.1(10)$ \\
\hline C40 & C35 & C36 & $\mathrm{Cl} 2$ & $-176.0(7)$ \\
\hline $\mathrm{C} 40$ & C35 & C36 & C37 & $0.8(13)$ \\
\hline C41 & C38 & C39 & C40 & $-178.6(9)$ \\
\hline C41 & $\mathrm{C} 42$ & $\mathrm{C} 43$ & C44 & $0.1(17)$ \\
\hline C42 & C41 & C46 & C45 & $5.3(16)$ \\
\hline C42 & C43 & C44 & C45 & $3.5(16)$ \\
\hline C42 & C43 & C44 & C47 & $-176.1(10)$ \\
\hline $\mathrm{C} 43$ & $\mathrm{C} 44$ & $\mathrm{C} 45$ & C46 & $-2.8(15)$ \\
\hline $\mathrm{C} 43$ & $\mathrm{C} 44$ & $\mathrm{C} 47$ & C48 & $176.0(10)$ \\
\hline C43 & $\mathrm{C} 44$ & C47 & C49 & $-65.4(13)$ \\
\hline C43 & $\mathrm{C} 44$ & C47 & $\mathrm{C} 50$ & $53.0(12)$ \\
\hline C44 & C45 & C46 & C41 & $-1.7(16)$ \\
\hline C45 & $\mathrm{C} 44$ & C47 & C48 & $-3.6(14)$ \\
\hline $\mathrm{C} 45$ & $\mathrm{C} 44$ & $\mathrm{C} 47$ & C49 & $115.0(11)$ \\
\hline $\mathrm{C} 45$ & $\mathrm{C} 44$ & $\mathrm{C} 47$ & C50 & $-126.6(10)$ \\
\hline C46 & C41 & C42 & C43 & $-4.4(16)$ \\
\hline
\end{tabular}




\begin{tabular}{|c|c|c|c|c|}
\hline Atom & Atom & Atom & Atom & Angle $/{ }^{\circ}$ \\
\hline$\overline{\mathrm{C} 47}$ & $\mathrm{C} 44$ & $\mathrm{C} 45$ & $\mathrm{C} 46$ & $176.8(9)$ \\
\hline C51 & C34 & C35 & C36 & 78.7(10) \\
\hline C51 & C34 & C35 & C40 & $-103.3(10)$ \\
\hline C51 & C53 & C54 & C55 & $-179.4(8)$ \\
\hline C51 & C53 & C58 & C57 & 179.7(8) \\
\hline C51 & C59 & C60 & C61 & 179.4(10) \\
\hline C51 & C59 & C64 & C63 & $-179.4(12)$ \\
\hline C52 & C34 & C35 & C36 & 144.1(8) \\
\hline C52 & C34 & C35 & $\mathrm{C} 40$ & $-37.9(12)$ \\
\hline C52 & C34 & C51 & C53 & $105.2(9)$ \\
\hline C52 & C34 & C51 & C59 & $-110.6(8)$ \\
\hline C52 & C51 & C53 & C54 & $-59.5(11)$ \\
\hline C52 & C51 & C53 & C58 & $122.9(9)$ \\
\hline C52 & C51 & C59 & C60 & $-171.7(9)$ \\
\hline C52 & C51 & C59 & C64 & $7.0(14)$ \\
\hline C53 & C51 & C52 & C34 & $-112.1(8)$ \\
\hline C53 & C51 & C59 & C60 & $42.6(12)$ \\
\hline C53 & C51 & C59 & C64 & $-138.6(10)$ \\
\hline C53 & C54 & C55 & C56 & $0.7(14)$ \\
\hline C54 & C53 & C58 & C57 & $2.1(13)$ \\
\hline C54 & C55 & C56 & C57 & $-0.1(13)$ \\
\hline C55 & C56 & C57 & C58 & $0.5(13)$ \\
\hline C56 & C57 & C58 & C53 & $-1.5(13)$ \\
\hline C58 & C53 & C54 & C55 & $-1.7(14)$ \\
\hline C59 & C51 & C52 & C34 & 103.0(8) \\
\hline C59 & C51 & C53 & C54 & $86.9(10)$ \\
\hline C59 & C51 & C53 & C58 & $-90.7(10)$ \\
\hline C59 & C60 & C61 & C62 & $0.3(17)$ \\
\hline C60 & C59 & C64 & C63 & $-0.6(18)$ \\
\hline $\mathrm{C} 60$ & C61 & C62 & C63 & $-1.1(19)$ \\
\hline C61 & C62 & C63 & C64 & $1(2)$ \\
\hline C62 & C63 & C64 & C59 & $0(2)$ \\
\hline C64 & C59 & C60 & C61 & $0.6(17)$ \\
\hline Rh3 & 07 & C69 & 06 & $7.9(12)$ \\
\hline Rh3 & 07 & C69 & C70 & $-173.8(6)$ \\
\hline Rh4 & 06 & C69 & 07 & $2.0(12)$ \\
\hline Rh4 & 06 & C69 & C70 & $-176.3(6)$ \\
\hline $\mathrm{Cl} 3$ & C72 & C73 & C74 & 178.3(8) \\
\hline 06 & C69 & C70 & C71 & $-9.0(12)$ \\
\hline 06 & C69 & C70 & C87 & $-163.2(8)$ \\
\hline 06 & C69 & C70 & C88 & 130.8(9) \\
\hline 07 & C69 & C70 & C71 & 172.5(8) \\
\hline 07 & C69 & C70 & C87 & 18.4(13) \\
\hline 07 & C69 & $\mathrm{C} 70$ & C88 & $-47.7(12)$ \\
\hline COAA & C95 & C96 & C97 & $-1.6(16)$ \\
\hline C69 & C70 & C71 & C72 & $-66.8(12)$ \\
\hline C69 & C70 & C71 & C76 & $109.0(11)$ \\
\hline C69 & C70 & C87 & C88 & $-98.8(9)$ \\
\hline C69 & C70 & C87 & C89 & $6.2(14)$ \\
\hline C69 & C70 & C87 & C95 & 155.4(8) \\
\hline C69 & C70 & C88 & C87 & $114.2(9)$ \\
\hline C70 & C71 & C72 & $\mathrm{Cl} 3$ & 2.7(13) \\
\hline C70 & C71 & C72 & C73 & 179.4(9) \\
\hline
\end{tabular}




\begin{tabular}{|c|c|c|c|c|}
\hline Atom & Atom & Atom & Atom & Angle $/{ }^{\circ}$ \\
\hline$\overline{\mathrm{C} 70}$ & C71 & C76 & C75 & $179.9(9)$ \\
\hline C70 & C87 & C89 & C90 & $-122.7(10)$ \\
\hline C70 & C87 & C89 & C94 & 66.8(13) \\
\hline C70 & C87 & C95 & $\mathrm{COAA}$ & $112.4(11)$ \\
\hline C70 & C87 & C95 & C96 & $-72.2(13)$ \\
\hline C71 & C70 & C87 & C88 & $107.6(9)$ \\
\hline C71 & C70 & C87 & C89 & $-147.4(9)$ \\
\hline C71 & C70 & C87 & C95 & $1.8(13)$ \\
\hline C71 & C70 & C88 & C87 & $-107.0(10)$ \\
\hline C71 & C72 & C73 & C74 & $1.5(16)$ \\
\hline C72 & C71 & C76 & C75 & $-4.1(15)$ \\
\hline C72 & C73 & C74 & C75 & $-5.5(16)$ \\
\hline C72 & C73 & C74 & C77 & $178.5(9)$ \\
\hline C73 & C74 & C75 & C76 & $4.6(15)$ \\
\hline C73 & C74 & C77 & C78 & 131.4(11) \\
\hline C73 & C74 & $\mathrm{C} 77$ & C82 & $-44.4(15)$ \\
\hline C74 & C75 & C76 & C71 & $0.3(16)$ \\
\hline C74 & $\mathrm{C} 77$ & $\mathrm{C} 78$ & C79 & $-177.2(10)$ \\
\hline C74 & C77 & C82 & C81 & $177.9(10)$ \\
\hline C75 & C74 & C77 & C78 & $-44.5(15)$ \\
\hline C75 & C74 & C77 & C82 & 139.7(11) \\
\hline C76 & C71 & C72 & $\mathrm{Cl} 3$ & $-173.5(8)$ \\
\hline C76 & C71 & C72 & $\mathrm{C} 73$ & $3.2(14)$ \\
\hline C77 & C74 & C75 & C76 & $-179.4(9)$ \\
\hline C77 & C78 & C79 & $\mathrm{C} 80$ & $3.5(17)$ \\
\hline C78 & C77 & C82 & C81 & $1.8(17)$ \\
\hline C78 & C79 & C80 & C81 & $-6.3(17)$ \\
\hline C78 & C79 & C80 & C83 & 178.3(11) \\
\hline C79 & $\mathrm{C} 80$ & C81 & C82 & 7.1(18) \\
\hline C79 & C80 & C83 & $\mathrm{C} 84$ & $56.6(14)$ \\
\hline C79 & $\mathrm{C} 80$ & C83 & $\mathrm{C} 85$ & $178.4(11)$ \\
\hline C79 & $\mathrm{C} 80$ & C83 & C86 & $-60.6(15)$ \\
\hline C80 & C81 & C82 & C77 & $-5.1(19)$ \\
\hline C81 & C80 & C83 & C84 & $-118.4(13)$ \\
\hline C81 & C80 & C83 & C85 & $3.4(17)$ \\
\hline C81 & $\mathrm{C} 80$ & C83 & C86 & $124.4(13)$ \\
\hline C82 & C77 & C78 & C79 & $-1.2(16)$ \\
\hline C83 & $\mathrm{C} 80$ & C81 & C82 & $-177.8(12)$ \\
\hline C87 & C70 & C71 & C72 & 88.3(11) \\
\hline C87 & C70 & C71 & C76 & $-95.8(12)$ \\
\hline C87 & C89 & C90 & C91 & $-176.2(10)$ \\
\hline C87 & C89 & C94 & C93 & $175.4(10)$ \\
\hline C87 & C95 & C96 & C97 & $-177.2(10)$ \\
\hline C88 & $\mathrm{C} 70$ & C71 & C72 & $155.6(9)$ \\
\hline C88 & $\mathrm{C} 70$ & C71 & C76 & $-28.5(14)$ \\
\hline C88 & $\mathrm{C} 70$ & C87 & C89 & $105.0(10)$ \\
\hline C88 & C70 & C87 & C95 & $-105.8(10)$ \\
\hline C88 & C87 & C89 & C90 & $-52.9(13)$ \\
\hline C88 & C87 & C89 & C94 & $136.6(10)$ \\
\hline C88 & C87 & C95 & COAA & $44.0(14)$ \\
\hline C88 & C87 & C95 & C96 & $-140.5(10)$ \\
\hline C89 & C87 & C88 & C70 & $-112.7(9)$ \\
\hline C89 & C87 & C95 & COAA & $-96.1(12)$ \\
\hline
\end{tabular}




\begin{tabular}{|c|c|c|c|c|}
\hline Atom & Atom & Atom & Atom & Angle/ ${ }^{\circ}$ \\
\hline $\mathrm{C89}$ & C87 & C95 & C96 & $79.3(12)$ \\
\hline C89 & $\mathrm{C} 90$ & C91 & C92 & 4.1(18) \\
\hline C90 & C89 & C94 & C93 & $4.5(15)$ \\
\hline C90 & C91 & C92 & C93 & $-1.2(19)$ \\
\hline C91 & C92 & C93 & C94 & $0(2)$ \\
\hline C92 & C93 & C94 & C89 & $-1.8(19)$ \\
\hline C94 & C89 & C90 & C91 & $-5.7(16)$ \\
\hline C95 & COAA & C99 & C98 & $2.5(19)$ \\
\hline C95 & C87 & C88 & C70 & $108.5(9)$ \\
\hline C95 & $\mathrm{C} 87$ & C89 & C90 & $86.9(12)$ \\
\hline C95 & C87 & C89 & C94 & $-83.6(11)$ \\
\hline C95 & C96 & C97 & C98 & $-1.5(18)$ \\
\hline C96 & C97 & C98 & C99 & $4.8(18)$ \\
\hline C97 & C98 & C99 & COAA & $-5.2(18)$ \\
\hline C99 & COAA & C95 & C87 & $176.6(11)$ \\
\hline C99 & COAA & C95 & C96 & $0.9(18)$ \\
\hline Rh4B & 06B & C69B & O7B & 2.1(10) \\
\hline Rh4B & O6B & C69B & C70B & $-172.9(4)$ \\
\hline Rh3B & 07B & C69B & 06B & 13.1(10) \\
\hline Rh3B & O7B & C69B & C70B & $-172.0(5)$ \\
\hline Cl3B & C72B & C73B & C74B & $174.3(7)$ \\
\hline C70B & C71B & C72B & Cl3B & $4.5(11)$ \\
\hline C70B & C71B & C72B & C73B & $179.9(8)$ \\
\hline C70B & C71B & C76B & C75B & $-179.8(7)$ \\
\hline C70B & C87B & C89B & C90B & $-127.0(8)$ \\
\hline C70B & C87B & C89B & C94B & 54.1(11) \\
\hline C70B & C87B & C95B & C1A & 83.3(11) \\
\hline C70B & C87B & C95B & C96B & $-94.9(9)$ \\
\hline C69B & C70B & C71B & C72B & $-73.6(9)$ \\
\hline C69B & C70B & C71B & C76B & $102.6(8)$ \\
\hline C69B & C70B & C87B & C88B & $-103.1(8)$ \\
\hline C69B & C70B & C87B & C89B & $2.2(10)$ \\
\hline C69B & C70B & C87B & C95B & $147.9(7)$ \\
\hline C69B & C70B & C88B & C87B & $111.6(7)$ \\
\hline C71B & C70B & C69B & 07B & $-176.5(6)$ \\
\hline C71B & C70B & C69B & 06B & $-1.0(9)$ \\
\hline C71B & C70B & C87B & C88B & 107.1(7) \\
\hline C71B & C70B & C87B & C89B & $-147.6(7)$ \\
\hline C71B & C70B & C87B & C95B & $-1.9(10)$ \\
\hline C71B & C70B & C88B & C87B & $-106.6(7)$ \\
\hline C71B & C72B & C73B & C74B & $-1.2(14)$ \\
\hline C72B & C71B & C76B & C75B & $-3.4(12)$ \\
\hline C72B & C73B & C74B & C75B & $-1.4(14)$ \\
\hline C72B & C73B & C74B & C77B & 178.1(8) \\
\hline C73B & C74B & C75B & C76B & 1.6(13) \\
\hline C73B & C74B & C77B & C78B & $125.3(10)$ \\
\hline C73B & C74B & C77B & C82B & $-54.7(14)$ \\
\hline C74B & C75B & C76B & C71B & $0.9(13)$ \\
\hline C74B & C77B & C78B & C79B & $175.8(9)$ \\
\hline C74B & C77B & C82B & C81B & $-177.3(12)$ \\
\hline C75B & C74B & C77B & C78B & $-55.2(13)$ \\
\hline C75B & C74B & C77B & C82B & $124.9(11)$ \\
\hline C76B & C71B & C72B & $\mathrm{Cl} 3 \mathrm{~B}$ & $-171.9(6)$ \\
\hline
\end{tabular}




\begin{tabular}{|c|c|c|c|c|}
\hline Atom & Atom & Atom & Atom & Angle/ ${ }^{\circ}$ \\
\hline$\overline{\mathrm{C} 76 \mathrm{~B}}$ & C71B & C72B & C73B & $3.5(12)$ \\
\hline C77B & C74B & C75B & C76B & $-177.9(8)$ \\
\hline C77B & C78B & C79B & C80B & $4.8(16)$ \\
\hline C78B & C77B & C82B & C81B & $2.7(19)$ \\
\hline C78B & C79B & C80B & C81B & $-3.3(16)$ \\
\hline C78B & C79B & C80B & C83B & $177.1(10)$ \\
\hline C79B & $\mathrm{C} 80 \mathrm{~B}$ & C81B & C82B & $2(2)$ \\
\hline C79B & C80B & C83B & C84B & $29.2(14)$ \\
\hline C79B & C80B & C83B & C86B & $-87.3(14)$ \\
\hline C79B & C80B & C83B & C85B & $149.0(11)$ \\
\hline C80B & C81B & C82B & C77B & $-2(2)$ \\
\hline C81B & C80B & C83B & C84B & $-150.3(12)$ \\
\hline C81B & C80B & C83B & C86B & 93.2(14) \\
\hline C81B & C80B & C83B & C85B & $-30.5(16)$ \\
\hline C82B & C77B & C78B & C79B & $-4.3(15)$ \\
\hline C83B & C80B & C81B & C82B & $-178.6(14)$ \\
\hline C87B & C70B & C69B & 07B & $33.1(9)$ \\
\hline C87B & C70B & C69B & O6B & $-151.3(7)$ \\
\hline C87B & C70B & C71B & C72B & $77.8(9)$ \\
\hline C87B & C70B & C71B & C76B & $-106.0(8)$ \\
\hline C87B & C89B & C90B & C91B & $-176.5(8)$ \\
\hline C87B & C89B & C94B & C93B & 178.1(7) \\
\hline C87B & C95B & C1A & C99B & $179.1(9)$ \\
\hline C87B & C95B & C96B & C97B & $-179.5(9)$ \\
\hline C88B & C70B & C69B & 07B & $-33.8(9)$ \\
\hline C88B & C70B & C69B & O6B & 141.7(7) \\
\hline C88B & C70B & C71B & C72B & $144.4(8)$ \\
\hline C88B & C70B & C71B & C76B & $-39.4(10)$ \\
\hline C88B & C70B & C87B & C89B & $105.3(8)$ \\
\hline C88B & C70B & C87B & C95B & $-109.0(8)$ \\
\hline C88B & C87B & C89B & C90B & $-57.2(10)$ \\
\hline C88B & C87B & C89B & C94B & $123.9(8)$ \\
\hline C88B & C87B & C95B & C1A & $13.8(12)$ \\
\hline C88B & C87B & C95B & C96B & $-164.4(8)$ \\
\hline C89B & C87B & C88B & C70B & $-112.4(7)$ \\
\hline C89B & C87B & C95B & C1A & $-127.8(9)$ \\
\hline C89B & C87B & C95B & C96B & $54.0(10)$ \\
\hline C89B & C90B & C91B & C92B & $-1.8(14)$ \\
\hline С90B & C89B & C94B & C93B & $-0.9(12)$ \\
\hline C90B & C91B & C92B & C93B & $-0.6(15)$ \\
\hline C91B & C92B & C93B & C94B & $2.2(14)$ \\
\hline C92B & C93B & C94B & C89B & $-1.4(13)$ \\
\hline C94B & C89B & C90B & C91B & $2.5(13)$ \\
\hline C95B & C87B & C88B & C70B & $108.0(8)$ \\
\hline C95B & C87B & C89B & C90B & 85.3(9) \\
\hline C95B & C87B & C89B & C94B & $-93.7(9)$ \\
\hline C95B & C1A & C99B & C98B & $-0.1(18)$ \\
\hline C1A & C95B & C96B & C97B & $2.3(15)$ \\
\hline C1A & C99B & C98B & C97B & $3.3(18)$ \\
\hline C99B & C98B & C97B & C96B & $-3.7(17)$ \\
\hline C98B & C97B & C96B & C95B & $1.0(17)$ \\
\hline C96B & C95B & $\mathrm{C} 1 \mathrm{~A}$ & C99B & $-2.7(15)$ \\
\hline
\end{tabular}


Table 6: Hydrogen Fractional Atomic Coordinates $\left(\times 10^{4}\right)$ and Equivalent Isotropic Displacement Parameters $\left(\AA^{2} \times 10^{3}\right)$ for 2cl4tbuph_twin. $U_{e q}$ is defined as $1 / 3$ of the trace of the orthogonalised $U_{i j}$.

\begin{tabular}{lcccc}
\hline Atom & $\mathbf{x}$ & $\mathbf{y}$ & $\mathbf{z}$ & $\boldsymbol{U}_{\boldsymbol{e q}}$ \\
\hline H3A & 5065.33 & 1947.88 & 5370.69 & 19 \\
H3B & 4882.58 & 1519.31 & 5973.78 & 19 \\
H7 & 6375.74 & 1394.04 & 2568.01 & 31 \\
H9 & 5322.52 & 2247.63 & 2484.82 & 44 \\
H10 & 5063.86 & 1958.36 & 3844.11 & 34 \\
H12 & 5490.17 & 2061.97 & 799.09 & 57 \\
H13 & 5786.73 & 2331.08 & -585.47 & 55 \\
H15 & 7010.66 & 2406.58 & 560.6 & 45 \\
H16 & 6737.36 & 2128.18 & 1919.53 & 42 \\
H18A & 6085.83 & 2902.29 & -1503.66 & 71 \\
H18B & 6532.21 & 3043.58 & -2008.9 & 71 \\
H18C & 6462.03 & 3161.24 & -955.39 & 71 \\
H19A & 7224.71 & 2627.36 & -527.94 & 74 \\
H19B & 7214.25 & 2809.82 & -1551.42 & 74 \\
H19C & 7265.27 & 2291.71 & -1366.14 & 74 \\
H20A & 6616.36 & 1857.36 & -1617.36 & 71 \\
H20B & 6677.49 & 2201.75 & -2434.86 & 71 \\
H20C & 6200.13 & 2126.76 & -1993.97 & 71 \\
H22 & 6034.56 & 757.39 & 6247.45 & 25 \\
H23 & 6145.89 & 378.79 & 7638.29 & 28 \\
H24 & 5844.17 & 652.39 & 9000.8 & 30 \\
H25 & 5475.97 & 1338.61 & 9013.29 & 32 \\
H26 & 5365.22 & 1727.6 & 7605.92 & 28 \\
H28 & 6472.49 & 1494.43 & 5504.9 & 30 \\
H29 & 7013.85 & 2045.32 & 5281.41 & 38 \\
H30 & 6824.71 & 2797.83 & 5454.26 & 55 \\
H31 & 6085.12 & 2986.93 & 5869.25 & 60 \\
H32 & 5541.98 & 2440.45 & 6019.52 & 50 \\
H37 & 3577.93 & 1322.34 & 2475.24 & 28 \\
H39 & 2855.01 & 168.24 & 2364.76 & 27 \\
H40 & 3126.97 & -31.57 & 3796.6 & 31 \\
H42 & 2370.14 & 681.82 & 1569.62 & 43 \\
H43 & 2064.24 & 922.56 & 187.14 & 38 \\
H45 & 3268.82 & 1351.29 & -625.88 & 36 \\
H46 & 3565.97 & 1093.42 & 743.31 & 39 \\
H48A & 3032.29 & 1470.44 & -1795.7 & 62 \\
H48B & 2769.23 & 1923.19 & -1651.1 & 62 \\
H48C & 2655.73 & 1612.78 & -2502.08 & 62 \\
H49A & 2074.66 & 1101.89 & -2424.97 & 73 \\
H49B & 1991.26 & 819.9 & -1518.75 & 73 \\
H49C & 2453.49 & 783.88 & -2041.68 & 73 \\
H50A & 2125.01 & 1910.52 & -607.57 & 63 \\
H50B & 1796.01 & 1497.81 & -654.65 & 63 \\
H50C & 1873.96 & 1797.44 & -1538.53 & 63 \\
H52A & 3048.01 & 76.12 & 5387.23 & 20 \\
H52B & 3472.78 & -98.09 & 6011.62 & 20 \\
H54 & 3271.5 & 381.44 & 7582.03 & 28 \\
H55 & 3654.67 & 477.37 & 8981.8 & 31
\end{tabular}




\begin{tabular}{|c|c|c|c|c|}
\hline Atom & $\mathbf{x}$ & $\mathbf{y}$ & $\mathbf{z}$ & $U_{e q}$ \\
\hline$\overline{\mathrm{H} 56}$ & 4337.5 & 849.07 & 8980.05 & 31 \\
\hline H57 & 4638.11 & 1114.53 & 7606.01 & 27 \\
\hline H58 & 4242.26 & 1020.62 & 6218.28 & 24 \\
\hline $\mathrm{H} 60$ & 3512.48 & 1486.85 & 5989.74 & 39 \\
\hline H61 & 3020.02 & 2077.55 & 5685.42 & 48 \\
\hline H62 & 2286.79 & 1896.73 & 5172.71 & 56 \\
\hline H63 & 2102.36 & 1174.71 & 4952.26 & 79 \\
\hline H64 & 2599.05 & 583.45 & 5257.04 & 58 \\
\hline H66A & 4921.61 & 294.76 & 9308.5 & 113 \\
\hline H66B & 5294.47 & -79.49 & 9308.5 & 113 \\
\hline H66C & 4783.92 & -215.27 & 9308.5 & 113 \\
\hline H68A & 4704.36 & 75.01 & 517.76 & 113 \\
\hline H68B & 5082.86 & -293.54 & 517.76 & 113 \\
\hline H68C & 5212.78 & 218.53 & 517.76 & 113 \\
\hline HOAA & 4980.45 & 2526.61 & 8575.65 & 48 \\
\hline H73 & 3822.85 & 3458.17 & 5064.15 & 43 \\
\hline H75 & 4996.55 & 2838.94 & 4702.99 & 37 \\
\hline H76 & 5242.72 & 3134.08 & 6125.62 & 39 \\
\hline H78 & 4768.8 & 3033.67 & 3075.83 & 39 \\
\hline H79 & 4522.14 & 2760.65 & 1712.52 & 54 \\
\hline H81 & 3345 & 2430.01 & 2912.9 & 48 \\
\hline H82 & 3587.04 & 2718.03 & 4277.29 & 47 \\
\hline H84A & 4307.54 & 2188.58 & 621.83 & 79 \\
\hline H84B & 3882.12 & 1938.14 & 233.34 & 79 \\
\hline H84C & 4056.11 & 1833.41 & 1240.36 & 79 \\
\hline H85A & 3228.96 & 1979.04 & 1512.75 & 77 \\
\hline H85B & 3127.35 & 2225.38 & 570.84 & 77 \\
\hline H85C & 3049.6 & 2475.96 & 1517.64 & 77 \\
\hline H86A & 3657.87 & 3096.25 & 785.63 & 80 \\
\hline H86B & 3544.23 & 2763.99 & -29.34 & 80 \\
\hline H86C & 4047.18 & 2847.58 & 256.4 & 80 \\
\hline H88A & 5320.56 & 3108.32 & 7670.68 & 32 \\
\hline H88B & 5411.65 & 3557.41 & 8300.98 & 32 \\
\hline H90 & 5099.22 & 3311.13 & 9950.62 & 46 \\
\hline H91 & 4923.31 & 3659.26 & 11368.84 & 67 \\
\hline H92 & 4265.83 & 4114.42 & 11442.37 & 60 \\
\hline H93 & 3861.71 & 4225.71 & 10157.6 & 62 \\
\hline H94 & 4036.08 & 3909.91 & 8756.69 & 47 \\
\hline H96 & 3862.94 & 3152.21 & 7792.2 & 37 \\
\hline H97 & 3497.84 & 2481.28 & 7704.67 & 46 \\
\hline H98 & 3856.69 & 1813.05 & 7936.96 & 45 \\
\hline H99 & 4625.79 & 1837.76 & 8524.47 & 56 \\
\hline H73B & 1683.67 & -1098.84 & 3921.65 & 29 \\
\hline H75B & 2139.71 & 174.5 & 3800.13 & 27 \\
\hline H76B & 1817.36 & 311.6 & 5226.53 & 19 \\
\hline H78B & 1864.83 & -85.73 & 2064.21 & 34 \\
\hline H79B & 2257.32 & -178.2 & 693.07 & 39 \\
\hline H81B & 2901.93 & -1220.62 & 1774.69 & 65 \\
\hline H82B & 2546.97 & -1127.61 & 3175.01 & 60 \\
\hline H84D & 2625.46 & -218.13 & -477.01 & 91 \\
\hline H84E & 3032.58 & -427.04 & -1022.16 & 91 \\
\hline H84F & 3116 & -170.63 & -84.12 & 91 \\
\hline H86D & 2276.55 & -965.6 & -595.09 & 99 \\
\hline
\end{tabular}




\begin{tabular}{lllll}
\hline Atom & \multicolumn{1}{c}{$\mathbf{x}$} & \multicolumn{1}{c}{$\mathbf{y}$} & \multicolumn{1}{c}{$\mathbf{z}$} & $\boldsymbol{U}_{\boldsymbol{e q}}$ \\
\hline H86E & 2470.91 & -1386.8 & -73.09 & 99 \\
H86F & 2700.38 & -1216.24 & -990.37 & 99 \\
H85D & 3432.36 & -1100.3 & -343.19 & 79 \\
H85E & 3290.73 & -1286.57 & 634.81 & 79 \\
H85F & 3507.78 & -806.67 & 550.15 & 79 \\
H88C & 1919.7 & 238.51 & 6825.9 & 17 \\
H88D & 1466.86 & 326.45 & 7445.93 & 17 \\
H90B & 1710.92 & -107.44 & 9040.65 & 25 \\
H91B & 1361.05 & -337.28 & 10393.72 & 35 \\
H92B & 783.2 & -853.2 & 10348 & 32 \\
H93B & 542.89 & -1135.63 & 8925.84 & 27 \\
H94B & 908.6 & -937.53 & 7575.63 & 22 \\
H1A & 2483.55 & -164.99 & 6992.36 & 40 \\
H99B & 3105.07 & -632.68 & 6814.5 & 55 \\
H98B & 3043.01 & -1399.95 & 6899.37 & 47 \\
H97B & 2333.93 & -1719.31 & 7018.23 & 51 \\
H96B & 1700.6 & -1258.46 & 7219.92 & 33 \\
H10A & -111.5 & -283.9 & 10770.85 & 80 \\
H10B & -190.11 & 238.51 & 10770.85 & 80 \\
H10C & 301.61 & 45.39 & 10770.85 & 80 \\
H10D & -43.5 & 301.89 & 2048.7 & 80 \\
H10E & -239.7 & -188.62 & 2048.7 & 80 \\
H10F & 283.2 & -113.27 & 2048.7 & 80 \\
H1DA & 5123.9 & 5278.71 & 3147.34 & 162 \\
H1DB & 4696.68 & 4967.95 & 3147.33 & 162 \\
H1DC & 5179.42 & 4753.34 & 3147.34 & 162 \\
H1EA & 5284.21 & 5110.7 & 11761.42 & 162 \\
H1EB & 4953.77 & 4698.51 & 11761.42 & 162 \\
H1EC & 4762.02 & 5190.78 & 11761.42 & 162 \\
& & & &
\end{tabular}

Table 7: Atomic Occupancies for all atoms that are not fully occupied in 2cl4tbuph_twin.

\begin{tabular}{|c|c|c|c|c|c|c|c|}
\hline & & & \\
\hline Atom & Occupancy & Atom & Occupancy & Atom & Occupancy & Atom & Occupancy \\
\hline H66A & 0.5 & H68C & 0.5 & $\overline{\mathrm{H} 10 \mathrm{E}}$ & 0.25 & $\overline{\mathrm{H} 1 \mathrm{EA}}$ & 0.25 \\
\hline H66B & 0.5 & $\mathrm{H} 10 \mathrm{~A}$ & 0.25 & $\mathrm{H} 10 \mathrm{~F}$ & 0.25 & H1EB & 0.25 \\
\hline H66C & 0.5 & H10B & 0.25 & H1DA & 0.25 & H1EC & 0.25 \\
\hline H68A & 0.5 & $\mathrm{H} 10 \mathrm{C}$ & 0.25 & H1DB & 0.25 & & \\
\hline H68B & 0.5 & H10D & 0.25 & H1DC & 0.25 & & \\
\hline
\end{tabular}

Table 8: Solvent masking (PLATON/SQUEEZE) information for 2cl4tbuph_twin.

\begin{tabular}{llllrrl}
\hline No & $\mathbf{x}$ & $\mathbf{y}$ & $\mathbf{z}$ & $\mathbf{V}$ & $\mathbf{e}$ & Content \\
\hline 1 & -0.214 & -0.324 & 0.294 & 2036.2 & 91.2 & $?$ \\
2 & 0.000 & 0.500 & 0.139 & 337.4 & 47.6 & $?$ \\
3 & 0.238 & 0.649 & 0.614 & 54.6 & 0.0 & $?$ \\
4 & 0.351 & 0.238 & 0.614 & 54.6 & 0.0 & $?$ \\
5 & 0.500 & 1.000 & 0.139 & 337.4 & 47.6 & $?$ \\
6 & 0.649 & 0.762 & 0.614 & 54.6 & 0.0 & $?$ \\
7 & 0.762 & 0.351 & 0.614 & 54.6 & 0.0 & $?$
\end{tabular}




\section{Citations}

APEX2 suite for crystallographic software, Bruker axs, Madison, WI ().

O.V. Dolomanov and L.J. Bourhis and R.J. Gildea and J.A.K. Howard and H. Puschmann, Olex2: A complete structure solution, refinement and analysis program, J. Appl. Cryst., (2009), 42, 339-341.

SADABS-2014/5, Bruker axs, Madison, WI (2014).

SAINT - Software for the Integration of CCD Detector System Bruker Analytical X-ray Systems, Bruker axs, Madison, WI (?).

Sheldrick, G.M., Crystal structure refinement with ShelXL, Acta Cryst., (2015), C71, 3-8.

Sheldrick, G.M., ShelXT-Integrated space-group and crystal-structure determination, Acta Cryst., (2015), A71, 3-8. 


\section{Crystal Data and Experimental}

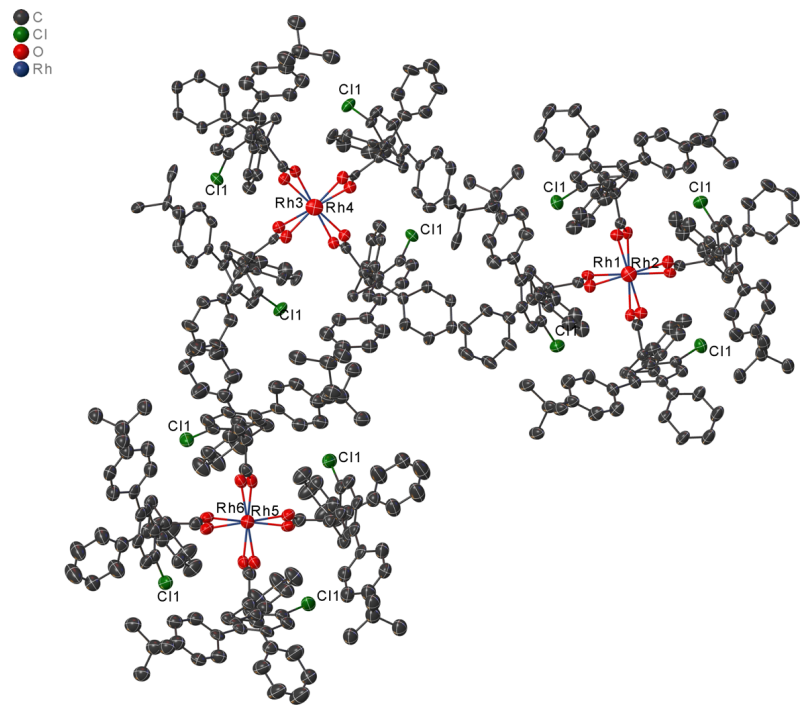

Experimental. Single green needle-shaped crystals of Rh$\mathbf{2 C l 5 t B u P h}$ were chosen from the sample as supplied. A suitable crystal with dimensions $0.55 \times 0.07 \times 0.03 \mathrm{~mm}^{3}$ was selected and mounted on a loop on a XtaLAB Synergy$\mathrm{S}$ diffractometer. The crystal was kept at a steady $T=$ 100(2) K during data collection. The structure was solved with the ShelXT (Sheldrick, 2015) solution program using dual methods and by using Olex2 1.3-alpha (Dolomanov et al., 2009) as the graphical interface. The model was refined with ShelXL 2018/3 (Sheldrick, 2015) using full matrix least squares minimisation on $\boldsymbol{F}^{2}$.

Crystal Data. $\mathrm{C}_{128} \mathrm{H}_{116} \mathrm{Cl}_{4} \mathrm{O}_{10} \mathrm{Rh}_{2}, M_{r}=2161.83$, tetragonal, $P 4$ (No. 75), $\mathrm{a}=32.0493(5) \AA, \mathrm{b}=32.0493(5) \AA, \mathrm{c}=$ 11.9878(3) $\AA, \alpha=\beta=\gamma=90^{\circ}, \quad V=12313.3(5) \AA^{3}, \quad T=$ 100(2) $\mathrm{K}, Z=4, Z^{\prime}=1, \mu\left(\mathrm{CuK}_{\alpha}\right)=3.384,69630$ reflections measured, 19495 unique $\left(R_{\text {int }}=0.0730\right)$ which were used in all calculations. The final $w R_{2}$ was 0.1804 (all data) and $R_{1}$ was $0.0648(\mathrm{I} \geq 2 \sigma(\mathrm{I}))$.

\begin{tabular}{|c|c|}
\hline Compound & Rh-2Cl5tBuPh \\
\hline Formula & $\mathrm{C}_{128} \mathrm{H}_{116} \mathrm{Cl}_{4} \mathrm{O}_{10} \mathrm{Rh}_{2}$ \\
\hline$D_{\text {calc. }} / \mathrm{g} \mathrm{cm}^{-3}$ & 1.166 \\
\hline$\mu / \mathrm{mm}^{-1}$ & 3.384 \\
\hline Formula Weight & 2161.83 \\
\hline Colour & green \\
\hline Shape & needle-shaped \\
\hline Size $/ \mathrm{mm}^{3}$ & $0.55 \times 0.07 \times 0.03$ \\
\hline$T / K^{\prime}$ & $100(2)$ \\
\hline Crystal System & tetragonal \\
\hline Flack Parameter & $0.305(10)$ \\
\hline Hooft Parameter & $0.049(5)$ \\
\hline Space Group & $P 4$ \\
\hline$a / \AA ̊$ & $32.0493(5)$ \\
\hline$b / \AA ̊$ & $32.0493(5)$ \\
\hline$c / \AA ̊$ & $11.9878(3)$ \\
\hline$\alpha /^{\circ}$ & 90 \\
\hline$\beta /^{\circ}$ & 90 \\
\hline$\gamma /^{\circ}$ & 90 \\
\hline $\mathrm{V} / \AA^{3}$ & $12313.3(5)$ \\
\hline Z & 4 \\
\hline$Z^{\prime}$ & 1 \\
\hline Wavelength/Å & 1.54184 \\
\hline Radiation type & $\mathrm{CuK}_{\alpha}$ \\
\hline$\Theta_{\min } /^{\circ}$ & 3.083 \\
\hline$\Theta_{\max } /^{\circ}$ & 65.079 \\
\hline Measured Refl's. & 69630 \\
\hline Indep't Refl's & 19495 \\
\hline Refl's I $\geq 2 \sigma(I)$ & 15871 \\
\hline$R_{\text {int }}$ & 0.0730 \\
\hline Parameters & 1415 \\
\hline Restraints & 3411 \\
\hline Largest Peak & 1.954 \\
\hline Deepest Hole & -1.782 \\
\hline GooF & 1.067 \\
\hline$w R_{2}$ (all data) & 0.1804 \\
\hline$w R_{2}$ & 0.1700 \\
\hline$R_{1}$ (all data) & 0.0791 \\
\hline$R_{1}$ & 0.0648 \\
\hline
\end{tabular}




\section{Structure Quality Indicators}

\begin{tabular}{|c|c|c|c|c|c|c|}
\hline efle & $\begin{array}{l}d \min \left(\mathrm{Cula}_{2}\right) \\
2 \theta=130.2^{\circ}\end{array}$ & $\sigma(I)$ & 14.9 & Rint & $7.30 \%$ & CAP $130.2^{\circ} 97.6$ \\
\hline & 0.004 & & 1.9 & & & GooF \\
\hline
\end{tabular}

A green needle-shaped-shaped crystal with dimensions $0.55 \times 0.07 \times 0.03 \mathrm{~mm}^{3}$ was mounted on a loop with paratone. Data were collected using a XtaLAB Synergy, Dualflex, HyPix diffractometer operating at $T=$ $100(2) \mathrm{K}$.

Data were measured using $\omega$ scans using $\mathrm{CuK}_{\alpha}$ radiation. The diffraction pattern was indexed and the total number of runs and images was based on the strategy calculation from the program CrysAlisPro 1.171.39.43c (Rigaku OD, 2018). The maximum resolution that was achieved was $\Theta=65.079^{\circ}(0.85 \AA)$.

The unit cell was refined using CrysAlisPro 1.171.39.43c (Rigaku OD, 2018) on 11234 reflections, $16 \%$ of the observed reflections.

Data reduction, scaling and absorption corrections were performed using CrysAlisPro 1.171.39.43c (Rigaku OD, 2018). The final completeness is $97.60 \%$ out to $65.079^{\circ}$ in $\Theta$. A numerical absorption correction based on gaussian integration over a multifaceted crystal model was performed using CrysAlisPro 1.171.41.108a (Rigaku Oxford Diffraction, 2021). An empirical absorption correction using spherical harmonics, implemented in SCALE3 ABSPACK scaling algorithm was also applied. The absorption coefficient $\mu$ of this material is $3.384 \mathrm{~mm}^{-1}$ at this wavelength $(\lambda=1.54184 \AA)$ and the minimum and maximum transmissions are 0.413 and 1.000 .

The structure was solved and the space group P4 (\# 75) determined by the ShelXT (Sheldrick, 2015) structure solution program using using dual methods and refined by full matrix least squares minimisation on $\boldsymbol{F}^{2}$ using version 2018/3 of ShelXL 2018/3 (Sheldrick, 2015). All non-hydrogen atoms were refined anisotropically. Hydrogen atom positions were calculated geometrically and refined using the riding model. Most hydrogen atom positions were calculated geometrically and refined using the riding model, but some hydrogen atoms were refined freely.

_refine_special_details: Refined as a 2-component inversion twin.

There is a single molecule in the asymmetric unit, which is represented by the reported sum formula. In other words: $\mathrm{Z}$ is 4 and $\mathrm{Z}^{\prime}$ is 1 .

The Flack parameter was refined to 0.305(10). Determination of absolute structure using Bayesian statistics on Bijvoet differences using the Olex2 results in 0.049(5). Note: The Flack parameter is used to determine chirality of the crystal studied, the value should be near 0 , a value of 1 means that the stereochemistry is wrong and the model should be inverted. A value of 0.5 means that the crystal consists of a racemic mixture of the two enantiomers. 
${ }^{\circ}$
$8_{\mathrm{Cl}}$
$\mathrm{O}_{\mathrm{Rh}}$

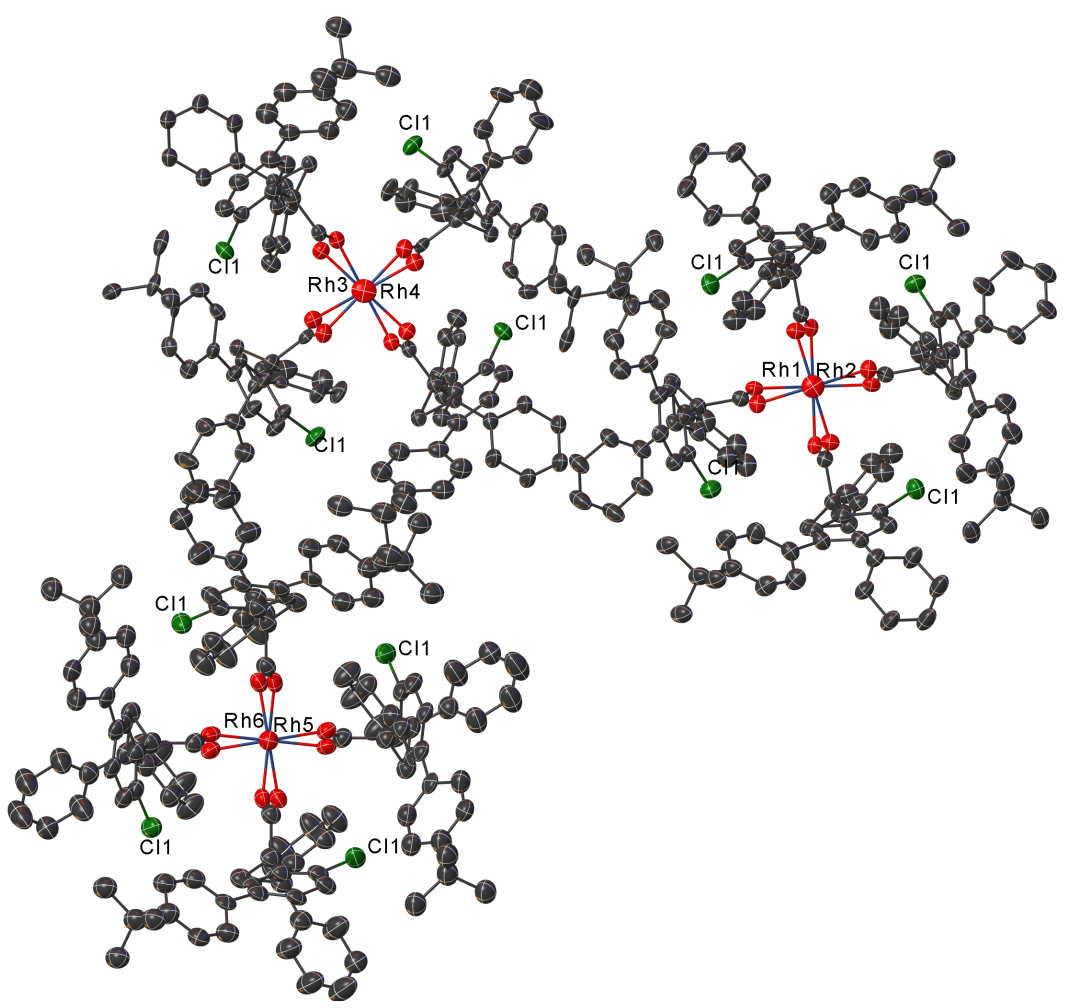

Figure 1:

$8{ }^{\circ}$
$8{ }^{\circ}$
$8 \mathrm{Rh}$

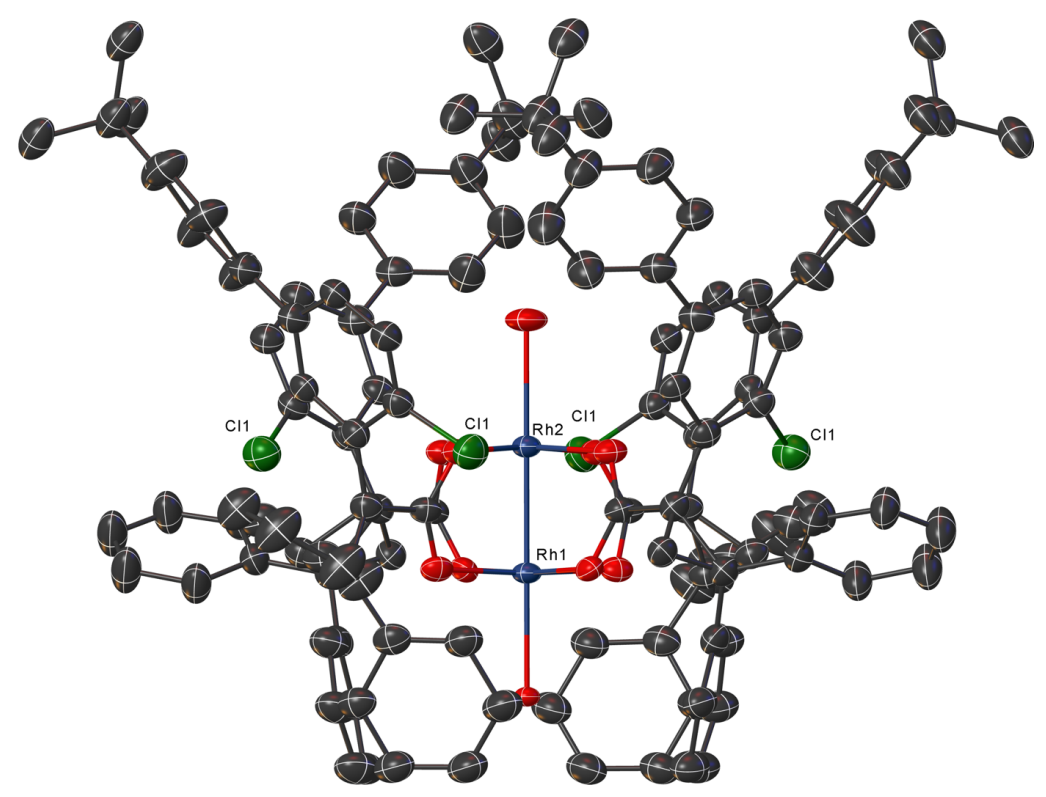

Figure 2: 


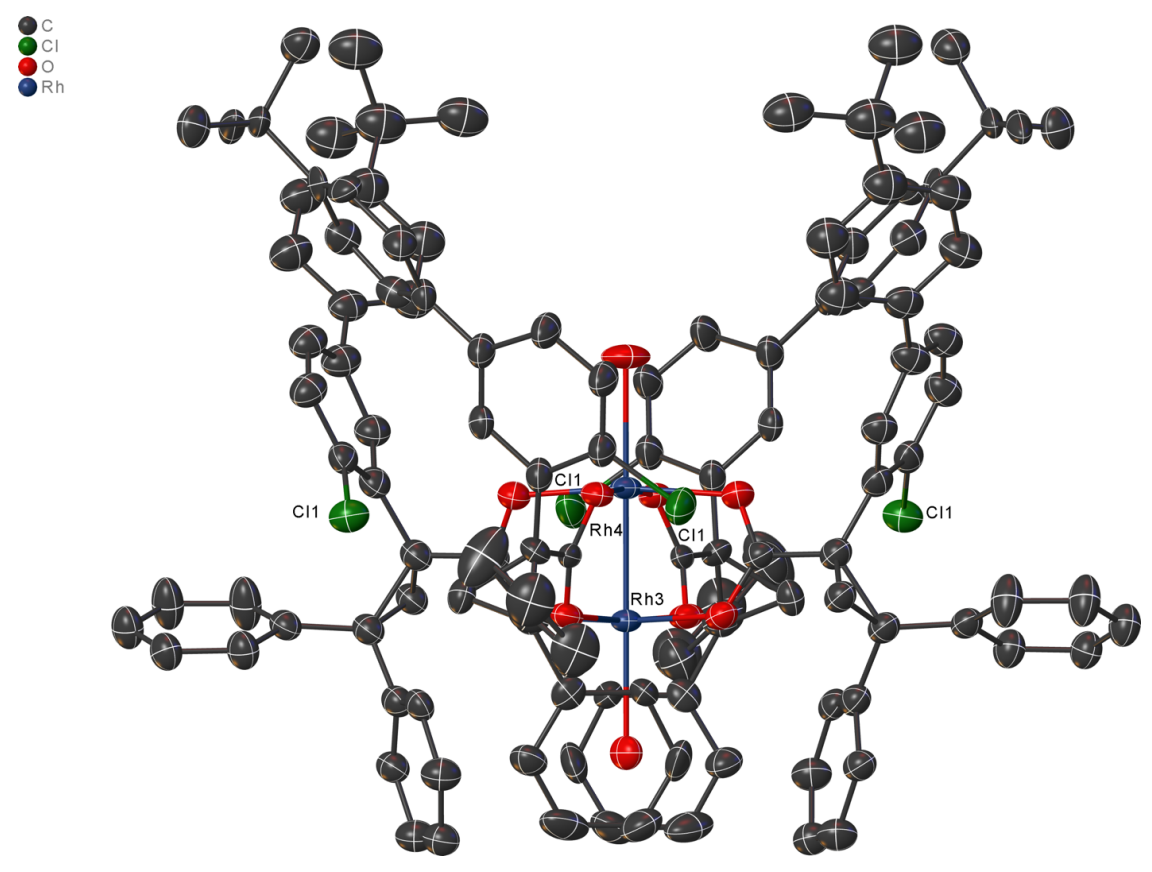

Figure 3:

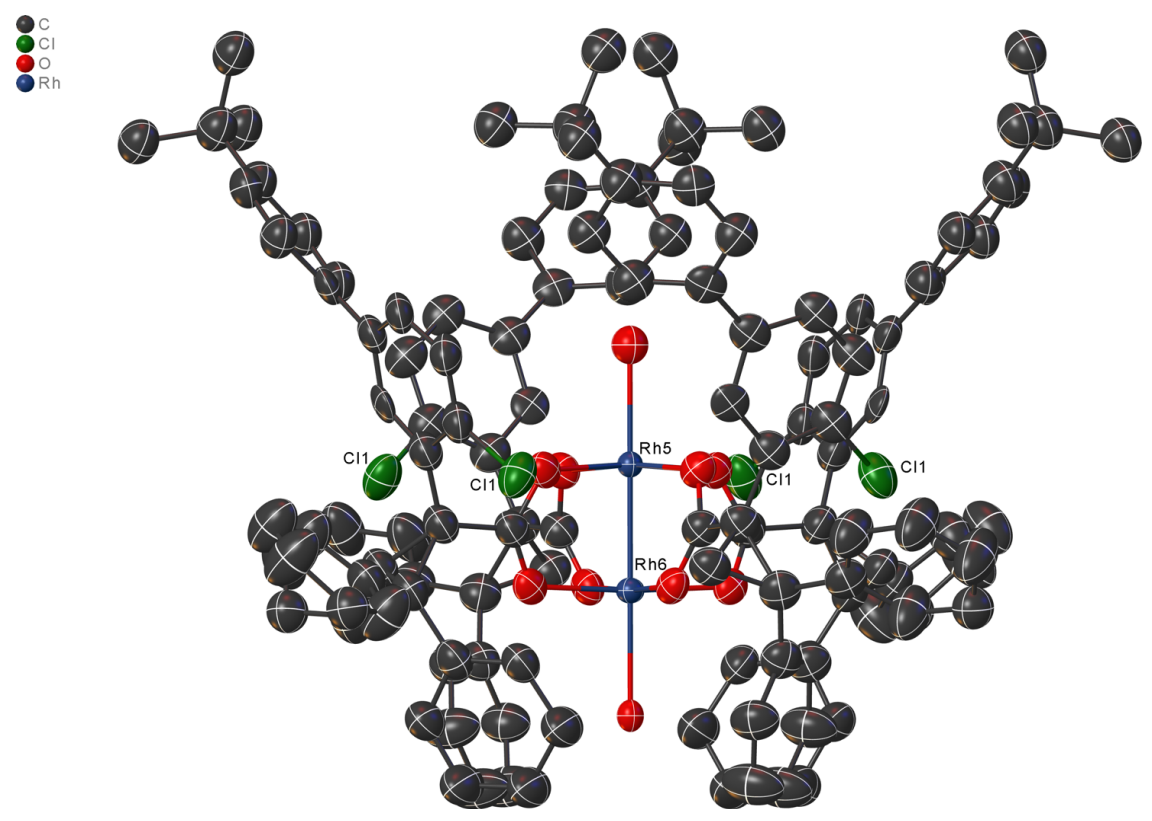

Figure 4: 
Data Plots: Diffraction Data
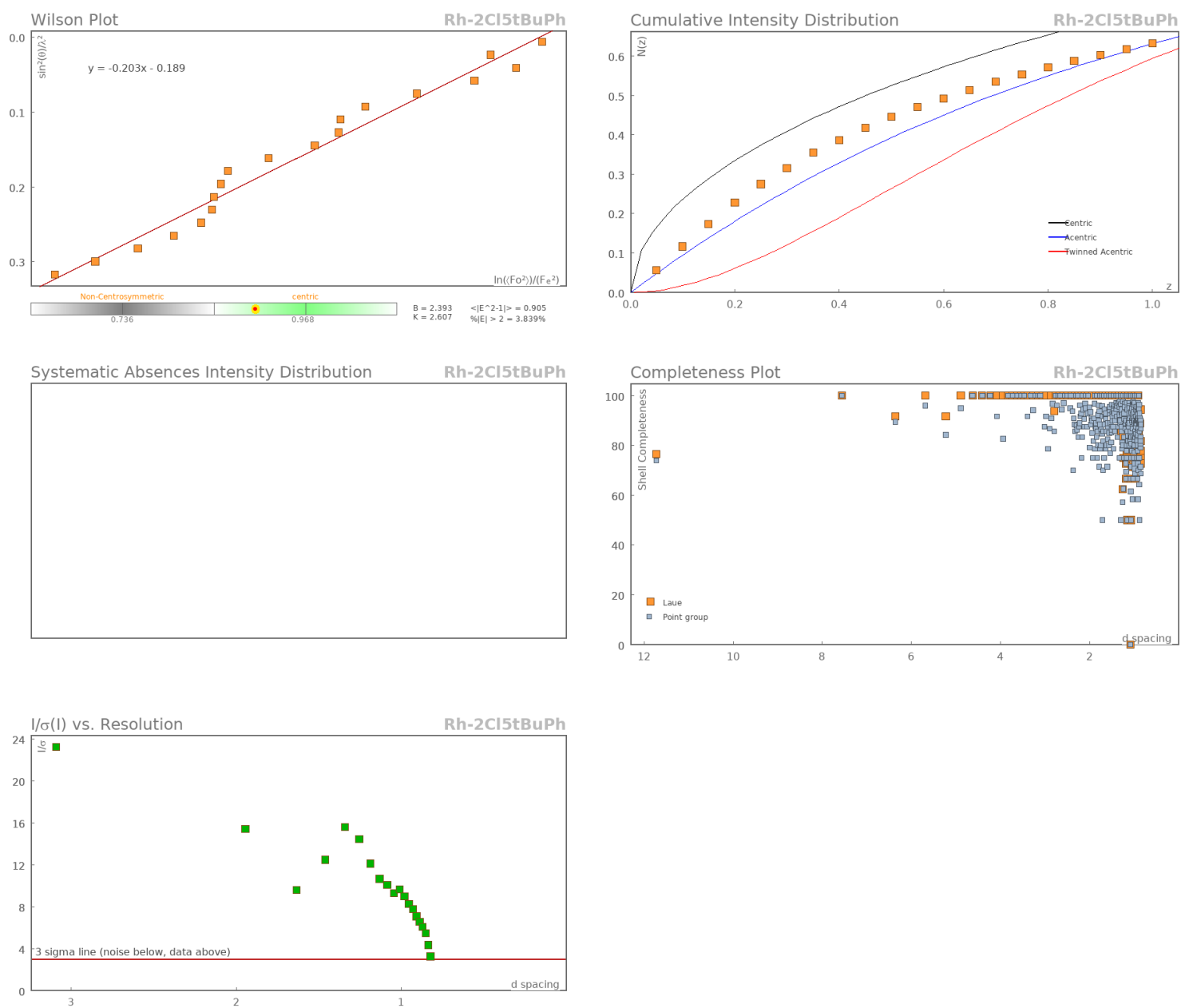

\section{Data Plots: Refinement and Data}
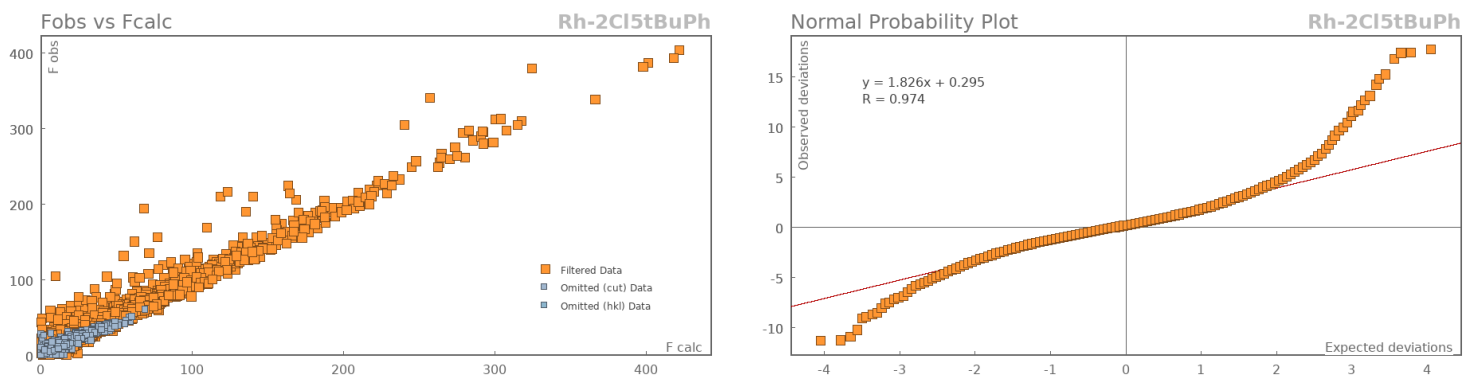

\section{Images of the Crystal on the Diffractometer}

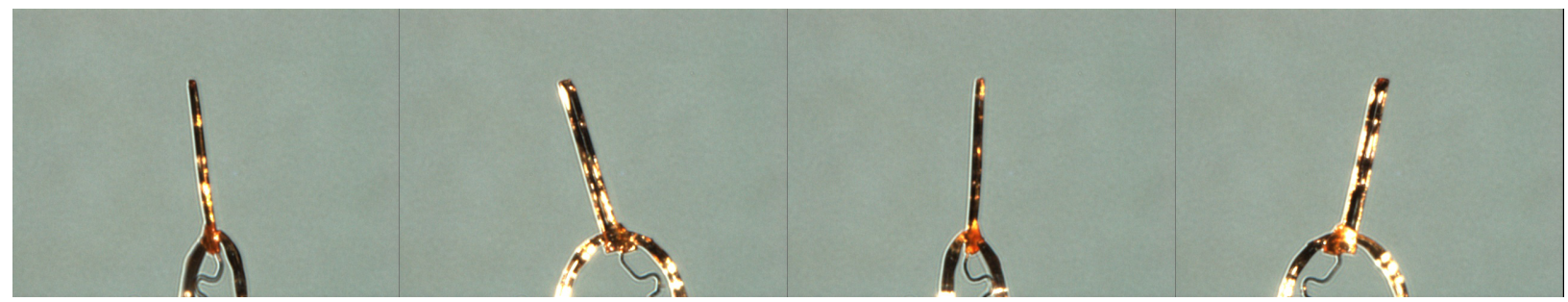

Table 9: Fractional Atomic Coordinates $\left(\times 10^{4}\right)$ and Equivalent Isotropic Displacement Parameters $\left(\AA^{2} \times 10^{3}\right)$ for 
Rh-2Cl5tBuPh. $U_{e q}$ is defined as $1 / 3$ of the trace of the orthogonalised $U_{i j}$.

\begin{tabular}{|c|c|c|c|c|}
\hline Atom & $\mathbf{x}$ & $\mathbf{y}$ & $\mathbf{z}$ & $U_{e q}$ \\
\hline$\overline{\mathrm{Cl} 1}$ & $1661.5(9)$ & $4810.1(8)$ & $5138(3)$ & $50.8(7)$ \\
\hline 01 & $542(2)$ & $4660.5(19)$ & $5324(6)$ & $33.0(14)$ \\
\hline 02 & $421(2)$ & $4525(2)$ & $3517(6)$ & $35.7(15)$ \\
\hline C1 & $980(3)$ & $4173(3)$ & $4475(10)$ & $38(2)$ \\
\hline $\mathrm{C} 2$ & $1235(3)$ & $4060(3)$ & $3405(9)$ & $37(2)$ \\
\hline $\mathrm{C} 3$ & $897(4)$ & $3777(3)$ & $3805(10)$ & $45(3)$ \\
\hline $\mathrm{C} 4$ & $614(3)$ & $4485(3)$ & $4383(8)$ & $30(2)$ \\
\hline $\mathrm{C} 5$ & $1165(3)$ & $4156(3)$ & $5612(10)$ & $38(2)$ \\
\hline C6 & $1482(4)$ & $4410(3)$ & $5975(9)$ & $43(2)$ \\
\hline $\mathrm{C} 7$ & $1652(3)$ & $4365(3)$ & $7023(11)$ & $50(3)$ \\
\hline $\mathrm{C} 8$ & $1509(4)$ & $4063(4)$ & $7740(11)$ & $53(3)$ \\
\hline $\mathrm{C} 9$ & $1172(3)$ & $3808(3)$ & $7457(10)$ & $42(2)$ \\
\hline C10 & $1021(3)$ & $3843(3)$ & $6390(9)$ & $33(2)$ \\
\hline C11 & $1690(3)$ & $3916(4)$ & $3504(12)$ & $52(3)$ \\
\hline C12 & $1984(4)$ & $4092(4)$ & $2893(12)$ & $56(3)$ \\
\hline C13 & $2401(4)$ & $3969(5)$ & $2970(14)$ & $67(4)$ \\
\hline C14 & $2518(4)$ & $3676(6)$ & $3672(19)$ & $98(7)$ \\
\hline C15 & $2217(4)$ & $3489(4)$ & $4410(16)$ & $73(4)$ \\
\hline C16 & $1813(3)$ & $3616(4)$ & 4311(11) & $53(3)$ \\
\hline C17 & $1160(3)$ & $4318(3)$ & 2334(9) & $35(2)$ \\
\hline C18 & $1084(4)$ & $4111(3)$ & $1338(10)$ & $45(2)$ \\
\hline C19 & $1015(4)$ & $4318(5)$ & $403(11)$ & $66(4)$ \\
\hline $\mathrm{C} 20$ & $1060(5)$ & $4766(4)$ & $373(13)$ & $70(4)$ \\
\hline C21 & $1140(5)$ & $4957(3)$ & $1336(13)$ & $67(4)$ \\
\hline $\mathrm{C} 22$ & $1210(3)$ & $4739(3)$ & $2335(9)$ & $39(2)$ \\
\hline C23 & $980(3)$ & $3522(3)$ & $8265(10)$ & $38(2)$ \\
\hline C24 & $552(3)$ & $3480(3)$ & 8320(9) & $37(2)$ \\
\hline C25 & $365(4)$ & $3253(4)$ & 9131(9) & $47(3)$ \\
\hline C26 & $612(4)$ & $3018(3)$ & 9954(9) & $42(3)$ \\
\hline C27 & $1039(4)$ & $3053(3)$ & $9846(8)$ & $40(2)$ \\
\hline C28 & $1225(4)$ & $3312(3)$ & $9026(10)$ & $45(3)$ \\
\hline C29 & $418(4)$ & $2744(3)$ & 10858(9) & $46(3)$ \\
\hline C30 & $-53(4)$ & $2735(3)$ & $10787(11)$ & $62(4)$ \\
\hline C31 & $573(3)$ & $2321(4)$ & $10783(12)$ & $55(3)$ \\
\hline C32 & $520(4)$ & $2954(4)$ & $11994(12)$ & $59(3)$ \\
\hline 01W_5 & 0 & 10000 & $-2917(7)$ & $34.3(18)$ \\
\hline $01 W \_6$ & 0 & 10000 & $2976(7)$ & $33(3)$ \\
\hline 01W_9 & 0 & 5000 & $7264(7)$ & $25.5(19)$ \\
\hline 01W_11 & 5000 & 5000 & $2472(7)$ & $14(2)$ \\
\hline 01W_12 & 5000 & 5000 & $-3419(7)$ & $34.3(18)$ \\
\hline 01W_13 & 0 & 5000 & $1353(6)$ & $34.3(18)$ \\
\hline Cl1_2 & $3950.0(10)$ & $3807.2(9)$ & 448(3) & $67.8(9)$ \\
\hline 01_2 & $4407.9(17)$ & $4763(2)$ & $536(5)$ & $39.1(14)$ \\
\hline $02 \_2$ & $4366.9(19)$ & $4925(2)$ & $-1286(5)$ & $47.1(16)$ \\
\hline C1_2 & $3759(2)$ & $4710(2)$ & $-318(6)$ & $56.8(17)$ \\
\hline C2_2 & $3518(2)$ & $4542(2)$ & $-1348(6)$ & 61.1(19) \\
\hline C3_2 & $3476(2)$ & $4984(2)$ & $-992(7)$ & $61(2)$ \\
\hline C4_2 & $4220(2)$ & $4809(3)$ & $-379(6)$ & $44.3(19)$ \\
\hline C5_2 & $3623(2)$ & $4565(2)$ & $799(6)$ & $56(2)$ \\
\hline C6_2 & $3696(3)$ & $4169(2)$ & $1258(7)$ & $52(2)$ \\
\hline C7_2 & $3596(4)$ & $4057(3)$ & $2307(7)$ & $62(3)$ \\
\hline C8_2 & $3390(3)$ & $4352(2)$ & $2994(7)$ & $55(2)$ \\
\hline C9_2 & $3347(3)$ & $4768(2)$ & $2664(6)$ & 49.1(19) \\
\hline C10_2 & $3457(3)$ & $4866(2)$ & $1572(6)$ & $42.6(19)$ \\
\hline C11_2 & $3163(3)$ & $4244(3)$ & $-1190(9)$ & $74(3)$ \\
\hline C12_2 & $3155(4)$ & $3862(4)$ & $-1801(14)$ & $98(5)$ \\
\hline
\end{tabular}




\begin{tabular}{|c|c|c|c|c|}
\hline Atom & $\mathbf{x}$ & $\mathbf{y}$ & $\mathbf{z}$ & $U_{e q}$ \\
\hline$\overline{C 13 \_2}$ & $2823(4)$ & $3595(3)$ & $-1728(11)$ & $76(3)$ \\
\hline C14_2 & $2528(4)$ & $3640(4)$ & $-889(13)$ & $92(4)$ \\
\hline C15_2 & $2521(5)$ & $4006(4)$ & $-350(16)$ & $110(6)$ \\
\hline C16_2 & $2823(3)$ & $4310(4)$ & $-519(13)$ & $90(4)$ \\
\hline C17_2 & $3764(3)$ & $4459(3)$ & $-2431(6)$ & $57(2)$ \\
\hline C18_2 & $3635(4)$ & $4647(4)$ & $-3403(7)$ & $69(3)$ \\
\hline C19_2 & $3841(5)$ & $4597(5)$ & $-4401(8)$ & $92(5)$ \\
\hline C20_2 & 4168(5) & $4315(5)$ & $-4465(9)$ & $95(5)$ \\
\hline C21_2 & $4300(4)$ & $4119(5)$ & $-3541(9)$ & $91(5)$ \\
\hline C22_2 & 4081(3) & 4181(4) & $-2511(8)$ & $68(3)$ \\
\hline C23_2 & $3182(3)$ & $5094(2)$ & $3424(7)$ & $55(2)$ \\
\hline C24_2 & $3356(3)$ & $5483(3)$ & $3472(9)$ & $64(3)$ \\
\hline C25_2 & $3250(3)$ & $5776(3)$ & $4254(9)$ & $60(3)$ \\
\hline C26_2 & $2926(3)$ & $5681(3)$ & $5037(9)$ & $65(3)$ \\
\hline C27_2 & $2747(3)$ & $5298(3)$ & 4963(8) & $53(2)$ \\
\hline C28_2 & $2862(3)$ & $5008(3)$ & $4175(9)$ & $61(3)$ \\
\hline C29_2 & 2793(3) & $6015(3)$ & $5912(9)$ & $76(3)$ \\
\hline C30_2 & $3056(4)$ & $6416(3)$ & $5880(11)$ & $77(4)$ \\
\hline C31_2 & $2349(3)$ & $6123(4)$ & $5700(12)$ & $82(4)$ \\
\hline C32_2 & $2831(4)$ & $5814(4)$ & $7062(9)$ & $80(4)$ \\
\hline Cl1_3 & 115.5(8) & 6576.3(8) & $5029(2)$ & $41.9(6)$ \\
\hline 01_3 & $333.3(18)$ & 5551.5(18) & $5316(5)$ & $32.6(13)$ \\
\hline 02_3 & 481.2(19) & $5407.6(18)$ & $3524(5)$ & $34.5(13)$ \\
\hline C1_3 & $834(2)$ & $5961(2)$ & $4423(6)$ & $33.8(12)$ \\
\hline C2_3 & $955(2)$ & $6202(2)$ & $3347(6)$ & $33.5(12)$ \\
\hline C3_3 & $1229(2)$ & $5868(2)$ & $3801(6)$ & $34.2(17)$ \\
\hline C4_3 & $514(2)$ & $5614(2)$ & 4399(6) & $29.8(14)$ \\
\hline C5_3 & $846(2)$ & $6197(2)$ & $5486(6)$ & $39.0(16)$ \\
\hline C6_3 & $538(2)$ & $6475(3)$ & $5870(6)$ & $38.8(18)$ \\
\hline C7_3 & $559(3)$ & $6678(3)$ & $6851(7)$ & $46(2)$ \\
\hline C8_3 & 883(3) & $6577(3)$ & $7608(7)$ & $48(2)$ \\
\hline C9_3 & 1192(3) & $6287(3)$ & 7336(7) & $46(2)$ \\
\hline C10_3 & $1170(3)$ & $6107(3)$ & $6282(7)$ & $49(2)$ \\
\hline C11_3 & $1102(2)$ & $6645(2)$ & $3418(7)$ & $33.9(16)$ \\
\hline C12_3 & 841(3) & $6975(2)$ & $3038(10)$ & $42(2)$ \\
\hline C13_3 & 981(3) & $7378(2)$ & $3000(11)$ & $48(2)$ \\
\hline C14_3 & $1380(3)$ & $7482(3)$ & $3345(11)$ & $49(2)$ \\
\hline C15_3 & $1620(3)$ & 7177(3) & $3756(13)$ & $61(3)$ \\
\hline C16_3 & 1495(3) & $6762(2)$ & $3744(13)$ & $63(4)$ \\
\hline C17_3 & $713(2)$ & $6109(2)$ & $2263(6)$ & $36.8(17)$ \\
\hline C18_3 & 933(2) & $6069(3)$ & $1283(6)$ & $39.2(18)$ \\
\hline C19_3 & 743(3) & $6004(3)$ & 270(7) & $43(2)$ \\
\hline C20_3 & $311(3)$ & $5980(3)$ & $218(8)$ & $47(2)$ \\
\hline C21_3 & $84(3)$ & $6058(3)$ & 1135(7) & $45(2)$ \\
\hline C22_3 & 291(2) & 6115(3) & 2183(7) & $38.0(18)$ \\
\hline C23_3 & $1513(2)$ & $6153(3)$ & $8156(7)$ & $44.8(18)$ \\
\hline C24_3 & $1639(3)$ & $5746(3)$ & 8241(8) & $55(2)$ \\
\hline C25_3 & $1886(4)$ & $5598(3)$ & $9086(9)$ & $62(3)$ \\
\hline C26_3 & $2045(3)$ & $5885(3)$ & 9898(8) & $62(3)$ \\
\hline C27_3 & $1935(4)$ & $6292(3)$ & $9775(9)$ & $66(3)$ \\
\hline C28_3 & $1665(3)$ & $6428(3)$ & $8960(9)$ & $60(3)$ \\
\hline C29_3 & $2325(3)$ & $5718(3)$ & 10861(8) & $80(3)$ \\
\hline C30_3 & $2340(4)$ & $5240(3)$ & $10950(10)$ & $78(3)$ \\
\hline C31_3 & $2756(3)$ & $5874(4)$ & 10673(11) & $77(4)$ \\
\hline C32_3 & 2158(4) & $5901(4)$ & 11950(9) & $83(4)$ \\
\hline Cl1_4 & $1324.9(8)$ & $9142.5(9)$ & 1043(2) & $51.5(7)$ \\
\hline 01_4 & $310(2)$ & $9449.6(16)$ & $1029(4)$ & $33.9(14)$ \\
\hline 02_4 & $155.4(18)$ & $9380.6(18)$ & $-788(4)$ & $34.6(14)$ \\
\hline
\end{tabular}




\begin{tabular}{lrrrl}
\hline Atom & $\mathbf{x}$ & $\mathbf{y}$ & $\mathbf{z}$ & \multicolumn{1}{c}{$\boldsymbol{U}$ eq } \\
\hline C1_4 & $470(2)$ & $8817(2)$ & $180(6)$ & $37.8(13)$ \\
C2_4 & $694(2)$ & $8612(2)$ & $-831(6)$ & $39.2(14)$ \\
C3_4 & $259(2)$ & $8494(2)$ & $-529(7)$ & $40.6(18)$ \\
C4_4 & $294(2)$ & $9253(2)$ & $120(6)$ & $31.9(16)$ \\
C5_4 & $621(2)$ & $8698(2)$ & $1306(6)$ & $38.0(17)$ \\
C6_4 & $1003(2)$ & $8815(3)$ & $1785(6)$ & $36.5(17)$ \\
C7_4 & $1128(3)$ & $8702(3)$ & $2814(7)$ & $44(2)$ \\
C8_4 & $849(2)$ & $8474(3)$ & $3496(7)$ & $38.1(18)$ \\
C9_4 & $447(2)$ & $8372(3)$ & $3132(6)$ & $43(2)$ \\
C10_4 & $345(2)$ & $8476(3)$ & $2039(6)$ & $37.4(18)$ \\
C11_4 & $1045(2)$ & $8313(2)$ & $-628(8)$ & $45(2)$ \\
C12_4 & $1456(2)$ & $8413(3)$ & $-1002(11)$ & $56(3)$ \\
C13_4 & $1791(3)$ & $8167(3)$ & $-744(11)$ & $62(3)$ \\
C14_4 & $1741(3)$ & $7791(4)$ & $-190(13)$ & $75(4)$ \\
C15_4 & $1355(3)$ & $7688(3)$ & $136(12)$ & $62(3)$ \\
C16_4 & $1013(3)$ & $7946(3)$ & $-49(8)$ & $44(2)$ \\
C1744 & $749(2)$ & $8870(2)$ & $-1905(6)$ & $42.8(18)$ \\
C18_4 & $592(3)$ & $8716(3)$ & $-2891(6)$ & $41.4(19)$ \\
C19_4 & $611(3)$ & $8934(3)$ & $-3877(7)$ & $55(2)$ \\
C20_4 & $803(4)$ & $9322(3)$ & $-3897(8)$ & $61(3)$ \\
C21_4 & $981(4)$ & $9473(3)$ & $-2968(8)$ & $60(3)$ \\
C22_4 & $952(3)$ & $9241(3)$ & $-1950(7)$ & $49(2)$ \\
C23_4 & $131(2)$ & $8191(3)$ & $3895(7)$ & $45(2)$ \\
C24_4 & $-279(3)$ & $8306(3)$ & $3848(9)$ & $51(2)$ \\
C25_4 & $-577(3)$ & $8163(4)$ & $4571(11)$ & $64(3)$ \\
C26_4 & $-456(3)$ & $7883(3)$ & $5446(8)$ & $51(2)$ \\
C27_4 & $-48(3)$ & $7771(4)$ & $5486(9)$ & $59(3)$ \\
C28_4 & $243(3)$ & $7910(4)$ & $4728(9)$ & $57(3)$ \\
C29_4 & $-793(3)$ & $7718(3)$ & $6270(8)$ & $59(2)$ \\
C3044 & $-1200(3)$ & $7973(3)$ & $6267(11)$ & $64(3)$ \\
C31_4 & $-890(3)$ & $7280(3)$ & $5960(10)$ & $57(3)$ \\
C32_4 & $-603(3)$ & $7726(3)$ & $7439(8)$ & $60(3)$ \\
Rh1_7 & 0 & 10000 & $1150.9(14)$ & $28.7(3)$ \\
Rh2_7 & 0 & 10000 & $-823.4(14)$ & $31.1(4)$ \\
Rh1_8 & 0 & 5000 & $5439.7(9)$ & $27.1(2)$ \\
Rh2_8 & 0 & 5000 & 3455.2 & $28.3(2)$ \\
Rh1_10 & 5000 & 5000 & $655.4(13)$ & $21.2(3)$ \\
Rh2_10 & 5000 & 5000 & $-1320.6(14)$ & $26.7(3)$ \\
& & & &
\end{tabular}

Table 10: Anisotropic Displacement Parameters $\left(\times 10^{4}\right)$ for $\mathbf{R h}-\mathbf{2 C l 5 t B u P h}$. The anisotropic displacement factor exponent takes the form: $-2 \pi^{2}\left[h^{2} a^{* 2} \times U_{11}+\ldots+2 h k a^{*} \times b^{*} \times U_{12}\right]$

\begin{tabular}{llllrrr}
\hline Atom & \multicolumn{1}{c}{$\boldsymbol{U}_{\mathbf{1 1}}$} & \multicolumn{1}{c}{$\boldsymbol{U}_{\mathbf{2 2}}$} & \multicolumn{1}{c}{$\boldsymbol{U}_{\mathbf{3 3}}$} & $\boldsymbol{U}_{\mathbf{2 3}}$ & $\boldsymbol{U}_{\mathbf{1 3}}$ & $\boldsymbol{U}_{\mathbf{1 2}}$ \\
\hline Cl1 & $59.5(17)$ & $44.7(14)$ & $48.3(16)$ & $9.5(11)$ & $-4.4(12)$ & $-21.5(12)$ \\
01 & $45(4)$ & $33(3)$ & $22(4)$ & $-3(3)$ & $-3(3)$ & $1(3)$ \\
O2 & $42(4)$ & $43(4)$ & $23(4)$ & $-9(3)$ & $-2(3)$ & $-1(3)$ \\
C1 & $51(6)$ & $27(5)$ & $37(6)$ & $-4(4)$ & $-13(5)$ & $-2(4)$ \\
C2 & $45(6)$ & $40(6)$ & $27(6)$ & $3(4)$ & $0(4)$ & $1(4)$ \\
C3 & $61(7)$ & $29(5)$ & $46(7)$ & $5(4)$ & $-7(5)$ & $-2(5)$ \\
C4 & $31(5)$ & $28(4)$ & $32(6)$ & $-1(4)$ & $4(4)$ & $-17(3)$ \\
C5 & $41(5)$ & $24(5)$ & $49(7)$ & $5(4)$ & $-17(5)$ & $-9(4)$ \\
C6 & $51(6)$ & $34(5)$ & $43(7)$ & $-3(4)$ & $-1(5)$ & $-3(5)$ \\
C7 & $45(6)$ & $45(6)$ & $58(8)$ & $3(5)$ & $-15(5)$ & $-16(5)$ \\
C8 & $50(7)$ & $62(8)$ & $47(8)$ & $5(5)$ & $-24(5)$ & $-15(6)$ \\
C9 & $37(6)$ & $27(5)$ & $63(8)$ & $3(4)$ & $-7(5)$ & $-7(4)$ \\
& & & & 5357 & &
\end{tabular}




\begin{tabular}{|c|c|c|c|c|c|c|}
\hline Atom & $U_{11}$ & $U_{22}$ & $U_{33}$ & $U_{23}$ & $U_{13}$ & $U_{12}$ \\
\hline C10 & $35(5)$ & $19(4)$ & $46(6)$ & $3(4)$ & $-9(4)$ & $-9(4)$ \\
\hline C11 & $37(6)$ & $47(7)$ & $73(9)$ & $-16(6)$ & $-8(5)$ & $3(5)$ \\
\hline C12 & $49(7)$ & $41(6)$ & $78(9)$ & $16(6)$ & $8(6)$ & $2(5)$ \\
\hline C13 & $29(6)$ & $81(10)$ & $91(11)$ & $-6(8)$ & $0(6)$ & $23(6)$ \\
\hline C14 & $27(7)$ & 102(13) & $160(20)$ & $-3(12)$ & $4(9)$ & $28(8)$ \\
\hline C15 & $50(7)$ & 66(8) & 102(13) & $17(8)$ & $16(8)$ & $11(6)$ \\
\hline C16 & $34(6)$ & $62(7)$ & 64(9) & $9(6)$ & $-12(5)$ & $-4(5)$ \\
\hline C17 & $29(5)$ & $46(6)$ & $31(6)$ & $14(4)$ & $9(4)$ & $6(4)$ \\
\hline C18 & $58(7)$ & $44(6)$ & $34(6)$ & $-12(5)$ & $-3(5)$ & $4(5)$ \\
\hline C19 & $50(7)$ & $108(11)$ & $39(8)$ & $-16(7)$ & $4(5)$ & $-17(7)$ \\
\hline $\mathrm{C} 20$ & 103(11) & $48(7)$ & $60(10)$ & $21(6)$ & $24(8)$ & $5(7)$ \\
\hline C21 & $100(11)$ & $27(6)$ & 75(10) & $9(6)$ & $47(8)$ & $-10(6)$ \\
\hline $\mathrm{C} 22$ & $47(6)$ & $34(5)$ & $36(6)$ & $-5(4)$ & $6(4)$ & $1(4)$ \\
\hline $\mathrm{C} 23$ & $41(6)$ & $24(5)$ & $50(7)$ & $-2(4)$ & $-4(4)$ & $8(4)$ \\
\hline $\mathrm{C} 24$ & $27(5)$ & $32(5)$ & $51(7)$ & $0(4)$ & $-7(4)$ & $4(4)$ \\
\hline C25 & $44(6)$ & $60(7)$ & $36(7)$ & $-4(5)$ & $1(4)$ & $-4(5)$ \\
\hline C26 & $58(7)$ & $11(4)$ & $56(8)$ & $-7(4)$ & $-6(5)$ & $-1(4)$ \\
\hline C27 & $56(7)$ & $30(5)$ & $34(6)$ & $9(4)$ & $-9(4)$ & $9(5)$ \\
\hline $\mathrm{C} 28$ & $46(6)$ & $31(5)$ & $57(7)$ & $-4(4)$ & $-14(5)$ & $8(5)$ \\
\hline $\mathrm{C} 29$ & $78(8)$ & $23(5)$ & $38(7)$ & $0(4)$ & $10(5)$ & $-7(5)$ \\
\hline C30 & $102(11)$ & $26(5)$ & $58(8)$ & $8(5)$ & $38(7)$ & $6(6)$ \\
\hline C31 & $38(6)$ & $56(7)$ & $72(9)$ & $9(6)$ & $9(5)$ & $-8(5)$ \\
\hline C32 & $78(9)$ & $48(7)$ & $52(8)$ & $-5(5)$ & $12(6)$ & $-3(6)$ \\
\hline 01W_5 & $47(3)$ & $47(3)$ & $9(4)$ & 0 & 0 & 0 \\
\hline 01W_6 & $23(4)$ & $23(4)$ & $54(9)$ & 0 & 0 & 0 \\
\hline 01W_9 & $18(4)$ & $45(5)$ & $13(4)$ & 0 & 0 & $0(3)$ \\
\hline 01W_11 & $9(3)$ & $9(3)$ & $23(6)$ & 0 & 0 & 0 \\
\hline 01W_12 & $47(3)$ & $47(3)$ & $9(4)$ & 0 & 0 & 0 \\
\hline 01W_13 & $47(3)$ & $47(3)$ & $9(4)$ & 0 & 0 & 0 \\
\hline Cl1_2 & $58.0(17)$ & $57.8(17)$ & $87(2)$ & $10.9(16)$ & $25.1(17)$ & $0.2(13)$ \\
\hline 01_2 & $36(3)$ & $44(4)$ & $37(3)$ & $-6(2)$ & $2(2)$ & $1(3)$ \\
\hline 02_2 & $54(4)$ & $44(4)$ & $43(3)$ & $-4(2)$ & $1(2)$ & $6(3)$ \\
\hline C1_2 & $47(3)$ & $69(4)$ & $54(3)$ & $4(2)$ & $-4.8(19)$ & $-2(3)$ \\
\hline C2_2 & $51(3)$ & $74(4)$ & $58(3)$ & $1(3)$ & $-8(2)$ & $-1(3)$ \\
\hline C3_2 & $53(5)$ & $74(3)$ & $56(5)$ & $3(3)$ & $-10(4)$ & $1(3)$ \\
\hline C4_2 & $46(3)$ & $47(5)$ & $40(3)$ & $-6(2)$ & $-3.6(18)$ & $2(3)$ \\
\hline C5_2 & $49(5)$ & $61(3)$ & $58(3)$ & $6(2)$ & $0(3)$ & $7(3)$ \\
\hline C6_2 & $26(4)$ & $62(3)$ & $69(4)$ & $11(3)$ & $2(3)$ & $3(3)$ \\
\hline C7_2 & $47(6)$ & $67(4)$ & $71(4)$ & $12(3)$ & $8(3)$ & $3(4)$ \\
\hline C8_2 & $34(5)$ & $68(3)$ & $64(5)$ & $10(3)$ & $-2(4)$ & $3(3)$ \\
\hline C9_2 & $25(4)$ & $67(3)$ & $55(3)$ & $7(2)$ & $-5(3)$ & $1(3)$ \\
\hline C10_2 & $15(4)$ & $59(4)$ & $54(3)$ & $5(2)$ & $-9(2)$ & $-2(3)$ \\
\hline C11_2 & $58(4)$ & $83(5)$ & $83(8)$ & $1(5)$ & $-4(4)$ & $-8(4)$ \\
\hline C12_2 & $90(4)$ & $93(5)$ & 111(11) & $-16(6)$ & $14(6)$ & $-18(5)$ \\
\hline C13_2 & $76(3)$ & $76(3)$ & 77(3) & $-0.5(14)$ & $-0.3(14)$ & $-2.1(14)$ \\
\hline C14_2 & $76(6)$ & $117(8)$ & $85(6)$ & $-26(6)$ & $3(5)$ & $-24(5)$ \\
\hline C15_2 & $84(8)$ & 127(7) & $119(13)$ & $-45(7)$ & $28(8)$ & $-38(6)$ \\
\hline C16_2 & $59(5)$ & 113(8) & 97(9) & $-22(7)$ & $2(6)$ & $-19(5)$ \\
\hline C17_2 & $52(4)$ & $62(6)$ & $57(3)$ & $0(3)$ & $-8(3)$ & $-6(4)$ \\
\hline C18_2 & $88(8)$ & $65(7)$ & $55(4)$ & $-6(4)$ & $-10(3)$ & $16(6)$ \\
\hline C19_2 & 104(8) & $115(10)$ & $57(4)$ & $-1(5)$ & $-5(5)$ & $38(7)$ \\
\hline C20_2 & 101(9) & $109(10)$ & $76(7)$ & $7(6)$ & $5(6)$ & $33(7)$ \\
\hline C21_2 & 102(9) & 97(11) & $74(6)$ & $2(5)$ & $4(5)$ & $32(8)$ \\
\hline C22_2 & $62(5)$ & $76(6)$ & $66(6)$ & $-8(5)$ & $-14(4)$ & $6(5)$ \\
\hline C23_2 & $34(4)$ & $74(3)$ & $56(4)$ & $4(3)$ & $-11(3)$ & $9(3)$ \\
\hline C24_2 & $58(6)$ & $79(4)$ & $54(6)$ & $0(3)$ & $-2(4)$ & $-2(3)$ \\
\hline C25_2 & $42(4)$ & $84(5)$ & $56(5)$ & $-4(4)$ & $-9(3)$ & $-1(4)$ \\
\hline \multirow[t]{2}{*}{ C26_2 } & $50(5)$ & $82(4)$ & $64(5)$ & $-6(3)$ & $-1(4)$ & $-3(3)$ \\
\hline & & & & S358 & & \\
\hline
\end{tabular}




\begin{tabular}{|c|c|c|c|c|c|c|}
\hline Atom & $U_{11}$ & $U_{22}$ & $U_{33}$ & $U_{23}$ & $U_{13}$ & $U_{12}$ \\
\hline$\overline{\text { C27_2 }}$ & $33(5)$ & $77(4)$ & $48(5)$ & $2(3)$ & $-14(3)$ & $6(3)$ \\
\hline C28_2 & $43(4)$ & $82(5)$ & $60(5)$ & $-6(4)$ & $-4(3)$ & $-1(4)$ \\
\hline C29_2 & $61(5)$ & $92(5)$ & $76(5)$ & $-16(4)$ & $2(4)$ & $-1(4)$ \\
\hline C30_2 & $59(6)$ & $91(5)$ & $82(11)$ & $-17(5)$ & $1(6)$ & $1(5)$ \\
\hline C31_2 & $61(5)$ & $95(10)$ & $90(11)$ & $-1(8)$ & $5(4)$ & $-2(4)$ \\
\hline C32_2 & $69(9)$ & $96(9)$ & $76(5)$ & $-15(5)$ & $0(5)$ & $-5(7)$ \\
\hline $\mathrm{Cl} 1 \_3$ & $41.4(13)$ & $49.9(15)$ & $34.4(14)$ & $-3.9(10)$ & $-4.1(9)$ & $13.5(11)$ \\
\hline 01_3 & $36(3)$ & $35(3)$ & $27(2)$ & $1.2(19)$ & $4(2)$ & $-3(2)$ \\
\hline 02_3 & $37(3)$ & $38(3)$ & $28(2)$ & $2(2)$ & $6(2)$ & $0(2)$ \\
\hline C1_3 & $34(2)$ & $34(2)$ & $33(2)$ & $6.6(16)$ & $1.8(17)$ & $2.9(17)$ \\
\hline C2_3 & $37(2)$ & $33(2)$ & $31(2)$ & $4.3(18)$ & $3.4(17)$ & $4.0(17)$ \\
\hline C3_3 & $35(3)$ & $33(3)$ & $35(3)$ & $6(2)$ & $3(2)$ & $2(2)$ \\
\hline C4_3 & $31(3)$ & $32(3)$ & $27(2)$ & 5.3(17) & $2.5(18)$ & $6(2)$ \\
\hline C5_3 & $46(3)$ & $37(3)$ & $34(2)$ & $5(2)$ & $4(2)$ & $-2(3)$ \\
\hline C6_3 & $45(3)$ & $35(4)$ & $36(3)$ & $5(2)$ & $4(2)$ & $-3(2)$ \\
\hline C7_3 & $44(4)$ & $52(5)$ & $41(3)$ & $-5(3)$ & $3(3)$ & $-2(3)$ \\
\hline C8_3 & $45(4)$ & $58(5)$ & $42(4)$ & $-3(3)$ & $2(3)$ & $-1(3)$ \\
\hline C9_3 & $42(3)$ & $55(4)$ & $41(3)$ & $-2(3)$ & $2(2)$ & $-4(3)$ \\
\hline C10_3 & $55(4)$ & $51(5)$ & $40(3)$ & $0(3)$ & $-3(3)$ & $7(4)$ \\
\hline C11_3 & $34(3)$ & $32(2)$ & $36(5)$ & $3(2)$ & $7(3)$ & $4.8(17)$ \\
\hline C12_3 & $40(3)$ & $32(2)$ & $56(6)$ & $2(3)$ & $-5(4)$ & $5(2)$ \\
\hline C13_3 & $48(4)$ & $32(3)$ & $64(7)$ & $2(3)$ & $-4(4)$ & $2(3)$ \\
\hline C14_3 & $49(4)$ & $33(4)$ & $67(7)$ & $3(4)$ & $-5(4)$ & $2(3)$ \\
\hline C15_3 & $48(4)$ & $36(3)$ & $99(9)$ & $14(4)$ & $-12(5)$ & $-2(2)$ \\
\hline C16_3 & $44(3)$ & $36(3)$ & $110(10)$ & $17(4)$ & $-21(4)$ & $0(2)$ \\
\hline C17_3 & $45(3)$ & $34(4)$ & $32(2)$ & $8(2)$ & $0.7(19)$ & $-3(2)$ \\
\hline C18_3 & $46(3)$ & $39(5)$ & $32(3)$ & $8(2)$ & $1(2)$ & $-2(3)$ \\
\hline C19_3 & $47(3)$ & $49(6)$ & $33(3)$ & $4(3)$ & $0(2)$ & $2(3)$ \\
\hline C20_3 & $47(3)$ & $55(6)$ & $39(4)$ & $2(4)$ & $0(2)$ & $2(3)$ \\
\hline C21_3 & $44(3)$ & $53(6)$ & $38(3)$ & $3(3)$ & $-1(2)$ & $3(4)$ \\
\hline C22_3 & $45(3)$ & $33(5)$ & $36(4)$ & $10(3)$ & $0(2)$ & $-4(2)$ \\
\hline C23_3 & $32(3)$ & $69(4)$ & $33(3)$ & $4(3)$ & $13(2)$ & $-2(3)$ \\
\hline C24_3 & $40(5)$ & $70(4)$ & $54(5)$ & $3(3)$ & $4(4)$ & 1(3) \\
\hline C25_3 & $51(6)$ & $76(5)$ & $60(5)$ & $4(3)$ & $-3(4)$ & $3(4)$ \\
\hline C26_3 & $47(5)$ & $82(4)$ & $57(5)$ & $2(3)$ & $4(4)$ & $-4(4)$ \\
\hline C27_3 & $65(6)$ & $82(5)$ & $51(6)$ & $-2(3)$ & $-6(4)$ & $-1(4)$ \\
\hline C28_3 & $58(6)$ & $76(5)$ & $45(4)$ & $-2(3)$ & $0(4)$ & $-2(4)$ \\
\hline C29_3 & $56(4)$ & $121(6)$ & $64(5)$ & $13(4)$ & $2(3)$ & $7(4)$ \\
\hline C30_3 & $52(8)$ & 121(6) & $60(9)$ & $15(4)$ & $-5(6)$ & $8(4)$ \\
\hline C31_3 & $53(4)$ & 118(10) & 61(9) & $30(8)$ & $-4(4)$ & $12(5)$ \\
\hline C32_3 & $42(6)$ & $144(11)$ & $63(5)$ & $6(6)$ & $-7(4)$ & $16(7)$ \\
\hline Cl1_4 & $48.5(15)$ & $58.8(16)$ & $47.2(16)$ & $4.3(11)$ & $4.2(11)$ & $-12.4(12)$ \\
\hline 01_4 & $57(4)$ & $27(3)$ & $19(2)$ & $7.5(17)$ & $1(2)$ & 1(3) \\
\hline $02 \_4$ & $30(3)$ & $50(4)$ & $24(2)$ & $4(2)$ & $3.2(19)$ & $-2(3)$ \\
\hline C1_4 & $35(3)$ & $41(2)$ & $38(2)$ & $0.2(16)$ & $3.1(18)$ & $0.4(19)$ \\
\hline C2__4 & $35(2)$ & $44(3)$ & $39(2)$ & $-1.0(19)$ & $3.0(19)$ & $1.0(19)$ \\
\hline C3_4 & $35(2)$ & $44(3)$ & $42(4)$ & $-4(3)$ & $4(2)$ & $0(2)$ \\
\hline C4_4 & $32(4)$ & $40(2)$ & $23(2)$ & $-0.4(15)$ & 2.7(19) & $0(2)$ \\
\hline C5_4 & $42(3)$ & $32(4)$ & $40(2)$ & $0(2)$ & $-1(2)$ & $-4(2)$ \\
\hline C6_4 & $38(3)$ & $35(3)$ & $36(3)$ & $-9(2)$ & $4.0(19)$ & $-1(3)$ \\
\hline C7__4 & $46(3)$ & $47(5)$ & $38(3)$ & $-4(3)$ & $0(2)$ & $-5(3)$ \\
\hline C8_4 & $41(3)$ & $36(4)$ & $38(4)$ & $-6(3)$ & $1(2)$ & $2(3)$ \\
\hline C9_4 & $43(3)$ & $49(5)$ & $38(3)$ & $2(3)$ & $-1(2)$ & $-4(3)$ \\
\hline C10_4 & $43(3)$ & $32(4)$ & $37(3)$ & $-1(2)$ & $-2(2)$ & $-5(3)$ \\
\hline C11_4 & $42(3)$ & $54(3)$ & $40(5)$ & $1(3)$ & $4(3)$ & $10(2)$ \\
\hline C12_4 & $41(3)$ & $68(6)$ & $60(7)$ & $24(5)$ & $5(3)$ & $14(3)$ \\
\hline C13_4 & $45(3)$ & $72(5)$ & $69(8)$ & $19(5)$ & $-1(4)$ & $18(4)$ \\
\hline \multirow[t]{2}{*}{ C14_4 } & $50(4)$ & $78(6)$ & $98(11)$ & $33(6)$ & $18(5)$ & $28(4)$ \\
\hline & & & & S359 & & \\
\hline
\end{tabular}




\begin{tabular}{llllrrr}
\hline Atom & $\boldsymbol{U}_{\mathbf{1 1}}$ & $\boldsymbol{U}_{\boldsymbol{2 2}}$ & $\boldsymbol{U}_{\boldsymbol{3 3}}$ & $\boldsymbol{U}_{\boldsymbol{2 3}}$ & $\boldsymbol{U}_{\mathbf{1 3}}$ & $\boldsymbol{U}_{\mathbf{1 2}}$ \\
\hline C15_4 & $50(4)$ & $71(5)$ & $64(8)$ & $12(5)$ & $14(3)$ & $24(3)$ \\
C16_4 & $42(4)$ & $53(3)$ & $37(5)$ & $-1(3)$ & $9(3)$ & $12(3)$ \\
C17_4 & $41(4)$ & $46(3)$ & $41(3)$ & $1(2)$ & $8(2)$ & $6(3)$ \\
C18_4 & $36(4)$ & $47(4)$ & $41(3)$ & $-1(2)$ & $8(3)$ & $10(3)$ \\
C19_4 & $61(6)$ & $58(4)$ & $45(3)$ & $6(3)$ & $11(3)$ & $11(4)$ \\
C20_4 & $70(6)$ & $60(4)$ & $51(5)$ & $8(3)$ & $9(4)$ & $6(4)$ \\
C21_4 & $67(7)$ & $62(5)$ & $50(4)$ & $8(3)$ & $13(3)$ & $2(4)$ \\
C22_4 & $52(5)$ & $49(3)$ & $46(5)$ & $0(3)$ & $11(3)$ & $1(3)$ \\
C23_4 & $46(3)$ & $51(5)$ & $38(4)$ & $1(3)$ & $0(2)$ & $-6(3)$ \\
C24_4 & $46(3)$ & $54(6)$ & $54(6)$ & $11(4)$ & $2(3)$ & $-6(3)$ \\
C25_4 & $52(3)$ & $69(6)$ & $72(5)$ & $25(5)$ & $11(4)$ & $-1(4)$ \\
C26_4 & $63(3)$ & $39(4)$ & $51(5)$ & $0(3)$ & $9(3)$ & $0(3)$ \\
C27_4 & $65(3)$ & $68(7)$ & $45(6)$ & $8(4)$ & $12(3)$ & $8(3)$ \\
C28_4 & $61(4)$ & $67(6)$ & $43(4)$ & $10(4)$ & $9(3)$ & $9(4)$ \\
C29_4 & $70(4)$ & $42(4)$ & $65(4)$ & $7(3)$ & $19(3)$ & $6(3)$ \\
C30_4 & $69(4)$ & $42(5)$ & $82(9)$ & $20(6)$ & $26(5)$ & $5(4)$ \\
C31_4 & $64(7)$ & $43(4)$ & $65(7)$ & $5(4)$ & $24(5)$ & $6(3)$ \\
C32_4 & $73(6)$ & $41(6)$ & $65(4)$ & $11(4)$ & $18(4)$ & $3(5)$ \\
Rh1_7 & $32.9(5)$ & $32.9(5)$ & $20.1(8)$ & 0 & 0 & 0 \\
Rh2_7 & $37.3(5)$ & $37.3(5)$ & $18.6(9)$ & 0 & 0 & 0 \\
Rh1_8 & $30.9(5)$ & $34.3(5)$ & $16.1(5)$ & 0 & 0 & $-1.4(4)$ \\
Rh2_8 & $34.8(5)$ & $35.5(5)$ & $14.7(5)$ & 0 & 0 & $0.0(4)$ \\
Rh1_10 & $21.6(4)$ & $21.6(4)$ & $20.6(7)$ & 0 & 0 & 0 \\
Rh2_10 & $30.5(5)$ & $30.5(5)$ & $19.1(8)$ & 0 & 0 & 0 \\
& & & & & &
\end{tabular}

Table 11: Bond Lengths in $\AA$ for Rh-2Cl5tBuPh.

\begin{tabular}{lll}
\hline Atom & Atom & Length/Å \\
\hline Cl1 & C6 & $1.726(11)$ \\
O1 & C4 & $1.282(12)$ \\
O1 & Rh1_8 & $2.054(6)$ \\
O2 & C4 & $1.215(12)$ \\
O2 & Rh2_8 & $2.036(7)$ \\
C1 & C2 & $1.564(16)$ \\
C1 & C3 & $1.526(14)$ \\
C1 & C4 & $1.543(14)$ \\
C1 & C5 & $1.488(15)$ \\
C2 & C3 & $1.492(15)$ \\
C2 & C11 & $1.535(15)$ \\
C2 & C17 & $1.545(14)$ \\
C5 & C6 & $1.372(15)$ \\
C5 & C10 & $1.447(14)$ \\
C6 & C7 & $1.377(17)$ \\
C7 & C8 & $1.371(17)$ \\
C8 & C9 & $1.398(15)$ \\
C9 & C10 & $1.372(16)$ \\
C9 & C23 & $1.468(15)$ \\
C11 & C12 & $1.319(18)$ \\
C11 & C16 & $1.419(18)$ \\
C12 & C13 & $1.395(17)$ \\
C13 & C14 & $1.31(2)$ \\
C14 & C15 & $1.44(2)$ \\
C15 & C16 & $1.363(17)$ \\
C17 & C18 & $1.387(15)$ \\
C17 & C22 & $1.360(15)$ \\
& &
\end{tabular}

\begin{tabular}{lll}
\hline Atom & Atom & Length/Å \\
\hline C18 & C19 & $1.321(19)$ \\
C19 & C20 & $1.446(19)$ \\
C20 & C21 & $1.33(2)$ \\
C21 & C22 & $1.403(18)$ \\
C23 & C24 & $1.382(14)$ \\
C23 & C28 & $1.378(15)$ \\
C24 & C25 & $1.354(15)$ \\
C25 & C26 & $1.471(16)$ \\
C26 & C27 & $1.380(16)$ \\
C26 & C29 & $1.528(15)$ \\
C27 & C28 & $1.417(16)$ \\
C29 & C30 & $1.510(19)$ \\
C29 & C31 & $1.447(16)$ \\
C29 & C32 & $1.553(17)$ \\
01W_5 & Rh2_7 & $2.510(8)$ \\
01W_6 & Rh1_7 & $2.188(9)$ \\
01W_9 & Rh1_8 & $2.186(8)$ \\
01W_11 & Rh1_10 & $2.178(8)$ \\
01W_12 & Rh2_10 & $2.515(8)$ \\
01W_13 & Rh2_8 & $2.520(8)$ \\
Cl1_2 & C6_2 & $1.717(7)$ \\
01_2 & C4_2 & $1.260(7)$ \\
01_2 & Rh1_10 & $2.049(6)$ \\
O2_2 & C4_2 & $1.242(8)$ \\
O2_2 & Rh2_10 & $2.043(6)$ \\
C1_2 & C2_2 & $1.552(9)$ \\
C1_2 & C3_2 & $1.499(9)$ \\
& &
\end{tabular}




\begin{tabular}{|c|c|c|}
\hline Atom & Atom & Length/Å \\
\hline$\overline{\mathrm{C} 1 \_2}$ & C4_2 & $1.513(8)$ \\
\hline C1_2 & $\mathrm{C} 5 \_2$ & $1.484(9)$ \\
\hline $\mathrm{C} 2 \_2$ & C3_2 & $1.485(9)$ \\
\hline C2_2 & C11_2 & $1.497(9)$ \\
\hline $\mathrm{C} 2 \_2$ & C17_2 & $1.541(9)$ \\
\hline C5_2 & C6_2 & $1.404(9)$ \\
\hline C5_2 & C10_2 & $1.438(9)$ \\
\hline C6_2 & C7_2 & $1.345(10)$ \\
\hline C7_2 & C8_2 & $1.418(10)$ \\
\hline C8_2 & C9_2 & $1.396(9)$ \\
\hline C9_2 & C10_2 & $1.392(9)$ \\
\hline C9_2 & C23_2 & $1.485(9)$ \\
\hline C11_2 & C12_2 & $1.428(10)$ \\
\hline C11_2 & C16_2 & $1.370(10)$ \\
\hline C12_2 & C13_2 & $1.368(10)$ \\
\hline C13_2 & C14_2 & $1.387(11)$ \\
\hline C14_2 & C15_2 & $1.339(11)$ \\
\hline C15_2 & C16_2 & $1.391(10)$ \\
\hline C17_2 & C18_2 & $1.375(10)$ \\
\hline C17_2 & C22_2 & $1.356(10)$ \\
\hline C18_2 & C19_2 & $1.375(10)$ \\
\hline C19_2 & C20_2 & $1.386(11)$ \\
\hline C20_2 & C21_2 & $1.342(11)$ \\
\hline C21_2 & C22_2 & $1.434(10)$ \\
\hline C23_2 & C24_2 & $1.367(10)$ \\
\hline C23_2 & C28_2 & $1.392(10)$ \\
\hline C24_2 & C25_2 & $1.371(11)$ \\
\hline C25_2 & C26_2 & $1.432(11)$ \\
\hline C26_2 & C27_2 & $1.358(11)$ \\
\hline C26_2 & C29_2 & $1.557(11)$ \\
\hline C27_2 & C28_2 & $1.376(11)$ \\
\hline C29_2 & C30_2 & $1.538(12)$ \\
\hline C29_2 & C31_2 & $1.486(11)$ \\
\hline C29_2 & C32_2 & $1.528(12)$ \\
\hline Cl1_3 & C6_3 & $1.720(7)$ \\
\hline 01_3 & C4_3 & $1.258(7)$ \\
\hline 01_3 & Rh1_8 & $2.071(5)$ \\
\hline 02_3 & C4_3 & $1.245(8)$ \\
\hline $02 \_3$ & Rh2_8 & $2.023(5)$ \\
\hline C1_3 & C2_3 & $1.552(9)$ \\
\hline C1_3 & C3_3 & $1.500(9)$ \\
\hline C1_3 & C4_3 & $1.514(8)$ \\
\hline C1_3 & C5_3 & $1.482(9)$ \\
\hline C2_3 & C3_3 & $1.488(9)$ \\
\hline C2_3 & C11_3 & $1.498(9)$ \\
\hline C2_3 & C17_3 & $1.540(9)$ \\
\hline C5_3 & C6_3 & $1.405(9)$ \\
\hline C5_3 & C10_3 & $1.439(9)$ \\
\hline C6_3 & C7_3 & $1.346(10)$ \\
\hline C7_3 & C8_3 & $1.416(10)$ \\
\hline C8_3 & C9_3 & $1.397(9)$ \\
\hline C9_3 & C10_3 & $1.391(9)$ \\
\hline C9_3 & C23_3 & $1.486(9)$ \\
\hline C11_3 & C12_3 & $1.426(9)$ \\
\hline C11_3 & C16_3 & $1.371(10)$ \\
\hline C12_3 & C13_3 & $1.366(10)$ \\
\hline C13_3 & C14_3 & $1.385(11)$ \\
\hline C14_3 & C15_3 & $1.339(11)$ \\
\hline
\end{tabular}

\begin{tabular}{|c|c|c|}
\hline Atom & Atom & Length/Å \\
\hline$\overline{C 15 \_3}$ & C16_3 & $1.390(10)$ \\
\hline C17_3 & C18_3 & $1.375(9)$ \\
\hline C17_3 & C22_3 & $1.356(9)$ \\
\hline C18_3 & C19_3 & $1.375(10)$ \\
\hline C19_3 & C20_3 & $1.387(10)$ \\
\hline C20_3 & C21_3 & $1.343(11)$ \\
\hline C21_3 & C22_3 & $1.433(10)$ \\
\hline C23_3 & C24_3 & $1.367(10)$ \\
\hline C23_3 & C28_3 & $1.393(10)$ \\
\hline C24_3 & C25_3 & $1.370(11)$ \\
\hline C25_3 & C26_3 & $1.434(11)$ \\
\hline C26_3 & C27_3 & $1.358(11)$ \\
\hline C26_3 & C29_3 & $1.557(11)$ \\
\hline C27_3 & C28_3 & $1.377(11)$ \\
\hline C29_3 & C30_3 & $1.539(12)$ \\
\hline C29_3 & C31_3 & $1.487(11)$ \\
\hline C29_3 & C32_3 & $1.527(12)$ \\
\hline Cl1_4 & C6_4 & $1.719(7)$ \\
\hline 01_4 & C4_4 & $1.260(7)$ \\
\hline 01_4 & Rh1_7 & $2.030(5)$ \\
\hline 02_4 & C4_4 & $1.244(8)$ \\
\hline 02_4 & Rh2_7 & $2.047(6)$ \\
\hline C1_4 & C2_4 & $1.553(9)$ \\
\hline C1_4 & C3_4 & $1.499(9)$ \\
\hline C1_4 & C4_4 & $1.511(8)$ \\
\hline C1_4 & C5_4 & $1.484(9)$ \\
\hline C2_4 & C3_4 & $1.486(9)$ \\
\hline C2_4 & C11_4 & $1.498(9)$ \\
\hline C2_4 & C17_4 & $1.540(9)$ \\
\hline C5_4 & C6_4 & $1.403(9)$ \\
\hline C5_4 & C10_4 & $1.437(9)$ \\
\hline C6_4 & C7_4 & $1.346(10)$ \\
\hline C7_4 & C8_4 & $1.417(10)$ \\
\hline C8_4 & C9_4 & $1.397(9)$ \\
\hline C9_4 & C10_4 & $1.391(9)$ \\
\hline C9_4 & C23_4 & $1.484(9)$ \\
\hline C11_4 & C12_4 & $1.427(10)$ \\
\hline C11_4 & C16_4 & $1.371(10)$ \\
\hline C12_4 & C13_4 & $1.366(10)$ \\
\hline C13_4 & C14_4 & $1.385(11)$ \\
\hline C14_4 & C15_4 & $1.339(11)$ \\
\hline C15_4 & C16_4 & $1.390(10)$ \\
\hline C17_4 & C18_4 & $1.376(9)$ \\
\hline C17_4 & C22_4 & $1.355(9)$ \\
\hline C18_4 & C19_4 & $1.375(10)$ \\
\hline C19_4 & C20_4 & $1.387(10)$ \\
\hline C20_4 & C21_4 & $1.342(11)$ \\
\hline C21_4 & C22_4 & $1.433(10)$ \\
\hline C23_4 & C24_4 & $1.366(10)$ \\
\hline C23_4 & C28_4 & $1.392(10)$ \\
\hline C24_4 & C25_4 & $1.370(11)$ \\
\hline C25_4 & C26_4 & $1.433(11)$ \\
\hline C26_4 & C27_4 & $1.357(11)$ \\
\hline C26_4 & C29_4 & $1.557(11)$ \\
\hline C27_4 & C28_4 & $1.376(11)$ \\
\hline C29_4 & C30_4 & $1.539(12)$ \\
\hline C29_4 & C31_4 & $1.486(11)$ \\
\hline C29_4 & C32_4 & $1.527(12)$ \\
\hline
\end{tabular}




\begin{tabular}{lll}
\hline Atom & Atom & Length/A \\
\hline Rh1_7 & Rh2_7 & $2.3667(17)$ \\
Rh1_8 & Rh2_8 & $2.3790(10)$
\end{tabular}

Table 12: Bond Angles in ${ }^{\circ}$ for Rh-2Cl5tBuPh.

\begin{tabular}{|c|c|c|c|}
\hline Atom & Atom & Atom & Angle ${ }^{\circ}$ \\
\hline$\overline{C 4}$ & 01 & Rh1_8 & $116.5(6)$ \\
\hline $\mathrm{C} 4$ & $\mathrm{O} 2$ & $\mathrm{Rh} 2 \_8$ & $116.6(6)$ \\
\hline C3 & C1 & $\mathrm{C} 2$ & $57.7(7)$ \\
\hline C3 & C1 & C4 & $111.6(8)$ \\
\hline C4 & $\mathrm{C} 1$ & C2 & 119.2(9) \\
\hline C5 & $\mathrm{C} 1$ & C2 & $122.2(9)$ \\
\hline C5 & $\mathrm{C} 1$ & C3 & $121.4(8)$ \\
\hline C5 & C1 & $\mathrm{C} 4$ & $113.2(9)$ \\
\hline C3 & C2 & C1 & $59.9(7)$ \\
\hline C3 & C2 & C11 & $118.9(9)$ \\
\hline C3 & C2 & C17 & 118.7(9) \\
\hline C11 & C2 & C1 & $120.2(9)$ \\
\hline C11 & C2 & C17 & $111.9(9)$ \\
\hline C17 & $\mathrm{C} 2$ & $\mathrm{C} 1$ & 118.4(8) \\
\hline C2 & C3 & C1 & $62.4(7)$ \\
\hline 01 & C4 & C1 & $111.0(8)$ \\
\hline 02 & $\mathrm{C} 4$ & 01 & $127.8(9)$ \\
\hline 02 & C4 & $\mathrm{C} 1$ & 121.1(9) \\
\hline C6 & $\mathrm{C} 5$ & C1 & 124.3(10) \\
\hline $\mathrm{C} 6$ & $\mathrm{C} 5$ & $\mathrm{C} 10$ & $116.4(10)$ \\
\hline C10 & C5 & C1 & $119.2(8)$ \\
\hline C5 & C6 & $\mathrm{Cl} 1$ & $120.2(9)$ \\
\hline C5 & C6 & C7 & $121.3(10)$ \\
\hline C7 & C6 & $\mathrm{Cl} 1$ & 118.5(8) \\
\hline C8 & C7 & C6 & $120.9(10)$ \\
\hline C7 & $\mathrm{C} 8$ & C9 & $121.2(11)$ \\
\hline C8 & C9 & $\mathrm{C} 23$ & $122.0(11)$ \\
\hline C10 & C9 & $\mathrm{C} 8$ & $116.8(10)$ \\
\hline C10 & C9 & $\mathrm{C} 23$ & $121.2(9)$ \\
\hline C9 & C10 & $\mathrm{C} 5$ & $123.0(9)$ \\
\hline C12 & C11 & $\mathrm{C} 2$ & $120.5(12)$ \\
\hline C12 & C11 & C16 & $118.0(11)$ \\
\hline C16 & C11 & $\mathrm{C} 2$ & $121.3(11)$ \\
\hline C11 & C12 & $\mathrm{C} 13$ & $121.8(13)$ \\
\hline C14 & C13 & C12 & $121.2(14)$ \\
\hline C13 & C14 & C15 & $119.9(12)$ \\
\hline C16 & C15 & C14 & $117.3(14)$ \\
\hline C15 & C16 & C11 & $121.7(12)$ \\
\hline C18 & C17 & $\mathrm{C} 2$ & 119.1(9) \\
\hline $\mathrm{C} 22$ & C17 & $\mathrm{C} 2$ & $120.8(10)$ \\
\hline C22 & C17 & C18 & $119.7(9)$ \\
\hline C19 & C18 & C17 & $121.4(11)$ \\
\hline C18 & C19 & $\mathrm{C} 20$ & $120.1(12)$ \\
\hline $\mathrm{C} 21$ & $\mathrm{C} 20$ & C19 & $117.0(12)$ \\
\hline $\mathrm{C} 20$ & $\mathrm{C} 21$ & $\mathrm{C} 22$ & $122.9(11)$ \\
\hline C17 & $\mathrm{C} 22$ & $\mathrm{C} 21$ & $118.3(10)$ \\
\hline $\mathrm{C} 24$ & $\mathrm{C} 23$ & C9 & $120.5(9)$ \\
\hline $\mathrm{C} 28$ & $\mathrm{C} 23$ & C9 & $120.2(10)$ \\
\hline $\mathrm{C} 28$ & $\mathrm{C} 23$ & C24 & $119.1(11)$ \\
\hline C25 & C24 & C23 & $121.8(10)$ \\
\hline
\end{tabular}

\begin{tabular}{lll}
\hline Atom & Atom & Length/Å \\
\hline Rh1_10 & Rh2_10 & $2.3687(17)$
\end{tabular}

\begin{tabular}{|c|c|c|c|}
\hline Atom & Atom & Atom & Angle $/^{\circ}$ \\
\hline$\overline{\mathrm{C} 24}$ & $\mathrm{C} 25$ & $\mathrm{C} 26$ & $121.2(10)$ \\
\hline $\mathrm{C} 25$ & C26 & C29 & $123.4(11)$ \\
\hline $\mathrm{C} 27$ & C26 & $\mathrm{C} 25$ & $115.5(10)$ \\
\hline $\mathrm{C} 27$ & C26 & C29 & $121.1(10)$ \\
\hline C26 & C27 & C28 & $121.9(9)$ \\
\hline $\mathrm{C} 23$ & C28 & C27 & $120.4(11)$ \\
\hline C26 & C29 & C32 & $106.7(9)$ \\
\hline C30 & C29 & C26 & $112.2(10)$ \\
\hline C30 & C29 & C32 & $105.6(10)$ \\
\hline C31 & C29 & C26 & $110.7(9)$ \\
\hline C31 & C29 & C30 & $108.8(9)$ \\
\hline C31 & C29 & C32 & $112.8(11)$ \\
\hline C4_2 & 01_2 & Rh1_10 & $117.5(5)$ \\
\hline C4_2 & $02 \_2$ & $\mathrm{Rh} 2 \_10$ & $115.5(5)$ \\
\hline C3_2 & C1_2 & C2_2 & $58.2(4)$ \\
\hline C3_2 & C1_2 & C4_2 & $116.2(6)$ \\
\hline C4_2 & C1_2 & $\mathrm{C} 2 \_2$ & $121.3(6)$ \\
\hline C5_2 & C1_2 & C2_2 & $117.6(6)$ \\
\hline C5_2 & C1_2 & C3_2 & $119.5(6)$ \\
\hline C5_2 & $\mathrm{C} 1 \_2$ & C4_2 & $113.4(6)$ \\
\hline C3_2 & C2_2 & C1_2 & $59.1(4)$ \\
\hline C3_2 & C2_2 & C11_2 & $120.2(6)$ \\
\hline C3_2 & C2_2 & C17_2 & $116.9(6)$ \\
\hline C11_2 & $\mathrm{C} 2 \_2$ & C1_2 & $119.8(6)$ \\
\hline C11_2 & C2_2 & C17_2 & $112.6(6)$ \\
\hline C17_2 & C2_2 & C1_2 & $118.4(6)$ \\
\hline C2_2 & C3_2 & C1_2 & $62.7(4)$ \\
\hline 01_2 & C4_2 & C1_2 & $113.6(6)$ \\
\hline 02_2 & C4_2 & 01_2 & $127.9(6)$ \\
\hline $02 \_2$ & C4_2 & C1_2 & $118.4(6)$ \\
\hline C6_2 & C5_2 & C1_2 & $126.0(6)$ \\
\hline C6_2 & C5_2 & C10_2 & $114.5(6)$ \\
\hline C10_2 & C5_2 & $\mathrm{C} 1 \_\overline{2}$ & $118.8(6)$ \\
\hline C5_2 & C6_2 & Cl1_2 & $118.0(5)$ \\
\hline C7_2 & C6_2 & Cl1_2 & $117.5(5)$ \\
\hline C7_2 & C6_2 & C5_2 & $124.5(7)$ \\
\hline C6_2 & C7_2 & C8_2 & $118.5(7)$ \\
\hline C9_2 & C8_2 & C7_2 & 121.1(7) \\
\hline C8_2 & C9_2 & C23_2 & $122.2(7)$ \\
\hline C10_2 & C9_2 & C8_2 & $117.2(7)$ \\
\hline C10_2 & C9_2 & C23_2 & $120.6(6)$ \\
\hline C9_2 & C10_2 & C5_2 & $123.3(6)$ \\
\hline C12_2 & C11_2 & C2_2 & $119.8(6)$ \\
\hline C16_2 & C11_2 & C2_2 & $125.4(7)$ \\
\hline C16_2 & C11_2 & C12_2 & $114.7(6)$ \\
\hline C13_2 & C12_2 & C11_2 & $121.2(7)$ \\
\hline C12_2 & C13_2 & C14_2 & $120.7(8)$ \\
\hline C15_2 & C14_2 & C13_2 & $116.9(8)$ \\
\hline C14_2 & C15_2 & C16_2 & 122.1(8) \\
\hline C11_2 & C16_2 & C15_2 & 122.1(7) \\
\hline
\end{tabular}




\begin{tabular}{|c|c|c|c|}
\hline Atom & Atom & Atom & Angle $/^{\circ}$ \\
\hline$\overline{\text { C18_2 }}$ & C17_2 & C2_2 & $119.0(6)$ \\
\hline C22_2 & C17_2 & C2_2 & $123.9(6)$ \\
\hline C22_2 & C17_2 & C18_2 & $116.9(7)$ \\
\hline C19_2 & C18_2 & C17_2 & $122.9(7)$ \\
\hline C18_2 & C19_2 & C20_2 & $119.1(8)$ \\
\hline C21_2 & C20_2 & C19_2 & $119.9(8)$ \\
\hline C20_2 & C21_2 & C22_2 & $119.4(8)$ \\
\hline C17_2 & C22_2 & C21_2 & $121.3(7)$ \\
\hline C24_2 & C23_2 & C9_2 & 121.5(7) \\
\hline C24_2 & C23_2 & C28_2 & $117.0(7)$ \\
\hline C28_2 & C23_2 & C9_2 & $121.4(7)$ \\
\hline C23_2 & C24_2 & C25_2 & $123.6(8)$ \\
\hline C24_2 & C25_2 & C26_2 & $118.8(8)$ \\
\hline C25_2 & C26_2 & C29_2 & $119.6(7)$ \\
\hline C27_2 & C26_2 & C25_2 & 117.1(7) \\
\hline C27_2 & C26_2 & C29_2 & $123.4(7)$ \\
\hline C26_2 & C27_2 & C28_2 & $123.0(8)$ \\
\hline C27_2 & C28_2 & C23_2 & $120.4(8)$ \\
\hline C30_2 & C29_2 & C26_2 & 114.1(7) \\
\hline C31_2 & C29_2 & C26_2 & $107.9(7)$ \\
\hline C31_2 & C29_2 & C30_2 & $109.0(8)$ \\
\hline C31_2 & C29_2 & C32_2 & $109.2(8)$ \\
\hline C32_2 & C29_2 & C26_2 & $107.2(7)$ \\
\hline C32_2 & C29_2 & C30_2 & $109.3(8)$ \\
\hline C4_3 & 01_3 & Rh1_8 & $115.8(4)$ \\
\hline C4_3 & 02_3 & Rh2_8 & $116.2(4)$ \\
\hline C3_3 & C1_3 & C2_3 & $58.3(4)$ \\
\hline C3_3 & C1_3 & C4_3 & $114.5(5)$ \\
\hline C4_3 & C1_3 & C2_3 & $121.2(6)$ \\
\hline C5_3 & C1_3 & C2_3 & $117.1(5)$ \\
\hline C5_3 & C1_3 & C3_3 & $120.5(6)$ \\
\hline C5_3 & C1_3 & C4_3 & $114.2(5)$ \\
\hline C3_3 & C2_3 & C1_3 & 59.1(4) \\
\hline C3_3 & C2_3 & C11_3 & $118.4(6)$ \\
\hline C3_3 & C2_3 & C17_3 & $117.8(6)$ \\
\hline C11_3 & C2_3 & C1_3 & $120.1(6)$ \\
\hline C11_3 & C2_3 & C17_3 & $112.9(5)$ \\
\hline C17_3 & C2_3 & C1_3 & $118.7(5)$ \\
\hline C2_3 & C3_3 & C1_3 & $62.6(4)$ \\
\hline 01_3 & C4_3 & C1_3 & $114.3(6)$ \\
\hline 02_3 & C4_3 & 01_3 & $127.9(6)$ \\
\hline 02_3 & C4_3 & C1_3 & $117.6(6)$ \\
\hline C6_3 & C5_3 & C1_3 & $125.9(6)$ \\
\hline C6_3 & C5_3 & C10_3 & $114.6(6)$ \\
\hline C10_3 & C5_3 & C1_3 & $119.1(6)$ \\
\hline C5_3 & C6_3 & Cl1_3 & $118.7(5)$ \\
\hline C7_3 & C6_3 & Cl1_3 & $117.4(5)$ \\
\hline C7_3 & C6_3 & C5_3 & $123.9(7)$ \\
\hline C6_3 & C7_3 & C8_3 & $119.0(7)$ \\
\hline C9_3 & C8_3 & C7_3 & $121.5(7)$ \\
\hline C8_3 & C9_3 & C23_3 & $121.9(6)$ \\
\hline C10_3 & C9_3 & C8_3 & $116.8(6)$ \\
\hline C10_3 & C9_3 & C23_3 & $121.2(6)$ \\
\hline C9_3 & C10_3 & C5_3 & $123.8(6)$ \\
\hline C12_3 & C11_3 & C2_3 & $120.1(6)$ \\
\hline C16_3 & C11_3 & C2_3 & $124.4(6)$ \\
\hline C16_3 & C11_3 & C12_3 & $115.2(6)$ \\
\hline C13_3 & C12_3 & C11_3 & $121.3(7)$ \\
\hline
\end{tabular}

\begin{tabular}{|c|c|c|c|}
\hline Atom & Atom & Atom & Angle $/^{\circ}$ \\
\hline$\overline{\mathrm{C} 12 \_3}$ & C13_3 & C14_3 & 121.5(7) \\
\hline C15_3 & C14_3 & C13_3 & $117.6(7)$ \\
\hline C14_3 & C15_3 & C16_3 & $122.0(8)$ \\
\hline C11_3 & C16_3 & C15_3 & $122.0(7)$ \\
\hline C18_3 & C17_3 & C2_3 & $118.8(6)$ \\
\hline C22_3 & C17_3 & C2_3 & $123.9(6)$ \\
\hline C22_3 & C17_3 & C18_3 & $116.8(6)$ \\
\hline C19_3 & C18_3 & C17_3 & $122.9(7)$ \\
\hline C18_3 & C19_3 & C20_3 & 119.3(7) \\
\hline C21_3 & C20_3 & C19_3 & $119.6(8)$ \\
\hline C20_3 & C21_3 & C22_3 & $119.3(7)$ \\
\hline C17_3 & C22_3 & C21_3 & $121.6(7)$ \\
\hline C24_3 & C23_3 & C9_3 & $121.9(7)$ \\
\hline C24_3 & C23_3 & C28_3 & $116.6(7)$ \\
\hline C28_3 & C23_3 & C9_3 & 121.1(7) \\
\hline C23_3 & C24_3 & C25_3 & $123.7(8)$ \\
\hline C24_3 & C25_3 & C26_3 & 119.1(8) \\
\hline C25_3 & C26_3 & C29_3 & $119.2(7)$ \\
\hline C27_3 & C26_3 & C25_3 & $116.7(7)$ \\
\hline C27_3 & C26_3 & C29_3 & $124.1(7)$ \\
\hline C26_3 & C27_3 & C28_3 & $123.0(8)$ \\
\hline C27_3 & C28_3 & C23_3 & $120.7(8)$ \\
\hline C30_3 & C29_3 & C26_3 & 114.3(7) \\
\hline C31_3 & C29_3 & C26_3 & $107.9(7)$ \\
\hline C31_3 & C29_3 & C30_3 & $108.4(7)$ \\
\hline C31_3 & C29_3 & C32_3 & $109.1(7)$ \\
\hline C32_3 & C29_3 & C26_3 & $107.5(7)$ \\
\hline C32_3 & C29_3 & C30_3 & $109.5(8)$ \\
\hline C4_4 & 01_4 & Rh1_7 & $118.4(4)$ \\
\hline C4_4 & $02 \_4$ & Rh2_7 & $115.0(4)$ \\
\hline C3_4 & C1_4 & C2_4 & $58.2(4)$ \\
\hline C3_4 & C1_4 & C4_4 & $116.3(6)$ \\
\hline C4_4 & C1_4 & C2_4 & $121.8(6)$ \\
\hline C5_4 & C1_4 & $\mathrm{C} 2 \_4$ & $116.8(5)$ \\
\hline C5_4 & C1_4 & C3_4 & $119.1(6)$ \\
\hline C5_4 & C1_4 & C4_4 & $113.8(5)$ \\
\hline C3_4 & C2_4 & C1_4 & $59.1(4)$ \\
\hline C3_4 & C2_4 & C11_4 & $120.2(6)$ \\
\hline C3_4 & C2_4 & C17_4 & $116.6(6)$ \\
\hline C11_4 & C2_4 & C1_4 & $119.3(6)$ \\
\hline C11_4 & C2_4 & C17_4 & $113.1(5)$ \\
\hline C17_4 & C2_4 & C1_4 & $118.6(5)$ \\
\hline C2_4 & C3_4 & C1_4 & $62.7(4)$ \\
\hline 01_4 & C4_4 & C1_4 & $113.9(6)$ \\
\hline 02_4 & C4_4 & 01_4 & $127.5(6)$ \\
\hline 02_4 & C4_4 & C1_4 & $118.6(6)$ \\
\hline C6_4 & C5_4 & C1_4 & $126.0(6)$ \\
\hline C6_4 & C5_4 & C10_4 & $114.9(6)$ \\
\hline C10_4 & C5_4 & C1_4 & $118.8(6)$ \\
\hline C5_4 4 & C6_4 & $\mathrm{Cl} 1{ }_{1} 44$ & $118.4(5)$ \\
\hline C7_4 & C6_4 & Cl1_4 & $117.4(5)$ \\
\hline C7_4 & C6_4 & C5_4 & $124.2(7)$ \\
\hline C6_4 & C7_4 & C8_4 & $118.7(7)$ \\
\hline C9_4 & C8_4 & C7_4 & $121.5(7)$ \\
\hline C8_4 & C9_4 & C23_4 & $121.9(7)$ \\
\hline C10_4 & C9_4 & C8_4 & $117.1(6)$ \\
\hline C10_4 & C9_4 & C23_4 & $120.9(6)$ \\
\hline C9_4 & C10_4 & C5_4 & $123.3(6)$ \\
\hline
\end{tabular}




\begin{tabular}{|c|c|c|c|}
\hline Atom & Atom & Atom & Angle/ ${ }^{\circ}$ \\
\hline C12_4 & C11_4 & C2_4 & $120.1(6)$ \\
\hline C16_4 & C11_4 & C2_4 & $125.0(6)$ \\
\hline C16_4 & C11_4 & C12_4 & $114.8(6)$ \\
\hline C13_4 & C12_4 & C11_4 & $121.6(7)$ \\
\hline C12_4 & C13_4 & C14_4 & 121.3(7) \\
\hline C15_4 & C14_4 & C13_4 & $117.5(7)$ \\
\hline C14_4 & C15_4 & C16_4 & $122.3(8)$ \\
\hline C11_4 & C16_4 & C15_4 & $122.2(7)$ \\
\hline C18_4 & C17_4 & C2_4 & $118.9(6)$ \\
\hline C22_4 & C17_4 & C2_4 & $124.1(6)$ \\
\hline C22_4 & C17_4 & C18_4 & $117.0(7)$ \\
\hline C19_4 & C18_4 & C17_4 & $122.8(7)$ \\
\hline C18_4 & C19_4 & C20_4 & 119.3(7) \\
\hline C21_4 & C20_4 & C19_4 & $119.9(8)$ \\
\hline C20_4 & C21_4 & C22_4 & 119.3(7) \\
\hline C17_4 & C22_4 & C21_4 & $121.5(7)$ \\
\hline C24_4 & C23_4 & C9_4 & $121.7(7)$ \\
\hline C24_4 & C23_4 & C28_4 & $117.0(7)$ \\
\hline C28_4 & C23_4 & C9_4 & 121.2(7) \\
\hline C23_4 & C24_4 & C25_4 & $123.6(8)$ \\
\hline C24_4 & C25_4 & C26_4 & 118.8(8) \\
\hline C25_4 & C26_4 & C29_4 & $119.2(7)$ \\
\hline C27_4 & C26_4 & C25_4 & $117.0(7)$ \\
\hline C27_4 & C26_4 & C29_4 & $123.7(7)$ \\
\hline C26_4 & C27_4 & C28_4 & $122.9(8)$ \\
\hline C27_4 & C28_4 & C23_4 & $120.4(8)$ \\
\hline C30_4 & C29_4 & C26_4 & $114.0(7)$ \\
\hline C31_4 & C29_4 & C26_4 & $108.0(7)$ \\
\hline C31_4 & C29_4 & C30_4 & 108.8(7) \\
\hline C31_4 & C29_4 & C32_4 & 109.1(7) \\
\hline C32_4 & C29_4 & C26_4 & $107.5(7)$ \\
\hline C32_4 & C29_4 & C30_4 & 109.4(7) \\
\hline 01W_6 & Rh1_7 & Rh2_7 & 180.0 \\
\hline 01_41 & Rh1_7 & 01W_6 & $94.11(15)$ \\
\hline 01_4 & Rh1_7 & 01W_6 & $94.11(15)$ \\
\hline $01 \_4^{2}$ & Rh1_7 & 01W_6 & $94.11(15)$ \\
\hline $01 \_4^{3}$ & Rh1_7 & 01W_6 & $94.11(15)$ \\
\hline 01_4 & Rh1_7 & $014^{2}$ & 89.71(2) \\
\hline $01 \_4^{2}$ & Rh1_7 & $014^{3}$ & $89.71(2)$ \\
\hline 01_4 & Rh1_7 & $01 \_41$ & $89.71(2)$ \\
\hline $01 \_4^{1}$ & Rh1_7 & $014^{2}$ & $171.8(3)$ \\
\hline 01_4 & Rh1_7 & $014^{3}$ & $171.8(3)$ \\
\hline 01_41 & Rh1_7 & $014^{3}$ & $89.71(2)$ \\
\hline 01_41 & Rh1_7 & Rh2_7 & $85.89(15)$ \\
\hline 01_4 & Rh1_7 & Rh2_7 & $85.89(15)$ \\
\hline $01 \_4^{2}$ & Rh1_7 & Rh2_7 & $85.89(15)$ \\
\hline $01 \_4^{3}$ & Rh1_7 & Rh2_7 & $85.89(15)$ \\
\hline $02 \_4^{2}$ & Rh2_7 & 01W_5 & $91.20(15)$ \\
\hline O2_4 & Rh2_7 & 01W_5 & $91.20(15)$ \\
\hline $02 \_4^{3}$ & Rh2_7 & 01W_5 & $91.20(15)$ \\
\hline O2_41 & Rh2_7 & 01W_5 & $91.20(15)$ \\
\hline O2_41 & Rh2_7 & $024^{3}$ & $89.975(8)$ \\
\hline $02 \_4^{2}$ & Rh2_7 & $024^{3}$ & $89.975(8)$ \\
\hline O2_4 & Rh2_7 & $024^{2}$ & $89.975(9)$ \\
\hline $02 \_4$ & Rh2_7 & $02 \_41$ & $89.975(9)$ \\
\hline $02 \_4$ & Rh2_7 & $024^{3}$ & 177.6(3) \\
\hline $02 \_4^{2}$ & Rh2_7 & O2_41 & $177.6(3)$ \\
\hline O2_4 & Rh2_7 & Rh1_7 & $88.80(15)$ \\
\hline
\end{tabular}

\begin{tabular}{|c|c|c|c|}
\hline Atom & Atom & Atom & Angle $/^{\circ}$ \\
\hline$\overline{02 \_41}$ & Rh2_7 & Rh1_7 & $88.80(15)$ \\
\hline $02 \_4^{3}$ & Rh2_7 & Rh1_7 & $88.80(15)$ \\
\hline $02 \_4^{2}$ & Rh2_7 & Rh1_7 & $88.80(15)$ \\
\hline Rh1_7 & Rh2_7 & 01W_5 & 180.0 \\
\hline 01 & Rh1_8 & $01^{4}$ & $172.2(4)$ \\
\hline $01^{4}$ & Rh1_8 & 01W_9 & $93.89(19)$ \\
\hline 01 & Rh1_8 & 01W_9 & $93.89(19)$ \\
\hline $01^{4}$ & Rh1_8 & 01_34 & $90.6(2)$ \\
\hline $01^{4}$ & Rh1_8 & 01_3 & $88.8(2)$ \\
\hline 01 & Rh1_8 & 01_3 & $90.6(2)$ \\
\hline 01 & Rh1_8 & $01 \_3^{4}$ & $88.8(2)$ \\
\hline $01^{4}$ & Rh1_8 & Rh2_8 & 86.11(19) \\
\hline 01 & Rh1_8 & Rh2_8 & $86.11(19)$ \\
\hline 01W_9 & Rh1_8 & Rh2_8 & 180.0 \\
\hline 01_3 & Rh1_8 & 01W_9 & $94.11(15)$ \\
\hline $01 \_3^{4}$ & Rh1_8 & 01W_9 & $94.11(15)$ \\
\hline 01_3 & Rh1_8 & $01 \_3^{4}$ & 171.8(3) \\
\hline 01_3 & Rh1_8 & Rh2_8 & $85.89(15)$ \\
\hline $01 \_3^{4}$ & Rh1_8 & Rh2_8 & $85.89(15)$ \\
\hline $02^{4}$ & Rh2_8 & 02 & $175.8(4)$ \\
\hline 02 & Rh2_8 & 01W_13 & $92.09(19)$ \\
\hline $02^{4}$ & Rh2_8 & 01W_13 & $92.09(19)$ \\
\hline $02^{4}$ & Rh2_8 & Rh1_8 & 87.91(19) \\
\hline 02 & Rh2_8 & Rh1_8 & 87.91(19) \\
\hline $02 \_3^{4}$ & $\mathrm{Rh} 2 \_8$ & 02 & $91.2(2)$ \\
\hline 02_3 & $\mathrm{Rh} 2 \_8$ & 02 & $88.6(2)$ \\
\hline $02 \_3^{4}$ & Rh2_8 & $\mathrm{O} 2^{4}$ & $88.6(2)$ \\
\hline 02_3 & Rh2_8 & $\mathrm{O} 2^{4}$ & $91.2(2)$ \\
\hline $02 \_3^{4}$ & Rh2_8 & 01W_13 & $92.34(16)$ \\
\hline 02_3 & Rh2_8 & 01W_13 & $92.34(16)$ \\
\hline $02 \_3^{4}$ & Rh2_8 & 02_3 & 175.3(3) \\
\hline $02 \_3^{4}$ & Rh2_8 & Rh1_8 & $87.66(16)$ \\
\hline 02_3 & Rh2_8 & Rh1_8 & $87.66(16)$ \\
\hline Rh1_8 & Rh2_8 & 01W_13 & 180.0 \\
\hline 01W_11 & Rh1_10 & Rh2_10 & 180.0 \\
\hline 01_25 & Rh1_10 & 01W_11 & $94.02(17)$ \\
\hline $01 \_2^{6}$ & Rh1_10 & 01W_11 & $94.02(17)$ \\
\hline 01_2 & Rh1_10 & 01W_11 & $94.02(17)$ \\
\hline $01 \_2^{7}$ & Rh1_10 & 01W_11 & $94.02(17)$ \\
\hline $01 \_2^{7}$ & Rh1_10 & 01_25 & $172.0(3)$ \\
\hline $01 \_2^{6}$ & Rh1_10 & $012^{5}$ & $89.72(2)$ \\
\hline 01_2 & Rh1_10 & 01_25 & $89.72(2)$ \\
\hline 01_2 & Rh1_10 & 01_26 & $172.0(3)$ \\
\hline 01_2 & Rh1_10 & 01_27 & $89.71(2)$ \\
\hline $01 \_2^{6}$ & Rh1_10 & $01 \_2^{7}$ & $89.72(2)$ \\
\hline $01 \_2^{5}$ & Rh1_10 & Rh2_10 & $85.98(17)$ \\
\hline $01 \_2^{6}$ & Rh1_10 & Rh2_10 & $85.98(17)$ \\
\hline 01_2 & Rh1_10 & Rh2_10 & $85.98(17)$ \\
\hline $01 \_2^{7}$ & Rh1_10 & Rh2_10 & $85.98(17)$ \\
\hline 02_2 & Rh2_10 & 01W_12 & $91.17(18)$ \\
\hline $02 \_2^{7}$ & Rh2_10 & 01W_12 & $91.17(18)$ \\
\hline $02 \_2^{6}$ & Rh2_10 & 01W_12 & $91.17(18)$ \\
\hline $02 \_2^{5}$ & Rh2_10 & 01W_12 & $91.17(18)$ \\
\hline $02 \_2^{5}$ & Rh2_10 & 02_27 & $177.7(4)$ \\
\hline 02_26 & Rh2_10 & 02_2 ${ }^{7}$ & $89.976(8)$ \\
\hline 02_2 & Rh2_10 & $02 \_2^{5}$ & $89.975(8)$ \\
\hline 02_2 & Rh2_10 & $02 \_2^{7}$ & $89.978(8)$ \\
\hline 02_2 & Rh2_10 & 02_26 & $177.7(4)$ \\
\hline
\end{tabular}




\begin{tabular}{lllc}
\hline Atom & Atom & Atom & Angle $^{\circ}$ \\
\hline O2_26 & Rh2_10 & O2_2 & $89.976(8)$ \\
O2_2 & Rh2_10 & Rh1_10 & $88.83(18)$ \\
O2_25 & Rh2_10 & Rh1_10 & $88.83(18)$ \\
O2_27 & Rh2_10 & Rh1_10 & $88.83(18)$ \\
O2_26 & Rh2_10 & Rh1_10 & $88.83(18)$
\end{tabular}

\begin{tabular}{|c|c|c|c|}
\hline Atom & Atom & Atom & Angle $/^{\circ}$ \\
\hline Rh1_10 & $\mathrm{Rh} 2 \_10$ & 01W_12 & 180.0 \\
\hline
\end{tabular}

Table 13: Torsion Angles in ${ }^{\circ}$ for Rh-2Cl5tBuPh.

\begin{tabular}{|c|c|c|c|c|}
\hline Atom & Atom & Atom & Atom & Angle $/^{\circ}$ \\
\hline$\overline{\mathrm{Cl} 1}$ & C6 & C7 & C8 & $177.3(10)$ \\
\hline $\mathrm{C} 1$ & $\mathrm{C} 2$ & C11 & $\mathrm{C} 12$ & $-129.6(13)$ \\
\hline $\mathrm{C} 1$ & $\mathrm{C} 2$ & C11 & C16 & $45.5(15)$ \\
\hline $\mathrm{C} 1$ & $\mathrm{C} 2$ & C17 & C18 & $-130.0(10)$ \\
\hline C1 & $\mathrm{C} 2$ & C17 & $\mathrm{C} 22$ & $56.9(13)$ \\
\hline $\mathrm{C} 1$ & $\mathrm{C} 5$ & C6 & Cl1 & $5.5(15)$ \\
\hline $\mathrm{C} 1$ & $\mathrm{C} 5$ & C6 & $\mathrm{C} 7$ & $-177.0(11)$ \\
\hline $\mathrm{C} 1$ & $\mathrm{C} 5$ & C10 & C9 & $-179.2(10)$ \\
\hline $\mathrm{C} 2$ & C1 & C4 & 01 & $-155.9(8)$ \\
\hline $\mathrm{C} 2$ & C1 & C4 & 02 & 27.7(12) \\
\hline $\mathrm{C} 2$ & C1 & C5 & C6 & $65.6(13)$ \\
\hline $\mathrm{C} 2$ & C1 & C5 & C10 & $-111.8(11)$ \\
\hline $\mathrm{C} 2$ & C11 & C12 & C13 & 179.6(12) \\
\hline $\mathrm{C} 2$ & C11 & C16 & C15 & $-179.6(13)$ \\
\hline $\mathrm{C} 2$ & C17 & C18 & C19 & 179.5(10) \\
\hline $\mathrm{C} 2$ & C17 & $\mathrm{C} 22$ & $\mathrm{C} 21$ & $179.7(10)$ \\
\hline C3 & C1 & C2 & C11 & $-108.0(11)$ \\
\hline C3 & C1 & C2 & C17 & 108.5(11) \\
\hline C3 & $\mathrm{C} 1$ & $\mathrm{C} 4$ & 01 & $140.0(9)$ \\
\hline C3 & C1 & C4 & 02 & $-36.4(13)$ \\
\hline $\mathrm{C} 3$ & $\mathrm{C} 1$ & $\mathrm{C} 5$ & $\mathrm{C} 6$ & $134.8(11)$ \\
\hline $\mathrm{C} 3$ & C1 & $\mathrm{C} 5$ & C10 & $-42.6(15)$ \\
\hline $\mathrm{C} 3$ & $\mathrm{C} 2$ & C11 & $\mathrm{C} 12$ & $160.4(12)$ \\
\hline C3 & $\mathrm{C} 2$ & C11 & C16 & $-24.5(16)$ \\
\hline C3 & C2 & C17 & C18 & $-60.8(13)$ \\
\hline C3 & C2 & C17 & $\mathrm{C} 22$ & 126.1(11) \\
\hline C4 & C1 & $\mathrm{C} 2$ & C3 & $-98.4(9)$ \\
\hline C4 & C1 & $\mathrm{C} 2$ & C11 & $153.6(9)$ \\
\hline C4 & C1 & C2 & C17 & 10.1(13) \\
\hline $\mathrm{C} 4$ & C1 & C3 & $\mathrm{C} 2$ & $111.8(10)$ \\
\hline $\mathrm{C} 4$ & C1 & $\mathrm{C} 5$ & C6 & $-88.3(13)$ \\
\hline $\mathrm{C} 4$ & C1 & $\mathrm{C} 5$ & C10 & $94.3(10)$ \\
\hline C5 & C1 & C2 & C3 & $109.2(10)$ \\
\hline C5 & C1 & $\mathrm{C} 2$ & C11 & $1.2(14)$ \\
\hline C5 & C1 & $\mathrm{C} 2$ & C17 & $-142.3(9)$ \\
\hline C5 & C1 & C3 & $\mathrm{C} 2$ & $-110.7(11)$ \\
\hline C5 & C1 & C4 & 01 & $-1.2(11)$ \\
\hline C5 & C1 & C4 & 02 & $-177.5(8)$ \\
\hline C5 & C6 & C7 & C8 & $-0.2(19)$ \\
\hline C6 & C5 & C10 & C9 & $3.2(16)$ \\
\hline C6 & C7 & C8 & C9 & $-4(2)$ \\
\hline C7 & C8 & C9 & C10 & $6.9(19)$ \\
\hline C7 & C8 & C9 & $\mathrm{C} 23$ & $-171.9(12)$ \\
\hline C8 & C9 & C10 & C5 & $-6.7(17)$ \\
\hline C8 & C9 & $\mathrm{C} 23$ & $\mathrm{C} 24$ & $138.3(12)$ \\
\hline C8 & C9 & $\mathrm{C} 23$ & $\mathrm{C} 28$ & $-37.5(17)$ \\
\hline C9 & $\mathrm{C} 23$ & $\mathrm{C} 24$ & $\mathrm{C} 25$ & $-173.0(10)$ \\
\hline
\end{tabular}




\begin{tabular}{|c|c|c|c|c|}
\hline Atom & Atom & Atom & Atom & Angle ${ }^{\circ}$ \\
\hline$\overline{C 9}$ & C23 & C28 & $\mathrm{C} 27$ & $176.6(10)$ \\
\hline C10 & $\mathrm{C} 5$ & C6 & Cl1 & $-177.0(8)$ \\
\hline C10 & $\mathrm{C} 5$ & C6 & $\mathrm{C} 7$ & $0.5(16)$ \\
\hline C10 & C9 & $\mathrm{C} 23$ & C24 & $-40.4(15)$ \\
\hline C10 & C9 & $\mathrm{C} 23$ & $\mathrm{C} 28$ & $143.8(11)$ \\
\hline C11 & $\mathrm{C} 2$ & $\mathrm{C} 3$ & $\mathrm{C} 1$ & $110.1(11)$ \\
\hline C11 & $\mathrm{C} 2$ & $\mathrm{C} 17$ & C18 & $83.6(12)$ \\
\hline C11 & $\mathrm{C} 2$ & C17 & $\mathrm{C} 22$ & $-89.5(11)$ \\
\hline C11 & C12 & $\mathrm{C} 13$ & C14 & $-1(3)$ \\
\hline C12 & C11 & C16 & C15 & $-4(2)$ \\
\hline C12 & $\mathrm{C} 13$ & C14 & C15 & $-3(3)$ \\
\hline C13 & C14 & C15 & C16 & $3(3)$ \\
\hline C14 & C15 & $\mathrm{C} 16$ & C11 & $1(2)$ \\
\hline C16 & C11 & $\mathrm{C} 12$ & $\mathrm{C} 13$ & $4(2)$ \\
\hline C17 & $\mathrm{C} 2$ & C3 & C1 & $-108.0(10)$ \\
\hline C17 & $\mathrm{C} 2$ & C11 & C12 & 16.1(16) \\
\hline C17 & $\mathrm{C} 2$ & C11 & C16 & $-168.8(10)$ \\
\hline C17 & C18 & C19 & $\mathrm{C} 20$ & 6.4(19) \\
\hline C18 & C17 & $\mathrm{C} 22$ & $\mathrm{C} 21$ & $6.6(15)$ \\
\hline C18 & C19 & $\mathrm{C} 20$ & $\mathrm{C} 21$ & $-5(2)$ \\
\hline C19 & $\mathrm{C} 20$ & $\mathrm{C} 21$ & $\mathrm{C} 22$ & $5(2)$ \\
\hline C20 & $\mathrm{C} 21$ & $\mathrm{C} 22$ & C17 & $-5.7(19)$ \\
\hline C22 & C17 & C18 & C19 & $-7.4(17)$ \\
\hline $\mathrm{C} 23$ & C9 & $\mathrm{C} 10$ & C5 & $172.1(10)$ \\
\hline $\mathrm{C} 23$ & $\mathrm{C} 24$ & $\mathrm{C} 25$ & $\mathrm{C} 26$ & $-3.8(16)$ \\
\hline $\mathrm{C} 24$ & $\mathrm{C} 23$ & $\mathrm{C} 28$ & $\mathrm{C} 27$ & $0.7(16)$ \\
\hline $\mathrm{C} 24$ & $\mathrm{C} 25$ & $\mathrm{C} 26$ & $\mathrm{C} 27$ & $1.0(15)$ \\
\hline $\mathrm{C} 24$ & $\mathrm{C} 25$ & $\mathrm{C} 26$ & C29 & $-177.7(10)$ \\
\hline $\mathrm{C} 25$ & $\mathrm{C} 26$ & $\mathrm{C} 27$ & C28 & $2.6(15)$ \\
\hline $\mathrm{C} 25$ & $\mathrm{C} 26$ & $\mathrm{C} 29$ & C30 & $1.5(14)$ \\
\hline $\mathrm{C} 25$ & $\mathrm{C} 26$ & $\mathrm{C} 29$ & C31 & $123.2(12)$ \\
\hline $\mathrm{C} 25$ & $\mathrm{C} 26$ & $\mathrm{C} 29$ & C32 & $-113.8(12)$ \\
\hline $\mathrm{C} 26$ & $\mathrm{C} 27$ & $\mathrm{C} 28$ & $\mathrm{C} 23$ & $-3.5(16)$ \\
\hline $\mathrm{C} 27$ & $\mathrm{C} 26$ & $\mathrm{C} 29$ & C30 & $-177.2(9)$ \\
\hline $\mathrm{C} 27$ & $\mathrm{C} 26$ & $\mathrm{C} 29$ & C31 & $-55.4(14)$ \\
\hline $\mathrm{C} 27$ & $\mathrm{C} 26$ & C29 & C32 & $67.6(13)$ \\
\hline C28 & $\mathrm{C} 23$ & $\mathrm{C} 24$ & C25 & $2.9(16)$ \\
\hline C29 & C26 & C27 & C28 & $-178.7(10)$ \\
\hline Cl1_2 & C6_2 & C7_2 & C8_2 & $179.5(9)$ \\
\hline $\mathrm{C} 1 \_2$ & C2_2 & C11_2 & C12_2 & $-128.4(13)$ \\
\hline C1_2 & C2_2 & C11_2 & C16_2 & $54.3(15)$ \\
\hline C1_2 & C2_2 & C17_2 & C18_2 & $-125.0(10)$ \\
\hline C1_2 & C2_2 & C17_2 & C22_2 & $61.0(11)$ \\
\hline C1_2 & C5_2 & C6_2 & Cl1_2 & $-2.6(13)$ \\
\hline C1_2 & C5_2 & C6_2 & C7_2 & 174.7(10) \\
\hline C1_2 & C5_2 & C10_2 & C9_2 & $-175.5(8)$ \\
\hline C2_2 & C1_2 & C4_2 & 01_2 & $-150.6(8)$ \\
\hline C2_2 & C1_2 & C4_2 & 02_2 & $29.9(11)$ \\
\hline C2_2 & C1_2 & C5_2 & C6_2 & $74.7(10)$ \\
\hline C2_2 & C1_2 & C5_2 & C10_2 & $-115.8(8)$ \\
\hline C2_2 & C11_2 & C12_2 & C13_2 & $-175.8(13)$ \\
\hline $\mathrm{C} 2 \_2$ & C11_2 & C16_2 & C15_2 & $-175.2(15)$ \\
\hline $\mathrm{C} 2 \_2$ & C17_2 & C18_2 & C19_2 & $178.7(14)$ \\
\hline $\mathrm{C} 2 \_2$ & C17_2 & $\mathrm{C} 22 \_2$ & C21_2 & $-179.6(12)$ \\
\hline C3_2 & C1_2 & C2_2 & C11_2 & $-109.5(8)$ \\
\hline C3_2 & C1_2 & C2_2 & C17_2 & $106.1(7)$ \\
\hline C3_2 & C1_2 & C4_2 & 01_2 & $142.2(8)$ \\
\hline C3_2 & C1_2 & C4_2 & $02 \_2$ & $-37.3(11)$ \\
\hline
\end{tabular}




\begin{tabular}{|c|c|c|c|c|}
\hline Atom & Atom & Atom & Atom & Angle $/^{\circ}$ \\
\hline$\overline{C 3 \_2}$ & C1_2 & C5_2 & C6_2 & $141.9(9)$ \\
\hline C3_2 & C1_2 & C5_2 & C10_2 & $-48.5(10)$ \\
\hline C3_2 & $\mathrm{C} 2 \_2$ & C11__2 & C12_2 & $162.2(13)$ \\
\hline C3_2 & $\mathrm{C} 2 \_2$ & C11_2 & C16_2 & $-15.1(16)$ \\
\hline C3_2 & C2_2 & C17_2 & C18_2 & $-57.4(12)$ \\
\hline C3_2 & C2_2 & C17_2 & C22_2 & 128.7(10) \\
\hline C4_2 & C1_2 & $\mathrm{C} 2 \_\overline{2}$ & C3_2 & $-103.3(7)$ \\
\hline C4_2 & C1_2 & $\mathrm{C} 2 \_2$ & C11_2 & $147.2(7)$ \\
\hline C4_2 & C1_2 & C2_2 & C17_2 & $2.7(9)$ \\
\hline C4_2 & C1_2 & C3_2 & C2_2 & $112.1(7)$ \\
\hline C4_2 & C1_2 & C5_2 & C6_2 & $-75.3(10)$ \\
\hline C4_2 & $\mathrm{C} 1 \_2$ & C5_2 & C10_2 & $94.3(8)$ \\
\hline C5_2 & C1_2 & C2_2 & C3_2 & 109.2(7) \\
\hline C5_2 & C1_2 & $\mathrm{C} 2 \_2$ & C11_2 & $-0.3(10)$ \\
\hline C5_2 & C1_2 & C2_2 & C17_2 & $-144.7(6)$ \\
\hline C5_2 & C1_2 & C3_2 & C2_2 & $-106.1(7)$ \\
\hline C5_2 & C1_2 & C4_2 & 01_2 & $-1.9(10)$ \\
\hline C5_2 & C1_2 & C4_2 & 02_2 & 178.7(8) \\
\hline C5_2 & C6_2 & C7_2 & C8_2 & $2.2(18)$ \\
\hline C6_2 & C5_2 & C10_2 & C9_2 & $-4.8(13)$ \\
\hline C6_2 & C7_2 & C8_2 & C9_2 & $-9.8(18)$ \\
\hline C7_2 & C8_2 & C9_2 & C10_2 & $9.6(15)$ \\
\hline C7_2 & C8_2 & C9_2 & C23_2 & $-172.0(10)$ \\
\hline C8_2 & C9_2 & C10_2 & C5_2 & $-2.1(14)$ \\
\hline C8_2 & C9_2 & C23_2 & C24_2 & $138.4(11)$ \\
\hline C8_2 & C9_2 & C23_2 & C28_2 & $-36.9(14)$ \\
\hline C9_2 & C23_2 & C24_2 & C25_2 & $-171.4(11)$ \\
\hline C9_2 & C23_2 & C28_2 & C27_2 & $172.1(9)$ \\
\hline C10_2 & C5_2 & C6_2 & Cl1_2 & $-172.5(7)$ \\
\hline C10_2 & C5_2 & C6_2 & C7_2 & $4.8(15)$ \\
\hline C10_2 & C9_2 & C23_2 & C24_2 & $-43.2(14)$ \\
\hline C10_2 & C9_2 & C23_2 & C28_2 & $141.5(10)$ \\
\hline C11_2 & C2_2 & C3_2 & C1_2 & 108.8(7) \\
\hline C11_2 & C2_2 & C17_2 & C18_2 & 88.1(11) \\
\hline C11_2 & C2_2 & C17_2 & C22_2 & $-85.8(11)$ \\
\hline C11_2 & C12_2 & C13_2 & C14_2 & $-14(3)$ \\
\hline C12_2 & C11_2 & C16_2 & C15_2 & $7(2)$ \\
\hline C12_2 & C13_2 & C14_2 & C15_2 & $17(3)$ \\
\hline C13_2 & C14_2 & C15_2 & C16_2 & $-8(3)$ \\
\hline C14_2 & C15_2 & C16_2 & C11_2 & $-5(3)$ \\
\hline C16_2 & C11_2 & C12_2 & C13_2 & $2(2)$ \\
\hline C17_2 & C2_2 & C3_2 & C1_2 & $-108.5(7)$ \\
\hline C17_2 & C2_2 & C11_2 & C12_2 & $17.9(15)$ \\
\hline C17_2 & C2_2 & C11_2 & C16_2 & $-159.3(13)$ \\
\hline C17_2 & C18_2 & C19_2 & C20_2 & $6(3)$ \\
\hline C18_2 & C17_2 & C22_2 & C21_2 & $6.3(19)$ \\
\hline C18_2 & C19_2 & C20_2 & C21_2 & $-5(3)$ \\
\hline C19_2 & C20_2 & C21_2 & C22_2 & $5(3)$ \\
\hline C20_2 & C21_2 & C22_2 & C17_2 & $-5(3)$ \\
\hline C22_2 & C17_2 & C18_2 & C19_2 & $-7(2)$ \\
\hline C23_2 & C9_2 & C10_2 & C5_2 & $179.4(8)$ \\
\hline C23_2 & C24_2 & C25_2 & C26_2 & $-3(2)$ \\
\hline C24_2 & C23_2 & C28_2 & C27_2 & $-3.4(17)$ \\
\hline C24_2 & C25_2 & C26_2 & C27_2 & $1.0(18)$ \\
\hline C24_2 & C25_2 & C26_2 & C29_2 & $-178.4(11)$ \\
\hline C25_2 & C26_2 & C27_2 & C28_2 & $-0.5(18)$ \\
\hline C25_2 & C26_2 & C29_2 & C30_2 & $-3.9(14)$ \\
\hline C25_2 & C26_2 & C29_2 & C31_2 & $117.4(12)$ \\
\hline
\end{tabular}




\begin{tabular}{|c|c|c|c|c|}
\hline Atom & Atom & Atom & Atom & Angle/ ${ }^{\circ}$ \\
\hline$\overline{C 25 \_2}$ & C26_2 & C29_2 & C32_2 & $-125.1(11)$ \\
\hline C26_2 & C27_2 & C28_2 & C23_2 & $1.7(18)$ \\
\hline C27_2 & C26_2 & C29_2 & C30_2 & $176.7(11)$ \\
\hline C27_2 & C26_2 & C29_2 & C31_2 & $-62.0(14)$ \\
\hline C27_2 & C26_2 & C29_2 & C32_2 & $55.5(13)$ \\
\hline C28_2 & C23_2 & C24_2 & C25_2 & 4.1(18) \\
\hline C29_2 & C26_2 & C27_2 & C28_2 & $178.9(10)$ \\
\hline Cl1_3 & C6_3 & C7_3 & C8_3 & $174.7(9)$ \\
\hline C1_3 & C2_3 & C11_3 & C12_3 & $-108.5(9)$ \\
\hline C1_3 & C2_3 & C11_3 & C16_3 & $77.4(12)$ \\
\hline C1_3 & C2_3 & C17_3 & C18_3 & $-136.9(8)$ \\
\hline C1_3 & $\mathrm{C} 2 \_3$ & C17_3 & C22_3 & 51.8(10) \\
\hline C1_3 & C5_3 & C6_3 & Cl1_3 & $-3.2(12)$ \\
\hline C1_3 & C5_3 & C6_3 & C7_3 & $178.9(9)$ \\
\hline C1_3 & C5_3 & C10_3 & C9_3 & $-175.1(9)$ \\
\hline C2_3 & C1_3 & C4_3 & 01_3 & $-155.1(7)$ \\
\hline $\mathrm{C} 2 \_3$ & C1_3 & C4_3 & $02 \_3$ & $29.9(10)$ \\
\hline C2_3 & C1_3 & C5_3 & C6_3 & $79.4(9)$ \\
\hline C2_3 & C1_3 & C5_3 & C10_3 & $-108.8(9)$ \\
\hline $\mathrm{C} 2 \_3$ & C11_3 & C12_3 & C13_3 & $-174.1(10)$ \\
\hline C2_3 & C11_3 & C16_3 & C15_3 & $177.6(12)$ \\
\hline $\mathrm{C} 2 \_3$ & C17_3 & C18_3 & C19_3 & $-177.3(8)$ \\
\hline C2_3 & C17_3 & C22_3 & C21_3 & $175.1(8)$ \\
\hline C3_3 & C1_3 & C2_3 & C11_3 & $-107.0(7)$ \\
\hline C3_3 & $\mathrm{C} 1 \_3$ & C2_3 & C17_3 & $106.9(7)$ \\
\hline C3_3 & C1_3 & C4_3 & 01_3 & 138.4(7) \\
\hline C3_3 & C1_3 & C4_3 & 02_3 & $-36.6(9)$ \\
\hline C3_3 & C1_3 & C5_3 & C6_3 & $146.9(8)$ \\
\hline C3_3 & C1_3 & C5_3 & C10_3 & $-41.3(10)$ \\
\hline C3_3 & $\mathrm{C} 2 \_3$ & C11__3 & C12_3 & $-177.2(8)$ \\
\hline C3_3 & C2_3 & C11_3 & C16_3 & 8.7(13) \\
\hline C3_3 & C2_3 & C17_3 & C18_3 & $-68.9(9)$ \\
\hline C3_3 & C2_3 & C17_3 & C22_3 & $119.9(8)$ \\
\hline C4_3 & C1_3 & C2_3 & C3_3 & $-101.3(7)$ \\
\hline C4_3 & C1_3 & C2_3 & C11_3 & $151.7(6)$ \\
\hline C4_3 & C1_3 & C2_3 & C17_3 & $5.6(9)$ \\
\hline C4_3 & C1_3 & C3_3 & C2_3 & $112.8(6)$ \\
\hline C4_3 & C1_3 & C5_3 & C6_3 & $-70.7(10)$ \\
\hline C4_3 & C1_3 & C5_3 & C10_3 & 101.1(9) \\
\hline C5_3 & C1_3 & C2_3 & C3_3 & $110.8(7)$ \\
\hline C5_3 & C1_3 & C2_3 & C11_3 & $3.7(9)$ \\
\hline C5_3 & C1_3 & C2_3 & C17_3 & $-142.3(6)$ \\
\hline C5_3 & C1_3 & C3_3 & C2_3 & $-104.9(7)$ \\
\hline C5_3 & C1_3 & C4_3 & 01_3 & $-6.3(9)$ \\
\hline C5_3 & C1_3 & C4_3 & 02_3 & 178.7(7) \\
\hline C5_3 & C6_3 & C7_3 & C8_3 & $-7.4(16)$ \\
\hline C6_3 & C5_3 & C10_3 & C9_3 & $-2.4(15)$ \\
\hline C6_3 & C7_3 & C8_3 & C9_3 & $3.3(17)$ \\
\hline C7_3 & C8_3 & C9_3 & C10_3 & $0.8(17)$ \\
\hline C7_3 & C8_3 & C9_3 & C23_3 & $-174.9(10)$ \\
\hline C8_3 & C9_3 & C10_3 & C5_3 & $-1.1(17)$ \\
\hline C8_3 & C9_3 & C23_3 & C24_3 & $139.3(11)$ \\
\hline C8_3 & C9_3 & C23_3 & C28_3 & $-33.3(15)$ \\
\hline C9_3 & C23_3 & C24_3 & C25_3 & $-170.0(11)$ \\
\hline C9_3 & C23_3 & C28_3 & C27_3 & $174.5(11)$ \\
\hline C10_3 & C5_3 & C6_3 & Cl1_3 & $-175.3(7)$ \\
\hline C10_3 & C5_3 & C6_3 & C7_3 & $6.8(14)$ \\
\hline C10_3 & C9_3 & C23_3 & C24_3 & $-36.2(14)$ \\
\hline
\end{tabular}




\begin{tabular}{|c|c|c|c|c|}
\hline Atom & Atom & Atom & Atom & Angle ${ }^{\circ}$ \\
\hline$\overline{C 10 \_3}$ & C9_3 & C23_3 & C28_3 & $151.2(11)$ \\
\hline C11_3 & C2_3 & C3_3 & C1_3 & $109.9(7)$ \\
\hline C11_3 & C2_3 & C17_3 & C18_3 & $74.7(9)$ \\
\hline C11_3 & C2_3 & C17_3 & C22_3 & $-96.5(8)$ \\
\hline C11_3 & C12_3 & C13_3 & C14_3 & $-0.9(19)$ \\
\hline C12_3 & C11_3 & C16_3 & C15_3 & $3(2)$ \\
\hline C12_3 & C13_3 & C14_3 & C15_3 & $-2(2)$ \\
\hline C13_3 & C14_3 & C15_3 & C16_3 & $6(2)$ \\
\hline C14_3 & C15_3 & C16_3 & C11_3 & $-7(2)$ \\
\hline C16_3 & C11_3 & C12_3 & C13_3 & $0.5(17)$ \\
\hline C17_3 & C2_3 & C3_3 & C1_3 & $-108.5(6)$ \\
\hline C17_3 & C2_3 & C11_3 & C12_3 & $39.4(10)$ \\
\hline C17_3 & C2_3 & C11_3 & C16_3 & $-134.7(11)$ \\
\hline C17_3 & C18_3 & C19_3 & C20_3 & $0.8(16)$ \\
\hline C18_3 & C17_3 & C22_3 & C21_3 & $3.6(13)$ \\
\hline C18_3 & C19_3 & C20_3 & C21_3 & $5.9(16)$ \\
\hline C19_3 & C20_3 & C21_3 & C22_3 & $-7.5(16)$ \\
\hline C20_3 & C21_3 & C22_3 & C17_3 & $2.7(15)$ \\
\hline C22_3 & C17_3 & C18_3 & C19_3 & $-5.4(14)$ \\
\hline C23_3 & C9_3 & C10_3 & C5_3 & $174.6(9)$ \\
\hline C23_3 & C24_3 & C25_3 & C26_3 & $-3.9(19)$ \\
\hline C24_3 & C23_3 & C28_3 & C27_3 & $1.5(17)$ \\
\hline C24_3 & C25_3 & C26_3 & C27_3 & $0.5(19)$ \\
\hline C24_3 & C25_3 & C26_3 & C29_3 & 179.8(10) \\
\hline C25_3 & C26_3 & C27_3 & C28_3 & $4(2)$ \\
\hline C25_3 & C26_3 & C29_3 & C30_3 & $-10.0(14)$ \\
\hline C25_3 & C26_3 & C29_3 & C31_3 & $110.7(12)$ \\
\hline C25_3 & C26_3 & C29_3 & C32_3 & $-131.8(11)$ \\
\hline C26_3 & C27_3 & C28_3 & C23_3 & $-5(2)$ \\
\hline C27_3 & C26_3 & C29_3 & C30_3 & $169.3(12)$ \\
\hline C27_3 & C26_3 & C29_3 & C31_3 & $-70.0(14)$ \\
\hline C27_3 & C26_3 & C29_3 & C32_3 & $47.5(14)$ \\
\hline C28_3 & C23_3 & C24_3 & C25_3 & $2.9(17)$ \\
\hline C29_3 & C26_3 & C27_3 & C28_3 & $-175.4(11)$ \\
\hline Cl1_4 & C6_4 & C7_4 & C8_4 & $172.7(8)$ \\
\hline C1_4 & C2_4 & C11_4 & C12_4 & $-116.0(10)$ \\
\hline C1_4 & C2_4 & C11_4 & C16_4 & $60.9(11)$ \\
\hline C1_4 & C2_4 & C17_4 & C18_4 & $-123.3(8)$ \\
\hline C1_4 & C2_4 & C17_44 & C22_4 & $58.0(10)$ \\
\hline C1_4 & C5_4 & C6_4 & Cl1_4 & $2.5(11)$ \\
\hline C1_4 & C5_4 & C6_4 & C7_4 & $179.6(9)$ \\
\hline C1_4 & C5_4 & C10_4 & C9_4 & $-176.0(8)$ \\
\hline C2_4 & C1_4 & C4_4 & 01_4 & $-147.3(7)$ \\
\hline C2_4 & C1_4 & C4_4 & O2_4 & $32.1(10)$ \\
\hline C2_4 & C1_4 & C5_4 & C6_4 & 71.2(10) \\
\hline C2__4 & C1_4 & C5_4 & C10_4 & $-115.4(8)$ \\
\hline C2__4 & C11_4 & C12_4 & C13_4 & $174.0(11)$ \\
\hline C2__4 & C11_4 & C16_4 & C15_4 & $-178.3(10)$ \\
\hline C2_4 & C17_4 & C18_4 & C19_4 & $176.9(9)$ \\
\hline C2_4 & C17_4 & C22_4 & C21_4 & $-177.9(9)$ \\
\hline C3_4 & C1_4 & C2_4 & C11_4 & $-109.6(7)$ \\
\hline C3_4 & C1_4 & C2_4 & C17_4 & 105.5(7) \\
\hline C3_4 & C1_4 & C4_4 & 01_4 & $145.3(7)$ \\
\hline C3_4 & C1_4 & C4_4 & 02_4 & $-35.2(10)$ \\
\hline C3_4 & C1_4 & C5_4 & C6_4 & $137.9(8)$ \\
\hline C3_4 & C1_4 & C5_4 & C10_4 & $-48.6(9)$ \\
\hline C3_4 & C2_4 & C11_4 & C12_4 & 174.8(9) \\
\hline C3_4 & C2_4 & C11_4 & C16_4 & $-8.3(13)$ \\
\hline
\end{tabular}




\begin{tabular}{|c|c|c|c|c|}
\hline Atom & Atom & Atom & Atom & Angle $/^{\circ}$ \\
\hline$\overline{C 3 \_4}$ & C2_4 & C17_4 & C18_4 & $-55.8(9)$ \\
\hline C3_4 & C2_4 & C17_4 & C22_4 & $125.5(9)$ \\
\hline C4_4 & C1_4 & C2_4 & C3_4 & $-103.2(7)$ \\
\hline C4_4 & C1_4 & $\mathrm{C} 2 \_4$ & C11__4 & 147.1(7) \\
\hline C4_4 & C1_4 & C2_4 & C17_4 & $2.3(9)$ \\
\hline C4_4 & C1_4 & C3_4 & C2_4 & $112.7(7)$ \\
\hline C4_4 & C1_4 & C5_4 & C6_4 & $-79.0(9)$ \\
\hline C4_4 & C1_4 & C5_4 & C10_4 & $94.4(8)$ \\
\hline C5_4 & C1_4 & C2_4 & C3_4 & $109.1(7)$ \\
\hline C5_4 & C1_4 & C2_4 & C11_4 & $-0.5(9)$ \\
\hline C5_4 & C1_4 & C2_4 & C17_4 & $-145.4(6)$ \\
\hline C5_4 & C1_4 & C3_4 & C2_4 & $-105.2(6)$ \\
\hline C5_4 & C1_4 & C4_4 & 01_4 & $1.2(9)$ \\
\hline C5_4 & C1_4 & C4_4 & O2_4 & $-179.3(7)$ \\
\hline C5_4 & C6_4 & C7_4 & C8_4 & $-4.5(15)$ \\
\hline C6_4 & C5_4 & C10_4 & C9_4 & $-1.8(13)$ \\
\hline C6_4 & C7_4 & C8_4 & C9_4 & $-1.3(15)$ \\
\hline C7_4 & C8_4 & C9_4 & C10_4 & $4.9(15)$ \\
\hline C7_4 & C8_4 & C9_4 & C23_4 & $-170.5(9)$ \\
\hline C8_4 & C9_4 & C10_4 & C5_4 & $-3.3(15)$ \\
\hline C8_4 & C9_4 & C23_4 & C24_4 & $140.5(11)$ \\
\hline C8_4 & C9_4 & C23_4 & C28_4 & $-36.3(15)$ \\
\hline C9_4 & C23_4 & C24_4 & C25_4 & $-176.6(12)$ \\
\hline C9_4 & C23_4 & C28_4 & C27_4 & $174.6(11)$ \\
\hline C10_4 & C5_4 & C6_4 & Cl1_4 & $-171.2(7)$ \\
\hline C10_4 & C5_4 & C6_4 & C7_4 & $5.9(13)$ \\
\hline C10_4 & C9_4 & C23_4 & C24_4 & $-34.8(15)$ \\
\hline C10_4 & C9_4 & C23_4 & C28_4 & $148.4(11)$ \\
\hline C11_4 & C2_4 & C3_4 & $\mathrm{C} 1 \_4$ & $108.2(7)$ \\
\hline C11_4 & C2_4 & C17_4 & C18_4 & 89.8(9) \\
\hline C11_4 & C2_4 & C17_4 & C22_4 & $-88.9(10)$ \\
\hline C11_4 & C12_4 & C13_4 & C14_4 & $6(2)$ \\
\hline C12_4 & C11_4 & C16_4 & C15_4 & $-1.2(16)$ \\
\hline C12_4 & C13_4 & C14_4 & C15_4 & $-4(2)$ \\
\hline C13_4 & C14_4 & C15_4 & C16_4 & $-1(2)$ \\
\hline C14_4 & C15_4 & C16_4 & C11_4 & $3(2)$ \\
\hline C16_4 & C11_4 & C12_4 & C13_4 & $-3.3(18)$ \\
\hline C17_4 & C2_4 & C3_4 & C1_4 & $-108.9(6)$ \\
\hline C17_4 & C2_4 & C11_4 & C12_4 & $30.6(12)$ \\
\hline C17_4 & C2_4 & C11_4 & C16_4 & $-152.5(9)$ \\
\hline C17_4 & C18_4 & C19_4 & C20_4 & $1.5(17)$ \\
\hline C18_4 & C17_4 & C22_4 & C21_4 & $3.4(15)$ \\
\hline C18_4 & C19_4 & C20_4 & C21_4 & $2.3(19)$ \\
\hline C19_4 & C20_4 & C21_4 & C22_4 & $-3(2)$ \\
\hline C20_4 & C21_4 & C22_4 & C17_4 & $0.2(18)$ \\
\hline C22_4 & C17_4 & C18_4 & C19_4 & $-4.3(14)$ \\
\hline C23_4 & C9_4 & C10_4 & C5_4 & $172.2(8)$ \\
\hline C23_4 & C24_4 & C25_4 & C26_4 & 1(2) \\
\hline C24_4 & C23_4 & C28_4 & C27_4 & $-2.3(18)$ \\
\hline C24_4 & C25_4 & C26_4 & C27_4 & $-1.3(19)$ \\
\hline C24_4 & C25_4 & C26_4 & C29_4 & $-179.9(11)$ \\
\hline C25_4 & C26_4 & C27_4 & C28_4 & $-0.7(19)$ \\
\hline C25_4 & C26_4 & C29_4 & C30_4 & $-17.0(14)$ \\
\hline C25_4 & C26_4 & C29_4 & C31_4 & $104.0(12)$ \\
\hline C25_4 & C26_4 & C29_4 & C32_4 & $-138.4(11)$ \\
\hline C26_4 & C27_4 & C28_4 & C23_4 & $3(2)$ \\
\hline C27_4 & C26_4 & C29_4 & C30_4 & $164.4(11)$ \\
\hline C27_4 & C26_4 & C29_4 & C31_4 & $-74.5(13)$ \\
\hline
\end{tabular}




\begin{tabular}{llllc}
\hline Atom & Atom & Atom & Atom & Angle ${ }^{\circ}$ \\
\hline C27_4 & C26_4 & C29_4 & C32_4 & $43.1(13)$ \\
C28_4 & C23_4 & C24_4 & C25_4 & $0.3(19)$ \\
C29_4 & C26_4 & C27_4 & C28_4 & $177.9(11)$ \\
Rh1_7 & 01_4 & C4_4 & O2_4 & $10.7(12)$ \\
Rh1_7 & O1_4 & C4_4 & C1_4 & $-169.9(5)$ \\
Rh2_7 & O2_4 & C4_4 & 01_4 & $7.4(11)$ \\
Rh2_7 & O2_4 & C4_4 & C1_4 & $-171.9(5)$ \\
Rh1_8 & O1 & C4 & O2 & $7.2(12)$ \\
Rh1_8 & 01 & C4 & C1 & $-168.8(5)$ \\
Rh1_8 & O1_3 & C4_3 & O2_3 & $8.2(11)$ \\
Rh1_8 & O1_3 & C4_3 & C1_3 & $-166.2(4)$ \\
Rh2_8 & O2 & C4 & O1 & $12.2(12)$ \\
Rh2_8 & O2 & C4 & C1 & $-172.1(6)$ \\
Rh2_8 & O2_3 & C4_3 & O1_3 & $13.7(11)$ \\
Rh2_8 & O2_3 & C4_3 & C1_3 & $-172.1(5)$ \\
Rh1_10 & 01_2 & C4_2 & O2_2 & $11.8(13)$ \\
Rh1_10 & 01_2 & C4_2 & C1_2 & $-167.6(5)$ \\
Rh2_10 & O2_2 & C4_2 & 01_2 & $6.0(13)$ \\
Rh2_10 & O2_2 & C4_2 & C1_2 & $-174.6(5)$
\end{tabular}

Table 14: Hydrogen Fractional Atomic Coordinates $\left(\times 10^{4}\right)$ and Equivalent Isotropic Displacement Parameters $\left(\AA^{2} \times 10^{3}\right)$ for $\mathbf{R h}-\mathbf{2 C l 5 t B u P h} . U_{e q}$ is defined as $1 / 3$ of the trace of the orthogonalised $U_{i j}$.

\begin{tabular}{lclll}
\hline Atom & \multicolumn{1}{c}{$\mathbf{x}$} & \multicolumn{1}{c}{$\mathbf{z}$} & $\boldsymbol{U}_{\boldsymbol{e q}}$ \\
\hline H3A & 979.6 & 3513.53 & 4135.58 & 54 \\
H3B & 641.94 & 3765.31 & 3369.67 & 54 \\
H7 & $1881(4)$ & $4552(4)$ & $7262(11)$ & 59 \\
H8 & $1648(4)$ & $4026(4)$ & $8471(12)$ & 64 \\
H10 & $803(3)$ & $3644(3)$ & $6141(9)$ & 40 \\
H12 & $1907(4)$ & $4316(4)$ & $2364(13)$ & 67 \\
H13 & $2612(4)$ & $4105(5)$ & $2489(15)$ & 80 \\
H14 & $2813(5)$ & $3585(6)$ & $3693(19)$ & 117 \\
H15 & $2300(4)$ & $3276(5)$ & $4966(17)$ & 87 \\
H16 & $1599(4)$ & $3496(4)$ & $4815(12)$ & 64 \\
H18 & $1082(4)$ & $3802(4)$ & $1329(10)$ & 54 \\
H19 & $933(4)$ & $4165(5)$ & $-280(12)$ & 79 \\
H20 & $1033(5)$ & $4925(4)$ & $-332(14)$ & 84 \\
H21 & $1150(5)$ & $5266(4)$ & $1349(13)$ & 81 \\
H22 & $1295(3)$ & $4889(3)$ & $3022(10)$ & 47 \\
H24 & $376(3)$ & $3620(3)$ & $7752(10)$ & 44 \\
H25 & $56(4)$ & $3244(4)$ & $9171(9)$ & 56 \\
H27 & $1222(4)$ & $2893(3)$ & $10357(9)$ & 48 \\
H28 & $1532(4)$ & $3343(3)$ & $9000(10)$ & 54 \\
H30A & -156.27 & 3014.38 & 10703.4 & 93 \\
H30B & -164.39 & 2614.58 & 11456.52 & 93 \\
H30C & -135.96 & 2570.56 & 10156.32 & 93 \\
H31A & 529.19 & 2217.16 & 10041.18 & 83 \\
H31B & 427.85 & 2148.1 & 11307.14 & 83 \\
H31C & 866.38 & 2318.44 & 10949.03 & 83 \\
H32A & 815.77 & 2942.07 & 12123.54 & 89 \\
H32B & 377.32 & 2809.31 & 12581.89 & 89 \\
H32C & 431.58 & 3239.94 & 11978.88 & 89 \\
H1WA_5 & $100(40)$ & $10280(20)$ & $-3000(400)$ & 51 \\
H1WB_5 & $240(30)$ & $9820(30)$ & $-3000(400)$ & 51 \\
H1WA_6 & $-230(20)$ & $9850(40)$ & $3340(50)$ & 49
\end{tabular}




\begin{tabular}{|c|c|c|c|c|}
\hline Atom & $\mathbf{x}$ & $\mathbf{y}$ & $\mathbf{z}$ & $U_{e q}$ \\
\hline H1WB_6 & $255(16)$ & $9900(50)$ & $3340(50)$ & 49 \\
\hline H1WA_9 & $-242(16)$ & $4880(40)$ & $7620(50)$ & 38 \\
\hline H1WB_9 & $244(16)$ & $4880(40)$ & $7620(50)$ & 38 \\
\hline H1WA_11 & $5030(60)$ & $4712(18)$ & $2720(170)$ & 21 \\
\hline H1WB_11 & $5230(30)$ & $5160(40)$ & $2810(140)$ & 21 \\
\hline H1WA_12 & $5270(20)$ & $5120(40)$ & $-3610(70)$ & 51 \\
\hline H1WB_12 & $5010(50)$ & $4704(6)$ & $-3610(70)$ & 51 \\
\hline H1WA_13 & $-120(40)$ & 4728(17) & $1170(70)$ & 51 \\
\hline H1WB_13 & $296(6)$ & $4990(40)$ & $1170(70)$ & 51 \\
\hline H3A_2 & 3213.77 & 5067.76 & -655.58 & 73 \\
\hline H3B_2 & 3605 & 5195.3 & -1457.91 & 73 \\
\hline H7_2 & $3666(4)$ & $3776(3)$ & 2592(8) & 74 \\
\hline H8_2 & $3274(3)$ & $4262(3)$ & $3721(9)$ & 66 \\
\hline H10_2 & $3420(3)$ & $5157(3)$ & 1315(7) & 51 \\
\hline H12_2 & $3394(4)$ & $3788(4)$ & $-2287(15)$ & 118 \\
\hline H13_2 & $2792(4)$ & $3367(4)$ & $-2280(12)$ & 92 \\
\hline H14_2 & $2332(4)$ & $3412(4)$ & $-697(13)$ & 111 \\
\hline H15_2 & $2292(5)$ & $4061(4)$ & $187(17)$ & 132 \\
\hline H16_2 & $2792(3)$ & $4583(4)$ & $-144(13)$ & 108 \\
\hline H18_2 & $3384(4)$ & $4826(4)$ & $-3384(7)$ & 83 \\
\hline H19_2 & $3756(5)$ & $4760(5)$ & $-5065(10)$ & 110 \\
\hline H20_2 & 4303(5) & $4257(5)$ & $-5192(11)$ & 115 \\
\hline H21_2 & $4547(5)$ & $3933(5)$ & $-3570(9)$ & 109 \\
\hline H22_2 & $4163(3)$ & $4017(4)$ & $-1846(9)$ & 82 \\
\hline H24_2 & $3570(4)$ & $5557(3)$ & 2911(10) & 77 \\
\hline H25_2 & $3394(3)$ & $6049(4)$ & $4277(9)$ & 72 \\
\hline H27_2 & $2524(3)$ & $5225(3)$ & $5499(9)$ & 63 \\
\hline H28_2 & $2716(3)$ & $4736(4)$ & $4142(9)$ & 74 \\
\hline H30A_2 & 3342.63 & 6349.33 & 6032.15 & 116 \\
\hline H30B_2 & 2955.5 & 6607.78 & 6434.02 & 116 \\
\hline H30C_2 & 3034.81 & 6541.8 & 5155.72 & 116 \\
\hline H31A_2 & 2187.5 & 5872.17 & 5640.18 & 123 \\
\hline H31B_2 & 2328.07 & 6278.83 & 5018.14 & 123 \\
\hline H31C_2 & 2244.81 & 6289.14 & 6306.48 & 123 \\
\hline H32A_2 & 2679.44 & 5555.57 & 7070.87 & 120 \\
\hline H32B_2 & 2717.97 & 5998.58 & 7615.63 & 120 \\
\hline H32C_2 & 3119.65 & 5761.02 & 7223.74 & 120 \\
\hline H3A_3 & 1489.53 & 5952.35 & 4142.1 & 41 \\
\hline H3B_3 & 1242.04 & 5606.73 & 3392.01 & 41 \\
\hline H7_3 & 351(3) & $6895(3)$ & 7041(7) & 55 \\
\hline H8_3 & $890(3)$ & $6715(3)$ & $8347(9)$ & 58 \\
\hline H10_3 & $1389(3)$ & $5904(3)$ & $6067(7)$ & 58 \\
\hline H12_3 & $552(3)$ & $6912(2)$ & $2796(10)$ & 51 \\
\hline H13_3 & 793(3) & $7600(3)$ & 2721(11) & 58 \\
\hline H14_3 & 1484(3) & $7772(3)$ & $3288(11)$ & 59 \\
\hline H15_3 & $1895(4)$ & $7249(3)$ & $4079(13)$ & 73 \\
\hline H16_3 & 1696(3) & $6544(3)$ & $3979(13)$ & 76 \\
\hline H18_3 & $1290(30)$ & $6092(4)$ & 1312(7) & 47 \\
\hline H19_3 & $912(3)$ & $5973(3)$ & $-416(9)$ & 52 \\
\hline H20_3 & 171(3) & $5906(3)$ & $-492(10)$ & 56 \\
\hline H21_3 & $-224(4)$ & $6077(3)$ & 1088(7) & 54 \\
\hline H22_3 & 123(3) & $6160(3)$ & $2865(8)$ & 46 \\
\hline H24_3 & 1546(3) & $5548(3)$ & $7659(9)$ & 65 \\
\hline H25_3 & 1953(4) & $5297(4)$ & $9135(9)$ & 74 \\
\hline H27_3 & $2055(4)$ & $6500(3)$ & $10294(10)$ & 79 \\
\hline H28_3 & $1577(4)$ & $6724(4)$ & 8945(9) & 72 \\
\hline H30A_3 & 2061.95 & 5129.57 & 10899.1 & 117 \\
\hline H30B_3 & 2460.62 & 5161.35 & 11652.02 & 117 \\
\hline
\end{tabular}




\begin{tabular}{|c|c|c|c|c|}
\hline Atom & $\mathbf{x}$ & $\mathbf{y}$ & $\mathbf{z}$ & $U_{e q}$ \\
\hline H30C_3 & 2506.38 & 5129.18 & 10352.87 & 117 \\
\hline H31A_3 & 2824.51 & 5851.13 & 9895.31 & 116 \\
\hline H31B_3 & 2949.1 & 5709.99 & 11102.29 & 116 \\
\hline H31C_3 & 2774.32 & 6160.49 & 10899.34 & 116 \\
\hline H32A_3 & 2175.21 & 6199.76 & 11922.61 & 124 \\
\hline H32B_3 & 2322.29 & 5799.5 & 12563.25 & 124 \\
\hline H32C_3 & 1872.91 & 5818.14 & 12049.2 & 124 \\
\hline H3A_4 & 215.88 & 8220.28 & -205.14 & 49 \\
\hline H3B_4 & 37.27 & 8587.44 & -1019.02 & 49 \\
\hline H7_4 & $1409(3)$ & $8777(3)$ & $3088(7)$ & 52 \\
\hline H8_4 & $940(2)$ & $8384(3)$ & $4248(9)$ & 46 \\
\hline $\mathrm{H} 10 \_4$ & $67(3)$ & $8392(3)$ & 1752(7) & 45 \\
\hline H12_4 & $1500(2)$ & $8666(4)$ & $-1461(11)$ & 67 \\
\hline H13_4 & $2075(3)$ & $8259(3)$ & $-957(11)$ & 74 \\
\hline H14_4 & $1982(3)$ & $7605(4)$ & $-41(13)$ & 91 \\
\hline H15_4 & 1311(3) & $7418(4)$ & $522(12)$ & 74 \\
\hline H16_4 & $738(3)$ & $7862(3)$ & $248(8)$ & 53 \\
\hline H18_4 & $460(3)$ & $8437(3)$ & $-2890(6)$ & 50 \\
\hline H19_4 & $488(4)$ & $8816(3)$ & $-4566(9)$ & 66 \\
\hline H20_4 & $809(4)$ & $9487(3)$ & $-4595(10)$ & 73 \\
\hline H21_4 & $1131(4)$ & $9743(4)$ & $-2985(8)$ & 72 \\
\hline H22_4 & $1083(3)$ & $9353(3)$ & $-1265(9)$ & 59 \\
\hline H24_4 & $-365(3)$ & $8505(4)$ & $3259(10)$ & 61 \\
\hline H25_4 & $-872(4)$ & $8251(4)$ & 4491(11) & 77 \\
\hline H27_4 & $45(3)$ & $7580(4)$ & $6088(10)$ & 71 \\
\hline H28_4 & $535(4)$ & $7809(4)$ & $4776(9)$ & 68 \\
\hline H30A_4 & -1138.66 & 8259.09 & 6439.69 & 96 \\
\hline H30B_4 & -1387.4 & 7862.2 & 6816.52 & 96 \\
\hline H30C_4 & -1326.99 & 7956.67 & 5543.63 & 96 \\
\hline H31A_4 & -641.2 & 7144.57 & 5702.38 & 86 \\
\hline H31B_4 & -1095.98 & 7277.79 & 5378.31 & 86 \\
\hline H31C_4 & -996.64 & 7134.84 & 6600.42 & 86 \\
\hline H32A_4 & -385.66 & 7520.29 & 7486.73 & 89 \\
\hline H32B_4 & -815.35 & 7664.22 & 7979.11 & 89 \\
\hline H32C_4 & -488.63 & 7997.06 & 7585 & 89 \\
\hline
\end{tabular}

Table 15: Atomic Occupancies for all atoms that are not fully occupied in Rh-2Cl5tBuPh.

\begin{tabular}{lrllr}
\hline Atom & Occupancy & & Atom & Occupancy \\
\cline { 5 - 5 } H1WA_5 & 0.25 & & H1WB_1 & 0.5 \\
H1WB_5 & 0.25 & & 3 \\
H1WA_6 & 0.25 & & \\
H1WB_6 & 0.25 & & \\
H1WA_9 & 0.5 & & \\
H1WB_9 & 0.5 & & \\
H1WA_1 & 0.25 & & \\
1 & & & \\
H1WB_1 & 0.25 & & \\
1 & & & \\
H1WA_1 & 0.25 & \\
H1WB_1 & 0.25 & \\
2 & & & \\
H1WA_1 & 0.5 & \\
3 & &
\end{tabular}




\section{Citations}

CrysAlisPro (Rigaku, V1.171.39.43c, 2018)

CrysAlisPro (ROD), Rigaku Oxford Diffraction, Poland (?).

O.V. Dolomanov and L.J. Bourhis and R.J. Gildea and J.A.K. Howard and H. Puschmann, Olex2: A complete structure solution, refinement and analysis program, J. Appl. Cryst., (2009), 42, 339-341.

Sheldrick, G.M., Crystal structure refinement with ShelXL, Acta Cryst., (2015), C71, 3-8.

Sheldrick, G.M., ShelXT-Integrated space-group and crystal-structure determination, Acta Cryst., (2015), A71, 3-8. 


\subsection{X-Ray Crystallographic Data for $\mathrm{Rh}_{2}(S-2-\mathrm{Cl}-4-\mathrm{MesTPCP})_{4}$}

$R_{1}=7.14 \%$

\section{Crystal Data and Experimental}

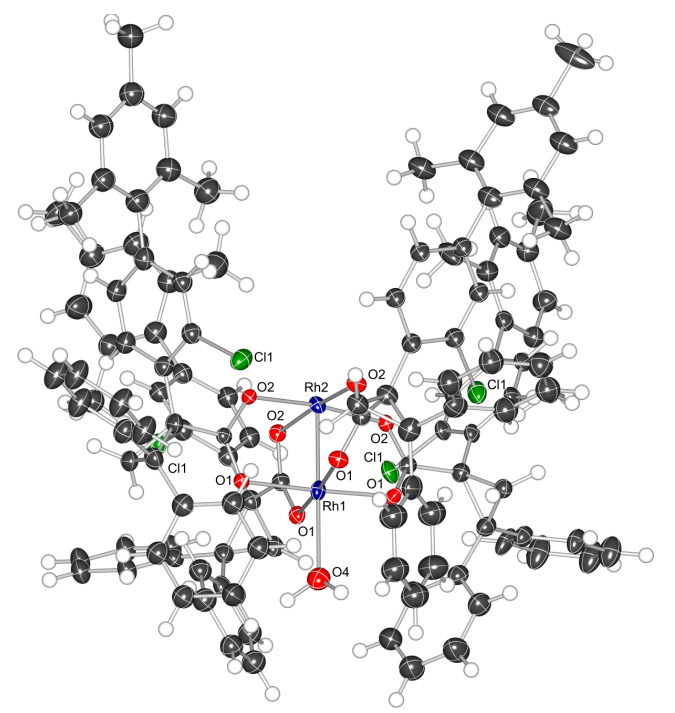

Experimental. Single, violet, block-shaped crystals of 2Cl4triMePh were chosen from the sample as supplied. A suitable crystal with dimensions $0.45 \times 0.42 \times 0.23 \mathrm{~mm}^{3}$ was selected and mounted on a loop with paratone oil on a Rigaku Synergy-S diffractometer. The crystal was kept at a steady $T=100.0(1) \mathrm{K}$ during data collection. The structure was solved with the XT (Sheldrick, 2015) solution program using dual methods and by using 0lex2 1.3-alpha (Dolomanov et al., 2009) as the graphical interface. The model was refined with ShelXL 2018/3 (Sheldrick, 2015) using full matrix least squares minimisation on $\boldsymbol{F}^{2}$.

Crystal Data. $\mathrm{C}_{124} \mathrm{H}_{106} \mathrm{Cl}_{4} \mathrm{O}_{9} \mathrm{Rh}_{2}, M_{r}=2087.70$, tetragonal, $P 4_{1}$ (No. 76), $\mathrm{a}=19.3558(3) \AA, \mathrm{b}=19.3558(3) \AA, \mathrm{c}=$ 31.1374(10) $\AA, \alpha=\beta=\gamma=90^{\circ}, V=11665.5(5) \AA^{3}, \quad T=$ $100.00(10) \mathrm{K}, Z=4, Z^{\prime}=1, \mu\left(\right.$ Mo $\left._{\alpha}\right)=0.428,88953$ reflections measured, 19677 unique $\left(\mathrm{R}_{\mathrm{int}}=0.0387\right)$ which were used in all calculations. The final $w R_{2}$ was 0.1981 (all data) and $R_{1}$ was $0.0714(\mathrm{I} \geq 2 \sigma(\mathrm{I})$ ).

\begin{tabular}{|c|c|}
\hline Compound & 2Cl4triMePh \\
\hline Formula & $\mathrm{C}_{124} \mathrm{H}_{106} \mathrm{Cl}_{4} \mathrm{O}_{9} \mathrm{Rh}$ \\
\hline$D_{\text {calc. }} / \mathrm{g} \mathrm{cm}^{-3}$ & 1.189 \\
\hline$\mu / \mathrm{mm}^{-1}$ & 0.428 \\
\hline Formula Weight & 2087.70 \\
\hline Colour & violet \\
\hline Shape & block-shaped \\
\hline Size $/ \mathrm{mm}^{3}$ & $0.45 \times 0.42 \times 0.23$ \\
\hline$T / \mathrm{K}$ & $100.00(10)$ \\
\hline Crystal System & tetragonal \\
\hline Flack Parameter & $0.08(5)$ \\
\hline Hooft Parameter & $0.113(5)$ \\
\hline Space Group & $P 4_{1}$ \\
\hline$a / \AA$ & $19.3558(3)$ \\
\hline$b / \AA$ & $19.3558(3)$ \\
\hline$c / \AA ̊$ & $31.1374(10)$ \\
\hline$\left.\alpha\right|^{\circ}$ & 90 \\
\hline$\beta /^{\circ}$ & 90 \\
\hline$\gamma /^{\circ}$ & 90 \\
\hline $\mathrm{V} / \AA^{3}$ & $11665.5(5)$ \\
\hline$Z^{\prime}$ & 4 \\
\hline$Z^{\prime}$ & 1 \\
\hline Wavelength/Å & 0.71073 \\
\hline Radiation type & Mo $\mathrm{K}_{\alpha}$ \\
\hline$\Theta_{\min } /^{\circ}$ & 1.625 \\
\hline$\Theta_{\max } /^{\circ}$ & 24.712 \\
\hline Measured Refl's. & 88953 \\
\hline Indep't Refl's & 19677 \\
\hline Refl's I $\geq 2 \sigma(\mathrm{I})$ & 18756 \\
\hline$R_{\text {int }}$ & 0.0387 \\
\hline Parameters & 1643 \\
\hline Restraints & 5450 \\
\hline Largest Peak & 2.550 \\
\hline Deepest Hole & -0.879 \\
\hline GooF & 1.048 \\
\hline$w R_{2}$ (all data) & 0.1981 \\
\hline$w R_{2}$ & 0.1961 \\
\hline$R_{1}$ (all data) & 0.0737 \\
\hline$R_{1}$ & 0.0714 \\
\hline
\end{tabular}


Structure Quality Indicators

Reflections:

Refinement:

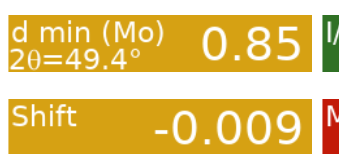

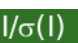

Maxpeak
34.6

\section{Rint}

2.5
$3.87 \%$

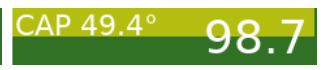

Min Peak

A violet block-shaped-shaped crystal with dimensions $0.45 \times 0.42 \times 0.23 \mathrm{~mm}^{3}$ was mounted on a loop with paratone oil. Data were collected using a XtaLAB Synergy, Dualflex, HyPix diffractometer operating at $T=$ $100.00(10) \mathrm{K}$.

Data were measured using $\omega$ scans using $\mathrm{Mo} \mathrm{K}_{\alpha}$ radiation. The diffraction pattern was indexed and the total number of runs and images was based on the strategy calculation from the program CrysAlisPro 1.171.39.29d (Rigaku OD, 2017). The maximum resolution that was achieved was $\Theta=24.712^{\circ}(0.85 \AA$ ).

The unit cell was refined using CrysAlisPro 1.171.39.29d (Rigaku OD, 2017) on 56021 reflections, 63\% of the observed reflections.

Data reduction, scaling and absorption corrections were performed using CrysAlisPro 1.171.39.29d (Rigaku OD, 2017). The final completeness is $98.90 \%$ out to $24.712^{\circ}$ in $\Theta$. No absorption correction was performed. The absorption coefficient $\mu$ of this material is $0.428 \mathrm{~mm}^{-1}$ at this wavelength $(\lambda=0.71073 \AA)$ and the minimum and maximum transmissions are 0.288 and 1.000 .

The structure was solved and the space group $P 4_{1}$ (\# 76) determined by the XT (Sheldrick, 2015) structure solution program using using dual methods and refined by full matrix least squares minimisation on $\boldsymbol{F}^{2}$ using version 2018/3 of ShelXL 2018/3 (Sheldrick, 2015). All non-hydrogen atoms were refined anisotropically. Hydrogen atom positions were calculated geometrically and refined using the riding model. Hydrogen atom positions were calculated geometrically and refined using the riding model. 


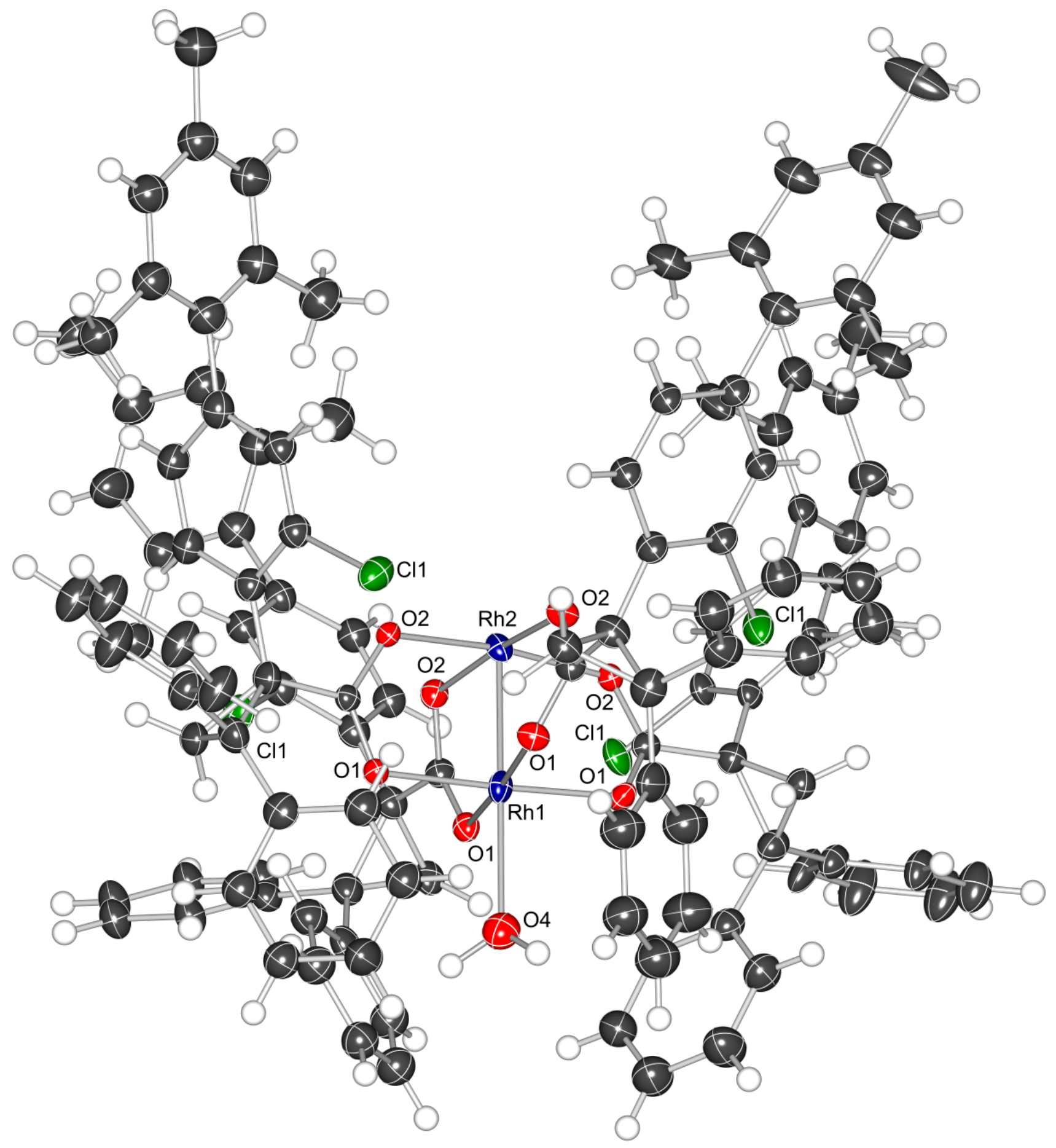

Figure 5:

_refine_special_details: Refined as a 2-component inversion twin. The structure has whole molecule disorder.

_exptl_absorpt_process_details: CrysAlisPro 1.171.39.29d (Rigaku Oxford Diffraction, 2017)Numerical absorption correction based on gaussian integration over a multifaceted crystal modelEmpirical absorption correction using spherical harmonics, implemented in SCALE3 ABSPACK scaling algorithm. 
Data Plots: Diffraction Data
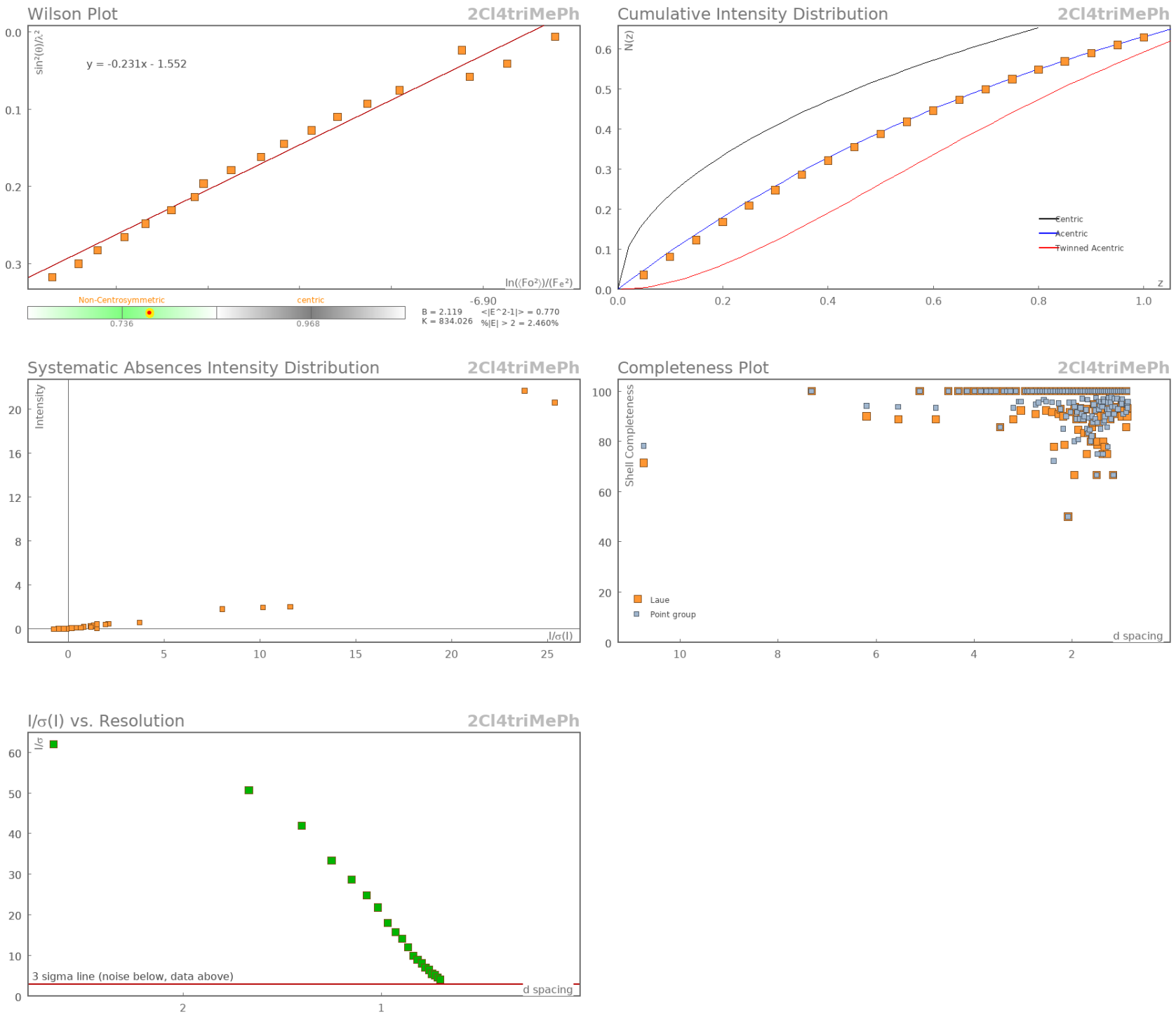

Data Plots: Refinement and Data
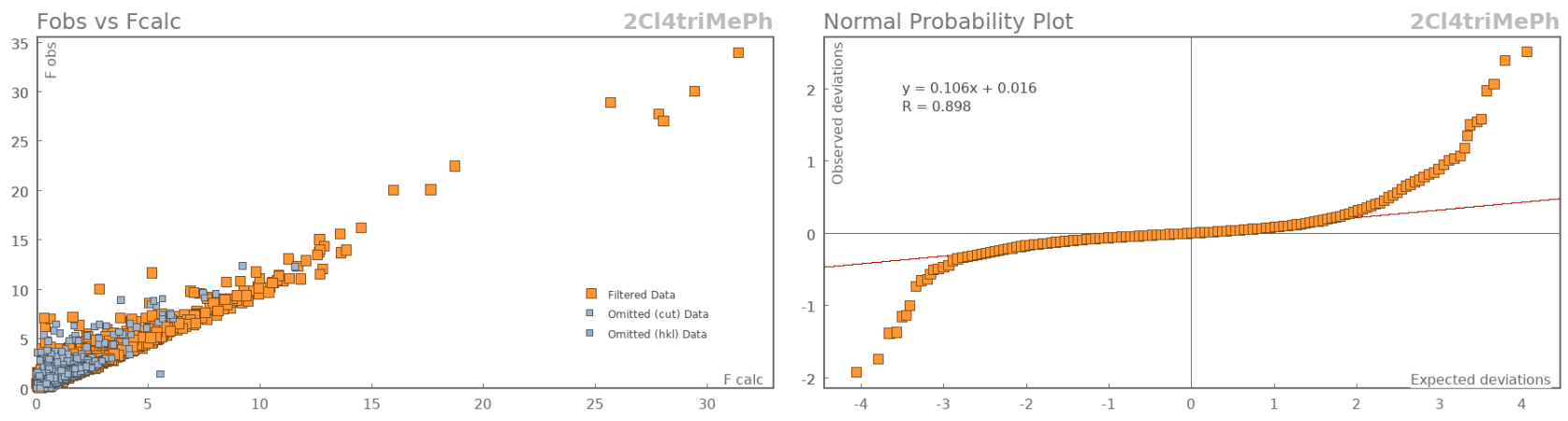

Images of the Crystal on the Diffractometer 

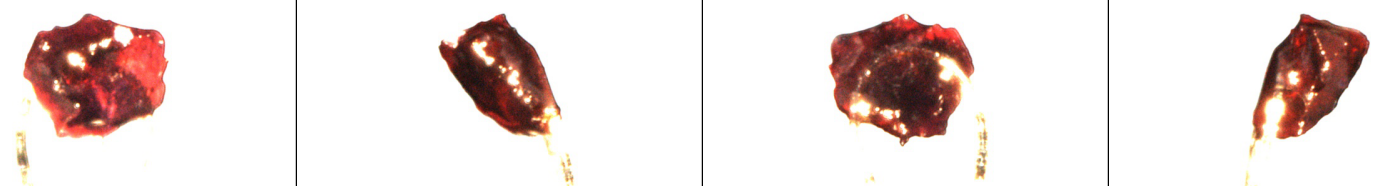

Table 16: Fractional Atomic Coordinates $\left(\times 10^{4}\right)$ and Equivalent Isotropic Displacement Parameters $\left(\AA^{2} \times 10^{3}\right)$ for 2Cl4triMePh. $U_{e q}$ is defined as $1 / 3$ of the trace of the orthogonalised $U_{i j}$.

\begin{tabular}{|c|c|c|c|c|}
\hline Atom & $\mathbf{x}$ & $\mathbf{y}$ & $\mathbf{z}$ & $U_{e q}$ \\
\hline$\overline{\mathrm{Rh} 1}$ & $4995(4)$ & $9676(4)$ & $6110.2(16)$ & $20.3(4)$ \\
\hline Rh2 & $4864(4)$ & $9786(3)$ & $5351(2)$ & $20.4(4)$ \\
\hline 04 & $5066.7(10)$ & $9445(9)$ & $6853.9(15)$ & $36(3)$ \\
\hline Rh1A & $5034(4)$ & $9669(4)$ & $6052.3(15)$ & $20.3(4)$ \\
\hline Rh2A & $4887(4)$ & $9780(3)$ & $5297(2)$ & $20.4(4)$ \\
\hline $04 \mathrm{~A}$ & $5210(5)$ & $9543(9)$ & $6800.5(7)$ & $36(3)$ \\
\hline Cl1_3 & $2474(8)$ & $9709(5)$ & $5543(3)$ & $31.0(8)$ \\
\hline 01_3 & $4328(8)$ & 8891(11) & $6021(4)$ & $21.4(16)$ \\
\hline 02_3 & $4035(8)$ & $9173(10)$ & $5343(4)$ & 22.1(17) \\
\hline C1_3 & $3946(5)$ & $8861(8)$ & $5697(3)$ & $23.2(15)$ \\
\hline C2_3 & $3316(4)$ & $8401(5)$ & $5708(3)$ & $27.5(11)$ \\
\hline C3_3 & $2945(4)$ & $8250(5)$ & $6134(2)$ & $29.3(11)$ \\
\hline C4_3 & $3413(5)$ & $7718(5)$ & $5943(3)$ & $27.9(17)$ \\
\hline C5_3 & $2919(3)$ & $8431(5)$ & $5296(2)$ & $27.9(11)$ \\
\hline C6_3 & $2513(4)$ & $9012(5)$ & $5187(2)$ & $30(2)$ \\
\hline C7_3 & $2152(5)$ & $9055(5)$ & $4819(3)$ & $25(4)$ \\
\hline C8_3 & $2164(5)$ & $8510(5)$ & $4519(3)$ & $27.9(11)$ \\
\hline C9_3 & $2561(6)$ & $7936(5)$ & $4623(3)$ & $29.3(12)$ \\
\hline C10_3 & $2925(6)$ & $7891(5)$ & $4994(3)$ & $29.3(12)$ \\
\hline C11_3 & $1807(4)$ & $8593(5)$ & $4096(3)$ & $34.8(16)$ \\
\hline C12_3 & $1087(4)$ & $8573(5)$ & $4090(2)$ & $37.9(17)$ \\
\hline C13_3 & $740(5)$ & $8717(6)$ & $3709(3)$ & $44.1(16)$ \\
\hline C14_3 & $1096(6)$ & $8876(6)$ & $3340(3)$ & $44.1(16)$ \\
\hline C15_3 & $1788(6)$ & 8891(7) & $3359(3)$ & $44.1(16)$ \\
\hline C16_3 & $2169(4)$ & $8751(7)$ & $3733(3)$ & $37.6(17)$ \\
\hline C17_3 & $678(5)$ & $8397(11)$ & $4485(3)$ & $36(2)$ \\
\hline C18_3 & $733(8)$ & $9033(10)$ & $2925(4)$ & $51(3)$ \\
\hline C19_3 & $2942(4)$ & $8824(13)$ & $3734(5)$ & $51(6)$ \\
\hline C20_3 & $3155(3)$ & $8612(5)$ & $6541(3)$ & $34(5)$ \\
\hline C21_3 & $3147(5)$ & $9320(5)$ & $6582(3)$ & $39.0(13)$ \\
\hline C22_3 & $3346(6)$ & $9638(4)$ & $6964(2)$ & $39.0(13)$ \\
\hline C23_3 & $3557(6)$ & $9244(3)$ & $7308(2)$ & $37.7(17)$ \\
\hline C24_3 & $3564(8)$ & $8536(3)$ & $7265(3)$ & $39.0(13)$ \\
\hline C25_3 & $3369(7)$ & $8216(5)$ & 6891(3) & $39.0(13)$ \\
\hline C26_3 & $2168(4)$ & $8126(6)$ & $6110(3)$ & $30(4)$ \\
\hline C27_3 & $1874(5)$ & $7662(6)$ & $5829(3)$ & $34.2(14)$ \\
\hline C28_3 & $1172(4)$ & $7562(5)$ & $5801(3)$ & $34.2(14)$ \\
\hline C29_3 & $735(4)$ & $7936(4)$ & $6062(2)$ & $37(2)$ \\
\hline C30_3 & $1008(5)$ & $8405(4)$ & $6348(3)$ & $34.2(14)$ \\
\hline C31_3 & $1713(5)$ & $8494(6)$ & $6369(3)$ & $34.2(14)$ \\
\hline Cl1_9 & $4915(6)$ & $12187(11)$ & $5506(5)$ & $33.7(11)$ \\
\hline 01_9 & $4265(8)$ & $10407(7)$ & $6100(3)$ & $20.2(15)$ \\
\hline 02_9 & $4323(9)$ & $10640(7)$ & $5393(3)$ & $18.5(15)$ \\
\hline C1_9 & $4114(7)$ & $10753(5)$ & $5773(3)$ & $18.3(16)$ \\
\hline C2_9 & $3662(5)$ & $11383(4)$ & $5820(3)$ & $28.4(13)$ \\
\hline
\end{tabular}




\begin{tabular}{|c|c|c|c|c|}
\hline Atom & $\mathbf{x}$ & $\mathbf{y}$ & $\mathbf{z}$ & $U_{e q}$ \\
\hline C3_9 & $3620(5)$ & $11782(4)$ & $6245(3)$ & $32.3(12)$ \\
\hline C4_9 & $3046(5)$ & $11304(5)$ & $6121(3)$ & $29.2(17)$ \\
\hline C5_9 & $3582(5)$ & $11764(5)$ & $5404(3)$ & 28.8(19) \\
\hline C6_9 & $4125(5)$ & $12167(5)$ & $5234(3)$ & $29.2(17)$ \\
\hline C7_9 & $4068(6)$ & $12522(6)$ & $4864(3)$ & $30.0(18)$ \\
\hline C8_9 & $3450(6)$ & $12507(6)$ & $4626(3)$ & $35(2)$ \\
\hline C9_9 & $2912(6)$ & 12112(8) & 4791(4) & $33(2)$ \\
\hline C10_9 & $2967(5)$ & 11753(7) & $5163(3)$ & $33(2)$ \\
\hline C11_9 & $3424(7)$ & $12834(5)$ & 4191(3) & $41(2)$ \\
\hline C12_9 & $3332(12)$ & $13547(6)$ & $4167(3)$ & $40(3)$ \\
\hline C13_9 & 3395(13) & $13875(6)$ & $3771(4)$ & $40(3)$ \\
\hline C14_9 & $3575(14)$ & 13513(7) & $3409(4)$ & $44(2)$ \\
\hline C15_9 & $3654(13)$ & 12827(7) & $3444(4)$ & $40(3)$ \\
\hline C16_9 & $3580(13)$ & $12462(5)$ & $3831(4)$ & $40(3)$ \\
\hline C17_9 & $3090(16)$ & 13957(8) & $4549(4)$ & $43(3)$ \\
\hline C18_9 & $3630(20)$ & $13855(10)$ & 2977(5) & $51(5)$ \\
\hline C19_9 & $3647(16)$ & 11689(6) & $3839(6)$ & $48(3)$ \\
\hline C20_9 & $4059(6)$ & $11603(5)$ & $6627(3)$ & $32.7(18)$ \\
\hline C21_9 & $4763(6)$ & $11519(6)$ & $6604(4)$ & $37.1(14)$ \\
\hline C22_9 & $5144(6)$ & $11320(7)$ & $6963(4)$ & $37.1(14)$ \\
\hline C23_9 & $4817(7)$ & 11204(10) & $7349(4)$ & $46(3)$ \\
\hline C24_9 & 4111(8) & 11289(11) & $7370(4)$ & 37.1(14) \\
\hline C25_9 & $3730(7)$ & 11484(9) & $7019(3)$ & $37.1(14)$ \\
\hline C26_9 & $3494(6)$ & $12559(5)$ & $6208(3)$ & $36(2)$ \\
\hline C27_9 & $2910(6)$ & $12832(5)$ & $6022(4)$ & $44.4(19)$ \\
\hline C28_9 & 2814(7) & 13532(5) & $5972(5)$ & $44.4(19)$ \\
\hline C29_9 & $3315(8)$ & 13985(5) & $6111(6)$ & $45(3)$ \\
\hline C30_9 & $3908(8)$ & 13732(5) & $6299(6)$ & $44.4(19)$ \\
\hline C31_9 & 3991(7) & $13030(5)$ & $6345(5)$ & $44.4(19)$ \\
\hline Cl1_7 & $7185(8)$ & $9707(4)$ & $5206(3)$ & $34.7(16)$ \\
\hline 01_7 & $5792(7)$ & 10441(9) & $6009(3)$ & $24.1(12)$ \\
\hline 02_7 & $5699(9)$ & 10389(11) & $5289(3)$ & $24.1(12)$ \\
\hline C1_7 & $5987(5)$ & 10581(7) & $5635(3)$ & $21(2)$ \\
\hline C2__7 & $6597(4)$ & 11055(5) & $5569(3)$ & $28.5(12)$ \\
\hline C3_7 & $7124(4)$ & $11203(5)$ & $5924(3)$ & $35.4(13)$ \\
\hline C4_7 & $6575(5)$ & $11724(5)$ & $5826(3)$ & 29.7(18) \\
\hline C5_7 & $6826(5)$ & $11062(5)$ & $5109(2)$ & $26.7(13)$ \\
\hline C6_7 & 7161(6) & $10486(5)$ & 4923(3) & $26.6(13)$ \\
\hline C7_7 & $7439(6)$ & 10501(5) & $4527(3)$ & $34(4)$ \\
\hline C8_7 & 7411(4) & $11105(5)$ & $4275(3)$ & $26.7(13)$ \\
\hline C9_7 & $7080(6)$ & 11673(5) & $4454(3)$ & $26.6(13)$ \\
\hline C10_7 & $6799(6)$ & 11661(5) & $4853(3)$ & $26.6(13)$ \\
\hline C11_7 & $7730(5)$ & 11111(4) & $3838(3)$ & $36(4)$ \\
\hline C12_7 & $8437(5)$ & $11238(5)$ & $3807(3)$ & $40.1(18)$ \\
\hline C13_7 & $8747(6)$ & $11244(6)$ & $3404(4)$ & $44(2)$ \\
\hline C14_7 & $8369(7)$ & 11128(5) & $3038(3)$ & $39(2)$ \\
\hline C15_7 & $7690(7)$ & 11008(7) & $3081(3)$ & $44(2)$ \\
\hline C16_7 & $7345(6)$ & 10995(7) & $3478(3)$ & 40.1(18) \\
\hline C17_7 & $8865(7)$ & $11400(15)$ & $4197(4)$ & $44(2)$ \\
\hline C18_7 & $8695(9)$ & 11132(8) & $2600(4)$ & $53(8)$ \\
\hline C19_7 & 6612(7) & 10741(14) & $3504(5)$ & $44(2)$ \\
\hline C20_7 & $7120(4)$ & 10852(6) & $6354(3)$ & $41(2)$ \\
\hline C21_7 & 7088(8) & $10147(6)$ & $6405(4)$ & $50.9(17)$ \\
\hline C22_7 & $7089(9)$ & 9849(7) & $6812(4)$ & $50.9(17)$ \\
\hline C23_7 & 7123(8) & 10259(8) & $7173(3)$ & $50.9(17)$ \\
\hline C24_7 & $7154(10)$ & $10965(8)$ & $7120(3)$ & $50.9(17)$ \\
\hline C25_7 & $7149(9)$ & $11265(7)$ & $6721(3)$ & $50.9(17)$ \\
\hline C26_7 & $7858(4)$ & 11358(5) & $5772(3)$ & $38(3)$ \\
\hline
\end{tabular}




\begin{tabular}{|c|c|c|c|c|}
\hline Atom & $\mathbf{x}$ & $\mathbf{y}$ & $\mathbf{z}$ & $U_{e q}$ \\
\hline$\overline{C 27 \_7}$ & $8099(5)$ & $12020(5)$ & $5713(3)$ & $44.2(16)$ \\
\hline C28_7 & $8748(6)$ & $12157(6)$ & $5554(4)$ & $44.2(16)$ \\
\hline C29_7 & $9181(5)$ & 11618(7) & $5448(5)$ & 45.1(19) \\
\hline C30_7 & $8958(6)$ & $10948(7)$ & $5502(6)$ & $44.2(16)$ \\
\hline C31_7 & $8307(5)$ & $10825(6)$ & $5661(5)$ & $44.2(16)$ \\
\hline Cl1_4 & $2509(8)$ & $9757(5)$ & $5608(4)$ & $31.0(8)$ \\
\hline 01_4 & $4292(8)$ & $8895(11)$ & $6086(4)$ & $21.4(16)$ \\
\hline 02_4 & $3997(8)$ & $9203(10)$ & $5415(4)$ & $22.1(17)$ \\
\hline C1_4 & $3942(5)$ & $8831(7)$ & $5749(3)$ & $23.2(15)$ \\
\hline C2_4 & $3287(4)$ & $8406(5)$ & $5766(3)$ & $27.6(11)$ \\
\hline C3_4 & 2921(4) & $8276(5)$ & $6197(3)$ & $29.2(11)$ \\
\hline C4_4 & $3352(5)$ & $7718(5)$ & $6001(3)$ & $27.9(17)$ \\
\hline C5_4 & $2877(5)$ & $8455(5)$ & $5361(3)$ & $27.9(11)$ \\
\hline C6_4 & $2467(9)$ & $9041(6)$ & $5266(4)$ & $30(3)$ \\
\hline C7_4 & $2060(11)$ & $9082(7)$ & $4917(5)$ & $29(3)$ \\
\hline C8_4 & $2073(6)$ & $8560(5)$ & $4602(3)$ & $27.9(11)$ \\
\hline C9_4 & 2492(9) & 7992(7) & $4684(4)$ & $29.3(12)$ \\
\hline C10_4 & $2872(10)$ & $7930(7)$ & $5048(4)$ & $29.3(12)$ \\
\hline C11_4 & $1606(5)$ & $8607(6)$ & $4220(3)$ & $34.8(16)$ \\
\hline C12_4 & $898(5)$ & $8521(6)$ & $4286(3)$ & $37.9(17)$ \\
\hline C13_4 & $440(5)$ & $8687(6)$ & $3958(4)$ & $52(6)$ \\
\hline C14_4 & $672(6)$ & $8934(6)$ & $3571(4)$ & $44.1(16)$ \\
\hline C15_4 & $1356(6)$ & $9011(7)$ & $3518(4)$ & $44.1(16)$ \\
\hline C16_4 & $1845(5)$ & $8852(7)$ & $3836(3)$ & $37.6(17)$ \\
\hline C17_4 & $619(7)$ & $8260(12)$ & $4706(4)$ & $36(2)$ \\
\hline C18_4 & 185(8) & $9122(10)$ & $3213(5)$ & $51(3)$ \\
\hline C19_4 & $2598(6)$ & $9000(14)$ & $3762(6)$ & $51(6)$ \\
\hline C20_4 & $3161(5)$ & $8616(5)$ & $6605(3)$ & $32(4)$ \\
\hline C21_4 & $3188(7)$ & $9322(5)$ & $6654(4)$ & $36(2)$ \\
\hline C22_4 & $3483(8)$ & $9619(6)$ & $7017(4)$ & $39.0(13)$ \\
\hline C23_4 & $3756(8)$ & $9206(7)$ & $7336(4)$ & $37.7(17)$ \\
\hline C24_4 & $3727(9)$ & $8500(7)$ & $7285(4)$ & $39.0(13)$ \\
\hline C25_4 & $3437(7)$ & $8201(6)$ & $6928(3)$ & $39.0(13)$ \\
\hline C26_4 & $2137(4)$ & $8205(6)$ & $6179(3)$ & $28(4)$ \\
\hline C27_4 & $1806(5)$ & $7713(7)$ & $5935(4)$ & $34.2(14)$ \\
\hline C28_4 & $1097(5)$ & $7675(7)$ & $5906(5)$ & $34.2(14)$ \\
\hline C29_4 & 694(5) & $8143(7)$ & $6127(5)$ & $37(2)$ \\
\hline C30_4 & $1005(5)$ & 8644(8) & $6375(5)$ & $34.2(14)$ \\
\hline C31_4 & 1715(5) & $8669(7)$ & 6398(5) & $34.2(14)$ \\
\hline Cl1_6 & $4941(5)$ & $12132(11)$ & $5594(5)$ & $33.7(11)$ \\
\hline 01_6 & $4226(8)$ & $10405(7)$ & $6192(3)$ & $20.2(15)$ \\
\hline 02_6 & $4285(9)$ & $10623(7)$ & $5484(3)$ & $18.5(15)$ \\
\hline C1_6 & $4052(7)$ & $10729(5)$ & $5860(3)$ & $18.3(16)$ \\
\hline C2_6 & $3646(5)$ & $11390(4)$ & $5909(3)$ & $28.4(13)$ \\
\hline C3_6 & $3633(5)$ & $11787(4)$ & $6336(3)$ & $32.3(12)$ \\
\hline C4_6 & $3026(5)$ & 11353(5) & $6209(3)$ & 29.2(17) \\
\hline C5_6 & $3587(5)$ & $11777(4)$ & $5494(3)$ & 28.2(19) \\
\hline C6_6 & $4147(5)$ & $12160(5)$ & $5326(3)$ & 29.2(17) \\
\hline C7_6 & $4105(5)$ & $12524(6)$ & $4960(3)$ & $30.0(18)$ \\
\hline C8_6 & $3487(5)$ & $12541(6)$ & $4721(3)$ & $33(3)$ \\
\hline C9_6 & 2933(5) & 12166(8) & $4883(4)$ & $33(2)$ \\
\hline C10_6 & 2973(5) & 11798(7) & $5253(3)$ & $32(2)$ \\
\hline C11_6 & $3469(6)$ & $12899(5)$ & $4296(3)$ & $41(2)$ \\
\hline C12_6 & $3301(6)$ & $13599(6)$ & $4288(3)$ & $42(2)$ \\
\hline C13_6 & $3357(7)$ & $13963(6)$ & $3904(4)$ & $43(2)$ \\
\hline C14_6 & $3574(9)$ & $13644(7)$ & $3534(4)$ & $44(2)$ \\
\hline C15_6 & $3731(9)$ & $12969(7)$ & $3554(4)$ & $44(2)$ \\
\hline C16_6 & $3686(8)$ & $12573(5)$ & 3931(3) & $43(2)$ \\
\hline
\end{tabular}




\begin{tabular}{|c|c|c|c|c|}
\hline Atom & $\mathbf{x}$ & $\mathbf{y}$ & $\mathbf{z}$ & $U_{e q}$ \\
\hline$\overline{C 17 \_6}$ & $3080(16)$ & $13974(8)$ & $4688(4)$ & $43(3)$ \\
\hline C18_6 & $3650(20)$ & $14028(10)$ & $3117(5)$ & $51(5)$ \\
\hline C19_6 & $3899(15)$ & $11827(7)$ & $3929(6)$ & $48(3)$ \\
\hline C20_6 & $4051(6)$ & $11571(5)$ & $6720(3)$ & $32.7(18)$ \\
\hline C21_6 & $4755(6)$ & $11480(6)$ & $6708(4)$ & $37.1(14)$ \\
\hline C22_6 & $5116(6)$ & $11269(7)$ & $7072(4)$ & $37.1(14)$ \\
\hline C23_6 & $4770(7)$ & 11147(10) & 7451(4) & $46(3)$ \\
\hline C24_6 & $4065(8)$ & 11239(11) & $7460(4)$ & $37.1(14)$ \\
\hline C25_6 & $3703(7)$ & $11447(9)$ & $7104(3)$ & $37.1(14)$ \\
\hline C26_6 & $3570(6)$ & $12571(4)$ & $6300(3)$ & $36(2)$ \\
\hline C27_6 & $3020(6)$ & 12892(5) & $6104(3)$ & $44.4(19)$ \\
\hline C28_6 & 2994(6) & $13596(5)$ & $6041(4)$ & $44.4(19)$ \\
\hline C29_6 & $3534(7)$ & $14004(5)$ & $6179(4)$ & $45(3)$ \\
\hline C30_6 & 4094(7) & $13702(6)$ & $6377(5)$ & $44.4(19)$ \\
\hline C31_6 & $4107(6)$ & 12998(5) & $6435(4)$ & $44.4(19)$ \\
\hline Cl1_2 & $7202(8)$ & $9701(4)$ & $5328(3)$ & $34.7(16)$ \\
\hline 01_2 & $5698(7)$ & $10497(8)$ & $6087(3)$ & $24.1(12)$ \\
\hline 02_2 & $5744(9)$ & 10378(11) & $5369(3)$ & $24.1(12)$ \\
\hline C1_2 & $5993(5)$ & 10558(7) & $5730(3)$ & $22(2)$ \\
\hline C2_2 & $6604(4)$ & $11039(5)$ & $5692(3)$ & $28.7(13)$ \\
\hline C3_2 & $7095(4)$ & 11185(5) & $6068(3)$ & $35.4(13)$ \\
\hline C4_2 & $6548(5)$ & 11701(5) & $5954(3)$ & $29.7(18)$ \\
\hline C5_2 & $6876(5)$ & $11065(5)$ & $5242(2)$ & $26.7(13)$ \\
\hline C6_2 & 7186(5) & 10482(5) & $5047(3)$ & $26.6(13)$ \\
\hline C7_2 & $7470(6)$ & $10502(5)$ & $4653(3)$ & $26.6(13)$ \\
\hline C8_2 & $7470(6)$ & 11118(5) & $4412(3)$ & $26.7(13)$ \\
\hline C9_2 & 7166(7) & 11694(5) & $4600(3)$ & $26.6(13)$ \\
\hline C10_2 & $6880(6)$ & $11676(5)$ & 4997(3) & $26.6(13)$ \\
\hline C11_2 & 7798(5) & $11137(5)$ & $3977(3)$ & $35(4)$ \\
\hline C12_2 & $8514(5)$ & $11200(6)$ & $3952(3)$ & $40.1(18)$ \\
\hline C13_2 & $8831(6)$ & $11204(6)$ & $3551(4)$ & $44(2)$ \\
\hline C14_2 & $8450(7)$ & 11148(7) & $3181(4)$ & $39(2)$ \\
\hline C15_2 & 7761(7) & 11092(7) & $3217(3)$ & $44(2)$ \\
\hline C16_2 & $7410(5)$ & 11085(7) & $3612(3)$ & $40.1(18)$ \\
\hline C17_2 & 8951(7) & 11261(10) & $4350(4)$ & $44(2)$ \\
\hline C18_2 & 8783(10) & 11149(11) & $2745(4)$ & $64(9)$ \\
\hline C19_2 & $6637(6)$ & 11017(10) & $3625(5)$ & $44(2)$ \\
\hline C20_2 & $7052(3)$ & $10819(6)$ & $6492(3)$ & $41(2)$ \\
\hline C21_2 & $7009(5)$ & 10113(6) & 6531(4) & $50.9(17)$ \\
\hline C22_2 & $6970(6)$ & $9800(7)$ & $6933(4)$ & $56(6)$ \\
\hline C23_2 & 6974(6) & 10197(8) & 7301(4) & $50.9(17)$ \\
\hline C24_2 & 7016(9) & 10904(8) & $7259(3)$ & $50.9(17)$ \\
\hline C25_2 & $7054(8)$ & 11218(7) & $6865(3)$ & $50.9(17)$ \\
\hline C26_2 & $7838(4)$ & $11359(5)$ & $5943(2)$ & $38(3)$ \\
\hline C27_2 & $8076(6)$ & $12027(5)$ & $5915(4)$ & $44.2(16)$ \\
\hline C28_2 & $8745(6)$ & 12183(6) & $5802(5)$ & $44.2(16)$ \\
\hline C29_2 & $9202(5)$ & 11657(7) & 5711(5) & 45.1(19) \\
\hline C30_2 & $8984(5)$ & 10981(7) & $5736(6)$ & $44.2(16)$ \\
\hline C31_2 & $8313(5)$ & $10840(6)$ & $5851(5)$ & $44.2(16)$ \\
\hline Cl1_8 & $4790(8)$ & 7475(19) & $5248(5)$ & $29.6(18)$ \\
\hline 01_8 & $5765(9)$ & 8954(8) & $5928(3)$ & $22.5(15)$ \\
\hline 02_8 & $5478(9)$ & 8924(8) & $5227(3)$ & $19.5(15)$ \\
\hline C1_8 & $5782(7)$ & $8697(5)$ & $5558(3)$ & $21.5(15)$ \\
\hline C2_8 & $6222(5)$ & $8065(4)$ & $5480(3)$ & $25.6(13)$ \\
\hline C3_8 & $6441(5)$ & $7589(4)$ & $5850(3)$ & $28.7(14)$ \\
\hline C4_8 & $6946(5)$ & $8102(5)$ & $5672(3)$ & $25.0(16)$ \\
\hline C5_8 & $6125(5)$ & 7781(5) & $5036(3)$ & $21.9(16)$ \\
\hline C6_8 & $5505(5)$ & $7458(6)$ & $4905(3)$ & $23.7(19)$ \\
\hline
\end{tabular}




\begin{tabular}{|c|c|c|c|c|}
\hline Atom & $\mathbf{x}$ & $\mathbf{y}$ & $\mathbf{z}$ & $U_{e q}$ \\
\hline$\overline{C 7 \_8}$ & $5417(5)$ & 7194(6) & $4509(3)$ & $25.4(19)$ \\
\hline C8_8 & $5949(5)$ & $7227(6)$ & $4202(3)$ & $24.6(18)$ \\
\hline C9_8 & $6561(6)$ & $7543(8)$ & $4327(3)$ & $27(2)$ \\
\hline C10_8 & $6653(5)$ & $7810(8)$ & $4725(3)$ & $29(2)$ \\
\hline C11_8 & $5834(6)$ & 6951(5) & $3759(3)$ & $28.4(19)$ \\
\hline C12_8 & 5921(5) & $6244(5)$ & $3692(3)$ & $32.0(19)$ \\
\hline C13_8 & $5899(6)$ & $5987(6)$ & $3275(4)$ & $34(3)$ \\
\hline C14_8 & $5794(6)$ & $6417(7)$ & 2931(3) & $38(2)$ \\
\hline C15_8 & $5712(8)$ & $7094(7)$ & $3007(3)$ & $36(2)$ \\
\hline C16_8 & $5729(9)$ & $7387(6)$ & $3420(3)$ & $35(2)$ \\
\hline C17_8 & 6082(17) & $5762(7)$ & $4057(4)$ & $38(3)$ \\
\hline C18_8 & $5768(10)$ & $6149(10)$ & $2478(4)$ & $58(5)$ \\
\hline C19_8 & $5720(19)$ & $8158(6)$ & $3476(5)$ & $45(4)$ \\
\hline C20_8 & $6182(6)$ & $7683(5)$ & $6301(3)$ & $30.3(18)$ \\
\hline C21_8 & $5502(7)$ & 7594(6) & $6415(4)$ & $35(2)$ \\
\hline C22_8 & $5295(8)$ & $7637(8)$ & $6842(4)$ & $40(2)$ \\
\hline C23_8 & $5773(10)$ & 7771(11) & $7160(3)$ & $47(3)$ \\
\hline C24_8 & 6453(9) & $7861(12)$ & 7043(3) & $42(3)$ \\
\hline C25_8 & $6663(7)$ & $7819(9)$ & $6623(3)$ & $39(3)$ \\
\hline C26_8 & $6552(6)$ & $6830(4)$ & $5737(3)$ & $29(2)$ \\
\hline C27_8 & $7195(6)$ & $6526(6)$ & $5740(4)$ & $34(3)$ \\
\hline C28_8 & $7294(8)$ & $5839(6)$ & $5642(5)$ & $48(4)$ \\
\hline C29_8 & $6735(9)$ & $5433(6)$ & $5536(7)$ & $51(5)$ \\
\hline C30_8 & 6083(8) & $5718(6)$ & $5529(8)$ & $55(5)$ \\
\hline C31_8 & $5999(7)$ & $6405(6)$ & $5629(6)$ & $38(4)$ \\
\hline Cl1_1 & $4807(8)$ & 7472(19) & $5325(5)$ & $29.6(18)$ \\
\hline 01_1 & $5752(9)$ & $8980(8)$ & $6007(4)$ & $22.5(15)$ \\
\hline $02 \_1$ & $5449(9)$ & 8921(7) & $5310(3)$ & $19.5(15)$ \\
\hline C1_1 & $5747(7)$ & $8697(5)$ & $5645(3)$ & $21.5(15)$ \\
\hline C2_1 & $6220(5)$ & $8088(4)$ & $5568(3)$ & $25.6(13)$ \\
\hline C3_1 & 6458(5) & $7625(4)$ & $5939(3)$ & $28.7(14)$ \\
\hline C4_1 & $6937(5)$ & $8164(5)$ & $5766(3)$ & $25.0(16)$ \\
\hline C5_1 & $6142(5)$ & $7797(4)$ & $5125(3)$ & $21.9(16)$ \\
\hline C6_1 & 5523(5) & $7487(6)$ & 4983(3) & 23.7(19) \\
\hline C7_1 & $5449(5)$ & $7216(6)$ & $4588(3)$ & $25.4(19)$ \\
\hline C8_1 & $5997(5)$ & $7229(4)$ & 4291(3) & $24.6(18)$ \\
\hline C9_1 & $6610(5)$ & 7534(8) & $4427(3)$ & $27(2)$ \\
\hline C10_1 & $6686(5)$ & $7807(8)$ & $4825(3)$ & $29(2)$ \\
\hline C11_1 & $5907(6)$ & $6925(5)$ & $3853(3)$ & $28.4(19)$ \\
\hline C12_1 & $5980(6)$ & $6212(5)$ & $3806(3)$ & $32.0(19)$ \\
\hline C13_1 & $5913(6)$ & $5921(6)$ & $3400(4)$ & $38(5)$ \\
\hline C14_1 & $5776(6)$ & $6322(7)$ & $3047(3)$ & $37(2)$ \\
\hline C15_1 & $5709(8)$ & $7006(7)$ & $3105(3)$ & $36(2)$ \\
\hline C16_1 & 5771(9) & $7333(5)$ & $3506(3)$ & $35(2)$ \\
\hline C17_1 & $6070(17)$ & 5748(7) & $4189(4)$ & $38(3)$ \\
\hline C18_1 & $5702(10)$ & $6018(10)$ & $2605(4)$ & $58(5)$ \\
\hline C19_1 & $5605(18)$ & $8088(6)$ & $3552(5)$ & $45(4)$ \\
\hline C20_1 & $6180(6)$ & $7705(5)$ & $6387(3)$ & $30.3(18)$ \\
\hline C21_1 & $5495(7)$ & $7610(6)$ & $6490(4)$ & $35(2)$ \\
\hline C22_1 & $5268(8)$ & $7654(8)$ & $6913(4)$ & $40(2)$ \\
\hline C23_1 & $5731(10)$ & $7795(12)$ & $7238(4)$ & $47(3)$ \\
\hline C24_1 & 6415(9) & $7889(13)$ & 7133(3) & $50(6)$ \\
\hline C25_1 & $6645(7)$ & $7847(10)$ & $6718(3)$ & $39(3)$ \\
\hline C26_1 & $6620(6)$ & 6874(5) & $5829(3)$ & $29(2)$ \\
\hline C27_1 & $7283(6)$ & $6615(6)$ & $5837(4)$ & $34(3)$ \\
\hline C28_1 & $7430(8)$ & $5934(6)$ & $5750(5)$ & $48(4)$ \\
\hline C29_1 & $6900(9)$ & $5485(6)$ & $5651(7)$ & $51(5)$ \\
\hline \multirow[t]{2}{*}{ C30_1 } & $6230(8)$ & $5724(6)$ & 5641(8) & $55(5)$ \\
\hline & & & S383 & \\
\hline
\end{tabular}




\begin{tabular}{lcccc}
\hline Atom & $\mathbf{x}$ & $\mathbf{y}$ & $\mathbf{z}$ & $\boldsymbol{U}_{\text {eq }}$ \\
\hline C31_1 & $6098(7)$ & $6408(6)$ & $5729(6)$ & $38(4)$
\end{tabular}

Table 17: Anisotropic Displacement Parameters $\left(\times 10^{4}\right)$ for 2Cl4triMePh. The anisotropic displacement factor exponent takes the form: $-2 \pi^{2}\left[h^{2} a^{* 2} \times U_{11}+\ldots+2 h k a^{*} \times b^{*} \times U_{12}\right]$

\begin{tabular}{|c|c|c|c|c|c|c|}
\hline Atom & $U_{11}$ & $U_{22}$ & $\boldsymbol{U}_{33}$ & $\boldsymbol{U}_{23}$ & $U_{13}$ & $U_{12}$ \\
\hline$\overline{\mathrm{Rh} 1}$ & $13.7(7)$ & $17.4(4)$ & $29.8(9)$ & $-0.7(8)$ & $-0.2(8)$ & $-1.6(4)$ \\
\hline $\mathrm{Rh} 2$ & $18.6(5)$ & $14.5(4)$ & $28.2(11)$ & $-1.4(6)$ & $3.9(7)$ & $-4.3(3)$ \\
\hline 04 & $36(4)$ & $32(4)$ & $41(4)$ & $4(3)$ & $0(3)$ & $-3(3)$ \\
\hline Rh1A & $13.7(7)$ & $17.4(4)$ & 29.8(9) & $-0.7(8)$ & $-0.2(8)$ & $-1.6(4)$ \\
\hline Rh2A & $18.6(5)$ & $14.5(4)$ & $28.2(11)$ & $-1.4(6)$ & $3.9(7)$ & $-4.3(3)$ \\
\hline $04 \mathrm{~A}$ & $36(4)$ & $32(4)$ & $41(4)$ & $4(3)$ & $0(3)$ & $-3(3)$ \\
\hline Cl1_3 & $35.5(18)$ & $22.6(14)$ & $35(2)$ & $-9.4(14)$ & $-5.5(18)$ & $6.1(13)$ \\
\hline 01_3 & $22(2)$ & $17(2)$ & $25(2)$ & $0.1(19)$ & 2.1(18) & $1.7(19)$ \\
\hline 02_3 & $20(3)$ & $20(2)$ & $26(2)$ & $1(2)$ & $0.0(17)$ & $-1.0(19)$ \\
\hline C1_3 & $24.0(17)$ & $20(3)$ & $26(2)$ & $1.1(17)$ & $1.0(14)$ & $0(2)$ \\
\hline C2_3 & $26.8(16)$ & $24.7(19)$ & $31.1(13)$ & $-0.5(12)$ & $2.8(10)$ & $-4.0(13)$ \\
\hline C3_3 & $25.9(17)$ & 29.6(19) & $32.3(15)$ & $3.5(13)$ & $3.1(12)$ & $0.1(14)$ \\
\hline C4_3 & $21(3)$ & $26.3(18)$ & $36(2)$ & $2.5(15)$ & $4(2)$ & $-4.2(18)$ \\
\hline C5_3 & $26.9(17)$ & $25.7(14)$ & $31.1(12)$ & $-1.3(10)$ & $2.9(11)$ & $-3.0(12)$ \\
\hline C6_3 & $30(3)$ & $26(2)$ & $33(2)$ & $-2.0(15)$ & $1(2)$ & $-1.6(19)$ \\
\hline C7_3 & $22(7)$ & $24(3)$ & $30(3)$ & $0(3)$ & $5(4)$ & $-5(4)$ \\
\hline C8_3 & $26.9(17)$ & $25.7(14)$ & $31.1(12)$ & $-1.3(10)$ & $2.9(11)$ & $-3.0(12)$ \\
\hline C9_3 & $29.3(18)$ & $26.6(14)$ & $32.0(14)$ & $-2.2(10)$ & $1.1(12)$ & $-1.6(12)$ \\
\hline C10_3 & $29.3(18)$ & $26.6(14)$ & $32.0(14)$ & $-2.2(10)$ & $1.1(12)$ & $-1.6(12)$ \\
\hline C11_3 & $35.9(17)$ & $34(3)$ & $34.6(13)$ & $-2.4(14)$ & $-2.6(12)$ & $-1.3(15)$ \\
\hline C12_3 & $36.1(18)$ & $38(3)$ & $39.5(17)$ & $-0.6(18)$ & $-1.5(12)$ & $-1.1(15)$ \\
\hline C13_3 & $45.7(19)$ & $44(2)$ & $42.6(16)$ & $0.6(13)$ & $-6.0(12)$ & $-0.3(15)$ \\
\hline C14_3 & $45.7(19)$ & $44(2)$ & $42.6(16)$ & $0.6(13)$ & $-6.0(12)$ & $-0.3(15)$ \\
\hline C15_3 & $45.7(19)$ & $44(2)$ & $42.6(16)$ & $0.6(13)$ & $-6.0(12)$ & $-0.3(15)$ \\
\hline C16_3 & $38.8(19)$ & $37(3)$ & $37.2(15)$ & $-0.7(16)$ & $-0.1(13)$ & $-0.8(18)$ \\
\hline C17_3 & $32(4)$ & $38(4)$ & $38(2)$ & $-1(2)$ & $-3(2)$ & $-1(3)$ \\
\hline C18_3 & $53(4)$ & $53(5)$ & $47(2)$ & $3(3)$ & $-11(2)$ & $0(3)$ \\
\hline C19_3 & $38.8(19)$ & $42(15)$ & $73(18)$ & $-21(13)$ & $2(2)$ & $-1(3)$ \\
\hline C20_3 & $28(13)$ & $39.0(18)$ & $36(2)$ & $-1.3(14)$ & $2(5)$ & $-0.6(18)$ \\
\hline C21_3 & $40(3)$ & $39.0(17)$ & $38.4(15)$ & $-0.7(13)$ & $-2.2(15)$ & $-0.1(15)$ \\
\hline C22_3 & $40(3)$ & $39.0(17)$ & $38.4(15)$ & $-0.7(13)$ & $-2.2(15)$ & $-0.1(15)$ \\
\hline C23_3 & $36(3)$ & $39.0(17)$ & $37.8(18)$ & $-0.7(13)$ & $-0.7(18)$ & $-0.2(15)$ \\
\hline C24_3 & $40(3)$ & $39.0(17)$ & $38.4(15)$ & $-0.7(13)$ & $-2.2(15)$ & $-0.1(15)$ \\
\hline C25_3 & $40(3)$ & $39.0(17)$ & $38.4(15)$ & $-0.7(13)$ & $-2.2(15)$ & $-0.1(15)$ \\
\hline C26_3 & $25.9(17)$ & $31(7)$ & $34(7)$ & $0(7)$ & $4.2(15)$ & $0.1(16)$ \\
\hline C27_3 & $26.4(16)$ & $37(3)$ & $39(2)$ & $-6(2)$ & $4.9(13)$ & $-0.6(14)$ \\
\hline C28_3 & $26.4(16)$ & $37(3)$ & $39(2)$ & $-6(2)$ & $4.9(13)$ & $-0.6(14)$ \\
\hline C29_3 & $26.4(18)$ & $41(3)$ & $45(4)$ & $-10(3)$ & $5.2(15)$ & $-0.9(16)$ \\
\hline C30_3 & $26.4(16)$ & $37(3)$ & $39(2)$ & $-6(2)$ & $4.9(13)$ & $-0.6(14)$ \\
\hline C31_3 & $26.4(16)$ & $37(3)$ & $39(2)$ & $-6(2)$ & $4.9(13)$ & $-0.6(14)$ \\
\hline Cl1_9 & $27.8(14)$ & $33(3)$ & $41(5)$ & $9(3)$ & $-5.6(18)$ & $-4.6(13)$ \\
\hline 01_9 & $20(2)$ & $22(2)$ & $19(2)$ & $-0.3(15)$ & $1.4(16)$ & $2.7(16)$ \\
\hline 02_9 & $19(2)$ & $18(2)$ & $19.1(19)$ & $2.0(14)$ & $0.5(16)$ & $2.7(16)$ \\
\hline C1_9 & $15(3)$ & $20(2)$ & $19.3(18)$ & $-0.2(13)$ & $0.0(15)$ & $-2(2)$ \\
\hline C2_9 & $29(3)$ & $26(2)$ & $30.6(18)$ & $0.0(13)$ & $3.1(14)$ & $7.0(16)$ \\
\hline C3_9 & $31(2)$ & $33(2)$ & $33.1(18)$ & $-4.0(16)$ & $5.0(16)$ & $4.6(17)$ \\
\hline C4_9 & $29(2)$ & $28(3)$ & $31(3)$ & $0(2)$ & $3.5(19)$ & $7(2)$ \\
\hline C5_9 & $27.8(19)$ & $27(3)$ & $31(2)$ & $1(2)$ & $-0.2(15)$ & $0.8(17)$ \\
\hline C6_9 & $28.7(19)$ & $27(3)$ & $32(2)$ & $-0.8(19)$ & $1.2(14)$ & $0.3(16)$ \\
\hline
\end{tabular}




\begin{tabular}{|c|c|c|c|c|c|c|}
\hline Atom & $U_{11}$ & $U_{22}$ & $U_{33}$ & $U_{23}$ & $U_{13}$ & $U_{12}$ \\
\hline C7_9 & $30(2)$ & $28(3)$ & $32(2)$ & $-0.1(19)$ & $1.4(16)$ & $0.2(18)$ \\
\hline C8_9 & $32(2)$ & $36(3)$ & $38(2)$ & $-2(2)$ & $-1.9(16)$ & $1(2)$ \\
\hline C9_9 & $30(2)$ & $33(4)$ & $35(3)$ & $-2(3)$ & $-2.9(19)$ & $3(3)$ \\
\hline C10_9 & $30(2)$ & $33(3)$ & $35(3)$ & $-1(2)$ & $-3.0(17)$ & $2.2(19)$ \\
\hline C11_9 & $41(6)$ & $43(2)$ & $39.5(18)$ & $1.3(14)$ & $-2.2(18)$ & $1(2)$ \\
\hline C12_9 & $37(8)$ & $43(2)$ & $40.5(19)$ & $0.4(14)$ & $-2(2)$ & $0(2)$ \\
\hline C13_9 & $34(8)$ & $45(2)$ & $41(2)$ & $1.0(15)$ & $-3(2)$ & $-1(2)$ \\
\hline C14_9 & $44(4)$ & $45(2)$ & $42(2)$ & $1.1(15)$ & $-0.4(19)$ & $0.0(18)$ \\
\hline C15_9 & $34(8)$ & $45(2)$ & $41(2)$ & $1.0(15)$ & $-3(2)$ & $-1(2)$ \\
\hline C16_9 & $37(8)$ & $43(2)$ & $40.5(19)$ & $0.4(14)$ & $-2(2)$ & $0(2)$ \\
\hline C17_9 & $45(5)$ & $43(4)$ & $40(3)$ & $2(3)$ & $-1(3)$ & $3(4)$ \\
\hline C18_9 & $52(9)$ & $55(11)$ & $45(4)$ & $7(6)$ & $3(6)$ & $6(11)$ \\
\hline C19_9 & $51(5)$ & $44(2)$ & $51(5)$ & $0.0(18)$ & $-2(4)$ & $1(2)$ \\
\hline C20_9 & $37.5(18)$ & $23(5)$ & $37.3(19)$ & $-4(2)$ & $-1.1(17)$ & $-0.8(16)$ \\
\hline C21_9 & $37.6(18)$ & $35(2)$ & $38.1(19)$ & $-0.6(16)$ & $-0.7(16)$ & $0.6(15)$ \\
\hline C22_9 & $37.6(18)$ & $35(2)$ & $38.1(19)$ & $-0.6(16)$ & $-0.7(16)$ & $0.6(15)$ \\
\hline C23_9 & 38.1(19) & $59(8)$ & $40(2)$ & $6(3)$ & $0.1(18)$ & $3.8(19)$ \\
\hline C24_9 & $37.6(18)$ & $35(2)$ & $38.1(19)$ & $-0.6(16)$ & $-0.7(16)$ & $0.6(15)$ \\
\hline C25_9 & $37.6(18)$ & $35(2)$ & $38.1(19)$ & $-0.6(16)$ & $-0.7(16)$ & $0.6(15)$ \\
\hline C26_9 & $37(4)$ & $33(2)$ & $39(6)$ & $-5.1(18)$ & $-6(4)$ & $4.6(18)$ \\
\hline C27_9 & $42(4)$ & $33(2)$ & $59(5)$ & $-3.9(19)$ & $-16(3)$ & $4.0(18)$ \\
\hline C28_9 & $42(4)$ & $33(2)$ & $59(5)$ & $-3.9(19)$ & $-16(3)$ & $4.0(18)$ \\
\hline C29_9 & $42(4)$ & $32(2)$ & $61(7)$ & $-4(2)$ & $-17(4)$ & $4.2(19)$ \\
\hline C30_9 & $42(4)$ & $33(2)$ & $59(5)$ & $-3.9(19)$ & $-16(3)$ & $4.0(18)$ \\
\hline C31_9 & $42(4)$ & $33(2)$ & $59(5)$ & $-3.9(19)$ & $-16(3)$ & $4.0(18)$ \\
\hline Cl1_7 & $32.5(16)$ & 23.8(13) & $48(4)$ & $6(2)$ & $10(3)$ & $4.4(10)$ \\
\hline 01_7 & $25.8(18)$ & $24(2)$ & $22(3)$ & $-0.6(18)$ & $0.0(16)$ & $-4.6(19)$ \\
\hline 02_7 & $25.8(18)$ & $24(2)$ & $22(3)$ & $-0.6(18)$ & $0.0(16)$ & $-4.6(19)$ \\
\hline C1_7 & $23(2)$ & $19(3)$ & $22(3)$ & $-0.6(18)$ & $-0.1(16)$ & $1(2)$ \\
\hline $\mathrm{C} 2 \_7$ & $28.7(17)$ & $26.3(19)$ & $30.4(16)$ & $-0.8(13)$ & $1.9(11)$ & $-5.5(15)$ \\
\hline $\mathrm{C}_{3}{ }_{-} 7$ & $36.9(15)$ & $31(2)$ & $38.0(18)$ & $-6.7(14)$ & $-5.4(12)$ & $-1.1(12)$ \\
\hline C4_7 & $31(2)$ & $27.4(19)$ & $31(3)$ & $-2.0(16)$ & $1(2)$ & $-5.8(16)$ \\
\hline C5_7 & $30(2)$ & $20.0(16)$ & $30.4(16)$ & $0.6(13)$ & $1.7(14)$ & $-0.1(14)$ \\
\hline C6_7 & $29(2)$ & $19.9(16)$ & $30.6(17)$ & $0.6(13)$ & $1.7(15)$ & $-0.1(14)$ \\
\hline C7_7 & $46(10)$ & $22(2)$ & $34(3)$ & $3(2)$ & $9(5)$ & $6(4)$ \\
\hline C8_7 & $30(2)$ & $20.0(16)$ & $30.4(16)$ & $0.6(13)$ & $1.7(14)$ & $-0.1(14)$ \\
\hline C9_7 & $29(2)$ & $19.9(16)$ & $30.6(17)$ & $0.6(13)$ & $1.7(15)$ & $-0.1(14)$ \\
\hline C10_7 & $29(2)$ & $19.9(16)$ & $30.6(17)$ & $0.6(13)$ & $1.7(15)$ & $-0.1(14)$ \\
\hline C11_7 & $47(3)$ & $28(12)$ & $33.8(18)$ & $-1(2)$ & $9(2)$ & $-5(4)$ \\
\hline C12_7 & $48(3)$ & $38(5)$ & $34.3(19)$ & $-1(2)$ & $9(2)$ & $-7(3)$ \\
\hline C13_7 & $58(4)$ & $38(5)$ & $37(2)$ & $-2(3)$ & $14(3)$ & $-9(4)$ \\
\hline C14_7 & $58(4)$ & $22(5)$ & $37(2)$ & $-1(3)$ & $14(3)$ & $-7(4)$ \\
\hline C15_7 & $58(4)$ & $38(5)$ & $37(2)$ & $-2(3)$ & $14(3)$ & $-9(4)$ \\
\hline C16_7 & $48(3)$ & $38(5)$ & $34.3(19)$ & $-1(2)$ & $9(2)$ & $-7(3)$ \\
\hline C17_7 & $48(3)$ & $49(6)$ & $35(2)$ & $-1(3)$ & $8(2)$ & $-10(3)$ \\
\hline C18_7 & 79(16) & $40(18)$ & $41(4)$ & $-6(7)$ & $23(8)$ & $-17(14)$ \\
\hline C19_7 & $48(3)$ & $49(6)$ & $35(2)$ & $-1(3)$ & $8(2)$ & $-10(3)$ \\
\hline C20_7 & $24(6)$ & $54(2)$ & $44.9(19)$ & $5.6(17)$ & $-4.1(17)$ & $1(2)$ \\
\hline C21_7 & $53(3)$ & $54(2)$ & $46(2)$ & $5.2(18)$ & $-3.2(18)$ & $-1(2)$ \\
\hline C22_7 & $53(3)$ & $54(2)$ & $46(2)$ & $5.2(18)$ & $-3.2(18)$ & $-1(2)$ \\
\hline C23_7 & $53(3)$ & $54(2)$ & $46(2)$ & $5.2(18)$ & $-3.2(18)$ & $-1(2)$ \\
\hline C24_7 & $53(3)$ & $54(2)$ & $46(2)$ & $5.2(18)$ & $-3.2(18)$ & $-1(2)$ \\
\hline C25_7 & $53(3)$ & $54(2)$ & $46(2)$ & $5.2(18)$ & $-3.2(18)$ & $-1(2)$ \\
\hline C26_7 & $37.0(17)$ & $45.9(19)$ & $31(9)$ & $0.2(15)$ & $-7(3)$ & $-1.7(11)$ \\
\hline C27_7 & $41.3(16)$ & $45.6(18)$ & $46(2)$ & $0.3(13)$ & $-0.5(12)$ & $-1.5(11)$ \\
\hline C28_7 & $41.3(16)$ & $45.6(18)$ & $46(2)$ & $0.3(13)$ & $-0.5(12)$ & $-1.5(11)$ \\
\hline C29_7 & $42.2(19)$ & $45.6(19)$ & $47(4)$ & $0.4(15)$ & $0.6(18)$ & $-1.4(11)$ \\
\hline C30_7 & $41.3(16)$ & $45.6(18)$ & $46(2)$ & $0.3(13)$ & $-0.5(12)$ & $-1.5(11)$ \\
\hline
\end{tabular}




\begin{tabular}{|c|c|c|c|c|c|c|}
\hline Atom & $U_{11}$ & $U_{22}$ & $U_{33}$ & $U_{23}$ & $U_{13}$ & $U_{12}$ \\
\hline$\overline{C 31 \_7}$ & $41.3(16)$ & $45.6(18)$ & $46(2)$ & $0.3(13)$ & $-0.5(12)$ & $-1.5(11)$ \\
\hline $\mathrm{Cl} 1 \_4$ & $35.5(18)$ & $22.6(14)$ & $35(2)$ & $-9.4(14)$ & $-5.5(18)$ & 6.1(13) \\
\hline 01_4 & $22(2)$ & $17(2)$ & $25(2)$ & $0.1(19)$ & 2.1(18) & $1.7(19)$ \\
\hline 02_4 & $20(3)$ & $20(2)$ & $26(2)$ & $1(2)$ & $0.0(17)$ & $-1.0(19)$ \\
\hline C1_4 & $24.0(17)$ & $20(3)$ & $26(2)$ & 1.1(17) & $1.0(14)$ & $0(2)$ \\
\hline $\mathrm{C} 2{ }_{-}^{-} 4$ & $26.5(16)$ & 25.1(19) & $31.2(13)$ & $-0.1(13)$ & $2.9(11)$ & $-4.0(13)$ \\
\hline C3 34 & $26.1(18)$ & $28(2)$ & $33.2(16)$ & $3.7(14)$ & $4.1(13)$ & $-0.6(15)$ \\
\hline C4_4 & $21(3)$ & $26.3(18)$ & $36(2)$ & $2.5(15)$ & $4(2)$ & $-4.2(18)$ \\
\hline C5_4 & $26.9(17)$ & $25.7(14)$ & $31.1(12)$ & $-1.3(10)$ & $2.9(11)$ & $-3.0(12)$ \\
\hline C6_4 & $31(6)$ & $27(2)$ & $32(4)$ & $-3(3)$ & $1(5)$ & $-1(3)$ \\
\hline C7_4 & $30(6)$ & $26(3)$ & $32(3)$ & $-2(3)$ & $2(4)$ & $-2(4)$ \\
\hline C8_4 & $26.9(17)$ & $25.7(14)$ & $31.1(12)$ & $-1.3(10)$ & $2.9(11)$ & $-3.0(12)$ \\
\hline C9 4 & $29.3(18)$ & $26.6(14)$ & $32.0(14)$ & $-2.2(10)$ & $1.1(12)$ & $-1.6(12)$ \\
\hline C10_4 & $29.3(18)$ & $26.6(14)$ & $32.0(14)$ & $-2.2(10)$ & $1.1(12)$ & $-1.6(12)$ \\
\hline C11_4 & $35.9(17)$ & $34(3)$ & $34.6(13)$ & $-2.4(14)$ & $-2.6(12)$ & $-1.3(15)$ \\
\hline C12_4 & $36.1(18)$ & $38(3)$ & $39.5(17)$ & $-0.6(18)$ & $-1.5(12)$ & $-1.1(15)$ \\
\hline C13_4 & $43(2)$ & $67(15)$ & $47(3)$ & $10(6)$ & $-6.9(15)$ & $-2(3)$ \\
\hline C14_4 & $45.7(19)$ & $44(2)$ & $42.6(16)$ & $0.6(13)$ & $-6.0(12)$ & $-0.3(15)$ \\
\hline C15_4 & $45.7(19)$ & $44(2)$ & $42.6(16)$ & $0.6(13)$ & $-6.0(12)$ & $-0.3(15)$ \\
\hline C16_4 & $38.8(19)$ & $37(3)$ & $37.2(15)$ & $-0.7(16)$ & $-0.1(13)$ & $-0.8(18)$ \\
\hline C17_4 & $32(4)$ & $38(4)$ & $38(2)$ & $-1(2)$ & $-3(2)$ & $-1(3)$ \\
\hline C18_4 & $53(4)$ & $53(5)$ & $47(2)$ & $3(3)$ & $-11(2)$ & $0(3)$ \\
\hline C19_4 & $39(2)$ & $50(15)$ & $63(16)$ & $9(13)$ & $2(4)$ & $-2(4)$ \\
\hline C20_4 & $23(11)$ & $37(2)$ & $34(3)$ & $-0.5(15)$ & $6(5)$ & $-1.5(18)$ \\
\hline C21_4 & $35(4)$ & $37(2)$ & $36(2)$ & $0.3(16)$ & $1(2)$ & $-0.9(19)$ \\
\hline $\mathrm{C} 22^{-} 4$ & $40(3)$ & $39.0(17)$ & $38.4(15)$ & $-0.7(13)$ & $-2.2(15)$ & $-0.1(15)$ \\
\hline C23_4 & $36(3)$ & $39.0(17)$ & $37.8(18)$ & $-0.7(13)$ & $-0.7(18)$ & $-0.2(15)$ \\
\hline C24_4 & $40(3)$ & $39.0(17)$ & $38.4(15)$ & $-0.7(13)$ & $-2.2(15)$ & $-0.1(15)$ \\
\hline C25_4 & $40(3)$ & $39.0(17)$ & $38.4(15)$ & $-0.7(13)$ & $-2.2(15)$ & $-0.1(15)$ \\
\hline C26_4 & $26.1(18)$ & $30(5)$ & $29(8)$ & $3(6)$ & $4.9(14)$ & $-0.6(15)$ \\
\hline C27_4 & $26.4(16)$ & $37(3)$ & $39(2)$ & $-6(2)$ & $4.9(13)$ & $-0.6(14)$ \\
\hline C28_4 & $26.4(16)$ & $37(3)$ & $39(2)$ & $-6(2)$ & $4.9(13)$ & $-0.6(14)$ \\
\hline C29_4 & $26.4(18)$ & $41(3)$ & $45(4)$ & $-10(3)$ & $5.2(15)$ & $-0.9(16)$ \\
\hline C30_4 & $26.4(16)$ & $37(3)$ & $39(2)$ & $-6(2)$ & $4.9(13)$ & $-0.6(14)$ \\
\hline C31_4 & $26.4(16)$ & $37(3)$ & $39(2)$ & $-6(2)$ & $4.9(13)$ & $-0.6(14)$ \\
\hline Cl1_6 & $27.8(14)$ & $33(3)$ & $41(5)$ & $9(3)$ & $-5.6(18)$ & $-4.6(13)$ \\
\hline 01_6 & $20(2)$ & $22(2)$ & $19(2)$ & $-0.3(15)$ & $1.4(16)$ & $2.7(16)$ \\
\hline $02 \_6$ & $19(2)$ & $18(2)$ & $19.1(19)$ & $2.0(14)$ & $0.5(16)$ & $2.7(16)$ \\
\hline C1_6 & $15(3)$ & $20(2)$ & $19.3(18)$ & $-0.2(13)$ & $0.0(15)$ & $-2(2)$ \\
\hline $\mathrm{C} 26$ & $29(3)$ & $26(2)$ & $30.6(18)$ & $0.0(13)$ & $3.1(14)$ & $7.0(16)$ \\
\hline C3_6 & $31(2)$ & $33(2)$ & $33.1(18)$ & $-4.0(16)$ & $5.0(16)$ & $4.6(17)$ \\
\hline C4_6 & $29(2)$ & $28(3)$ & $31(3)$ & $0(2)$ & $3.5(19)$ & $7(2)$ \\
\hline C5_6 & $27.5(19)$ & $26(3)$ & $31(2)$ & $0(2)$ & $-0.2(15)$ & $1.0(17)$ \\
\hline C6_6 & 28.7(19) & $27(3)$ & $32(2)$ & $-0.8(19)$ & $1.2(14)$ & $0.3(16)$ \\
\hline C7_6 & $30(2)$ & $28(3)$ & $32(2)$ & $-0.1(19)$ & $1.4(16)$ & $0.2(18)$ \\
\hline C8_6 & $31(2)$ & $32(7)$ & $37(3)$ & $-4(4)$ & $-1(2)$ & $2(3)$ \\
\hline C9_6 & $30(2)$ & $33(4)$ & $35(3)$ & $-2(3)$ & $-2.9(19)$ & $3(3)$ \\
\hline C10_6 & $29(2)$ & $32(3)$ & $35(2)$ & $-2(2)$ & $-2.7(17)$ & $2.8(19)$ \\
\hline C11_6 & $41(6)$ & $43(2)$ & $39.5(18)$ & $1.3(14)$ & $-2.2(18)$ & $1(2)$ \\
\hline C12_6 & $41(4)$ & $43(2)$ & $41(2)$ & $1.1(15)$ & $-1(2)$ & $0.4(19)$ \\
\hline C13_6 & $43(4)$ & $44(3)$ & $41(2)$ & $1.6(16)$ & $-0.8(18)$ & $0(2)$ \\
\hline C14_6 & $44(4)$ & $45(2)$ & $42(2)$ & $1.1(15)$ & $-0.4(19)$ & $0.0(18)$ \\
\hline C15_6 & $44(4)$ & $45(2)$ & $42(3)$ & $1.3(17)$ & $1(2)$ & $-0.1(19)$ \\
\hline C16_6 & $43(4)$ & $43(2)$ & $41(2)$ & $0.3(15)$ & $0.0(19)$ & $-1(2)$ \\
\hline C17_6 & $45(5)$ & $43(4)$ & $40(3)$ & $2(3)$ & $-1(3)$ & $3(4)$ \\
\hline C18_6 & $52(9)$ & $55(11)$ & $45(4)$ & $7(6)$ & $3(6)$ & $6(11)$ \\
\hline C19_6 & $51(5)$ & $44(2)$ & $51(5)$ & $0.0(18)$ & $-2(4)$ & $1(2)$ \\
\hline C20_6 & $37.5(18)$ & $23(5)$ & $37.3(19)$ & $-4(2)$ & $-1.1(17)$ & $-0.8(16)$ \\
\hline
\end{tabular}




\begin{tabular}{|c|c|c|c|c|c|c|}
\hline Atom & $U_{11}$ & $U_{22}$ & $\boldsymbol{U}_{33}$ & $U_{23}$ & $U_{13}$ & $U_{12}$ \\
\hline$\overline{C 21 \_6}$ & $37.6(18)$ & $35(2)$ & $38.1(19)$ & $-0.6(16)$ & $-0.7(16)$ & $0.6(15)$ \\
\hline C22_6 & $37.6(18)$ & $35(2)$ & $38.1(19)$ & $-0.6(16)$ & $-0.7(16)$ & $0.6(15)$ \\
\hline C23_6 & $38.1(19)$ & $59(8)$ & $40(2)$ & $6(3)$ & $0.1(18)$ & $3.8(19)$ \\
\hline C24_6 & $37.6(18)$ & $35(2)$ & $38.1(19)$ & $-0.6(16)$ & $-0.7(16)$ & $0.6(15)$ \\
\hline C25_6 & $37.6(18)$ & $35(2)$ & $38.1(19)$ & $-0.6(16)$ & $-0.7(16)$ & $0.6(15)$ \\
\hline C26_6 & $37(4)$ & $33(2)$ & $39(6)$ & $-5.1(18)$ & $-6(4)$ & $4.6(18)$ \\
\hline C27_6 & $42(4)$ & $33(2)$ & $59(5)$ & $-3.9(19)$ & $-16(3)$ & $4.0(18)$ \\
\hline C28_6 & $42(4)$ & $33(2)$ & $59(5)$ & $-3.9(19)$ & $-16(3)$ & $4.0(18)$ \\
\hline C29_6 & $42(4)$ & $32(2)$ & $61(7)$ & $-4(2)$ & $-17(4)$ & $4.2(19)$ \\
\hline C30_6 & $42(4)$ & $33(2)$ & $59(5)$ & $-3.9(19)$ & $-16(3)$ & $4.0(18)$ \\
\hline C31_6 & $42(4)$ & $33(2)$ & $59(5)$ & $-3.9(19)$ & $-16(3)$ & $4.0(18)$ \\
\hline Cl1_2 & $32.5(16)$ & 23.8(13) & $48(4)$ & $6(2)$ & $10(3)$ & $4.4(10)$ \\
\hline 01_2 & $25.8(18)$ & $24(2)$ & $22(3)$ & $-0.6(18)$ & $0.0(16)$ & $-4.6(19)$ \\
\hline $02 \_2$ & $25.8(18)$ & $24(2)$ & $22(3)$ & $-0.6(18)$ & $0.0(16)$ & $-4.6(19)$ \\
\hline C1_2 & $25(2)$ & $19(5)$ & $22(3)$ & $0(2)$ & $-0.4(16)$ & $-2(3)$ \\
\hline C2_2 & $29.2(19)$ & $26(2)$ & $30.7(16)$ & $-1.2(14)$ & $2.1(12)$ & $-6.9(17)$ \\
\hline C3_2 & $36.9(15)$ & $31(2)$ & $38.0(18)$ & $-6.7(14)$ & $-5.4(12)$ & $-1.1(12)$ \\
\hline C4_2 & $31(2)$ & 27.4(19) & $31(3)$ & $-2.0(16)$ & $1(2)$ & $-5.8(16)$ \\
\hline C5_2 & $30(2)$ & $20.0(16)$ & $30.4(16)$ & $0.6(13)$ & $1.7(14)$ & $-0.1(14)$ \\
\hline C6_2 & $29(2)$ & $19.9(16)$ & $30.6(17)$ & $0.6(13)$ & $1.7(15)$ & $-0.1(14)$ \\
\hline C7_2 & $29(2)$ & $19.9(16)$ & $30.6(17)$ & $0.6(13)$ & $1.7(15)$ & $-0.1(14)$ \\
\hline C8_2 & $30(2)$ & $20.0(16)$ & $30.4(16)$ & $0.6(13)$ & $1.7(14)$ & $-0.1(14)$ \\
\hline C9_2 & $29(2)$ & $19.9(16)$ & $30.6(17)$ & $0.6(13)$ & $1.7(15)$ & $-0.1(14)$ \\
\hline C10_2 & $29(2)$ & $19.9(16)$ & $30.6(17)$ & $0.6(13)$ & $1.7(15)$ & $-0.1(14)$ \\
\hline C11_2 & $47(3)$ & $23(11)$ & $33.8(18)$ & $-2(2)$ & $9(2)$ & $-5(3)$ \\
\hline C12_2 & $48(3)$ & $38(5)$ & $34.3(19)$ & $-1(2)$ & $9(2)$ & $-7(3)$ \\
\hline C13_2 & $58(4)$ & $38(5)$ & $37(2)$ & $-2(3)$ & $14(3)$ & $-9(4)$ \\
\hline C14_2 & $58(4)$ & $22(5)$ & $37(2)$ & $-1(3)$ & $14(3)$ & $-7(4)$ \\
\hline C15_2 & $58(4)$ & $38(5)$ & $37(2)$ & $-2(3)$ & $14(3)$ & $-9(4)$ \\
\hline C16_2 & $48(3)$ & $38(5)$ & $34.3(19)$ & $-1(2)$ & $9(2)$ & $-7(3)$ \\
\hline C17_2 & 48(3) & $49(6)$ & $35(2)$ & $-1(3)$ & $8(2)$ & $-10(3)$ \\
\hline C18_2 & 105(18) & 40(18) & $48(5)$ & $-17(8)$ & $36(9)$ & $-40(16)$ \\
\hline C19_2 & $48(3)$ & $49(6)$ & $35(2)$ & $-1(3)$ & $8(2)$ & $-10(3)$ \\
\hline C20_2 & $24(6)$ & $54(2)$ & $44.9(19)$ & $5.6(17)$ & $-4.1(17)$ & $1(2)$ \\
\hline C21_2 & $53(3)$ & $54(2)$ & $46(2)$ & $5.2(18)$ & $-3.2(18)$ & $-1(2)$ \\
\hline C22_2 & $69(19)$ & $54(3)$ & $46(2)$ & $5.1(18)$ & $-3(2)$ & $-1(3)$ \\
\hline C23_2 & $53(3)$ & $54(2)$ & $46(2)$ & $5.2(18)$ & $-3.2(18)$ & $-1(2)$ \\
\hline C24_2 & $53(3)$ & $54(2)$ & $46(2)$ & $5.2(18)$ & $-3.2(18)$ & $-1(2)$ \\
\hline C25_2 & $53(3)$ & $54(2)$ & $46(2)$ & $5.2(18)$ & $-3.2(18)$ & $-1(2)$ \\
\hline C26_2 & $37.0(17)$ & $45.9(19)$ & $31(9)$ & $0.2(15)$ & $-7(3)$ & $-1.7(11)$ \\
\hline C27_2 & $41.3(16)$ & $45.6(18)$ & $46(2)$ & $0.3(13)$ & $-0.5(12)$ & $-1.5(11)$ \\
\hline C28_2 & $41.3(16)$ & $45.6(18)$ & $46(2)$ & $0.3(13)$ & $-0.5(12)$ & $-1.5(11)$ \\
\hline C29_2 & $42.2(19)$ & $45.6(19)$ & $47(4)$ & $0.4(15)$ & $0.6(18)$ & $-1.4(11)$ \\
\hline C30_2 & $41.3(16)$ & $45.6(18)$ & $46(2)$ & $0.3(13)$ & $-0.5(12)$ & $-1.5(11)$ \\
\hline C31_2 & $41.3(16)$ & $45.6(18)$ & $46(2)$ & $0.3(13)$ & $-0.5(12)$ & $-1.5(11)$ \\
\hline Cl1_8 & 19.3(13) & $32.7(14)$ & $37(5)$ & $-4(5)$ & $8(2)$ & $-4.4(12)$ \\
\hline 01_8 & $24(4)$ & $22(3)$ & $21.9(19)$ & $1.0(19)$ & $-4.0(18)$ & $5(3)$ \\
\hline 02_8 & $18(2)$ & $20(2)$ & $20(2)$ & $-1.6(14)$ & $1.2(16)$ & $0.4(15)$ \\
\hline C1_8 & $20(2)$ & $22(2)$ & $22.1(19)$ & $0.6(13)$ & $-0.7(14)$ & $-1.9(15)$ \\
\hline C2_8 & $24.5(18)$ & $24.8(18)$ & $27(2)$ & $0.2(14)$ & $-0.4(14)$ & $1.4(14)$ \\
\hline C3_8 & $26(2)$ & $29(2)$ & $31(2)$ & $4.3(17)$ & $-2.8(16)$ & $-0.7(15)$ \\
\hline C4_8 & $24.6(19)$ & $24(2)$ & $26(2)$ & $-0.9(17)$ & $-0.1(14)$ & $1.5(14)$ \\
\hline C5_8 & $16(2)$ & $23(3)$ & $27(2)$ & 1.1(18) & $1.7(16)$ & $-1(2)$ \\
\hline C6_8 & $16(2)$ & $27(4)$ & $28(3)$ & $-2(3)$ & $2.9(19)$ & $-2(2)$ \\
\hline C7_8 & $18(3)$ & $29(5)$ & $29(3)$ & $-2(2)$ & $2(2)$ & $-1(3)$ \\
\hline C8_8 & $19(3)$ & $25(3)$ & $30(3)$ & $3.0(19)$ & $3(2)$ & $4(2)$ \\
\hline C9_8 & $21(3)$ & $32(5)$ & $29(3)$ & $4(4)$ & $3(2)$ & $0(3)$ \\
\hline C10_8 & $18(3)$ & $40(6)$ & $30(3)$ & $1(3)$ & $4(2)$ & $-2(3)$ \\
\hline
\end{tabular}




\begin{tabular}{|c|c|c|c|c|c|c|}
\hline Atom & $U_{11}$ & $U_{22}$ & $U_{33}$ & $U_{23}$ & $U_{13}$ & $U_{12}$ \\
\hline$\overline{C 11 \_8}$ & $23(5)$ & $31(2)$ & $31(3)$ & $0.7(17)$ & $4(2)$ & $0(2)$ \\
\hline C12_8 & $32(3)$ & $31(2)$ & $33(2)$ & $0.0(16)$ & $0(2)$ & $2(2)$ \\
\hline C13_8 & $34(4)$ & $36(3)$ & $34(2)$ & $-1.9(16)$ & $0.4(17)$ & $1(2)$ \\
\hline C14_8 & $38(4)$ & $40(3)$ & $36(2)$ & $1.2(17)$ & $-1(2)$ & $1.0(18)$ \\
\hline C15_8 & $32(6)$ & $40(2)$ & $35(3)$ & $2.4(18)$ & $4(4)$ & $0(2)$ \\
\hline C16_8 & $36(5)$ & $36(3)$ & $35(3)$ & $4.3(17)$ & $3(3)$ & $2(3)$ \\
\hline C17_8 & $46(7)$ & $37(5)$ & $32(4)$ & $1(4)$ & $10(5)$ & $18(5)$ \\
\hline C18_8 & $64(11)$ & $68(11)$ & $41(3)$ & $-11(5)$ & $-10(5)$ & $21(9)$ \\
\hline C19_8 & $53(8)$ & $35(3)$ & $48(9)$ & $6(2)$ & $26(7)$ & $2(3)$ \\
\hline C20_8 & $30(3)$ & $29(4)$ & $32(2)$ & $4(2)$ & $-1.3(18)$ & $-1(2)$ \\
\hline C21_8 & $31(3)$ & $38(6)$ & $36(4)$ & $1(4)$ & $0(2)$ & $-3(3)$ \\
\hline C22_8 & $40(4)$ & $43(7)$ & $37(4)$ & $2(3)$ & $3(2)$ & $2(4)$ \\
\hline C23_8 & $45(3)$ & $54(8)$ & $41(4)$ & $0(5)$ & $-2(3)$ & $1(3)$ \\
\hline C24_8 & $44(4)$ & $44(6)$ & $38(3)$ & $0(3)$ & $-3(3)$ & $3(3)$ \\
\hline C25_8 & $35(3)$ & $44(7)$ & $37(3)$ & $-1(3)$ & $-6(2)$ & $0(4)$ \\
\hline C26_8 & $22(3)$ & $29(2)$ & $34(6)$ & $4(2)$ & $-3(4)$ & $-0.9(17)$ \\
\hline C27_8 & $22(4)$ & $29(4)$ & $51(9)$ & $3(5)$ & $-3(4)$ & $-1(2)$ \\
\hline C28_8 & $39(6)$ & $31(4)$ & 74(11) & $-2(5)$ & $-4(7)$ & $2(3)$ \\
\hline C29_8 & $41(6)$ & $30(4)$ & 81(14) & $2(6)$ & $-10(6)$ & $2(3)$ \\
\hline C30_8 & $41(6)$ & $28(3)$ & $95(15)$ & $3(5)$ & $-10(6)$ & $1(3)$ \\
\hline C31_8 & $25(4)$ & $27(3)$ & $63(10)$ & $9(4)$ & $-16(4)$ & $0(2)$ \\
\hline Cl1_1 & $19.3(13)$ & $32.7(14)$ & $37(5)$ & $-4(5)$ & $8(2)$ & $-4.4(12)$ \\
\hline 01_1 & $24(4)$ & $22(3)$ & $21.9(19)$ & $1.0(19)$ & $-4.0(18)$ & $5(3)$ \\
\hline $02 \_1$ & $18(2)$ & $20(2)$ & $20(2)$ & $-1.6(14)$ & $1.2(16)$ & $0.4(15)$ \\
\hline C1_1 & $20(2)$ & $22(2)$ & 22.1(19) & $0.6(13)$ & $-0.7(14)$ & $-1.9(15)$ \\
\hline C2_1 & $24.5(18)$ & $24.8(18)$ & $27(2)$ & $0.2(14)$ & $-0.4(14)$ & $1.4(14)$ \\
\hline C3_1 & $26(2)$ & $29(2)$ & $31(2)$ & $4.3(17)$ & $-2.8(16)$ & $-0.7(15)$ \\
\hline C4_1 & $24.6(19)$ & $24(2)$ & $26(2)$ & $-0.9(17)$ & $-0.1(14)$ & $1.5(14)$ \\
\hline C5_1 & $16(2)$ & $23(3)$ & $27(2)$ & 1.1(18) & $1.7(16)$ & $-1(2)$ \\
\hline C6_1 & $16(2)$ & $27(4)$ & $28(3)$ & $-2(3)$ & $2.9(19)$ & $-2(2)$ \\
\hline C7_1 & $18(3)$ & $29(5)$ & $29(3)$ & $-2(2)$ & $2(2)$ & $-1(3)$ \\
\hline C8_1 & $19(3)$ & $25(3)$ & $30(3)$ & $3.0(19)$ & $3(2)$ & $4(2)$ \\
\hline C9_1 & $21(3)$ & $32(5)$ & $29(3)$ & $4(4)$ & $3(2)$ & $0(3)$ \\
\hline C10_1 & 18(3) & $40(6)$ & $30(3)$ & $1(3)$ & $4(2)$ & $-2(3)$ \\
\hline C11_1 & $23(5)$ & $31(2)$ & $31(3)$ & $0.7(17)$ & $4(2)$ & $0(2)$ \\
\hline C12_1 & $32(3)$ & $31(2)$ & $33(2)$ & $0.0(16)$ & $0(2)$ & $2(2)$ \\
\hline C13_1 & $47(13)$ & $35(3)$ & $33(2)$ & $-1.4(17)$ & $-1(3)$ & $2(3)$ \\
\hline C14_1 & $37(4)$ & $40(3)$ & $35(3)$ & 2.1(18) & $1(2)$ & $0.7(19)$ \\
\hline C15_1 & $32(6)$ & $40(2)$ & $35(3)$ & $2.4(18)$ & $4(4)$ & $0(2)$ \\
\hline C16_1 & $36(5)$ & $36(3)$ & $35(3)$ & $4.3(17)$ & $3(3)$ & $2(3)$ \\
\hline C17_1 & $46(7)$ & $37(5)$ & $32(4)$ & $1(4)$ & $10(5)$ & $18(5)$ \\
\hline C18_1 & $64(11)$ & $68(11)$ & $41(3)$ & $-11(5)$ & $-10(5)$ & $21(9)$ \\
\hline C19_1 & $53(8)$ & $35(3)$ & $48(9)$ & $6(2)$ & $26(7)$ & $2(3)$ \\
\hline C20_1 & $30(3)$ & $29(4)$ & $32(2)$ & $4(2)$ & $-1.3(18)$ & $-1(2)$ \\
\hline C21_1 & $31(3)$ & $38(6)$ & $36(4)$ & $1(4)$ & $0(2)$ & $-3(3)$ \\
\hline C22_1 & $40(4)$ & $43(7)$ & $37(4)$ & $2(3)$ & $3(2)$ & $2(4)$ \\
\hline C23_1 & $45(3)$ & $54(8)$ & $41(4)$ & $0(5)$ & $-2(3)$ & $1(3)$ \\
\hline C24_1 & $45(3)$ & $67(18)$ & $38(3)$ & $-2(4)$ & $-2(3)$ & $-1(5)$ \\
\hline C25_1 & $35(3)$ & $44(7)$ & $37(3)$ & $-1(3)$ & $-6(2)$ & $0(4)$ \\
\hline C26_1 & $22(3)$ & $29(2)$ & $34(6)$ & $4(2)$ & $-3(4)$ & $-0.9(17)$ \\
\hline C27_1 & $22(4)$ & $29(4)$ & 51(9) & $3(5)$ & $-3(4)$ & $-1(2)$ \\
\hline C28_1 & $39(6)$ & $31(4)$ & 74(11) & $-2(5)$ & $-4(7)$ & $2(3)$ \\
\hline C29_1 & $41(6)$ & $30(4)$ & 81(14) & $2(6)$ & $-10(6)$ & $2(3)$ \\
\hline C30_1 & $41(6)$ & $28(3)$ & $95(15)$ & $3(5)$ & $-10(6)$ & $1(3)$ \\
\hline C31_1 & $25(4)$ & $27(3)$ & $63(10)$ & $9(4)$ & $-16(4)$ & $0(2)$ \\
\hline
\end{tabular}


Table 18: Bond Lengths in Å for 2Cl4triMePh.

\begin{tabular}{|c|c|c|c|c|c|}
\hline Atom & Atom & Length/Å & Atom & Atom & Length/Å \\
\hline$\overline{\mathrm{Rh} 1}$ & Rh2 & $2.388(6)$ & C29_3 & C30_3 & $1.378(4)$ \\
\hline Rh1 & 04 & $2.363(5)$ & C30_3 & C31_3 & $1.376(9)$ \\
\hline Rh1 & 01_4 & $2.035(5)$ & Cl1_9 & C6_9 9 & $1.748(6)$ \\
\hline Rh1 & $01 \_6$ & $2.067(5)$ & 01_9 & C1_9 & $1.253(7)$ \\
\hline Rh1 & 01_2 & $2.093(13)$ & 02_9 & C1_9 & $1.269(7)$ \\
\hline Rh1 & 01_1 & $2.016(5)$ & C1_9 & C2_9 & $1.509(7)$ \\
\hline $\mathrm{Rh} 2$ & 02_4 & $2.031(5)$ & $\mathrm{C} 2 \_9$ & C3_9 & $1.536(8)$ \\
\hline $\mathrm{Rh} 2$ & $02 \_6$ & $2.014(5)$ & C2_9 & C4_9 & $1.523(8)$ \\
\hline $\mathrm{Rh} 2$ & 02_2 & $2.054(12)$ & C2_9 & C5_9 & $1.498(8)$ \\
\hline Rh2 & 02_1 & $2.026(5)$ & C3_9 & C4_9 & $1.497(8)$ \\
\hline Rh1A & $\operatorname{Rh} \overline{2} \mathrm{~A}$ & $2.378(6)$ & C3_9 & C20_9 & $1.502(9)$ \\
\hline Rh1A & $04 \mathrm{~A}$ & $2.367(5)$ & C3_9 & C26_9 & $1.526(8)$ \\
\hline Rh1A & 01_3 & $2.035(5)$ & C5_9 & C6_9 & $1.413(7)$ \\
\hline Rh1A & 01_9 & $2.068(5)$ & C5_9 & C10_9 & $1.406(8)$ \\
\hline Rh1A & 01_7 & $2.099(13)$ & C6_9 & C7_9 & $1.344(8)$ \\
\hline Rh1A & 01_8 & $2.017(5)$ & C7_9 & C8_9 & $1.408(8)$ \\
\hline $\mathrm{Rh} 2 \mathrm{~A}$ & 02_3 & $2.030(5)$ & C8_9 & C9_9 & $1.390(8)$ \\
\hline Rh2A & 02_9 & $2.013(5)$ & C8_9 & C11_9 & $1.497(9)$ \\
\hline $\mathrm{Rh} 2 \mathrm{~A}$ & $02 \_7$ & $1.964(12)$ & C9_9 & C10_9 & $1.357(9)$ \\
\hline Rh2A & $02 \_8$ & $2.025(5)$ & C11_9 & C12_9 & $1.394(9)$ \\
\hline Cl1_3 & C6_3 & $1.748(6)$ & C11_9 & C16_9 & $1.364(10)$ \\
\hline $01 \_3$ & C1_3 & $1.252(7)$ & C12_9 & C13_9 & $1.391(10)$ \\
\hline $02 \_3$ & $\mathrm{C} 1 \_3$ & $1.268(7)$ & C12_9 & C17_9 & $1.503(7)$ \\
\hline C1_3 & $\mathrm{C} 2 \_3$ & $1.512(7)$ & C13_9 & C14_9 & $1.372(12)$ \\
\hline C2_3 & C3_3 & $1.537(8)$ & C14_9 & C15_9 & $1.342(11)$ \\
\hline C2_3 & C4_3 & $1.523(8)$ & C14_9 & C18_9 & $1.503(11)$ \\
\hline C2_3 & C5_3 & $1.495(8)$ & C15_9 & C16_9 & $1.405(10)$ \\
\hline C3_3 & C4_3 & $1.496(8)$ & C16_9 & C19_9 & $1.503(7)$ \\
\hline C3_3 & C20_3 & $1.504(9)$ & C20_9 & C21_9 & $1.376(9)$ \\
\hline C3_3 & C26_3 & $1.525(8)$ & C20_9 & C25_9 & $1.395(9)$ \\
\hline C5_3 & C6_3 & $1.414(7)$ & C21_9 & C22_9 & $1.392(9)$ \\
\hline C5_3 & C10_3 & $1.407(8)$ & C22_9 & C23_9 & $1.378(5)$ \\
\hline C6_3 & C7_ $\overline{3}$ & $1.344(8)$ & C23_9 & C24_9 & $1.377(5)$ \\
\hline C7_3 & C8_3 & $1.408(8)$ & C24_9 & C25_9 & $1.371(10)$ \\
\hline C8_3 & C9_3 & $1.390(8)$ & C26_9 & C27_9 & $1.376(9)$ \\
\hline C8_3 & C11_3 & $1.496(9)$ & C26_9 & C31_9 & $1.392(9)$ \\
\hline C9_3 & C10_3 & $1.357(9)$ & C27_9 & C28_9 & $1.377(9)$ \\
\hline C11_3 & C12_3 & $1.394(9)$ & C28_9 & C29_9 & $1.378(5)$ \\
\hline C11_3 & C16_3 & $1.364(10)$ & C29_9 & C30_9 & $1.378(5)$ \\
\hline C12_3 & C13_3 & $1.391(10)$ & C30_9 & C31_9 & $1.376(9)$ \\
\hline C12_3 & C17_3 & $1.503(7)$ & Cl1_7 & C6_7 & $1.748(6)$ \\
\hline C13_3 & C14_3 & $1.372(12)$ & 01_7 & C1_7 & $1.252(7)$ \\
\hline C14_3 & C15_3 & $1.342(11)$ & $02 \_7$ & C1_7 & $1.269(7)$ \\
\hline C14_3 & C18_3 & $1.503(11)$ & C1_7 & C2_7 & $1.509(7)$ \\
\hline C15_3 & C16_3 & $1.405(10)$ & C2_7 & C3_7 & $1.533(8)$ \\
\hline C16_3 & C19_3 & $1.503(7)$ & C2_7 & C4_7 & $1.524(8)$ \\
\hline C20_3 & C21_3 & $1.376(9)$ & C2_7 & C5_7 & $1.500(8)$ \\
\hline C20_3 & C25_3 & $1.394(9)$ & C3_7 & $\mathrm{C} 4 \_7$ & $1.497(8)$ \\
\hline C21_3 & C22_3 & $1.392(9)$ & C3_7 & C20_7 & $1.501(9)$ \\
\hline C22_3 & C23_3 & $1.378(4)$ & C3_7 & C26_7 & $1.528(8)$ \\
\hline C23_3 & C24_3 & $1.378(4)$ & C5_7 & C6_7 & $1.414(7)$ \\
\hline C24_3 & C25_3 & $1.372(10)$ & C5_7 & C10_7 & $1.407(8)$ \\
\hline C26_3 & C27_3 & $1.376(9)$ & C6_7 & C7_7 & $1.345(8)$ \\
\hline C26_3 & C31_3 & $1.392(9)$ & C7_7 & C8_7 & 1.408(8) \\
\hline C27_3 & C28_3 & $1.377(9)$ & C8_7 & C9_7 & $1.390(8)$ \\
\hline C28_3 & C29_3 & $1.378(4)$ & C8_7 & C11_7 & $1.496(9)$ \\
\hline
\end{tabular}




\begin{tabular}{|c|c|c|c|c|c|}
\hline Atom & Atom & Length/Å & Atom & Atom & Length/Å \\
\hline C9_7 & C10_7 & $1.357(9)$ & $\overline{C 29 \_4}$ & C30_4 & $1.378(5)$ \\
\hline C11_7 & C12_7 & $1.394(9)$ & C30_4 & C31_4 & $1.375(9)$ \\
\hline C11_7 & C16_7 & $1.364(10)$ & Cl1_6 & C6_6 & $1.748(6)$ \\
\hline C12_7 & C13_7 & $1.391(10)$ & $01 \_6$ & C1_6 & $1.254(7)$ \\
\hline C12_7 & C17_7 & $1.503(7)$ & 02_6 & C1_6 & $1.270(7)$ \\
\hline C13_7 & C14_7 & $1.372(12)$ & C1_6 & C2_6 & $1.510(7)$ \\
\hline C14_7 & C15_7 & $1.342(11)$ & C2_6 & C3_6 & $1.536(8)$ \\
\hline C14_7 & C18_7 & $1.503(11)$ & C2_6 & C4_6 & $1.524(8)$ \\
\hline C15_7 & C16_7 & $1.406(10)$ & C2_6 & C5_6 & $1.498(8)$ \\
\hline C16_7 & C19_7 & $1.504(7)$ & C3_6 & C4_6 & $1.497(8)$ \\
\hline C20_7 & C21_7 & $1.375(9)$ & C3_6 & C20_6 & $1.503(9)$ \\
\hline C20_7 & C25_7 & $1.395(9)$ & C3_6 & C26_6 & $1.526(8)$ \\
\hline C21_7 & C22_7 & $1.392(9)$ & C5_6 & C6_6 & $1.413(7)$ \\
\hline C22_7 & C23_7 & $1.378(5)$ & C5_6 & C10_6 & $1.407(8)$ \\
\hline C23_7 & C24_7 & $1.377(5)$ & C6_6 & C7_6 & $1.344(8)$ \\
\hline C24_7 & C25_7 & $1.371(10)$ & C7_6 & C8_6 & $1.408(8)$ \\
\hline C26_7 & C27_7 & $1.376(9)$ & C8_6 & C9_6 & $1.390(8)$ \\
\hline C26_7 & C31_7 & 1.391(9) & C8_6 & C11_6 & $1.496(9)$ \\
\hline C27_7 & C28_7 & $1.377(9)$ & C9_6 & C10_6 & $1.357(9)$ \\
\hline C28_7 & C29_7 & $1.377(5)$ & C11_6 & C12_6 & $1.394(9)$ \\
\hline C29_7 & C30_7 & $1.378(5)$ & C11_6 & C16_6 & $1.364(10)$ \\
\hline C30_7 & C31_7 & $1.376(9)$ & C12_6 & C13_6 & $1.391(10)$ \\
\hline Cl1_4 & C6_4 & $1.748(6)$ & C12_6 & C17_6 & $1.503(7)$ \\
\hline 01_4 & C1_4 & $1.255(7)$ & C13_6 & C14_6 & $1.372(12)$ \\
\hline 02_4 & C1_4 & $1.269(7)$ & C14_6 & C15_6 & $1.342(11)$ \\
\hline C1_4 & C2_4 & $1.512(7)$ & C14_6 & C18_6 & $1.503(11)$ \\
\hline $\mathrm{C} 2 \_4$ & C3_4 & $1.537(8)$ & C15_6 & C16_6 & $1.405(10)$ \\
\hline $\mathrm{C} 2 \_4$ & C4_4 & $1.523(8)$ & C16_6 & C19_6 & $1.503(7)$ \\
\hline $\mathrm{C} 2 \_4$ & C5_4 & $1.496(8)$ & C20_6 & C21_6 & $1.375(9)$ \\
\hline C3_4 & C4_4 & $1.495(8)$ & C20_6 & C25_6 & $1.395(9)$ \\
\hline C3_4 & C20_4 & $1.504(9)$ & C21_6 & C22_6 & $1.392(9)$ \\
\hline C3_4 & C26_4 & $1.525(8)$ & C22_6 & C23_6 & $1.378(5)$ \\
\hline C5_4 & C6_4 & 1.414(7) & C23_6 & C24_6 & $1.377(5)$ \\
\hline C5_4 & C10_4 & $1.407(8)$ & C24_6 & C25_6 & $1.371(10)$ \\
\hline C6_4 & C7_4 & $1.345(8)$ & C26_6 & C27_6 & $1.376(9)$ \\
\hline C7_4 & C8_4 & $1.408(8)$ & C26_6 & C31_6 & $1.392(9)$ \\
\hline C8_4 & C9_4 & $1.390(8)$ & C27_6 & C28_6 & $1.377(9)$ \\
\hline C8_4 & C11_4 & $1.497(9)$ & C28_6 & C29_6 & $1.378(5)$ \\
\hline C9_4 & C10_4 & $1.357(9)$ & C29_6 & C30_6 & $1.378(5)$ \\
\hline C11_4 & C12_4 & $1.395(9)$ & C30_6 & C31_6 & $1.375(9)$ \\
\hline C11_4 & C16_4 & $1.365(10)$ & Cl1_2 & C6_2 & $1.748(6)$ \\
\hline C12_4 & C13_4 & $1.391(10)$ & 01_2 & C1_2 & $1.254(7)$ \\
\hline C12_4 & C17_4 & $1.503(7)$ & 02_2 & C1_2 & $1.272(7)$ \\
\hline C13_4 & C14_4 & $1.372(12)$ & C1_2 & C2_2 & $1.510(7)$ \\
\hline C14_4 & C15_4 & $1.342(11)$ & C2_2 & C3_2 & 1.533(8) \\
\hline C14_4 & C18_4 & $1.503(11)$ & C2_2 & C4_2 & $1.524(8)$ \\
\hline C15_4 & C16_4 & $1.405(10)$ & C2_2 & C5_2 & 1.499(8) \\
\hline C16_4 & C19_4 & $1.503(7)$ & C3_2 & C4_2 & $1.498(8)$ \\
\hline C20_4 & C21_4 & $1.376(9)$ & C3_2 & C20_2 & $1.501(9)$ \\
\hline C20_4 & C25_4 & $1.394(9)$ & C3_2 & C26_2 & $1.528(8)$ \\
\hline C21_4 & C22_4 & $1.392(9)$ & C5_2 & C6_2 & $1.413(7)$ \\
\hline C22_4 & C23_4 & $1.378(5)$ & C5_2 & C10_2 & 1.407(8) \\
\hline C23_4 & C24_4 & $1.377(5)$ & C6_2 & C7_2 & $1.345(8)$ \\
\hline C24_4 & C25_4 & $1.371(10)$ & C7_2 & C8_2 & $1.408(8)$ \\
\hline C26_4 & C27_4 & $1.376(9)$ & C8_2 & C9_2 & $1.390(8)$ \\
\hline C26_4 & C31_4 & $1.392(9)$ & C8_2 & C11_2 & $1.496(9)$ \\
\hline C27_4 & C28_4 & $1.377(9)$ & C9_2 & C10_2 & $1.357(9)$ \\
\hline C28_4 & C29_4 & $1.378(5)$ & C11_2 & C12_2 & $1.394(9)$ \\
\hline
\end{tabular}




\begin{tabular}{lll}
\hline Atom & Atom & Length/Å \\
\hline C11_2 & C16_2 & $1.364(10)$ \\
C12_2 & C13_2 & $1.391(10)$ \\
C12_2 & C17_2 & $1.503(7)$ \\
C13_2 & C14_2 & $1.372(12)$ \\
C14_2 & C15_2 & $1.342(11)$ \\
C14_2 & C18_2 & $1.503(11)$ \\
C15_2 & C16_2 & $1.405(10)$ \\
C16_2 & C19_2 & $1.503(7)$ \\
C20_2 & C21_2 & $1.376(9)$ \\
C20_2 & C25_2 & $1.395(9)$ \\
C21_2 & C22_2 & $1.392(9)$ \\
C22_2 & C23_2 & $1.378(5)$ \\
C23_2 & C24_2 & $1.377(5)$ \\
C24_2 & C25_2 & $1.371(10)$ \\
C26_2 & C27_2 & $1.376(9)$ \\
C26_2 & C31_2 & $1.391(9)$ \\
C27_2 & C28_2 & $1.377(9)$ \\
C28_2 & C29_2 & $1.378(5)$ \\
C29_2 & C30_2 & $1.378(5)$ \\
C30_2 & C31_2 & $1.376(9)$ \\
Cl1_8 & C6_8 & $1.748(6)$ \\
01_8 & C1_8 & $1.253(7)$ \\
O2_8 & C1_8 & $1.268(7)$ \\
C1_8 & C2_8 & $1.511(7)$ \\
C2_8 & C3_8 & $1.534(8)$ \\
C2_8 & C4_8 & $1.526(8)$ \\
C2_8 & C5_8 & $1.498(8)$ \\
C3_8 & C4_8 & $1.498(8)$ \\
C3_8 & C20_8 & $1.503(9)$ \\
C3_8 & C26_8 & $1.526(8)$ \\
C5_8 & C6_8 & $1.413(7)$ \\
C5_8 & C10_8 & $1.408(8)$ \\
C6_8 & C7_8 & $1.346(8)$ \\
C7_8 & C8_8 & $1.407(8)$ \\
C8_8 & C9_8 & $1.390(8)$ \\
C8_8 & C11_8 & $1.496(9)$ \\
C9_8 & C10_8 & $1.356(9)$ \\
C11_8 & C12_8 & $1.394(9)$ \\
C11_8 & C16_8 & $1.365(10)$ \\
C12_8 & C13_8 & $1.391(10)$ \\
C12_8 & C17_8 & $1.503(7)$ \\
C13_8 & C14_8 & $1.372(12)$ \\
C14_8 & C15_8 & $1.342(11)$ \\
C14_8 & C18_8 & $1.503(11)$ \\
C15_8 & C16_8 & $1.405(10)$ \\
C16_8 & C19_8 & $1.503(7)$ \\
C20_8 & C21_8 & $1.376(9)$ \\
C20_8 & C25_8 & $1.395(9)$ \\
& &
\end{tabular}

\begin{tabular}{lll}
\hline Atom & Atom & Length/A \\
\hline C21_8 & C22_8 & $1.393(9)$ \\
C22_8 & C23_8 & $1.378(5)$ \\
C23_8 & C24_8 & $1.377(5)$ \\
C24_8 & C25_8 & $1.371(10)$ \\
C26_8 & C27_8 & $1.376(9)$ \\
C26_8 & C31_8 & $1.391(9)$ \\
C27_8 & C28_8 & $1.377(9)$ \\
C28_8 & C29_8 & $1.378(5)$ \\
C29_8 & C30_8 & $1.378(5)$ \\
C30_8 & C31_8 & $1.376(9)$ \\
Cl1_1 & C6_1 & $1.748(6)$ \\
01_1 & C1_1 & $1.254(7)$ \\
O2_1 & C1_1 & $1.268(7)$ \\
C1_1 & C2_1 & $1.512(7)$ \\
C2_1 & C3_1 & $1.534(8)$ \\
C2_1 & C4_1 & $1.525(8)$ \\
C2_1 & C5_1 & $1.497(8)$ \\
C3_1 & C4_1 & $1.498(8)$ \\
C3_1 & C20_1 & $1.503(9)$ \\
C3_1 & C26_1 & $1.526(8)$ \\
C5_1 & C6_1 & $1.413(7)$ \\
C5_1 & C10_1 & $1.408(8)$ \\
C6_1 & C7_1 & $1.345(8)$ \\
C7_1 & C8_1 & $1.408(8)$ \\
C8_1 & C9_1 & $1.390(8)$ \\
C8_1 & C11_1 & $1.496(9)$ \\
C9_1 & C10_1 & $1.356(9)$ \\
C11_1 & C12_1 & $1.394(9)$ \\
C11_1 & C16_1 & $1.365(10)$ \\
C12_1 & C13_1 & $1.391(10)$ \\
C12_1 & C17_1 & $1.503(7)$ \\
C13_1 & C14_1 & $1.372(12)$ \\
C14_1 & C15_1 & $1.342(11)$ \\
C14_1 & C18_1 & $1.503(11)$ \\
C15_1 & C16_1 & $1.406(10)$ \\
C16_1 & C19_1 & $1.503(7)$ \\
C20_1 & C21_1 & $1.376(9)$ \\
C20_1 & C25_1 & $1.394(9)$ \\
C21_1 & C22_1 & $1.392(9)$ \\
C22_1 & C23_1 & $1.378(5)$ \\
C23_1 & C24_1 & $1.377(5)$ \\
C24_1 & C25_1 & $1.371(10)$ \\
C26_1 & C27_1 & $1.377(9)$ \\
C26_1 & C31_1 & $1.391(9)$ \\
C27_1 & C28_1 & $1.376(9)$ \\
C28_1 & C29_1 & $1.378(5)$ \\
C29_1 & C30_1 & $1.378(5)$ \\
C30_1 & C31_1 & $1.376(9)$ \\
& &
\end{tabular}

Table 19: Bond Angles in ${ }^{\circ}$ for 2Cl4triMePh.

\begin{tabular}{llll}
\hline Atom & Atom & Atom & \multicolumn{1}{c}{ Angle $^{\circ}$} \\
\hline O4 & $\mathrm{Rh} 1$ & $\mathrm{Rh} 2$ & $173.6(6)$ \\
01_4 & $\mathrm{Rh} 1$ & $\mathrm{Rh} 2$ & $87.6(5)$ \\
01_4 & $\mathrm{Rh} 1$ & $\mathrm{O} 4$ & $86.3(4)$
\end{tabular}

\begin{tabular}{llll}
\hline Atom & Atom & Atom & \multicolumn{1}{c}{ Angle $^{\circ}$} \\
\hline 01_4 & Rh1 & 01_6 & $91.8(10)$ \\
01_4 & Rh1 & 01_2 & $175.6(6)$ \\
01_6 & Rh1 & Rh2 & 89.1(4)
\end{tabular}




\begin{tabular}{|c|c|c|c|}
\hline Atom & Atom & Atom & Angle $/^{\circ}$ \\
\hline$\overline{01 \_6}$ & Rh1 & 04 & 93.0(5) \\
\hline $01 \_6$ & Rh1 & 01_2 & $87.4(8)$ \\
\hline 01_2 & $\mathrm{Rh} 1$ & $\mathrm{Rh} 2$ & 88.1(4) \\
\hline 01_2 & Rh1 & 04 & $98.0(6)$ \\
\hline 01_1 & Rh1 & Rh2 & $88.8(4)$ \\
\hline 01_1 & Rh1 & 04 & $89.2(4)$ \\
\hline 01_1 & Rh1 & 01_4 & $89.0(9)$ \\
\hline 01_1 & Rh1 & 01_6 & $177.7(6)$ \\
\hline 01_1 & Rh1 & 01_2 & $91.7(8)$ \\
\hline $02 \_4$ & $\mathrm{Rh} 2$ & $\mathrm{Rh} 1$ & $86.6(5)$ \\
\hline 02_4 & $\mathrm{Rh} 2$ & 02_2 & $172.7(6)$ \\
\hline $02 \_6$ & $\mathrm{Rh} 2$ & $\mathrm{Rh} 1$ & $85.8(4)$ \\
\hline $02 \_6$ & $\mathrm{Rh} 2$ & O2_4 & 88.1(9) \\
\hline $02 \_6$ & $\mathrm{Rh} 2$ & 02_2 & $90.4(9)$ \\
\hline 02_6 & $\mathrm{Rh} 2$ & 02_1 & $171.7(6)$ \\
\hline 02_2 & $\mathrm{Rh} 2$ & $\mathrm{Rh} 1$ & $86.2(4)$ \\
\hline 02_1 & $\mathrm{Rh} 2$ & Rh1 & $85.9(4)$ \\
\hline 02_1 & $\mathrm{Rh} 2$ & 02_4 & $90.5(9)$ \\
\hline 02_1 & Rh2 & 02_2 & $89.9(10)$ \\
\hline $04 \mathrm{~A}$ & Rh1A & $\mathrm{Rh} 2 \mathrm{~A}$ & $178.4(3)$ \\
\hline 01_3 & Rh1A & $\mathrm{Rh} 2 \mathrm{~A}$ & $86.5(5)$ \\
\hline 01_3 & Rh1A & $04 \mathrm{~A}$ & $93.9(6)$ \\
\hline 01_3 & Rh1A & 01_9 & $91.8(10)$ \\
\hline 01_3 & Rh1A & $01 \_7$ & $173.1(7)$ \\
\hline 01_9 & Rh1A & Rh2A & $85.5(4)$ \\
\hline 01_9 & Rh1A & $04 \mathrm{~A}$ & $96.0(4)$ \\
\hline 01_9 & Rh1A & 01_7 & $90.9(9)$ \\
\hline 01_7 & Rh1A & Rh2A & $87.4(4)$ \\
\hline 01_7 & Rh1A & $04 \mathrm{~A}$ & $92.0(6)$ \\
\hline $01 \_8$ & Rh1A & Rh2A & $87.4(4)$ \\
\hline 01_8 & Rh1A & 04A & $91.0(3)$ \\
\hline 01_8 & Rh1A & 01_3 & 87.4(9) \\
\hline 01_8 & Rh1A & 01_9 & $173.0(5)$ \\
\hline 01_8 & Rh1A & 01_7 & $89.1(8)$ \\
\hline 02_3 & $\mathrm{Rh} 2 \mathrm{~A}$ & Rh1A & $88.6(5)$ \\
\hline 02_9 & Rh2A & Rh1A & $89.6(4)$ \\
\hline 02_9 & Rh2A & 02_3 & $91.5(9)$ \\
\hline 02_9 & Rh2A & 02_8 & 177.3(7) \\
\hline 02_7 & Rh2A & Rh1A & $88.4(4)$ \\
\hline 02_7 & Rh2A & 02_3 & $176.4(8)$ \\
\hline 02_7 & $\mathrm{Rh} 2 \mathrm{~A}$ & 02_9 & $86.5(10)$ \\
\hline 02_7 & $\mathrm{Rh} 2 \mathrm{~A}$ & 02_8 & $92.2(10)$ \\
\hline 02_8 & Rh2A & Rh1A & $88.0(4)$ \\
\hline 02_8 & Rh2A & 02_3 & $89.6(9)$ \\
\hline C1_3 & 01_3 & Rh1A & 118.0(7) \\
\hline C1_3 & 02_3 & Rh2A & $116.6(6)$ \\
\hline 01_3 & C1_3 & 02_3 & $126.7(5)$ \\
\hline 01_3 & C1_3 & C2_3 & $119.0(6)$ \\
\hline 02_3 & C1_3 & C2_3 & $114.3(5)$ \\
\hline C1_3 & $\mathrm{C} 2 \_3$ & C3_3 & $120.6(6)$ \\
\hline C1_3 & C2_3 & C4_3 & $115.0(5)$ \\
\hline C4_3 & C2_3 & C3_3 & $58.5(4)$ \\
\hline C5_3 & C2_3 & C1_3 & $111.7(4)$ \\
\hline C5_3 & C2_3 & C3_3 & $120.6(4)$ \\
\hline C5_3 & C2_3 & C4_3 & $120.7(5)$ \\
\hline C4_3 & C3_3 & C2_3 & $60.2(4)$ \\
\hline C4_3 & C3_3 & C20_3 & $119.4(5)$ \\
\hline C4_3 & C3_3 & C26_3 & $118.0(5)$ \\
\hline
\end{tabular}

\begin{tabular}{|c|c|c|c|}
\hline Atom & Atom & Atom & Angle $/^{\circ}$ \\
\hline$\overline{\text { C20_3 }}$ & C3_3 & C2_3 & $120.8(5)$ \\
\hline C20_3 & C3_3 & C26_3 & $112.5(4)$ \\
\hline C26_3 & C3_3 & C2_3 & $116.6(5)$ \\
\hline C3_3 & C4_3 & $\mathrm{C} 2 \_3$ & $61.2(4)$ \\
\hline C6_3 & C5_3 & C2_3 & $121.6(5)$ \\
\hline C10_3 & C5_3 & C2_3 & $122.7(5)$ \\
\hline C10_3 & C5_3 & C6_3 & $115.7(5)$ \\
\hline C5_3 & C6_3 & Cl1_3 & $119.0(5)$ \\
\hline C7_3 & C6_3 & Cl1_3 & $118.0(5)$ \\
\hline C7_3 & C6_3 & C5_3 & $122.9(6)$ \\
\hline C6_3 & C7_3 & C8_3 & $120.7(6)$ \\
\hline C7_3 & C8_3 & C11_3 & $119.8(5)$ \\
\hline C9_3 & C8_3 & C7_3 & $117.0(6)$ \\
\hline C9_3 & C8_3 & C11_3 & $123.1(5)$ \\
\hline C10_3 & C9_3 & C8_3 & $122.5(6)$ \\
\hline C9_3 & C10_3 & C5_3 & $121.2(6)$ \\
\hline C12_3 & C11_3 & C8_3 & 118.1(6) \\
\hline C16_3 & C11_3 & C8_3 & 121.1(5) \\
\hline C16_3 & C11_3 & C12_3 & $120.5(6)$ \\
\hline C11_3 & C12_3 & C17_3 & $121.4(6)$ \\
\hline C13_3 & C12_3 & C11_3 & $119.2(7)$ \\
\hline C13_3 & C12_3 & C17_3 & 119.4(7) \\
\hline C14_3 & C13_3 & C12_3 & $121.1(7)$ \\
\hline C13_3 & C14_3 & C18_3 & $122.1(8)$ \\
\hline C15_3 & C14_3 & C13_3 & $118.0(7)$ \\
\hline C15_3 & C14_3 & C18_3 & $119.9(9)$ \\
\hline C14_3 & C15_3 & C16_3 & $123.7(8)$ \\
\hline C11_3 & C16_3 & C15_3 & $117.4(6)$ \\
\hline C11_3 & C16_3 & C19_3 & 122.1(7) \\
\hline C15_3 & C16_3 & C19_3 & 120.3(8) \\
\hline C21_3 & C20_3 & C3_3 & $122.6(6)$ \\
\hline C21_3 & C20_3 & C25_3 & 118.6(6) \\
\hline C25_3 & C20_3 & C3_3 & 118.8(6) \\
\hline C20_3 & C21_3 & C22_3 & 121.1(7) \\
\hline C23_3 & C22_3 & C21_3 & 120.1(6) \\
\hline C24_3 & C23_3 & C22_3 & 118.5(6) \\
\hline C25_3 & C24_3 & C23_3 & $121.9(7)$ \\
\hline C24_3 & C25_3 & C20_3 & $119.8(7)$ \\
\hline C27_3 & C26_3 & C3_3 & $122.8(6)$ \\
\hline C27_3 & C26_3 & C31_3 & $116.2(6)$ \\
\hline C31_3 & C26_3 & C3_3 & $120.9(6)$ \\
\hline C26_3 & C27_3 & C28_3 & $122.7(7)$ \\
\hline C27_3 & C28_3 & C29_3 & 119.6(6) \\
\hline C30_3 & C29_3 & C28_3 & $119.5(6)$ \\
\hline C31_3 & C30_3 & C29_3 & 119.6(7) \\
\hline C30_3 & C31_3 & C26_3 & $122.4(7)$ \\
\hline C1_9 & 01_9 & Rh1A & $118.6(6)$ \\
\hline C1_9 & 02_9 & $\mathrm{Rh} 2 \mathrm{~A}$ & $117.0(6)$ \\
\hline 01_9 & C1_9 & 02_9 & $126.2(5)$ \\
\hline 01_9 & C1_9 & C2_9 & $119.2(6)$ \\
\hline 02_9 & C1_9 & C2_9 & $114.5(5)$ \\
\hline C1_9 & C2_9 & C3_9 & $121.4(6)$ \\
\hline C1_9 & C2_9 & C4_9 & $115.6(5)$ \\
\hline C4_9 & C2_9 & C3_9 & $58.6(4)$ \\
\hline C5_9 & C2_9 & C1_9 & $112.0(5)$ \\
\hline C5_9 & C2_9 & C3_9 & 119.5(5) \\
\hline C5_9 & C2_9 & C4_9 & $120.0(5)$ \\
\hline C4_9 & C3_9 & C2_9 & $60.3(4)$ \\
\hline
\end{tabular}




\begin{tabular}{|c|c|c|c|}
\hline Atom & Atom & Atom & Angle $/^{\circ}$ \\
\hline C4_9 & C3_9 & C20_9 & $118.8(5)$ \\
\hline C4_9 & C3_9 & C26_9 & 118.1(5) \\
\hline C20_9 & C3_9 & C2_9 & $122.4(5)$ \\
\hline C20_9 & C3_9 & C26_9 & $112.2(4)$ \\
\hline C26_9 & C3_9 & C2_9 & $116.0(5)$ \\
\hline C3_9 & C4_9 & C2_9 & $61.1(4)$ \\
\hline C6_9 & C5_9 & C2_9 & $121.3(5)$ \\
\hline C10_9 & C5_9 & C2_9 & $122.8(5)$ \\
\hline C10_9 & C5_9 & C6_9 & $115.9(5)$ \\
\hline C5_9 & C6_9 & Cl1_9 & $118.7(5)$ \\
\hline C7_-9 & C6_9 & $\mathrm{Cl} 1 \_9$ & $118.4(5)$ \\
\hline C7_9 & C6_9 & C5_9 & $122.9(6)$ \\
\hline C6_9 & C7_9 & C8_9 & $120.7(6)$ \\
\hline C7_9 & C8_9 & C11_9 & $119.8(5)$ \\
\hline C9_9 & C8_9 & C7_9 & $117.0(6)$ \\
\hline C9_9 & C8_9 & C11_9 & $122.8(5)$ \\
\hline C10_9 & C9_9 & C8_9 & $122.5(6)$ \\
\hline C9_9 & C10_9 & C5_9 & $121.0(6)$ \\
\hline C12_9 & C11_9 & C8_9 & $118.1(6)$ \\
\hline C16_9 & C11_9 & C8_9 & $120.9(6)$ \\
\hline C16_9 & C11_9 & C12_9 & $120.5(7)$ \\
\hline C11_9 & C12_9 & C17_9 & 121.3(7) \\
\hline C13_9 & C12_9 & C11_9 & 119.1(7) \\
\hline C13_9 & C12_9 & C17_9 & 119.2(7) \\
\hline C14_9 & C13_9 & C12_9 & $121.2(7)$ \\
\hline C13_9 & C14_9 & C18_9 & $121.9(8)$ \\
\hline C15_9 & C14_9 & C13_9 & $118.0(8)$ \\
\hline C15_9 & C14_9 & C18_9 & $120.0(9)$ \\
\hline C14_9 & C15_9 & C16_9 & $123.6(8)$ \\
\hline C11_9 & C16_9 & C15_9 & $117.6(6)$ \\
\hline C11_9 & C16_9 & C19_9 & 122.1(7) \\
\hline C15_9 & C16_9 & C19_9 & $120.3(8)$ \\
\hline C21_9 & C20_9 & C3_9 & 123.1(6) \\
\hline C21_9 & C20_9 & C25_9 & $118.6(7)$ \\
\hline C25_9 & C20_9 & C3_9 & $118.2(6)$ \\
\hline C20_9 & C21_9 & C22_9 & $121.0(7)$ \\
\hline C23_9 & C22_9 & C21_9 & $120.2(6)$ \\
\hline C24_9 & C23_9 & C22_9 & 118.5(7) \\
\hline C25_9 & C24_9 & C23_9 & $121.9(7)$ \\
\hline C24_9 & C25_9 & C20_9 & $119.8(7)$ \\
\hline C27_9 & C26_9 & C3_9 & $122.8(6)$ \\
\hline C27_9 & C26_9 & C31_9 & $116.3(6)$ \\
\hline C31_9 & C26_9 & C3_9 & $120.8(6)$ \\
\hline C26_9 & C27_9 & C28_9 & $122.5(7)$ \\
\hline C27_9 & C28_9 & C29_9 & $119.7(6)$ \\
\hline C28_9 & C29_9 & C30_9 & 119.6(7) \\
\hline C31_9 & C30_9 & C29_9 & 119.5(7) \\
\hline C30_9 & C31_9 & C26_9 & $122.4(7)$ \\
\hline C1_7 & 01_7 & Rh1A & 115.1(7) \\
\hline C1_7 & 02_7 & Rh2A & 121.1(7) \\
\hline 01_7 & C1_7 & 02_7 & $126.4(5)$ \\
\hline 01_7 & C1_7 & C2_7 & $119.6(6)$ \\
\hline 02_7 & C1_7 & C2_7 & $113.9(5)$ \\
\hline C1_7 & C2_7 & C3_7 & $122.4(6)$ \\
\hline C1_7 & C2_7 & C4_7 & 115.1(5) \\
\hline C4_7 & C2_7 & C3_7 & $58.7(4)$ \\
\hline C5_7 & C2_7 & C1_7 & $111.6(5)$ \\
\hline C5_7 & C2_7 & C3_7 & $119.4(5)$ \\
\hline
\end{tabular}

\begin{tabular}{|c|c|c|c|}
\hline Atom & Atom & Atom & Angle $/^{\circ}$ \\
\hline$\overline{C 5} 7$ & C2_7 & C4_7 & $120.1(5)$ \\
\hline C4_7 & C3_7 & C2_7 & $60.4(4)$ \\
\hline C4_7 & C3_7 & C20_7 & $118.9(5)$ \\
\hline C4_7 & C3_7 & C26_7 & $117.6(6)$ \\
\hline C20_7 & C3_7 & C2_7 & $123.7(5)$ \\
\hline C20_7 & C3_7 & C26_7 & $111.8(4)$ \\
\hline C26_7 & C3_7 & C2_-7 & $115.6(5)$ \\
\hline C3_7 & C4_7 & C2_7 & $61.0(4)$ \\
\hline C6_7 & C5_7 & C2_7 & 121.3(5) \\
\hline C10_7 & C5_7 & C2_7 & $122.5(5)$ \\
\hline C10_7 & C5_7 & C6_7 & $115.7(5)$ \\
\hline C5_7 & C6_7 & Cl1_7 & $119.0(5)$ \\
\hline C7_7 & C6_7 & Cl1_7 & 118.1(5) \\
\hline C7_7 & C6_7 & C5_7 & $122.9(6)$ \\
\hline C6_7 & C7_7 & C8_7 & $120.8(6)$ \\
\hline C7_7 & C8_7 & C11_7 & 119.8(5) \\
\hline C9_7 & C8_7 & C7_7 & $116.9(6)$ \\
\hline C9_7 & C8_7 & C11_7 & $123.3(5)$ \\
\hline C10_7 & C9_7 & C8_7 & $122.5(6)$ \\
\hline C9_7 & C10_7 & C5_7 & $121.2(6)$ \\
\hline C12_7 & C11_7 & C8_7 & $118.1(5)$ \\
\hline C16_7 & C11_7 & C8_7 & 121.4(5) \\
\hline C16_7 & C11_7 & C12_7 & $120.6(7)$ \\
\hline C11_7 & C12_7 & C17_7 & $121.4(7)$ \\
\hline C13_7 & C12_7 & C11_7 & $119.2(7)$ \\
\hline C13_7 & C12_7 & C17_7 & $119.4(7)$ \\
\hline C14_7 & C13_7 & C12_7 & 121.1(7) \\
\hline C13_7 & C14_7 & C18_7 & $121.9(8)$ \\
\hline C15_7 & C14_7 & C13_7 & $118.0(7)$ \\
\hline C15_7 & C14_7 & C18_7 & 120.1(9) \\
\hline C14_7 & C15_7 & C16_7 & $123.7(8)$ \\
\hline C11_7 & C16_7 & C15_7 & 117.4(6) \\
\hline C11_7 & C16_7 & C19_7 & $121.7(7)$ \\
\hline C15_7 & C16_7 & C19_7 & $120.0(8)$ \\
\hline C21_7 & C20_7 & C3_7 & 123.4(6) \\
\hline C21_7 & C20_7 & C25_7 & 118.5(7) \\
\hline C25_7 & C20_7 & C3_7 & 118.1(6) \\
\hline C20_7 & C21_7 & C22_7 & $121.0(7)$ \\
\hline C23_7 & C22_7 & C21_7 & $120.2(6)$ \\
\hline C24_7 & C23_7 & C22_7 & 118.5(7) \\
\hline C25_7 & C24_7 & C23_7 & $121.9(7)$ \\
\hline C24_7 & C25_7 & C20_7 & $119.9(7)$ \\
\hline C27_7 & C26_7 & C3_7 & $122.6(6)$ \\
\hline C27_7 & C26_7 & C31_7 & $116.5(6)$ \\
\hline C31_7 & C26_7 & C3_7 & $120.8(6)$ \\
\hline C26_7 & C27_7 & C28_7 & $122.4(7)$ \\
\hline C27_7 & C28_7 & C29_7 & $119.6(6)$ \\
\hline C28_7 & C29_7 & C30_7 & 119.7(7) \\
\hline C31_7 & C30_7 & C29_7 & 119.5(7) \\
\hline C30_7 & C31_7 & C26_7 & 122.3(7) \\
\hline C1_4 & 01_4 & Rh1 & 117.8(7) \\
\hline C1_4 & 02_4 & $\mathrm{Rh} 2$ & $117.7(7)$ \\
\hline 01_4 & C1_4 & 02_4 & $125.6(6)$ \\
\hline 01_4 & C1_4 & C2_4 & $118.4(6)$ \\
\hline 02_4 & C1_4 & C2_4 & $114.2(6)$ \\
\hline C1_4 & C2_4 & C3_4 & $120.5(6)$ \\
\hline C1_4 & C2_4 & C4_4 & 115.1(5) \\
\hline C4_4 & C2_4 & C3_4 & $58.5(4)$ \\
\hline
\end{tabular}




\begin{tabular}{|c|c|c|c|}
\hline Atom & Atom & Atom & Angle $/^{\circ}$ \\
\hline$\overline{\mathrm{C} 5 \_4}$ & C2_4 & C1_4 & $112.3(5)$ \\
\hline C5_4 & C2_4 & C3_4 & $120.1(5)$ \\
\hline C5_4 & $\mathrm{C} 2 \_4$ & C4_4 & $120.3(5)$ \\
\hline C4_4 & C3_4 & C2_4 & $60.3(4)$ \\
\hline C4_4 & C3_4 & C20_4 & $119.3(5)$ \\
\hline C4_4 & C3_4 & C26_4 & $118.4(5)$ \\
\hline C20_4 & C3_4 & C2_4 & $121.5(5)$ \\
\hline C20_4 & C3_4 & C26_4 & $112.1(4)$ \\
\hline C26_4 & C3_4 & C2_4 & $116.2(5)$ \\
\hline C3_4 & C4_4 & C2_4 & $61.2(4)$ \\
\hline C6_4 & C5_4 & $\mathrm{C} 2 \_4$ & $121.7(5)$ \\
\hline C10_4 & C5_4 & C2_4 & $122.8(5)$ \\
\hline C10_4 & C5_4 & C6_4 & $115.6(5)$ \\
\hline C5_4 & C6_4 & $\mathrm{Cl}_{1}{ }_{-} 4$ & $118.9(5)$ \\
\hline C7_4 & C6_4 & $\mathrm{Cl}_{1} 44$ & $118.2(5)$ \\
\hline C7_4 & C6_4 & C5_4 & $122.9(6)$ \\
\hline C6_4 & C7_4 & C8_4 & $120.7(6)$ \\
\hline C7_4 & C8_4 & C11_4 & $120.0(5)$ \\
\hline C9_4 & C8_4 & C7_4 & $116.7(6)$ \\
\hline C9_4 & C8_4 & C11_4 & $123.1(5)$ \\
\hline C10_4 & C9_4 & C8_4 & $122.6(6)$ \\
\hline C9_4 & C10_4 & C5_4 & $121.2(6)$ \\
\hline C12_4 & C11_4 & C8_4 & $117.9(6)$ \\
\hline C16_4 & C11_4 & C8_4 & $120.7(6)$ \\
\hline C16_4 & C11_4 & C12_4 & $120.2(7)$ \\
\hline C11_4 & C12_4 & C17_4 & $121.4(7)$ \\
\hline C13_4 & C12_4 & C11_4 & $119.3(7)$ \\
\hline C13_4 & C12_4 & C17_4 & $119.2(7)$ \\
\hline C14_4 & C13_4 & C12_4 & $121.2(7)$ \\
\hline C13_4 & C14_4 & C18_4 & $122.0(8)$ \\
\hline C15_4 & C14_4 & C13_4 & $117.9(8)$ \\
\hline C15_4 & C14_4 & C18_4 & 120.1(9) \\
\hline C14_4 & C15_4 & C16_4 & $123.7(8)$ \\
\hline C11_4 & C16_4 & C15_4 & $117.7(6)$ \\
\hline C11_4 & C16_4 & C19_4 & $122.0(7)$ \\
\hline C15_4 & C16_4 & C19_4 & $120.2(8)$ \\
\hline C21_4 & C20_4 & C3_4 & $122.7(6)$ \\
\hline C21_4 & C20_4 & C25_4 & $118.5(7)$ \\
\hline C25_4 & C20_4 & C3_4 & $118.5(6)$ \\
\hline C20_4 & C21_4 & C22_4 & $121.1(7)$ \\
\hline C23_4 & C22_4 & C21_4 & $120.2(6)$ \\
\hline C24_4 & C23_4 & C22_4 & $118.4(7)$ \\
\hline C25_4 & C24_4 & C23_4 & $121.9(7)$ \\
\hline C24_4 & C25_4 & C20_4 & $119.9(7)$ \\
\hline C27_4 & C26_4 & C3_4 & $123.0(6)$ \\
\hline C27_4 & C26_4 & C31_4 & $116.3(6)$ \\
\hline C31_4 & C26_4 & C3_4 & $120.6(6)$ \\
\hline C26_4 & C27_4 & C28_4 & $122.5(7)$ \\
\hline C27_4 & C28_4 & C29_4 & $119.7(6)$ \\
\hline C28_4 & C29_4 & C30_4 & $119.6(7)$ \\
\hline C31_4 & C30_4 & C29_4 & $119.4(7)$ \\
\hline C30_4 & C31_4 & C26_4 & $122.4(7)$ \\
\hline C1_6 & 01_6 & Rh1 & $115.6(6)$ \\
\hline C1_6 & 02_6 & Rh2 & $121.2(6)$ \\
\hline 01_6 & C1_6 & 02_6 & $125.7(6)$ \\
\hline $01 \_6$ & C1_6 & C2_6 & $118.7(6)$ \\
\hline $02 \_6$ & C1_6 & C2_6 & $114.5(5)$ \\
\hline C1_6 & C2_6 & C3_6 & $121.4(6)$ \\
\hline
\end{tabular}

\begin{tabular}{|c|c|c|c|}
\hline Atom & Atom & Atom & Angle $/^{\circ}$ \\
\hline$\overline{\mathrm{C} 1 \_6}$ & C2_6 & C4_6 & $115.6(5)$ \\
\hline C4_6 & C2_6 & C3_6 & $58.6(4)$ \\
\hline C5_6 & C2_6 & C1_6 & $112.1(5)$ \\
\hline C5_6 & C2_6 & C3_6 & 119.7(5) \\
\hline C5_6 & C2_6 & C4_6 & 119.6(5) \\
\hline C4_6 & C3_6 & C2_6 & $60.3(4)$ \\
\hline C4_6 & C3_6 & C20_6 & $118.4(5)$ \\
\hline C4_6 & C3_6 & C26_6 & 118.4(5) \\
\hline C20_6 & C3_6 & C2_6 & $122.7(5)$ \\
\hline C20_6 & C3_6 & C26_6 & $112.2(4)$ \\
\hline C26_6 & C3_6 & C2_6 & 115.8(5) \\
\hline C3_6 & C4_6 & C2_6 & $61.1(4)$ \\
\hline C6_6 & C5_6 & C2_6 & $121.5(5)$ \\
\hline C10_6 & C5_6 & C2_6 & $122.6(5)$ \\
\hline C10_6 & C5_6 & C6_6 & $115.9(5)$ \\
\hline C5_6 & C6_6 & Cl1_6 & 118.8(5) \\
\hline C7_6 & C6_6 & Cl1_6 & 118.3(5) \\
\hline C7_6 & C6_6 & C5_6 & $122.9(6)$ \\
\hline C6_6 & C7_6 & C8_6 & $120.7(6)$ \\
\hline C7_6 & C8_6 & C11_6 & $119.8(5)$ \\
\hline C9_6 & C8_6 & C7_6 & $117.0(6)$ \\
\hline C9_6 & C8_6 & C11_6 & $123.0(5)$ \\
\hline C10_6 & C9_6 & C8_6 & $122.5(6)$ \\
\hline C9_6 & C10_6 & C5_6 & 121.1(6) \\
\hline C12_6 & C11_6 & C8_6 & $118.1(6)$ \\
\hline C16_6 & C11_6 & C8_6 & $121.1(5)$ \\
\hline C16_6 & C11_6 & C12_6 & $120.4(7)$ \\
\hline C11_6 & C12_6 & C17_6 & $121.4(7)$ \\
\hline C13_6 & C12_6 & C11_6 & 119.2(7) \\
\hline C13_6 & C12_6 & C17_6 & $119.3(7)$ \\
\hline C14_6 & C13_6 & C12_6 & 121.1(7) \\
\hline C13_6 & C14_6 & C18_6 & 122.1(8) \\
\hline C15_6 & C14_6 & C13_6 & $118.0(7)$ \\
\hline C15_6 & C14_6 & C18_6 & $119.9(9)$ \\
\hline C14_6 & C15_6 & C16_6 & 123.7(8) \\
\hline C11_6 & C16_6 & C15_6 & 117.5(6) \\
\hline C11_6 & C16_6 & C19_6 & $122.2(7)$ \\
\hline C15_6 & C16_6 & C19_6 & 120.2(8) \\
\hline C21_6 & C20_6 & C3_6 & $123.3(6)$ \\
\hline C21_6 & C20_6 & C25_6 & $118.6(7)$ \\
\hline C25_6 & C20_6 & C3_6 & $118.1(6)$ \\
\hline C20_6 & C21_6 & C22_6 & $120.9(7)$ \\
\hline C23_6 & C22_6 & C21_6 & $120.2(6)$ \\
\hline C24_6 & C23_6 & C22_6 & 118.5(7) \\
\hline C25_6 & C24_6 & C23_6 & 121.8(7) \\
\hline C24_6 & C25_6 & C20_6 & $119.9(7)$ \\
\hline C27_6 & C26_6 & C3_6 & $122.9(6)$ \\
\hline C27_6 & C26_6 & C31_6 & 116.3(6) \\
\hline C31_6 & C26_6 & C3_6 & $120.6(6)$ \\
\hline C26_6 & C27_6 & C28_6 & $122.6(7)$ \\
\hline C27_6 & C28_6 & C29_6 & $119.7(6)$ \\
\hline C30_6 & C29_6 & C28_6 & 119.6(7) \\
\hline C31_6 & C30_6 & C29_6 & $119.5(7)$ \\
\hline C30_6 & C31_6 & C26_6 & $122.4(7)$ \\
\hline C1_2 & 01_2 & Rh1 & $113.6(7)$ \\
\hline C1_2 & 02_2 & $\mathrm{Rh} 2$ & 119.5(7) \\
\hline $01 \_2$ & C1_2 & 02_2 & $125.6(6)$ \\
\hline 01_2 & C1_2 & C2_2 & $119.0(6)$ \\
\hline
\end{tabular}




\begin{tabular}{|c|c|c|c|}
\hline Atom & Atom & Atom & Angle $/^{\circ}$ \\
\hline$\overline{02 \_2}$ & C1_2 & C2_2 & $113.4(6)$ \\
\hline C1_2 & C2_2 & C3_2 & $122.6(6)$ \\
\hline C1_2 & C2_2 & C4_2 & $114.9(5)$ \\
\hline C4_2 & C2_2 & C3_2 & $58.7(4)$ \\
\hline C5_2 & C2_2 & C1_2 & $111.7(5)$ \\
\hline C5_2 & C2_2 & C3_2 & $119.4(5)$ \\
\hline C5_2 & C2_2 & C4_2 & $119.9(5)$ \\
\hline C4_2 & C3_2 & C2_2 & $60.3(4)$ \\
\hline C4_2 & C3_2 & C20_2 & $118.9(5)$ \\
\hline C4_2 & C3_2 & C26_2 & $117.3(5)$ \\
\hline C20_2 & C3_2 & C2_z & $123.4(5)$ \\
\hline C20_2 & C3_2 & C26_2 & $112.3(3)$ \\
\hline C26_2 & C3_2 & C2_2 & $115.5(4)$ \\
\hline C3_2 & C4_2 & C2_2 & $61.0(4)$ \\
\hline C6_2 & C5_2 & C2_2 & $121.6(5)$ \\
\hline C10_2 & C5_2 & C2_2 & $122.3(5)$ \\
\hline C10_2 & C5_2 & C6_2 & $115.9(5)$ \\
\hline C5_2 & C6_2 & Cl1_2 & $118.9(5)$ \\
\hline C7_2 & C6_2 & Cl1_2 & $118.3(5)$ \\
\hline C7_2 & C6_2 & C5_2 & $122.8(5)$ \\
\hline C6_2 & C7_2 & C8_2 & $120.7(6)$ \\
\hline C7_2 & C8_2 & C11_2 & $120.3(5)$ \\
\hline C9_2 & C8_2 & C7_2 & $117.0(6)$ \\
\hline C9_2 & C8_2 & C11_2 & $122.7(5)$ \\
\hline C10_2 & C9_2 & C8_2 & $122.5(6)$ \\
\hline C9_2 & C10_2 & C5_2 & $121.1(6)$ \\
\hline C12_2 & C11_2 & C8_2 & $118.3(6)$ \\
\hline C16_2 & C11_2 & C8_2 & $121.2(5)$ \\
\hline C16_2 & C11_2 & C12_2 & $120.5(7)$ \\
\hline C11_2 & C12_2 & C17_2 & 121.4(7) \\
\hline C13_2 & C12_2 & C11_2 & 119.2(7) \\
\hline C13_2 & C12_2 & C17_2 & 119.4(7) \\
\hline C14_2 & C13_2 & C12_2 & 121.1(7) \\
\hline C13_2 & C14_2 & C18_2 & $121.9(8)$ \\
\hline C15_2 & C14_2 & C13_2 & $118.0(7)$ \\
\hline C15_2 & C14_2 & C18_2 & 120.1(9) \\
\hline C14_2 & C15_2 & C16_2 & 123.7(8) \\
\hline C11_2 & C16_2 & C15_2 & 117.5(6) \\
\hline C11_2 & C16_2 & C19_2 & $122.1(7)$ \\
\hline C15_2 & C16_2 & C19_2 & $120.4(8)$ \\
\hline C21_2 & C20_2 & C3_2 & $123.4(6)$ \\
\hline C21_2 & C20_2 & C25_2 & 118.4(7) \\
\hline C25_2 & C20_2 & C3_2 & 118.2(6) \\
\hline C20_2 & C21_2 & C22_2 & 121.0(7) \\
\hline C23_2 & C22_2 & C21_2 & $120.2(6)$ \\
\hline C24_2 & C23_2 & C22_2 & 118.4(7) \\
\hline C25_2 & C24_2 & C23_2 & $121.9(7)$ \\
\hline C24_2 & C25_2 & C20_2 & $120.0(7)$ \\
\hline C27_2 & C26_2 & C3_2 & $122.5(6)$ \\
\hline C27_2 & C26_2 & C31_2 & 116.5(6) \\
\hline C31_2 & C26_2 & C3_2 & $121.0(6)$ \\
\hline C26_2 & C27_2 & C28_2 & $122.5(7)$ \\
\hline C27_2 & C28_2 & C29_2 & $119.7(6)$ \\
\hline C28_2 & C29_2 & C30_2 & $119.6(7)$ \\
\hline C31_2 & C30_2 & C29_2 & $119.5(7)$ \\
\hline C30_2 & C31_2 & C26_2 & $122.3(7)$ \\
\hline C1_8 & 01_8 & Rh1A & $117.9(6)$ \\
\hline C1_8 & 02_8 & $\mathrm{Rh} 2 \mathrm{~A}$ & $117.2(6)$ \\
\hline
\end{tabular}

\begin{tabular}{|c|c|c|c|}
\hline Atom & Atom & Atom & Angle $/^{\circ}$ \\
\hline$\overline{01 \_8}$ & C1_8 & 02_8 & $126.7(5)$ \\
\hline 01_8 & C1_8 & C2_8 & $119.0(6)$ \\
\hline 02_8 & C1_8 & C2_8 & $114.2(5)$ \\
\hline C1_8 & C2_8 & C3_8 & 121.4(6) \\
\hline C1_8 & C2_8 & C4_8 & $114.6(5)$ \\
\hline C4_8 & C2_8 & C3_8 & $58.6(4)$ \\
\hline C5_8 & C2_8 & C1_8 & $112.1(5)$ \\
\hline C5_8 & C2_8 & C3_8 & $120.4(5)$ \\
\hline C5_8 & C2_8 & C4_8 & $119.6(5)$ \\
\hline C4_8 & C3_8 & C2_8 & $60.4(4)$ \\
\hline C4_8 & C3_8 & C20_8 & 118.8(5) \\
\hline C4_8 & C3_8 & C26_8 & $117.5(6)$ \\
\hline C20_8 & C3_8 & C2_8 & $122.4(5)$ \\
\hline C20_8 & C3_8 & C26_8 & $112.2(4)$ \\
\hline C26_8 & C3_8 & C2_8 & $116.4(5)$ \\
\hline C3_8 & C4_8 & C2_8 & $61.0(4)$ \\
\hline C6_8 & C5_8 & C2_8 & 122.3(5) \\
\hline C10_8 & C5_8 & C2_8 & $121.9(5)$ \\
\hline C10_8 & C5_8 & C6_8 & 115.7(5) \\
\hline C5_8 & C6_8 & Cl1_8 & $119.2(5)$ \\
\hline C7_8 & C6_8 & Cl1_8 & $117.7(5)$ \\
\hline C7_8 & C6_8 & C5_8 & $122.8(6)$ \\
\hline C6_8 & C7_8 & C8_8 & $120.8(6)$ \\
\hline C7_8 & C8_8 & C11_8 & $120.2(5)$ \\
\hline C9_8 & C8_8 & C7_8 & $117.0(6)$ \\
\hline C9_8 & C8_8 & C11_8 & $122.8(5)$ \\
\hline C10_8 & C9_8 & C8_8 & $122.4(6)$ \\
\hline C9_8 & C10_8 & C5_8 & $121.3(6)$ \\
\hline C12_8 & C11_8 & C8_8 & 118.1(6) \\
\hline C16_8 & C11_8 & C8_8 & $120.9(5)$ \\
\hline C16_8 & C11_8 & C12_8 & $120.6(7)$ \\
\hline C11_8 & C12_8 & C17_8 & 121.4(7) \\
\hline C13_8 & C12_8 & C11_8 & 119.2(7) \\
\hline C13_8 & C12_8 & C17_8 & 119.3(7) \\
\hline C14_8 & C13_8 & C12_8 & 121.1(7) \\
\hline C13_8 & C14_8 & C18_8 & $121.9(8)$ \\
\hline C15_8 & C14_8 & C13_8 & 118.1(7) \\
\hline C15_8 & C14_8 & C18_8 & $120.0(9)$ \\
\hline C14_8 & C15_8 & C16_8 & $123.6(8)$ \\
\hline C11_8 & C16_8 & C15_8 & $117.4(6)$ \\
\hline C11_8 & C16_8 & C19_8 & $121.9(7)$ \\
\hline C15_8 & C16_8 & C19_8 & $120.3(8)$ \\
\hline C21_8 & C20_8 & C3_8 & 123.1(6) \\
\hline C21_8 & C20_8 & C25_8 & 118.5(7) \\
\hline C25_8 & C20_8 & C3_8 & 118.3(6) \\
\hline C20_8 & C21_8 & C22_8 & $121.0(7)$ \\
\hline C23_8 & C22_8 & C21_8 & $120.2(6)$ \\
\hline C24_8 & C23_8 & C22_8 & 118.5(7) \\
\hline C25_8 & C24_8 & C23_8 & 121.8(7) \\
\hline C24_8 & C25_8 & C20_8 & $120.0(7)$ \\
\hline C27_8 & C26_8 & C3_8 & $122.5(6)$ \\
\hline C27_8 & C26_8 & C31_8 & $116.4(6)$ \\
\hline C31_8 & C26_8 & C3_8 & $121.1(6)$ \\
\hline C26_8 & C27_8 & C28_8 & $122.5(7)$ \\
\hline C27_8 & C28_8 & C29_8 & $119.7(6)$ \\
\hline C28_8 & C29_8 & C30_8 & $119.6(7)$ \\
\hline C31_8 & C30_8 & C29_8 & 119.5(7) \\
\hline C30_8 & C31_8 & C26_8 & 122.3(7) \\
\hline
\end{tabular}




\begin{tabular}{llll}
\hline Atom & Atom & Atom & \multicolumn{1}{|c}{ Angle/ } \\
\hline C1_1 & O1_1 & Rh1 & $115.4(6)$ \\
C1_1 & O2_1 & Rh2 & $119.0(6)$ \\
01_1 & C1_1 & O2_1 & $126.4(6)$ \\
01_1 & C1_1 & C2_1 & $118.6(6)$ \\
O2_1 & C1_1 & C2_1 & $114.3(5)$ \\
C1_1 & C2_1 & C3_1 & $121.2(6)$ \\
C1_1 & C2_1 & C4_1 & $114.3(5)$ \\
C4_1 & C2_1 & C3_1 & $58.6(4)$ \\
C5_1 & C2_1 & C1_1 & $112.2(5)$ \\
C5_1 & C2_1 & C3_1 & $120.3(5)$ \\
C5_1 & C2_1 & C4_1 & $120.0(5)$ \\
C4_1 & C3_1 & C2_1 & $60.4(4)$ \\
C4_1 & C3_1 & C20_1 & $119.0(5)$ \\
C4_1 & C3_1 & C26_1 & $117.1(6)$ \\
C20_1 & C3_1 & C2_1 & $122.2(5)$ \\
C20_1 & C3_1 & C26_1 & $112.3(4)$ \\
C26_1 & C3_1 & C2_1 & $116.7(5)$ \\
C3_1 & C4_1 & C2_1 & $61.0(4)$ \\
C6_1 & C5_1 & C2_1 & $122.3(5)$ \\
C10_1 & C5_1 & C2_1 & $122.1(5)$ \\
C10_1 & C5_1 & C6_1 & $115.6(5)$ \\
C5_1 & C6_1 & Cl1_1 & $119.2(5)$ \\
C7_1 & C6_1 & Cl1_1 & $117.9(5)$ \\
C7_1 & C6_1 & C5_1 & $122.9(5)$ \\
C6_1 & C7_1 & C8_1 & $120.9(6)$ \\
C7_1 & C8_1 & C11_1 & $120.2(5)$ \\
C9_1 & C8_1 & C7_1 & $116.8(6)$ \\
C9_1 & C8_1 & C11_1 & $123.0(5)$ \\
C10_1 & C9_1 & C8_1 & $122.5(6)$ \\
C9_1 & C10_1 & C5_1 & $121.3(6)$ \\
& & & \\
\end{tabular}

\begin{tabular}{|c|c|c|c|}
\hline Atom & Atom & Atom & Angle ${ }^{\circ}$ \\
\hline$\overline{C 12 \_1}$ & C11_1 & C8_1 & $118.3(5)$ \\
\hline C16_1 & C11_1 & C8_1 & $121.1(5)$ \\
\hline C16_1 & C11_1 & C12_1 & $120.6(7)$ \\
\hline C11_1 & C12_1 & C17_1 & $121.3(6)$ \\
\hline C13_1 & C12_1 & C11_1 & $119.2(7)$ \\
\hline C13_1 & C12_1 & C17_1 & $119.3(7)$ \\
\hline C14_1 & C13_1 & C12_1 & $121.1(7)$ \\
\hline C13_1 & C14_1 & C18_1 & $122.0(8)$ \\
\hline C15_1 & C14_1 & C13_1 & $118.0(7)$ \\
\hline C15_1 & C14_1 & C18_1 & $120.0(9)$ \\
\hline C14_1 & C15_1 & C16_1 & $123.7(8)$ \\
\hline C11_1 & C16_1 & C15_1 & $117.4(6)$ \\
\hline C11_1 & C16_1 & C19_1 & $121.9(7)$ \\
\hline C15_1 & C16_1 & C19_1 & $120.2(8)$ \\
\hline C21_1 & C20_1 & C3_1 & $123.1(6)$ \\
\hline C21_1 & C20_1 & C25_1 & $118.5(7)$ \\
\hline C25_1 & C20_1 & C3_1 & $118.3(6)$ \\
\hline C20_1 & C21_1 & C22_1 & $121.0(7)$ \\
\hline C23_1 & C22_1 & C21_1 & $120.2(6)$ \\
\hline C24_1 & C23_1 & C22_1 & $118.5(7)$ \\
\hline C25_1 & C24_1 & C23_1 & $121.9(7)$ \\
\hline C24_1 & C25_1 & C20_1 & $119.9(7)$ \\
\hline C27_1 & C26_1 & C3_1 & $122.3(6)$ \\
\hline C27_1 & C26_1 & C31_1 & $116.4(6)$ \\
\hline C31_1 & C26_1 & C3_1 & $121.2(6)$ \\
\hline C28_1 & C27_1 & C26_1 & $122.5(7)$ \\
\hline C27_1 & C28_1 & C29_1 & $119.6(6)$ \\
\hline C28_1 & C29_1 & C30_1 & $119.6(7)$ \\
\hline C31_1 & C30_1 & C29_1 & $119.5(7)$ \\
\hline C30_1 & C31_1 & C26_1 & $122.3(7)$ \\
\hline
\end{tabular}

Table 20: Torsion Angles in ${ }^{\circ}$ for 2Cl4triMePh.

\begin{tabular}{|c|c|c|c|c|}
\hline Atom & Atom & Atom & Atom & Angle $/^{\circ}$ \\
\hline$\overline{\mathrm{Rh} 1}$ & 01_4 & C1_4 & O2_4 & $2(3)$ \\
\hline Rh1 & 01_4 & C1_4 & C2_4 & $-162.3(11)$ \\
\hline Rh1 & 01_6 & C1_6 & $02 \_6$ & $3(2)$ \\
\hline Rh1 & 01_6 & C1_6 & C2_6 & $-163.5(9)$ \\
\hline Rh1 & 01_2 & C1_2 & 02_2 & $28(2)$ \\
\hline Rh1 & 01_2 & C1_2 & C2_2 & $-168.8(8)$ \\
\hline Rh1 & 01_1 & C1_1 & 02_1 & $20(3)$ \\
\hline Rh1 & 01_1 & C1_1 & C2_1 & $-170.1(10)$ \\
\hline $\mathrm{Rh} 2$ & 02_4 & C1_4 & 01_4 & 16(3) \\
\hline Rh2 & 02_4 & C1_4 & C2_4 & $-179.0(11)$ \\
\hline Rh2 & 02_6 & C1_6 & 01_6 & 11(3) \\
\hline Rh2 & $02 \_6$ & C1_6 & C2_6 & $178.2(10)$ \\
\hline Rh2 & 02_2 & C1_2 & 01_2 & $-11(3)$ \\
\hline $\mathrm{Rh} 2$ & 02_2 & C1_2 & C2_2 & $-175.2(11)$ \\
\hline $\mathrm{Rh} 2$ & 02_1 & C1_1 & 01_1 & $-4(3)$ \\
\hline $\mathrm{Rh} 2$ & 02_1 & C1_1 & C2_1 & $-174.7(10)$ \\
\hline Rh1A & 01_3 & C1_3 & 02_3 & 16(3) \\
\hline Rh1A & 01_3 & C1_3 & C2_3 & $-166.0(10)$ \\
\hline Rh1A & 01_9 & C1_9 & 02_9 & $10(3)$ \\
\hline Rh1A & 01_9 & C1_9 & C2_9 & $-167.9(9)$ \\
\hline Rh1A & 01_7 & C1_7 & 02_7 & $12(3)$ \\
\hline
\end{tabular}




\begin{tabular}{|c|c|c|c|c|}
\hline Atom & Atom & Atom & Atom & Angle/ ${ }^{\circ}$ \\
\hline$\overline{R h 1 A}$ & 01_7 & C1_7 & C2_7 & $-171.6(8)$ \\
\hline Rh1A & 01_8 & C1_8 & $02 \_8$ & $15(3)$ \\
\hline Rh1A & 01_8 & C1_8 & $\mathrm{C} 2 \_8$ & $-167.7(10)$ \\
\hline $\mathrm{Rh} 2 \mathrm{~A}$ & $02 \_3$ & C1_3 & 01_3 & $0(3)$ \\
\hline Rh2A & 02_3 & C1_3 & C2_3 & $-178.3(10)$ \\
\hline $\mathrm{Rh} 2 \mathrm{~A}$ & 02_9 & C1_9 & 01_9 & $5(3)$ \\
\hline $\mathrm{Rh} 2 \mathrm{~A}$ & 02_9 & C1_9 & C2_9 & $-176.9(9)$ \\
\hline $\mathrm{Rh} 2 \mathrm{~A}$ & $02 \_7$ & $\mathrm{C} 1 \_7$ & $01 \_7$ & $-3(3)$ \\
\hline Rh2A & 02_7 & C1_7 & C2_7 & $-179.3(12)$ \\
\hline Rh2A & 02_8 & C1_8 & 01_8 & $-1(3)$ \\
\hline $\mathrm{Rh} 2 \mathrm{~A}$ & 02_8 & C1_8 & C2_8 & $-179.1(10)$ \\
\hline Cl1_3 & C6_3 & C7_3 & C8_3 & $179.96(17)$ \\
\hline 01_3 & C1_3 & C2_3 & C3_3 & $29.0(19)$ \\
\hline $01 \_3$ & C1_3 & $\mathrm{C} 2 \_3$ & C4_3 & $-37.9(19)$ \\
\hline $01 \_3$ & C1_3 & $\mathrm{C} 2 \_3$ & C5_3 & $179.7(17)$ \\
\hline 02_3 & C1_3 & C2_3 & C3_3 & $-152.3(15)$ \\
\hline $02 \_3$ & C1_3 & $\mathrm{C} 2 \_3$ & C4_3 & $140.8(15)$ \\
\hline 02_3 & C1_3 & C2_3 & C5_3 & $-1.6(16)$ \\
\hline C1_3 & C2_3 & C3_3 & C4_3 & $-102.3(7)$ \\
\hline C1_3 & $\mathrm{C} 2 \_3$ & C3_3 & C20̄_3 & $6.2(9)$ \\
\hline C1_3 & $\mathrm{C} 2 \_3$ & C3_3 & C26_3 & $149.1(7)$ \\
\hline C1_3 & C2_3 & C4_3 & C3_3 & $112.0(7)$ \\
\hline C1_3 & C2_3 & C5_3 & C6_3 & $-73.9(7)$ \\
\hline C1_3 & C2_3 & C5_3 & C10_3 & $106.0(7)$ \\
\hline C2_3 & C3_3 & C20_3 & C21_3 & 58.4(8) \\
\hline C2_3 & C3_3 & C20_3 & C25_3 & $-121.5(8)$ \\
\hline $\mathrm{C} 2 \_3$ & C3_3 & C26_3 & C27_3 & $50.7(9)$ \\
\hline $\mathrm{C} 2 \_3$ & C3_3 & C26_3 & C31_3 & $-127.7(7)$ \\
\hline C2_3 & C5_3 & C6_3 & Cl1_3 & $-0.1(2)$ \\
\hline $\mathrm{C} 2 \_3$ & C5_3 & C6_3 & C7_3 & $179.88(14)$ \\
\hline $\mathrm{C} 2 \_3$ & C5_3 & C10_3 & C9_3 & $-179.8(2)$ \\
\hline C3_3 & C2_3 & C5_3 & C6_3 & 76.9(7) \\
\hline C3_3 & C2_3 & C5_3 & C10_3 & $-103.2(7)$ \\
\hline C3_3 & C20-3 & C $2 \overline{1} \_3$ & C22_3 & $-179.92(13)$ \\
\hline C3_3 & C20_3 & C25_3 & C24_3 & $179.91(17)$ \\
\hline C3_3 & C26_3 & C27_3 & C28_3 & $-178.5(8)$ \\
\hline C3_3 & C26_3 & C31_3 & C30_3 & $178.5(8)$ \\
\hline C4_3 & C2_3 & C3_3 & C20_3 & $108.5(6)$ \\
\hline C4_3 & C2_3 & C3_3 & C26_3 & $-108.6(7)$ \\
\hline C4_3 & $\mathrm{C} 2 \_3$ & C5_3 & C6_ $\overline{3}$ & $146.1(7)$ \\
\hline C4_3 & $\mathrm{C} 2 \_3$ & C5_3 & C10_3 & $-34.0(7)$ \\
\hline C4_3 & C3_3 & C20_3 & C21_3 & $129.3(7)$ \\
\hline C4_3 & C3_3 & C20_3 & C25_3 & $-50.6(7)$ \\
\hline C4_3 & C3_3 & C26_3 & C27_3 & $-18.0(9)$ \\
\hline C4_3 & C3_3 & C26_3 & C31_3 & $163.5(7)$ \\
\hline C5_3 & C2_3 & C3_3 & C4_3 & $109.5(6)$ \\
\hline C5_3 & C2_3 & C3_3 & C20_3 & $-142.0(6)$ \\
\hline C5_3 & C2_3 & C3_3 & C26_3 & $0.9(9)$ \\
\hline C5_3 & C2_3 & C4_3 & C3_3 & $-109.3(5)$ \\
\hline C5_3 & C6_3 & C7_3 & C8_3 & $-0.02(9)$ \\
\hline C6_3 & C5_3 & C10_3 & C9_3 & $0.1(2)$ \\
\hline C6_3 & C7_3 & C8_3 & C9_3 & $0.0(2)$ \\
\hline C6_3 & C7_3 & C8_3 & C11_3 & $-174.9(6)$ \\
\hline C7_3 & C8_3 & C9_3 & C10_3 & $0.0(3)$ \\
\hline C7_3 & C8_3 & C11_3 & C12_3 & $-73.4(8)$ \\
\hline C7_3 & C8_3 & C11_3 & C16_3 & $100.3(7)$ \\
\hline C8_3 & C9_3 & C10_3 & C5_3근 & $-0.1(3)$ \\
\hline C8_3 & C11_3 & C12_3 & C13_3 & $173.7(6)$ \\
\hline
\end{tabular}




\begin{tabular}{|c|c|c|c|c|}
\hline Atom & Atom & Atom & Atom & Angle ${ }^{\circ}$ \\
\hline$\overline{C 8 \_3}$ & C11_3 & C12_3 & C17_3 & $-6.6(13)$ \\
\hline C8_3 & C11_3 & C16_3 & C15_3 & $-173.5(6)$ \\
\hline C8_3 & C11_3 & C16_3 & C19_3 & $2.3(16)$ \\
\hline C9_3 & C8_3 & C11_3 & C12_3 & $112.1(7)$ \\
\hline C9_3 & C8_3 & C11_3 & C16_3 & $-74.3(8)$ \\
\hline C10_3 & C5_3 & C6_3 & Cl1_3 & 179.98(17) \\
\hline C10_3 & C5_3 & C6_3 & C7_3 & $-0.04(9)$ \\
\hline C11_3 & C8_3 & C9_3 & C10_3 & $174.7(6)$ \\
\hline C11_3 & C12_3 & C13_3 & C14_3 & $0.00(9)$ \\
\hline C12_3 & C11_3 & C16_3 & C15_3 & $-0.01(19)$ \\
\hline C12_3 & C11_3 & C16_3 & C19_3 & $175.8(16)$ \\
\hline C12_3 & C13_3 & C14_3 & C15_3 & 0.01(19) \\
\hline C12_3 & C13_3 & C14_3 & C18_3 & $-180.0(2)$ \\
\hline C13_3 & C14_3 & C15_3 & C16_3 & $0.0(3)$ \\
\hline C14_3 & C15_3 & C16_3 & C11_3 & $0.0(3)$ \\
\hline C14_3 & C15_3 & C16_3 & C19_3 & $-175.9(16)$ \\
\hline C16_3 & C11_3 & C12_3 & C13_3 & $0.00(9)$ \\
\hline C16_3 & C11_3 & C12_3 & C17_3 & $179.7(13)$ \\
\hline C17_3 & C12_3 & C13_3 & C14_3 & $-179.7(13)$ \\
\hline C18_3 & C14_3 & C15_3 & C16_3 & $180.0(3)$ \\
\hline C20_3 & C3_3 & C4_3 & C2_3 & $-110.8(6)$ \\
\hline C20_3 & C3_3 & C26_3 & C27_3 & $-163.4(6)$ \\
\hline C20_3 & C3_3 & C26_3 & C31_3 & 18.2(9) \\
\hline C20_3 & C21_3 & C22_3 & C23_3 & $0.01(9)$ \\
\hline C21_3 & C20_3 & C25_3 & C24_3 & $-0.02(13)$ \\
\hline C21_3 & C22_3 & C23_3 & C24_3 & $-0.01(18)$ \\
\hline C22_3 & C23_3 & C24_3 & C25_3 & $0.0(2)$ \\
\hline C23_3 & C24_3 & C25_3 & C20_3 & $0.0(2)$ \\
\hline C25_3 & C20_3 & C21_3 & C22_3 & $0.01(6)$ \\
\hline C26_3 & C3_3 & C4_ $\overline{3}$ & $\mathrm{C} 2 \_\overline{3}$ & $106.3(6)$ \\
\hline C26_3 & C3_3 & C20_3 & C21_3 & $-85.9(7)$ \\
\hline C26_3 & C3_3 & C20_3 & C25_3 & $94.2(7)$ \\
\hline C26_3 & C27̄_3 & C28_3 & C29_3 & $0.02(14)$ \\
\hline C27_3 & C26_3 & C31_3 & C30_3 & $0.0(2)$ \\
\hline C27_3 & C28_3 & C29_3 & C30_3 & $0.0(2)$ \\
\hline C28_3 & C29_3 & C30_3 & C31_3 & $0.0(2)$ \\
\hline C29_3 & C30_3 & C31_3 & C26_3 & $0.0(2)$ \\
\hline C31_3 & C26_3 & C27_3 & C28_3 & $0.00(11)$ \\
\hline Cl1_9 & C6_9 & C7_9 & C8_9 & $178.2(14)$ \\
\hline 01_9 & C1_9 & C2_9 & C3_9 & $25.0(16)$ \\
\hline 01_9 & C1_9 & C2_9 & C4_9 & $-42.3(16)$ \\
\hline 01_9 & C1_9 & C2_9 & C5_9 & $175.4(14)$ \\
\hline 02_9 & C1_9 & C2_9 & C3_9 & $-152.9(14)$ \\
\hline 02_9 & C1_9 & C2_9 & C4_9 & 139.7(13) \\
\hline 02_9 & C1_9 & C2_9 & C5_9 & $-2.6(15)$ \\
\hline C1_9 & C2_9 & C3_9 & C4_9 & $-102.7(7)$ \\
\hline C1_9 & C2_9 & C3_9 & C20_9 & $4.5(9)$ \\
\hline C1_9 & C2_9 & C3_9 & C26_9 & $148.3(7)$ \\
\hline C1_9 & C2_9 & C4_9 & C3_9 & $112.7(7)$ \\
\hline C1_9 & C2_9 & C5_9 & C6_9 & $-74.2(9)$ \\
\hline C1_9 & C2_9 & C5_9 & C10_9 & $106.7(9)$ \\
\hline C2_9 & C3_9 & C20_9 & C21_9 & $50.4(9)$ \\
\hline C2_9 & C3_9 & C20_9 & C25_9 & $-126.5(9)$ \\
\hline C2_9 & C3_9 & C26_9 & C27_9 & 61.4(10) \\
\hline C2_9 & C3_9 & C26_9 & C31_9 & $-115.5(9)$ \\
\hline C2_9 & C5_9 & C6_9 & Cl1_9 & $2.6(14)$ \\
\hline C2_9 & C5_9 & C6_9 & C7_9 & $-179.2(7)$ \\
\hline C2_9 & C5_9 & C10_9 & C9_9 & 179.2(7) \\
\hline
\end{tabular}




\begin{tabular}{|c|c|c|c|c|}
\hline Atom & Atom & Atom & Atom & Angle $/^{\circ}$ \\
\hline C3_9 & C2_9 & C5_9 & C6_9 & $76.8(9)$ \\
\hline C3_9 & C2_9 & C5_9 & C10_9 & $-102.3(9)$ \\
\hline C3_9 & C20_9 & C21_9 & C22_9 & $-176.9(8)$ \\
\hline C3_9 & C20_9 & C25_9 & C24_9 & 177.1(7) \\
\hline C3_9 & C26_9 & C27_9 & C28_9 & $-177.0(9)$ \\
\hline C3_9 & C26_9 & C31_9 & C30_9 & 177.1(9) \\
\hline C4_9 & C2_9 & C3_9 & C20_9 & $107.1(7)$ \\
\hline C4_9 & C2_9 & C3_9 & C26_9 & $-109.0(7)$ \\
\hline C4_9 & $\mathrm{C} 2 \_9$ & C5_9 & C6_ē & $145.4(8)$ \\
\hline C4_9 & C2_9 & C5_9 & C10_9 & $-33.7(9)$ \\
\hline C4_9 & C3_9 & C20_9 & C21_9 & $121.6(8)$ \\
\hline C4_9 & C3_9 & C20_9 & C25_9 & $-55.3(9)$ \\
\hline C4_9 & C3_9 & C26_9 & C27_9 & $-7.2(10)$ \\
\hline C4_9 & C3_9 & C26_9 & C31_9 & $175.9(8)$ \\
\hline C5_9 & C2_9 & C3_9 & C4_9 & $109.1(6)$ \\
\hline C5_9 & C2_9 & C3_9 & C20_9 & $-143.8(6)$ \\
\hline C5_9 & C2_9 & C3_9 & C26_9 & $0.1(8)$ \\
\hline C5_9 & C2_9 & C4_9 & C3_9 & $-108.3(6)$ \\
\hline C5_9 & C6_9 & C7_9 & C8_9 & $0.00(9)$ \\
\hline C6_9 & C5_9 & C10_9 & C9_9 & $0.0(2)$ \\
\hline C6_9 & C7_9 & C8_9 & C9_9 & $0.0(2)$ \\
\hline C6_9 & C7_9 & C8_9 & C11_9 & $-172.6(7)$ \\
\hline C7_9 & C8_9 & C9_9 & C10_9 & $0.0(3)$ \\
\hline C7_9 & C8_9 & C11_9 & C12_9 & $-81.2(15)$ \\
\hline C7_9 & C8_9 & C11_9 & C16_9 & $90.0(16)$ \\
\hline C8_9 & C9_9 & C10_9 & C5_ & $0.0(3)$ \\
\hline C8_9 & C11_9 & C12_9 & C13_9 & $171.8(16)$ \\
\hline C8_9 & C11_9 & C12_9 & C17_9 & $-15(3)$ \\
\hline C8_9 & C11_9 & C16_9 & C15_9 & $-170.0(16)$ \\
\hline C8_9 & C11_9 & C16_9 & C19_9 & $12(3)$ \\
\hline C9_9 & C8_9 & C11_9 & C12_9 & $106.7(14)$ \\
\hline C9_9 & C8_9 & C11_9 & C16_9 & -82.1(16) \\
\hline C10_9 & C5_9 & C6_9 9 & Cl1_9 & $-178.2(14)$ \\
\hline C10_9 & C5_9 & C6_9 & C7_9 & $-0.01(9)$ \\
\hline C11_9 & C8_9 & C9_9 & C10_9 & $172.3(8)$ \\
\hline C11_9 & C12_9 & C13_9 & C14_9 & $-3(3)$ \\
\hline C12_9 & C11_9 & C16_9 & C15_9 & 1(3) \\
\hline C12_9 & C11_9 & C16_9 & C19_9 & $-177(2)$ \\
\hline C12_9 & C13_9 & C14_9 & C15_9 & $4(4)$ \\
\hline C12_9 & C13_9 & C14_9 & C18_9 & $178(3)$ \\
\hline C13_9 & C14_9 & C15_9 & C16_9 & $-2(4)$ \\
\hline C14_9 & C15_9 & C16_9 & C11_9 & $0(4)$ \\
\hline C14_9 & C15_9 & C16_9 & C19_9 & 177(3) \\
\hline C16_9 & C11_9 & C12_9 & C13_9 & 1(3) \\
\hline C16_9 & C11_9 & C12_9 & C17_9 & $174(2)$ \\
\hline C17_9 & C12_9 & C13_9 & C14_9 & $-176(2)$ \\
\hline C18_9 & C14_9 & C15_9 & C16_9 & $-177(3)$ \\
\hline C20_9 & C3_9 & C4_9 & C2_9 & $-113.0(6)$ \\
\hline C20_9 & C3_9 & C26_9 & C27_9 & $-151.1(8)$ \\
\hline C20_9 & C3_9 & C26_9 & C31_9 & $31.9(9)$ \\
\hline C20_9 & C21_9 & C22_9 & C23_9 & $0.00(9)$ \\
\hline C21_9 & C20_9 & C25_9 & C24_9 & $0.0(2)$ \\
\hline C21_9 & C22_9 & C23_9 & C24_9 & $0.0(2)$ \\
\hline C22_9 & C23_9 & C24_9 & C25_9 & $0.0(3)$ \\
\hline C23_9 & C24_9 & C25_9 & C20_9 & $0.0(3)$ \\
\hline C25_9 & C20_9 & C21_9 & C22_9 & $0.00(9)$ \\
\hline C26_9 & C3_9 & C4_9 & C2_9 & $105.4(6)$ \\
\hline C26_9 & C3_9 & C20_9 & C21_9 & $-94.6(8)$ \\
\hline
\end{tabular}




\begin{tabular}{|c|c|c|c|c|}
\hline Atom & Atom & Atom & Atom & Angle $/^{\circ}$ \\
\hline C26_9 & C3_9 & C20_9 & C25_9 & $88.4(9)$ \\
\hline C26_9 & C27̄_9 & C28_9 & C29_9 & $0.00(9)$ \\
\hline C27_9 & C26_9 & C31_9 & C30_9 & $0.0(2)$ \\
\hline C27_9 & C28_9 & C29_9 & C30_9 & $0.0(2)$ \\
\hline C28_9 & C29_9 & C30_9 & C31_9 & $0.0(3)$ \\
\hline C29_9 & C30_9 & C31_9 & C26_9 & $0.0(3)$ \\
\hline C31_9 & C26_9 & C27_9 & C28_9 & $0.00(9)$ \\
\hline Cl1_7 & C6_7 & C7_7 & C8_7 & $177.4(11)$ \\
\hline 01_7 & C1_7 & C2_7 & C3_7 & $18.9(16)$ \\
\hline 01_7 & C1_7 & C2_7 & C4_7 & $-48.6(16)$ \\
\hline 01_7 & C1_7 & C2_7 & C5_7 & $170.1(14)$ \\
\hline 02_7 & C1_7 & C2_7 & C3_7 & $-164.5(15)$ \\
\hline $02 \_7$ & C1_7 & C2_7 & C4_7 & $128.1(15)$ \\
\hline $02 \_7$ & C1_7 & C2_7 & C5_7 & $-13.3(16)$ \\
\hline C1_7 & C2_7 & C3_7 & C4_7 & $-101.6(7)$ \\
\hline C1_7 & C2_7 & C3_7 & C20_7 & $5.2(9)$ \\
\hline C1_7 & C2_7 & C3_7 & C26_7 & $149.7(7)$ \\
\hline C1_7 & C2_7 & C4_7 & C3_7 & 114.1(7) \\
\hline C1_7 & C2_7 & C5_7 & C6_7 & $-71.3(9)$ \\
\hline C1_7 & C2_7 & C5_7 & C10_7 & $116.7(8)$ \\
\hline C2_7 & C3_7 & C20_7 & C21_7 & $50.8(9)$ \\
\hline C2_7 & C3_7 & C20_7 & C25_7 & $-129.2(9)$ \\
\hline C2_7 & C3_7 & C26_7 & C27_7 & $95.4(8)$ \\
\hline $\mathrm{C} 2 \_7$ & C3_7 & C26_7 & C31_7 & $-80.9(8)$ \\
\hline C2_7 & C5_7 & C6_7 & Cl1_7 & $10.3(11)$ \\
\hline $\mathrm{C} 2 \_7$ & C5_7 & C6_7 & C7_7 & $-172.6(6)$ \\
\hline $\mathrm{C} 2 \_7$ & C5_7 & C10_7 & C9_7 & $172.6(6)$ \\
\hline C3_7 & C2_7 & C5_7 & C6_7 & 80.9(8) \\
\hline C3_7 & $\mathrm{C} 2 \_7$ & C5_7 & C10_7 & $-91.1(8)$ \\
\hline C3_7 & C20_7 & C21_7 & C22_7 & $179.6(2)$ \\
\hline C3_7 & C20_7 & C25_7 & C24_7 & $-179.3(5)$ \\
\hline C3_7 & C26_7 & C27_7 & C28_7 & $-176.5(7)$ \\
\hline C3_7 & C26_7 & C31_7 & C30_7 & 176.6(7) \\
\hline C4_7 & C2_7 & C3_7 & C20_7 & $106.8(7)$ \\
\hline C4_7 & C2_7 & C3_7 & C26_7 & $-108.6(6)$ \\
\hline C4_7 & C2_7 & C5_7 & C6_7 & $149.6(7)$ \\
\hline C4_7 & C2_7 & C5_7 & C10_7 & $-22.4(9)$ \\
\hline C4_7 & C3_7 & C20_7 & C21_7 & $122.7(9)$ \\
\hline C4__7 & C3_7 & C20_7 & C25_7 & $-57.3(9)$ \\
\hline C4_7 & C3_7 & C26_7 & C27_7 & 27.1(9) \\
\hline C4_7 & C3_7 & C26_7 & C31_7 & $-149.3(8)$ \\
\hline C5_7 & C2_7 & C3_7 & C4_7 & $109.3(6)$ \\
\hline C5_7 & C2_7 & C3_7 & C20_7 & $-143.9(6)$ \\
\hline C5_7 & C2_7 & C3_7 & C26_7 & $0.7(8)$ \\
\hline C5_7 & C2_7 & C4_7 & C3_7 & $-108.1(6)$ \\
\hline C5_7 & C6_7 & C7_7 & C8_7 & $0.18(13)$ \\
\hline C6_7 & C5_7 & C10_7 & C9_7 & $0.2(3)$ \\
\hline C6_7 & C7_7 & C8_7 & C9_7 & $-0.5(3)$ \\
\hline C6_7 & C7_7 & C8_7 & C11_7 & $179.4(3)$ \\
\hline C7_7 & C8_7 & C9_7 & C10_7 & $0.7(4)$ \\
\hline C7_7 & C8_7 & C11_7 & C12_7 & $-83.5(7)$ \\
\hline C7_7 & C8_7 & C11_7 & C16_7 & $96.4(7)$ \\
\hline C8_7 & C9_7 & C10_7 & C5_7 & $-0.5(4)$ \\
\hline C8_7 & C11_7 & C12_7 & C13_7 & $179.8(2)$ \\
\hline C8_7 & C11_7 & C12_7 & C17_7 & $-3.0(15)$ \\
\hline C8_7 & C11_7 & C16_7 & C15_7 & $-179.8(3)$ \\
\hline C8_7 & C11_7 & C16_7 & C19_7 & $-10.2(16)$ \\
\hline C9_7 & C8_7 & C11_7 & C12_7 & $96.4(7)$ \\
\hline
\end{tabular}




\begin{tabular}{|c|c|c|c|c|}
\hline Atom & Atom & Atom & Atom & Angle/ ${ }^{\circ}$ \\
\hline$\overline{C 9 \_7}$ & C8_7 & C11_7 & C16_7 & $-83.6(7)$ \\
\hline C10_7 & C5_7 & C6_7 & $\mathrm{Cl} 1 \_7$ & $-177.2(11)$ \\
\hline C10_7 & C5_7 & C6_7 & C7_7 & $-0.02(13)$ \\
\hline C11_7 & C8_7 & C9_7 & C10_7 & $-179.3(4)$ \\
\hline C11_7 & C12_7 & C13_7 & C14_7 & $0.05(9)$ \\
\hline C12_7 & C11_7 & C16_7 & C15_7 & $0.2(3)$ \\
\hline C12_7 & C11_7 & C16_7 & C19_7 & $169.7(16)$ \\
\hline C12_7 & C13_7 & C14_7 & C15_7 & $0.01(9)$ \\
\hline C12_7 & C13_7 & C14_7 & C18_7 & $-179.98(16)$ \\
\hline C13_7 & C14_7 & C15_7 & C16_7 & $0.0(2)$ \\
\hline C14_7 & C15_7 & C16_7 & C11_7 & $-0.1(3)$ \\
\hline C14_7 & C15_7 & C16_7 & C19_7 & $-169.8(16)$ \\
\hline C16_7 & C11_7 & C12_7 & C13_7 & $-0.14(19)$ \\
\hline C16_7 & C11_7 & C12_7 & C17_7 & 177.1(16) \\
\hline C17_7 & C12_7 & C13_7 & C14_7 & $-177.2(15)$ \\
\hline C18_7 & C14_7 & C15_7 & C16_7 & $-180.0(2)$ \\
\hline C20_7 & C3_7 & C4_7 & C2_7 & $-114.5(6)$ \\
\hline C20_7 & C3_7 & C26_6_7 & C27﹎7 & $-115.8(8)$ \\
\hline C20_7 & C3_7 & C26_7 & C31_7 & 67.8(8) \\
\hline C20_7 & C21_7 & C22_7 & C23_7 & $-0.1(2)$ \\
\hline C21_7 & C20_7 & C25_7 & C24_7 & $0.7(5)$ \\
\hline C21_7 & C22_7 & C23_7 & C24_7 & $0.2(5)$ \\
\hline C22_7 & C23_7 & C24_7 & C25_7 & $0.2(7)$ \\
\hline C23_7 & C24_7 & C25_7 & C20_7 & $-0.6(7)$ \\
\hline C25_7 & C20_7 & C21_7 & C22_7 & $-0.3(2)$ \\
\hline C26_7 & C3_7 & C4_7 & C2_7 & $105.2(6)$ \\
\hline C26_7 & C3_7 & C20_7 & C21_7 & $-94.9(8)$ \\
\hline C26_7 & C3_7 & C20_7 & C25_7 & $85.1(8)$ \\
\hline C26_7 & C27_7 & C28_7 & C29_7 & $-0.01(9)$ \\
\hline C27_7 & C26_7 & C31_7 & C30_7 & $0.0(2)$ \\
\hline C27_7 & C28_7 & C29_7 & C30_7 & $0.0(2)$ \\
\hline C28_7 & C29_7 & C30_7 & C31_7 & $0.0(3)$ \\
\hline C29_7 & C30_7 & C31_7 & C26_7 & $0.0(3)$ \\
\hline C31_7 & C26_7 & C27_7 & C28_7 & $0.00(9)$ \\
\hline Cl1_4 & C6_4 & C7_4 & C8_4 & $171.8(17)$ \\
\hline 01_4 & C1_4 & C2_4 & C3_4 & 21.6(17) \\
\hline 01_4 & C1_4 & C2_4 & C4_4 & $-45.2(16)$ \\
\hline 01_4 & C1_4 & C2_4 & C5_4 & $172.2(15)$ \\
\hline $02 \_4$ & C1_4 & C2_4 & C3_4 & $-144.3(14)$ \\
\hline 02_4 & C1_4 & C2_4 & C4_4 & $148.9(14)$ \\
\hline 02_4 & C1_4 & C2_4 & C5_4 & 6.3(15) \\
\hline C1_4 & C2_4 & C3_4 & C4_4 & $-102.6(7)$ \\
\hline C1_4 & C2_4 & C3_4 & C20_4 & $5.6(9)$ \\
\hline C1_4 & $\mathrm{C} 2 \_4$ & C3_4 & C26_4 & $148.2(7)$ \\
\hline C1_4 & C2_4 & C4_4 & C3_4 & $111.7(6)$ \\
\hline C1_4 & C2_4 & C5_4 & C6_4 & $-77.3(14)$ \\
\hline C1_4 & C2_4 & C5_4 & C10_4 & $102.5(14)$ \\
\hline C2_4 & C3_4 & C20_4 & C21_4 & 59.4(10) \\
\hline C2_4 & C3_4 & C20_4 & C25_4 & $-113.4(9)$ \\
\hline C2_4 & C3_4 & C26_4 & C27_4 & $58.4(10)$ \\
\hline C2_4 & C3_4 & C26_4 & C31_4 & $-118.4(9)$ \\
\hline $\mathrm{C} 2 \_4$ & C5_4 & C6_4 & $\mathrm{Cl} 1 \_4$ & $6(2)$ \\
\hline C2_4 & C5_4 & C6_4 & C7_4 & $-175.9(17)$ \\
\hline C2_4 & C5_4 & C10_4 & C9_4 & $179.9(15)$ \\
\hline C3_4 & C2_4 & C5_4 & C6_4 & 73.4(14) \\
\hline C3_4 & C2_4 & C5_4 & C10_4 & $-106.8(14)$ \\
\hline C3_4 & C20_4 & C21_4 & C22_4 & $-172.8(8)$ \\
\hline C3_4 & C20_4 & C25_4 & C24_4 & 173.1(7) \\
\hline
\end{tabular}




\begin{tabular}{|c|c|c|c|c|}
\hline Atom & Atom & Atom & Atom & Angle $/{ }^{\circ}$ \\
\hline$\overline{C 3 \_4}$ & C26_4 & C27_4 & C28_4 & $-176.9(9)$ \\
\hline C3_4 & C26_4 & C31_4 & C30_4 & $176.9(10)$ \\
\hline C4_4 & $\mathrm{C} 2 \_4$ & C3_4 & C20_4 & $108.1(6)$ \\
\hline C4_4 & C2_4 & C3_4 & C26_4 & $-109.2(7)$ \\
\hline C4_4 & C2_4 & C5_4 & C6_4 & $142.2(13)$ \\
\hline C4_4 & $\mathrm{C} 2 \_4$ & C5_4 & C10_4 & $-38.0(15)$ \\
\hline C4_4 & C3_4 & C20_4 & C21_4 & $130.6(9)$ \\
\hline C4_4 & C3_4 & C20_4 & C25_4 & $-42.2(9)$ \\
\hline C4_4 & C3_4 & C26_4 & C27_4 & $-10.4(11)$ \\
\hline C4_4 & C3_4 & C26_4 & C31_4 & 172.8(9) \\
\hline C5_4 & C2_4 & C3_4 & C4_4 & 109.1(6) \\
\hline C5_4 & $\mathrm{C} 2 \_4$ & C3_4 & C20_4 & $-142.8(6)$ \\
\hline C5_4 & $\mathrm{C} 2 \_4$ & C3_4 & C26_4 & $-0.1(9)$ \\
\hline C5_4 & C2_4 & C4_4 & C3_4 & $-108.9(6)$ \\
\hline C5_4 & C6_4 & C7_4 & C8_4 & $-6(3)$ \\
\hline C6_4 & C5_4 & C10_4 & C9_4 & $0(3)$ \\
\hline C6_4 & C7_4 & C8_4 & C9_4 & $3(3)$ \\
\hline C6_4 & C7_4 & C8_4 & C11_4 & 178.7(17) \\
\hline C7__4 & C8_4 & C9_4 & C10_4 & $0(3)$ \\
\hline C7_4 & C8_4 & C11_4 & C12_4 & $-69.5(16)$ \\
\hline C7_4 & C8_4 & C11_4 & C16_4 & $98.5(16)$ \\
\hline C8_4 & C9_4 & C10_4 & C5_4 & $-2(3)$ \\
\hline C8_4 & C11_4 & C12_4 & C13_4 & $168.0(7)$ \\
\hline C8_4 & C11_4 & C12_4 & C17_4 & $-11.2(14)$ \\
\hline C8_4 & C11_4 & C16_4 & C15_4 & $-167.7(7)$ \\
\hline C8_4 & C11_4 & C16_4 & C19_4 & $7.8(16)$ \\
\hline C9_4 & C8_4 & C11_4 & C12_4 & $105.4(15)$ \\
\hline C9_4 & C8_4 & C11_4 & C16_4 & $-86.6(15)$ \\
\hline C10_4 & C5_4 & C6_4 & Cl1_4 & $-173.5(14)$ \\
\hline C10_4 & C5_4 & C6_4 & C7_4 & $4(3)$ \\
\hline C11_4 & C8_4 & C9_4 & C10_4 & $-174.6(16)$ \\
\hline C11_4 & C12_4 & C13_4 & C14_4 & $0.02(9)$ \\
\hline C12_4 & C11_4 & C16_4 & C15_4 & 0.1(3) \\
\hline C12_4 & C11_4 & C16_4 & C19_4 & $175.5(17)$ \\
\hline C12_4 & C13_4 & C14_4 & C15_4 & $0.01(9)$ \\
\hline C12_4 & C13_4 & C14_4 & C18_4 & $-179.6(8)$ \\
\hline C13_4 & C14_4 & C15_4 & C16_4 & $0.0(2)$ \\
\hline C14_4 & C15_4 & C16_4 & C11_4 & $0.0(3)$ \\
\hline C14_4 & C15_4 & C16_4 & C19_4 & $-175.6(17)$ \\
\hline C16_4 & C11_4 & C12_4 & C13_4 & $-0.1(2)$ \\
\hline C16_4 & C11_4 & C12_4 & C17_4 & $-179.2(14)$ \\
\hline C17_4 & C12_4 & C13_4 & C14_4 & $179.2(14)$ \\
\hline C18_4 & C14_4 & C15_4 & C16_4 & $179.6(8)$ \\
\hline C20_4 & C3_4 & C4_4 & C2_4 & $-111.7(6)$ \\
\hline C20_4 & C3_4 & C26_4 & C27_4 & $-155.6(8)$ \\
\hline C20_4 & C3_4 & C26_4 & C31_4 & 27.6(10) \\
\hline C20_4 & C21_4 & C22_4 & C23_4 & $0.00(16)$ \\
\hline C21_4 & C20_4 & C25_4 & C24_4 & $-0.01(12)$ \\
\hline C21_4 & C22_4 & C23_4 & C24_4 & $0.0(3)$ \\
\hline C22_4 & C23_4 & C24_4 & C25_4 & $0.0(3)$ \\
\hline C23_4 & C24_4 & C25_4 & C20_4 & $0.0(3)$ \\
\hline C25_4 & C20_4 & C21_4 & C22_4 & $0.00(7)$ \\
\hline C26_4 & C3_4 & C4_4 & C2_4 & $105.7(6)$ \\
\hline C26_4 & C3_4 & C20_4 & C21_4 & $-84.6(9)$ \\
\hline C26_4 & C3_4 & C20_4 & C25_4 & $102.6(8)$ \\
\hline C26_4 & C27_4 & C28_4 & C29_4 & $0.0(2)$ \\
\hline C27_4 & C26_4 & C31_4 & C30_4 & $-0.1(5)$ \\
\hline C27_4 & C28_4 & C29_4 & C30_4 & $0.0(5)$ \\
\hline
\end{tabular}




\begin{tabular}{|c|c|c|c|c|}
\hline Atom & Atom & Atom & Atom & Angle ${ }^{\circ}$ \\
\hline$\overline{C 28 \_4}$ & C29_4 & C30_4 & C31_4 & $0.0(7)$ \\
\hline C29_4 & C30_4 & C31_4 & C26_4 & $0.1(7)$ \\
\hline C31_4 & C26_4 & C27_4 & C28_4 & $0.0(2)$ \\
\hline Cl1_6 & C6_6 & C7_6 & C8_6 & $177.3(14)$ \\
\hline 01_6 & C1_6 & C2_6 & C3_6 & $18.9(15)$ \\
\hline 01_6 & C1_6 & C2_6 & C4_6 & $-48.4(15)$ \\
\hline $01 \_6$ & C1_6 & $\mathrm{C} 2{ }_{-}^{-} 6$ & C5_6 & $169.9(13)$ \\
\hline $02 \_6$ & C1_6 & C2_6 & C3_6 & $-149.3(13)$ \\
\hline $02 \_6$ & C1_6 & $\mathrm{C} 2 \_6$ & C4_6 & $143.4(13)$ \\
\hline 02_6 & C1_6 & C2_6 & C5_6 & $1.7(14)$ \\
\hline C1_6 & C2_6 & C3_6 & C4_6 & $-102.7(7)$ \\
\hline C1_6 & $\mathrm{C} 2 \_6$ & C3_6 & C20̄_6 & $3.8(9)$ \\
\hline C1_6 & C2_6 & C3_6 & C26_6 & $147.8(7)$ \\
\hline C1_6 & $\mathrm{C} 2 \_6$ & C4_6 & C3_6 & $112.6(6)$ \\
\hline C1_6 & C2_6 & C5_6 & C6_6 & $-76.2(9)$ \\
\hline C1_6 & $\mathrm{C} 2 \_6$ & C5_6 & C10_6 & $105.4(8)$ \\
\hline C2_6 & C3_6 & C20_6 & C21_6 & $55.4(9)$ \\
\hline C2_6 & C3_6 & C20_6 & C25_6 & $-123.6(9)$ \\
\hline C2_6 & C3_6 & C26_6 & C27_6 & $59.6(9)$ \\
\hline C2_6 & C3_6 & C26_6 & C31_6 & $-114.7(8)$ \\
\hline C2_6 & C5_6 & C6_6 & Cl1_6 & $4.2(14)$ \\
\hline C2_6 & C5_6 & C6_6 & C7_6 & $-178.5(7)$ \\
\hline C2_6 & C5_6 & C10_6 & C9_6 & $178.5(7)$ \\
\hline C3_6 & C2_6 & C5_6 & C6_6 & $75.2(9)$ \\
\hline C3_6 & $\mathrm{C} 2 \_6$ & C5_6 & C10̄6 & $-103.1(9)$ \\
\hline C3_6 & C20̄6 6 & C21﹎6 & C22_6 & $-179.0(8)$ \\
\hline C3_6 & C20_6 & C25_6 & C24_6 & $179.0(7)$ \\
\hline C3_6 & C26_6 & C27_6 & C28_6 & $-174.5(8)$ \\
\hline C3_6 & C26_6 & C31_6 & C30_6 & $174.7(8)$ \\
\hline C4_6 & C2_6 & C3_6 & C20_6 & $106.5(7)$ \\
\hline C4_6 & C2_6 & C3_6 & C26_6 & $-109.5(7)$ \\
\hline C4_6 & C2_6 & C5_6 & C6_6 & 143.8(8) \\
\hline C4_6 & C2_6 & C5_6 & C10_6 & $-34.6(9)$ \\
\hline C4_6 & C3_6 & C20_6 & C21_6 & $126.6(8)$ \\
\hline C4_6 & C3_6 & C20_6 & C25_6 & $-52.4(9)$ \\
\hline C4_6 & C3_6 & C26_6 & C27_6 & $-9.0(10)$ \\
\hline C4_6 & C3_6 & C26_6 & C31_6 & $176.6(7)$ \\
\hline C5_6 & C2_6 & C3_6 & C4_6 & $108.5(6)$ \\
\hline C5_6 & $\mathrm{C} 2 \_6$ & C3_6 & C20̄6 & $-144.9(6)$ \\
\hline C5_6 & C2_6 & C3_6 & C26_6 & $-1.0(8)$ \\
\hline C5_6 & C2_6 & C4_6 & C3_6 & $-108.7(6)$ \\
\hline C5_6 & C6_6 & C7_6 & C8_6 & $-0.01(9)$ \\
\hline C6_6 & C5_6 & C10_6 & C9_6 & $0.0(2)$ \\
\hline C6_6 & C7_6 & C8_6 & C9_6 & $0.0(2)$ \\
\hline C6_6 & C7_6 & C8_6 & C11__6 & $-174.7(7)$ \\
\hline C7_6 & C8_6 & C9_6 & C10_6 & $0.0(3)$ \\
\hline C7_6 & C8_6 & C11_6 & C12_6 & $-89.7(9)$ \\
\hline C7_6 & C8_6 & C11_6 & C16_6 & $83.1(10)$ \\
\hline C8_6 & C9_6 & C10_6 & C5_6 & $0.0(3)$ \\
\hline C8_6 & C11_6 & C12_6 & C13_6 & $172.8(8)$ \\
\hline C8_6 & C11_6 & C12_6 & C17_6 & $-5.6(18)$ \\
\hline C8_6 & C11_6 & C16_6 & C15_6 & $-172.6(8)$ \\
\hline C8_6 & C11_6 & C16_6 & C19_6 & $4.9(19)$ \\
\hline C9_6 & C8_6 & C11_6 & C12_6 & $96.0(9)$ \\
\hline C9_6 & C8_6 & C11_6 & C16_6 & $-91.3(9)$ \\
\hline C10_6 & C5_6 & C6_6 & Cl1_6 & $-177.3(14)$ \\
\hline C10_6 & C5_6 & C6_6 & C7_6 & $-0.01(9)$ \\
\hline C11_6 & C8_6 & C9_6 & C10_6 & $174.5(8)$ \\
\hline
\end{tabular}




\begin{tabular}{|c|c|c|c|c|}
\hline Atom & Atom & Atom & Atom & Angle ${ }^{\circ}$ \\
\hline$\overline{C 11 \_6}$ & C12_6 & C13_6 & C14_6 & $0.00(9)$ \\
\hline C12_6 & C11_6 & C16_6 & C15_6 & $-0.01(19)$ \\
\hline C12_6 & C11_6 & C16_6 & C19_6 & $177.5(19)$ \\
\hline C12_6 & C13_6 & C14_6 & C15_6 & $0.00(19)$ \\
\hline C12_6 & C13_6 & C14_6 & C18_6 & $-179(2)$ \\
\hline C13_6 & C14_6 & C15_6 & C16_6 & $0.0(3)$ \\
\hline C14_6 & C15_6 & C16_6 & C11_6 & $0.0(3)$ \\
\hline C14_6 & C15_6 & C16_6 & C19_6 & $-177.5(18)$ \\
\hline C16_6 & C11_6 & C12_6 & C13_6 & $0.00(9)$ \\
\hline C16_6 & C11_6 & C12_6 & C17_6 & $-178.3(18)$ \\
\hline C17_6 & C12_6 & C13_6 & C14_6 & 178.4(18) \\
\hline C18_6 & C14_6 & C15_6 & C16_6 & $179(2)$ \\
\hline C20_6 & C3_6 & C4_6 & $\mathrm{C} 2{ }_{-} \overline{6}$ & $-113.5(6)$ \\
\hline C20_6 & C3_6 & C26_6 & C27_6 & $-152.7(7)$ \\
\hline C20_6 & C3_6 & C26_6 & C31_6 & $33.0(9)$ \\
\hline C20_6 & C21﹎ㅇ & C22_6 & C23_6 & $0.00(9)$ \\
\hline C21_6 & C20_6 & C25_6 & C24_6 & $0.0(2)$ \\
\hline C21_6 & C22_6 & C23_6 & C24_6 & $0.0(2)$ \\
\hline C22_6 & C23_6 & C24_6 & C25_6 & $0.0(3)$ \\
\hline C23_6 & C24_6 & C25_6 & C20_6 & $0.0(3)$ \\
\hline C25_6 & C20_6 & C21_6 & C22_6 & $0.00(9)$ \\
\hline C26_6 & C3_6 & C4_6 & C2_6 & $105.1(6)$ \\
\hline C26_6 & C3_6 & C20_6 6 & C21﹎6 & $-89.8(8)$ \\
\hline C26_6 & C3_6 & C20_6 & C25_6 & $91.3(9)$ \\
\hline C26_6 & C27_6 & C28_6 & C29_6 & $0.00(5)$ \\
\hline C27_6 & C26_6 & C31_6 & C30_6 & $0.00(9)$ \\
\hline C27_6 & C28_6 & C29_6 & C30_6 & $0.00(8)$ \\
\hline C28_6 & C29_6 & C30_6 & C31_6 & $0.00(9)$ \\
\hline C29_6 & C30_6 & C31_6 & C26_6 & $0.00(9)$ \\
\hline C31_6 & C26_6 & C27_6 & C28_6 & $0.00(6)$ \\
\hline Cl1_2 & C6_2 & C7_2 & C8_2 & $179.9(10)$ \\
\hline 01_2 & C1_2 & C2_2 & C3_2 & $30.2(15)$ \\
\hline 01_2 & C1_2 & $\mathrm{C} 2 \_2$ & C4_2 & $-37.3(14)$ \\
\hline 01_2 & C1_2 & $\mathrm{C} 2 \_2$ & C5_2 & $-178.2(13)$ \\
\hline 02_2 & C1_2 & C2_2 & C3_2 & $-164.7(14)$ \\
\hline 02_2 & C1_2 & C2_2 & C4_2 & $127.8(14)$ \\
\hline 02_2 & C1_2 & C2_2 & C5_2 & $-13.1(15)$ \\
\hline C1_2 & C2_2 & C3_2 & C4_2 & $-101.3(7)$ \\
\hline C1_2 & $\mathrm{C} 2 \_2$ & C3_2 & C20_2 & $5.5(9)$ \\
\hline C1_2 & $\mathrm{C} 2 \_2$ & C3_2 & C26_2 & $150.5(7)$ \\
\hline C1_2 & $\mathrm{C} 2 \_2$ & C4_2 & C3_2 & $114.4(7)$ \\
\hline C1_2 & C2_2 & C5_2 & C6_2 & $-66.2(9)$ \\
\hline C1_2 & C2_2 & C5_2 & C10_2 & $117.9(7)$ \\
\hline C2_2 & C3_2 & C20_2 & C21_2 & 49.3(8) \\
\hline C2_2 & C3_2 & C20_2 & C25_2 & $-130.7(8)$ \\
\hline C2_2 & C3_2 & C26_2 & C27_2 & 97.5(8) \\
\hline C2_2 & C3_2 & C26_2 & C31_2 & $-82.4(8)$ \\
\hline C2_2 & C5_2 & C6_2 & Cl1_2 & 4.1(11) \\
\hline C2_2 & C5_2 & C6_2 & C7_2 & $-176.1(7)$ \\
\hline C2_2 & C5_2 & C10_2 & C9_2 & $176.1(7)$ \\
\hline C3_2 & C2_2 & C5_2 & C6_2 & 86.4(8) \\
\hline C3_2 & $\mathrm{C} 2 \_2$ & C5_2 & C10_2 & $-89.4(8)$ \\
\hline C3_2 & C20_2 & C21_2 & C22_2 & $179.96(17)$ \\
\hline C3_2 & C20_2 & C25_2 & C24_2 & $-179.9(2)$ \\
\hline C3_2 & C26_2 & C27_2 & C28_2 & $-179.8(2)$ \\
\hline C3_2 & C26_2 & C31_2 & C30_2 & $179.6(5)$ \\
\hline C4_2 & C2_2 & C3_2 & C20_2 & $106.8(7)$ \\
\hline C4_2 & C2_2 & C3_2 & C26_2 & $-108.2(6)$ \\
\hline
\end{tabular}




\begin{tabular}{|c|c|c|c|c|}
\hline Atom & Atom & Atom & Atom & Angle $/^{\circ}$ \\
\hline$\overline{C 4 \_2}$ & C2_2 & C5_2 & C6_2 & $155.0(7)$ \\
\hline C4_2 & $\mathrm{C} 2 \_2$ & C5_2 & C10_2 & $-20.8(9)$ \\
\hline C4_2 & C3_2 & C20_2 & C21_2 & $121.0(8)$ \\
\hline C4_2 & C3_2 & C20_2 & C25_2 & $-59.0(8)$ \\
\hline C4_2 & C3_2 & C26_2 & C27_2 & 29.3(8) \\
\hline C4_2 & C3_2 & C26_2 & C31_2 & $-150.6(8)$ \\
\hline C5_2 & $\mathrm{C} 2 \_2$ & C3_2 & C4_2 & $109.2(6)$ \\
\hline C5_2 & C2_2 & C3_2 & C20_2 & $-144.0(6)$ \\
\hline C5_2 & C2_2 & C3_2 & C26_2 & $0.9(8)$ \\
\hline C5_2 & C2_2 & C4_2 & C3_2 & $-108.2(6)$ \\
\hline C5_2 & C6_2 & C7_2 & C8_2 & $0.00(9)$ \\
\hline C6_2 & C5_2 & C10_2 & C9_2 & $0.0(2)$ \\
\hline C6_2 & C7_2 & C8_2 & C9_2 & $0.0(2)$ \\
\hline C6_2 & C7_2 & C8_2 & C11_2 & $178.9(7)$ \\
\hline C7_2 & C8_2 & C9_2 & C10_2 & $0.0(3)$ \\
\hline C7_2 & C8_2 & C11_2 & C12_2 & $-79.7(8)$ \\
\hline C7_2 & C8_2 & C11_2 & C16_2 & 99.3(9) \\
\hline C8_2 & C9_2 & C10_2 & C5_2 & $0.0(3)$ \\
\hline C8_2 & C11_2 & C12_2 & C13_2 & 178.5(7) \\
\hline C8_2 & C11_2 & C12_2 & C17_2 & $-1.2(8)$ \\
\hline C8_2 & C11_2 & C16_2 & C15_2 & $-178.5(8)$ \\
\hline C8_2 & C11_2 & C16_2 & C19_2 & 1.1(10) \\
\hline C9_2 & C8_2 & C11_2 & C12_2 & $99.1(8)$ \\
\hline C9_2 & C8_2 & C11_2 & C16_2 & $-81.9(9)$ \\
\hline C10_2 & C5_2 & C6_2 & Cl1_2 & $-179.9(10)$ \\
\hline C10_2 & C5_2 & C6_2 & C7_2 & $-0.01(9)$ \\
\hline C11_2 & C8_2 & C9_2 & C10_2 & $-178.9(7)$ \\
\hline C11_2 & C12_2 & C13_2 & C14_2 & $0.2(3)$ \\
\hline C12_2 & C11_2 & C16_2 & C15_2 & $0.4(9)$ \\
\hline C12_2 & C11_2 & C16_2 & C19_2 & $-180.0(5)$ \\
\hline C12_2 & C13_2 & C14_2 & C15_2 & $0.1(3)$ \\
\hline C12_2 & C13_2 & C14_2 & C18_2 & $-179.9(3)$ \\
\hline C13_2 & C14_2 & C15_2 & C16_2 & $-0.1(7)$ \\
\hline C14_2 & C15_2 & C16_2 & C11_2 & $-0.1(9)$ \\
\hline C14_2 & C15_2 & C16_2 & C19_2 & $-179.8(5)$ \\
\hline C16_2 & C11_2 & C12_2 & C13_2 & $-0.4(7)$ \\
\hline C16_2 & C11_2 & C12_2 & C17_2 & $179.8(5)$ \\
\hline C17_2 & C12_2 & C13_2 & C14_2 & $179.9(3)$ \\
\hline C18_2 & C14_2 & C15_2 & C16_2 & 179.8(5) \\
\hline C20_2 & C3_2 & C4_2 & C2_2 & $-114.2(6)$ \\
\hline C20_2 & C3_2 & C26_2 & C27_2 & $-113.7(8)$ \\
\hline C20_2 & C3_2 & C26_2 & C31_2 & $66.4(8)$ \\
\hline C20_2 & C21_2 & C22_2 & C23_2 & $0.00(6)$ \\
\hline C21_2 & C20_2 & C25_2 & C24_2 & $0.0(2)$ \\
\hline C21_2 & C22_2 & C23_2 & C24_2 & $-0.01(8)$ \\
\hline C22_2 & C23_2 & C24_2 & C25_2 & $0.03(19)$ \\
\hline C23_2 & C24_2 & C25_2 & C20_2 & $0.0(2)$ \\
\hline C25_2 & C20_2 & C21_2 & C22_2 & $-0.02(13)$ \\
\hline C26_2 & C3_2 & C4_2 & C2_2 & $105.3(5)$ \\
\hline C26_2 & C3_2 & C20_2 & C21_2 & $-96.6(7)$ \\
\hline C26_2 & C3_2 & C20_2 & C25_2 & 83.4(7) \\
\hline C26_2 & C27_2 & C28_2 & C29_2 & $0.1(2)$ \\
\hline C27_2 & C26_2 & C31_2 & C30_2 & $-0.3(5)$ \\
\hline C27_2 & C28_2 & C29_2 & C30_2 & $-0.1(5)$ \\
\hline C28_2 & C29_2 & C30_2 & C31_2 & $0.0(7)$ \\
\hline C29_2 & C30_2 & C31_2 & C26_2 & $0.3(7)$ \\
\hline C31_2 & C26_2 & C27_2 & C28_2 & $0.1(2)$ \\
\hline Cl1_8 & C6_8 & C7_8 & C8_8 & $173.6(17)$ \\
\hline
\end{tabular}




\begin{tabular}{|c|c|c|c|c|}
\hline Atom & Atom & Atom & Atom & Angle ${ }^{\circ}$ \\
\hline$\overline{01 \_8}$ & C1_8 & C2_8 & C3_8 & $21.3(17)$ \\
\hline 01_8 & C1_8 & C2_8 & C4_8 & $-45.6(17)$ \\
\hline 01_8 & C1_8 & C2_8 & C5_8 & $173.9(15)$ \\
\hline $02 \_8$ & C1_8 & $\mathrm{C} 2 \_8$ & C3_8 & $-160.7(15)$ \\
\hline 02_8 & C1_8 & C2_8 & C4_8 & $132.4(15)$ \\
\hline 02_8 & C1_8 & C2_8 & C5_8 & $-8.1(16)$ \\
\hline C1_8 & C2_8 & C3_8 & C4_8 & $-101.4(7)$ \\
\hline C1_8 & $\mathrm{C} 2 \_8$ & C3_8 & C20_8 & $5.8(9)$ \\
\hline C1_8 & C2_8 & C3_8 & C26_8 & $150.4(7)$ \\
\hline C1_8 & C2_8 & C4_8 & C3_8 & $113.1(6)$ \\
\hline C1_8 & C2_8 & C5_8 & C6_8 & $-69.5(10)$ \\
\hline C1_8 & C2_8 & C5_8 & C10_8 & $111.5(9)$ \\
\hline C2_8 & C3_8 & C20_8 & C21_8 & $66.0(10)$ \\
\hline C2_8 & C3_8 & C20_8 & C25_8 & $-118.8(9)$ \\
\hline $\mathrm{C} 2 \_8$ & C3_8 & C26_8 & C27_8 & $111.2(9)$ \\
\hline C2_8 & C3_8 & C26_8 & C31_8 & $-69.3(10)$ \\
\hline C2_8 & C5_8 & C6_8 & Cl1_8 & 7.5(17) \\
\hline C2_8 & C5_8 & C6_8 & C7_8 & $-179.1(7)$ \\
\hline C2_8 & C5_8 & C10_8 & C9_8 & 179.1(7) \\
\hline C3_8 & C2_8 & C5_8 & C6_8 & 83.4(9) \\
\hline C3_8 & C2_8 & C5_8 & C10_8 & $-95.6(9)$ \\
\hline C3_8 & C20_8 & C21_8 & C22_8 & $175.2(8)$ \\
\hline C3_8 & C20_8 & C25_8 & C24_8 & $-175.4(8)$ \\
\hline C3_8 & C26_8 & C27_8 & C28_8 & $179.5(9)$ \\
\hline C3_8 & C26_8 & C31_8 & C30_8 & $-179.5(9)$ \\
\hline C4_8 & C2_8 & C3_8 & C20_8 & 107.2(7) \\
\hline C4_8 & C2_8 & C3_8 & C26_8 & $-108.2(7)$ \\
\hline C4_8 & $\mathrm{C} 2 \_8$ & C5_8 & C6_8 & $152.2(8)$ \\
\hline C4_8 & C2_8 & C5_8 & C10_8 & $-26.8(9)$ \\
\hline C4_8 & C3_8 & C20_8 & C21_8 & 137.4(9) \\
\hline C4_8 & C3_8 & C20_8 & C25_8 & $-47.4(10)$ \\
\hline C4_8 & C3_8 & C26_8 & C27_8 & $42.4(10)$ \\
\hline C4_8 & C3_8 & C26_8 & C31_8 & $-138.1(9)$ \\
\hline C5_8 & C2_8 & C3_8 & C4_8 & 108.2(6) \\
\hline C5_8 & C2_8 & C3_8 & C20_8 & $-144.6(6)$ \\
\hline C5_8 & C2_8 & C3_8 & C26_8 & $0.1(9)$ \\
\hline C5_8 & C2_8 & C4_8 & C3_8 & $-109.6(6)$ \\
\hline C5_8 & C6_8 & C7_8 & C8_8 & $0.01(9)$ \\
\hline C6_8 & C5_8 & C10_8 & C9_8 & $0.0(2)$ \\
\hline C6_8 & C7_8 & C8_8 & C9_8 & $0.0(2)$ \\
\hline C6_8 & C7_8 & C8_8 & C11_8 & $-178.0(8)$ \\
\hline C7_8 & C8_8 & C9_8 & C10_8 & $0.0(3)$ \\
\hline C7_8 & C8_8 & C11_8 & C12_8 & $-83.1(10)$ \\
\hline C7_8 & C8_8 & C11_8 & C16_8 & $104.5(9)$ \\
\hline C8_8 & C9_8 & C10_8 & C5_8 & $0.0(3)$ \\
\hline C8_8 & C11_8 & C12_8 & C13_8 & $-172.4(6)$ \\
\hline C8_8 & C11_8 & C12_8 & C17_8 & 3.7(18) \\
\hline C8_8 & C11_8 & C16_8 & C15_8 & $172.2(7)$ \\
\hline C8_8 & C11_8 & C16_8 & C19_8 & $0(2)$ \\
\hline C9_8 & C8_8 & C11_8 & C12_8 & $99.0(9)$ \\
\hline C9_8 & C8_8 & C11_8 & C16_8 & $-73.3(10)$ \\
\hline C10_8 & C5_8 & C6_8 & Cl1_8 & $-173.5(17)$ \\
\hline C10_8 & C5_8 & C6_8 & C7_8 & $0.01(9)$ \\
\hline C11_8 & C8_8 & C9_8 & C10_8 & $177.9(8)$ \\
\hline C11_8 & C12_8 & C13_8 & C14_8 & $0.00(9)$ \\
\hline C12_8 & C11_8 & C16_8 & C15_8 & $0.01(19)$ \\
\hline C12_8 & C11_8 & C16_8 & C19_8 & $-172(2)$ \\
\hline C12_8 & C13_8 & C14_8 & C15_8 & $0.01(19)$ \\
\hline
\end{tabular}




\begin{tabular}{|c|c|c|c|c|}
\hline Atom & Atom & Atom & Atom & Angle/ ${ }^{\circ}$ \\
\hline$\overline{\mathrm{C} 12 \_8}$ & C13_8 & C14_8 & C18_8 & $-180.0(2)$ \\
\hline C13_8 & C14_8 & C15_8 & C16_8 & $0.0(3)$ \\
\hline C14_8 & C15_8 & C16_8 & C11_8 & $0.0(3)$ \\
\hline C14_8 & C15_8 & C16_8 & C19_8 & $173(2)$ \\
\hline C16_8 & C11_8 & C12_8 & C13_8 & $-0.01(9)$ \\
\hline C16_8 & C11_8 & C12_8 & C17_8 & $176.0(18)$ \\
\hline C17_8 & C12_8 & C13_8 & C14_8 & $-176.1(17)$ \\
\hline C18_8 & C14_8 & C15_8 & C16_8 & $180.0(3)$ \\
\hline C20_8 & C3_8 & C4_8 & C2_8 & $-113.0(6)$ \\
\hline C20_8 & C3_8 & C26_8 & C27_8 & $-100.7(9)$ \\
\hline C20_8 & C3_8 & C26_8 & C31_8 & 78.8(9) \\
\hline C20_8 & C21_8 & C22_8 & C23_8 & $0.00(9)$ \\
\hline C21_8 & C20_8 & C25_8 & C24_8 & $0.0(2)$ \\
\hline C21_8 & C22_8 & C23_8 & C24_8 & $0.0(2)$ \\
\hline C22_8 & C23_8 & C24_8 & C25_8 & $0.0(3)$ \\
\hline C23_8 & C24_8 & C25_8 & C20_8 & $0.0(3)$ \\
\hline C25_8 & C20_8 & C21_8 & C22_8 & $-0.01(9)$ \\
\hline C26_8 & C3_8 & C4_8 & C2_8 & $106.3(6)$ \\
\hline C26_8 & C3_8 & C20_8 & C21_8 & $-80.0(9)$ \\
\hline C26_8 & C3_8 & C20_8 & C25_8 & 95.2(9) \\
\hline C26_8 & C27_8 & C28_8 & C29_8 & $0.00(9)$ \\
\hline C27_8 & C26_8 & C31_8 & C30_8 & $0.0(2)$ \\
\hline C27_8 & C28_8 & C29_8 & C30_8 & $0.0(2)$ \\
\hline C28_8 & C29_8 & C30_8 & C31_8 & $0.0(3)$ \\
\hline C29_8 & C30_8 & C31_8 & C26_8 & $0.0(3)$ \\
\hline C31_8 & C26_8 & C27_8 & C28_8 & $0.00(9)$ \\
\hline Cl1_1 & C6_1 & C7_1 & C8_1 & $178.0(17)$ \\
\hline 01_1 & C1_1 & C2_1 & C3_1 & 26.5(17) \\
\hline 01_1 & C1_1 & C2_1 & C4_1 & $-40.3(16)$ \\
\hline 01_1 & C1_1 & C2_1 & C5_1 & $178.6(15)$ \\
\hline $02 \_1$ & C1_1 & C2_1 & C3_1 & $-162.4(14)$ \\
\hline 02_1 & C1_1 & C2_1 & C4_1 & $130.8(14)$ \\
\hline 02_1 & C1_1 & C2_1 & C5_1 & $-10.2(15)$ \\
\hline C1_1 & C2_1 & C3_1 & C4_1 & $-101.3(7)$ \\
\hline C1_1 & C2_1 & C3_1 & C20_1 & $6.2(9)$ \\
\hline C1_1 & C2_1 & C3_1 & C26_1 & $151.2(7)$ \\
\hline C1_1 & C2_1 & C4_1 & C3_1 & $112.9(6)$ \\
\hline C1_1 & C2_1 & C5_1 & C6_1 & $-64.6(9)$ \\
\hline C1_1 & $\mathrm{C} 2 \_1$ & C5_1 & C10_1 & $116.3(8)$ \\
\hline C2_1 & C3_1 & C20_1 & C21_1 & $62.6(10)$ \\
\hline $\mathrm{C} 2 \_1$ & C3_1 & C20_1 & C25_1 & $-120.8(10)$ \\
\hline C2_1 & C3_1 & C26_1 & C27_1 & $110.4(9)$ \\
\hline C2_1 & C3_1 & C26_1 & C31_1 & $-71.6(10)$ \\
\hline $\mathrm{C} 2 \_1$ & C5_1 & C6_1 & Cl1_1 & $2.9(17)$ \\
\hline C2_1 & C5_1 & C6_1 & C7_1 & $-179.1(5)$ \\
\hline C2_1 & C5_1 & C10_1 & C9_1 & 179.1(6) \\
\hline C3_1 & C2_1 & C5_1 & C6_1 & 87.8(9) \\
\hline C3_1 & C2_1 & C5_1 & C10_1 & $-91.3(9)$ \\
\hline C3_1 & C20_1 & C21_1 & C22_1 & $176.6(9)$ \\
\hline C3_1 & C20_1 & C25_1 & C24_1 & $-176.8(8)$ \\
\hline C3_1 & C26_1 & C27_1 & C28_1 & 178.1(9) \\
\hline C3_1 & C26_1 & C31_1 & C30_1 & $-178.1(9)$ \\
\hline C4_1 & C2_1 & C3_1 & C20_1 & 107.5(7) \\
\hline C4_1 & C2_1 & C3_1 & C26_1 & $-107.5(7)$ \\
\hline C4_1 & C2_1 & C5_1 & C6_1 & $156.8(8)$ \\
\hline C4_1 & C2_1 & C5_1 & C10_1 & $-22.3(9)$ \\
\hline C4_1 & C3_1 & C20_1 & C21_1 & $134.0(9)$ \\
\hline C4_1 & C3_1 & C20_1 & C25_1 & $-49.4(10)$ \\
\hline
\end{tabular}




\begin{tabular}{|c|c|c|c|c|}
\hline Atom & Atom & Atom & Atom & Angle ${ }^{\circ}$ \\
\hline$\overline{C 4 \_1}$ & C3_1 & C26_1 & C27_1 & $41.8(10)$ \\
\hline C4_1 & C3_1 & C26_1 & C31_1 & $-140.2(9)$ \\
\hline C5_1 & C2_1 & C3_1 & C4_1 & $108.8(6)$ \\
\hline C5_1 & C2_1 & C3_1 & C20_1 & $-143.7(6)$ \\
\hline C5_1 & C2_1 & C3_1 & C26_1 & $1.3(9)$ \\
\hline C5_1 & C2_1 & C4_1 & C3_1 & $-109.3(6)$ \\
\hline C5_1 & C6_1 & C7_1 & C8_1 & $0.01(9)$ \\
\hline C6_1 & C5_1 & C10_1 & C9_1 & $0.0(2)$ \\
\hline C6_1 & C7_1 & C8_1 & C9_1 & $-0.04(19)$ \\
\hline C6_1 & C7_1 & C8_1 & C11_1 & $180.0(2)$ \\
\hline C7_1 & C8_1 & C9_1 & C10_1 & $0.0(3)$ \\
\hline C7_1 & C8_1 & C11_1 & C12_1 & $-81.7(9)$ \\
\hline C7_1 & C8_1 & C11_1 & C16_1 & $99.8(8)$ \\
\hline C8_1 & C9_1 & C10_1 & C5_1 & $0.0(3)$ \\
\hline C8_1 & C11_1 & C12_1 & C13_1 & $-178.6(6)$ \\
\hline C8_1 & C11_1 & C12_1 & C17_1 & $6.6(18)$ \\
\hline C8_1 & C11_1 & C16_1 & C15_1 & $178.6(7)$ \\
\hline C8_1 & C11_1 & C16_1 & C19_1 & $-8.9(19)$ \\
\hline C9_1 & C8_1 & C11_1 & C12_1 & $98.3(9)$ \\
\hline C9_1 & C8_1 & C11_1 & C16_1 & $-80.2(8)$ \\
\hline C10_1 & C5_1 & C6_1 & Cl1_1 & $-178.0(17)$ \\
\hline C10_1 & C5_1 & C6_1 & C7_1 & $0.01(9)$ \\
\hline C11_1 & C8_1 & C9_1 & C10_1 & $-180.0(3)$ \\
\hline C11_1 & C12_1 & C13_1 & C14_1 & $0.00(9)$ \\
\hline C12_1 & C11_1 & C16_1 & C15_1 & $0.02(19)$ \\
\hline C12_1 & C11_1 & C16_1 & C19_1 & 173(2) \\
\hline C12_1 & C13_1 & C14_1 & C15_1 & $0.00(19)$ \\
\hline C12_1 & C13_1 & C14_1 & C18_1 & $180.0(2)$ \\
\hline C13_1 & C14_1 & C15_1 & C16_1 & $0.0(3)$ \\
\hline C14_1 & C15_1 & C16_1 & C11_1 & $0.0(3)$ \\
\hline C14_1 & C15_1 & C16_1 & C19_1 & $-173(2)$ \\
\hline C16_1 & C11_1 & C12_1 & C13_1 & $-0.01(9)$ \\
\hline C16_1 & C11_1 & C12_1 & C17_1 & $-174.8(18)$ \\
\hline C17_1 & C12_1 & C13_1 & C14_1 & $174.9(17)$ \\
\hline C18_1 & C14_1 & C15_1 & C16_1 & $-180.0(3)$ \\
\hline C20_1 & C3_1 & C4_1 & C2_1 & $-112.7(6)$ \\
\hline C20_1 & C3_1 & C26_1 & C27_1 & $-101.2(9)$ \\
\hline C20_1 & C3_1 & C26_1 & C31_1 & 76.8(10) \\
\hline C20_1 & C21_1 & C22_1 & C23_1 & $0.00(9)$ \\
\hline C21_1 & C20_1 & C25_1 & C24_1 & $0.0(2)$ \\
\hline C21_1 & C22_1 & C23_1 & C24_1 & $0.0(2)$ \\
\hline C22_1 & C23_1 & C24_1 & C25_1 & $0.0(3)$ \\
\hline C23_1 & C24_1 & C25_1 & C20_1 & $0.0(3)$ \\
\hline C25_1 & C20_1 & C21_1 & C22_1 & $-0.01(9)$ \\
\hline C26_1 & C3_1 & C4_1 & C2_1 & $106.9(6)$ \\
\hline C26_1 & C3_1 & C20_1 & C21_1 & $-83.8(9)$ \\
\hline C26_1 & C3_1 & C20_1 & C25_1 & $92.8(9)$ \\
\hline C26_1 & C27_1 & C28_1 & C29_1 & $0.00(9)$ \\
\hline C27_1 & C26_1 & C31_1 & C30_1 & $0.0(2)$ \\
\hline C27_1 & C28_1 & C29_1 & C30_1 & $0.0(2)$ \\
\hline C28_1 & C29_1 & C30_1 & C31_1 & $0.0(3)$ \\
\hline C29_1 & C30_1 & C31_1 & C26_1 & $0.0(3)$ \\
\hline C31_1 & C26_1 & C27_1 & C28_1 & $0.01(9)$ \\
\hline
\end{tabular}

Table 21: Hydrogen Fractional Atomic Coordinates $\left(\times 10^{4}\right)$ and Equivalent Isotropic Displacement Parameters 
$\left(\AA^{2} \times 10^{3}\right)$ for 2 Cl4triMePh. $U_{e q}$ is defined as $1 / 3$ of the trace of the orthogonalised $U_{i j}$.

\begin{tabular}{|c|c|c|c|c|}
\hline Atom & $\mathbf{x}$ & $\mathbf{y}$ & $\mathbf{z}$ & $U_{e q}$ \\
\hline$\overline{\mathrm{H}} 4 \mathrm{~A}$ & 4657.6 & 9480.5 & 7036.29 & 54 \\
\hline H4B & 5482.81 & 9542.89 & 7020.49 & 54 \\
\hline H4AA & 4914.49 & 9705.89 & 7034 & 54 \\
\hline $\mathrm{H} 4 \mathrm{AB}$ & 5636.01 & 9296.49 & 6871.5 & 54 \\
\hline H4A_3 & 3198.08 & 7301.19 & 5817.04 & 33 \\
\hline H4B_3 & 3863.03 & 7636.61 & 6086.3 & 33 \\
\hline H7_3 & 1886.94 & 9456.94 & 4760.37 & 31 \\
\hline H9_3 & 2578.29 & 7561.06 & 4426.17 & 35 \\
\hline H10_3 & 3188.81 & 7487.26 & 5050.89 & 35 \\
\hline H13_3 & 249.94 & 8703.44 & 3703.14 & 53 \\
\hline H15_3 & 2035.34 & 9001.15 & 3104.06 & 53 \\
\hline H17A_3 & 834.74 & 7951.98 & 4599.04 & 54 \\
\hline H17B_3 & 186.89 & 8368.23 & 4411.92 & 54 \\
\hline H17C_3 & 747.15 & 8757.12 & 4702.46 & 54 \\
\hline H18A_3 & 960.11 & 8783.87 & 2690.19 & 76 \\
\hline H18B_3 & 753.57 & 9531.11 & 2868.81 & 76 \\
\hline H18C_3 & 249.44 & 8887.31 & 2944.87 & 76 \\
\hline H19A_3 & 3065.66 & 9311.92 & 3697.81 & 77 \\
\hline H19B_3 & 3137.67 & 8554.5 & 3496.65 & 77 \\
\hline H19C_3 & 3127.19 & 8654.12 & 4006.82 & 77 \\
\hline H21_3 & 3002.35 & 9596.12 & 6346.74 & 47 \\
\hline H22_3 & 3336.67 & 10127.3 & 6986.54 & 47 \\
\hline H23_3 & 3693.6 & 9456.27 & 7570 & 45 \\
\hline H24_3 & 3708.41 & 8260.58 & 7501 & 47 \\
\hline H25_3 & 3378.83 & 7726.78 & 6870.23 & 47 \\
\hline H27_3 & 2168.76 & 7400.55 & 5646.59 & 41 \\
\hline H28_3 & 988.75 & 7236.8 & 5602.65 & 41 \\
\hline H29_3 & 249.72 & 7870.21 & 6045.01 & 45 \\
\hline H30_3 & 712.24 & 8665.09 & 6529.89 & 41 \\
\hline H31_3 & 1894.91 & 8819.4 & 6567.78 & 41 \\
\hline H4A_9 & 2602.51 & 11512.58 & 6030.64 & 35 \\
\hline H4B_9 & 3002.62 & 10864.44 & 6280.99 & 35 \\
\hline H7_9 & 4448.71 & 12785.71 & 4762.95 & 36 \\
\hline H9_9 & 2488.65 & 12093.12 & 4636.34 & 39 \\
\hline H10_9 & 2584.84 & 11490.69 & 5263.29 & 39 \\
\hline H13_9 & 3310.97 & 14357.62 & 3751.23 & 48 \\
\hline H15_9 & 3764.64 & 12572.68 & 3191.69 & 48 \\
\hline H17A_9 & 2796.93 & 13665.18 & 4730.57 & 64 \\
\hline H17B_9 & 2825.12 & 14357.95 & 4450.59 & 64 \\
\hline H17C_9 & 3491.05 & 14113.67 & 4714.65 & 64 \\
\hline H18A_9 & 3226.19 & 13730.84 & 2802.7 & 76 \\
\hline H18B_9 & 4051 & 13700.18 & 2832.77 & 76 \\
\hline H18C_9 & 3643.92 & 14357.86 & 3014.37 & 76 \\
\hline H19A_9 & 3929.55 & 11550.11 & 4085.22 & 73 \\
\hline H19B_9 & 3867.72 & 11531.76 & 3572.82 & 73 \\
\hline H19C_9 & 3187.26 & 11480.57 & 3863.02 & 73 \\
\hline H21_9 & 4993.97 & 11598.22 & 6339.25 & 44 \\
\hline H22_9 & 5630.51 & 11265.04 & 6941.21 & 44 \\
\hline H23_9 & 5072.44 & 11068.29 & 7595.68 & 55 \\
\hline H24_9 & 3881.74 & 11209.53 & 7635.09 & 44 \\
\hline H25_9 & 3244.2 & 11539 & 7042.98 & 44 \\
\hline H27_9 & 2559.51 & 12526.4 & 5925.16 & 53 \\
\hline H28_9 & 2403.05 & 13702.53 & 5842.64 & 53 \\
\hline H29_9 & 3252.84 & 14469.01 & 6077.64 & 54 \\
\hline H30_9 & 4257.3 & 14039.92 & 6395.52 & 53 \\
\hline H31_9 & 4401.49 & 12860.87 & 6474.38 & 53 \\
\hline
\end{tabular}




\begin{tabular}{|c|c|c|c|c|}
\hline Atom & $\mathbf{x}$ & $\mathbf{y}$ & $\mathbf{z}$ & $\boldsymbol{U}_{e q}$ \\
\hline H4A_7 & 6717.75 & 12154.36 & 5678.44 & 36 \\
\hline H4B_7 & 6197.25 & 11781.79 & 6037.04 & 36 \\
\hline H7_7 & 7658.05 & 10099.53 & 4416.26 & 41 \\
\hline H9_7 & 7048.22 & 12086.43 & 4290.29 & 32 \\
\hline H10_7 & 6581.6 & 12064.26 & 4962.68 & 32 \\
\hline H13_7 & 9229.14 & 11330.17 & 3381.38 & 53 \\
\hline H15_7 & 7426.8 & 10926.75 & 2828.23 & 53 \\
\hline H17A_7 & 8604.53 & 11707.78 & 4387.61 & 66 \\
\hline H17B_7 & 9294.21 & 11627.47 & 4108.58 & 66 \\
\hline H17C_7 & 8974.3 & 10970.83 & 4349.4 & 66 \\
\hline H18A_7 & 8510.11 & 11518.78 & 2432.85 & 80 \\
\hline H18B_7 & 8590.99 & 10696.59 & 2453.03 & 80 \\
\hline H18C_7 & 9196.69 & 11183.07 & 2628.92 & 80 \\
\hline H19A_7 & 6603.76 & 10237.98 & 3470.95 & 66 \\
\hline H19B_7 & 6338.61 & 10954.27 & 3274.15 & 66 \\
\hline H19C_7 & 6414.58 & 10867.05 & 3783.05 & 66 \\
\hline H21_7 & 7065.52 & 9858.65 & 6158.12 & 61 \\
\hline H22_7 & 7066.79 & 9360.48 & 6840.59 & 61 \\
\hline H23_7 & 7125.9 & 10059.79 & 7451.5 & 61 \\
\hline H24_7 & 7178.88 & 11252.01 & 7366.99 & 61 \\
\hline H25_7 & 7165.7 & 11753.72 & 6694.56 & 61 \\
\hline H27_7 & 7806.38 & 12396.44 & 5785.51 & 53 \\
\hline H28_7 & 8897.76 & 12621.07 & 5518.29 & 53 \\
\hline H29_7 & 9629.52 & 11708.57 & 5338.38 & 54 \\
\hline H30_7 & 9252.77 & 10572.54 & 5429.2 & 53 \\
\hline H31_7 & 8158.58 & 10361.11 & 5697.3 & 53 \\
\hline H4A_4 & 3108.72 & 7314.46 & 5876.71 & 33 \\
\hline H4B_4 & 3799.92 & 7609.82 & 6138.44 & 33 \\
\hline H7_4 & 1761.93 & 9467.63 & 4882.24 & 35 \\
\hline H9_4 & 2513.32 & 7632.63 & 4476.33 & 35 \\
\hline H10_4 & 3139.31 & 7525.06 & 5093 & 35 \\
\hline H13_4 & -41.79 & 8629.22 & 4002.24 & 63 \\
\hline H15_4 & 1518.44 & 9181.55 & 3250.53 & 53 \\
\hline H17A_4 & 919.78 & 7893.62 & 4816.08 & 54 \\
\hline H17B_4 & 152.2 & 8076.92 & 4662.78 & 54 \\
\hline H17C_4 & 602.6 & 8640.91 & 4913.48 & 54 \\
\hline H18A_4 & -116.56 & 8728.21 & 3150.49 & 76 \\
\hline H18B_4 & 451.56 & 9240.65 & 2955.66 & 76 \\
\hline H18C_4 & -95.83 & 9519.09 & 3299.77 & 76 \\
\hline H19A_4 & 2738.01 & 9397.93 & 3936.69 & 76 \\
\hline H19B_4 & 2673.89 & 9104.28 & 3457.9 & 76 \\
\hline H19C_4 & 2872.74 & 8595.95 & 3843.93 & 76 \\
\hline H21_4 & 3001.23 & 9611.2 & 6436.39 & 44 \\
\hline H22_4 & 3497.04 & 10107.42 & 7045.73 & 47 \\
\hline H23_4 & 3958.4 & 9404.62 & 7584.94 & 45 \\
\hline H24_4 & 3914 & 8211.9 & 7502.39 & 47 \\
\hline H25_4 & 3425.15 & 7712.49 & 6901.79 & 47 \\
\hline H27_4 & 2076.88 & 7387.75 & 5781.23 & 41 \\
\hline H28_4 & 886.77 & 7328.33 & 5734.31 & 41 \\
\hline H29_4 & 205.09 & 8119.8 & 6107.83 & 45 \\
\hline H30_4 & 732.51 & 8968.46 & 6527.92 & 41 \\
\hline H31_4 & 1924.15 & 9016.11 & 6570.11 & 41 \\
\hline H4A_6 & 2598.47 & 11595.05 & 6120.49 & 35 \\
\hline H4B_6 & 2949.83 & 10916.79 & 6368.27 & 35 \\
\hline H7_6 & 4496.81 & 12773.58 & 4860.32 & 36 \\
\hline H9_6 & 2509.05 & 12168.61 & 4728.95 & 39 \\
\hline H10_6 & 2579.51 & 11549.96 & 5350.99 & 38 \\
\hline H13_6 & 3242.86 & 14439.95 & 3897.81 & 51 \\
\hline
\end{tabular}




\begin{tabular}{|c|c|c|c|c|}
\hline Atom & $\mathbf{x}$ & $\mathbf{y}$ & $\mathbf{z}$ & $U_{e q}$ \\
\hline H15_6 & 3880.93 & 12746.94 & 3298.22 & 52 \\
\hline H17A_6 & 2584.73 & 13901.04 & 4734.76 & 64 \\
\hline H17B_6 & 3171.99 & 14468.72 & 4655 & 64 \\
\hline H17C_6 & 3339.21 & 13795.24 & 4934.42 & 64 \\
\hline H18A_6 & 3640.98 & 14526.18 & 3172.69 & 76 \\
\hline H18B_6 & 3261.26 & 13907.18 & 2926.97 & 76 \\
\hline H18C_6 & 4083.63 & 13900.47 & 2979.94 & 76 \\
\hline H19A_6 & 4401.94 & 11794.63 & 3960.68 & 73 \\
\hline H19B_6 & 3760.73 & 11613.77 & 3656.31 & 73 \\
\hline H19C_6 & 3674.42 & 11584.88 & 4167.15 & 73 \\
\hline H21_6 & 4999 & 11562.96 & 6448.28 & 44 \\
\hline H22_6 & 5602.96 & 11208.47 & 7058.52 & 44 \\
\hline H23_6 & 5012.99 & 11002.24 & 7700.7 & 55 \\
\hline H24_6 & 3822.14 & 11155.85 & 7720.5 & 44 \\
\hline H25_6 & 3216.85 & 11506.12 & 7119.87 & 44 \\
\hline H27_6 & 2643.82 & 12617.73 & 6007.44 & 53 \\
\hline H28_6 & 2604.98 & 13799.36 & 5904.38 & 53 \\
\hline H29_6 & 3519.44 & 14489.9 & 6136.76 & 54 \\
\hline H30_6 & 4469.14 & 13979.03 & 6472.55 & 53 \\
\hline H31_6 & 4495.55 & 12795.79 & 6571.82 & 53 \\
\hline H4A_2 & 6698.02 & 12137.59 & 5816.77 & 36 \\
\hline H4B_2 & 6149.97 & 11747.97 & 6151.74 & 36 \\
\hline H7_2 & 7672.03 & 10096.63 & 4535.77 & 32 \\
\hline H9_2 & 7158.59 & 12116.05 & 4444.34 & 32 \\
\hline H10_2 & 6678.11 & 12083.31 & 5112.87 & 32 \\
\hline H13_2 & 9318.84 & 11245 & 3533.51 & 53 \\
\hline H15_2 & 7496.25 & 11054.05 & 2961.69 & 53 \\
\hline H17A_2 & 8745.66 & 11602.59 & 4544.03 & 66 \\
\hline H17B_2 & 9417.68 & 11408.11 & 4270.12 & 66 \\
\hline H17C_2 & 8972.9 & 10811.67 & 4494.27 & 66 \\
\hline H18A_2 & 9118.98 & 11526.75 & 2728.68 & 96 \\
\hline H18B_2 & 8427.5 & 11214.26 & 2524.58 & 96 \\
\hline H18C_2 & 9018.17 & 10707.59 & 2698.19 & 96 \\
\hline H19A_2 & 6512.47 & 10566.42 & 3746.93 & 66 \\
\hline H19B_2 & 6451.69 & 11053.71 & 3333.45 & 66 \\
\hline H19C_2 & 6442.92 & 11385.1 & 3804.28 & 66 \\
\hline H21_2 & 7006.55 & 9833.57 & 6280.48 & 61 \\
\hline H22_2 & 6941.63 & 9311.18 & 6954.55 & 67 \\
\hline H23_2 & 6947.43 & 9987.86 & 7576.41 & 61 \\
\hline H24_2 & 7019.09 & 11182.25 & 7510.58 & 61 \\
\hline H25_2 & 7083.01 & 11707.16 & 6846.34 & 61 \\
\hline H27_2 & 7766.07 & 12395.19 & 5976.63 & 53 \\
\hline H28_2 & 8891.54 & 12651.2 & 5786.01 & 53 \\
\hline H29_2 & 9664.88 & 11760.52 & 5632.36 & 54 \\
\hline H30_2 & 9295.44 & 10614.7 & 5674.22 & 53 \\
\hline H31_2 & 8168.08 & 10371.64 & 5868.69 & 53 \\
\hline H4A_8 & 7333.7 & 7922.13 & 5497.54 & 30 \\
\hline H4B_8 & 7060.35 & 8508.08 & 5851.65 & 30 \\
\hline H7_8 & 4989.95 & 6981.7 & 4436.68 & 30 \\
\hline H9_8 & 6929.27 & 7572.5 & 4125.86 & 33 \\
\hline H10_8 & 7080.71 & 8021.75 & 4796.14 & 35 \\
\hline H13_8 & 5957.49 & 5505.32 & 3227.52 & 41 \\
\hline H15_8 & 5639.46 & 7392.6 & 2769.5 & 43 \\
\hline H17A_8 & 6312.95 & 6018.65 & 4286.97 & 57 \\
\hline H17B_8 & 6386.33 & 5392.03 & 3954.78 & 57 \\
\hline H17C_8 & 5652.12 & 5561.15 & 4166.43 & 57 \\
\hline H18A_8 & 5915.78 & 5664.53 & 2473.6 & 87 \\
\hline H18B_8 & 6077.09 & 6423.72 & 2296.47 & 87 \\
\hline
\end{tabular}




\begin{tabular}{lllll}
\hline Atom & \multicolumn{1}{c}{$\mathbf{x}$} & \multicolumn{1}{c}{$\mathbf{z}$} & $\boldsymbol{U}_{\boldsymbol{e q}}$ \\
\hline H18C_8 & 5294.56 & 6183.28 & 2368.51 & 87 \\
H19A_8 & 5350.53 & 8286.45 & 3675.69 & 68 \\
H19B_8 & 5636.85 & 8378.44 & 3196.94 & 68 \\
H19C_8 & 6165.36 & 8312.3 & 3590.04 & 68 \\
H21_8 & 5167.52 & 7500.86 & 6199 & 42 \\
H22_8 & 4822.56 & 7573.71 & 6915.73 & 48 \\
H23_8 & 5636.15 & 7801.77 & 7452.14 & 56 \\
H24_8 & 6786.61 & 7953.58 & 7259.44 & 51 \\
H25_8 & 7136.32 & 7883.33 & 6552.14 & 46 \\
H27_8 & 7584.74 & 6799.78 & 5812.61 & 41 \\
H28_8 & 7745.35 & 5646.94 & 5647.03 & 58 \\
H29_8 & 6798.98 & 4958.88 & 5467.55 & 61 \\
H30_8 & 5694.01 & 5441.97 & 5456.6 & 66 \\
H31_8 & 5547.04 & 6596.84 & 5623.7 & 46 \\
H4A_1 & 7337.56 & 8006.29 & 5594.14 & 30 \\
H4B_1 & 7025.72 & 8576.27 & 5946.01 & 30 \\
H7_1 & 5021.04 & 7012.35 & 4507.97 & 30 \\
H9_1 & 6989.33 & 7550.62 & 4233.55 & 33 \\
H10_1 & 7115.63 & 8010.01 & 4902.95 & 35 \\
H13_1 & 5962.16 & 5435.65 & 3366.14 & 46 \\
H15_1 & 5614.3 & 7285.48 & 2860.72 & 43 \\
H17A_1 & 6504.43 & 5861.26 & 4334.67 & 57 \\
H17B_1 & 6082.51 & 5265.29 & 4093.6 & 57 \\
H17C_1 & 5683.38 & 5813.78 & 4387.45 & 57 \\
H18A_1 & 6088.55 & 5704.36 & 2548.65 & 87 \\
H18B_1 & 5701.61 & 6389.78 & 2391.3 & 87 \\
H18C_1 & 5266.04 & 5761.51 & 2587.04 & 87 \\
H19A_1 & 5296.5 & 8155.54 & 3797.78 & 68 \\
H19B_1 & 5377.49 & 8252.91 & 3289.88 & 68 \\
H19C_1 & 6032.97 & 8348.7 & 3597.42 & 68 \\
H21_1 & 5171.83 & 7512.57 & 6268.18 & 42 \\
H22_1 & 4793.46 & 7587.26 & 6978.32 & 48 \\
H23_1 & 5580.49 & 7825.95 & 7528.12 & 56 \\
H24_1 & 6737.73 & 7986.38 & 7355.28 & 60 \\
H25_1 & 7120.48 & 7914.5 & 6654.67 & 46 \\
H27_1 & 7652.36 & 6919.25 & 5904.4 & 41 \\
H28_1 & 7893.41 & 5773.71 & 5758.21 & 58 \\
H29_1 & 6997.13 & 5013.86 & 5591.13 & 61 \\
H30_1 & 5861.61 & 5418.26 & 5573.1 & 66 \\
H31_1 & 5633.59 & 6566.91 & 5720.71 & 46 \\
& & & &
\end{tabular}

Table 22: Atomic Occupancies for all atoms that are not fully occupied in 2Cl4triMePh.

\begin{tabular}{|c|c|c|c|}
\hline Atom & Occupancy & Atom & Occupancy \\
\hline $\mathrm{Rh1}$ & 0.5 & $\overline{01 \_3}$ & 0.5 \\
\hline $\mathrm{Rh} 2$ & 0.5 & 02_3 & 0.5 \\
\hline 04 & 0.5 & C1_3 & 0.5 \\
\hline $\mathrm{H} 4 \mathrm{~A}$ & 0.5 & C2_3 & 0.5 \\
\hline H4B & 0.5 & C3_3 & 0.5 \\
\hline Rh1A & 0.5 & C4_3 & 0.5 \\
\hline $\mathrm{Rh} 2 \mathrm{~A}$ & 0.5 & H4A_3 & 0.5 \\
\hline $04 \mathrm{~A}$ & 0.5 & H4B_3 & 0.5 \\
\hline H4AA & 0.5 & C5_3 & 0.5 \\
\hline $\mathrm{H} 4 \mathrm{AB}$ & 0.5 & C6_3 & 0.5 \\
\hline Cl1_3 & 0.5 & C7_3 & 0.5 \\
\hline
\end{tabular}

\begin{tabular}{lr}
\hline Atom & Occupancy \\
\hline H7_3 & 0.5 \\
C8_3 & 0.5 \\
C9_3 & 0.5 \\
H9_3 & 0.5 \\
C10_3 & 0.5 \\
H10_3 & 0.5 \\
C11_3 & 0.5 \\
C12_3 & 0.5 \\
C13_3 & 0.5 \\
H13_3 & 0.5 \\
C14_3 & 0.5 \\
&
\end{tabular}

\begin{tabular}{lr}
\hline Atom & Occupancy \\
\hline C15_3 & 0.5 \\
H15_3 & 0.5 \\
C16_3 & 0.5 \\
C17_3 & 0.5 \\
H17A_3 & 0.5 \\
H17B_3 & 0.5 \\
H17C_3 & 0.5 \\
C18_3 & 0.5 \\
H18A_3 & 0.5 \\
H18B_3 & 0.5 \\
H18C_3 & 0.5
\end{tabular}




\begin{tabular}{|c|c|c|c|c|c|c|c|}
\hline & & & & \\
\hline Atom & Occupancy & Atom & Occupancy & Atom & Occupancy & Atom & Occupancy \\
\hline$\overline{\text { C19_3 }}$ & 0.5 & $\overline{\mathrm{H} 18 \mathrm{~B} \_9}$ & 0.5 & $\overline{\text { C18_7 }}$ & 0.5 & $\overline{\mathrm{H} 17 \mathrm{~B} \_4}$ & 0.5 \\
\hline H19A_3 & 0.5 & H18C_9 & 0.5 & H18A_7 & 0.5 & H17C_4 & 0.5 \\
\hline H19B_3 & 0.5 & C19_9 & 0.5 & H18B_7 & 0.5 & C18_4 & 0.5 \\
\hline H19C_3 & 0.5 & H19A_9 & 0.5 & H18C_7 & 0.5 & H18A_4 & 0.5 \\
\hline C20_3 & 0.5 & H19B_9 & 0.5 & C19_7 & 0.5 & H18B_4 & 0.5 \\
\hline C21_3 & 0.5 & H19C_9 & 0.5 & H19A_7 & 0.5 & H18C_4 & 0.5 \\
\hline $\mathrm{H} 21 \_3$ & 0.5 & C20_9 & 0.5 & H19B_7 & 0.5 & C19_4 & 0.5 \\
\hline C22_3 & 0.5 & C21_9 & 0.5 & H19C_7 & 0.5 & H19A_4 & 0.5 \\
\hline H22_3 & 0.5 & H21_9 & 0.5 & C20_7 & 0.5 & H19B_4 & 0.5 \\
\hline C23_3 & 0.5 & C22_9 & 0.5 & C21_7 & 0.5 & H19C_4 & 0.5 \\
\hline H23_3 & 0.5 & H22_9 & 0.5 & H21_7 & 0.5 & C20_4 & 0.5 \\
\hline C24_3 & 0.5 & C23_9 & 0.5 & C22_7 & 0.5 & C21_4 & 0.5 \\
\hline H24_3 & 0.5 & H23_9 & 0.5 & H22_7 & 0.5 & H21_4 & 0.5 \\
\hline C25_3 & 0.5 & C24_9 & 0.5 & C23_7 & 0.5 & $\mathrm{C} 22{ }_{-} 4$ & 0.5 \\
\hline H25_3 & 0.5 & H24_9 & 0.5 & H23_7 & 0.5 & H22_4 & 0.5 \\
\hline C26_3 & 0.5 & C25_9 & 0.5 & C24_7 & 0.5 & C23_4 & 0.5 \\
\hline C27_3 & 0.5 & H25_9 & 0.5 & $\mathrm{H} 24 \_7$ & 0.5 & H23_4 & 0.5 \\
\hline H27_3 & 0.5 & C26_9 & 0.5 & C25_7 & 0.5 & C24_4 & 0.5 \\
\hline C28_3 & 0.5 & C27_9 & 0.5 & H25_7 & 0.5 & H24_4 & 0.5 \\
\hline H28_3 & 0.5 & H27_9 & 0.5 & C26_7 & 0.5 & C25_4 & 0.5 \\
\hline C29_3 & 0.5 & C28_9 & 0.5 & C27_7 & 0.5 & H25_4 & 0.5 \\
\hline H29_3 & 0.5 & H28_9 & 0.5 & H27_7 & 0.5 & C26_4 & 0.5 \\
\hline C30_3 & 0.5 & C29_9 & 0.5 & C28_7 & 0.5 & C27_4 & 0.5 \\
\hline H30_3 & 0.5 & H29_9 & 0.5 & H28_7 & 0.5 & H27_4 & 0.5 \\
\hline C31_3 & 0.5 & C30_9 & 0.5 & C29_7 & 0.5 & C28_4 & 0.5 \\
\hline H31_3 & 0.5 & H30_9 & 0.5 & H29_7 & 0.5 & H28_4 & 0.5 \\
\hline Cl1_9 & 0.5 & C31_9 & 0.5 & C30_7 & 0.5 & C29_4 & 0.5 \\
\hline 01_9 & 0.5 & H31_9 & 0.5 & H30_7 & 0.5 & H29_4 & 0.5 \\
\hline 02_9 & 0.5 & Cl1_7 & 0.5 & C31_7 & 0.5 & C30_4 & 0.5 \\
\hline C1_9 & 0.5 & $01 \_7$ & 0.5 & H31_7 & 0.5 & H30_4 & 0.5 \\
\hline C2_9 & 0.5 & 02_7 & 0.5 & Cl1_4 & 0.5 & C31_4 & 0.5 \\
\hline C3_9 & 0.5 & C1_7 & 0.5 & $01 \_4$ & 0.5 & H31_4 & 0.5 \\
\hline C4_9 & 0.5 & $\mathrm{C} 2 \_7$ & 0.5 & $02 \_4$ & 0.5 & Cl1_k & 0.5 \\
\hline H4A_9 & 0.5 & C3_7 & 0.5 & C1_4 & 0.5 & $01 \_6$ & 0.5 \\
\hline H4B_9 & 0.5 & C4_7 & 0.5 & C2_4 & 0.5 & $02 \_6$ & 0.5 \\
\hline C5_9 & 0.5 & H4A_7 & 0.5 & C3_4 & 0.5 & C1_6 & 0.5 \\
\hline C6_9 & 0.5 & H4B_7 & 0.5 & C4_4 & 0.5 & $\mathrm{C} 2 \_6$ & 0.5 \\
\hline C7_99 & 0.5 & C5_7 & 0.5 & H4A_4 & 0.5 & C3_6 & 0.5 \\
\hline H7_9 & 0.5 & C6_7 & 0.5 & H4B_4 & 0.5 & C4_6 & 0.5 \\
\hline C8_9 & 0.5 & C7_7 & 0.5 & C5_4 & 0.5 & H4A_6 & 0.5 \\
\hline C9_9 & 0.5 & H7_7 & 0.5 & C6_4 & 0.5 & H4B_6 & 0.5 \\
\hline H9_9 & 0.5 & C8_7 & 0.5 & C7_4 & 0.5 & C5_6 & 0.5 \\
\hline C10_9 & 0.5 & C9_7 & 0.5 & $\mathrm{H}^{-}{ }_{-}^{-} 4$ & 0.5 & C6_6 & 0.5 \\
\hline H10_9 & 0.5 & H9_7 & 0.5 & C8_4 & 0.5 & C7_6 & 0.5 \\
\hline C11_9 & 0.5 & C10_7 & 0.5 & C9_4 & 0.5 & H7_6 & 0.5 \\
\hline C12_9 & 0.5 & H10_7 & 0.5 & H9_4 & 0.5 & C8_6 & 0.5 \\
\hline C13_9 & 0.5 & C11_7 & 0.5 & C10_4 & 0.5 & C9_6 & 0.5 \\
\hline H13_9 & 0.5 & $\mathrm{C} 12 \_7$ & 0.5 & H10_4 & 0.5 & H9_6 & 0.5 \\
\hline C14_9 & 0.5 & C13_7 & 0.5 & C11_4 & 0.5 & C10_6 & 0.5 \\
\hline C15_9 & 0.5 & H13_7 & 0.5 & C12_4 & 0.5 & H10_6 & 0.5 \\
\hline H15_9 & 0.5 & C14_7 & 0.5 & C13_4 & 0.5 & C11_6 & 0.5 \\
\hline C16_9 & 0.5 & C15_7 & 0.5 & H13_4 & 0.5 & C12_6 & 0.5 \\
\hline C17_9 & 0.5 & H15_7 & 0.5 & C14_4 & 0.5 & C13_6 & 0.5 \\
\hline H17A_9 & 0.5 & C16_7 & 0.5 & C15_4 & 0.5 & H13_6 & 0.5 \\
\hline H17B_9 & 0.5 & C17_7 & 0.5 & H15_4 & 0.5 & C14_6 & 0.5 \\
\hline H17C_9 & 0.5 & H17A_7 & 0.5 & C16_4 & 0.5 & C15_6 & 0.5 \\
\hline C18_9 & 0.5 & H17B_7 & 0.5 & C17_4 & 0.5 & H15_6 & 0.5 \\
\hline H18A_9 & 0.5 & H17C_7 & 0.5 & H17A_4 & 0.5 & C16_6 & 0.5 \\
\hline
\end{tabular}




\begin{tabular}{|c|c|c|c|c|c|c|c|}
\hline \multirow{2}{*}{\multicolumn{2}{|c|}{ Occupancy }} & & & \\
\hline & & Atom & Occupancy & Atom & Occupancy & Atom & Occupancy \\
\hline C17_6 & 0.5 & $\overline{\mathrm{C} 13 \_2}$ & 0.5 & $\overline{\text { C9_8 }}$ & 0.5 & H4B_1 & 0.5 \\
\hline H17A_6 & 0.5 & H13_2 & 0.5 & H9_8 & 0.5 & C5_1 & 0.5 \\
\hline H17B_6 & 0.5 & C14_2 & 0.5 & C10_8 & 0.5 & C6_1 & 0.5 \\
\hline H17C_6 & 0.5 & C15_2 & 0.5 & H10_8 & 0.5 & C7_1 & 0.5 \\
\hline C18_6 & 0.5 & H15_2 & 0.5 & C11_8 & 0.5 & H7_1 & 0.5 \\
\hline H18A_6 & 0.5 & C16_2 & 0.5 & C12_8 & 0.5 & C8_1 & 0.5 \\
\hline H18B_6 & 0.5 & C17_2 & 0.5 & C13_8 & 0.5 & C9_1 & 0.5 \\
\hline H18C_6 & 0.5 & H17A_2 & 0.5 & H13_8 & 0.5 & H9_1 & 0.5 \\
\hline C19_6 & 0.5 & H17B_2 & 0.5 & C14_8 & 0.5 & C10_1 & 0.5 \\
\hline H19A_6 & 0.5 & H17C_2 & 0.5 & C15_8 & 0.5 & H10_1 & 0.5 \\
\hline H19B_6 & 0.5 & C18_2 & 0.5 & H15_8 & 0.5 & C11_1 & 0.5 \\
\hline H19C_6 & 0.5 & H18A_2 & 0.5 & C16_8 & 0.5 & C12_1 & 0.5 \\
\hline C20_6 & 0.5 & H18B_2 & 0.5 & C17_8 & 0.5 & C13_1 & 0.5 \\
\hline C21_6 & 0.5 & H18C_2 & 0.5 & H17A_8 & 0.5 & H13_1 & 0.5 \\
\hline H21_6 & 0.5 & C19_2 & 0.5 & H17B_8 & 0.5 & C14_1 & 0.5 \\
\hline C22_6 & 0.5 & H19A_2 & 0.5 & H17C_8 & 0.5 & C15_1 & 0.5 \\
\hline $\mathrm{H} 22 \_6$ & 0.5 & H19B_2 & 0.5 & C18_8 & 0.5 & H15_1 & 0.5 \\
\hline C23_6 & 0.5 & H19C_2 & 0.5 & H18A_8 & 0.5 & C16_1 & 0.5 \\
\hline H23_6 & 0.5 & C20_2 & 0.5 & H18B_8 & 0.5 & C17_1 & 0.5 \\
\hline C24_6 & 0.5 & C21_2 & 0.5 & H18C_8 & 0.5 & H17A_1 & 0.5 \\
\hline H24_6 & 0.5 & H21_2 & 0.5 & C19_8 & 0.5 & H17B_1 & 0.5 \\
\hline C25_6 & 0.5 & $\mathrm{C} 22 \_2$ & 0.5 & H19A_8 & 0.5 & H17C_1 & 0.5 \\
\hline H25_6 & 0.5 & H22_2 & 0.5 & H19B_8 & 0.5 & C18_1 & 0.5 \\
\hline C26_6 & 0.5 & C23_2 & 0.5 & H19C_8 & 0.5 & H18A_1 & 0.5 \\
\hline C27_6 & 0.5 & H23_2 & 0.5 & C20_8 & 0.5 & H18B_1 & 0.5 \\
\hline H27_6 & 0.5 & C24_2 & 0.5 & C21_8 & 0.5 & H18C_1 & 0.5 \\
\hline C28_6 & 0.5 & H24_2 & 0.5 & H21_8 & 0.5 & C19_1 & 0.5 \\
\hline H28_6 & 0.5 & C25_2 & 0.5 & C22_8 & 0.5 & H19A_1 & 0.5 \\
\hline C29_6 & 0.5 & H25_2 & 0.5 & $\mathrm{H} 222_{-}^{-} 8$ & 0.5 & H19B_1 & 0.5 \\
\hline H29_6 & 0.5 & C26_2 & 0.5 & C23_8 & 0.5 & H19C_1 & 0.5 \\
\hline C30_6 & 0.5 & C27_2 & 0.5 & H23_8 & 0.5 & C20_1 & 0.5 \\
\hline H30_6 & 0.5 & H27_2 & 0.5 & C24_8 & 0.5 & C21_1 & 0.5 \\
\hline C31_6 & 0.5 & C28_2 & 0.5 & H24_8 & 0.5 & H21_1 & 0.5 \\
\hline H31_6 & 0.5 & H28_2 & 0.5 & C25_8 & 0.5 & C22_1 & 0.5 \\
\hline Cl1_2 & 0.5 & C29_2 & 0.5 & H25_8 & 0.5 & H22_1 & 0.5 \\
\hline 01_2 & 0.5 & H29_2 & 0.5 & C26_8 & 0.5 & C23_1 & 0.5 \\
\hline $02 \_2$ & 0.5 & C30_2 & 0.5 & C27_8 & 0.5 & H23_1 & 0.5 \\
\hline C1_2 & 0.5 & H30_2 & 0.5 & H27_8 & 0.5 & C24_1 & 0.5 \\
\hline C2_2 & 0.5 & C31_2 & 0.5 & C28_8 & 0.5 & H24_1 & 0.5 \\
\hline C3_2 & 0.5 & H31_2 & 0.5 & H28_8 & 0.5 & C25_1 & 0.5 \\
\hline C4_2 & 0.5 & Cl1_8 & 0.5 & C29_8 & 0.5 & H25_1 & 0.5 \\
\hline H4A_2 & 0.5 & 01_8 & 0.5 & H29_8 & 0.5 & C26_1 & 0.5 \\
\hline H4B_2 & 0.5 & 02_8 & 0.5 & C30_8 & 0.5 & C27_1 & 0.5 \\
\hline C5_2 & 0.5 & C1_8 & 0.5 & H30_8 & 0.5 & H27_1 & 0.5 \\
\hline C6_2 & 0.5 & C2_8 & 0.5 & C31_8 & 0.5 & C28_1 & 0.5 \\
\hline C7_2 & 0.5 & C3_8 & 0.5 & H31_8 & 0.5 & H28_1 & 0.5 \\
\hline H7_2 & 0.5 & C4_8 & 0.5 & Cl1_1 & 0.5 & C29_1 & 0.5 \\
\hline C8_2 & 0.5 & H4A_8 & 0.5 & 01_1 & 0.5 & H29_1 & 0.5 \\
\hline C9_2 & 0.5 & H4B_8 & 0.5 & 02_1 & 0.5 & C30_1 & 0.5 \\
\hline H9_2 & 0.5 & C5_8 & 0.5 & C1_1 & 0.5 & H30_1 & 0.5 \\
\hline C10_2 & 0.5 & C6_8 & 0.5 & C2_1 & 0.5 & C31_1 & 0.5 \\
\hline H10_2 & 0.5 & C7_8 & 0.5 & C3_1 & 0.5 & H31_1 & 0.5 \\
\hline C11_2 & 0.5 & H7_8 & 0.5 & C4_1 & 0.5 & & \\
\hline C12_2 & 0.5 & C8_8 & 0.5 & H4A_1 & 0.5 & & \\
\hline
\end{tabular}

Table 23: Solvent masking (PLATON/SQUEEZE) information for 2Cl4triMePh. 


\begin{tabular}{lllllll}
\hline No & $\mathbf{x}$ & $\mathbf{y}$ & $\mathbf{z}$ & $\mathbf{V}$ & $\mathbf{e}$ & Content \\
\hline 1 & -0.500 & -0.500 & -0.093 & 2604.9 & 476.4 & $?$
\end{tabular}




\section{Citations}

CrysAlisPro (Rigaku, V1.171.39.29d, 2017)

CrysAlisPro (ROD), Rigaku Oxford Diffraction, Poland (?).

O.V. Dolomanov and L.J. Bourhis and R.J. Gildea and J.A.K. Howard and H. Puschmann, Olex2: A complete structure solution, refinement and analysis program, J. Appl. Cryst., (2009), 42, 339-341.

Sheldrick, G.M., Crystal structure refinement with ShelXL, Acta Cryst., (2015), C71, 3-8.

Sheldrick, G.M., ShelXT-Integrated space-group and crystal-structure determination, Acta Cryst., (2015), A71, 3-8.

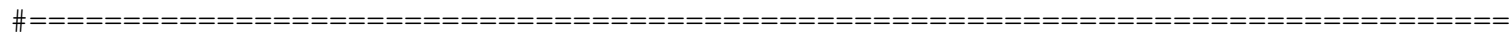

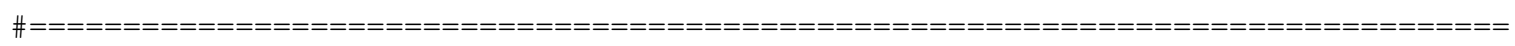

!! Congratulations !! : No (A, B, C) ALERT Conditions were Detected

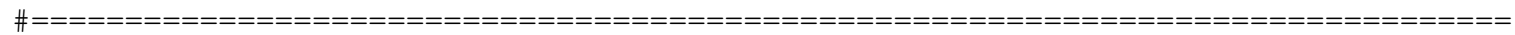




\section{Crystal Data and Experimental}

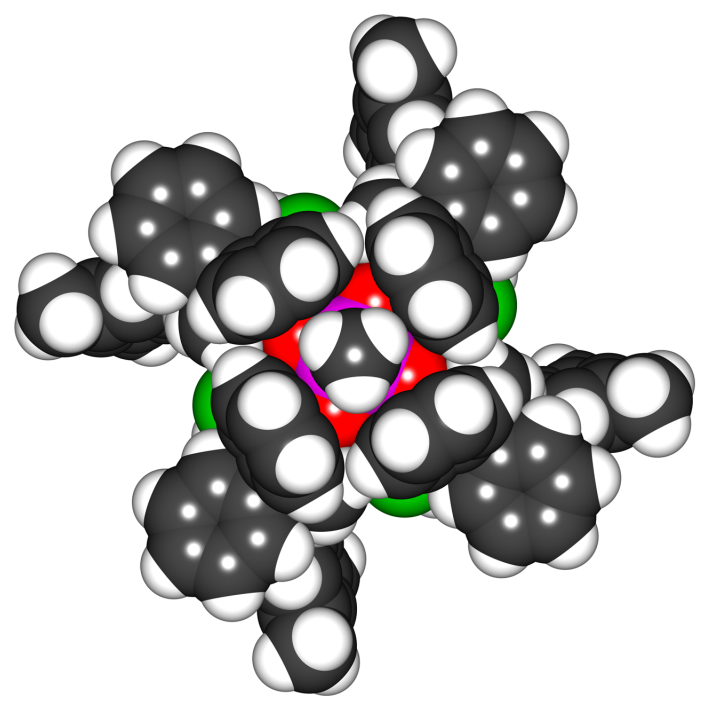

Experimental. Single violet prism-shaped crystals of WLELN-0051-115 were chosen from the sample as supplied. A suitable crystal with dimensions $0.41 \times 0.26 \times 0.18 \mathrm{~mm}^{3}$ was selected and mounted on a loop with paratone on a XtaLAB Synergy-S diffractometer. The crystal was kept at a steady $T=100.01(10) \mathrm{K}$ during data collection. The structure was solved with the ShelXT (Sheldrick, 2015) solution program using dual methods and by using 0lex2 1.3-alpha (Dolomanov et al., 2009) as the graphical interface. The model was refined with ShelXL 2018/3 (Sheldrick, 2015) using full matrix least squares minimisation on $\boldsymbol{F}^{2}$.

Crystal Data. $\mathrm{C}_{122} \mathrm{H}_{99} \mathrm{Cl}_{4} \mathrm{NO}_{8} \mathrm{Rh}_{2}, M_{r}=2054.64$, tetragonal, I4 (No. 79), $\mathrm{a}=18.38221(15) \AA \AA \mathrm{d}=18.38221(15) \AA, \mathrm{c}=$ 16.4051(2) $\AA, \alpha=\beta=\gamma=90^{\circ}, V=5543.38(12) \AA^{3}, \quad T=$ 100.01(10) $\mathrm{K}, Z=2, Z^{\prime}=0.25, \mu(\mathrm{Cu} \mathrm{K})=3.723,18735$ reflections measured, 5299 unique $\left(\mathrm{R}_{\text {int }}=0.0293\right)$ which were used in all calculations. The final $w R_{2}$ was 0.1220 (all data) and $R_{1}$ was 0.0444 (I $\geq 2 \sigma(\mathrm{I})$ ).

\begin{tabular}{|c|c|}
\hline Compound & WL-ELN-0051-115 \\
\hline Formula & $\mathrm{C}_{122} \mathrm{H}_{99} \mathrm{Cl}_{4} \mathrm{NO}_{8} \mathrm{Rh}_{2}$ \\
\hline$D_{\text {calc. }} / \mathrm{g} \mathrm{cm}^{-3}$ & 1.231 \\
\hline$\mu / \mathrm{mm}^{-1}$ & 3.723 \\
\hline Formula Weight & 2054.64 \\
\hline Colour & violet \\
\hline Shape & prism-shaped \\
\hline Size $/ \mathrm{mm}^{3}$ & $0.41 \times 0.26 \times 0.18$ \\
\hline$T / \mathrm{K}$ & $100.01(10)$ \\
\hline Crystal System & tetragonal \\
\hline Flack Parameter & $-0.007(6)$ \\
\hline Hooft Parameter & $0.049(2)$ \\
\hline Space Group & I4 \\
\hline$a / \AA$ & $18.38221(15)$ \\
\hline$b / \AA$ & $18.38221(15)$ \\
\hline$c / \AA ̊$ & $16.4051(2)$ \\
\hline$\alpha /^{\circ}$ & 90 \\
\hline$\beta /^{\circ}$ & 90 \\
\hline$\gamma 1^{\circ}$ & 90 \\
\hline $\mathrm{V} / \AA^{3}$ & $5543.38(12)$ \\
\hline$Z$ & 2 \\
\hline$Z^{\prime}$ & 0.25 \\
\hline Wavelength/Å & 1.54184 \\
\hline Radiation type & $\mathrm{Cu} \mathrm{K} \alpha$ \\
\hline$\Theta_{\min } /^{\circ}$ & 3.400 \\
\hline$\Theta_{\max } /^{\circ}$ & 73.468 \\
\hline Measured Refl's. & 18735 \\
\hline Indep't Refl's & 5299 \\
\hline Refl's I $\geq 2 \sigma(I)$ & 5110 \\
\hline$R_{\text {int }}$ & 0.0293 \\
\hline Parameters & 338 \\
\hline Restraints & 205 \\
\hline Largest Peak & 0.696 \\
\hline Deepest Hole & -0.817 \\
\hline GooF & 1.030 \\
\hline$w R_{2}$ (all data) & 0.1220 \\
\hline$w R_{2}$ & 0.1207 \\
\hline$R_{1}$ (all data) & 0.0460 \\
\hline$R_{1}$ & 0.0444 \\
\hline
\end{tabular}




\section{Structure Quality Indicators}

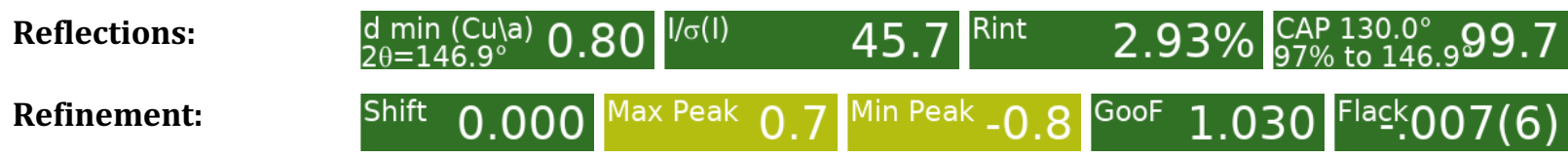

A violet prism-shaped-shaped crystal with dimensions $0.41 \times 0.26 \times 0.18 \mathrm{~mm}^{3}$ was mounted on a loop with paratone. Data were collected using a XtaLAB Synergy, Dualflex, HyPix diffractometer equipped with an Oxford Cryosystems low-temperature device operating at $T=100.01(10) \mathrm{K}$.

Data were measured using $\omega$ scans using $\mathrm{Cu} \mathrm{K}_{\alpha}$ radiation. The diffraction pattern was indexed and the total number of runs and images was based on the strategy calculation from the program CrysAlisPro 1.171.40.67a (Rigaku OD, 2019). The maximum resolution that was achieved was $\Theta=73.468^{\circ}(0.80 \AA)$.

The unit cell was refined using CrysAlisPro 1.171.40.67a (Rigaku OD, 2019) on 15500 reflections, 83\% of the observed reflections.

Data reduction, scaling and absorption corrections were performed using CrysAlisPro 1.171.40.67a (Rigaku OD, 2019). The final completeness is $99.80 \%$ out to $73.468^{\circ}$ in $\Theta$. numerical absorption correction based on gaussian integration over a multifaceted crystal model was performed using CrysAlisPro 1.171.41.108a (Rigaku Oxford Diffraction, 2021). An empirical absorption correction using spherical harmonics, implemented in SCALE3 ABSPACK scaling algorithm was also applied. The absorption coefficient $\mu$ of this material is $3.723 \mathrm{~mm}^{-1}$ at this wavelength $(\lambda=1.54184 \AA)$ and the minimum and maximum transmissions are 0.350 and 1.000 .

The structure was solved and the space group I4 (\# 79) determined by the ShelXT (Sheldrick, 2015) structure solution program using using dual methods and refined by full matrix least squares minimisation on $\boldsymbol{F}^{2}$ using version 2018/3 of ShelXL 2018/3 (Sheldrick, 2015). All non-hydrogen atoms were refined anisotropically. Hydrogen atom positions were calculated geometrically and refined using the riding model.

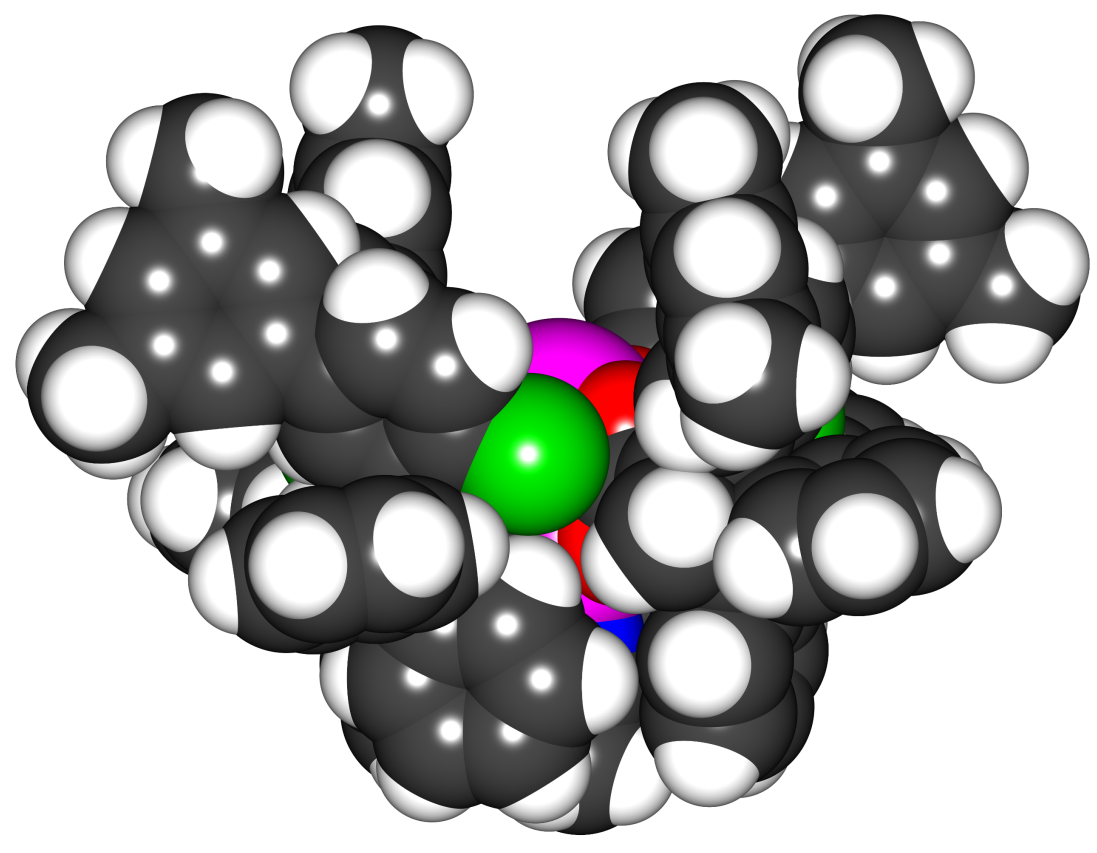

Figure 6: 


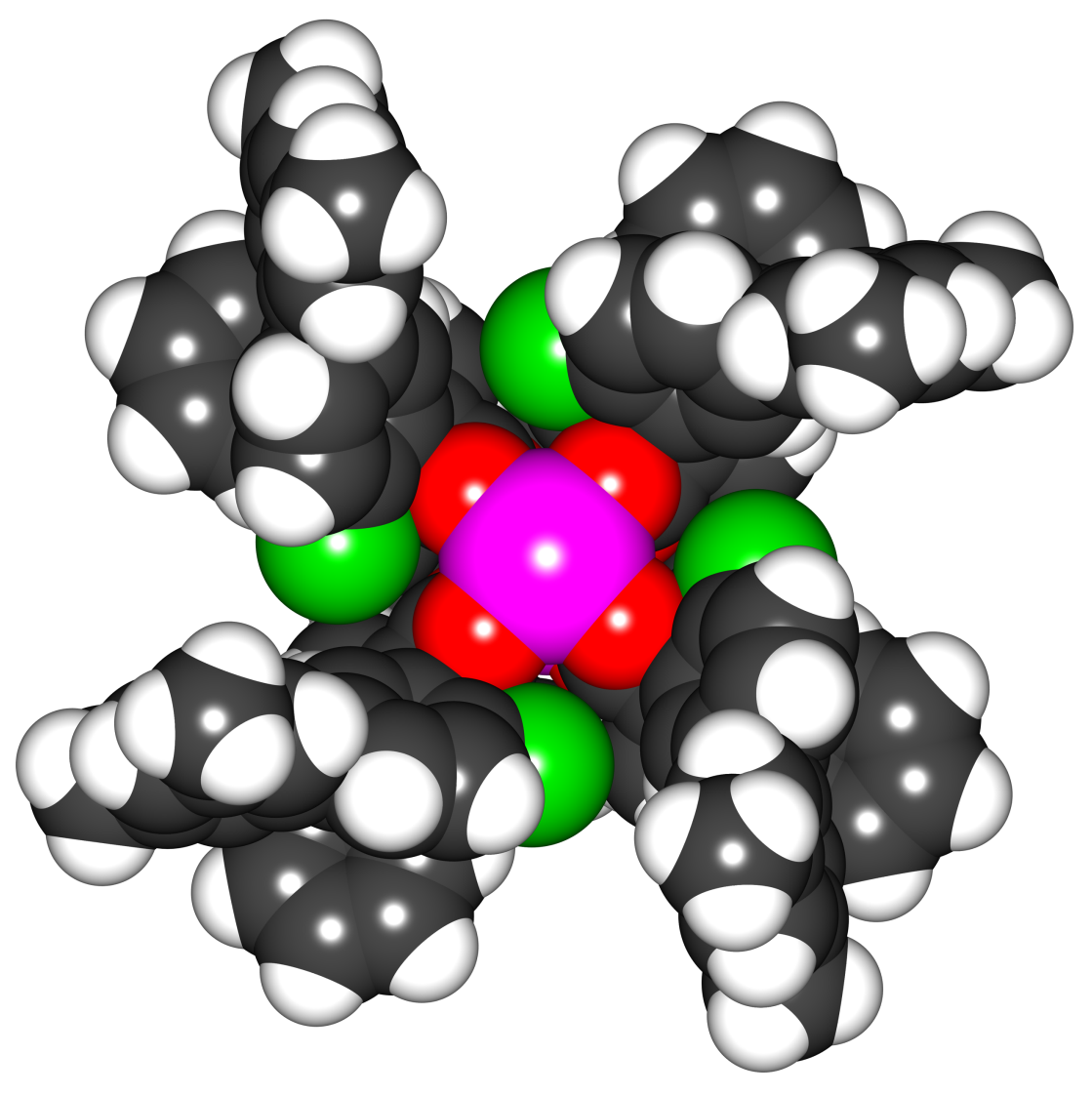

Figure 7: 


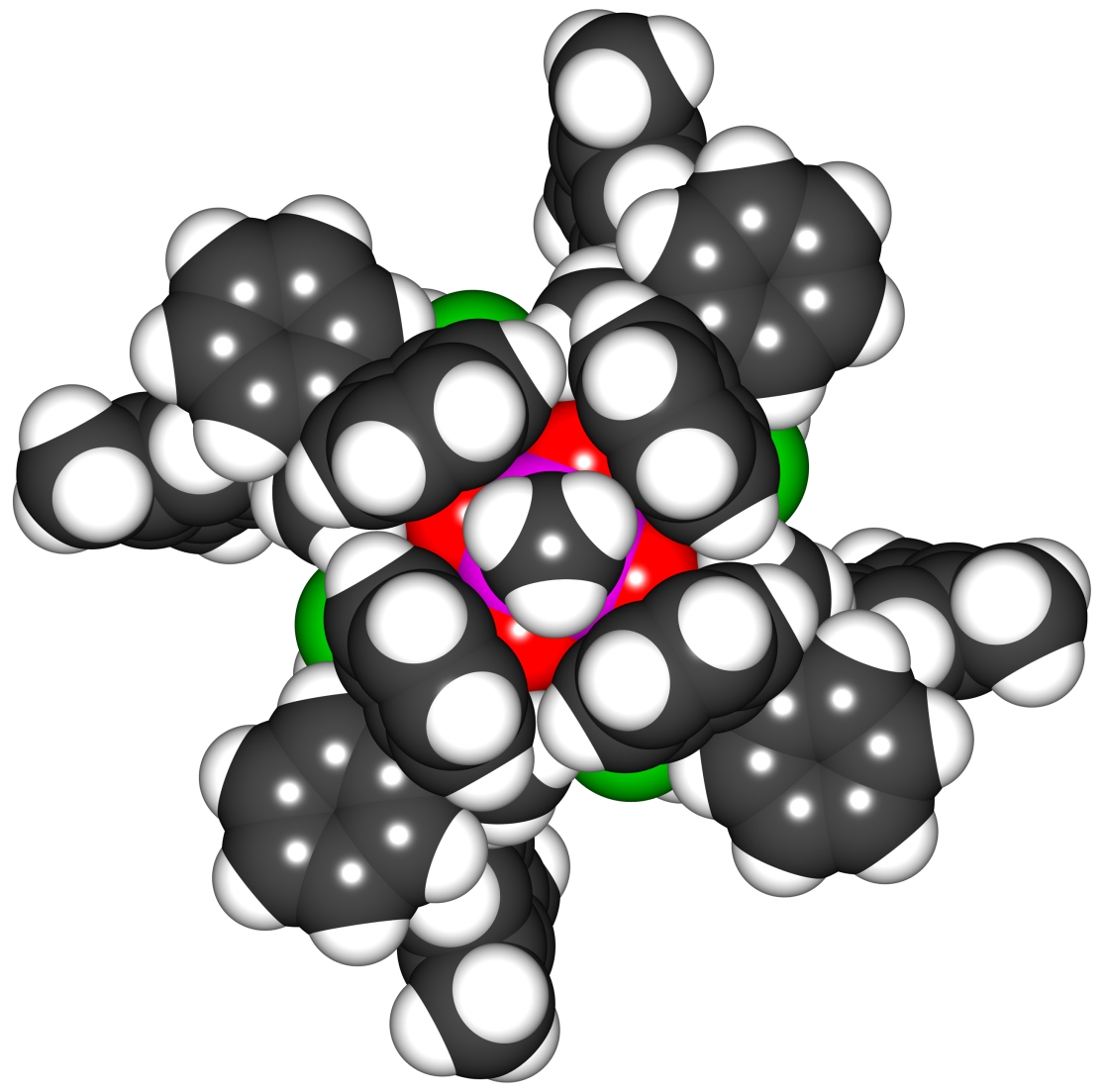

Figure 8:

_exptl_absorpt_process_details: CrysAlisPro 1.171.40.67a (Rigaku Oxford Diffraction, 2019)Numerical absorption correction based on gaussian integration over a multifaceted crystal modelEmpirical absorption correction using spherical harmonics, implemented in SCALE3 ABSPACK scaling algorithm.

\section{Data Plots: Diffraction Data}
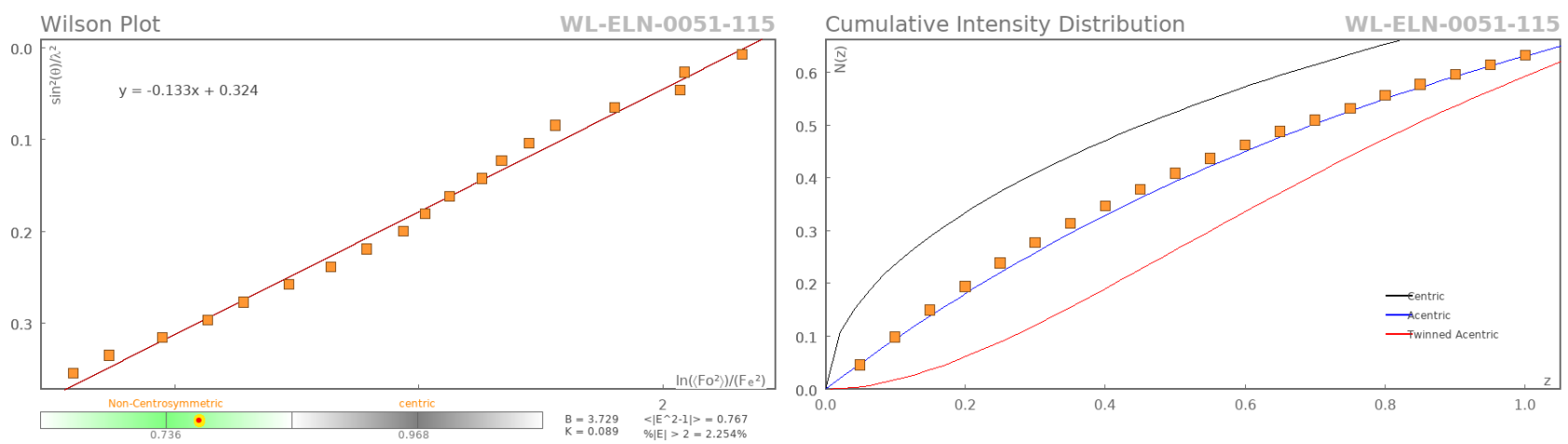

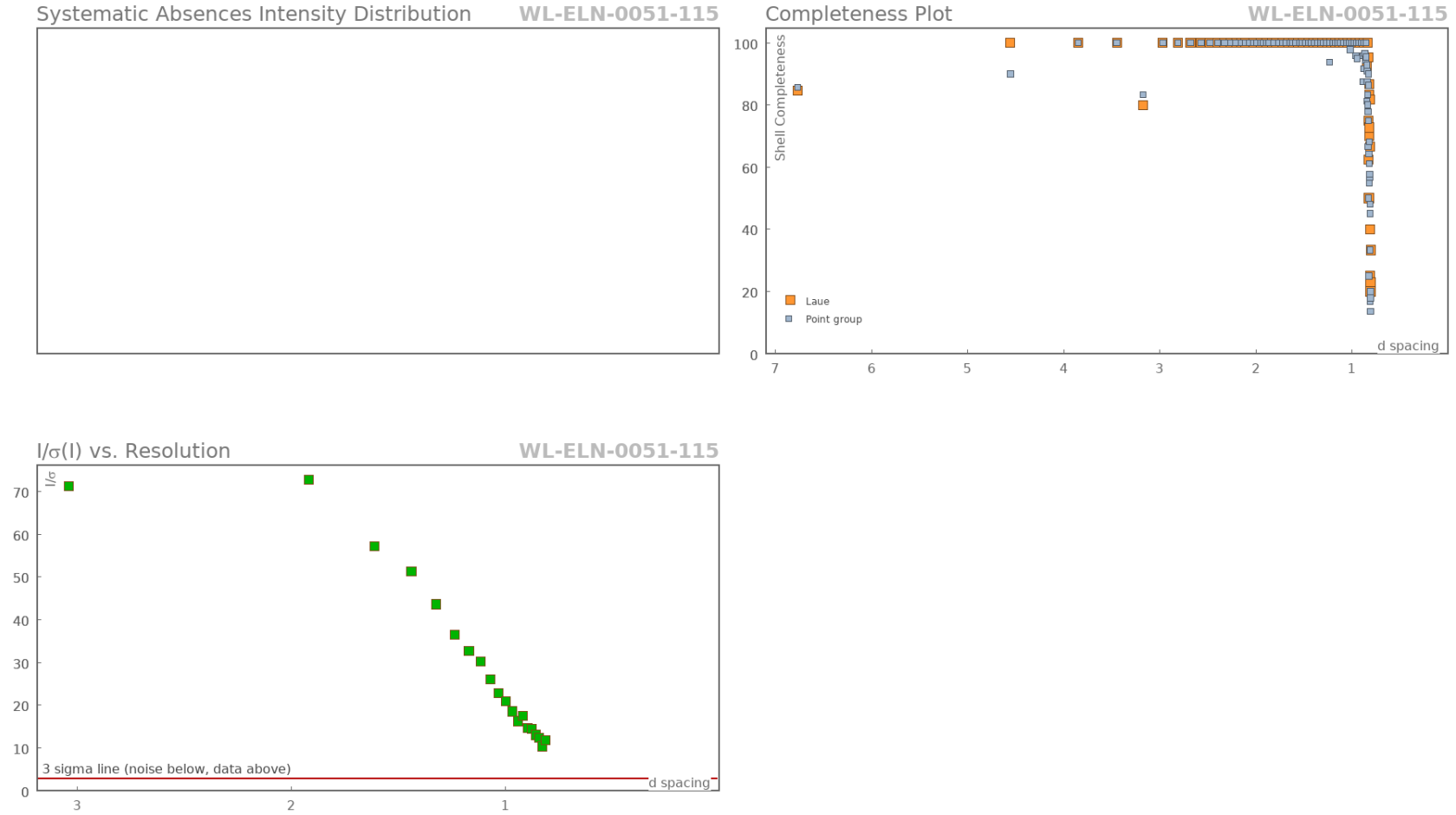

\section{Data Plots: Refinement and Data}
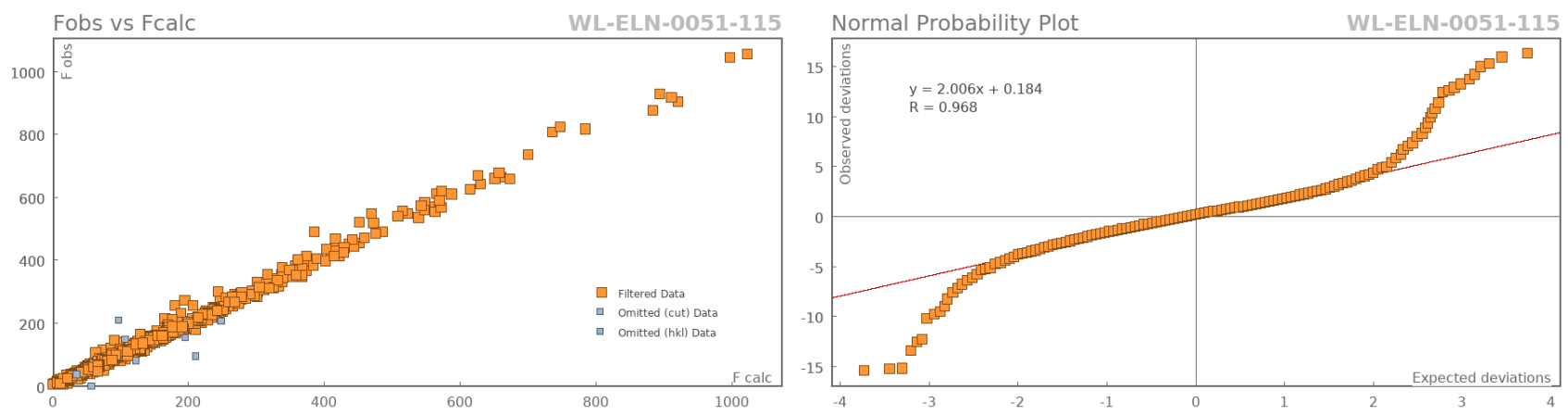

\section{Images of the Crystal on the Diffractometer}
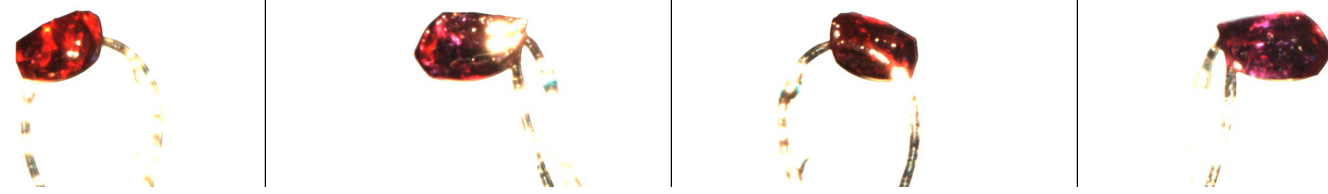

Table 24: Fractional Atomic Coordinates $\left(\times 10^{4}\right)$ and Equivalent Isotropic Displacement Parameters $\left(\AA^{2} \times 10^{3}\right)$ for WL-ELN-0051-115. $U_{e q}$ is defined as $1 / 3$ of the trace of the orthogonalised $U_{i j}$.

\begin{tabular}{lllll}
\hline Atom & \multicolumn{1}{c}{$\mathbf{x}$} & \multicolumn{1}{c}{$\mathbf{y}$} & \multicolumn{1}{c}{$\mathbf{z}$} & \multicolumn{1}{c}{$\boldsymbol{U}_{\boldsymbol{e q}}$} \\
\hline Rh1 & 5000 & 5000 & $4817.6(5)$ & $43.4(2)$ \\
$\mathrm{Rh} 2$ & 5000 & 5000 & 6278.51 & $44.0(2)$ \\
$\mathrm{Cl} 1$ & $7212.2(8)$ & $5972.7(8)$ & $4870.3(12)$ & $60.9(4)$ \\
01 & $5539(2)$ & $5965(2)$ & $4848(3)$ & $47.7(8)$
\end{tabular}




\begin{tabular}{|c|c|c|c|c|}
\hline Atom & $\mathbf{x}$ & $\mathbf{y}$ & $\mathbf{z}$ & $U_{e q}$ \\
\hline 02 & $5399(2)$ & $6035(2)$ & $6219(3)$ & $47.8(8)$ \\
\hline C5 & $6342(3)$ & $7180(3)$ & $4721(4)$ & $51.2(12)$ \\
\hline C25 & $6400(4)$ & $6902(3)$ & $7096(4)$ & $54.8(13)$ \\
\hline C2 & $5998(3)$ & 6992(3) & $5525(5)$ & $52.1(9)$ \\
\hline C30 & 6781(4) & $6258(4)$ & $7142(4)$ & $62.6(16)$ \\
\hline C6 & 6891(3) & $6747(3)$ & $4362(4)$ & $54.2(13)$ \\
\hline C29 & $6881(4)$ & $5899(4)$ & 7881(4) & $70.2(18)$ \\
\hline C26 & $6127(4)$ & 7193(4) & 7788(4) & 62.1(15) \\
\hline C12 & $6138(4)$ & $8679(4)$ & 2291(5) & 68.1(17) \\
\hline C1 & $5620(2)$ & 6268(3) & $5532(4)$ & $45.8(9)$ \\
\hline C3 & $6340(3)$ & $7317(3)$ & $6297(5)$ & $54.3(10)$ \\
\hline C10 & $6137(3)$ & 7799(4) & $4286(4)$ & $52.1(13)$ \\
\hline C13 & $5955(4)$ & $9302(6)$ & 1874(6) & $83(3)$ \\
\hline C19 & $6978(4)$ & $7834(4)$ & $6167(4)$ & $64.9(13)$ \\
\hline $\mathrm{C} 23$ & $8243(5)$ & $8032(4)$ & $5864(5)$ & $87.4(19)$ \\
\hline C8 & $6972(3)$ & 7531(4) & $3218(4)$ & $61.6(15)$ \\
\hline C11 & $6245(4)$ & $8662(4)$ & $3110(4)$ & $65.3(16)$ \\
\hline C7 & 7187(3) & $6908(4)$ & $3618(4)$ & $60.7(15)$ \\
\hline $\mathrm{C} 22$ & $8145(5)$ & 8771(4) & $5940(5)$ & $92.8(19)$ \\
\hline C4 & $5613(4)$ & 7572(3) & $6025(4)$ & $55.7(11)$ \\
\hline C14 & $5876(4)$ & $9942(6)$ & $2320(6)$ & $87(3)$ \\
\hline C9 & $6452(4)$ & 7988(4) & $3554(4)$ & $60.2(15)$ \\
\hline C24 & $7656(4)$ & 7573(5) & $5982(4)$ & $73.0(15)$ \\
\hline C18 & $5890(6)$ & $10664(5)$ & $3638(7)$ & $100(3)$ \\
\hline $\mathrm{C} 20$ & $6890(4)$ & $8576(4)$ & $6239(5)$ & 77.3(16) \\
\hline C15 & $5984(4)$ & $9966(5)$ & $3162(5)$ & $76(2)$ \\
\hline C28 & $6587(4)$ & $6208(4)$ & $8571(4)$ & $76(2)$ \\
\hline C21 & $7467(4)$ & $9047(5)$ & $6120(6)$ & $100(2)$ \\
\hline C16 & 6171(3) & $9324(4)$ & $3560(5)$ & 67.2(17) \\
\hline C17 & $5815(5)$ & $9285(6)$ & 961(6) & $99(3)$ \\
\hline $\mathrm{C} 27$ & $6209(4)$ & $6854(4)$ & $8528(4)$ & 70.4(17) \\
\hline C31 & 5000 & 5000 & 8293(10) & $67(3)$ \\
\hline C32 & 5000 & 5000 & $9189(12)$ & 116(8) \\
\hline N1 & 5000 & 5000 & 7590(7) & $51(2)$ \\
\hline
\end{tabular}

Table 25: Anisotropic Displacement Parameters $\left(\times 10^{4}\right)$ for WL-ELN-0051-115. The anisotropic displacement factor exponent takes the form: $-2 \pi^{2}\left[h^{2} a^{* 2} \times U_{11}+\ldots+2 h k a^{*} \times b^{*} \times U_{12}\right]$

\begin{tabular}{lllllll}
\hline Atom & $\boldsymbol{U}_{\mathbf{1 1}}$ & $\boldsymbol{U}_{\mathbf{2 2}}$ & \multicolumn{1}{c}{$\boldsymbol{U}_{\mathbf{3 3}}$} & \multicolumn{1}{c}{$\boldsymbol{U}_{\mathbf{2 3}}$} & \multicolumn{1}{c}{$\boldsymbol{U}_{\mathbf{1 3}}$} & \multicolumn{1}{c}{$\boldsymbol{U}_{\mathbf{1 2}}$} \\
\hline Rh1 & $48.5(3)$ & $48.5(3)$ & $33.0(4)$ & 0 & 0 & 0 \\
Rh2 & $49.0(3)$ & $49.0(3)$ & $34.2(4)$ & 0 & 0 & 0 \\
Cl1 & $54.7(7)$ & $70.3(8)$ & $57.7(8)$ & $7.5(7)$ & $-1.8(7)$ & $5.2(6)$ \\
O1 & $50.6(18)$ & $56.0(19)$ & $36.6(17)$ & $0(2)$ & $1.8(19)$ & $-0.7(15)$ \\
O2 & $52.3(18)$ & $50.6(18)$ & $41(2)$ & $0.6(19)$ & $1.6(19)$ & $-6.8(15)$ \\
C5 & $50(2)$ & $61(3)$ & $43(2)$ & $-2(2)$ & $-3.0(19)$ & $-8(2)$ \\
C25 & $62(3)$ & $57(3)$ & $46(2)$ & $0(2)$ & $-3.8(19)$ & $-13(3)$ \\
C2 & $58(2)$ & $54(2)$ & $44.3(15)$ & $-2.2(18)$ & $1.4(17)$ & $-4.4(17)$ \\
C30 & $73(4)$ & $65(4)$ & $49(3)$ & $0(3)$ & $-3(3)$ & $-1(3)$ \\
C6 & $47(3)$ & $61(3)$ & $55(3)$ & $4(3)$ & $2(3)$ & $-6(2)$ \\
C29 & $87(5)$ & $73(4)$ & $51(3)$ & $5(3)$ & $-23(3)$ & $-9(4)$ \\
C26 & $65(4)$ & $72(4)$ & $50(3)$ & $1(3)$ & $-4(3)$ & $-9(3)$ \\
C12 & $55(3)$ & $86(5)$ & $63(4)$ & $18(4)$ & $10(3)$ & $8(3)$ \\
C1 & $43(2)$ & $49(2)$ & $45(2)$ & $-2(3)$ & $-5(3)$ & $1.3(18)$ \\
C3 & $66(2)$ & $52(2)$ & $44.9(17)$ & $-1.8(18)$ & $-0.4(17)$ & $-6.2(17)$ \\
C10 & $50(3)$ & $62(3)$ & $44(3)$ & $1(3)$ & $-1(2)$ & $-8(3)$ \\
& & & & & &
\end{tabular}




\begin{tabular}{lcccccc}
\hline Atom & $\boldsymbol{U}_{\mathbf{1 1}}$ & $\boldsymbol{U}_{\mathbf{2 2}}$ & $\boldsymbol{U}_{\mathbf{3 3}}$ & $\boldsymbol{U}_{\mathbf{2 3}}$ & $\boldsymbol{U}_{\mathbf{1 3}}$ & $\boldsymbol{U}_{\mathbf{1 2}}$ \\
\hline C13 & $54(4)$ & $114(7)$ & $82(5)$ & $41(5)$ & $8(4)$ & $-1(4)$ \\
C19 & $73(2)$ & $75(2)$ & $46(3)$ & $-7(2)$ & $-4(2)$ & $-25(2)$ \\
C23 & $92(3)$ & $116(3)$ & $55(4)$ & $1(3)$ & $2(3)$ & $-41(3)$ \\
C8 & $58(3)$ & $77(4)$ & $50(3)$ & $11(3)$ & $6(3)$ & $-5(3)$ \\
C11 & $55(3)$ & $76(4)$ & $65(4)$ & $15(3)$ & $8(3)$ & $-3(3)$ \\
C7 & $57(3)$ & $73(4)$ & $52(3)$ & $4(3)$ & $6(3)$ & $-1(3)$ \\
C22 & $108(4)$ & $115(3)$ & $55(4)$ & $1(3)$ & $-2(3)$ & $-39(3)$ \\
C4 & $67(2)$ & $54(2)$ & $46(2)$ & $-2.6(19)$ & $2.1(17)$ & $-1.8(18)$ \\
C14 & $68(5)$ & $97(6)$ & $95(6)$ & $44(5)$ & $3(4)$ & $4(4)$ \\
C9 & $58(3)$ & $70(4)$ & $53(3)$ & $14(3)$ & $0(3)$ & $-8(3)$ \\
C24 & $76(2)$ & $88(3)$ & $56(3)$ & $0(3)$ & $1(2)$ & $-20(2)$ \\
C18 & $95(6)$ & $89(6)$ & $115(8)$ & $30(5)$ & $31(6)$ & $7(5)$ \\
C20 & $89(3)$ & $75(2)$ & $67(4)$ & $-10(2)$ & $0(3)$ & $-25(2)$ \\
C15 & $54(3)$ & $81(5)$ & $93(6)$ & $27(4)$ & $8(3)$ & $-3(3)$ \\
C28 & $85(5)$ & $86(5)$ & $56(4)$ & $8(3)$ & $-18(4)$ & $-9(4)$ \\
C21 & $108(3)$ & $99(4)$ & $94(6)$ & $-11(4)$ & $4(3)$ & $-46(3)$ \\
C16 & $55(3)$ & $73(4)$ & $73(4)$ & $19(3)$ & $4(3)$ & $-8(3)$ \\
C17 & $69(5)$ & $141(8)$ & $88(6)$ & $51(6)$ & $6(4)$ & $5(5)$ \\
C27 & $75(4)$ & $83(5)$ & $53(4)$ & $-2(3)$ & $4(3)$ & $-3(4)$ \\
C31 & $69(4)$ & $69(4)$ & $63(4)$ & 0 & 0 & 0 \\
C32 & $142(12)$ & $142(12)$ & $63(4)$ & 0 & 0 & 0 \\
N1 & $46(3)$ & $46(3)$ & $63(4)$ & 0 & 0 & 0
\end{tabular}

Table 26: Bond Lengths in Å for WL-ELN-0051-115.

\begin{tabular}{|c|c|c|c|c|c|}
\hline Atom & Atom & Length/Å & Atom & Atom & Length/Å \\
\hline$\overline{\mathrm{Rh} 1}$ & Rh2 & $2.3966(9)$ & $\overline{\mathrm{C} 12}$ & C13 & $1.377(11)$ \\
\hline Rh1 & $01^{1}$ & $2.032(4)$ & C12 & C11 & $1.359(10)$ \\
\hline Rh1 & 01 & $2.032(4)$ & $\mathrm{C} 3$ & C19 & $1.525(8)$ \\
\hline Rh1 & $01^{2}$ & $2.032(4)$ & $\mathrm{C} 3$ & $\mathrm{C} 4$ & $1.485(9)$ \\
\hline Rh1 & $01^{3}$ & $2.032(4)$ & C10 & $\mathrm{C} 9$ & $1.377(8)$ \\
\hline $\mathrm{Rh} 2$ & $02^{3}$ & $2.042(4)$ & C13 & C14 & $1.392(14)$ \\
\hline Rh2 & $02^{1}$ & $2.042(4)$ & C13 & C17 & $1.519(13)$ \\
\hline Rh2 & $\mathrm{O} 2^{2}$ & $2.042(4)$ & C19 & $\mathrm{C} 24$ & $1.371(11)$ \\
\hline $\mathrm{Rh} 2$ & 02 & $2.042(4)$ & C19 & $\mathrm{C} 20$ & $1.378(11)$ \\
\hline $\mathrm{Rh} 2$ & N1 & $2.151(11)$ & $\mathrm{C} 23$ & $\mathrm{C} 22$ & $1.377(6)$ \\
\hline $\mathrm{Cl} 1$ & C6 & $1.753(7)$ & $\mathrm{C} 23$ & $\mathrm{C} 24$ & $1.382(8)$ \\
\hline 01 & $\mathrm{C} 1$ & $1.261(8)$ & C8 & $\mathrm{C} 7$ & $1.379(10)$ \\
\hline 02 & $\mathrm{C} 1$ & $1.272(8)$ & C8 & C9 & $1.387(10)$ \\
\hline C5 & $\mathrm{C} 2$ & $1.502(10)$ & C11 & C9 & $1.487(9)$ \\
\hline C5 & C6 & $1.414(9)$ & C11 & C16 & $1.430(11)$ \\
\hline C5 & C10 & $1.395(9)$ & $\mathrm{C} 22$ & $\mathrm{C} 21$ & $1.376(6)$ \\
\hline $\mathrm{C} 25$ & C30 & $1.378(10)$ & C14 & C15 & $1.397(14)$ \\
\hline $\mathrm{C} 25$ & C26 & $1.350(10)$ & C18 & C15 & $1.513(14)$ \\
\hline $\mathrm{C} 25$ & C3 & $1.521(9)$ & $\mathrm{C} 20$ & $\mathrm{C} 21$ & $1.383(8)$ \\
\hline $\mathrm{C} 2$ & C1 & $1.501(7)$ & C15 & C16 & $1.391(10)$ \\
\hline $\mathrm{C} 2$ & C3 & $1.535(10)$ & $\mathrm{C} 28$ & $\mathrm{C} 27$ & $1.378(6)$ \\
\hline $\mathrm{C} 2$ & $\mathrm{C} 4$ & $1.521(9)$ & C31 & $\mathrm{C} 32$ & $1.47(2)$ \\
\hline C30 & C29 & $1.391(9)$ & $\mathrm{C} 31$ & N1 & $1.154(19)$ \\
\hline C6 & C7 & $1.368(9)$ & ---- & & \\
\hline C29 & C28 & $1.377(6)$ & \multirow{2}{*}{\multicolumn{3}{|c|}{${ }^{1}+\mathrm{y}, 1-\mathrm{x},+\mathrm{z} ;{ }^{2} 1-\mathrm{y},+\mathrm{x},+\mathrm{z} ;{ }^{3} 1-\mathrm{x}, 1-\mathrm{y},+\mathrm{z}$}} \\
\hline C26 & $\mathrm{C} 27$ & $1.373(10)$ & & & \\
\hline
\end{tabular}

Table 27: Bond Angles in ${ }^{\circ}$ for WL-ELN-0051-115. 


\begin{tabular}{|c|c|c|c|}
\hline Atom & Atom & Atom & Angle ${ }^{\circ}$ \\
\hline$\overline{01^{1}}$ & Rh1 & Rh2 & $88.58(14)$ \\
\hline $01^{2}$ & $\mathrm{Rh} 1$ & $\mathrm{Rh} 2$ & $88.58(14)$ \\
\hline $01^{3}$ & $\mathrm{Rh} 1$ & $\mathrm{Rh} 2$ & $88.58(14)$ \\
\hline 01 & Rh1 & $\mathrm{Rh} 2$ & $88.58(14)$ \\
\hline $01^{3}$ & Rh1 & 01 & $89.964(8)$ \\
\hline $01^{1}$ & $\mathrm{Rh} 1$ & 01 & $177.2(3)$ \\
\hline $01^{1}$ & $\mathrm{Rh} 1$ & $01^{2}$ & $89.965(8)$ \\
\hline $01^{2}$ & $\mathrm{Rh} 1$ & 01 & $89.965(8)$ \\
\hline $01^{1}$ & Rh1 & $01^{3}$ & $89.965(7)$ \\
\hline $01^{3}$ & $\mathrm{Rh} 1$ & $01^{2}$ & $177.2(3)$ \\
\hline $\mathrm{O} 2^{2}$ & $\mathrm{Rh} 2$ & Rh1 & $87.25(13)$ \\
\hline 02 & $\mathrm{Rh} 2$ & $\mathrm{Rh} 1$ & $87.25(13)$ \\
\hline $02^{1}$ & $\mathrm{Rh} 2$ & Rh1 & $87.25(13)$ \\
\hline $\mathrm{O} 2^{3}$ & $\mathrm{Rh} 2$ & $\mathrm{Rh} 1$ & $87.25(13)$ \\
\hline $\mathrm{O} 2^{3}$ & $\mathrm{Rh} 2$ & 02 & $89.867(14)$ \\
\hline $\mathrm{O} 2^{2}$ & Rh2 & 02 & $89.869(13)$ \\
\hline $\mathrm{O} 2^{1}$ & $\mathrm{Rh} 2$ & 02 & $174.5(3)$ \\
\hline $02^{1}$ & $\mathrm{Rh} 2$ & $\mathrm{O} 2^{2}$ & $89.868(14)$ \\
\hline $\mathrm{O} 2^{1}$ & $\mathrm{Rh} 2$ & $\mathrm{O} 2^{3}$ & $89.868(13)$ \\
\hline $\mathrm{O} 2^{2}$ & $\mathrm{Rh} 2$ & $\mathrm{O} 2^{3}$ & $174.5(3)$ \\
\hline $\mathrm{O} 2^{1}$ & $\mathrm{Rh} 2$ & $\mathrm{~N} 1$ & $92.75(13)$ \\
\hline $\mathrm{O} 2^{3}$ & $\mathrm{Rh} 2$ & N1 & $92.75(13)$ \\
\hline 02 & Rh2 & N1 & $92.75(13)$ \\
\hline $\mathrm{O} 2^{2}$ & $\mathrm{Rh} 2$ & $\mathrm{~N} 1$ & $92.75(13)$ \\
\hline N1 & $\mathrm{Rh} 2$ & Rh1 & 180.0 \\
\hline $\mathrm{C} 1$ & 01 & $\mathrm{Rh} 1$ & $117.7(4)$ \\
\hline $\mathrm{C} 1$ & 02 & $\mathrm{Rh} 2$ & $118.1(4)$ \\
\hline C6 & C5 & $\mathrm{C} 2$ & $122.5(6)$ \\
\hline C10 & C5 & $\mathrm{C} 2$ & $121.5(5)$ \\
\hline C10 & C5 & C6 & $116.0(6)$ \\
\hline $\mathrm{C} 30$ & $\mathrm{C} 25$ & C3 & $121.0(6)$ \\
\hline C26 & $\mathrm{C} 25$ & C30 & $118.9(6)$ \\
\hline C26 & C25 & C3 & $120.0(6)$ \\
\hline C5 & $\mathrm{C} 2$ & C3 & $117.6(4)$ \\
\hline C5 & $\mathrm{C} 2$ & $\mathrm{C} 4$ & $120.6(5)$ \\
\hline $\mathrm{C} 1$ & $\mathrm{C} 2$ & C5 & $113.9(6)$ \\
\hline $\mathrm{C} 1$ & $\mathrm{C} 2$ & C3 & $121.9(6)$ \\
\hline C1 & C2 & C4 & $113.7(5)$ \\
\hline $\mathrm{C} 4$ & $\mathrm{C} 2$ & C3 & $58.1(4)$ \\
\hline $\mathrm{C} 25$ & C30 & C29 & $121.5(7)$ \\
\hline C5 & C6 & $\mathrm{Cl} 1$ & $119.9(5)$ \\
\hline C7 & C6 & $\mathrm{Cl} 1$ & $117.8(5)$ \\
\hline C7 & C6 & C5 & $122.3(6)$ \\
\hline $\mathrm{C} 28$ & C29 & C30 & $117.9(7)$ \\
\hline
\end{tabular}

\begin{tabular}{|c|c|c|c|}
\hline Atom & Atom & Atom & Angle ${ }^{\circ}$ \\
\hline$\overline{\mathrm{C} 25}$ & $\mathrm{C} 26$ & $\mathrm{C} 27$ & $121.6(7)$ \\
\hline C11 & C12 & $\mathrm{C} 13$ & $123.1(9)$ \\
\hline 01 & $\mathrm{C} 1$ & 02 & $127.0(4)$ \\
\hline 01 & C1 & $\mathrm{C} 2$ & 116.1(6) \\
\hline 02 & $\mathrm{C} 1$ & $\mathrm{C} 2$ & $116.9(6)$ \\
\hline C25 & C3 & $\mathrm{C} 2$ & $123.1(5)$ \\
\hline $\mathrm{C} 25$ & C3 & C19 & $112.2(5)$ \\
\hline C19 & $\mathrm{C} 3$ & $\mathrm{C} 2$ & $116.2(6)$ \\
\hline $\mathrm{C} 4$ & $\mathrm{C} 3$ & $\mathrm{C} 25$ & $118.9(6)$ \\
\hline $\mathrm{C} 4$ & C3 & C2 & $60.4(4)$ \\
\hline $\mathrm{C} 4$ & $\mathrm{C} 3$ & C19 & $116.9(6)$ \\
\hline C9 & C10 & C5 & $122.5(6)$ \\
\hline C12 & C13 & C14 & $117.9(8)$ \\
\hline C12 & C13 & C17 & $120.9(10)$ \\
\hline C14 & $\mathrm{C} 13$ & $\mathrm{C} 17$ & $121.2(8)$ \\
\hline C24 & C19 & C3 & $120.8(6)$ \\
\hline C24 & C19 & $\mathrm{C} 20$ & 118.1(7) \\
\hline $\mathrm{C} 20$ & C19 & C3 & $121.0(7)$ \\
\hline $\mathrm{C} 22$ & $\mathrm{C} 23$ & C24 & 119.1(8) \\
\hline C7 & C8 & C9 & $120.7(6)$ \\
\hline C12 & C11 & C9 & $122.7(7)$ \\
\hline C12 & C11 & C16 & 118.5(7) \\
\hline C16 & C11 & C9 & 118.8(6) \\
\hline C6 & $\mathrm{C} 7$ & C8 & $119.3(6)$ \\
\hline $\mathrm{C} 21$ & $\mathrm{C} 22$ & $\mathrm{C} 23$ & $120.1(8)$ \\
\hline C3 & $\mathrm{C} 4$ & C2 & $61.4(4)$ \\
\hline C13 & C14 & C15 & $122.1(8)$ \\
\hline C10 & C9 & C8 & $119.0(6)$ \\
\hline $\mathrm{C} 10$ & C9 & C11 & $122.0(6)$ \\
\hline C8 & C9 & C11 & $119.0(6)$ \\
\hline C19 & $\mathrm{C} 24$ & $\mathrm{C} 23$ & $121.9(8)$ \\
\hline C19 & $\mathrm{C} 20$ & C21 & $121.2(8)$ \\
\hline C14 & C15 & C18 & $121.4(8)$ \\
\hline C16 & C15 & C14 & 118.2(9) \\
\hline C16 & C15 & C18 & $120.3(8)$ \\
\hline $\mathrm{C} 29$ & C28 & C27 & $120.8(7)$ \\
\hline $\mathrm{C} 22$ & $\mathrm{C} 21$ & $\mathrm{C} 20$ & $119.6(8)$ \\
\hline C15 & C16 & C11 & $120.2(7)$ \\
\hline $\mathrm{C} 26$ & $\mathrm{C} 27$ & $\mathrm{C} 28$ & $119.4(7)$ \\
\hline N1 & C31 & C32 & 180.0 \\
\hline C31 & N1 & $\mathrm{Rh} 2$ & 180.0 \\
\hline
\end{tabular}

Table 28: Torsion Angles in ${ }^{\circ}$ for WL-ELN-0051-115.

\begin{tabular}{llllr}
\hline Atom & Atom & Atom & Atom & Angle $^{\circ}$ \\
\hline Rh1 & 01 & C1 & 02 & $-0.1(6)$ \\
Rh1 & 01 & C1 & C2 & $-178.0(3)$ \\
Rh2 & O2 & C1 & 01 & $9.9(6)$ \\
Rh2 & O2 & C1 & C2 & $-172.3(3)$ \\
Cl1 & C6 & C7 & C8 & $-177.0(5)$ \\
C5 & C2 & C1 & 01 & $-10.6(7)$ \\
C5 & C2 & C1 & 02 & $171.4(5)$
\end{tabular}




\begin{tabular}{|c|c|c|c|c|}
\hline Atom & Atom & Atom & Atom & Angle $/{ }^{\circ}$ \\
\hline$\overline{\mathrm{C} 5}$ & $\mathrm{C} 2$ & C3 & $\mathrm{C} 25$ & $-142.4(6)$ \\
\hline $\mathrm{C} 5$ & $\mathrm{C} 2$ & C3 & C19 & $3.0(8)$ \\
\hline C5 & $\mathrm{C} 2$ & C3 & $\mathrm{C} 4$ & $110.5(6)$ \\
\hline C5 & $\mathrm{C} 2$ & C4 & C3 & $-105.5(6)$ \\
\hline C5 & C6 & C7 & C8 & $3.3(10)$ \\
\hline C5 & C10 & C9 & $\mathrm{C} 8$ & $2.7(10)$ \\
\hline C5 & C10 & C9 & C11 & $-178.0(6)$ \\
\hline C25 & C30 & C29 & $\mathrm{C} 28$ & $0.1(11)$ \\
\hline $\mathrm{C} 25$ & C26 & C27 & $\mathrm{C} 28$ & $1.0(11)$ \\
\hline C25 & C3 & C19 & $\mathrm{C} 24$ & $72.1(8)$ \\
\hline $\mathrm{C} 25$ & C3 & C19 & $\mathrm{C} 20$ & $-107.3(8)$ \\
\hline $\mathrm{C} 25$ & C3 & C4 & $\mathrm{C} 2$ & $-113.9(6)$ \\
\hline $\mathrm{C} 2$ & $\mathrm{C} 5$ & C6 & $\mathrm{Cl} 1$ & $-1.7(8)$ \\
\hline $\mathrm{C} 2$ & $\mathrm{C} 5$ & C6 & $\mathrm{C} 7$ & $178.0(6)$ \\
\hline $\mathrm{C} 2$ & $\mathrm{C} 5$ & C10 & $\mathrm{C} 9$ & $179.0(6)$ \\
\hline $\mathrm{C} 2$ & C3 & C19 & $\mathrm{C} 24$ & $-77.0(8)$ \\
\hline $\mathrm{C} 2$ & C3 & C19 & $\mathrm{C} 20$ & $103.6(7)$ \\
\hline C30 & $\mathrm{C} 25$ & C26 & $\mathrm{C} 27$ & $-1.2(10)$ \\
\hline C30 & $\mathrm{C} 25$ & C3 & $\mathrm{C} 2$ & $59.6(9)$ \\
\hline C30 & $\mathrm{C} 25$ & C3 & C19 & $-87.1(7)$ \\
\hline C30 & $\mathrm{C} 25$ & C3 & C4 & $131.4(7)$ \\
\hline C30 & $\mathrm{C} 29$ & C28 & $\mathrm{C} 27$ & $-0.4(11)$ \\
\hline C6 & $\mathrm{C} 5$ & C2 & C1 & $-60.3(7)$ \\
\hline C6 & $\mathrm{C} 5$ & $\mathrm{C} 2$ & $\mathrm{C} 3$ & 91.7(7) \\
\hline C6 & $\mathrm{C} 5$ & $\mathrm{C} 2$ & $\mathrm{C} 4$ & 159.1(6) \\
\hline $\mathrm{C} 6$ & $\mathrm{C} 5$ & C10 & $\mathrm{C} 9$ & $-0.6(9)$ \\
\hline $\mathrm{C} 29$ & C28 & $\mathrm{C} 27$ & $\mathrm{C} 26$ & $-0.2(11)$ \\
\hline $\mathrm{C} 26$ & $\mathrm{C} 25$ & C30 & $\mathrm{C} 29$ & $0.7(10)$ \\
\hline $\mathrm{C} 26$ & $\mathrm{C} 25$ & C3 & $\mathrm{C} 2$ & $-125.6(7)$ \\
\hline $\mathrm{C} 26$ & $\mathrm{C} 25$ & C3 & C19 & $87.8(8)$ \\
\hline C26 & $\mathrm{C} 25$ & C3 & C4 & $-53.8(8)$ \\
\hline C12 & C13 & C14 & C15 & $0.6(12)$ \\
\hline $\mathrm{C} 12$ & C11 & C9 & $\mathrm{C} 10$ & $-136.9(7)$ \\
\hline C12 & C11 & C9 & C8 & 42.4(9) \\
\hline $\mathrm{C} 12$ & C11 & C16 & C15 & $0.8(9)$ \\
\hline C1 & $\mathrm{C} 2$ & C3 & $\mathrm{C} 25$ & $7.2(9)$ \\
\hline C1 & $\mathrm{C} 2$ & C3 & C19 & $152.6(5)$ \\
\hline $\mathrm{C} 1$ & $\mathrm{C} 2$ & $\mathrm{C} 3$ & $\mathrm{C} 4$ & $-99.8(6)$ \\
\hline $\mathrm{C} 1$ & $\mathrm{C} 2$ & $\mathrm{C} 4$ & C3 & $113.9(7)$ \\
\hline $\mathrm{C} 3$ & $\mathrm{C} 25$ & C30 & $\mathrm{C} 29$ & $175.5(6)$ \\
\hline C3 & $\mathrm{C} 25$ & $\mathrm{C} 26$ & $\mathrm{C} 27$ & $-176.1(6)$ \\
\hline $\mathrm{C} 3$ & $\mathrm{C} 2$ & $\mathrm{C} 1$ & 01 & $-161.3(5)$ \\
\hline $\mathrm{C} 3$ & $\mathrm{C} 2$ & $\mathrm{C} 1$ & 02 & 20.7(7) \\
\hline C3 & C19 & $\mathrm{C} 24$ & $\mathrm{C} 23$ & $-179.8(6)$ \\
\hline C3 & C19 & $\mathrm{C} 20$ & $\mathrm{C} 21$ & $-179.9(8)$ \\
\hline C10 & $\mathrm{C} 5$ & C2 & C1 & $120.1(6)$ \\
\hline C10 & $\mathrm{C} 5$ & C2 & C3 & $-87.9(7)$ \\
\hline C10 & $\mathrm{C} 5$ & C2 & C4 & $-20.4(8)$ \\
\hline C10 & $\mathrm{C} 5$ & C6 & $\mathrm{Cl} 1$ & $177.9(4)$ \\
\hline C10 & $\mathrm{C} 5$ & C6 & C7 & $-2.4(9)$ \\
\hline C13 & C12 & C11 & C9 & $-179.2(7)$ \\
\hline C13 & C12 & C11 & C16 & $-0.6(10)$ \\
\hline C13 & C14 & C15 & C18 & $-179.5(8)$ \\
\hline C13 & $\mathrm{C} 14$ & C15 & C16 & $-0.4(11)$ \\
\hline C19 & $\mathrm{C} 3$ & $\mathrm{C} 4$ & $\mathrm{C} 2$ & $106.4(7)$ \\
\hline C19 & $\mathrm{C} 20$ & C21 & $\mathrm{C} 22$ & $-1.2(14)$ \\
\hline $\mathrm{C} 23$ & $\mathrm{C} 22$ & $\mathrm{C} 21$ & $\mathrm{C} 20$ & $1.3(14)$ \\
\hline C11 & $\mathrm{C} 12$ & C13 & C14 & $-0.1(11)$ \\
\hline
\end{tabular}




\begin{tabular}{llllc}
\hline Atom & Atom & Atom & Atom & Angle $^{\circ}$ \\
\hline C11 & C12 & C13 & C17 & $-177.8(7)$ \\
C7 & C8 & C9 & C10 & $-1.8(10)$ \\
C7 & C8 & C9 & C11 & $178.9(6)$ \\
C22 & C23 & C24 & C19 & $0.5(12)$ \\
C4 & C2 & C1 & O1 & $132.7(6)$ \\
C4 & C2 & C1 & O2 & $-45.4(7)$ \\
C4 & C2 & C3 & C25 & $107.0(7)$ \\
C4 & C2 & C3 & C19 & $-107.5(6)$ \\
C4 & C3 & C19 & C24 & $-145.5(7)$ \\
C4 & C3 & C19 & C20 & $35.1(10)$ \\
C14 & C15 & C16 & C11 & $-0.3(10)$ \\
C9 & C8 & C7 & C6 & $-1.2(10)$ \\
C9 & C11 & C16 & C15 & $179.5(6)$ \\
C24 & C19 & C20 & C21 & $0.7(12)$ \\
C24 & C23 & C22 & C21 & $-0.9(13)$ \\
C18 & C15 & C16 & C11 & $178.8(7)$ \\
C20 & C19 & C24 & C23 & $-0.4(11)$ \\
C16 & C11 & C9 & C10 & $44.5(9)$ \\
C16 & C11 & C9 & C8 & $-136.2(7)$ \\
C17 & C13 & C14 & C15 & $178.3(7)$
\end{tabular}

Table 29: Hydrogen Fractional Atomic Coordinates $\left(\times 10^{4}\right)$ and Equivalent Isotropic Displacement Parameters $\left(\AA^{2} \times 10^{3}\right)$ for WL-ELN-0051-115. $U_{e q}$ is defined as $1 / 3$ of the trace of the orthogonalised $U_{i j .}$.

\begin{tabular}{llllr}
\hline Atom & \multicolumn{1}{c}{$\mathbf{x}$} & \multicolumn{1}{c}{$\mathbf{y}$} & $\boldsymbol{U}_{\boldsymbol{e q}}$ \\
\hline H30 & $6980(5)$ & $6052(5)$ & $6655(9)$ & 75 \\
H29 & $7147(6)$ & $5452(8)$ & $7908(4)$ & 84 \\
H26 & $5870(6)$ & $7645(9)$ & $7761(4)$ & 75 \\
H12 & $6193(4)$ & $8236(9)$ & $1989(7)$ & 82 \\
H10 & $5763(7)$ & $8104(6)$ & $4505(5)$ & 63 \\
H23 & $8712(9)$ & $7838(5)$ & $5731(5)$ & 105 \\
H8 & $7184(5)$ & $7651(4)$ & $2702(9)$ & 74 \\
H7 & $7540(7)$ & $6590(7)$ & $3377(6)$ & 73 \\
H22 & $8549(8)$ & $9094(7)$ & $5868(5)$ & 111 \\
H14 & $5745(5)$ & $10380(9)$ & $2040(8)$ & 104 \\
H24 & $7726(4)$ & $7059(10)$ & $5933(4)$ & 88 \\
H18A & $6302(14)$ & $10961(11)$ & $3564(7)$ & 150 \\
H18B & $5835(6)$ & $10555(6)$ & $4195(19)$ & 150 \\
H18C & $5474(14)$ & $10911(9)$ & $3451(9)$ & 150 \\
H20 & $6421(9)$ & $8769(5)$ & $6374(5)$ & 93 \\
H28 & $6646(4)$ & $5971(5)$ & $9087(9)$ & 91 \\
H21 & $7396(4)$ & $9561(10)$ & $6162(6)$ & 120 \\
H16 & $6250(4)$ & $9326(4)$ & $4138(11)$ & 81 \\
H17A & $5706(6)$ & $9758(16)$ & $777(9)$ & 149 \\
H17B & $5421(13)$ & $8975(12)$ & $850(7)$ & 149 \\
H17C & $6232(14)$ & $9113(8)$ & $691(11)$ & 149 \\
H27 & $6005(5)$ & $7065(5)$ & $9011(9)$ & 85 \\
H32B & $5039.3(12)$ & $4519(15)$ & $9380(14)$ & 174 \\
H32A & $4564(14)$ & $5206(6)$ & $9380(14)$ & 174 \\
H32C & $5397(12)$ & $5274(9)$ & $9380(14)$ & 174 \\
H4A & $5160(20)$ & $7370(30)$ & $6320(30)$ & $51(11)$ \\
H4B & $5540(30)$ & $8085(14)$ & $5780(30)$ & $51(11)$ \\
& & & &
\end{tabular}


Table 30: Atomic Occupancies for all atoms that are not fully occupied in WL-ELN-0051-115.

\begin{tabular}{|c|c|c|c|c|c|}
\hline & & & & & \\
\hline Atom & Occupancy & Atom & Occupancy & Atom & Occupancy \\
\hline & 0.25 & H32A & 0.25 & H32C & 0.25 \\
\hline
\end{tabular}

Table 31: Solvent masking (PLATON/SQUEEZE) information for WL-ELN-0051-115.

\begin{tabular}{lllllll}
\hline No & $\mathbf{x}$ & $\mathbf{y}$ & $\mathbf{z}$ & $\mathbf{V}$ & $\mathbf{e}$ & Content \\
\hline 1 & -0.032 & 0.603 & 0.637 & 787.9 & 29.0 & $?$ \\
2 & -0.945 & -0.266 & 0.137 & 787.9 & 29.0 & $?$
\end{tabular}

\section{Citations}

CrysAlisPro (Rigaku, V1.171.40.67a, 2019)

CrysAlisPro (ROD), Rigaku Oxford Diffraction, Poland (?).

O.V. Dolomanov and L.J. Bourhis and R.J. Gildea and J.A.K. Howard and H. Puschmann, Olex2: A complete structure solution, refinement and analysis program, J. Appl. Cryst., (2009), 42, 339-341.

Sheldrick, G.M., Crystal structure refinement with ShelXL, Acta Cryst., (2015), C71, 3-8.

Sheldrick, G.M., ShelXT-Integrated space-group and crystal-structure determination, Acta Cryst., (2015), A71, 3-8. 